\title{
Consumption Estimates
}

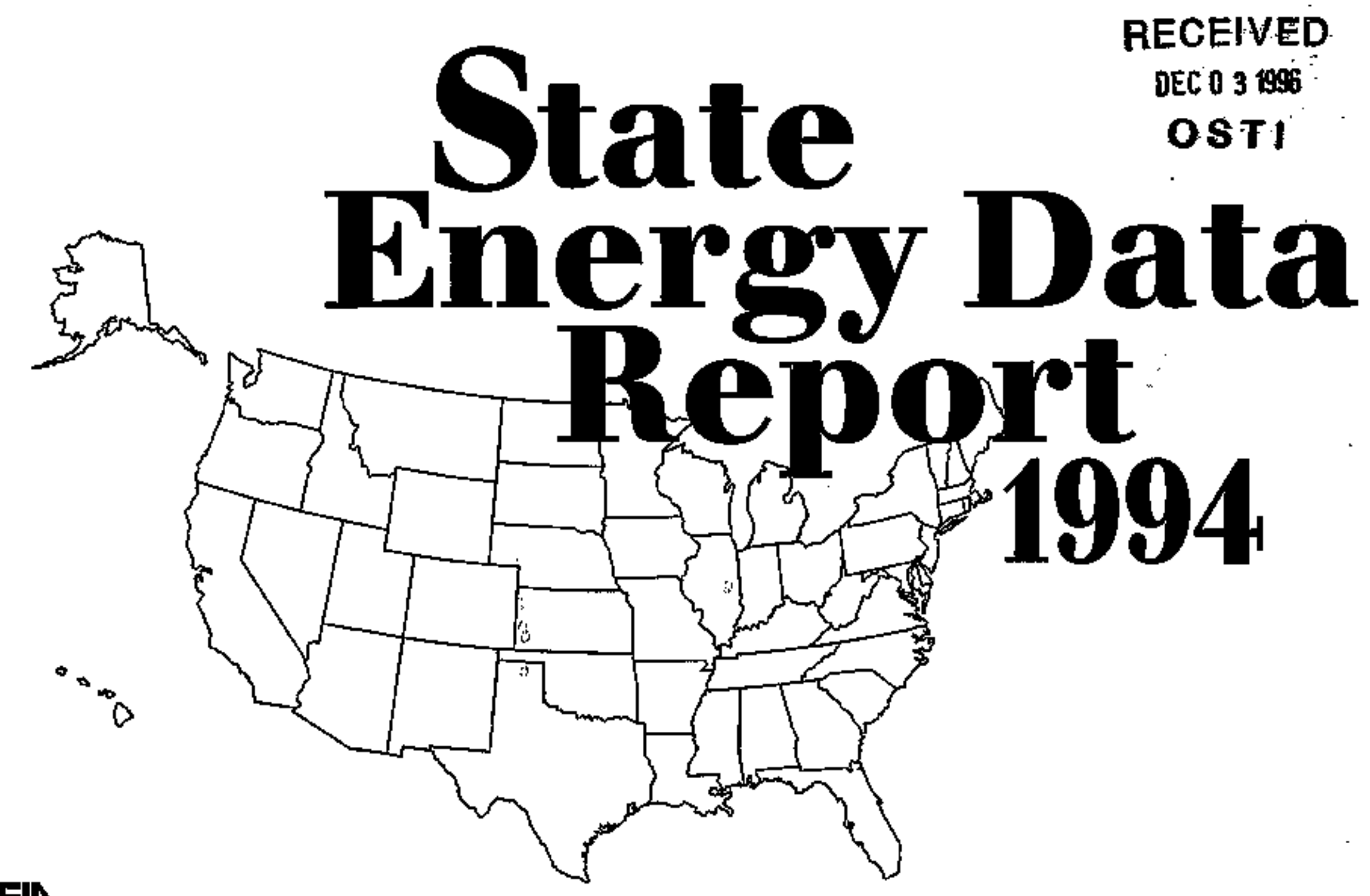

EA

Energy Information Administration 


\section{State Energy Data Report 1994 Consumption Estimates}

The State Entrgy Data Report 1994 presents estimates of antual energy consumption at the State and national levels by major economic sector and by principal energy' type for 1960, 1965, and 1970 through 1994. Included in the report are documentation describing how the estinates were made for each energy source, the sources of all input data, and a summary of changes from the State Energy Data Report publjshed in July 1995.

Publication of this report is in keeping with responsibilities given to the Energy Information Admisistration (ELA) in Rublic Law 95-91 (Department of Energy Organization Act), which states, in part, in Section 205(a)(2) that

The Administrator shall be responsible for carrying out a central, comprehersive, and unffled energy data and information program which will colleck, evaluate, assemble, anelyze, and disseminate data and information .r. .

\section{Contacts}

The State Energy Data Report is prepared by the Energy Information Administration, under the direction of W. Calvin Kilgore, Director of the Office of Energy Markets and End Lise, 202-586-1617; Lynda T. Carlson, Director of the Energy End Use and Integrated Statistice Division, 202-586-1112; and Kathertine E. Seiferlein, Chief of the integrated Statistics Brandh, 202-5865695 .

Questions concerning the contents of the State Energy Data Report may be referred to Julia F. Hutchins (202-586-5138 or jhutchingleia.doe.gov). Thomas J. Leckey (202-586-9413 or tleckey@eda.doegov), or Roy M. Stanley (202-5865839 or rstanley oieia.doe.gov). The Division fox number is 2012-586-0018.

\section{General Information}

General questions on energy statistics may be directed to the National Energy Information Center at the address, telephone, and teleconumunications numbers shown under Ordering Information.

Released for Printing: Ockober 29, 1996

GPO Stock Na. 061-003-00972-7

6 Printed with soy ink on recycled paper.

\section{Ordering Information}

This publication and other Energy Information Administration (ELA) publjcations may be purchased from the Superintendent of Documents, U.S. Government Ptinting Office. An order form for this publication is provided in the back of this report. Orders may be directed to:

\section{Superintendent of Documents \\ U.S. Government Printing Office \\ P.O. Box 371954 \\ Pittsburgh, PA 15250-7954}

$202-512-1800$

Fax: 202-512-2250

8 a.m. to $4: 30$ p.m., eastern time, M-F

Complimentary subscriptions and single issues are available to certain groups of subscribers, such as public and academic lbrarles; Pederal, State, local, and foreigrt zoyernments; ElA survey respondents; and the media. For further information and for answers to questions on energy statistics, contact EIA's National Energy Information Center at:

\author{
National Energy Information Center, EI-231 \\ Energy Information Administration \\ Forrestal Building, Room 1F-048 \\ Washington, DC 20585 \\ 202-586-8800 \\ Fax: $202-586-0727$ \\ Internet E-Mafl: infoctroia.doe.gov \\ TTY: For people who are deat \\ or hard of hearing: 202-586-1181 \\ 9 a.m. to 5 p.m., eastern time, $M-F$
}

\section{Electronic Access}

State Energy Data Report 1994 data are available electronically. See the inside back cover of this report for information about persontal computer diskettes or reach EIA at the following lnternet addresses;

$$
\begin{array}{ll}
\text { E-mail: } & \text { infoctroeia.doe.gov } \\
\text { World Wide Web: } & \text { http: } / / \text { www.eia.doe-gov } \\
\text { Gopher Site: } & \text { gopher.//gopher.eia.doe.gov } \\
\text { FTP Site: } & \text { ftp://ftp.eia.doe.gov }
\end{array}
$$

Orer 180 EIA reports, databases, and models are available on a CD-ROM. For information about the Energy lnfoDist, call 1-\$00-\$TAT-USA. 


\title{
State Energy Data Report 1994
}

\section{Consumption Estimates}

\author{
October 1996
}

\author{
Energy Information Adminlstration \\ Office of Energy Markets and End Use \\ U.S. Department of Energy \\ Washington, DC 20585
}

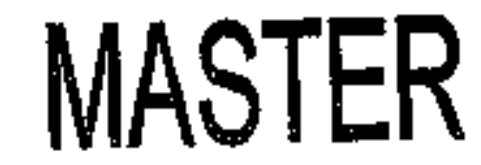

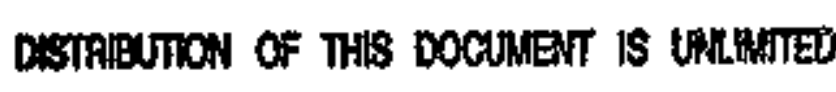

Ths report was prepared by lhe Energy Iniormation Administration, the independent stazhelical and analyweal

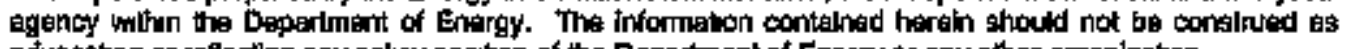
advoceatung or reflectling any policy posthon of the Department of Energy or any other coganization. 


\section{DISCLAMER}

Portions of this document may be illegible in electronic image products. Images are produced from the best available original document. 


\section{DISCI.AMYER}

This report was prepared as an accopnt of work sportored by an agency of the United States Government. Neither the United States Government noe any agency

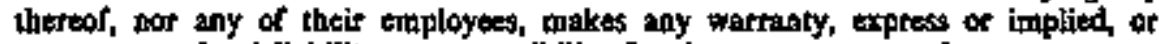
assumes any legal liability of responsibitity for the accoracy, complestentess, or usefulness of any information, appacatus, product, of process ofsclosed, of represents that its use would not inftinge privately owned rights. Reference herein to any speciflic commerciat produet, process, or service by trade nans, trademark, manuface turer, or otherwise doss not necessarily constitute or fmply its endersement, recommendstion, or favoting by the Umited States Govemment or any agency therest. The views and opunions of anthors expressed herein do not necessarify state or reflect those of the Unted States Coremment of any agency thereof. 


\section{Contents}

Intraduction $\ldots \ldots \ldots \ldots \ldots \ldots \ldots \ldots \ldots \ldots \ldots \ldots \ldots \ldots \ldots \ldots \ldots \ldots$

Tables

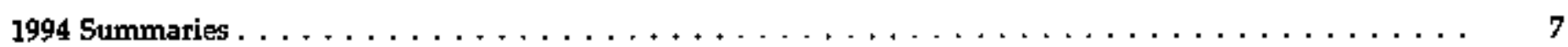

Unted States Summartest 1960,1965 , and 1970 through $1994 \ldots \ldots \ldots \ldots \ldots \ldots \ldots \ldots \ldots \ldots$

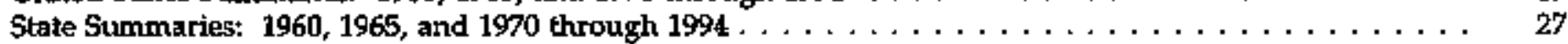

Appendices

(Listed alphabetically by State)

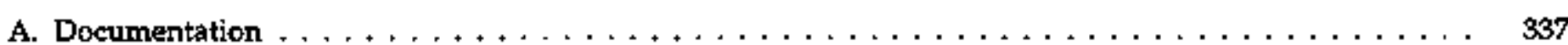

Section l Documentation Guide

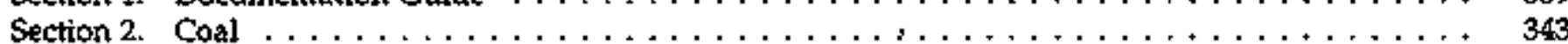

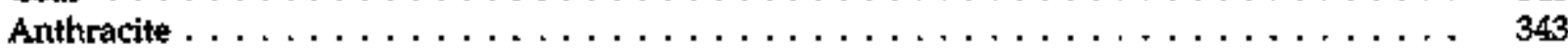

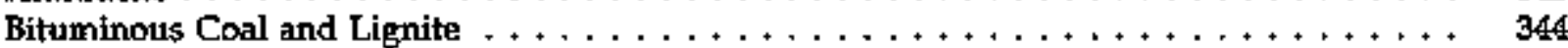

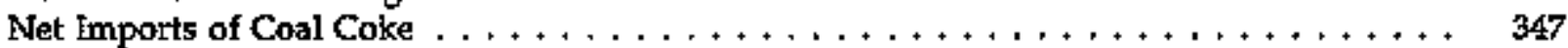

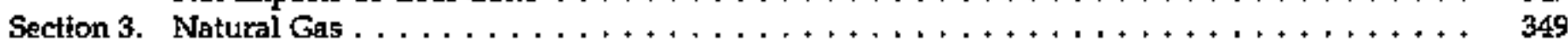

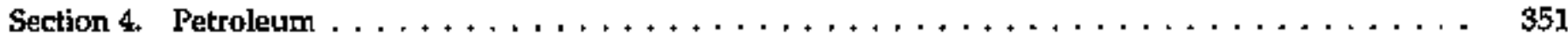

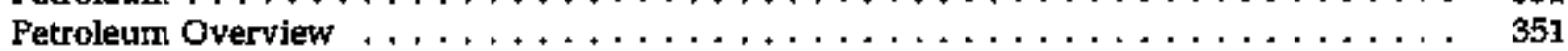

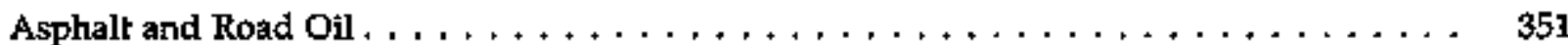

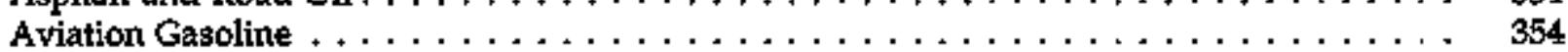

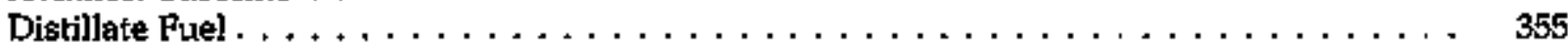

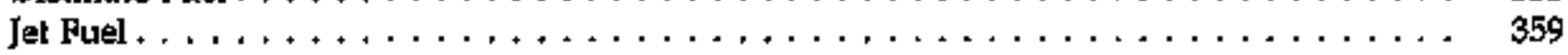

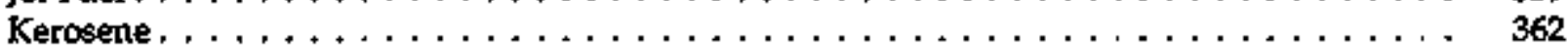

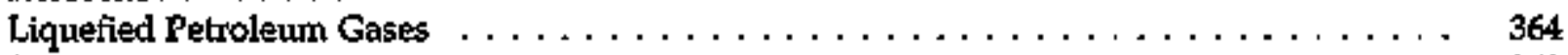

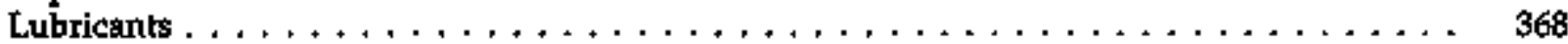

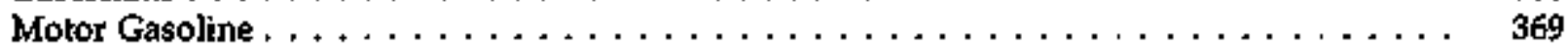

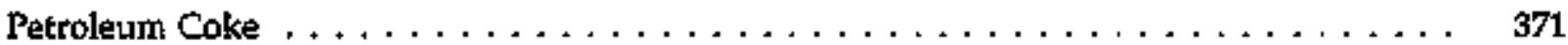

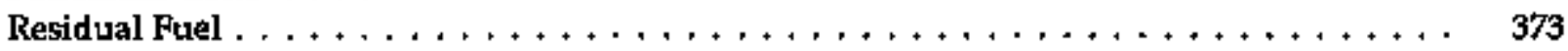

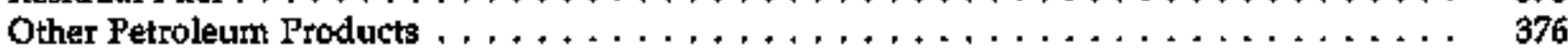

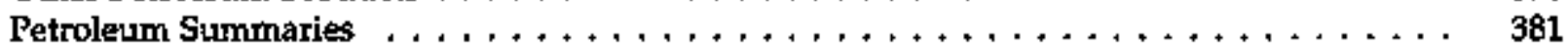




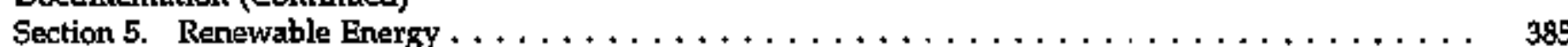

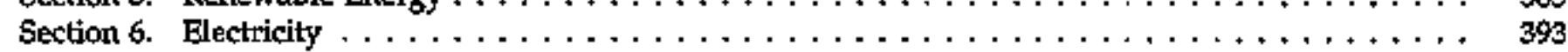

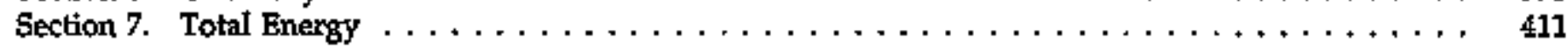

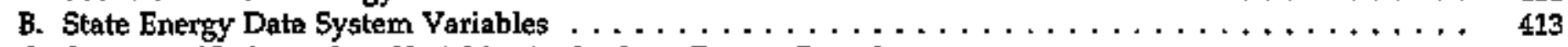
C. Sources of Independent Variables in the State Energy Data System $\ldots \ldots \ldots \ldots \ldots \ldots \ldots \ldots \ldots \ldots \ldots$

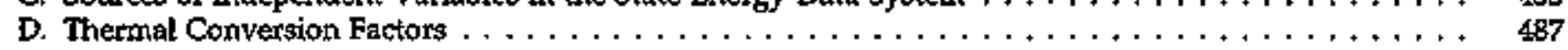
E. Metric and Other Physical Convergion Factors $\ldots \ldots \ldots \ldots \ldots \ldots \ldots \ldots \ldots \ldots \ldots \ldots$

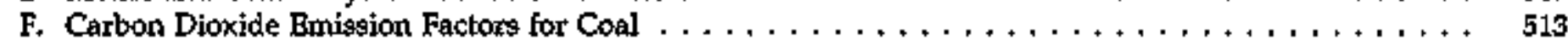
G. Summary of Changes Since the State Energy Data Report $1993 \ldots \ldots \ldots \ldots \ldots \ldots \ldots \ldots \ldots \ldots \ldots$

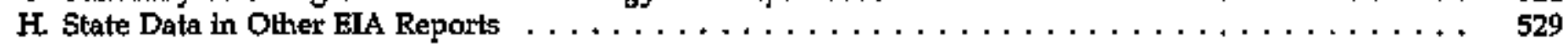

A. Documentation (Continued)

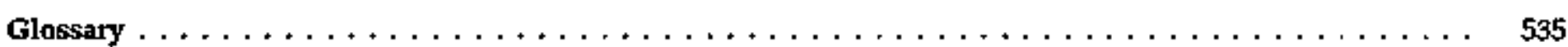




\section{Introduction}

\section{Purpose}

The State Energy Data Report (SEDR) provides annual time series estimates of State-level energy consumption by major economic sector. The estimates are developed in the State Energy Data System (SEDS), which is maintained and operated by the Energy Information Administration (EIA). The goal in maintaining SEDS is to create historical time series of energy consumption by State that are defined as consistently as possible over time and across sectors. SEDS exists for two principal reasons: (1) to provide State energy consumption estimates to Members of Congress, Federal and State agencies, and the general public; and (2) to provide the historical series necessary for ELA's energy models.

\section{System and Report}

During the development of the State Energy Data System, efforts are made lo ensure that the sums of the State data equal the national totals as closely as possible for each energy type and end-use sector as published in other EIA publications. Estimates in this State Energy Data Report (SEDR) are generally comparable to the statistics shown in the Annual Energy Review 1995 and the Monthly Energy Review, March 1996.

Due to page-size constraints, SEDR tables do not show data from 1961 through 1964 and 1966 through 1969; however, those data are maintained in SEDS, are included on the personal computer diskettes, and are covered by the documentation in this report.

Extensive documentation follows the data tables in this report. Appendix A describes how the estimates were derived in SEDS by each individual energy source. Appendix B lists alphabetically all of the varlable names and formulas used in the system. Appendix $C$ lists the sources of all data series entered into SEDS. Appendix D lists the conversion factors used in SEDS to convert physical units into British thermal units and cites the sources for those factors. Appendix $\mathrm{E}$ provides metric and other physical conversion factors for measures used in energy analyses. Appendix $F$ contains carbon dioxide emission factors. Appendix $G$ summarizes the changes made in SEDS since the last report, whith was released in July 1995. Appendix $\mathrm{H}$ lists other EIA reports containing State-level data.

\section{Improvements}

Renewable Energy. The coverage of renewable energy data is significantly expanded in this edition of SEDS and SEDR. In previous editions, renewable energy was fully covered in only the electric utility sector. This year, estimates of renewable energy used in the other sectors are incorporated beginning with 1990. This change adds about three quadrillion Btu to the total amount of energy consurned annually in the United States. While this change gives a more comprehensive total of U.S. energy usage, it does cause a notable break in the time series between 1989 and 1990.

Specifically, the residential sector now includes biofuels (i.e., wood) and solar energy estimates. The industrial sector is expanded to report biofuels and other energy sources (which comprise geothermal, wind, photovoltaic, and solar energy-all separately available in the data system) and incorporates augmented hydroelectric power data. The transportation sector now displays estimates of ethanol consumption. 
Electric utilities' coverage remains the same but biofuels are now separately displayed on the SEDR tables. Within SEDS, beginning with the 1990 data, conventional power generation and pumped storage generation from hydroelectric resources are available separately, and traded electricity, previously assumed to be exclusively hydroelectric, is now classified as hydroelectric-based, geothermal-based, or nonrenewable-based. Also within SEDS from 1982 forward, data on electricity generation from wood, waste, solar, and wind energy sources are available separately.
Vehicle Fuel. Natural gas consumed as vebicle fuel has been removed from the commercial sector, where it was traditionally reported and has been added to the transportation sector consumption for 1990 forward.

Appendix G, Detatled information about all data revisions in this edition of SEDR is contained in Appendix $G$. All data values that have been revised since the last edition are preceded with an " $\mathrm{R}$ " in the report tables.

\section{Energy Consumption Measures-Total and Site}

Sources of energy can be categorized as primary and secondary. Primary sounces of energy, such as coal, petroleum, and natural gas are consumed directly. Electriclty is a secondary form of energy that is created from primary energy sources. The amount of electricity actually consumed by the end user (site consump. tion) does not include the energy lost in the generation and delivery of the electricity to the point of tase.

Primary sources of energy are measured in applicable physical units. Coal is measured by the short ton (equal to 2,000 pounds); petroleum, by the barret (equivalent to 42 gallons); and natural gas, by the cubic foot. Energy sources are also measured by their heat content, generally expressed in Brittish thermal units (Btu). As shown in the tables in Appendix D, in 1994 the average short ton of bituminous coal and lignite consumed at electric utilities contained 20.7 million Btu, the average barrel of distillate fuel contained 5.825 million Btu, and the average cubic foot of natural gas consumed at electric utilities contained 1,023 Btu.

Electricity, a secondary form of energy, can also be measured in physical units, commonly in kilowatthours, and by heat content. The conventional thermal conversion factor for electricity consumed by the end user (site consumption) is 3,412 Btu per kilowathour.

Tabie A9 on page 401 shows that electric utilities consumed 30.4 quadrillion Btu of primary energy sources in 1994 in order to generate 9.9 quadrillion Btu of electricity. These data indicate that 67 percent of the primary (embodied) energy in the fuels consumed to generate the electricity was used or lost in the conversion to electricity and transmission of it to the consumers, and 33 percent was used as site (point-of-use) electricity by consumers.

In evaluating energy consumption in this report, tables titled "Total Energy Consumption" inciude all primary energy sources, tncluding those ubed to generate electricity; the electricity generated is not included. Tables showing "Total End-Use Sector Consumption" include columns for the primary sources and electricity that are consumed by the sector, as well as a column for the estimated energy lost in the electrical system processes. The "Total" column in those tables tncludes all energy consumed by the sector and the associated energy lost in the generation and transmission of electricity. The column titled "Net" is site energy consumption-that is, the sum of the primary sources and electricity, excluding the electrical system energy losses. 


\section{Data}

Estimation Methodologies. SEDS develops estimates of energy consumption by principal energy sources and major end-use sectors, by State, for a 35-year period. Energy consumption is estimated by using data from existing surveys of energy suppliens that report con- sumption, sales, or distribution of energy at the State level. Most of the SEDS estimates rely directly on collected State-jevel cansumption data. (See box below that summarizes the status of current data sources used.) Some consumption estimates in SEDS are based on a variety of surrogate measures. The measures were selected prixipally on the basis of applicability as an indicator of consumption,

\section{Collected Data and Estimated Values in SEDS}

Coal. U.S. anthracite, bituminous coal and lignite, and total coal consumption data by sector are taken directly from ElA's $Q u a r-$ terly Conl Report ( $Q C R$ ) or are unpublished data from EIA's Weekly Coal Production database. Total coal consumption by State and for most sectors is from the $Q C R$, except where values are withheld and must be estimated. The State-level disaggregation of the $Q C R$ 's combined residential and commercial sector and the combined anthracite and bituminous coal and binite use in all sectors (except electric utllitles) are estlmates. Data on electric uttlity coal consumption by State and coal type are data from the Form ElA-759, 'Monthly Power Plant Report," database.

Natural Gas. Natural gas consumption by State and sector are taken directly from the EIA's Naturol Gas Annual (NGA). The data series from the NGA, natural gas consumed as lease fuel and plant fuel and natural gas delivered to industral consumers, are combined in SEDS as industrial sector consumption. Natural gas consumed as vehicle fuel and pipeline fuel are combined in SEDS as transportation sector consumption.

Petroleum. U.S. total consumption for each petroleum product is the "product supplied" data from ElA's Petroleum Supply Annuthl. State values for distillate fuel and resldual fuel consumption at electric utilities are unpublished data from EIA's Form EIA.759 database. All other State and sector values for consumption of petroleum products are estimates based on sales data seties from several sources.
Renewable Energy, * Residential and commercial sector con* sumption of biofuels and solar energy are estimated. - Industrial consumption of biofuels is also estimated. Industrial consumption of hydroelectric power is data collected by the Federal Power Commission for 1960 through 1978, SEDS' estimates for 1979 through 1989, and data collected by EIA on Form. EIA867, "Annual Nonutility Power Producer Report," for nonutility power production for 1990 forward. Industrial consumption of geothermal, wind, solar thermal, and photovoltaic energy is oollected on the Form ETAn867. - State-level transportation use of biofuels is estimated, although the U.S. data are collected on several forms and reported in the Renewable Energy Annual. - All sources of renewable energy used for electricity genetation at electric utilities (i.e., biofuels, hydroelectric power, geothermal, wind, solar thermal, and photovoltaic energy) by State are from ELA's Electric Povter Antual (EPA) or are unpublished data from the Form EIA-759 database.

Nuclear Electric Power. Nuclear electricity generation by State is from the EPA.

Electrictity. Electricity consumption is sales data by sector and State from the EPA with one exception. The EPA "Other" category is allocated to the transportation and commercial sectors in each State by estimation.

Electrical System Energy Losses and Net Interstate Flow of Electricity. These series are estimated in SEDS. 
availability, continuity over time, and consistency. For instance, for petroleum, product supplied is a surrogate for consumption and is derived by summing field and refinery production, plus imports, minus exports, plus or minus change in stocks. State-level sales survey data are used to disaggregate the national petroleum product supplied totals to the States. The measures of consumption and estimation methodologies are explained in detail under each energy source in Appendix A.

Methods are also created to estimate State electrical system energy losses that are not available from any surveys. See the box on page 2 for a discussion of electrical system energy losses and how they are reflected in SEDR tables. U.S. total electrical system energy losses are ailocated to each individual State's end-use sectors in proportion to the sectors' electricity sales. The method for estimating does not separately identify electrical system energy losses from interstate flow of electricity. Therefore, speciftc estimates are developed for Alaska and Hawaii and for the 48 contiguous States. Section 5 of Appendix A explains the methodology in detail. EIA is examining a method to disaggregate the estimates of net interstate flow of electricity and electrical system energy losses that are currently combined in SEDS. The explanation and Tables A10 through A18 in the Electricity section of Appendix A (pages 401-409) discuss and iliustrate the alternattve methodology.

Data Sources. The original source documents cited in Appendix C include descriptions of the data collection methodologies, universes, imputation or adjustment techniques (if any), and errors assoctated with the processes. Dut to the numerous collection forms and procedures associated with those reports, it is not possible to develop a meaningful numerical estimate of the overall ertors of the integrated data published here.

Reliable, consistent series for long periods of time-especially in the earlier years-are difficult to develop, and estimates and assumptions must be applied to fill data gaps and to maintain defiritional consistency. Although SEDS incorporates the most consistent series and procedures possible, users of this report should recognize the limitations of the data that are due to changing and inadequate data soutces.
In reports prepared by the Bureau of Mines in the late 1960's and early 1970 's, petroleum consumption was equated to demand. Later, consumption was equated to apparent demand and, more recently, to praduct supplied. Changes in surveys and reduction of data collections, especially after 1978, disturbed the continuity of some petroleum consumption series, most notably for distillate fuel, residual fuel, kerosene, and liquefied petroleum gases. These and other date series' inconsistencies are explained in detail under the "Additional Notes" for each energy source in Appendix A. All data series with recognized data inconsistencies are footnoted in the SEDR tables.

\section{Comparison with Other Energy Consumption Reports}

EIA conducts numerous energy-related surveys. In general, the surveys can be divided into two broad groups. One group of surveys, called supply surveys, is directed to the suppliers and maxketers of specific energy sources. Those surveys measure the quantities of spectific fuels supplied to the market. The results of supply surveys are combined and published in a number of EIA publications, in cluding the Monthiy Entergy Reoiew and SEDR. The Becond group of surveys, called energy consumption surveys, gathers information directly from end users of energy. Although there are some elements in common, the supply survey data and the consumption survey data have substantially different approaches, capabilities, and objectives. Thus, care must be taken in analyzing SEDR data in conjunction with consumption survey data, for the following reasons:

- SEDR is designed to be a broad accounting of energy consumption, covering all energy use and splitting it thto major sectors as clearly as possible. The energy consumption surveys are designed to be comprehensive and representative within individual sectors. However, the sectors are restricted for purposes of creating relatively homogeneotss, well-deftned populations and aiding in sampling and data collection. For example, the Residenttal Transportation Energy Consumption Survey covers only household vehtcles; SEDS covers all uses of energy for transportation of persons and commoditles. Similarly, the Commerctal Buildings Energy Consumption Survey covers only energy consumption in commercial bujldings, while SEDS includes other commercial consumption. 
such as street lighting and public services; and the Manufacturing Energy Consumpion Survey covers only manufachuring establishments, while SEDS includes other industrial energy consumption (i.e., mining, construction, agriculture, fisheries, and forestry). Further, the consumption surveys do not cover all energy-using sectors. Therefore, energy consumption surveys cannot be summed together to account for all energy use.

- Energy consumption surveys provide user characteristics that allow for bath macro-level (for major sectoral sub-populations) and micro-level (at the unit of data collection) interpretive analysis. The surveys of energy consumption by residential households from the Residential Energy Consumption Survey (Form EIA-457 series), for personal transportation from the Residenttal Transportation Energy Consumption Survey (Form EIA-876 series), and by commercial butldings from the Commercial Buildings Energy Consumption Survey (Form EIA-871 series) provide detailed information about the energy end users, their size, their stock of energy-consuming equipment and appliances, and their total energy consumption and expenditures. The Manufacturing Energy Consumption Survey (Form EIA-846 series) collects consumption, fuel switching capabitity, and limited end-user data from manufacturing establishments. SEDS, on the other hand, provides limited characterization of the end users of energy but much greater geographic and energy product detall, as well as extensive annual historical time series.

- Sectoral classification in SEDS is generally based on supplier classifications of customer accounts, by whatever means suppliers choose to use. (See discussion in next section.) Sectoral classification for the energy consumption surveys is based upon a categorization, verified by end user, of the primary economic activity of the data collection unit (household, vehicle, building, or establishment).

- The energy consumption surveys provide data at national and Census region and/or Census division levels, whereas the estimates in SEDS are on national and State levels.
- The reference periods are also different in that SEDS covers calendar years from 1960 through 1994, while the consumption surveys are for selected years, and the residential end-use surveys taken proor to 1987 cover a heating season year (i.e., April through March). Beginning with the 1987 residential end-use survey, the reference period is a calendar year.

For a more detailed description of the differences between SEDS and the energy consumption surveys, see the EIA analysis report Energy Consuntyfion by Erd-Lise Sector: A Contprrison of Mersures by Consumptiont and Supply Suroeys, DOE/EIA-0533, April 1990.

\section{Energy Consuming Sectors}

The consumption estimates in SEDS are based on data collected by various surveys that do not necessarily define the consuming sectors exactly the same way. Appendix A of this report describes in detail for each energy source how the collected data series are combined and assigned to SEDS consuming sectors. To the degree possible, energy consumption in this report has been assigned to the five sectors according to the following general definitions:

- Residential Sector: The residential bector is considered to consist of all private residences, whether occupied or vacant, owned or rented, including single-family homes, multifamily housing units, and moblle homes. Secondary homes, such as stummer homes, are also included. Insttzutional housing, such as school dormitories, hospitals, and military barracks, generally are not included in the residential sector; they are in. cluded in the commercial sector.

- Commercial Sector: The commercial sector, as defined economically, consists of business establishments that are not engaged in transportation or in manufacturing or other types of industrial activity (agriculture, mining, or construction). Commercial establishments include hotels, motels, restaurants, wholesale businesses, retail stores, laundries, and other service enterprises; religious and nonprofit organizations; health, social, and educational institutions; and Federal, State, and local governments. Street lights, pumps, bridges, and 
public services are also included if the establishment opetating them fs considered commercial.

- Industrial Sector: The industrial sector comprises manufacturing industries, which make up the largest part of the eector, along with mining, construction, agziculture, fisheries, and for" estry. Establishments in this sector range from steel mills, to small farms, to companies assembling electronic components.

- Transportation Sector: The transportation sector consists of private and public vehicles that move people and commodities. Included are automobiles, trucks, buses, motorcycles, tailroads and railways (including streetcars), aixcraitt, shlps, barges, and natural gas pipelines.

- Electric Utility Sectoc: The electric utłlity sector consists of privately and publicly owned establishments that generate, transmit, distribute, or sell electricity primarily for use by the public and that meet the definition of an electric utility. Nonutility power producers are not included in the electric utitity sector.
Sector Definition Discrepancies. Although the end-use allocations are made according to these aggregations as closely as possible, some data are collected by using different classifications. For example, electric utilities may classify commercial and industrial users by the quantity of electricity purchased rather than by the business activity of the purchaser. Agricultural use of natural gas is collected and reported in the commercial sector, rather than in the industrial sector. Because agricuItural use of natural gas cannot be identified separately, it is included in the commercial sector in this report. Another example is master-metered condominiums and apartments, and buildings with a combination of residential and commercial units. In many cases, the metering and billing practices cause residential energy usage of electricity, natural gas, or fuel oil to be included in the commercial sector. No adjustments for these discrepancies were made.

SEDS does not provtde further disaggregated end-use consumption estimates. For example, the industrial sectot cannot be broken down into the chemtcal of rubber industries, or all manufacturing, or agriculture. The input series for the system are provided in broad enduse categories from the data collection forms and are not available by the individual components. Further disaggregated regional information, such as counties or cities, are also not available from SEDS. 


\section{Summaries}


Table 1. Energy Consumption Estimales by \$ource and Endolise Sector, 1994 (Tri.Ion Biut)

\begin{tabular}{|c|c|c|c|c|c|c|c|c|c|c|c|c|c|}
\hline \multirow[b]{2}{*}{ stons } & \multirow[b]{2}{*}{ 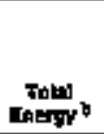 } & \multicolumn{8}{|c|}{ Serree: } & \multicolumn{4}{|c|}{ Eratunn Secters" } \\
\hline & & Ceses & 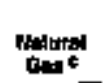 & Ritroturen & 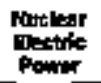 & 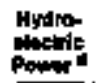 & Bdolvalp " & Onnr 1 & 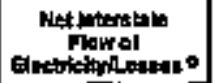 & Aouldendtid & Cominureta & theoputal " & Tramepontalion \\
\hline "1. & 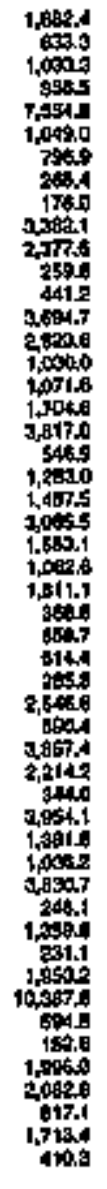 & 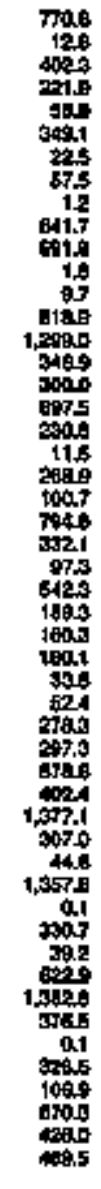 & 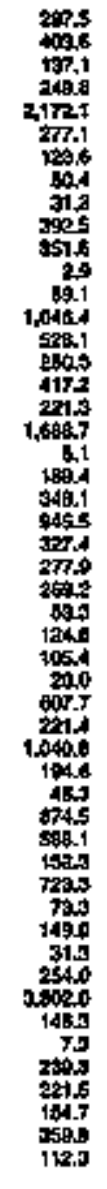 & 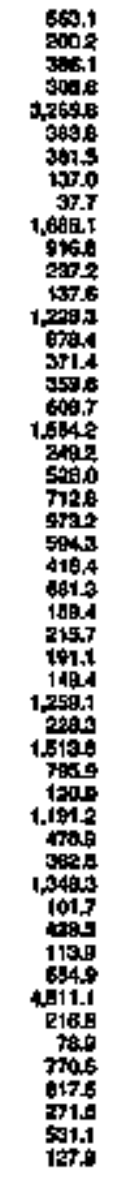 & 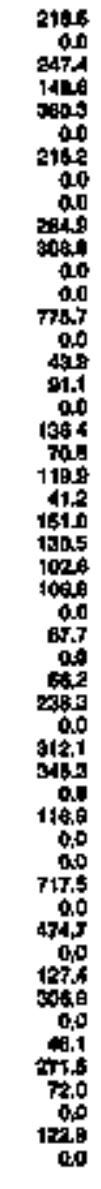 & 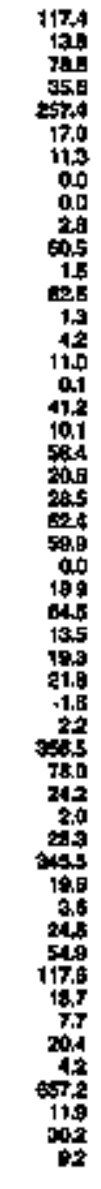 & 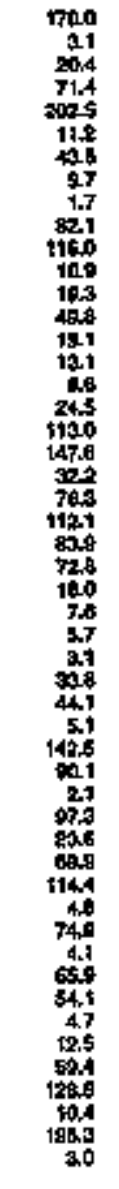 & 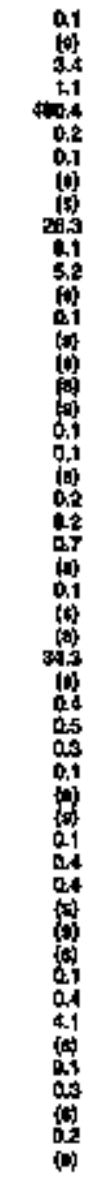 & 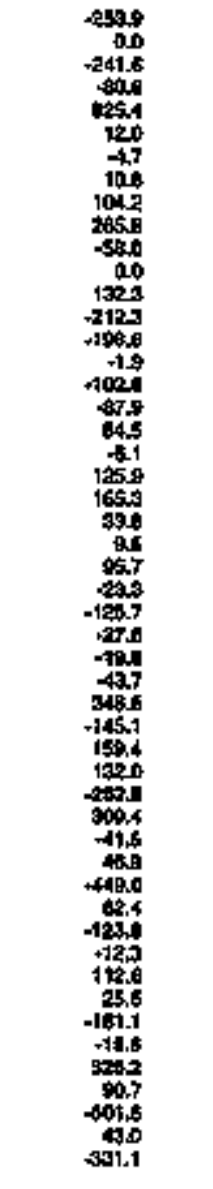 & 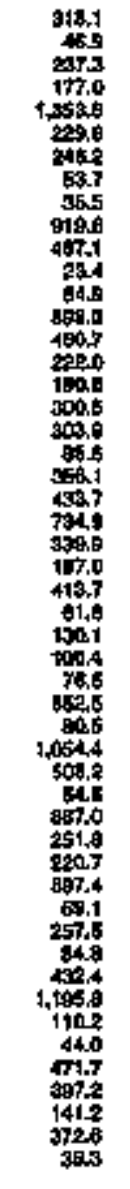 & 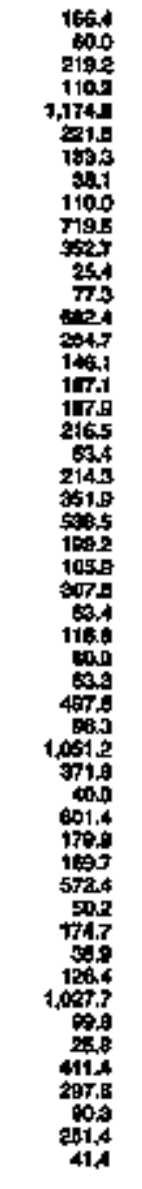 & 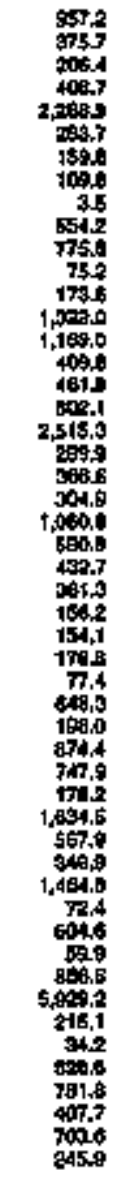 & 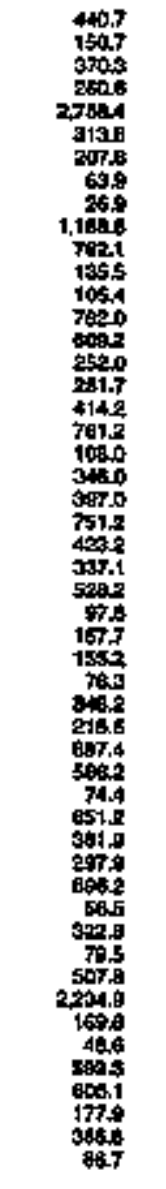 \\
\hline 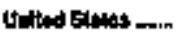 & so,7ased & 18E:1,1 & $21,31,0$ & 34,73:0 & $4 \times 973$ & 202035 & $2,052.2$ & 4800 & 0.0 & 17,6292 & 13,4312 & 3, $161 . \$$ & $23,572 x$ \\
\hline
\end{tabular}

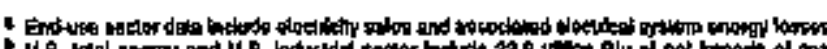

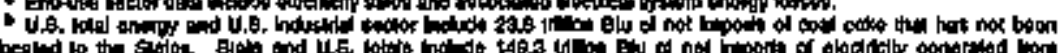

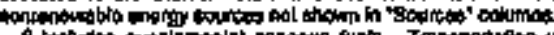

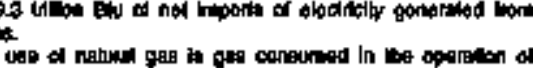

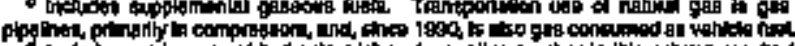

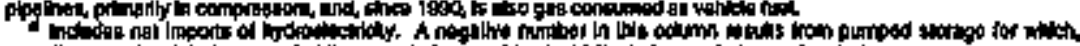

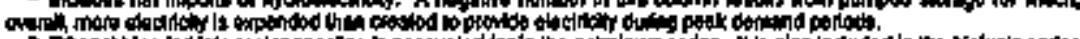

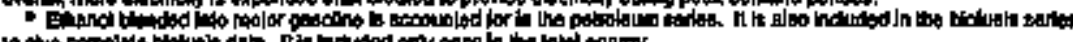

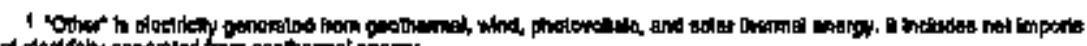

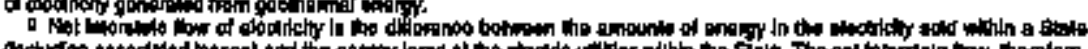

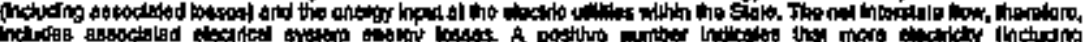

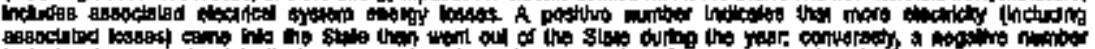

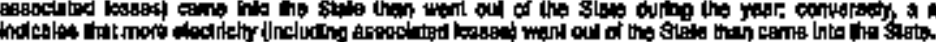

(l)

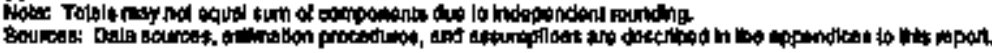


Table 2. Energy Consumpllon Esulmates in Physlcal Units, 1994

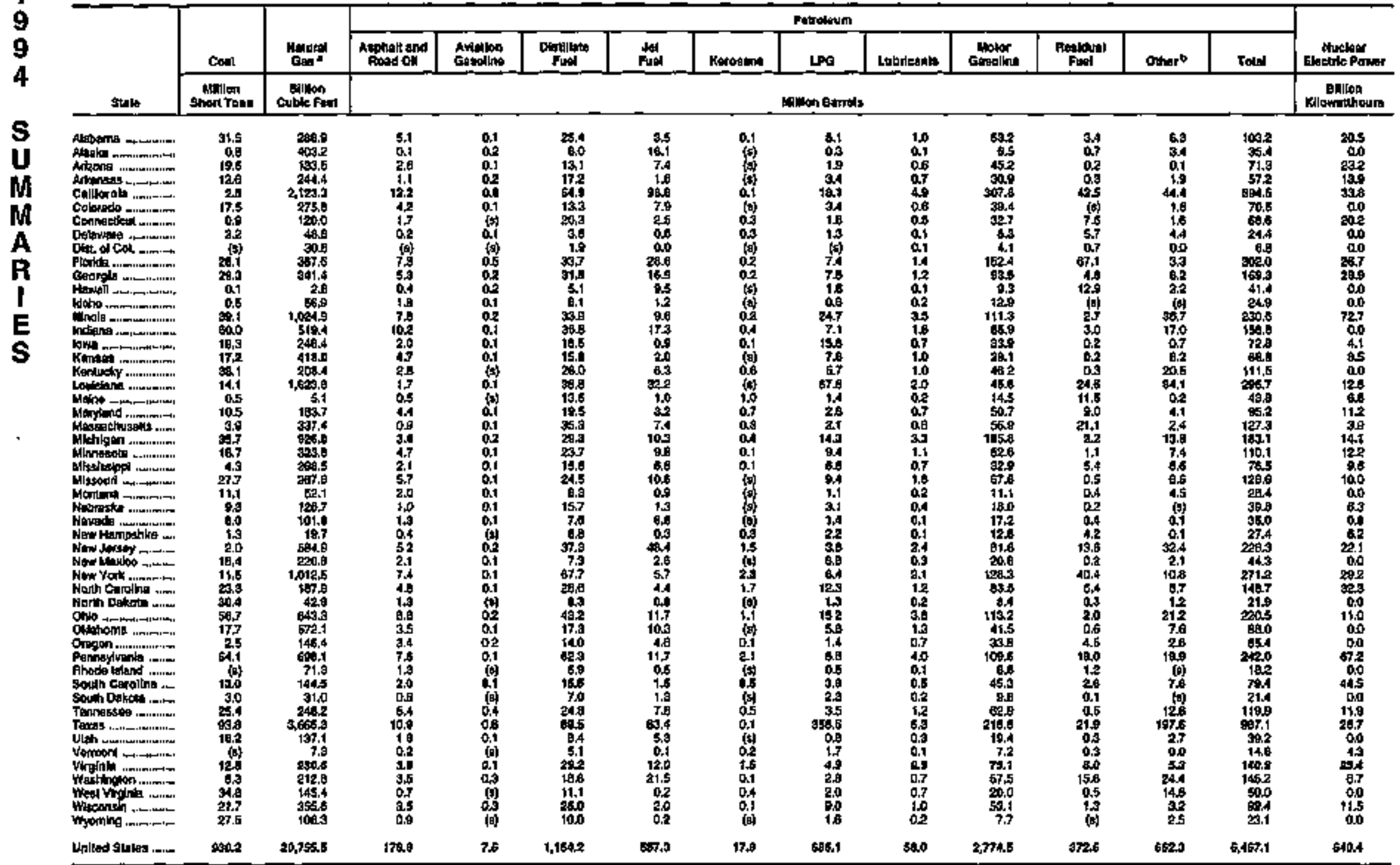

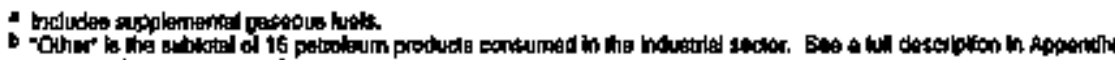

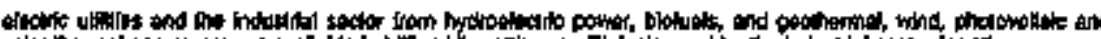

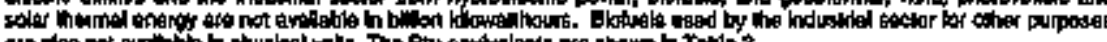

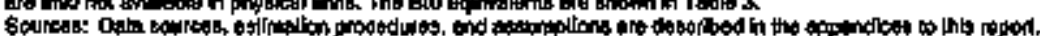


Tablo 3. Energy Consumption Estimates by Source, 1994 (Trillion Exu)

\begin{tabular}{|c|c|c|c|c|c|c|c|c|c|c|c|c|c|c|c|c|c|c|c|}
\hline \multirow[b]{2}{*}{ state } & \multirow[b]{2}{*}{$\cos$} & \multirow[b]{2}{*}{$\begin{array}{c}\text { Manural } \\
\text { coise: }\end{array}$} & \multicolumn{11}{|c|}{ Pelru|teun } & \multirow{2}{*}{ 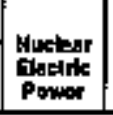 } & \multirow{2}{*}{ 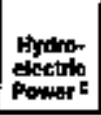 } & \multirow[b]{2}{*}{ Dbruokt of } & \multirow[b]{2}{*}{ Olber * } & \multirow{2}{*}{ 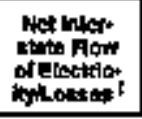 } & \multirow[b]{2}{*}{ Totow I } \\
\hline & & & resphant and & 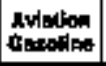 & 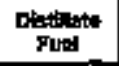 & fles & Karos: & LFo & $\begin{array}{l}\text { Letorit } \\
\text { cantx }\end{array}$ & Motor & Fontwel & atren & Fold & & & & & & \\
\hline . & 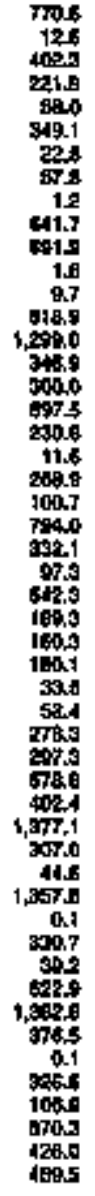 & 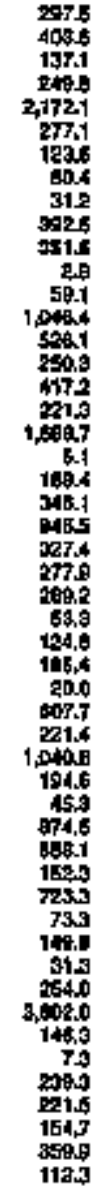 & 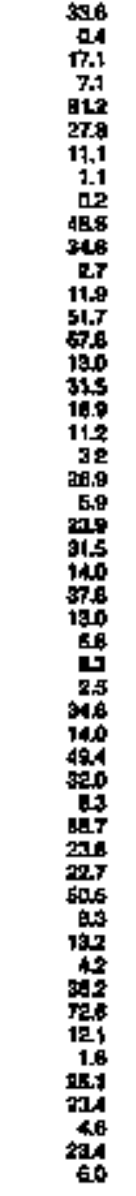 & 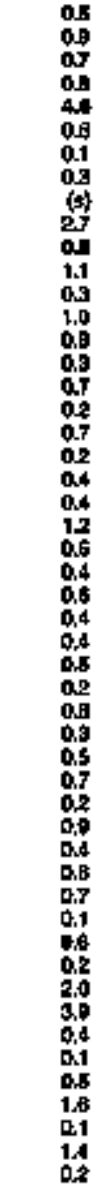 & 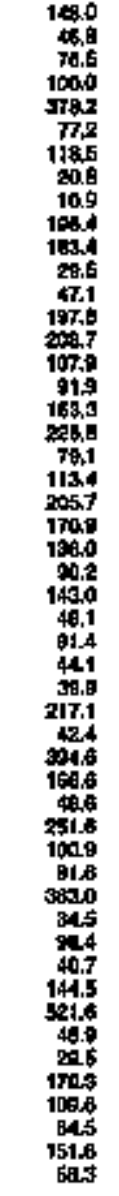 & 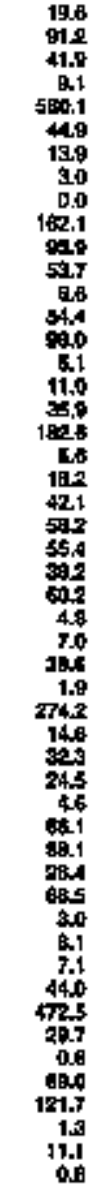 & 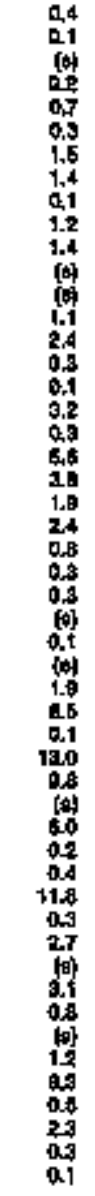 & 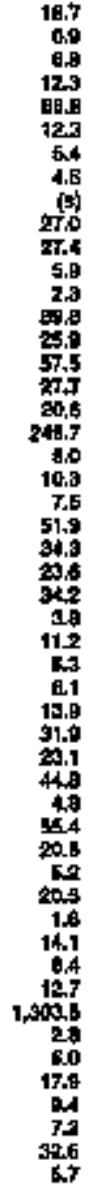 & 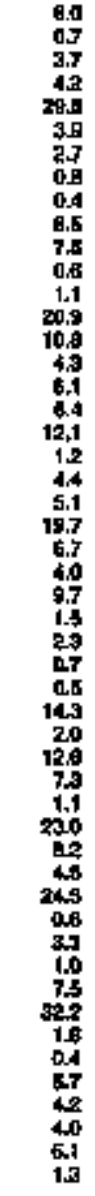 & 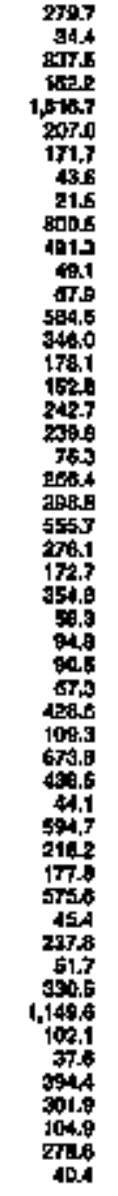 & 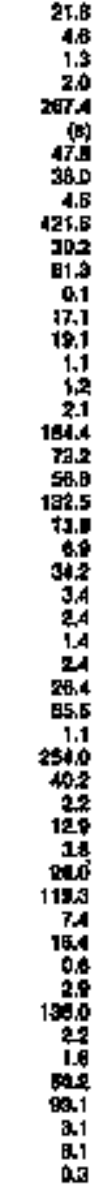 & 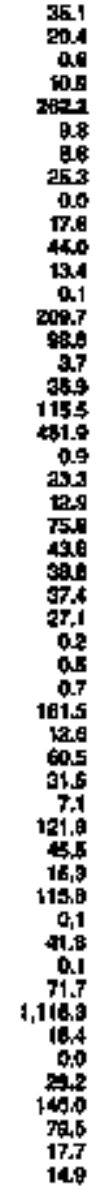 & 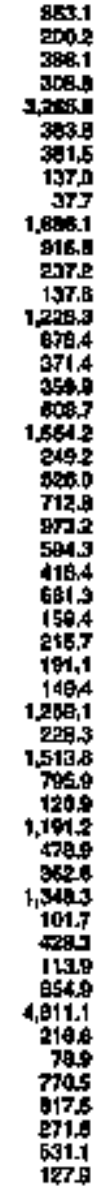 & 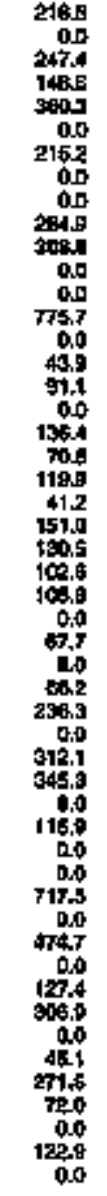 & 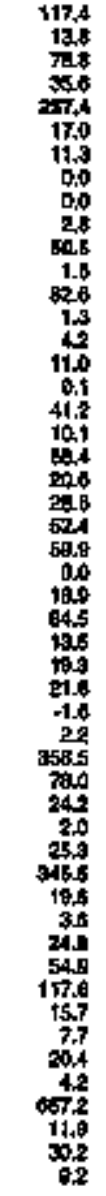 & 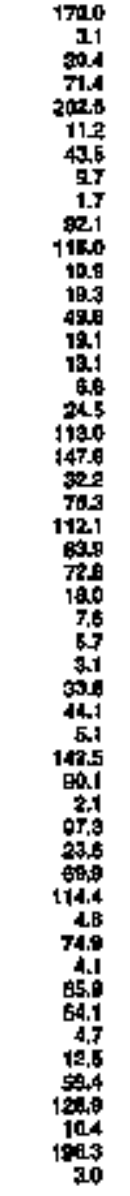 & 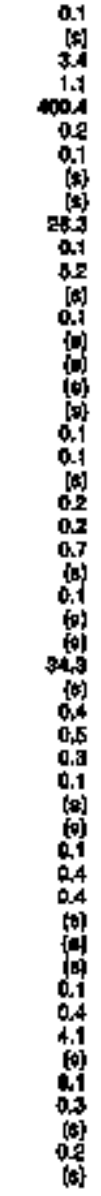 & 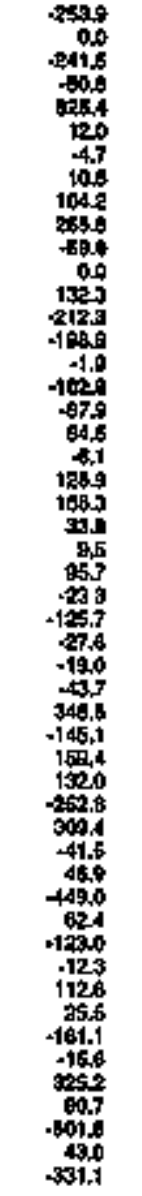 & 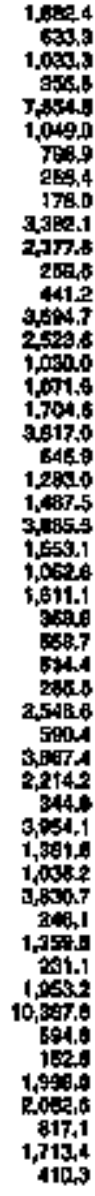 \\
\hline 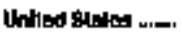 & $19,51 t .1$ & 21. Aง & 1,1729 & sa.1 & 6,7203 & $3,154,5$ & 101.1 & $2, \infty 9.9$ & $\mathbf{3 1 . 7}$ & 21,574, & 20ath.5 & 3, & $34 \pi \times 3$ & 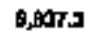 & 2,92313 & $2,42,2$ & exts & ab & 19,7\%6 \\
\hline
\end{tabular}

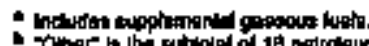

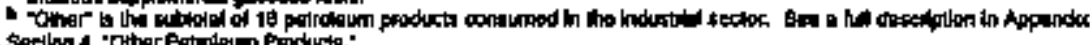

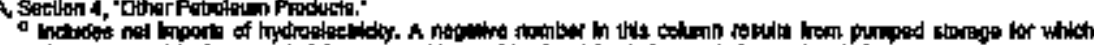

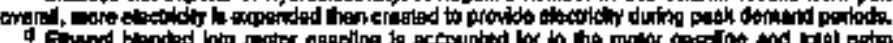

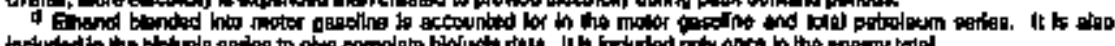
hectudedin

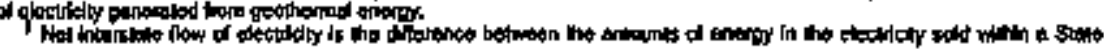

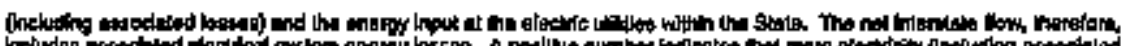

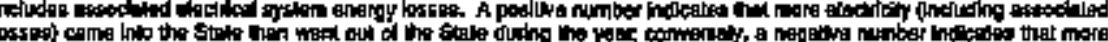

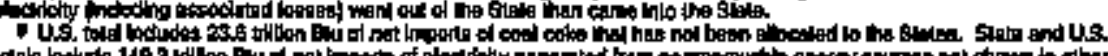

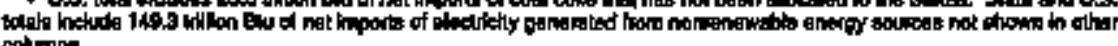

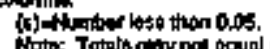

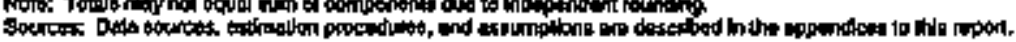


Table 4. Residential Energy Consumption Estimates, 1994 (Trillion Bhu)

\begin{tabular}{|c|c|c|c|c|c|c|c|c|c|c|c|c|c|c|}
\hline \multirow[b]{2}{*}{ gatn } & \multicolumn{3}{|c|}{ Cool 1} & \multirow[b]{2}{*}{$\begin{array}{l}\text { Munral } \\
\text { Gest }\end{array}$} & \multicolumn{4}{|c|}{ Pofrowitim } & \multirow[b]{2}{*}{ Elorlupats" } & \multirow[b]{2}{*}{ Sotar" } & \multirow[b]{2}{*}{ Asedingly } & \multirow[b]{2}{*}{ Enot } & \multirow[b]{2}{*}{ 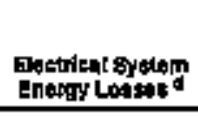 } & \multirow[b]{2}{*}{ Toldet bet } \\
\hline & 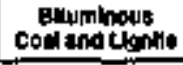 & Anuractive & Totopl 1 & & $\begin{array}{c}\text { Stallanit } \\
\text { Fued } \\
\end{array}$ & Karrosine & LPG & TOHI & & & & & & \\
\hline 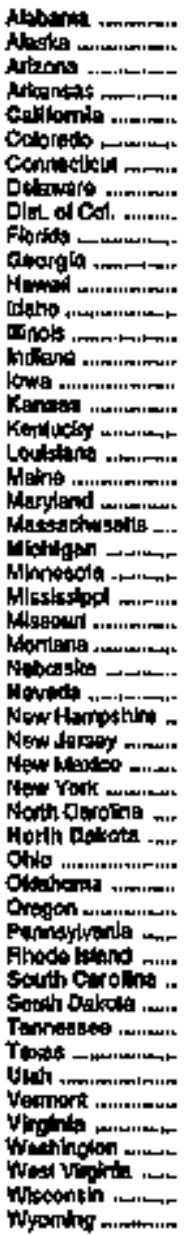 & 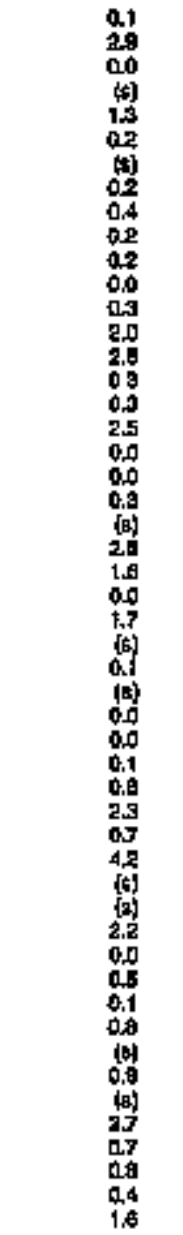 & 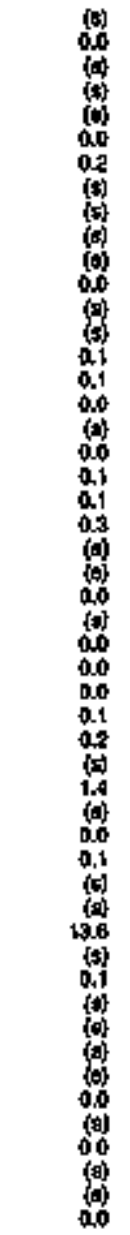 & 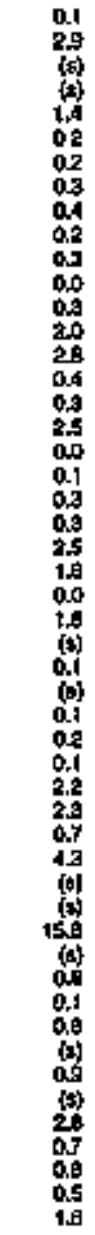 & 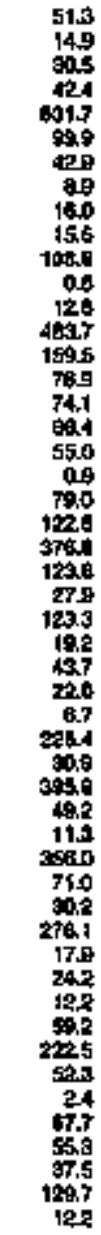 & 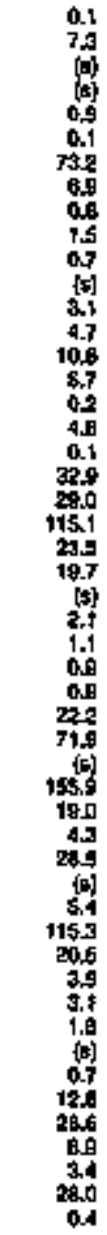 & 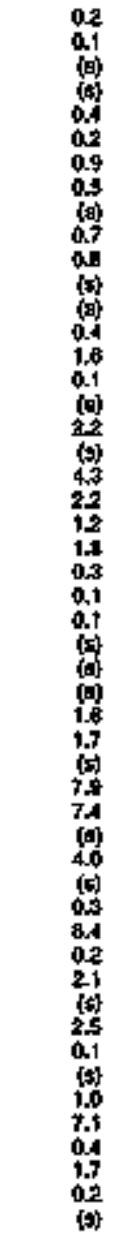 & 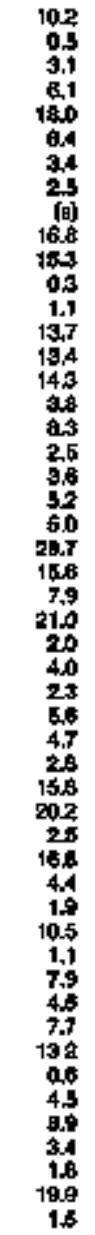 & 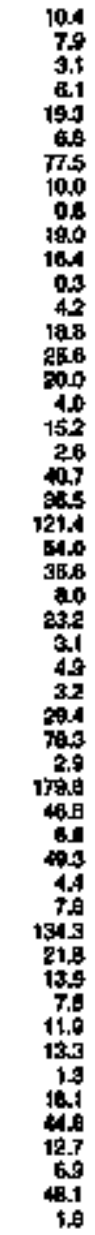 & 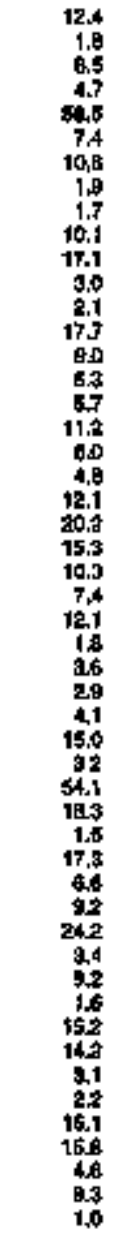 & 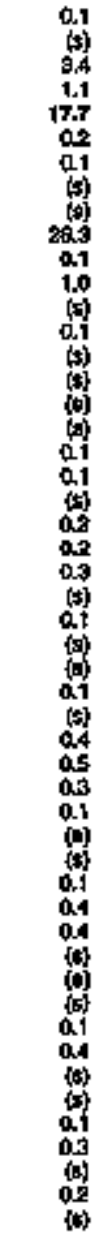 & 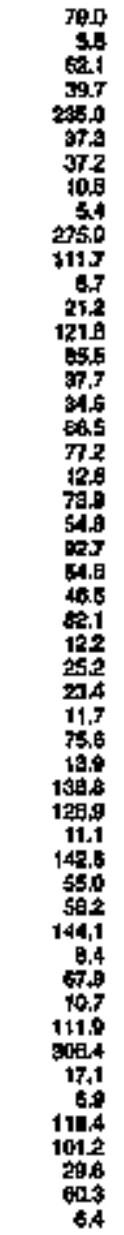 & 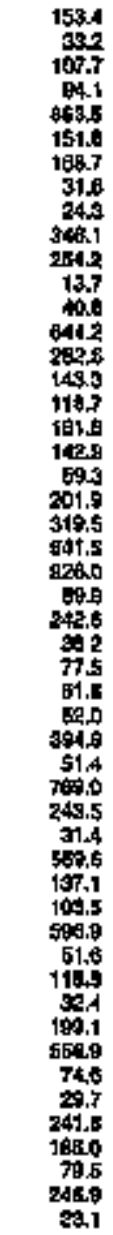 & 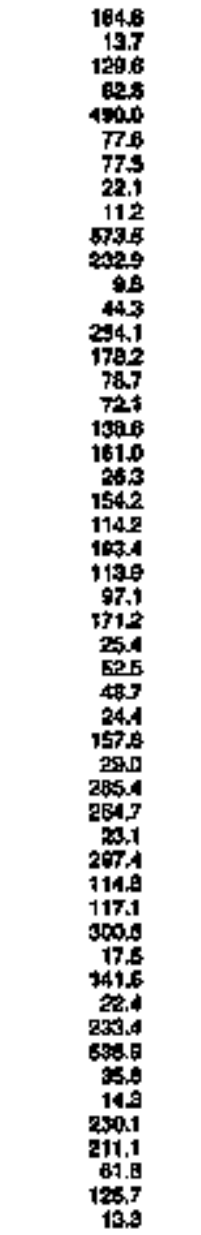 & 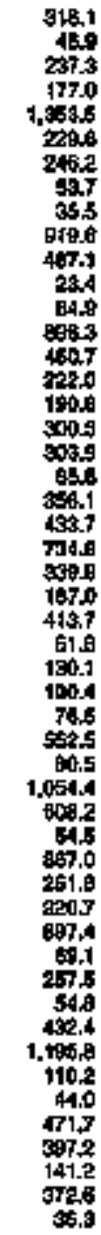 \\
\hline Unflod Shaleg L & 346 & $15 \mathrm{~A}$ & $\$ .1$ & $4,4+0, A$ & 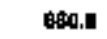 & 64.8 & 3855 & $1,340.4$ & $\operatorname{sen} 0$ & 55.2 & J,Aeass & $10,45 d, 0$. & $3,160,2$ & 17,0 ent \\
\hline
\end{tabular}

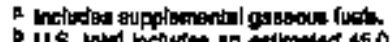

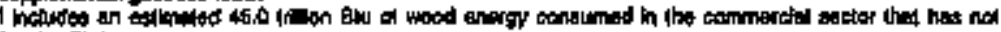

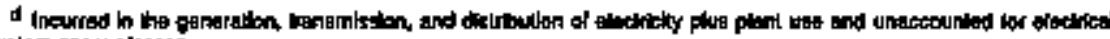

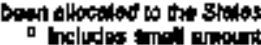

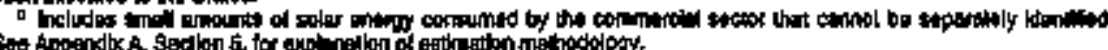

Inewrised in the

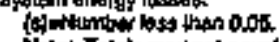

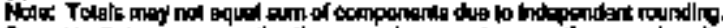

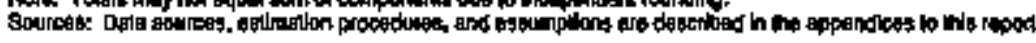


Table 5. Cornmereial Energy Consumption Estimates, 1994 (Tridtion BiU)

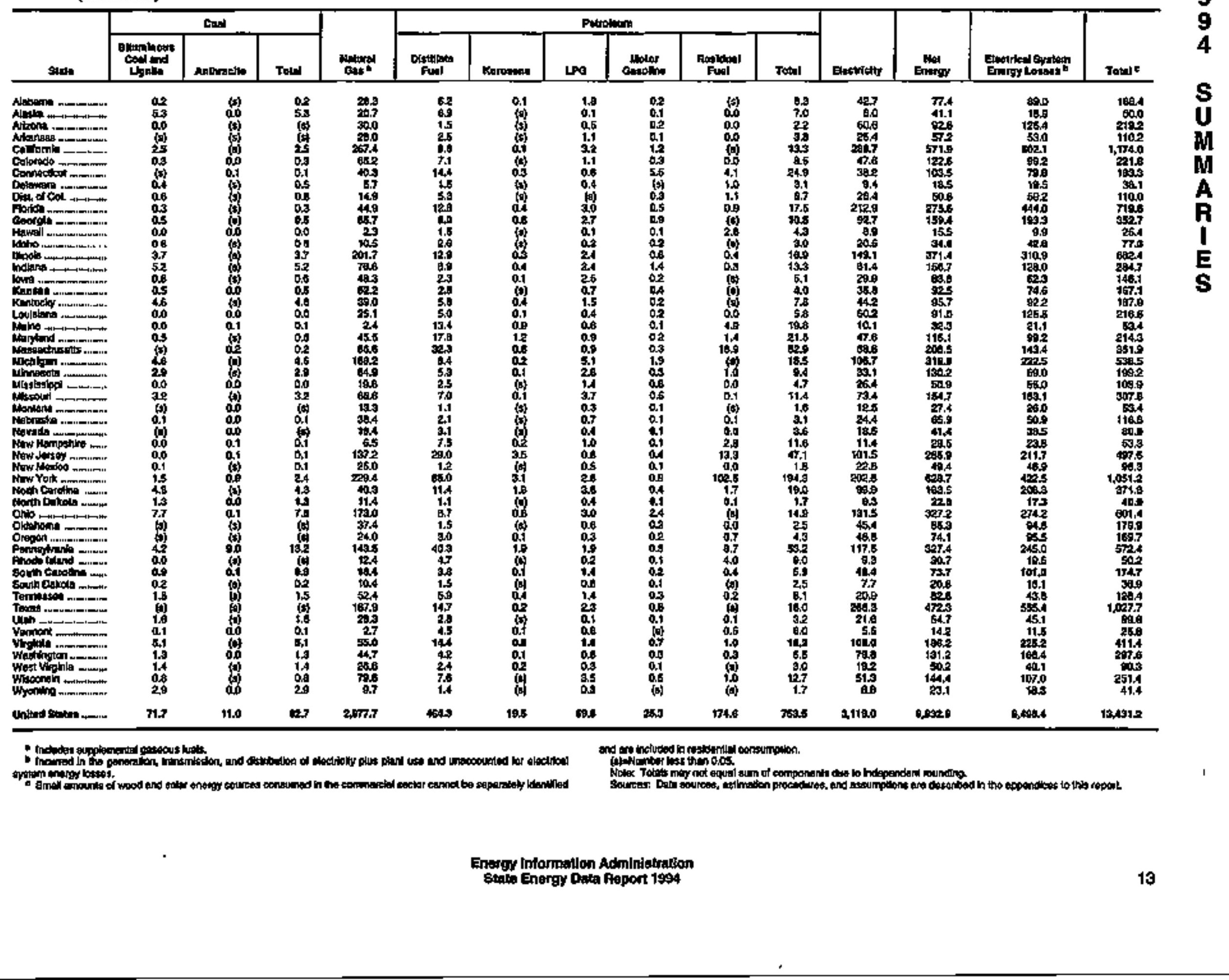


Teble 6. Indusblal Energy Consumption Estimales, 1994 (Trillion But)

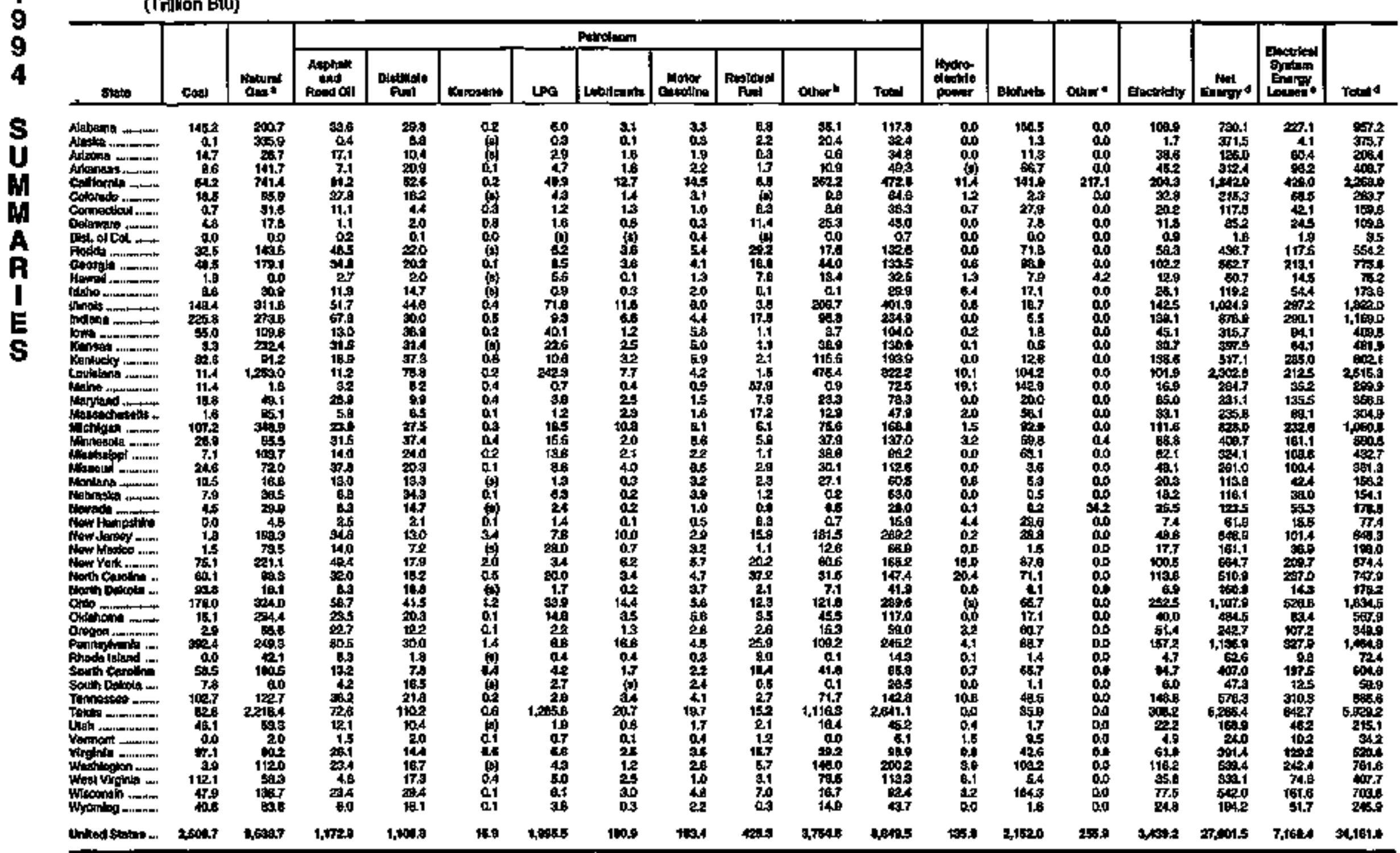

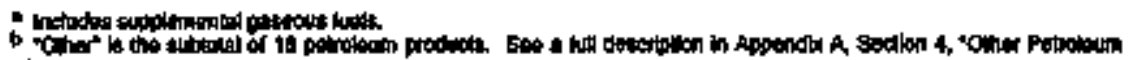
Produce"

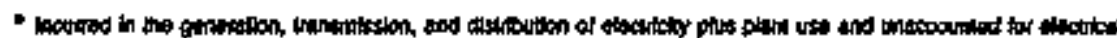

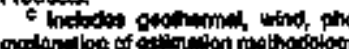


Tuble 7. Transportatlon Energy Consumption Esthmates, 1994 (Trillion B(u)

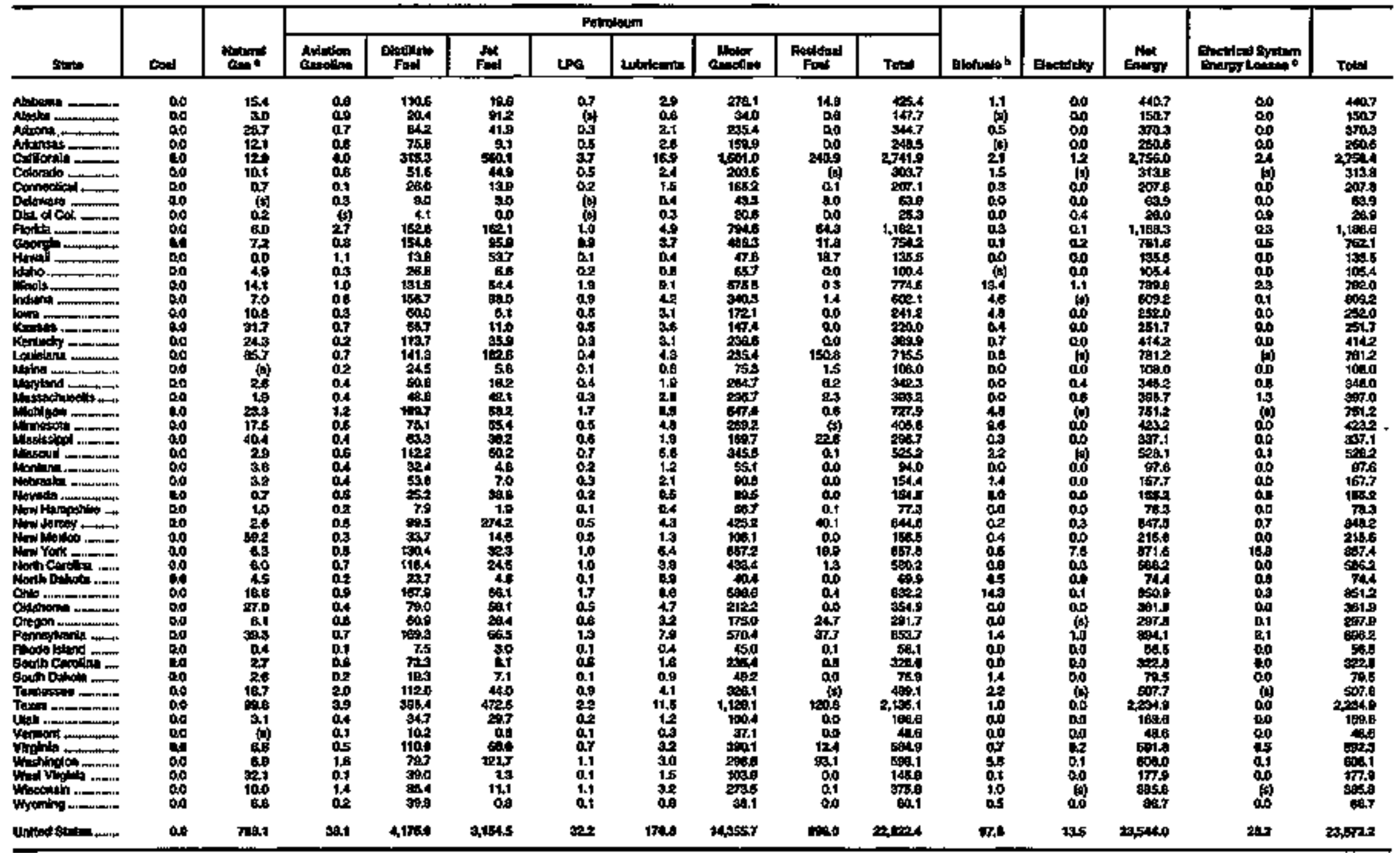

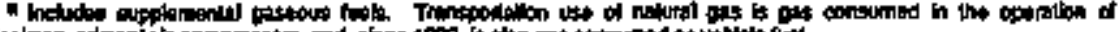

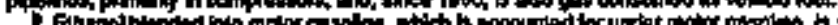

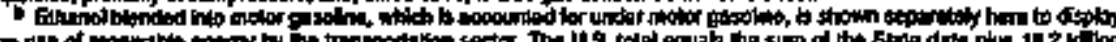

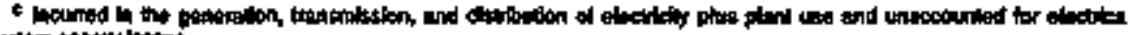

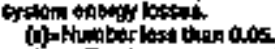

(1). The

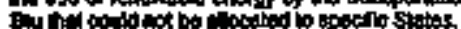

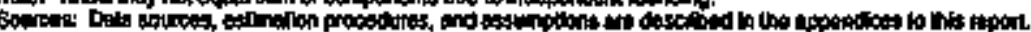




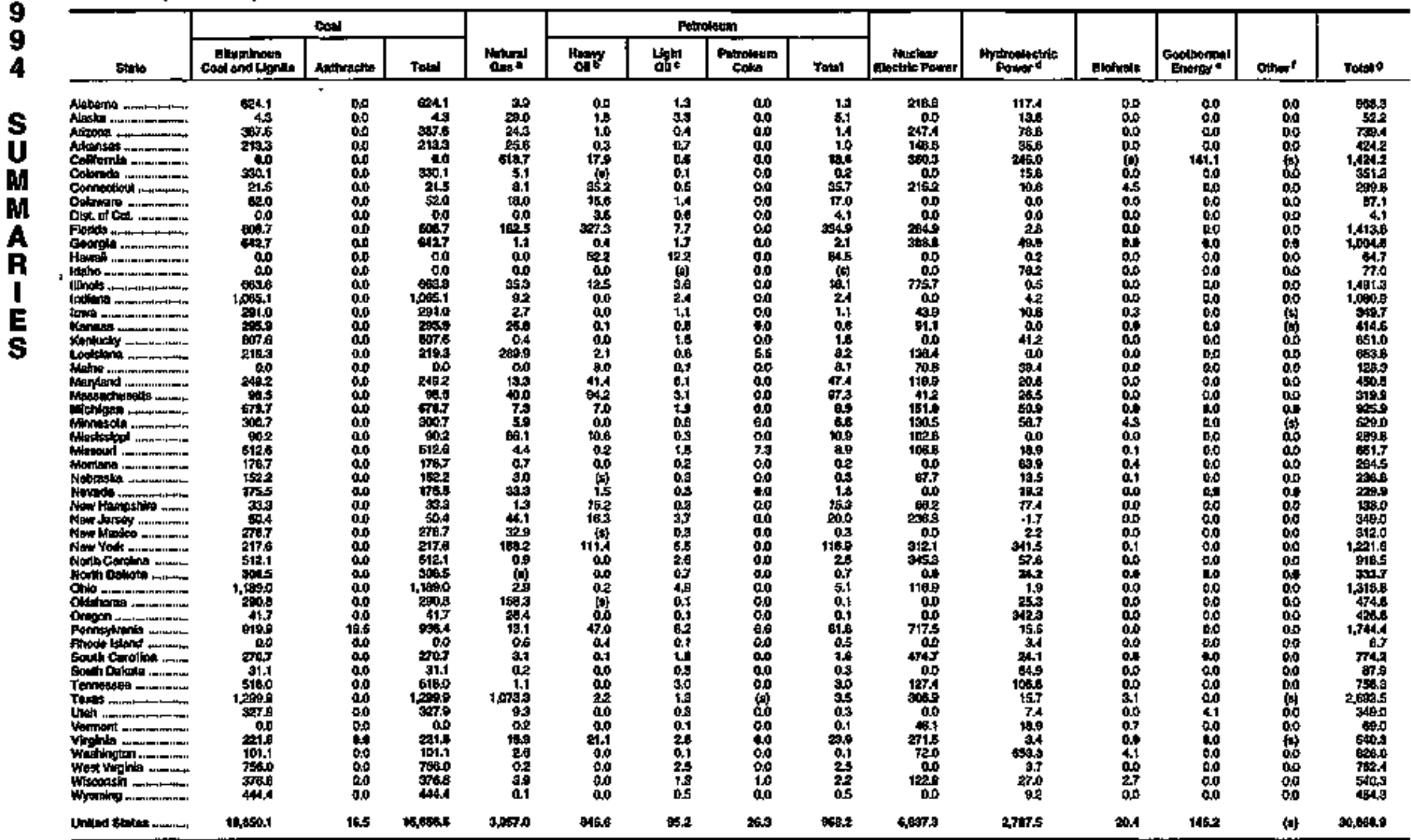

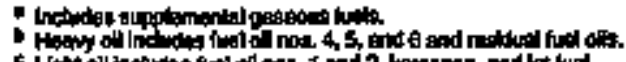

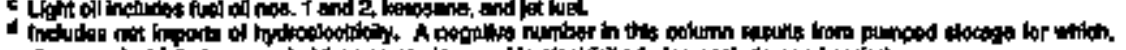

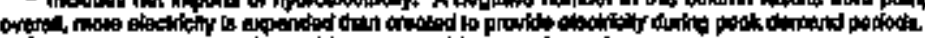

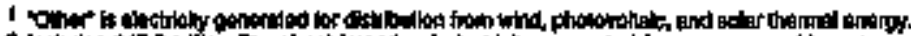

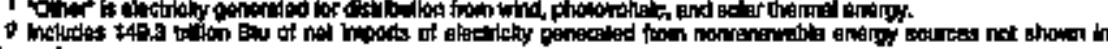

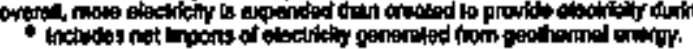

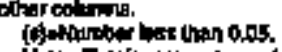

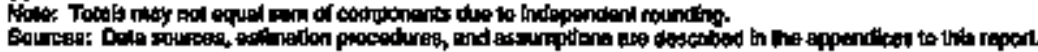




\begin{tabular}{|c|c|c|c|c|c|c|c|c|c|c|}
\hline \multirow{2}{*}{ Pank } & \multicolumn{2}{|c|}{ Fiesidentival Sector } & \multicolumn{2}{|c|}{ Compitirelal Settar } & \multicolumn{2}{|c|}{ Itodustrial Sogtur } & \multicolumn{2}{|c|}{ Tansportidion sector } & \multicolumn{2}{|c|}{ Toles Concumpition } \\
\hline & stalo & Thllion Btu & Snte & THIlon Bky & serate & Trakon Biy & stats & Tritilon Em & Stets & Trillon Bto \\
\hline 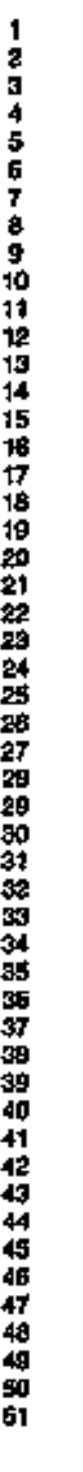 & 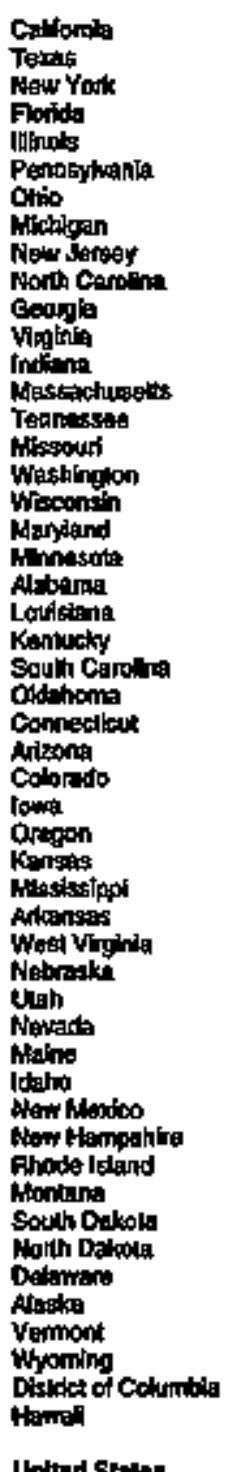 & 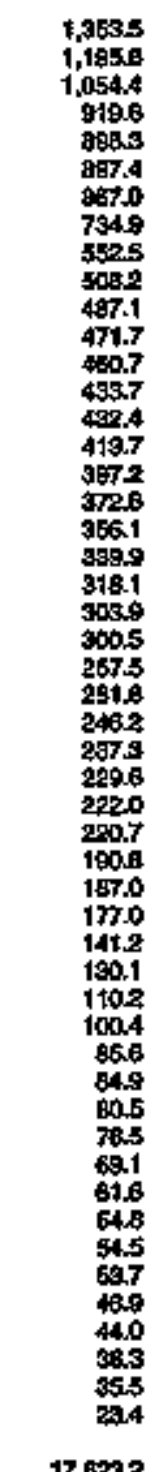 & 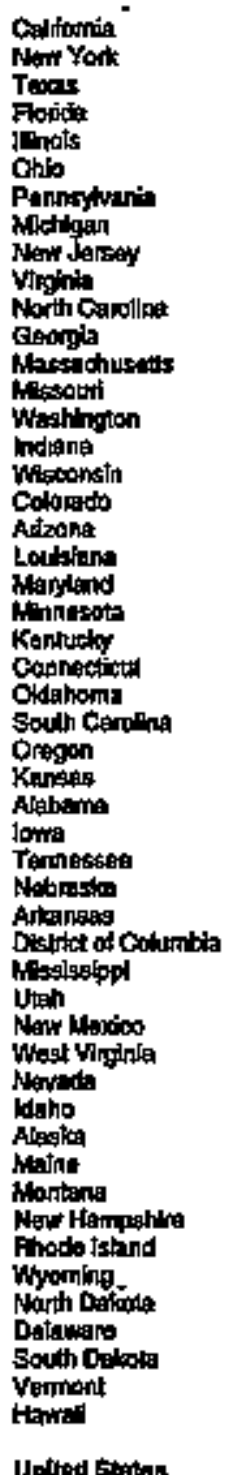 & 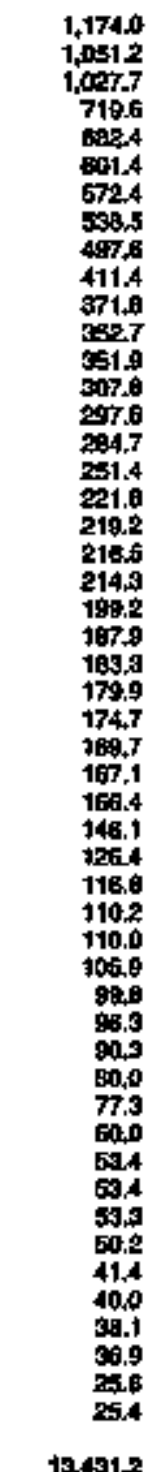 & 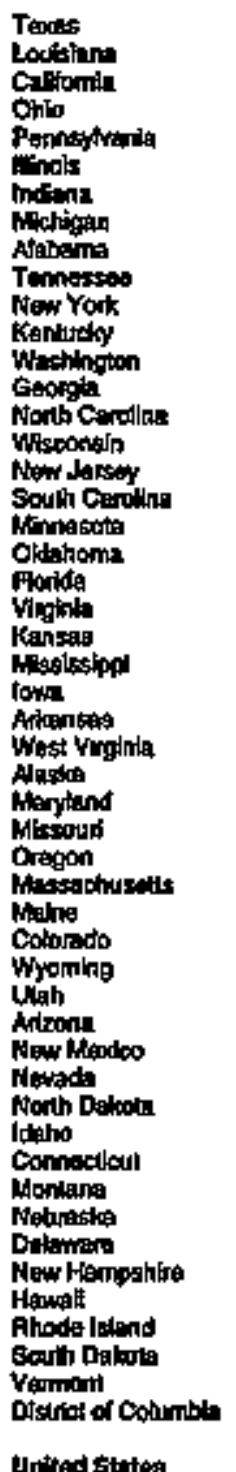 & 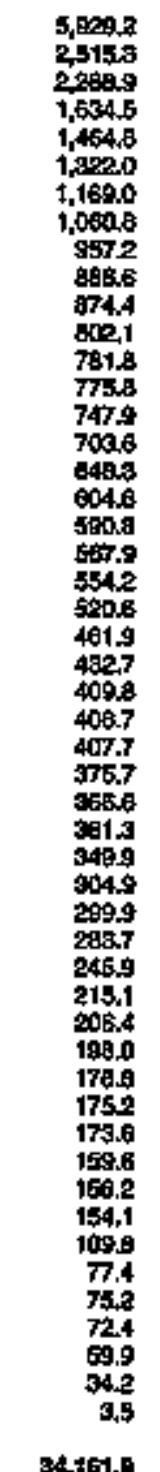 & 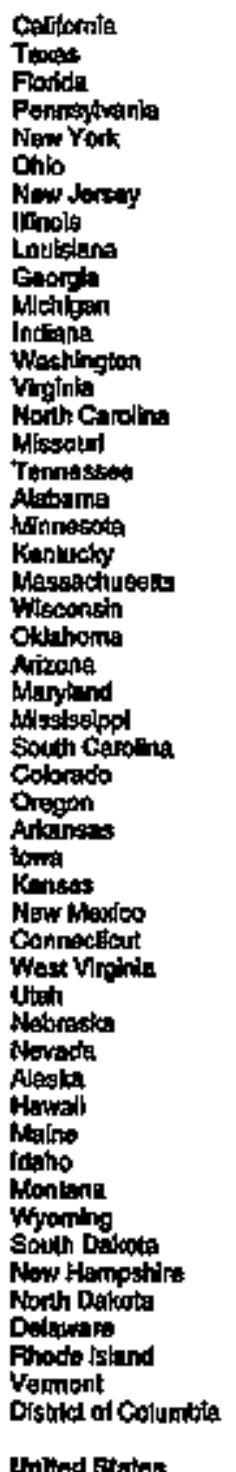 & 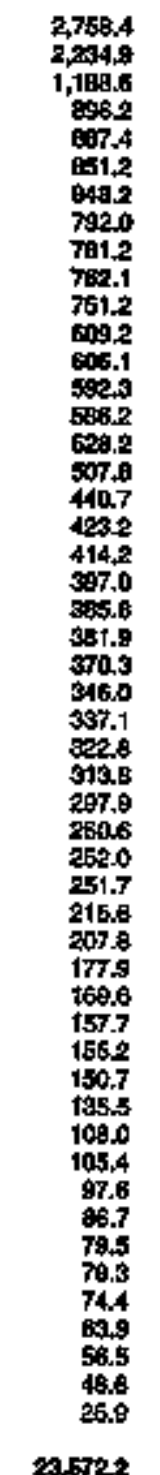 & 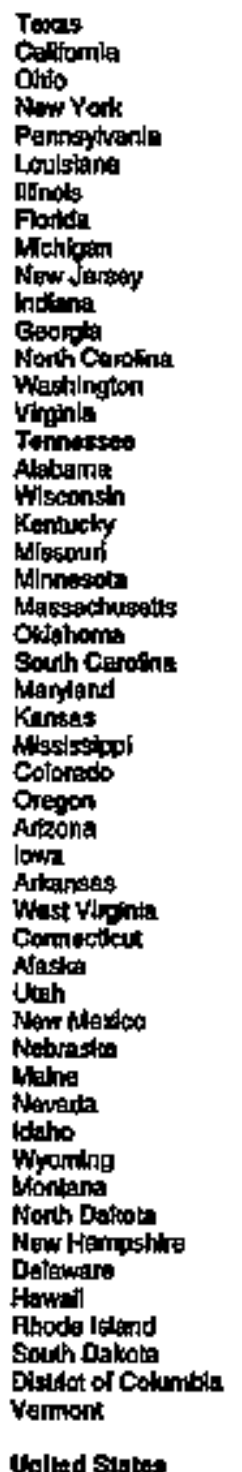 & 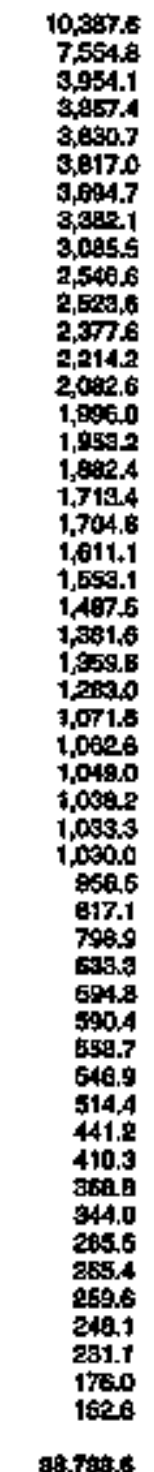 \\
\hline & Untted Stalas & 17,6022 & Untiod boptos & $13,431,2$ & Unitud Stites & $34,169.0$ & Unlived Btotes: & 20,6722 & Calted Siabes & as, ratas \\
\hline
\end{tabular}

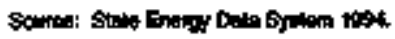




\begin{tabular}{|c|c|c|c|c|c|c|c|c|c|c|}
\hline \multirow{2}{*}{ Fiank } & \multicolumn{2}{|c|}{$\operatorname{Cos} 1$} & \multicolumn{2}{|c|}{ Natural Gas: } & \multicolumn{2}{|c|}{ Petroleun } & \multicolumn{2}{|c|}{ Eleatsoly' } & \multicolumn{2}{|c|}{$\begin{array}{l}\text { Totul Congumpition } \\
\text { par Cyplat }\end{array}$} \\
\hline & stote & Triflon Bita & Stents & Trillian 日tu & Stanto & Trlilon Bin & B*atg & Trialon Ban & State & Metion Bu \\
\hline \multirow[t]{2}{*}{ 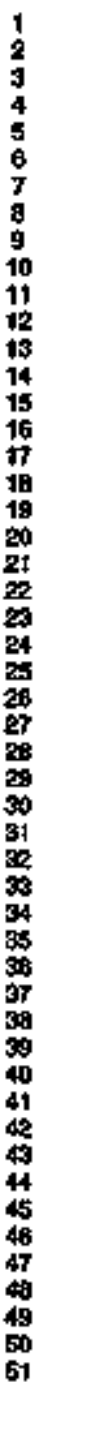 } & 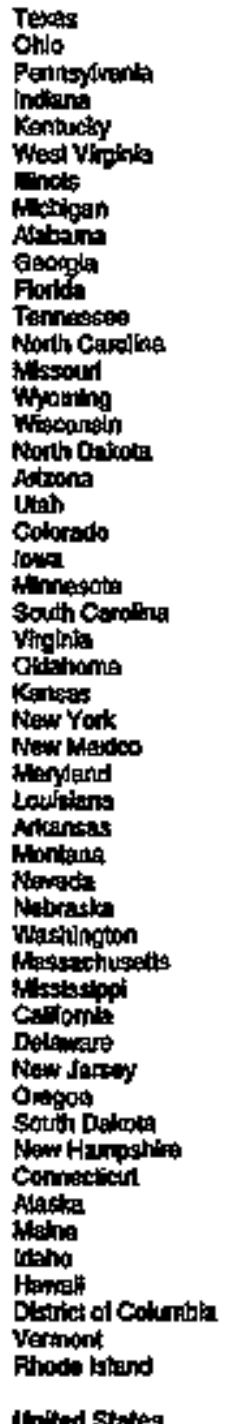 & 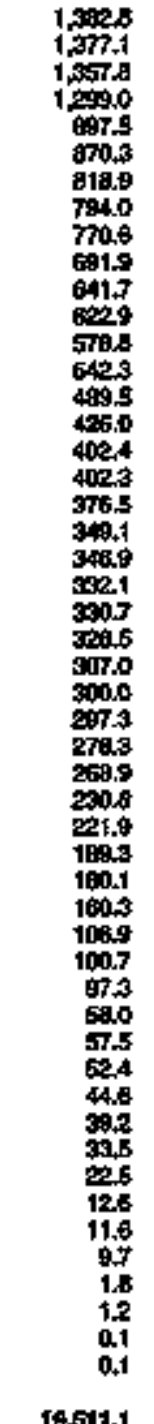 & 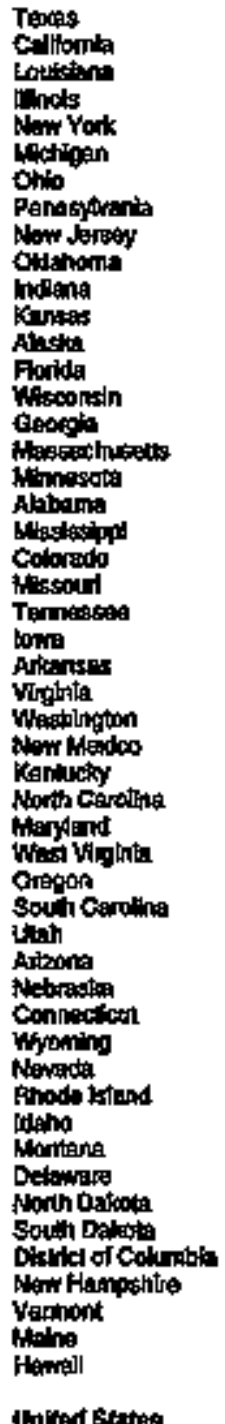 & 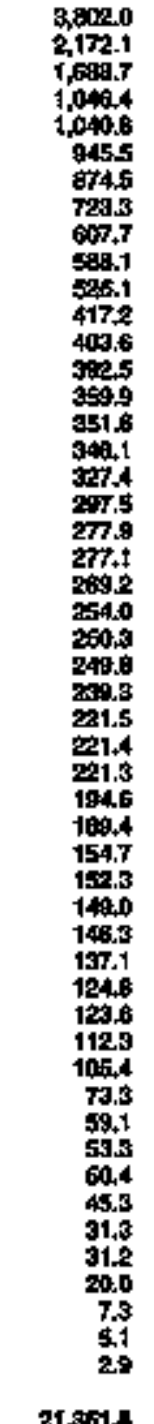 & 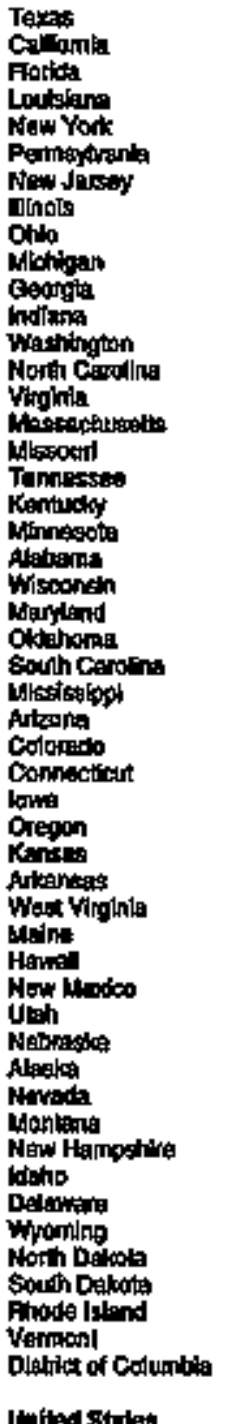 & 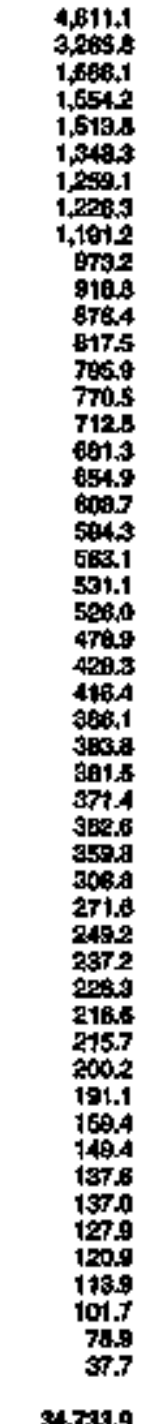 & 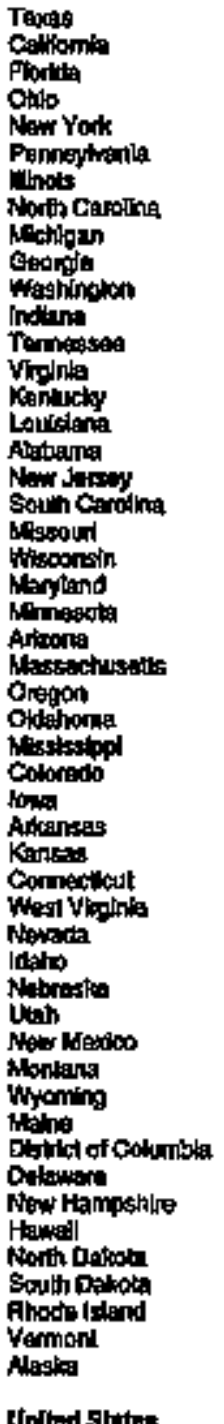 & 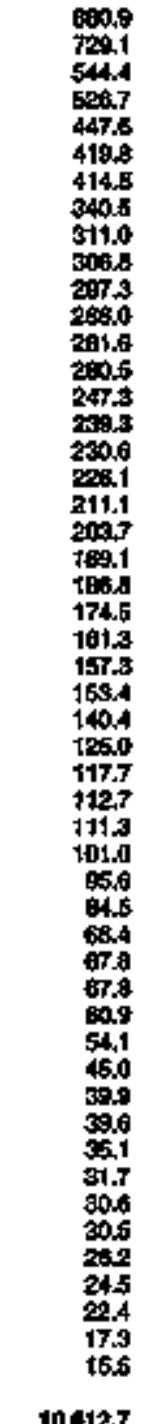 & 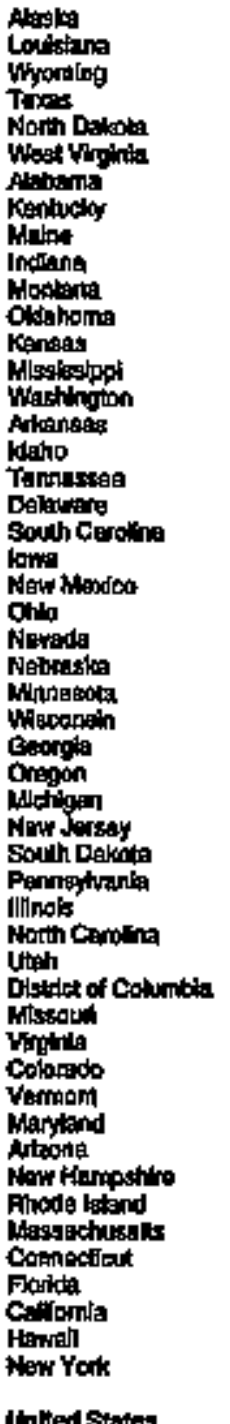 & 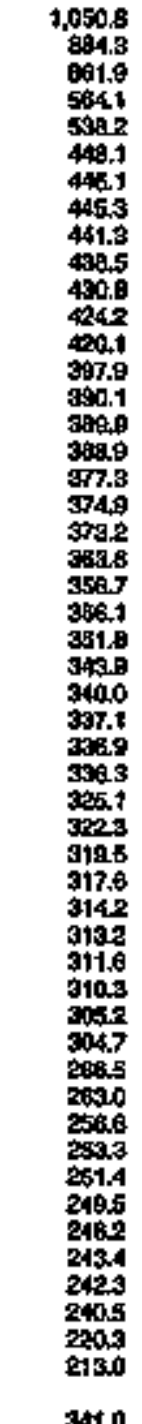 \\
\hline & Whilied Stateg & ofstt.1 & ilted sentes & I\$51. & Ifted Strilot & 1,7339 & Shtes: & 0,4127 & led Stateg & 3+10 \\
\hline
\end{tabular}

enoctertar in andictiys 


\section{United States Summaries}




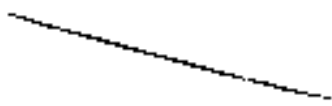

$\cdots$

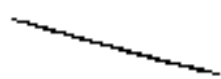


Table 11. Energy Constionplion Estimates by Soumb, 1960, 1965, 1970-1994, United Stales

\begin{tabular}{|c|c|c|c|c|c|c|c|c|c|c|c|c|c|c|c|c|c|c|c|}
\hline \multirow[b]{3}{*}{ Yo: } & \multirow{2}{*}{\multicolumn{2}{|c|}{ 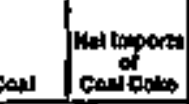 }} & \multirow{3}{*}{ 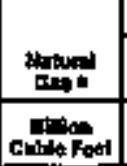 } & \multicolumn{11}{|c|}{ Pansm } & \multirow[b]{2}{*}{ 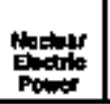 } & \multirow[b]{2}{*}{ 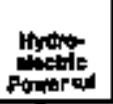 } & \multirow[b]{2}{*}{ Elonisols - } & \multirow[b]{2}{*}{ Otheres } & \multirow[b]{3}{*}{ Tould } \\
\hline & & & & 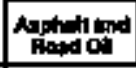 & 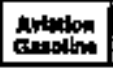 & 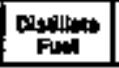 & Fin & Noto- & $\mathbf{L P g}$ & \begin{tabular}{|l|l|} 
wht: \\
cant
\end{tabular} & 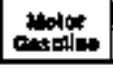 & mollow & ones: & 704pl & & & & & \\
\hline & 5 & $\min _{10 n}$ & & \multicolumn{11}{|c|}{ Hin Eimite } & \multicolumn{4}{|c|}{ 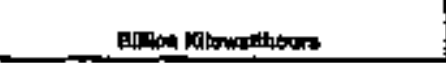 } & \\
\hline 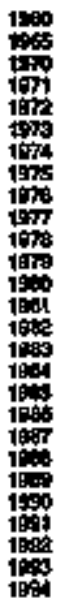 & 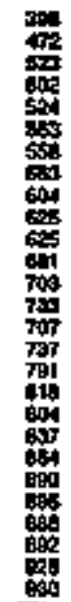 & 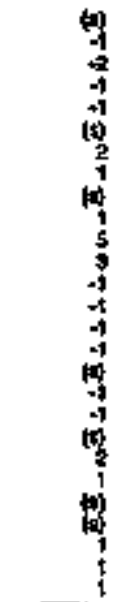 & 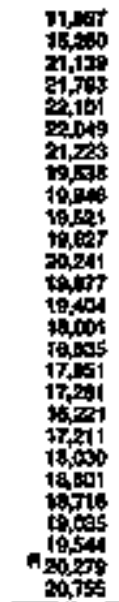 & 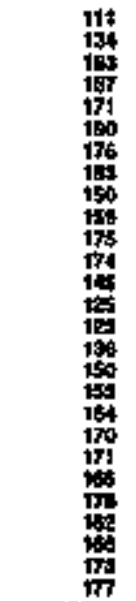 & 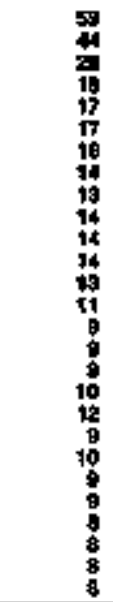 & 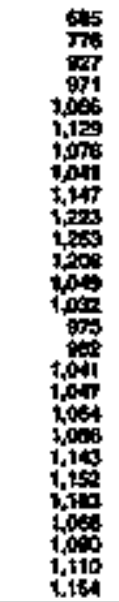 & 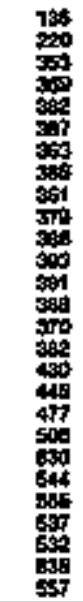 & 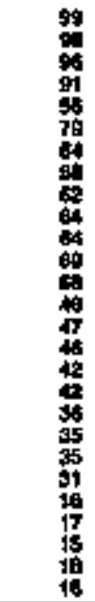 & 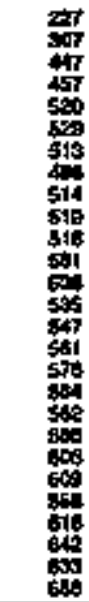 & 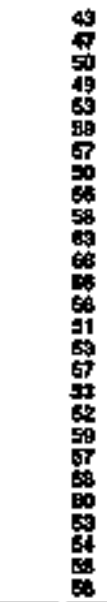 & 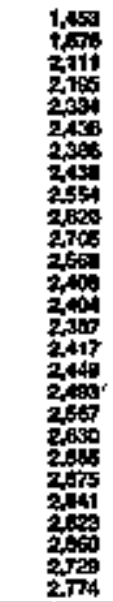 & 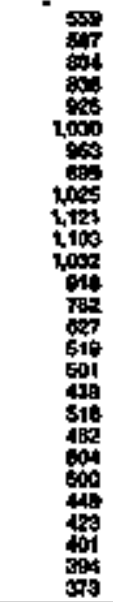 & 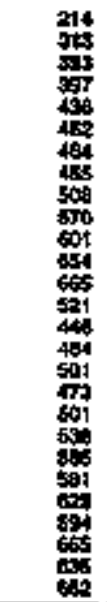 & 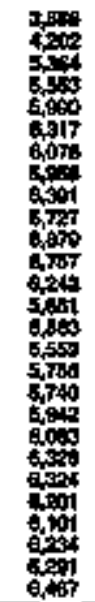 & 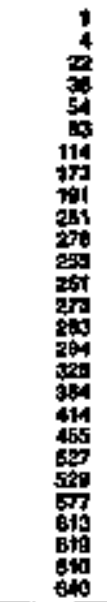 & 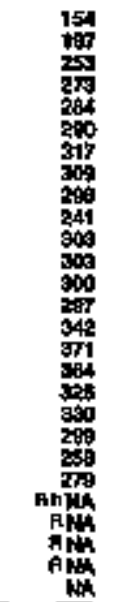 & 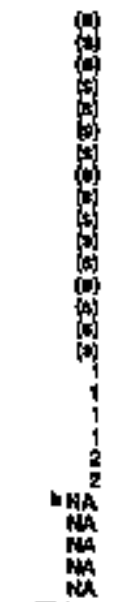 & 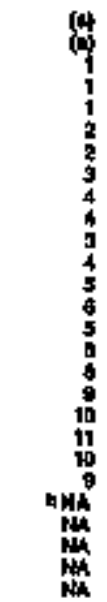 & $\begin{array}{l}\bar{z} \\
\bar{z} \\
\bar{z} \\
z \\
\bar{z} \\
\bar{z}\end{array}$ \\
\hline
\end{tabular}

\begin{tabular}{|c|c|c|c|c|c|c|c|c|c|c|c|c|c|c|c|c|c|c|c|}
\hline (1900 & 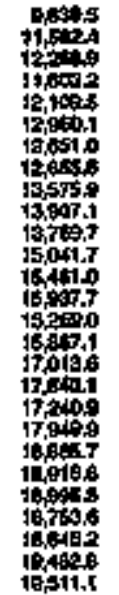 & 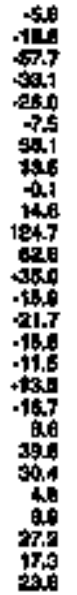 & 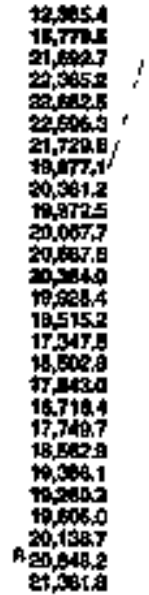 & 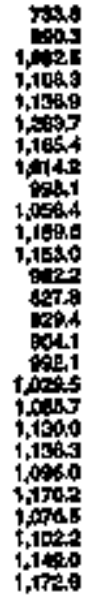 & 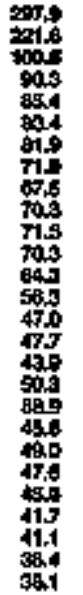 & 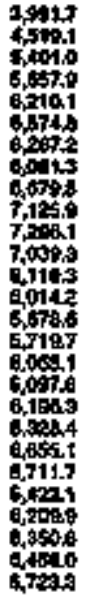 & 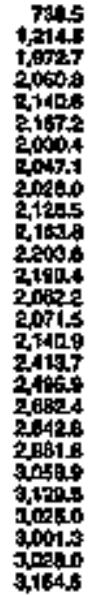 & 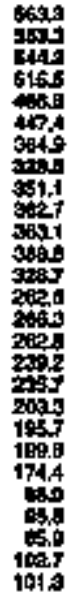 & 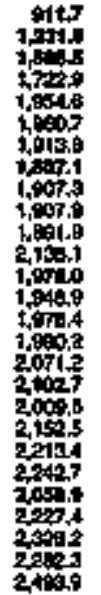 & 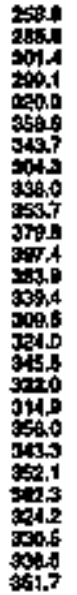 & 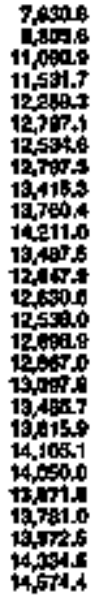 & 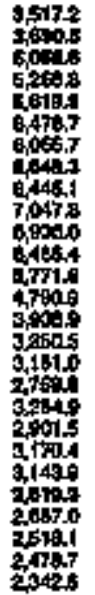 & 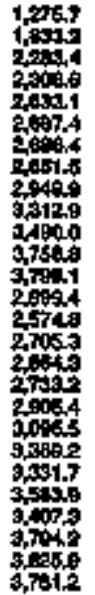 & 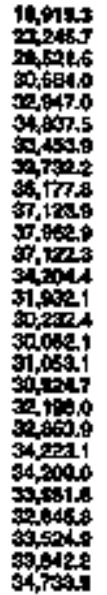 & 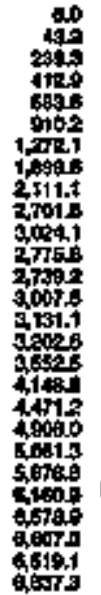 & 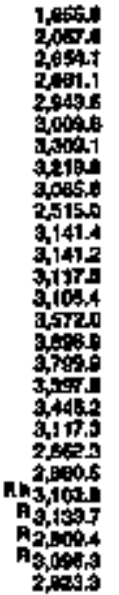 & 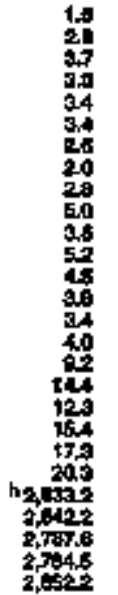 & 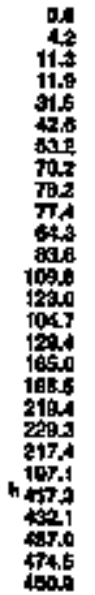 & 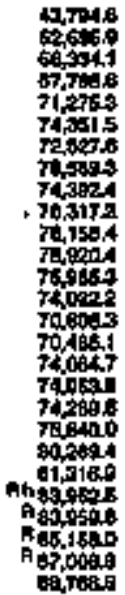 \\
\hline
\end{tabular}

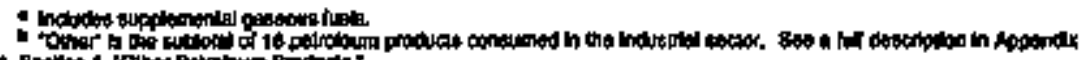

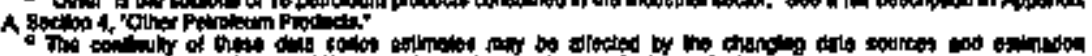

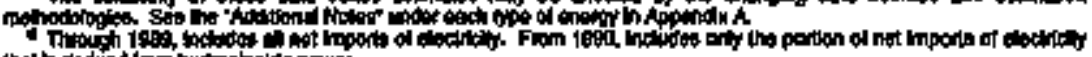

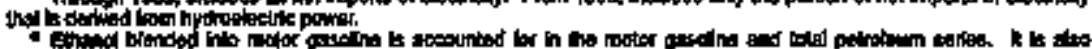

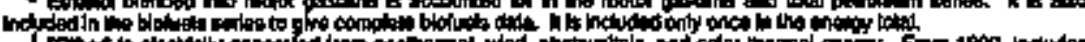

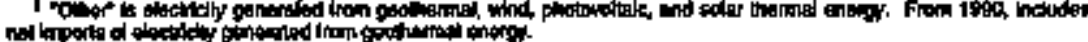

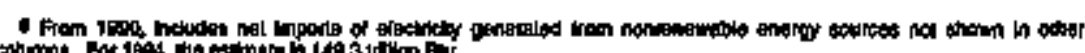

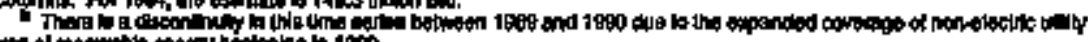
oto 에

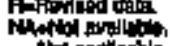

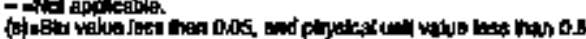

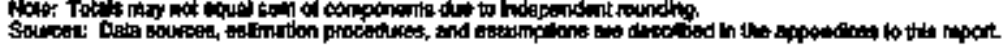


U Table 12. Residential Energy Consumption Estiqnates, 1960, 1965, 1970-1994, Unitad States

\begin{tabular}{|c|c|c|c|c|c|c|c|c|c|c|c|c|c|c|}
\hline \multirow[b]{3}{*}{ Yearl } & \multicolumn{3}{|c|}{$\operatorname{con}$} & \multirow{3}{*}{ 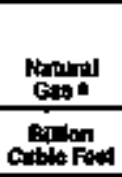 } & \multicolumn{4}{|c|}{ Potrosing } & \multirow{3}{*}{ 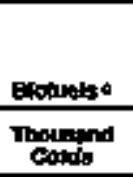 } & & \multirow[b]{2}{*}{ Dectrichys } & \multirow[b]{2}{*}{ theng } & \multirow{3}{*}{ 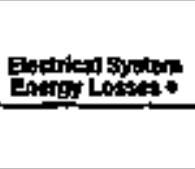 } & \multirow[b]{3}{*}{ Towr } \\
\hline & 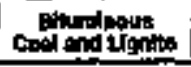 & Antrinach & Totely & & 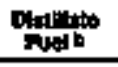 & Kerpatenes & Lpeqb & Totat & & Solatrd & & & & \\
\hline & \multicolumn{3}{|c|}{ Mon Shat Tone } & & \multicolumn{4}{|c|}{ 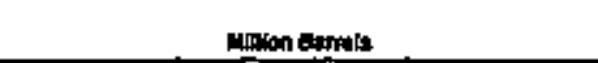 } & & \multicolumn{3}{|c|}{ 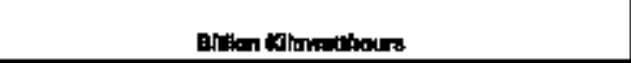 } & & \\
\hline 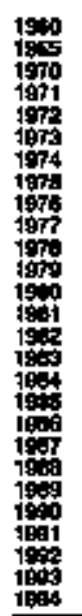 & $\begin{array}{l}11 \\
7 \\
4 \\
4 \\
3 \\
3 \\
3 \\
3 \\
\frac{1}{2} \\
\frac{2}{2} \\
3 \\
\frac{2}{2} \\
2 \\
2 \\
2 \\
2 \\
3 \\
\frac{2}{2} \\
2 \\
2 \\
\frac{2}{2} \\
2 \\
2 \\
2 \\
2 \\
\frac{2}{2}\end{array}$ & $\begin{array}{l}1 \\
4 \\
2 \\
2 \\
2 \\
2 \\
2 \\
2 \\
1 \\
1 \\
1 \\
\vdots \\
1 \\
1 \\
\vdots \\
1 \\
1 \\
1 \\
1 \\
1 \\
1 \\
1\end{array}$ & 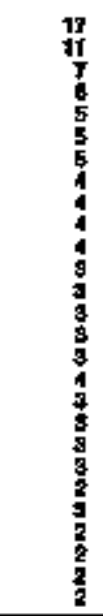 & 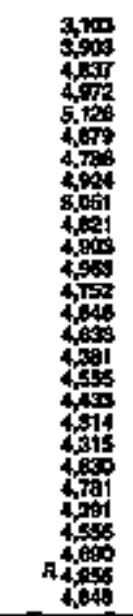 & 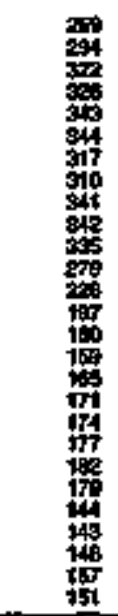 & 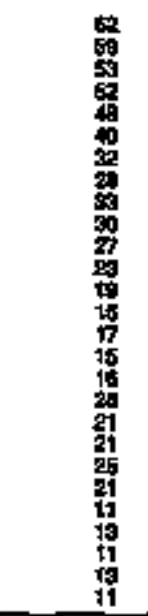 & 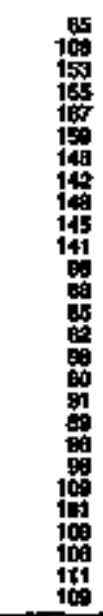 & 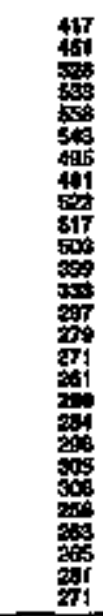 & 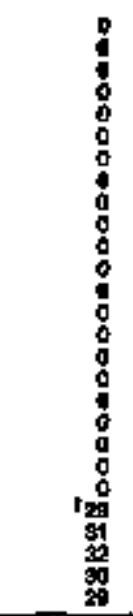 & 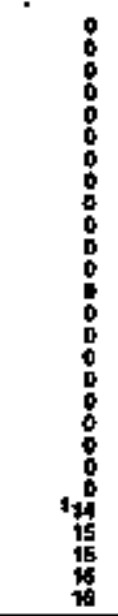 & 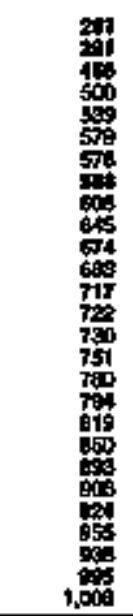 & $\begin{array}{l}= \\
\vdots \\
z \\
z \\
z \\
z \\
z \\
z \\
z \\
z \\
z\end{array}$ & 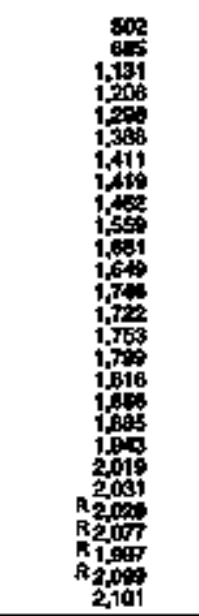 & $\begin{array}{l}z \\
\vdots \\
z \\
z \\
\vdots \\
z \\
\vdots \\
z \\
z \\
z \\
z \\
z \\
z\end{array}$ \\
\hline & & & & & & & Tringen & & & & & & & \\
\hline 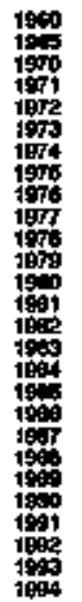 & 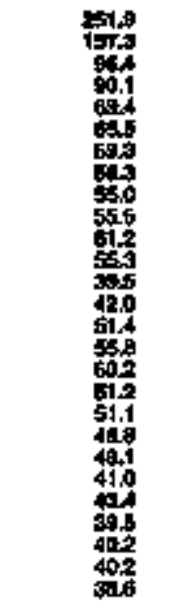 & 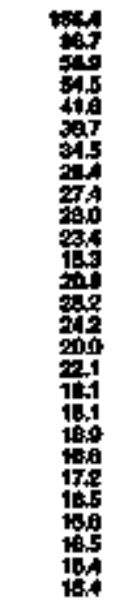 & 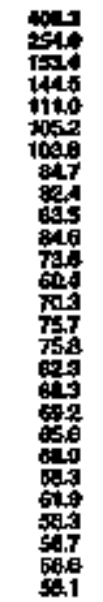 & 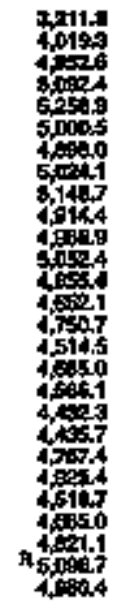 & 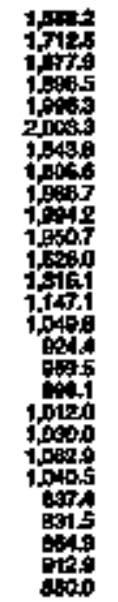 & 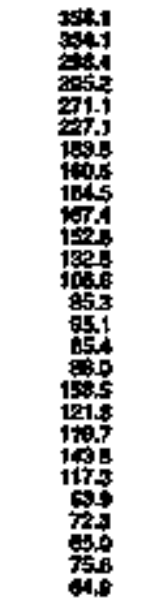 & 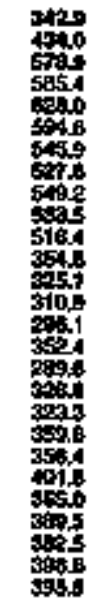 & 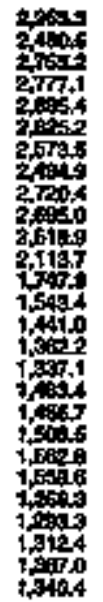 & 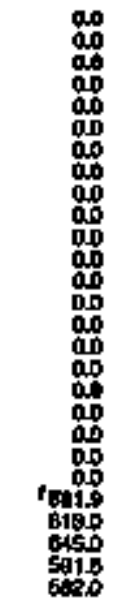 & 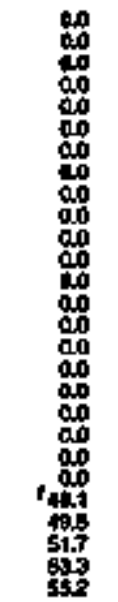 & 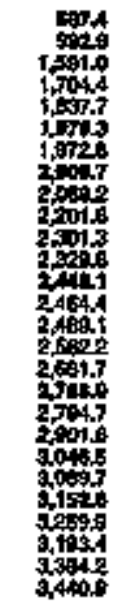 & 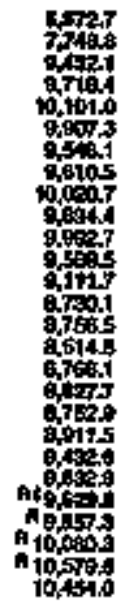 & 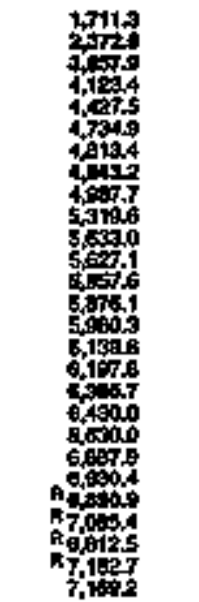 & 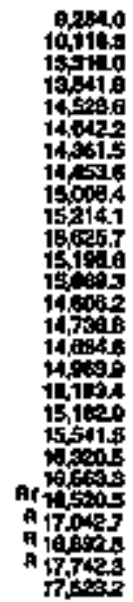 \\
\hline
\end{tabular}

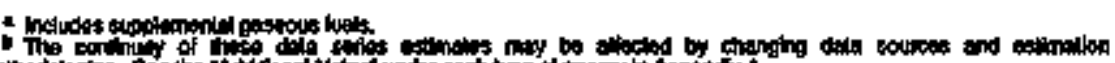

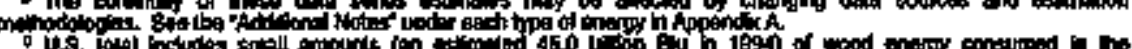

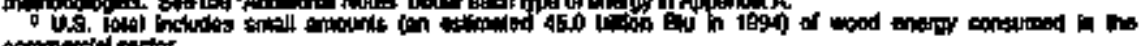

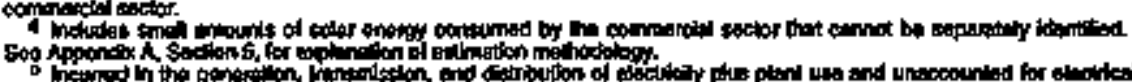

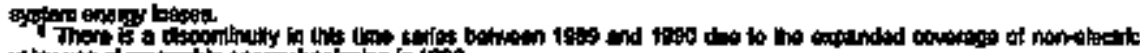

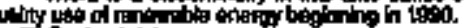

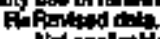

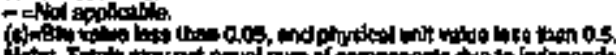

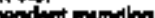

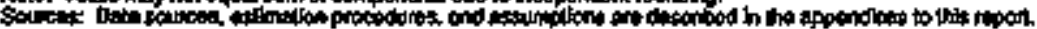


Table 13. Commercial Energy Consumptlon Estimales, 1960, 1965, 1970-1994, United Stales

\begin{tabular}{|c|c|c|c|c|c|c|c|c|c|c|c|c|c|c|}
\hline \multirow[b]{3}{*}{ Yos } & \multicolumn{3}{|c|}{ cow } & \multirow[b]{2}{*}{ Molduris } & \multicolumn{6}{|c|}{ A } & \multirow[b]{2}{*}{ Entritay b } & \multirow[b]{2}{*}{ 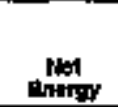 } & \multirow[b]{2}{*}{ 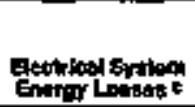 } & \multirow[b]{3}{*}{ Totand } \\
\hline & consininount. & Natherste & row & & 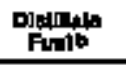 & Karomant & Lab & tholor. & 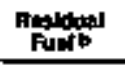 & Fow & & & & \\
\hline & \multicolumn{3}{|c|}{ mons sheart tions } & 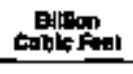 & \multicolumn{6}{|c|}{ 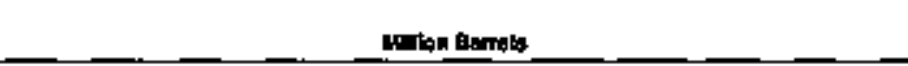 } & \multicolumn{3}{|c|}{ Equn kntrowathowrs } & \\
\hline 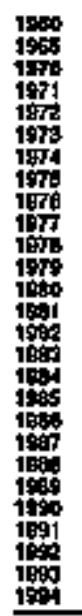 & 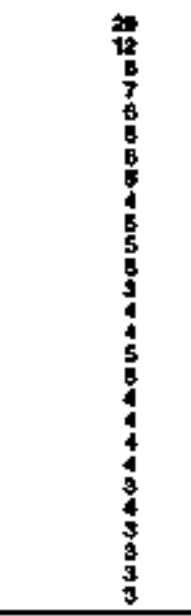 & 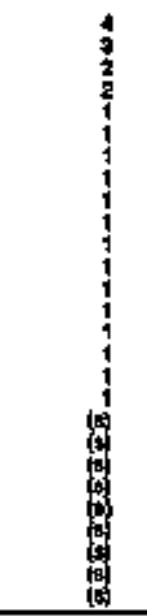 & $\begin{array}{l}24 \\
14 \\
3 \\
7 \\
7 \\
5 \\
5 \\
5 \\
5 \\
6 \\
5 \\
4 \\
5 \\
5 \\
5 \\
5 \\
4 \\
4 \\
4 \\
4 \\
4 \\
4\end{array}$ & 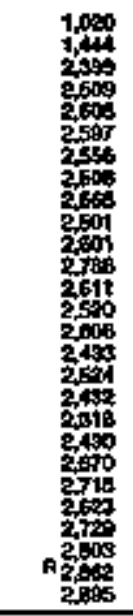 & 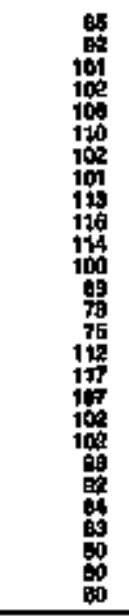 & 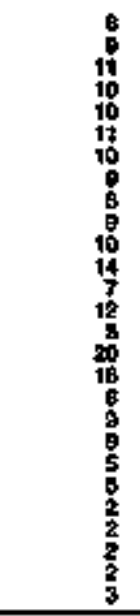 & 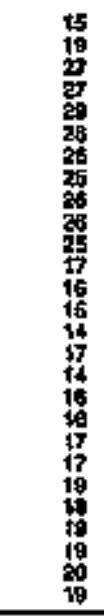 & 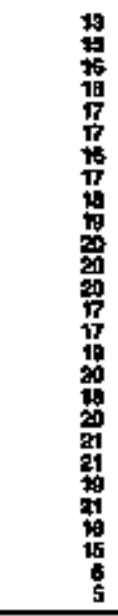 & 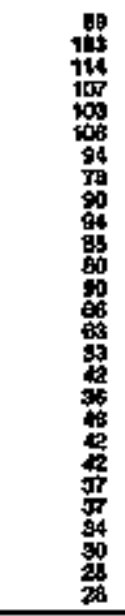 & 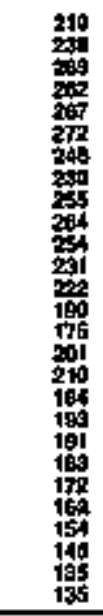 & 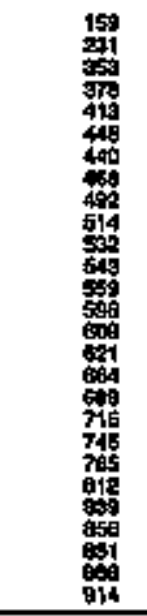 & $\begin{array}{l}\vdots \\
\vdots \\
\vdots \\
\vdots \\
\vdots \\
\vdots \\
\vdots \\
\vdots \\
\vdots \\
\vdots \\
\vdots \\
\vdots \\
\vdots \\
\vdots\end{array}$ & 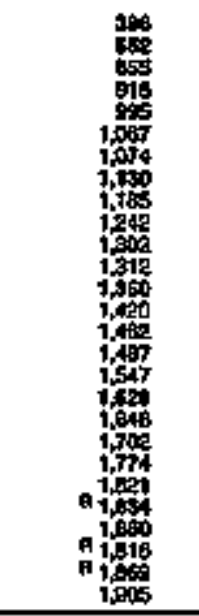 & $\begin{array}{l}\vdots \\
\vdots \\
\vdots \\
\vdots \\
\vdots \\
\vdots \\
\vdots \\
\vdots \\
\vdots \\
\vdots \\
\vdots \\
\vdots \\
\vdots \\
\vdots\end{array}$ \\
\hline \multicolumn{15}{|c|}{ nnmen } \\
\hline 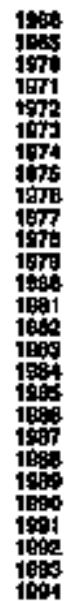 & 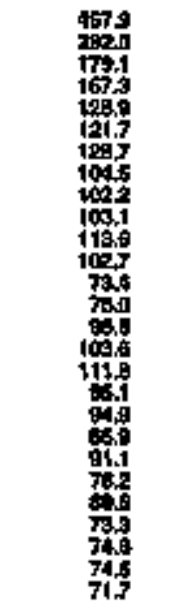 & 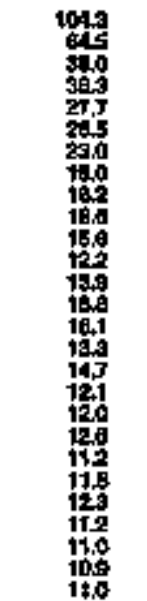 & 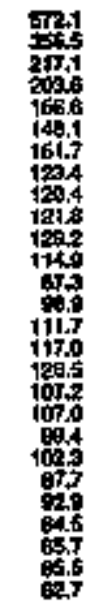 & 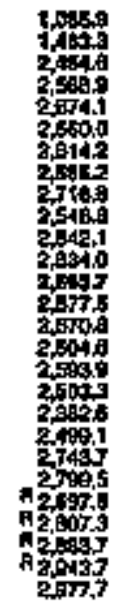 & 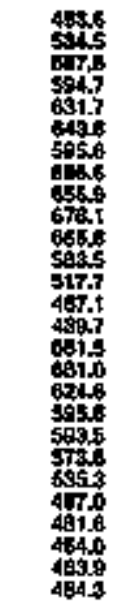 & 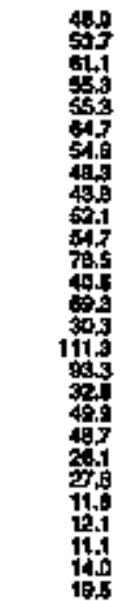 & 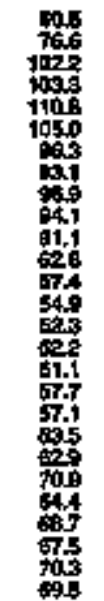 & 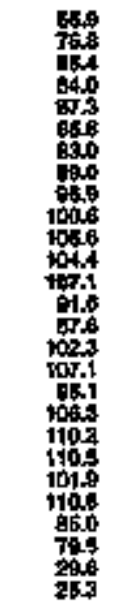 & 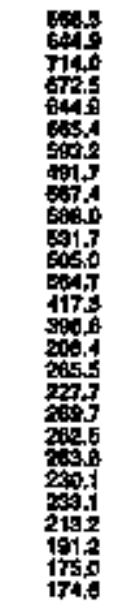 & 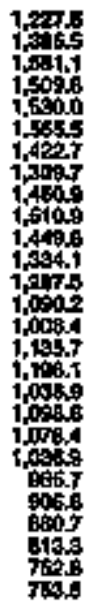 & 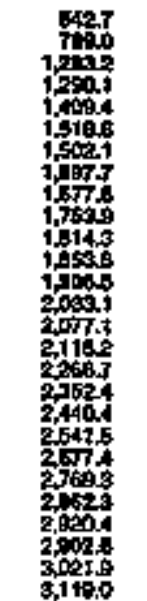 & 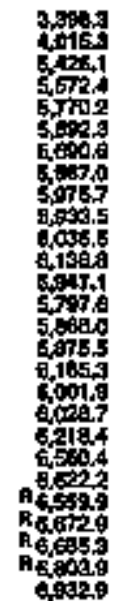 & 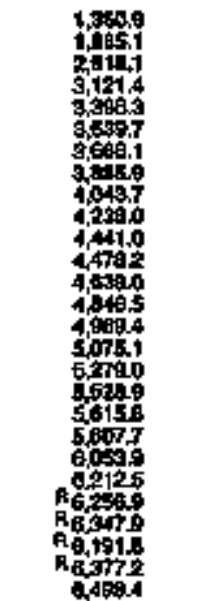 & 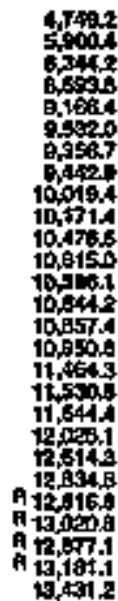 \\
\hline
\end{tabular}




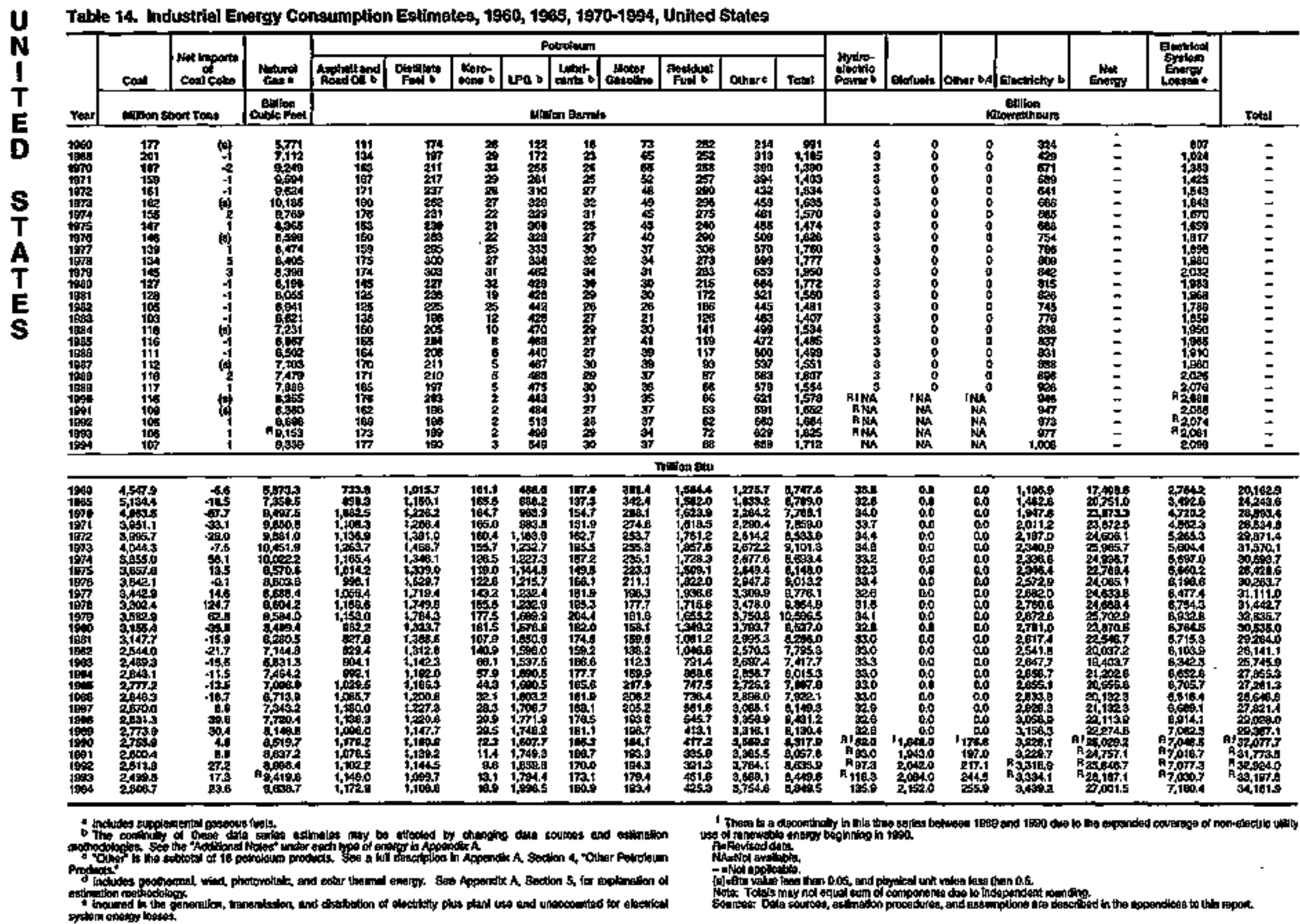


Table 15. Transporlation Energy Consumpilon Estimates, 1960, 1865, 1970-1994, Unifed States

\begin{tabular}{|c|c|c|c|c|c|c|c|c|c|c|c|c|c|c|c|}
\hline \multirow[b]{3}{*}{ yar } & \multirow[b]{2}{*}{ teat } & \multirow[b]{2}{*}{ Hencer } & \multicolumn{8}{|c|}{ Palocieurn } & \multirow{3}{*}{ 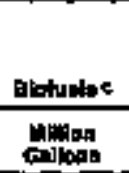 } & & \multirow[b]{2}{*}{ 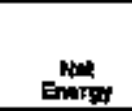 } & \multirow[b]{2}{*}{ Cloctples Spring } & \multirow[b]{3}{*}{ Thatal } \\
\hline & & & Avtrition & 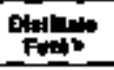 & D. & IFta & Lonkeants & Alowh & 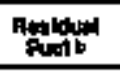 & Total & & Electikny & & & \\
\hline & shortion & 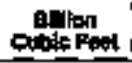 & \multicolumn{8}{|c|}{ andin Butats } & & \multicolumn{3}{|c|}{ 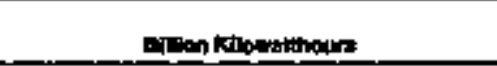 } & \\
\hline 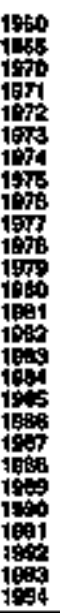 & $\begin{array}{l}3 \\
0 \\
0 \\
0 \\
9 \\
0 \\
0 \\
0 \\
0 \\
0 \\
0 \\
0 \\
0 \\
0 \\
0 \\
0 \\
0 \\
0 \\
0 \\
0 \\
0 \\
0 \\
0 \\
0 \\
0\end{array}$ & 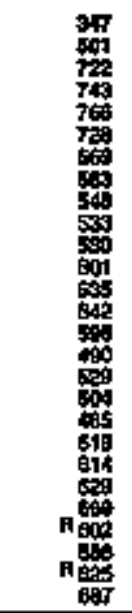 & $\begin{array}{l}9 \\
4 \\
24 \\
19 \\
17 \\
7 \\
16 \\
14 \\
19 \\
14 \\
14 \\
10 \\
11 \\
8 \\
0 \\
10 \\
12 \\
6 \\
10 \\
8 \\
8 \\
8 \\
8 \\
8 \\
8\end{array}$ & 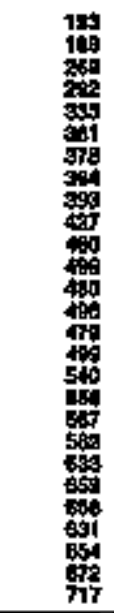 & 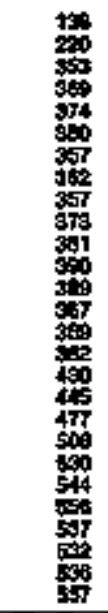 & $\begin{array}{c}5 \\
5 \\
12 \\
13 \\
14 \\
15 \\
12 \\
14 \\
12 \\
19 \\
14 \\
6 \\
5 \\
5 \\
11 \\
11 \\
8 \\
7 \\
8 \\
6 \\
6 \\
5 \\
5 \\
5 \\
5\end{array}$ & 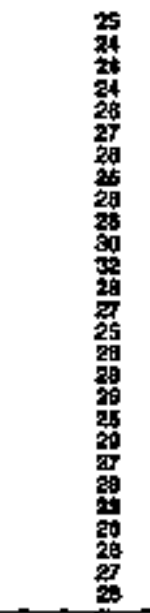 & 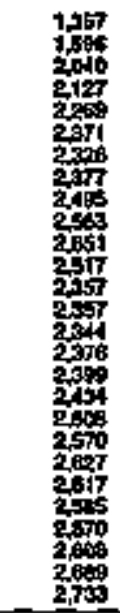 & 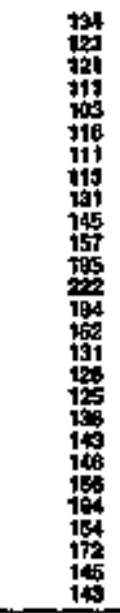 & 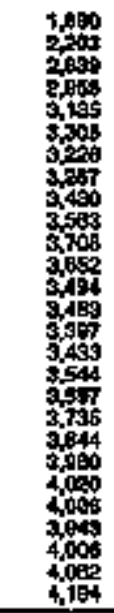 & 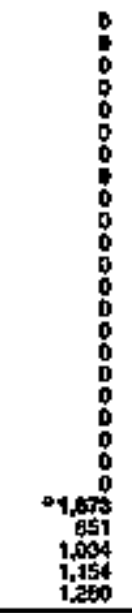 & $\begin{array}{l}3 \\
3 \\
3 \\
2 \\
2 \\
2 \\
2 \\
3 \\
3 \\
3 \\
3 \\
3 \\
3 \\
3 \\
3 \\
3 \\
3 \\
4 \\
4 \\
4 \\
4 \\
4 \\
4 \\
4 \\
4 \\
4 \\
4\end{array}$ & $\begin{array}{l}= \\
= \\
= \\
= \\
= \\
= \\
= \\
= \\
= \\
= \\
= \\
= \\
= \\
=\end{array}$ & $\begin{array}{l}5 \\
7 \\
6 \\
6 \\
6 \\
6 \\
6 \\
7 \\
7 \\
7 \\
6 \\
7 \\
6 \\
6 \\
8 \\
6 \\
9 \\
9 \\
9 \\
9 \\
9 \\
9 \\
9 \\
9 \\
9 \\
\end{array}$ & $\begin{array}{l}= \\
= \\
z \\
z \\
z \\
z \\
= \\
z \\
= \\
= \\
= \\
z \\
z \\
=\end{array}$ \\
\hline \multicolumn{16}{|c|}{ MriEgan Eu } \\
\hline 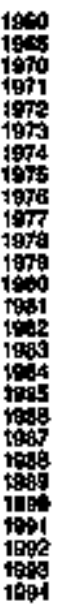 & 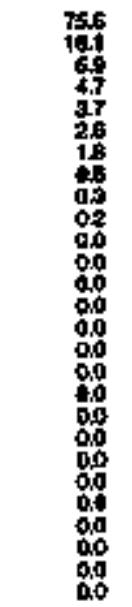 & 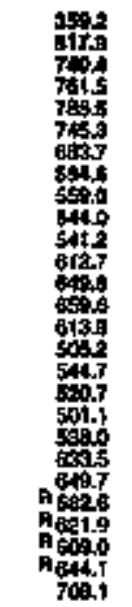 & 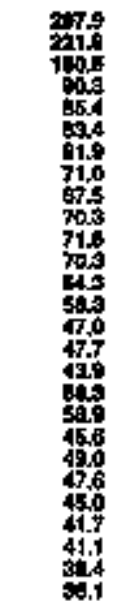 & 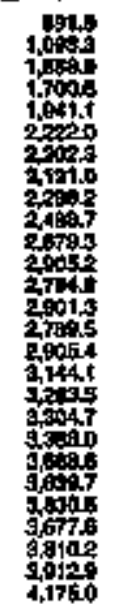 & 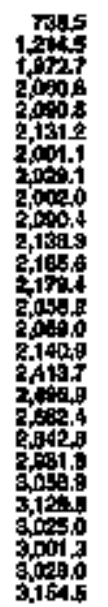 & 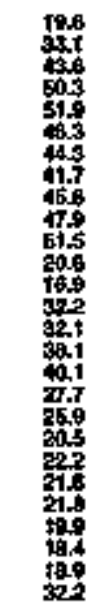 & 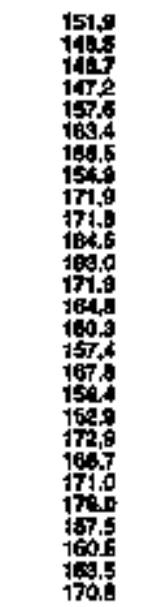 & 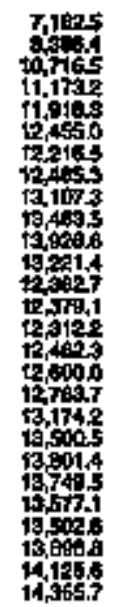 & 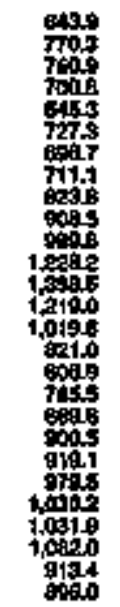 & 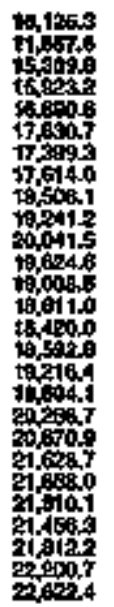 & 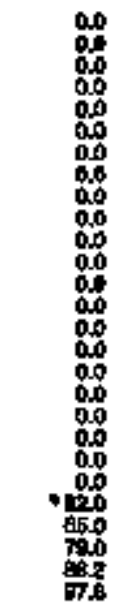 & 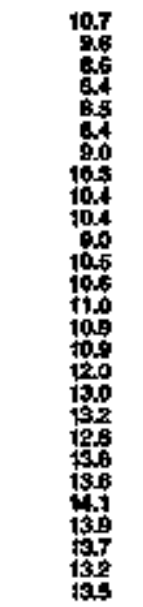 & 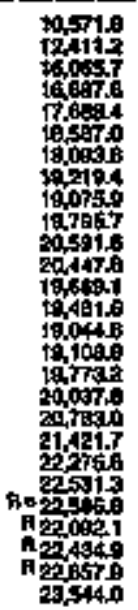 & 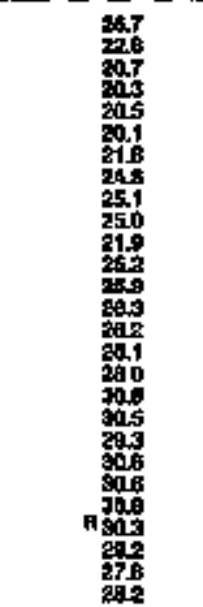 & 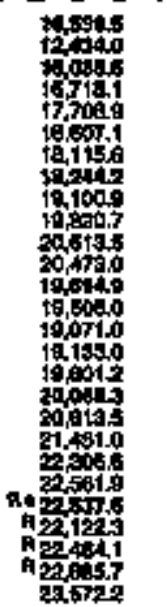 \\
\hline & & & & & & 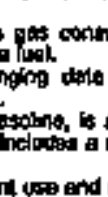 & thest & a & & & & 100 & thent & the apoundoet & of non-atocits; \\
\hline
\end{tabular}


Table 16. Estimates of Energy Input at 피ectric Uticites, 1960, 1965, 1970-1994, United States

\begin{tabular}{|c|c|c|c|c|c|c|c|c|c|c|c|c|c|c|}
\hline \multirow[b]{3}{*}{ [ } & \multicolumn{3}{|c|}{ coen } & \multirow{3}{*}{ 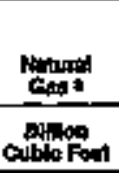 } & \multicolumn{4}{|c|}{ Pulnotuen } & \multirow[b]{2}{*}{ 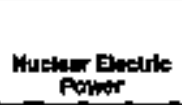 } & \multirow[b]{2}{*}{ Bindroniesterse } & \multirow[b]{2}{*}{ Bloknals } & \multirow[b]{2}{*}{ Eeolbermpil } & \multirow{3}{*}{ Othurbog } & \multirow{3}{*}{ Toth } \\
\hline & consmintion & Anthinallos & Tolol & & Hatur & 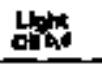 & Dotrolebrit & Ta햐이 & & & & & & \\
\hline & \multicolumn{3}{|c|}{ 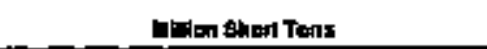 } & & \multicolumn{4}{|c|}{ 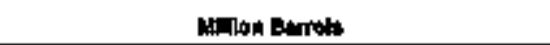 } & \multicolumn{4}{|c|}{ 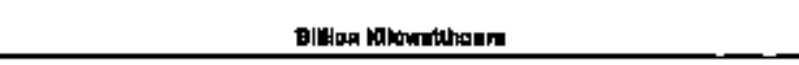 } & & \\
\hline 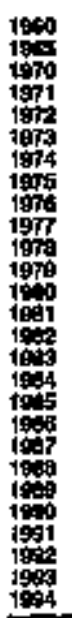 & 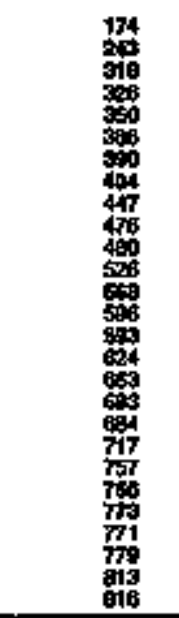 & $\begin{array}{l}3 \\
3 \\
2 \\
2 \\
2 \\
2 \\
1 \\
1 \\
1 \\
1 \\
1 \\
1 \\
1 \\
1 \\
1 \\
1 \\
1 \\
1 \\
1 \\
1 \\
1 \\
1 \\
1 \\
1 \\
1\end{array}$ & 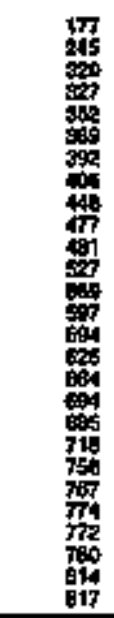 & 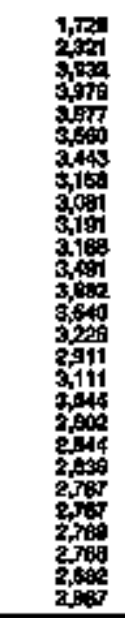 & 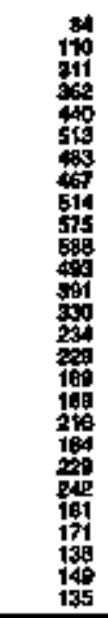 & 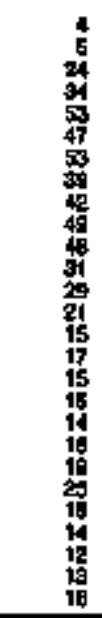 & 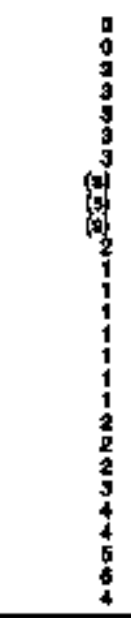 & 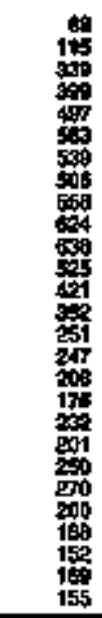 & 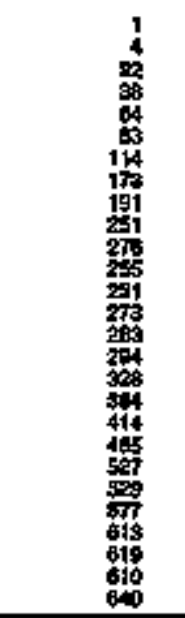 & 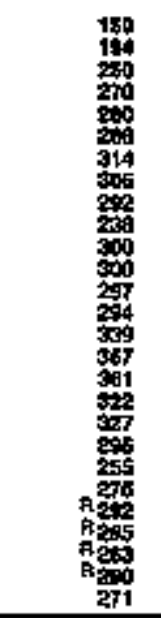 & 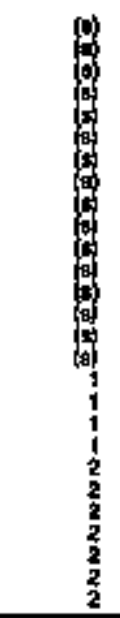 & 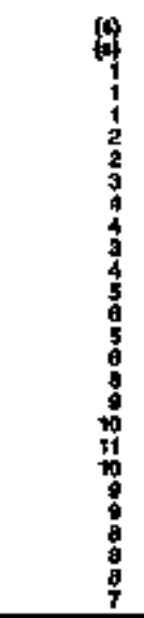 & 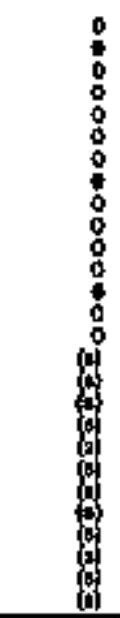 & $\begin{array}{l}z \\
z \\
z \\
z \\
z \\
z \\
z \\
z \\
z \\
z \\
z \\
z \\
z\end{array}$ \\
\hline \multicolumn{15}{|c|}{ Tralloon Bro } \\
\hline 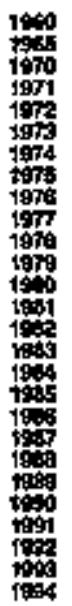 & 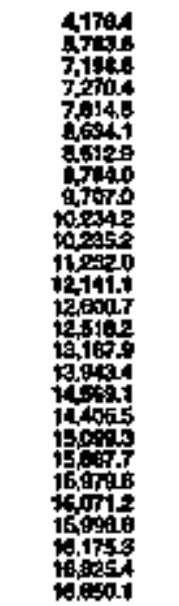 & 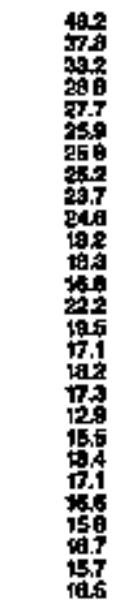 & 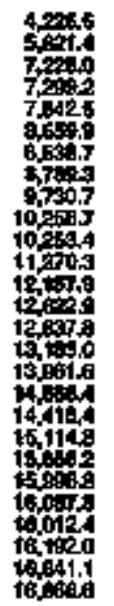 & 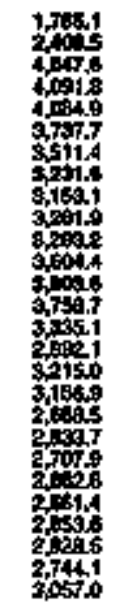 & 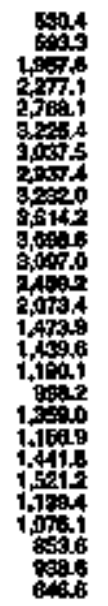 & 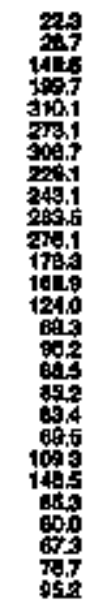 & 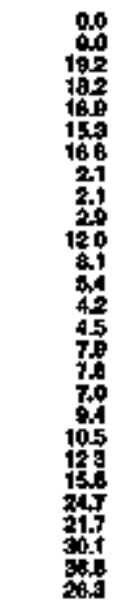 & 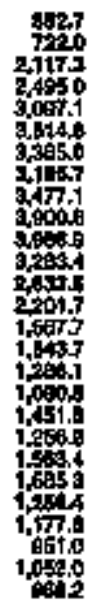 & 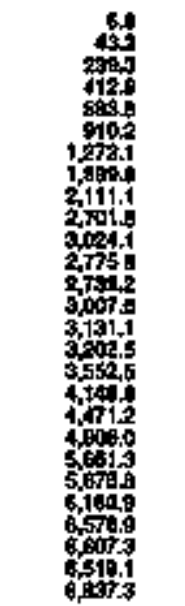 & 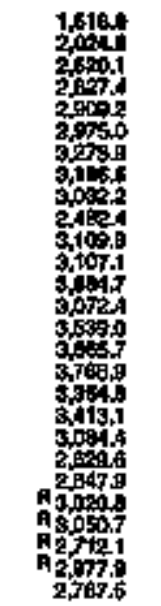 & 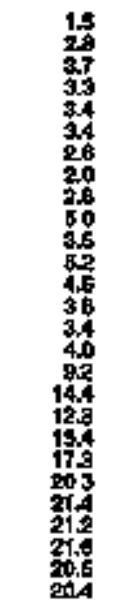 & 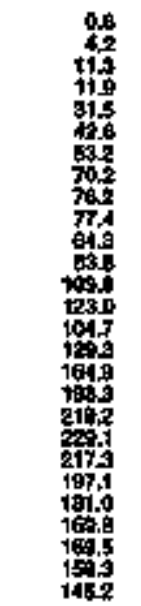 & 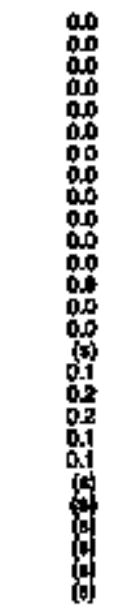 & 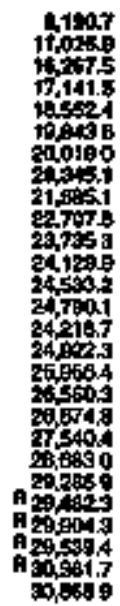 \\
\hline
\end{tabular}

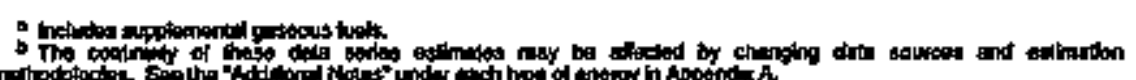

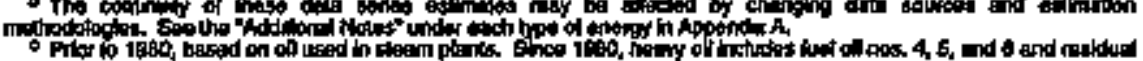

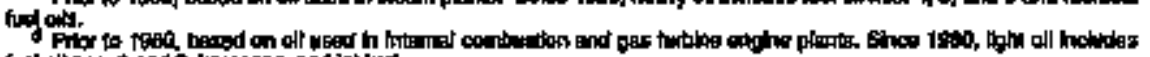

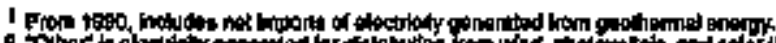

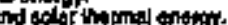

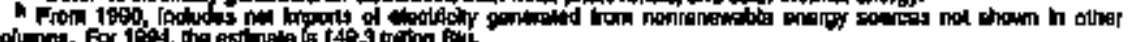

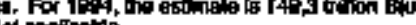

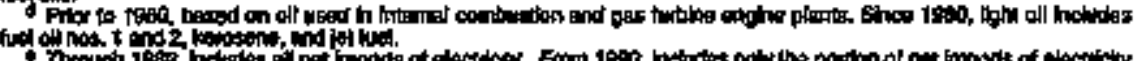

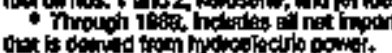

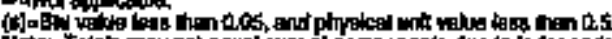

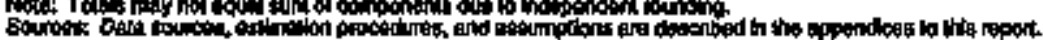




\section{State Summaries}




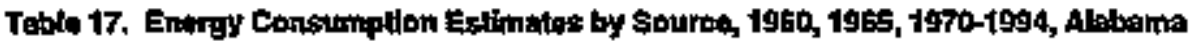

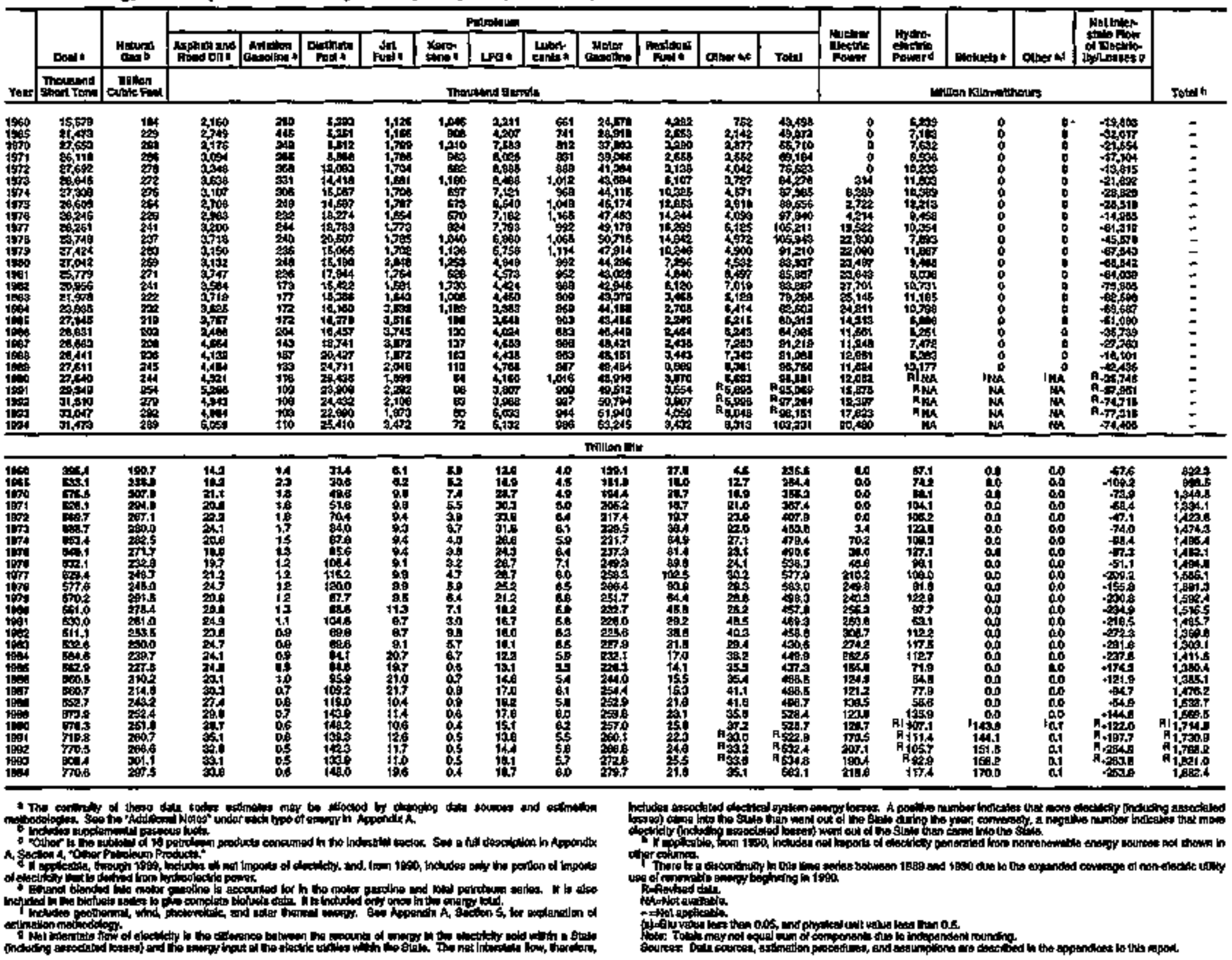


A Table 18. Aesidential Energy Consumaption Estinates, 1960, 1965, 1970-1994, Alabanara

\begin{tabular}{|c|c|c|c|c|c|c|c|c|c|c|c|c|c|c|}
\hline \multirow[b]{3}{*}{ Yerr } & \multicolumn{3}{|c|}{ "הenct } & \multirow{3}{*}{ 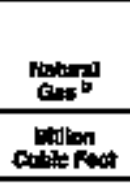 } & & $P$ PH & & & \multirow{3}{*}{ 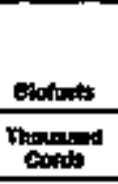 } & & \multirow[b]{2}{*}{ Exestehy" } & \multirow[b]{2}{*}{ Exing } & \multirow{3}{*}{ 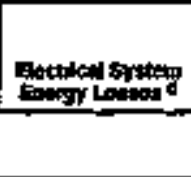 } & \multirow[b]{3}{*}{ Fats } \\
\hline & 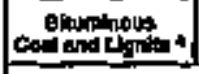 & 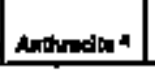 & Tored & & Fot & 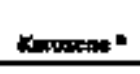 & 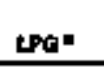 & Tow & & 50h-1" & & & & \\
\hline & \multicolumn{3}{|c|}{ Phoverand Stoon Tont } & & \multicolumn{4}{|c|}{ Thourand Bumpen } & & \multicolumn{3}{|c|}{ 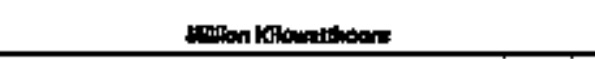 } & & \\
\hline 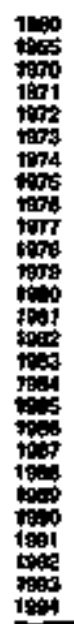 & 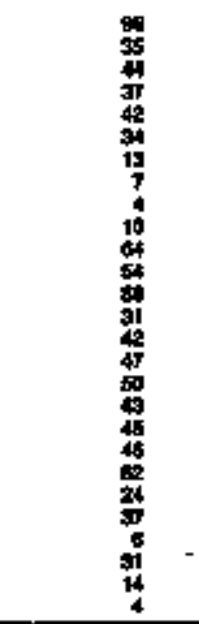 & 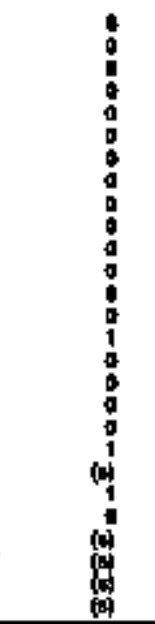 & 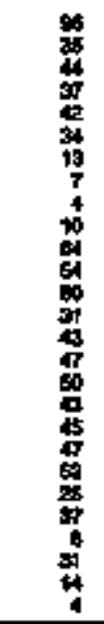 & 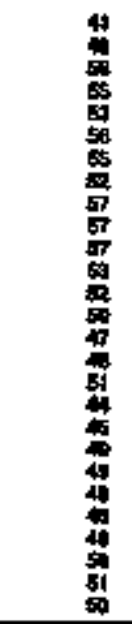 & 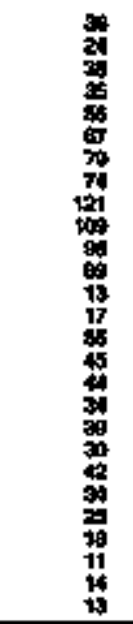 & 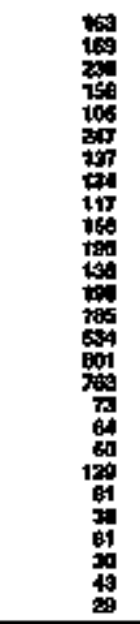 & 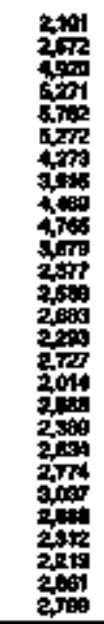 & 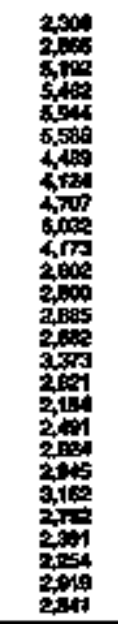 & 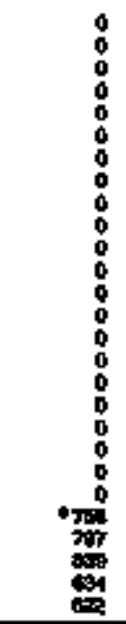 & 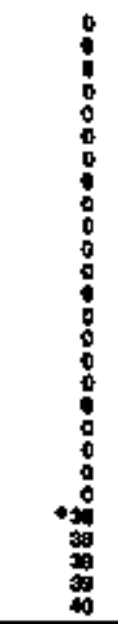 & 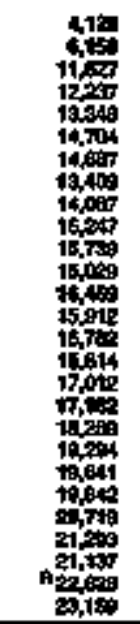 & $\begin{array}{l}\vdots \\
\vdots \\
\vdots \\
\vdots \\
\vdots \\
\vdots \\
\vdots \\
\vdots \\
\vdots \\
\vdots \\
\vdots \\
\vdots \\
\vdots \\
\vdots\end{array}$ & 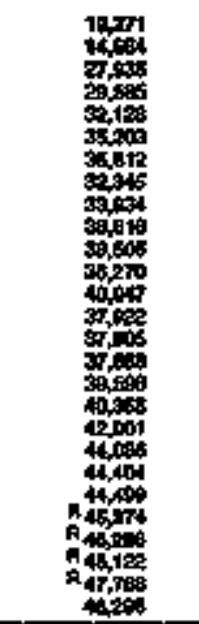 & $\begin{array}{l}\vdots \\
\vdots \\
\vdots \\
\vdots \\
\vdots \\
\end{array}$ \\
\hline & & & & & & & $y_{n=m}$ & & & & & & & \\
\hline 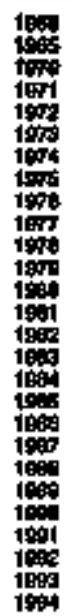 & 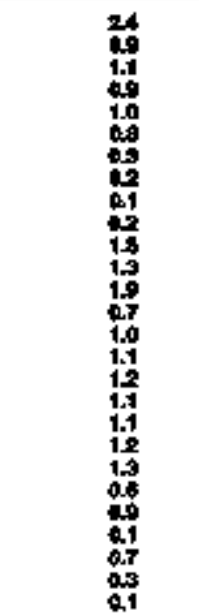 & 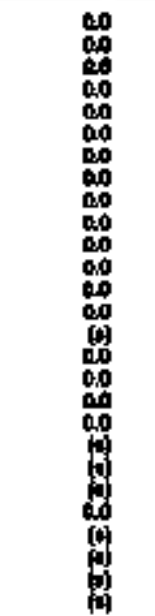 & 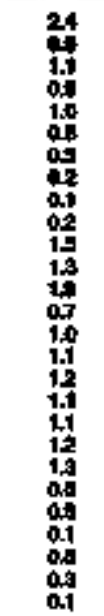 & 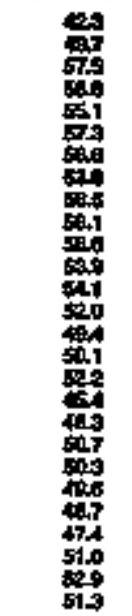 & 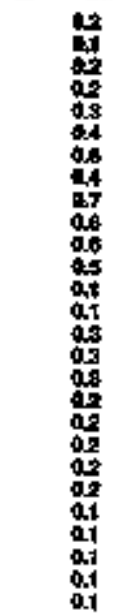 & 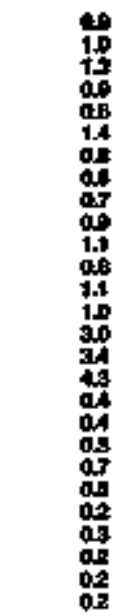 & 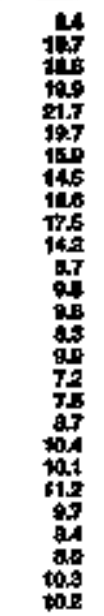 & 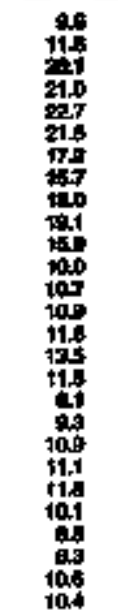 & 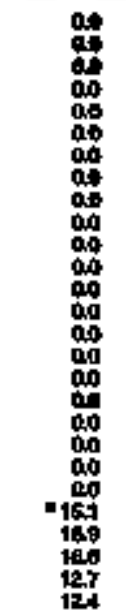 & 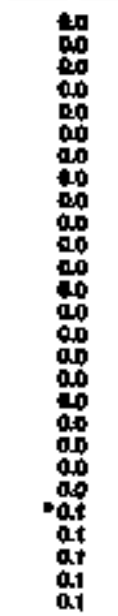 & 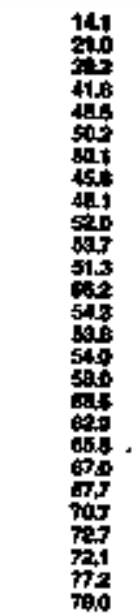 & 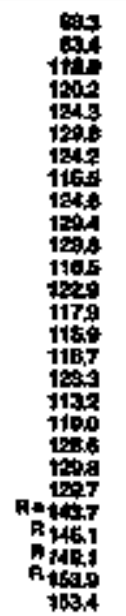 & 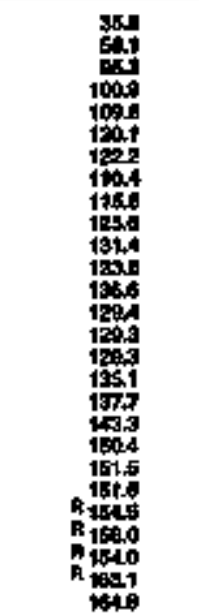 & 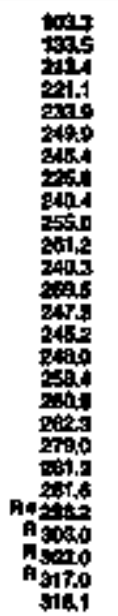 \\
\hline
\end{tabular}


Table 19. Commercid Energy Consumption Estimates, 1960, 1965, 1970-1994, Alabana

\begin{tabular}{|c|c|c|c|c|c|c|c|c|c|c|c|c|c|c|}
\hline \multirow[b]{3}{*}{ Yar } & \multicolumn{3}{|c|}{ Co: } & \multirow{3}{*}{ 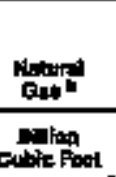 } & \multicolumn{6}{|c|}{ Pelrolons } & \multirow[b]{2}{*}{ Intelaty } & \multirow[b]{2}{*}{ Net } & \multirow[b]{2}{*}{ 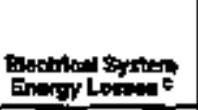 } & \multirow[b]{3}{*}{4} \\
\hline & " & Anthasith 4 & Tot: & & 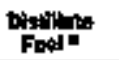 & Rtrosempt & LPG 4 & moter & melay" & 7ot & & & & \\
\hline & \multicolumn{3}{|c|}{ Thowand Groor Tone } & & \multicolumn{6}{|c|}{ Thousuns antels } & \multicolumn{3}{|c|}{ 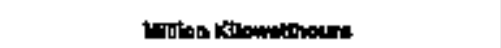 } & \\
\hline 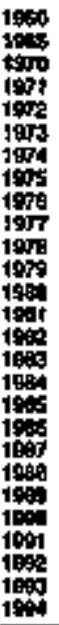 & 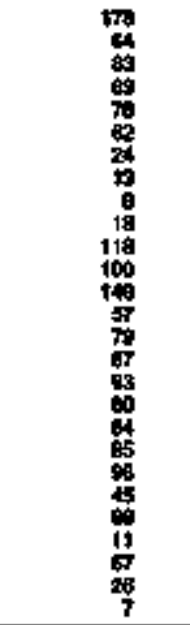 & $\begin{array}{l}0 \\
0 \\
0 \\
0 \\
0 \\
0 \\
0 \\
0 \\
0 \\
0 \\
0 \\
0 \\
0 \\
0 \\
0 \\
0 \\
0 \\
0 \\
0 \\
0 \\
0 \\
0 \\
0 \\
0 \\
0 \\
0 \\
0 \\
0 \\
0\end{array}$ & 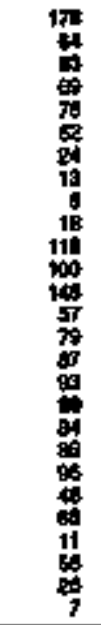 & 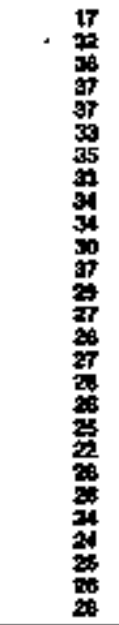 & 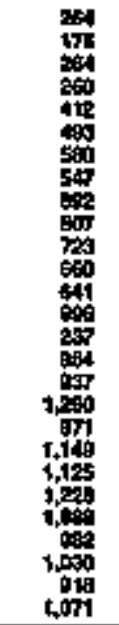 & 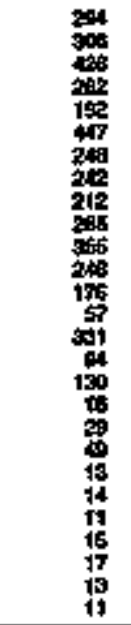 & 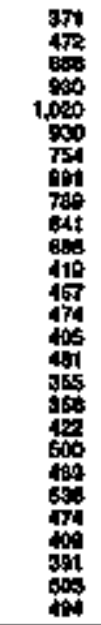 & 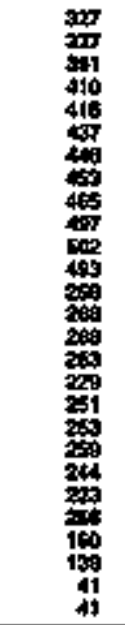 & 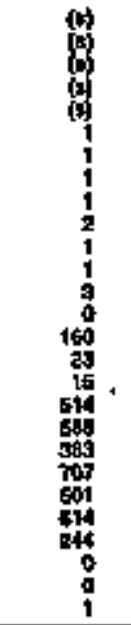 & 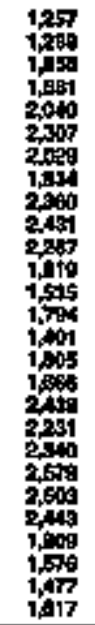 & 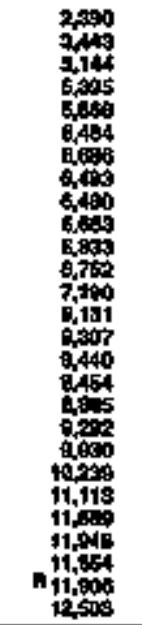 & $\begin{array}{l}= \\
z \\
z \\
z \\
z \\
z \\
z \\
z \\
z \\
z \\
z \\
z \\
z \\
z \\
z \\
z\end{array}$ & 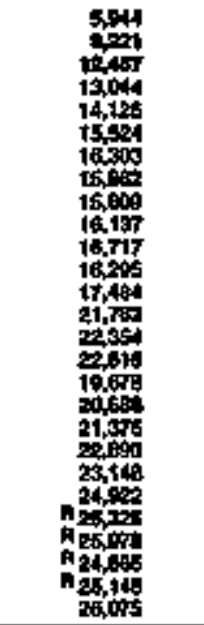 & $\begin{array}{l}= \\
z \\
z \\
z \\
z \\
z \\
z \\
= \\
= \\
= \\
z \\
= \\
z \\
= \\
=\end{array}$ \\
\hline \multicolumn{15}{|c|}{ tamion otu } \\
\hline 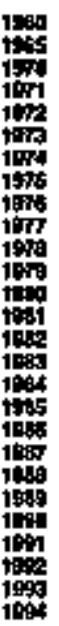 & $\begin{array}{l}44 \\
10 \\
20 \\
16 \\
10 \\
11 \\
06 \\
04 \\
02 \\
04 \\
20 \\
24 \\
314 \\
14 \\
21 \\
23 \\
20 \\
21 \\
21 \\
25 \\
1.1 \\
103 \\
14 \\
06 \\
02\end{array}$ & 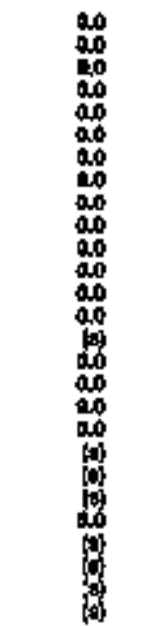 & 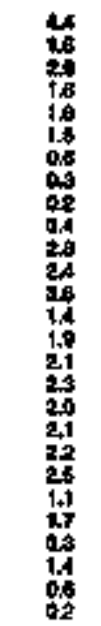 & 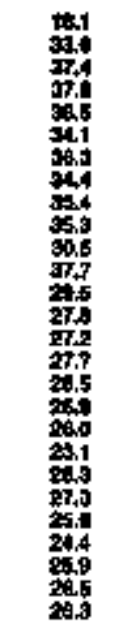 & 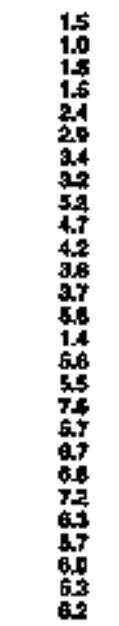 & 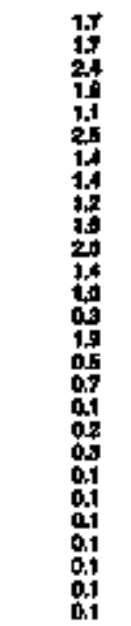 & 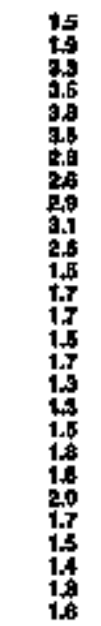 & 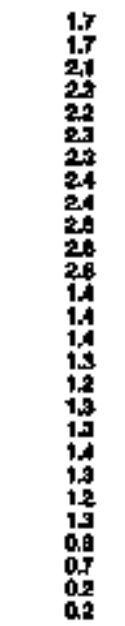 & 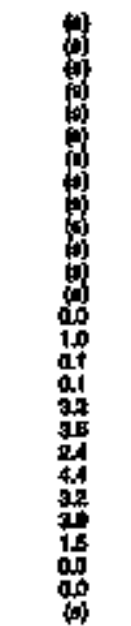 & 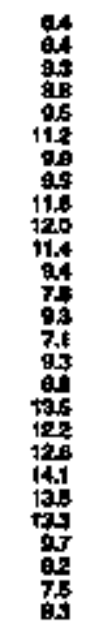 & 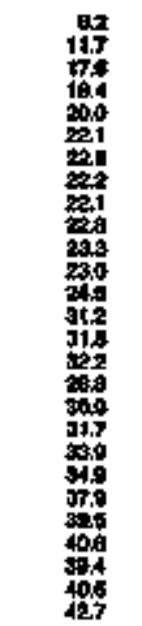 & 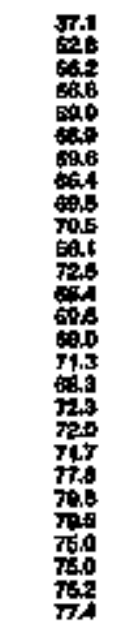 & 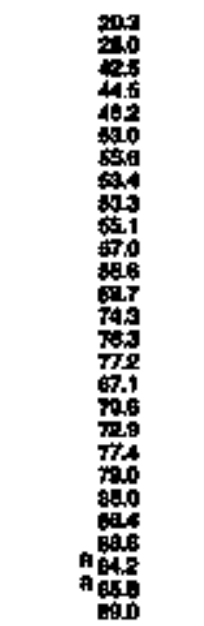 & 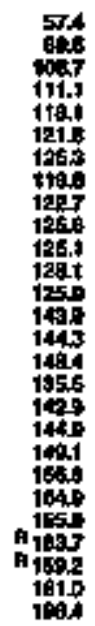 \\
\hline & & wats & $n$ & tectost & & & & & & & & 16 & & \\
\hline
\end{tabular}




\begin{tabular}{|c|c|c|c|c|c|c|c|c|c|c|c|c|c|c|c|c|c|c|}
\hline & \multirow[b]{2}{*}{ can } & \multirow[b]{2}{*}{ 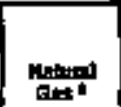 } & \multicolumn{9}{|c|}{ Potroshitm } & \multirow{2}{*}{ 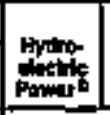 } & \multirow[b]{2}{*}{ Dofines I I } & \multirow[b]{2}{*}{ oster 40} & \multirow[b]{2}{*}{ 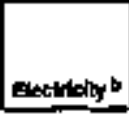 } & \multirow[b]{2}{*}{ totiont } & \multirow{2}{*}{ 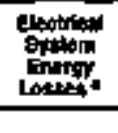 } & \multirow[b]{3}{*}{ Toll } \\
\hline & & & 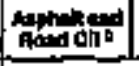 & Detullot & 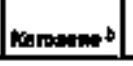 & 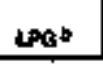 & " - & 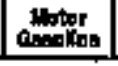 & Antuind & olsensen & Totald & & & & & & & \\
\hline$v_{m}$ & 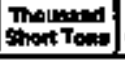 & allstion & \multicolumn{9}{|c|}{ 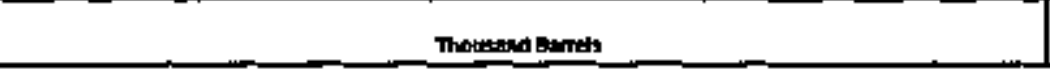 } & \multicolumn{6}{|c|}{ mon knowithoun } & \\
\hline 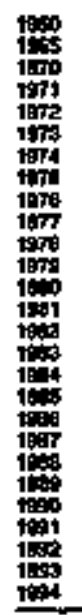 & 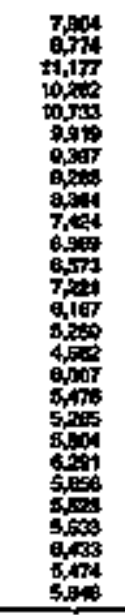 & 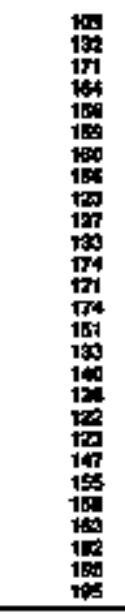 & 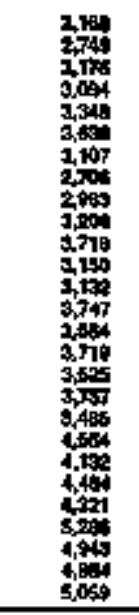 & 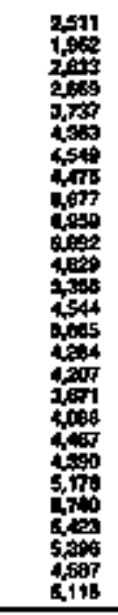 & 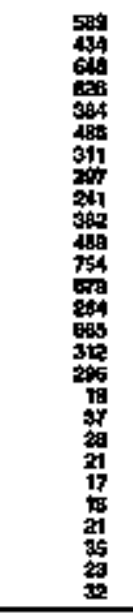 & 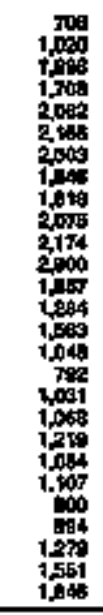 & 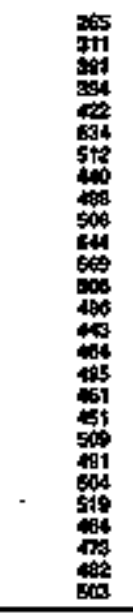 & 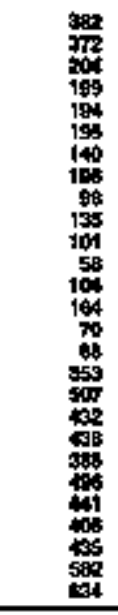 & 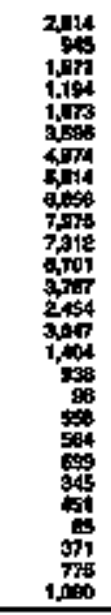 & 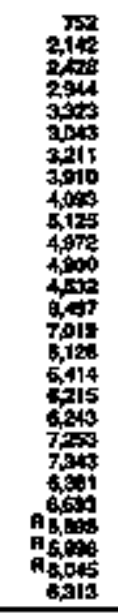 & 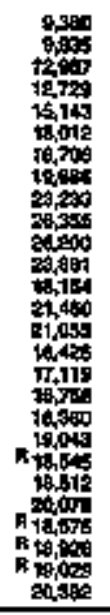 & 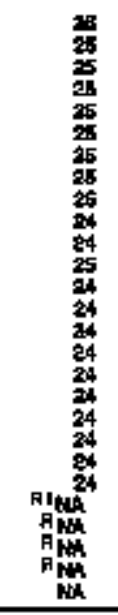 & 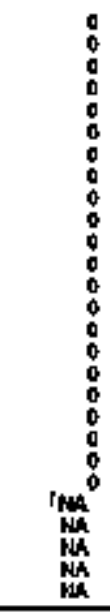 & 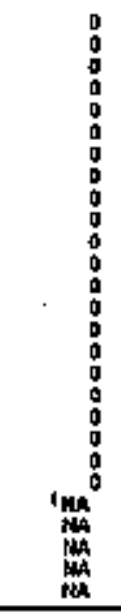 & 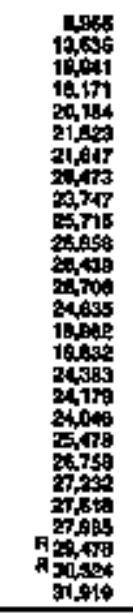 & $\begin{array}{l}\vdots \\
\vdots \\
\vdots \\
\vdots \\
\vdots \\
\vdots \\
\vdots \\
\vdots \\
\vdots \\
\vdots \\
\vdots \\
\vdots \\
\end{array}$ & 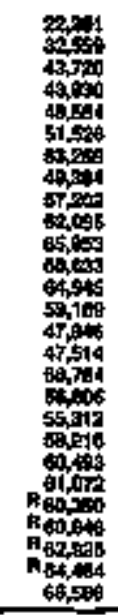 & $\begin{array}{l}z \\
z \\
z \\
z \\
z\end{array}$ \\
\hline \multicolumn{18}{|c|}{ 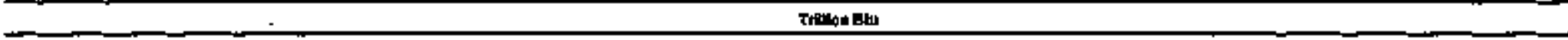 } & \\
\hline 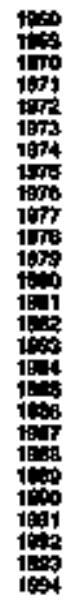 & 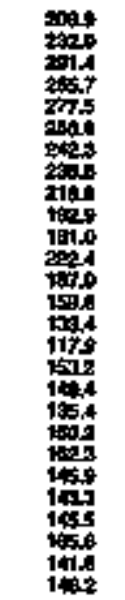 & 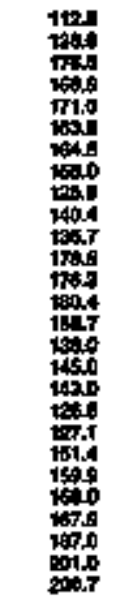 & 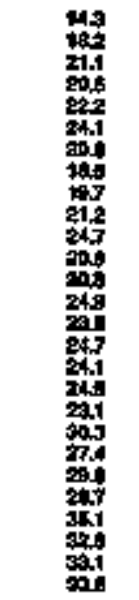 & 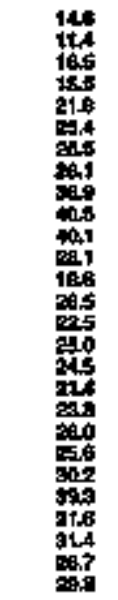 & 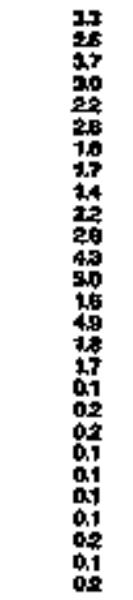 & 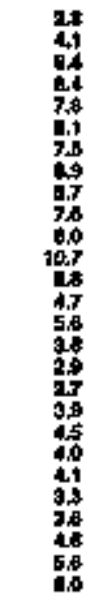 & 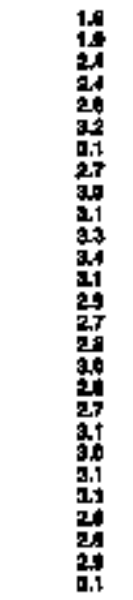 & 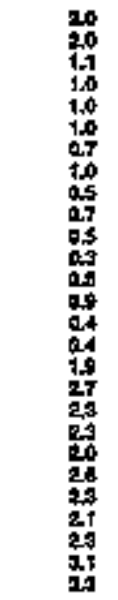 & 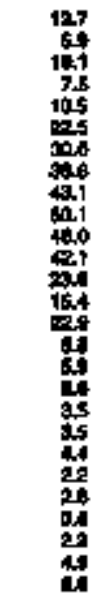 & 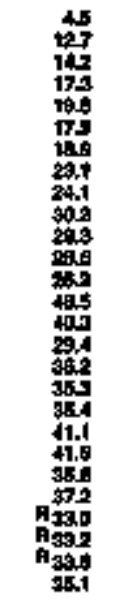 & 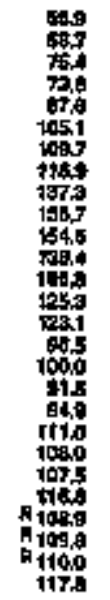 & 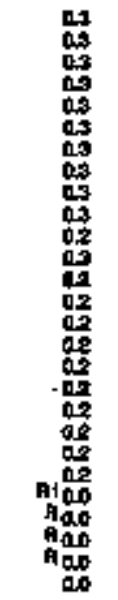 & 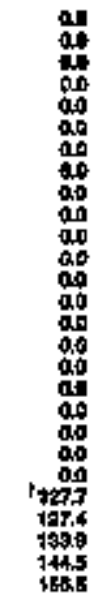 & 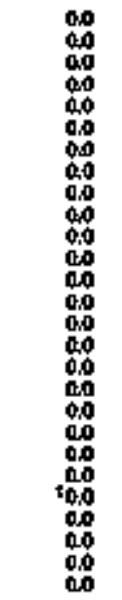 & 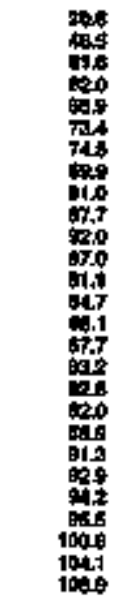 & 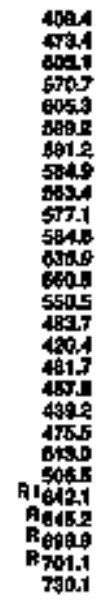 & 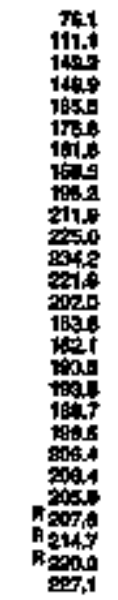 & 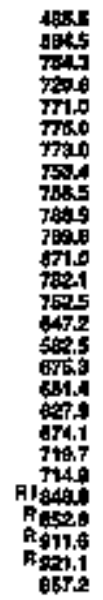 \\
\hline
\end{tabular}


Table 21. Transportation Energy Consumpition Eestimalts, 1960, 1855, 1970-1994, Alabama

\begin{tabular}{|c|c|c|c|c|c|c|c|c|c|c|c|c|c|c|c|}
\hline \multirow[b]{3}{*}{$r$} & \multirow[b]{2}{*}{ Dow I } & \multirow[b]{2}{*}{ Not } & \multicolumn{8}{|c|}{ Potrolugen } & \multirow{3}{*}{ 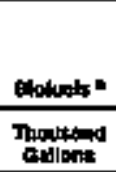 } & \multirow[b]{2}{*}{ 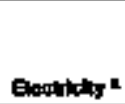 } & \multirow[b]{2}{*}{ Enoryt } & \multirow[b]{2}{*}{ 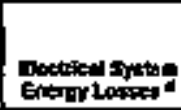 } & \multirow[b]{3}{*}{ 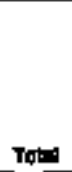 } \\
\hline & & & Antellon. & tonstom & Pod & LPQ: & 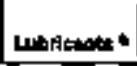 & mation & Fopetid & (1) & & & & & \\
\hline & $\begin{array}{l}\text { Trootsand } \\
\text { Shent Tera: }\end{array}$ & atiof fax & \multicolumn{8}{|c|}{ Thousand Gurnt: } & & \multicolumn{3}{|c|}{ 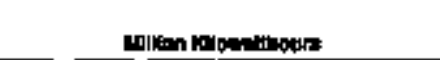 } & \\
\hline 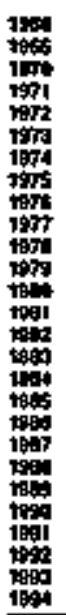 & 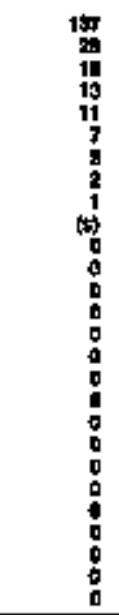 & 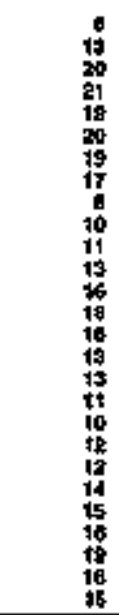 & 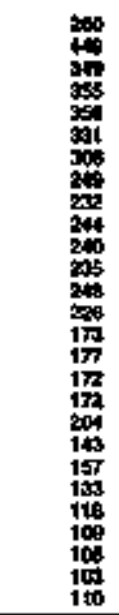 & 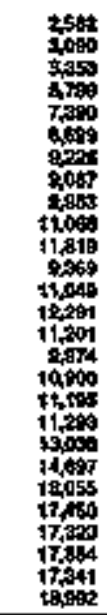 & 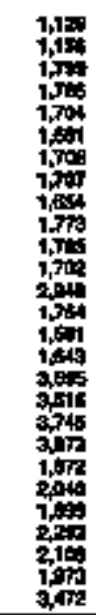 & 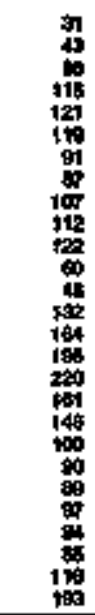 & 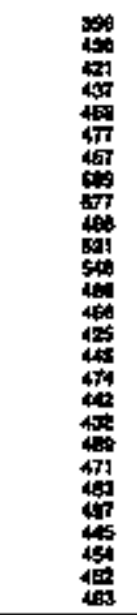 & 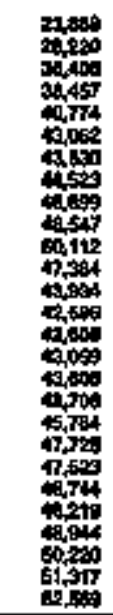 & 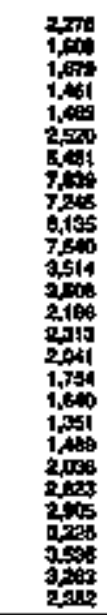 & 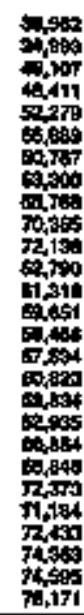 & 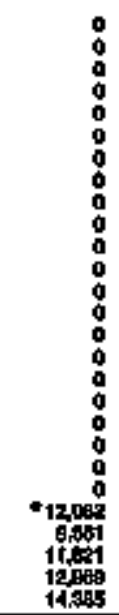 & $\begin{array}{l}0 \\
0 \\
0 \\
0 \\
0 \\
0 \\
0 \\
0 \\
0 \\
0 \\
0 \\
0 \\
0 \\
0 \\
0 \\
0 \\
0 \\
0 \\
0 \\
0 \\
0 \\
0 \\
0 \\
0 \\
0 \\
0 \\
0\end{array}$ & $\begin{array}{l}= \\
= \\
= \\
= \\
= \\
= \\
= \\
= \\
= \\
= \\
= \\
= \\
= \\
=\end{array}$ & $\begin{array}{l}: \\
: \\
0 \\
0 \\
0 \\
: \\
: \\
0 \\
0 \\
0 \\
0 \\
0 \\
0 \\
0 \\
0 \\
0 \\
0 \\
0 \\
0 \\
0 \\
0\end{array}$ & $\begin{array}{l}= \\
= \\
= \\
= \\
= \\
= \\
= \\
= \\
= \\
= \\
= \\
= \\
= \\
=\end{array}$ \\
\hline \multicolumn{16}{|c|}{ Mingon tow } \\
\hline 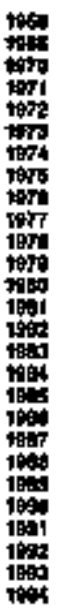 & 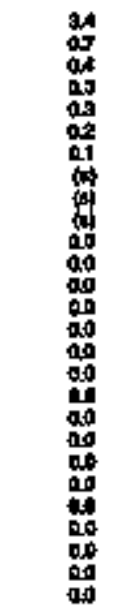 & 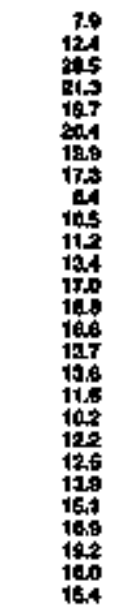 & 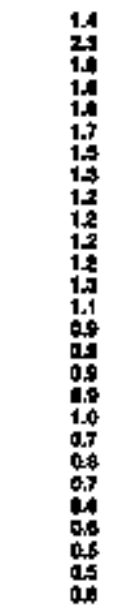 & 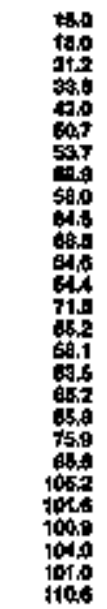 & 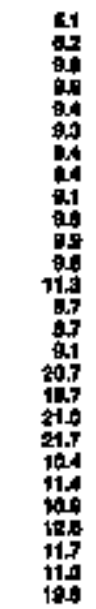 & 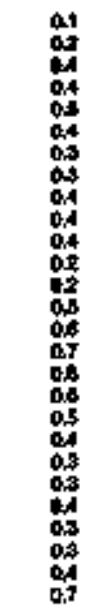 & 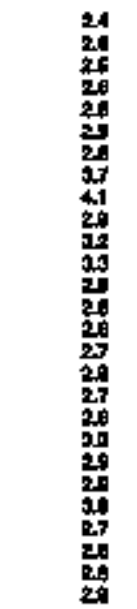 & 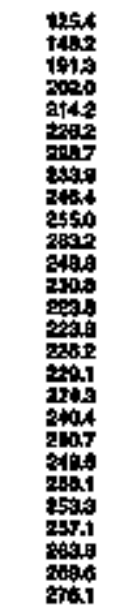 & 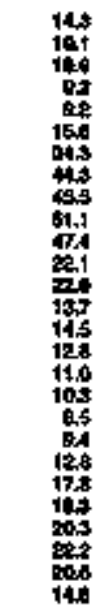 & 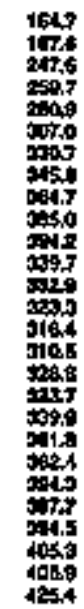 & 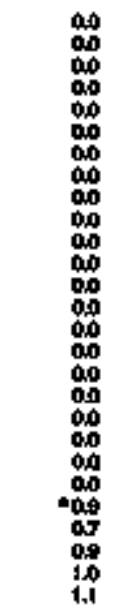 & $\begin{array}{l}00 \\
00 \\
00 \\
00 \\
00 \\
00 \\
00 \\
000 \\
00 \\
00 \\
00 \\
00 \\
000 \\
00 \\
000 \\
00 \\
00 \\
00 \\
00 \\
00 \\
00 \\
00 \\
00 \\
00 \\
00 \\
000 \\
00\end{array}$ & 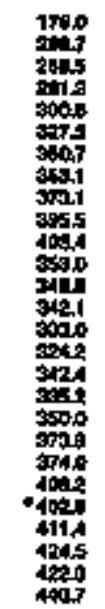 & $\begin{array}{l}00 \\
00 \\
00 \\
00 \\
00 \\
00 \\
00 \\
00 \\
00 \\
00 \\
00 \\
00 \\
100 \\
0.0 \\
00 \\
00 \\
00 \\
00 \\
00 \\
00 \\
00 \\
00 \\
00 \\
00 \\
00 \\
00 \\
00\end{array}$ & 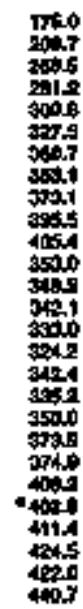 \\
\hline
\end{tabular}

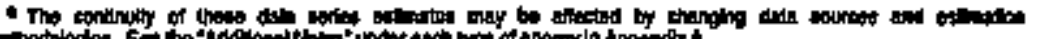

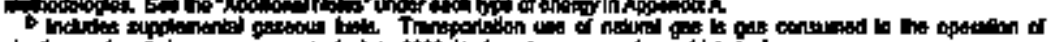

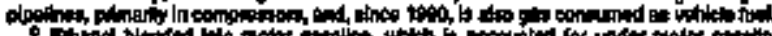

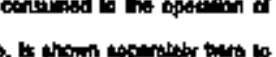

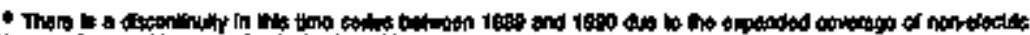

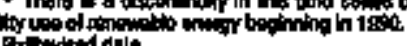
$\rightarrow$ that apolete.

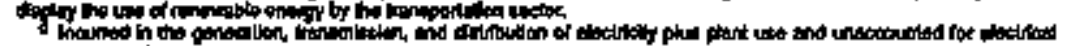

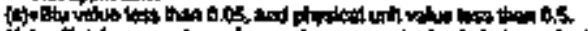
inconom 


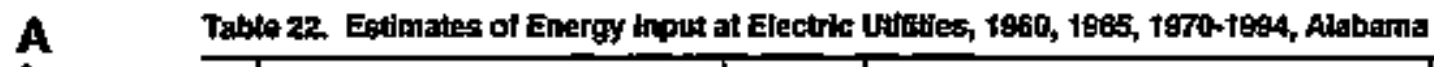

$\mathbf{L}$
$\mathbf{A}$
$\mathbf{B}$
$\mathbf{A}$
$\mathbf{A}$

\begin{tabular}{|c|c|c|c|c|c|c|c|c|c|c|c|c|c|c|}
\hline \multirow[b]{3}{*}{ yosr] } & \multicolumn{3}{|c|}{ cons } & \multirow[b]{2}{*}{ mentint } & \multicolumn{4}{|c|}{ Potrobutin } & \multirow[b]{2}{*}{ 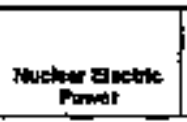 } & \multirow[b]{2}{*}{ 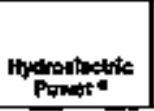 } & \multirow[b]{2}{*}{ eblowers, } & \multirow[b]{2}{*}{ antarmas } & \multirow[b]{2}{*}{ Onter bu } & \multirow[b]{3}{*}{ тоны: } \\
\hline & 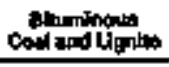 & Antring the & Tolod & & maty & 岸施 & monotent" & The & & & & & & \\
\hline & \multicolumn{3}{|c|}{ Theurand and Tons } & 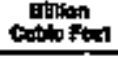 & \multicolumn{4}{|c|}{ Thentawd Mards } & \multicolumn{5}{|c|}{ 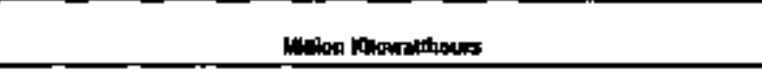 } & \\
\hline 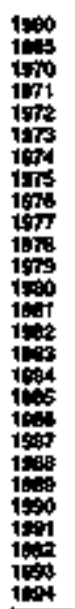 & 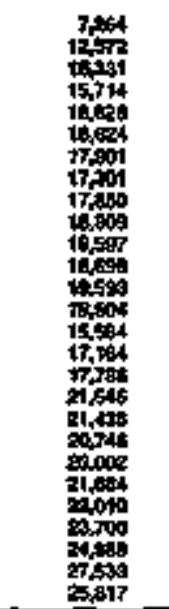 & $\begin{array}{l}: \\
0 \\
0 \\
0 \\
0 \\
0 \\
0 \\
0 \\
0 \\
0 \\
0 \\
0 \\
0 \\
0 \\
0\end{array}$ & 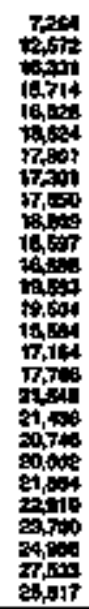 & 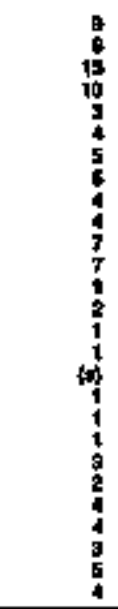 & 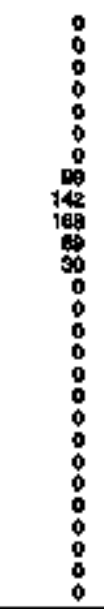 & 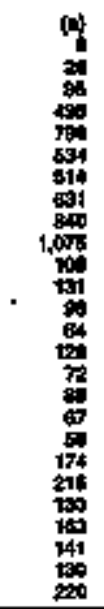 & 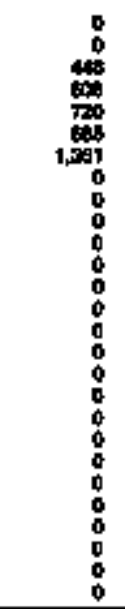 & 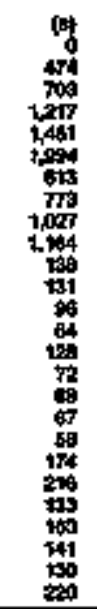 & 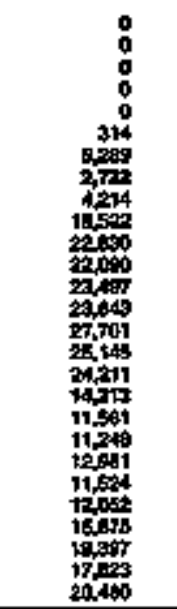 & 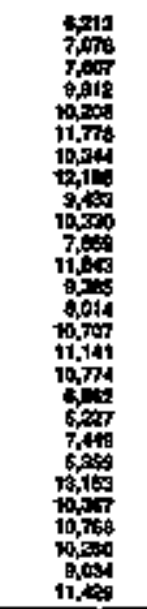 & $\begin{array}{l}0 \\
0 \\
0 \\
0 \\
0 \\
0 \\
0 \\
0 \\
0 \\
0 \\
0 \\
0 \\
0 \\
0 \\
0 \\
0 \\
0 \\
0 \\
0 \\
0 \\
0 \\
0 \\
0 \\
0 \\
0 \\
0 \\
0 \\
0 \\
0 \\
0 \\
0 \\
0\end{array}$ & $\begin{array}{l}0 \\
0 \\
0 \\
0 \\
0 \\
0 \\
0 \\
0 \\
0 \\
0 \\
0 \\
0 \\
0 \\
0 \\
0 \\
0 \\
0 \\
0 \\
0 \\
0 \\
0 \\
0 \\
0 \\
0\end{array}$ & $\begin{array}{l}0 \\
0 \\
0 \\
0 \\
0 \\
0 \\
0 \\
0 \\
0 \\
0 \\
0 \\
0 \\
0 \\
0 \\
0 \\
0 \\
0 \\
0 \\
0 \\
0 \\
0 \\
0 \\
0 \\
0 \\
0 \\
0 \\
0 \\
0 \\
0\end{array}$ & 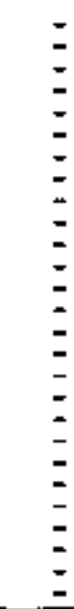 \\
\hline
\end{tabular}

\begin{tabular}{|c|c|c|}
\hline 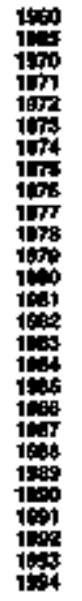 & 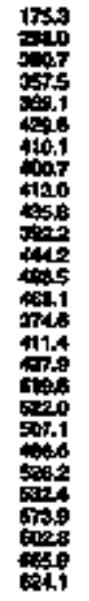 & $\begin{array}{l}a \\
0 \\
d\end{array}$ \\
\hline
\end{tabular}

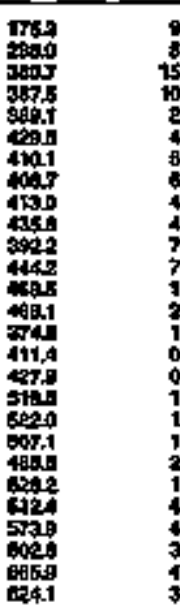

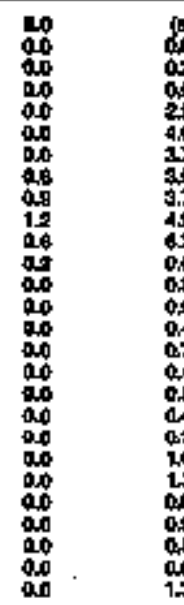

io

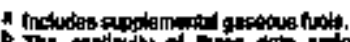

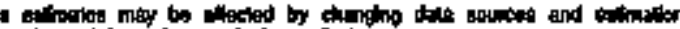

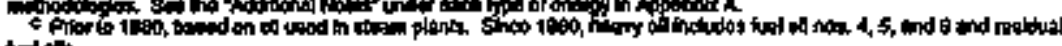

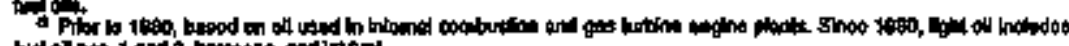

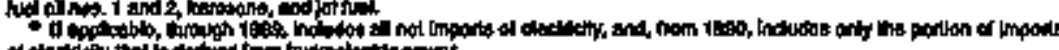

\begin{tabular}{|c|c|c|c|c|c|c|}
\hline 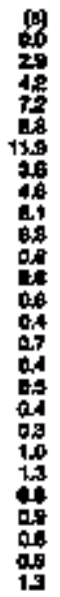 & 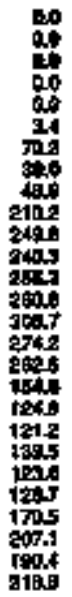 & 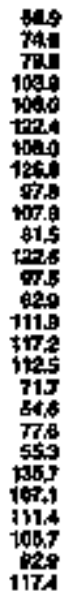 & 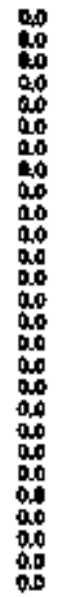 & 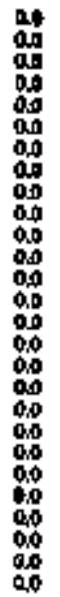 & 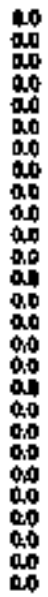 & 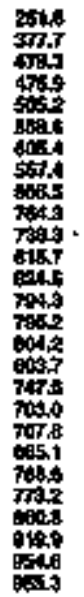 \\
\hline
\end{tabular}

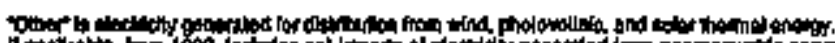
- th in odtar column

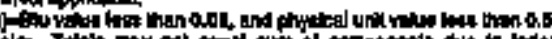

moponant rounding

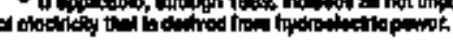




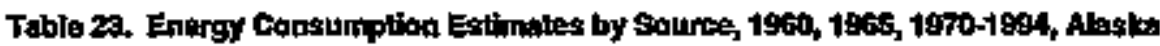

\begin{tabular}{|c|c|c|c|c|c|c|c|c|c|c|c|c|c|c|c|c|c|c|c|}
\hline \multirow[b]{3}{*}{$\mathrm{Ym}$} & \multirow[b]{2}{*}{$\cos 1$} & \multirow[b]{2}{*}{$\operatorname{man}_{\substack{0 \\
0}}$} & \multicolumn{11}{|c|}{ 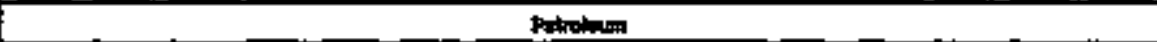 } & \multirow[b]{2}{*}{ 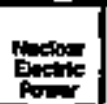 } & \multirow[b]{2}{*}{ 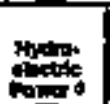 } & \multirow[b]{2}{*}{ Gtotneste } & \multirow[b]{2}{*}{ | } & \multirow{2}{*}{ ה } & \multirow[b]{3}{*}{ Toted } \\
\hline & & & Apen: & 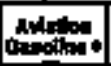 & 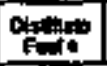 & 영. & 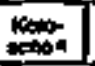 & a. ? & lutis. & Conotition & 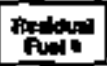 & $\tan c$ & tot & & & & & & \\
\hline & Thousind & ation & \multicolumn{11}{|c|}{ 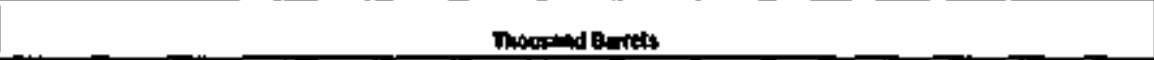 } & \multicolumn{5}{|c|}{ 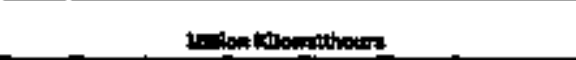 } & \\
\hline 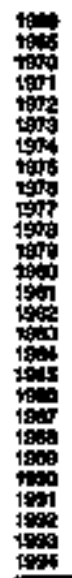 & 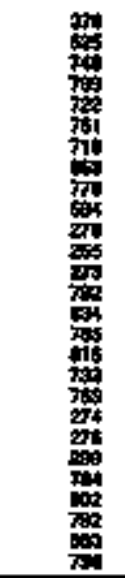 & 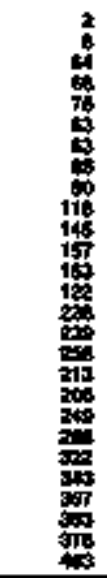 & 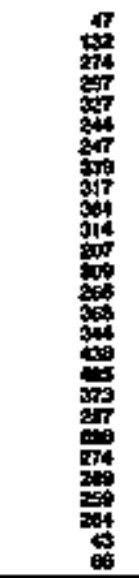 & 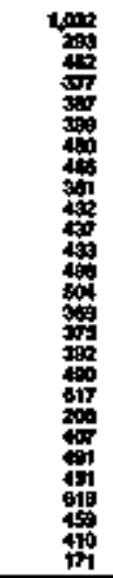 & 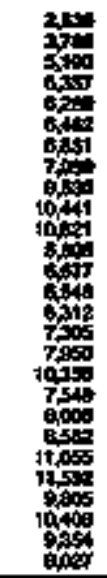 & 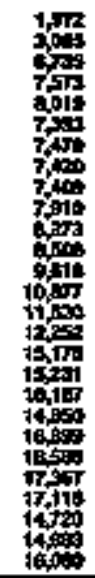 & 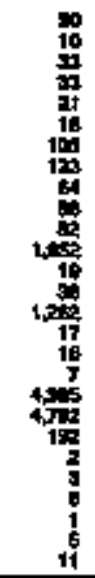 & 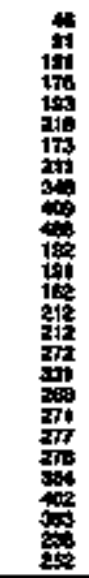 & 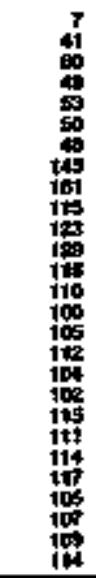 & 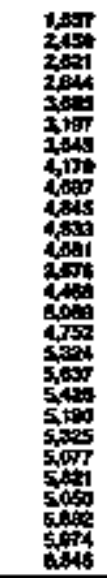 & 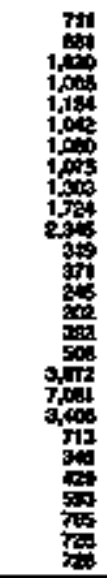 & 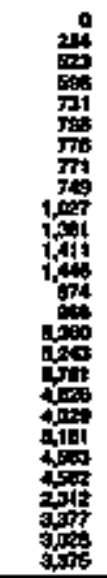 & 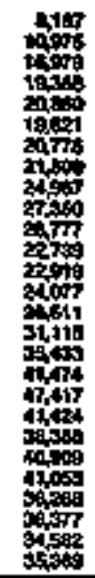 & $\begin{array}{l}0 \\
0 \\
0 \\
0 \\
0 \\
0 \\
0 \\
0 \\
0 \\
0 \\
0 \\
0 \\
0 \\
0 \\
0 \\
0 \\
0 \\
0 \\
0 \\
0 \\
0 \\
0 \\
0 \\
0 \\
0\end{array}$ & 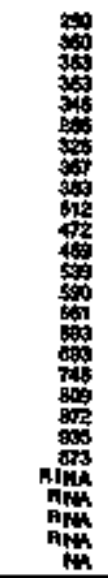 & 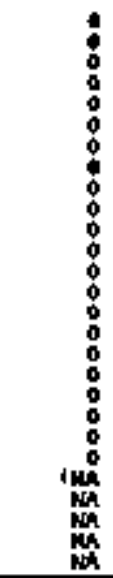 & 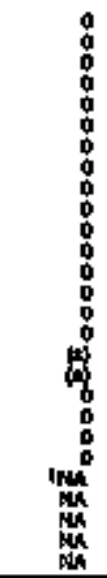 & $\begin{array}{l}0 \\
0 \\
0 \\
0 \\
0 \\
0 \\
0 \\
0 \\
0 \\
0 \\
0 \\
0 \\
0 \\
0 \\
0 \\
0 \\
0 \\
0 \\
0 \\
0 \\
0 \\
0 \\
0 \\
0\end{array}$ & $\begin{array}{l}z \\
z \\
z \\
\vdots \\
\vdots \\
z \\
z \\
z \\
z \\
z \\
z \\
z \\
z \\
z\end{array}$ \\
\hline \multicolumn{20}{|c|}{ In noeveth } \\
\hline 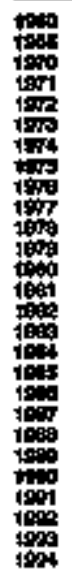 & 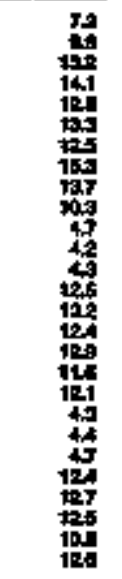 & 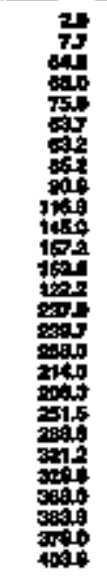 & 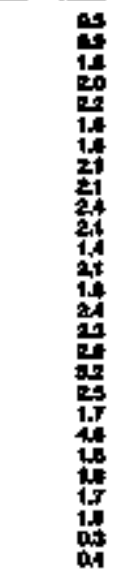 & 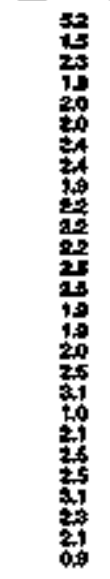 & 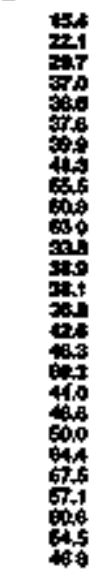 & 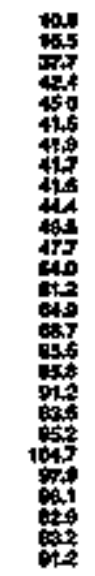 & 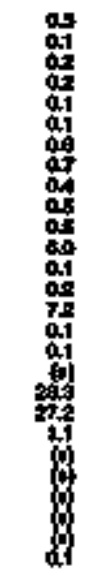 & 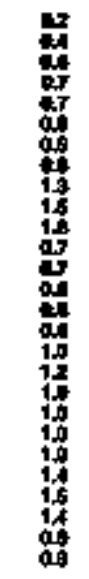 & 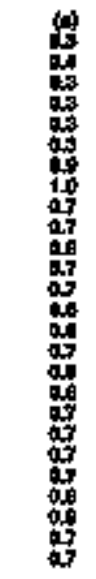 & 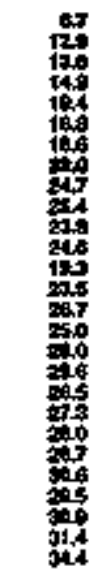 & 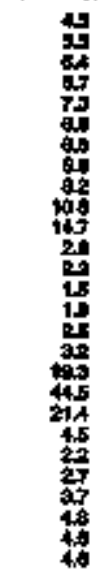 & 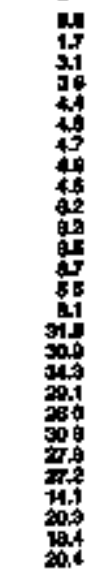 & 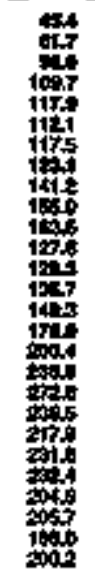 & 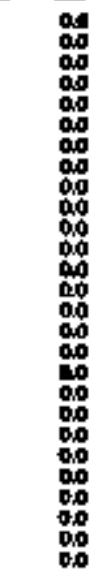 & 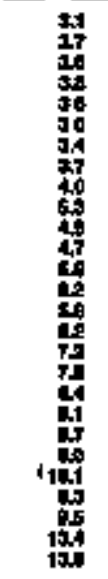 & 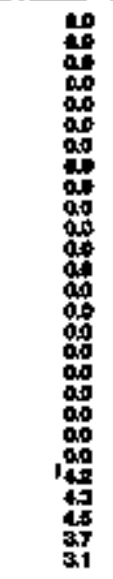 & 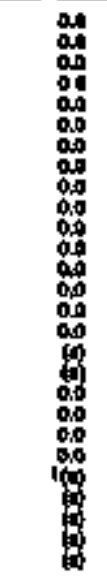 & 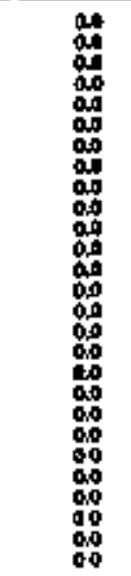 & 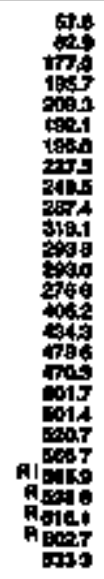 \\
\hline
\end{tabular}

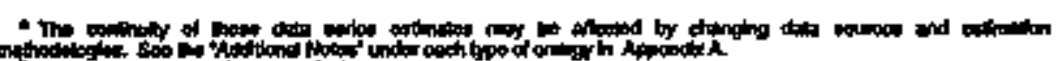

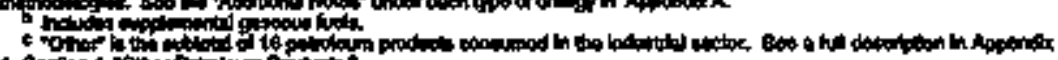

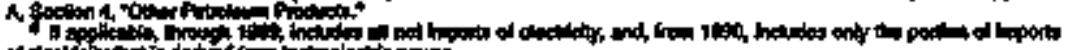

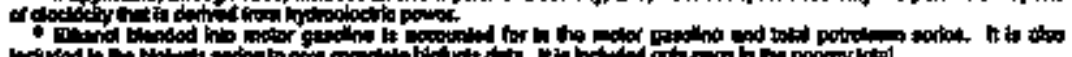

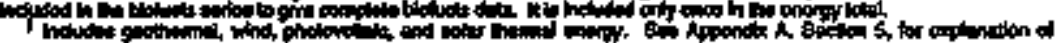

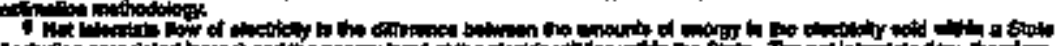

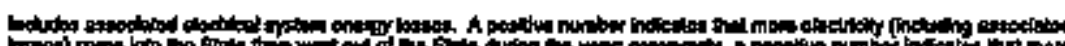
of

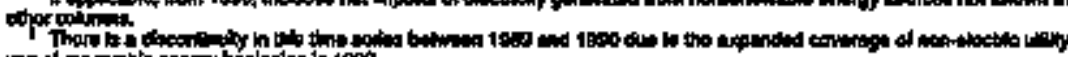

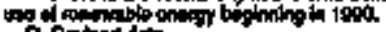


$\mathbf{A}$
$\mathbf{L}$
$\mathbf{S}$
$\mathbf{K}$
$\mathbf{A}$

Table 24. Residenlial Energy Consunnption Estimates, 1960, 196B, 1970-1994, Alaska

\begin{tabular}{|c|c|c|c|c|c|c|c|c|c|c|c|c|c|c|}
\hline \multirow[b]{3}{*}{ Yatr } & \multicolumn{3}{|c|}{$\cos$} & \multirow{3}{*}{ 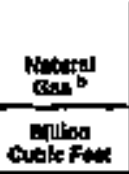 } & & & & & \multirow{3}{*}{ 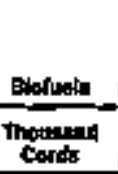 } & & \multirow[b]{2}{*}{ 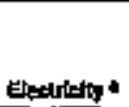 } & \multirow[b]{2}{*}{ Fonoty } & \multirow{3}{*}{ 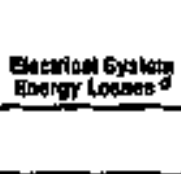 } & \multirow[b]{3}{*}{ Tots } \\
\hline & A & anding" & roun & & Destung & 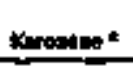 & Los: & Jotal & & Batrar & & & & \\
\hline & \multicolumn{3}{|c|}{ hoowend Short tond } & & \multicolumn{4}{|c|}{ Thounnd format } & & \multicolumn{3}{|c|}{ 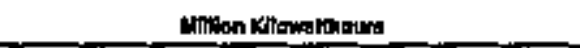 } & & \\
\hline 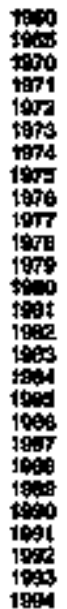 & 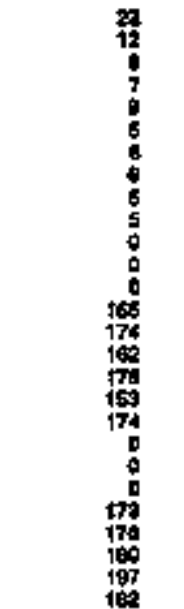 & $\begin{array}{l}0 \\
\vdots \\
0 \\
0 \\
\vdots \\
\vdots \\
\vdots \\
\vdots \\
\vdots \\
\vdots \\
\vdots \\
\vdots \\
\vdots \\
\vdots \\
\vdots \\
\vdots \\
\vdots \\
0\end{array}$ & 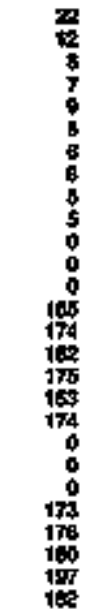 & 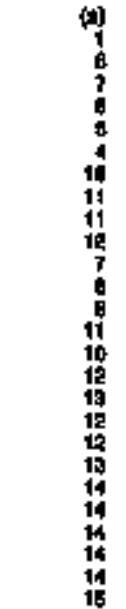 & 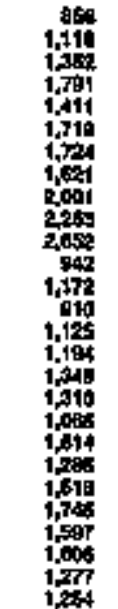 & 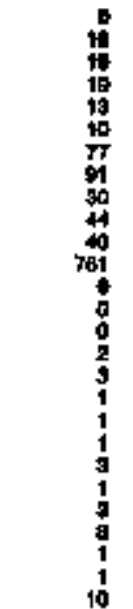 & 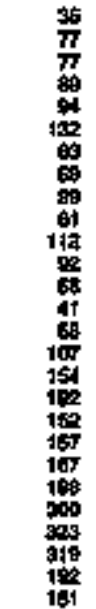 & 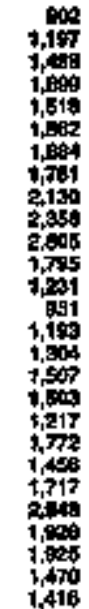 & $\begin{array}{l}0 \\
0 \\
0 \\
0 \\
0 \\
0 \\
0 \\
0 \\
0 \\
0 \\
0 \\
0 \\
0 \\
0 \\
0 \\
0 \\
0 \\
0 \\
0 \\
0 \\
0 \\
0 \\
0 \\
0 \\
0 \\
0 \\
0 \\
0 \\
0 \\
100 \\
10 \\
0\end{array}$ & $\begin{array}{l}0 \\
0 \\
0 \\
0 \\
0 \\
0 \\
0 \\
0 \\
0 \\
0 \\
0 \\
0 \\
0 \\
0 \\
0 \\
0 \\
0 \\
0 \\
9 \\
9 \\
9 \\
9\end{array}$ & 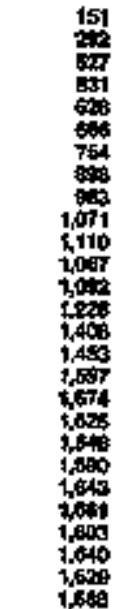 & 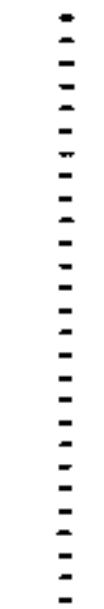 & 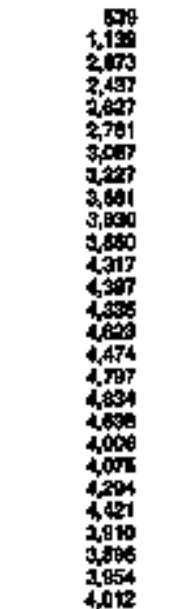 & $\bar{z}$ \\
\hline
\end{tabular}

\begin{tabular}{|c|c|c|c|c|c|}
\hline & 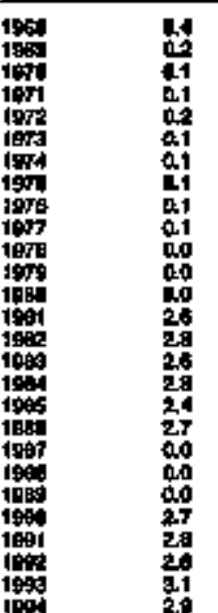 & 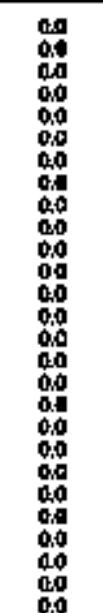 & 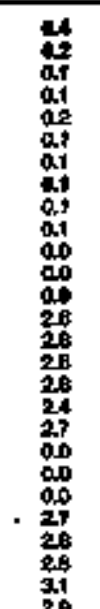 & 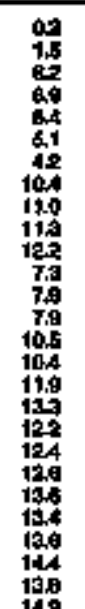 & 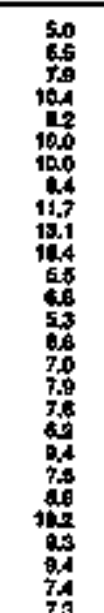 \\
\hline
\end{tabular}

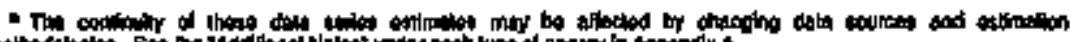

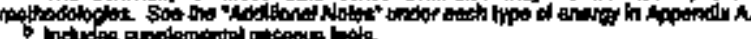

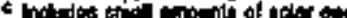

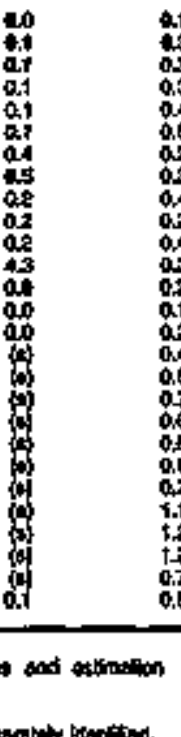

.

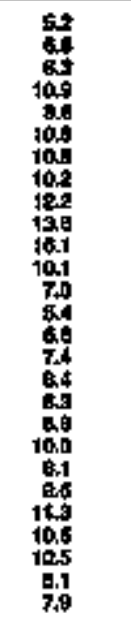

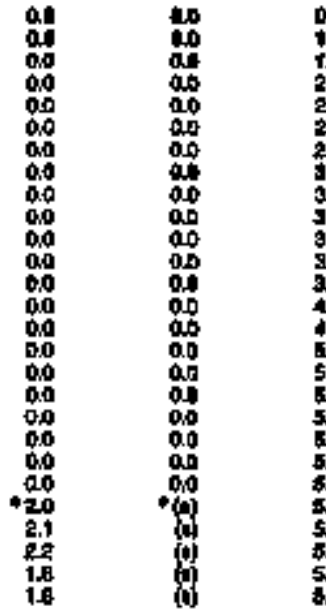

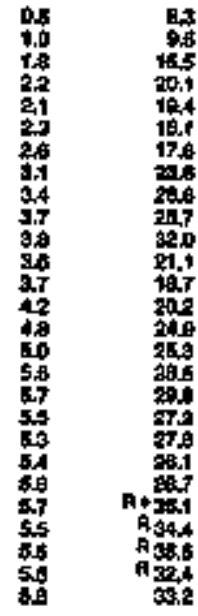

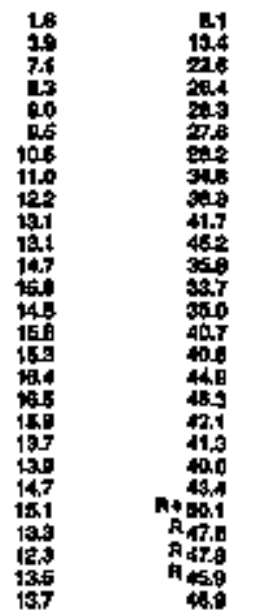

Sog Appond A soctions lor

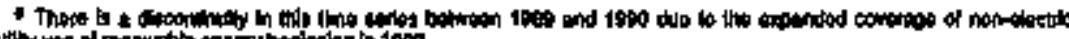

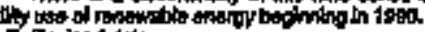

- litot appliats

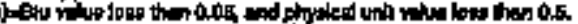

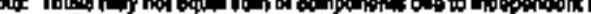

cyotimentind on 
Table 25. Commercial Energy Consumptlon Estlmates, 1950, 1965, 1970-1994, Alaska

\begin{tabular}{|c|c|c|c|c|c|c|c|c|c|c|c|c|c|c|}
\hline \multirow[b]{3}{*}{$\mathrm{rem}$} & \multicolumn{3}{|c|}{ cow } & \multirow{3}{*}{ 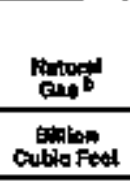 } & \multicolumn{6}{|c|}{ Putolewn } & \multirow[b]{2}{*}{ 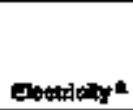 } & \multirow[b]{2}{*}{ Eis } & \multirow[b]{2}{*}{ 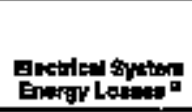 } & \multirow[b]{3}{*}{ Tatal 4} \\
\hline & 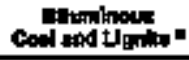 & Anthostap= & Toptow & & Fidento & Kerobenat & LFG & 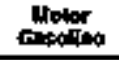 & Propidual & Tolut & & & & \\
\hline & \multicolumn{2}{|c|}{ Thounend Shent Tons } & & & \multicolumn{6}{|c|}{ Thoos:-id Barifs } & \multicolumn{3}{|c|}{ Hinstrlowatinoure } & \\
\hline 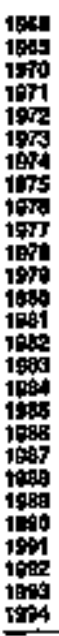 & 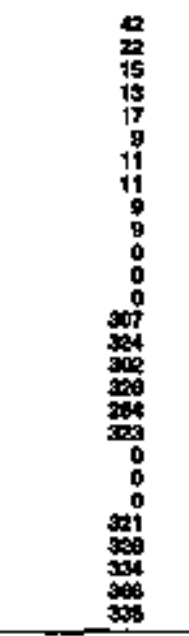 & $\begin{array}{l}0 \\
0 \\
0 \\
0 \\
0 \\
0 \\
0 \\
0 \\
0 \\
0 \\
0 \\
0 \\
0 \\
0 \\
0 \\
0 \\
0 \\
0 \\
0 \\
0 \\
0 \\
0 \\
0 \\
0 \\
0 \\
0 \\
0\end{array}$ & 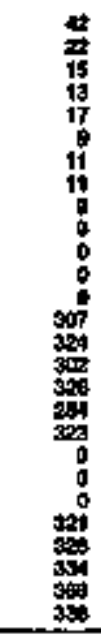 & 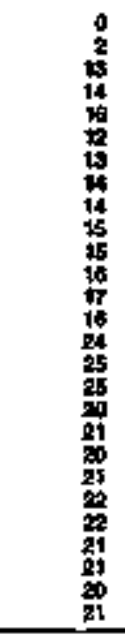 & 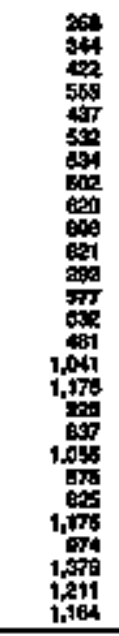 & 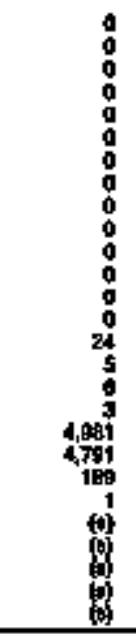 & 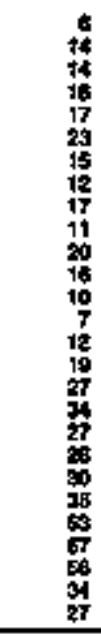 & 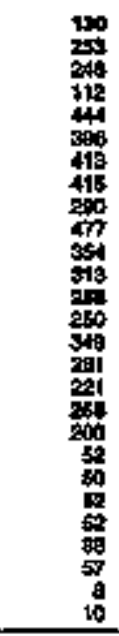 & 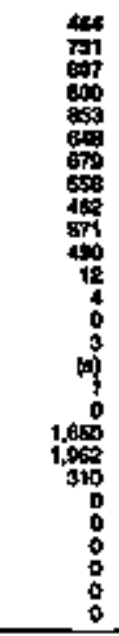 & 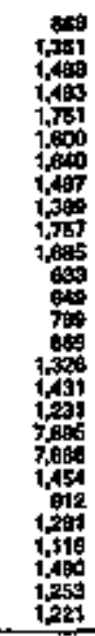 & 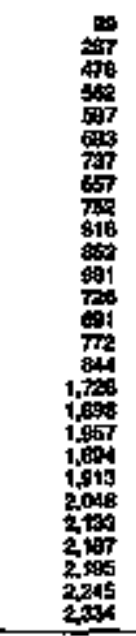 & $\begin{array}{l}= \\
= \\
= \\
= \\
= \\
= \\
= \\
= \\
= \\
= \\
= \\
= \\
= \\
= \\
= \\
= \\
=\end{array}$ & 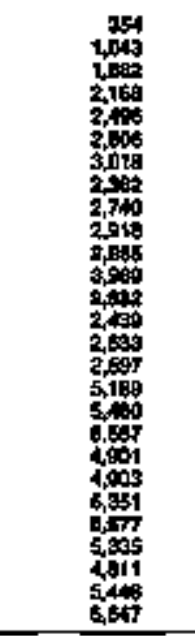 & $\begin{array}{l}= \\
= \\
= \\
= \\
= \\
= \\
= \\
= \\
= \\
= \\
z \\
= \\
= \\
=\end{array}$ \\
\hline \multicolumn{15}{|c|}{ 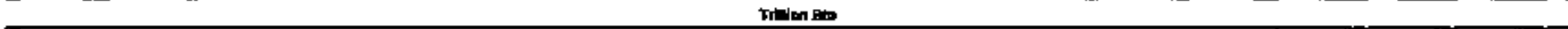 } \\
\hline 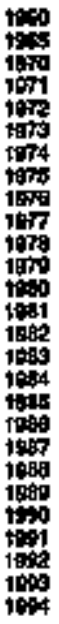 & 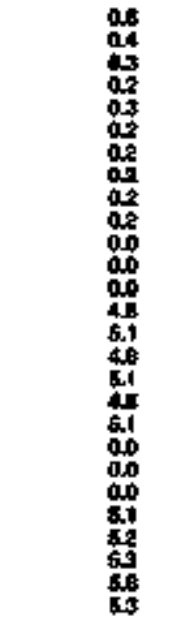 & 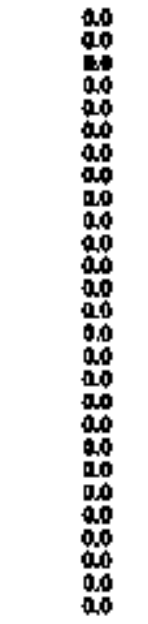 & 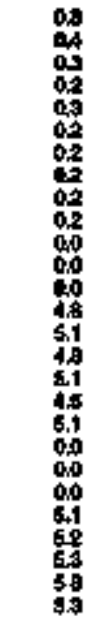 & 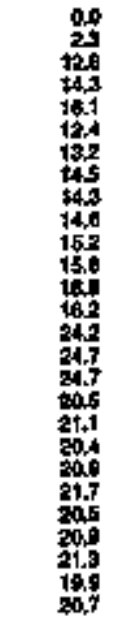 & 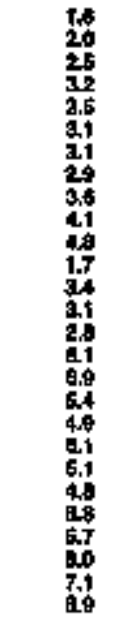 & 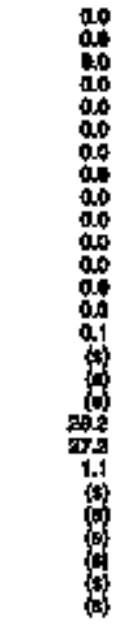 & $\begin{array}{l}10 \\
0.1 \\
0.1 \\
0.1 \\
0.1 \\
0.1 \\
0.1 \\
0.1 \\
0.0 \\
0.1 \\
0.1 \\
0.1 \\
0.9 \\
1.1 \\
0.1 \\
0.1 \\
0.1 \\
0.1 \\
0.1 \\
0.1 \\
0.1 \\
0.2 \\
0.2 \\
0.2 \\
0.1 \\
0.1\end{array}$ & 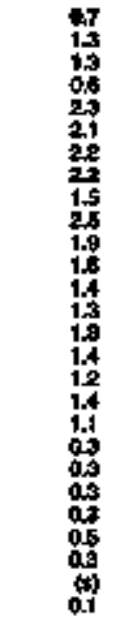 & 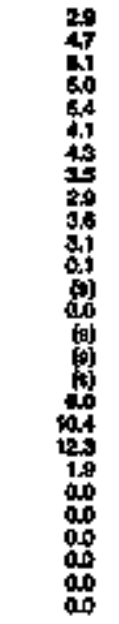 & 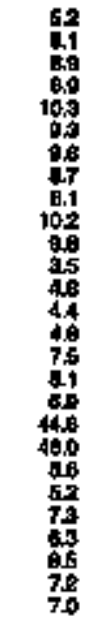 & 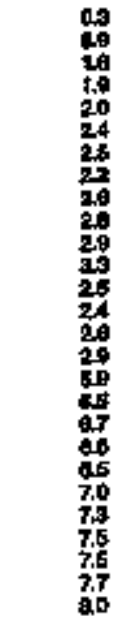 & 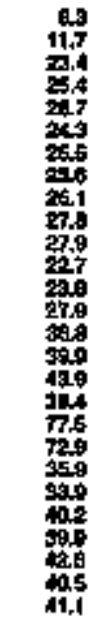 & 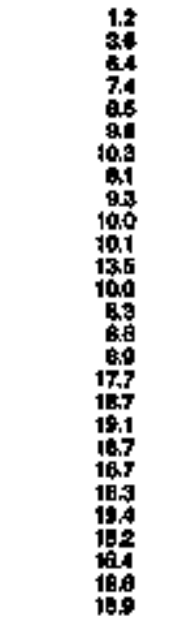 & 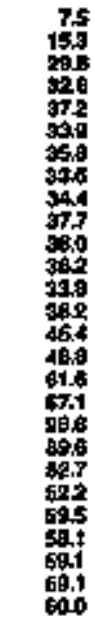 \\
\hline & & 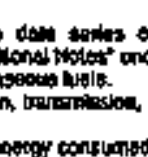 & 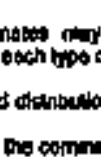 & & & & & & $\lim$. & & I & 10 & 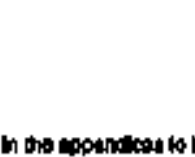 & \\
\hline
\end{tabular}

$\mathbf{A}$
$\mathbf{L}$
$\mathbf{A}$
$\mathbf{S}$
$\mathbf{K}$
$\mathbf{A}$ 
Tabte 25. Industriad Energy Consumption Estimates, 1960, 1965, 1970-1994, Alasice

\begin{tabular}{|c|c|c|c|c|c|c|c|c|c|c|c|c|c|c|c|c|c|c|}
\hline \multirow[b]{3}{*}{ Yer. } & \multirow[b]{2}{*}{ Con } & \multirow[b]{2}{*}{ 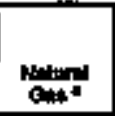 } & \multicolumn{9}{|c|}{ Fetrolum } & \multirow[b]{2}{*}{ 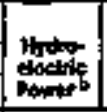 } & \multirow[b]{2}{*}{ 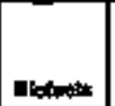 } & \multirow[b]{2}{*}{ Onerest } & \multirow[b]{2}{*}{ Exerikhy } & \multirow[b]{2}{*}{ Enerpint } & \multirow{2}{*}{ 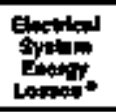 } & \multirow[b]{3}{*}{ Total } \\
\hline & & & repposing in & 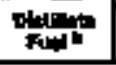 & Kenche & LPQ b & 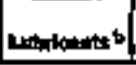 & onsor & Futed & Othas bot & Total & & & & & & & \\
\hline & Thertind & 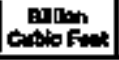 & \multicolumn{9}{|c|}{ Trovgund Bunth } & \multicolumn{6}{|c|}{ 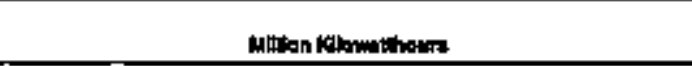 } & \\
\hline 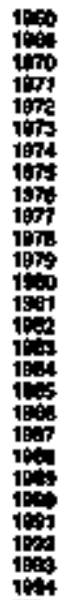 & 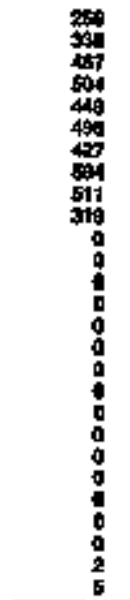 & 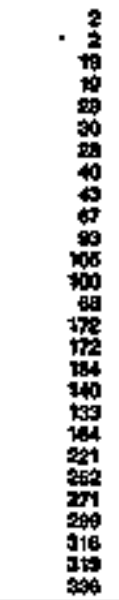 & 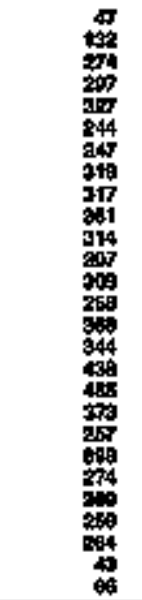 & 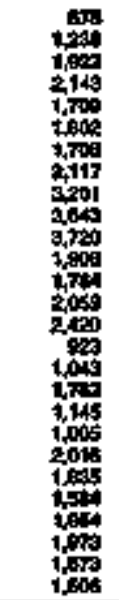 & 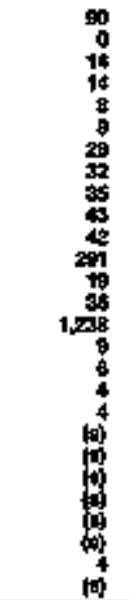 & 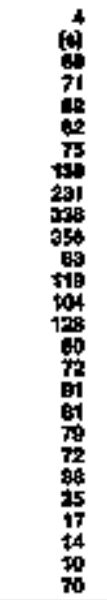 & $\begin{array}{l}1 \\
1 \\
1 \\
1 \\
1 \\
1 \\
1 \\
21 \\
27 \\
21 \\
21 \\
23 \\
31 \\
20 \\
11 \\
10 \\
20 \\
11 \\
10 \\
21 \\
21 \\
21 \\
31 \\
11 \\
10 \\
90 \\
20\end{array}$ & 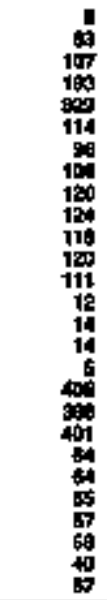 & 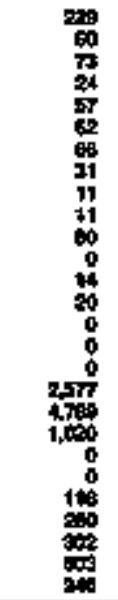 & 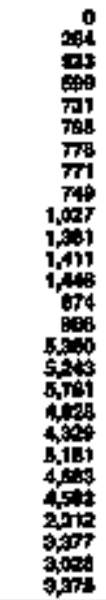 & 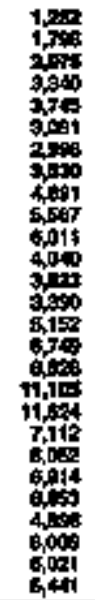 & 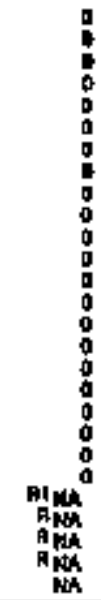 & 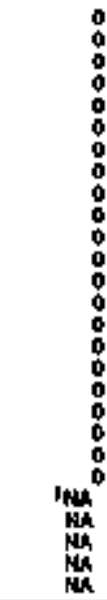 & 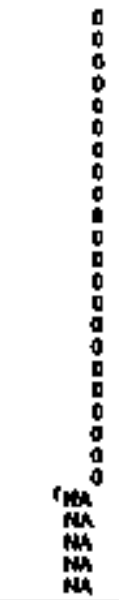 & 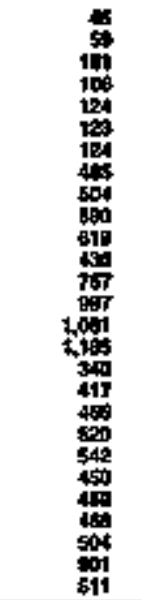 & $\begin{array}{l}= \\
= \\
z \\
= \\
\vdots \\
= \\
= \\
= \\
= \\
= \\
z \\
z \\
z \\
z \\
z\end{array}$ & 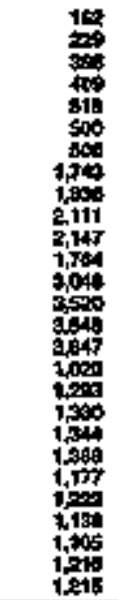 & 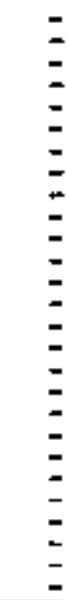 \\
\hline
\end{tabular}

\begin{tabular}{|c|c|c|c|c|c|c|c|c|c|c|c|c|c|c|c|c|}
\hline 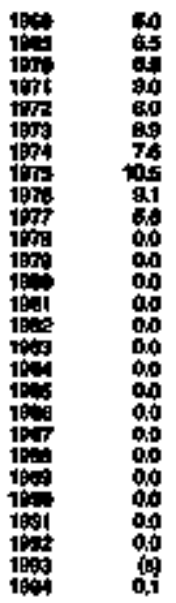 & 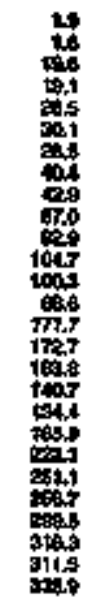 & 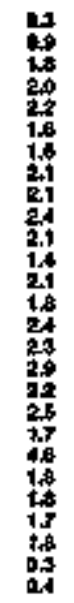 & 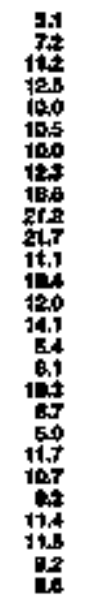 & 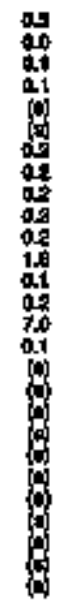 & 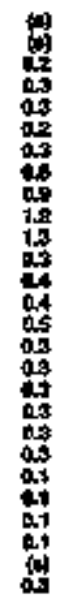 & 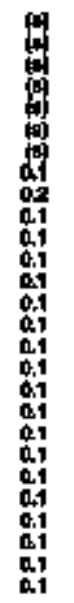 & 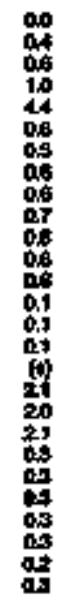 & 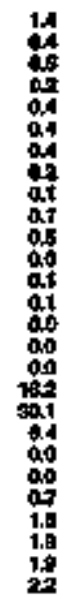 & 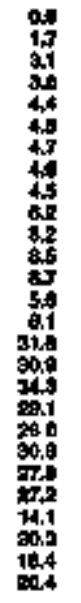 & 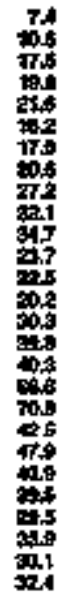 & 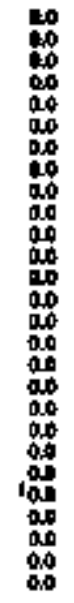 & 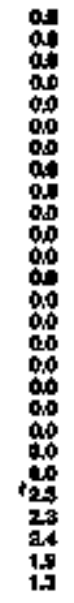 & 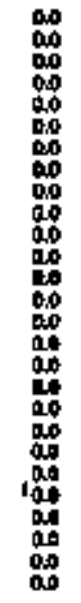 & 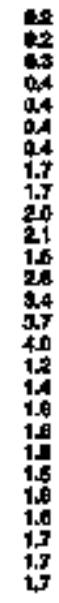 & 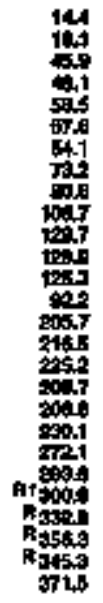 & 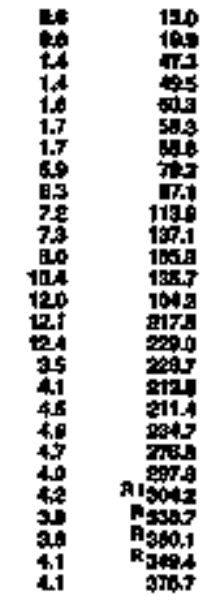 \\
\hline
\end{tabular}

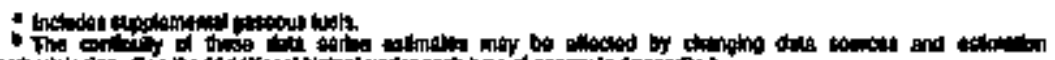

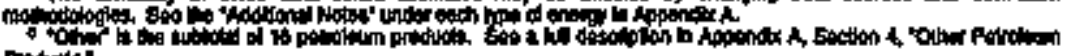
Progute

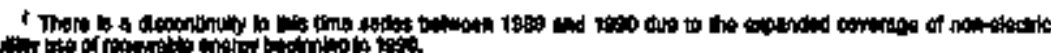
Protion

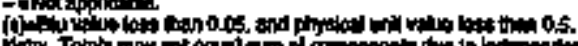

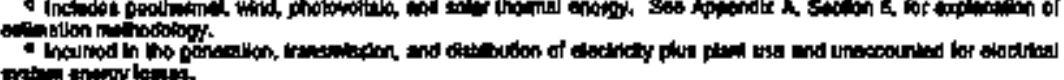

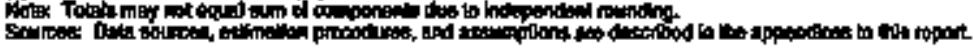


Table 27. Transportalion Energy Consuripition Estimalas; 1950, 1985, 1970-1994, Alasks

\begin{tabular}{|c|c|c|c|c|c|c|c|c|c|c|c|c|c|c|c|}
\hline \multirow[b]{3}{*}{ ven } & \multirow[b]{2}{*}{ Dow" } & \multirow[b]{2}{*}{$\ln _{n \rightarrow \infty}$} & \multicolumn{8}{|c|}{ Petolone } & \multirow{3}{*}{ Bothes: } & \multirow[b]{2}{*}{ toochithy " } & \multirow[b]{2}{*}{ th } & \multirow[b]{2}{*}{ 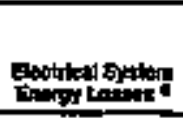 } & \multirow[b]{3}{*}{ Towt } \\
\hline & & & 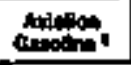 & Rention & int. & IPQ: & Lobribente: & Golor & Funtion & Jot & & & & & \\
\hline & noventint & 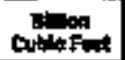 & \multicolumn{8}{|c|}{ Thourend Derrel, } & & \multicolumn{3}{|c|}{ 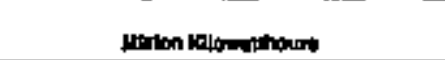 } & \\
\hline 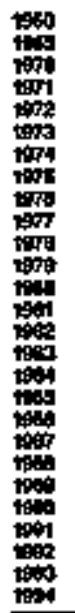 & 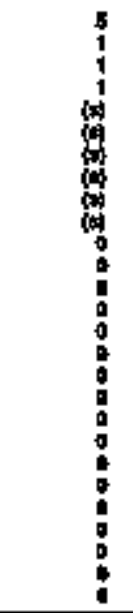 & 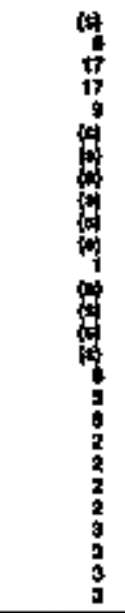 & 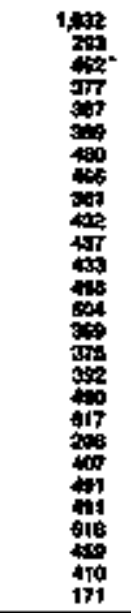 & 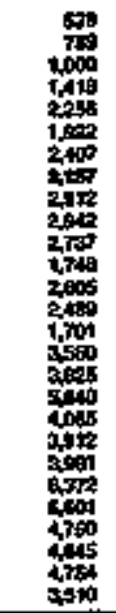 & 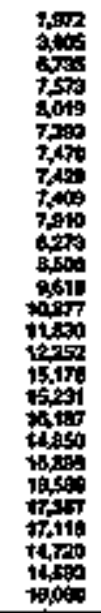 & $\begin{array}{l}0 \\
0 \\
1 \\
1 \\
0 \\
0 \\
0 \\
0 \\
0 \\
0 \\
0 \\
1 \\
8 \\
0 \\
8 \\
0 \\
4 \\
0 \\
8 \\
7 \\
0 \\
4 \\
1 \\
2 \\
4\end{array}$ & 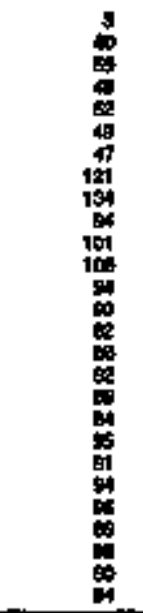 & 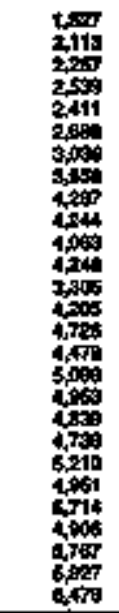 & 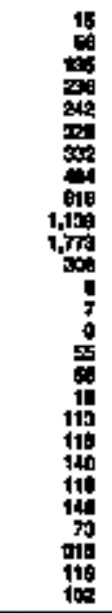 & 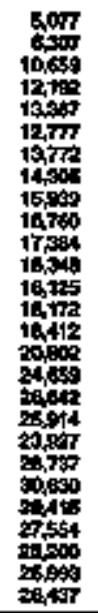 & 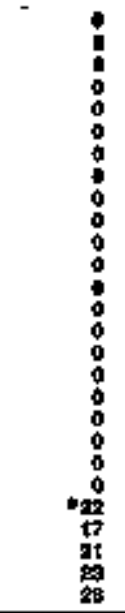 & $\begin{array}{l}0 \\
0 \\
0 \\
0 \\
0 \\
0 \\
0 \\
0 \\
0 \\
0 \\
0 \\
0 \\
0 \\
0 \\
0 \\
0 \\
0 \\
0 \\
0 \\
0 \\
0 \\
0 \\
0\end{array}$ & $\begin{array}{l}= \\
= \\
= \\
= \\
= \\
= \\
= \\
= \\
= \\
= \\
= \\
= \\
= \\
=\end{array}$ & $\begin{array}{l}0 \\
0 \\
0 \\
0 \\
0 \\
0 \\
0 \\
0 \\
0 \\
0 \\
0 \\
0 \\
0 \\
0 \\
0 \\
0 \\
0 \\
0 \\
0 \\
0 \\
0 \\
0 \\
0 \\
0 \\
0 \\
0 \\
0\end{array}$ & $\begin{array}{l}= \\
= \\
= \\
= \\
= \\
= \\
= \\
= \\
= \\
= \\
= \\
= \\
= \\
=\end{array}$ \\
\hline \multicolumn{16}{|c|}{ Thes at } \\
\hline 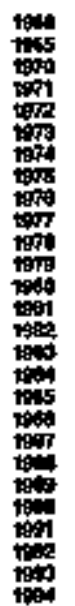 & 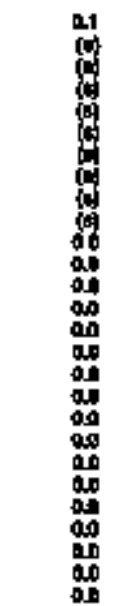 & 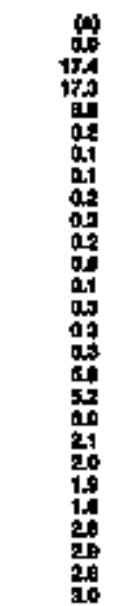 & 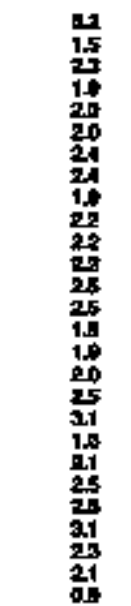 & 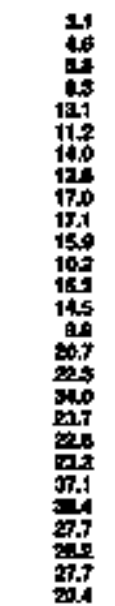 & 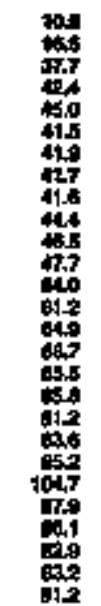 & 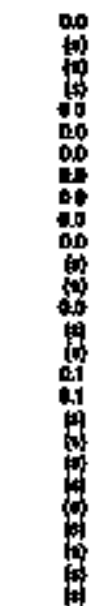 & 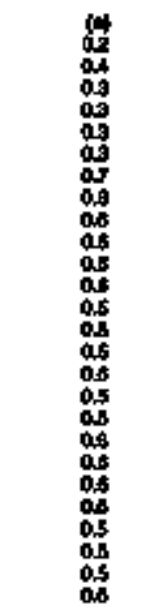 & 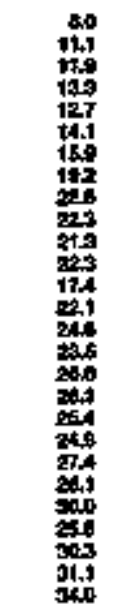 & 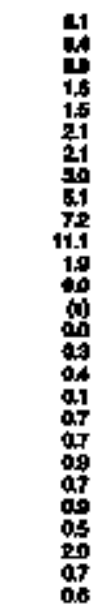 & 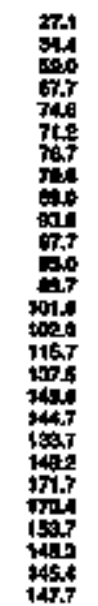 & $\begin{array}{l}00 \\
00 \\
00 \\
00 \\
00 \\
00 \\
00 \\
00 \\
00 \\
00 \\
00 \\
00 \\
00 \\
00 \\
00 \\
00 \\
00 \\
00 \\
00 \\
00 \\
00 \\
00 \\
00 \\
0 \\
0 \\
0 \\
09\end{array}$ & $\begin{array}{l}a 0 \\
00 \\
00 \\
00 \\
00 \\
000 \\
000 \\
00 \\
00 \\
00 \\
00 \\
00 \\
00 \\
00 \\
00 \\
00 \\
00 \\
00 \\
00 \\
00 \\
00 \\
00 \\
10 \\
00 \\
00 \\
00 \\
00\end{array}$ & 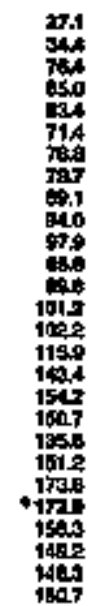 & 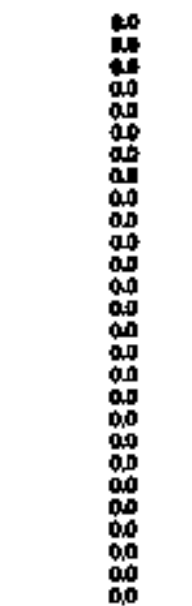 & 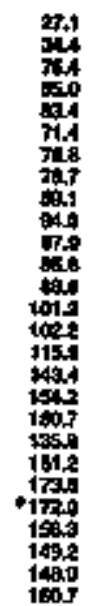 \\
\hline
\end{tabular}

$\mathbf{A}$
$\mathbf{L}$
$\mathbf{A}$
$\mathbf{S}$
$\mathbf{K}$
$\mathbf{A}$ 
Table 28. Estlmales of Energy Input at Electric Utitites, 1960, 1965, 1970-1994, Alaska

A

\begin{tabular}{|c|c|c|c|c|c|c|c|c|c|c|c|c|c|c|}
\hline & \multicolumn{3}{|c|}{ cons } & \multirow[b]{2}{*}{ 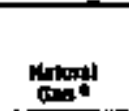 } & \multicolumn{4}{|c|}{ Pmodetsm } & \multirow[b]{2}{*}{ mecherste } & \multirow[b]{2}{*}{ 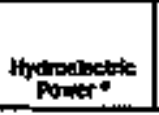 } & \multirow[b]{2}{*}{ Himents } & \multirow[b]{2}{*}{ 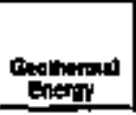 } & \multirow[b]{2}{*}{ 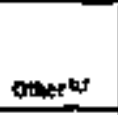 } & \multirow[b]{3}{*}{ Tot:1 } \\
\hline & 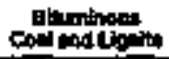 & 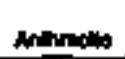 & 7040 & & wat & 唩㛎 & Potoluty & To년 & & & & & & \\
\hline $\mathrm{Yu}$ & \multicolumn{3}{|c|}{ Thoenund Ghat Teat } & 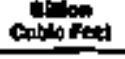 & \multicolumn{4}{|c|}{ 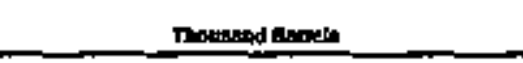 } & \multicolumn{5}{|c|}{ 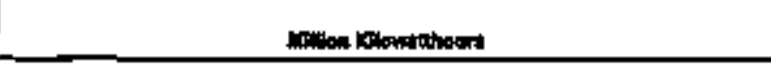 } & \\
\hline 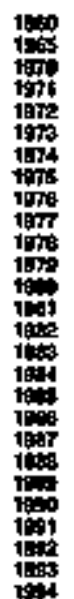 & 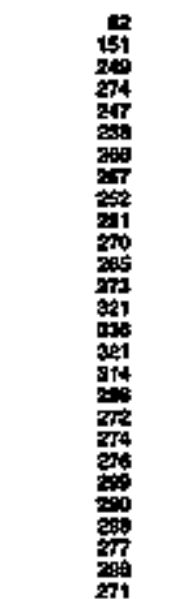 & $\begin{array}{l}0 \\
0 \\
0 \\
0 \\
0 \\
0 \\
0 \\
0 \\
0 \\
0 \\
0 \\
0 \\
0 \\
0 \\
0 \\
0 \\
0 \\
0 \\
0 \\
0 \\
0 \\
0 \\
0 \\
0 \\
0 \\
0 \\
0 \\
0 \\
0\end{array}$ & 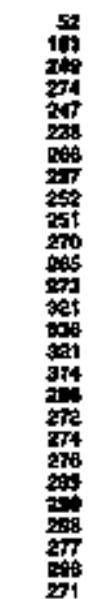 & 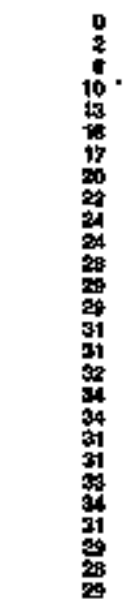 & 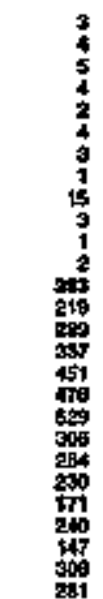 & 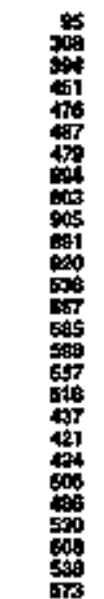 & $\begin{array}{l}: \\
: \\
0 \\
0 \\
0 \\
0 \\
0 \\
0 \\
0 \\
0 \\
0 \\
0 \\
0 \\
0 \\
0 \\
0 \\
0 \\
0 \\
0 \\
0 \\
0 \\
0 \\
0\end{array}$ & 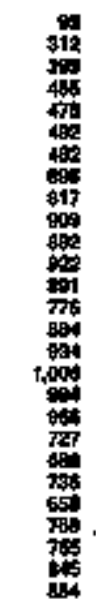 & $\begin{array}{l}0 \\
0 \\
0 \\
0 \\
0 \\
0 \\
0 \\
0 \\
0 \\
0 \\
0 \\
0 \\
0 \\
0 \\
0 \\
0 \\
0 \\
0 \\
0 \\
0 \\
0 \\
0 \\
0 \\
0 \\
0 \\
0 \\
0\end{array}$ & 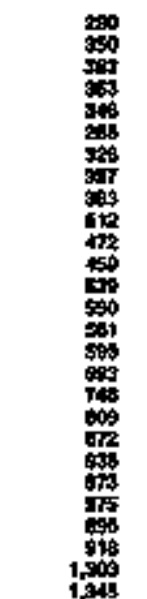 & $\begin{array}{l}0 \\
0 \\
0 \\
0 \\
0 \\
0 \\
0 \\
0 \\
0 \\
0 \\
0 \\
0 \\
0 \\
0 \\
0 \\
0 \\
0 \\
0 \\
0 \\
0 \\
0 \\
0 \\
0 \\
0 \\
0 \\
0 \\
0 \\
0\end{array}$ & $\begin{array}{l}0 \\
0 \\
0 \\
0 \\
0 \\
0 \\
0 \\
0 \\
0 \\
0 \\
0 \\
0 \\
0 \\
0 \\
0 \\
0 \\
0 \\
0 \\
0 \\
0 \\
0 \\
0 \\
0 \\
0 \\
0\end{array}$ & $\begin{array}{l}0 \\
0 \\
0 \\
0 \\
0 \\
0 \\
0 \\
0 \\
0 \\
0 \\
0 \\
0 \\
0 \\
0 \\
0 \\
0 \\
0 \\
0 \\
0 \\
0 \\
0 \\
0 \\
0 \\
0 \\
0 \\
0 \\
0 \\
0 \\
0 \\
0 \\
0\end{array}$ & $\begin{array}{l}z \\
z \\
z \\
z \\
z \\
z \\
z \\
z \\
\vdots \\
\vdots \\
z \\
z \\
z\end{array}$ \\
\hline
\end{tabular}

\begin{tabular}{|c|c|c|c|c|c|c|}
\hline 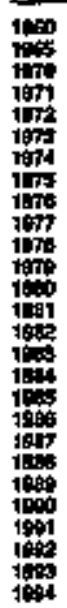 & 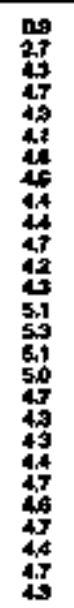 & 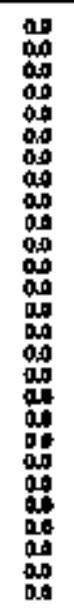 & 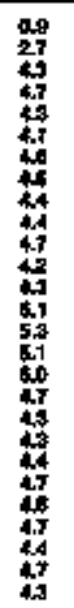 & 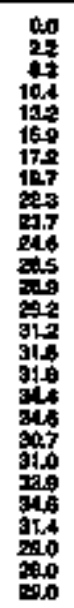 & 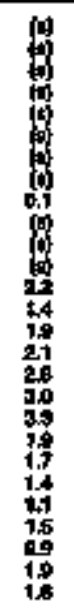 & 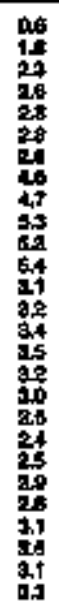 \\
\hline
\end{tabular}

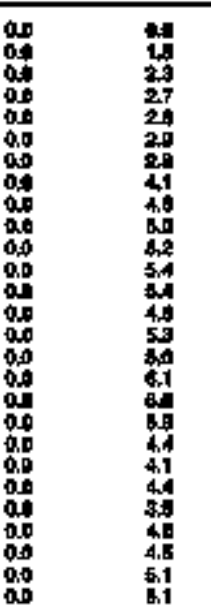

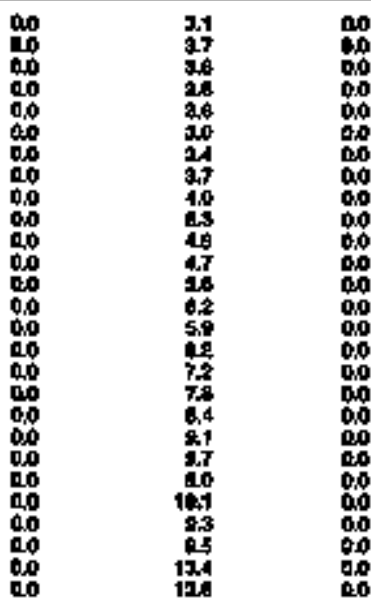

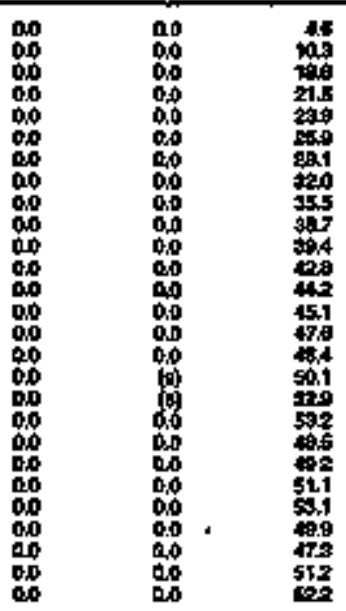

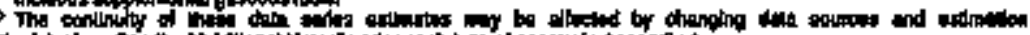

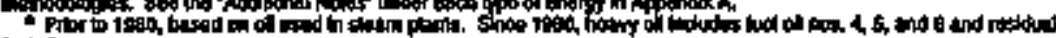

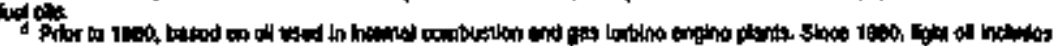

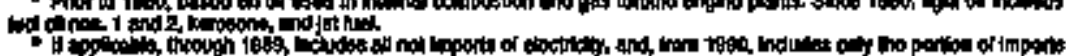

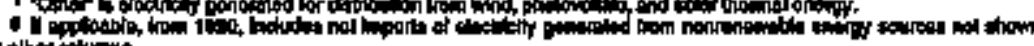

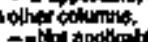

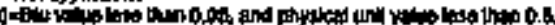

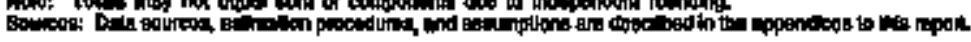

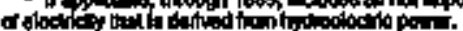


Table 29. Energy Consumptkon Estlmatos by Soure, 1960, 1965, 1970-1994, Arkona

\begin{tabular}{|c|c|c|c|c|c|c|c|c|c|c|c|c|c|c|c|c|c|c|c|}
\hline \multirow[b]{3}{*}{$Y_{i=0}$} & \multirow[b]{2}{*}{ Cowl- } & \multirow[b]{2}{*}{ vaturats } & \multicolumn{11}{|c|}{ 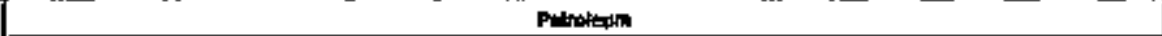 } & \multirow[b]{2}{*}{ 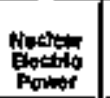 } & \multirow[b]{2}{*}{ Dot } & \multirow[b]{2}{*}{ Exolveta I I } & \multirow[b]{2}{*}{ Onet ad } & \multirow{2}{*}{ 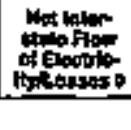 } & \multirow[b]{3}{*}{ tolde } \\
\hline & & & Asplatt ad & morlefion & Dinterstions & Let. & ind & LPE. & Eubt: & 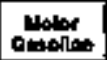 & 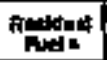 & | onen an a & 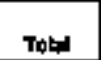 & & & & & & \\
\hline & 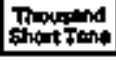 & 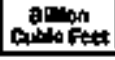 & \multicolumn{11}{|c|}{ 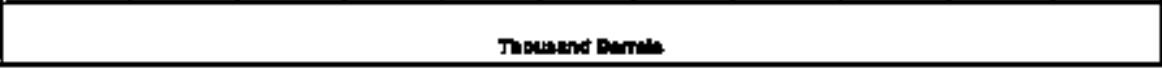 } & \multicolumn{5}{|c|}{ 些 } & \\
\hline 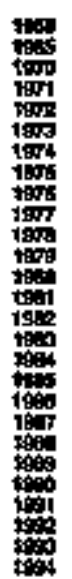 & 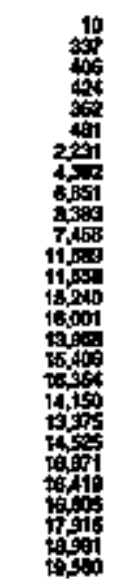 & 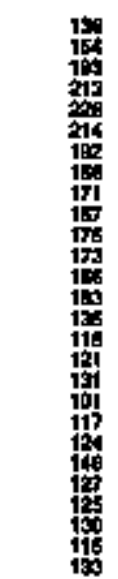 & 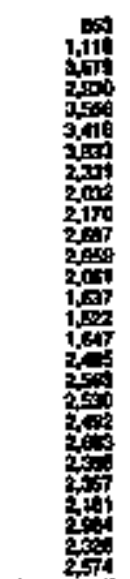 & 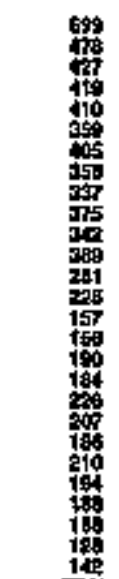 & 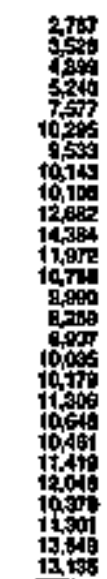 & 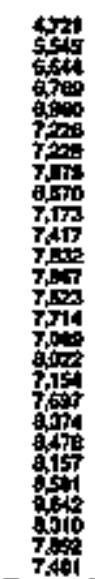 & 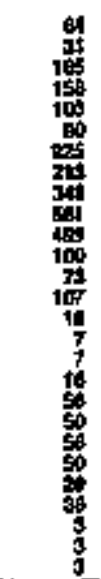 & 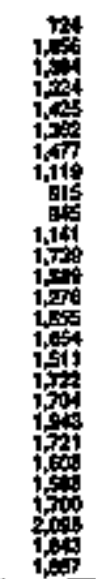 & 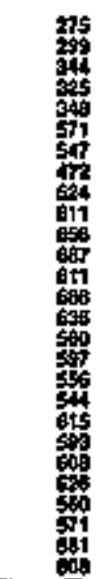 & 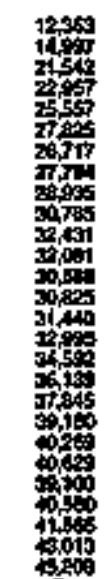 & 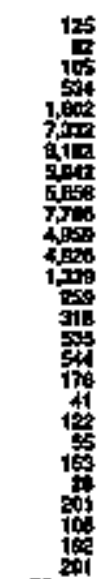 & 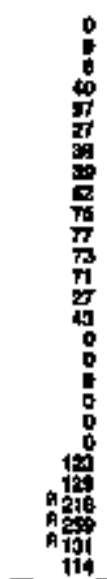 & 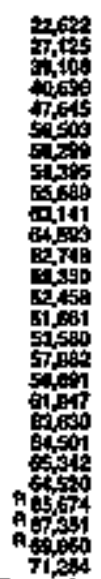 & 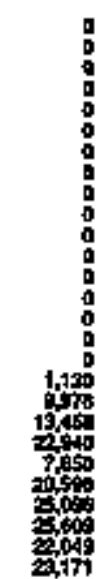 & 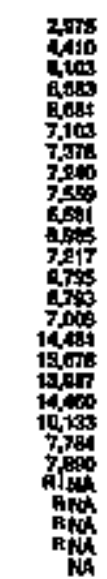 & 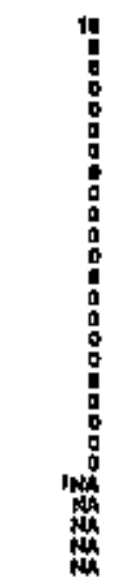 & 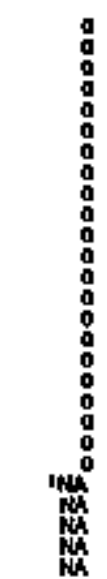 & 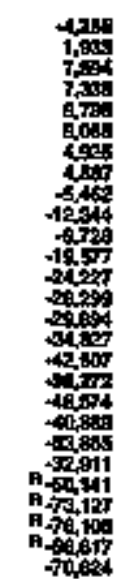 & $\begin{array}{l}z \\
z \\
z \\
z \\
z \\
z \\
z \\
z \\
z\end{array}$ \\
\hline & & & & & & & & & & 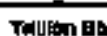 & & & & & & & & & \\
\hline
\end{tabular}

\begin{tabular}{|c|c|c|c|c|c|c|c|c|c|c|c|c|c|c|c|c|c|c|c|}
\hline 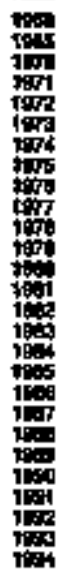 & 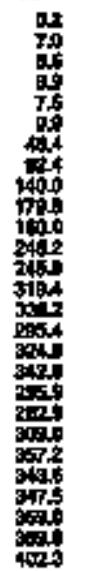 & 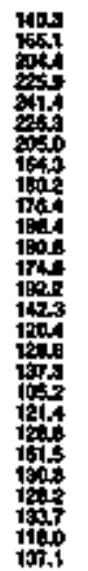 & 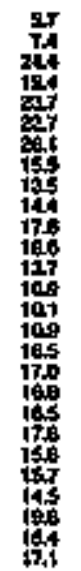 & 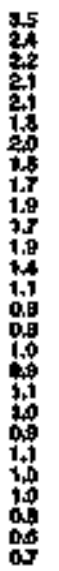 & 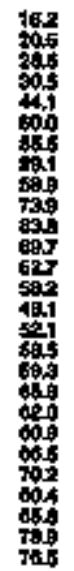 & 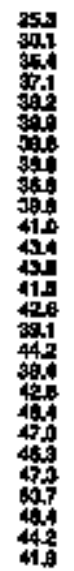 & 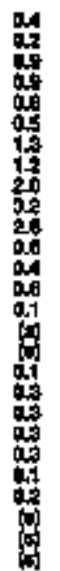 & 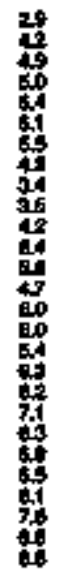 & 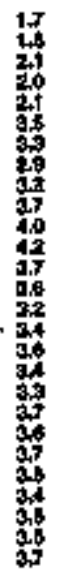 & 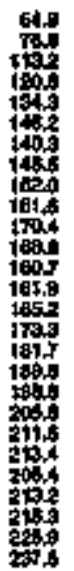 & 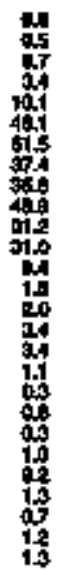 & 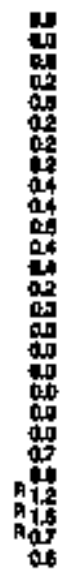 & 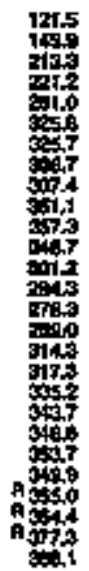 & 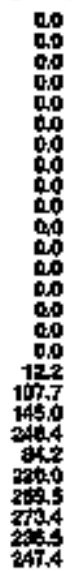 & 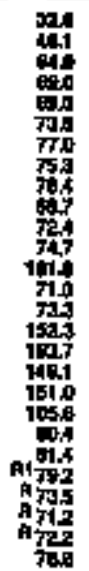 & 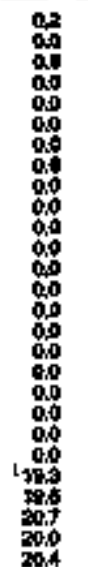 & 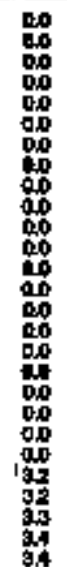 & 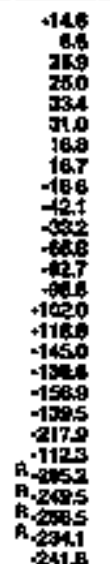 & 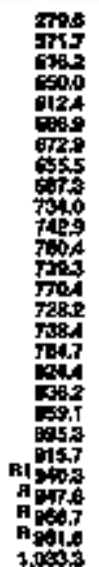 \\
\hline
\end{tabular}

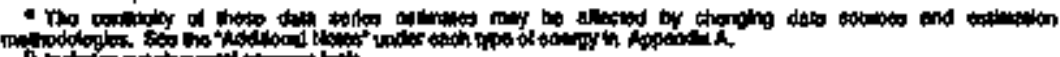

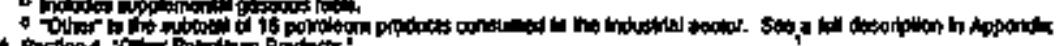

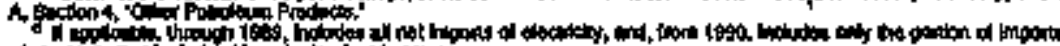
dd of

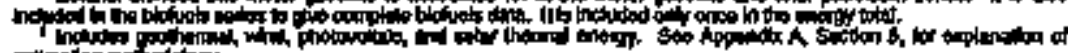

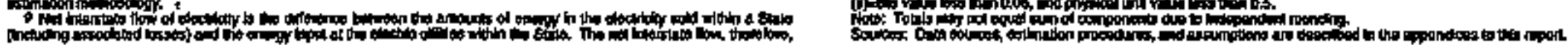

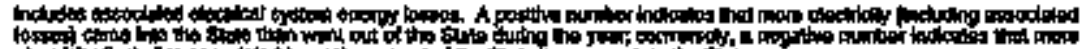

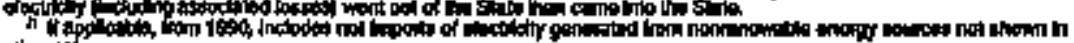

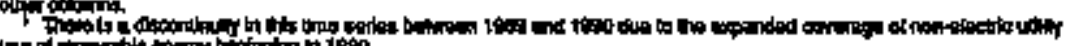
cog of

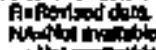

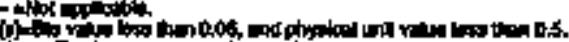


A Table 30. Residential Energy Constumption Estimates, 1960, 1965, 1970-1994, Arizona

\begin{tabular}{|c|c|c|c|c|c|c|c|c|c|c|c|c|c|c|}
\hline \multirow[b]{3}{*}{ Yest } & \multicolumn{3}{|c|}{ (1) } & \multirow[b]{2}{*}{ 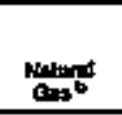 } & \multicolumn{4}{|c|}{ Parroterm } & \multirow{3}{*}{ 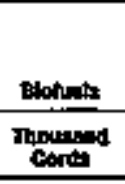 } & & \multirow[b]{2}{*}{ Enertistots" } & \multirow[b]{2}{*}{ thet } & \multirow{3}{*}{ 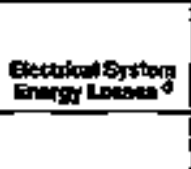 } & \multirow[b]{3}{*}{ Fotal } \\
\hline & 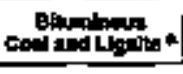 & Nituralasen & Tower & & 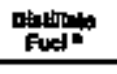 & Karrean: & $\mathrm{PQ}=$ & Tonel & & Sower & & & & \\
\hline & \multicolumn{2}{|c|}{ Thounad Sher Tores } & & 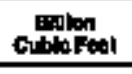 & \multicolumn{4}{|c|}{ Thouthod Bume } & & \multicolumn{3}{|c|}{ 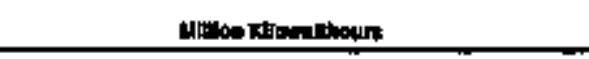 } & & \\
\hline 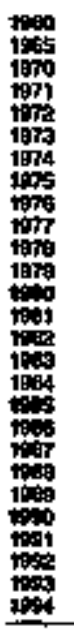 & $\begin{array}{l}0 \\
0 \\
0 \\
0 \\
0 \\
0 \\
0 \\
0 \\
0 \\
0 \\
0 \\
0 \\
0 \\
0 \\
0 \\
0 \\
0 \\
0 \\
0 \\
0 \\
0 \\
0 \\
0\end{array}$ & $\begin{array}{l}0 \\
0 \\
0 \\
0 \\
0 \\
0 \\
0 \\
0 \\
0 \\
\vdots \\
0 \\
0 \\
0 \\
0 \\
0 \\
0 \\
0 \\
0 \\
0\end{array}$ & $\begin{array}{l}0 \\
0 \\
0 \\
0 \\
0 \\
0 \\
0 \\
0 \\
0 \\
0 \\
0 \\
0 \\
0 \\
0 \\
0 j \\
0 \\
0 \\
0 \\
0 \\
0 \\
0 \\
0 \\
0 \\
0 \\
0 \\
0\end{array}$ & 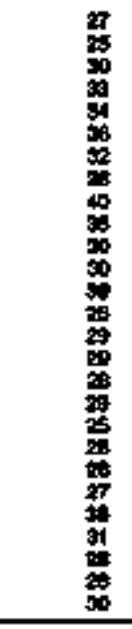 & 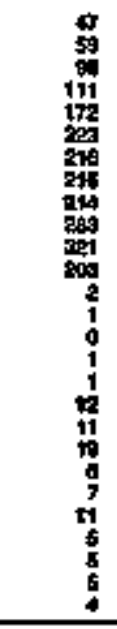 & 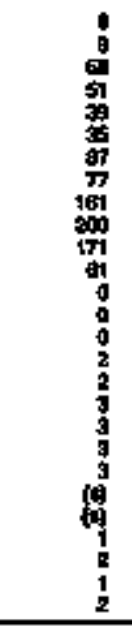 & 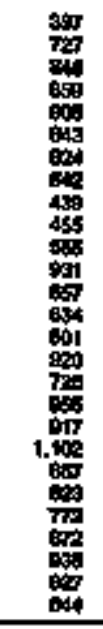 & 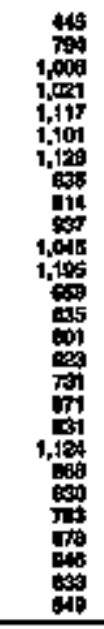 & 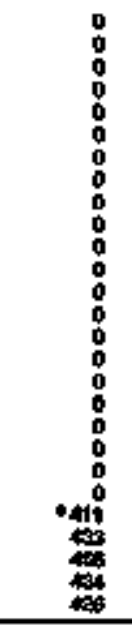 & 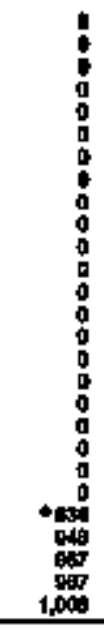 & 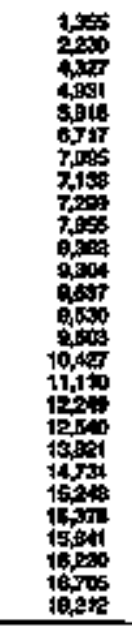 & $\begin{array}{l}\vdots \\
\vdots \\
\vdots \\
\vdots \\
\vdots \\
\vdots \\
\vdots \\
\vdots \\
\vdots \\
\vdots \\
\vdots \\
\vdots \\
\vdots\end{array}$ & 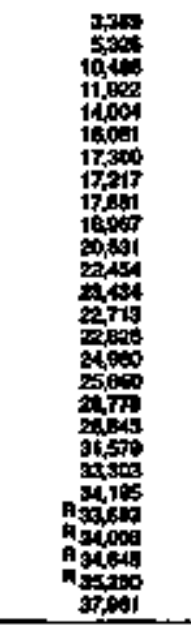 & $\begin{array}{l}z \\
\bar{z} \\
\bar{z} \\
\bar{z} \\
\bar{z} \\
\bar{z}\end{array}$ \\
\hline \multicolumn{15}{|c|}{ Antron the } \\
\hline 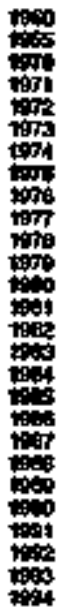 & 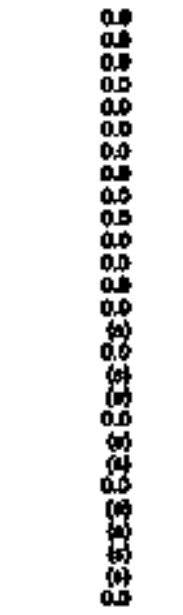 & 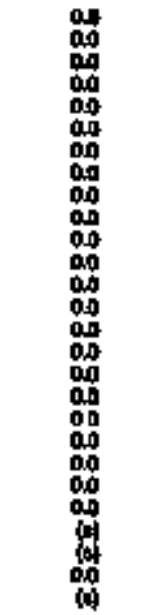 & 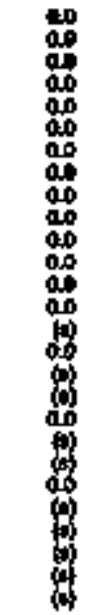 & 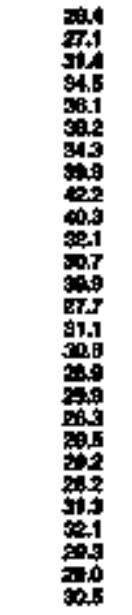 & 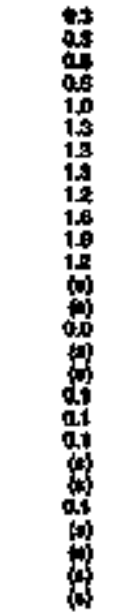 & 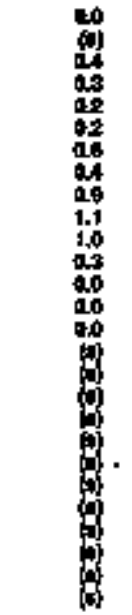 & 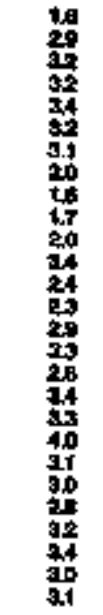 & 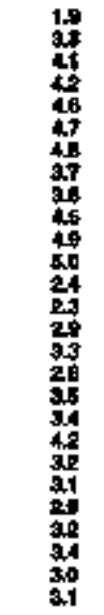 & 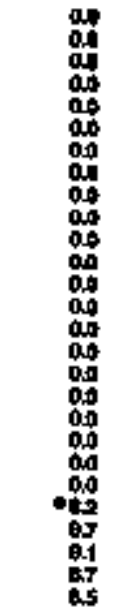 & 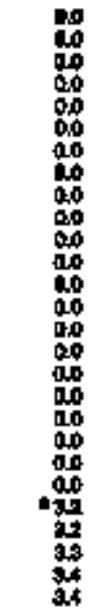 & 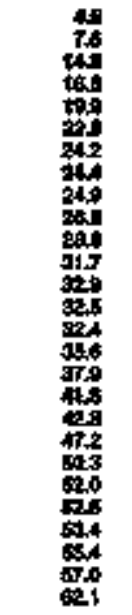 & 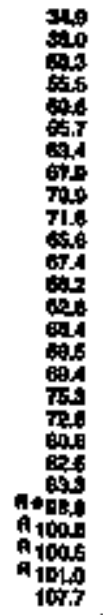 & 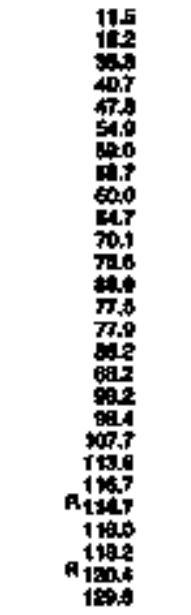 & 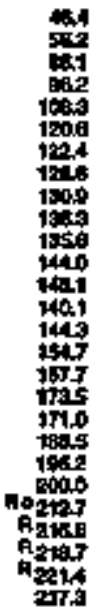 \\
\hline
\end{tabular}

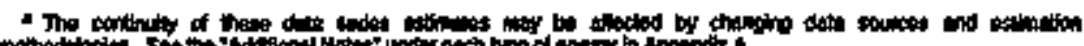

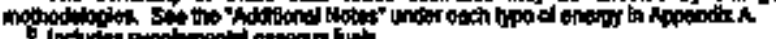

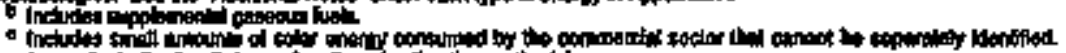

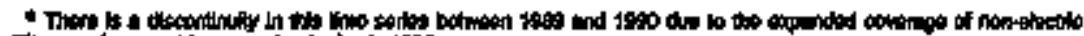

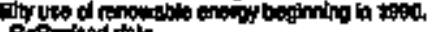

ranisod thin

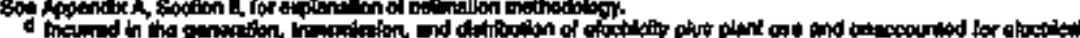

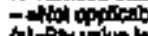

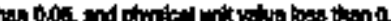

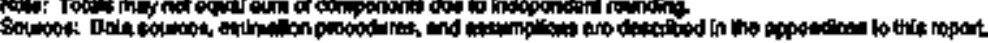

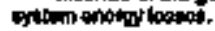


Table 91. Commoncial Energy Consumption Estimates, 1960, 1985, 1970-1994, Arlzona

\begin{tabular}{|c|c|c|c|c|c|c|c|c|c|c|c|c|c|c|}
\hline \multirow[b]{3}{*}{$\mathrm{Ye}$} & \multicolumn{3}{|c|}{ coll } & \multirow{3}{*}{ 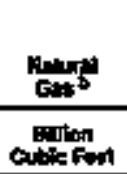 } & \multicolumn{6}{|c|}{ Atching } & \multirow[b]{2}{*}{ Exetrlefly" } & \multirow[b]{2}{*}{ Not } & \multirow[b]{2}{*}{ 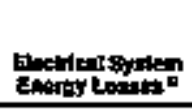 } & \multirow[b]{3}{*}{ Total 4} \\
\hline & 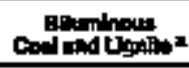 & Antracine & Tow & & Dont & Kervesen:" & LPG = & Motertict & Retater & Tow & & & & \\
\hline & \multicolumn{2}{|c|}{ Thous: netshent Ton: } & & & \multicolumn{6}{|c|}{ Thoos:matimits } & \multicolumn{3}{|c|}{ Anten XHEwnithoun } & \\
\hline 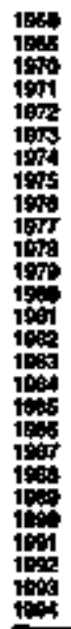 & 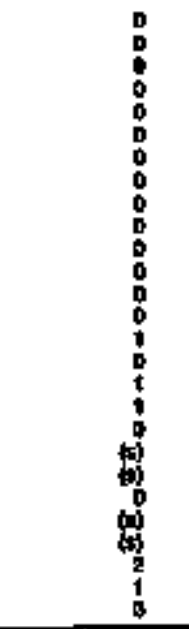 & $\begin{array}{l}0 \\
0 \\
0 \\
0 \\
0 \\
0 \\
0 \\
0 \\
0 \\
0 \\
0 \\
0 \\
0 \\
0 \\
0 \\
0 \\
0 \\
0 \\
0 \\
0 \\
0 \\
0 \\
0 \\
0 \\
0 \\
0 \\
0\end{array}$ & 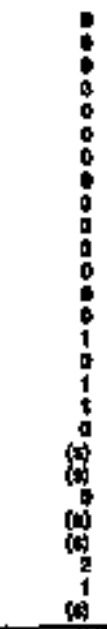 & 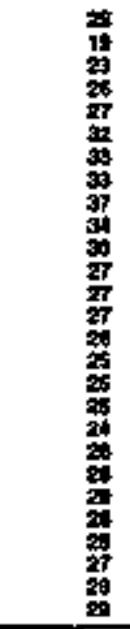 & 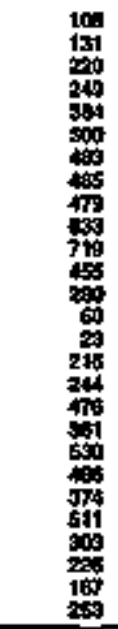 & 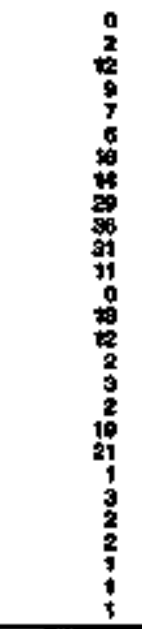 & 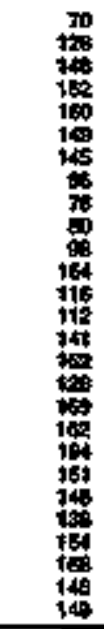 & 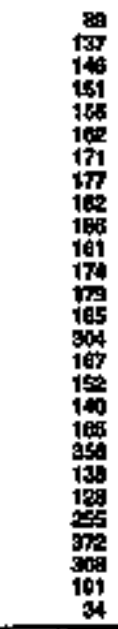 & 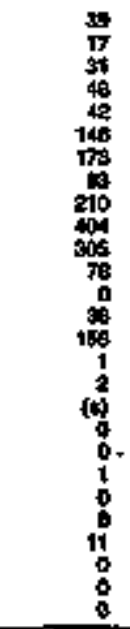 & 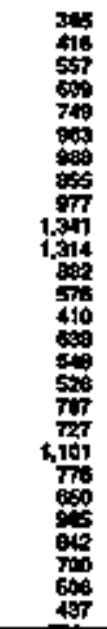 & 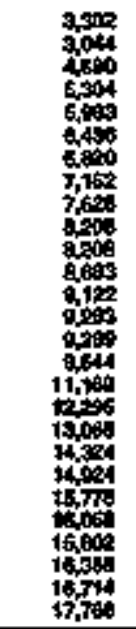 & $\begin{array}{l}= \\
= \\
= \\
= \\
= \\
= \\
= \\
= \\
= \\
= \\
= \\
= \\
= \\
= \\
=\end{array}$ & 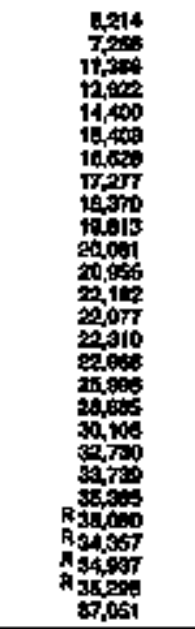 & $\begin{array}{l}= \\
= \\
= \\
= \\
= \\
= \\
= \\
= \\
= \\
= \\
= \\
= \\
= \\
=\end{array}$ \\
\hline \multicolumn{15}{|c|}{ Diten end } \\
\hline 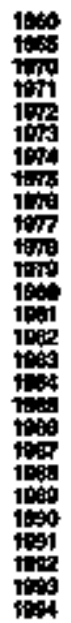 & 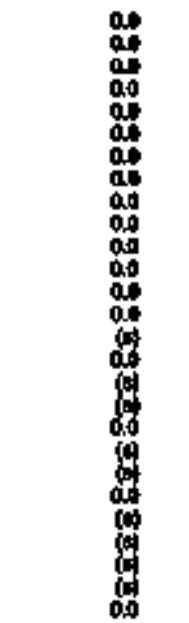 & $\begin{array}{l}00 \\
00 \\
00 \\
00 \\
00 \\
00 \\
00 \\
00 \\
000 \\
00 \\
00 \\
00 \\
00 \\
00 \\
00 \\
00 \\
00 \\
00 \\
00 \\
00 \\
00 \\
00 \\
00 \\
00 \\
00 \\
00\end{array}$ & 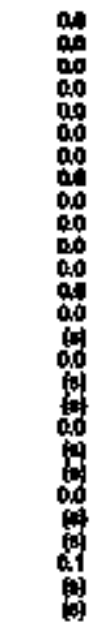 & 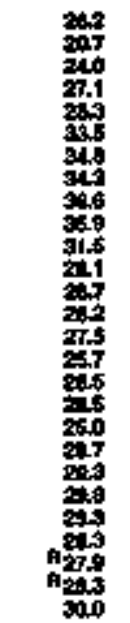 & 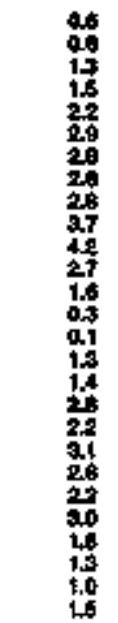 & 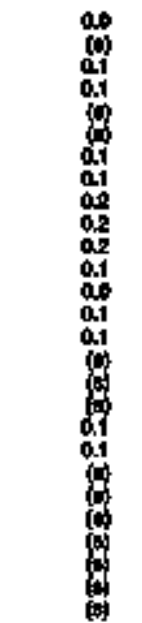 & 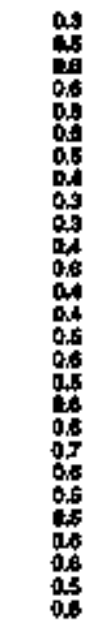 & 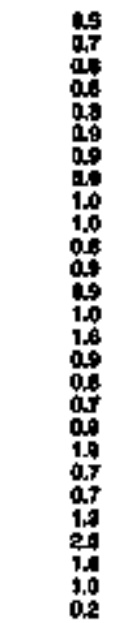 & 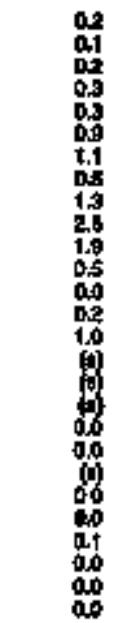 & 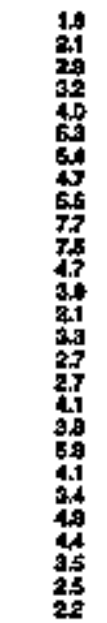 & 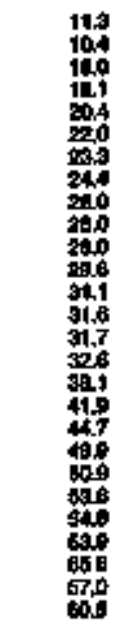 & 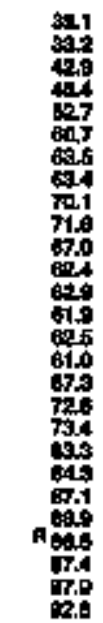 & 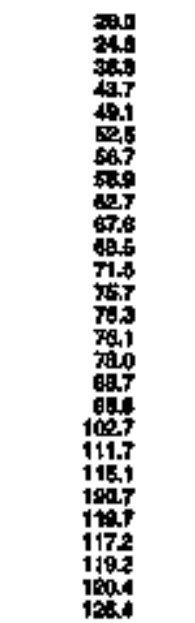 & 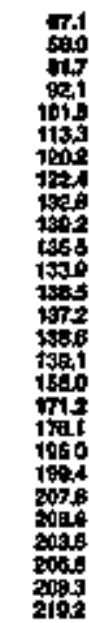 \\
\hline
\end{tabular}

$\mathbf{A}$
$\mathbf{R}$
$\mathbf{I}$
$\mathbf{Z}$
$\mathbf{O}$
$\mathbf{N}$

A 


\begin{tabular}{|c|c|c|c|c|c|c|c|c|c|c|c|c|c|c|c|c|c|c|}
\hline & \multirow[b]{2}{*}{ Cod } & \multirow[b]{2}{*}{ Mrapti } & \multicolumn{9}{|c|}{ Potroteenn } & \multirow[b]{2}{*}{ 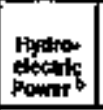 } & \multirow[b]{2}{*}{ Biotusb } & \multirow[b]{2}{*}{ 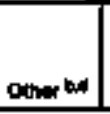 } & \multirow[b]{2}{*}{ Enctrally } & \multirow[b]{2}{*}{ 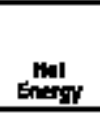 } & \multirow{2}{*}{ 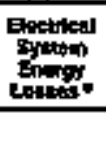 } & \multirow[b]{3}{*}{ Fotell } \\
\hline & & & 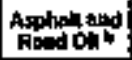 & 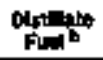 & KWOAHE: & Lpq'b & tontransts & ansorint & Propind & Others & Tow & & & & & & & \\
\hline res & 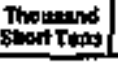 & 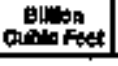 & \multicolumn{9}{|c|}{ Mocound Eamek } & \multicolumn{6}{|c|}{ 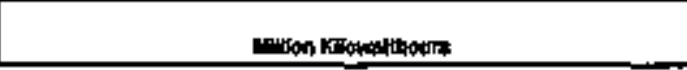 } & \\
\hline 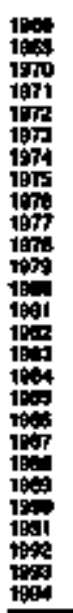 & 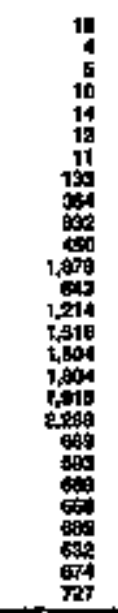 & 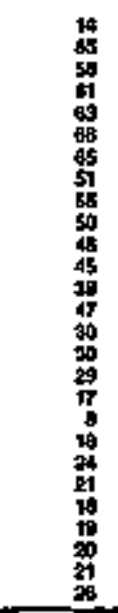 & 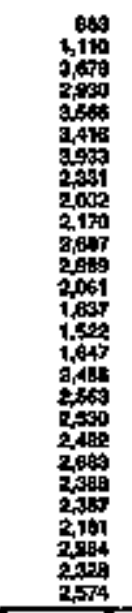 & 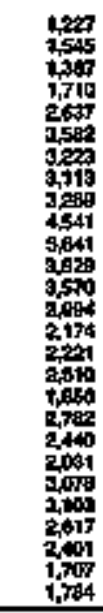 & 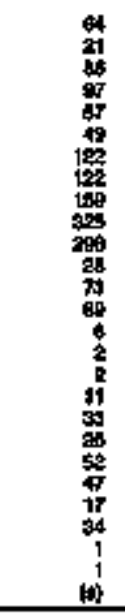 & 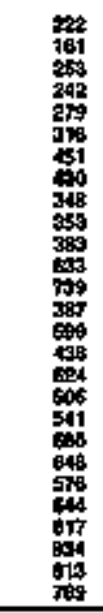 & 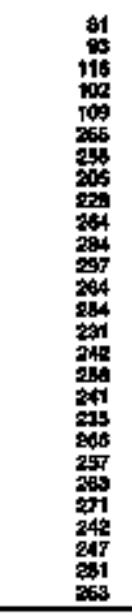 & 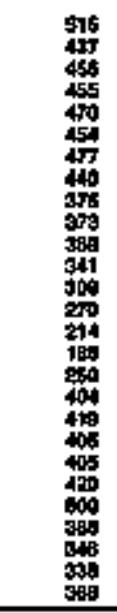 & 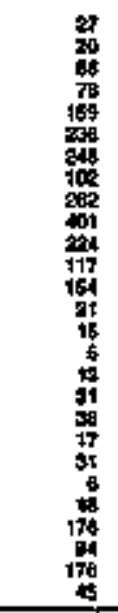 & 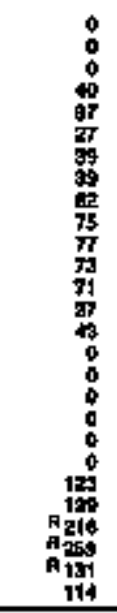 & 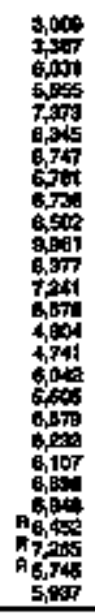 & 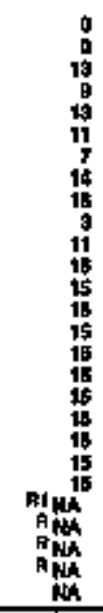 & 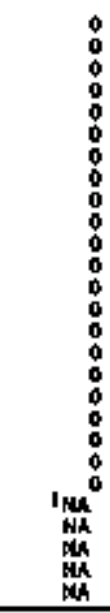 & 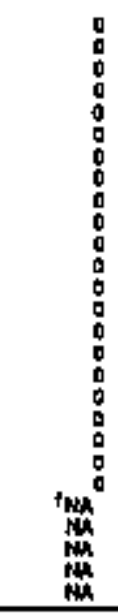 & 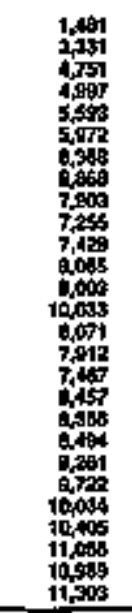 & $\begin{array}{l}\bar{z} \\
\bar{z} \\
z\end{array}$ & 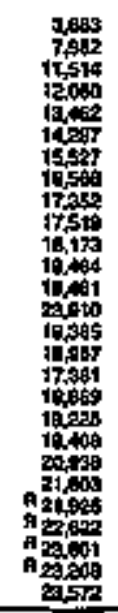 & $\begin{array}{l}z \\
z\end{array}$ \\
\hline \multicolumn{19}{|c|}{ rillowew } \\
\hline 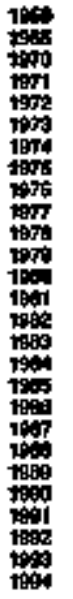 & 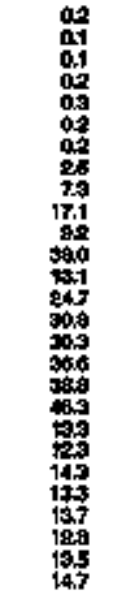 & 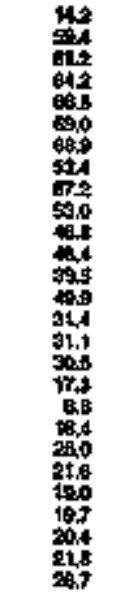 & 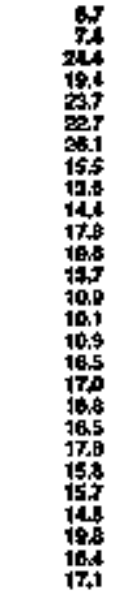 & 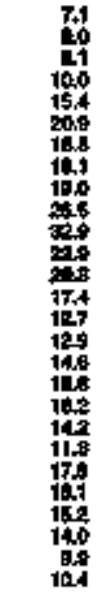 & 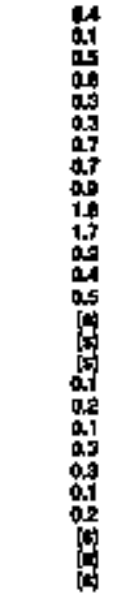 & 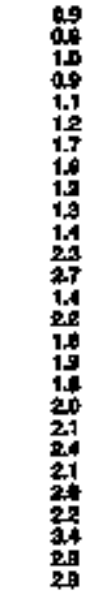 & 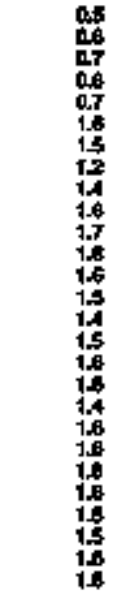 & 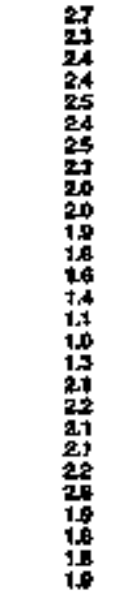 & 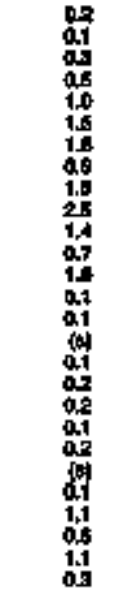 & 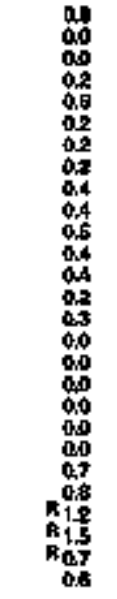 & 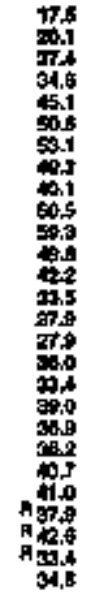 & 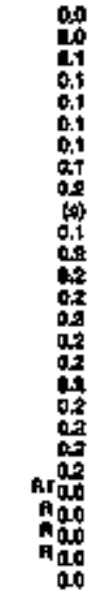 & 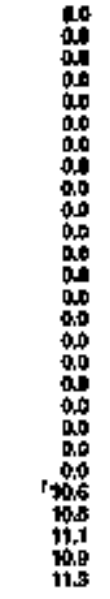 & 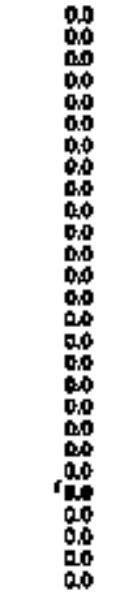 & 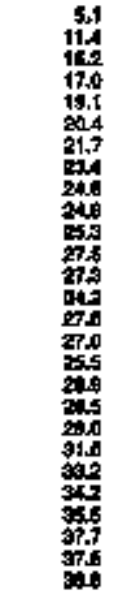 & 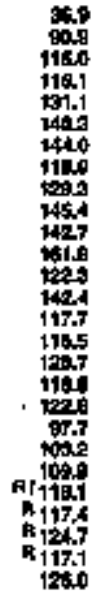 & 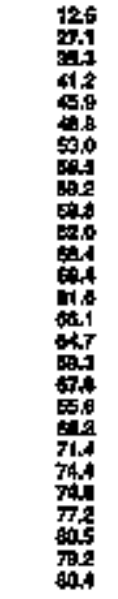 & 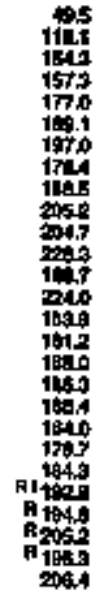 \\
\hline
\end{tabular}

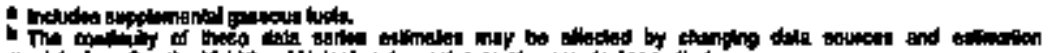

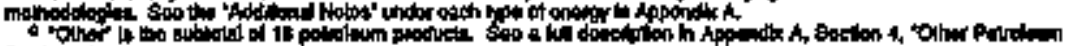

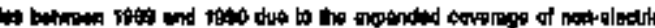

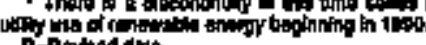

P.hom

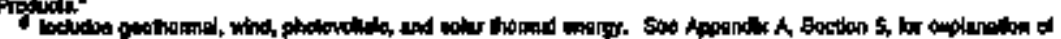
Nathot

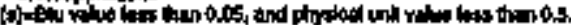

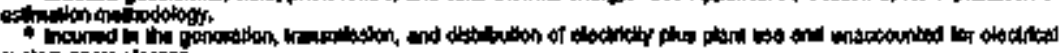

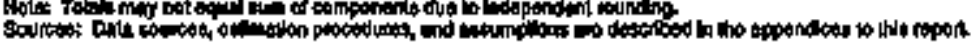

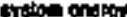


Table 39. Transportation Energy Consumption Estlmales, 1960, 1865, 1970-1994, Arlzon:

\begin{tabular}{|c|c|c|c|c|c|c|c|c|c|c|c|c|c|c|c|}
\hline & \multirow[b]{2}{*}{ Cons } & \multirow[b]{2}{*}{ Nintiont" } & \multicolumn{8}{|c|}{ Peiroteter } & \multirow{3}{*}{ Butathe } & & \multirow[b]{2}{*}{ Enet } & \multirow[b]{2}{*}{ 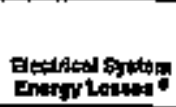 } & \multirow[b]{3}{*}{ Jotom } \\
\hline & & & Autronting. & 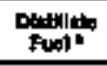 & thet. & tors: & 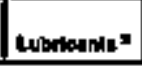 & Motor & Fusteyt" & Tols & & Eoctikely" & & & \\
\hline שומר & Thospond & 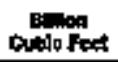 & \multicolumn{8}{|c|}{ 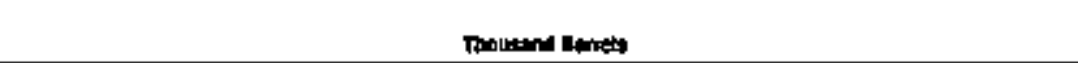 } & & \multicolumn{3}{|c|}{ 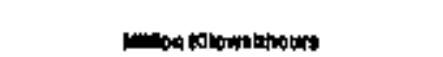 } & \\
\hline 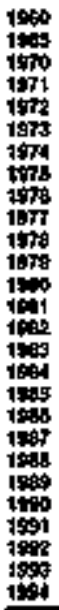 & $\begin{array}{l}0 \\
0 \\
0 \\
0 \\
0 \\
0 \\
0 \\
0 \\
0 \\
0 \\
0 \\
0 \\
0 \\
0 \\
0 \\
0 \\
0 \\
0 \\
0 \\
0 \\
0 \\
0\end{array}$ & 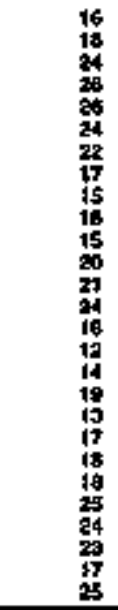 & 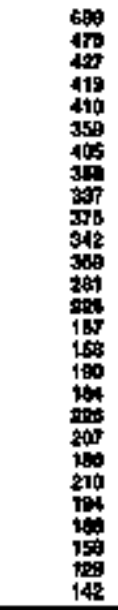 & 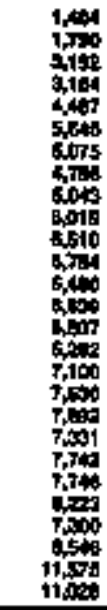 & 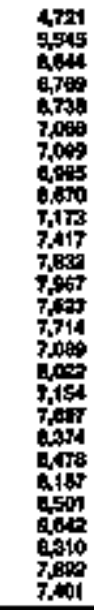 & 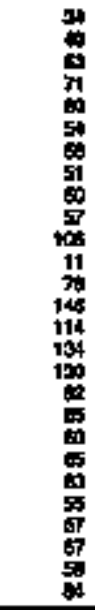 & 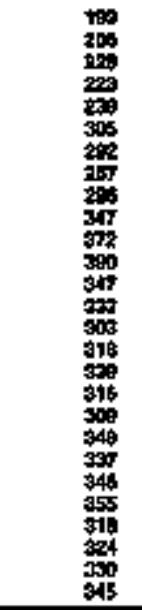 & 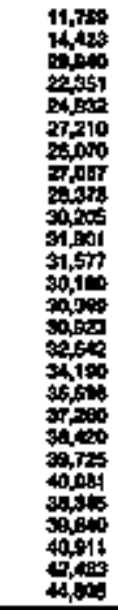 & $\begin{array}{l}7 \\
0 \\
0 \\
0 \\
0 \\
0 \\
0 \\
0 \\
0 \\
0 \\
0 \\
0 \\
0 \\
0 \\
0 \\
0 \\
0 \\
0 \\
0 \\
0 \\
0 \\
0 \\
0 \\
0\end{array}$ & 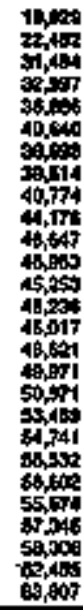 & 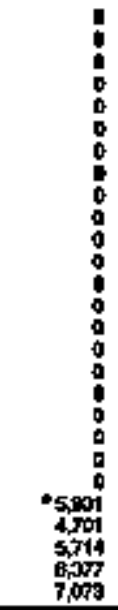 & $\begin{array}{l}0 \\
0 \\
0 \\
0 \\
0 \\
0 \\
0 \\
0 \\
0 \\
0 \\
0 \\
0 \\
0 \\
0 \\
0 \\
0 \\
0 \\
0 \\
0 \\
0 \\
0 \\
0 \\
0 \\
0 \\
0\end{array}$ & $\begin{array}{l}= \\
= \\
= \\
= \\
= \\
= \\
= \\
= \\
= \\
= \\
= \\
= \\
= \\
= \\
= \\
=\end{array}$ & $\begin{array}{l}0 \\
0 \\
0 \\
0 \\
0 \\
0 \\
0 \\
0 \\
0 \\
0 \\
0 \\
0 \\
0 \\
0 \\
0 \\
0 \\
0 \\
0 \\
0 \\
0 \\
0 \\
0 \\
0 \\
0 \\
0 \\
0 \\
0 \\
0 \\
0 \\
0 \\
\end{array}$ & $\begin{array}{l}= \\
= \\
= \\
= \\
= \\
= \\
= \\
= \\
= \\
= \\
= \\
= \\
= \\
=\end{array}$ \\
\hline \multicolumn{16}{|c|}{ T秘的 } \\
\hline 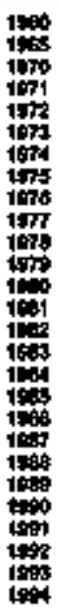 & 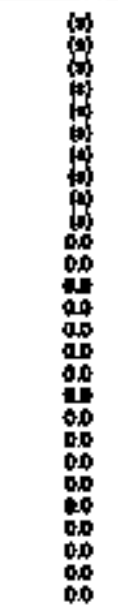 & 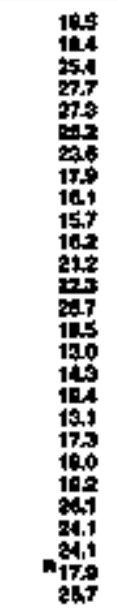 & 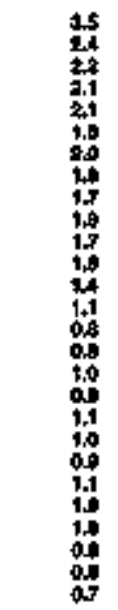 & 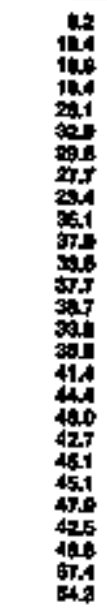 & 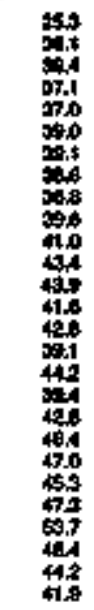 & 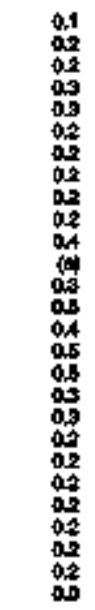 & 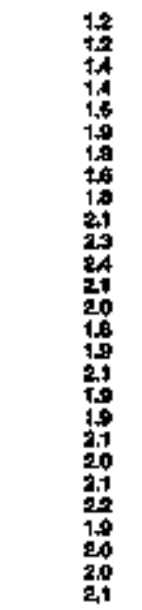 & 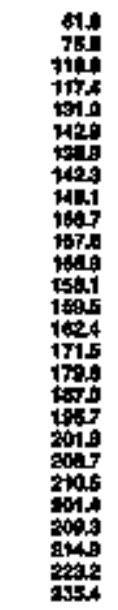 & 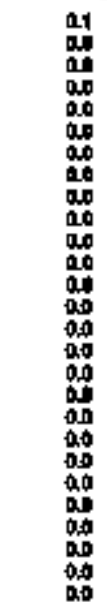 & 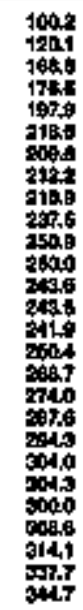 & 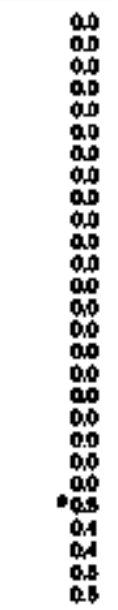 & $\begin{array}{l}00 \\
00 \\
00 \\
00 \\
00 \\
00 \\
000 \\
00 \\
00 \\
00 \\
00 \\
000 \\
00 \\
000 \\
000 \\
00 \\
00 \\
00 \\
00 \\
00 \\
00 \\
00 \\
00 \\
00 \\
00 \\
0.0\end{array}$ & 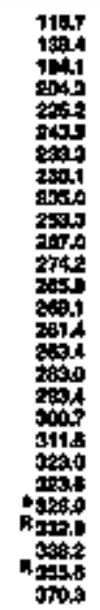 & $\begin{array}{l}00 \\
00 \\
00 \\
00 \\
00 \\
00 \\
00 \\
00 \\
00 \\
00 \\
00 \\
00 \\
00 \\
00 \\
00 \\
00 \\
00 \\
000 \\
00 \\
00 \\
00 \\
00 \\
00 \\
00 \\
00\end{array}$ & 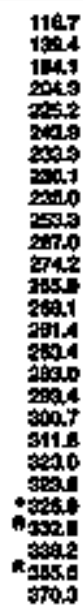 \\
\hline
\end{tabular}

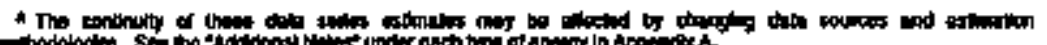

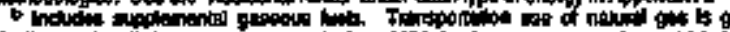

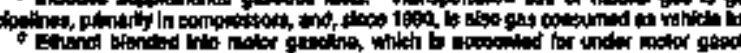

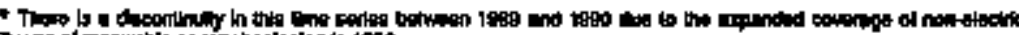

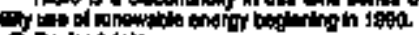

Fin antododin

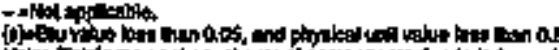

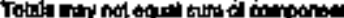




\begin{tabular}{|c|c|c|c|c|c|c|c|c|c|c|c|c|c|c|}
\hline \multirow[b]{3}{*}{$\underline{x \in=}$} & \multicolumn{3}{|c|}{$\cos$} & \multirow[b]{2}{*}{ 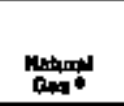 } & \multicolumn{4}{|c|}{ Putrotolm } & \multirow[b]{2}{*}{ mener Electite } & \multirow[b]{2}{*}{ introtute } & \multirow[b]{2}{*}{ 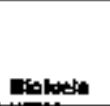 } & \multirow[b]{2}{*}{ 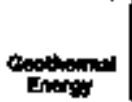 } & \multirow[b]{2}{*}{ othes } & \multirow[b]{3}{*}{ Totat d } \\
\hline & 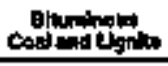 & Anthenchy & Tow & & ontox & 践始 & 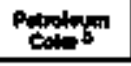 & Totet & & & & & & \\
\hline & \multicolumn{3}{|c|}{ Twousud Short Tons } & $\operatorname{minhon}_{1}$ & \multicolumn{4}{|c|}{ mowend tenth } & \multicolumn{5}{|c|}{ 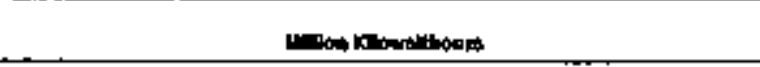 } & \\
\hline 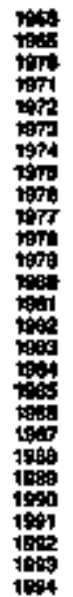 & 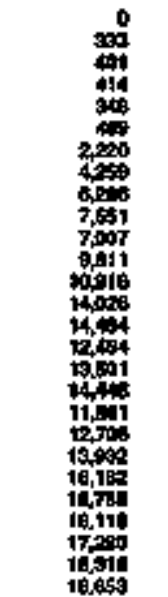 & $\begin{array}{l}0 \\
0 \\
0 \\
0 \\
0 \\
0 \\
0 \\
0 \\
0 \\
0 \\
0 \\
0 \\
0 \\
0 \\
0 \\
0 \\
0 \\
0 \\
0 \\
0 \\
0 \\
0 \\
0 \\
0\end{array}$ & 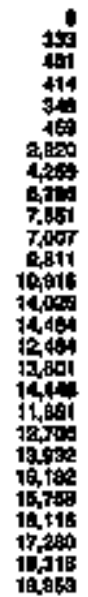 & 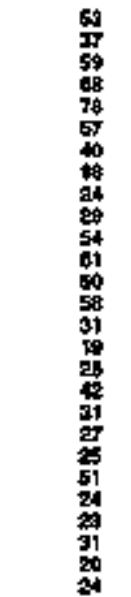 & 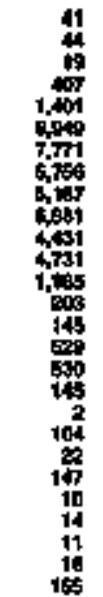 & 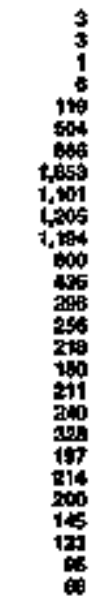 & $\begin{array}{l}0 \\
0 \\
0 \\
0 \\
0 \\
0 \\
0 \\
0 \\
0 \\
0 \\
0 \\
0 \\
0 \\
0 \\
0 \\
0 \\
\vdots \\
0 \\
0 \\
0 \\
0 \\
0 \\
0 \\
0 \\
0\end{array}$ & 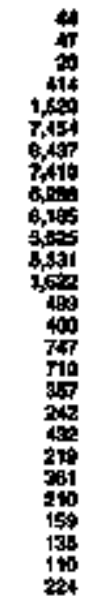 & 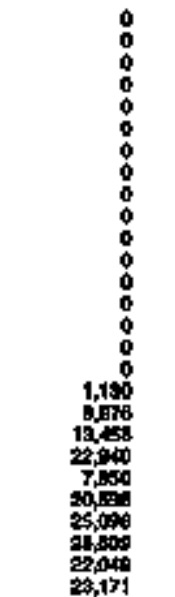 & 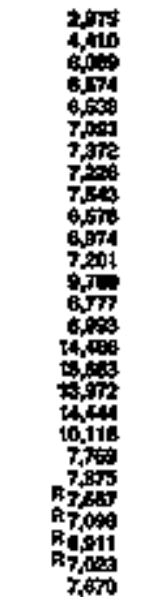 & $\begin{array}{l}\text { to } \\
0 \\
0 \\
0 \\
0 \\
0 \\
0 \\
0 \\
0 \\
0 \\
0 \\
0 \\
0 \\
0 \\
0 \\
0 \\
0 \\
0 \\
0 \\
0 \\
0 \\
0 \\
0 \\
0 \\
0\end{array}$ & $\begin{array}{l}1 \\
: \\
0 \\
0 \\
0 \\
0 \\
0 \\
0 \\
0 \\
0 \\
0 \\
0 \\
0 \\
0 \\
0 \\
0 \\
0 \\
0 \\
0 \\
0\end{array}$ & $\begin{array}{l}0 \\
\vdots \\
0 \\
0 \\
0 \\
0 \\
0 \\
0 \\
0 \\
0 \\
0 \\
0 \\
0 \\
0 \\
0 \\
0 \\
0 \\
0 \\
0 \\
0 \\
0 \\
0 \\
0\end{array}$ & $\begin{array}{l}\vdots \\
\vdots \\
\vdots \\
\vdots \\
\vdots \\
\vdots \\
\vdots \\
\vdots \\
\vdots \\
\vdots \\
\vdots \\
\vdots \\
\vdots \\
\vdots\end{array}$ \\
\hline
\end{tabular}

\begin{tabular}{|c|c|c|c|c|c|c|c|}
\hline 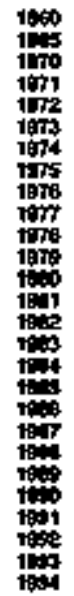 & 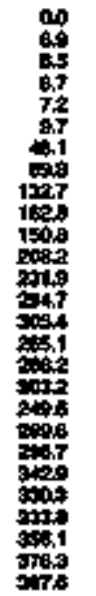 & 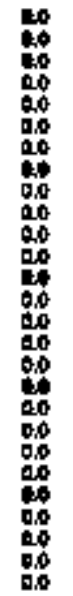 & 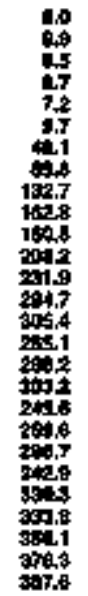 & 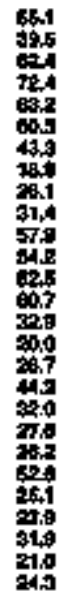 & 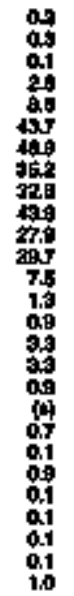 & 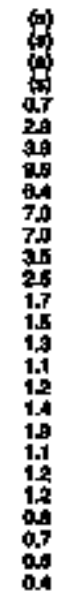 & 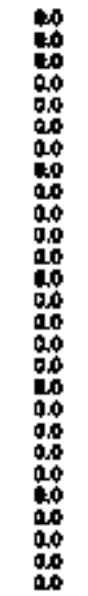 \\
\hline
\end{tabular}

\begin{tabular}{|c|c|c|c|c|c|c|}
\hline 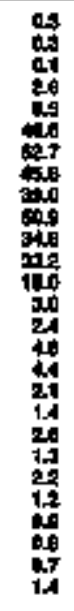 & 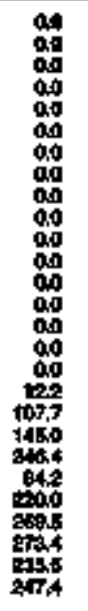 & 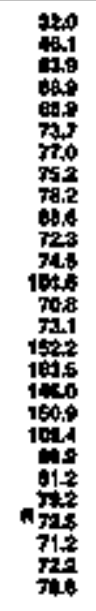 & 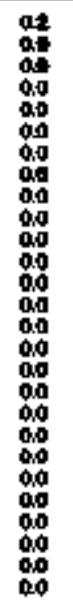 & 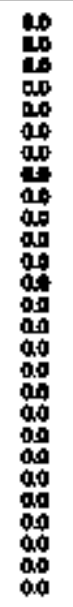 & 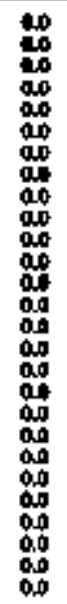 & 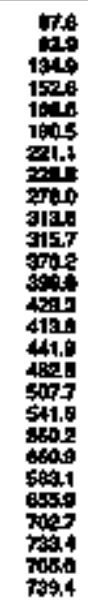 \\
\hline
\end{tabular}

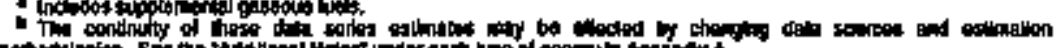

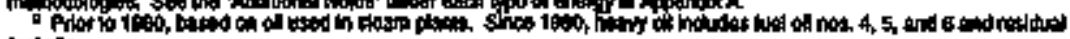

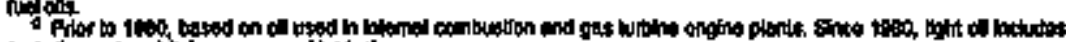

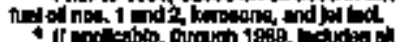

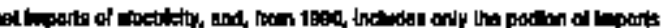

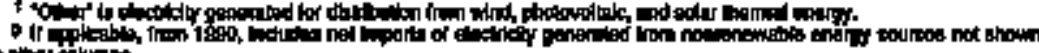

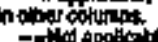

(1)

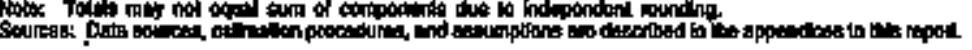




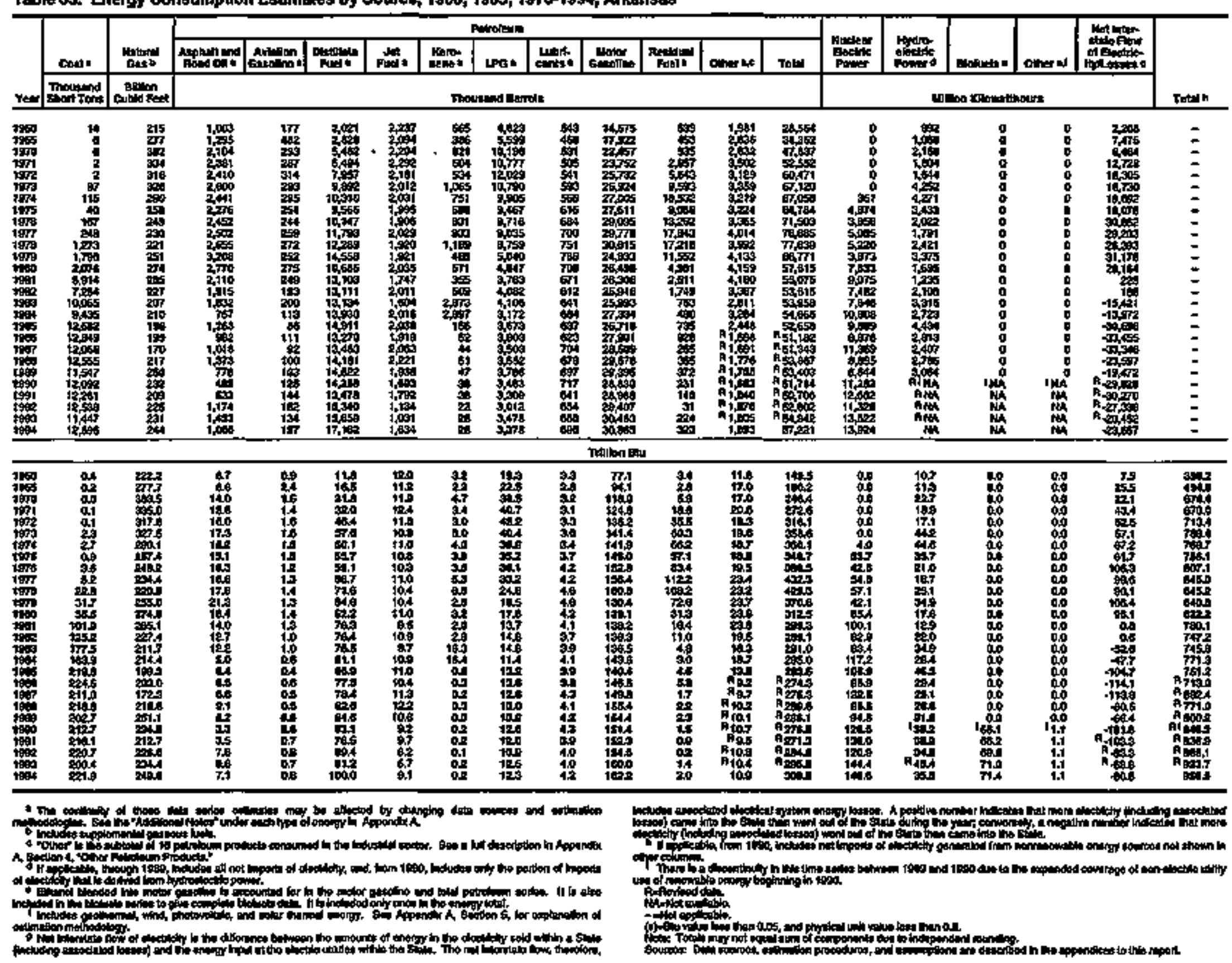


A Table 36. Aesidential Energy Consumplion Estimates, 1960, 1965, 1970-1994, Arkansas

\begin{tabular}{|c|c|c|c|c|c|c|c|c|c|c|c|c|c|c|}
\hline \multirow[b]{3}{*}{ rof } & \multicolumn{3}{|c|}{ cont } & \multirow{3}{*}{ 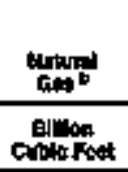 } & \multicolumn{4}{|c|}{ Patroknan } & \multirow{3}{*}{ Thotocin } & & \multirow[b]{2}{*}{ Exctid/t" } & \multirow[b]{2}{*}{ mang } & \multirow{3}{*}{ 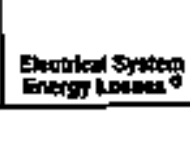 } & \multirow[b]{3}{*}{$T+x$} \\
\hline & conduston: & Avanote & Tomall & & 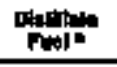 & Kerosenne" & LPG: & $\tau$ & & solk-" & & & & \\
\hline & \multicolumn{3}{|c|}{ Theosund Surt Tone } & & \multicolumn{4}{|c|}{ Thoos:me Bumels: } & & \multicolumn{3}{|c|}{ 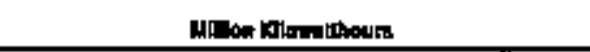 } & & \\
\hline 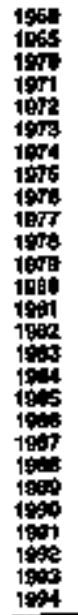 & 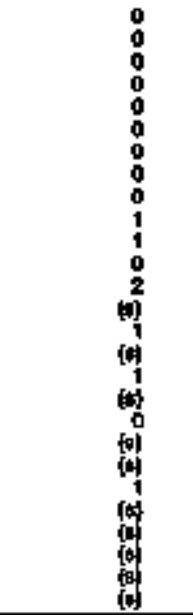 & $\begin{array}{l}: \\
: \\
: \\
: \\
: \\
0 \\
0 \\
\vdots \\
0 \\
\vdots \\
0 \\
0 \\
0 \\
0 \\
0 \\
0 \\
0 \\
0 \\
0\end{array}$ & 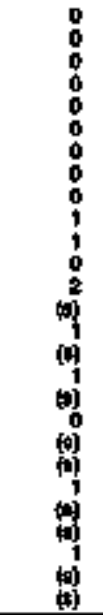 & 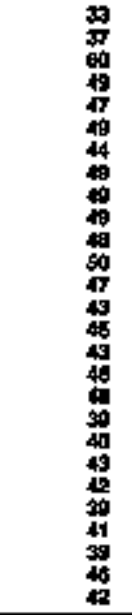 & 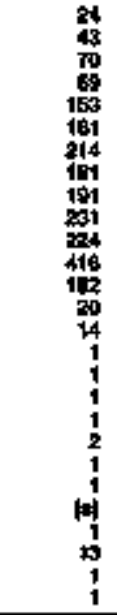 & 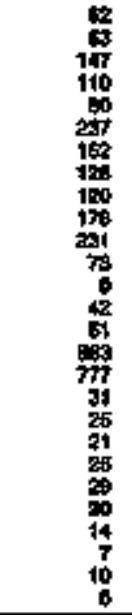 & 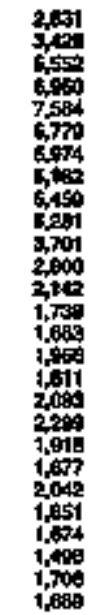 & 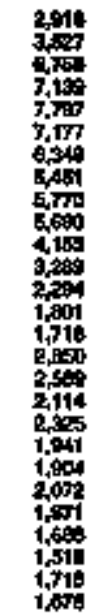 & 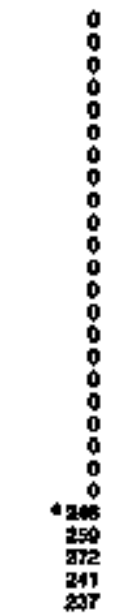 & 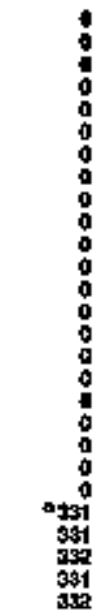 & 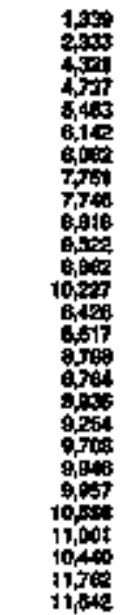 & $\begin{array}{l}\vdots \\
\vdots \\
\vdots \\
\vdots \\
\vdots \\
\vdots \\
\vdots \\
\vdots \\
\vdots \\
\vdots \\
\vdots\end{array}$ & 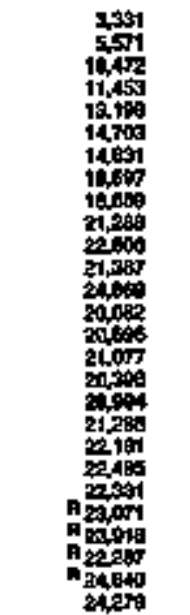 & $\begin{array}{l}\vdots \\
\vdots \\
\vdots \\
\vdots \\
\vdots \\
\vdots \\
\vdots \\
\vdots \\
\vdots \\
\vdots \\
\vdots \\
z \\
z\end{array}$ \\
\hline \multicolumn{15}{|c|}{ Tridlon Bts } \\
\hline 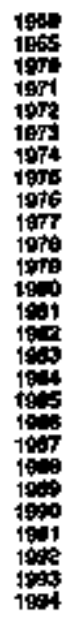 & 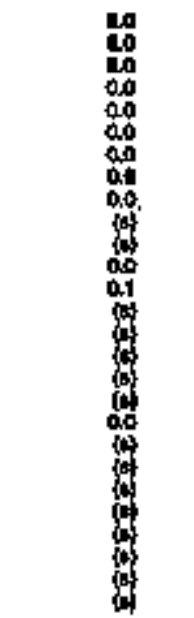 & 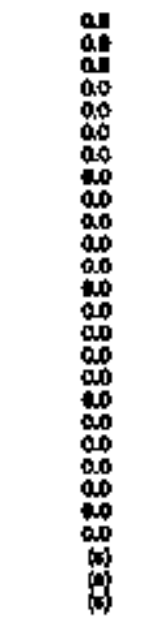 & 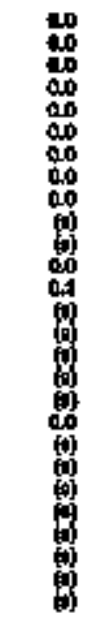 & 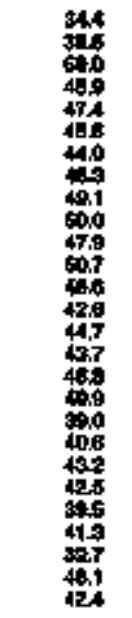 & 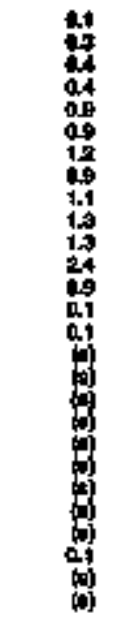 & 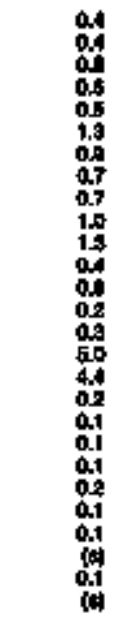 & 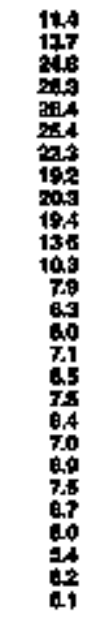 & 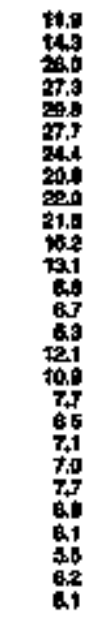 & 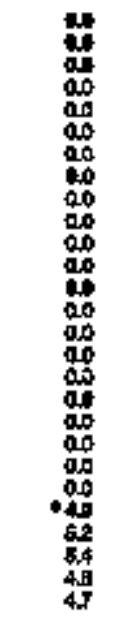 & 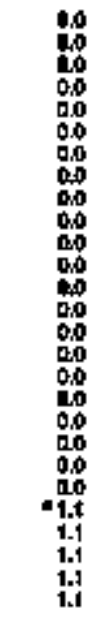 & 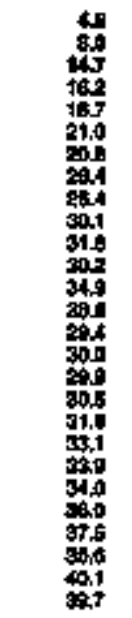 & 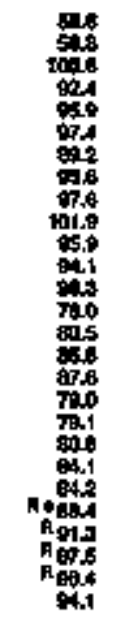 & 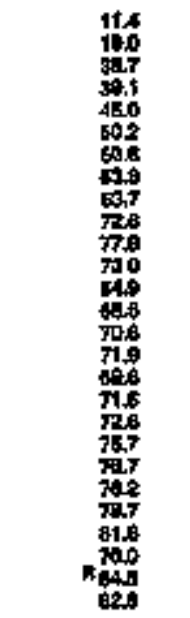 & 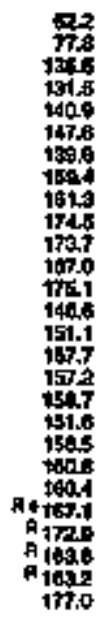 \\
\hline
\end{tabular}

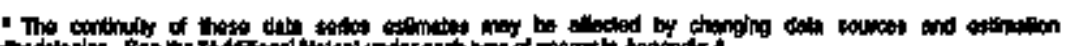

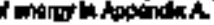

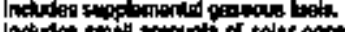

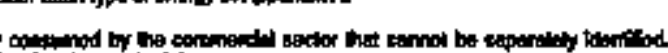

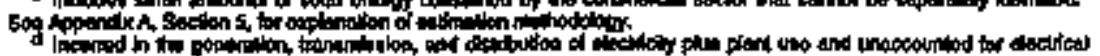

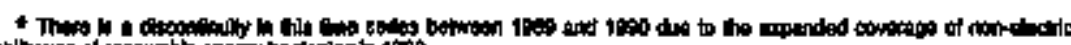

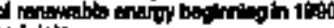

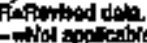

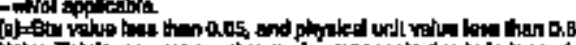

Cover The 
Table 37. Commerciad Energy Consumption Estimates, 1950, 19k5, 1970-1994, Arkansas

\begin{tabular}{|c|c|c|c|c|c|c|c|c|c|c|c|c|c|c|}
\hline \multirow[b]{3}{*}{ Yot } & \multicolumn{3}{|c|}{ cosid } & \multirow{3}{*}{ 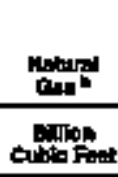 } & \multicolumn{6}{|c|}{ Potrolate } & \multirow[b]{2}{*}{ anctatyo" } & \multirow[b]{2}{*}{ Finerof } & \multirow[b]{2}{*}{ 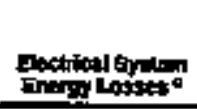 } & \multirow[b]{3}{*}{ Totult } \\
\hline & 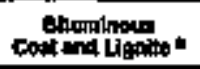 & Andingerily" & 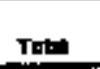 & & moed & Kerocanes & LPEn & Fingor & Find & Total & & & & \\
\hline & \multicolumn{3}{|c|}{ Thoweted short Tont } & & \multicolumn{6}{|c|}{ 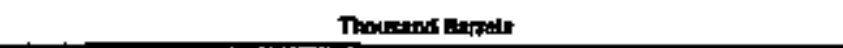 } & \multicolumn{3}{|c|}{ Written Kancithotin } & \\
\hline 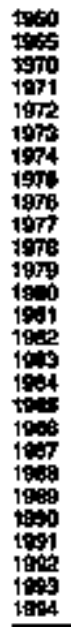 & 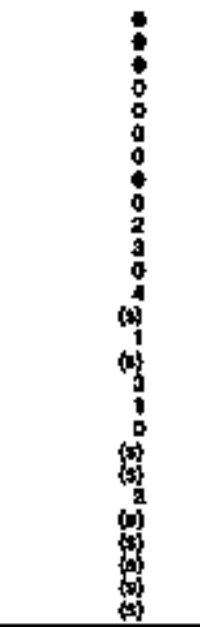 & $\begin{array}{l}0 \\
0 \\
0 \\
0 \\
0 \\
0 \\
0 \\
0 \\
0 \\
0 \\
0 \\
0 \\
0 \\
0 \\
0 \\
0 \\
0 \\
0 \\
0 \\
0 \\
0 \\
0\end{array}$ & 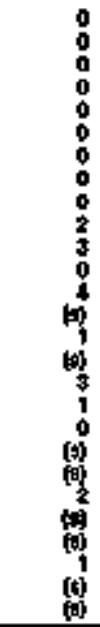 & 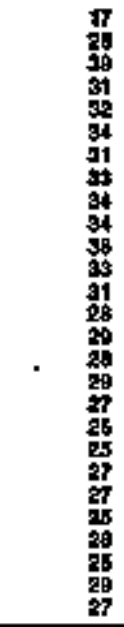 & 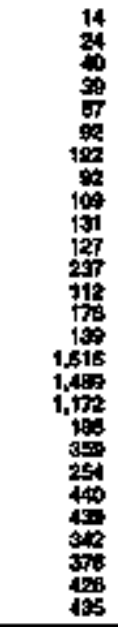 & 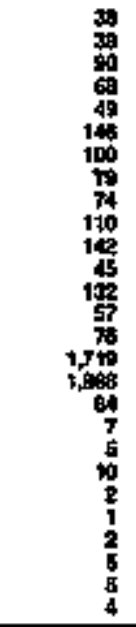 & 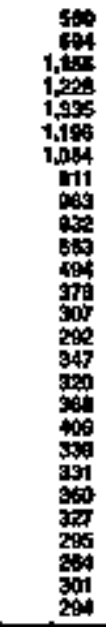 & 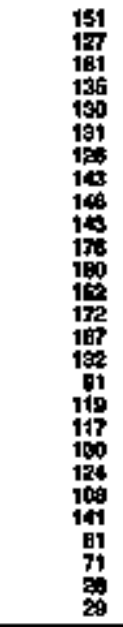 & 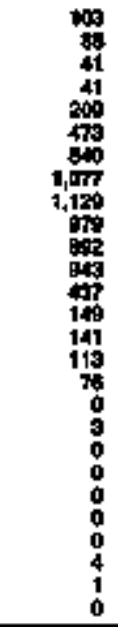 & 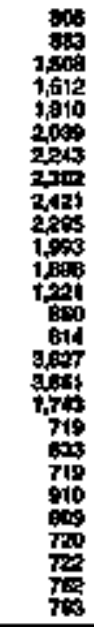 & 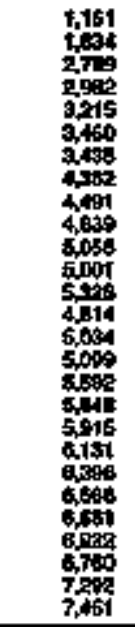 & $\begin{array}{l}= \\
= \\
= \\
= \\
= \\
= \\
= \\
= \\
= \\
= \\
= \\
= \\
= \\
=\end{array}$ & 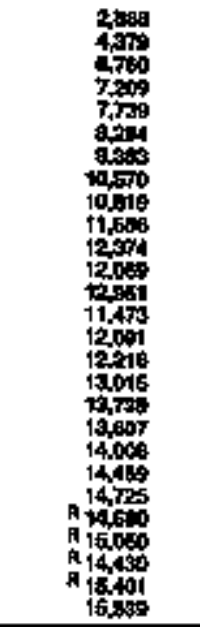 & $\begin{array}{l}= \\
= \\
= \\
= \\
= \\
= \\
= \\
= \\
= \\
= \\
= \\
= \\
= \\
= \\
=\end{array}$ \\
\hline \multicolumn{15}{|c|}{ Trdilon Bu } \\
\hline 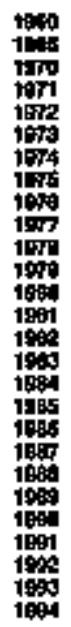 & 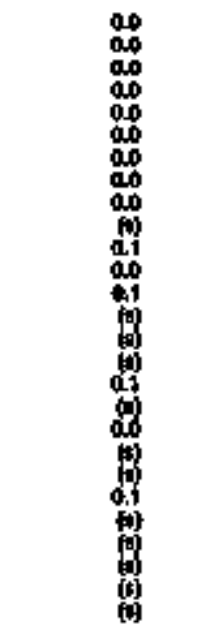 & 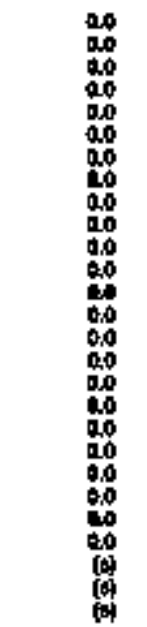 & 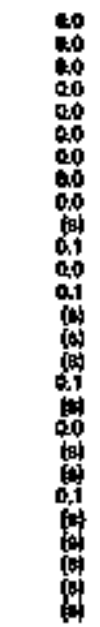 & 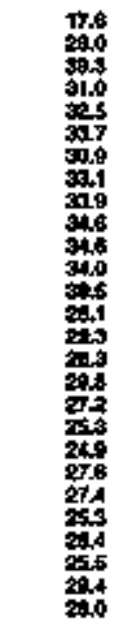 & 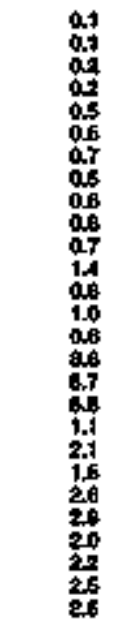 & 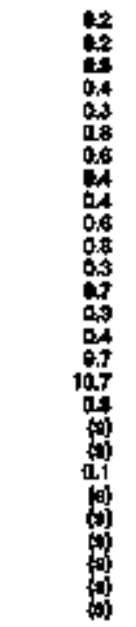 & 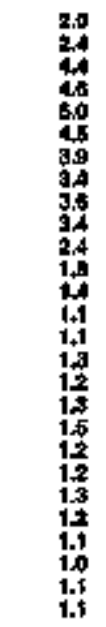 & 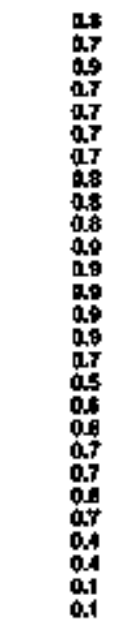 & 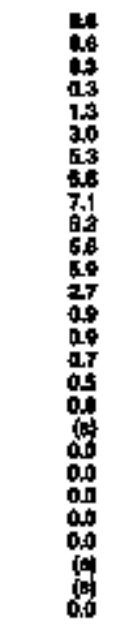 & 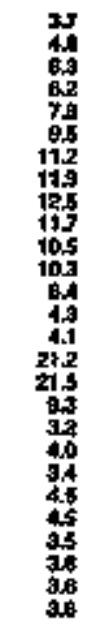 & 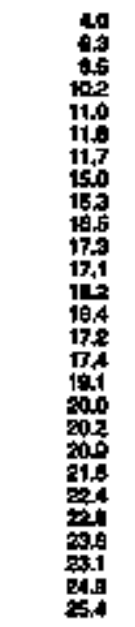 & 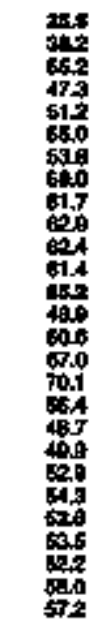 & 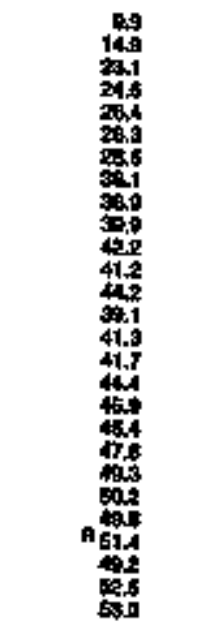 & 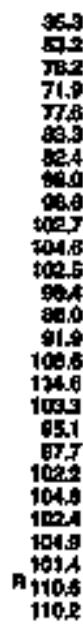 \\
\hline
\end{tabular}

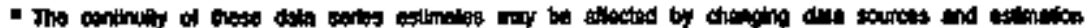

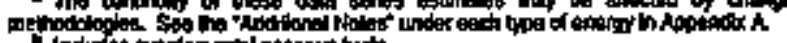

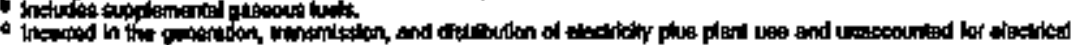

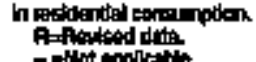

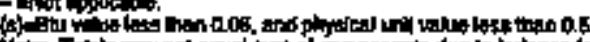

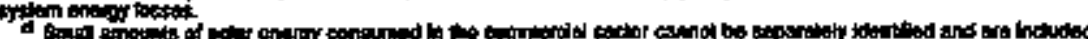

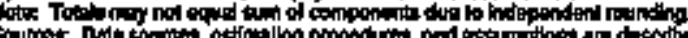


Table 38. Industrial Energy Consumption Esilinates, t960, 1065, 1970-1994, Arkansas

\begin{tabular}{|c|c|c|c|c|c|c|c|c|c|c|c|c|c|c|c|c|c|c|}
\hline \multirow[b]{3}{*}{ vest: } & \multirow[b]{2}{*}{ إل } & \multirow[b]{2}{*}{ and } & \multicolumn{9}{|c|}{ Petpolar } & \multirow[b]{2}{*}{$\mid$} & \multirow[b]{2}{*}{ Ints } & \multirow[b]{2}{*}{ Dherende } & \multirow[b]{2}{*}{ eloctrotion } & \multirow[b]{2}{*}{ Exty } & \multirow{3}{*}{ 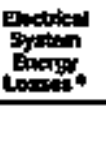 } & \multirow[b]{3}{*}{ 1) } \\
\hline & & & 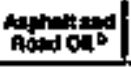 & entats & 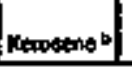 & Q6: & $4=0$ & motor & $\frac{8}{90}$ & olnerese & Towat & & & & & & & \\
\hline & Thoosud & in & \multicolumn{9}{|c|}{ Thoosind Bunth } & \multicolumn{5}{|c|}{ 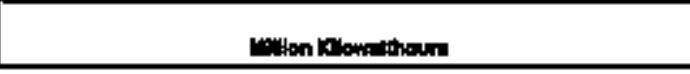 } & & \\
\hline 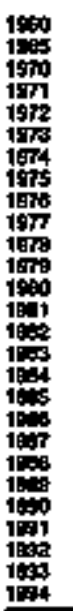 & 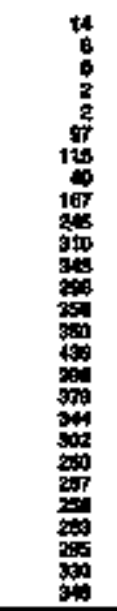 & 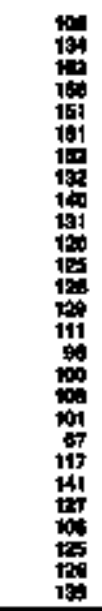 & 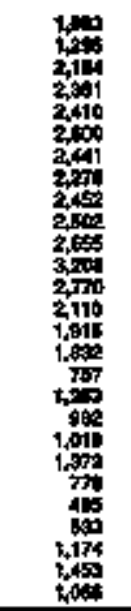 & 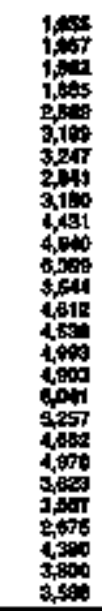 & 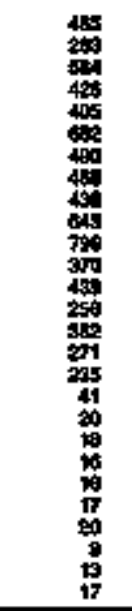 & 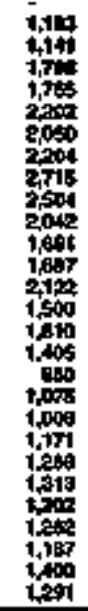 & 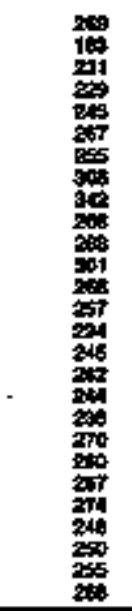 & 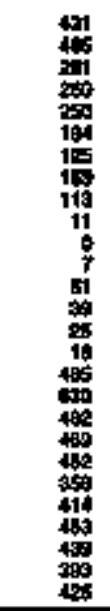 & 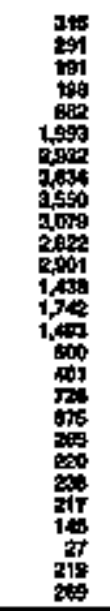 & 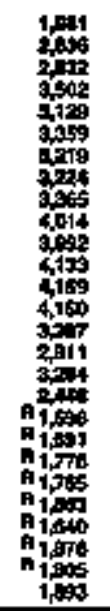 & 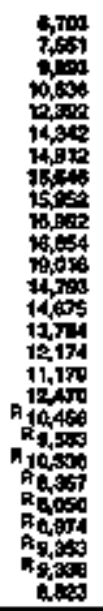 & 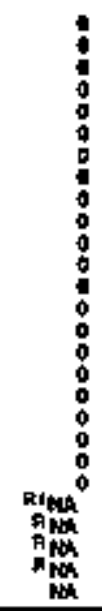 & $\begin{array}{l}0 \\
\vdots \\
\vdots \\
\vdots \\
\vdots \\
\vdots \\
\vdots \\
: \\
\vdots \\
\vdots \\
\vdots \\
: \\
: \\
:\end{array}$ & $\begin{array}{l}0 \\
0 \\
0 \\
0 \\
0 \\
0 \\
0 \\
0 \\
0 \\
0 \\
0 \\
0 \\
0 \\
0 \\
0 \\
0 \\
0 \\
0 \\
0 \\
0 \\
0 \\
0\end{array}$ & 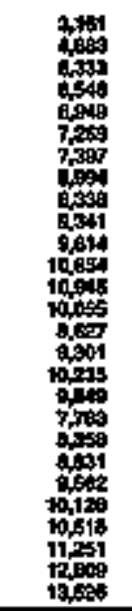 & $\bar{z} \bar{z}=$ & 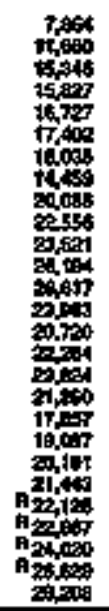 & \\
\hline \multicolumn{19}{|c|}{ Dollop Em } \\
\hline 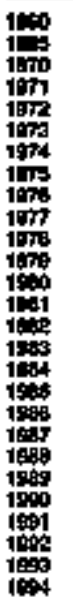 & 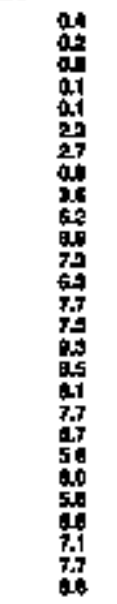 & 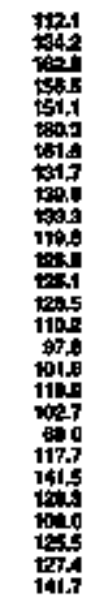 & 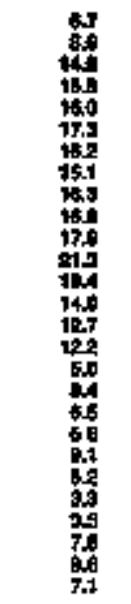 & 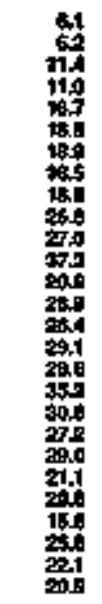 & 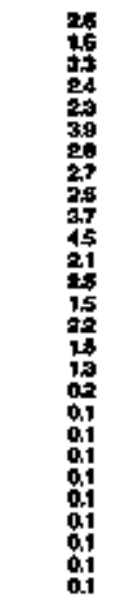 & 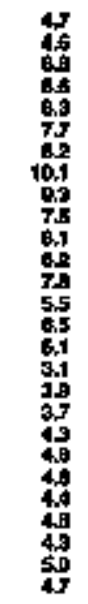 & 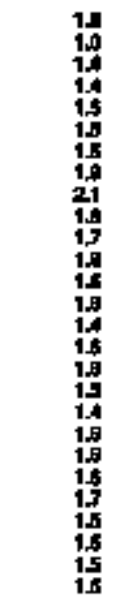 & 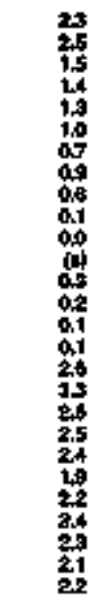 & 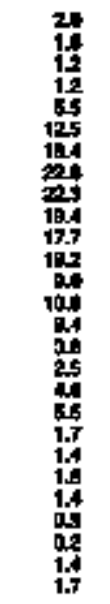 & 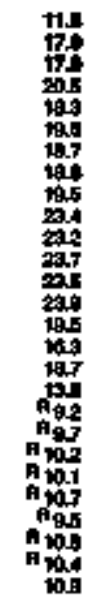 & 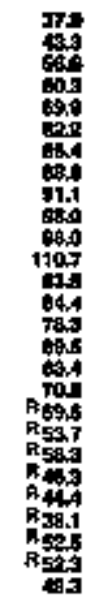 & 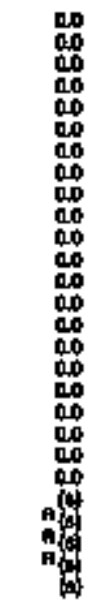 & 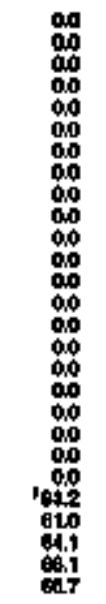 & 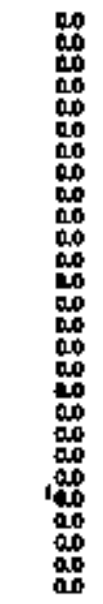 & 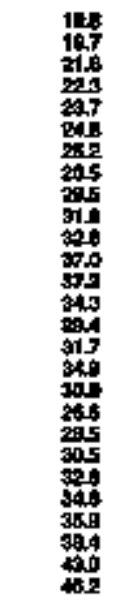 & 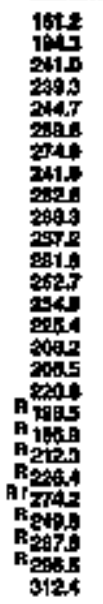 & 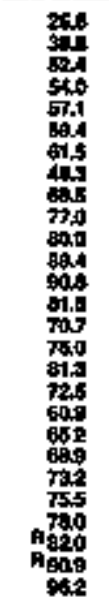 & 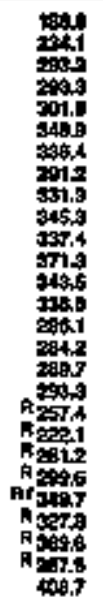 \\
\hline
\end{tabular}

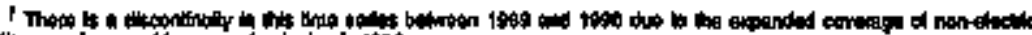

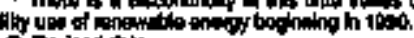

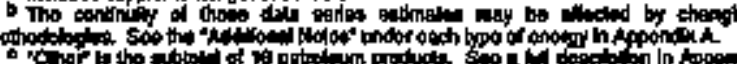

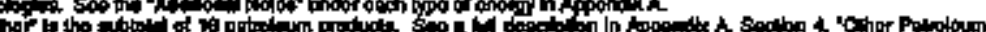

Produst

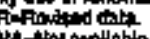

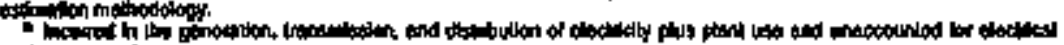

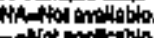

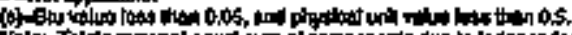

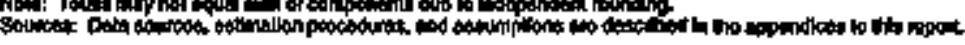

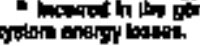


Table as. Transportation Energy Coinsurpition Estimates, 1960, 1965, 1970-1994, Arkansas

\begin{tabular}{|c|c|c|c|c|c|c|c|}
\hline \multirow[b]{3}{*}{$k=$} & \multirow[b]{2}{*}{ cont } & \multirow[b]{2}{*}{ 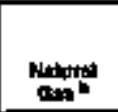 } & \multicolumn{5}{|c|}{ 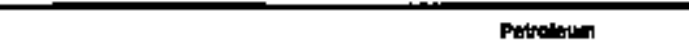 } \\
\hline & & & Ansontion. & Daspente & "أlit & Wa" & Lobrapante" \\
\hline & $\begin{array}{l}\text { Theuspod } \\
\text { Shent Tain }\end{array}$ & optistext & & & \multicolumn{3}{|c|}{ mowitend semest } \\
\hline 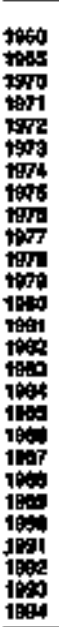 & 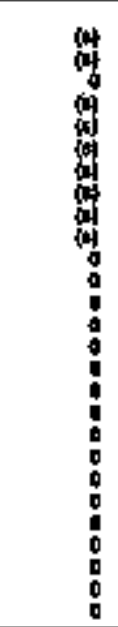 & 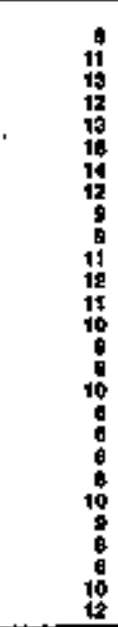 & 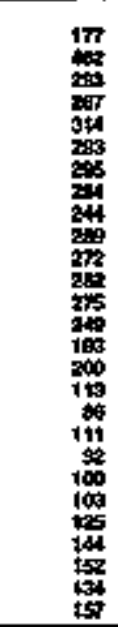 & 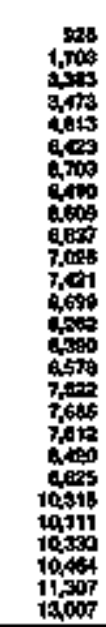 & 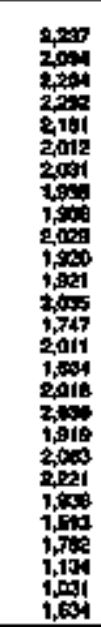 & 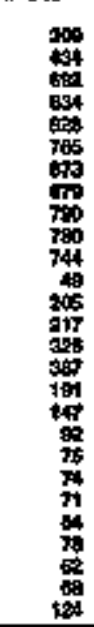 & 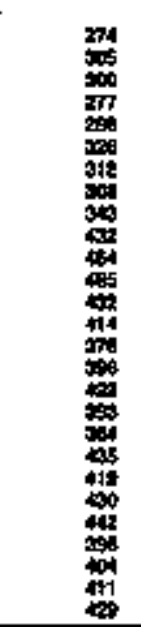 \\
\hline 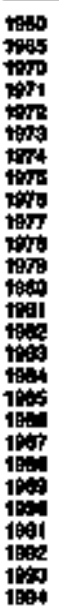 & 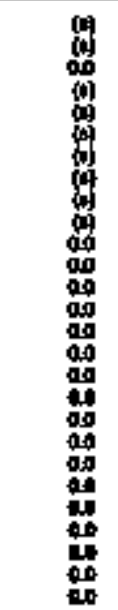 & 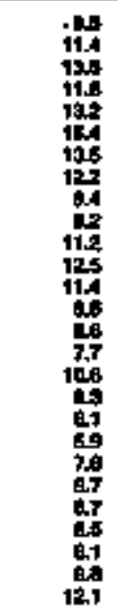 & 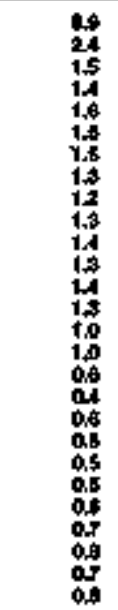 & 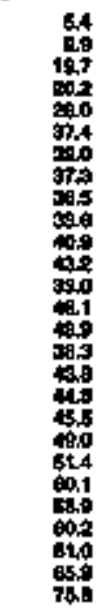 & 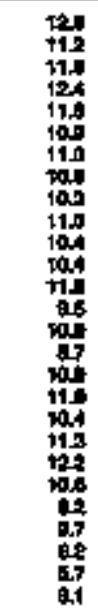 & 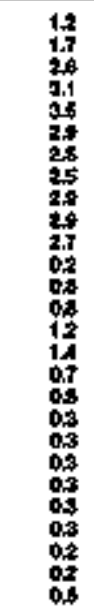 & 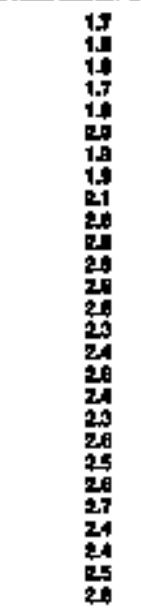 \\
\hline
\end{tabular}

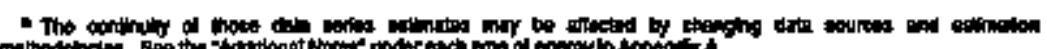

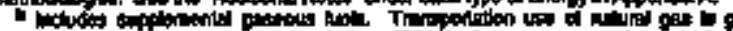

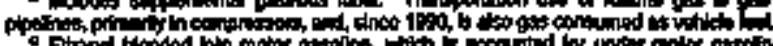

(1)

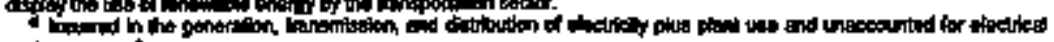

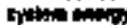

$\mathbf{A}$
$\mathbf{R}$
$\mathbf{K}$
$\mathbf{A}$
$\mathbf{N}$
$\mathbf{S}$
$\mathbf{A}$
$\mathbf{S}$

\begin{tabular}{|c|c|c|c|c|c|c|c|}
\hline 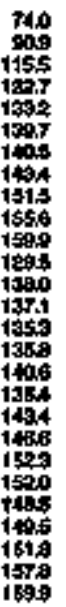 & 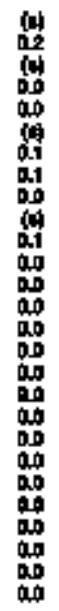 & 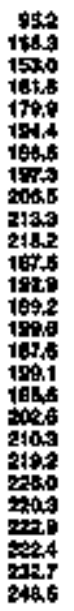 & 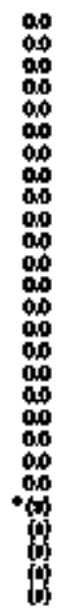 & 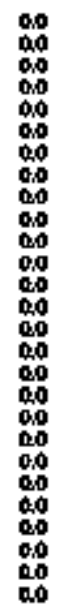 & 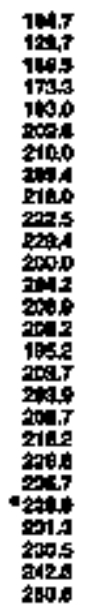 & 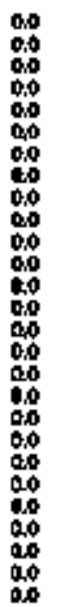 & 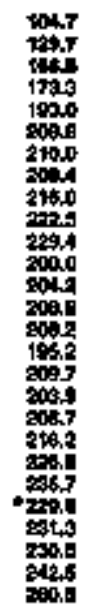 \\
\hline
\end{tabular}

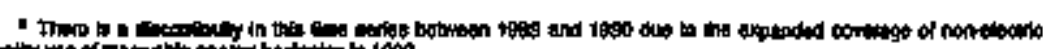

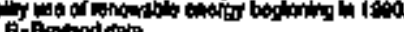

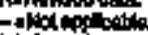

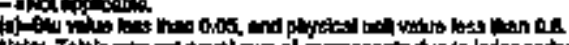

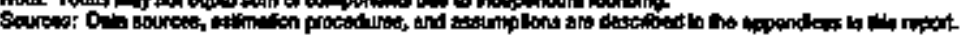


Tabla 40. Estimales of Energy Input at Electric Utilltes, 1980, 1965, 1970-1994, Arkansas

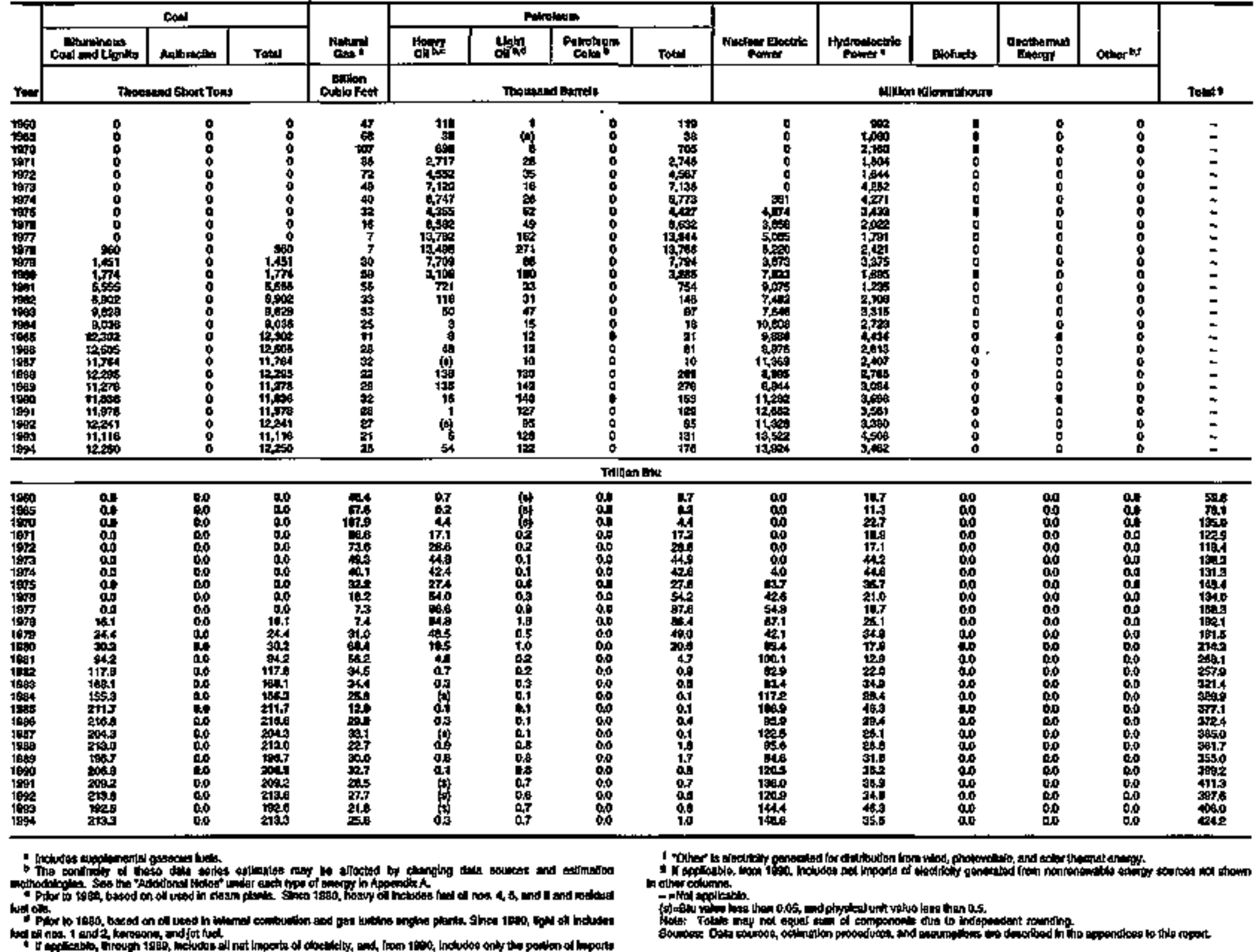

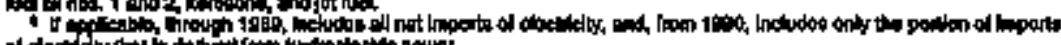




\begin{tabular}{|c|c|c|c|c|c|c|c|c|c|c|c|c|c|c|c|c|c|c|c|}
\hline \multirow[b]{3}{*}{$r \in=$} & \multirow[b]{2}{*}{ cont } & \multirow[b]{2}{*}{ chand } & \multicolumn{11}{|c|}{ Poivolum } & \multirow[b]{2}{*}{ 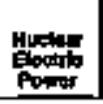 } & \multirow[b]{2}{*}{ 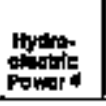 } & \multirow[b]{2}{*}{ eloturas } & \multirow[b]{2}{*}{ onem in } & \multirow{3}{*}{ 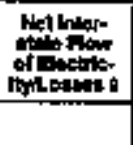 } & \multirow[b]{3}{*}{ That } \\
\hline & & & Naphan ind & Anstom & popto & tell. & 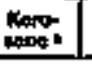 & EPQR & tit. & and & ats & her $\infty$ & Jold & & & & & & \\
\hline & | tond & siblopfot & \multicolumn{11}{|c|}{ 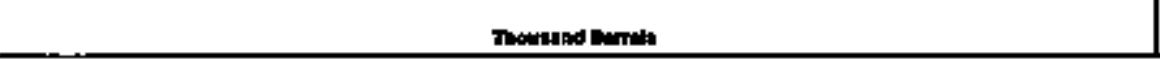 } & \multicolumn{4}{|c|}{ 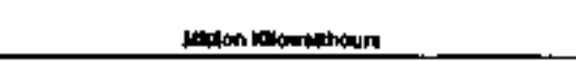 } & & \\
\hline 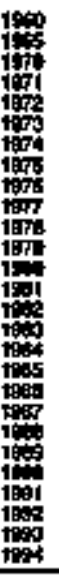 & 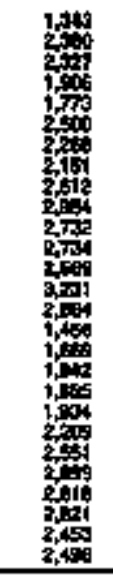 & 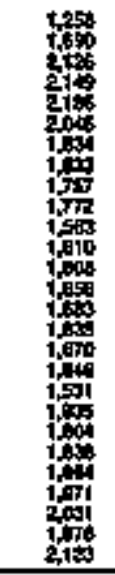 & 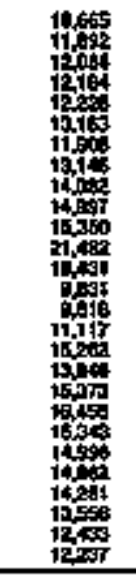 & 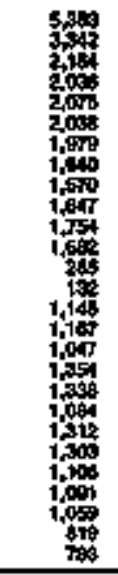 & 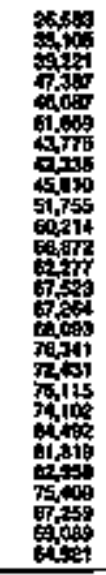 & 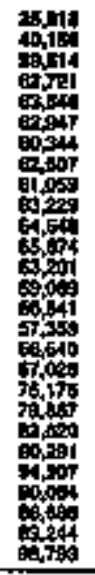 & 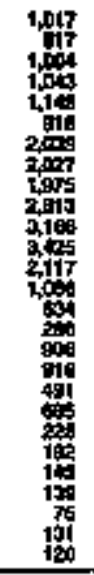 & 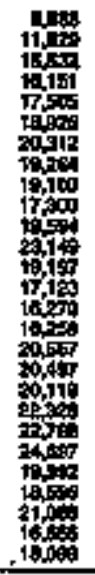 & 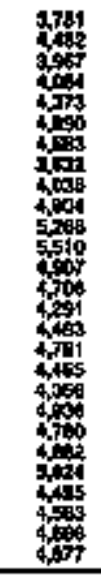 & 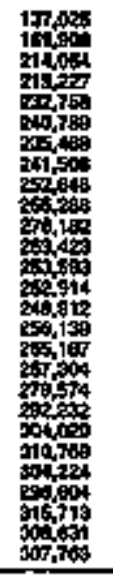 & 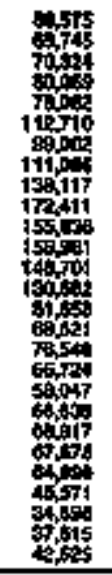 & 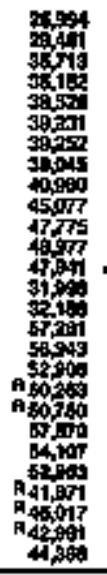 & 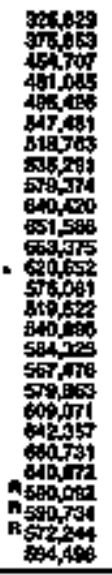 & 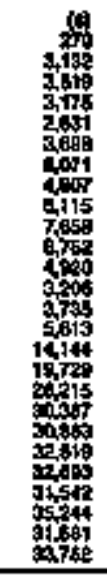 & 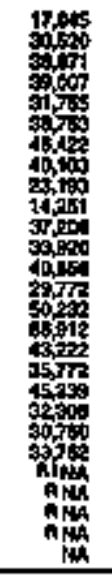 & 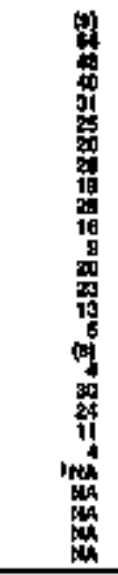 & 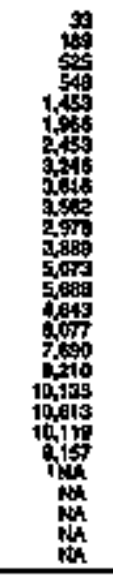 & 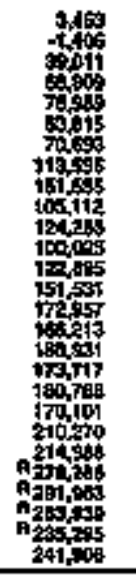 & $\begin{array}{l}z \\
z \\
z \\
z \\
z \\
\vdots \\
z \\
z \\
z \\
z \\
z \\
z \\
z\end{array}$ \\
\hline \multicolumn{20}{|c|}{ mingen bu } \\
\hline 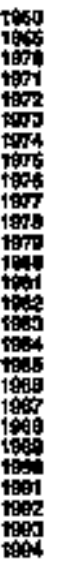 & 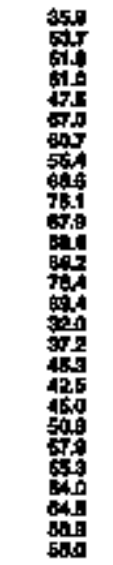 & 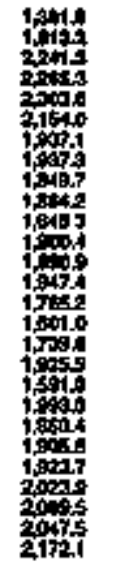 & 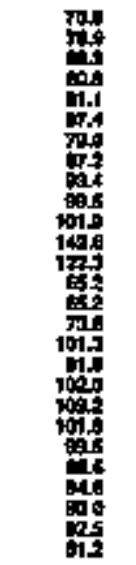 & 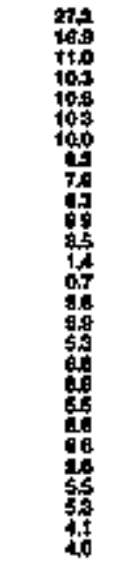 & 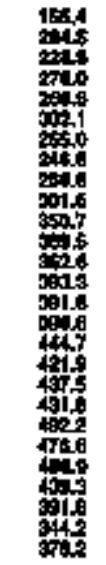 & 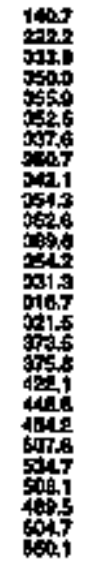 & 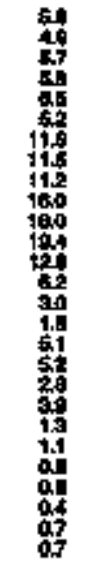 & 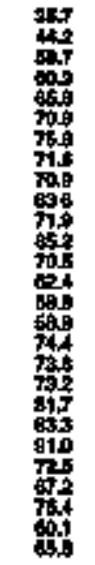 & 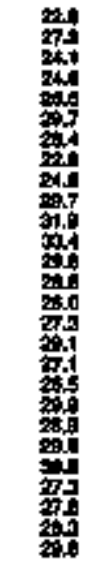 & 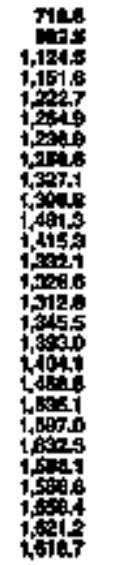 & 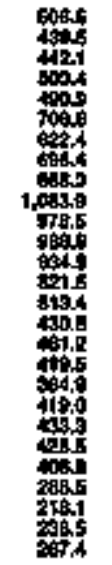 & 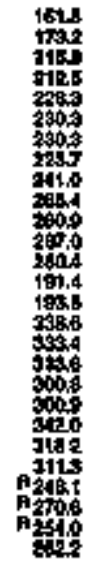 & 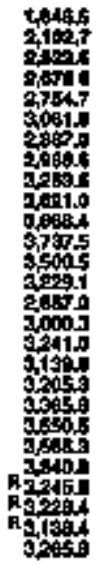 & 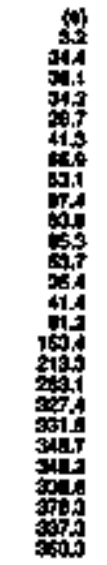 & 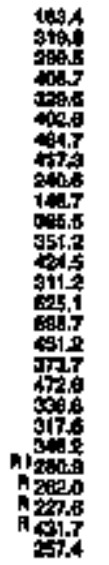 & 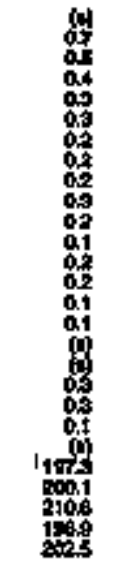 & 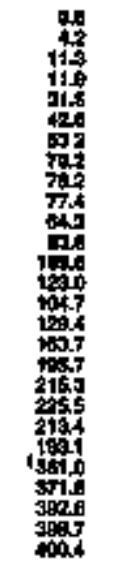 & 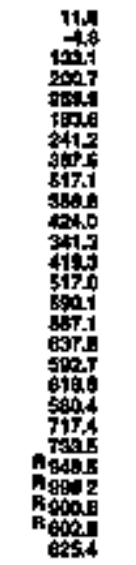 & 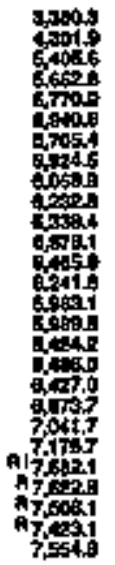 \\
\hline
\end{tabular}

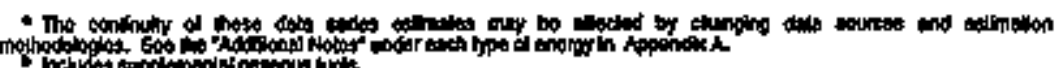

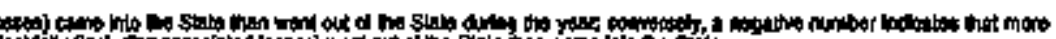

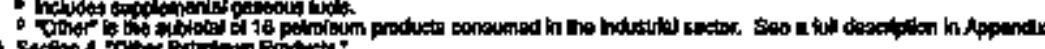

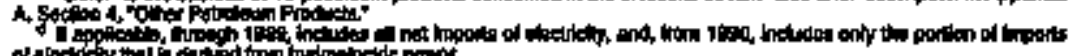

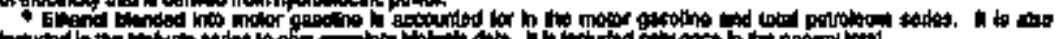

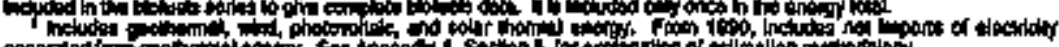

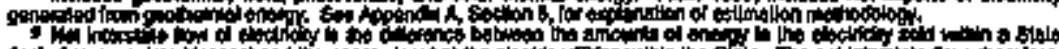

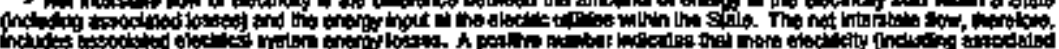

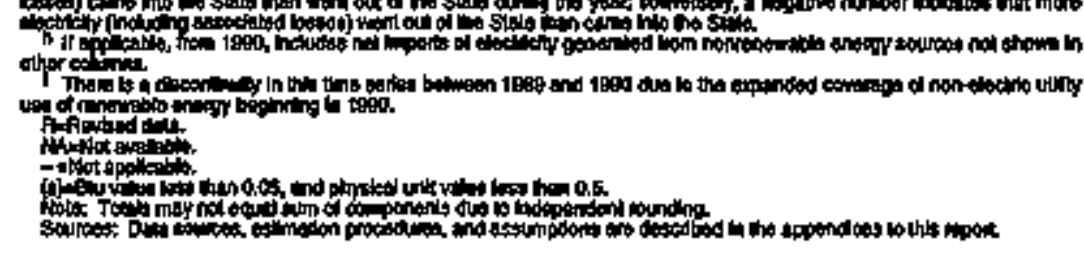

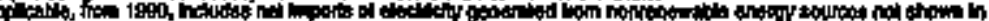

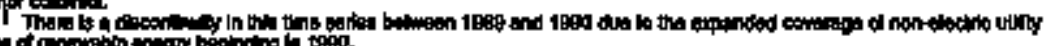

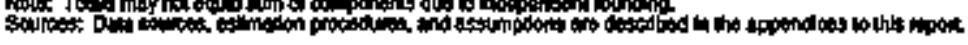


C Teble 42. Resldentlal Energy Conaumption Estimales, 1960, 1965, 1970-1994, Califiornla

\begin{tabular}{|c|c|c|c|c|c|c|c|c|c|c|c|c|c|c|}
\hline \multirow[b]{3}{*}{ rasl } & \multicolumn{3}{|c|}{ coos } & \multirow[b]{2}{*}{$\log ^{2}$} & \multicolumn{4}{|c|}{ Detrmberis: } & \multirow{3}{*}{ 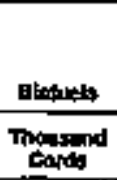 } & & & \multirow[b]{2}{*}{ mor } & \multirow{3}{*}{ 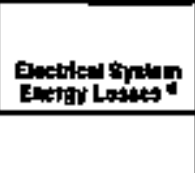 } & \multirow[b]{3}{*}{ Totet } \\
\hline & 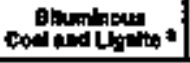 & Antrmatastat & Tol & & 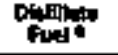 & kroesent & LE: & noten & & $500 \times 5$ & andetis Ry & & & \\
\hline & \multicolumn{3}{|c|}{ 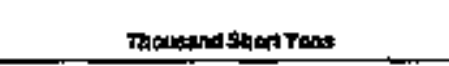 } & 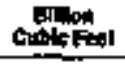 & \multicolumn{4}{|c|}{ 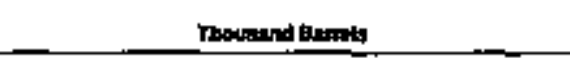 } & & \multicolumn{3}{|c|}{ 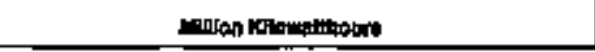 } & & \\
\hline 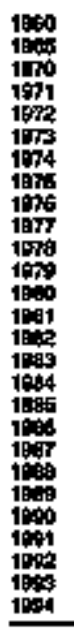 & 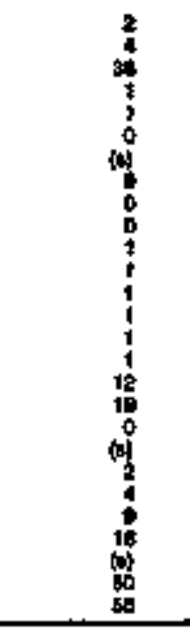 & $\begin{array}{c}0 \\
0 \\
0 \\
0 \\
0 \\
0 \\
0 \\
0 \\
0 \\
0 \\
0 \\
0 \\
0 \\
0 \\
0 \\
0 \\
0 \\
0 \\
0 \\
0 \\
0 \\
0\end{array}$ & 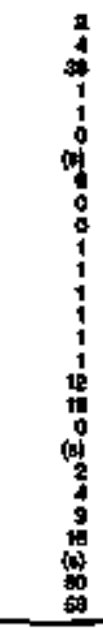 & 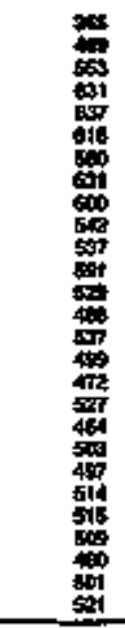 & 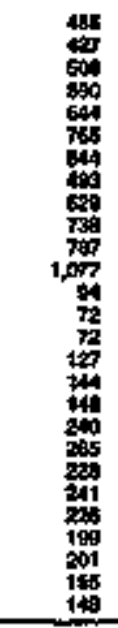 & 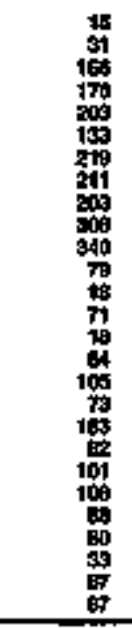 & 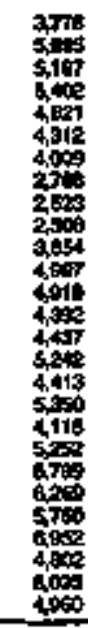 & 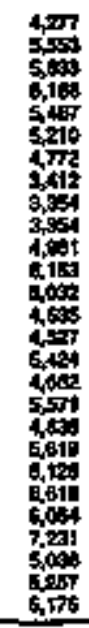 & 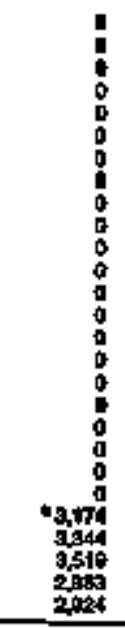 & 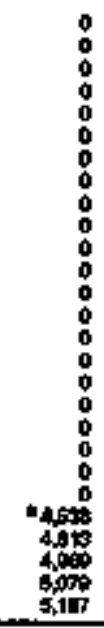 & 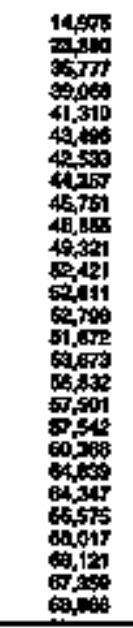 & $\begin{array}{l}\vdots \\
\vdots \\
\vdots \\
\vdots \\
\vdots \\
\vdots \\
\vdots \\
\vdots \\
\vdots \\
\vdots \\
\vdots \\
\vdots \\
\vdots\end{array}$ & 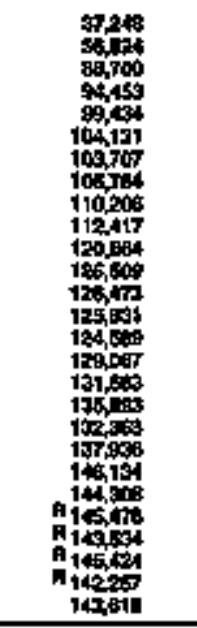 & $\begin{array}{l}z \\
z \\
\vdots \\
z \\
\vdots \\
z \\
\bar{z} \\
\bar{z} \\
\bar{z} \\
\bar{z}\end{array}$ \\
\hline \multicolumn{15}{|c|}{$T$} \\
\hline 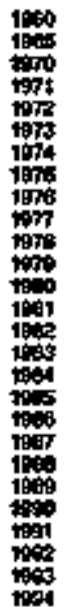 & 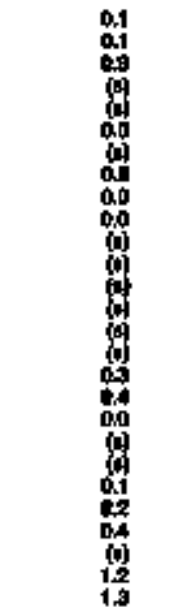 & 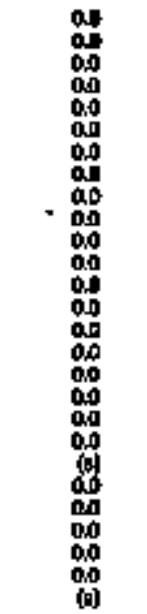 & 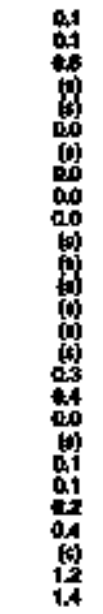 & 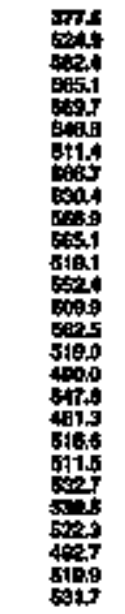 & 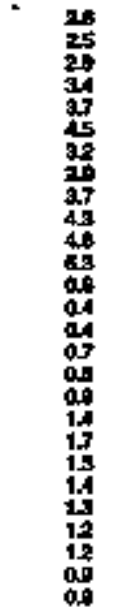 & 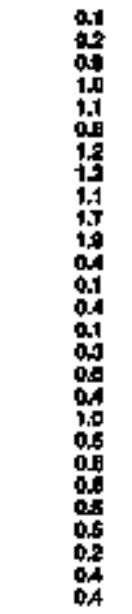 & 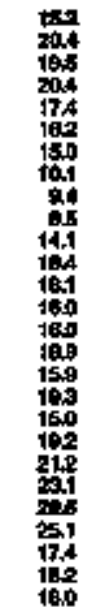 & 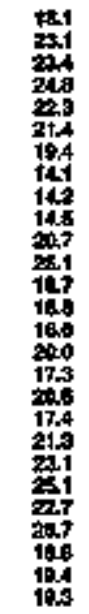 & 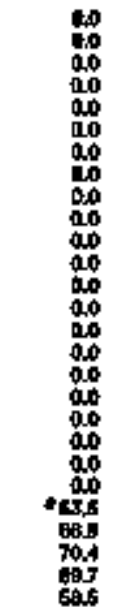 & 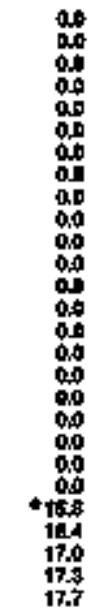 & 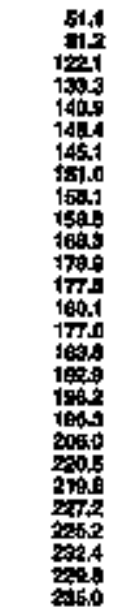 & 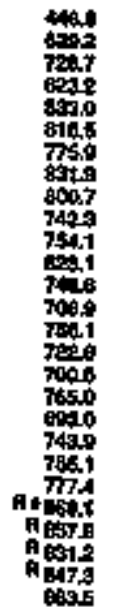 & 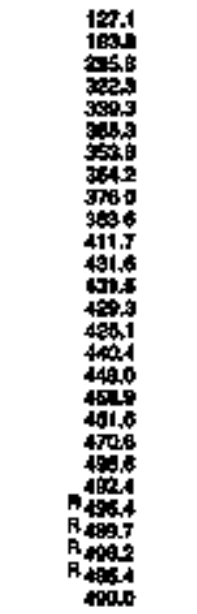 & 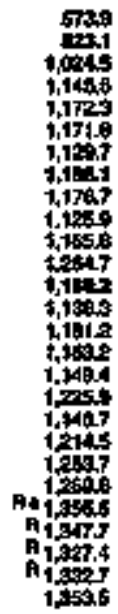 \\
\hline
\end{tabular}

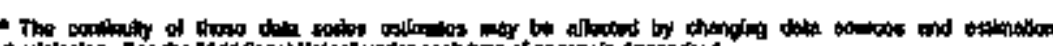

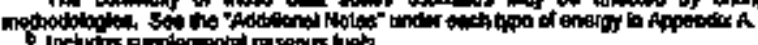

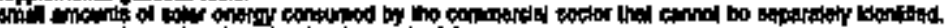

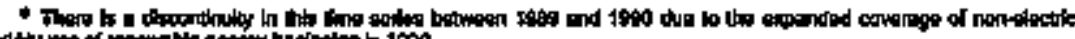

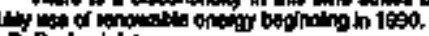


Table 43. Commerclal Energy Consumption Estimates, 1960, 1965, 1970-1994, Calitornla

\begin{tabular}{|c|c|c|c|c|c|c|c|c|c|c|c|c|c|c|}
\hline \multirow[b]{3}{*}{ Yop } & \multicolumn{3}{|c|}{ هat } & \multirow{3}{*}{ 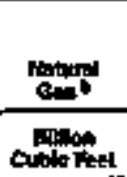 } & \multicolumn{6}{|c|}{ Potolien } & \multirow[b]{2}{*}{ Daterutienty } & \multirow[b]{2}{*}{ Het } & \multirow[b]{2}{*}{ 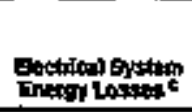 } & \multirow[b]{3}{*}{ Totwd } \\
\hline & condingond & Antures. & Totet & & $\begin{array}{c}\text { Ditalinin } \\
\text { Putal }\end{array}$ & Kurobonat & LLPG: & Gotor & mosionde & Totod & & & & \\
\hline & \multicolumn{3}{|c|}{ Moureand short tond } & & \multicolumn{6}{|c|}{ Mourind barnets } & \multicolumn{3}{|c|}{ 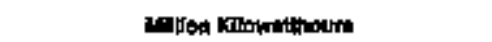 } & \\
\hline 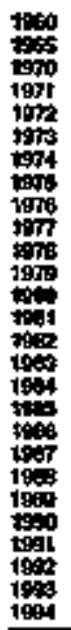 & 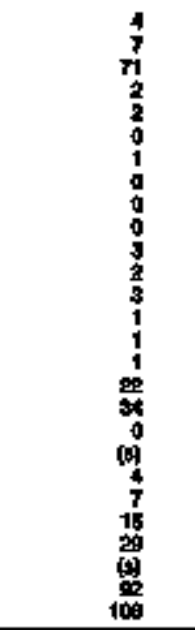 & $\begin{array}{l}0 \\
0 \\
0 \\
0 \\
0 \\
0 \\
0 \\
0 \\
0 \\
0 \\
0 \\
0 \\
0 \\
0 \\
0 \\
0 \\
0 \\
0 \\
0 \\
0 \\
0 \\
0\end{array}$ & 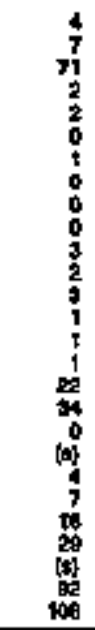 & 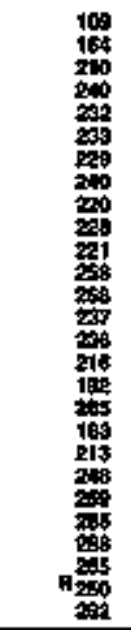 & 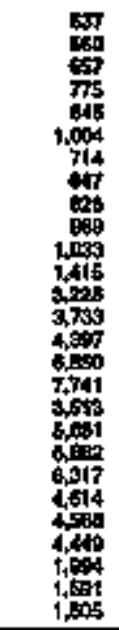 & 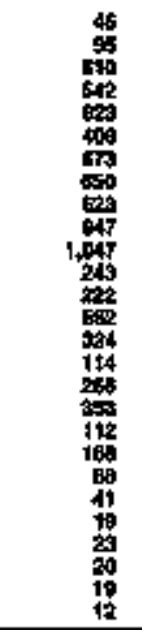 & 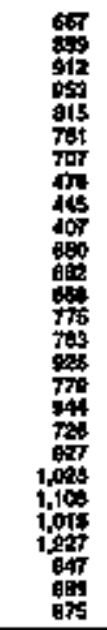 & 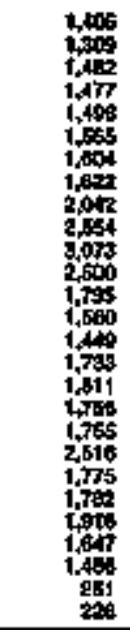 & 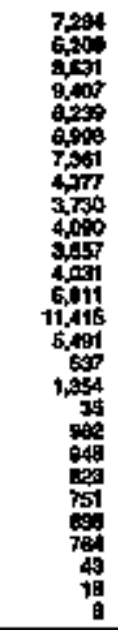 & 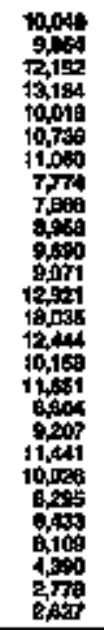 & 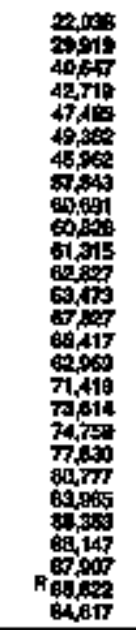 & $\begin{array}{l}= \\
= \\
z \\
= \\
= \\
= \\
= \\
= \\
= \\
= \\
= \\
= \\
= \\
= \\
= \\
=\end{array}$ & 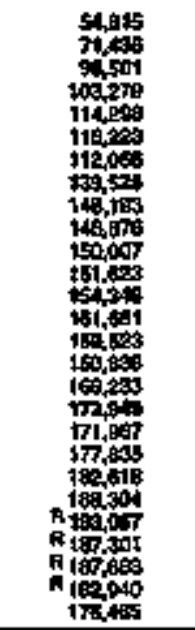 & $\begin{array}{l}= \\
= \\
= \\
= \\
z \\
= \\
= \\
= \\
z \\
= \\
z \\
z \\
=\end{array}$ \\
\hline \multicolumn{15}{|c|}{ 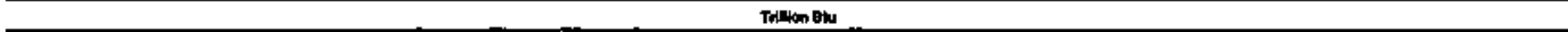 } \\
\hline 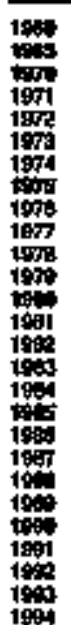 & 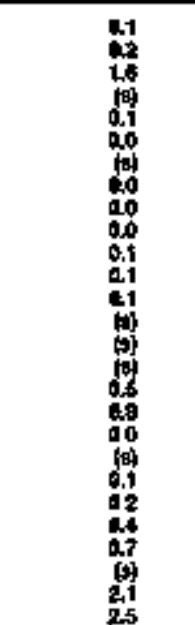 & 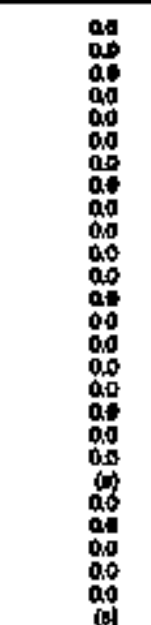 & 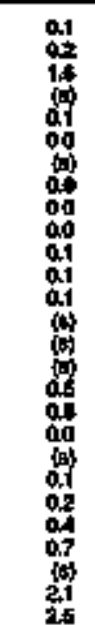 & 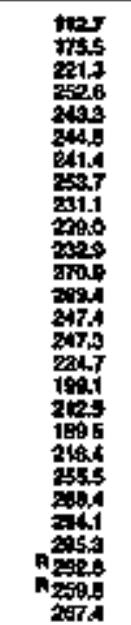 & 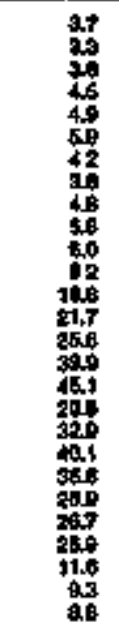 & 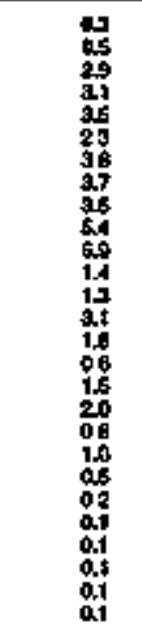 & 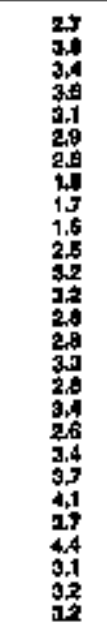 & 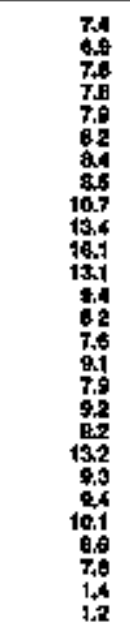 & 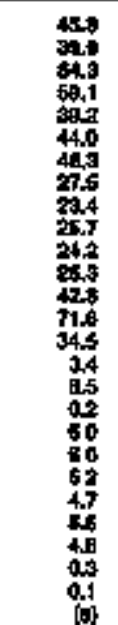 & 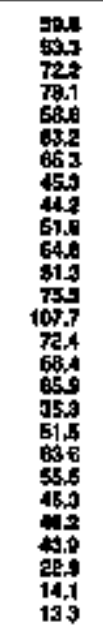 & 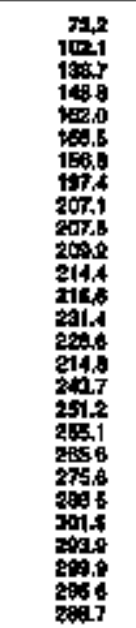 & 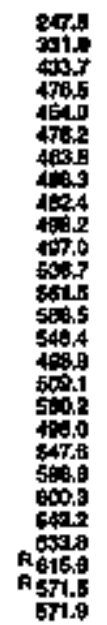 & 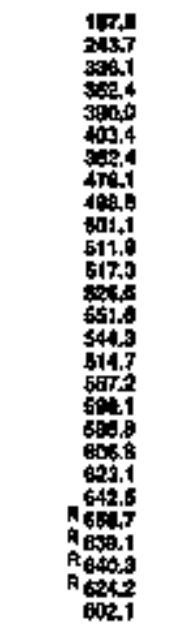 & 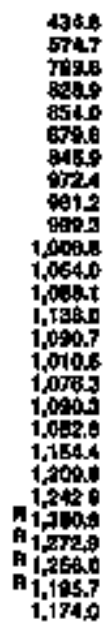 \\
\hline
\end{tabular}

$\mathbf{C}$
$\mathbf{A}$
$\mathbf{L}$
$\mathbf{1}$
$\mathbf{F}$
$\mathbf{O}$
$\mathbf{R}$
$\mathbf{N}$
$\mathbf{L}$
$\mathbf{A}$ 


\begin{tabular}{|c|c|c|c|c|c|c|c|c|c|c|c|c|c|c|c|c|c|c|}
\hline \multirow[b]{2}{*}{ Year } & Eon & ast & \multicolumn{9}{|c|}{ Netrolewn } & 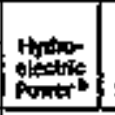 & slower & intert & elowitesty & thespos & \multirow{2}{*}{ 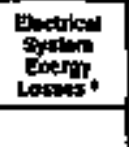 } & \multirow[b]{2}{*}{ Tola } \\
\hline & thentrants & 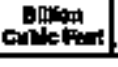 & \multicolumn{9}{|c|}{ Thouthd Buth } & \multicolumn{5}{|c|}{ 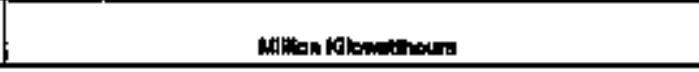 } & & \\
\hline 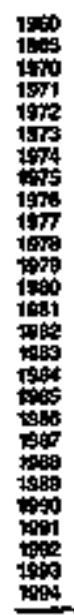 & 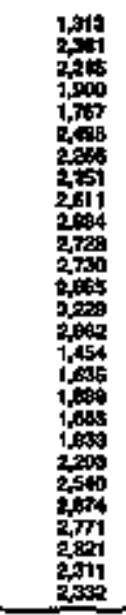 & 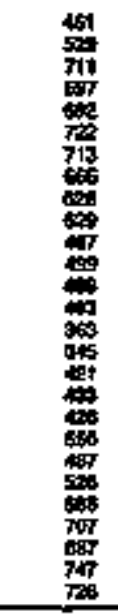 & 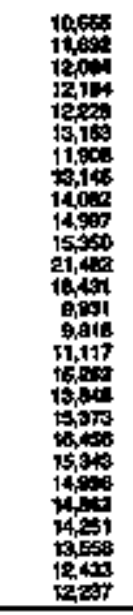 & 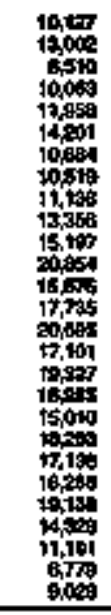 & 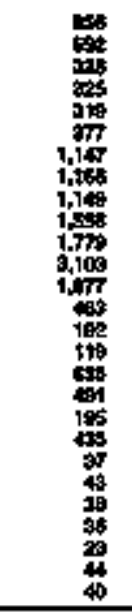 & 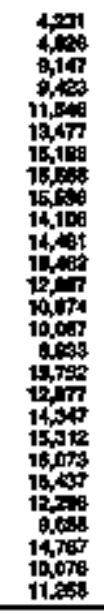 & 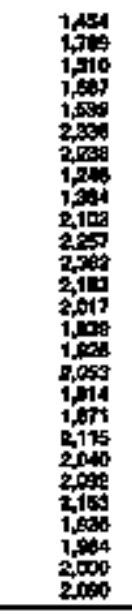 & 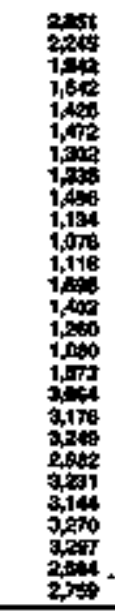 & 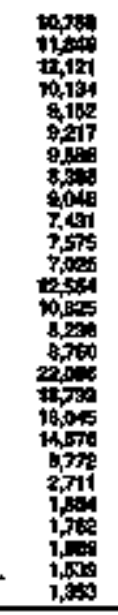 & 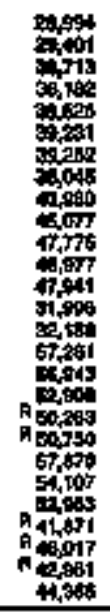 & 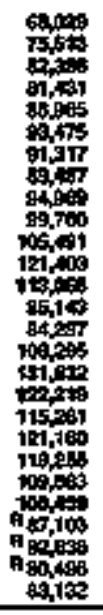 & $\begin{array}{c}8 \\
9 \\
\vdots \\
\vdots \\
\vdots \\
\vdots \\
\vdots \\
\vdots \\
\vdots \\
\vdots \\
\vdots \\
\vdots \\
\vdots \\
\vdots \\
0 \\
0\end{array}$ & 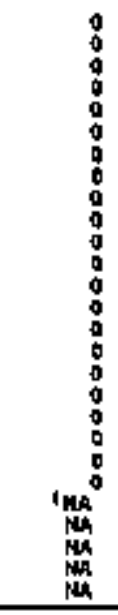 & 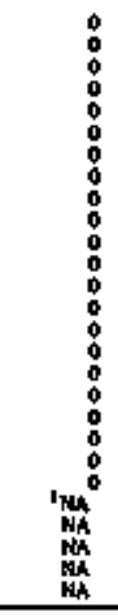 & 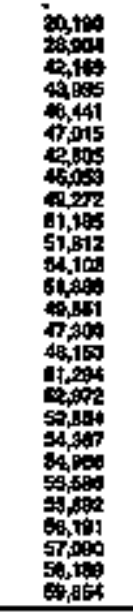 & $\begin{array}{l}z \\
z \\
z \\
z \\
z \\
z \\
z \\
z\end{array}$ & 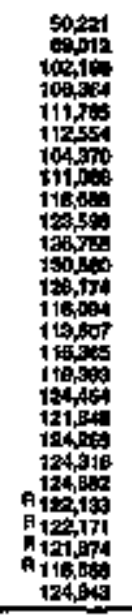 & $\begin{array}{l}z \\
z \\
z \\
z \\
z \\
z \\
z\end{array}$ \\
\hline
\end{tabular}

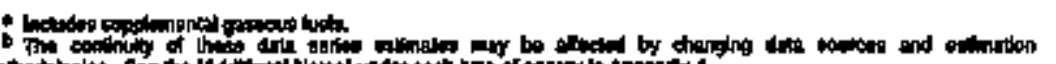

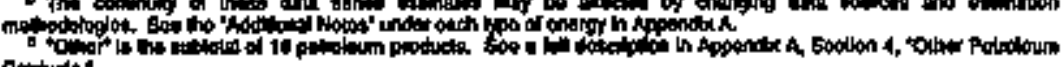
Findet:

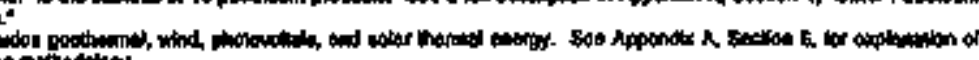

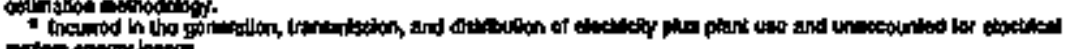

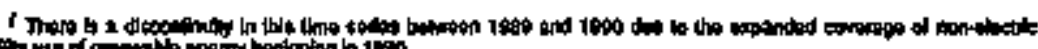

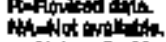

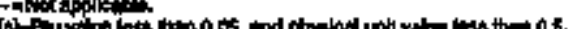


Table 45. Transportetion Energy Consunption Estimeles, 1950, 1965, 1974-1994, Cerfornle

\begin{tabular}{|c|c|c|c|c|c|c|c|c|c|c|c|c|c|c|c|}
\hline \multirow[b]{3}{*}{$k_{w}$} & \multirow[b]{2}{*}{$\cos 1$} & \multirow[b]{2}{*}{ Noting } & \multicolumn{8}{|c|}{ Patrolener } & \multirow{3}{*}{ 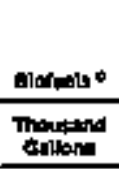 } & \multirow[b]{2}{*}{ Boctikety " } & \multirow[b]{2}{*}{ Enit } & \multirow[b]{2}{*}{ 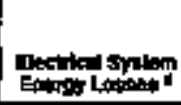 } & \multirow[b]{3}{*}{ Tato } \\
\hline & & & 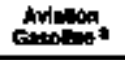 & andide & . & $\operatorname{Leg} \cdot$ & uneriemas a & Gaterin & Resilenal & Total & & & & & \\
\hline & Thent rond & cuttian Fer & \multicolumn{8}{|c|}{ Thooland Gurd: } & & \multicolumn{3}{|c|}{ 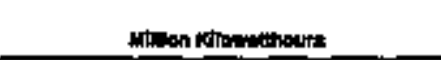 } & \\
\hline 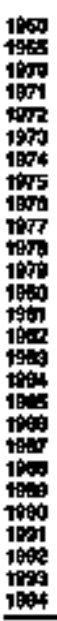 & $\begin{array}{r}29 \\
0 \\
4 \\
3 \\
2 \\
2 \\
1 \\
01 \\
09 \\
0 \\
0 \\
0 \\
0 \\
0 \\
0 \\
0 \\
0 \\
0 \\
0 \\
0 \\
0 \\
0 \\
0 \\
0 \\
0 \\
\end{array}$ & 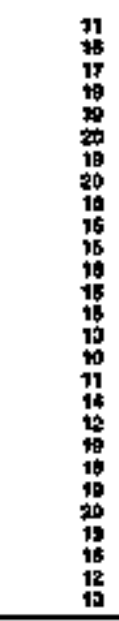 & 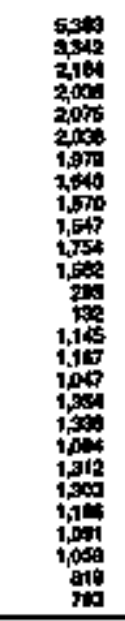 & 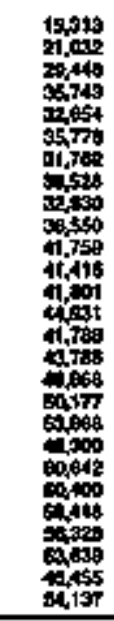 & 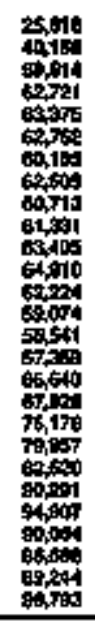 & 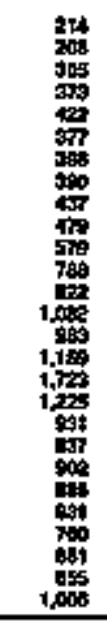 & 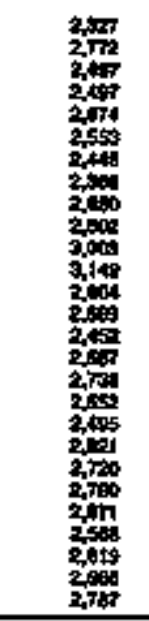 & 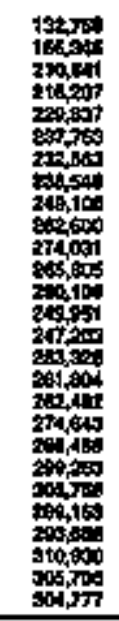 & 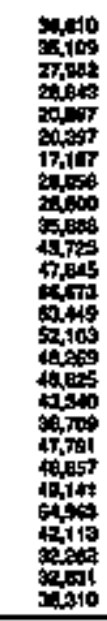 & 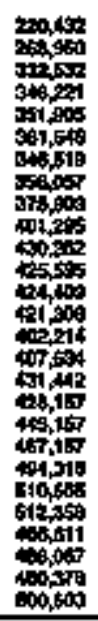 & 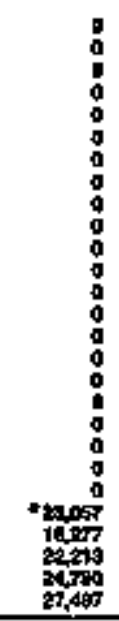 & 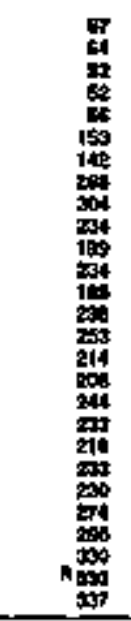 & $\begin{array}{l}= \\
= \\
= \\
= \\
= \\
= \\
= \\
= \\
= \\
= \\
= \\
= \\
= \\
=\end{array}$ & 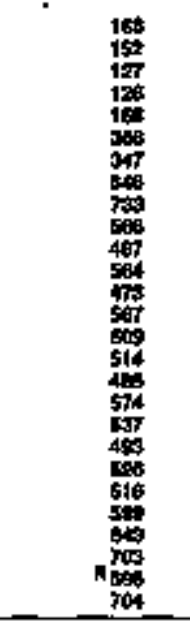 & $\begin{array}{l}= \\
= \\
= \\
= \\
= \\
= \\
= \\
= \\
= \\
= \\
= \\
= \\
= \\
=\end{array}$ \\
\hline \multicolumn{16}{|c|}{ 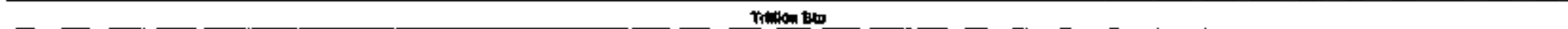 } \\
\hline 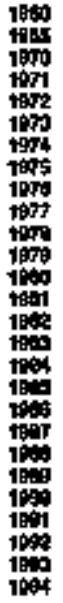 & 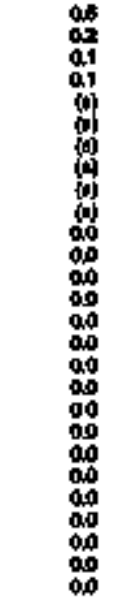 & 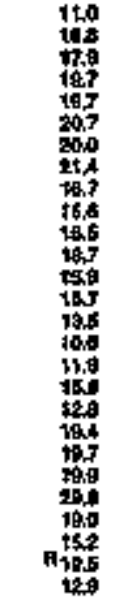 & 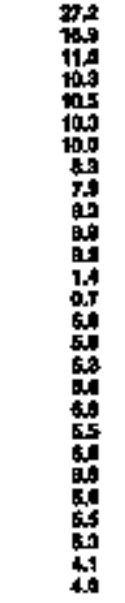 & 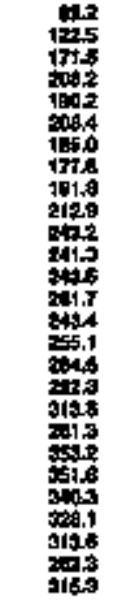 & 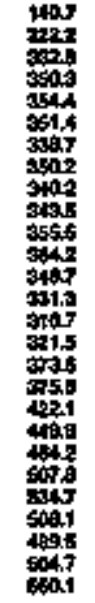 & 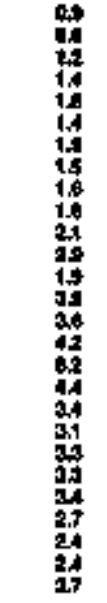 & 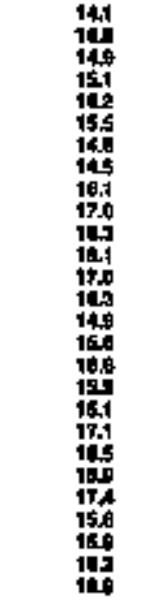 & 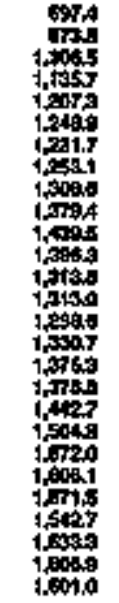 & 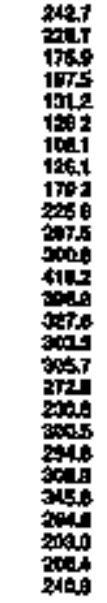 & 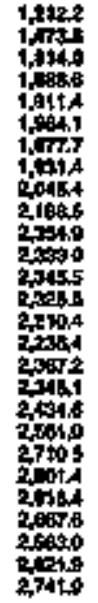 & $\begin{array}{l}00 \\
00 \\
00 \\
00 \\
00 \\
00 \\
00 \\
00 \\
00 \\
00 \\
00 \\
00 \\
00 \\
00 \\
00 \\
00 \\
00 \\
00 \\
00 \\
00 \\
00 \\
00 \\
00 \\
098 \\
74 \\
74 \\
7.9 \\
2.9\end{array}$ & 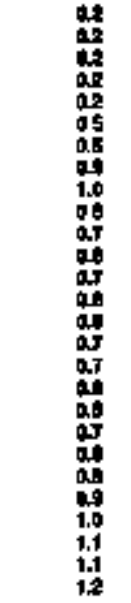 & 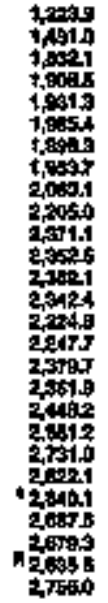 & 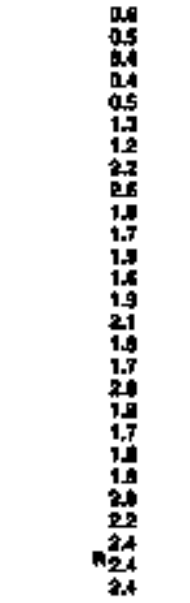 & 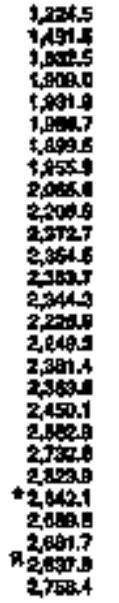 \\
\hline
\end{tabular}

$\mathbf{C}$
$\mathbf{A}$
$\mathbf{L}$
$\mathbf{I}$
$\mathbf{F}$
$\mathbf{O}$
$\mathbf{R}$
$\mathbf{N}$
$\mathbf{I}$
$\mathbf{A}$ 


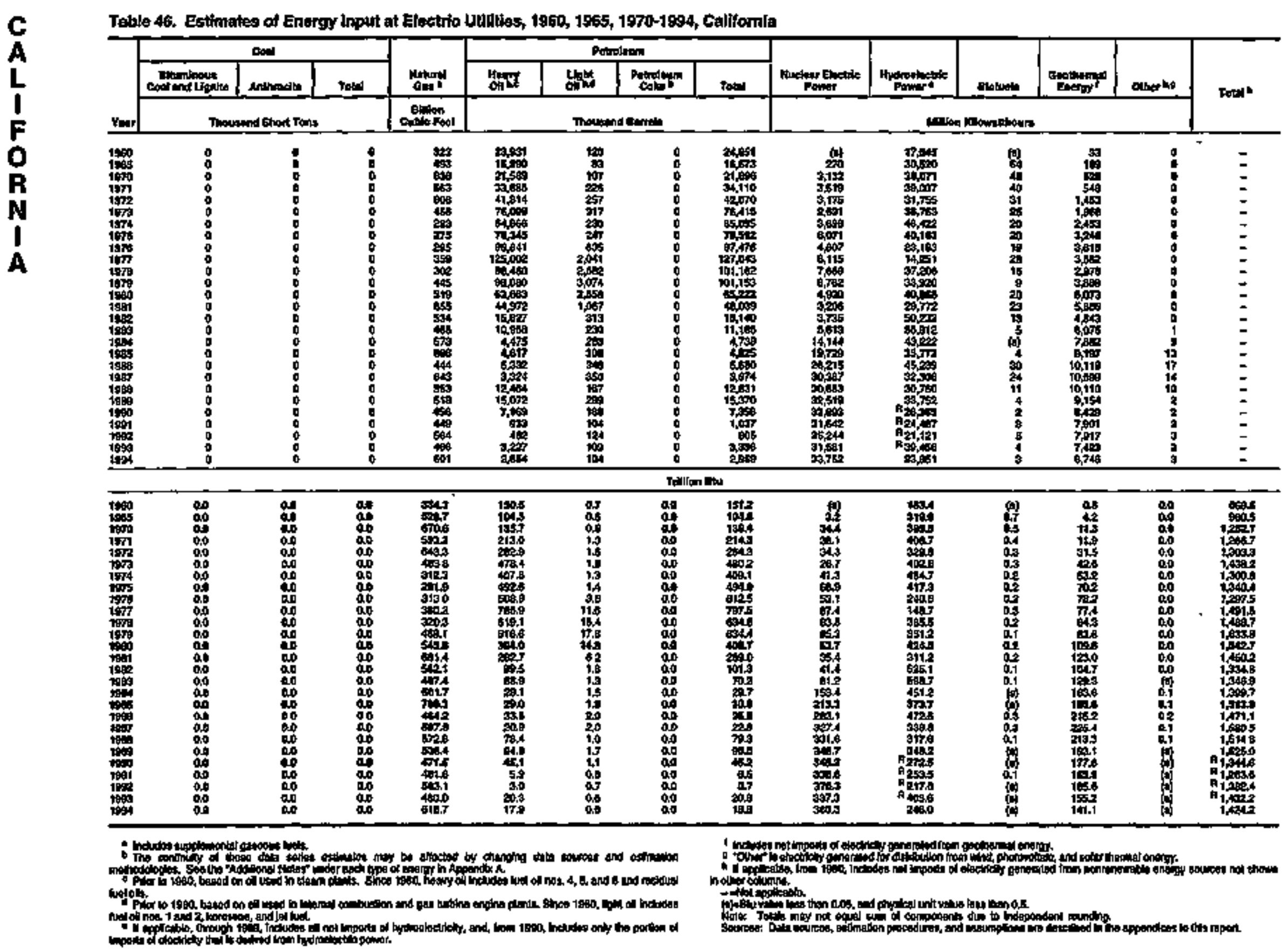




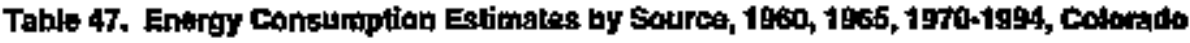

\begin{tabular}{|c|c|c|c|c|c|c|c|c|c|c|c|c|c|c|c|c|c|c|c|}
\hline \multirow[b]{3}{*}{ Der } & \multirow[b]{2}{*}{$\cos 1$} & \multirow[b]{2}{*}{ Mutping } & \multicolumn{11}{|c|}{ Pctrolanen } & \multirow{2}{*}{ 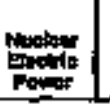 } & \multirow{2}{*}{ 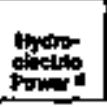 } & \multirow[b]{2}{*}{ Batholet } & \multirow[b]{2}{*}{ 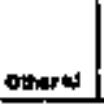 } & \multirow{3}{*}{ 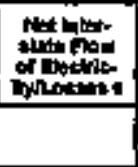 } & \multirow[b]{3}{*}{ Touns } \\
\hline & & & 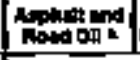 & Antion & Fure & Fit. & 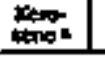 & Luen: & 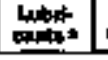 & | & | & oones 4 & Toto & & & & & & \\
\hline & Thore Tod & conterin & \multicolumn{11}{|c|}{ 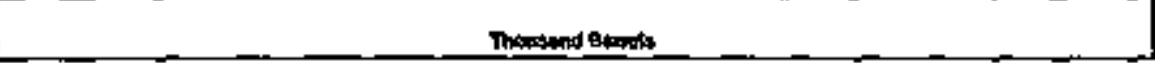 } & \multicolumn{4}{|c|}{ 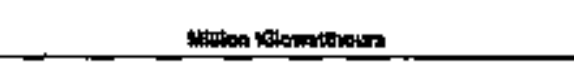 } & & \\
\hline 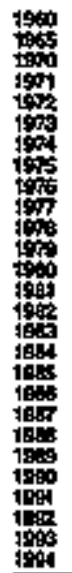 & 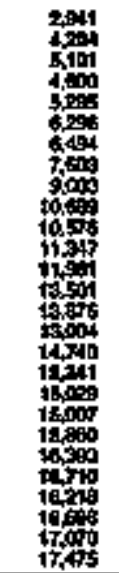 & 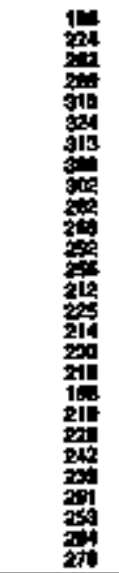 & 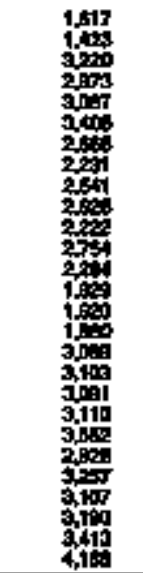 & 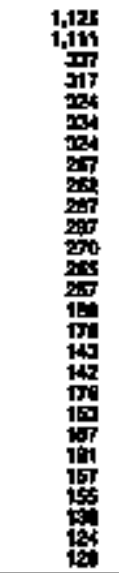 & 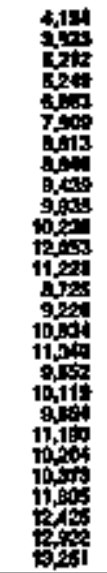 & 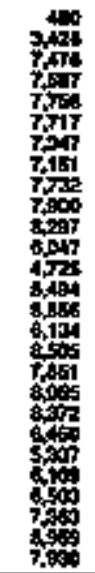 & 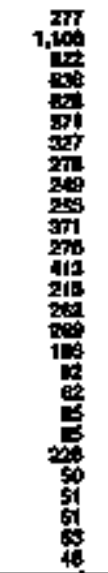 & 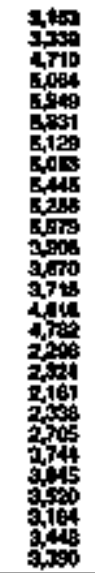 & 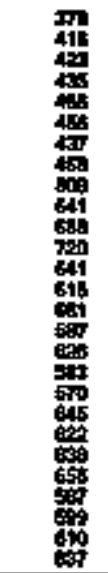 & 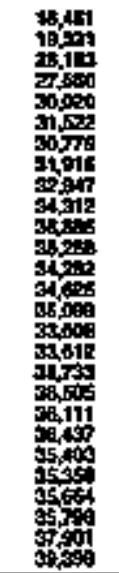 & 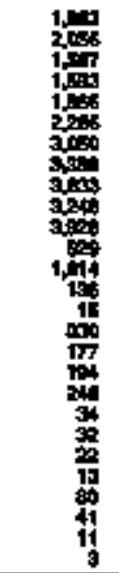 & 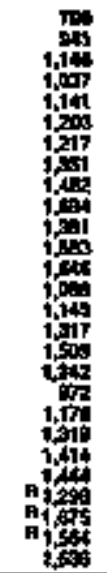 & 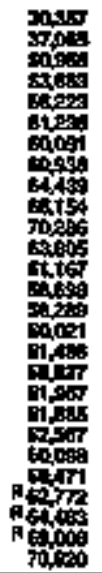 & 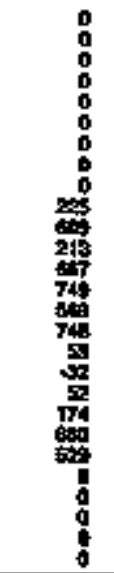 & 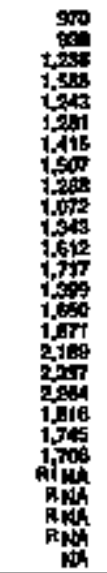 & 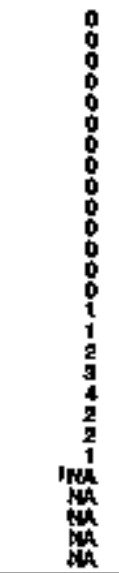 & $\begin{array}{l}0 \\
0 \\
0 \\
0 \\
0 \\
0 \\
0 \\
0 \\
0 \\
0 \\
0 \\
0 \\
0 \\
0 \\
0 \\
0 \\
0 \\
a \\
\text { a } \\
\text { M } \\
\text { M }\end{array}$ & 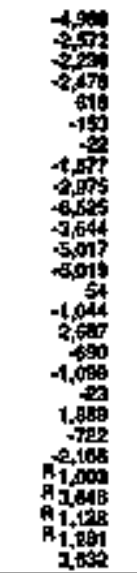 & $\begin{array}{l}E \\
z \\
z \\
z \\
z \\
z\end{array}$ \\
\hline
\end{tabular}

\begin{tabular}{|c|c|c|c|c|c|c|c|c|c|c|c|c|c|c|c|c|c|c|c|}
\hline 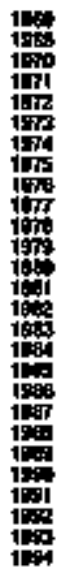 & 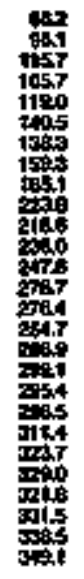 & 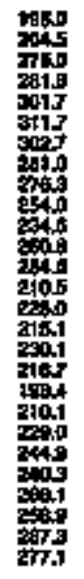 & 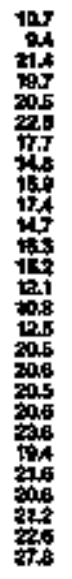 & 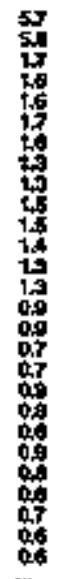 & 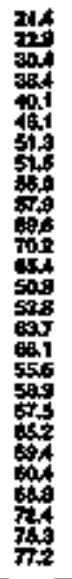 & 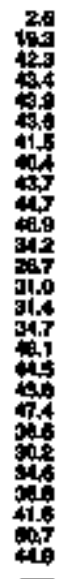 & 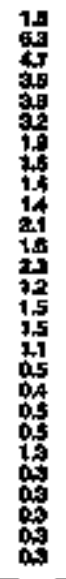 & 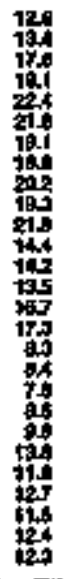 & 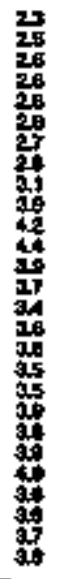 & 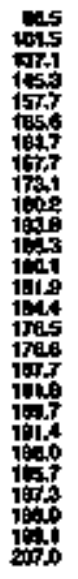 & 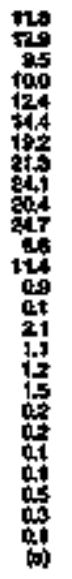 & 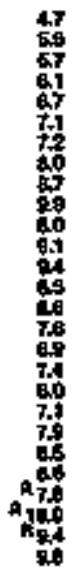 & 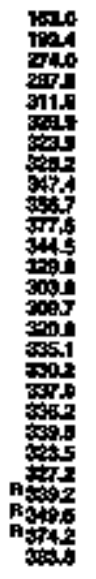 & 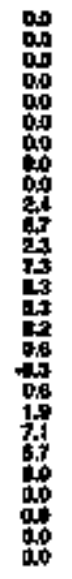 & 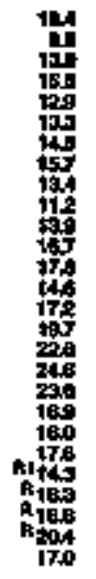 & 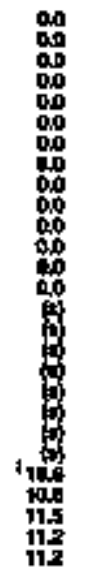 & 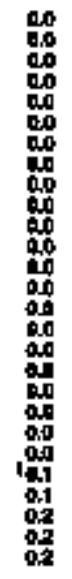 & 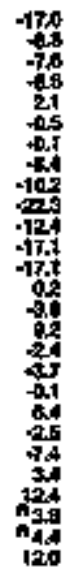 & 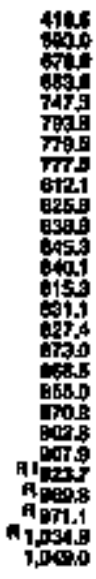 \\
\hline
\end{tabular}

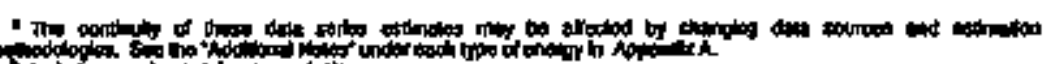

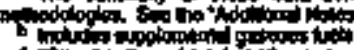

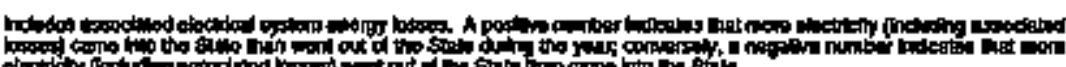

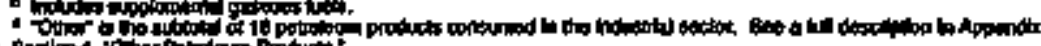

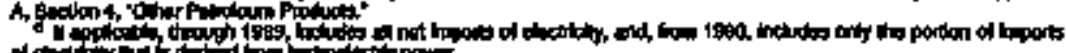

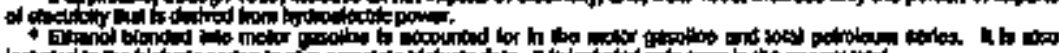

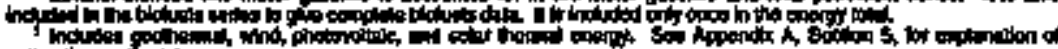

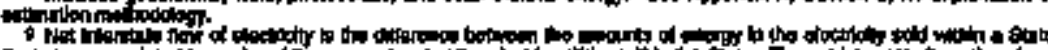

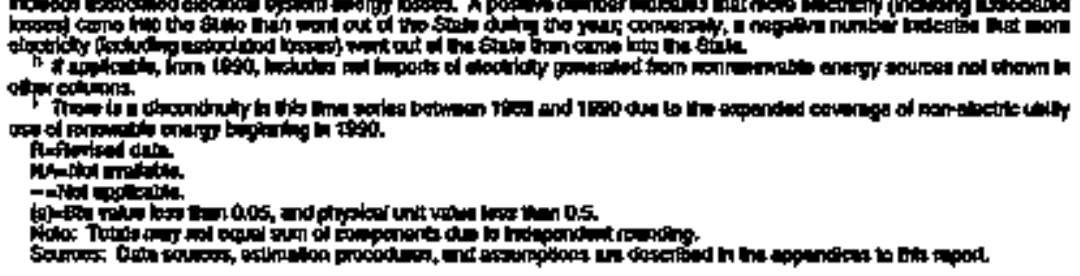
Fion

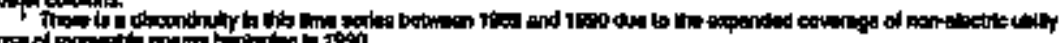
.

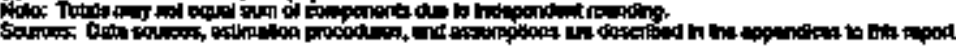


C Table 49. Rusidenlial Entrgy Consumplion Estimates, 1950, 1955, 1970-1994, Colorado

O
L
O
A
D
O

\begin{tabular}{|c|c|c|c|c|c|c|c|c|c|c|c|c|c|c|}
\hline & & Eov & & & & & & & & & & & & \\
\hline & 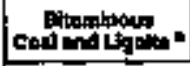 & wethaston: & - tot & Meturi & $\begin{array}{l}\text { Dhetenals } \\
\text { Fued " }\end{array}$ & Morotinto" & LPG: & Toth & Btofun an & Ootar & Eloctitiky & norsy & 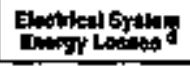 & \\
\hline ratr & Thov & and fleot ton & & chathon Fon & & Thoen: & mats & & $\begin{array}{l}\text { Thevalad } \\
\text { Conts }\end{array}$ & & Natlon & lowathoun & & Total \\
\hline 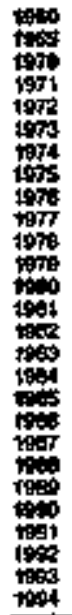 & 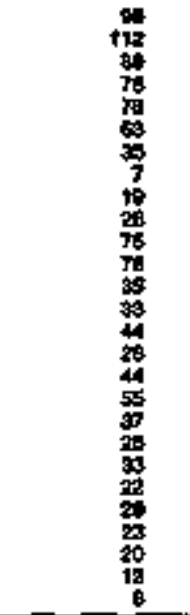 & $\begin{array}{l}0 \\
0 \\
0 \\
0 \\
0 \\
0 \\
0 \\
0 \\
0 \\
0 \\
0 \\
0 \\
0 \\
0 \\
0 \\
0 \\
0 \\
0 \\
0 \\
0 \\
0 \\
0 \\
0 \\
0 \\
0 \\
0 \\
0 \\
0\end{array}$ & 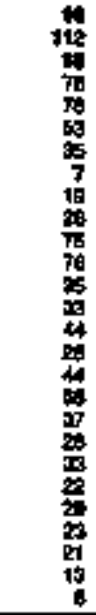 & 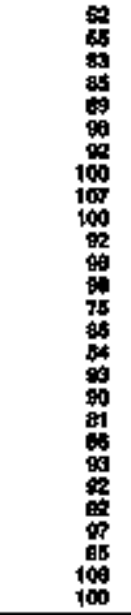 & 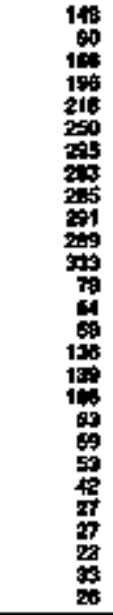 & 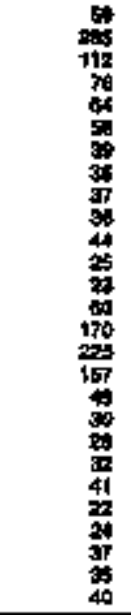 & 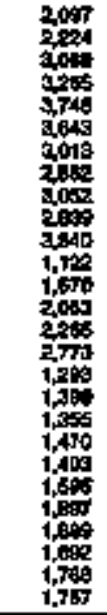 & 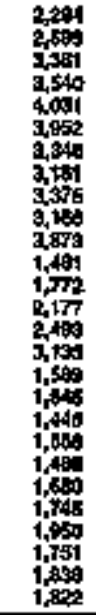 & 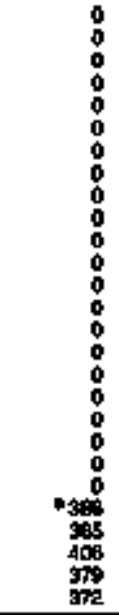 & $\begin{array}{r}0 \\
8 \\
0 \\
0 \\
0 \\
0 \\
0 \\
0 \\
0 \\
0 \\
0 \\
0 \\
0 \\
0 \\
0 \\
0 \\
0 \\
0 \\
0 \\
0 \\
4 \\
48 \\
49 \\
0\end{array}$ & 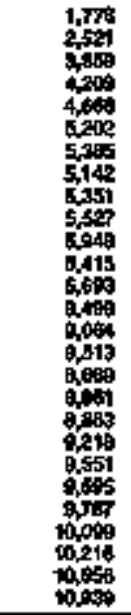 & $\begin{array}{l}z \\
z \\
z \\
z \\
z \\
z \\
= \\
z \\
= \\
= \\
z \\
z \\
z \\
z \\
=\end{array}$ & 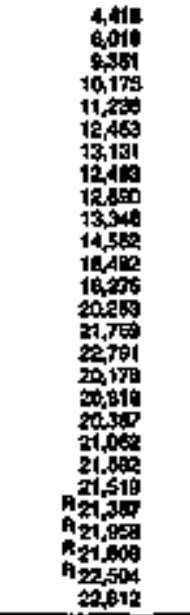 & $\begin{array}{l}\overline{ } \\
\bar{z} \\
\bar{z} \\
\bar{z} \\
\bar{z} \\
\bar{z}\end{array}$ \\
\hline
\end{tabular}

\begin{tabular}{|c|c|c|c|c|c|c|c|c|c|c|c|c|c|c|}
\hline 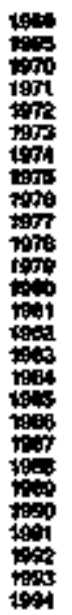 & 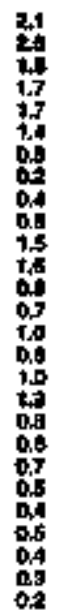 & 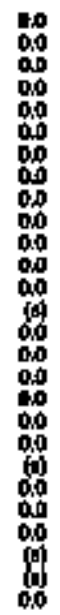 & 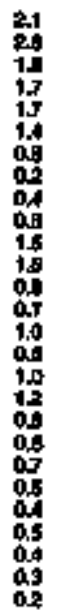 & 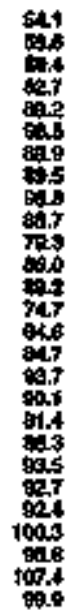 & 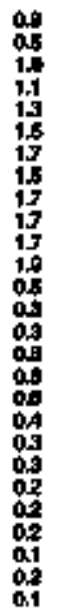 & 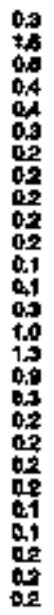 & 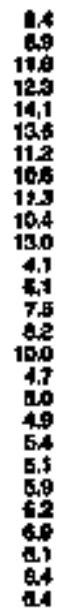 & 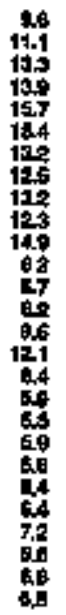 & 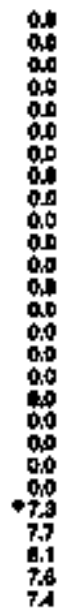 & 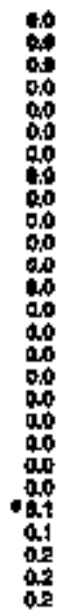 & 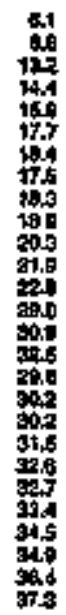 & 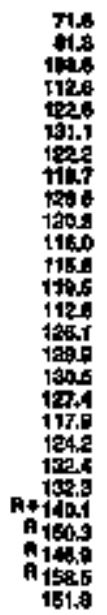 & 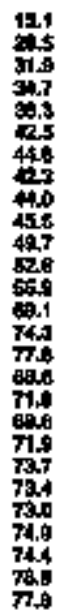 & 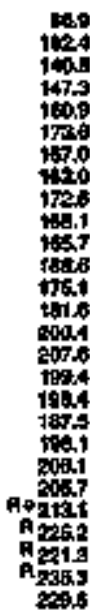 \\
\hline
\end{tabular}

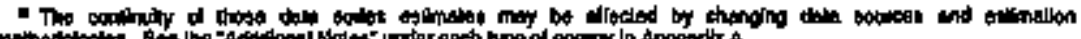

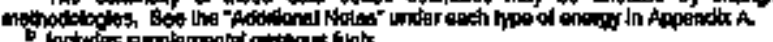

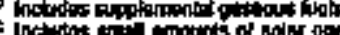

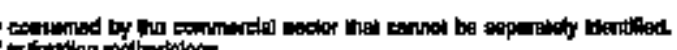

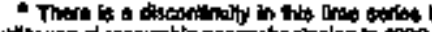

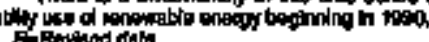

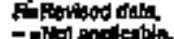

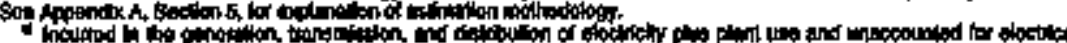

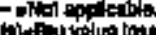

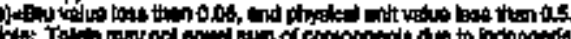

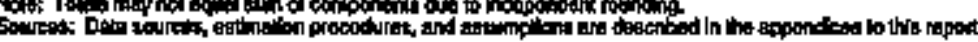


Table 49. Commercial Energy Consumption Estimates, 19:0, 1965, t970-1994, Colorado

\begin{tabular}{|c|c|c|c|c|c|c|c|c|c|c|c|c|c|c|}
\hline \multirow[b]{3}{*}{ Ye: } & \multicolumn{3}{|c|}{ cald } & \multirow{3}{*}{$\frac{c}{\text { Gas: }}$} & \multicolumn{6}{|c|}{ Potrolenn } & \multirow[b]{2}{*}{ Detritehy " } & \multirow[b]{2}{*}{ enert } & \multirow[b]{2}{*}{ 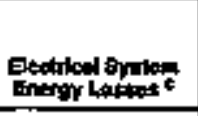 } & \multirow[b]{3}{*}{ Towald } \\
\hline & 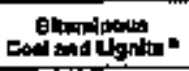 & Andwation: & Totat & & $\begin{array}{l}\text { Duthats } \\
\text { Funt }\end{array}$ & Kercosinox & Lere. & Gotor & $\begin{array}{l}\text { Pajdun } \\
\text { Pend }\end{array}$ & Tow & & & & \\
\hline & \multicolumn{3}{|c|}{ Thouspind shert fons: } & & \multicolumn{6}{|c|}{ Thougend Dorests } & \multicolumn{3}{|c|}{ 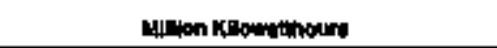 } & \\
\hline 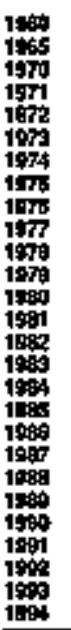 & 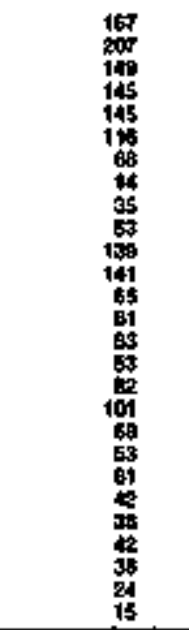 & $\begin{array}{l}0 \\
0 \\
0 \\
0 \\
0 \\
0 \\
0 \\
0 \\
0 \\
0 \\
0 \\
0 \\
0 \\
09 \\
0 \\
0 \\
0 \\
0 \\
0 \\
0 \\
0 \\
0 \\
0 \\
0 \\
0 \\
0\end{array}$ & 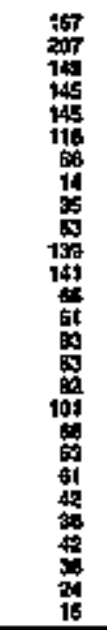 & 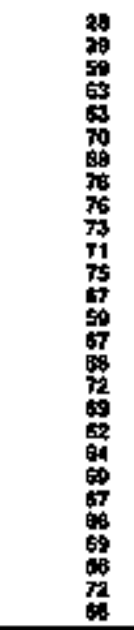 & 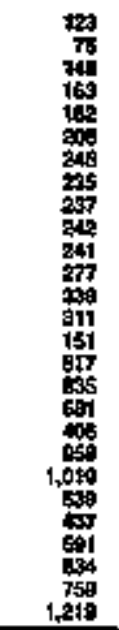 & 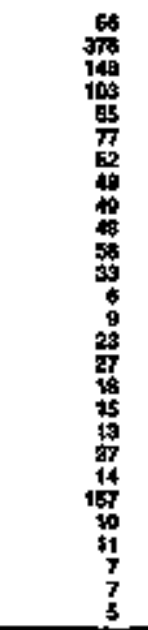 & 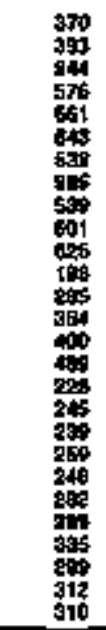 & 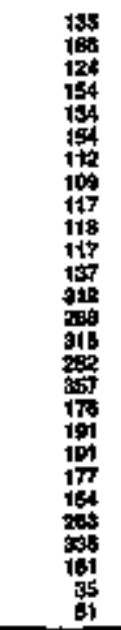 & 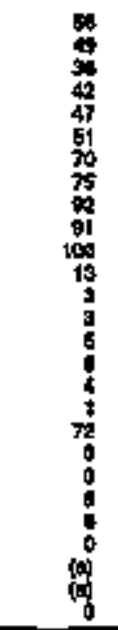 & 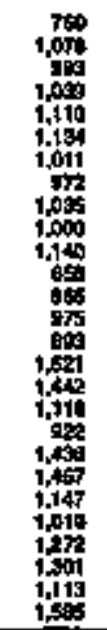 & 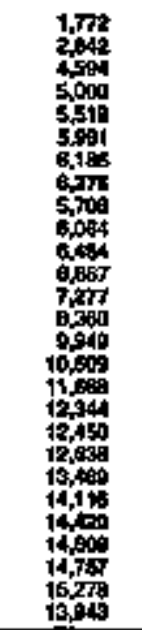 & $\begin{array}{l}= \\
= \\
= \\
= \\
z \\
= \\
= \\
= \\
= \\
z \\
z \\
z \\
z \\
z\end{array}$ & 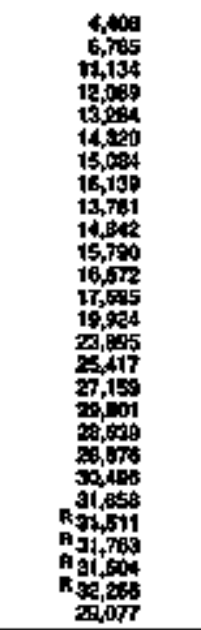 & $\begin{array}{l}= \\
= \\
= \\
= \\
= \\
= \\
= \\
= \\
= \\
= \\
z \\
= \\
= \\
= \\
=\end{array}$ \\
\hline \multicolumn{15}{|c|}{ Tfllon Eid } \\
\hline 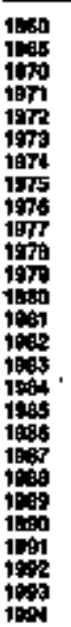 & 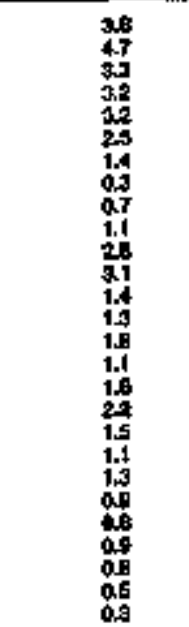 & 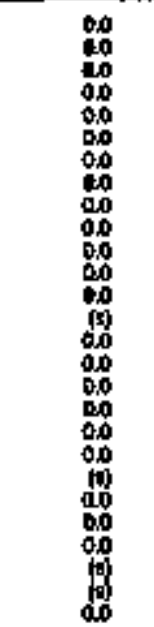 & 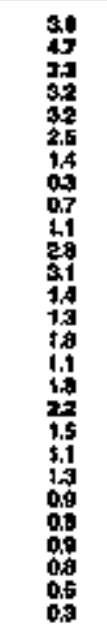 & 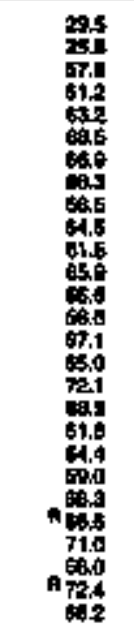 & 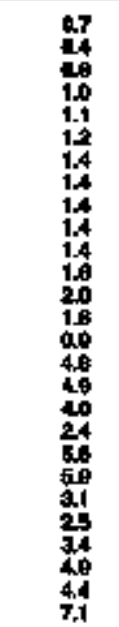 & 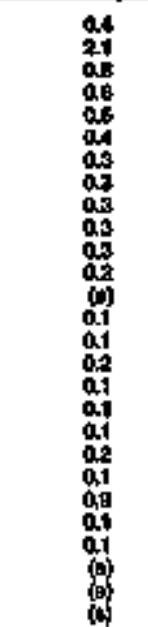 & 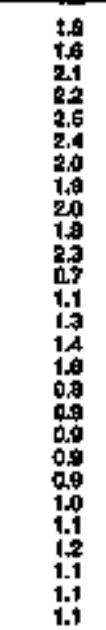 & 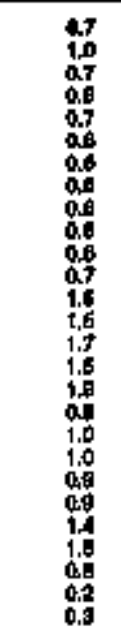 & 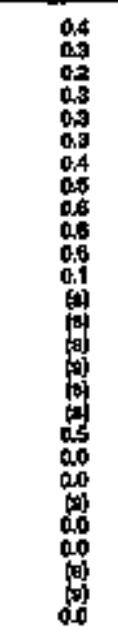 & 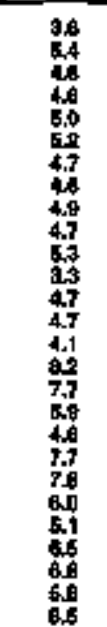 & 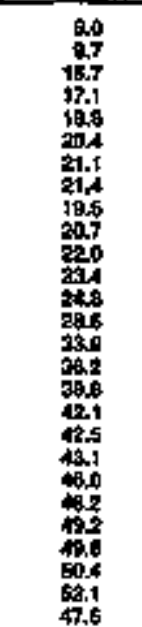 & 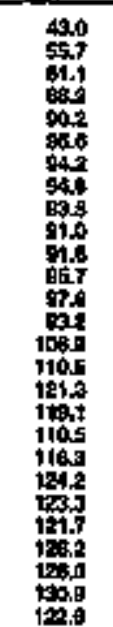 & 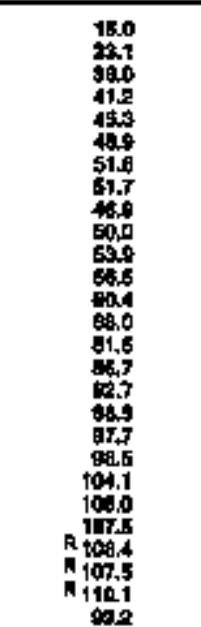 & 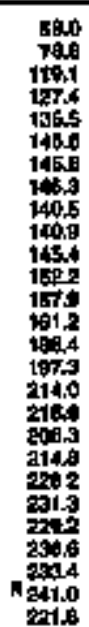 \\
\hline
\end{tabular}

C
O
$\mathbf{L}$
$\mathbf{0}$
$\mathbf{R}$
$\mathbf{A}$
$\mathbf{D}$
$\mathbf{0}$ 


\begin{tabular}{|c|c|c|c|c|c|c|c|c|c|c|c|c|c|c|c|c|c|c|}
\hline \multirow[b]{3}{*}{ Your } & \multirow[b]{2}{*}{ Coste } & \multirow[b]{2}{*}{ anter } & \multicolumn{9}{|c|}{ Potrocounn } & \multirow[b]{2}{*}{ 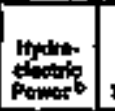 } & \multirow[b]{2}{*}{ Bofines | } & \multirow[b]{2}{*}{ Oener bat } & \multirow[b]{2}{*}{ 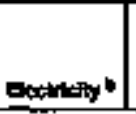 } & \multirow{3}{*}{ 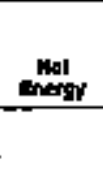 } & \multirow{3}{*}{ 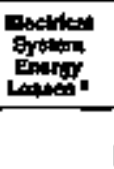 } & \multirow[b]{3}{*}{ Totel } \\
\hline & & & 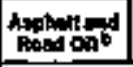 & 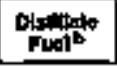 & 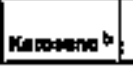 & $\mathbf{L P O}$ & 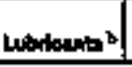 & 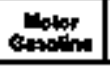 & 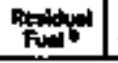 & onaros & 7olv & & & & & & & \\
\hline & Thomenond & 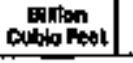 & \multicolumn{9}{|c|}{ 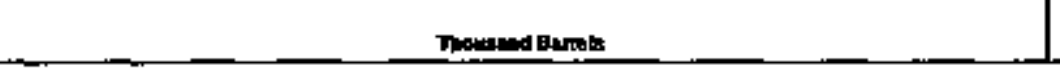 } & \multicolumn{4}{|c|}{ Mplon } & & & \\
\hline 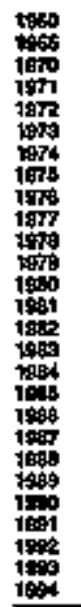 & 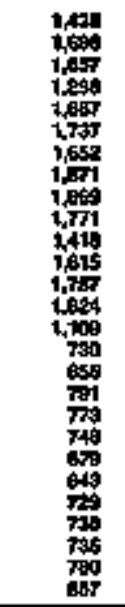 & 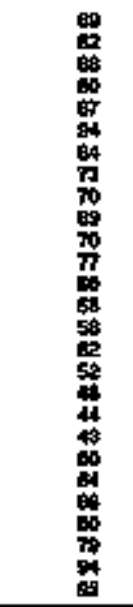 & 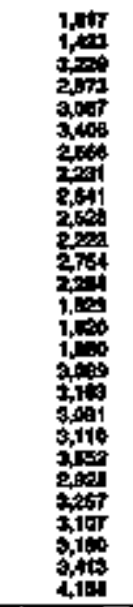 & 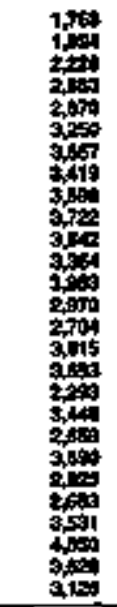 & 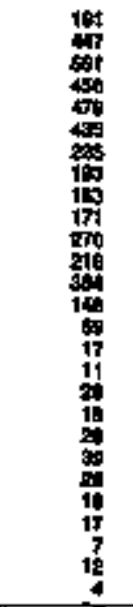 & 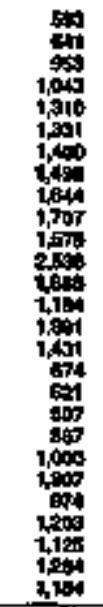 & 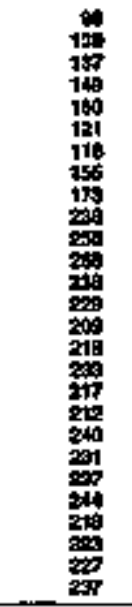 & 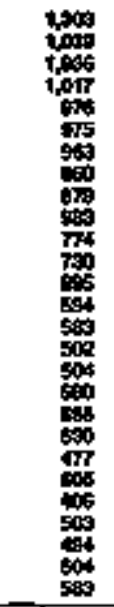 & 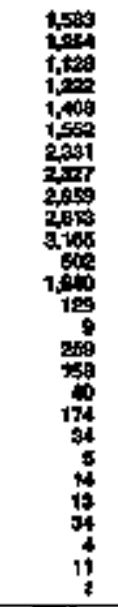 & 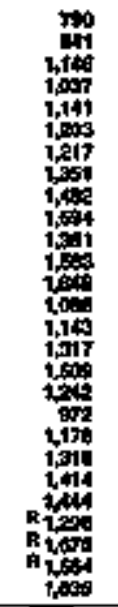 & 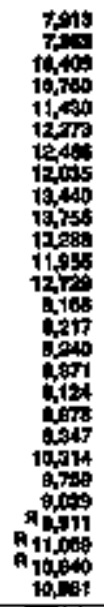 & 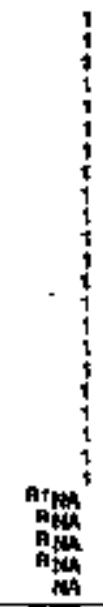 & 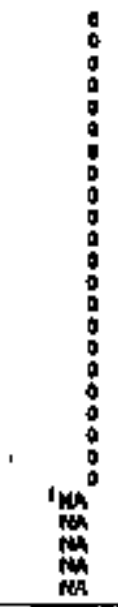 & 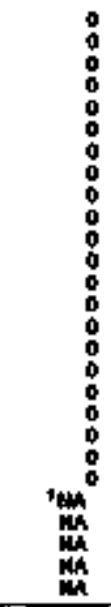 & 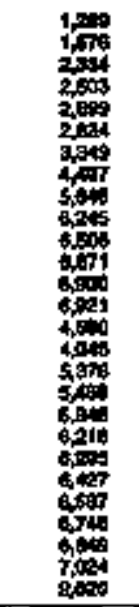 & $\begin{array}{l}\vdots \\
\vdots \\
\vdots \\
\vdots \\
\vdots \\
\vdots \\
\vdots \\
\vdots \\
\vdots \\
\vdots \\
\vdots \\
\vdots \\
\vdots\end{array}$ & 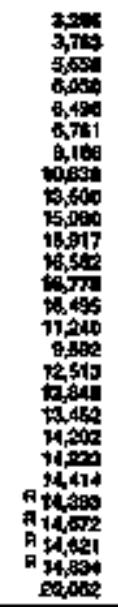 & $\begin{array}{l}\bar{z} \\
\vdots \\
\vdots \\
\vdots \\
\vdots \\
\vdots \\
z \\
\vdots \\
\vdots \\
\vdots \\
\vdots \\
z \\
z \\
z\end{array}$ \\
\hline & & & & & & & & & Then & & & & & & & & & \\
\hline 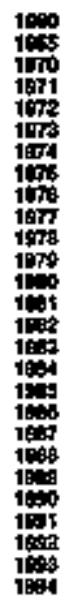 & 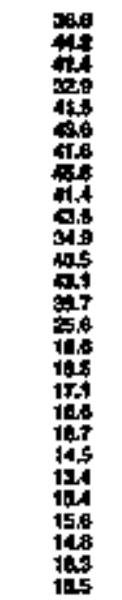 & 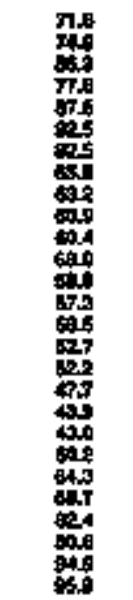 & 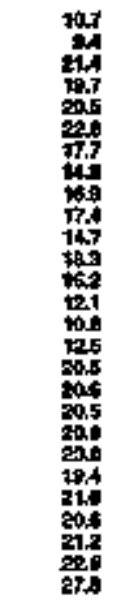 & 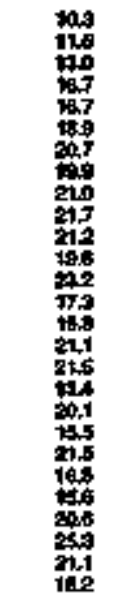 & 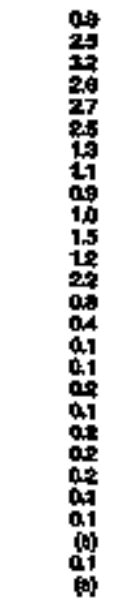 & 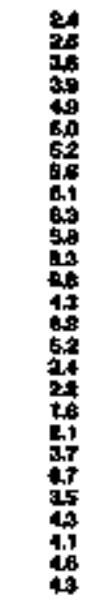 & 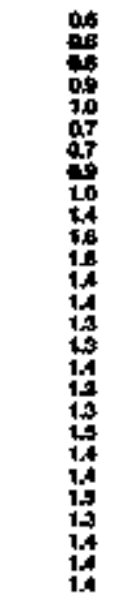 & 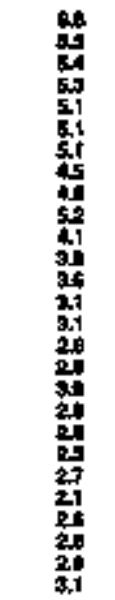 & 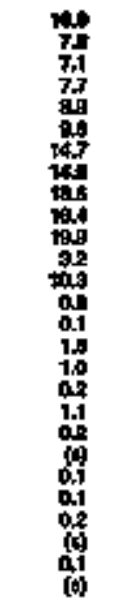 & 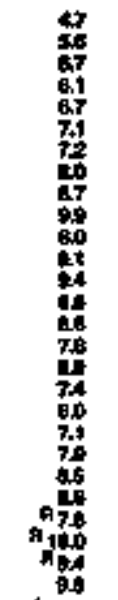 & 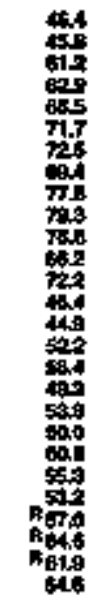 & 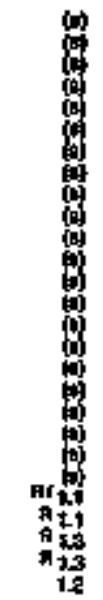 & 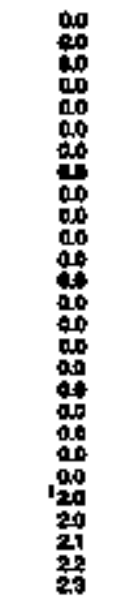 & 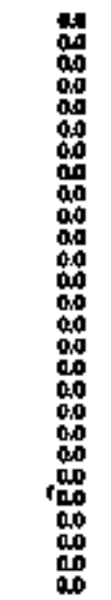 & 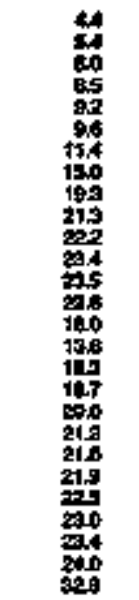 & 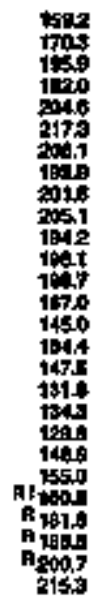 & 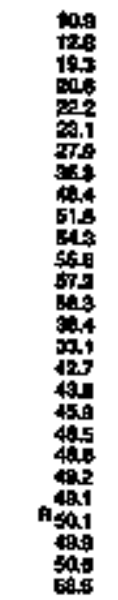 & 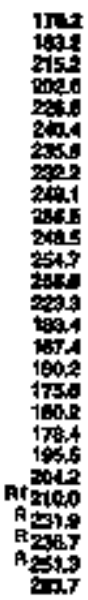 \\
\hline
\end{tabular}

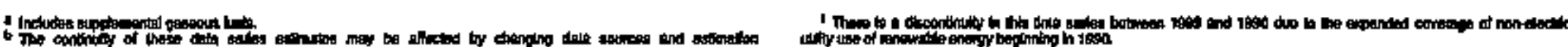

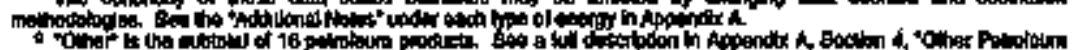
Proguelt: thet

That

Plest

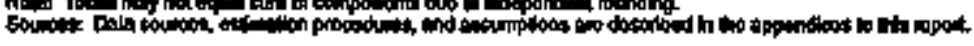

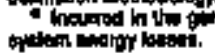


Table 51. Transportation Energy Consumption Estingites, 7960, 1965, 1970-1894, Colorado

\begin{tabular}{|c|c|c|c|c|c|c|c|c|c|c|c|c|c|c|c|}
\hline \multirow[b]{3}{*}{ Yowat } & \multirow[b]{2}{*}{$\cos 1$} & \multirow[b]{2}{*}{ thents } & \multicolumn{8}{|c|}{ Patrotata } & \multirow{3}{*}{ Drollonit: } & & \multirow[b]{2}{*}{ intipt } & \multirow[b]{2}{*}{ 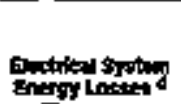 } & \multirow[b]{3}{*}{ Toke } \\
\hline & & & Antons & 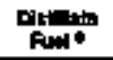 & fo: & Lat & 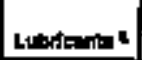 & chowing & Prold & Toa타 & & nostricty" & & & \\
\hline & Thowesad & condon & \multicolumn{8}{|c|}{ 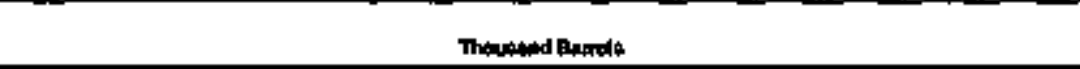 } & & \multicolumn{3}{|c|}{ 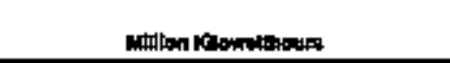 } & \\
\hline 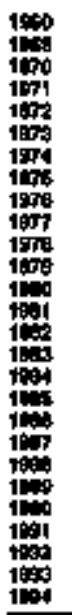 & $\begin{array}{l}3 \\
0 \\
0 \\
0 \\
2 \\
1 \\
1 \\
0 \\
0 \\
0 \\
0 \\
0 \\
0 \\
0 \\
0 \\
0 \\
0 \\
0 \\
0 \\
0 \\
0 \\
0 \\
0\end{array}$ & $\begin{array}{r}1 \\
3 \\
2 \\
2 \\
3 \\
3 \\
2 \\
5 \\
7 \\
7 \\
5 \\
7 \\
7 \\
8 \\
7 \\
7 \\
7 \\
6 \\
17 \\
8 \\
8 \\
10\end{array}$ & 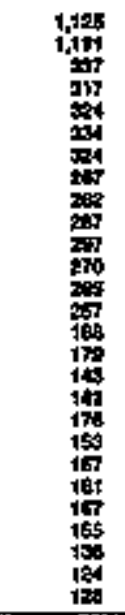 & 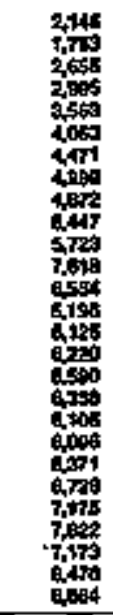 & 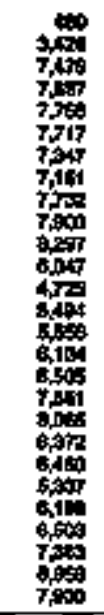 & 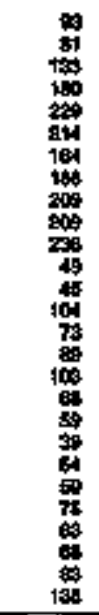 & 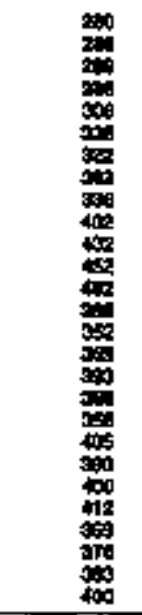 & 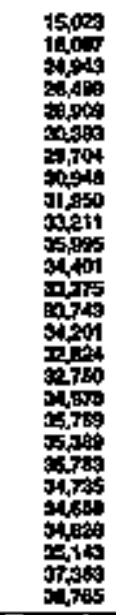 & 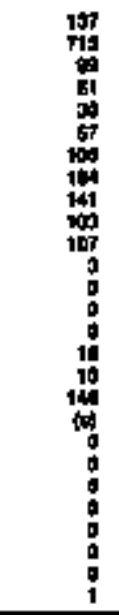 & 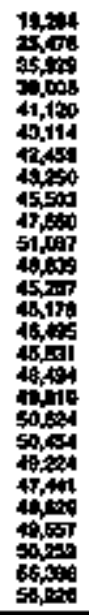 & 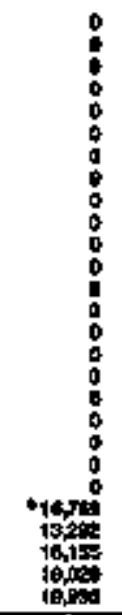 & $\begin{array}{l}0 \\
0 \\
b \\
0 \\
0 \\
0 \\
0 \\
0 \\
0 \\
0 \\
0 \\
0 \\
a \\
0 \\
0 \\
0 \\
0 \\
0 \\
0 \\
0 \\
0 \\
0 \\
a \\
a \\
0 \\
0 \\
0 \\
0 \\
1\end{array}$ & $\begin{array}{l}= \\
= \\
= \\
= \\
= \\
= \\
= \\
= \\
= \\
= \\
= \\
= \\
= \\
=\end{array}$ & $\begin{array}{l}0 \\
: \\
: \\
: \\
0 \\
0 \\
0 \\
0 \\
0 \\
0 \\
0 \\
: \\
0 \\
0 \\
0 \\
0 \\
0\end{array}$ & $\begin{array}{l}z \\
z \\
z \\
z \\
z \\
z \\
z \\
= \\
= \\
z \\
z \\
z \\
z \\
z\end{array}$ \\
\hline \multicolumn{16}{|c|}{ Thomentu } \\
\hline 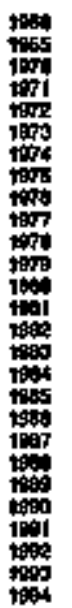 & 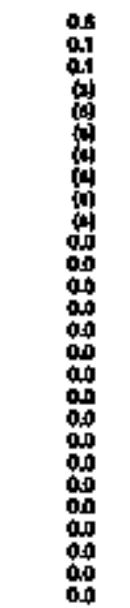 & 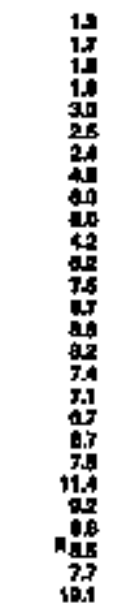 & 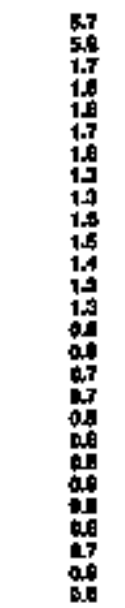 & 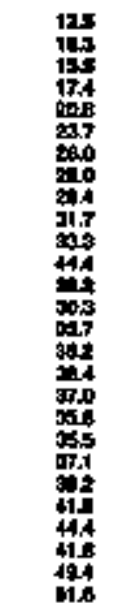 & 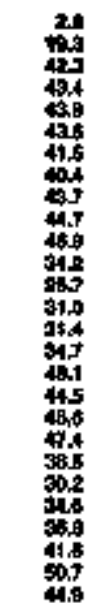 & 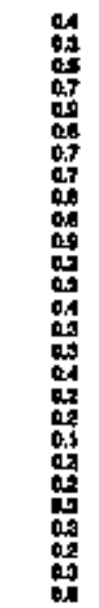 & $\begin{array}{l}13 \\
17 \\
19 \\
17 \\
19 \\
20 \\
20 \\
19 \\
20 \\
24 \\
24 \\
27 \\
24 \\
23 \\
21 \\
22 \\
24 \\
29 \\
28 \\
28 \\
24 \\
24 \\
22 \\
23 \\
23 \\
24\end{array}$ & 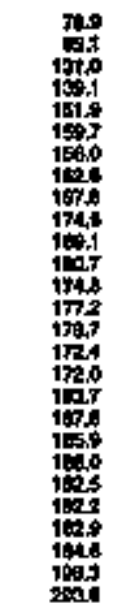 & 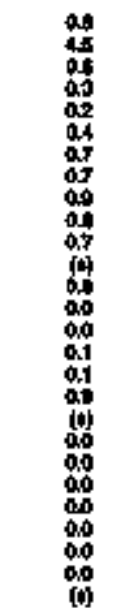 & 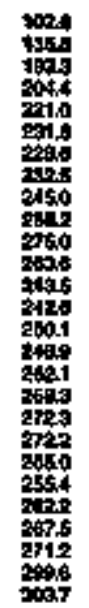 & 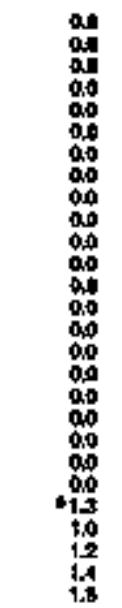 & $\begin{array}{l}00 \\
00 \\
00 \\
00 \\
00 \\
00 \\
00 \\
00 \\
00 \\
00 \\
00 \\
00 \\
00 \\
00 \\
00 \\
00 \\
00 \\
00 \\
00 \\
00 \\
00 \\
00 \\
00 \\
00 \\
00 \\
00 \\
00\end{array}$ & 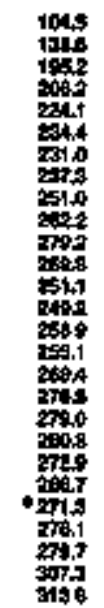 & 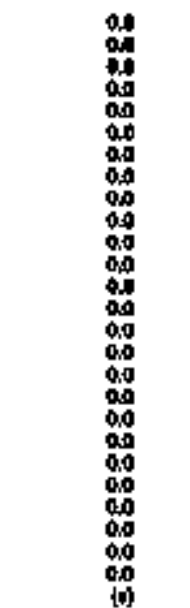 & 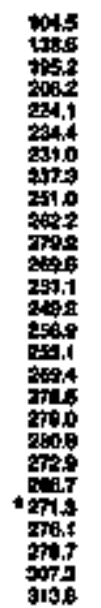 \\
\hline
\end{tabular}

C
L
O
$\mathbf{R}$
$\mathbf{A}$
$\mathbf{D}$
$\mathbf{0}$ 


\begin{tabular}{|c|c|c|c|c|c|c|c|c|c|c|c|c|c|c|}
\hline \multirow{3}{*}{ rear } & \multicolumn{3}{|c|}{ cont } & \multirow[b]{2}{*}{ stown } & \multicolumn{4}{|c|}{ Fellowinn } & \multirow[b]{2}{*}{ 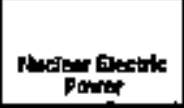 } & \multirow[b]{2}{*}{ Aropolective } & \multirow[b]{2}{*}{ Boweth } & \multirow[b]{2}{*}{ 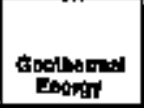 } & \multirow[b]{2}{*}{ Optor ht } & \multirow[b]{3}{*}{ Jotn' } \\
\hline & 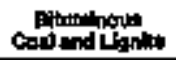 & Anthocis & Tost & & togs & 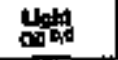 & mothen & Trote & & & & & & \\
\hline & \multicolumn{3}{|c|}{ Thowand Gtont Tome } & mition & \multicolumn{4}{|c|}{ 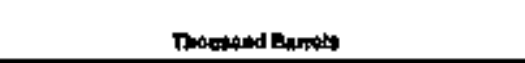 } & \multicolumn{5}{|c|}{ 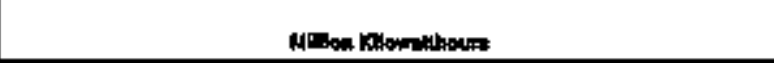 } & \\
\hline 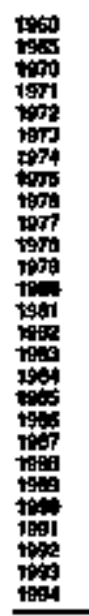 & 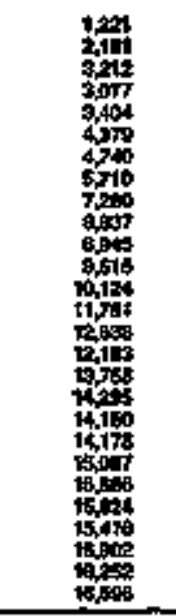 & $\begin{array}{l}0 \\
0 \\
0 \\
0 \\
0 \\
0 \\
0 \\
0 \\
0 \\
0 \\
0 \\
0 \\
0 \\
0 \\
0 \\
0 \\
0 \\
0 \\
0 \\
0 \\
0 \\
0 \\
0 \\
0 \\
0 \\
0 \\
0 \\
0 \\
0 \\
0 \\
0 \\
0 \\
0 \\
\end{array}$ & 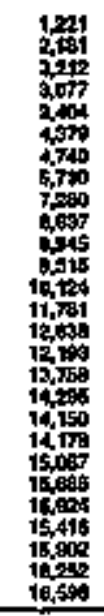 & 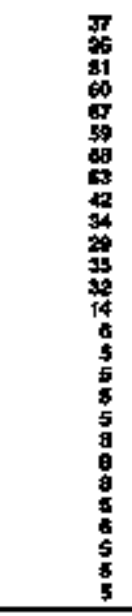 & 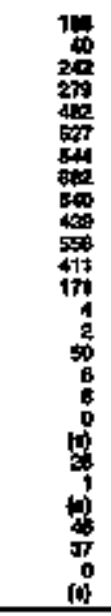 & 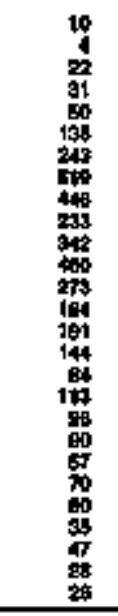 & $\begin{array}{l}: \\
\vdots \\
\vdots \\
\vdots \\
\vdots \\
\vdots \\
\vdots \\
\vdots \\
\vdots \\
0 \\
\vdots \\
\vdots \\
\vdots \\
\vdots \\
\vdots \\
0 \\
0\end{array}$ & 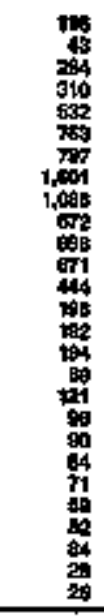 & 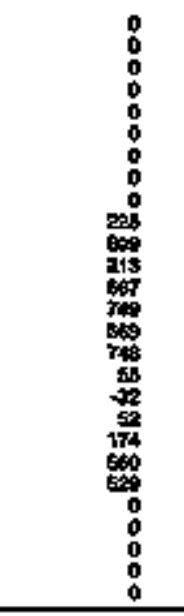 & 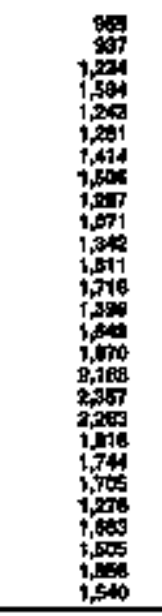 & 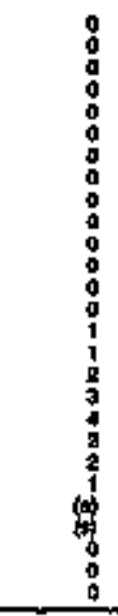 & $\begin{array}{l}0 \\
0 \\
0 \\
0 \\
0 \\
\vdots \\
\vdots \\
\vdots \\
0 \\
0 \\
0 \\
0 \\
0 \\
0 \\
0 \\
0 \\
0 \\
0 \\
0 \\
0 \\
0 \\
0 \\
0 \\
0 \\
0 \\
\end{array}$ & $\begin{array}{l}0 \\
0 \\
0 \\
0 \\
0 \\
0 \\
0 \\
0 \\
0 \\
0 \\
0 \\
0 \\
0 \\
0 \\
0 \\
0 \\
0 \\
0 \\
0 \\
0 \\
0 \\
0 \\
0 \\
0 \\
0 \\
\end{array}$ & $\begin{array}{l}z \\
\vdots \\
z \\
z \\
z \\
\vdots \\
\vdots \\
\vdots \\
\vdots \\
\vdots \\
\vdots \\
z \\
z\end{array}$ \\
\hline 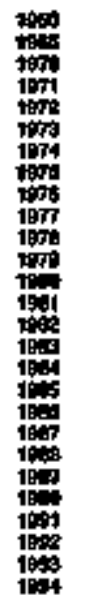 & 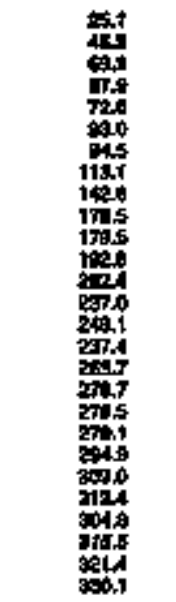 & 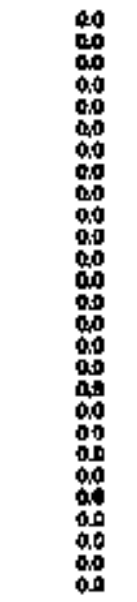 & 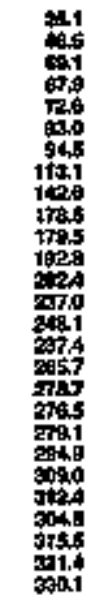 & 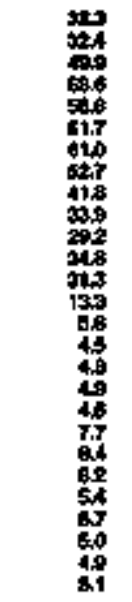 & 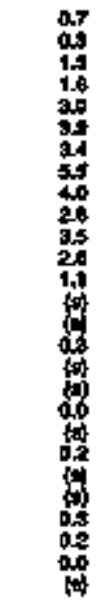 & 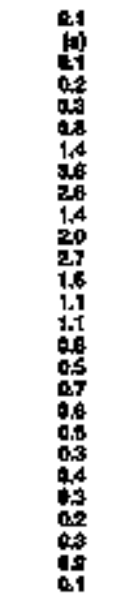 & 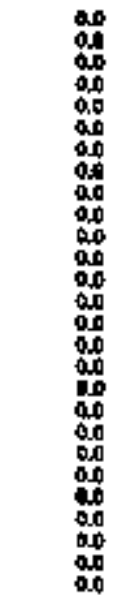 & 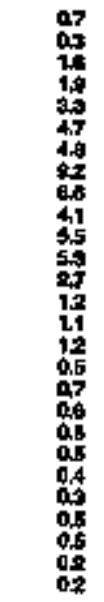 & 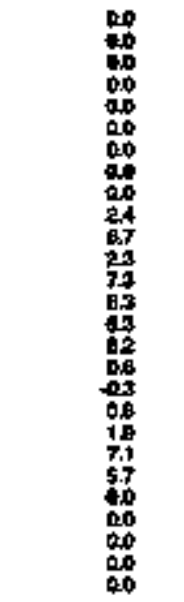 & 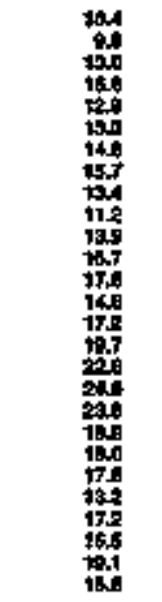 & 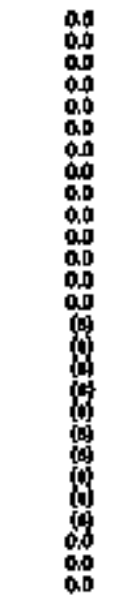 & 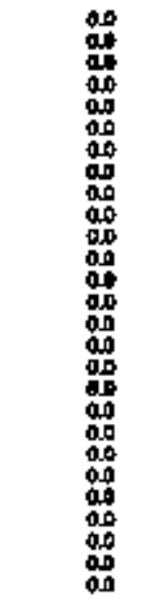 & 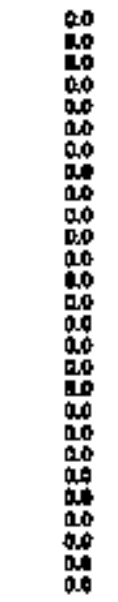 & 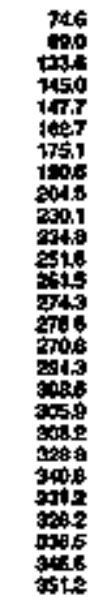 \\
\hline
\end{tabular}




\begin{tabular}{|c|c|c|c|c|c|c|c|c|c|c|c|c|c|c|c|c|c|c|c|}
\hline & \multirow[b]{2}{*}{ Cons } & \multirow[b]{2}{*}{$\operatorname{matan}_{\cos }^{\log }$} & \multicolumn{11}{|c|}{ Mitrowa } & \multirow{2}{*}{ Pover } & \multirow{2}{*}{ 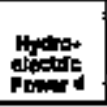 } & \multirow[b]{2}{*}{ nontente. } & \multirow[b]{2}{*}{ Onner | } & \multirow{2}{*}{ 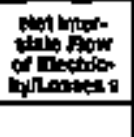 } & \multirow[b]{3}{*}{ Tote리 } \\
\hline & & & 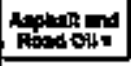 & Amilen: & Daflats: & P. & Kero: & $\operatorname{tog} \times$ & wort. & 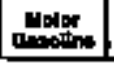 & Fand: & Ooner. & Toted & & & & & & \\
\hline rex & Thont Toud & oulaken & \multicolumn{11}{|c|}{ 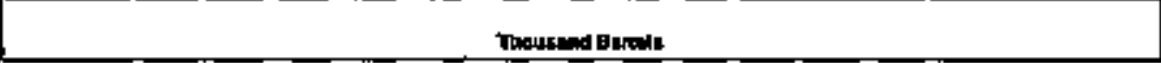 } & \multicolumn{5}{|c|}{ 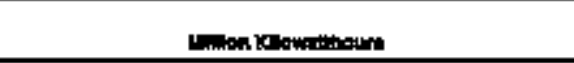 } & \\
\hline 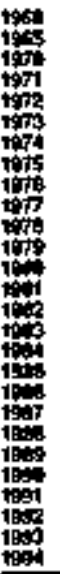 & 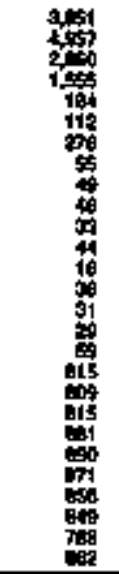 & 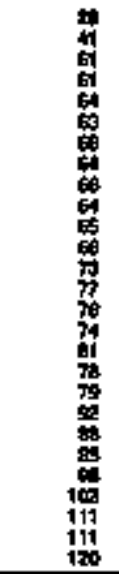 & 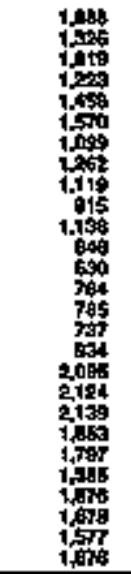 & 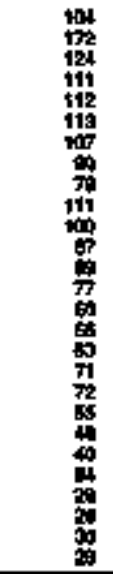 & 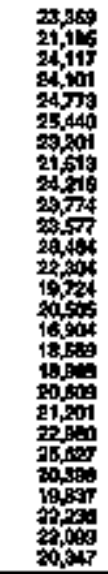 & 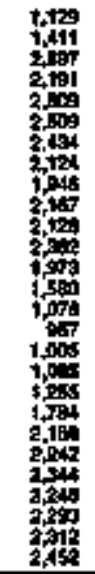 & 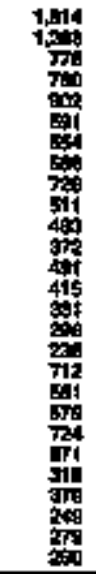 & 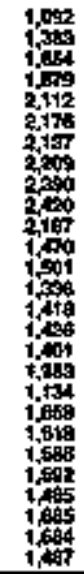 & 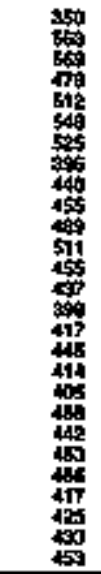 & 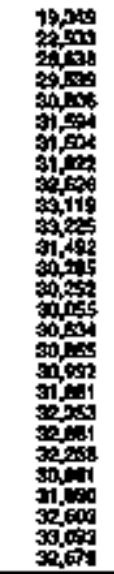 & 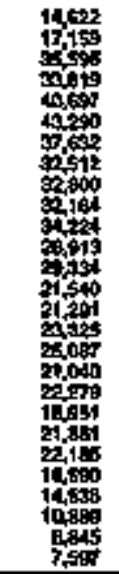 & 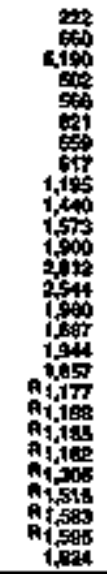 & 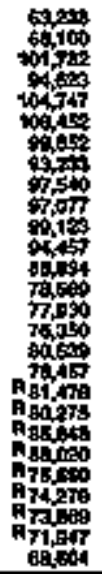 & 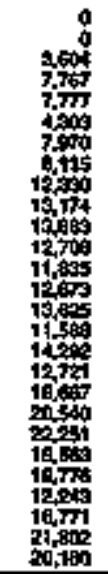 & 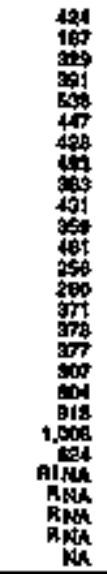 & 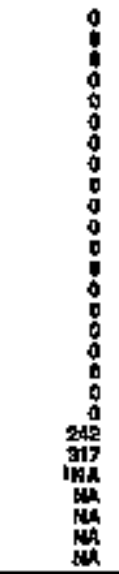 & 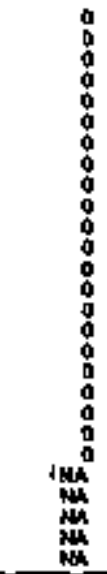 & 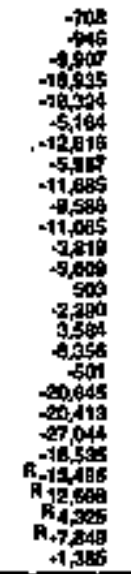 & $\begin{array}{l}\text { z } \\
\text { z }\end{array}$ \\
\hline & & & & & & & & & & THenon aty & & & & & & & & & \\
\hline
\end{tabular}

\begin{tabular}{|c|c|c|c|c|c|c|c|c|c|c|c|c|c|c|c|c|c|c|}
\hline 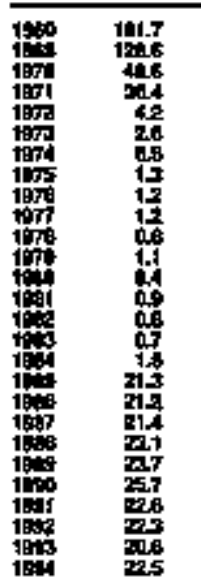 & 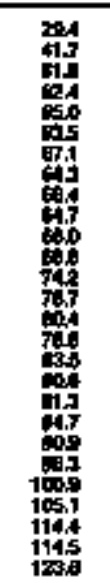 & 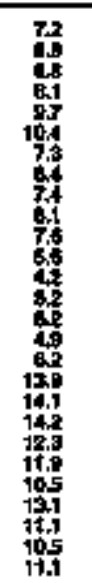 & 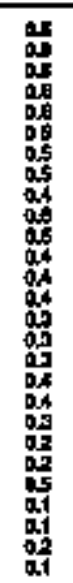 & 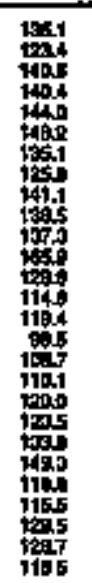 & 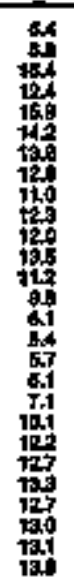 & 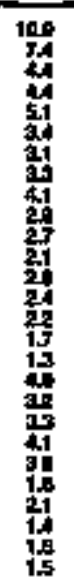 & 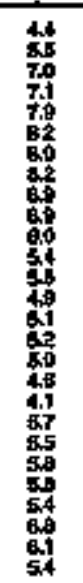 & 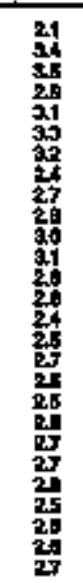 & 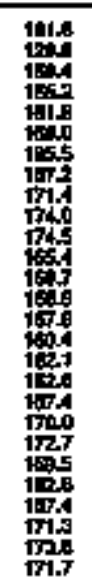 & 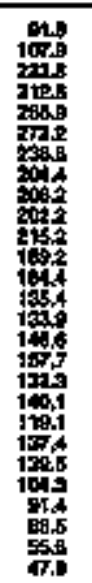 & 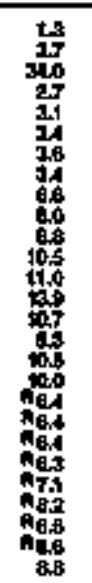 & 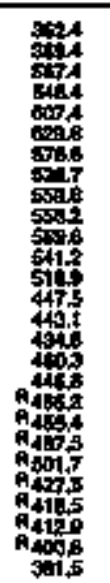 & 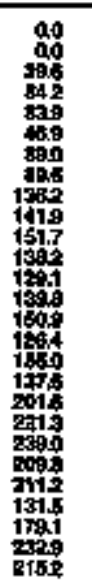 & 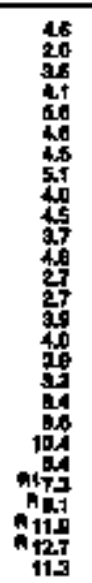 & 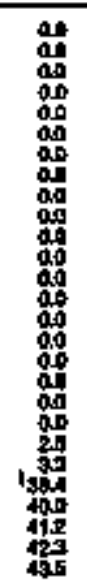 & 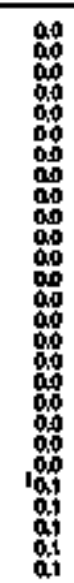 & 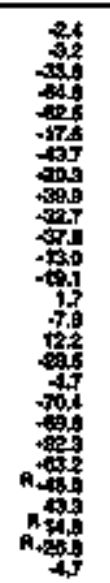 & 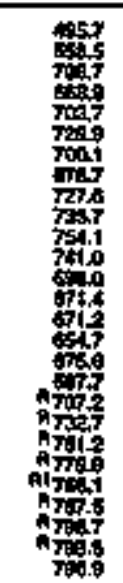 \\
\hline
\end{tabular}

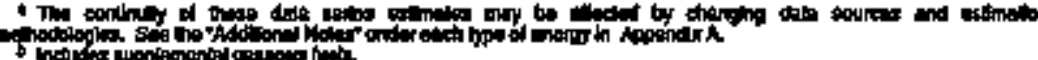

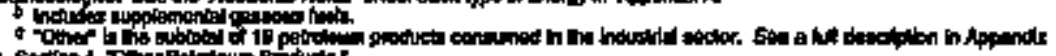

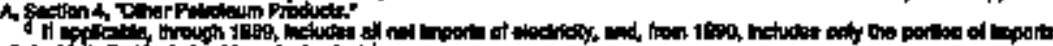

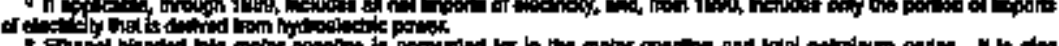

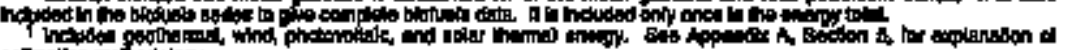

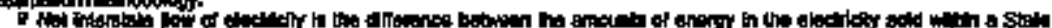

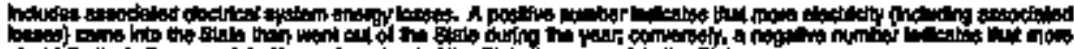

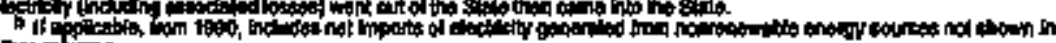

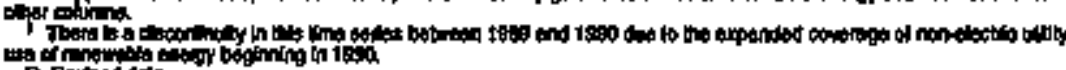

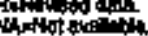

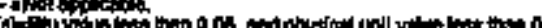

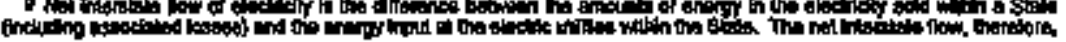




\begin{tabular}{|c|c|c|c|c|c|c|c|c|c|c|c|c|c|c|}
\hline \multirow[b]{3}{*}{ nam } & \multicolumn{3}{|c|}{ cosin } & \multirow{3}{*}{ 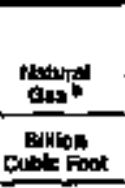 } & \multicolumn{4}{|c|}{ Patrolem } & \multirow{3}{*}{ Dotiven } & \multirow[b]{2}{*}{ Solver } & \multirow[b]{2}{*}{ secentialy: } & \multirow[b]{2}{*}{ trin } & \multirow{3}{*}{ 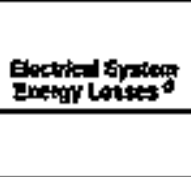 } & \multirow[b]{3}{*}{ Total } \\
\hline & 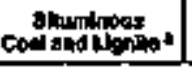 & 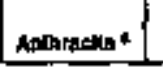 & row & & $\begin{array}{c}\text { Diturusta } \\
\text { Fuet }\end{array}$ & Kcrosenen: & LPo: & Totan & & & & & & \\
\hline & \multicolumn{3}{|c|}{ 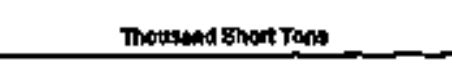 } & & \multicolumn{4}{|c|}{ 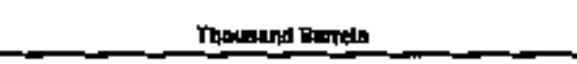 } & & \multicolumn{3}{|c|}{ 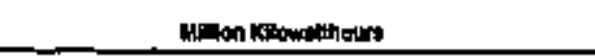 } & & \\
\hline 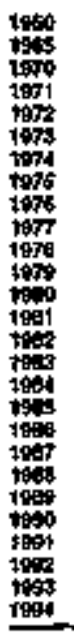 & $\begin{array}{r}20 \\
4 \\
0 \\
0 \\
0 \\
0 \\
0 \\
0 \\
0 \\
0 \\
0 \\
0 \\
0 \\
0 \\
0 \\
0 \\
0 \\
0 \\
0 \\
0 \\
0 \\
0 \\
0 \\
0 \\
3 \\
0 \\
0 \\
0\end{array}$ & 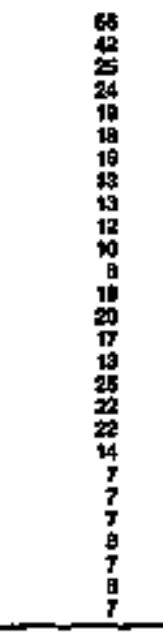 & 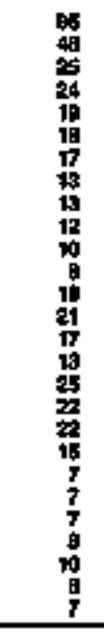 & 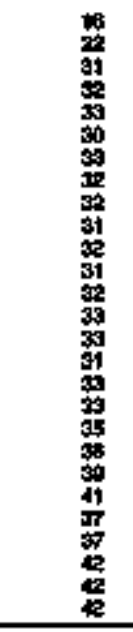 & 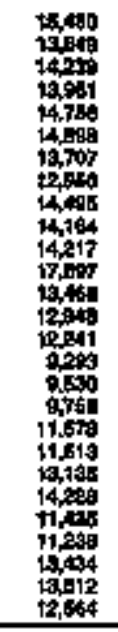 & 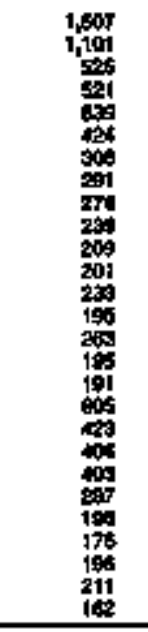 & 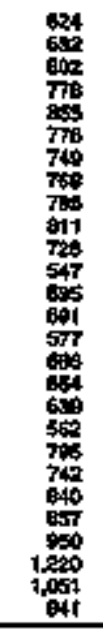 & 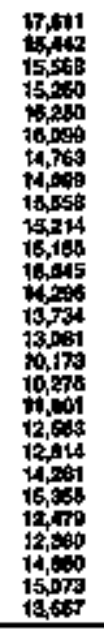 & 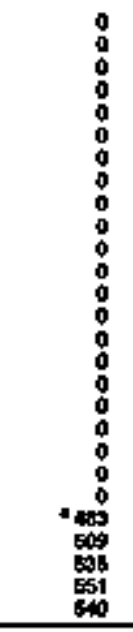 & $\begin{array}{c}0 \\
0 \\
0 \\
0 \\
0 \\
0 \\
0 \\
0 \\
0 \\
0 \\
0 \\
0 \\
0 \\
0 \\
0 \\
0 \\
0 \\
0 \\
0 \\
0 \\
0 \\
0 \\
0 \\
0 \\
+17 \\
10 \\
10 \\
00 \\
3 \\
0\end{array}$ & 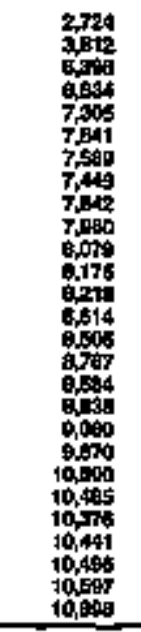 & $\begin{array}{l}\vdots \\
\vdots \\
\vdots \\
\vdots \\
\vdots \\
\vdots \\
\vdots \\
\vdots \\
\vdots \\
\vdots \\
\vdots \\
\vdots \\
\vdots\end{array}$ & 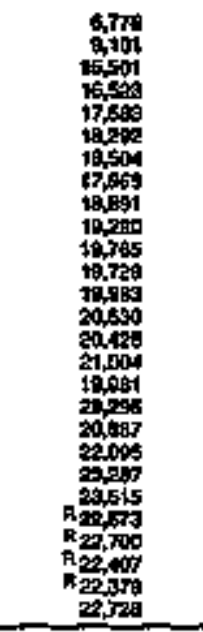 & $\begin{array}{l}\vdots \\
\vdots \\
\vdots \\
\vdots \\
\vdots \\
\vdots \\
\vdots \\
\vdots \\
\vdots \\
\vdots \\
\vdots \\
\vdots \\
\vdots\end{array}$ \\
\hline \multicolumn{15}{|c|}{ Thleon Bux } \\
\hline 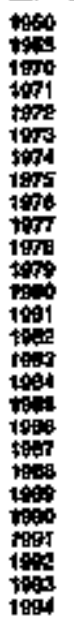 & 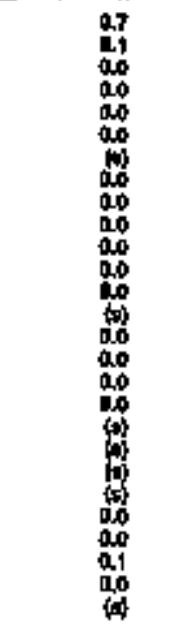 & 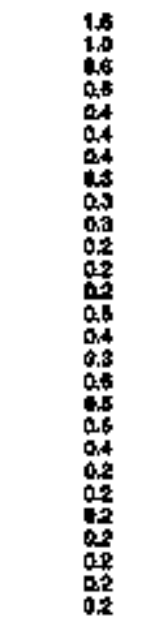 & 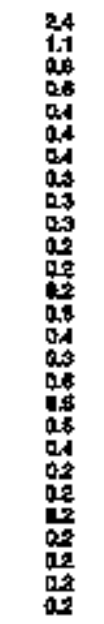 & 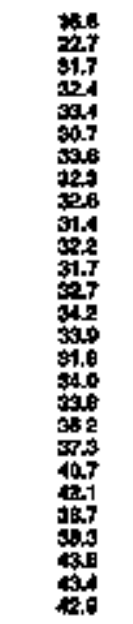 & 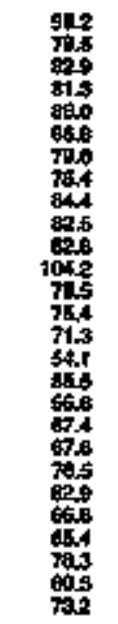 & 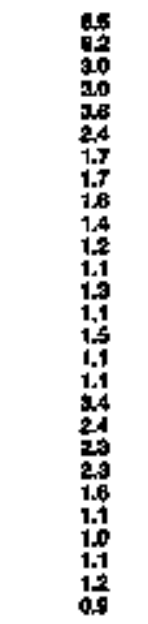 & 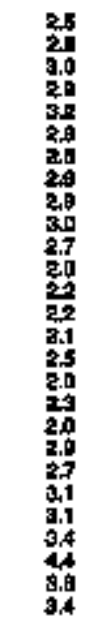 & 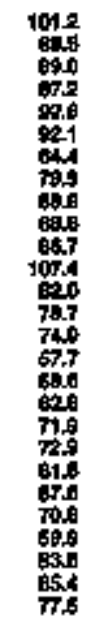 & 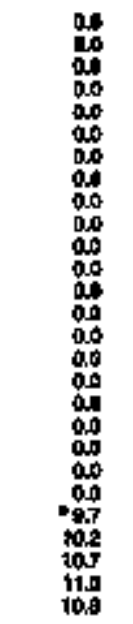 & 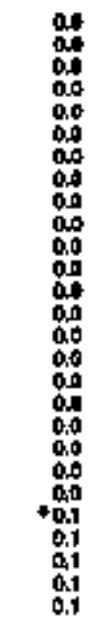 & 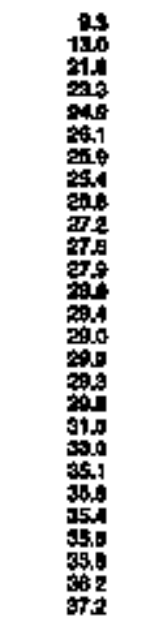 & 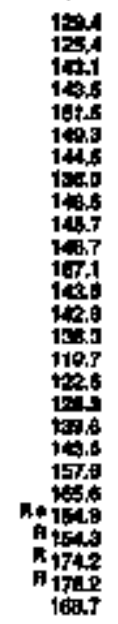 & 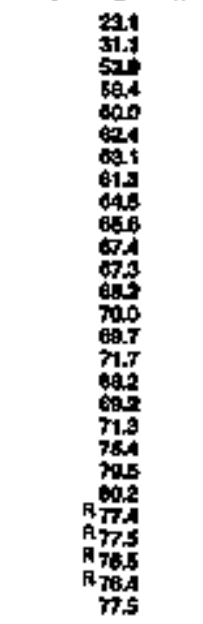 & 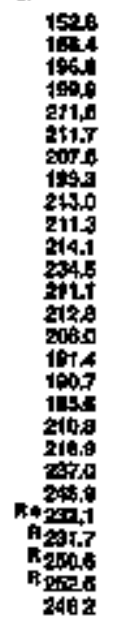 \\
\hline
\end{tabular}


Table 53, Cornmerclal Energy Consumption Estimales, 1960, 1985, 1970-1994, connectlout

\begin{tabular}{|c|c|c|c|c|c|c|c|c|c|c|c|c|c|c|}
\hline \multirow[b]{3}{*}{$\mathrm{Vaw}$} & \multicolumn{3}{|c|}{$\operatorname{sen}$} & \multirow{3}{*}{ 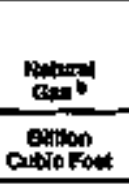 } & \multicolumn{6}{|c|}{ Potroton } & \multirow[b]{2}{*}{ Dotitely } & \multirow[b]{2}{*}{ Enecty } & \multirow[b]{2}{*}{ 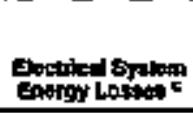 } & \multirow[b]{3}{*}{ Totw " } \\
\hline & 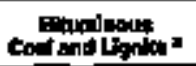 & Nontratilon & Told & & Dotancing & Kinconte & LPa: & moror & Fontent & Tokt & & & & \\
\hline & \multicolumn{2}{|c|}{ Thou:and short Tane } & \multirow[b]{2}{*}{ 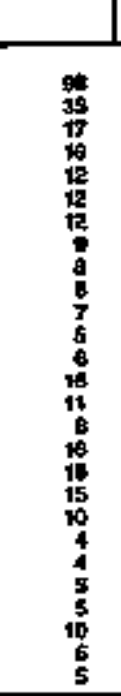 } & & \multicolumn{6}{|c|}{ Thoutand Barmil } & \multicolumn{3}{|c|}{ 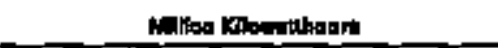 } & \\
\hline 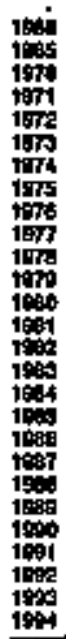 & $\begin{array}{l}54 \\
7 \\
0 \\
0 \\
0 \\
1 \\
0 \\
0 \\
0 \\
0 \\
0 \\
0 \\
0 \\
0 \\
0 \\
0 \\
0 \\
0 \\
1 \\
0 \\
0 \\
0 \\
0 \\
5 \\
0 \\
0\end{array}$ & 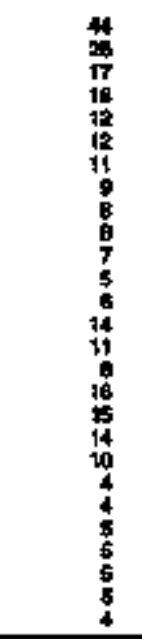 & & 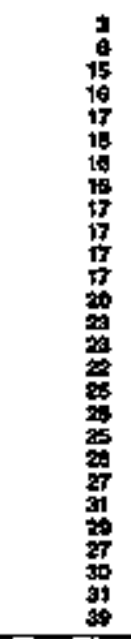 & 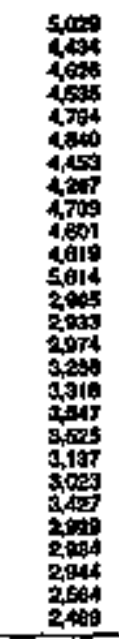 & 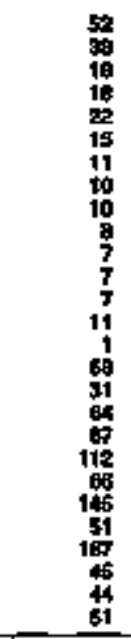 & 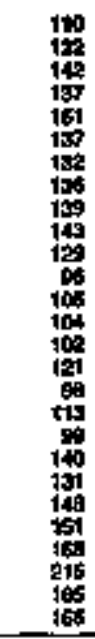 & 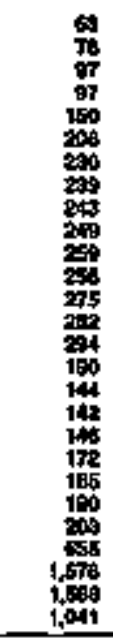 & 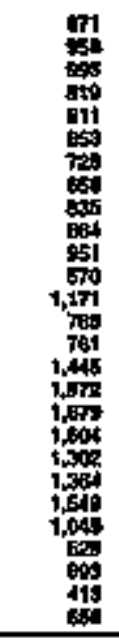 & 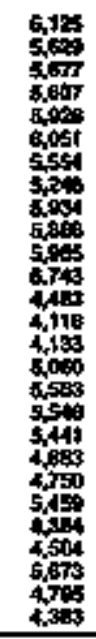 & 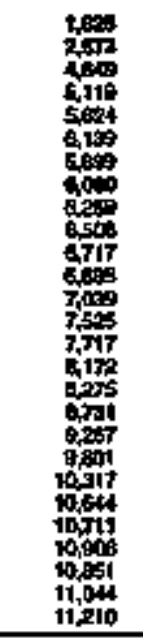 & $\begin{array}{l}= \\
= \\
= \\
= \\
= \\
= \\
= \\
= \\
= \\
= \\
= \\
= \\
= \\
=\end{array}$ & 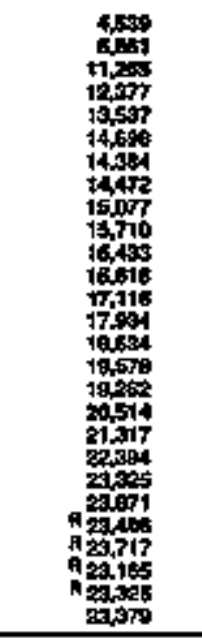 & $\begin{array}{l}= \\
= \\
= \\
= \\
= \\
= \\
= \\
z \\
= \\
= \\
= \\
= \\
z \\
z \\
=\end{array}$ \\
\hline \multicolumn{15}{|c|}{$\pi$ Thing Bur } \\
\hline 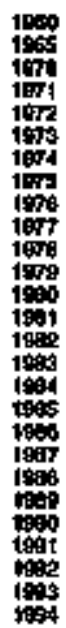 & 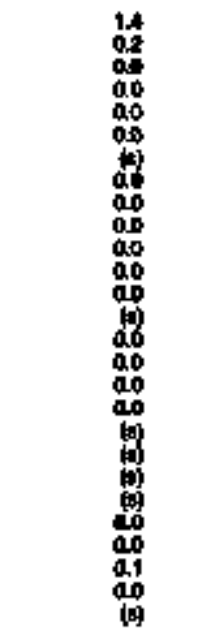 & $\begin{array}{l}t .1 \\
0.7 \\
0.4 \\
0.3 \\
0.3 \\
0.3 \\
0.2 \\
0.2 \\
0.8 \\
0.2 \\
0.2 \\
0.1 \\
0.1 \\
0.3 \\
0.3 \\
0.2 \\
0.4 \\
0.4 \\
0.4 \\
0.3 \\
0.1 \\
0.1 \\
0.1 \\
0.1 \\
0.1 \\
0.1 \\
0.1\end{array}$ & 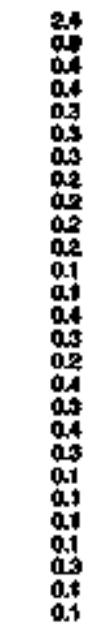 & 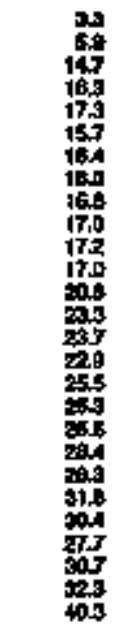 & 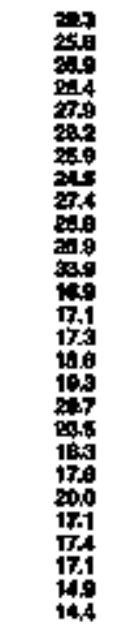 & 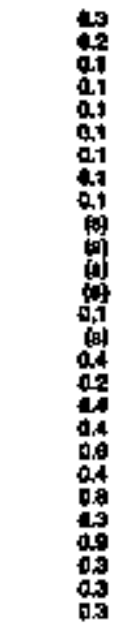 & 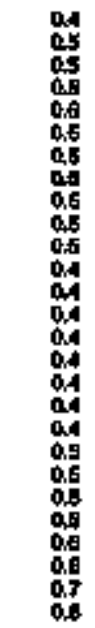 & 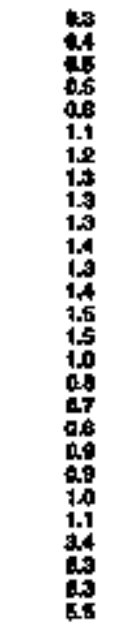 & 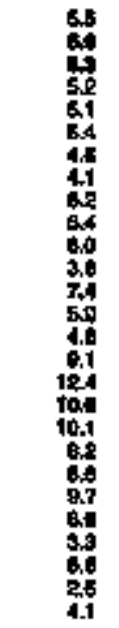 & 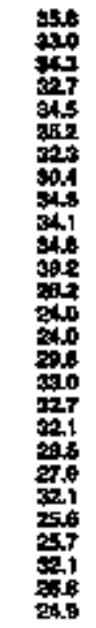 & 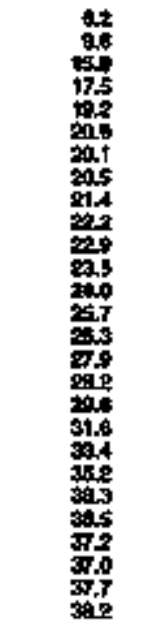 & 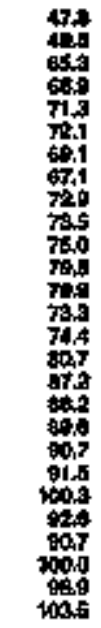 & 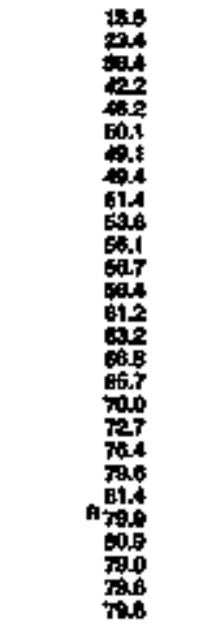 & 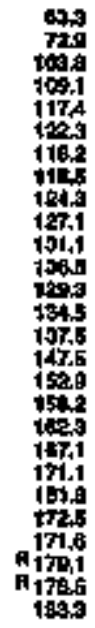 \\
\hline
\end{tabular}

$\mathbf{C}$
$\mathbf{O}$
$\mathbf{N}$
$\mathbf{N}$
$\mathbf{E}$
$\mathbf{C}$
$\mathbf{T}$
$\mathbf{C}$
$\mathbf{C}$
$\mathbf{U}$

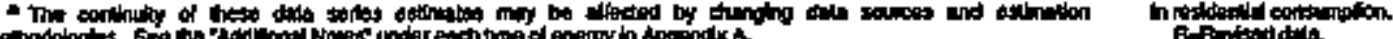

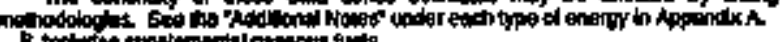

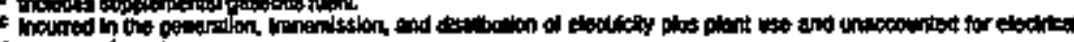

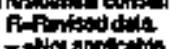

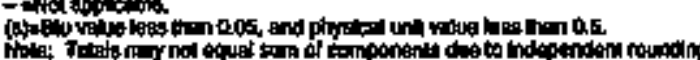

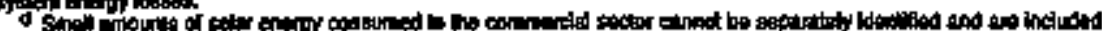

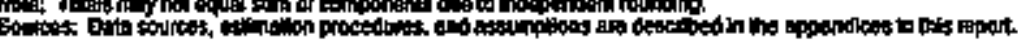




\begin{tabular}{|c|c|c|c|c|c|c|c|c|c|c|c|c|c|c|c|c|c|c|}
\hline \multirow[b]{3}{*}{ 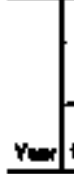 } & \multirow[b]{2}{*}{ dent } & \multirow[b]{2}{*}{ "avares } & \multicolumn{9}{|c|}{ Mrovenn } & \multirow[b]{2}{*}{ testion } & \multirow[b]{2}{*}{ Eloterts } & \multirow[b]{2}{*}{ oneros } & \multirow[b]{2}{*}{ Alecindery 4} & \multirow[b]{2}{*}{ Het } & \multirow{3}{*}{ 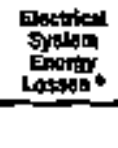 } & \multirow[b]{3}{*}{ Tois } \\
\hline & & & Andat: & 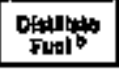 & Kerrokm: & Log" & Lworkantas & 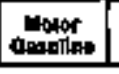 & Fondy & 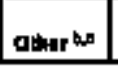 & Toter & & & & & & & \\
\hline & Thounend & croblen $\mathrm{Fen}$ & \multicolumn{9}{|c|}{ 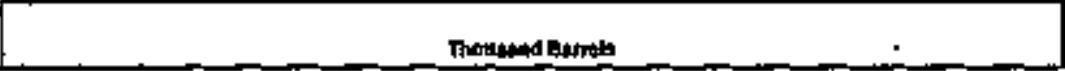 } & \multicolumn{5}{|c|}{ 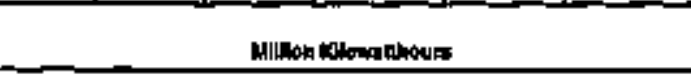 } & & \\
\hline 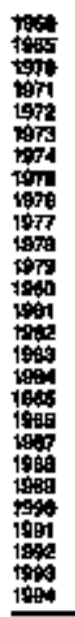 & 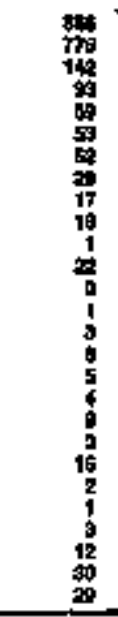 & 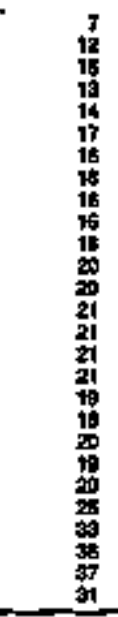 & 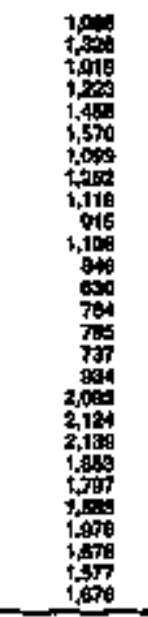 & 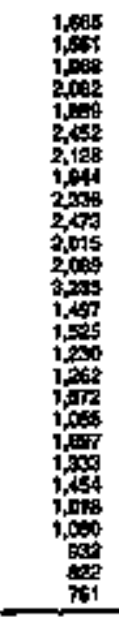 & 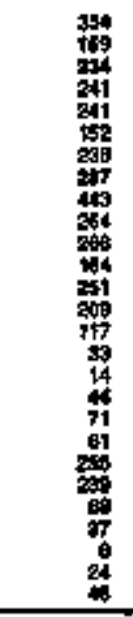 & 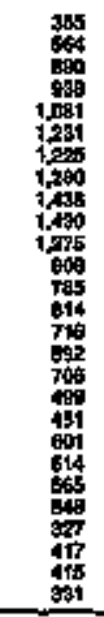 & 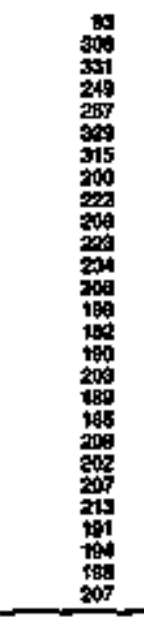 & 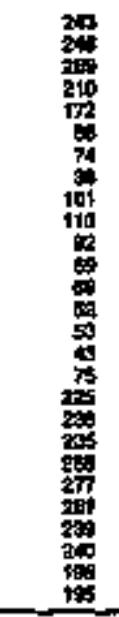 & 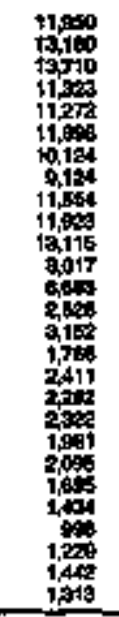 & 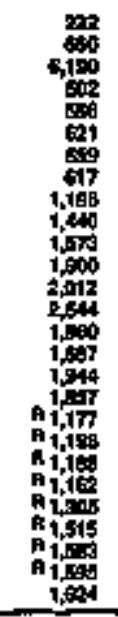 & 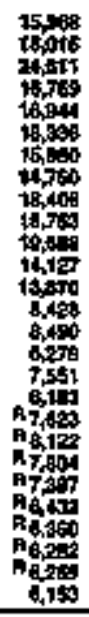 & 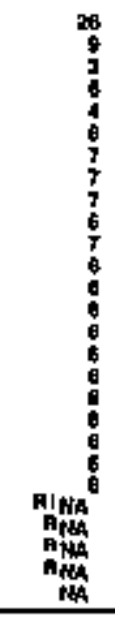 & $\begin{array}{l}0 \\
0 \\
0 \\
0 \\
0 \\
0 \\
0 \\
0 \\
0 \\
0 \\
0 \\
0 \\
0 \\
0 \\
0 \\
0 \\
0\end{array}$ & 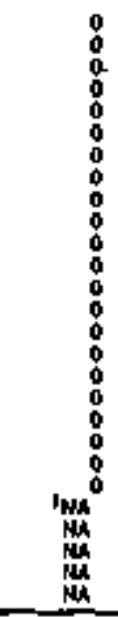 & 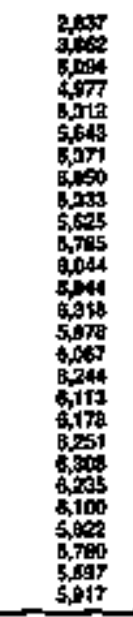 & $\begin{array}{l}z \\
\vdots \\
z \\
z \\
z\end{array}$ & 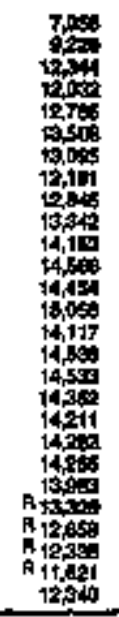 & \\
\hline 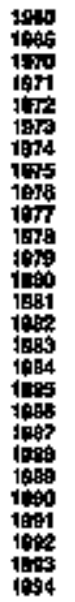 & 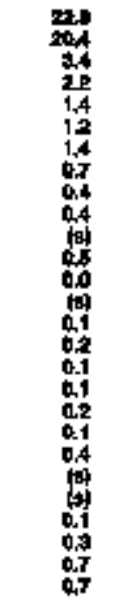 & 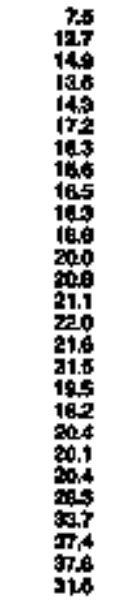 & 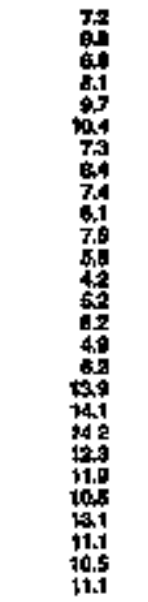 & 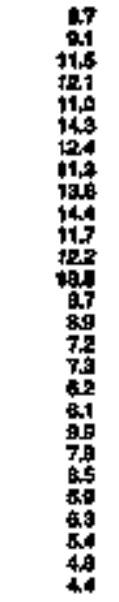 & 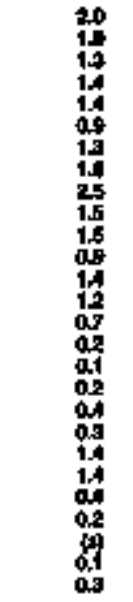 & 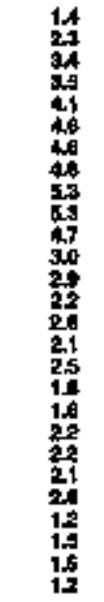 & 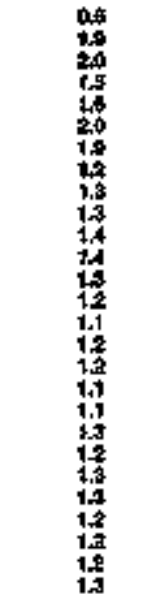 & 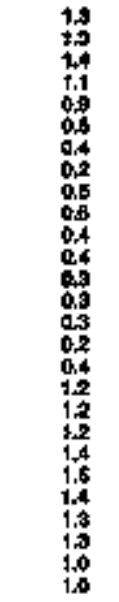 & 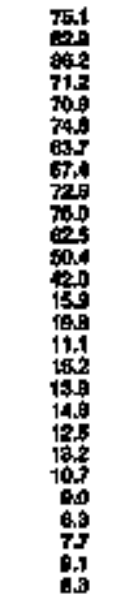 & 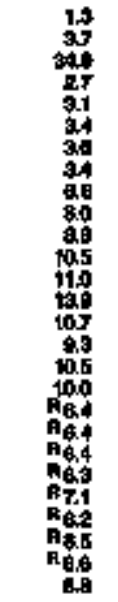 & 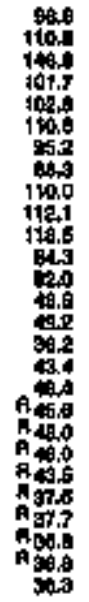 & 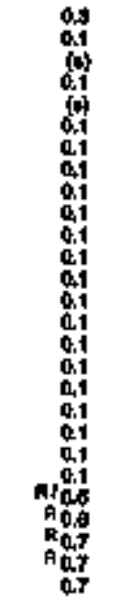 & 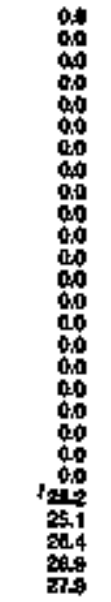 & 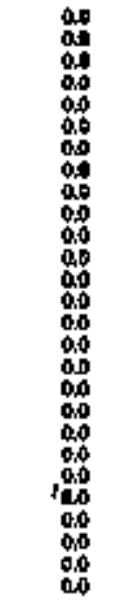 & 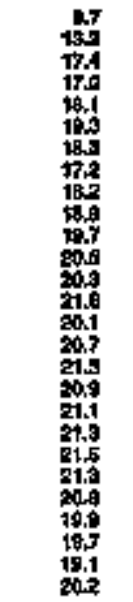 & 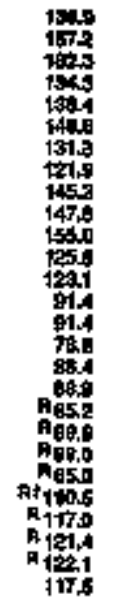 & 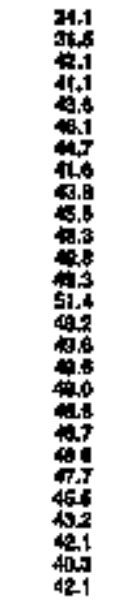 & 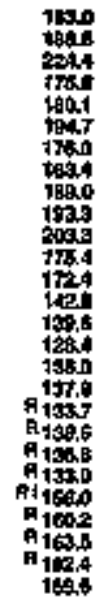 \\
\hline
\end{tabular}

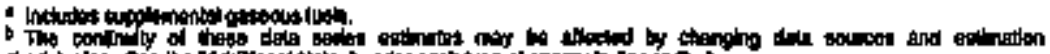

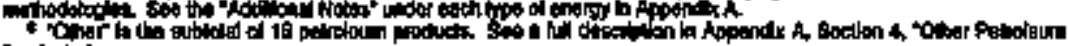
Pingente.

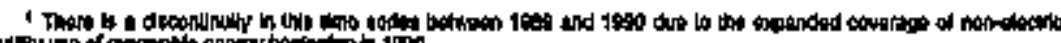

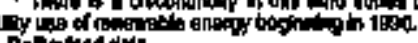

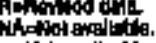

ath toolletion

tit

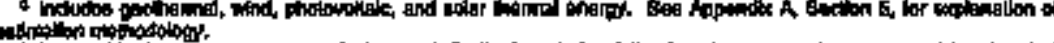

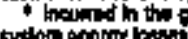

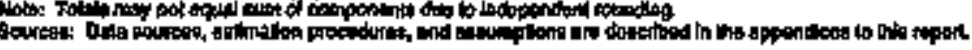


Table 57. Trensportation Enorgy Consumption Estimatits, 1960, 1985, 1970-1994, Conmmatieut

\begin{tabular}{|c|c|c|c|c|c|c|c|c|c|c|c|c|c|c|c|}
\hline \multirow[b]{3}{*}{$\mathrm{ren}$} & \multirow[b]{2}{*}{ cons } & \multirow[b]{2}{*}{ Matures } & \multicolumn{8}{|c|}{ 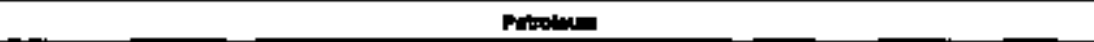 } & \multirow[b]{2}{*}{ Blotuels:" } & \multirow[b]{2}{*}{ Eectrtely 6} & \multirow[b]{2}{*}{ tinery } & \multirow[b]{2}{*}{ 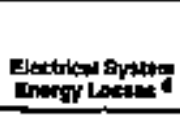 } & \multirow[b]{3}{*}{ ודוt } \\
\hline & & & Anvoline & $\begin{array}{l}\text { Detrins } \\
\text { Fund }\end{array}$ & sil. & $\mathrm{LPC}_{\mathrm{C}}=$ & 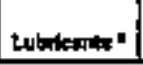 & Motor & Reapouid & Foled & & & & & \\
\hline & Thout tand & Gomplon fort & \multicolumn{8}{|c|}{ 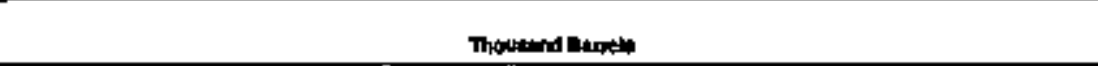 } & $\begin{array}{l}\text { Fhovesnd } \\
\text { cuitione }\end{array}$ & \multicolumn{3}{|c|}{ 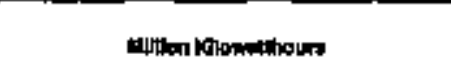 } & \\
\hline 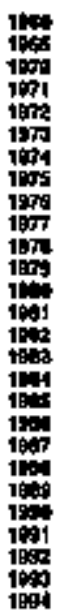 & $\begin{array}{l}5 \\
3 \\
0 \\
09 \\
60 \\
0 \\
60 \\
0 \\
0 \\
0 \\
0 \\
0 \\
0 \\
0 \\
0 \\
0 \\
0 \\
0 \\
0 \\
0 \\
0 \\
0 \\
0 \\
0\end{array}$ & 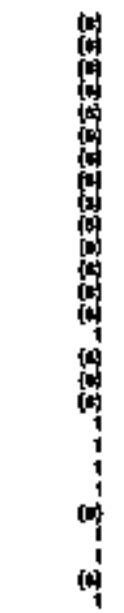 & 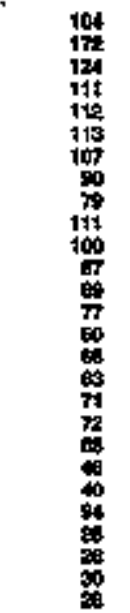 & 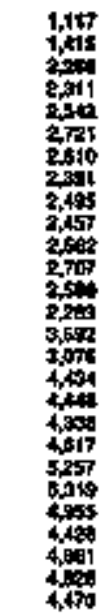 & 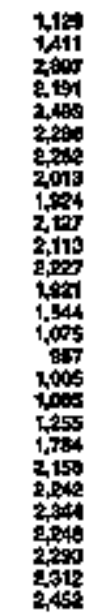 & 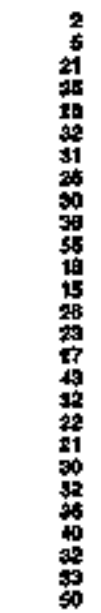 & 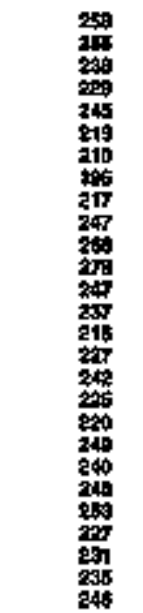 & 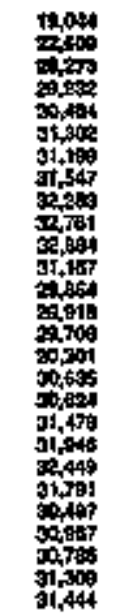 & 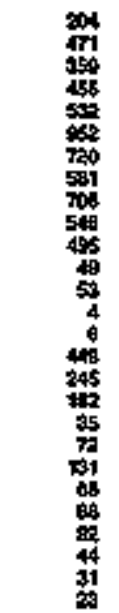 & 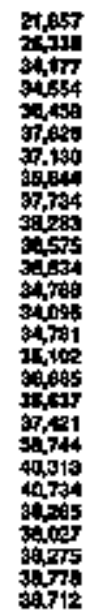 & 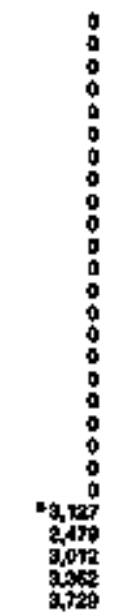 & $\begin{array}{l}0 \\
0 \\
0 \\
0 \\
0 \\
0 \\
0 \\
0 \\
0 \\
0 \\
0 \\
0 \\
0 \\
0 \\
0 \\
0 \\
0 \\
0 \\
0 \\
0 \\
0 \\
0 \\
0 \\
0 \\
0 \\
0\end{array}$ & $\begin{array}{l}= \\
z \\
= \\
z \\
z \\
z \\
z \\
z \\
= \\
z \\
z \\
z \\
z \\
z\end{array}$ & $\begin{array}{l}0 \\
0 \\
0 \\
0 \\
0 \\
0 \\
0 \\
0 \\
0 \\
0 \\
0 \\
0 \\
0 \\
0 \\
0 \\
0 \\
0 \\
0 \\
0 \\
0 \\
0 \\
0 \\
0 \\
0 \\
0 \\
0 \\
0\end{array}$ & $\begin{array}{l}= \\
= \\
= \\
= \\
= \\
= \\
= \\
= \\
= \\
= \\
= \\
= \\
5 \\
=\end{array}$ \\
\hline
\end{tabular}

\begin{tabular}{|c|c|c|c|c|c|c|c|c|c|c|c|c|c|c|c|}
\hline 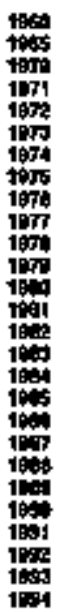 & 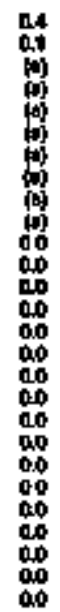 & 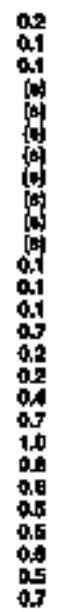 & 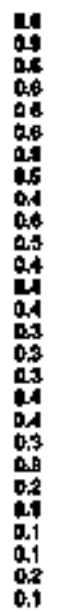 & 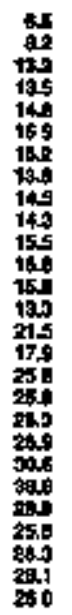 & 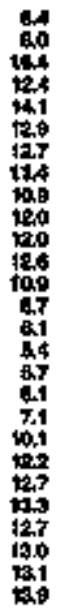 & 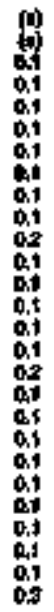 & 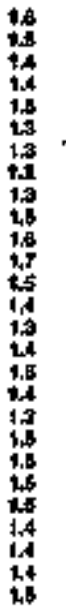 & 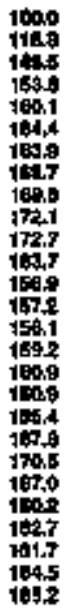 & 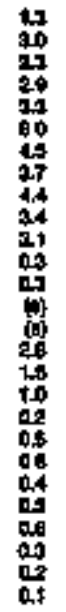 & 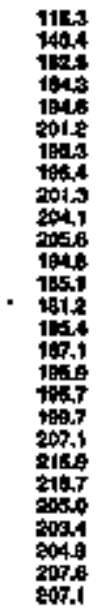 & $\begin{array}{l}00 \\
00 \\
00 \\
00 \\
00 \\
00 \\
00 \\
00 \\
00 \\
00 \\
00 \\
00 \\
100 \\
00 \\
00 \\
00 \\
00 \\
00 \\
00 \\
00 \\
00 \\
00 \\
00 \\
02 \\
02 \\
02 \\
03 \\
03\end{array}$ & 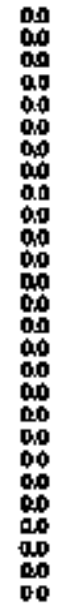 & 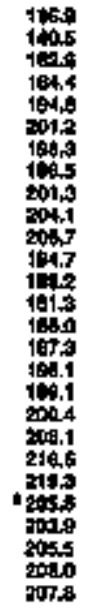 & 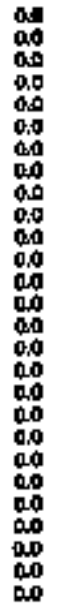 & 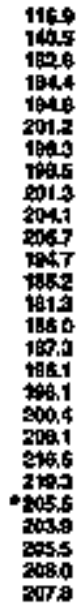 \\
\hline
\end{tabular}

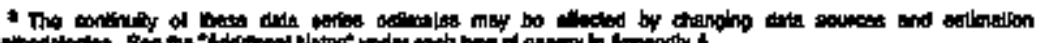

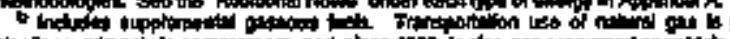

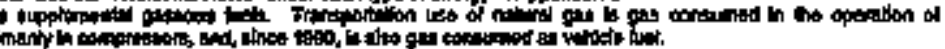

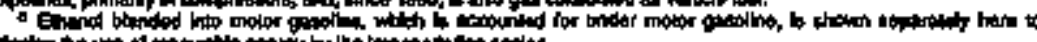

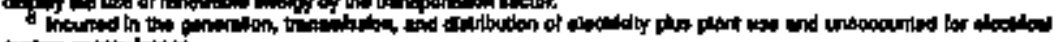

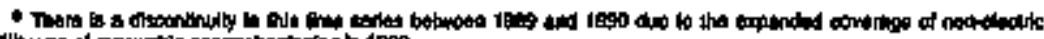

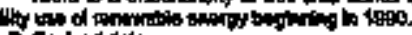

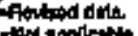

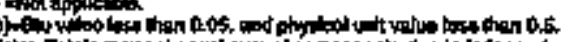

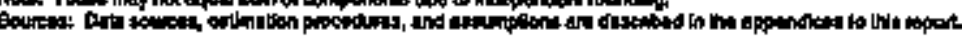


Table 58. Esthinates of Enorgy Input at Electric Utilitios, 1960, 1968, 1970-1994, Connecticut

\begin{tabular}{|c|c|c|c|c|c|c|c|c|c|c|c|c|c|c|}
\hline \multirow[b]{3}{*}{ Vom } & \multicolumn{3}{|c|}{ Coll } & \multirow{3}{*}{ (1) } & \multicolumn{4}{|c|}{ atementin } & \multirow[b]{2}{*}{ 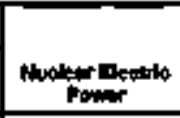 } & \multirow[b]{2}{*}{ 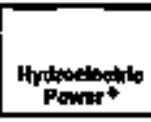 } & \multirow[b]{2}{*}{ Etoruth } & \multirow[b]{2}{*}{ 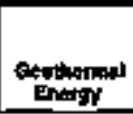 } & \multirow[b]{2}{*}{ Oather Wh } & \multirow[b]{3}{*}{ Tow: } \\
\hline & 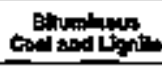 & rannosen & Tolle & & Honth & 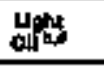 & Putheyn & Toll & & & & & & \\
\hline & \multicolumn{3}{|c|}{ 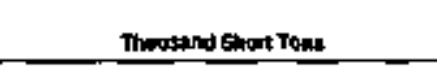 } & & \multicolumn{4}{|c|}{ 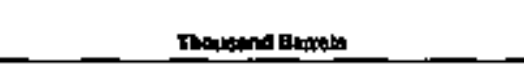 } & \multicolumn{5}{|c|}{ 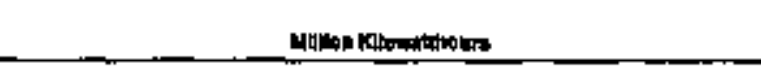 } & \\
\hline 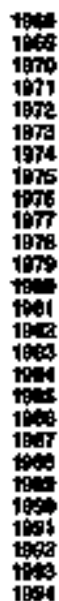 & 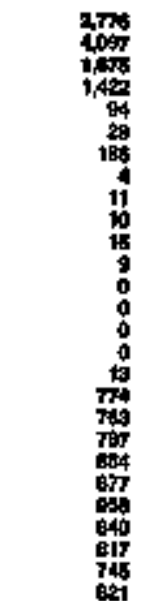 & $\begin{array}{l}0 \\
0 \\
0 \\
0 \\
0 \\
0 \\
0 \\
0 \\
0 \\
0 \\
0 \\
0 \\
0 \\
0 \\
0 \\
0 \\
0 \\
0 \\
0 \\
0 \\
0 \\
0 \\
0 \\
0 \\
0 \\
0 \\
0 \\
0\end{array}$ & 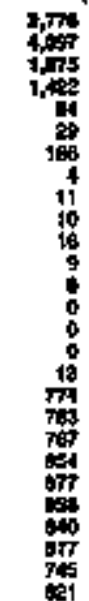 & 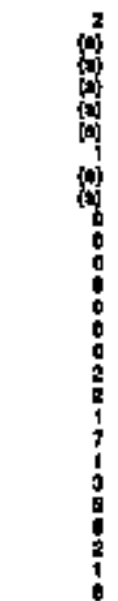 & 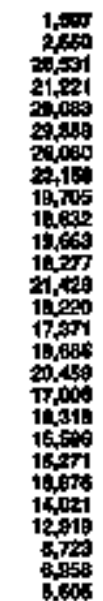 & 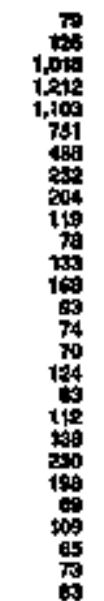 & $\begin{array}{l}0 \\
0 \\
0 \\
0 \\
0 \\
0 \\
0 \\
0 \\
0 \\
0 \\
0 \\
0 \\
0 \\
0 \\
0 \\
0 \\
0 \\
0 \\
0 \\
0 \\
0 \\
0 \\
0 \\
0 \\
0\end{array}$ & 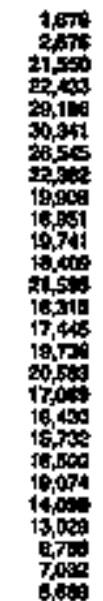 & 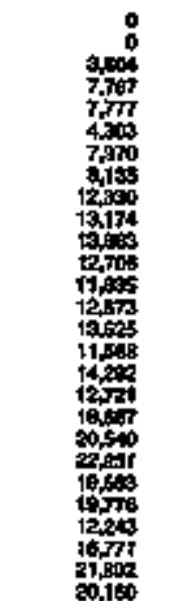 & 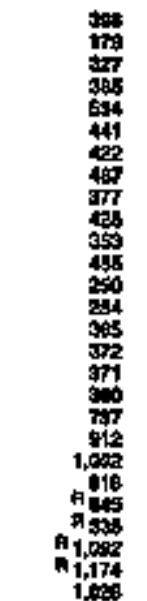 & 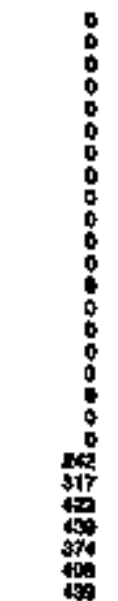 & $\begin{array}{l}: \\
\vdots \\
\vdots \\
\vdots \\
\vdots \\
\vdots \\
\vdots \\
\vdots \\
\vdots \\
\vdots \\
\vdots \\
\vdots \\
0 \\
\vdots \\
\vdots \\
0\end{array}$ & $\begin{array}{l}0 \\
0 \\
0 \\
0 \\
0 \\
0 \\
0 \\
0 \\
0 \\
0 \\
0 \\
0 \\
0 \\
0 \\
0 \\
0 \\
0 \\
0 \\
0 \\
0 \\
0 \\
0 \\
0 \\
0 \\
0\end{array}$ & $\begin{array}{l}z \\
\vdots \\
\vdots \\
\vdots \\
\vdots \\
\vdots \\
\vdots \\
\vdots \\
\vdots \\
\vdots \\
\vdots \\
z\end{array}$ \\
\hline
\end{tabular}

\begin{tabular}{|c|c|c|c|c|c|}
\hline 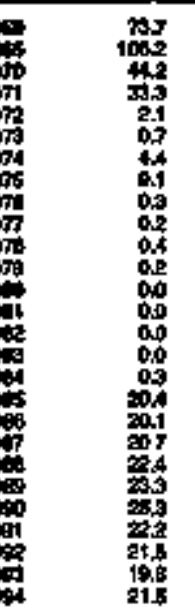 & 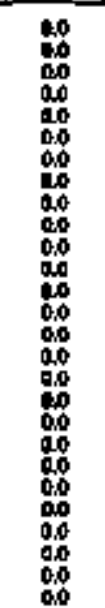 & 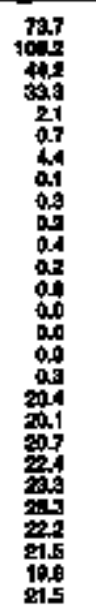 & 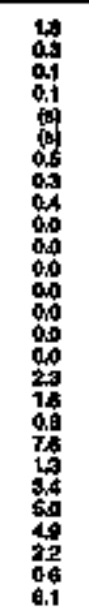 & 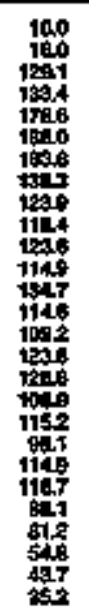 & 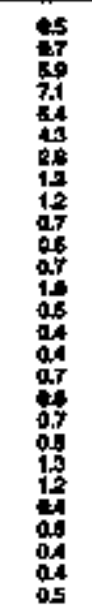 \\
\hline
\end{tabular}

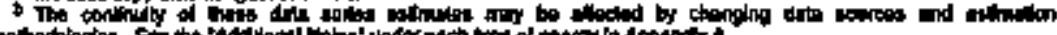

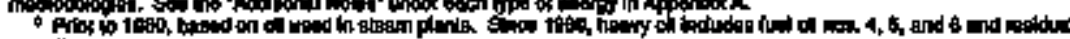

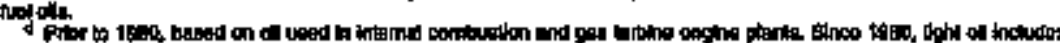

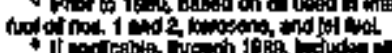

\begin{tabular}{|c|c|c|c|c|c|c|}
\hline 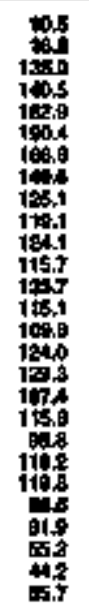 & 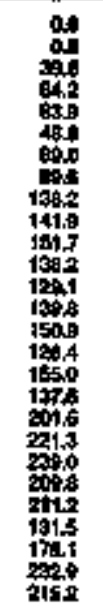 & 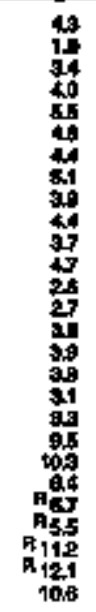 & 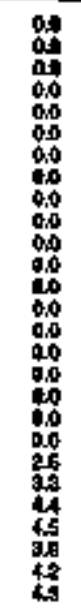 & 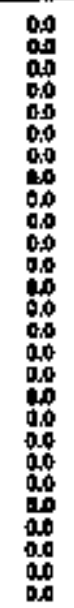 & 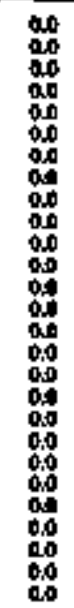 & 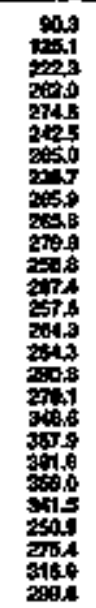 \\
\hline
\end{tabular}

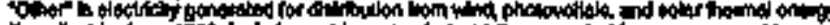

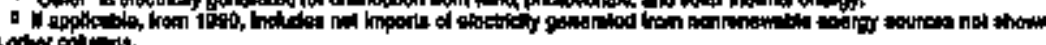
$\rightarrow 0$

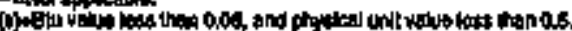

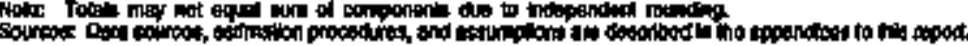

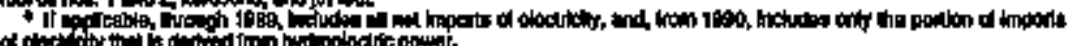


Table 59. Energy Consumption Estimates by Sourca, 1960, 1985, 1970-1994, Delaware

\begin{tabular}{|c|c|c|c|c|c|c|c|c|c|c|c|c|c|c|c|c|c|c|c|}
\hline \multirow[b]{3}{*}{ Yant } & \multirow[b]{2}{*}{ Cont: } & \multirow[b]{2}{*}{ Notons } & \multicolumn{11}{|c|}{ Porrolowis } & \multirow[b]{2}{*}{ 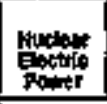 } & \multirow{2}{*}{ 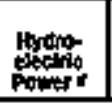 } & \multirow[b]{2}{*}{ 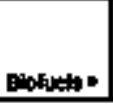 } & \multirow[b]{2}{*}{ Otherme } & \multirow{3}{*}{ 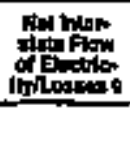 } & \multirow[b]{3}{*}{ Tots h } \\
\hline & & & Alowatind & Antions & 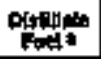 & 熄. & $\lim _{100}$ & Leat & untit. & 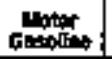 & 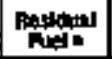 & Onter an & Tad & & & & & & \\
\hline & Thostind & 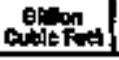 & \multicolumn{11}{|c|}{ Thosend lappoth } & \multicolumn{4}{|c|}{ 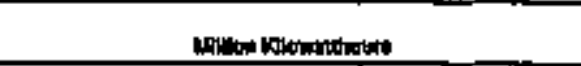 } & & \\
\hline 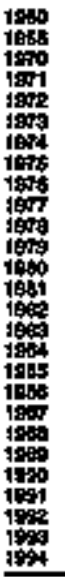 & 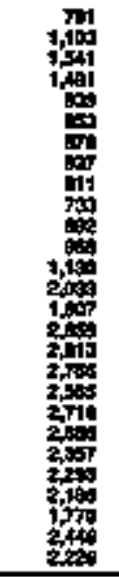 & 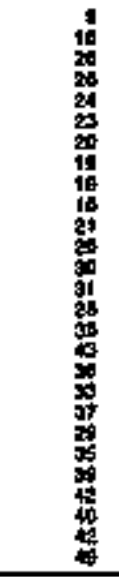 & 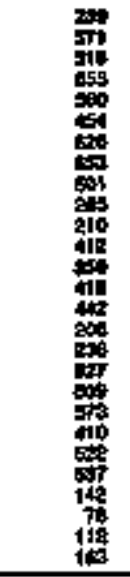 & 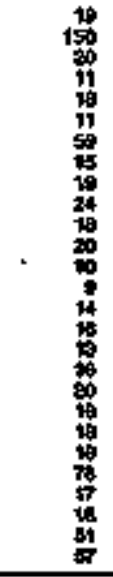 & 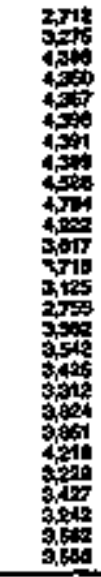 & 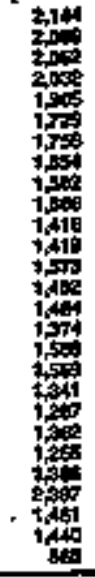 & 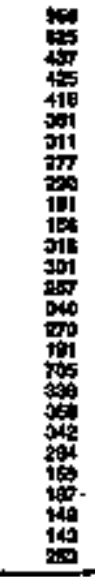 & 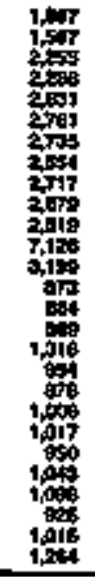 & 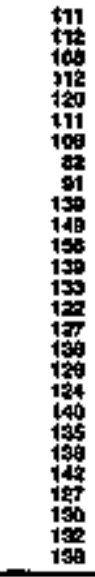 & 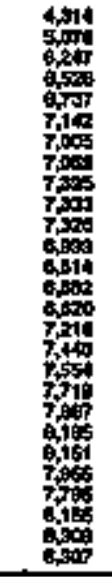 & 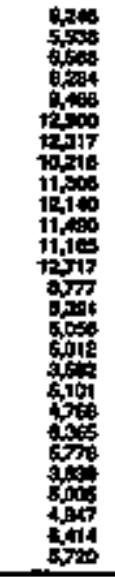 & 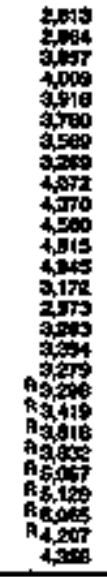 & 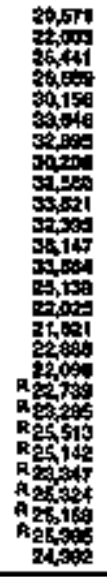 & $\begin{array}{l}0 \\
8 \\
0 \\
0 \\
0 \\
0 \\
0 \\
0 \\
0 \\
0 \\
0 \\
0\end{array}$ & 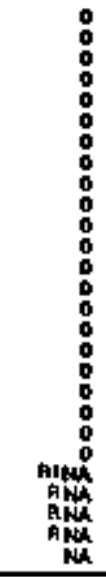 & 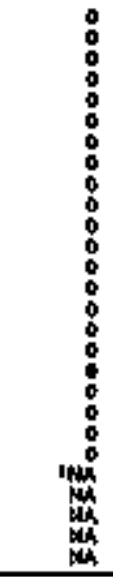 & 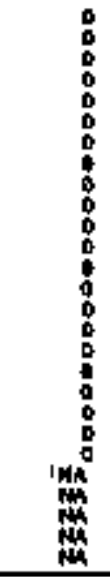 & 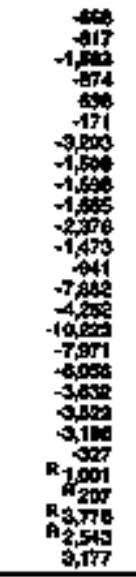 & $\begin{array}{l}z \\
z \\
z \\
z \\
z \\
z \\
z \\
z \\
z \\
z \\
z \\
z \\
z \\
z \\
z \\
\end{array}$ \\
\hline \multicolumn{20}{|c|}{ ntillow Bu } \\
\hline 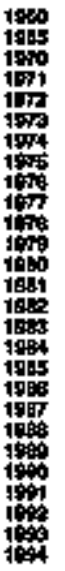 & 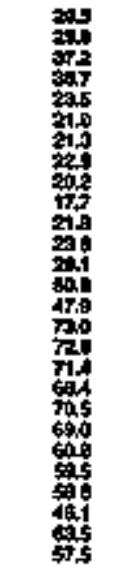 & 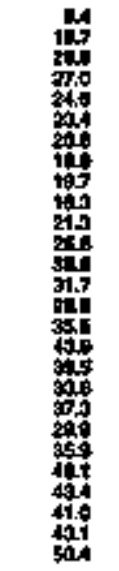 & 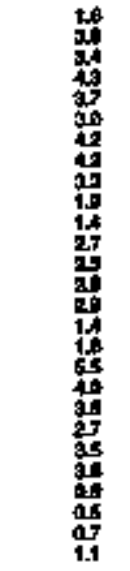 & 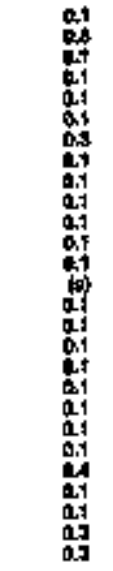 & 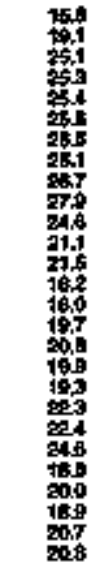 & 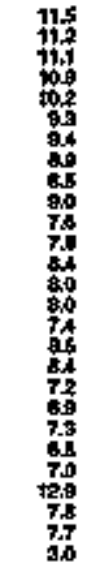 & 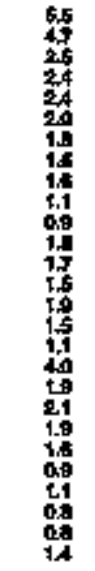 & 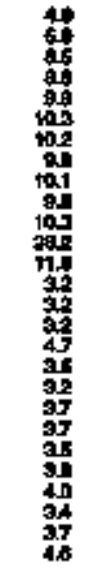 & 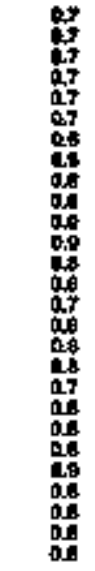 & 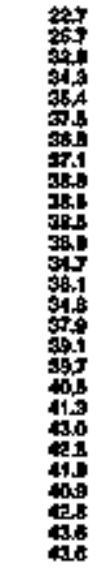 & 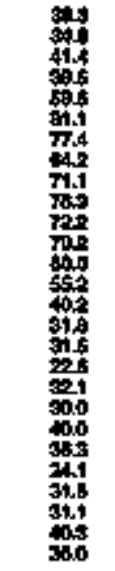 & 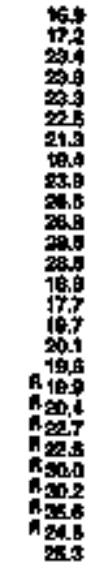 & 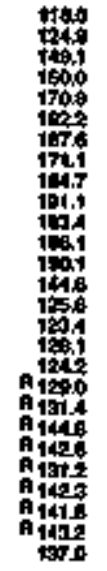 & 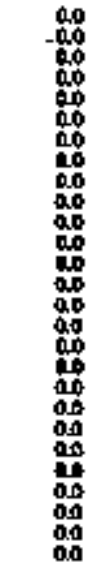 & 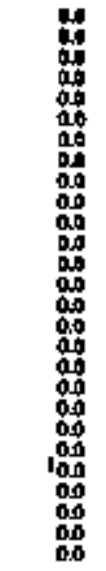 & 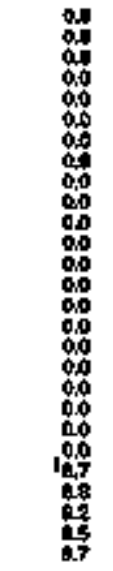 & 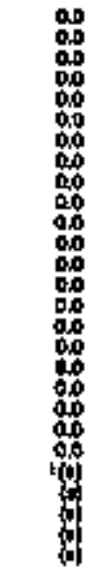 & 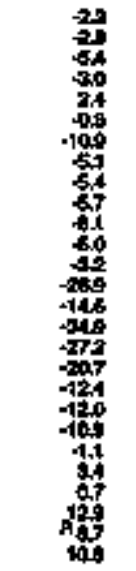 & 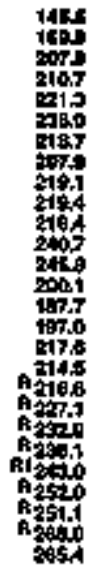 \\
\hline
\end{tabular}

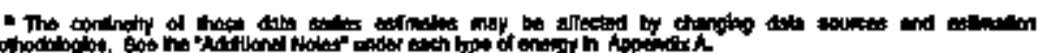

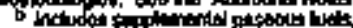

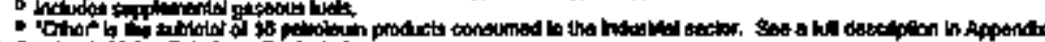

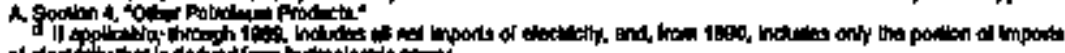

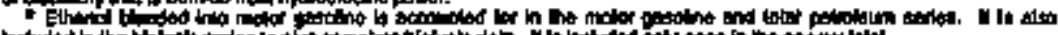

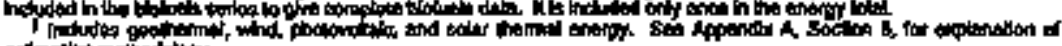

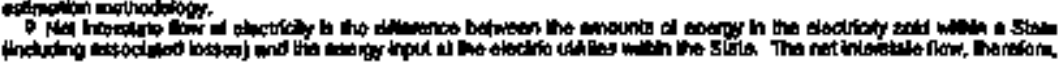

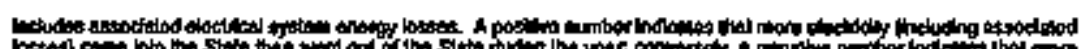

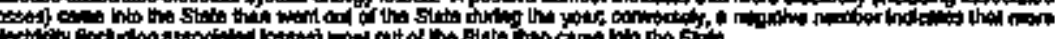

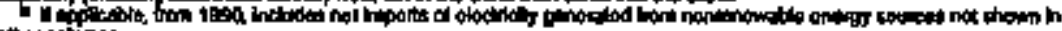

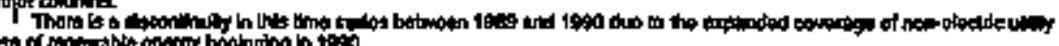
Rifluntod ditition.

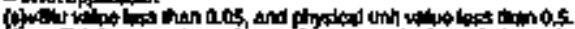

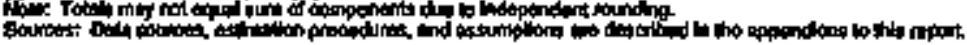


Table 60. Fesidential Energy Consumption Estimattes, 1960, 1968, 1970-1994, Delaware

\begin{tabular}{|c|c|c|c|c|c|c|c|c|c|c|c|c|c|c|}
\hline \multirow[b]{3}{*}{ Yearl } & \multicolumn{3}{|c|}{$\operatorname{con}$} & \multirow{3}{*}{ - } & & Pats & & & \multirow{3}{*}{ Bboveds } & & & & & \multirow{3}{*}{ rotal } \\
\hline & 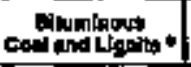 & Anthere " & Toter & & $\begin{array}{l}\text { Dunghet } \\
\text { funt: }\end{array}$ & Kersene : & wo: & Total & & solur" & Evetility " & thent & 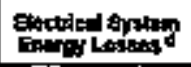 & \\
\hline & \multicolumn{3}{|c|}{ 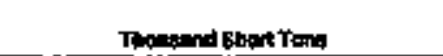 } & & \multicolumn{4}{|c|}{ Thowend Bunolt } & & \multicolumn{4}{|c|}{ in } & \\
\hline 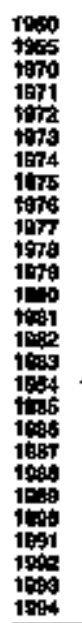 & $\begin{array}{l}: \\
\vdots \\
0 \\
0 \\
0 \\
0 \\
0 \\
0 \\
0 \\
0 \\
7 \\
1 \\
0 \\
1 \\
\frac{2}{13} \\
\frac{1}{8} \\
7 \\
7 \\
7 \\
17 \\
10\end{array}$ & $\begin{array}{l}12 \\
\vdots \\
5 \\
5 \\
3 \\
3 \\
3 \\
9 \\
2 \\
2 \\
2 \\
1 \\
2 \\
2 \\
2 \\
1 \\
2 \\
1 \\
2 \\
2 \\
2 \\
4 \\
0 \\
9 \\
9 \\
9 \\
1 \\
1\end{array}$ & 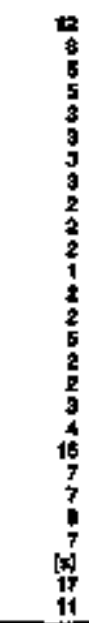 & $\begin{array}{l}1 \\
6 \\
8 \\
8 \\
8 \\
7 \\
7 \\
7 \\
7 \\
7 \\
7 \\
7 \\
6 \\
7 \\
6 \\
7 \\
8 \\
6 \\
7 \\
7 \\
8 \\
0 \\
8\end{array}$ & 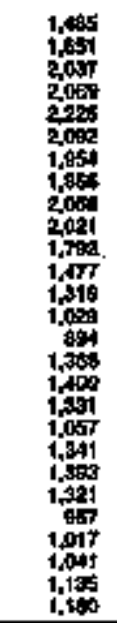 & 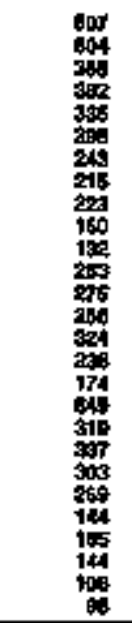 & 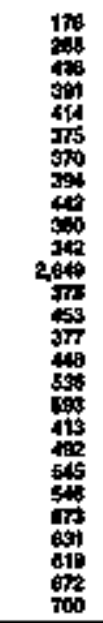 & 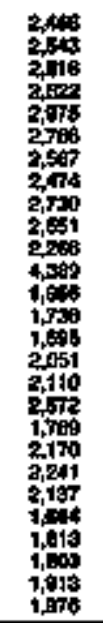 & 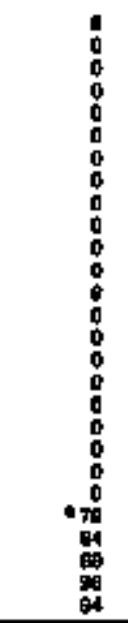 & $\begin{array}{l}0 \\
0 \\
0 \\
0 \\
0 \\
0 \\
0 \\
0 \\
0 \\
0 \\
0 \\
0 \\
0 \\
0 \\
0 \\
0 \\
0 \\
0 \\
0 \\
0 \\
0 \\
0 \\
0 \\
0 \\
7 \\
7 \\
7 \\
7 \\
7\end{array}$ & 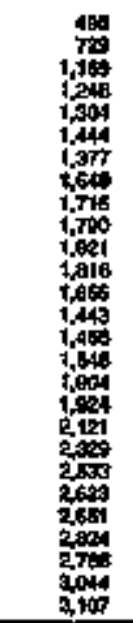 & $\begin{array}{l}\vdots \\
\vdots \\
\vdots \\
\vdots \\
\vdots \\
\vdots \\
\vdots \\
\vdots \\
\vdots \\
\vdots \\
\vdots \\
\vdots\end{array}$ & 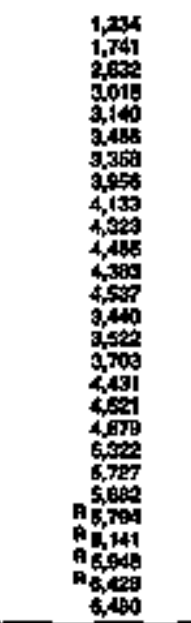 & $\begin{array}{l}z \\
\vdots \\
\vdots \\
z \\
z \\
z \\
z \\
z \\
z \\
z \\
z \\
z \\
z\end{array}$ \\
\hline & & & & & & & Tralliton & & & & & & & \\
\hline 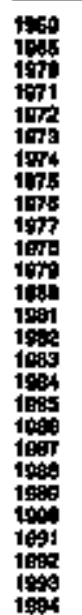 & 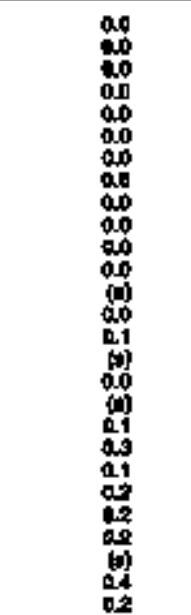 & 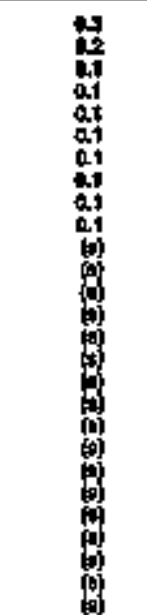 & 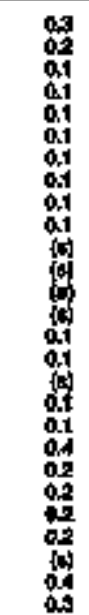 & 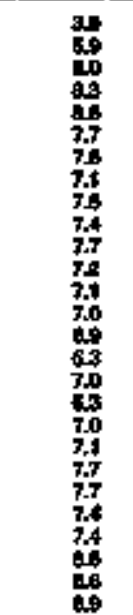 & 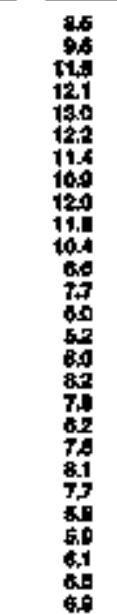 & 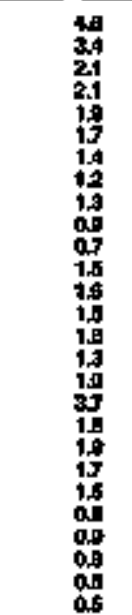 & 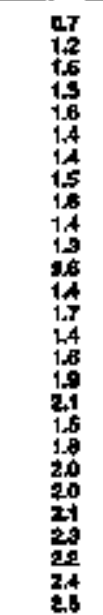 & 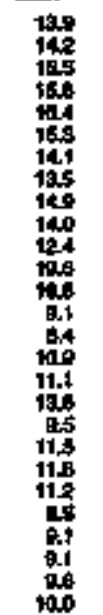 & 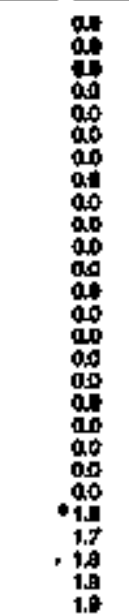 & 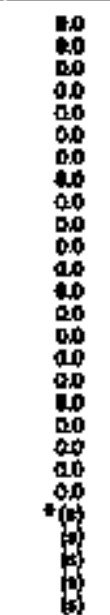 & 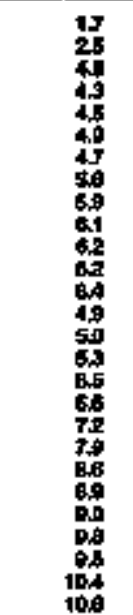 & 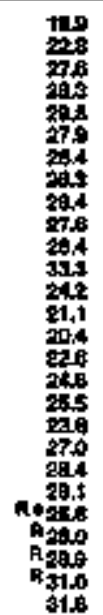 & 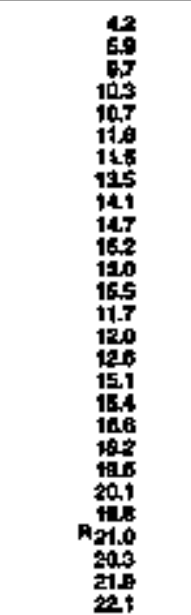 & 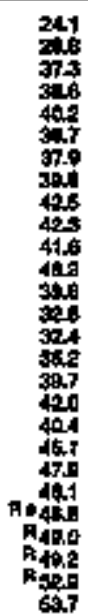 \\
\hline
\end{tabular}

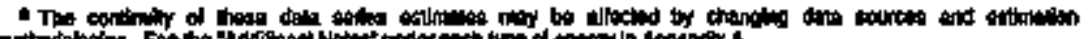

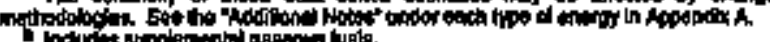

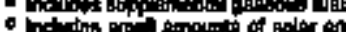

然

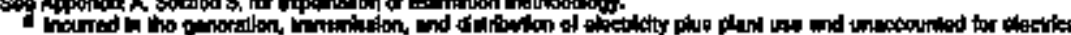

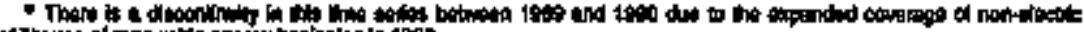

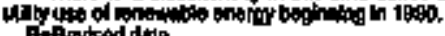

Hinfortod dra 
Table 61. Commexcial Energy Consumpilon Esilmates, 1960, 1965, 1970-1994, Delaware

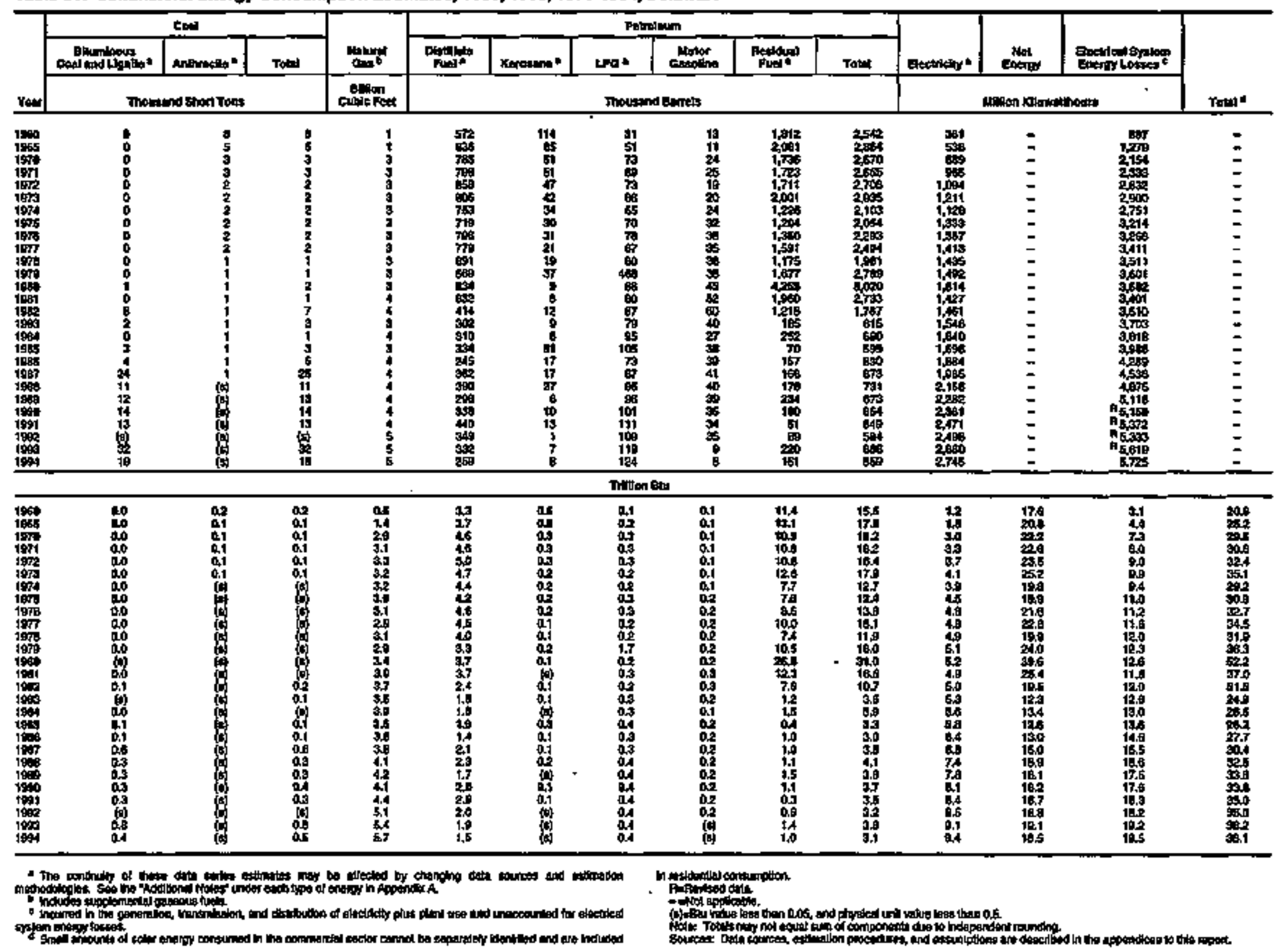

$\mathbf{D}$
$\mathbf{E}$
$\mathbf{L}$
$\mathbf{A}$
$\mathbf{W}$
$\mathbf{A}$
$\mathbf{R}$
$\mathbf{E}$ 
Table 62 incustrial Energy Consumpiton Estlmates, 1960, 1965, 1970-1994, Delawrare

\begin{tabular}{|c|c|c|c|c|c|c|c|c|c|c|c|c|c|c|c|c|c|c|}
\hline \multirow[b]{3}{*}{$\mathrm{r}=$} & \multirow[b]{2}{*}{$\cos$} & \multirow[b]{2}{*}{ mater } & \multicolumn{9}{|c|}{ Patroloten } & \multirow[b]{2}{*}{ 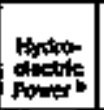 } & \multirow[b]{2}{*}{ wantsols } & \multirow[b]{2}{*}{ Oatwer in I I } & \multirow[b]{2}{*}{ Evostation } & \multirow[b]{2}{*}{ entrits } & \multirow{2}{*}{ 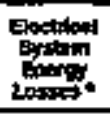 } & \multirow[b]{3}{*}{ Totol } \\
\hline & & & Motenging & atander & Kreasenet & at & 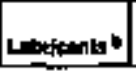 & Anotions & Pealone & | & 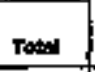 & & & & & & & \\
\hline & Thoming & 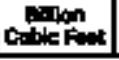 & \multicolumn{9}{|c|}{ 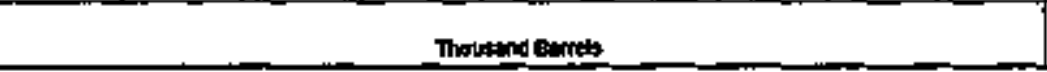 } & \multicolumn{6}{|c|}{ Maflion Ktrometheran } & \\
\hline 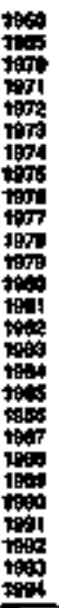 & 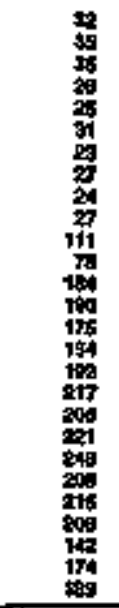 & 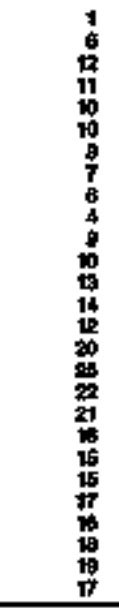 & 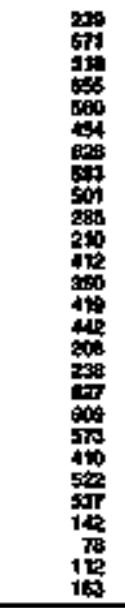 & 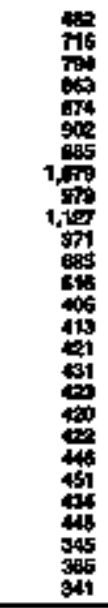 & 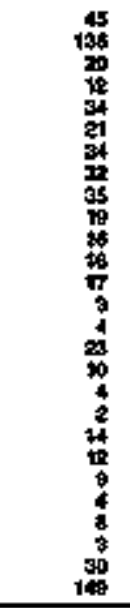 & 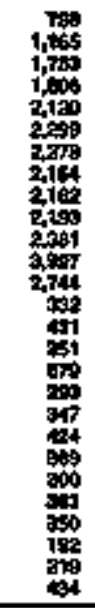 & 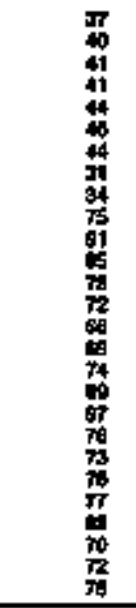 & 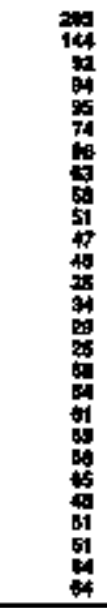 & 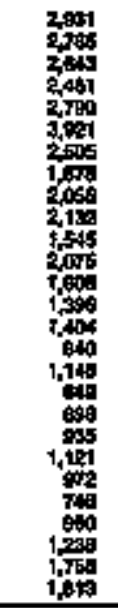 & 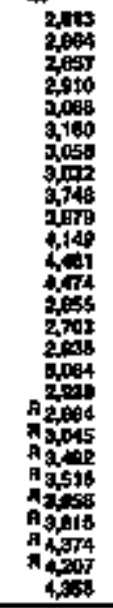 & 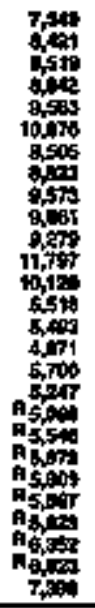 & 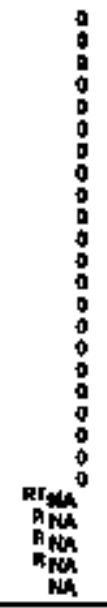 & $\begin{array}{l}0 \\
0 \\
0 \\
0 \\
0 \\
0 \\
0 \\
0 \\
0 \\
0 \\
0 \\
0 \\
0 \\
0 \\
0 \\
0 \\
0 \\
0 \\
0 \\
0 \\
0 \\
0 \\
0 \\
0 \\
0\end{array}$ & 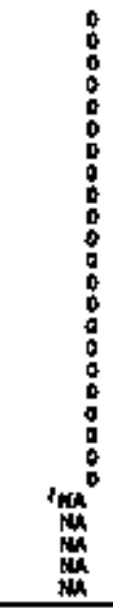 & 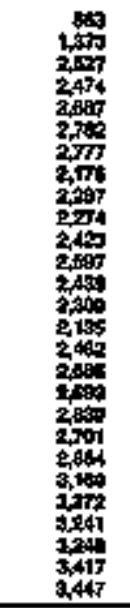 & $\begin{array}{l}= \\
z \\
z \\
z \\
z \\
z \\
= \\
z \\
z \\
z \\
z \\
z \\
z \\
z\end{array}$ & 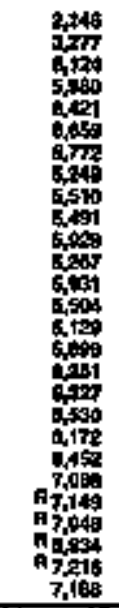 & $\begin{array}{l}= \\
= \\
z \\
z \\
z \\
= \\
= \\
z \\
z \\
z \\
z \\
z \\
z \\
z\end{array}$ \\
\hline \multicolumn{19}{|c|}{ Trimenen } \\
\hline 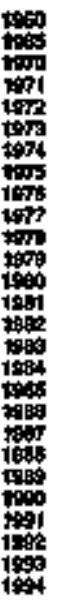 & 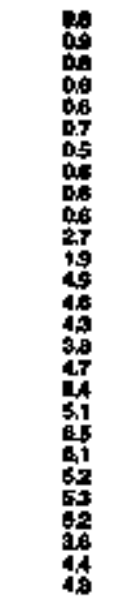 & 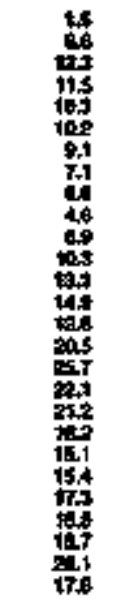 & 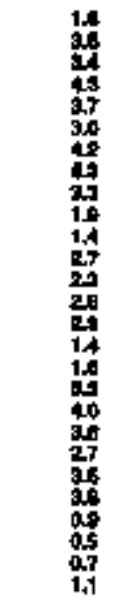 & 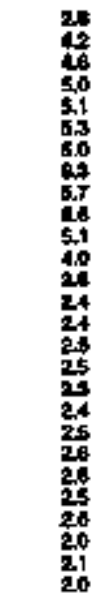 & 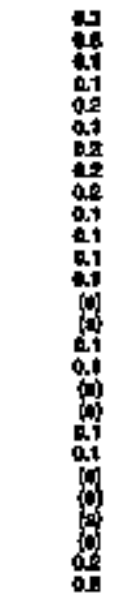 & 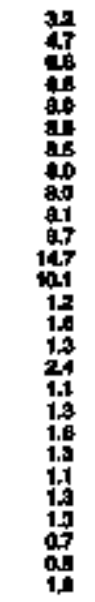 & 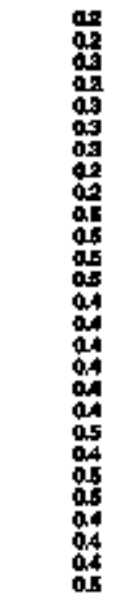 & 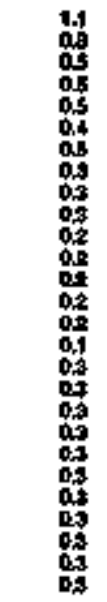 & 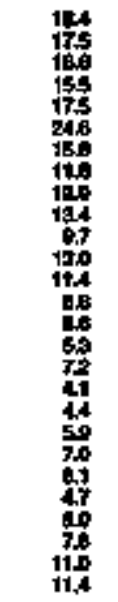 & 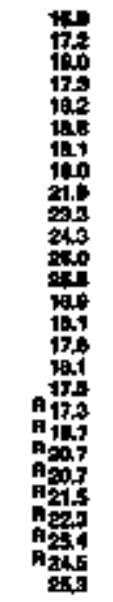 & 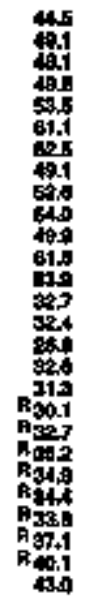 & $\begin{array}{l}10 \\
00 \\
00 \\
00 \\
00 \\
00 \\
00 \\
00 \\
00 \\
00 \\
00 \\
00 \\
00 \\
00 \\
00 \\
00 \\
00 \\
00 \\
00 \\
00 \\
00 \\
00 \\
100 \\
00 \\
00 \\
00 \\
00\end{array}$ & 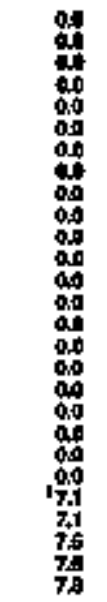 & $\begin{array}{l}00 \\
00 \\
00 \\
00 \\
00 \\
00 \\
0.0 \\
00 \\
00 \\
00 \\
00 \\
00 \\
00 \\
00 \\
00 \\
00 \\
00 \\
00 \\
00 \\
00 \\
00 \\
000 \\
00 \\
00 \\
00 \\
0.0\end{array}$ & 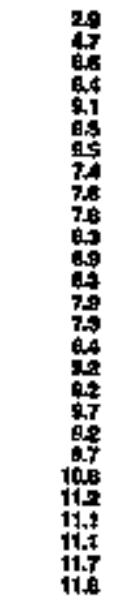 & 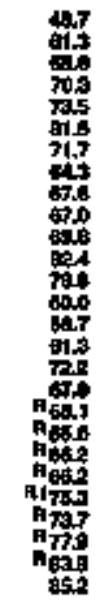 & 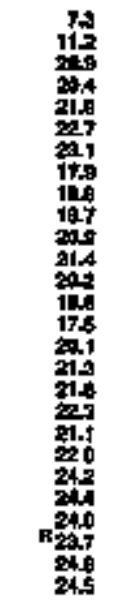 & 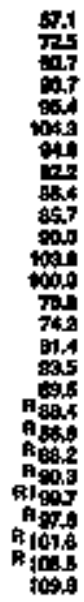 \\
\hline
\end{tabular}

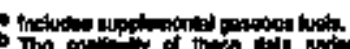

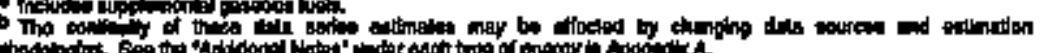

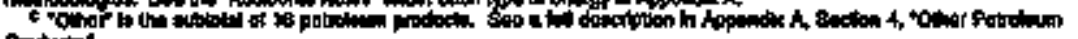

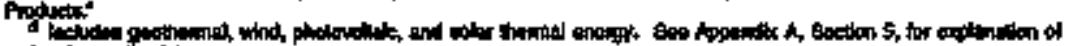

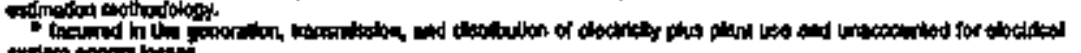

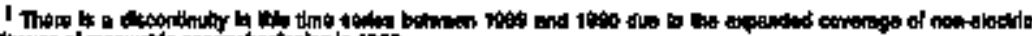

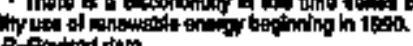
fimitod

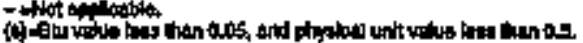




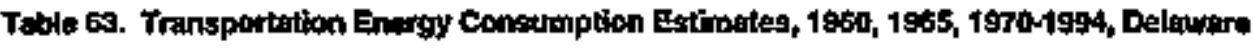

\begin{tabular}{|c|c|c|c|c|c|c|c|c|c|c|c|c|c|c|c|}
\hline \multirow[b]{3}{*}{ Yar| } & \multirow{3}{*}{ 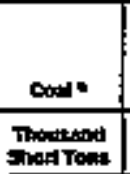 } & \multirow{3}{*}{ 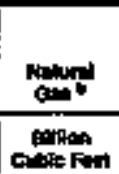 } & \multicolumn{8}{|c|}{ Patroinen } & \multirow{3}{*}{ moknte" } & \multirow[b]{2}{*}{ Enevinety } & \multirow[b]{2}{*}{ Enol } & \multirow[b]{2}{*}{ Enetreal syats } & \multirow[b]{3}{*}{ Tots } \\
\hline & & & Avitifon. & Oniming & 证, & $-100 *$ & undeank ' & Notor & Reglesul & Toll & & & & & \\
\hline & & & \multicolumn{8}{|c|}{ thomene Burth } & & \multicolumn{3}{|c|}{ Doo Kowallbours: } & \\
\hline 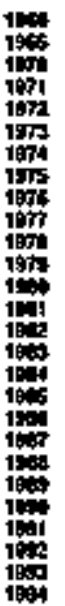 & $\begin{array}{l}0 \\
0 \\
0 \\
0 \\
0 \\
0 \\
0 \\
0 \\
0 \\
0 \\
0 \\
0 \\
0 \\
0 \\
0 \\
0 \\
0 \\
0 \\
0 \\
0 \\
0 \\
0 \\
0\end{array}$ & 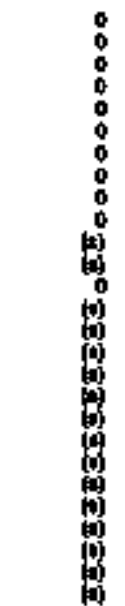 & 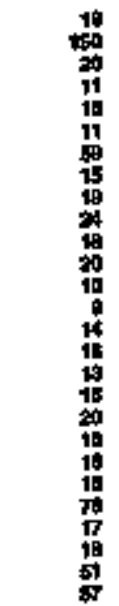 & 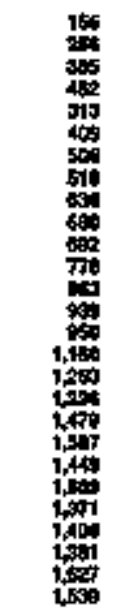 & 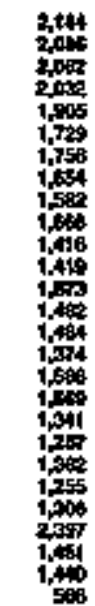 & 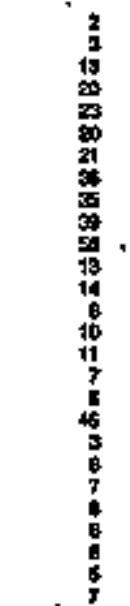 & 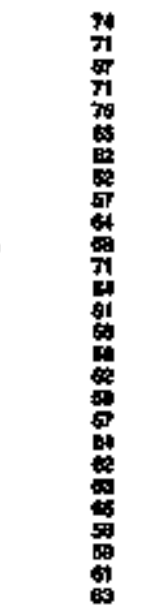 & 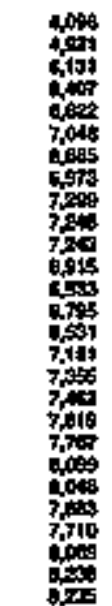 & 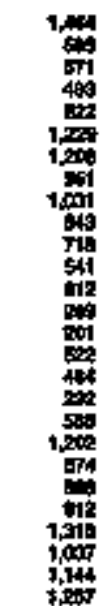 & 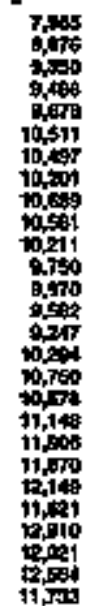 & $\begin{array}{l}0 \\
0 \\
0 \\
0 \\
0 \\
0 \\
0 \\
0 \\
0 \\
0 \\
0 \\
0 \\
0 \\
0 \\
0 \\
0 \\
0 \\
0 \\
0 \\
0 \\
0 \\
0 \\
0 \\
0 \\
0 \\
0 \\
0 \\
0\end{array}$ & $\begin{array}{l}0 \\
0 \\
0 \\
0 \\
0 \\
0 \\
0 \\
0 \\
0 \\
0 \\
0 \\
0 \\
0 \\
0 \\
0 \\
0 \\
0 \\
0 \\
0 \\
0 \\
0 \\
0 \\
0 \\
0 \\
0\end{array}$ & $\begin{array}{l}z \\
z \\
z \\
z \\
z \\
z \\
z \\
z \\
z \\
z \\
z \\
z \\
z \\
z\end{array}$ & $\begin{array}{l}0 \\
0 \\
0 \\
0 \\
0 \\
0 \\
0 \\
0 \\
0 \\
0 \\
0 \\
0 \\
0 \\
0 \\
0 \\
0 \\
0 \\
0 \\
0 \\
0 \\
0 \\
0 \\
0 \\
0\end{array}$ & 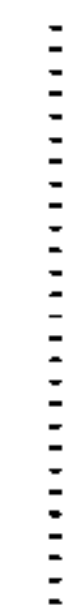 \\
\hline
\end{tabular}

\begin{tabular}{|c|c|c|c|c|c|c|c|c|c|c|c|c|c|c|c|}
\hline 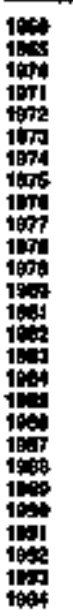 & 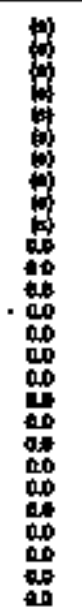 & 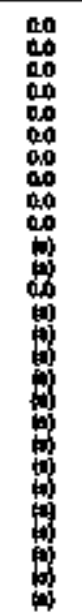 & 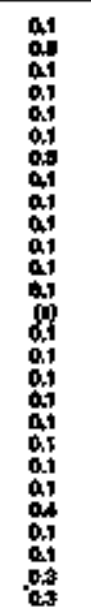 & 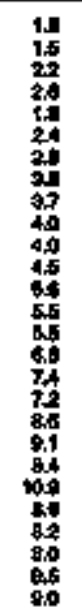 & 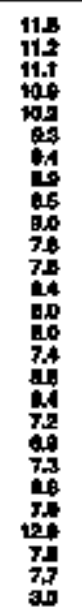 & 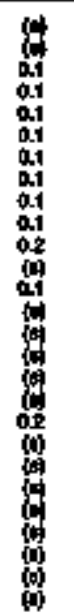 & 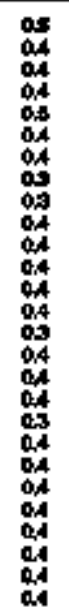 & 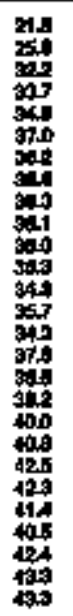 & 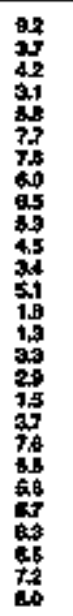 & 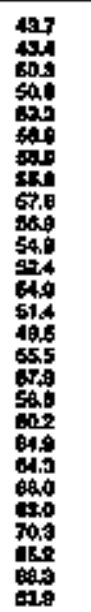 & 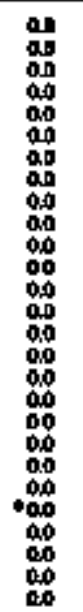 & 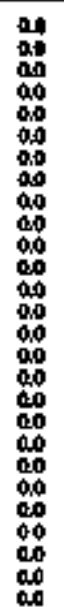 & 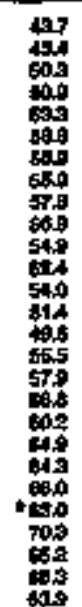 & 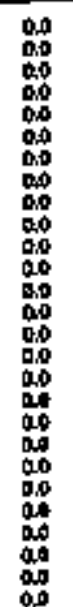 & 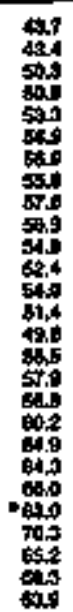 \\
\hline
\end{tabular}

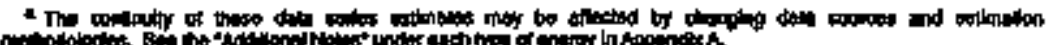

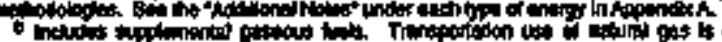

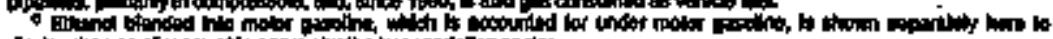

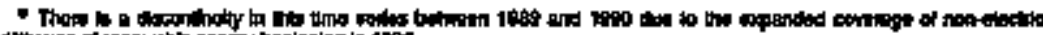

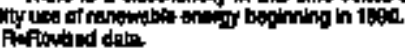

Amingad digh

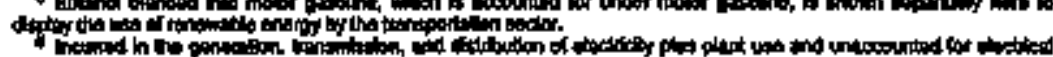

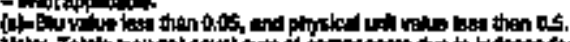

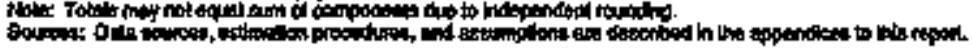

pation the 
$\mathbf{D}$
$\mathbf{E}$
$\mathbf{L}$
$\mathbf{A}$
$\mathbf{W}$
$\mathbf{A}$
$\mathbf{R}$
$\mathbf{E}$

Table 64. Estimates of Energy Input at Electric Uübthes, 1860, 1985, 1970-1994, Delaware

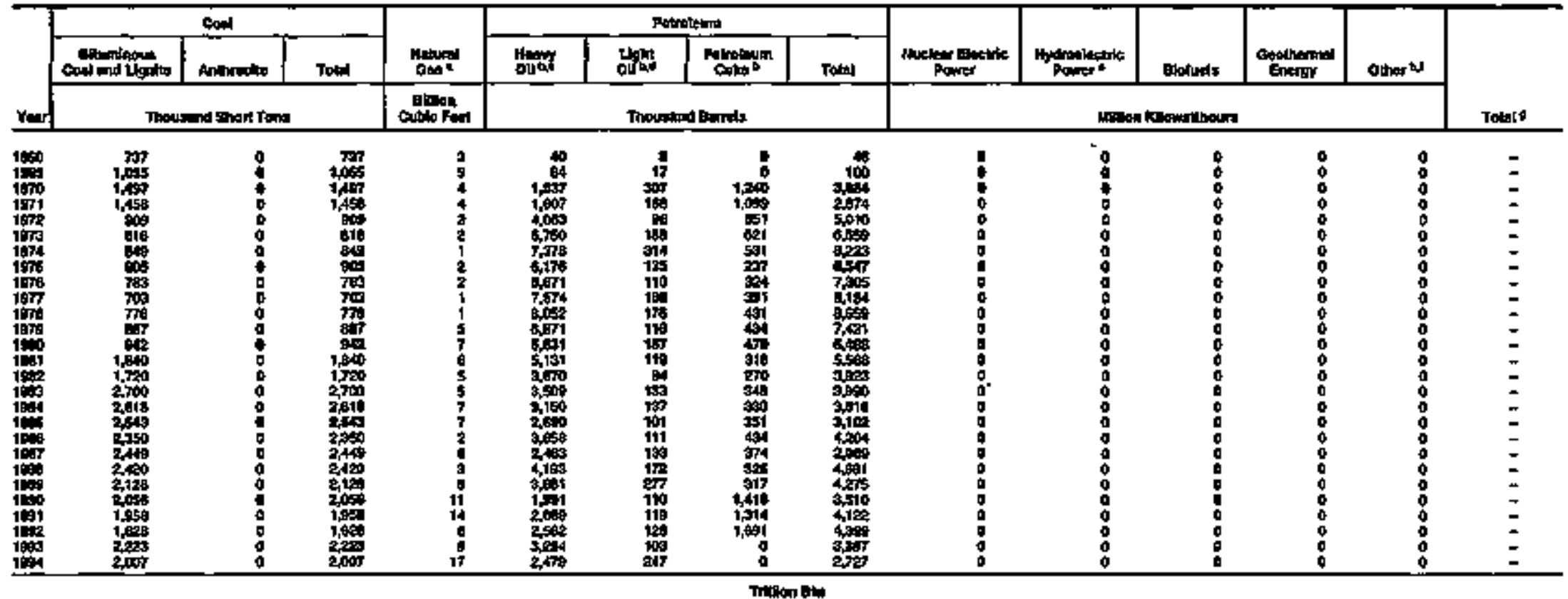

\begin{tabular}{|c|c|c|c|c|c|c|c|c|c|c|c|c|c|c|}
\hline 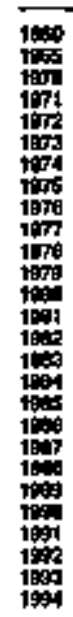 & 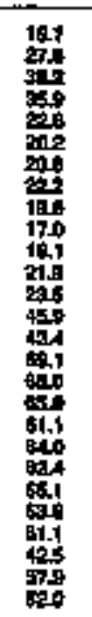 & 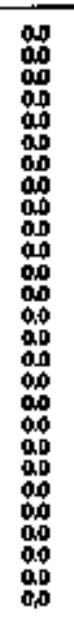 & 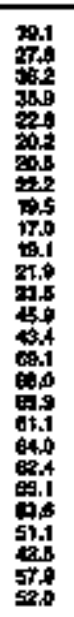 & 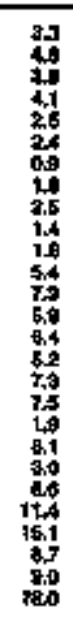 & 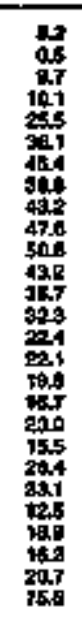 & 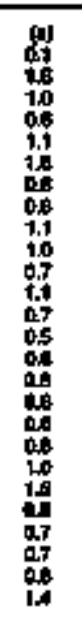 & 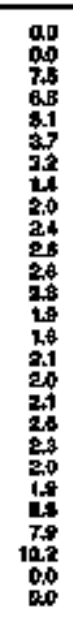 & 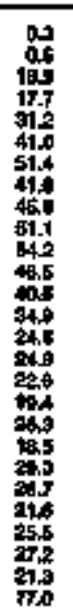 & 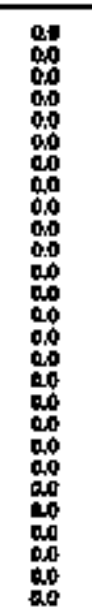 & 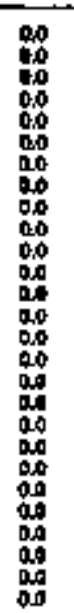 & 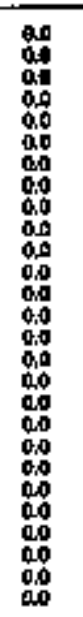 & 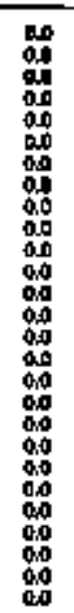 & 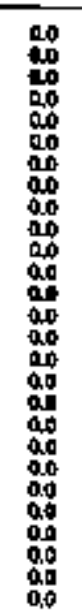 & 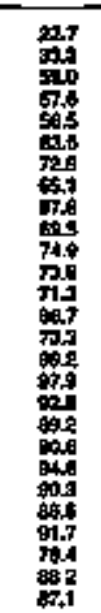 \\
\hline
\end{tabular}

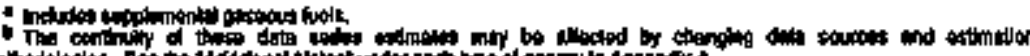

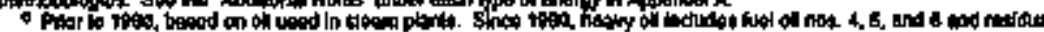

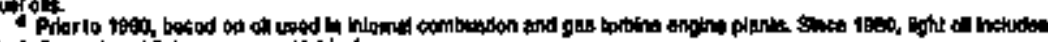

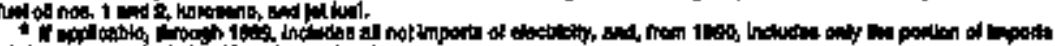

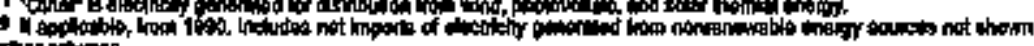

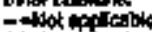

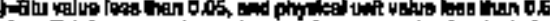

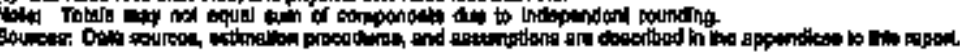


Table 65. Energy Consumption Estimalas by Sowre, 1960, 1965, 1970-1994, Distritot of Cohumble

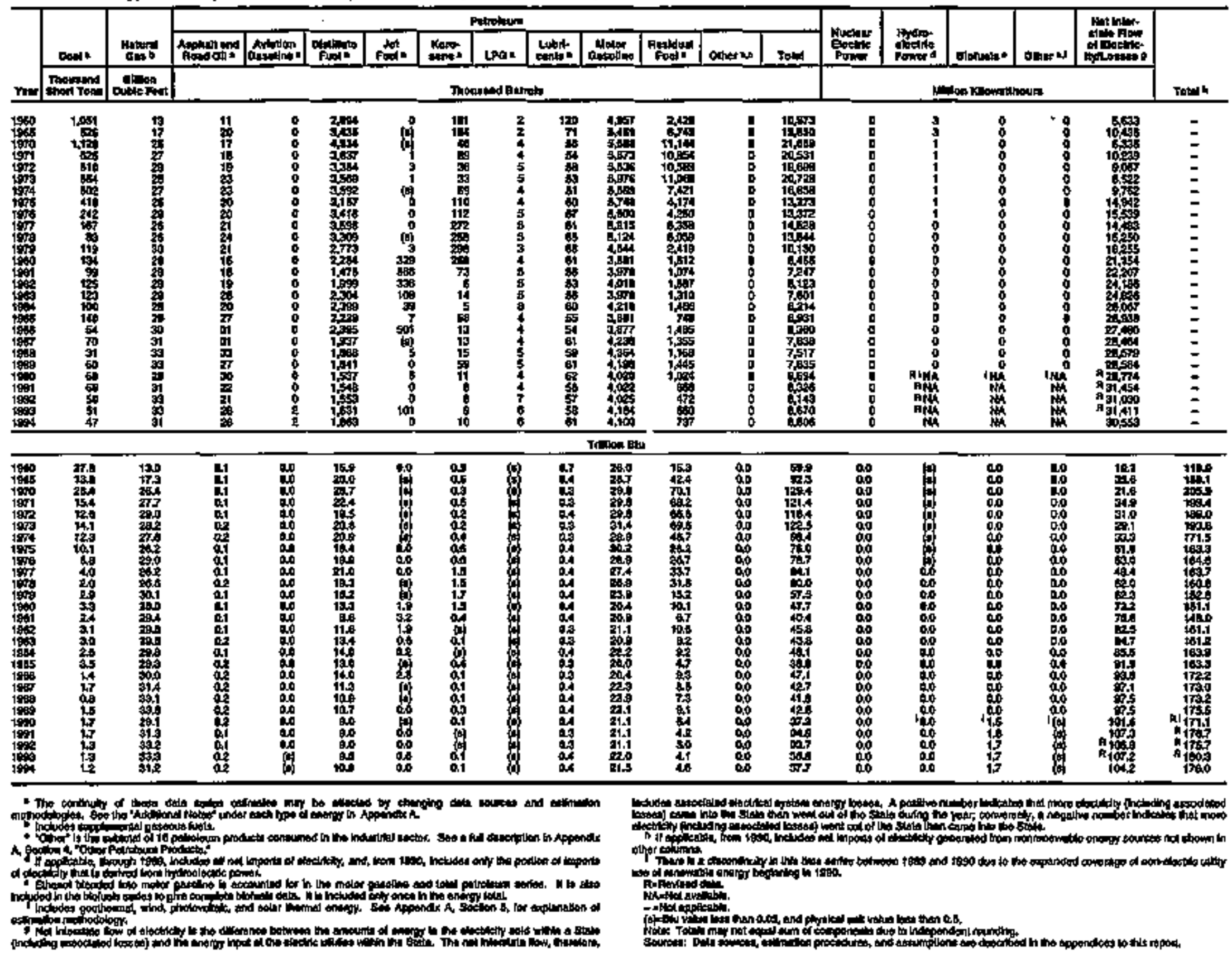


Table 66. Res/dentlal Energy Consumption Estimates, 1950, 1965, 1970-1994, Dlstrict of Columbla

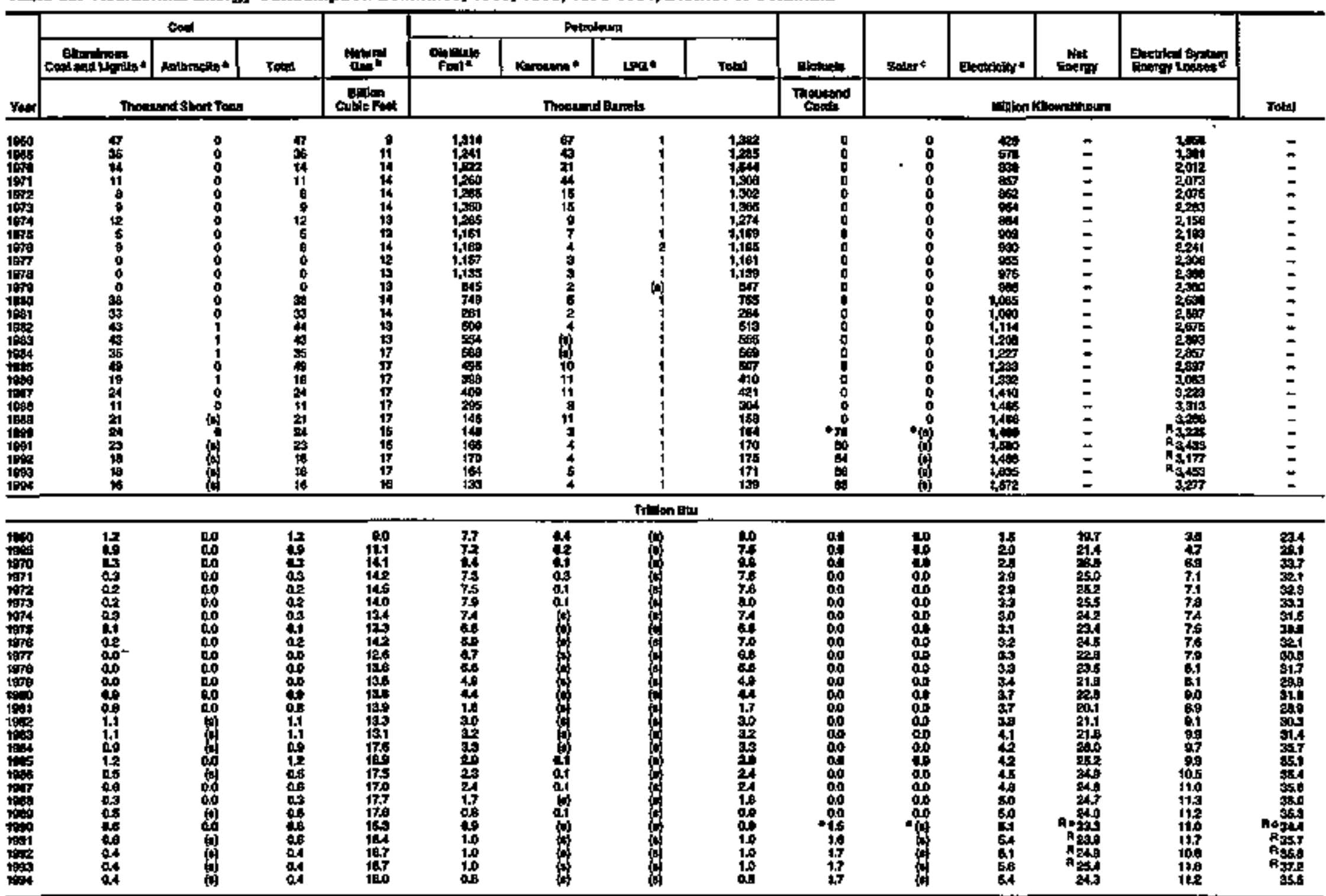

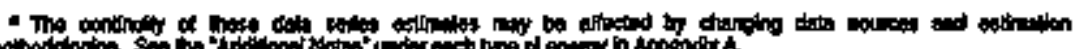

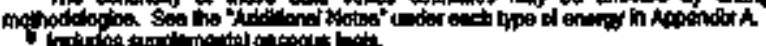

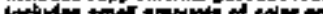

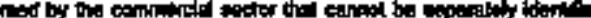

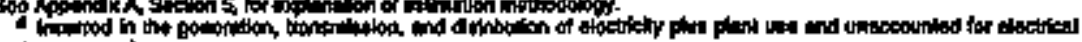

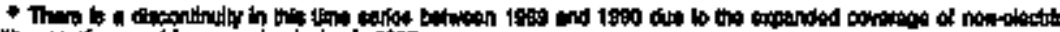

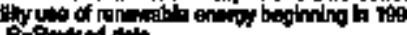


Table 67. Commercial Enongy Consumption Estimates, 1960, 1965, 1970-1994, Dtstrict of Columbia

\begin{tabular}{|c|c|c|c|c|c|c|c|c|c|c|c|c|c|c|}
\hline \multirow[b]{3}{*}{ Yom } & \multicolumn{3}{|c|}{ toe } & \multirow{3}{*}{ 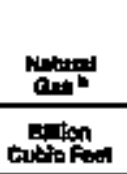 } & \multicolumn{6}{|c|}{ 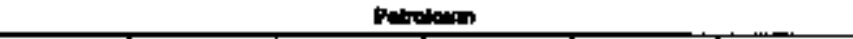 } & \multirow[b]{2}{*}{ Intaldsy } & \multirow[b]{2}{*}{ Enet } & \multirow[b]{2}{*}{ 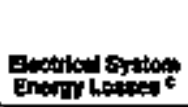 } & \multirow[b]{3}{*}{ Taten } \\
\hline & 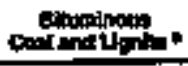 & " & Toted & & Foil: & noteren $n$ & LPo" & motor & Fille & Totol & & & & \\
\hline & \multicolumn{2}{|c|}{ Thouend Shent Tars } & & & \multicolumn{6}{|c|}{ rownsnd tearets } & \multicolumn{3}{|c|}{ 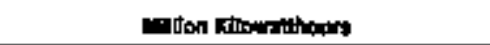 } & \\
\hline 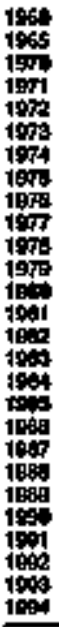 & 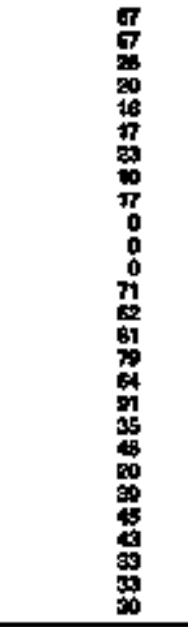 & 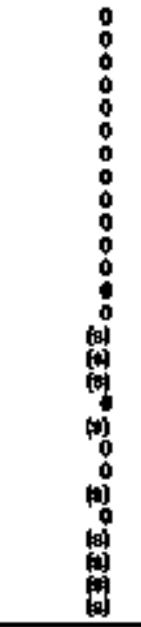 & 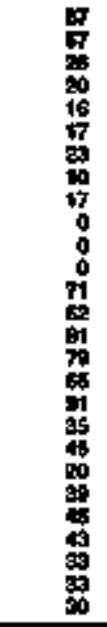 & $\begin{array}{l}4 \\
5 \\
12 \\
13 \\
14 \\
13 \\
13 \\
12 \\
14 \\
13 \\
12 \\
16 \\
14 \\
18 \\
16 \\
16 \\
12 \\
18 \\
18 \\
14 \\
15 \\
16 \\
17 \\
16 \\
16 \\
16 \\
15 \\
\end{array}$ & 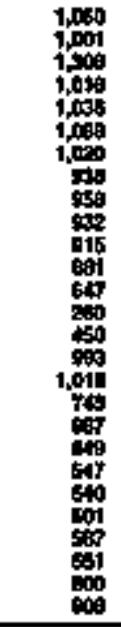 & 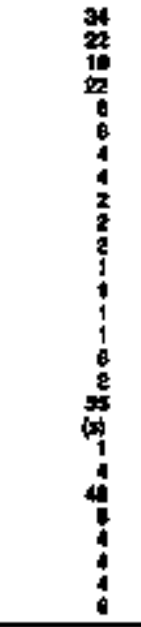 & 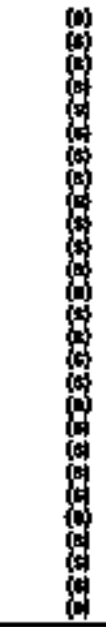 & 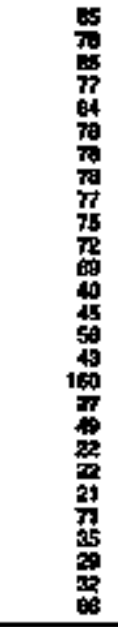 & 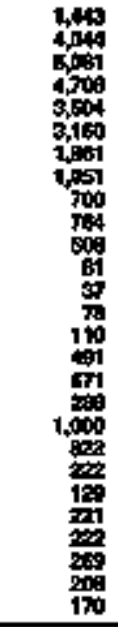 & 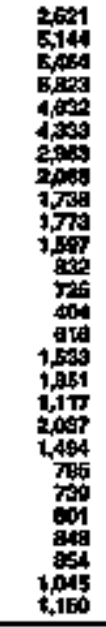 & 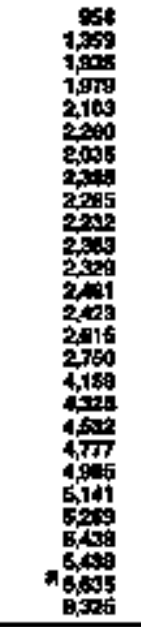 & $\begin{array}{l}= \\
= \\
= \\
= \\
= \\
= \\
= \\
= \\
= \\
= \\
= \\
= \\
= \\
= \\
= \\
\end{array}$ & 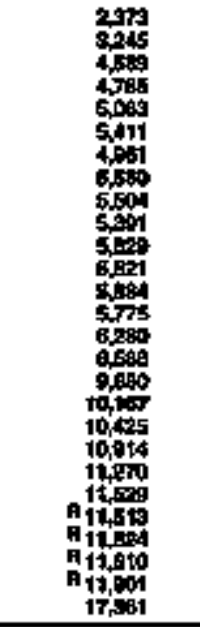 & $\begin{array}{l}= \\
= \\
= \\
= \\
= \\
= \\
= \\
= \\
= \\
= \\
= \\
= \\
= \\
= \\
=\end{array}$ \\
\hline \multicolumn{15}{|c|}{ Tdilon Bu } \\
\hline 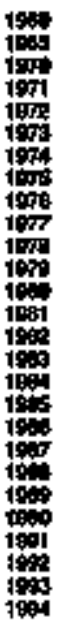 & 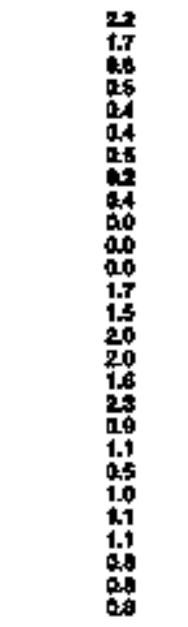 & 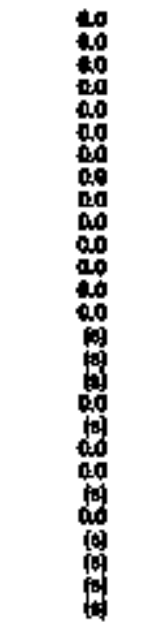 & 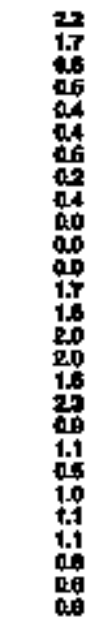 & 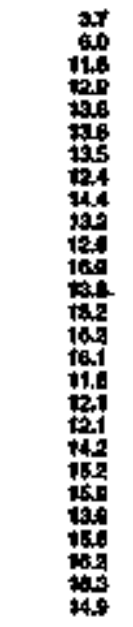 & 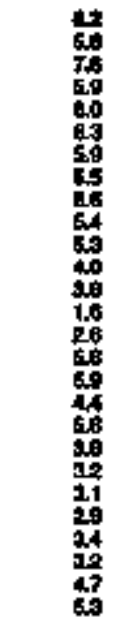 & 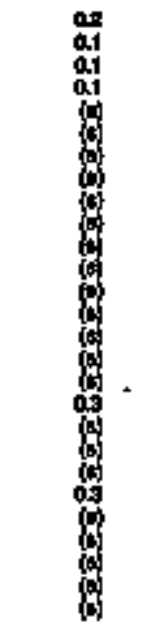 & 8 & 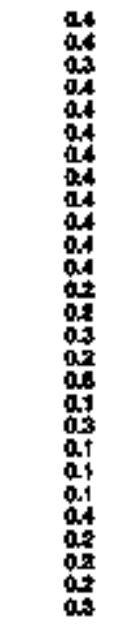 & 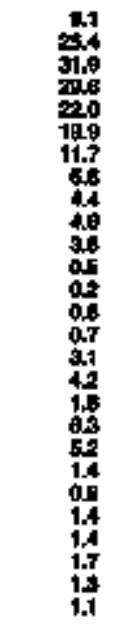 & 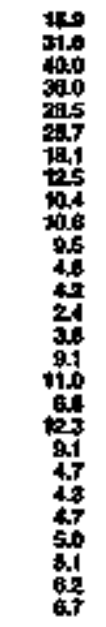 & 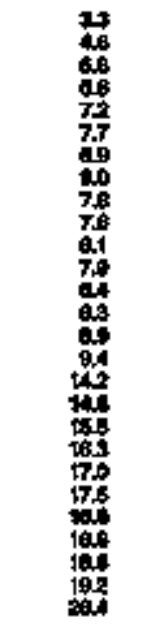 & 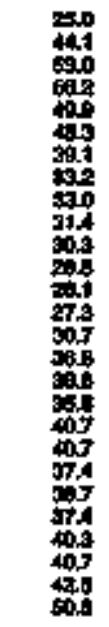 & 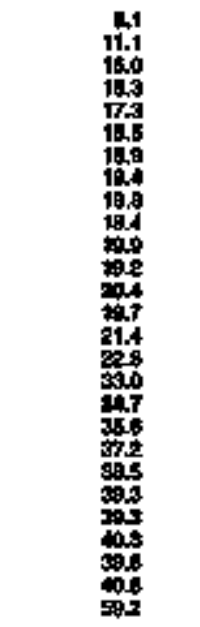 & 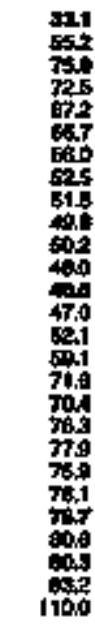 \\
\hline
\end{tabular}

D
$\mathbf{1}$
$\mathbf{S}$
$\mathbf{T}$
$\mathbf{R}$
$\mathbf{C}$
$\mathbf{C}$
$\mathbf{T}$
$\mathbf{0}$
$\mathbf{F}$
$\mathbf{C}$
$\mathbf{0}$
$\mathbf{L}$
$\mathbf{M}$
$\mathbf{B}$
$\mathbf{A}$
$\mathbf{A}$ 
Table 66. Industrlal Eaergy Consumption Eallmates, 19:4, 1955, 1970-1994, Dlstrict of Columbla

\begin{tabular}{|c|c|c|c|c|c|c|c|c|c|c|c|c|c|c|c|c|c|c|}
\hline \multirow[b]{3}{*}{ Yex } & \multirow[b]{2}{*}{ cont } & \multirow[b]{2}{*}{ thent } & \multicolumn{9}{|c|}{ 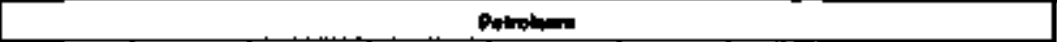 } & \multirow[b]{2}{*}{ 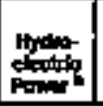 } & \multirow[b]{2}{*}{ elatwet } & \multirow[b]{2}{*}{ Othor hed | } & \multirow[b]{2}{*}{ anesolks b } & \multirow[b]{2}{*}{ Entror } & \multirow{3}{*}{ 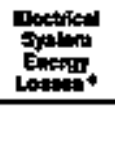 } & \multirow[b]{3}{*}{ Tomap } \\
\hline & & & Andition & 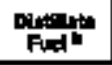 & $x+4000$ & LPQ" & 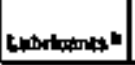 & ontat & Fostall & Oon & Yotw & & & & & & & \\
\hline & $\begin{array}{l}\text { Thoupend } \\
\text { steont thats }\end{array}$ & 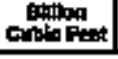 & \multicolumn{9}{|c|}{ 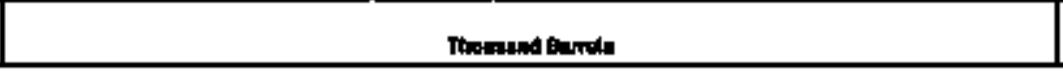 } & \multicolumn{5}{|c|}{ 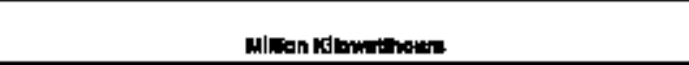 } & & \\
\hline 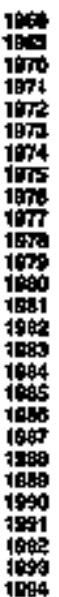 & 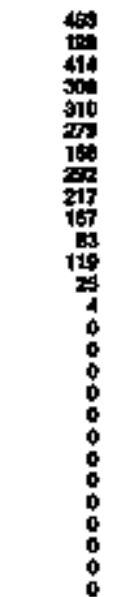 & 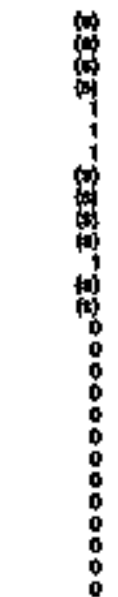 & 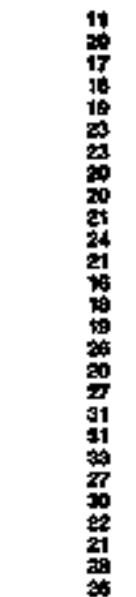 & 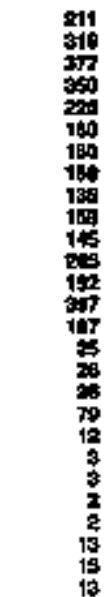 & 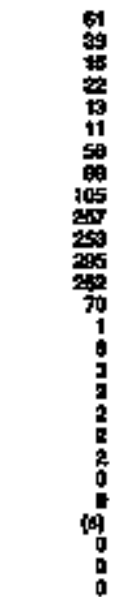 & $\begin{array}{l}1 \\
1 \\
2 \\
2 \\
3 \\
2 \\
2 \\
2 \\
2 \\
2 \\
2 \\
3 \\
3 \\
3 \\
3 \\
4 \\
4 \\
1 \\
2 \\
2 \\
3 \\
3 \\
3 \\
2 \\
2 \\
5 \\
3 \\
2\end{array}$ & $\begin{array}{c}1 \\
11 \\
5 \\
0 \\
0 \\
1 \\
1 \\
4 \\
15 \\
7 \\
1 \\
7 \\
7 \\
7 \\
7 \\
7 \\
7 \\
7 \\
7 \\
7 \\
7 \\
7 \\
7\end{array}$ & $\begin{array}{l}0 \\
0 \\
0 \\
0 \\
0 \\
0 \\
0 \\
0 \\
0 \\
0 \\
0 \\
0 \\
0 \\
0 \\
0 \\
0 \\
0 \\
0 \\
50 \\
40 \\
00 \\
60 \\
65 \\
50 \\
50 \\
50 \\
30 \\
30 \\
70\end{array}$ & 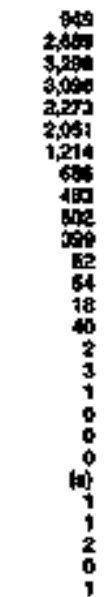 & $\begin{array}{l}0 \\
0 \\
0 \\
0 \\
0 \\
0 \\
0 \\
0 \\
0 \\
0 \\
0 \\
0 \\
0 \\
0 \\
0 \\
0 \\
0 \\
0 \\
0 \\
0 \\
0 \\
0\end{array}$ & 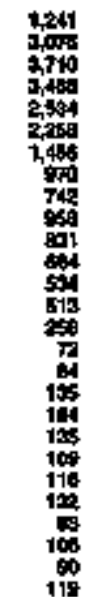 & 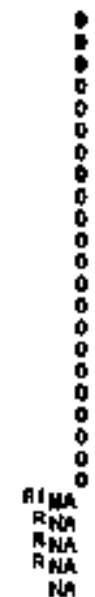 & 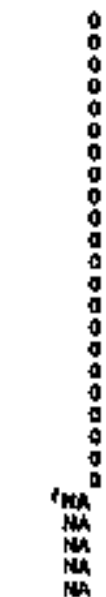 & 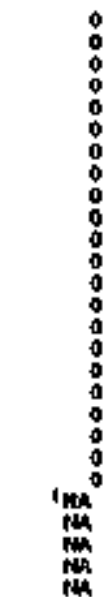 & 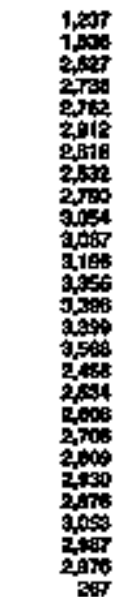 & $\begin{array}{l}= \\
= \\
z \\
= \\
= \\
z \\
= \\
z \\
z \\
z \\
z \\
z \\
z \\
z\end{array}$ & 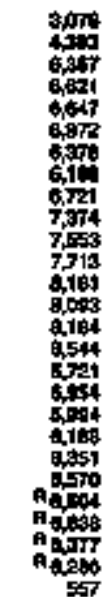 & 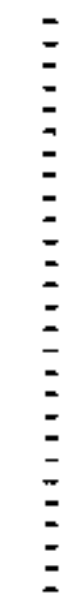 \\
\hline
\end{tabular}

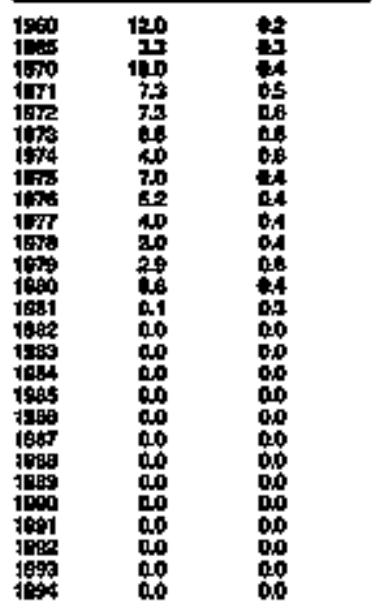

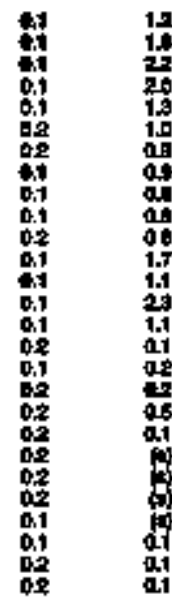

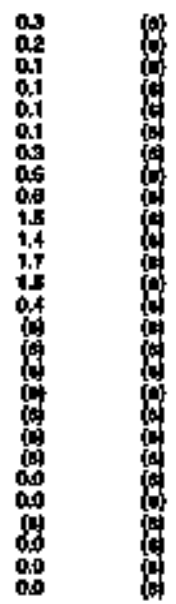

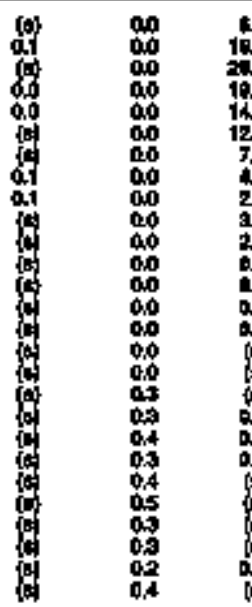

\begin{tabular}{|c|c|}
\hline $\begin{array}{l}00 \\
00 \\
00 \\
00 \\
00 \\
00 \\
000 \\
000 \\
000 \\
00 \\
00 \\
00 \\
00 \\
00 \\
00 \\
00 \\
00 \\
00 \\
00 \\
00 \\
00 \\
00 \\
00 \\
00 \\
00 \\
00 \\
00 \\
00\end{array}$ & 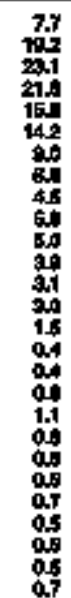 \\
\hline
\end{tabular}

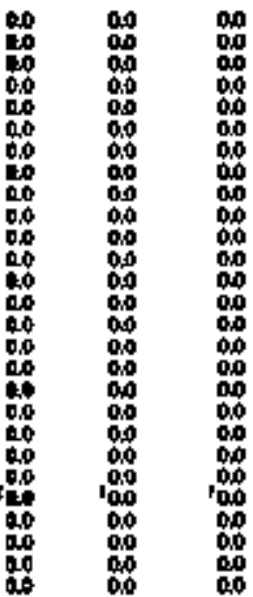

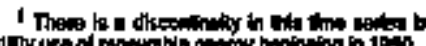

10
0
0

\begin{tabular}{|c|c|}
\hline 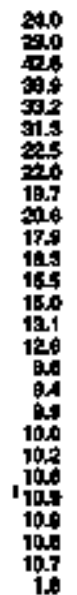 & 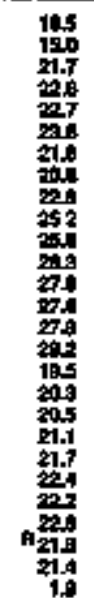 \\
\hline
\end{tabular}

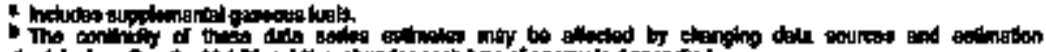

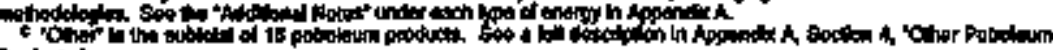
Profthich

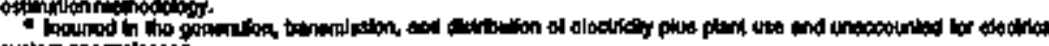

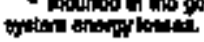


Table 68. Transportation Enorgy Consumption Estimates, 1950, 1965, 1970-1994, olshict pi Columbla

\begin{tabular}{|c|c|c|c|c|c|c|c|c|c|c|c|c|c|c|c|}
\hline \multirow[b]{3}{*}{$\mathrm{Yur}$} & \multirow[b]{2}{*}{ sone" } & \multirow[b]{2}{*}{ inting } & \multicolumn{8}{|c|}{ Potrolous } & \multirow{3}{*}{ Thoulate } & \multirow[b]{2}{*}{ Etatioty } & \multirow[b]{2}{*}{$\lim _{\ln }$} & \multirow[b]{2}{*}{ 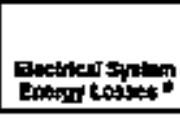 } & \multirow[b]{3}{*}{ The } \\
\hline & & & midnion. & 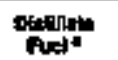 & int. & EPT: : & undanom: & $\underset{\text { mintor }}{\log }$ & 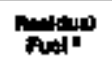 & Totold & & & & & \\
\hline & Thoution & cimpian & \multicolumn{8}{|c|}{ 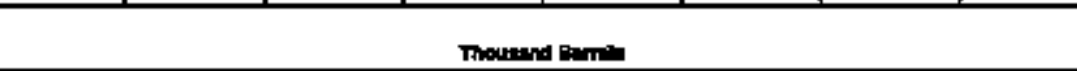 } & & \multicolumn{3}{|c|}{ | } & \\
\hline 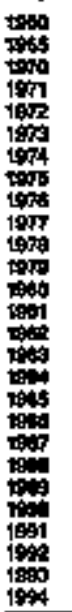 & 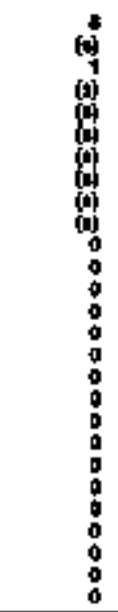 & 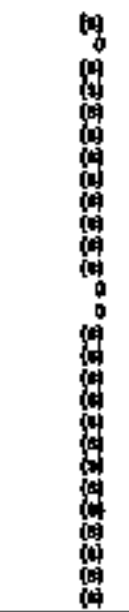 & $\begin{array}{l}0 \\
: \\
0 \\
0 \\
0 \\
0 \\
0 \\
0 \\
0 \\
0 \\
0 \\
0 \\
0 \\
0 \\
0 \\
0 \\
0 \\
0 \\
0 \\
0 \\
0 \\
0 \\
0 \\
2 \\
2\end{array}$ & 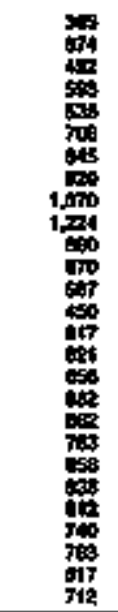 & 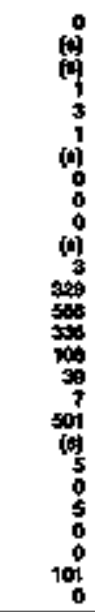 & 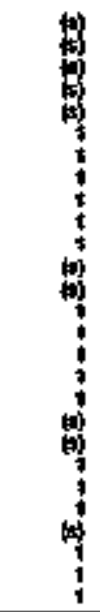 & 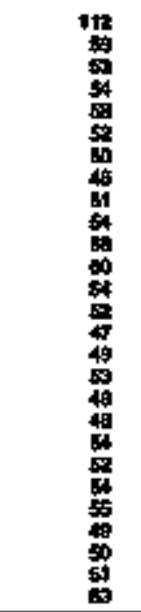 & 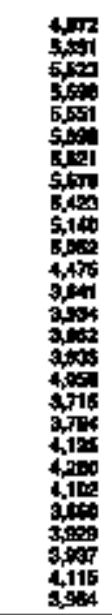 & 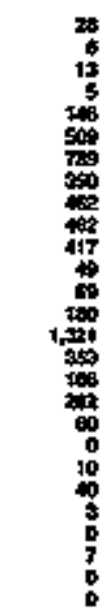 & 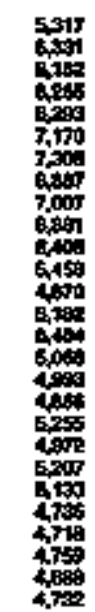 & $\begin{array}{c}0 \\
0 \\
0 \\
0 \\
0 \\
0 \\
0 \\
0 \\
0 \\
0 \\
0 \\
0 \\
0 \\
0 \\
0 \\
0 \\
0 \\
0 \\
0 \\
0 \\
0 \\
0 \\
0 \\
0 \\
0 \\
0 \\
0 \\
0\end{array}$ & 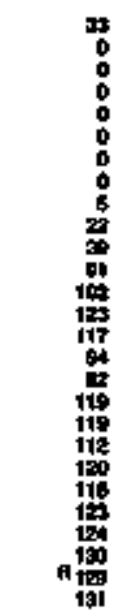 & $\begin{array}{l}= \\
= \\
= \\
= \\
= \\
= \\
= \\
= \\
= \\
= \\
= \\
= \\
= \\
=\end{array}$ & 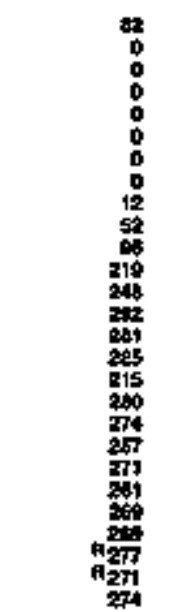 & 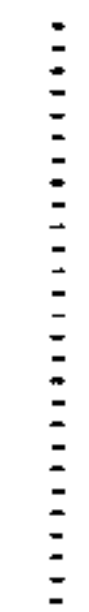 \\
\hline \multicolumn{16}{|c|}{ Thing } \\
\hline 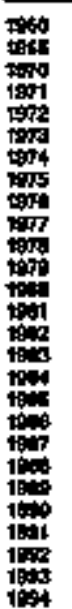 & 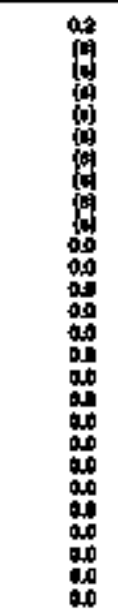 & 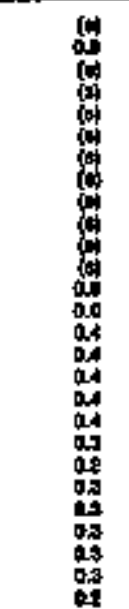 & 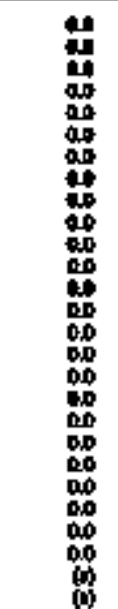 & 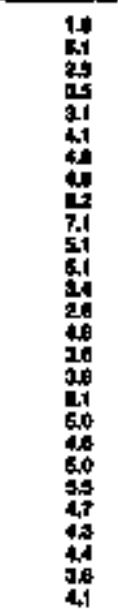 & 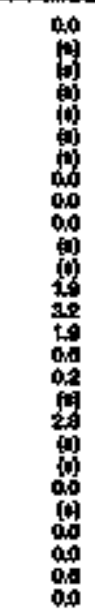 & 團 & 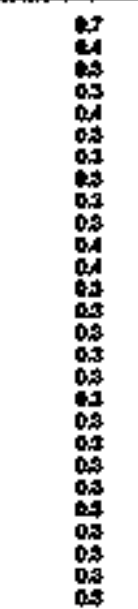 & 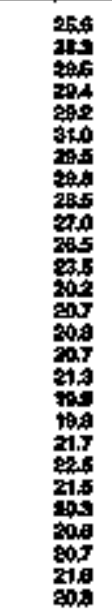 & 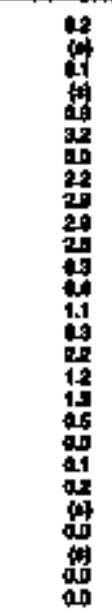 & 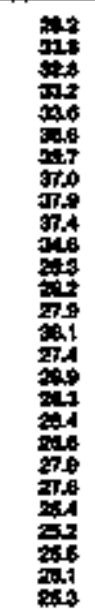 & 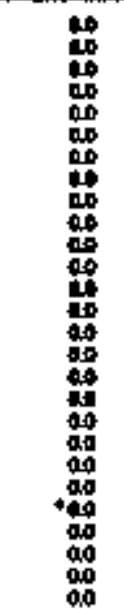 & 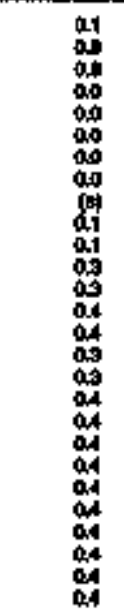 & 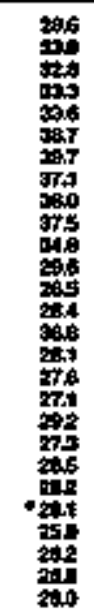 & 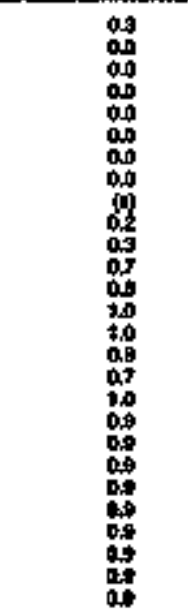 & 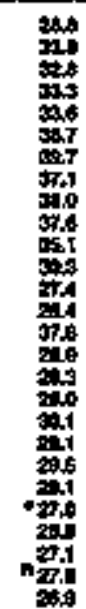 \\
\hline
\end{tabular}

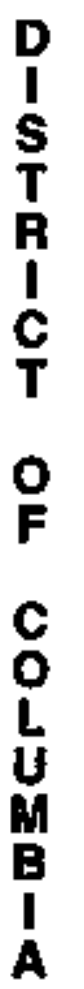

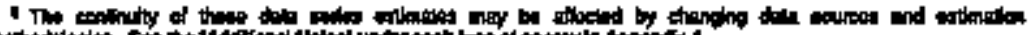

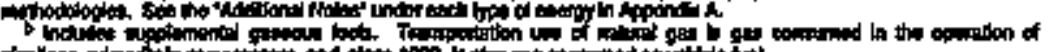

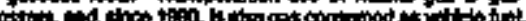

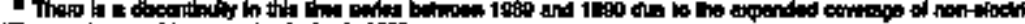

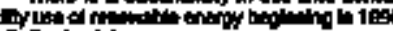
Aflatod dic

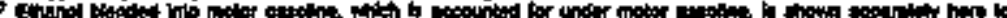

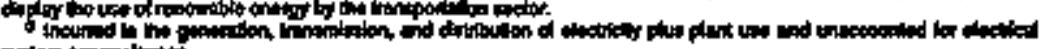

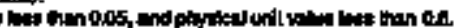

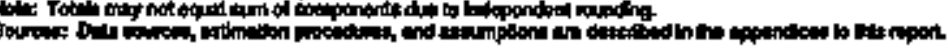


Table 70. Esthmales of Energy Input at Electric tolities, 1960, 1965, 1970-1994, Oistolot of Columbla

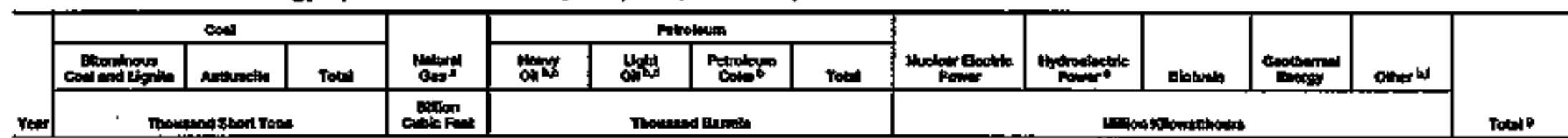

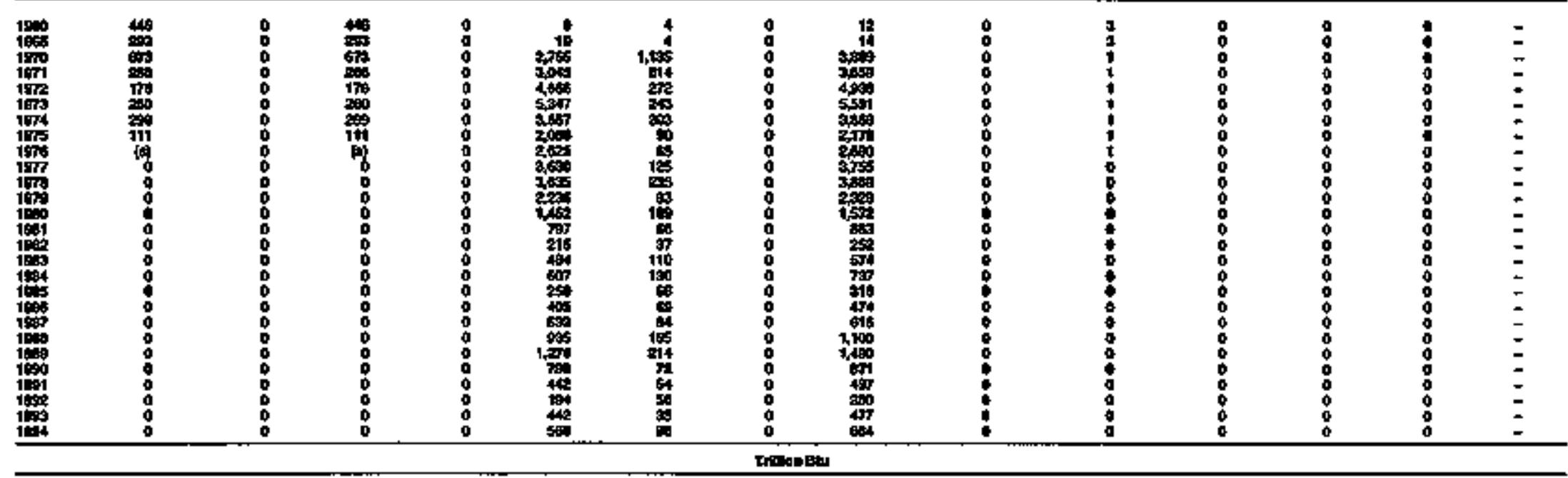

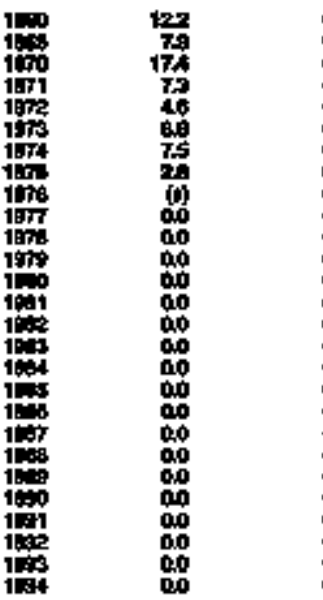

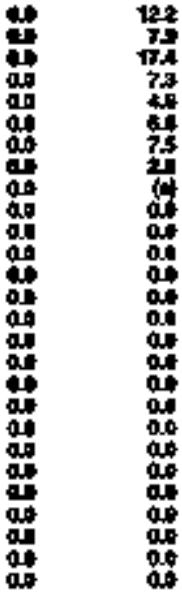

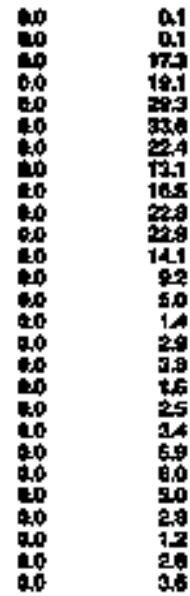

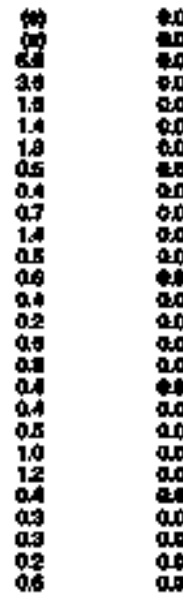

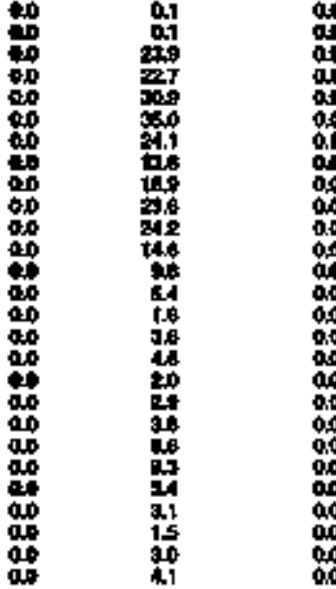

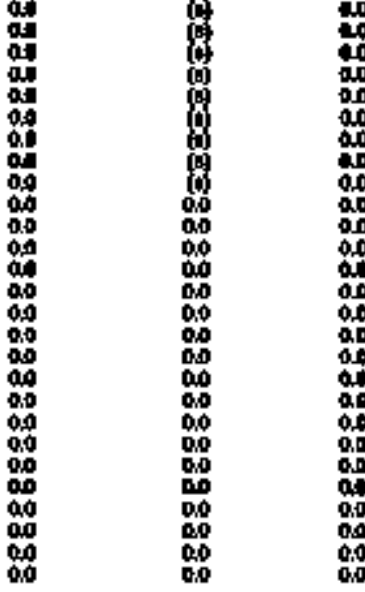

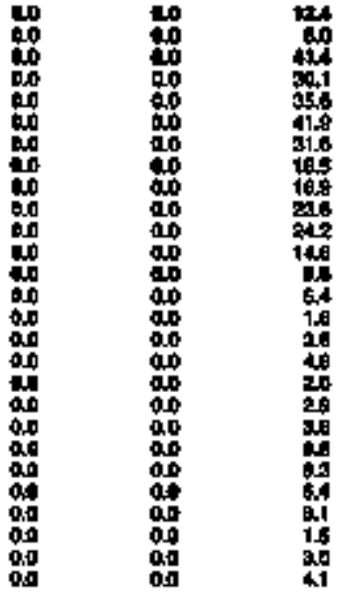

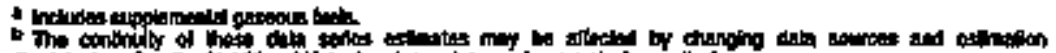

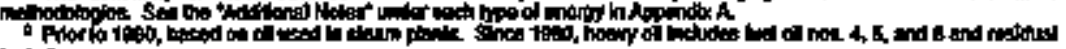

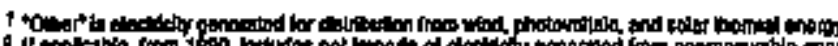

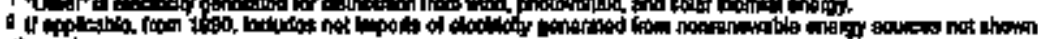

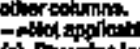

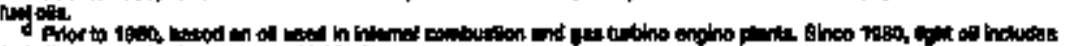

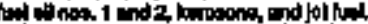

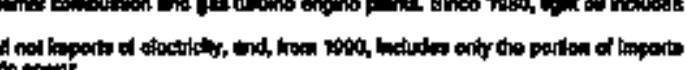

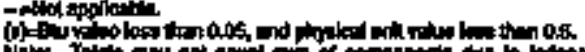

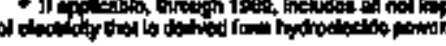


Table 71. Energy Consumption Estimates by Source, 1960, 1965, 1970-1994, Florida

\begin{tabular}{|c|c|c|c|c|c|c|c|c|c|c|c|c|c|c|c|c|c|c|c|}
\hline \multirow[b]{3}{*}{ : } & \multirow[b]{2}{*}{ cost. } & \multirow[b]{2}{*}{ maner } & \multicolumn{11}{|c|}{ Pyopolen } & \multirow{2}{*}{ 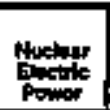 } & \multirow[b]{2}{*}{ 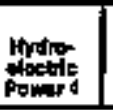 } & \multirow[b]{2}{*}{ Blohwis: } & \multirow[b]{2}{*}{ 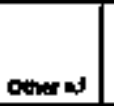 } & \multirow{3}{*}{ 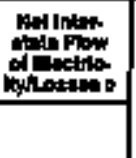 } & \multirow[b]{3}{*}{ יו- } \\
\hline & & & Mupheyind & Answitar & 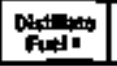 & Fon. & $\lim _{\min }$ & LPQ: & thit. & ansolition & 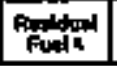 & Oalyos & Tot & & & & & & \\
\hline & Thanitad & 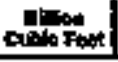 & + & \multicolumn{10}{|c|}{ 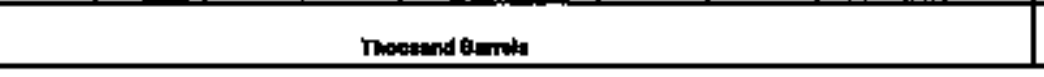 } & \multicolumn{4}{|c|}{ 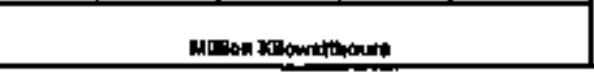 } & & \\
\hline 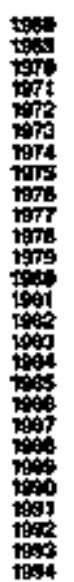 & 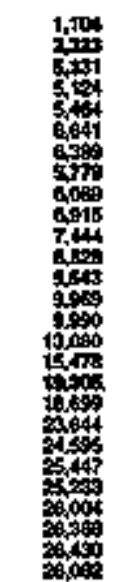 & 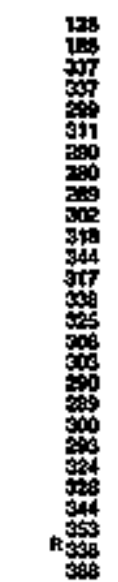 & 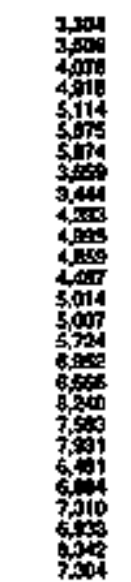 & 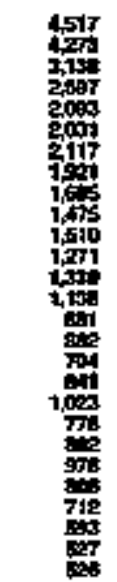 & 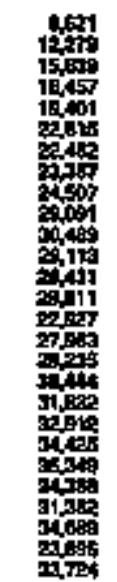 & 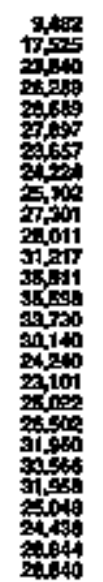 & 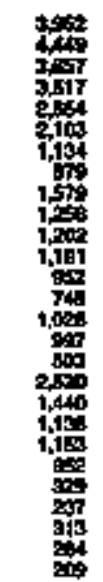 & 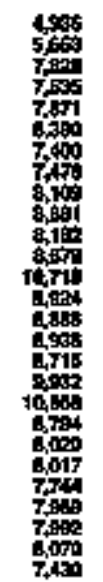 & 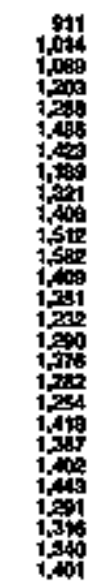 & 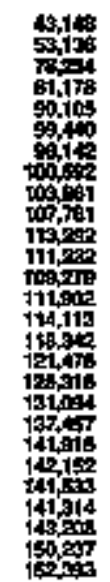 & 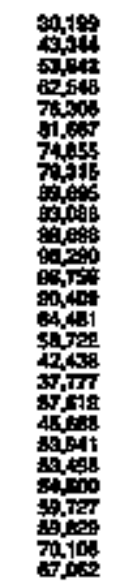 & 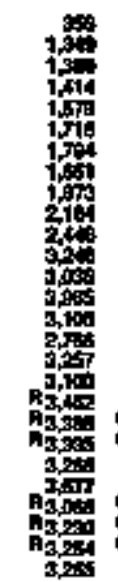 & 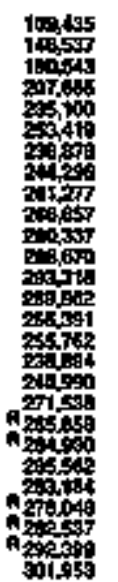 & 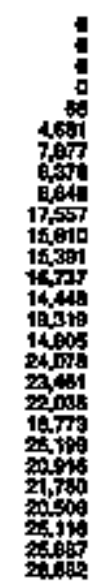 & 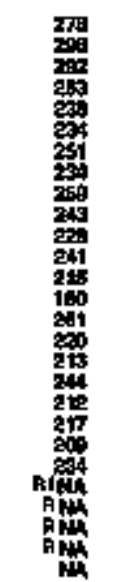 & 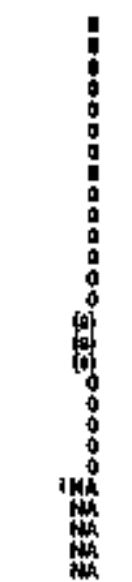 & 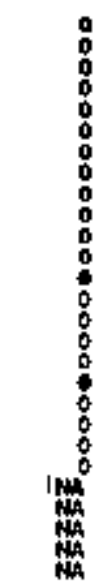 & 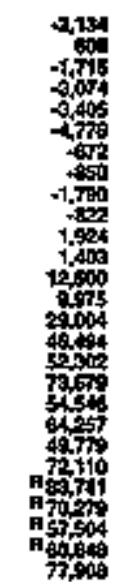 & $=$ \\
\hline & & & & & & & & & & & & & & & & & & & \\
\hline
\end{tabular}

\begin{tabular}{|c|c|c|c|c|c|c|c|c|c|c|c|c|c|c|c|c|c|c|c|}
\hline 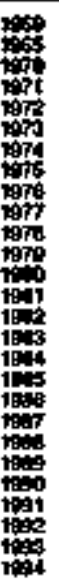 & 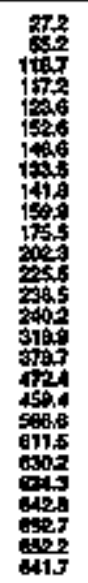 & 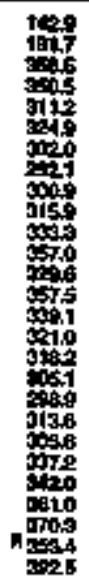 & 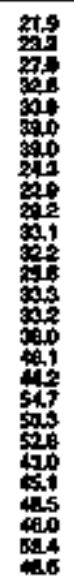 & 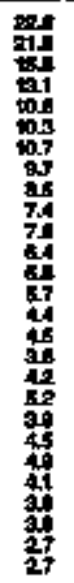 & 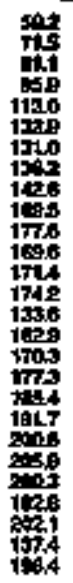 & 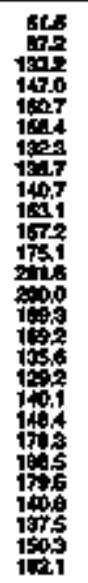 & 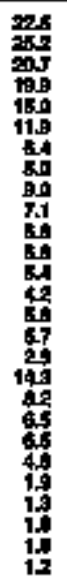 & 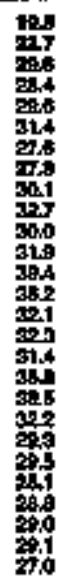 & 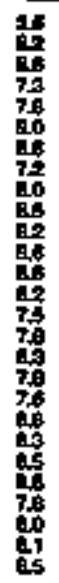 & 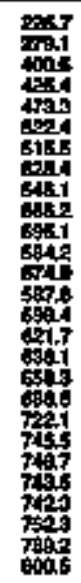 & 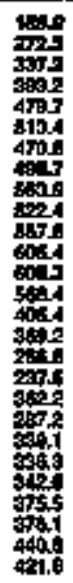 & 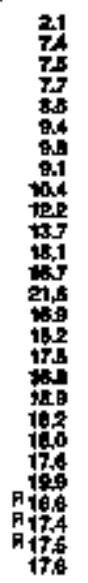 & 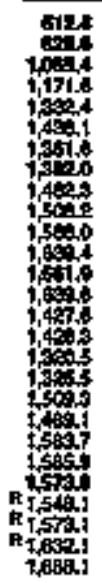 & 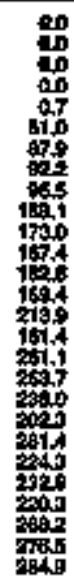 & 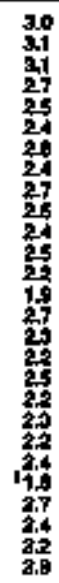 & 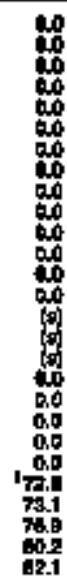 & 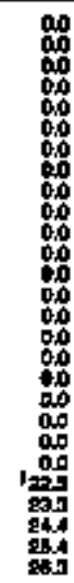 & 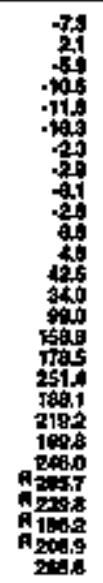 & 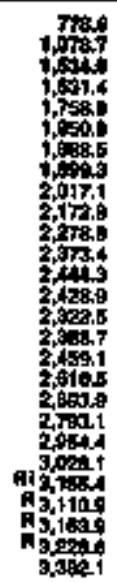 \\
\hline
\end{tabular}

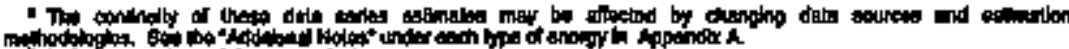

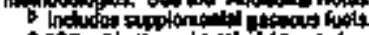

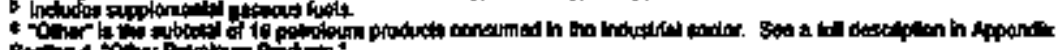

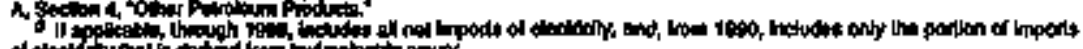

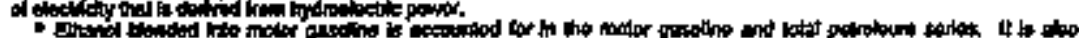

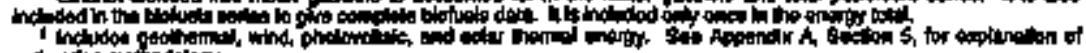

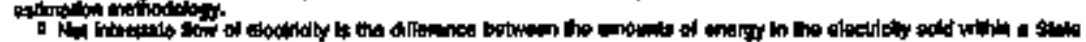

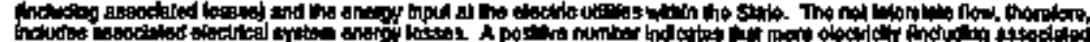

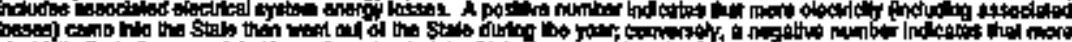

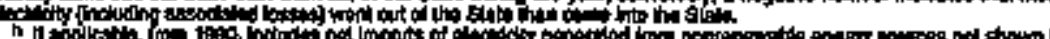

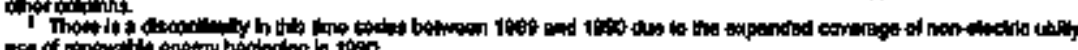
$\rightarrow x$ Hot

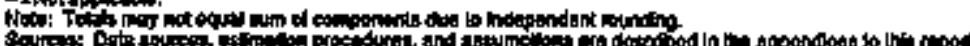




\begin{tabular}{|c|c|c|c|c|c|c|c|c|c|c|c|c|c|c|}
\hline \multirow[b]{3}{*}{ Your } & \multicolumn{3}{|c|}{ Cos } & \multirow[b]{2}{*}{ Mand } & \multicolumn{4}{|c|}{ Potrolum } & \multirow{3}{*}{ 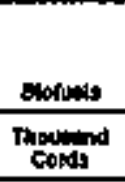 } & & \multirow[b]{2}{*}{ Enation=" } & \multirow[b]{2}{*}{ 些1 } & \multirow{3}{*}{ 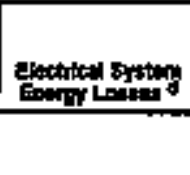 } & \multirow[b]{3}{*}{ Tots: } \\
\hline & 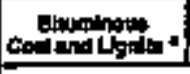 & 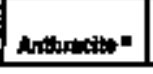 & Jold & & 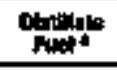 & Kompenens" & LPA: & Tots & & solure & & & & \\
\hline & \multicolumn{3}{|c|}{ Thoutind sinort Tond } & axtifion & \multicolumn{4}{|c|}{ 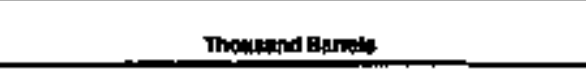 } & & \multicolumn{3}{|c|}{ Whon Kllowatition } & & \\
\hline 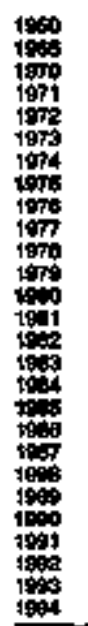 & 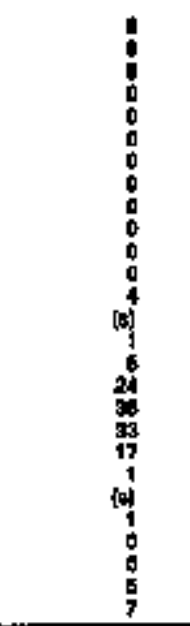 & 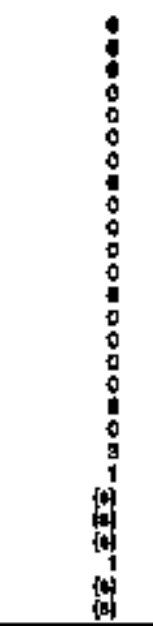 & 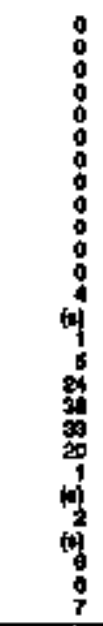 & 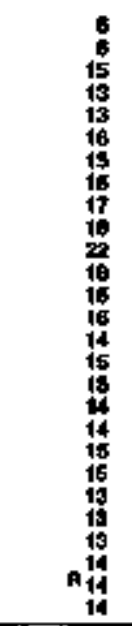 & 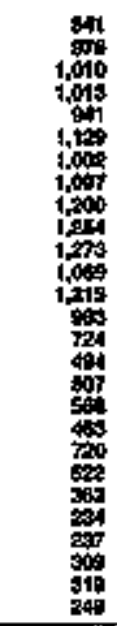 & 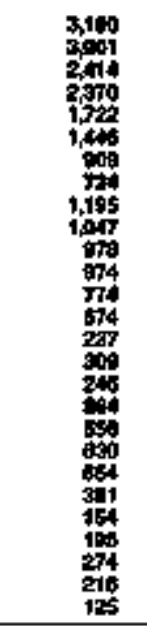 & 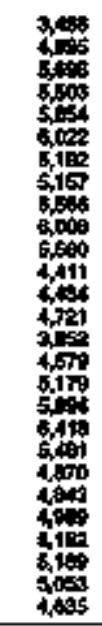 & 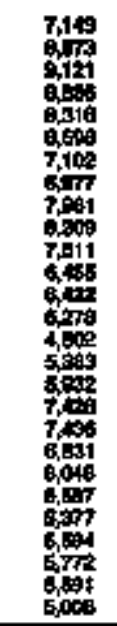 & 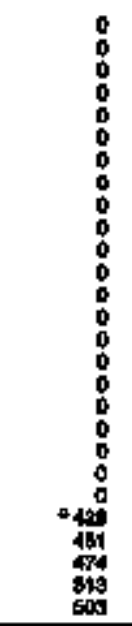 & 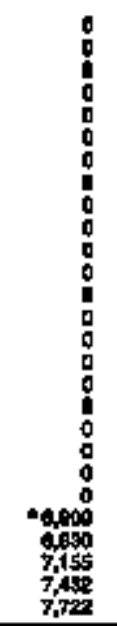 & 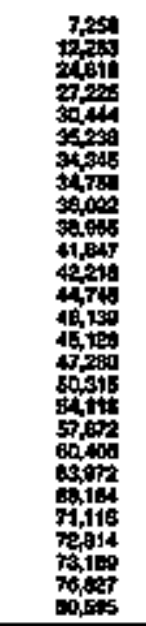 & $\begin{array}{l}\vdots \\
\vdots \\
\vdots \\
\vdots \\
\vdots \\
\vdots \\
\vdots \\
\vdots \\
\vdots \\
\vdots \\
\vdots \\
\vdots \\
\vdots\end{array}$ & 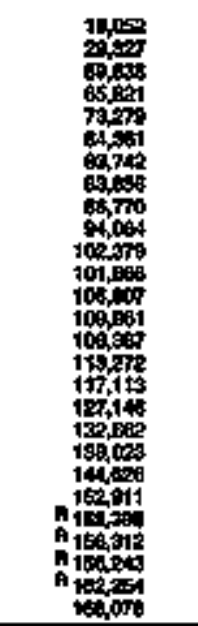 & $\begin{array}{l}\vdots \\
\vdots \\
\vdots \\
\vdots \\
\vdots \\
\vdots \\
\vdots \\
\vdots \\
\vdots \\
\vdots \\
\vdots \\
\vdots \\
\vdots\end{array}$ \\
\hline \multicolumn{15}{|c|}{$\operatorname{Tin}=0$} \\
\hline 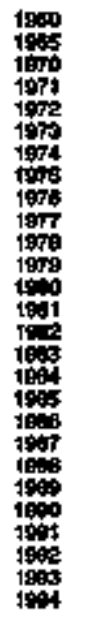 & 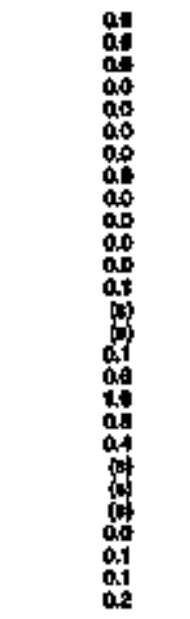 & 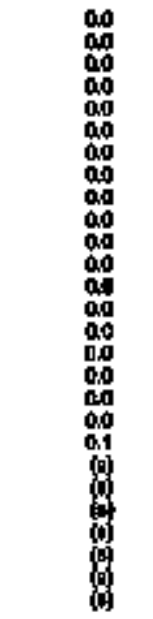 & 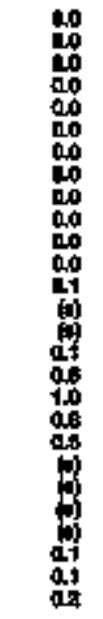 & 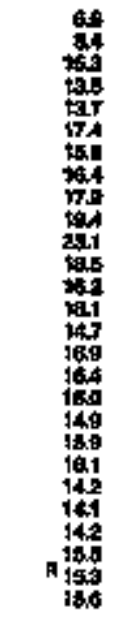 & 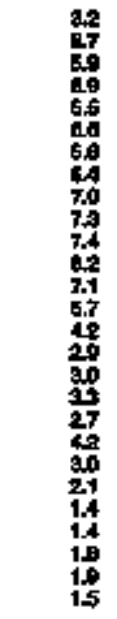 & 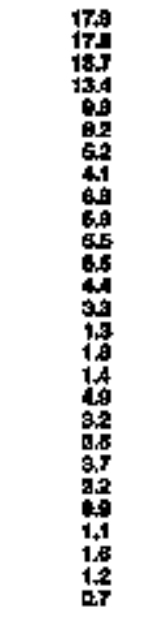 & 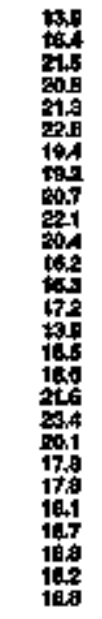 & 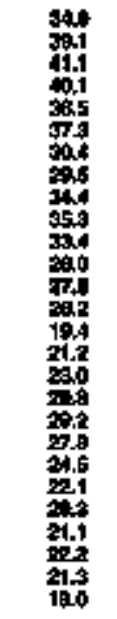 & 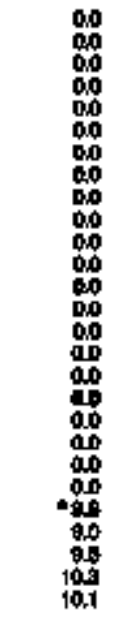 & 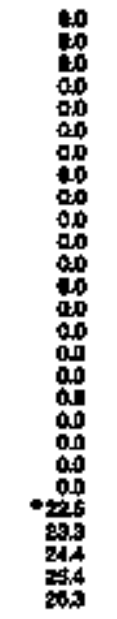 & 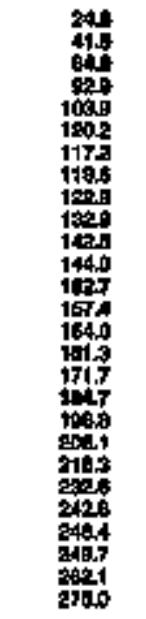 & 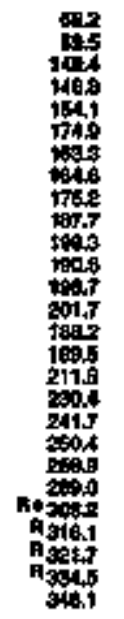 & 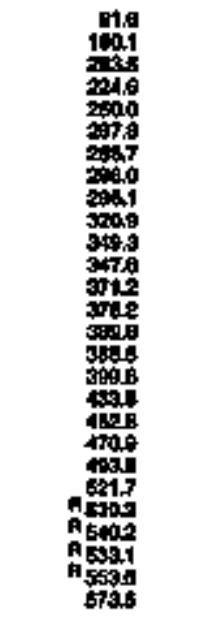 & 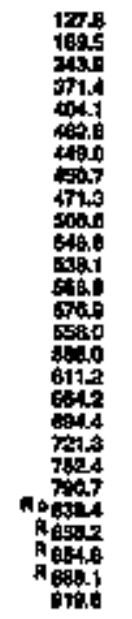 \\
\hline
\end{tabular}


Toble 73. Commerclal Energy Consumption Estimates, 1960, 1965, 1970-1994, Florida

\begin{tabular}{|c|c|c|c|c|c|c|c|c|c|c|c|c|c|c|}
\hline \multirow[b]{3}{*}{ Your } & \multicolumn{3}{|c|}{ cole } & \multirow{3}{*}{ 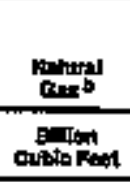 } & \multicolumn{6}{|c|}{ Foprobioum } & \multirow[b]{2}{*}{ Eopetricter } & \multirow[b]{2}{*}{ 161 } & \multirow{3}{*}{ 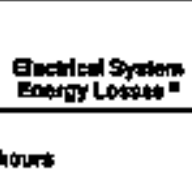 } & \multirow[b]{3}{*}{ Tordd } \\
\hline & Etuninow: & Anmerita: & Tated & & Ditellot: & Kerosene" & LPE: & motore & Antapd & Tots & & & & \\
\hline & \multicolumn{3}{|c|}{ 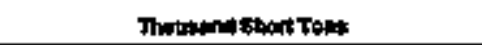 } & & \multicolumn{6}{|c|}{ 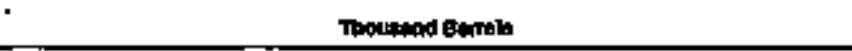 } & \multicolumn{2}{|c|}{ 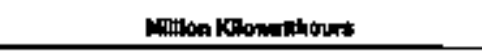 } & & \\
\hline 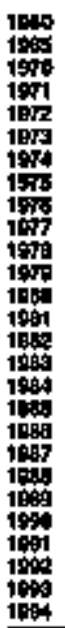 & 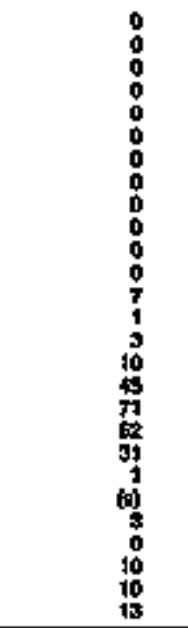 & $\begin{array}{l}0 \\
0 \\
0 \\
0 \\
0 \\
0 \\
0 \\
0 \\
0 \\
0 \\
0 \\
0 \\
0 \\
0 \\
0 \\
0 \\
0 \\
0 \\
0 \\
0 \\
0 \\
0 \\
0 \\
0 \\
0\end{array}$ & $\begin{array}{l}0 \\
0 \\
0 \\
0 \\
0 \\
0 \\
0 \\
0 \\
0 \\
0 \\
7 \\
1 \\
10 \\
45 \\
71 \\
02 \\
39 \\
19 \\
4 \\
40 \\
10 \\
13\end{array}$ & 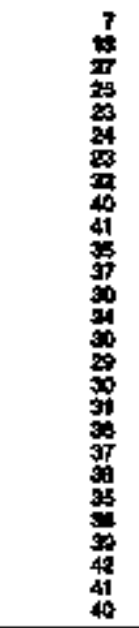 & 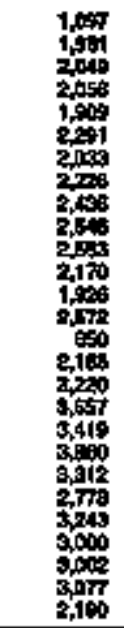 & 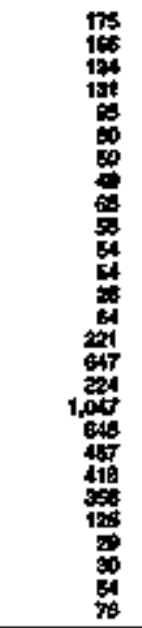 & 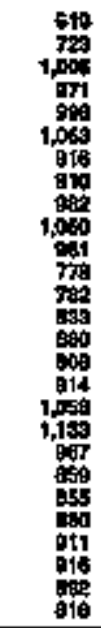 & 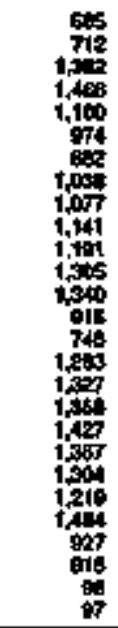 & 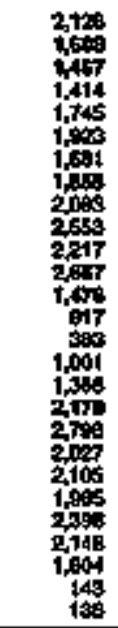 & 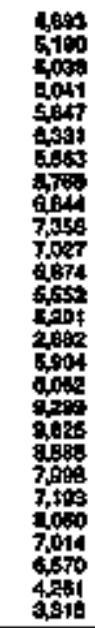 & 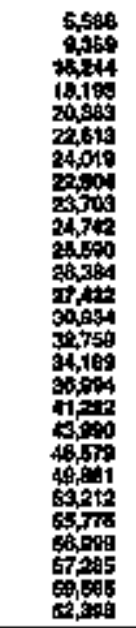 & $\begin{array}{l}= \\
= \\
= \\
= \\
= \\
= \\
= \\
= \\
= \\
= \\
= \\
= \\
= \\
=\end{array}$ & 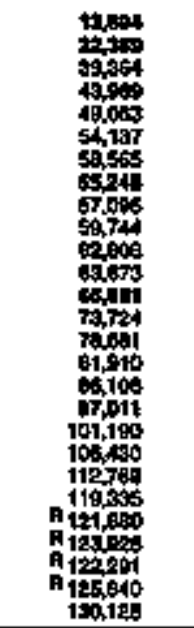 & $\begin{array}{l}z \\
= \\
z \\
z \\
= \\
= \\
= \\
= \\
= \\
z \\
z \\
z \\
z \\
z\end{array}$ \\
\hline \multicolumn{15}{|c|}{ Thtold ben } \\
\hline 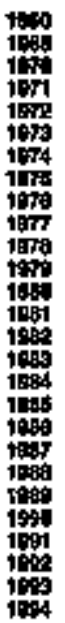 & 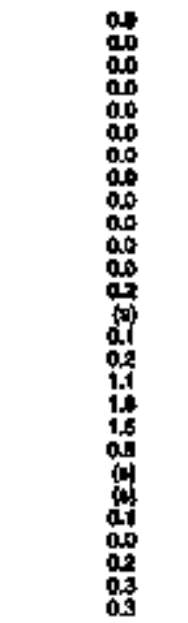 & 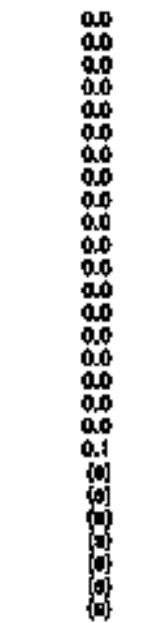 & 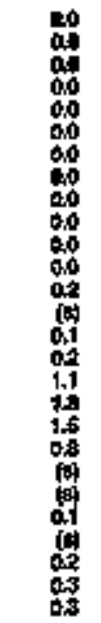 & 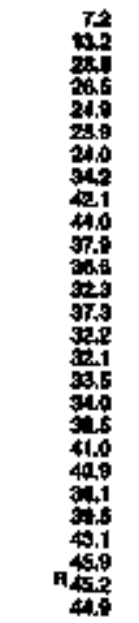 & 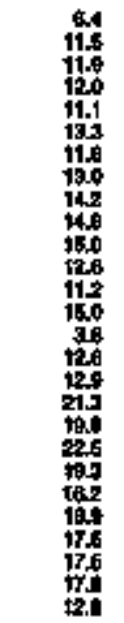 & 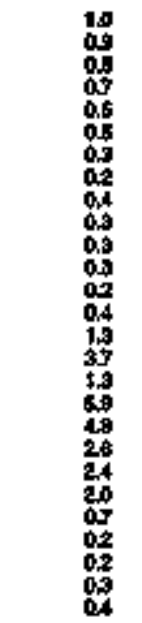 & 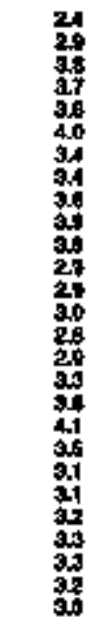 & 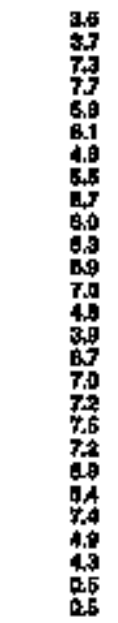 & 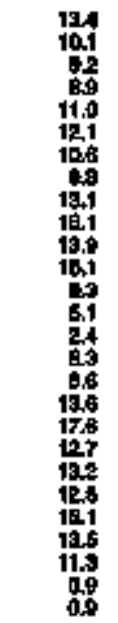 & 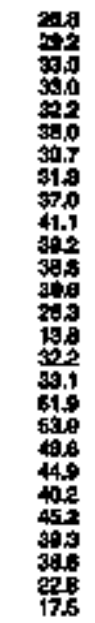 & 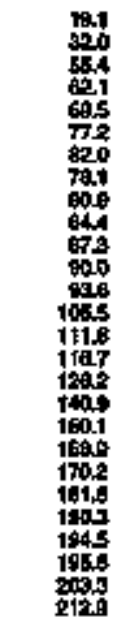 & 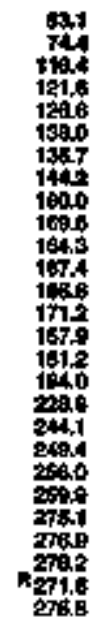 & 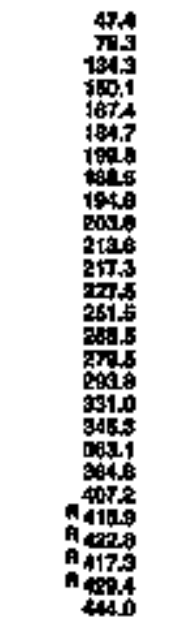 & 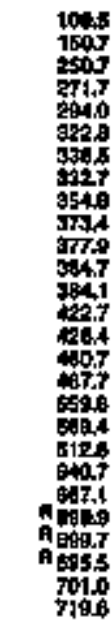 \\
\hline & & & 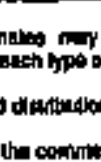 & alloctad & קיis & & & & & & $\boldsymbol{w}$ & (16) & & \\
\hline
\end{tabular}


Table 74. Industriat Energy Consumption Estrmates, 1960, 1965, 1970-1994, Florida

\begin{tabular}{|c|c|c|c|c|c|c|c|c|c|c|c|c|c|c|c|c|c|c|}
\hline \multirow[b]{3}{*}{5} & \multirow[b]{2}{*}{ cont } & \multirow[b]{2}{*}{ Gast } & \multicolumn{9}{|c|}{ Potrothesen } & \multirow[b]{2}{*}{. } & \multirow[b]{2}{*}{ Bropetat } & \multirow[b]{2}{*}{ onested } & \multirow[b]{2}{*}{ Detationy } & \multirow[b]{2}{*}{ Entrot } & \multirow{3}{*}{ 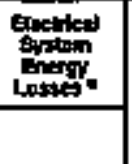 } & \multirow[b]{3}{*}{ Towe } \\
\hline & & & 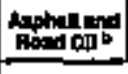 & Dented & tersomos & $L \mathrm{LG}^{\circ}$ & enterst: & 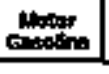 & 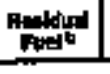 & Qens & reter & & & & & & & \\
\hline & 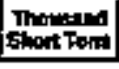 & 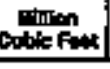 & \multicolumn{9}{|c|}{ Meanmanement } & \multicolumn{5}{|c|}{ 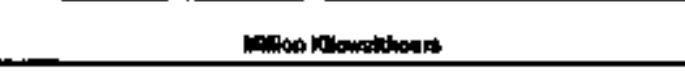 } & & \\
\hline 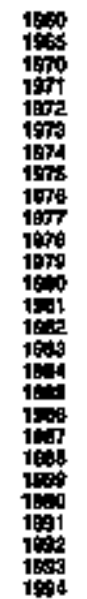 & 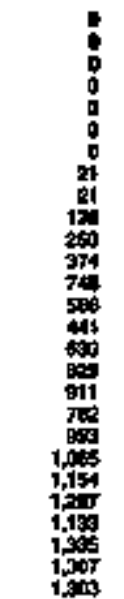 & 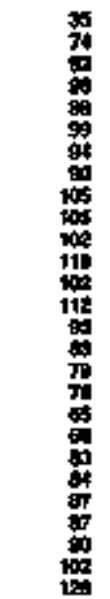 & 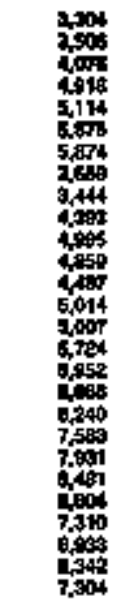 & 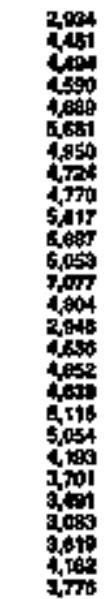 & 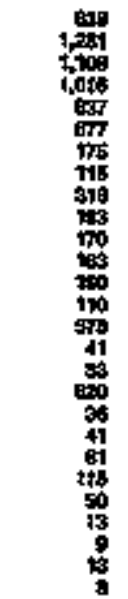 & 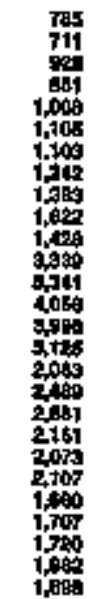 & 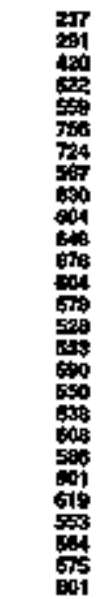 & 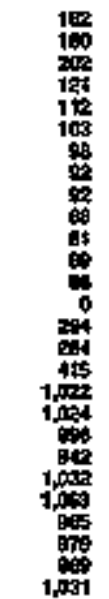 & 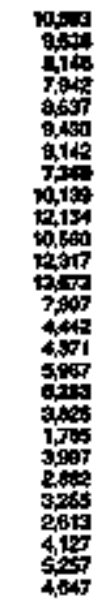 & 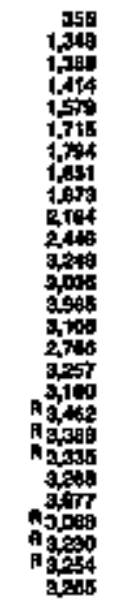 & 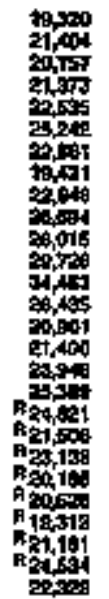 & 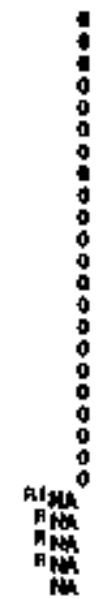 & 篗 & 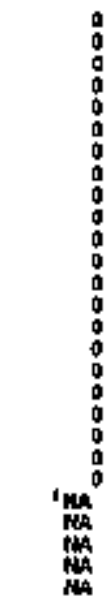 & 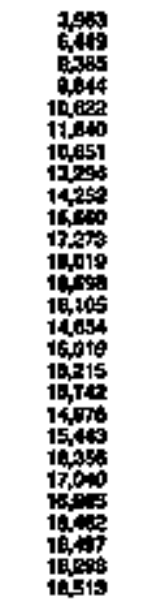 & $=$ & 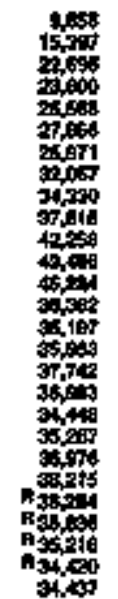 & $=$ \\
\hline
\end{tabular}

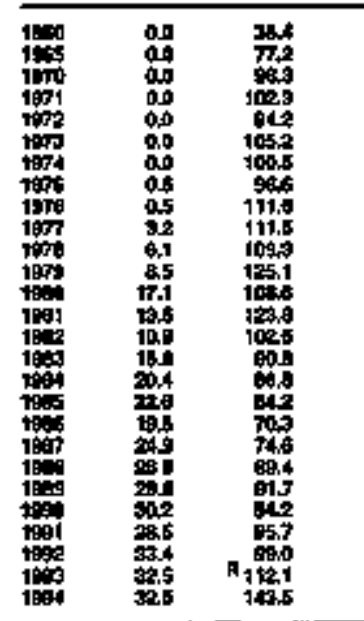

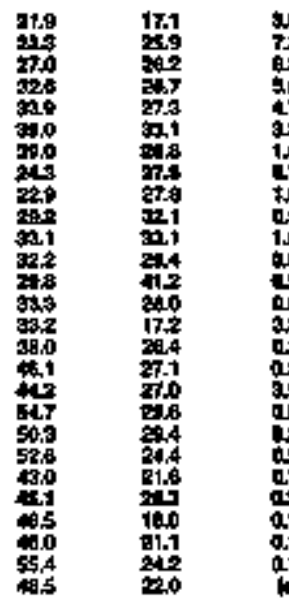

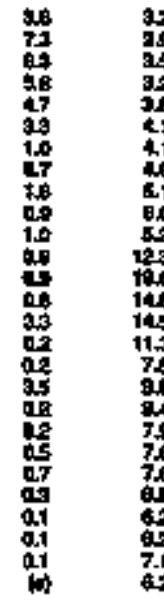

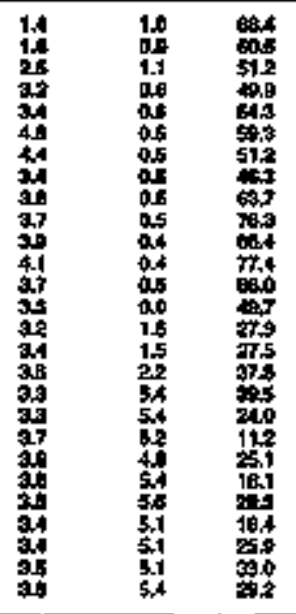

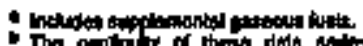

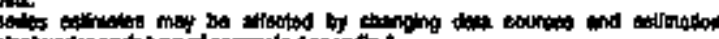

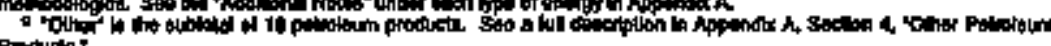

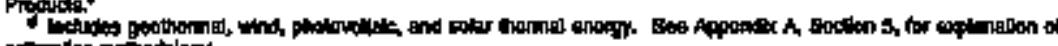

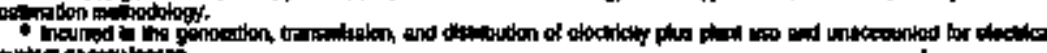

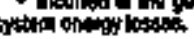


Table 76. Tranopoltation Energy Consumption Estimates, 1960, 1965, 1870-1994, Finida

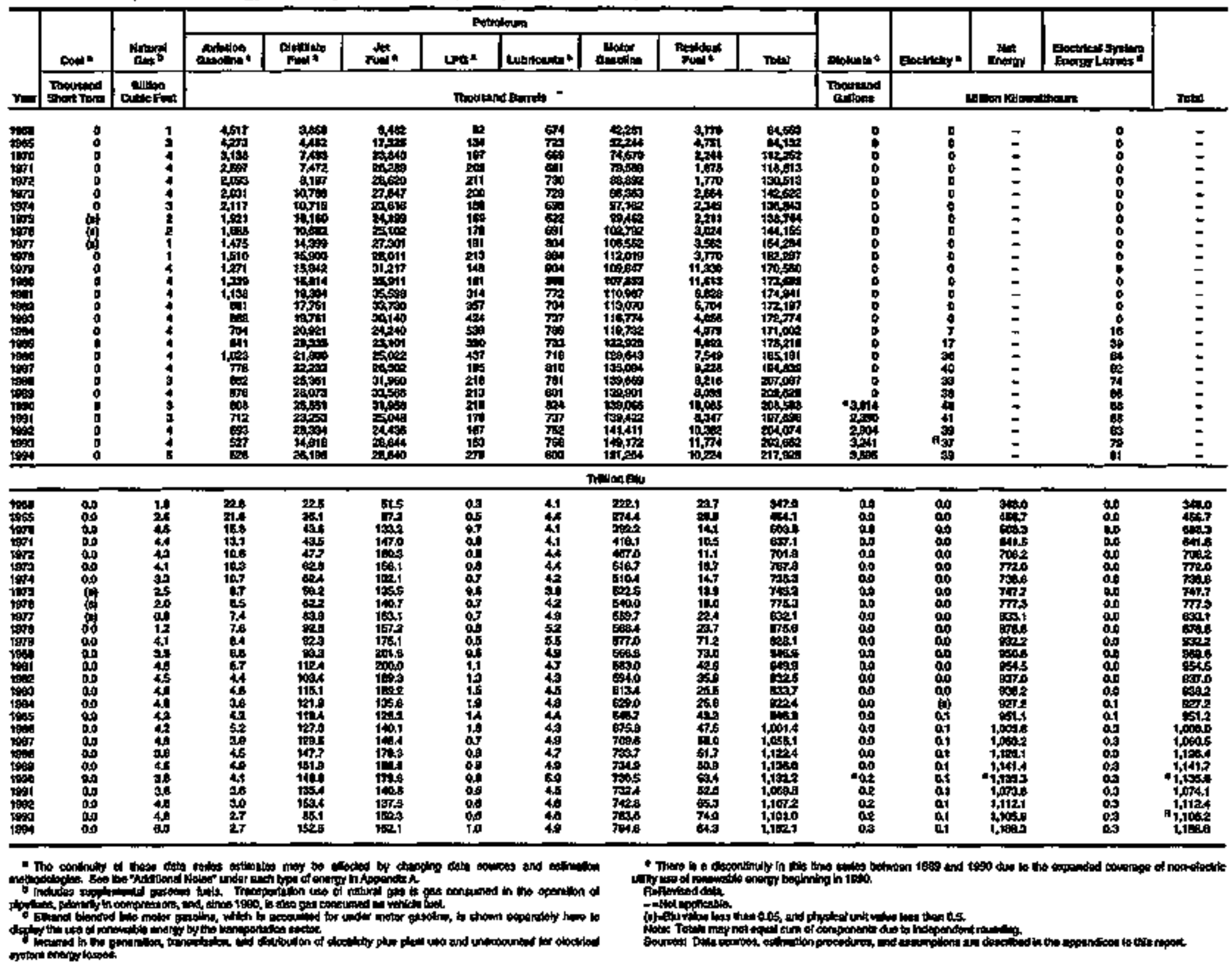


Table 7t. Estimates of Energy Input at Electric Utulties, 1960, 1865, t970-1994, Florlda

\begin{tabular}{|c|c|c|c|c|c|c|c|c|c|c|c|c|c|c|}
\hline \multirow[b]{3}{*}{ Yerp } & \multicolumn{3}{|c|}{$\operatorname{sen}$} & \multirow{3}{*}{ ins } & \multicolumn{4}{|c|}{ Putrating } & \multirow[b]{2}{*}{ 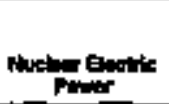 } & \multirow[b]{2}{*}{ 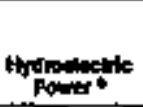 } & \multirow[b]{2}{*}{ mofinds } & \multirow[b]{2}{*}{ 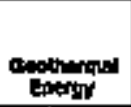 } & \multirow[b]{2}{*}{ Otherent } & \multirow[b]{3}{*}{ Totalp } \\
\hline & 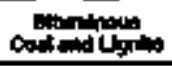 & 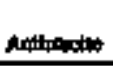 & Thed & & 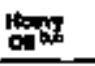 & 㟧蔯 & Potolump & Tat느 - & & & & & & \\
\hline & \multicolumn{3}{|c|}{ 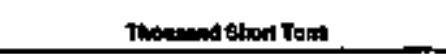 } & & \multicolumn{4}{|c|}{ 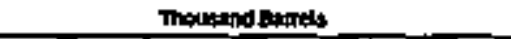 } & \multicolumn{5}{|c|}{ 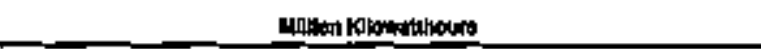 } & \\
\hline 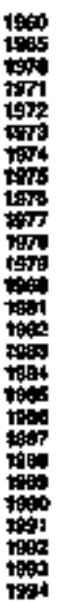 & 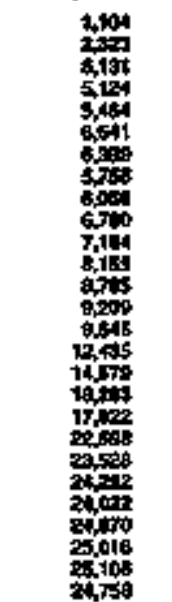 & $\begin{array}{l}0 \\
0 \\
0 \\
0 \\
0 \\
0 \\
0 \\
0 \\
0 \\
0 \\
0 \\
0 \\
0 \\
0 \\
0 \\
0 \\
0 \\
0 \\
0 \\
0 \\
0 \\
0 \\
0 \\
0 \\
0 \\
0 \\
0\end{array}$ & 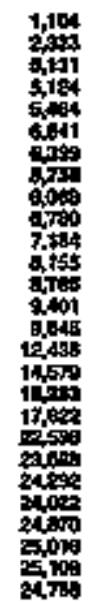 & 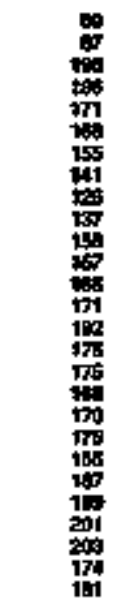 & 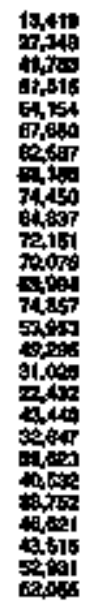 & 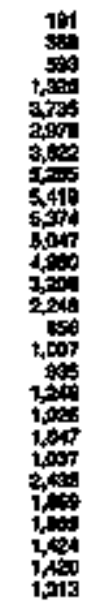 & $\begin{array}{l}0 \\
0 \\
0 \\
0 \\
0 \\
0 \\
0 \\
0 \\
0 \\
0 \\
0 \\
0 \\
0 \\
0 \\
0 \\
0 \\
0 \\
0 \\
0 \\
0 \\
0 \\
0 \\
0 \\
0 \\
0 \\
0 \\
0\end{array}$ & 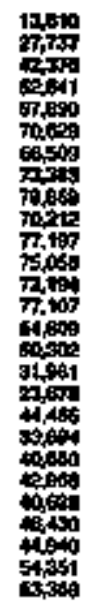 & 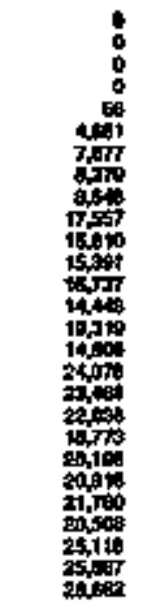 & 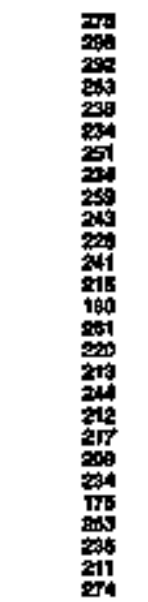 & 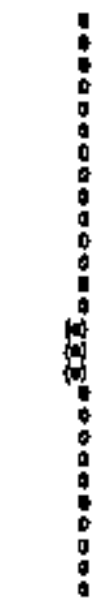 & $\begin{array}{l}0 \\
0 \\
0 \\
0 \\
0 \\
0 \\
0 \\
0 \\
0 \\
0 \\
0 \\
0 \\
0 \\
0 \\
0 \\
0 \\
0 \\
0 \\
0 \\
0 \\
0\end{array}$ & $\begin{array}{l}0 \\
0 \\
0 \\
0 \\
0 \\
0 \\
0 \\
0 \\
0 \\
0 \\
0 \\
0 \\
0 \\
0 \\
0 \\
0 \\
0 \\
0 \\
0 \\
0 \\
0 \\
0 \\
0 \\
0\end{array}$ & 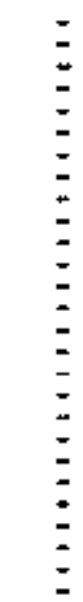 \\
\hline
\end{tabular}

\begin{tabular}{|c|c|c|c|c|c|c|c|c|c|c|c|c|c|c|}
\hline 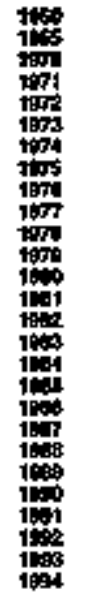 & 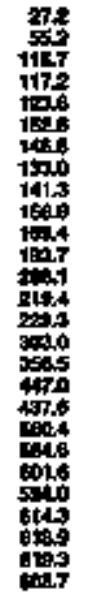 & 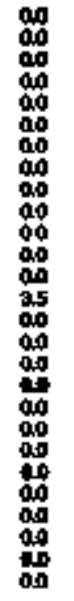 & 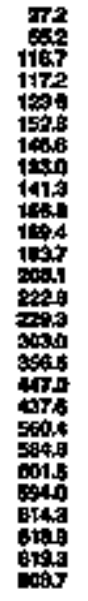 & 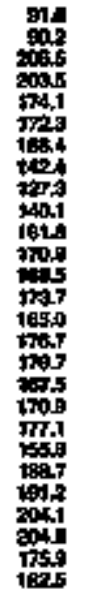 & 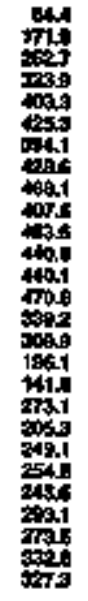 & 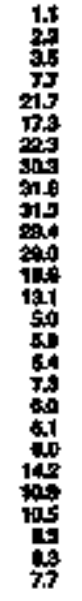 & 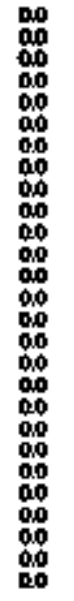 & 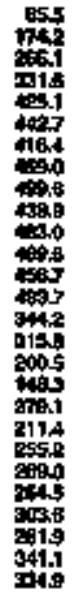 & 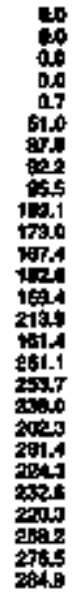 & 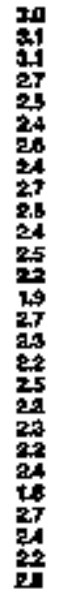 & 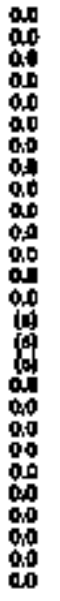 & 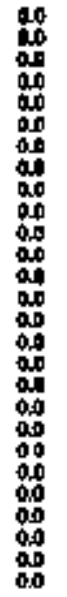 & 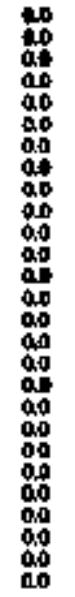 & 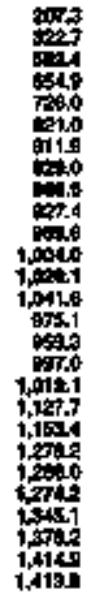 \\
\hline
\end{tabular}

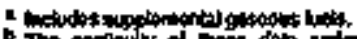

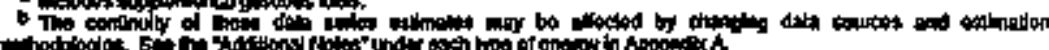

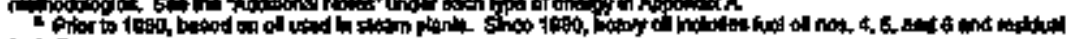

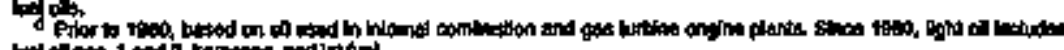

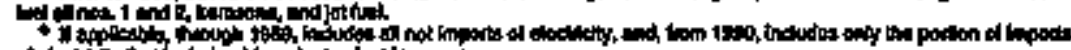

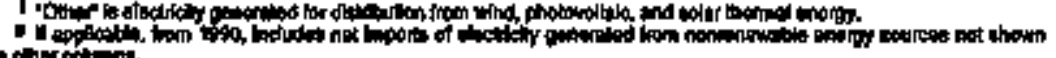
- Whit unisabto.

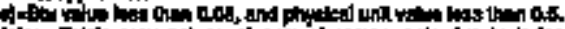

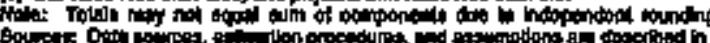

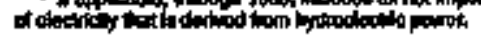




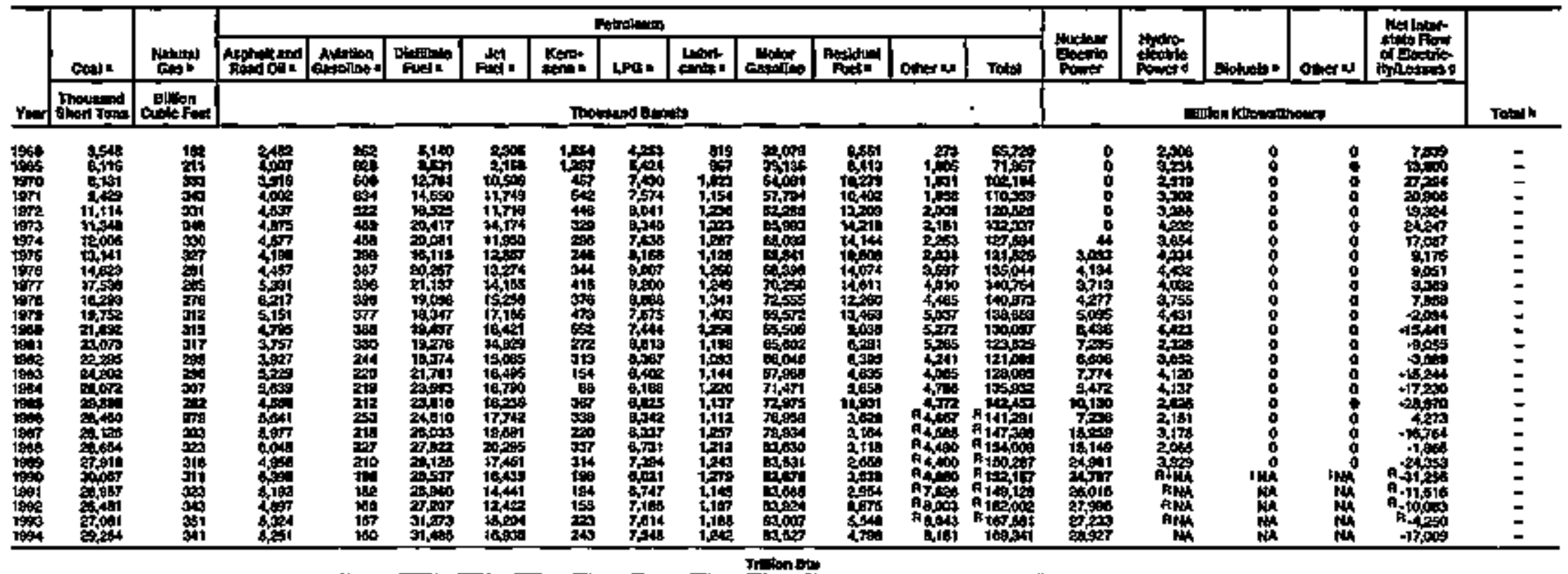

\begin{tabular}{|c|c|c|c|c|c|c|c|c|c|c|c|c|c|c|c|c|c|c|c|}
\hline & 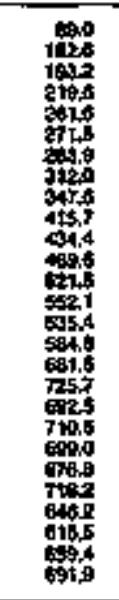 & 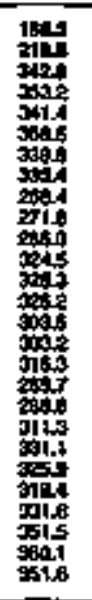 & 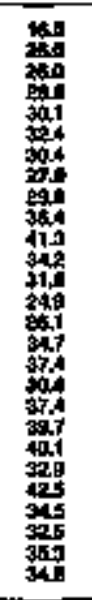 & 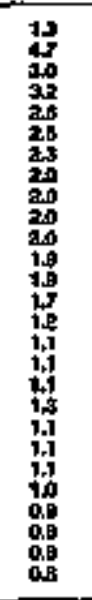 & 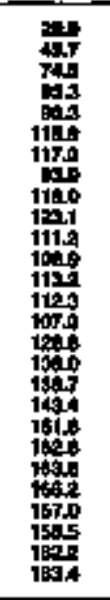 & 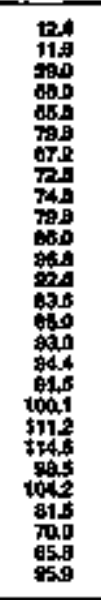 & 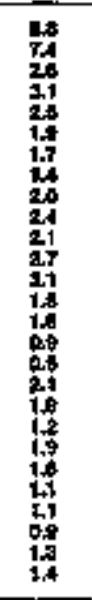 & 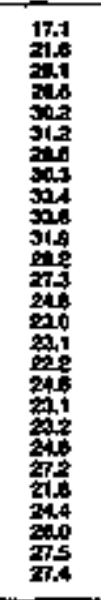 & $\begin{array}{l}\text { 竞 } \\
7 \\
7 \\
7 \\
7\end{array}$ & 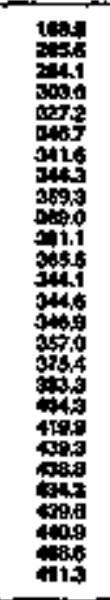 & 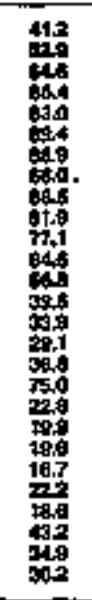 & 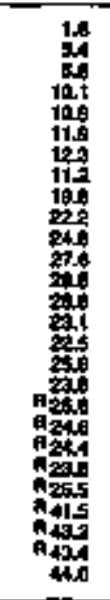 & 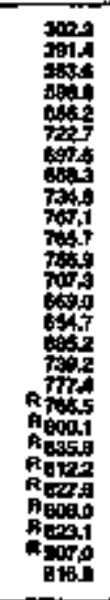 & 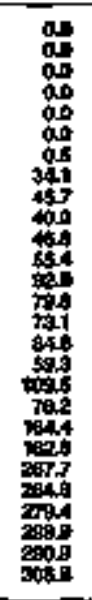 & 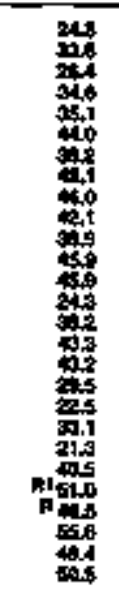 & 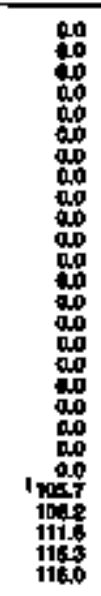 & 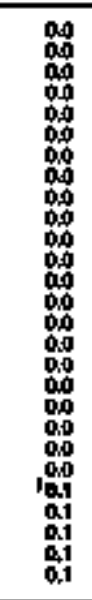 & 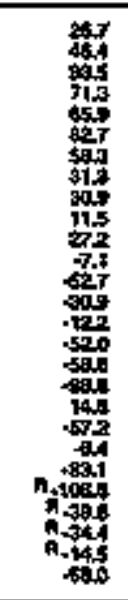 & 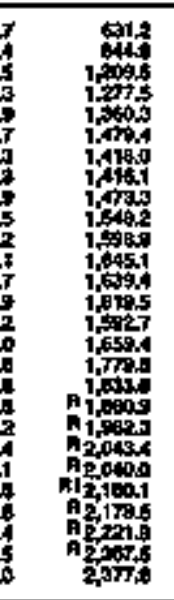 \\
\hline
\end{tabular}

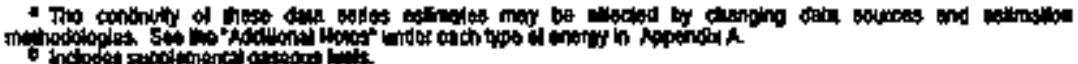

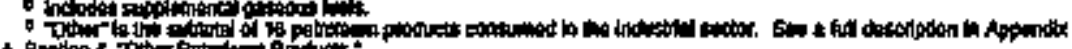

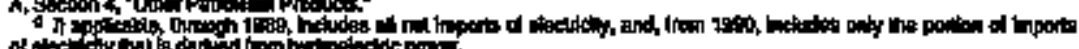

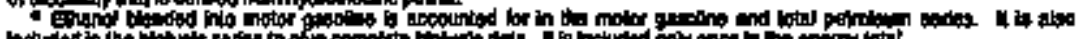

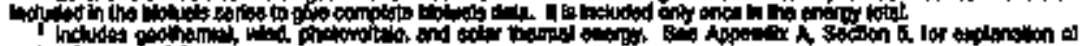

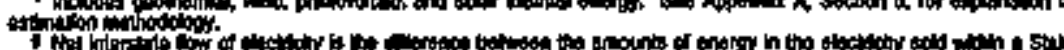

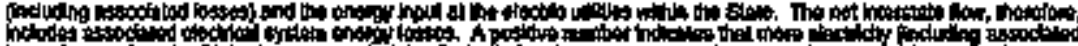

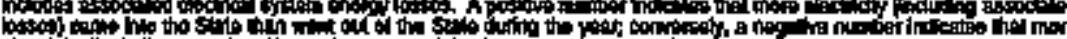

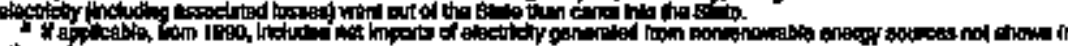

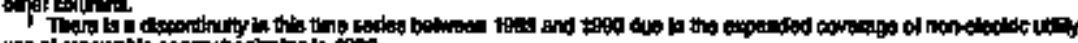
100 ind is

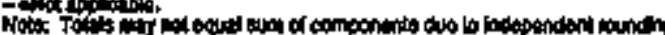

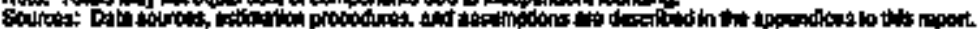


$\mathbf{G}$
$\mathbf{E}$
$\mathbf{O}$
$\mathbf{R}$
$\mathbf{G}$
$\mathbf{I}$
$\mathbf{A}$

Table 76. Rosidential Encrgy Comsumption Estlmates, 1950, 1965, 1970-1994, Ceorgia

\begin{tabular}{|c|c|c|c|c|c|c|c|c|c|c|c|c|c|c|}
\hline & & tert & & & & & & & \multirow[b]{2}{*}{ Batofino: } & \multirow[b]{2}{*}{ Soline } & \multirow[b]{2}{*}{ Enctelen " } & \multirow[b]{2}{*}{ 华 } & \multirow[b]{2}{*}{ 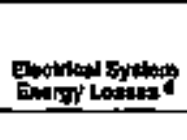 } & \multirow[b]{3}{*}{ Toll } \\
\hline & 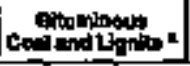 & Antarakin=" & Tow & Molumer & Donite & Notenting * & LPS* & Totin & & & & & & \\
\hline Yarl & \multicolumn{3}{|c|}{ Thoriend athert Tona } & cillow & \multicolumn{4}{|c|}{ Thouland owing } & Tlousasd & \multicolumn{4}{|c|}{ 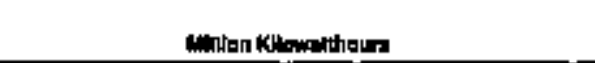 } & \\
\hline
\end{tabular}

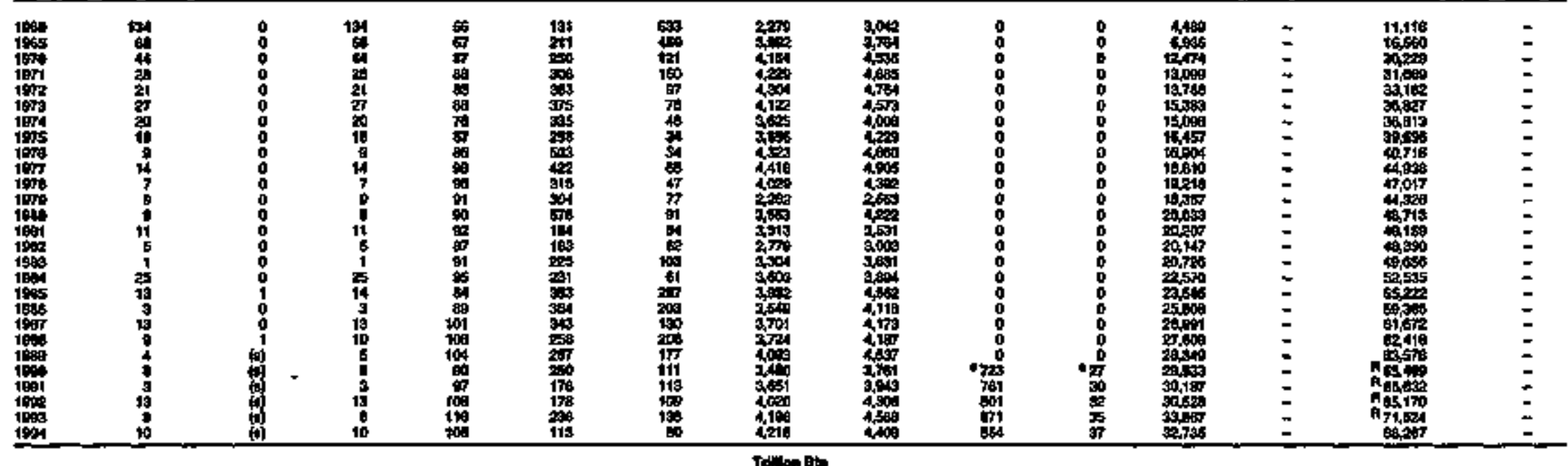

\begin{tabular}{|c|c|c|c|c|c|c|}
\hline 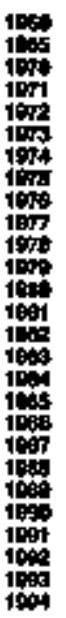 & 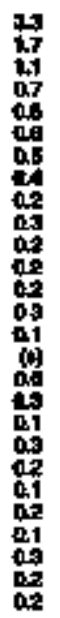 & 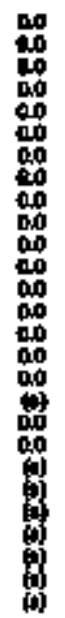 & 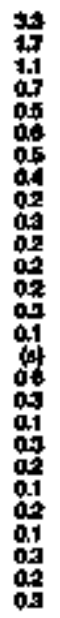 & 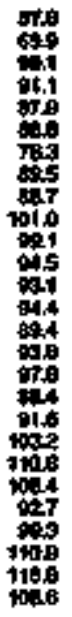 & 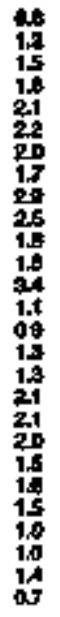 & 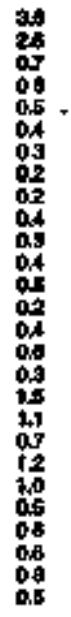 \\
\hline
\end{tabular}

*

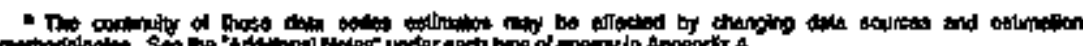

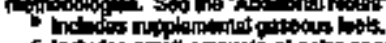


Table 79. Commerclal Energy Consumptlon Estimates, 1960, 1965, 1970-1994, Eeorgia

\begin{tabular}{|c|c|c|c|c|c|c|c|c|c|c|c|c|c|c|}
\hline \multirow[b]{3}{*}{ You } & \multicolumn{3}{|c|}{$\cos$} & \multirow{3}{*}{ 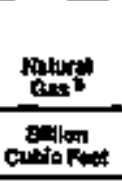 } & \multicolumn{6}{|c|}{ Petrolevap } & \multirow[b]{2}{*}{ Eotovelty 1} & \multirow[b]{2}{*}{ Entrot } & \multirow[b]{2}{*}{ 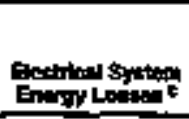 } & \multirow[b]{3}{*}{ Toted d } \\
\hline & 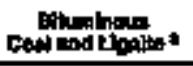 & Nothonime: & Toti & & Detende & 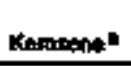 & LPE: : & centertins & 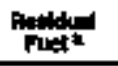 & Tot: & & & & \\
\hline & \multicolumn{3}{|c|}{ 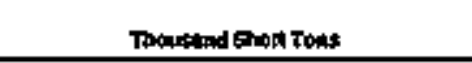 } & & \multicolumn{6}{|c|}{ Thougnod Bumis } & \multicolumn{3}{|c|}{ 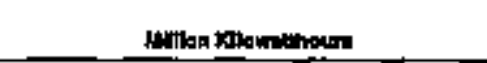 } & \\
\hline 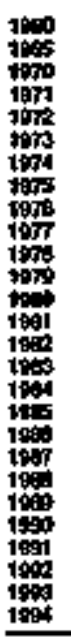 & 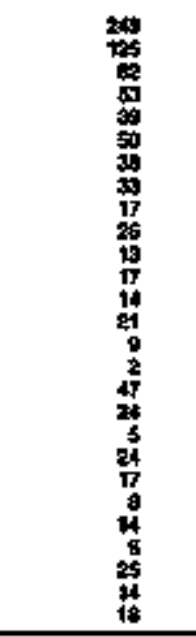 & $\begin{array}{l}0 \\
0 \\
0 \\
0 \\
0 \\
0 \\
0 \\
0 \\
0 \\
0 \\
0 \\
0 \\
0 \\
0 \\
0 \\
0 \\
0 \\
0 \\
0 \\
0 \\
9 \\
0 \\
0 \\
0 \\
0 \\
0 \\
0 \\
0\end{array}$ & 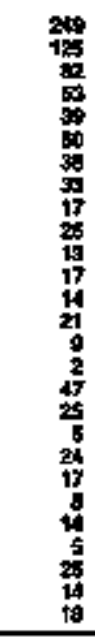 & 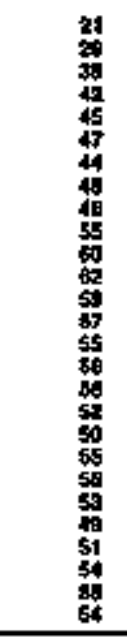 & 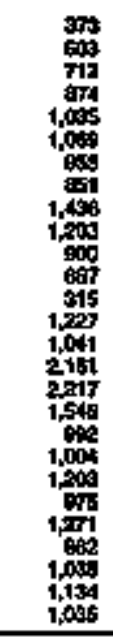 & 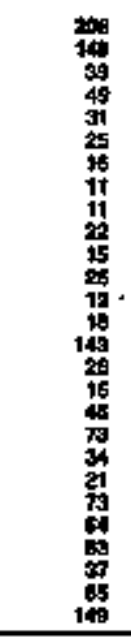 & 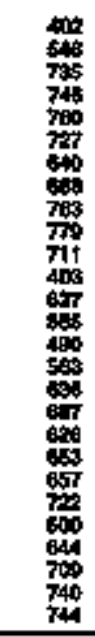 & 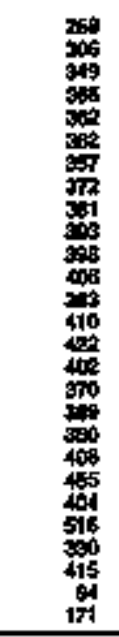 & 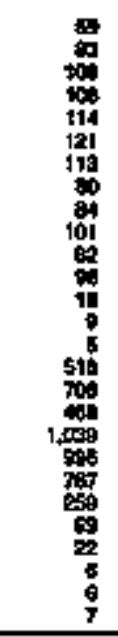 & 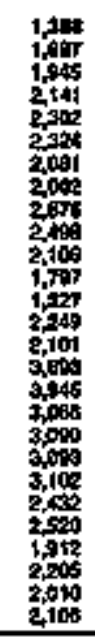 & 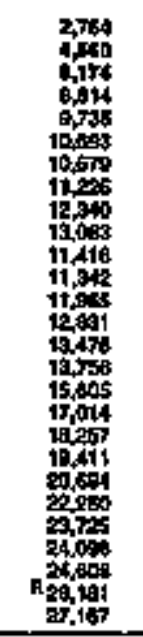 & $\begin{array}{l}= \\
= \\
= \\
= \\
= \\
= \\
= \\
= \\
= \\
= \\
= \\
= \\
= \\
= \\
=\end{array}$ & 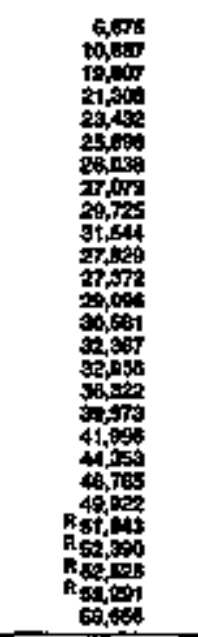 & $\begin{array}{l}= \\
z \\
z \\
z \\
z \\
z \\
z \\
z \\
z \\
z \\
z \\
z \\
z \\
z \\
z\end{array}$ \\
\hline \multicolumn{15}{|c|}{ Triden Bar } \\
\hline 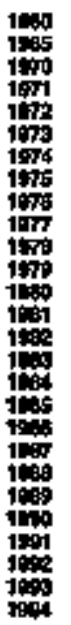 & 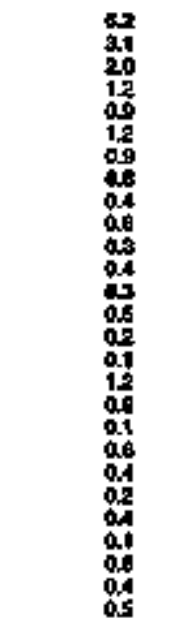 & 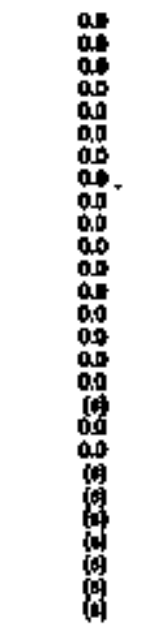 & 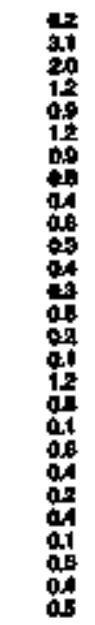 & 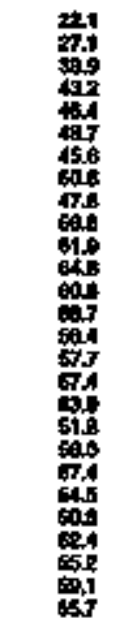 & 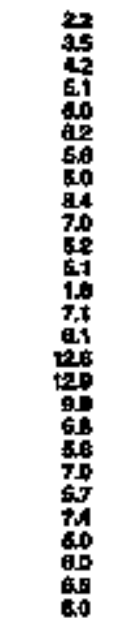 & 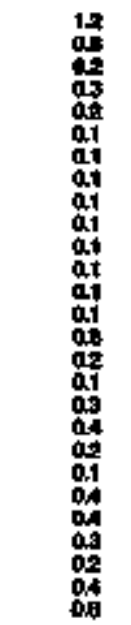 & 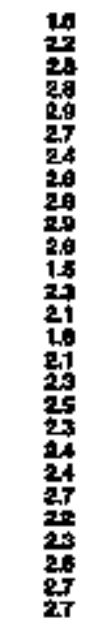 & 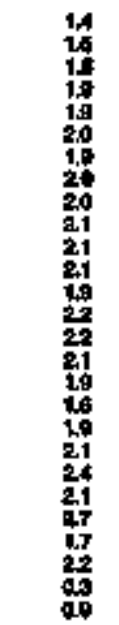 & 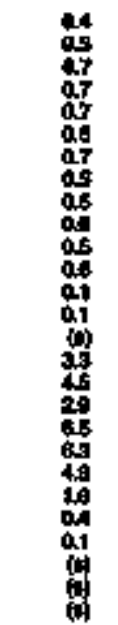 & 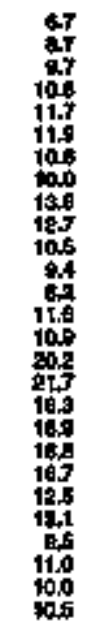 & 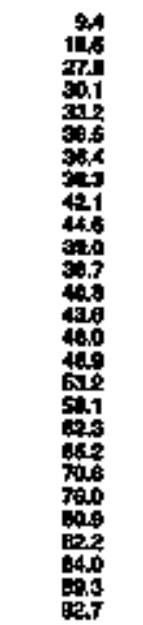 & 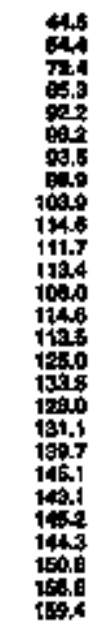 & 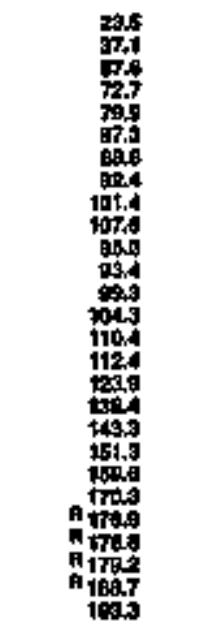 & 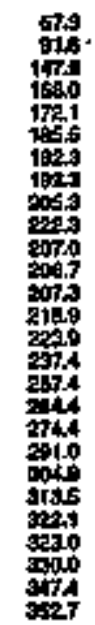 \\
\hline
\end{tabular}

$\mathbf{G}$
$\mathbf{E}$
$\mathbf{0}$
$\mathbf{R}$
$\mathbf{G}$
$\mathbf{I}$
$\mathbf{A}$

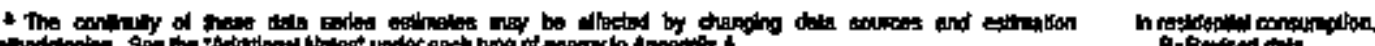

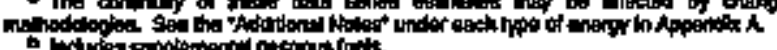

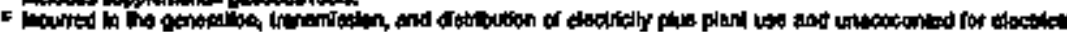
Anfinitiod dein

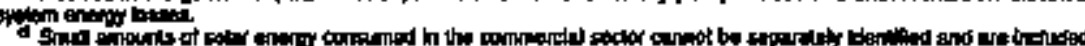

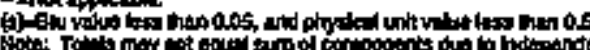

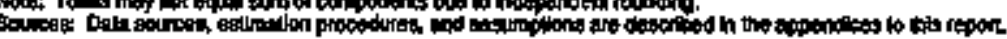




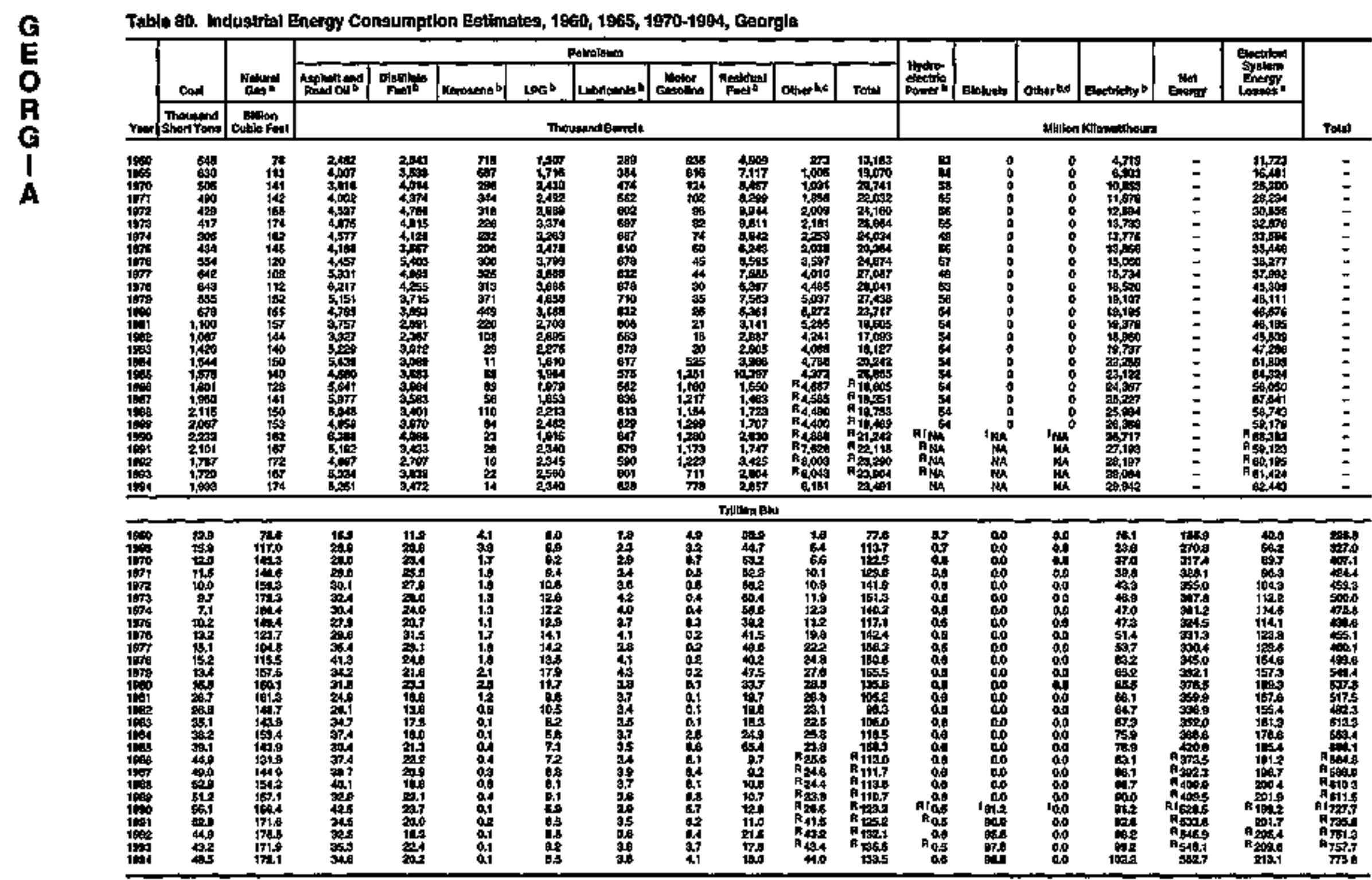

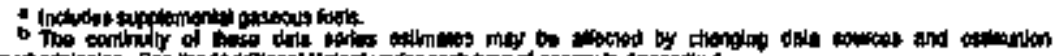

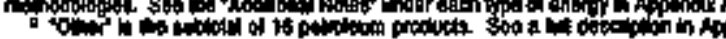

然

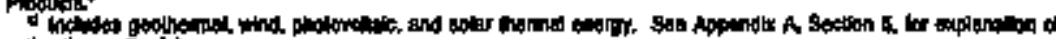

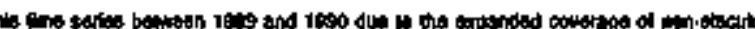
aty

Nhathot solition

C.

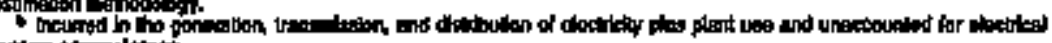
arres of

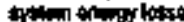


Thbip 61. Transportetion Energy Cansumption Estimates, 1960, 1855, 1970-1994, teargia

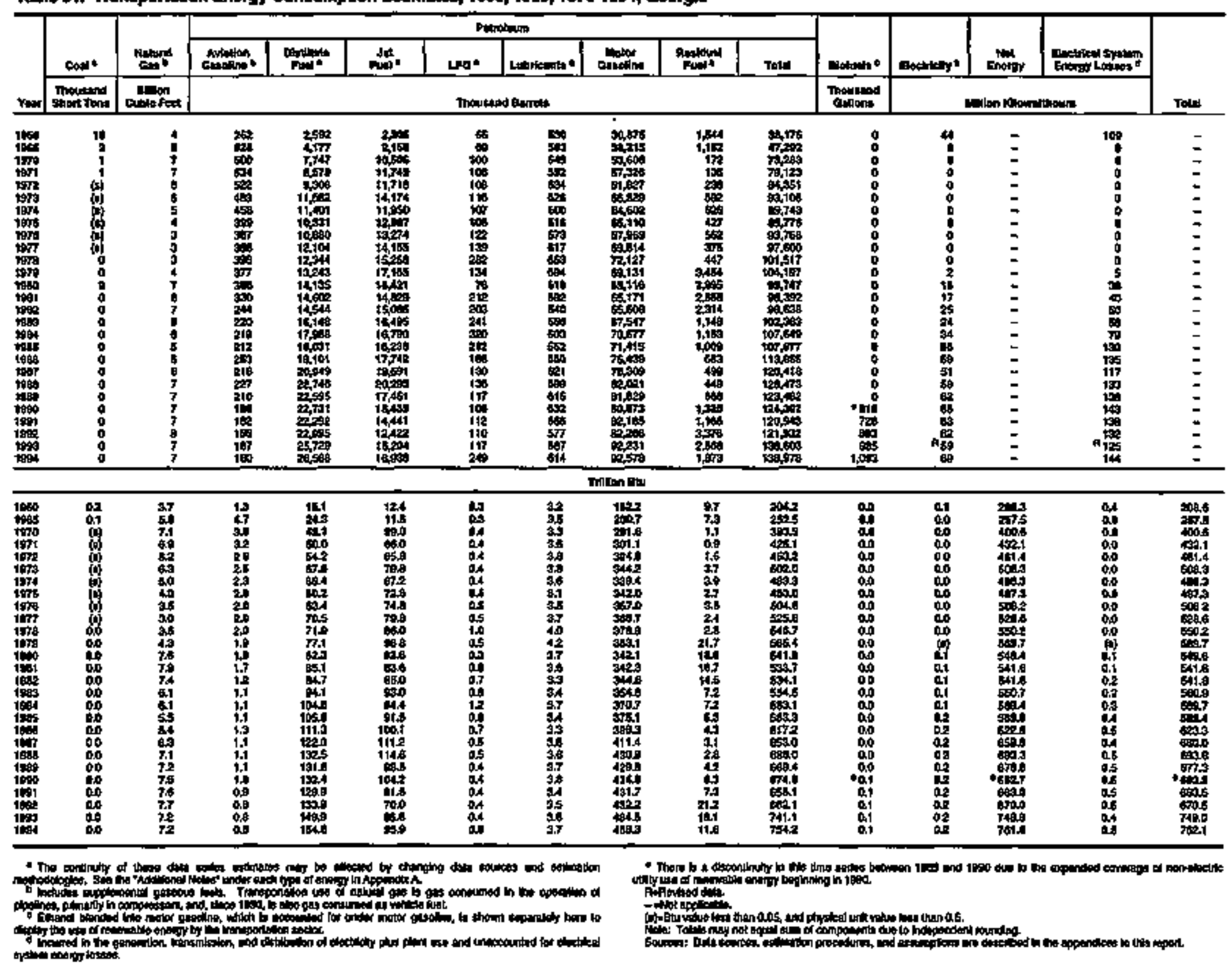


Table 82 Estimates of Energy Input al Electrio Litilitios, 1650, 1945, 1970-1994, 6ecorgla

\begin{tabular}{|c|c|c|c|c|c|c|c|c|c|c|c|c|c|c|}
\hline & \multicolumn{3}{|c|}{ con } & \multirow[b]{2}{*}{ Gest. } & \multicolumn{4}{|c|}{ Petrotipym } & \multirow[b]{2}{*}{ 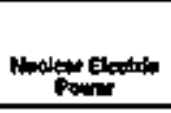 } & \multirow[b]{2}{*}{ 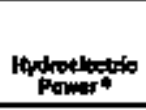 } & \multirow[b]{2}{*}{ Elofunte } & \multirow[b]{2}{*}{ 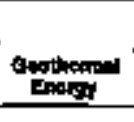 } & \multirow[b]{2}{*}{ our } & \multirow[b]{3}{*}{ Totod } \\
\hline & colliningon & Mantine & 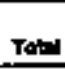 & & ont & Labto & 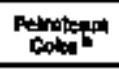 & Pour & & & & & & \\
\hline$k=1$ & \multicolumn{3}{|c|}{ Movandstan Ton: } & 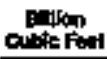 & \multicolumn{4}{|c|}{ Inavand Bannest } & \multicolumn{5}{|c|}{ 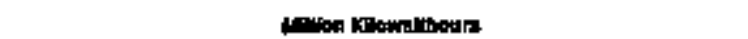 } & \\
\hline
\end{tabular}

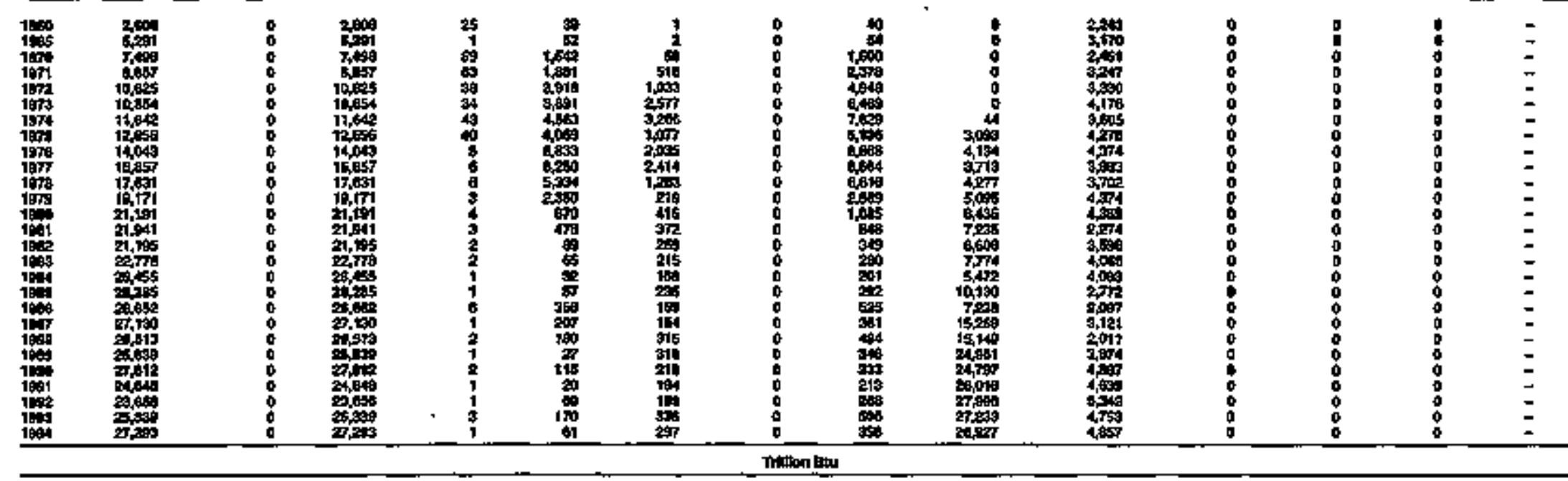

\begin{tabular}{|c|c|c|c|c|c|c|c|c|c|c|c|c|c|c|}
\hline 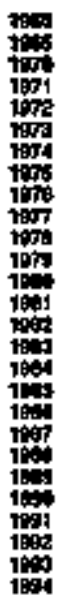 & 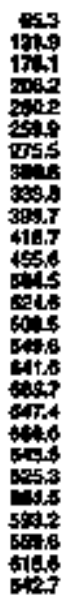 & 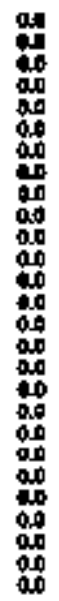 & 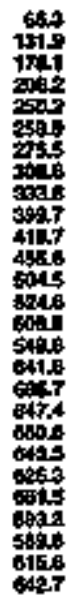 & 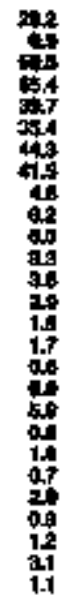 & 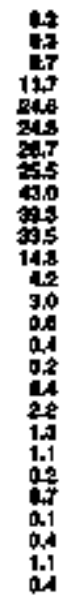 & 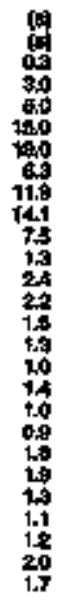 & 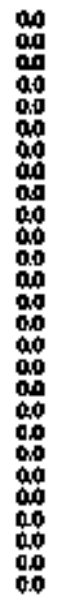 & 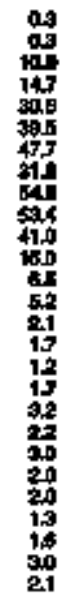 & 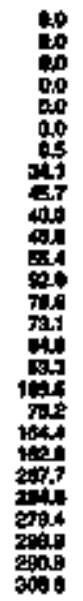 & 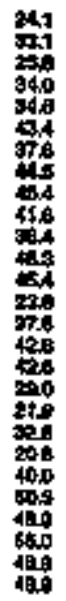 & 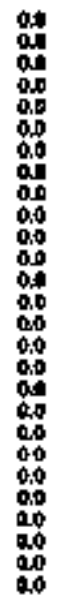 & 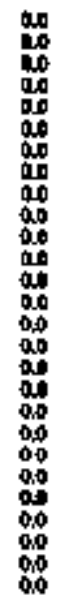 & 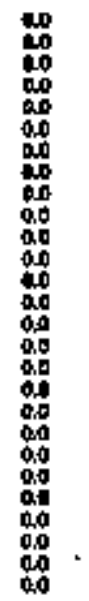 & 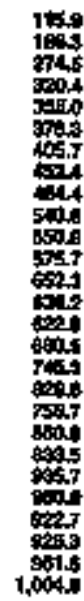 \\
\hline
\end{tabular}

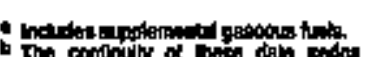

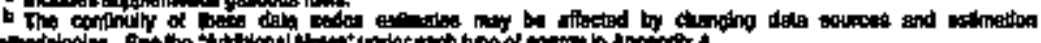

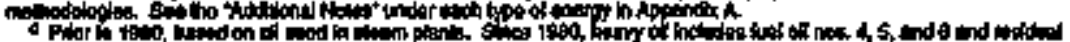

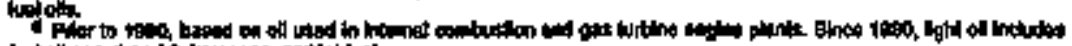

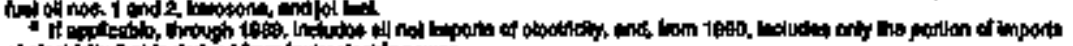

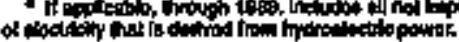

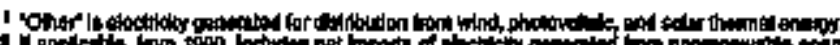

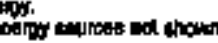

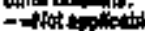

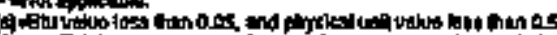

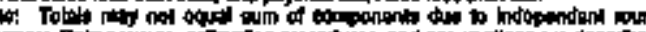


Tablu 93. Enargy Consumplion Estimates by Solwee, 1660, 1965, 1970-1994, Hawaif

\begin{tabular}{|c|c|c|c|c|c|c|c|c|c|c|c|c|c|c|c|c|c|c|c|}
\hline \multirow[b]{3}{*}{ val } & \multirow[b]{2}{*}{ Cand } & \multirow[b]{2}{*}{ Mations } & \multicolumn{11}{|c|}{ Patrinin: } & \multirow{2}{*}{ manter } & \multirow{2}{*}{ 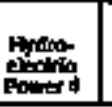 } & \multirow[b]{2}{*}{ Dohnele: | } & \multirow[b]{2}{*}{ Other at } & \multirow{2}{*}{ 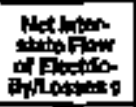 } & \multirow[b]{3}{*}{ Toted Is } \\
\hline & & & 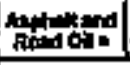 & 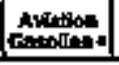 & 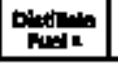 & fat. & Kent & t5ot: & Lathe & ating & Finder & atseras & Thell & & & & & & \\
\hline & Thendind & anderent & \multicolumn{11}{|c|}{ 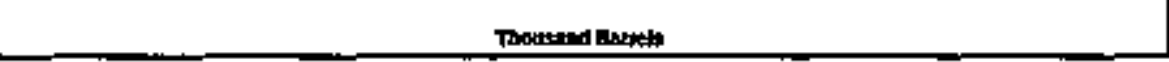 } & \multicolumn{5}{|c|}{ 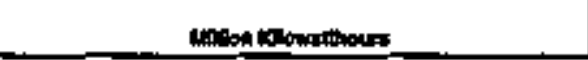 } & \\
\hline 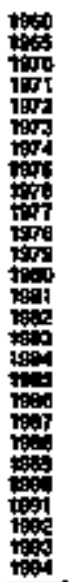 & 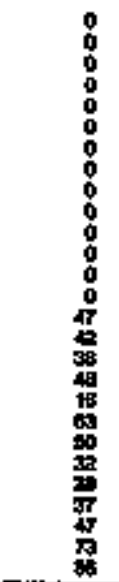 & $\begin{array}{l}0 \\
0 \\
0 \\
\vdots \\
\vdots \\
\vdots \\
0 \\
0 \\
0 \\
0 \\
5 \\
3 \\
5 \\
2 \\
2 \\
2 \\
\frac{2}{3} \\
3 \\
3 \\
3 \\
3 \\
3\end{array}$ & 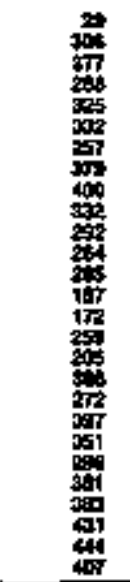 & 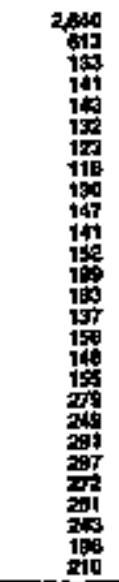 & 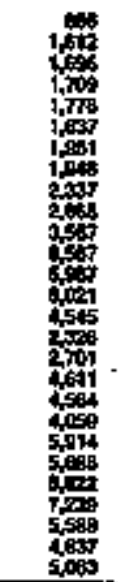 & 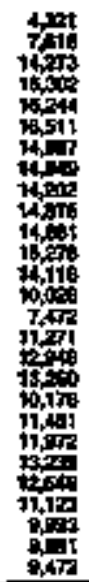 & 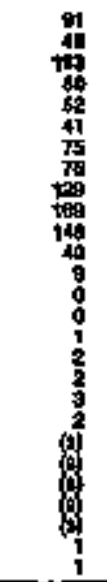 & 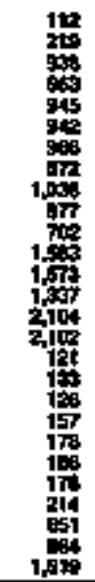 & 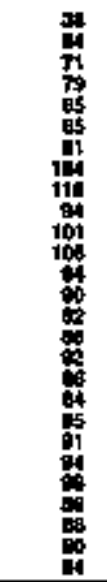 & 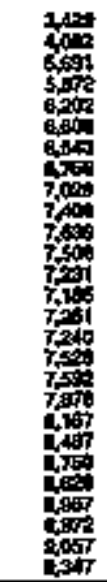 & 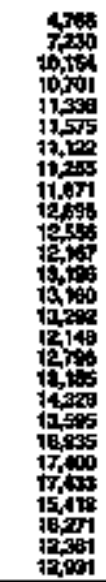 & 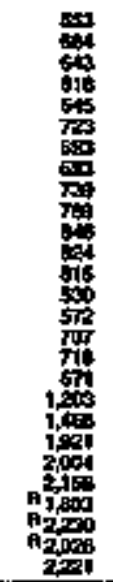 & 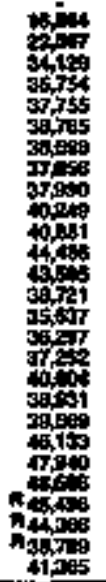 & $\begin{array}{l}0 \\
0 \\
0 \\
0 \\
0 \\
0 \\
0 \\
0 \\
0 \\
0 \\
0 \\
0 \\
0 \\
0 \\
0 \\
0 \\
0 \\
0 \\
0 \\
0 \\
0 \\
0 \\
0 \\
0 \\
0\end{array}$ & 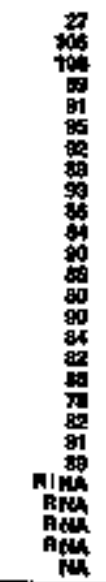 & 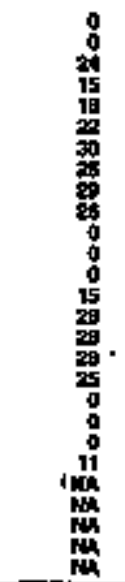 & 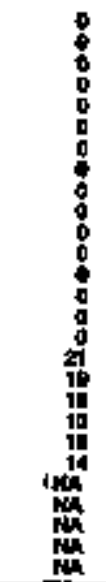 & $\begin{array}{l}0 \\
0 \\
0 \\
0 \\
0 \\
0 \\
0 \\
0 \\
0 \\
0 \\
0 \\
0 \\
0 \\
0 \\
0 \\
0 \\
0 \\
0 \\
0 \\
0 \\
0 \\
0 \\
0 \\
0 \\
0 \\
0\end{array}$ & $\begin{array}{l}= \\
= \\
z \\
= \\
= \\
= \\
= \\
= \\
z \\
z\end{array}$ \\
\hline
\end{tabular}

\begin{tabular}{|c|c|c|c|c|c|c|c|c|c|c|c|c|c|c|c|c|c|c|c|}
\hline 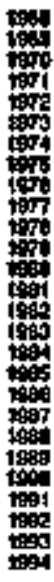 & 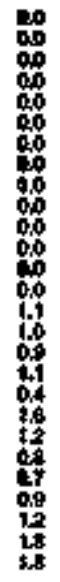 & 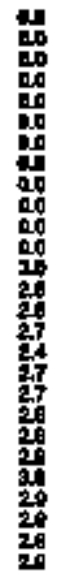 & 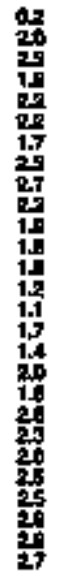 & 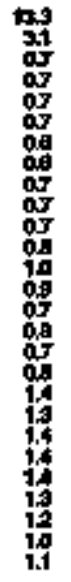 & 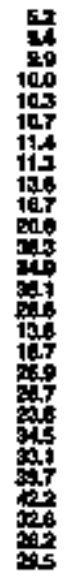 & 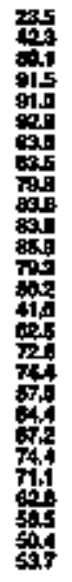 & 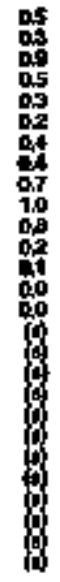 & 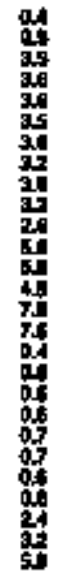 & 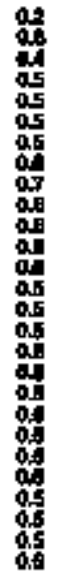 & 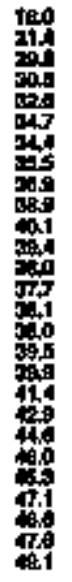 & 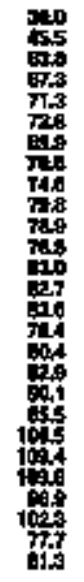 & 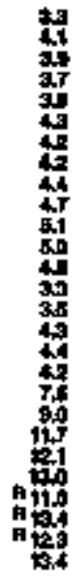 & 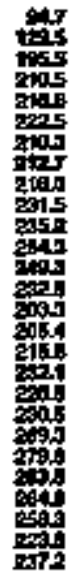 & 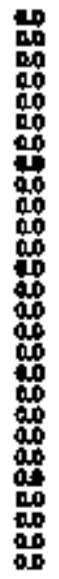 & 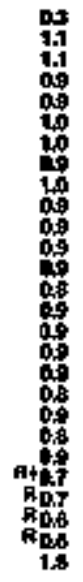 & 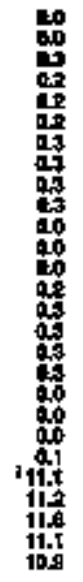 & 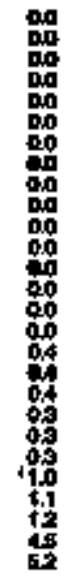 & 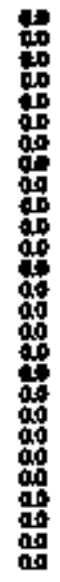 & 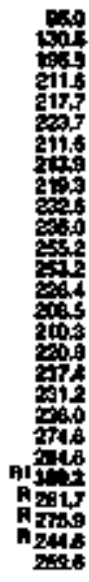 \\
\hline
\end{tabular}

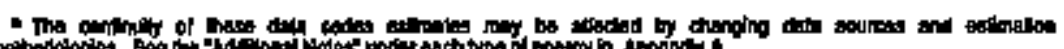

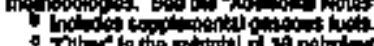

47.

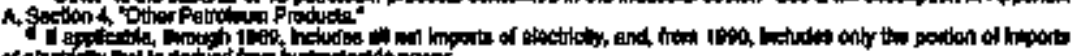

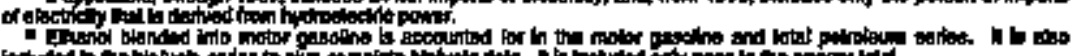

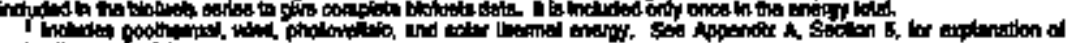

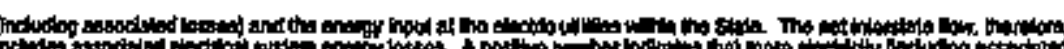

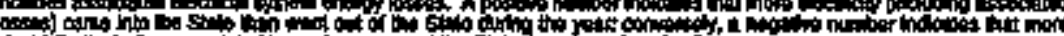
actrif

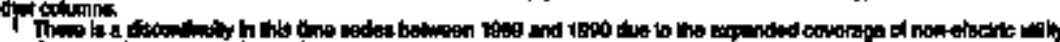

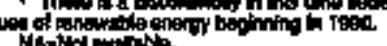

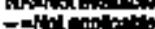

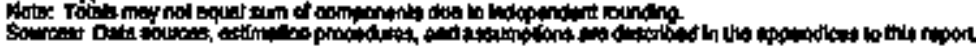

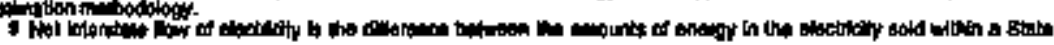


Teblo 94. Residential Enerw Consumpilon Eetlmates, 1960, 1965, 1970-1894, Hawall

\begin{tabular}{|c|c|c|c|c|c|c|c|c|c|c|c|c|c|c|}
\hline \multirow[b]{3}{*}{ Vers } & \multicolumn{3}{|c|}{$\cos$} & \multirow{3}{*}{ 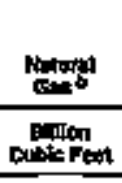 } & & & & & \multirow{3}{*}{ Thoostis } & & \multirow[b]{2}{*}{ Eoctitelyty } & \multirow[b]{2}{*}{ Emoldor } & \multirow{3}{*}{ 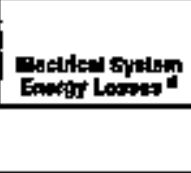 } & \multirow[b]{3}{*}{ TTotol } \\
\hline & 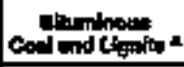 & Anthreine & Tot & & 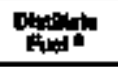 & 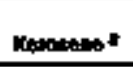 & LPA. & Totat & & Solves: & & & & \\
\hline & \multicolumn{3}{|c|}{ 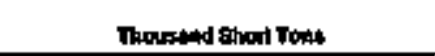 } & & \multicolumn{4}{|c|}{ Thounand Bemests } & & \multicolumn{3}{|c|}{ 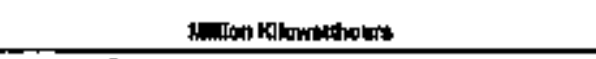 } & & \\
\hline 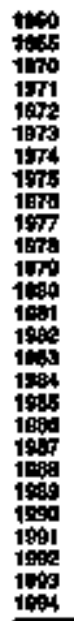 & $\begin{array}{l}: \\
0 \\
0 \\
0 \\
0 \\
0 \\
0 \\
0 \\
0 \\
0 \\
0 \\
0 \\
0 \\
0 \\
0 \\
0 \\
0 \\
0 \\
0 \\
0 \\
0 \\
0 \\
0 \\
0 \\
0 \\
0 \\
0\end{array}$ & $\begin{array}{l}0 \\
0 \\
0 \\
0 \\
0 \\
0 \\
0 \\
0 \\
0 \\
0 \\
0 \\
0 \\
0 \\
0 \\
0 \\
0 \\
0 \\
0 \\
0 \\
0 \\
0 \\
0 \\
0 \\
0 \\
0 \\
0 \\
0\end{array}$ & $\begin{array}{l}0 \\
0 \\
0 \\
0 \\
0 \\
0 \\
0 \\
0 \\
0 \\
0 \\
0 \\
0 \\
0 \\
0 \\
0 \\
0 \\
0 \\
0 \\
0 \\
0 \\
0 \\
0 \\
0 \\
0 \\
0 \\
0 \\
0 \\
0 \\
0 \\
0 \\
0 \\
0 \\
0\end{array}$ & $\begin{array}{l}0 \\
0 \\
0 \\
0 \\
0 \\
0 \\
0 \\
0 \\
0 \\
0 \\
1 \\
1 \\
1 \\
1 \\
1 \\
1 \\
\vdots \\
1 \\
1 \\
1 \\
1\end{array}$ & 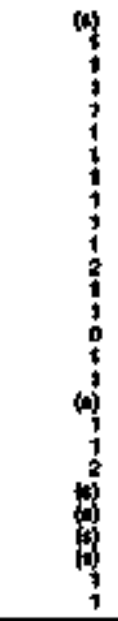 & $\begin{array}{l}: \\
: \\
0 \\
0 \\
0 \\
0 \\
0 \\
0 \\
0 \\
0 \\
0 \\
0 \\
0 \\
0 \\
0 \\
0 \\
0 \\
0 \\
0 \\
0 \\
0 \\
0 \\
0\end{array}$ & 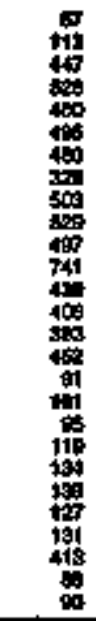 & 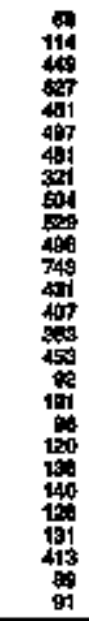 & $\begin{array}{r}0 \\
0 \\
0 \\
0 \\
0 \\
0 \\
0 \\
0 \\
0 \\
0 \\
0 \\
0 \\
0 \\
0 \\
0 \\
0 \\
0 \\
0 \\
0 \\
0 \\
0 \\
0 \\
0 \\
0 \\
919 \\
170 \\
194 \\
191\end{array}$ & 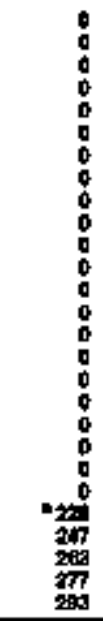 & 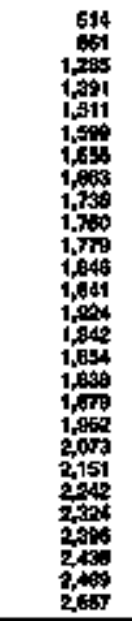 & $\begin{array}{l}\vdots \\
\vdots \\
\vdots \\
\vdots \\
\vdots \\
\vdots \\
\vdots \\
\vdots \\
\vdots \\
\vdots \\
\vdots \\
\vdots\end{array}$ & 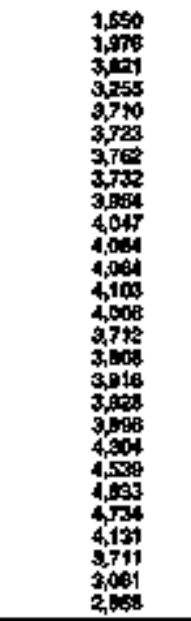 & $\begin{array}{l}\vdots \\
\vdots \\
\vdots \\
\vdots \\
\vdots \\
\vdots \\
\vdots \\
\vdots \\
\vdots \\
\vdots \\
\vdots \\
\vdots \\
\vdots\end{array}$ \\
\hline \multicolumn{15}{|c|}{ 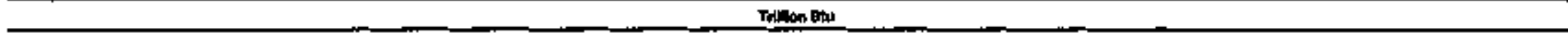 } \\
\hline 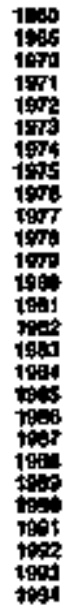 & 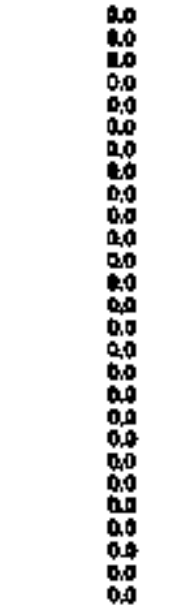 & 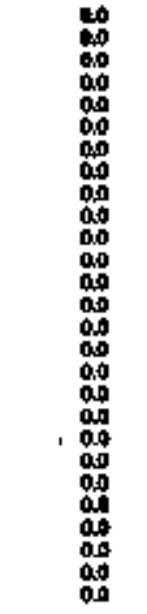 & 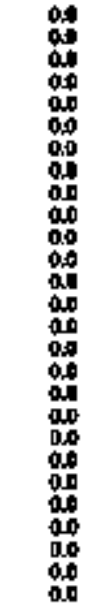 & 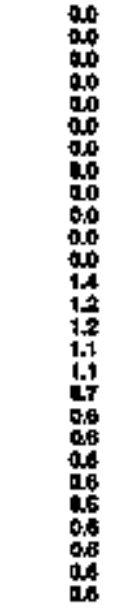 & $\begin{array}{l}8 \\
8 \\
8 \\
8 \\
8 \\
8 \\
8 \\
80 \\
8 \\
8 \\
8 \\
8 \\
8 \\
8\end{array}$ & 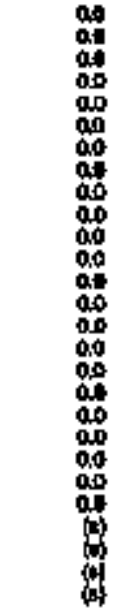 & 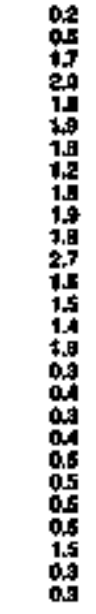 & 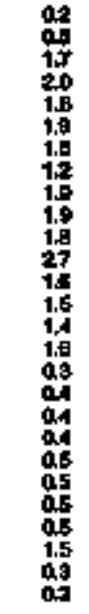 & 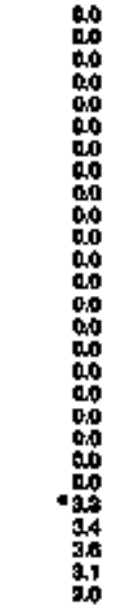 & 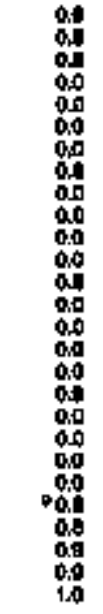 & 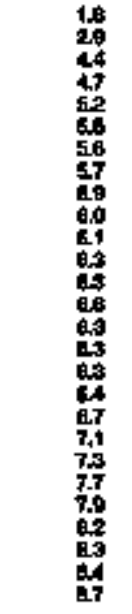 & 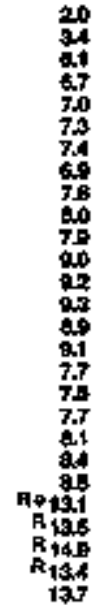 & 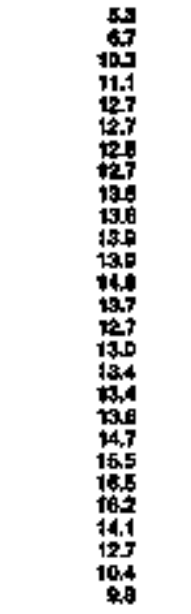 & 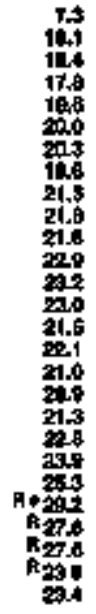 \\
\hline
\end{tabular}

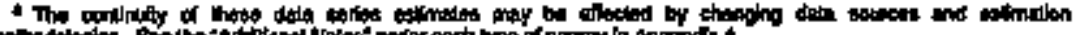

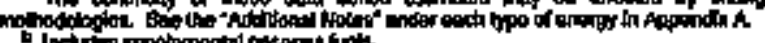

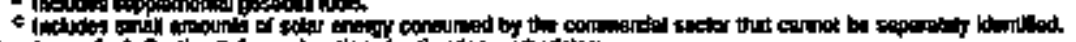

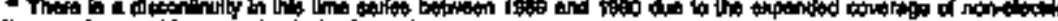

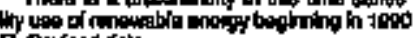


Table 85. Conmercial Enorgy Consumption Estimates, 1960, 1965, 1970-1994, Hawaili

\begin{tabular}{|c|c|c|c|c|c|c|c|c|c|c|c|c|c|c|}
\hline \multirow[b]{3}{*}{ 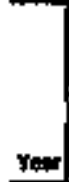 } & \multicolumn{3}{|c|}{ Cad } & \multirow{3}{*}{ 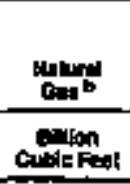 } & \multicolumn{6}{|c|}{ Proliwam } & \multirow[b]{2}{*}{ 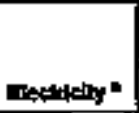 } & \multirow[b]{2}{*}{ 的 } & \multirow[b]{2}{*}{ 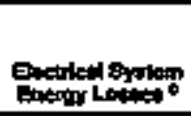 } & \multirow[b]{3}{*}{ Totw 1} \\
\hline & 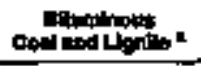 & Anthnelan 4 & Tow & & Futernate & Kropanan:" & LPo" & Molor & 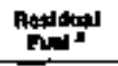 & Totol & & & & \\
\hline & \multicolumn{2}{|c|}{ Mous:ned Stant Toas } & \multirow[b]{2}{*}{$\begin{array}{l}0 \\
0 \\
0 \\
0 \\
0 \\
0 \\
0 \\
0 \\
0 \\
0 \\
0 \\
0 \\
0 \\
0 \\
0 \\
0 \\
0 \\
0 \\
0 \\
0 \\
0 \\
0 \\
0 \\
0 \\
0 \\
0\end{array}$} & & \multicolumn{6}{|c|}{ Thompandewnth } & \multicolumn{3}{|c|}{ 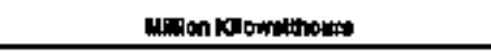 } & \\
\hline 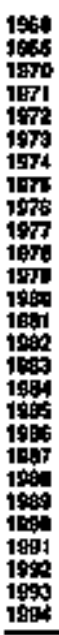 & $\begin{array}{l}0 \\
0 \\
0 \\
0 \\
0 \\
0 \\
0 \\
0 \\
0 \\
0 \\
0 \\
0 \\
0 \\
0 \\
0 \\
0 \\
0 \\
0 \\
0 \\
0 \\
0 \\
0 \\
0 \\
0 \\
0 \\
0 \\
0 \\
0 \\
\end{array}$ & $\begin{array}{l}0 \\
0 \\
0 \\
0 \\
0 \\
0 \\
0 \\
0 \\
0 \\
0 \\
0 \\
0 \\
0 \\
0 \\
0 \\
0 \\
0 \\
0 \\
0 \\
0 \\
0 \\
0 \\
0 \\
0 \\
0 \\
0 \\
0 \\
\end{array}$ & & $\begin{array}{l}0 \\
0 \\
0 \\
0 \\
0 \\
0 \\
0 \\
0 \\
0 \\
0 \\
0 \\
0 \\
2 \\
2 \\
2 \\
2 \\
1 \\
2 \\
2 \\
2 \\
2 \\
2 \\
2 \\
2 \\
2 \\
2 \\
2 \\
2 \\
\end{array}$ & 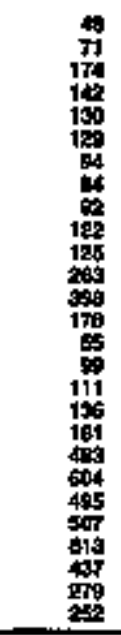 & 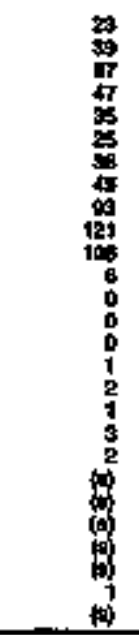 & 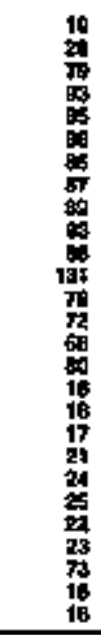 & 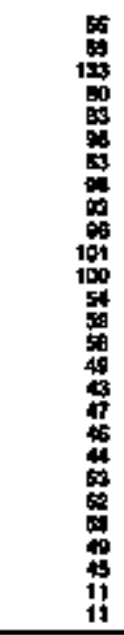 & 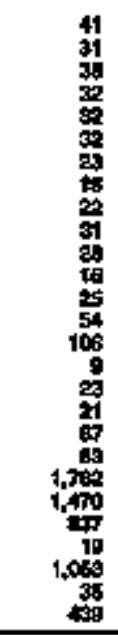 & 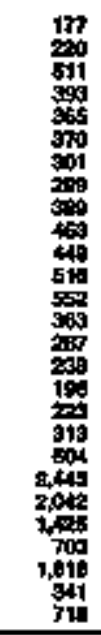 & 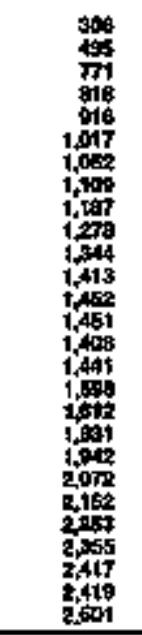 & $\begin{array}{l}= \\
z \\
5 \\
z \\
= \\
= \\
= \\
= \\
= \\
= \\
= \\
z \\
z \\
z\end{array}$ & 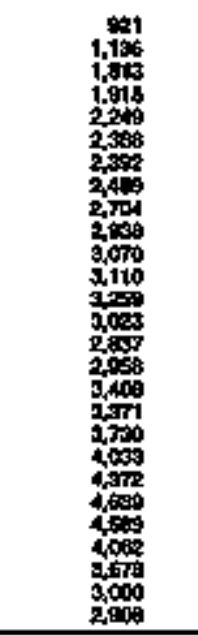 & $\begin{array}{l}= \\
z \\
z \\
z \\
= \\
z \\
z \\
= \\
= \\
= \\
= \\
= \\
=\end{array}$ \\
\hline \multicolumn{15}{|c|}{ Trinton Bus } \\
\hline 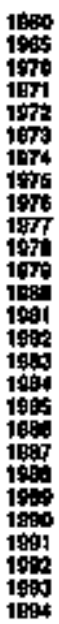 & $\begin{array}{l}00 \\
00 \\
00 \\
00 \\
000 \\
00 \\
00 \\
000 \\
000 \\
000 \\
00 \\
00 \\
000 \\
00 \\
00 \\
00 \\
000 \\
00 \\
00 \\
000 \\
00 \\
00 \\
00 \\
00 \\
00 \\
00\end{array}$ & 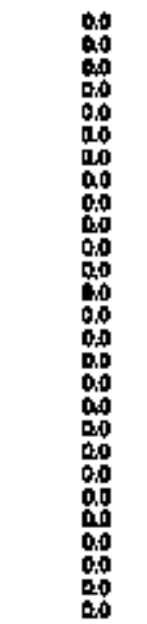 & 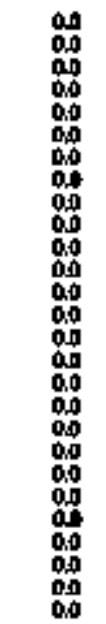 & 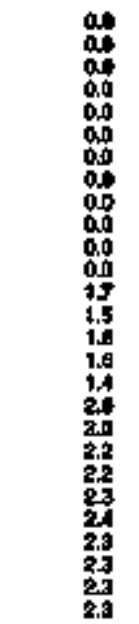 & 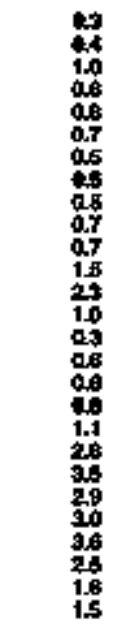 & 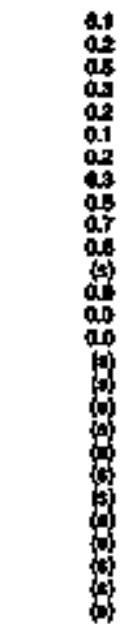 & $\begin{array}{l}0.9 \\
0.1 \\
0.3 \\
0.4 \\
0.3 \\
0.3 \\
0.3 \\
0.2 \\
0.3 \\
0.3 \\
0.3 \\
0.5 \\
0.4 \\
0.3 \\
0.3 \\
0.3 \\
0.1 \\
0.1 \\
0.1 \\
0.1 \\
0.1 \\
0.1 \\
0.1 \\
0.1 \\
0.3 \\
0.1 \\
0.1 \\
0.1\end{array}$ & 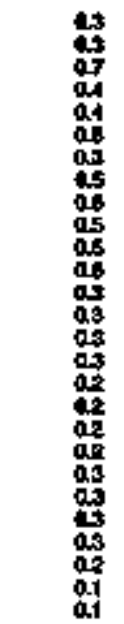 & 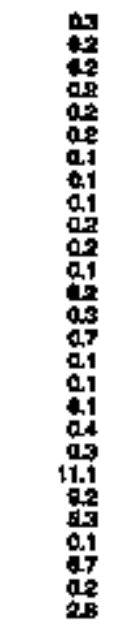 & 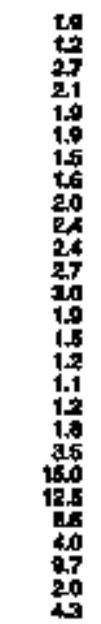 & 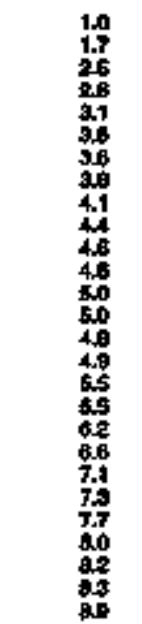 & 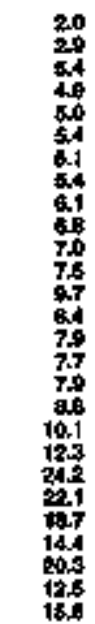 & 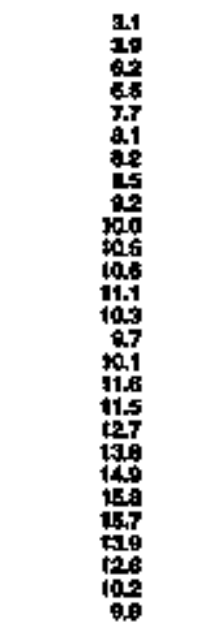 & 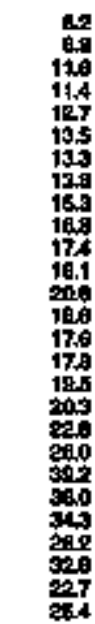 \\
\hline
\end{tabular}


Table 86. Industrial Enargy Consumplion Exthnsios, 1950, 1965, 1970-1984, Hawaii

\begin{tabular}{|c|c|c|c|c|c|c|c|c|c|c|c|c|c|c|c|c|c|c|}
\hline \multirow[b]{3}{*}{ reat } & \multirow[b]{2}{*}{ i } & \multirow[b]{2}{*}{$\operatorname{mos}_{a}$} & \multicolumn{9}{|c|}{ Polpodoun } & \multirow[b]{2}{*}{ 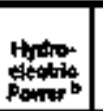 } & \multirow[b]{2}{*}{ 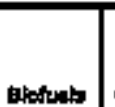 } & \multirow[b]{2}{*}{ Onese of ? } & \multirow[b]{2}{*}{ 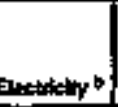 } & \multirow[b]{2}{*}{ then } & \multirow{2}{*}{ 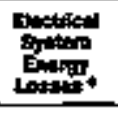 } & \multirow[b]{3}{*}{ Tow } \\
\hline & & & 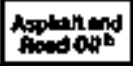 & 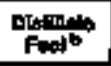 & Konosenes & LPa' & 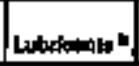 & anditions & 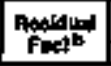 & other $b$ & Totat & & & & & & & \\
\hline & Thoosind & 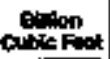 & \multicolumn{9}{|c|}{ 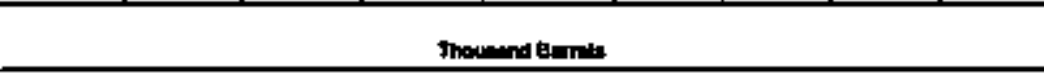 } & \multicolumn{6}{|c|}{ 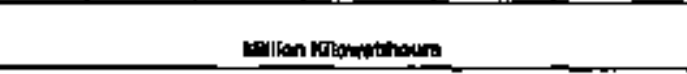 } & \\
\hline 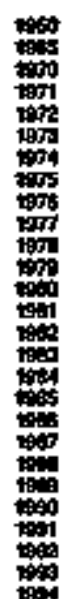 & 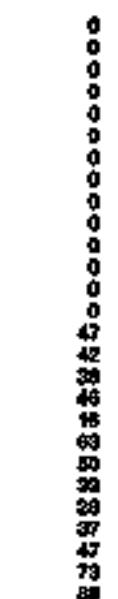 & $\begin{array}{l}0 \\
0 \\
0 \\
0 \\
0 \\
0 \\
0 \\
0 \\
0 \\
0 \\
0 \\
0 \\
0 \\
0 \\
0 \\
0 \\
0 \\
0 \\
0 \\
0 \\
0 \\
0 \\
0 \\
0 \\
0 \\
0 \\
0 \\
0\end{array}$ & 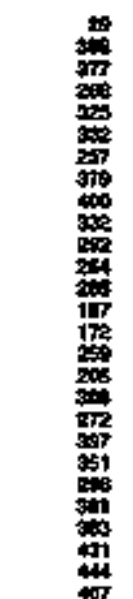 & 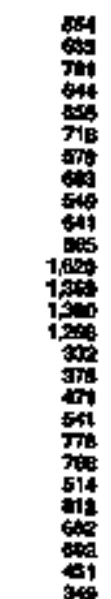 & 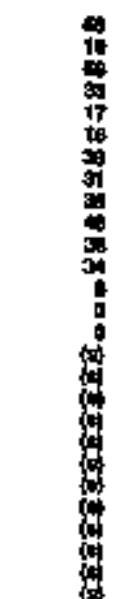 & 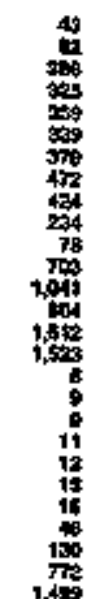 & 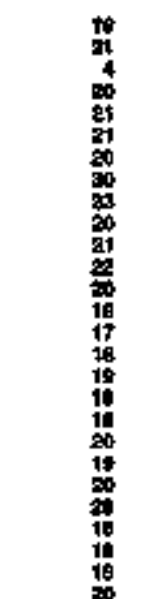 & 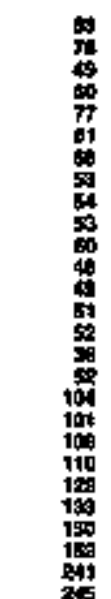 & 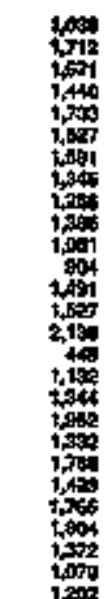 & 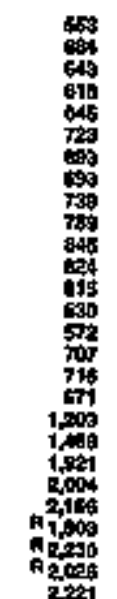 & 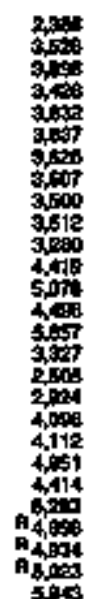 & 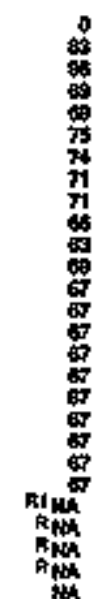 & 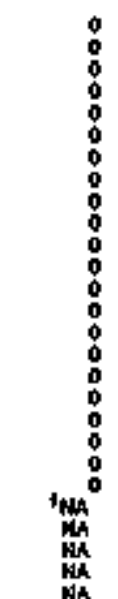 & 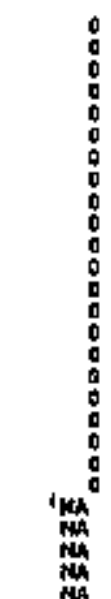 & 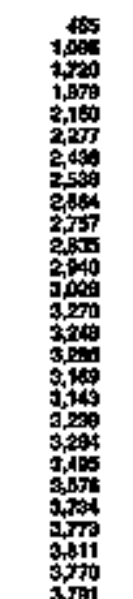 & $\bar{z}$ & 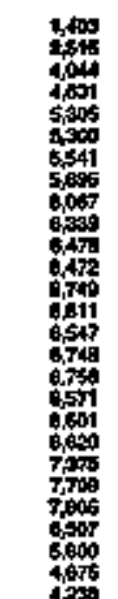 & $=$ \\
\hline
\end{tabular}

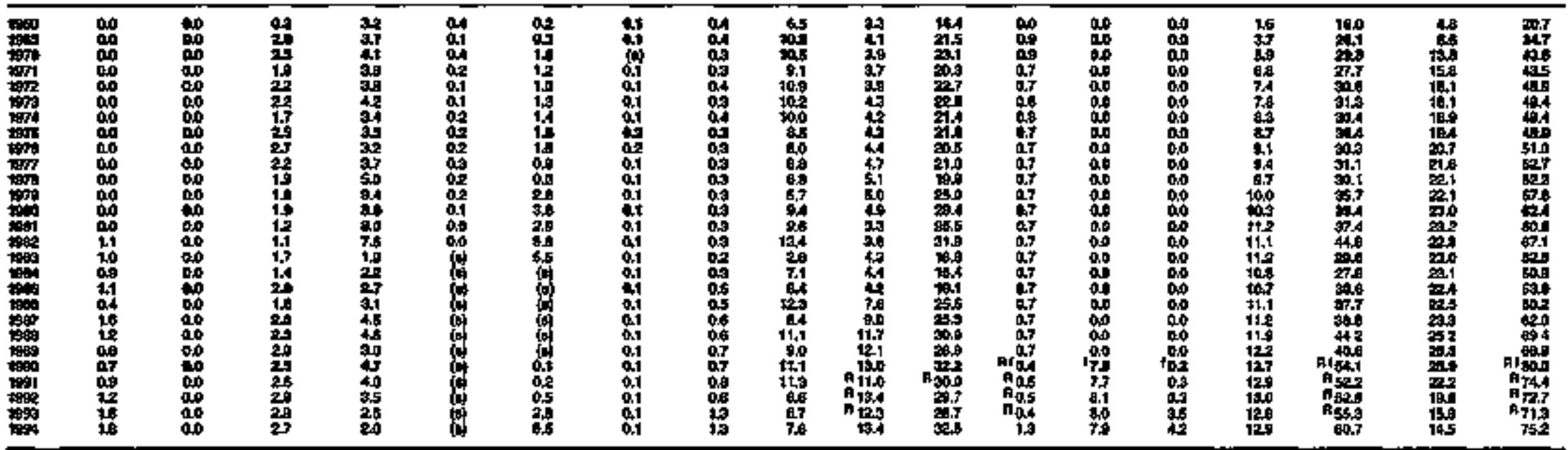

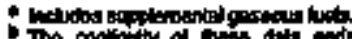

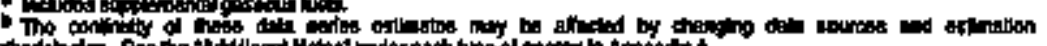

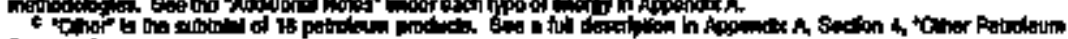

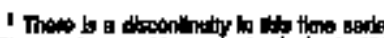

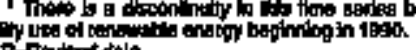

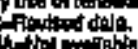

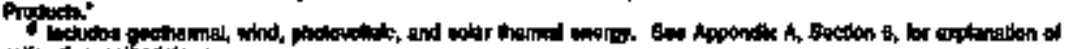

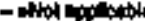

(1)

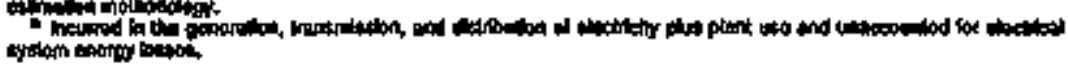


Table 87. Transportation Emargy Consinption Estimales, 1960, 1965, 1870-1994, Hansei

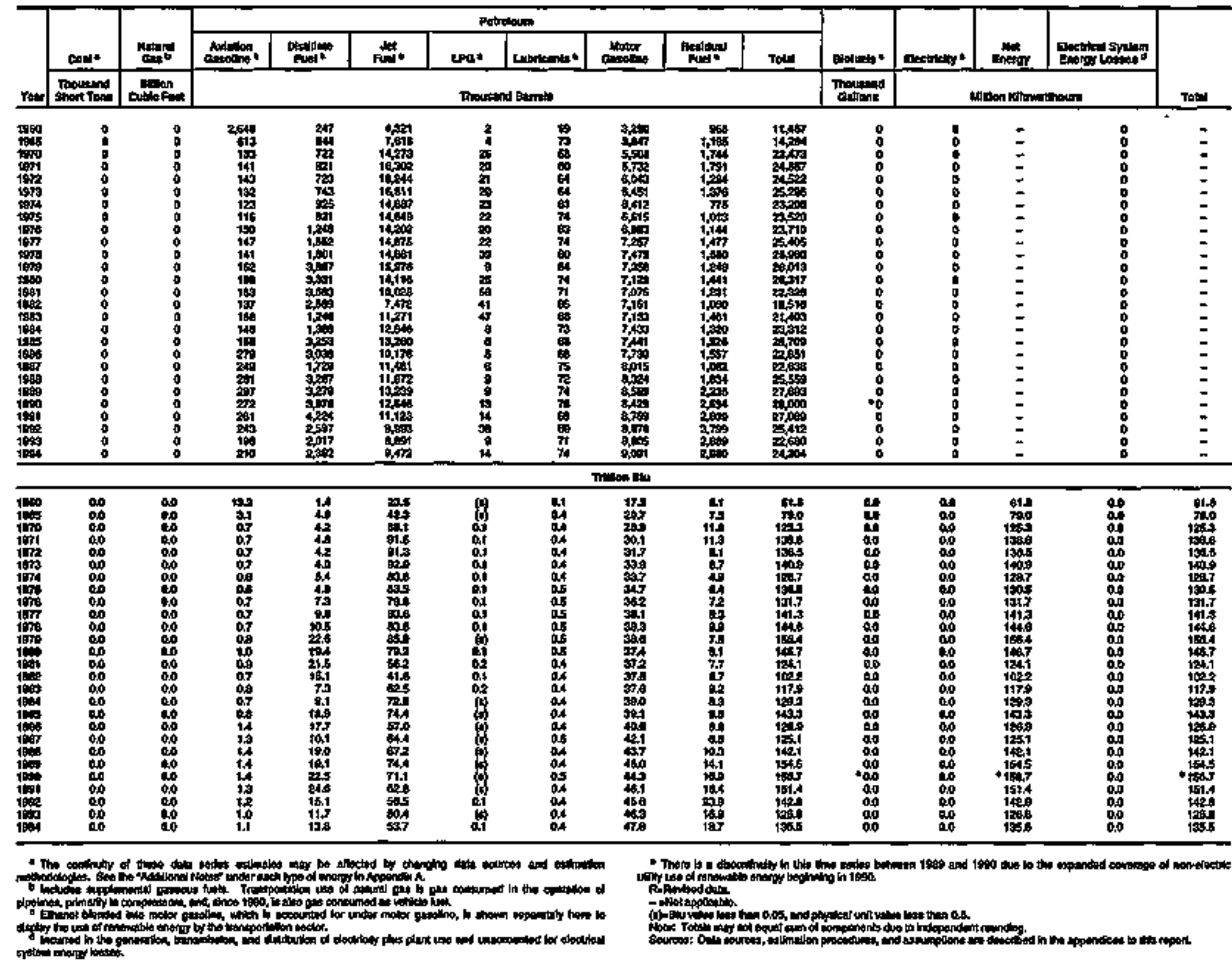


Table 88. Eestimates of Energy input at Electric Uuliles, 1960, 1965, 1970-1994, Hawali

\begin{tabular}{|c|c|c|c|c|c|c|c|c|c|c|c|c|c|c|}
\hline \multirow[b]{3}{*}{ tari } & \multicolumn{3}{|c|}{ cont } & \multirow{3}{*}{ 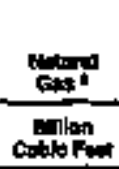 } & \multicolumn{4}{|c|}{ 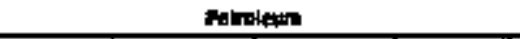 } & \multirow[b]{2}{*}{ 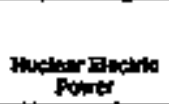 } & \multirow[b]{2}{*}{ 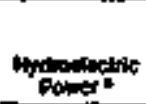 } & \multirow[b]{2}{*}{ Biscols } & \multirow[b]{2}{*}{ Ecotbersis } & \multirow{3}{*}{ olherts } & \multirow[b]{3}{*}{ Total 10} \\
\hline & 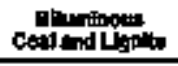 & Anthineto & Tow & & ond & 나에 & 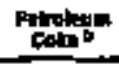 & Tatel & & & & & & \\
\hline & \multicolumn{3}{|c|}{ Thouasend Shont Tons } & & \multicolumn{4}{|c|}{ 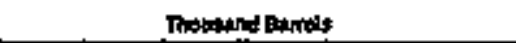 } & \multicolumn{4}{|c|}{ 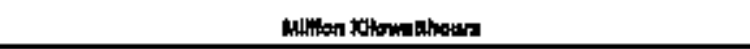 } & & \\
\hline 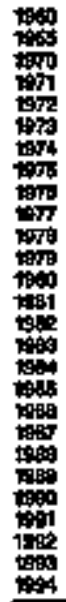 & $\begin{array}{l}0 \\
0 \\
0 \\
0 \\
0 \\
0 \\
0 \\
0 \\
0 \\
0 \\
0 \\
0 \\
0 \\
0 \\
0 \\
0 \\
0 \\
0 \\
0 \\
0\end{array}$ & $\begin{array}{l}0 \\
0 \\
0 \\
0 \\
0 \\
0 \\
0 \\
0 \\
0 \\
0 \\
0 \\
0 \\
0 \\
0 \\
0 \\
0 \\
0 \\
0 \\
0 \\
0 \\
0 \\
0 \\
0 \\
0 \\
0\end{array}$ & $\begin{array}{l}0 \\
0 \\
0 \\
0 \\
0 \\
0 \\
0 \\
0 \\
0 \\
0 \\
0 \\
0 \\
0 \\
0 \\
0 \\
0 \\
0 \\
0 \\
0 \\
0 \\
0\end{array}$ & $\begin{array}{l}: \\
0 \\
0 \\
0 \\
0 \\
0 \\
0 \\
0 \\
0 \\
0 \\
0 \\
0 \\
0 \\
0 \\
0 \\
0 \\
0 \\
0 \\
0 \\
0 \\
0 \\
0 \\
0 \\
0 \\
0\end{array}$ & 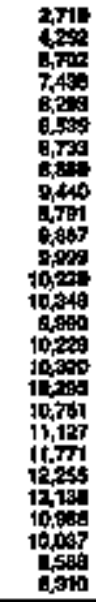 & 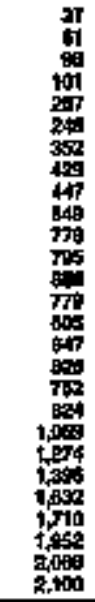 & $\begin{array}{l}0 \\
0 \\
0 \\
0 \\
0 \\
0 \\
0 \\
0 \\
0 \\
0 \\
: \\
0 \\
0 \\
0 \\
0 \\
0 \\
0 \\
0 \\
0 \\
0 \\
0 \\
0\end{array}$ & 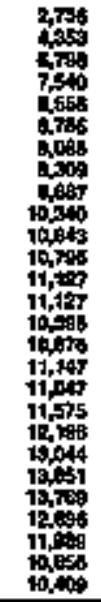 & $\begin{array}{l}0 \\
0 \\
0 \\
0 \\
0 \\
0 \\
0 \\
0 \\
0 \\
0 \\
0 \\
0 \\
0 \\
0 \\
0 \\
0 \\
0 \\
0 \\
0 \\
0 \\
0 \\
0 \\
0 \\
0 \\
0 \\
0\end{array}$ & 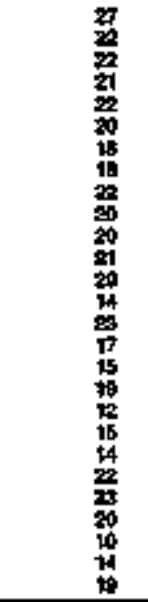 & 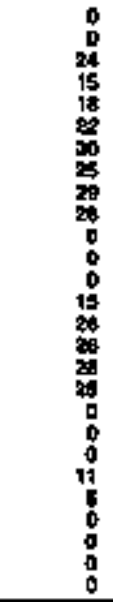 & $\begin{array}{l}0 \\
0 \\
0 \\
0 \\
0 \\
0 \\
0 \\
0 \\
0 \\
0 \\
0 \\
0 \\
0 \\
0 \\
0 \\
0 \\
11 \\
19 \\
18 \\
19 \\
10 \\
14 \\
0 \\
0 \\
0 \\
0 \\
0\end{array}$ & $\begin{array}{l}0 \\
0 \\
0 \\
0 \\
0 \\
0 \\
0 \\
0 \\
0 \\
0 \\
0 \\
0 \\
0 \\
0 \\
0 \\
0 \\
0 \\
0 \\
0 \\
0 \\
0 \\
0 \\
0 \\
0 \\
0 \\
0 \\
0\end{array}$ & $\begin{array}{l}= \\
= \\
= \\
= \\
= \\
= \\
= \\
= \\
= \\
= \\
= \\
z \\
= \\
z\end{array}$ \\
\hline
\end{tabular}

\begin{tabular}{|c|c|c|c|c|c|c|c|c|c|c|c|c|c|c|}
\hline 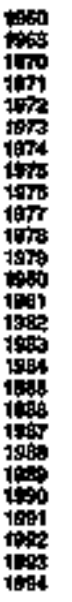 & 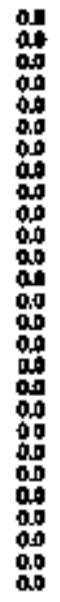 & 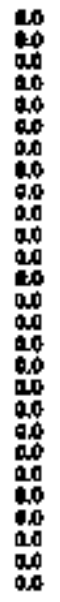 & $\begin{array}{l}00 \\
00 \\
00 \\
0.0 \\
00 \\
00 \\
00 \\
00 \\
00 \\
0.0 \\
000 \\
00 \\
00 \\
00 \\
00 \\
00 \\
0.0 \\
00 \\
00 \\
00 \\
000 \\
00 \\
00 \\
00 \\
00 \\
00 \\
00\end{array}$ & 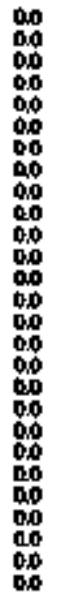 & 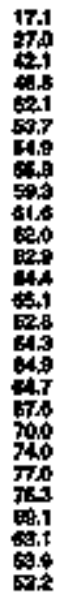 & 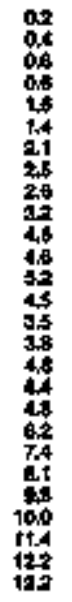 & 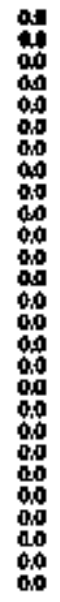 & 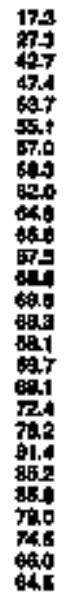 & 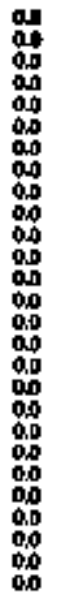 & 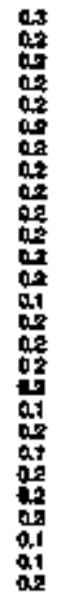 & 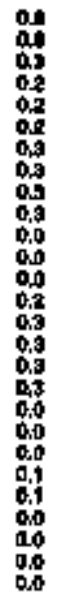 & 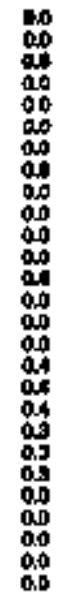 & 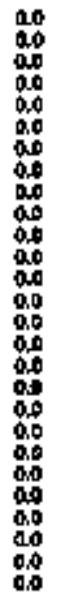 & 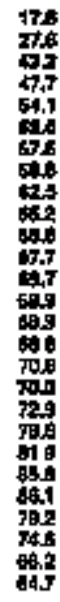 \\
\hline
\end{tabular}

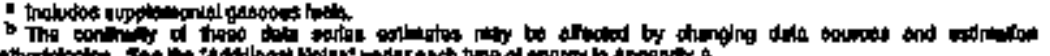

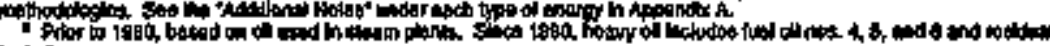

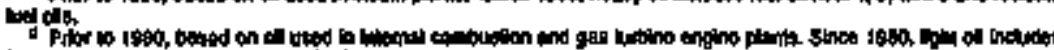

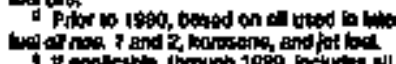

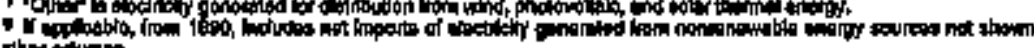

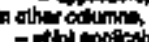

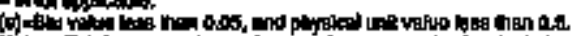

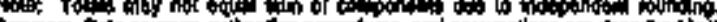

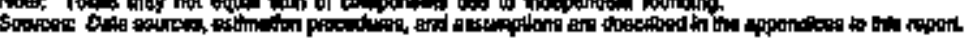


Table 89. Energy Consumption Estimales by Soumcle, 1960, 1965, 1970-1894, Utaho

\begin{tabular}{|c|c|c|c|c|c|c|c|c|c|c|c|c|c|c|c|c|c|c|c|}
\hline \multirow[b]{3}{*}{ Ylapt } & \multirow[b]{2}{*}{ const. } & \multirow[b]{2}{*}{ Find } & \multicolumn{11}{|c|}{ Parolan: } & \multirow{2}{*}{ 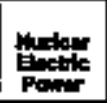 } & \multirow{2}{*}{ pested } & \multirow[b]{2}{*}{ ㅂighanated } & \multirow[b]{2}{*}{ Dever at } & \multirow{3}{*}{ Let } & \multirow[b]{3}{*}{ Tots } \\
\hline & & & 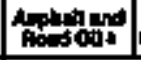 & 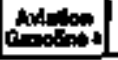 & 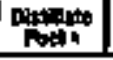 & Finn & 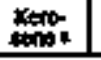 & ERE. & | & 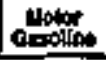 & Fanteris & Otwer at & Tond & & & & & & \\
\hline & 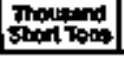 & 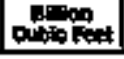 & \multicolumn{11}{|c|}{ Thoon:and Bumb } & \multicolumn{4}{|c|}{ 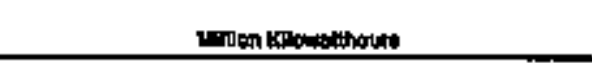 } & & \\
\hline 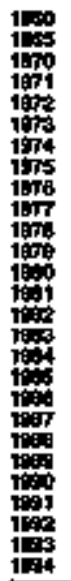 & 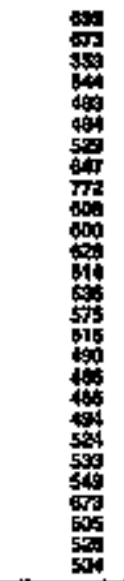 & 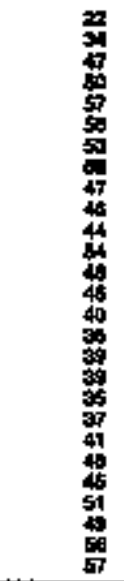 & 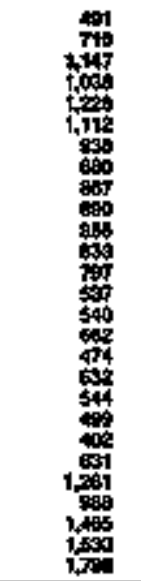 & 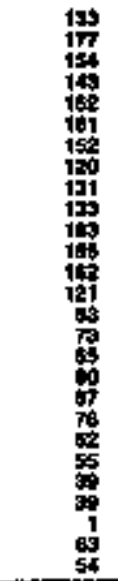 & 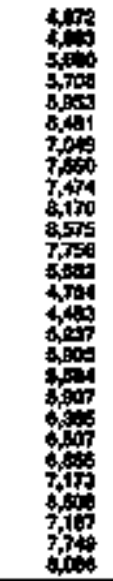 & 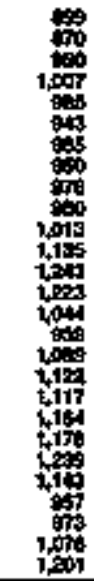 & 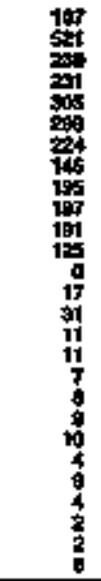 & 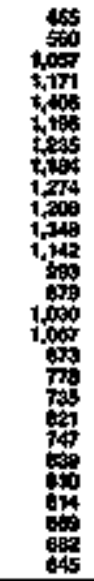 & 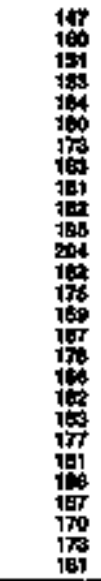 & 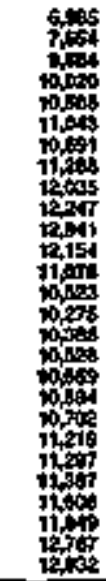 & 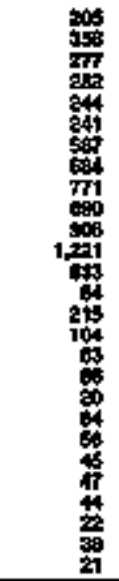 & 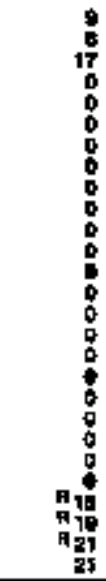 & 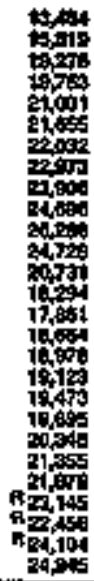 & $\begin{array}{l}: \\
0 \\
0 \\
0 \\
0 \\
0 \\
0 \\
0 \\
0 \\
0 \\
0 \\
0 \\
0 \\
0 \\
0 \\
0 \\
0 \\
0 \\
0 \\
0 \\
0 \\
0\end{array}$ & 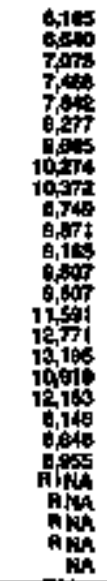 & 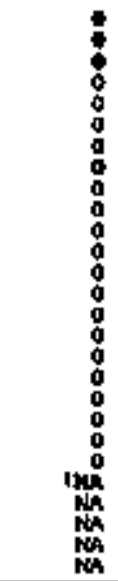 & 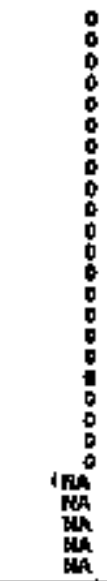 & 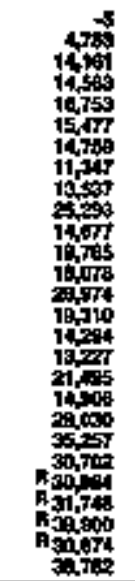 & $\begin{array}{l}\bar{z} \\
z \\
z \\
\vdots \\
z \\
z \\
z \\
z\end{array}$ \\
\hline
\end{tabular}

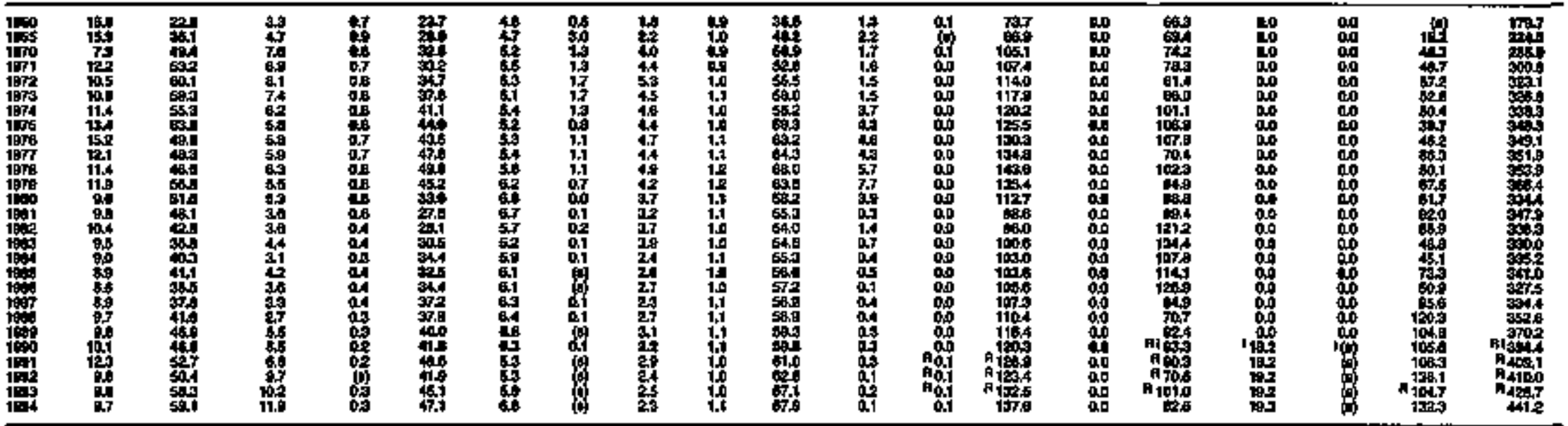

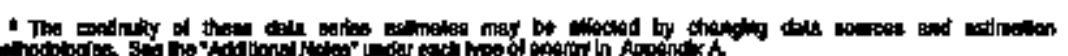

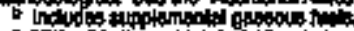

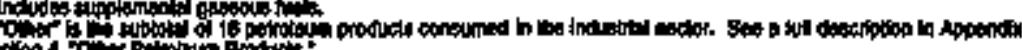

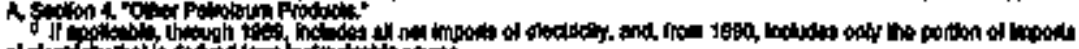

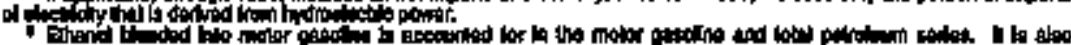

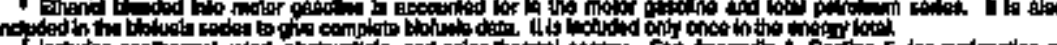

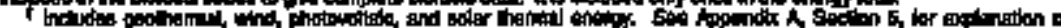

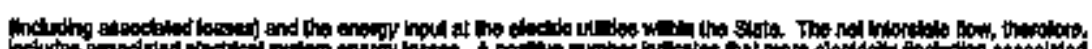

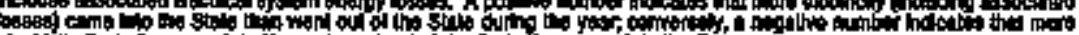

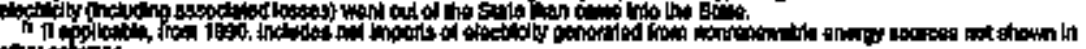

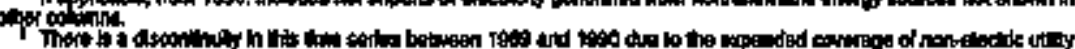
然

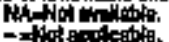

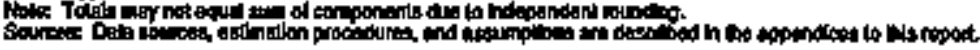

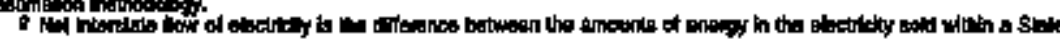


Table 90. Besidentlal Energy Consumption Estimates, 1960, 1965, 1970-1994, Idaho

$\mathrm{A}$
$\mathrm{H}$

O

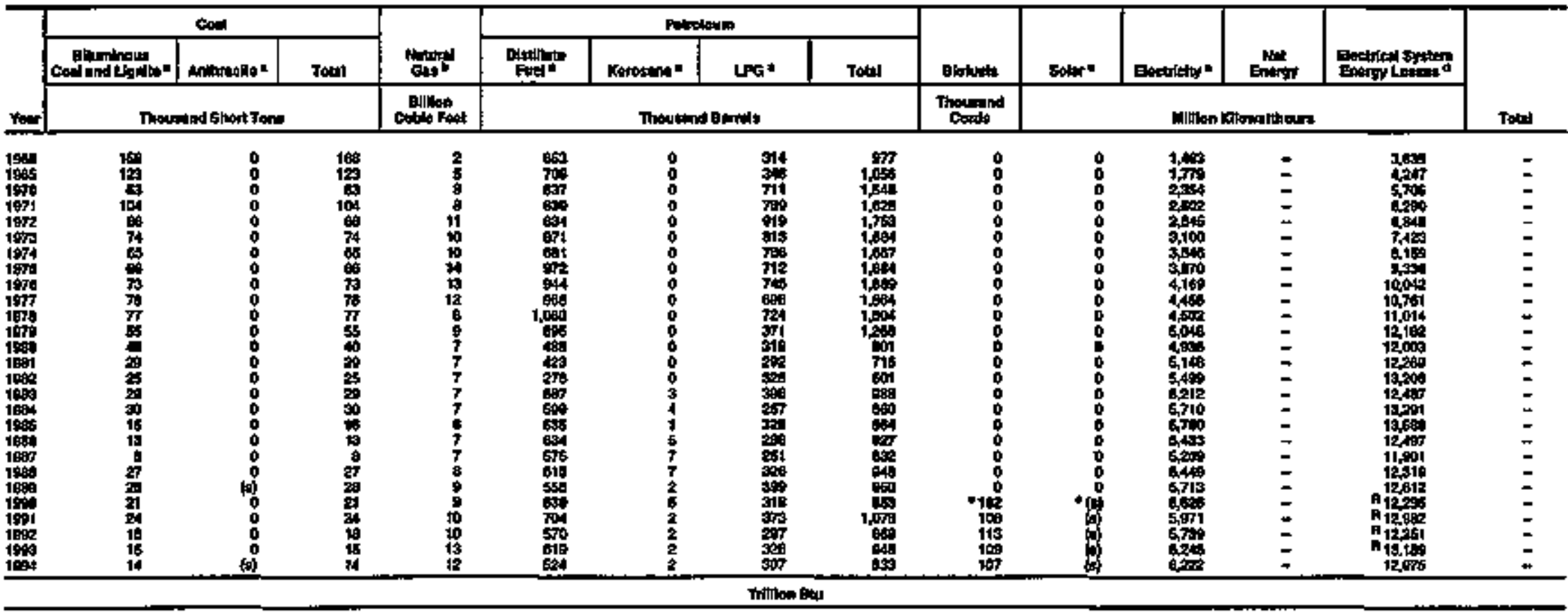

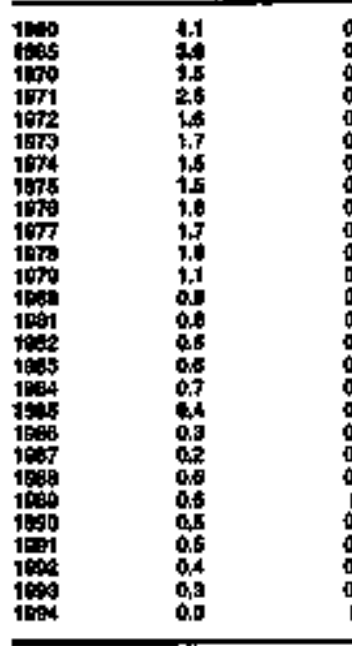

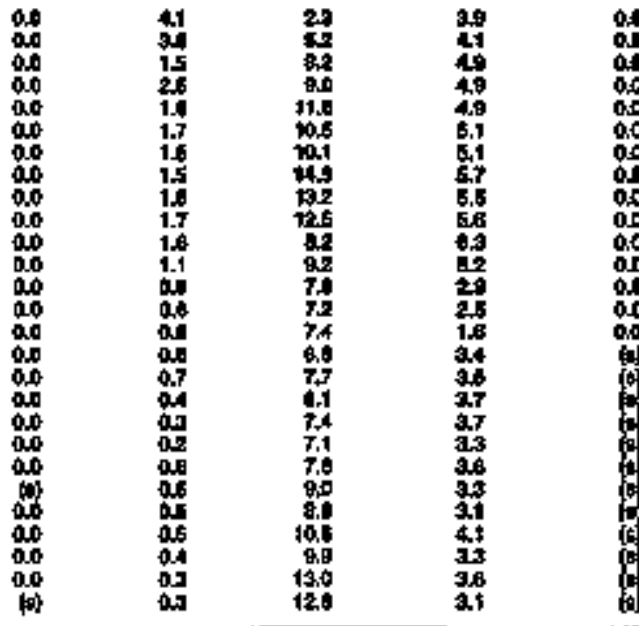

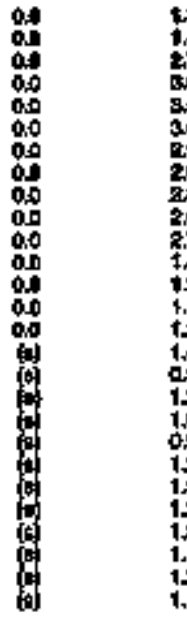

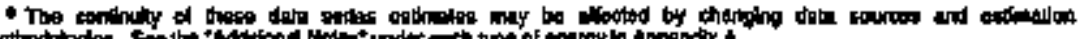

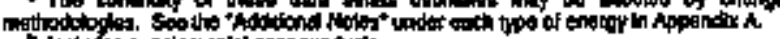

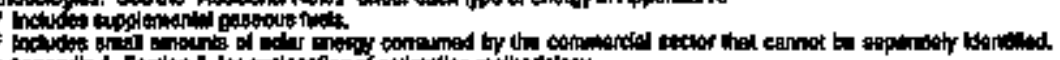

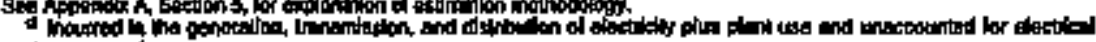

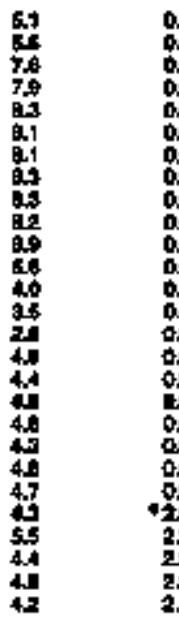

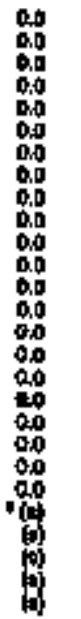

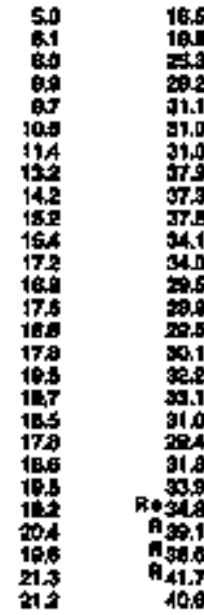

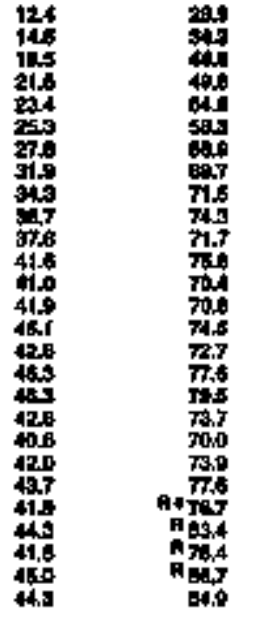

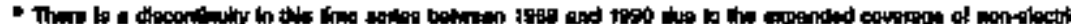

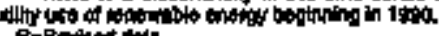

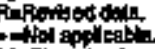

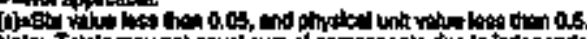

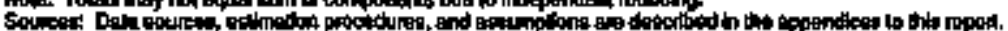


Table 9t. Comanenclal Energy Consunption Estimates, 1960, 1965, 1970-1994, Idaho

\begin{tabular}{|c|c|c|c|c|c|c|c|c|c|c|c|c|c|c|}
\hline \multirow[b]{3}{*}{ Yat } & \multicolumn{3}{|c|}{ tant } & \multirow[b]{2}{*}{ (1) } & \multicolumn{6}{|c|}{ Potrodoon } & \multirow[b]{2}{*}{ mactikty" } & \multirow[b]{2}{*}{$\min _{0 \rightarrow 0}$} & \multirow[b]{2}{*}{ 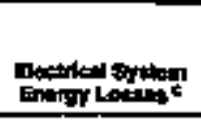 } & \multirow[b]{3}{*}{ Toldid d } \\
\hline & 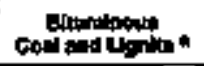 & Anturastio: & Totol & & $\begin{array}{l}\text { Dansente } \\
\text { hude }\end{array}$ & Whosene* & tPan & molor & 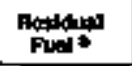 & Total & & & & \\
\hline & \multicolumn{3}{|c|}{ Thoo:uld short tons } & 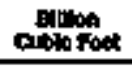 & \multicolumn{6}{|c|}{ Thousand Eamath } & \multicolumn{3}{|c|}{ 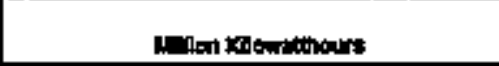 } & \\
\hline 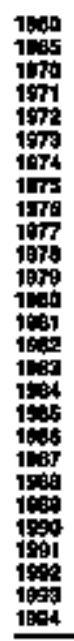 & 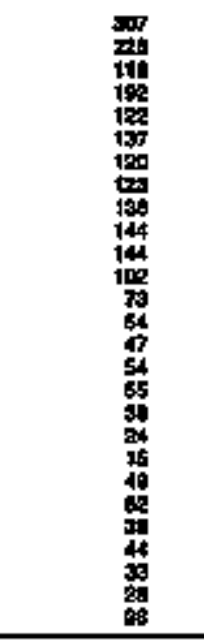 & $\begin{array}{l}0 \\
0 \\
0 \\
0 \\
0 \\
0 \\
0 \\
0 \\
0 \\
0 \\
0 \\
0 \\
0 \\
0 \\
0 \\
0 \\
0 \\
0 \\
0 \\
0 \\
0 \\
0 \\
0 \\
0 \\
0 \\
0 \\
0 \\
0 \\
0 \\
0 . j \\
j\end{array}$ & 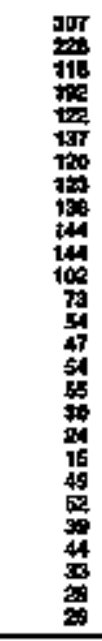 & 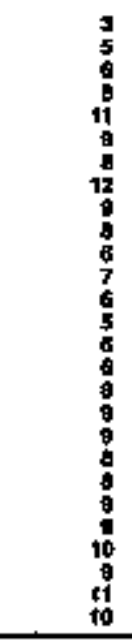 & 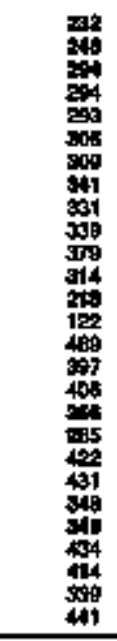 & 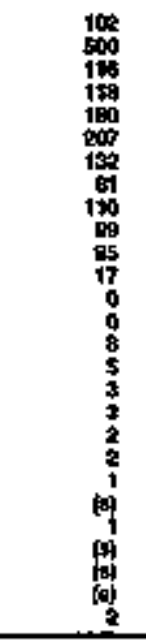 & 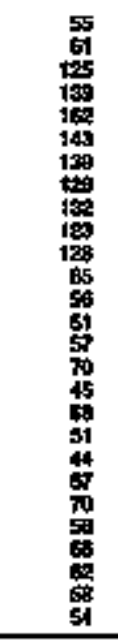 & 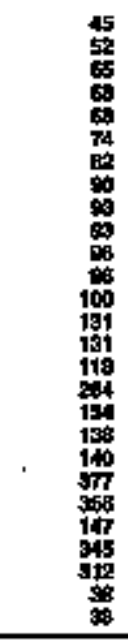 & 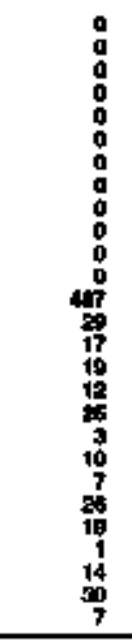 & 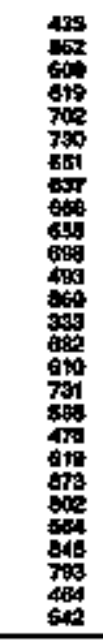 & 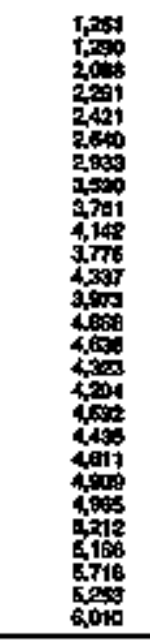 & $\begin{array}{l}\vdots \\
\vdots \\
\vdots \\
\vdots \\
\vdots \\
\vdots \\
\vdots \\
\vdots \\
\vdots \\
\vdots \\
\vdots \\
\vdots \\
\end{array}$ & 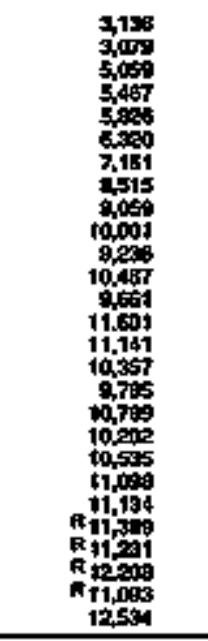 & $\begin{array}{l}\vdots \\
\vdots \\
\vdots \\
\vdots \\
\vdots \\
\vdots \\
\vdots \\
\vdots \\
\vdots \\
\vdots \\
\vdots \\
\vdots \\
\end{array}$ \\
\hline \multicolumn{15}{|c|}{ Tritlan ew } \\
\hline 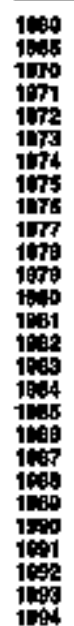 & 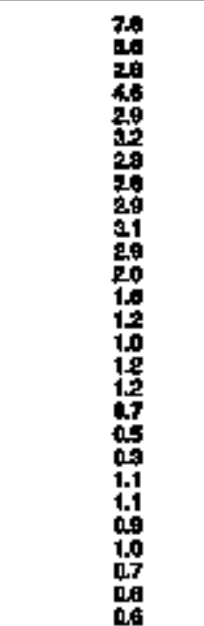 & 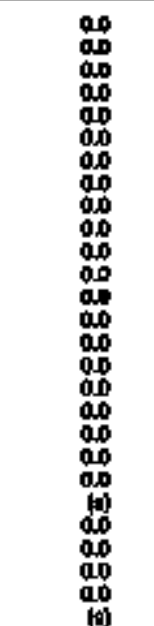 & 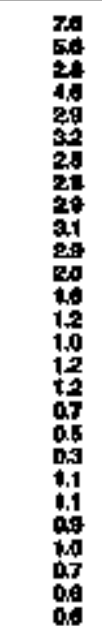 & 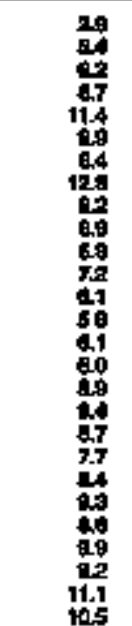 & 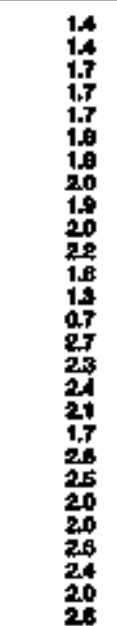 & 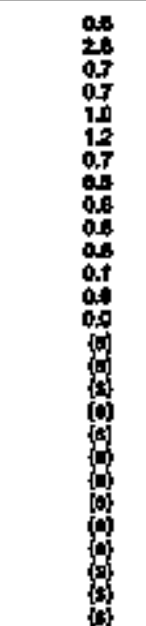 & 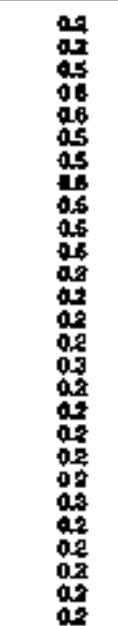 & 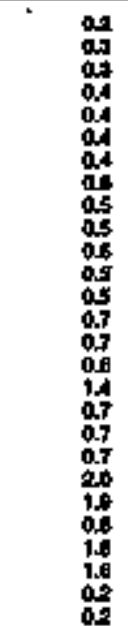 & 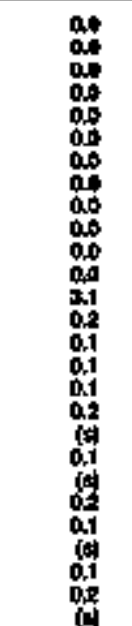 & 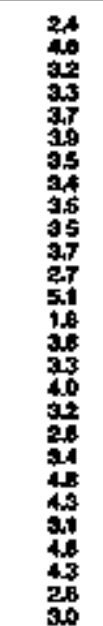 & 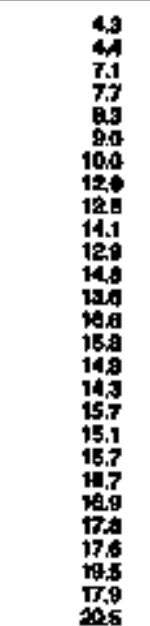 & 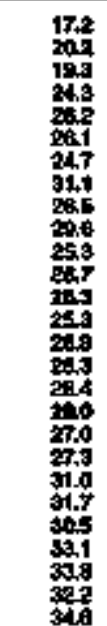 & 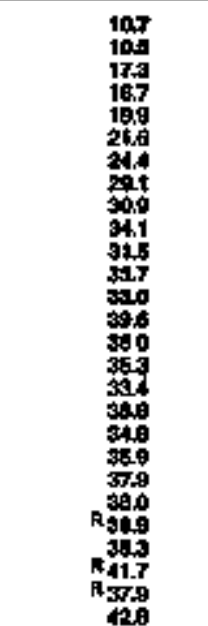 & 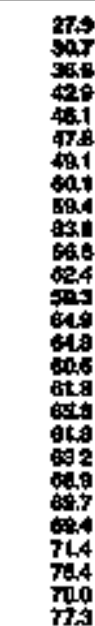 \\
\hline
\end{tabular}

$\mathbf{J}$
$\mathbf{D}$
$\mathbf{A}$
$\mathbf{H}$
$\mathbf{0}$ 
Table 92 Industrial Enongy Consumplion Estinales, 1550, 1965, 1970-1994, Waho

I
A
H
O

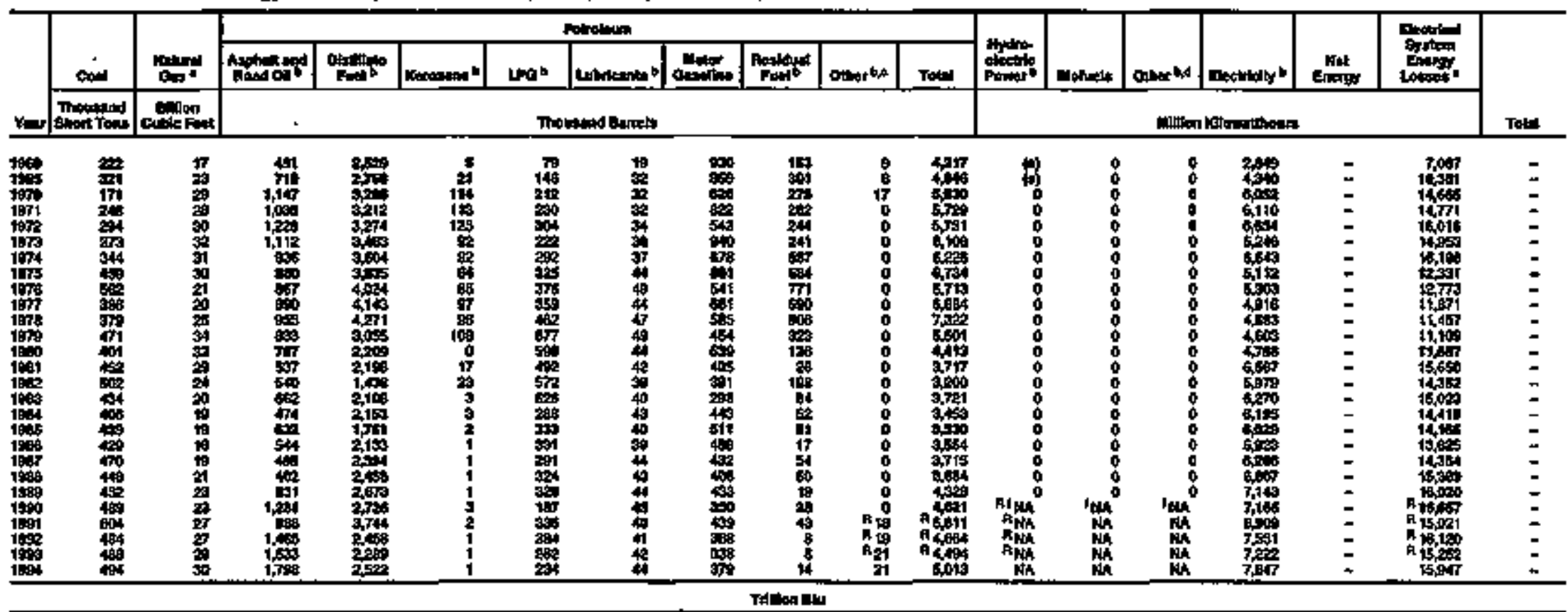

\begin{tabular}{|c|c|c|}
\hline 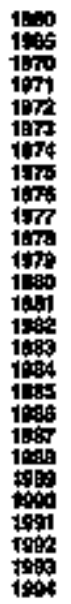 & 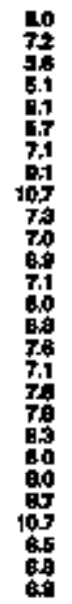 & 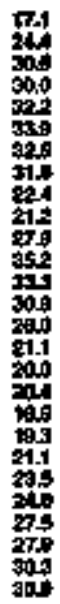 \\
\hline
\end{tabular}

14.

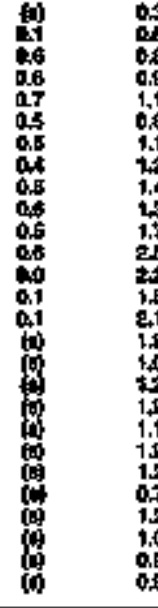

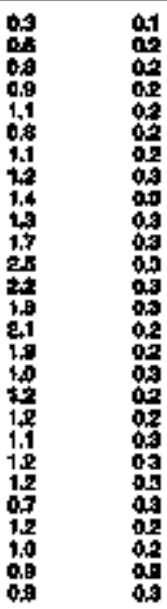

点

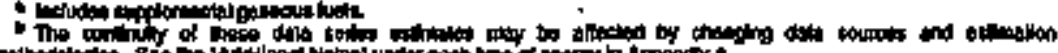

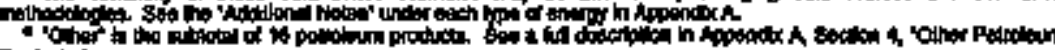

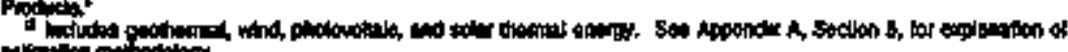

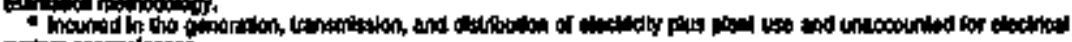

\begin{tabular}{|c|c|c|c|c|c|c|c|c|}
\hline 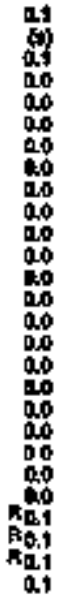 & 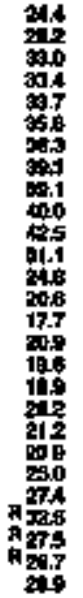 & 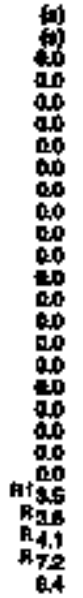 & 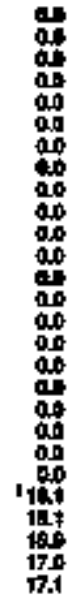 & 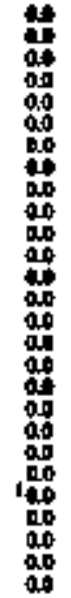 & 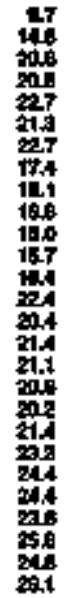 & 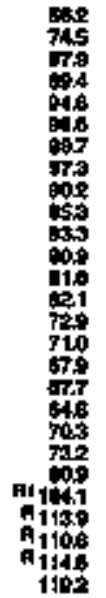 & 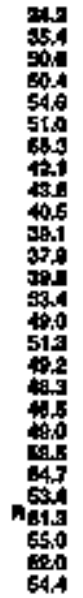 & 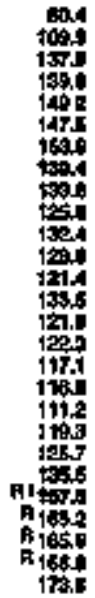 \\
\hline
\end{tabular}

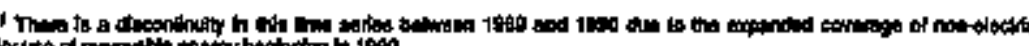
管

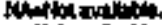

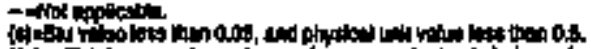

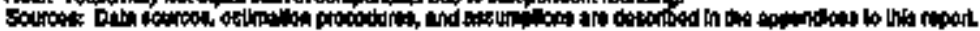


Table 93. Transportation Enwgy Consumption Estimates, 1960, 1955, 1970-1994, ktaho

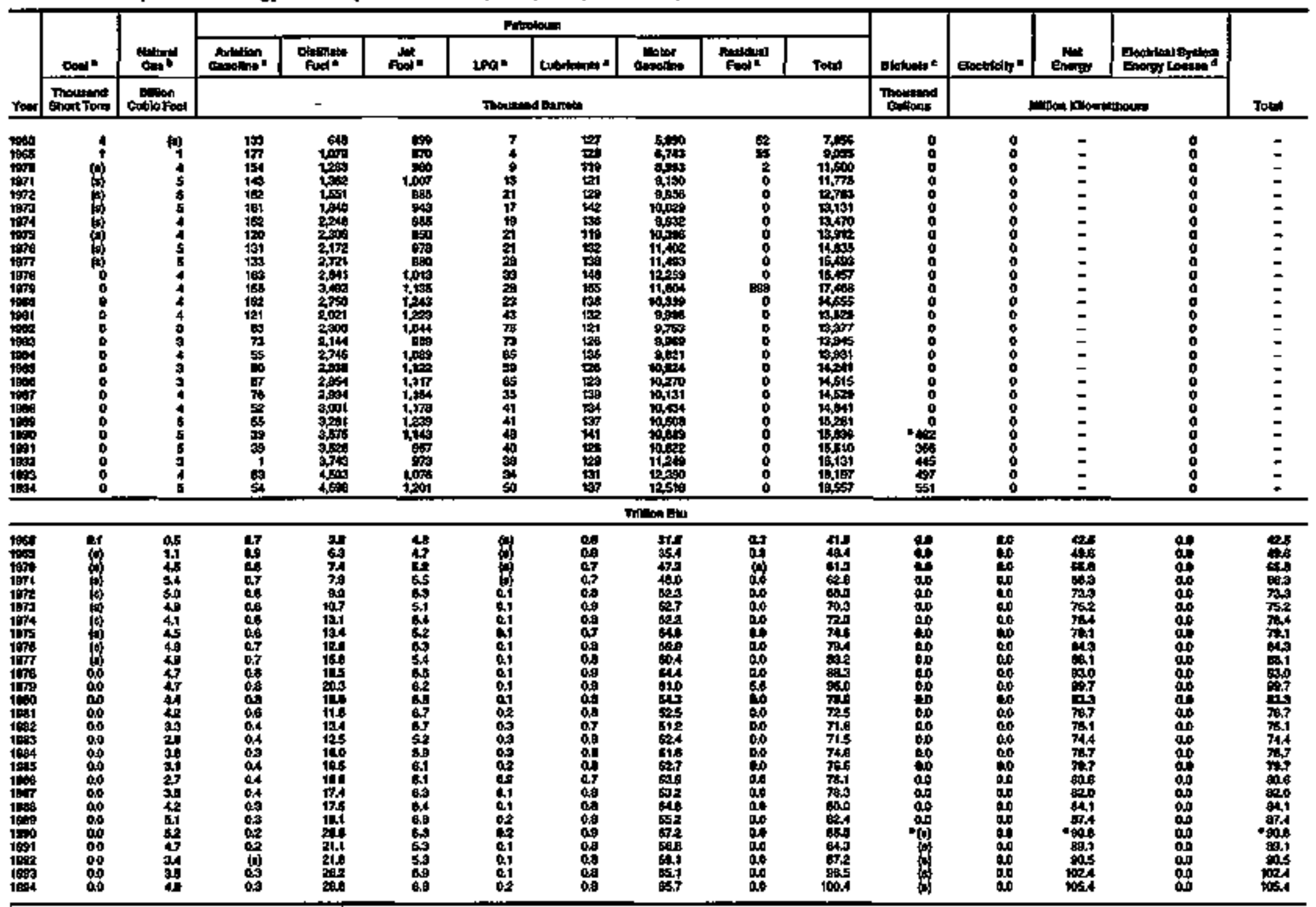

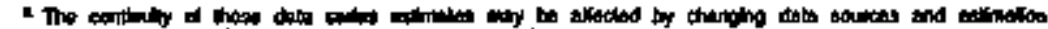

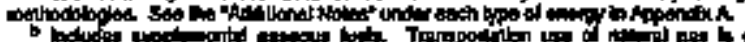

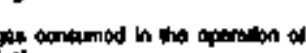

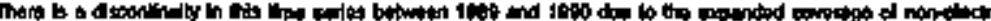

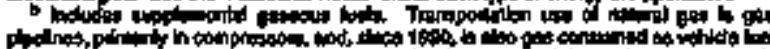

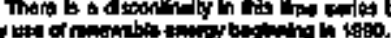

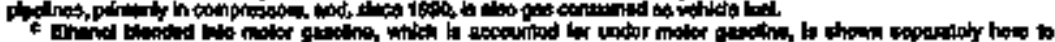

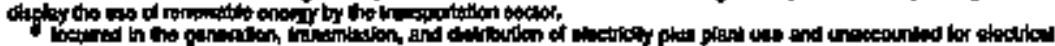

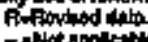

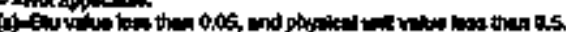

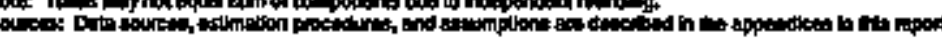

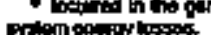




\begin{tabular}{|c|c|c|c|c|c|c|c|c|c|c|c|c|c|}
\hline \multicolumn{3}{|c|}{ कos } & \multirow[b]{2}{*}{ 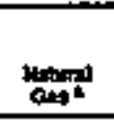 } & \multicolumn{4}{|c|}{ Potrotemat } & \multirow[b]{2}{*}{ 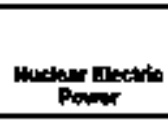 } & \multirow[b]{2}{*}{ 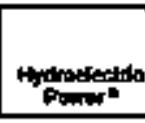 } & \multirow[b]{2}{*}{ Bonneste } & \multirow[b]{2}{*}{ test } & \multirow{3}{*}{ atoross } & \\
\hline 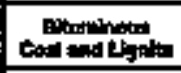 & Antreation & rot & & 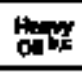 & $\log _{0,40}$ & 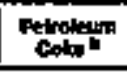 & Jow & & & & & & \multirow[b]{2}{*}{ Toustist } \\
\hline \multicolumn{3}{|c|}{ Trow } & antion & \multicolumn{4}{|c|}{ 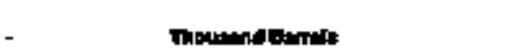 } & \multicolumn{4}{|c|}{ atest } & & \\
\hline
\end{tabular}

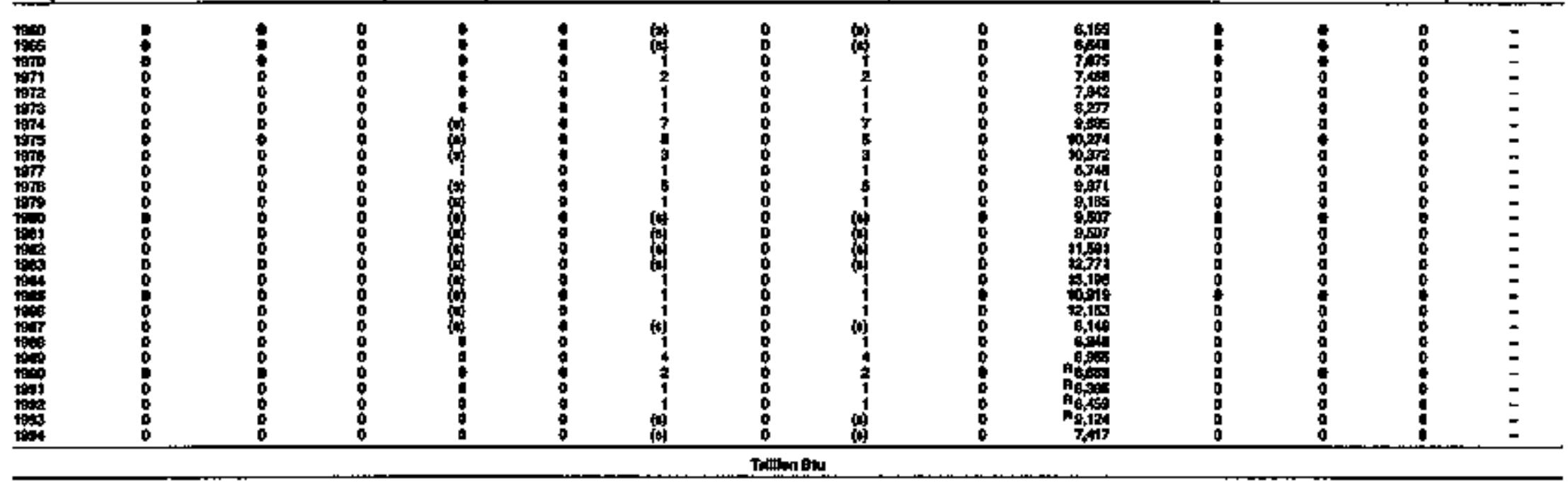

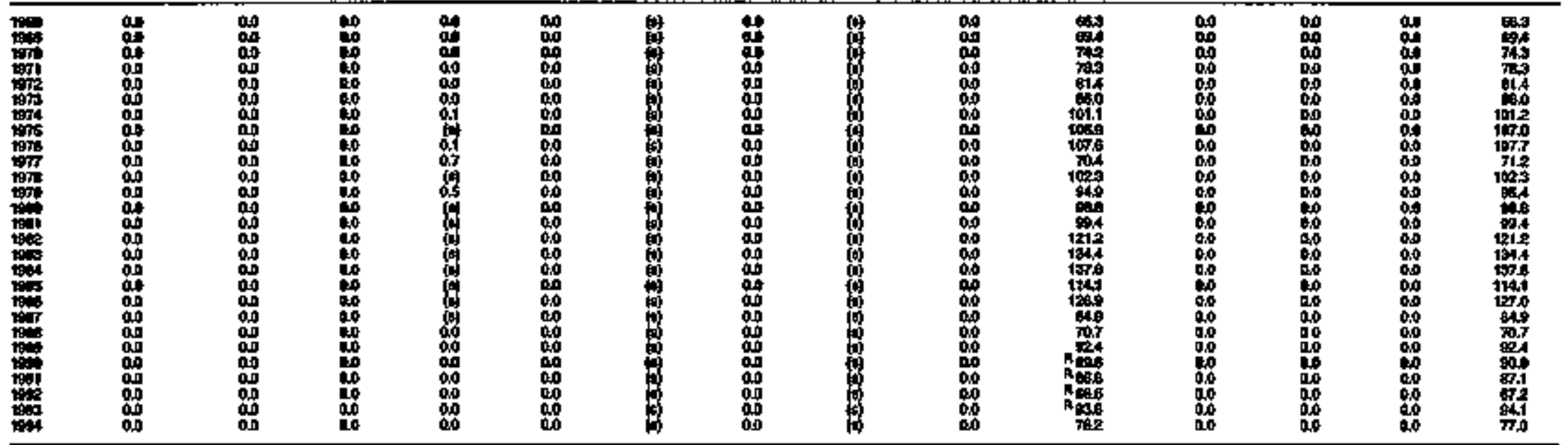

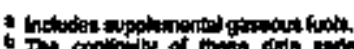

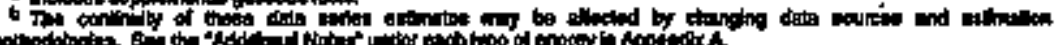

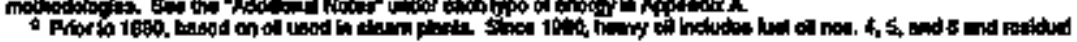

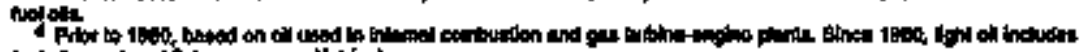

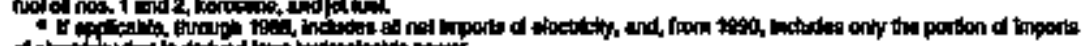

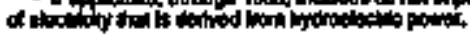

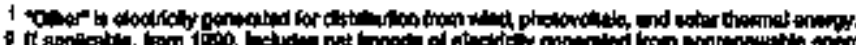

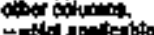

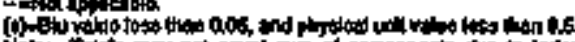

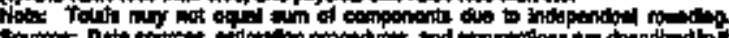

\section{$:$}


Table 95. Energy Consumption Estimates by Source, 1960, 1965, 1970-1994, tainols

\begin{tabular}{|c|c|c|c|c|c|c|c|c|c|c|c|c|c|c|c|c|c|c|c|}
\hline & \multirow[b]{2}{*}{ Cool 1} & \multirow[b]{2}{*}{ 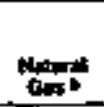 } & \multicolumn{11}{|c|}{ Morions } & \multirow[b]{2}{*}{ 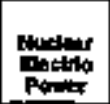 } & \multirow[b]{2}{*}{ mand } & \multirow[b]{2}{*}{ 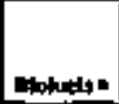 } & \multirow[b]{2}{*}{ Doter } & \multirow{2}{*}{ 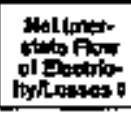 } & \multirow[b]{3}{*}{ Tots an } \\
\hline & & & Arofoctind & 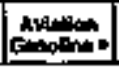 & Dratme & $\lim _{10}$ & $\infty$ & Lార: & Wort. & 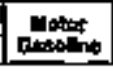 & 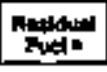 & Onter 5 & Tolow & & & & & & \\
\hline res & theorint & 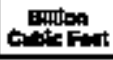 & \multicolumn{11}{|c|}{ Trousend tericts } & \multicolumn{5}{|c|}{ 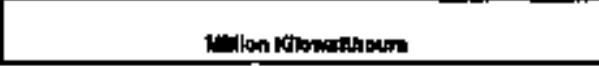 } & \\
\hline 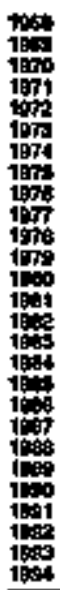 & 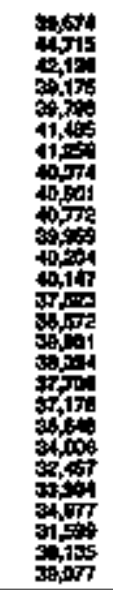 & 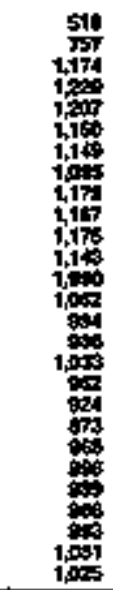 & 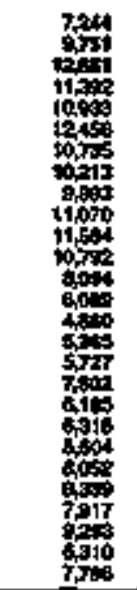 & 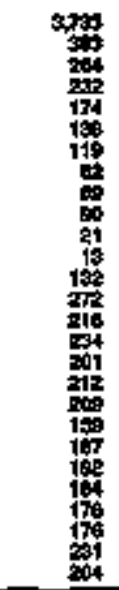 & 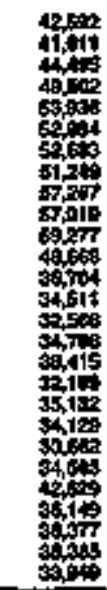 & 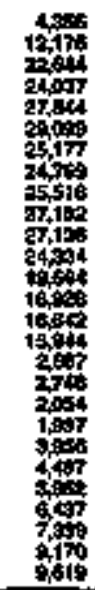 & 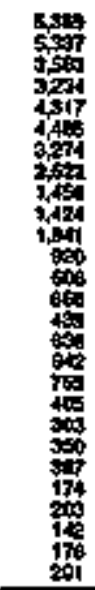 & 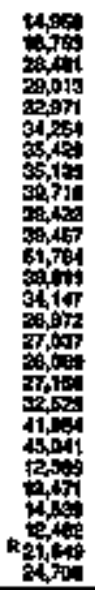 & 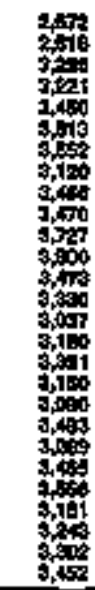 & 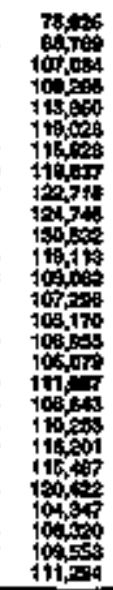 & 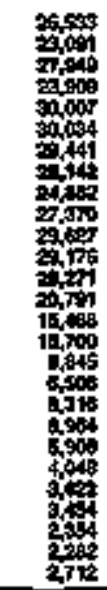 & 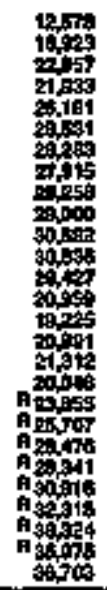 & 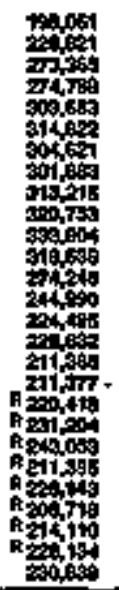 & 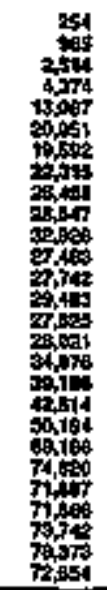 & 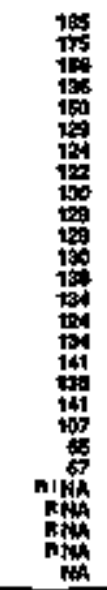 & 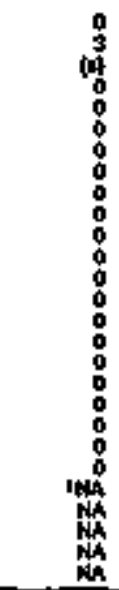 & 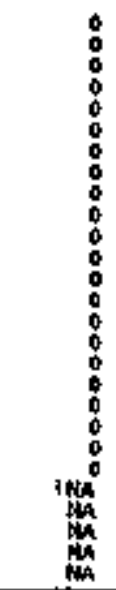 & 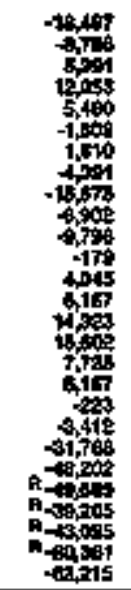 & $\begin{array}{l}= \\
= \\
= \\
= \\
\vdots \\
= \\
= \\
5 \\
= \\
5 \\
= \\
= \\
= \\
=\end{array}$ \\
\hline \multicolumn{20}{|c|}{ Intwing } \\
\hline 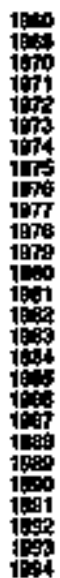 & 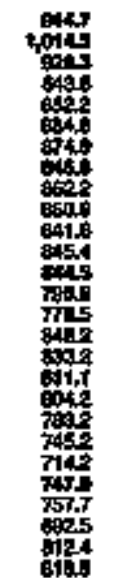 & 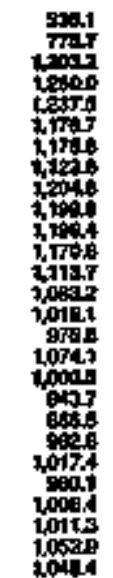 & 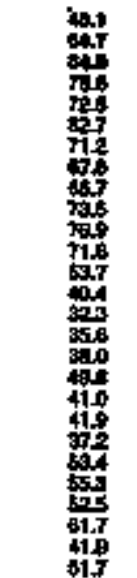 & 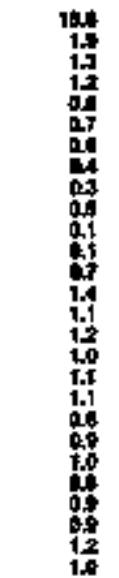 & 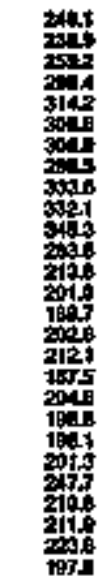 & 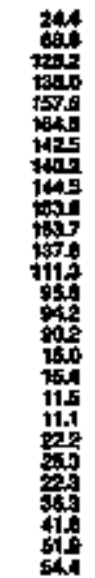 & 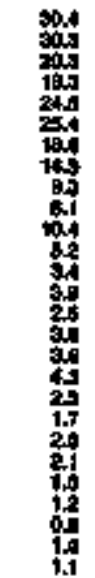 & 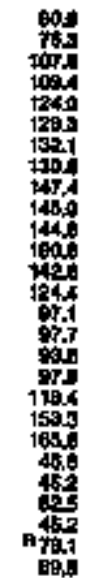 & 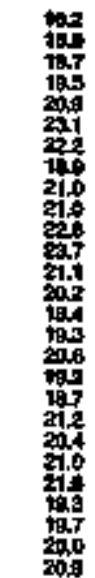 & 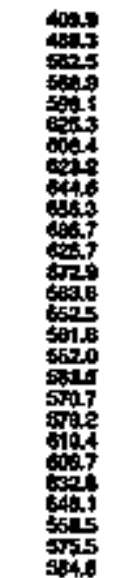 & 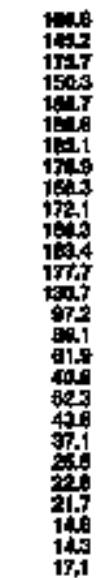 & 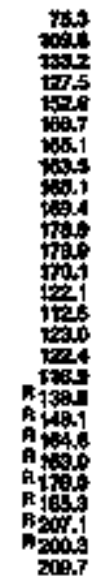 & 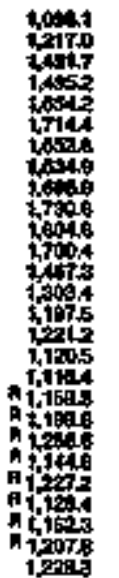 & 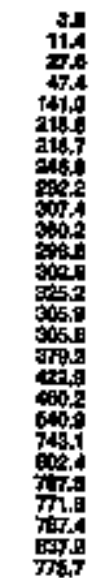 & 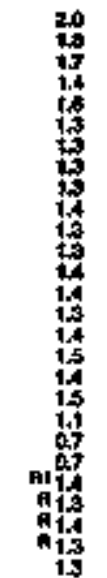 & 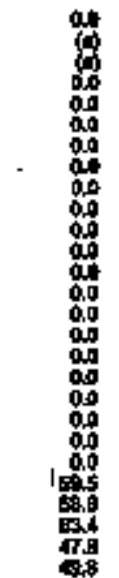 & 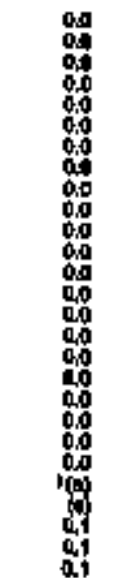 & 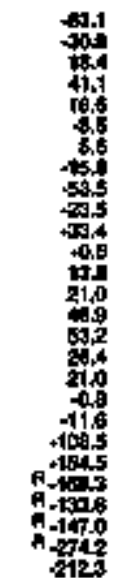 & 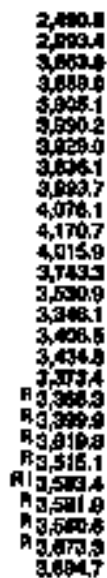 \\
\hline
\end{tabular}

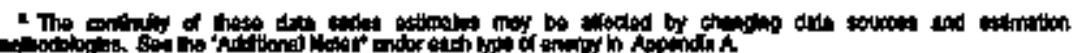

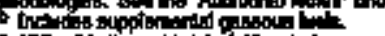

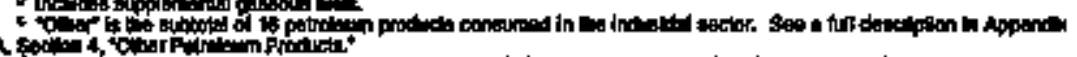

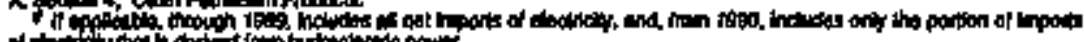

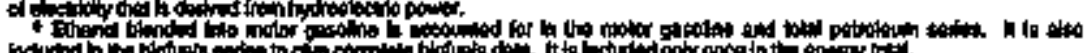

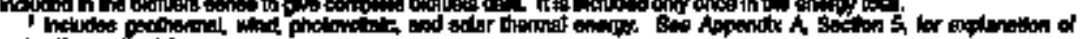

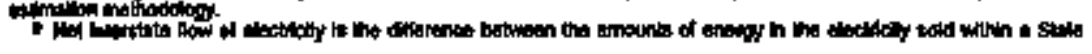

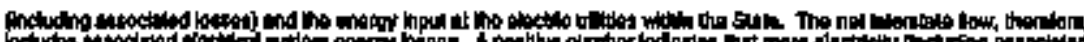

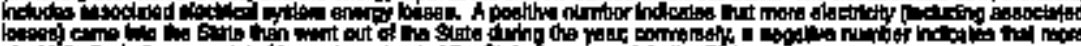

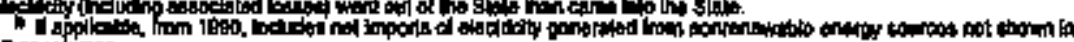

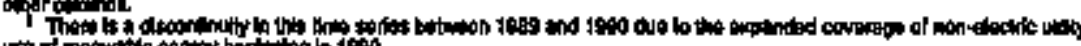

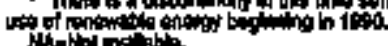

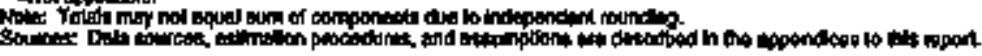


Table 86. Restdentiti Energy Consumplion Eslinates, 1960, 1955, 1970-1894, Iltinols

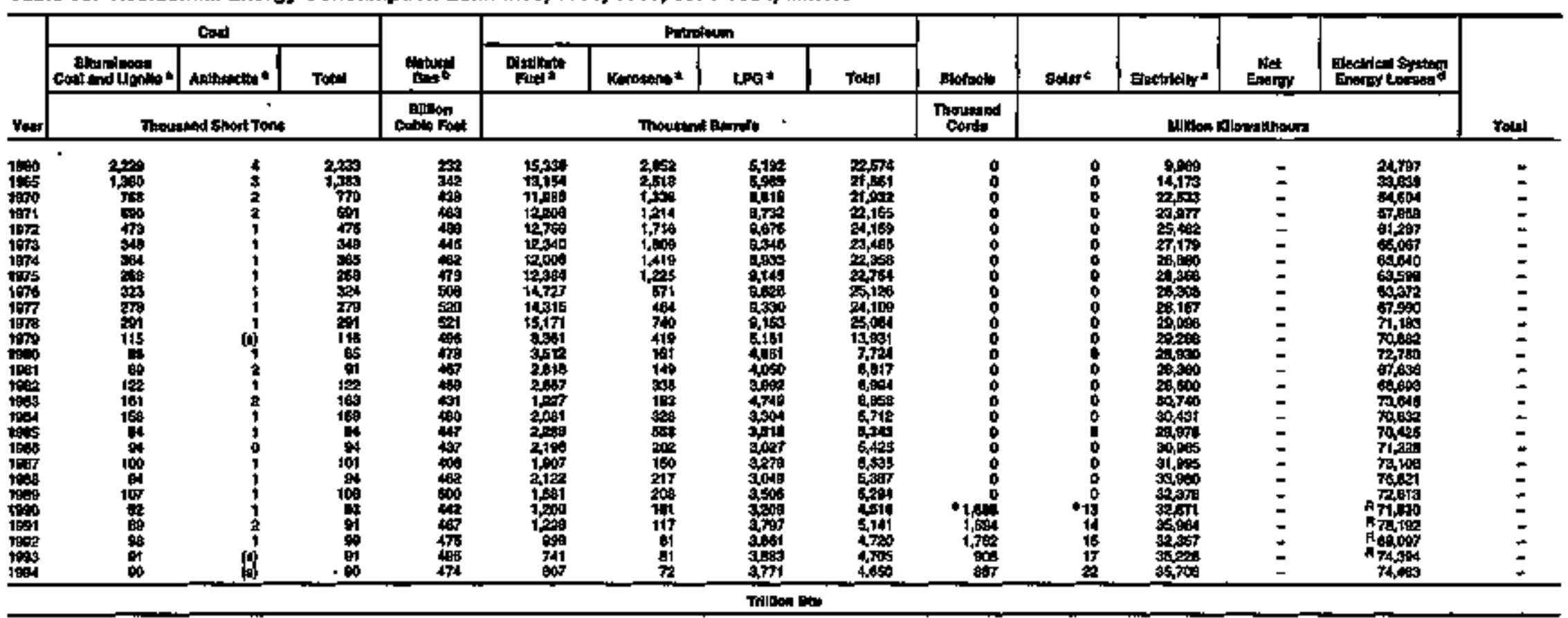

\begin{tabular}{|c|c|c|c|c|c|c|}
\hline 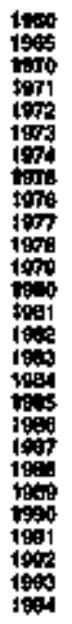 & 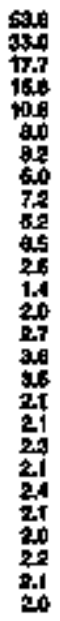 & 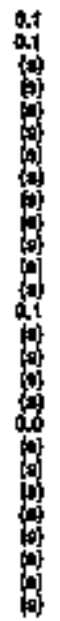 & 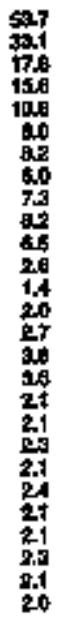 & 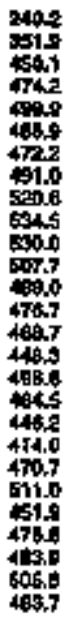 & 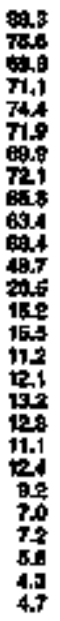 & 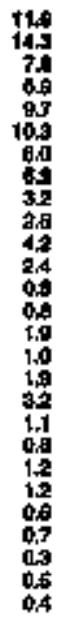 \\
\hline
\end{tabular}

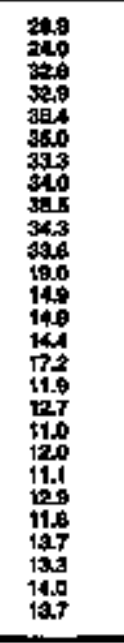

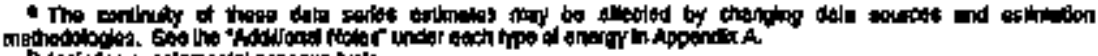

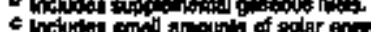

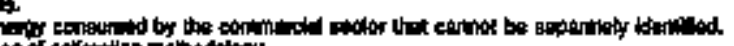

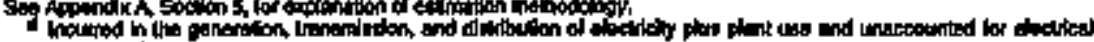

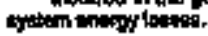

\begin{tabular}{|c|c|c|c|c|c|c|}
\hline 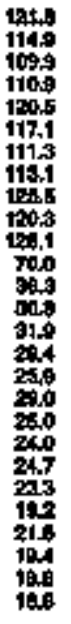 & 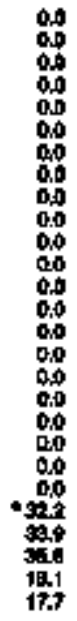 & 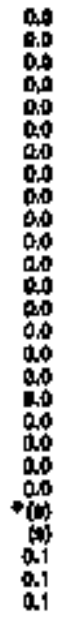 & 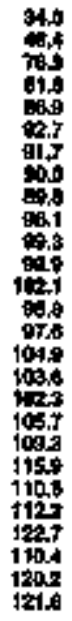 & 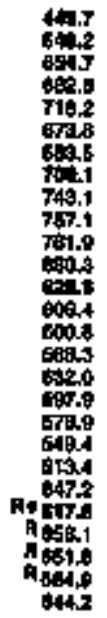 & 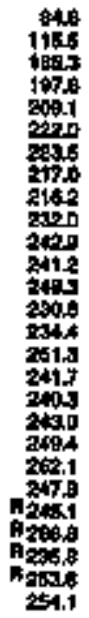 & 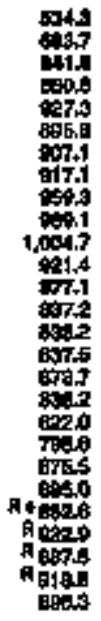 \\
\hline
\end{tabular}


Talble 97. Commercial Entrgy Constumption Estimates, 1960, 1965, 1970-1994, Illinois

\begin{tabular}{|c|c|c|c|c|c|c|c|c|c|c|c|c|c|c|}
\hline \multirow[b]{3}{*}{ Yow } & \multicolumn{3}{|c|}{ Cod } & \multirow{3}{*}{ 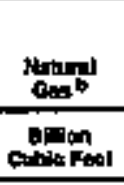 } & \multicolumn{6}{|c|}{ Potrodedn } & \multirow[b]{2}{*}{ Enotidiky" } & \multirow[b]{2}{*}{ Enem } & \multirow[b]{2}{*}{ 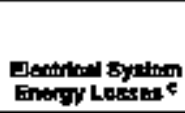 } & \multirow[b]{3}{*}{ Fotat } \\
\hline & A & Antration & Pats & & 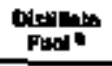 & Kerosena" & Let: & $\begin{array}{c}\text { Motor } \\
\text { Gasolins }\end{array}$ & $\begin{array}{l}\text { Musidtur } \\
\text { Fuld }\end{array}$ & rode & & & & \\
\hline & \multicolumn{3}{|c|}{ Thounend Bhont Tamb } & & \multicolumn{6}{|c|}{ 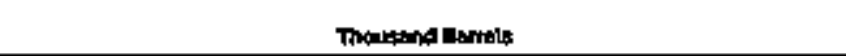 } & \multicolumn{3}{|c|}{ 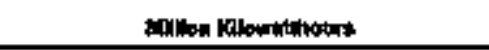 } & \\
\hline 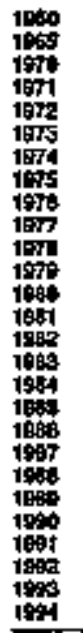 & 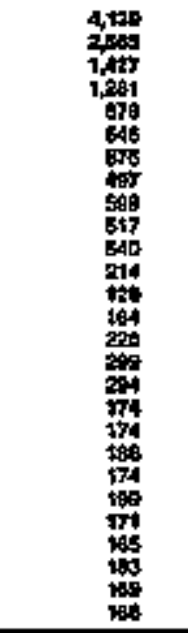 & 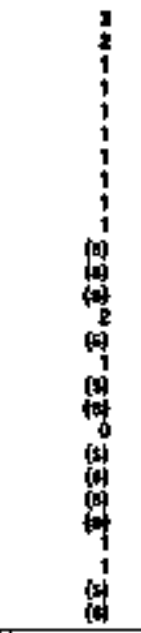 & 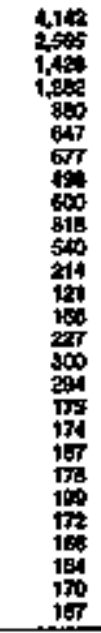 & 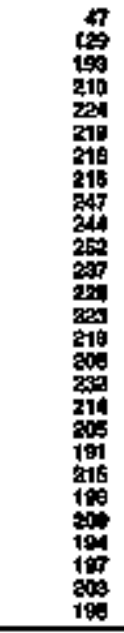 & 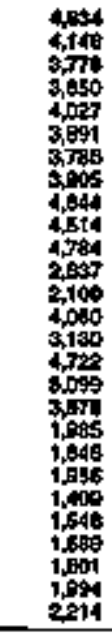 & 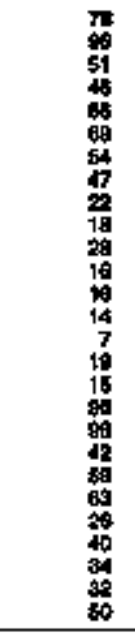 & 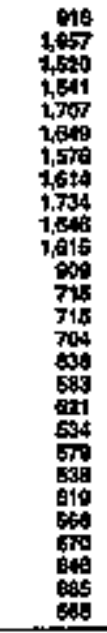 & 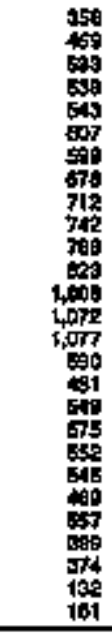 & 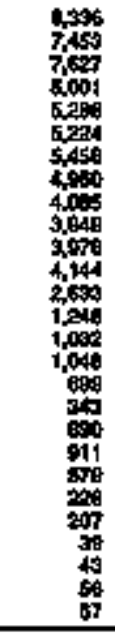 & 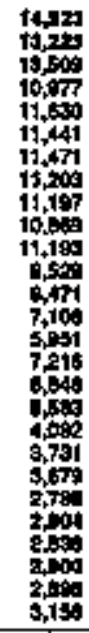 & 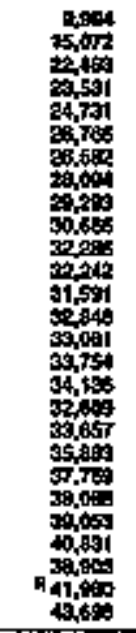 & $\begin{array}{l}= \\
= \\
= \\
\vdots \\
= \\
= \\
= \\
= \\
= \\
= \\
= \\
= \\
= \\
= \\
=\end{array}$ & 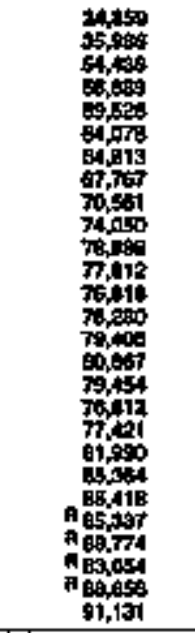 & $\begin{array}{l}= \\
z \\
z \\
z \\
z \\
z \\
z \\
z \\
z \\
z \\
z \\
z \\
z \\
z \\
z\end{array}$ \\
\hline \multicolumn{15}{|c|}{ Trillson Bu } \\
\hline 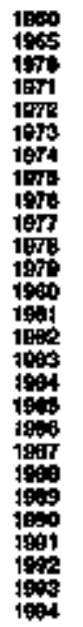 & 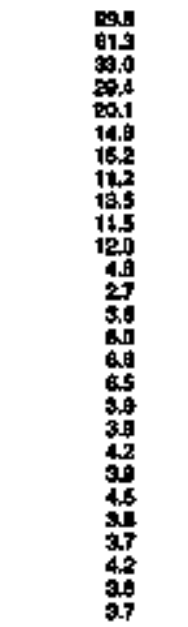 & 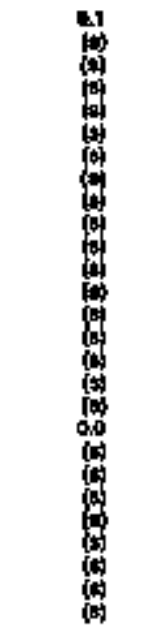 & 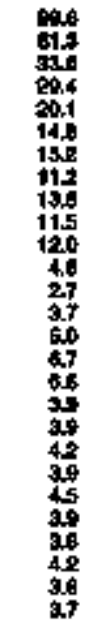 & 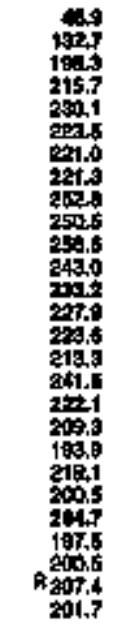 & 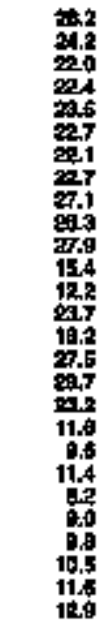 & 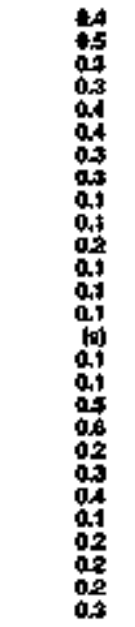 & 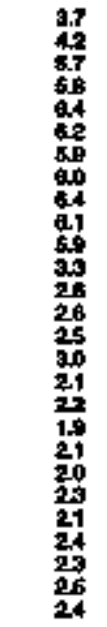 & 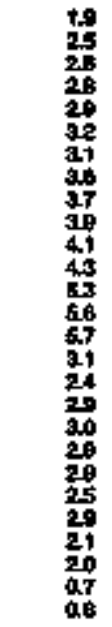 & 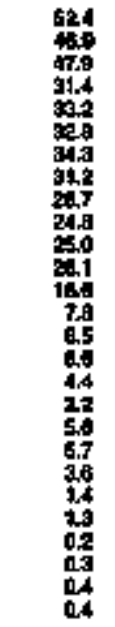 & 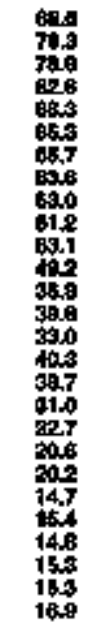 & 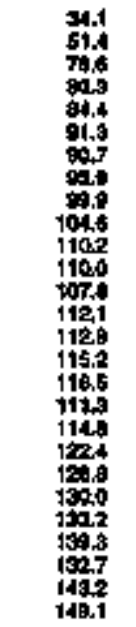 & 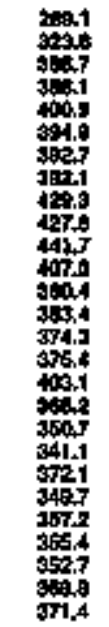 & 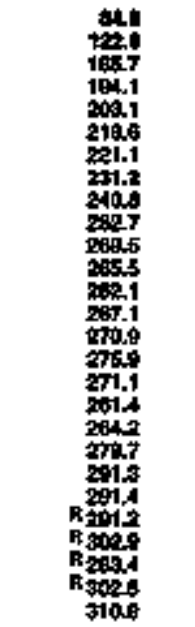 & 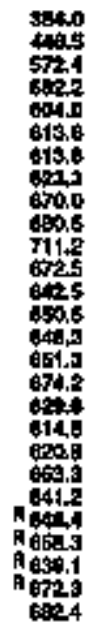 \\
\hline
\end{tabular}

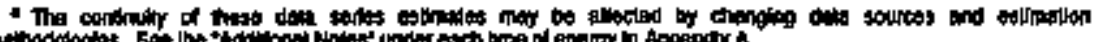

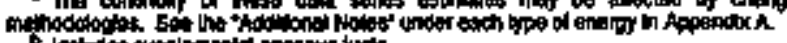

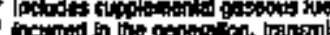

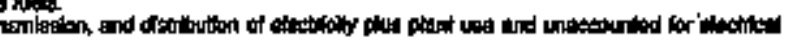

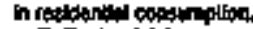

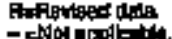

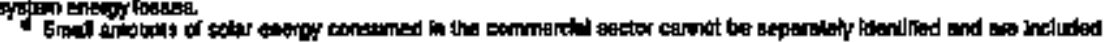


Table 88. Industrlat Enargy Corsumption Eatimatist, 19ert, 1965, 1970-1994, lilinois

\begin{tabular}{|c|c|c|c|c|c|c|c|c|c|c|c|c|c|c|c|c|c|c|}
\hline \multirow[b]{3}{*}{$r=r$} & \multirow[b]{2}{*}{ الس } & \multirow[b]{2}{*}{ What: } & \multicolumn{9}{|c|}{ Pastotenter } & \multirow{2}{*}{ 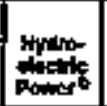 } & \multirow[b]{2}{*}{ Elature } & \multirow[b]{2}{*}{ Ether 201} & \multirow[b]{2}{*}{ Denting } & \multirow[b]{2}{*}{ Entrot } & \multirow{2}{*}{ Dinted } & \multirow[b]{3}{*}{ Tota } \\
\hline & & & 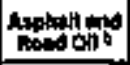 & Dentoly & Xureann & LPB & tubrikantaly & choption & Proplate & onter ac & Totot & & & & & & & \\
\hline & Thertions & callowisor & \multicolumn{9}{|c|}{ 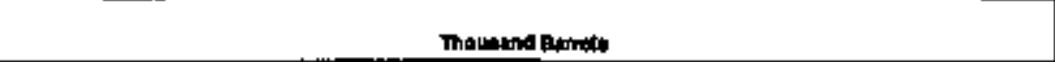 } & \multicolumn{6}{|c|}{ Inelon KClownethern } & \\
\hline 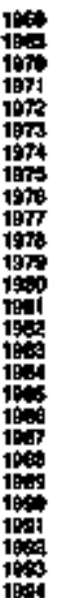 & 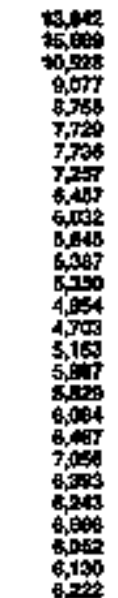 & 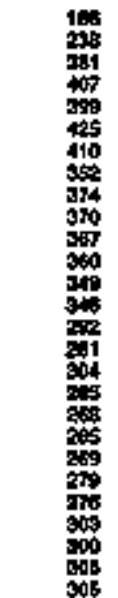 & 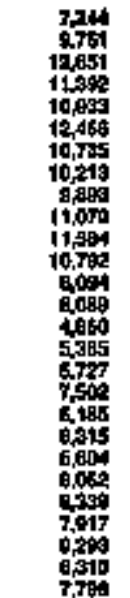 & 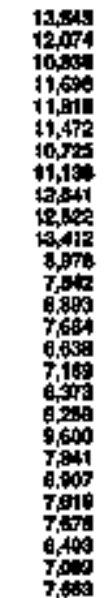 & 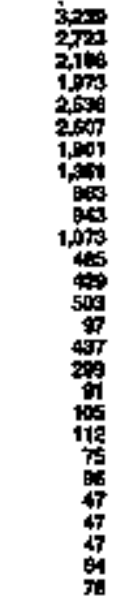 & 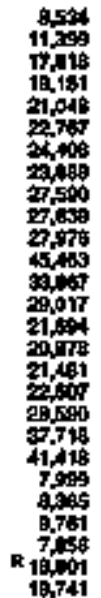 & 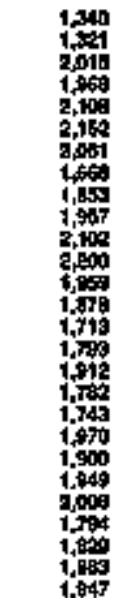 & 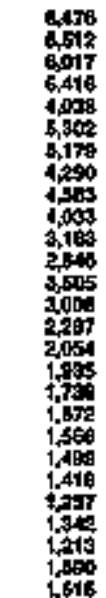 & 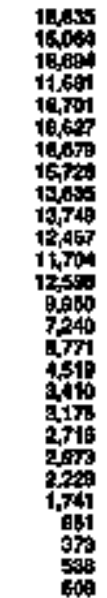 & 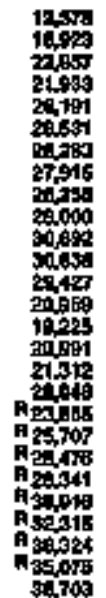 & 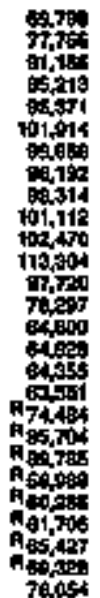 & 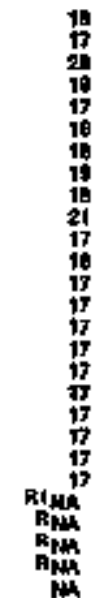 & 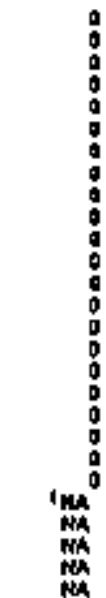 & 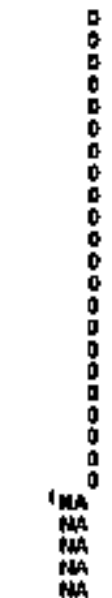 & 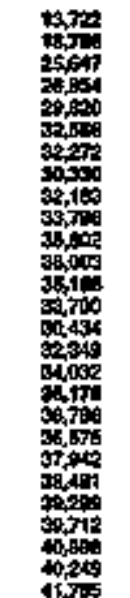 & $\begin{array}{l}= \\
= \\
= \\
z \\
z \\
= \\
= \\
= \\
= \\
= \\
= \\
= \\
z \\
z \\
z \\
z\end{array}$ & 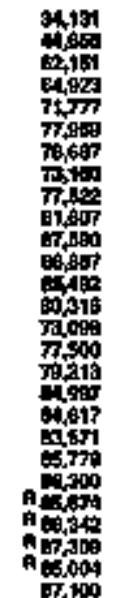 & $\begin{array}{l}= \\
= \\
= \\
z \\
= \\
= \\
= \\
= \\
= \\
= \\
= \\
= \\
= \\
= \\
=\end{array}$ \\
\hline
\end{tabular}

\begin{tabular}{|c|c|c|c|c|}
\hline 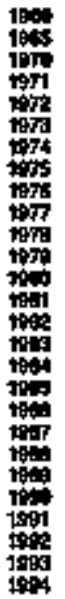 & 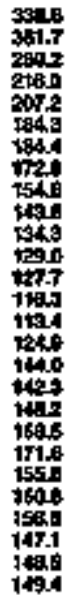 & 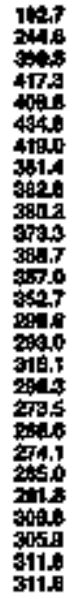 & 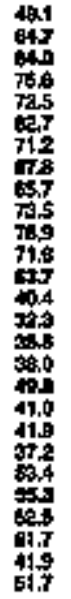 & 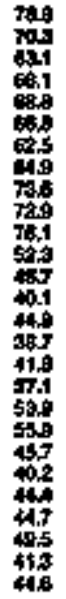 \\
\hline
\end{tabular}

\begin{tabular}{|c|}
\hline 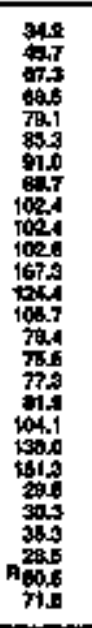 \\
\hline
\end{tabular}

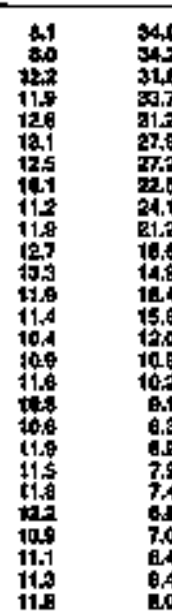

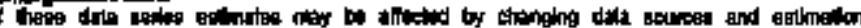

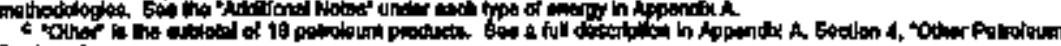

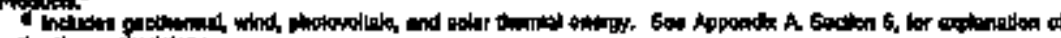

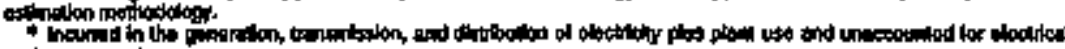

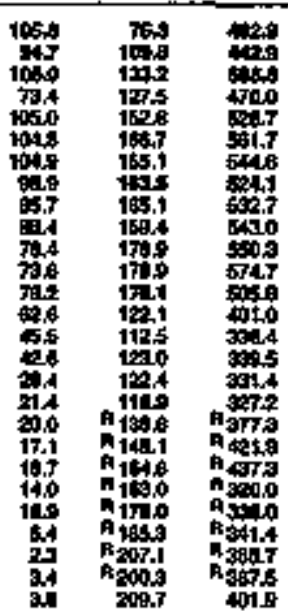

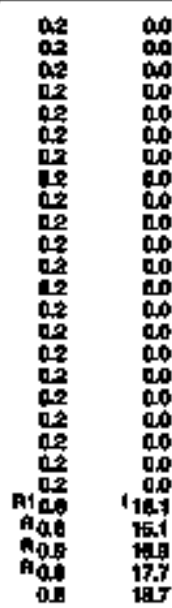

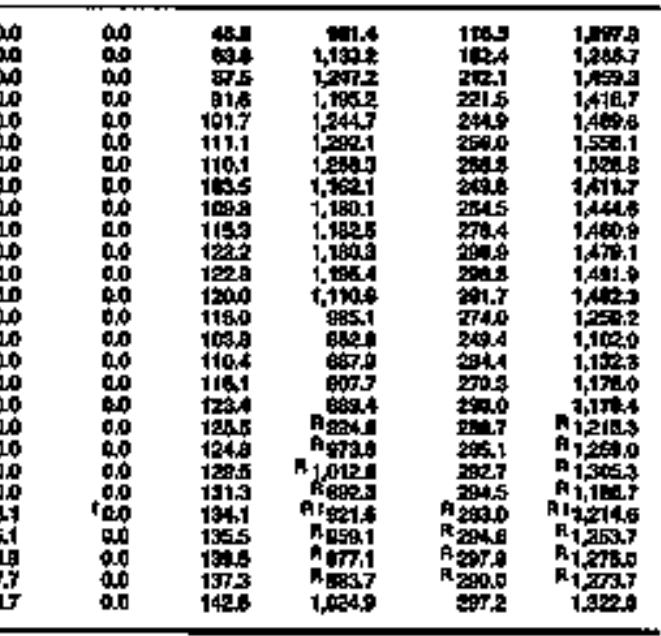

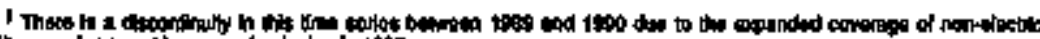

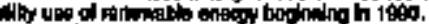
mathed in

ThBop

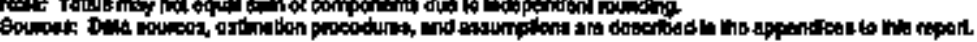

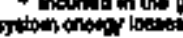


Table 99. Transportation Energy Coneumption Estimates, 1980, 1965, 1970-1994, Illinois

\begin{tabular}{|c|c|c|c|c|c|c|c|c|c|c|c|c|c|c|c|}
\hline \multirow[b]{3}{*}{ Yar| } & \multirow[b]{2}{*}{ 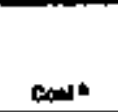 } & \multirow[b]{2}{*}{ Thing } & \multicolumn{8}{|c|}{ Pescolenal } & \multirow{3}{*}{ 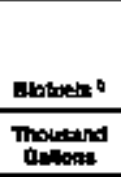 } & \multirow[b]{2}{*}{ anectritity " } & \multirow[b]{2}{*}{ Enery } & \multirow[b]{2}{*}{ 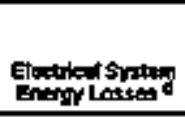 } & \multirow[b]{3}{*}{ Toted } \\
\hline & & & 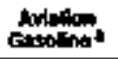 & $x^{\infty}$ & Nof. & wor & Lubreants: & 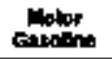 & 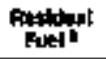 & Totos & & & & & \\
\hline & Thert Tos: & ontion & \multicolumn{8}{|c|}{ 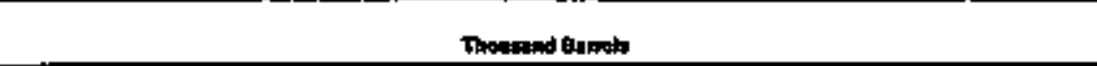 } & & \multicolumn{3}{|c|}{ 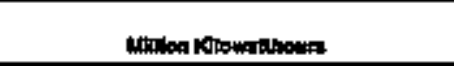 } & \\
\hline 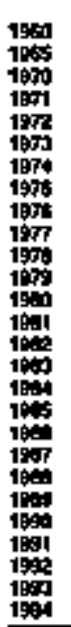 & $\begin{array}{c}20 \\
51 \\
7 \\
12 \\
0 \\
1 \\
1 \\
1 \\
0 \\
0 \\
0 \\
0 \\
0 \\
0 \\
0 \\
0 \\
0 \\
0 \\
0 \\
0 \\
0 \\
0 \\
0 \\
0\end{array}$ & $\begin{array}{l}10 \\
13 \\
29 \\
29 \\
29 \\
21 \\
19 \\
14 \\
17 \\
19 \\
19 \\
19 \\
19 \\
12 \\
14 \\
11 \\
11 \\
11 \\
7 \\
19 \\
14 \\
12 \\
11 \\
11 \\
12 \\
14\end{array}$ & 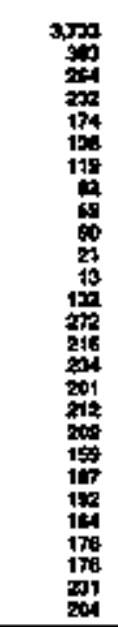 & 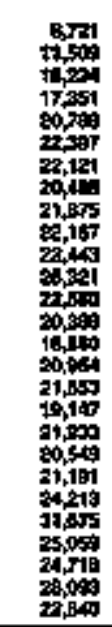 & 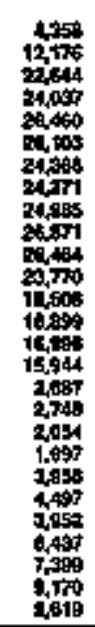 & 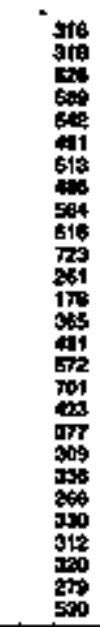 & 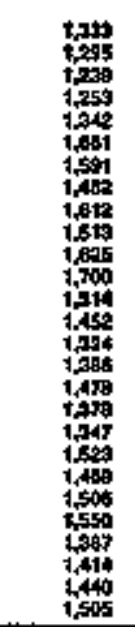 & 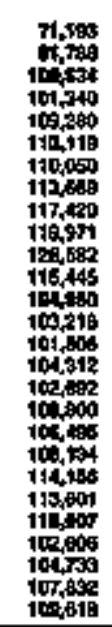 & 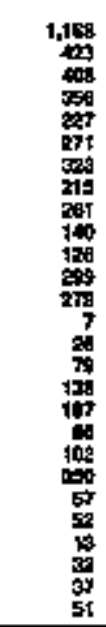 & 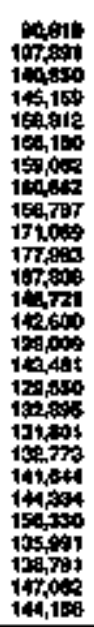 & 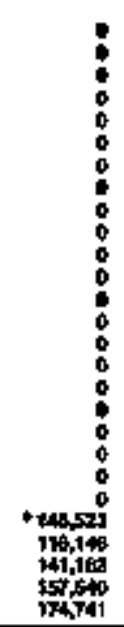 & 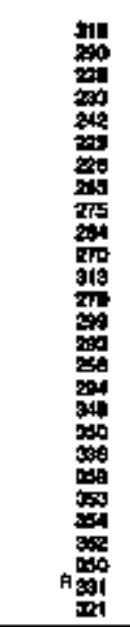 & $\begin{array}{l}= \\
= \\
= \\
= \\
= \\
= \\
= \\
= \\
= \\
= \\
= \\
= \\
= \\
= \\
= \\
=\end{array}$ & 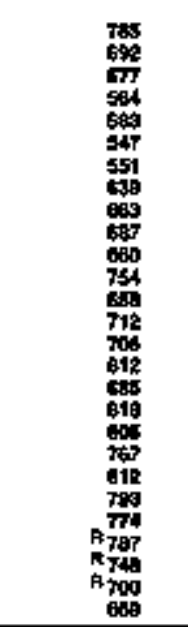 & $\begin{array}{l}= \\
z \\
= \\
z \\
z \\
= \\
= \\
= \\
z \\
z \\
z \\
z \\
z \\
=\end{array}$ \\
\hline \multicolumn{16}{|c|}{ Tranom } \\
\hline 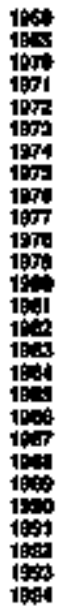 & $\begin{array}{l}57 \\
1.0 \\
01 \\
03 \\
02 \\
0.4 \\
01 \\
0 \\
0 \\
00 \\
00 \\
00 \\
00 \\
00 \\
00 \\
00 \\
00 \\
00 \\
00 \\
00 \\
00 \\
00 \\
00 \\
00 \\
00 \\
00 \\
00 \\
00\end{array}$ & 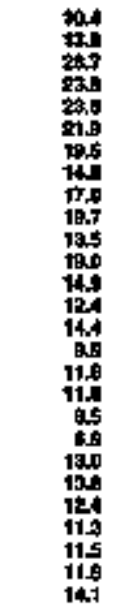 & 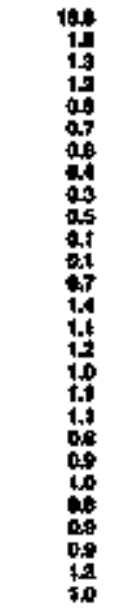 & 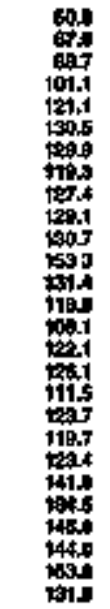 & 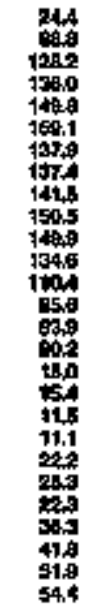 & 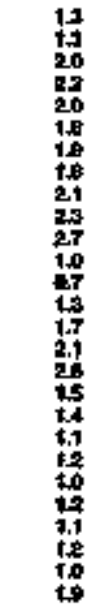 & 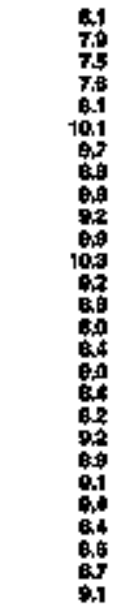 & 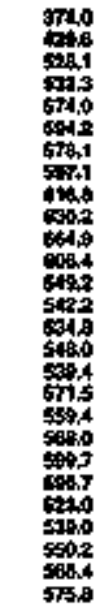 & 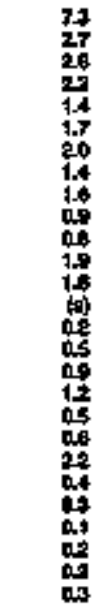 & 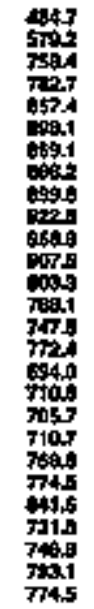 & 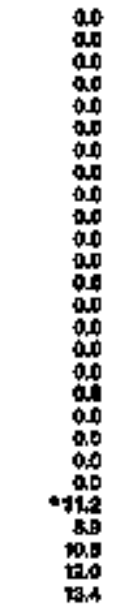 & 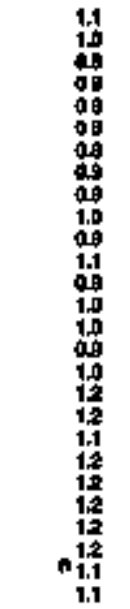 & 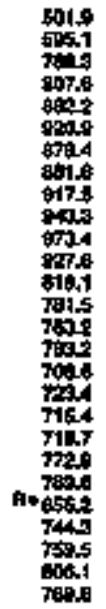 & 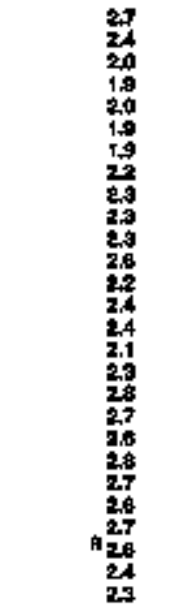 & 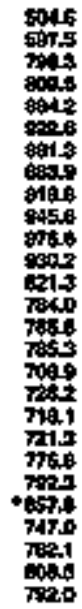 \\
\hline
\end{tabular}

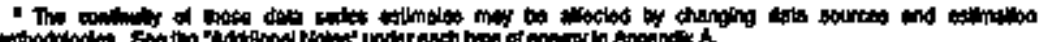

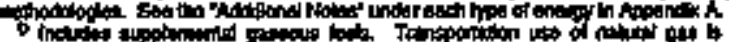

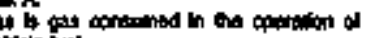

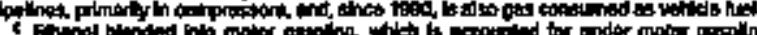

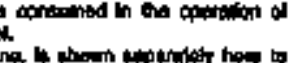

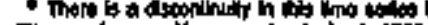

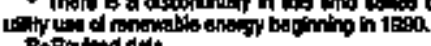

r.

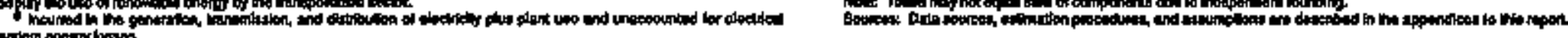


Tabłe 100. Ealimalas of Energy Input at Eectric Utikties, t980, 1965, 1970-1994, Illinois

\begin{tabular}{|c|c|c|c|c|c|c|c|c|c|c|c|c|c|c|}
\hline \multirow[b]{3}{*}{ Yard } & \multicolumn{3}{|c|}{ conl } & \multirow{3}{*}{ 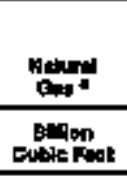 } & \multicolumn{4}{|c|}{ Putrolon } & \multirow[b]{2}{*}{ 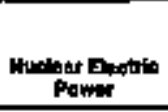 } & \multirow[b]{2}{*}{ 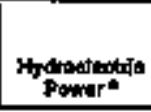 } & \multirow[b]{2}{*}{ Bloweh } & \multirow[b]{2}{*}{ 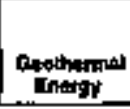 } & \multirow{3}{*}{ oner } & \multirow[b]{3}{*}{ 70난 N } \\
\hline & 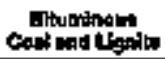 & Anterresth & Teld & & ond & Lats & 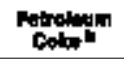 & Told & & & & & & \\
\hline & \multicolumn{3}{|c|}{ 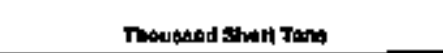 } & & \multicolumn{4}{|c|}{ Thwend Berrols } & \multicolumn{4}{|c|}{ 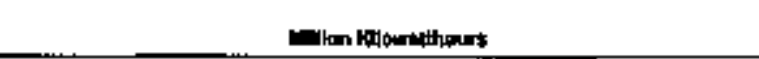 } & & \\
\hline 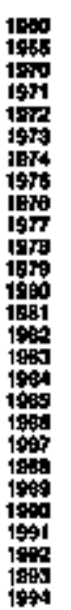 & 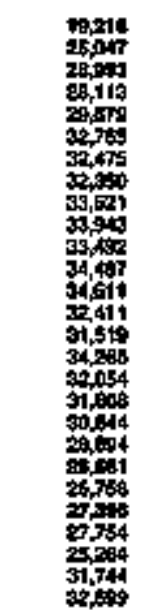 & $\begin{array}{l}0 \\
0 \\
0 \\
0 \\
0 \\
0 \\
0 \\
0 \\
0 \\
0 \\
0 \\
0 \\
0 \\
0 \\
0 \\
0 \\
0 \\
0 \\
0 \\
0 \\
0 \\
0 \\
0 \\
0 \\
0\end{array}$ & 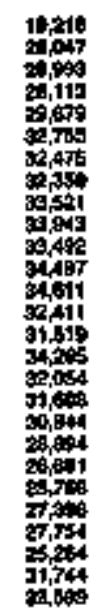 & 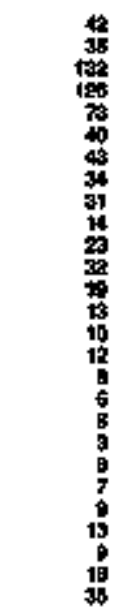 & 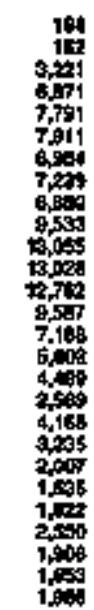 & 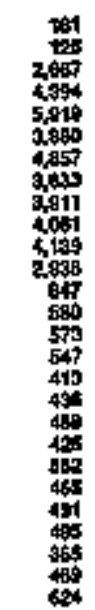 & $\begin{array}{r}0 \\
0 \\
0 \\
0 \\
0 \\
0 \\
0 \\
0 \\
0 \\
0 \\
0 \\
0 \\
0 \\
0 \\
0 \\
0 \\
0 \\
0 \\
0 \\
0 \\
0 \\
0 \\
0 \\
0 \\
0 \\
0 \\
0\end{array}$ & 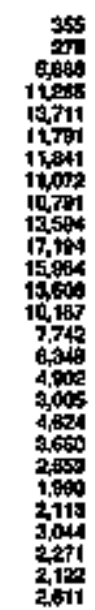 & 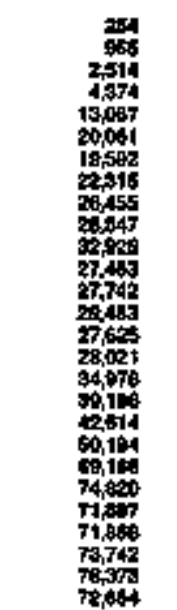 & 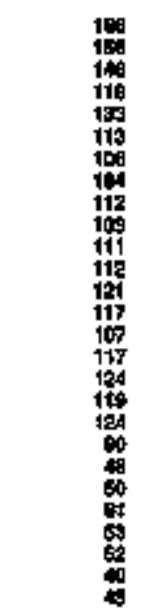 & $\begin{array}{r}0 \\
3 \\
0 \\
0 \\
0 \\
0 \\
0 \\
0 \\
0 \\
0 \\
0 \\
0 \\
0 \\
0 \\
0 \\
0 \\
0 \\
0 \\
0 \\
0 \\
0 \\
0 \\
0\end{array}$ & $\begin{array}{l}0 \\
0 \\
0 \\
0 \\
0 \\
0 \\
0 \\
0 \\
0 \\
0 \\
0 \\
0 \\
0 \\
0 \\
0 \\
0 \\
0 \\
0 \\
0 \\
0 \\
0 \\
0 \\
0 \\
0 \\
0 \\
0 \\
0\end{array}$ & $\begin{array}{l}0 \\
0 \\
0 \\
0 \\
0 \\
0 \\
0 \\
0 \\
0 \\
0 \\
0 \\
0 \\
0 \\
0 \\
0 \\
0 \\
0 \\
0 \\
0 \\
0 \\
0 \\
0 \\
0 \\
0 \\
0 \\
0\end{array}$ & $\begin{array}{l}\bar{z} \\
= \\
= \\
z \\
z \\
= \\
= \\
= \\
= \\
= \\
z \\
= \\
z \\
= \\
=\end{array}$ \\
\hline \multicolumn{15}{|c|}{ Thenengen } \\
\hline
\end{tabular}

\begin{tabular}{|c|c|}
\hline 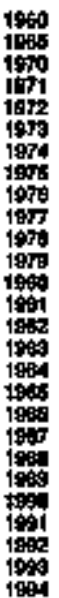 & 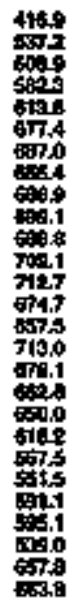 \\
\hline
\end{tabular}

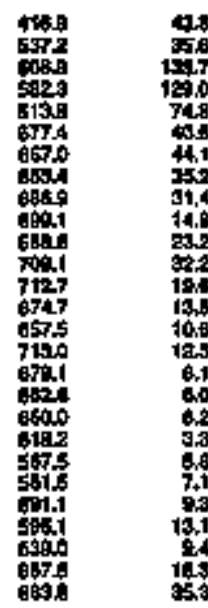

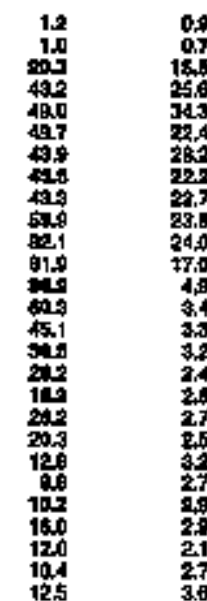

00
10
00
00
0.0
00
00
00
00
00
00
00
00
00
00
00
00
00
00
00
00
00
00
00
00

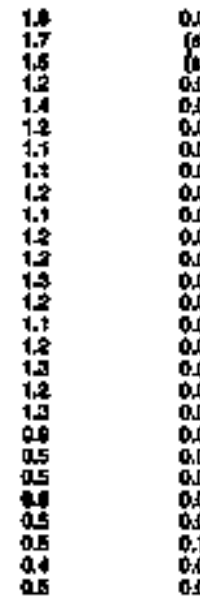

\begin{tabular}{|c|c|c|}
\hline 祭 & 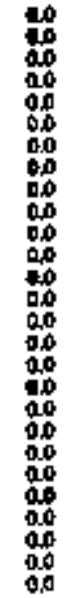 & 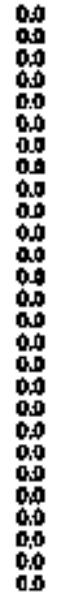 \\
\hline
\end{tabular}

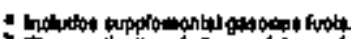

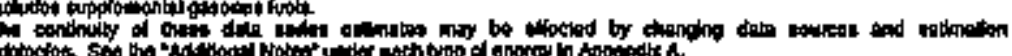

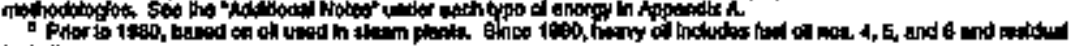
wod pod

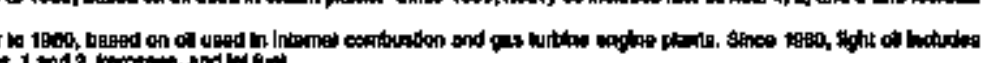

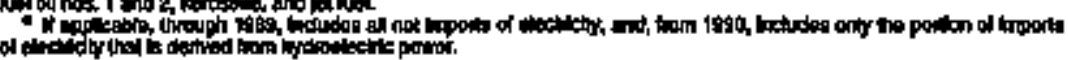

\begin{tabular}{|c|c|}
\hline 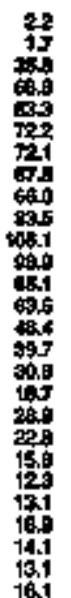 & 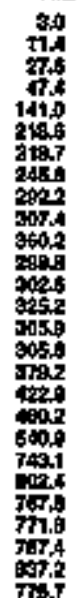 \\
\hline
\end{tabular}

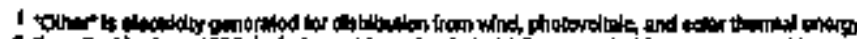

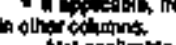

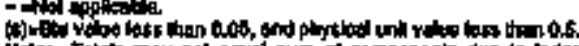

(1) 
Table to1. Engrgy Consumplion Estimates by Sourcen, 1960, 1958, 1970-1994, Indian:a

\begin{tabular}{|c|c|c|c|c|c|c|c|c|c|c|c|c|c|c|c|c|c|c|c|}
\hline \multirow[b]{3}{*}{$\mathrm{Yan}$} & \multirow[b]{2}{*}{ Conts } & \multirow[b]{2}{*}{ (a) } & \multicolumn{11}{|c|}{ Potroben } & \multirow{2}{*}{ 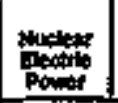 } & \multirow[b]{2}{*}{ Pontor } & \multirow[b]{2}{*}{ Elotodsts. } & \multirow[b]{2}{*}{ Ooher \&1 } & \multirow{2}{*}{ 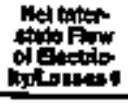 } & \multirow[b]{3}{*}{ Toten h } \\
\hline & & & 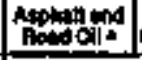 & Avistion & 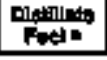 & 년. & מ. & LPE: & Listit. & : & pestos & then 4 & Tore & & & & & & \\
\hline & Drouspat & curbie Find & \multicolumn{11}{|c|}{ Thous: } & \multicolumn{5}{|c|}{ Milki Klowathons } & \\
\hline 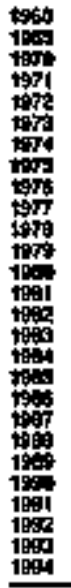 & 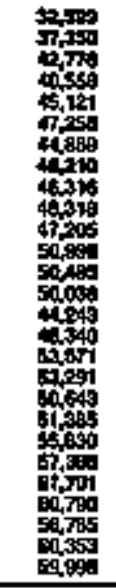 & 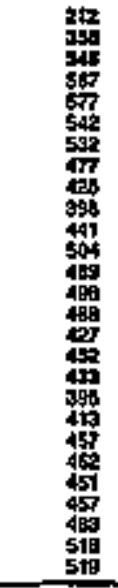 & 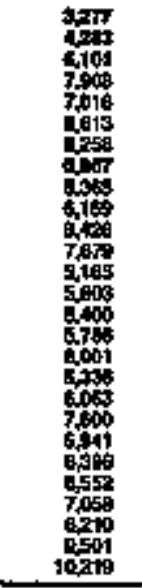 & 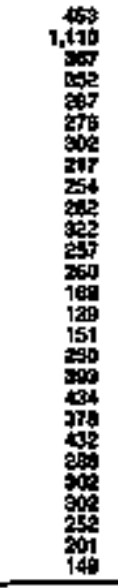 & 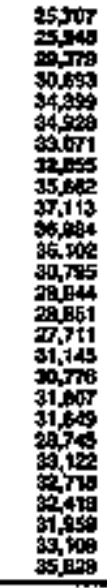 & 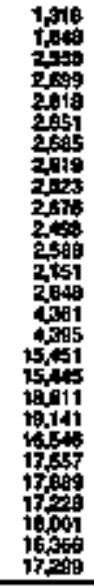 & 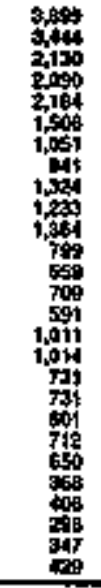 & 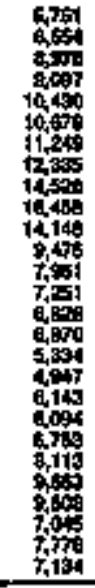 & 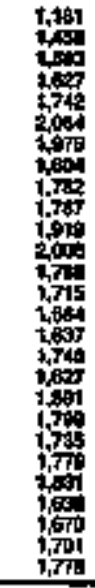 & 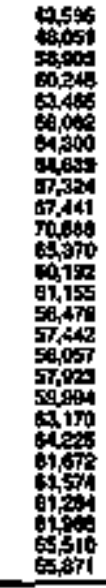 & 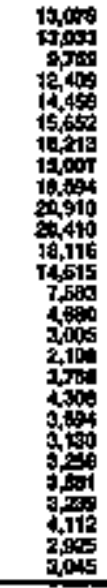 & 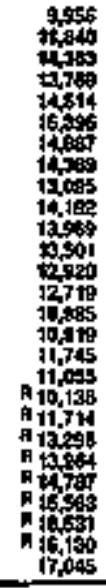 & 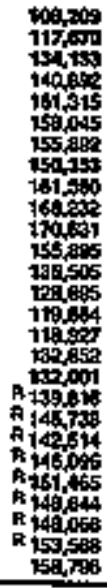 & $\begin{array}{l}8 \\
0 \\
0 \\
0 \\
0 \\
0 \\
0 \\
0 \\
0 \\
0 \\
0 \\
0 \\
0 \\
0 \\
0 \\
0 \\
0 \\
0 \\
0 \\
0 \\
0 \\
0 \\
0\end{array}$ & 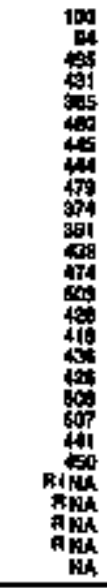 & 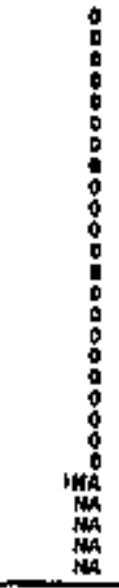 & 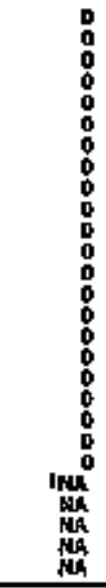 & 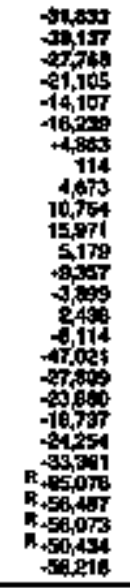 & 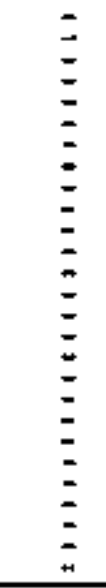 \\
\hline \multicolumn{20}{|c|}{ Thiten Bs: } \\
\hline 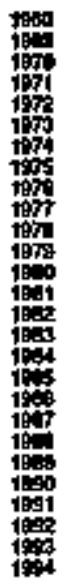 & 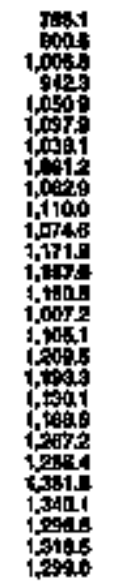 & 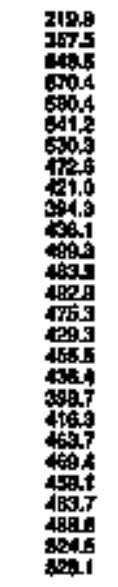 & 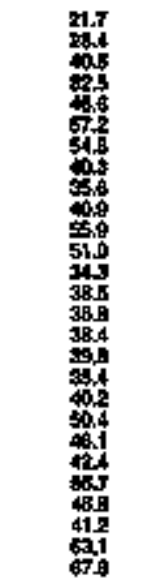 & 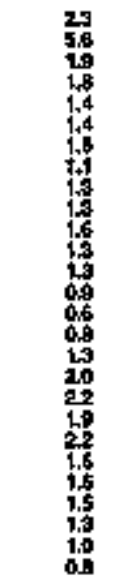 & 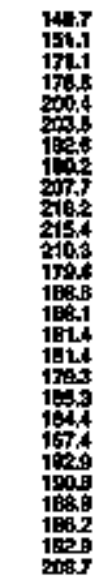 & 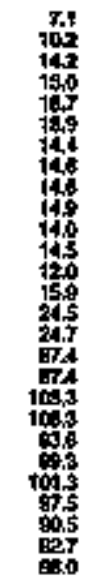 & 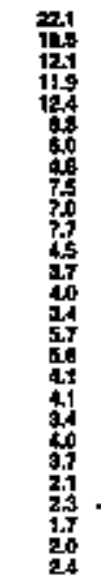 & 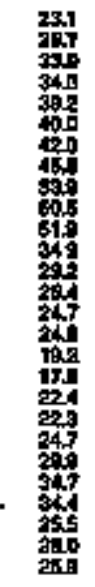 & 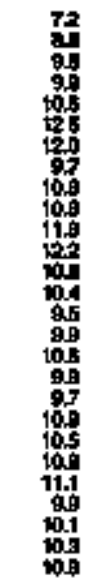 & 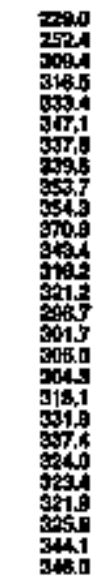 & 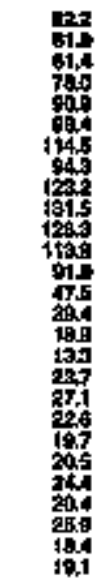 & 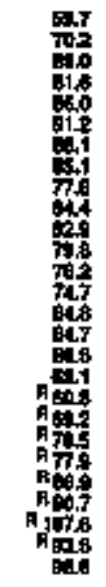 & 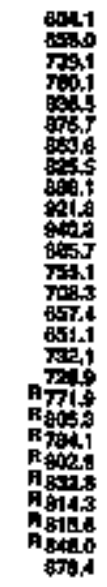 & 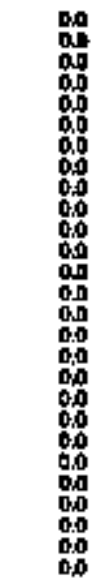 & 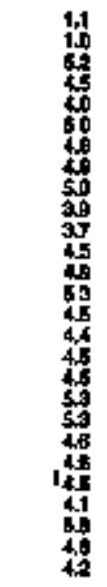 & 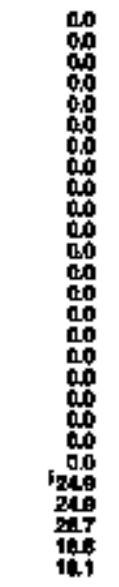 & 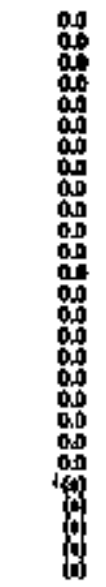 & 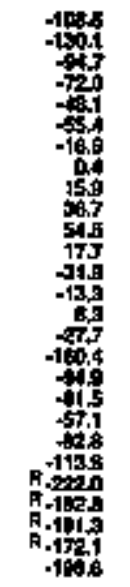 & 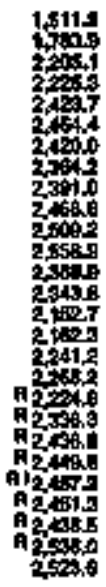 \\
\hline
\end{tabular}

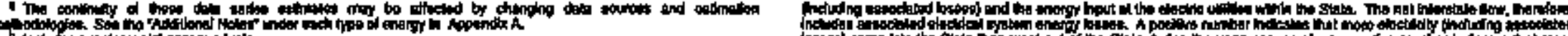

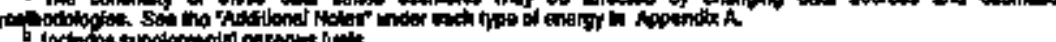

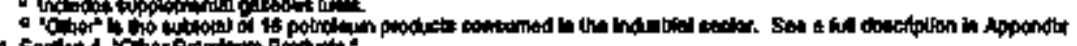

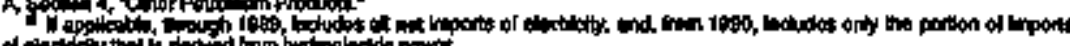

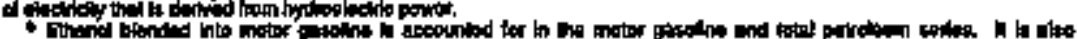

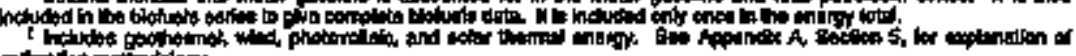

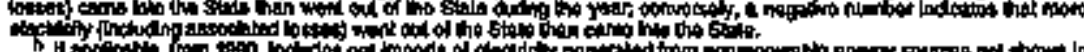

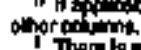

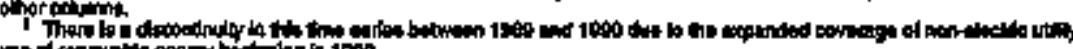

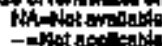

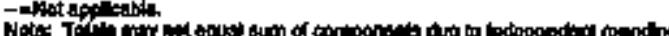

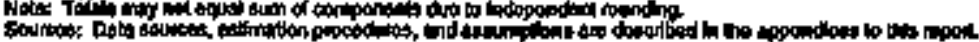


Table 102. Resldential Energy Constumptlon Estimates, 1950, 1965, 1970-1994, Indiana

\begin{tabular}{|c|c|c|c|c|c|c|c|c|c|c|c|c|c|c|}
\hline & & Eo: & & & & Petr & & & \multirow[b]{2}{*}{ Eabofuels: } & \multirow[b]{2}{*}{ soly. } & \multirow[b]{2}{*}{ Bouturfth" } & \multirow[b]{2}{*}{$e_{1}^{\min }$} & \multirow[b]{2}{*}{ 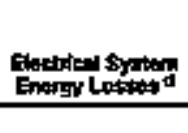 } & \multirow[b]{3}{*}{ Tota: } \\
\hline & 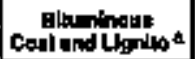 & Ambrastion & Toted & that & Distines & Kerosenes: & LF: & Tow: & & & & & & \\
\hline Your & \multicolumn{3}{|c|}{ 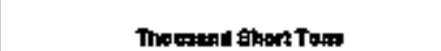 } & cillon & \multicolumn{4}{|c|}{ Thoutind Bupphe } & Thorand & \multicolumn{4}{|c|}{ Mlliton Klonathorn } & \\
\hline
\end{tabular}

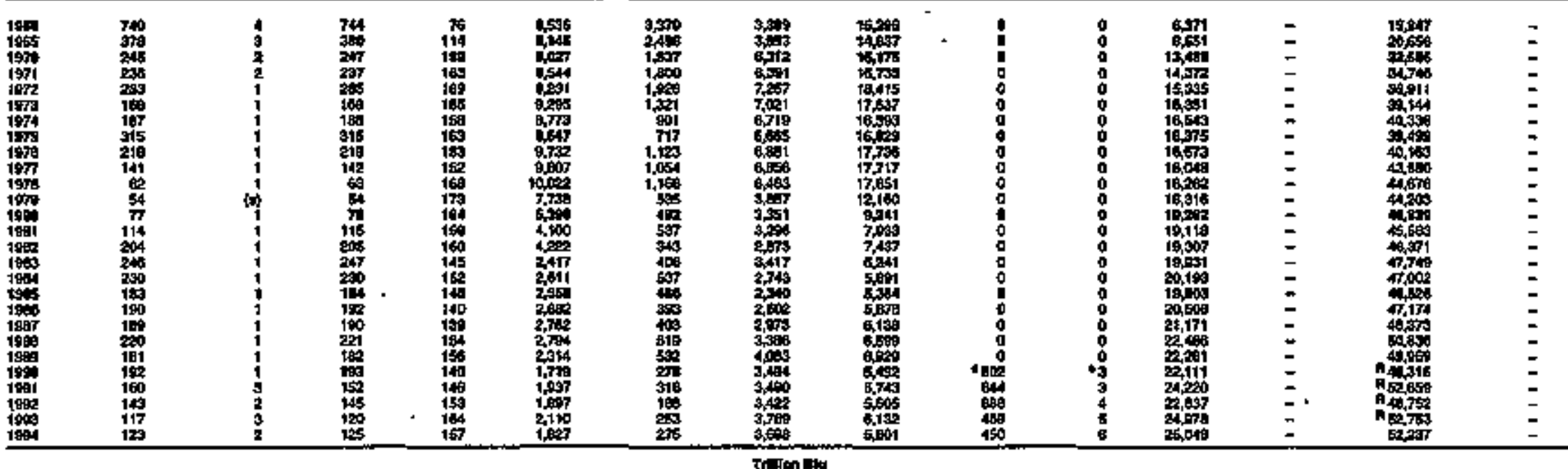

\begin{tabular}{|c|c|c|c|c|c|c|}
\hline 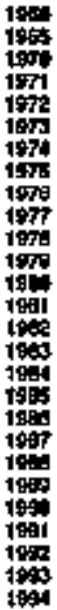 & 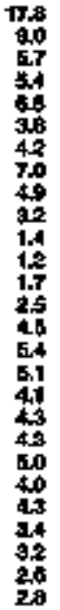 & 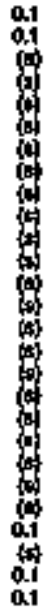 & 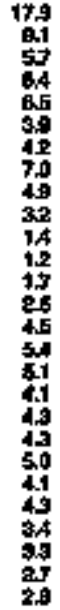 & 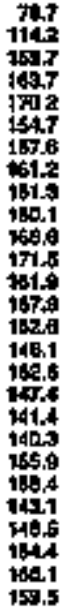 & 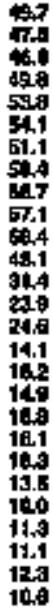 & 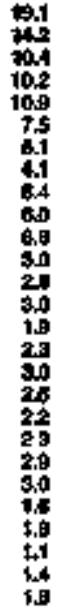 \\
\hline
\end{tabular}

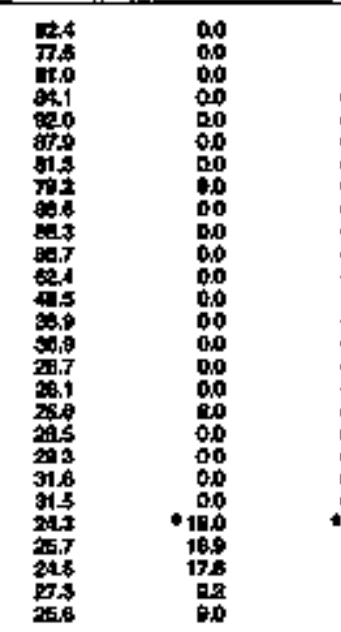

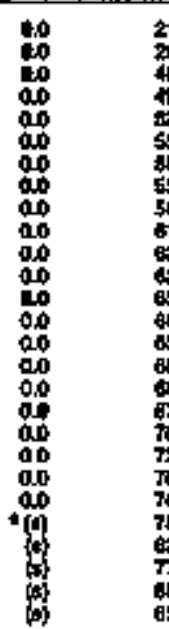

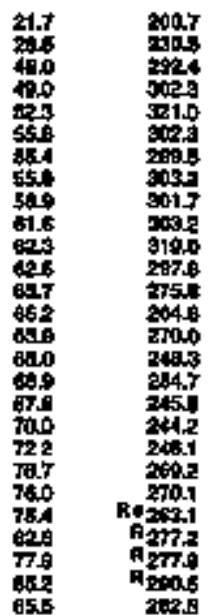

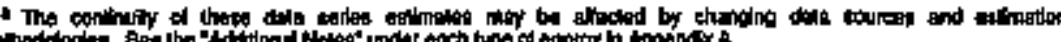

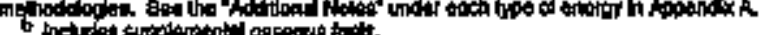

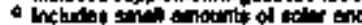

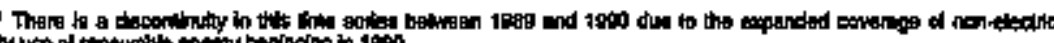

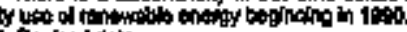

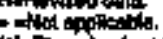

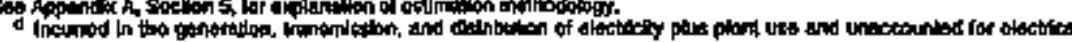

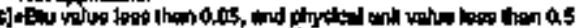


Table 103. Commorcial Energy Consumption Estimates, 1980, 1965, 1970-1994, Inctiana

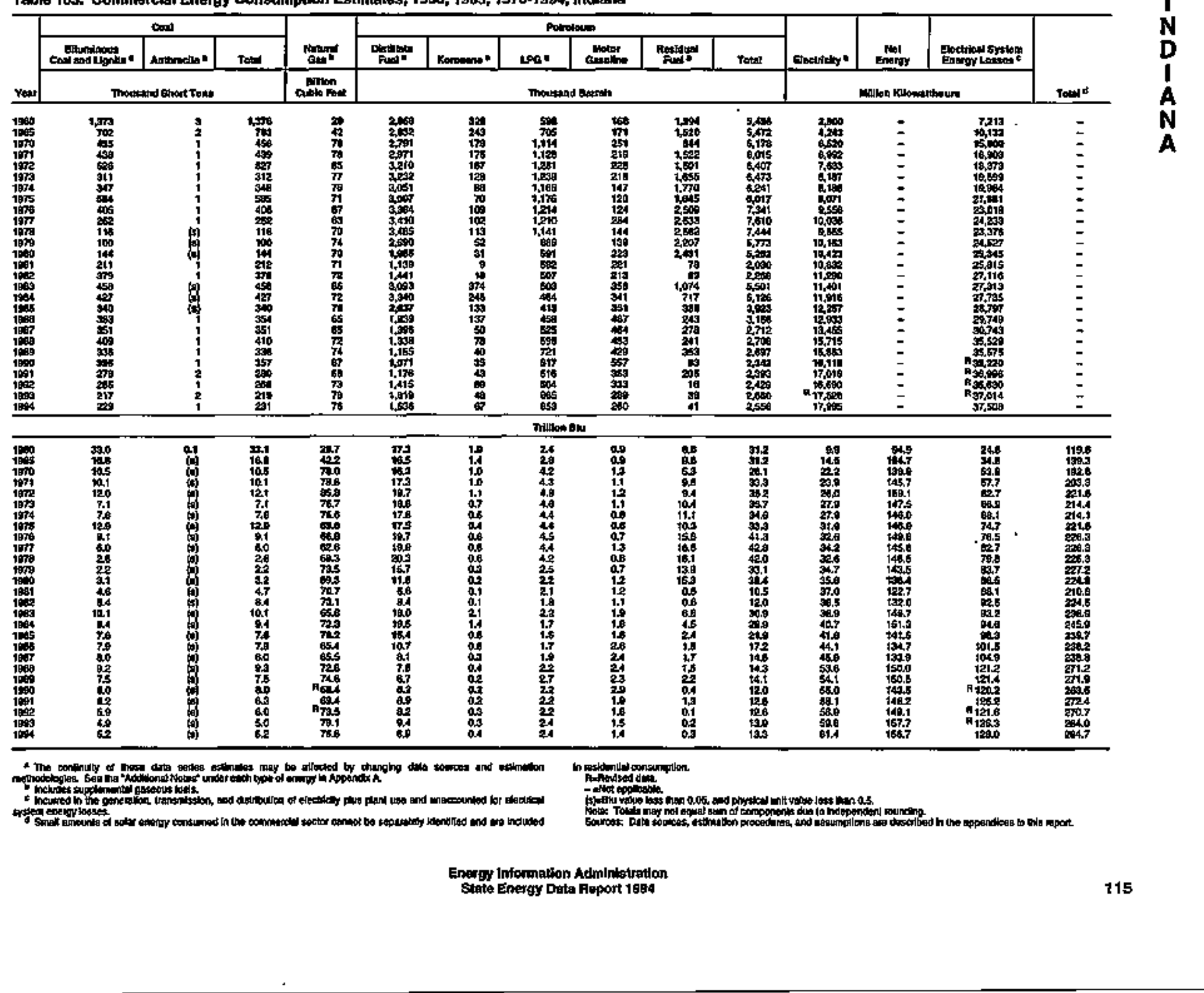




\begin{tabular}{|c|c|c|c|c|c|c|c|c|c|c|c|c|c|c|c|c|c|c|}
\hline \multirow[b]{3}{*}{$\operatorname{man}$} & \multirow[b]{2}{*}{ الهيc } & \multirow[b]{2}{*}{ tentis } & \multicolumn{9}{|c|}{ Potroletem } & \multirow{2}{*}{ 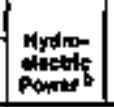 } & \multirow[b]{2}{*}{ 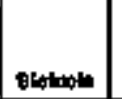 } & \multirow[b]{2}{*}{ Onterend } & \multirow[b]{2}{*}{ 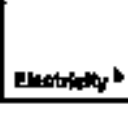 } & \multirow[b]{2}{*}{ Entrir } & \multirow{2}{*}{ 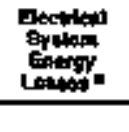 } & \multirow[b]{3}{*}{ Tot } \\
\hline & & & 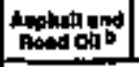 & 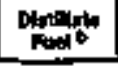 & 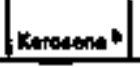 & Las: & 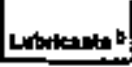 & Etator & 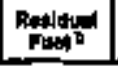 & 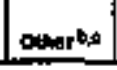 & Tolves & & & & & & & \\
\hline & Thantind & $\begin{array}{l}\text { citron } \\
\text { cutie foes }\end{array}$ & \multicolumn{9}{|c|}{ Thentant Bareolo } & \multicolumn{6}{|c|}{ 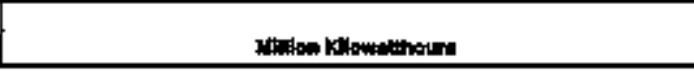 } & \\
\hline 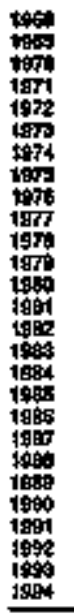 & 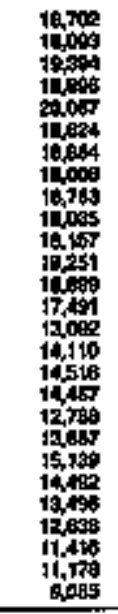 & 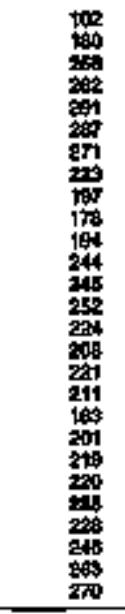 & 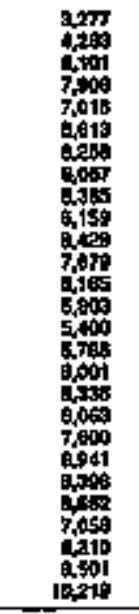 & 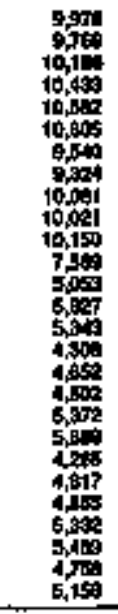 & 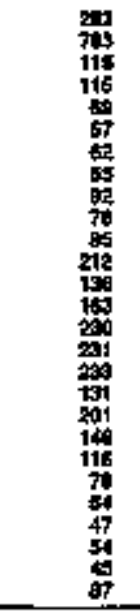 & 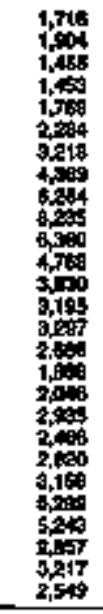 & 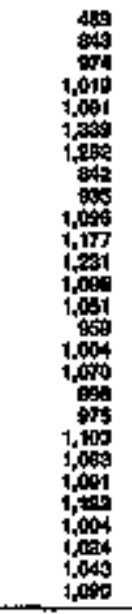 & 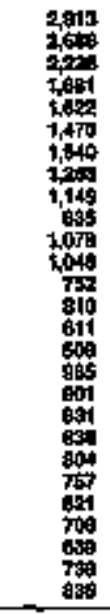 & 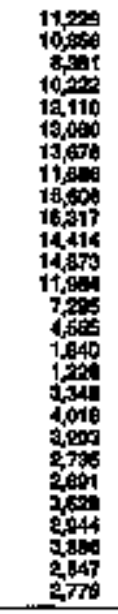 & 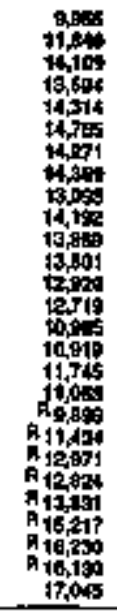 & 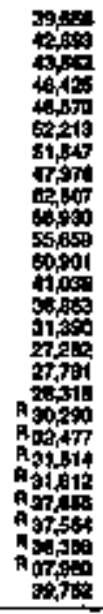 & 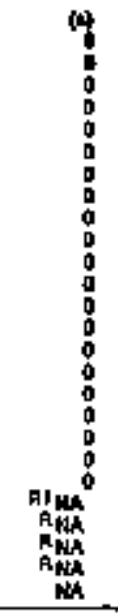 & 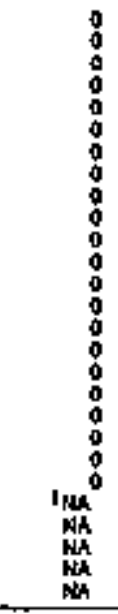 & $\begin{array}{l}0 \\
0 \\
0 \\
0 \\
0 \\
0 \\
0 \\
0 \\
0 \\
0 \\
0 \\
0 \\
0 \\
0 \\
0 \\
0 \\
0 \\
0 \\
0 \\
0 \\
0 \\
\operatorname{con}^{0} \\
\mathrm{~m} \\
\mathrm{~m} \\
\mathrm{~m} \\
\mathrm{~m} \\
\mathrm{~m}\end{array}$ & 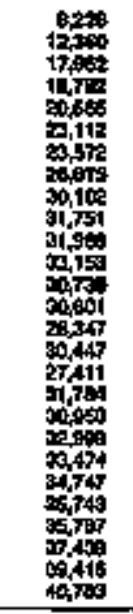 & $\begin{array}{l}\bar{z} \\
\bar{z} \\
\bar{z} \\
\bar{z} \\
\bar{z} \\
\bar{z}\end{array}$ & 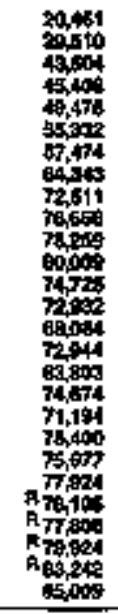 & \\
\hline
\end{tabular}


Table 105. Transportation Energy Constumption Estlmates, 1960, 1965, 1970-1994, Indiana

\begin{tabular}{|c|c|c|c|c|c|c|c|c|c|c|c|c|c|c|c|}
\hline \multirow[b]{3}{*}{ vear } & \multirow[b]{2}{*}{$\cos 11$} & \multirow[b]{2}{*}{ Mande } & \multicolumn{8}{|c|}{ Fotrotan: } & \multirow{3}{*}{ 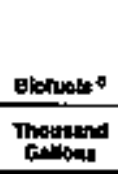 } & & \multirow[b]{2}{*}{ matrit } & \multirow[b]{2}{*}{ 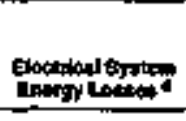 } & \multirow[b]{3}{*}{ Total } \\
\hline & & & 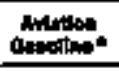 & $\begin{array}{l}\text { Duthent } \\
\text { furd }\end{array}$ & f녕. & LPR: & Luterats = & borotion & 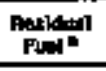 & Toted & & Dextriky * & & & \\
\hline & 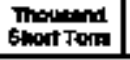 & colvilofor & \multicolumn{8}{|c|}{ 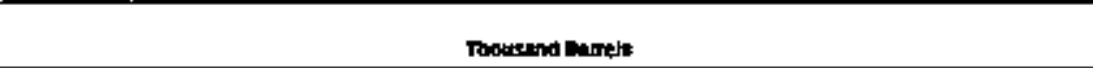 } & & \multicolumn{3}{|c|}{ 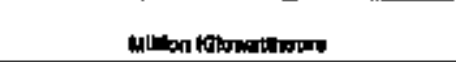 } & \\
\hline 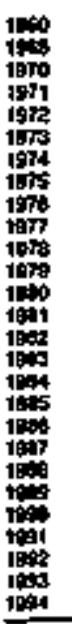 & 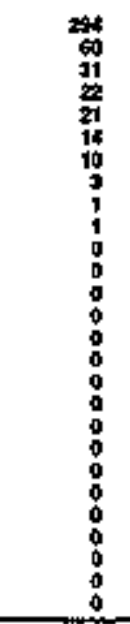 & 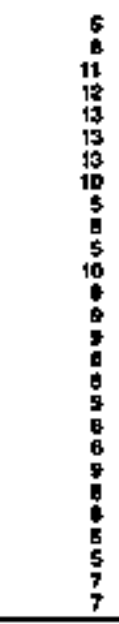 & 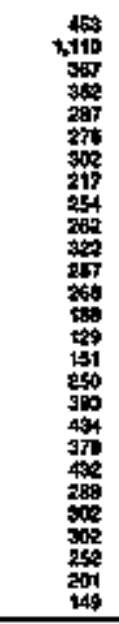 & 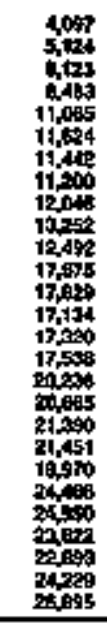 & 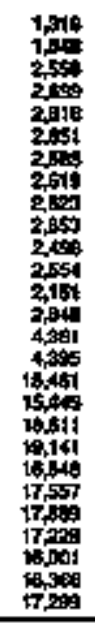 & 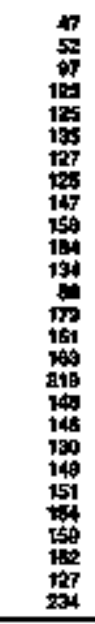 & 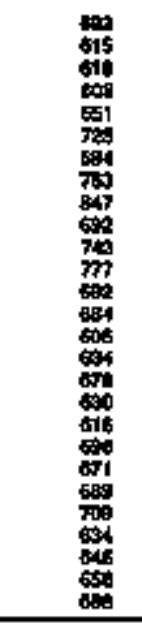 & 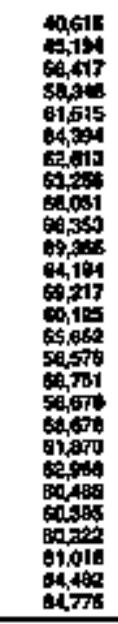 & 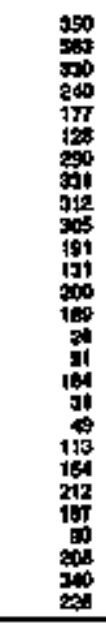 & 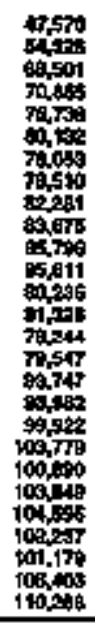 & 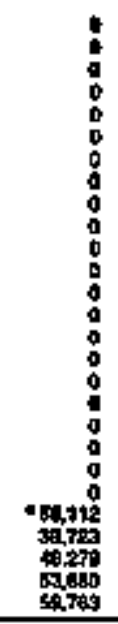 & $\begin{array}{r}1 \\
0 \\
0 \\
0 \\
0 \\
0 \\
0 \\
0 \\
0 \\
0 \\
0 \\
0 \\
0 \\
0 \\
0 \\
0 \\
11 \\
11 \\
11 \\
11 \\
11 \\
\end{array}$ & 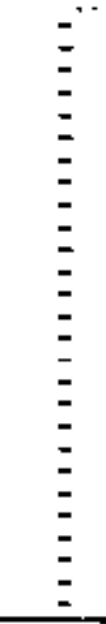 & $\begin{array}{r}z \\
0 \\
0 \\
0 \\
0 \\
0 \\
0 \\
0 \\
0 \\
0 \\
0 \\
0 \\
a \\
0 \\
0 \\
0 \\
0 \\
0 \\
0 \\
0 \\
0 \\
0 \\
20 \\
20 \\
23 \\
24 \\
24 \\
24\end{array}$ & $\begin{array}{l}= \\
z \\
= \\
= \\
= \\
= \\
= \\
= \\
= \\
z \\
z \\
z \\
z \\
z \\
=\end{array}$ \\
\hline \multicolumn{16}{|c|}{ 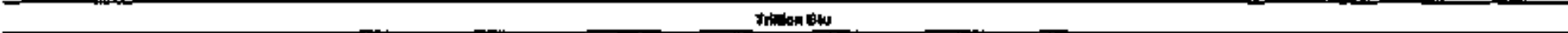 } \\
\hline 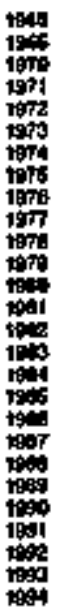 & 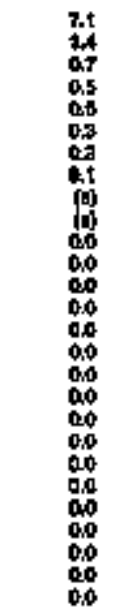 & 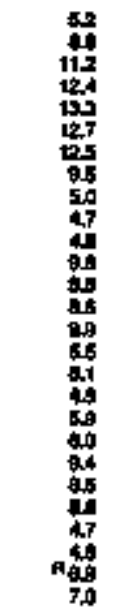 & 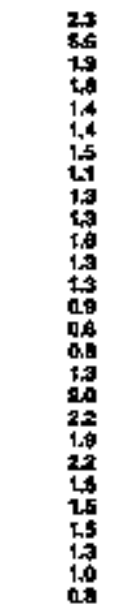 & 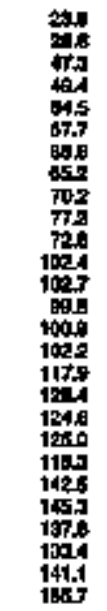 & 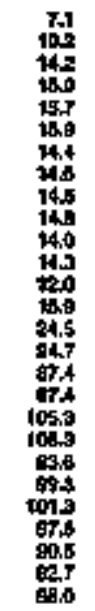 & 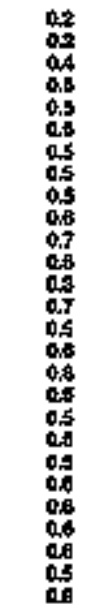 & 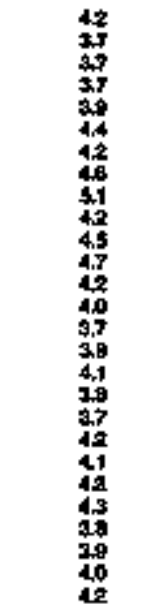 & 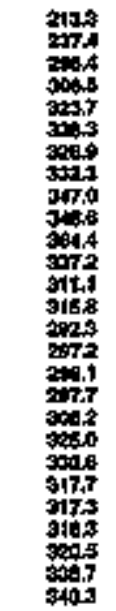 & 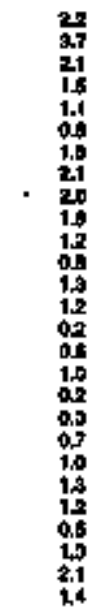 & 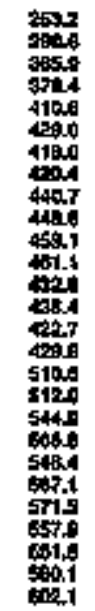 & 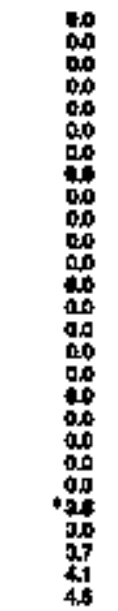 & 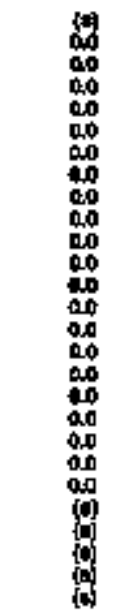 & 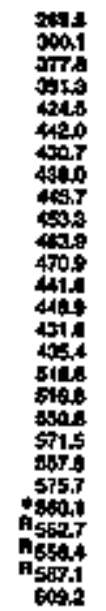 & $\begin{array}{l}0 \\
00 \\
0.0 \\
0.0 \\
00 \\
0.0 \\
0.0 \\
0.0 \\
0.0 \\
0.0 \\
0.0 \\
00 \\
0.0 \\
0.0 \\
0.0 \\
0.0 \\
00 \\
0.0 \\
0.0 \\
0.0 \\
0.0 \\
00 \\
0.1 \\
0.1 \\
0.1 \\
0.1 \\
0.1\end{array}$ & 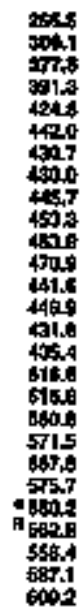 \\
\hline
\end{tabular}

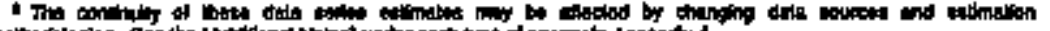

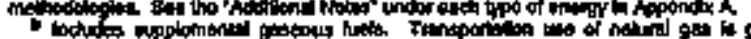

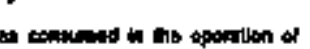

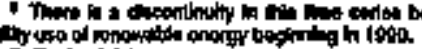

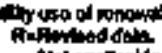

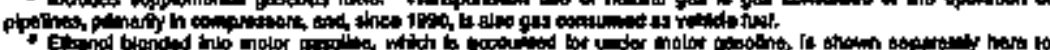

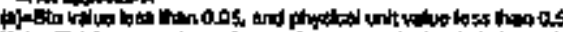

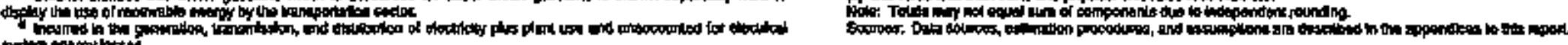

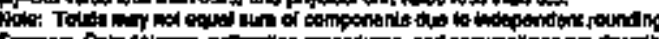


Table 106. Estimates of Energy Input at Electrte Utilittes, 1960, 1965, 1970-1994, Indiana

\begin{tabular}{|c|c|c|c|c|c|c|c|c|c|c|c|c|c|c|}
\hline & \multicolumn{3}{|c|}{ Com } & \multirow[b]{2}{*}{ 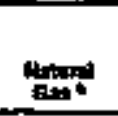 } & \multicolumn{4}{|c|}{ Parolearn } & \multirow[b]{2}{*}{ 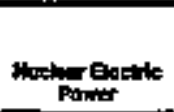 } & \multirow[b]{2}{*}{ 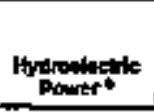 } & \multirow[b]{2}{*}{ Etohnt } & \multirow[b]{2}{*}{ 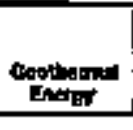 } & \multirow[b]{2}{*}{ Detsent } & \multirow[b]{3}{*}{ Toldelp } \\
\hline & Foningus & 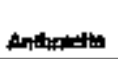 & Tolta & & 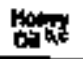 & 籍视 & Podpon & Tots & & & & & & \\
\hline 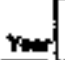 & \multicolumn{3}{|c|}{ Thordact thent tons } & criblefor for & \multicolumn{4}{|c|}{ 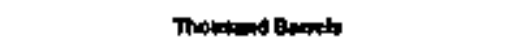 } & \multicolumn{5}{|c|}{ 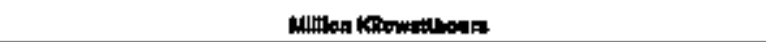 } & \\
\hline
\end{tabular}

\begin{tabular}{|c|c|c|c|c|}
\hline 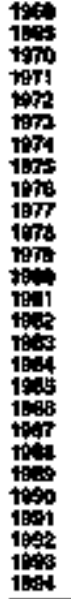 & 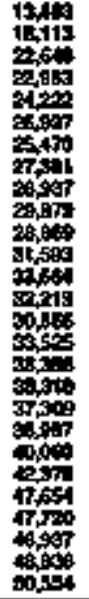 & $\begin{array}{l}: \\
: \\
0 \\
: \\
: \\
: \\
: \\
: \\
: \\
: \\
: \\
: \\
: \\
: \\
:\end{array}$ & 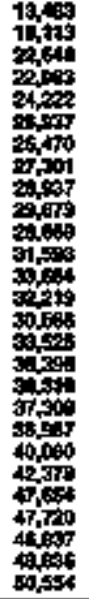 & $\begin{array}{r}5 \\
13 \\
30 \\
39 \\
10 \\
10 \\
14 \\
11 \\
3 \\
1 \\
3 \\
3 \\
2 \\
6 \\
3 \\
3 \\
1 \\
1 \\
1 \\
3 \\
5 \\
7 \\
10 \\
8 \\
6 \\
9\end{array}$ \\
\hline
\end{tabular}

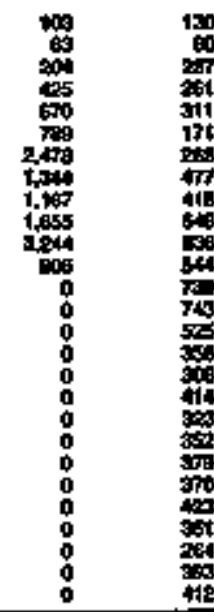

(1)

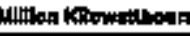

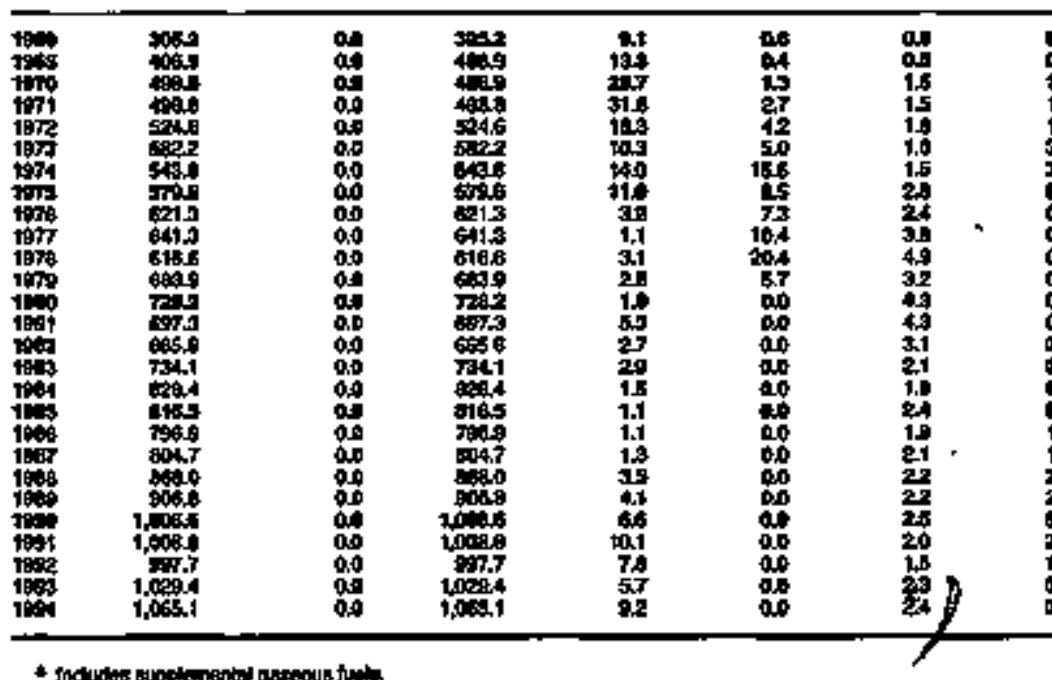

nition

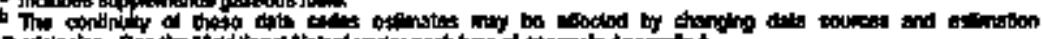

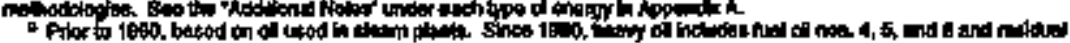
nong ala

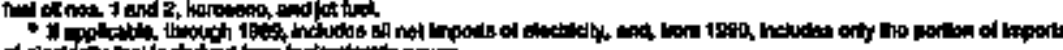

\begin{tabular}{|c|c|c|c|c|c|c|}
\hline 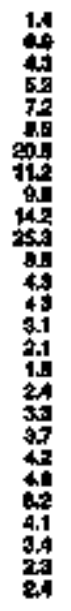 & 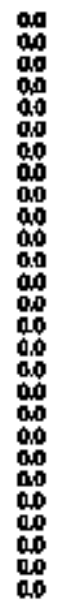 & 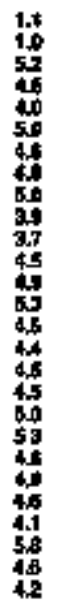 & 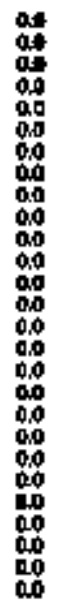 & 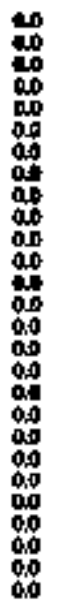 & 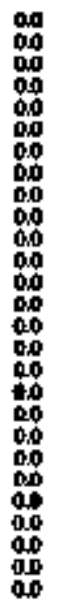 & 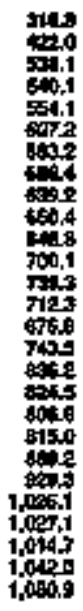 \\
\hline
\end{tabular}

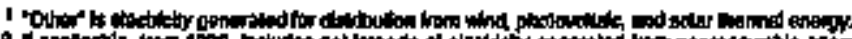

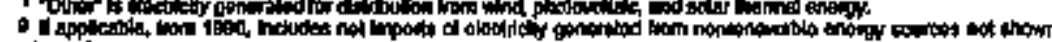

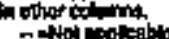

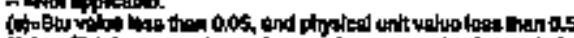

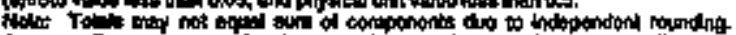

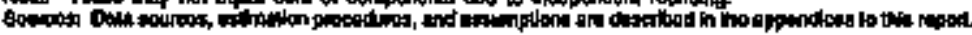


Table 107. Energy Consumption Estimates by \$ource, 1950, 1955, 1970-1994, lowa

\begin{tabular}{|c|c|c|c|c|c|c|c|c|c|c|c|c|c|c|c|c|c|c|c|}
\hline \multirow[b]{3}{*}{ Yene } & \multirow[b]{2}{*}{ Con. } & \multirow[b]{2}{*}{ Mrthons } & \multicolumn{11}{|c|}{ Patrolom } & \multirow{2}{*}{ 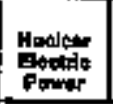 } & \multirow[b]{2}{*}{ 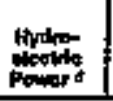 } & \multirow[b]{2}{*}{ Glokionet } & \multirow[b]{2}{*}{ Gour of ? } & \multirow{3}{*}{ 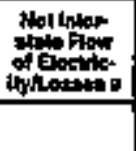 } & \multirow[b]{3}{*}{ Tratedn } \\
\hline & & & 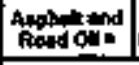 & 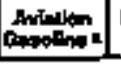 & 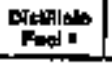 & tor. & Kans: & LPE. & Litit. & 烈 & mationd & Doner an & Tot & & & & & & \\
\hline & mowned & 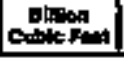 & \multicolumn{11}{|c|}{ Movisnd Eants } & \multicolumn{4}{|c|}{ N-Ton KThmathoume } & & \\
\hline 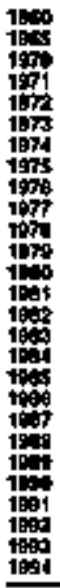 & 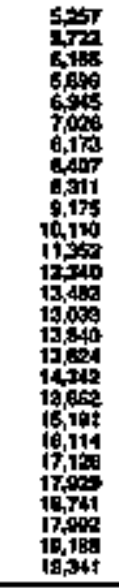 & 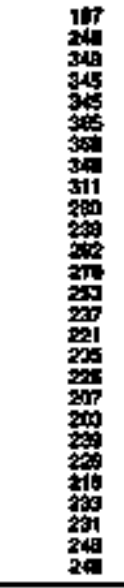 & 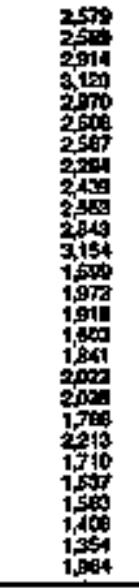 & 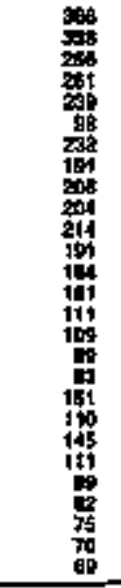 & 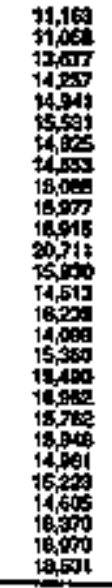 & 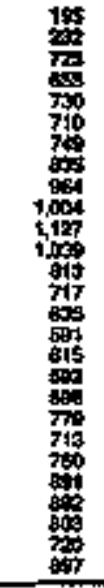 & 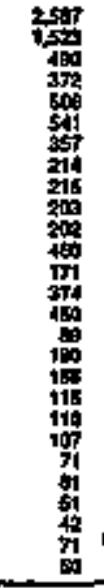 & 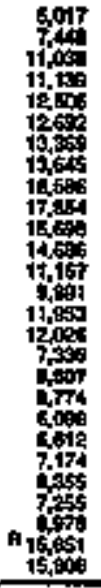 & 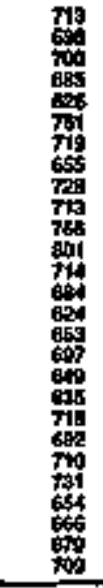 & 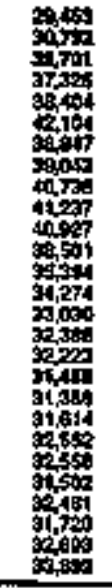 & 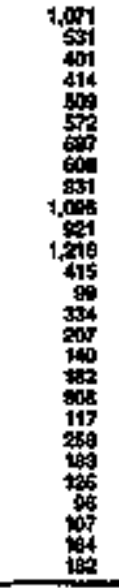 & 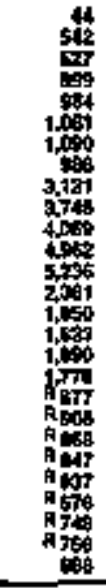 & 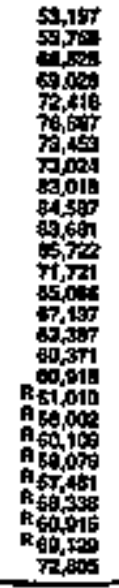 & 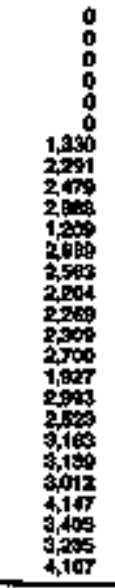 & 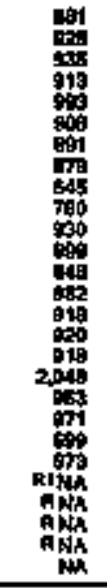 & 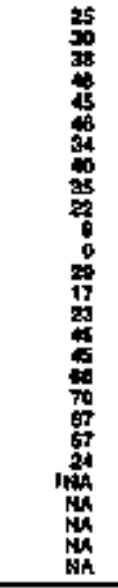 & 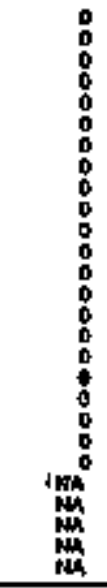 & 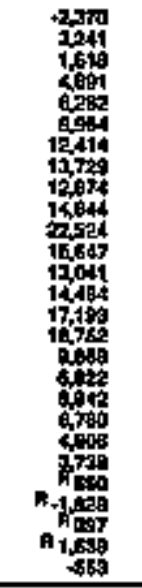 & $\begin{array}{l}z \\
z \\
z \\
z \\
z \\
z \\
z \\
z \\
z \\
z \\
z \\
z \\
z \\
z\end{array}$ \\
\hline \multicolumn{20}{|c|}{ Thiten Bth } \\
\hline 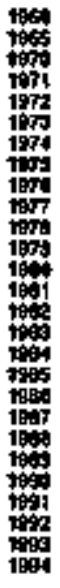 & 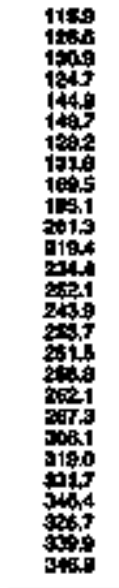 & 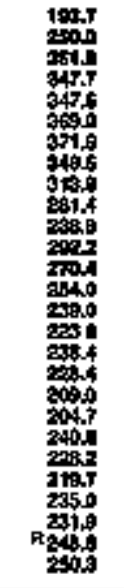 & 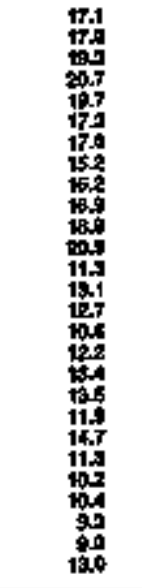 & 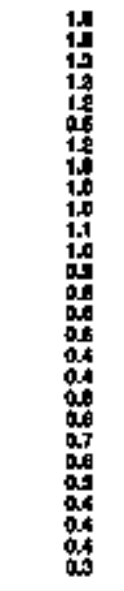 & 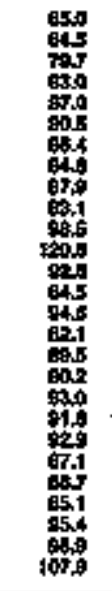 & 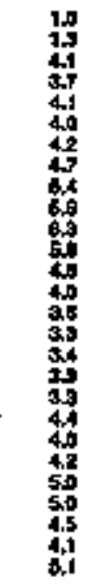 & 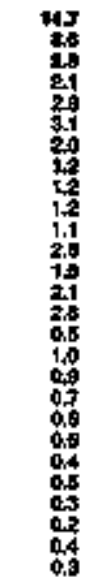 & 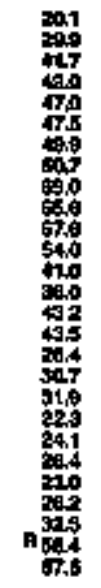 & 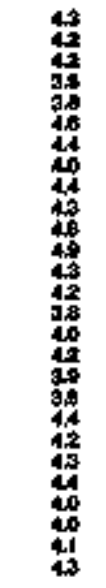 & 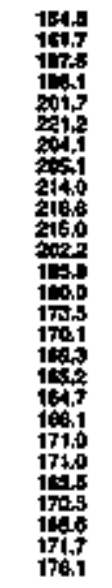 & 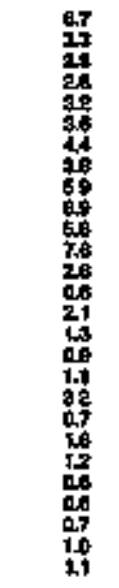 & 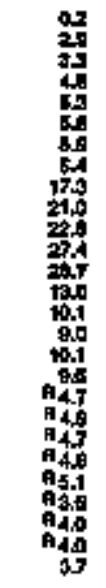 & 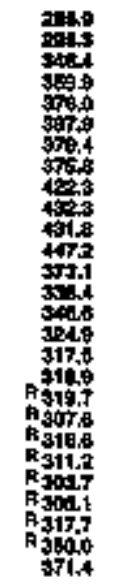 & 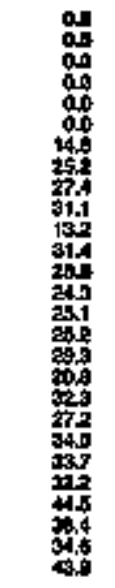 & 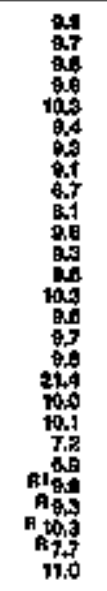 & 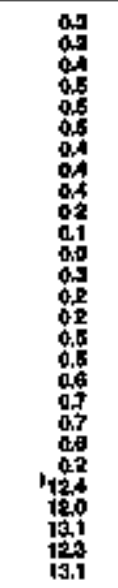 & 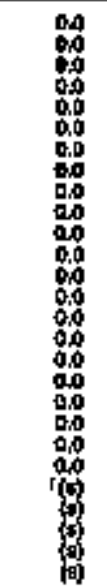 & 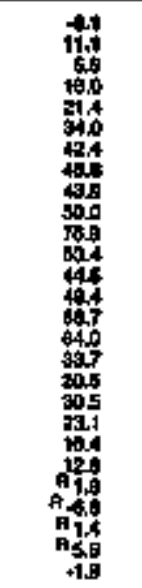 & 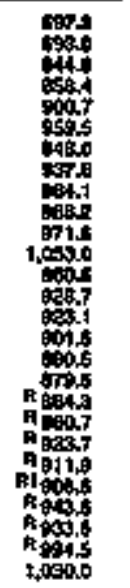 \\
\hline & & & & & & & 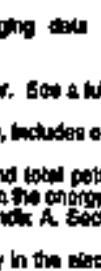 & al. & 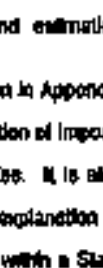 & & & & & . & . & 9000 & 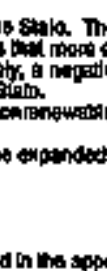 & 每 & 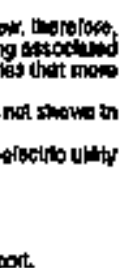 \\
\hline
\end{tabular}


Table 106. Aesidential Energy Consumption Estimates, t960, 1965, 1970-1994, lowa

\begin{tabular}{|c|c|c|c|c|c|c|c|c|c|c|c|c|c|c|}
\hline \multirow[b]{3}{*}{ Year } & \multicolumn{3}{|c|}{ cond } & \multirow{3}{*}{ 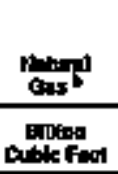 } & \multicolumn{4}{|c|}{ 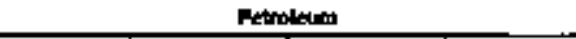 } & \multirow{3}{*}{ 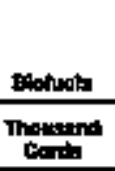 } & & \multirow[b]{2}{*}{ Eevirily } & \multirow[b]{2}{*}{ enter } & \multirow{3}{*}{ 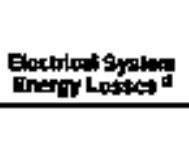 } & \multirow[b]{3}{*}{ Total } \\
\hline & 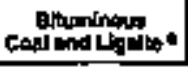 & Anthecis: & Towt & & Distinge & Krowen: & Lerex & 7olin & & Sotare & & & & \\
\hline & \multicolumn{3}{|c|}{ Thoupd Shont Tona } & & \multicolumn{4}{|c|}{ 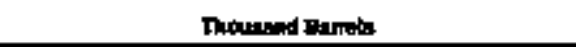 } & & \multicolumn{3}{|c|}{ 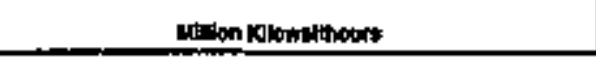 } & & \\
\hline 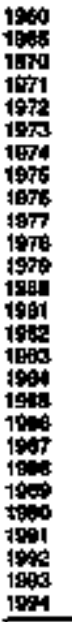 & 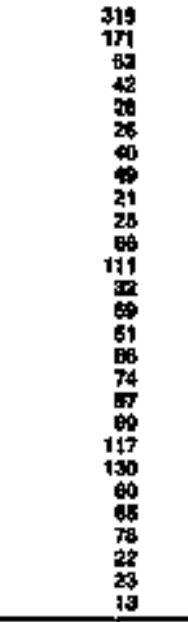 & $\begin{array}{l}0 \\
0 \\
0 \\
0 \\
0 \\
0 \\
0 \\
0 \\
0 \\
0 \\
0 \\
0 \\
0 \\
1 \\
3 \\
0 \\
1 \\
1 \\
1 \\
1 \\
0 \\
2 \\
1 \\
001 \\
1 \\
3 \\
2 \\
\end{array}$ & 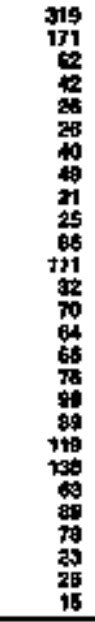 & 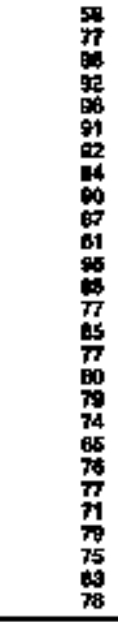 & 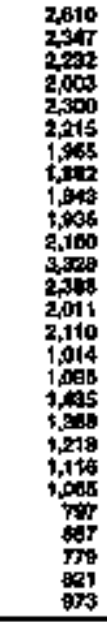 & 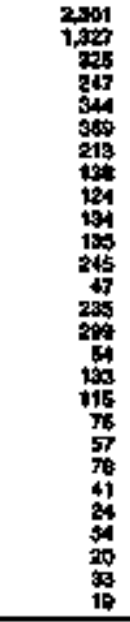 & 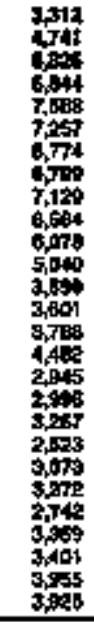 & 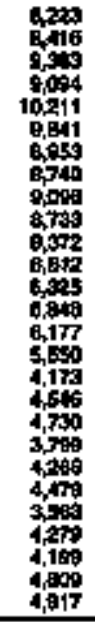 & 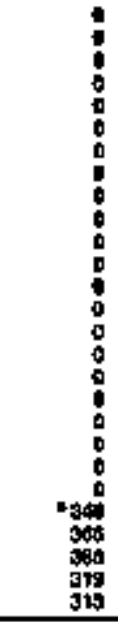 & $\begin{array}{r}0 \\
0 \\
0 \\
0 \\
0 \\
0 \\
0 \\
0 \\
0 \\
0 \\
0 \\
0 \\
0 \\
0 \\
0 \\
0 \\
0 \\
0 \\
0 \\
0 \\
0 \\
0 \\
02 \\
2 \\
8 \\
2 \\
2 \\
2\end{array}$ & 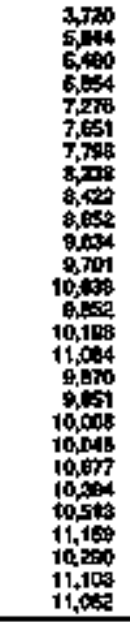 & $\begin{array}{l}z \\
z \\
z \\
z \\
z \\
z \\
z \\
z \\
z \\
z \\
z \\
z \\
z \\
z \\
z\end{array}$ & 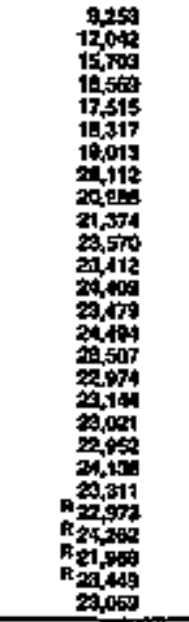 & $\begin{array}{l}z \\
z \\
z \\
z \\
z \\
z \\
z \\
z \\
z \\
z \\
z \\
z \\
z \\
z \\
=\end{array}$ \\
\hline \multicolumn{15}{|c|}{ 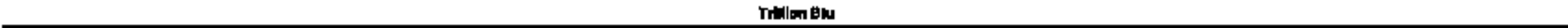 } \\
\hline 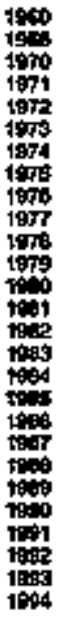 & 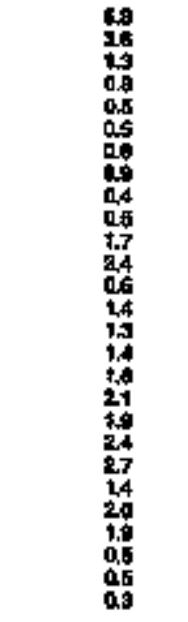 & 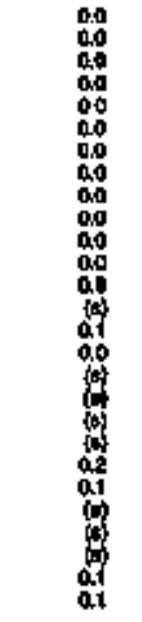 & 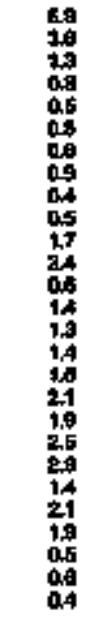 & 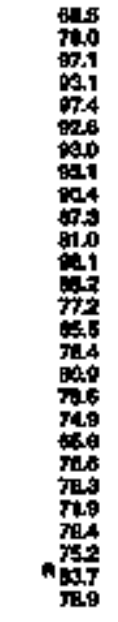 & 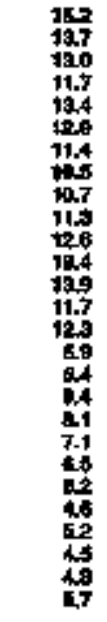 & 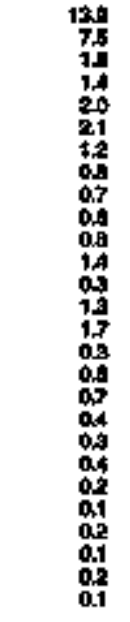 & 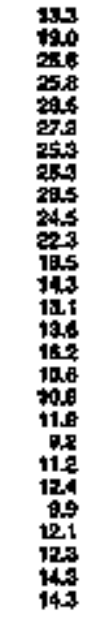 & 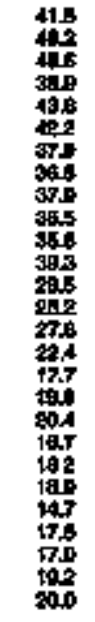 & 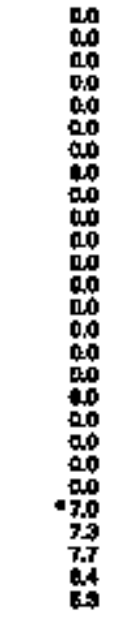 & 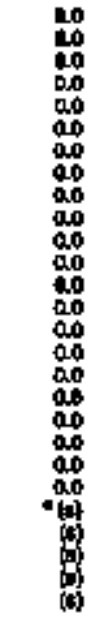 & 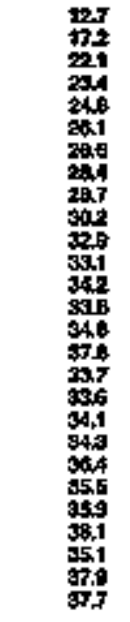 & 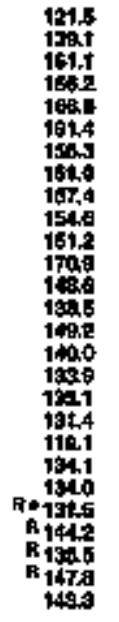 & 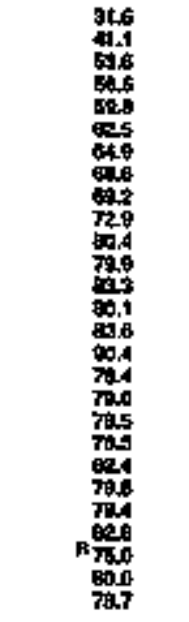 & 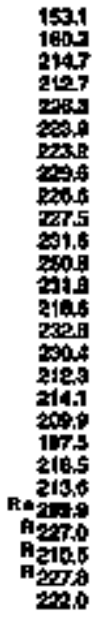 \\
\hline
\end{tabular}

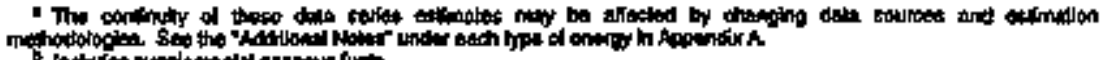

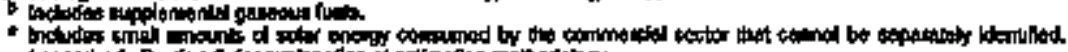

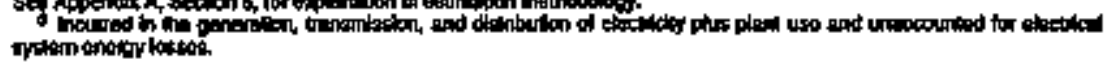

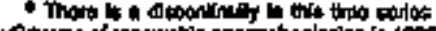

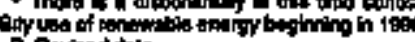
Rifinations.

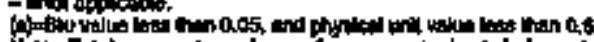

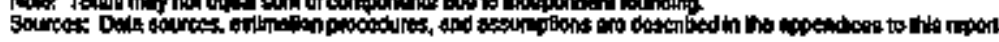


Table 109. Commerctal Energy Consumplion Estlmates, 1950, 1955, 1970-1994, lowra

\begin{tabular}{|c|c|c|c|c|c|c|c|c|c|c|c|c|c|c|}
\hline \multirow[b]{3}{*}{ Your } & \multicolumn{3}{|c|}{ Eal } & \multirow{3}{*}{ 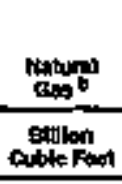 } & \multicolumn{6}{|c|}{ Parrotarn } & \multirow[b]{2}{*}{ Exemeath" } & \multirow[b]{2}{*}{ not } & \multirow[b]{2}{*}{ 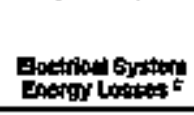 } & \multirow[b]{3}{*}{ Told } \\
\hline & 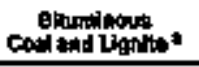 & Antronolise & 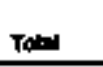 & & 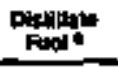 & Kersemp: & ton* & Molet & mosigey & Tolot & & & & \\
\hline & \multicolumn{3}{|c|}{ Thounead stant Tong } & & \multicolumn{6}{|c|}{ Thowand Barrole } & \multicolumn{3}{|c|}{ 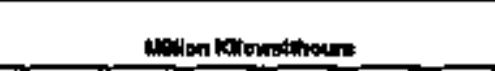 } & \\
\hline 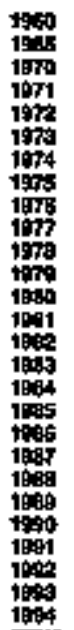 & 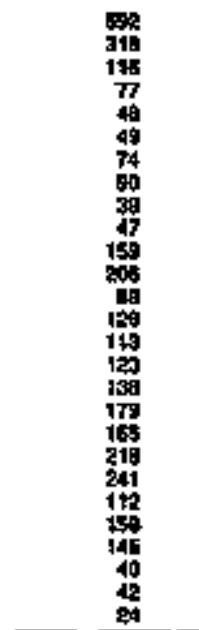 & $\begin{array}{c}0 \\
0 \\
0 \\
0 \\
0 \\
0 \\
0 \\
0 \\
0 \\
0 \\
0 \\
0 \\
0 \\
0 \\
1 \\
2 \\
0 \\
0 \\
1 \\
1 \\
1 \\
1 \\
0 \\
0 \\
1 \\
1 \\
1 \\
1 \\
1 \\
1 \\
1 \\
1\end{array}$ & 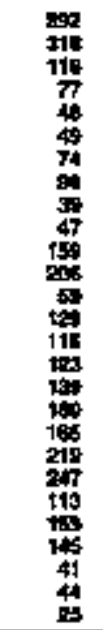 & 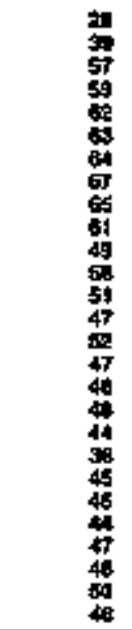 & 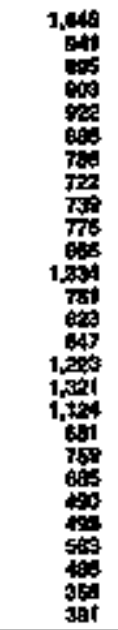 & 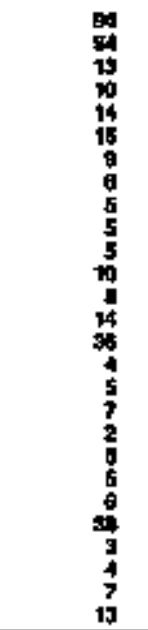 & 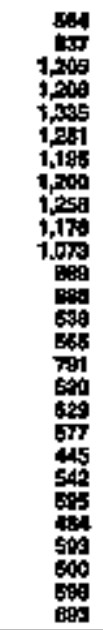 & 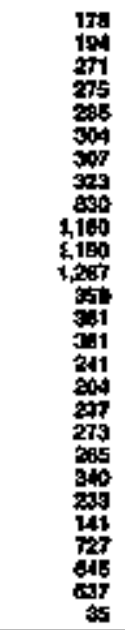 & 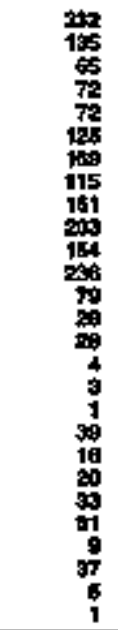 & 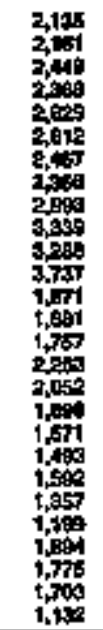 & 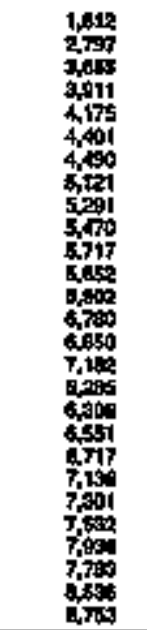 & $\begin{array}{l}\vdots \\
\vdots \\
\vdots \\
\vdots \\
\vdots \\
\vdots \\
\vdots \\
\vdots \\
\vdots \\
\vdots \\
\vdots \\
\vdots \\
\vdots \\
z\end{array}$ & 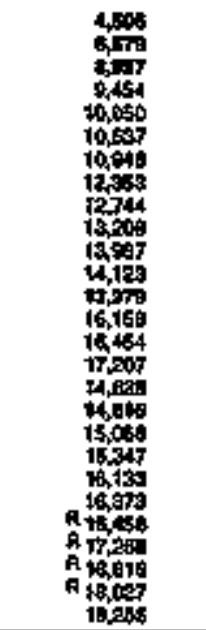 & $\begin{array}{l}z \\
z \\
z \\
z \\
\vdots \\
z \\
\vdots \\
\vdots \\
\vdots \\
z \\
z \\
z \\
z \\
z\end{array}$ \\
\hline \multicolumn{15}{|c|}{ Whthon alu } \\
\hline 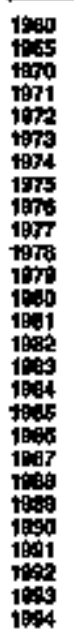 & 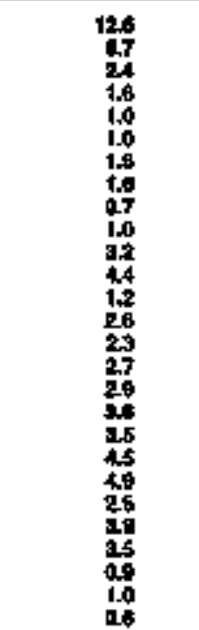 & 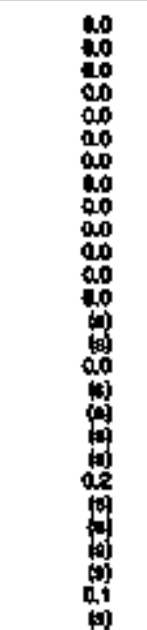 & 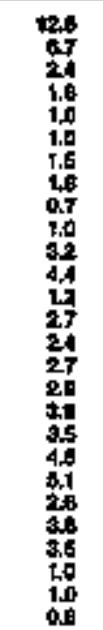 & 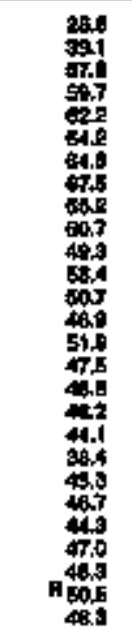 & 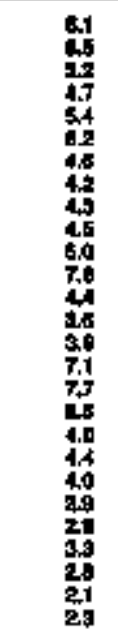 & 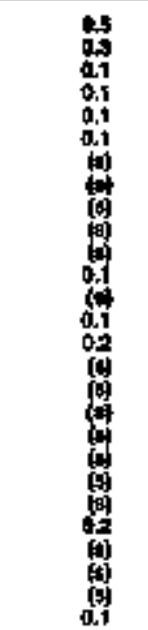 & 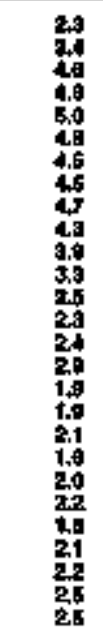 & 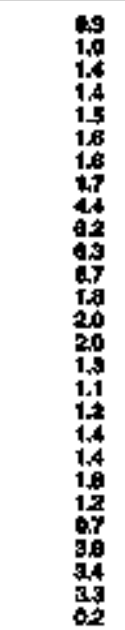 & 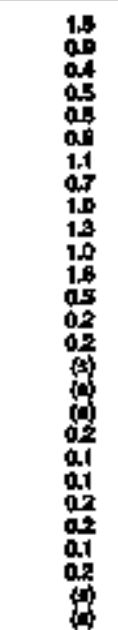 & 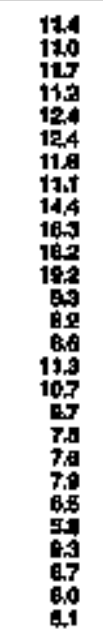 & 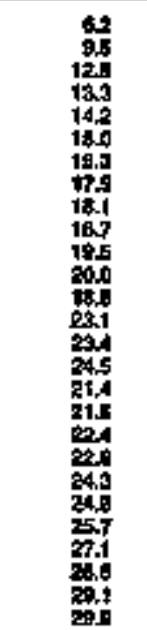 & 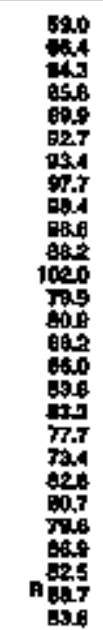 & 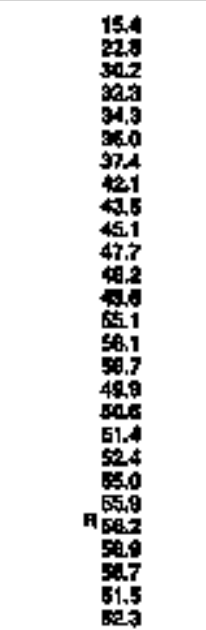 & 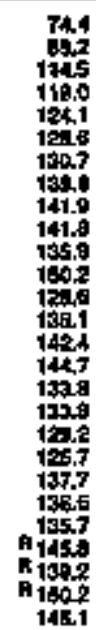 \\
\hline
\end{tabular}


Tabio 1f0. Indusirial Energy Consumption Estimates, 1960, 1965, 1970-1994, lowa

\begin{tabular}{|c|c|c|c|c|c|c|c|c|c|c|c|c|c|c|c|c|c|c|}
\hline \multirow[b]{3}{*}{ Yew } & \multirow[b]{2}{*}{ cont } & \multirow[b]{2}{*}{ Nonst } & \multicolumn{9}{|c|}{ Putroloum } & \multirow[b]{2}{*}{ 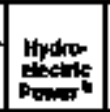 } & \multirow[b]{2}{*}{ Doturts } & \multirow[b]{2}{*}{ Goun the } & \multirow[b]{2}{*}{ electidy" } & \multirow[b]{2}{*}{ Enes } & \multirow{2}{*}{ 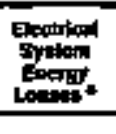 } & \multirow[b]{3}{*}{ Thit } \\
\hline & & & Alphangent & ated & kemasenot & LPQ: & 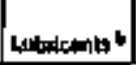 & mover & Fucles & Other the & - & & & & & & & \\
\hline & Theorind: & 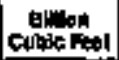 & \multicolumn{9}{|c|}{ Thex. anc Bimits } & \multicolumn{6}{|c|}{ 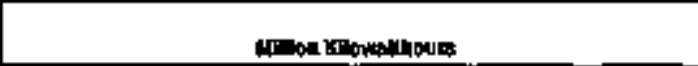 } & \\
\hline 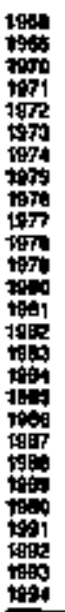 & 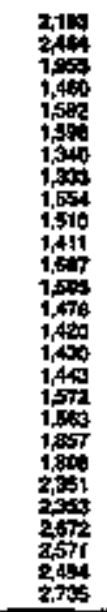 & 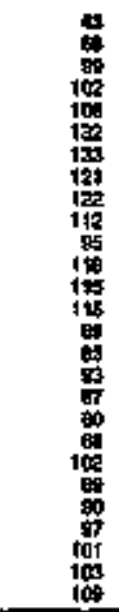 & 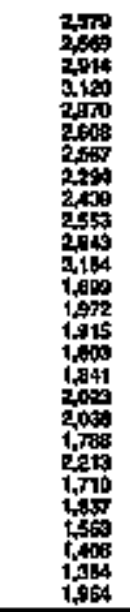 & 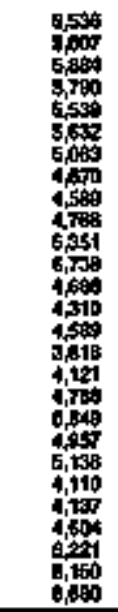 & 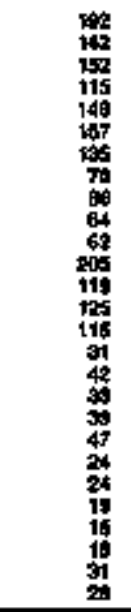 & 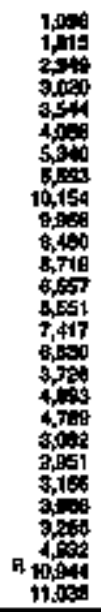 & 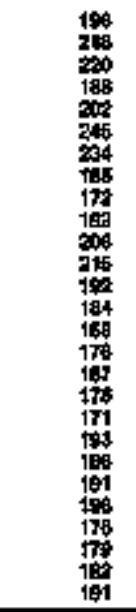 & 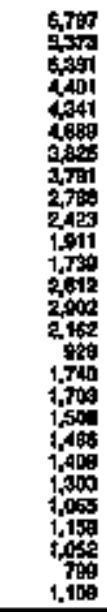 & 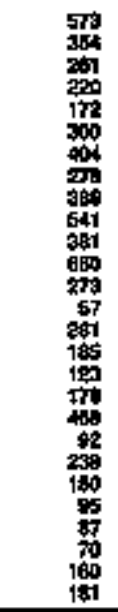 & 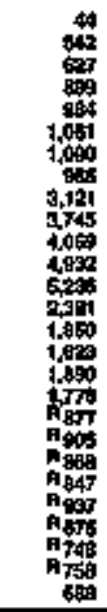 & 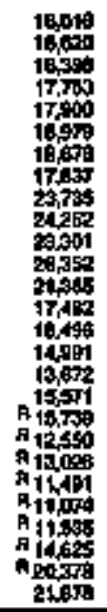 & $\begin{array}{r}\mathbf{2} \\
\mathbf{1} \\
\mathbf{1} \\
\mathbf{1} \\
\mathbf{1} \\
\mathbf{1} \\
\mathbf{1} \\
\mathbf{1} \\
\mathbf{1} \\
\mathbf{1} \\
\mathbf{1} \\
\mathbf{1} \\
\mathbf{1} \\
\mathbf{1} \\
\mathbf{1} \\
\mathbf{1}\end{array}$ & 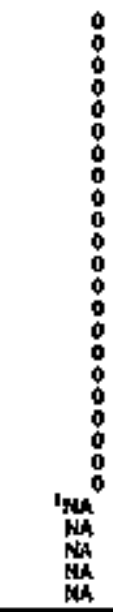 & $\begin{array}{r}0 \\
0 \\
0 \\
0 \\
0 \\
0 \\
0 \\
0 \\
0 \\
0 \\
0 \\
0 \\
0 \\
0 \\
0 \\
0 \\
0 \\
0 \\
4 \\
4 \\
4 \\
4\end{array}$ & 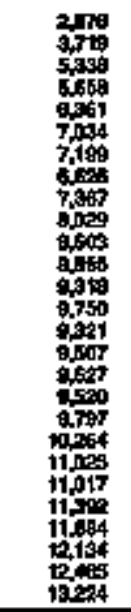 & $\begin{array}{l}= \\
= \\
= \\
= \\
= \\
= \\
= \\
= \\
= \\
= \\
= \\
= \\
= \\
= \\
=\end{array}$ & 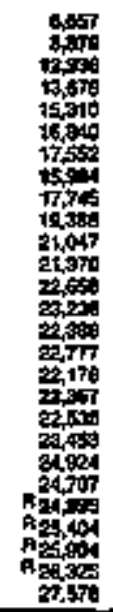 & $\begin{array}{l}= \\
= \\
= \\
= \\
= \\
= \\
= \\
= \\
= \\
= \\
= \\
= \\
= \\
z \\
=\end{array}$ \\
\hline \multicolumn{19}{|c|}{ Trition Bin } \\
\hline 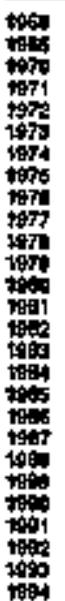 & 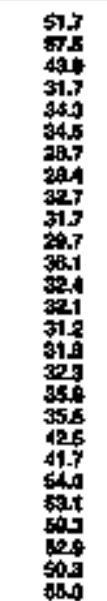 & 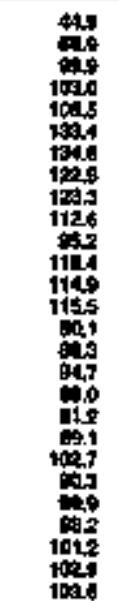 & 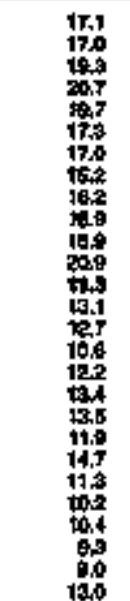 & 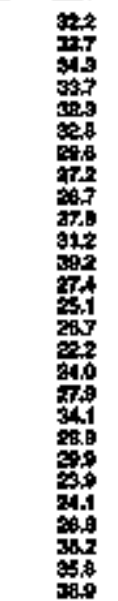 & 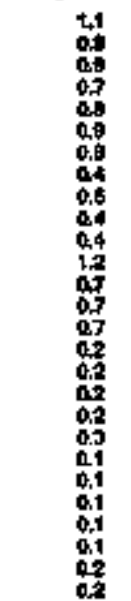 & 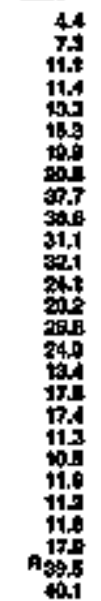 & 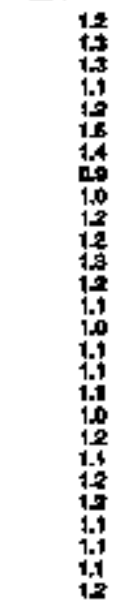 & 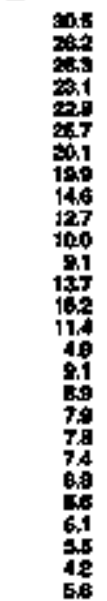 & 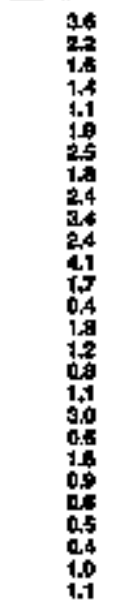 & 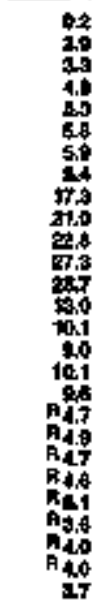 & 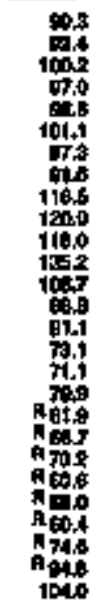 & 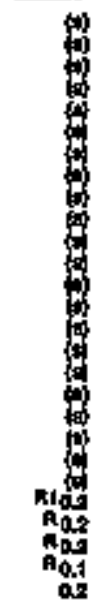 & 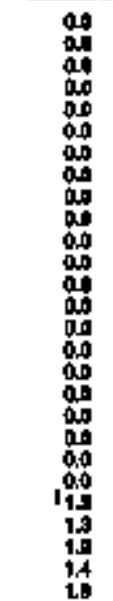 & 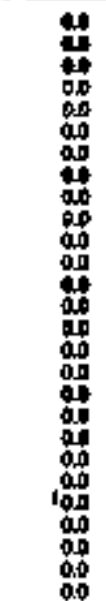 & 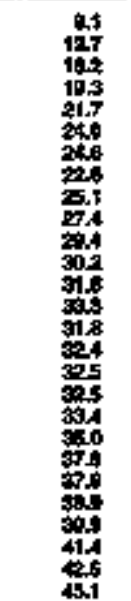 & 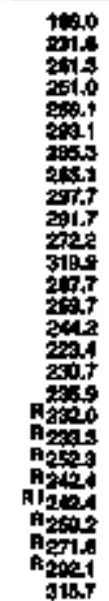 & 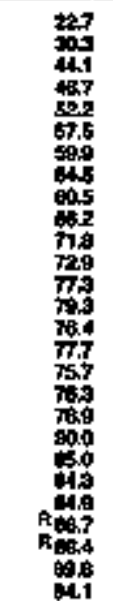 & 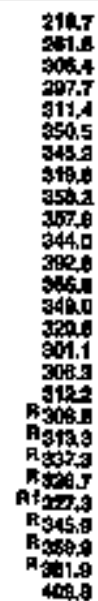 \\
\hline
\end{tabular}

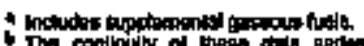

Ins conving of

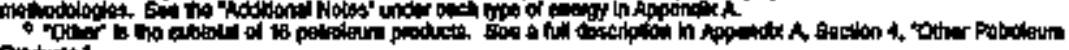

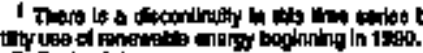

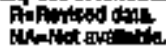
Whated and

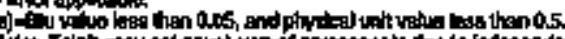

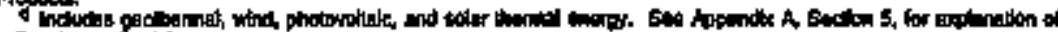
lo hisponos.

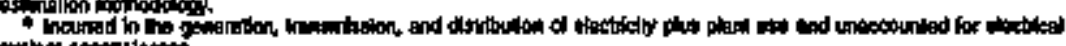

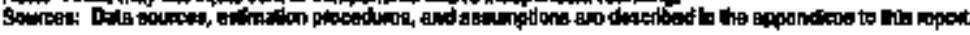

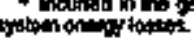


Tatbe 111. Transportalion Energy Consumption Estimates, 7860, 1965, 1970-1994, bwa

\begin{tabular}{|c|c|c|c|c|c|c|c|c|c|c|c|c|c|c|c|}
\hline \multirow[b]{3}{*}{ rear } & \multirow[b]{2}{*}{ cost. } & \multirow[b]{2}{*}{ athent } & \multicolumn{8}{|c|}{ Potodention } & \multirow{3}{*}{ 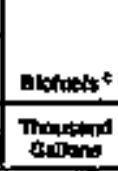 } & & \multirow[b]{2}{*}{ 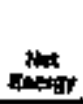 } & \multirow{3}{*}{ 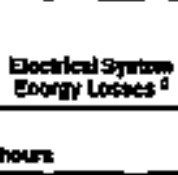 } & \multirow[b]{3}{*}{ Tote } \\
\hline & & & Antions. & Fontento & Int. & LMa" & 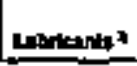 & 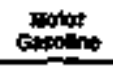 & 1) & Told & & 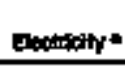 & & & \\
\hline & $\begin{array}{l}\text { Thoutend } \\
\text { seod Tous }\end{array}$ & contion Fon & \multicolumn{8}{|c|}{ 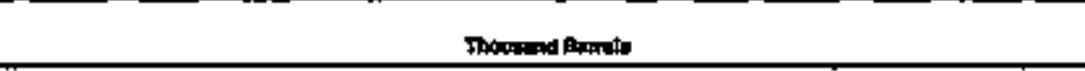 } & & \multicolumn{2}{|c|}{ 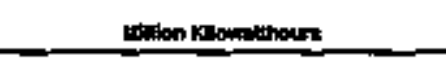 } & & \\
\hline 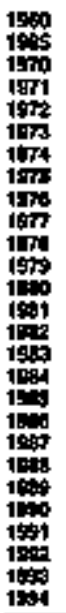 & 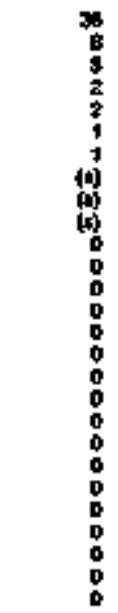 & 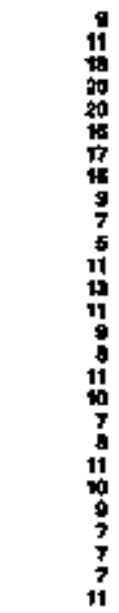 & 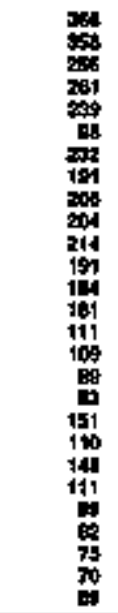 & 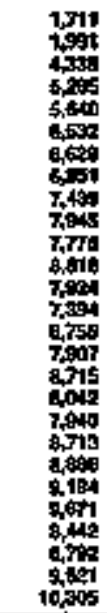 & 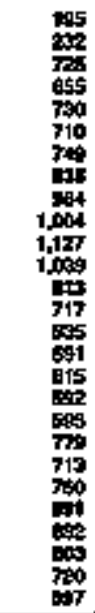 & 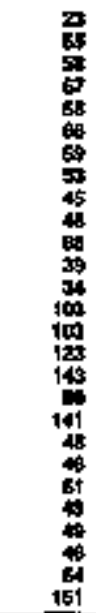 & 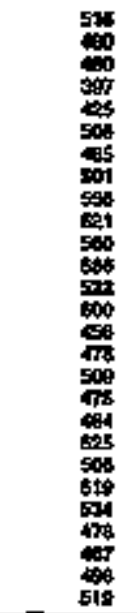 & 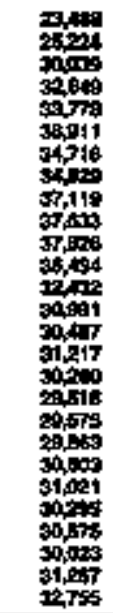 & 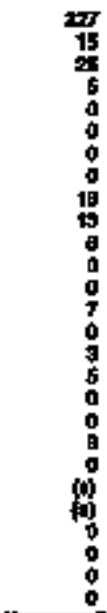 & 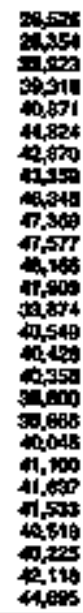 & 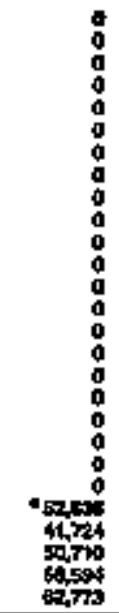 & $\begin{array}{l}0 \\
0 \\
0 \\
0 \\
0 \\
0 \\
0 \\
0 \\
0 \\
0 \\
0 \\
0 \\
0 \\
0 \\
0 \\
0 \\
0 \\
0 \\
0 \\
0 \\
0 \\
0 \\
0 \\
0 \\
0 \\
0 \\
0\end{array}$ & $\begin{array}{l}= \\
= \\
= \\
= \\
= \\
= \\
= \\
= \\
= \\
= \\
= \\
z \\
= \\
= \\
=\end{array}$ & $\begin{array}{l}0 \\
0 \\
0 \\
0 \\
0 \\
0 \\
0 \\
0 \\
0 \\
0 \\
0 \\
0 \\
0 \\
0 \\
0 \\
0 \\
0 \\
0 \\
0 \\
0 \\
0 \\
0 \\
0 \\
0 \\
0\end{array}$ & 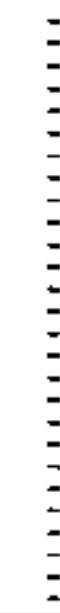 \\
\hline & & & & & & & & Hing & & & & & & & \\
\hline
\end{tabular}

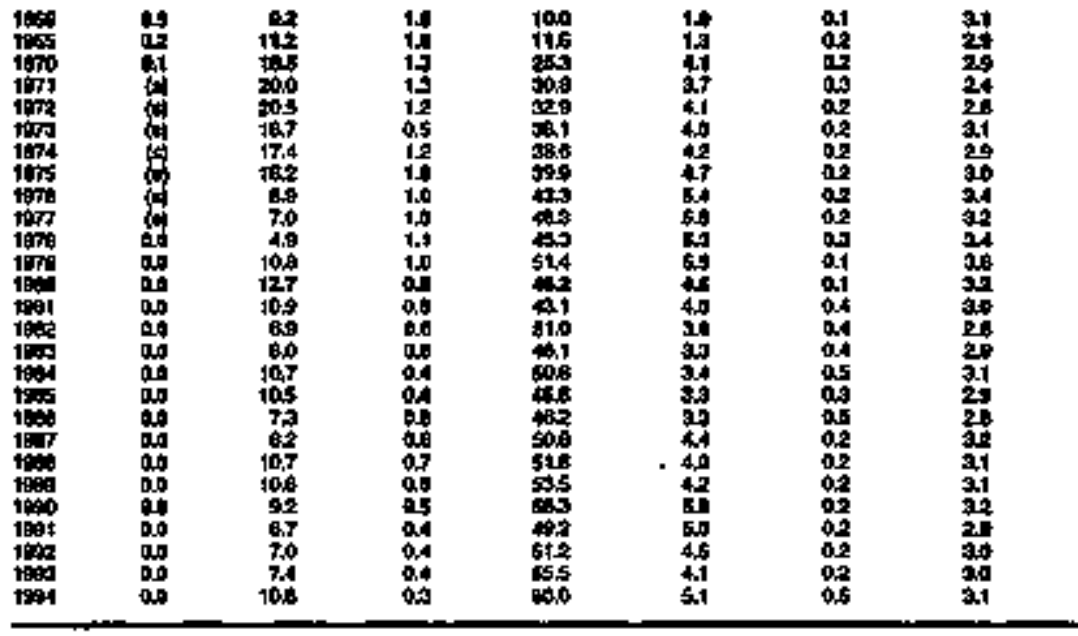

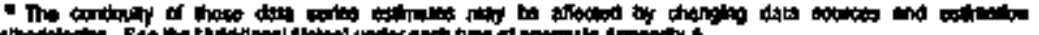

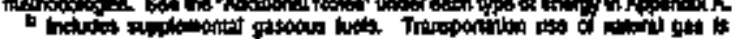

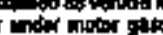

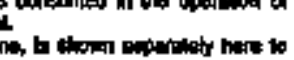

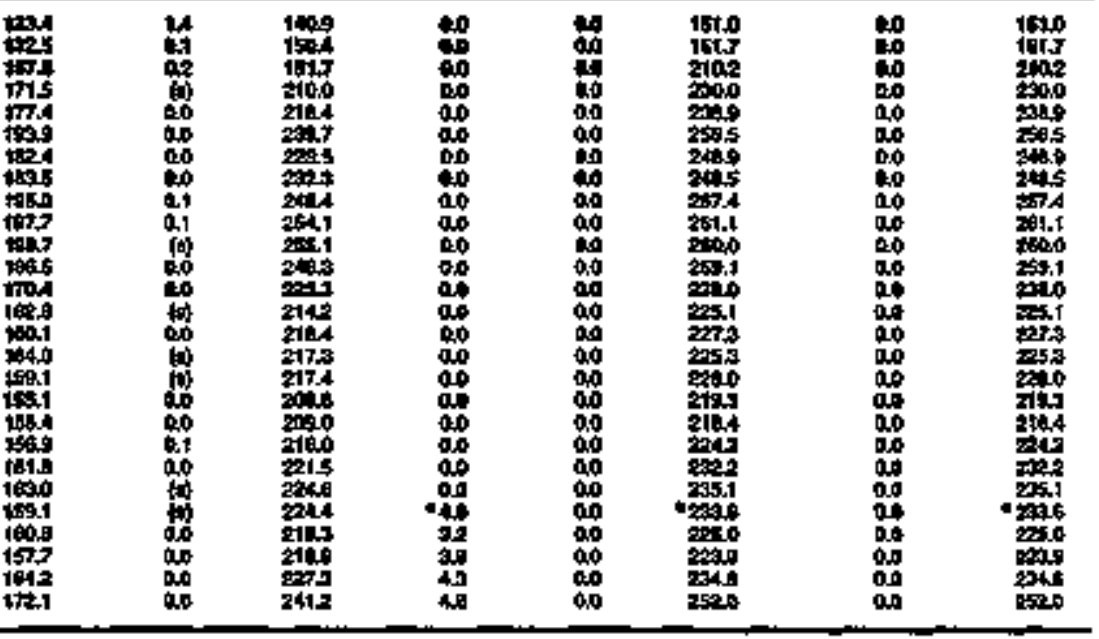

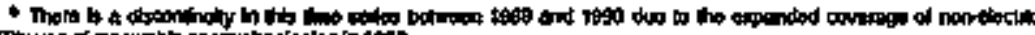

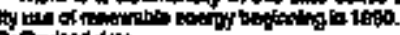


Table 112. Estimates of Energy Input at Electric Uniktieg, 1980, 1965, 1970-1994, 10wa

\begin{tabular}{|c|c|c|c|c|c|c|c|c|c|c|c|c|c|c|}
\hline \multirow[b]{3}{*}{$Y_{\Delta \infty}$} & \multicolumn{3}{|c|}{ con } & \multirow{3}{*}{ 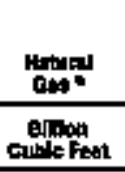 } & \multicolumn{4}{|c|}{ 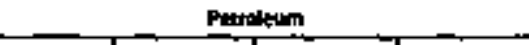 } & \multirow[b]{2}{*}{ 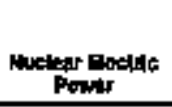 } & \multirow[b]{2}{*}{ 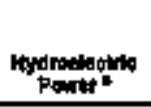 } & \multirow[b]{2}{*}{ Biofola } & \multirow[b]{2}{*}{$\begin{array}{l}\text { Ceophomil } \\
\text { Eningy }\end{array}$} & \multirow[b]{2}{*}{ Ounerl } & \multirow[b]{3}{*}{ 7하버 항 } \\
\hline & 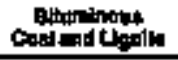 & Anthesele & $\pi+\infty$ & & Hency & 㟧㗪 & Prtrolate & Tot & & & & & & \\
\hline & \multicolumn{2}{|c|}{ Therend Shen Toon } & - & & \multicolumn{4}{|c|}{ Fllow:und anmests } & \multicolumn{5}{|c|}{ 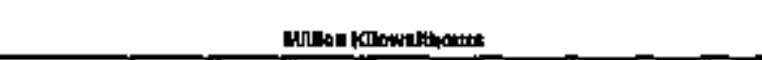 } & \\
\hline 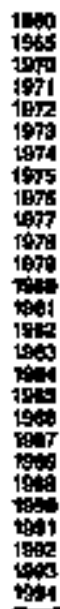 & 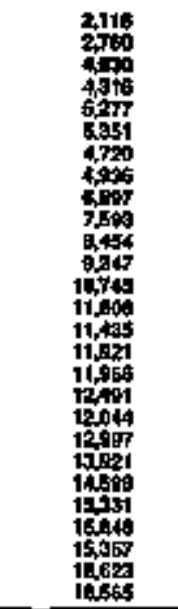 & $\begin{array}{l}0 \\
0 \\
0 \\
0 \\
0 \\
0 \\
0 \\
0 \\
0 \\
0 \\
0 \\
0 \\
0 \\
0 \\
0 \\
0 \\
0 \\
0 \\
0 \\
0 \\
0 \\
0 \\
0 \\
0 \\
0 \\
0 \\
0\end{array}$ & 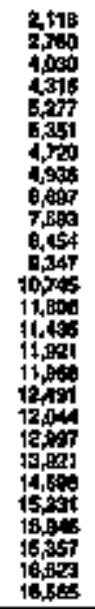 & 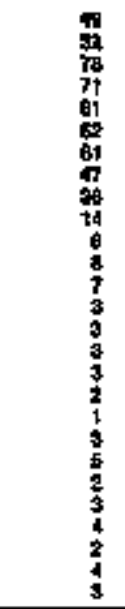 & 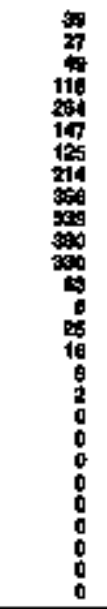 & 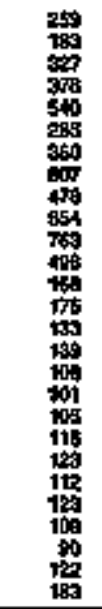 & $\begin{array}{l}0 \\
0 \\
0 \\
0 \\
0 \\
0 \\
0 \\
0 \\
0 \\
00 \\
0 \\
0 \\
0 \\
0 \\
0 \\
0 \\
0 \\
0 \\
0 \\
0 \\
0 \\
0 \\
0\end{array}$ & 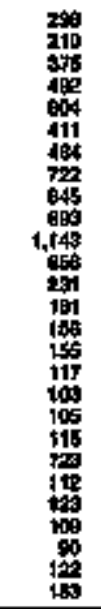 & 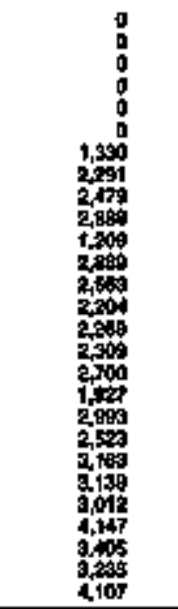 & 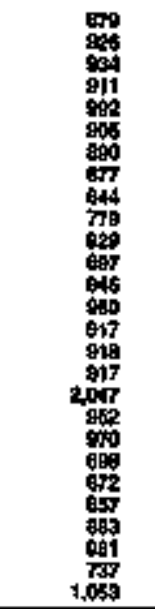 & 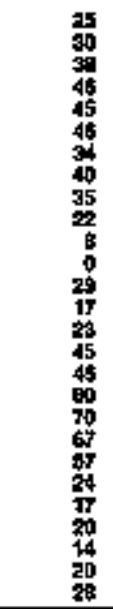 & $\begin{array}{l}0 \\
0 \\
0 \\
0 \\
0 \\
0 \\
0 \\
0 \\
0 \\
0 \\
0 \\
0 \\
0 \\
0 \\
0 \\
0 \\
0 \\
0 \\
0 \\
0 \\
0 \\
0 \\
0 \\
0 \\
0 \\
0 \\
0\end{array}$ & $\begin{array}{l}: \\
: \\
0 \\
0 \\
0 \\
0 \\
0 \\
0 \\
: \\
0 \\
0 \\
0 \\
0 \\
0 \\
0 \\
0 \\
0 \\
0 \\
0 \\
0 \\
0\end{array}$ & 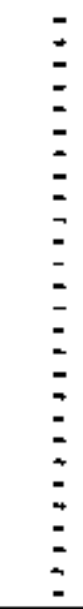 \\
\hline
\end{tabular}

\begin{tabular}{|c|c|}
\hline 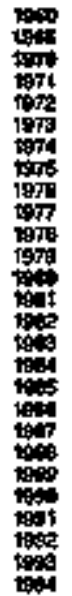 & 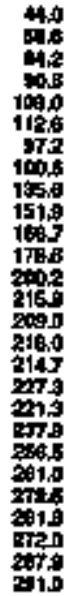 \\
\hline
\end{tabular}

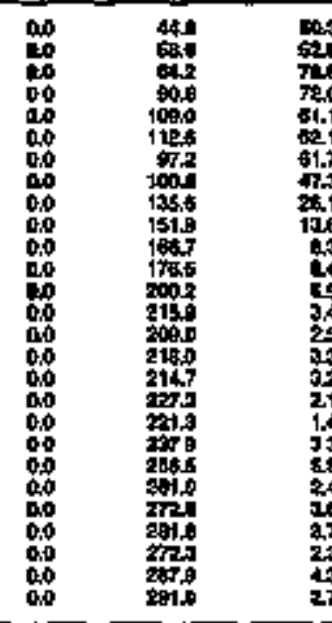

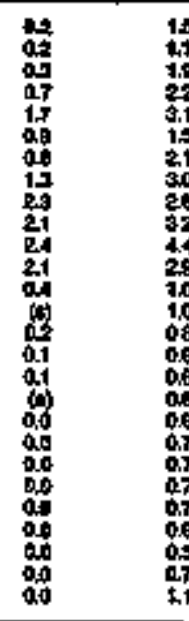

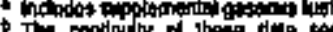

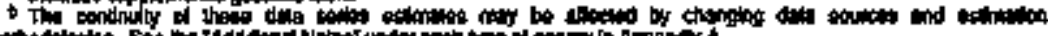

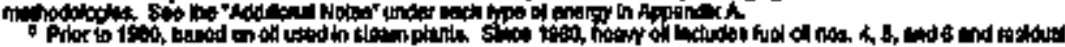

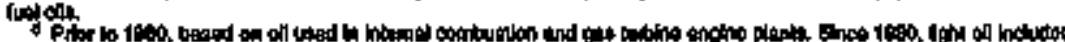

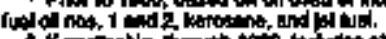

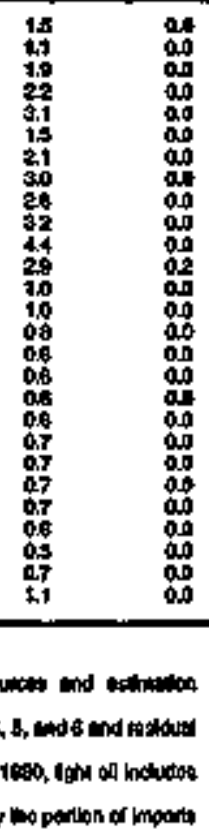

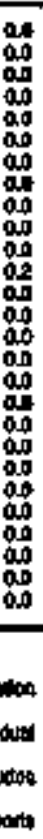

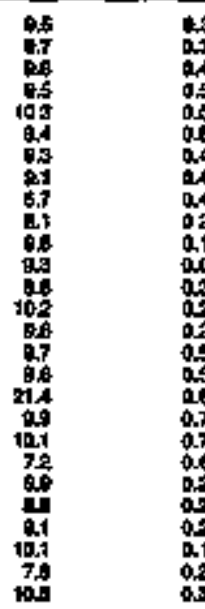

\begin{tabular}{|c|c|c|c|}
\hline 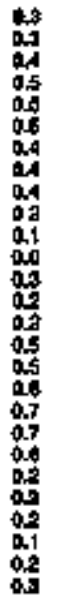 & $\begin{array}{l}00 \\
00 \\
00 \\
00 \\
00 \\
00 \\
00 \\
00 \\
00 \\
000 \\
000 \\
00 \\
00 \\
00 \\
00 \\
00 \\
00 \\
00 \\
00 \\
00 \\
00 \\
00 \\
00 \\
00 \\
00 \\
00 \\
00 \\
00\end{array}$ & 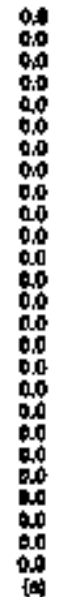 & 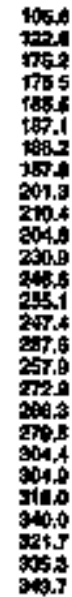 \\
\hline
\end{tabular}

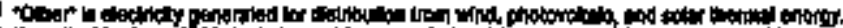

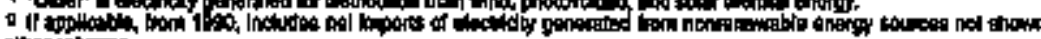
tometem

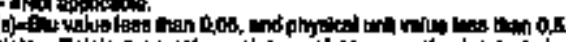

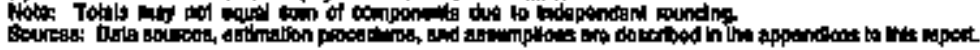


Table 1t3. Enargy Consumplion Estimates by Source, t960, 1865, 1970-1894, Kansas

\begin{tabular}{|c|c|c|c|c|c|c|c|c|c|c|c|c|c|c|c|c|c|c|c|}
\hline \multirow[b]{3}{*}{ Yeur } & \multirow[b]{2}{*}{ Cowe } & \multirow[b]{2}{*}{$\begin{array}{c}\text { Mrtatai } \\
\text { Cuts: }\end{array}$} & \multicolumn{11}{|c|}{ Putroun } & \multirow[b]{2}{*}{ 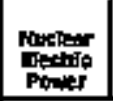 } & \multirow[b]{2}{*}{ 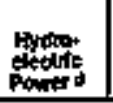 } & \multirow[b]{2}{*}{ 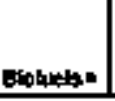 } & \multirow[b]{2}{*}{ ONher as } & \multirow{2}{*}{ 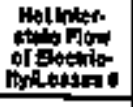 } & \multirow[b]{3}{*}{ Tolnt } \\
\hline & & & Alphentind & Antiofon & Otelfirin: & Fot. & 40 & LFa: & workt & molor & 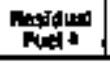 & 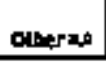 & Toptat & & & & & & \\
\hline & $\begin{array}{l}\text { Then:und } \\
\text { short Tona }\end{array}$ & 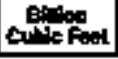 & \multicolumn{11}{|c|}{ 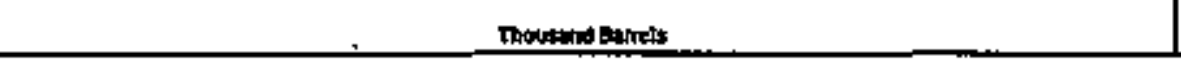 } & \multicolumn{5}{|c|}{ 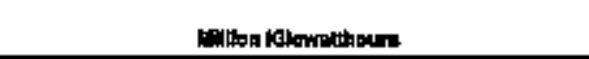 } & \\
\hline 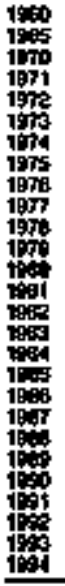 & 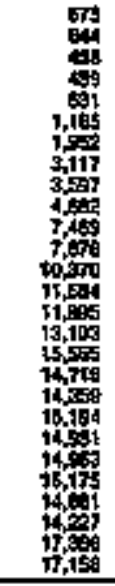 & 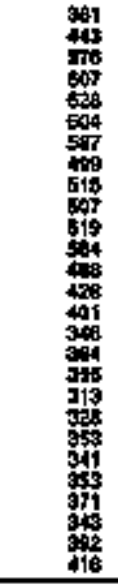 & 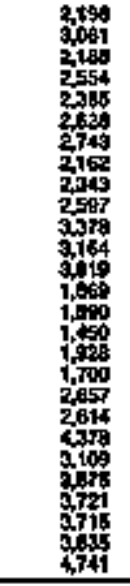 & 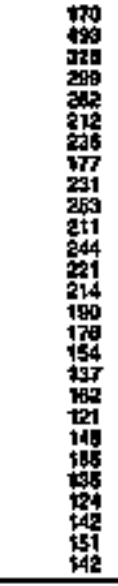 & 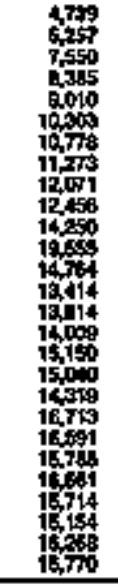 & 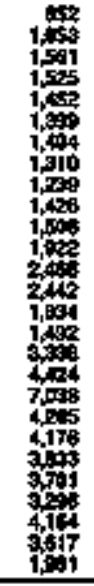 & 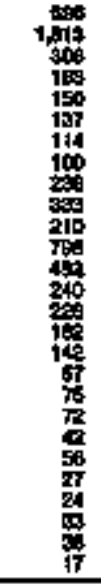 & 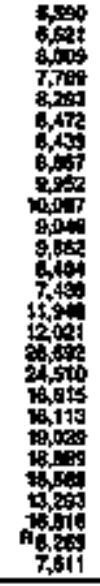 & 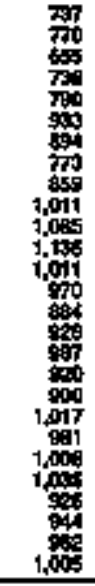 & 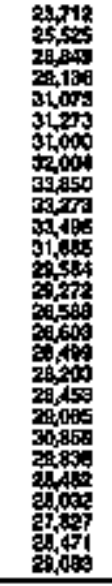 & 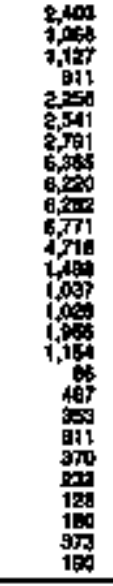 & 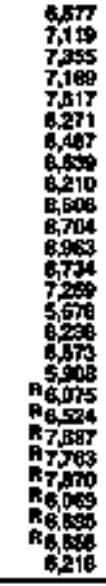 & 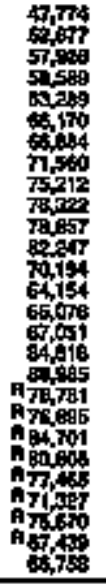 & 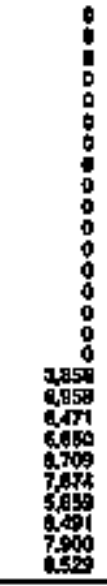 & 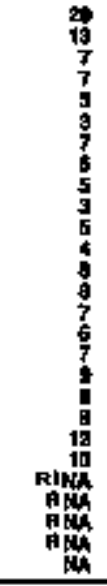 & 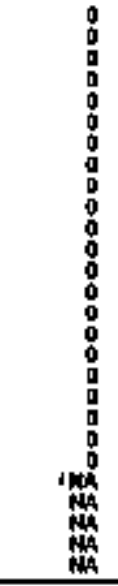 & $\begin{array}{l}0 \\
0 \\
0 \\
0 \\
0 \\
0 \\
0 \\
0 \\
0 \\
0 \\
0 \\
0 \\
0 \\
0 \\
9 \\
0 \\
0 \\
0\end{array}$ & 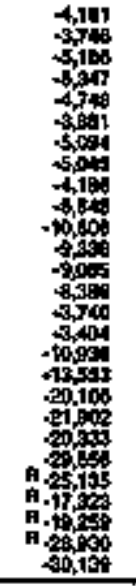 & $\begin{array}{l}z \\
\vdots \\
\vdots \\
\vdots \\
\vdots \\
\vdots \\
\vdots \\
\vdots \\
\vdots \\
\vdots \\
z \\
z \\
z \\
z\end{array}$ \\
\hline \multicolumn{20}{|c|}{ Then } \\
\hline 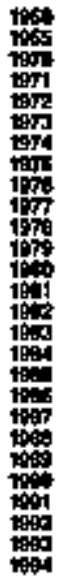 & 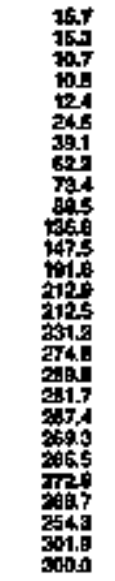 & 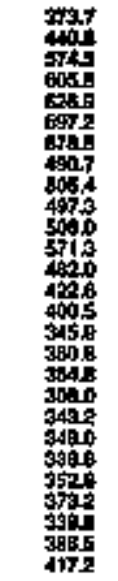 & 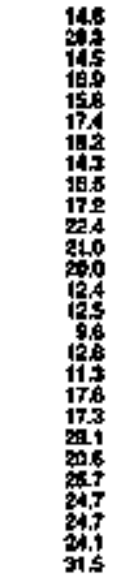 & 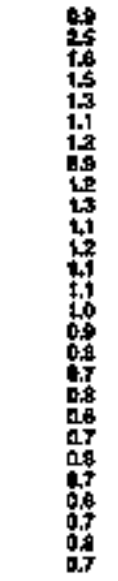 & 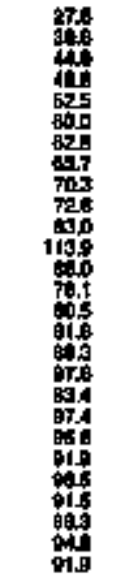 & 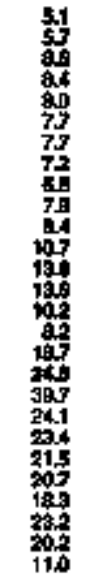 & 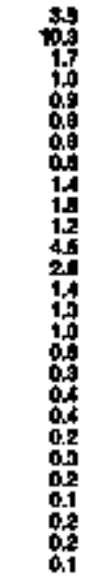 & 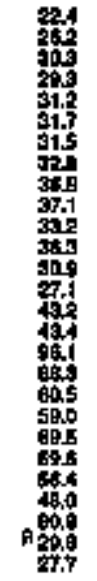 & 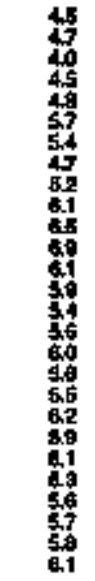 & 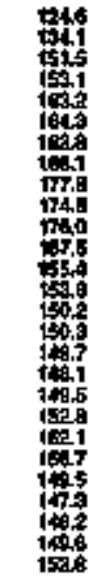 & 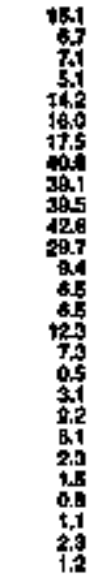 & 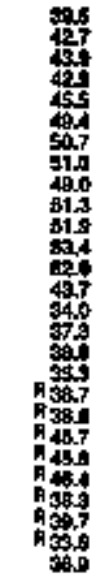 & 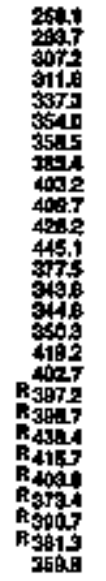 & 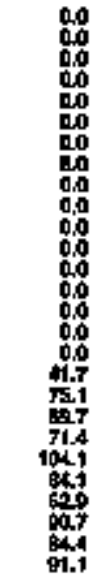 & 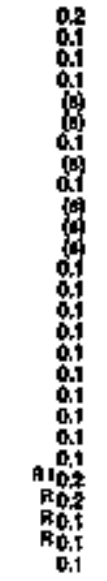 & 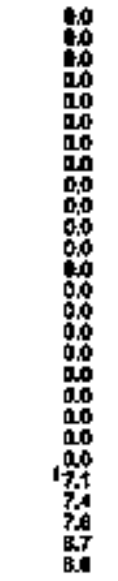 & 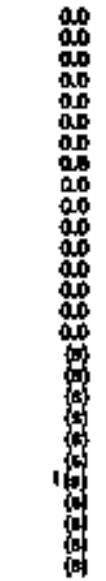 & 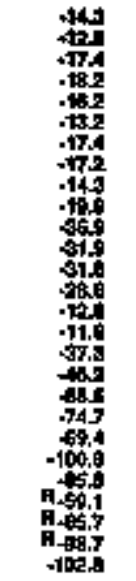 & 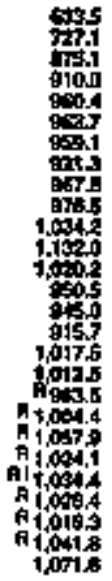 \\
\hline
\end{tabular}

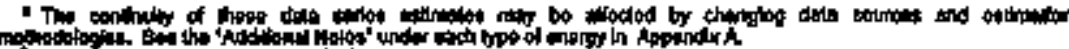

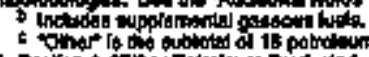

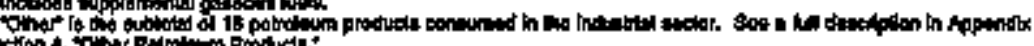

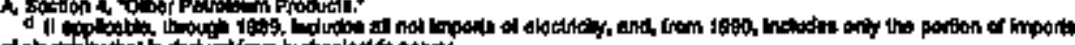

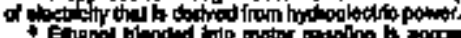

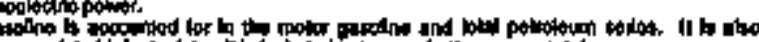

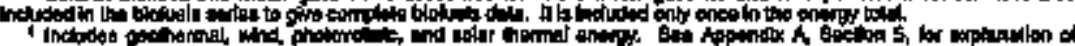

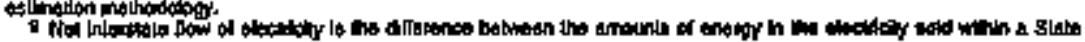

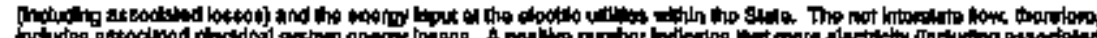

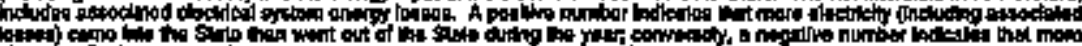

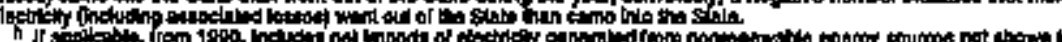

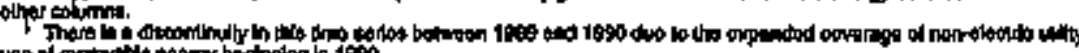

20. 
K Table 114. Festidentlal Energy Consumption Estimatos, 1960, 1965, 1970-1994, Kansas

A

N

\begin{tabular}{|c|c|c|c|c|c|c|c|c|c|c|c|c|c|c|}
\hline & & $\cos$ & & & & & & & & & & & & \\
\hline & 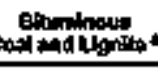 & I & Tratas & 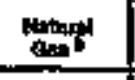 & 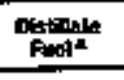 & Knomenes & trat & Toon & Einoterts & soure & 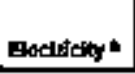 & thex & 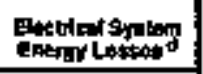 & \\
\hline $\boldsymbol{\omega}$ & & Astantitions & & 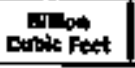 & 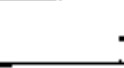 & theneses & & & 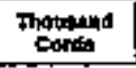 & & MIItument: & 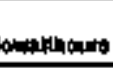 & & Troat \\
\hline 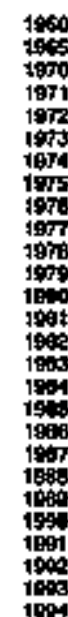 & 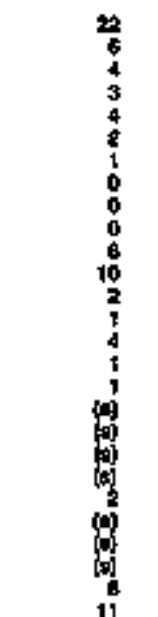 & $\begin{array}{c}: \\
\vdots \\
0 \\
0 \\
0 \\
0 \\
0 \\
\vdots \\
0 \\
\vdots \\
0 \\
\vdots \\
0 \\
0 \\
0 \\
0 \\
0 \\
0 \\
0 \\
0 \\
0\end{array}$ & 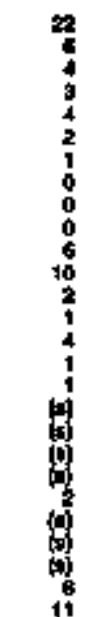 & 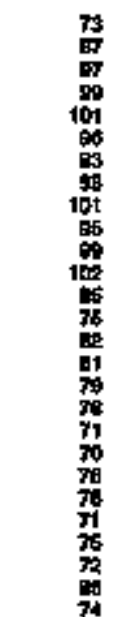 & 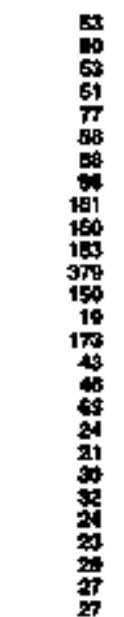 & 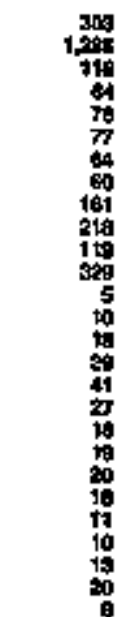 & 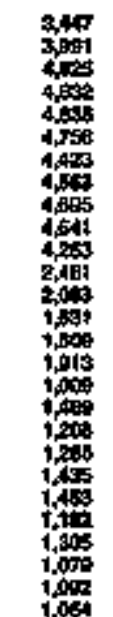 & 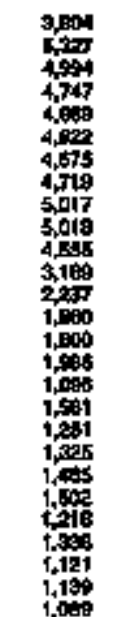 & 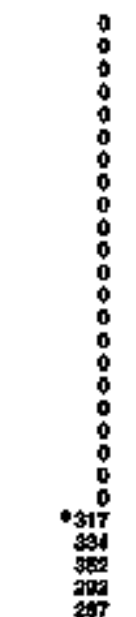 & 象 & 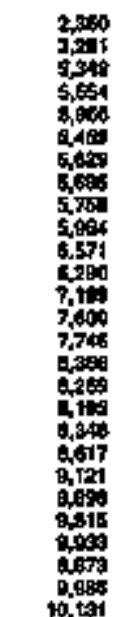 & $\Xi$ & 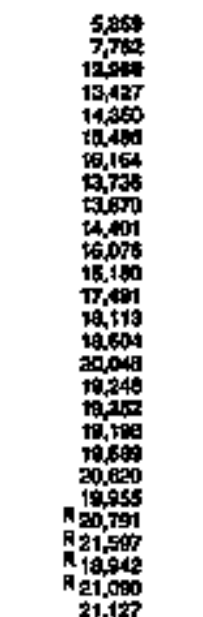 & \\
\hline
\end{tabular}

\begin{tabular}{|c|c|c|c|c|c|}
\hline & 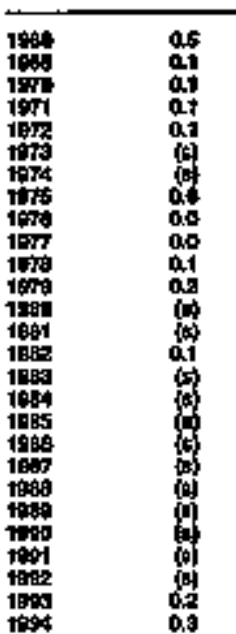 & 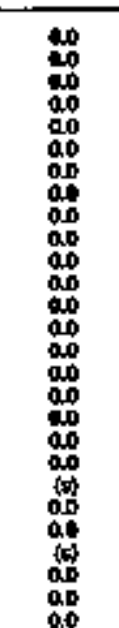 & 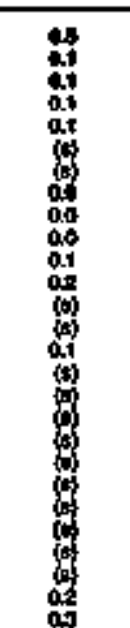 & 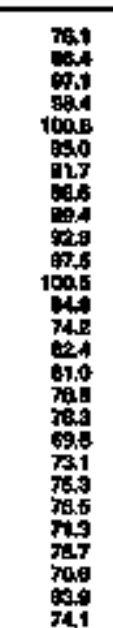 & 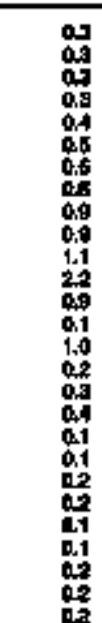 \\
\hline
\end{tabular}

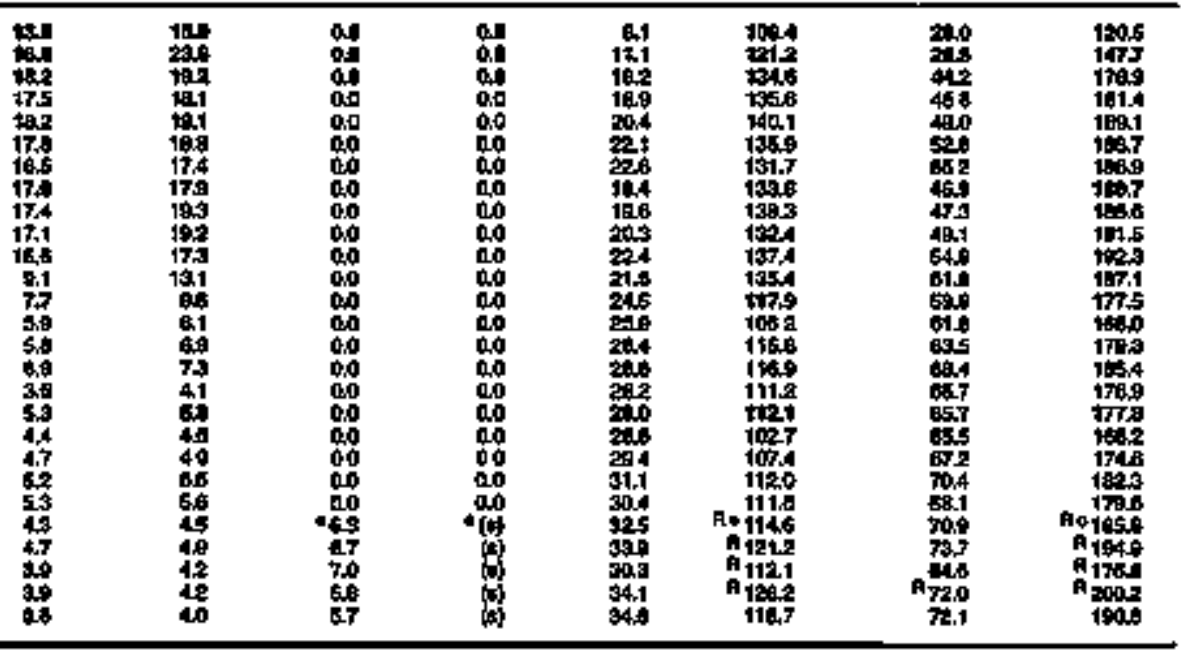

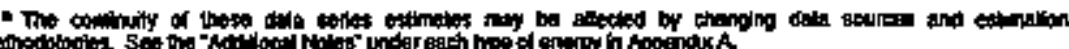

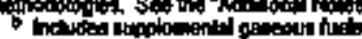

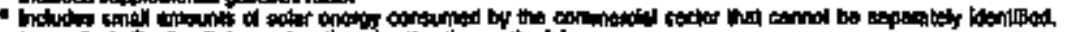

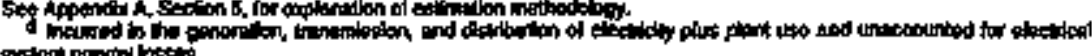

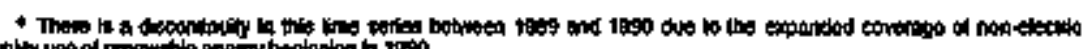

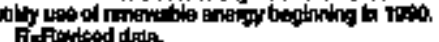

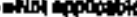

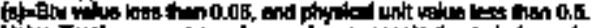

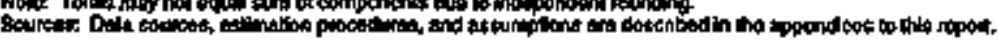


Table 115. Commercial Energy Consumption Estimates, 1950, 1995, 1970-1994, Kansas:

\begin{tabular}{|c|c|c|c|c|c|c|c|c|c|c|c|c|c|c|}
\hline \multirow[b]{3}{*}{ her } & \multicolumn{3}{|c|}{ con } & \multirow{3}{*}{ 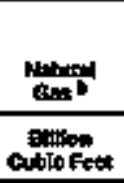 } & \multicolumn{6}{|c|}{ Potrofivin } & \multirow[b]{2}{*}{ Extrithy } & \multirow[b]{2}{*}{ Enotigy } & \multirow[b]{2}{*}{ 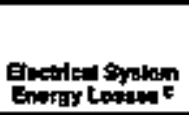 } & \multirow[b]{3}{*}{ Totud } \\
\hline & 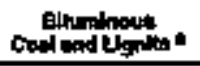 & Anthracis: & Tototion & & 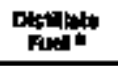 & Kemosina" & 2FG* & coloex & Fidtons & Total & & & & \\
\hline & \multicolumn{2}{|c|}{ Droesced Ghort Ton: } & & & \multicolumn{6}{|c|}{ 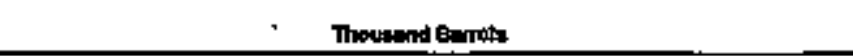 } & \multicolumn{3}{|c|}{ 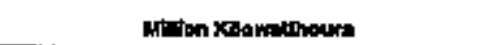 } & \\
\hline 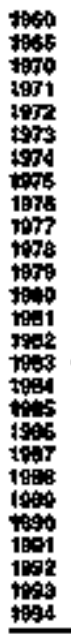 & 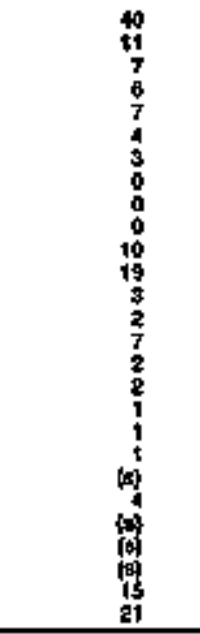 & $\begin{array}{l}0 \\
0 \\
0 \\
0 \\
0 \\
0 \\
0 \\
0 \\
0 \\
0 \\
0 \\
0 \\
0 \\
0 \\
0 \\
0 \\
0 \\
0 \\
0 \\
0 \\
0 \\
0 \\
0 \\
0 \\
0 \\
0 \\
\end{array}$ & 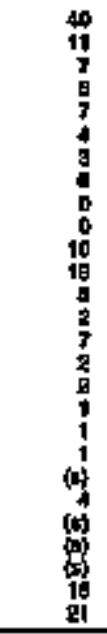 & 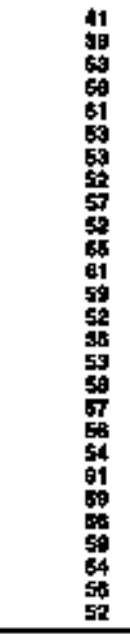 & 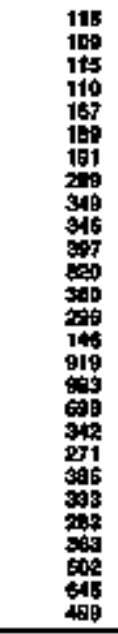 & 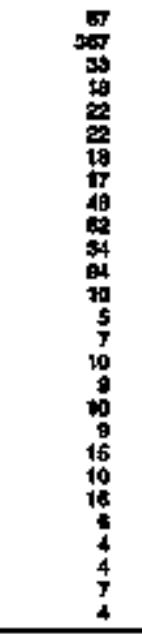 & 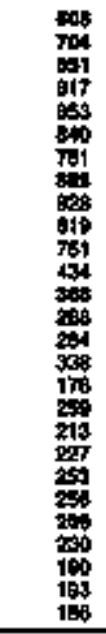 & 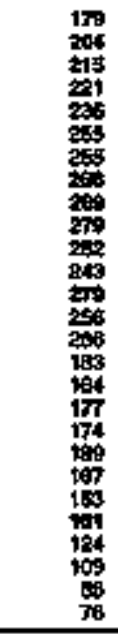 & 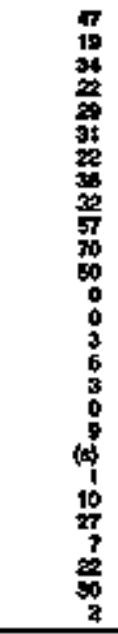 & 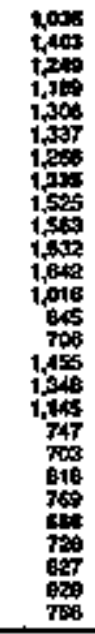 & 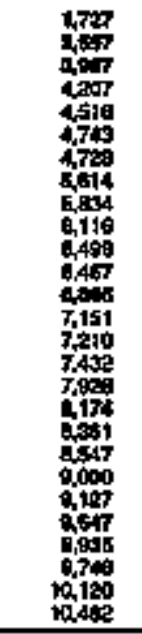 & $\begin{array}{l}= \\
= \\
z \\
= \\
= \\
= \\
= \\
= \\
z \\
= \\
z \\
z \\
z \\
=\end{array}$ & 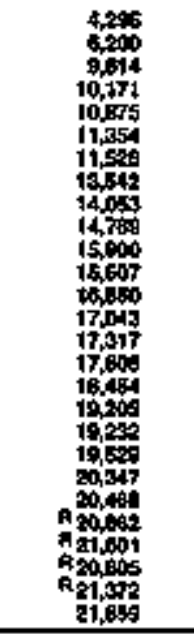 & 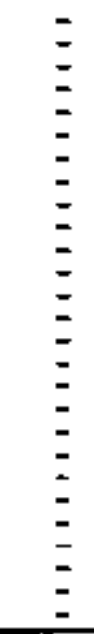 \\
\hline \multicolumn{15}{|c|}{ 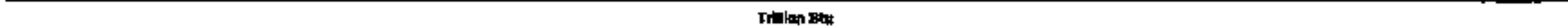 } \\
\hline 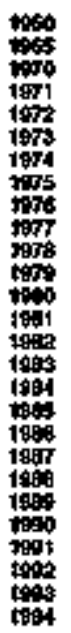 & 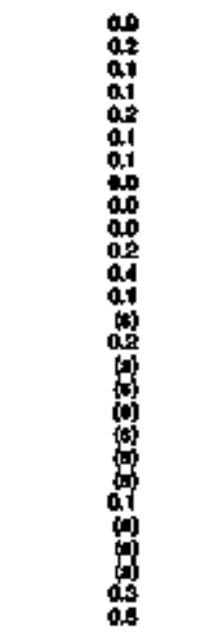 & 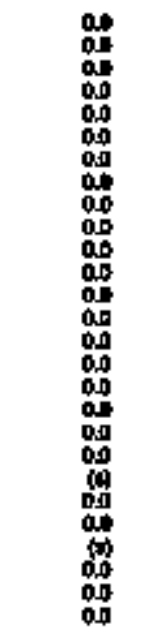 & 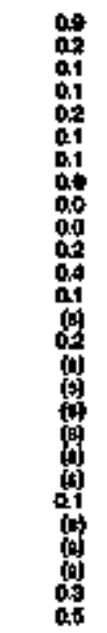 & 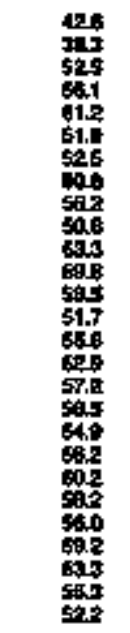 & 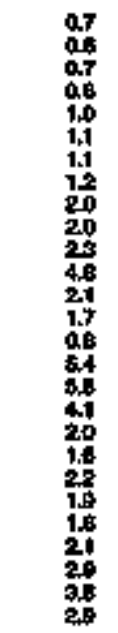 & 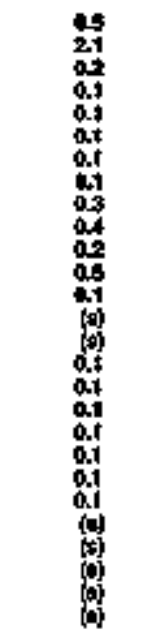 & 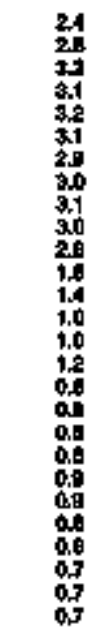 & 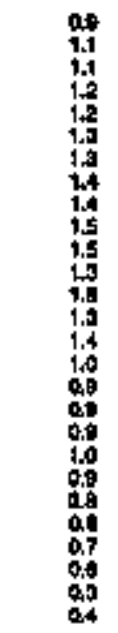 & 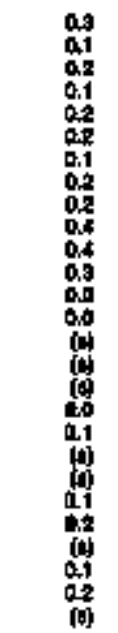 & 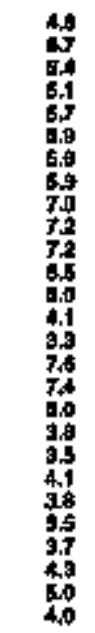 & 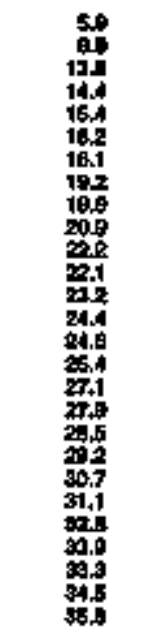 & 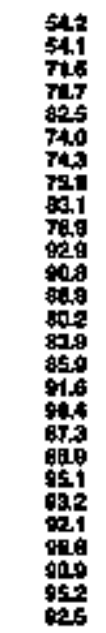 & 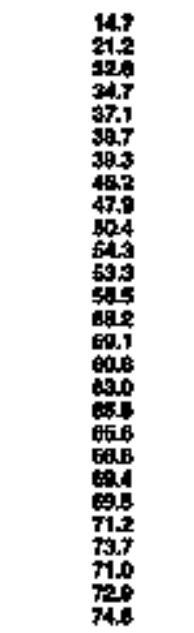 & 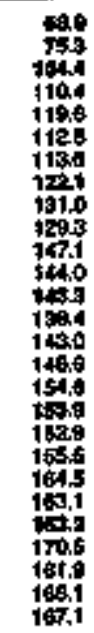 \\
\hline
\end{tabular}


Table 116. Industrial Energy Consumpllon Estimates, 1850, 1965, 1970-1994, Kansas

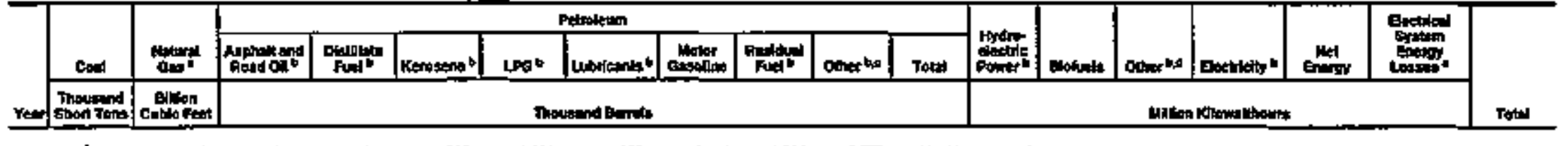

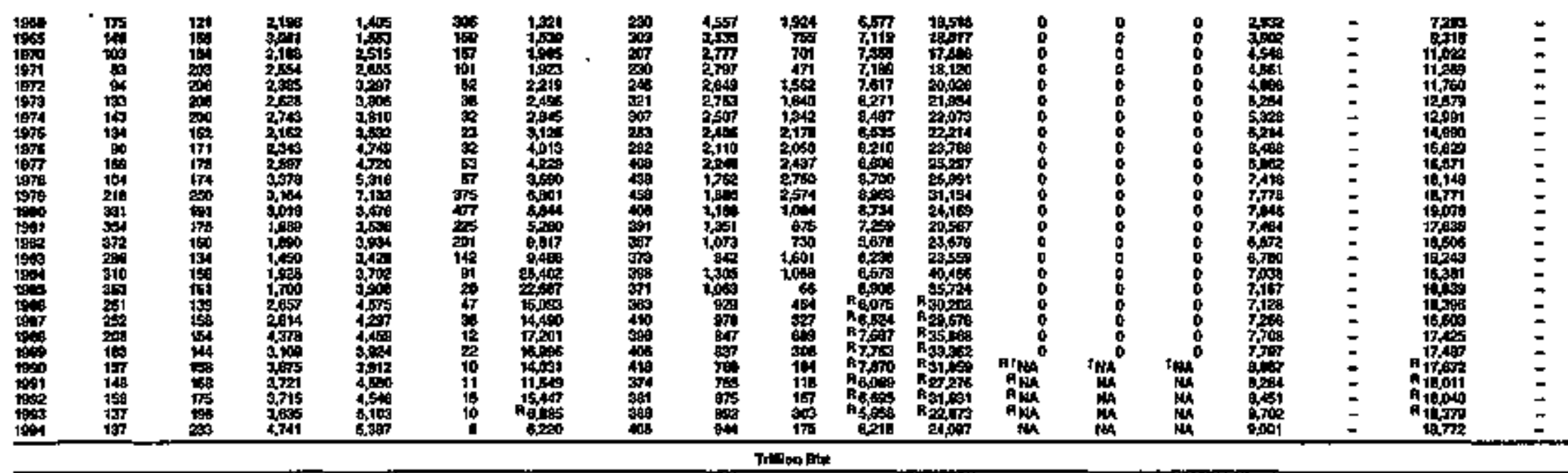

\begin{tabular}{|c|c|c|c|c|c|c|c|c|}
\hline 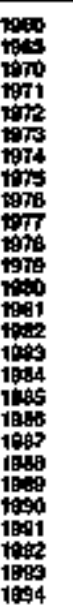 & 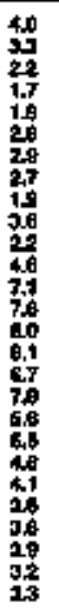 & 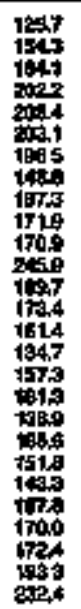 & 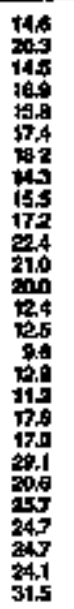 & 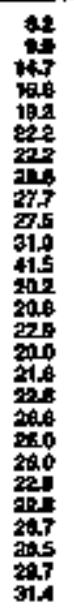 & 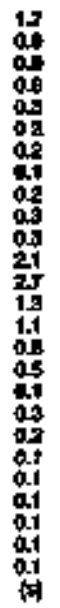 & 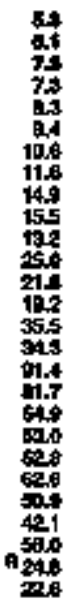 & 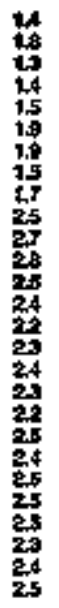 & 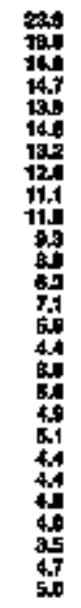 \\
\hline
\end{tabular}

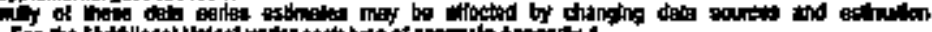

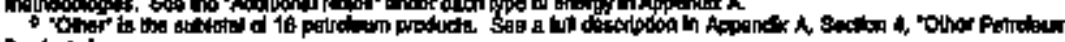

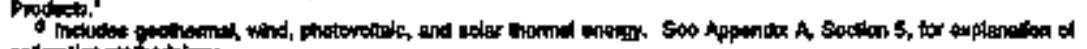

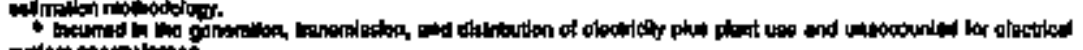

\begin{tabular}{|c|c|c|c|}
\hline 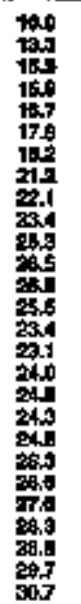 & 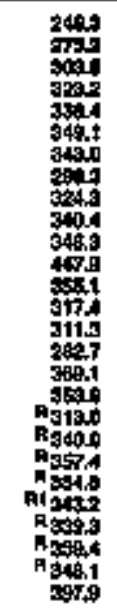 & 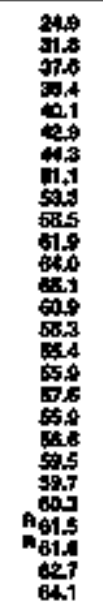 & 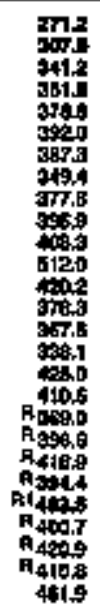 \\
\hline
\end{tabular}

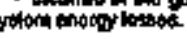


Tabid 117. Transportation Enargy Consumplion Estimates, 1960, 1965, 1970-1994, Kansas

\begin{tabular}{|c|c|c|c|c|c|c|c|c|c|c|c|c|c|c|c|}
\hline \multirow[b]{3}{*}{ Ven } & \multirow[b]{2}{*}{ " } & \multirow[b]{2}{*}{ Thath } & \multicolumn{8}{|c|}{ Prtolan } & \multirow{3}{*}{ 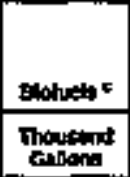 } & \multirow[b]{2}{*}{ 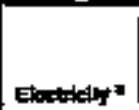 } & \multirow[b]{2}{*}{ Exty } & \multirow[b]{2}{*}{ 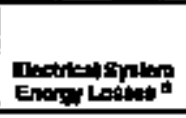 } & \multirow[b]{3}{*}{ Total } \\
\hline & & & andesos. & ming & then & LPG: & Lubromis: & capoinor & 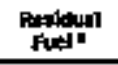 & Totil & & & & & \\
\hline & $\begin{array}{l}\text { Thounens } \\
\text { entit Tous }\end{array}$ & 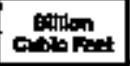 & \multicolumn{8}{|c|}{ 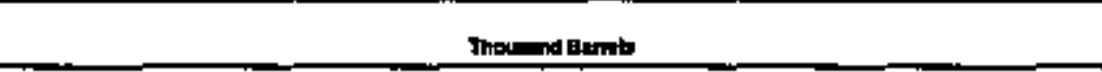 } & & \multicolumn{3}{|c|}{ 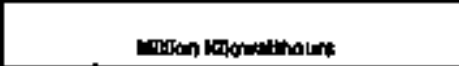 } & \\
\hline 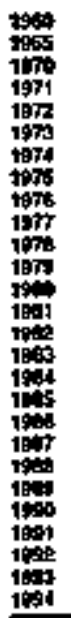 & $\begin{array}{l}0 \\
0 \\
0 \\
0 \\
0 \\
0 \\
0 \\
0 \\
0 \\
0 \\
0 \\
0 \\
0 \\
0 \\
0 \\
0 \\
0 \\
0 \\
0 \\
0 \\
0 \\
0 \\
0\end{array}$ & 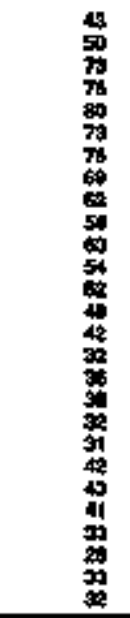 & 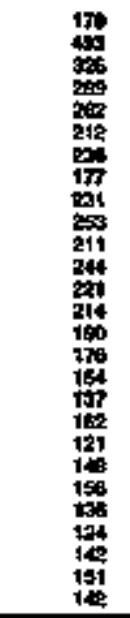 & 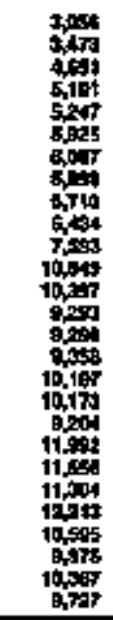 & 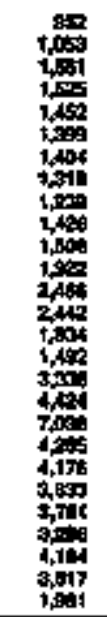 & 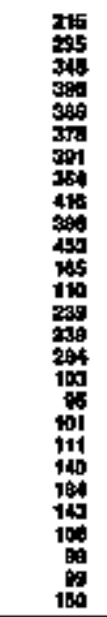 & 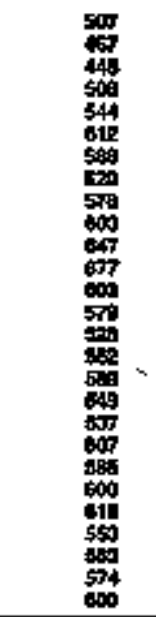 & 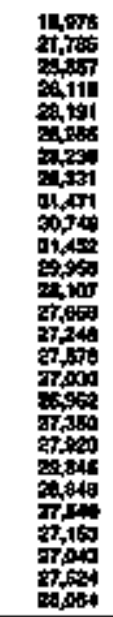 & 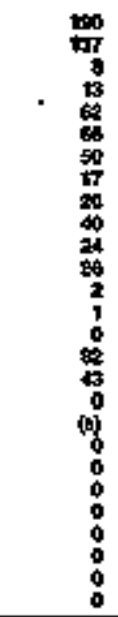 & 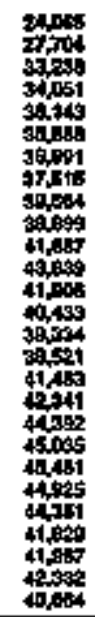 & 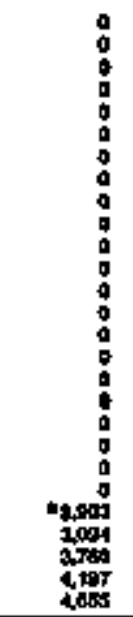 & $\begin{array}{l}0 \\
0 \\
0 \\
0 \\
0 \\
0 \\
0 \\
0 \\
0 \\
0 \\
0 \\
0 \\
0 \\
0 \\
0 \\
0 \\
0 \\
0 \\
0 \\
0 \\
0 \\
0 \\
0 \\
0 \\
0 \\
0 \\
0 \\
0 \\
0\end{array}$ & $\begin{array}{l}= \\
= \\
= \\
= \\
= \\
= \\
= \\
= \\
= \\
= \\
= \\
= \\
= \\
=\end{array}$ & $\begin{array}{l}0 \\
0 \\
0 \\
0 \\
0 \\
0 \\
0 \\
0 \\
0 \\
0 \\
0 \\
0 \\
0 \\
0 \\
0 \\
0 \\
0 \\
0 \\
0 \\
0 \\
0 \\
0 \\
0 \\
0 \\
0 \\
0\end{array}$ & $\begin{array}{l}= \\
= \\
= \\
= \\
= \\
= \\
= \\
= \\
= \\
= \\
= \\
= \\
= \\
= \\
=\end{array}$ \\
\hline \multicolumn{16}{|c|}{ Vhimentu } \\
\hline 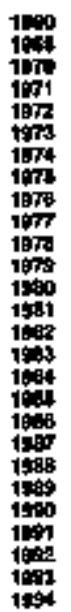 & $\begin{array}{l}01 \\
0 \\
00 \\
0 \\
0 \\
00 \\
00 \\
0 \\
00 \\
00 \\
00 \\
00 \\
00 \\
00 \\
00 \\
00 \\
00 \\
00 \\
00 \\
00 \\
00 \\
00 \\
00 \\
00 \\
00 \\
00\end{array}$ & 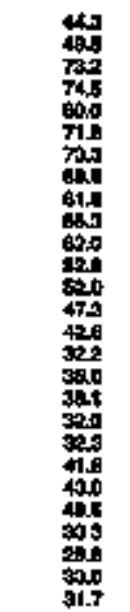 & 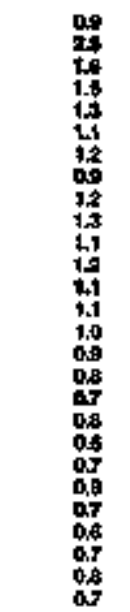 & 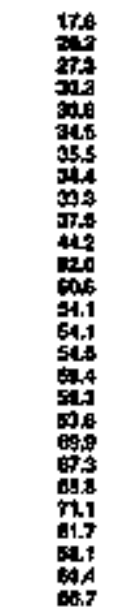 & 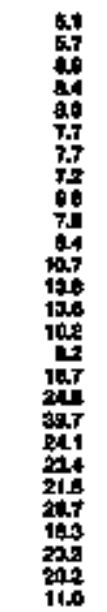 & 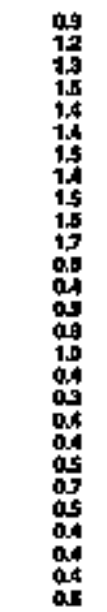 & 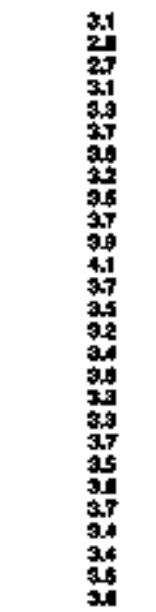 & 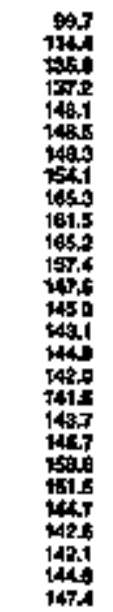 & 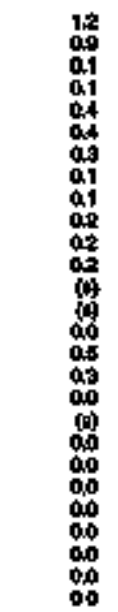 & 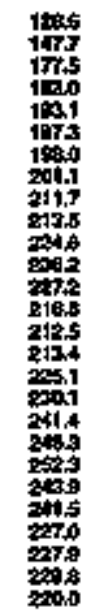 & 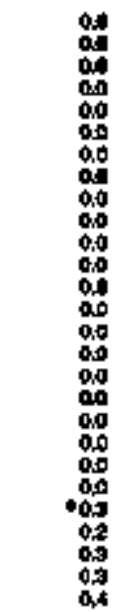 & 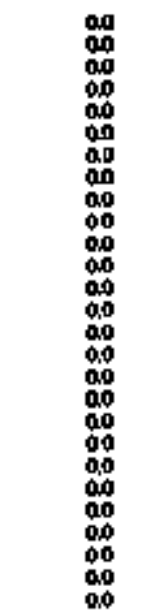 & 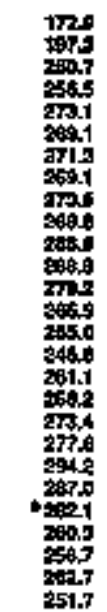 & 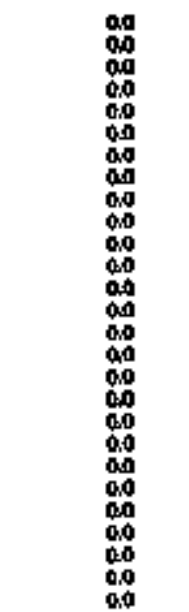 & 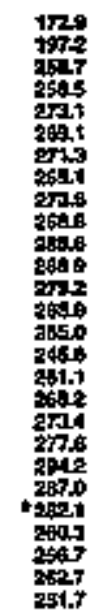 \\
\hline
\end{tabular}


Tabke 1fg. Estimates of Energy Input at Electric Utiltes, 1960, 1965, 1970-1994, Kansas

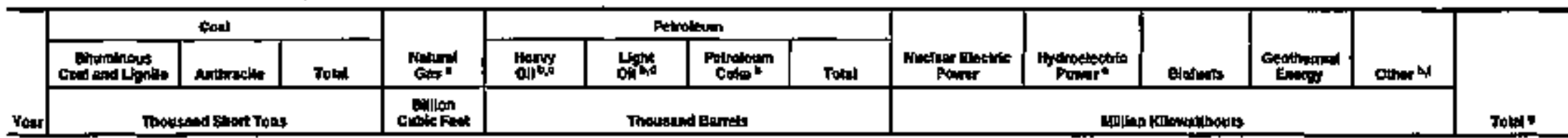

\begin{tabular}{|c|c|c|c|c|c|}
\hline 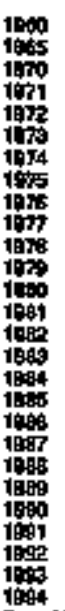 & 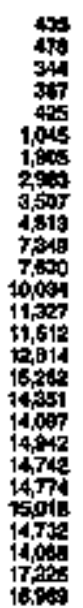 & $\begin{array}{l}0 \\
0 \\
0 \\
0 \\
0 \\
0 \\
0 \\
0 \\
0 \\
0 \\
0 \\
0 \\
0 \\
0 \\
0 \\
0 \\
0 \\
0 \\
0 \\
0 \\
0 \\
0 \\
0 \\
0 \\
0 \\
0\end{array}$ & 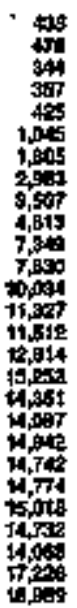 & 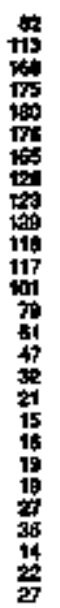 & 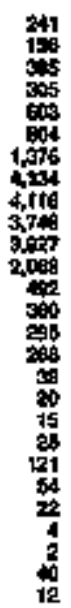 \\
\hline
\end{tabular}

\section{0} Toming

\begin{tabular}{|c|c|c|c|c|c|c|c|c|c|c|c|c|c|c|}
\hline & & & & & & & & & & & & & & \\
\hline 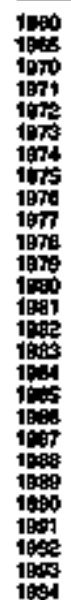 & 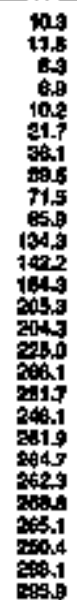 & $\begin{array}{l}00 \\
00 \\
00 \\
00 \\
00 \\
00 \\
00 \\
00 \\
00 \\
00 \\
00 \\
00 \\
00 \\
00 \\
00 \\
00 \\
00 \\
00 \\
00 \\
00 \\
00 \\
00 \\
00 \\
00 \\
00 \\
00 \\
00\end{array}$ & 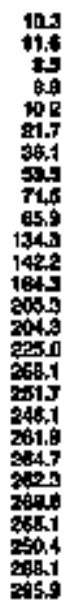 & 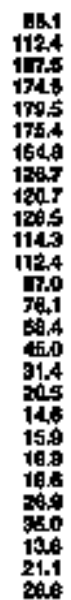 & 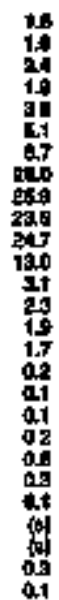 & 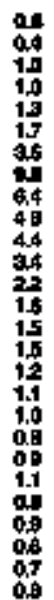 & 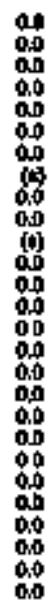 & 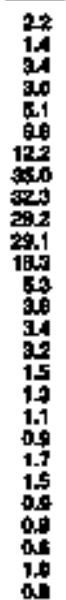 & 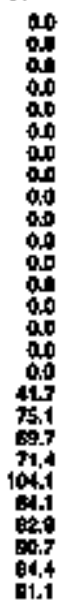 & 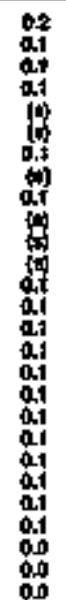 & 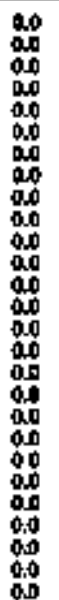 & 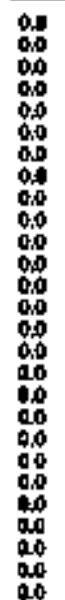 & 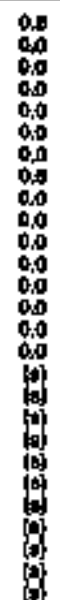 & 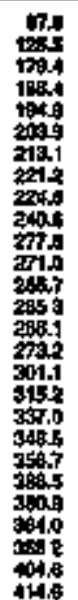 \\
\hline
\end{tabular}

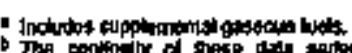

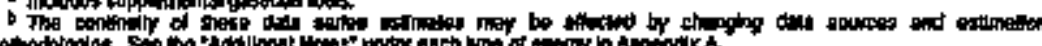

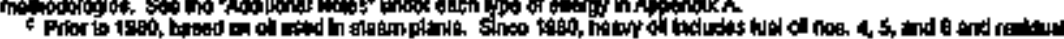

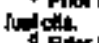

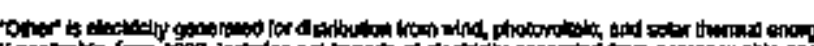

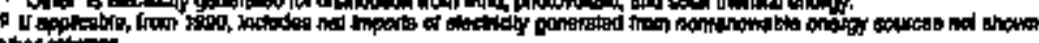

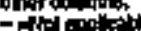

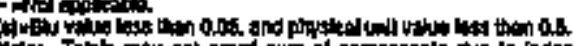

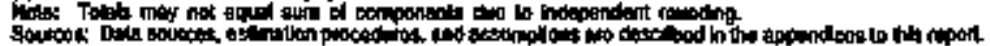

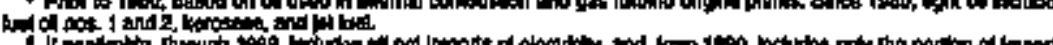

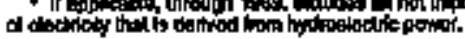


Tabls 119. Enargy Cansemplion Estimates by \$ource, 1960, 1965, 1970-1994, Kantubly

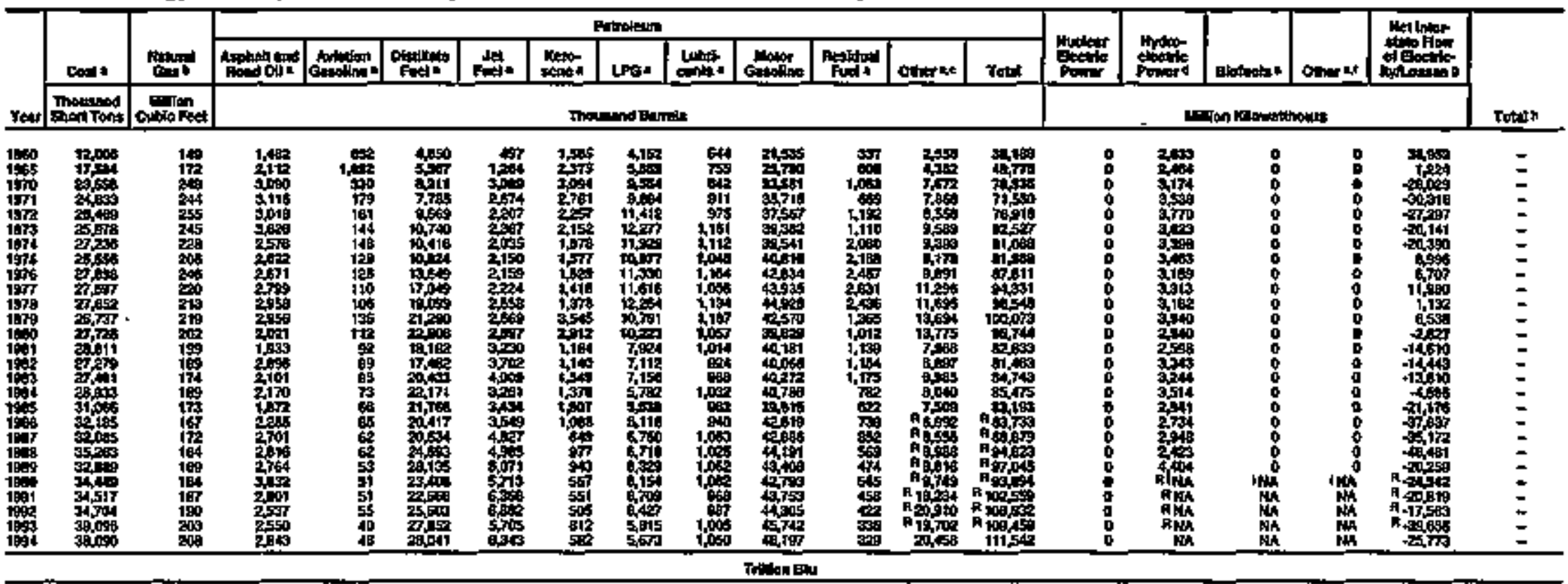

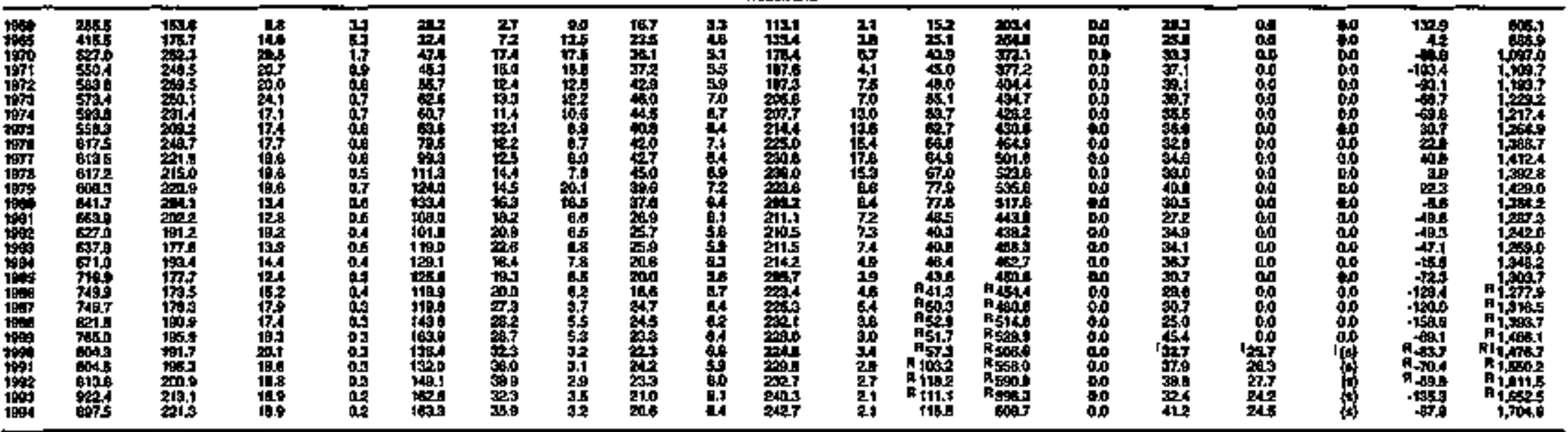

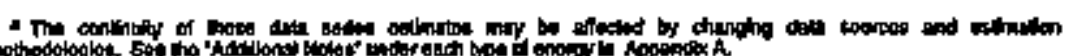

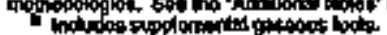

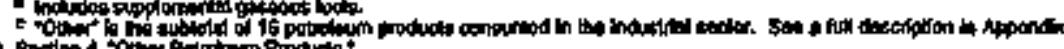

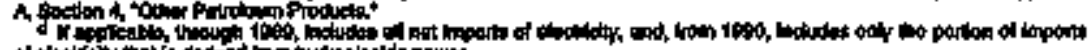

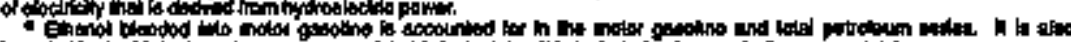

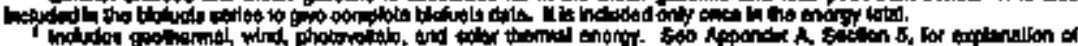

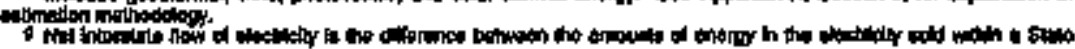

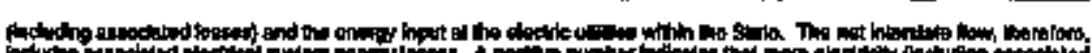

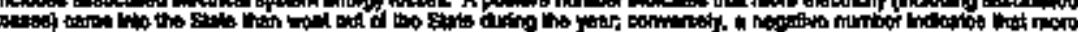

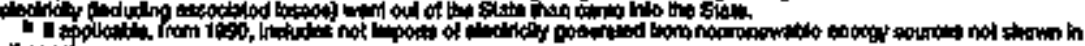

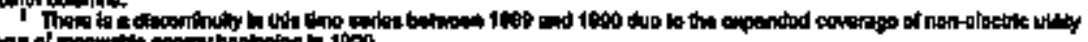

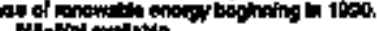

-

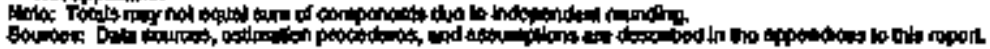


Tabbe 120. Fesidential Energy Consumptlon Estimates, 1960, 1965, 1970-1994, Kentucky

\begin{tabular}{|c|c|c|c|c|c|c|c|c|c|c|c|c|c|c|}
\hline \multirow[b]{3}{*}{ Yost } & \multicolumn{3}{|c|}{$\cos 1$} & \multirow{3}{*}{ 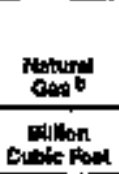 } & \multicolumn{4}{|c|}{ Pabrotoun } & \multirow{3}{*}{ 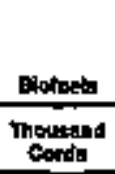 } & & \multirow[b]{2}{*}{ Euetritein 2} & \multirow[b]{2}{*}{ Net } & \multirow{3}{*}{ 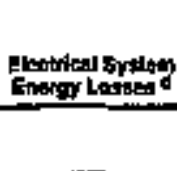 } & \multirow[b]{3}{*}{ Tats } \\
\hline & 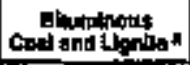 & Anthrastin 1 & Totil & & $\begin{array}{l}\text { Ointiling } \\
\text { Funt: }\end{array}$ & Kerosino" & Los: & Totw & & Setor: & & & & \\
\hline & \multicolumn{3}{|c|}{ Thoupand Shert Jons } & & \multicolumn{4}{|c|}{ 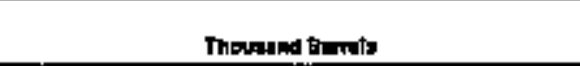 } & & \multicolumn{3}{|c|}{ Henlen Krowathoun } & & \\
\hline 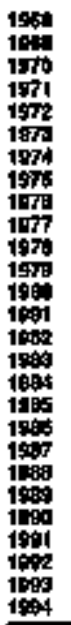 & 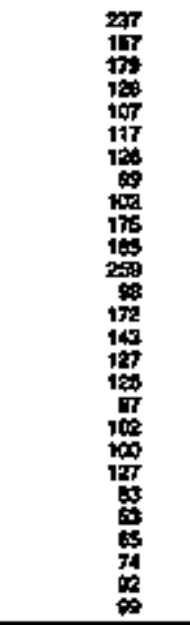 & 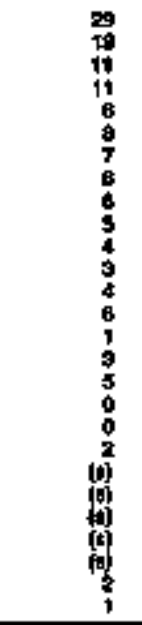 & 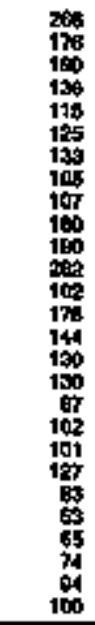 & 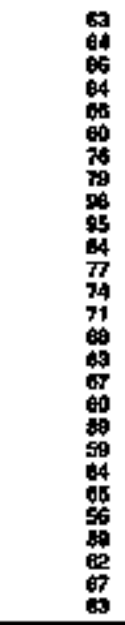 & 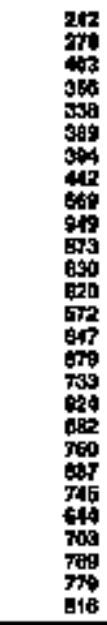 & 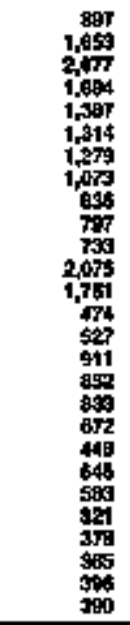 & 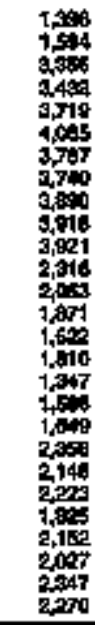 & 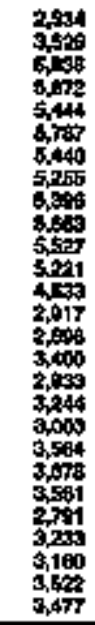 & 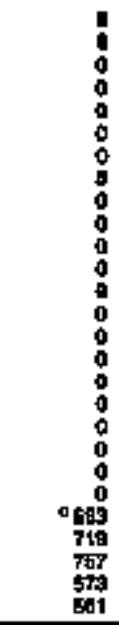 & $\begin{array}{l}0 \\
0 \\
0 \\
0 \\
0 \\
0 \\
0 \\
0 \\
0 \\
0 \\
0 \\
0 \\
0 \\
0 \\
0 \\
0 \\
0 \\
0 \\
1 \\
1 \\
\mathbf{1} \\
\mathbf{1}\end{array}$ & 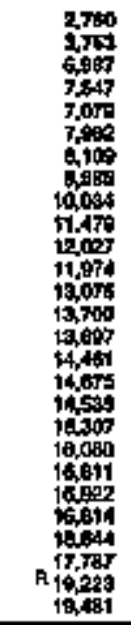 & $\begin{array}{l}= \\
= \\
= \\
= \\
= \\
= \\
= \\
= \\
= \\
= \\
= \\
= \\
= \\
= \\
= \\
=\end{array}$ & 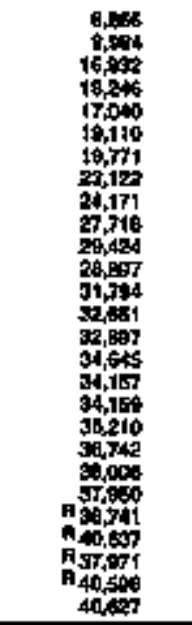 & $\begin{array}{l}= \\
= \\
z \\
= \\
z \\
= \\
= \\
= \\
= \\
= \\
z \\
= \\
= \\
z \\
z\end{array}$ \\
\hline \multicolumn{15}{|c|}{ Trian en } \\
\hline 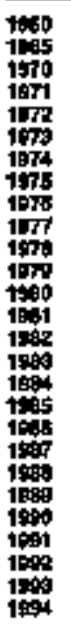 & 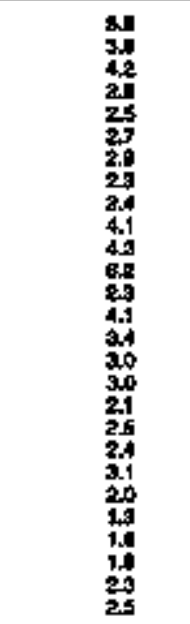 & 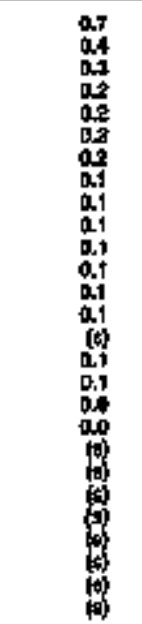 & 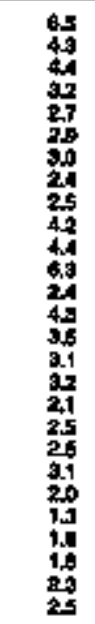 & 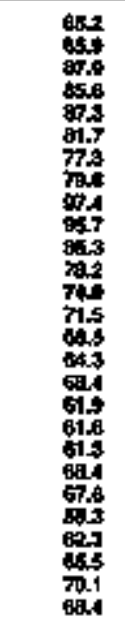 & 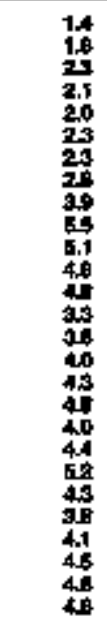 & 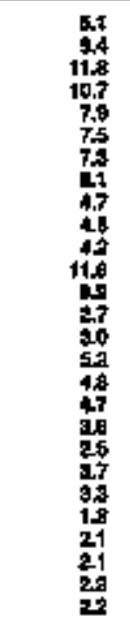 & 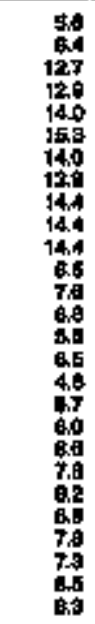 & 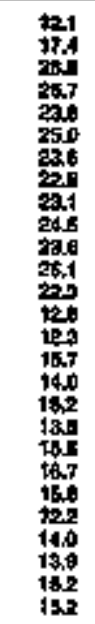 & 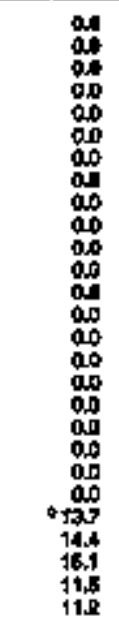 & 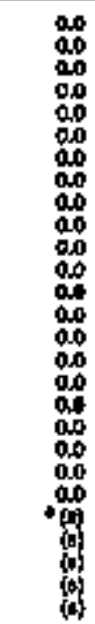 & 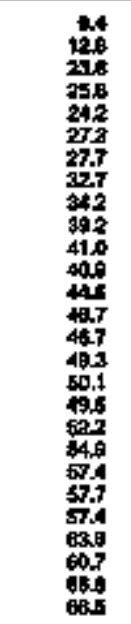 & 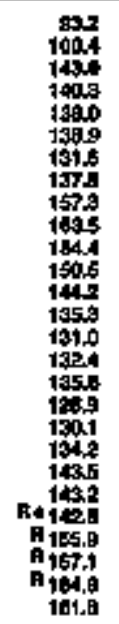 & 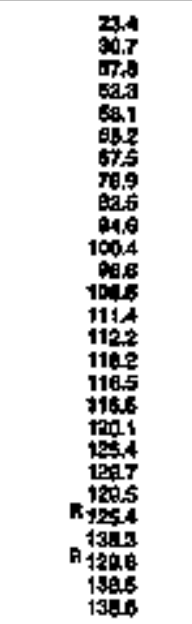 & 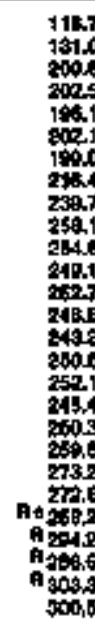 \\
\hline
\end{tabular}

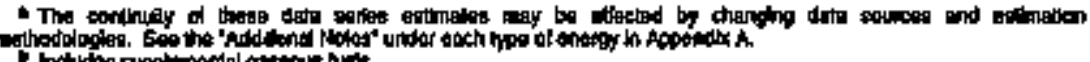

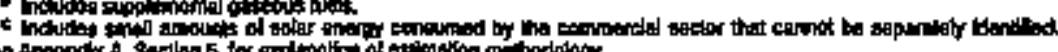

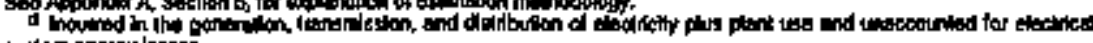

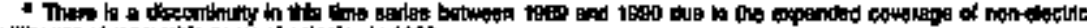

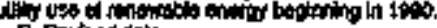

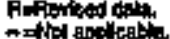

(0)

tytion onongy losed.

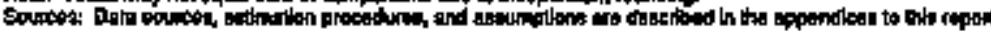


Tabto 121. Corramercial Energy Consumption Estimates, 1960, 1965, 1970-1994, Kentuoky

\begin{tabular}{|c|c|c|c|c|c|c|c|c|c|c|c|c|c|c|}
\hline \multirow[b]{3}{*}{ Yound } & \multicolumn{3}{|c|}{ cones } & \multirow[b]{2}{*}{ Puheret } & \multicolumn{6}{|c|}{ Provolown } & \multirow[b]{2}{*}{ Emortath" } & \multirow[b]{2}{*}{ t } & \multirow[b]{2}{*}{ 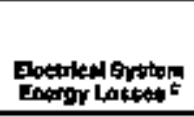 } & \multirow[b]{3}{*}{ Towa d } \\
\hline & 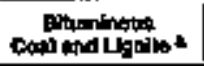 & Anoratede" & Thol & & 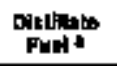 & Kerosens: & LPA: & $\begin{array}{l}\text { Metor } \\
\text { Gaspollap }\end{array}$ & mostonter & Toted & & & & \\
\hline & \multicolumn{3}{|c|}{ Thousend Shant Tons } & 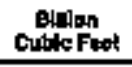 & \multicolumn{6}{|c|}{ 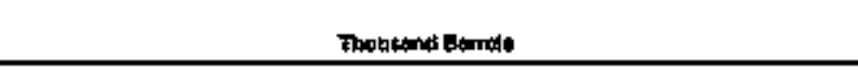 } & \multicolumn{3}{|c|}{ WHon Xlownithowr } & \\
\hline 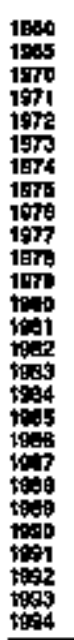 & 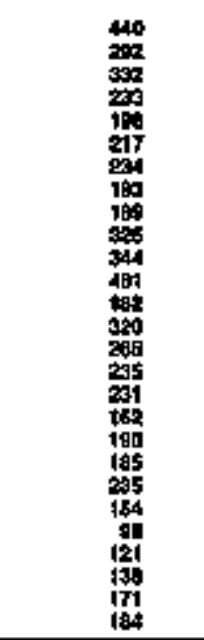 & 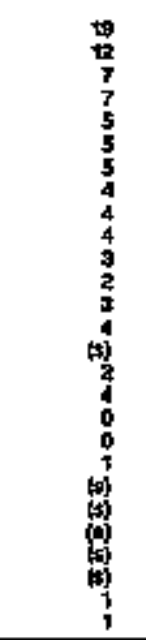 & 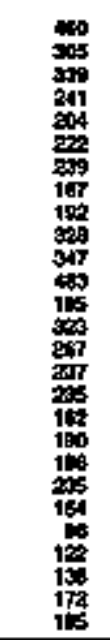 & 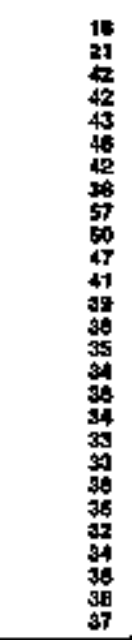 & 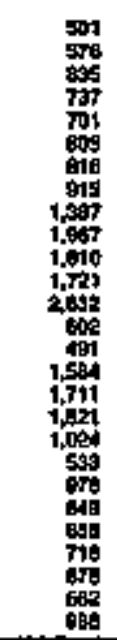 & 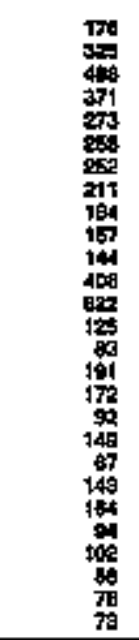 & 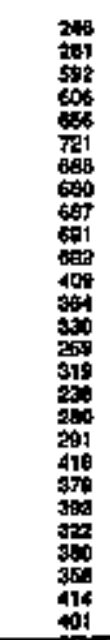 & 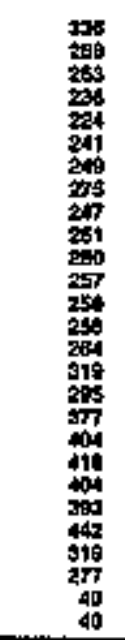 & 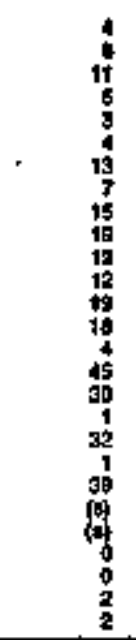 & 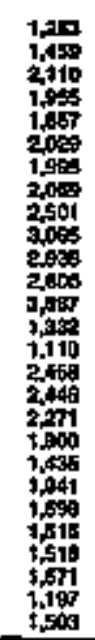 & 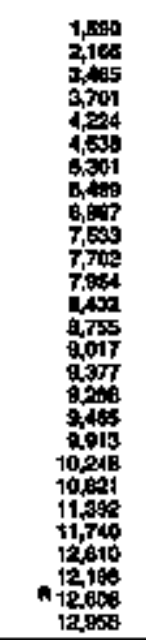 & $\begin{array}{l}\vdots \\
\vdots \\
\vdots \\
\vdots \\
\vdots \\
\vdots \\
\vdots \\
\vdots \\
\vdots \\
\vdots \\
\vdots \\
\vdots\end{array}$ & 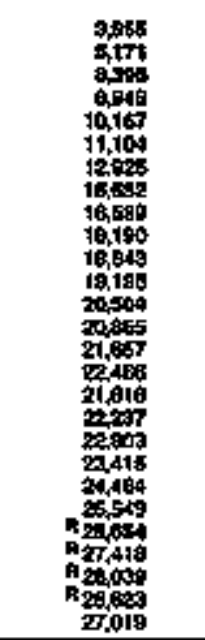 & $\begin{array}{l}\vdots \\
\vdots \\
\vdots \\
\vdots \\
\vdots \\
\vdots \\
\vdots \\
\vdots \\
\vdots \\
\vdots \\
\vdots \\
\vdots \\
\vdots \\
\vdots\end{array}$ \\
\hline \multicolumn{15}{|c|}{ Telloos Ex } \\
\hline 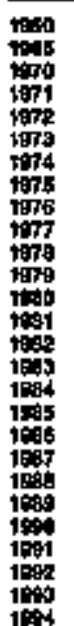 & 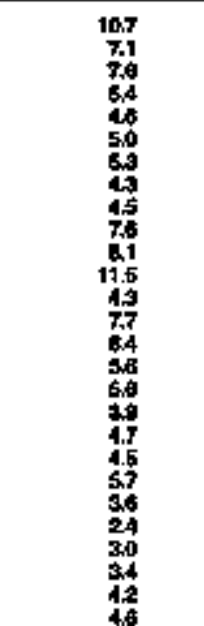 & 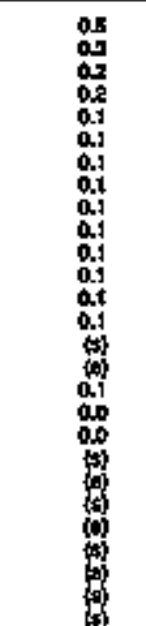 & 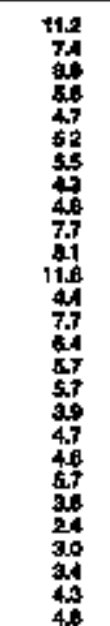 & 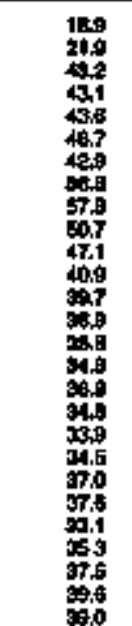 & 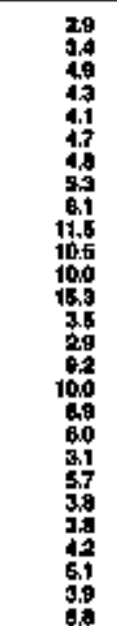 & 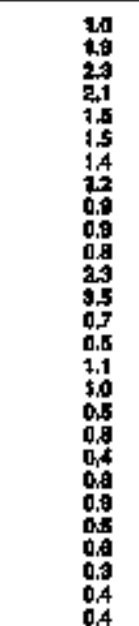 & 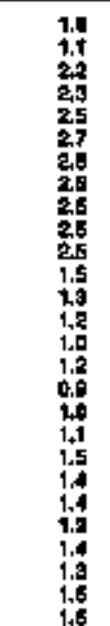 & 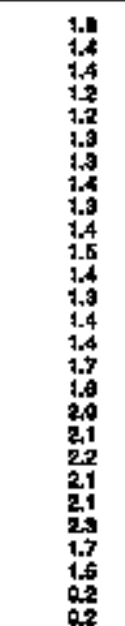 & 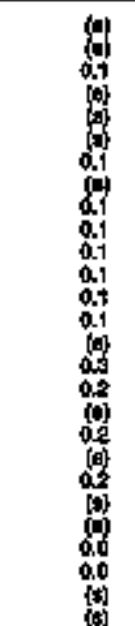 & 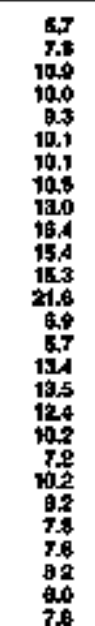 & 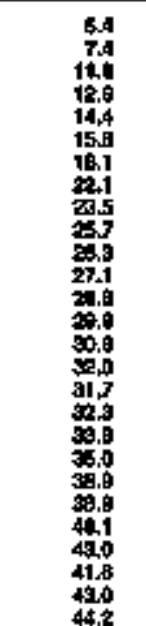 & 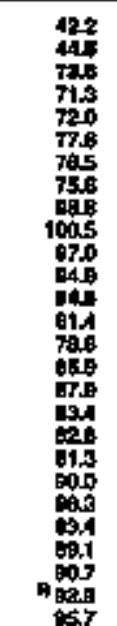 & 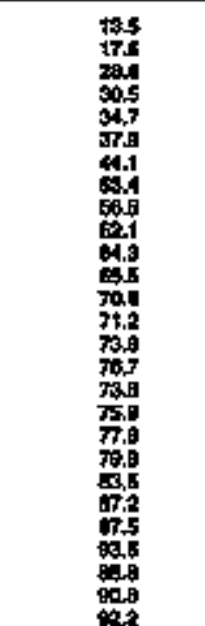 & 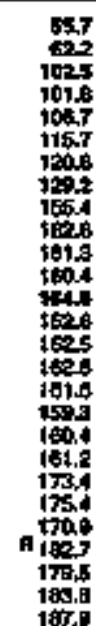 \\
\hline
\end{tabular}




\begin{tabular}{|c|c|c|c|c|c|c|c|c|c|c|c|c|c|c|c|c|c|c|}
\hline \multirow[b]{3}{*}{ Year } & \multirow[b]{2}{*}{ con } & \multirow[b]{2}{*}{ matur } & \multicolumn{9}{|c|}{ Pomoticuan } & \multirow[b]{2}{*}{ 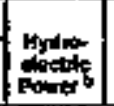 } & \multirow[b]{2}{*}{ Dosing } & \multirow[b]{2}{*}{ Other and } & \multirow[b]{2}{*}{$\cos$} & \multirow[b]{2}{*}{ Entol } & \multirow{2}{*}{ 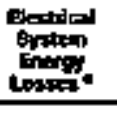 } & \multirow[b]{3}{*}{$-\operatorname{tot}$} \\
\hline & & & Aaphentend & 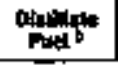 & Kanoment & $\mathrm{tpq} \mathbf{Q}^{-1}$ & | & 蓶 & 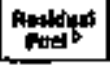 & | onersol & Tote르. & & & & & & & \\
\hline & $\begin{array}{l}\text { mounand } \\
\text { Shont Tonst }\end{array}$ & antive Fon & \multicolumn{9}{|c|}{ moowndouph } & \multicolumn{6}{|c|}{ 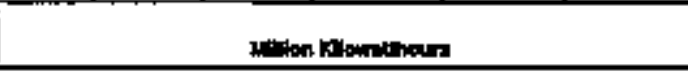 } & \\
\hline 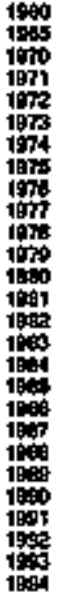 & 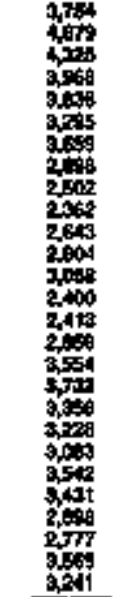 & 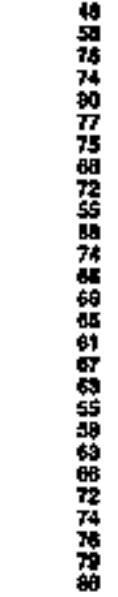 & 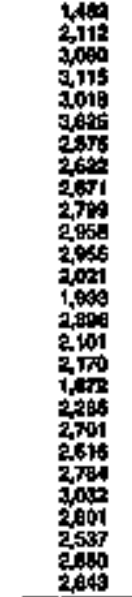 & 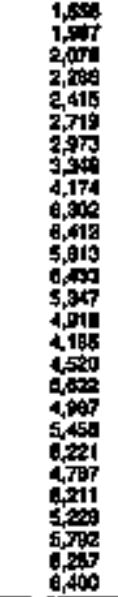 & 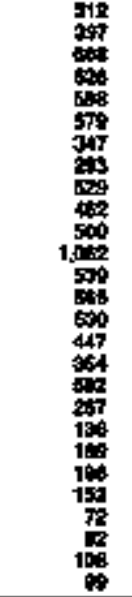 & 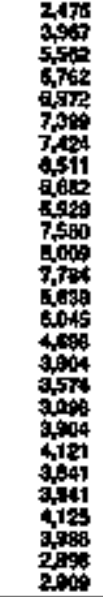 & 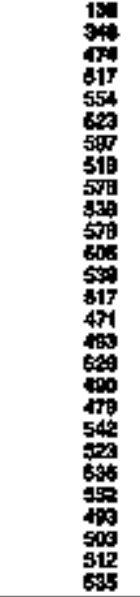 & 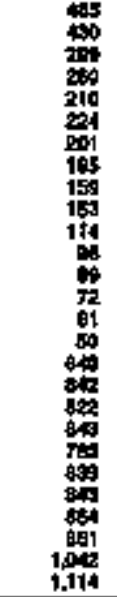 & 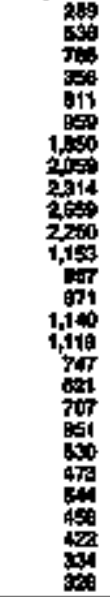 & 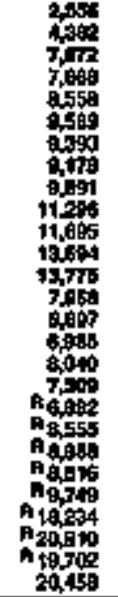 & 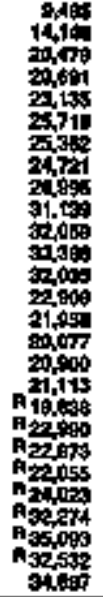 & : & 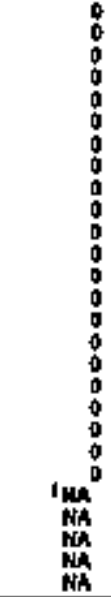 & $\begin{array}{r}0 \\
0 \\
0 \\
0 \\
0 \\
0 \\
0 \\
0 \\
0 \\
0 \\
0 \\
0 \\
0 \\
0 \\
0 \\
0 \\
0 \\
0 \\
0 \\
0 \\
0 \\
0 \\
\text { w } \\
4 \\
4\end{array}$ & 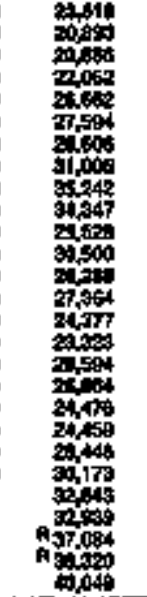 & $\begin{array}{l}z \\
\vdots \\
\vdots \\
\vdots \\
\vdots \\
\vdots \\
\vdots \\
z\end{array}$ & 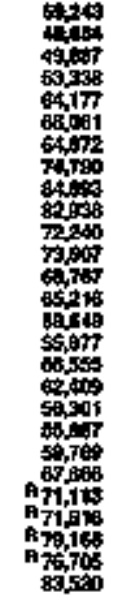 & $\begin{array}{l}\bar{z} \\
\bar{z} \\
\bar{z}\end{array}$ \\
\hline
\end{tabular}

\begin{tabular}{|c|c|c|c|c|c|c|c|c|c|c|c|c|c|c|c|c|c|c|}
\hline 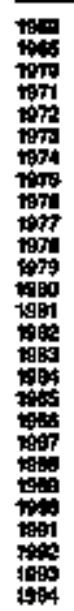 & 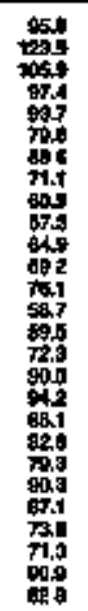 & 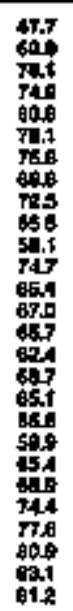 & 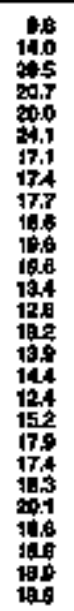 & 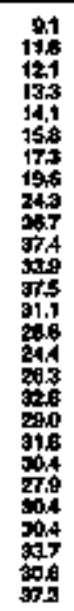 & 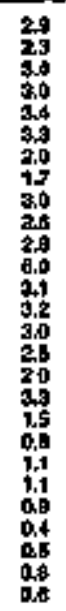 & 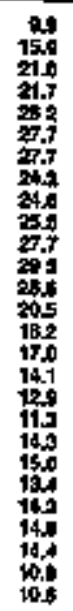 & 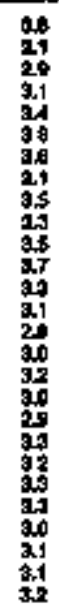 & 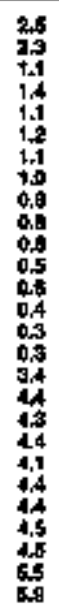 & 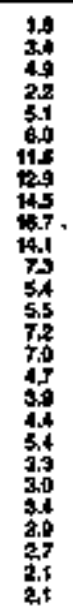 & 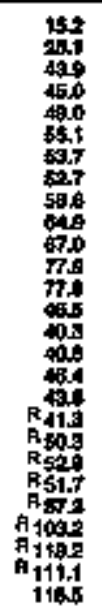 & 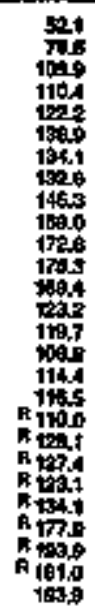 & 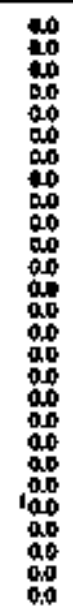 & 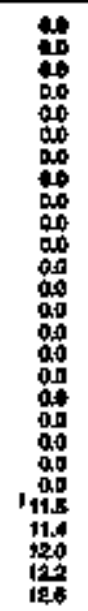 & 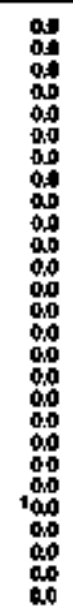 & 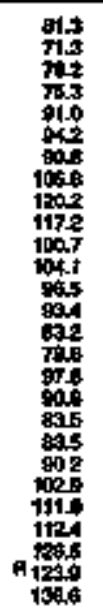 & 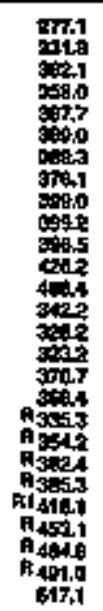 & 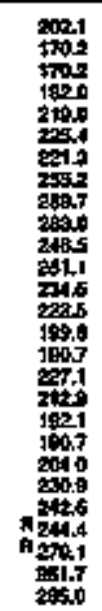 & 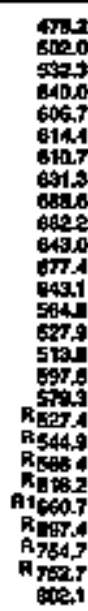 \\
\hline
\end{tabular}

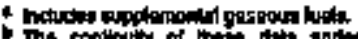

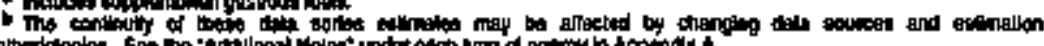

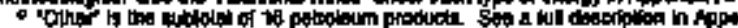

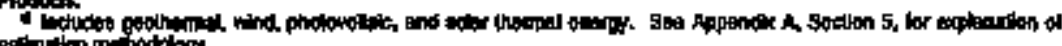

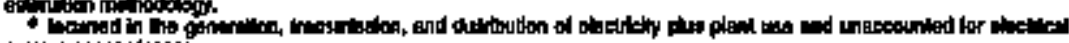

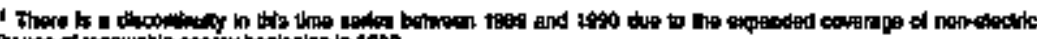

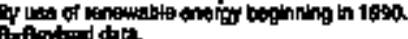

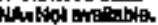

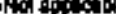

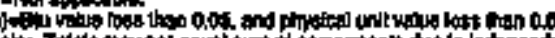

ation 
Table 123. Transportation Energy Consumption Estimates, 1960, 1965, 1974-1994, Kortheky

\begin{tabular}{|c|c|c|c|c|c|c|c|c|c|c|c|c|c|c|c|}
\hline \multirow[b]{3}{*}{$\mathrm{Ym}$} & \multirow[b]{2}{*}{$\cos 1$} & \multirow[b]{2}{*}{ 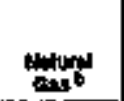 } & \multicolumn{8}{|c|}{ Petrolition } & \multirow{3}{*}{ 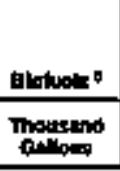 } & \multirow{2}{*}{\multicolumn{2}{|c|}{ 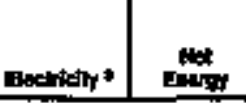 }} & \multirow{3}{*}{ 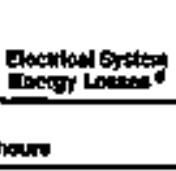 } & \multirow[b]{3}{*}{ Toba } \\
\hline & & & 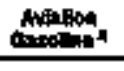 & 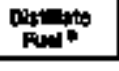 & fevel. & LPe: & Enseante " & chand & Rentalis & Totid & & & & & \\
\hline & $\begin{array}{l}\text { Thousand } \\
\text { ghort Tons }\end{array}$ & cinteran: & \multicolumn{8}{|c|}{ 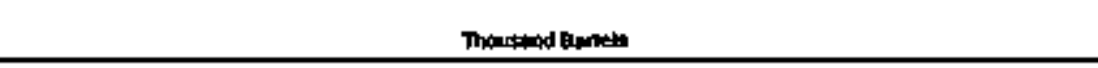 } & & \multicolumn{2}{|c|}{ 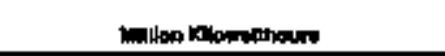 } & & \\
\hline 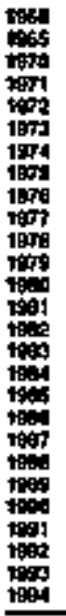 & $\begin{array}{c}10 \\
15 \\
7 \\
5 \\
4 \\
2 \\
2 \\
04 \\
0 \\
0 \\
0 \\
0 \\
0 \\
0 \\
0 \\
0 \\
0 \\
0 \\
0 \\
0 \\
0 \\
0 \\
0 \\
0 \\
0 \\
0 \\
0 \\
\end{array}$ & 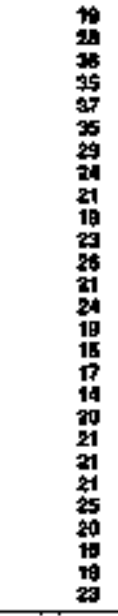 & 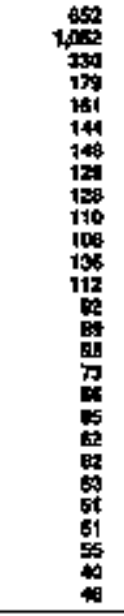 & 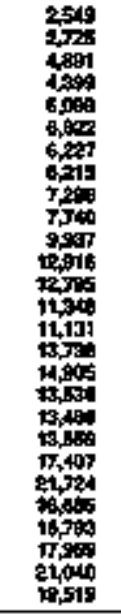 & 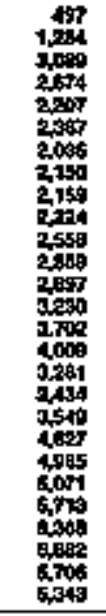 & 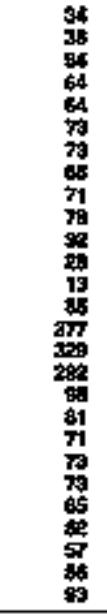 & 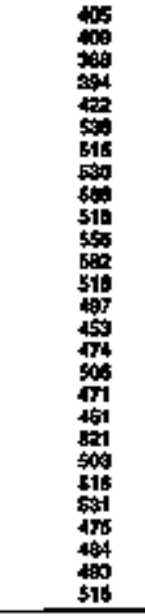 & 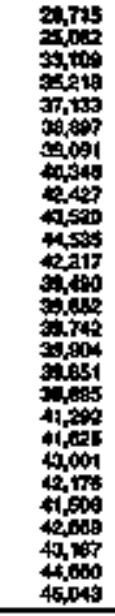 & 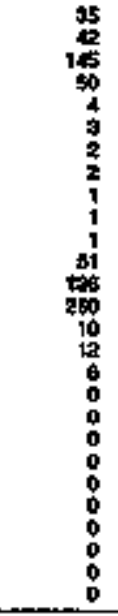 & 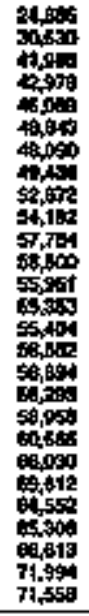 & 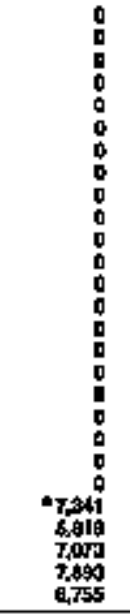 & $\begin{array}{l}0 \\
0 \\
0 \\
0 \\
0 \\
0 \\
0 \\
0 \\
0 \\
0 \\
0 \\
0 \\
0 \\
0 \\
0 \\
0 \\
0 \\
0 \\
0 \\
0 \\
0 \\
0 \\
0 \\
0 \\
0 \\
0 \\
0 \\
\end{array}$ & $\begin{array}{l}z \\
= \\
z \\
z \\
z \\
z \\
z \\
z \\
z \\
z \\
z \\
z \\
z \\
\vdots \\
z\end{array}$ & $\begin{array}{l}0 \\
0 \\
0 \\
0 \\
0 \\
0 \\
0 \\
0 \\
0 \\
0 \\
0 \\
0 \\
0 \\
0 \\
0 \\
0 \\
0 \\
0 \\
0 \\
0 \\
0 \\
0 \\
0 \\
0 \\
0 \\
0 \\
\end{array}$ & $\begin{array}{l}= \\
= \\
= \\
= \\
= \\
= \\
= \\
= \\
= \\
= \\
= \\
= \\
= \\
=\end{array}$ \\
\hline \multicolumn{16}{|c|}{ rabion Bh } \\
\hline 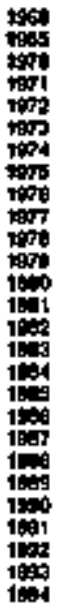 & 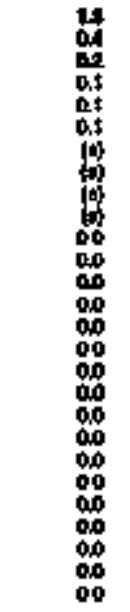 & 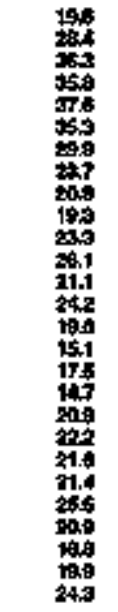 & 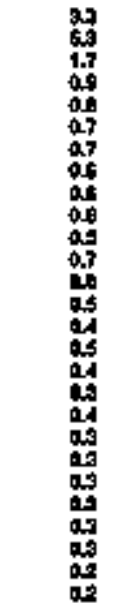 & 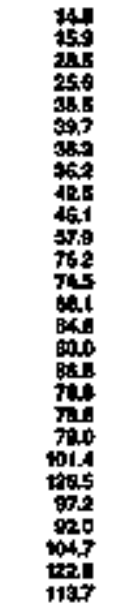 & 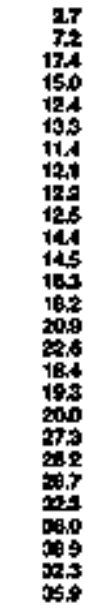 & 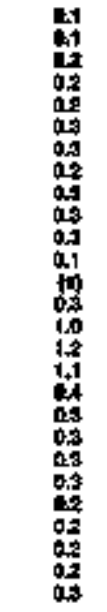 & 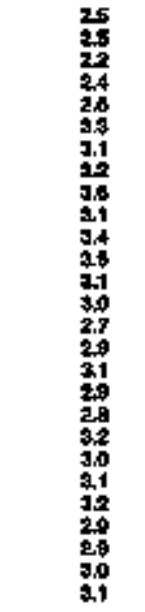 & 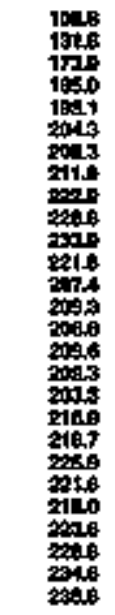 & 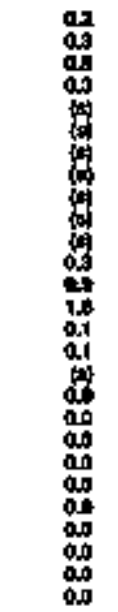 & 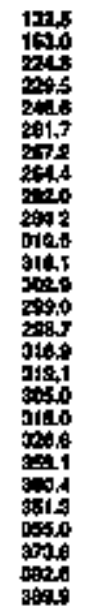 & 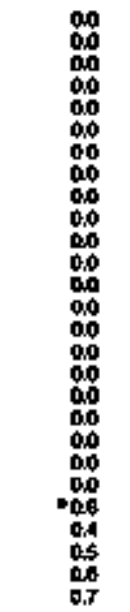 & 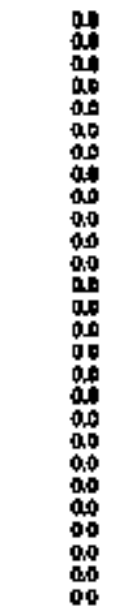 & 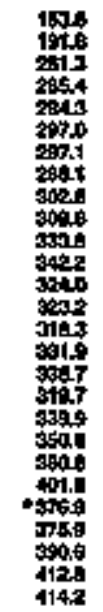 & $\begin{array}{l}00 \\
00 \\
000 \\
000 \\
000 \\
00 \\
00 \\
00 \\
06 \\
00 \\
00 \\
00 \\
00 \\
00 \\
00 \\
00 \\
00 \\
00 \\
00 \\
00 \\
00 \\
00 \\
00 \\
00 \\
0.0 \\
00 \\
0.0\end{array}$ & 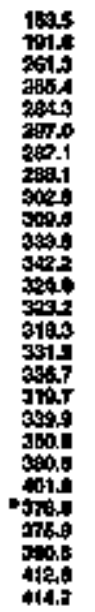 \\
\hline
\end{tabular}


Tabis 124. Estimates of Energy Input at Electuc Utittlos, 1960, 1965, 1970-1994, Kentucky

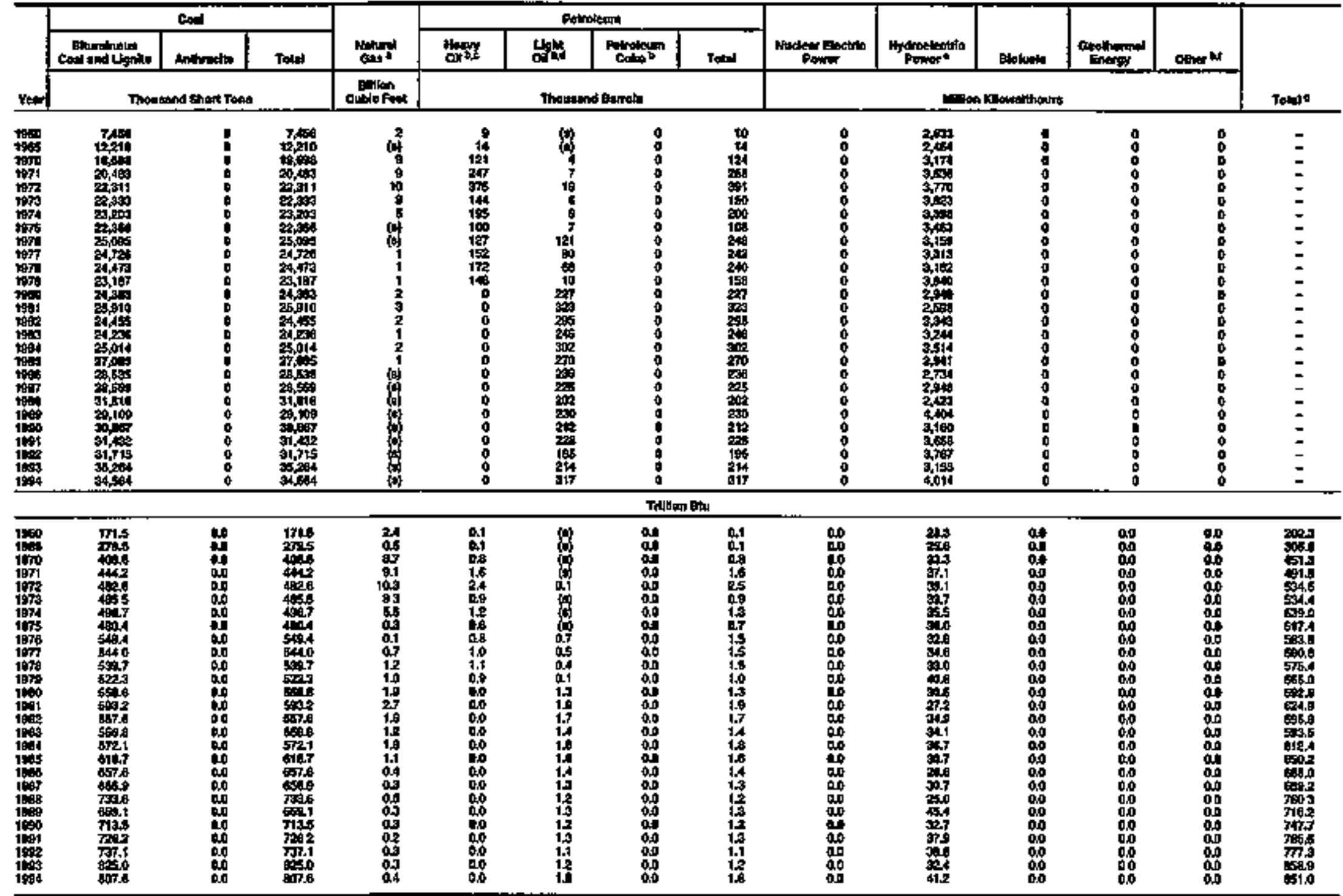

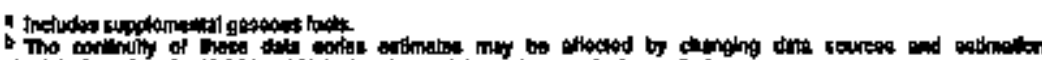

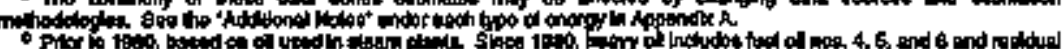

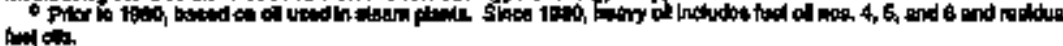

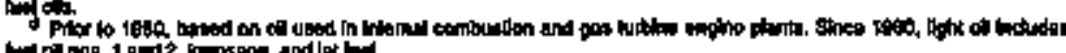

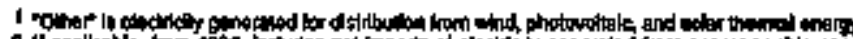

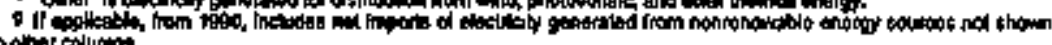

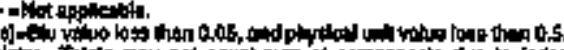

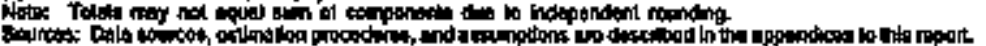

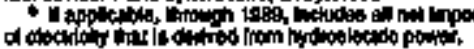


Table 125. Energy Consumption Estimates by Source, 1960, 1965, 1970-1994, Louisiana

\begin{tabular}{|c|c|c|c|c|c|c|c|c|c|c|c|c|c|c|c|c|c|c|c|}
\hline \multirow[b]{3}{*}{ Yan } & \multirow[b]{2}{*}{ Codj 1} & \multirow[b]{2}{*}{ tontion } & \multicolumn{11}{|c|}{ Petolen } & \multirow[b]{2}{*}{ Prectar: } & \multirow[b]{2}{*}{ 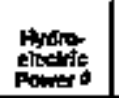 } & \multirow[b]{2}{*}{ Elotucle. | } & \multirow[b]{2}{*}{ Othert: } & \multirow{2}{*}{ Mether } & \multirow[b]{3}{*}{ Tothy } \\
\hline & & & 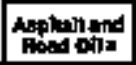 & Andation & 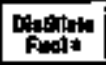 & 祡。 & Kerp: & IPG: & Lath & atominam & 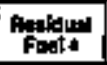 & the & Total & & & & & & \\
\hline & Thoutind & Duth Fed & \multicolumn{11}{|c|}{ moneasud Bumats } & \multicolumn{5}{|c|}{ 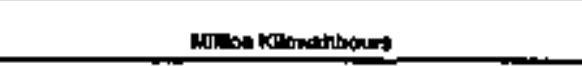 } & \\
\hline 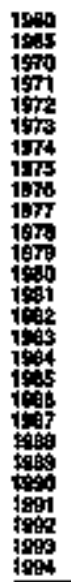 & 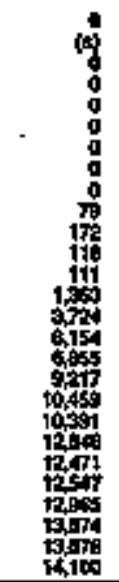 & 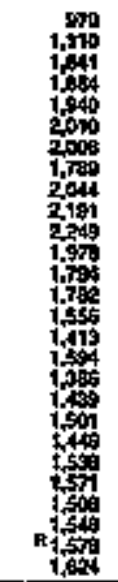 & 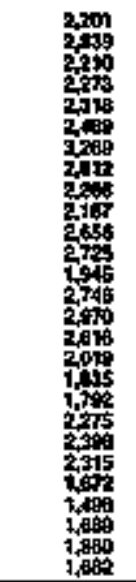 & 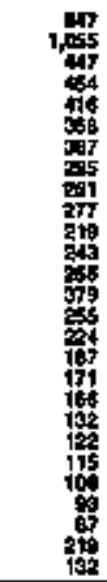 & 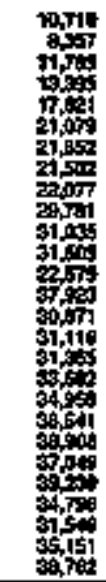 & 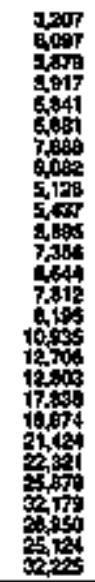 & 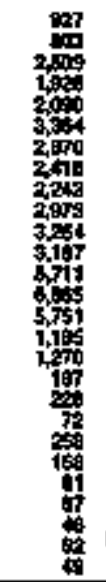 & 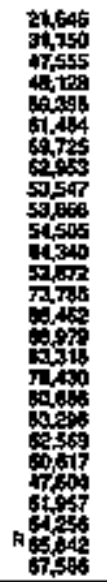 & 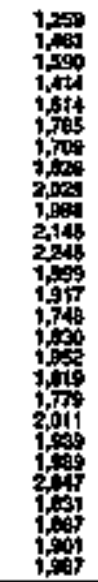 & 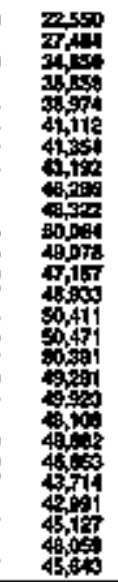 & 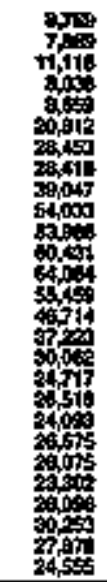 & 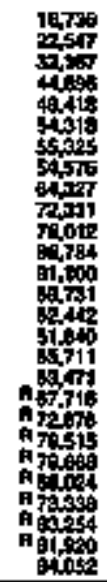 & 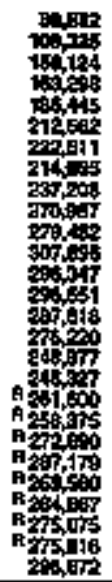 & 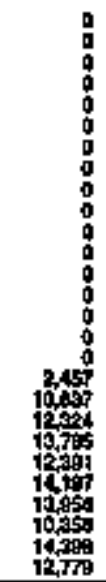 & 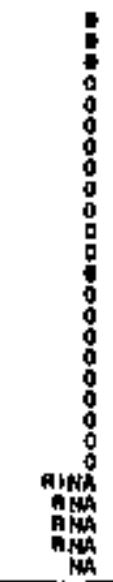 & 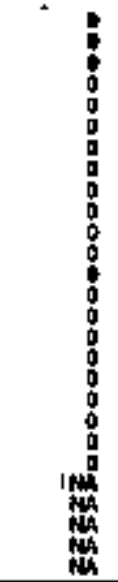 & $\begin{array}{l}0 \\
0 \\
0 \\
0 \\
0 \\
0 \\
0 \\
0 \\
0 \\
0 \\
0 \\
0 \\
0 \\
0 \\
0 \\
0 \\
0 \\
0 \\
0 \\
M \\
M\end{array}$ & 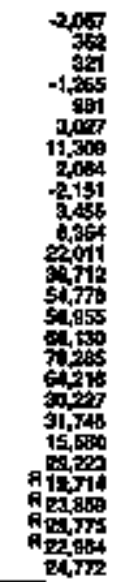 & 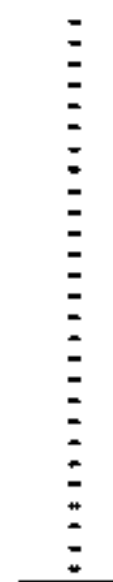 \\
\hline \multicolumn{20}{|c|}{ Trilum biv } \\
\hline 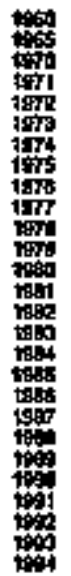 & 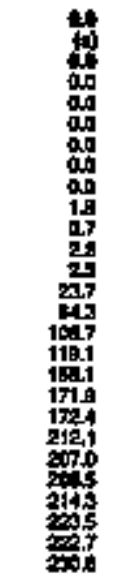 & 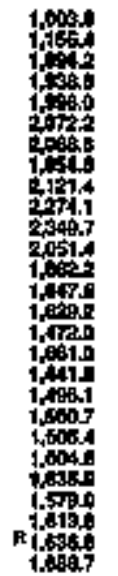 & 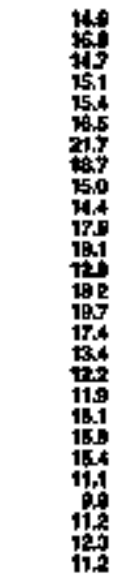 & 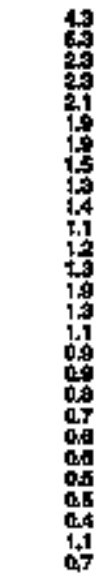 & 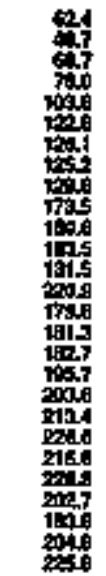 & 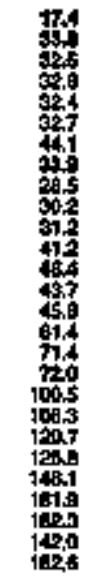 & 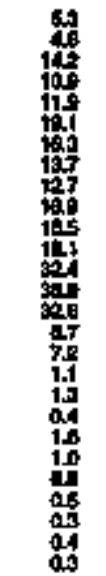 & 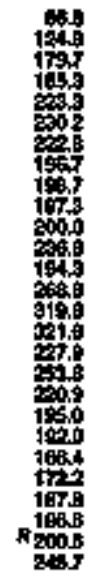 & 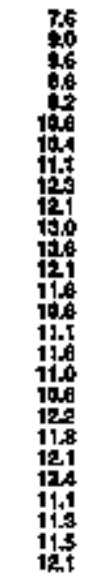 & 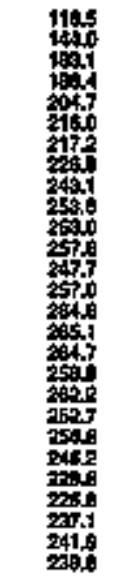 & 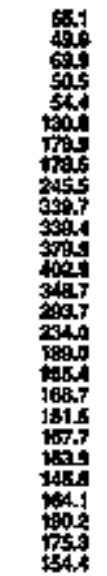 & 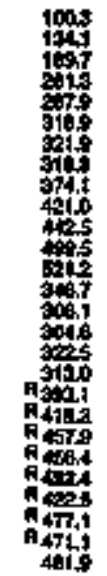 & 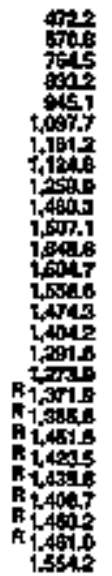 & 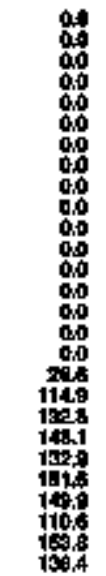 & 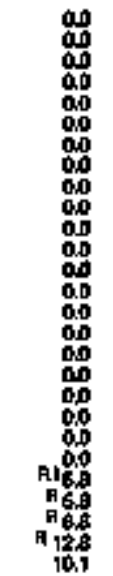 & 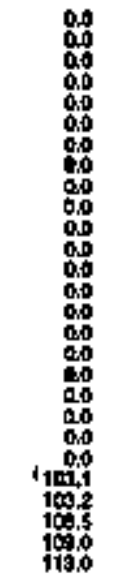 & 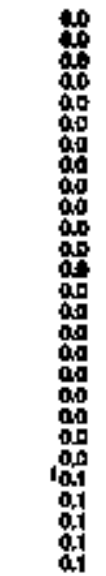 & 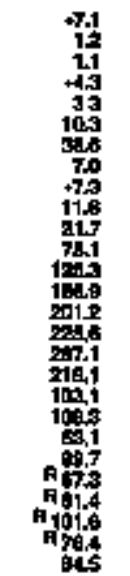 & 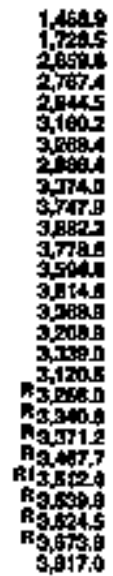 \\
\hline
\end{tabular}

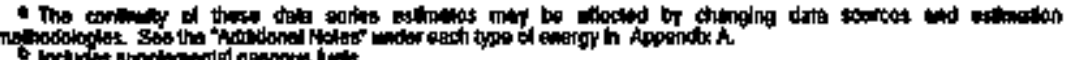

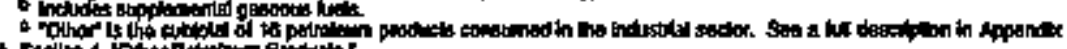

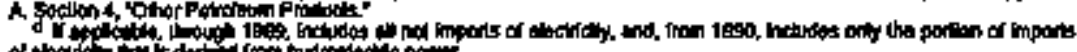

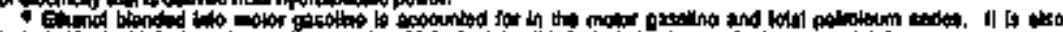

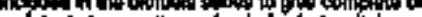

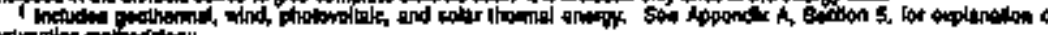

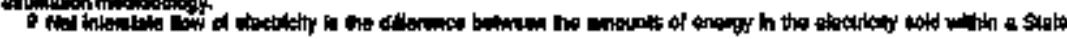

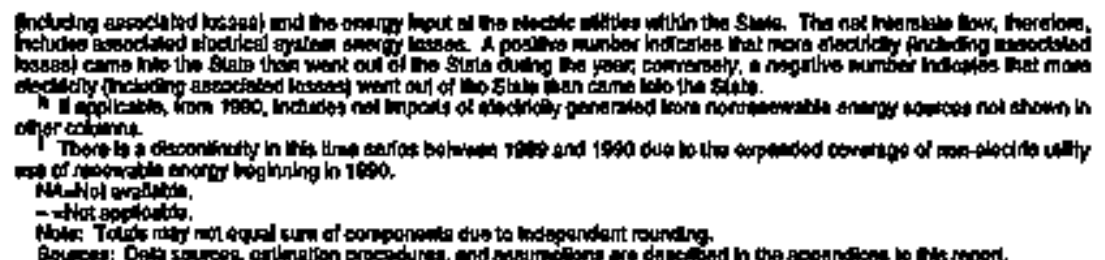


L Table 126. Fesidiential Energy Consumpilen Estlmates, 1960, 1965, 1970-1994, Louisiana

\begin{tabular}{|c|c|c|c|c|c|c|c|c|c|c|c|c|c|c|}
\hline & \multicolumn{3}{|c|}{ con } & \multirow[b]{2}{*}{ matsis } & \multicolumn{4}{|c|}{ Porration } & \multirow[b]{2}{*}{ Blobiens: } & \multirow[b]{2}{*}{ Solure } & \multirow[b]{2}{*}{ Eectitionst } & \multirow[b]{2}{*}{ Entaty } & \multirow[b]{2}{*}{ 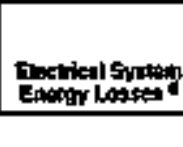 } & \multirow[b]{3}{*}{ Tow } \\
\hline & 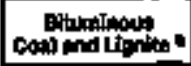 & Animelise & $\operatorname{tat}$ & & Futh & Kerrosent: & LPe A & rete & & & & & & \\
\hline Yaur & \multicolumn{3}{|c|}{ moutsenet short Tan } & 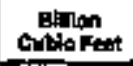 & \multicolumn{4}{|c|}{ thoumand IImel: } & Moousend & \multicolumn{4}{|c|}{ 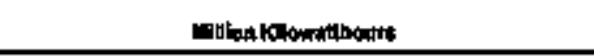 } & \\
\hline
\end{tabular}

\begin{tabular}{|c|c|c|c|}
\hline 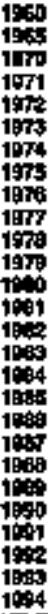 & $\begin{array}{l}0 \\
0 \\
0 \\
0 \\
0 \\
0 \\
0 \\
0 \\
0 \\
0 \\
0 \\
0 \\
0 \\
1 \\
0 \\
0 \\
0 \\
0 \\
0 \\
0 \\
0 \\
0 \\
0 \\
0 \\
0 \\
0 \\
0 \\
0 \\
0 \\
0\end{array}$ & $\begin{array}{l}0 \\
: \\
0 \\
0 \\
0 \\
0 \\
0 \\
0 \\
0 \\
0 \\
0 \\
0 \\
0 \\
0 \\
0 \\
0 \\
0 \\
0 \\
0 \\
0 \\
0 \\
0\end{array}$ & $\begin{array}{l}0 \\
0 \\
0 \\
0 \\
0 \\
0 \\
0 \\
0 \\
0 \\
0 \\
0 \\
0 \\
0 \\
0 \\
0 \\
0 \\
0 \\
0 \\
0 \\
0\end{array}$ \\
\hline
\end{tabular}

—1.

Tllongan

\begin{tabular}{|c|c|c|c|c|c|c|c|}
\hline 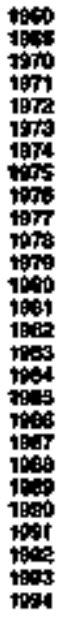 & $\begin{array}{l}00 \\
00 \\
00 \\
00 \\
000 \\
000 \\
00 \\
00 \\
00 \\
00 \\
00 \\
00 \\
00 \\
00 \\
00 \\
00 \\
0.1 \\
00 \\
00 \\
00 \\
00 \\
00 \\
00 \\
00 \\
00 \\
00 \\
0.0\end{array}$ & 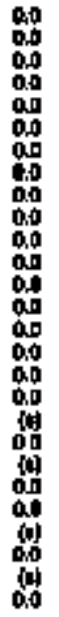 & 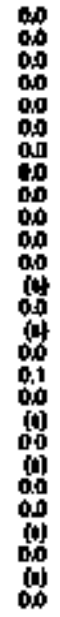 & 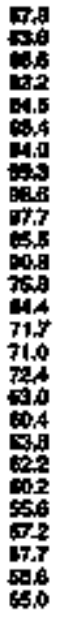 & 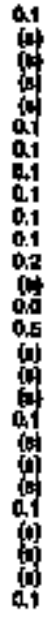 & 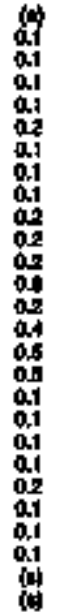 & 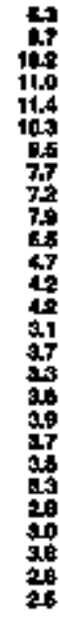 \\
\hline
\end{tabular}

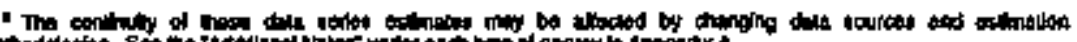

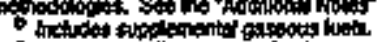

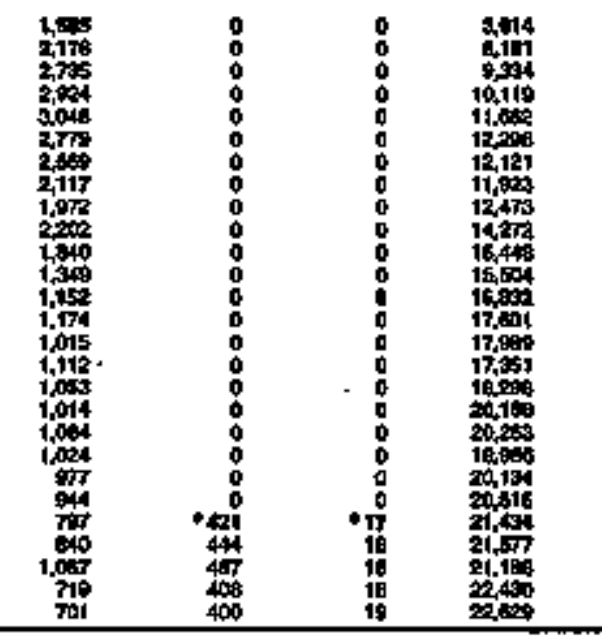

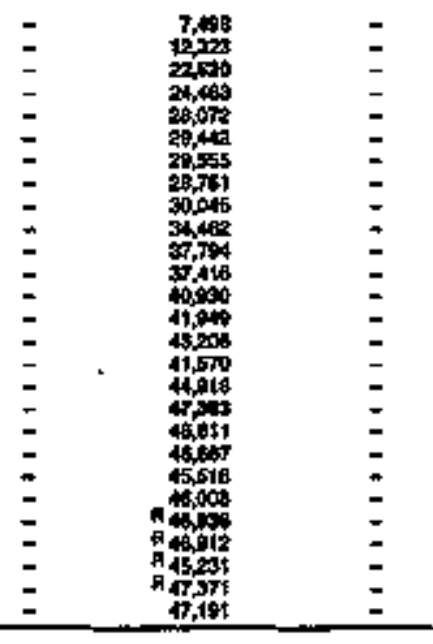

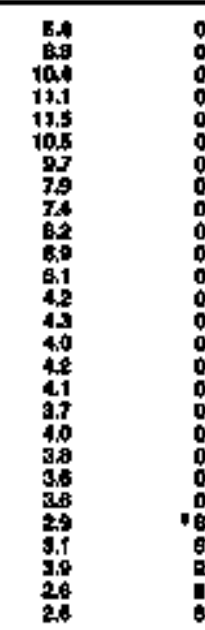

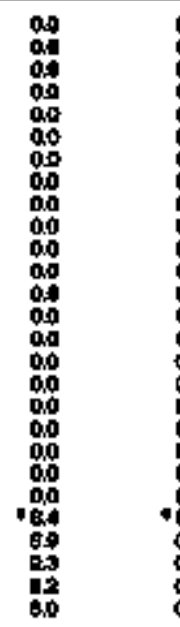

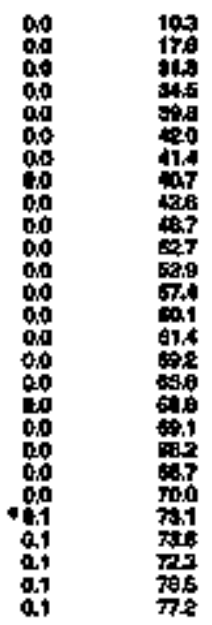

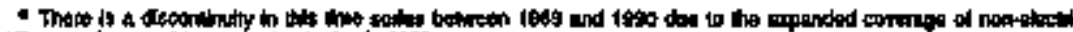

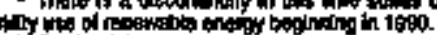
Arfievised dots. 
Table 127. Commerclal Energy Consumption Estimates, 1960, 1965, 1970-1994, Loulslana

\begin{tabular}{|c|c|c|c|c|c|c|c|c|c|c|c|c|c|c|}
\hline \multirow[b]{3}{*}{ Yee } & \multicolumn{3}{|c|}{$\cos$} & \multirow{3}{*}{ 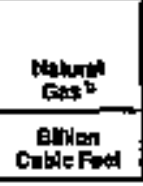 } & \multicolumn{6}{|c|}{ Podrotoun } & \multirow[b]{2}{*}{ Boterikent" } & \multirow[b]{2}{*}{ Enotrit } & \multirow{3}{*}{ 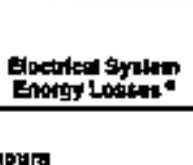 } & \multirow[b]{3}{*}{ Total of } \\
\hline & 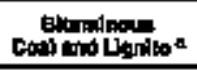 & Anmorache = 1 & Total & & Displupto & $x \cos 00001$ & LPQ $=$ & Gatolor & Regldert & Totod & & & & \\
\hline & \multicolumn{3}{|c|}{ Thousendesthen Teap } & & \multicolumn{6}{|c|}{ Mrowatad Barth } & \multicolumn{2}{|c|}{ 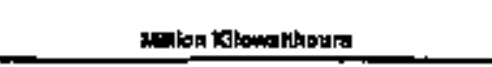 } & & \\
\hline 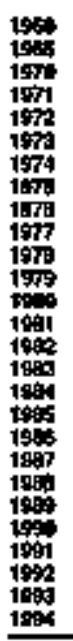 & 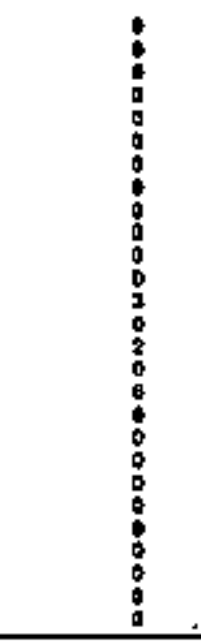 & $\begin{array}{l}0 \\
\vdots \\
0 \\
0 \\
0 \\
0 \\
0 \\
0 \\
0 \\
0 \\
0 \\
0 \\
0 \\
0 \\
0 \\
0 \\
0 \\
0 \\
0 \\
0 \\
0 \\
0 \\
0 \\
0 \\
0\end{array}$ & $\begin{array}{l}0 \\
0 \\
0 \\
0 \\
0 \\
0 \\
0 \\
0 \\
0 \\
0 \\
0 \\
3 \\
0 \\
2 \\
0 \\
0 \\
0 \\
0 \\
0 \\
0 \\
0 \\
0 \\
0 \\
0 \\
0 \\
0 \\
0 \\
0\end{array}$ & 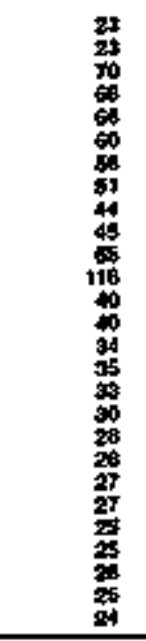 & 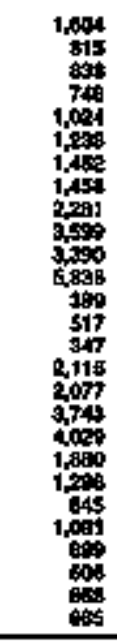 & 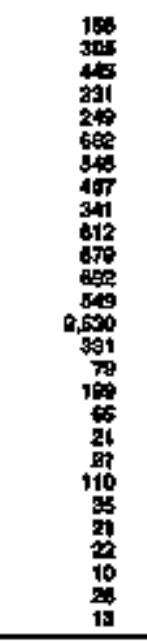 & 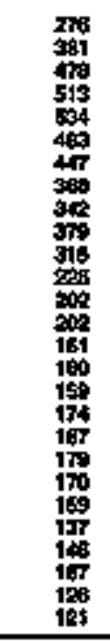 & 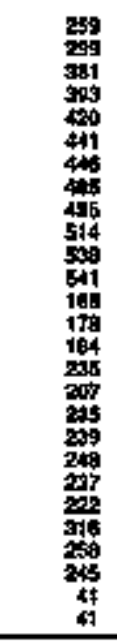 & 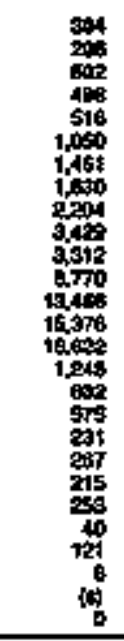 & 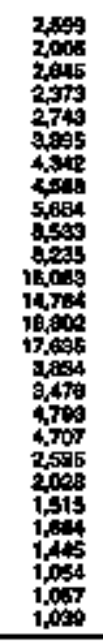 & 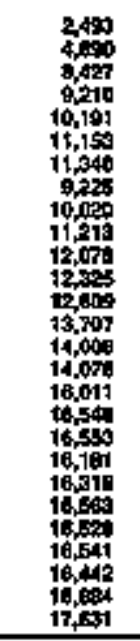 & $\begin{array}{l}\vdots \\
\vdots \\
\vdots \\
\vdots \\
\vdots \\
\vdots \\
\vdots \\
\vdots \\
\vdots \\
\vdots \\
\vdots \\
\vdots \\
\vdots \\
\end{array}$ & 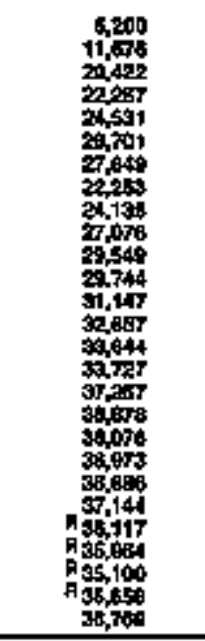 & $\begin{array}{l}\vdots \\
\vdots \\
\vdots \\
\vdots \\
\vdots \\
\vdots \\
\vdots \\
\vdots \\
\vdots \\
\vdots \\
\vdots \\
\vdots \\
\vdots \\
\end{array}$ \\
\hline \multicolumn{15}{|c|}{7 thillow and } \\
\hline 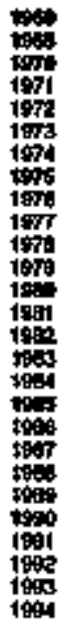 & 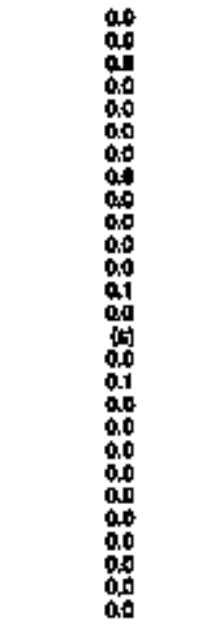 & 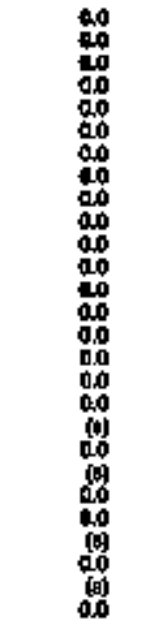 & 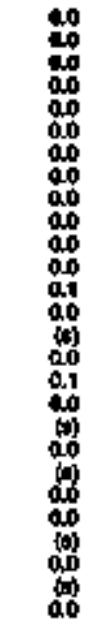 & 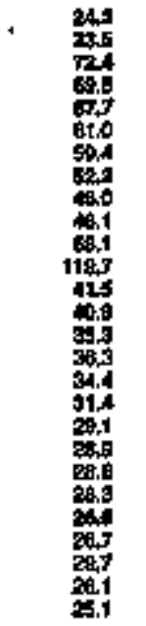 & 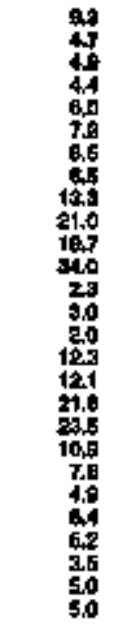 & 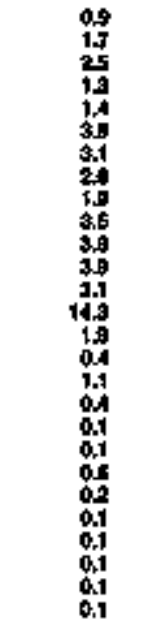 & 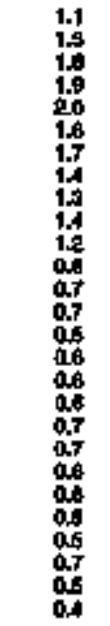 & 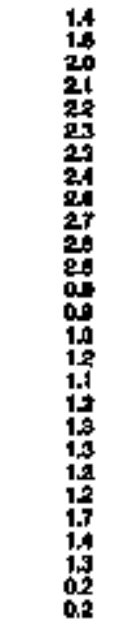 & 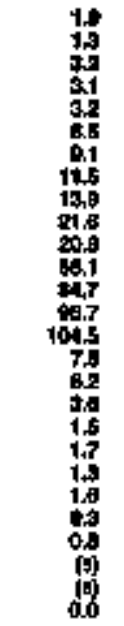 & 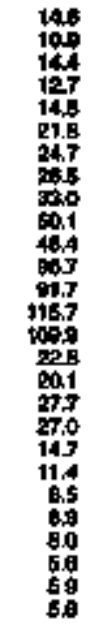 & 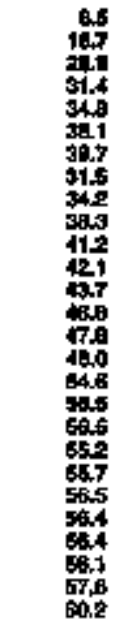 & 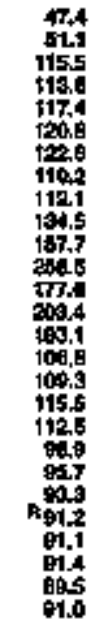 & 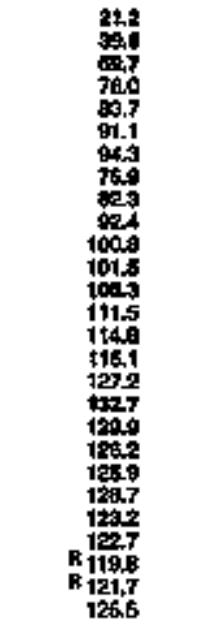 & 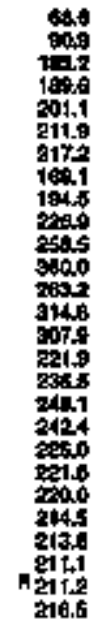 \\
\hline
\end{tabular}




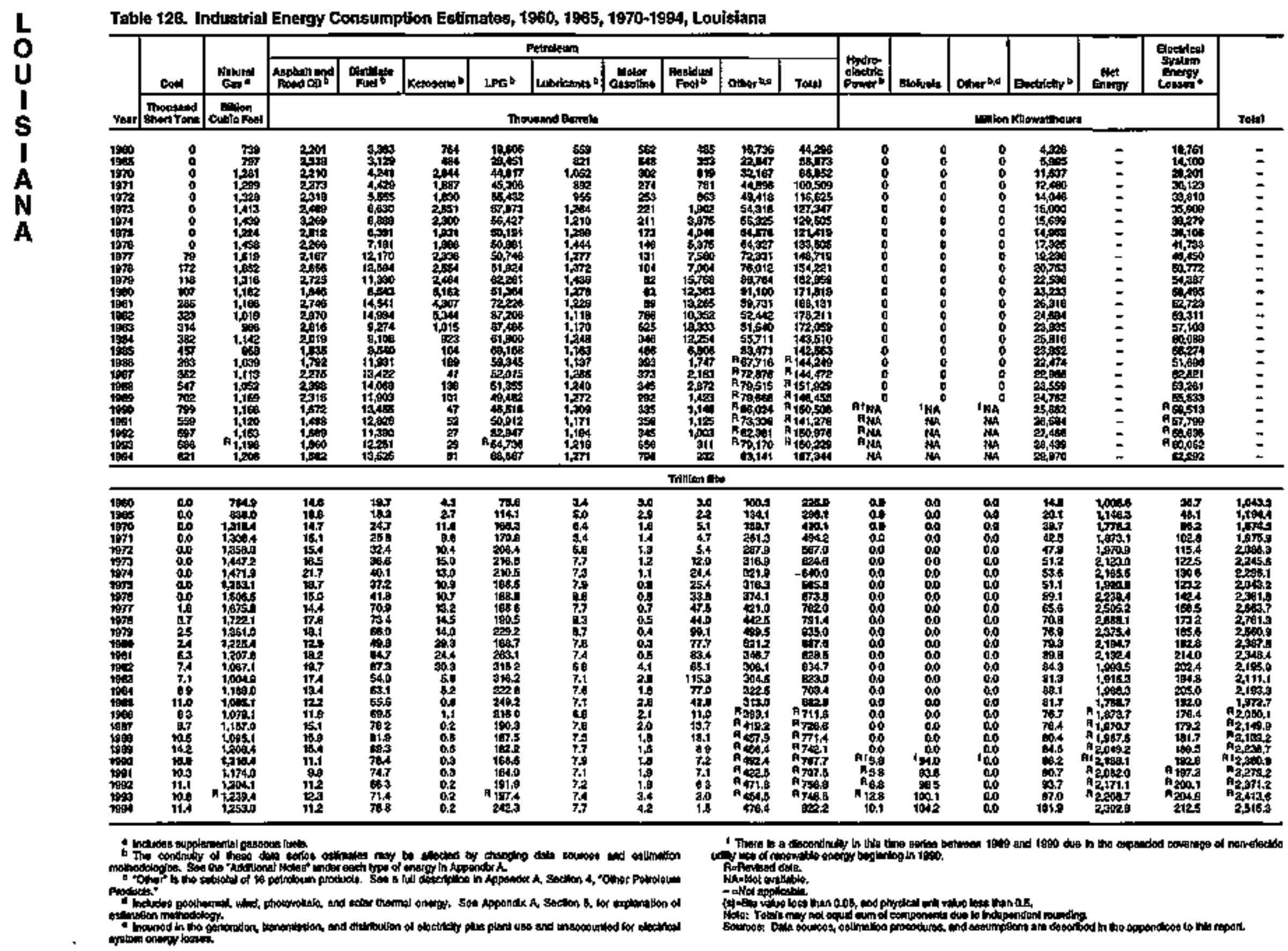


Table 129. Trumeportation Energy Consurption Estrmates, 1960, 1965, 1970-1994, Lousisine

\begin{tabular}{|c|c|c|c|c|c|c|c|c|c|c|c|c|c|c|c|}
\hline \multirow[b]{3}{*}{ - $x_{*=r}$} & \multirow[b]{2}{*}{ con: } & \multirow[b]{2}{*}{ Mutat } & \multicolumn{8}{|c|}{ Punowing } & \multirow{3}{*}{$\frac{\text { mitade: }}{\text { moureans }}$} & & \multirow[b]{2}{*}{ entist } & \multirow[b]{2}{*}{ 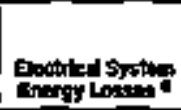 } & \multirow[b]{3}{*}{704} \\
\hline & & & Alation. & Detorto & Pat. & LPa: & Lwhikanas" & areor & 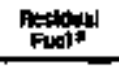 & Than & & Pecteley" & & & \\
\hline & 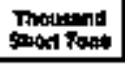 & andenton & \multicolumn{8}{|c|}{ 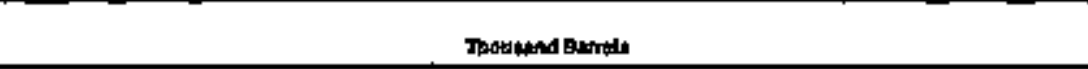 } & & \multicolumn{3}{|c|}{ 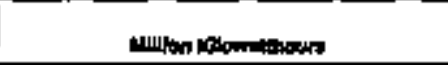 } & \\
\hline 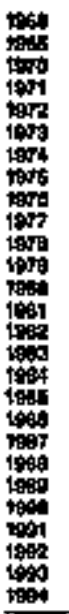 & $\begin{array}{l}0 \\
0 \\
0 \\
0 \\
0 \\
0 \\
0 \\
0 \\
0 \\
0 \\
0 \\
0 \\
0 \\
0 \\
0 \\
0 \\
0 \\
0 \\
0 \\
0 \\
0 \\
0 \\
0 \\
0 \\
0 \\
0 \\
0 \\
0 \\
0\end{array}$ & 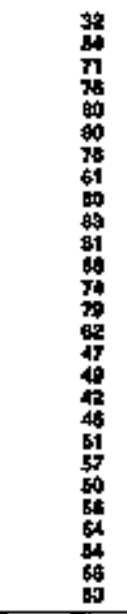 & 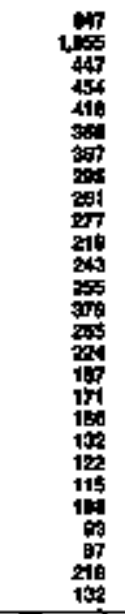 & 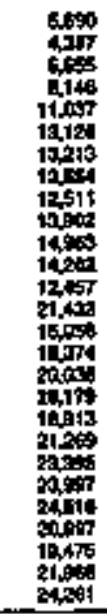 & 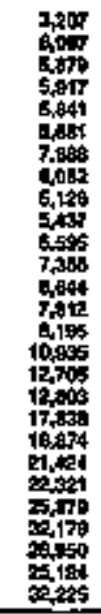 & 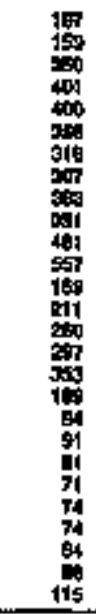 & 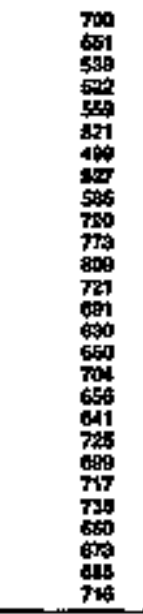 & 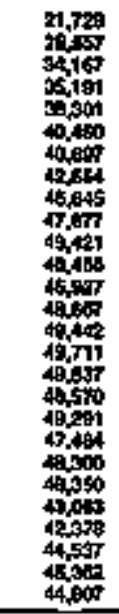 & 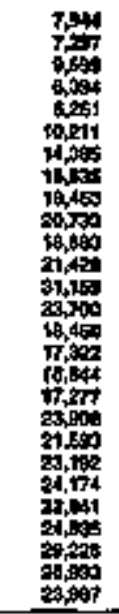 & 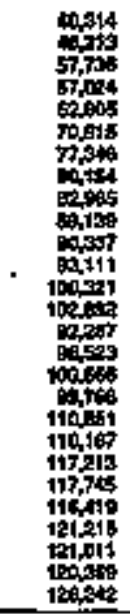 & 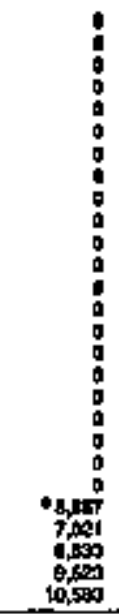 & $\begin{array}{l}26 \\
\mathbf{2} \\
3 \\
3 \\
3 \\
3 \\
3 \\
3 \\
3 \\
\mathbf{3} \\
\mathbf{3} \\
\mathbf{3} \\
\mathbf{3} \\
\mathbf{3} \\
\mathbf{3} \\
\mathbf{3} \\
\mathbf{2} \\
\mathbf{2} \\
\mathbf{3} \\
\mathbf{3} \\
\mathbf{2} \\
\mathbf{3} \\
\mathbf{2} \\
\mathbf{2} \\
\mathbf{3} \\
\mathbf{3} \\
\mathbf{2} \\
\mathbf{3} \\
\end{array}$ & $\begin{array}{l}= \\
z \\
z \\
z \\
z \\
z \\
z \\
z \\
z \\
z \\
z \\
z \\
z \\
z\end{array}$ & $\begin{array}{c}5 \\
17 \\
7 \\
5 \\
7 \\
7 \\
7 \\
8 \\
8 \\
7 \\
7 \\
6 \\
7 \\
5 \\
5 \\
8 \\
8 \\
8 \\
4 \\
5 \\
6 \\
54 \\
5 \\
5\end{array}$ & $\begin{array}{l}= \\
z \\
z \\
z \\
z \\
z \\
z \\
z \\
z \\
z \\
z \\
z \\
z \\
z\end{array}$ \\
\hline \multicolumn{16}{|c|}{$7 \ln \mid \operatorname{lon} \theta 0$} \\
\hline 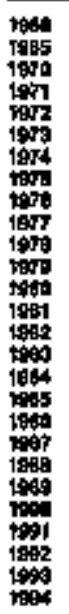 & 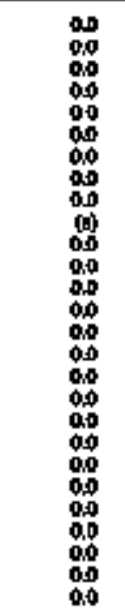 & 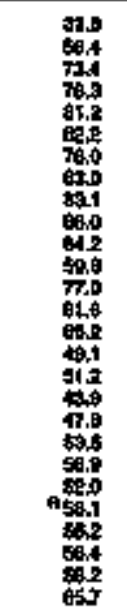 & 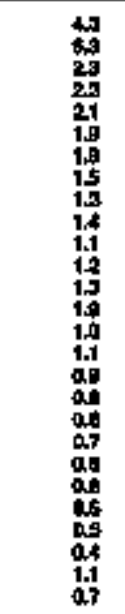 & 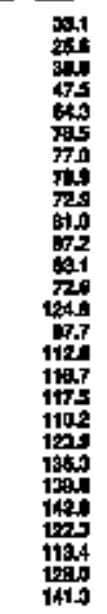 & 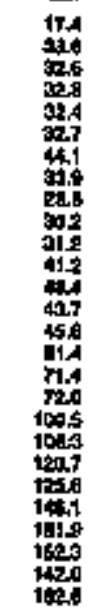 & 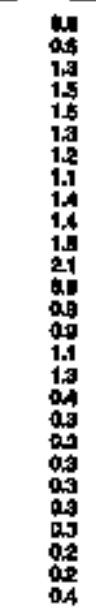 & 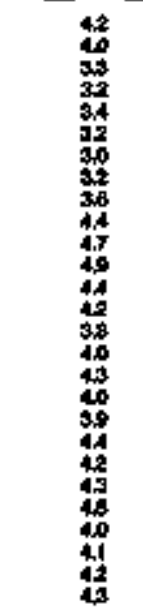 & 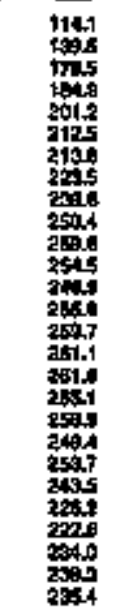 & 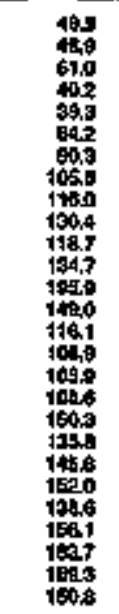 & 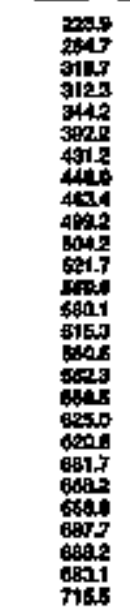 & 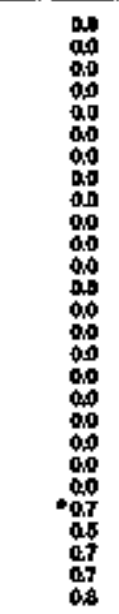 & 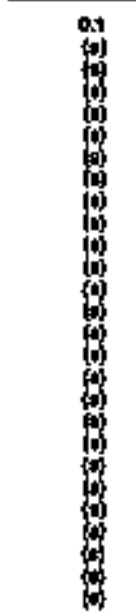 & 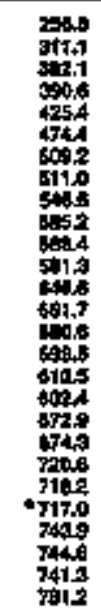 & 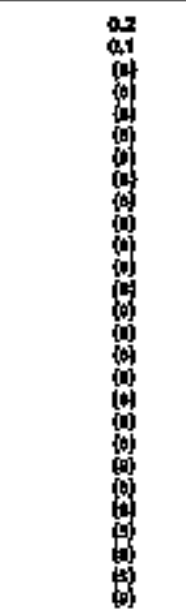 & 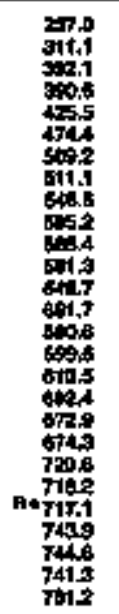 \\
\hline
\end{tabular}

L
$\mathbf{0}$
$\mathbf{U}$
$\mathbf{1}$
$\mathbf{S}$
$\mathbf{I}$
$\mathbf{A}$
$\mathbf{N}$
$\mathbf{A}$ 
L. Table 13D. Estimates of Energy Input at Electric Utilltes, 1960, 1968, t970-1994, Louisfana

\begin{tabular}{|c|c|c|c|c|c|c|c|c|c|c|c|c|c|c|}
\hline & \multicolumn{3}{|c|}{ Cond } & \multirow[b]{2}{*}{ Merl' } & \multicolumn{4}{|c|}{ 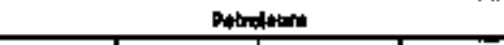 } & \multirow[b]{2}{*}{ 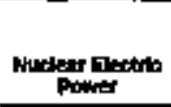 } & \multirow[b]{2}{*}{ 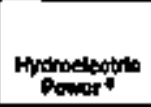 } & \multirow[b]{2}{*}{ Blokth } & \multirow[b]{2}{*}{ 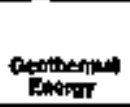 } & \multirow[b]{2}{*}{ ONm or } & \multirow[b]{3}{*}{ गоㅂㅁ } \\
\hline & 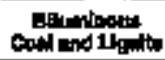 & Antriation & Tot:il & & 的和 & 战虫 & Protatar & Total & & & & & & \\
\hline vent & \multicolumn{3}{|c|}{ Thourend Shord Tom } & antion & \multicolumn{4}{|c|}{ 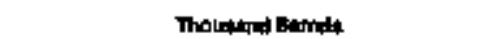 } & \multicolumn{5}{|c|}{ Withion Kfllownthoun } & \\
\hline
\end{tabular}

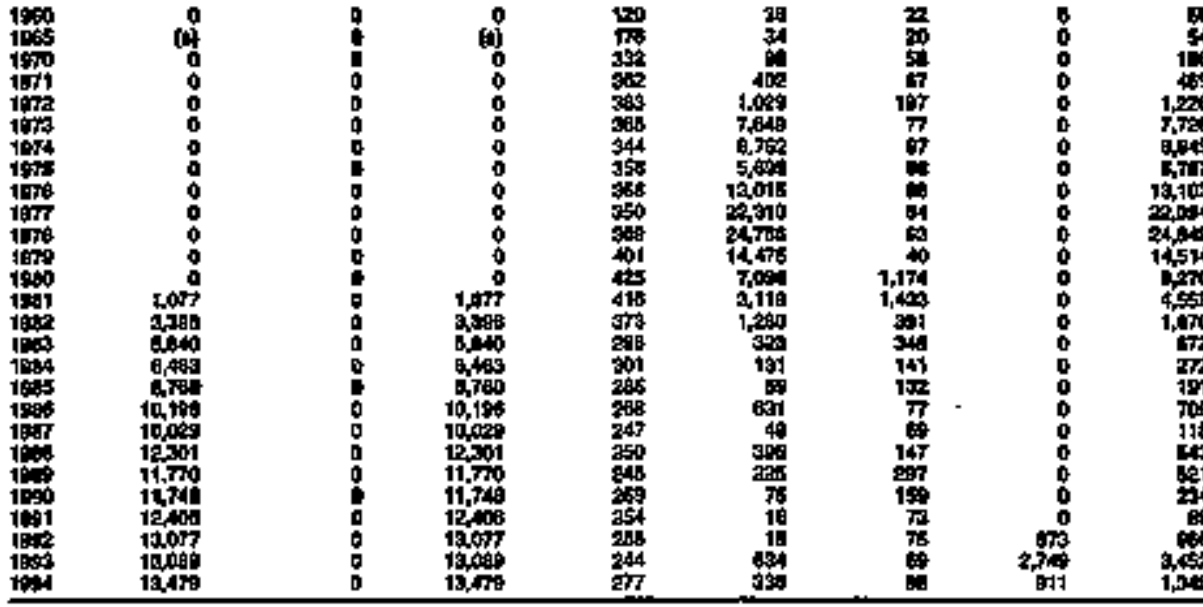

Tfiben aw

\begin{tabular}{|c|c|c|c|c|}
\hline 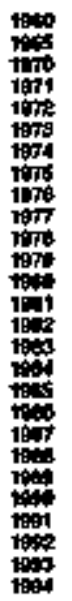 & 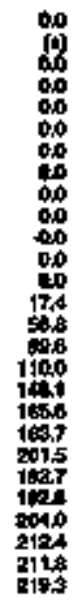 & 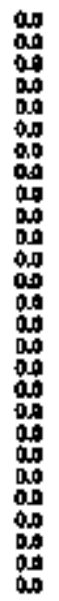 & 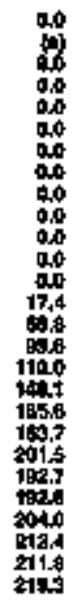 & 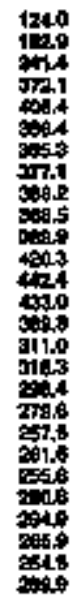 \\
\hline
\end{tabular}

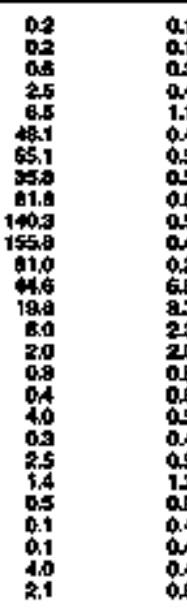

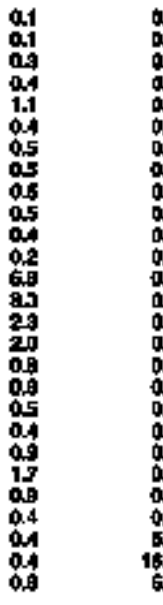

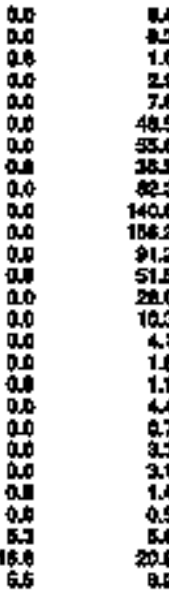

\begin{tabular}{|c|c|c|}
\hline 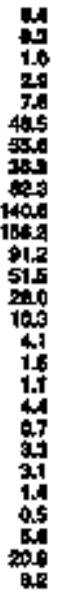 & 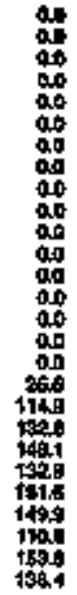 & $\begin{array}{l}00 \\
00 \\
00 \\
00 \\
00 \\
00 \\
00 \\
00 \\
00 \\
00 \\
00 \\
00 \\
000 \\
00 \\
00 \\
00 \\
00 \\
00 \\
00 \\
00 \\
00 \\
00 \\
000 \\
00\end{array}$ \\
\hline
\end{tabular}

\begin{tabular}{|c|c|c|c|}
\hline 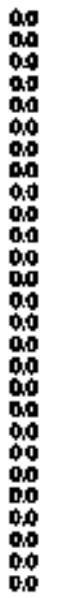 & 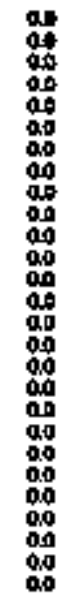 & 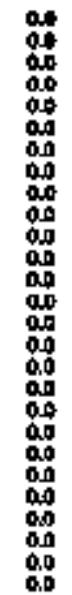 & 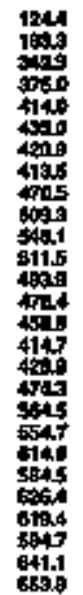 \\
\hline
\end{tabular}

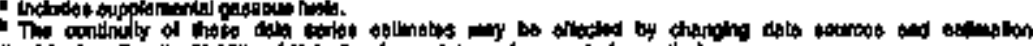

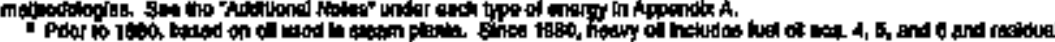
Deet ots

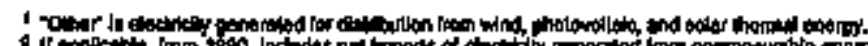

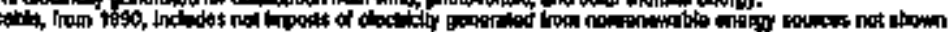

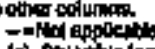

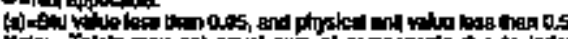

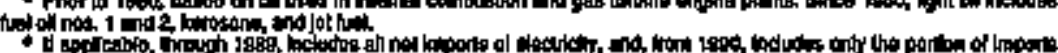

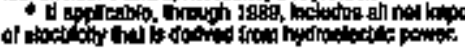


Tabla 131. Entrgy Consumplion Estimates by Source, 1960, 1985, 1970-1994, Waine

\begin{tabular}{|c|c|c|c|c|c|c|c|c|c|c|c|c|c|c|c|c|c|c|c|}
\hline \multirow[b]{3}{*}{ remets: } & \multirow[b]{2}{*}{$\cos }$. & \multirow[b]{2}{*}{$\frac{\operatorname{mat}}{\operatorname{mat}}$} & \multicolumn{11}{|c|}{ Parding } & \multirow[b]{2}{*}{ 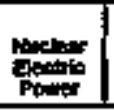 } & \multirow[b]{2}{*}{ extont } & \multirow[b]{2}{*}{ 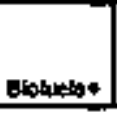 } & \multirow[b]{2}{*}{ Ooter an } & \multirow{3}{*}{ 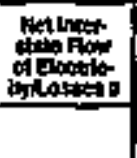 } & \multirow[b]{3}{*}{ Totol: } \\
\hline & & & casphentind & Aflution & and & is. & I. & LPG. & : & 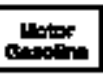 & sentis & | & Foted & & & & & & \\
\hline & Thoutind & 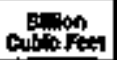 & \multicolumn{11}{|c|}{ 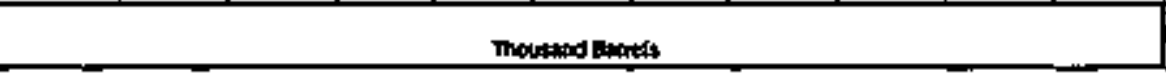 } & \multicolumn{4}{|c|}{ 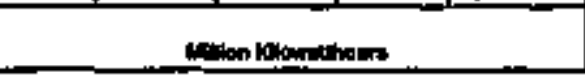 } & & \\
\hline & 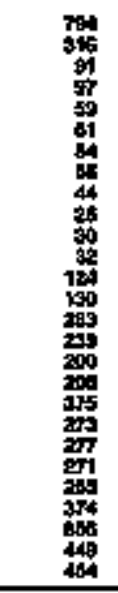 & $\begin{array}{l}\frac{2}{2} \\
\frac{2}{2} \\
\frac{2}{2} \\
\frac{2}{2} \\
2\end{array}$ & 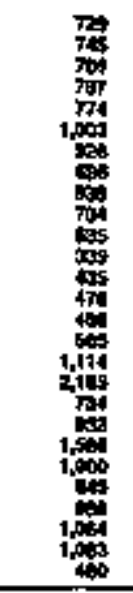 & 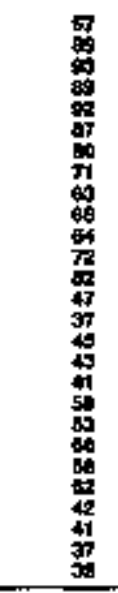 & 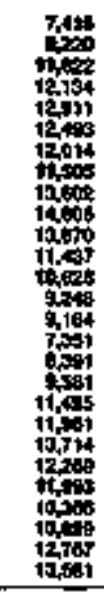 & 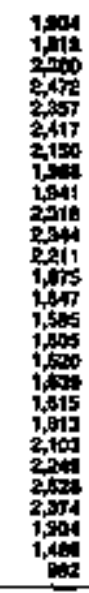 & 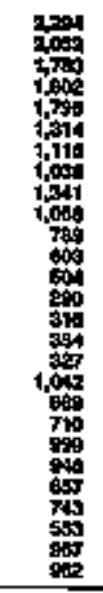 & 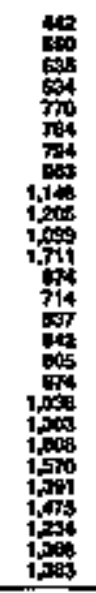 & 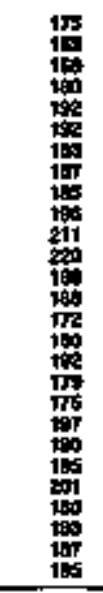 & 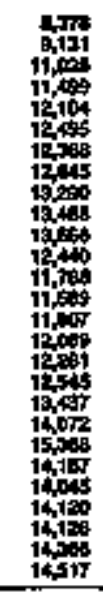 & 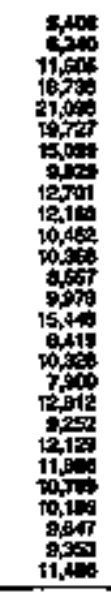 & 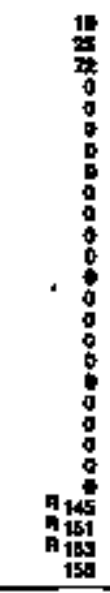 & 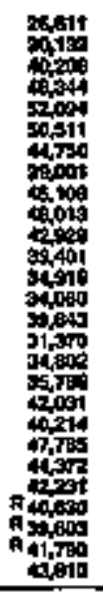 & 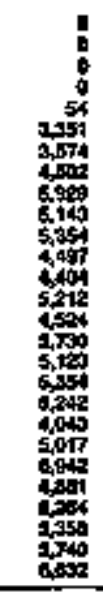 & 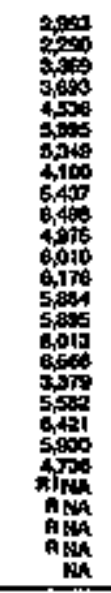 & 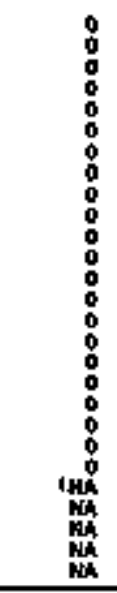 & 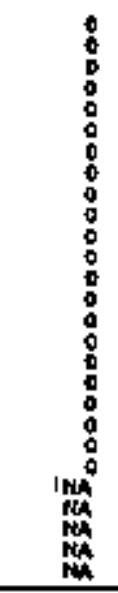 & 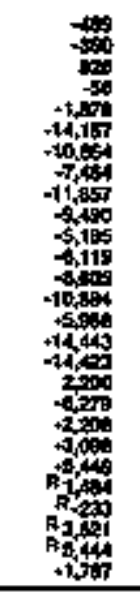 & \\
\hline \multicolumn{20}{|c|}{ Thianon bes } \\
\hline & 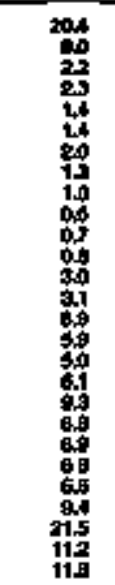 & 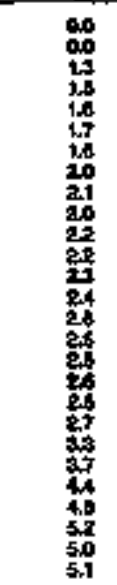 & 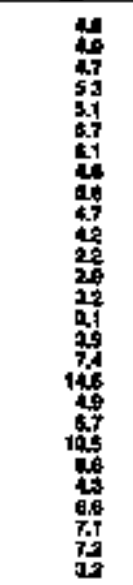 & 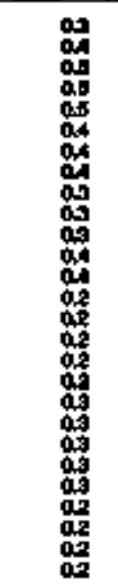 & 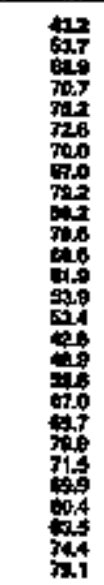 & 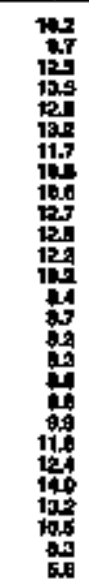 & 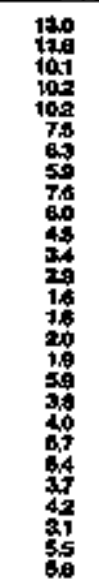 & 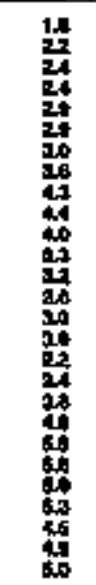 & 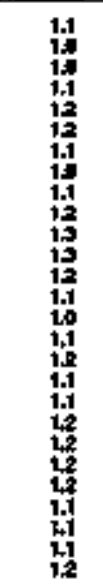 & 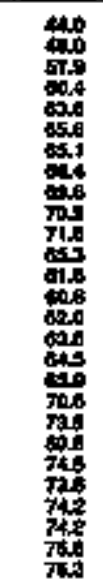 & 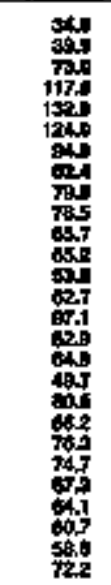 & 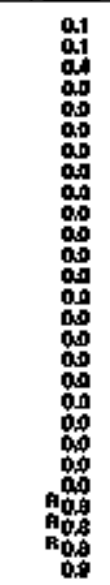 & 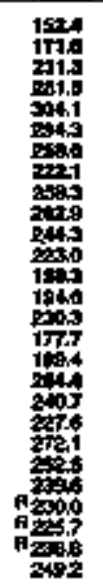 & 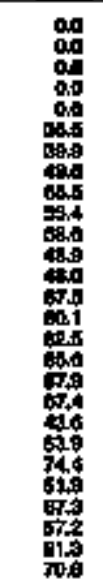 & 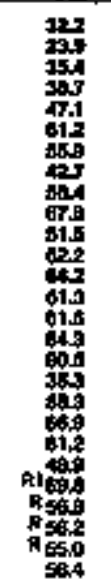 & 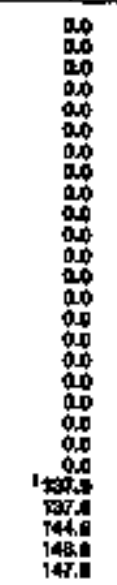 & 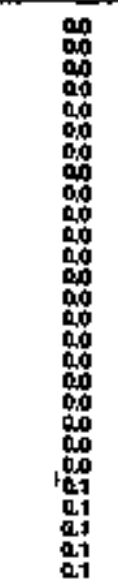 & 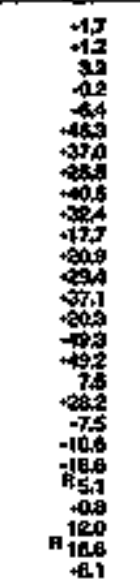 & 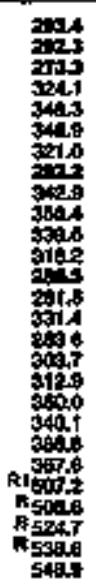 \\
\hline
\end{tabular}

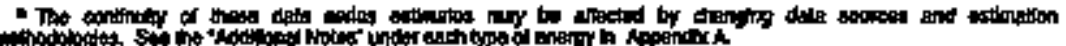

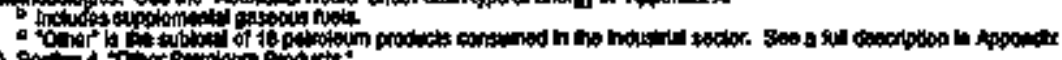

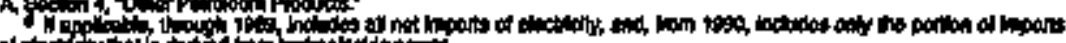

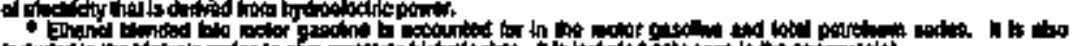

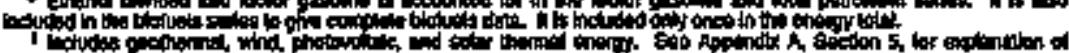

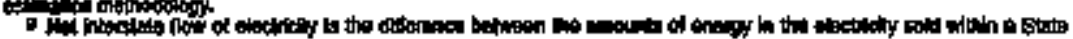

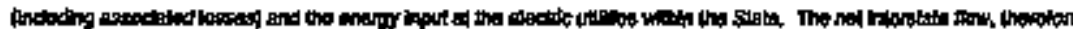

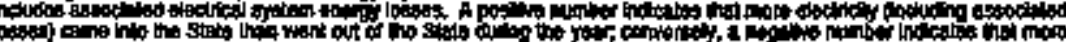
17 .

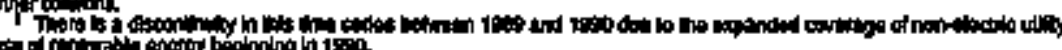


Table 132. Rostidential Energy Comatmplion Estimates, 1960, 1955, 1970-1994, Main

\begin{tabular}{|c|c|c|c|c|c|c|c|c|c|c|c|c|c|c|}
\hline \multirow[b]{3}{*}{ Yaus } & \multicolumn{3}{|c|}{$\cos 1$} & \multirow{3}{*}{ 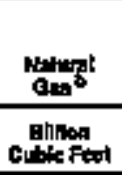 } & \multicolumn{4}{|c|}{ 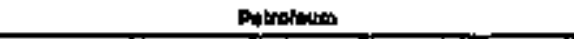 } & \multirow{3}{*}{ 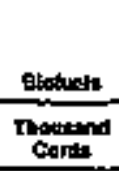 } & & \multirow[b]{2}{*}{ Tercutelys" } & \multirow[b]{2}{*}{$\operatorname{einery~}_{\text {end }}^{\mathrm{N}}$} & \multirow{3}{*}{ 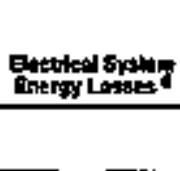 } & \multirow[b]{3}{*}{ Tols1 } \\
\hline & 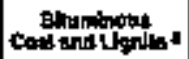 & Anctorseclus I & Total & & 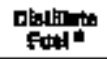 & Katwanst & LPQ: & Told & & Bolar" & & & & \\
\hline & \multicolumn{3}{|c|}{ Thetalad Shen Jon, } & & \multicolumn{4}{|c|}{ Thouswand Bomb } & & \multicolumn{3}{|c|}{ 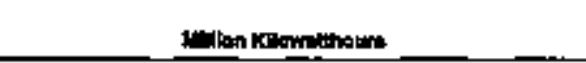 } & & \\
\hline 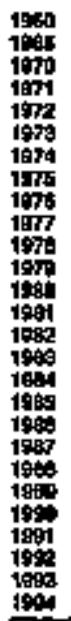 & 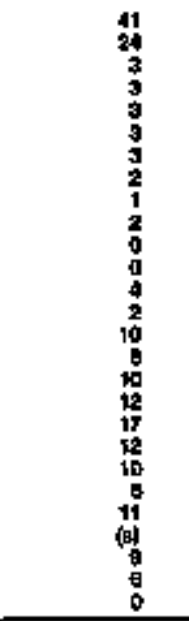 & $\begin{array}{l}54 \\
34 \\
24 \\
20 \\
15 \\
15 \\
19 \\
11 \\
10 \\
10 \\
0 \\
6 \\
9 \\
90 \\
15 \\
11 \\
15 \\
10 \\
0 \\
8 \\
5 \\
5 \\
5 \\
7 \\
5 \\
5 \\
4 \\
\end{array}$ & 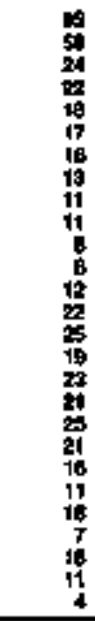 & $\begin{array}{l}0 \\
0 \\
\vdots \\
\vdots \\
\vdots \\
\vdots \\
1 \\
1 \\
1 \\
1 \\
1 \\
1 \\
1 \\
1 \\
1 \\
1 \\
1 \\
1\end{array}$ & 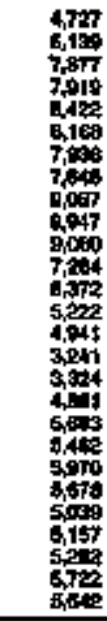 & 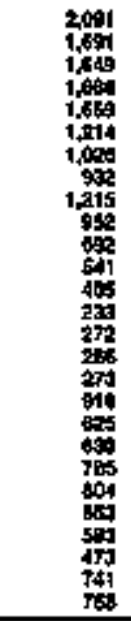 & 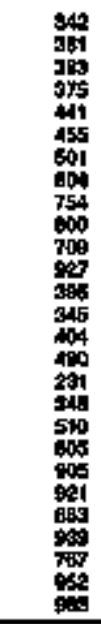 & 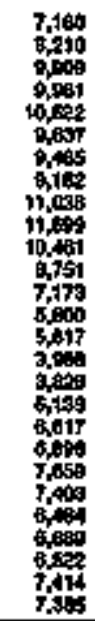 & 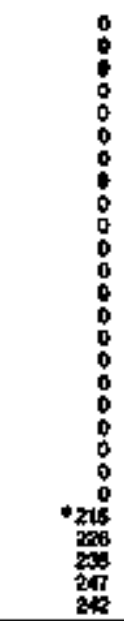 & $\begin{array}{r}0 \\
0 \\
0 \\
0 \\
0 \\
0 \\
0 \\
0 \\
0 \\
0 \\
0 \\
0 \\
0 \\
0 \\
0 \\
0 \\
0 \\
0 \\
0 \\
0 \\
0 \\
0 \\
-22 \\
23 \\
24 \\
25 \\
20\end{array}$ & 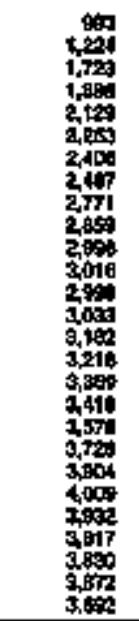 & $\begin{array}{l}= \\
= \\
= \\
= \\
= \\
= \\
= \\
= \\
= \\
= \\
= \\
= \\
= \\
=\end{array}$ & 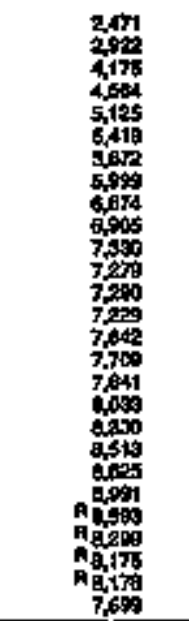 & $\begin{array}{l}= \\
= \\
= \\
= \\
= \\
= \\
= \\
= \\
= \\
= \\
= \\
= \\
= \\
=\end{array}$ \\
\hline \multicolumn{15}{|c|}{ TAlifon ta } \\
\hline 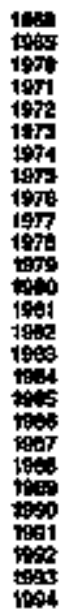 & 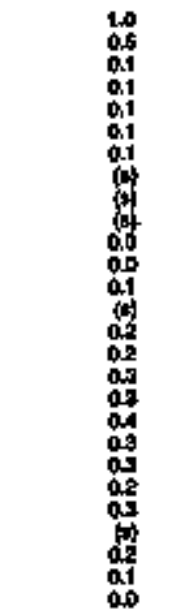 & $\begin{array}{l}1.3 \\
00 \\
0.5 \\
0.5 \\
04 \\
0.3 \\
03 \\
02 \\
02 \\
02 \\
02 \\
02 \\
0.2 \\
0.5 \\
04 \\
0.3 \\
0.3 \\
02 \\
00 \\
02 \\
0.1 \\
0.1 \\
0.3 \\
0.2 \\
0.1 \\
0.1 \\
0.1\end{array}$ & 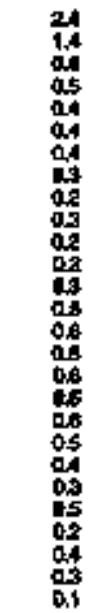 & 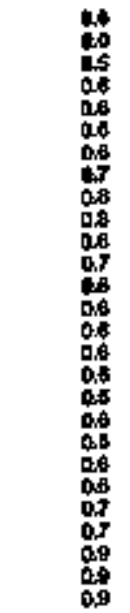 & 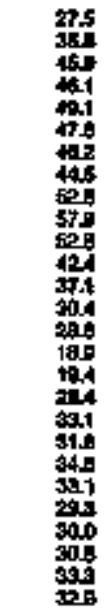 & 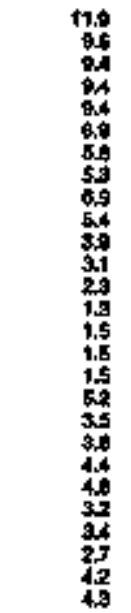 & 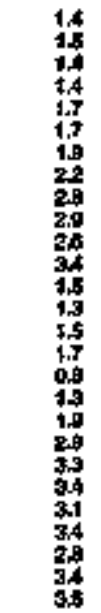 & 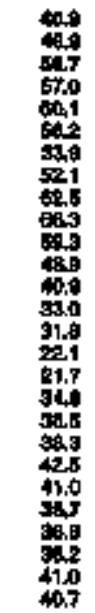 & $\begin{array}{l}00 \\
00 \\
00 \\
00 \\
00 \\
00 \\
00 \\
00 \\
00 \\
00 \\
00 \\
00 \\
00 \\
00 \\
00 \\
00 \\
00 \\
00 \\
00 \\
00 \\
00 \\
00 \\
00 \\
04 \\
45 \\
40 \\
40 \\
40\end{array}$ & 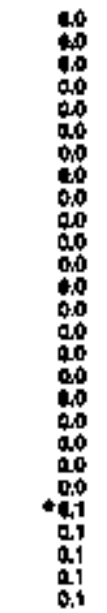 & 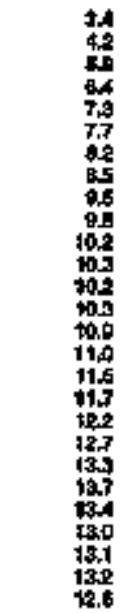 & 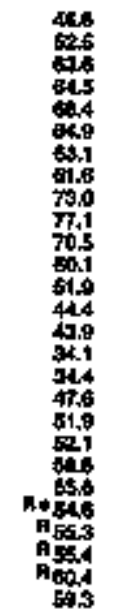 & 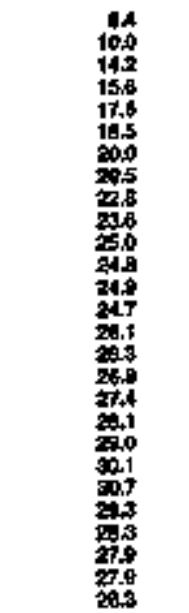 & 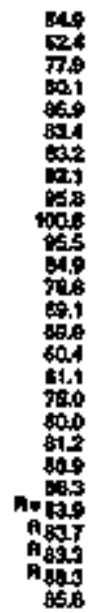 \\
\hline
\end{tabular}

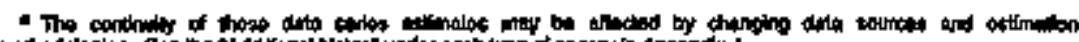

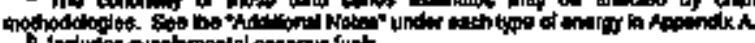

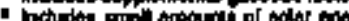

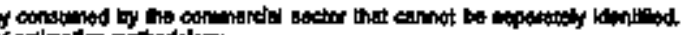

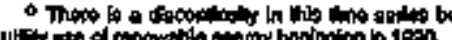

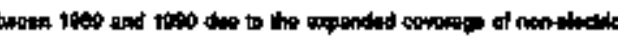
Phofiphoed din

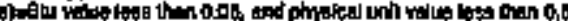

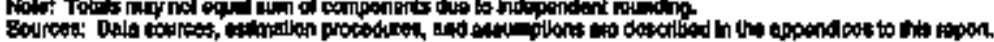

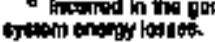


Teble 133. Commercial Energy Consumptlon Estimates, 1960, 1965, 1970-1994, Waine

\begin{tabular}{|c|c|c|c|c|c|c|c|c|c|c|c|c|c|c|}
\hline \multirow[b]{3}{*}{ YaW } & \multicolumn{3}{|c|}{ Con } & \multirow{3}{*}{ 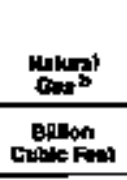 } & \multicolumn{6}{|c|}{ natroter } & \multirow[b]{2}{*}{ 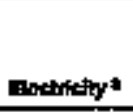 } & \multirow[b]{2}{*}{$\operatorname{mat}^{2}$} & \multirow[b]{2}{*}{ 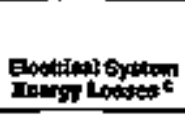 } & \multirow[b]{3}{*}{ Tot:ald } \\
\hline & 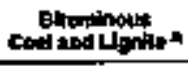 & Nollinestind & 70 to & & Fintion & Krouse" & LPan & artotine & $\begin{array}{l}\text { nothen } \\
\text { full }\end{array}$ & Yotat & & & & \\
\hline & \multicolumn{3}{|c|}{ 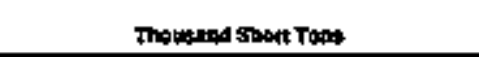 } & & \multicolumn{6}{|c|}{ Therandend barpets } & \multicolumn{3}{|c|}{ 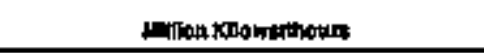 } & \\
\hline 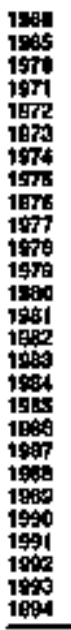 & $\begin{array}{l}76 \\
44 \\
5 \\
5 \\
5 \\
5 \\
5 \\
1 \\
1 \\
3 \\
0 \\
0 \\
9 \\
3 \\
19 \\
13 \\
19 \\
21 \\
32 \\
33 \\
19 \\
11 \\
20 \\
1 \\
17 \\
11 \\
0 \\
\end{array}$ & $\begin{array}{c}3 \\
2 \\
4 \\
13 \\
13 \\
70 \\
10 \\
9 \\
7 \\
7 \\
7 \\
5 \\
4 \\
5 \\
15 \\
10 \\
6 \\
6 \\
6 \\
5 \\
5 \\
4 \\
3 \\
5 \\
5 \\
4 \\
4 \\
2 \\
\end{array}$ & 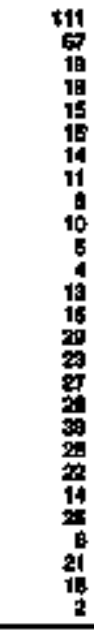 & 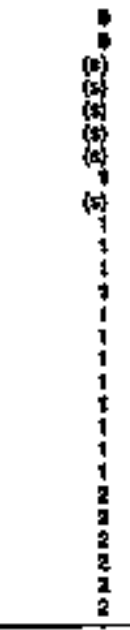 & 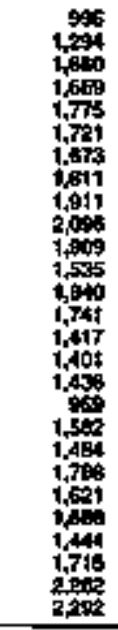 & 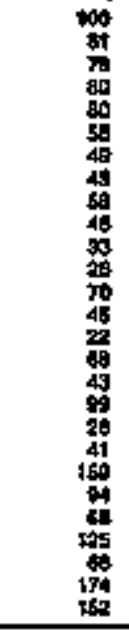 & 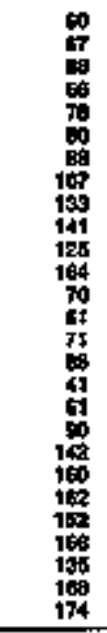 & 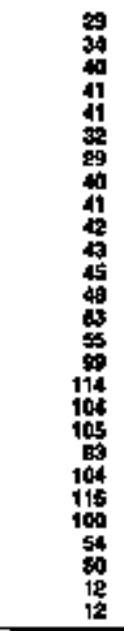 & 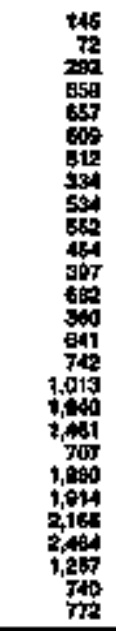 & 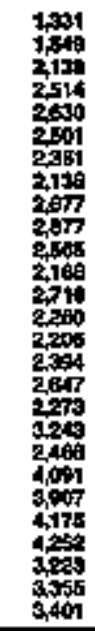 & 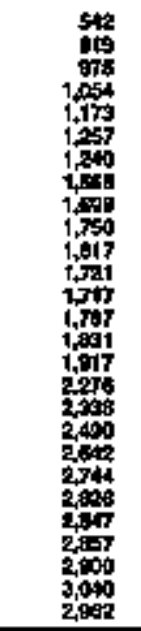 & $\begin{array}{l}= \\
= \\
= \\
= \\
= \\
= \\
= \\
= \\
= \\
= \\
= \\
= \\
= \\
= \\
=\end{array}$ & 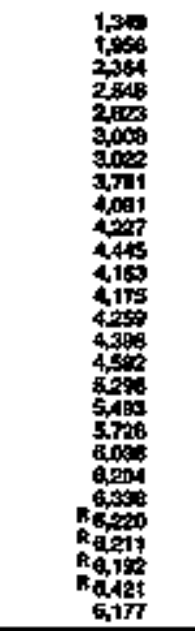 & $\begin{array}{l}= \\
= \\
= \\
= \\
= \\
= \\
= \\
= \\
= \\
= \\
= \\
= \\
= \\
=\end{array}$ \\
\hline \multicolumn{15}{|c|}{ 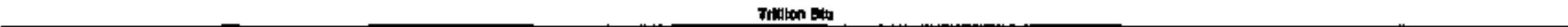 } \\
\hline 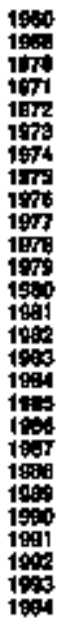 & 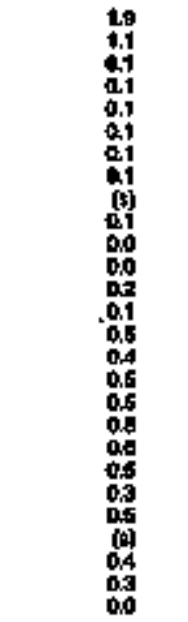 & $\begin{array}{l}1.9 \\
0.5 \\
0.3 \\
0.3 \\
0.3 \\
0.2 \\
0.4 \\
0.2 \\
0.2 \\
0.2 \\
0.1 \\
01 \\
0.1 \\
0.3 \\
0.2 \\
0.2 \\
0.2 \\
0.1 \\
0.1 \\
0.1 \\
0.1 \\
0.1 \\
01 \\
0.1 \\
0.1 \\
01 \\
0.1\end{array}$ & $\begin{array}{l}2.1 \\
1.7 \\
0.5 \\
0.4 \\
0.4 \\
0.3 \\
0.3 \\
0.3 \\
02 \\
0.2 \\
0.1 \\
0.1 \\
0.9 \\
0.4 \\
0.7 \\
0.6 \\
0.7 \\
0.7 \\
0.7 \\
0.7 \\
0.5 \\
0.4 \\
0.5 \\
0.1 \\
0.5 \\
0.4 \\
0.1\end{array}$ & 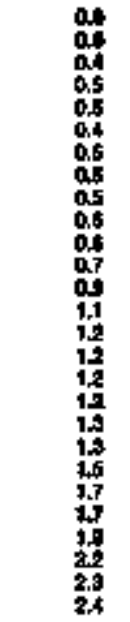 & 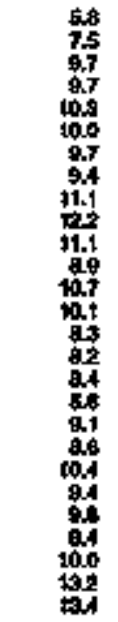 & 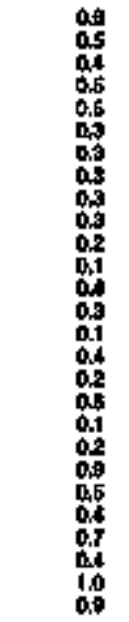 & 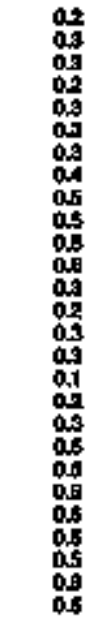 & 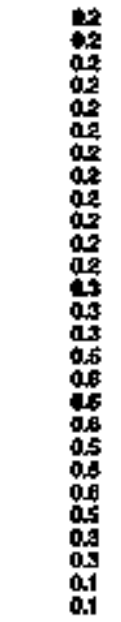 & 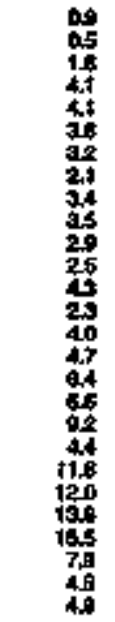 & 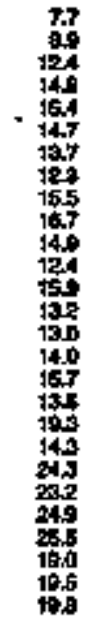 & 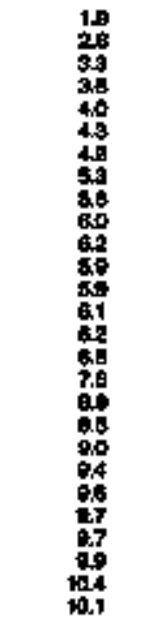 & 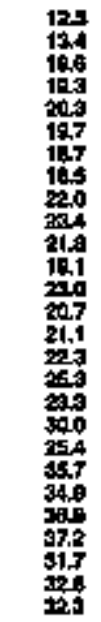 & 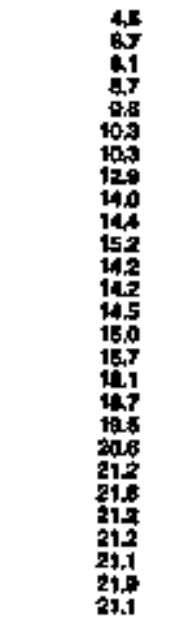 & 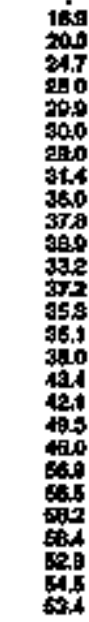 \\
\hline
\end{tabular}

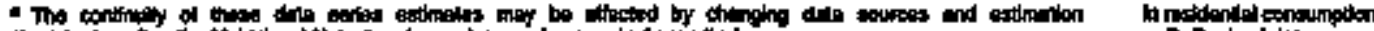

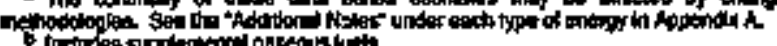

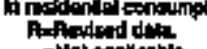

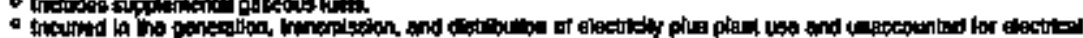

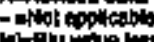

(1)

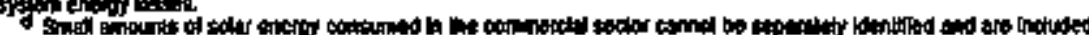


Tabbo 134. Industriad Energy Consumption Egellnates, 1960, 1965, 1970-1994, Malno

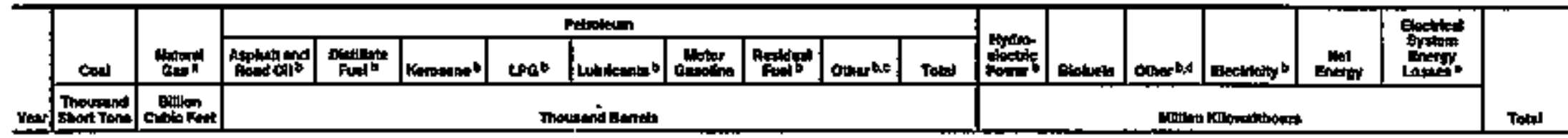

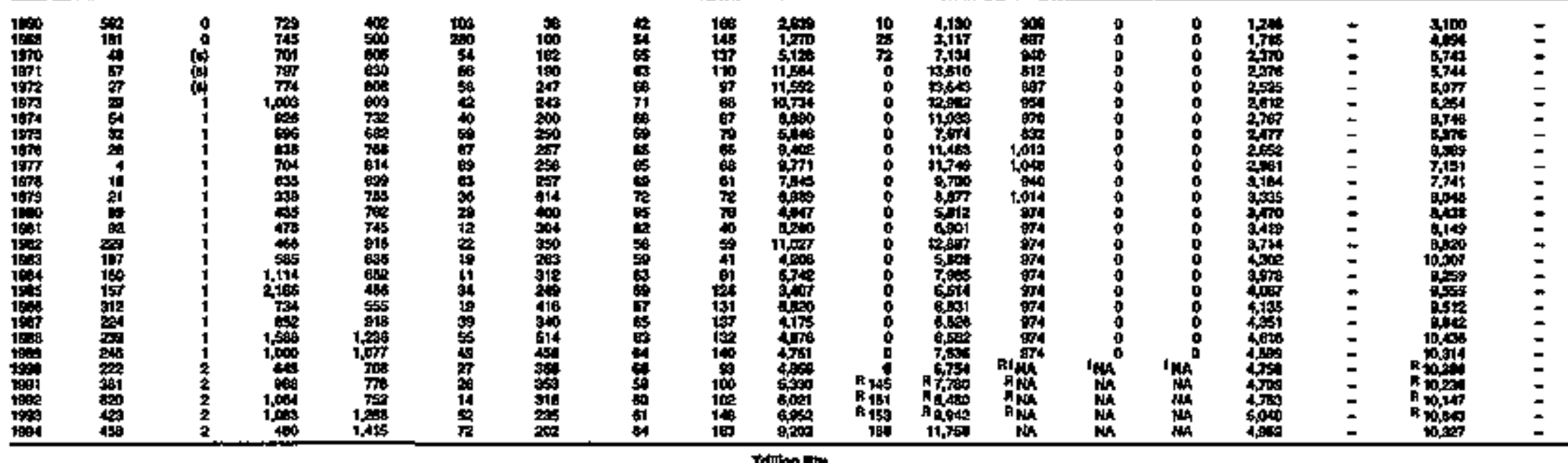

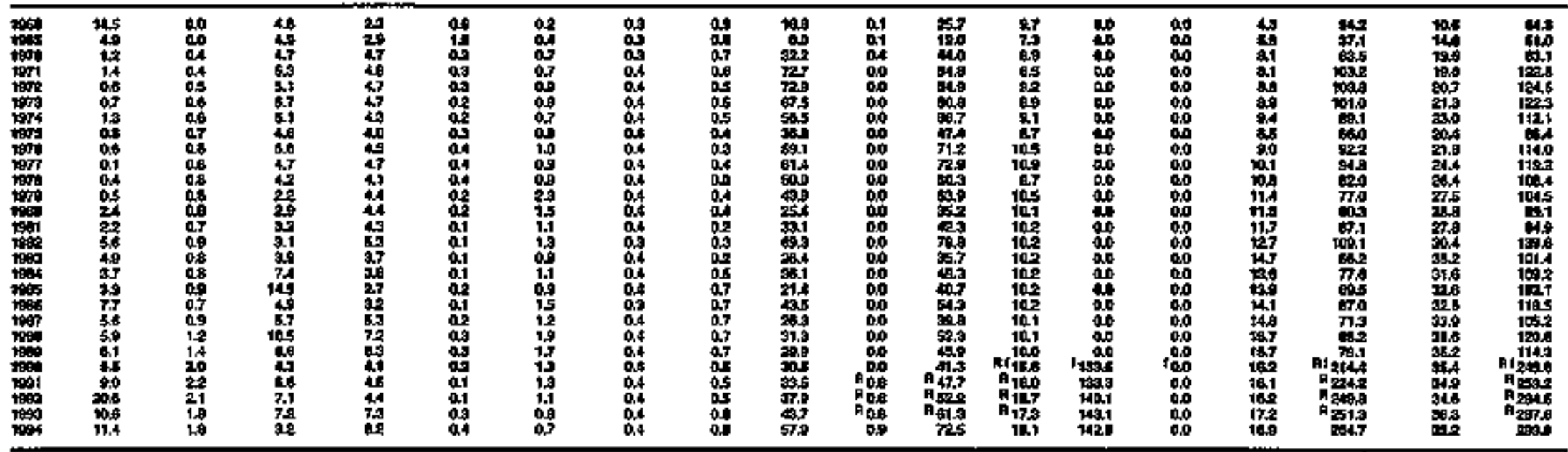

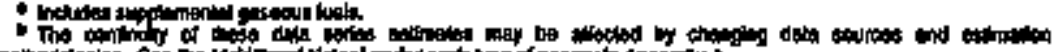

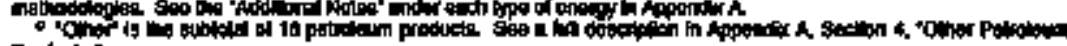
Protition tortion inalned ond pon

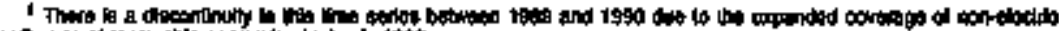
Cray

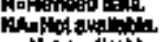

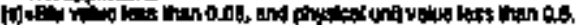

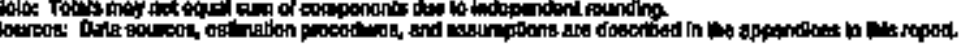


Table 135. Transportalion Energy Consumption Estimales, 1960, 1965, 1970-1994, Nalne

\begin{tabular}{|c|c|c|c|c|c|c|c|c|c|c|c|c|c|c|c|}
\hline \multirow[b]{3}{*}{$Y_{t-1}$} & \multirow[b]{2}{*}{ cowe } & \multirow[b]{2}{*}{ 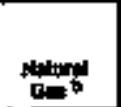 } & \multicolumn{8}{|c|}{ Proptopop } & \multirow{3}{*}{ 벼tonla } & & \multirow[b]{2}{*}{ Enel } & \multirow[b]{2}{*}{ 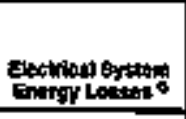 } & \multirow[b]{3}{*}{ Totat } \\
\hline & & & Andilon. & Dintions & Fuel. & $\omega_{0}=$ & 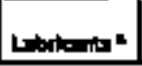 & Motor & 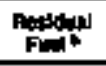 & Tat: & & Extraty" & & & \\
\hline & Thousund & 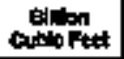 & \multicolumn{8}{|c|}{ Toutend Burpen } & & \multicolumn{3}{|c|}{ 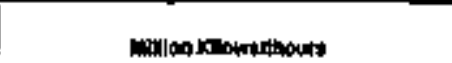 } & \\
\hline 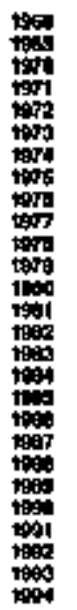 & 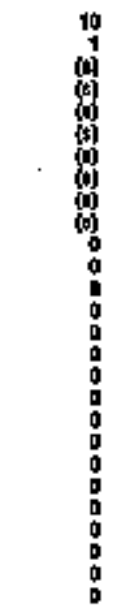 & 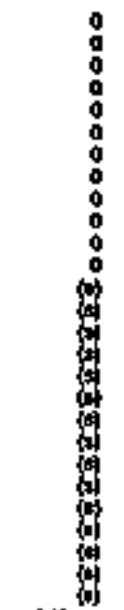 & 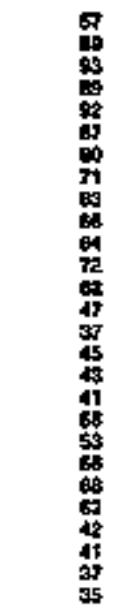 & 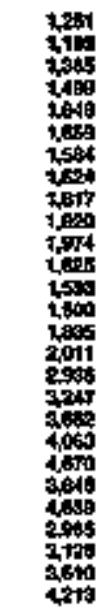 & 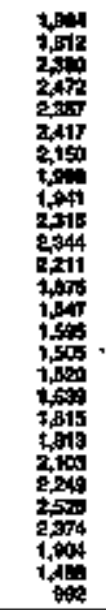 & $\begin{array}{l}1 \\
2 \\
3 \\
3 \\
4 \\
8 \\
5 \\
3 \\
4 \\
1 \\
6 \\
7 \\
9 \\
4 \\
78 \\
15 \\
24 \\
15 \\
38 \\
15 \\
30 \\
30 \\
77 \\
17 \\
15 \\
13 \\
25\end{array}$ & 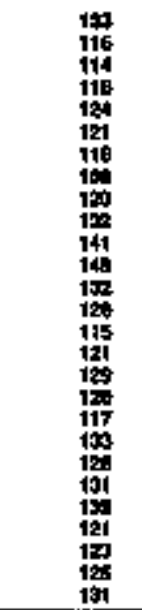 & 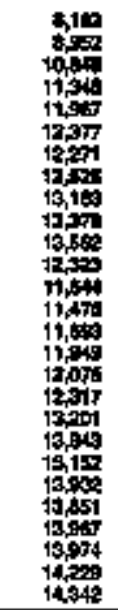 & 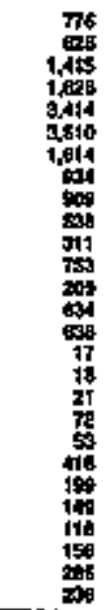 & 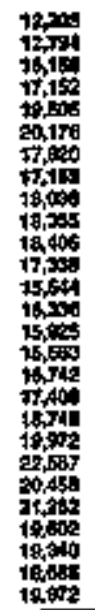 & $\begin{array}{l}0 \\
0 \\
0 \\
0 \\
0 \\
0 \\
0 \\
0 \\
0 \\
0 \\
0 \\
0 \\
0 \\
0 \\
0 \\
0 \\
0 \\
0 \\
0 \\
0 \\
0 \\
0 \\
0 \\
0 \\
0 \\
0 \\
0 \\
0\end{array}$ & $\begin{array}{l}0 \\
0 \\
0 \\
0 \\
0 \\
0 \\
0 \\
0 \\
0 \\
0 \\
0 \\
0 \\
0 \\
0 \\
0 \\
0 \\
0 \\
0 \\
0 \\
0 \\
0 \\
0 \\
0 \\
0 \\
0 \\
0 \\
0 \\
0 \\
0\end{array}$ & $\begin{array}{l}= \\
= \\
= \\
= \\
= \\
= \\
= \\
= \\
= \\
= \\
= \\
= \\
= \\
= \\
=\end{array}$ & $\begin{array}{l}0 \\
0 \\
0 \\
0 \\
0 \\
0 \\
0 \\
0 \\
0 \\
0 \\
0 \\
0 \\
0 \\
0 \\
0 \\
0 \\
0 \\
0 \\
0 \\
0 \\
0 \\
0 \\
0 \\
0 \\
0 \\
0\end{array}$ & $\begin{array}{l}z \\
z \\
z \\
z \\
z \\
z \\
z \\
z \\
z \\
z \\
z \\
z \\
z \\
z\end{array}$ \\
\hline \multicolumn{16}{|c|}{ Thimen Giv } \\
\hline 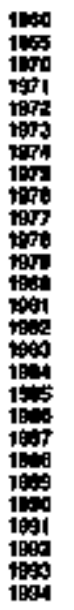 & 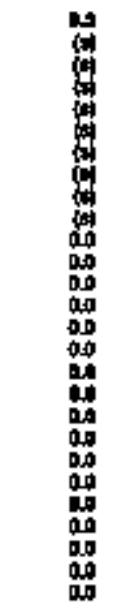 & 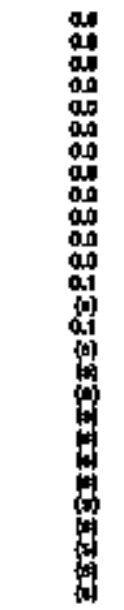 & $\begin{array}{l}0.9 \\
04 \\
05 \\
04 \\
05 \\
04 \\
04 \\
04 \\
03 \\
03 \\
03 \\
04 \\
04 \\
02 \\
02 \\
02 \\
02 \\
02 \\
03 \\
03 \\
03 \\
03 \\
03 \\
00 \\
02 \\
00 \\
02\end{array}$ & 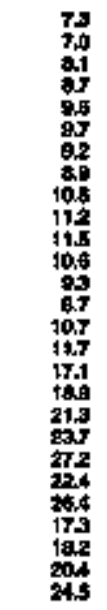 & 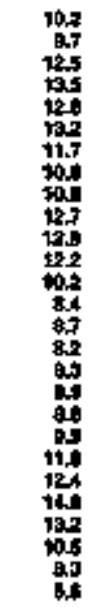 & 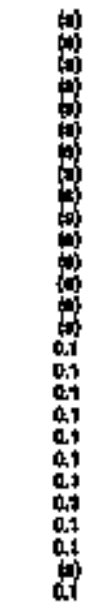 & 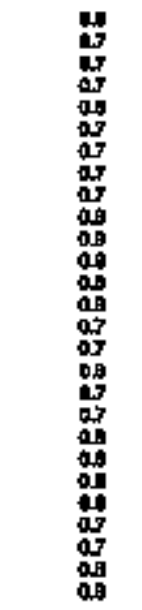 & 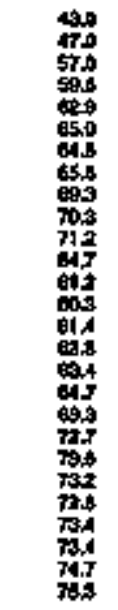 & 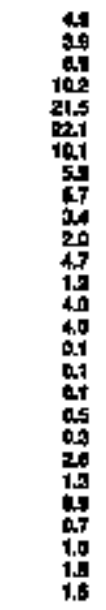 & 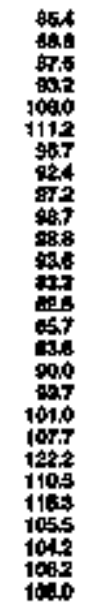 & 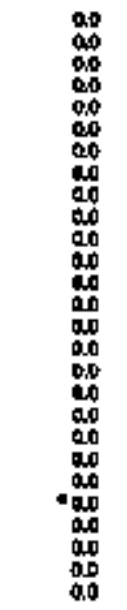 & 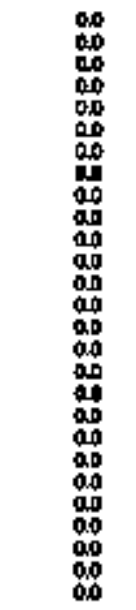 & 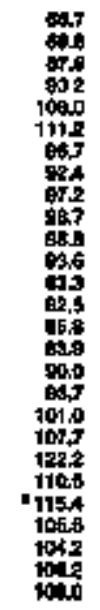 & 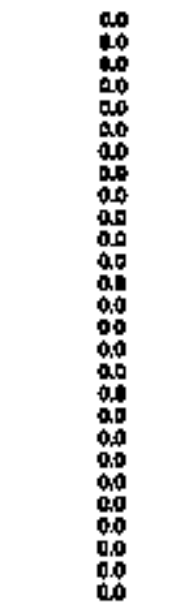 & 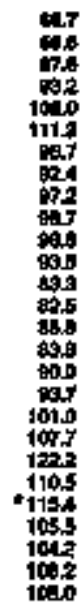 \\
\hline
\end{tabular}

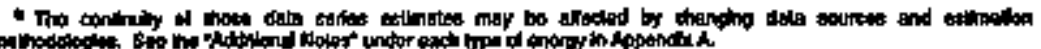

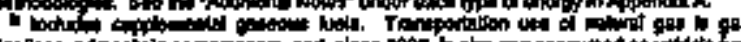

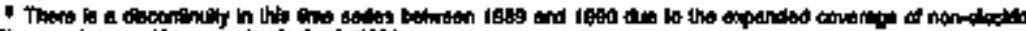

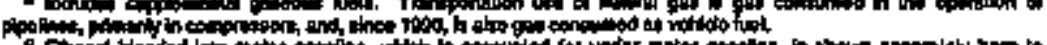

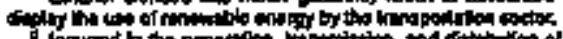

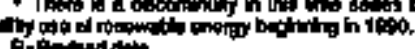

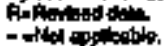

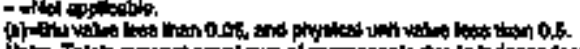

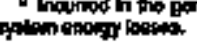


Table 136. Estomates of Enorgy Input at Eketric Utilities, 1960, 1965, 1970-1994, Maine

A

\begin{tabular}{|c|c|c|c|c|c|c|c|c|c|c|c|c|c|c|}
\hline 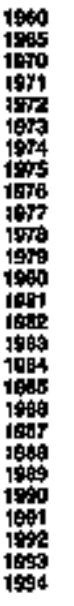 & 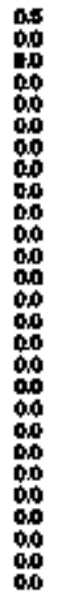 & 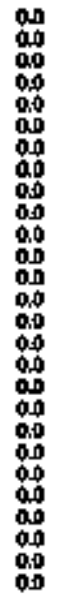 & 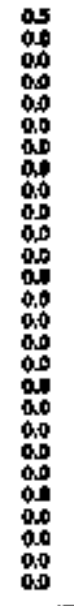 & 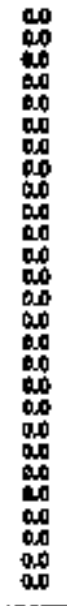 & 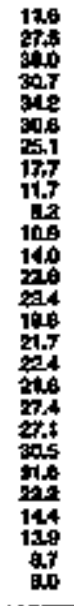 & 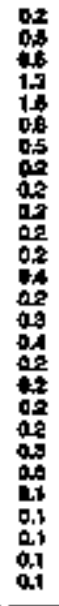 & 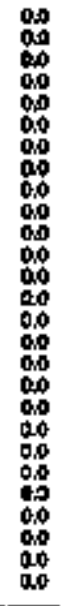 & 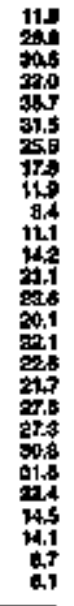 & 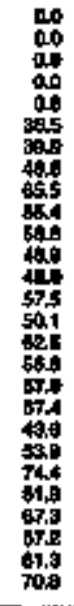 & 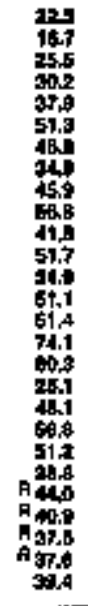 & 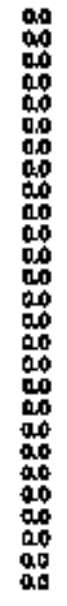 & 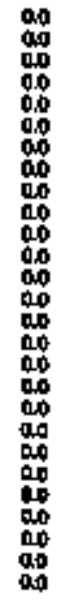 & 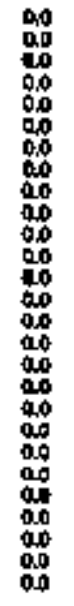 & 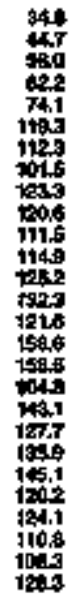 \\
\hline
\end{tabular}

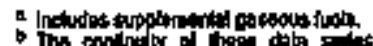

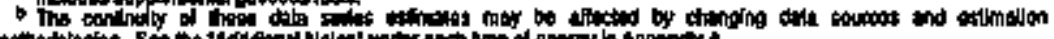

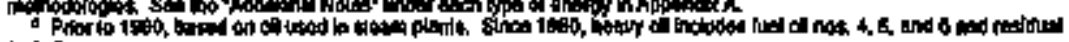

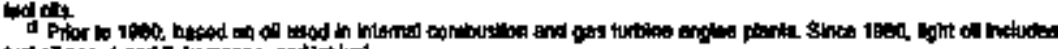

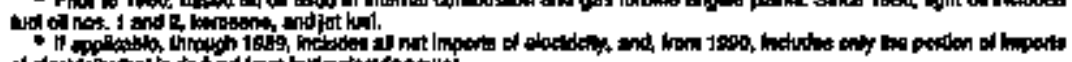

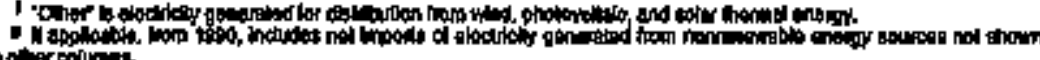

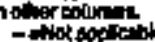

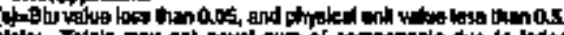

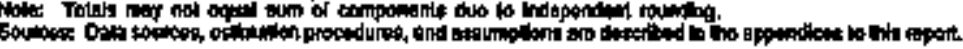

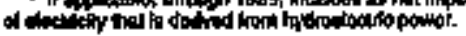


Tabla 137, Enurgy Consumption Estimales by Source, 1960, 1965, 1970-1994, Marylend

\begin{tabular}{|c|c|c|c|c|c|c|c|c|c|c|c|c|c|c|c|c|c|c|}
\hline \multirow[b]{2}{*}{ cons $=$} & \multirow[b]{2}{*}{$\operatorname{man}_{\mathrm{Bat}}$} & \multicolumn{11}{|c|}{ Potodum } & \multirow[b]{2}{*}{ 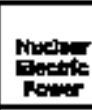 } & \multirow[b]{2}{*}{ Pristion } & \multirow[b]{2}{*}{ Bisoknth. } & \multirow[b]{2}{*}{ 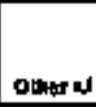 } & \multirow{3}{*}{ 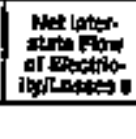 } & \multirow[b]{3}{*}{ 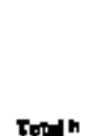 } \\
\hline & & 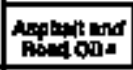 & 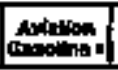 & 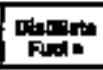 & Find. & koros: & Lpace & Loth: & 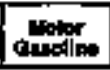 & 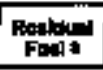 & Onm 40 & Tow & & & & & & \\
\hline Thend & $\sin 20$ & \multicolumn{11}{|c|}{ Tromerod Bunant } & \multicolumn{4}{|c|}{ 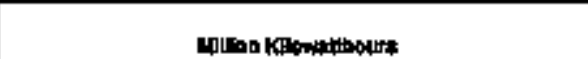 } & & \\
\hline
\end{tabular}

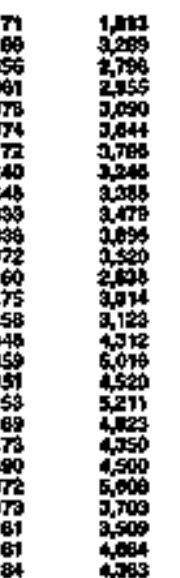

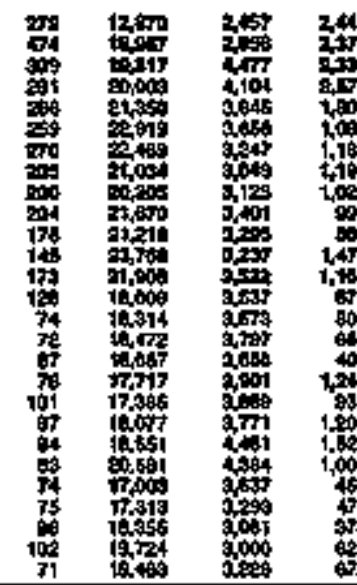

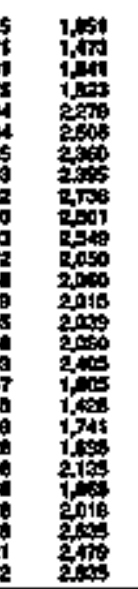

\begin{tabular}{|c|c|c|c|c|c|}
\hline 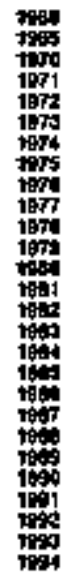 & 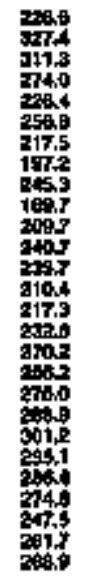 & 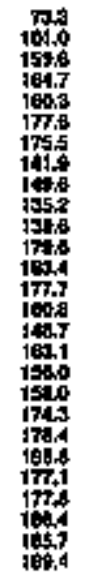 & 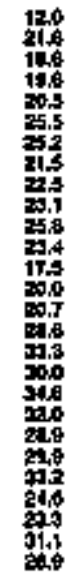 & 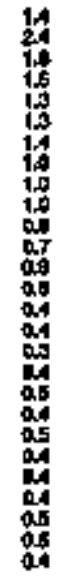 & 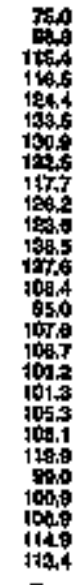 \\
\hline
\end{tabular}

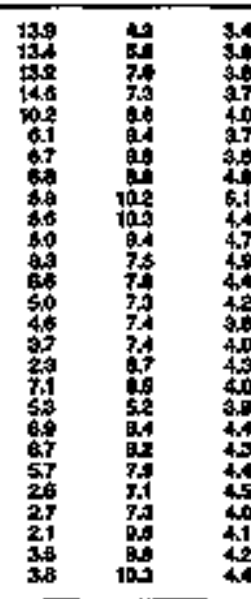

Thlont aty

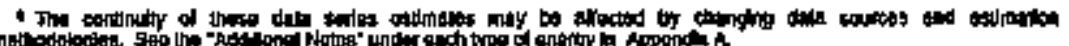

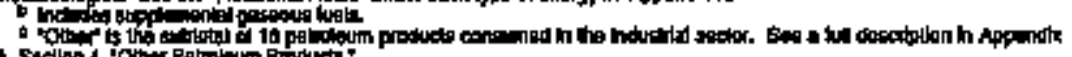

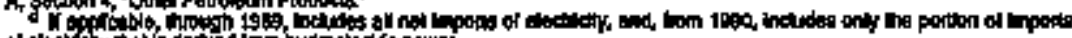

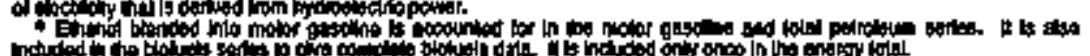

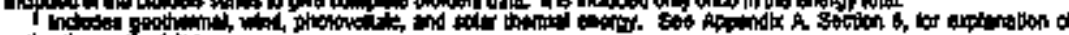

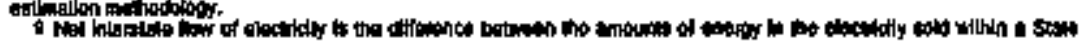

\begin{tabular}{|c|c|c|c|c|c|c|c|c|}
\hline 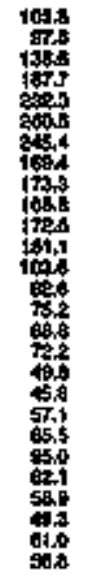 & 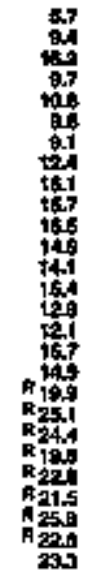 & 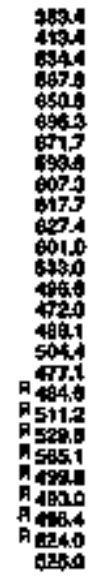 & 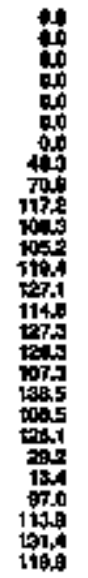 & 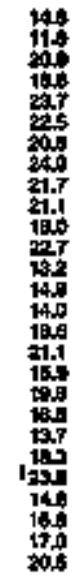 & 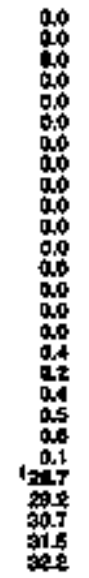 & 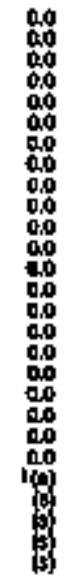 & 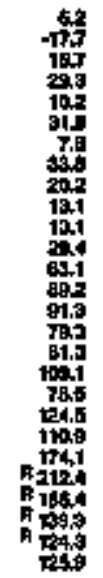 & 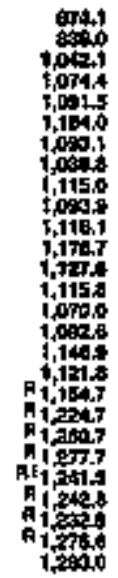 \\
\hline
\end{tabular}

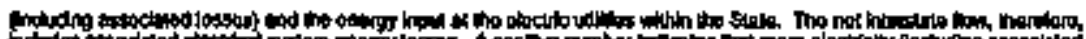

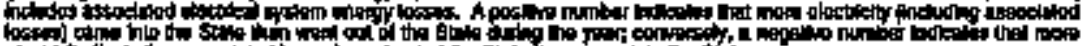
thing

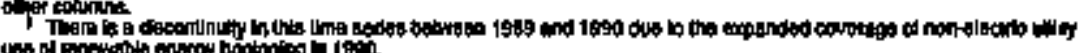

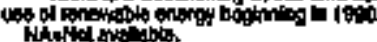

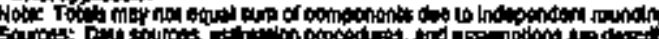


Tabie 138. Aesidential Energy Cons:umpllon Estimates, 1960, 1965, 1970-1994, Maryland

\begin{tabular}{|c|c|c|c|c|c|c|c|c|c|c|c|c|c|c|}
\hline & \multicolumn{3}{|c|}{$\cos 1$} & \multirow[b]{2}{*}{ mang } & \multicolumn{4}{|c|}{ Putrofinen } & \multirow[b]{2}{*}{ Biflub } & \multirow[b]{2}{*}{ solare } & \multirow[b]{2}{*}{ 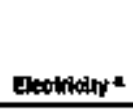 } & \multirow[b]{2}{*}{ enorisy } & \multirow[b]{2}{*}{ 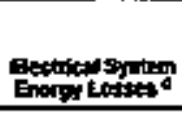 } & \multirow[b]{3}{*}{ 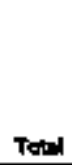 } \\
\hline & 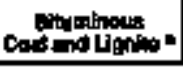 & Nothoshate & Totat & & Denter & temente & LPt: & Totol & & & & & & \\
\hline Your & \multicolumn{3}{|c|}{ Thousend short Tons } & 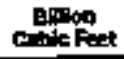 & \multicolumn{4}{|c|}{ Thounond Bande } & Thoostid & \multicolumn{4}{|c|}{ 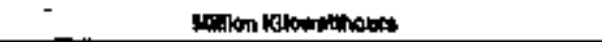 } & \\
\hline
\end{tabular}

\begin{tabular}{|c|c|c|c|c|c|c|c|c|c|c|c|c|c|c|}
\hline 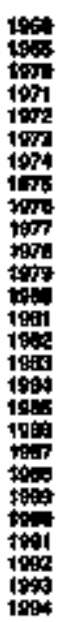 & 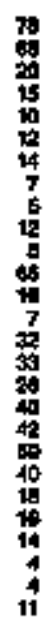 & $\begin{array}{l}37 \\
37 \\
14 \\
14 \\
10 \\
10 \\
8 \\
8 \\
7 \\
7 \\
4 \\
4 \\
4 \\
11 \\
7 \\
4 \\
4 \\
4 \\
4 \\
4 \\
5 \\
2 \\
2 \\
2 \\
1 \\
3 \\
3\end{array}$ & 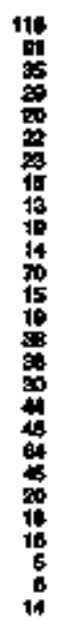 & 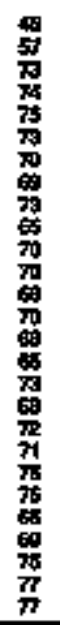 & 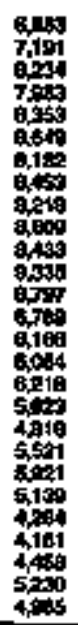 & 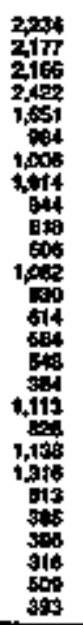 & 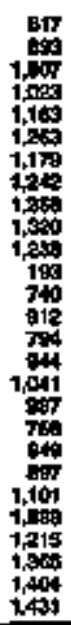 & 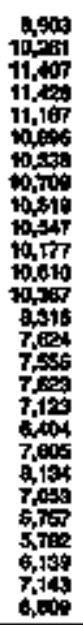 & 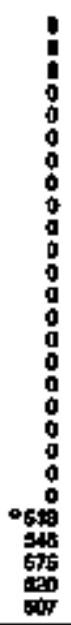 & $\begin{array}{r}0 \\
0 \\
0 \\
0 \\
0 \\
0 \\
0 \\
0 \\
0 \\
0 \\
0 \\
0 \\
0 \\
0 \\
0 \\
0 \\
0 \\
0 \\
0 \\
0 \\
-11 \\
11 \\
12 \\
13 \\
13 \\
13\end{array}$ & 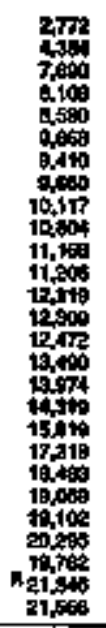 & $\begin{array}{l}= \\
= \\
= \\
= \\
= \\
= \\
= \\
= \\
= \\
= \\
= \\
z \\
= \\
= \\
=\end{array}$ & 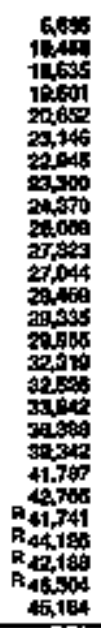 & $\begin{array}{l}= \\
= \\
= \\
= \\
= \\
= \\
= \\
= \\
= \\
= \\
= \\
= \\
= \\
= \\
=\end{array}$ \\
\hline
\end{tabular}

\begin{tabular}{|c|c|c|c|c|c|c|c|c|c|c|c|c|c|c|}
\hline 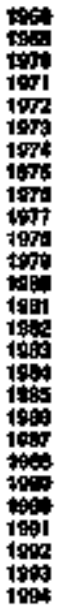 & $\begin{array}{l}20 \\
03 \\
05 \\
0.4 \\
0.2 \\
0.3 \\
0.3 \\
0.2 \\
0.1 \\
0.3 \\
0.2 \\
0.5 \\
02 \\
0.2 \\
0.9 \\
0.9 \\
0.0 \\
0.0 \\
1.0 \\
1.5 \\
0.0 \\
0.4 \\
0.4 \\
0.3 \\
0.1 \\
0.1 \\
0.3\end{array}$ & 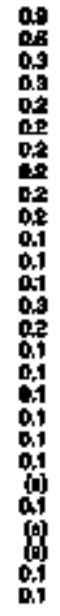 & 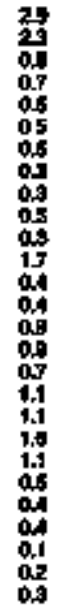 & 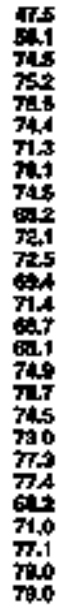 & 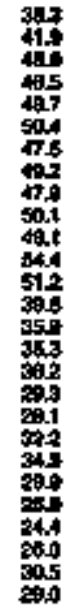 & 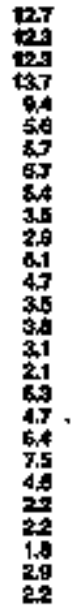 & 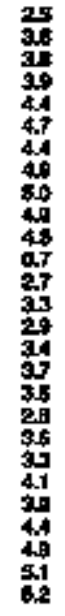 & 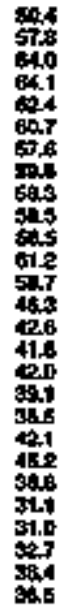 & 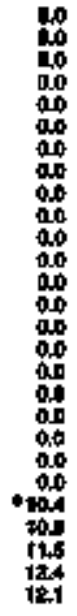 & $\begin{array}{l}00 \\
00 \\
00 \\
00 \\
00 \\
00 \\
00 \\
00 \\
00 \\
00 \\
00 \\
00 \\
00 \\
00 \\
000 \\
00 \\
000 \\
00 \\
00 \\
000 \\
00 \\
000 \\
00 \\
00 \\
000\end{array}$ & 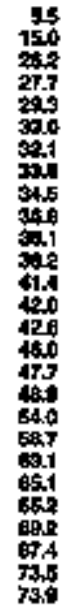 & 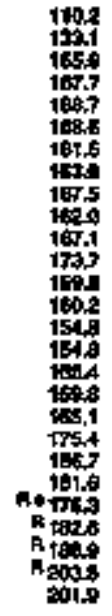 & 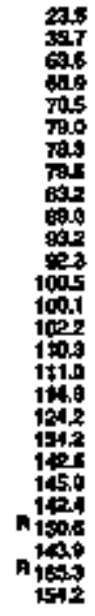 & 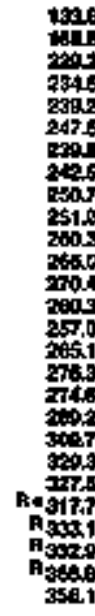 \\
\hline
\end{tabular}

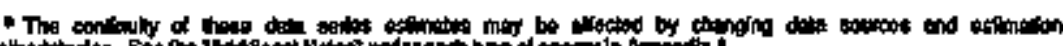

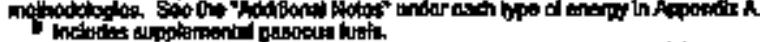

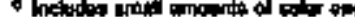

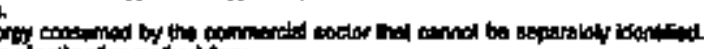

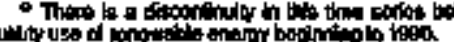

iutrugo ad

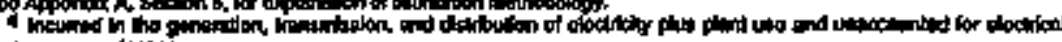


Table 139. Conmercial Energy Consumption Estimates, 1960, 1965, 1970-1994, Maryland

\begin{tabular}{|c|c|c|c|c|c|c|c|c|c|c|c|c|c|c|}
\hline \multirow[b]{3}{*}{$\underline{Y} \underline{x}$} & \multicolumn{3}{|c|}{ cow } & \multirow{3}{*}{ 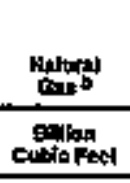 } & \multicolumn{6}{|c|}{ Petroletern } & \multirow[b]{2}{*}{ Dectitaxy } & \multirow[b]{2}{*}{ Hot } & \multirow[b]{2}{*}{ Bonthal Syeten } & \multirow[b]{3}{*}{ Toteld } \\
\hline & 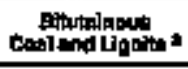 & Anthrastite & Fots & & 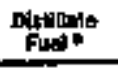 & Korsacene & LPG " & crestion & 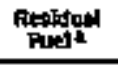 & Total & & & & \\
\hline & \multicolumn{3}{|c|}{ 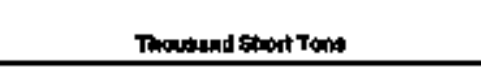 } & & \multicolumn{6}{|c|}{ Nortand Binche } & \multicolumn{3}{|c|}{ 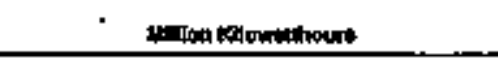 } & \\
\hline 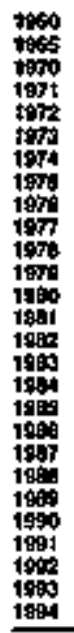 & 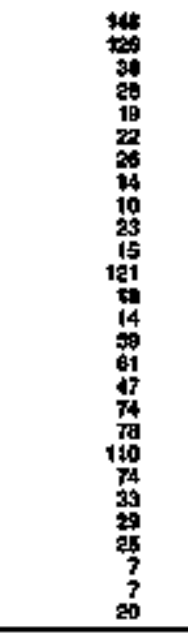 & $\begin{array}{l}25 \\
10 \\
10 \\
1 \\
7 \\
7 \\
6 \\
5 \\
5 \\
5 \\
4 \\
3 \\
4 \\
4 \\
2 \\
3 \\
3 \\
2 \\
3 \\
4 \\
1 \\
1 \\
1 \\
2 \\
2 \\
2\end{array}$ & 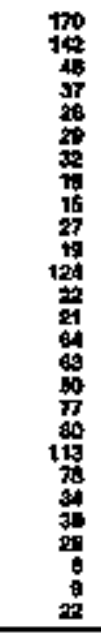 & 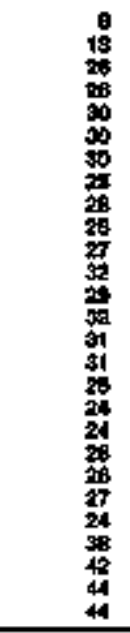 & 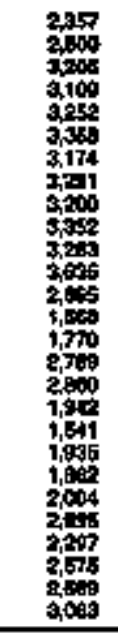 & 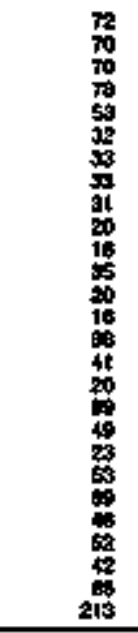 & 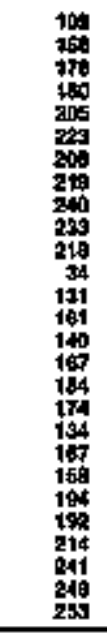 & 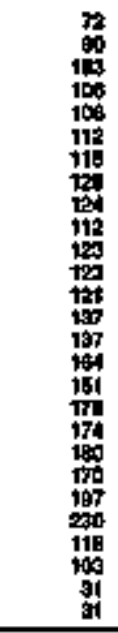 & 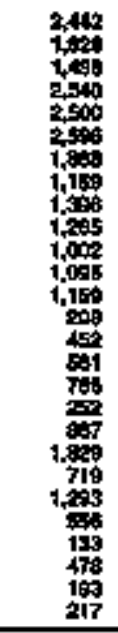 & 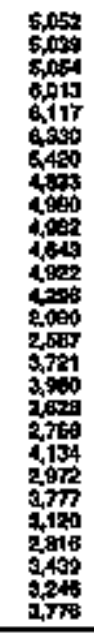 & 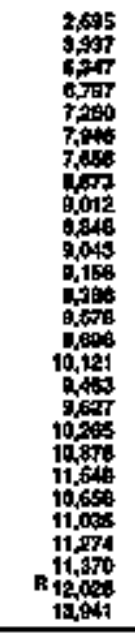 & $\begin{array}{l}= \\
= \\
= \\
= \\
= \\
= \\
= \\
5 \\
5 \\
= \\
= \\
= \\
= \\
= \\
= \\
=\end{array}$ & 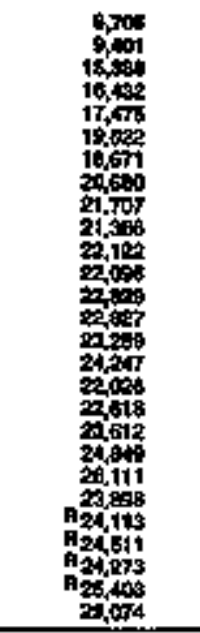 & $\begin{array}{l}z \\
z \\
z \\
z \\
z \\
z \\
z \\
z \\
z \\
z \\
z \\
z \\
z \\
z \\
=\end{array}$ \\
\hline \multicolumn{15}{|c|}{ MAlllow Gus } \\
\hline 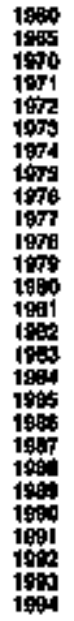 & 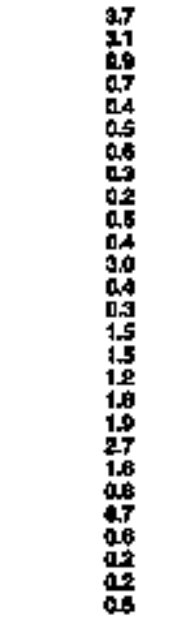 & 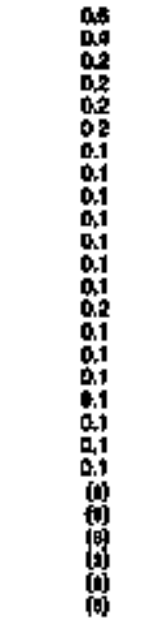 & 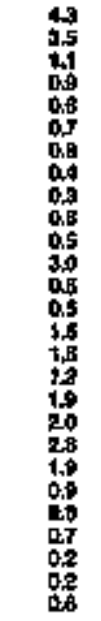 & 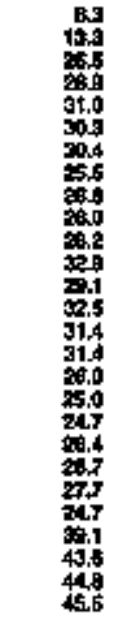 & 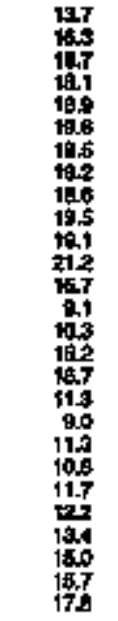 & 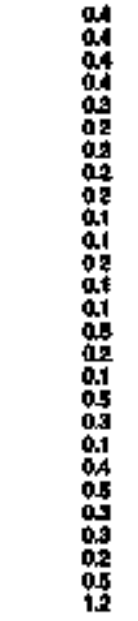 & 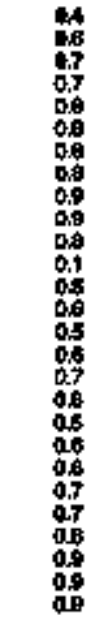 & 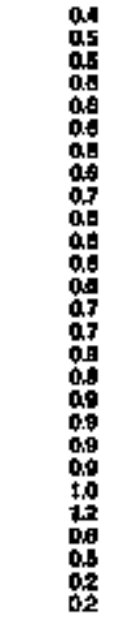 & 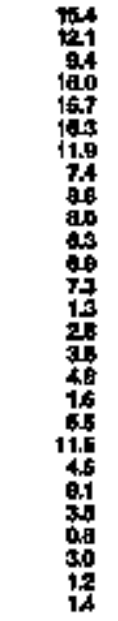 & 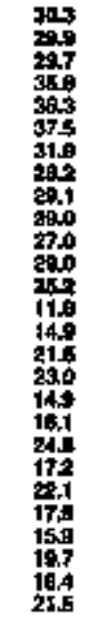 & 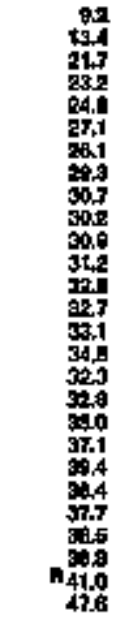 & 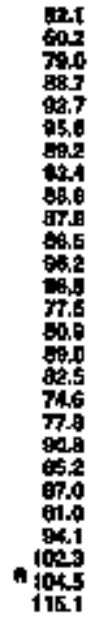 & 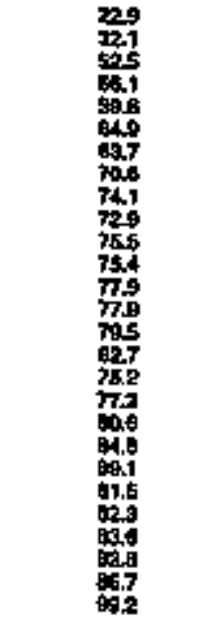 & 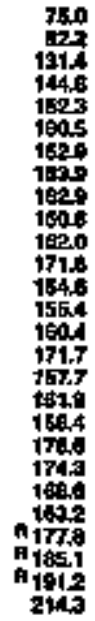 \\
\hline
\end{tabular}




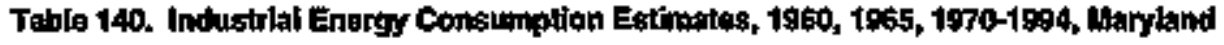

\begin{tabular}{|c|c|c|c|c|c|c|c|c|c|c|c|c|c|c|c|c|c|c|}
\hline \multirow[b]{3}{*}{ vat } & \multirow[b]{2}{*}{ Cand } & \multirow[b]{2}{*}{$\operatorname{lath}_{0 \rightarrow 4}$} & \multicolumn{9}{|c|}{ Patroven } & \multirow[b]{2}{*}{ 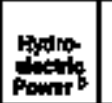 } & \multirow[b]{2}{*}{ molotendin } & \multirow[b]{2}{*}{ onem bed } & \multirow[b]{2}{*}{ nestetely } & \multirow[b]{2}{*}{ Enterts } & \multirow{2}{*}{ 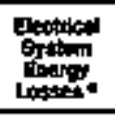 } & \multirow[b]{3}{*}{ " Tholl } \\
\hline & & & Aspond ont & 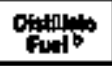 & $\cos$ 에 & a" & | & onsontion & 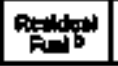 & oumens & Tot: & & & & & & & \\
\hline & Thot at & 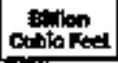 & \multicolumn{9}{|c|}{ Thousand harrta } & \multicolumn{6}{|c|}{ Mon wat } & \\
\hline 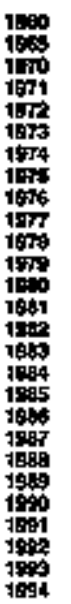 & 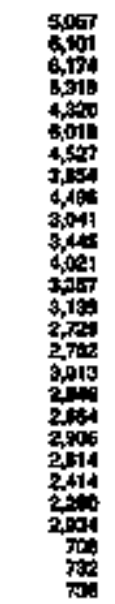 & 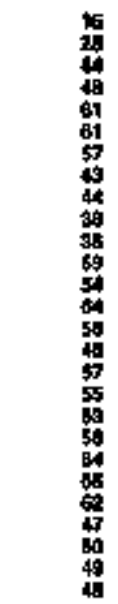 & 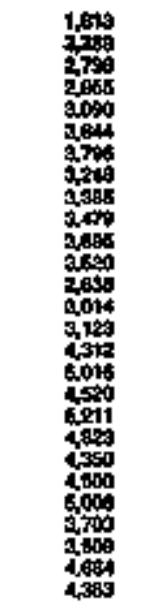 & 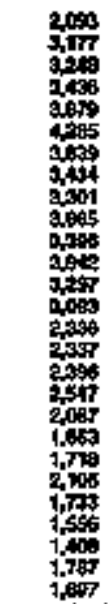 & 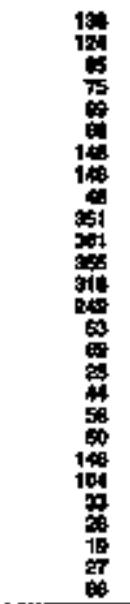 & 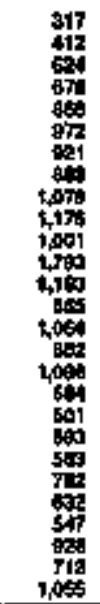 & 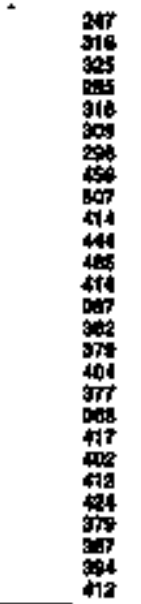 & 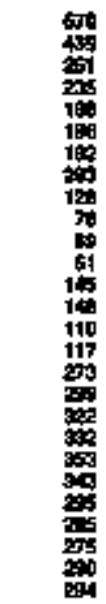 & 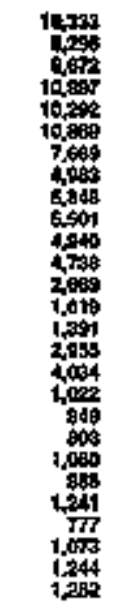 & 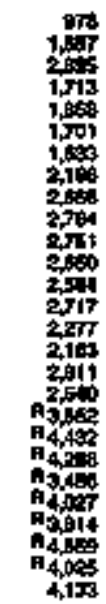 & 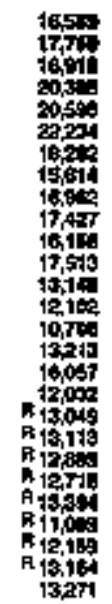 & 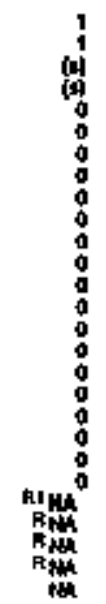 & 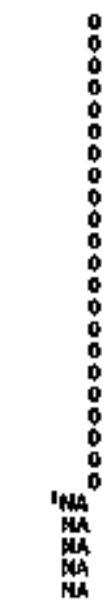 & $\begin{array}{l}0 \\
0 \\
0 \\
0 \\
0 \\
0 \\
0 \\
0 \\
0 \\
0 \\
0 \\
0 \\
0 \\
0 \\
0 \\
0 \\
0 \\
0 \\
0 \\
0 \\
0 \\
0 \\
4\end{array}$ & 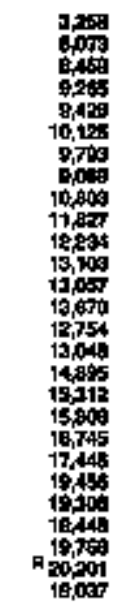 & 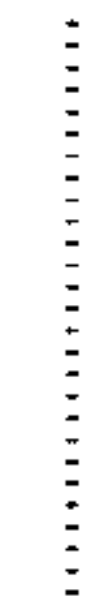 & 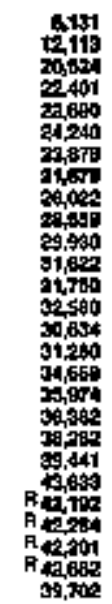 & $\begin{array}{l}= \\
z \\
z \\
z \\
z \\
z \\
z \\
z \\
z \\
z \\
z \\
z \\
z \\
z\end{array}$ \\
\hline \multicolumn{19}{|c|}{ Thoom EAy } \\
\hline 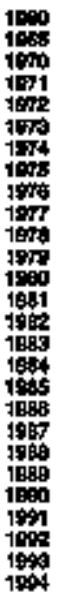 & 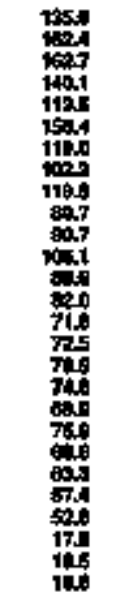 & 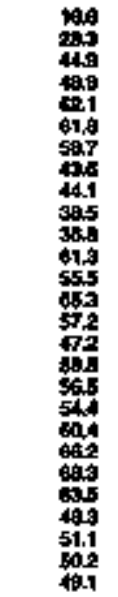 & 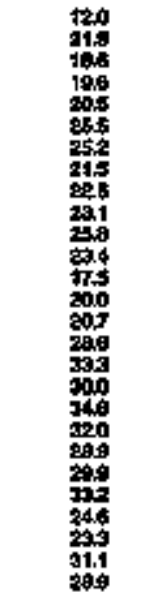 & 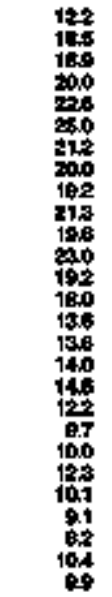 & 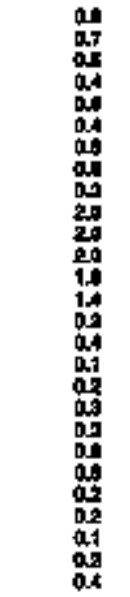 & 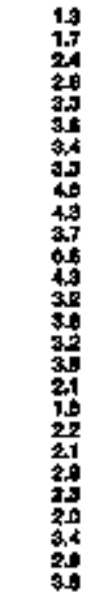 & $\begin{array}{l}15 \\
15 \\
20 \\
18 \\
19 \\
10 \\
10 \\
21 \\
34 \\
25 \\
27 \\
20 \\
25 \\
24 \\
20 \\
25 \\
25 \\
23 \\
25 \\
25 \\
24 \\
25 \\
24 \\
25 \\
23 \\
24 \\
25\end{array}$ & 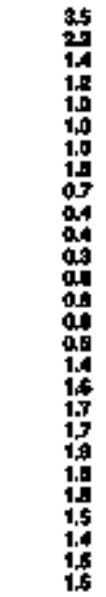 & 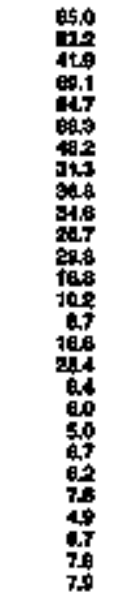 & 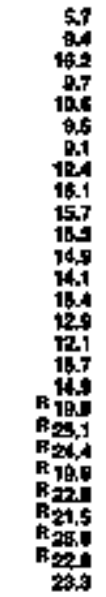 & 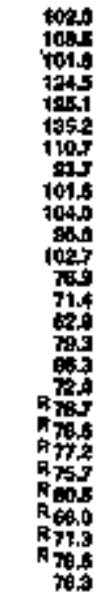 & 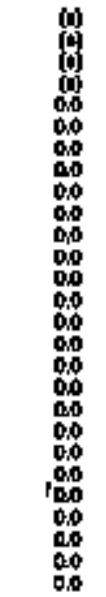 & 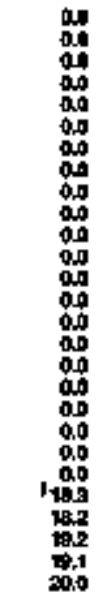 & $\begin{array}{l}00 \\
00 \\
00 \\
00 \\
00 \\
00 \\
00 \\
00 \\
000 \\
00 \\
00 \\
00 \\
00 \\
00 \\
00 \\
00 \\
00 \\
000 \\
00 \\
000 \\
000 \\
000 \\
00 \\
00 \\
00 \\
0.0\end{array}$ & 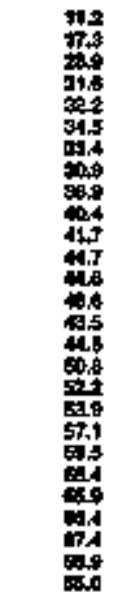 & 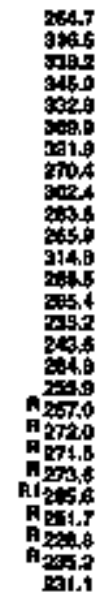 & 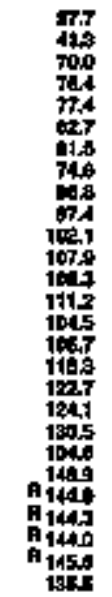 & 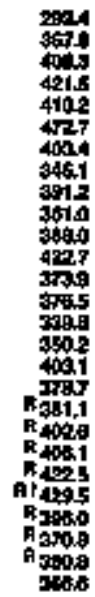 \\
\hline
\end{tabular}

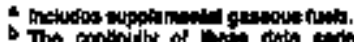

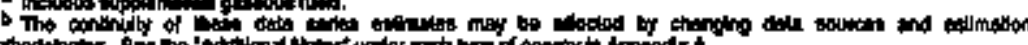

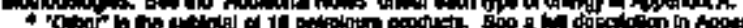

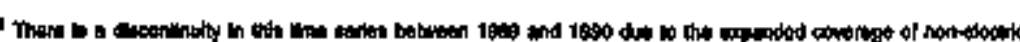

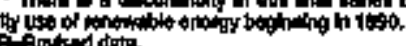

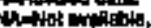

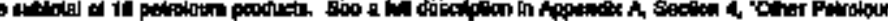

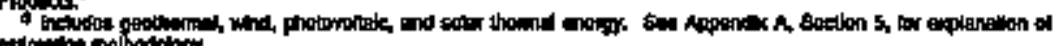

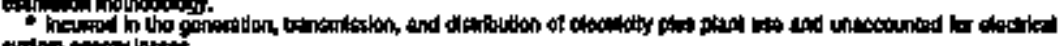

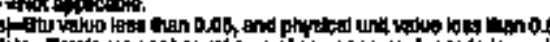

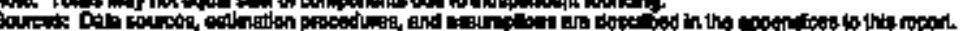
ordention 
Tabye 141. Transportilion Enorgy Consumption Estimetes, 1950, 1955, 1970-1994, Nexyland

\begin{tabular}{|c|c|c|c|c|c|c|c|c|c|c|c|c|c|c|c|}
\hline \multirow[b]{3}{*}{ rand } & \multirow[b]{2}{*}{ cont" } & \multirow[b]{2}{*}{ mantist } & \multicolumn{8}{|c|}{ Parowers } & \multirow{3}{*}{ Blokets" } & \multirow[b]{2}{*}{ Exctithy = } & \multirow[b]{2}{*}{ Exity } & \multirow[b]{2}{*}{ 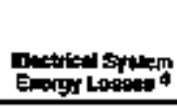 } & \multirow[b]{3}{*}{ rotal } \\
\hline & & & Aviation. & 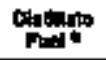 & Ind & LPG: & Lubtzanits" & ontom & 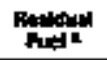 & Totat & & & & & \\
\hline & $\begin{array}{l}\text { Thoutiold } \\
\text { start Tont }\end{array}$ & cilite Fat & \multicolumn{8}{|c|}{ Thooupoo Durnts } & & \multicolumn{3}{|c|}{ 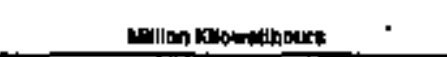 } & \\
\hline 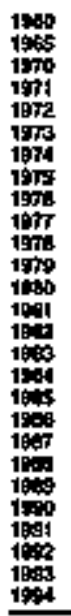 & $\begin{array}{c}0 \\
00 \\
10 \\
7 \\
5 \\
5 \\
1 \\
0 \\
06 \\
0 \\
0 \\
0 \\
0 \\
0 \\
0 \\
0 \\
0 \\
0 \\
0 \\
0 \\
0 \\
0 \\
0 \\
0\end{array}$ & $\begin{array}{l}1 \\
1 \\
2 \\
2 \\
2 \\
1 \\
2 \\
2 \\
2 \\
2 \\
2 \\
2 \\
2 \\
2 \\
4 \\
4 \\
1 \\
1 \\
3 \\
2 \\
2 \\
2 \\
2 \\
3 \\
2 \\
2 \\
2 \\
3 \\
2 \\
2 \\
3 \\
\end{array}$ & 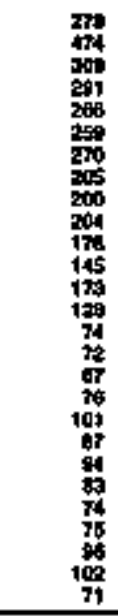 & 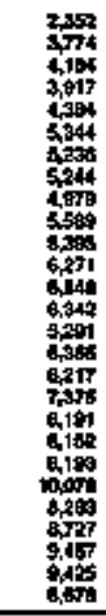 & 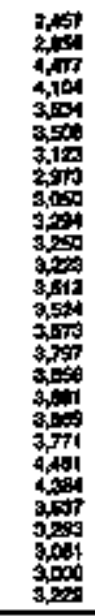 & 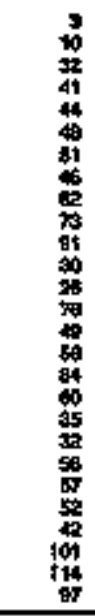 & 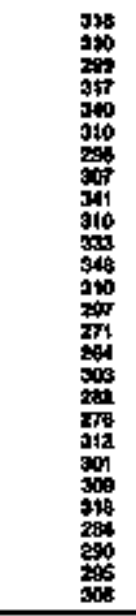 & 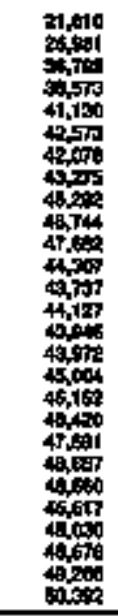 & 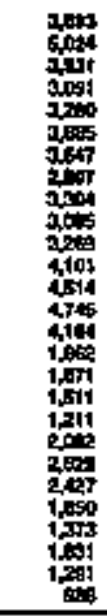 & 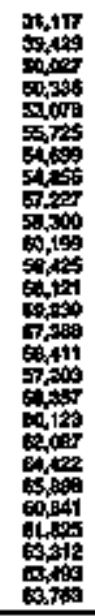 & $\begin{array}{l}0 \\
0 \\
0 \\
0 \\
0 \\
0 \\
0 \\
0 \\
0 \\
0 \\
0 \\
0 \\
0 \\
0 \\
0 \\
0 \\
0 \\
0 \\
0 \\
0 \\
0 \\
0 \\
0 \\
0 \\
0 \\
0 \\
0 \\
0\end{array}$ & 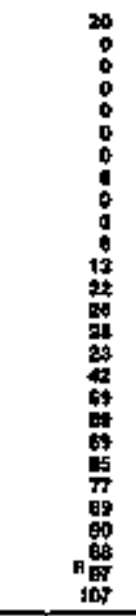 & $\begin{array}{l}z \\
z \\
z \\
z \\
z \\
= \\
z \\
z \\
= \\
z \\
z \\
z \\
z \\
z \\
z\end{array}$ & 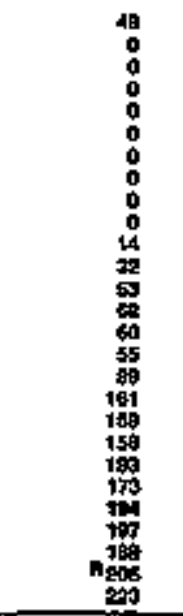 & $\begin{array}{l}z \\
z \\
z \\
z \\
z \\
z \\
z \\
z \\
z \\
z \\
z \\
z \\
z \\
z\end{array}$ \\
\hline \multicolumn{16}{|c|}{ 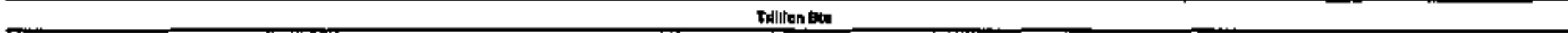 } \\
\hline 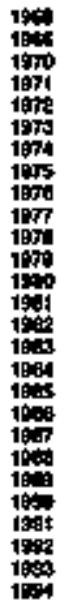 & $\begin{array}{l}23 \\
03 \\
02 \\
00 \\
01 \\
01 \\
01 \\
0 y \\
09 \\
00 \\
00 \\
00 \\
00 \\
00 \\
00 \\
00 \\
00 \\
00 \\
00 \\
00 \\
00 \\
00 \\
00 \\
00 \\
00\end{array}$ & 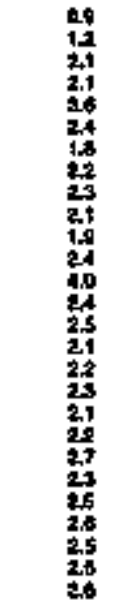 & 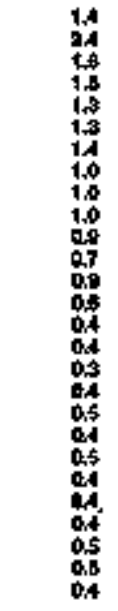 & 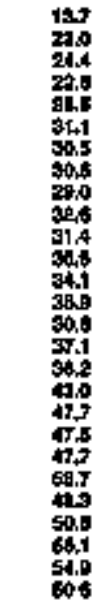 & 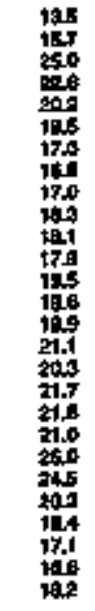 & 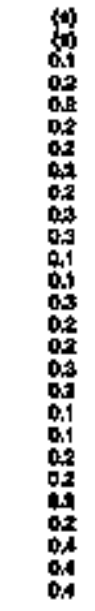 & 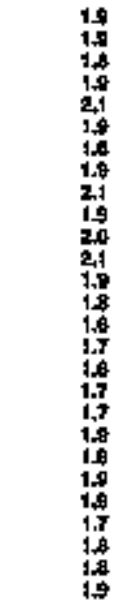 & 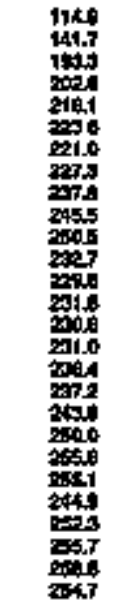 & 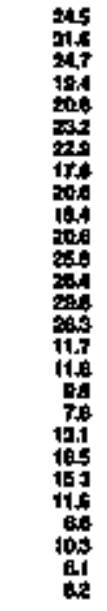 & 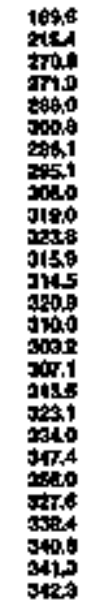 & $\begin{array}{l}00 \\
00 \\
00 \\
00 \\
00 \\
00 \\
00 \\
00 \\
00 \\
00 \\
00 \\
00 \\
00 \\
00 \\
000 \\
00 \\
00 \\
00 \\
00 \\
00 \\
00 \\
00 \\
00 \\
000 \\
00 \\
00 \\
00 \\
0.00\end{array}$ & 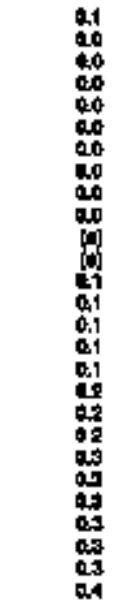 & 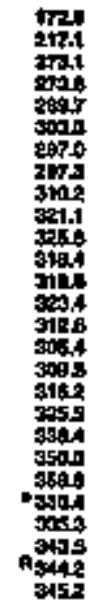 & 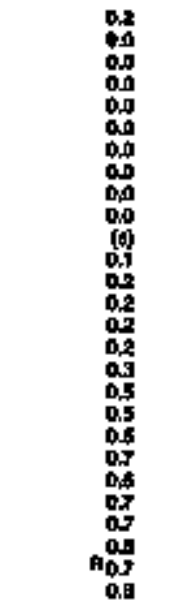 & 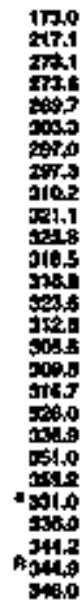 \\
\hline
\end{tabular}

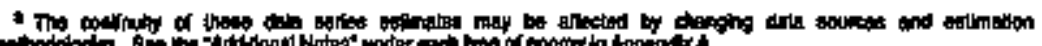

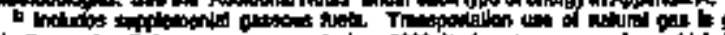

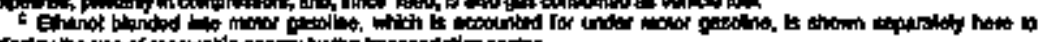

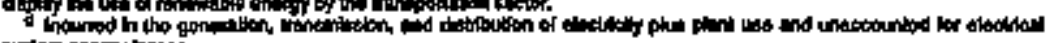

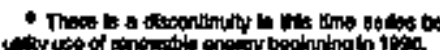

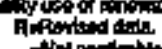
Afor appination.

10 J

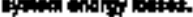


Teble 142. Estimates ol Energy Inpul at Elactrio Ltililies, 1960, 1965, 1970-1994, Maryland

$\mathbf{R}$
$\mathbf{Y}$
$\mathbf{D}$

\begin{tabular}{|c|c|c|c|c|c|c|c|c|c|c|c|c|c|c|}
\hline & \multicolumn{3}{|c|}{ cont } & \multirow[b]{2}{*}{ tatre } & \multicolumn{4}{|c|}{ Petrotumn } & \multirow[b]{2}{*}{ 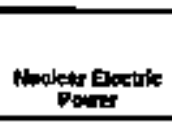 } & \multirow[b]{2}{*}{ Astrostectite } & \multirow[b]{2}{*}{ Broluent: } & \multirow[b]{2}{*}{ Gonemest } & \multirow[b]{2}{*}{ ontertous } & \multirow[b]{3}{*}{ Treaty } \\
\hline & 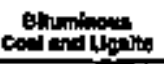 & anonemens & Tow & & 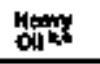 & 敂 & Nexpotang & Tare & & & & & & \\
\hline$w$ & \multicolumn{3}{|c|}{ Thonsenasmat Tone } & aton & \multicolumn{4}{|c|}{ Momanad Bapels } & \multicolumn{5}{|c|}{ 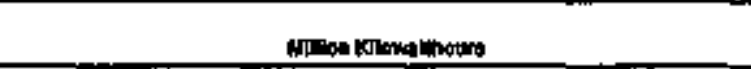 } & \\
\hline 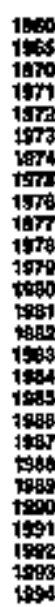 & 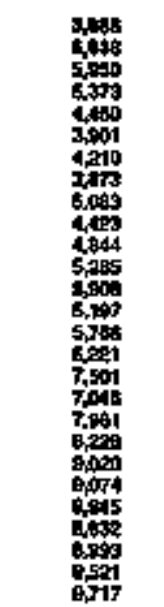 & $:$ & 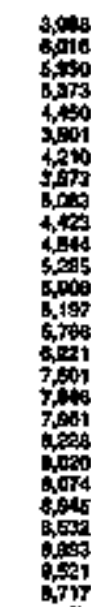 & 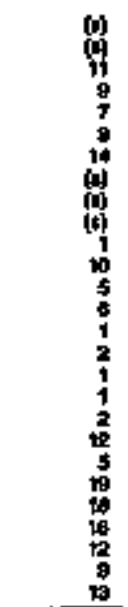 & 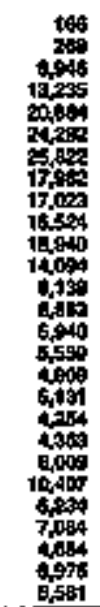 & 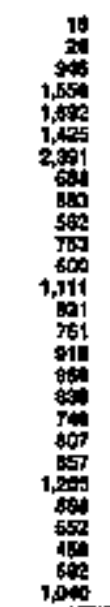 & 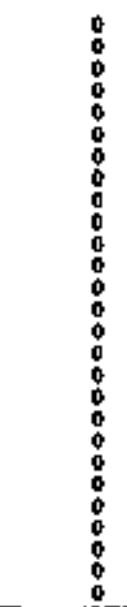 & 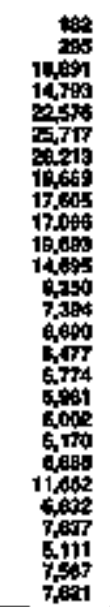 & 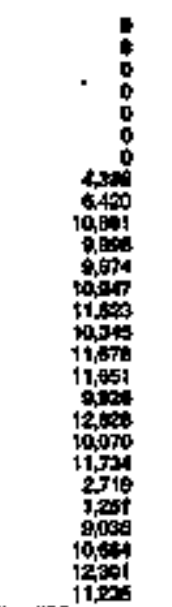 & 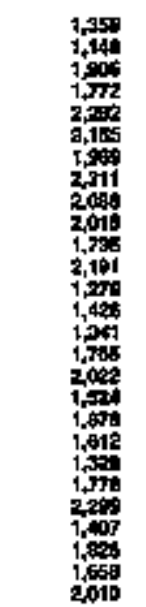 & 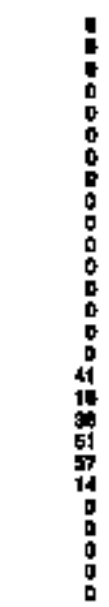 & $:$ & $:$ & $\Xi$ \\
\hline
\end{tabular}

\begin{tabular}{|c|c|c|c|c|c|}
\hline 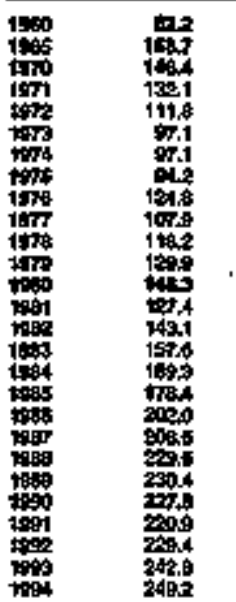 & 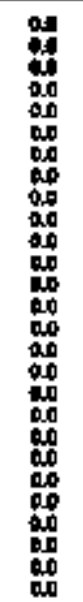 & 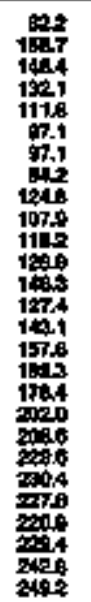 & 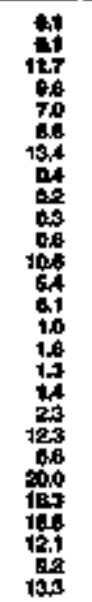 & 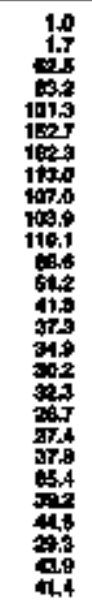 & 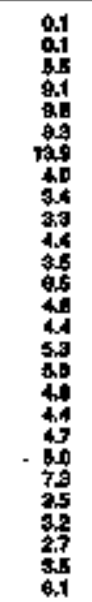 \\
\hline
\end{tabular}

.

\begin{tabular}{|c|c|c|c|c|c|c|c|}
\hline 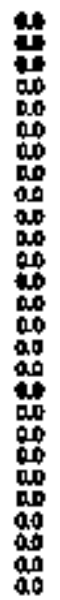 & 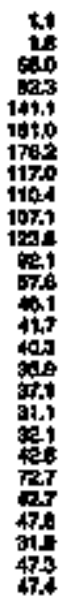 & 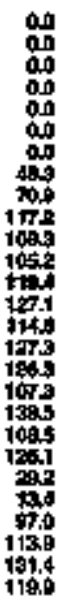 & 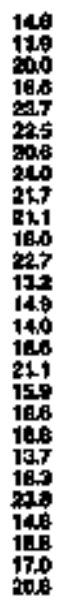 & 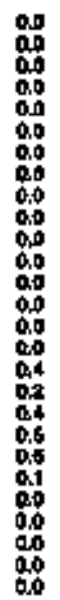 & 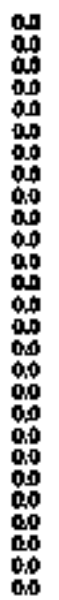 & 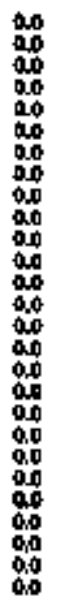 & 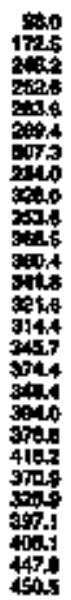 \\
\hline
\end{tabular}

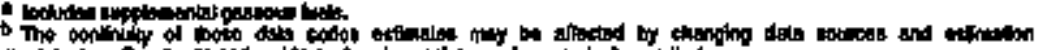

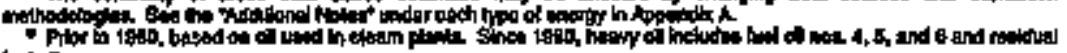

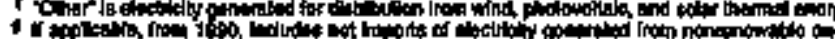

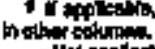

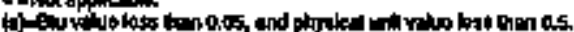

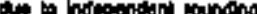

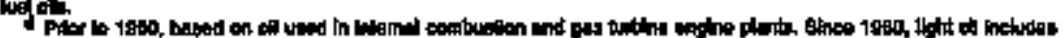

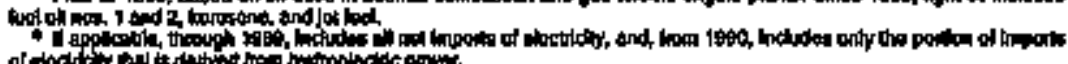

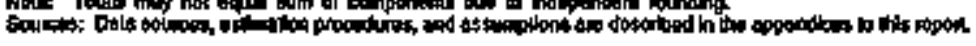


Table 149, Energy Consemption Eshimates by Source, 1960, 1965, 1670-1994, Mlassachusents

\begin{tabular}{|c|c|c|c|c|c|c|c|c|c|c|c|c|c|c|c|c|c|c|c|}
\hline \multirow[b]{3}{*}{$Y \Leftrightarrow$} & \multirow[b]{2}{*}{ "البه } & \multirow[b]{2}{*}{ Matint } & \multicolumn{11}{|c|}{ Putroten } & \multirow[b]{2}{*}{ 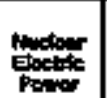 } & \multirow[b]{2}{*}{ 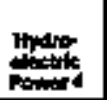 } & \multirow[b]{2}{*}{ Erofinele } & \multirow[b]{2}{*}{ atser 4} & \multirow{2}{*}{ ס } & \multirow[b]{3}{*}{ Tots } \\
\hline & & & 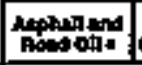 & A & 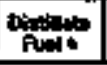 & Pit. & ima. & tets: & Let & | & Pathes & other at & Totoll & & & & & & \\
\hline & $\begin{array}{l}\text { Thougal } \\
\text { ghout Tout }\end{array}$ & nospons & \multicolumn{11}{|c|}{ 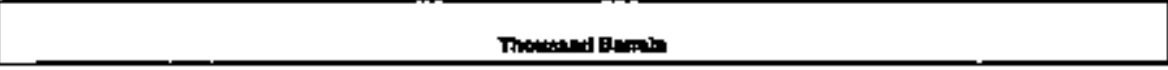 } & \multicolumn{5}{|c|}{ : } & \\
\hline 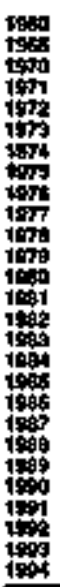 & 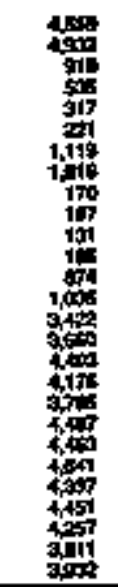 & 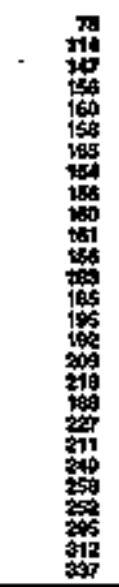 & 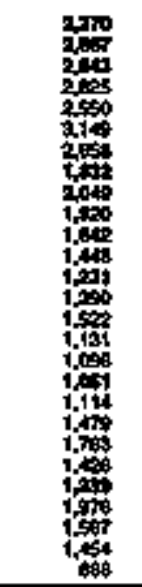 & 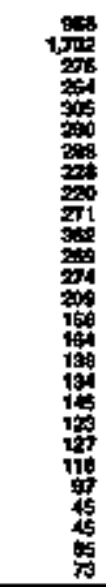 & 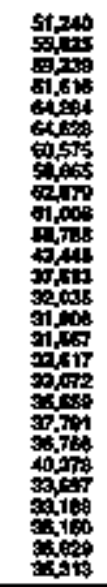 & 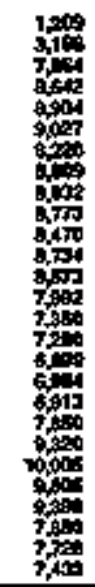 & 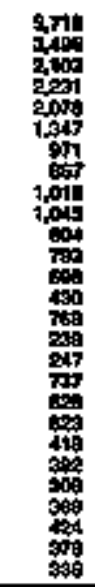 & 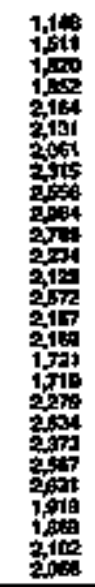 & 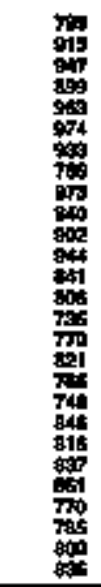 & 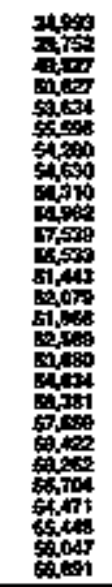 & 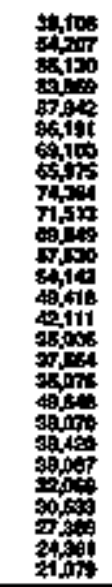 & 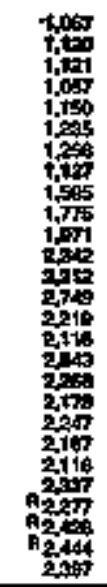 & 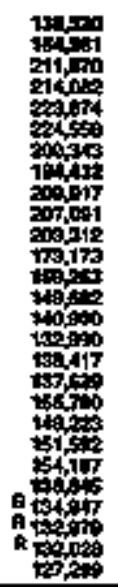 & 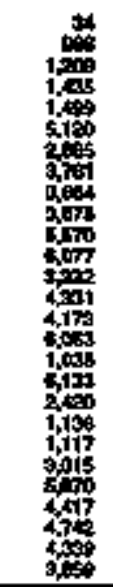 & 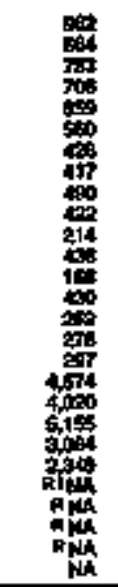 & 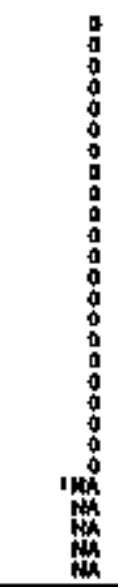 & 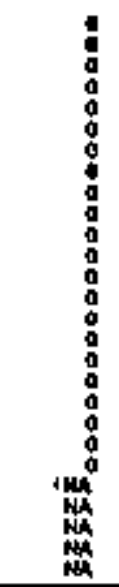 & 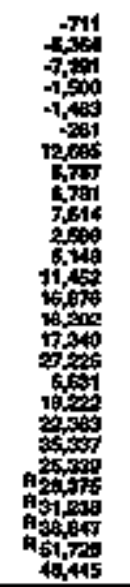 & $\begin{array}{l}z \\
z \\
z \\
z \\
z \\
z \\
z \\
\vdots \\
\vdots \\
z \\
\vdots \\
\vdots \\
z \\
z \\
z\end{array}$ \\
\hline \multicolumn{20}{|c|}{ 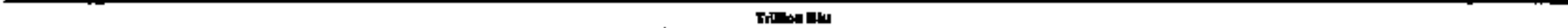 } \\
\hline 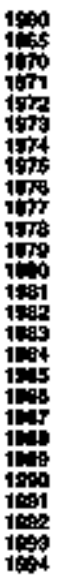 & 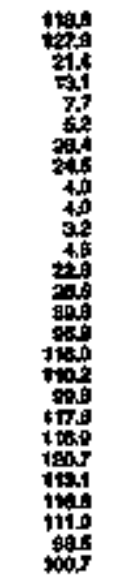 & 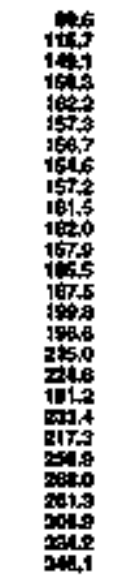 & 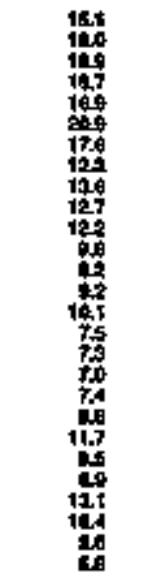 & 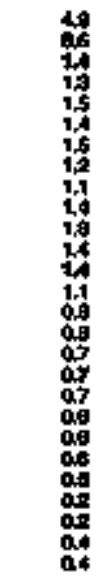 & 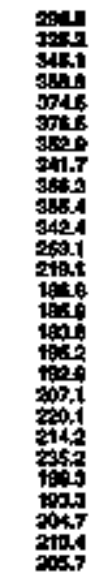 & 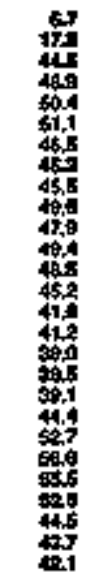 & 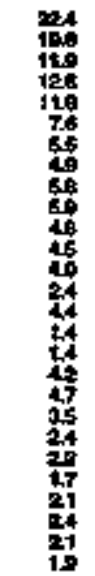 & 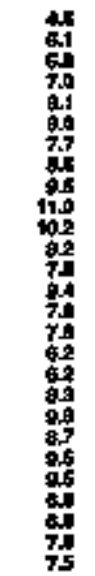 & 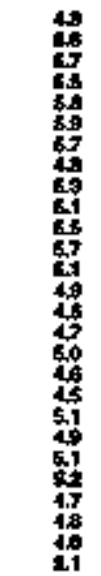 & 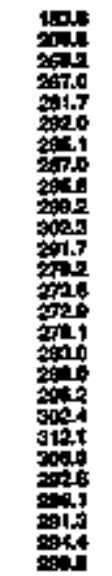 & 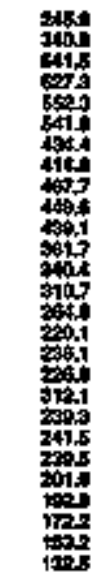 & 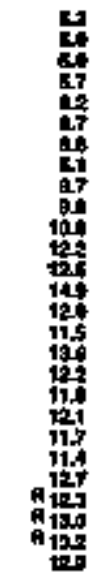 & 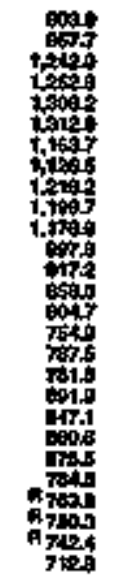 & 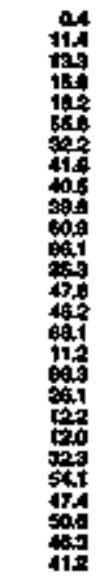 & 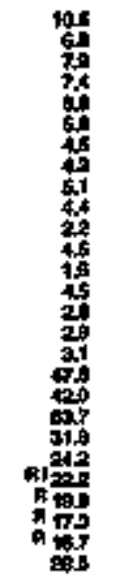 & 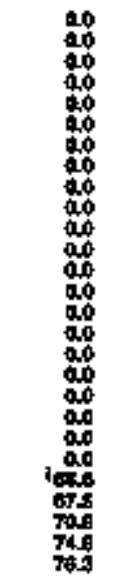 & 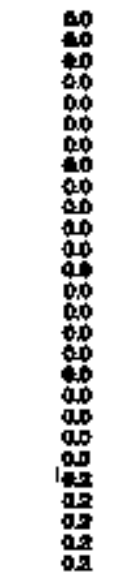 & 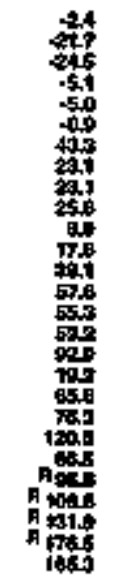 & 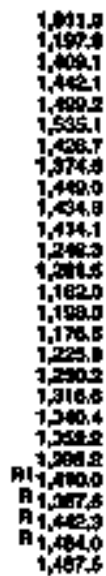 \\
\hline
\end{tabular}

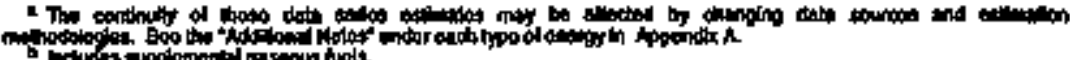

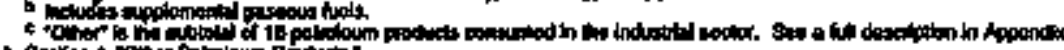

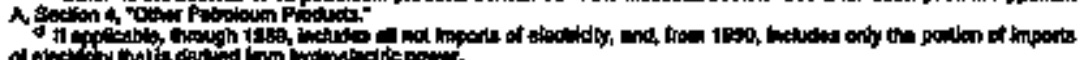

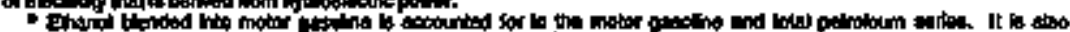

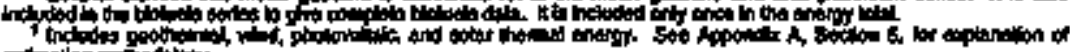

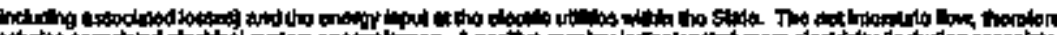

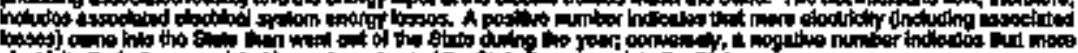
in

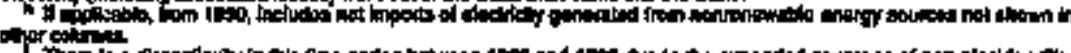

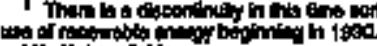

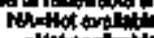

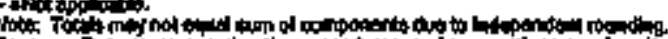

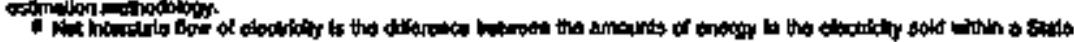

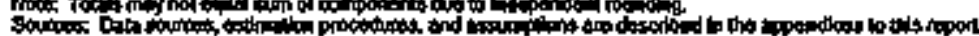


Table 144. Residential Energy Consumption Estimates, 1960, 1965, 1970-1994, Massachusetts

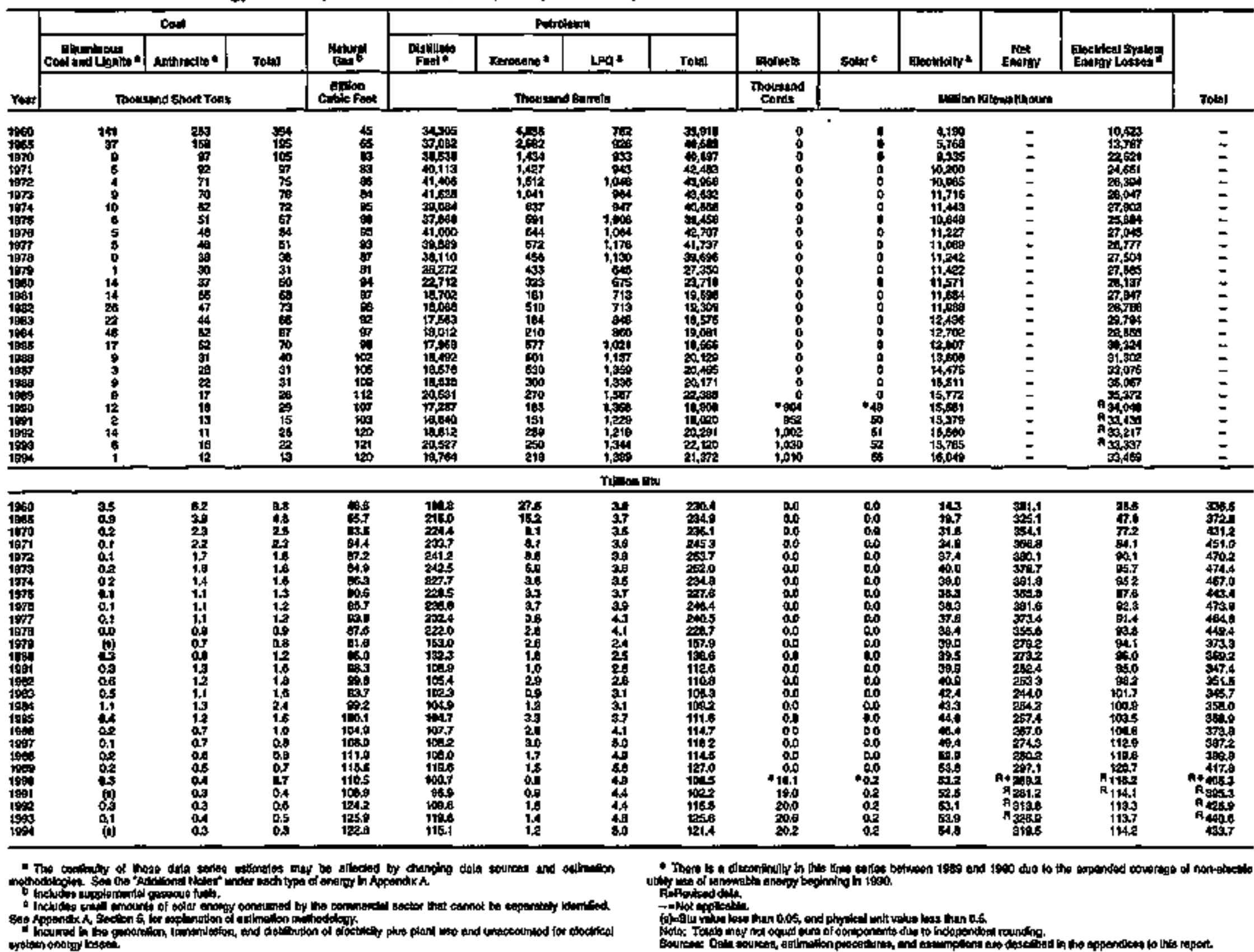

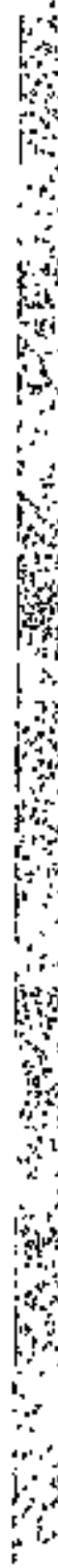


Table 145. Commerelal Energy Consumption Estimates, 1980, 1955, 1970-1994, Mas-8achusetts

\begin{tabular}{|c|c|c|c|c|c|c|c|c|c|c|c|c|c|c|c|}
\hline \multirow[b]{3}{*}{ Yer } & \multicolumn{3}{|c|}{ Cool } & \multirow{3}{*}{$\frac{\text { Gaptipl }}{\text { ablon }}$} & \multicolumn{6}{|c|}{ Petrolotant } & \multirow[b]{2}{*}{ Elotrolity ${ }^{2}$} & \multirow[b]{2}{*}{ Enerost } & \multirow[b]{2}{*}{ Twetiol syoten } & \multirow[b]{3}{*}{ Tolatd } & \\
\hline & 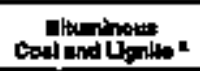 & Anturethe: & Tow & & 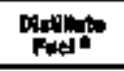 & Kanosenas & LPA: & 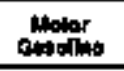 & Renlend & Towd & & & & & \\
\hline & \multicolumn{3}{|c|}{ Thous:and short Tone. } & & \multicolumn{6}{|c|}{ 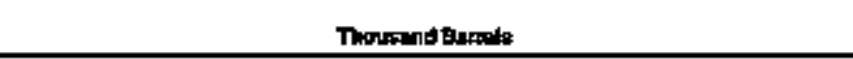 } & \multicolumn{3}{|c|}{ 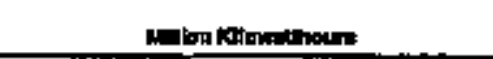 } & & \\
\hline 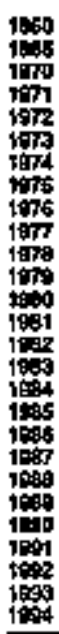 & 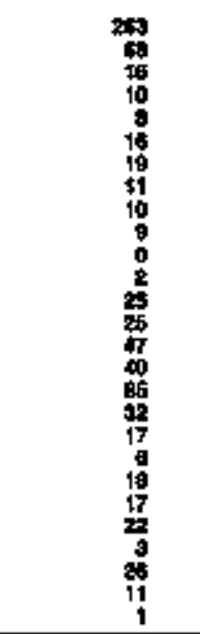 & 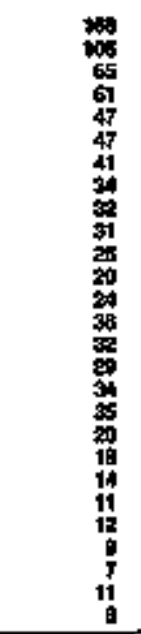 & 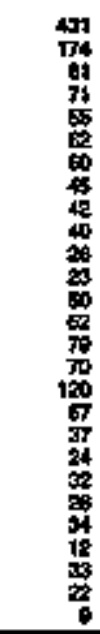 & 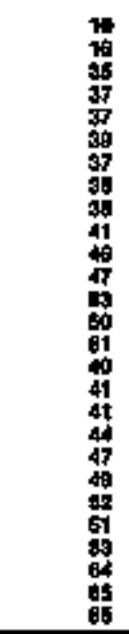 & 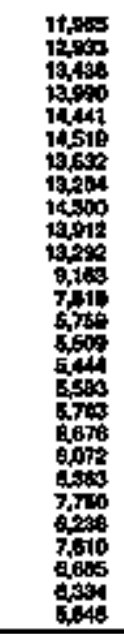 & 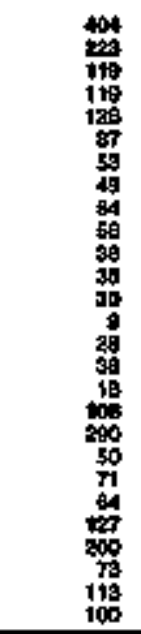 & 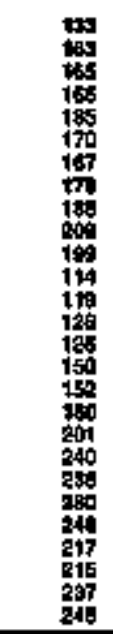 & 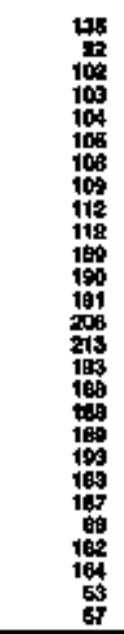 & 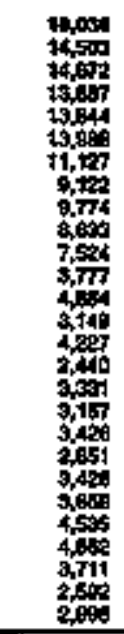 & 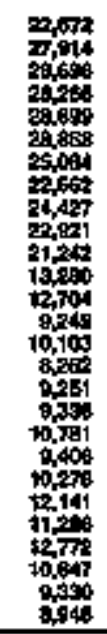 & 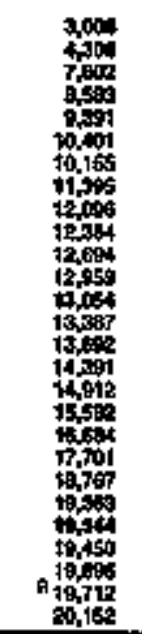 & $\begin{array}{l}= \\
= \\
= \\
= \\
= \\
= \\
= \\
= \\
= \\
= \\
= \\
= \\
= \\
= \\
=\end{array}$ & 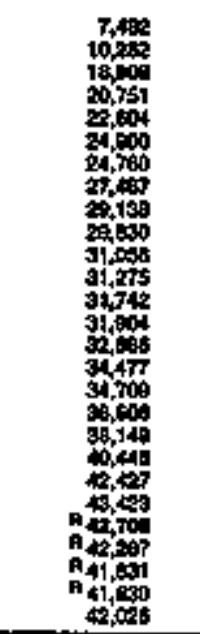 & $\begin{array}{l}= \\
z \\
z \\
z \\
z \\
z \\
= \\
= \\
z \\
z \\
z \\
z \\
z \\
z\end{array}$ & \\
\hline \multicolumn{15}{|c|}{ Mrlon Ban } & \\
\hline 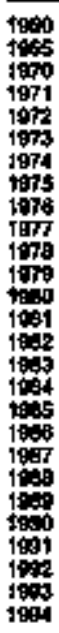 & 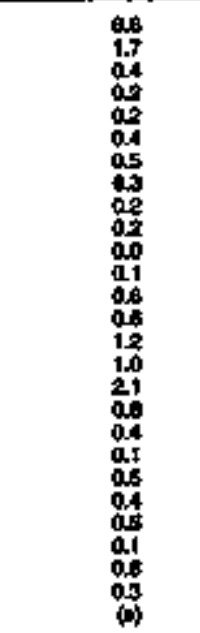 & $\begin{array}{l}4.2 \\
2.6 \\
1.8 \\
1.4 \\
1.1 \\
1.1 \\
0.9 \\
0.9 \\
0.7 \\
0.9 \\
0.5 \\
0.5 \\
0.9 \\
0.9 \\
0.9 \\
0.9 \\
0.9 \\
0.5 \\
0.5 \\
0.4 \\
0.5 \\
03 \\
0.4 \\
0.2 \\
0.3 \\
0.2\end{array}$ & 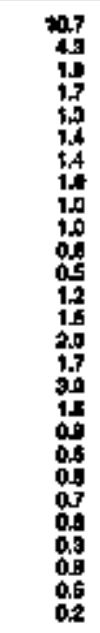 & 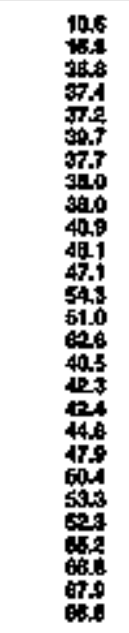 & 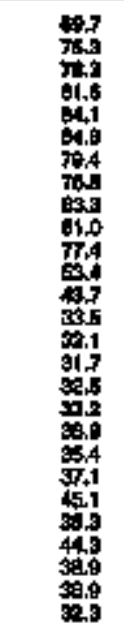 & 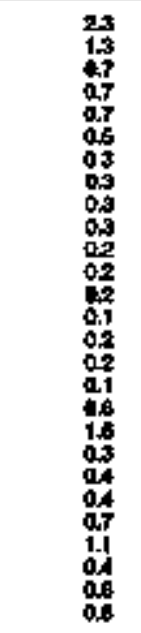 & 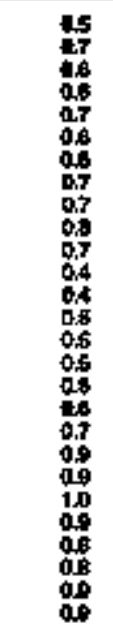 & 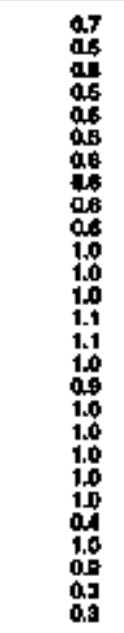 & 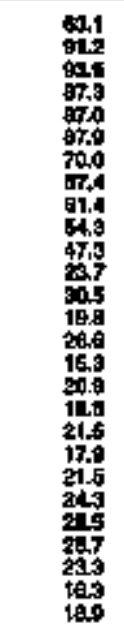 & 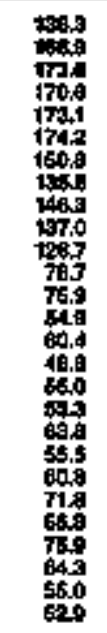 & 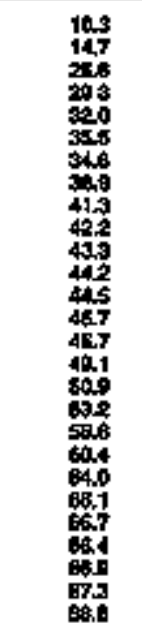 & 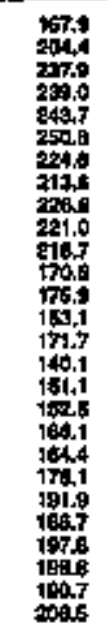 & 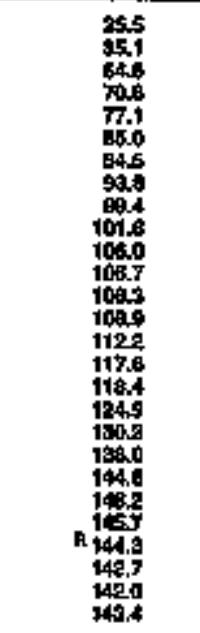 & 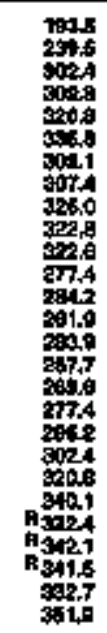 & \\
\hline \multirow{2}{*}{\multicolumn{15}{|c|}{ 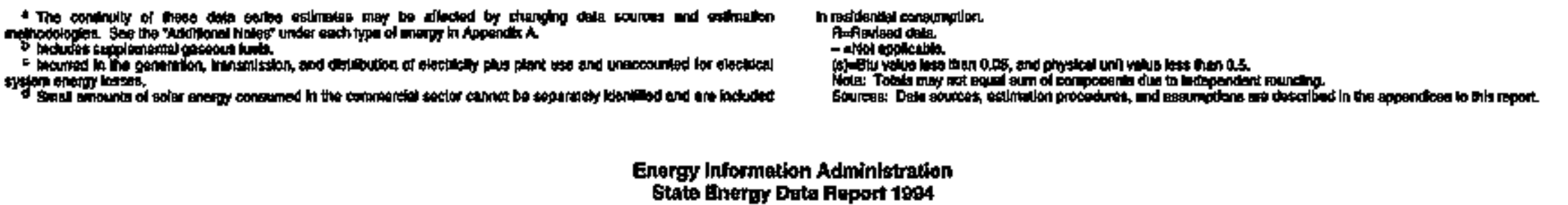 }} & \\
\hline & & & & & & & & & & & & & & & 157 \\
\hline
\end{tabular}




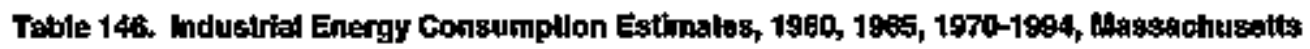

\begin{tabular}{|c|c|c|c|c|c|c|c|c|c|c|c|c|c|c|c|c|c|c|}
\hline \multirow[b]{3}{*}{ Your } & \multirow[b]{2}{*}{ Da } & \multirow[b]{2}{*}{ mane: } & \multicolumn{9}{|c|}{ Furoleven } & \multirow[b]{2}{*}{ ponto- } & \multirow[b]{2}{*}{ Bishole } & \multirow[b]{2}{*}{ onarad } & \multirow[b]{2}{*}{ Dowataty } & \multirow[b]{2}{*}{$\min _{\operatorname{mon}}$} & \multirow{2}{*}{ 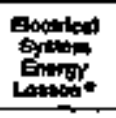 } & \multirow[b]{3}{*}{ Tolpt: } \\
\hline & & & 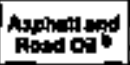 & Plom & 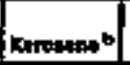 & Lo9: & 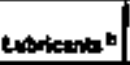 & antoper & Ploldid & Othor at: & Total & & & & & & & \\
\hline & 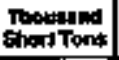 & cillop & \multicolumn{9}{|c|}{ 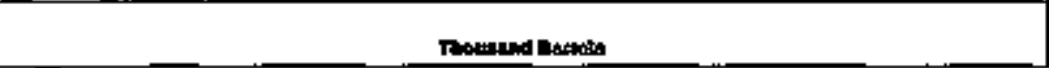 } & \multicolumn{6}{|c|}{ 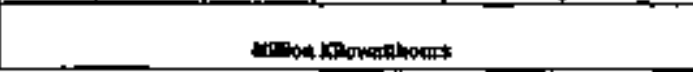 } & \\
\hline 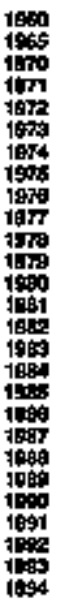 & 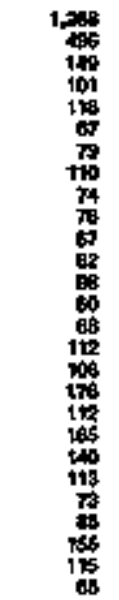 & 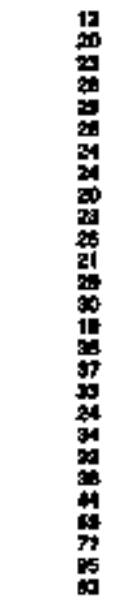 & 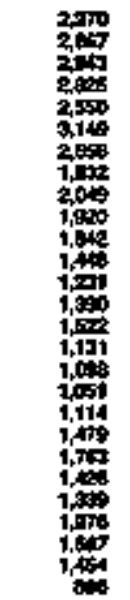 & 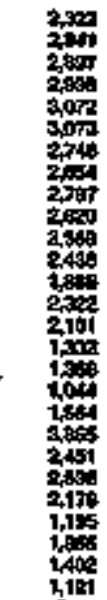 & 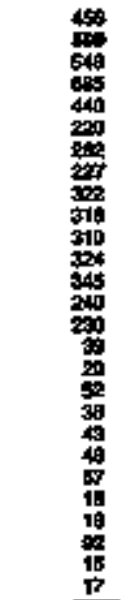 & 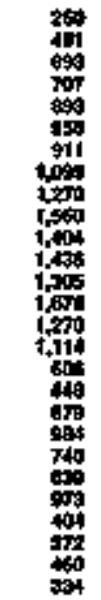 & 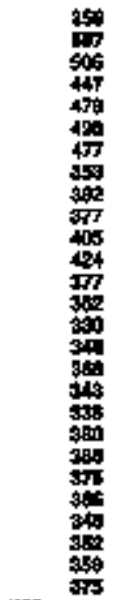 & 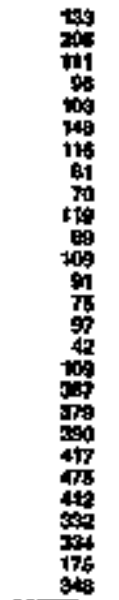 & 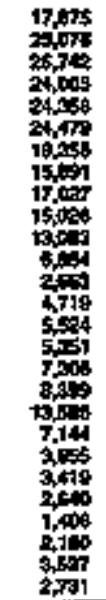 & 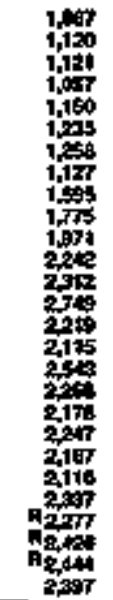 & 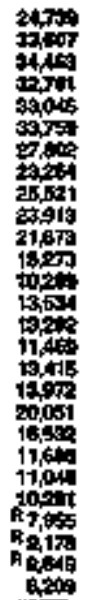 & 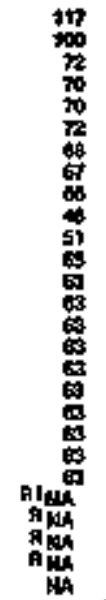 & 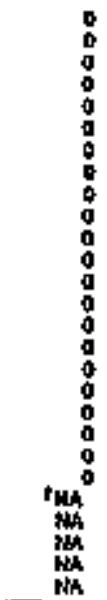 & 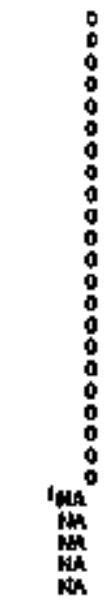 & 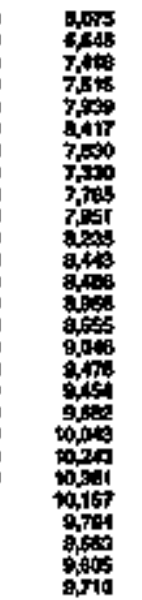 & $\begin{array}{l}= \\
= \\
z \\
z \\
= \\
= \\
= \\
= \\
z \\
= \\
z \\
= \\
z\end{array}$ & 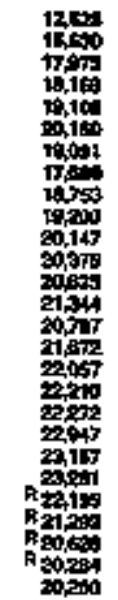 & 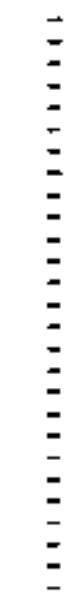 \\
\hline
\end{tabular}

\begin{tabular}{|c|c|c|c|c|c|c|c|c|c|c|c|c|c|c|c|c|c|c|}
\hline 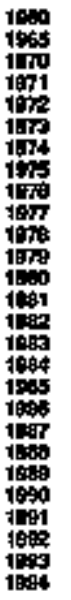 & 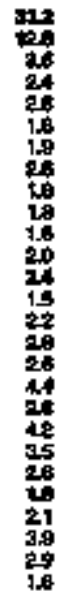 & 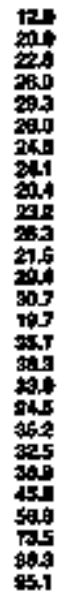 & 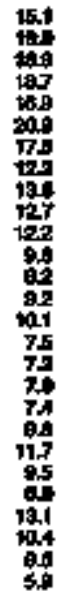 & 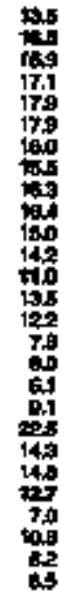 & 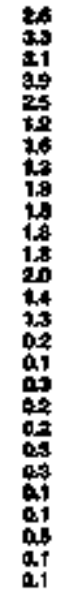 & 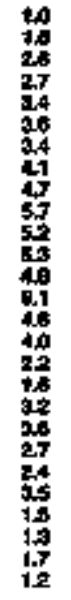 & $\begin{array}{l}22 \\
71 \\
21 \\
27 \\
29 \\
20 \\
20 \\
23 \\
21 \\
24 \\
23 \\
25 \\
28 \\
23 \\
20 \\
20 \\
21 \\
21 \\
21 \\
20 \\
23 \\
28 \\
25 \\
23 \\
21 \\
21 \\
28 \\
20\end{array}$ & 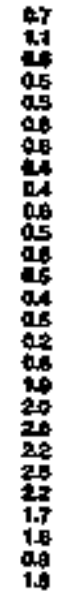 & 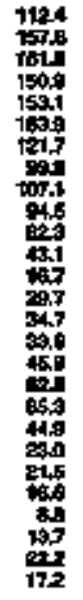 & 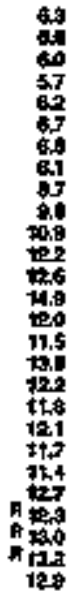 & 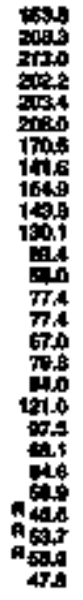 & 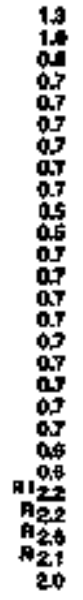 & 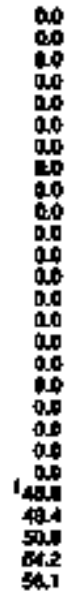 & 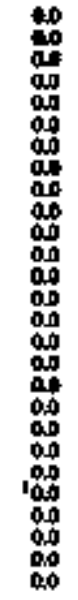 & 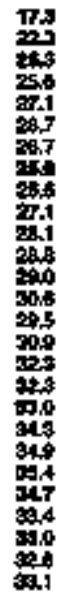 & 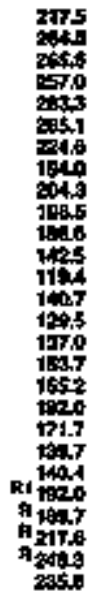 & 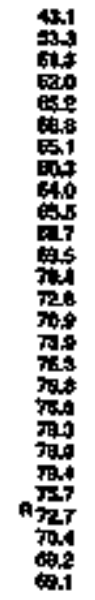 & 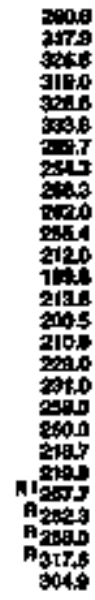 \\
\hline
\end{tabular}

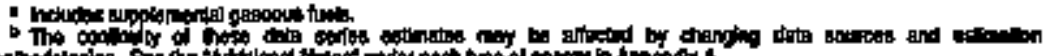

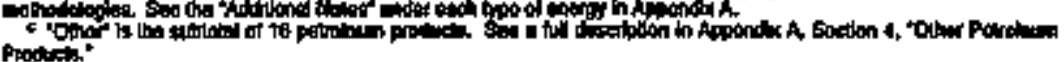

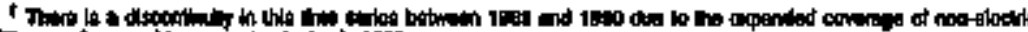

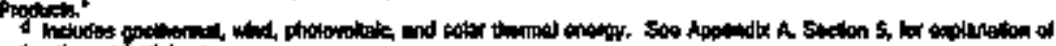

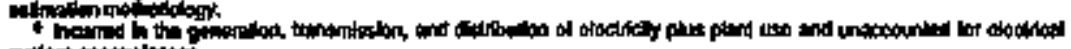

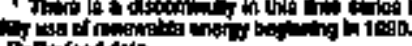

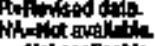

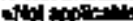

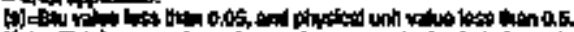

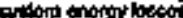




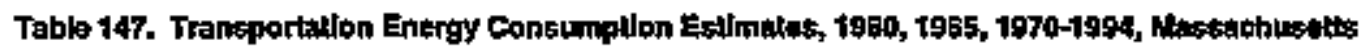

\begin{tabular}{|c|c|c|c|c|c|c|c|c|c|c|c|c|c|c|c|}
\hline \multirow[b]{3}{*}{ Your } & \multirow[b]{2}{*}{ Aod } & \multirow[b]{2}{*}{ Mather } & \multicolumn{8}{|c|}{ 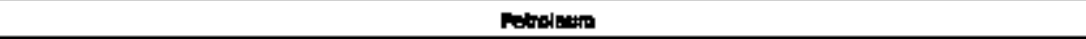 } & \multirow{3}{*}{ 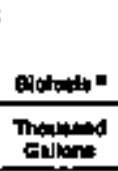 } & \multirow[b]{2}{*}{ Expitity 1} & \multirow[b]{2}{*}{ Enopist } & \multirow[b]{2}{*}{ 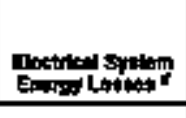 } & \multirow[b]{3}{*}{ Tota } \\
\hline & & & Antan & Plinte & 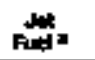 & UPE: & Litotanot: & Getporition & Fodery & Toth & & & & & \\
\hline & $\begin{array}{l}\text { Maptod } \\
\text { shoof time }\end{array}$ & $\begin{array}{c}\text { Allion } \\
\text { cuble Fine }\end{array}$ & \multicolumn{8}{|c|}{ 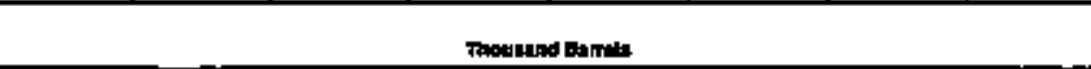 } & & \multicolumn{3}{|c|}{ 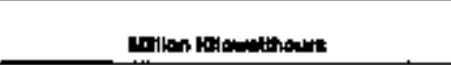 } & \\
\hline 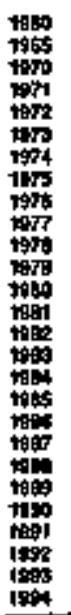 & $\begin{array}{l}n \\
0 \\
0 \\
0 \\
00 \\
0 \\
0 \\
0 \\
0 \\
0 \\
0 \\
0 \\
0 \\
0 \\
0 \\
0 \\
0 \\
0 \\
0 \\
0 \\
0 \\
0 \\
0 \\
0\end{array}$ & 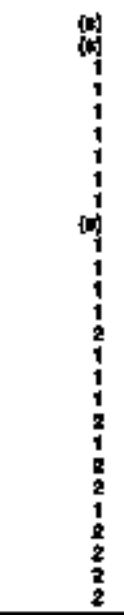 & 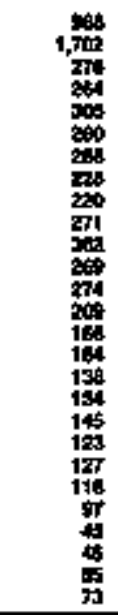 & 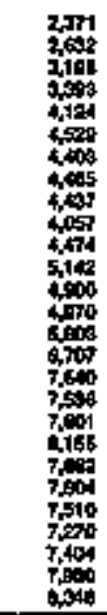 & 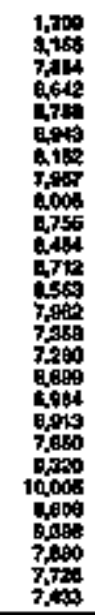 & 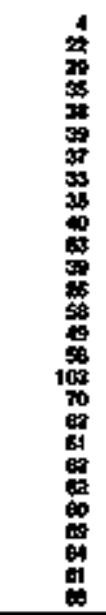 & 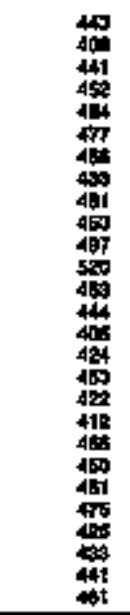 & 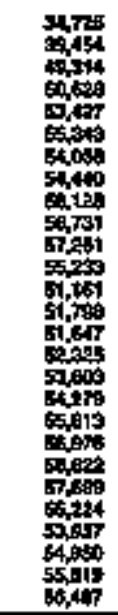 & 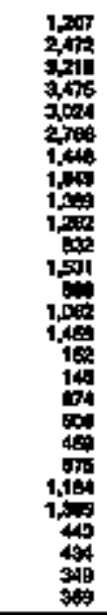 & 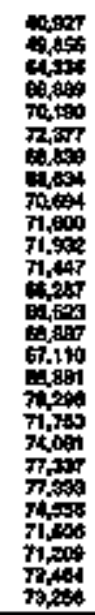 & $\begin{array}{l}0 \\
0 \\
0 \\
0 \\
0 \\
0 \\
0 \\
0 \\
0 \\
0 \\
0 \\
0 \\
0 \\
0 \\
0 \\
0 \\
0 \\
0 \\
0 \\
0 \\
0 \\
0 \\
0 \\
0 \\
0 \\
0\end{array}$ & 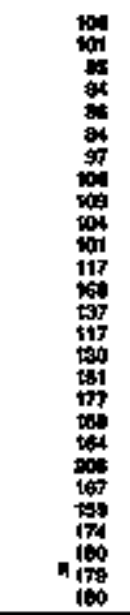 & $\begin{array}{l}z \\
z \\
z \\
z \\
z \\
z \\
z \\
z \\
z \\
z \\
z \\
z \\
z \\
z\end{array}$ & 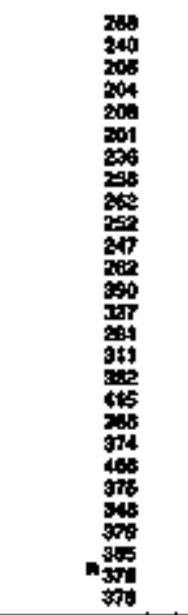 & 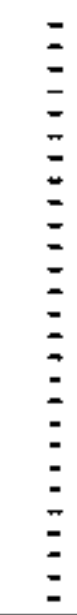 \\
\hline \multicolumn{16}{|c|}{ Tanlon Big } \\
\hline 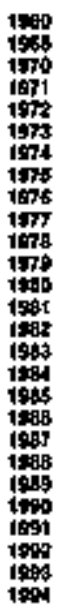 & 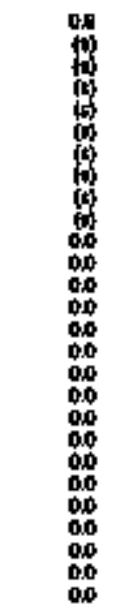 & 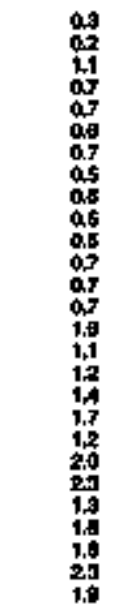 & 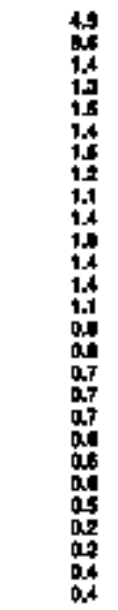 & 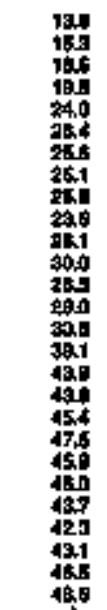 & 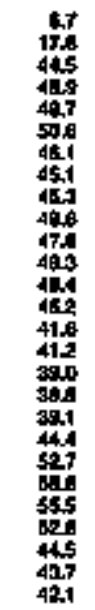 & 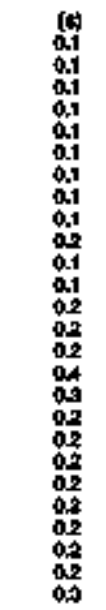 & 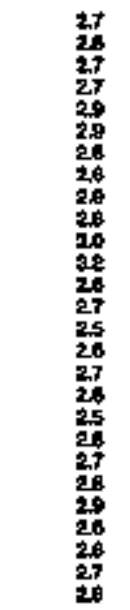 & 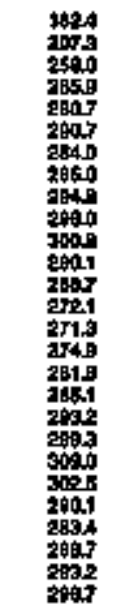 & 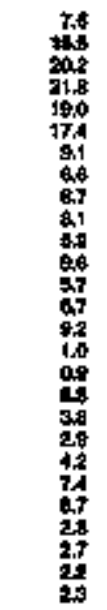 & 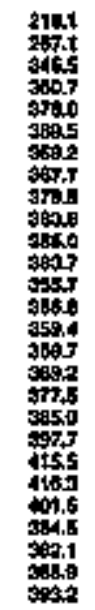 & $\begin{array}{l}00 \\
00 \\
00 \\
00 \\
00 \\
00 \\
00 \\
00 \\
00 \\
00 \\
00 \\
00 \\
00 \\
00 \\
00 \\
00 \\
00 \\
00 \\
00 \\
00 \\
00 \\
00 \\
00 \\
-00 \\
00 \\
00 \\
00 \\
00\end{array}$ & 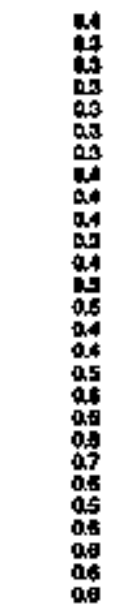 & 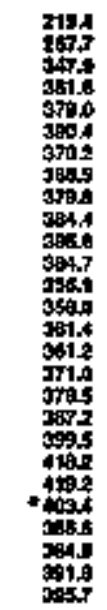 & 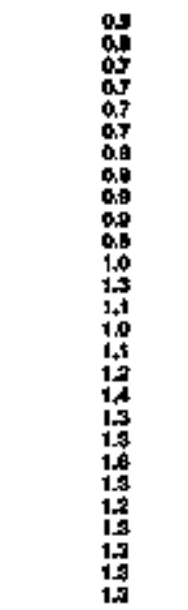 & 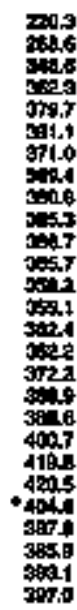 \\
\hline
\end{tabular}

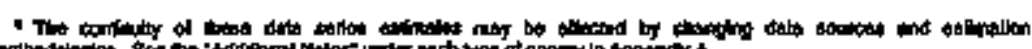

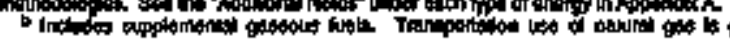

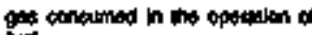

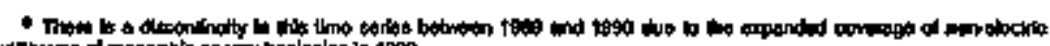

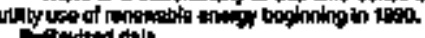

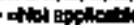

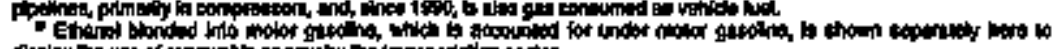

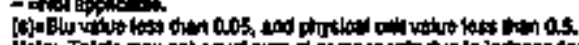

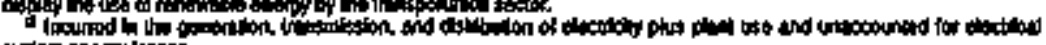

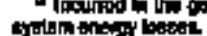


Table 148. Eslimales of Energy tnput at Elettro Uiltites, 1960, 1955, 1970-1994, Missachuselts

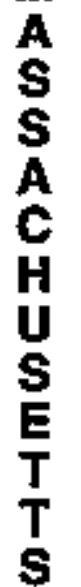

\begin{tabular}{|c|c|c|c|c|c|c|c|c|c|c|c|c|c|c|}
\hline \multirow[b]{3}{*}{ Yem } & \multicolumn{3}{|c|}{ Cas } & \multirow{3}{*}{ 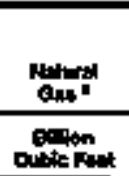 } & \multicolumn{4}{|c|}{ Delrokeum } & \multirow[b]{2}{*}{ 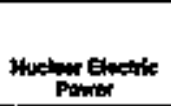 } & \multirow[b]{2}{*}{ Hodvatedite } & \multirow[b]{2}{*}{ Dotala } & \multirow[b]{2}{*}{ Onotherme } & \multirow[b]{2}{*}{ odser bl } & \multirow[b]{3}{*}{$T+\infty, 10$} \\
\hline & 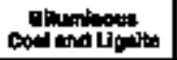 & 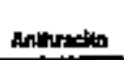 & $\operatorname{tatat}$ & & $\operatorname{ton} x$ & 比皆 & 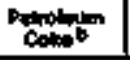 & Tolat & & & & & & \\
\hline & \multicolumn{3}{|c|}{ Thonted Shout Thos } & & \multicolumn{4}{|c|}{ 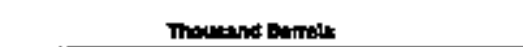 } & \multicolumn{5}{|c|}{ 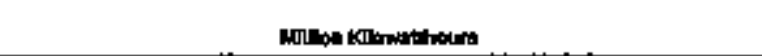 } & \\
\hline 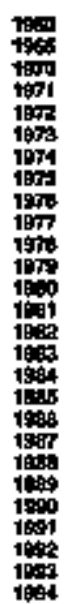 & 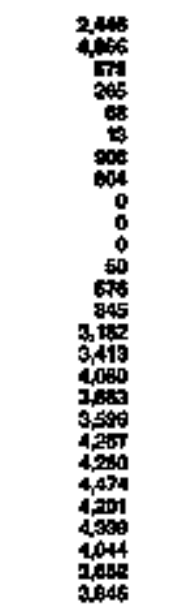 & $\begin{array}{l}0 \\
0 \\
0 \\
0 \\
0 \\
0 \\
0 \\
0 \\
0 \\
0 \\
0 \\
0 \\
0 \\
0 \\
0 \\
0 \\
0 \\
0 \\
0 \\
0 \\
0 \\
0 \\
0 \\
0 \\
0 \\
0 \\
0\end{array}$ & 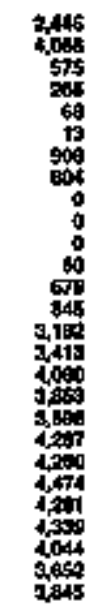 & 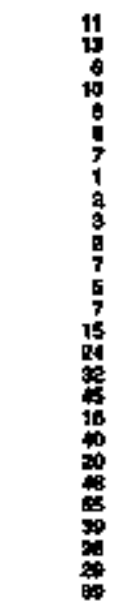 & 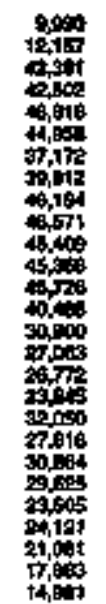 & 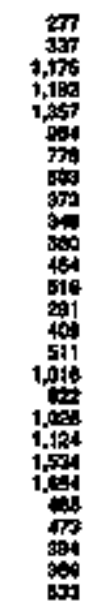 & $\begin{array}{l}0 \\
0 \\
0 \\
0 \\
0 \\
0 \\
0 \\
0 \\
0 \\
0 \\
0 \\
0 \\
0 \\
0 \\
0 \\
0 \\
0 \\
0 \\
0 \\
0 \\
0 \\
0 \\
0 \\
0 \\
0 \\
0\end{array}$ & 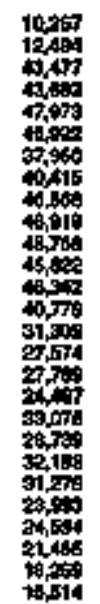 & 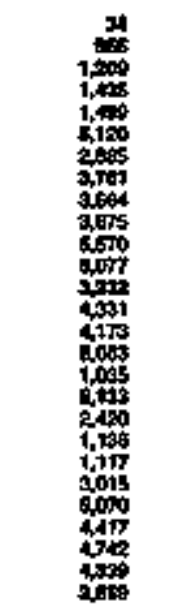 & 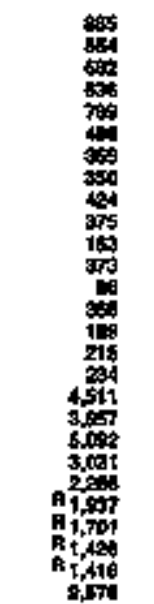 & $\begin{array}{l}0 \\
0 \\
0 \\
0 \\
0 \\
0 \\
0 \\
0 \\
0 \\
0 \\
0 \\
0 \\
0 \\
0 \\
0 \\
0 \\
0 \\
0 \\
0 \\
0 \\
0 \\
0 \\
0 \\
0 \\
0 \\
0 \\
0 \\
0\end{array}$ & $\begin{array}{l}0 \\
0 \\
0 \\
0 \\
0 \\
0 \\
0 \\
0 \\
0 \\
0 \\
0 \\
0 \\
0 \\
0 \\
0 \\
0 \\
0 \\
0 \\
0 \\
0 \\
0 \\
0 \\
0 \\
0 \\
0 \\
0 \\
0\end{array}$ & $\begin{array}{l}0 \\
a \\
0 \\
0 \\
0 \\
0 \\
0 \\
0 \\
0 \\
0 \\
0 \\
0 \\
0 \\
0 \\
0 \\
0 \\
0 \\
0 \\
0 \\
0 \\
0 \\
0 \\
0 \\
0 \\
0 \\
0\end{array}$ & $\begin{array}{l}= \\
\vdots \\
\vdots \\
\vdots \\
\vdots \\
\vdots \\
\vdots \\
\vdots \\
\vdots \\
z \\
z \\
z \\
z \\
z \\
z\end{array}$ \\
\hline
\end{tabular}

\begin{tabular}{|c|c|c|c|c|c|c|}
\hline 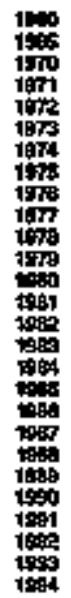 & 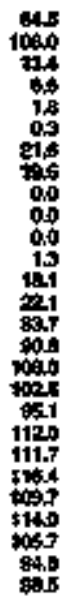 & 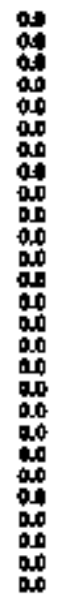 & 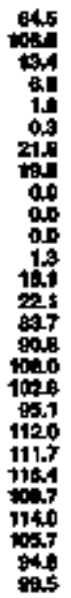 & 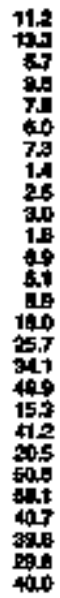 & 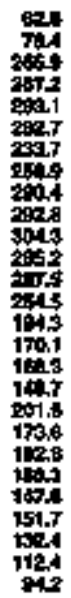 & 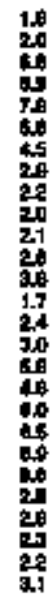 \\
\hline
\end{tabular}

\begin{tabular}{|c|c|c|c|c|c|c|}
\hline & 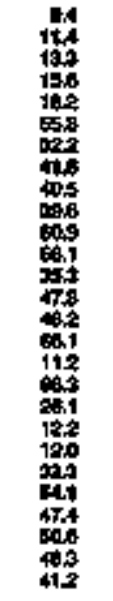 & 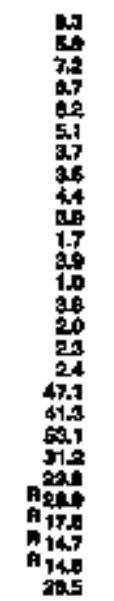 & 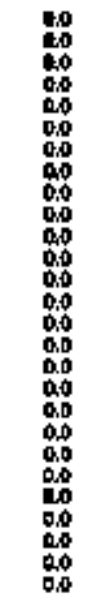 & \multicolumn{2}{|c|}{ 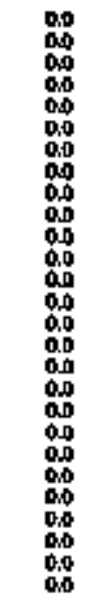 } & 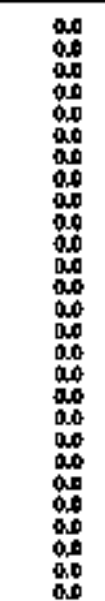 \\
\hline
\end{tabular}

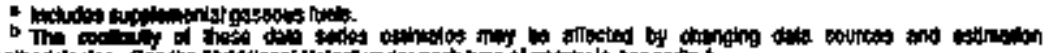

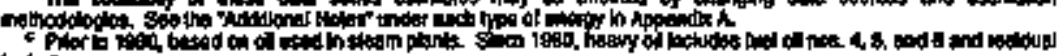

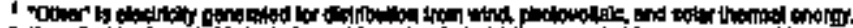

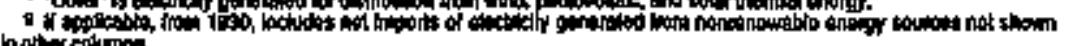
notwer colum

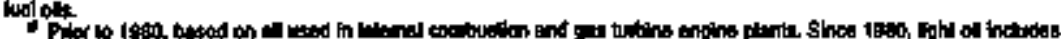

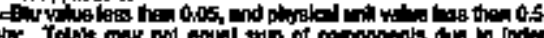

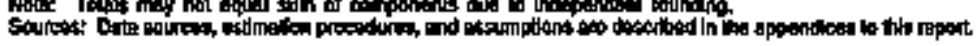


Table 149. Energy Consuraption Estimatas by Sourco, 1960, 1965, 1970-1994, Mischlgan

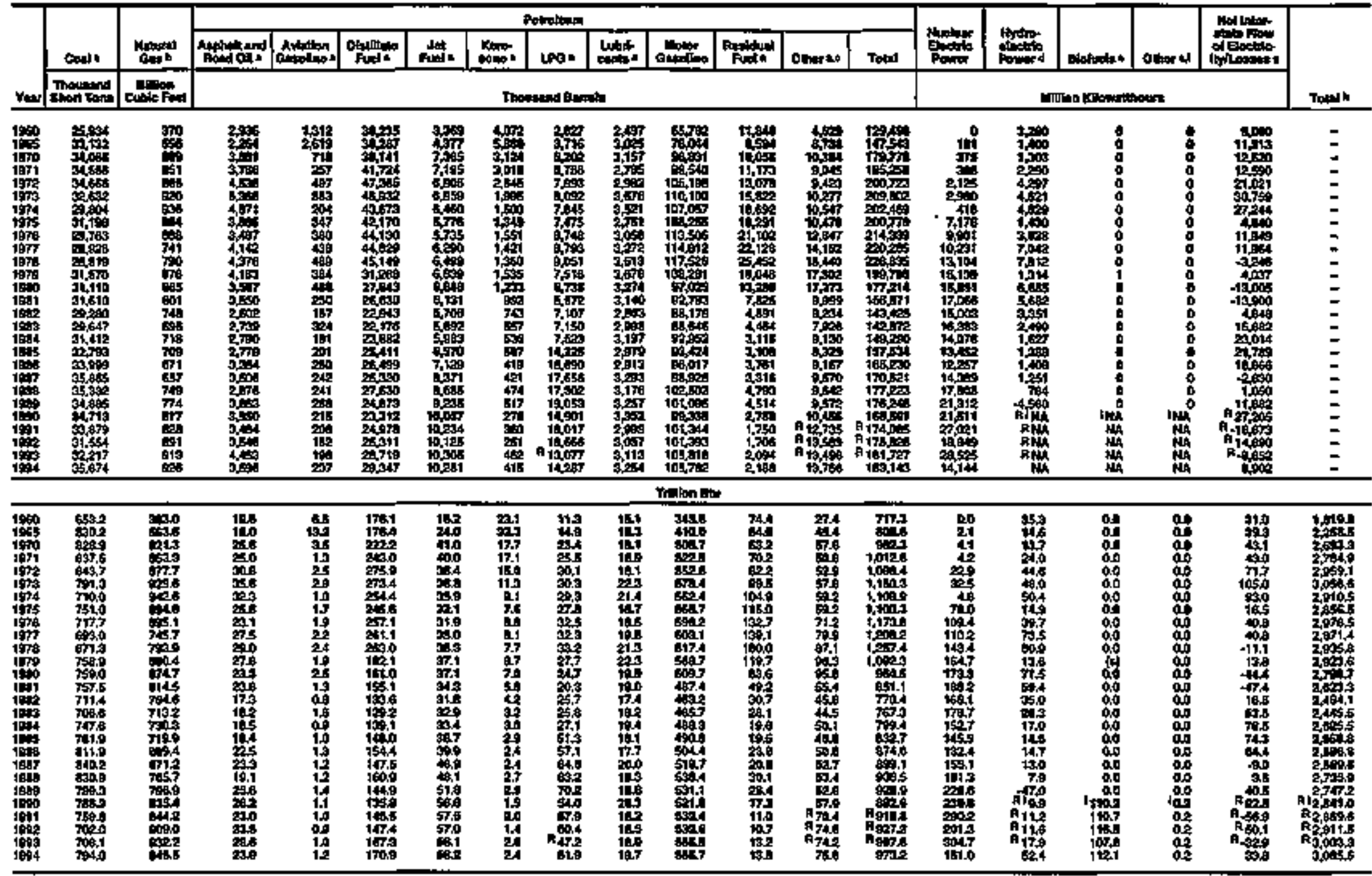

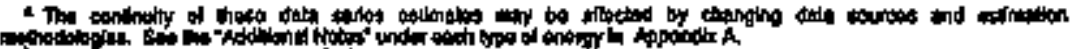

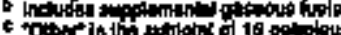

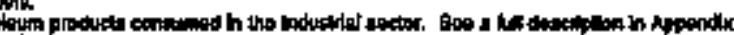

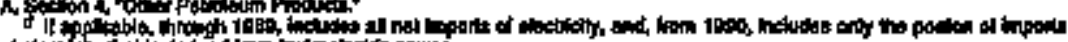

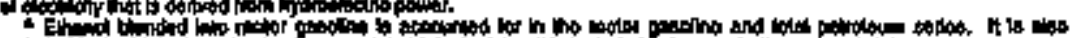

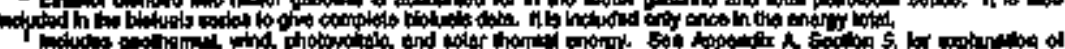

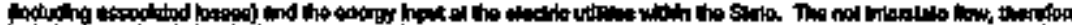

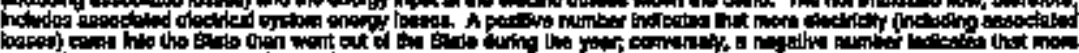

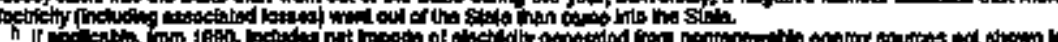

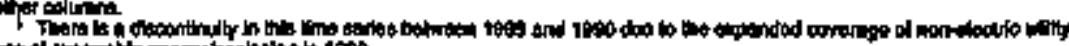
the

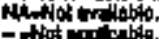

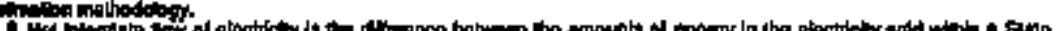




\begin{tabular}{|c|c|c|c|c|c|c|c|c|c|c|c|c|c|c|}
\hline \multirow{3}{*}{ Ywr } & \multicolumn{3}{|c|}{ Cons } & \multirow{3}{*}{ 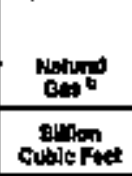 } & \multicolumn{4}{|c|}{ Povelangm } & \multirow{3}{*}{ 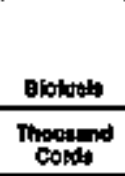 } & & \multirow[b]{2}{*}{ Enetrieldy } & \multirow[b]{2}{*}{ 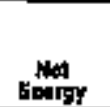 } & \multirow{3}{*}{ 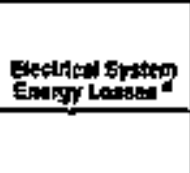 } & \multirow[b]{3}{*}{ Tood } \\
\hline & costuringen: & 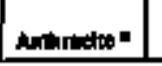 & Folal & & 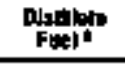 & Kamosente & Lea: & Tolatal & & Solut ${ }^{\circ}$ & & & & \\
\hline & \multicolumn{3}{|c|}{ Nows:ind Shent Tona } & & \multicolumn{4}{|c|}{ 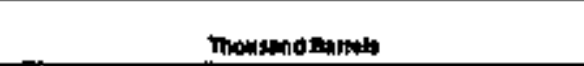 } & & \multicolumn{3}{|c|}{ 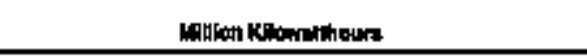 } & & \\
\hline 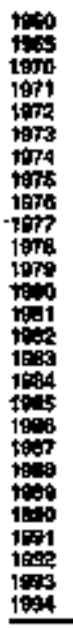 & 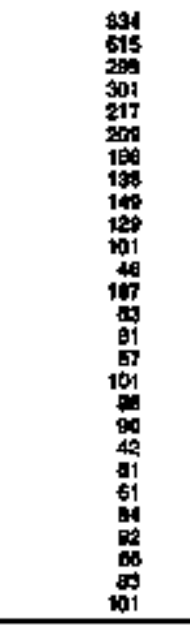 & 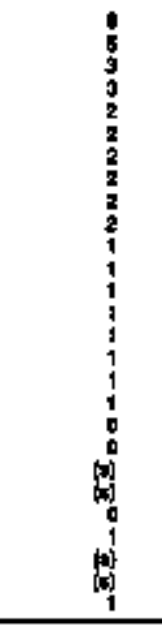 & 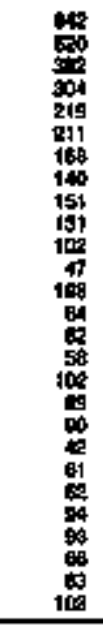 & 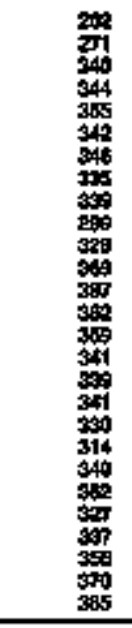 & 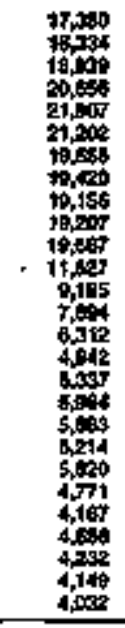 & 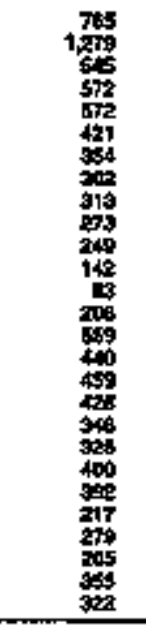 & 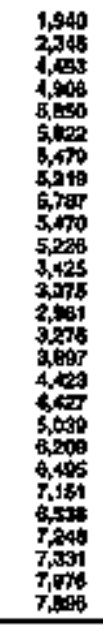 & 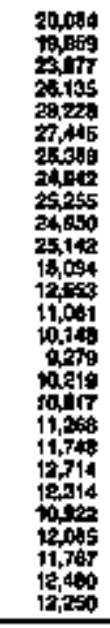 & 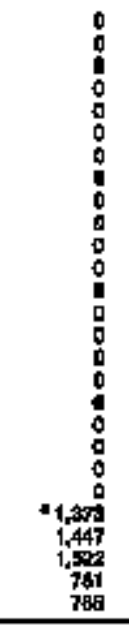 & 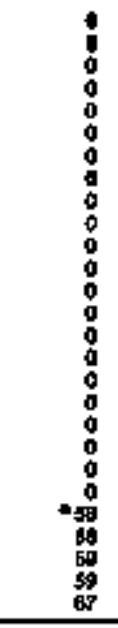 & 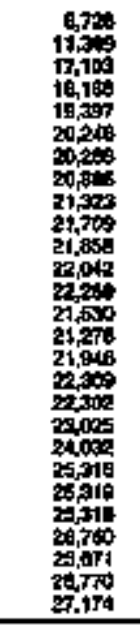 & $\begin{array}{l}z \\
\vdots \\
\vdots \\
\vdots \\
\vdots \\
\vdots \\
\vdots \\
\vdots \\
\vdots \\
\vdots \\
\vdots \\
\vdots \\
\vdots \\
\vdots\end{array}$ & 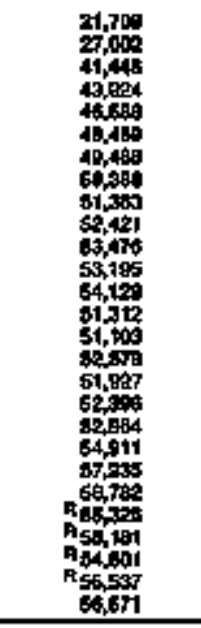 & $\begin{array}{l}\vdots \\
\vdots \\
\vdots \\
\vdots \\
\vdots \\
\vdots \\
\vdots \\
\vdots \\
\vdots \\
\vdots\end{array}$ \\
\hline \multicolumn{15}{|c|}{ 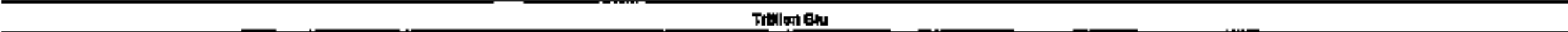 } \\
\hline 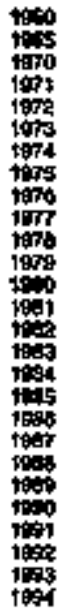 & 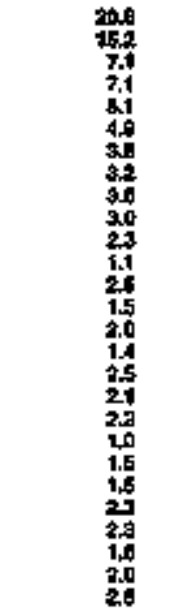 & 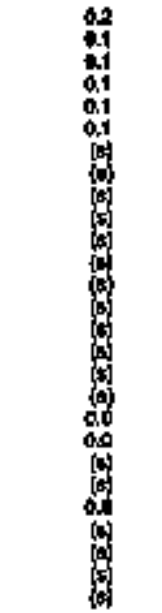 & 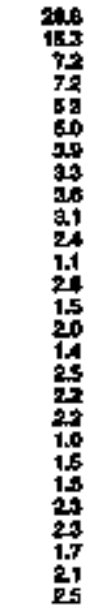 & 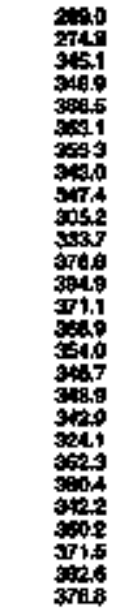 & 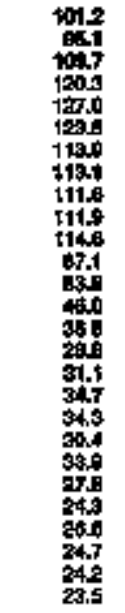 & 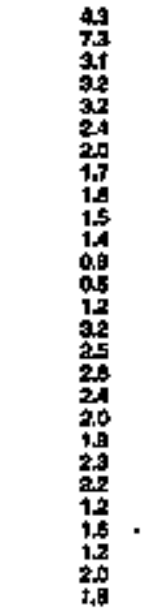 & 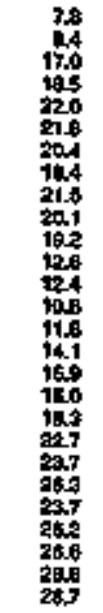 & 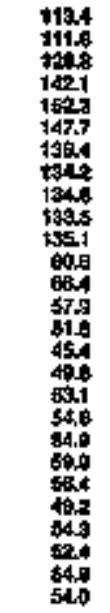 & 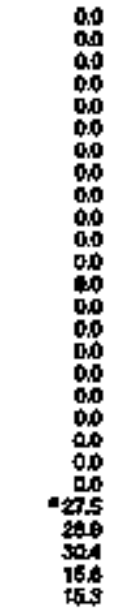 & 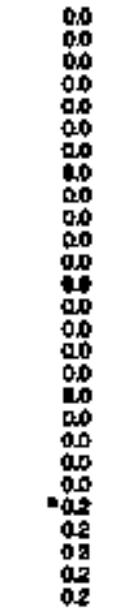 & 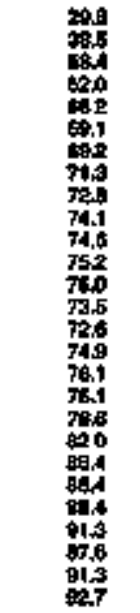 & 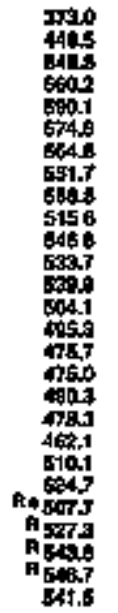 & 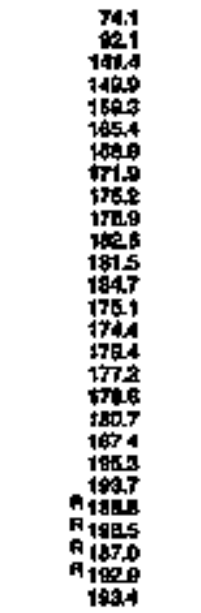 & 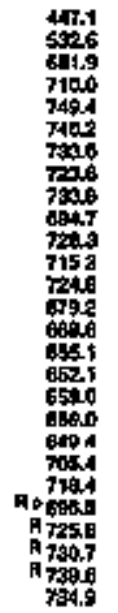 \\
\hline
\end{tabular}


Table 151. Commerclal Energy Consumption Estlmates, 1960, 1965, 1970-1994, Hiohigan

\begin{tabular}{|c|c|c|c|c|c|c|c|c|c|c|c|c|c|c|}
\hline \multirow[b]{3}{*}{$r=$} & \multicolumn{3}{|c|}{ Cost } & \multirow{3}{*}{ 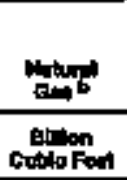 } & \multicolumn{6}{|c|}{ petroluan } & \multirow[b]{2}{*}{ Eoctionty } & \multirow[b]{2}{*}{ Enting } & \multirow[b]{2}{*}{ 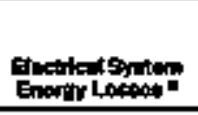 } & \multirow[b]{3}{*}{ Tot:-1 } \\
\hline & 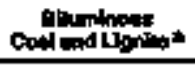 & 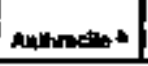 & товы & & Potenthote & Karoetena" & LPG $\mathbf{X}^{2}$ & Morot & Ponted & Tintid & & & & \\
\hline & \multicolumn{2}{|c|}{ 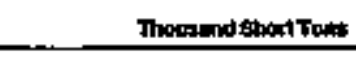 } & & & \multicolumn{6}{|c|}{ Moustand Bunts } & \multicolumn{3}{|c|}{ 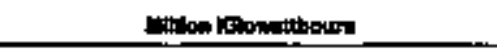 } & \\
\hline 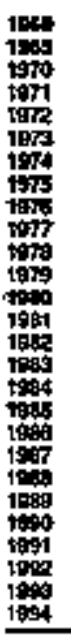 & 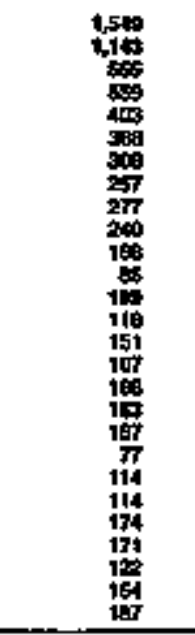 & 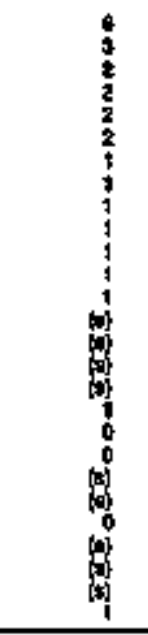 & 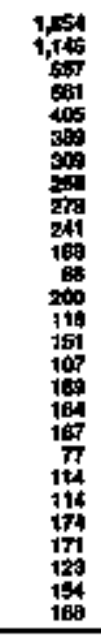 & 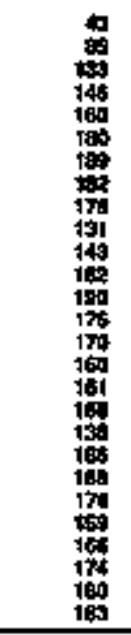 & 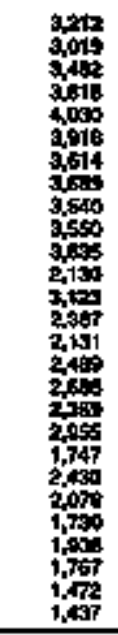 & 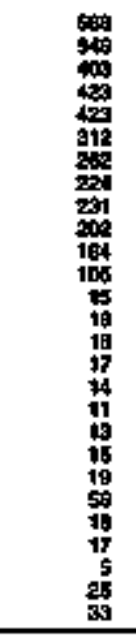 & 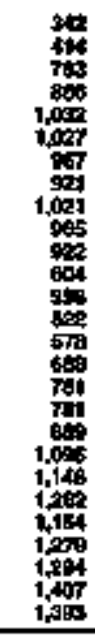 & 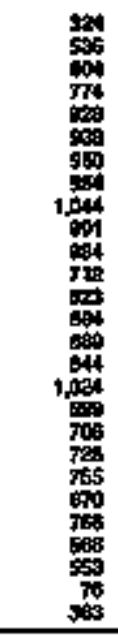 & 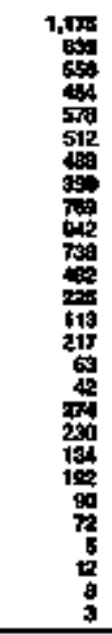 & 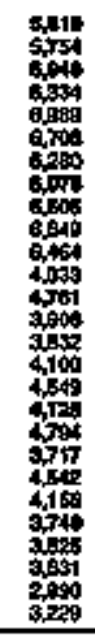 & 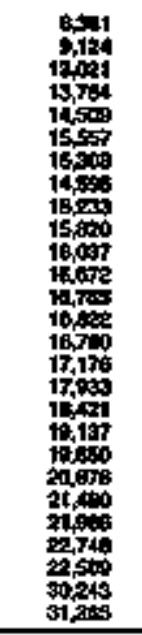 & $\begin{array}{l}= \\
= \\
= \\
= \\
= \\
= \\
= \\
= \\
= \\
= \\
= \\
= \\
= \\
=\end{array}$ & 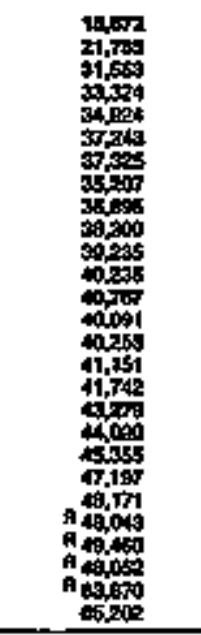 & $\begin{array}{l}= \\
= \\
z \\
= \\
= \\
= \\
= \\
= \\
= \\
z \\
= \\
= \\
= \\
=\end{array}$ \\
\hline \multicolumn{15}{|c|}{ TdEon El } \\
\hline 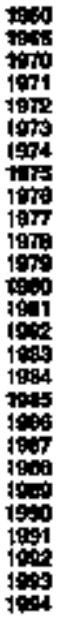 & 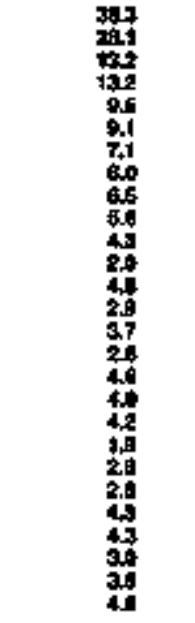 & 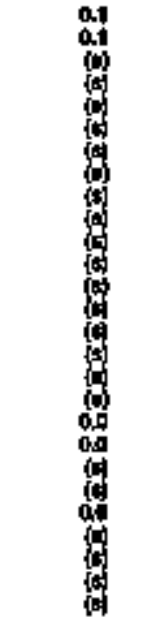 & 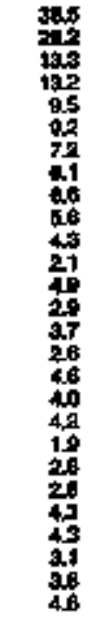 & 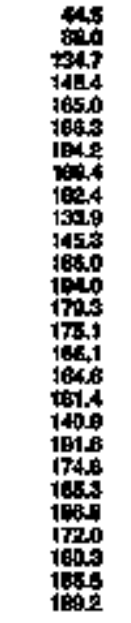 & 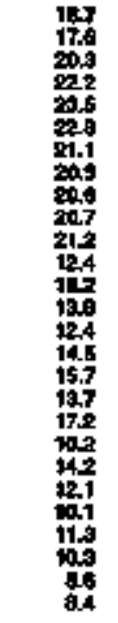 & 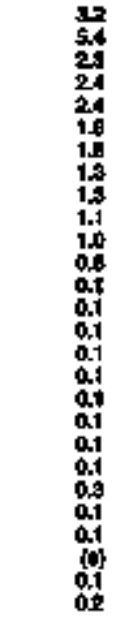 & 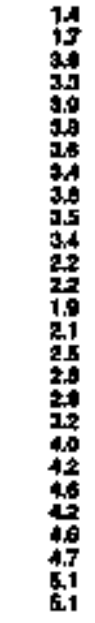 & 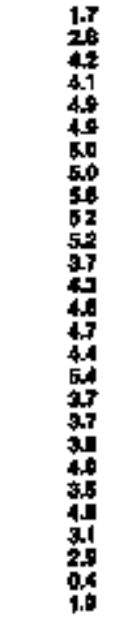 & 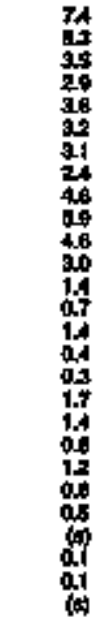 & 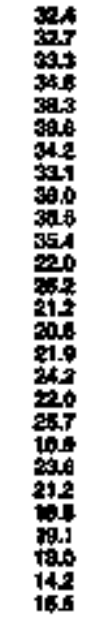 & 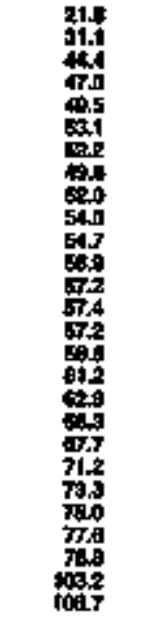 & 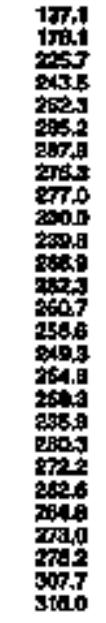 & 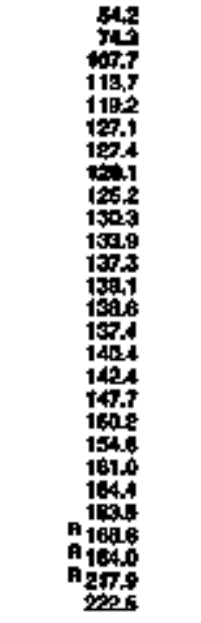 & 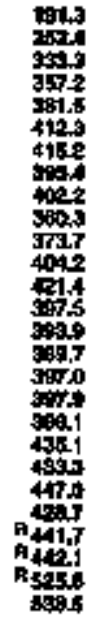 \\
\hline & & setsens & & $x_{i=0}$ & & & & & & & & & & \\
\hline
\end{tabular}


Table 152. Industrial Energy Consumption Estrmates, 1960, 1965, t970-1994, Michlgap

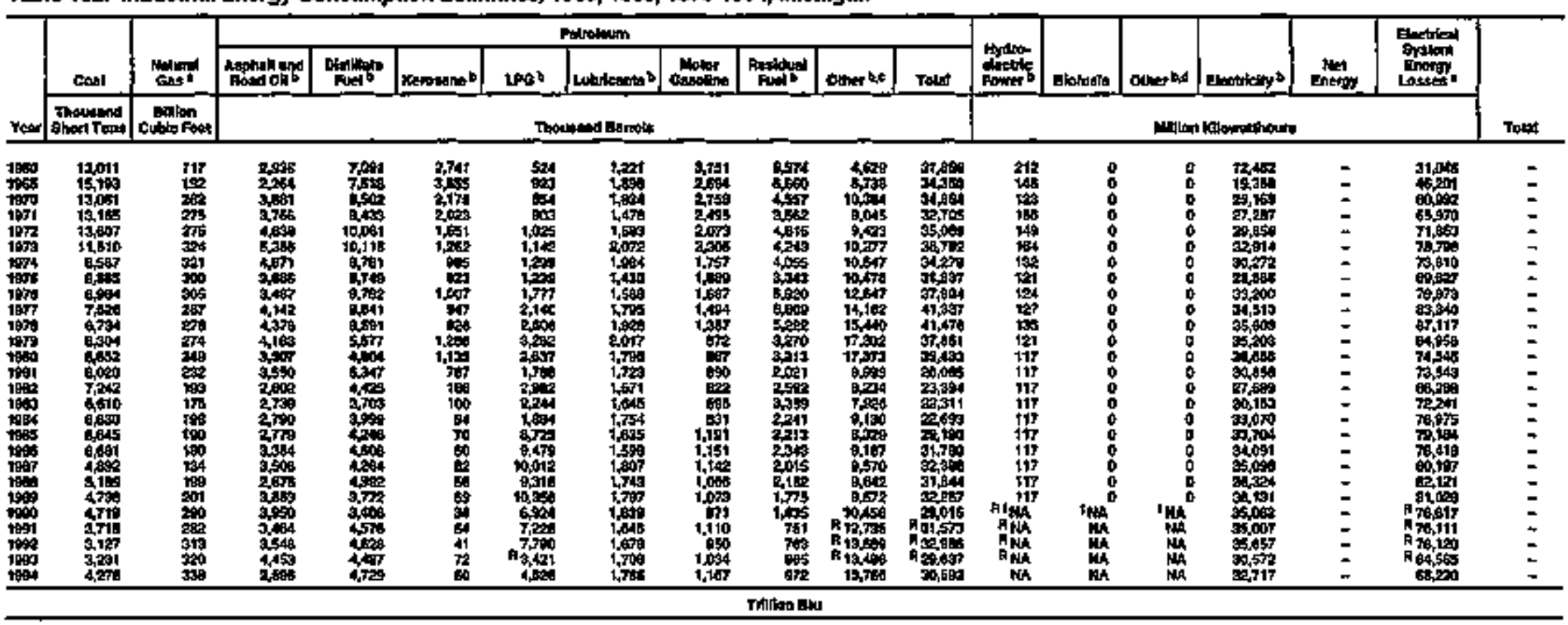

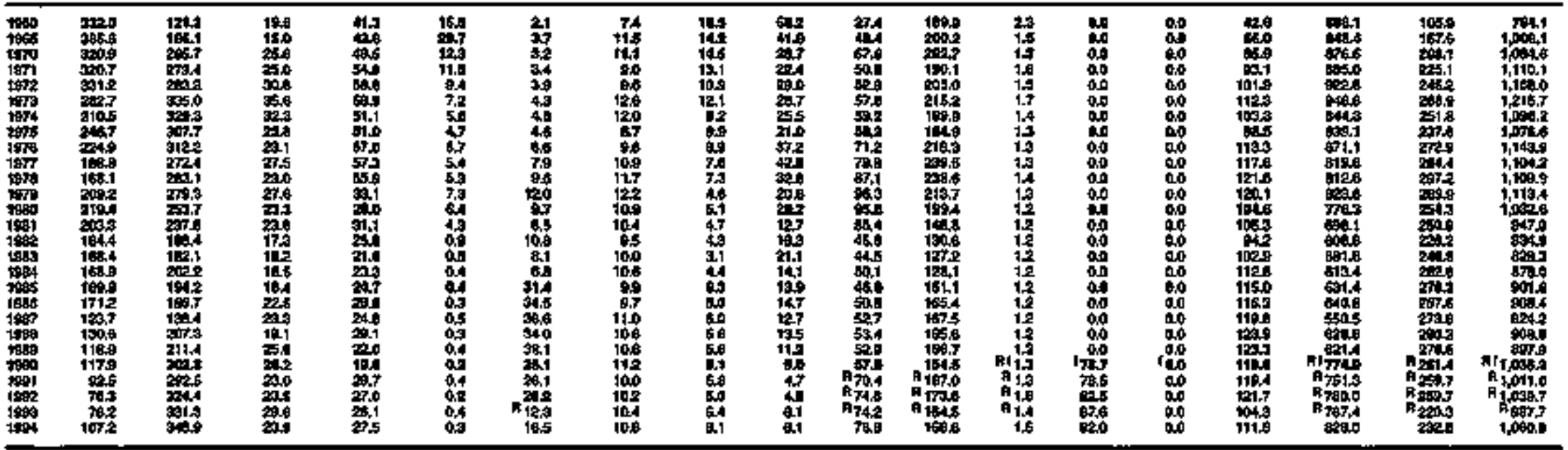

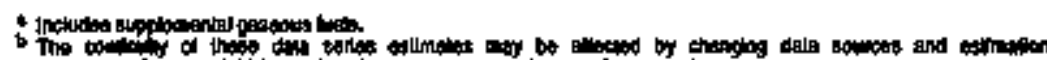

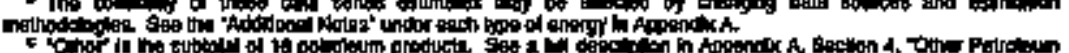
Pequect-

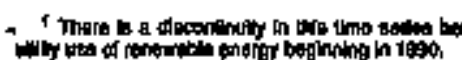

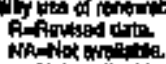

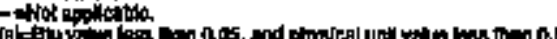

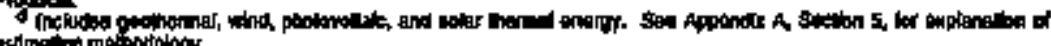

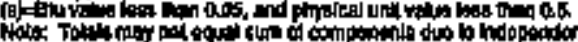

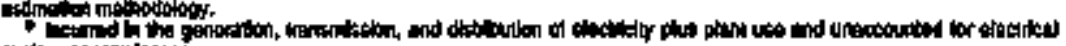
oxtom morat kntos. 
Table 153. Transportation Energy Consumption Estimates, 1960, 1985, 1970-1994, Nichigan

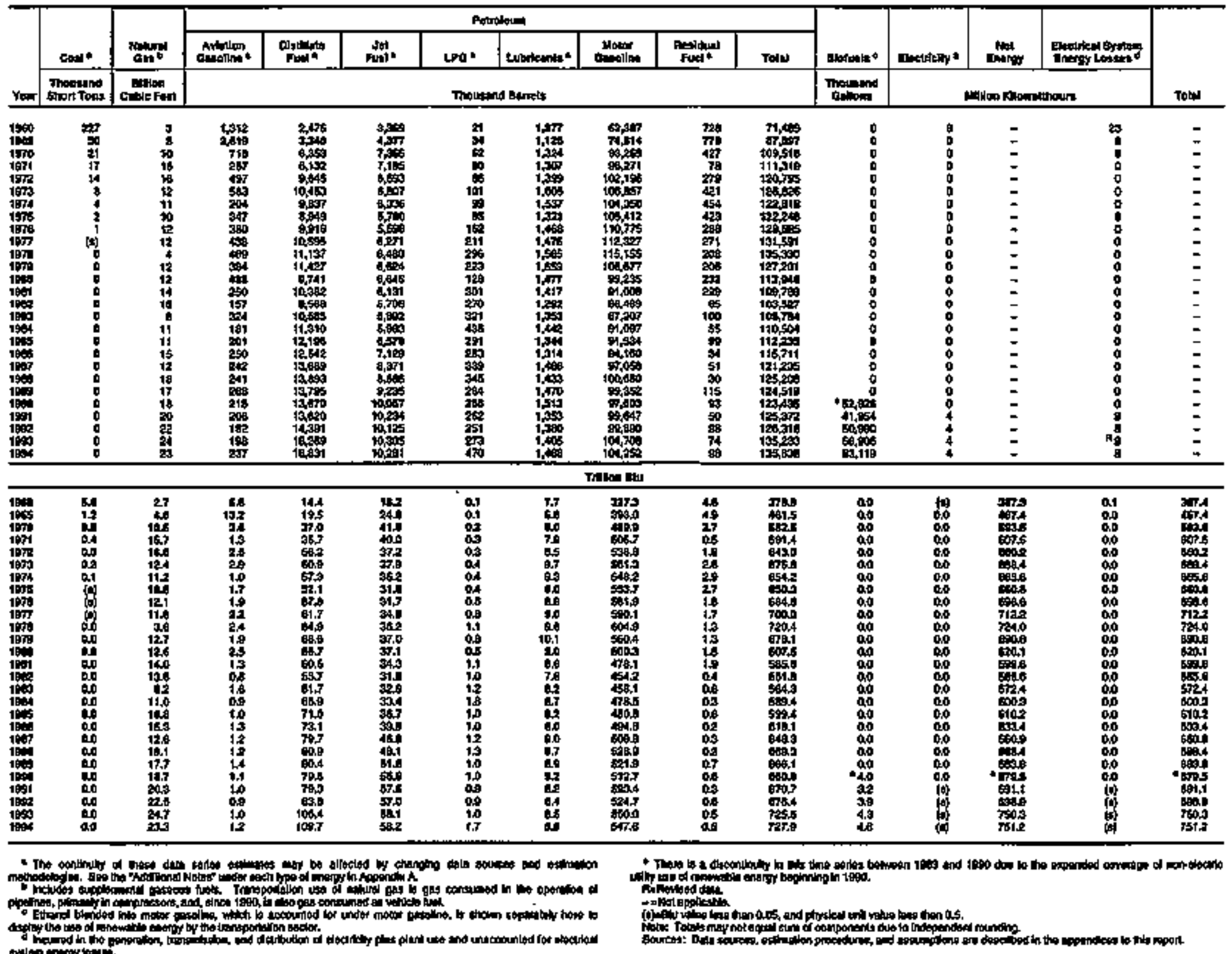




\begin{tabular}{|c|c|c|c|c|c|c|c|c|c|c|c|c|c|c|}
\hline \multirow[b]{3}{*}{ Vond } & \multicolumn{3}{|c|}{$\operatorname{con}$} & \multirow{3}{*}{ 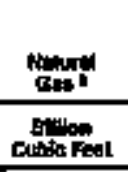 } & \multicolumn{4}{|c|}{ 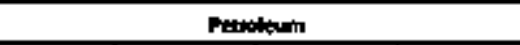 } & \multirow[b]{2}{*}{ Whelen Bocks } & \multirow[b]{2}{*}{ 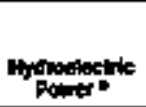 } & \multirow[b]{2}{*}{ arotolets } & \multirow[b]{2}{*}{ acothen } & \multirow{3}{*}{ anestid } & \multirow[b]{3}{*}{ Towelp } \\
\hline & costimintising & Matapenat & $T$ & & ond & 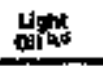 & 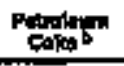 & row & & & & & & \\
\hline & \multicolumn{3}{|c|}{ Thourend Ghent Tonst } & & - & \multicolumn{3}{|c|}{ 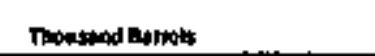 } & \multicolumn{4}{|c|}{ 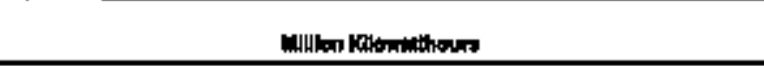 } & & \\
\hline 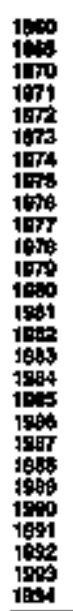 & 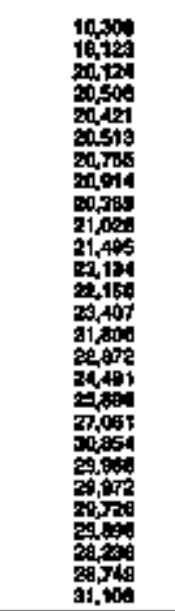 & $\begin{array}{l}0 \\
0 \\
0 \\
0 \\
0 \\
0 \\
0 \\
0 \\
0 \\
0 \\
0 \\
0 \\
0 \\
0 \\
0 \\
0 \\
0 \\
0 \\
0 \\
0 \\
0 \\
0 \\
0 \\
0 \\
0 \\
0\end{array}$ & 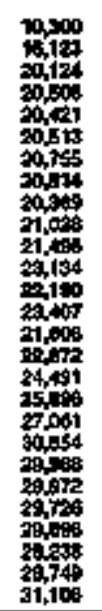 & 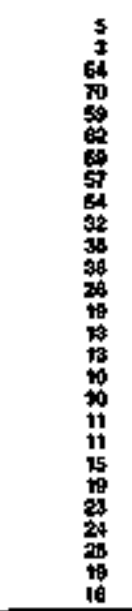 & 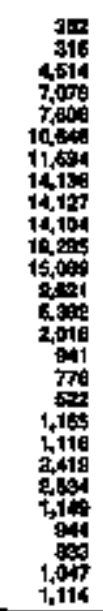 & 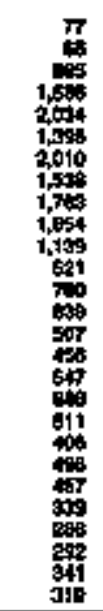 & $\begin{array}{l}\mathbf{g} \\
\mathbf{0} \\
\mathbf{g} \\
0 \\
0 \\
0 \\
0 \\
0 \\
0 \\
0 \\
0 \\
0 \\
0 \\
0 \\
0 \\
0 \\
0 \\
\vdots \\
0 \\
0 \\
0 \\
: \\
0 \\
0 \\
0 \\
0 \\
0\end{array}$ & 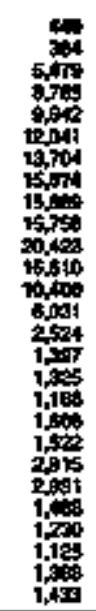 & 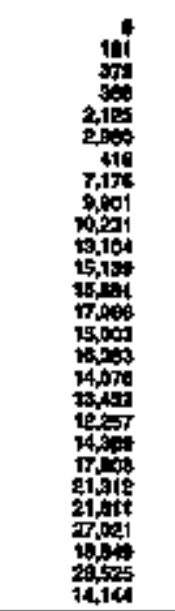 & 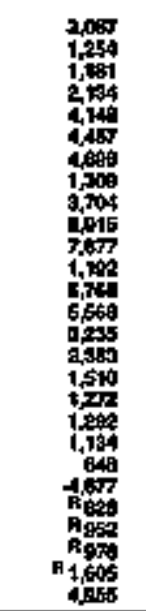 & 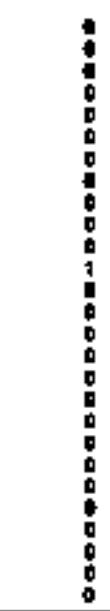 & $\begin{array}{l}0 \\
0 \\
0 \\
0 \\
0 \\
0 \\
0 \\
0 \\
0 \\
0 \\
0 \\
0 \\
0 \\
0 \\
0 \\
0 \\
0 \\
0 \\
0 \\
0 \\
0 \\
0 \\
0 \\
0 \\
0 \\
0\end{array}$ & $\begin{array}{l}: \\
0 \\
0 \\
0 \\
0 \\
0 \\
0 \\
0 \\
0 \\
0 \\
0 \\
0 \\
0 \\
0 \\
0 \\
0 \\
0 \\
0 \\
0 \\
0 \\
0 \\
0 \\
0 \\
0\end{array}$ & $\begin{array}{l}\vdots \\
\vdots \\
\vdots \\
\vdots \\
\vdots \\
\vdots \\
\vdots \\
\vdots \\
\vdots \\
\vdots \\
\vdots \\
\vdots \\
\vdots\end{array}$ \\
\hline \multicolumn{15}{|c|}{ 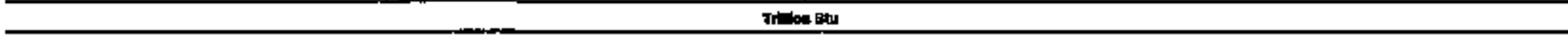 } \\
\hline 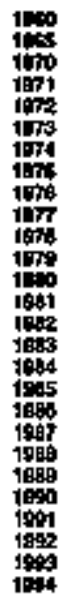 & 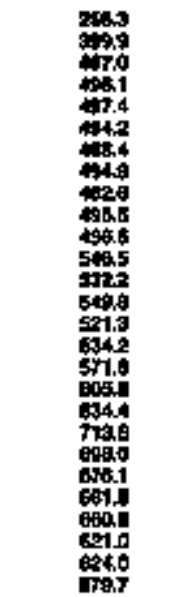 & 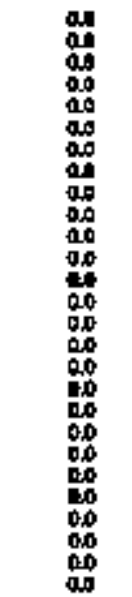 & 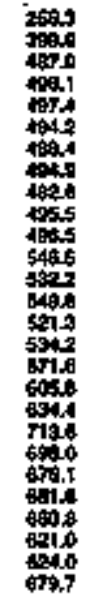 & 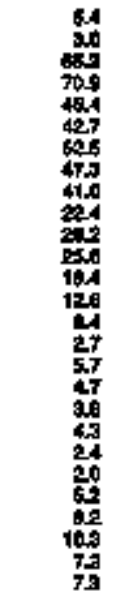 & 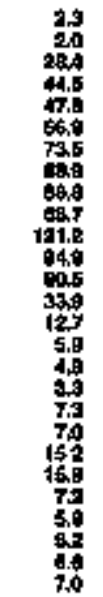 & 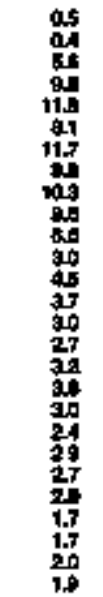 & 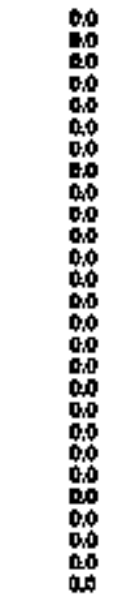 & 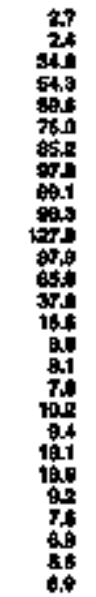 & 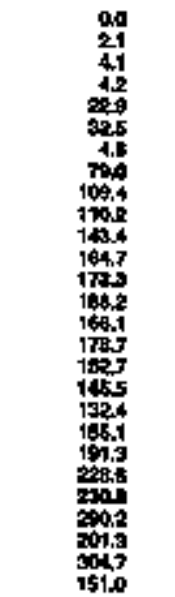 & 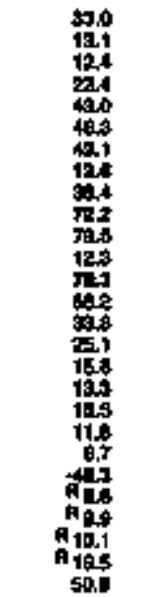 & 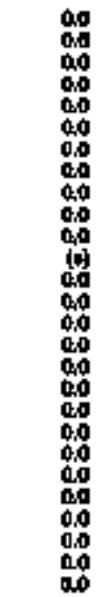 & 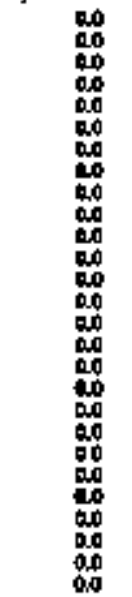 & 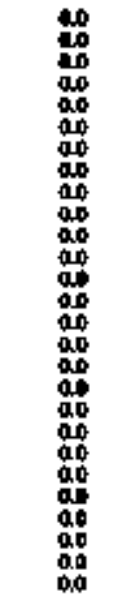 & 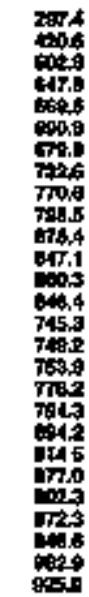 \\
\hline
\end{tabular}

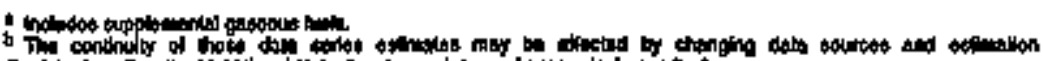

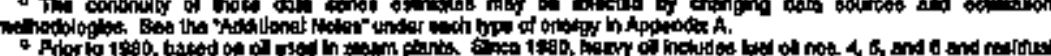

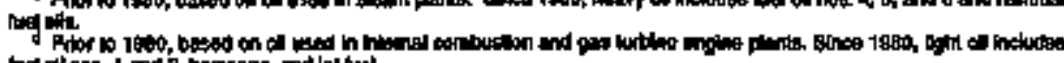

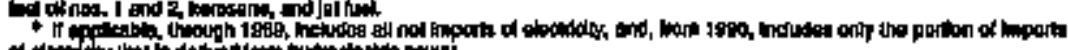

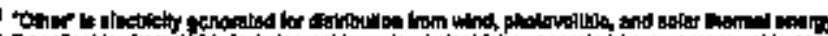

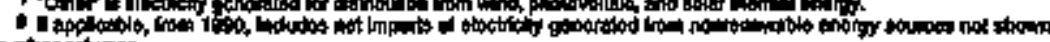

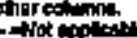

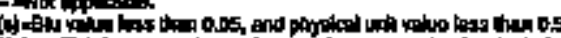

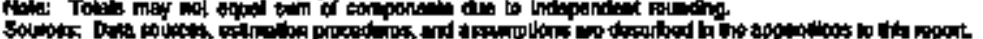


Table 1S5. Energy Conswmplion Estimales by Source, 1969, 1965, 1970-1994, Minnesota

\begin{tabular}{|c|c|c|c|c|c|c|c|c|c|c|c|c|c|c|c|c|c|c|c|}
\hline \multirow[b]{3}{*}{ סt: } & \multirow[b]{2}{*}{ Cor. } & \multirow[b]{2}{*}{ tons } & \multicolumn{11}{|c|}{ Papdrutre } & \multirow[b]{2}{*}{ 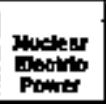 } & \multirow[b]{2}{*}{ 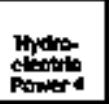 } & \multirow[b]{2}{*}{ Dibluebs" } & \multirow[b]{2}{*}{ oser an | } & \multirow{2}{*}{ 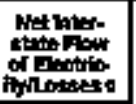 } & \multirow[b]{3}{*}{ Totwa n } \\
\hline & & & 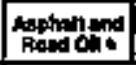 & Avesom & Ponto & foid. & 1. & LPA. & Loth & ations & 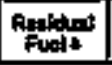 & Oober ac & Tot & & & & & & \\
\hline & mant Tand & coubte Foed & \multicolumn{11}{|c|}{ molvend Rondt } & \multicolumn{5}{|c|}{ 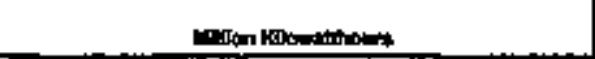 } & \\
\hline 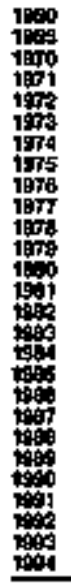 & 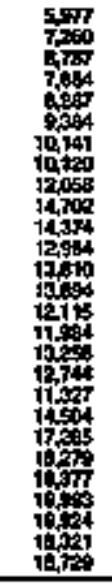 & 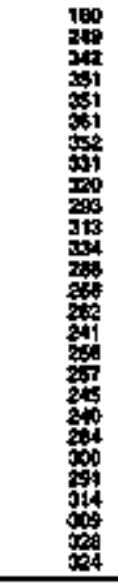 & 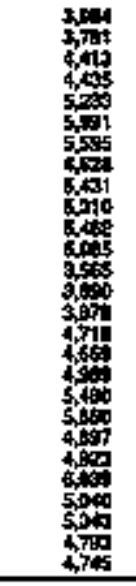 & 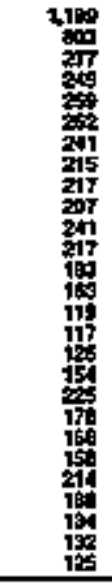 & 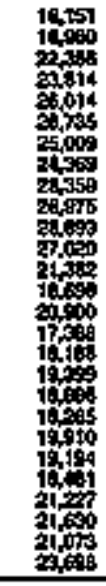 & 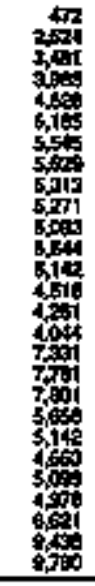 & 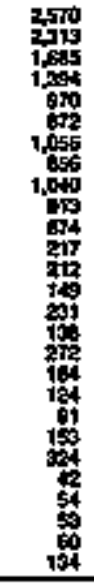 & 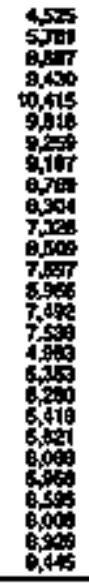 & 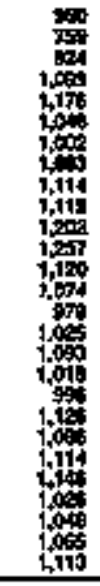 & 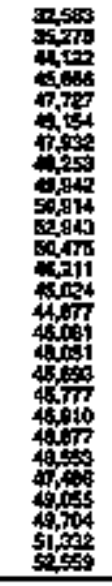 & 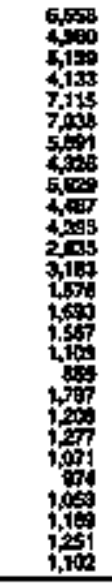 & 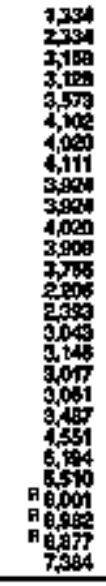 & 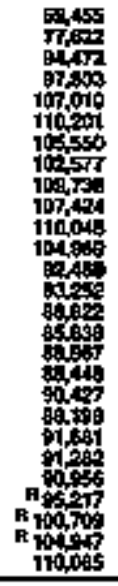 & 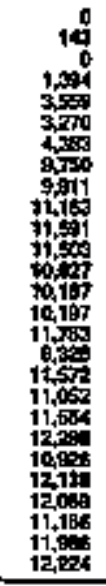 & 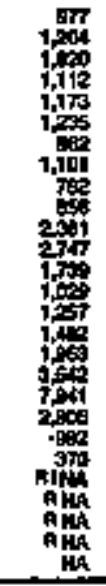 & 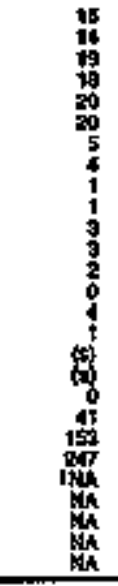 & 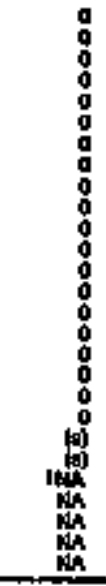 & 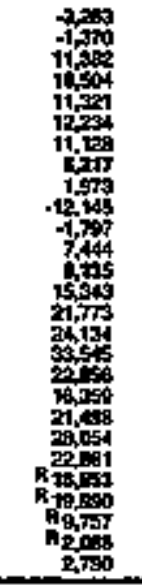 & $\begin{array}{l}z \\
\vdots \\
\vdots \\
\vdots \\
\vdots \\
\vdots \\
\vdots \\
\vdots \\
= \\
\vdots \\
z \\
\vdots \\
z \\
z\end{array}$ \\
\hline \multicolumn{20}{|c|}{ Tnimon en } \\
\hline 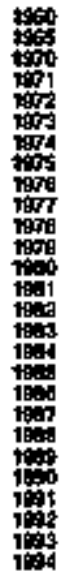 & 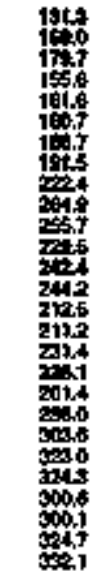 & 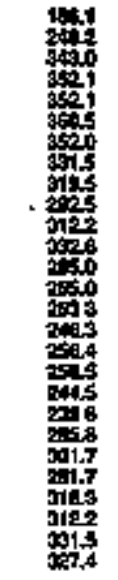 & 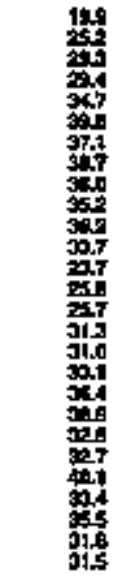 & 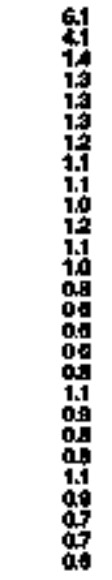 & 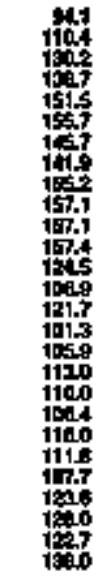 & 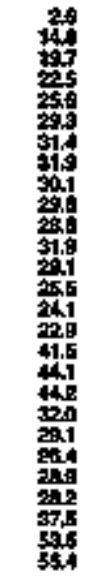 & 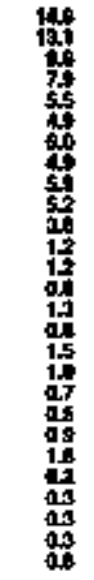 & 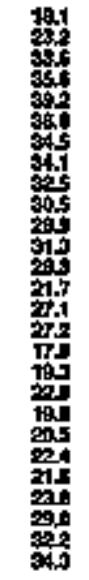 & 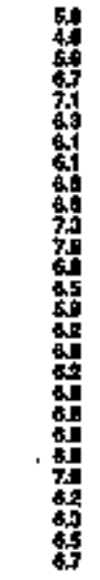 & 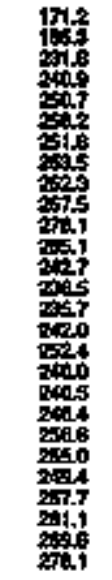 & 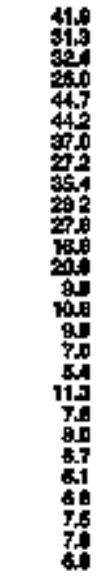 & 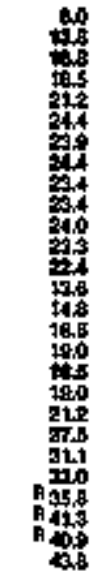 & 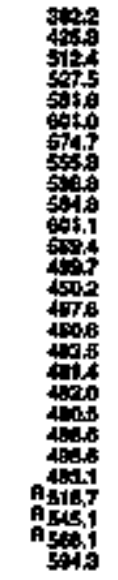 & 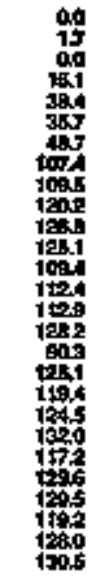 & 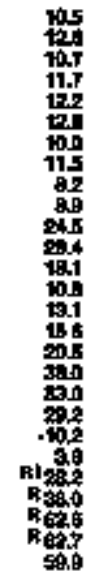 & 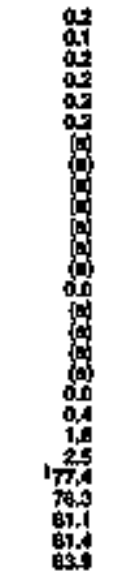 & 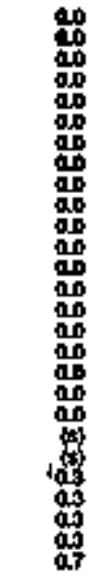 & 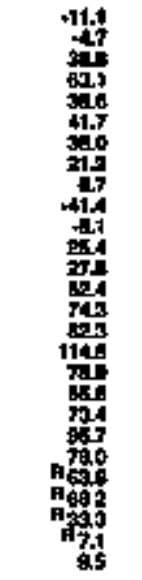 & 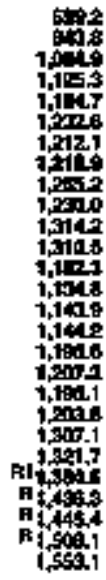 \\
\hline
\end{tabular}

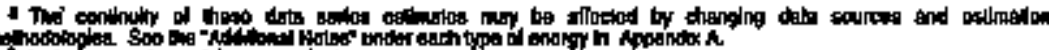

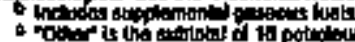

A getion s.

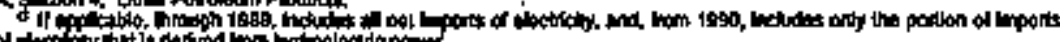

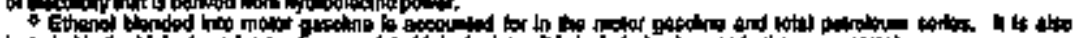

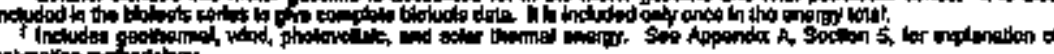

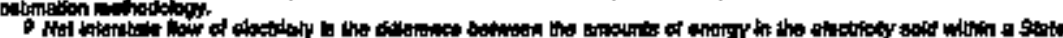

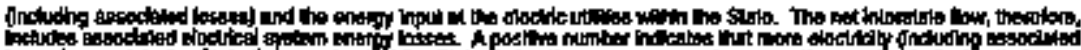
betsed a

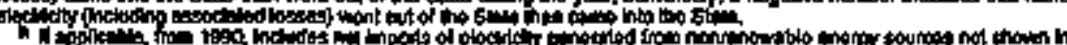

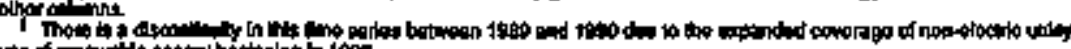
teof

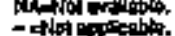

Hot Tof 
Tabie 153. Residentlal Energy Consumption Eshiolatog, 1960, 1965, 1970-1934, Minnessola

\begin{tabular}{|c|c|c|c|c|c|c|c|c|c|c|c|c|c|c|}
\hline \multirow[b]{3}{*}{ tom } & \multicolumn{3}{|c|}{ and } & \multirow{3}{*}{ 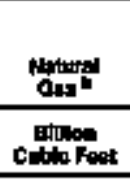 } & \multicolumn{4}{|c|}{ Patroiloun } & \multirow{3}{*}{ Alutula } & \multirow[b]{2}{*}{ Bolur" } & \multirow[b]{2}{*}{ Emethely" } & \multirow[b]{2}{*}{ thersy } & \multirow{3}{*}{ 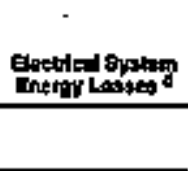 } & \multirow[b]{3}{*}{ Totod } \\
\hline & 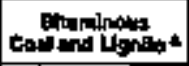 & Antorato" & Tote & & 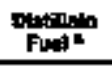 & trovanen & 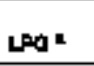 & Totat & & & & & & \\
\hline & \multicolumn{3}{|c|}{ 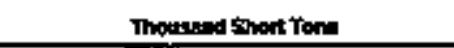 } & & \multicolumn{4}{|c|}{ 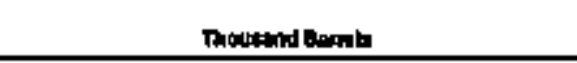 } & & \multicolumn{3}{|c|}{ 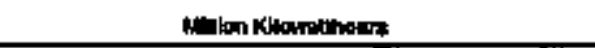 } & & \\
\hline 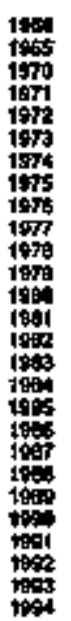 & 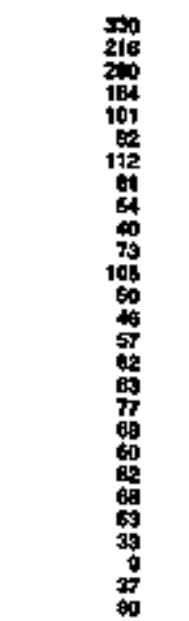 & $\begin{array}{l}0 \\
0 \\
0 \\
0 \\
0 \\
0 \\
0 \\
0 \\
0 \\
0 \\
0 \\
0 \\
0 \\
0 \\
0 \\
0 \\
0 \\
0 \\
0 \\
0 \\
0 \\
0 \\
0 \\
0 \\
0 \\
0 \\
0\end{array}$ & 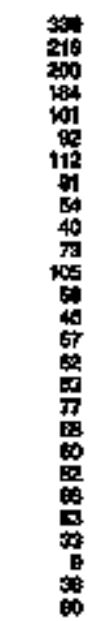 & 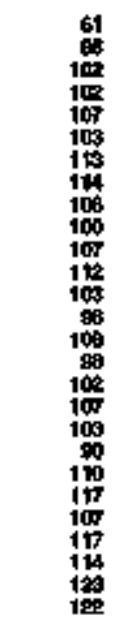 & 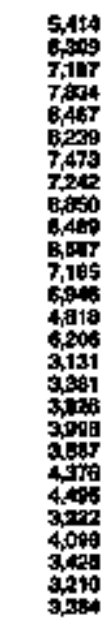 & 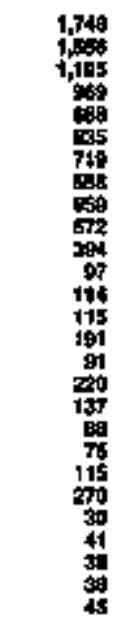 & 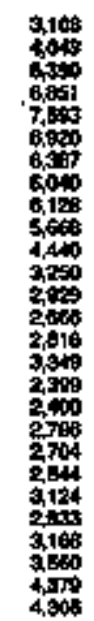 & 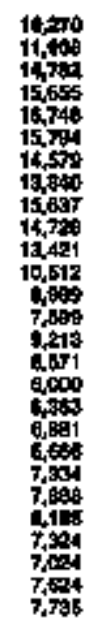 & 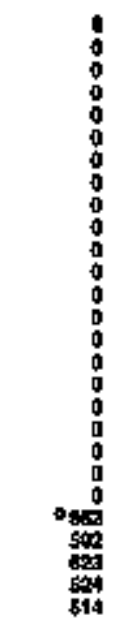 & $\begin{array}{l}0 \\
0 \\
0 \\
0 \\
0 \\
0 \\
0 \\
0 \\
0 \\
0 \\
0 \\
0 \\
0 \\
0 \\
0 \\
0 \\
0\end{array}$ & 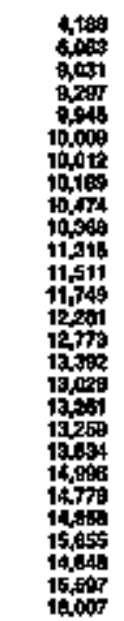 & $\begin{array}{l}= \\
= \\
z \\
z \\
z \\
z \\
= \\
= \\
z \\
z \\
z \\
z \\
z \\
z\end{array}$ & 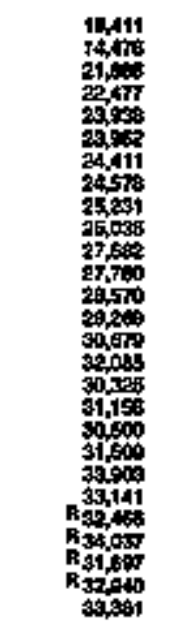 & $\begin{array}{l}= \\
z \\
= \\
= \\
= \\
z \\
= \\
z \\
z \\
= \\
= \\
z \\
z \\
z\end{array}$ \\
\hline
\end{tabular}

\begin{tabular}{|c|c|}
\hline 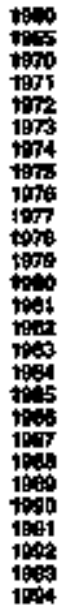 & 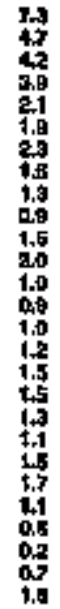 \\
\hline
\end{tabular}

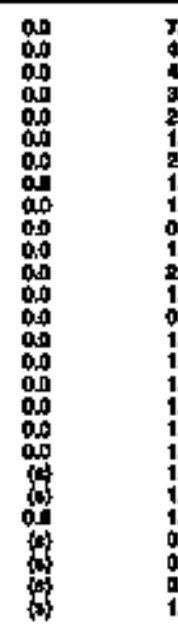

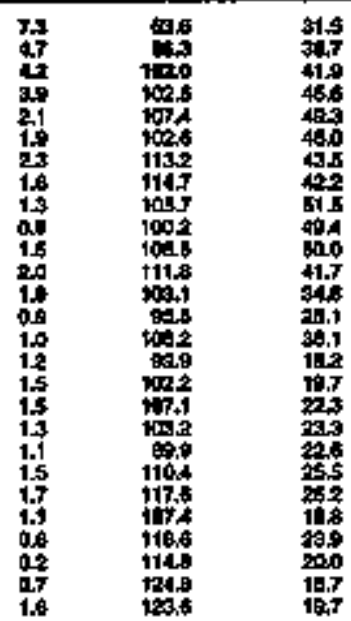

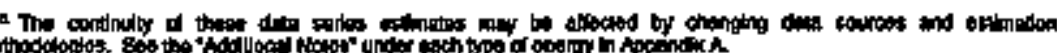

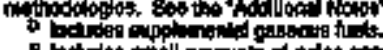

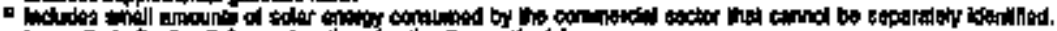

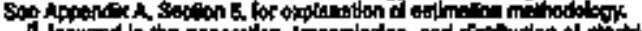

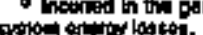

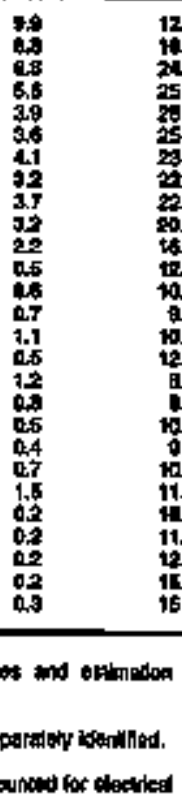

\begin{tabular}{|c|c|c|c|c|c|c|c|}
\hline 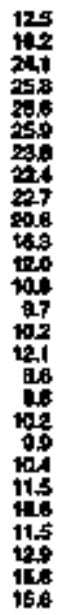 & 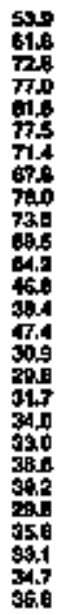 & 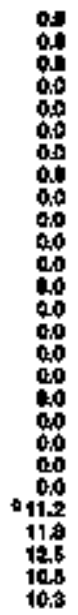 & $\begin{array}{l}00 \\
00 \\
000 \\
00 \\
000 \\
00 \\
00 \\
00 \\
00 \\
00 \\
0.0 \\
0.0 \\
0.0 \\
00 \\
0.0 \\
0.0 \\
0.0 \\
0.0 \\
0.0 \\
00 \\
0.0 \\
00.3 \\
0.3 \\
0.3 \\
0.9 \\
0.3\end{array}$ & 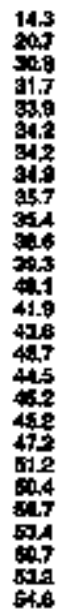 & 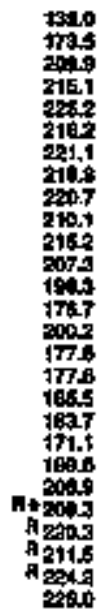 & 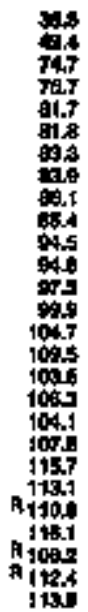 & 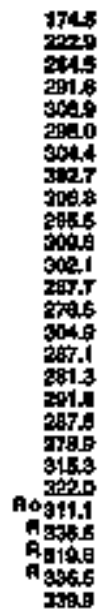 \\
\hline
\end{tabular}

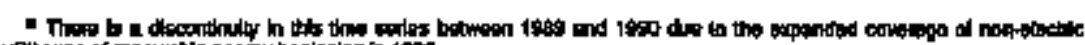
Propord

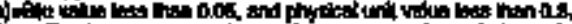

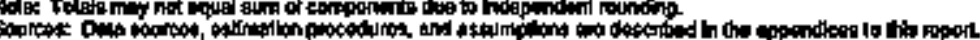


Table 157. Commorclal Enorgy Consumption Estimates, 1950, 1965, 1979-1994, Minnesota

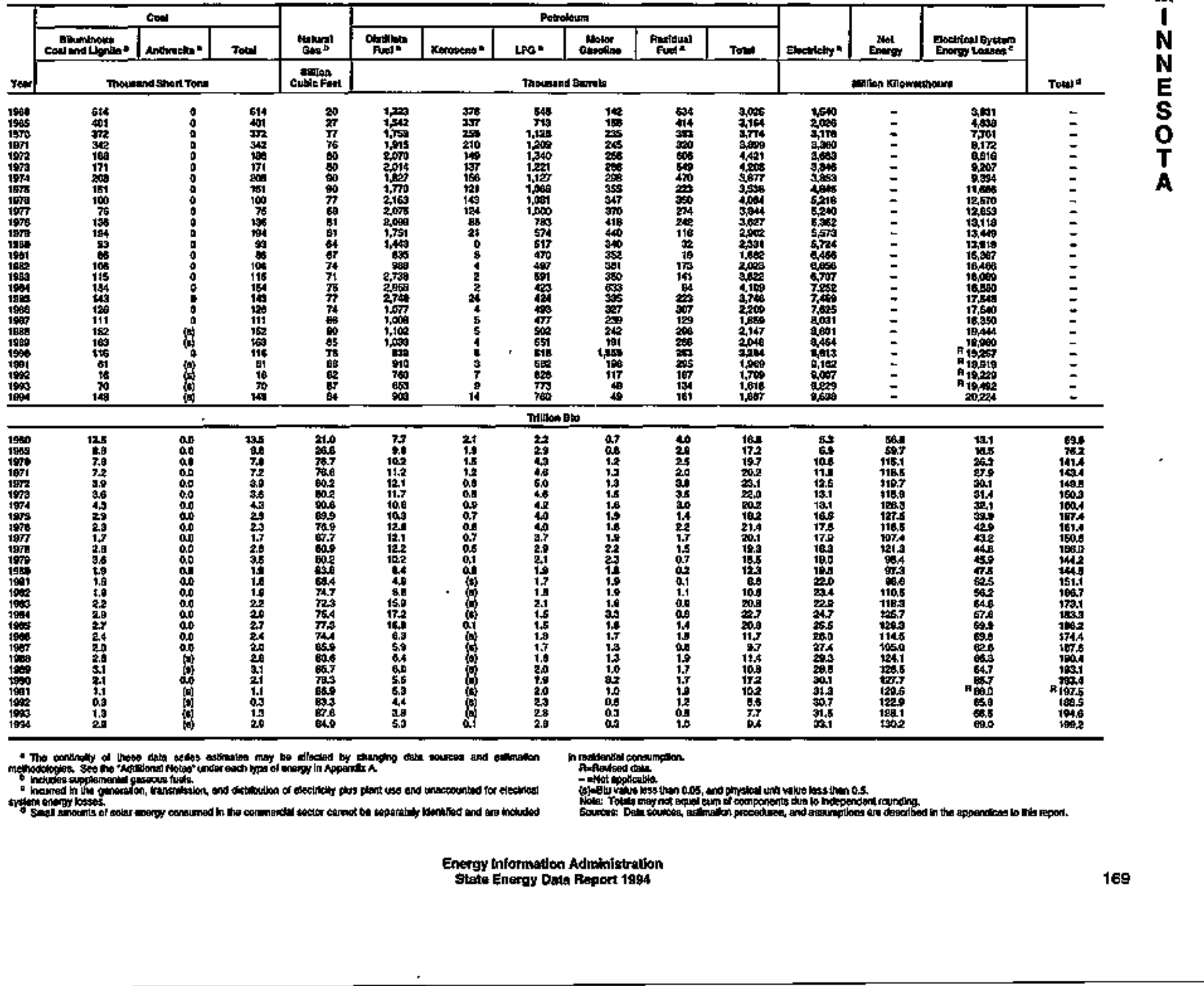




\begin{tabular}{|c|c|c|c|c|c|c|c|c|c|c|c|c|c|c|c|c|c|c|}
\hline \multirow[b]{3}{*}{ 然 } & \multirow[b]{2}{*}{ cont } & \multirow[b]{2}{*}{$\operatorname{mog}_{2}$} & \multicolumn{9}{|c|}{ Prowen } & \multirow[b]{2}{*}{$\mid$} & \multirow[b]{2}{*}{ Elothal } & \multirow[b]{2}{*}{ tomeras a } & \multirow[b]{2}{*}{ Anetrienge } & \multirow[b]{2}{*}{ 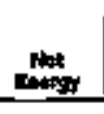 } & \multirow{2}{*}{ 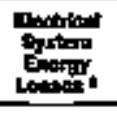 } & \multirow[b]{3}{*}{ Tow } \\
\hline & & & 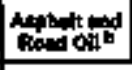 & 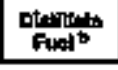 & 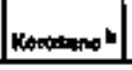 & tpat & Lent & 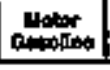 & 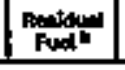 & Otherer & Tow & & & & & & & \\
\hline & mownod & 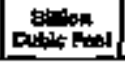 & \multicolumn{9}{|c|}{ 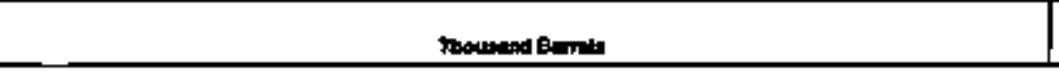 } & \multicolumn{6}{|c|}{ 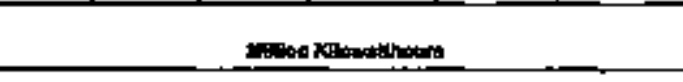 } & \\
\hline 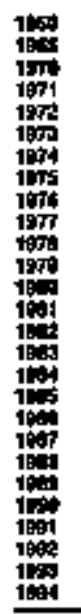 & 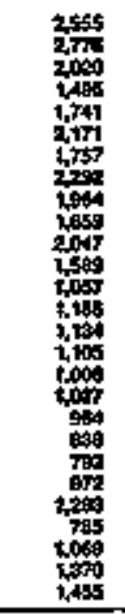 & 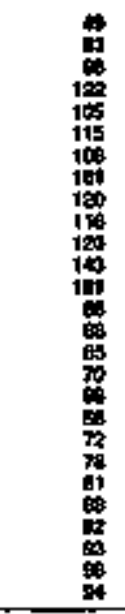 & 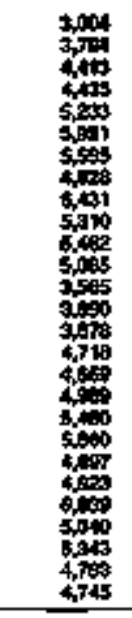 & 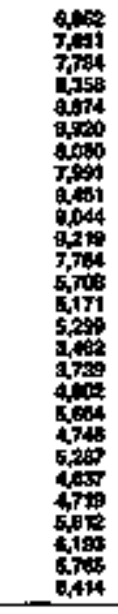 & 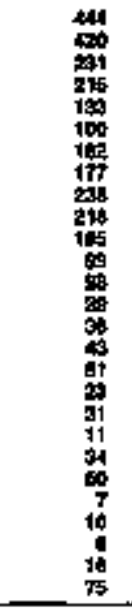 & 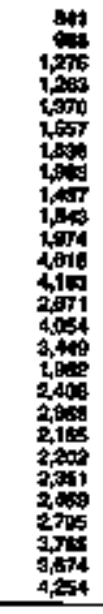 & 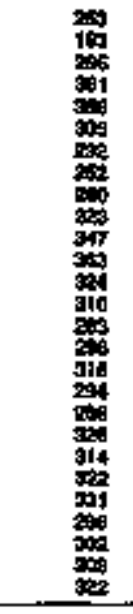 & 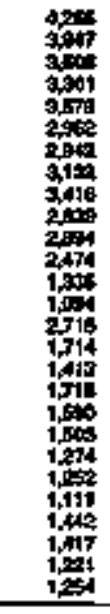 & 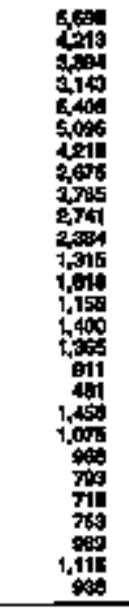 & 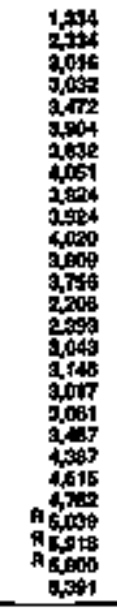 & 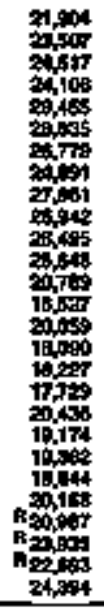 & 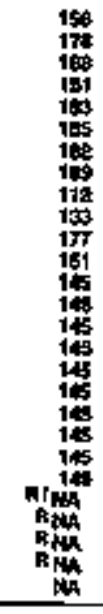 & 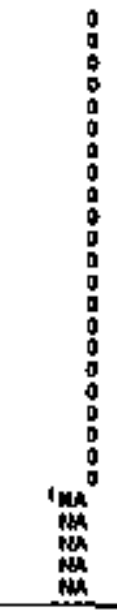 & 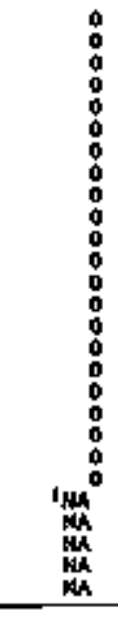 & 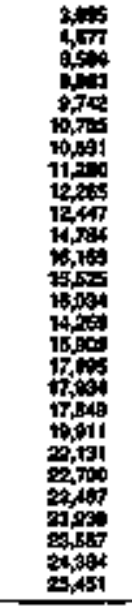 & $\begin{array}{l}\vdots \\
\vdots \\
\vdots \\
\vdots \\
\vdots \\
\vdots \\
\vdots \\
\vdots \\
\vdots \\
\vdots \\
\vdots \\
\vdots \\
\vdots \\
\end{array}$ & 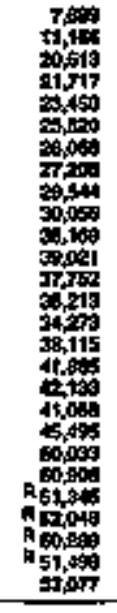 & $\begin{array}{l}z \\
\bar{z} \\
\bar{z} \\
\bar{z} \\
\bar{z}\end{array}$ \\
\hline 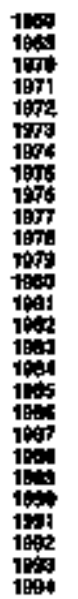 & 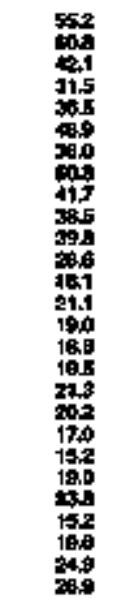 & 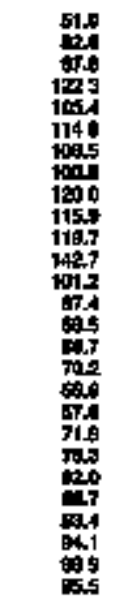 & 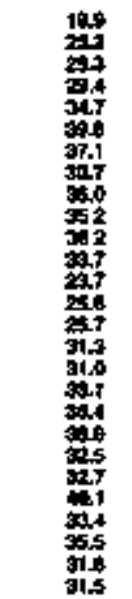 & 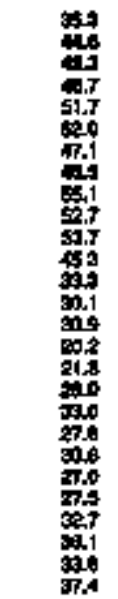 & 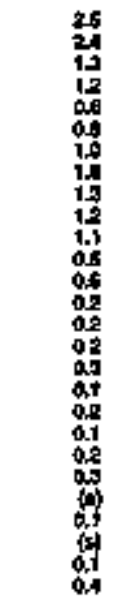 & 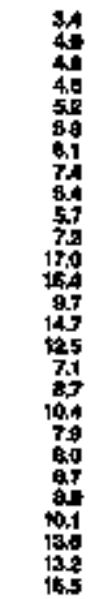 & 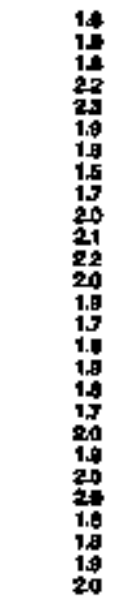 & 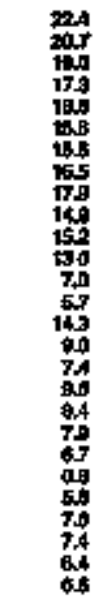 & 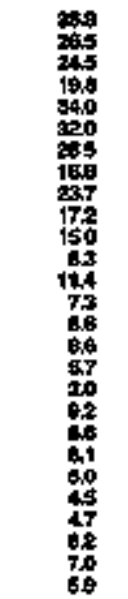 & 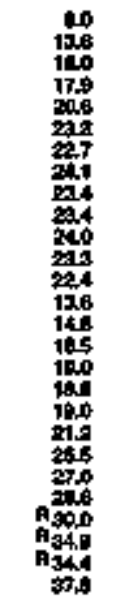 & 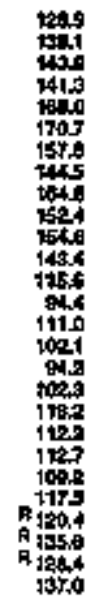 & 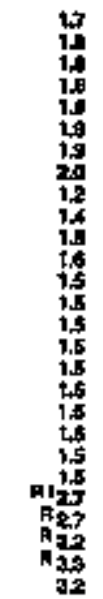 & 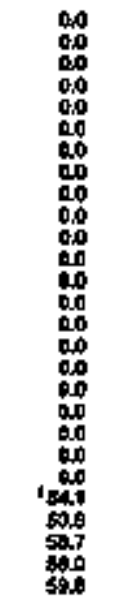 & 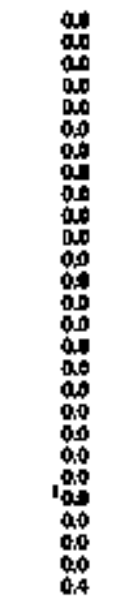 & 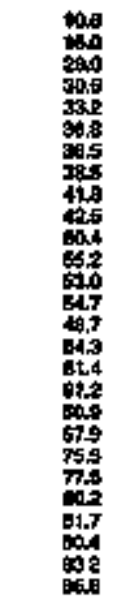 & 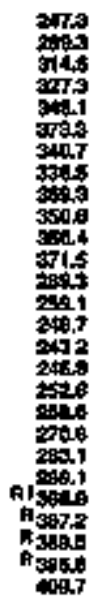 & 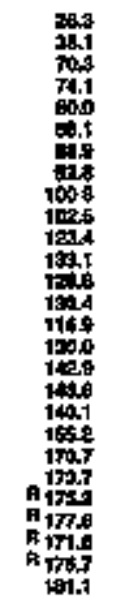 & 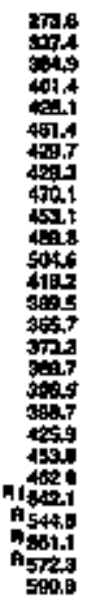 \\
\hline
\end{tabular}

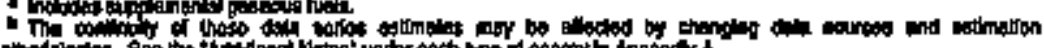

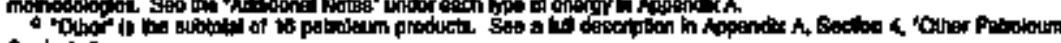

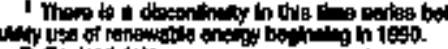

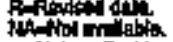
-

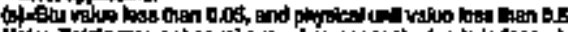

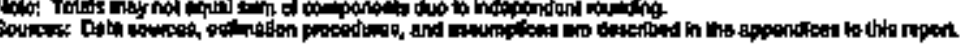

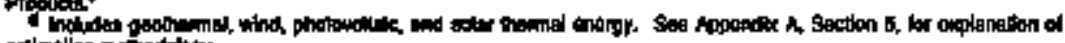

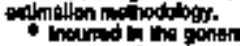
( now 
Table 159. Transportalion Enargy Consumplion Estimoles, 1950, 1965, 1970-1994, Minnosalk

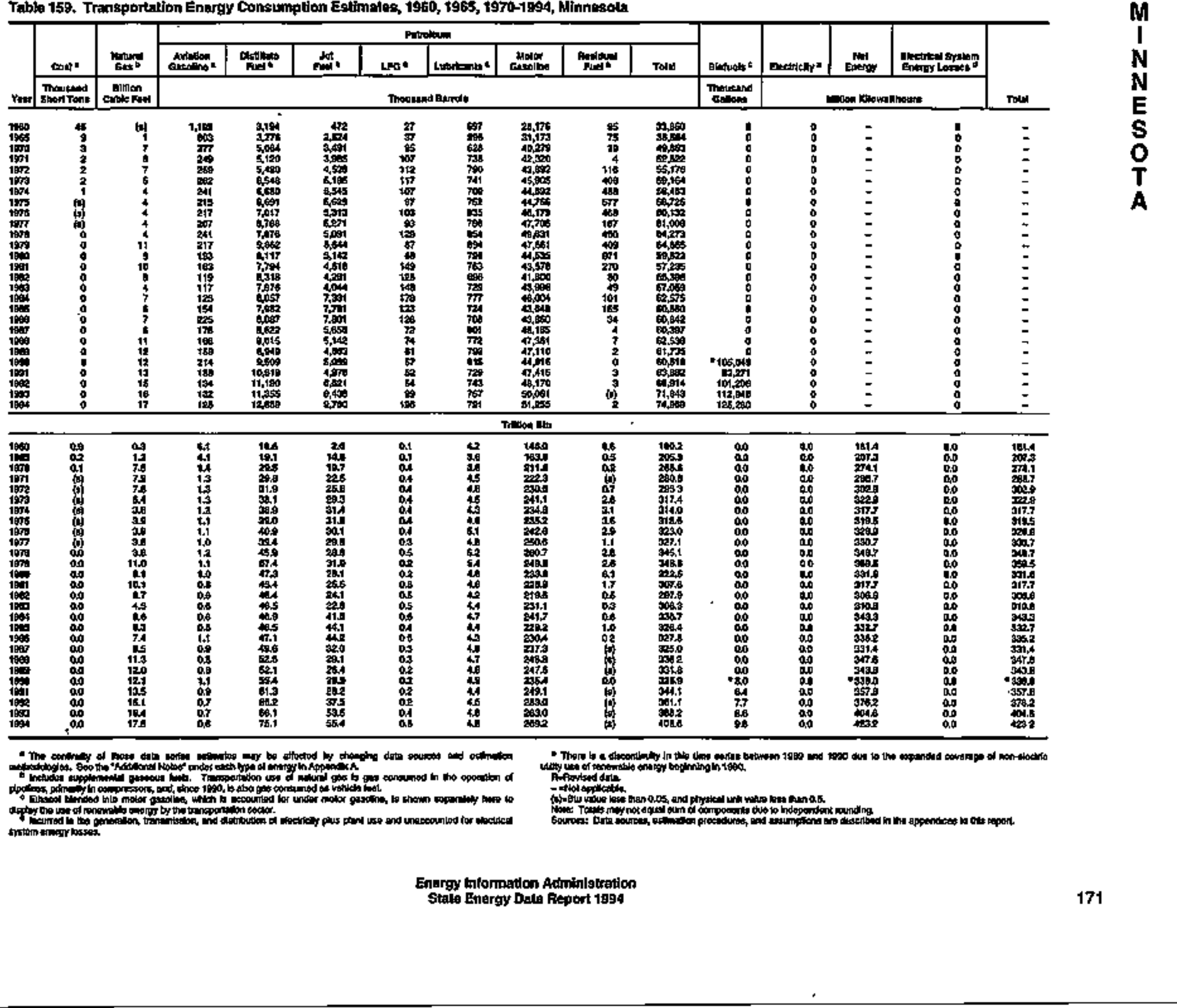


Table 160. Estlmates of Engrgy Jnput at Electric Ulilities, 1960, 1655, 1970-1994, Minnesoth

\begin{tabular}{|c|c|c|c|c|c|c|c|c|c|c|c|c|c|c|}
\hline \multirow[b]{3}{*}{$Y \notin$} & \multicolumn{3}{|c|}{ coled } & \multirow[b]{2}{*}{ 梠 } & \multicolumn{4}{|c|}{ 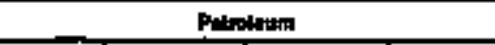 } & \multirow[b]{2}{*}{ 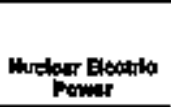 } & \multirow[b]{2}{*}{ Indodecte } & \multirow[b]{2}{*}{ Blatualn } & \multirow[b]{2}{*}{ 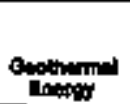 } & \multirow[b]{2}{*}{ Other } & \multirow[b]{3}{*}{ Total } \\
\hline & Altion & Antrache & Tot!l & & $\cos$ & Lofot & Fenters & Toto & & & & & & \\
\hline & \multicolumn{3}{|c|}{ Thomand sorl Tous } & 19: & \multicolumn{4}{|c|}{ Jwovand Bunts } & \multicolumn{5}{|c|}{ 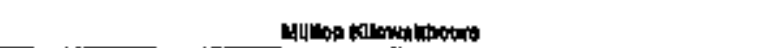 } & \\
\hline
\end{tabular}

\begin{tabular}{|c|c|c|c|c|c|c|c|c|c|c|c|c|c|c|}
\hline 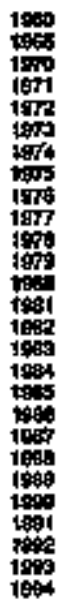 & 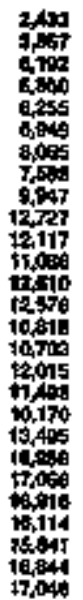 & $\begin{array}{l}0 \\
0 \\
0 \\
0 \\
0 \\
0 \\
0 \\
0 \\
0 \\
0 \\
0 \\
0 \\
0 \\
0 \\
0 \\
0 \\
0 \\
0 \\
0 \\
0 \\
0 \\
0 \\
0 \\
0 \\
0 \\
0 \\
0\end{array}$ & 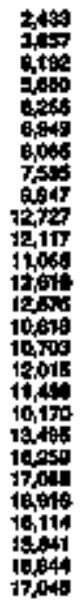 & 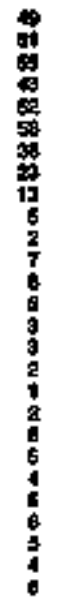 & 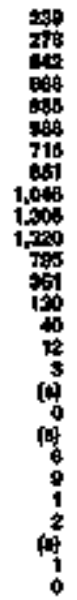 & 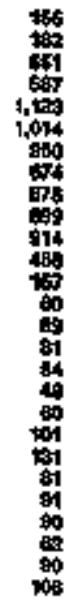 & 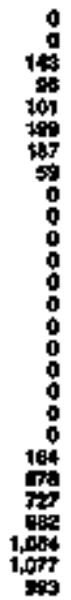 & 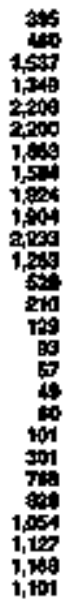 & 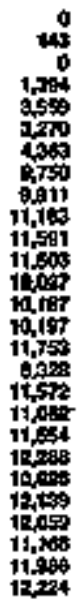 & 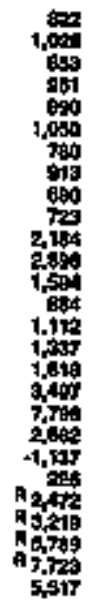 & 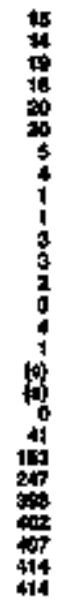 & $\begin{array}{l}0 \\
0 \\
0 \\
0 \\
0 \\
0 \\
0 \\
0 \\
0 \\
0 \\
0 \\
0 \\
0 \\
0 \\
0 \\
0 \\
0 \\
0 \\
0 \\
0 \\
0 \\
0 \\
0 \\
0 \\
0 \\
0 \\
0\end{array}$ & 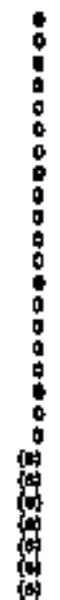 & 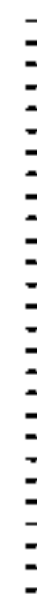 \\
\hline
\end{tabular}

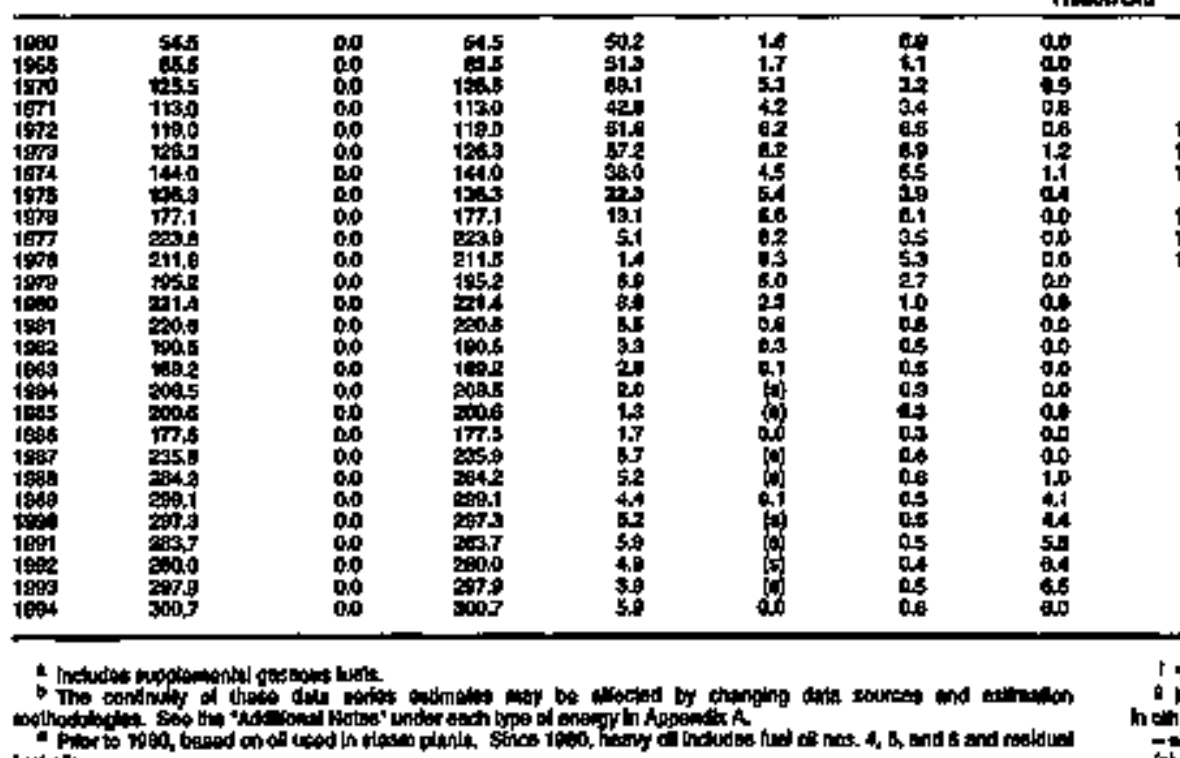

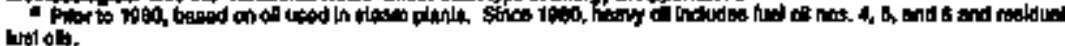

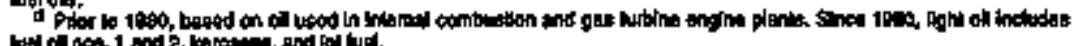

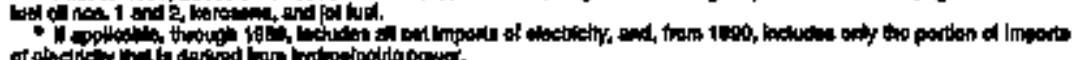

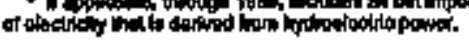


Tablo 161. Energy Consamption Estimates by Source, 1960, 1965, 1970-1984, Alsastesippd

\begin{tabular}{|c|c|c|c|c|c|c|c|c|c|c|c|c|c|c|c|c|c|c|c|}
\hline \multirow[b]{3}{*}{$Y \in+$} & \multirow[b]{2}{*}{ Cond } & \multirow[b]{2}{*}{ tets } & \multicolumn{11}{|c|}{ Putpon } & \multirow[b]{2}{*}{ 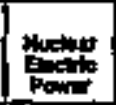 } & \multirow[b]{2}{*}{ 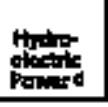 } & \multirow[b]{2}{*}{ Blotherine } & \multirow[b]{2}{*}{ | لب } & \multirow{3}{*}{ 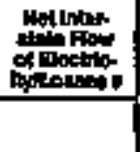 } & \multirow[b]{3}{*}{ 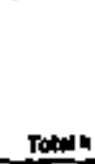 } \\
\hline & & & Aaphatinged & Antolsons & 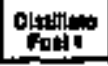 & Fen, & 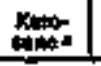 & LFa. & chast. & 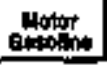 & Preationt & 告 & Tokley & & & & & & \\
\hline & Thoostion & crivion & \multicolumn{11}{|c|}{ 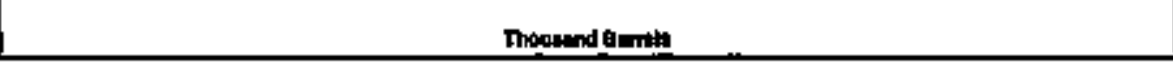 } & \multicolumn{4}{|c|}{ 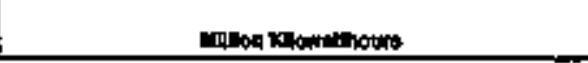 } & & \\
\hline 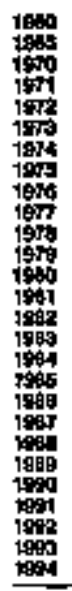 & 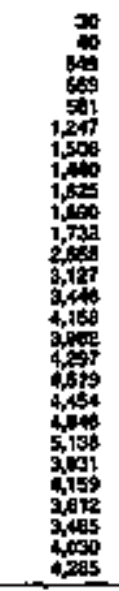 & 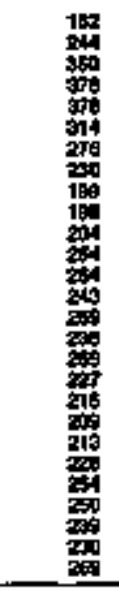 & 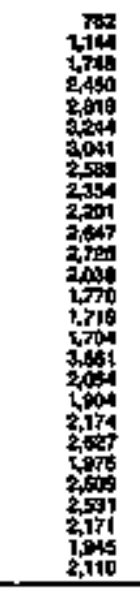 & 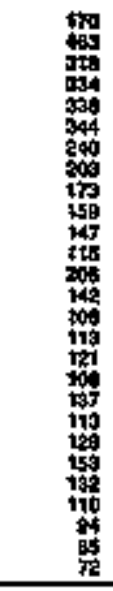 & 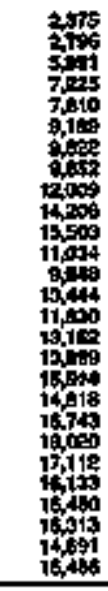 & 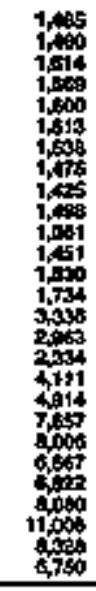 & 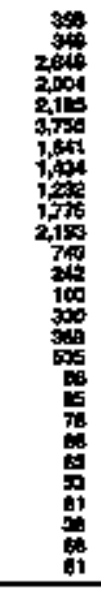 & 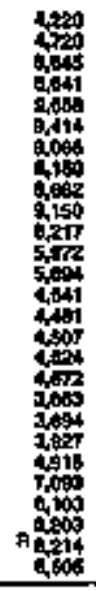 & 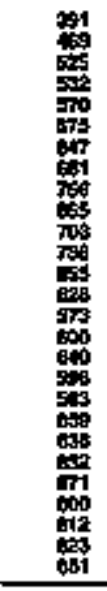 & 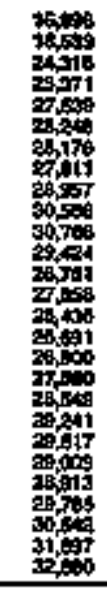 & 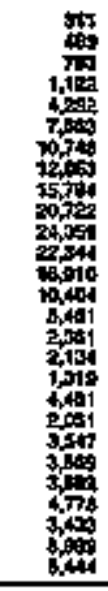 & 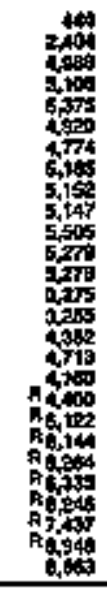 & 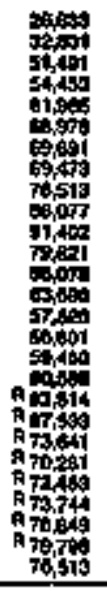 & 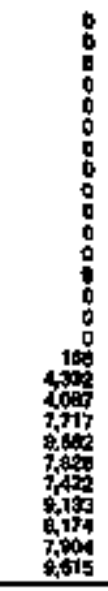 & 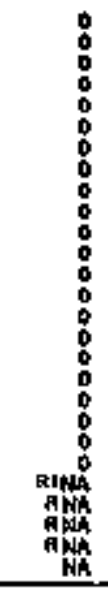 & 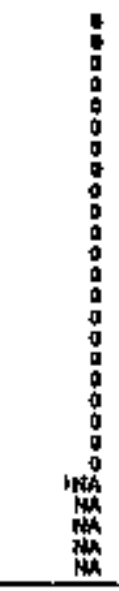 & $\begin{array}{l}0 \\
0 \\
0 \\
0 \\
0 \\
0 \\
0 \\
0 \\
0 \\
0 \\
0 \\
0 \\
0 \\
0 \\
0 \\
0 \\
0 \\
0 \\
0 \\
0 \\
0 \\
4 \\
4 \\
M \\
M \\
M\end{array}$ & 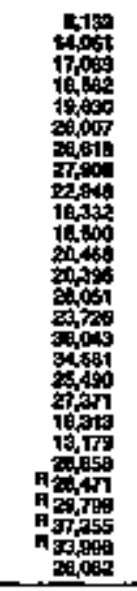 & $\begin{array}{l}= \\
= \\
= \\
= \\
5 \\
= \\
= \\
= \\
= \\
= \\
= \\
= \\
\vdots \\
=\end{array}$ \\
\hline \multicolumn{20}{|c|}{ Thallop an } \\
\hline 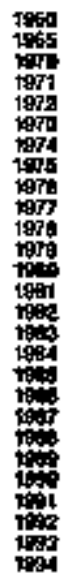 & 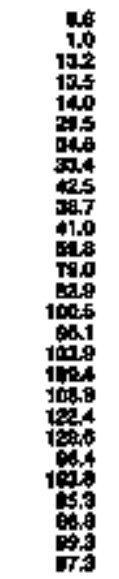 & 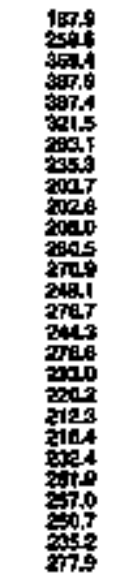 & 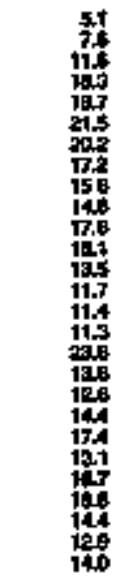 & 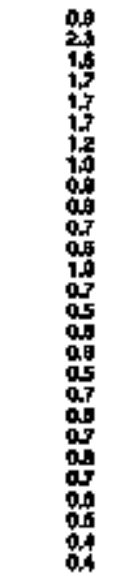 & 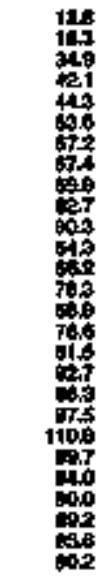 & 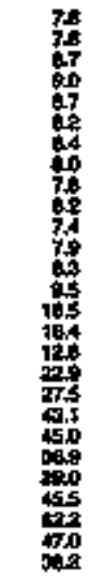 & 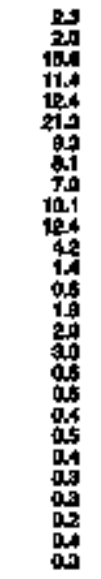 & 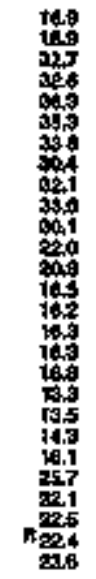 & 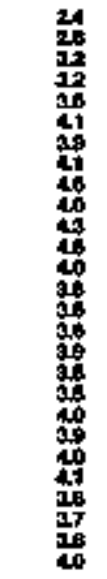 & 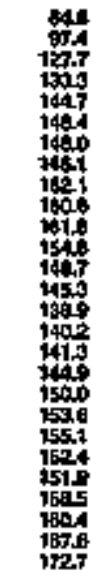 & 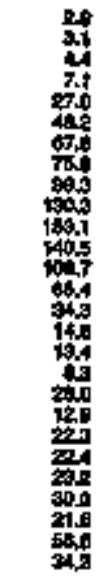 & 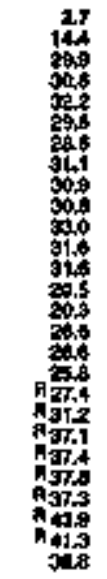 & 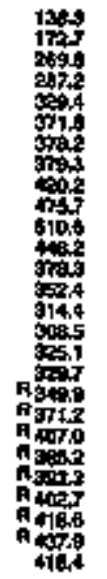 & 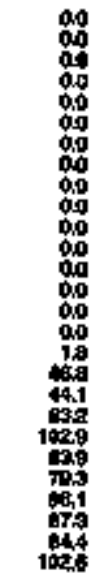 & 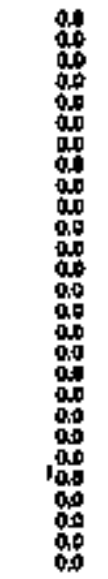 & 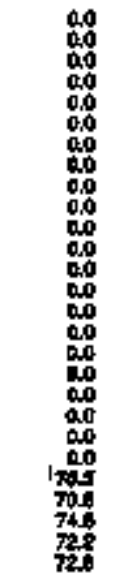 & 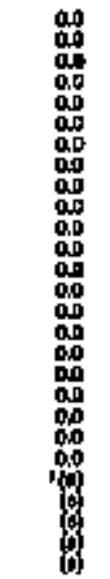 & 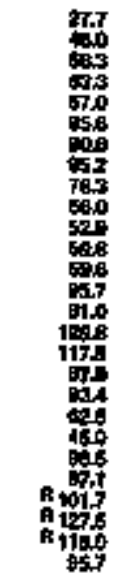 & 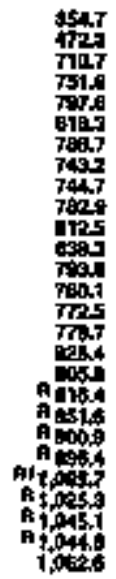 \\
\hline
\end{tabular}

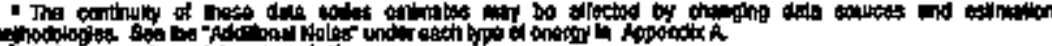

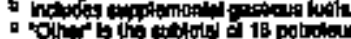

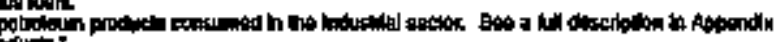

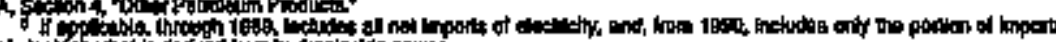

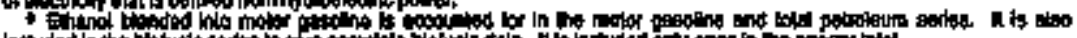

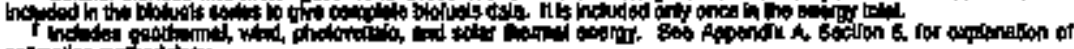

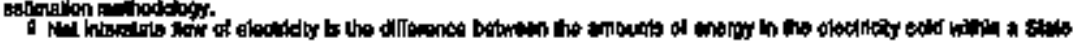

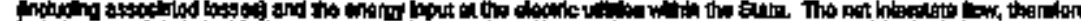

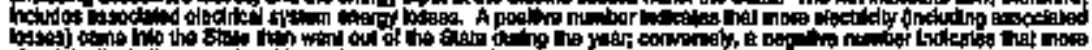

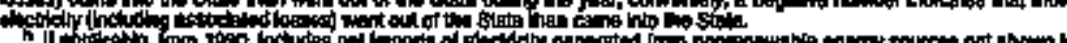

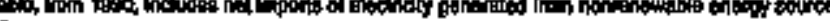

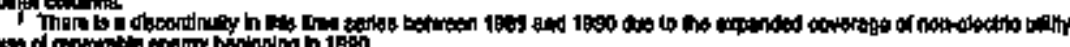

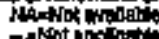

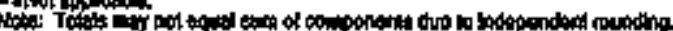

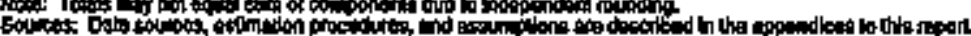




\begin{tabular}{|c|c|c|c|c|c|c|c|c|c|c|c|c|c|c|}
\hline \multirow[b]{3}{*}{$x=$} & \multicolumn{3}{|c|}{ Cod } & \multirow[b]{2}{*}{ 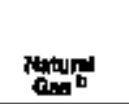 } & \multicolumn{4}{|c|}{ Petroleven } & \multirow{3}{*}{ 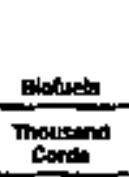 } & & \multirow[b]{2}{*}{ 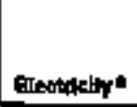 } & \multirow[b]{2}{*}{ thet } & \multirow{3}{*}{ 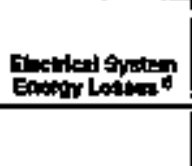 } & \multirow[b]{3}{*}{ Toto } \\
\hline & 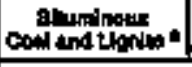 & Antinnotios & rowa & & Fitede & Xwrobent: & Len & Total & & satar: & & & & \\
\hline & \multicolumn{3}{|c|}{ 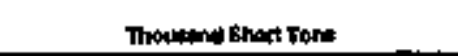 } & $\begin{array}{c}\text { Dision } \\
\text { curbefert }\end{array}$ & \multicolumn{4}{|c|}{ 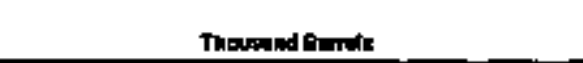 } & & \multicolumn{3}{|c|}{ 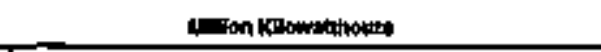 } & & \\
\hline 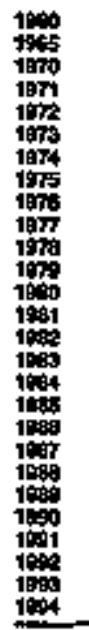 & $\begin{array}{c}0 \\
0 \\
0 \\
0 \\
0 \\
0 \\
0 \\
0 \\
0 \\
1 \\
1 \\
0 \\
0 \\
0 \\
1 \\
04 \\
1 \\
2 \\
5 \\
0 \\
0 \\
0 \\
0 \\
0 \\
\end{array}$ & $\begin{array}{l}0 \\
0 \\
0 \\
0 \\
0 \\
0 \\
0 \\
0 \\
0 \\
0 \\
0 \\
0 \\
0 \\
0 \\
0 \\
0 \\
0 \\
0 \\
0 \\
0 \\
0 \\
0 \\
0 \\
9 \\
0 \\
0 \\
0\end{array}$ & 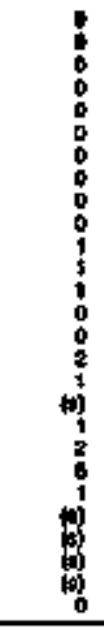 & 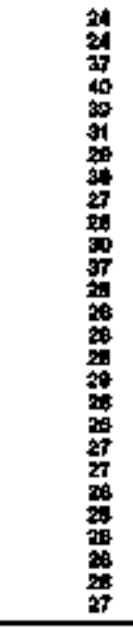 & 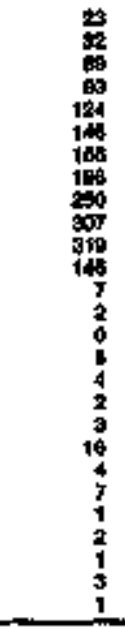 & 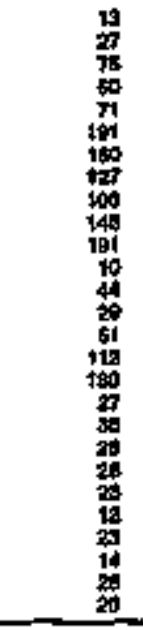 & 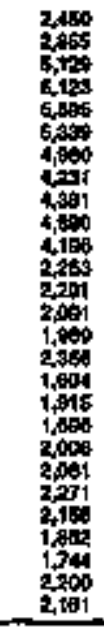 & 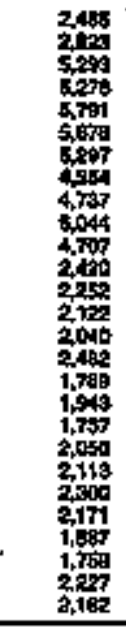 & 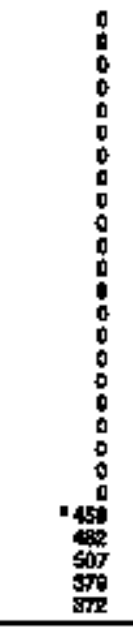 & $\begin{array}{l}0 \\
0 \\
0 \\
0 \\
0 \\
0 \\
0 \\
0 \\
0 \\
0 \\
0 \\
0 \\
0 \\
0 \\
0 \\
-1 \\
1 \\
1 \\
2 \\
\end{array}$ & 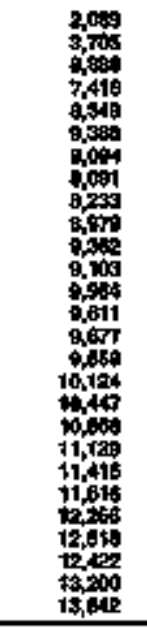 & $\begin{array}{l}\vdots \\
\vdots \\
\vdots \\
\vdots \\
\vdots \\
\vdots \\
\vdots \\
\vdots \\
\vdots \\
\vdots \\
\vdots \\
\vdots \\
\vdots\end{array}$ & 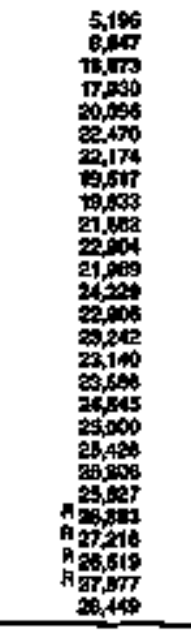 & $\begin{array}{l}\vdots \\
\vdots \\
\vdots \\
\vdots \\
\vdots \\
\vdots \\
\vdots \\
\vdots \\
\vdots \\
\vdots \\
\vdots \\
\vdots \\
\vdots\end{array}$ \\
\hline \multicolumn{15}{|c|}{ Titergan ala } \\
\hline 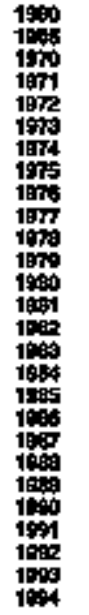 & 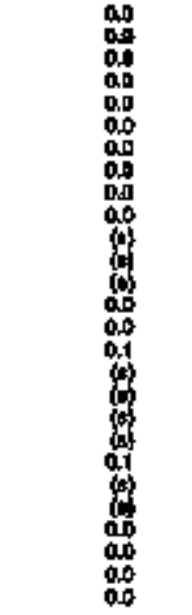 & 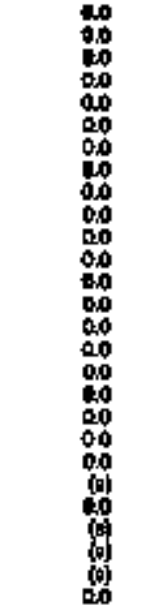 & 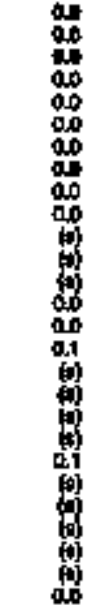 & 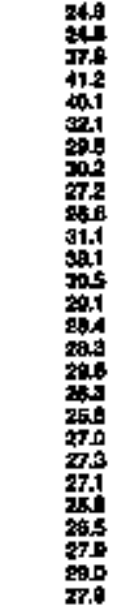 & 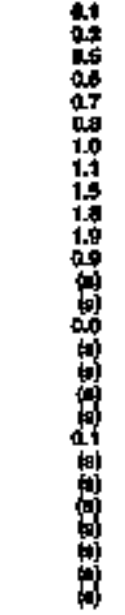 & 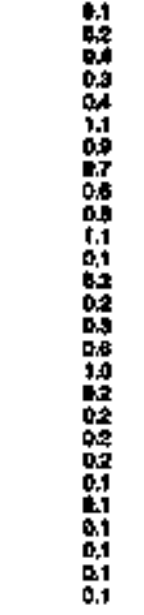 & 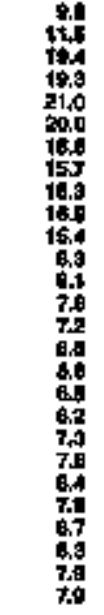 & 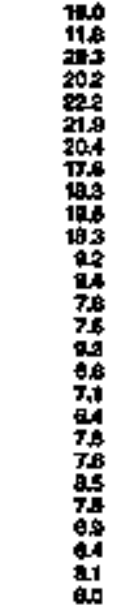 & 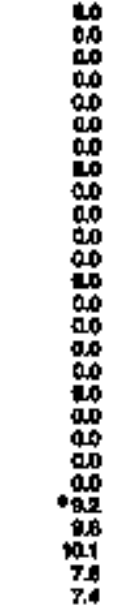 & 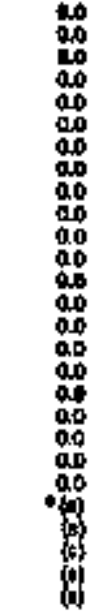 & 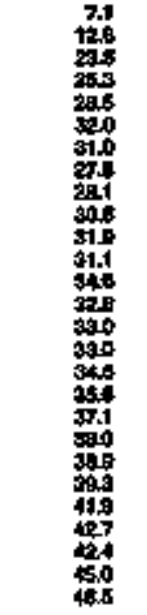 & 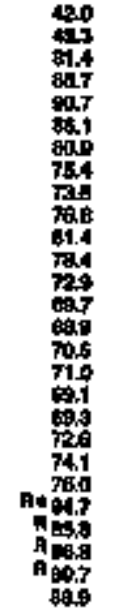 & 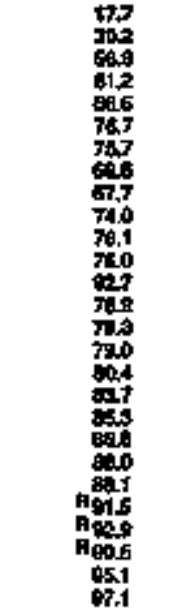 & 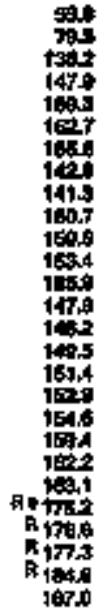 \\
\hline
\end{tabular}

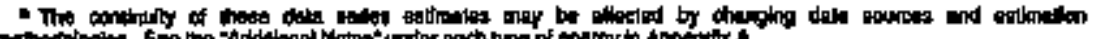

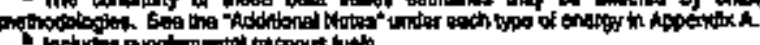

(a)

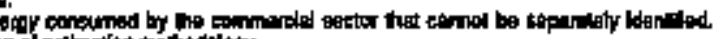

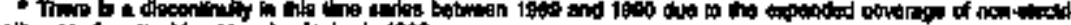

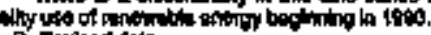

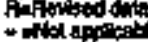

(t)

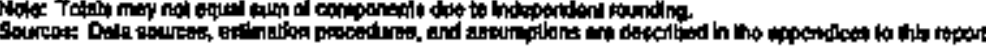


Table 163. Commerclal Energy Consumption Estimales, 1960, 1965, 1970-1994, Mississippi

\begin{tabular}{|c|c|c|c|c|c|c|c|c|c|c|c|c|c|c|}
\hline \multirow[b]{3}{*}{ Vin } & \multicolumn{3}{|c|}{ con } & \multirow{3}{*}{ 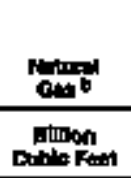 } & \multicolumn{6}{|c|}{ Futroledrd } & \multirow[b]{2}{*}{ 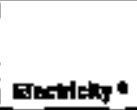 } & \multirow[b]{2}{*}{ Enetry } & \multirow[b]{2}{*}{ 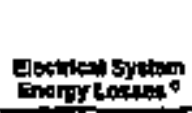 } & \multirow[b]{3}{*}{ 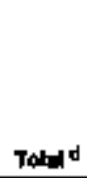 } \\
\hline & 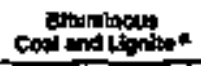 & Antracto: & tot & & 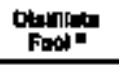 & Kerosuane & 100" & atsoline & 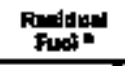 & Tout & & & & \\
\hline & \multicolumn{3}{|c|}{ 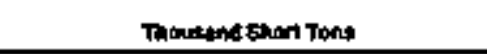 } & & \multicolumn{6}{|c|}{ Thoorand Eared: } & \multicolumn{3}{|c|}{ 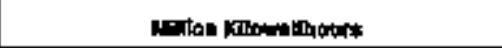 } & \\
\hline 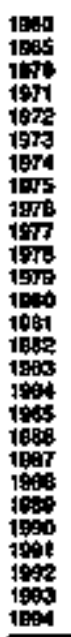 & $\begin{array}{l}0 \\
0 \\
0 \\
0 \\
0 \\
0 \\
0 \\
0 \\
0 \\
3 \\
2 \\
0 \\
0 \\
5 \\
1 \\
1 \\
3 \\
0 \\
0 \\
0 \\
0 \\
0 \\
0\end{array}$ & $\begin{array}{l}a \\
0 \\
0 \\
0 \\
0 \\
0 \\
0 \\
0 \\
0 \\
0 \\
0 \\
0 \\
0 \\
0 \\
0 \\
0 \\
0 \\
0 \\
0 \\
0 \\
0 \\
0 \\
0 \\
0 \\
0\end{array}$ & $\begin{array}{l}0 \\
0 \\
0 \\
0 \\
0 \\
0 \\
0 \\
0 \\
0 \\
3 \\
2 \\
1 \\
0 \\
5 \\
1 \\
\vdots \\
3 \\
0 \\
1 \\
0 \\
0 \\
0 \\
0 \\
0\end{array}$ & 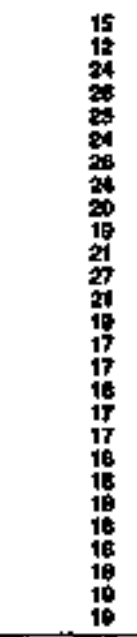 & 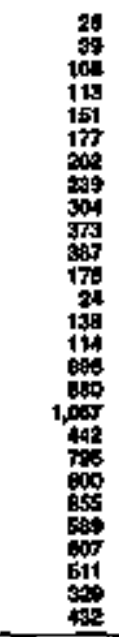 & 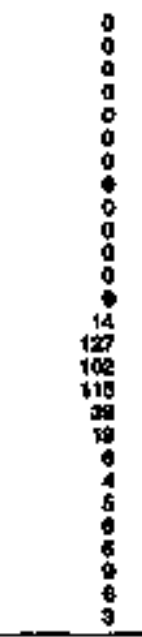 & 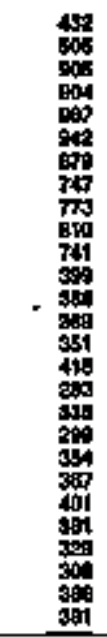 & 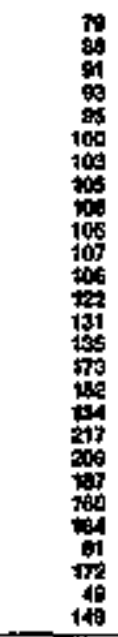 & 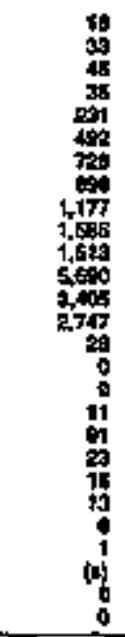 & 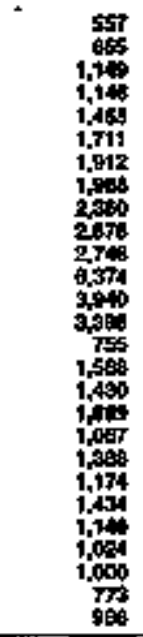 & 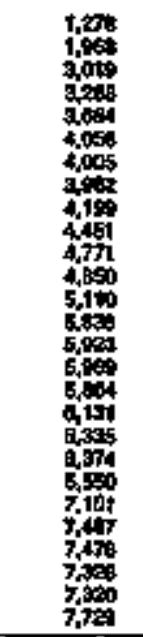 & $\begin{array}{l}= \\
= \\
= \\
= \\
= \\
= \\
= \\
= \\
= \\
= \\
= \\
= \\
=\end{array}$ & 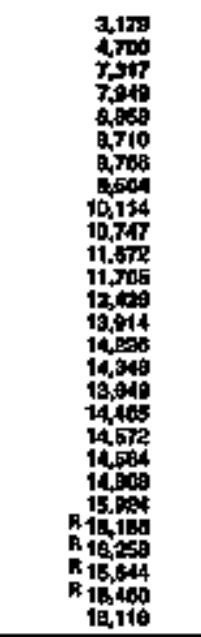 & $\begin{array}{l}z \\
z \\
z \\
z \\
z \\
z \\
z \\
z \\
z \\
z \\
z \\
z \\
z \\
z \\
z\end{array}$ \\
\hline \multicolumn{15}{|c|}{ 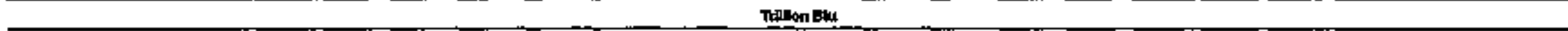 } \\
\hline 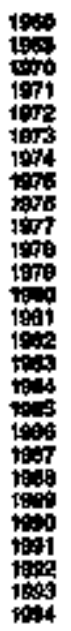 & 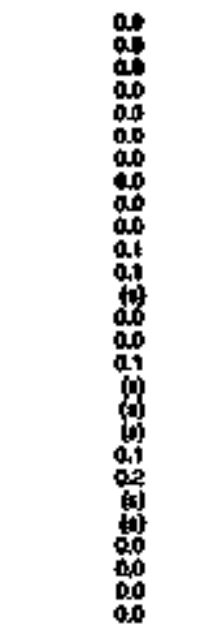 & $\begin{array}{l}0 \\
01 \\
00 \\
00 \\
00 \\
00 \\
00 \\
00 \\
00 \\
00 \\
000 \\
00 \\
00 \\
00 \\
00 \\
00 \\
00 \\
00 \\
00 \\
00 \\
00 \\
00 \\
00 \\
00\end{array}$ & 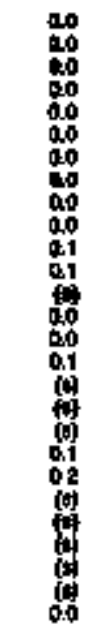 & 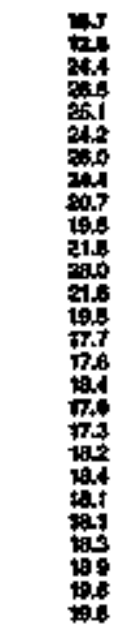 & 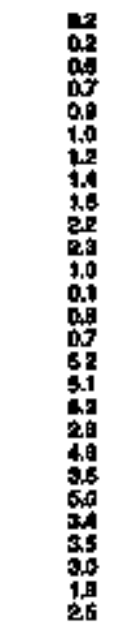 & 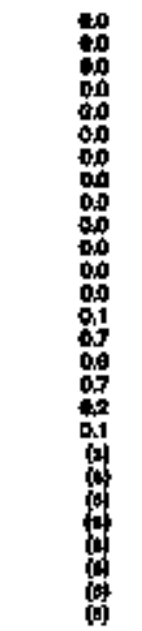 & 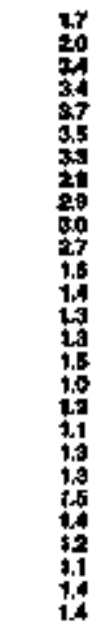 & 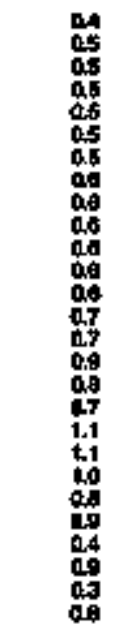 & 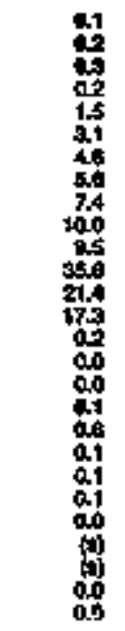 & 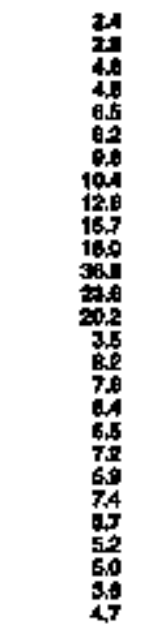 & 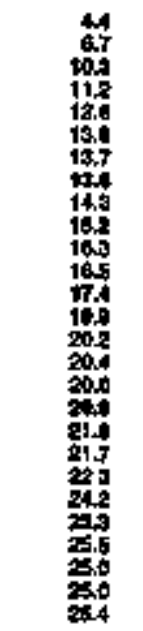 & 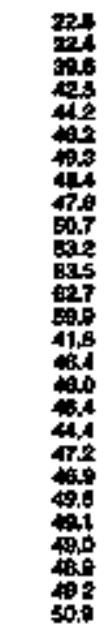 & 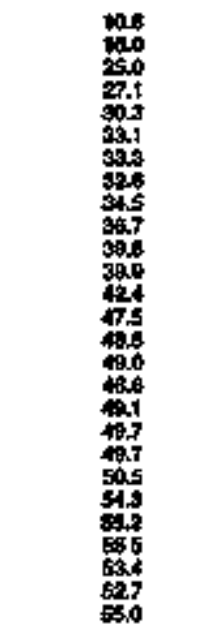 & 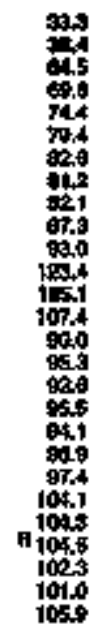 \\
\hline
\end{tabular}




\begin{tabular}{|c|c|c|c|c|c|c|c|c|c|c|c|c|c|c|c|c|c|c|}
\hline \multirow[b]{3}{*}{$\mathrm{rem}$} & \multirow[b]{2}{*}{ con } & \multirow[b]{2}{*}{ Motart } & \multicolumn{9}{|c|}{ Potorolum } & \multirow[b]{2}{*}{ 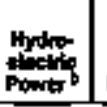 } & \multirow[b]{2}{*}{ 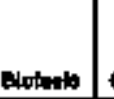 } & \multirow[b]{2}{*}{ Other 20} & \multirow[b]{2}{*}{ 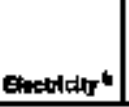 } & \multirow[b]{2}{*}{ Exergy } & \multirow{2}{*}{ 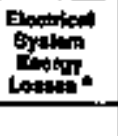 } & \multirow[b]{3}{*}{ Todin } \\
\hline & & & 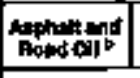 & OAsalmo & Kenomenol of & Lpes & 至 & motos & 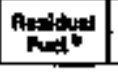 & 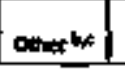 & 政 & & & & & & & \\
\hline & Thoon & 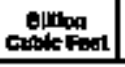 & \multicolumn{9}{|c|}{ 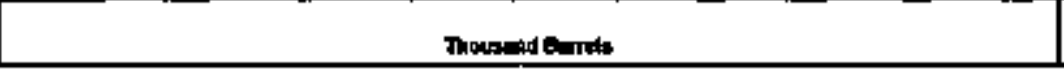 } & \multicolumn{6}{|c|}{ Hoben KTompenthoure } & \\
\hline 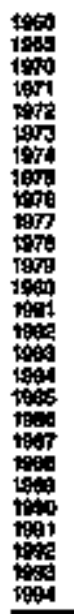 & 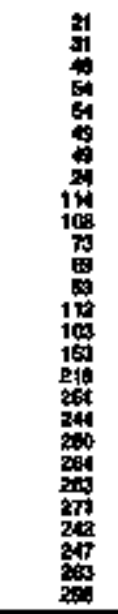 & 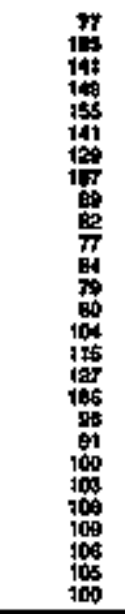 & 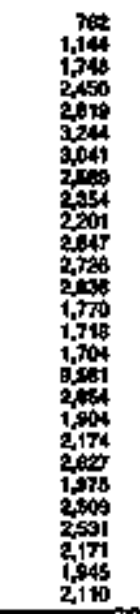 & 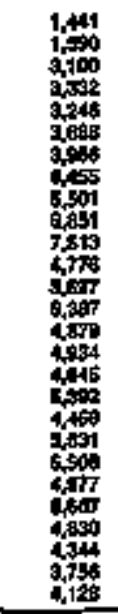 & 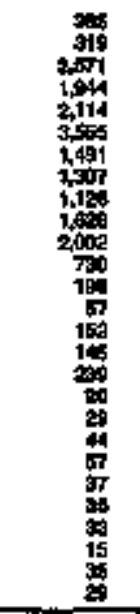 & 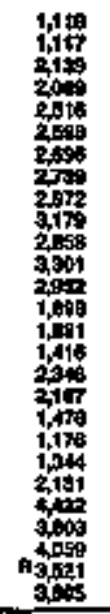 & 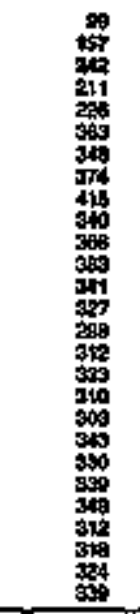 & 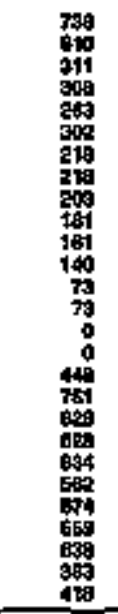 & 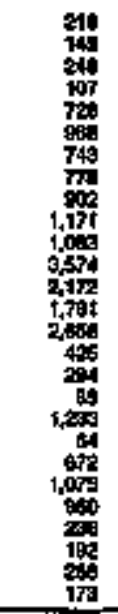 & 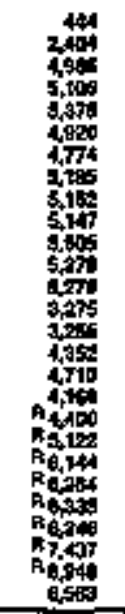 & 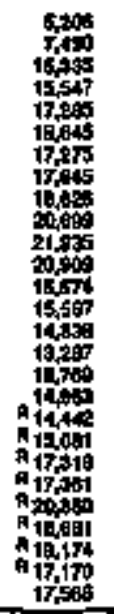 & 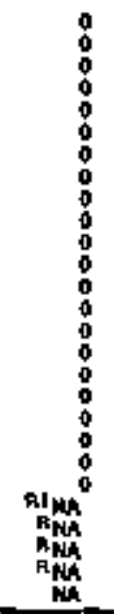 & 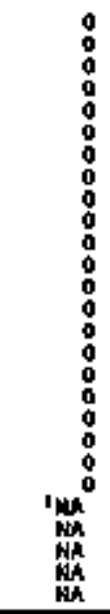 & 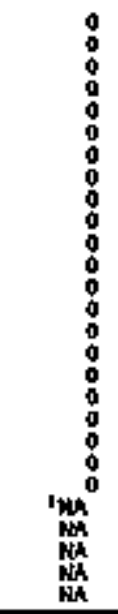 & 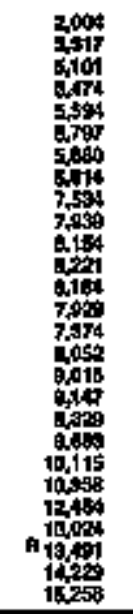 & $\begin{array}{l}z \\
\vdots \\
\vdots \\
\vdots \\
\vdots \\
\vdots \\
\vdots \\
\vdots\end{array}$ & 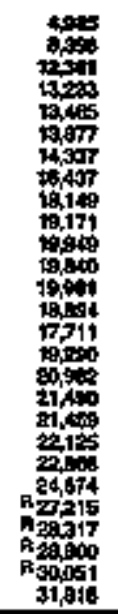 & $=$ \\
\hline \multicolumn{19}{|c|}{ mintan mate } \\
\hline 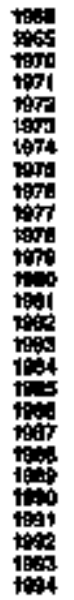 & 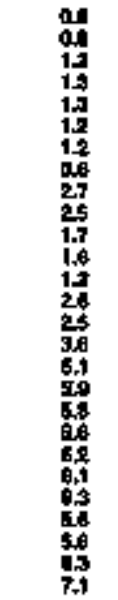 & 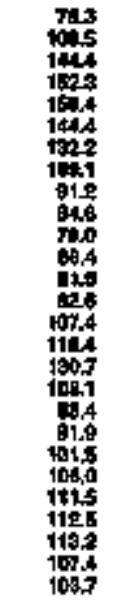 & 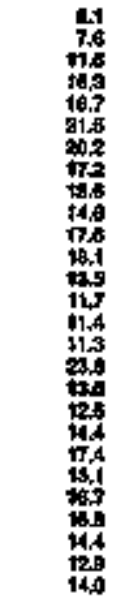 & 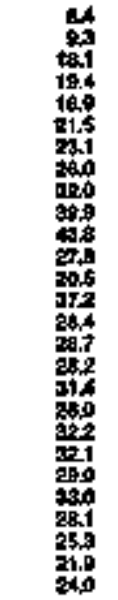 & 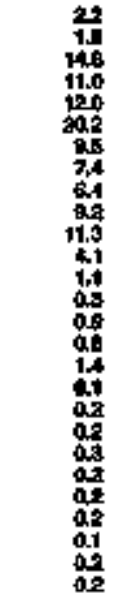 & 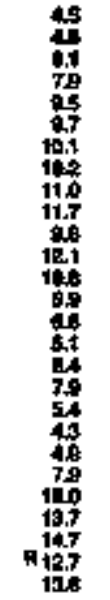 & 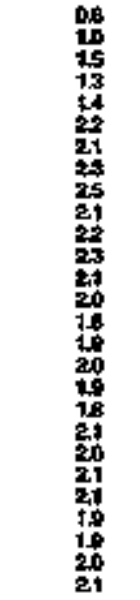 & 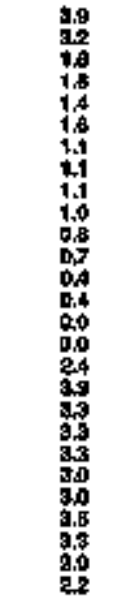 & 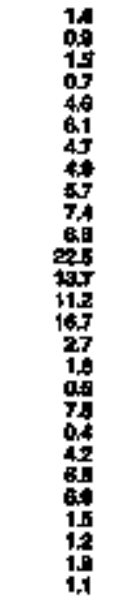 & 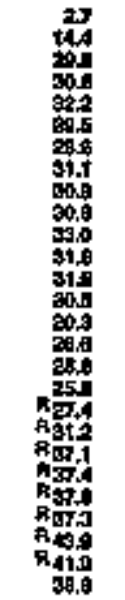 & 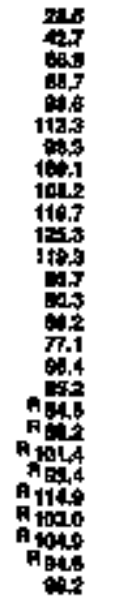 & 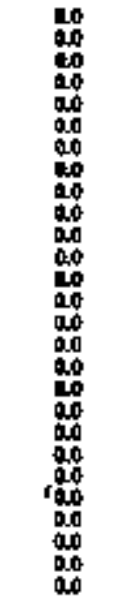 & 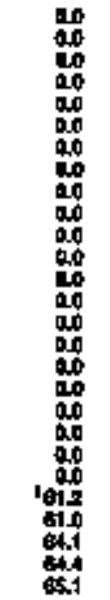 & 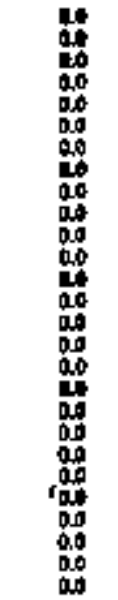 & 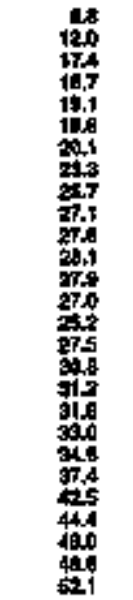 & 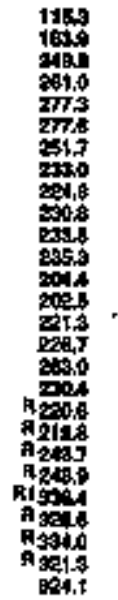 & 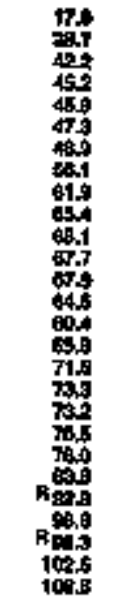 & 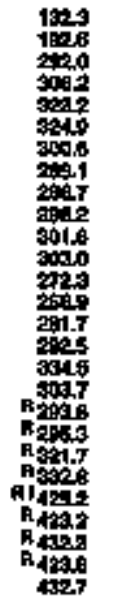 \\
\hline
\end{tabular}


Tabte 165. Transportation Energy Consumption Esthatos, 1950, 1965, 1970-1994, Mlississippi

\begin{tabular}{|c|c|c|c|c|c|c|c|c|c|c|c|c|c|c|c|}
\hline \multirow[b]{3}{*}{ rem } & \multirow[b]{2}{*}{$\cos *$} & \multirow[b]{2}{*}{ 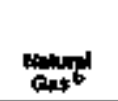 } & \multicolumn{8}{|c|}{ 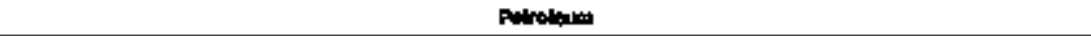 } & \multirow{3}{*}{ mates } & & \multirow[b]{2}{*}{ tions } & \multirow[b]{2}{*}{ 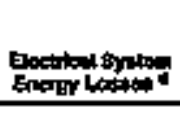 } & \multirow[b]{3}{*}{ Totor } \\
\hline & & & 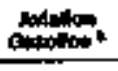 & 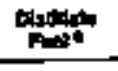 & 急. & Lrax & 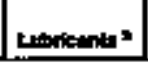 & 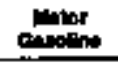 & 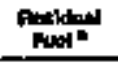 & This & & 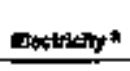 & & & \\
\hline & thent Tow & otilon & \multicolumn{8}{|c|}{ 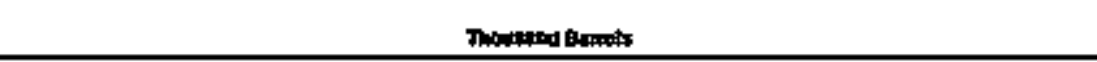 } & & \multicolumn{3}{|c|}{ 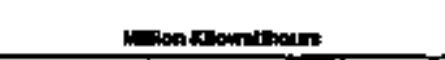 } & \\
\hline 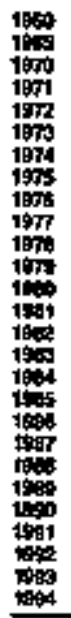 & 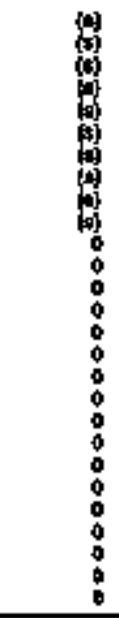 & 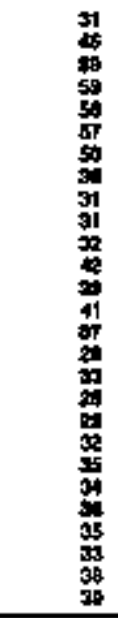 & 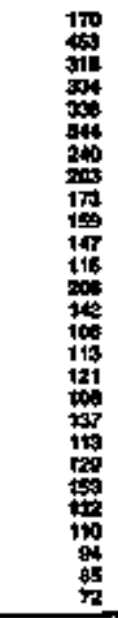 & 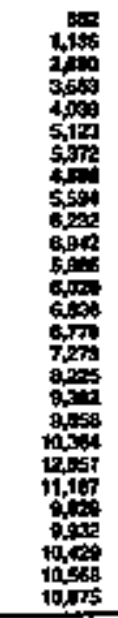 & 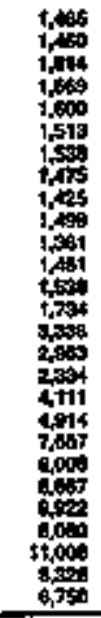 & 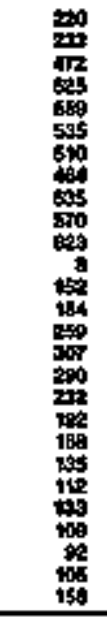 & 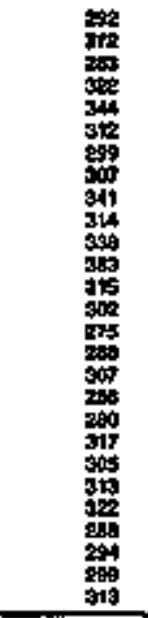 & 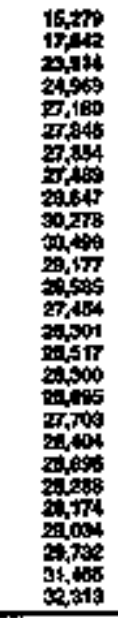 & 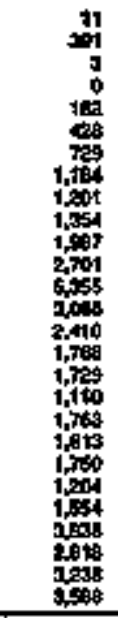 & 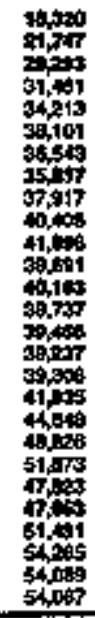 & 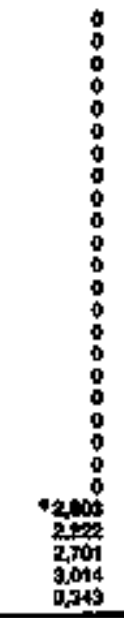 & $\begin{array}{l}0 \\
0 \\
0 \\
0 \\
0 \\
0 \\
0 \\
0 \\
0 \\
0 \\
0 \\
0 \\
0 \\
0 \\
0 \\
0 \\
0 \\
0 \\
0 \\
0 \\
0 \\
0 \\
0 \\
0 \\
0 \\
0 \\
0\end{array}$ & 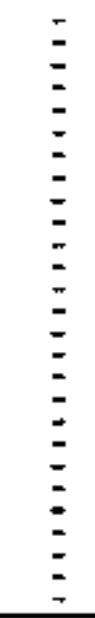 & $\begin{array}{l}0 \\
0 \\
0 \\
0 \\
0 \\
0 \\
0 \\
0 \\
0 \\
0 \\
0 \\
0 \\
0 \\
0 \\
0 \\
0 \\
0 \\
0 \\
0 \\
0 \\
0 \\
0 \\
0 \\
0 \\
0\end{array}$ & $\begin{array}{l}= \\
= \\
z \\
z \\
z \\
z \\
z \\
z \\
z \\
z \\
z \\
z \\
z \\
z \\
z\end{array}$ \\
\hline \multicolumn{16}{|c|}{ thera nlu } \\
\hline 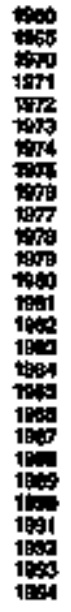 & 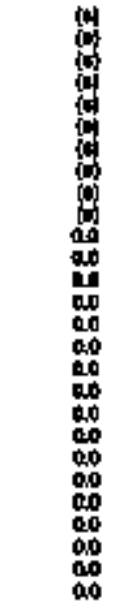 & 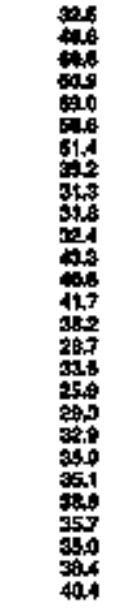 & 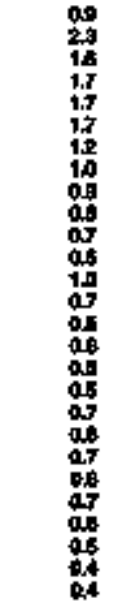 & 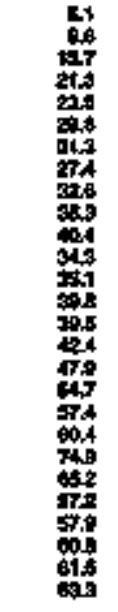 & 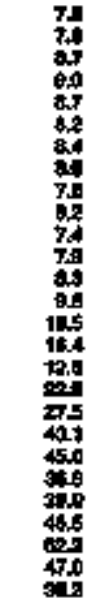 & 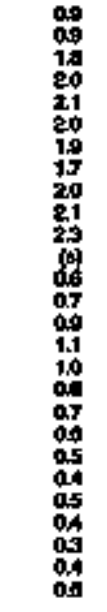 & 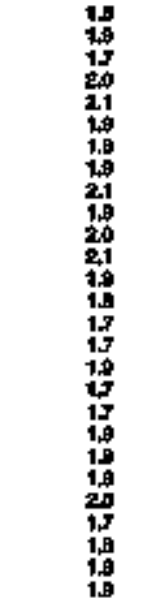 & 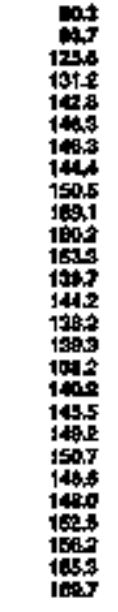 & 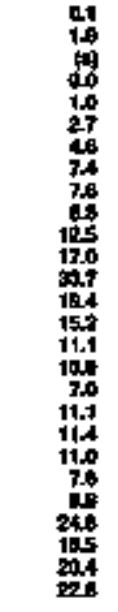 & 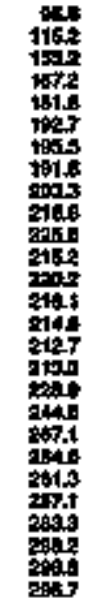 & 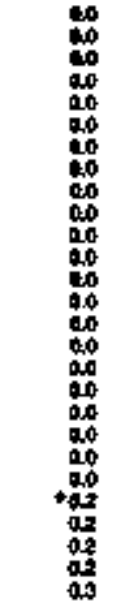 & 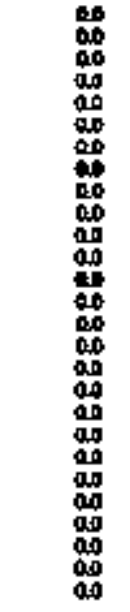 & 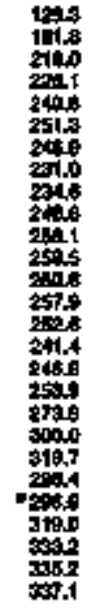 & 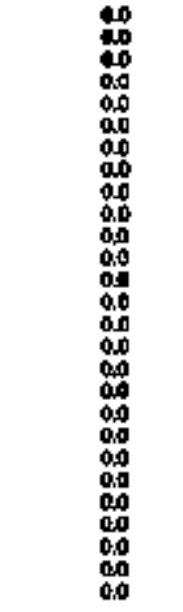 & 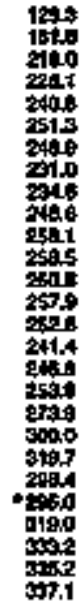 \\
\hline
\end{tabular}

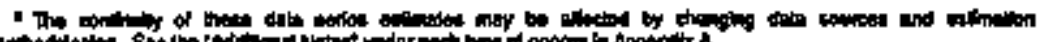

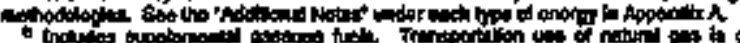

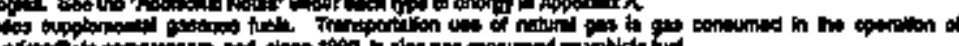

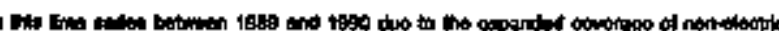

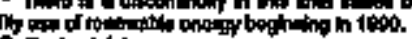

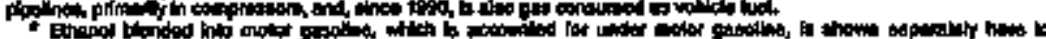

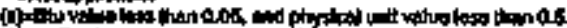

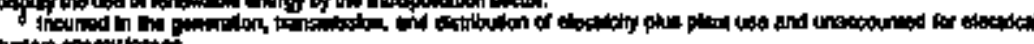




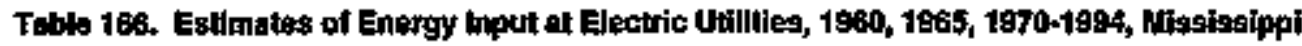

\begin{tabular}{|c|c|c|c|c|c|c|c|c|c|c|c|c|c|c|}
\hline & \multicolumn{3}{|c|}{$\cos$} & \multirow[b]{2}{*}{ 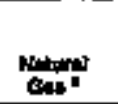 } & \multicolumn{4}{|c|}{ Porrokitan } & \multirow[b]{2}{*}{ 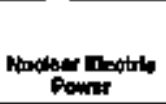 } & \multirow[b]{2}{*}{ 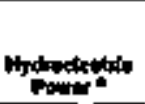 } & \multirow[b]{2}{*}{ Eyotimeitip } & \multirow[b]{2}{*}{ Anothanis } & \multirow[b]{2}{*}{ olber 4} & \multirow[b]{3}{*}{$T a t=9$} \\
\hline & A & Antriedse & Toted & & tonty & 敬幽 & 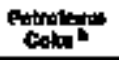 & Tolnt & & & & & & \\
\hline Yex| & \multicolumn{3}{|c|}{ 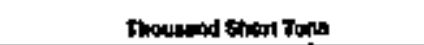 } & oidien & \multicolumn{4}{|c|}{ 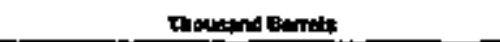 } & \multicolumn{5}{|c|}{ 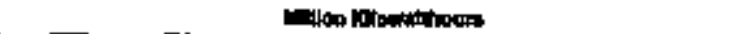 } & \\
\hline
\end{tabular}

\begin{tabular}{|c|c|c|c|c|c|c|c|c|c|c|c|c|c|c|}
\hline 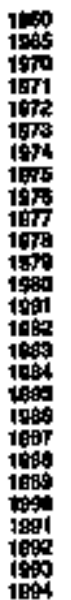 & 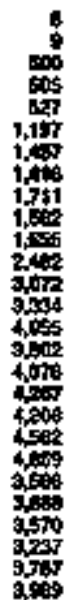 & $\begin{array}{l}0 \\
0 \\
0 \\
0 \\
0 \\
0 \\
0 \\
0 \\
0 \\
0 \\
0 \\
0 \\
0 \\
0 \\
0 \\
0 \\
0 \\
0 \\
0 \\
0 \\
0 \\
0\end{array}$ & 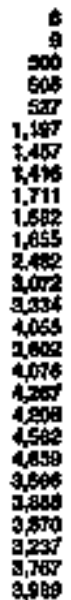 & 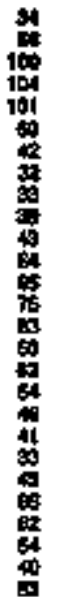 & 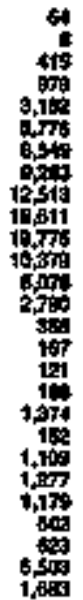 & 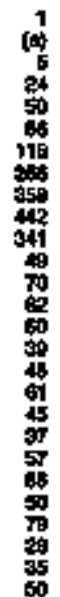 & $\begin{array}{l}0 \\
0 \\
0 \\
0 \\
0 \\
0 \\
0 \\
0 \\
0 \\
0 \\
0 \\
0 \\
0 \\
0 \\
0 \\
0 \\
0 \\
0 \\
0 \\
0 \\
0 \\
0 \\
0 \\
0\end{array}$ & 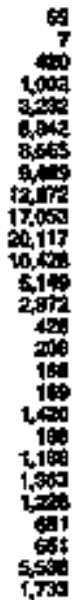 & 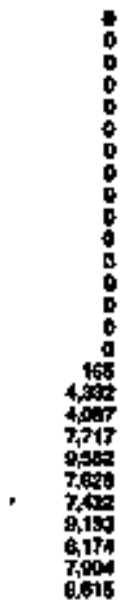 & $\begin{array}{l}0 \\
0 \\
0 \\
0 \\
0 \\
0 \\
0 \\
0 \\
0 \\
0 \\
0 \\
0 \\
0 \\
0 \\
0 \\
0 \\
0 \\
0 \\
0\end{array}$ & $\begin{array}{l}0 \\
0 \\
0 \\
0 \\
0 \\
0 \\
0 \\
0 \\
0 \\
0 \\
0 \\
0 \\
0 \\
0 \\
0 \\
0 \\
0 \\
0 \\
0 \\
0 \\
0 \\
0 \\
0 \\
0 \\
0 \\
0 \\
0 \\
0\end{array}$ & $\begin{array}{l}0 \\
0 \\
0 \\
0 \\
0 \\
0 \\
0 \\
0 \\
0 \\
0 \\
0 \\
0 \\
0 \\
0 \\
0 \\
0 \\
0 \\
0 \\
0 \\
0 \\
0 \\
0 \\
0 \\
0\end{array}$ & $\begin{array}{l}0 \\
0 \\
0 \\
0 \\
0 \\
0 \\
0 \\
0 \\
0 \\
0 \\
0 \\
0 \\
0 \\
0 \\
0 \\
0 \\
0 \\
0 \\
0 \\
0 \\
0 \\
0 \\
0 \\
0 \\
0 \\
0 \\
0\end{array}$ & 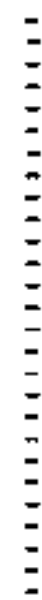 \\
\hline
\end{tabular}

\begin{tabular}{|c|c|}
\hline 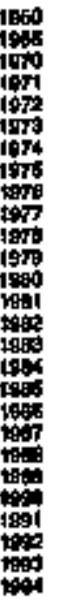 & 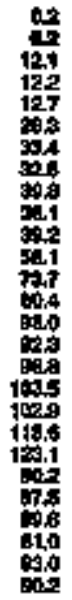 \\
\hline
\end{tabular}

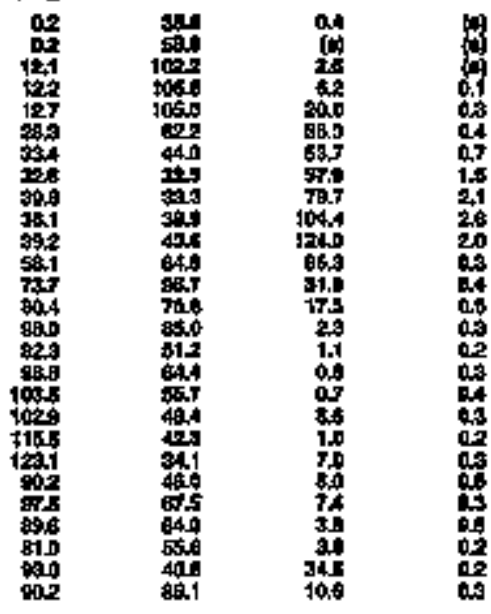

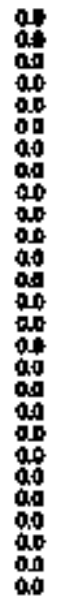

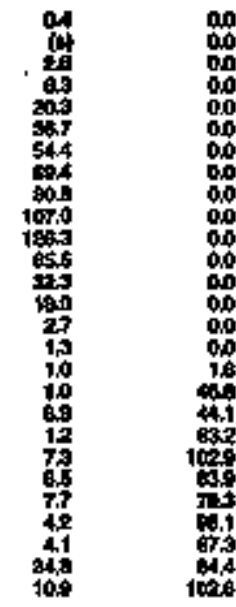

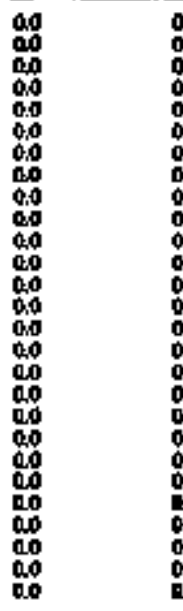

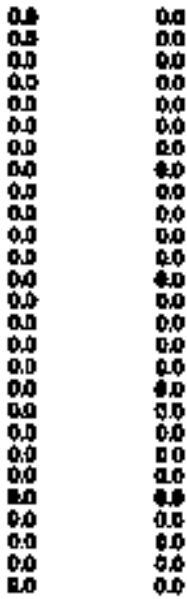

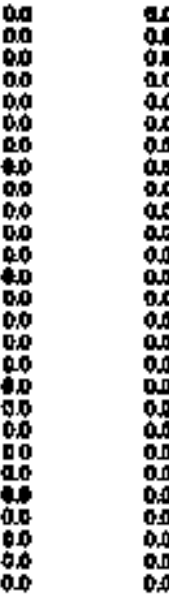

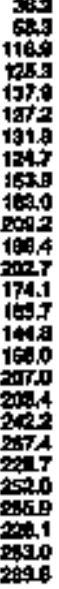

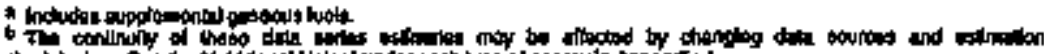

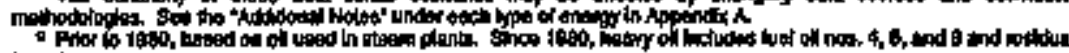

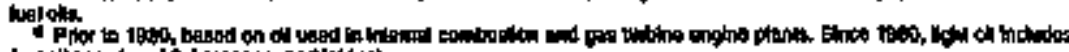

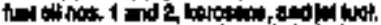

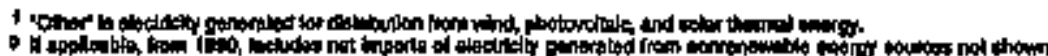

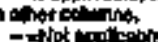

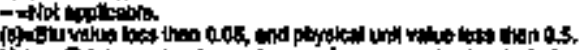

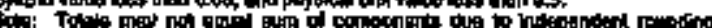

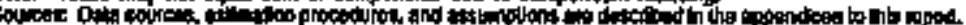


Table 167. Energy Consumption Eslmates by Source, 1960, 1965, 1970-1984, Mlssouri

\begin{tabular}{|c|c|c|c|c|c|c|c|c|c|c|c|c|c|c|c|c|c|c|c|}
\hline \multirow[b]{3}{*}{ Yent } & \multirow[b]{2}{*}{ Cow. } & \multirow[b]{2}{*}{ 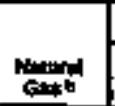 } & \multicolumn{11}{|c|}{ Pandorm } & \multirow{2}{*}{ 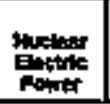 } & \multirow{2}{*}{ 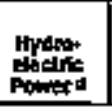 } & \multirow[b]{2}{*}{ Otohnt:4 } & \multirow[b]{2}{*}{ 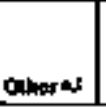 } & \multirow{2}{*}{ 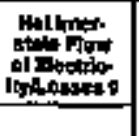 } & \multirow[b]{3}{*}{ Toten A } \\
\hline & & & Aspand and & Antions & Patel & Folit. & Kans. & LPD: & twatis. & motom & 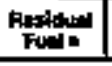 & Odner ax & Total & & & & & & \\
\hline & $\begin{array}{l}\text { Thou: nd } \\
\text { sherifond }\end{array}$ & 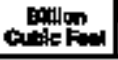 & \multicolumn{11}{|c|}{ 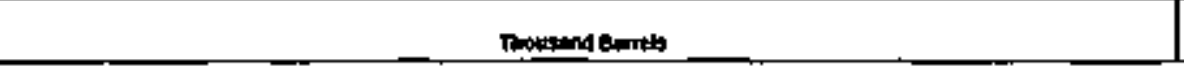 } & \multicolumn{5}{|c|}{ 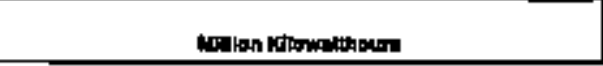 } & \\
\hline 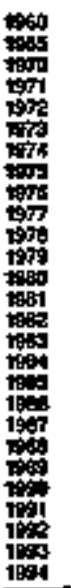 & 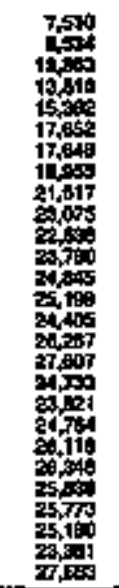 & 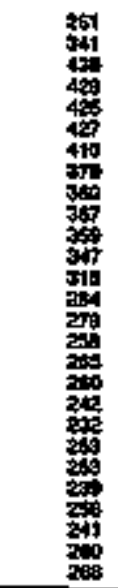 & 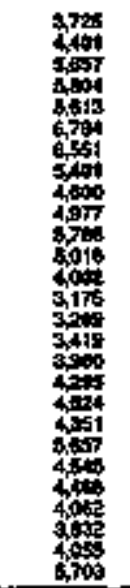 & 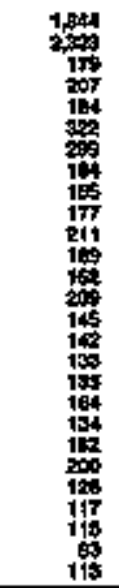 & 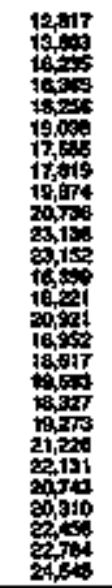 & 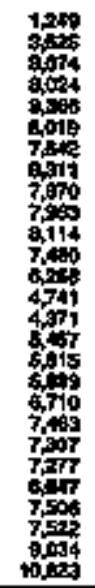 & 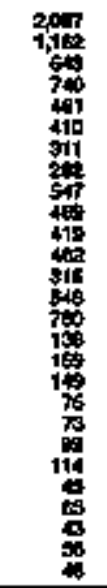 & 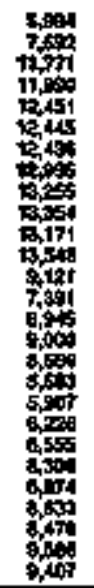 & 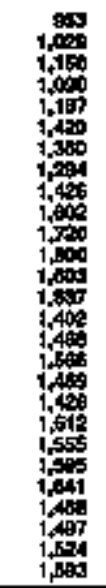 & 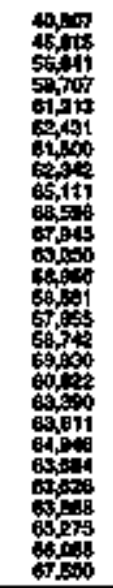 & 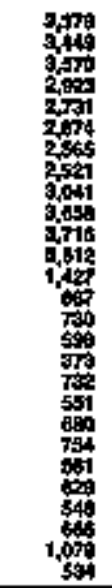 & 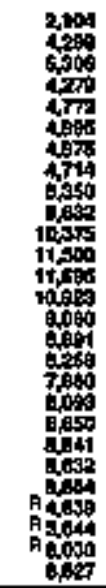 & 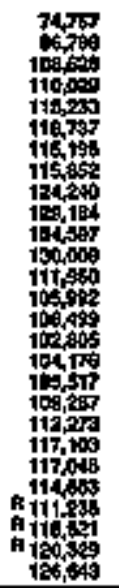 & 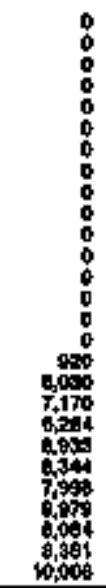 & 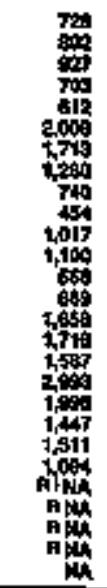 & 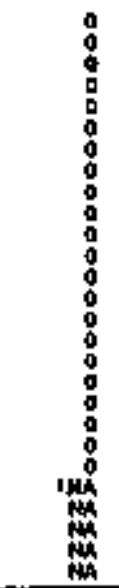 & $\begin{array}{l}0 \\
\vdots \\
0 \\
0 \\
0 \\
\vdots \\
0 \\
0 \\
\vdots \\
\vdots \\
\vdots \\
\vdots \\
0 \\
0 \\
0\end{array}$ & 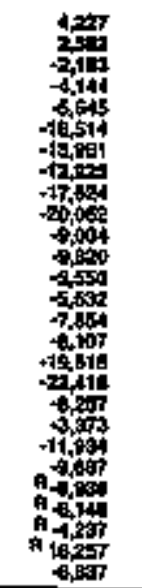 & $\begin{array}{l}\bar{z} \\
\bar{z} \\
\bar{z} \\
\bar{z} \\
\bar{z} \\
\bar{z} \\
\bar{z} \\
\bar{z} \\
z \\
z \\
z \\
z\end{array}$ \\
\hline & & & & & & & & & & Thlltan In & & & & & & & & & \\
\hline
\end{tabular}

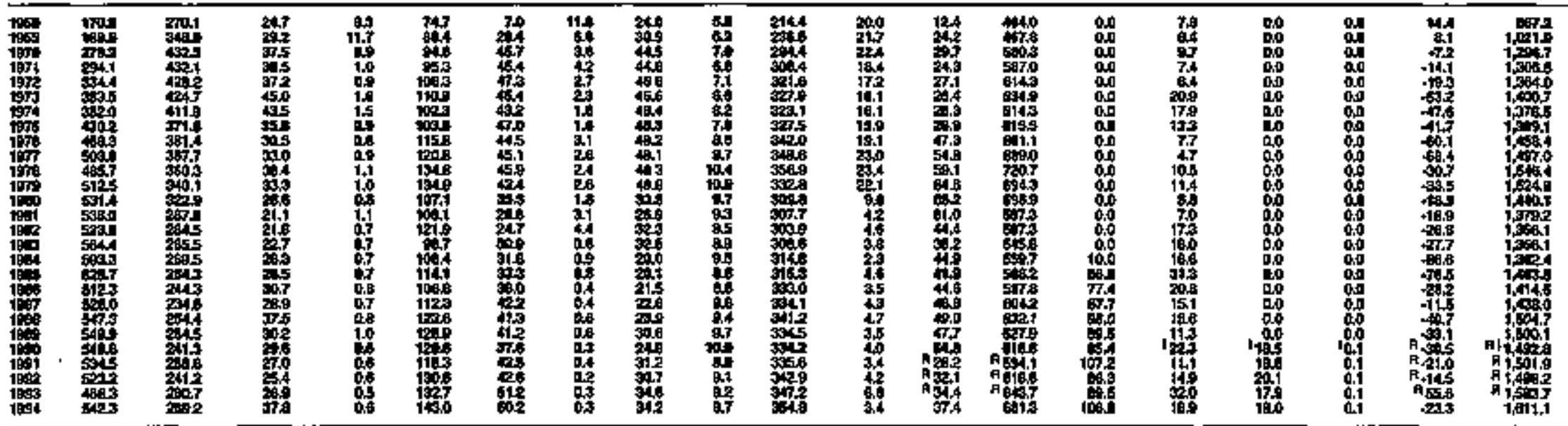

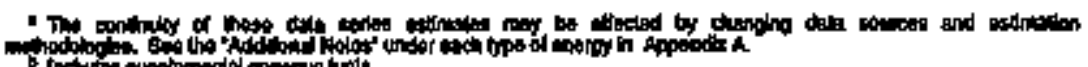

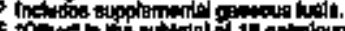

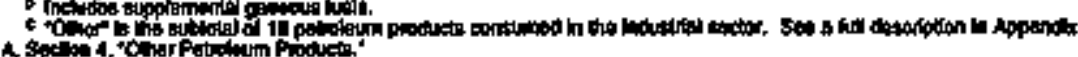

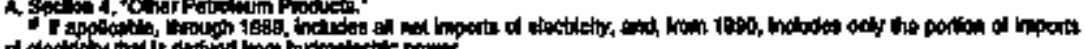

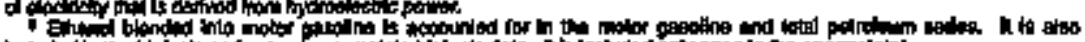

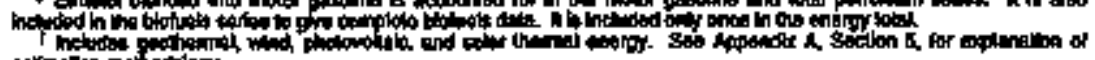

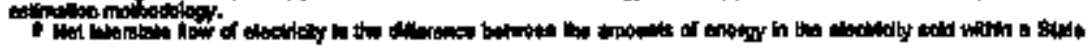

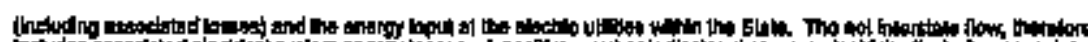

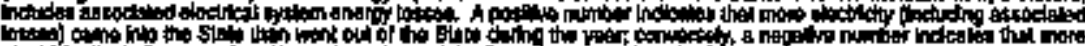

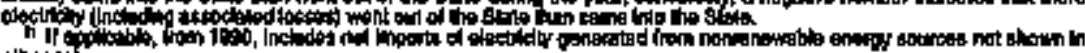

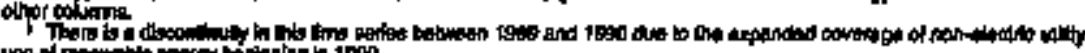

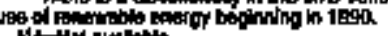

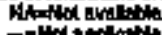

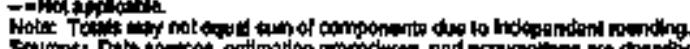

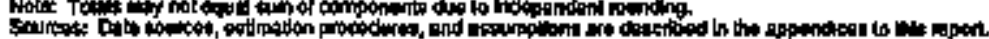


Table 169. ResIdential Energy Consumption Estimates, 1960, 1985, 1970-1994, Khssouri

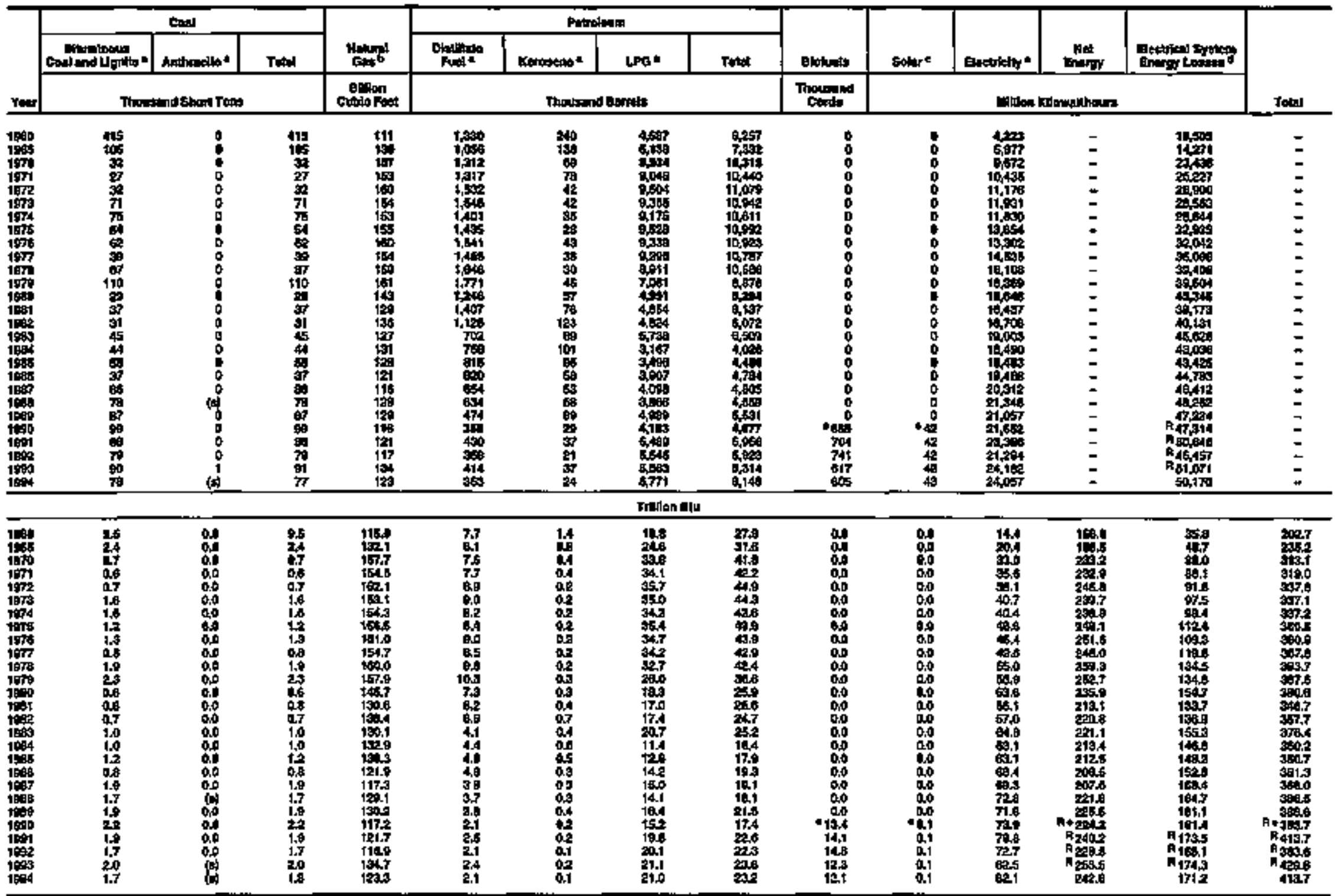

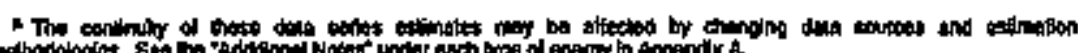

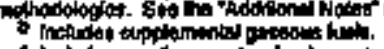

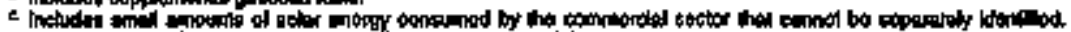

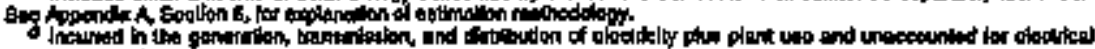

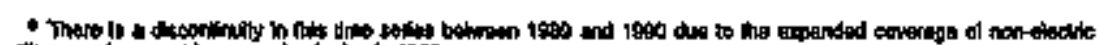

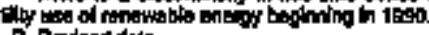


Table 169. Comunerciel Energy Consumption Estimates, 1960, 1985, 1970-1994, Mssouri

\begin{tabular}{|c|c|c|c|c|c|c|c|c|c|c|c|c|c|c|}
\hline \multirow[b]{3}{*}{ Yas l } & \multicolumn{3}{|c|}{ 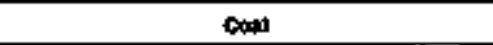 } & \multirow{3}{*}{ 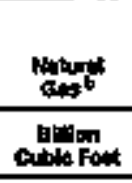 } & \multicolumn{6}{|c|}{ Postrobum } & \multirow[b]{2}{*}{ Boctikly : } & \multirow[b]{2}{*}{ Stot } & \multirow[b]{2}{*}{ 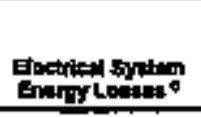 } & \multirow[b]{3}{*}{ Tots d } \\
\hline & coluntionsu & Antirestolo: & Total & & 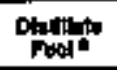 & Koropenas. & LPOE & Matoriture & Powdyed & Todel & & & & \\
\hline & \multicolumn{3}{|c|}{ Thousund Shert tant } & & \multicolumn{6}{|c|}{ Mhounod Bmete } & \multicolumn{3}{|c|}{ Whiton Kromemethour: } & \\
\hline 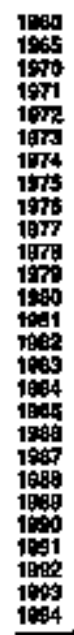 & 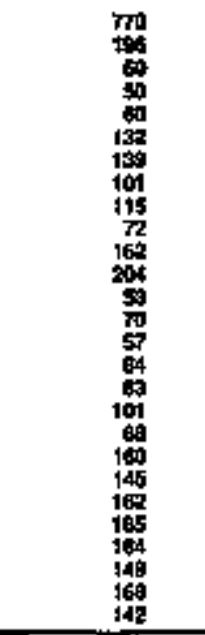 & 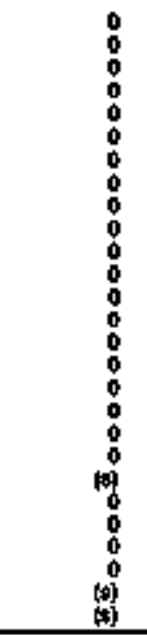 & 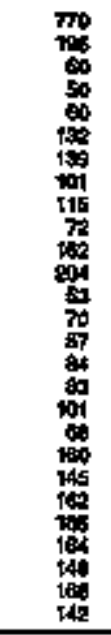 & 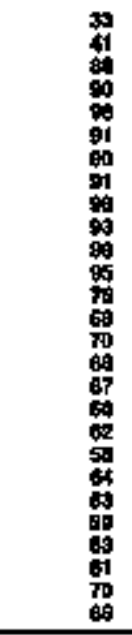 & 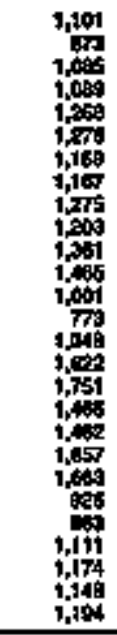 & 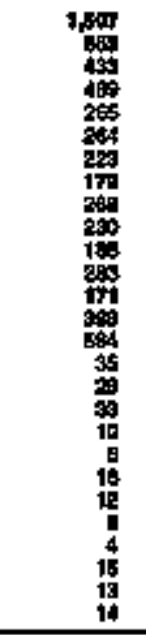 & 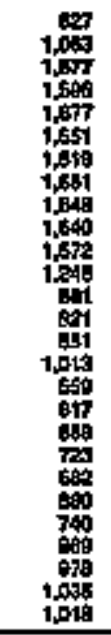 & 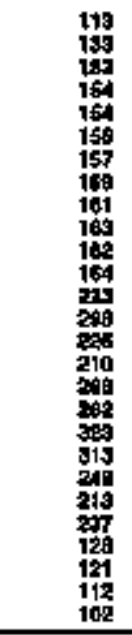 & 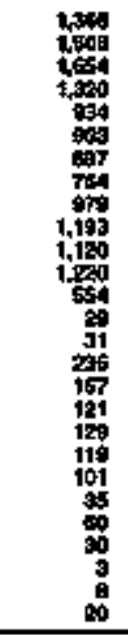 & 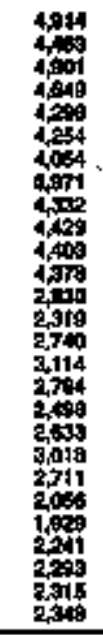 & 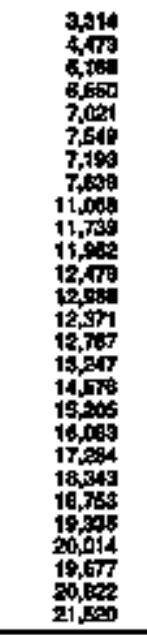 & $\begin{array}{l}\vdots \\
\vdots \\
\vdots \\
\vdots \\
\vdots \\
\vdots \\
\vdots \\
\vdots \\
\vdots \\
\vdots \\
\vdots \\
\vdots \\
\vdots \\
\end{array}$ & 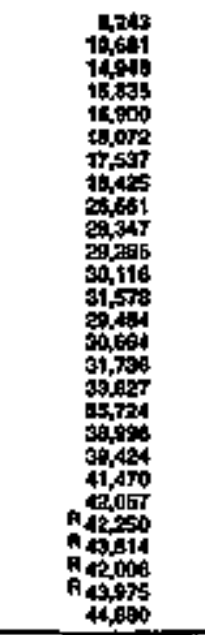 & $\begin{array}{l}\vdots \\
\vdots \\
\vdots \\
\vdots \\
\vdots \\
\vdots \\
\vdots \\
\vdots \\
\vdots \\
\vdots \\
\vdots \\
\vdots \\
\vdots \\
\vdots\end{array}$ \\
\hline \multicolumn{15}{|c|}{ Ninom an } \\
\hline 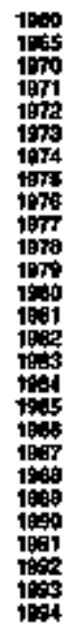 & 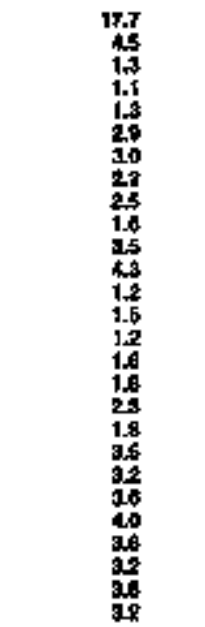 & 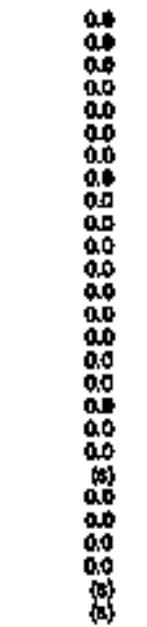 & 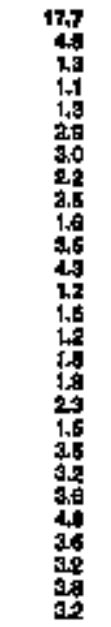 & 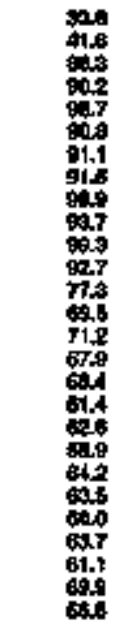 & 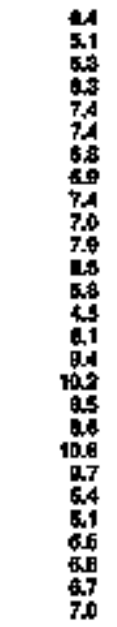 & 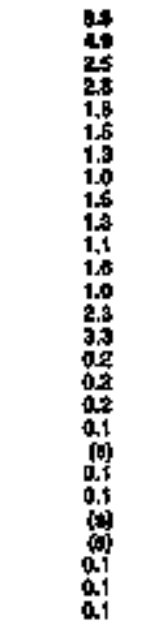 & 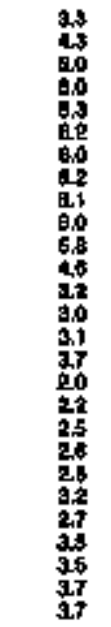 & 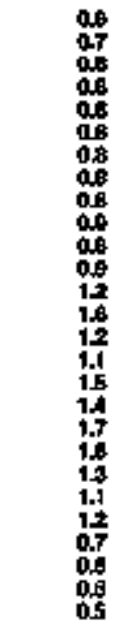 & 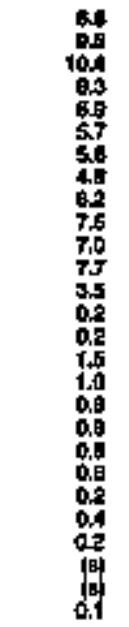 & 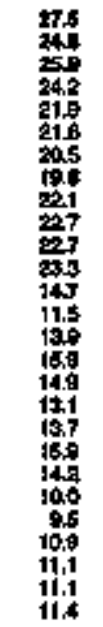 & 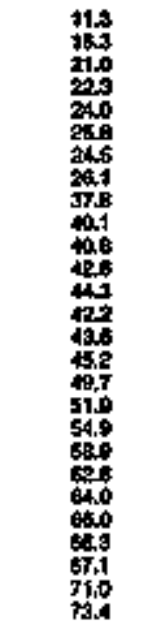 & 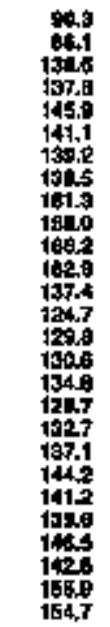 & 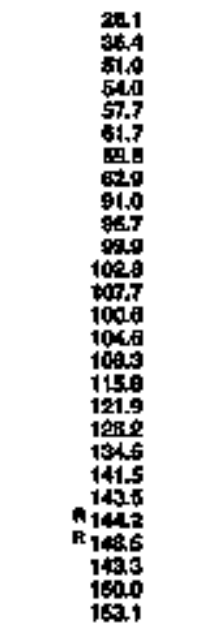 & 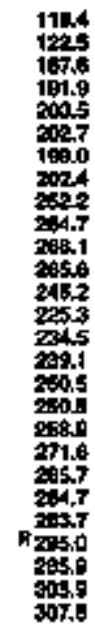 \\
\hline
\end{tabular}


Tablo 170. Industral Energy Coneumption Eallimates, 1960, 1965, 1970-1994, Mistauri

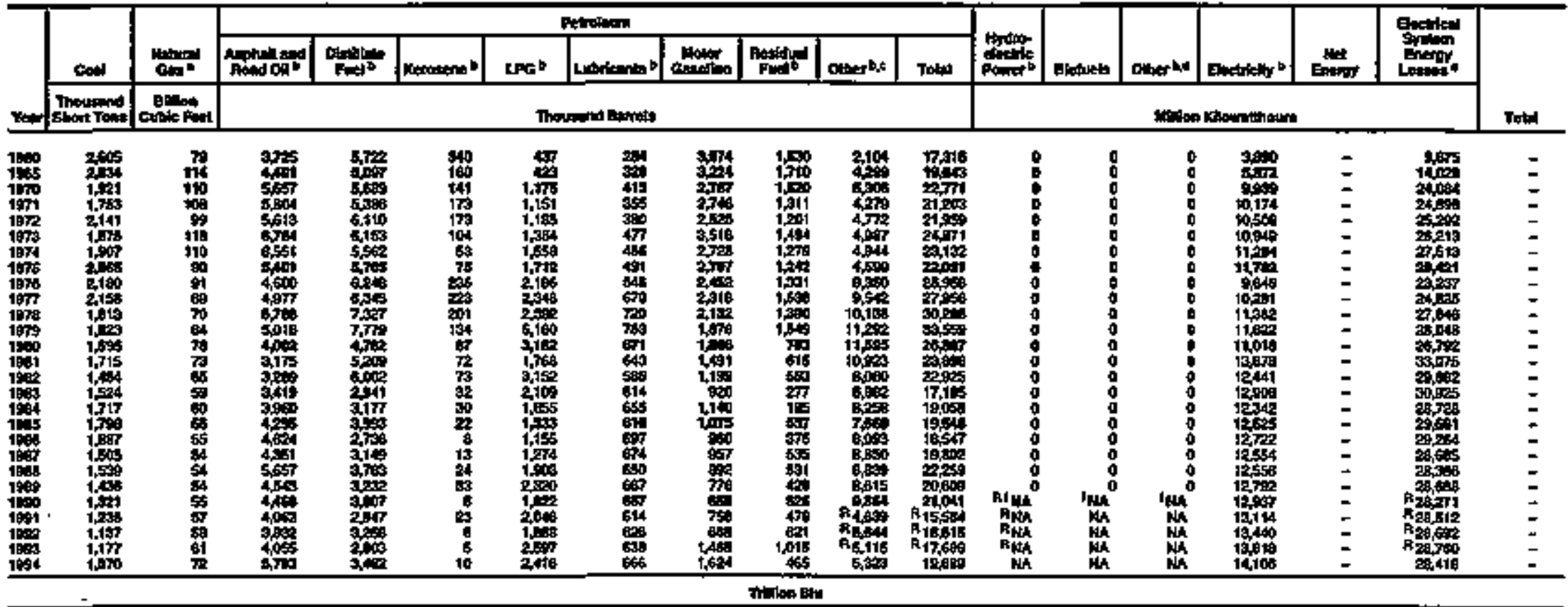

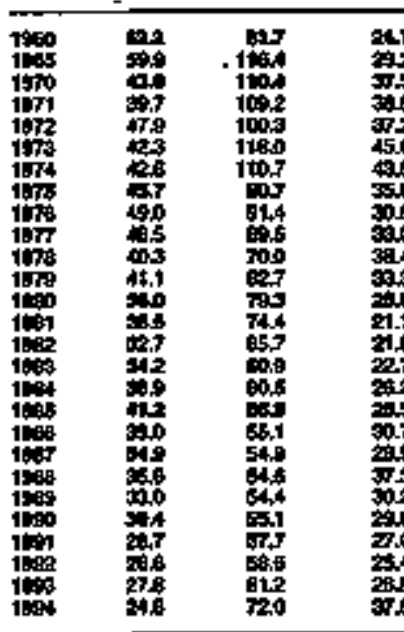

\begin{tabular}{|c|c|}
\hline & 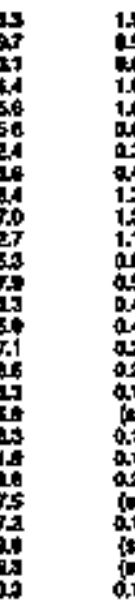 \\
\hline
\end{tabular}

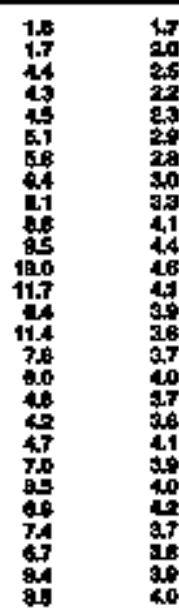

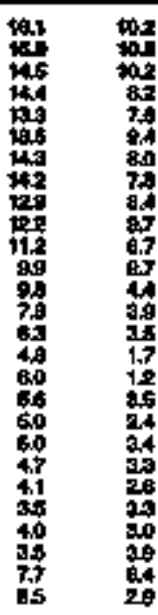

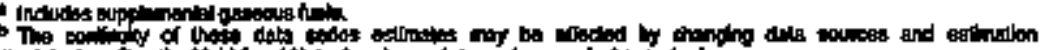

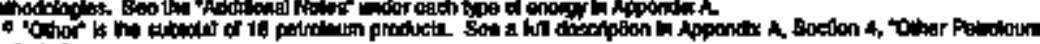

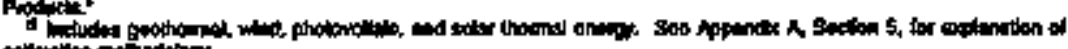

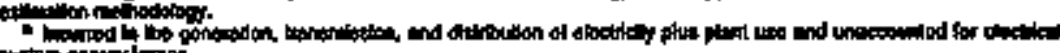

\begin{tabular}{|c|c|}
\hline 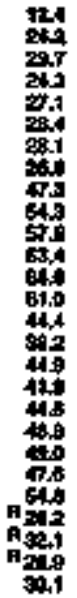 & 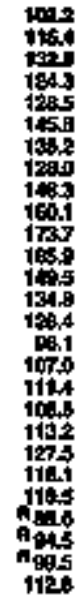 \\
\hline
\end{tabular}

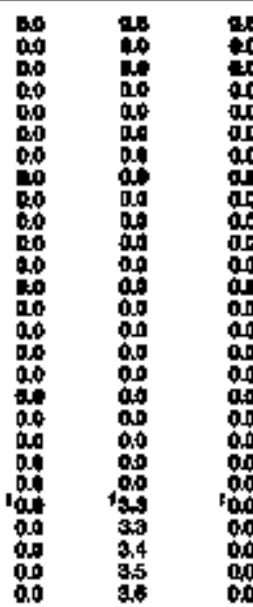

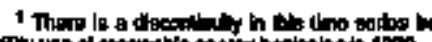

\begin{tabular}{|c|c|c|c|}
\hline 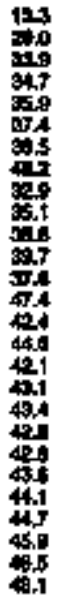 & 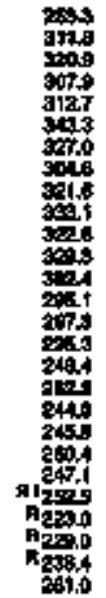 & 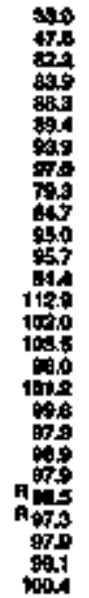 & 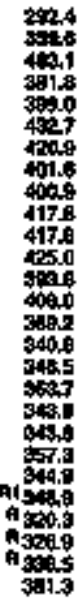 \\
\hline
\end{tabular}

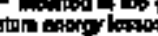


Tabte 17t. Transportation Enesoy Consumpltor Estintatos, t960, 1965, 1970-1994, MIssourt

\begin{tabular}{|c|c|c|c|c|c|c|c|c|c|c|c|c|c|c|c|}
\hline & \multirow[b]{2}{*}{ coll } & \multirow[b]{2}{*}{$\min _{\min }$} & \multicolumn{8}{|c|}{ 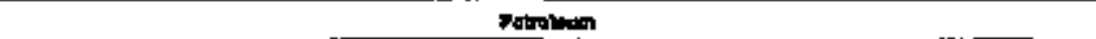 } & \multirow[b]{2}{*}{ Blotumite" } & \multirow[b]{2}{*}{ Eoctikely" } & \multirow[b]{2}{*}{ Engy } & \multirow[b]{2}{*}{ 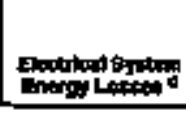 } & \multirow[b]{3}{*}{ Tato } \\
\hline & & & 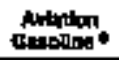 & Deponters & Al. & LPB: & 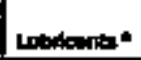 & 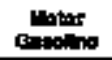 & Fund" & Total & & & & & \\
\hline Yase & Shoutand & 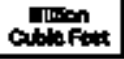 & \multicolumn{8}{|c|}{ 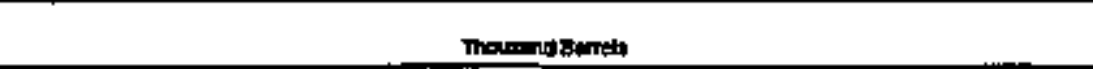 } & $\begin{array}{l}\text { Thousund } \\
\text { oflons }\end{array}$ & \multicolumn{3}{|c|}{ 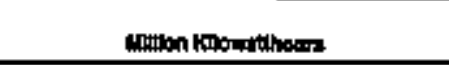 } & \\
\hline 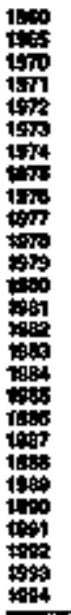 & $\begin{array}{l}4 \\
8 \\
3 \\
2 \\
2 \\
2 \\
1 \\
1 \\
0 \\
0 \\
0 \\
0 \\
0 \\
0 \\
0 \\
0 \\
0 \\
0 \\
0 \\
0 \\
0 \\
0 \\
0 \\
0 \\
0 \\
0 \\
0 \\
0 \\
0 \\
0\end{array}$ & $\begin{array}{r}6 \\
13 \\
19 \\
40 \\
5 \\
7 \\
5 \\
5 \\
4 \\
5 \\
6 \\
5 \\
4 \\
5 \\
4 \\
2 \\
5 \\
8 \\
5 \\
3 \\
2 \\
40 \\
3 \\
\end{array}$ & 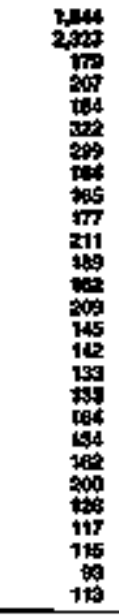 & 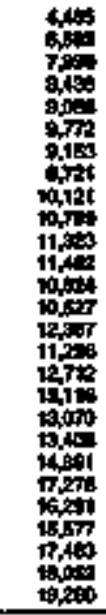 & 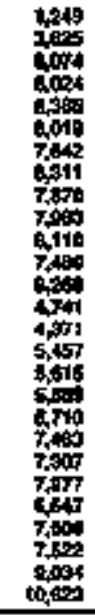 & 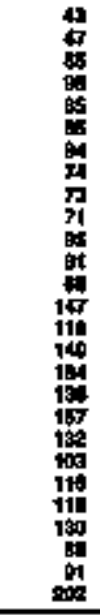 & 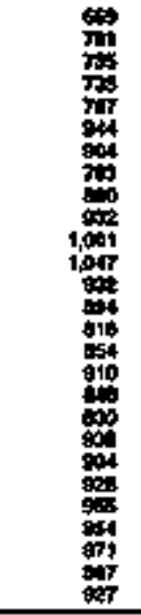 & 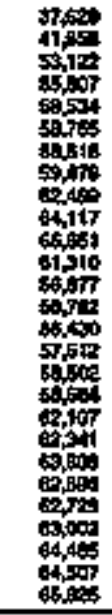 & 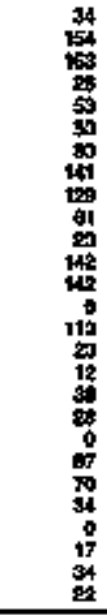 & 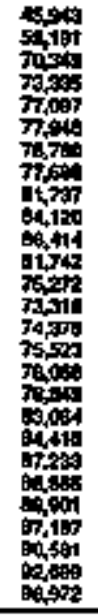 & 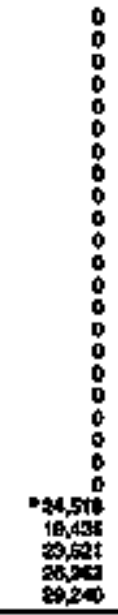 & $\begin{array}{l}2 \\
0 \\
0 \\
0 \\
0 \\
0 \\
0 \\
0 \\
0 \\
0 \\
0 \\
0 \\
0 \\
0 \\
0 \\
0 \\
0 \\
0 \\
0 \\
0 \\
0 \\
0 \\
0 \\
0 \\
0 \\
0 \\
0\end{array}$ & $\begin{array}{l}= \\
= \\
= \\
= \\
= \\
= \\
= \\
= \\
= \\
= \\
= \\
= \\
= \\
= \\
= \\
=\end{array}$ & $\begin{array}{l}8 \\
0 \\
0 \\
0 \\
0 \\
0 \\
0 \\
0 \\
0 \\
0 \\
0 \\
0 \\
0 \\
0 \\
0 \\
0 \\
0 \\
0 \\
0 \\
0 \\
0 \\
0 \\
0 \\
0 \\
0 \\
0\end{array}$ & $\begin{array}{l}= \\
= \\
z \\
z \\
z \\
z \\
z \\
z \\
z \\
z \\
z \\
z \\
z \\
z \\
z \\
\end{array}$ \\
\hline \multicolumn{16}{|c|}{ Todullon Ben } \\
\hline 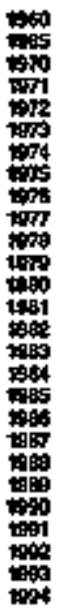 & 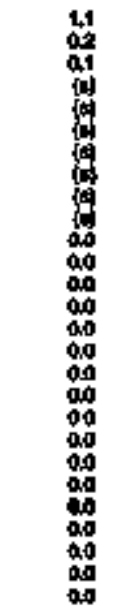 & 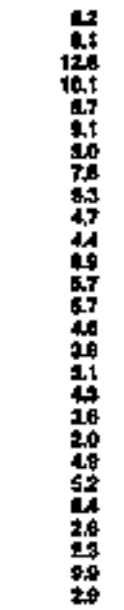 & 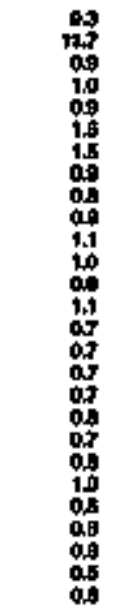 & 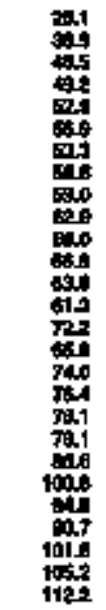 & 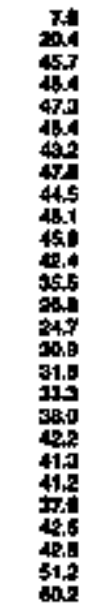 & 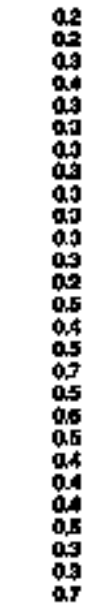 & 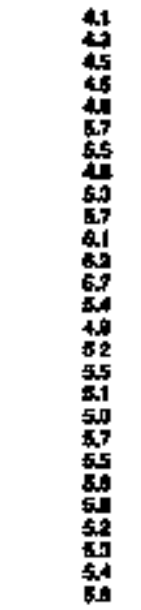 & 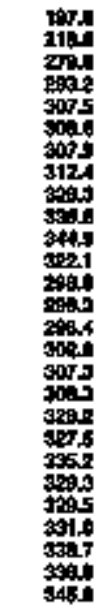 & 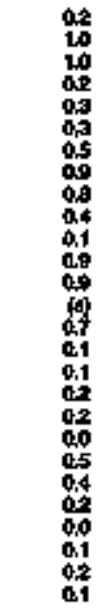 & 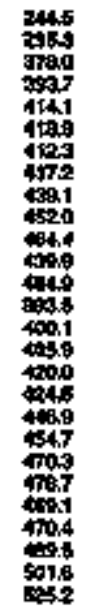 & 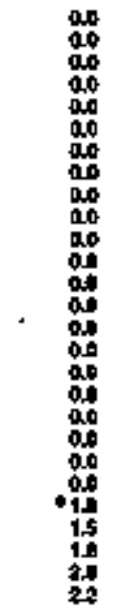 & 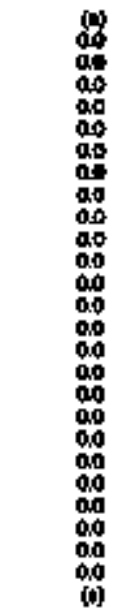 & 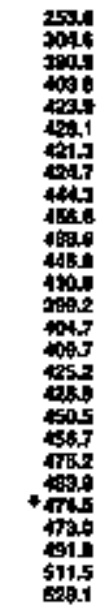 & 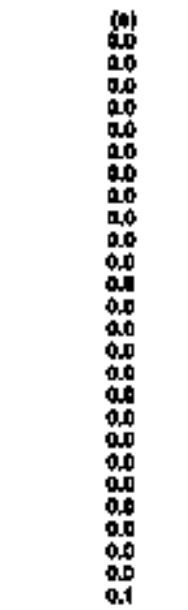 & 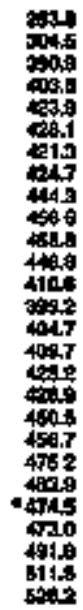 \\
\hline
\end{tabular}

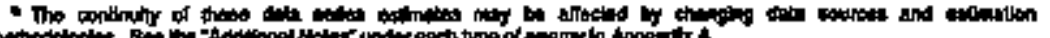

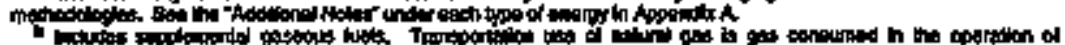

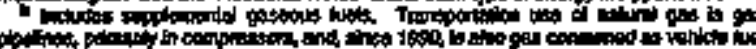

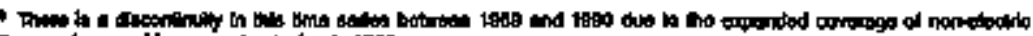

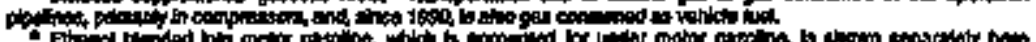

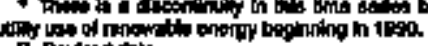

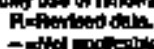

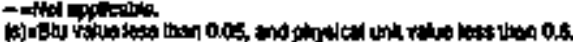

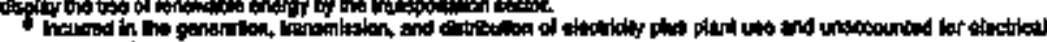

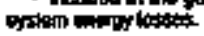


Table 172, Estimates of Energy Input at Electrio Utilities, 1960, 1865, 1970-1994, Missourl

\begin{tabular}{|c|c|c|c|c|c|c|c|c|c|c|c|c|c|c|}
\hline \multirow[b]{3}{*}{$y=0$} & \multicolumn{3}{|c|}{ cont } & \multirow{3}{*}{ 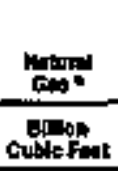 } & \multicolumn{4}{|c|}{ Pusolinum } & \multirow[b]{2}{*}{ 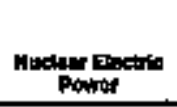 } & \multirow[b]{2}{*}{ 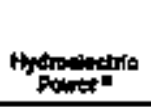 } & \multirow[b]{2}{*}{ Bofinsts } & \multirow[b]{2}{*}{ Exingming } & \multirow[b]{2}{*}{ ots=n } & \multirow[b]{3}{*}{ Totod I } \\
\hline & 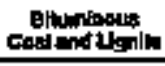 & 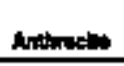 & Thent & & of & Lبsit & Anorienth & Toted & & & & & & \\
\hline & \multicolumn{3}{|c|}{ moukend short Tond } & & \multicolumn{4}{|c|}{ 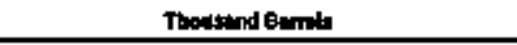 } & \multicolumn{5}{|c|}{ 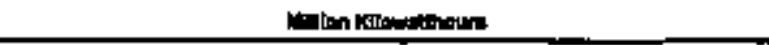 } & \\
\hline 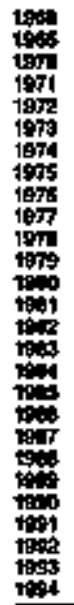 & 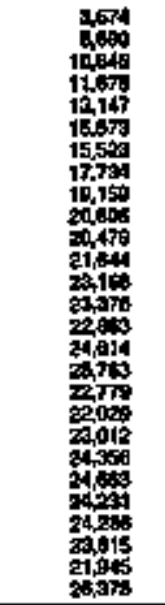 & $\begin{array}{l}0 \\
0 \\
0 \\
0 \\
0 \\
0 \\
0 \\
0 \\
0 \\
0 \\
0 \\
0 \\
0 \\
0 \\
0 \\
0 \\
0 \\
0 \\
0 \\
0 \\
0 \\
0\end{array}$ & 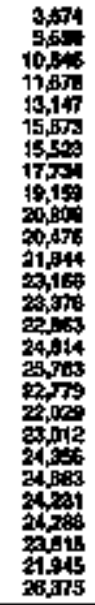 & 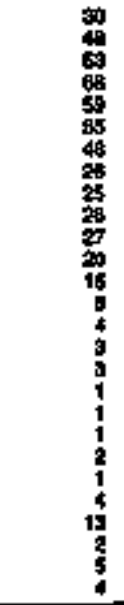 & 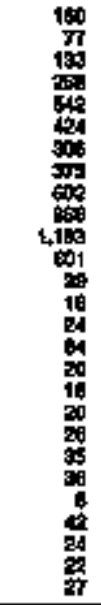 & 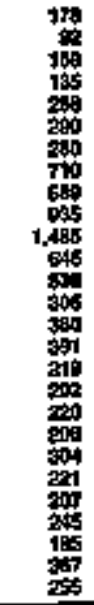 & 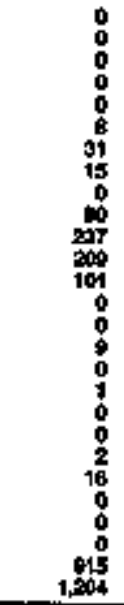 & 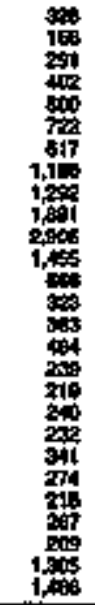 & 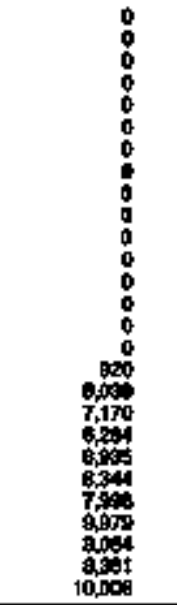 & 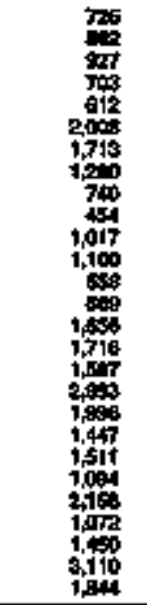 & $\begin{array}{l}0 \\
0 \\
0 \\
0 \\
0 \\
0 \\
0 \\
0 \\
0 \\
0 \\
0 \\
0 \\
0 \\
0 \\
0 \\
0 \\
0 \\
0 \\
0 \\
0 \\
0 \\
0 \\
1 \\
7\end{array}$ & $\begin{array}{l}0 \\
0 \\
0 \\
0 \\
0 \\
0 \\
0 \\
0 \\
0 \\
0 \\
0 \\
0 \\
0 \\
0 \\
0 \\
0 \\
0 \\
0 \\
0 \\
0 \\
0 \\
0 \\
0 \\
0 \\
0 \\
0 \\
0 \\
0 \\
0 \\
0 \\
0\end{array}$ & $\begin{array}{l}0 \\
0 \\
0 \\
0 \\
0 \\
0 \\
0 \\
0 \\
0 \\
0 \\
0 \\
0 \\
0 \\
0 \\
0 \\
0 \\
0 \\
0 \\
0 \\
0\end{array}$ & 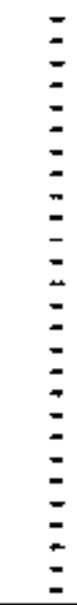 \\
\hline
\end{tabular}

\begin{tabular}{|c|c|c|c|c|c|c|c|c|c|c|c|c|c|c|}
\hline 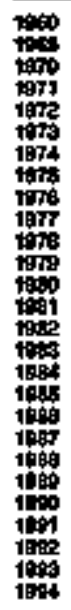 & 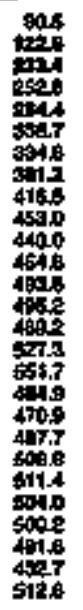 & 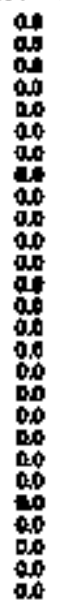 & 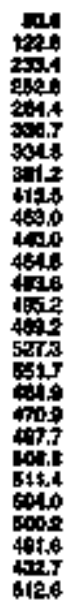 & 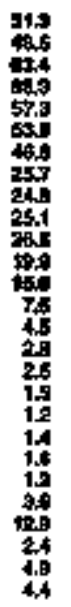 & 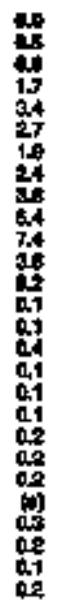 & 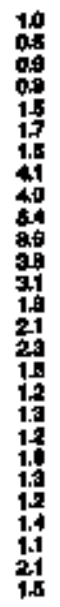 & 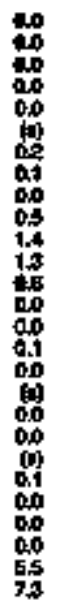 & 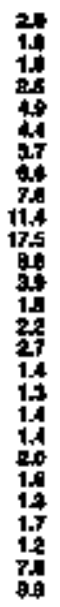 & 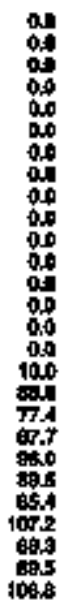 & 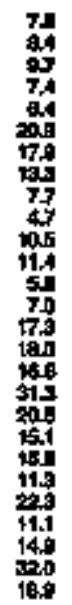 & 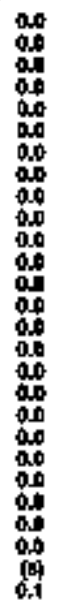 & 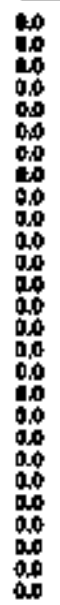 & 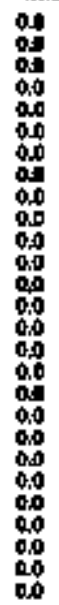 & 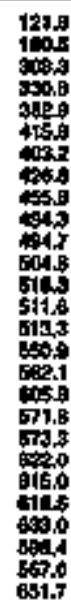 \\
\hline
\end{tabular}

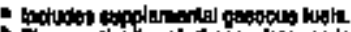

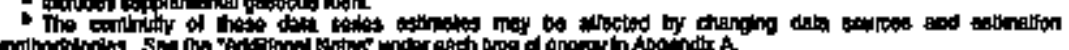

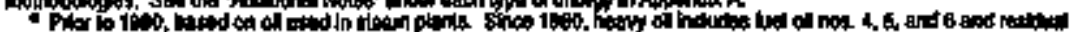

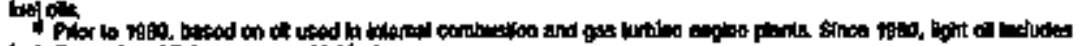

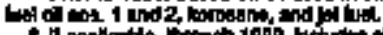

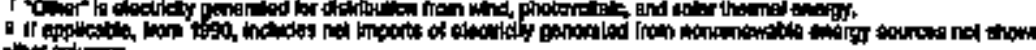

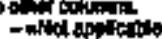

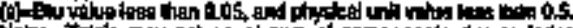

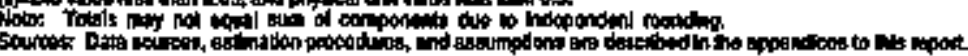

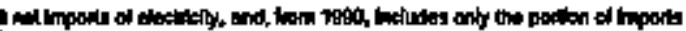


Tablo 173. Envorg Consumption Eetlmalos by Sowce, 1950, 1965, 1970-1994, Montana

\begin{tabular}{|c|c|c|c|c|c|c|c|c|c|c|c|c|c|c|c|c|c|c|c|}
\hline \multirow[b]{3}{*}{ rest: } & \multirow[b]{2}{*}{ sans: } & \multirow[b]{2}{*}{$\operatorname{lom} 6$} & \multicolumn{11}{|c|}{ ח } & \multirow[b]{2}{*}{ 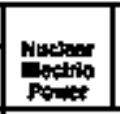 } & \multirow[b]{2}{*}{ 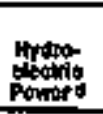 } & \multirow[b]{2}{*}{ Etolutits" } & \multirow[b]{2}{*}{ 年 } & \multirow{3}{*}{ 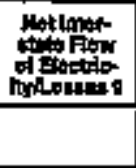 } & \multirow[b]{3}{*}{ Tons: } \\
\hline & & & Anohus:ond & Antostions & 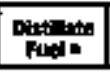 & rete. & $\cos _{x \rightarrow \infty}$ & 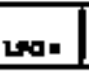 & Lats. & 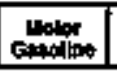 & 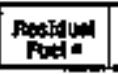 & ones an & Totien & & & & & & \\
\hline & Thenund & clapteon & \multicolumn{11}{|c|}{ 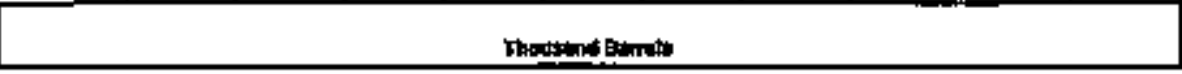 } & \multicolumn{4}{|c|}{ 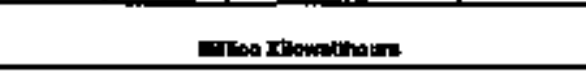 } & & \\
\hline 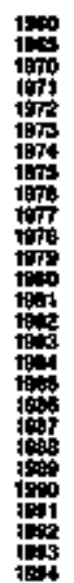 & 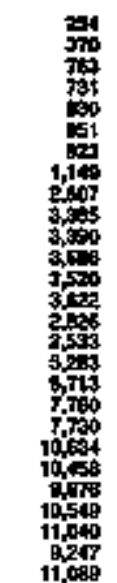 & 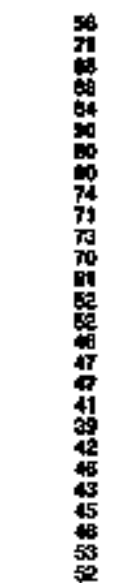 & 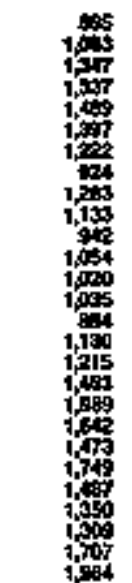 & 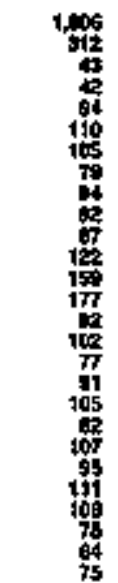 & 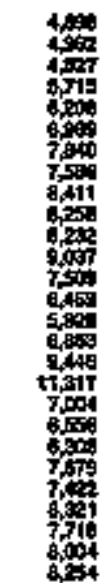 & 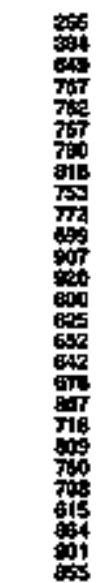 & 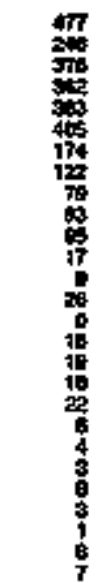 & 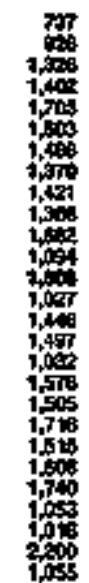 & 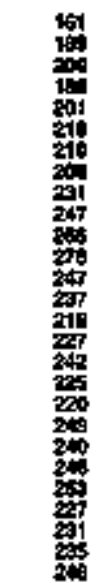 & 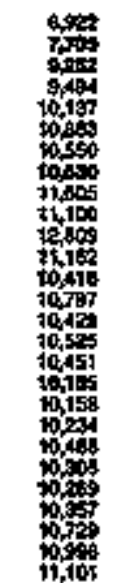 & 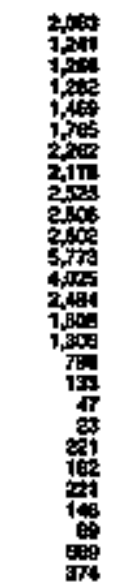 & 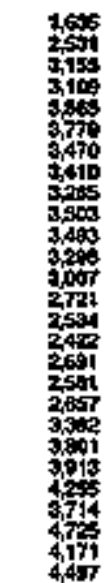 & 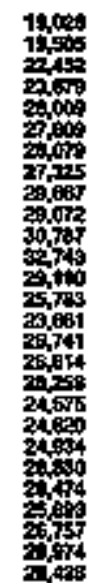 & $\begin{array}{l}0 \\
0 \\
0 \\
0 \\
0 \\
0 \\
0 \\
0 \\
0 \\
0 \\
0 \\
0 \\
0 \\
0 \\
0 \\
0 \\
0 \\
0\end{array}$ & 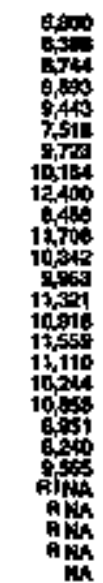 & 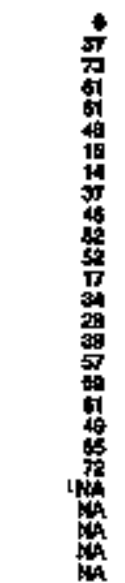 & 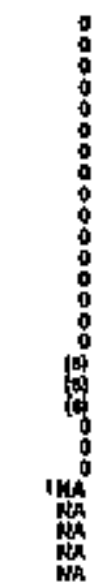 & 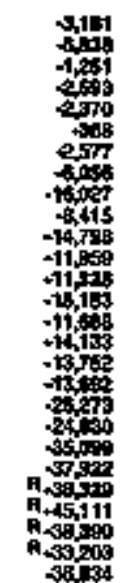 & $=$ \\
\hline & & & & & & & & & & Trillon ew & & & & & & & & & \\
\hline
\end{tabular}

\begin{tabular}{|c|c|c|c|c|c|c|c|c|c|c|c|c|c|c|c|c|c|c|c|}
\hline 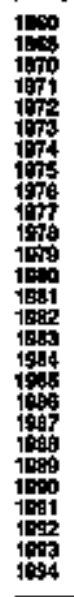 & 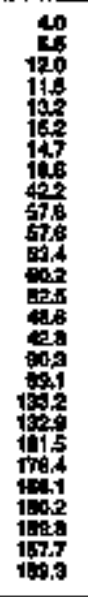 & 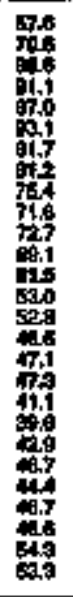 & 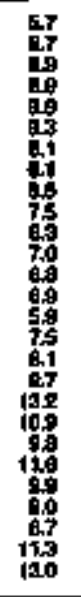 & 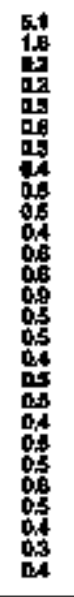 & 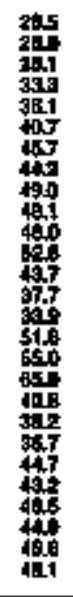 & 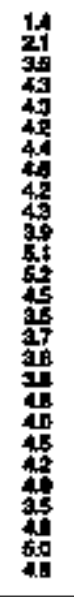 & 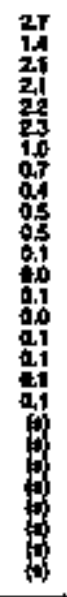 & 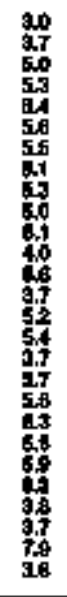 & 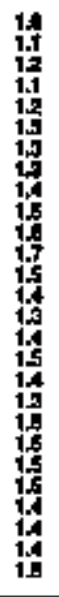 & 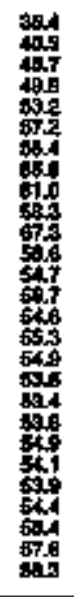 & 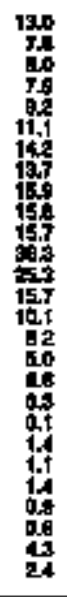 & 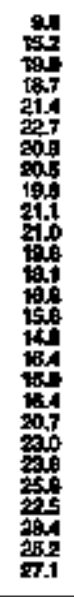 & 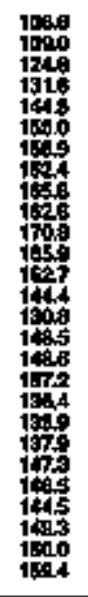 & 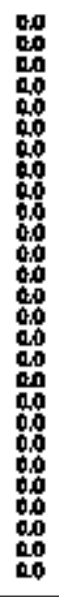 & 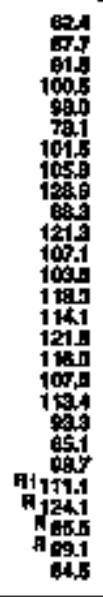 & 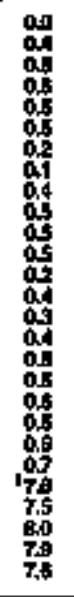 & 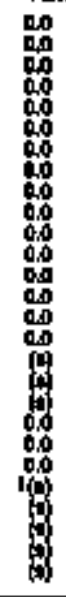 & 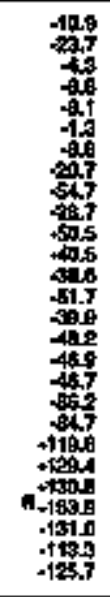 & 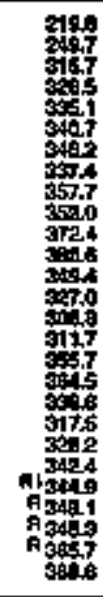 \\
\hline
\end{tabular}

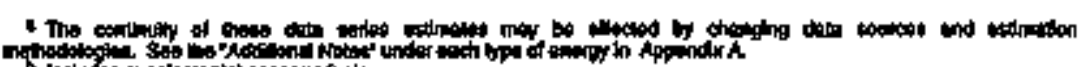

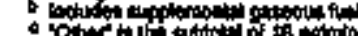

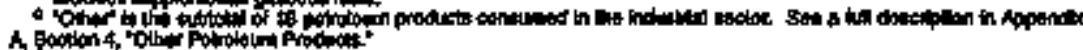

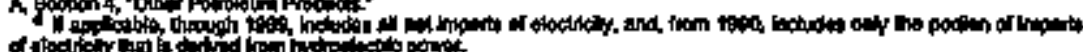

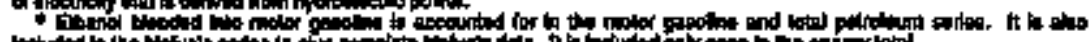

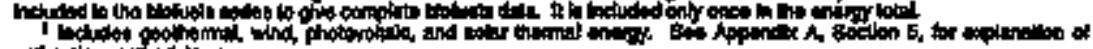

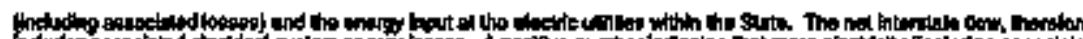

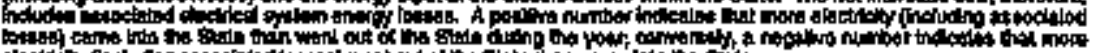

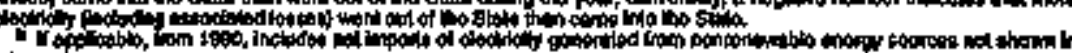

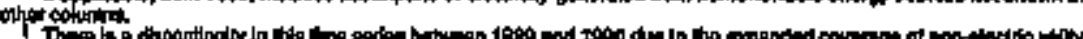

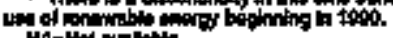

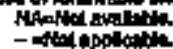

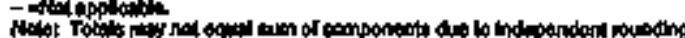

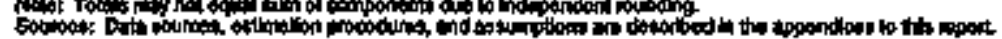


Tubse 174. Residential Energy Consumption Estimales, 1930, 1965, 1970-1994, Monlena

\begin{tabular}{|c|c|c|c|c|c|c|c|c|c|c|c|c|c|c|}
\hline \multirow[b]{3}{*}{ Yem } & \multicolumn{3}{|c|}{ con } & \multirow[b]{2}{*}{ 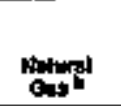 } & \multicolumn{4}{|c|}{ Potrotum } & \multirow{3}{*}{ 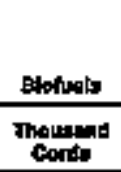 } & & \multirow[b]{2}{*}{ mostinkly a } & \multirow[b]{2}{*}{ tinery } & \multirow{3}{*}{ 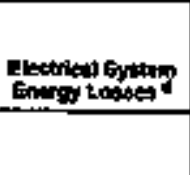 } & \multirow[b]{3}{*}{ Totot } \\
\hline & 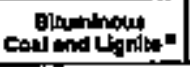 & 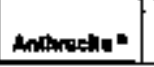 & Tomin & & Otinterse & Konsonst & EPQ: & Total & & Bolura & & & & \\
\hline & \multicolumn{3}{|c|}{ Thowsend Sthort Tons } & 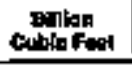 & \multicolumn{4}{|c|}{ Thousund Bands } & & \multicolumn{3}{|c|}{ 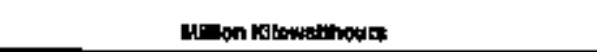 } & & \\
\hline 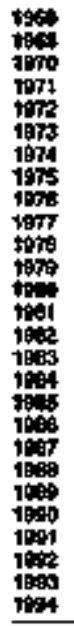 & 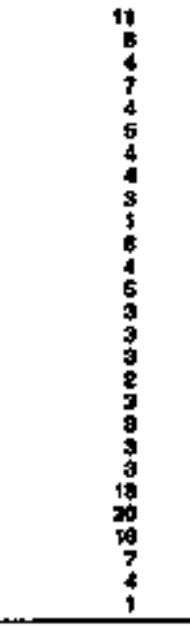 & $\begin{array}{l}0 \\
: \\
0 \\
0 \\
0 \\
0 \\
0 \\
0 \\
0 \\
0 \\
0 \\
0 \\
0 \\
0 \\
0 \\
0 \\
0 \\
0 \\
0 \\
0 \\
0 \\
0 \\
0 \\
0 \\
0 \\
0 \\
0\end{array}$ & 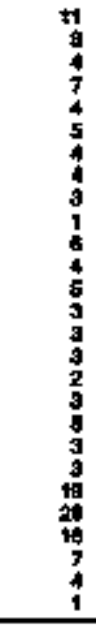 & 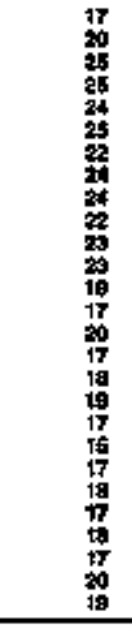 & 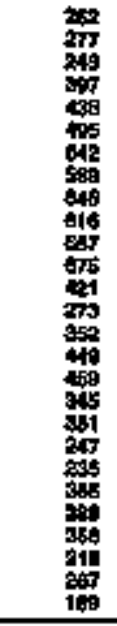 & 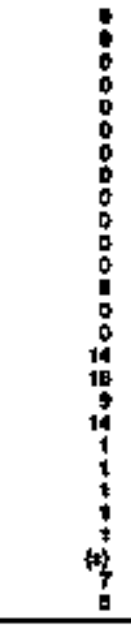 & 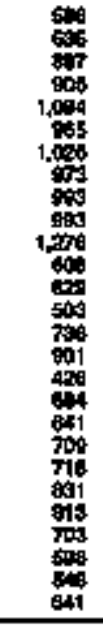 & 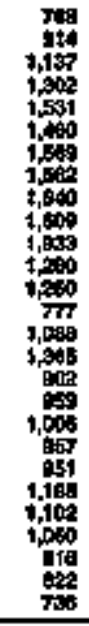 & 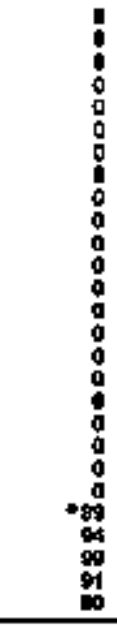 & $\begin{array}{l}0 \\
0 \\
0 \\
0 \\
0 \\
0 \\
0 \\
0 \\
0 \\
0 \\
0 \\
0 \\
0 \\
0 \\
0 \\
0 \\
0 \\
0 \\
0 \\
0 \\
9 \\
9 \\
6 \\
6 \\
6\end{array}$ & 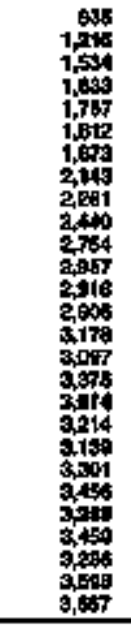 & $\begin{array}{l}\vdots \\
\vdots \\
\vdots \\
\vdots \\
\vdots \\
\vdots \\
\vdots \\
\vdots \\
\vdots \\
\vdots \\
\vdots \\
\vdots \\
\vdots \\
\end{array}$ & 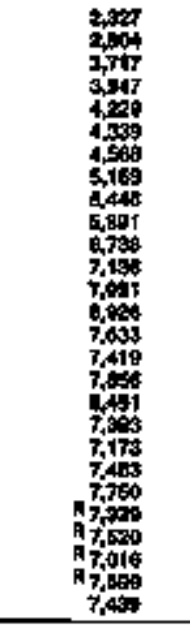 & 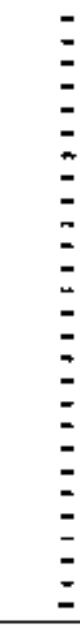 \\
\hline \multicolumn{15}{|c|}{ Tallit on Ban } \\
\hline 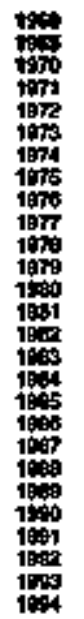 & 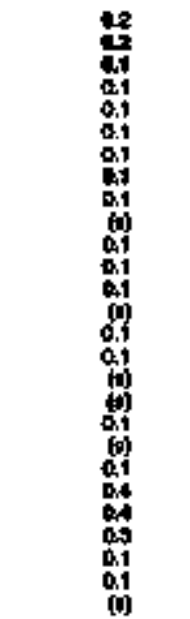 & 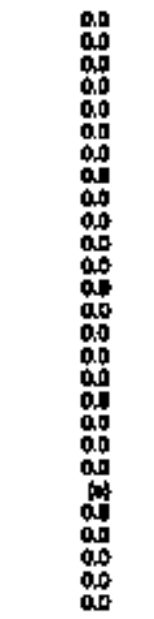 & 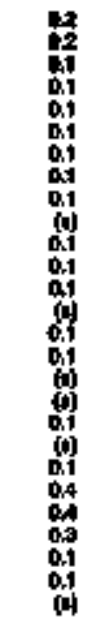 & 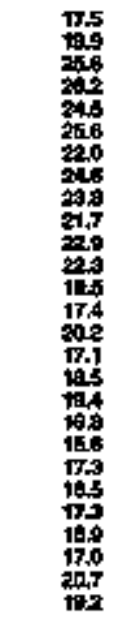 & 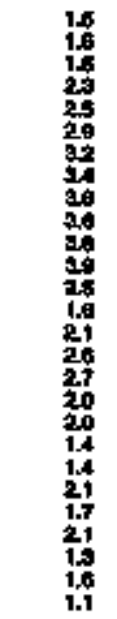 & 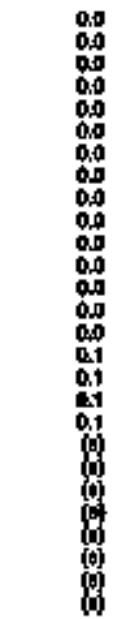 & 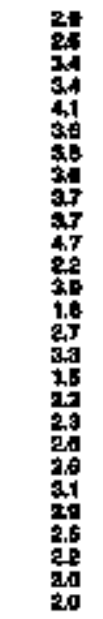 & 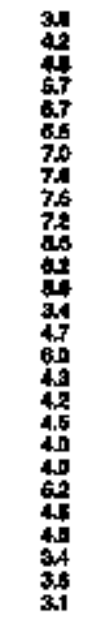 & 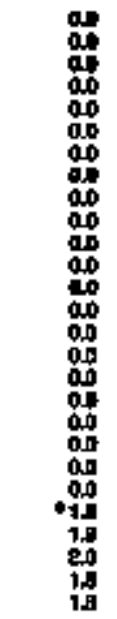 & 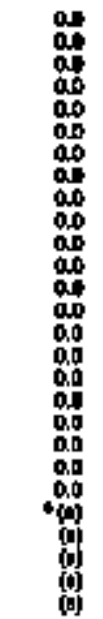 & 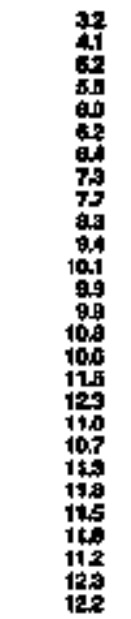 & 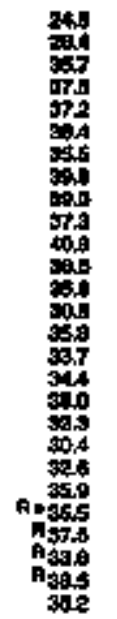 & 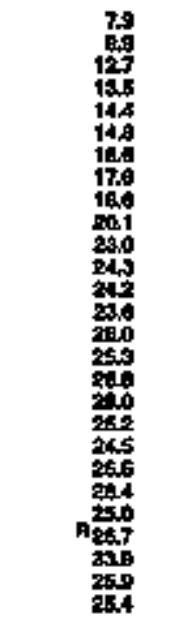 & 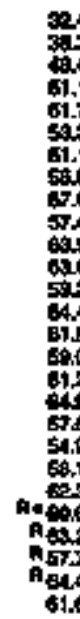 \\
\hline
\end{tabular}

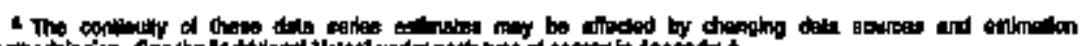

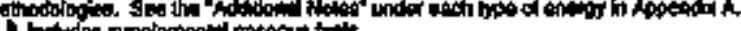

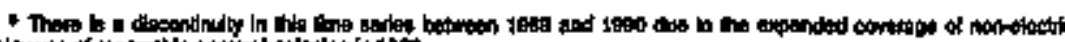

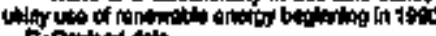

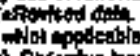

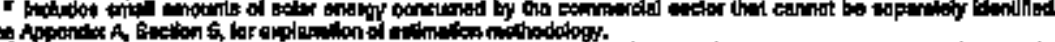

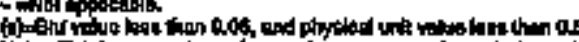

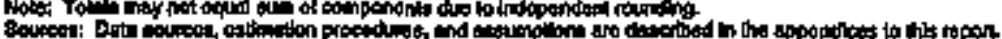
tyoum 
Table 175. Cornmercial Energy Consumption Estimalos, 1960, 1965, 1970-1994, Montana

\begin{tabular}{|c|c|c|c|c|c|c|c|c|c|c|c|c|c|c|c|}
\hline \multirow[b]{3}{*}{ Tat } & \multicolumn{3}{|c|}{ con } & \multirow{3}{*}{ andingen } & \multicolumn{6}{|c|}{ Potrolan } & \multirow[b]{2}{*}{ roctitenty } & \multirow[b]{2}{*}{ Entrity } & \multirow[b]{2}{*}{ 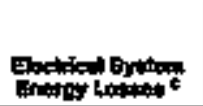 } & \multirow[b]{3}{*}{ Toted d } & \\
\hline & 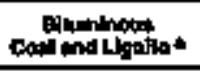 & Anstangont & 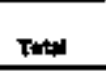 & & Pation & Kareanen & ePan & thesere & 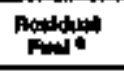 & Tatal & & & & & \\
\hline & \multicolumn{3}{|c|}{ 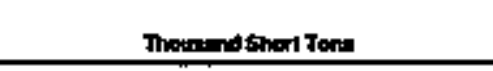 } & & \multicolumn{6}{|c|}{ Thontapd Bimple } & \multicolumn{3}{|c|}{ 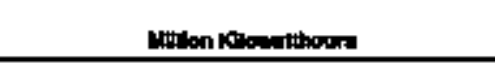 } & & \\
\hline 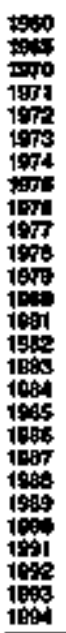 & 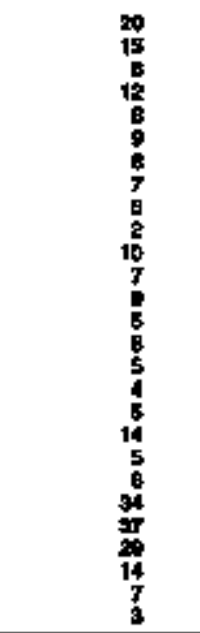 & $\begin{array}{l}0 \\
0 \\
0 \\
0 \\
0 \\
0 \\
0 \\
0 \\
0 \\
0 \\
0 \\
0 \\
0 \\
0 \\
0 \\
0 \\
0 \\
0 \\
0 \\
0 \\
0 \\
0 \\
0 \\
0\end{array}$ & 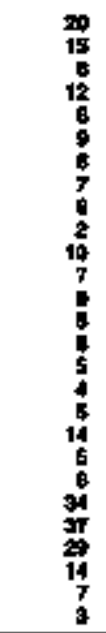 & 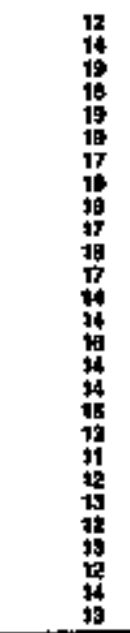 & 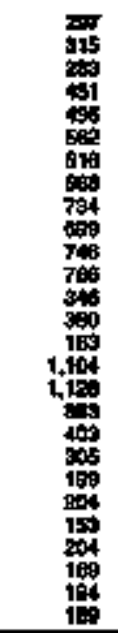 & 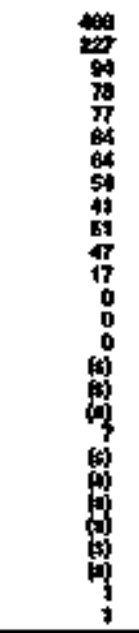 & 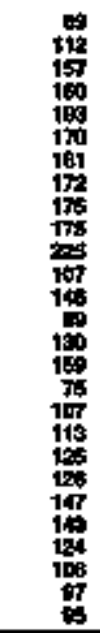 & 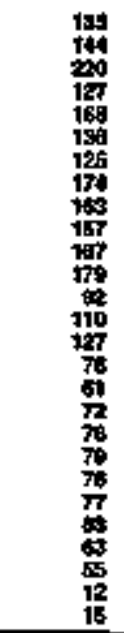 & 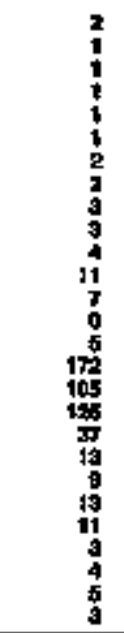 & 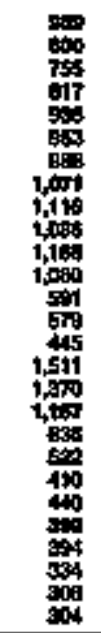 & 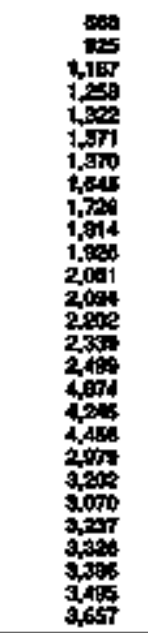 & $\begin{array}{l}= \\
= \\
= \\
= \\
= \\
= \\
= \\
= \\
= \\
= \\
z \\
z \\
z \\
z \\
=\end{array}$ & 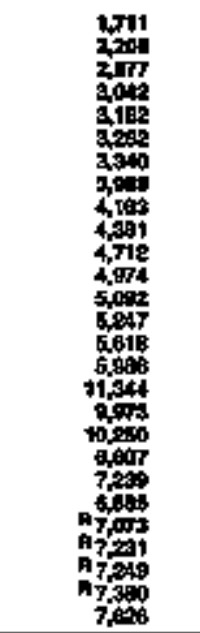 & $\begin{array}{l}= \\
= \\
= \\
= \\
= \\
= \\
= \\
= \\
= \\
= \\
= \\
= \\
=\end{array}$ & \\
\hline \multicolumn{15}{|c|}{ Dimin Ex: } & \\
\hline 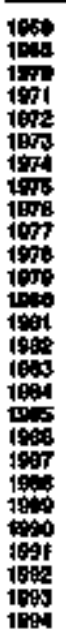 & 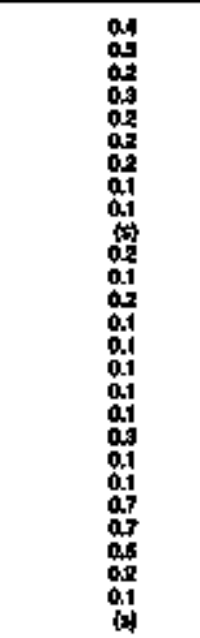 & 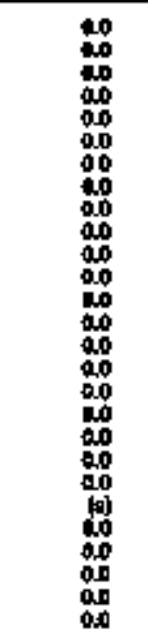 & 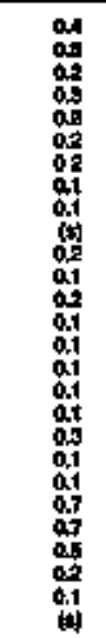 & 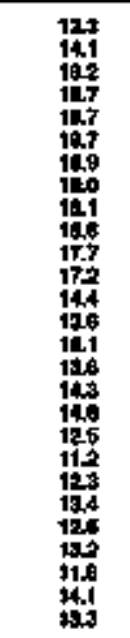 & 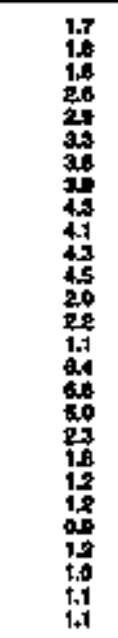 & 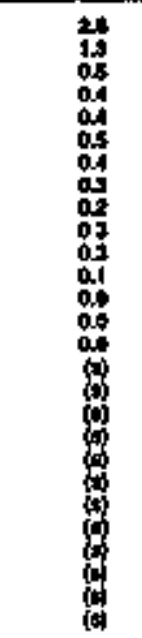 & 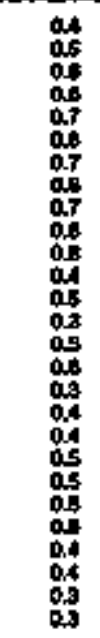 & 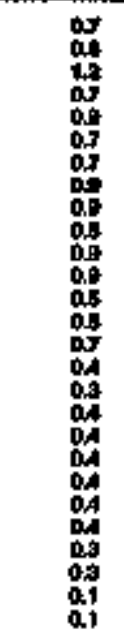 & 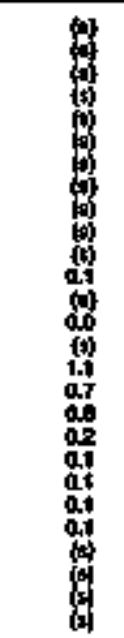 & 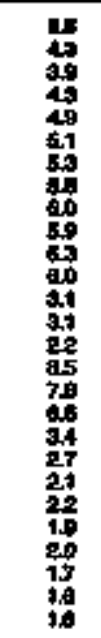 & 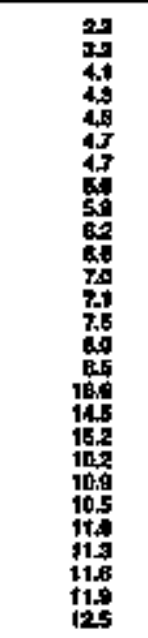 & 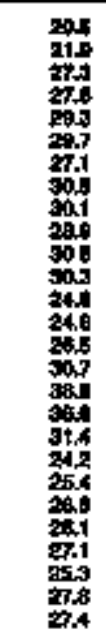 & 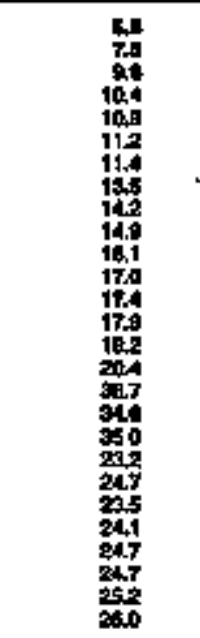 & 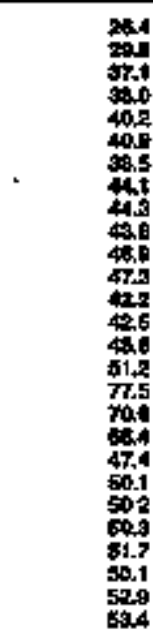 & \\
\hline \multirow{2}{*}{\multicolumn{15}{|c|}{ 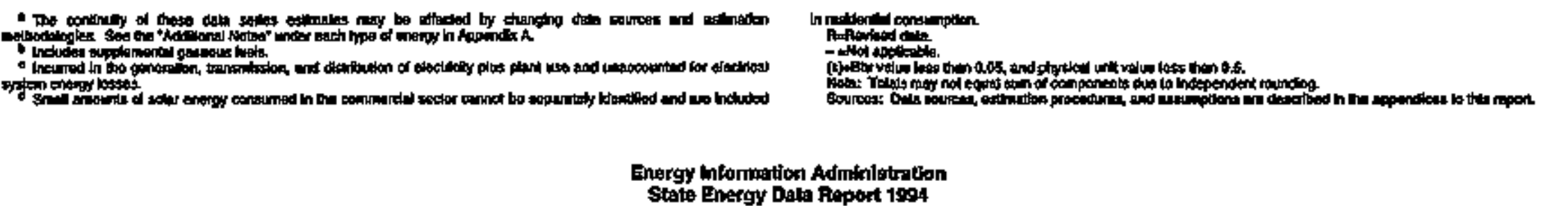 }} & \\
\hline & & & & & & & & & & & & & & & 187 \\
\hline
\end{tabular}


Table 176. Intugtrlal Energy Consumption Eellmates, 1960, 1955, 1970-1994, Montana

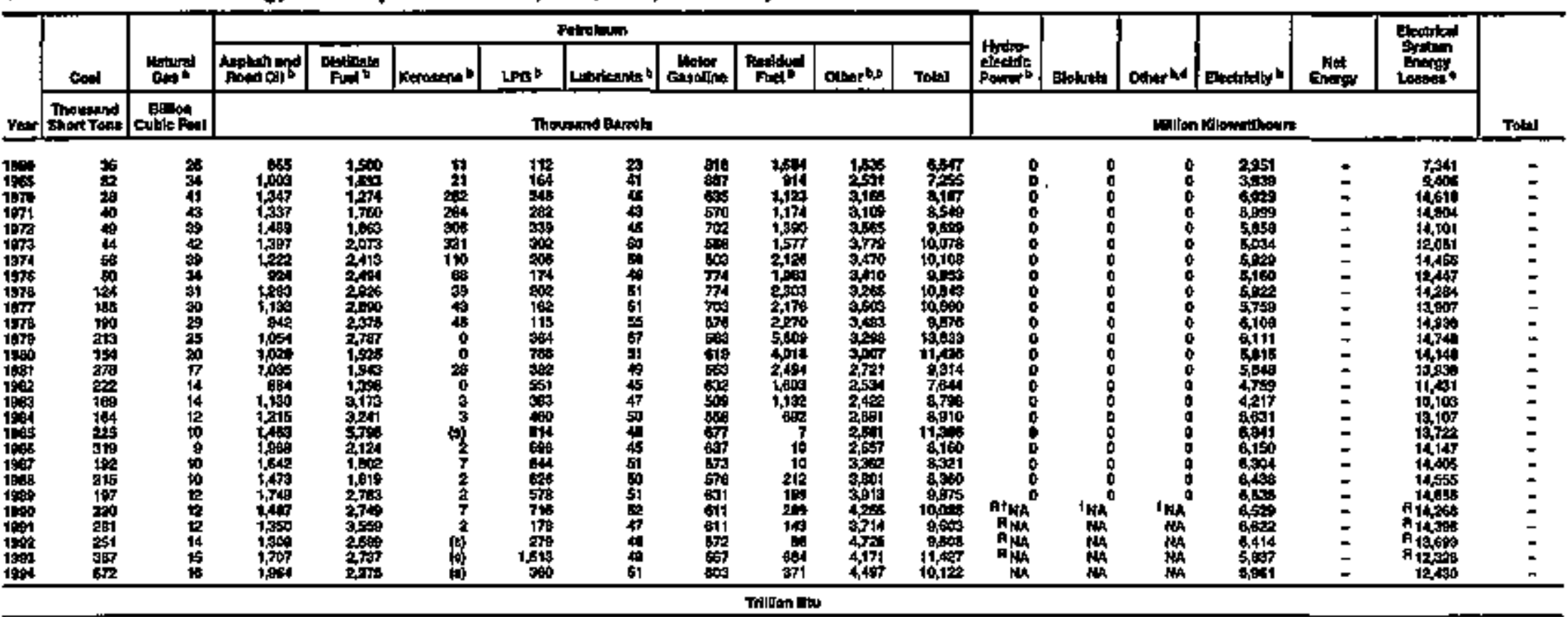

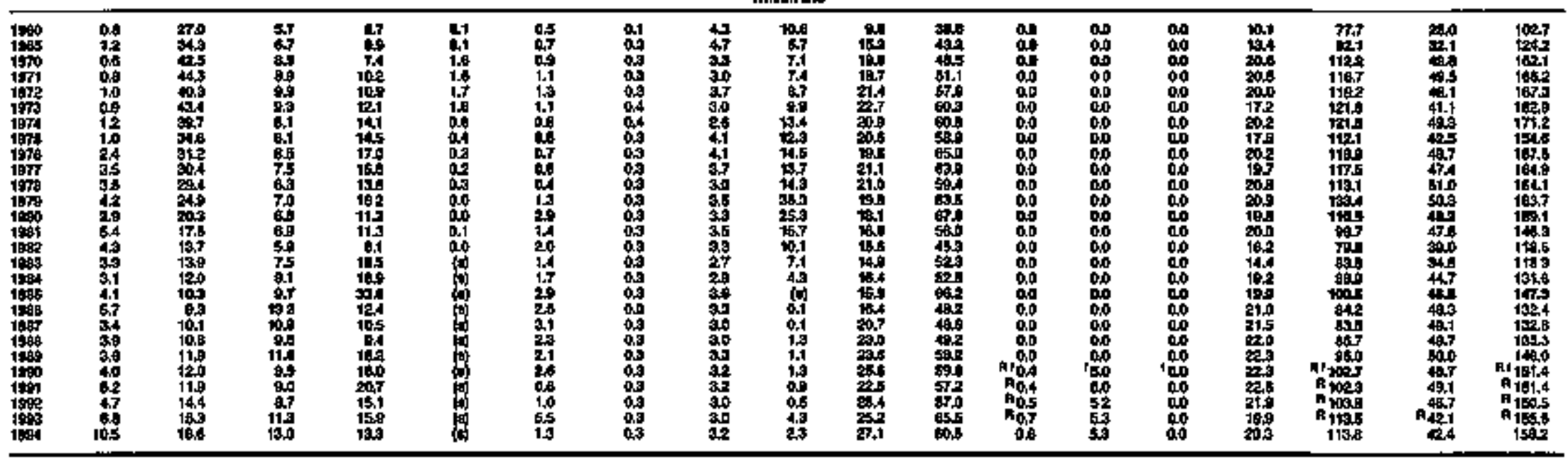

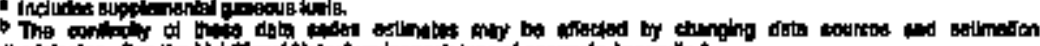

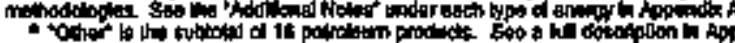
a

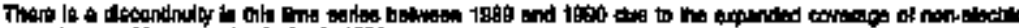

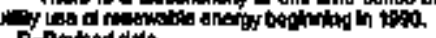

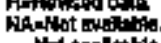

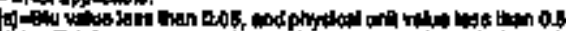

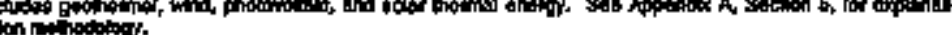

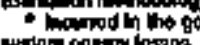


Tabłe 177. Transportallon Energy Consumplion Estimates, 1960, 1955, 1970-1994, Montara

\begin{tabular}{|c|c|c|c|c|c|c|c|c|c|c|c|c|c|c|c|}
\hline \multirow[b]{3}{*}{ Y } & \multirow[b]{2}{*}{ Conte } & \multirow[b]{2}{*}{ 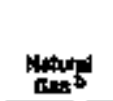 } & \multicolumn{8}{|c|}{ 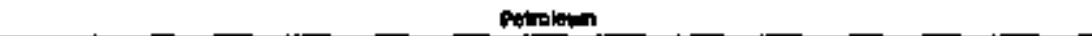 } & \multirow{3}{*}{ montes } & & \multirow[b]{2}{*}{$\lim _{n=0}^{n}$} & \multirow[b]{2}{*}{ 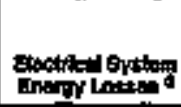 } & \multirow[b]{3}{*}{ тоңы } \\
\hline & & & Antion. & Detarist: & feret" & LPG: & Ltrleater & unopotion & ratum & Tote & & Electidty * & & & \\
\hline & $\begin{array}{l}\text { Thourtind } \\
\text { Slopt Touth }\end{array}$ & 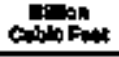 & \multicolumn{8}{|c|}{ 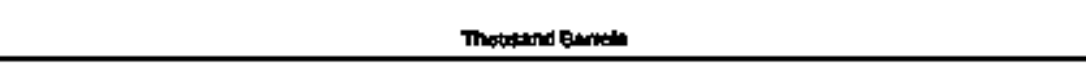 } & & \multicolumn{3}{|c|}{ 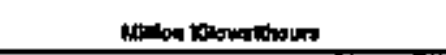 } & \\
\hline 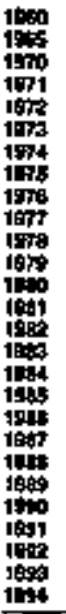 & $\begin{array}{l}1 \\
0 \\
0 \\
0 \\
0 \\
0 \\
60 \\
0 \\
0 \\
0 \\
0 \\
0 \\
0 \\
0 \\
0 \\
0 \\
0 \\
0 \\
0 \\
0 \\
0 \\
0 \\
0\end{array}$ & 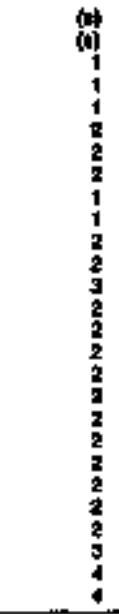 & 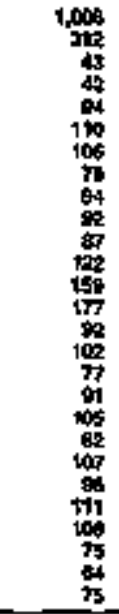 & 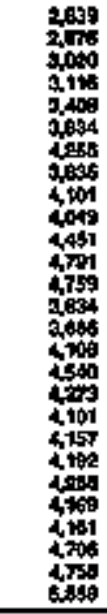 & 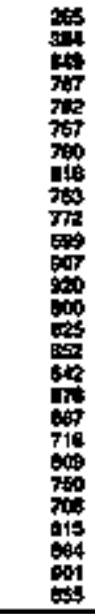 & 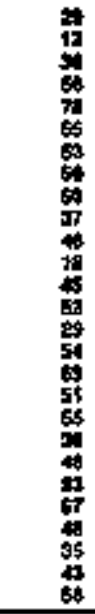 & 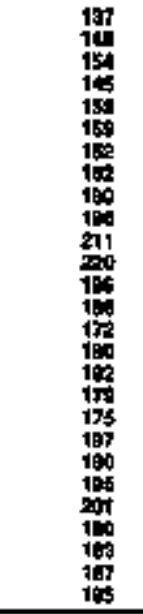 & 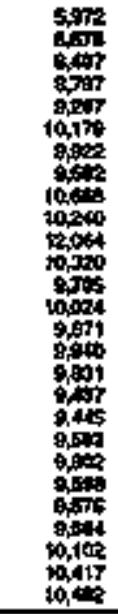 & 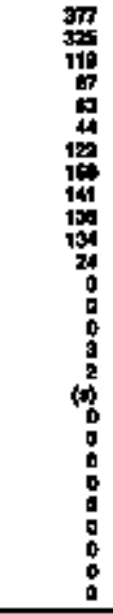 & 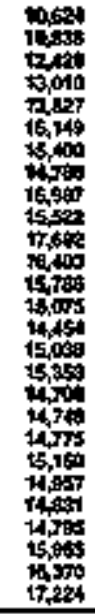 & $\begin{array}{l}0 \\
0 \\
0 \\
0 \\
0 \\
0 \\
0 \\
0 \\
0 \\
0 \\
0 \\
0 \\
0 \\
0 \\
0 \\
0 \\
0 \\
0 \\
0 \\
0 \\
0\end{array}$ & $\begin{array}{l}0 \\
0 \\
0 \\
0 \\
0 \\
0 \\
0 \\
0 \\
0 \\
0 \\
0 \\
0 \\
0 \\
0 \\
0 \\
0 \\
0 \\
0 \\
0 \\
0 \\
0 \\
0 \\
0 \\
0 \\
0 \\
0 \\
0 \\
0\end{array}$ & $\begin{array}{l}= \\
= \\
= \\
= \\
= \\
= \\
= \\
= \\
= \\
= \\
= \\
= \\
= \\
= \\
=\end{array}$ & 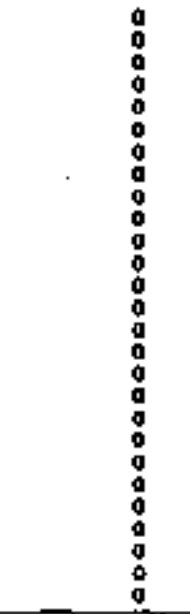 & $\begin{array}{l}= \\
= \\
= \\
= \\
= \\
= \\
= \\
= \\
= \\
= \\
= \\
= \\
=\end{array}$ \\
\hline \multicolumn{16}{|c|}{ relllion Btu } \\
\hline 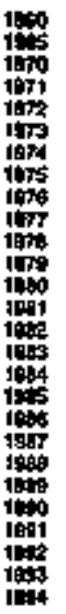 & 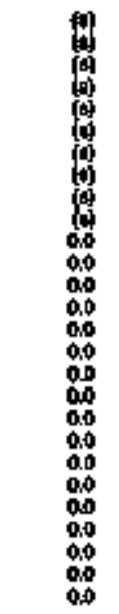 & 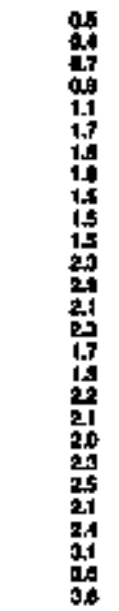 & $\begin{array}{l}5.1 \\
1.5 \\
0.3 \\
0.2 \\
0.5 \\
0.6 \\
0.5 \\
0.4 \\
0.5 \\
0.5 \\
0.4 \\
0.6 \\
0.4 \\
0.9 \\
0.5 \\
0.5 \\
0.4 \\
0.5 \\
0.5 \\
0.4 \\
0.5 \\
0.5 \\
0.5 \\
0.5 \\
0.4 \\
0.3 \\
0.4\end{array}$ & 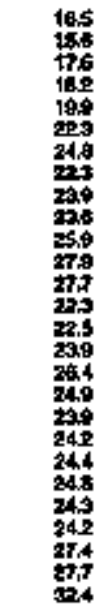 & 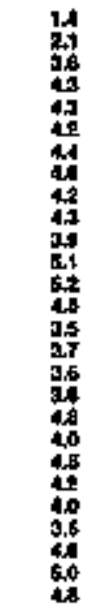 & 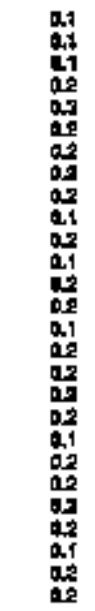 & 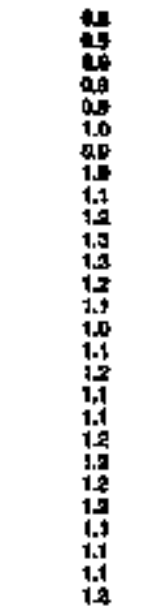 & 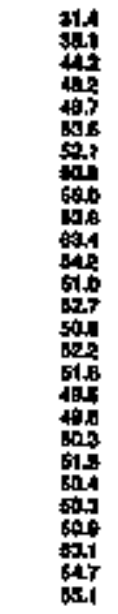 & 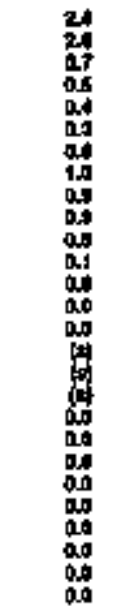 & 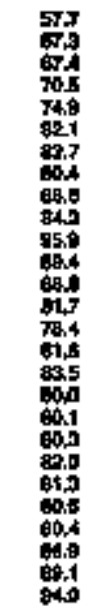 & 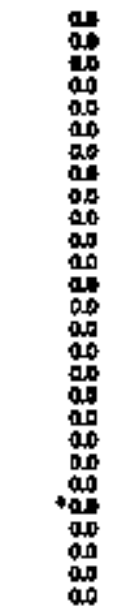 & $\begin{array}{l}00 \\
00 \\
00 \\
00 \\
00 \\
0.0 \\
00 \\
00 \\
00 \\
000 \\
000 \\
00 \\
000 \\
00 \\
000 \\
00 \\
00 \\
00 \\
000 \\
0.0 \\
00 \\
00 \\
00 \\
00 \\
000 \\
00\end{array}$ & 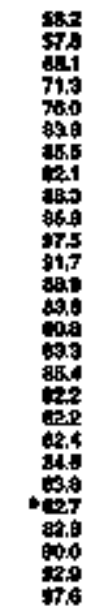 & 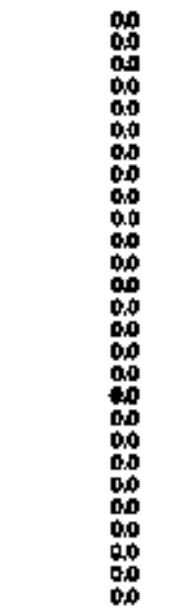 & 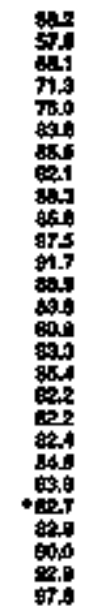 \\
\hline
\end{tabular}

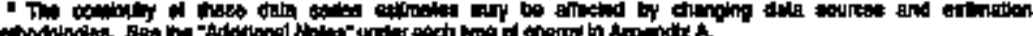

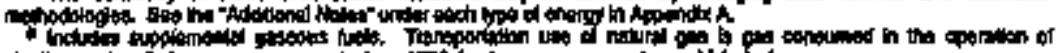

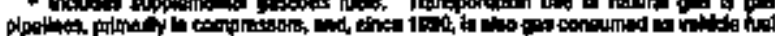

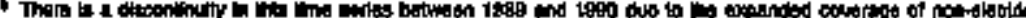

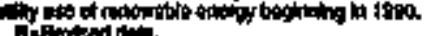

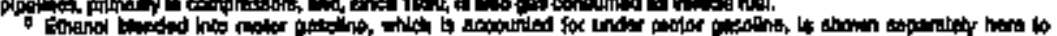

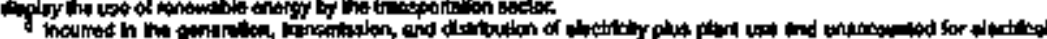

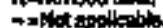

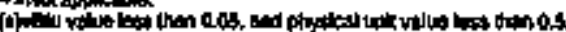

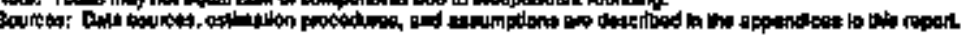

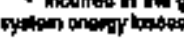


Tablo 179. Estimates of Energy Input at Electric Utibtites, 1960, 1865, 1970-1994, Montans

\begin{tabular}{|c|c|c|c|c|c|c|c|c|c|c|c|c|c|c|}
\hline & \multicolumn{3}{|c|}{$\cos$} & \multirow[b]{2}{*}{ Nemint } & \multicolumn{4}{|c|}{ Proplaner } & \multirow[b]{2}{*}{ 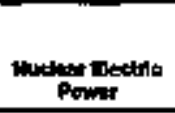 } & \multirow[b]{2}{*}{ Mydratoutste } & \multirow[b]{2}{*}{ Ebotunk } & \multirow[b]{2}{*}{ 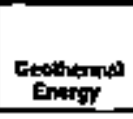 } & \multirow[b]{2}{*}{ Other bل } & \multirow[b]{3}{*}{ Taten } \\
\hline & 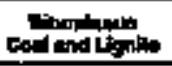 & Arthosine & Tolat & & 4 & 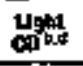 & Pothering & Total & & & & & & \\
\hline Yere & \multicolumn{3}{|c|}{ Thenenthent Thent } & 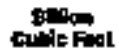 & \multicolumn{4}{|c|}{ 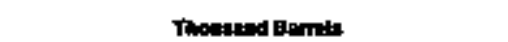 } & \multicolumn{5}{|c|}{ 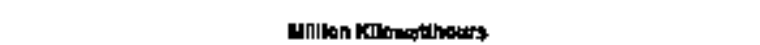 } & \\
\hline
\end{tabular}

\begin{tabular}{|c|c|c|c|c|c|c|c|c|c|c|c|c|c|c|}
\hline 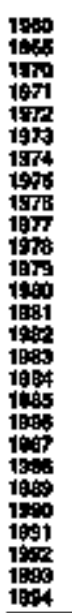 & 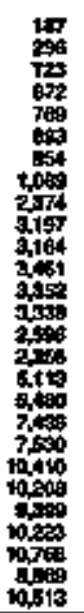 & $\begin{array}{l}0 \\
0 \\
0 \\
0 \\
0 \\
0 \\
0 \\
0 \\
0 \\
0 \\
0 \\
0 \\
0 \\
0 \\
0 \\
0 \\
0 \\
0 \\
0 \\
0 \\
0\end{array}$ & 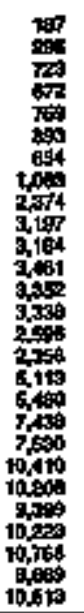 & 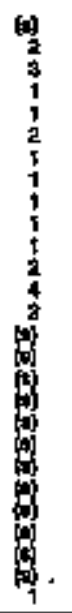 & 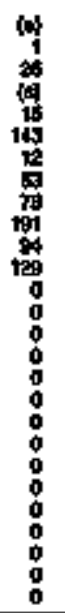 & 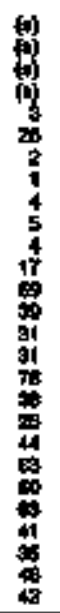 & $\begin{array}{l}0 \\
0 \\
0 \\
0 \\
0 \\
0 \\
0 \\
0 \\
0 \\
0 \\
8 \\
0 \\
0 \\
0 \\
0 \\
0 \\
0 \\
0 \\
0 \\
0 \\
0 \\
0 \\
0 \\
0\end{array}$ & 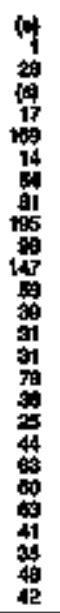 & $\begin{array}{l}0 \\
0 \\
0 \\
0 \\
0 \\
0 \\
0 \\
0 \\
0 \\
0 \\
0 \\
0 \\
0 \\
0 \\
0 \\
0 \\
0 \\
0 \\
0 \\
0 \\
0 \\
0 \\
0 \\
0 \\
0 \\
0 \\
0\end{array}$ & 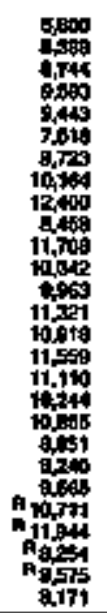 & 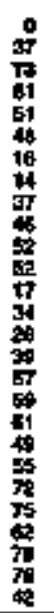 & $\begin{array}{l}0 \\
\vdots \\
0 \\
0 \\
0 \\
: \\
0 \\
0 \\
0 \\
0 \\
0 \\
0 \\
0 \\
0 \\
0 \\
0 \\
0 \\
0 \\
0 \\
0 \\
0 \\
0 \\
0\end{array}$ & $\begin{array}{r}0 \\
0 \\
0 \\
0 \\
0 \\
0 \\
0 \\
0 \\
0 \\
0 \\
0 \\
0 \\
0 \\
0 \\
0 \\
0 \\
00 \\
0 \\
0 \\
0 \\
0 \\
0 \\
0 \\
0 \\
0 \\
0\end{array}$ & $\begin{array}{l}z \\
z \\
= \\
= \\
z \\
= \\
= \\
= \\
= \\
z \\
z \\
= \\
z \\
z \\
=\end{array}$ \\
\hline \multicolumn{15}{|c|}{ Jittan but } \\
\hline
\end{tabular}

\begin{tabular}{|c|c|c|c|c|c|c|c|c|c|c|c|c|c|c|}
\hline 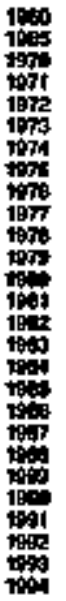 & 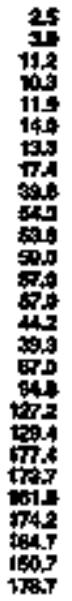 & $\begin{array}{l}04 \\
00 \\
00 \\
00 \\
00 \\
00 \\
00 \\
00 \\
09 \\
000 \\
00 \\
00 \\
000 \\
000 \\
000 \\
00 \\
00 \\
00 \\
00 \\
00 \\
00 \\
00 \\
00 \\
00 \\
00 \\
00\end{array}$ & 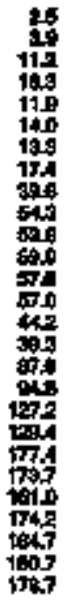 & 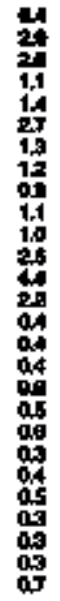 & 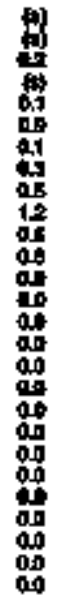 & 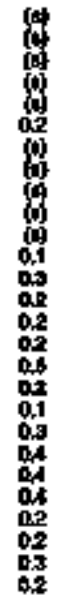 & 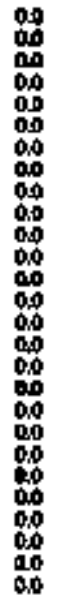 & 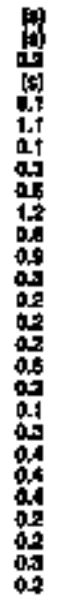 & 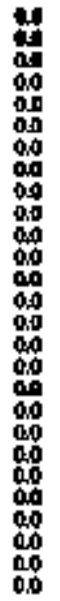 & 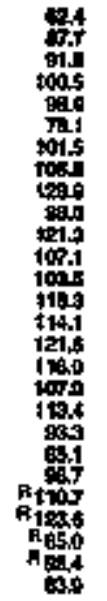 & 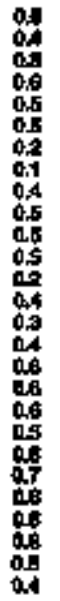 & 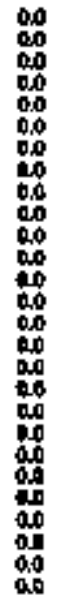 & 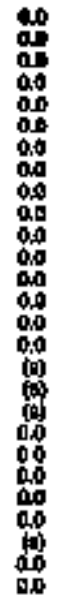 & 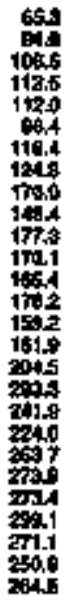 \\
\hline
\end{tabular}

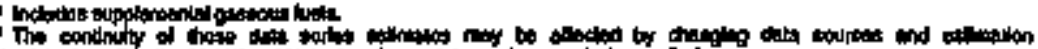

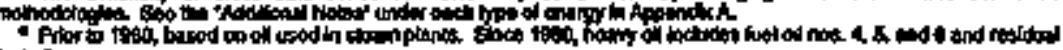

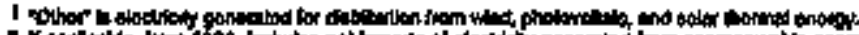

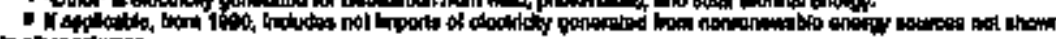

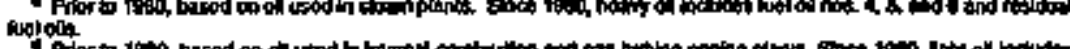
mations

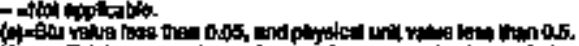

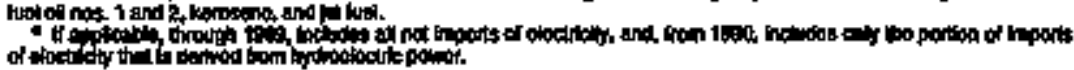


Table 179. Energy Consumption Eotimated by Sourct, 1960, 1985, 1970-1994, Kiebraska

\begin{tabular}{|c|c|c|c|c|c|c|c|c|c|c|c|c|c|c|c|c|c|c|c|}
\hline \multirow[b]{3}{*}{ Y. } & \multirow[b]{2}{*}{ Co: - } & \multirow[b]{2}{*}{ Mmer } & \multicolumn{11}{|c|}{ 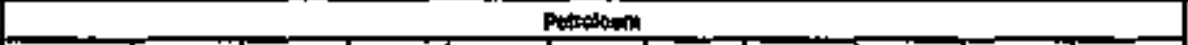 } & \multirow[b]{2}{*}{\begin{tabular}{|l|} 
Mocterer \\
Eefter \\
Promer
\end{tabular}} & \multirow[b]{2}{*}{ tats } & \multirow[b]{2}{*}{ ablent:- } & \multirow[b]{2}{*}{ ond ot } & \multirow{2}{*}{ 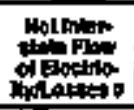 } & \multirow[b]{3}{*}{ toten } \\
\hline & & & Rophentand & Antons & 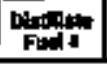 & Pats. & (4). & 노요 & conth & ativor & 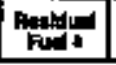 & Dathere & Tot: & & & & & & \\
\hline & Rowed & $\cos 10 F_{0}$ & \multicolumn{11}{|c|}{ 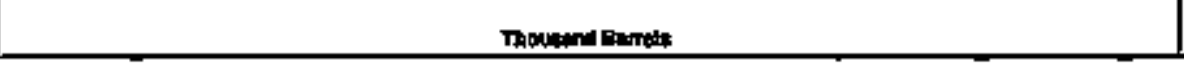 } & \multicolumn{5}{|c|}{ 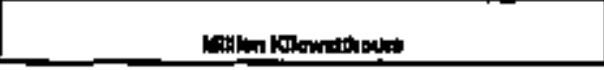 } & \\
\hline 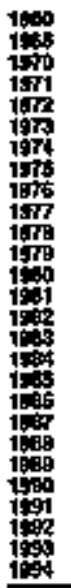 & 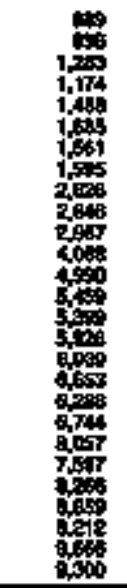 & 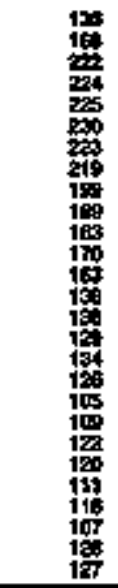 & 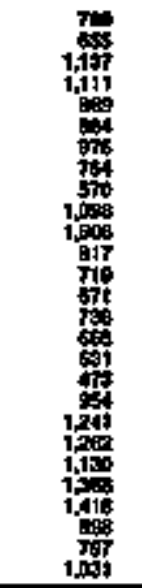 & 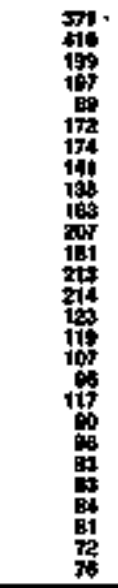 & 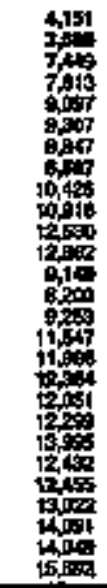 & 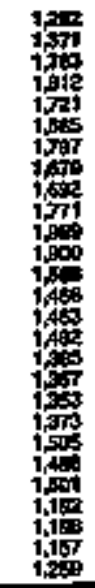 & 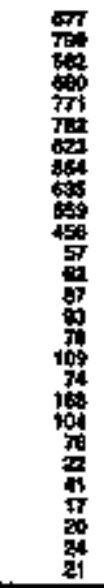 & 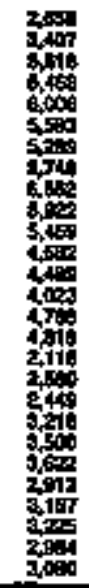 & 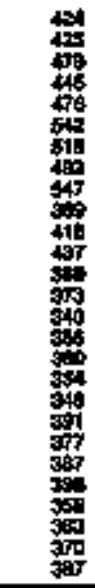 & 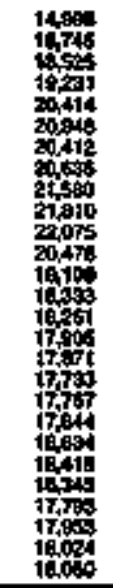 & 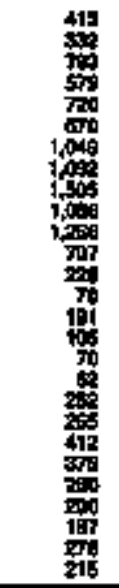 & 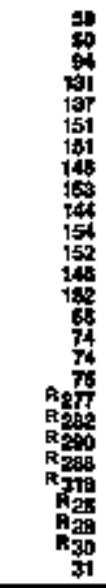 & 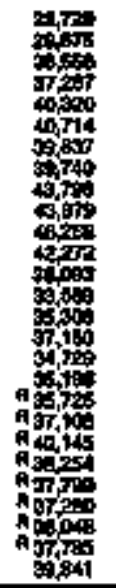 & 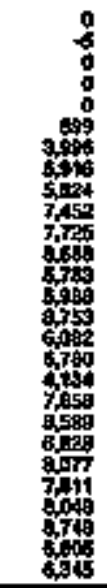 & 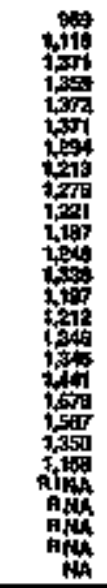 & 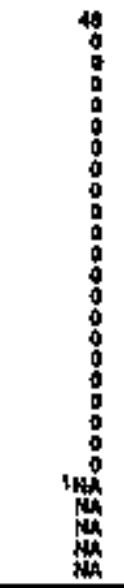 & $\begin{array}{l}0 \\
0 \\
0 \\
0 \\
0 \\
0 \\
0 \\
0 \\
0 \\
0 \\
0 \\
0 \\
0 \\
0 \\
0 \\
0 \\
0 \\
0 \\
0 \\
0 \\
0 \\
4 \\
4 \\
\text { M } \\
\end{array}$ & 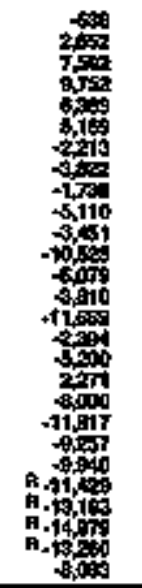 & $\begin{array}{l}= \\
z \\
z \\
z \\
z \\
z \\
= \\
z \\
z \\
z \\
z \\
=\end{array}$ \\
\hline \multicolumn{20}{|c|}{ 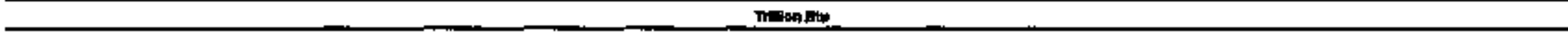 } \\
\hline 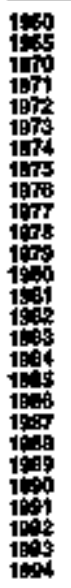 & 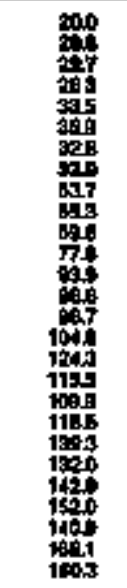 & 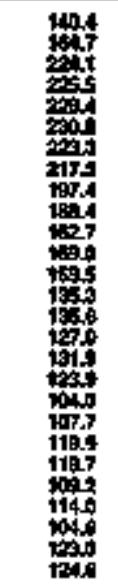 & 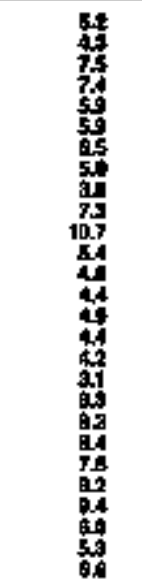 & 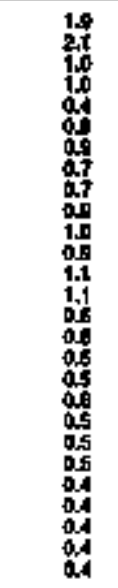 & 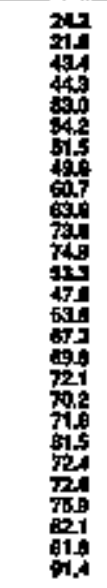 & 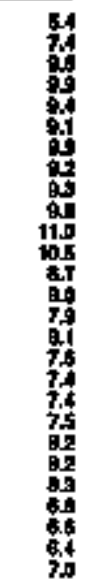 & 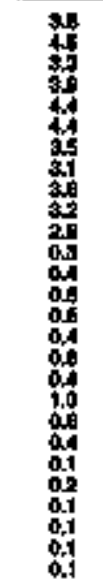 & 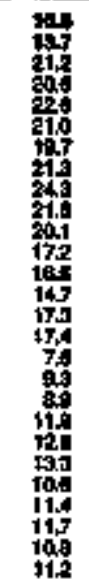 & 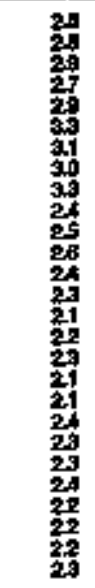 & 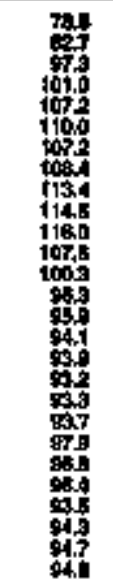 & 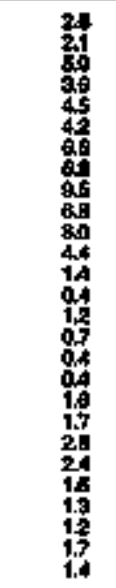 & 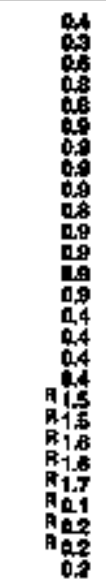 & 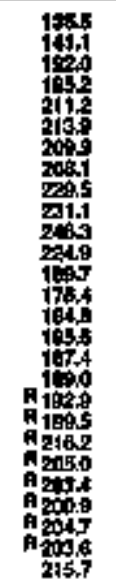 & 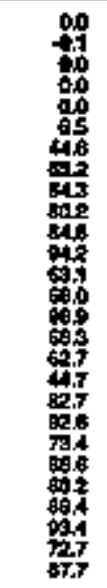 & 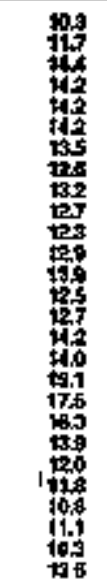 & 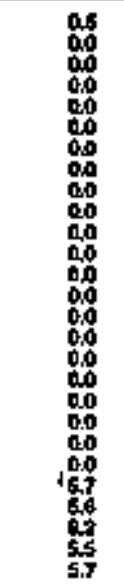 & 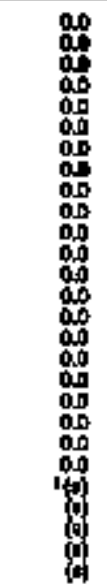 & 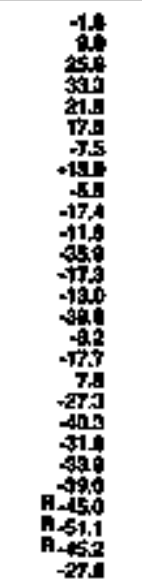 & 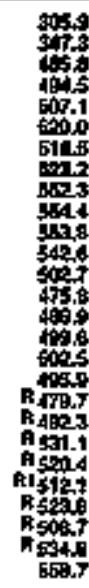 \\
\hline
\end{tabular}

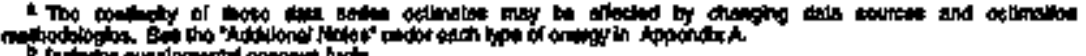

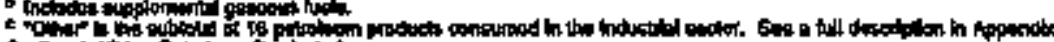

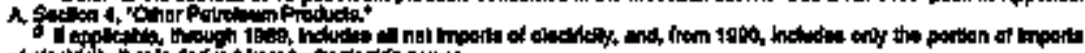

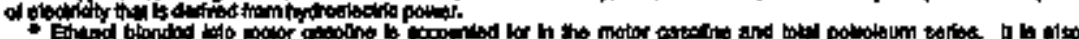

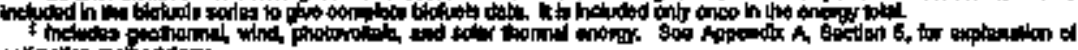

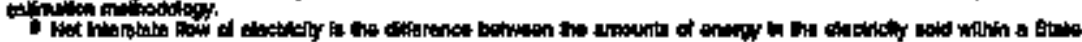

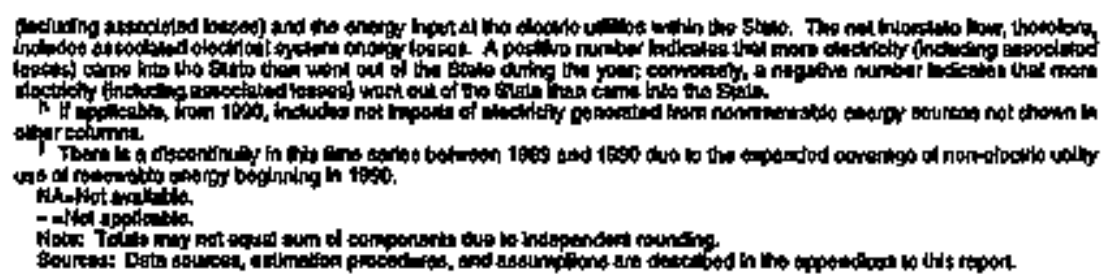


Table 180. Fesidential Entrgy Consumption Estimates, 1960, 1965, 1970-1994, Nobraska

\begin{tabular}{|c|c|c|c|c|c|c|c|c|c|c|c|c|c|c|}
\hline \multirow[b]{3}{*}{ Vow } & \multicolumn{3}{|c|}{ cond } & \multirow{3}{*}{ 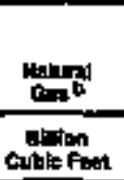 } & \multicolumn{4}{|c|}{ Petrosentm } & \multirow{3}{*}{ Diotuet } & & \multirow[b]{2}{*}{ Dectritethy a } & \multirow[b]{2}{*}{ 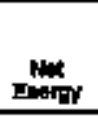 } & \multirow{3}{*}{ 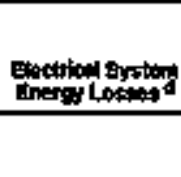 } & \multirow[b]{3}{*}{ Tow } \\
\hline & 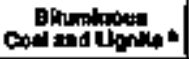 & Antheothat I & Toter & & 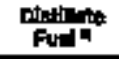 & Kessenenen & LPE: & Tol:01 & & sotur $c$ & & & & \\
\hline & \multicolumn{3}{|c|}{ Tironended shert Ton: } & & \multicolumn{4}{|c|}{ Thousind Amits } & & \multicolumn{3}{|c|}{ 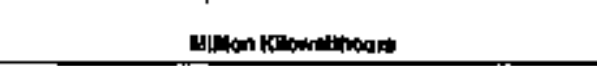 } & & \\
\hline 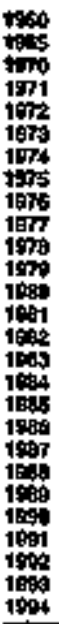 & 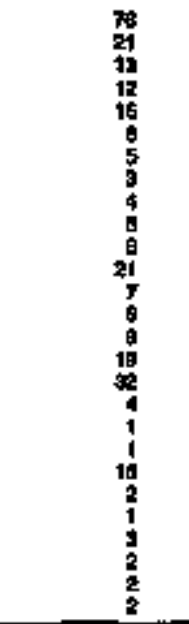 & $\begin{array}{l}0 \\
0 \\
0 \\
0 \\
0 \\
0 \\
0 \\
0 \\
0 \\
0 \\
0 \\
0 \\
0 \\
0 \\
1 \\
0 \\
0 \\
0 \\
0 \\
0 \\
0 \\
0 \\
2 \\
1 \\
0 \\
0 \\
0\end{array}$ & $\begin{array}{r}76 \\
21 \\
19 \\
15 \\
16 \\
6 \\
5 \\
3 \\
4 \\
6 \\
6 \\
21 \\
7 \\
6 \\
6 \\
30 \\
3 \\
4 \\
1 \\
1 \\
18 \\
2 \\
1 \\
5 \\
3 \\
2 \\
2\end{array}$ & 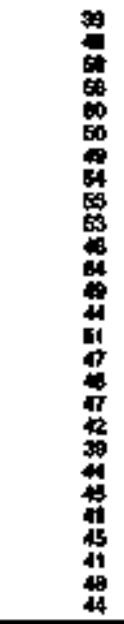 & 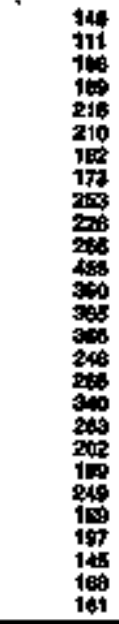 & 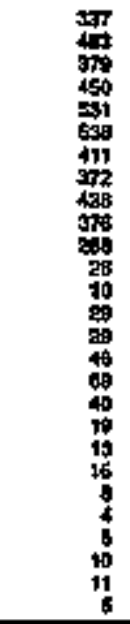 & 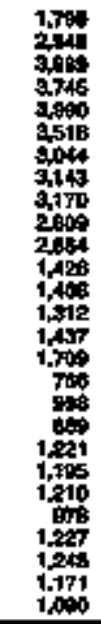 & 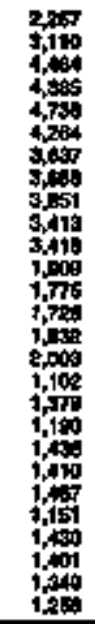 & $\begin{array}{r}0 \\
0 \\
0 \\
0 \\
0 \\
0 \\
0 \\
0 \\
0 \\
0 \\
0 \\
0 \\
0 \\
0 \\
0 \\
0 \\
0 \\
0 \\
0 \\
0 \\
0 \\
-201 \\
212 \\
200 \\
109 \\
102 \\
102\end{array}$ & $\begin{array}{r}0 \\
0 \\
0 \\
0 \\
0 \\
0 \\
0 \\
0 \\
0 \\
0 \\
0 \\
0 \\
0 \\
0 \\
0 \\
0 \\
0 \\
0 \\
2 \\
2 \\
2 \\
3 \\
4\end{array}$ & 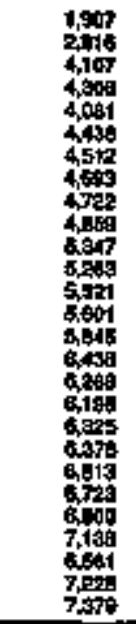 & $\begin{array}{l}= \\
= \\
= \\
= \\
= \\
= \\
= \\
= \\
= \\
= \\
= \\
= \\
= \\
= \\
=\end{array}$ & 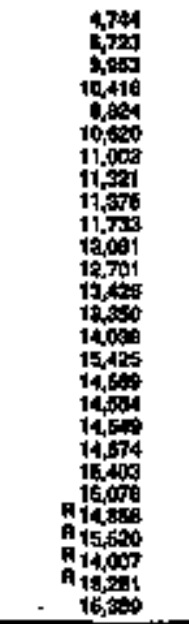 & $\begin{array}{l}= \\
= \\
= \\
= \\
= \\
= \\
= \\
= \\
= \\
= \\
= \\
= \\
= \\
=\end{array}$ \\
\hline \multicolumn{15}{|c|}{ TARan Gu } \\
\hline 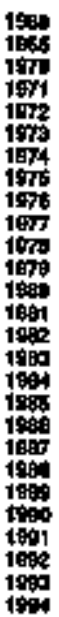 & 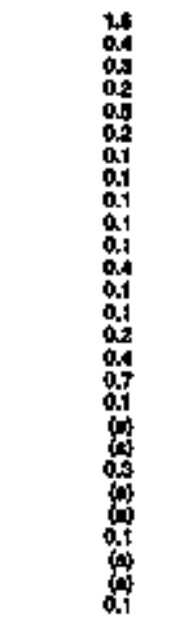 & 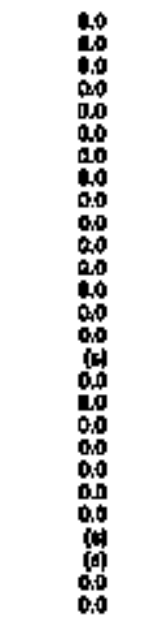 & 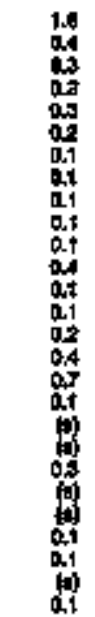 & 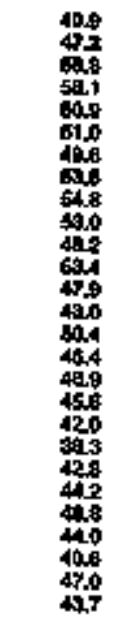 & 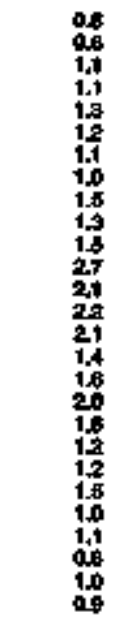 & 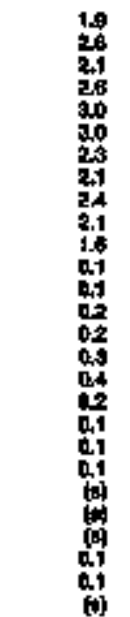 & 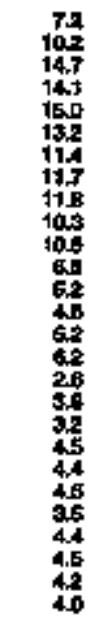 & 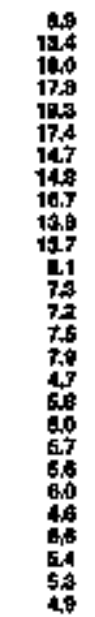 & 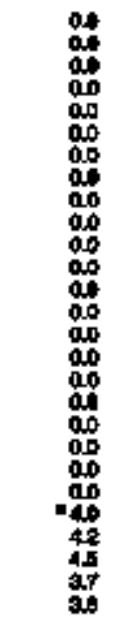 & 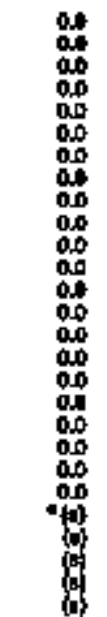 & 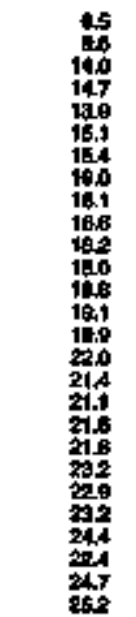 & 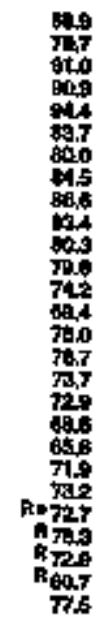 & 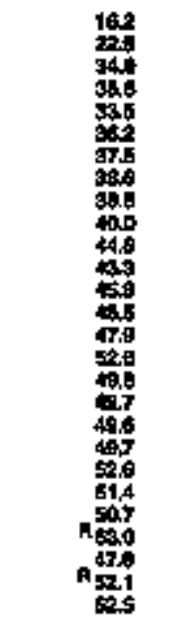 & 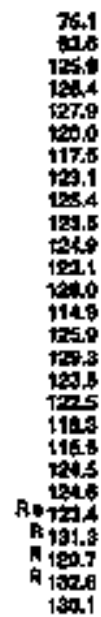 \\
\hline
\end{tabular}

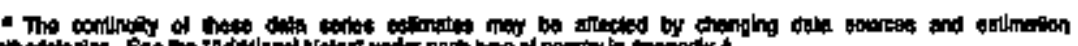

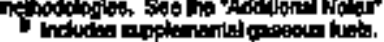


Table 181. Commercial Energy Consumption Estimales, 1980, 1955, 1970-1994, Nebraska

\begin{tabular}{|c|c|c|c|c|c|c|c|c|c|c|c|c|c|c|}
\hline \multirow[b]{3}{*}{ rear } & \multicolumn{3}{|c|}{ Dal } & \multirow[b]{2}{*}{ 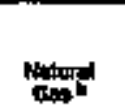 } & \multicolumn{6}{|c|}{ Petrolieun } & \multirow[b]{2}{*}{ Eartely " } & \multirow[b]{2}{*}{ ting } & \multirow[b]{2}{*}{ 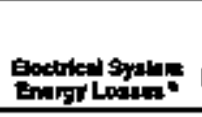 } & \multirow[b]{3}{*}{ Trow } \\
\hline & costuningous & Antadian-1 & Tot: & & 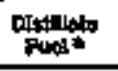 & xamanos & LPQ: & (1) & Prostunt & 7otat & & & & \\
\hline & \multicolumn{3}{|c|}{ Theorand Shant 7 ort } & pillon, & \multicolumn{6}{|c|}{ 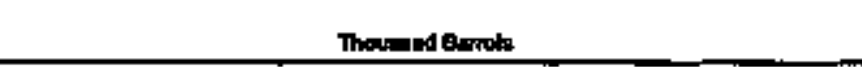 } & \multicolumn{3}{|c|}{ 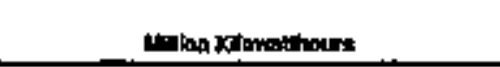 } & \\
\hline 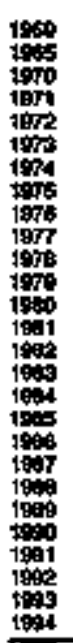 & 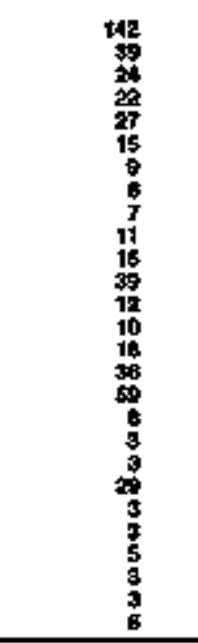 & $\begin{array}{l}0 \\
0 \\
0 \\
0 \\
0 \\
0 \\
0 \\
0 \\
0 \\
0 \\
0 \\
0 \\
0 \\
0 \\
0 \\
0 \\
0 \\
0 \\
0 \\
0 \\
0 \\
0 \\
0 \\
0 \\
0 \\
0 \\
1 \\
1 \\
0 \\
0 \\
0\end{array}$ & 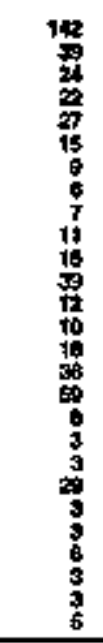 & 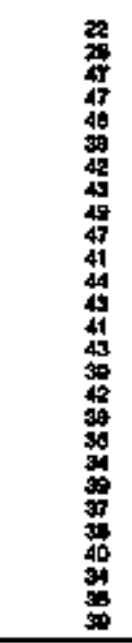 & 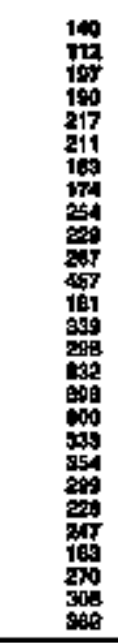 & 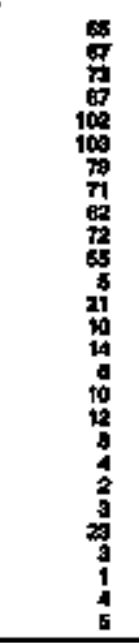 & 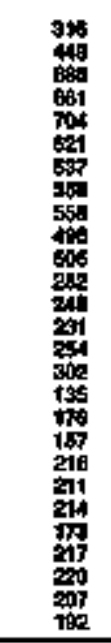 & 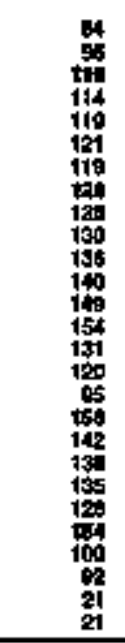 & 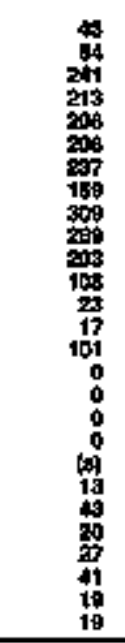 & 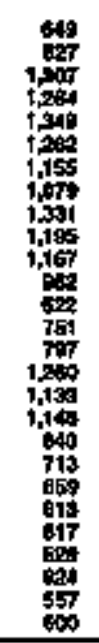 & 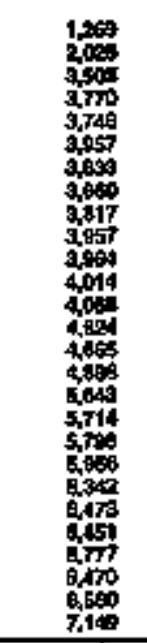 & $\begin{array}{l}\vdots \\
\vdots \\
\vdots \\
\vdots \\
\vdots \\
\vdots \\
\vdots \\
\vdots \\
\vdots \\
\vdots \\
\vdots \\
\vdots \\
\vdots \\
\vdots\end{array}$ & 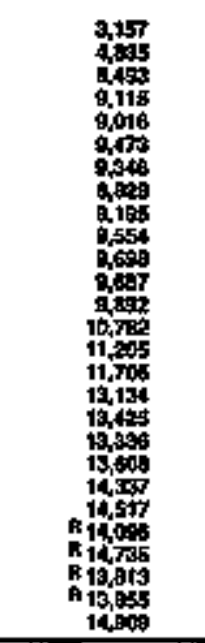 & $\begin{array}{l}z \\
\vdots \\
z \\
\vdots \\
\vdots \\
z \\
\vdots \\
\vdots \\
\vdots \\
\vdots \\
\vdots \\
\vdots \\
z\end{array}$ \\
\hline \multicolumn{15}{|c|}{ natition $\cos$} \\
\hline 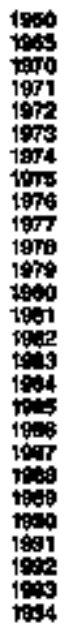 & 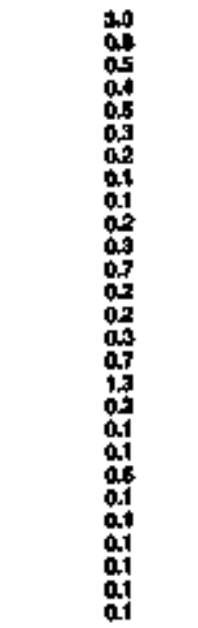 & 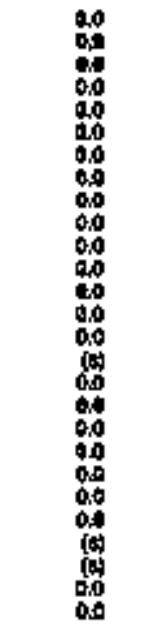 & 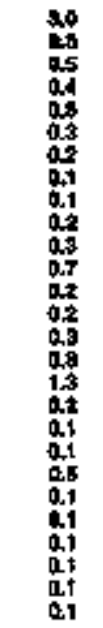 & 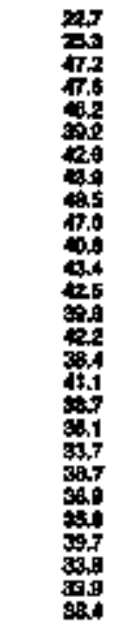 & 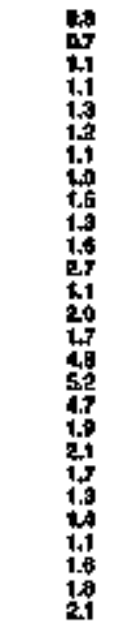 & 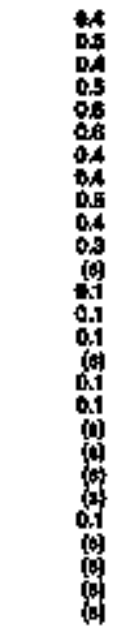 & 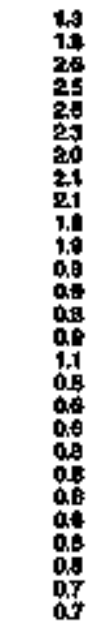 & 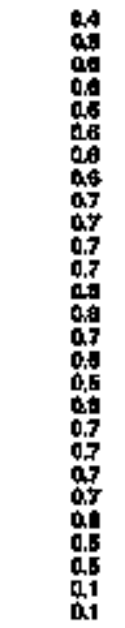 & 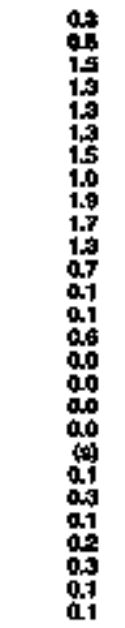 & 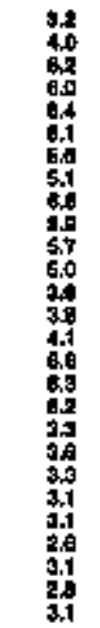 & 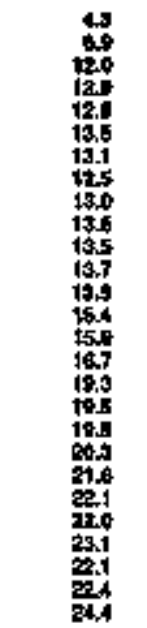 & 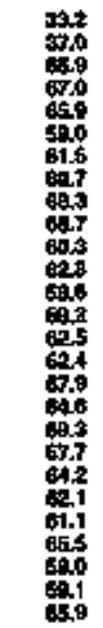 & 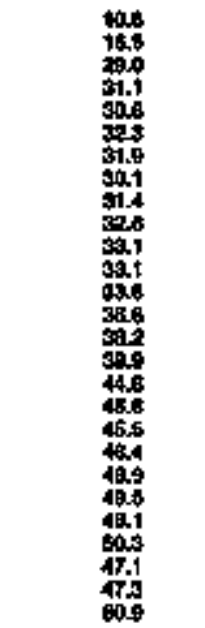 & 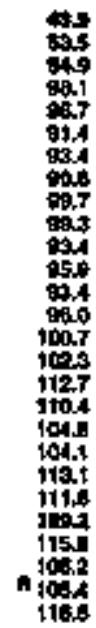 \\
\hline
\end{tabular}

$\mathbf{N}$
$\mathbf{E}$
$\mathbf{B}$
$\mathbf{R}$
$\mathbf{A}$
$\mathbf{S}$
$\mathbf{K}$
$\mathbf{A}$ 
Table t92. Industrial Energy Cansumplion Eatimates, 1960, 1985, 1970-1994, Nebraska

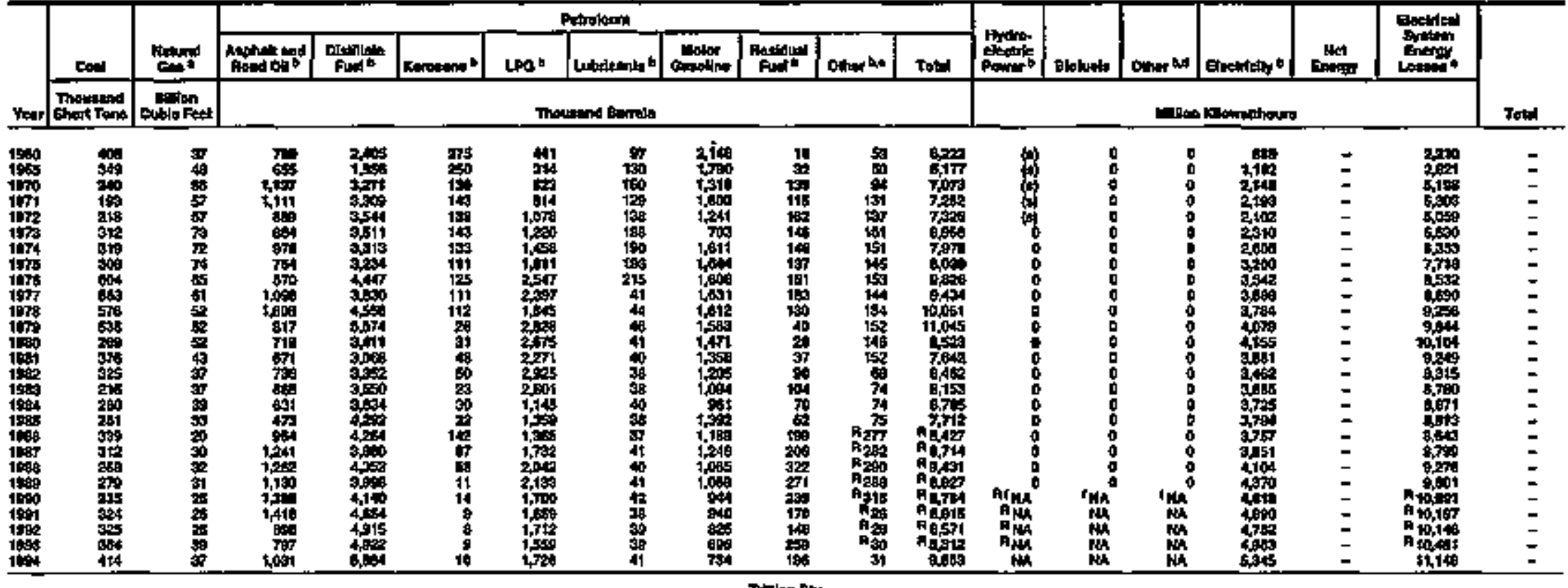

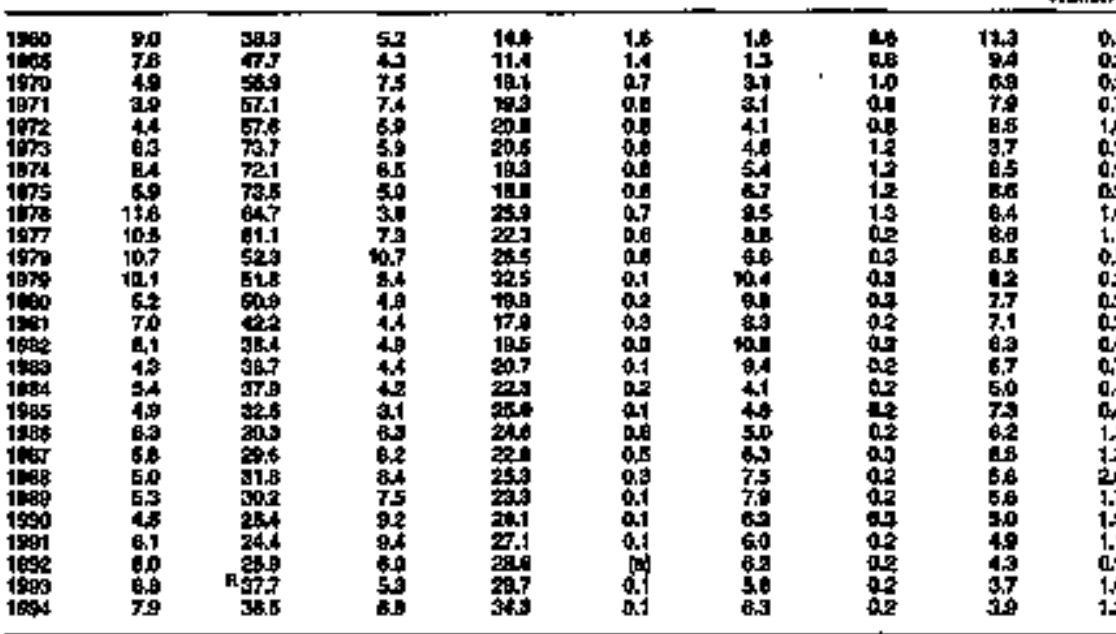

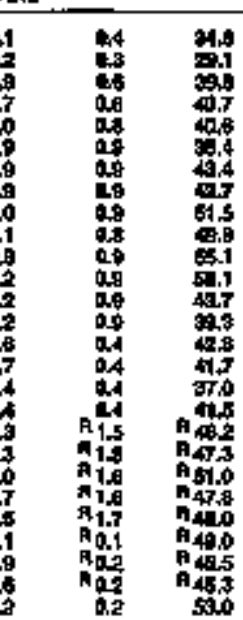

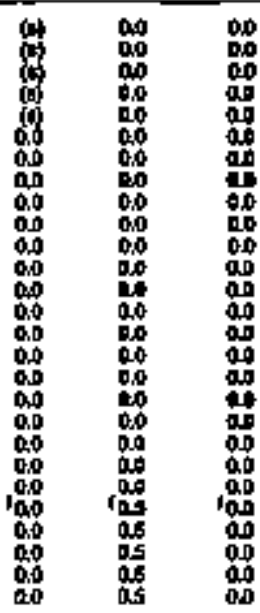

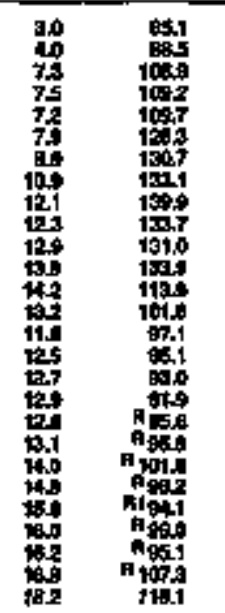

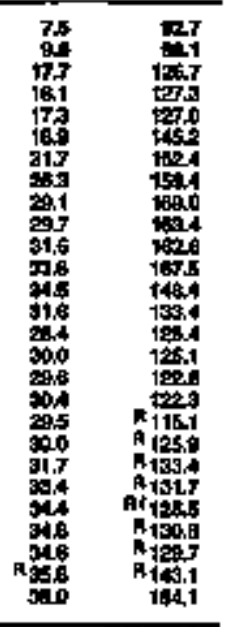

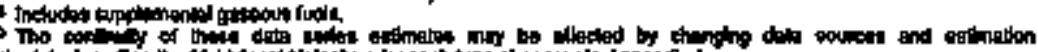

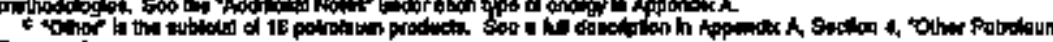

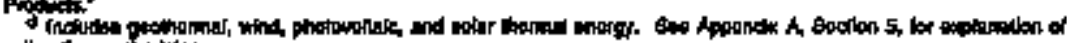

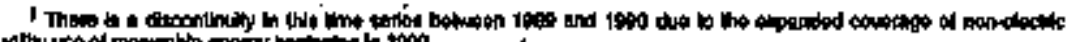

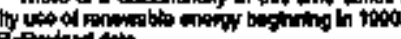

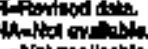

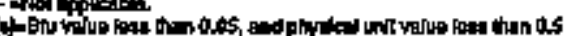

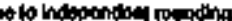

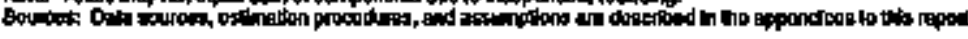

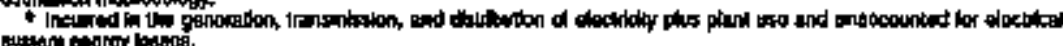


Table t63. Transportation Encrgy Cansumption Estimates, 1960, 1955, 1970-1994, Hehrasks

\begin{tabular}{|c|c|c|c|c|c|c|c|c|c|c|c|c|c|c|c|}
\hline \multirow[b]{3}{*}{ rat } & \multirow[b]{2}{*}{ Cost 1} & \multirow[b]{2}{*}{ Mationt } & \multicolumn{8}{|c|}{ Purrotion } & \multirow{3}{*}{ 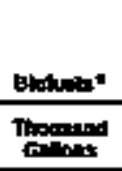 } & & \multirow[b]{2}{*}{ Esty } & \multirow[b]{2}{*}{ 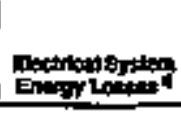 } & \multirow[b]{3}{*}{ Toto } \\
\hline & & & Awation. & Dathe & nit. & APG: & 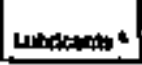 & motom & Fented & rest & & Eoctidif " & & & \\
\hline & mousind & 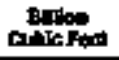 & \multicolumn{8}{|c|}{ 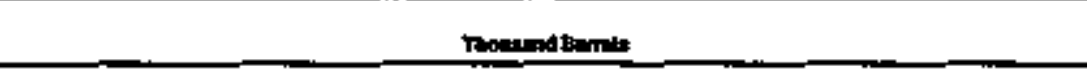 } & & \multicolumn{3}{|c|}{ 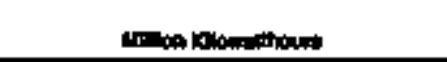 } & \\
\hline 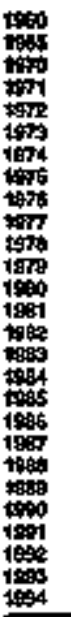 & $\begin{array}{l}7 \\
1 \\
0 \\
90 \\
0 \\
0 \\
0 \\
0 \\
090 \\
0 \\
0 \\
0 \\
0 \\
0 \\
0 \\
0 \\
0 \\
0 \\
0 \\
0 \\
0 \\
0 \\
0 \\
0 \\
0\end{array}$ & 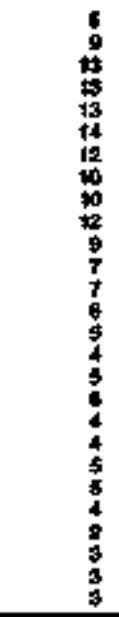 & 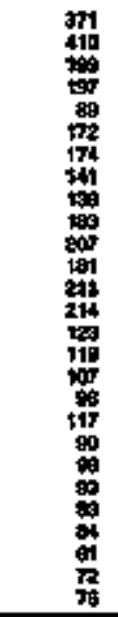 & 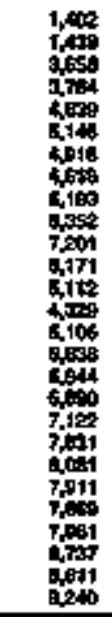 & 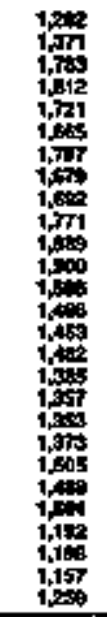 & 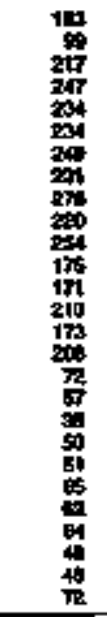 & 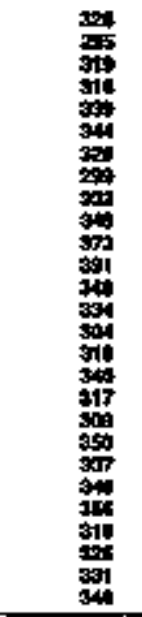 & 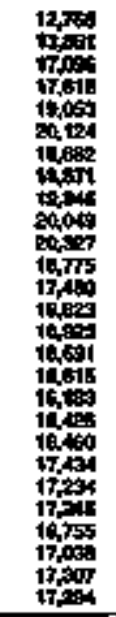 & 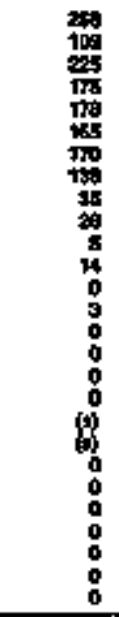 & 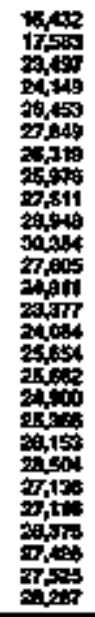 & 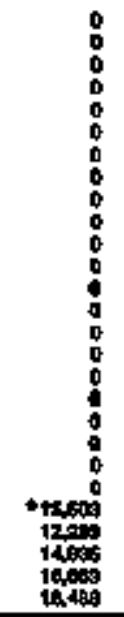 & $\begin{array}{l}0 \\
0 \\
0 \\
0 \\
0 \\
0 \\
0 \\
0 \\
0 \\
0 \\
0 \\
0 \\
0 \\
0 \\
0 \\
0 \\
0 \\
0 \\
0 \\
0 \\
0 \\
0 \\
0 \\
0 \\
0\end{array}$ & $\begin{array}{l}= \\
= \\
= \\
= \\
= \\
= \\
= \\
= \\
= \\
z \\
z \\
z \\
= \\
=\end{array}$ & $\begin{array}{l}0 \\
0 \\
0 \\
0 \\
0 \\
0 \\
0 \\
0 \\
0 \\
0 \\
0 \\
0 \\
0 \\
0 \\
0 \\
0 \\
0 \\
0 \\
0 \\
0 \\
0 \\
0 \\
0 \\
0 \\
0\end{array}$ & $\begin{array}{l}= \\
z \\
z \\
z \\
z \\
z \\
z \\
z \\
z \\
z \\
z \\
z \\
z \\
z\end{array}$ \\
\hline \multicolumn{16}{|c|}{$m$ ming ben } \\
\hline 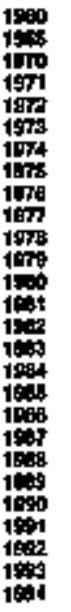 & $\begin{array}{l}02 \\
00 \\
60 \\
09 \\
0 \\
60 \\
0 \\
0 \\
00 \\
00 \\
00 \\
00 \\
00 \\
00 \\
00 \\
00 \\
00 \\
00 \\
00 \\
00 \\
00 \\
00 \\
00 \\
00 \\
00 \\
00\end{array}$ & 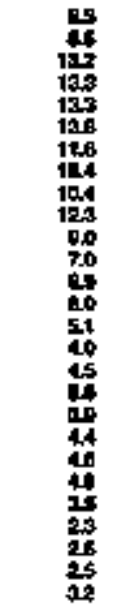 & 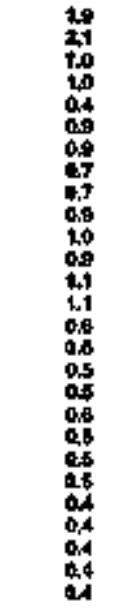 & 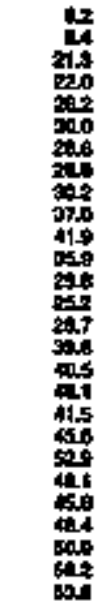 & 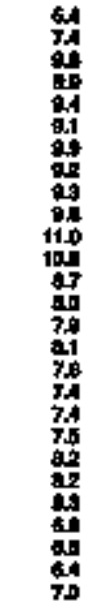 & 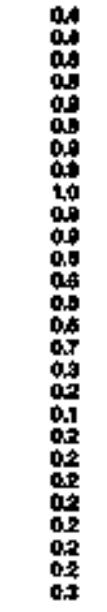 & 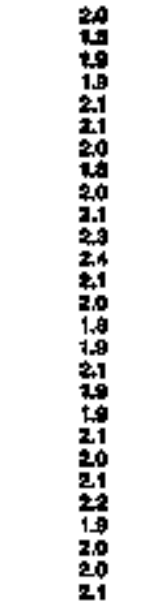 & 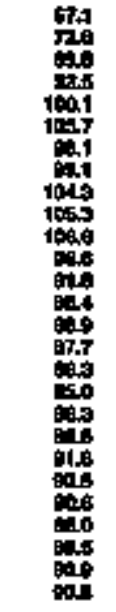 & 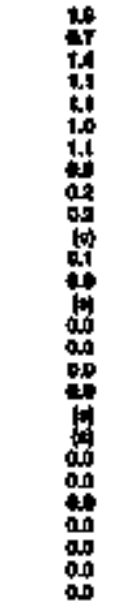 & 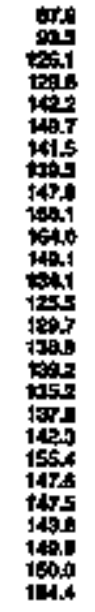 & $\begin{array}{l}00 \\
00 \\
00 \\
00 \\
000 \\
00 \\
00 \\
00 \\
00 \\
00 \\
00 \\
00 \\
000 \\
00 \\
00 \\
00 \\
00 \\
00 \\
00 \\
00 \\
00 \\
00 \\
0.0 \\
0.0 \\
1.9 \\
1.4 \\
1.4\end{array}$ & $\begin{array}{l}00 \\
00 \\
000 \\
000 \\
000 \\
000 \\
000 \\
000 \\
00 \\
000 \\
000 \\
000 \\
000 \\
00 \\
00 \\
00 \\
00 \\
00 \\
00 \\
00 \\
000 \\
00 \\
00 \\
00 \\
00 \\
00\end{array}$ & 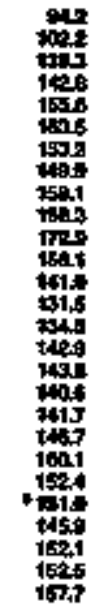 & 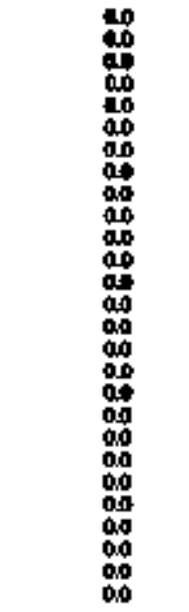 & 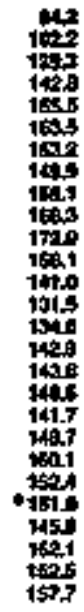 \\
\hline
\end{tabular}

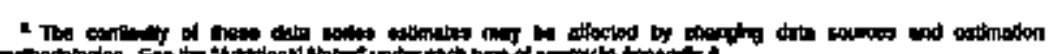

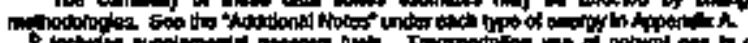
ges conanod the the peration of

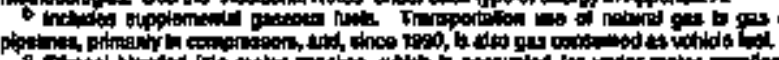

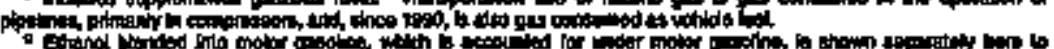

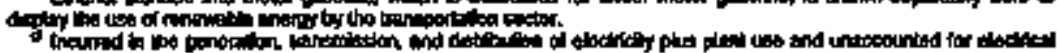

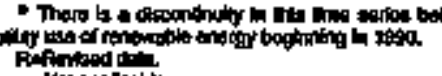

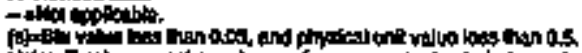

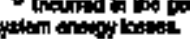

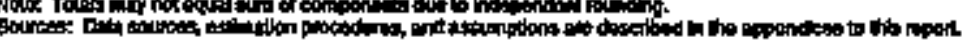


Table t94. Estimates of Energy Input at Eeotrto Uilities, 1960, 1965, 1970-1994, Nabraska

\begin{tabular}{|c|c|c|c|c|c|c|c|c|c|c|c|c|c|c|}
\hline & \multicolumn{3}{|c|}{$\cos$} & \multirow[b]{2}{*}{ Hrturd } & \multicolumn{4}{|c|}{ Pulliterate } & \multirow[b]{2}{*}{ 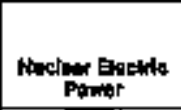 } & \multirow[b]{2}{*}{ Mydrowlocite } & \multirow[b]{2}{*}{ Binfuop } & \multirow[b]{2}{*}{ 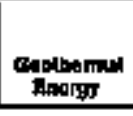 } & \multirow{3}{*}{ Obenof } & \multirow[b]{3}{*}{ Total I } \\
\hline & 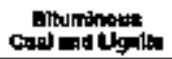 & Anturation & Tot & & thes & 넝 & Potroluin & Tolal & & & & & & \\
\hline vear & \multicolumn{3}{|c|}{ Thovand Shent Tond } & culderefoul & \multicolumn{4}{|c|}{ 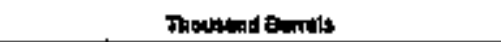 } & \multicolumn{4}{|c|}{ 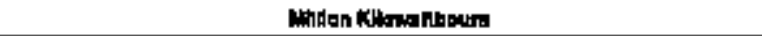 } & & \\
\hline
\end{tabular}

\begin{tabular}{|c|c|c|c|c|c|c|c|c|c|c|c|c|c|c|}
\hline 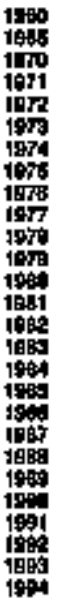 & 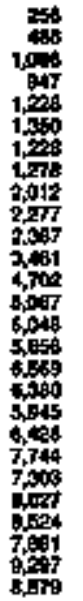 & $\begin{array}{l}0 \\
0 \\
0 \\
0 \\
0 \\
0 \\
0 \\
0 \\
0 \\
0 \\
0 \\
0 \\
0 \\
0 \\
0 \\
0 \\
0 \\
0 \\
0 \\
0 \\
0 \\
0 \\
0 \\
0 \\
0 \\
0 \\
0\end{array}$ & 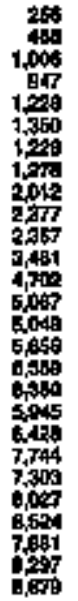 & 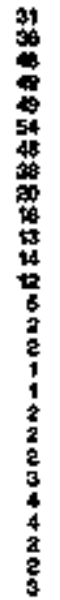 & 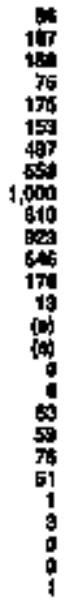 & 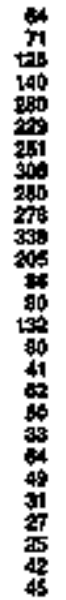 & $\begin{array}{l}0 \\
0 \\
0 \\
0 \\
0 \\
0 \\
0 \\
0 \\
0 \\
0 \\
0 \\
0 \\
0 \\
0 \\
0 \\
0 \\
0 \\
0 \\
0 \\
0 \\
0 \\
0 \\
0 \\
0 \\
0 \\
0 \\
0 \\
0\end{array}$ & 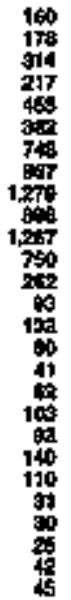 & 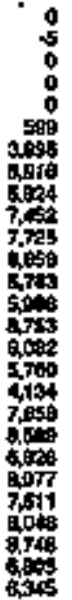 & 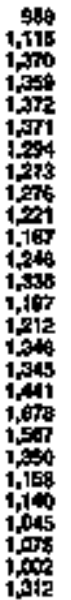 & $\begin{array}{l}40 \\
0 \\
0 \\
0 \\
0 \\
0 \\
0 \\
0 \\
0 \\
0 \\
0 \\
0 \\
0 \\
0 \\
0 \\
0 \\
0 \\
0 \\
0 \\
0 \\
0 \\
0 \\
0 \\
0 \\
0 \\
0 \\
0 \\
0\end{array}$ & $\begin{array}{l}0 \\
0 \\
0 \\
0 \\
0 \\
0 \\
0 \\
0 \\
0 \\
0 \\
0 \\
0 \\
0 \\
0 \\
0 \\
0 \\
0 \\
0 \\
0 \\
0 \\
0 \\
0 \\
0 \\
0 \\
0 \\
0\end{array}$ & $\begin{array}{l}0 \\
0 \\
0 \\
0 \\
0 \\
0 \\
0 \\
0 \\
0 \\
0 \\
0 \\
0 \\
0 \\
0 \\
0 \\
0 \\
0 \\
0 \\
0 \\
0 \\
0 \\
0 \\
0\end{array}$ & $\bar{z}$ \\
\hline
\end{tabular}

\begin{tabular}{|c|c|c|c|c|c|c|c|c|c|c|c|c|c|c|}
\hline 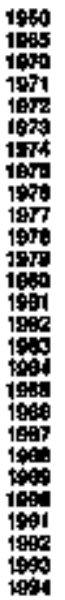 & 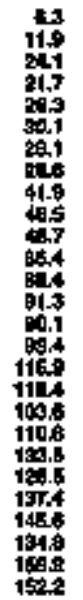 & 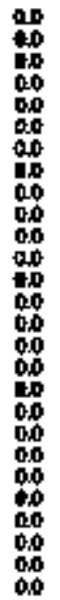 & 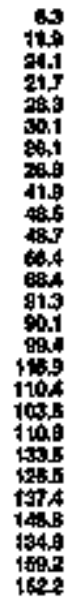 & 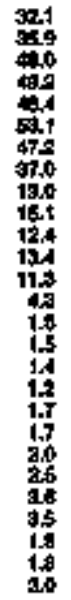 & 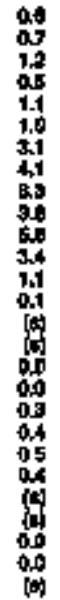 & 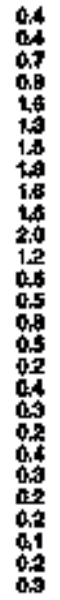 & $\begin{array}{l}10 \\
00 \\
00 \\
00 \\
00 \\
00 \\
00 \\
00 \\
00 \\
00 \\
00 \\
00 \\
000 \\
00 \\
00 \\
00 \\
00 \\
00 \\
00 \\
00 \\
00 \\
00 \\
00 \\
00 \\
00 \\
00 \\
00\end{array}$ & 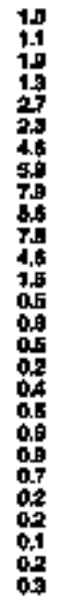 & 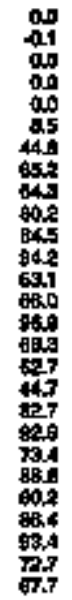 & 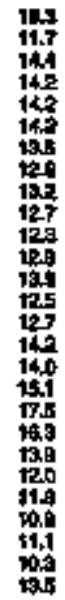 & 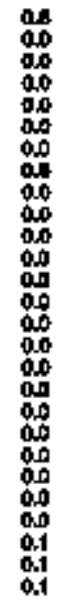 & 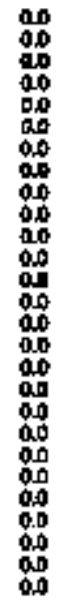 & 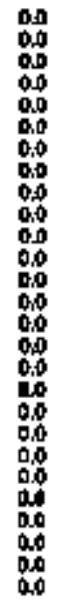 & 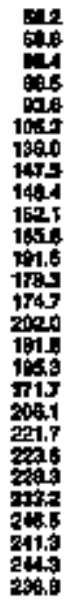 \\
\hline
\end{tabular}

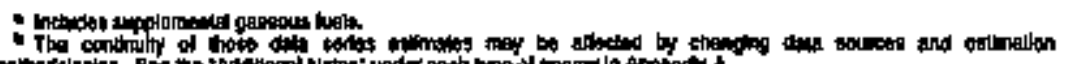

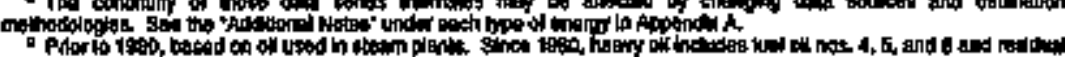

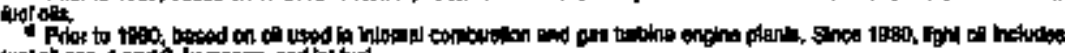

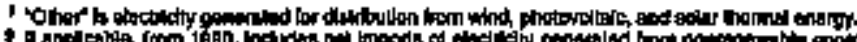

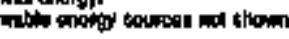

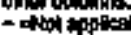

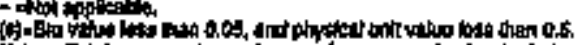

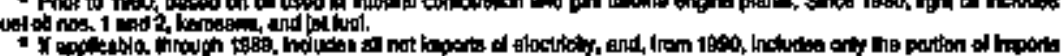

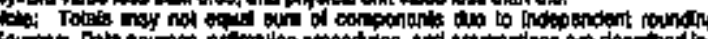

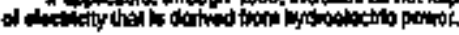


Table 1B3. Emargy Consumption Estimales by Scurce, 1960, 1965, 1970-1994, Nevada

\begin{tabular}{|c|c|c|c|c|c|c|c|c|c|c|c|c|c|c|c|c|c|c|c|}
\hline \multirow[b]{3}{*}{ You } & \multirow[b]{2}{*}{ Coods } & \multirow[b]{2}{*}{ 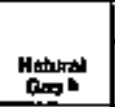 } & \multicolumn{11}{|c|}{ Potroleum } & \multirow{2}{*}{ 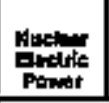 } & \multirow{2}{*}{ 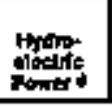 } & \multirow[b]{2}{*}{ mofielse } & \multirow[b]{2}{*}{ OAbet } & \multirow{3}{*}{ 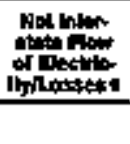 } & \multirow[b]{3}{*}{ Towat t } \\
\hline & & & 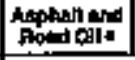 & Anters & Diaplonte & . & Kanos. & set & cerbit. & cholor & 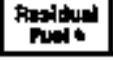 & Onem & Tolly & & & & & & \\
\hline & Thenentind & cition foel & \multicolumn{11}{|c|}{ 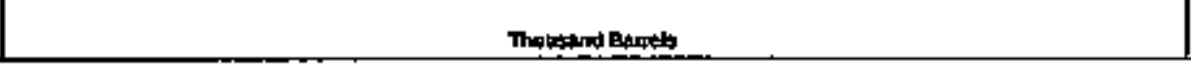 } & \multicolumn{4}{|c|}{ 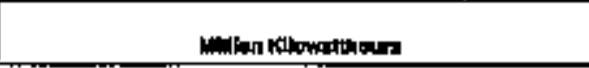 } & & \\
\hline 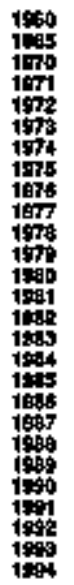 & 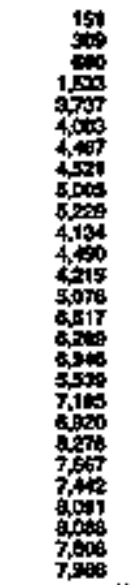 & 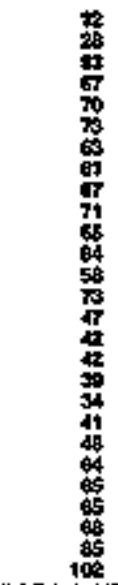 & 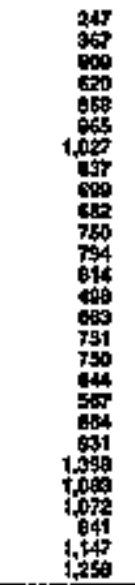 & 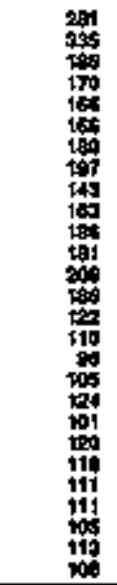 & 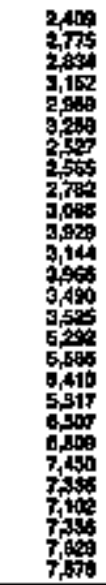 & 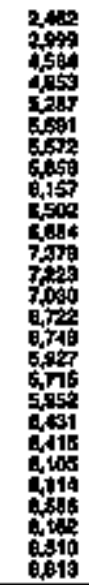 & 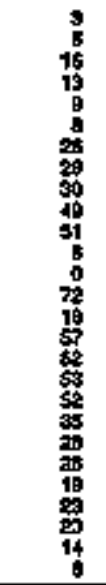 & 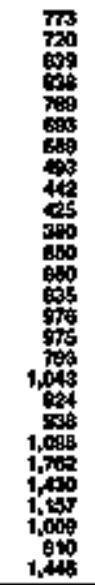 & 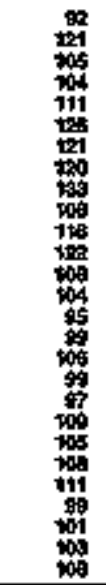 & 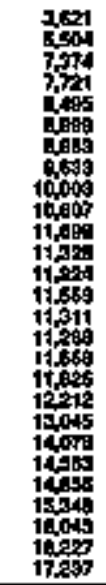 & 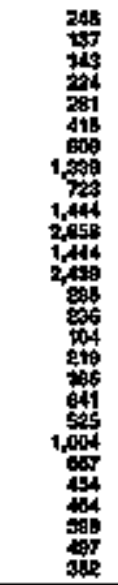 & 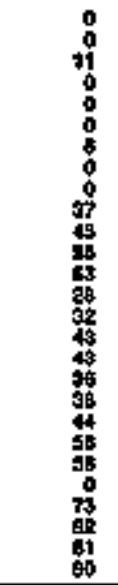 & 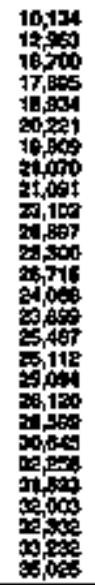 & $\begin{array}{l}0 \\
0 \\
0 \\
0 \\
0 \\
0 \\
0 \\
0 \\
0 \\
0 \\
0 \\
0 \\
0 \\
0 \\
0 \\
0 \\
0 \\
0 \\
0 \\
0 \\
0 \\
0 \\
0 \\
0 \\
0 \\
0 \\
0 \\
0\end{array}$ & 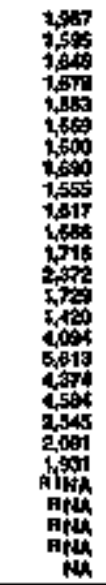 & $\begin{array}{l}0 \\
0 \\
0 \\
0 \\
0 \\
0 \\
0 \\
0 \\
0 \\
0 \\
0 \\
0 \\
0 \\
0 \\
0 \\
0 \\
0 \\
0 \\
0 \\
0 \\
0 \\
0 \\
1 \\
4 \\
4 \\
4 \\
4 \\
4\end{array}$ & $\begin{array}{l}0 \\
0 \\
0 \\
0 \\
0 \\
0 \\
0 \\
0 \\
0 \\
0 \\
0 \\
0 \\
0 \\
0 \\
0 \\
0 \\
0 \\
0 \\
0 \\
0 \\
4 \\
4 \\
4\end{array}$ & 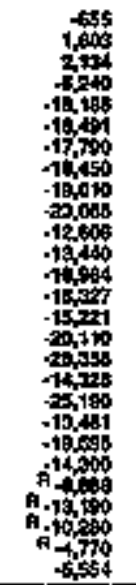 & $\begin{array}{l}z \\
z \\
z \\
\vdots \\
z \\
z \\
z \\
\vdots \\
z \\
z \\
z \\
z \\
z\end{array}$ \\
\hline
\end{tabular}

\begin{tabular}{|c|c|c|c|c|c|c|c|c|c|c|c|c|c|c|c|c|c|c|c|}
\hline 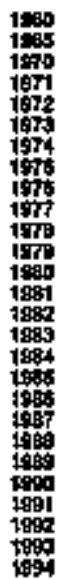 & 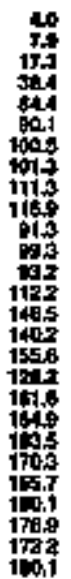 & 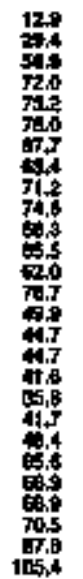 & 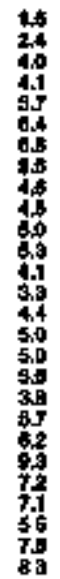 & 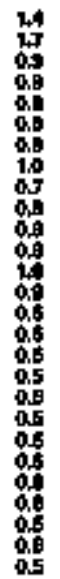 & 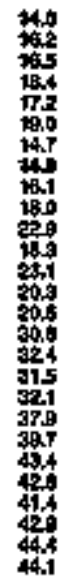 & 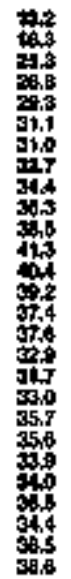 & 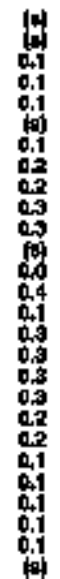 & 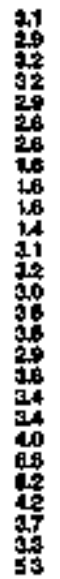 & 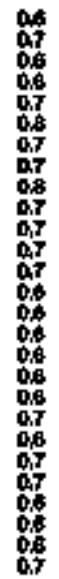 & 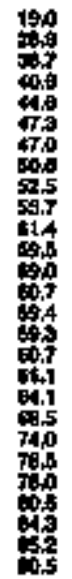 & 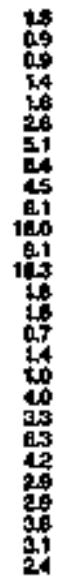 & 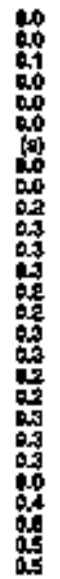 & 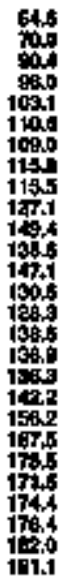 & 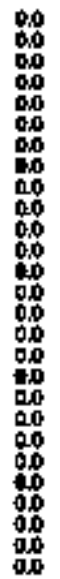 & 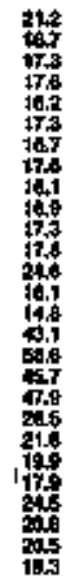 & 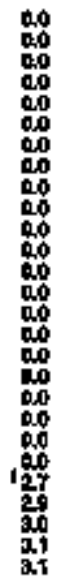 & 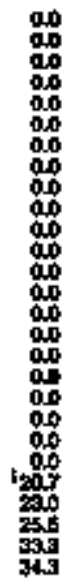 & 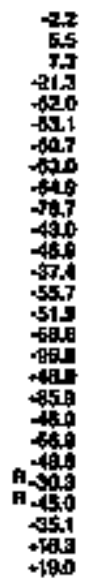 & 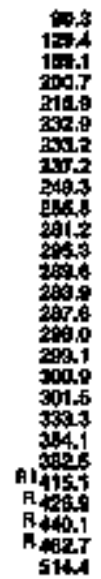 \\
\hline
\end{tabular}

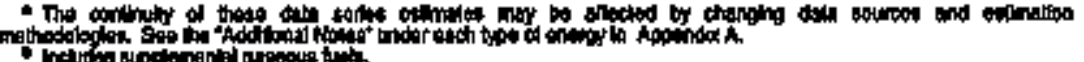

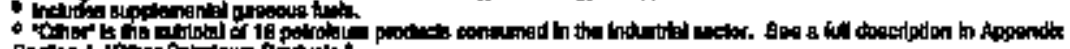

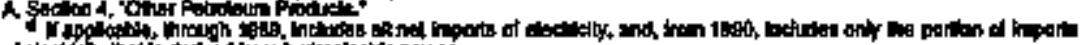

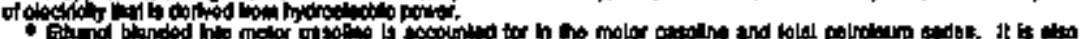

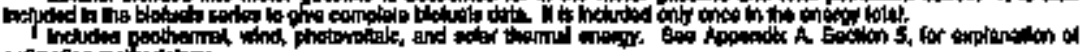

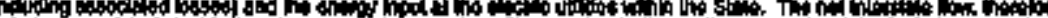

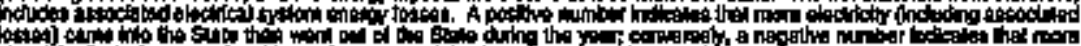

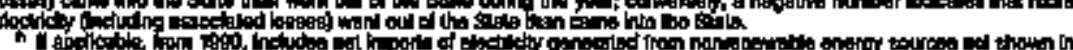

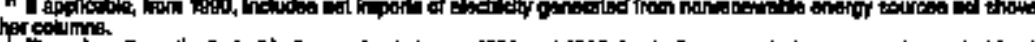

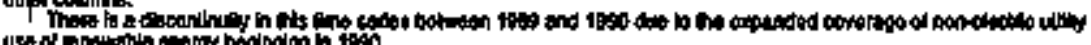

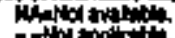

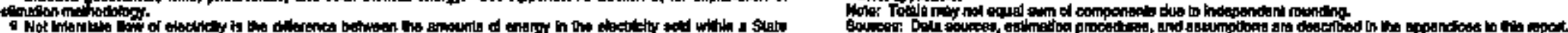


Table 186. Resldentlal Enorgy Consumption Estimatos, 1960, 1955, 1970-1994, Nevada

\begin{tabular}{|c|c|c|c|c|c|c|c|c|c|c|c|c|c|c|}
\hline \multirow[b]{3}{*}{ Yeat } & \multicolumn{3}{|c|}{ Cods } & \multirow{3}{*}{$\frac{\text { Nutura }}{\text { oteb }}$} & \multicolumn{4}{|c|}{ Potrolonen } & \multirow{3}{*}{ 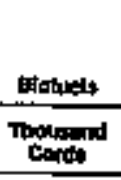 } & & \multirow[b]{2}{*}{ 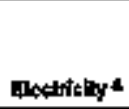 } & \multirow[b]{2}{*}{ Mot } & \multirow{3}{*}{ Extrich } & \multirow[b]{3}{*}{ Totol } \\
\hline & 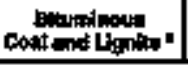 & Anthrantio: & The & & 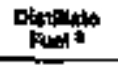 & Kerotoso I & 나무 & Toter. & & solate & & & & \\
\hline & \multicolumn{3}{|c|}{ Thoond thont Tond } & & \multicolumn{4}{|c|}{ 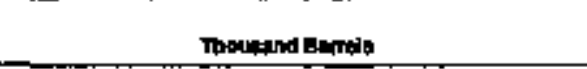 } & & \multicolumn{3}{|c|}{ Won Kisowethouts } & & \\
\hline 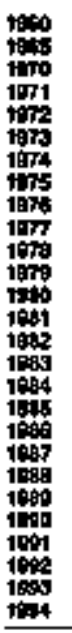 & 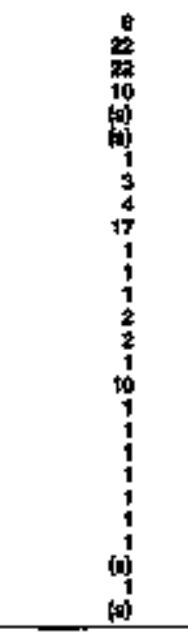 & $\begin{array}{l}1 \\
0 \\
i \\
2 \\
1 \\
1 \\
1 \\
1 \\
1 \\
1 \\
1 \\
01 \\
1 \\
0 \\
0 \\
0 \\
0 \\
0 \\
0 \\
0 \\
0 \\
1 \\
0 \\
0 \\
0 \\
0 \\
\end{array}$ & 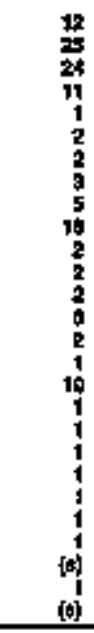 & 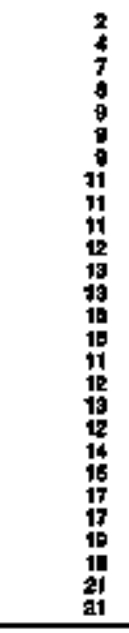 & 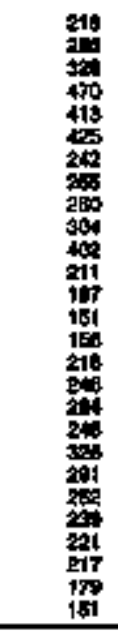 & $\begin{array}{l}0 \\
0 \\
0 \\
0 \\
0 \\
0 \\
0 \\
0 \\
0 \\
0 \\
0 \\
0 \\
36 \\
12 \\
43 \\
4 \\
40 \\
40 \\
17 \\
14 \\
10 \\
10 \\
11 \\
4 \\
\end{array}$ & 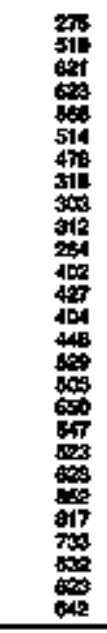 & 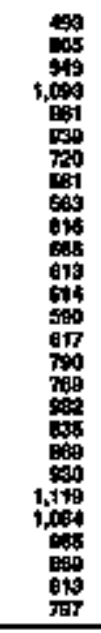 & 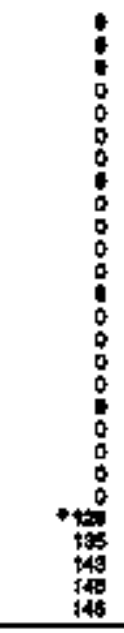 & 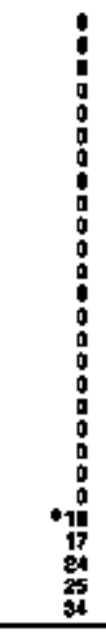 & 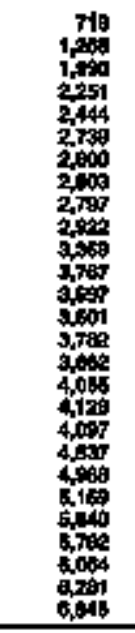 & $\begin{array}{l}= \\
= \\
= \\
= \\
= \\
= \\
= \\
= \\
= \\
= \\
= \\
= \\
= \\
= \\
=\end{array}$ & 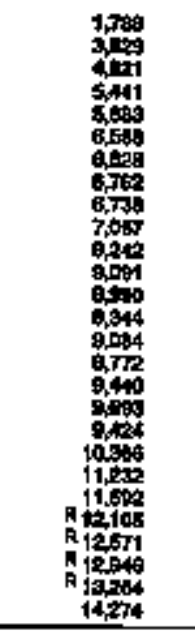 & $\begin{array}{l}= \\
= \\
z \\
z \\
= \\
= \\
z \\
= \\
= \\
z \\
z \\
z \\
z \\
z\end{array}$ \\
\hline \multicolumn{15}{|c|}{ Triston bu } \\
\hline 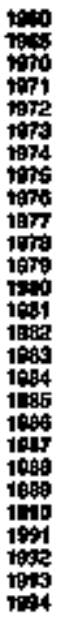 & 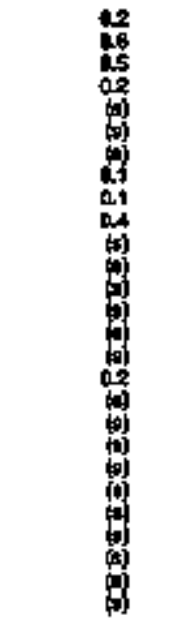 & 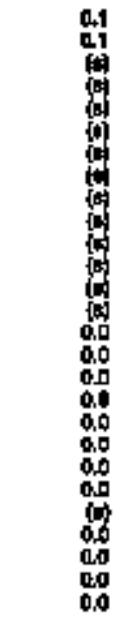 & 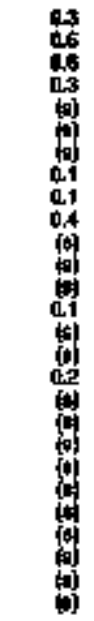 & 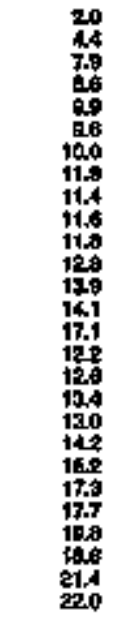 & 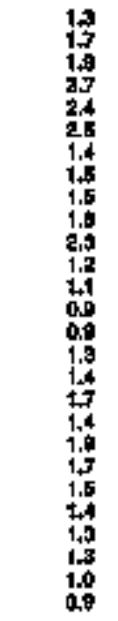 & 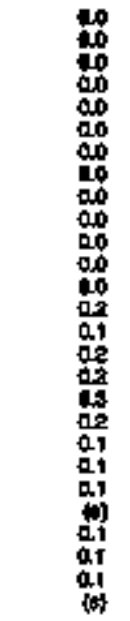 & 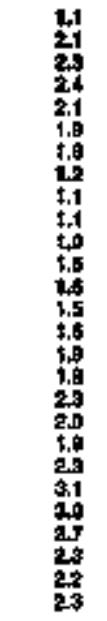 & 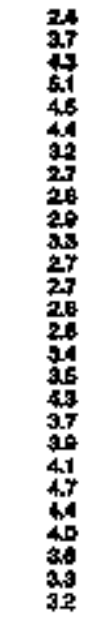 & $\begin{array}{l}00 \\
10 \\
00 \\
00 \\
00 \\
00 \\
00 \\
00 \\
00 \\
00 \\
00 \\
00 \\
00 \\
00 \\
00 \\
00 \\
00 \\
00 \\
00 \\
00 \\
00 \\
00 \\
00 \\
020 \\
27 \\
20 \\
30 \\
2.0\end{array}$ & $\begin{array}{l}00 \\
000 \\
00 \\
0.0 \\
00 \\
00 \\
00 \\
0.0 \\
00 \\
00 \\
0.0 \\
00 \\
00 \\
00 \\
00 \\
000 \\
0.0 \\
00 \\
00 \\
0.0 \\
00 \\
0.0 \\
0.1 \\
0.1 \\
0.1 \\
0.1 \\
0.1\end{array}$ & 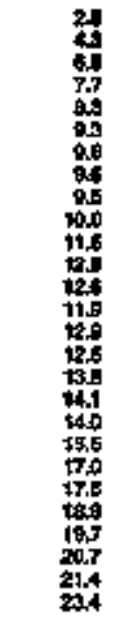 & 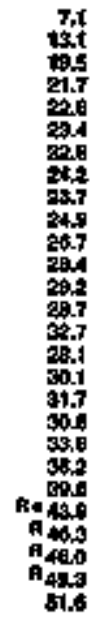 & 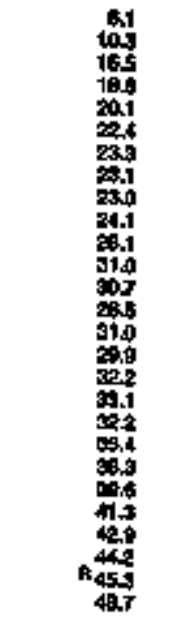 & 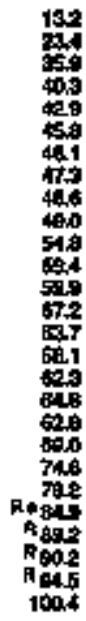 \\
\hline
\end{tabular}

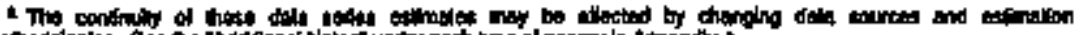

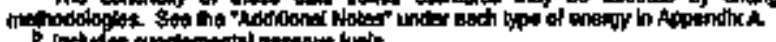

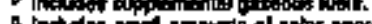

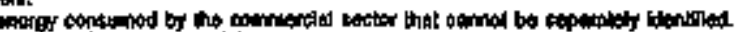

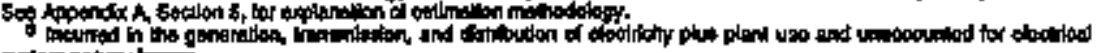

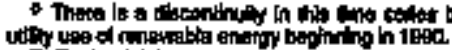
Rafiontepd doth

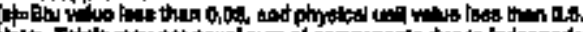

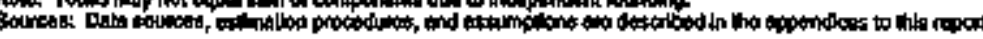


Table 187. Commerclal Energy Consumptlon Estimakes, 1960, 1965, 1970-1994, Nevada

\begin{tabular}{|c|c|c|c|c|c|c|c|c|c|c|c|c|c|c|}
\hline \multirow[b]{3}{*}{ rast } & \multicolumn{3}{|c|}{$\mathrm{Con}$} & \multirow{3}{*}{ 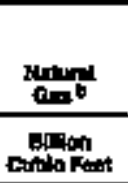 } & \multicolumn{6}{|c|}{ Potodoum } & \multirow[b]{2}{*}{ 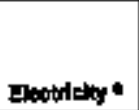 } & \multirow[b]{2}{*}{$e_{\text {notgry }}^{\text {nat }}$} & \multirow[b]{2}{*}{ 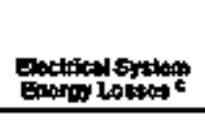 } & \multirow[b]{3}{*}{ Totol } \\
\hline & coniminifous & Antrofins: & Tow & & 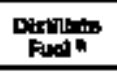 & Koroman:- & Lpe! & moter & 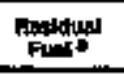 & rotes & & & & \\
\hline & \multicolumn{3}{|c|}{ Thourand gant Toun } & & \multicolumn{6}{|c|}{ Thoutand Barrals } & \multicolumn{3}{|c|}{ 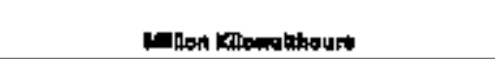 } & \\
\hline 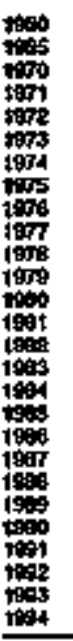 & 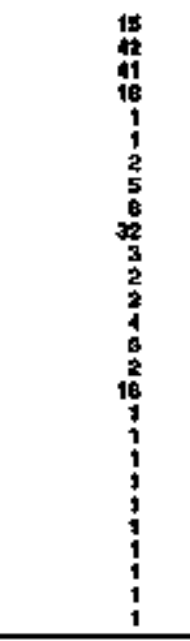 & $\begin{array}{r}3 \\
2 \\
1 \\
1 \\
1 \\
1 \\
1 \\
1 \\
9 \\
9 \\
0 \\
0 \\
0 \\
0 \\
0 \\
0 \\
0 \\
0 \\
0 \\
0 \\
0 \\
0 \\
0 \\
0 \\
0\end{array}$ & 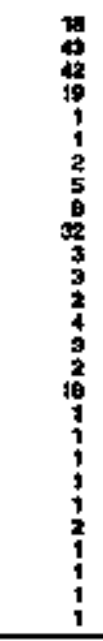 & 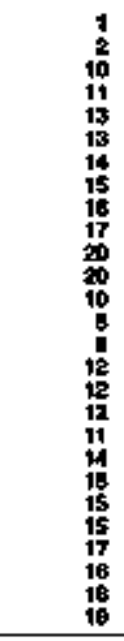 & 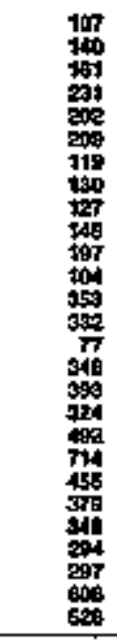 & 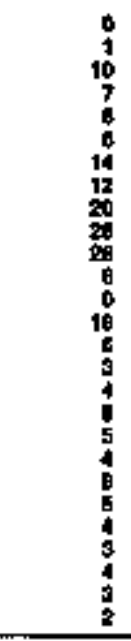 & 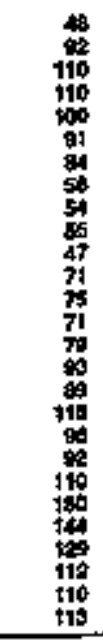 & 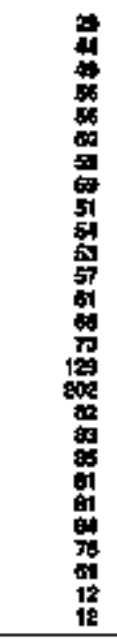 & 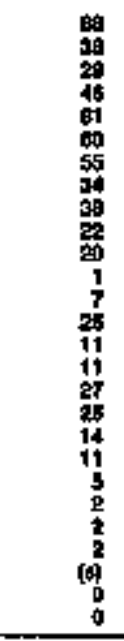 & 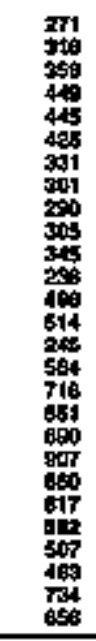 & 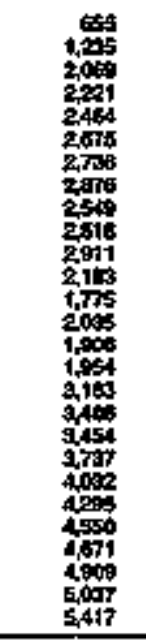 & $\begin{array}{l}\vdots \\
\vdots \\
\vdots \\
\vdots \\
\vdots \\
\vdots \\
\vdots \\
\vdots \\
\vdots \\
\vdots \\
\vdots \\
\vdots \\
\vdots \\
\end{array}$ & 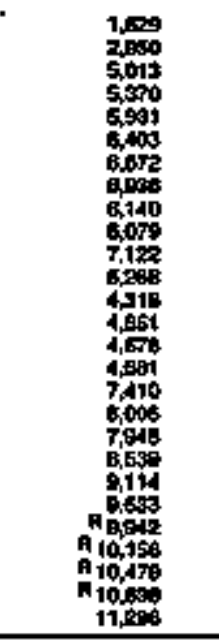 & $\begin{array}{l}z \\
\vdots \\
\vdots \\
\vdots \\
\vdots \\
\vdots \\
\vdots \\
\vdots \\
\vdots \\
\vdots \\
\vdots \\
\vdots \\
\vdots \\
\end{array}$ \\
\hline \multicolumn{15}{|c|}{ Trilution Eht } \\
\hline 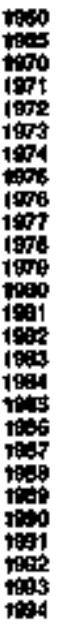 & 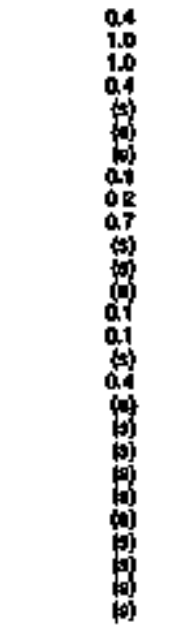 & 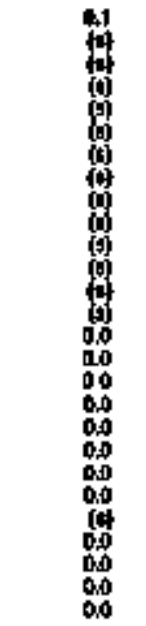 & 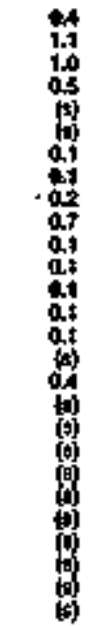 & 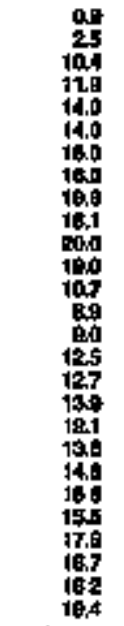 & 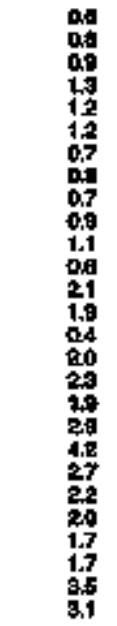 & 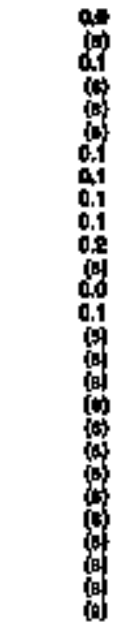 & 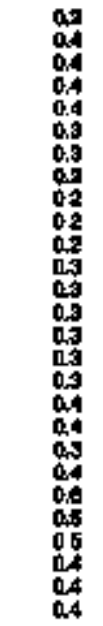 & 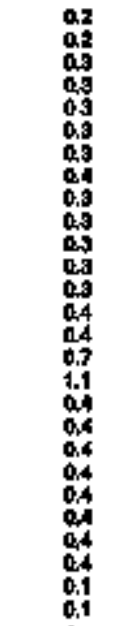 & 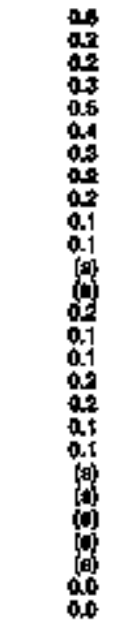 & 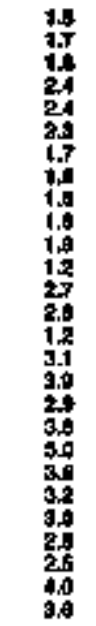 & 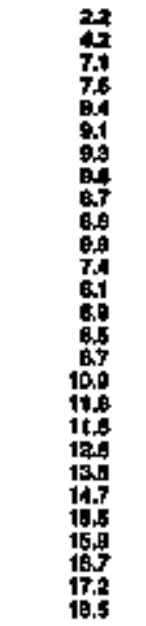 & 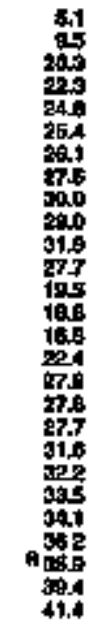 & 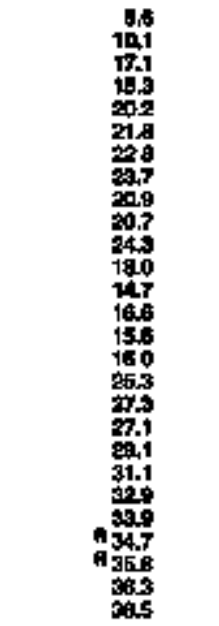 & 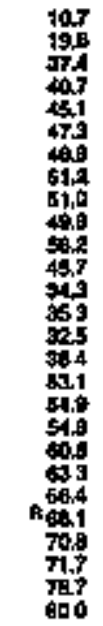 \\
\hline
\end{tabular}

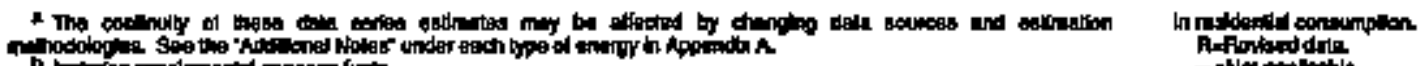

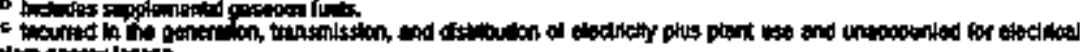

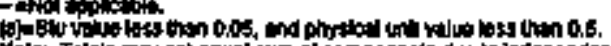

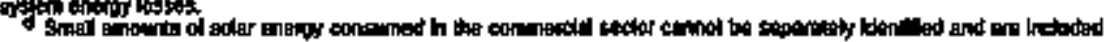

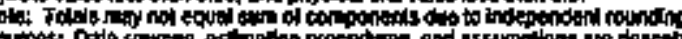

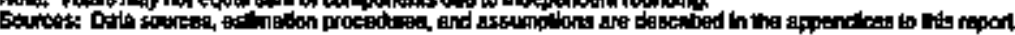




\begin{tabular}{|c|c|c|c|c|c|c|c|c|c|c|c|c|c|c|c|c|c|c|}
\hline \multirow[b]{3}{*}{ Yeses } & \multirow[b]{2}{*}{ Con } & \multirow[b]{2}{*}{ Nownt } & \multicolumn{9}{|c|}{ Petrotioun } & \multirow[b]{2}{*}{ 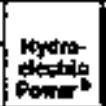 } & \multirow[b]{2}{*}{ Bolones: } & \multirow[b]{2}{*}{ | } & \multirow[b]{2}{*}{ Enstating of } & \multirow[b]{2}{*}{ trenty } & \multirow{2}{*}{ 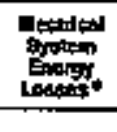 } & \multirow[b]{3}{*}{ Tou } \\
\hline & & & 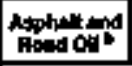 & 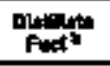 & Kerporene & $\cos$ & Letriaments st & mations & Fustol| & Oother al & Tols & & & & & & & \\
\hline & 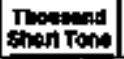 & $\mid$ & \multicolumn{9}{|c|}{ Thovand Burrob } & \multicolumn{6}{|c|}{ 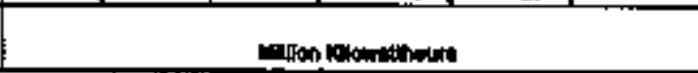 } & \\
\hline 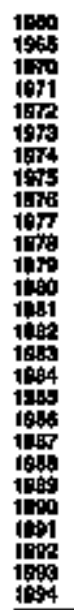 & 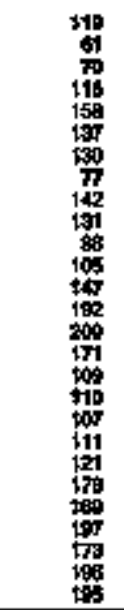 & 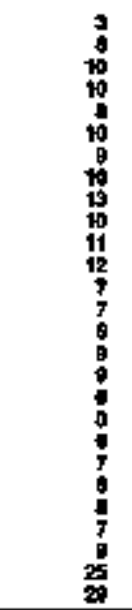 & 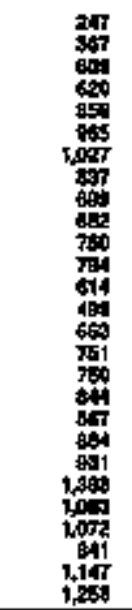 & 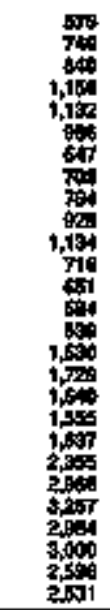 & 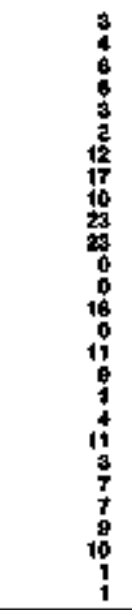 & 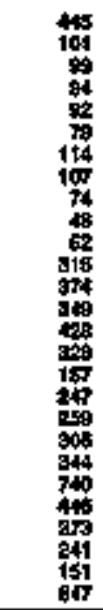 & 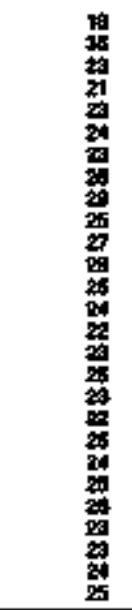 & 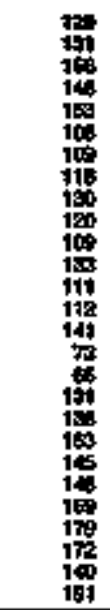 & 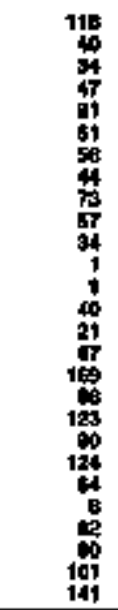 & 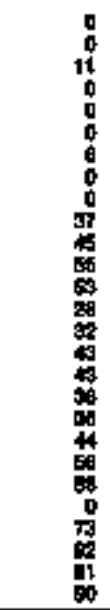 & 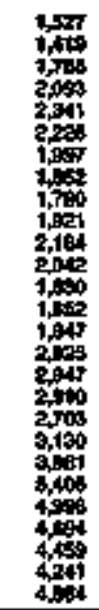 & $\begin{array}{l}9 \\
0 \\
\vdots \\
\vdots \\
\vdots\end{array}$ & 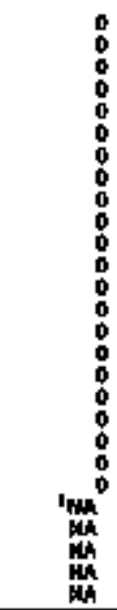 & 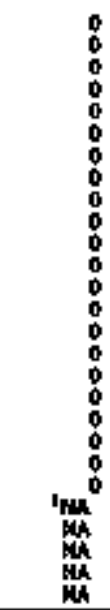 & 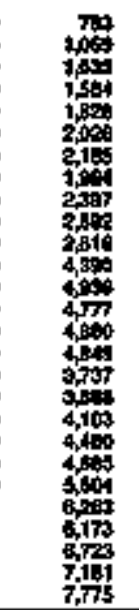 & $\begin{array}{l}\vdots \\
\vdots \\
\vdots \\
\vdots \\
\vdots \\
\vdots \\
\vdots \\
\vdots \\
\vdots \\
\vdots \\
\vdots \\
\vdots \\
\vdots\end{array}$ & 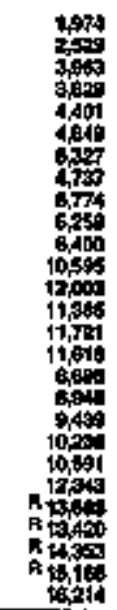 & $\begin{array}{l}\vdots \\
\vdots \\
\vdots \\
\vdots \\
\vdots \\
\vdots \\
\vdots \\
\vdots \\
\vdots \\
\vdots \\
\vdots \\
\vdots \\
\vdots\end{array}$ \\
\hline \multicolumn{19}{|c|}{ notllwat Bin } \\
\hline 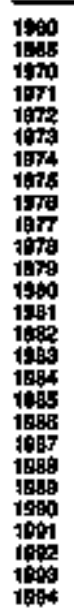 & 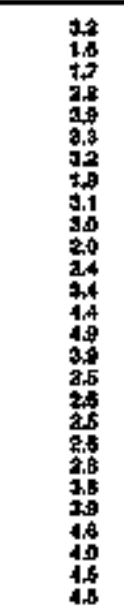 & 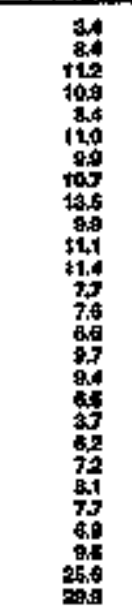 & 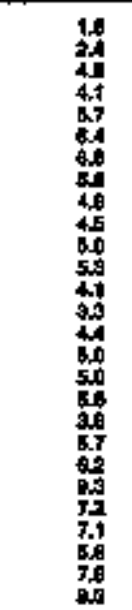 & 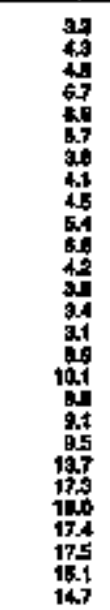 & 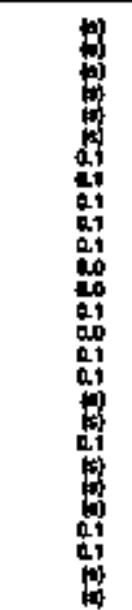 & 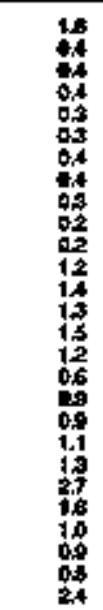 & 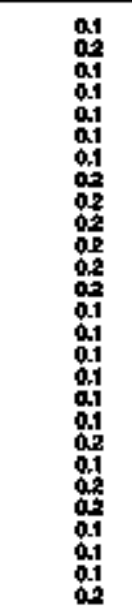 & 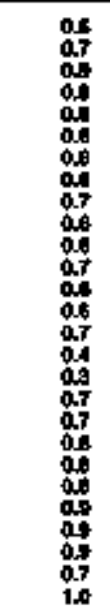 & 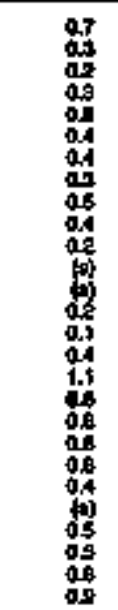 & 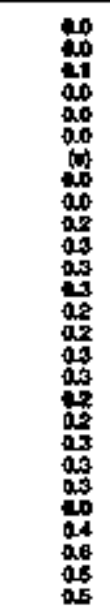 & 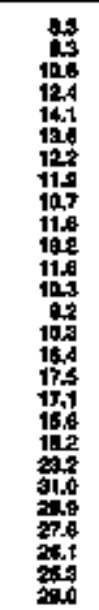 & 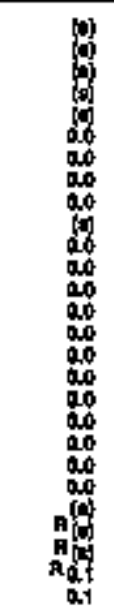 & 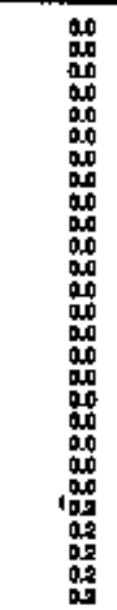 & 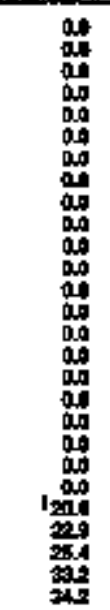 & 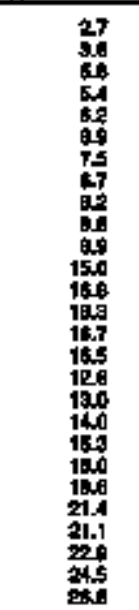 & 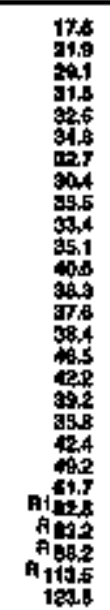 & 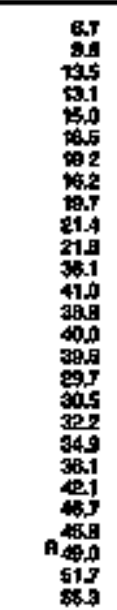 & 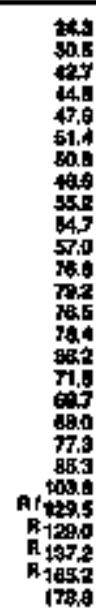 \\
\hline
\end{tabular}

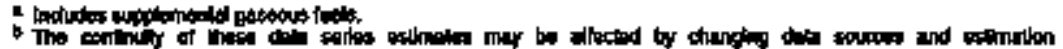
madt Probosin

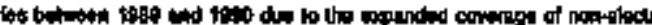

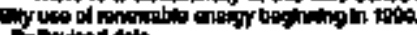

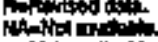

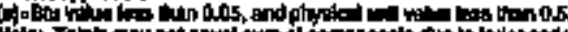

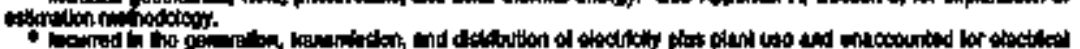

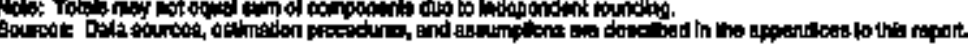

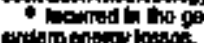


Table 199. Transportation Energy Consumption Estlmates, 1960, 1965, 1970-1994, Nevada

\begin{tabular}{|c|c|c|c|c|c|c|c|}
\hline \multirow[b]{3}{*}{ rev } & \multirow[b]{2}{*}{$\cos x=$} & \multirow[b]{2}{*}{ 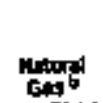 } & \multicolumn{5}{|c|}{ Paptan } \\
\hline & & & Antivion. & mistion & mide & Lot* & Mrmang \\
\hline & 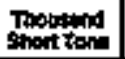 & Evilion & \multicolumn{5}{|c|}{ 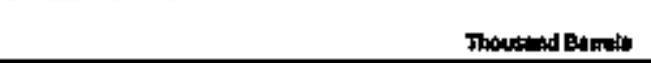 } \\
\hline 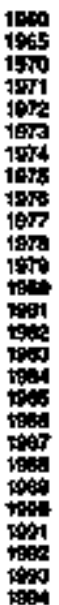 & 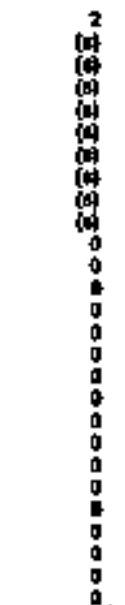 & 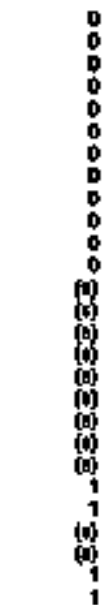 & 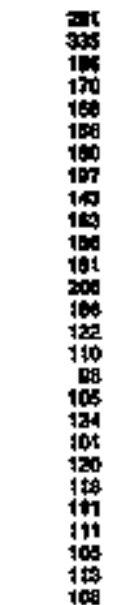 & 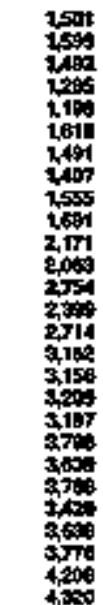 & 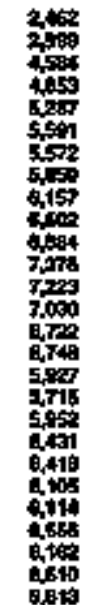 & 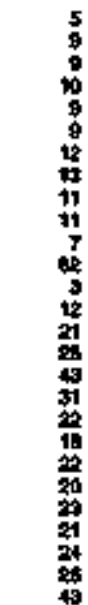 & 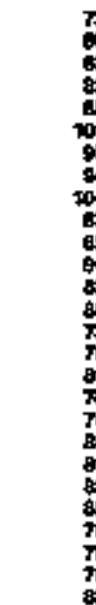 \\
\hline
\end{tabular}

\begin{tabular}{|c|c|c|c|c|c|c|c|c|c|c|c|c|c|c|c|}
\hline 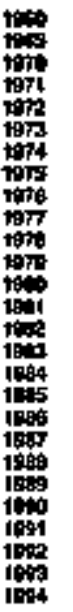 & 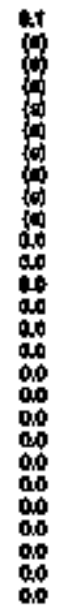 & 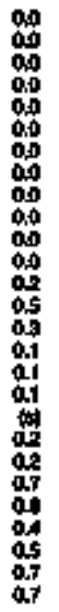 & 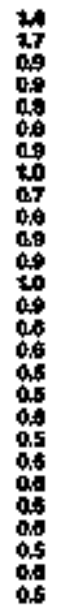 & 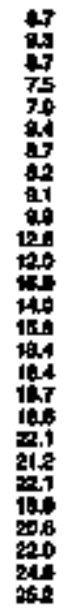 & 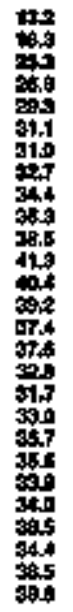 & 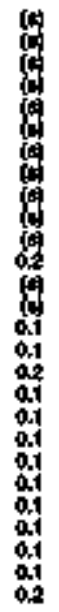 & 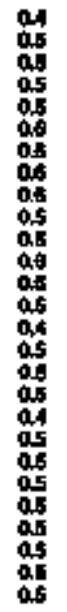 & 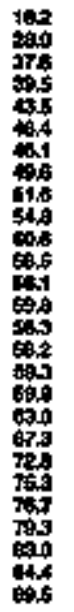 & 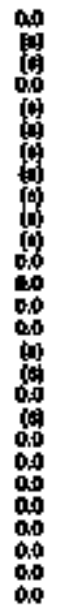 & 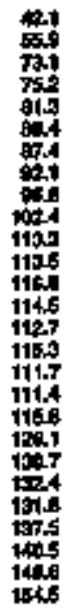 & 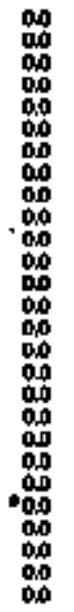 & 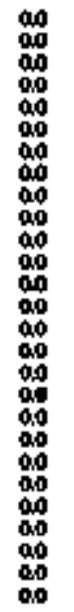 & 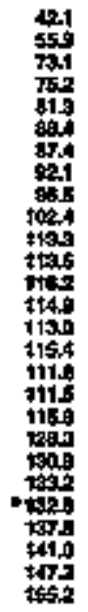 & 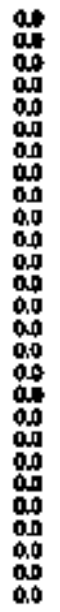 & 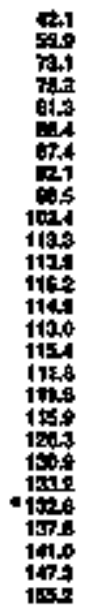 \\
\hline
\end{tabular}

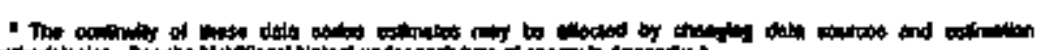

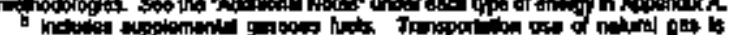

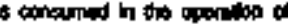

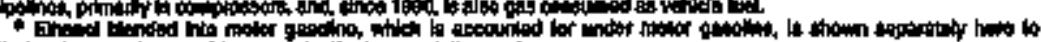

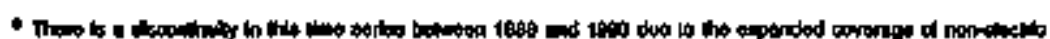

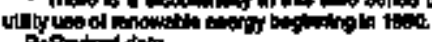

Arimations

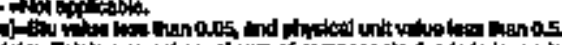

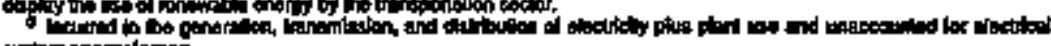

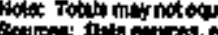

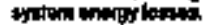


Teble 190. Esitimates of Energy lnpint at Electric Utillies, 1950, 1965, 1970-1994, Nevada

\begin{tabular}{|c|c|c|c|c|c|c|c|c|c|c|c|c|c|c|}
\hline \multirow[b]{3}{*}{ vent } & \multicolumn{3}{|c|}{ cons } & \multirow{3}{*}{ 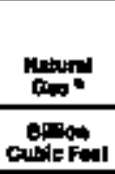 } & \multicolumn{4}{|c|}{ Puttaloin } & \multirow[b]{2}{*}{ 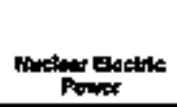 } & \multirow[b]{2}{*}{ 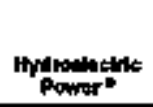 } & \multirow[b]{2}{*}{ elofush } & \multirow[b]{2}{*}{ Expenty } & \multirow{3}{*}{ other th } & \multirow[b]{3}{*}{ Tatente } \\
\hline & 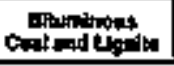 & Anchnothe & 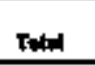 & & 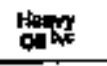 & 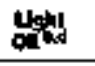 & Patroluentin & Totot & & & & & & \\
\hline & \multicolumn{3}{|c|}{ 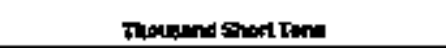 } & & \multicolumn{4}{|c|}{ Thoolind em mits } & \multicolumn{4}{|c|}{ 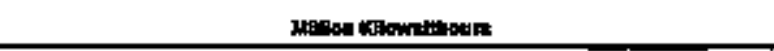 } & & \\
\hline 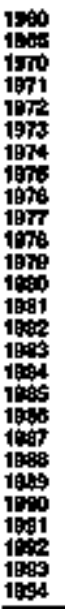 & 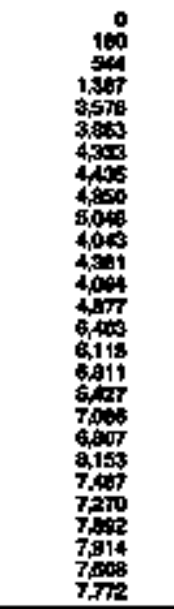 & $\begin{array}{l}0 \\
0 \\
0 \\
0 \\
0 \\
0 \\
0 \\
0 \\
0 \\
0 \\
0 \\
0 \\
0 \\
0 \\
0 \\
0 \\
0 \\
0 \\
0 \\
0 \\
0 \\
0\end{array}$ & 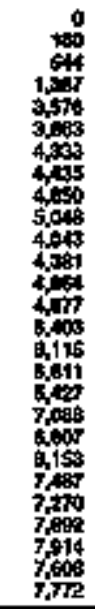 & 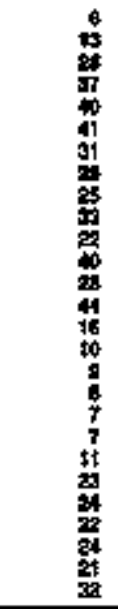 & 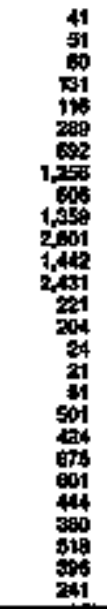 & 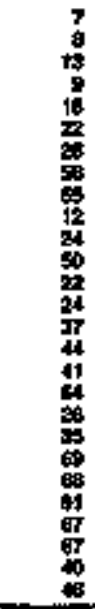 & $\begin{array}{l}0 \\
0 \\
0 \\
0 \\
0 \\
0 \\
0 \\
0 \\
0 \\
0 \\
0 \\
0 \\
0 \\
0 \\
0 \\
0 \\
0 \\
0 \\
0 \\
0 \\
0 \\
0 \\
0\end{array}$ & 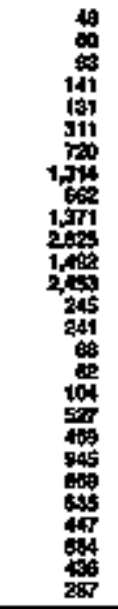 & $\begin{array}{l}0 \\
0 \\
0 \\
0 \\
0 \\
0 \\
0 \\
0 \\
0 \\
0 \\
0 \\
0 \\
0 \\
0 \\
0 \\
0 \\
0 \\
0 \\
0 \\
0 \\
0 \\
0 \\
0 \\
0 \\
0 \\
0 \\
0\end{array}$ & 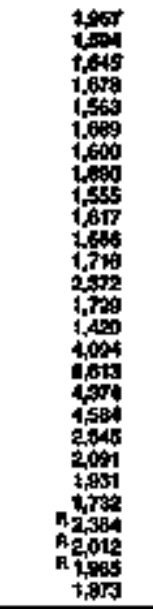 & $\begin{array}{l}0 \\
0 \\
0 \\
0 \\
0 \\
0 \\
0 \\
0 \\
0 \\
0 \\
0 \\
0 \\
0 \\
0 \\
0 \\
0 \\
0 \\
0 \\
0 \\
0 \\
0 \\
0 \\
0 \\
0 \\
0 \\
0 \\
0\end{array}$ & $\begin{array}{l}0 \\
0 \\
0 \\
0 \\
0 \\
0 \\
0 \\
0 \\
0 \\
0 \\
0 \\
0 \\
0 \\
0 \\
0 \\
0 \\
0 \\
0 \\
0 \\
0 \\
0 \\
0 \\
0 \\
0 \\
0 \\
0\end{array}$ & $\begin{array}{l}0 \\
0 \\
0 \\
0 \\
0 \\
0 \\
0 \\
0 \\
0 \\
0 \\
0 \\
0 \\
0 \\
0 \\
0 \\
0 \\
0 \\
0 \\
0 \\
0 \\
0 \\
0 \\
0 \\
0 \\
0 \\
0 \\
0\end{array}$ & $\begin{array}{l}z \\
z \\
z \\
z \\
z \\
z \\
z \\
z \\
z \\
z \\
z \\
z \\
z \\
z\end{array}$ \\
\hline \multicolumn{15}{|c|}{ Tamino ow } \\
\hline 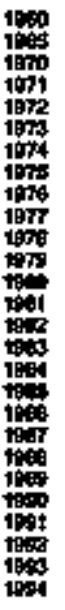 & 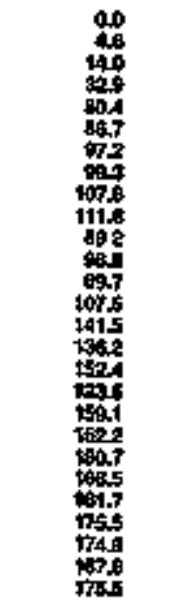 & 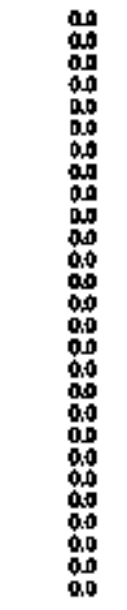 & 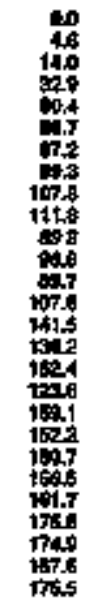 & 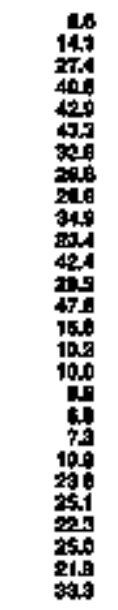 & 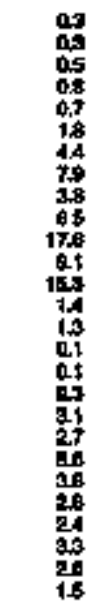 & 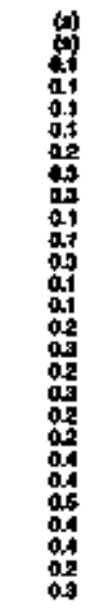 & 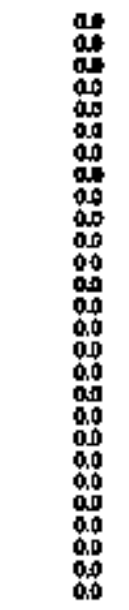 & 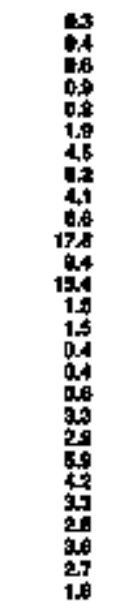 & $\begin{array}{l}00 \\
00 \\
00 \\
00 \\
00 \\
00 \\
00 \\
00 \\
00 \\
00 \\
00 \\
00 \\
00 \\
00 \\
00 \\
00 \\
00 \\
00 \\
00 \\
00 \\
000 \\
000 \\
00 \\
00 \\
00 \\
00\end{array}$ & 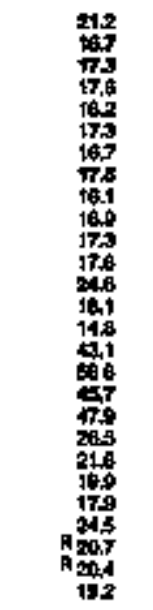 & $\begin{array}{l}00 \\
00 \\
00 \\
00 \\
00 \\
0.0 \\
00 \\
00 \\
00 \\
00 \\
00 \\
00 \\
000 \\
000 \\
000 \\
000 \\
000 \\
100 \\
0.0 \\
00 \\
000 \\
000 \\
000 \\
000 \\
000\end{array}$ & 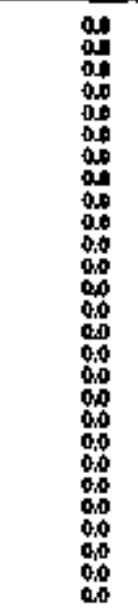 & 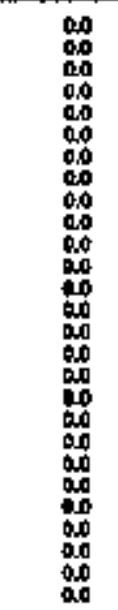 & 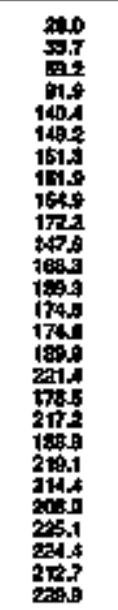 \\
\hline & 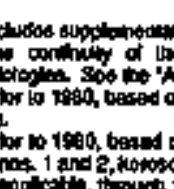 & 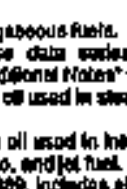 & 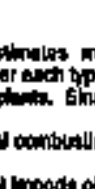 & Fom & etr & 1000 & atder & 50 & sourcose of & inethen & $\lim _{x \rightarrow 0}$ & atented & $\operatorname{man}$ & Dort. \\
\hline
\end{tabular}


Tablo 191. Entrgy Consumption Estimales by Source, 1960, 1965, 1970-1994, New Hampsilre

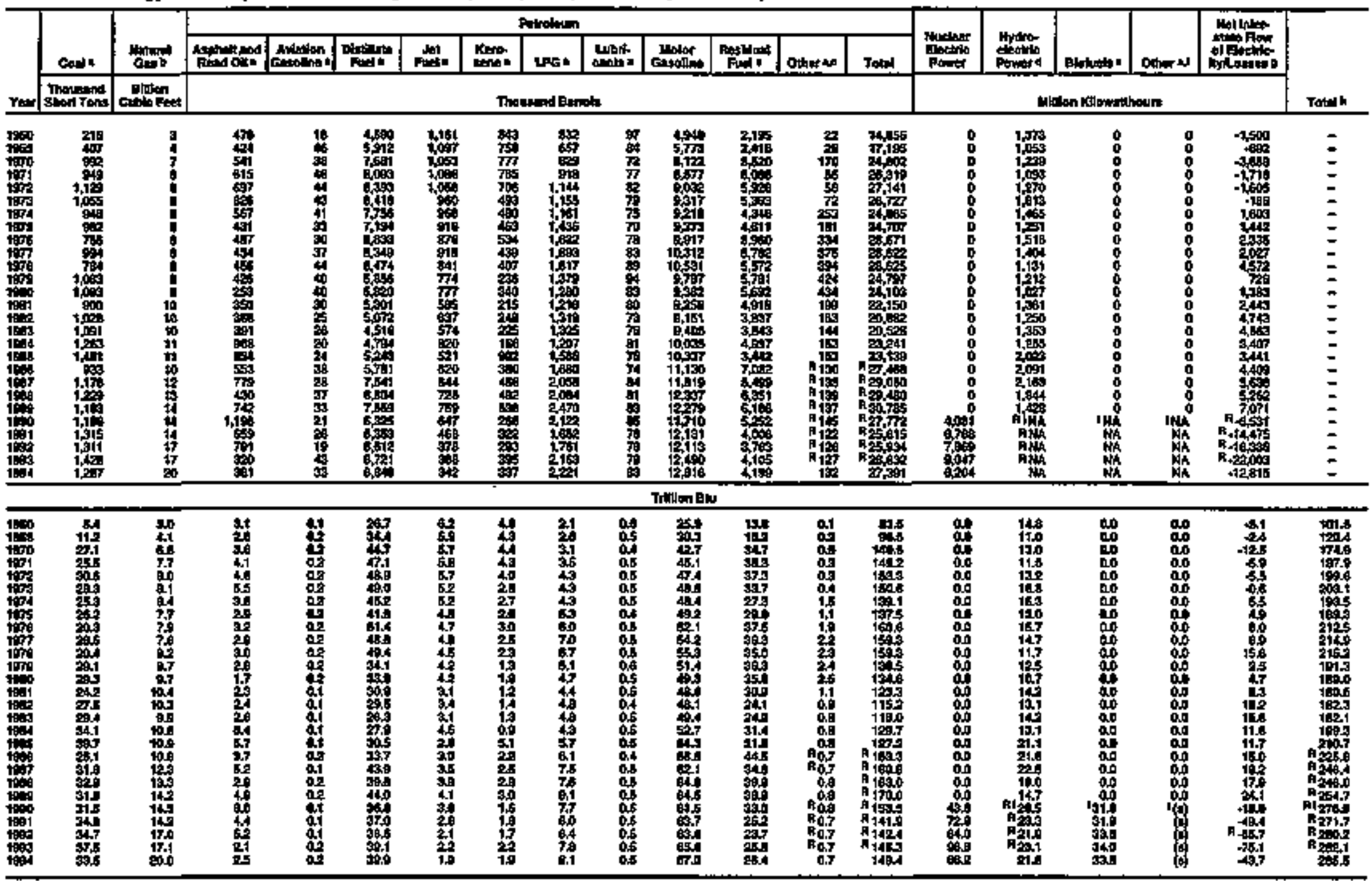

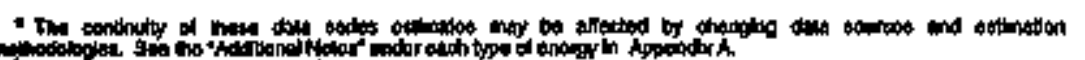

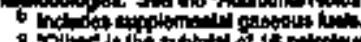

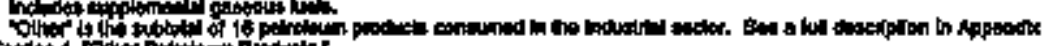

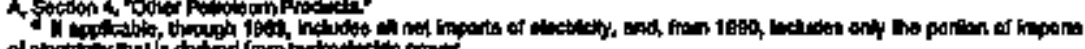

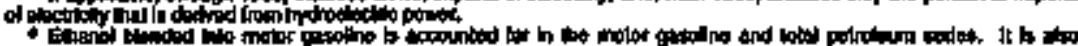

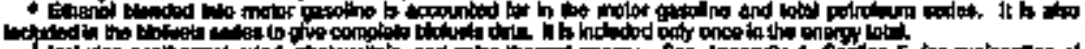

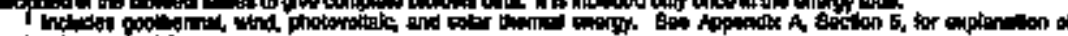

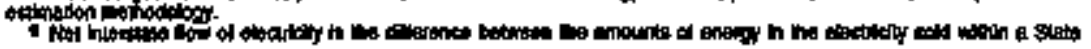

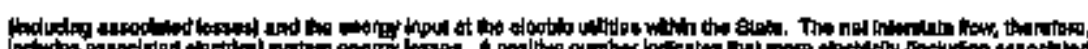

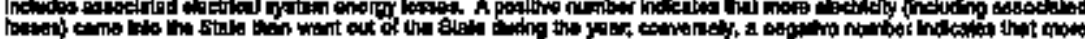

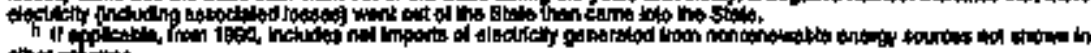

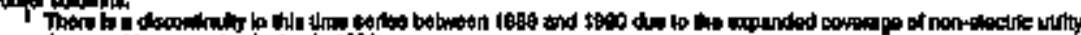

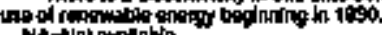

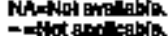

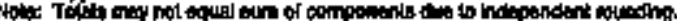

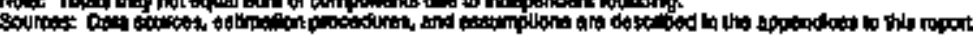


N Table 192. Rosidentlal Energy Gonsumpilon Estimales, 1960, 1965, 1970-1994, New Hampshire

\begin{tabular}{|c|c|c|c|c|c|c|c|c|c|c|c|c|c|c|}
\hline \multirow[b]{3}{*}{ ver } & \multicolumn{3}{|c|}{ con } & \multirow{3}{*}{ 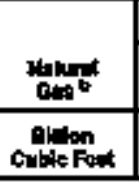 } & \multicolumn{4}{|c|}{ Pelrotern } & \multirow{3}{*}{ 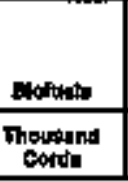 } & & \multirow[b]{2}{*}{ Bastillys" } & \multirow[b]{2}{*}{ EAtorsy } & \multirow{3}{*}{ 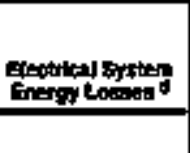 } & \multirow[b]{3}{*}{ Tolit } \\
\hline & 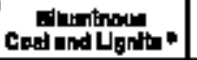 & Nithunetion & Tot & & 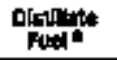 & Nenosenest & LPa= & Told & & Aolis ${ }^{\circ}$ & & & & \\
\hline & \multicolumn{3}{|c|}{ 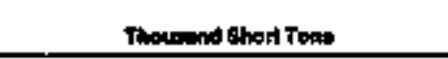 } & & \multicolumn{4}{|c|}{ 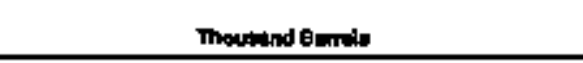 } & & \multicolumn{3}{|c|}{ Hillon Lalowathoor: } & & \\
\hline 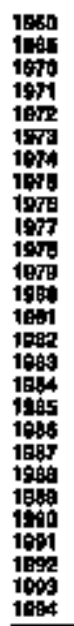 & 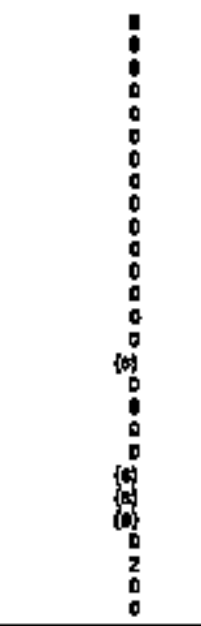 & 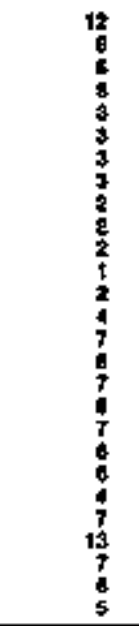 & 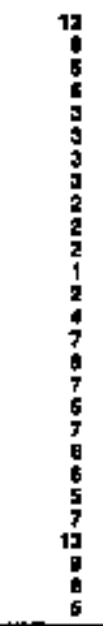 & 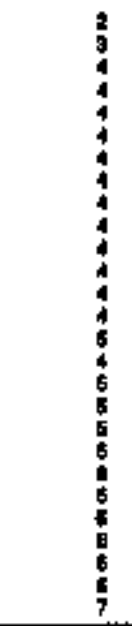 & 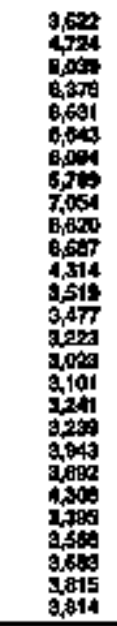 & 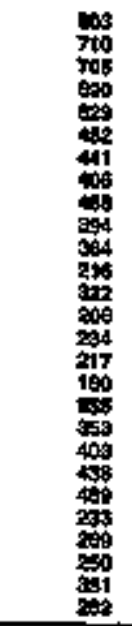 & 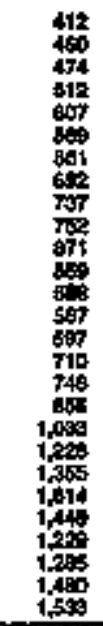 & 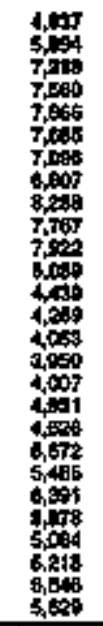 & 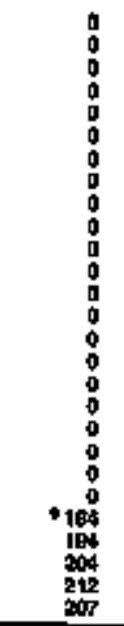 & $\begin{array}{l}0 \\
0 \\
0 \\
0 \\
0 \\
0 \\
0 \\
0 \\
0 \\
0 \\
0 \\
0 \\
0 \\
0 \\
0 \\
0 \\
0 \\
0 \\
0 \\
0 \\
0 \\
0 \\
0 \\
0 \\
0 \\
0 \\
7 \\
7 \\
7 \\
7 \\
7 \\
8\end{array}$ & 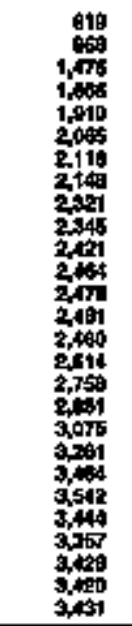 & $\begin{array}{l}\vdots \\
\vdots \\
\vdots \\
\vdots \\
\vdots \\
\vdots \\
\vdots \\
\vdots \\
\vdots \\
\vdots \\
\vdots \\
\vdots\end{array}$ & 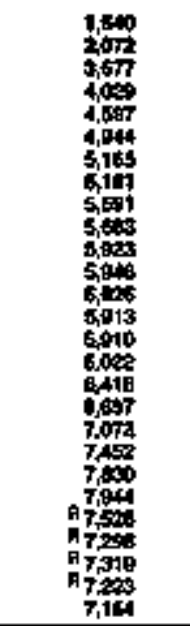 & $\begin{array}{l}z \\
\vdots \\
\vdots \\
z \\
\vdots \\
\vdots \\
\vdots \\
\vdots \\
\vdots \\
\vdots \\
\vdots \\
z \\
z\end{array}$ \\
\hline \multicolumn{15}{|c|}{ 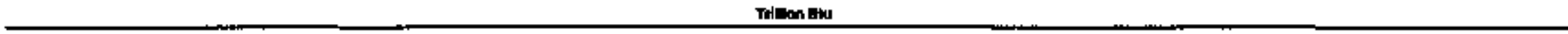 } \\
\hline 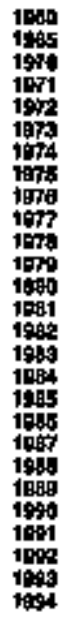 & 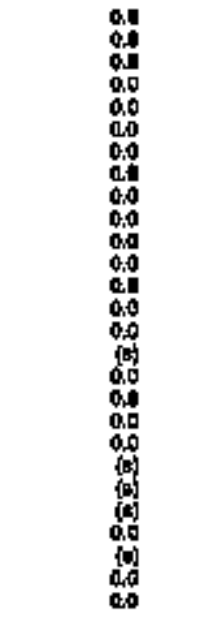 & 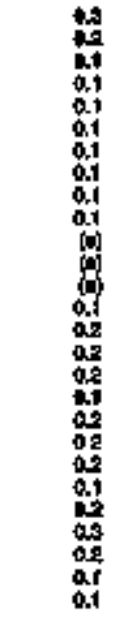 & 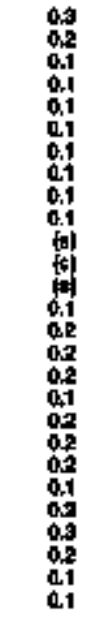 & 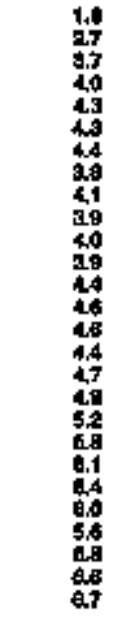 & 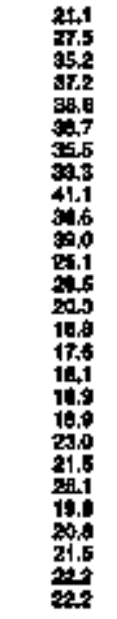 & 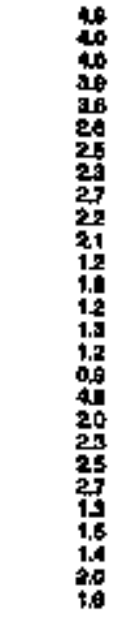 & 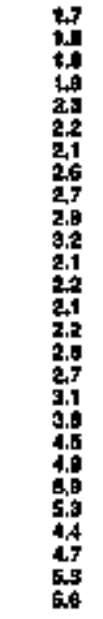 & 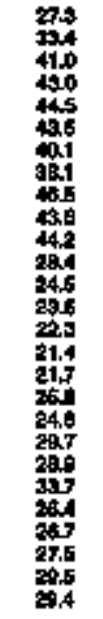 & 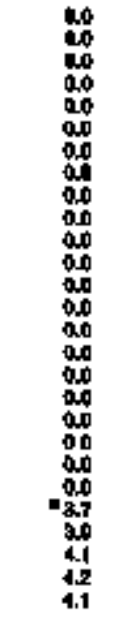 & 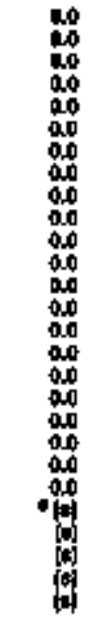 & 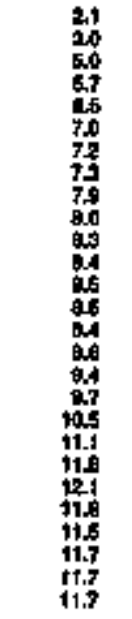 & 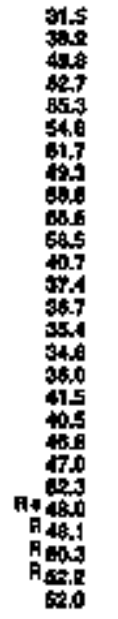 & 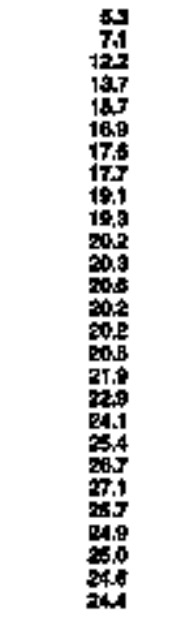 & 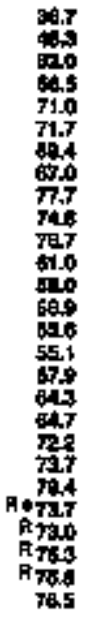 \\
\hline
\end{tabular}

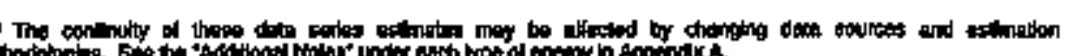

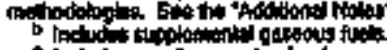

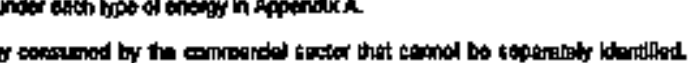

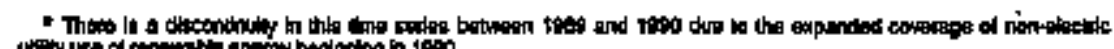

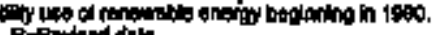

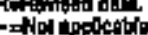

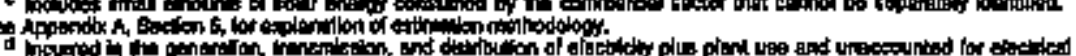

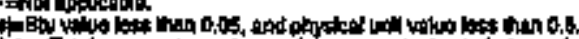


Tabla 193. Commerclal Energy Consumption Estimates, 1960, 1965, 1970-1994, Now Hampshire

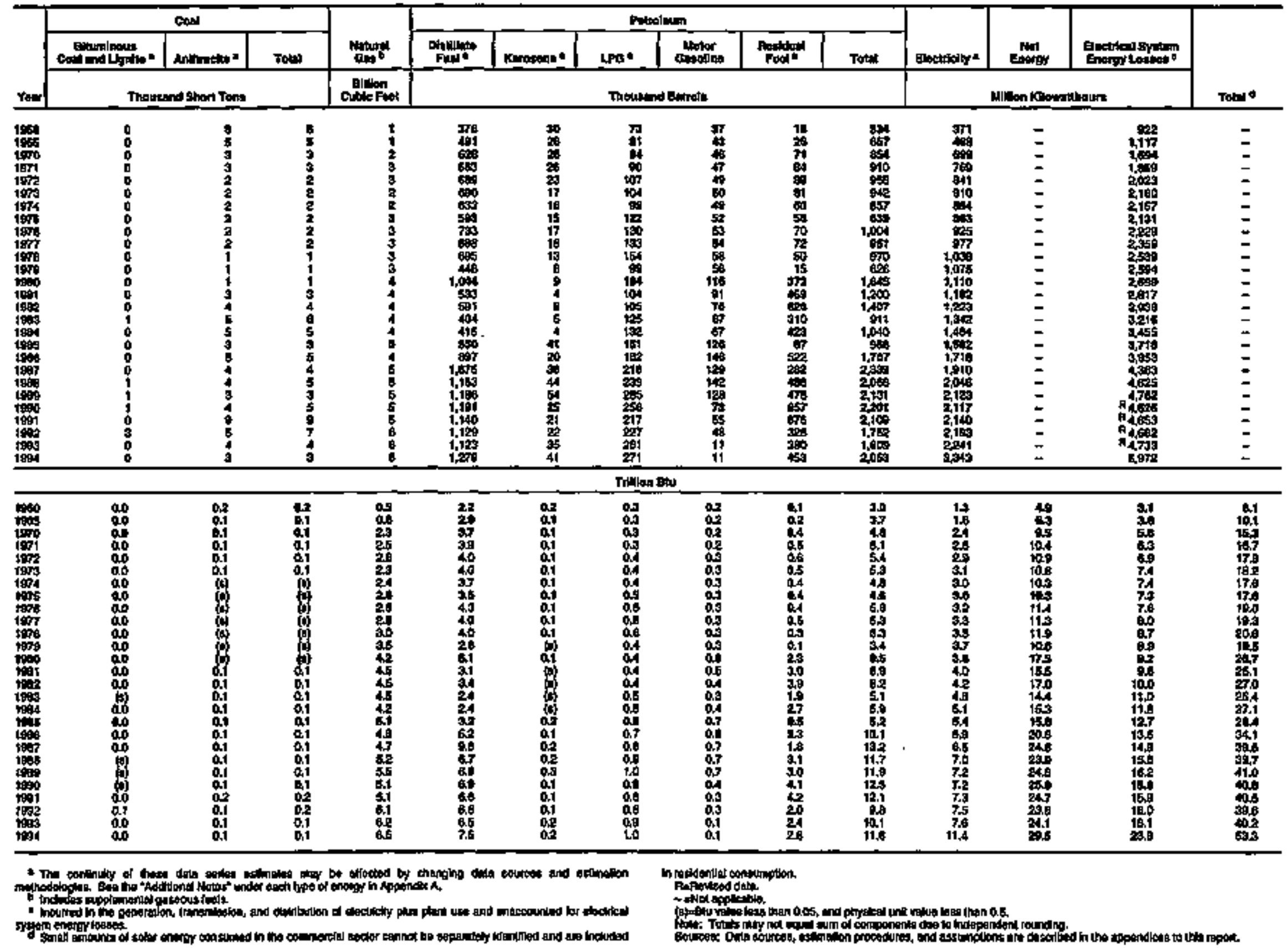




\begin{tabular}{|c|c|c|c|c|c|c|c|c|c|c|c|c|c|c|c|c|c|c|}
\hline & coss. & Merant & \multicolumn{9}{|c|}{ Perovioun } & 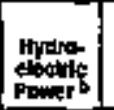 & Hofoum & ones tad & enowitely 4 & Enters & \multirow{2}{*}{ 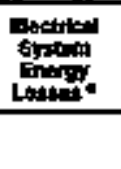 } & \multirow[b]{2}{*}{ 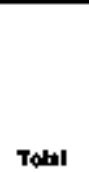 } \\
\hline mat & 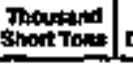 & pantion & \multicolumn{9}{|c|}{ Thontend Burets } & \multicolumn{5}{|c|}{ 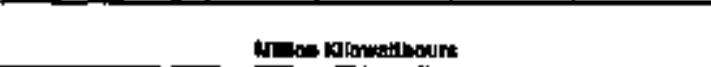 } & & \\
\hline 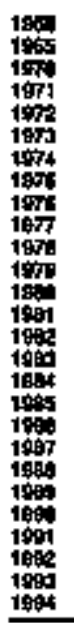 & 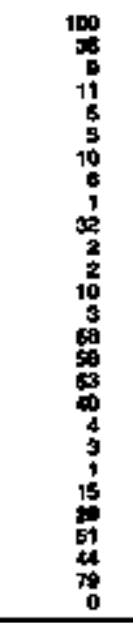 & 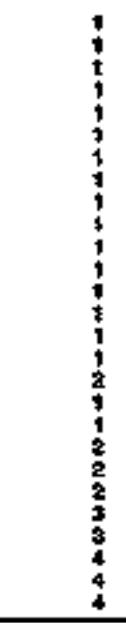 & 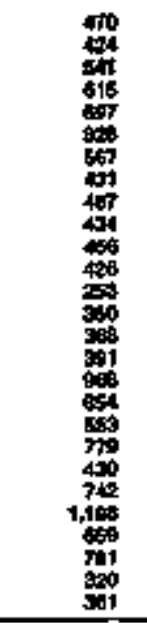 & 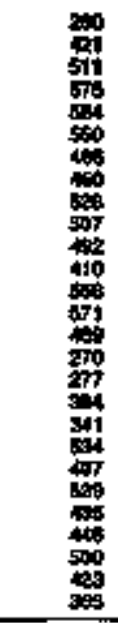 & 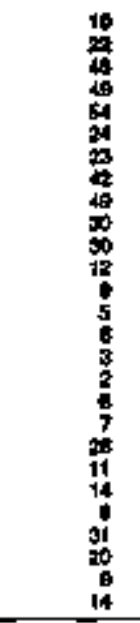 & 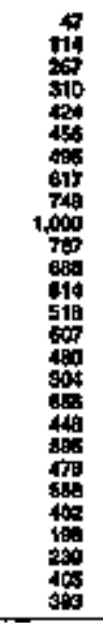 & 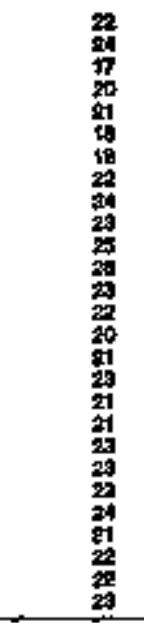 & 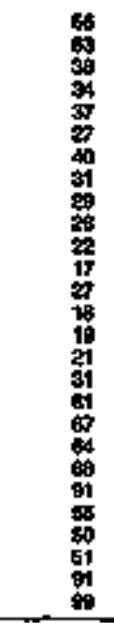 & 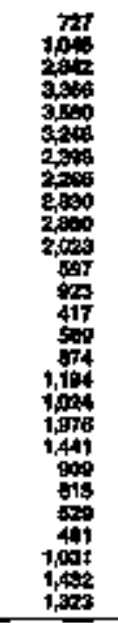 & 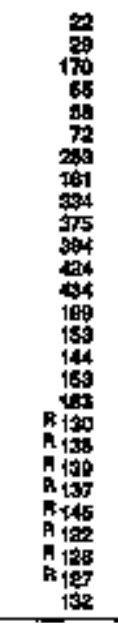 & 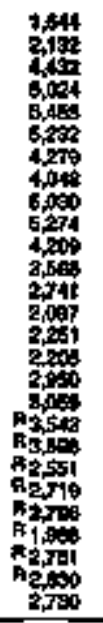 & 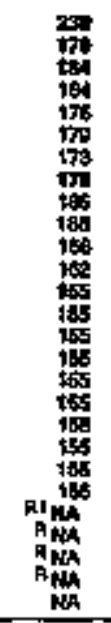 & 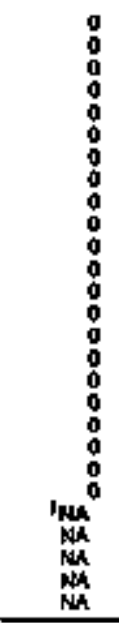 & 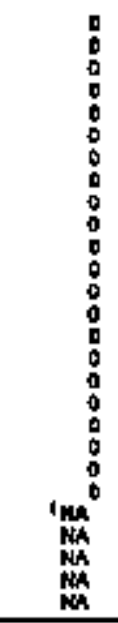 & 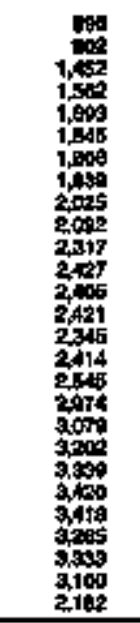 & $\begin{array}{l}\vdots \\
\vdots \\
\vdots \\
\vdots \\
\vdots \\
\vdots \\
\vdots \\
\vdots \\
\vdots \\
\vdots \\
\vdots \\
\vdots \\
\vdots \\
\vdots\end{array}$ & 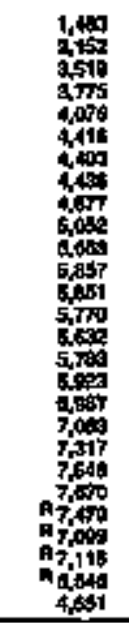 & $\begin{array}{l}z \\
z \\
z \\
z\end{array}$ \\
\hline
\end{tabular}


Table 185, Transportation Energy Consumption Estlmales, 1900, 1965, 1970-1994, New Hampshlre

\begin{tabular}{|c|c|c|c|c|c|c|c|c|c|c|c|c|c|c|c|}
\hline \multirow[b]{3}{*}{$\mathrm{xen}$} & \multirow[b]{2}{*}{$\cos 1=$} & \multirow[b]{2}{*}{ "4at: } & \multicolumn{8}{|c|}{ 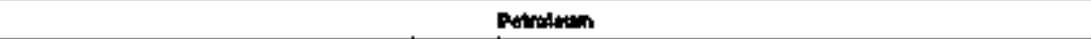 } & \multirow{3}{*}{ 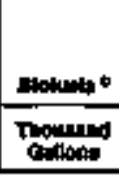 } & & \multirow[b]{2}{*}{$\lim _{\ln }$} & \multirow[b]{2}{*}{ 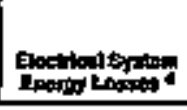 } & \multirow[b]{3}{*}{ Tato } \\
\hline & & & 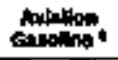 & 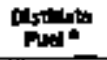 & Put. & $\mathbf{L P O}=$ & Loteantu- & ainoritin & 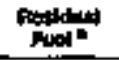 & Totel & & Eectively" & & & \\
\hline & Thoot Tout & Eimition & \multicolumn{8}{|c|}{ Tpomindentints } & & \multicolumn{3}{|c|}{ 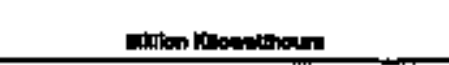 } & \\
\hline 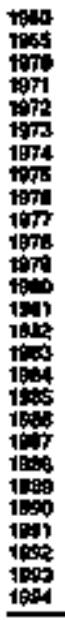 & 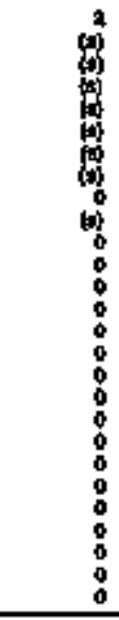 & 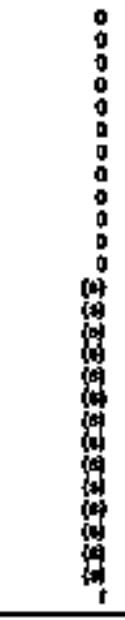 & 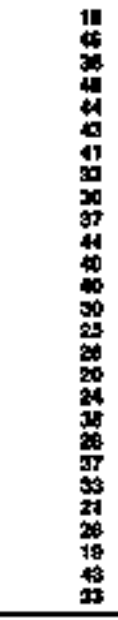 & 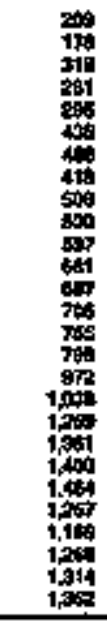 & 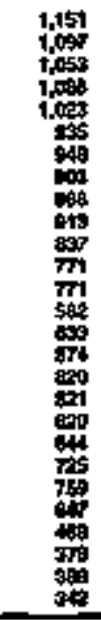 & 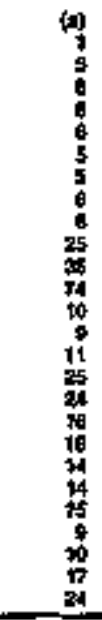 & 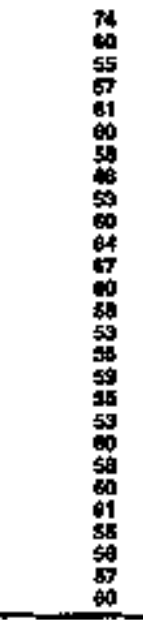 & 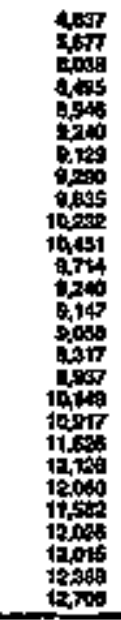 & 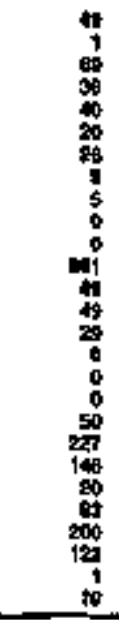 & 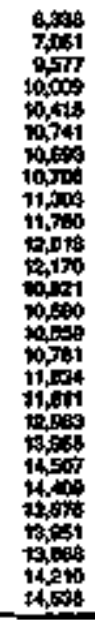 & $\begin{array}{l}0 \\
0 \\
0 \\
0 \\
0 \\
0 \\
0 \\
0 \\
0 \\
0 \\
0 \\
0 \\
0 \\
0 \\
0 \\
0 \\
0 \\
0 \\
0 \\
0 \\
0 \\
0 \\
0 \\
0 \\
0 \\
0 \\
0 \\
\end{array}$ & $\begin{array}{l}0 \\
0 \\
0 \\
0 \\
0 \\
0 \\
0 \\
0 \\
0 \\
0 \\
0 \\
0 \\
0 \\
0 \\
0 \\
0 \\
0 \\
0 \\
0 \\
0 \\
0 \\
0 \\
0 \\
0 \\
0 \\
0 \\
0 \\
0\end{array}$ & $\begin{array}{l}= \\
= \\
= \\
= \\
= \\
= \\
= \\
= \\
= \\
= \\
= \\
= \\
= \\
=\end{array}$ & $\begin{array}{l}0 \\
0 \\
0 \\
0 \\
0 \\
0 \\
0 \\
0 \\
0 \\
0 \\
0 \\
0 \\
0 \\
0 \\
0 \\
0 \\
0 \\
0 \\
0 \\
0 \\
0 \\
0 \\
0 \\
0 \\
0 \\
0 \\
0 \\
0 \\
0\end{array}$ & $\begin{array}{l}= \\
z \\
= \\
= \\
z \\
= \\
= \\
= \\
= \\
= \\
z \\
z \\
z \\
z\end{array}$ \\
\hline \multicolumn{16}{|c|}{ Thenen } \\
\hline 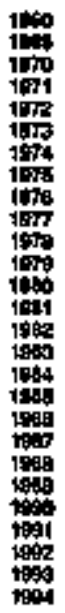 & 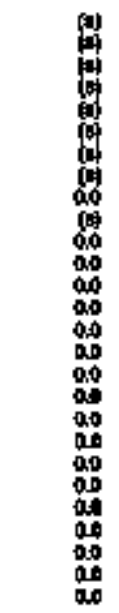 & 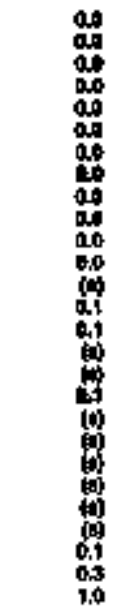 & 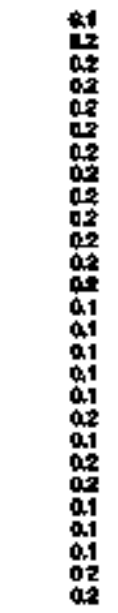 & 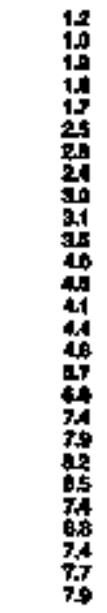 & 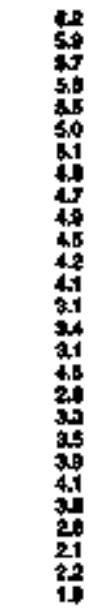 & 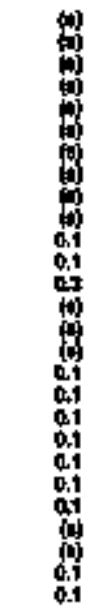 & 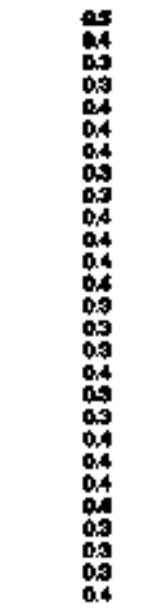 & 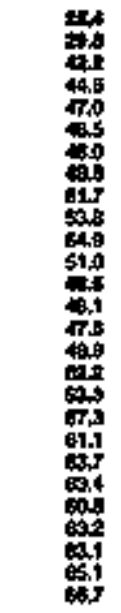 & 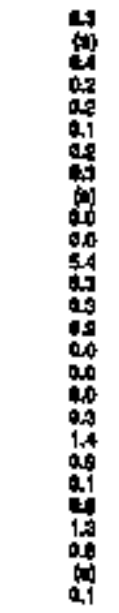 & 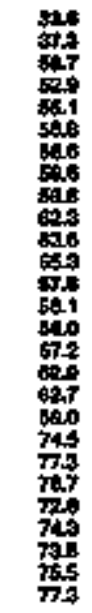 & $\begin{array}{l}00 \\
00 \\
00 \\
000 \\
00 \\
00 \\
00 \\
00 \\
00 \\
00 \\
00 \\
000 \\
00 \\
00 \\
00 \\
00 \\
00 \\
00 \\
00 \\
00 \\
00 \\
00 \\
00 \\
00 \\
00 \\
00 \\
00\end{array}$ & 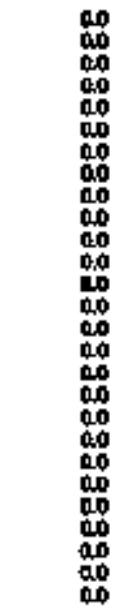 & 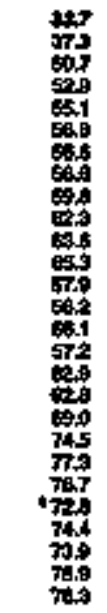 & $\begin{array}{l}00 \\
00 \\
0.0 \\
0.0 \\
00 \\
000 \\
0.0 \\
0.0 \\
0.0 \\
0.0 \\
0.0 \\
00 \\
00 \\
0.0 \\
0.0 \\
0.0 \\
0.0 \\
0.0 \\
0.0 \\
00 \\
00 \\
0.0 \\
00 \\
0.0 \\
0.0\end{array}$ & 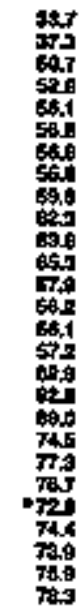 \\
\hline
\end{tabular}

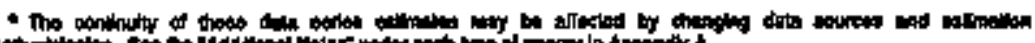

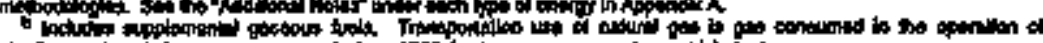

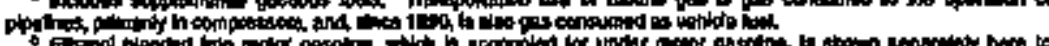

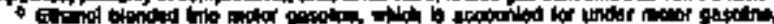

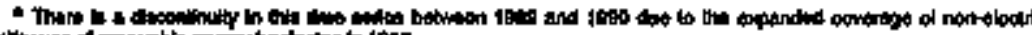

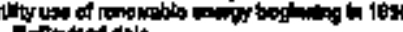

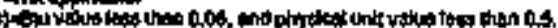

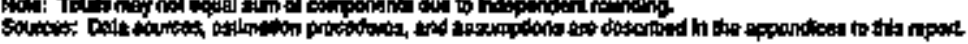

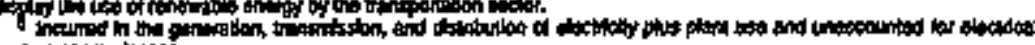




\begin{tabular}{|c|c|c|c|c|c|c|c|c|c|c|c|c|c|c|}
\hline \multirow[b]{2}{*}{ vom. } & \multicolumn{3}{|c|}{ and } & \multirow{2}{*}{ 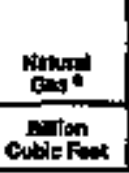 } & \multicolumn{4}{|c|}{ Fomienga } & mactor Bostik & Prometrit: & Botunde & 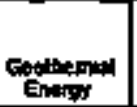 & Oan=m & \multirow[b]{2}{*}{ Totat } \\
\hline & \multicolumn{3}{|c|}{ Troneund Shont Tons } & & \multicolumn{4}{|c|}{ 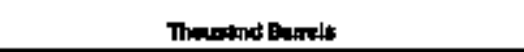 } & \multicolumn{5}{|c|}{ 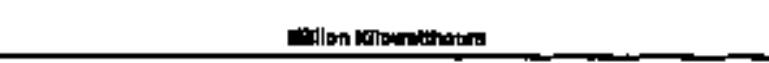 } & \\
\hline 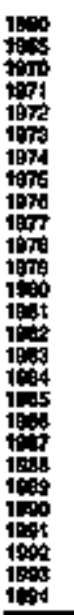 & 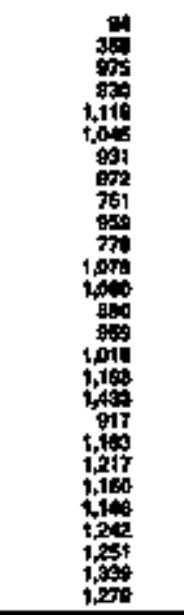 & $\begin{array}{l}1 \\
0 \\
0 \\
0 \\
0 \\
0 \\
0 \\
0 \\
0 \\
0 \\
0 \\
0 \\
0 \\
0 \\
0 \\
0 \\
0 \\
0 \\
0 \\
0 \\
0 \\
0 \\
0\end{array}$ & 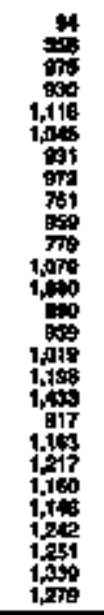 & 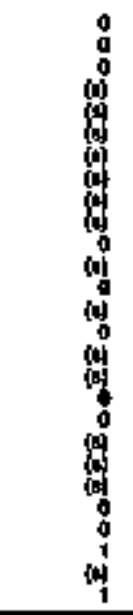 & 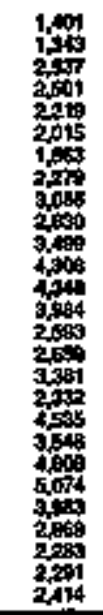 & 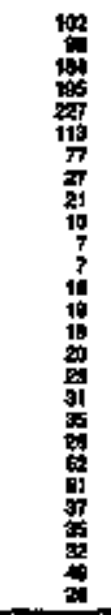 & $\begin{array}{l}0 \\
0 \\
0 \\
0 \\
0 \\
0 \\
0 \\
0 \\
0 \\
0 \\
0 \\
0 \\
0 \\
0 \\
0 \\
0 \\
0 \\
0 \\
0 \\
0 \\
0 \\
\end{array}$ & 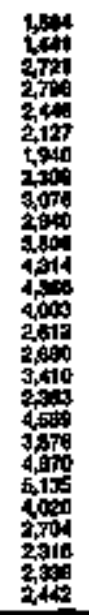 & 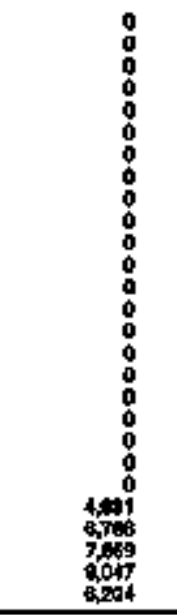 & 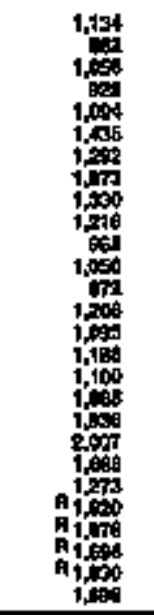 & 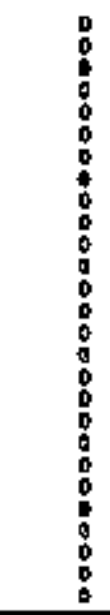 & $\begin{array}{l}\mathbf{b} \\
0 \\
0 \\
0 \\
0 \\
0 \\
0 \\
0 \\
0 \\
0 \\
0 \\
0 \\
0 \\
0 \\
0 \\
0 \\
0 \\
0 \\
0 \\
0 \\
0 \\
0 \\
0 \\
0 \\
0 \\
0 \\
0\end{array}$ & $\begin{array}{l}0 \\
0 \\
0 \\
0 \\
0 \\
0 \\
0 \\
0 \\
\vdots \\
0 \\
0 \\
\vdots \\
\vdots \\
0 \\
0 \\
0 \\
0 \\
0 \\
0 \\
0 \\
\vdots \\
0 \\
\vdots \\
0 \\
\end{array}$ & $\begin{array}{l}z \\
z \\
z \\
z \\
z \\
z \\
z \\
z \\
z \\
z \\
z \\
z \\
z \\
z \\
\end{array}$ \\
\hline 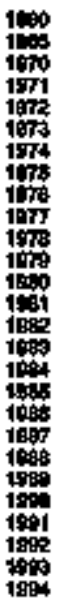 & 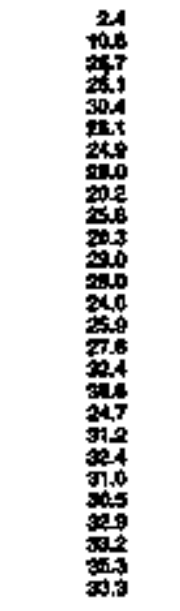 & 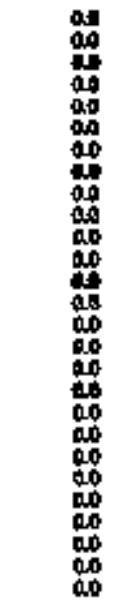 & 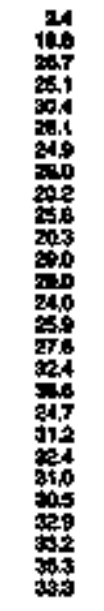 & 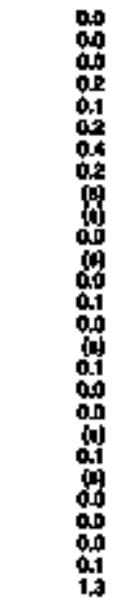 & 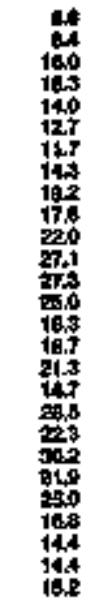 & 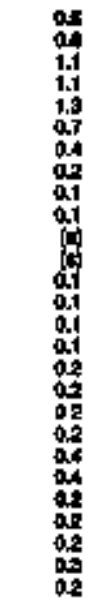 & 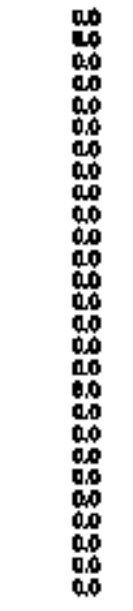 & 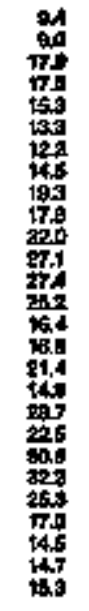 & 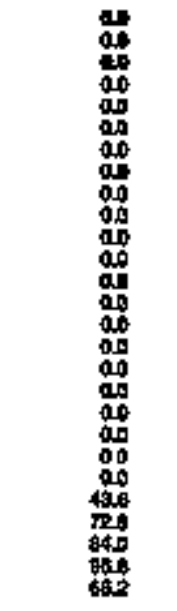 & 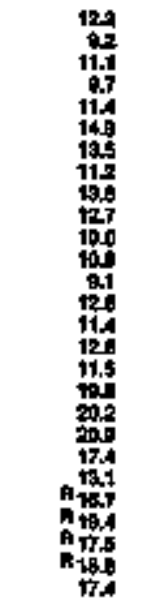 & 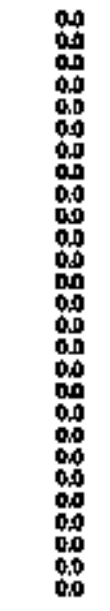 & 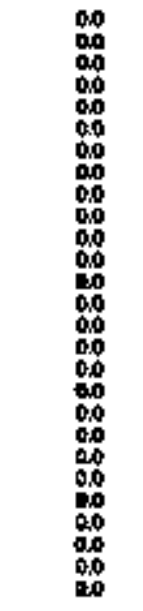 & 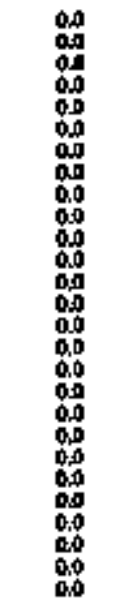 & 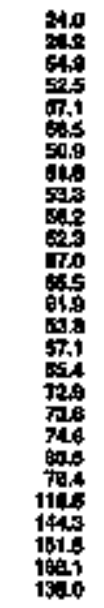 \\
\hline
\end{tabular}

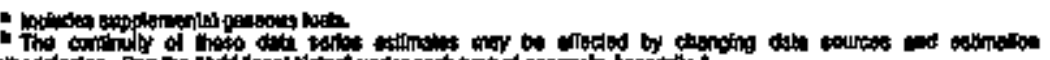

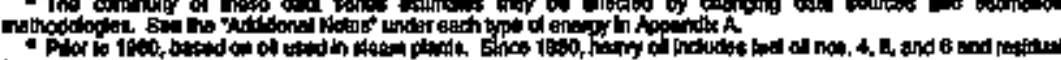

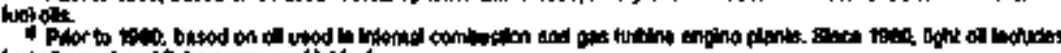

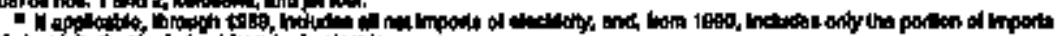

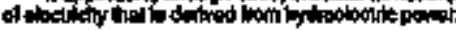

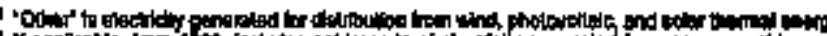

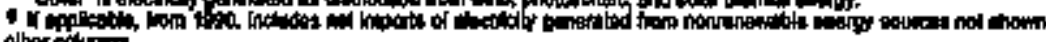
aliol and

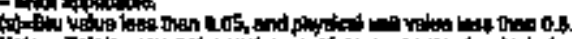

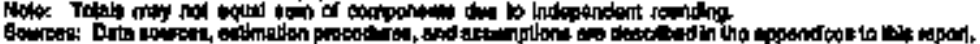


Toble 197. Energy Consumption Estimates by Sourcs, 1960, 1965, 1970-1994, How Jersey

\begin{tabular}{|c|c|c|c|c|c|c|c|c|c|c|c|c|c|c|c|c|c|c|c|}
\hline \multirow[b]{3}{*}{ 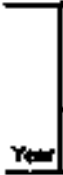 } & \multirow[b]{2}{*}{ cones 4} & \multirow[b]{2}{*}{ "Nonts } & \multicolumn{11}{|c|}{ 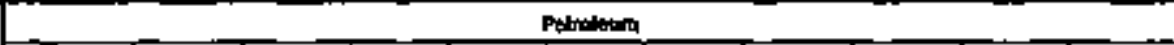 } & \multirow[b]{2}{*}{ perter } & \multirow[b]{2}{*}{ Pyoser } & \multirow[b]{2}{*}{ Botmin. } & \multirow[b]{2}{*}{ oner al } & \multirow{3}{*}{ 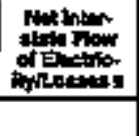 } & \multirow[b]{3}{*}{ Tot:all } \\
\hline & & & Arpayd ond & Antionsons & 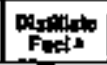 & Fut. & kno. & LPOA & $\operatorname{lop}_{0 \rightarrow 1}$ & 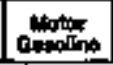 & 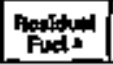 & ther ace & Tow & & & & & & \\
\hline & Tropens & cintion & \multicolumn{11}{|c|}{ 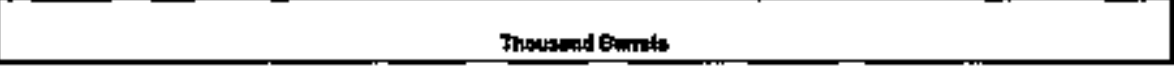 } & \multicolumn{4}{|c|}{ 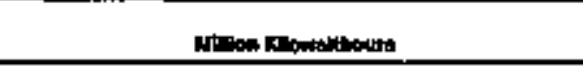 } & & \\
\hline 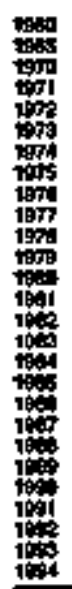 & 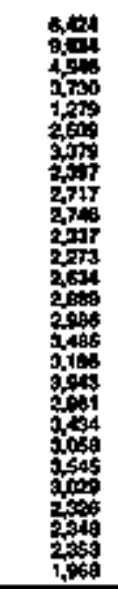 & 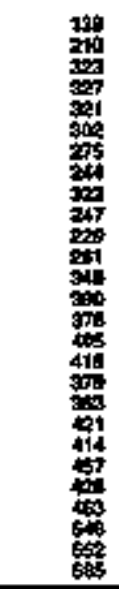 & 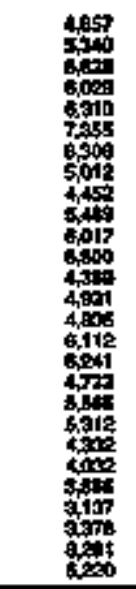 & 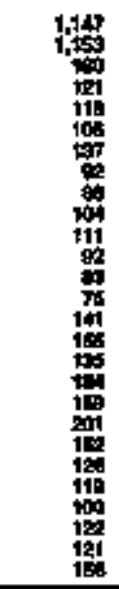 & 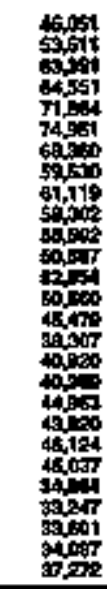 & 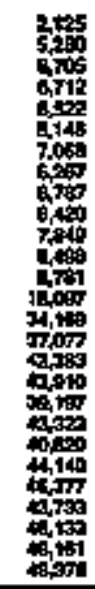 & 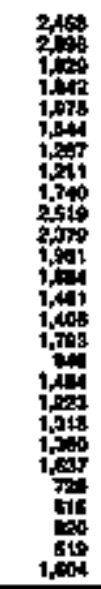 & 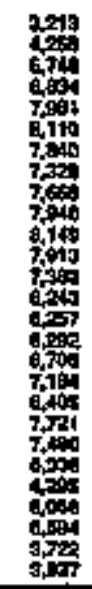 & 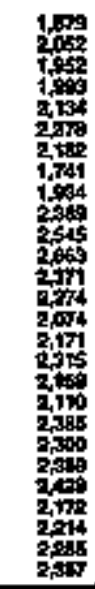 & 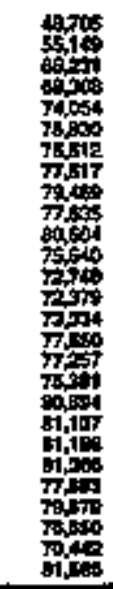 & 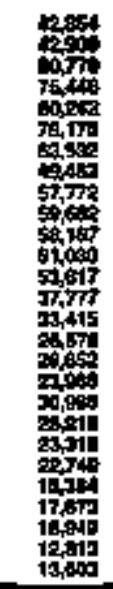 & 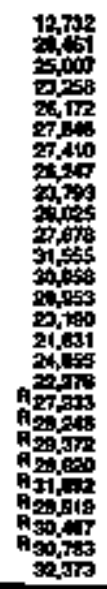 & 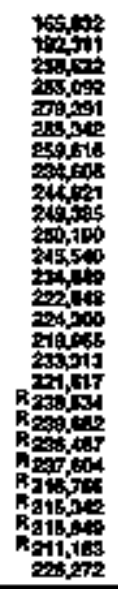 & 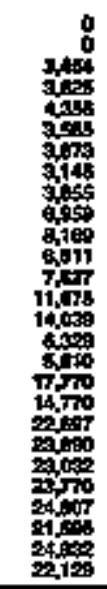 & 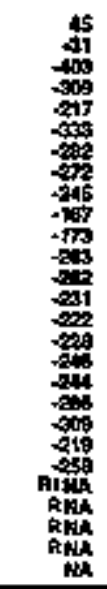 & 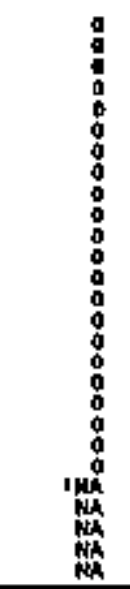 & 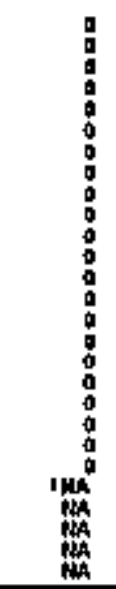 & 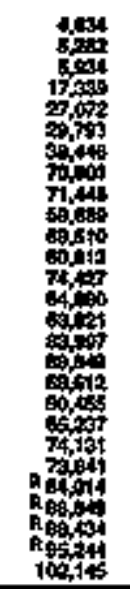 & $\begin{array}{l}z \\
z \\
z \\
z \\
\vdots \\
z \\
\vdots \\
z \\
z \\
z \\
z \\
z \\
z\end{array}$ \\
\hline \multicolumn{20}{|c|}{ nofluton in } \\
\hline 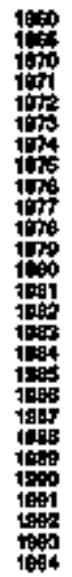 & 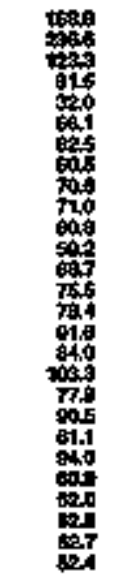 & 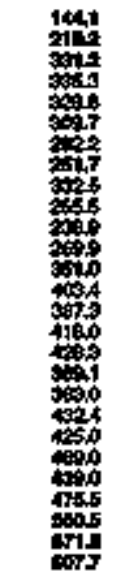 & 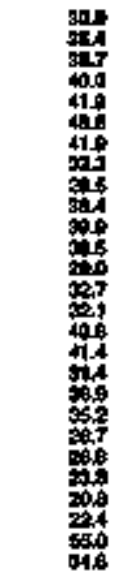 & 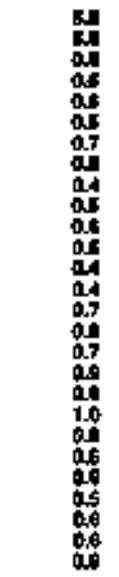 & 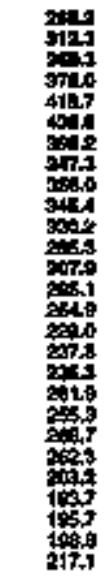 & 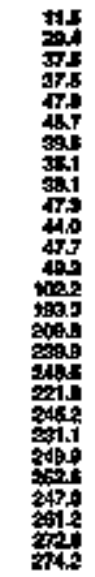 & 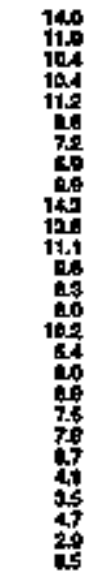 & 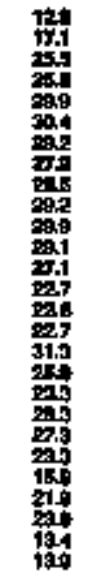 & 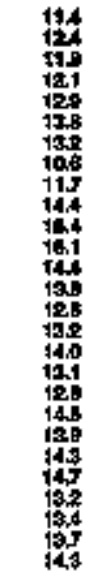 & 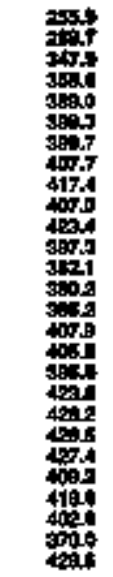 & 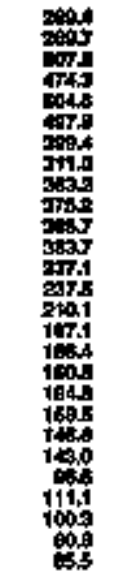 & 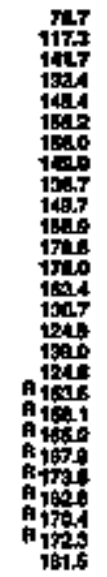 & 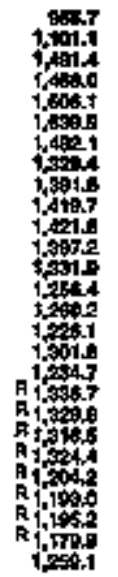 & 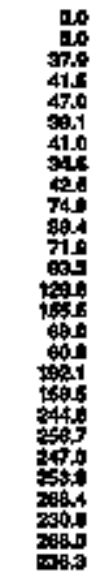 & 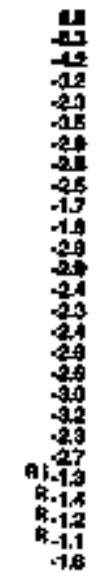 & 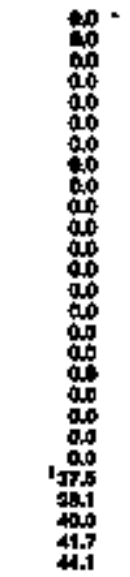 & 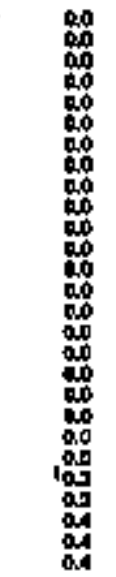 & 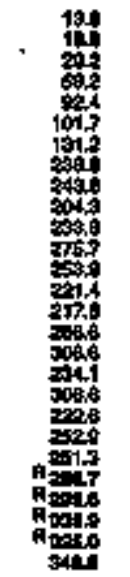 & 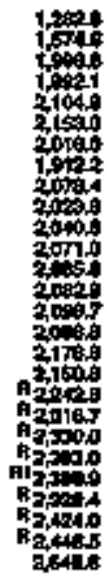 \\
\hline
\end{tabular}

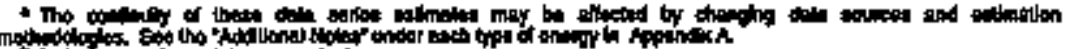

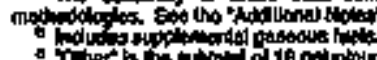

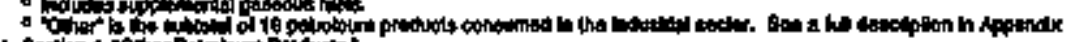

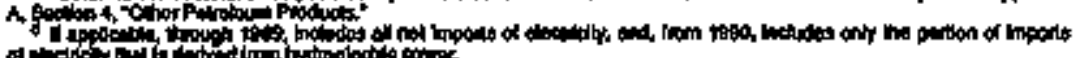

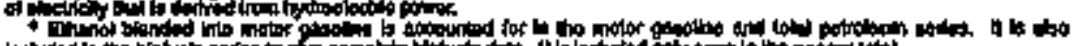

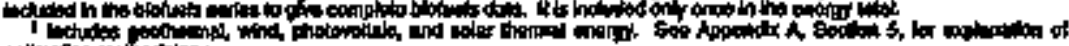

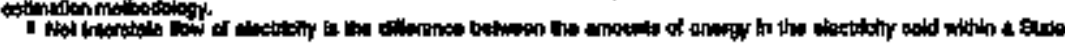

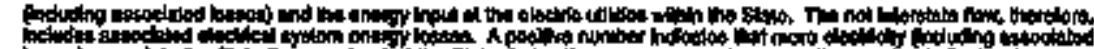

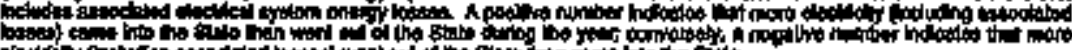

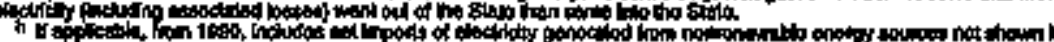

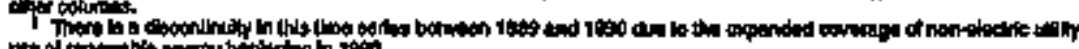

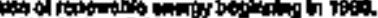
N4)

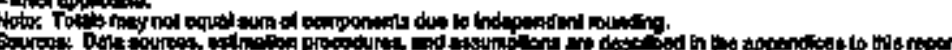


N Table 199. Residential Energy Consumption Estimales, 1960, 1965, 1970-1994, New Jersey

\begin{tabular}{|c|c|c|c|c|c|c|c|c|c|c|c|c|c|c|}
\hline \multirow[b]{3}{*}{ Yot } & \multicolumn{3}{|c|}{ Eow } & \multirow{3}{*}{ 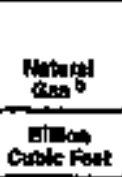 } & \multicolumn{4}{|c|}{ Purother } & \multirow{3}{*}{ 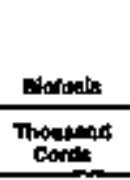 } & \multirow[b]{2}{*}{ golme } & \multirow[b]{2}{*}{ Eeotrieliny" } & \multirow[b]{2}{*}{ Enetrit } & \multirow{3}{*}{ 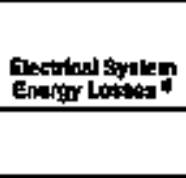 } & \multirow[b]{3}{*}{ Tata } \\
\hline & 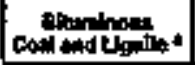 & Anthrecillat & Total & & 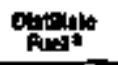 & Kamanna" & LPe: & Tol & & & & & & \\
\hline & \multicolumn{3}{|c|}{ Thentald Shoet Toak } & & \multicolumn{4}{|c|}{ Thousund Banoth } & & \multicolumn{3}{|c|}{ 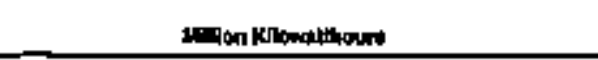 } & & \\
\hline 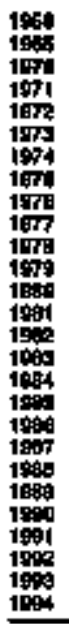 & 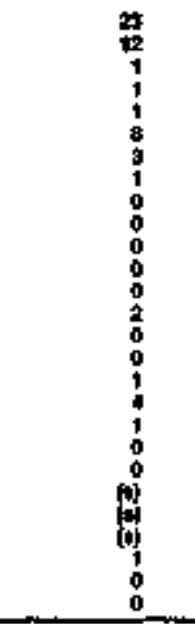 & 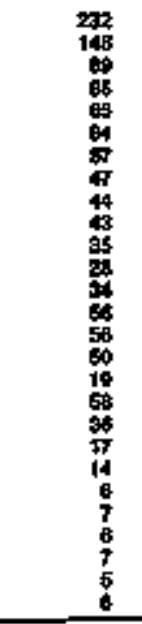 & 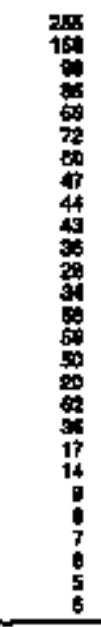 & 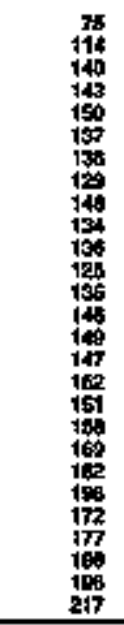 & 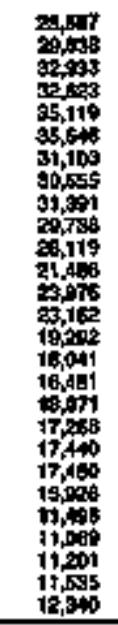 & 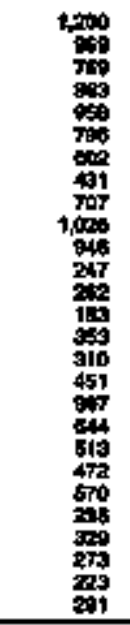 & 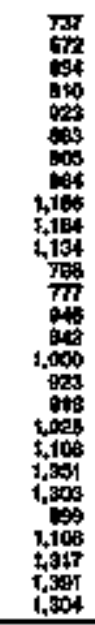 & 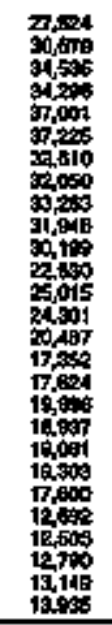 & 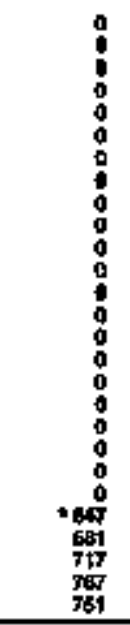 & 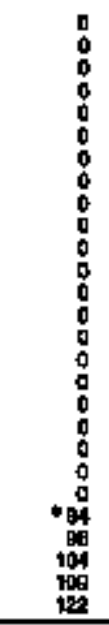 & 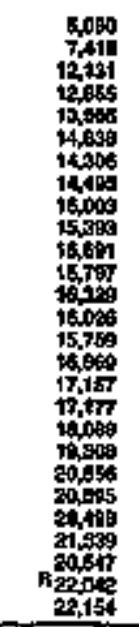 & 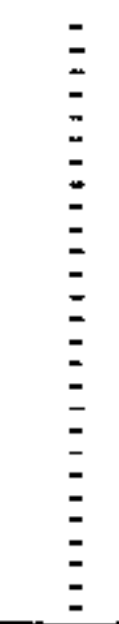 & 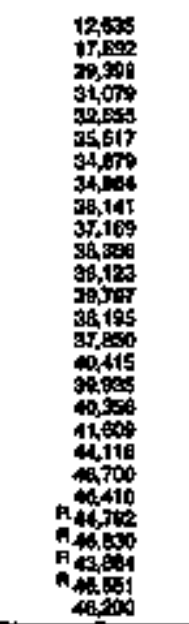 & $\begin{array}{l}= \\
z \\
z \\
z \\
z \\
z \\
z \\
z \\
z \\
z \\
z \\
z \\
z \\
z \\
z\end{array}$ \\
\hline \multicolumn{15}{|c|}{ fithten Eu } \\
\hline 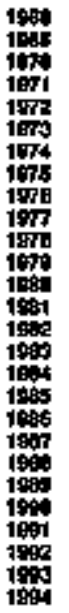 & 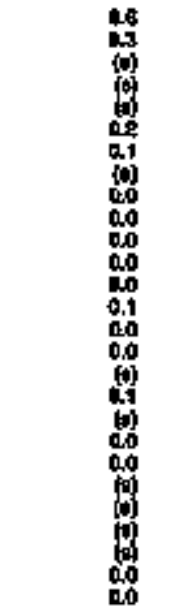 & 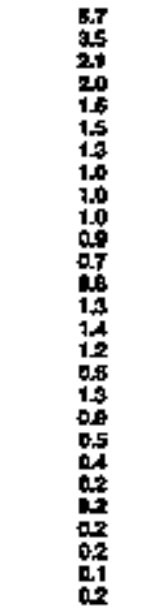 & 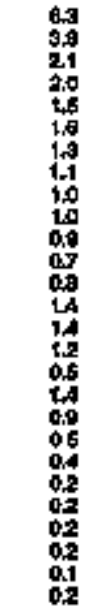 & 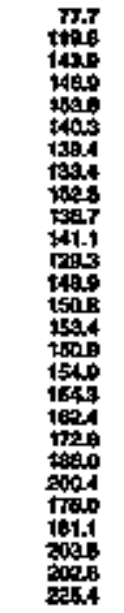 & 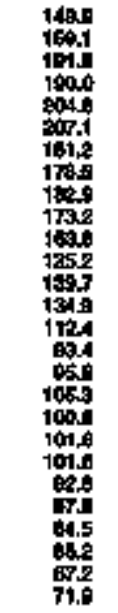 & 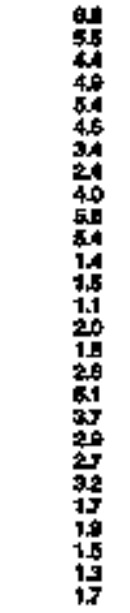 & 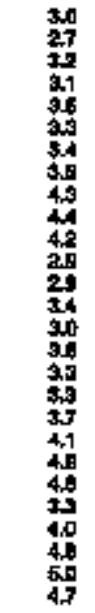 & 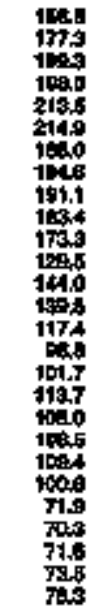 & 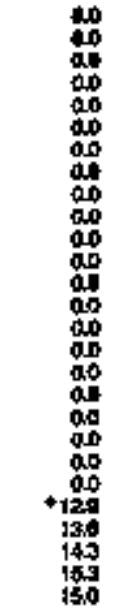 & 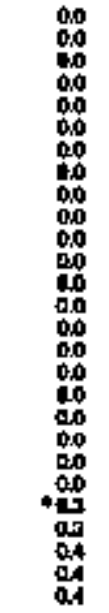 & 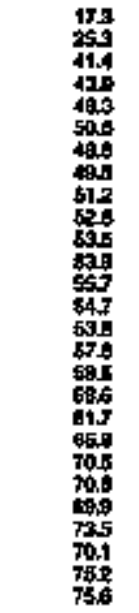 & 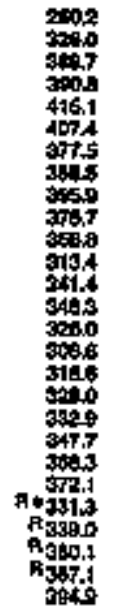 & 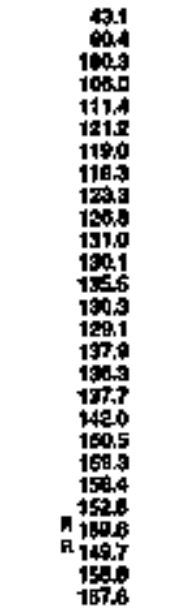 & 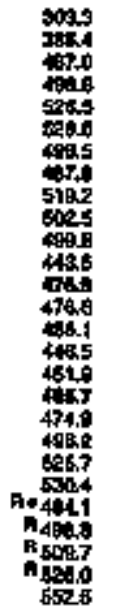 \\
\hline
\end{tabular}


Table 199. Commercial Energy Consumptlon Estimates, 1960, 1955, 1970-1994, Now Jersoy

\begin{tabular}{|c|c|c|c|c|c|c|c|c|c|c|c|c|c|c|}
\hline \multirow[b]{3}{*}{$x \in$} & \multicolumn{3}{|c|}{ Cod } & \multirow[b]{2}{*}{ Mandist } & \multicolumn{6}{|c|}{ Potroloten } & \multirow[b]{2}{*}{ antrifing } & \multirow[b]{2}{*}{ Mot } & \multirow[b]{2}{*}{ 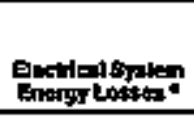 } & \multirow[b]{3}{*}{ Totold } \\
\hline & conturitions & antromitio & Tolat & & 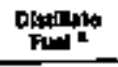 & Karosean:" & LLat. & tholor & 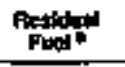 & Totol & & & & \\
\hline & \multicolumn{3}{|c|}{ Thominestorit Tons } & covilofin & \multicolumn{6}{|c|}{ Thousand Gath } & \multicolumn{3}{|c|}{ 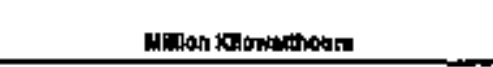 } & \\
\hline 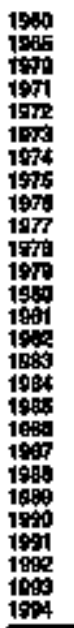 & $\begin{array}{c}0 \\
2 \\
2 \\
1 \\
1 \\
1 \\
15 \\
0 \\
1 \\
0 \\
0 \\
0 \\
0 \\
0 \\
0 \\
0 \\
0 \\
0 \\
3 \\
7 \\
1 \\
0 \\
0 \\
\vdots \\
1 \\
0 \\
0 \\
0 \\
0 \\
0\end{array}$ & 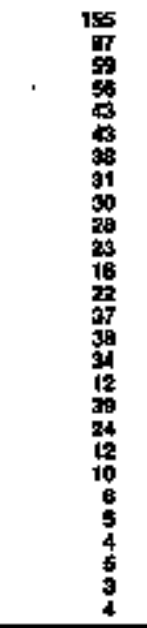 & 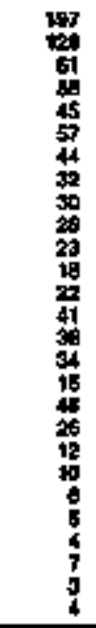 & 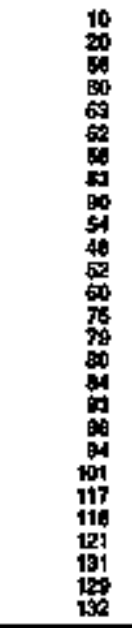 & 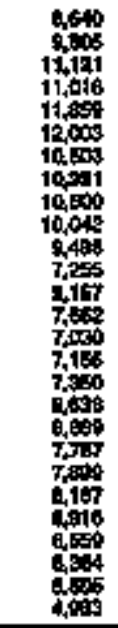 & 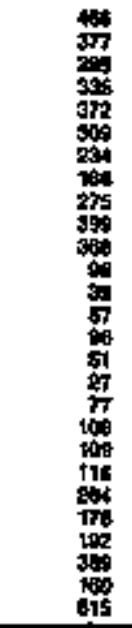 & 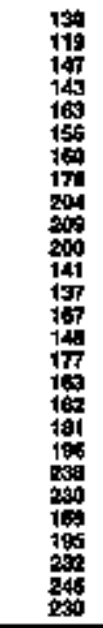 & 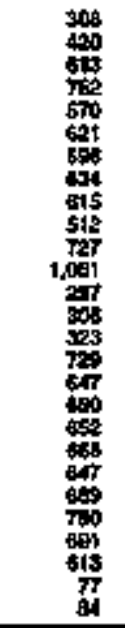 & 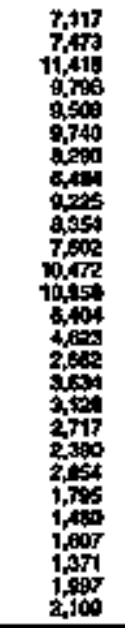 & 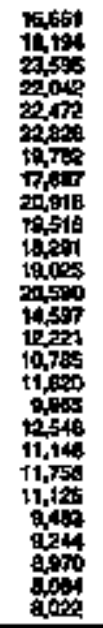 & 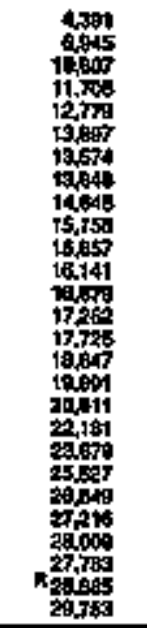 & $\begin{array}{l}z \\
z \\
z \\
z \\
z \\
z \\
z \\
z \\
z \\
\vdots \\
z \\
z \\
z \\
z\end{array}$ & 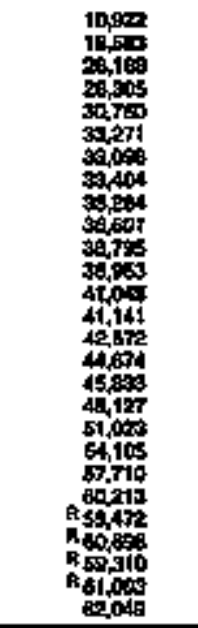 & $\begin{array}{l}\vdots \\
\vdots \\
\vdots \\
\vdots \\
\vdots \\
\vdots \\
\vdots \\
\vdots \\
\vdots \\
\vdots \\
\vdots \\
\vdots \\
\vdots \\
\vdots\end{array}$ \\
\hline \multicolumn{15}{|c|}{ Inflicon Bitu } \\
\hline 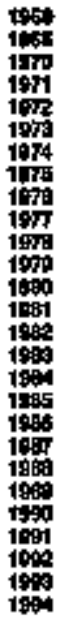 & 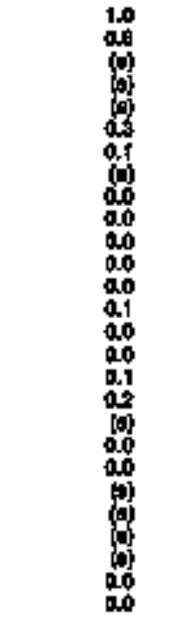 & 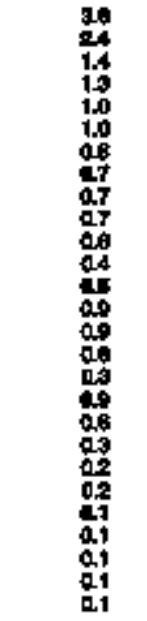 & 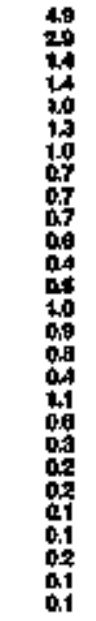 & 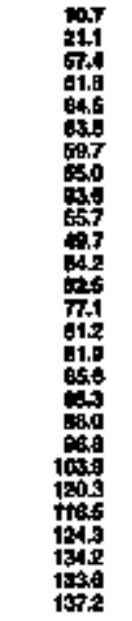 & 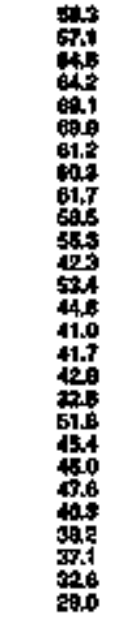 & 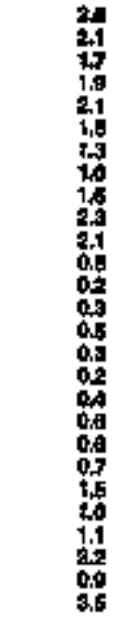 & 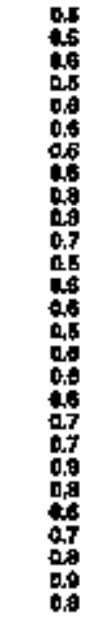 & 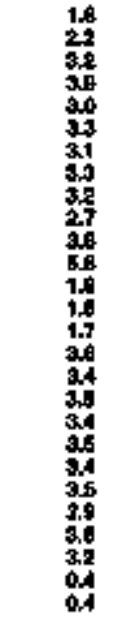 & 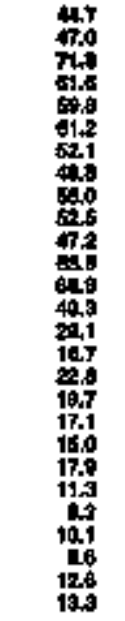 & 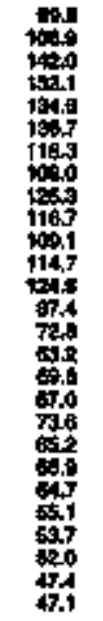 & 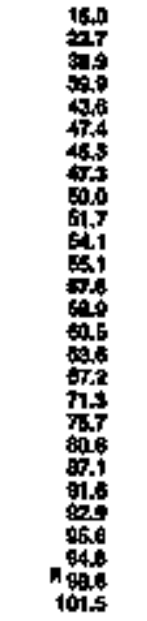 & 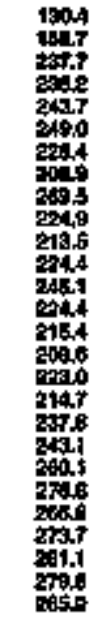 & 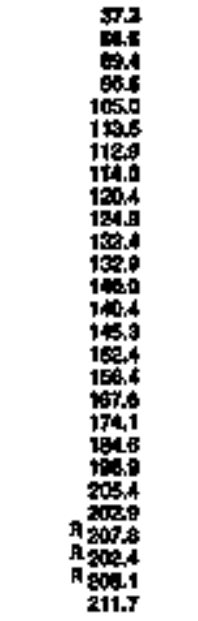 & 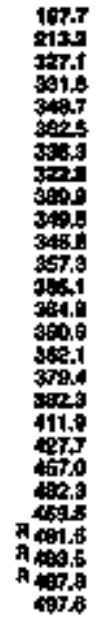 \\
\hline
\end{tabular}


Table 200. Indushtial Energy Consurnption Estimates, 1960, 1965, 1970-1994, New Jersey

\begin{tabular}{|c|c|c|c|c|c|c|c|c|c|c|c|c|c|c|c|c|c|c|}
\hline \multirow[b]{3}{*}{ two } & \multirow[b]{2}{*}{ cont } & \multirow[b]{2}{*}{ 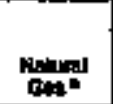 } & \multicolumn{9}{|c|}{ Petrosteran } & \multirow[b]{2}{*}{ Notho- } & \multirow[b]{2}{*}{ Dotusts: I } & \multirow[b]{2}{*}{ Otheren } & \multirow[b]{2}{*}{ Eextilethr| } & \multirow[b]{2}{*}{ Meter } & \multirow{2}{*}{ 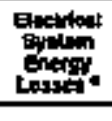 } & \multirow[b]{3}{*}{ Toto } \\
\hline & & & 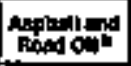 & 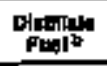 & Kemouna of & LNG: & 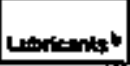 & Motor & Fudedst & Dither & Tabal & & & & & & & \\
\hline & nhorend & cilie Fin & \multicolumn{9}{|c|}{ 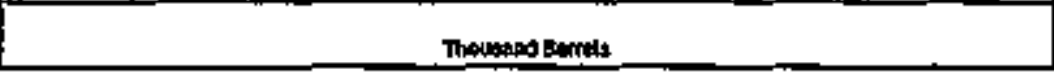 } & \multicolumn{6}{|c|}{ Wintion IStow thown } & \\
\hline 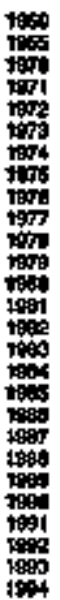 & 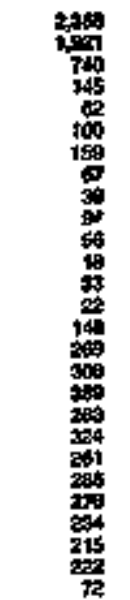 & 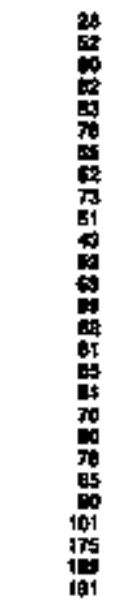 & 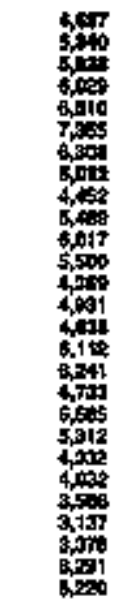 & 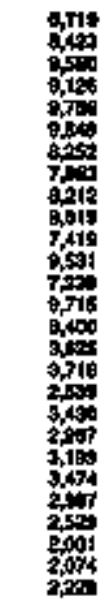 & 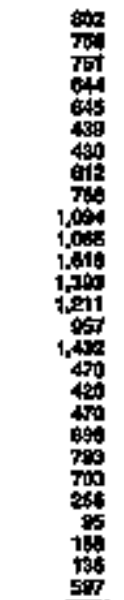 & 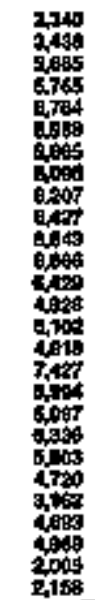 & 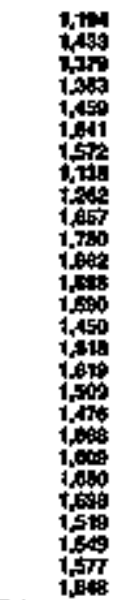 & 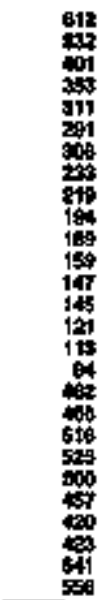 & 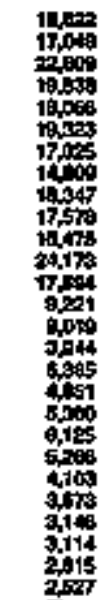 & 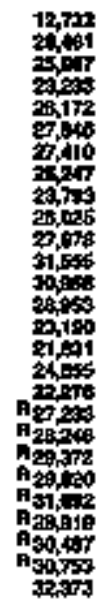 & 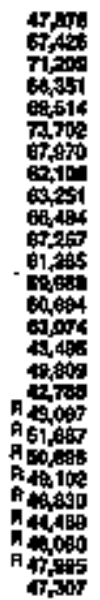 & 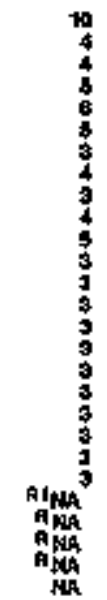 & 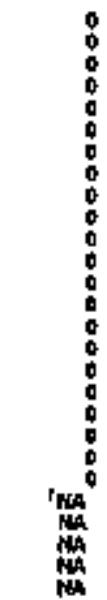 & 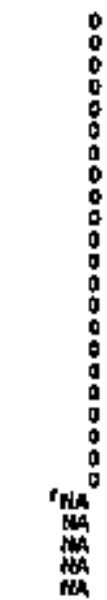 & 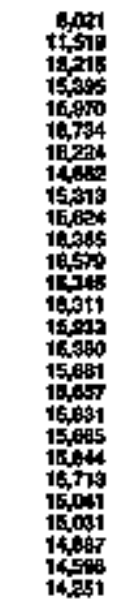 & $\begin{array}{l}z \\
z \\
z \\
z \\
z \\
z \\
z \\
z \\
z \\
z \\
z \\
z \\
z \\
z\end{array}$ & 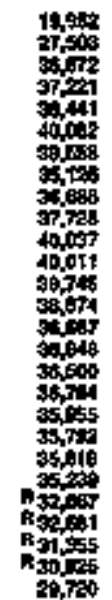 & $\begin{array}{l}\bar{z} \\
\overline{5} \\
\overline{ } \\
\overline{ } \\
\overline{ } \\
\bar{z}\end{array}$ \\
\hline
\end{tabular}

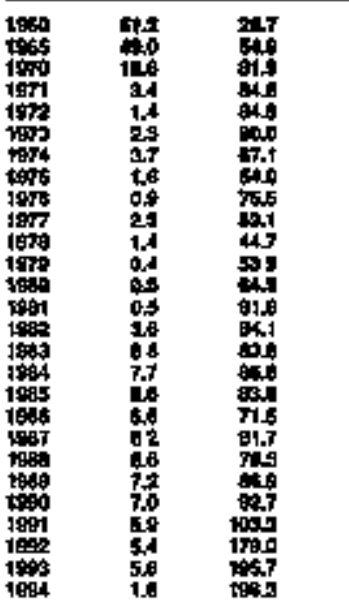

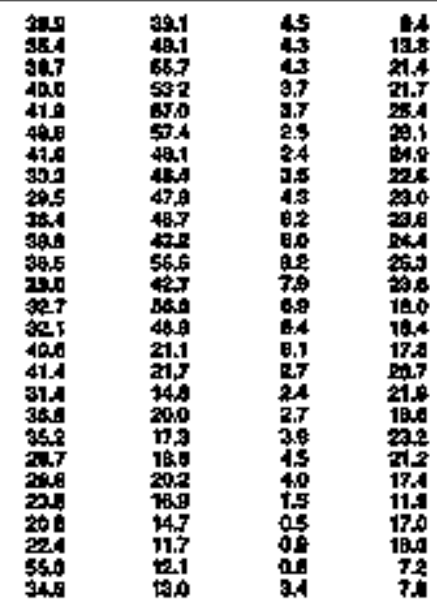

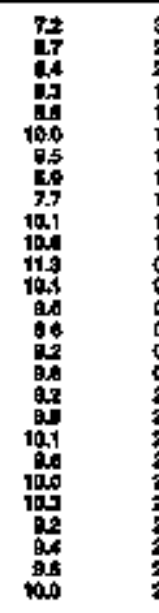

Tmand and

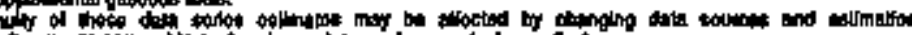

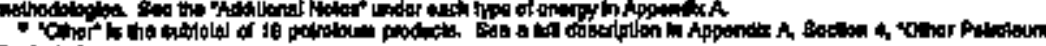

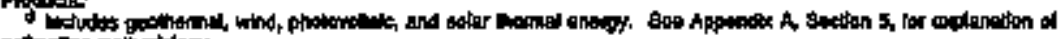

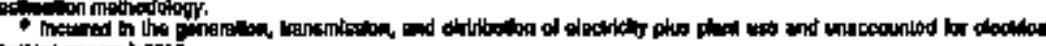

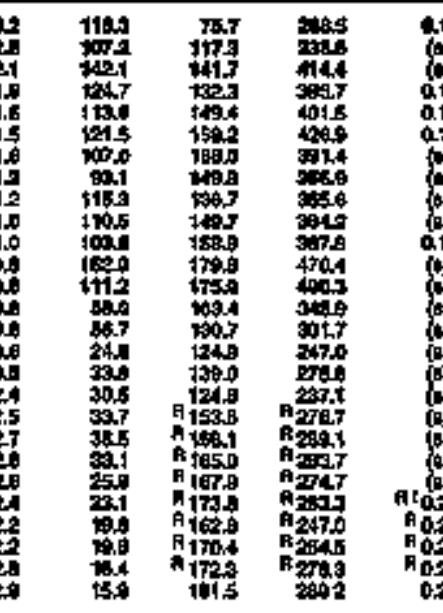

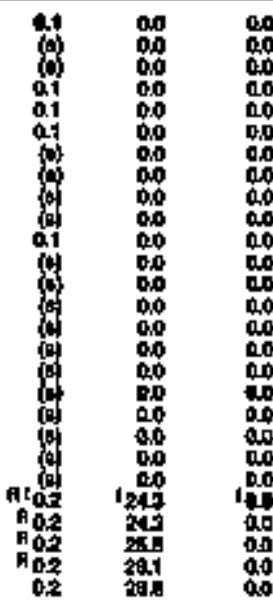

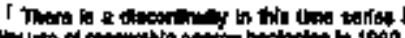

\begin{tabular}{|c|c|c|c|}
\hline 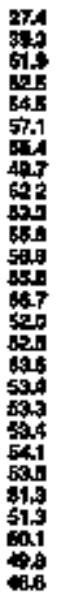 & 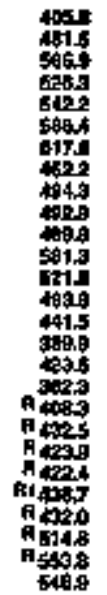 & 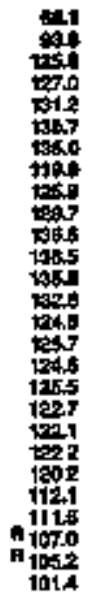 & 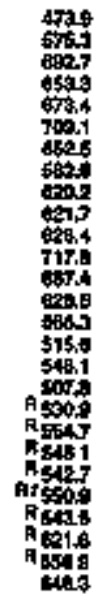 \\
\hline
\end{tabular}

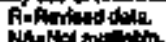
whenction.

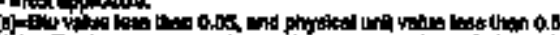

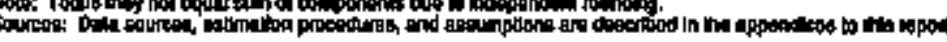


Table 20t. Transportation Energy Consianptian Estlmates, 1950, 1955, 1570-1994, Now dorsey

\begin{tabular}{|c|c|c|c|c|c|c|c|c|c|c|c|c|c|c|c|}
\hline \multirow[b]{3}{*}{ rem } & \multirow[b]{2}{*}{ can' } & \multirow[b]{2}{*}{ Mutorsts } & \multicolumn{8}{|c|}{ 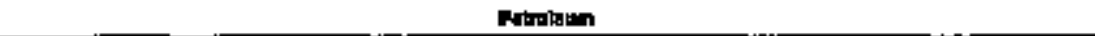 } & \multirow{3}{*}{ 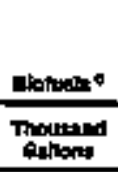 } & & \multirow[b]{2}{*}{ thent } & \multirow[b]{2}{*}{ 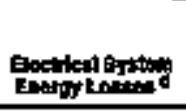 } & \multirow[b]{3}{*}{ Tolut } \\
\hline & & & notionicion. & Detplints & Dive & Lo: & Lbokante | & ationser & moenter & Tot & & Boctiven" & & & \\
\hline & Thandind & 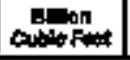 & \multicolumn{8}{|c|}{ moutmals:melr } & & \multicolumn{3}{|c|}{ 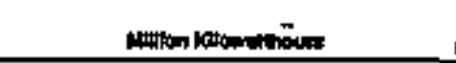 } & \\
\hline 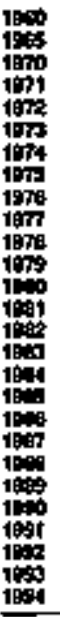 & $\begin{array}{l}40 \\
5 \\
1 \\
0 \\
0 \\
0 \\
0 \\
09 \\
90 \\
0 \\
0 \\
0 \\
0 \\
0 \\
0 \\
0 \\
0 \\
0 \\
0 \\
0 \\
0 \\
0 \\
0 \\
0 \\
0\end{array}$ & $\begin{array}{r}1 \\
09 \\
1 \\
1 \\
1 \\
1 \\
1 \\
0 \\
1 \\
1 \\
1 \\
1 \\
04 \\
1 \\
1 \\
1 \\
2 \\
2 \\
3 \\
3 \\
j \\
4 \\
3 \\
3 \\
5 \\
3 \\
3 \\
\end{array}$ & 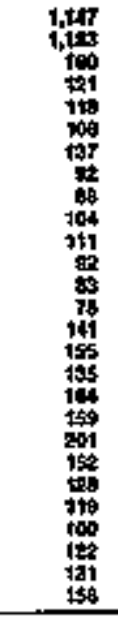 & 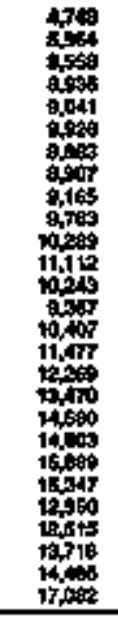 & 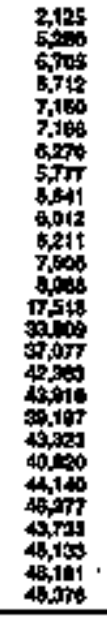 & 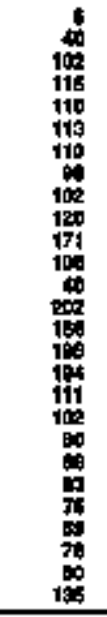 & 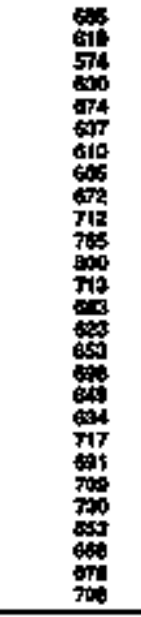 & 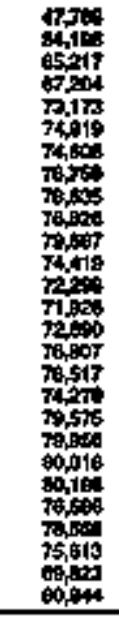 & 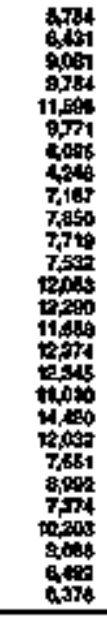 & 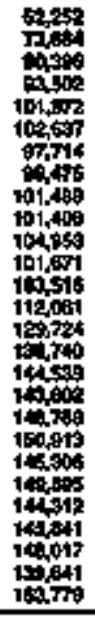 & 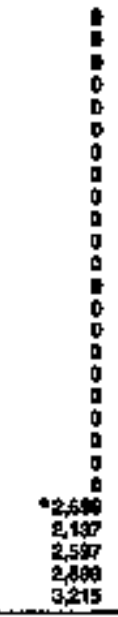 & 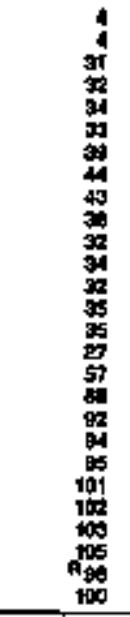 & $\begin{array}{l}= \\
= \\
= \\
= \\
= \\
= \\
= \\
= \\
= \\
= \\
= \\
= \\
= \\
= \\
\end{array}$ & 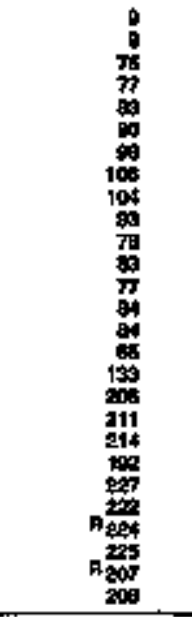 & 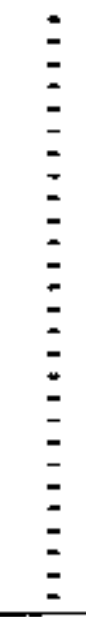 \\
\hline \multicolumn{16}{|c|}{ Todlison 助 } \\
\hline 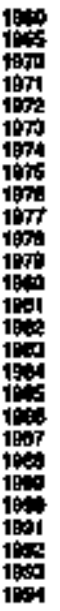 & 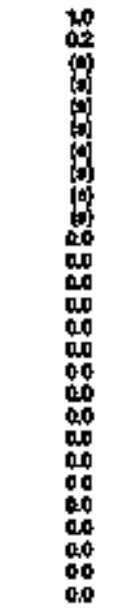 & 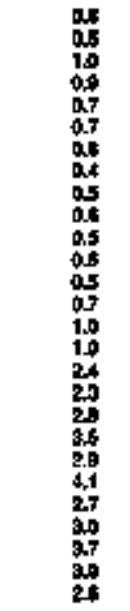 & 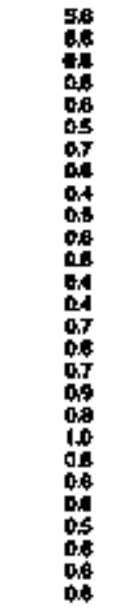 & 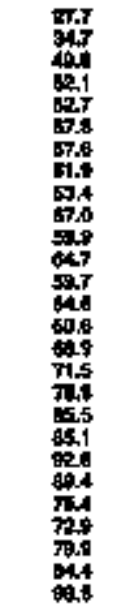 & 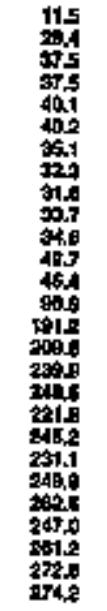 & 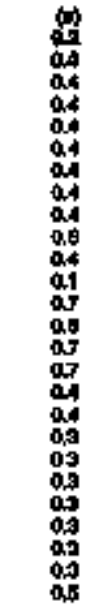 & 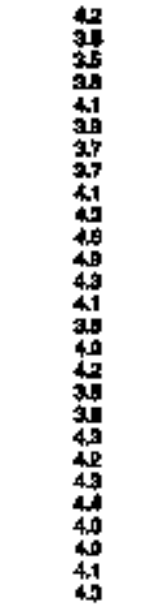 & 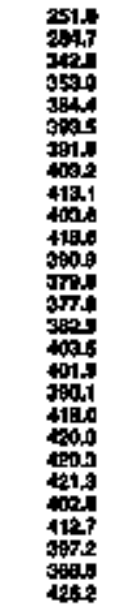 & 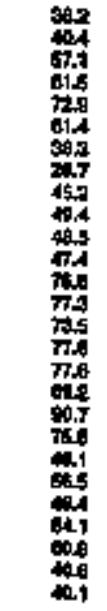 & 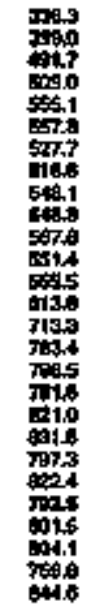 & 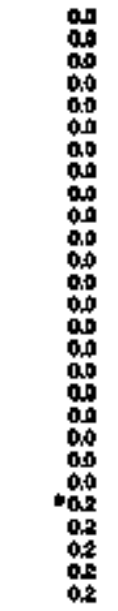 & 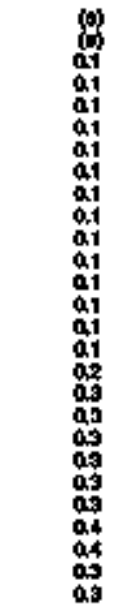 & 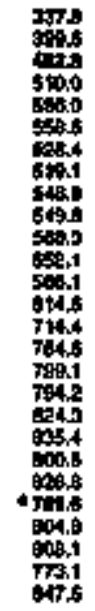 & 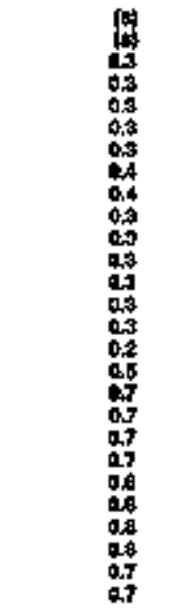 & 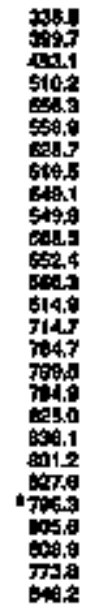 \\
\hline
\end{tabular}

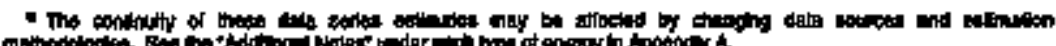

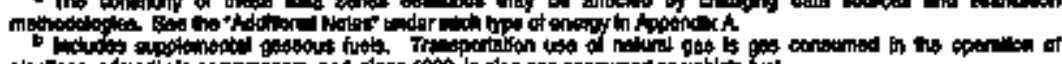

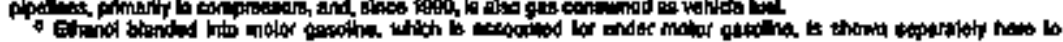

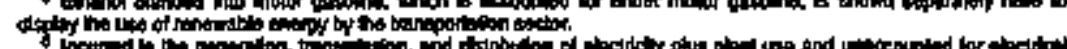

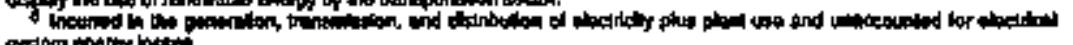

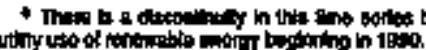

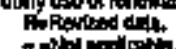




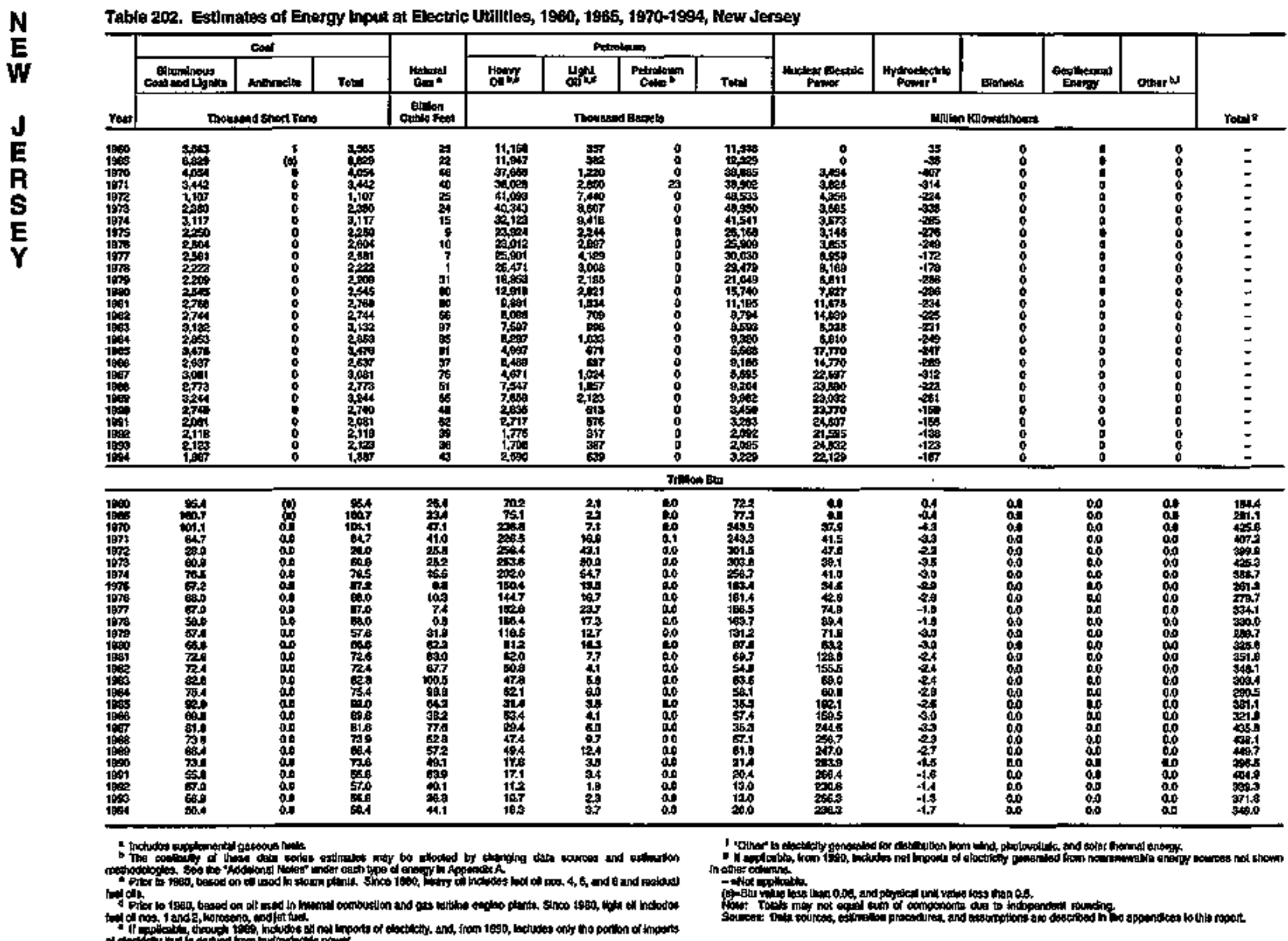


Table 203. Energy Consumption Estimates by 5ource, 1860, t965, 1970-1994, New Mexico

\begin{tabular}{|c|c|c|c|c|c|c|c|c|c|c|c|c|c|c|c|c|c|c|c|}
\hline \multirow[b]{3}{*}{ r } & \multirow[b]{2}{*}{ Conls } & \multirow[b]{2}{*}{ Existid } & \multicolumn{11}{|c|}{ Potroterin } & \multirow[b]{2}{*}{ 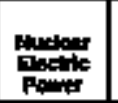 } & \multirow[b]{2}{*}{ 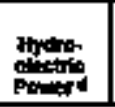 } & \multirow[b]{2}{*}{ Dofodelt: } & \multirow[b]{2}{*}{ onso } & \multirow{3}{*}{ 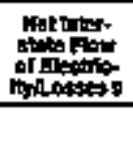 } & \multirow[b]{3}{*}{ Tol:t } \\
\hline & & & Atphatond & Arandos & 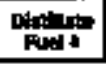 & Fet. & Kors & LPQD & cont. & 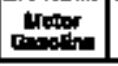 & Fatided & Otbaras & Tate & & & & & & \\
\hline & Thouttind & 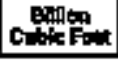 & \multicolumn{11}{|c|}{ Thoustad baneh } & \multicolumn{4}{|c|}{ 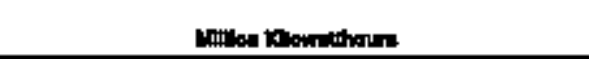 } & & \\
\hline 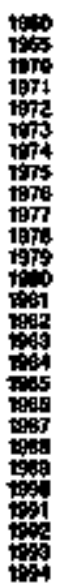 & 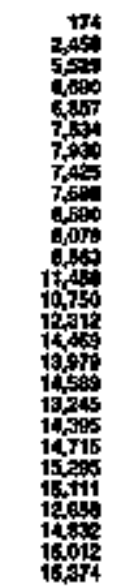 & 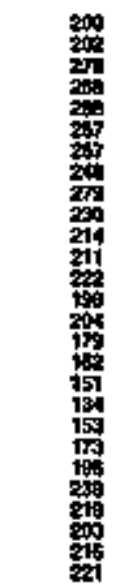 & 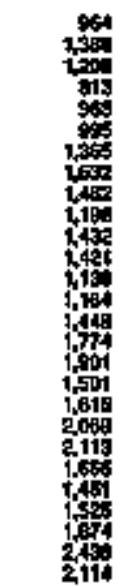 & 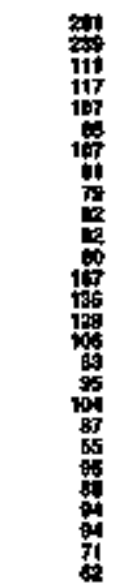 & 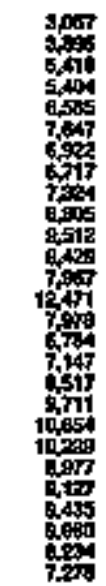 & 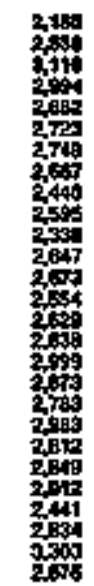 & 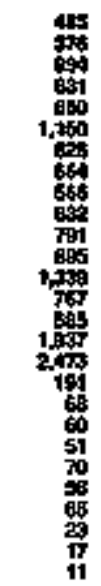 & 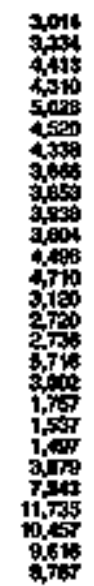 & 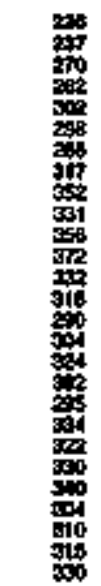 & 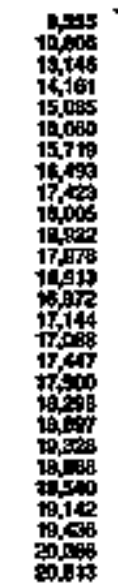 & 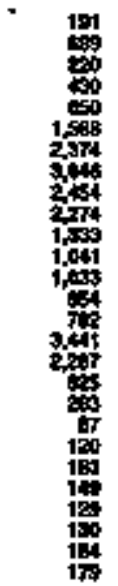 & 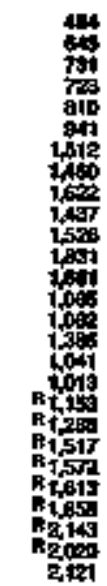 & 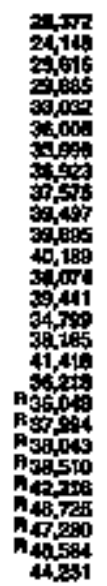 & 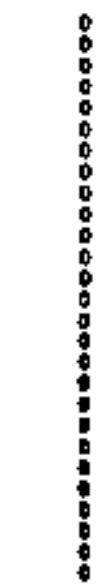 & 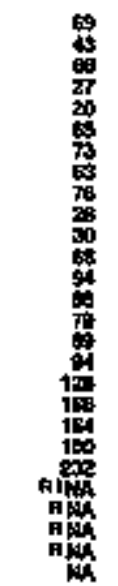 & 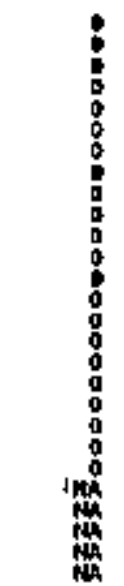 & 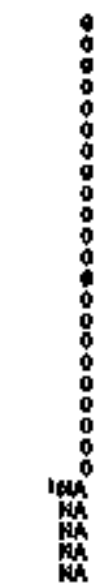 & 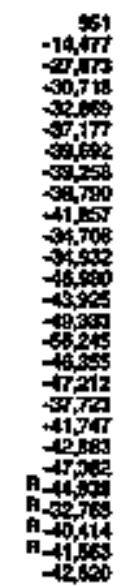 & $\begin{array}{l}z \\
\vdots \\
\vdots \\
\vdots \\
z \\
z \\
z \\
z \\
z \\
z \\
z \\
z \\
z\end{array}$ \\
\hline & & & & & & & & & & 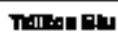 & & & & & & & & & \\
\hline
\end{tabular}

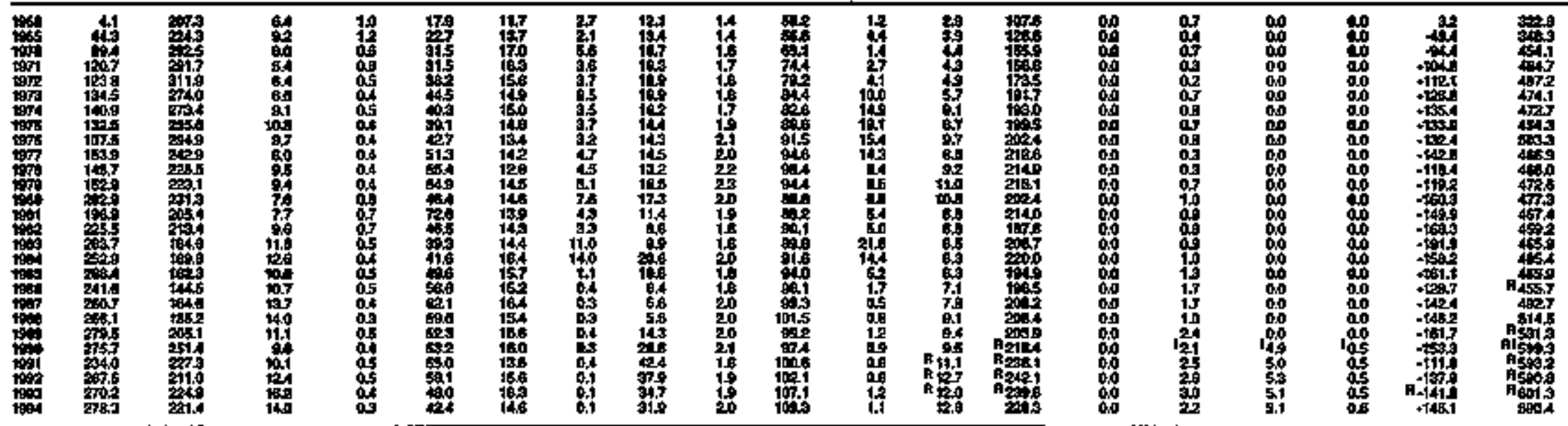

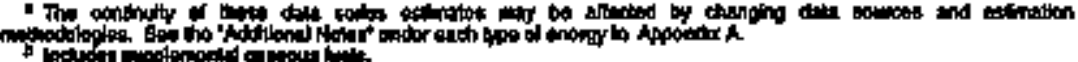

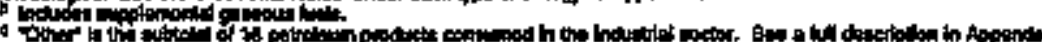

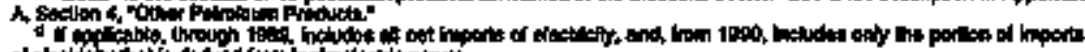

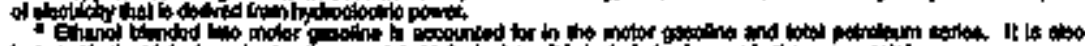

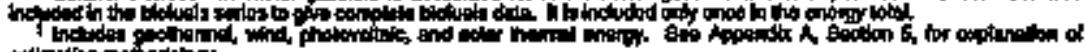

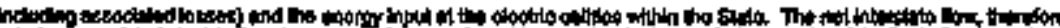

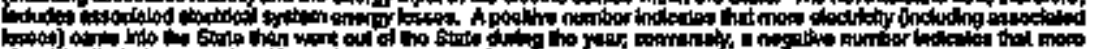

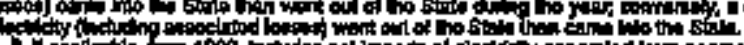

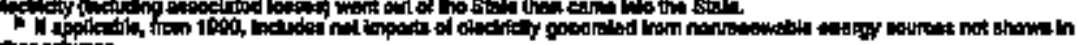

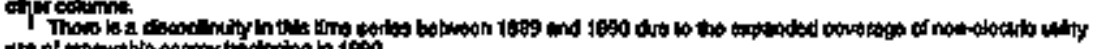

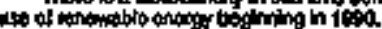
Muthot

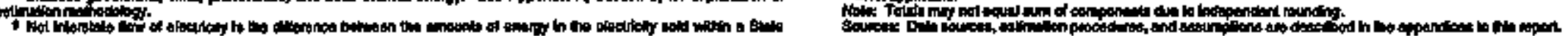


N Table 204. Aesidential Energy Consumption Estitnates, 1960, 1955, 1970-1994, New Mexico

\begin{tabular}{|c|c|c|c|c|c|c|c|c|c|c|c|c|c|c|}
\hline \multirow[b]{3}{*}{ Yepe } & \multicolumn{3}{|c|}{ Con } & \multirow{3}{*}{ 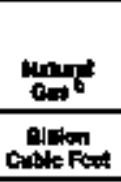 } & \multicolumn{4}{|c|}{ Purolom } & \multirow{3}{*}{ 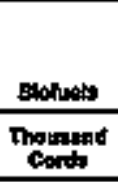 } & & \multirow[b]{2}{*}{ Euctileth ${ }^{2}$} & \multirow[b]{2}{*}{ Enet } & \multirow{3}{*}{ 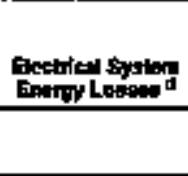 } & \multirow[b]{3}{*}{ Toull } \\
\hline & 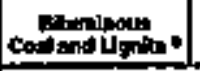 & 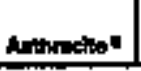 & Tots & & 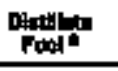 & Kitosinat & LPG: & Tokl & & Sotur & & & & \\
\hline & \multicolumn{3}{|c|}{ mousind short Toas: } & & \multicolumn{4}{|c|}{ 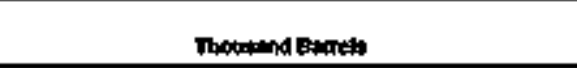 } & & \multicolumn{3}{|c|}{ 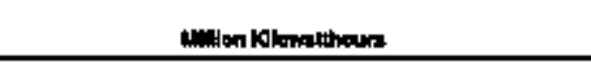 } & & \\
\hline 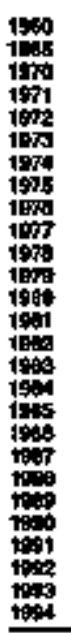 & $\begin{array}{c}15 \\
0 \\
4 \\
0 \\
0 \\
0 \\
0 \\
0 \\
0 \\
0 \\
1 \\
15 \\
2 \\
4 \\
4 \\
3 \\
3 \\
2 \\
2 \\
1 \\
3 \\
2 \\
3 \\
3 \\
3 \\
3\end{array}$ & $\begin{array}{l}0 \\
0 \\
0 \\
0 \\
0 \\
0 \\
0 \\
0 \\
0 \\
0 \\
0 \\
0 \\
0 \\
0 \\
0 \\
0 \\
0 \\
0 \\
0 \\
0 \\
0 \\
0 \\
0 \\
0 \\
0 \\
0\end{array}$ & $\begin{array}{c}15 \\
4 \\
00 \\
0 \\
0 \\
0 \\
0 \\
0 \\
0 \\
0 \\
0 \\
1 \\
15 \\
2 \\
4 \\
4 \\
3 \\
3 \\
2 \\
3 \\
1 \\
5 \\
2 \\
5 \\
3 \\
4 \\
\end{array}$ & 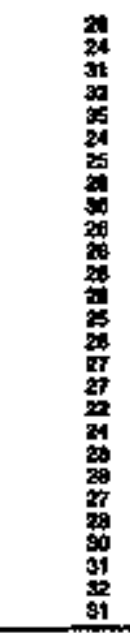 & 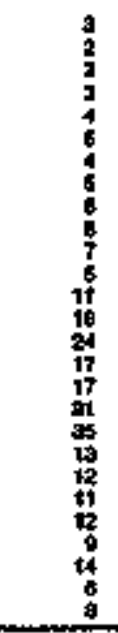 & 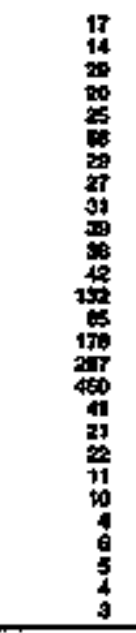 & 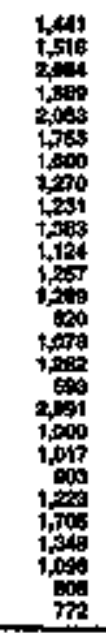 & 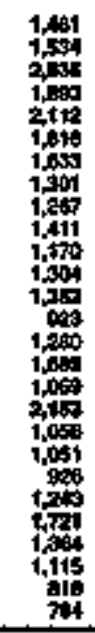 & 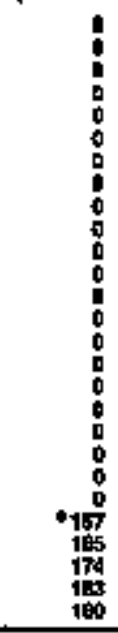 & $\begin{array}{r}1 \\
0 \\
0 \\
0 \\
0 \\
0 \\
0 \\
0 \\
0 \\
0 \\
0 \\
0 \\
0 \\
0 \\
0 \\
0 \\
0 \\
140 \\
150 \\
150 \\
105 \\
155 \\
\end{array}$ & 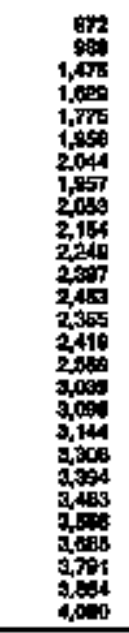 & $\begin{array}{l}= \\
= \\
= \\
z \\
= \\
= \\
= \\
= \\
= \\
= \\
= \\
= \\
= \\
=\end{array}$ & 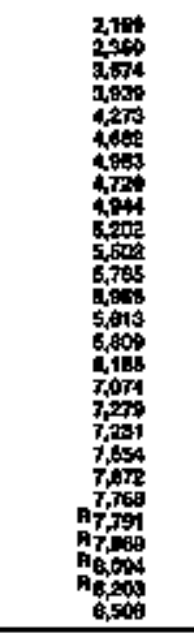 & $\begin{array}{l}z \\
z \\
z \\
z \\
z \\
z \\
z \\
z \\
z \\
z \\
z \\
z \\
z \\
z \\
z\end{array}$ \\
\hline \multicolumn{15}{|c|}{ Tfiflomentip } \\
\hline 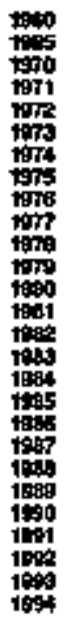 & 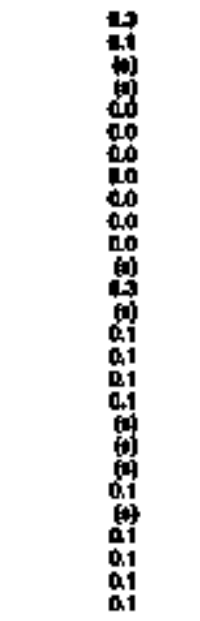 & 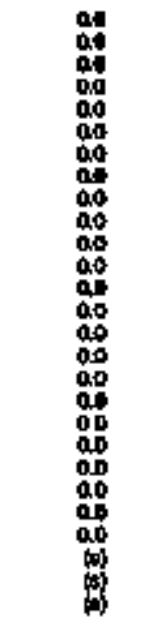 & 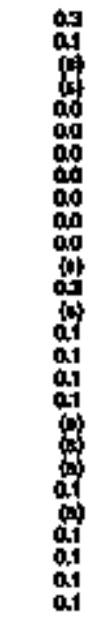 & 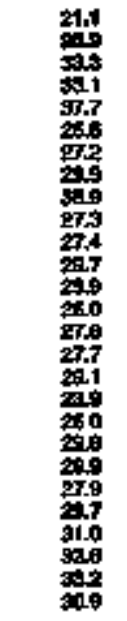 & 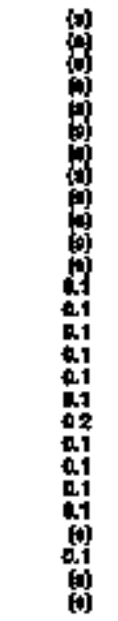 & 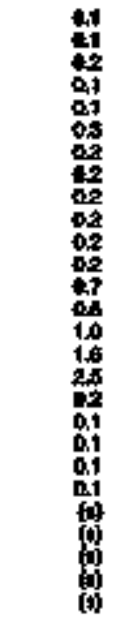 & 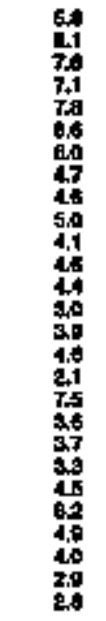 & 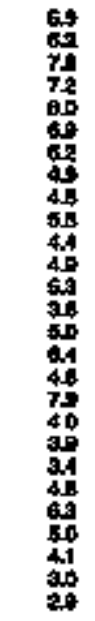 & 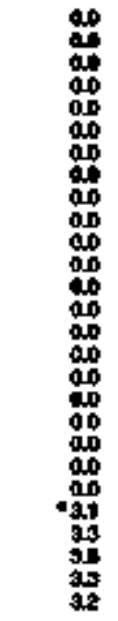 & 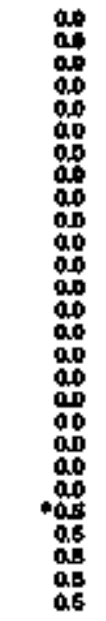 & 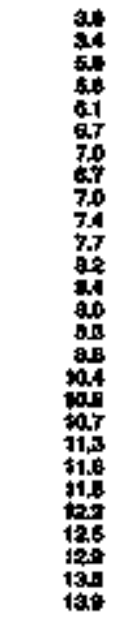 & 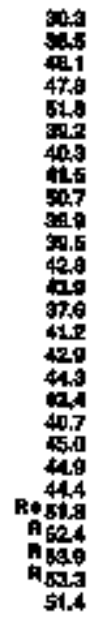 & 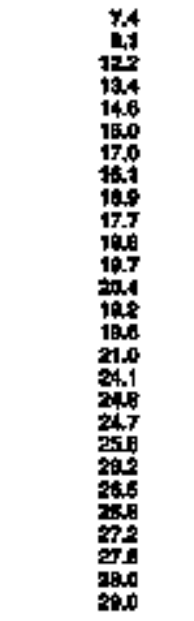 & 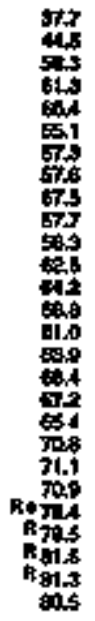 \\
\hline
\end{tabular}

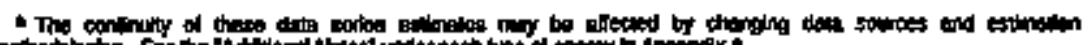

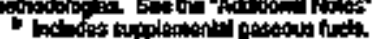

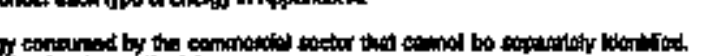

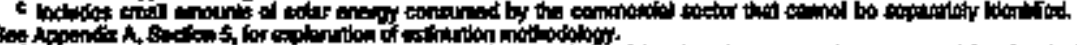

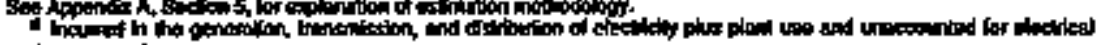

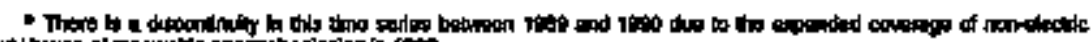

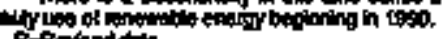

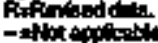


Table 205. Commerclal Energy Consumption Estimates, 1960, 1965, 1970-1994, Now Lexico

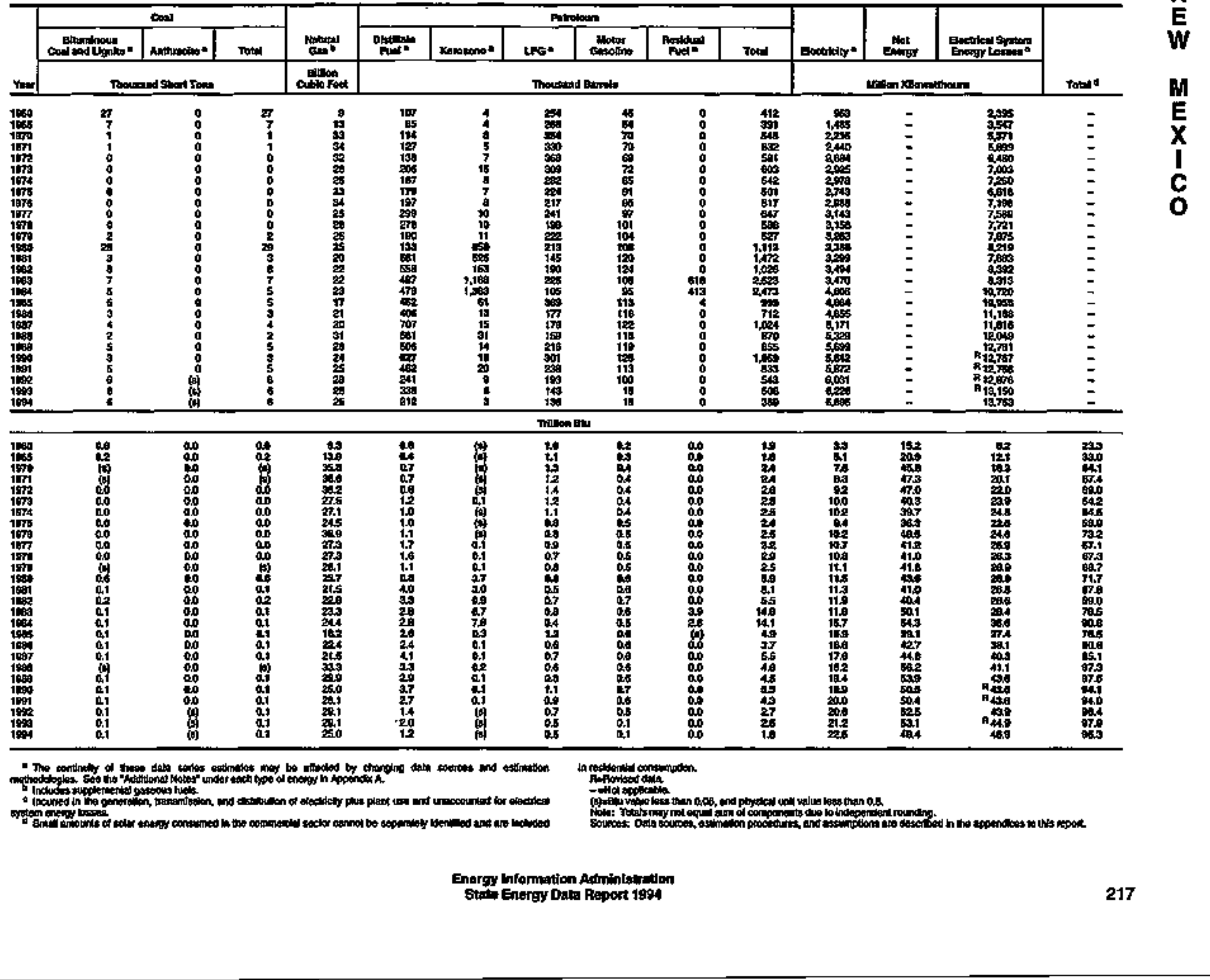


N Table 206. Industrfal Energy Consumption Estimates, 1969, 1965, 1970-1994, Naw Uaxito

$\mathbf{E}$
$\mathbf{W}$
$\mathbf{M}$
$\mathbf{X}$
$\mathbf{I}$
$\mathbf{C}$
$\mathbf{0}$

\begin{tabular}{|c|c|c|c|c|c|c|c|c|c|c|c|c|c|c|c|c|c|c|}
\hline \multirow{2}{*}{\multicolumn{2}{|c|}{ con }} & \multirow[b]{2}{*}{ thent } & \multicolumn{9}{|c|}{ Dathont } & \multirow[b]{2}{*}{ Pats } & \multirow[b]{2}{*}{ Datoment } & \multirow[b]{2}{*}{ otsons } & \multirow[b]{2}{*}{ andiong } & \multirow[b]{2}{*}{ Smentin } & \multirow{2}{*}{ 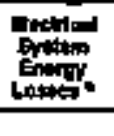 } & \multirow[b]{3}{*}{ The } \\
\hline & & & 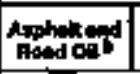 & 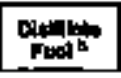 & Komananel| & $\theta$ & whoseme" & mations & 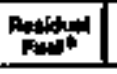 & onowat & Total & & & & & & & \\
\hline 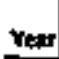 & 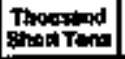 & אer & \multicolumn{9}{|c|}{ 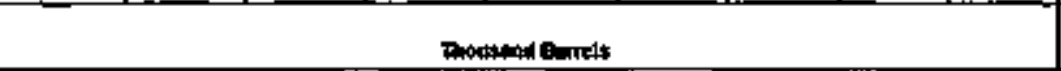 } & \multicolumn{6}{|c|}{ 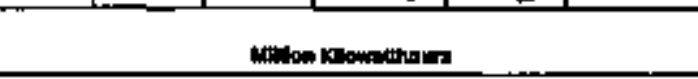 } & \\
\hline & 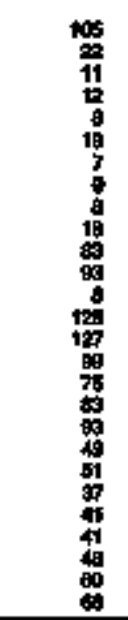 & 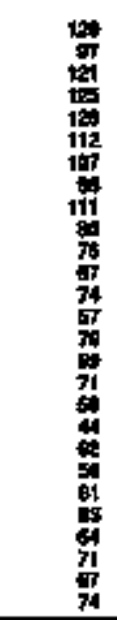 & 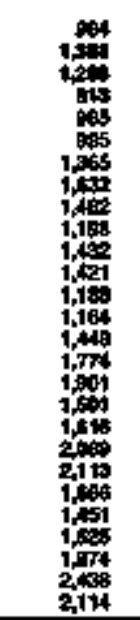 & 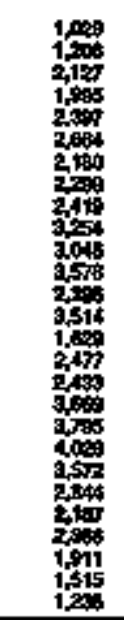 & 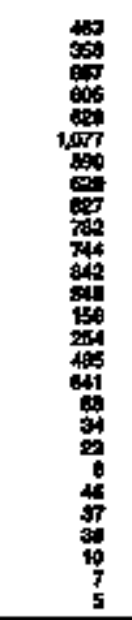 & 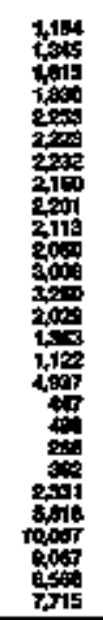 & 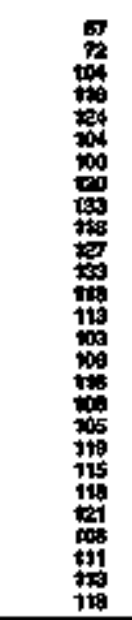 & 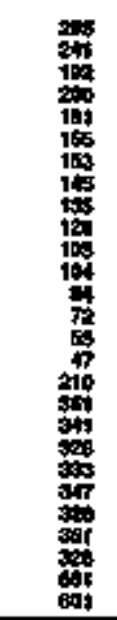 & 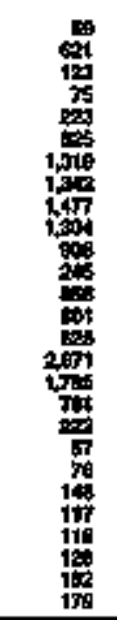 & 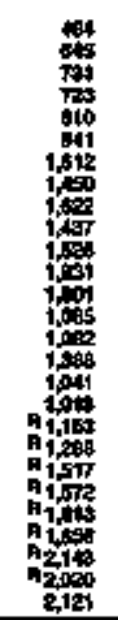 & 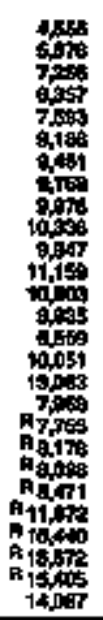 & 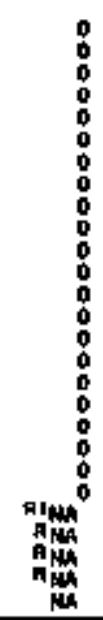 & $\begin{array}{r}0 \\
0 \\
0 \\
0 \\
0 \\
0 \\
0 \\
0 \\
0 \\
0 \\
0 \\
0 \\
\text { on }\end{array}$ & 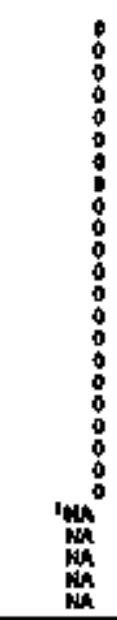 & 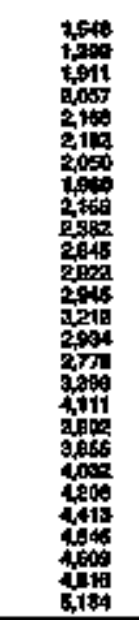 & $\Xi$ & 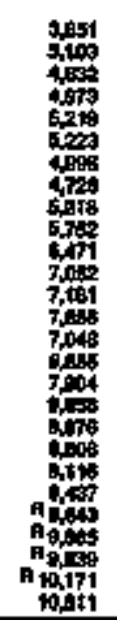 & $=$ \\
\hline \multicolumn{19}{|c|}{ naliman } \\
\hline & 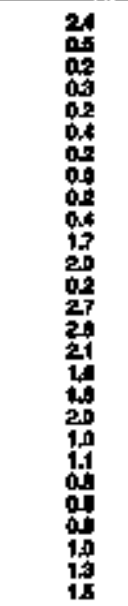 & 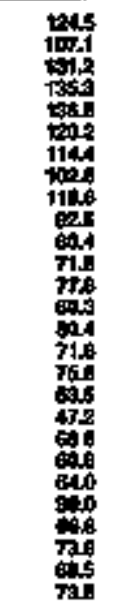 & 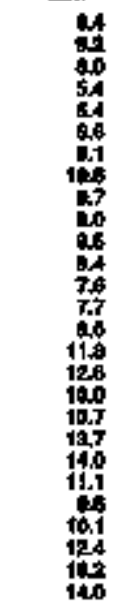 & 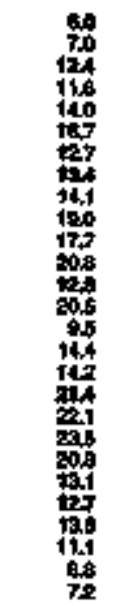 & 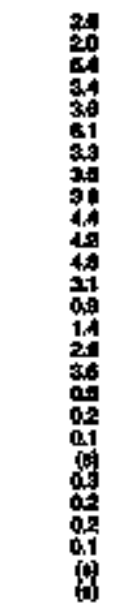 & 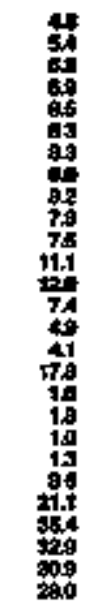 & 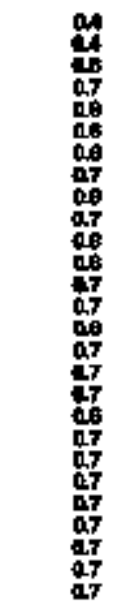 & 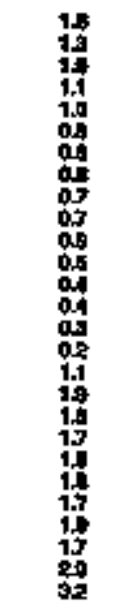 & 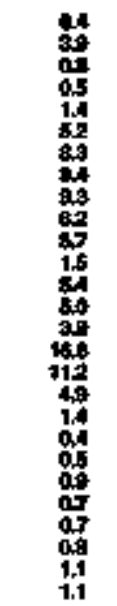 & 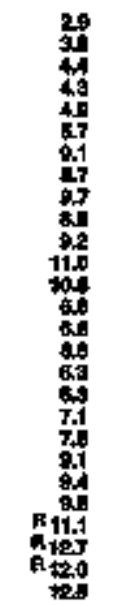 & 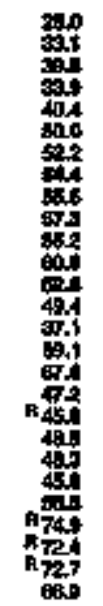 & 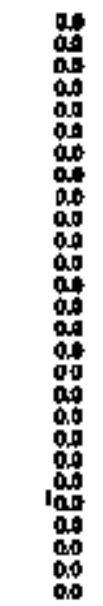 & 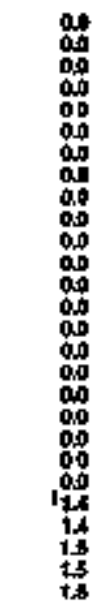 & 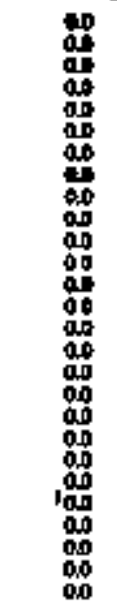 & 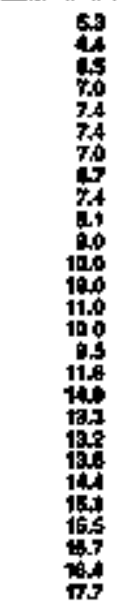 & 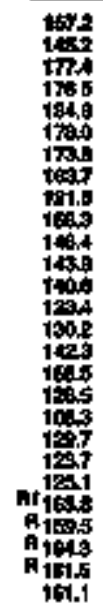 & 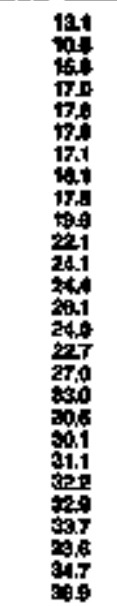 & 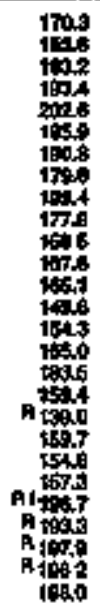 \\
\hline
\end{tabular}

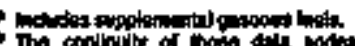

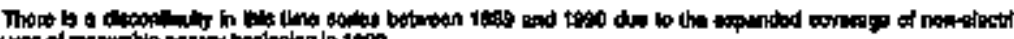

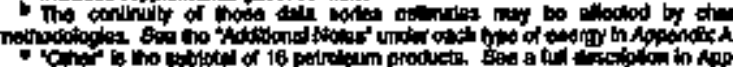

(1)

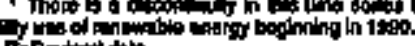

.

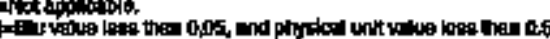

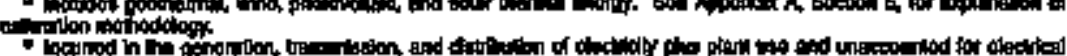

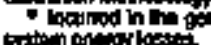

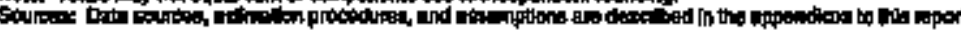


Table 207. Transportation Energy Consuchitlon Estlinales, 195D, 1965, 1970-1994, Now Hexico

\begin{tabular}{|c|c|c|c|c|c|c|c|c|c|c|c|c|c|c|c|}
\hline \multirow[b]{3}{*}{ إI } & \multirow[b]{2}{*}{ cons. } & \multirow[b]{2}{*}{ Mations } & \multicolumn{8}{|c|}{ Protamen } & \multirow[b]{2}{*}{ Diotucke" } & \multirow[b]{2}{*}{ Eoctraly" } & \multirow[b]{2}{*}{ Iten } & \multirow[b]{2}{*}{ 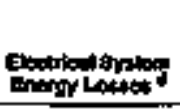 } & \multirow[b]{3}{*}{ Totor } \\
\hline & & & 1 & 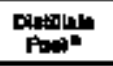 & Fool. & UP: & 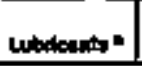 & wations & 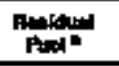 & Toket & & & & & \\
\hline & 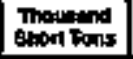 & 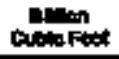 & \multicolumn{8}{|c|}{ 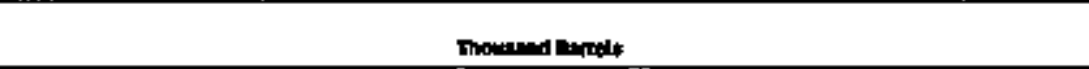 } & mound & \multicolumn{3}{|c|}{ 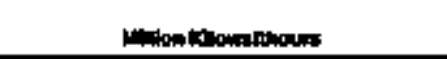 } & \\
\hline 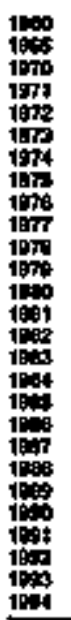 & 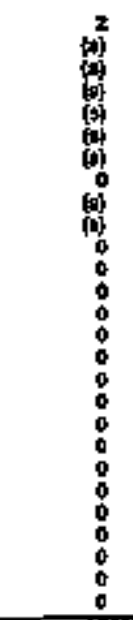 & 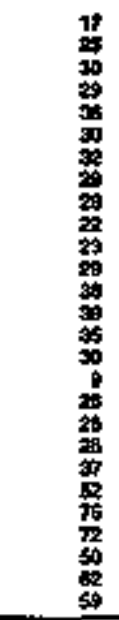 & 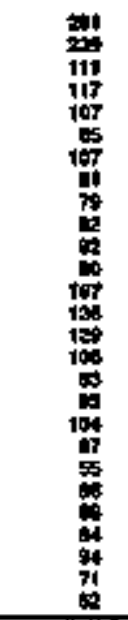 & 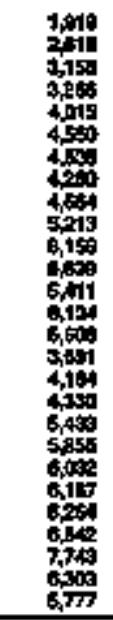 & 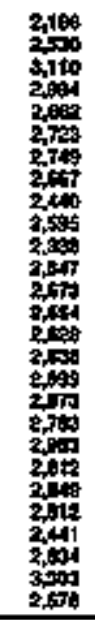 & 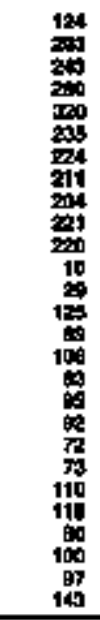 & 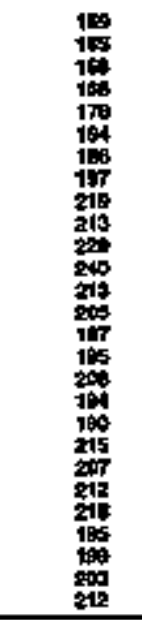 & 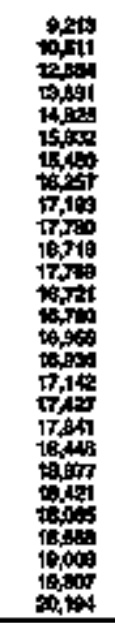 & $\begin{array}{c}30 \\
\text { 草 } \\
11 \\
0 \\
0 \\
0 \\
0 \\
0 \\
0 \\
0 \\
0 \\
0 \\
0 \\
0 \\
0 \\
00 \\
0 \\
0 \\
0 \\
0 \\
0 \\
0 \\
0 \\
0 \\
0 \\
0 \\
0\end{array}$ & 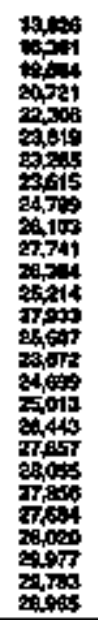 & 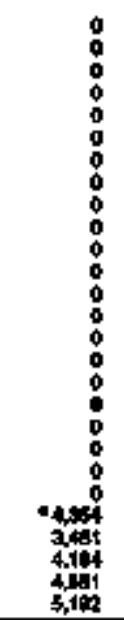 & $\begin{array}{l}0 \\
0 \\
0 \\
0 \\
0 \\
0 \\
0 \\
0 \\
0 \\
0 \\
0 \\
0 \\
0 \\
0 \\
0 \\
0 \\
0 \\
0 \\
0 \\
0 \\
0 \\
0 \\
0 \\
0\end{array}$ & $\begin{array}{l}= \\
= \\
= \\
z \\
= \\
z \\
= \\
= \\
z \\
= \\
z \\
z \\
z \\
z\end{array}$ & $\begin{array}{l}0 \\
0 \\
0 \\
0 \\
0 \\
0 \\
0 \\
0 \\
0 \\
0 \\
0 \\
0 \\
0 \\
0 \\
0 \\
0 \\
0 \\
0 \\
0 \\
0 \\
0 \\
0 \\
0 \\
0 \\
0 \\
0\end{array}$ & $\begin{array}{l}= \\
= \\
= \\
= \\
z \\
z \\
= \\
z \\
z \\
= \\
z \\
z \\
z \\
z \\
z\end{array}$ \\
\hline \multicolumn{16}{|c|}{ mution Ebu } \\
\hline 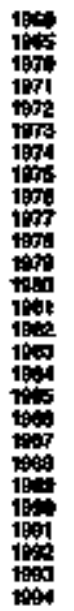 & $\begin{array}{l}8 \\
0 \\
0 \\
0 \\
0 \\
0 \\
0 \\
00 \\
00 \\
00 \\
00 \\
00 \\
00 \\
00 \\
00 \\
00 \\
00 \\
00 \\
00 \\
00 \\
00 \\
00\end{array}$ & 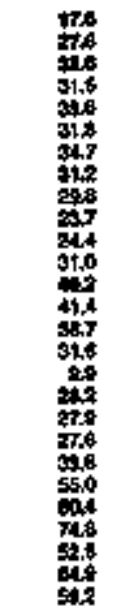 & 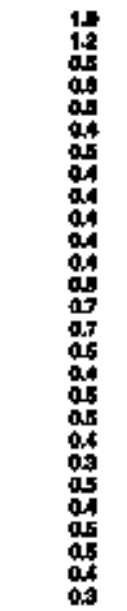 & 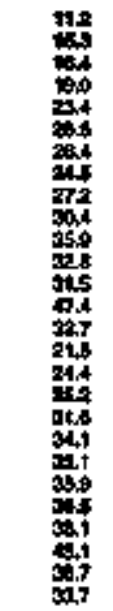 & 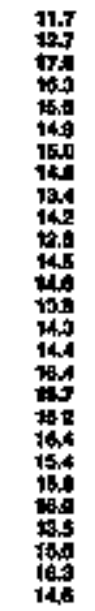 & 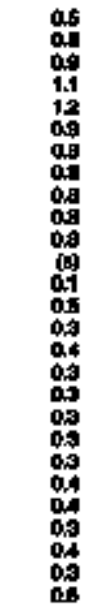 & 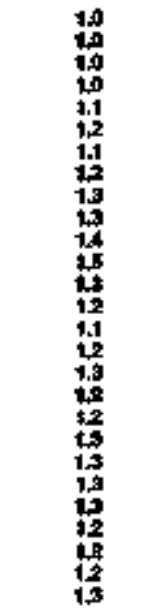 & 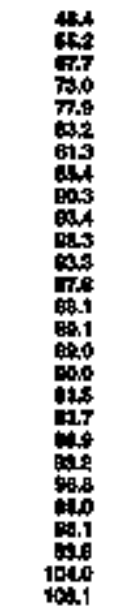 & 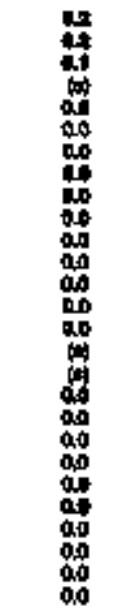 & 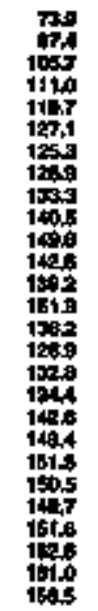 & 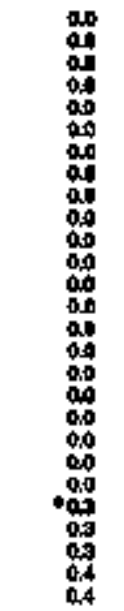 & $\begin{array}{l}00 \\
000 \\
000 \\
00 \\
00 \\
000 \\
00 \\
000 \\
00 \\
00 \\
00 \\
00 \\
00 \\
00 \\
00 \\
00 \\
00 \\
00 \\
00 \\
00 \\
00 \\
00 \\
00 \\
00 \\
00 \\
00 \\
00\end{array}$ & 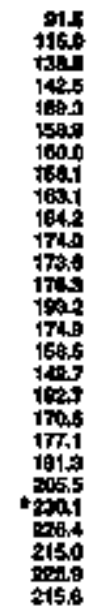 & 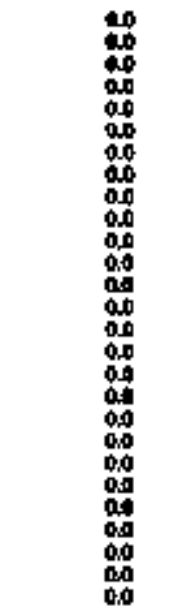 & 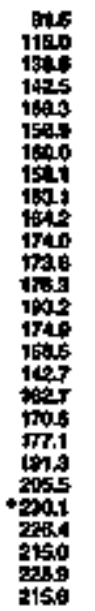 \\
\hline
\end{tabular}




\begin{tabular}{|c|c|c|c|c|c|c|c|c|c|c|c|c|c|c|}
\hline \multirow[b]{3}{*}{$\mathrm{rem}$} & \multicolumn{3}{|c|}{ cost } & \multirow[b]{2}{*}{ 至 } & \multicolumn{4}{|c|}{ 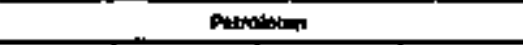 } & \multirow[b]{2}{*}{ Hoctine Enotion } & \multirow[b]{2}{*}{ Hydipodetoto } & \multirow[b]{2}{*}{ anwoth } & \multirow[b]{2}{*}{ 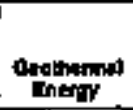 } & \multirow[b]{2}{*}{ 9xaces } & \multirow[b]{3}{*}{ Tams: } \\
\hline & 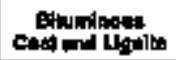 & enterneds & 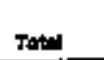 & & 然率 & 㟧出 & Dotrollation & Foter & & & & & & \\
\hline & \multicolumn{3}{|c|}{ Thousant Sthon 7 7ape } & axido Feot & \multicolumn{4}{|c|}{ 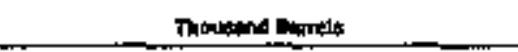 } & \multicolumn{5}{|c|}{ 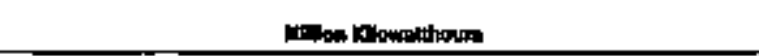 } & \\
\hline 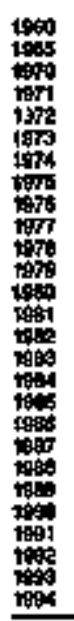 & 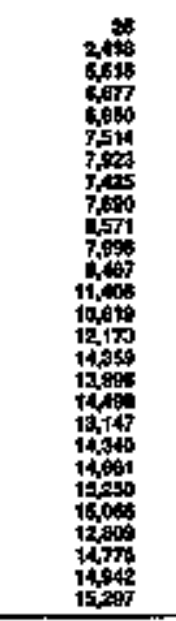 & $\begin{array}{l}0 \\
0 \\
8 \\
0 \\
8 \\
0 \\
0 \\
0 \\
0 \\
0 \\
0 \\
0 \\
0 \\
0 \\
0 \\
0 \\
0 \\
0 \\
0 \\
8 \\
8 \\
0 \\
0 \\
0 \\
0 \\
0 \\
0 \\
0\end{array}$ & 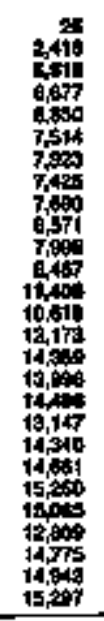 & 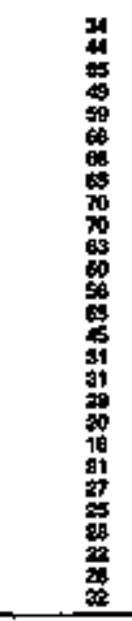 & 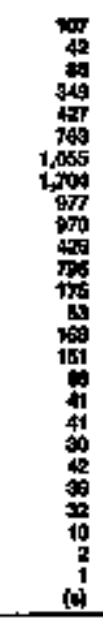 & 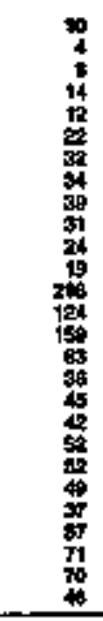 & $\begin{array}{l}: \\
0 \\
0 \\
0 \\
0 \\
0 \\
0 \\
0 \\
0 \\
0 \\
0 \\
0 \\
0 \\
0 \\
0 \\
0 \\
0 \\
0 \\
0 \\
0 \\
0 \\
0 \\
0 \\
0 \\
0 \\
0\end{array}$ & 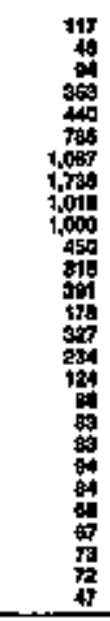 & $\begin{array}{l}: \\
\vdots \\
0 \\
0 \\
\vdots \\
0 \\
0 \\
0 \\
0 \\
0 \\
0 \\
0 \\
0 \\
0 \\
0 \\
0 \\
0 \\
0 \\
0 \\
0\end{array}$ & 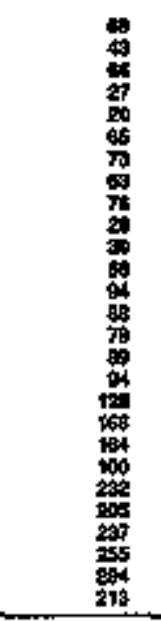 & $\begin{array}{l}: \\
0 \\
0 \\
0 \\
0 \\
0 \\
0 \\
0 \\
0 \\
0 \\
0 \\
0 \\
0 \\
0 \\
0 \\
0 \\
0 \\
0 \\
0 \\
0 \\
0\end{array}$ & $\begin{array}{l}0 \\
0 \\
0 \\
0 \\
0 \\
0 \\
0 \\
0 \\
0 \\
0 \\
0 \\
0 \\
0 \\
0 \\
0 \\
0 \\
0 \\
0 \\
0 \\
0 \\
0 \\
0 \\
0 \\
0\end{array}$ & $\begin{array}{l}0 \\
0 \\
0 \\
0 \\
0 \\
0 \\
0 \\
0 \\
0 \\
0 \\
0 \\
0 \\
0 \\
0 \\
0 \\
0 \\
0 \\
0 \\
0 \\
0 \\
0 \\
0 \\
0 \\
0\end{array}$ & $\begin{array}{l}\vdots \\
\vdots \\
\vdots \\
\vdots \\
\vdots \\
\vdots \\
\vdots \\
\vdots \\
\vdots \\
\vdots \\
\vdots \\
\vdots\end{array}$ \\
\hline \multicolumn{15}{|c|}{ 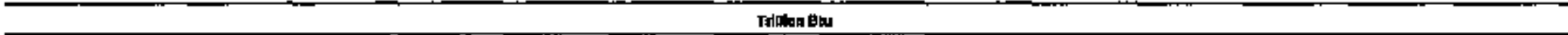 } \\
\hline 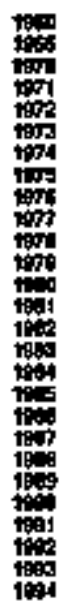 & 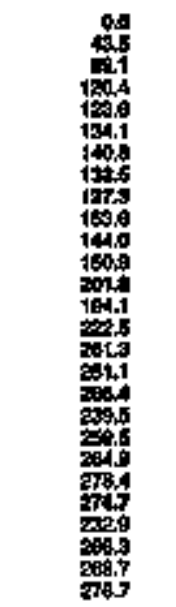 & 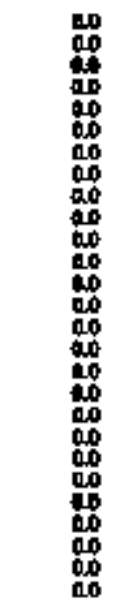 & 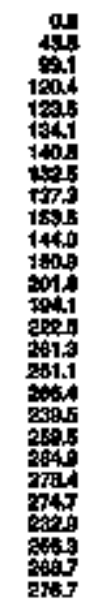 & 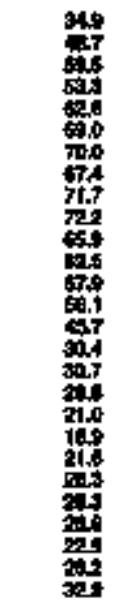 & 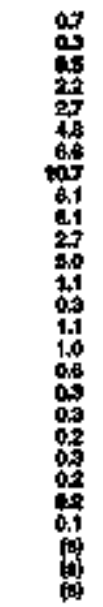 & 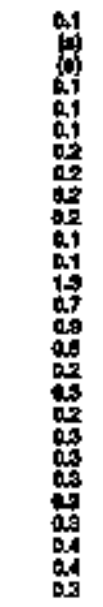 & 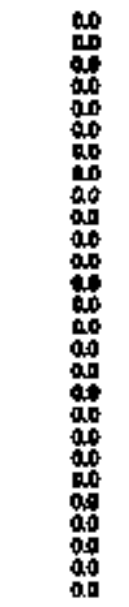 & 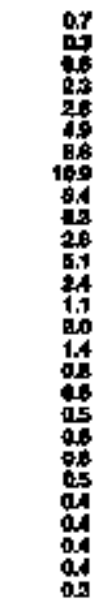 & $\begin{array}{l}00 \\
000 \\
00 \\
000 \\
000 \\
000 \\
000 \\
000 \\
000 \\
000 \\
000 \\
000 \\
000 \\
000 \\
000 \\
000 \\
000 \\
000 \\
000 \\
000 \\
000 \\
000 \\
000 \\
000 \\
000 \\
000 \\
000 \\
000\end{array}$ & 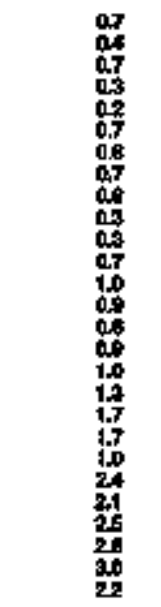 & 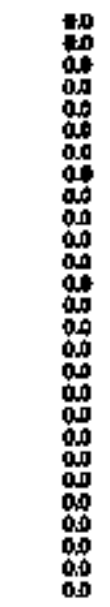 & 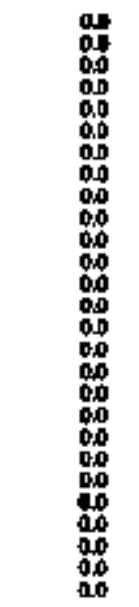 & 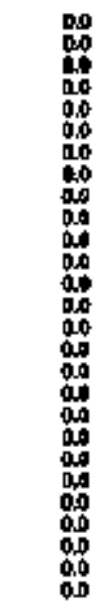 & 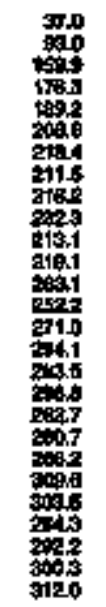 \\
\hline
\end{tabular}


Table 209. Energy Consumption Esthrates by Source, 1960, 1965, 1970-1999, New York

\begin{tabular}{|c|c|c|c|c|c|c|c|c|c|c|c|c|c|c|c|c|c|c|c|}
\hline \multirow[b]{3}{*}{ vert } & \multirow[b]{2}{*}{ conds } & \multirow[b]{2}{*}{ Motaral } & \multicolumn{11}{|c|}{ Petroloum } & \multirow[b]{2}{*}{ 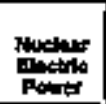 } & \multirow[b]{2}{*}{ 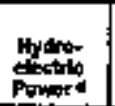 } & \multirow[b]{2}{*}{ Brotuch : } & \multirow[b]{2}{*}{ Other 4} & \multirow{2}{*}{ 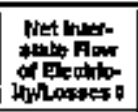 } & \multirow[b]{3}{*}{ Toten h } \\
\hline & & & 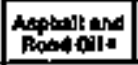 & 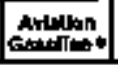 & 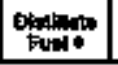 & boef. & Ener. & Intat & Dit. & |rot & 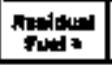 & Other an & Totoll & & & & & & \\
\hline & thoryans & 1 & \multicolumn{11}{|c|}{ Thoplusond Bonn } & \multicolumn{5}{|c|}{ 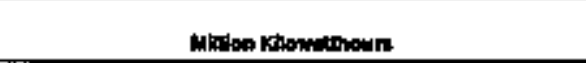 } & \\
\hline 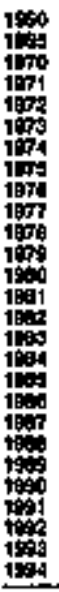 & 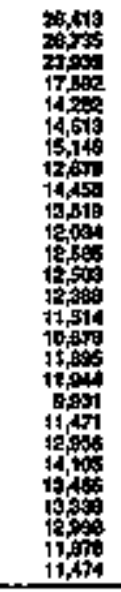 & 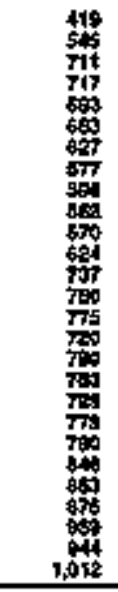 & 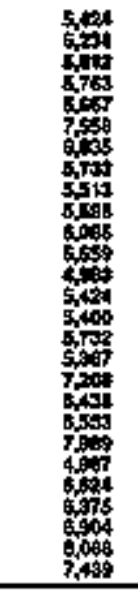 & 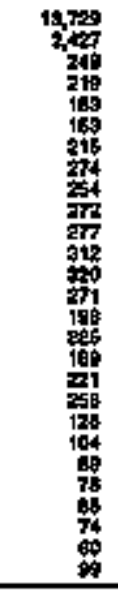 & 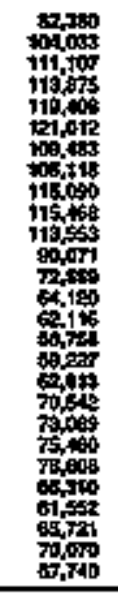 & 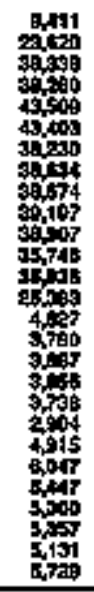 & 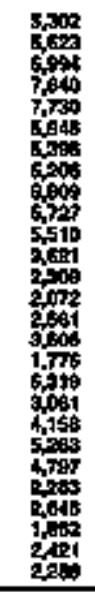 & 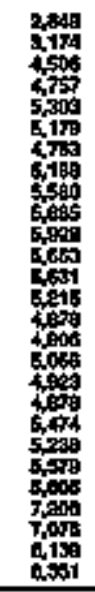 & 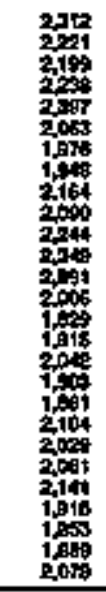 & 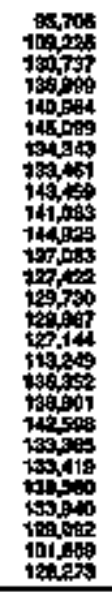 & 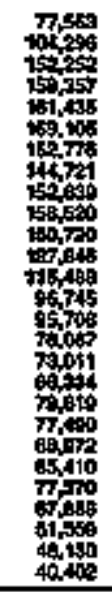 & 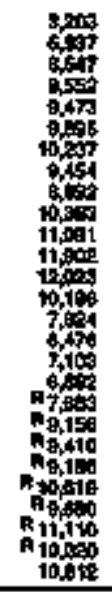 & 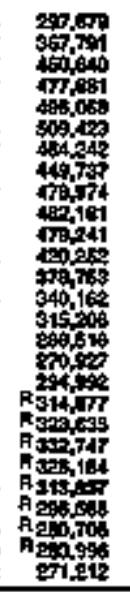 & 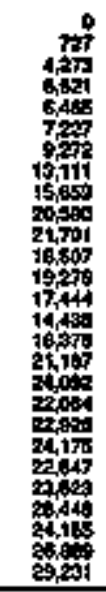 & 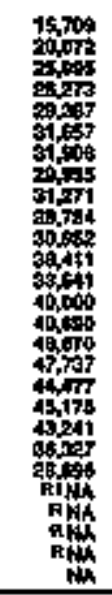 & 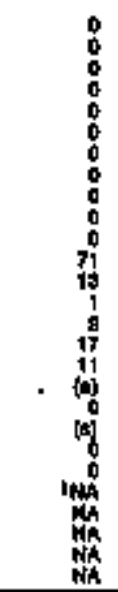 & 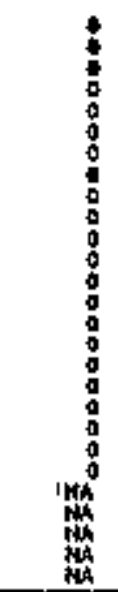 & 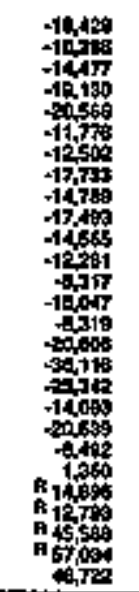 & 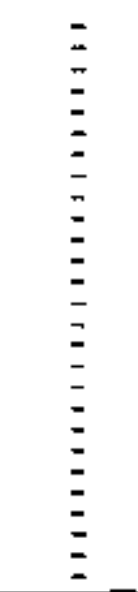 \\
\hline \multicolumn{20}{|c|}{ Tfinen $2: 4$} \\
\hline 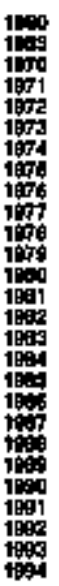 & 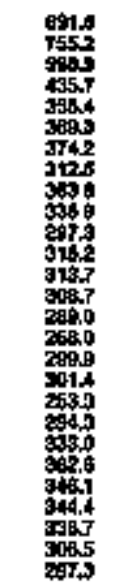 & 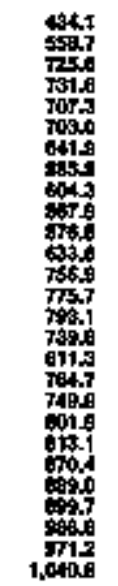 & 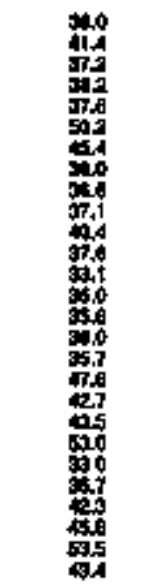 & 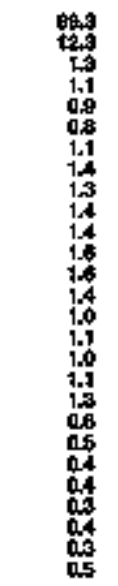 & 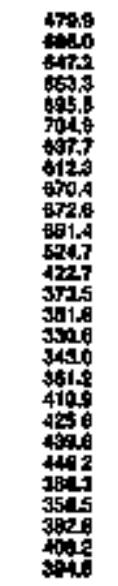 & 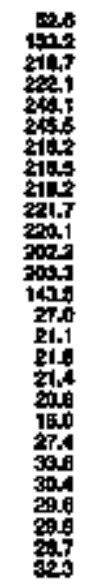 & 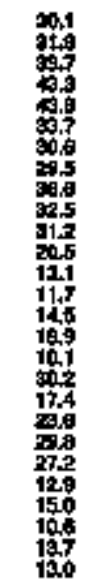 & 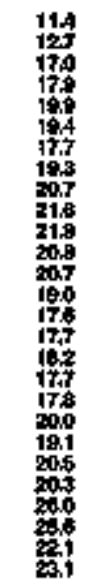 & 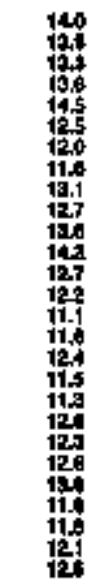 & 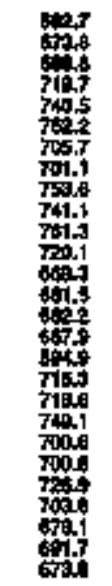 & 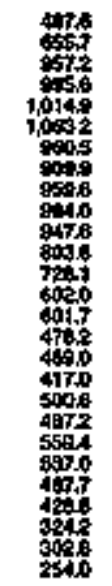 & 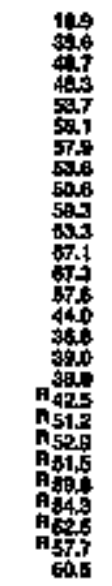 & 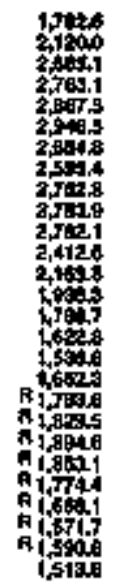 & 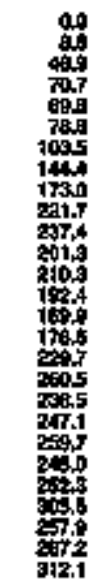 & 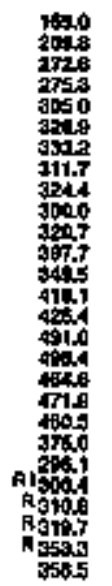 & 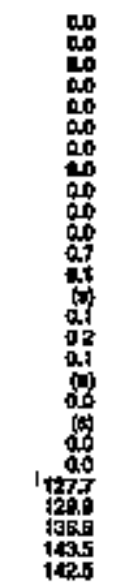 & 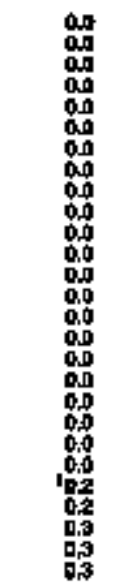 & 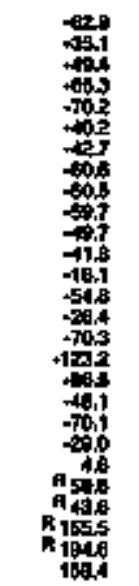 & 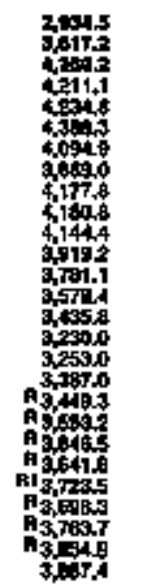 \\
\hline & & & & & & & & & & & & & & & & & a & & 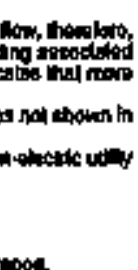 \\
\hline
\end{tabular}




\begin{tabular}{|c|c|c|c|c|c|c|c|c|c|c|c|c|c|c|}
\hline & & Cost & & & & & & & \multirow{3}{*}{ 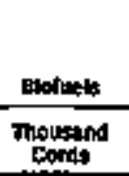 } & & \multirow[b]{2}{*}{ Eectidaly" } & \multirow[b]{2}{*}{ stortar } & \multirow{3}{*}{ 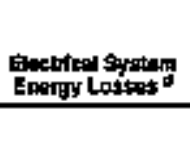 } & \multirow[b]{3}{*}{ Tots } \\
\hline & 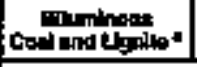 & Annteaction & Tohal & 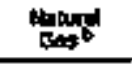 & 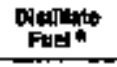 & Kerosentos & cost & Total & & Solate & & & & \\
\hline Y Youl & \multicolumn{3}{|c|}{ Therdend sinot Tons } & plimion & \multicolumn{4}{|c|}{ Thowand bank } & & \multicolumn{3}{|c|}{ 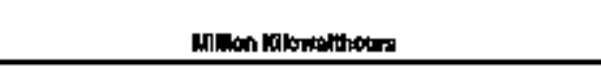 } & & \\
\hline 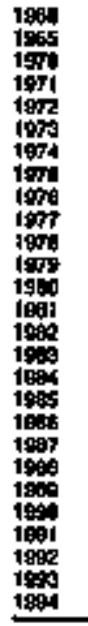 & 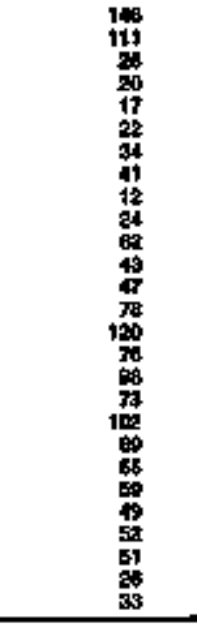 & 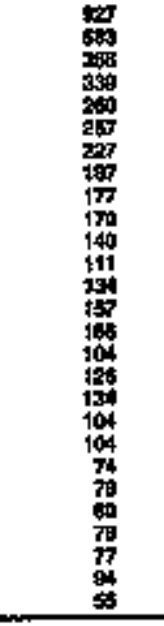 & 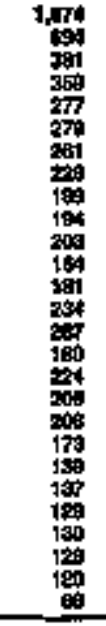 & 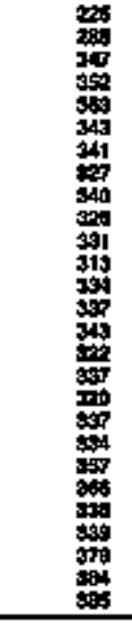 & 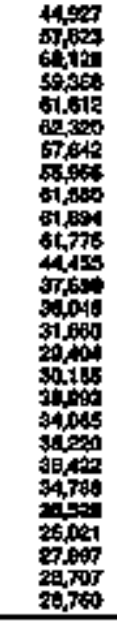 & 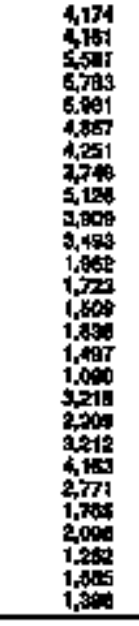 & 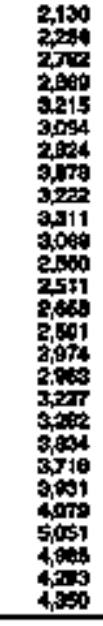 & 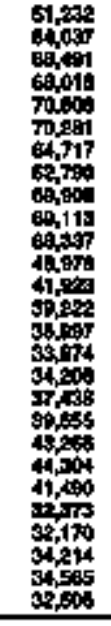 & 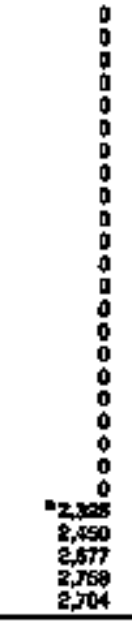 & 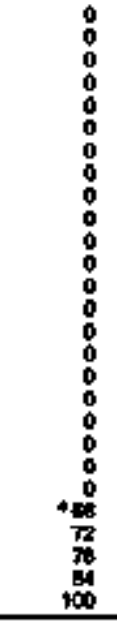 & 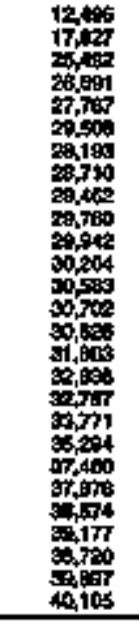 & $\begin{array}{l}\vdots \\
\vdots \\
\vdots \\
\vdots \\
\vdots \\
\vdots \\
\vdots \\
\vdots \\
\vdots \\
\vdots \\
\vdots \\
\vdots \\
\vdots \\
\vdots\end{array}$ & 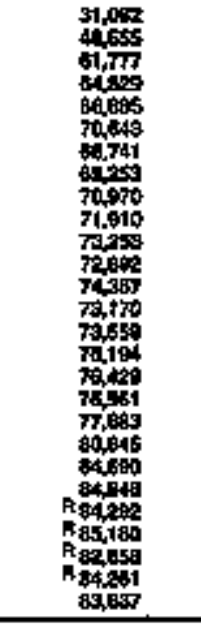 & $\begin{array}{l}\vdots \\
\vdots \\
\vdots \\
\vdots \\
\vdots \\
\vdots \\
\vdots \\
\vdots \\
\vdots \\
\vdots \\
\vdots \\
\vdots\end{array}$ \\
\hline \multicolumn{15}{|c|}{ Finden aw } \\
\hline 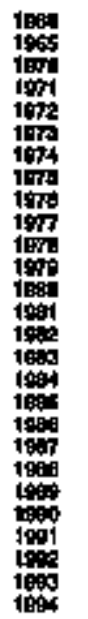 & 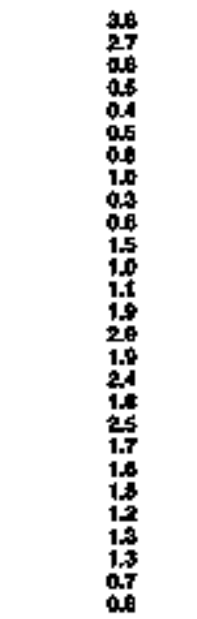 & 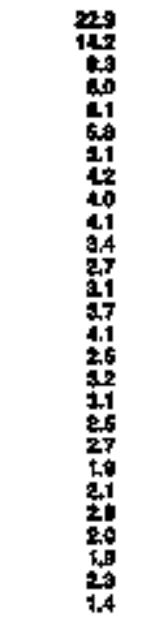 & 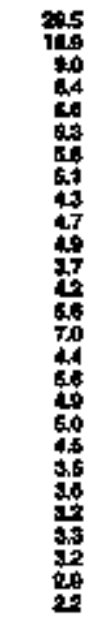 & 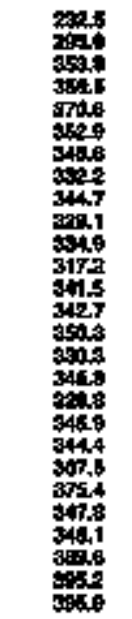 & 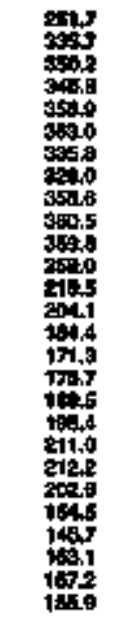 & 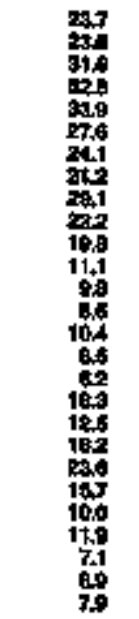 & 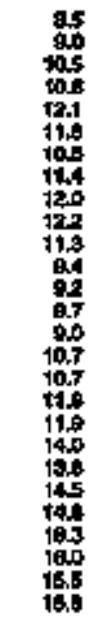 & 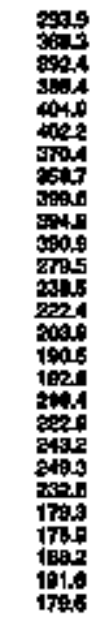 & 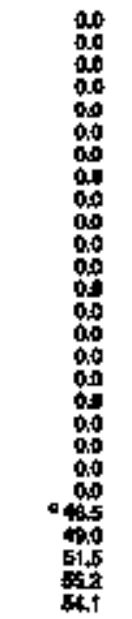 & 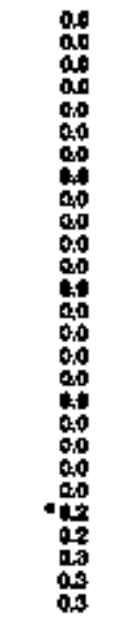 & 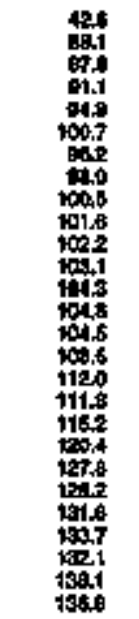 & 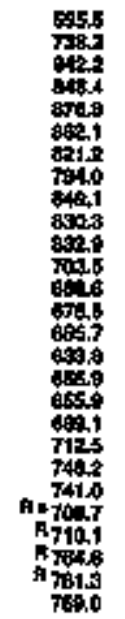 & 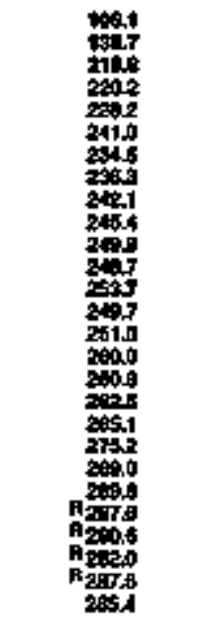 & 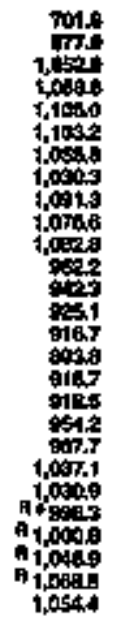 \\
\hline
\end{tabular}


Table ż1. Commereial Energy Consumption Estimates, 1960, 1965, 1970-1994, Naw York

\begin{tabular}{|c|c|c|c|c|c|c|c|c|c|c|c|c|c|c|}
\hline \multirow[b]{3}{*}{ Yas. } & \multicolumn{3}{|c|}{ Cow } & \multirow{3}{*}{ 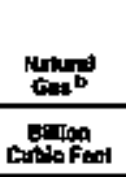 } & \multicolumn{6}{|c|}{ Protader } & \multirow[b]{2}{*}{ Exetstive * } & \multirow[b]{2}{*}{ Enety } & \multirow[b]{2}{*}{ 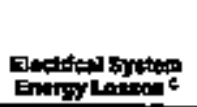 } & \multirow[b]{3}{*}{ Totot:ㅇ } \\
\hline & 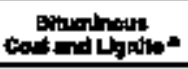 & Anturetin" & Tats & & 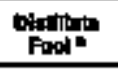 & Karogene" & LPQ" & notion & Foolder & Tat & & & & \\
\hline & \multicolumn{3}{|c|}{ Thouspand stonct tons } & & \multicolumn{6}{|c|}{ Thourand Eereth } & \multicolumn{3}{|c|}{ 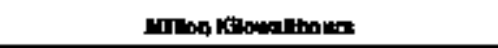 } & \\
\hline 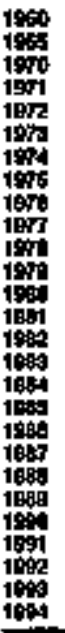 & 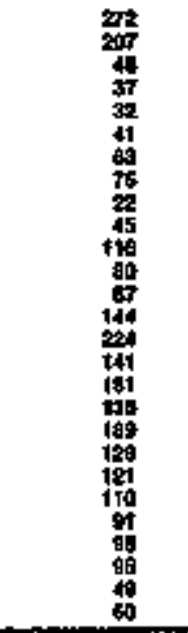 & 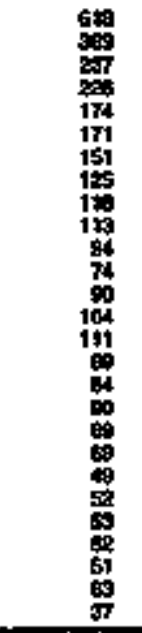 & 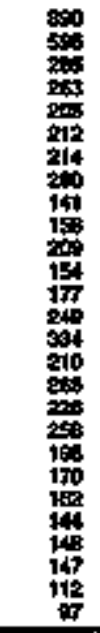 & 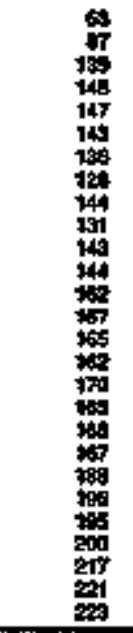 & 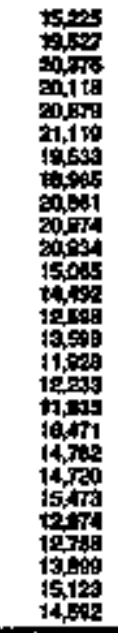 & 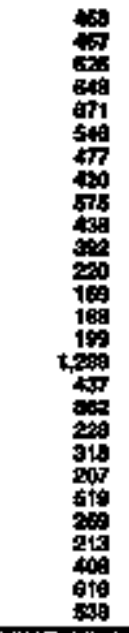 & 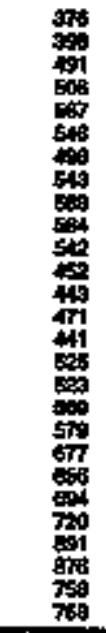 & 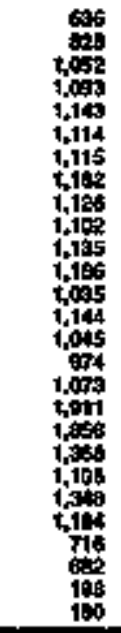 & 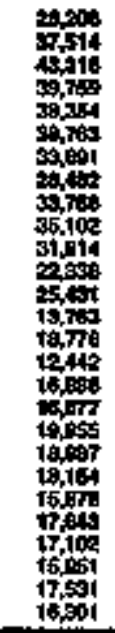 & 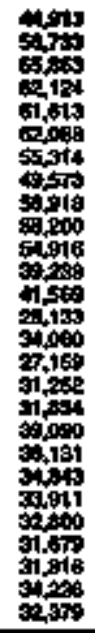 & 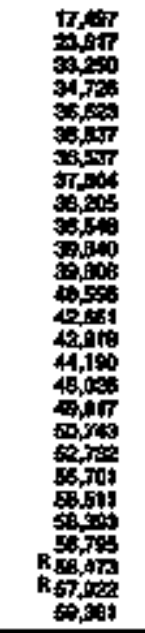 & $\begin{array}{l}= \\
= \\
z \\
z \\
= \\
= \\
= \\
= \\
= \\
= \\
= \\
z \\
= \\
=\end{array}$ & 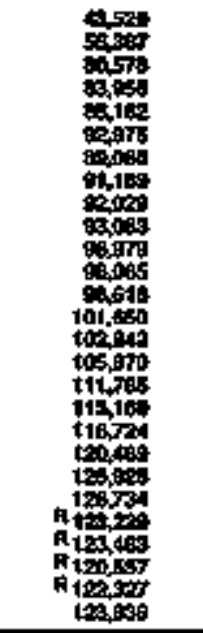 & $\begin{array}{l}= \\
= \\
= \\
= \\
= \\
= \\
= \\
= \\
= \\
= \\
= \\
= \\
= \\
=\end{array}$ \\
\hline \multicolumn{15}{|c|}{ Trinlin atu } \\
\hline 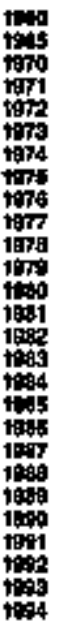 & 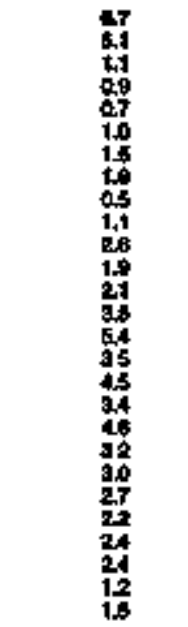 & 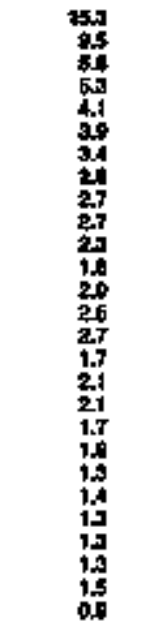 & 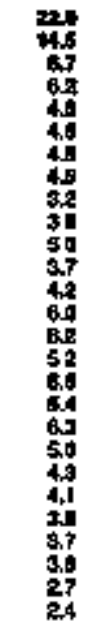 & 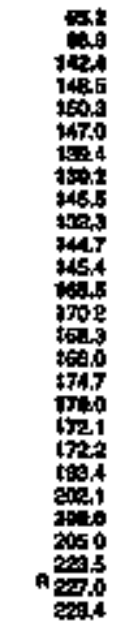 & 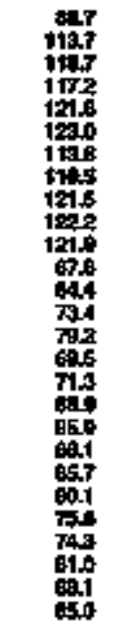 & 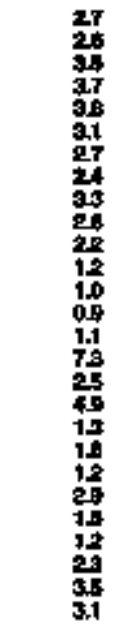 & 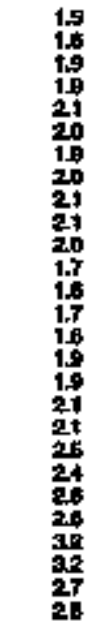 & 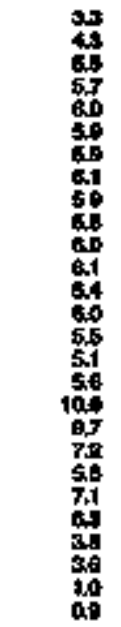 & 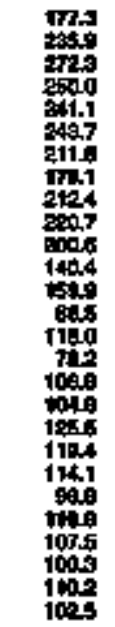 & 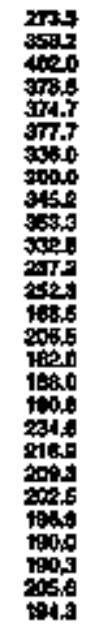 & 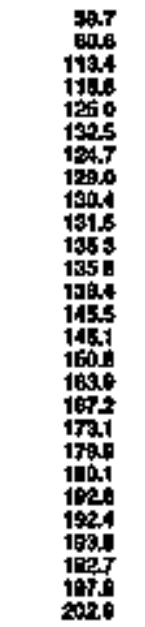 & 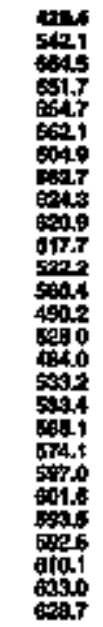 & 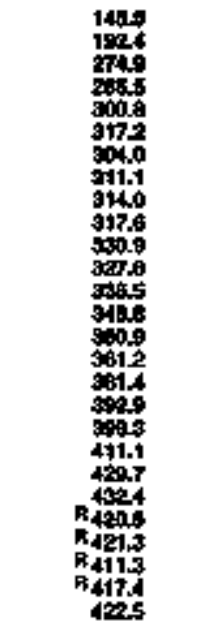 & 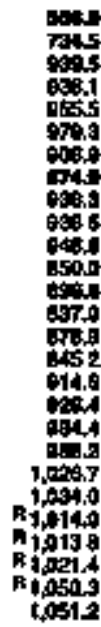 \\
\hline & 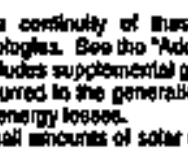 & lag & & & & & & 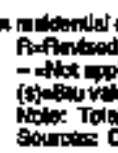 & Wen & & & & & \\
\hline
\end{tabular}


Table 212. Industrial Energy Consumplion Estimates, 1960, 1965, 1970-1994, Now York

\begin{tabular}{|c|c|c|c|c|c|c|c|c|c|c|c|c|c|c|c|c|c|c|}
\hline & \multirow[b]{2}{*}{ cont } & \multirow[b]{2}{*}{ 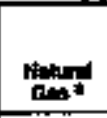 } & \multicolumn{9}{|c|}{ Putrowing } & \multirow[b]{2}{*}{ Proter } & \multirow[b]{2}{*}{ moskila } & \multirow[b]{2}{*}{ Otherend } & \multirow[b]{2}{*}{ Elochtolyt } & \multirow[b]{2}{*}{ Em: } & \multirow{2}{*}{ 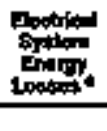 } & \multirow[b]{3}{*}{ Tots } \\
\hline & & & 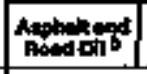 & 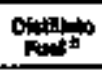 & monosento & LRE: & 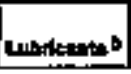 & 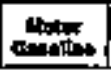 & 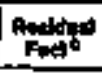 & other bes & Tot & & & & & & & \\
\hline & Thont ion & $\min _{10}$ & \multicolumn{9}{|c|}{ Thousend Bumph } & \multicolumn{6}{|c|}{ 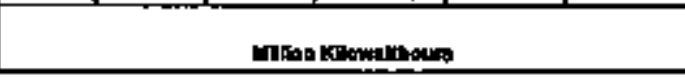 } & \\
\hline 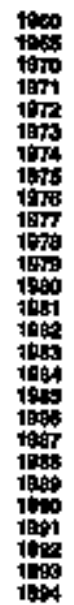 & 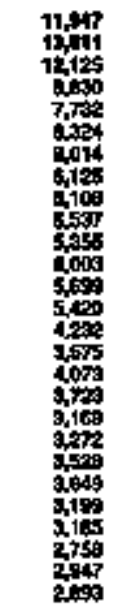 & 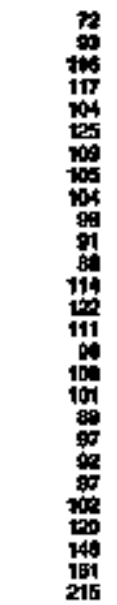 & 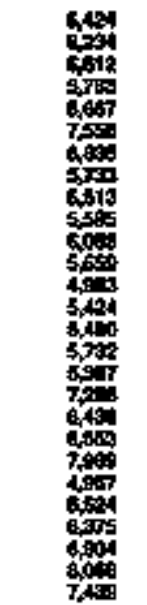 & 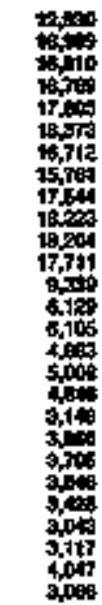 & 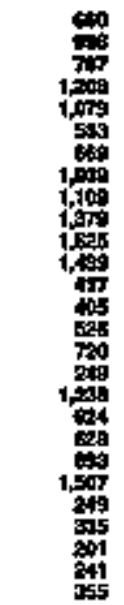 & 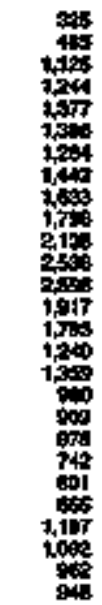 & 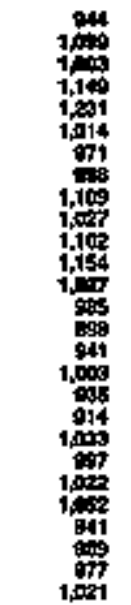 & 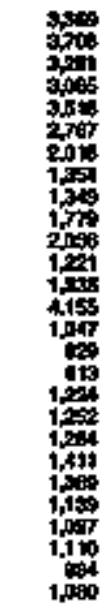 & 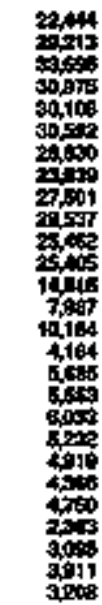 & 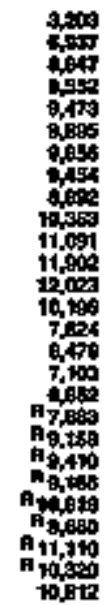 & 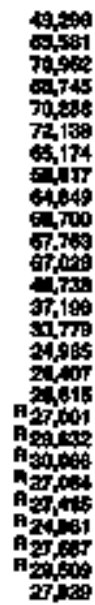 & 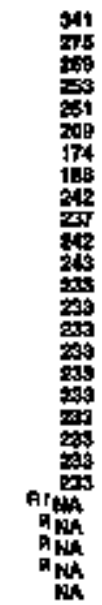 & $\begin{array}{l}0 \\
0 \\
0 \\
0 \\
0 \\
0 \\
0 \\
0 \\
0 \\
0 \\
0 \\
0 \\
0 \\
0 \\
0 \\
0 \\
0 \\
4 \\
4 \\
4\end{array}$ & 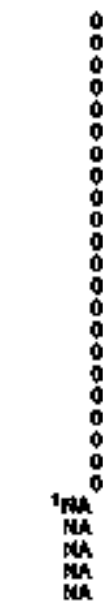 & 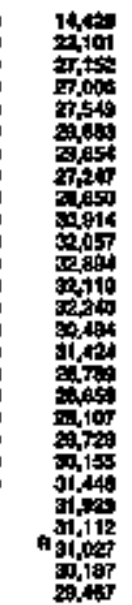 & $\begin{array}{l}\bar{z} \\
\bar{z}\end{array}$ & 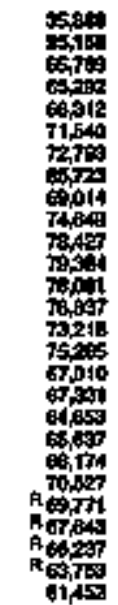 & - \\
\hline
\end{tabular}

\begin{tabular}{|c|c|c|c|c|c|c|}
\hline$\frac{190}{1906}$ & 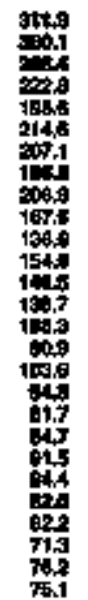 & 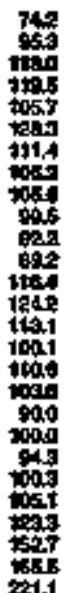 & 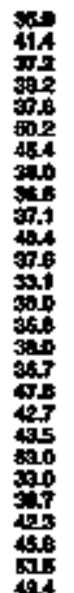 & 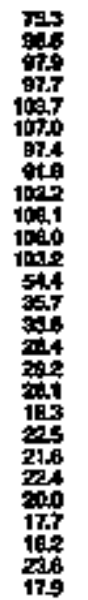 & 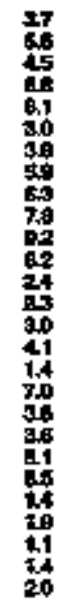 & 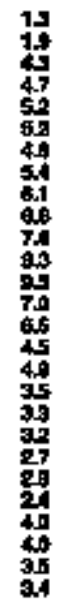 \\
\hline
\end{tabular}

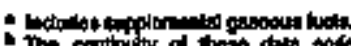

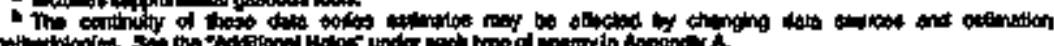

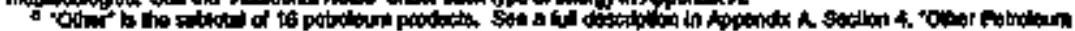

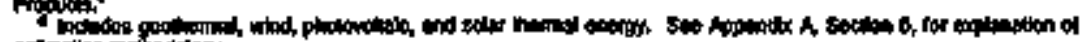

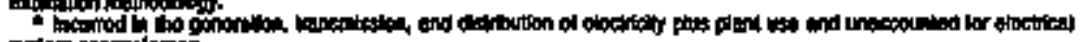

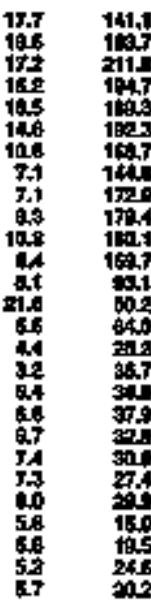

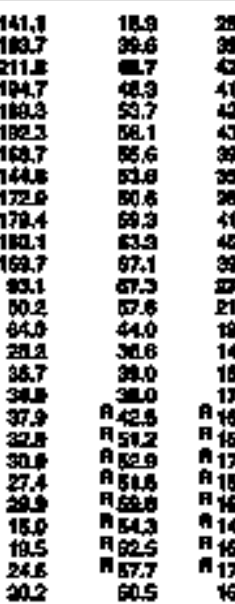

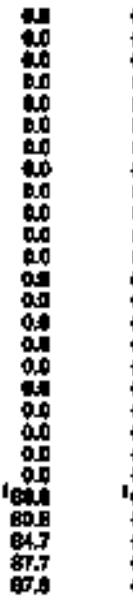

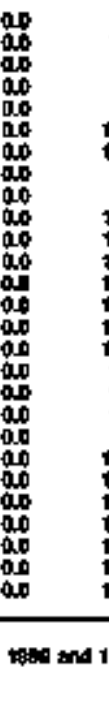

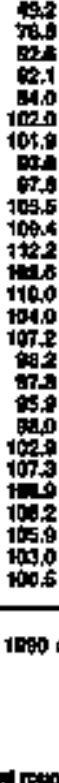

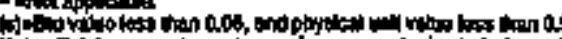

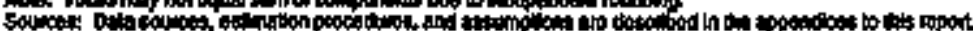


Table 219. Transportation Energy Consumption Rstimates, 1960, 1965, 1970-19g4, New Yark

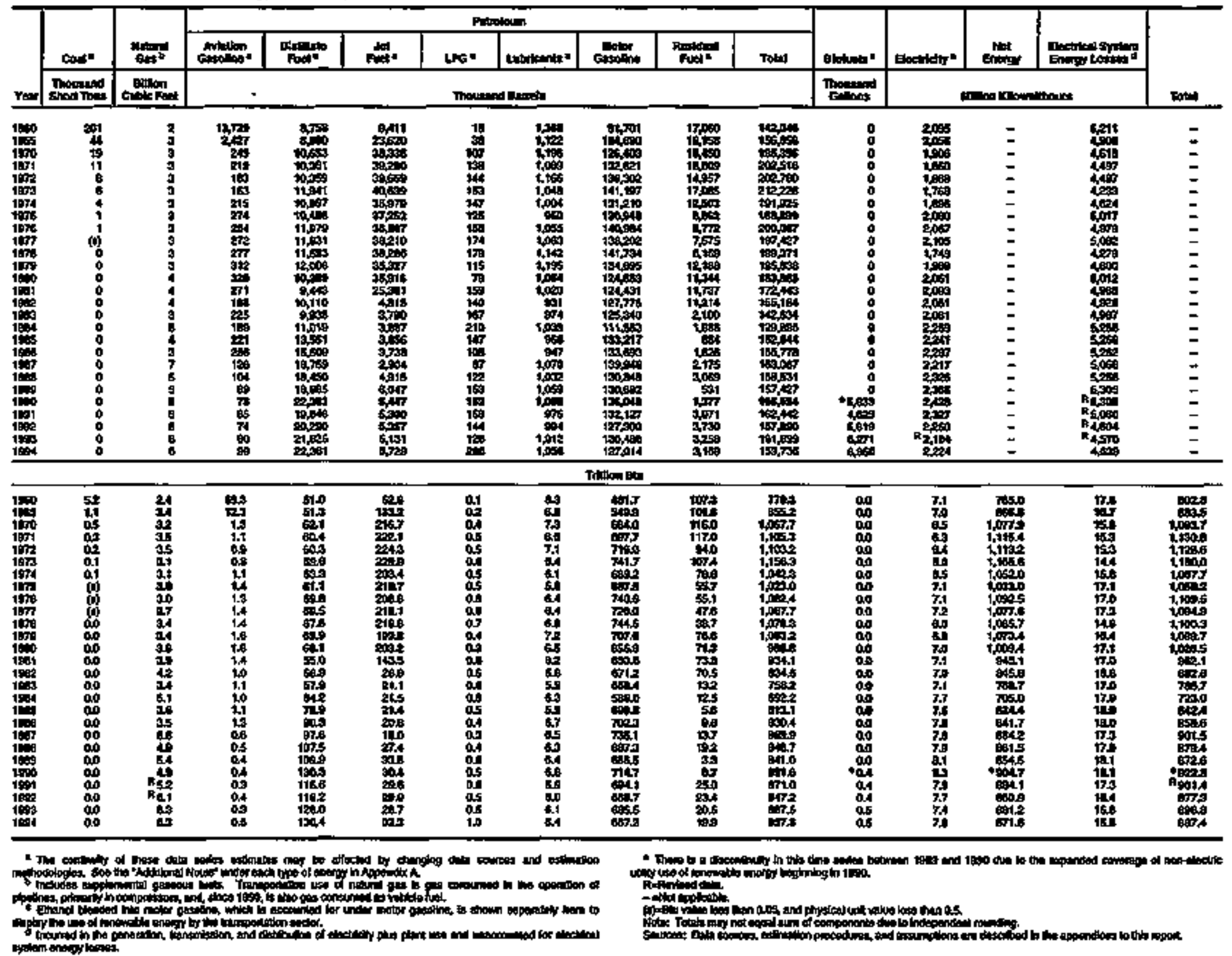

$\mathbf{N}$
$\mathbf{E}$
$\mathbf{W}$
$\mathbf{Y}$
$\mathbf{0}$
$\mathbf{R}$
$\mathbf{K}$ 
Tatule 214. Estimates of Energy Input at Electric Utillues, 1960, 1965, 1970-1994, New York

\begin{tabular}{|c|c|c|c|c|c|c|c|c|c|c|c|c|c|c|}
\hline \multirow[b]{3}{*}{ ren } & \multicolumn{3}{|c|}{ cort } & \multirow{3}{*}{ 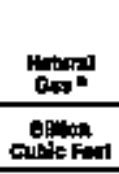 } & \multicolumn{4}{|c|}{ Petrikom } & \multirow[b]{2}{*}{ 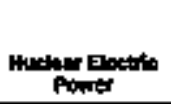 } & \multirow[b]{2}{*}{ 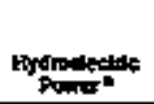 } & \multirow[b]{2}{*}{ Bhelkola } & \multirow[b]{2}{*}{ 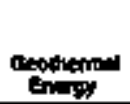 } & \multirow[b]{2}{*}{ ons } & \multirow[b]{3}{*}{ 70에에 8} \\
\hline & 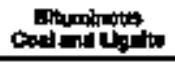 & Anternotio & Joht & & 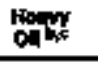 & 战被 & Notoment & 70 & & & & & & \\
\hline & \multicolumn{3}{|c|}{ Thenume Bhat Tor: } & & \multicolumn{4}{|c|}{ 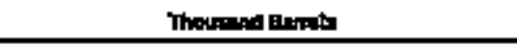 } & \multicolumn{5}{|c|}{ 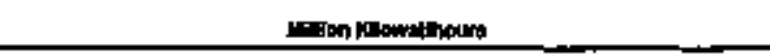 } & \\
\hline 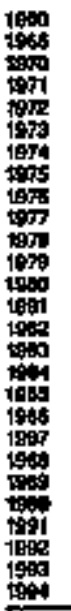 & 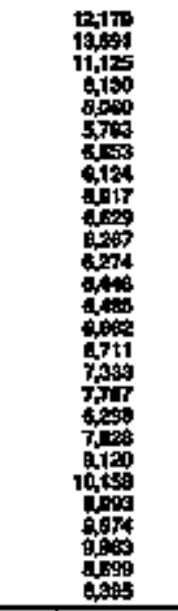 & $\begin{array}{r}18 \\
0 \\
0 \\
0 \\
0 \\
0 \\
0 \\
0 \\
0 \\
0 \\
0 \\
0 \\
0 \\
0 \\
0 \\
0 \\
0 \\
0 \\
0 \\
0 \\
0 \\
0 \\
0 \\
0 \\
0 \\
0 \\
0 \\
\end{array}$ & 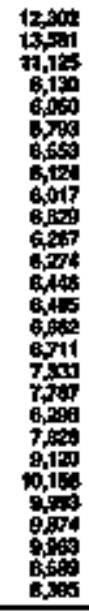 & 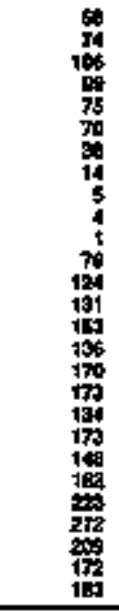 & 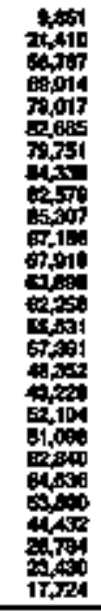 & 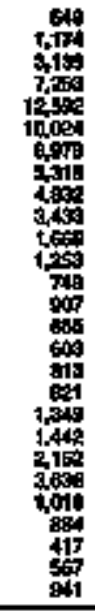 & $\begin{array}{l}0 \\
0 \\
0 \\
0 \\
0 \\
0 \\
0 \\
0 \\
0 \\
0 \\
0 \\
0 \\
0 \\
0 \\
0 \\
0 \\
0 \\
0 \\
0 \\
0 \\
0 \\
0 \\
0 \\
0 \\
\end{array}$ & 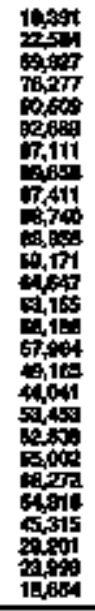 & 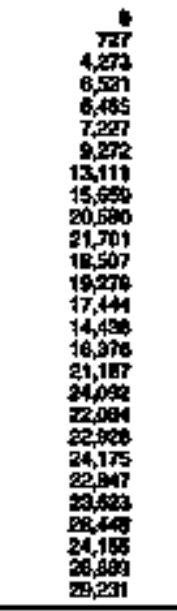 & 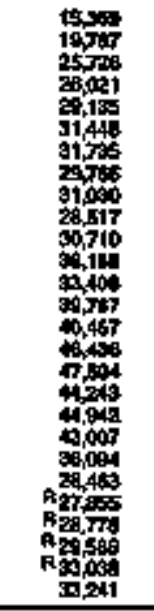 & 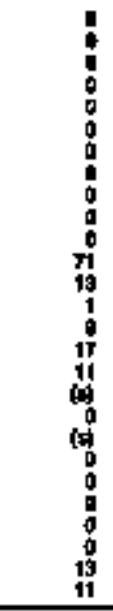 & $\begin{array}{l}0 \\
0 \\
0 \\
0 \\
0 \\
0 \\
0 \\
0 \\
0 \\
0 \\
0 \\
0 \\
0 \\
0 \\
0 \\
0 \\
0 \\
0 \\
0 \\
0 \\
0 \\
0 \\
0 \\
0 \\
0 \\
0 \\
0 \\
\end{array}$ & $\begin{array}{l}: \\
0 \\
0 \\
0 \\
: \\
0 \\
0 \\
0 \\
0 \\
0 \\
0 \\
0 \\
0 \\
0 \\
0 \\
: \\
0 \\
0 \\
0 \\
0\end{array}$ & $\begin{array}{l}= \\
z \\
z \\
z \\
z \\
z \\
z \\
z \\
z \\
z \\
z \\
z \\
z \\
z \\
z \\
\end{array}$ \\
\hline \multicolumn{15}{|c|}{ nimin } \\
\hline 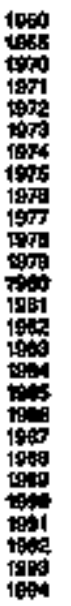 & 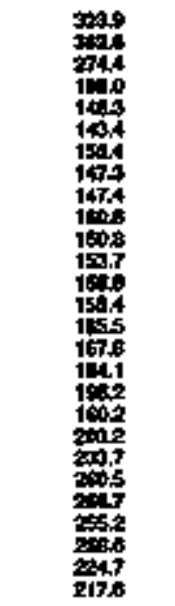 & 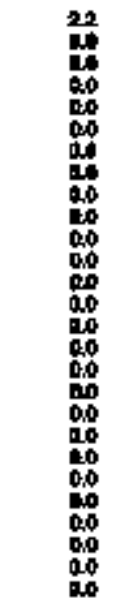 & 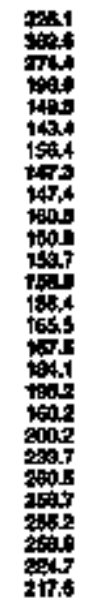 & 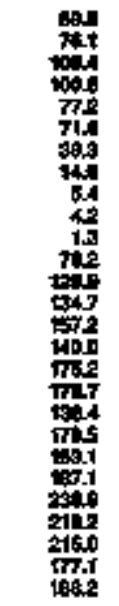 & 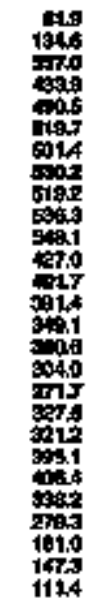 & 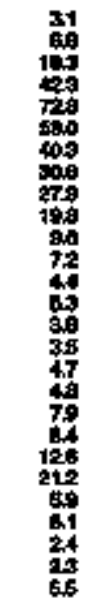 & 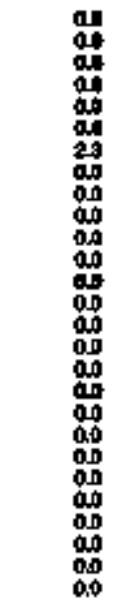 & 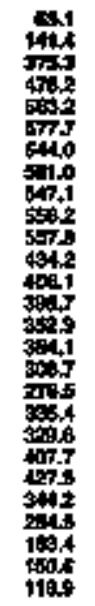 & 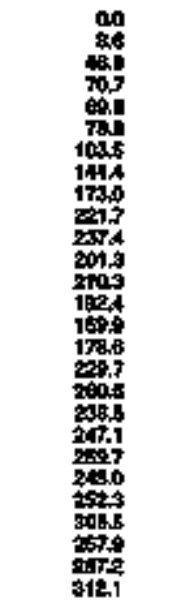 & 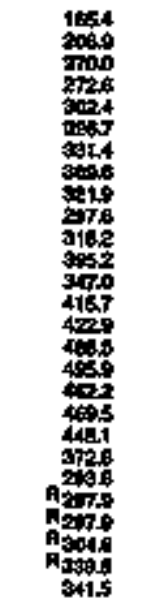 & 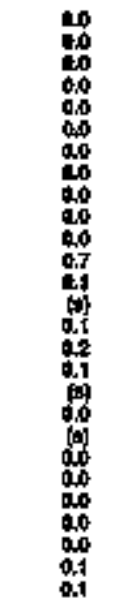 & $\begin{array}{l}00 \\
00 \\
00 \\
00 \\
00 \\
00 \\
00 \\
00 \\
00 \\
00 \\
00 \\
00 \\
00 \\
00 \\
000 \\
00 \\
000 \\
00 \\
00 \\
00 \\
00 \\
00 \\
00 \\
00 \\
00 \\
00 \\
00\end{array}$ & 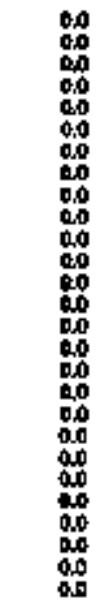 & 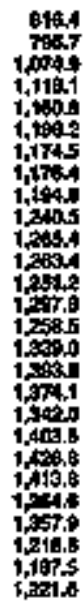 \\
\hline
\end{tabular}

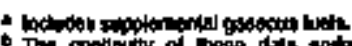

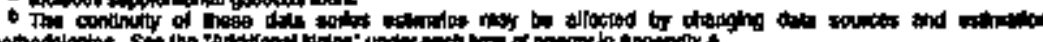

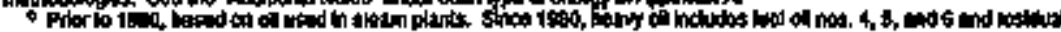

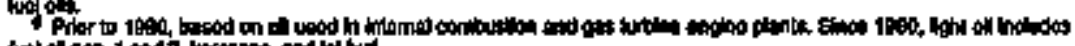

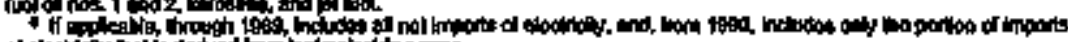

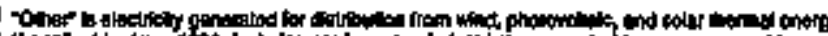

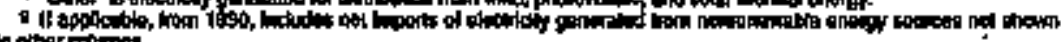

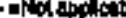

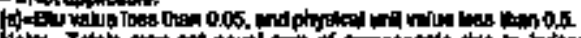

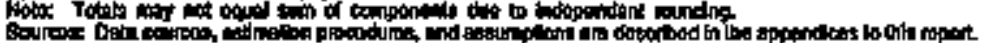


Table 215. Energy Consemption Estimates by Source, 1960, 1965, 1970-1984, North Carolint

\begin{tabular}{|c|c|c|c|c|c|c|c|c|c|c|c|c|c|c|c|c|c|c|c|}
\hline \multirow[b]{3}{*}{$Y=1$} & \multirow[b]{2}{*}{ Co:t: } & \multirow[b]{2}{*}{ Pats } & \multicolumn{11}{|c|}{ 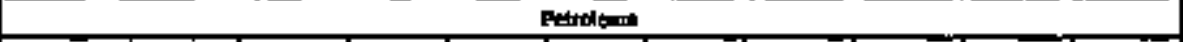 } & \multirow{2}{*}{ 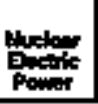 } & \multirow{2}{*}{ 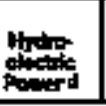 } & \multirow[b]{2}{*}{ 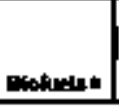 } & \multirow[b]{2}{*}{ oteres! | } & \multirow{3}{*}{ 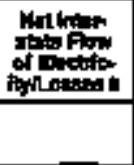 } & \multirow[b]{3}{*}{ Total b } \\
\hline & & & 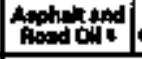 & Antropen & 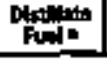 & chaf: & Kan: & Hot & tonst. & Mots & $\begin{array}{l}\text { Roglded } \\
\text { Fuol: }\end{array}$ & Other te & Tots & & & & & & \\
\hline & 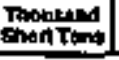 & Distom & \multicolumn{11}{|c|}{ - Thotsend Inerob } & \multicolumn{4}{|c|}{ 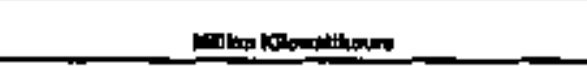 } & & \\
\hline 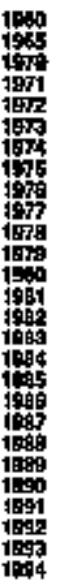 & 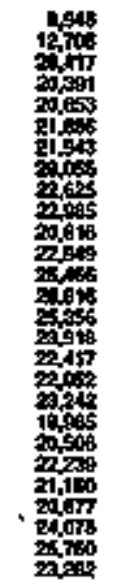 & 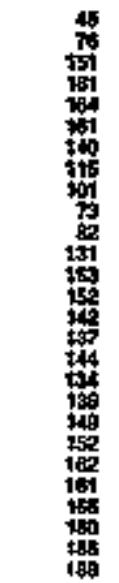 & 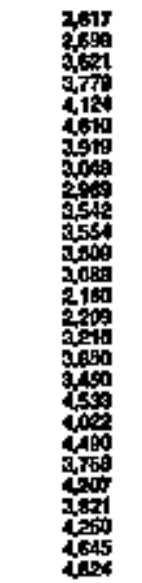 & 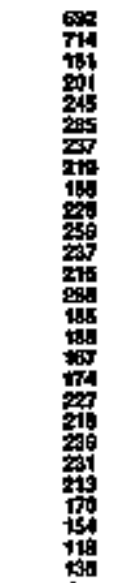 & 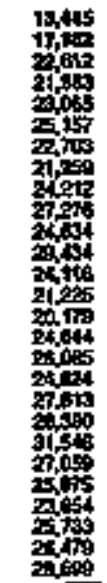 & 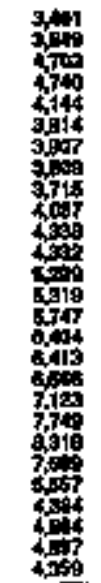 & 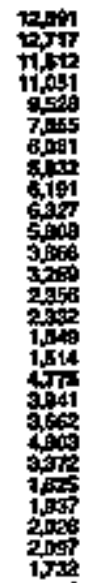 & 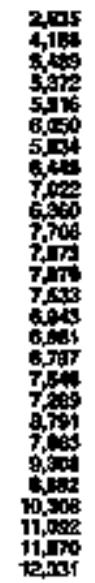 & 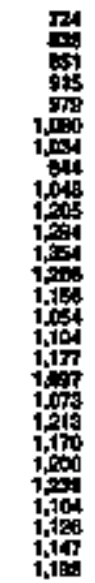 & 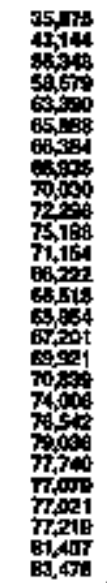 & 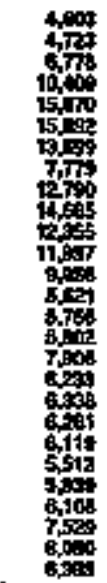 & 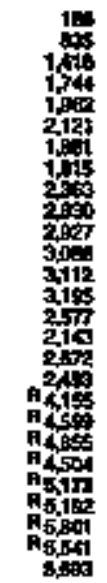 & 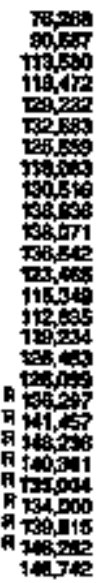 & 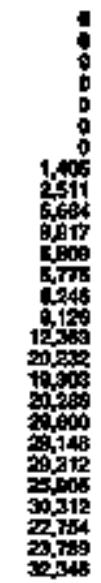 & 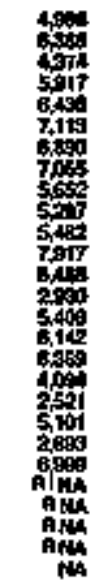 & 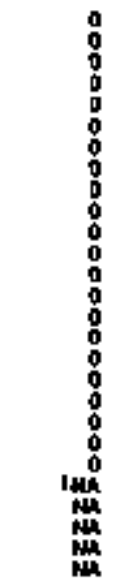 & 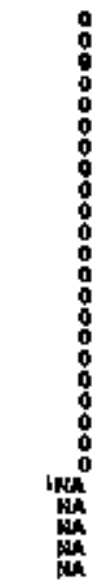 & 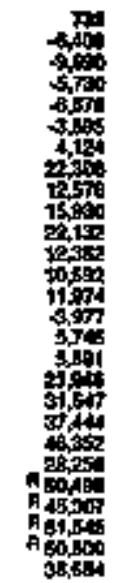 & $\begin{array}{l}z \\
z \\
z \\
z \\
z \\
z \\
z \\
z \\
z \\
z \\
z \\
z \\
z \\
z\end{array}$ \\
\hline
\end{tabular}

$\mathbf{N}$
$\mathbf{O}$
$\mathbf{R}$
$\mathbf{T}$
$\mathbf{H}$
$\mathbf{C}$
$\mathbf{A}$
$\mathbf{R}$
$\mathbf{O}$
$\mathbf{L}$
$\mathbf{I}$
$\mathbf{N}$
$\mathbf{A}$

\begin{tabular}{|c|c|c|c|c|c|c|c|c|c|c|c|c|c|c|c|c|c|c|c|}
\hline 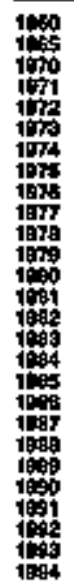 & 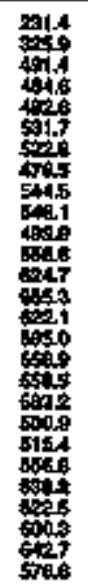 & 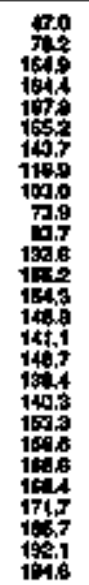 & 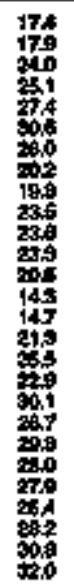 & 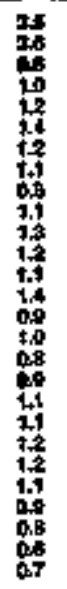 & 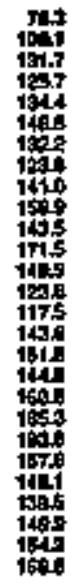 & 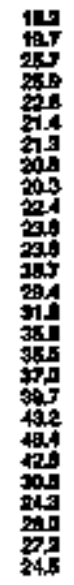 & 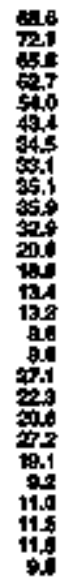 & 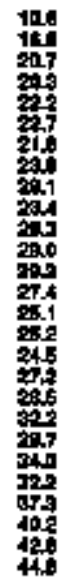 & 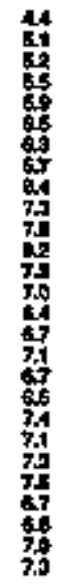 & 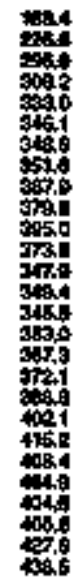 & 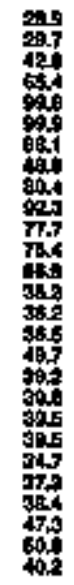 & 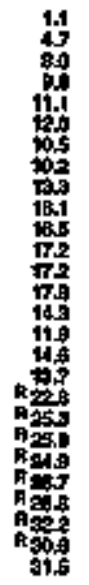 & 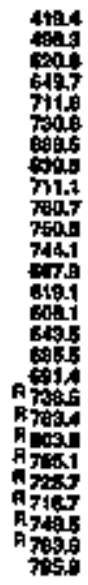 & 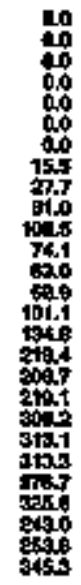 & 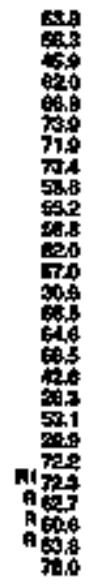 & 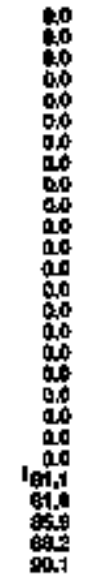 & 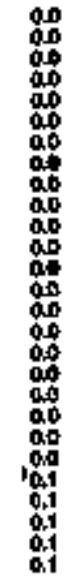 & 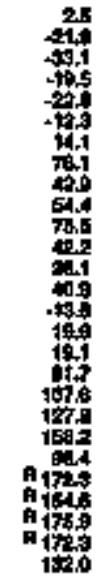 & 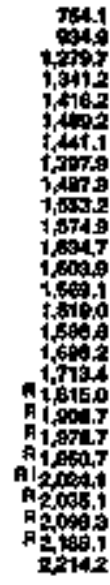 \\
\hline
\end{tabular}

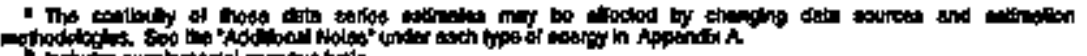

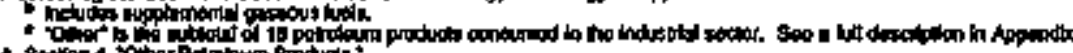

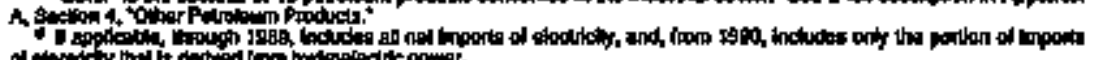

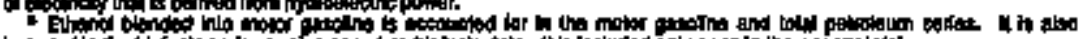

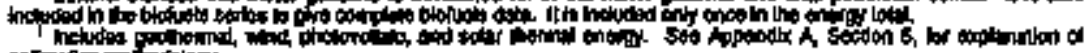

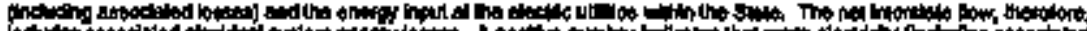
tow

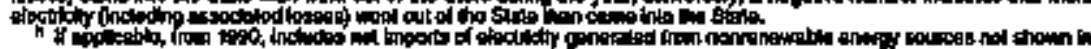

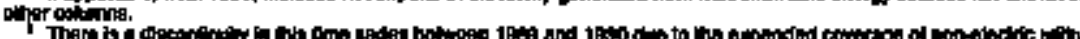

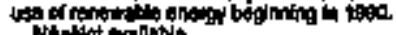

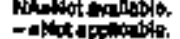

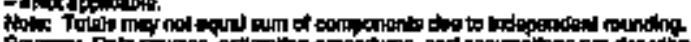

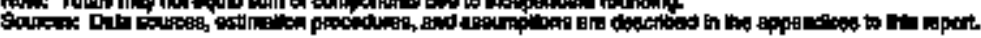




\begin{tabular}{|c|c|c|c|c|c|c|c|c|c|c|c|c|c|c|}
\hline \multirow[b]{3}{*}{$\mathrm{Y}_{\mathrm{Ex}}$} & \multicolumn{3}{|c|}{ cant } & \multirow[b]{2}{*}{ Montid } & \multicolumn{4}{|c|}{ Potrobsun } & \multirow{3}{*}{$\begin{array}{l}\text { Motuad, } \\
\text { Theusend } \\
\text { Tondil }\end{array}$} & & \multirow[b]{2}{*}{ Inestalys } & \multirow[b]{2}{*}{ Enoty } & \multirow{3}{*}{ 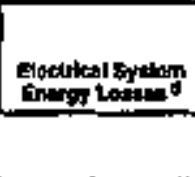 } & \multirow[b]{3}{*}{ Teted } \\
\hline & 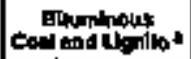 & 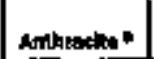 & row & & 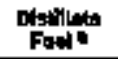 & 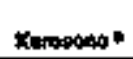 & LPQ: & Totat & & Solver $=$ & & & & \\
\hline & \multicolumn{3}{|c|}{ Thorespod Sbont Tons } & 最hen & \multicolumn{4}{|c|}{ Thoinghd Bannth } & & \multicolumn{3}{|c|}{ 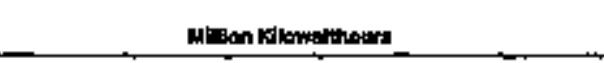 } & & \\
\hline 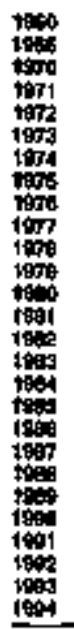 & 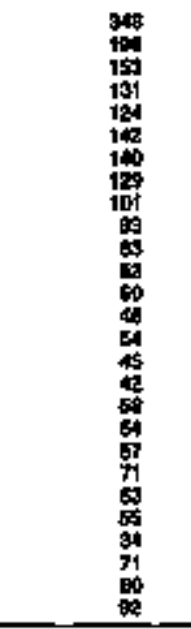 & $\begin{array}{l}0 \\
0 \\
0 \\
0 \\
0 \\
0 \\
0 \\
0 \\
0 \\
0 \\
0 \\
0 \\
0 \\
0 \\
0 \\
0 \\
0 \\
0 \\
0 \\
0 \\
0 \\
0 \\
0 \\
9 \\
0 \\
0 \\
0 \\
0 \\
0 \\
0\end{array}$ & 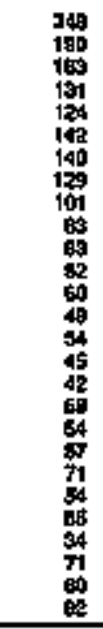 & 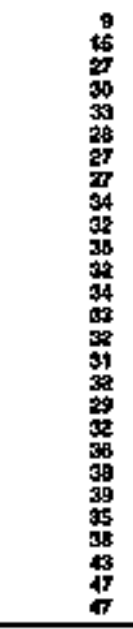 & 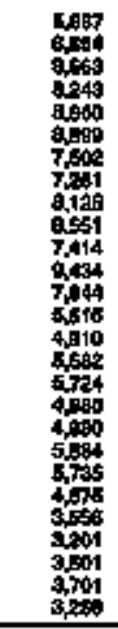 & 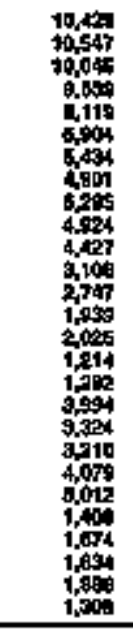 & 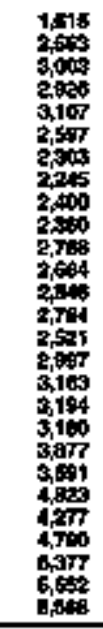 & 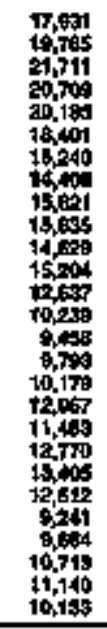 & 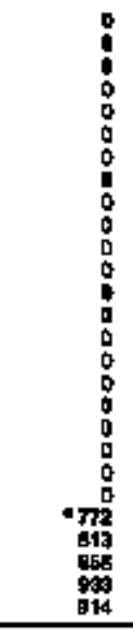 & 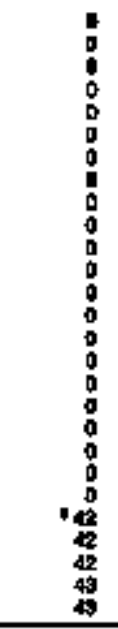 & 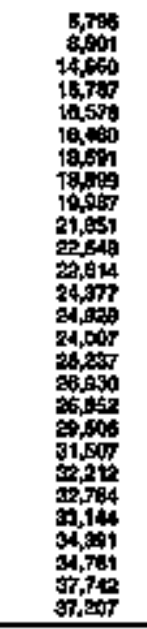 & $\begin{array}{l}z \\
\vdots \\
\vdots \\
\vdots \\
\vdots \\
\vdots \\
\vdots \\
\vdots \\
\vdots \\
\vdots \\
\vdots \\
\vdots \\
\vdots \\
\end{array}$ & 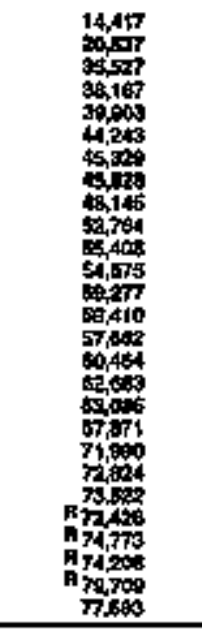 & $\begin{array}{l}\vdots \\
\vdots \\
\vdots \\
\vdots \\
\vdots \\
\vdots \\
\vdots \\
\vdots \\
\vdots\end{array}$ \\
\hline \multicolumn{15}{|c|}{ Trithen } \\
\hline 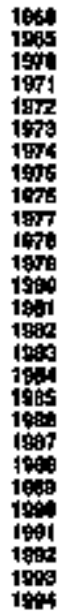 & 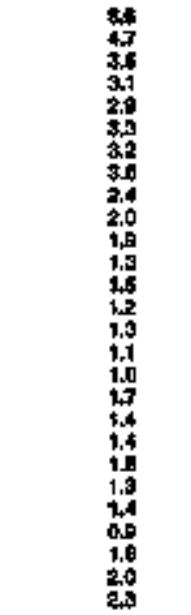 & 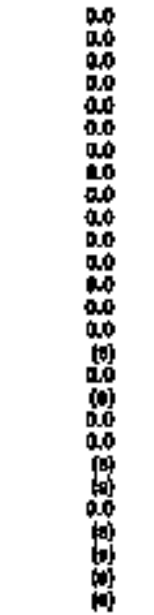 & 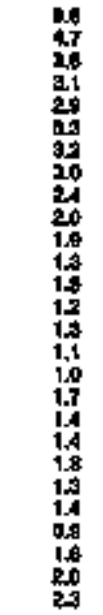 & 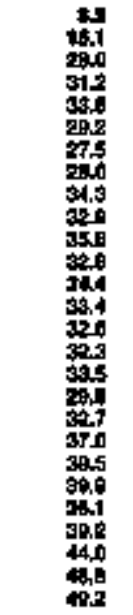 & 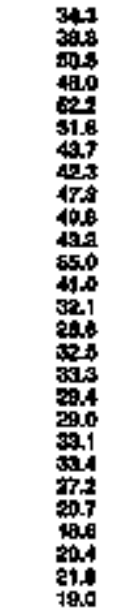 & 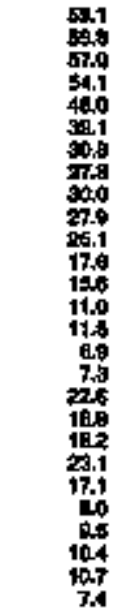 & 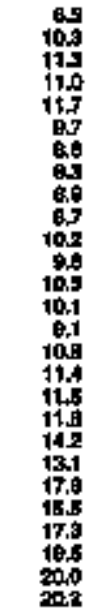 & 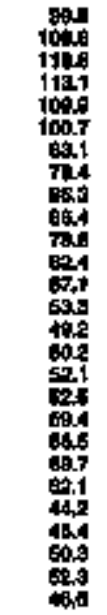 & 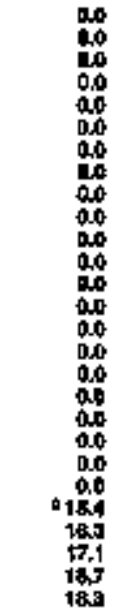 & 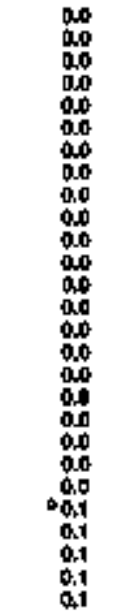 & 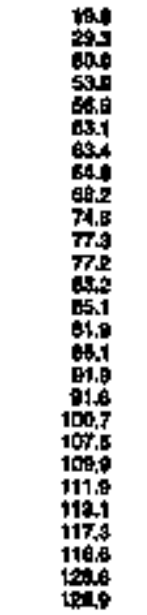 & 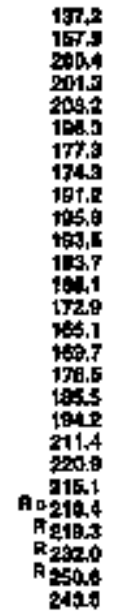 & 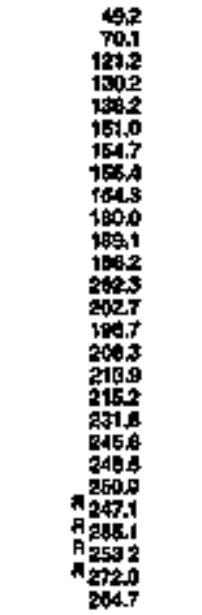 & 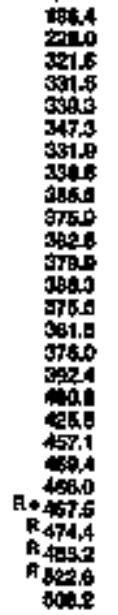 \\
\hline
\end{tabular}


Table 218. Industrial Energy Consumption Estimales, 1960, 1985, 1970-1994, North Cerolina

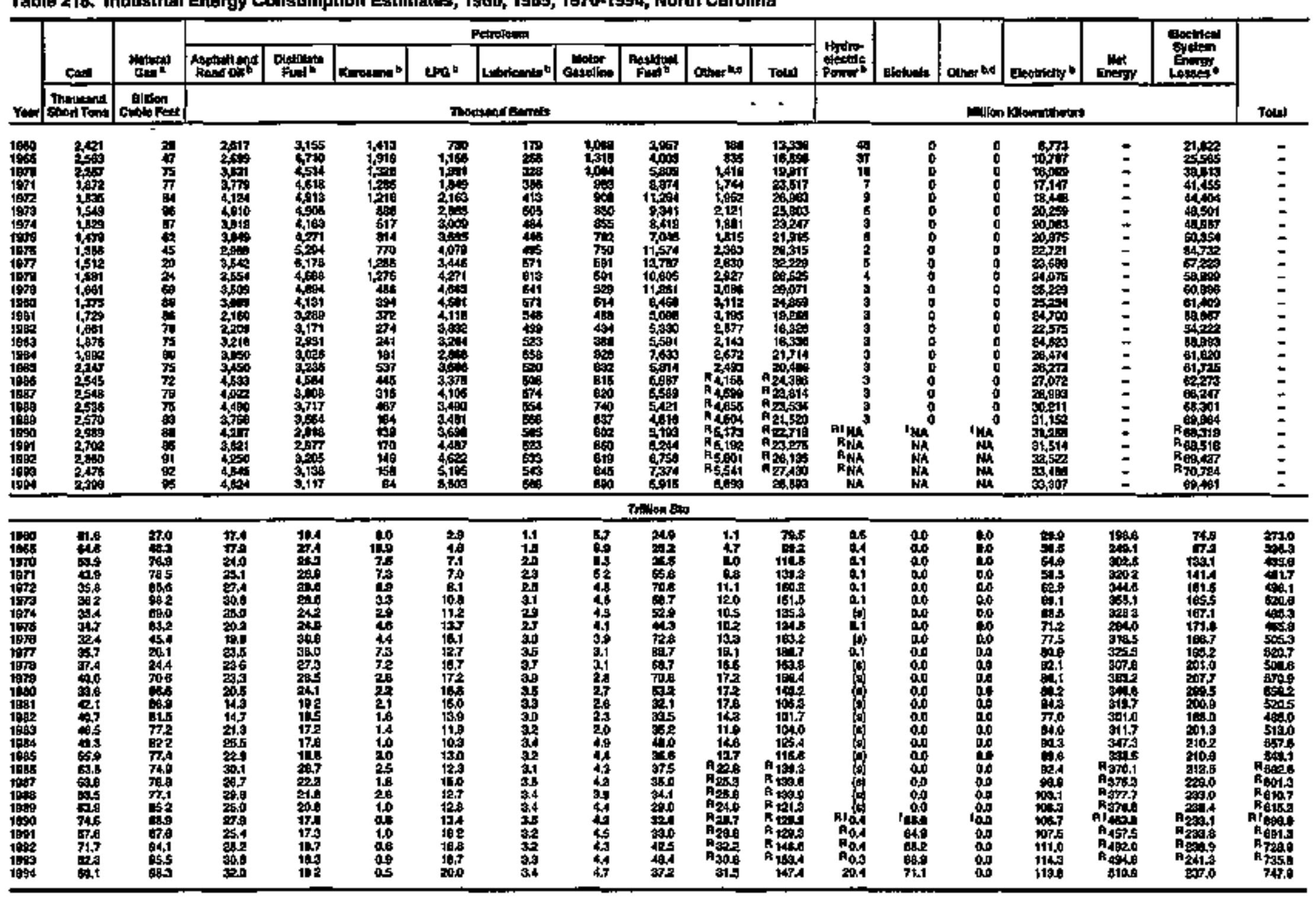

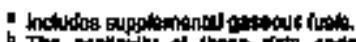

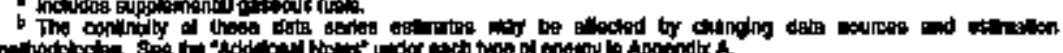

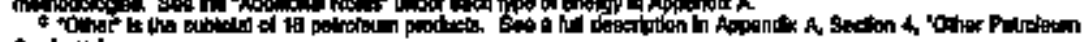

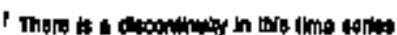

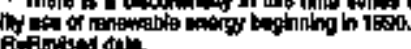

Axithox

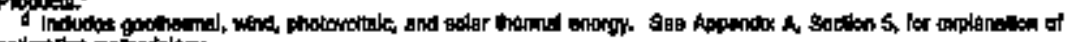

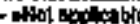




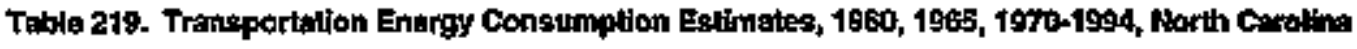

\begin{tabular}{|c|c|c|c|c|c|c|c|c|c|c|c|c|c|c|c|}
\hline \multirow[b]{3}{*}{ V } & \multirow{3}{*}{ 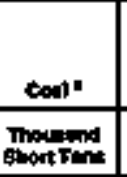 } & \multirow{3}{*}{ 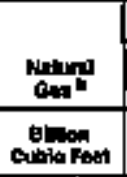 } & \multicolumn{8}{|c|}{ Potrolenn } & \multirow{3}{*}{ 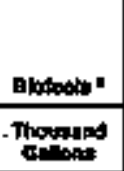 } & & \multirow[b]{2}{*}{ Enet } & \multirow{3}{*}{ 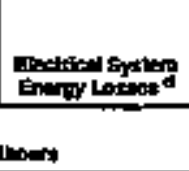 } & \multirow[b]{3}{*}{ Tơtell } \\
\hline & & & culation. & 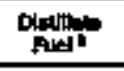 & 战。 & LPE: * & Lusteants & 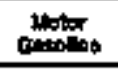 & Faldert & Toint & & Enctidy. & & & \\
\hline & & & \multicolumn{8}{|c|}{ Thougrad Bum } & & \multicolumn{2}{|c|}{ 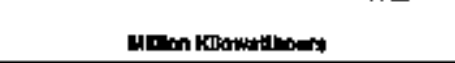 } & & \\
\hline 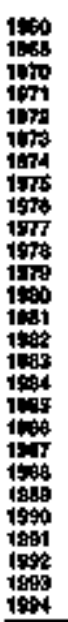 & $\begin{array}{l}4 \\
0 \\
0 \\
2 \\
2 \\
1 \\
1 \\
1 \\
0 \\
0 \\
0 \\
0 \\
0 \\
0 \\
0 \\
0 \\
0 \\
0 \\
0 \\
0 \\
0 \\
0 \\
0 \\
0 \\
0 \\
0 \\
0 \\
0 \\
0\end{array}$ & $\begin{array}{l}2 \\
4 \\
6 \\
6 \\
6 \\
6 \\
4 \\
4 \\
3 \\
2 \\
3 \\
5 \\
6 \\
6 \\
7 \\
6 \\
5 \\
5 \\
5 \\
5 \\
8 \\
8 \\
6 \\
6 \\
6 \\
4\end{array}$ & 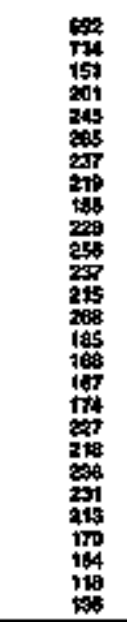 & 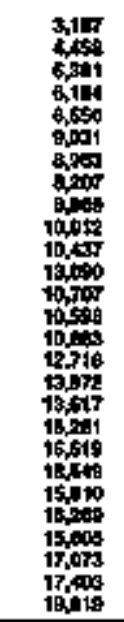 & 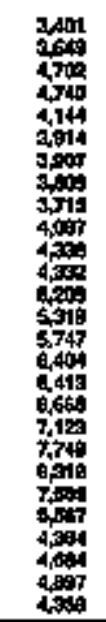 & 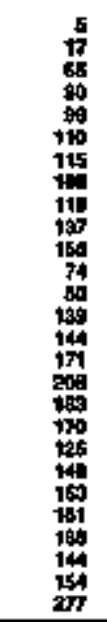 & 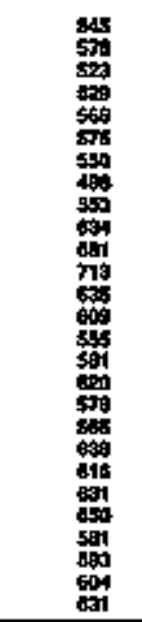 & 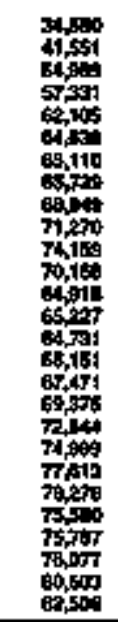 & 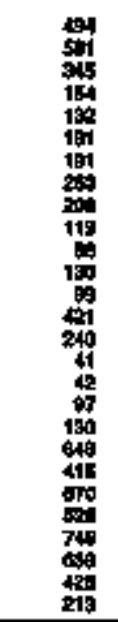 & 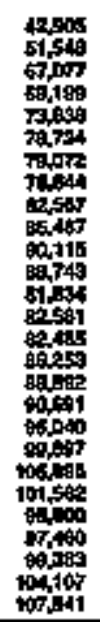 & 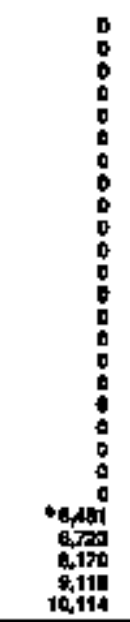 & 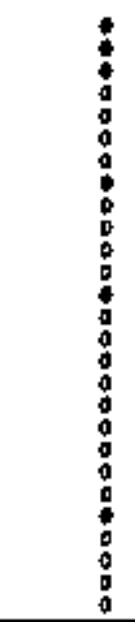 & $\begin{array}{l}= \\
= \\
= \\
= \\
= \\
= \\
= \\
= \\
= \\
= \\
= \\
z \\
= \\
=\end{array}$ & $\begin{array}{l}0 \\
0 \\
0 \\
0 \\
0 \\
0 \\
0 \\
0 \\
0 \\
0 \\
0 \\
0 \\
0 \\
0 \\
0 \\
0 \\
0 \\
0 \\
0 \\
0 \\
0 \\
0\end{array}$ & $\begin{array}{l}= \\
z \\
z \\
z \\
z \\
z \\
= \\
= \\
z \\
z \\
z \\
= \\
= \\
z\end{array}$ \\
\hline \multicolumn{16}{|c|}{ Hition on } \\
\hline 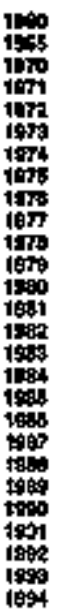 & 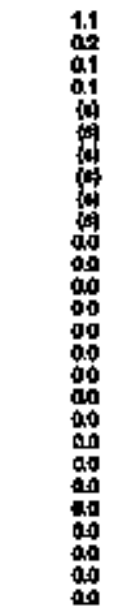 & 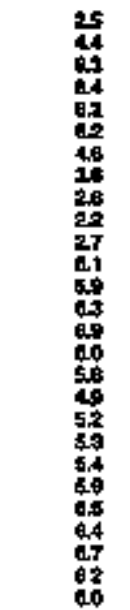 & 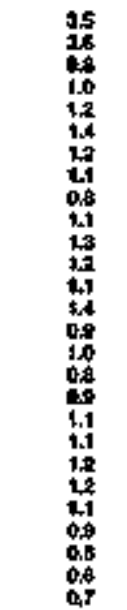 & 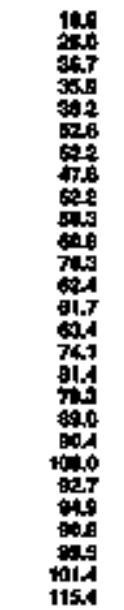 & 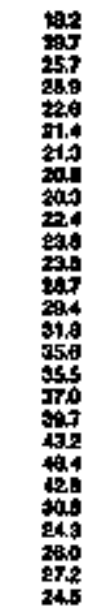 & 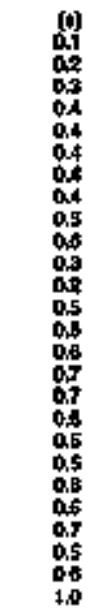 & 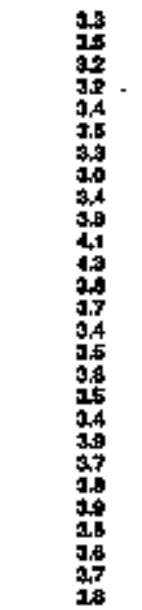 & 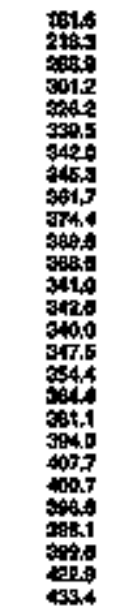 & 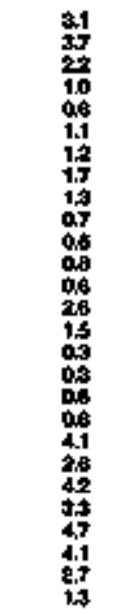 & 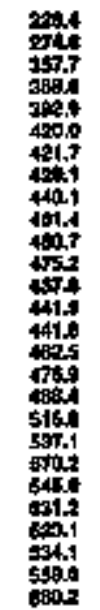 & 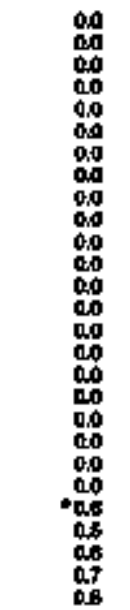 & 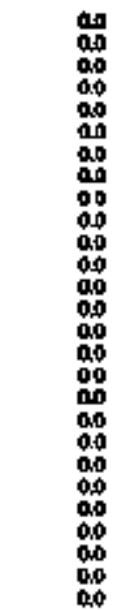 & 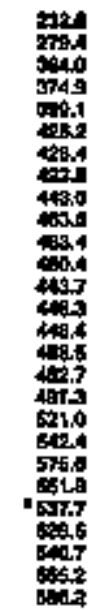 & 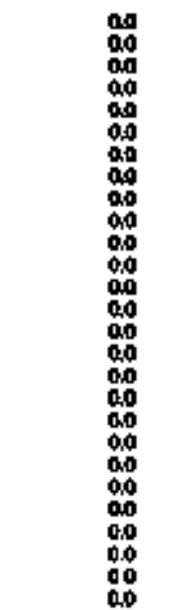 & 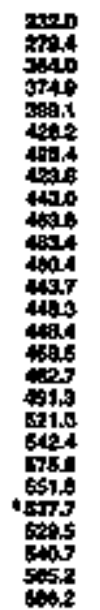 \\
\hline
\end{tabular}




\begin{tabular}{|c|c|c|c|c|c|c|c|c|c|c|c|c|c|c|}
\hline \multirow[b]{3}{*}{ Yand } & \multicolumn{3}{|c|}{ السبc } & \multirow{3}{*}{ 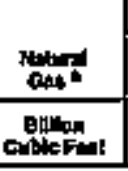 } & \multicolumn{4}{|c|}{ Pumping } & \multirow[b]{2}{*}{ 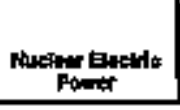 } & \multirow[b]{2}{*}{ 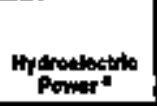 } & \multirow[b]{2}{*}{ eloforela } & \multirow[b]{2}{*}{ Cons } & \multirow{3}{*}{0 obersts } & \multirow[b]{3}{*}{ Tolath } \\
\hline & 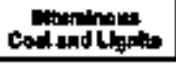 & Anthesefos & Totw & & tongt & 나요 & 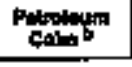 & $T \rightarrow \infty$ & & & & & & \\
\hline & \multicolumn{3}{|c|}{ Inowand shost Tont } & & \multicolumn{4}{|c|}{ 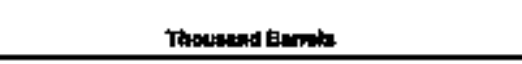 } & \multicolumn{4}{|c|}{ 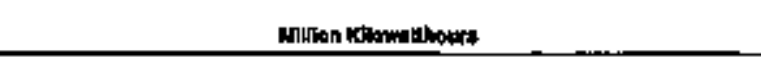 } & & \\
\hline 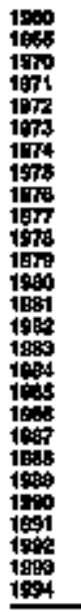 & 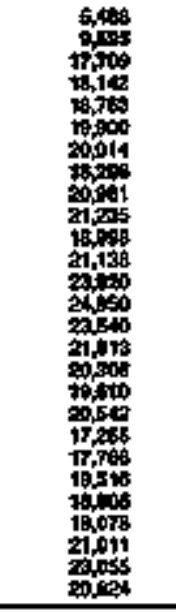 & $\begin{array}{l}0 \\
0 \\
0 \\
0 \\
0 \\
0 \\
0 \\
0 \\
0 \\
0 \\
0 \\
0 \\
0 \\
0 \\
0 \\
0 \\
0 \\
0 \\
0 \\
0 \\
0 \\
\end{array}$ & 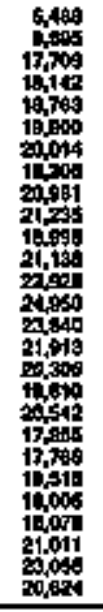 & 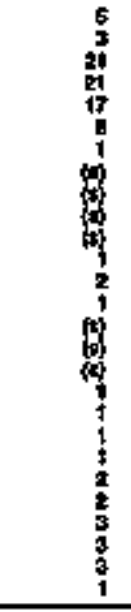 & 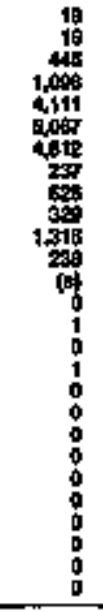 & 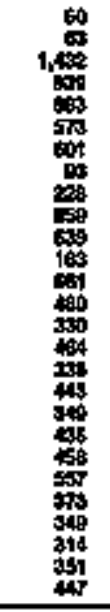 & $\begin{array}{l}0 \\
0 \\
0 \\
0 \\
0 \\
0 \\
0 \\
0 \\
0 \\
0 \\
0 \\
0 \\
0 \\
0 \\
0 \\
0 \\
0 \\
0 \\
0 \\
0 \\
0 \\
0 \\
0 \\
\end{array}$ & 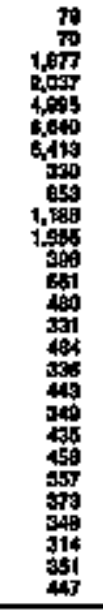 & 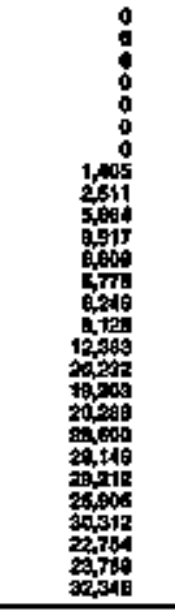 & 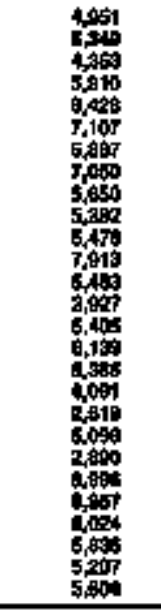 & $\begin{array}{l}0 \\
0 \\
0 \\
0 \\
0 \\
0 \\
0 \\
0 \\
0 \\
0 \\
0 \\
0 \\
0 \\
0 \\
0 \\
0 \\
0 \\
0 \\
0 \\
0 \\
0 \\
0 \\
0 \\
0 \\
0 \\
0 \\
0 \\
\end{array}$ & $\begin{array}{l}0 \\
0 \\
0 \\
0 \\
0 \\
0 \\
0 \\
0 \\
0 \\
0 \\
0 \\
0 \\
0 \\
0 \\
0 \\
0 \\
0 \\
0 \\
0 \\
0 \\
0\end{array}$ & $\begin{array}{l}0 \\
0 \\
0 \\
0 \\
0 \\
0 \\
0 \\
0 \\
0 \\
0 \\
0 \\
0 \\
0 \\
0 \\
0 \\
0 \\
0 \\
0 \\
0 \\
0 \\
0 \\
0 \\
0 \\
0 \\
0\end{array}$ & $\begin{array}{l}\vdots \\
\vdots \\
\vdots \\
\vdots \\
\vdots \\
\vdots \\
\vdots \\
\vdots \\
\vdots \\
\vdots \\
\vdots \\
\vdots \\
\vdots \\
\vdots\end{array}$ \\
\hline \multicolumn{15}{|c|}{ Trimenew } \\
\hline 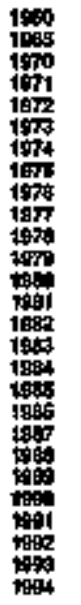 & 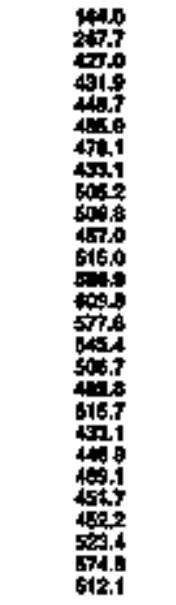 & 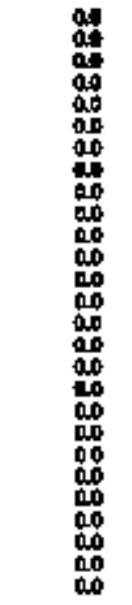 & 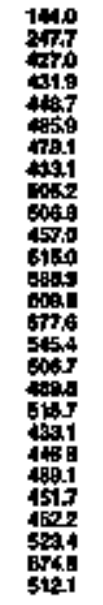 & 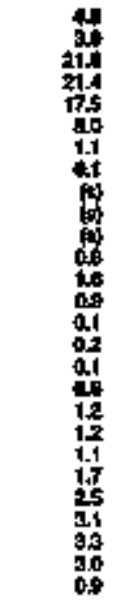 & 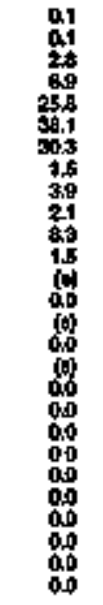 & 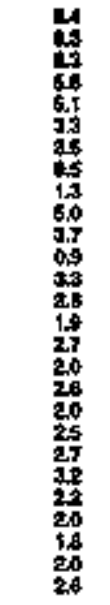 & 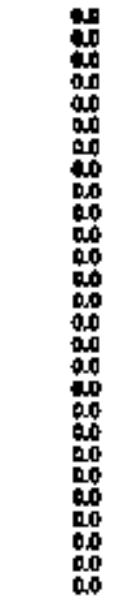 & 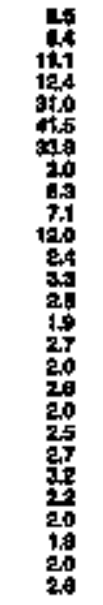 & 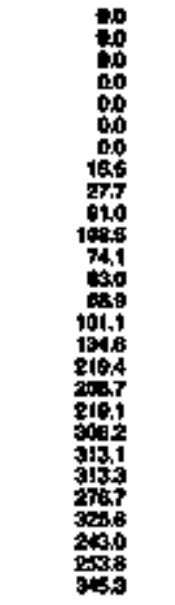 & 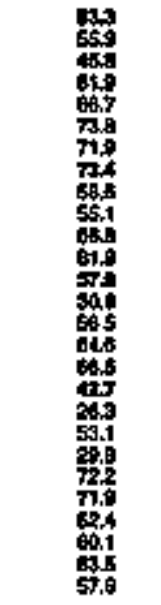 & 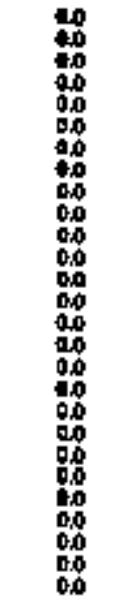 & 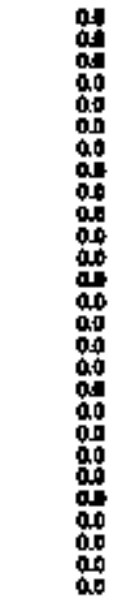 & 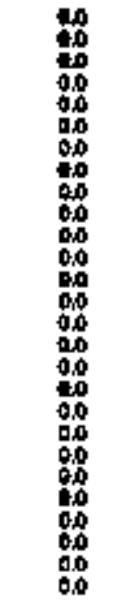 & 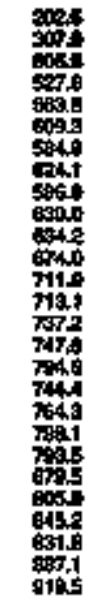 \\
\hline
\end{tabular}

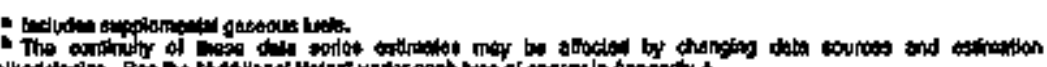

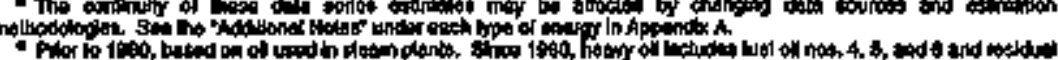

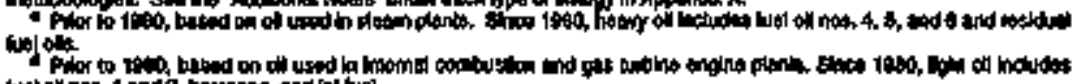

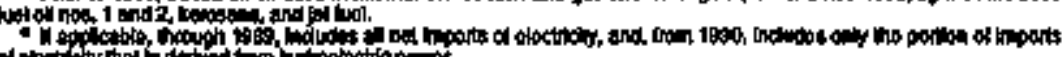

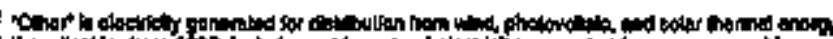

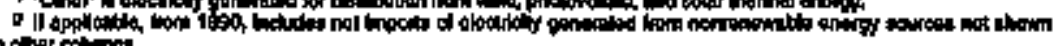

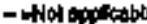

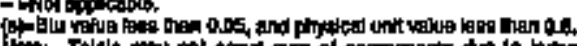

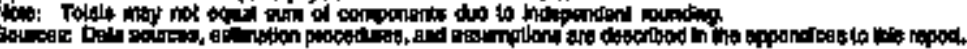

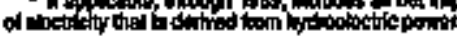


Table 221. Enwrgy Consumption Estimates by Source, 1960, t958, 1970-1694, North Dakota

\begin{tabular}{|c|c|c|c|c|c|c|c|c|c|c|c|c|c|c|c|c|c|c|c|}
\hline \multirow[b]{3}{*}{ Year } & \multirow[b]{2}{*}{ Cond } & \multirow[b]{2}{*}{ stod } & \multicolumn{11}{|c|}{ Fonotong } & \multirow{2}{*}{ 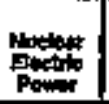 } & \multirow{2}{*}{ Powate } & \multirow[b]{2}{*}{ Blobtatios } & \multirow[b]{2}{*}{ Ghored | } & \multirow{3}{*}{ 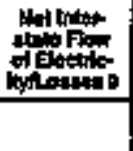 } & \multirow[b]{3}{*}{ Totath } \\
\hline & & & 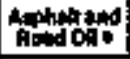 & Antion & 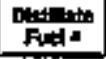 & Fit. & Knton: & LPE: & tomit. & Notint & Alestade & Other as & Toth & & & & & & \\
\hline & gantind & critian & \multicolumn{11}{|c|}{ 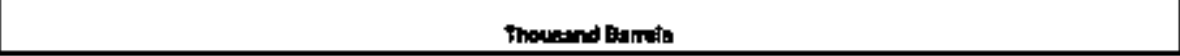 } & \multicolumn{4}{|c|}{ 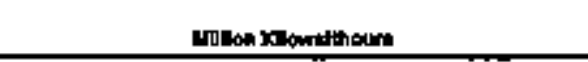 } & & \\
\hline 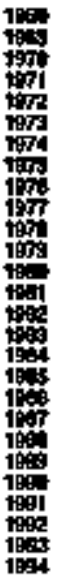 & 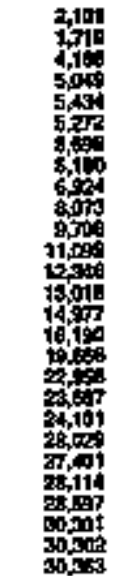 & 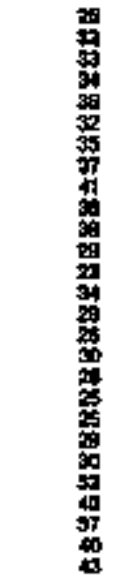 & 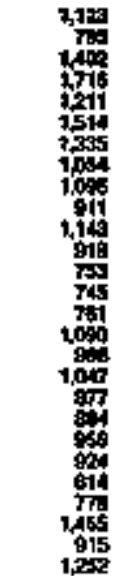 & 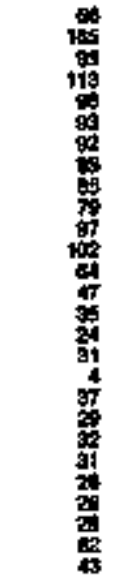 & 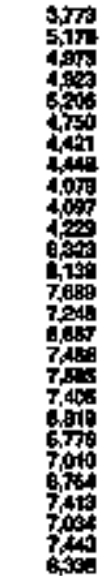 & 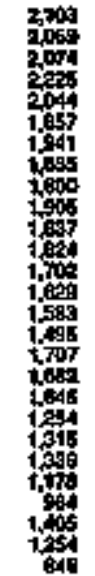 & 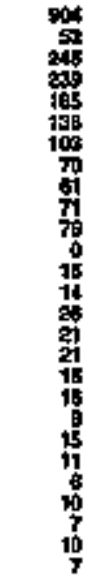 & 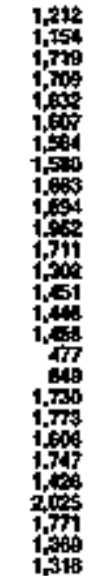 & 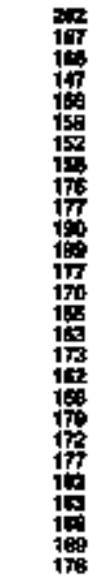 & 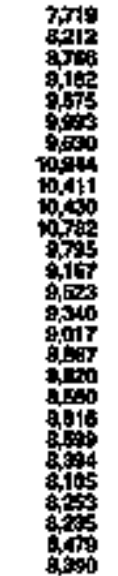 & 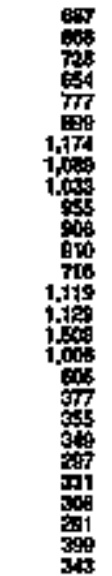 & 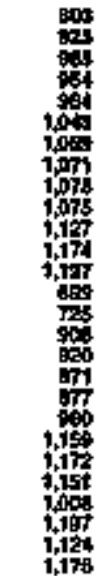 & 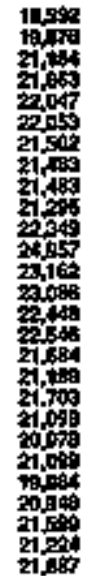 & $\begin{array}{l}0 \\
0 \\
0 \\
0 \\
0 \\
0 \\
0 \\
0 \\
0 \\
0 \\
0 \\
0 \\
0 \\
0 \\
0 \\
0 \\
0 \\
0 \\
0 \\
0 \\
0\end{array}$ & 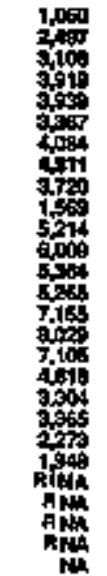 & 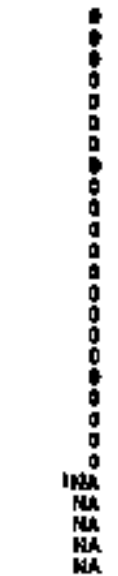 & 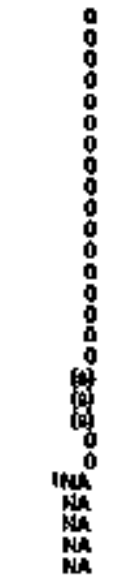 & 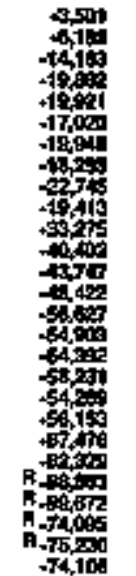 & $\begin{array}{l}\bar{z} \\
\bar{z} \\
\bar{z} \\
\bar{z} \\
\bar{z}\end{array}$ \\
\hline
\end{tabular}

$\mathbf{N}$
$\mathbf{O}$
$\mathbf{R}$
$\mathbf{T}$
$\mathbf{H}$
$\mathbf{D}$
$\mathbf{A}$
$\mathbf{K}$
$\mathbf{0}$
$\mathbf{T}$
$\mathbf{A}$

\begin{tabular}{|c|c|c|c|c|c|c|c|c|c|c|c|c|c|c|c|}
\hline 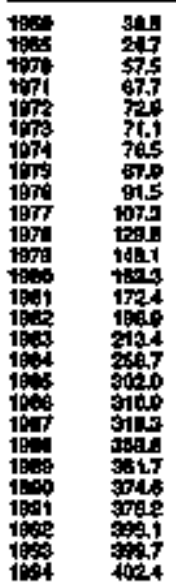 & 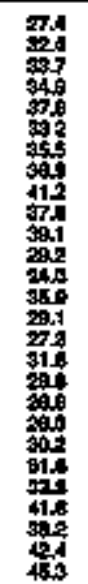 & 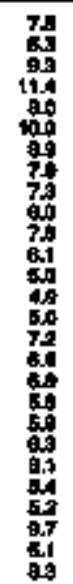 & 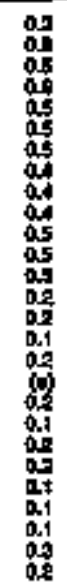 & 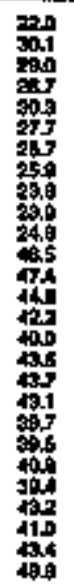 & 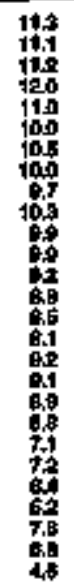 & 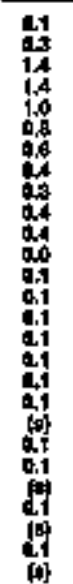 & 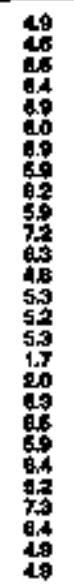 & 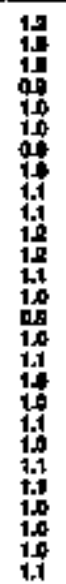 & 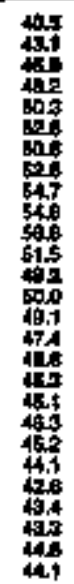 & 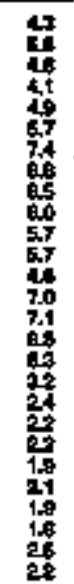 & 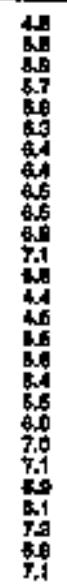 & 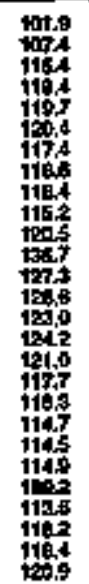 & 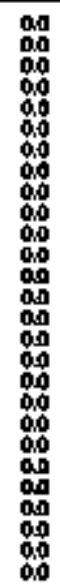 & 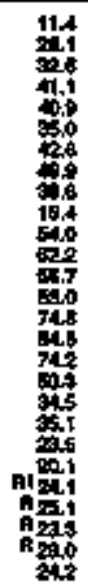 & \\
\hline
\end{tabular}

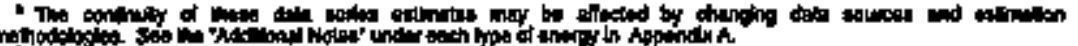

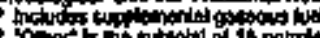

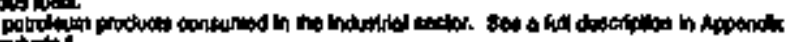

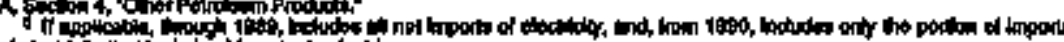

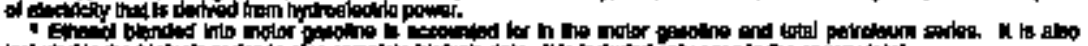

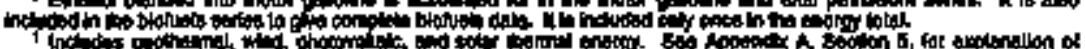

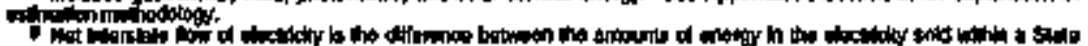

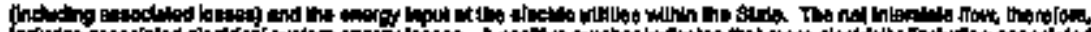

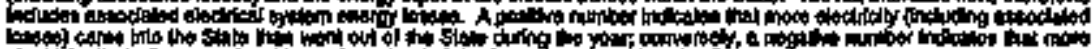

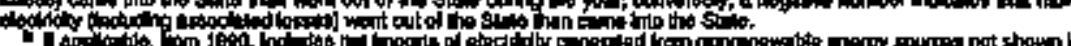

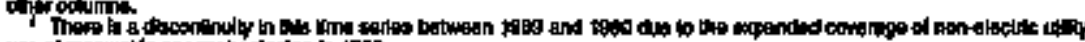

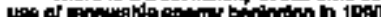

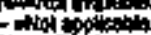

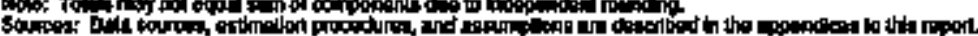




\begin{tabular}{|c|c|c|c|c|c|c|c|c|c|c|c|c|c|c|}
\hline \multirow[b]{3}{*}{$\underline{\text { rost }}$} & \multicolumn{3}{|c|}{ का } & \multirow[b]{2}{*}{ mented } & \multicolumn{4}{|c|}{ Pathenturn } & \multirow{3}{*}{ 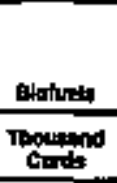 } & & \multirow[b]{2}{*}{ Goptreky 0} & \multirow[b]{2}{*}{ enery } & \multirow{3}{*}{ Entrateroteng } & \multirow[b]{3}{*}{ Total } \\
\hline & 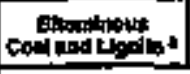 & Nontradn : | & trotat & & Pontinta & Krosene" & Lpa* & Tots & & Bon-" & & & & \\
\hline & \multicolumn{3}{|c|}{ Thougind Stront Tena } & athes & \multicolumn{4}{|c|}{ Imosuand Samm } & & \multicolumn{3}{|c|}{ 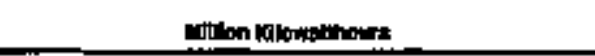 } & & \\
\hline 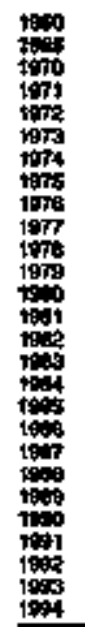 & 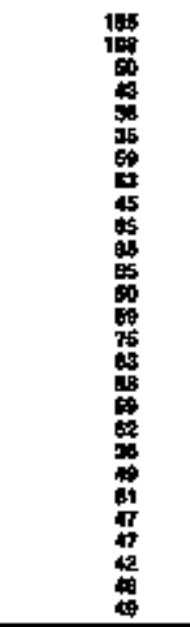 & $\begin{array}{l}0 \\
0 \\
0 \\
0 \\
0 \\
0 \\
0 \\
0 \\
0 \\
0 \\
0 \\
0 \\
0 \\
0 \\
0 \\
0 \\
0 \\
0 \\
0 \\
0 \\
0 \\
0 \\
9 \\
0 \\
0 \\
0 \\
0 \\
0\end{array}$ & 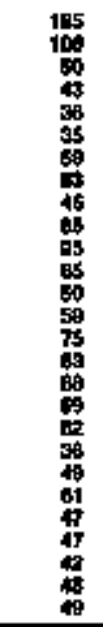 & 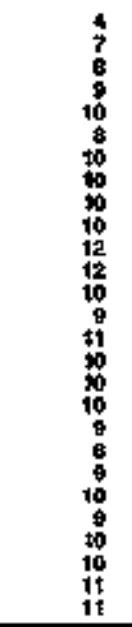 & 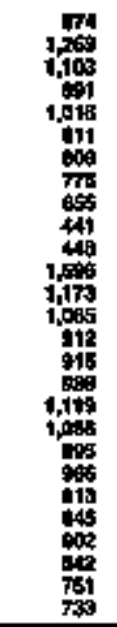 & 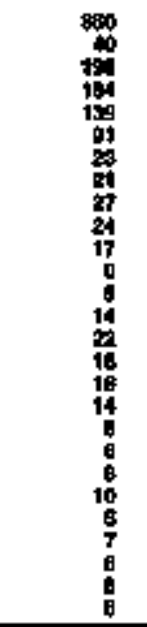 & 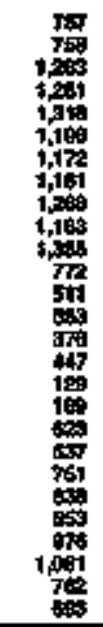 & 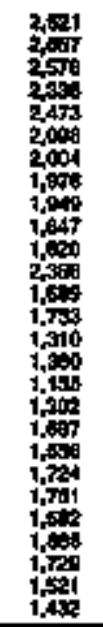 & $\begin{array}{c}0 \\
0 \\
\vdots \\
0 \\
0 \\
0 \\
0 \\
0 \\
0 \\
\vdots \\
0 \\
\vdots \\
\vdots \\
\vdots \\
\vdots \\
0 \\
0 \\
0 \\
0 \\
0 \\
7 \\
70\end{array}$ & $\begin{array}{l}0 \\
0 \\
0 \\
0 \\
0 \\
0 \\
0 \\
0 \\
0 \\
0 \\
0 \\
0 \\
0 \\
0 \\
0 \\
0 \\
0 \\
0 \\
0 \\
0 \\
0 \\
0 \\
6 \\
6\end{array}$ & 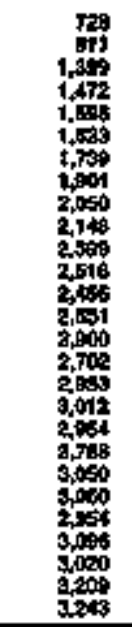 & $\begin{array}{l}\vdots \\
\vdots \\
\vdots \\
\vdots \\
\vdots \\
\vdots \\
\vdots \\
\vdots \\
\vdots \\
\vdots \\
\vdots \\
\vdots\end{array}$ & 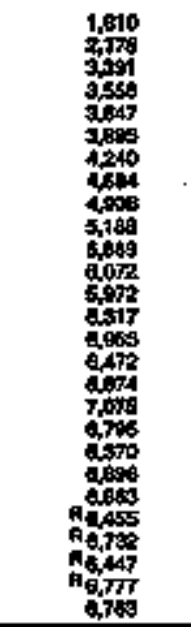 & $\begin{array}{l}\vdots \\
\vdots \\
\vdots \\
\vdots \\
\vdots \\
\vdots \\
\vdots \\
\vdots \\
\vdots \\
\vdots \\
\vdots \\
\vdots \\
\vdots\end{array}$ \\
\hline \multicolumn{15}{|c|}{ Thether Bin } \\
\hline 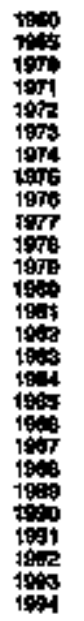 & 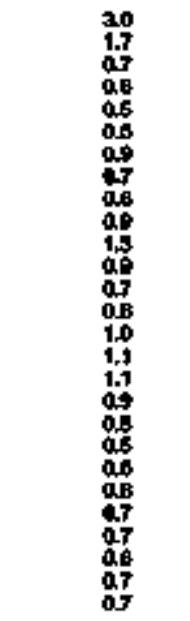 & 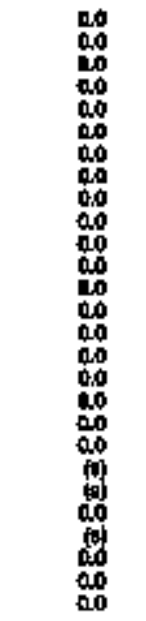 & 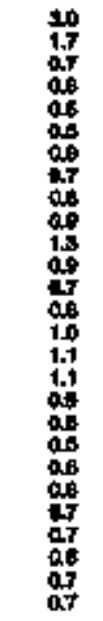 & 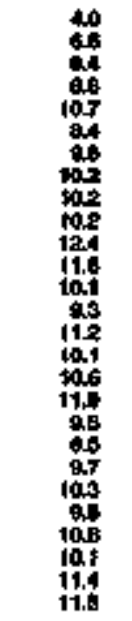 & 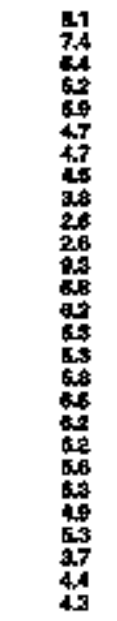 & 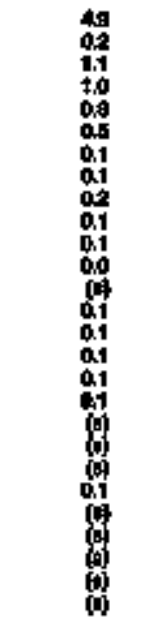 & 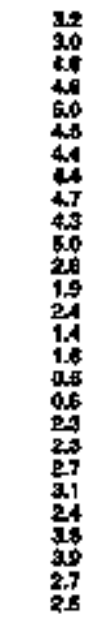 & 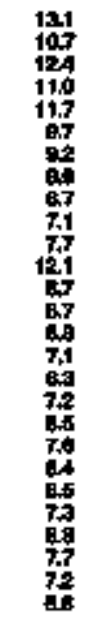 & 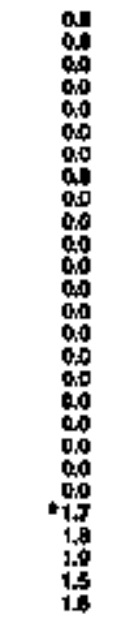 & 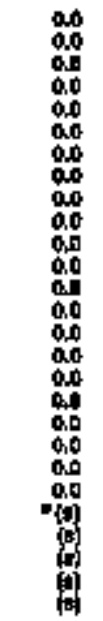 & 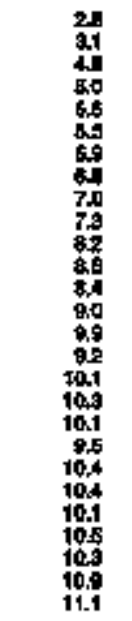 & 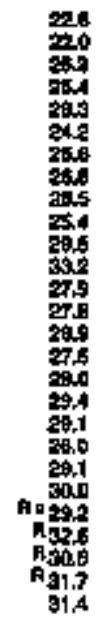 & 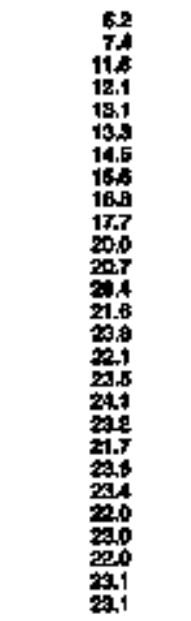 & 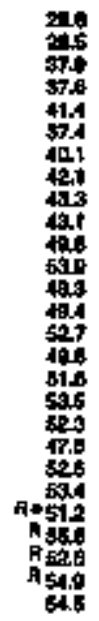 \\
\hline
\end{tabular}

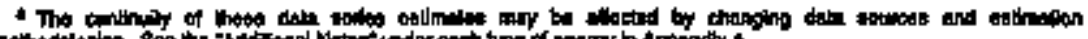

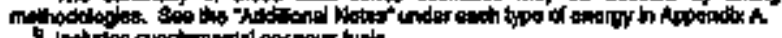

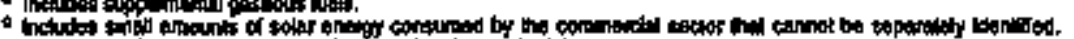

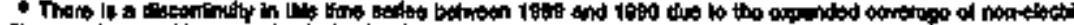

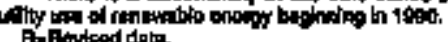

-

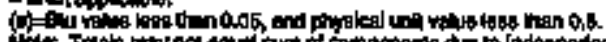

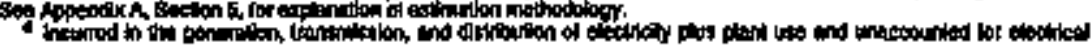

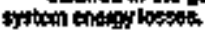

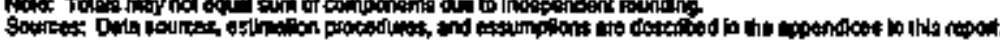


Table 229. Commerclel Energy Consumption Estimales, 1960, 1965, 1970-1994, Nortt Dakota

\begin{tabular}{|c|c|c|c|c|c|c|c|c|c|c|c|c|c|c|}
\hline \multirow[b]{3}{*}{ Yot: } & \multicolumn{3}{|c|}{$\cos$} & \multirow{3}{*}{ 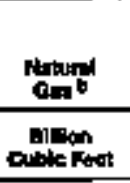 } & \multicolumn{6}{|c|}{ Folvedosm } & \multirow[b]{2}{*}{ Eectrdity" } & \multirow[b]{2}{*}{ nampon } & \multirow[b]{2}{*}{ 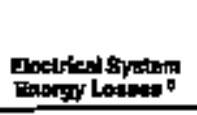 } & \multirow[b]{3}{*}{ Tates 1} \\
\hline & 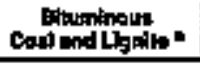 & Anthenelo: & Totol & & Datillotio & Korotone & Lot: & $\begin{array}{c}\text { Motor } \\
\text { Cupoifon } \\
\end{array}$ & 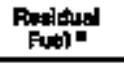 & Tot & & & & \\
\hline & \multicolumn{3}{|c|}{ Thowand shart Toul } & & \multicolumn{6}{|c|}{ moustand thands } & \multicolumn{3}{|c|}{ 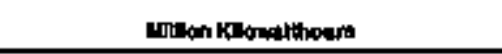 } & \\
\hline 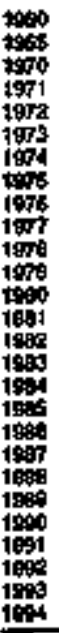 & 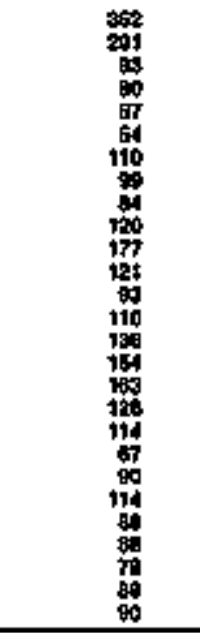 & $\begin{array}{l}0 \\
0 \\
0 \\
0 \\
0 \\
0 \\
0 \\
0 \\
0 \\
0 \\
0 \\
0 \\
0 \\
0 \\
0 \\
0 \\
0 \\
0 \\
0 \\
0 \\
0 \\
0 \\
0 \\
0 \\
0 \\
0 \\
0 \\
\end{array}$ & 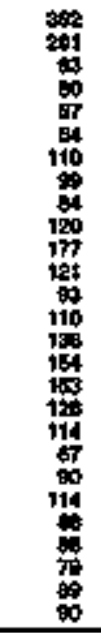 & $\begin{array}{c}3 \\
5 \\
5 \\
8 \\
10 \\
10 \\
12 \\
12 \\
12 \\
11 \\
12 \\
12 \\
11 \\
0 \\
11 \\
10 \\
10 \\
10 \\
0 \\
0 \\
10 \\
11 \\
10 \\
11 \\
10 \\
11 \\
11 \\
11\end{array}$ & 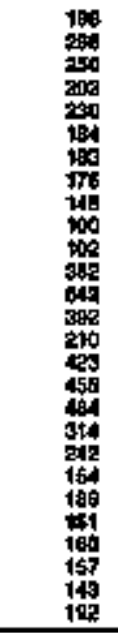 & 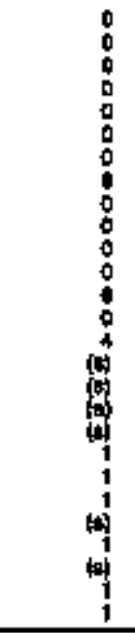 & 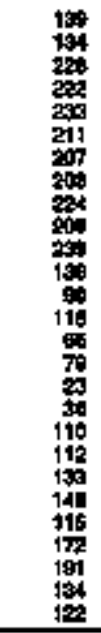 & 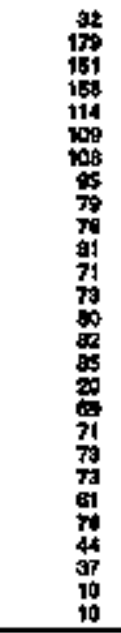 & 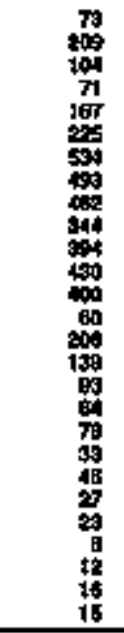 & 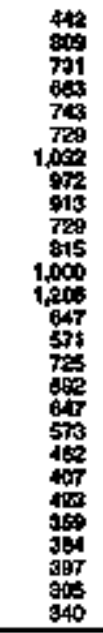 & 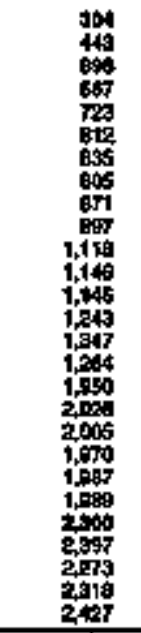 & $\begin{array}{l}= \\
= \\
= \\
= \\
= \\
= \\
= \\
= \\
= \\
= \\
= \\
= \\
= \\
= \\
=\end{array}$ & 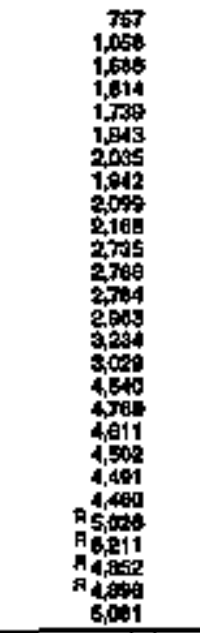 & $\begin{array}{l}= \\
= \\
z \\
= \\
z \\
z \\
= \\
= \\
= \\
z \\
z \\
z \\
z \\
z \\
z \\
\end{array}$ \\
\hline \multicolumn{15}{|c|}{ Tollion Ex } \\
\hline 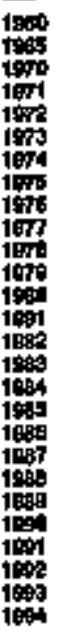 & 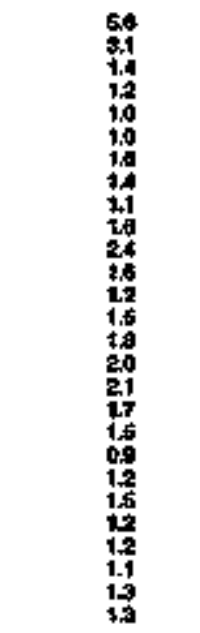 & $\begin{array}{l}00 \\
00 \\
00 \\
00 \\
00 \\
00 \\
00 \\
00 \\
00 \\
10 \\
00 \\
00 \\
100 \\
00 \\
00 \\
00 \\
00 \\
00 \\
00 \\
00 \\
00 \\
00 \\
00 \\
00 \\
00 \\
00\end{array}$ & 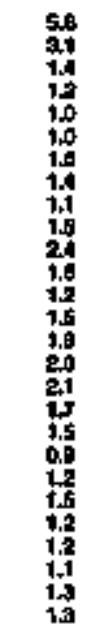 & 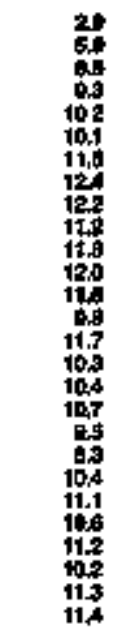 & 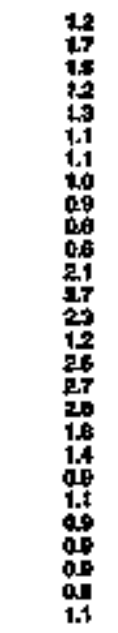 & 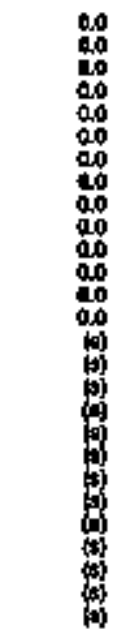 & 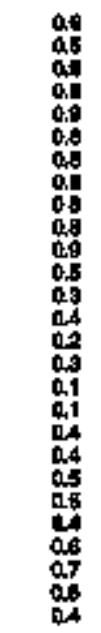 & 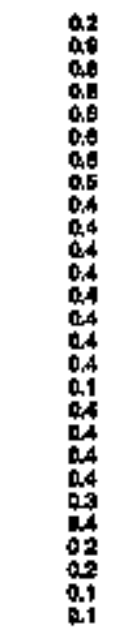 & 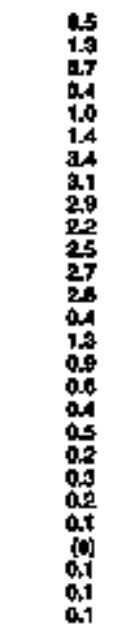 & 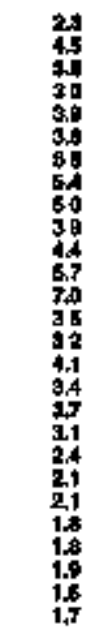 & 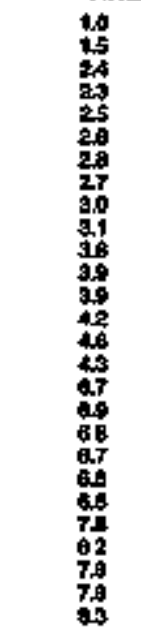 & 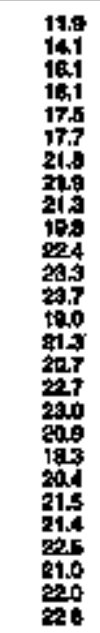 & 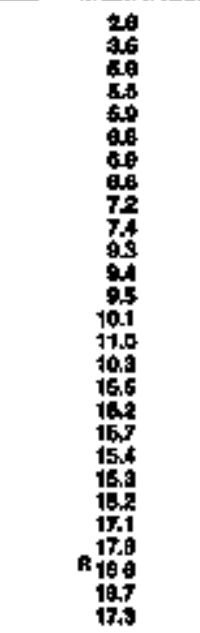 & 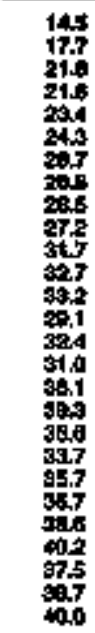 \\
\hline
\end{tabular}

$\mathbf{N}$
$\mathbf{O}$
$\mathbf{R}$
$\mathbf{T}$
$\mathbf{H}$

$\mathbf{D}$
$\mathbf{A}$
$\mathbf{K}$
0
$\mathbf{T}$
$\mathbf{A}$ 
Table 224. Industrlal Enongy Cansumplion Eatimetes, 1980, 1955, 1970-1964, Nonth Dakota

\begin{tabular}{|c|c|c|c|c|c|c|c|c|c|c|c|c|c|c|c|c|c|c|}
\hline \multirow[b]{3}{*}{ You } & \multirow[b]{2}{*}{ cost } & \multirow[b]{2}{*}{ Maturnil } & \multicolumn{9}{|c|}{ Petreftann } & \multirow{2}{*}{ 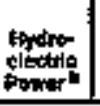 } & \multirow[b]{2}{*}{ Bofium: } & \multirow[b]{2}{*}{ oument } & \multirow[b]{2}{*}{ Enctidely of } & \multirow[b]{2}{*}{ inery } & \multirow{2}{*}{ 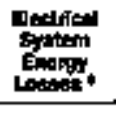 } & \multirow[b]{3}{*}{ Tow } \\
\hline & & & 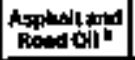 & Povent" & Kerosen: & LPo' & Lebrok=ms & botor & 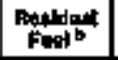 & andros & Tolon & & & & & & & \\
\hline & Drousan: & Contion Fot & \multicolumn{9}{|c|}{ 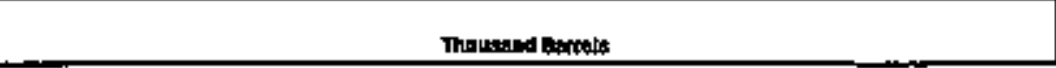 } & \multicolumn{6}{|c|}{ 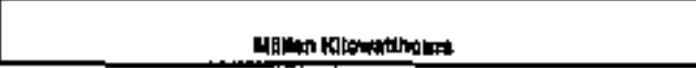 } & \\
\hline 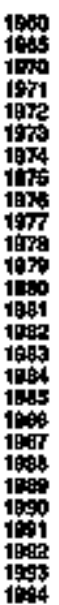 & 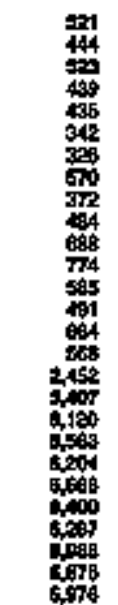 & 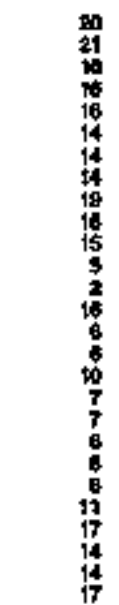 & 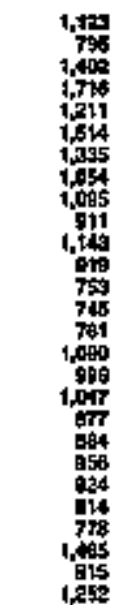 & 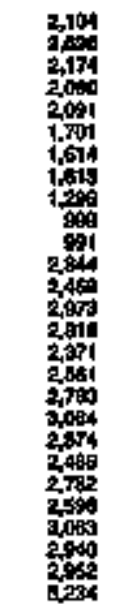 & 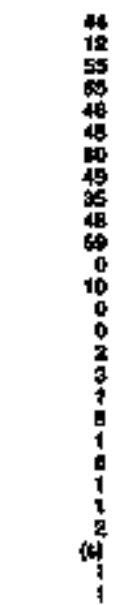 & 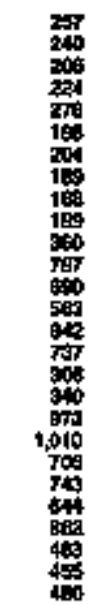 & 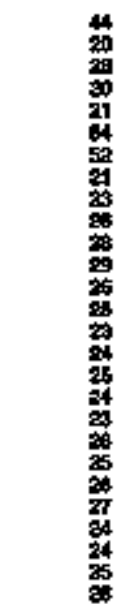 & 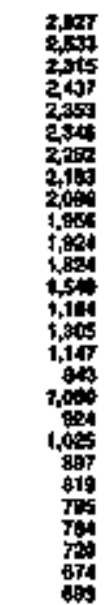 & 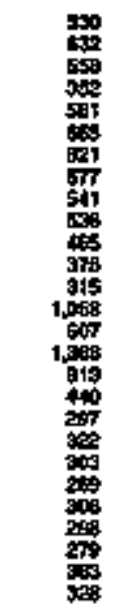 & 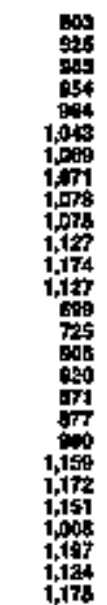 & 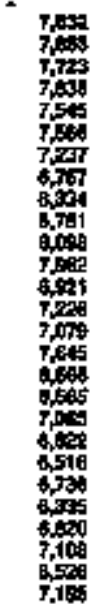 & 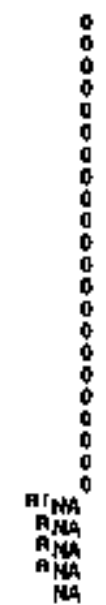 & 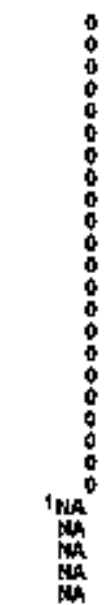 & $\begin{array}{l}0 \\
0 \\
0 \\
0 \\
0 \\
0 \\
0 \\
0 \\
0 \\
0 \\
0 \\
0 \\
0 \\
0 \\
0 \\
0 \\
0 \\
0 \\
0 \\
0 \\
4 \\
4 \\
4 \\
4\end{array}$ & 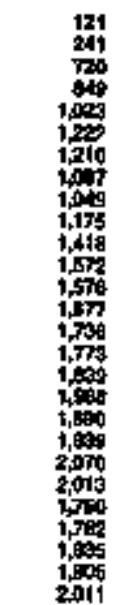 & $\begin{array}{l}= \\
z \\
z \\
z \\
z \\
z \\
= \\
z \\
z \\
z \\
z \\
z \\
z \\
z\end{array}$ & 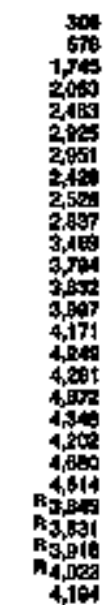 & 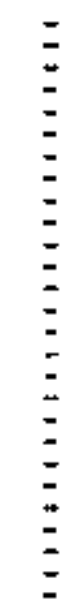 \\
\hline
\end{tabular}

\begin{tabular}{|c|c|c|c|c|c|c|c|c|c|c|c|c|c|c|c|c|c|c|}
\hline 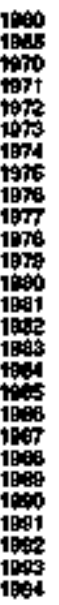 & 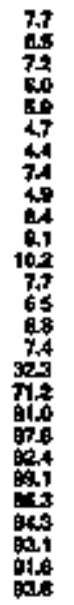 & 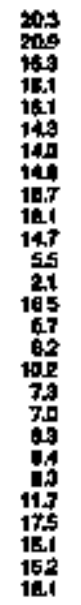 & 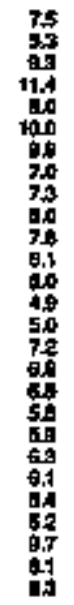 & 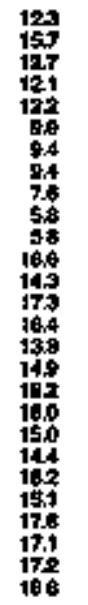 & 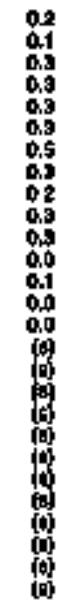 & 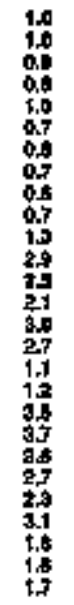 & 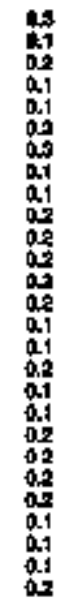 & 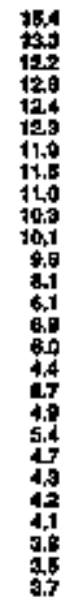 & 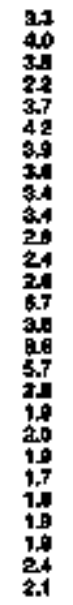 & 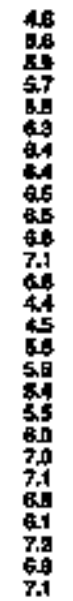 & 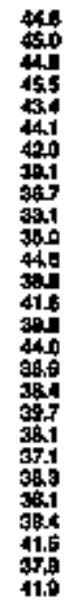 & 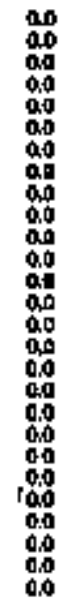 & 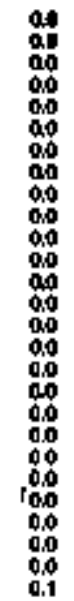 & 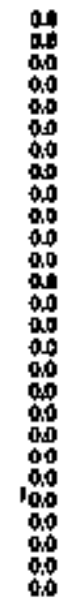 & 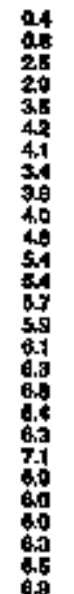 & 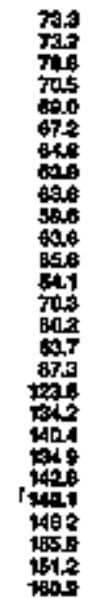 & 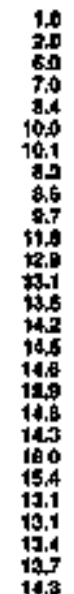 & 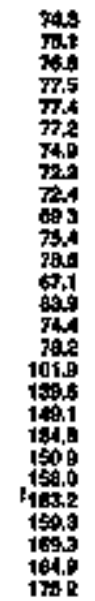 \\
\hline
\end{tabular}

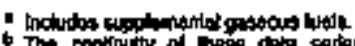

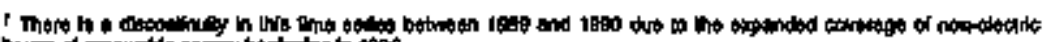

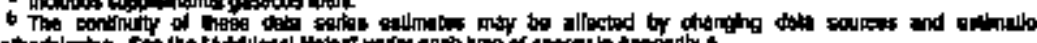

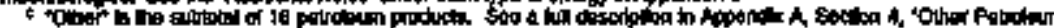
Prodicts:

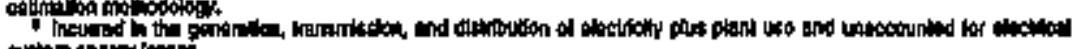

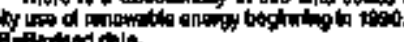
Matiot and

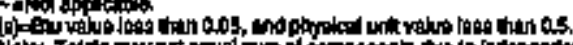


Table 225. Trensportation Energy Consumption Estingtes, 1980, 1965, 1970-1994, North Dakoth

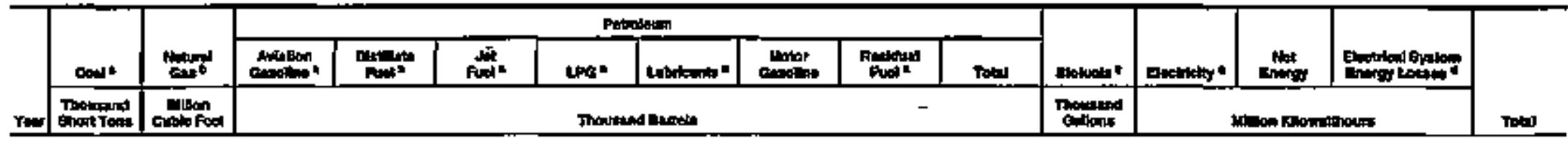

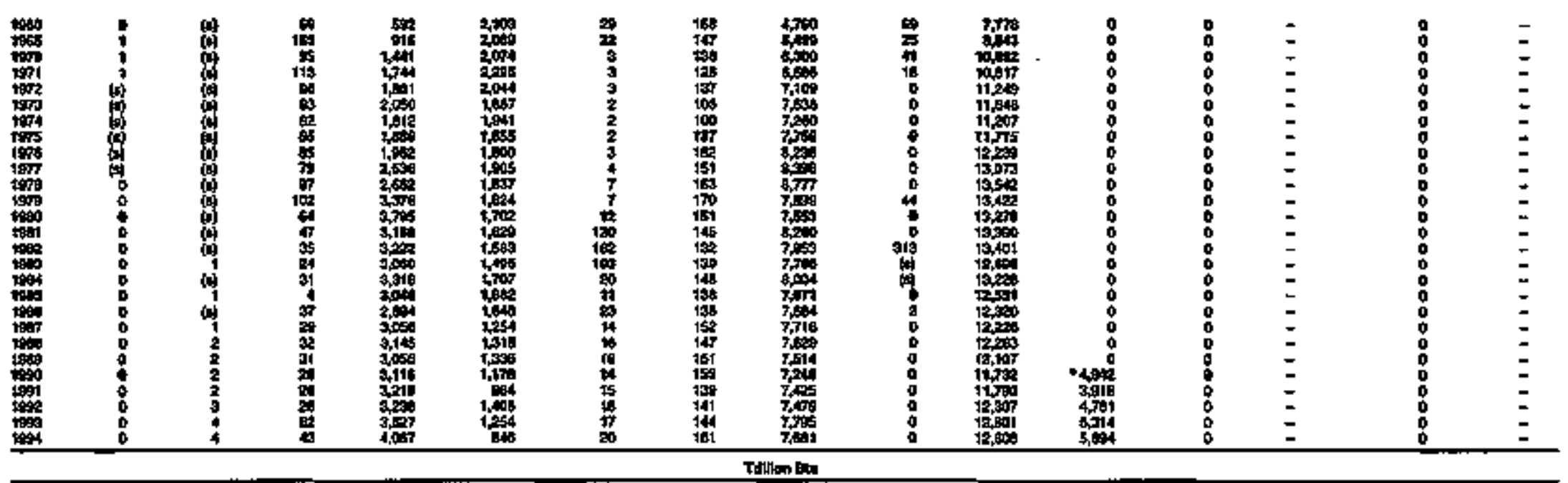

\begin{tabular}{|c|c|c|c|c|c|c|c|}
\hline 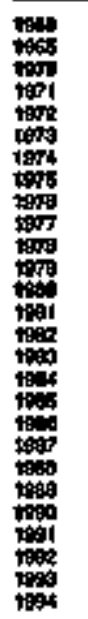 & 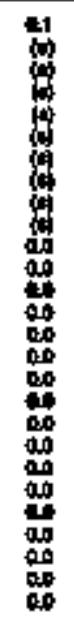 & 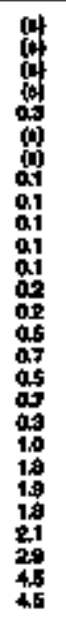 & 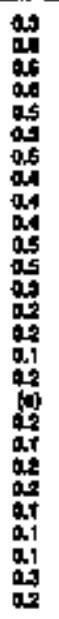 & 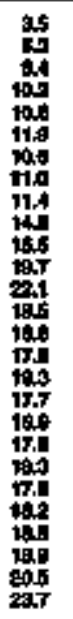 & 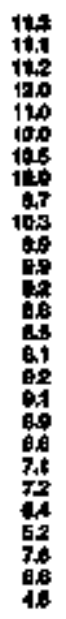 & 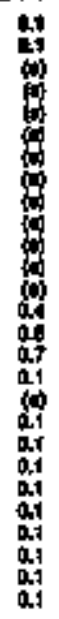 & 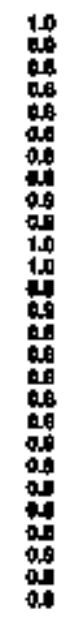 \\
\hline
\end{tabular}

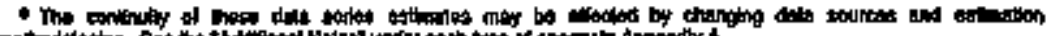

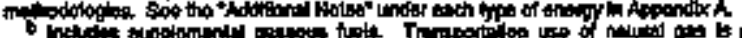

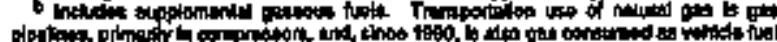

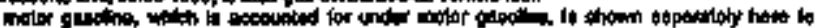

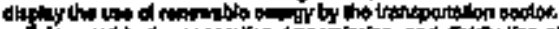

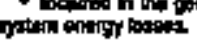

\begin{tabular}{|c|c|c|c|c|c|c|}
\hline 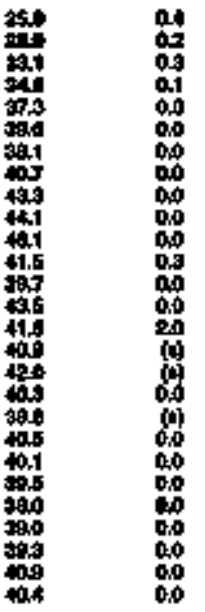 & 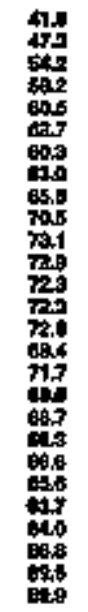 & 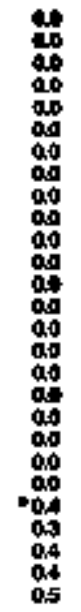 & 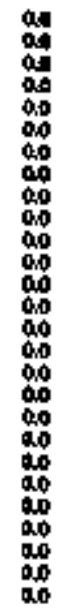 & 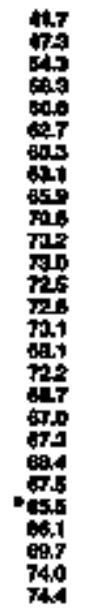 & 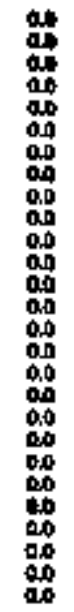 & 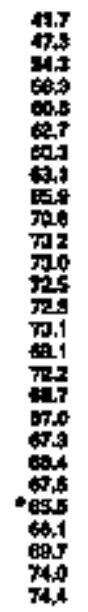 \\
\hline
\end{tabular}

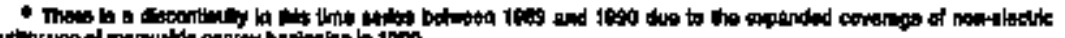

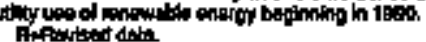

$\rightarrow \rightarrow \log$

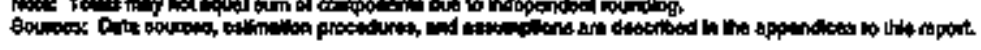

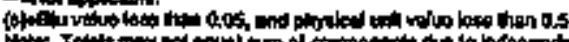




\begin{tabular}{|c|c|c|c|c|c|c|c|c|c|c|c|c|c|c|}
\hline \multirow[b]{3}{*}{ Yow } & \multicolumn{3}{|c|}{ Cons } & \multirow{3}{*}{ 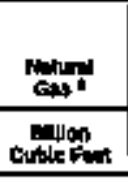 } & \multicolumn{4}{|c|}{ P } & \multirow[b]{2}{*}{ 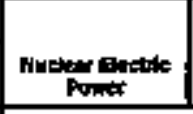 } & \multirow[b]{2}{*}{ 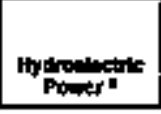 } & \multirow[b]{2}{*}{ Atohnth } & \multirow[b]{2}{*}{ 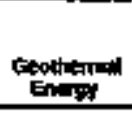 } & \multirow[b]{2}{*}{ ond w } & \multirow[b]{3}{*}{ Totst } \\
\hline & 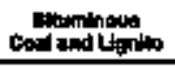 & Mntringetion & $\operatorname{Toth}$ & & 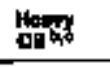 & 嵧復 & 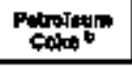 & Tote & & & & & & \\
\hline & \multicolumn{3}{|c|}{ Thoupand short tons } & & \multicolumn{4}{|c|}{ Thowand ends } & \multicolumn{5}{|c|}{ 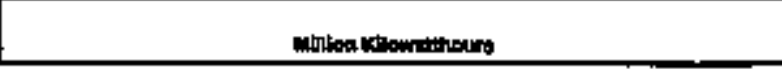 } & \\
\hline 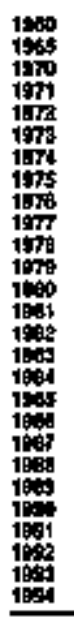 & 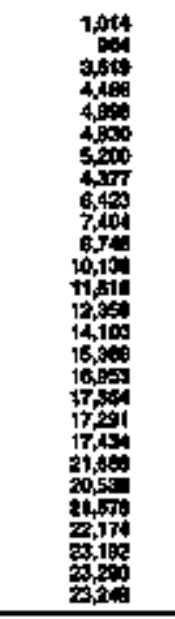 & 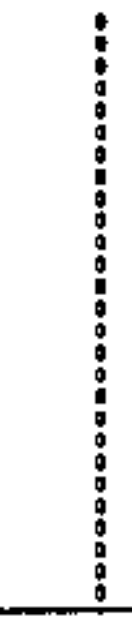 & 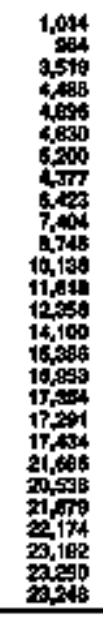 & 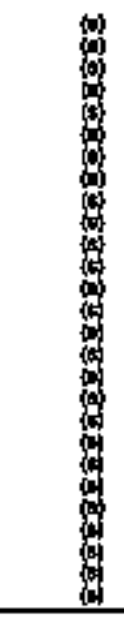 & 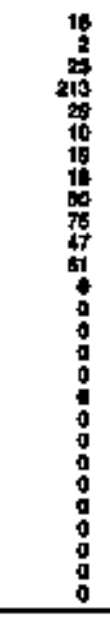 & 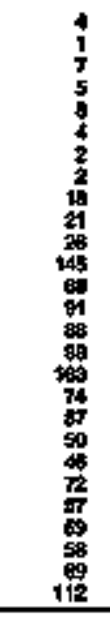 & $\begin{array}{l}0 \\
0 \\
0 \\
0 \\
0 \\
0 \\
0 \\
0 \\
0 \\
0 \\
0 \\
0 \\
0 \\
\vdots \\
\vdots \\
0 \\
0 \\
0 \\
0 \\
0 \\
0 \\
0 \\
\end{array}$ & 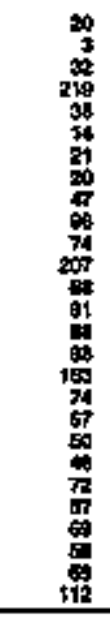 & $\begin{array}{l}: \\
0 \\
0 \\
0 \\
0 \\
0 \\
0 \\
0 \\
0 \\
0 \\
0 \\
0 \\
0 \\
0 \\
0 \\
0 \\
0 \\
0 \\
0 \\
0 \\
0 \\
0 \\
0 \\
0 \\
\end{array}$ & 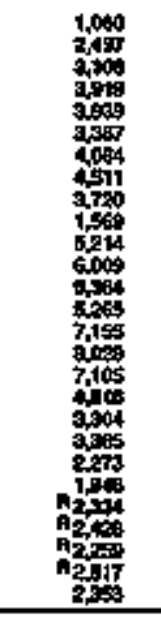 & $\begin{array}{l}: \\
\vdots \\
0 \\
\vdots \\
\vdots \\
\vdots \\
0 \\
0 \\
0 \\
0 \\
0 \\
0 \\
0 \\
0 \\
0 \\
0 \\
0 \\
0 \\
\end{array}$ & $\begin{array}{l}: \\
\vdots \\
0 \\
0 \\
0 \\
0 \\
0 \\
0 \\
0 \\
0 \\
0 \\
0 \\
0 \\
0 \\
0 \\
0 \\
0 \\
0 \\
0 \\
0 \\
0 \\
\end{array}$ & 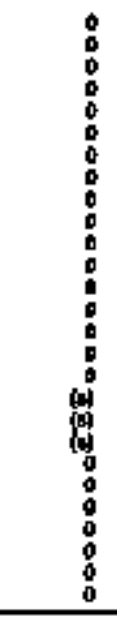 & $\begin{array}{l}\vdots \\
\vdots \\
\vdots \\
\vdots \\
\vdots \\
\vdots \\
\vdots \\
\vdots \\
\vdots \\
\vdots \\
\vdots \\
\vdots \\
\vdots\end{array}$ \\
\hline \multicolumn{15}{|c|}{ Trimen Btru } \\
\hline 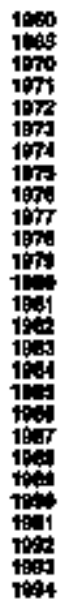 & 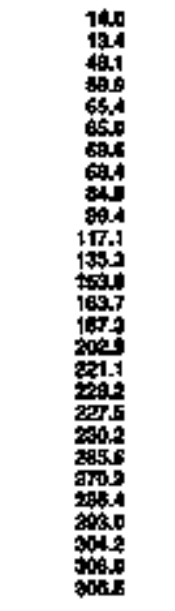 & 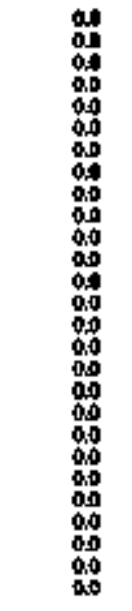 & 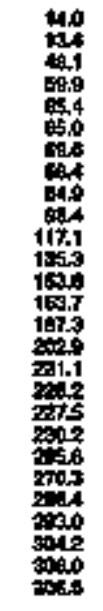 & 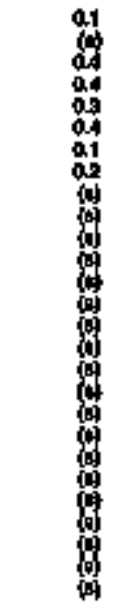 & 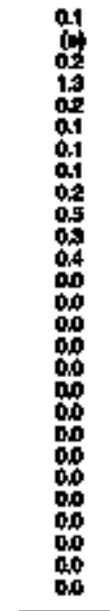 & 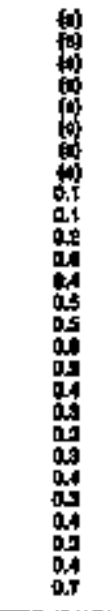 & 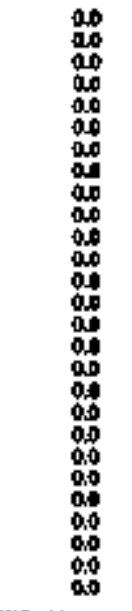 & 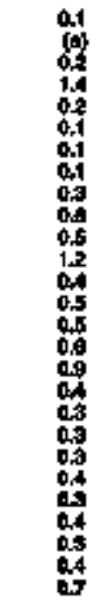 & 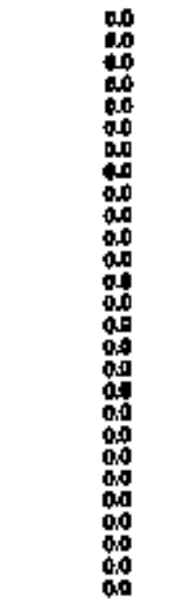 & 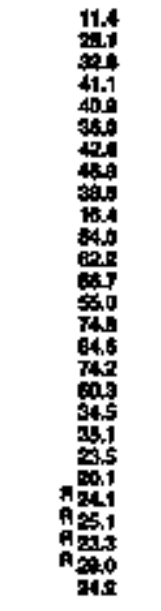 & 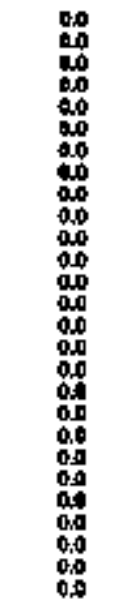 & 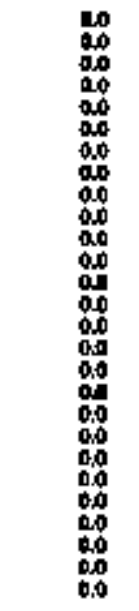 & 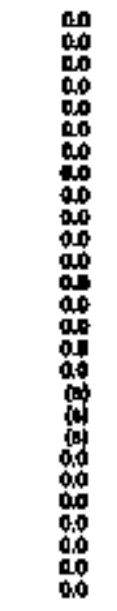 & 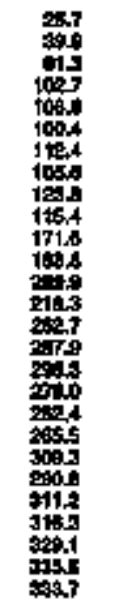 \\
\hline
\end{tabular}


Table 227. Energy Consumption Estinates by Source, 1950, 1565, 1970-1994, Ohio

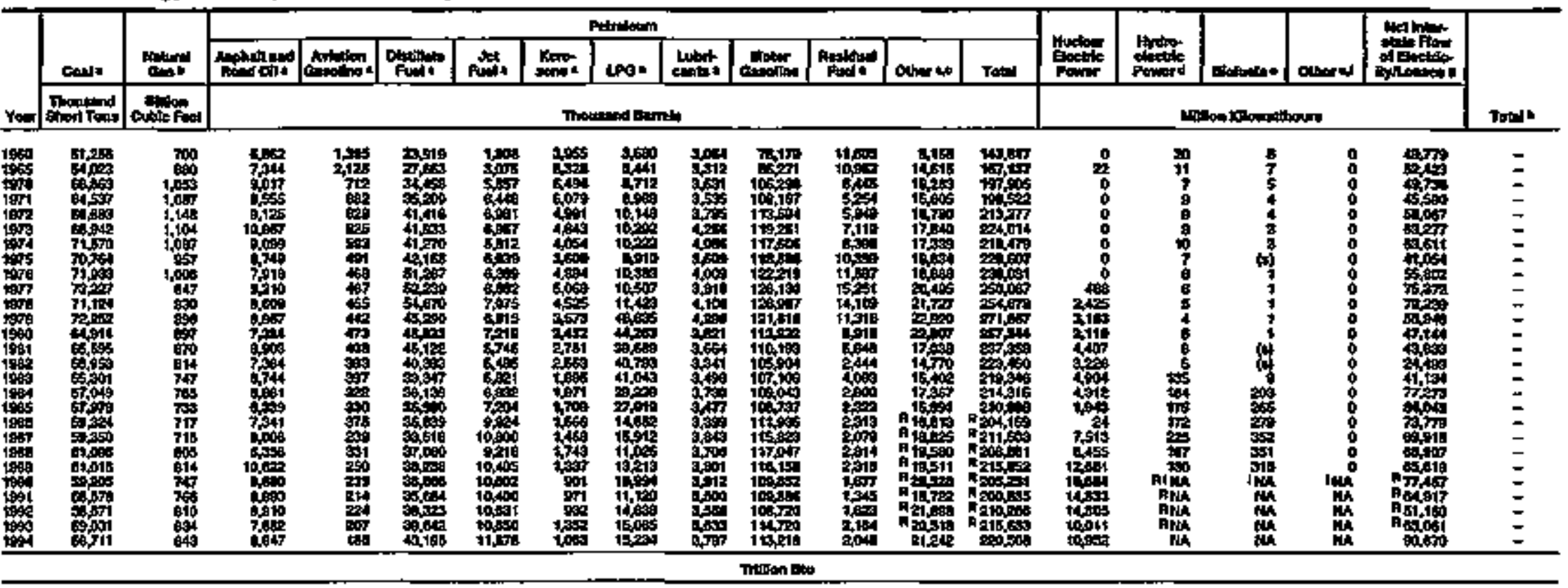

\begin{tabular}{|c|c|c|c|c|c|c|c|c|c|c|c|c|c|c|c|c|c|c|c|}
\hline 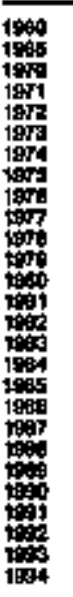 & 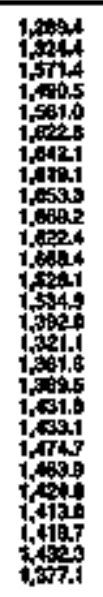 & 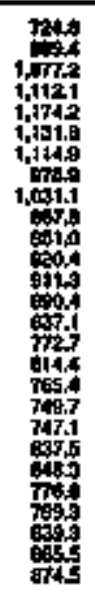 & 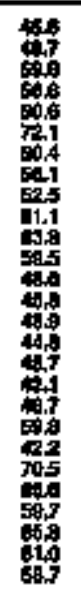 & 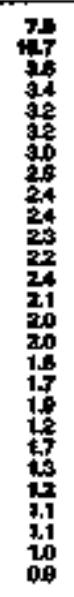 & 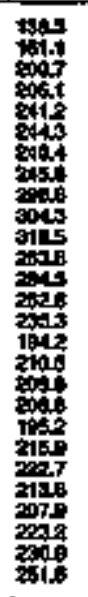 & 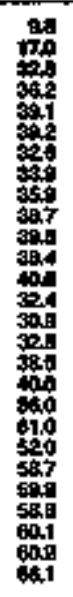 & 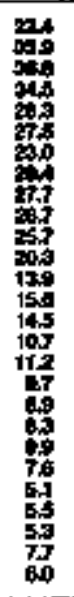 & 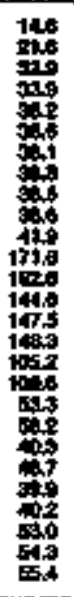 & 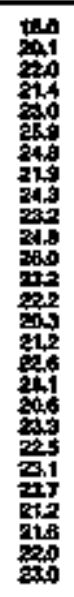 & 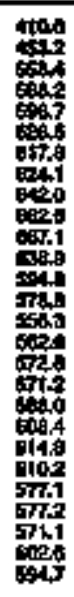 & 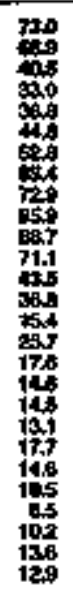 & 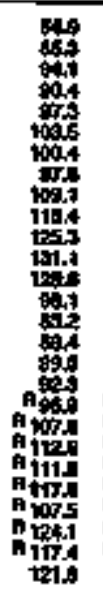 & 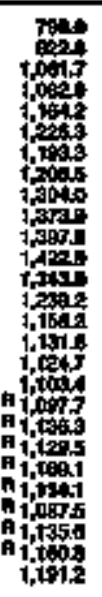 & 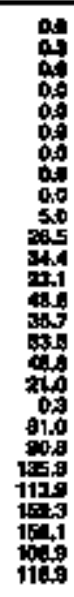 & 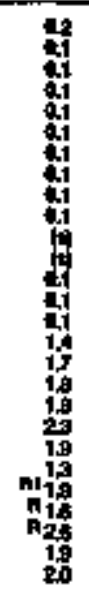 & 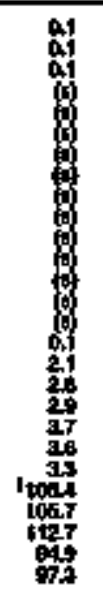 & 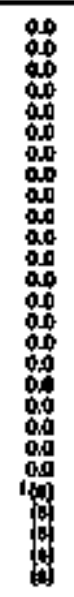 & 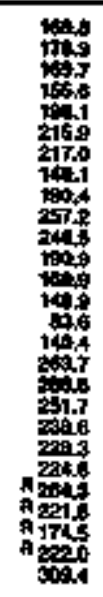 & 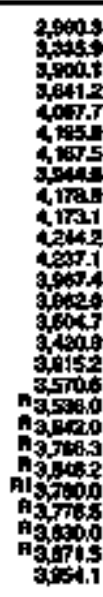 \\
\hline
\end{tabular}

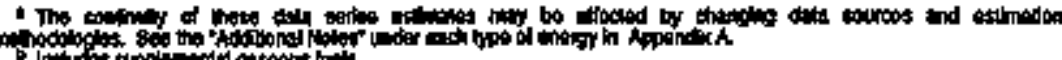

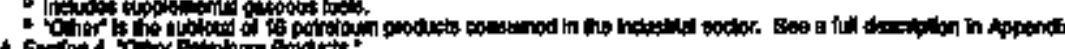

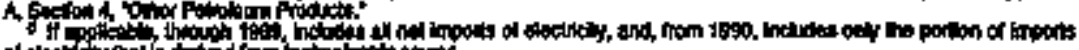

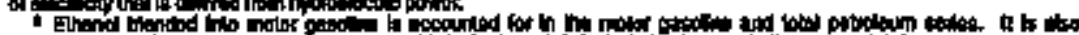

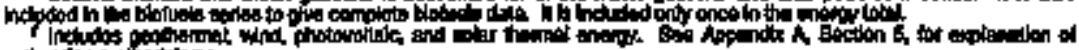

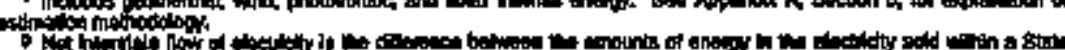

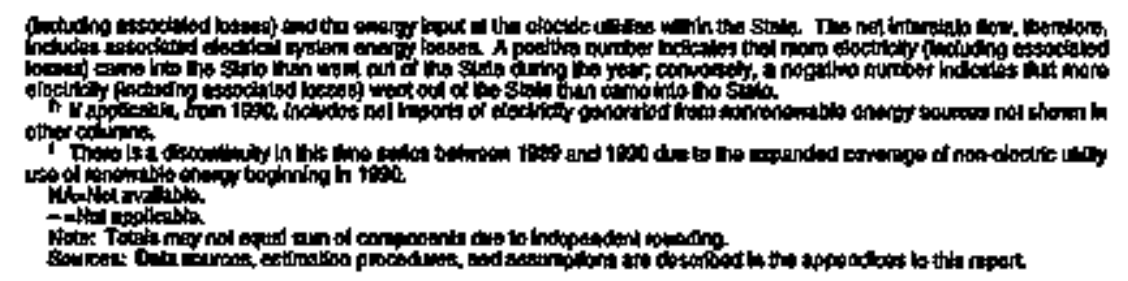


- Table 228. Residential Energy Consumption Estimales, 1960, 1965, 1970-1994, Ohlo

H

\begin{tabular}{|c|c|c|c|c|c|c|c|c|c|c|c|c|c|c|}
\hline & \multicolumn{3}{|c|}{ con } & \multirow[b]{2}{*}{ thang } & \multicolumn{4}{|c|}{ Putmonation } & \multirow[b]{2}{*}{ Bsorumels } & \multirow[b]{2}{*}{ solete } & \multirow[b]{2}{*}{ Eestiviont } & \multirow[b]{2}{*}{ Enil } & \multirow[b]{2}{*}{ 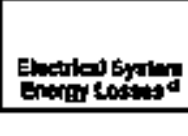 } & \multirow[b]{3}{*}{ Totil } \\
\hline & 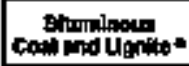 & " & ה & & 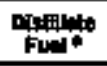 & Krono:men & LPQ: & Tot & & & & & & \\
\hline Your & \multicolumn{3}{|c|}{ Thootind shept Tove } & pillion & \multicolumn{4}{|c|}{ Thornad antst: } & mousand & \multicolumn{4}{|c|}{ 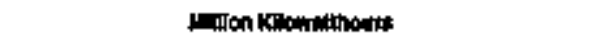 } & \\
\hline
\end{tabular}

\begin{tabular}{|c|c|c|c|c|c|c|c|c|c|c|c|c|c|c|}
\hline 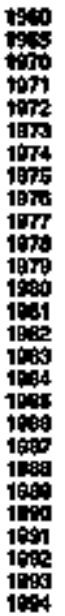 & 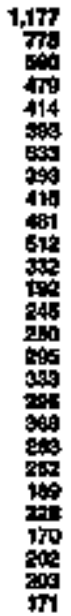 & $\begin{array}{c}20 \\
\text { 19 } \\
11 \\
11 \\
8 \\
8 \\
7 \\
6 \\
6 \\
5 \\
4 \\
3 \\
4 \\
2 \\
2 \\
2 \\
2 \\
7 \\
1 \\
8 \\
4 \\
4 \\
1 \\
8 \\
7 \\
3 \\
8\end{array}$ & 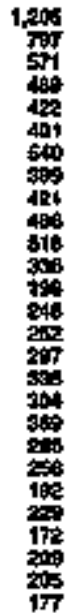 & 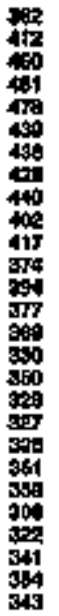 & 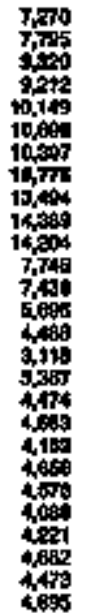 & 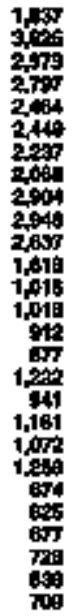 & 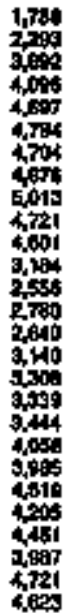 & 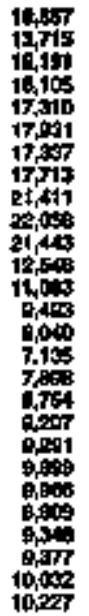 & 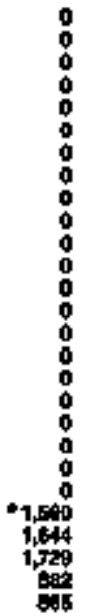 & $\begin{array}{l}0 \\
0 \\
0 \\
0 \\
0 \\
0 \\
0 \\
0 \\
0 \\
0 \\
0 \\
0 \\
0 \\
0 \\
0 \\
0 \\
0 \\
0 \\
0 \\
0 \\
0 \\
0 \\
0 \\
8 \\
8 \\
6 \\
7 \\
8\end{array}$ & 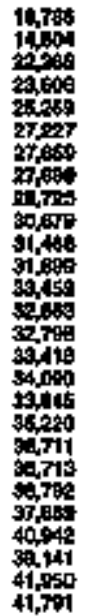 & 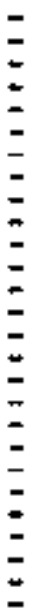 & 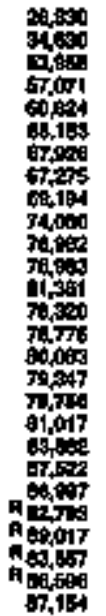 & $\begin{array}{l}= \\
= \\
= \\
= \\
= \\
= \\
= \\
= \\
= \\
= \\
= \\
= \\
= \\
=\end{array}$ \\
\hline
\end{tabular}

\begin{tabular}{|c|c|c|c|c|c|c|c|c|c|c|c|c|c|c|}
\hline 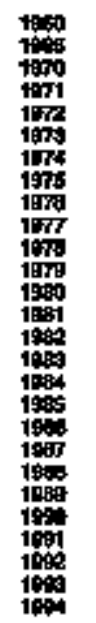 & 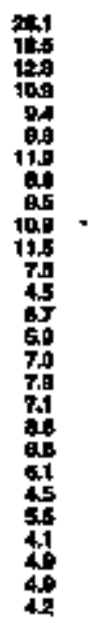 & 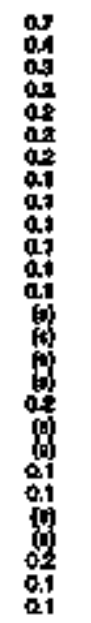 & 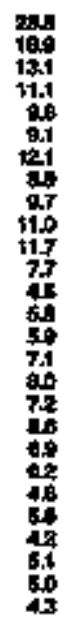 & 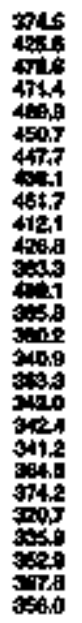 & 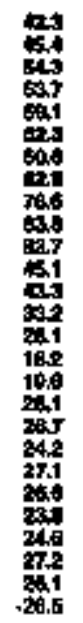 & 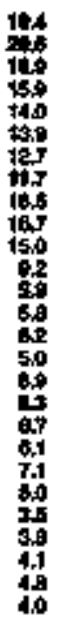 & 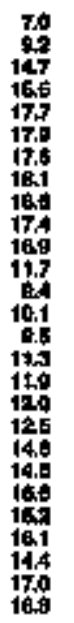 & 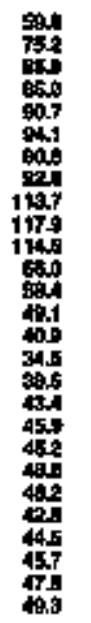 & 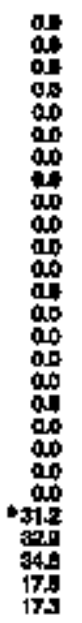 & 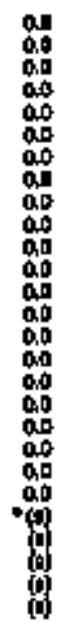 & 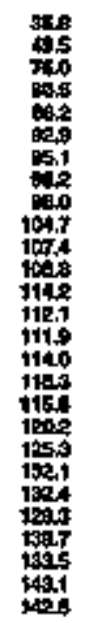 & 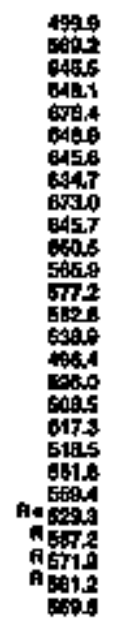 & 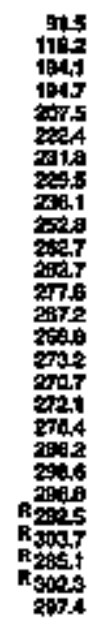 & 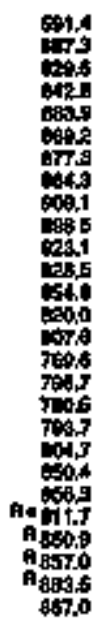 \\
\hline
\end{tabular}

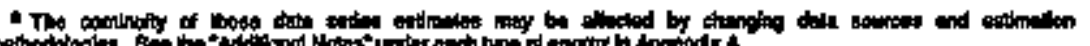

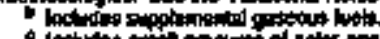

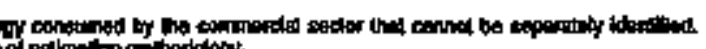

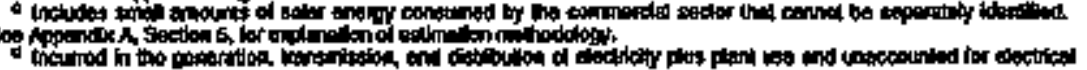

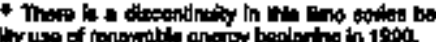

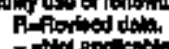

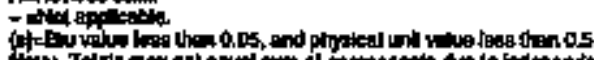

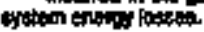

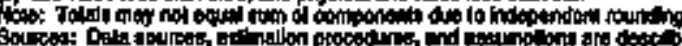


Teble 229. Commerctal Energy Consumptlon Estlmales, 1960, 1965, 1970-1994, Ohlo

\begin{tabular}{|c|c|c|c|c|c|c|c|c|c|c|c|c|c|c|}
\hline \multirow[b]{3}{*}{ reur } & \multicolumn{3}{|c|}{ Cont } & \multirow{3}{*}{ 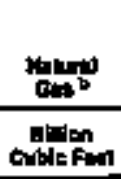 } & \multicolumn{6}{|c|}{ Parodown } & \multirow[b]{2}{*}{ Boctiklhy" } & \multirow[b]{2}{*}{ ting } & \multirow[b]{2}{*}{ 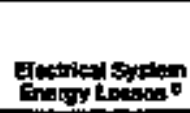 } & \multirow[b]{3}{*}{ Totol=10 } \\
\hline & costhrinon: & 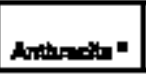 & Fots & & antions & Konosenose & LPG= & 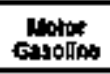 & Fetsons & Total & & & & \\
\hline & \multicolumn{3}{|c|}{ Thou:and Bhort Tons } & & \multicolumn{6}{|c|}{ 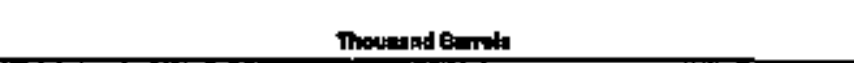 } & \multicolumn{3}{|c|}{ Wortan KB: } & \\
\hline 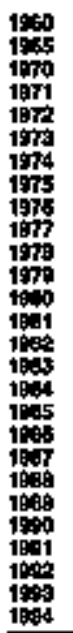 & 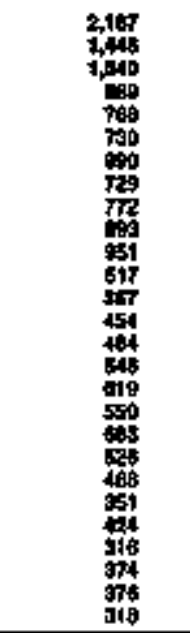 & $\begin{array}{r}19 \\
12 \\
7 \\
7 \\
5 \\
5 \\
5 \\
4 \\
4 \\
4 \\
3 \\
2 \\
3 \\
1 \\
1 \\
1 \\
1 \\
5 \\
41 \\
1 \\
2 \\
2 \\
0 \\
0 \\
5 \\
5 \\
2 \\
4\end{array}$ & 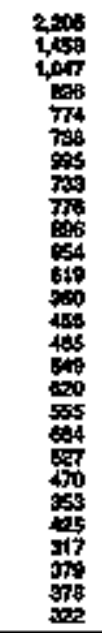 & 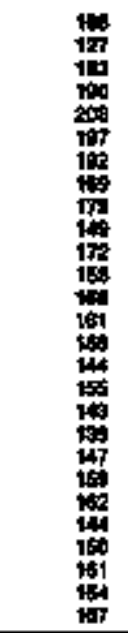 & 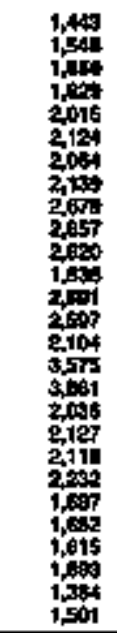 & 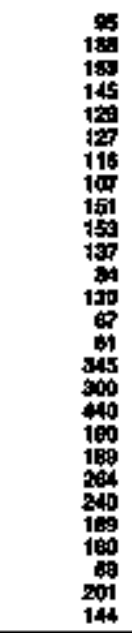 & 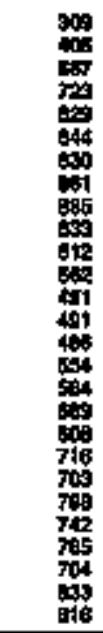 & 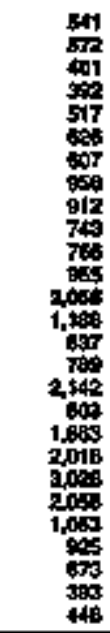 & 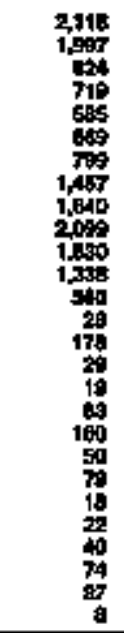 & 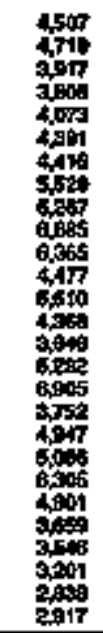 & 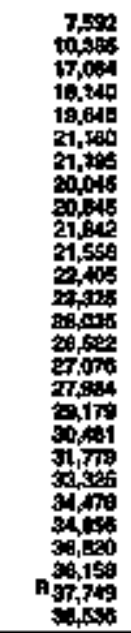 & $\begin{array}{l}\vdots \\
\vdots \\
\vdots \\
\vdots \\
\vdots \\
\vdots \\
\vdots \\
\vdots \\
\vdots \\
\vdots \\
\vdots \\
\vdots \\
\vdots\end{array}$ & 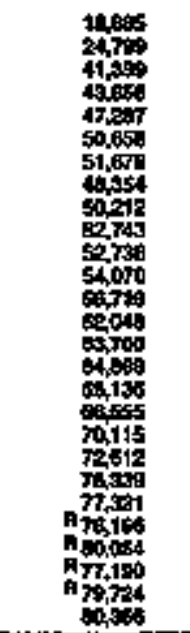 & $\begin{array}{l}z \\
\vdots \\
z \\
z \\
\vdots \\
\vdots \\
\vdots \\
z \\
\vdots \\
z \\
z \\
z \\
z\end{array}$ \\
\hline \multicolumn{15}{|c|}{ molliog Beow } \\
\hline 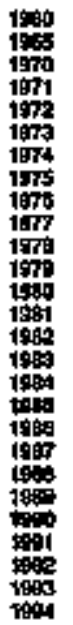 & 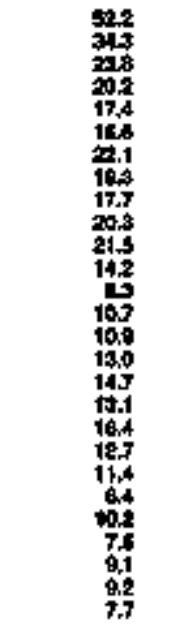 & 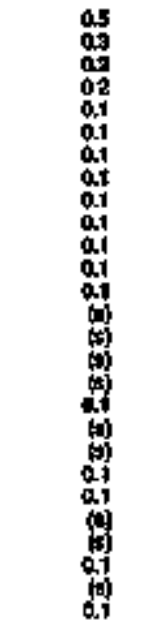 & 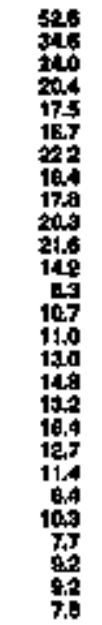 & 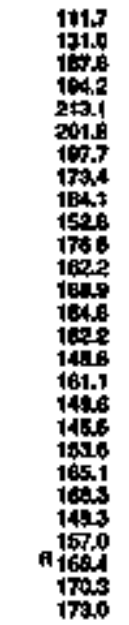 & 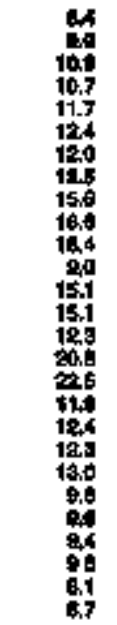 & 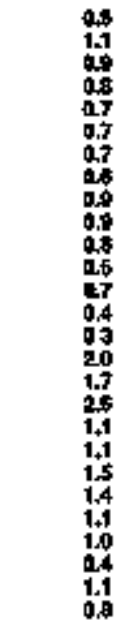 & 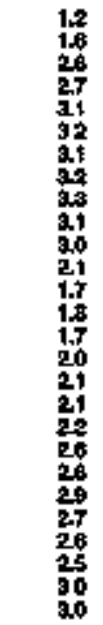 & 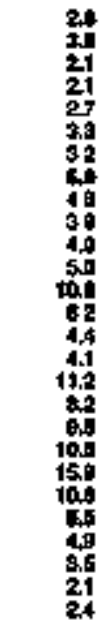 & 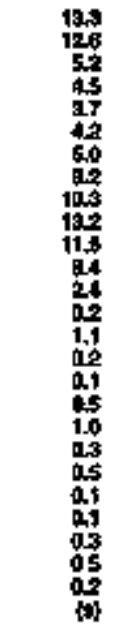 & 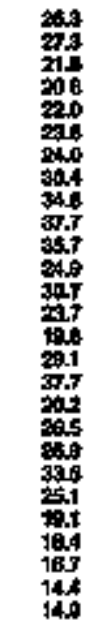 & 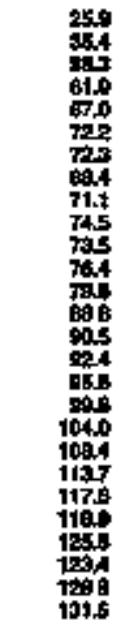 & 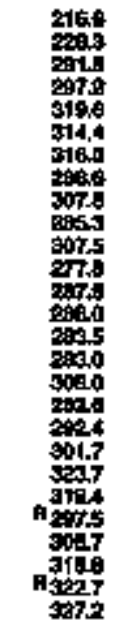 & 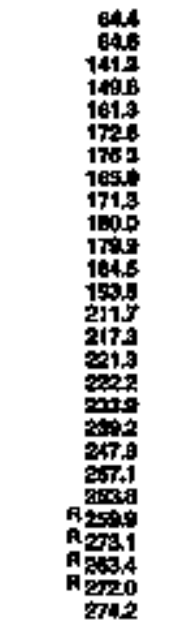 & 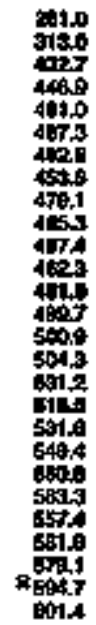 \\
\hline
\end{tabular}

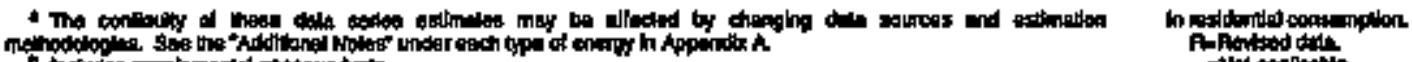

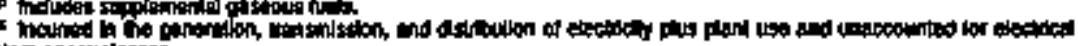

in

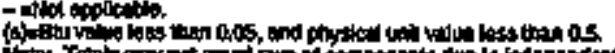

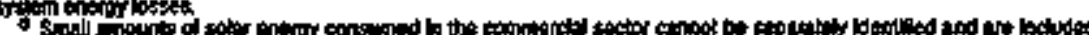

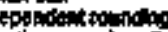

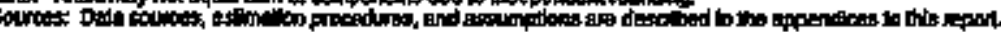


Table 230. Industrial Entwy Conotumption Estinates, 1550, 1965, 1970-1994, Ohio

\begin{tabular}{|c|c|c|c|c|c|c|c|c|c|c|c|c|c|c|c|c|c|c|}
\hline \multirow[b]{3}{*}{$\mathrm{ren}$} & \multirow[b]{2}{*}{ Cow } & \multirow[b]{2}{*}{ 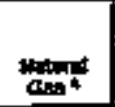 } & \multicolumn{9}{|c|}{ Patolnin } & \multirow[b]{2}{*}{ | } & \multirow[b]{2}{*}{ Edonuels } & \multirow[b]{2}{*}{ Onert } & \multirow[b]{2}{*}{ 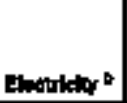 } & \multirow[b]{2}{*}{ Entrint } & \multirow{2}{*}{ 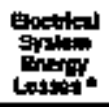 } & \multirow[b]{3}{*}{ Tota } \\
\hline & & & 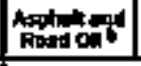 & 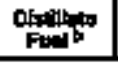 & rases & LPa" & 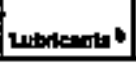 & atser & Pandiol & Otner & Totol & & & & & & & \\
\hline & shoort fond & colsten & \multicolumn{9}{|c|}{ Thontenderith } & \multicolumn{6}{|c|}{ 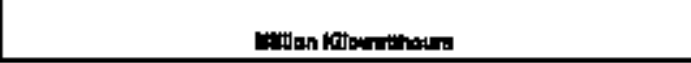 } & \\
\hline 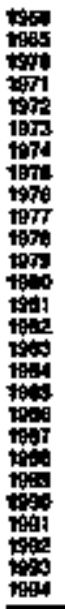 & 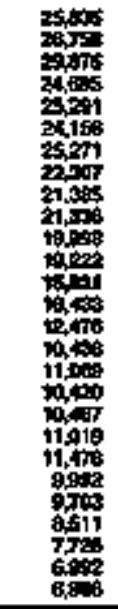 & 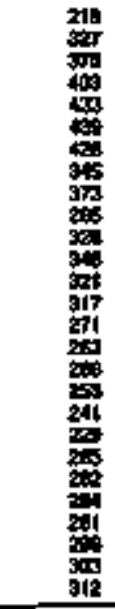 & 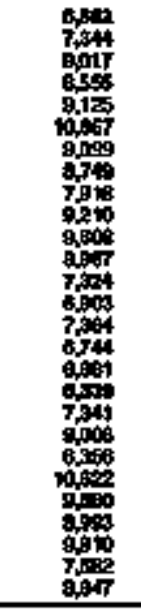 & 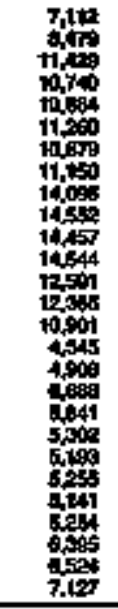 & 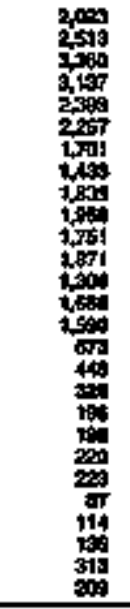 & 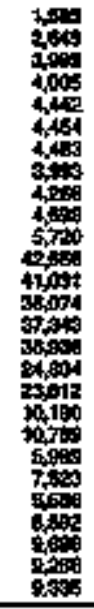 & 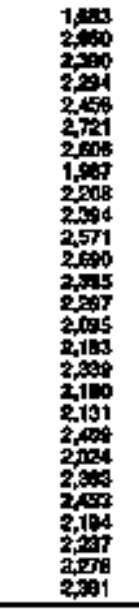 & 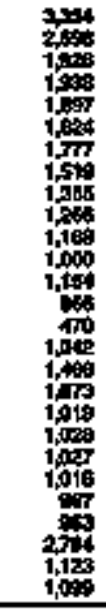 & 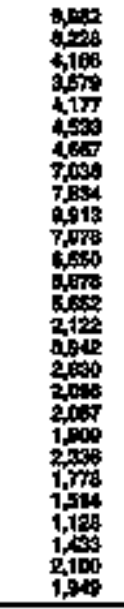 & 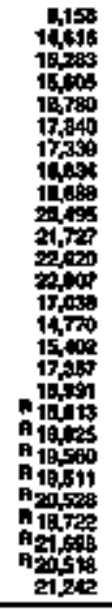 & 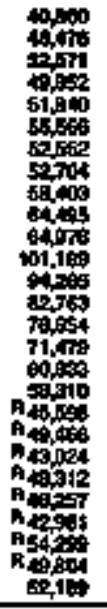 & 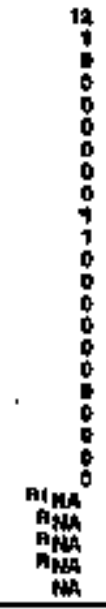 & 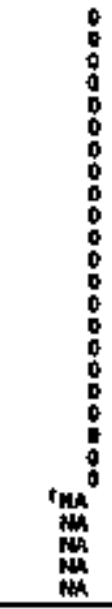 & 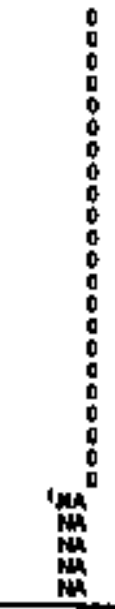 & 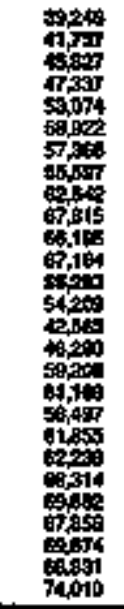 & $\begin{array}{l}= \\
= \\
= \\
= \\
z \\
z \\
z \\
= \\
z \\
z \\
z \\
z \\
z \\
z\end{array}$ & 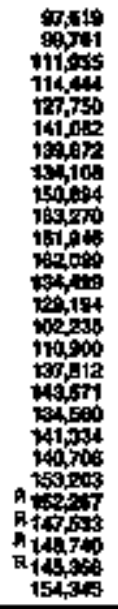 & $\begin{array}{l}z \\
z \\
z \\
z \\
z \\
z \\
z \\
z \\
z \\
z\end{array}$ \\
\hline \multicolumn{19}{|c|}{ Tollonseven } \\
\hline 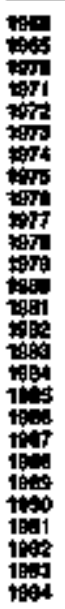 & 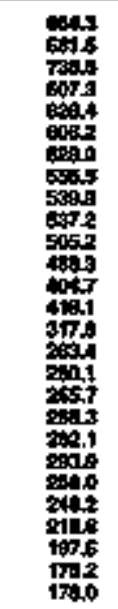 & 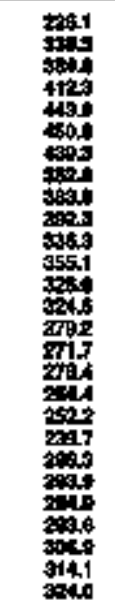 & 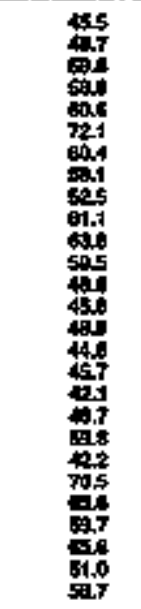 & 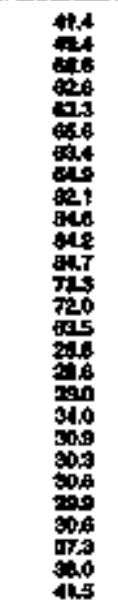 & 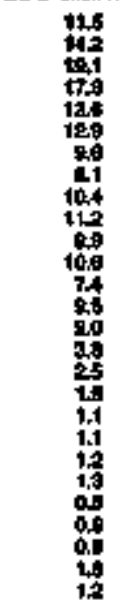 & 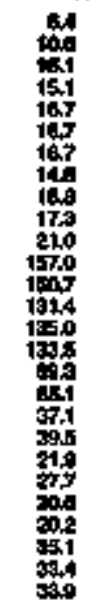 & 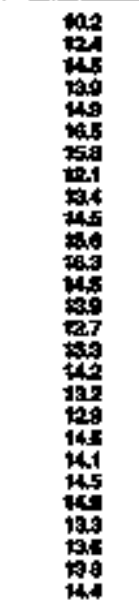 & 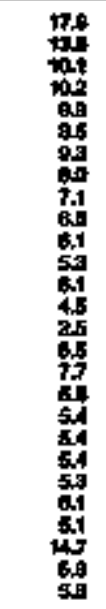 & 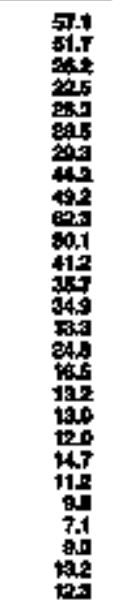 & 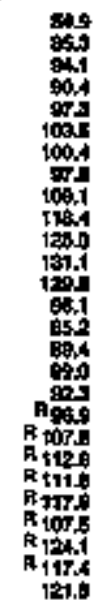 & 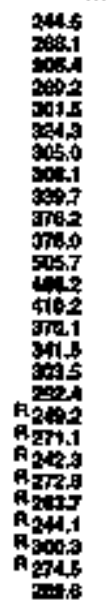 & 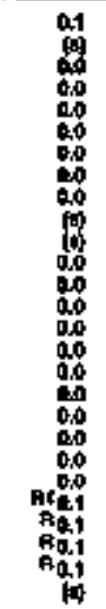 & 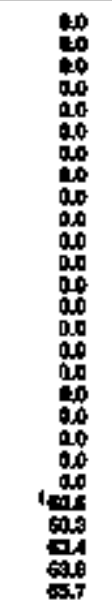 & $\begin{array}{l}00 \\
00 \\
00 \\
00 \\
000 \\
00 \\
00 \\
00 \\
00 \\
00 \\
00 \\
000 \\
000 \\
00 \\
00 \\
00 \\
00 \\
00 \\
00 \\
00 \\
00 \\
00 \\
00 \\
00\end{array}$ & 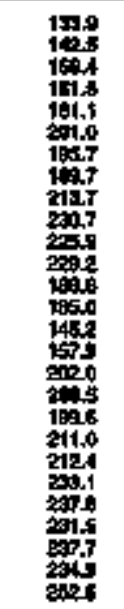 & 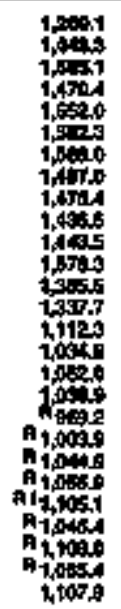 & 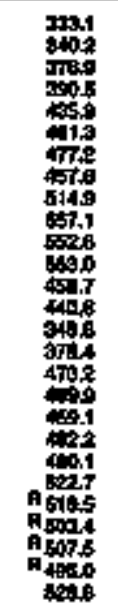 & 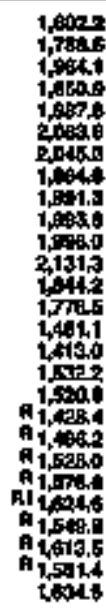 \\
\hline
\end{tabular}

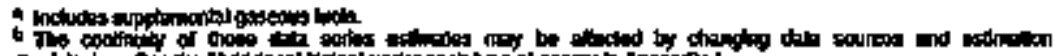

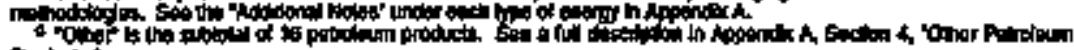
Prgductic

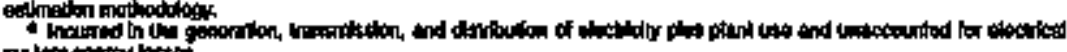

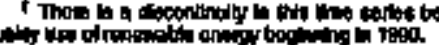
Rifinged difia.

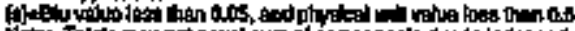

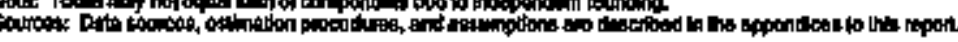

\section{等}

$\therefore$ 
Teble 231. Transportation Enargy Consumption Estlmates, 1980, 1965, 1970-1994, Ohto

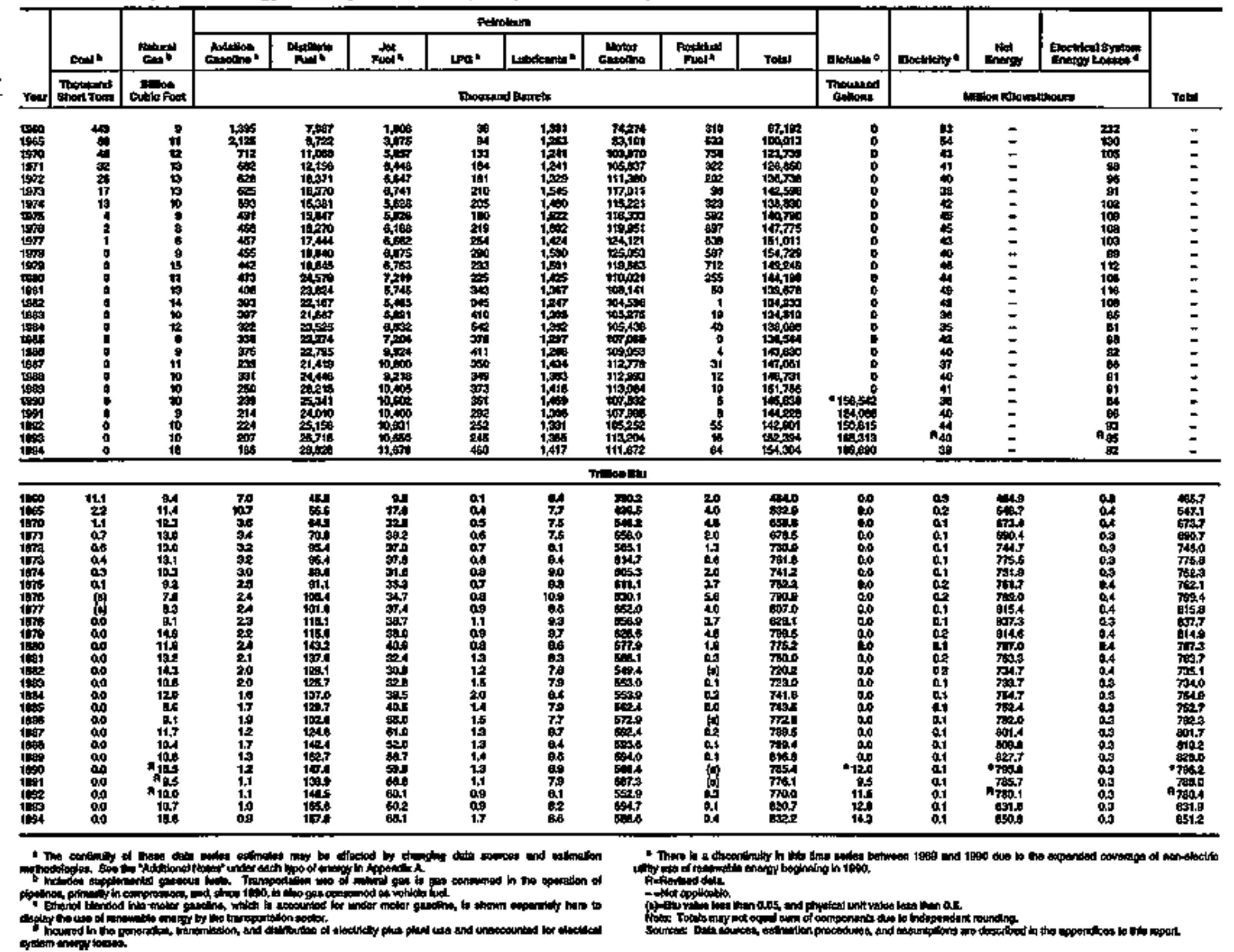


Y rable 232. Esulnates of Energy Input at Electric Utilitles, 1960, 1965, 1970-1994, Ohlo

\begin{tabular}{|c|c|c|c|c|c|c|c|c|c|c|c|c|c|c|}
\hline & \multicolumn{3}{|c|}{ ant } & \multirow[b]{2}{*}{ "tont: } & \multicolumn{4}{|c|}{ Putpolecun } & \multirow[b]{2}{*}{ Norelomente } & \multirow[b]{2}{*}{ 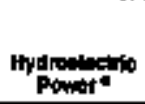 } & \multirow[b]{2}{*}{ Otornt } & \multirow[b]{2}{*}{ coptontion } & \multirow[b]{2}{*}{ other th } & \multirow[b]{3}{*}{ Total } \\
\hline & 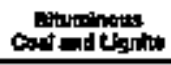 & Metroall & Ton & & oplont & 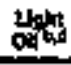 & Pations & Totol & & & & & & \\
\hline$Y \in=0$ & \multicolumn{3}{|c|}{ 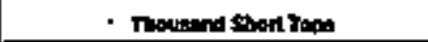 } & 10 & \multicolumn{4}{|c|}{ Thoseand Bermen } & \multicolumn{5}{|c|}{ 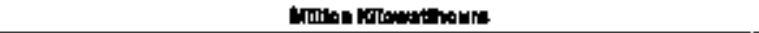 } & \\
\hline
\end{tabular}

\begin{tabular}{|c|c|c|c|c|c|c|c|c|c|c|c|c|c|c|}
\hline 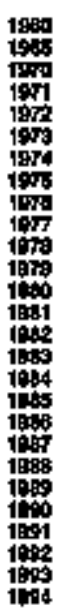 & 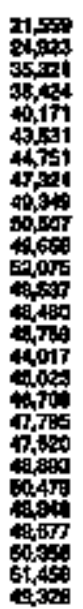 & $\begin{array}{l}0 \\
0 \\
0 \\
0 \\
0 \\
0 \\
0 \\
0 \\
0 \\
0 \\
0 \\
0 \\
0 \\
0 \\
0 \\
0 \\
0 \\
0 \\
0 \\
0 \\
0 \\
0\end{array}$ & 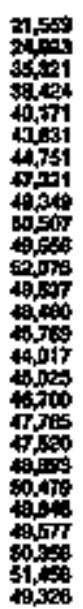 & 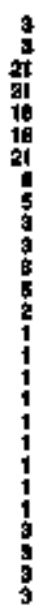 & 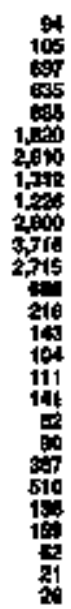 & 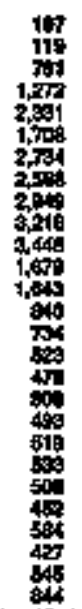 & $\begin{array}{l}1 \\
0 \\
0 \\
0 \\
0 \\
0 \\
0 \\
0 \\
0 \\
0 \\
0 \\
0 \\
0 \\
0 \\
0 \\
0 \\
0 \\
0 \\
0 \\
0 \\
0 \\
0 \\
0 \\
0 \\
0\end{array}$ & 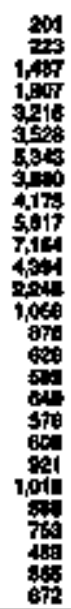 & 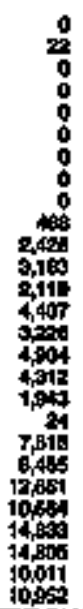 & 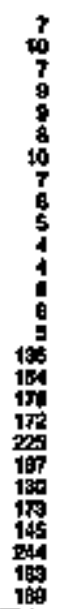 & 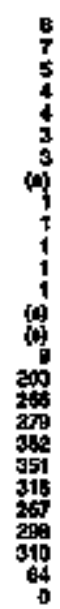 & $\begin{array}{l}0 \\
0 \\
0 \\
0 \\
0 \\
0 \\
0 \\
0 \\
0 \\
0 \\
0 \\
0 \\
0 \\
0 \\
0 \\
0 \\
0 \\
0 \\
0 \\
0 \\
0 \\
0 \\
0 \\
0 \\
0\end{array}$ & $\begin{array}{l}0 \\
0 \\
0 \\
0 \\
0 \\
0 \\
0 \\
0 \\
0 \\
0 \\
0 \\
0 \\
0 \\
0 \\
0 \\
0 \\
0 \\
0 \\
0 \\
0 \\
0 \\
0 \\
0 \\
0 \\
0 \\
0 \\
0\end{array}$ & $=$ \\
\hline
\end{tabular}

\begin{tabular}{|c|c|c|c|c|c|c|c|}
\hline 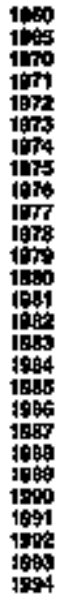 & 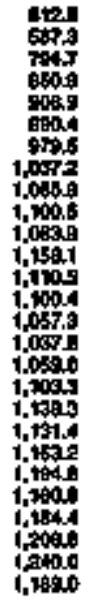 & 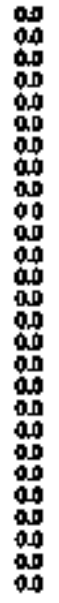 & 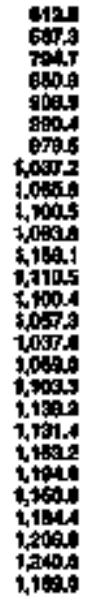 & 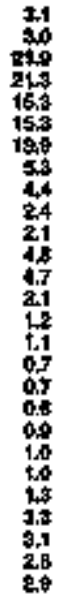 & 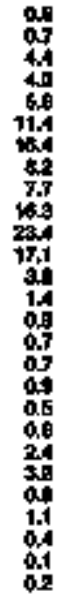 & 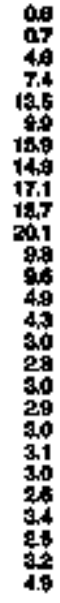 & $\begin{array}{l}00 \\
00 \\
00 \\
00 \\
00 \\
00 \\
00 \\
00 \\
00 \\
00 \\
00 \\
00 \\
00 \\
00 \\
00 \\
00 \\
00 \\
00 \\
00 \\
00 \\
00 \\
00 \\
00 \\
00 \\
00 \\
00 \\
00\end{array}$ \\
\hline
\end{tabular}

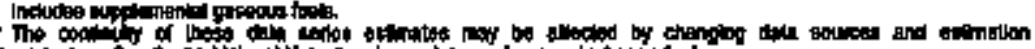

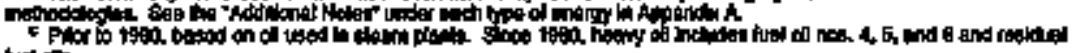

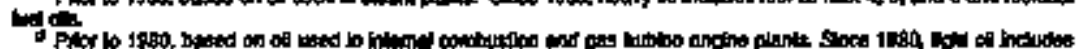

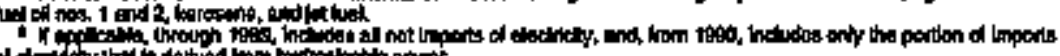

\begin{tabular}{|c|c|c|c|c|c|c|}
\hline 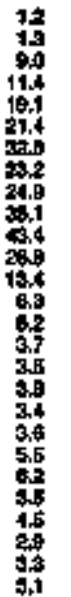 & 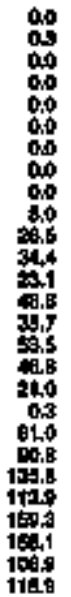 & 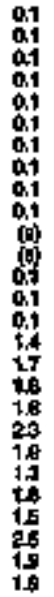 & 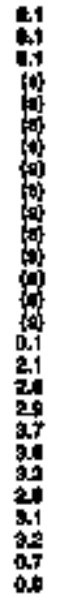 & 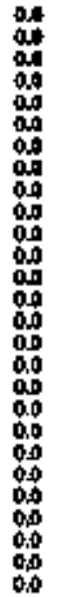 & 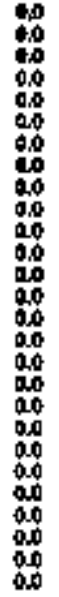 & 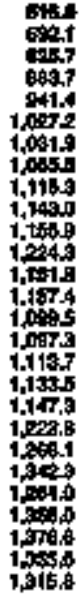 \\
\hline
\end{tabular}

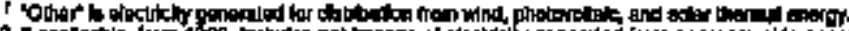
intioposection

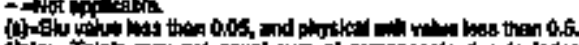

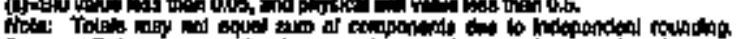

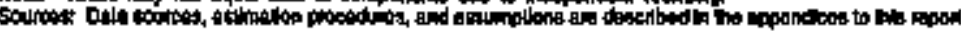


Table 235. Energy Consumpkton Estimates by Source, 1960, 1965, 1970-1994, Oktahoma

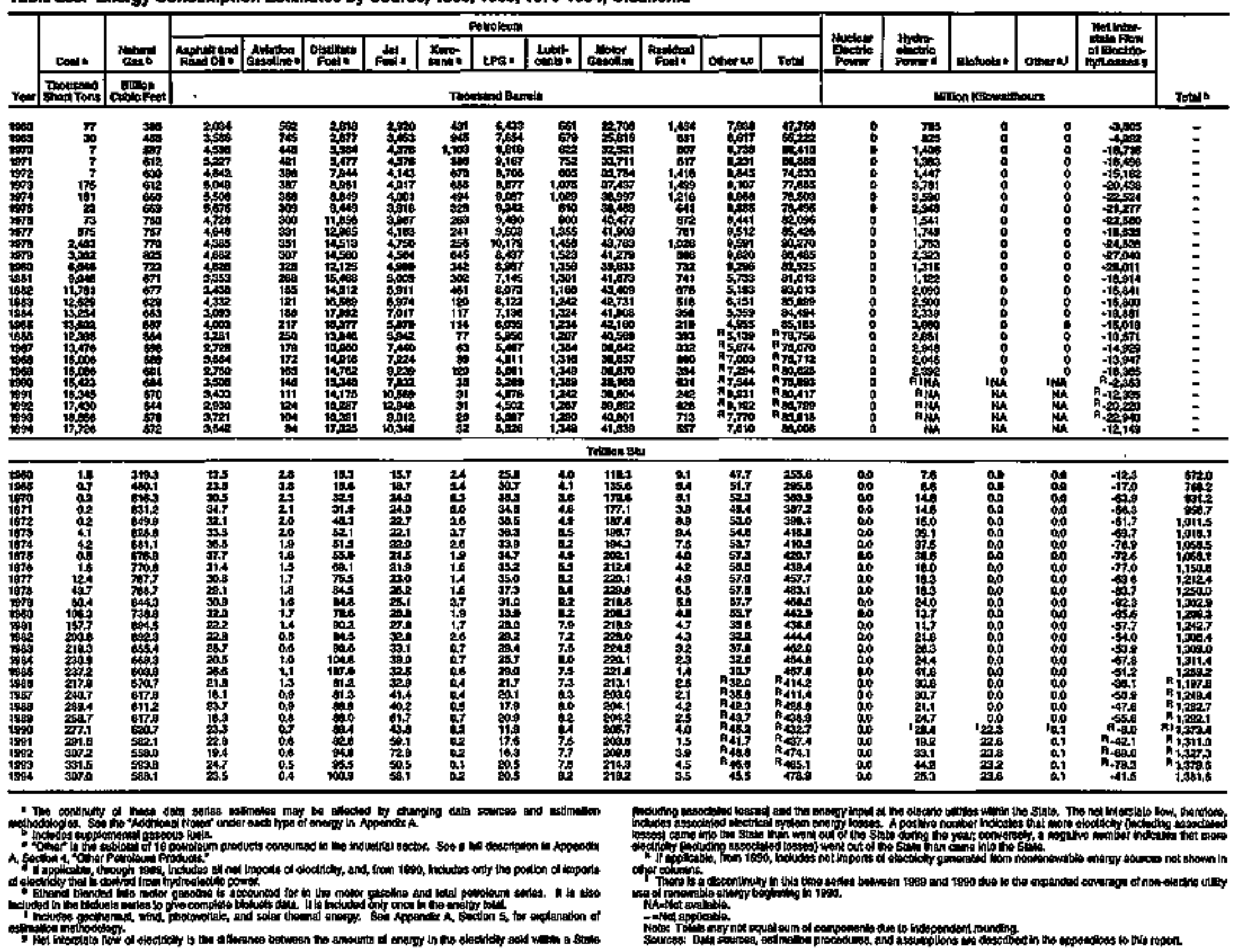


Table 234. Festitentia Energy Consumption Estimates, 1980, 1965, 1970-1994, OkLahoma

\begin{tabular}{|c|c|c|c|c|c|c|c|c|c|c|c|c|c|c|}
\hline \multirow[b]{3}{*}{ Yetr } & \multicolumn{3}{|c|}{ الئc } & \multirow{3}{*}{ 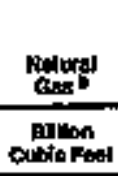 } & \multicolumn{4}{|c|}{ Putrotan } & \multirow{3}{*}{ 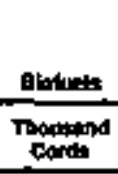 } & \multirow[b]{2}{*}{ Solare } & \multirow[b]{2}{*}{ Dectidn't } & \multirow[b]{2}{*}{ Expengy } & \multirow{3}{*}{ 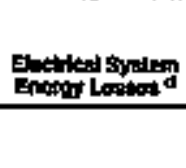 } & \multirow[b]{3}{*}{ Tateil } \\
\hline & 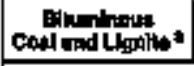 & Animasula & Iot & & 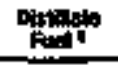 & Merocines & LPG & Total & & & & & & \\
\hline & \multicolumn{2}{|c|}{ 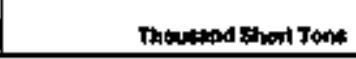 } & $\cdots$ & & \multicolumn{4}{|c|}{ Thowsend Eamels } & & \multicolumn{3}{|c|}{ 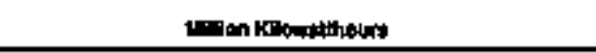 } & & \\
\hline 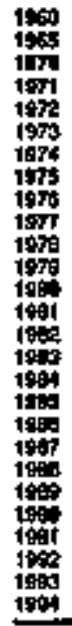 & 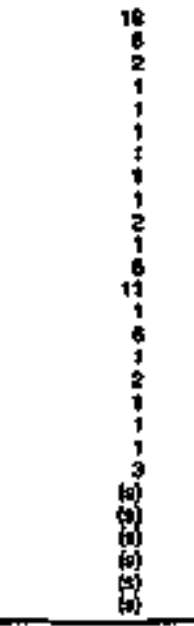 & $\begin{array}{l}0 \\
0 \\
0 \\
0 \\
0 \\
0 \\
0 \\
0 \\
0 \\
0 \\
0 \\
0 \\
0 \\
0 \\
0 \\
0 \\
0 \\
0 \\
0 \\
0 \\
0 \\
0 \\
0 \\
0 \\
0 \\
0 \\
0\end{array}$ & 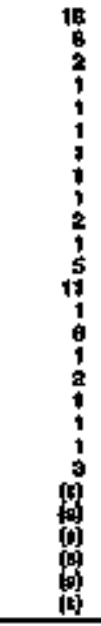 & 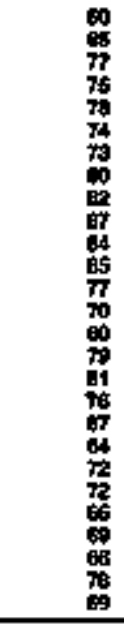 & 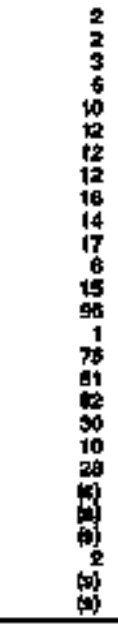 & 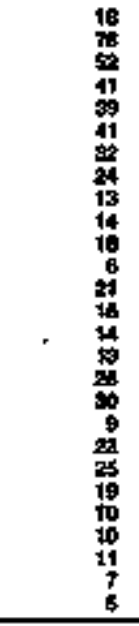 & 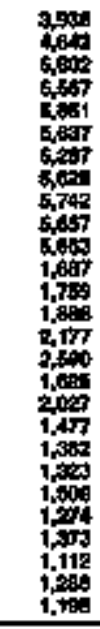 & 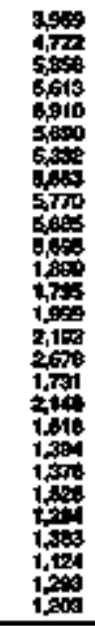 & 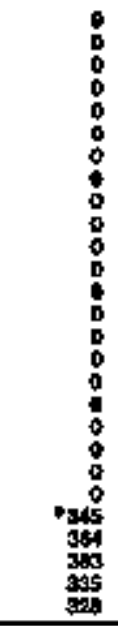 & $\begin{array}{r}0 \\
0 \\
0 \\
0 \\
0 \\
0 \\
0 \\
0 \\
0 \\
0 \\
0 \\
0 \\
0 \\
0 \\
0 \\
0 \\
0 \\
0 \\
0 \\
-20 \\
203 \\
23 \\
20 \\
2\end{array}$ & 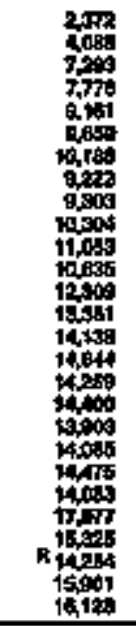 & $\begin{array}{l}= \\
= \\
= \\
= \\
= \\
= \\
= \\
= \\
= \\
= \\
= \\
= \\
= \\
= \\
= \\
=\end{array}$ & 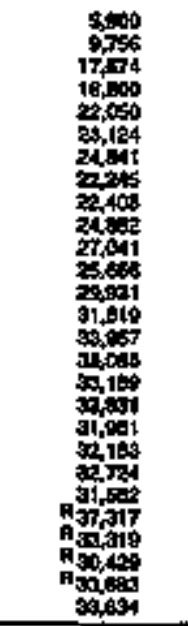 & $\begin{array}{l}\bar{z} \\
= \\
\bar{z} \\
= \\
= \\
= \\
= \\
= \\
= \\
= \\
= \\
= \\
= \\
= \\
= \\
=\end{array}$ \\
\hline \multicolumn{15}{|c|}{ Thlllow be } \\
\hline 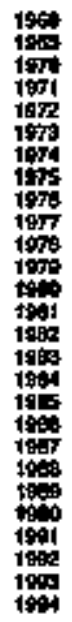 & 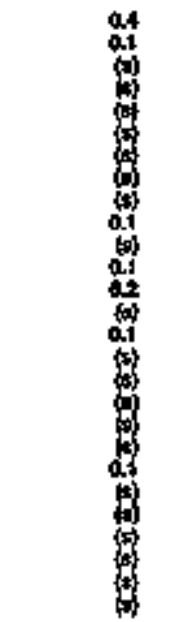 & 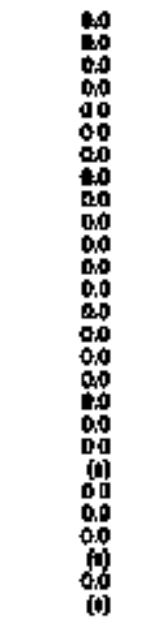 & 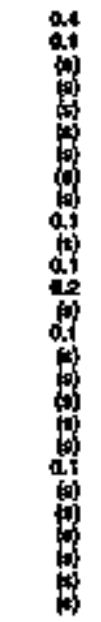 & 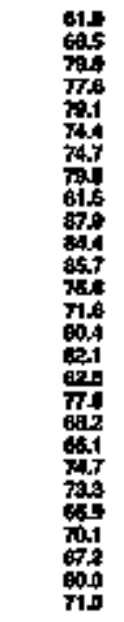 & 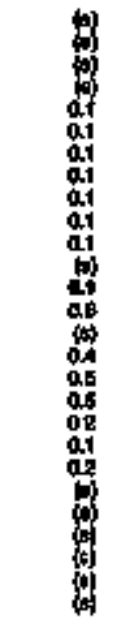 & $\begin{array}{l}0.1 \\
0.4 \\
0.4 \\
0.2 \\
0.2 \\
0.2 \\
0.2 \\
0.1 \\
0.1 \\
0.4 \\
0.1 \\
0.1 \\
0.1 \\
0.1 \\
0.1 \\
0.1 \\
0.1 \\
0.2 \\
0.1 \\
0.1 \\
0.1 \\
0.1 \\
0.1 \\
0.1 \\
0.1 \\
04 \\
0.91\end{array}$ & 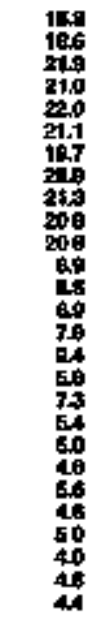 & 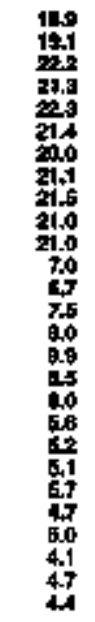 & 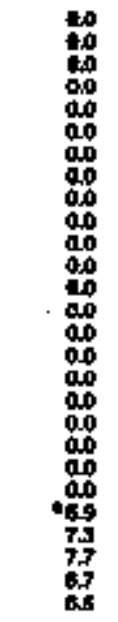 & $\begin{array}{l}00 \\
00 \\
00 \\
0.0 \\
00 \\
00 \\
00 \\
00 \\
00 \\
0.0 \\
00 \\
00 \\
100 \\
00 \\
00 \\
00 \\
00 \\
00 \\
00 \\
00 \\
00 \\
00 \\
0.4 \\
0.1 \\
0.1 \\
0.1 \\
0.1\end{array}$ & 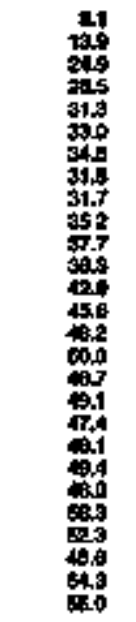 & 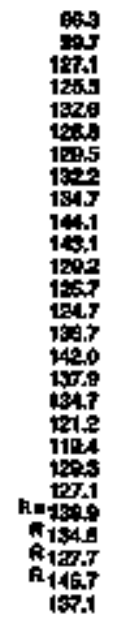 & 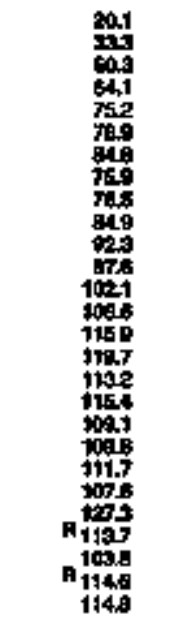 & 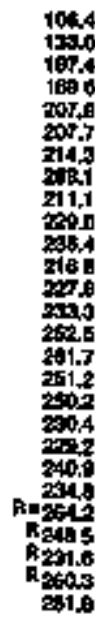 \\
\hline
\end{tabular}

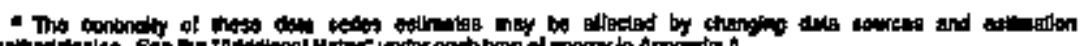

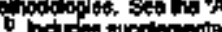

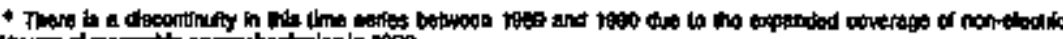

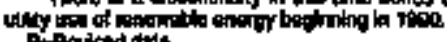

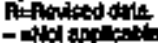

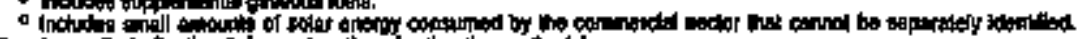

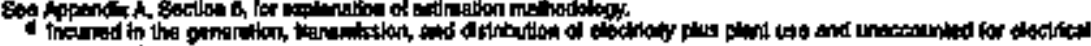

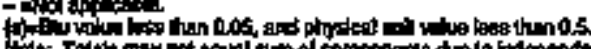

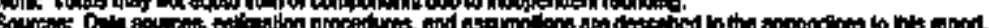

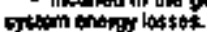


Table 235. Commertal Energy Consumption Estimate8, 19100, 1965, 1970-1994, Okahoma

\begin{tabular}{|c|c|c|c|c|c|c|c|c|c|c|c|c|c|c|}
\hline \multirow[b]{3}{*}{$\mathrm{nu}$} & \multicolumn{3}{|c|}{$\cos$} & \multirow{3}{*}{ 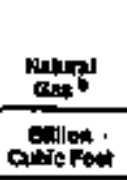 } & \multicolumn{6}{|c|}{ Potrotwn } & \multirow[b]{2}{*}{ enentibly. } & \multirow[b]{2}{*}{$\operatorname{lex}_{0}$} & \multirow[b]{2}{*}{ 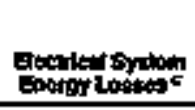 } & \multirow[b]{3}{*}{ Toter 4} \\
\hline & 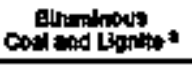 & Anthnopas & Tats & & Distorto & Karomean" & LPE * & stotor & Fistion & To:s & & & & \\
\hline & \multicolumn{3}{|c|}{ Thootaned Short Tons: } & & \multicolumn{6}{|c|}{ 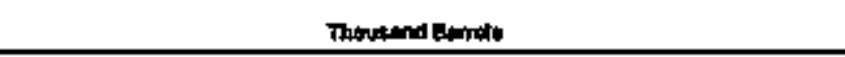 } & \multicolumn{3}{|c|}{ 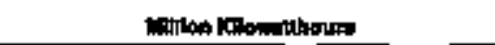 } & \\
\hline 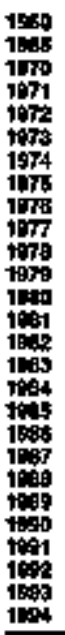 & 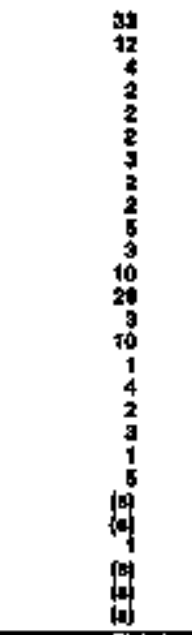 & $\begin{array}{l}0 \\
0 \\
0 \\
0 \\
0 \\
0 \\
0 \\
0 \\
0 \\
0 \\
0 \\
0 \\
0 \\
0 \\
0 \\
0 \\
0 \\
0 \\
0 \\
0 \\
01 \\
0 \\
0 \\
0 \\
01 \\
0 \\
0 \\
0\end{array}$ & 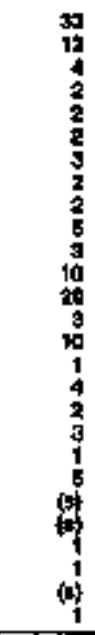 & 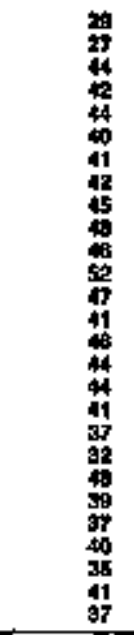 & 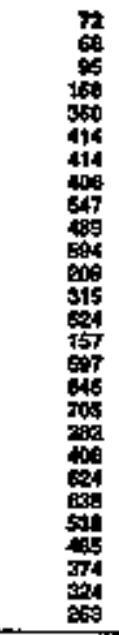 & 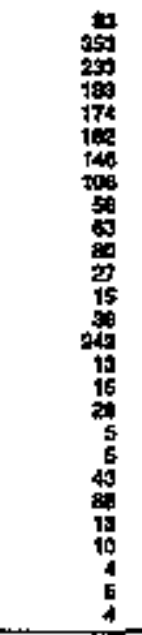 & 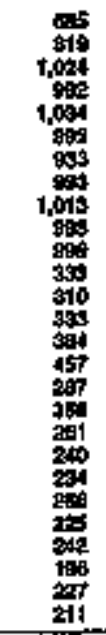 & 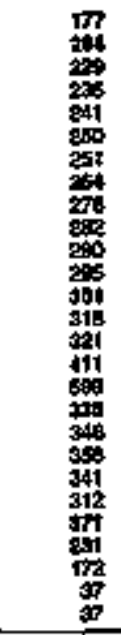 & 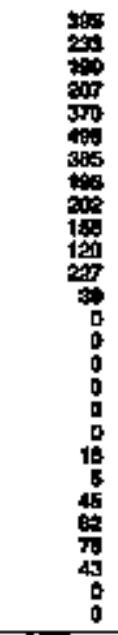 & 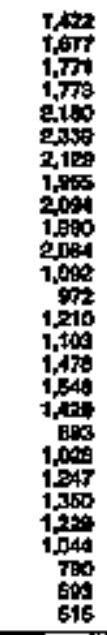 & 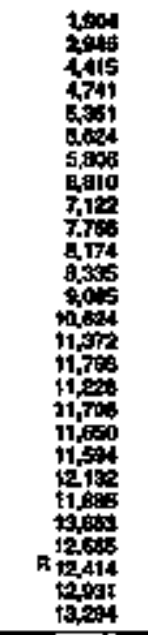 & $\begin{array}{l}= \\
= \\
= \\
= \\
= \\
= \\
= \\
= \\
= \\
= \\
= \\
= \\
= \\
=\end{array}$ & 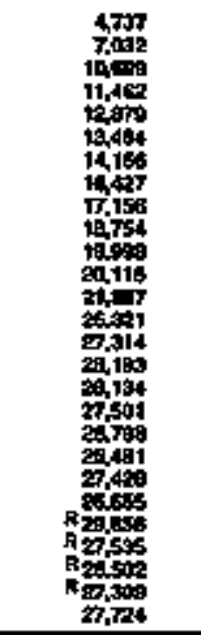 & $\begin{array}{l}= \\
z \\
z \\
z \\
z \\
z \\
z \\
z \\
z \\
z \\
z \\
z \\
z \\
z \\
=\end{array}$ \\
\hline \multicolumn{15}{|c|}{ Trlukn Eth } \\
\hline 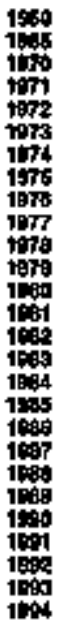 & 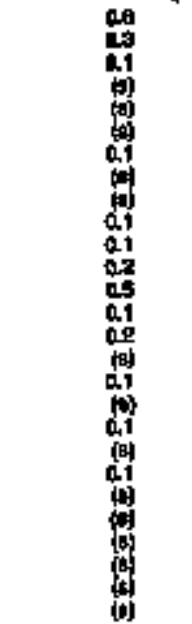 & 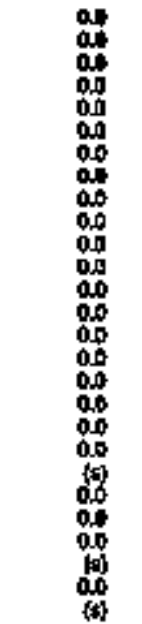 & 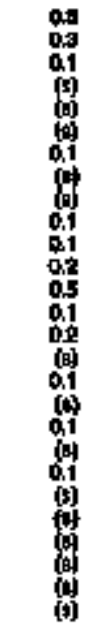 & 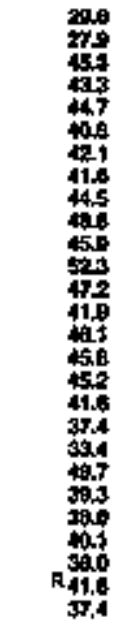 & 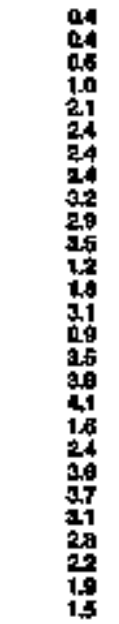 & 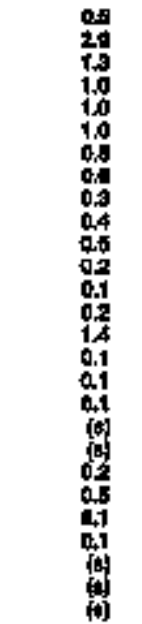 & 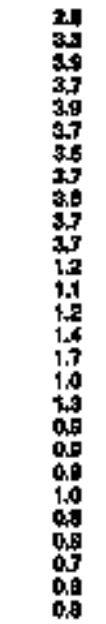 & 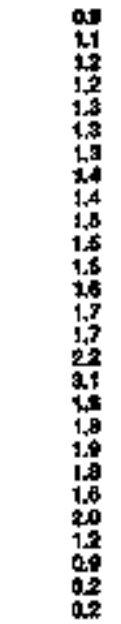 & 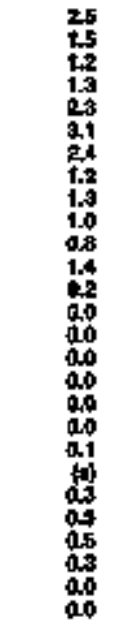 & 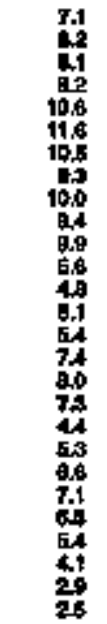 & 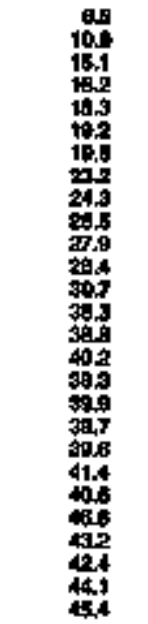 & 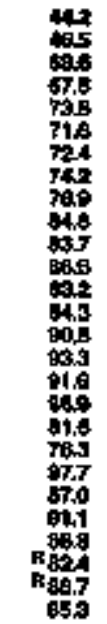 & 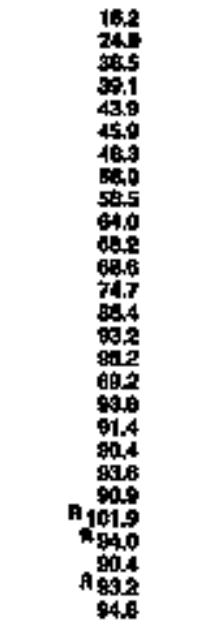 & 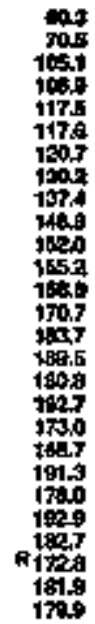 \\
\hline & inomitid & 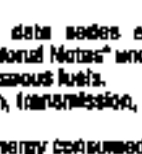 & $m$ & Ithered & $\mathbf{g}$ & $\infty$ & & 4 & & & 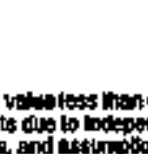 & 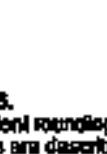 & & \\
\hline
\end{tabular}


$\mathbf{0}$

Table 236. Industrial Energy Consumption Estlmales, 1960, 1965, 1970-1954, OkJahama

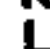

\begin{tabular}{|c|c|c|c|c|c|c|c|c|c|c|c|c|c|c|c|c|c|c|}
\hline & \multirow[b]{2}{*}{ Cesil } & \multirow[b]{2}{*}{ thent: } & \multicolumn{9}{|c|}{ |س } & \multirow{2}{*}{ 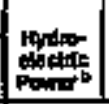 } & \multirow[b]{2}{*}{ Blotugh } & \multirow[b]{2}{*}{ Other bu } & \multirow[b]{2}{*}{ Enctidedy } & \multirow[b]{2}{*}{ Met } & \multirow{2}{*}{ 1. } & \multirow[b]{3}{*}{ Toted } \\
\hline & & & Aspentots & Fot: & |l| & $\operatorname{Ln} 0^{\circ}$ & Lorkimats & Mtor & Fonftis | & 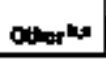 & Tow & & & & & & & \\
\hline$r=$ & Thowand & 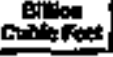 & \multicolumn{9}{|c|}{ 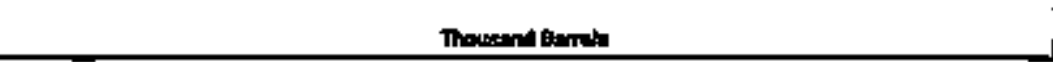 } & \multicolumn{6}{|c|}{ 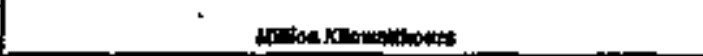 } & \\
\hline 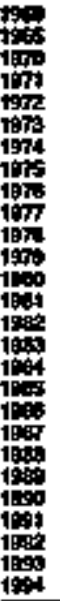 & 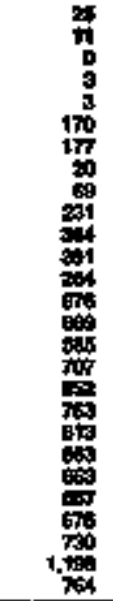 & 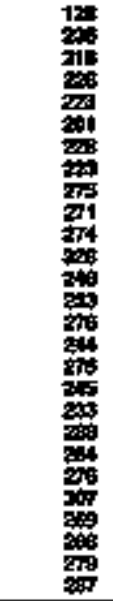 & 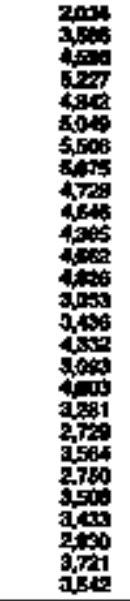 & 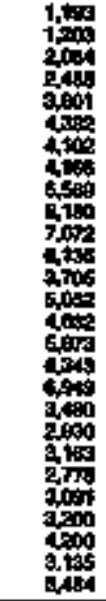 & 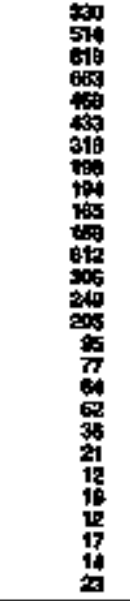 & 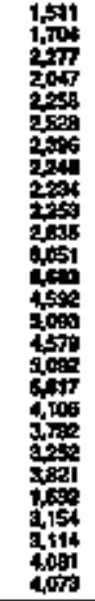 & 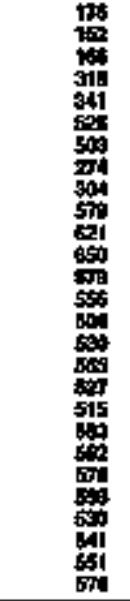 & 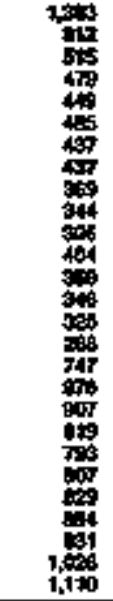 & 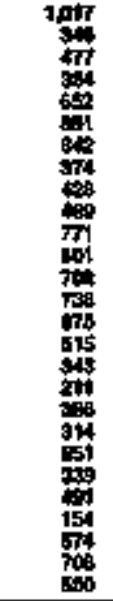 & 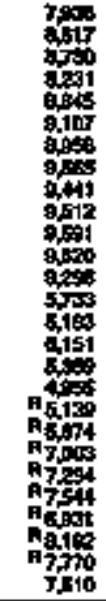 & 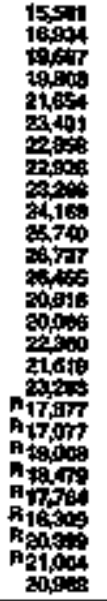 & 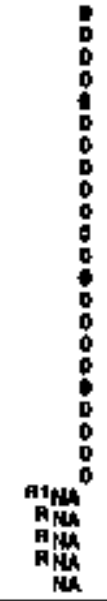 & 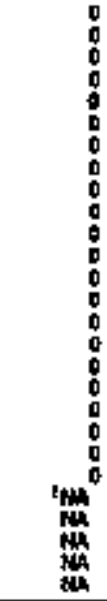 & 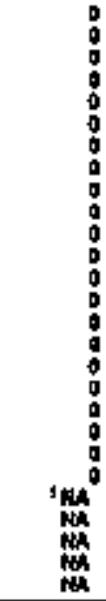 & 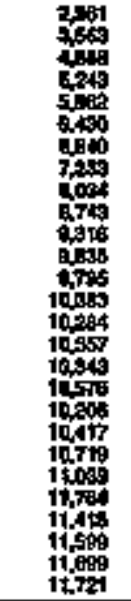 & $\begin{array}{l}= \\
= \\
= \\
= \\
z \\
= \\
= \\
= \\
= \\
= \\
= \\
z \\
z \\
z \\
z\end{array}$ & 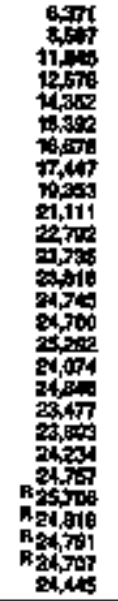 & $\begin{array}{l}\text { } \\
\end{array}$ \\
\hline & & & & & & & & & 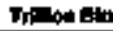 & & & & & & & & & \\
\hline
\end{tabular}

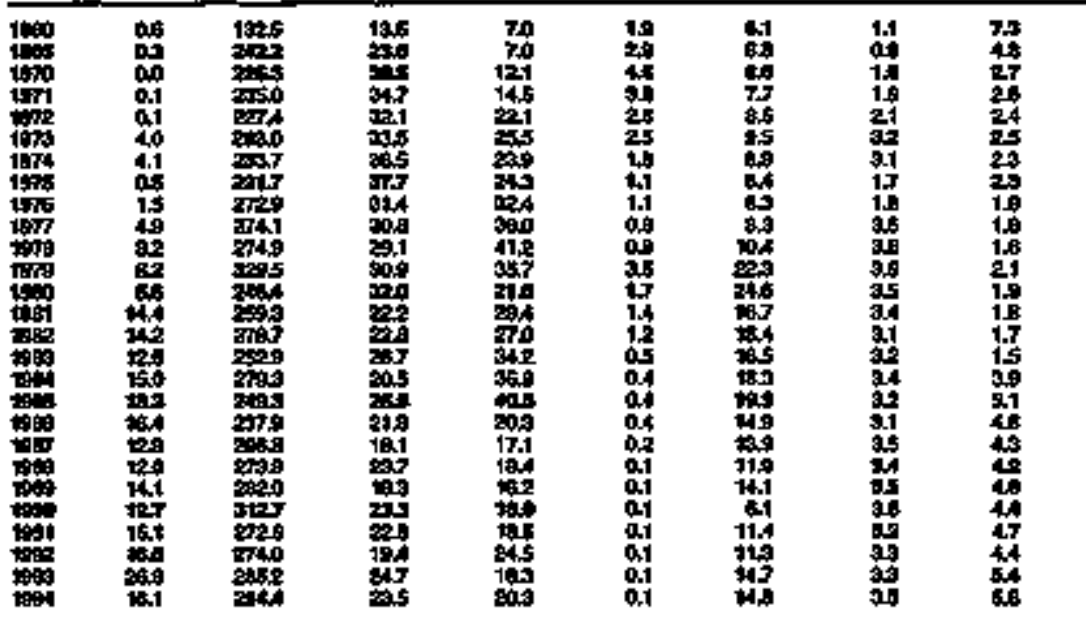

\begin{tabular}{|c|c|c|c|c|}
\hline 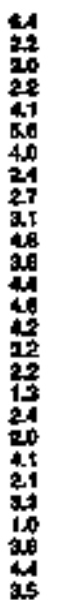 & 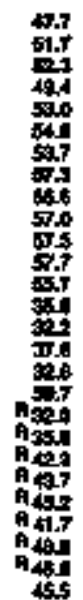 & 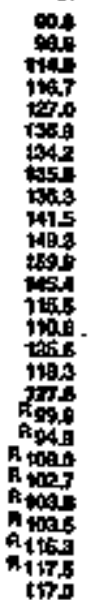 & 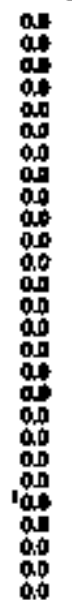 & 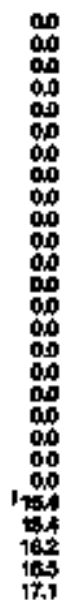 \\
\hline
\end{tabular}

\begin{tabular}{|c|c|c|c|c|}
\hline 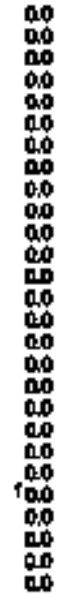 & 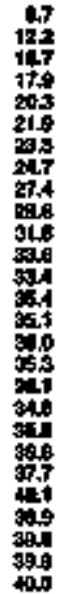 & 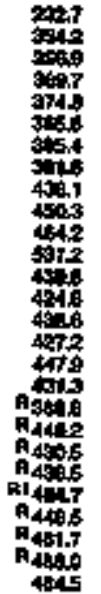 & 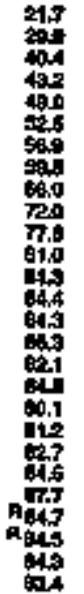 & 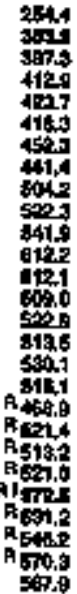 \\
\hline
\end{tabular}

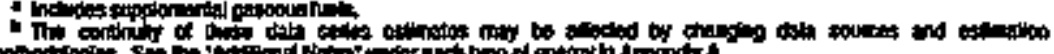

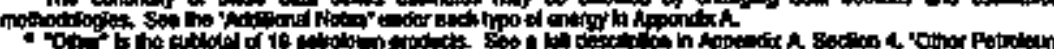
(n)

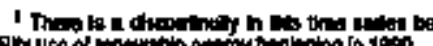

Pratrit.

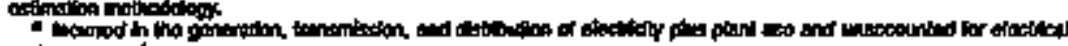

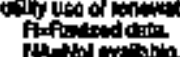

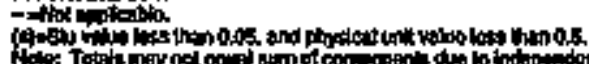




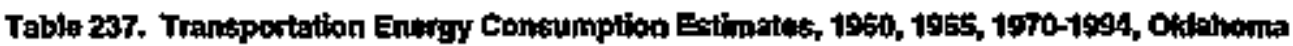

\begin{tabular}{|c|c|c|c|c|c|c|c|c|c|c|c|c|c|c|c|}
\hline \multirow[b]{3}{*}{ 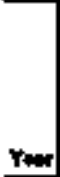 } & \multirow[b]{2}{*}{ Doos L } & \multirow[b]{2}{*}{ pann: } & \multicolumn{8}{|c|}{ Poding } & \multirow{3}{*}{ 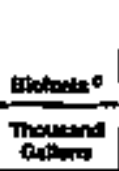 } & & & \multirow[b]{2}{*}{ 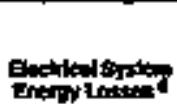 } & \multirow[b]{3}{*}{ Tobl } \\
\hline & & & Antition. & Dethe & sied. & $\mathbf{H a}=$ & 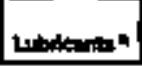 & (1) & moverter & Tobs & & 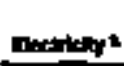 & Wint & & \\
\hline & Thentiond & onion & \multicolumn{8}{|c|}{ 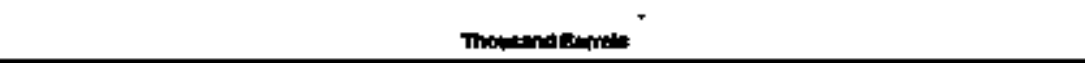 } & & \multicolumn{3}{|c|}{ החים } & \\
\hline 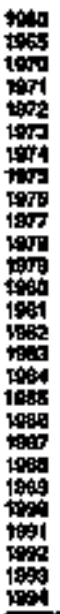 & 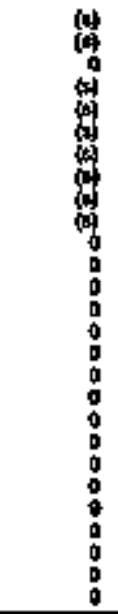 & 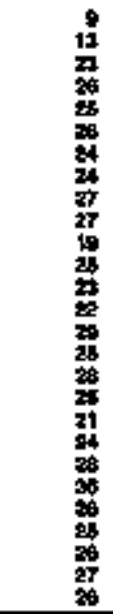 & 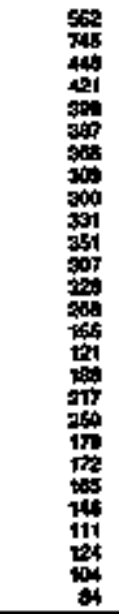 & 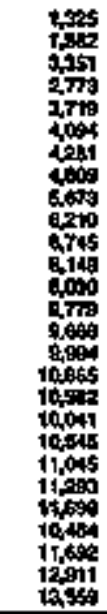 & 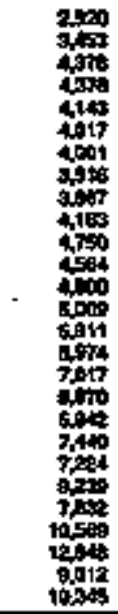 & 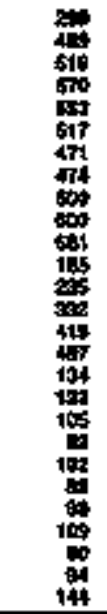 & 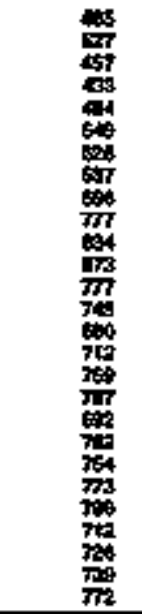 & 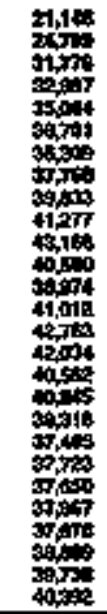 & 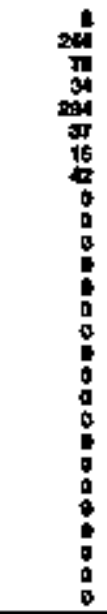 & 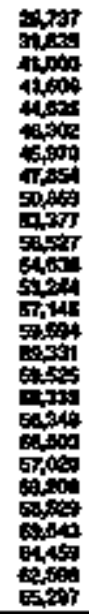 & $\begin{array}{l}0 \\
0 \\
0 \\
0 \\
0 \\
0 \\
0 \\
0 \\
0 \\
0 \\
0 \\
0 \\
0 \\
0 \\
0 \\
0 \\
0 \\
0 \\
0 \\
0 \\
0\end{array}$ & $\begin{array}{l}0 \\
0 \\
0 \\
0 \\
0 \\
0 \\
0 \\
0 \\
0 \\
0 \\
0 \\
0 \\
0 \\
0 \\
0 \\
0 \\
0 \\
0 \\
0 \\
0 \\
0 \\
0 \\
0 \\
0 \\
0\end{array}$ & $\begin{array}{l}= \\
= \\
= \\
= \\
= \\
= \\
= \\
= \\
= \\
= \\
= \\
= \\
= \\
= \\
= \\
=\end{array}$ & $\begin{array}{l}8 \\
8 \\
8 \\
8 \\
8 \\
8 \\
8 \\
8 \\
8 \\
8 \\
8 \\
8 \\
0\end{array}$ & $\begin{array}{l}= \\
= \\
= \\
z \\
= \\
= \\
= \\
= \\
z \\
= \\
z \\
z \\
z \\
z \\
z\end{array}$ \\
\hline \multicolumn{16}{|c|}{ ntwon } \\
\hline 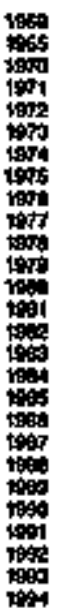 & 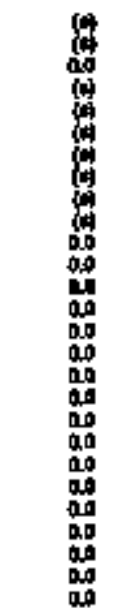 & 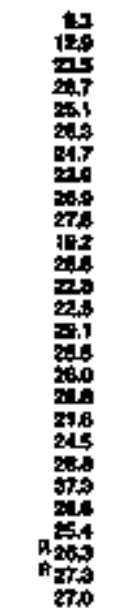 & 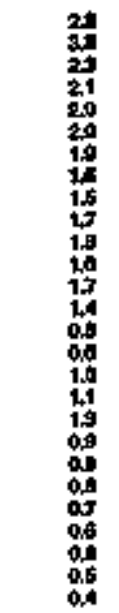 & 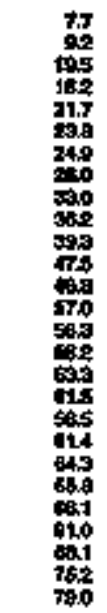 & 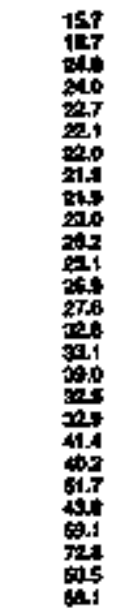 & 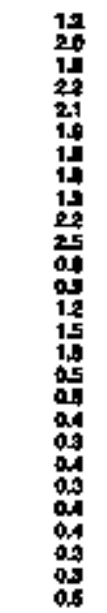 & 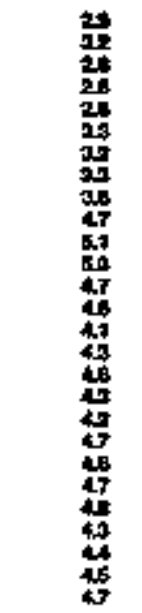 & 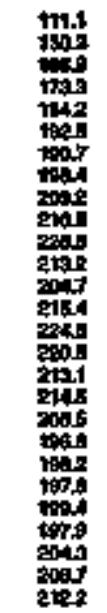 & 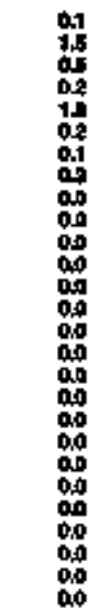 & 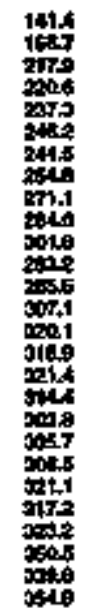 & $\begin{array}{l}00 \\
00 \\
00 \\
00 \\
00 \\
00 \\
00 \\
00 \\
00 \\
00 \\
00 \\
00 \\
00 \\
00 \\
00 \\
00 \\
00 \\
00 \\
00 \\
00 \\
00 \\
00 \\
00 \\
00 \\
00 \\
00 \\
00 \\
00 \\
00\end{array}$ & 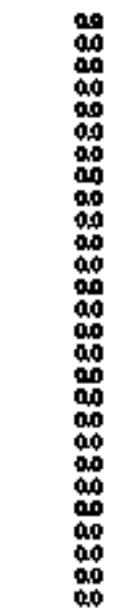 & 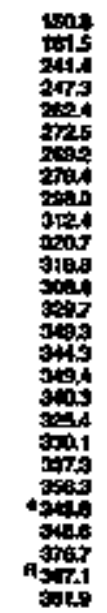 & 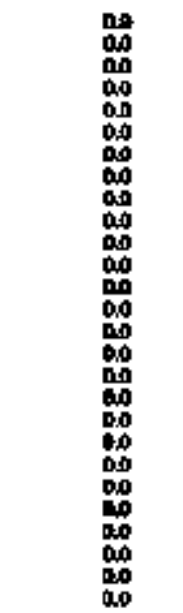 & 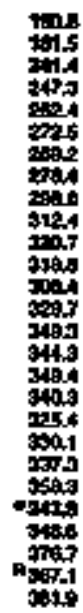 \\
\hline
\end{tabular}

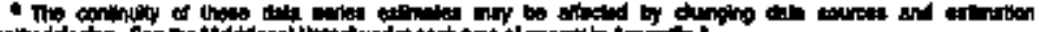

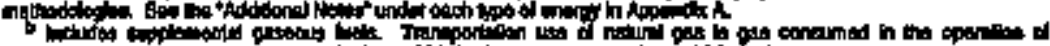

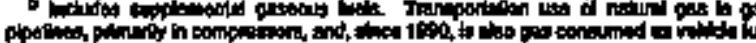

- Theo 110 a

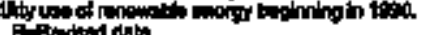

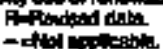

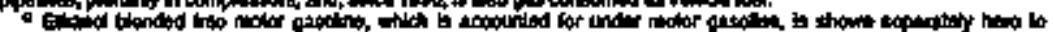

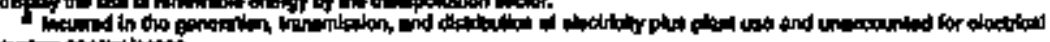

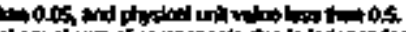

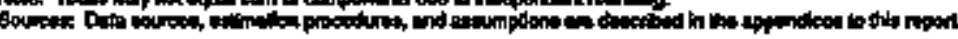


Teble 238. Estimates of Enengy Ingth al Electrio Utilities, 1960, 1968, 1970-1994, Oklahoma

\begin{tabular}{|c|c|c|c|c|c|c|c|c|c|c|c|c|c|c|}
\hline \multirow[b]{3}{*}{ إمبY } & \multicolumn{3}{|c|}{ Con } & \multirow{3}{*}{$\frac{\text { cotion }}{\text { cotio Rent }}$} & \multicolumn{4}{|c|}{ Arrown } & \multirow[b]{2}{*}{ 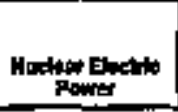 } & \multirow[b]{2}{*}{ 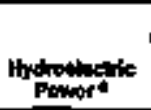 } & \multirow[b]{2}{*}{ Elotents } & \multirow[b]{2}{*}{ Copthern:d } & \multirow{3}{*}{ Alther bi } & \multirow[b]{3}{*}{ 70:4=1 4} \\
\hline & 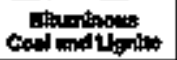 & 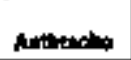 & Totol & & botid & 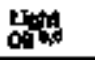 & 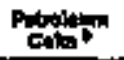 & Tolet & & & & & & \\
\hline & \multicolumn{3}{|c|}{ Mnund stant Toes } & & \multicolumn{4}{|c|}{ Theortad Benth } & \multicolumn{4}{|c|}{ Millen Kllowathoure } & & \\
\hline 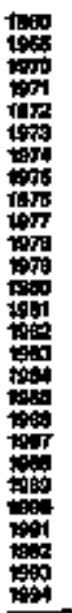 & 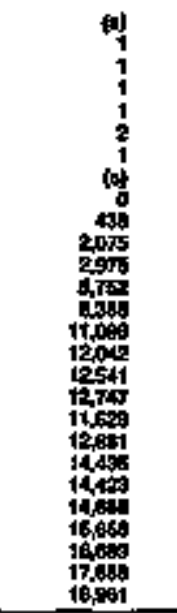 & $\begin{array}{l}0 \\
0 \\
0 \\
0 \\
0 \\
0 \\
0 \\
0 \\
0 \\
0 \\
0 \\
0 \\
0 \\
0 \\
0 \\
0 \\
0 \\
0 \\
0 \\
0 \\
0 \\
0 \\
0 \\
0 \\
0\end{array}$ & 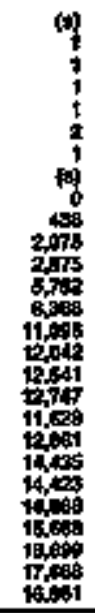 & 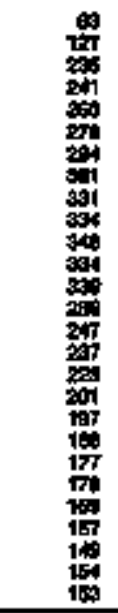 & 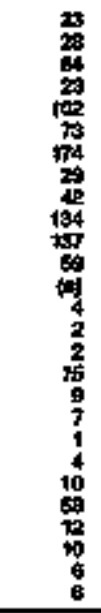 & 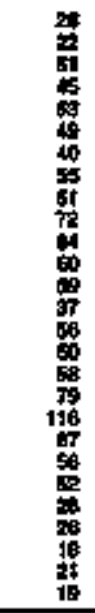 & $\begin{array}{l}6 \\
0 \\
0 \\
0 \\
0 \\
0 \\
0 \\
0 \\
0 \\
0 \\
0 \\
0 \\
0 \\
0 \\
0 \\
0 \\
0 \\
0 \\
0 \\
0 \\
0 \\
0\end{array}$ & 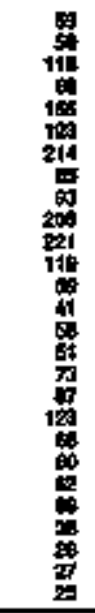 & $\begin{array}{l}0 \\
0 \\
0 \\
0 \\
0 \\
0 \\
0 \\
0 \\
0 \\
0 \\
0 \\
0 \\
0 \\
0 \\
0 \\
0 \\
0 \\
0 \\
0 \\
0\end{array}$ & 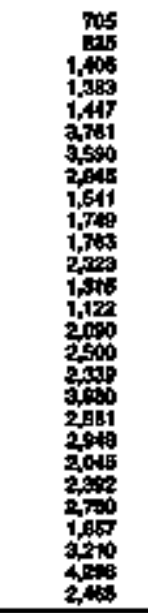 & $\begin{array}{l}0 \\
0 \\
0 \\
0 \\
0 \\
0 \\
0 \\
0 \\
0 \\
0 \\
0 \\
0 \\
0 \\
0 \\
0 \\
0 \\
0 \\
0 \\
0 \\
0\end{array}$ & $\begin{array}{l}0 \\
0 \\
0 \\
0 \\
0 \\
0 \\
0 \\
0 \\
0 \\
0 \\
0 \\
0 \\
0 \\
0 \\
0 \\
0 \\
0 \\
0 \\
0 \\
0 \\
0 \\
0 \\
0 \\
0 \\
0 \\
0 \\
0\end{array}$ & $\begin{array}{l}0 \\
0 \\
0 \\
0 \\
0 \\
0 \\
0 \\
0 \\
0 \\
0 \\
0 \\
0 \\
0 \\
0 \\
0 \\
0 \\
0 \\
0 \\
0 \\
0 \\
0 \\
0 \\
0 \\
0 \\
0 \\
0 \\
0 \\
0 \\
0 \\
0 \\
0 \\
0\end{array}$ & $\begin{array}{l}z \\
z \\
z \\
z \\
z \\
z \\
z \\
z \\
z \\
z \\
z \\
z \\
z \\
z \\
z \\
z\end{array}$ \\
\hline \multicolumn{15}{|c|}{ 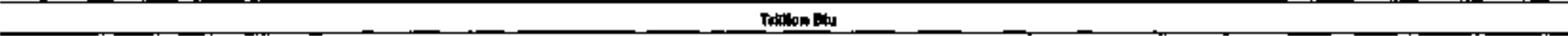 } \\
\hline 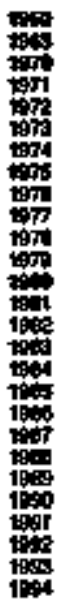 & 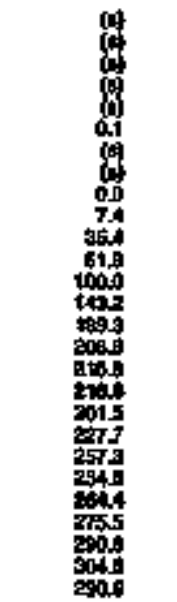 & 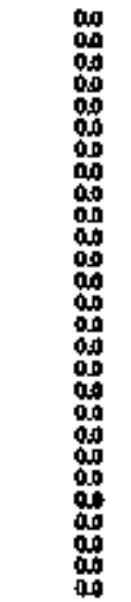 & 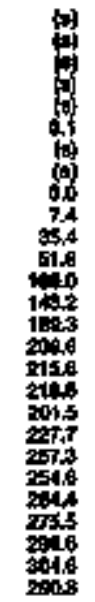 & 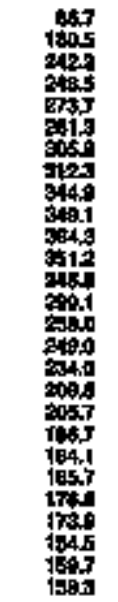 & 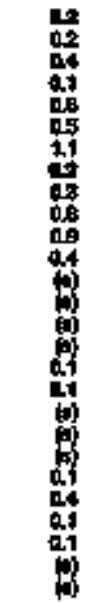 & 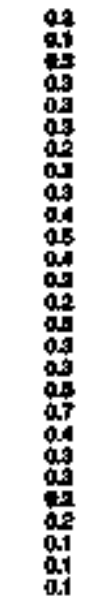 & 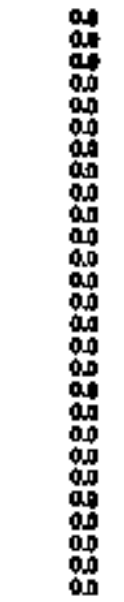 & 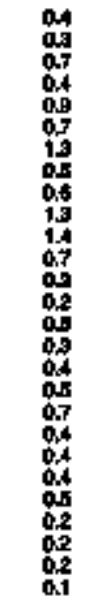 & 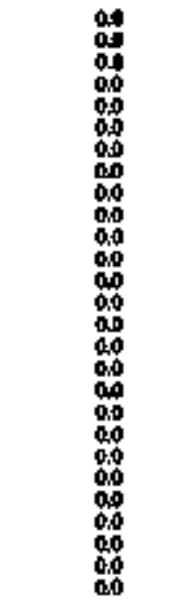 & 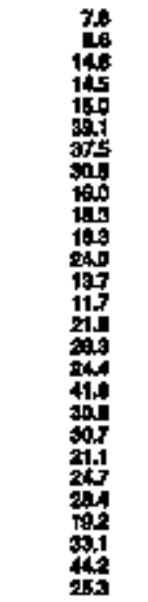 & 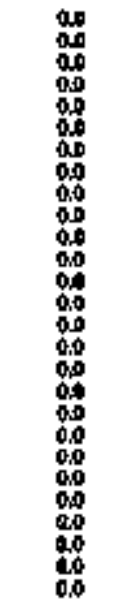 & 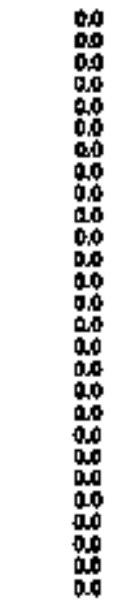 & 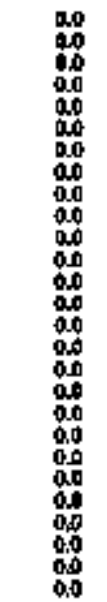 & 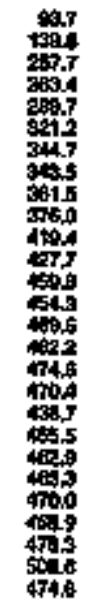 \\
\hline
\end{tabular}

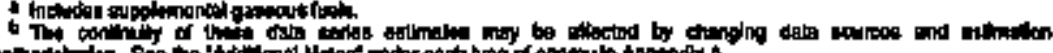

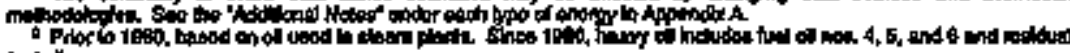

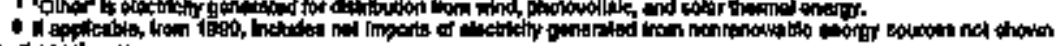

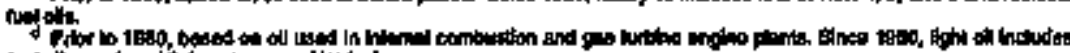

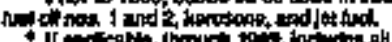
mothof agingit:

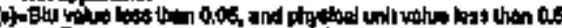

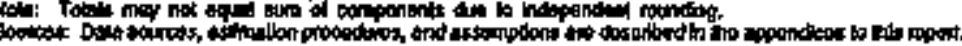

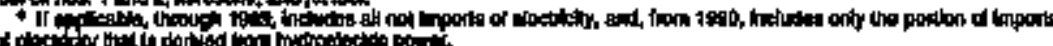


Table 259. Enargy Consumption Estimates by Source, 1960, 1965, 1970-1994, Omgon

\begin{tabular}{|c|c|c|c|c|c|c|c|c|c|c|c|c|c|c|c|c|c|c|c|}
\hline \multirow[b]{3}{*}{ שي } & \multirow[b]{2}{*}{ (1) } & \multirow[b]{2}{*}{ 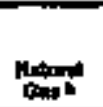 } & \multicolumn{11}{|c|}{ Parotom } & \multirow{2}{*}{ 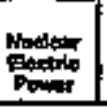 } & \multirow[b]{2}{*}{ petet } & \multirow[b]{2}{*}{ enotonth } & \multirow[b]{2}{*}{ Oener as ] } & \multirow{3}{*}{ 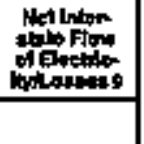 } & \multirow[b]{3}{*}{ Tot h h } \\
\hline & & & 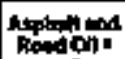 & Andiction & 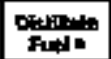 & Fon. & $\mathrm{km}$ & LFE: & - & , & Apentiont & STheras & Totat & & & & & & \\
\hline & Movinded & 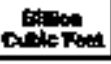 & & \multicolumn{10}{|c|}{ Proosmand Bumin } & \multicolumn{4}{|c|}{ 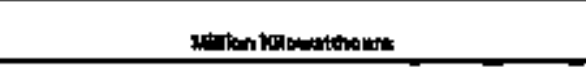 } & & \\
\hline 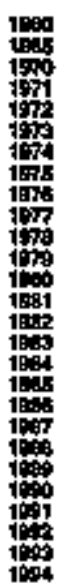 & 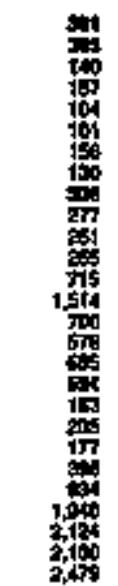 & 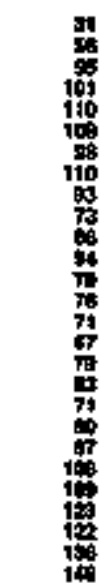 & 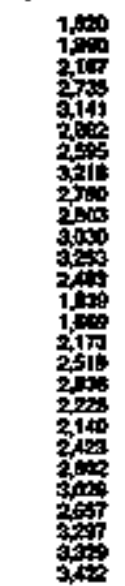 & 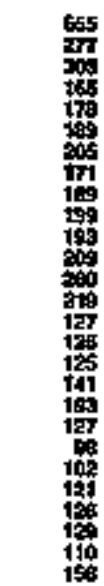 & 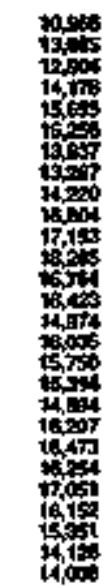 & 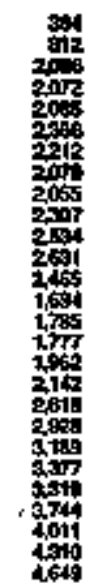 & 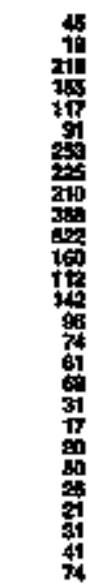 & 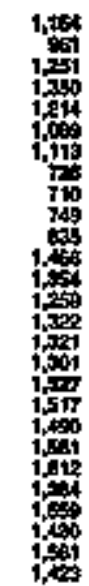 & 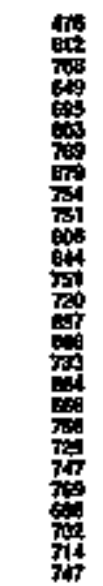 & 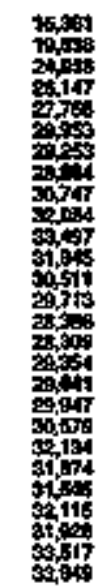 & 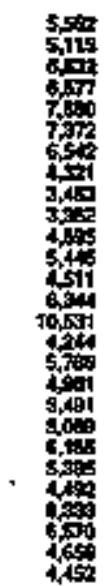 & 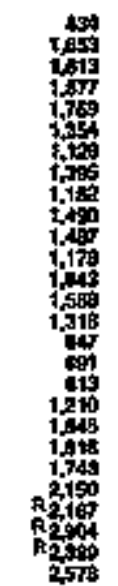 & 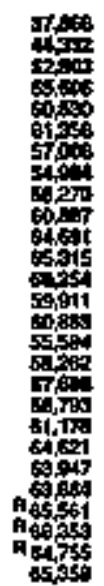 & 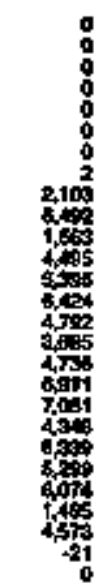 & 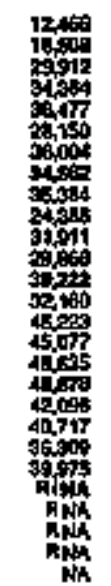 & 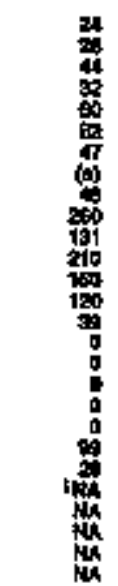 & 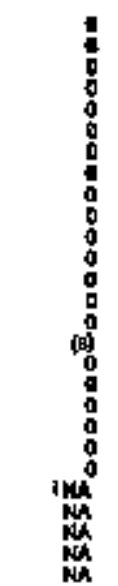 & 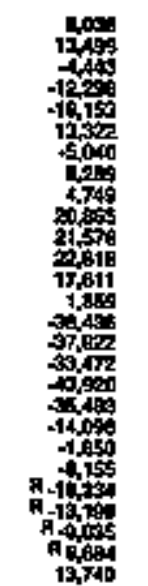 & $=$ \\
\hline & & & & & & & & & & ton & & & & & & & & & \\
\hline
\end{tabular}

\begin{tabular}{|c|c|c|c|c|c|c|c|c|c|c|c|c|c|c|c|c|c|c|}
\hline 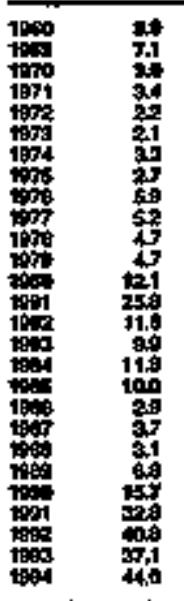 & 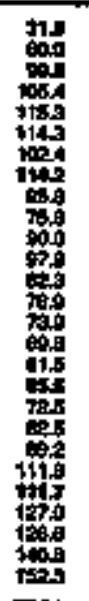 & 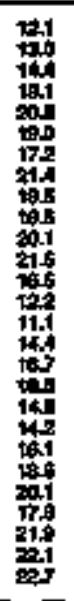 & 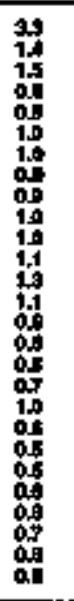 & 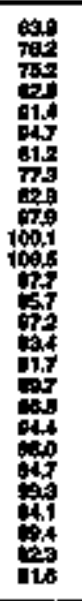 & 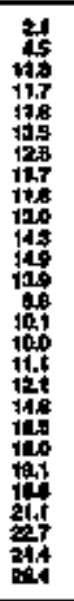 & 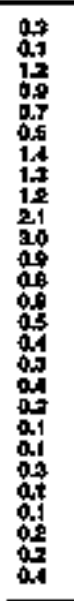 & 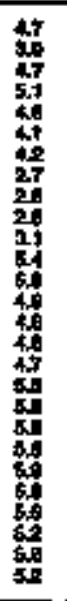 & 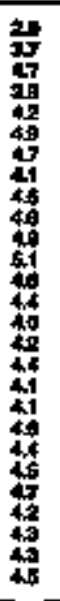 & 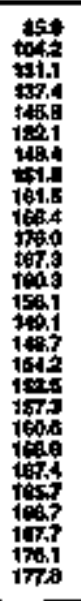 & 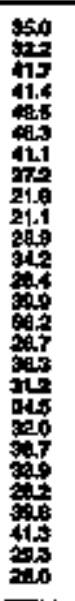 & 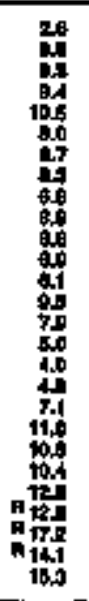 & 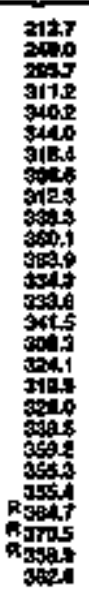 & 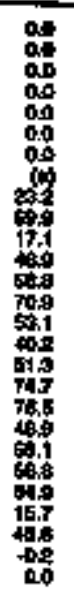 & 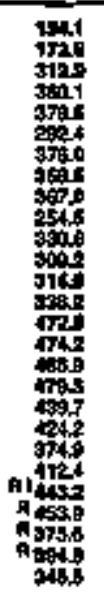 & 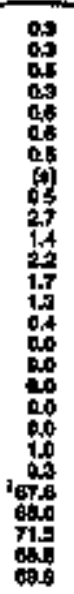 & 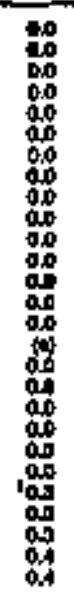 & 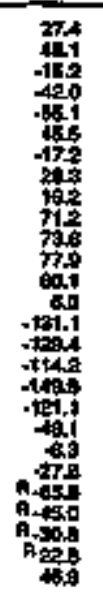 & 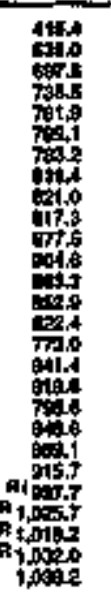 \\
\hline
\end{tabular}

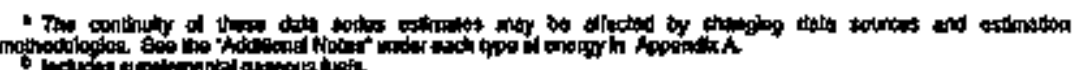

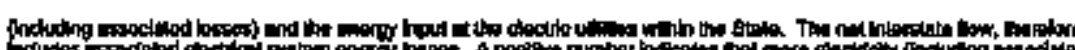

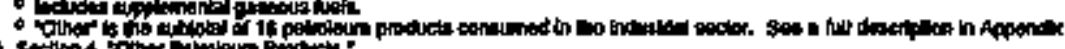

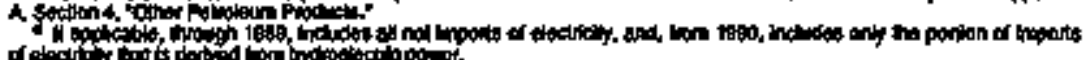

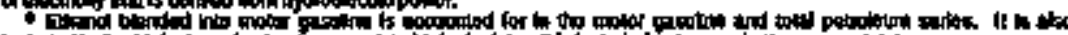

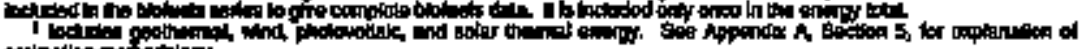

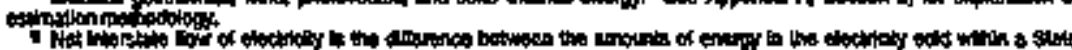

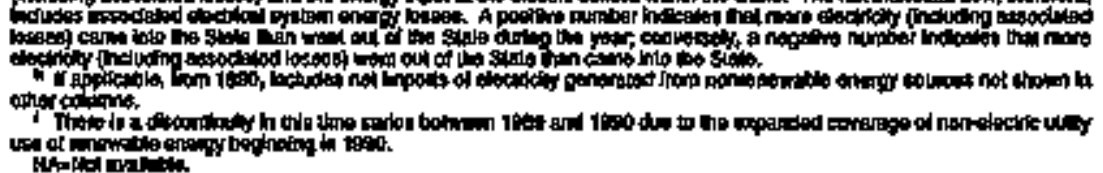
Mptor nim

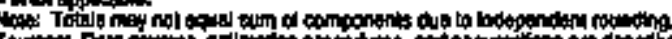

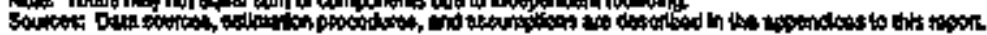


Teble 240. Residentlal Energy Consumption Eelfmates, 1960, 1865, 1970-1994, Oregon

\begin{tabular}{|c|c|c|c|c|c|c|c|c|c|c|c|c|c|c|}
\hline \multirow[b]{3}{*}{ Yeat } & \multicolumn{3}{|c|}{ con } & \multirow{3}{*}{ 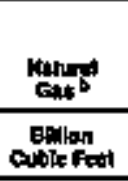 } & \multicolumn{4}{|c|}{ Patortom } & \multirow{3}{*}{$\begin{array}{l}\text { Dlototh } \\
\text { Thoosend } \\
\text { Conth }\end{array}$} & \multirow[b]{2}{*}{ polute } & \multirow[b]{2}{*}{ Eoptitelhy " } & \multirow[b]{2}{*}{ Enorgot } & \multirow{3}{*}{ 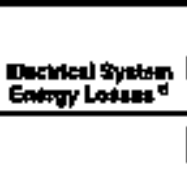 } & \multirow[b]{3}{*}{ Ton } \\
\hline & 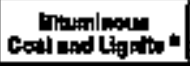 & Anthentotot & Toltal & & Dationt" & 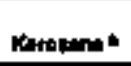 & LPR: & Tom & & & & & & \\
\hline & \multicolumn{3}{|c|}{ 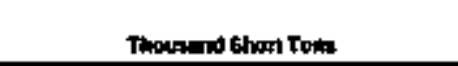 } & & \multicolumn{4}{|c|}{ 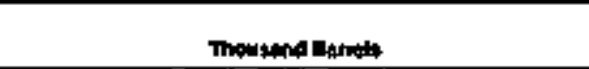 } & & \multicolumn{3}{|c|}{ 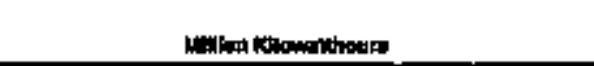 } & & \\
\hline 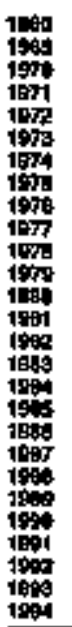 & 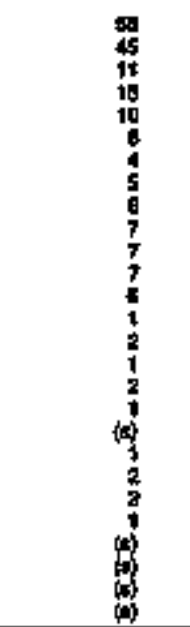 & $\begin{array}{l}0 \\
0 \\
0 \\
0 \\
0 \\
0 \\
0 \\
0 \\
0 \\
0 \\
0 \\
0 \\
0 \\
0 \\
0 \\
0 \\
0 \\
0 \\
0 \\
0 \\
0 \\
0 \\
0 \\
0 \\
0 \\
1 \\
10\end{array}$ & 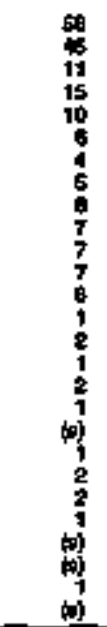 & 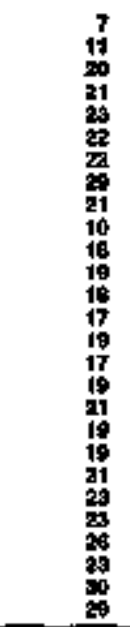 & 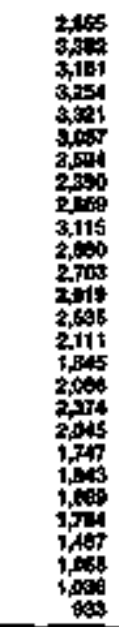 & 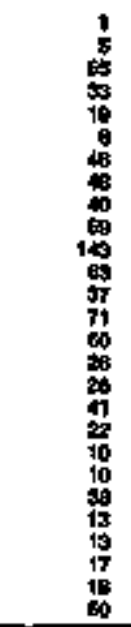 & 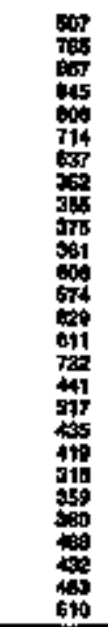 & 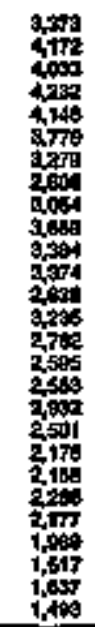 & 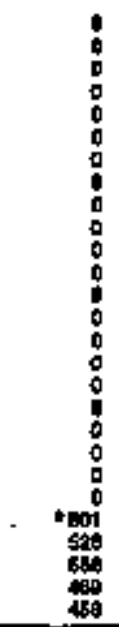 & 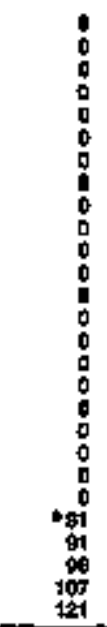 & 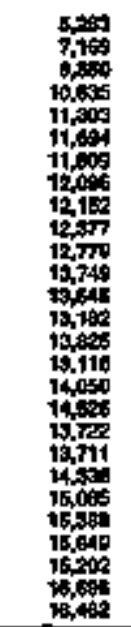 & $\begin{array}{l}z \\
z \\
z \\
z \\
z \\
z \\
z \\
z \\
z \\
z \\
z \\
z \\
z \\
z\end{array}$ & 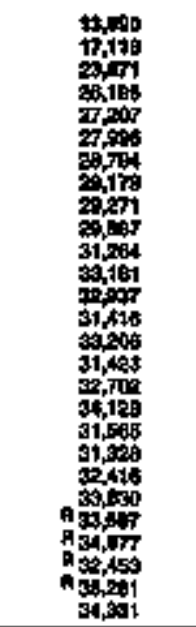 & $\begin{array}{l}= \\
= \\
z \\
z \\
z \\
z \\
z \\
= \\
z \\
z \\
z \\
z \\
z \\
z \\
z\end{array}$ \\
\hline \multicolumn{15}{|c|}{ Ftotor 텬, } \\
\hline 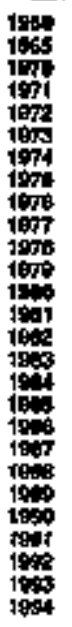 & 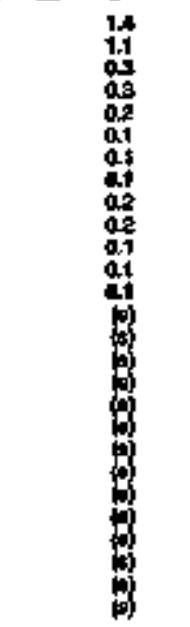 & 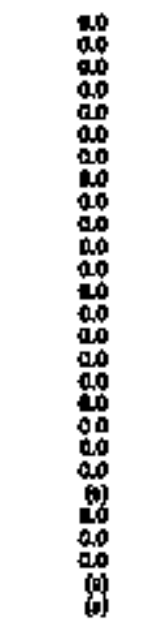 & 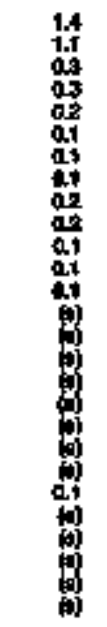 & 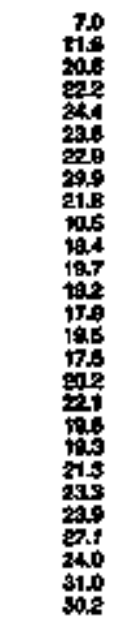 & 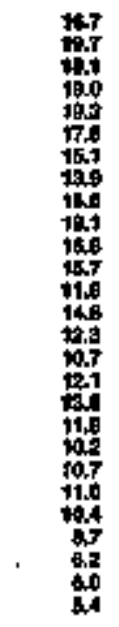 & 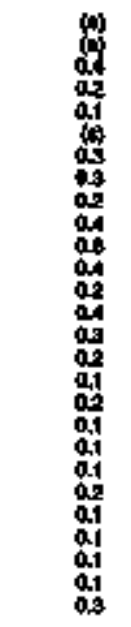 & 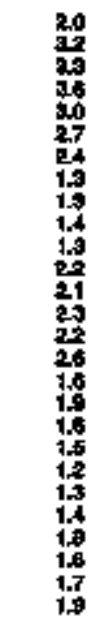 & 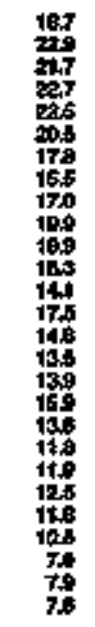 & 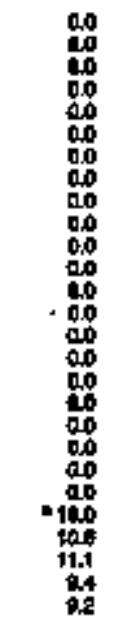 & 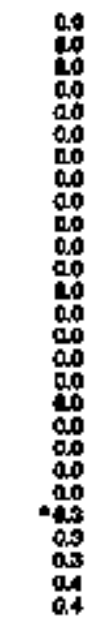 & 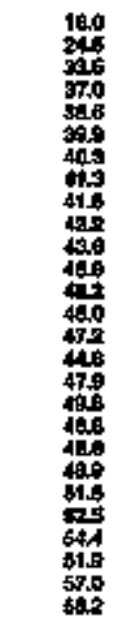 & 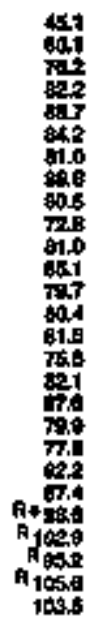 & 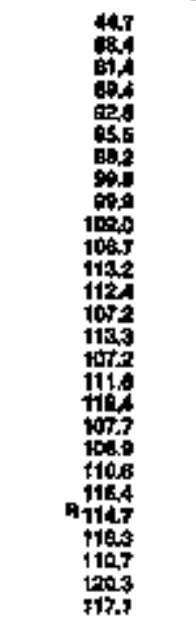 & 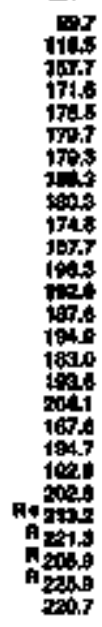 \\
\hline
\end{tabular}

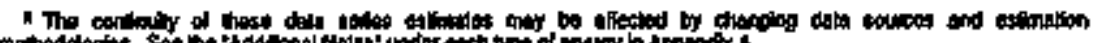

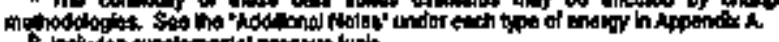

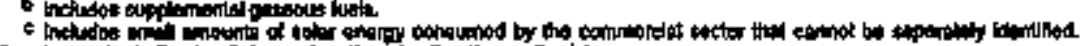

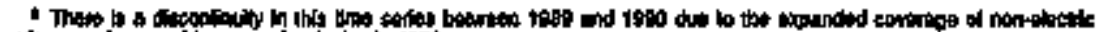

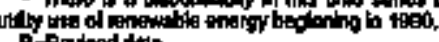

Paffordsed deits.

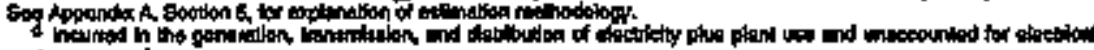
INod

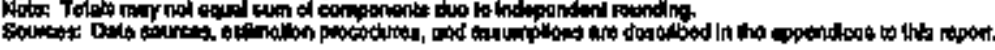

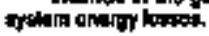


Table 241. Commercial Enargy Consumptlon Eslimates, 1960, 1965, 1970-1994, Oregon

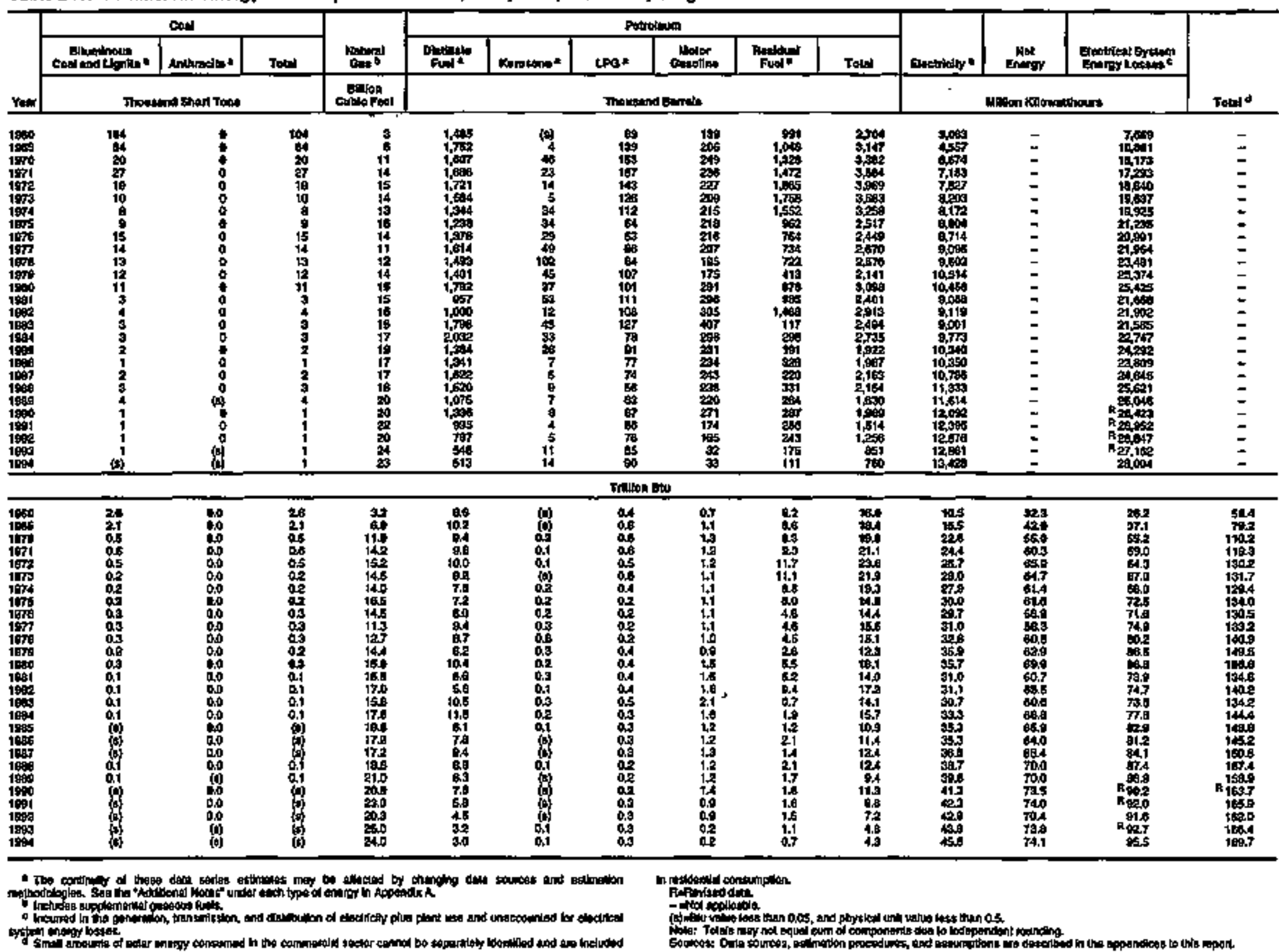

$\mathbf{O}$
$\mathbf{R}$
$\mathbf{E}$
$\mathbf{G}$
$\mathbf{O}$
$\mathbf{N}$ 
Table 242. Industrial Eneroy Consumption Estimates, 1960, 1955, 1970-1994, Oregon

\begin{tabular}{|c|c|c|c|c|c|c|c|c|c|c|c|c|c|c|c|c|c|c|}
\hline \multirow[b]{3}{*}{ nom } & \multirow[b]{2}{*}{ Con } & \multirow[b]{2}{*}{ Manter } & \multicolumn{9}{|c|}{ Adrolen } & \multirow{2}{*}{ 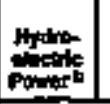 } & \multirow[b]{2}{*}{ 1 } & \multirow[b]{2}{*}{ Oather } & \multirow[b]{2}{*}{ Eloctilely } & \multirow[b]{2}{*}{ thengy } & \multirow{2}{*}{ 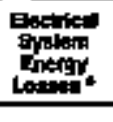 } & \multirow[b]{3}{*}{$\tau$} \\
\hline & & & Aaphat and & Foton & Menosent & $\cos$ & Cobropantes of & Asting & 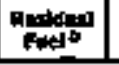 & Other so & Toted & & & & & & & \\
\hline & $\begin{array}{l}\text { Theurend } \\
\text { Sthert Tonk }\end{array}$ & 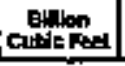 & \multicolumn{9}{|c|}{ Thousond Bumb } & \multicolumn{6}{|c|}{ 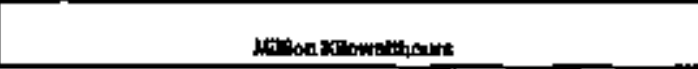 } & \\
\hline 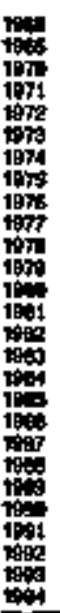 & 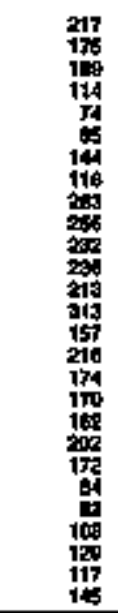 & 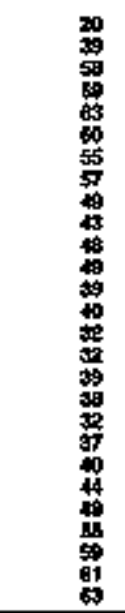 & 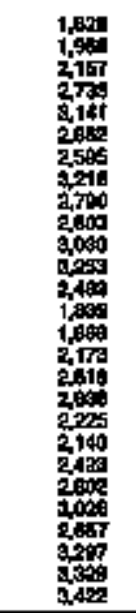 & 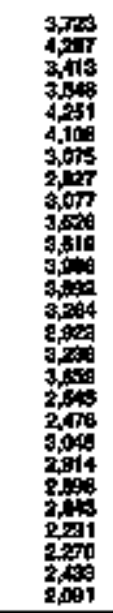 & 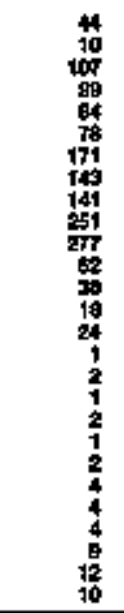 & 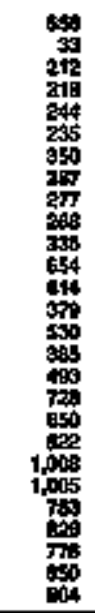 & 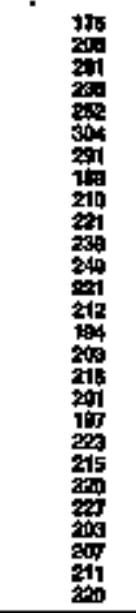 & 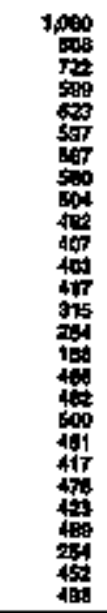 & 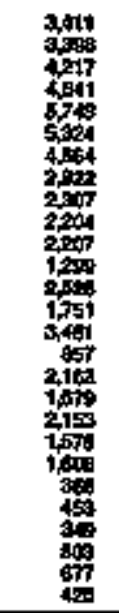 & 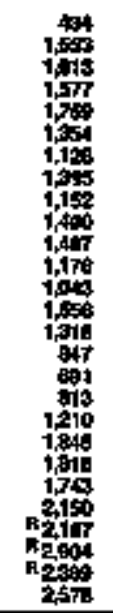 & 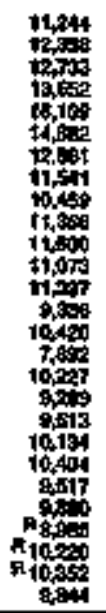 & 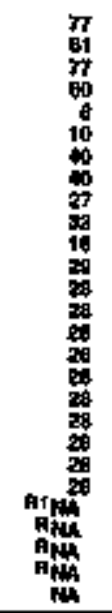 & $\begin{array}{r}0 \\
0 \\
0 \\
0 \\
0 \\
0 \\
0 \\
0 \\
0 \\
0 \\
0 \\
0 \\
0 \\
0 \\
0 \\
0 \\
0 \\
0 \\
0 \\
0 \\
0 \\
0\end{array}$ & $\begin{array}{l}0 \\
0 \\
0 \\
0 \\
0 \\
0 \\
0 \\
0 \\
0 \\
0 \\
0 \\
0 \\
0 \\
0 \\
0 \\
0 \\
0 \\
0 \\
0 \\
0 \\
0 \\
0 \\
0 \\
14 \\
4 \\
4 \\
4\end{array}$ & 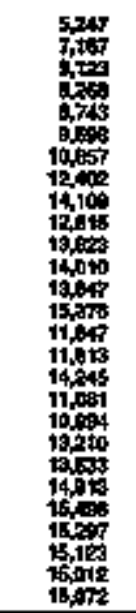 & $\begin{array}{l}z \\
z \\
z \\
z \\
z \\
z \\
z \\
z \\
z \\
z \\
z \\
z \\
z \\
z \\
z\end{array}$ & 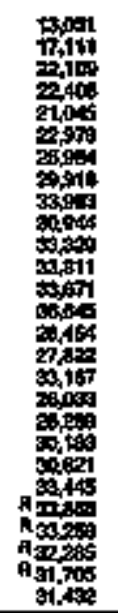 & $\begin{array}{l}= \\
= \\
= \\
= \\
= \\
= \\
= \\
= \\
= \\
= \\
= \\
= \\
= \\
=\end{array}$ \\
\hline & & & & & & & & & Tfill & & & & & & & & & \\
\hline
\end{tabular}

\begin{tabular}{|c|c|c|c|c|c|c|c|c|c|c|c|c|c|c|c|c|c|c|}
\hline 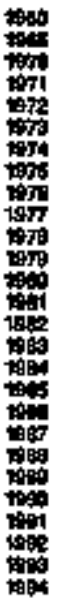 & 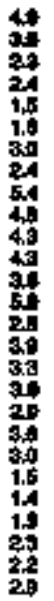 & 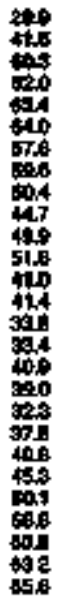 & 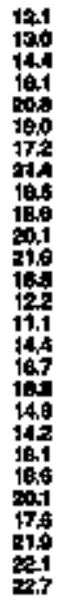 & 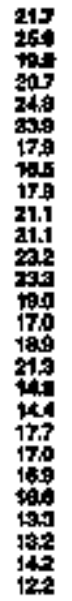 & 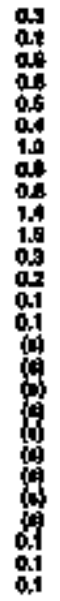 & 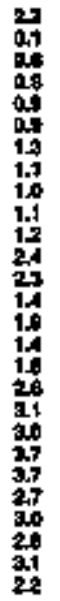 & 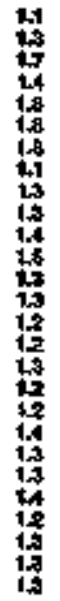 & 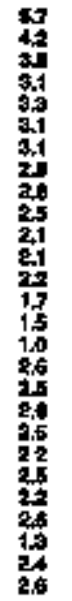 & 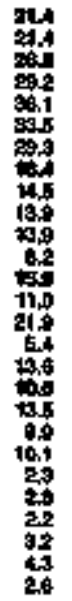 & 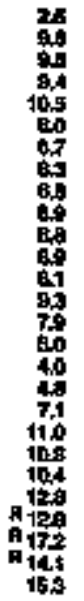 & 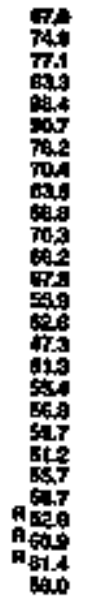 & 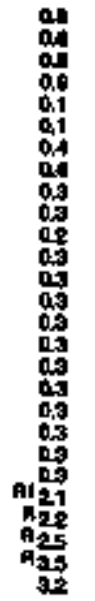 & 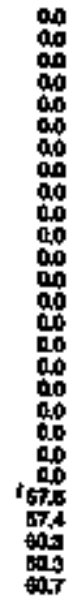 & $\begin{array}{l}00 \\
00 \\
00 \\
00 \\
00 \\
00 \\
00 \\
00 \\
00 \\
00 \\
00 \\
00 \\
00 \\
00 \\
00 \\
00 \\
00 \\
00 \\
00 \\
00 \\
00 \\
00 \\
00 \\
00 \\
00\end{array}$ & 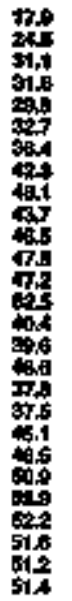 & 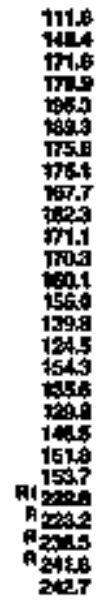 & 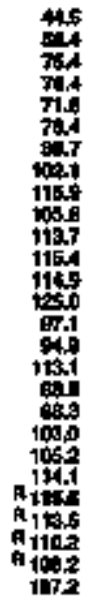 & 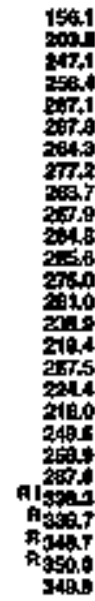 \\
\hline
\end{tabular}

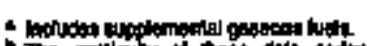

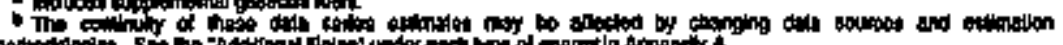

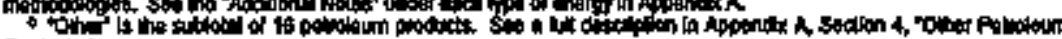
Anture.

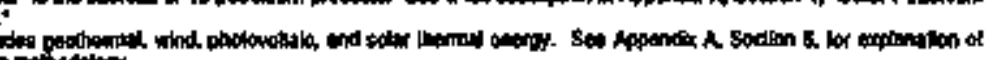

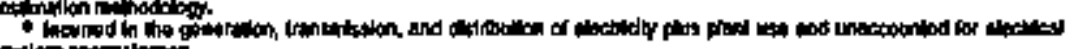

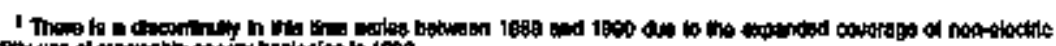
morot boginging in 1900.

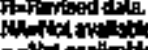

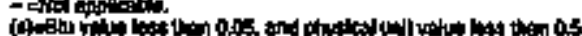

To Tot

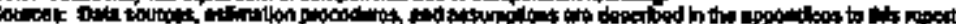


Table 243. Transportalion Energy Consumption Estumates, 1960, 1965, 1970-1994, Oregon

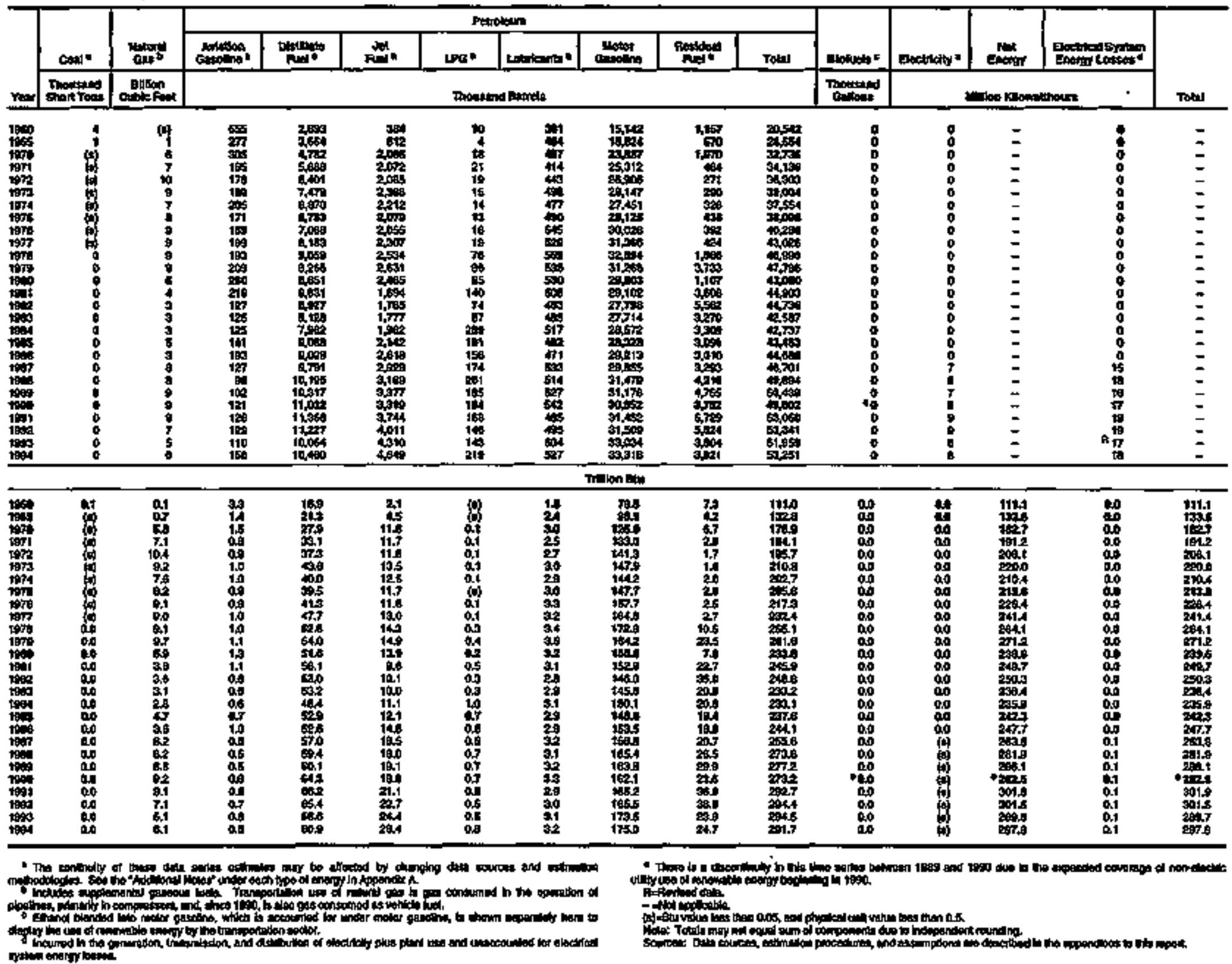


Table 244. Estimates of Energy lnpud a Electric Utifities, 1960, 1965, 1970-1994, Oregon

\begin{tabular}{|c|c|c|c|c|c|c|c|c|c|c|c|c|c|c|}
\hline \multirow[b]{3}{*}{ reat } & \multicolumn{3}{|c|}{ Dow } & \multirow{3}{*}{ 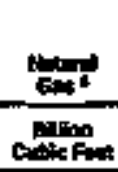 } & \multicolumn{4}{|c|}{ Poraten } & \multirow[b]{2}{*}{ Muclow Elocits } & \multirow[b]{2}{*}{ It) } & \multirow[b]{2}{*}{ Biploct } & \multirow[b]{2}{*}{ Denthermed } & \multirow[b]{2}{*}{ Other by } & \multirow[b]{3}{*}{ Totals } \\
\hline & collindus & Antrations & Totw & & ond & 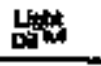 & Paten & Totol & & & & & & \\
\hline & \multicolumn{3}{|c|}{ Trowsend Sthen Tonal } & & \multicolumn{4}{|c|}{ 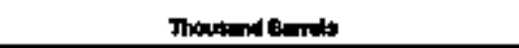 } & \multicolumn{5}{|c|}{ 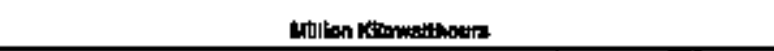 } & \\
\hline 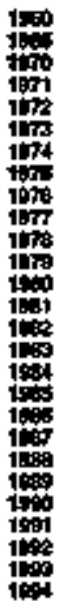 & 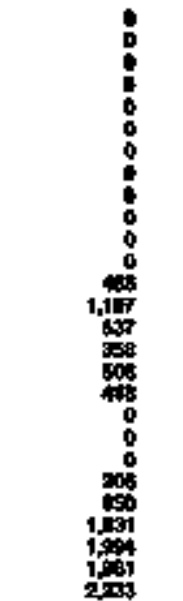 & $\begin{array}{l}0 \\
0 \\
0 \\
0 \\
0 \\
0 \\
0 \\
0 \\
0 \\
0 \\
0 \\
0 \\
0 \\
0 \\
0 \\
0 \\
0 \\
0 \\
0 \\
0 \\
0\end{array}$ & 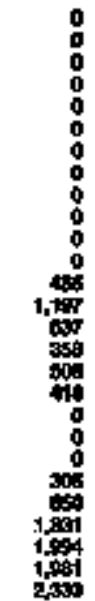 & 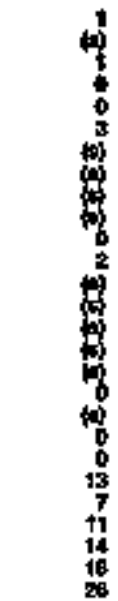 & $\begin{array}{r}3 \\
1 \\
10 \\
0 \\
0 \\
1 \\
0 \\
0 \\
0 \\
0 \\
0 \\
0 \\
0 \\
0 \\
0 \\
0 \\
0 \\
0 \\
0 \\
0 \\
0 \\
0 \\
0 \\
0 \\
0 \\
0\end{array}$ & 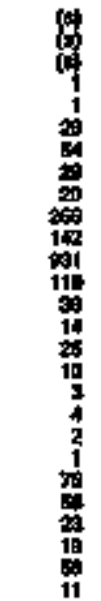 & $\begin{array}{l}0 \\
0 \\
0 \\
0 \\
0 \\
0 \\
0 \\
0 \\
0 \\
0 \\
0 \\
0 \\
0 \\
0 \\
0 \\
0 \\
0 \\
0 \\
0 \\
0 \\
0 \\
0 \\
0 \\
0 \\
0 \\
0 \\
0\end{array}$ & 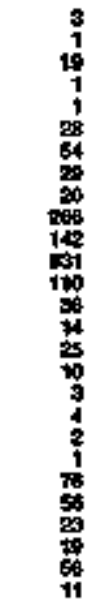 & 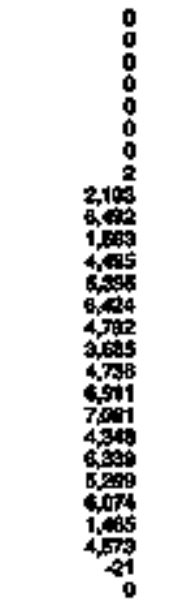 & 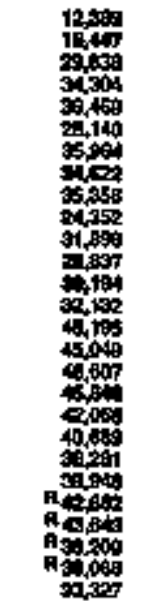 & 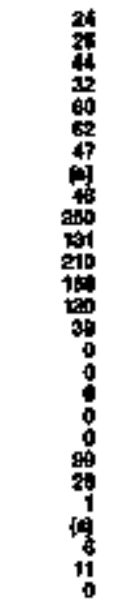 & $\begin{array}{l}0 \\
0 \\
0 \\
0 \\
0 \\
0 \\
0 \\
0 \\
0 \\
0 \\
0 \\
0 \\
0 \\
0 \\
0 \\
0 \\
0 \\
0 \\
0 \\
0 \\
0 \\
0\end{array}$ & $\begin{array}{l}1 \\
0 \\
0 \\
0 \\
0 \\
0 \\
0 \\
0 \\
0 \\
0 \\
0 \\
0 \\
0 \\
0 \\
0 \\
0 \\
0 \\
0 \\
0 \\
0 \\
0 \\
0 \\
0 \\
0 \\
0 \\
0\end{array}$ & 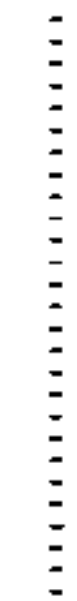 \\
\hline
\end{tabular}

\begin{tabular}{|c|c|c|c|c|c|c|}
\hline 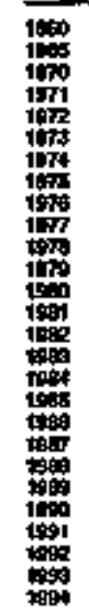 & 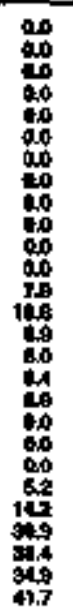 & 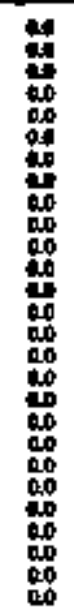 & 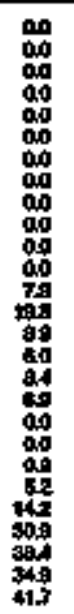 & 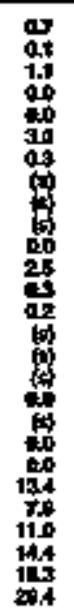 & 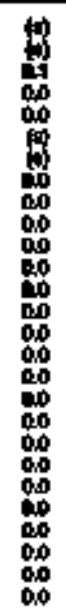 & 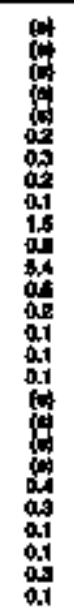 \\
\hline
\end{tabular}

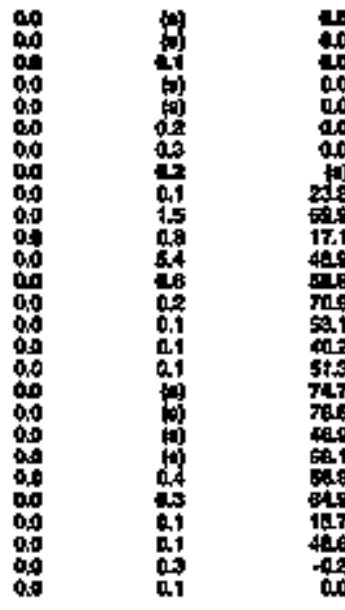

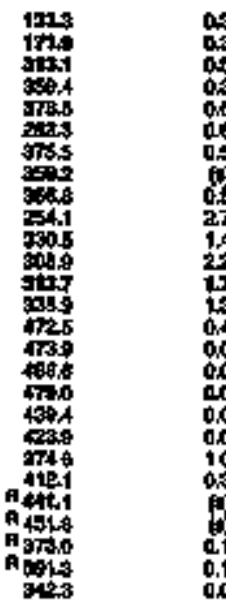

\begin{tabular}{|c|c|c|c|}
\hline$a$ & 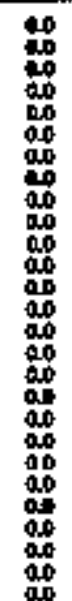 & 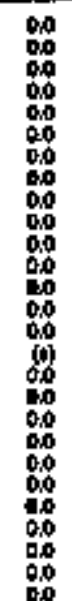 & 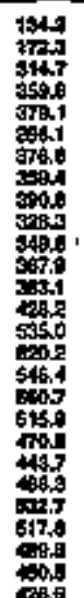 \\
\hline
\end{tabular}

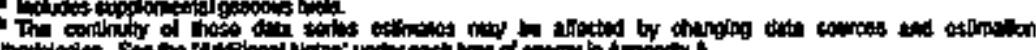

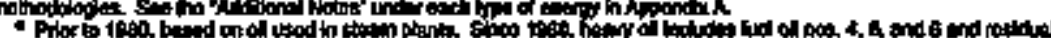

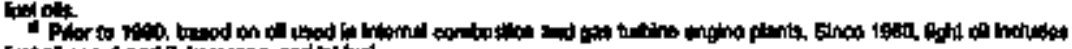

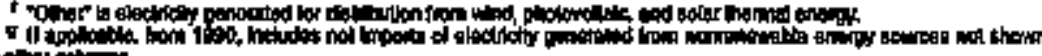

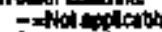

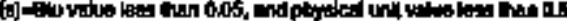

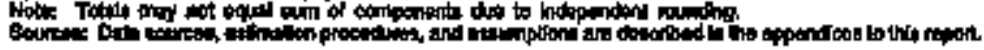

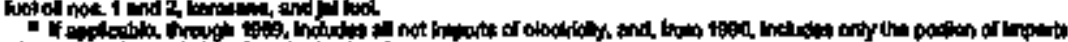

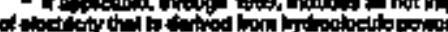


Tabje 245. Energy Consumption Estimates by Source, 1960, 1965, 1970-1994, Pennsyluenia

\begin{tabular}{|c|c|c|c|c|c|c|c|c|c|c|c|c|c|c|c|c|c|c|c|}
\hline \multirow[b]{3}{*}{$\mathrm{res}:$} & \multirow[b]{2}{*}{$\cos _{n}$} & \multirow[b]{2}{*}{ Natry } & \multicolumn{11}{|c|}{ porringm } & \multirow[b]{2}{*}{ 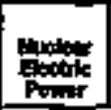 } & \multirow[b]{2}{*}{ 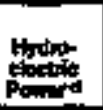 } & \multirow[b]{2}{*}{ Bdolvetie } & \multirow[b]{2}{*}{ 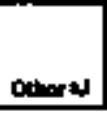 } & \multirow{3}{*}{ 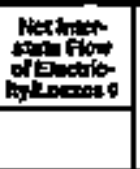 } & \multirow[b]{3}{*}{ Toten h } \\
\hline & & & Aspantind & Anteren & 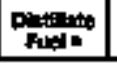 & स्र: & Kow: & wan: & Lent| & 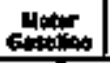 & Fontat & Oever an & Toth & & & & & & \\
\hline & nowath & 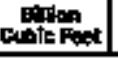 & \multicolumn{11}{|c|}{ Thoustor Bunch } & \multicolumn{4}{|c|}{ mank } & & \\
\hline 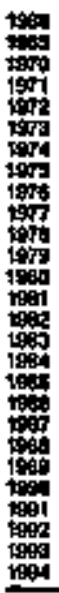 & 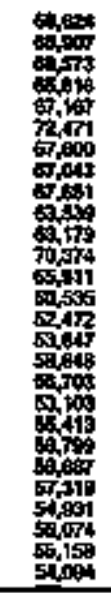 & 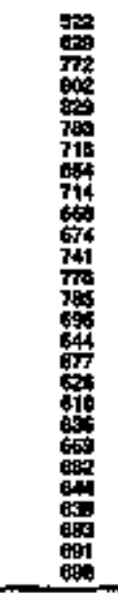 & 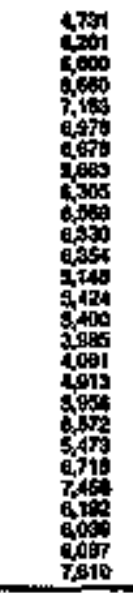 & 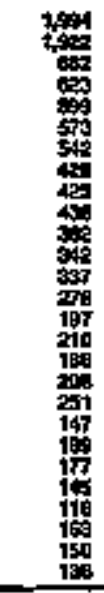 & 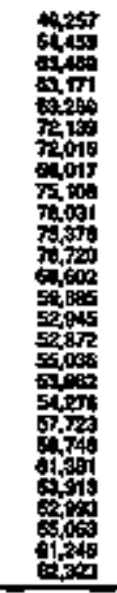 & 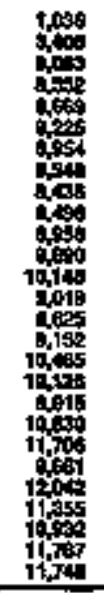 & 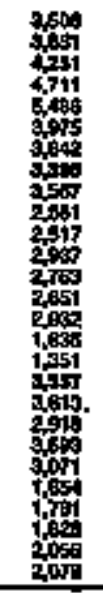 & 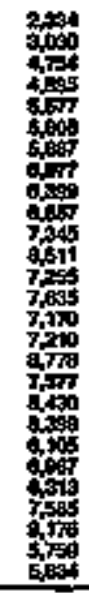 & 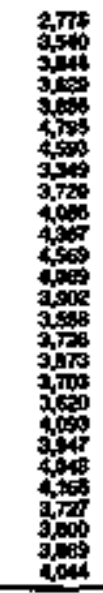 & 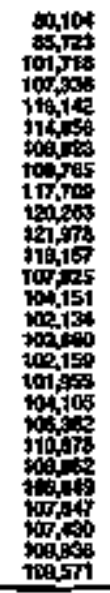 & 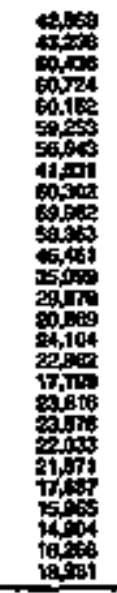 & 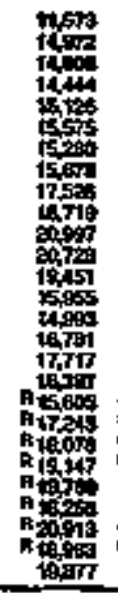 & 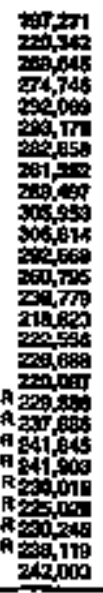 & 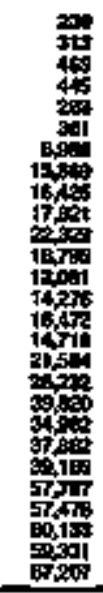 & 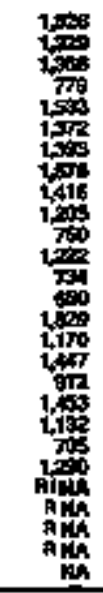 & 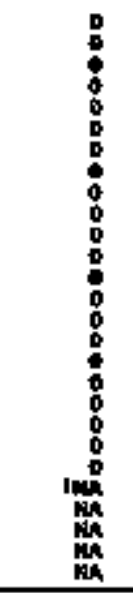 & 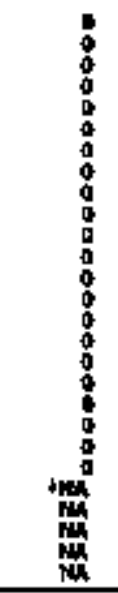 & 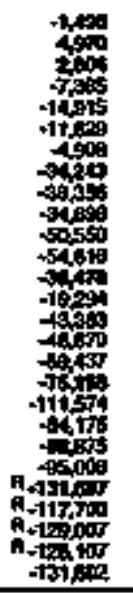 & 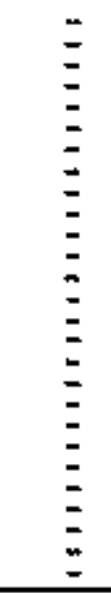 \\
\hline \multicolumn{20}{|c|}{$V_{f}$} \\
\hline 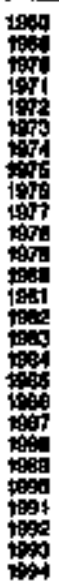 & 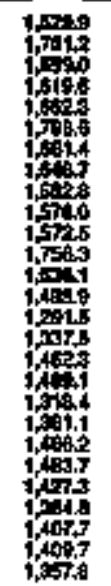 & 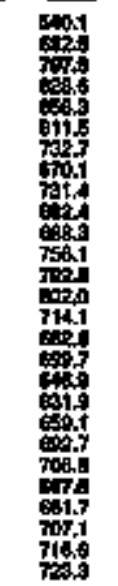 & 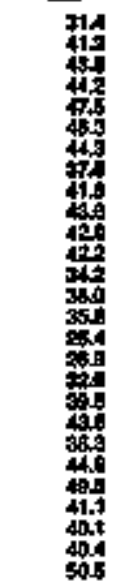 & 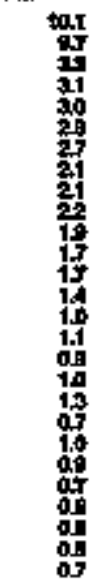 & 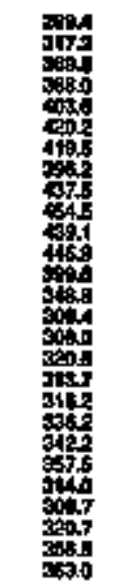 & 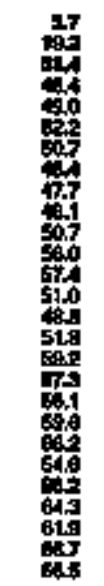 & 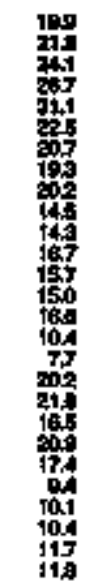 & 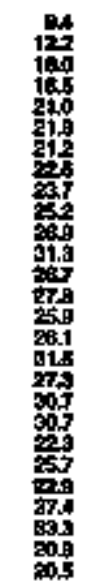 & 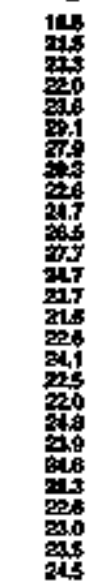 & 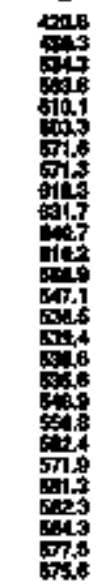 & 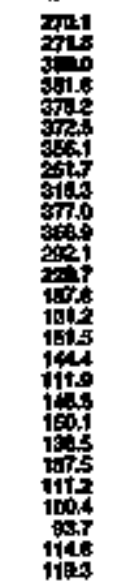 & 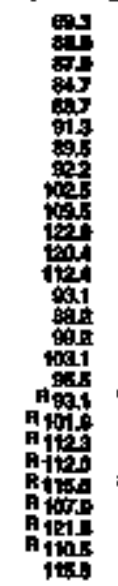 & 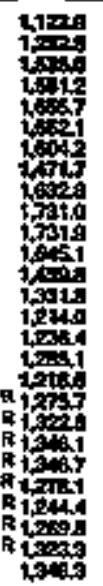 & 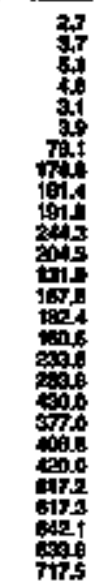 & 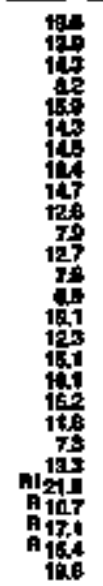 & 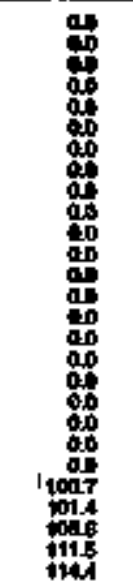 & 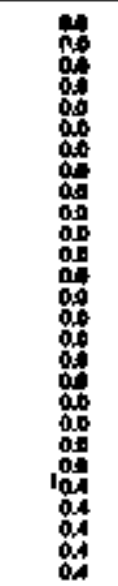 & 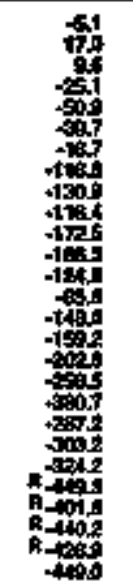 & 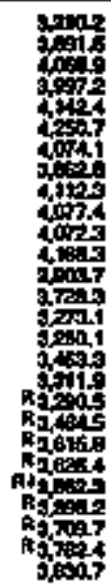 \\
\hline
\end{tabular}

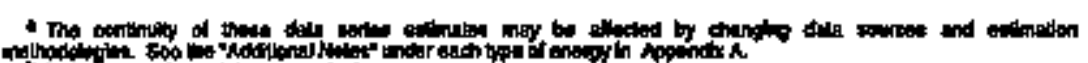

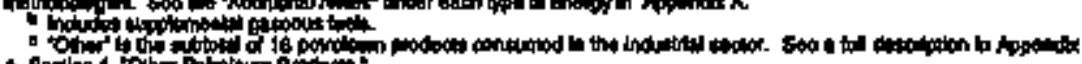

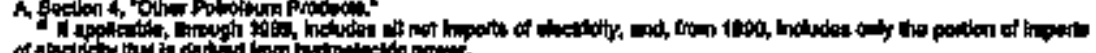

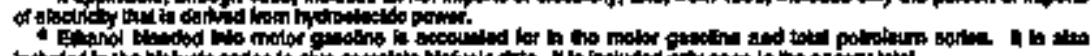

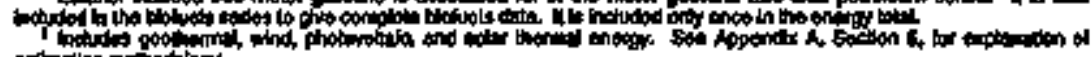

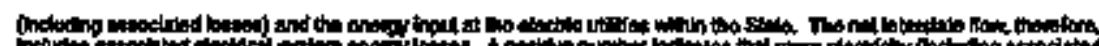

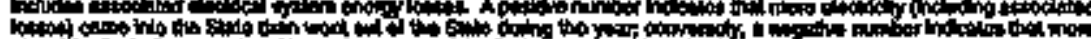

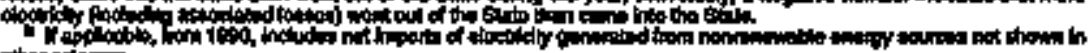

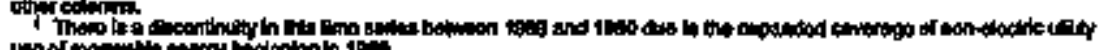
noortion

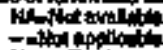

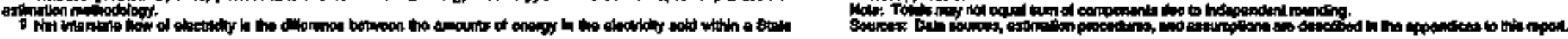


Table 246. Fesidentiat Energy Constimption Estinates, 1960, 1965, 1970-1994, Penmsyluania

트

\begin{tabular}{|c|c|c|c|c|c|c|c|c|c|c|c|c|c|c|}
\hline \multirow[b]{3}{*}{ Yest. } & \multicolumn{3}{|c|}{ Con } & \multirow{3}{*}{ 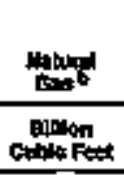 } & \multicolumn{4}{|c|}{ Putrolanin } & \multirow{3}{*}{ 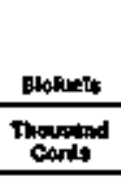 } & \multirow[b]{2}{*}{$504 x^{\circ}$} & \multirow[b]{2}{*}{ Enetritaly" } & \multirow[b]{2}{*}{ Emothr } & \multirow{3}{*}{ 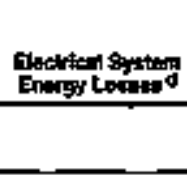 } & \multirow[b]{3}{*}{ Total } \\
\hline & 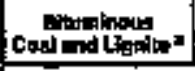 & Anthroelis " & Totat & & Dintifto & Kerpatinate & LQG & $70 t+1$ & & & & & & \\
\hline & \multicolumn{3}{|c|}{ Thoowand Sherd Tons } & & \multicolumn{4}{|c|}{ Thoutend operds } & & \multicolumn{3}{|c|}{ 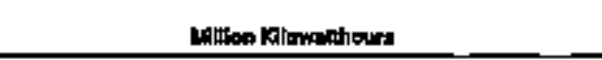 } & & \\
\hline 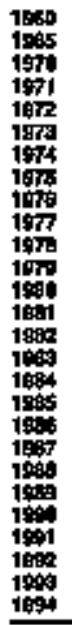 & 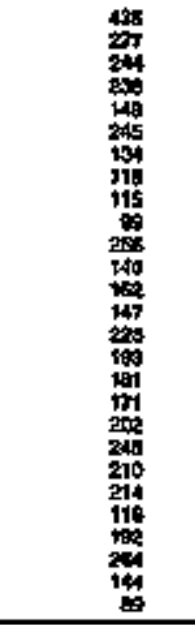 & 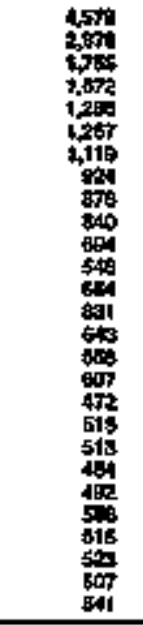 & 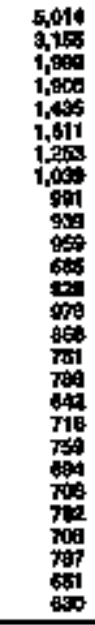 & 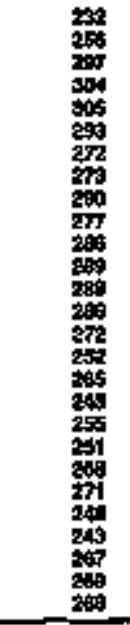 & 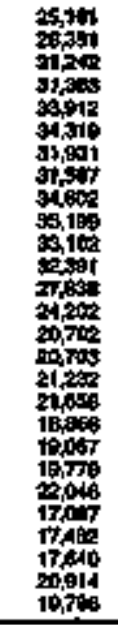 & 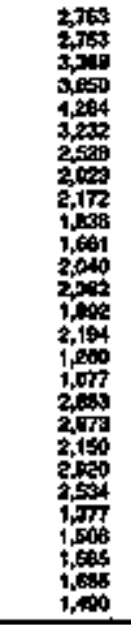 & 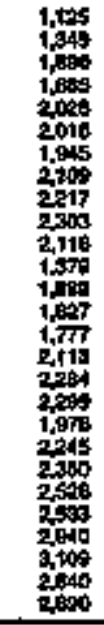 & 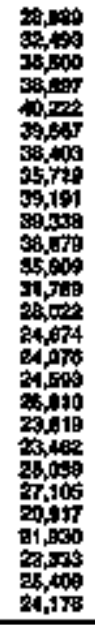 & 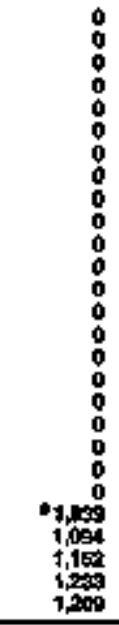 & $\begin{array}{r}0 \\
0 \\
0 \\
0 \\
0 \\
0 \\
0 \\
0 \\
0 \\
0 \\
0 \\
0 \\
0 \\
0 \\
0 \\
0 \\
0 \\
0 \\
0115 \\
117 \\
110 \\
10 \\
127 \\
\end{array}$ & 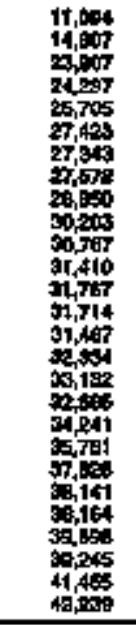 & $\begin{array}{l}= \\
= \\
z \\
= \\
= \\
= \\
z \\
= \\
= \\
z \\
z \\
z \\
z \\
=\end{array}$ & 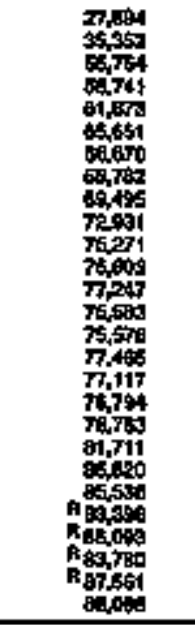 & $\begin{array}{l}= \\
= \\
= \\
= \\
= \\
= \\
= \\
= \\
= \\
= \\
= \\
= \\
z \\
=\end{array}$ \\
\hline \multicolumn{15}{|c|}{ Trillogen biv } \\
\hline 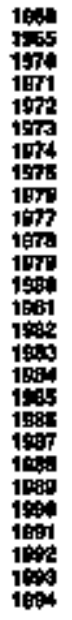 & 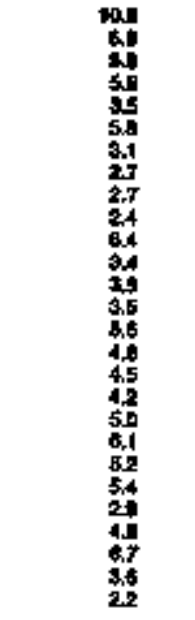 & 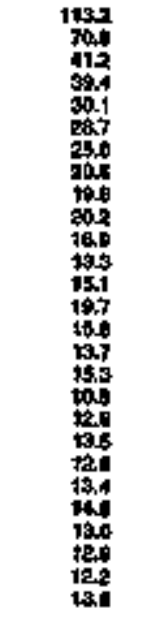 & 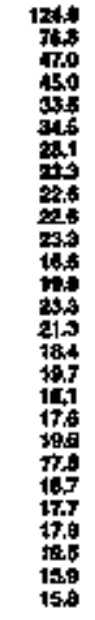 & 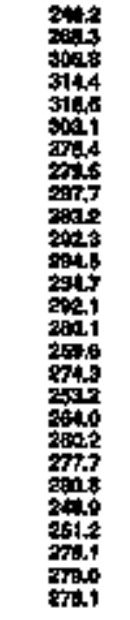 & 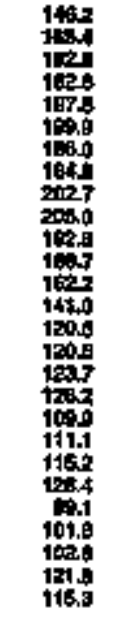 & 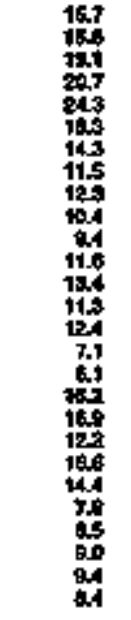 & 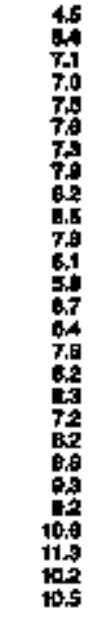 & 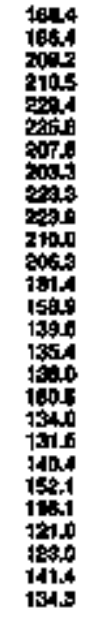 & 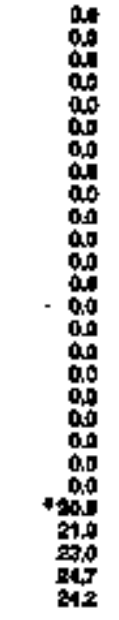 & 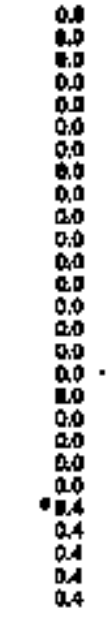 & 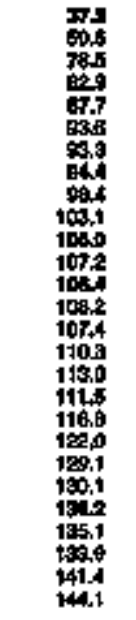 & 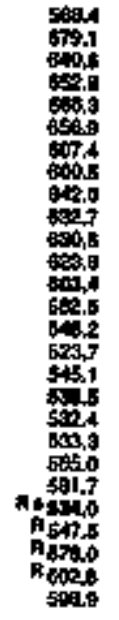 & 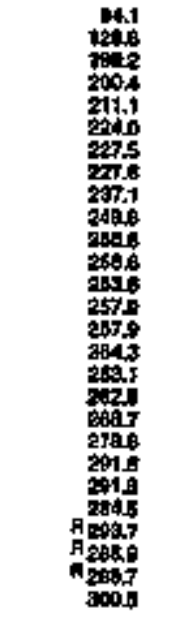 & 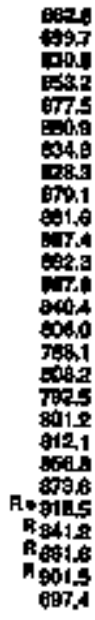 \\
\hline
\end{tabular}

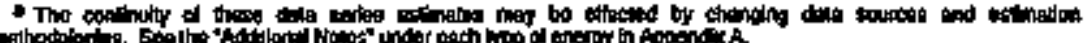

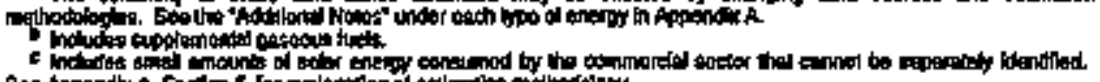

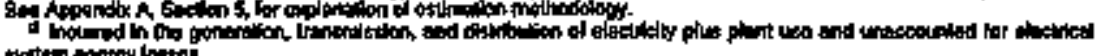

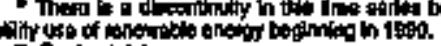

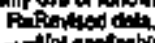

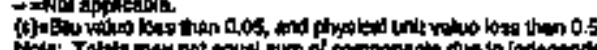


Table 247. Commerciad Eneryy Consumuption Estimates, 1950, 1965, 1970-1994, Pennsyivania

\begin{tabular}{|c|c|c|c|c|c|c|c|c|c|c|c|c|c|c|}
\hline \multirow[b]{3}{*}{ Vat } & \multicolumn{3}{|c|}{ ton } & \multirow{3}{*}{ 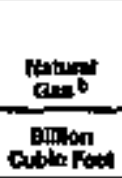 } & \multicolumn{6}{|c|}{ Patroloun } & \multirow[b]{2}{*}{ Eosiveling } & \multirow[b]{2}{*}{ 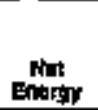 } & \multirow[b]{2}{*}{ 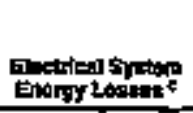 } & \multirow[b]{3}{*}{ Thald d } \\
\hline & 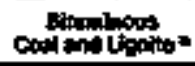 & 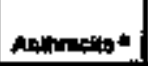 & Tot & & Daspont & 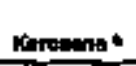 & epa. & cotor & 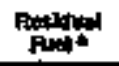 & 70tal & & & & \\
\hline & \multicolumn{3}{|c|}{ 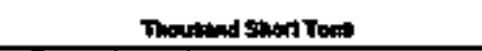 } & & \multicolumn{6}{|c|}{ modtend Britold } & \multicolumn{3}{|c|}{ Maten klomathaun } & \\
\hline 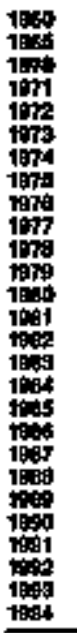 & 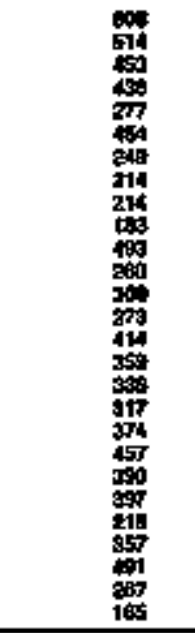 & 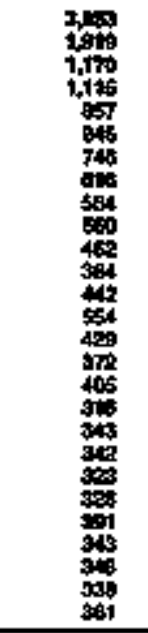 & 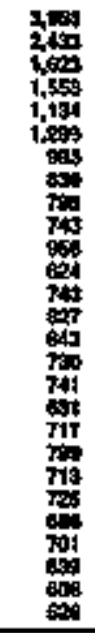 & 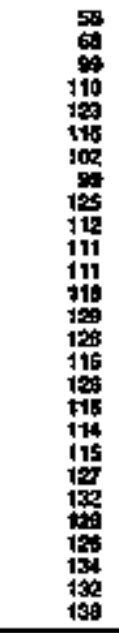 & 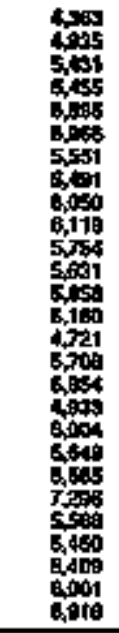 & 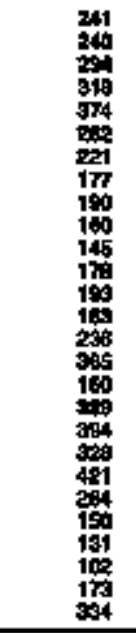 & 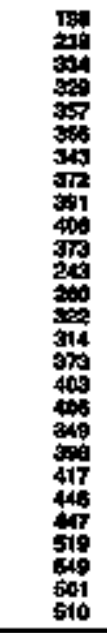 & 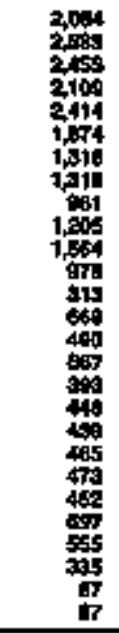 & 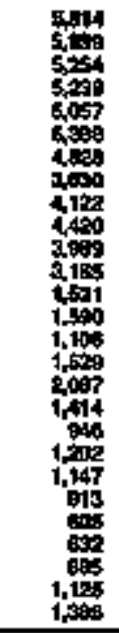 & 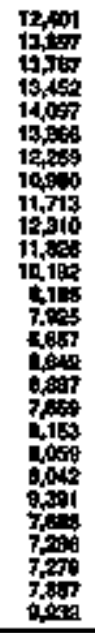 & 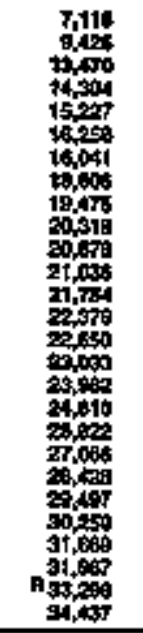 & $\begin{array}{l}= \\
= \\
= \\
= \\
= \\
= \\
= \\
= \\
= \\
= \\
z \\
= \\
= \\
= \\
=\end{array}$ & 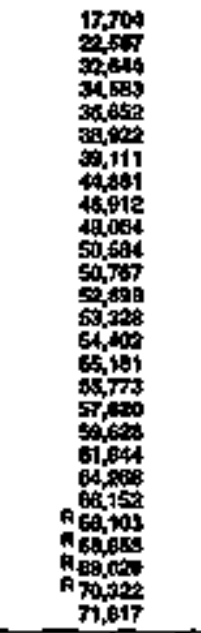 & $\begin{array}{l}z \\
= \\
z \\
= \\
= \\
= \\
= \\
= \\
= \\
z \\
z \\
= \\
= \\
=\end{array}$ \\
\hline \multicolumn{15}{|c|}{ Tnotleas bu } \\
\hline 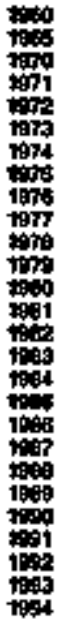 & 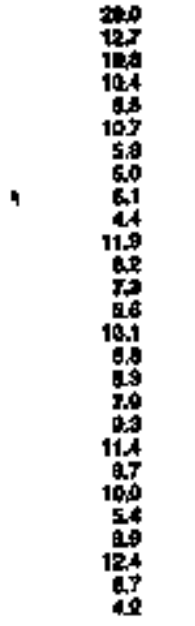 & 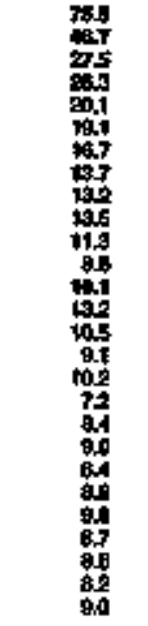 & 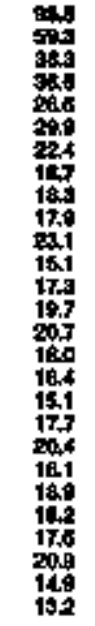 & 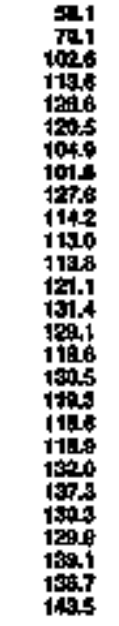 & 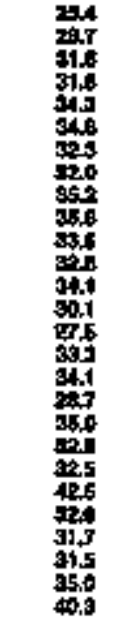 & 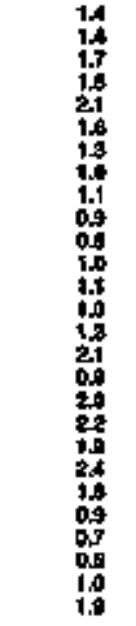 & 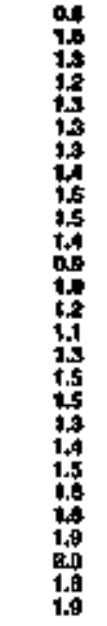 & 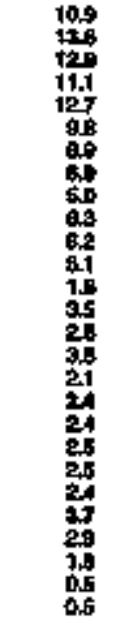 & 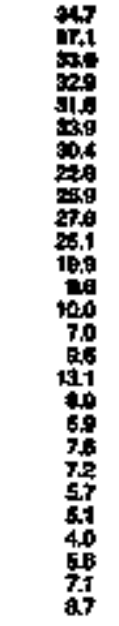 & 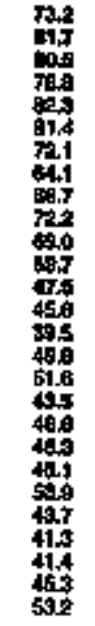 & 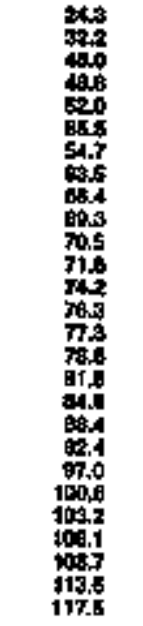 & 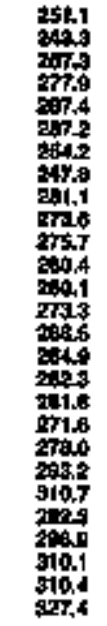 & 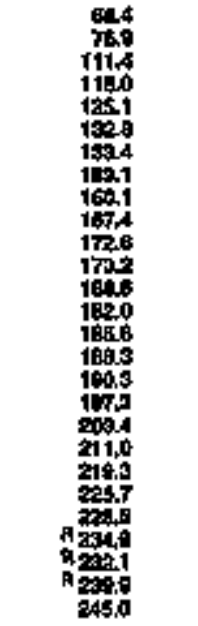 & 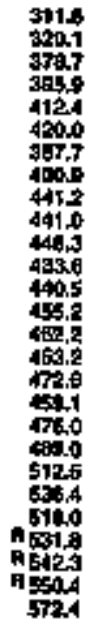 \\
\hline
\end{tabular}

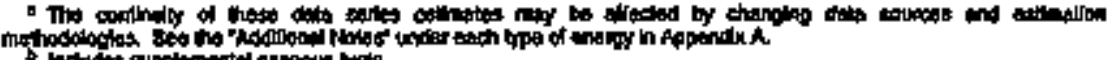

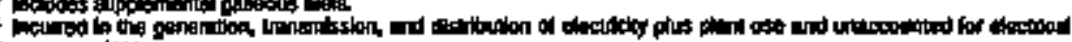

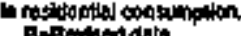

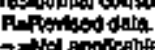

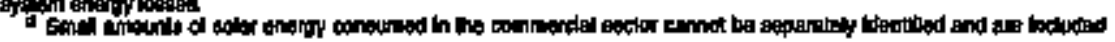

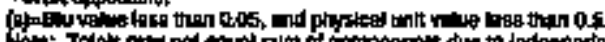

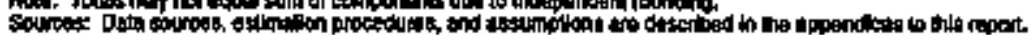


Table 249. Industrial Energy Consurnptipn Estimated, 1960, 1965, 1970-1994, Penusylvan|a

\begin{tabular}{|c|c|c|c|c|c|c|c|c|c|c|c|c|c|c|c|c|c|c|}
\hline \multirow[b]{3}{*}{$n=$} & \multirow[b]{2}{*}{ cont } & \multirow[b]{2}{*}{ Mature } & \multicolumn{9}{|c|}{ Potrofiann } & \multirow[b]{2}{*}{ | } & \multirow[b]{2}{*}{ alowere } & \multirow[b]{2}{*}{ onesul } & \multirow[b]{2}{*}{ | } & \multirow[b]{2}{*}{ int } & \multirow{3}{*}{ 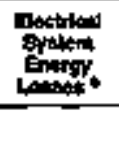 } & \multirow[b]{3}{*}{ Toent } \\
\hline & & & 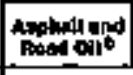 & 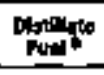 & Kenossana & 내: & LLenteands & 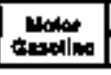 & 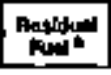 & tonerest & Toldi & & & & & & & \\
\hline & $\begin{array}{l}\text { Mrourtand } \\
\text { strot Toun }\end{array}$ & 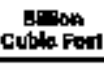 & \multicolumn{9}{|c|}{ 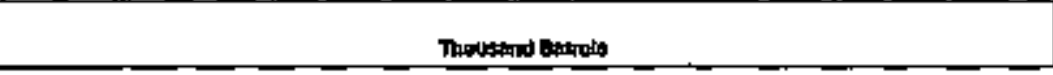 } & \multicolumn{5}{|c|}{ Hatoe Kllomithers } & & \\
\hline (1900 & 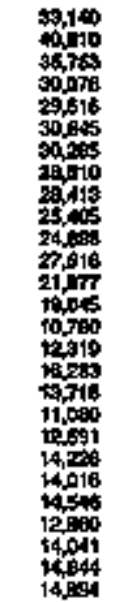 & 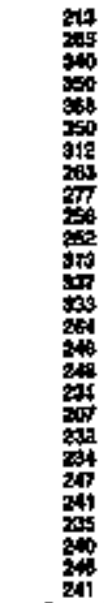 & 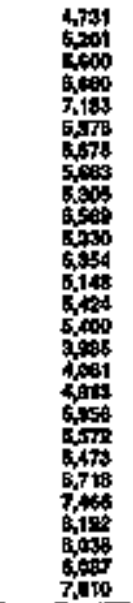 & 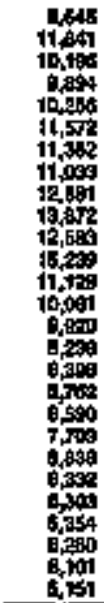 & 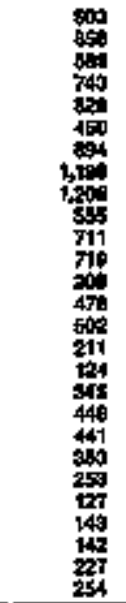 & 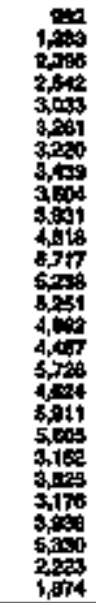 & 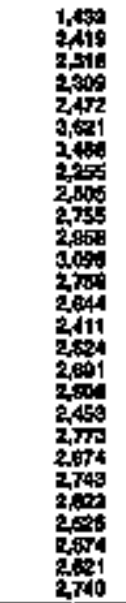 & 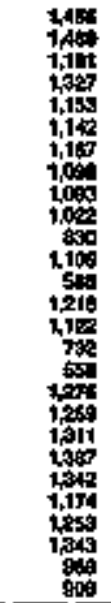 & 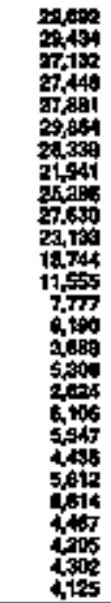 & 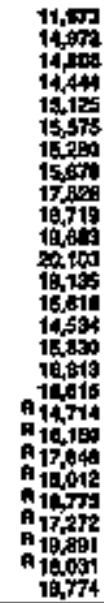 & 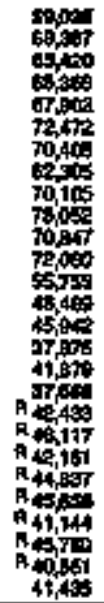 & 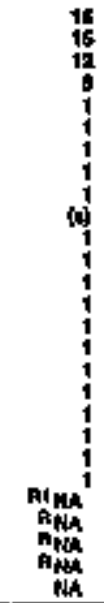 & 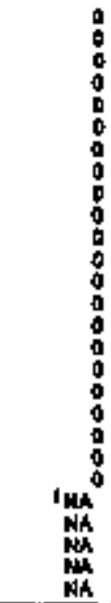 & 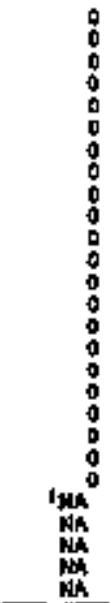 & 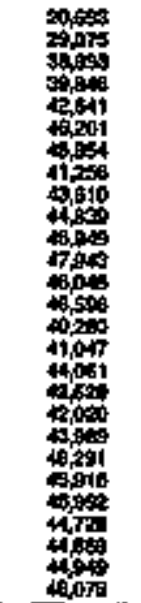 & $\begin{array}{l}z \\
\vdots \\
\vdots \\
\vdots \\
\vdots \\
z\end{array}$ & 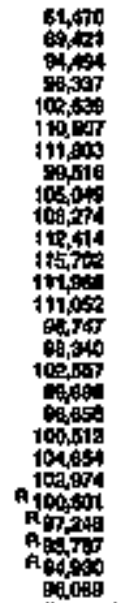 & \\
\hline
\end{tabular}

\begin{tabular}{|c|c|c|c|c|c|c|c|c|c|c|c|c|c|c|c|c|c|c|}
\hline 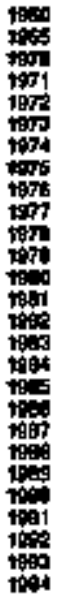 & 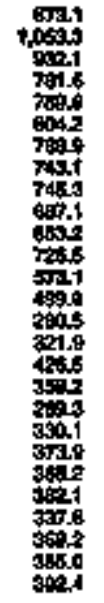 & 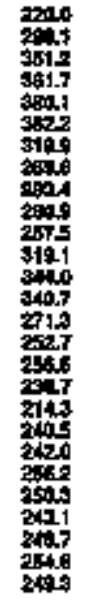 & 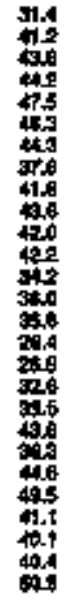 & 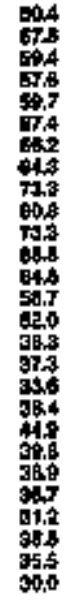 & 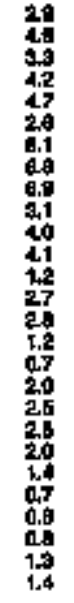 & 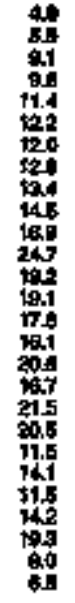 & 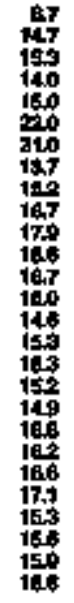 & 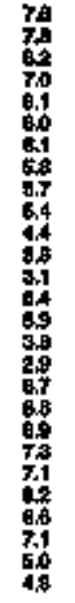 & 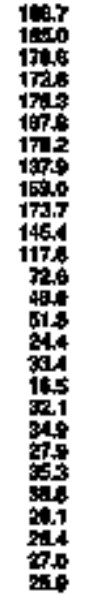 & 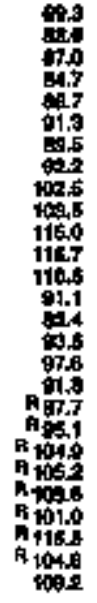 & 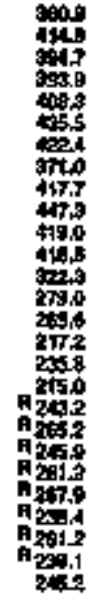 & 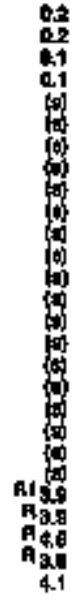 & 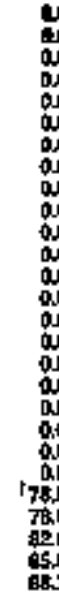 & & 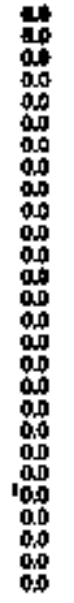 & 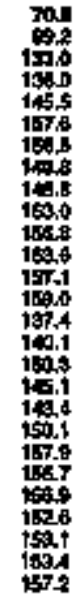 & 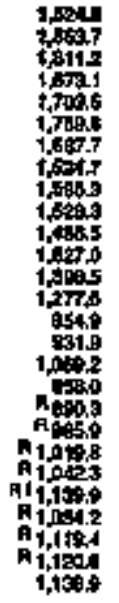 & 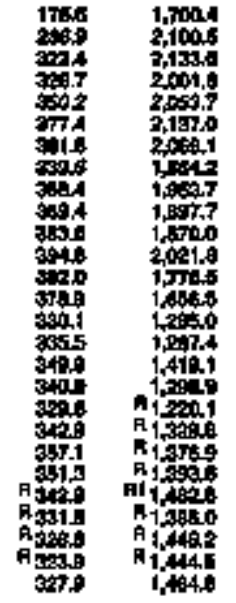 \\
\hline
\end{tabular}

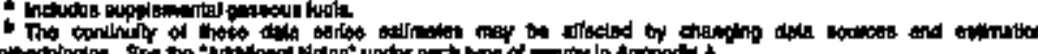

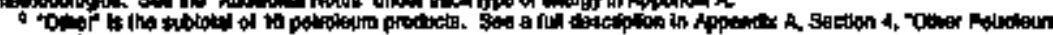

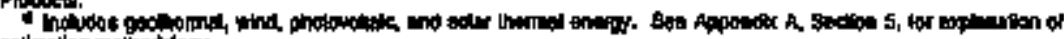

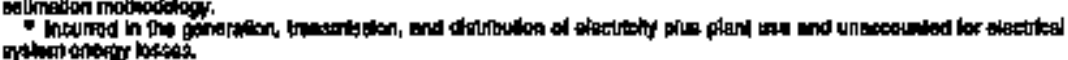

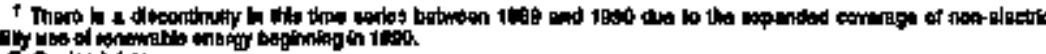

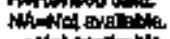

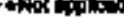

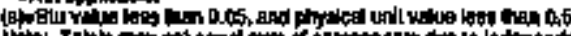

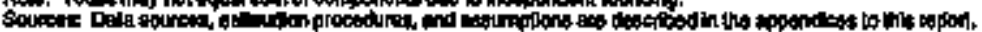


Teile 249. Transportation Energy Consumption Estifnatos, 1960, 1966, 1970-1994, Pennsylvanla

\begin{tabular}{|c|c|c|c|c|c|c|c|c|c|c|c|c|c|c|c|}
\hline \multirow[b]{3}{*}{ ras } & \multirow[b]{2}{*}{$\cos =$} & \multirow[b]{2}{*}{ Matit } & \multicolumn{8}{|c|}{ Promolusen } & \multirow{3}{*}{ 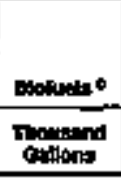 } & & \multirow[b]{2}{*}{ maty } & \multirow[b]{2}{*}{ 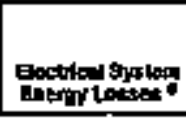 } & \multirow[b]{3}{*}{ rotal } \\
\hline & & & . & Matiluta & ret. & LPG* & workenins" & $\begin{array}{l}\text { Holwor } \\
\text { antom }\end{array}$ & 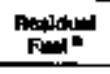 & Tod & & enstraty" & & & \\
\hline & stontinan: & 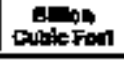 & \multicolumn{8}{|c|}{ mousmidemint } & & \multicolumn{3}{|c|}{ antion knowithour } & \\
\hline 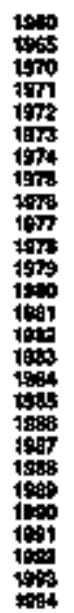 & 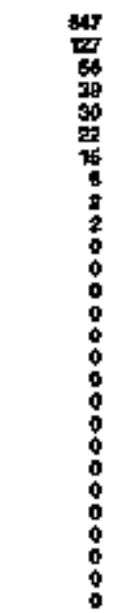 & 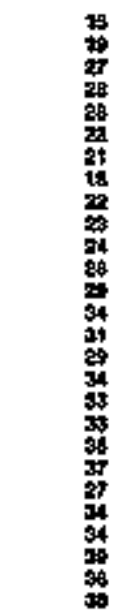 & 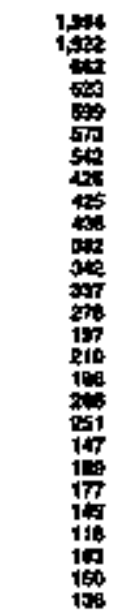 & 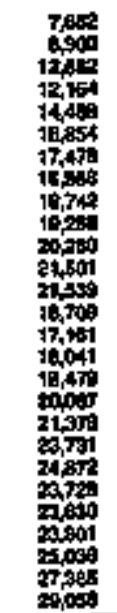 & 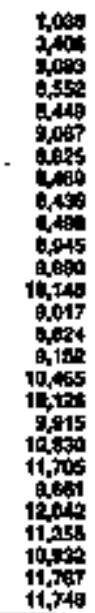 & 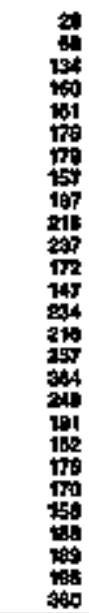 & 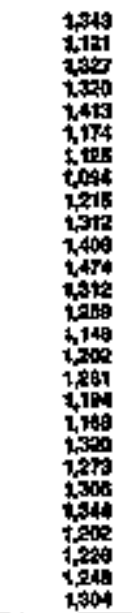 & 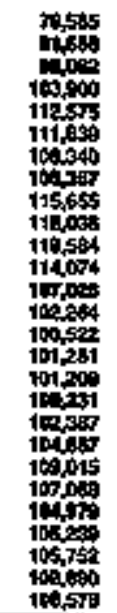 & 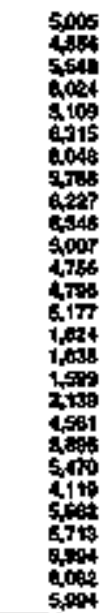 & 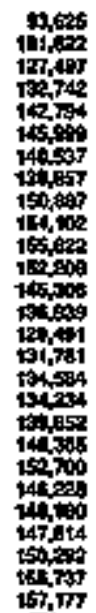 & 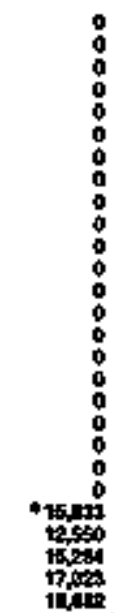 & 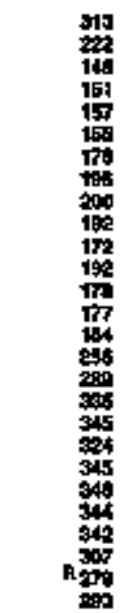 & $\begin{array}{l}\vdots \\
\vdots \\
\vdots \\
\vdots \\
\vdots \\
\vdots \\
\vdots \\
\vdots \\
\vdots \\
\vdots \\
\vdots \\
\vdots \\
\vdots \\
\vdots \\
-\end{array}$ & 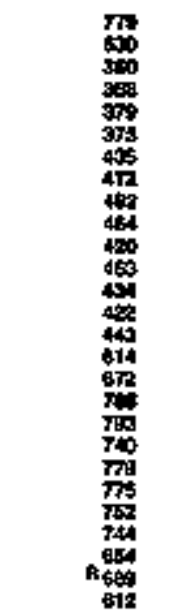 & $\begin{array}{l}\vdots \\
\vdots \\
\vdots \\
\vdots \\
\vdots \\
\vdots \\
\vdots \\
\vdots \\
\vdots \\
\vdots \\
\vdots\end{array}$ \\
\hline \multicolumn{16}{|c|}{ 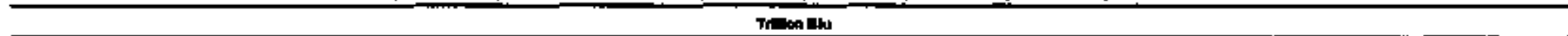 } \\
\hline 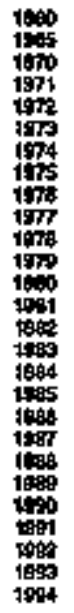 & 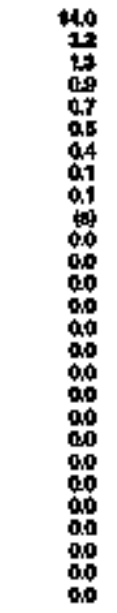 & 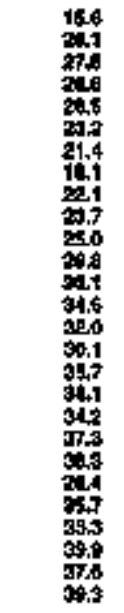 & 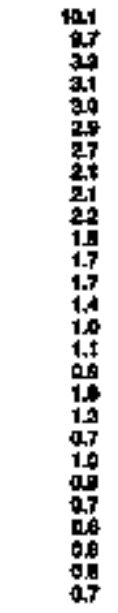 & 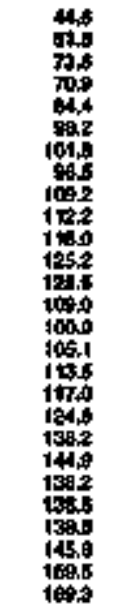 & 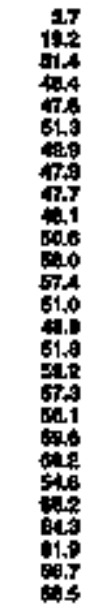 & 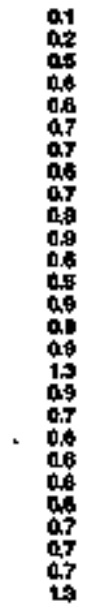 & 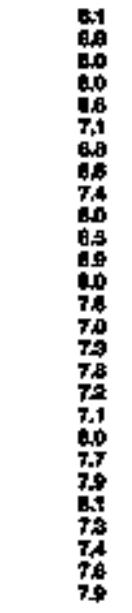 & 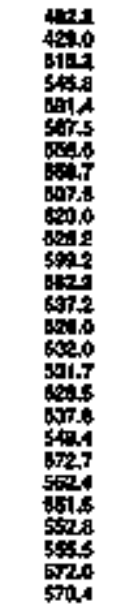 & 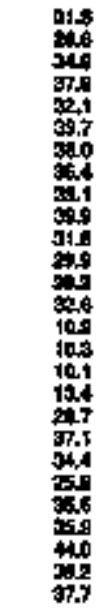 & 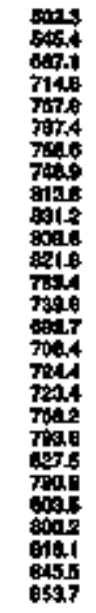 & 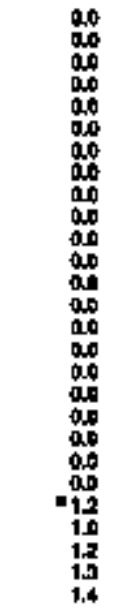 & 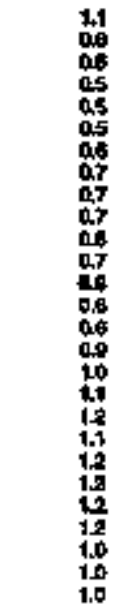 & 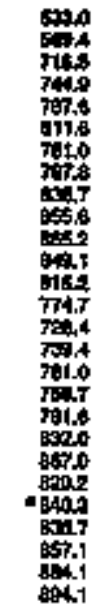 & 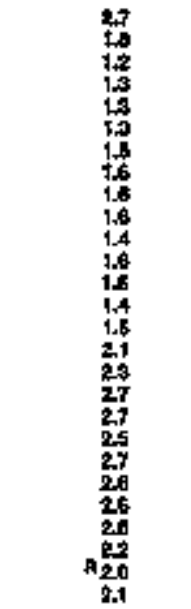 & 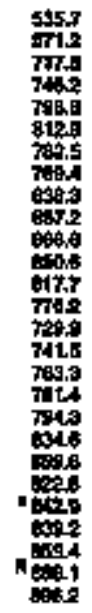 \\
\hline
\end{tabular}

$\mathbf{P}$
$\mathbf{E}$
$\mathbf{N}$
$\mathbf{N}$
$\mathbf{S}$
$\mathbf{Y}$
$\mathbf{L}$
$\mathbf{V}$
$\mathbf{A}$
$\mathbf{N}$
$\mathbf{I}$
$\mathbf{A}$

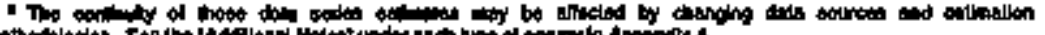

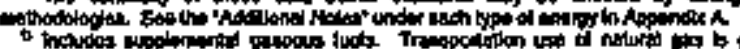

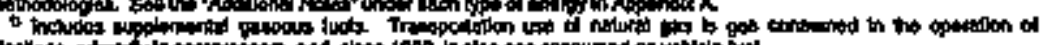

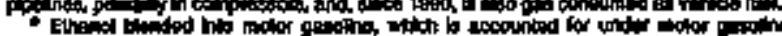

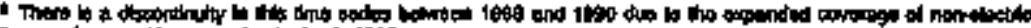

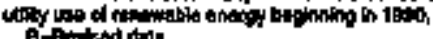

A-

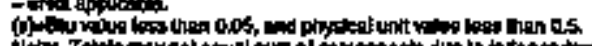

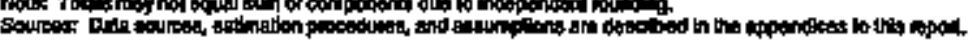

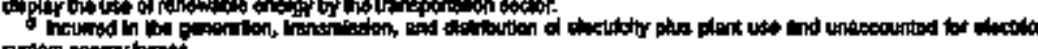


Table 250. Eshlmates ol Energy Input at Elects]c Utifitles, 1980, 1965, 1970-1994, Pennsylvania

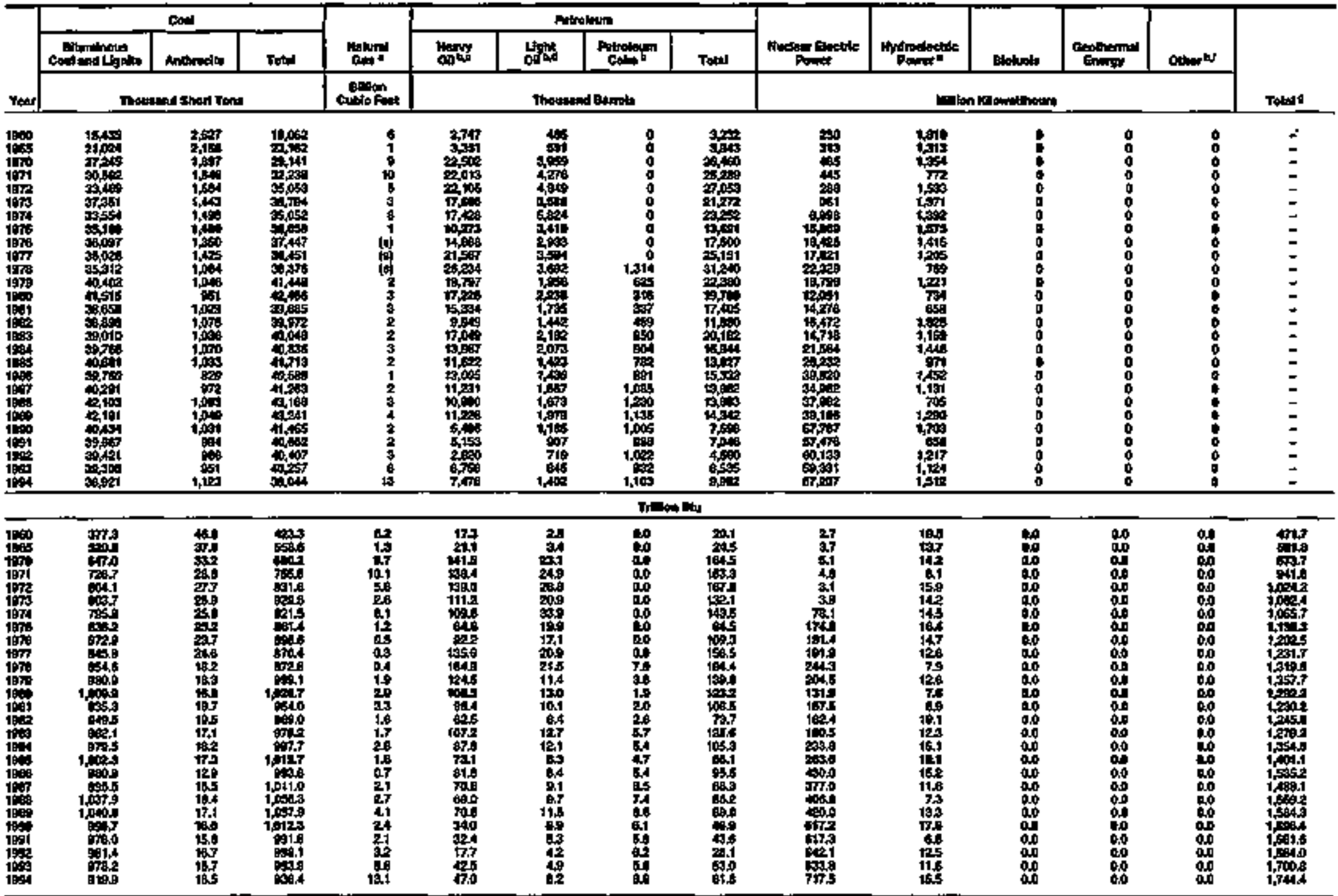

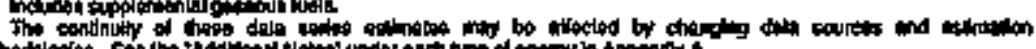

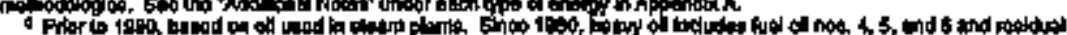

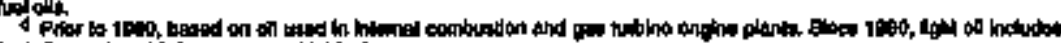

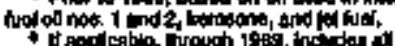

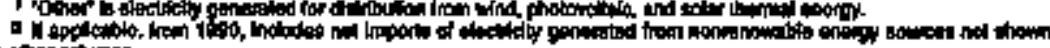
ind

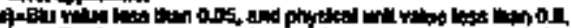

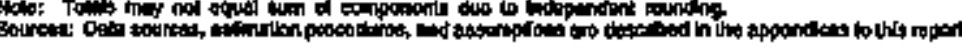


Table 251. Energy Consumption Estimattes by Spurce, 1960, 1965, 1970-1994, Rhode Istand

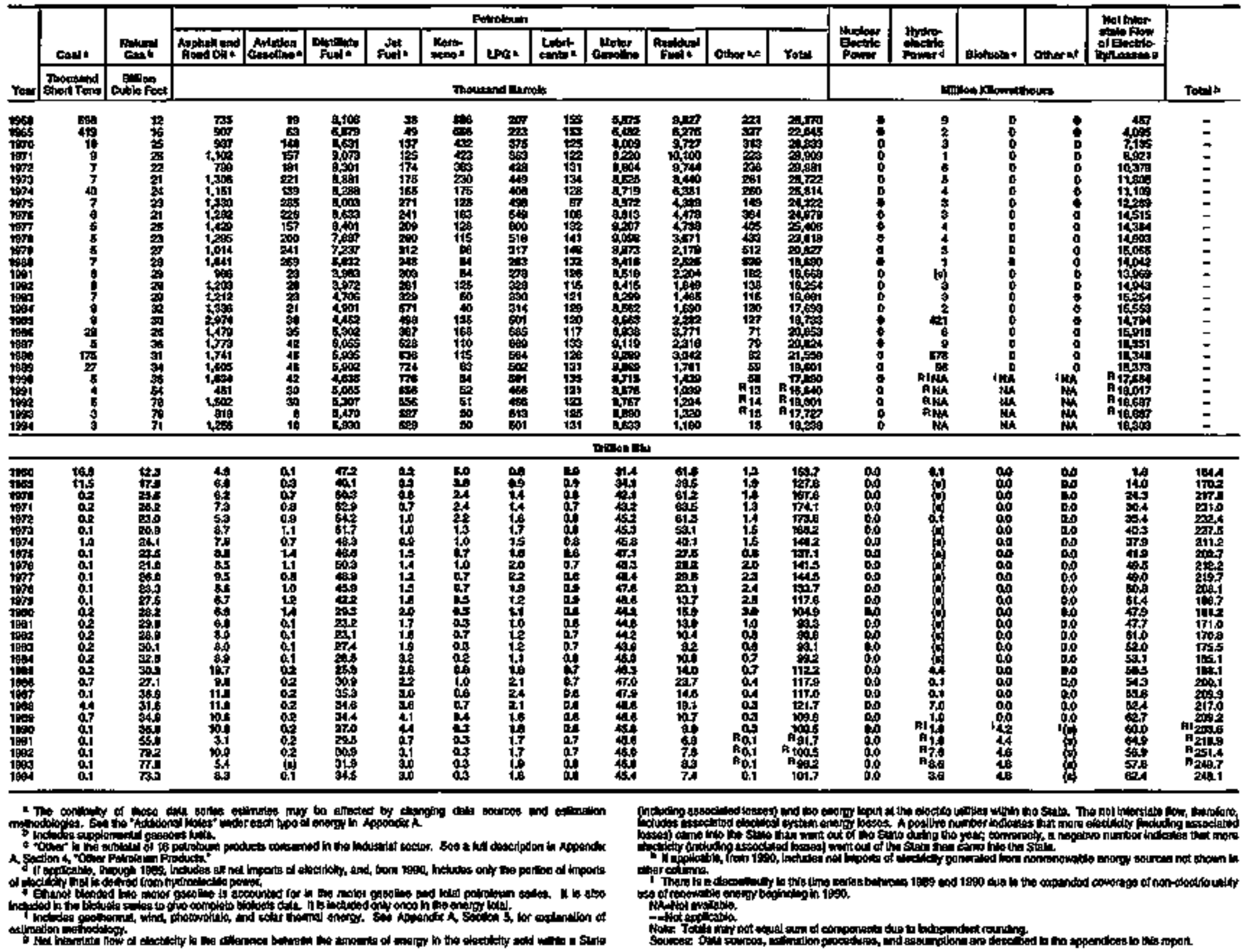


A Table 252. Rosidenllal Energy Consumption Estimales, 1960, 1965, 1970-1984, Rhode 1sland

\begin{tabular}{|c|c|c|c|c|c|c|c|c|c|c|c|c|c|c|}
\hline \multirow[b]{3}{*}{ real } & \multicolumn{3}{|c|}{ cont } & \multirow[b]{2}{*}{$\operatorname{lom}^{6}$} & \multicolumn{4}{|c|}{ Patrotount } & \multirow{3}{*}{ 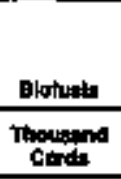 } & & \multirow[b]{2}{*}{ Bestritity an } & \multirow[b]{2}{*}{ Entrigr } & \multirow{3}{*}{ 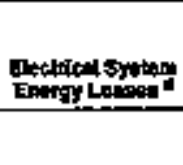 } & \multirow[b]{3}{*}{ Tont } \\
\hline & A & Andrateln: I & Tomat & & Pations & $x$ & Leat & Tort & & somen" & & & & \\
\hline & \multicolumn{2}{|c|}{ Thorand Stont Tone } & & a & \multicolumn{4}{|c|}{ Theoriend banth } & & \multicolumn{3}{|c|}{ 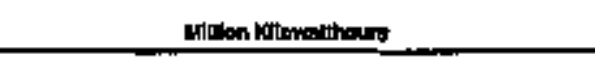 } & & \\
\hline 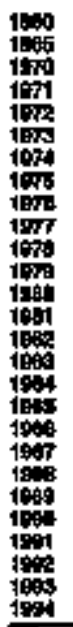 & $\begin{array}{l}0 \\
: \\
0 \\
0 \\
0 \\
0 \\
0 \\
0 \\
0 \\
0 \\
0 \\
0 \\
0 \\
0 \\
0 \\
0 \\
0 \\
0 \\
0 \\
0 \\
0 \\
0 \\
0\end{array}$ & $\begin{array}{c}\mathbf{1 2} \\
\mathbf{E} \\
\mathbf{5} \\
\mathbf{5} \\
\mathbf{3} \\
\mathbf{3} \\
\mathbf{3} \\
\mathbf{3} \\
\mathbf{2} \\
\mathbf{2} \\
\mathbf{2} \\
\mathbf{2} \\
\mathbf{2} \\
\mathbf{4} \\
\mathbf{5} \\
\mathbf{4} \\
\mathbf{3} \\
\mathbf{3} \\
\mathbf{3} \\
\mathbf{2} \\
\mathbf{2} \\
\mathbf{2} \\
\mathbf{3} \\
\mathbf{2} \\
\mathbf{3} \\
\mathbf{2} \\
\mathbf{2}\end{array}$ & $\begin{array}{c}12 \\
\mathbf{E} \\
5 \\
5 \\
3 \\
3 \\
3 \\
3 \\
3 \\
2 \\
2 \\
2 \\
2 \\
1 \\
2 \\
2 \\
5 \\
5 \\
4 \\
4 \\
3 \\
3 \\
2 \\
2 \\
2 \\
5 \\
2 \\
3 \\
3 \\
2 \\
2\end{array}$ & 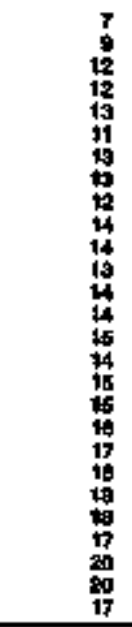 & 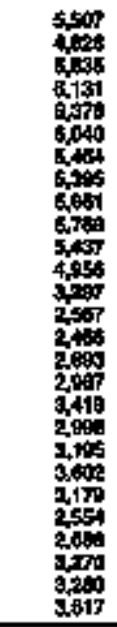 & 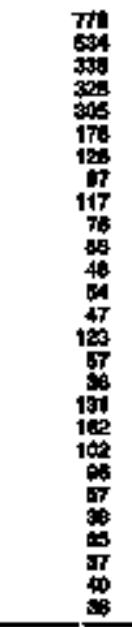 & 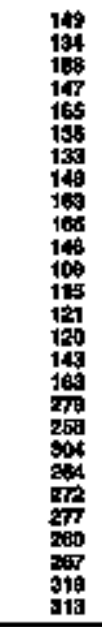 & 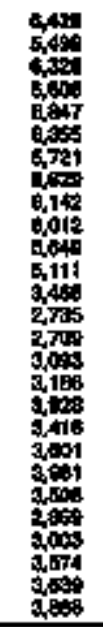 & 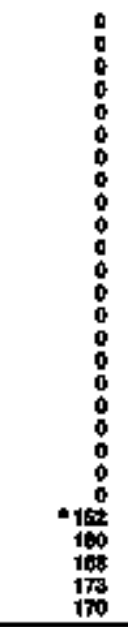 & $\begin{array}{r}0 \\
0 \\
0 \\
0 \\
0 \\
0 \\
0 \\
0 \\
0 \\
0 \\
0 \\
0 \\
0 \\
0 \\
0 \\
0 \\
0 \\
0 \\
0 \\
0 \\
0 \\
0 \\
0 \\
10 \\
11 \\
11 \\
11 \\
11\end{array}$ & 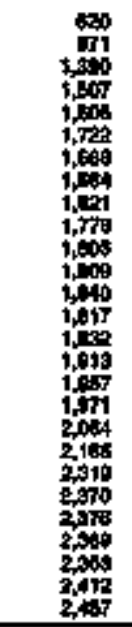 & $\begin{array}{l}\vdots \\
\vdots \\
\vdots \\
\vdots \\
\vdots \\
\vdots \\
\vdots \\
\vdots \\
\vdots \\
\vdots \\
\vdots \\
\vdots \\
\vdots\end{array}$ & 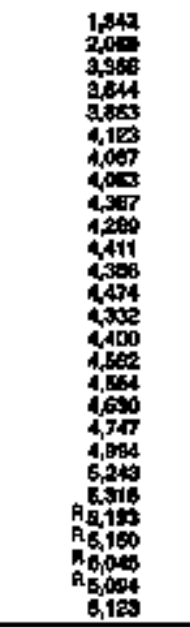 & $\begin{array}{l}\vdots \\
\vdots \\
\vdots \\
\vdots \\
\vdots \\
\vdots \\
\vdots \\
\vdots \\
\vdots \\
\vdots \\
\vdots \\
\vdots \\
\vdots\end{array}$ \\
\hline \multicolumn{15}{|c|}{ Tryon ben } \\
\hline 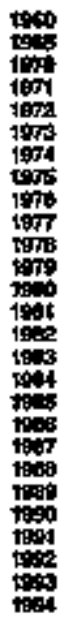 & 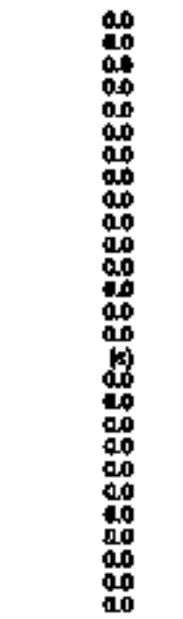 & 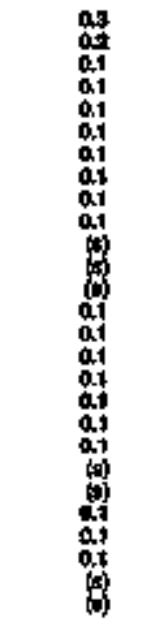 & 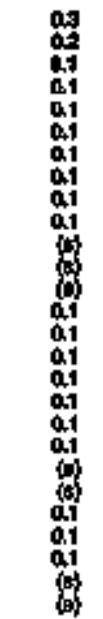 & 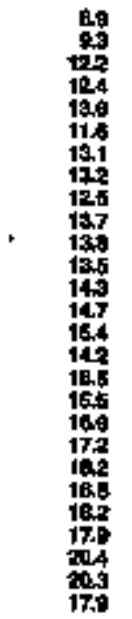 & 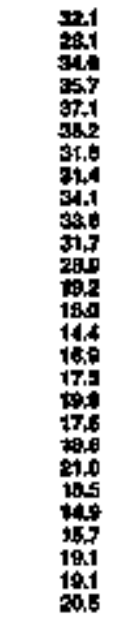 & 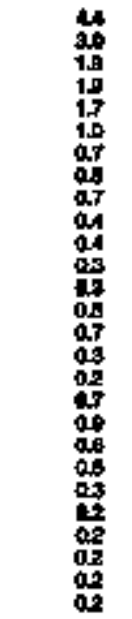 & 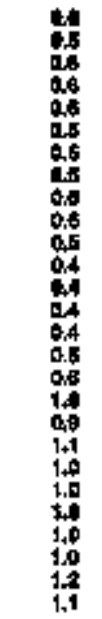 & 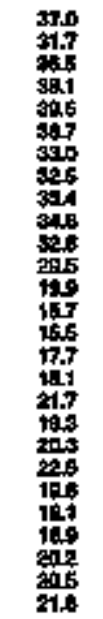 & 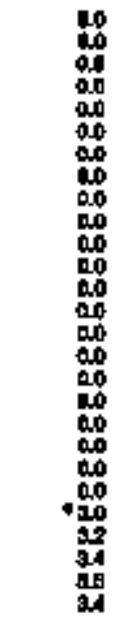 & 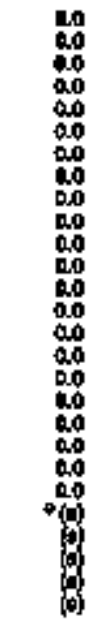 & 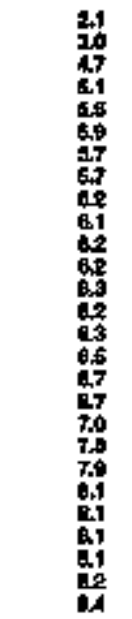 & 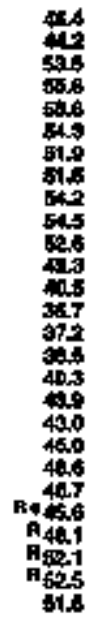 & 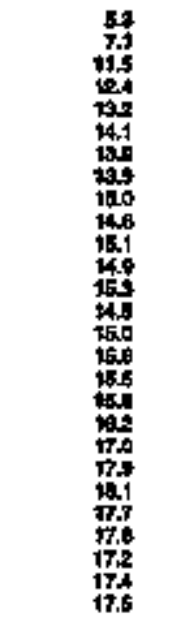 & 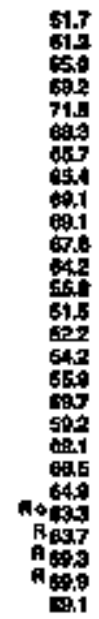 \\
\hline
\end{tabular}

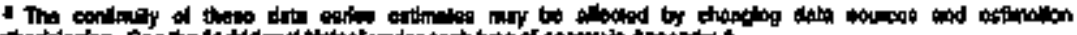

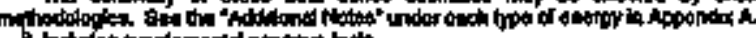

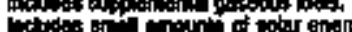

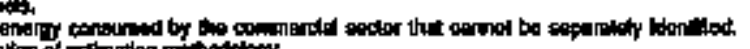

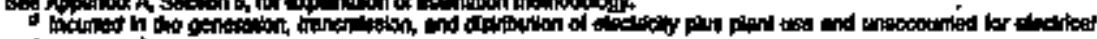

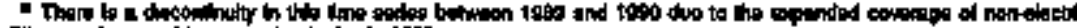

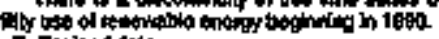

hinewiod diva

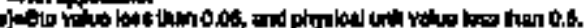


Table 253. Commerclal Energy Consumption Esilmates, 1960, 1965, 1970-1994, Rhode lsland

\begin{tabular}{|c|c|c|c|c|c|c|c|c|c|c|c|c|c|c|c|}
\hline \multirow[b]{3}{*}{ rat } & \multicolumn{3}{|c|}{ cond } & \multirow{3}{*}{ 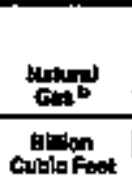 } & \multicolumn{6}{|c|}{ Mimoluman } & \multirow[b]{2}{*}{ Exethen" } & \multirow[b]{2}{*}{$\min$} & \multirow[b]{2}{*}{ 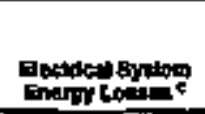 } & \multirow{3}{*}{\multicolumn{2}{|c|}{ Ton: }} \\
\hline & 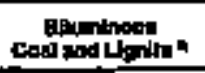 & Anementan" & $\operatorname{Tot}$ & & 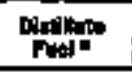 & Knoton: - & LPaA & 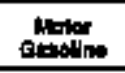 & Funtental & Totel & & & & & \\
\hline & \multicolumn{3}{|c|}{ mouvend ghort tons: } & & \multicolumn{6}{|c|}{ mounund Bamere } & \multicolumn{3}{|c|}{ 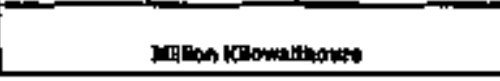 } & & \\
\hline 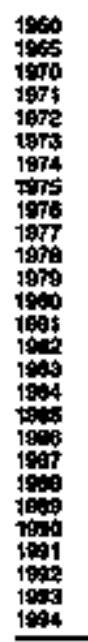 & $\begin{array}{l}0 \\
0 \\
0 \\
0 \\
0 \\
0 \\
0 \\
0 \\
0 \\
0 \\
0 \\
0 \\
0 \\
0 \\
1 \\
0 \\
0 \\
0 \\
0 \\
0 \\
0 \\
0 \\
0 \\
0 \\
0 \\
0\end{array}$ & 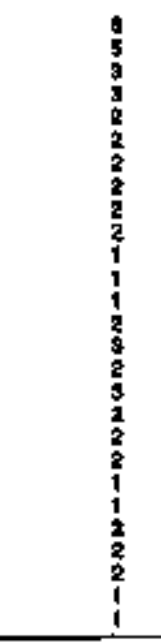 & $\begin{array}{l}9 \\
5 \\
3 \\
3 \\
2 \\
2 \\
2 \\
2 \\
2 \\
2 \\
2 \\
\frac{2}{1} \\
1 \\
1 \\
2 \\
3 \\
3 \\
3 \\
2 \\
2 \\
2 \\
2 \\
1 \\
2 \\
2 \\
2 \\
1 \\
1 \\
\end{array}$ & $\begin{array}{l}2 \\
3 \\
5 \\
5 \\
8 \\
5 \\
5 \\
4 \\
4 \\
3 \\
3 \\
5 \\
8 \\
7 \\
7 \\
7 \\
7 \\
7 \\
0 \\
6 \\
6 \\
6 \\
6 \\
0 \\
0 \\
0 \\
0 \\
12\end{array}$ & 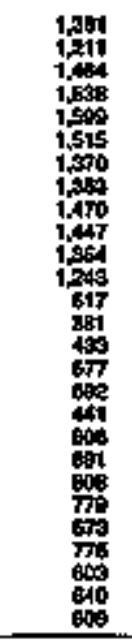 & $\begin{array}{l}17 \\
12 \\
7 \\
7 \\
7 \\
3 \\
3 \\
2 \\
3 \\
3 \\
7 \\
5 \\
0 \\
1 \\
2 \\
3 \\
3 \\
4 \\
4 \\
3 \\
3 \\
5 \\
2 \\
3 \\
3 \\
2 \\
6 \\
\end{array}$ & 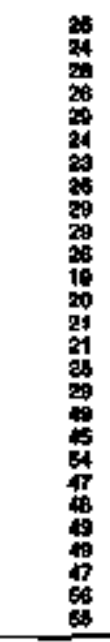 & 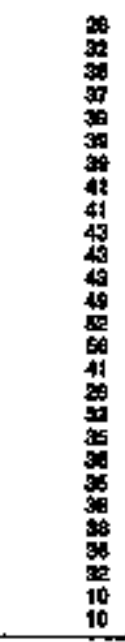 & 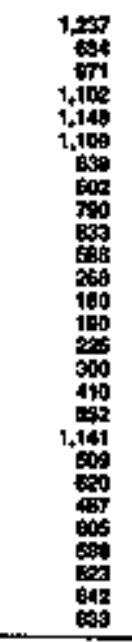 & 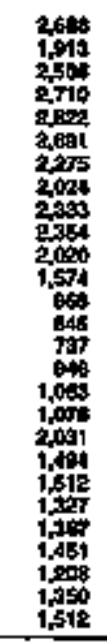 & 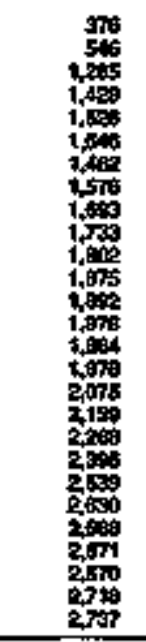 & $\begin{array}{l}\vdots \\
\vdots \\
\vdots \\
\vdots \\
\vdots \\
\vdots \\
\vdots \\
\vdots \\
\vdots \\
\vdots \\
\vdots \\
\vdots\end{array}$ & 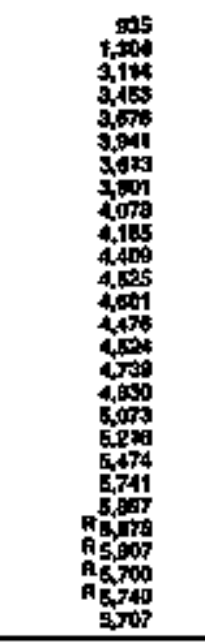 & $\begin{array}{l}\vdots \\
\vdots \\
\vdots \\
\vdots \\
\vdots \\
\vdots \\
\vdots \\
\vdots \\
\vdots \\
\vdots \\
\vdots \\
\vdots \\
\vdots\end{array}$ & \\
\hline \multicolumn{15}{|c|}{$\pi T_{10}$} & \\
\hline 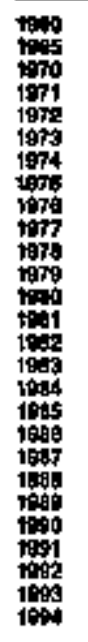 & 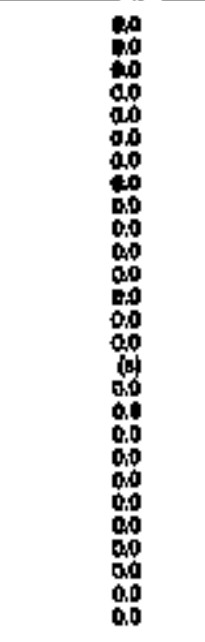 & 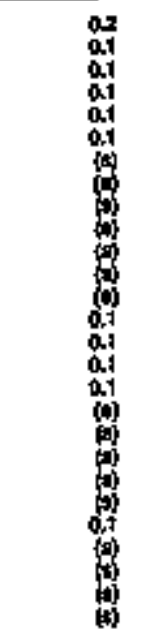 & 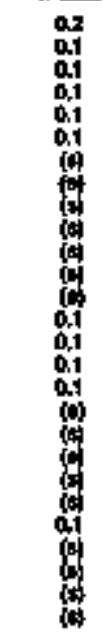 & 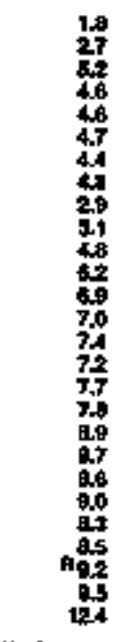 & 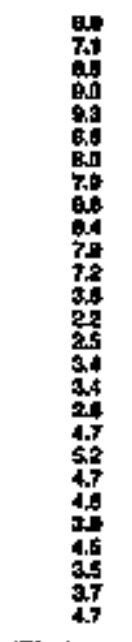 & 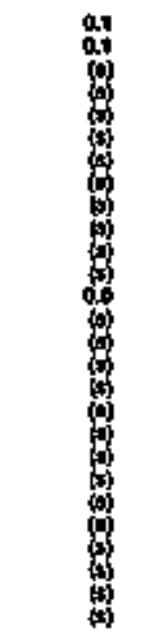 & 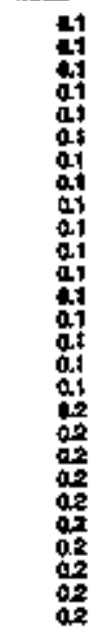 & 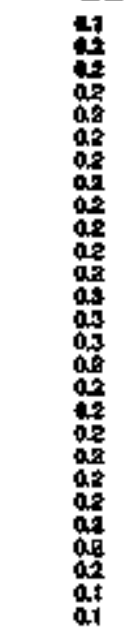 & 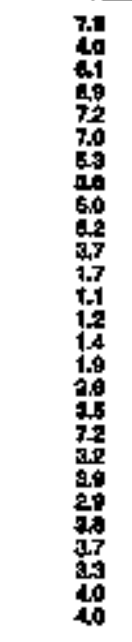 & 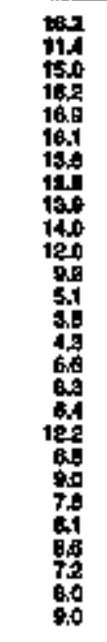 & 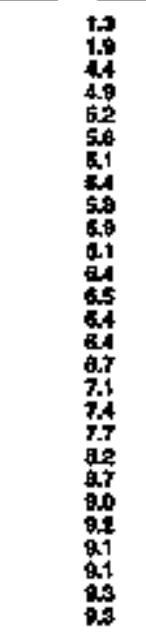 & 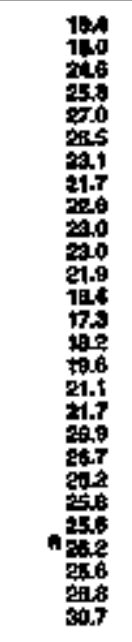 & 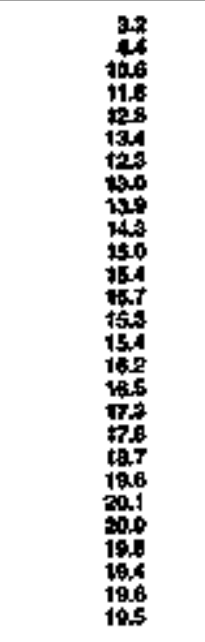 & 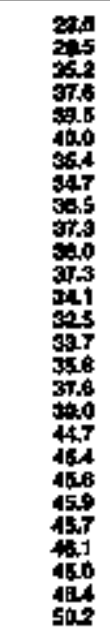 & \\
\hline \multirow{2}{*}{\multicolumn{15}{|c|}{ 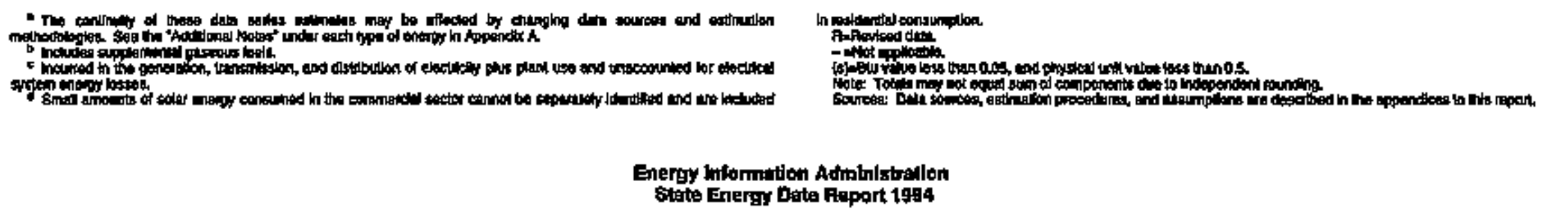 }} & \\
\hline & & & & & & & & & & & & & & & 265 \\
\hline
\end{tabular}




\begin{tabular}{|c|c|c|c|c|c|c|c|c|c|c|c|c|c|c|c|c|c|c|}
\hline \multirow[b]{3}{*}{ reat } & \multirow[b]{2}{*}{ cost } & \multirow[b]{2}{*}{ disti } & \multicolumn{9}{|c|}{ Nrollon: } & \multirow[b]{2}{*}{ 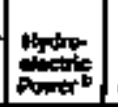 } & \multirow[b]{2}{*}{ Etoknh ! } & \multirow[b]{2}{*}{ 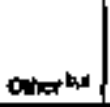 } & \multirow[b]{2}{*}{ 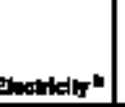 } & \multirow[b]{2}{*}{ Net } & \multirow{2}{*}{ 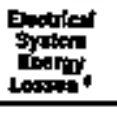 } & \multirow[b]{3}{*}{ Tat } \\
\hline & & & Aaphosiond & 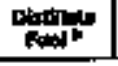 & 我 & swe & 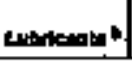 & 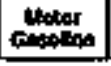 & Aosteted & Onter & Totes & & & & & & & \\
\hline & 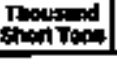 & 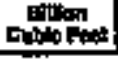 & \multicolumn{9}{|c|}{ 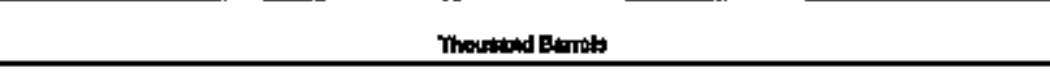 } & \multicolumn{6}{|c|}{ 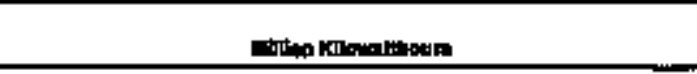 } & \\
\hline 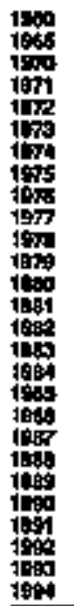 & 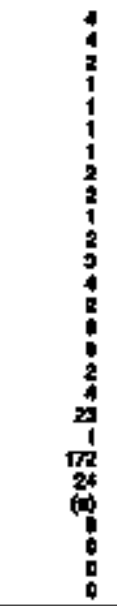 & 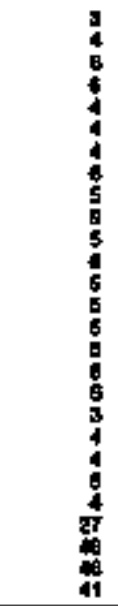 & 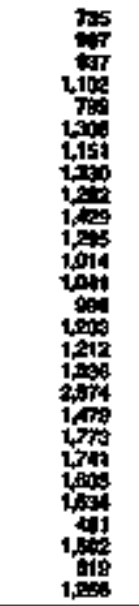 & 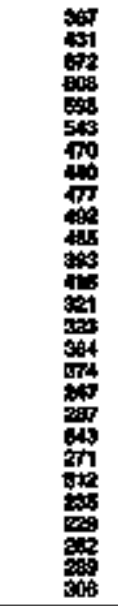 & 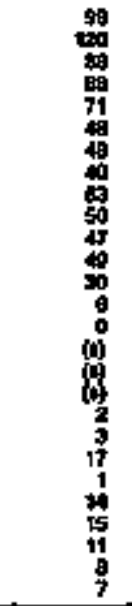 & 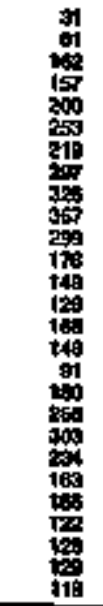 & 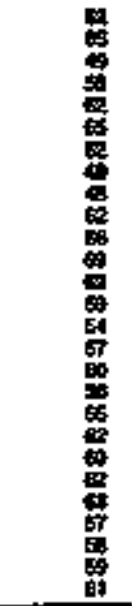 & 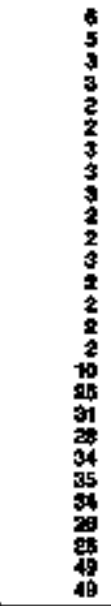 & 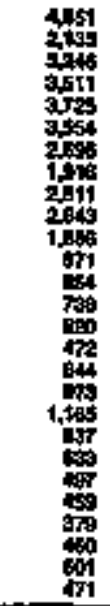 & 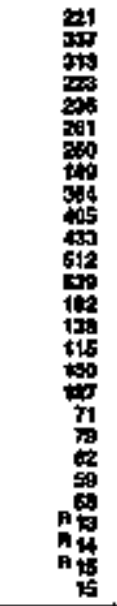 & 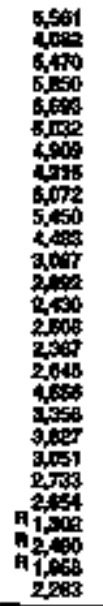 & 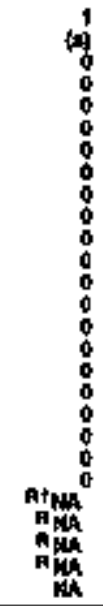 & $\begin{array}{l}0 \\
\vdots \\
0 \\
\vdots \\
\vdots\end{array}$ & 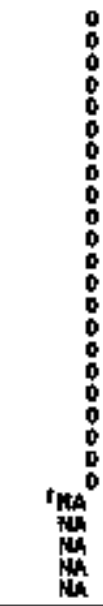 & 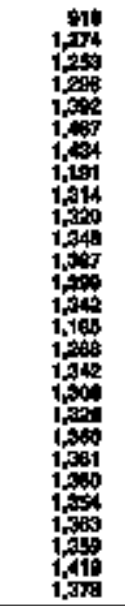 & $\begin{array}{l}z \\
z \\
z \\
z \\
z \\
z \\
z \\
z \\
\vdots \\
z \\
z \\
z\end{array}$ & 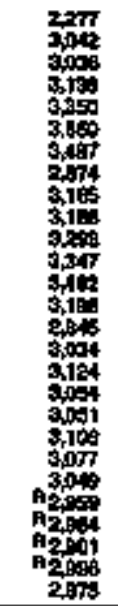 & z \\
\hline
\end{tabular}

\begin{tabular}{|c|c|c|c|c|c|c|c|c|c|c|c|c|c|c|c|c|c|c|}
\hline 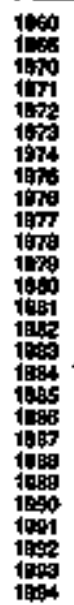 & 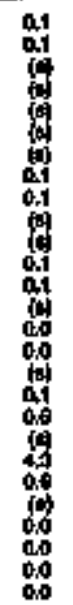 & 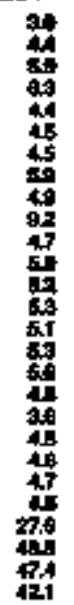 & 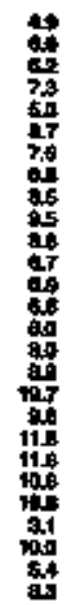 & 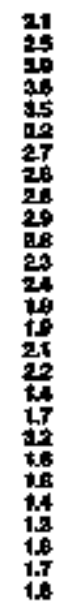 & 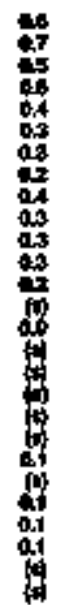 & 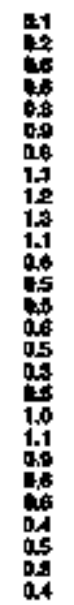 & 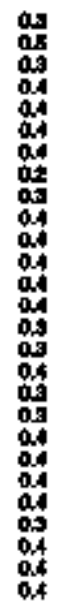 & 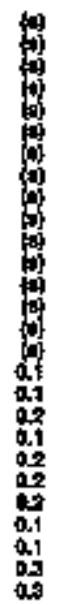 & 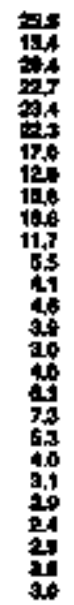 & 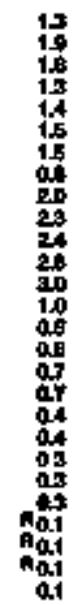 & 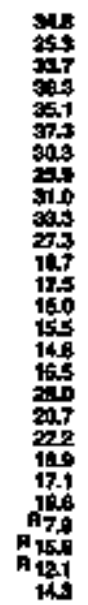 & 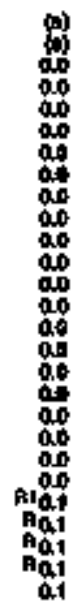 & 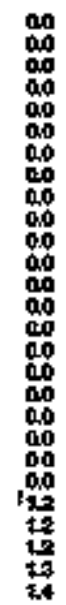 & 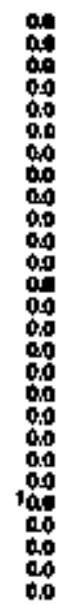 & 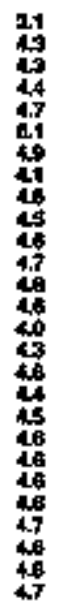 & 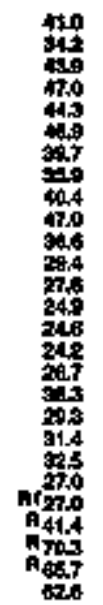 & 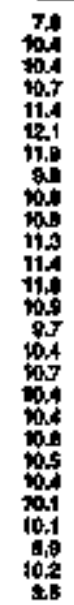 & 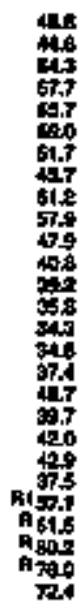 \\
\hline
\end{tabular}

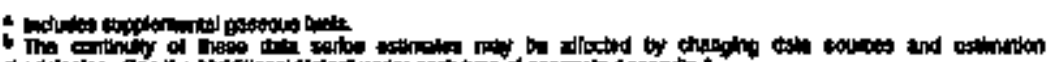

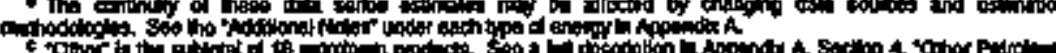

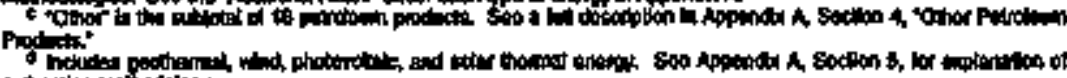

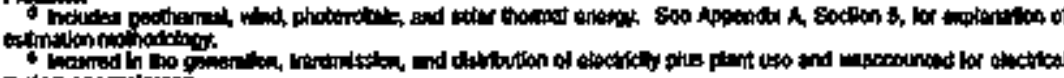

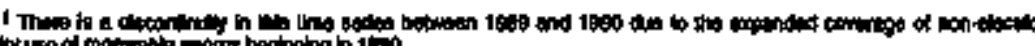

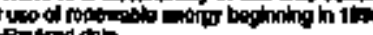

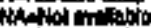

Hoc

$T$ Ton in

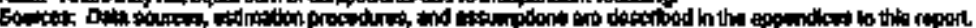


Teble 255. Transportation Energy Cansomption Estimates, 1960, 1965, 1670-1904, Fhota Ishand

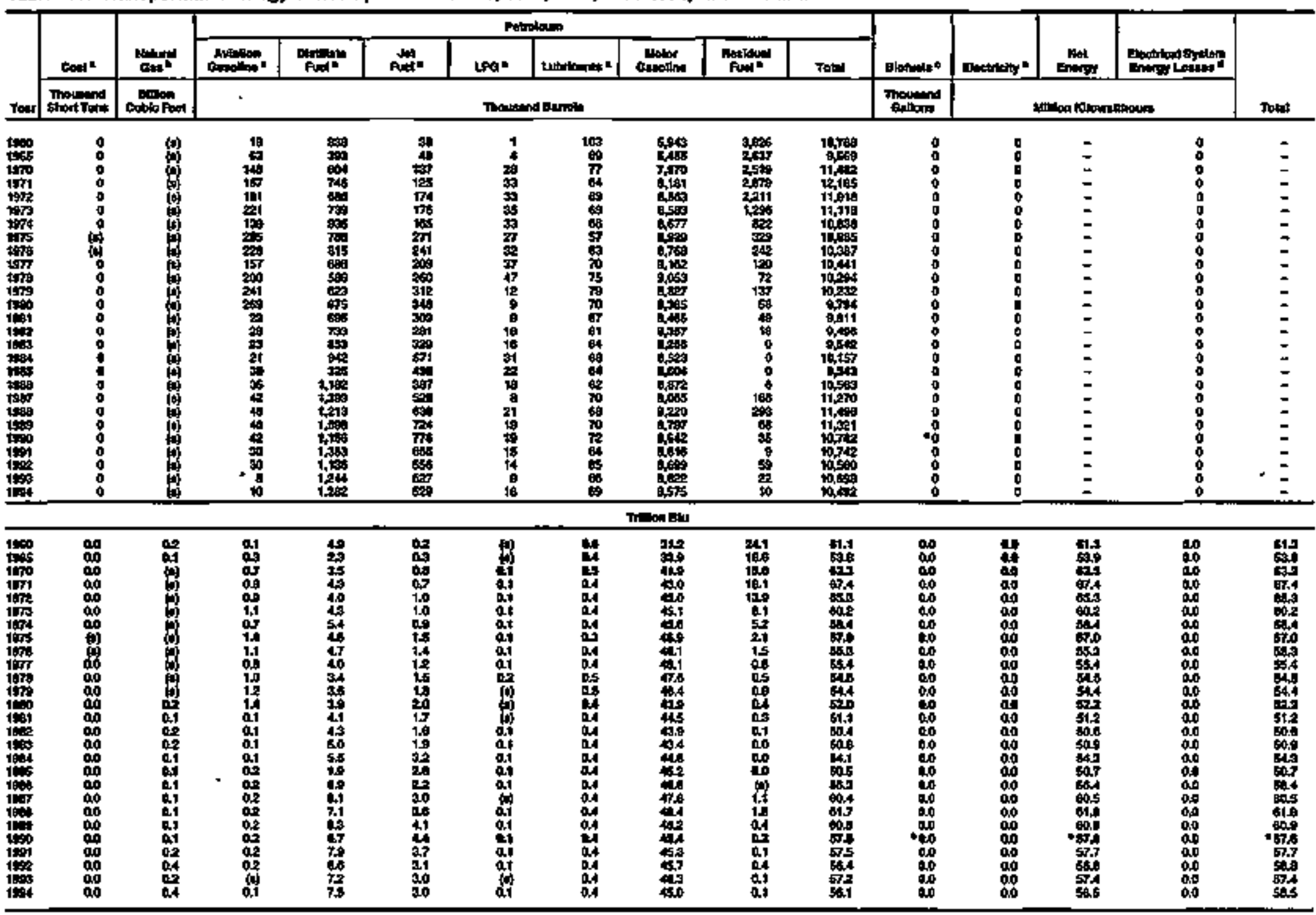

$\mathbf{R}$
$\mathbf{H}$
$\mathbf{O}$
$\mathbf{D}$
$\mathbf{E}$

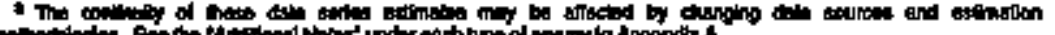

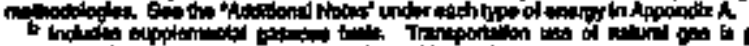

ate

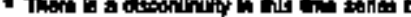
Hy

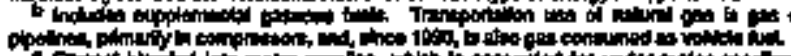

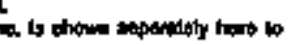

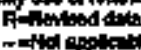

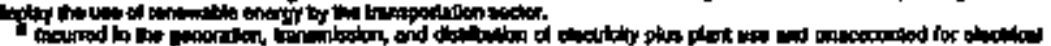

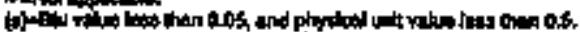

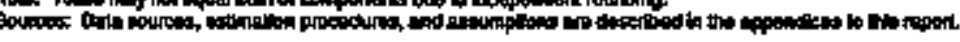

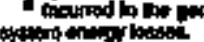




\begin{tabular}{|c|c|c|c|c|c|c|c|c|c|c|c|c|c|c|}
\hline \multirow[b]{3}{*}{ not } & \multicolumn{3}{|c|}{ cand } & \multirow[b]{2}{*}{ 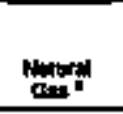 } & \multicolumn{4}{|c|}{ Potrotoum } & \multirow[b]{2}{*}{ 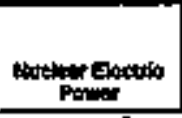 } & \multirow[b]{2}{*}{ Anderenterte } & \multirow[b]{2}{*}{ 西 } & \multirow[b]{2}{*}{ 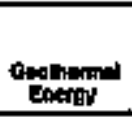 } & \multirow[b]{2}{*}{ 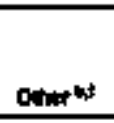 } & \multirow[b]{3}{*}{ Totold } \\
\hline & 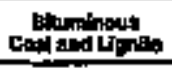 & Entrinasests & Tom & & todn & $\operatorname{los}_{n}$ & 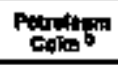 & row & & & & & & \\
\hline & \multicolumn{3}{|c|}{ Thousmassmont Tom } & comition & \multicolumn{4}{|c|}{ 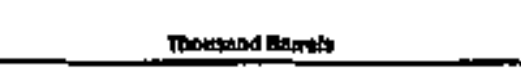 } & \multicolumn{5}{|c|}{ 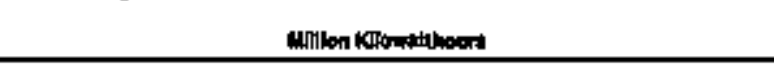 } & \\
\hline 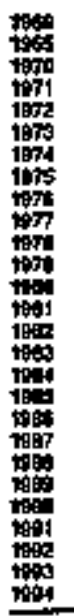 & $\begin{array}{c}\text { gat } \\
0 \\
0 \\
0 \\
0 \\
0 \\
3 \\
0 \\
0 \\
0 \\
0 \\
0 \\
0 \\
0 \\
0 \\
0 \\
0 \\
0 \\
0 \\
0 \\
0 \\
0 \\
0 \\
0 \\
0 \\
0 \\
0 \\
0 \\
0 \\
0\end{array}$ & 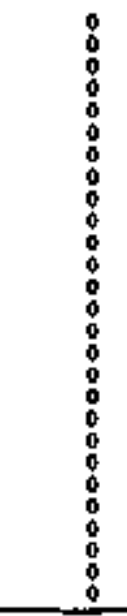 & 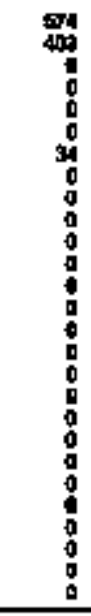 & 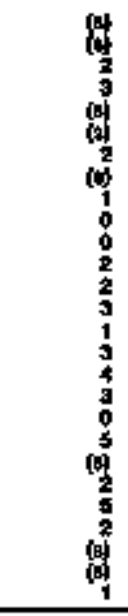 & 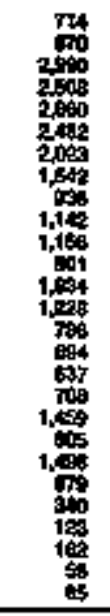 & 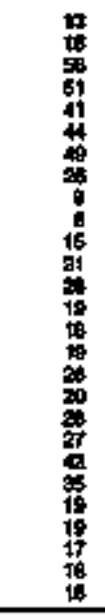 & & 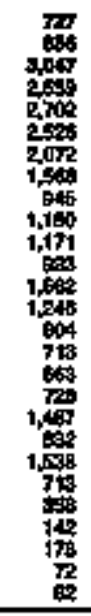 & $:$ & 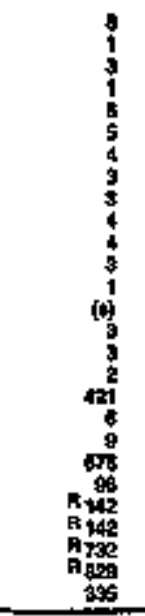 & $\begin{array}{l}0 \\
0 \\
0 \\
0 \\
0 \\
0 \\
0 \\
0 \\
0 \\
0 \\
0 \\
0 \\
0 \\
0 \\
0 \\
0 \\
0 \\
0 \\
0 \\
0 \\
0 \\
0 \\
0 \\
\end{array}$ & $\begin{array}{l}0 \\
0 \\
0 \\
0 \\
0 \\
0 \\
0 \\
\vdots \\
0 \\
\vdots \\
0 \\
0 \\
\vdots \\
0 \\
0 \\
0 \\
0 \\
0 \\
\vdots \\
0 \\
\end{array}$ & $\begin{array}{l}: \\
: \\
0 \\
0 \\
0 \\
0 \\
0 \\
0 \\
0 \\
0 \\
0 \\
0 \\
0 \\
0 \\
0 \\
0\end{array}$ & $\begin{array}{l}\vdots \\
\vdots \\
\vdots \\
\vdots \\
\vdots \\
\vdots \\
\vdots \\
\vdots \\
\vdots \\
\vdots \\
\end{array}$ \\
\hline \multicolumn{15}{|c|}{ Trethen Bat } \\
\hline 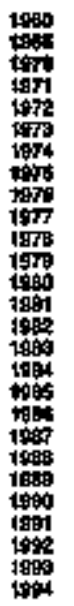 & 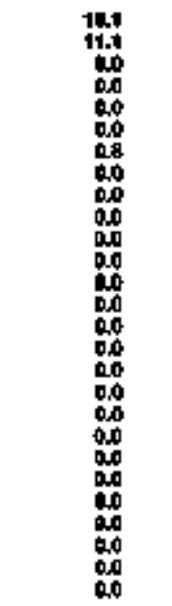 & 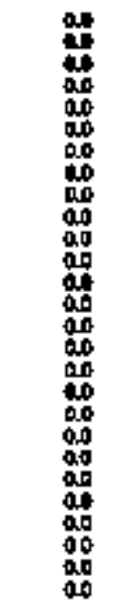 & 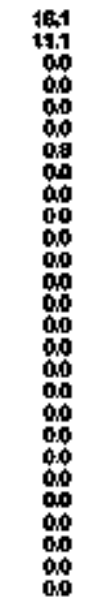 & 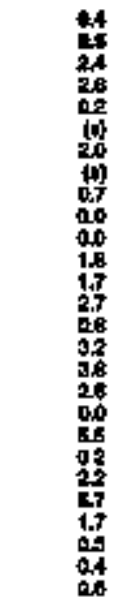 & 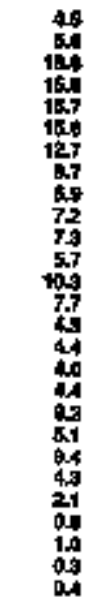 & 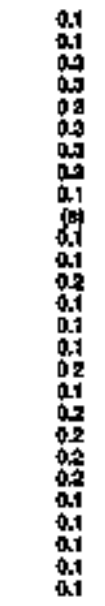 & 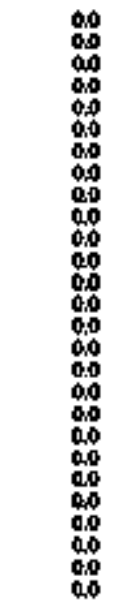 & 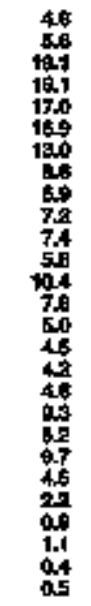 & 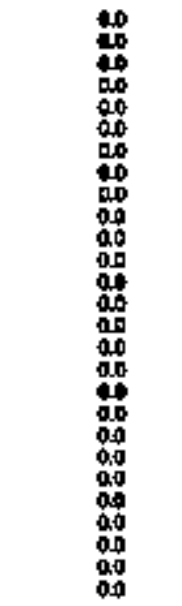 & 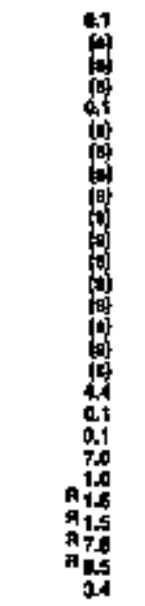 & 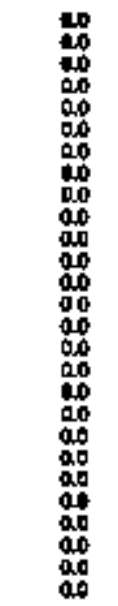 & 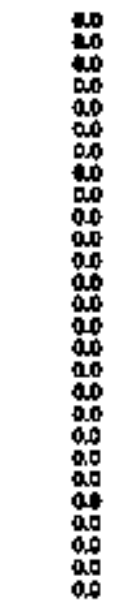 & 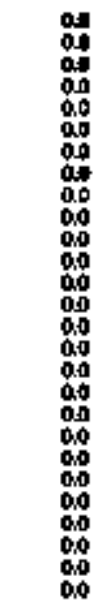 & 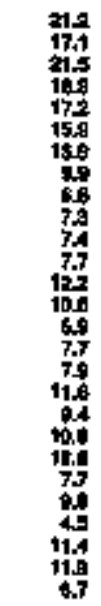 \\
\hline
\end{tabular}

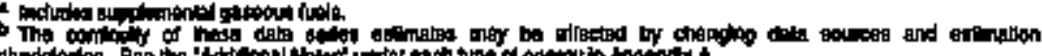

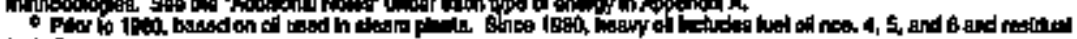

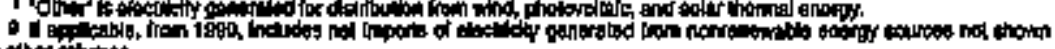

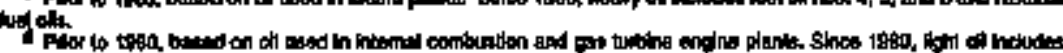

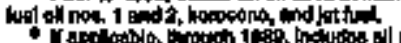

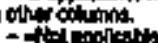

(j)

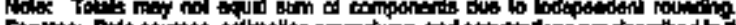

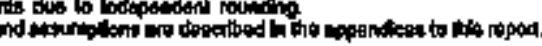


Table 257. Energy Consurnption Earlmates by Source, 1960, 1965, 1970-1994, South Carollna

\begin{tabular}{|c|c|c|c|c|c|c|c|c|c|c|c|c|c|c|c|c|c|c|c|}
\hline \multirow{2}{*}{\multicolumn{2}{|c|}{ cond I }} & \multirow[b]{2}{*}{ thats } & \multicolumn{11}{|c|}{ Polotann } & \multirow[b]{2}{*}{ 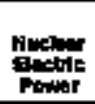 } & \multirow[b]{2}{*}{ 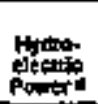 } & \multirow[b]{2}{*}{ Paturetont } & \multirow[b]{2}{*}{ Gother at } & \multirow{2}{*}{ 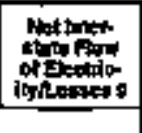 } & \multirow[b]{3}{*}{ Totan $\mathrm{h}$} \\
\hline & & & \begin{tabular}{|l|} 
Asphunin and \\
Rphad of I
\end{tabular} & Anstion & 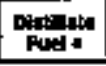 & Fued. & Kime & LNa: & unstit. & Gotor & Peitures & عبser & Tond & & & & & & \\
\hline rart & Mhould tod & colllon & \multicolumn{11}{|c|}{$\pi$ nowind ents } & \multicolumn{5}{|c|}{ 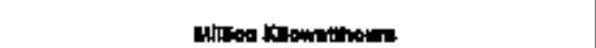 } & \\
\hline
\end{tabular}

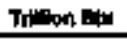

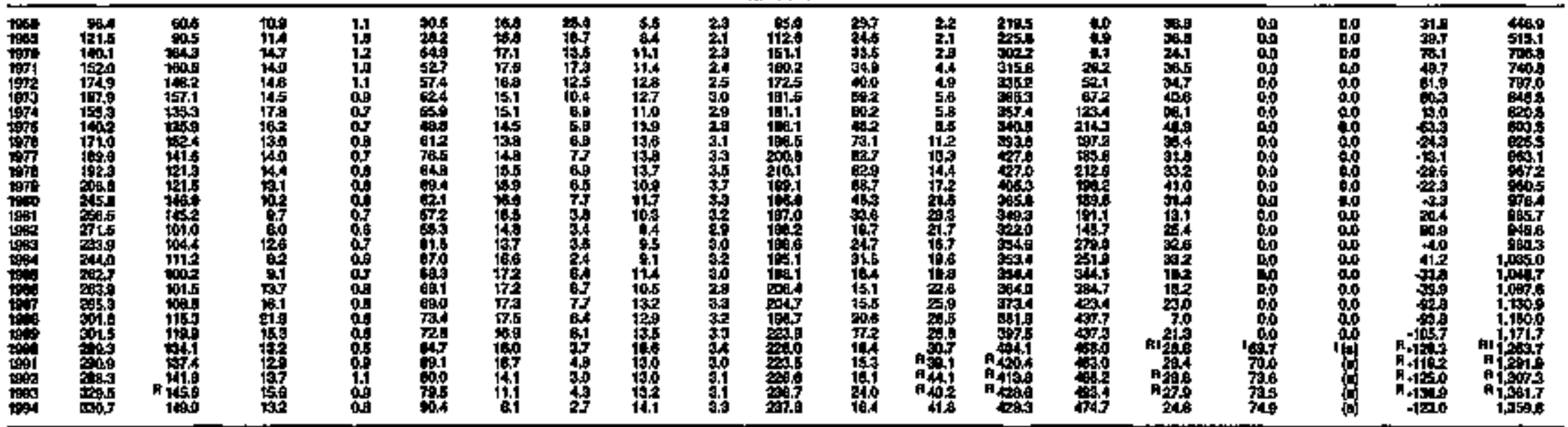

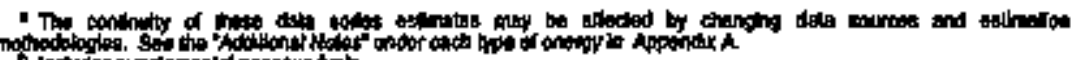

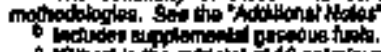

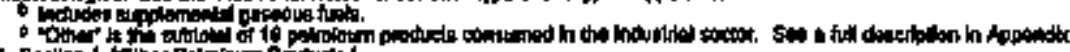

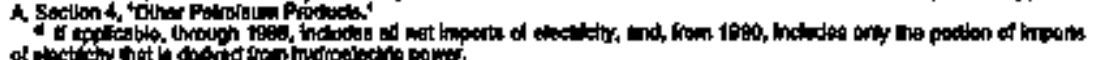

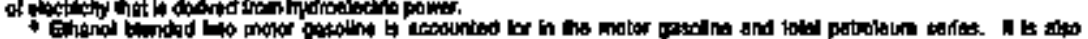

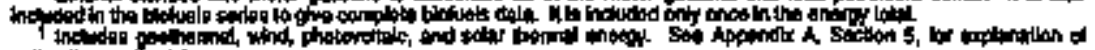

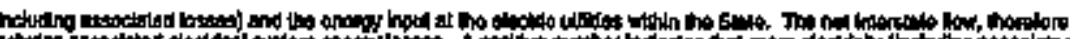

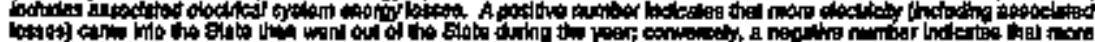

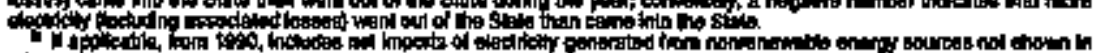

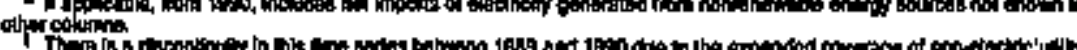

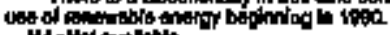

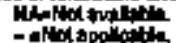

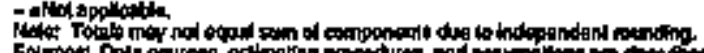

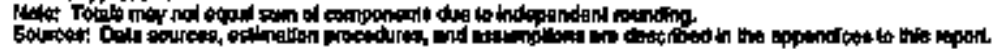


Teble 259. Resldential Engrgy Consumptian Estianates, 1960, 1965, 1970-1994, soulh Curolin:

\begin{tabular}{|c|c|c|c|c|c|c|c|c|c|c|c|c|c|}
\hline \multicolumn{3}{|c|}{$\operatorname{sen}$} & \multirow[b]{2}{*}{ Nations } & \multicolumn{4}{|c|}{ Puntolom } & \multirow[b]{2}{*}{ Elofitith } & \multirow[b]{2}{*}{ Golate } & \multirow[b]{2}{*}{ abotikfty } & \multirow[b]{2}{*}{ Everty } & \multirow[b]{2}{*}{ Emarkel syoten } & \\
\hline Coniminour & Anturatiant & $\tau+\infty$ & & 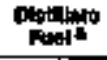 & Kerousone 1 & LPE* & Totol & & & & & & \multirow[b]{2}{*}{$T=0$} \\
\hline \multicolumn{3}{|c|}{ 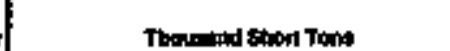 } & cintistin & \multicolumn{4}{|c|}{ 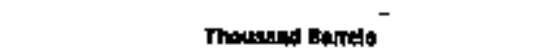 } & Thoonends & \multicolumn{4}{|c|}{ 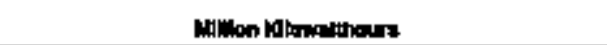 } & \\
\hline
\end{tabular}

\begin{tabular}{|c|c|c|}
\hline 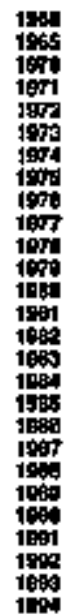 & 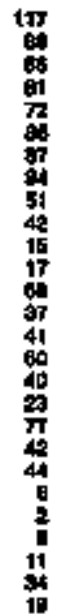 & $\begin{array}{l}0 \\
0 \\
0 \\
0 \\
0 \\
0 \\
0 \\
0 \\
0 \\
0 \\
0 \\
0 \\
0 \\
0 \\
0 \\
0 \\
1 \\
0 \\
0 \\
69 \\
9 \\
9 \\
0 \\
7 \\
7\end{array}$ \\
\hline
\end{tabular}

117
4

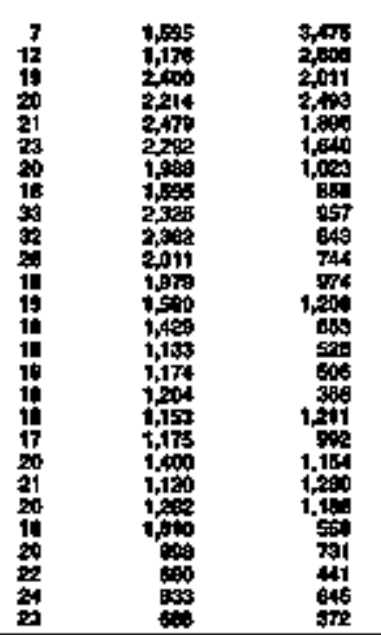

\begin{tabular}{|c|c|}
\hline 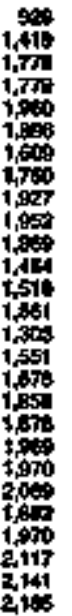 & 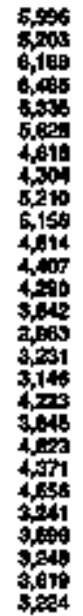 \\
\hline
\end{tabular}

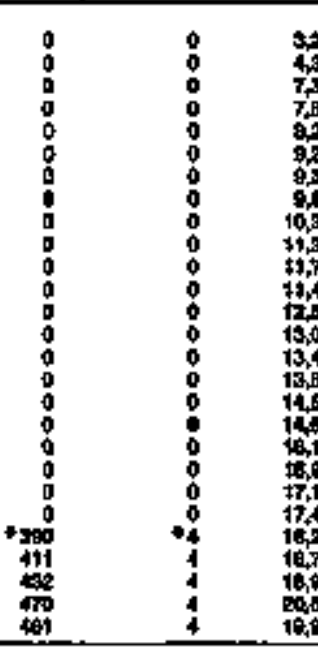

sate

\begin{tabular}{|c|}
\hline 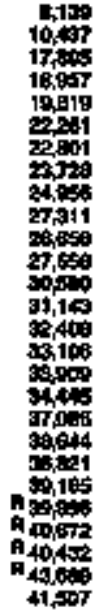 \\
\hline
\end{tabular}

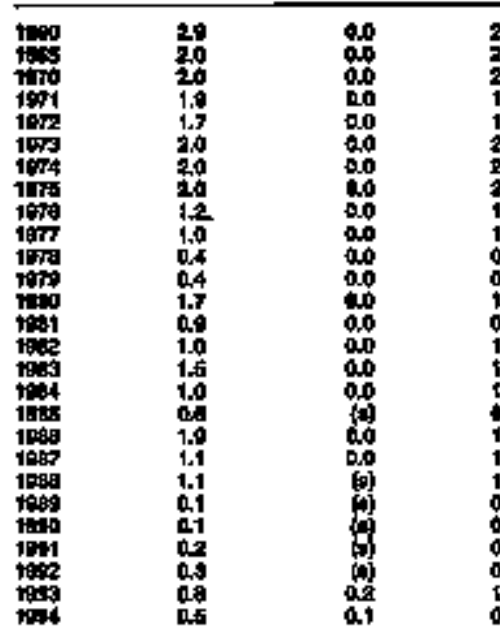

Tileon inu

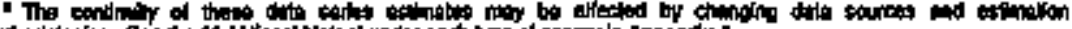

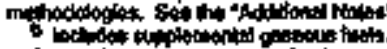

\begin{tabular}{|c|c|c|c|}
\hline 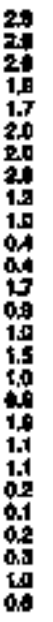 & 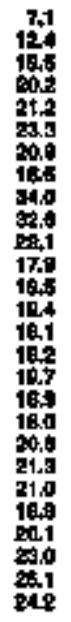 & 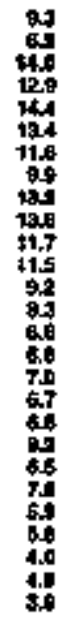 & 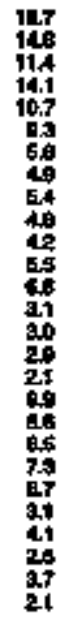 \\
\hline
\end{tabular}

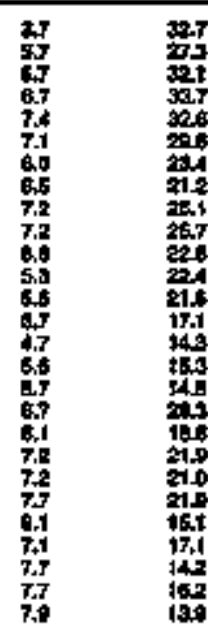

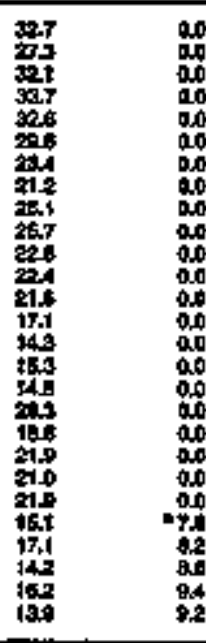

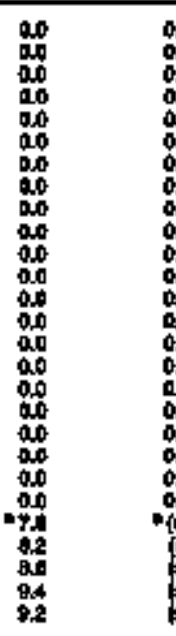

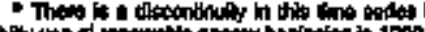

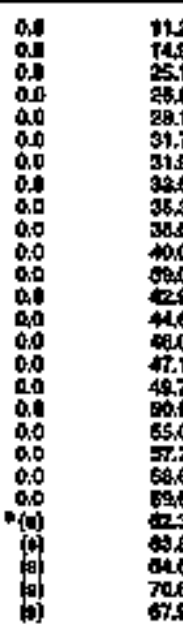

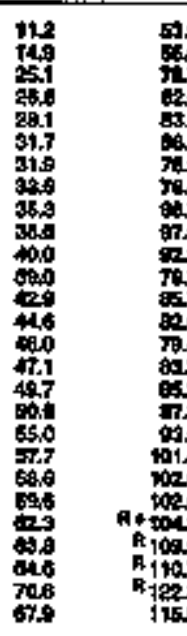

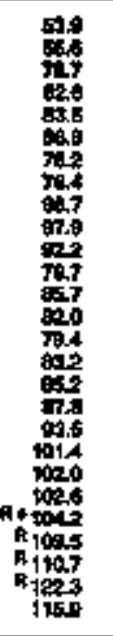

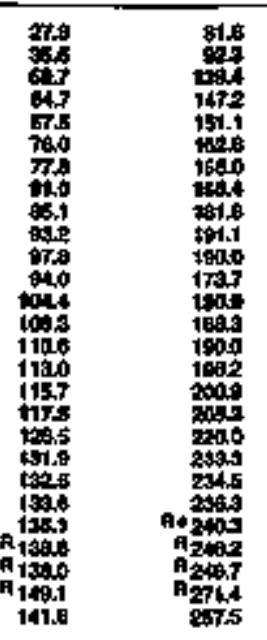

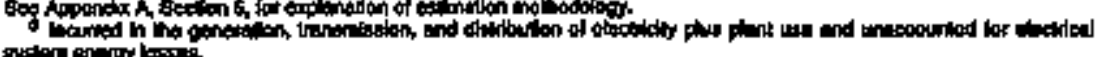

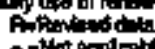

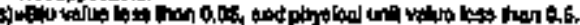

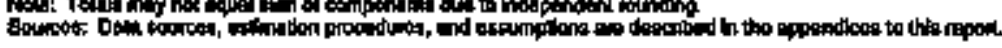


Table 259. Comonorclal Energy Constumption Estimates, 1960, 1965, 1970-1994, South Carelina

\begin{tabular}{|c|c|c|c|c|c|c|c|c|c|c|c|c|c|c|}
\hline \multirow[b]{3}{*}{ Yoof } & \multicolumn{3}{|c|}{ con } & \multirow{3}{*}{ 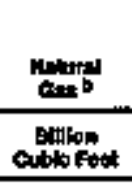 } & \multicolumn{6}{|c|}{ Perrotomat } & \multirow[b]{2}{*}{ Doetivithy } & \multirow[b]{2}{*}{ Enoty } & \multirow{3}{*}{ 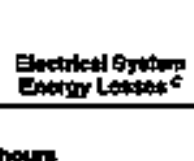 } & \multirow[b]{3}{*}{ Total" } \\
\hline & 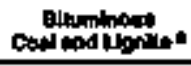 & Anthe & Folel & & 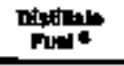 & Kromen: & LPE: & motor & 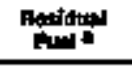 & $T$ & & & & \\
\hline & \multicolumn{3}{|c|}{ Thoupand Grent Tons } & & \multicolumn{6}{|c|}{ Thoutaind Bandoll } & \multicolumn{2}{|c|}{ 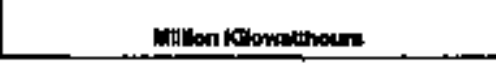 } & & \\
\hline
\end{tabular}

$\mathbf{S}$
$\mathbf{O}$
$\mathbf{U}$
$\mathbf{T}$
$\mathbf{H}$

$\mathbf{C}$
$\mathbf{A}$
$\mathbf{R}$
$\mathbf{0}$
$\mathbf{L}$
$\mathbf{I}$
$\mathbf{N}$
$\mathbf{A}$

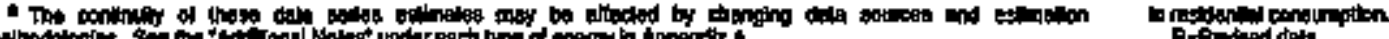

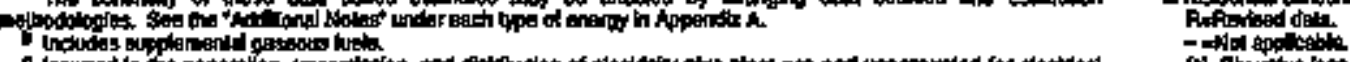

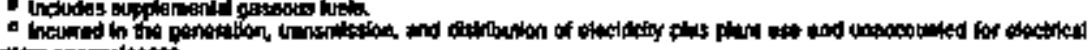

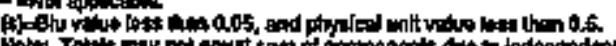

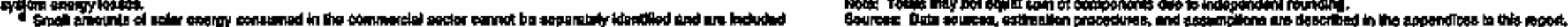




\begin{tabular}{|c|c|c|c|c|c|c|c|c|c|c|c|c|c|c|c|c|c|c|}
\hline \multirow[b]{3}{*}{ rat } & \multirow[b]{2}{*}{ 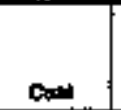 } & \multirow[b]{2}{*}{ nowed } & \multicolumn{9}{|c|}{ Puniturar } & \multirow[b]{2}{*}{ 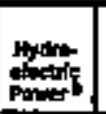 } & \multirow[b]{2}{*}{ monely if } & \multirow[b]{2}{*}{ | } & \multirow[b]{2}{*}{ Expotidy } & \multirow[b]{2}{*}{ Emetry } & \multirow{2}{*}{ 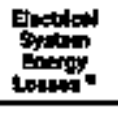 } & \multirow[b]{3}{*}{ Tolow } \\
\hline & & & Antintonst & "10 & Karowene of & LPa" & | & | & 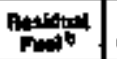 & asorber & 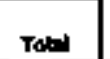 & & & & & & & \\
\hline & Thoovend & cullown & \multicolumn{9}{|c|}{ 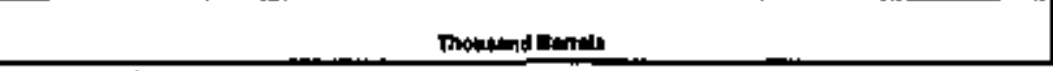 } & \multicolumn{5}{|c|}{ 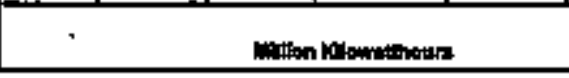 } & & \\
\hline 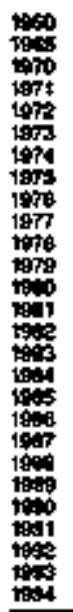 & 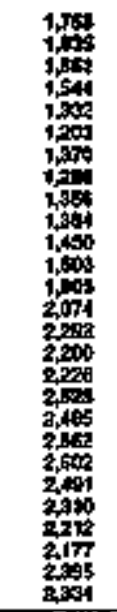 & 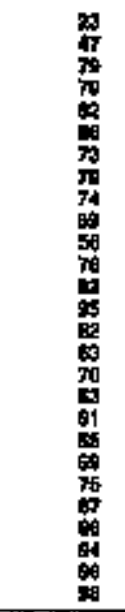 & 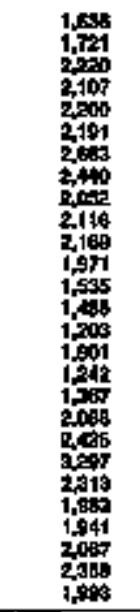 & 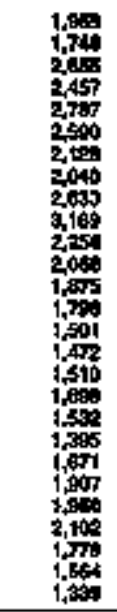 & 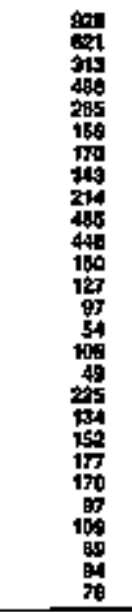 & 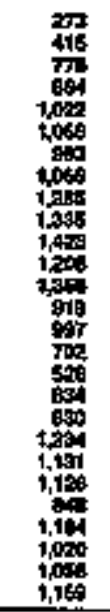 & 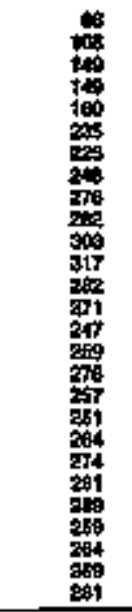 & 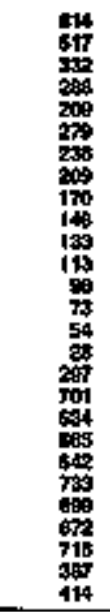 & 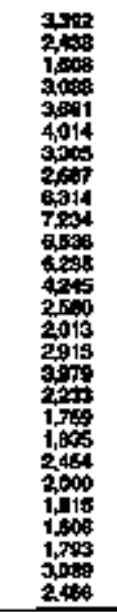 & 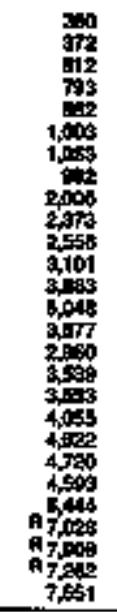 & 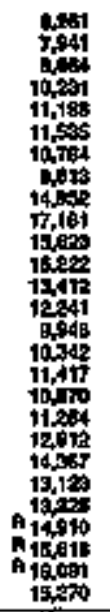 & 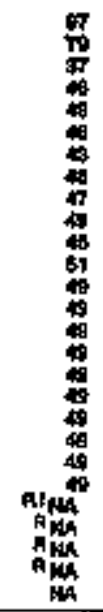 & 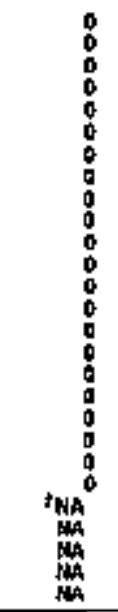 & 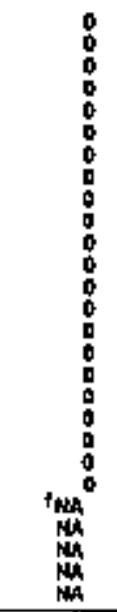 & 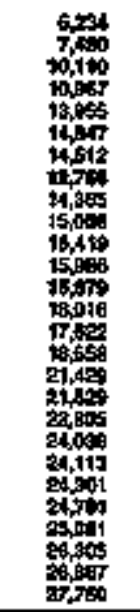 & $\begin{array}{l}\vdots \\
\vdots \\
\vdots \\
\vdots \\
\vdots \\
\vdots \\
\vdots \\
\vdots \\
\vdots \\
\vdots \\
\vdots \\
\vdots \\
\vdots\end{array}$ & 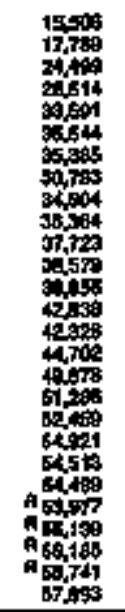 & $\begin{array}{l}\vdots \\
\vdots \\
\vdots \\
\vdots \\
\vdots \\
\vdots \\
\vdots \\
\vdots \\
\vdots \\
\vdots \\
\vdots \\
\vdots \\
\vdots \\
\vdots\end{array}$ \\
\hline \multicolumn{19}{|c|}{ Trillon Em } \\
\hline 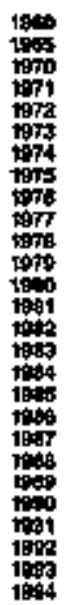 & 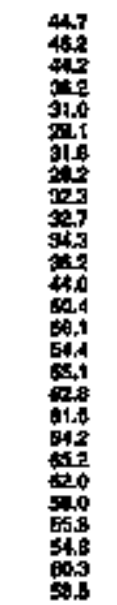 & 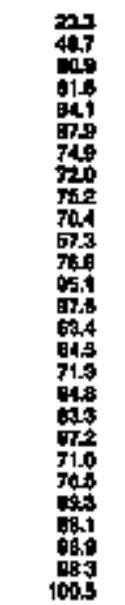 & 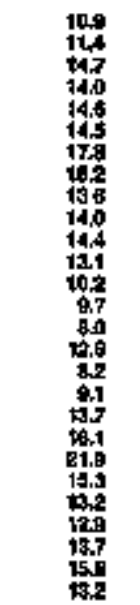 & 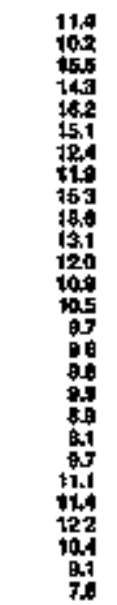 & 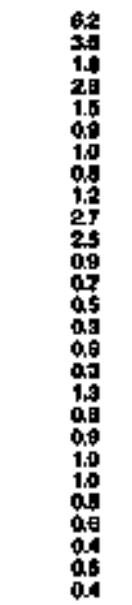 & 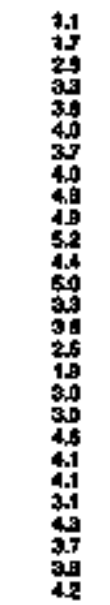 & 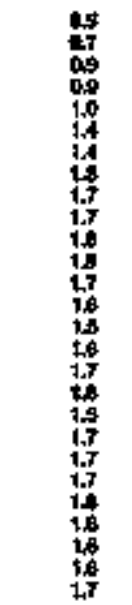 & 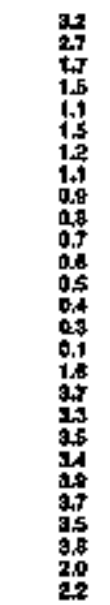 & 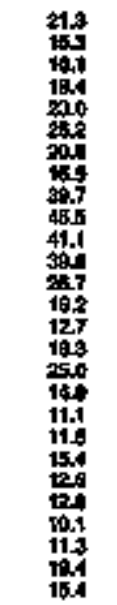 & 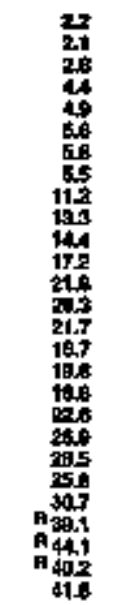 & 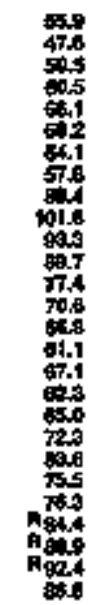 & 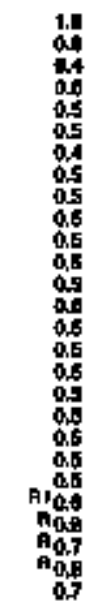 & 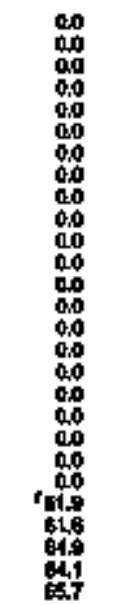 & 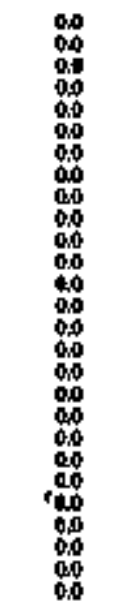 & 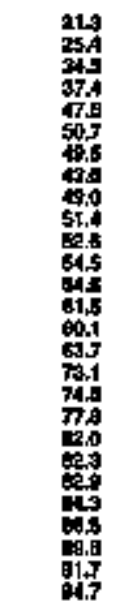 & 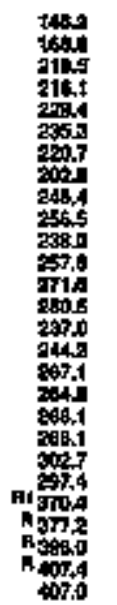 & 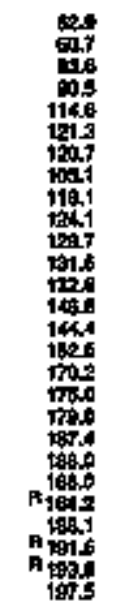 & 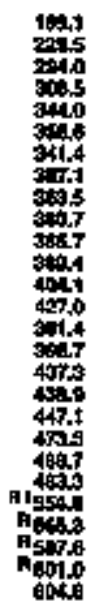 \\
\hline & & & & & & & & & & Bows & & & & mans 18 & $1 \mathrm{mat} 1890$ & & & \\
\hline
\end{tabular}


Table 261. Transportation Energy Consomption Esilmates, t860, 186, 1070-1994, South Carolina

\begin{tabular}{|c|c|c|c|c|c|c|c|c|c|c|c|c|c|c|c|}
\hline \multirow[b]{3}{*}{ Your } & \multirow[b]{2}{*}{ ") } & \multirow[b]{2}{*}{ lonter } & \multicolumn{8}{|c|}{ 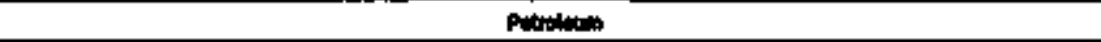 } & \multirow{3}{*}{ 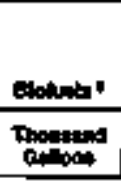 } & \multirow[b]{2}{*}{ Electikty " } & \multirow[b]{2}{*}{$\min _{5}^{m}$} & \multirow{3}{*}{ 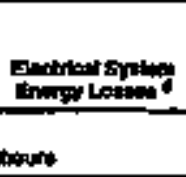 } & \multirow[b]{3}{*}{ Ton } \\
\hline & & & 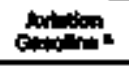 & Fint & Int. & LE: & 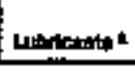 & cotation & 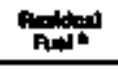 & Total & & & & & \\
\hline & Thoonand & 然 & \multicolumn{8}{|c|}{ 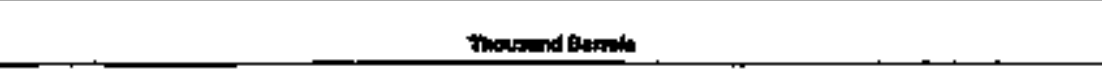 } & & \multicolumn{2}{|c|}{ 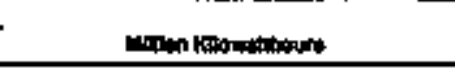 } & & \\
\hline 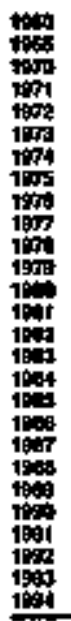 & $\begin{array}{l}30 \\
0 \\
8 \\
1 \\
1 \\
0 \\
0 \\
0 \\
0 \\
0 \\
0 \\
0 \\
0 \\
0 \\
0 \\
0 \\
0 \\
0 \\
0 \\
0 \\
0 \\
0 \\
0\end{array}$ & 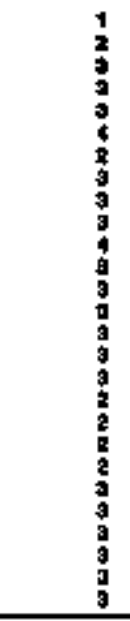 & 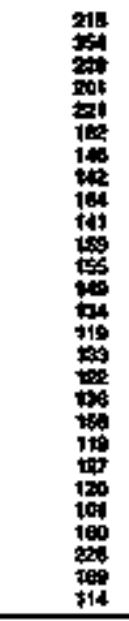 & 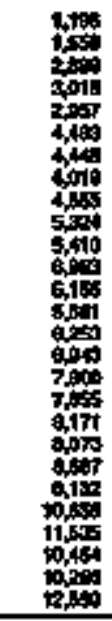 & 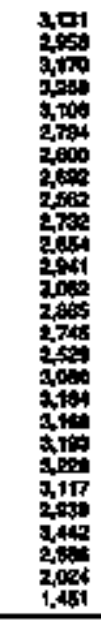 & 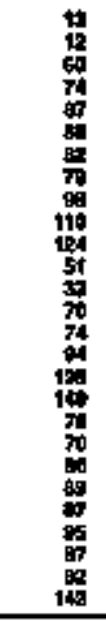 & 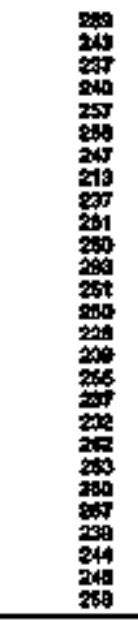 & 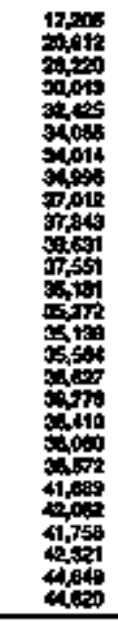 & 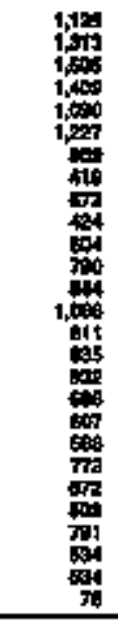 & 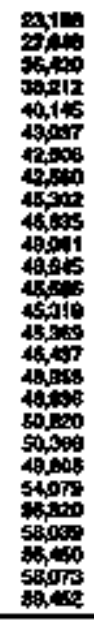 & $\begin{array}{l}: \\
: \\
: \\
: \\
: \\
: \\
: \\
0 \\
0 \\
0 \\
0 \\
0 \\
0 \\
0\end{array}$ & $\begin{array}{l}0 \\
0 \\
0 \\
0 \\
0 \\
0 \\
0 \\
0 \\
0 \\
0 \\
0 \\
0 \\
0 \\
0 \\
0 \\
0 \\
0 \\
0 \\
0 \\
0 \\
0 \\
0 \\
0 \\
0\end{array}$ & $\begin{array}{l}= \\
= \\
= \\
= \\
= \\
= \\
= \\
= \\
= \\
= \\
= \\
= \\
=\end{array}$ & $\begin{array}{l}0 \\
0 \\
0 \\
0 \\
0 \\
0 \\
0 \\
0 \\
0 \\
0 \\
0 \\
0 \\
0 \\
0 \\
0 \\
0 \\
0 \\
0 \\
0 \\
0 \\
0 \\
0 \\
0 \\
0 \\
0 \\
0\end{array}$ & $\begin{array}{l}= \\
= \\
= \\
= \\
= \\
= \\
= \\
= \\
= \\
= \\
= \\
= \\
=\end{array}$ \\
\hline \multicolumn{16}{|c|}{ Jiten en } \\
\hline 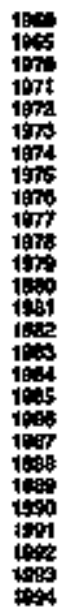 & 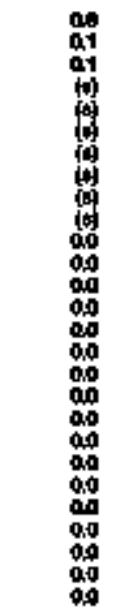 & 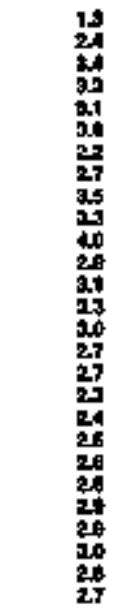 & 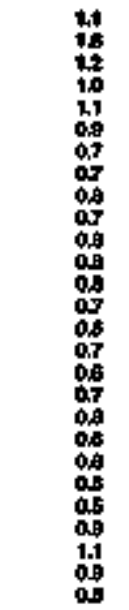 & 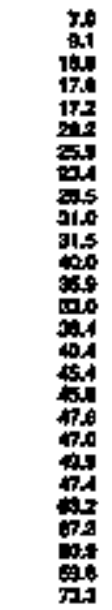 & 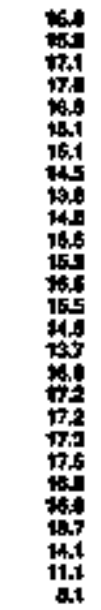 & 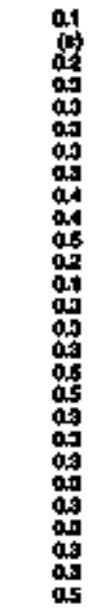 & $\begin{array}{l}1.5 \\
1.5 \\
1.4 \\
1.5 \\
1.5 \\
1.5 \\
1.4 \\
1.4 \\
1.7 \\
1.5 \\
1.5 \\
1.4 \\
1.5 \\
1.4 \\
1.5 \\
1.5 \\
1.5 \\
1.4 \\
1.5 \\
1.5 \\
1.5\end{array}$ & 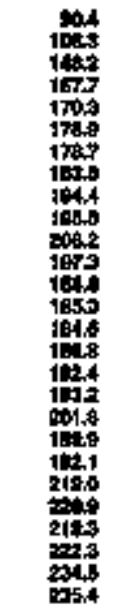 & 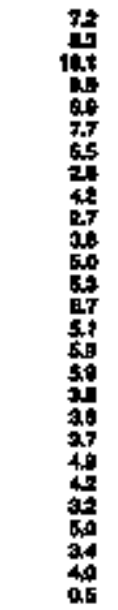 & 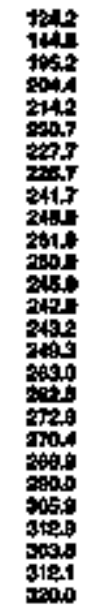 & 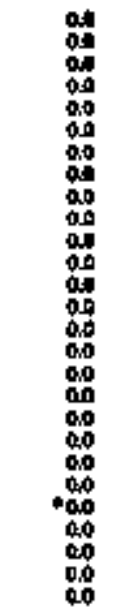 & $\begin{array}{l}00 \\
00 \\
000 \\
000 \\
000 \\
000 \\
000 \\
000 \\
000 \\
0.0 \\
000 \\
000 \\
000 \\
000 \\
000 \\
00 \\
000 \\
000 \\
000 \\
00 \\
000 \\
00 \\
00 \\
00 \\
00\end{array}$ & 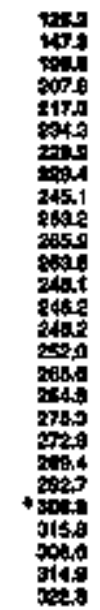 & 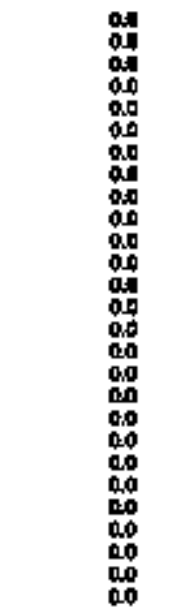 & 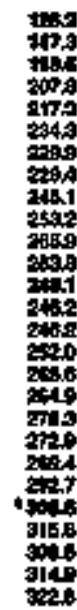 \\
\hline
\end{tabular}


Tablu 202. Estimates of Energy Input at Electric Uubities, 1960, 1965, 1970-1894, South Carchina

\begin{tabular}{|c|c|c|c|c|c|c|c|c|c|c|c|c|c|c|}
\hline \multirow[b]{3}{*}{ ( } & \multicolumn{3}{|c|}{$\cos$} & \multirow[b]{2}{*}{ 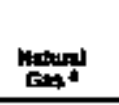 } & \multicolumn{4}{|c|}{ Pexpentank } & \multirow[b]{2}{*}{ 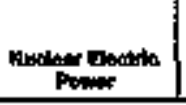 } & \multirow[b]{2}{*}{ 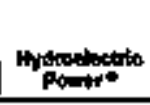 } & \multirow[b]{2}{*}{ eloturero } & \multirow[b]{2}{*}{ 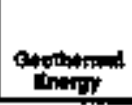 } & \multirow{2}{*}{ onerw } & \multirow[b]{3}{*}{$\operatorname{man}$} \\
\hline & 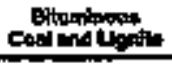 & entrocesto & Totot & & tons & 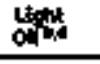 & restent & Tow & & & & & & \\
\hline & \multicolumn{3}{|c|}{ 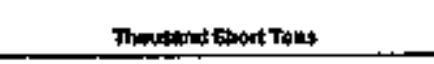 } & 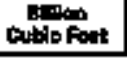 & \multicolumn{4}{|c|}{ monmanemb } & \multicolumn{5}{|c|}{ 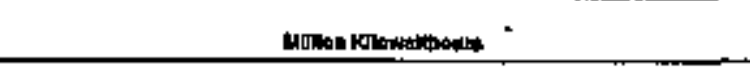 } & \\
\hline 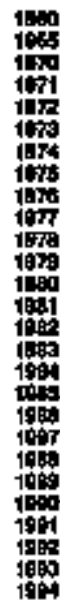 & 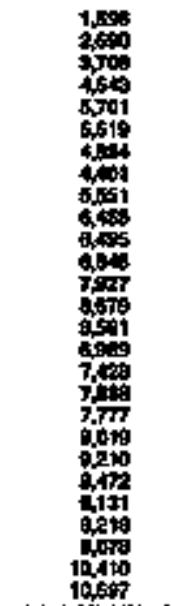 & $\begin{array}{l}0 \\
0 \\
0 \\
0 \\
0 \\
0 \\
0 \\
0 \\
0 \\
0 \\
0 \\
0 \\
0 \\
0 \\
0 \\
0 \\
0 \\
0 \\
0 \\
0 \\
0 \\
0 \\
0 \\
0 \\
0 \\
0 \\
0 \\
0 \\
0\end{array}$ & 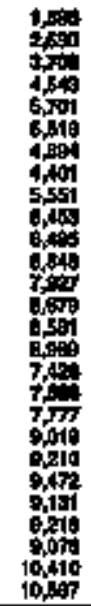 & 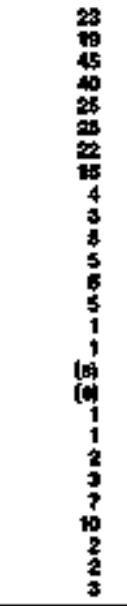 & 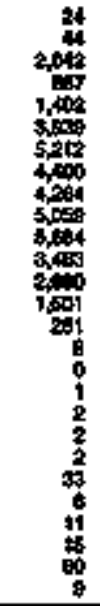 & 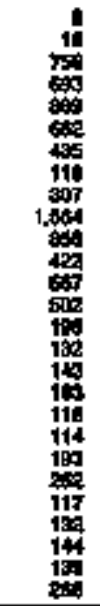 & 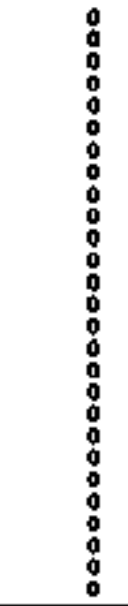 & 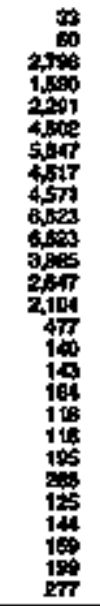 & 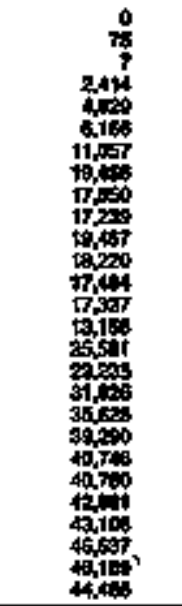 & 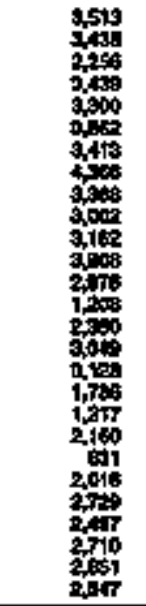 & $:$ & & & $\begin{array}{l}z \\
z \\
z \\
z\end{array}$ \\
\hline
\end{tabular}

\begin{tabular}{|c|c|c|c|c|c|c|c|c|c|c|c|c|c|c|}
\hline 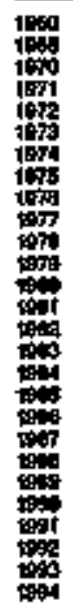 & 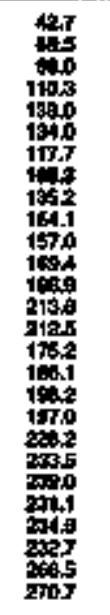 & 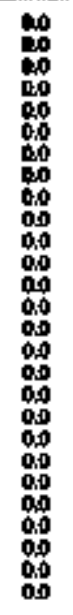 & 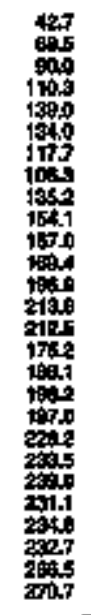 & 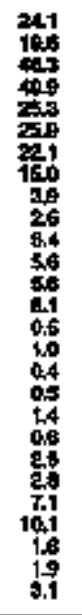 & 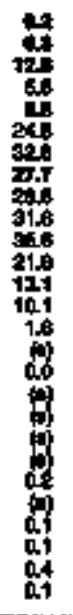 & 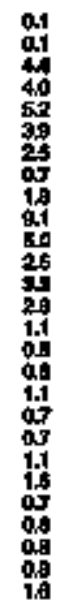 & 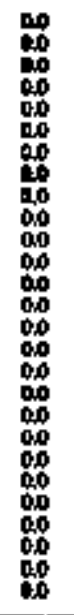 & 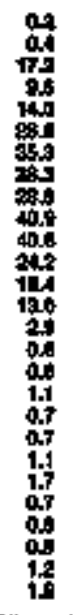 & 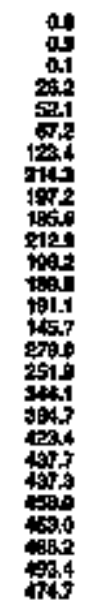 & 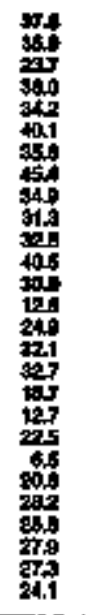 & 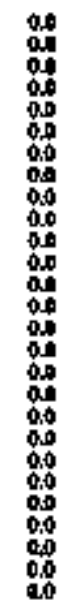 & 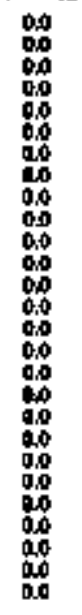 & 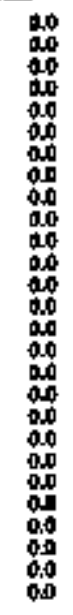 & 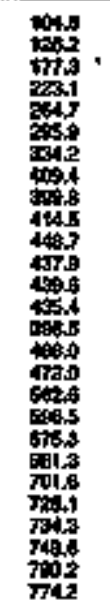 \\
\hline
\end{tabular}

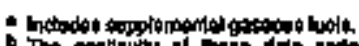

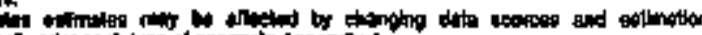

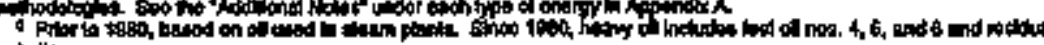

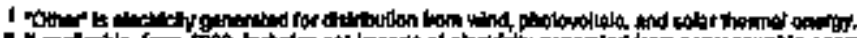

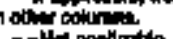

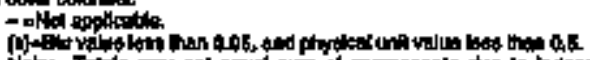

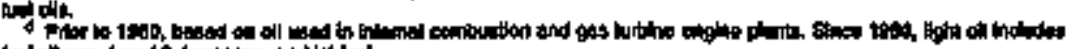

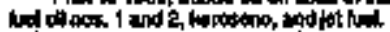

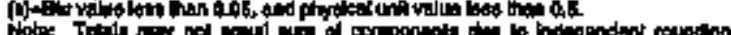


Tabla 263. Energy Consumption Estlmates by Source, 1960, 1965, 1970-1994, South Daknta

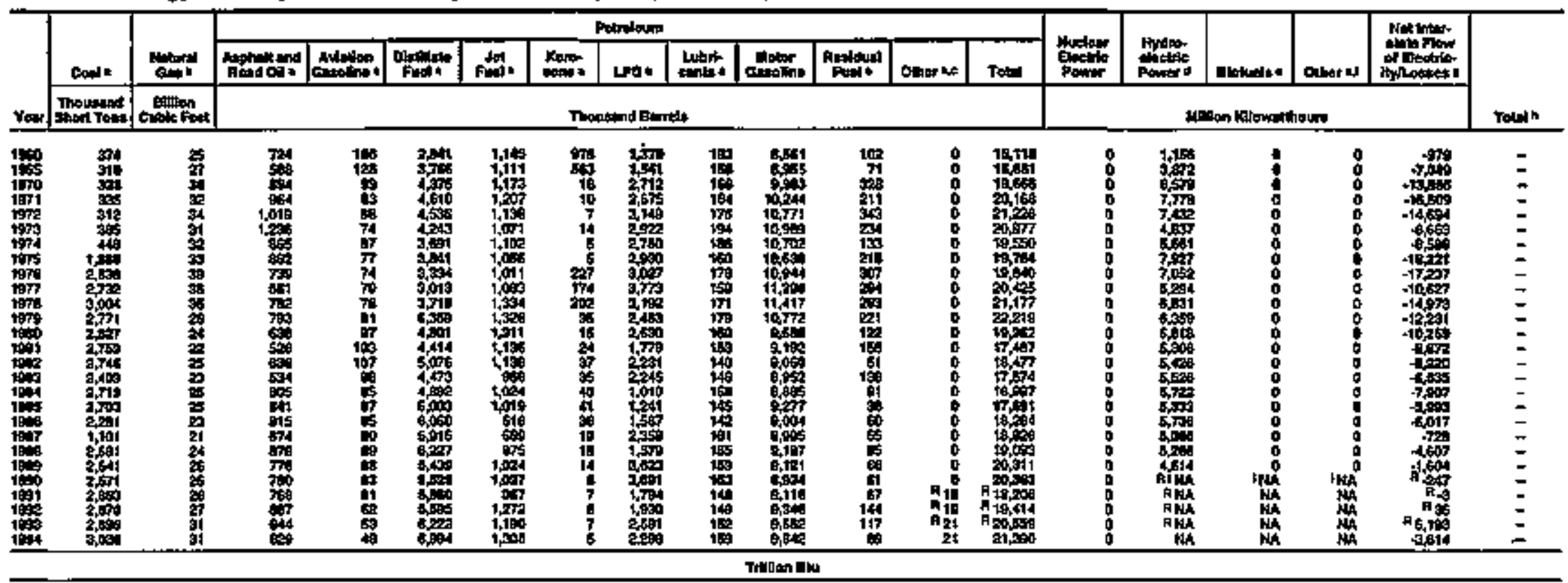

\begin{tabular}{|c|c|c|c|c|c|c|c|c|c|c|c|c|c|c|c|c|c|c|c|}
\hline 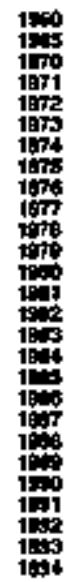 & 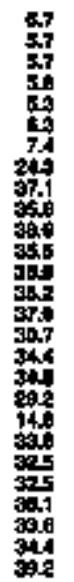 & 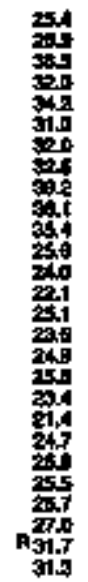 & 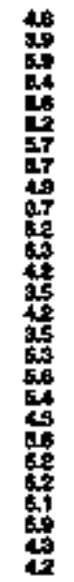 & 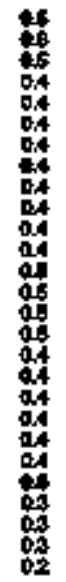 & 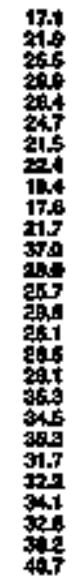 & 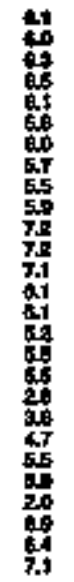 & 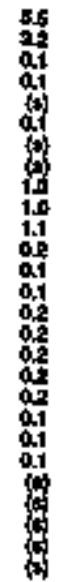 & 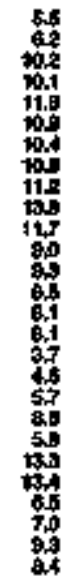 & 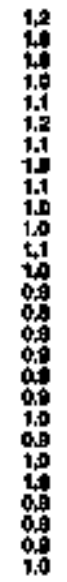 & 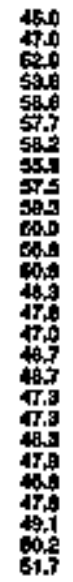 & 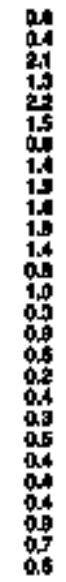 & 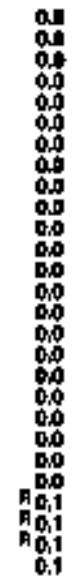 & 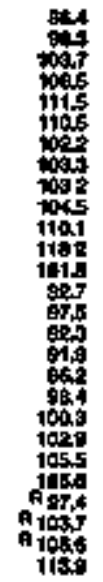 & 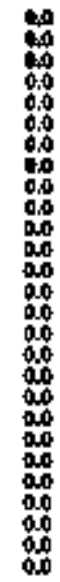 & 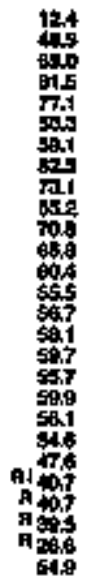 & $\begin{array}{l}0 \\
0 \\
0\end{array}$ & 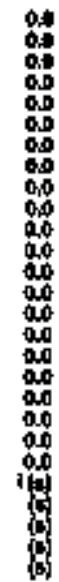 & 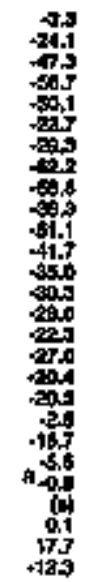 & 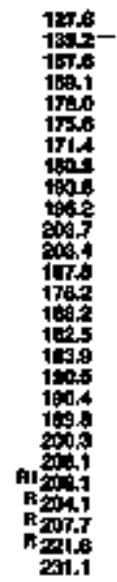 \\
\hline
\end{tabular}

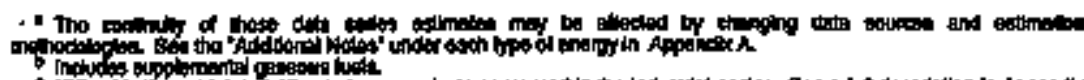

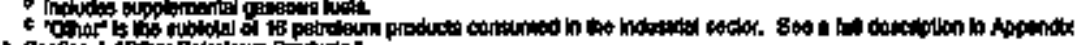
$\lambda$ S

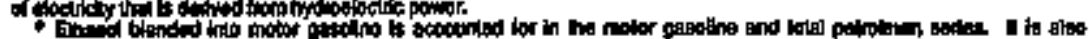

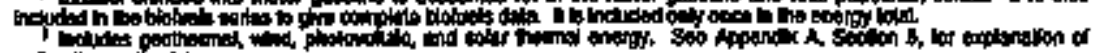

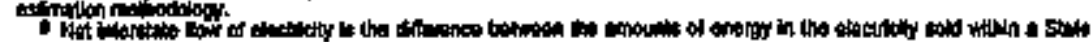

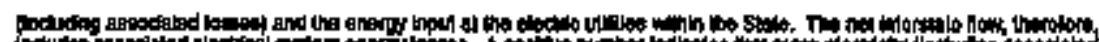

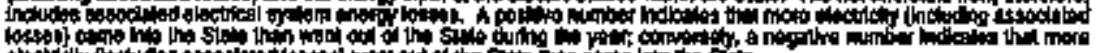

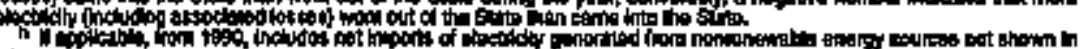

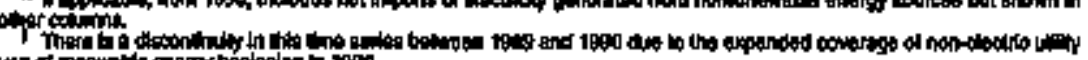

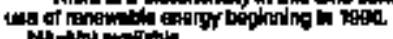

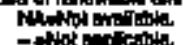

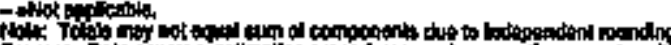

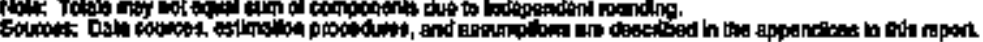


5 Table 264. Pesidential Energy Consumptlon Estimates, 1660, 1965, 1970-1994, Soulh Dakota

$\mathbf{O}$
$\mathbf{U}$
$\mathbf{T}$
$\mathbf{H}$
$\mathbf{D}$
$\mathbf{A}$
$\mathbf{K}$
$\mathbf{0}$
$\mathbf{T}$
$\mathbf{A}$

\begin{tabular}{|c|c|c|c|c|c|c|c|c|c|c|c|c|c|c|}
\hline & & call & & & & & & & & & & & & \\
\hline & martion: & Nonthailus & Totat & 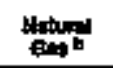 & Masultit & 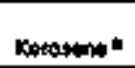 & LPQ & rom & Estront & sourt & Ementerer. & 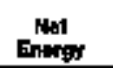 & 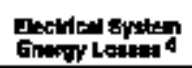 & \\
\hline & & mathos Trear & & 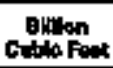 & & Moster & & & 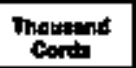 & & malont & andrown & & Tous \\
\hline 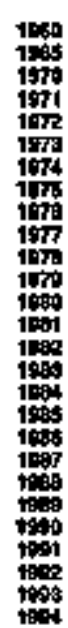 & 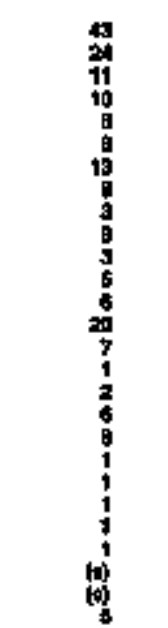 & 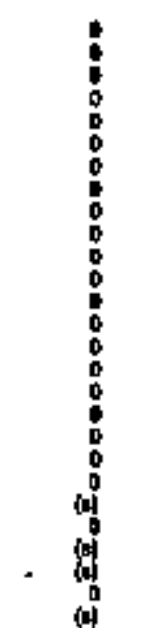 & נים & 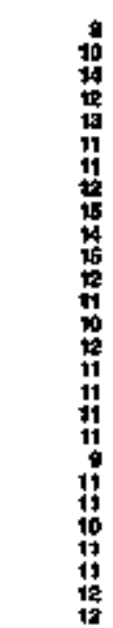 & 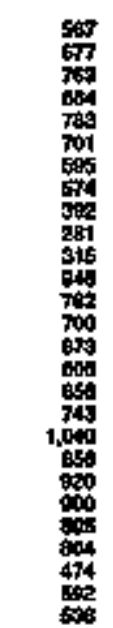 & 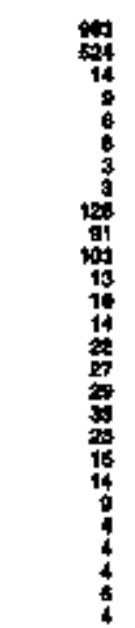 & 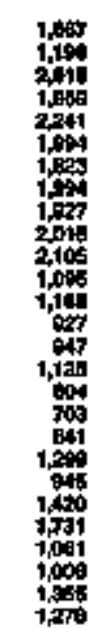 & 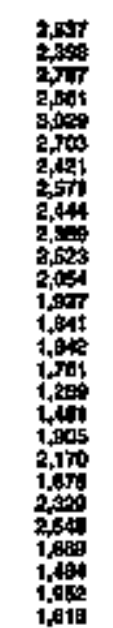 & 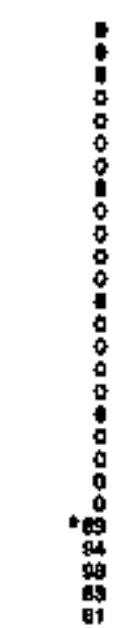 & 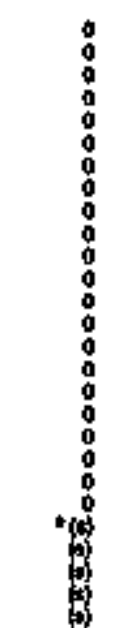 & 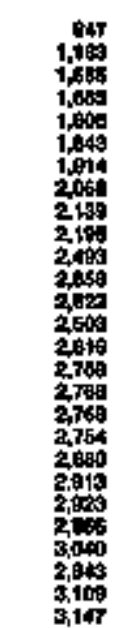 & $\Xi$ & 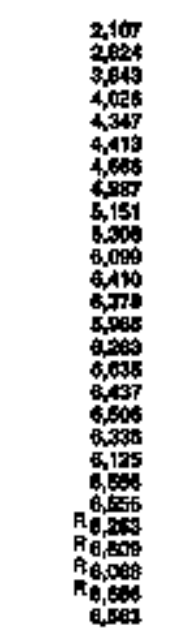 & \\
\hline
\end{tabular}

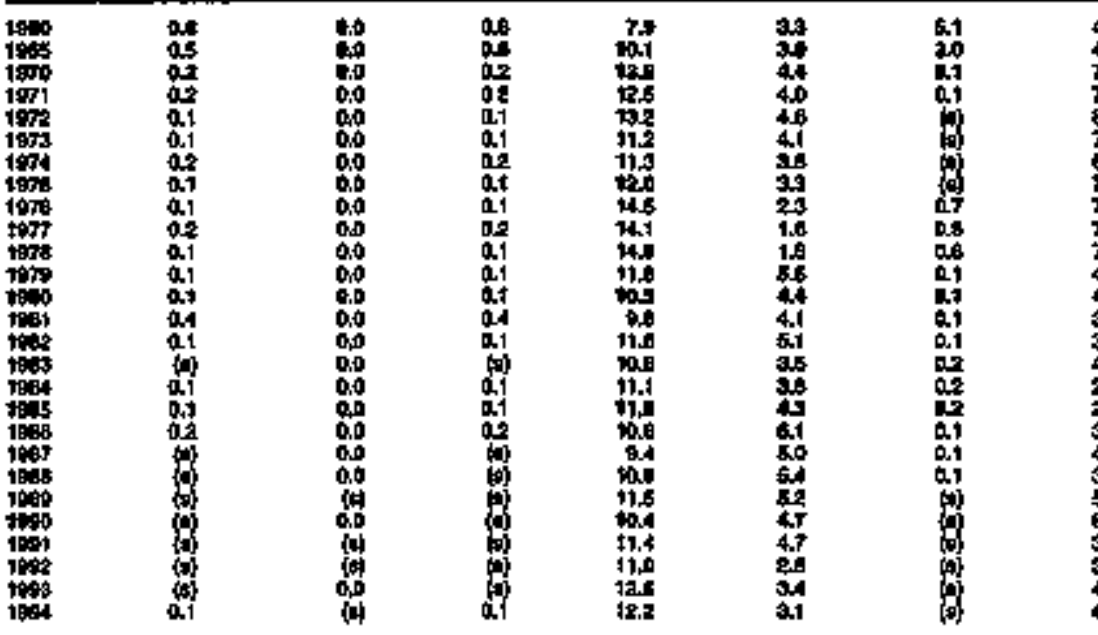

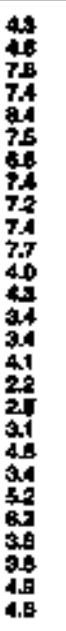

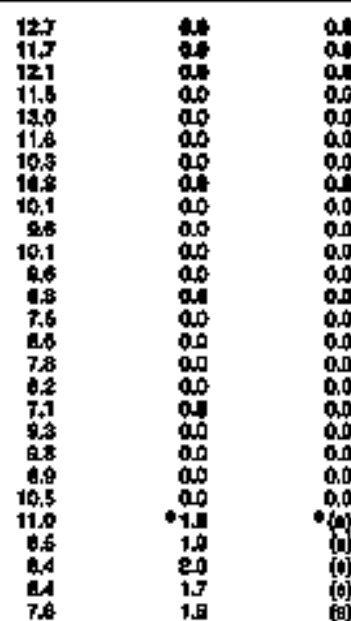

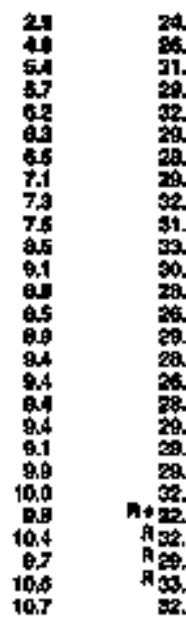

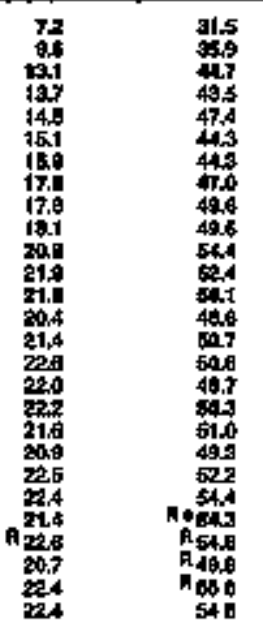

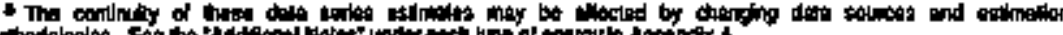

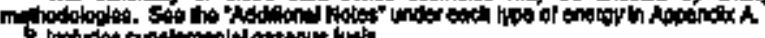

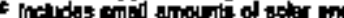

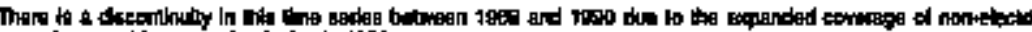

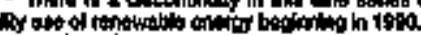
Propolped ditio

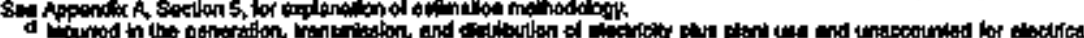

9.

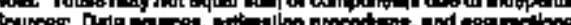

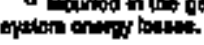


Table 265. Commerctal Energy Consumption Estlmates, 1960, 1965, 1970-1994, South Dakota

\begin{tabular}{|c|c|c|c|c|c|c|c|c|c|c|c|c|c|c|c|}
\hline \multirow[b]{3}{*}{ Your } & \multicolumn{3}{|c|}{ (1) } & \multirow{3}{*}{ 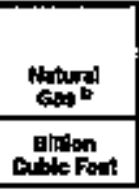 } & \multicolumn{6}{|c|}{ Portolum } & \multirow[b]{2}{*}{ Ementidy" } & \multirow[b]{2}{*}{ Extrols } & \multirow{3}{*}{ 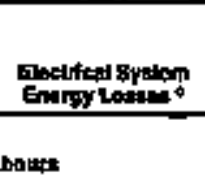 } & \multirow[b]{3}{*}{ Thond d } & \\
\hline & Comurdigover, & Anthercho: & Torat & & 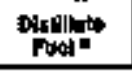 & Kamateng" & LPE: & Motor ar & Papoldure & 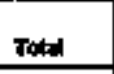 & & & & & \\
\hline & \multicolumn{3}{|c|}{ Thousend Shoort tias } & & \multicolumn{6}{|c|}{ Thourand Oarreth } & \multicolumn{2}{|c|}{ 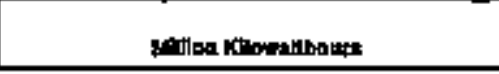 } & & & \\
\hline 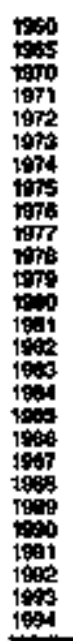 & 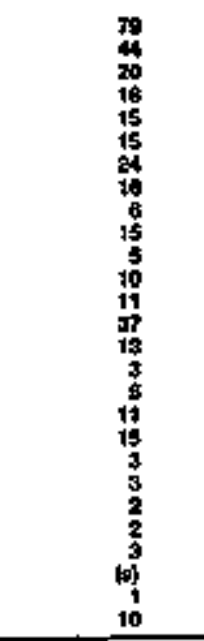 & 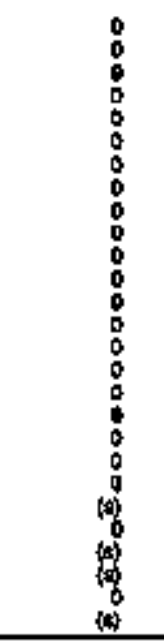 & 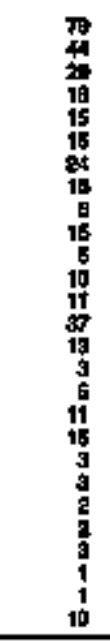 & 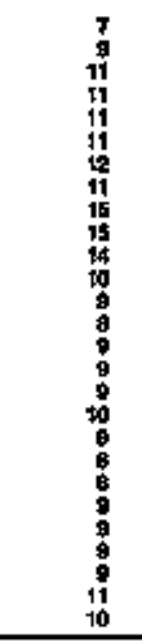 & 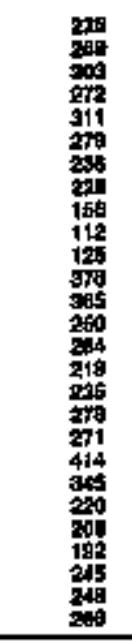 & 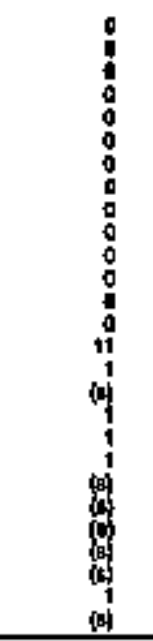 & 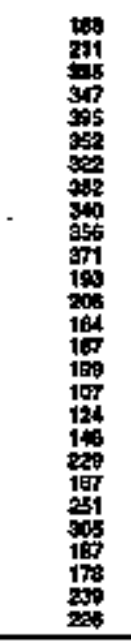 & 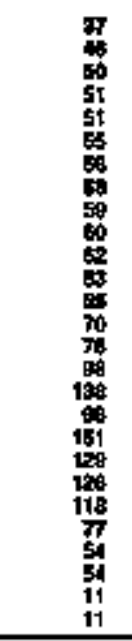 & 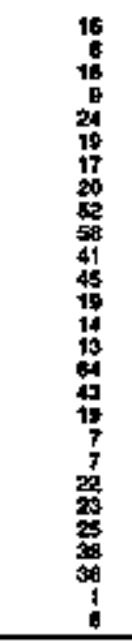 & 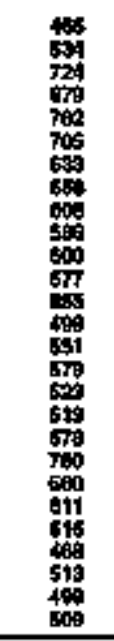 & 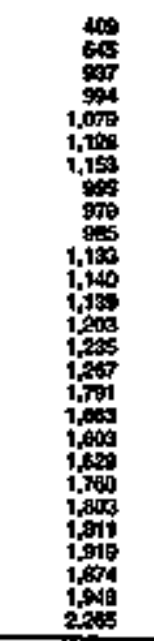 & $\begin{array}{l}\vdots \\
\vdots \\
\vdots \\
\vdots \\
\vdots \\
\vdots \\
\vdots \\
\vdots \\
\vdots \\
\vdots \\
\vdots \\
\vdots \\
\vdots\end{array}$ & 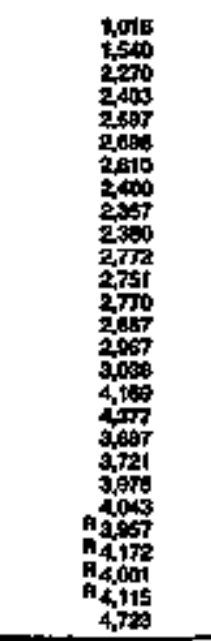 & $\begin{array}{l}\vdots \\
\vdots \\
\vdots \\
\vdots \\
\vdots \\
\vdots \\
\vdots \\
\vdots \\
\vdots \\
\vdots \\
\vdots \\
\vdots \\
\vdots\end{array}$ & \\
\hline \multicolumn{15}{|c|}{$m \lim \theta 00$} & \\
\hline 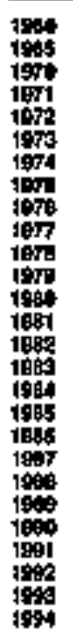 & 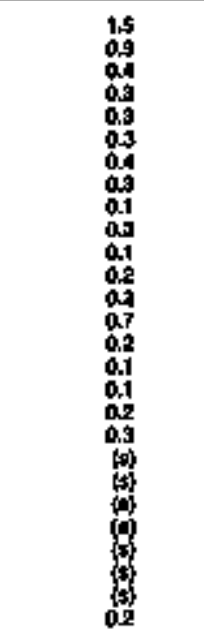 & 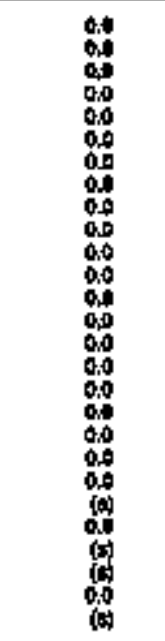 & 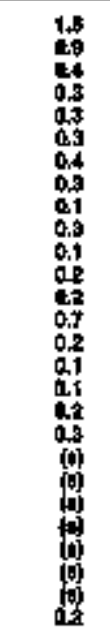 & 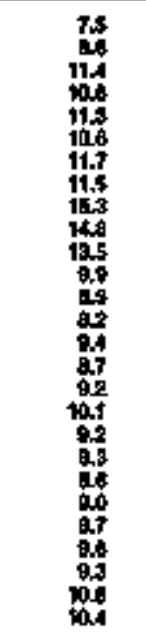 & 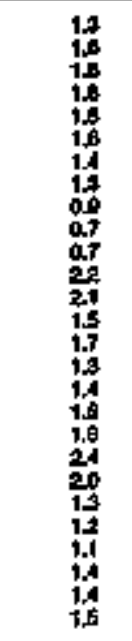 & 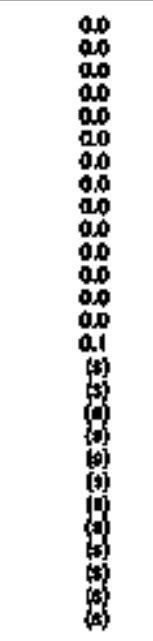 & 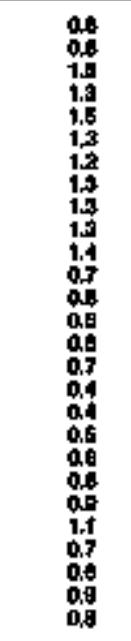 & 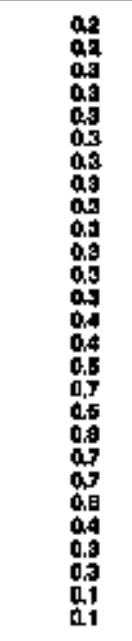 & 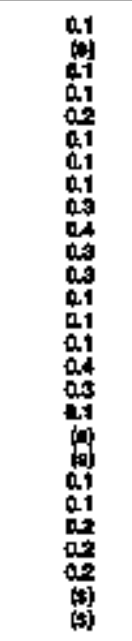 & 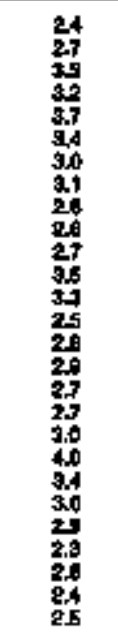 & 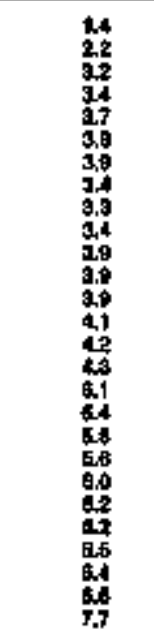 & 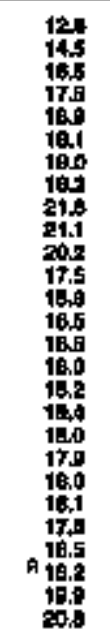 & 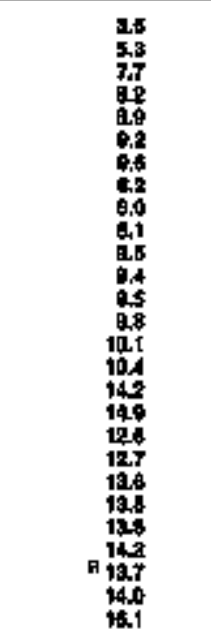 & 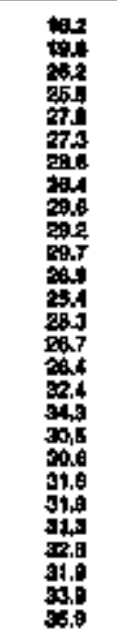 & \\
\hline \multirow{2}{*}{\multicolumn{15}{|c|}{ 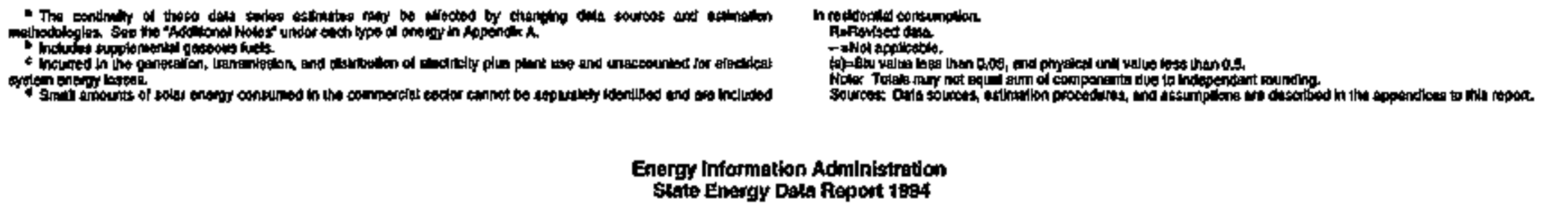 }} & \\
\hline & & & & & & & & & & & & & & & 277 \\
\hline
\end{tabular}


Table 266. Industrial Entugy Consumption Estimates, 1960, 1965, 1970-1994, South Dakata

\begin{tabular}{|c|c|c|c|c|c|c|c|c|c|c|c|c|c|c|c|c|c|c|}
\hline \multirow[b]{3}{*}{ Kar } & \multirow[b]{2}{*}{ cos } & \multirow[b]{2}{*}{ Nonft } & \multicolumn{9}{|c|}{ Porotan } & \multirow[b]{2}{*}{ 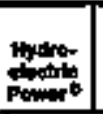 } & \multirow[b]{2}{*}{ Glonsele } & \multirow[b]{2}{*}{ ocmertad } & \multirow[b]{2}{*}{ Dostialy 6} & \multirow[b]{2}{*}{ 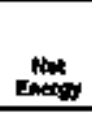 } & \multirow{2}{*}{ 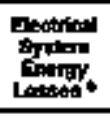 } & \multirow[b]{3}{*}{ Total } \\
\hline & & & Anothend & 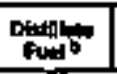 & Karosing b th & LPok & Lontemant & 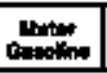 & 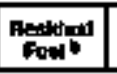 & ons & Toton & & & & & & & \\
\hline & Thent tons & contion & \multicolumn{9}{|c|}{ ח } & \multicolumn{6}{|c|}{ 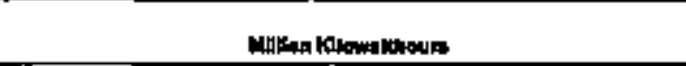 } & \\
\hline 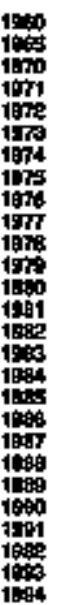 & 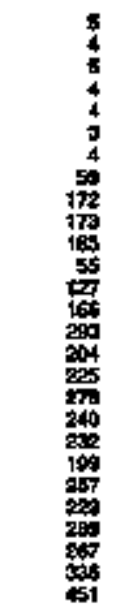 & $\begin{array}{l}8 \\
8 \\
8 \\
8 \\
5 \\
5\end{array}$ & 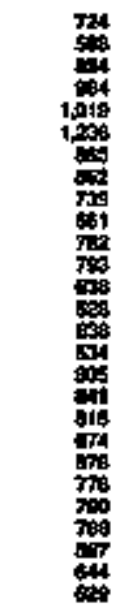 & 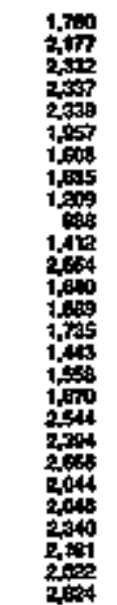 & 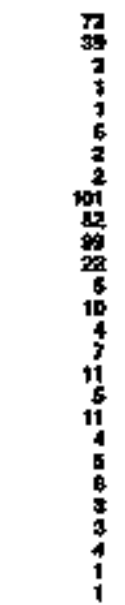 & 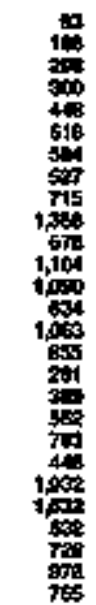 & 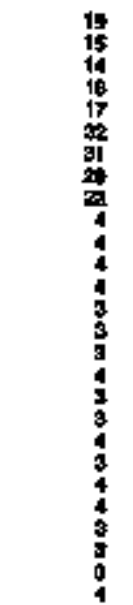 & 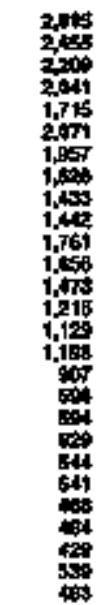 & 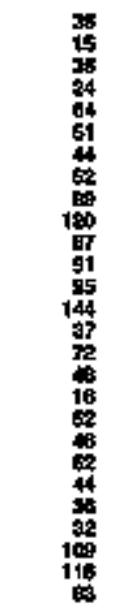 & 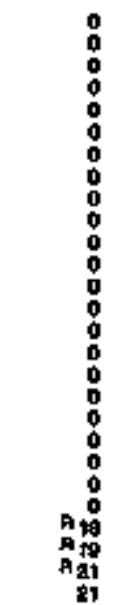 & 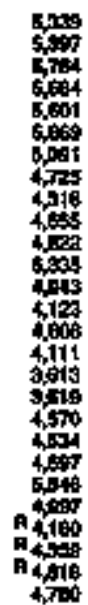 & 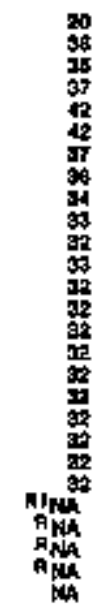 & $\begin{array}{l}0 \\
0 \\
0 \\
0 \\
0 \\
0 \\
0 \\
0 \\
0 \\
0 \\
0 \\
0 \\
0 \\
0 \\
0 \\
0 \\
0 \\
0 \\
0 \\
0 \\
0 \\
0 \\
4\end{array}$ & $\begin{array}{l}0 \\
0 \\
0 \\
0 \\
0 \\
0 \\
0 \\
0 \\
0 \\
0 \\
0 \\
0 \\
0 \\
0 \\
0 \\
0 \\
0 \\
0 \\
0 \\
0 \\
0 \\
0 \\
\text { ma }\end{array}$ & 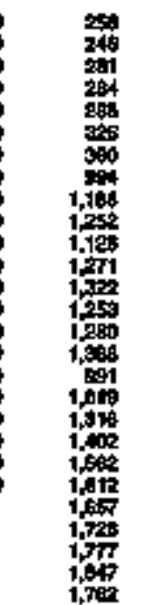 & $\begin{array}{l}\bar{z} \\
\bar{z} \\
\bar{z} \\
\bar{z} \\
\bar{z}\end{array}$ & 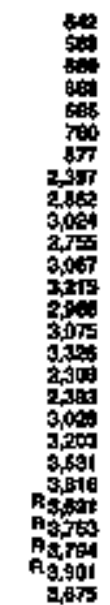 & $\begin{array}{l}\bar{z} \\
\bar{z} \\
\bar{z}\end{array}$ \\
\hline
\end{tabular}

\begin{tabular}{|c|c|c|c|c|c|c|c|c|c|c|c|c|c|c|c|c|c|c|}
\hline & & & & & & & & & אם & & & & & & & & & \\
\hline 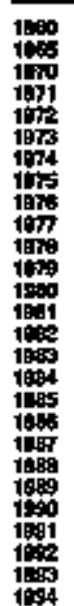 & 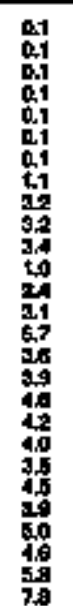 & 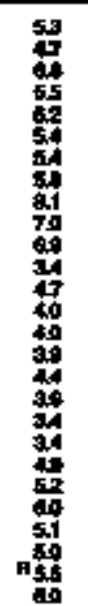 & 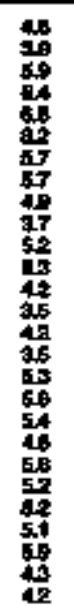 & 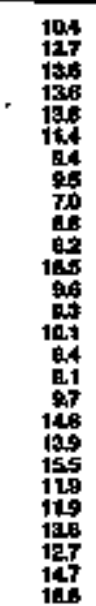 & 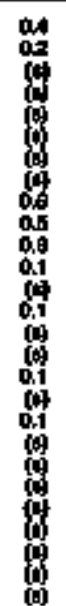 & 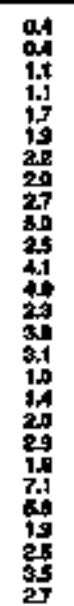 & 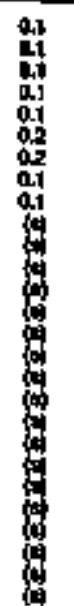 & 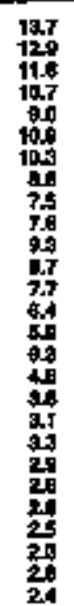 & 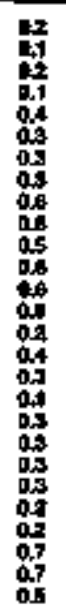 & 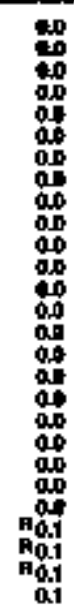 & 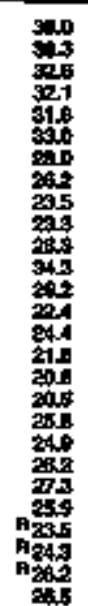 & 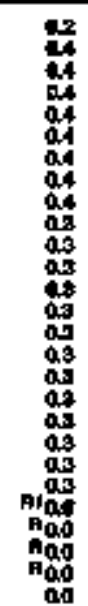 & 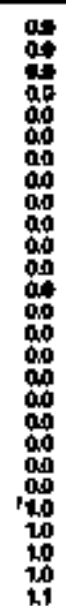 & 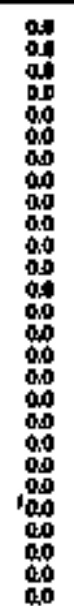 & 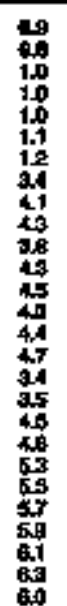 & 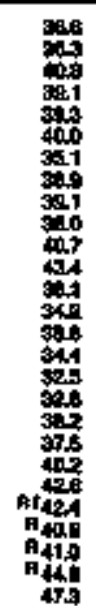 & 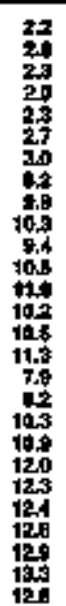 & 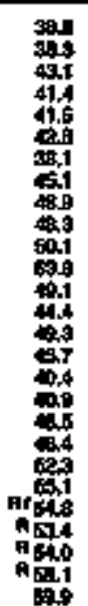 \\
\hline
\end{tabular}

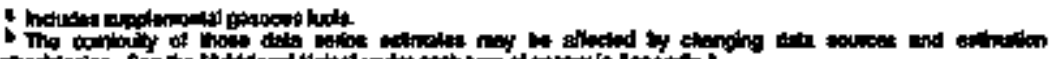

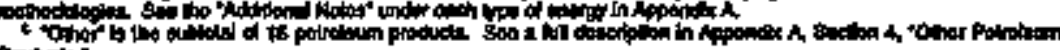
Prodictio.

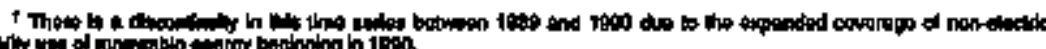

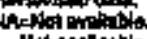

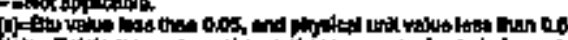

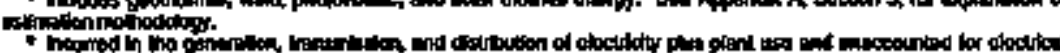

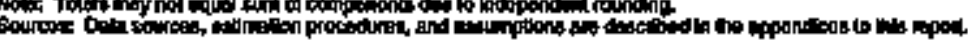

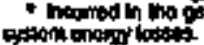


Teble 267. Transportalion Energy Consumption Estimatins, 1950, 1985, 1970-1994, 8outh Dakota

\begin{tabular}{|c|c|c|c|c|c|c|c|c|c|c|c|c|c|c|c|}
\hline \multirow[b]{3}{*}{$\mathrm{ren}$} & \multirow[b]{2}{*}{$\cos 10$} & \multirow[b]{2}{*}{ mates } & \multicolumn{8}{|c|}{ Potralem } & \multirow{3}{*}{ 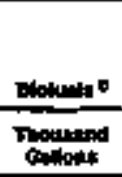 } & & & \multirow[b]{2}{*}{ 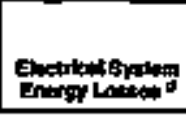 } & \multirow[b]{3}{*}{ roter } \\
\hline & & & cofacion. & Drtingt" & fort. & EPG: & 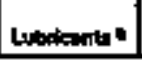 & andition & Fontontel & Toto & & Inetrefly 1 & reosty & & \\
\hline & $\begin{array}{l}\text { Proutents } \\
\text { thent Fons }\end{array}$ & 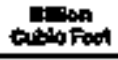 & \multicolumn{8}{|c|}{ mo demente } & & \multicolumn{3}{|c|}{ 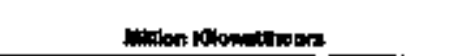 } & \\
\hline 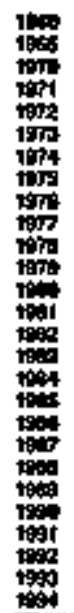 & 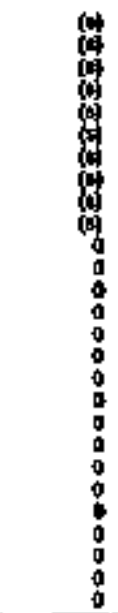 & 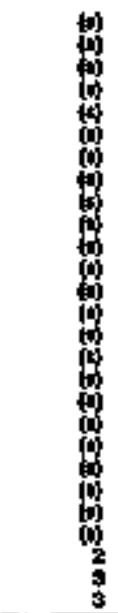 & 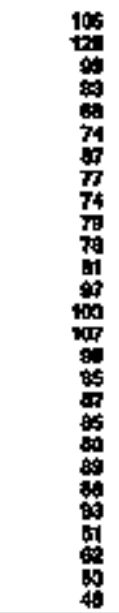 & 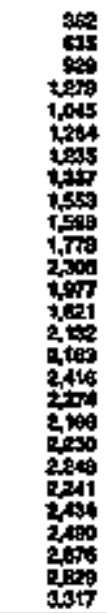 & 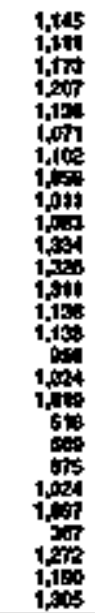 & 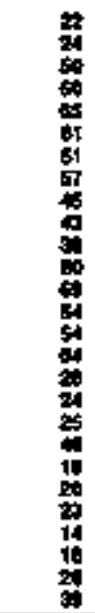 & 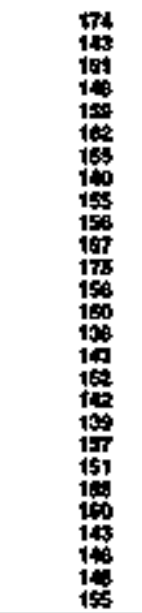 & 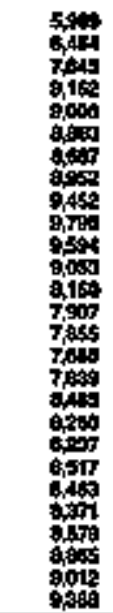 & $\begin{array}{r}11 \\
1 \\
1 \\
0 \\
0 \\
0 \\
\vdots \\
1 \\
0 \\
0 \\
0 \\
0 \\
0 \\
0 \\
0 \\
0 \\
0 \\
0 \\
0 \\
0\end{array}$ & 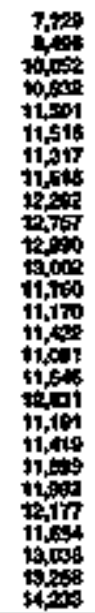 & 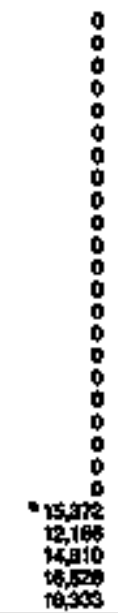 & $\begin{array}{l}0 \\
0 \\
0 \\
0 \\
0 \\
0 \\
0 \\
0 \\
0 \\
0 \\
0 \\
0 \\
0 \\
0 \\
0 \\
0 \\
0 \\
0 \\
0 \\
0 \\
0 \\
0 \\
0 \\
0 \\
0 \\
0\end{array}$ & $\begin{array}{l}z \\
z \\
z \\
z \\
z \\
z \\
z \\
z \\
z \\
z \\
z \\
z \\
z \\
z \\
z\end{array}$ & $\begin{array}{l}0 \\
0 \\
0 \\
0 \\
0 \\
0 \\
0 \\
0 \\
0 \\
0 \\
0 \\
0 \\
0 \\
0 \\
0 \\
0 \\
0 \\
0 \\
0 \\
0 \\
0\end{array}$ & 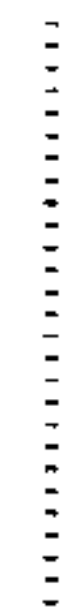 \\
\hline
\end{tabular}

\begin{tabular}{|c|c|c|c|c|c|c|c|c|c|c|c|c|c|c|c|}
\hline 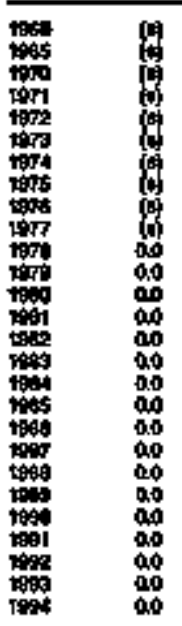 & 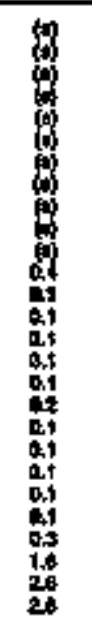 & 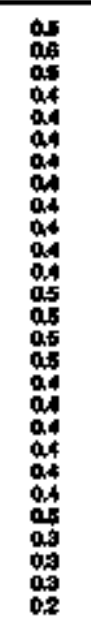 & 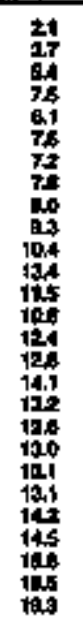 & 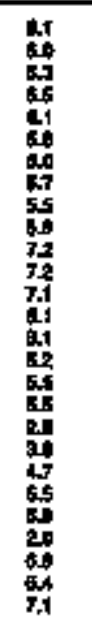 & 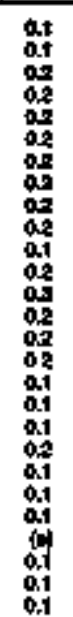 & 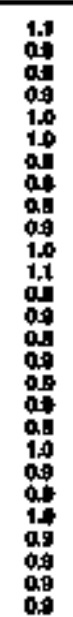 & 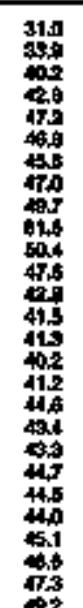 & 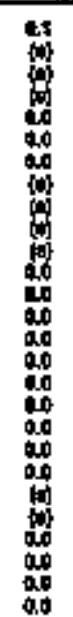 & 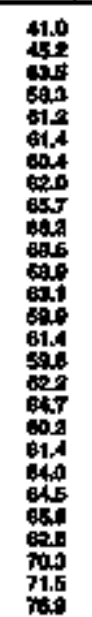 & 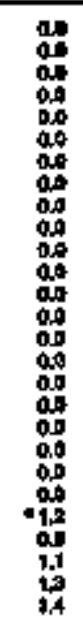 & & & 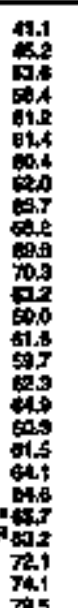 & 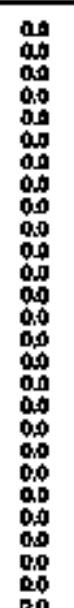 & 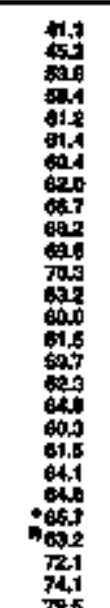 \\
\hline
\end{tabular}

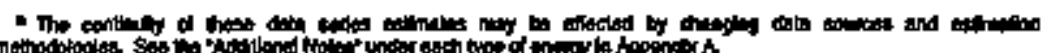

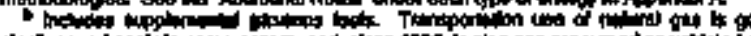

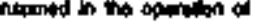
do

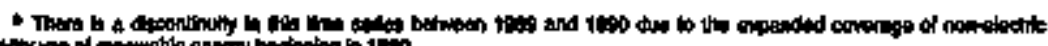

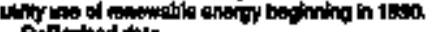
ariontiod

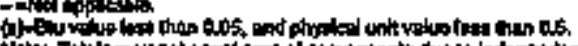

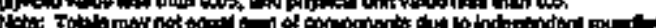

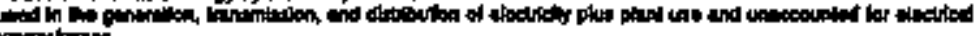

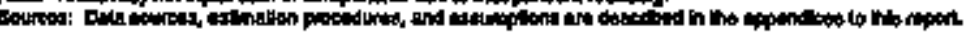




\begin{tabular}{|c|c|c|c|c|c|c|c|c|c|c|c|c|c|c|}
\hline \multirow[b]{3}{*}{ rout } & \multicolumn{3}{|c|}{$\omega_{0}$} & \multirow[b]{2}{*}{ tort } & \multicolumn{4}{|c|}{ From } & \multirow[b]{2}{*}{ 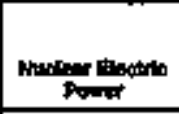 } & \multirow[b]{2}{*}{ 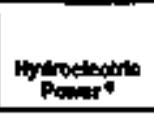 } & \multirow[b]{2}{*}{ Olotum } & \multirow[b]{2}{*}{ 1 } & \multirow[b]{2}{*}{ الt } & \multirow[b]{3}{*}{ D } \\
\hline & 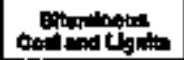 & Antwedn & Ta & & 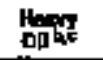 & 남하 & Putoleng & Tat & & & & & & \\
\hline & \multicolumn{3}{|c|}{ 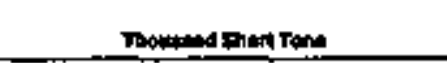 } & 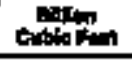 & \multicolumn{4}{|c|}{ 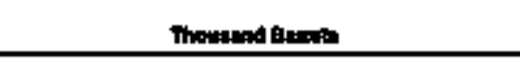 } & \multicolumn{5}{|c|}{ 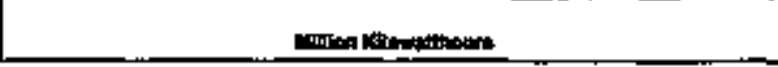 } & \\
\hline 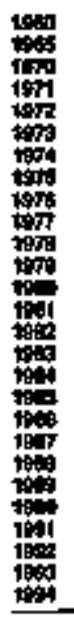 & 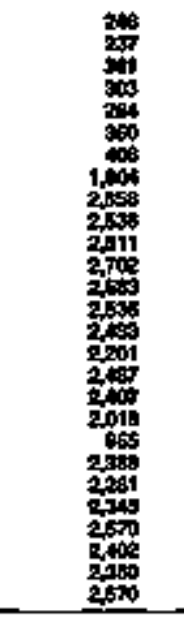 & $\begin{array}{l}0 \\
0 \\
0 \\
0 \\
0 \\
0 \\
0 \\
0 \\
0 \\
0 \\
0 \\
0 \\
0 \\
0 \\
0 \\
0 \\
0 \\
0 \\
0 \\
0 \\
0 \\
0 \\
0 \\
\end{array}$ & 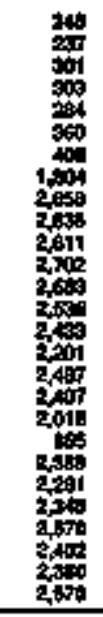 & 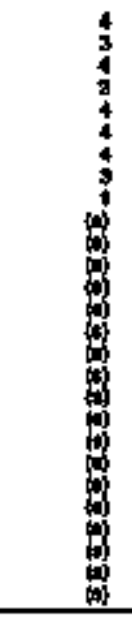 & 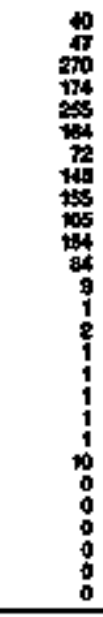 & 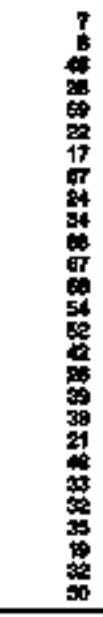 & $\begin{array}{l}0 \\
0 \\
0 \\
0 \\
0 \\
0 \\
0 \\
0 \\
0 \\
0 \\
0 \\
0 \\
0 \\
0 \\
0 \\
0 \\
0 \\
0 \\
0 \\
0 \\
0 \\
0 \\
0 \\
0 \\
0 \\
0 \\
0 \\
0\end{array}$ & 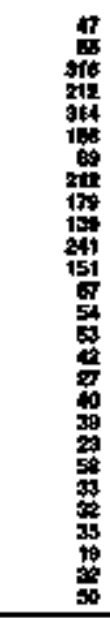 & $\begin{array}{l}0 \\
0 \\
0 \\
0 \\
0 \\
0 \\
0 \\
0 \\
0 \\
0 \\
0 \\
0 \\
0 \\
0 \\
0 \\
0 \\
0 \\
0 \\
0 \\
0 \\
0 \\
0 \\
0 \\
0 \\
0 \\
0 \\
0\end{array}$ & 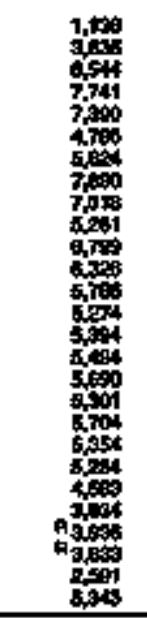 & 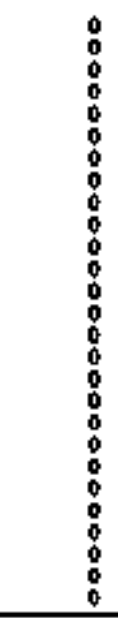 & $\begin{array}{l}0 \\
\vdots \\
\vdots \\
\vdots \\
\vdots \\
\vdots \\
\vdots \\
\vdots \\
\vdots \\
\vdots \\
\vdots \\
\vdots \\
\vdots \\
\vdots\end{array}$ & $\begin{array}{l}: \\
: \\
0 \\
0 \\
\vdots \\
0 \\
0 \\
0 \\
0 \\
0 \\
0 \\
0 \\
0 \\
0 \\
0 \\
0 \\
0 \\
0 \\
0 \\
0 \\
0 \\
0\end{array}$ & $\begin{array}{l}\vdots \\
\vdots \\
\vdots \\
\vdots \\
\vdots \\
\vdots \\
\vdots \\
\vdots \\
\vdots \\
\vdots \\
\vdots \\
\vdots\end{array}$ \\
\hline \multicolumn{15}{|c|}{ Trtation 0to } \\
\hline 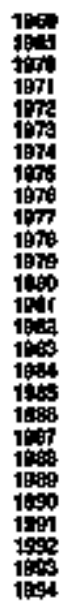 & 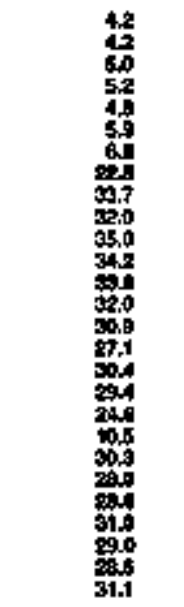 & 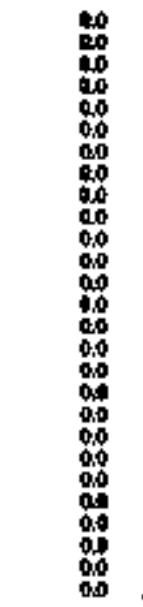 & 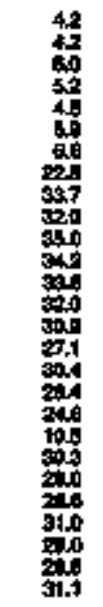 & 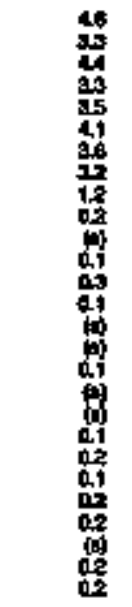 & 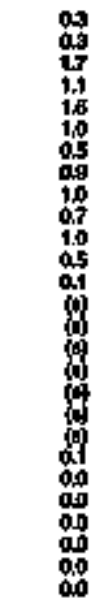 & 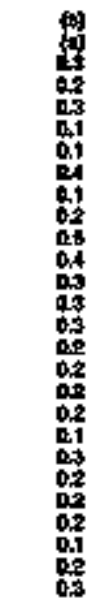 & 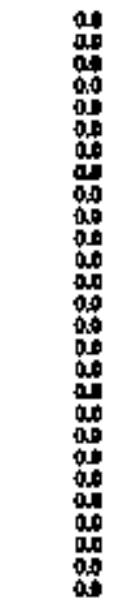 & 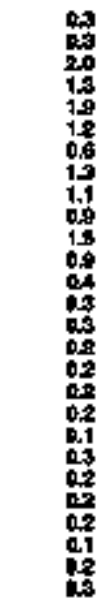 & 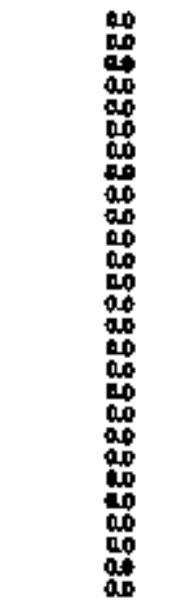 & 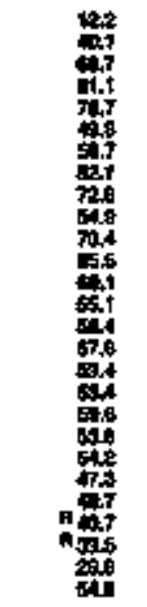 & 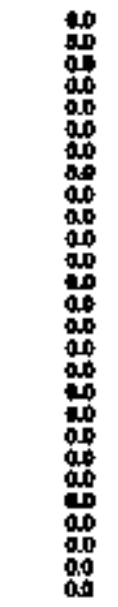 & 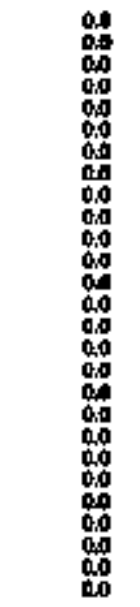 & 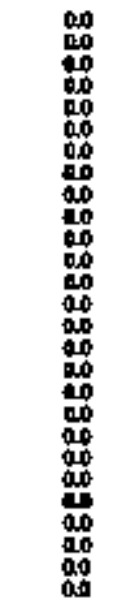 & 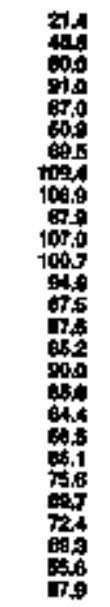 \\
\hline
\end{tabular}




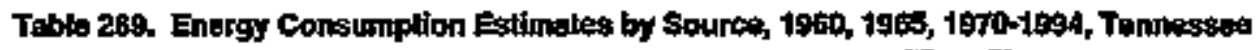

\begin{tabular}{|c|c|c|c|c|c|c|c|c|c|c|c|c|c|c|c|c|c|c|c|}
\hline \multirow[b]{3}{*}{ Yen } & \multirow[b]{2}{*}{ an: } & \multirow[b]{2}{*}{ 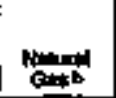 } & \multicolumn{11}{|c|}{ Putrolan } & \multirow{2}{*}{ 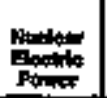 } & \multirow{2}{*}{ Aldos } & \multirow[b]{2}{*}{ Eaphudse | } & \multirow[b]{2}{*}{ Otwer of } & \multirow{3}{*}{ 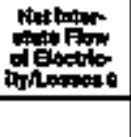 } & \multirow[b]{3}{*}{ Totat W } \\
\hline & & & 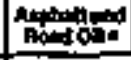 & 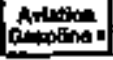 & 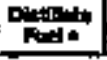 & Fit. & Kim. & Led & Latid & Holse & 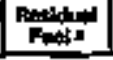 & anters & البئي & & & & & & \\
\hline & monend & astion & \multicolumn{11}{|c|}{ 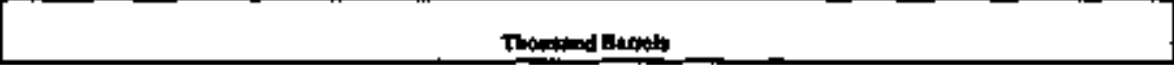 } & \multicolumn{4}{|c|}{ 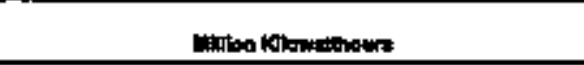 } & & \\
\hline 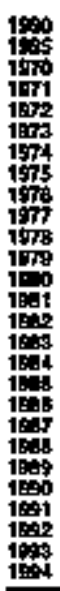 & 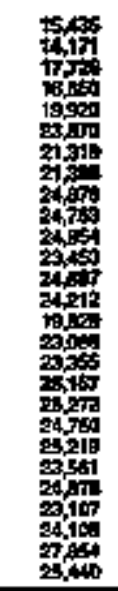 & 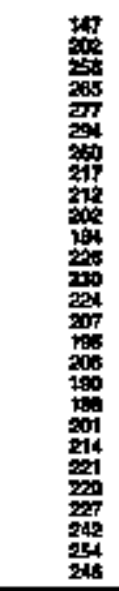 & 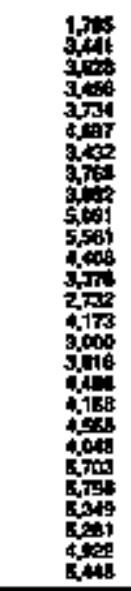 & 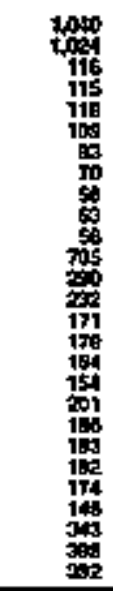 & 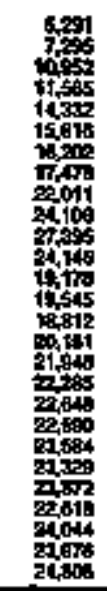 & 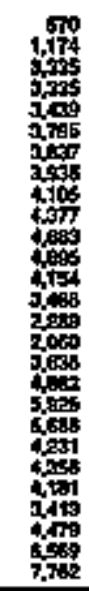 & 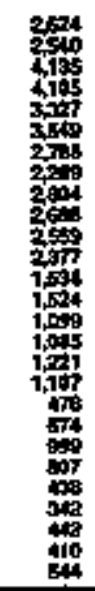 & 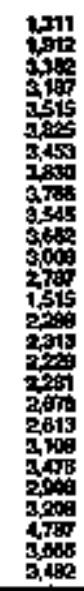 & 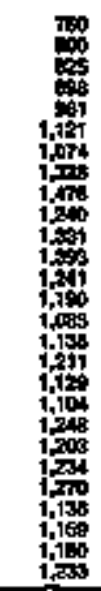 & 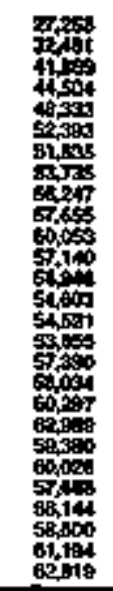 & 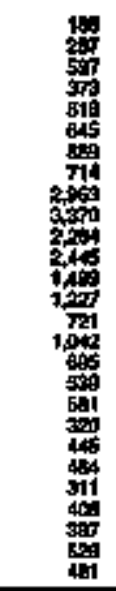 & 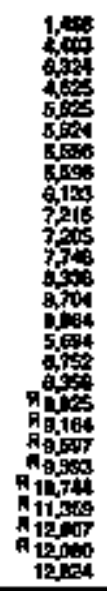 & 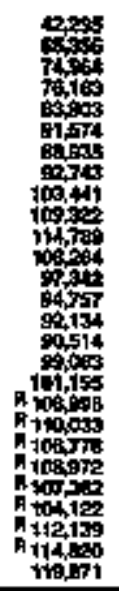 & 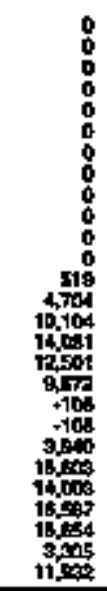 & 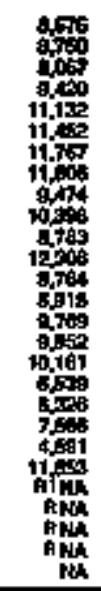 & 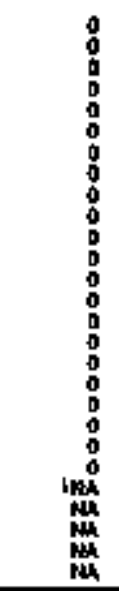 & 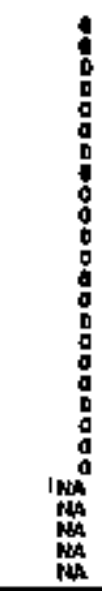 & 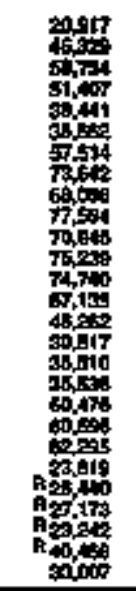 & $\begin{array}{l}z \\
z \\
z \\
z \\
z \\
z \\
z \\
z \\
z \\
z \\
z \\
z \\
z\end{array}$ \\
\hline \multicolumn{20}{|c|}{ Mithon aly } \\
\hline 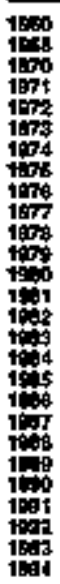 & 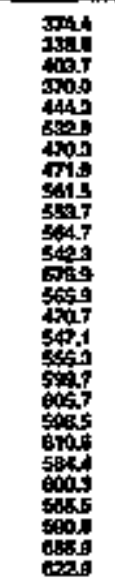 & 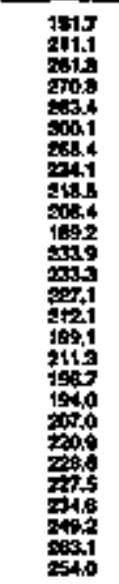 & 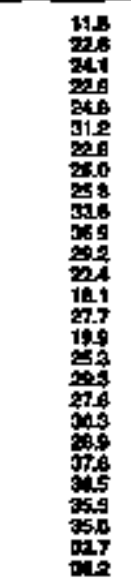 & 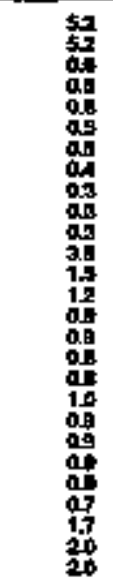 & 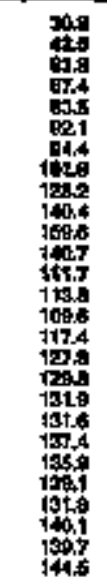 & 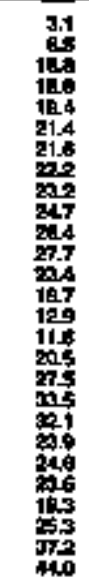 & 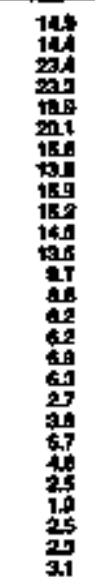 & 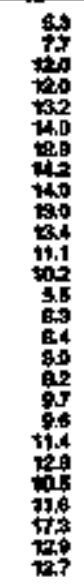 & 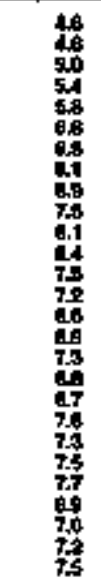 & 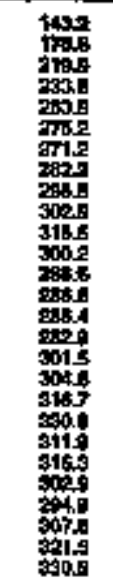 & 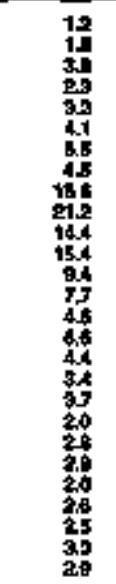 & 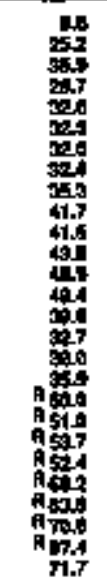 & 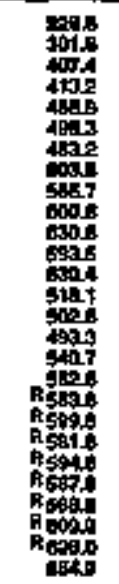 & 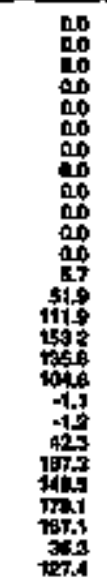 & 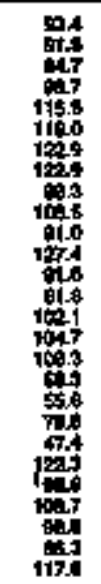 & 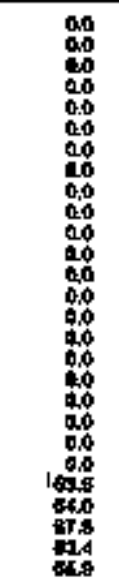 & 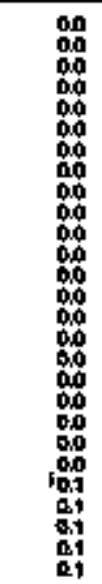 & 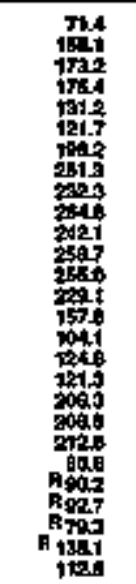 & 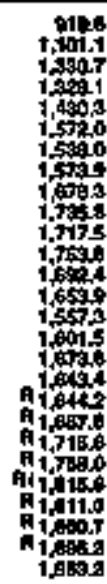 \\
\hline
\end{tabular}

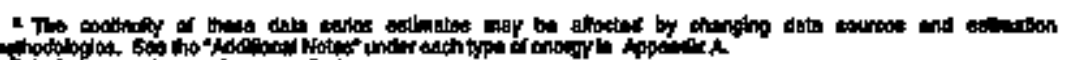

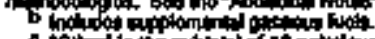

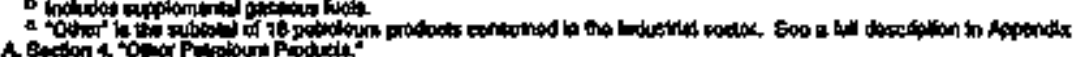

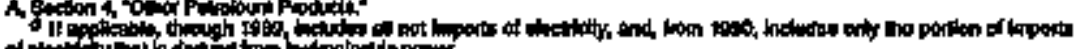

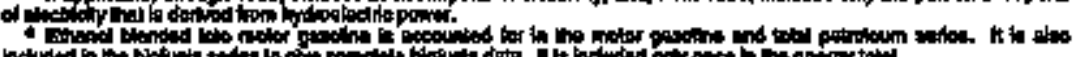

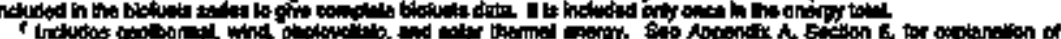

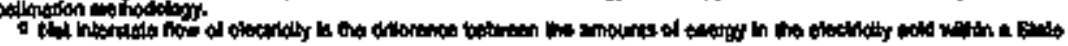

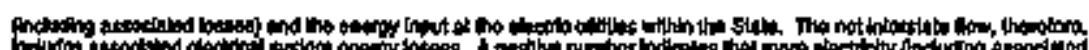

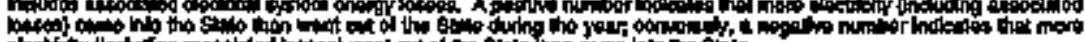

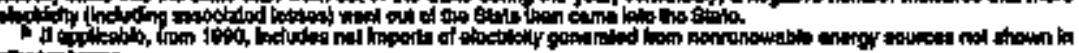

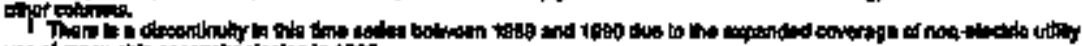

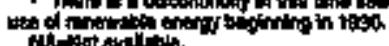

\section{-}

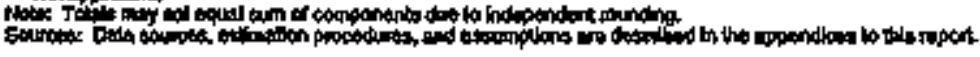


Table 270. Residential Energy Consumption Estimates, 1960, 1955, 1970-1994, Tennessede

\begin{tabular}{|c|c|c|c|c|c|c|c|c|c|c|c|c|c|c|}
\hline & \multicolumn{3}{|c|}{ con } & \multirow{3}{*}{ 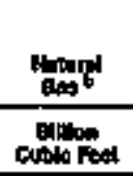 } & \multicolumn{4}{|c|}{ Petrotiven } & \multirow{3}{*}{ 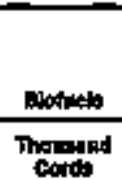 } & \multirow[b]{2}{*}{$\operatorname{sens}{ }^{\circ}$} & \multirow[b]{2}{*}{ Exotolithy" } & \multirow[b]{2}{*}{ Extry } & \multirow[b]{2}{*}{ 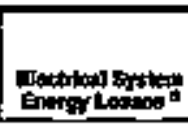 } & \multirow[b]{3}{*}{ Tobal } \\
\hline \multirow[b]{2}{*}{$\mathrm{rew}$} & 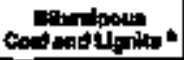 & Anthrimion & المئه & & 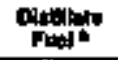 & 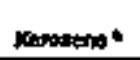 & LNGE & The dot & & & & & & \\
\hline & \multicolumn{3}{|c|}{ Thovo:ind short Tant } & & \multicolumn{4}{|c|}{ Thousand Banth } & & \multicolumn{4}{|c|}{ 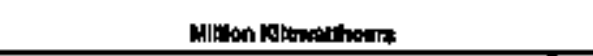 } & \\
\hline \multicolumn{15}{|c|}{ matmen } \\
\hline 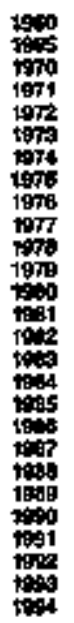 & 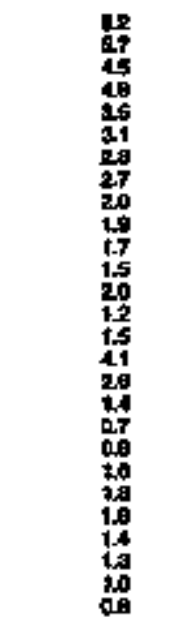 & 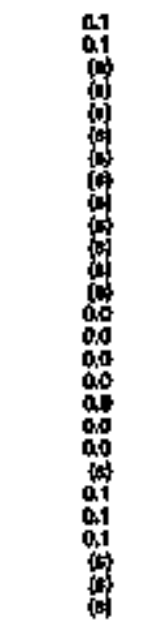 & 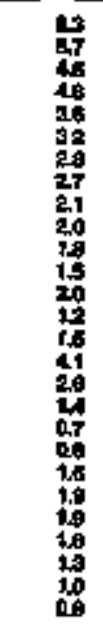 & 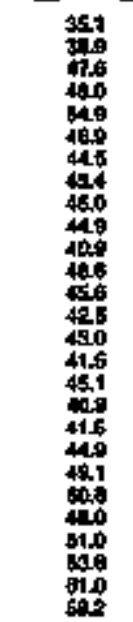 & 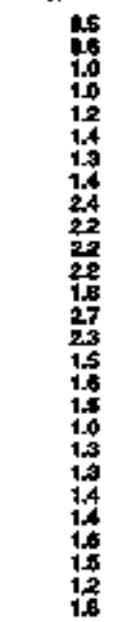 & 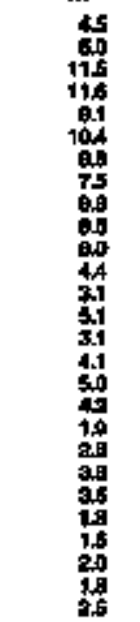 & 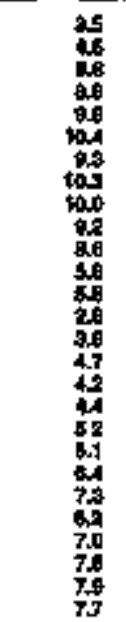 & 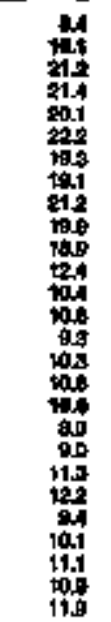 & 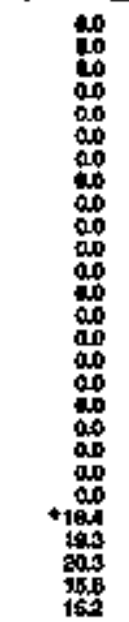 & 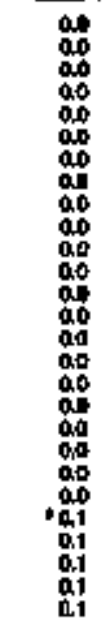 & 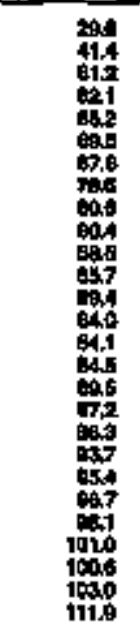 & 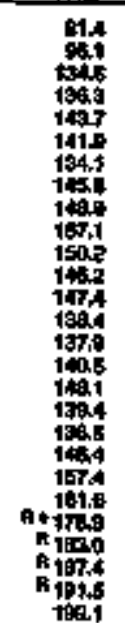 & 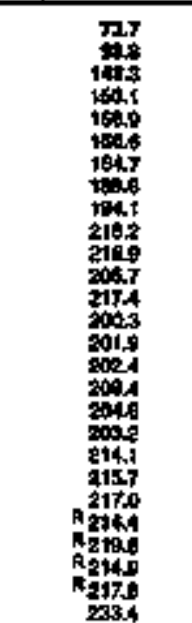 & 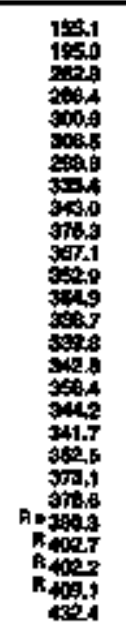 \\
\hline
\end{tabular}


Table 271. Commencial Energy Consumption Estimates, 1960, 1965, 1970-19\$4, Tennesses

\begin{tabular}{|c|c|c|c|c|c|c|c|c|c|c|c|c|c|c|}
\hline \multirow[b]{3}{*}{ rats } & \multicolumn{3}{|c|}{$\cos$} & \multirow{3}{*}{ 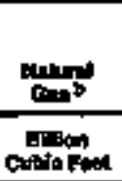 } & \multicolumn{6}{|c|}{ Potruloorn } & \multirow[b]{2}{*}{ Fectidily" } & \multirow[b]{2}{*}{ Het } & \multirow[b]{2}{*}{ 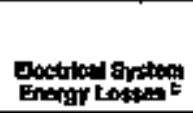 } & \multirow[b]{3}{*}{ Pot } \\
\hline & 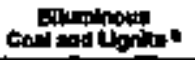 & 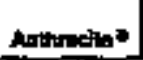 & Total & & 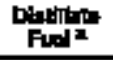 & 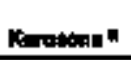 & Le: & Dolor & Aceloupl & Toted & & & & \\
\hline & \multicolumn{3}{|c|}{ 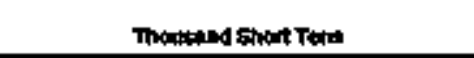 } & & \multicolumn{6}{|c|}{ moxpand hanels } & \multicolumn{3}{|c|}{ 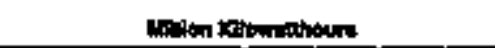 } & \\
\hline 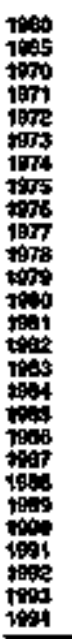 & 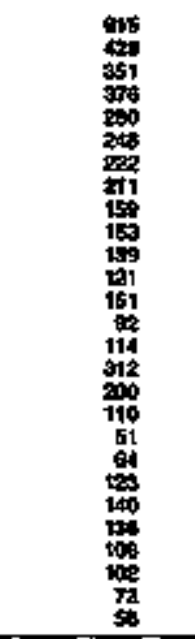 & 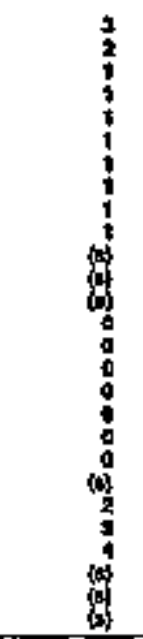 & 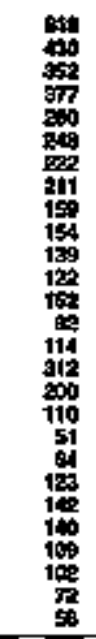 & 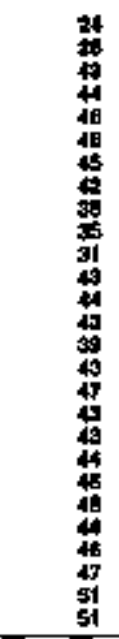 & 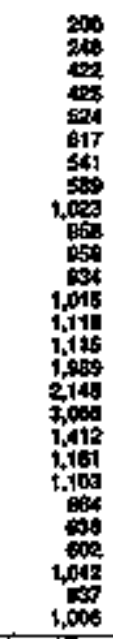 & 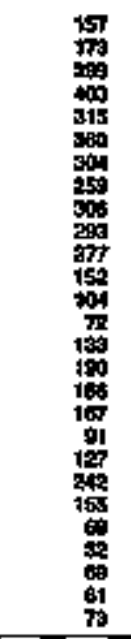 & 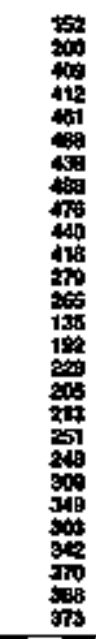 & 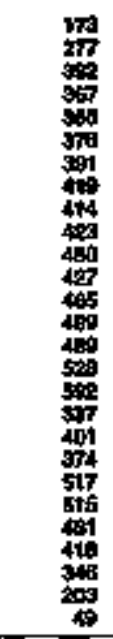 & 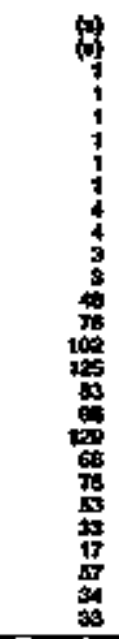 & 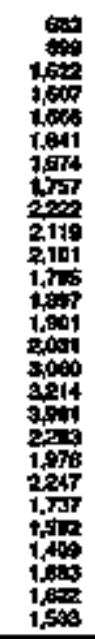 & 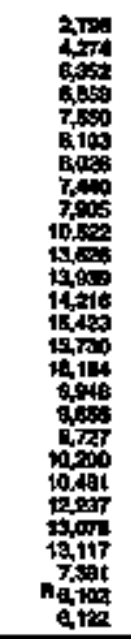 & $\begin{array}{l}= \\
= \\
= \\
= \\
= \\
= \\
= \\
= \\
= \\
= \\
= \\
= \\
= \\
=\end{array}$ & 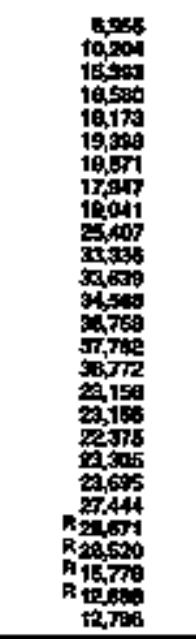 & $\begin{array}{l}= \\
= \\
= \\
= \\
= \\
= \\
= \\
= \\
= \\
= \\
= \\
= \\
=\end{array}$ \\
\hline \multicolumn{15}{|c|}{ nimlon ex: } \\
\hline 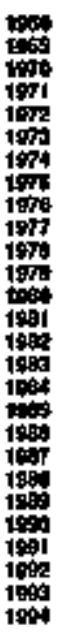 & 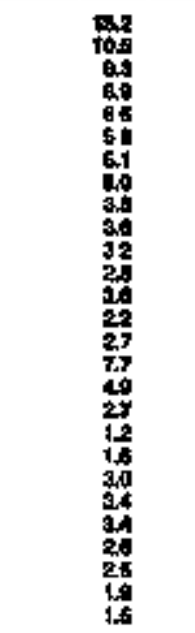 & 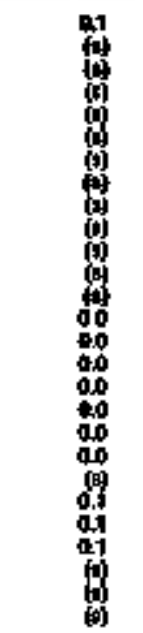 & 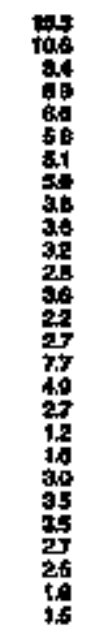 & 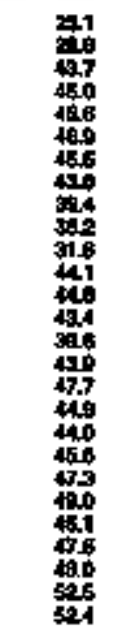 & 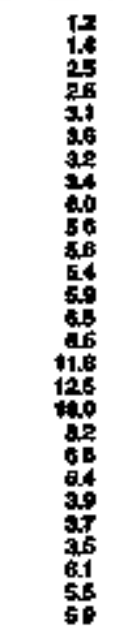 & 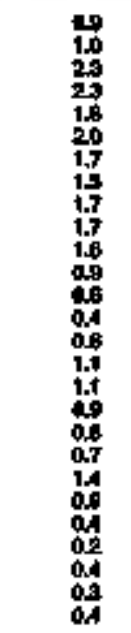 & 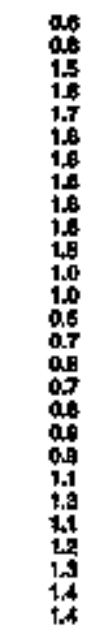 & 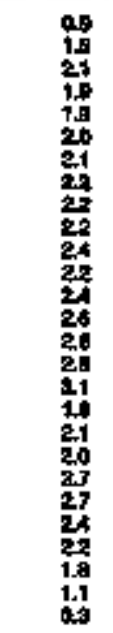 & 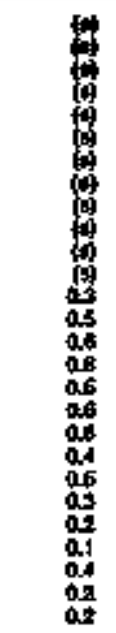 & 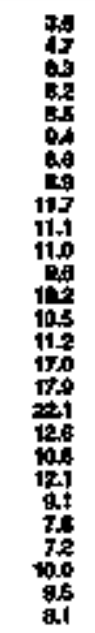 & 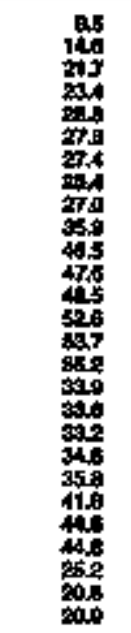 & 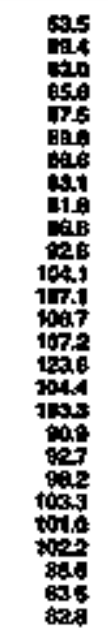 & 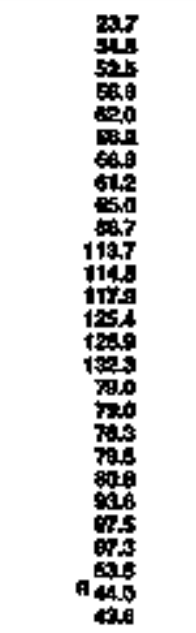 & 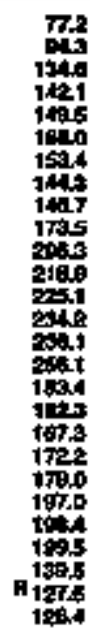 \\
\hline
\end{tabular}

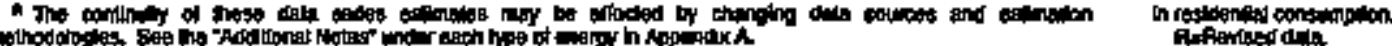

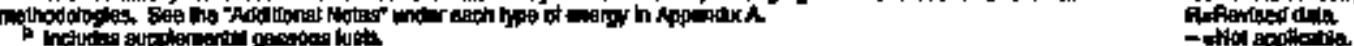

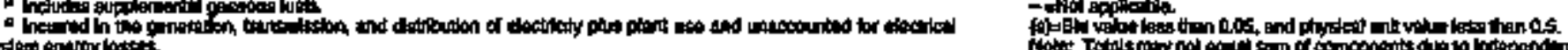

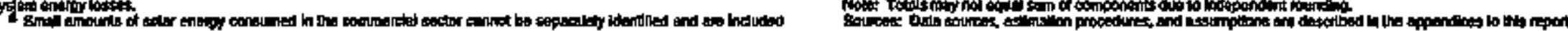




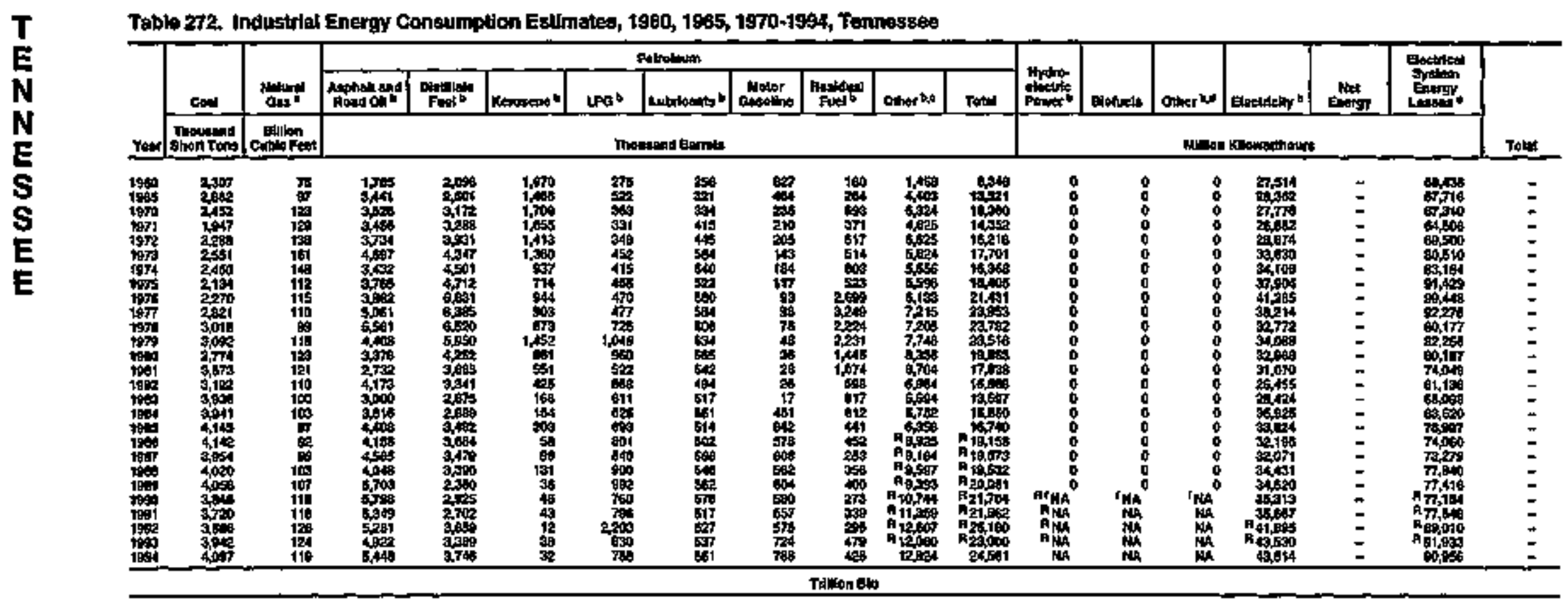

\begin{tabular}{|c|c|c|c|c|c|c|c|c|c|c|c|c|c|c|c|c|c|c|}
\hline 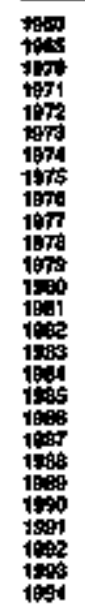 & 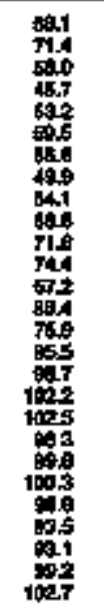 & 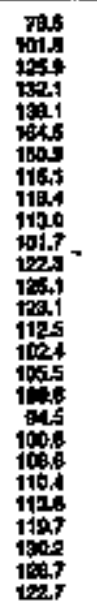 & 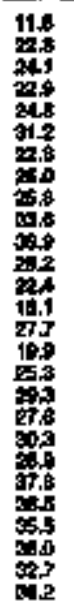 & 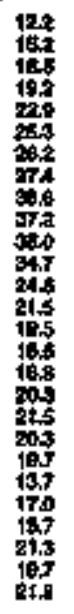 & 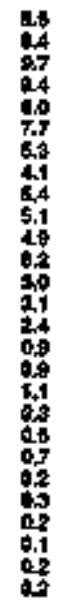 & 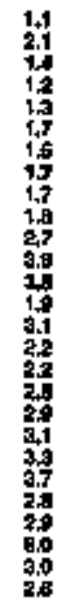 & 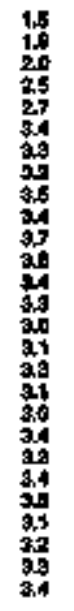 & 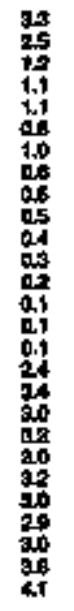 & 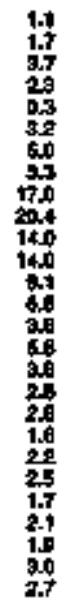 & 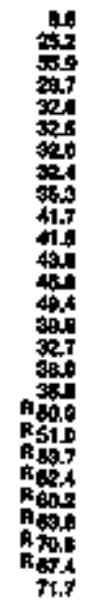 & 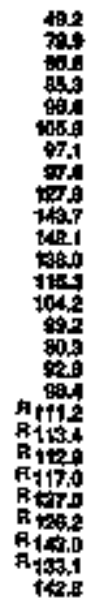 & 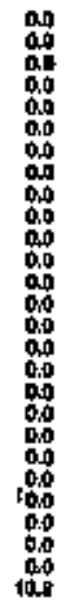 & 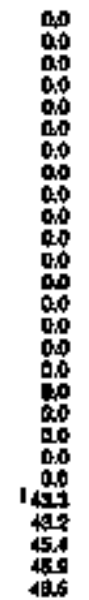 & 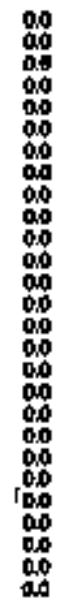 & 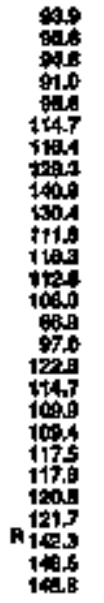 & 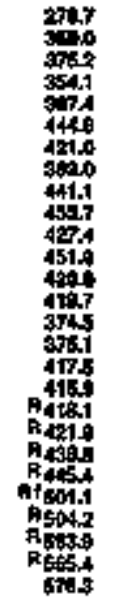 & 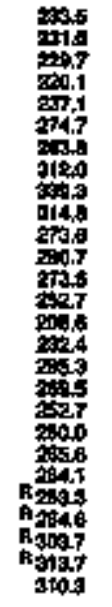 & 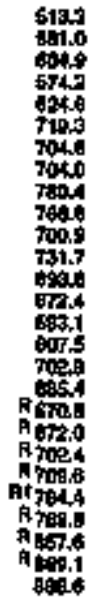 \\
\hline
\end{tabular}

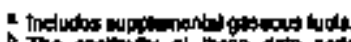

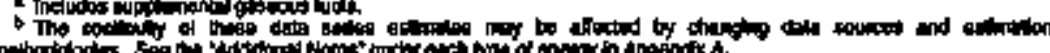

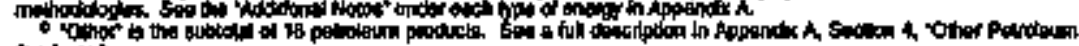

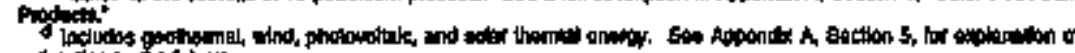

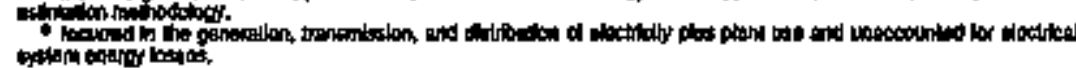

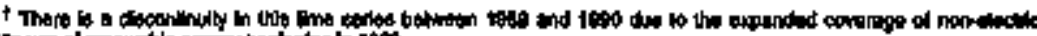

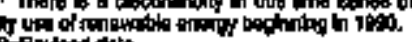

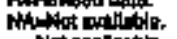

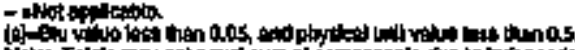

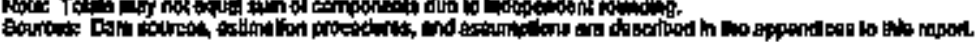


Table 273. Transportation Energy Consumption Esllmates, 1960, 1965, 1970-1994, Tennessitio

\begin{tabular}{|c|c|c|c|c|c|c|c|c|c|c|c|c|c|c|c|}
\hline \multirow[b]{3}{*}{$r=$} & \multirow[b]{2}{*}{ cont" } & \multirow[b]{2}{*}{ 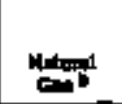 } & \multicolumn{8}{|c|}{ Patrolater } & \multirow{3}{*}{ 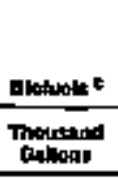 } & \multirow[b]{2}{*}{ nethaty. } & \multirow[b]{2}{*}{ 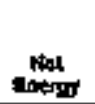 } & \multirow[b]{2}{*}{ 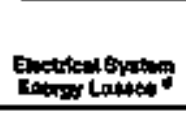 } & \multirow[b]{3}{*}{ Total } \\
\hline & & & Ation: & Ortens: & Net. & Lad: & " & 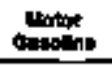 & 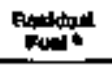 & Tot & & & & & \\
\hline & $\begin{array}{l}\text { Thenowid } \\
\text { Sylont Tons }\end{array}$ & arterent & \multicolumn{8}{|c|}{ 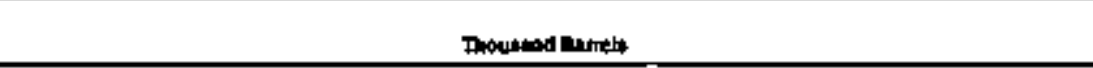 } & & \multicolumn{3}{|c|}{ 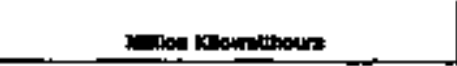 } & \\
\hline 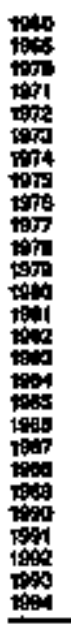 & $\begin{array}{r}19 \\
9 \\
4 \\
2 \\
2 \\
2 \\
2 \\
1 \\
0 \\
0 \\
010 \\
0 \\
0 \\
0 \\
0 \\
0 \\
0 \\
0 \\
0 \\
0 \\
0 \\
0 \\
0 \\
0 \\
0 \\
0 \\
0 \\
0 \\
\end{array}$ & 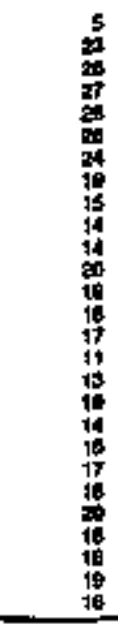 & 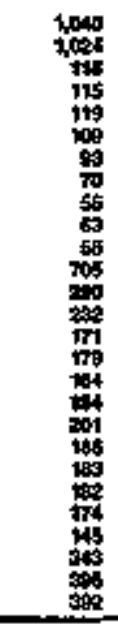 & 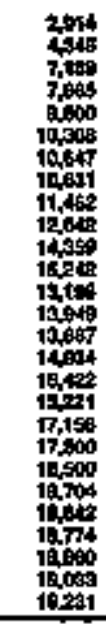 & 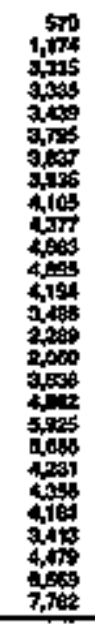 & 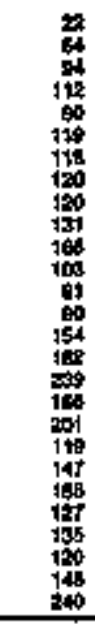 & 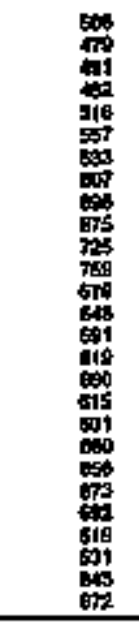 & 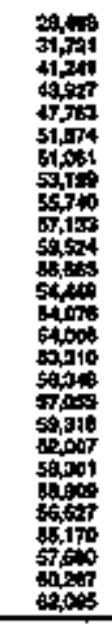 & 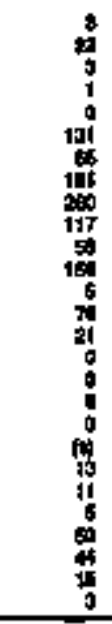 & 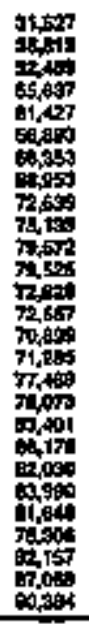 & 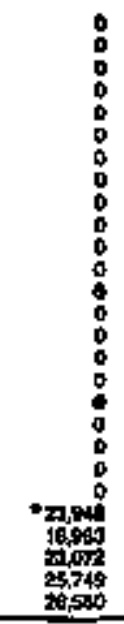 & 胻 & $\begin{array}{l}z \\
z \\
z \\
z \\
z \\
z \\
z \\
z \\
z \\
z \\
z \\
z \\
z \\
z\end{array}$ & 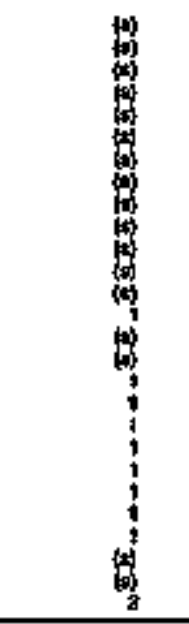 & $\begin{array}{l}= \\
= \\
= \\
= \\
= \\
= \\
= \\
= \\
= \\
= \\
z \\
z \\
z\end{array}$ \\
\hline \multicolumn{16}{|c|}{ Thallan Eit } \\
\hline 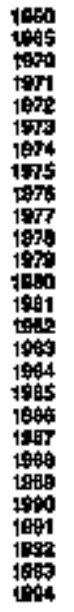 & $\begin{array}{l}40 \\
01 \\
01 \\
0.1 \\
0.1 \\
090 \\
00 \\
00 \\
00 \\
00 \\
00 \\
00 \\
00 \\
00 \\
00 \\
00 \\
00 \\
00 \\
00 \\
00 \\
00 \\
00 \\
40 \\
00\end{array}$ & 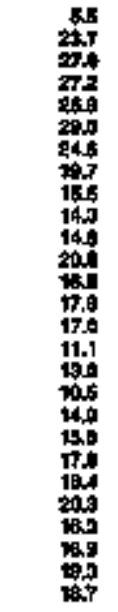 & 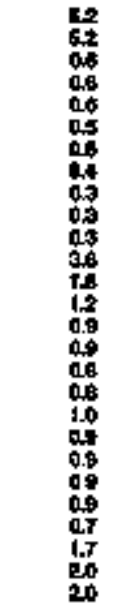 & 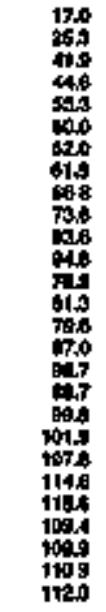 & 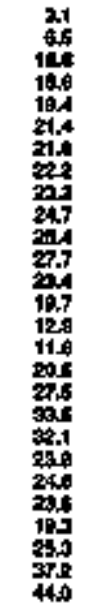 & 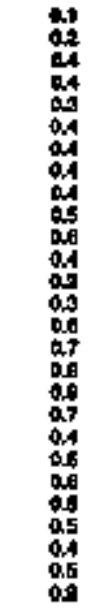 & 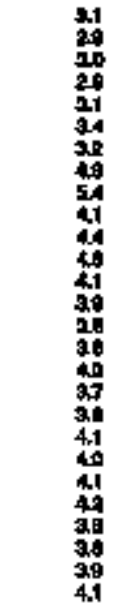 & 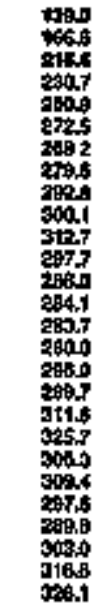 & 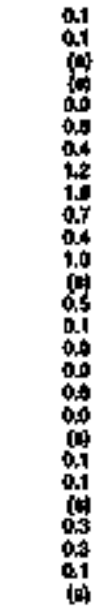 & 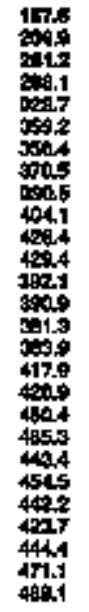 & 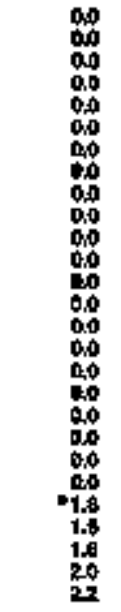 & 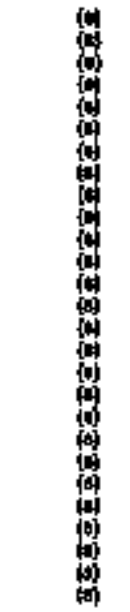 & 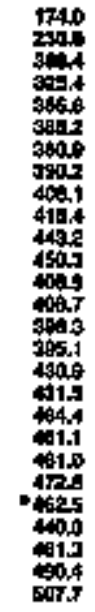 & 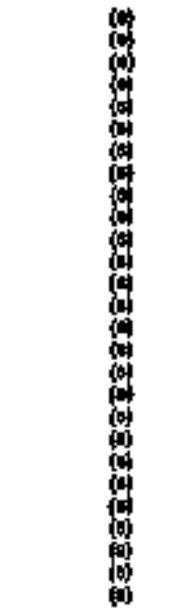 & 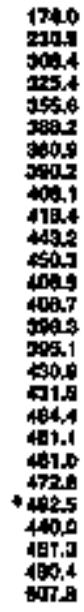 \\
\hline
\end{tabular}

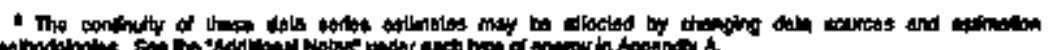

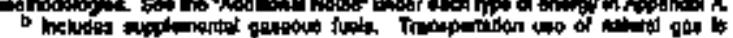

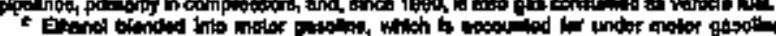

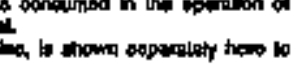

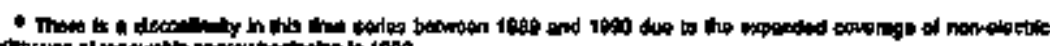

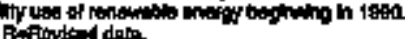

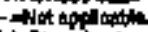

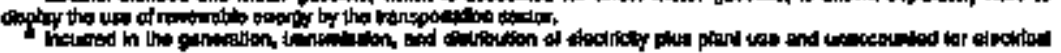

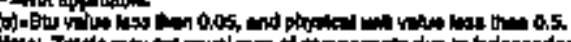

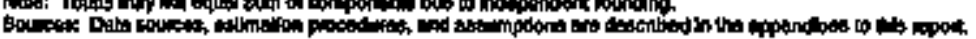

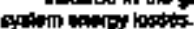


Table 274. Estimates of Enorgy Input at Gitutric Utitities, 1960, 1955, 1970-1994, Tennesseo

\begin{tabular}{|c|c|c|c|c|c|c|c|c|c|c|c|c|c|c|}
\hline \multirow[b]{3}{*}{ Ywe } & \multicolumn{3}{|c|}{ Cond } & \multirow{3}{*}{ 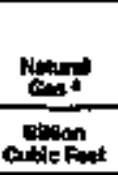 } & \multicolumn{4}{|c|}{ Putrofingm } & \multirow[b]{2}{*}{ 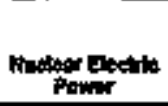 } & \multirow[b]{2}{*}{ 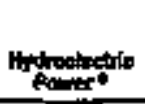 } & \multirow[b]{2}{*}{ Eobuell } & \multirow[b]{2}{*}{ anthem } & \multirow[b]{2}{*}{ Other ht } & \multirow[b]{3}{*}{ Tat: 1} \\
\hline & 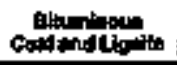 & Narecin & Tot & & sing & 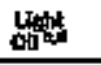 & Protom & Tata & & & & & & \\
\hline & \multicolumn{3}{|c|}{ Theusendstiont Tent } & & \multicolumn{4}{|c|}{ 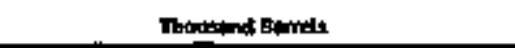 } & \multicolumn{5}{|c|}{ 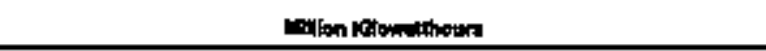 } & \\
\hline 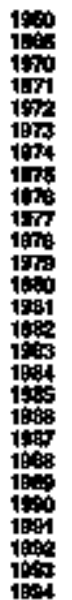 & 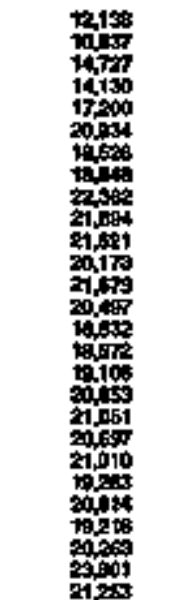 & $\begin{array}{l}0 \\
0 \\
0 \\
0 \\
0 \\
0 \\
0 \\
0 \\
0 \\
0 \\
0 \\
0 \\
0 \\
0 \\
0 \\
0 \\
0 \\
0 \\
0 \\
0 \\
0 \\
0 \\
0\end{array}$ & 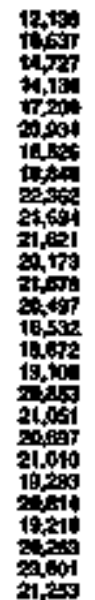 & 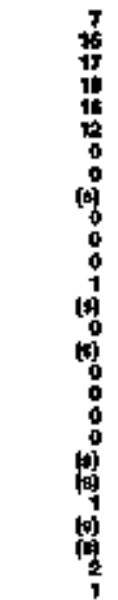 & $\begin{array}{l}0 \\
0 \\
0 \\
0 \\
0 \\
0 \\
0 \\
0 \\
0 \\
0 \\
0 \\
0 \\
0 \\
0 \\
0 \\
0 \\
0 \\
0 \\
0 \\
0 \\
0 \\
0\end{array}$ & 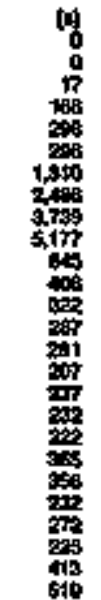 & $\begin{array}{l}0 \\
0 \\
0 \\
0 \\
0 \\
0 \\
0 \\
0 \\
0 \\
0 \\
0 \\
0 \\
0 \\
0 \\
0 \\
0 \\
0 \\
0 \\
0 \\
0 \\
0 \\
0 \\
0 \\
0 \\
0 \\
0\end{array}$ & 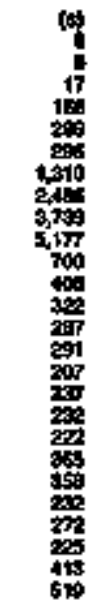 & 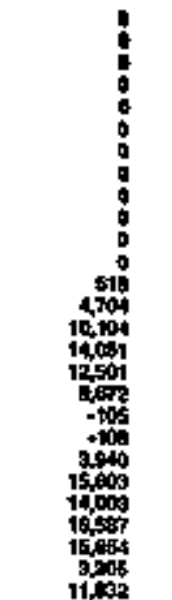 & 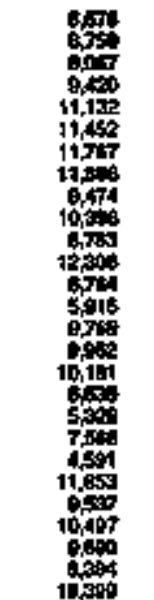 & $\begin{array}{l}0 \\
0 \\
0 \\
0 \\
0 \\
0 \\
0 \\
0 \\
0 \\
0 \\
0 \\
0 \\
0 \\
0 \\
0 \\
0 \\
0 \\
0 \\
0 \\
0 \\
0 \\
0\end{array}$ & $\begin{array}{l}0 \\
0 \\
0 \\
0 \\
0 \\
0 \\
0 \\
0 \\
0 \\
0 \\
0 \\
0 \\
0 \\
0 \\
0 \\
0 \\
0 \\
0 \\
0 \\
0 \\
0 \\
0 \\
0 \\
0 \\
0 \\
0 \\
0\end{array}$ & $\begin{array}{l}0 \\
0 \\
0 \\
0 \\
0 \\
0 \\
0 \\
0 \\
0 \\
0 \\
0 \\
0 \\
0 \\
0 \\
0 \\
0 \\
0 \\
0 \\
0 \\
0\end{array}$ & $\begin{array}{l}= \\
= \\
= \\
= \\
= \\
= \\
= \\
= \\
= \\
= \\
= \\
= \\
z \\
z\end{array}$ \\
\hline
\end{tabular}

\begin{tabular}{|c|c|c|c|c|c|}
\hline 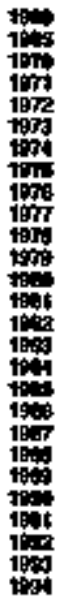 & 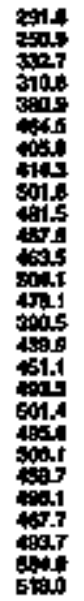 & 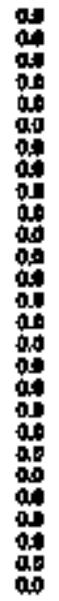 & 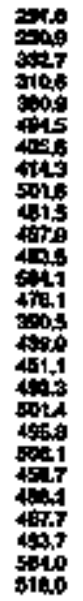 & 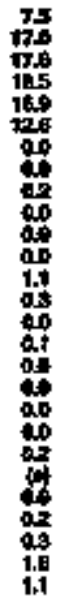 & 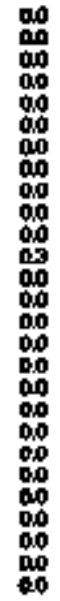 \\
\hline
\end{tabular}

00
00
00

\begin{tabular}{|c|c|c|c|c|}
\hline 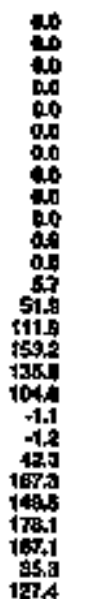 & 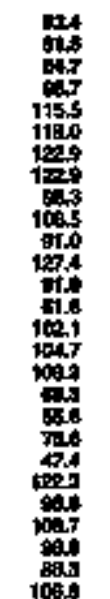 & 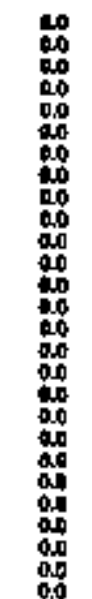 & 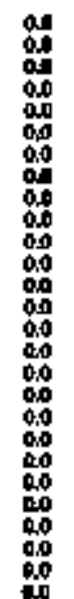 & 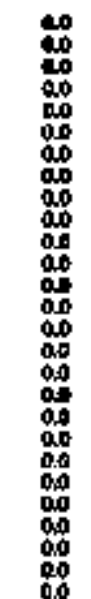 \\
\hline
\end{tabular}

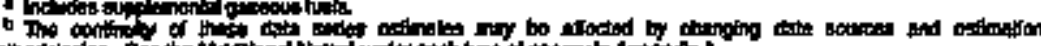

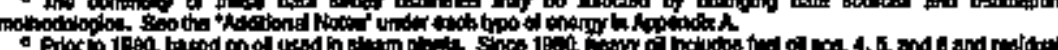

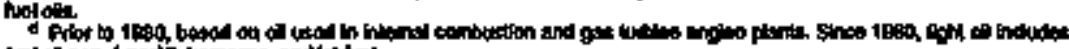
seylol nos 1 and

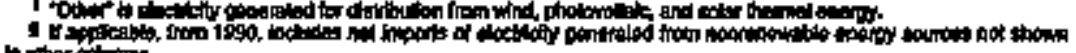
oftercingen

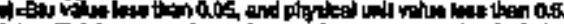

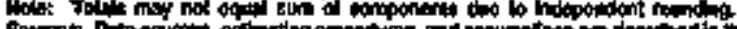

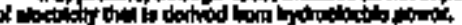


Toble 275. Enargy Consumption Estinates by Source, 1930, 1965, t970-1994, Texes

\begin{tabular}{|c|c|c|c|c|c|c|c|c|c|c|c|c|c|c|c|c|c|c|c|}
\hline \multirow[b]{3}{*}{ xen } & \multirow[b]{2}{*}{ Conte } & \multirow[b]{2}{*}{ Minions } & \multicolumn{11}{|c|}{ Patrolengm } & \multirow{2}{*}{ Powe } & \multirow{2}{*}{ Protion } & \multirow[b]{2}{*}{ Bifoteotate } & \multirow[b]{2}{*}{ Other in I } & \multirow{2}{*}{ 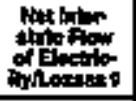 } & \multirow[b]{3}{*}{ Totsh h } \\
\hline & & & aphasind & 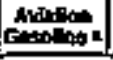 & Dintin & INAd. & . & LPa* & 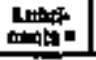 & Motion & Fontitions & Ointur on & Tot & & & & & & \\
\hline & $\begin{array}{l}\text { onsund } \\
\text { ot Tolat: }\end{array}$ & con & \multicolumn{11}{|c|}{ 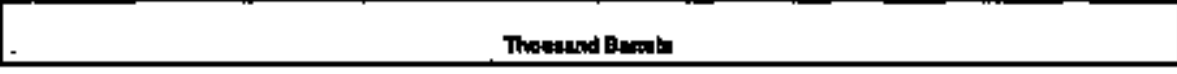 } & \multicolumn{5}{|c|}{ 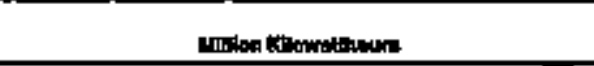 } & \\
\hline 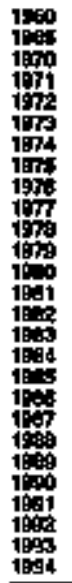 & 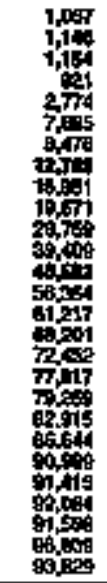 & 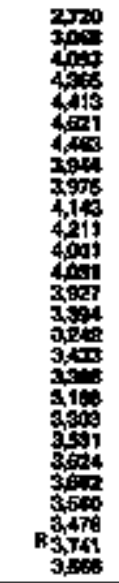 & 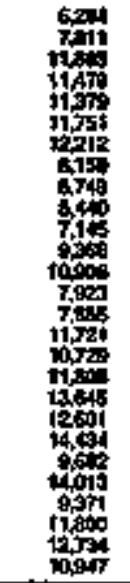 & 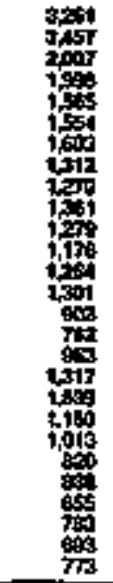 & 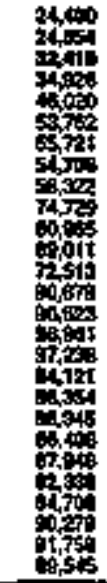 & 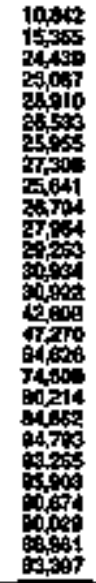 & 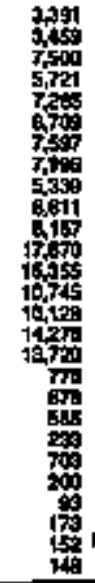 & 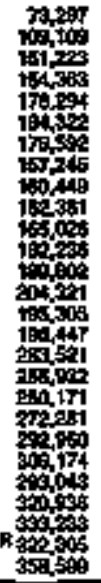 & 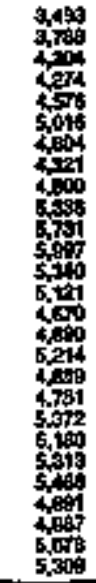 & 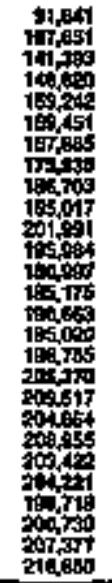 & 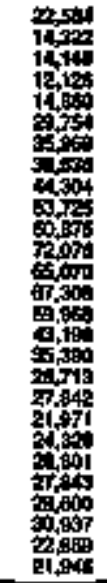 & 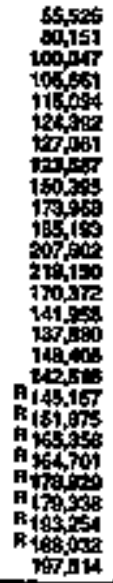 & 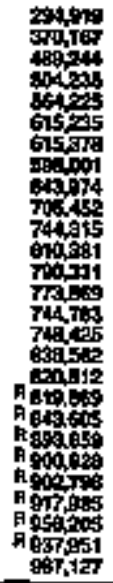 & 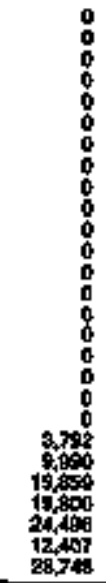 & 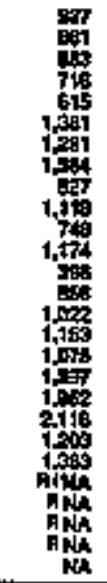 & 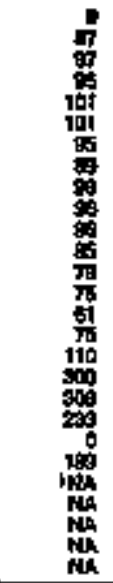 & 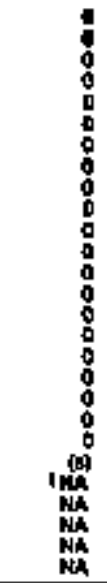 & 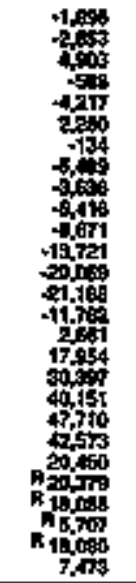 & $\begin{array}{l}z \\
z \\
z \\
z \\
z \\
z\end{array}$ \\
\hline \multicolumn{20}{|c|}{ Tition } \\
\hline 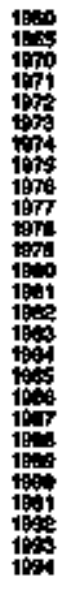 & 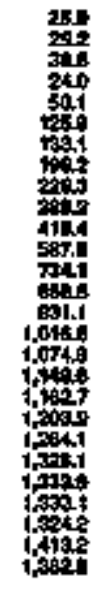 & 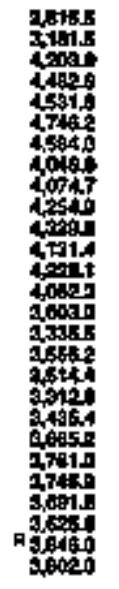 & 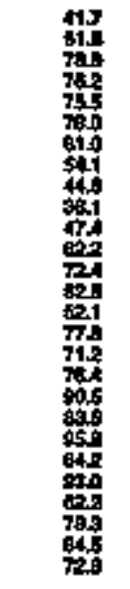 & 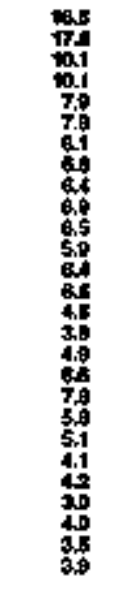 & 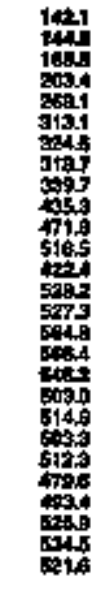 & 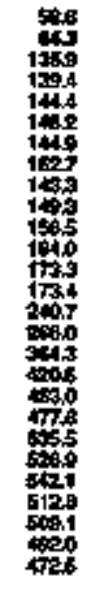 & 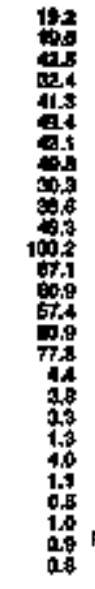 & 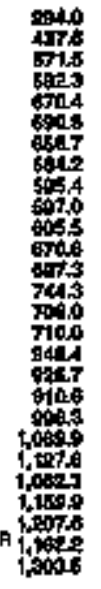 & 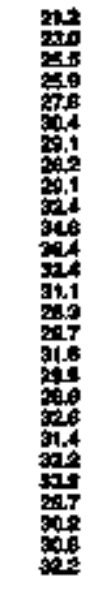 & 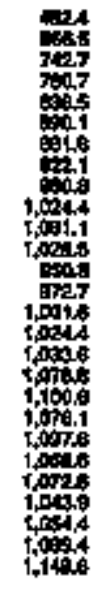 & 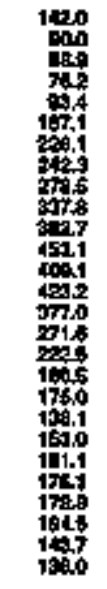 & 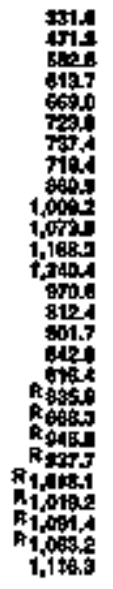 & 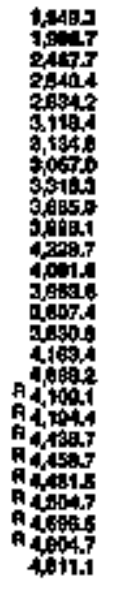 & 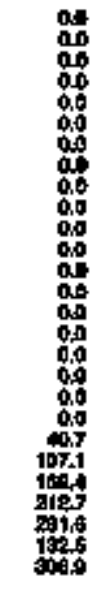 & 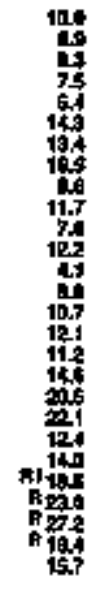 & 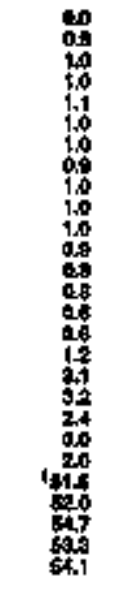 & 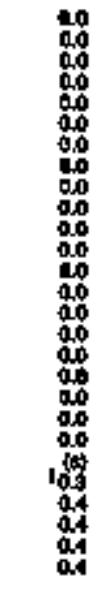 & 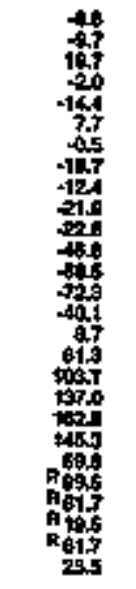 & 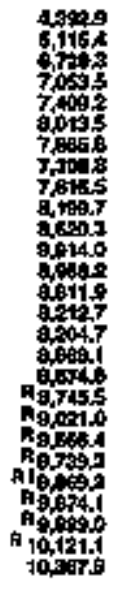 \\
\hline
\end{tabular}

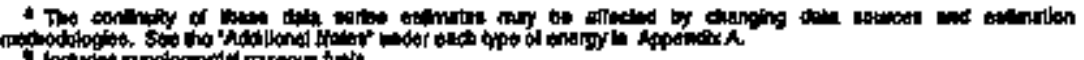

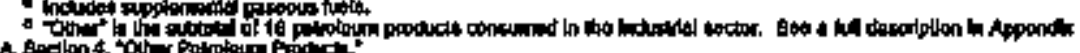

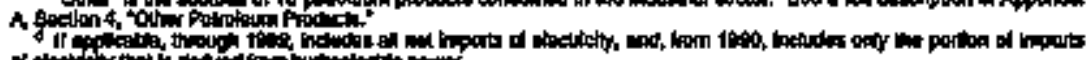

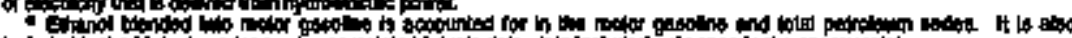

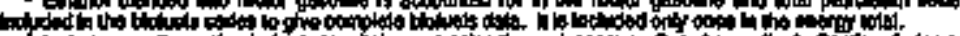

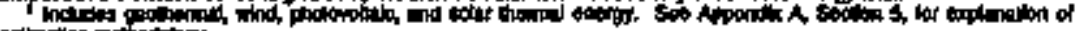

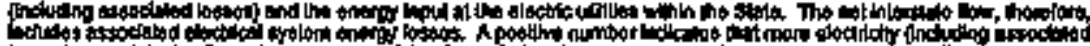
che

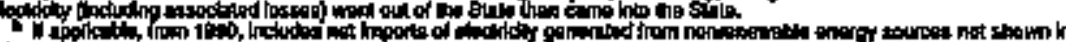

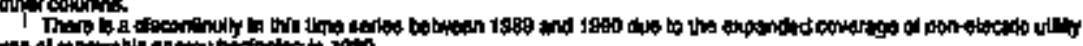

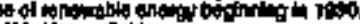

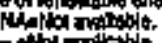

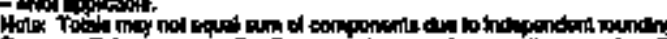

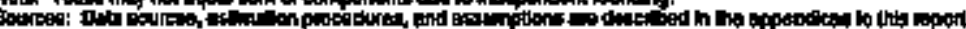


Table 276. Residential Energy Consurtption Estamabes, 1960, 1965, 1970-1994, Texas

\begin{tabular}{|c|c|c|c|c|c|c|c|c|c|c|c|c|c|c|}
\hline \multirow[b]{3}{*}{ Your } & \multicolumn{3}{|c|}{ Con } & \multirow{3}{*}{ 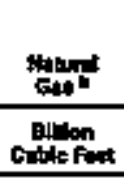 } & \multicolumn{4}{|c|}{ Potrolening } & \multirow{3}{*}{$\begin{array}{l}\text { Bights } \\
\text { Toowenns } \\
\text { toontil }\end{array}$} & & \multirow[b]{2}{*}{ Etectrildy" } & \multirow[b]{2}{*}{ 대 } & \multirow{3}{*}{ 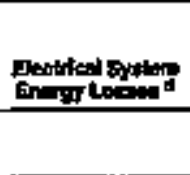 } & \multirow[b]{3}{*}{ Totel } \\
\hline & 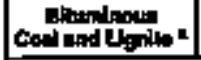 & Monthentione & Totad & & Dintillot: & Kerosonget & LPE. & Totall & & Bolen" & & & & \\
\hline & \multicolumn{3}{|c|}{ 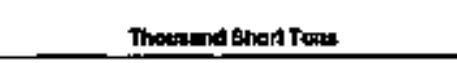 } & & \multicolumn{4}{|c|}{ Fhooumint Barah } & & \multicolumn{3}{|c|}{ 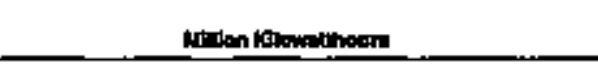 } & & \\
\hline 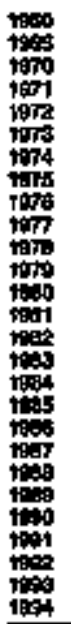 & 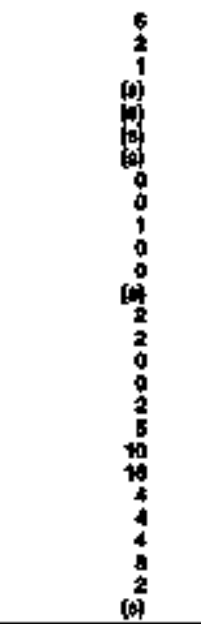 & $\begin{array}{l}: \\
\vdots \\
0 \\
0 \\
0 \\
\vdots \\
0 \\
0 \\
\vdots \\
0 \\
0 \\
0 \\
\vdots \\
0 \\
0 \\
0\end{array}$ & 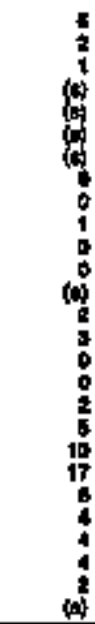 & 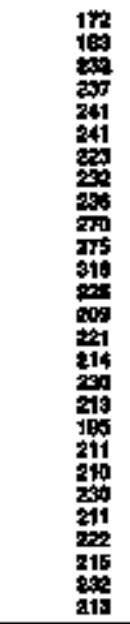 & 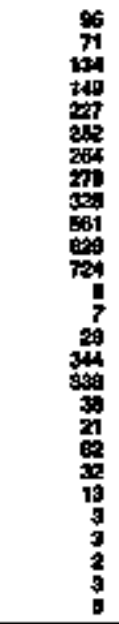 & 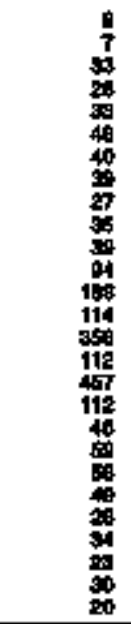 & 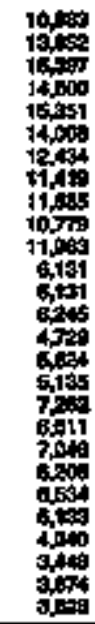 & 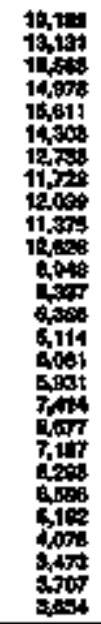 & 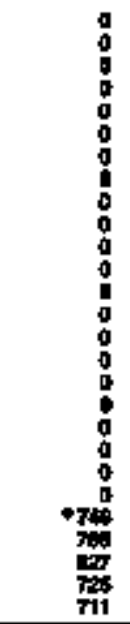 & 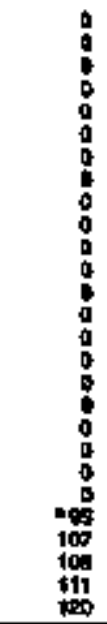 & 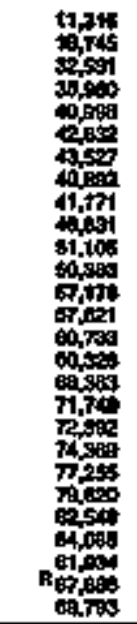 & $\begin{array}{l}= \\
= \\
= \\
= \\
= \\
= \\
= \\
= \\
= \\
= \\
= \\
= \\
= \\
=\end{array}$ & 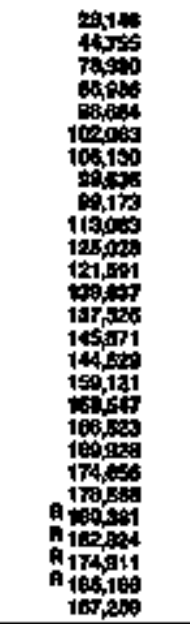 & 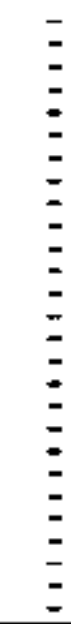 \\
\hline
\end{tabular}

\begin{tabular}{|c|c|c|c|c|c|c|}
\hline 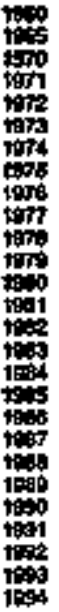 & 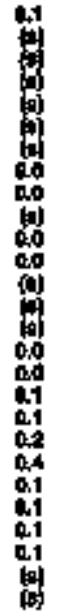 & 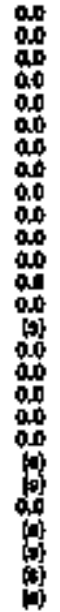 & 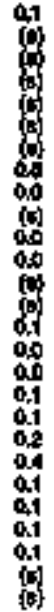 & 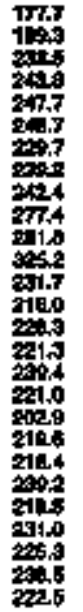 & 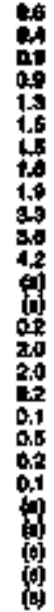 & 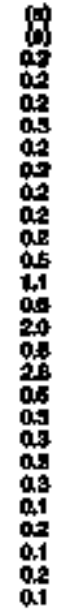 \\
\hline
\end{tabular}

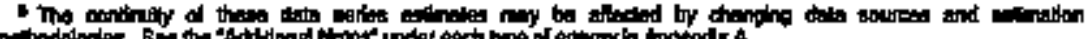

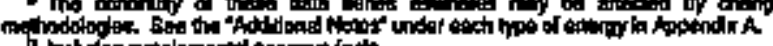

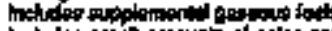

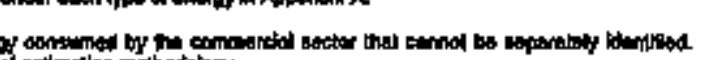

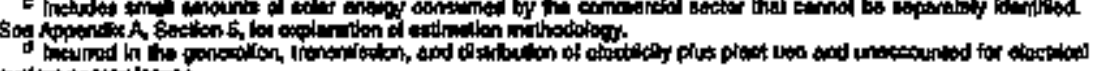


Table 277. Commerciel Energy Consumplion Estimatas, 1960, 1965, 1970-1994, Texas

\begin{tabular}{|c|c|c|c|c|c|c|c|c|c|c|c|c|c|c|}
\hline \multirow[b]{3}{*}{ 제 } & \multicolumn{3}{|c|}{$\cos$} & \multirow{3}{*}{ 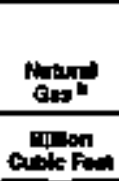 } & \multicolumn{6}{|c|}{ 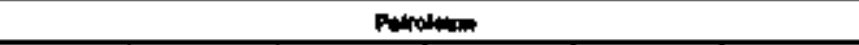 } & \multirow[b]{2}{*}{ Enctilely" } & \multirow[b]{2}{*}{ Nant } & \multirow[b]{2}{*}{ 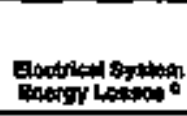 } & \multirow[b]{3}{*}{ Tokd d } \\
\hline & 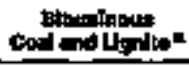 & 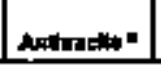 & Totet & & Dasilint: & Korotorale" & Lాa: & Gotor & Finder & Fold & & & & \\
\hline & \multicolumn{2}{|c|}{ Trangand Sabort Ton } & \multirow[b]{2}{*}{$\begin{array}{r}1 \\
1 \\
1 \\
1 \\
1 \\
1 \\
0 \\
0 \\
2 \\
0 \\
0 \\
1 \\
3 \\
4 \\
0 \\
0 \\
8 \\
10 \\
30 \\
6 \\
7 \\
7 \\
6 \\
1 \\
15]\end{array}$} & & \multicolumn{6}{|c|}{ Tronkend Bamit } & \multicolumn{3}{|c|}{ 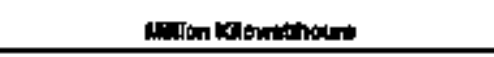 } & \\
\hline 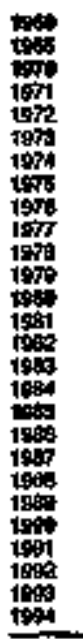 & 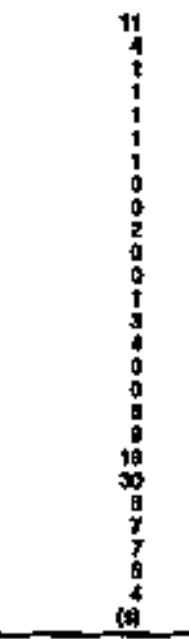 & $\begin{array}{l}0 \\
0 \\
0 \\
0 \\
0 \\
0 \\
0 \\
0 \\
0 \\
0 \\
0 \\
0 \\
0 \\
0 \\
0 \\
0 \\
0 \\
0 \\
0 \\
0 \\
0 \\
0 \\
0 \\
0\end{array}$ & & 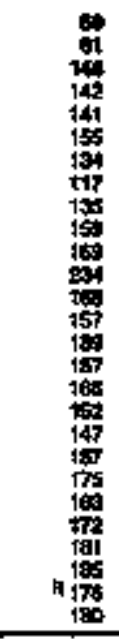 & 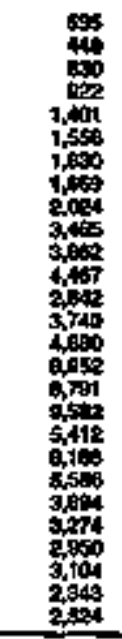 & 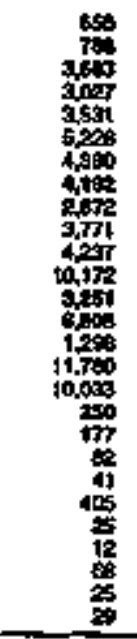 & 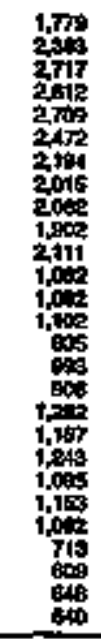 & 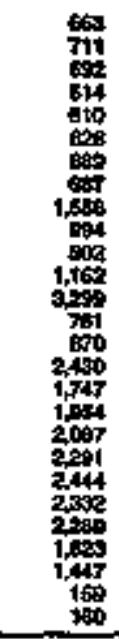 & 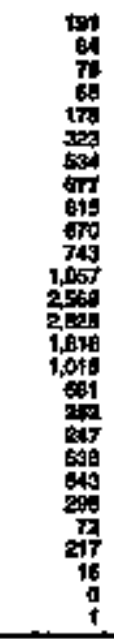 & 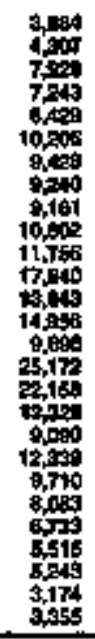 & 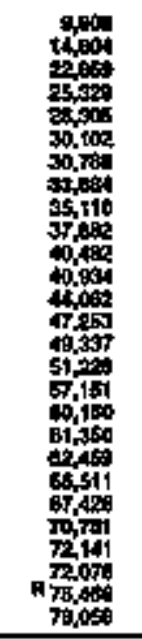 & $\begin{array}{l}= \\
z \\
= \\
= \\
= \\
= \\
= \\
5 \\
= \\
z \\
z \\
z \\
z \\
=\end{array}$ & 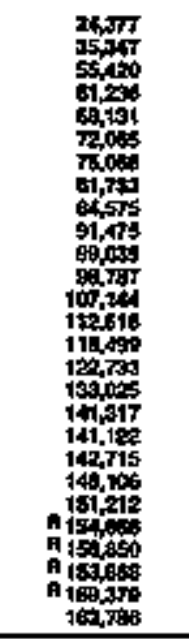 & $\begin{array}{l}z \\
= \\
= \\
z \\
= \\
z \\
= \\
= \\
z \\
= \\
z \\
= \\
z\end{array}$ \\
\hline \multicolumn{15}{|c|}{ ningon } \\
\hline 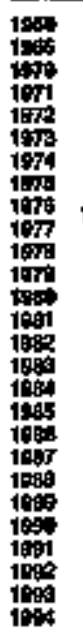 & 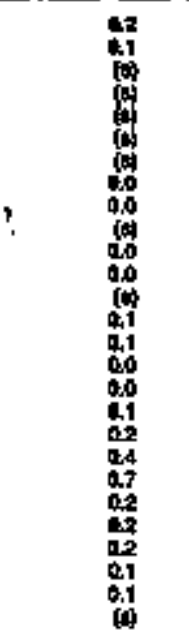 & 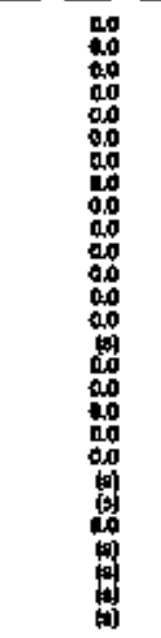 & 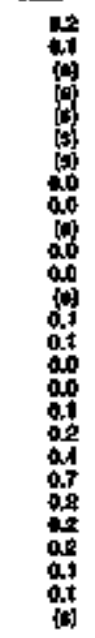 & 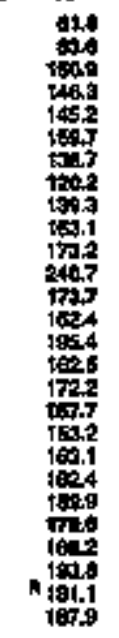 & 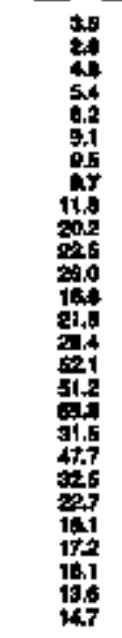 & 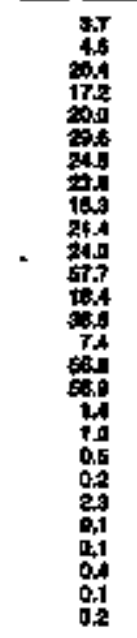 & 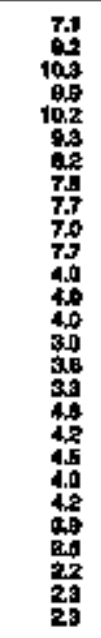 & 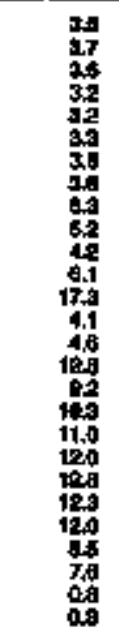 & 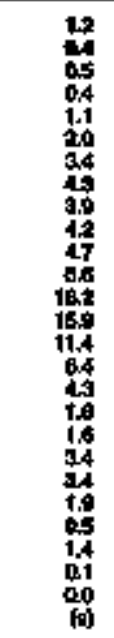 & 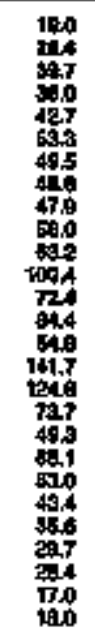 & 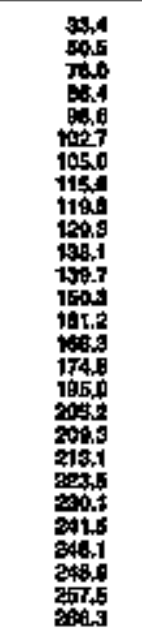 & 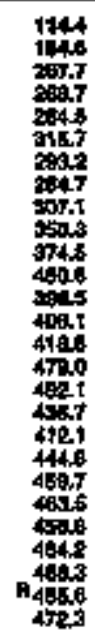 & 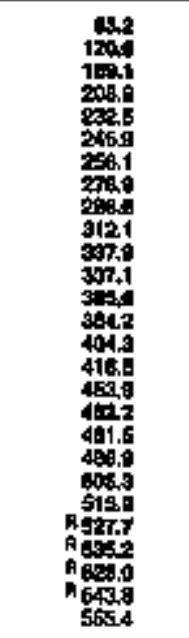 & 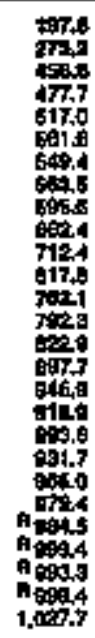 \\
\hline
\end{tabular}


Talate 278. Industrial Enargy Consumption Estimates, 1860, 1965, 1970-1994, Texa9

\begin{tabular}{|c|c|c|c|c|c|c|c|c|c|c|c|c|c|c|c|c|c|c|}
\hline \multirow[b]{3}{*}{ Yerr } & \multirow[b]{2}{*}{ Cod } & \multirow[b]{2}{*}{ chond } & \multicolumn{9}{|c|}{ Aatrition } & \multirow[b]{2}{*}{ 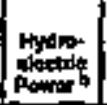 } & \multirow[b]{2}{*}{ abotinter } & \multirow[b]{2}{*}{ onent as } & \multirow[b]{2}{*}{ Doctidery } & \multirow[b]{2}{*}{ tit } & \multirow{2}{*}{ 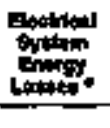 } & \multirow[b]{3}{*}{ Totet } \\
\hline & & & 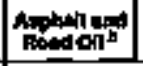 & 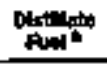 & Kureasen' & ton: & 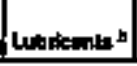 & motion & 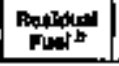 & ovestas & Toetad & & & & & & & \\
\hline & 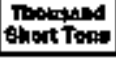 & cintor Fen & \multicolumn{9}{|c|}{ 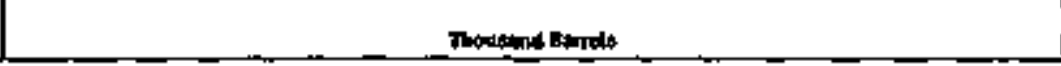 } & \multicolumn{6}{|c|}{ 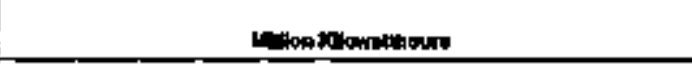 } & \\
\hline 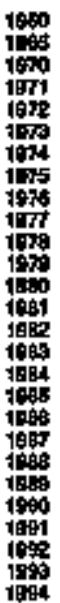 & 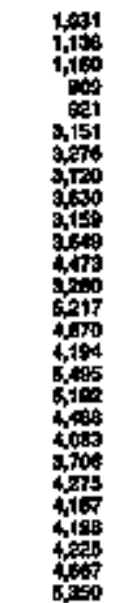 & 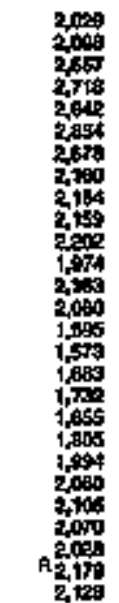 & 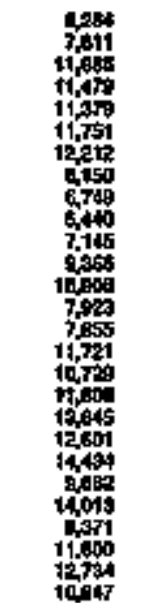 & 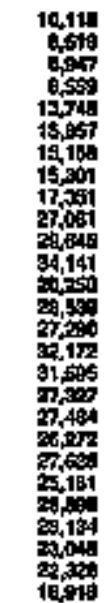 & 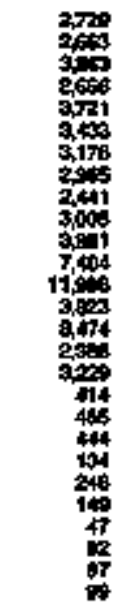 & 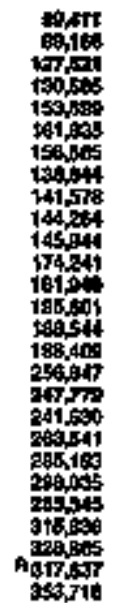 & 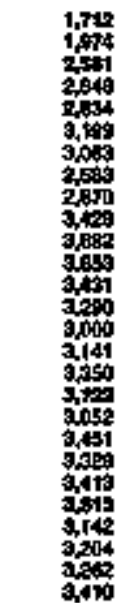 & 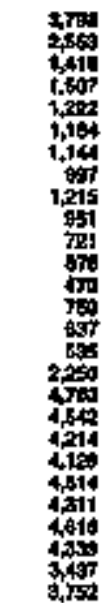 & 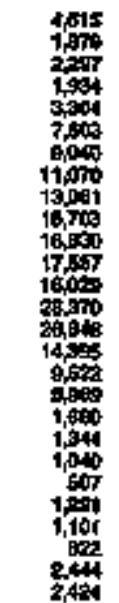 & 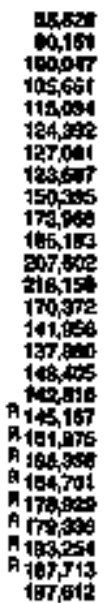 & 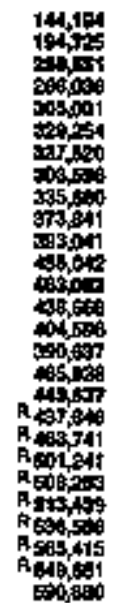 & 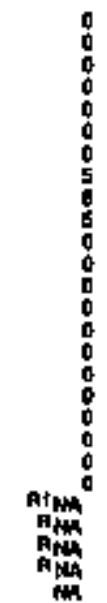 & 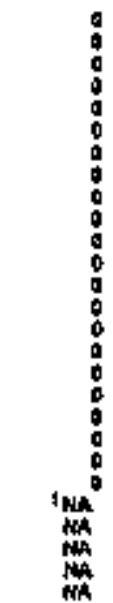 & 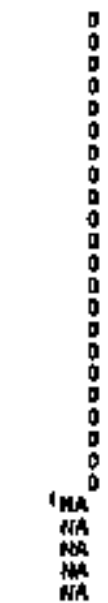 & 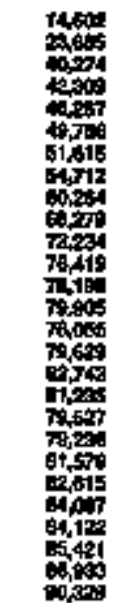 & $\begin{array}{l}= \\
\vdots \\
\vdots \\
\vdots \\
\vdots \\
\vdots \\
\vdots \\
\vdots \\
= \\
z \\
\vdots \\
z \\
z \\
z \\
z\end{array}$ & 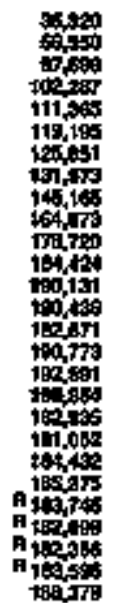 & $\begin{array}{l}z \\
z \\
z \\
= \\
z \\
= \\
z \\
= \\
= \\
z \\
= \\
z \\
z \\
z\end{array}$ \\
\hline
\end{tabular}

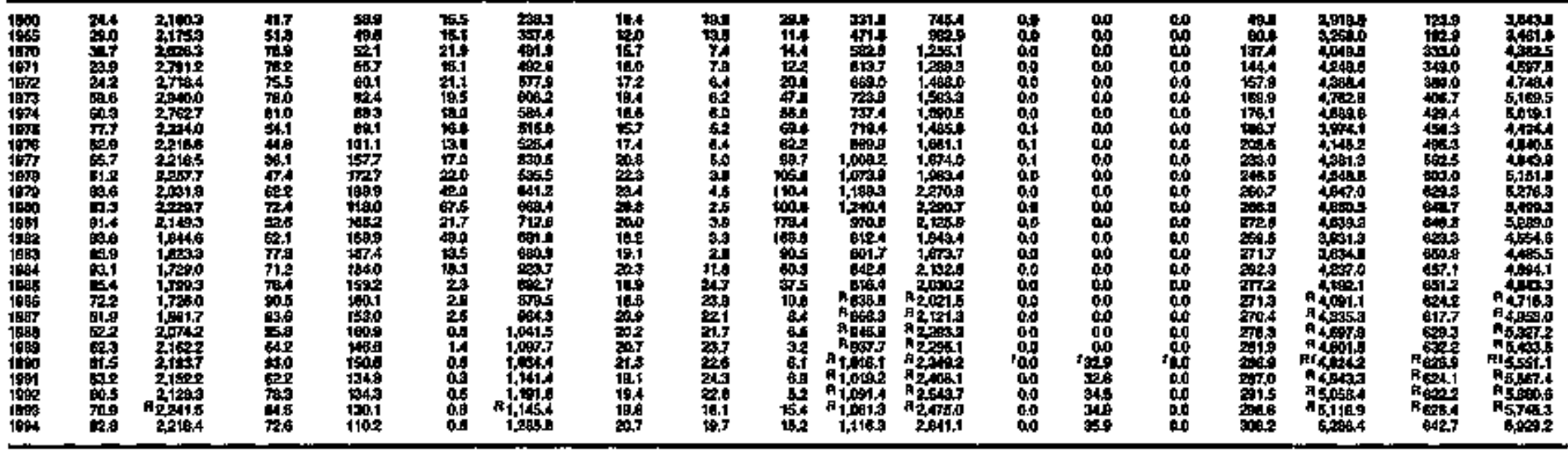

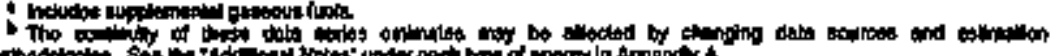

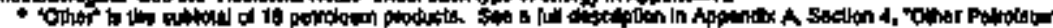

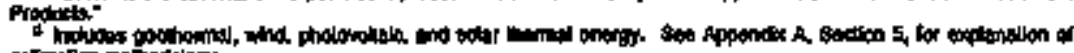

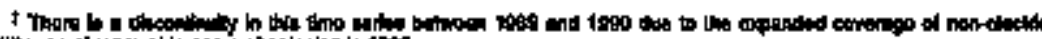
Ily abo of

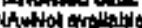

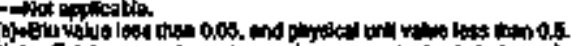

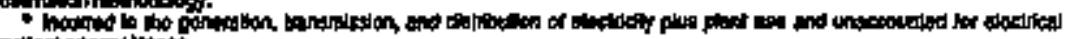

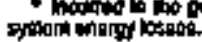




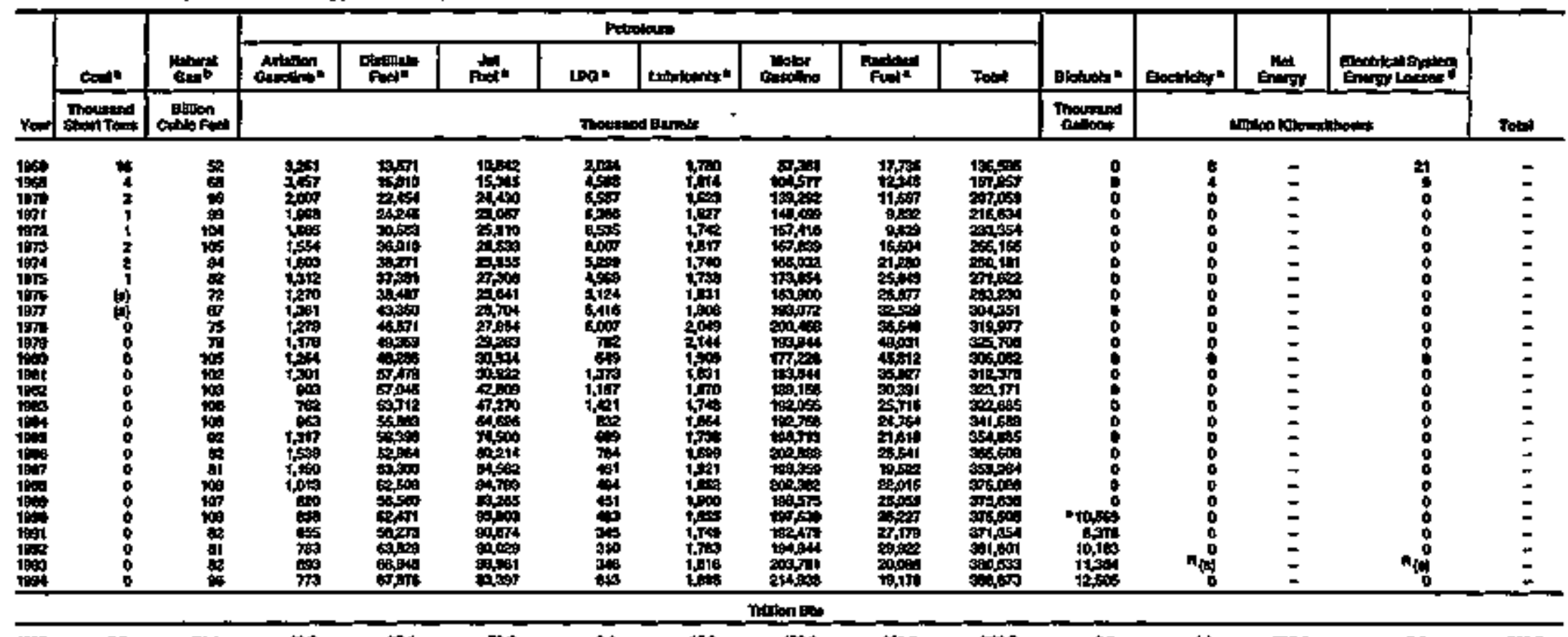

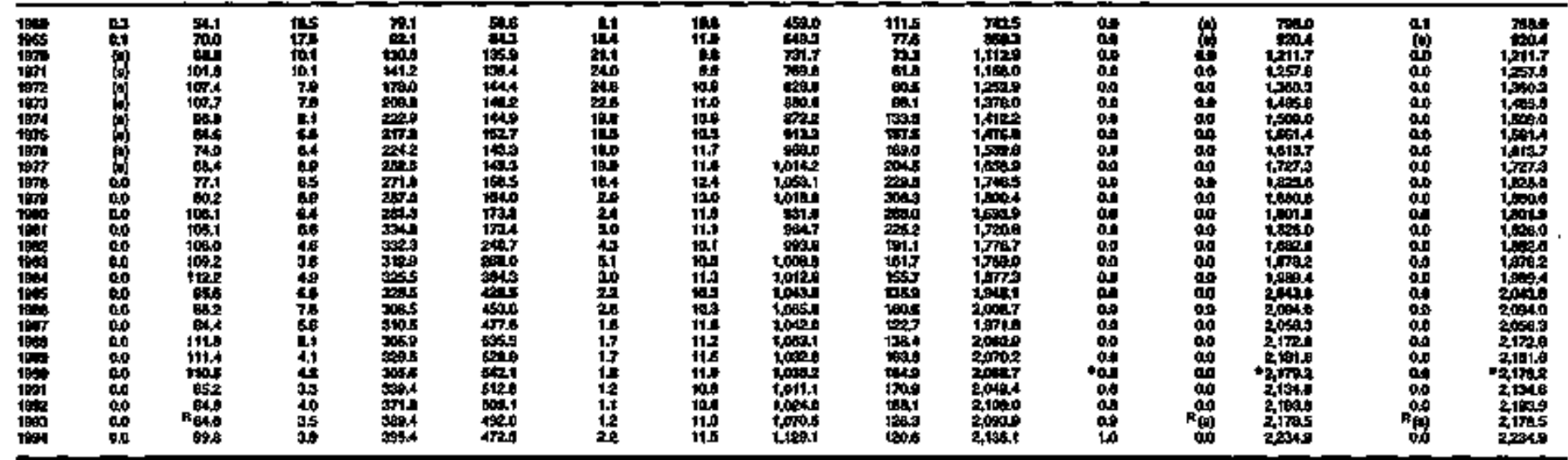

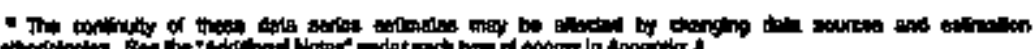

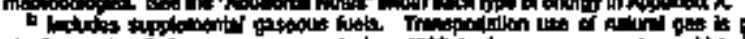

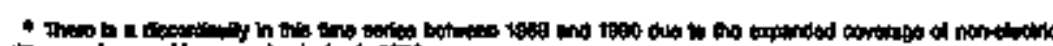

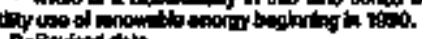

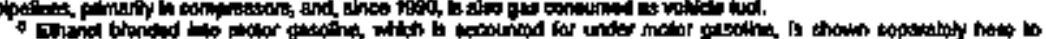

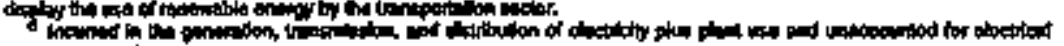

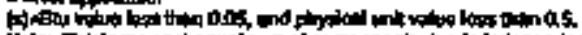

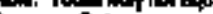

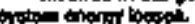


Tabis 290. Estumates of Energy Input at Electric Utilities, 1960, 1965, 1970-1994, Toxas

\begin{tabular}{|c|c|c|c|c|c|c|c|c|c|c|c|c|c|c|}
\hline \multirow[b]{3}{*}{ kear } & \multicolumn{3}{|c|}{$c_{\text {cond }}$} & \multirow[b]{2}{*}{ shangal } & \multicolumn{4}{|c|}{ mancoloum } & \multirow[b]{2}{*}{$\begin{array}{c}\text { Muschar Eledde } \\
\text { Ponwef }\end{array}$} & \multirow[b]{2}{*}{ Mydmolnetite } & \multirow[b]{2}{*}{ Diofouly } & \multirow[b]{2}{*}{ 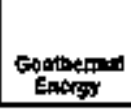 } & \multirow[b]{2}{*}{ cotoref } & \multirow[b]{3}{*}{ Totent } \\
\hline & 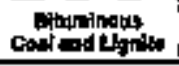 & Anthrestion & Tate & & Henty & 岁察 & 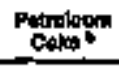 & $70+\infty$ & & & & & & \\
\hline & \multicolumn{3}{|c|}{ Thounend Ghers Tona } & 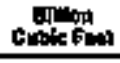 & \multicolumn{4}{|c|}{ Thousuad Alam't } & \multicolumn{5}{|c|}{ 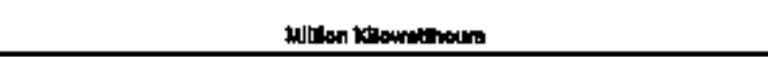 } & \\
\hline 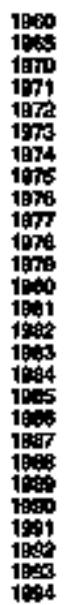 & 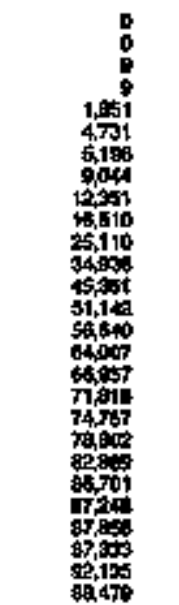 & $\begin{array}{l}0 \\
0 \\
0 \\
0 \\
0 \\
0 \\
0 \\
0 \\
0 \\
0 \\
0 \\
0 \\
0 \\
0 \\
0 \\
0 \\
0 \\
0 \\
0 \\
0 \\
0 \\
0 \\
0 \\
0 \\
0 \\
0\end{array}$ & 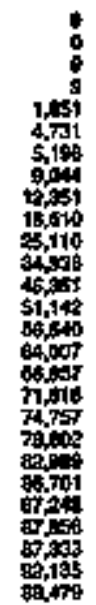 & 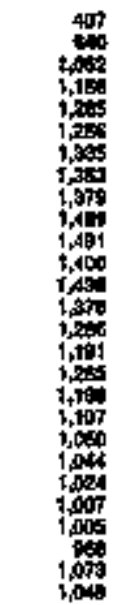 & 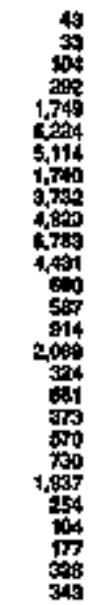 & 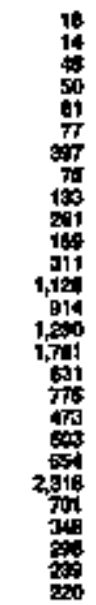 & $\begin{array}{l}0 \\
0 \\
0 \\
0 \\
0 \\
0 \\
0 \\
0 \\
0 \\
0 \\
0 \\
0 \\
0 \\
0 \\
0 \\
0 \\
0 \\
0 \\
0 \\
0 \\
0 \\
0 \\
0 \\
\text { 9o } \\
2\end{array}$ & 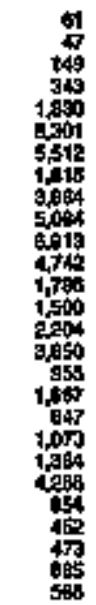 & 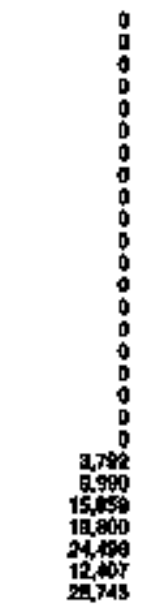 & 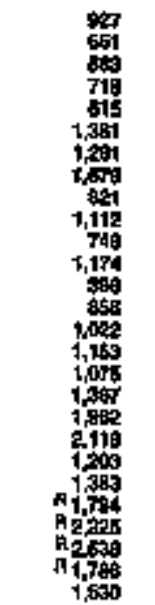 & 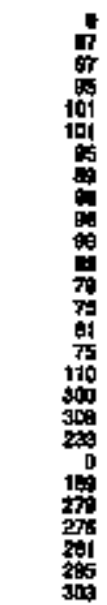 & $\begin{array}{l}0 \\
0 \\
0 \\
0 \\
0 \\
0 \\
0 \\
0 \\
0 \\
0 \\
0 \\
0 \\
0 \\
0 \\
0 \\
0 \\
0 \\
0 \\
0 \\
0 \\
0 \\
0 \\
0 \\
0 \\
0 \\
0 \\
0\end{array}$ & $\begin{array}{r}0 \\
0 \\
0 \\
0 \\
0 \\
0 \\
0 \\
0 \\
0 \\
0 \\
0 \\
0 \\
0 \\
0 \\
0 \\
0 \\
0 \\
0 \\
0 \\
0 \\
0 \\
0 \\
0 \\
0 \\
0\end{array}$ & $\begin{array}{l}z \\
= \\
= \\
= \\
= \\
= \\
= \\
= \\
z \\
= \\
z \\
z \\
= \\
z \\
z\end{array}$ \\
\hline
\end{tabular}

\begin{tabular}{|c|c|c|c|c|c|c|c|}
\hline 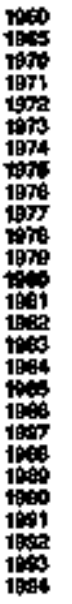 & 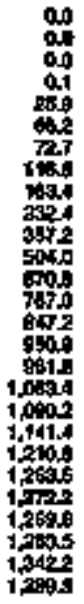 & 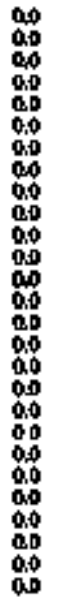 & 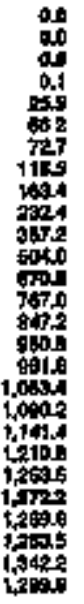 & 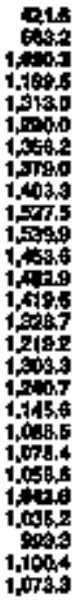 & 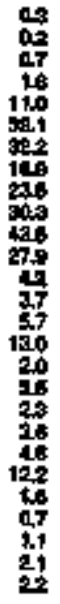 & 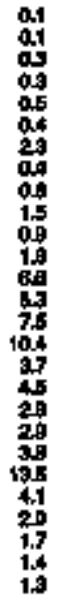 & 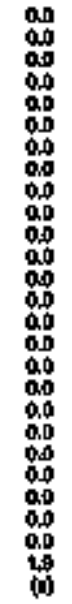 \\
\hline
\end{tabular}

\begin{tabular}{|c|c|c|c|c|c|c|}
\hline 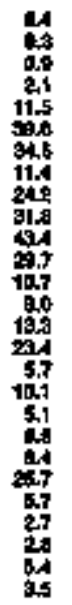 & 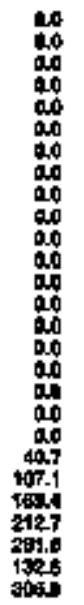 & 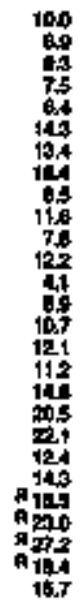 & 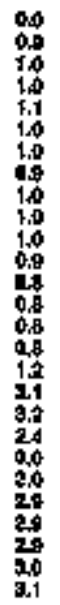 & 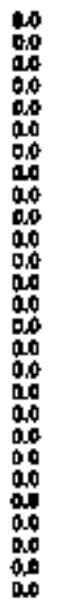 & 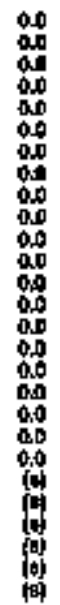 & 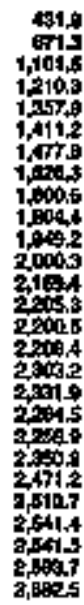 \\
\hline
\end{tabular}

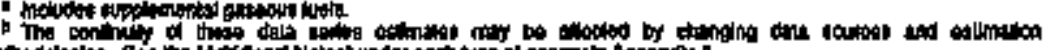

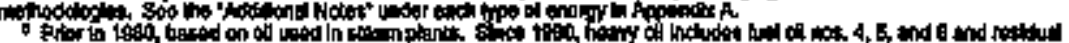

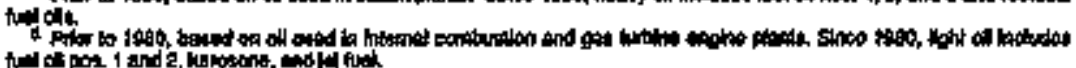

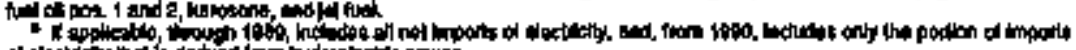

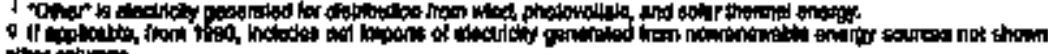
colunents.

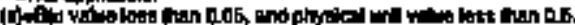

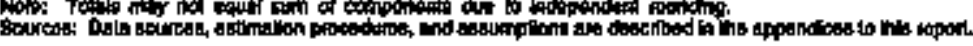


Table 281. Energy Consumption Estimates by Source, 1960, 1965, 1970-1994, Utah

\begin{tabular}{|c|c|c|c|c|c|c|c|c|c|c|c|c|c|c|c|c|c|c|c|}
\hline \multirow[b]{3}{*}{ Yw } & \multirow[b]{2}{*}{ Cowl: } & \multirow[b]{2}{*}{ Whangh } & \multicolumn{11}{|c|}{ Patrodawin } & \multirow{2}{*}{ 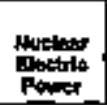 } & \multirow{2}{*}{ 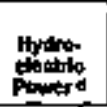 } & \multirow[b]{2}{*}{ 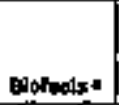 } & \multirow[b]{2}{*}{ ather is } & \multirow{3}{*}{ 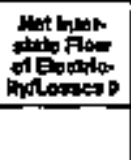 } & \multirow[b]{3}{*}{ I } \\
\hline & & & Arotentind & 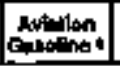 & 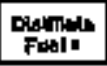 & Podl. & Karto: & Lئ. & Linst: & 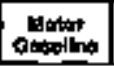 & Rmanoss & Oalwer w. & The & & & & & & \\
\hline & moverised & 1 & & & \multicolumn{9}{|c|}{ 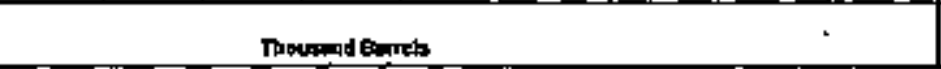 } & \multicolumn{4}{|c|}{ Whon Xlowathoura } & & \\
\hline 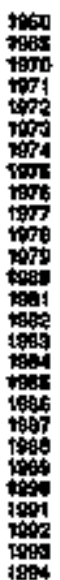 & 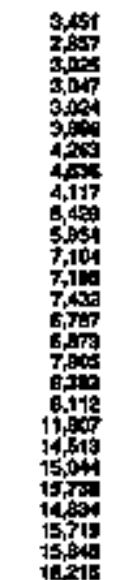 & 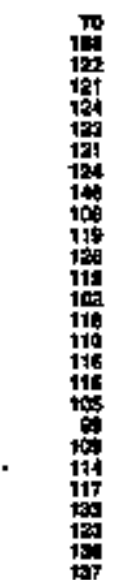 & 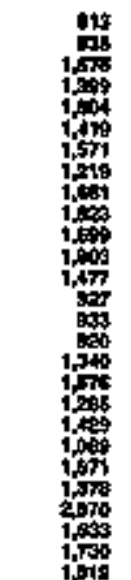 & 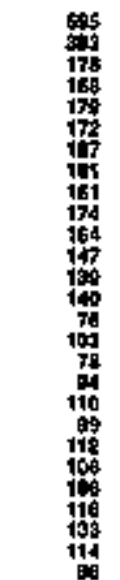 & 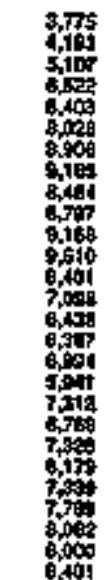 & 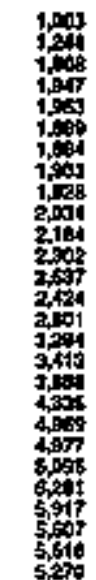 & 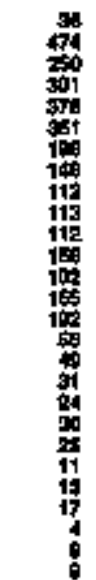 & 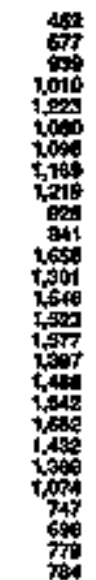 & 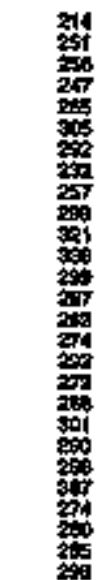 & 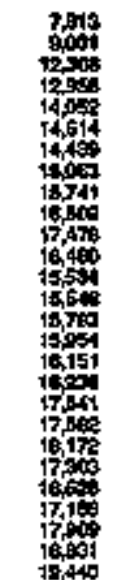 & 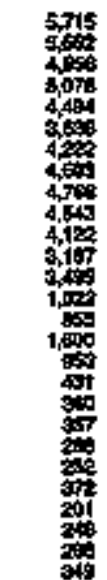 & 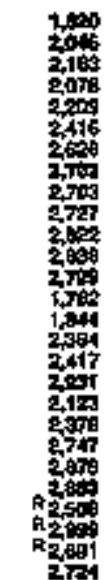 & 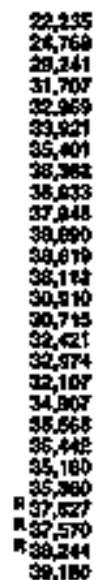 & $\begin{array}{l}0 \\
0 \\
0 \\
0 \\
0 \\
0 \\
0 \\
8 \\
0 \\
0 \\
0 \\
0 \\
0 \\
0 \\
0 \\
0 \\
0 \\
0 \\
0 \\
0 \\
0\end{array}$ & 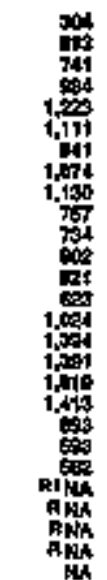 & $\begin{array}{l}0 \\
0 \\
0 \\
0 \\
0 \\
0 \\
0 \\
0 \\
0 \\
0 \\
0 \\
0 \\
0 \\
0 \\
0 \\
0 \\
0 \\
0 \\
1 M \\
14 \\
M \\
M\end{array}$ & 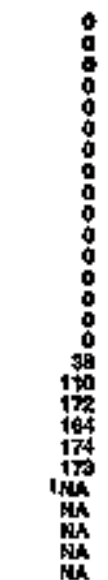 & 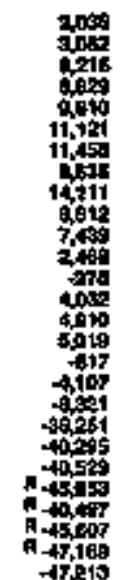 & $\begin{array}{l}\bar{z} \\
\bar{z} \\
\bar{z} \\
\bar{z} \\
\bar{z} \\
\bar{z} \\
\bar{z}\end{array}$ \\
\hline
\end{tabular}

\begin{tabular}{|c|c|c|c|c|c|c|c|c|c|c|c|c|c|c|c|c|c|c|c|c|}
\hline & 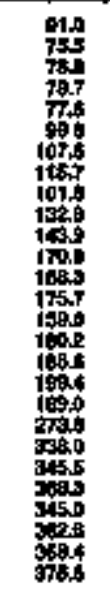 & 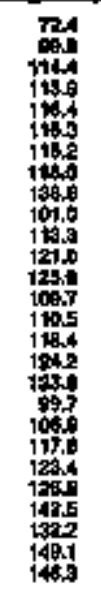 & 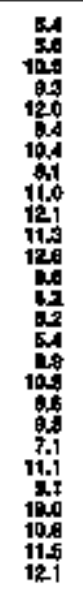 & 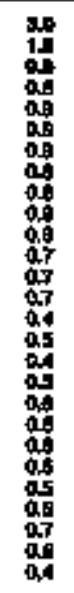 & 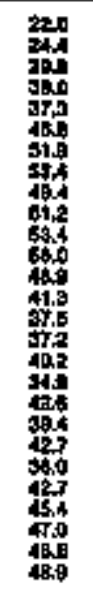 & 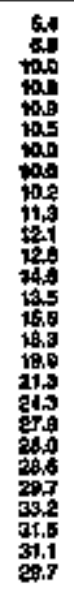 & 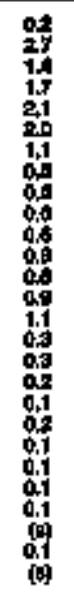 & 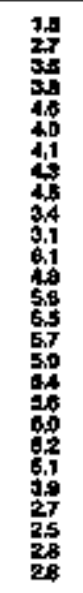 & 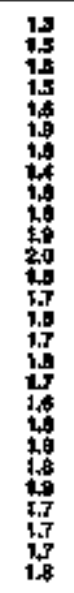 & 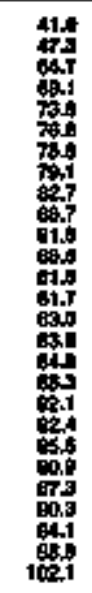 & 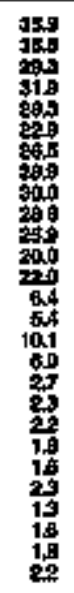 & 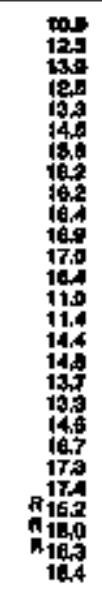 & 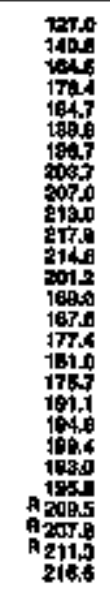 & 玨 & 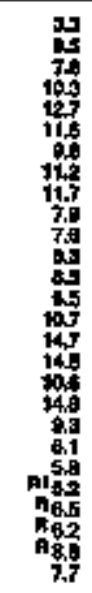 & 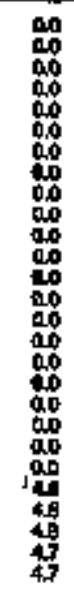 & & & 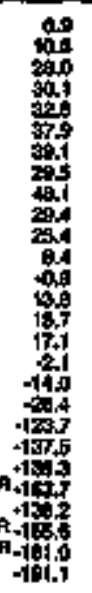 & 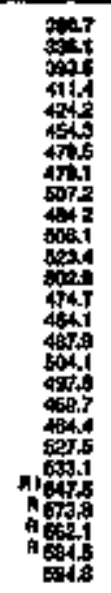 \\
\hline
\end{tabular}

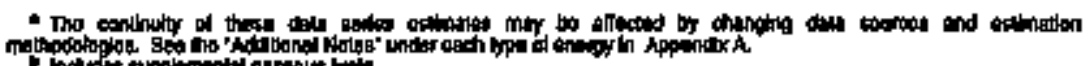

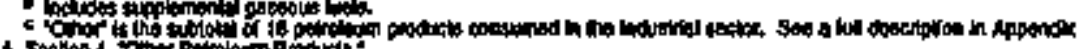

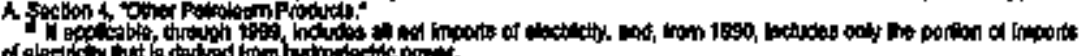

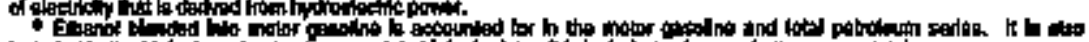

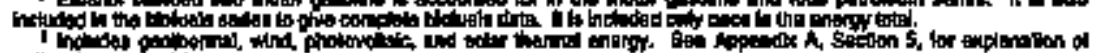

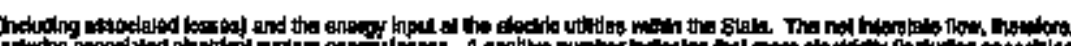

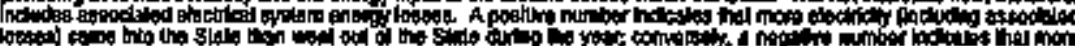

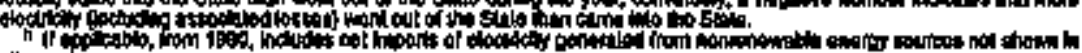

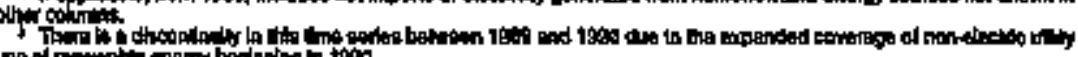

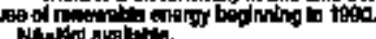

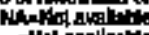

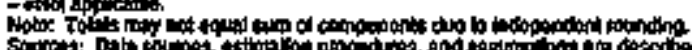

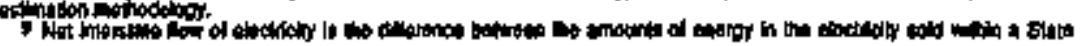

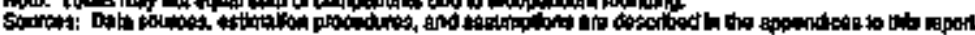


Table 292. Hasidential Energy Consthmption Estimates, 1960, 1968, 1970-1994, Utah

\begin{tabular}{|c|c|c|c|c|c|c|c|c|c|c|c|c|c|c|}
\hline \multirow[b]{3}{*}{$\mathrm{ran}$} & \multicolumn{3}{|c|}{ and } & \multirow[b]{2}{*}{$\lim _{0 \rightarrow 0}$} & \multicolumn{4}{|c|}{ Patrobeanin } & \multirow{3}{*}{ Thomentiph } & & \multirow[b]{2}{*}{ aetritaty } & \multirow[b]{2}{*}{ thenty } & \multirow{3}{*}{ 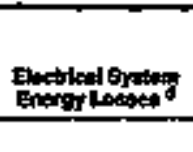 } & \multirow[b]{3}{*}{ Tot } \\
\hline & 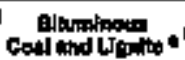 & 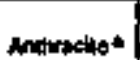 & Totol & & Fund in & Keroesma - & Lean & Trow & & solut $\mathrm{t}$ & & & & \\
\hline & \multicolumn{3}{|c|}{ 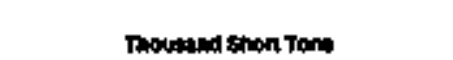 } & com & \multicolumn{4}{|c|}{ mockandemant: } & & \multicolumn{3}{|c|}{ 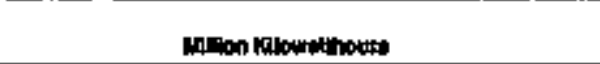 } & & \\
\hline 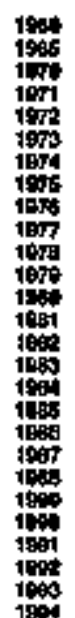 & 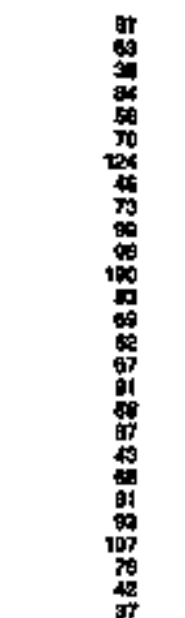 & $\begin{array}{l}0 \\
0 \\
0 \\
0 \\
0 \\
0 \\
0 \\
0 \\
0 \\
0 \\
0 \\
0 \\
0 \\
0 \\
0 \\
0 \\
0 \\
0 \\
0 \\
0 \\
0 \\
0 \\
0 \\
0 \\
0 \\
0 \\
0 \\
0\end{array}$ & 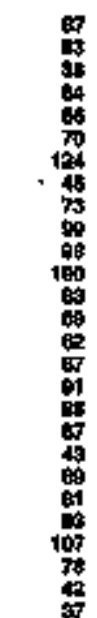 & 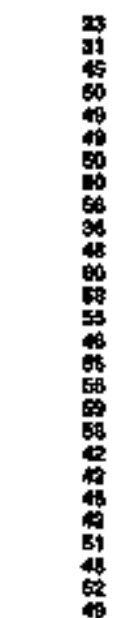 & 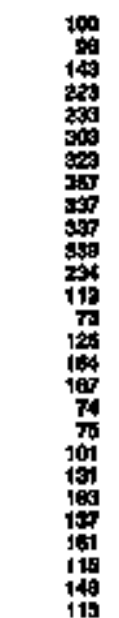 & 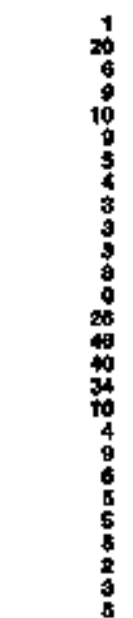 & 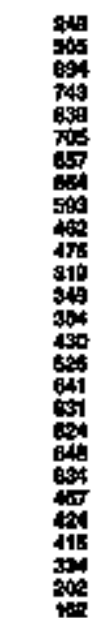 & 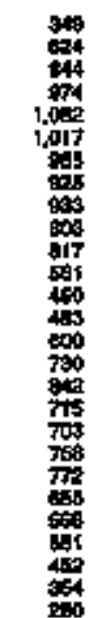 & 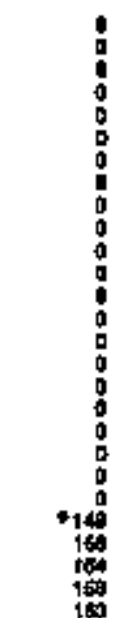 & 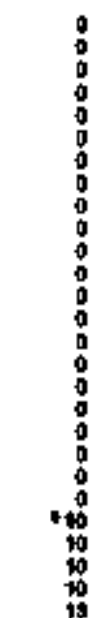 & 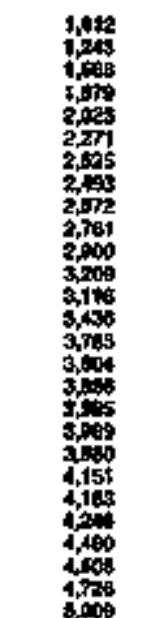 & $\begin{array}{l}\vdots \\
\vdots \\
\vdots \\
\vdots \\
\vdots \\
\vdots \\
\vdots \\
\vdots \\
\vdots \\
\vdots \\
\vdots \\
z\end{array}$ & 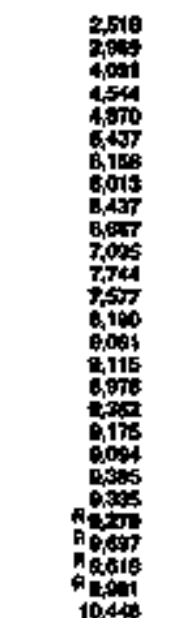 & 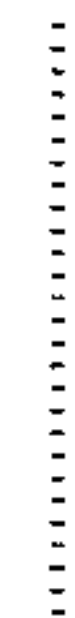 \\
\hline
\end{tabular}

\begin{tabular}{|c|c|c|c|c|c|c|c|c|c|c|c|c|c|c|}
\hline 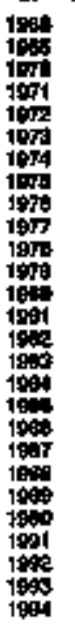 & 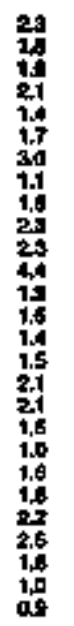 & 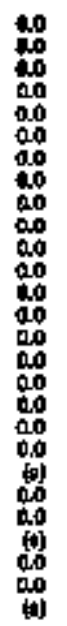 & 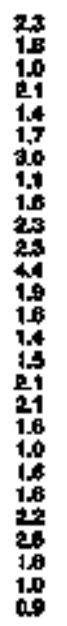 & 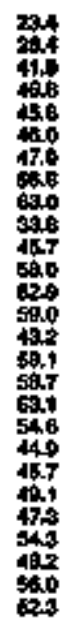 & 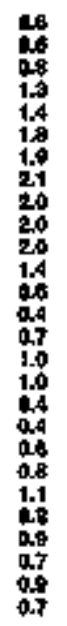 & 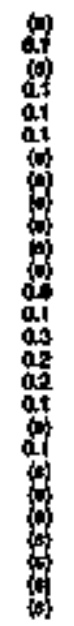 & 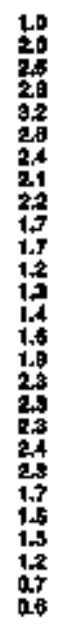 & 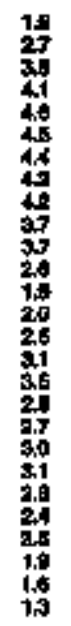 & 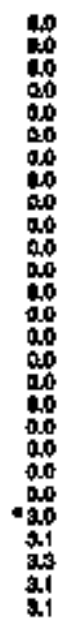 & 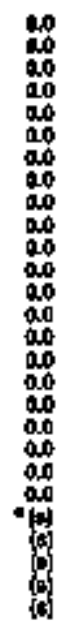 & 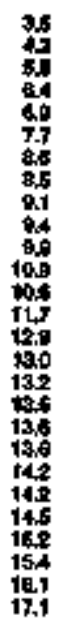 & 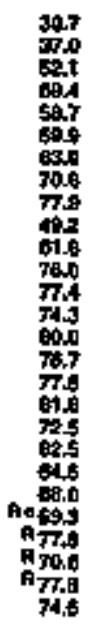 & 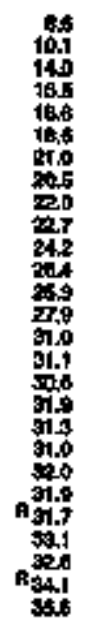 & 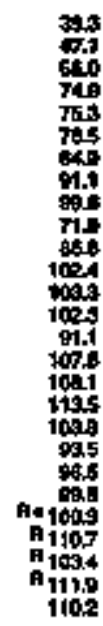 \\
\hline
\end{tabular}

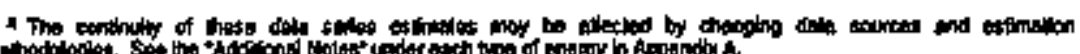

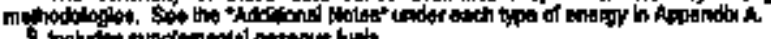

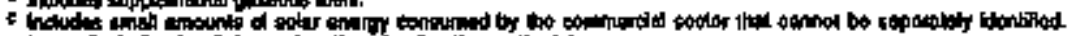

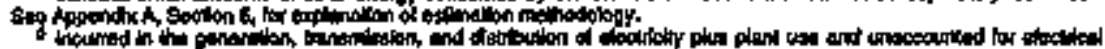

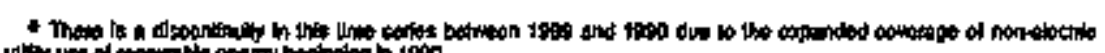

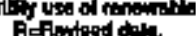

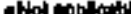


Table 269. Cornmertial Energy Consumption Estipates, 1960, 1965, 1970-1994, Utah

\begin{tabular}{|c|c|c|c|c|c|c|c|c|c|c|c|c|c|c|}
\hline \multirow[b]{3}{*}{ Yet: } & \multicolumn{3}{|c|}{ cond } & \multirow{3}{*}{$\frac{\text { thetrid }}{\text { cintion }}$} & \multicolumn{6}{|c|}{ Palmeloum } & \multirow[b]{2}{*}{ 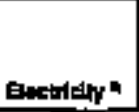 } & \multirow[b]{2}{*}{ Enimy } & \multirow[b]{2}{*}{ 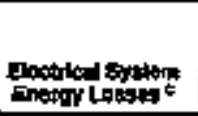 } & \multirow[b]{3}{*}{ Totid } \\
\hline & 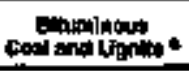 & Arthade = & Toter & & Dating & torponene & tope & moler & 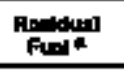 & rolv & & & & \\
\hline & \multicolumn{3}{|c|}{ Thotsind shont Toos } & & \multicolumn{6}{|c|}{ Thouesad Alamel: } & \multicolumn{3}{|c|}{ 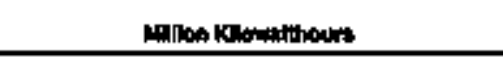 } & \\
\hline 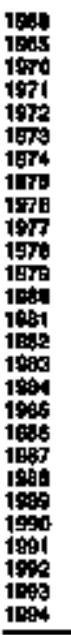 & 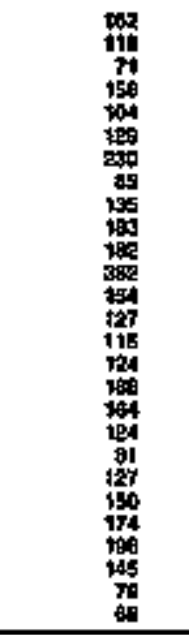 & $\begin{array}{r}0 \\
0 \\
0 \\
0 \\
0 \\
0 \\
0 \\
0 \\
0 \\
0 \\
0 \\
0 \\
0 \\
0 \\
0 \\
0 \\
0 \\
0 \\
0 \\
0 \\
09 \\
0 \\
0 \\
010 \\
0 \\
0 \\
0 \\
0\end{array}$ & 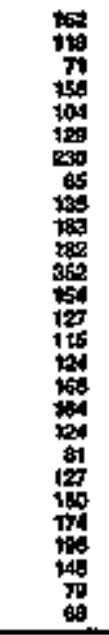 & 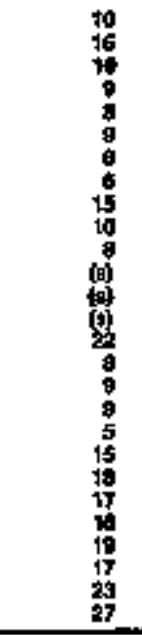 & 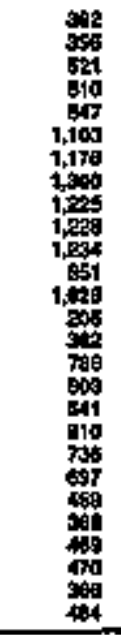 & 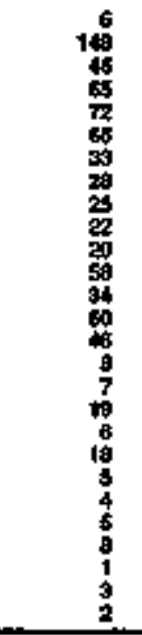 & 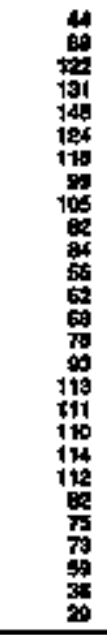 & 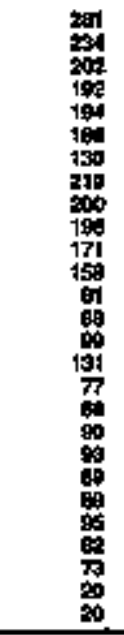 & 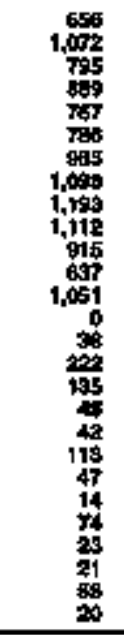 & 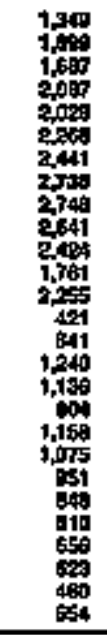 & 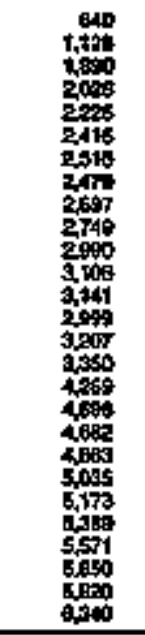 & $\begin{array}{l}= \\
= \\
= \\
= \\
= \\
= \\
= \\
= \\
= \\
= \\
= \\
= \\
= \\
z\end{array}$ & 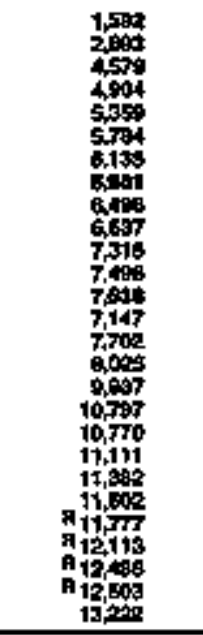 & $\begin{array}{l}= \\
= \\
= \\
= \\
= \\
= \\
= \\
= \\
z \\
= \\
z \\
= \\
z \\
z \\
=\end{array}$ \\
\hline \multicolumn{15}{|c|}{ Trtien Eta } \\
\hline 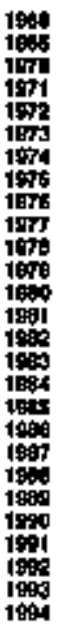 & 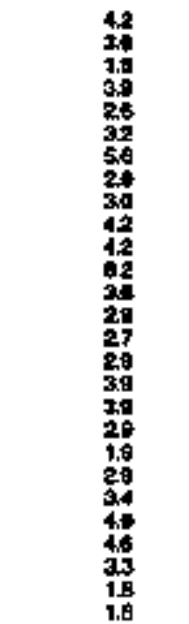 & 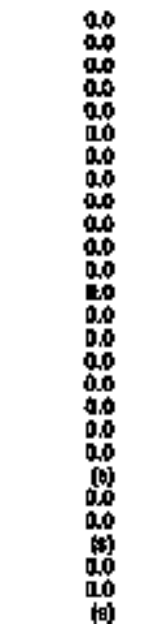 & 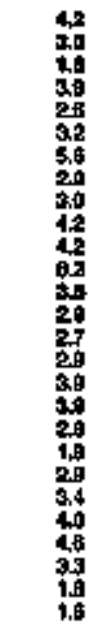 & 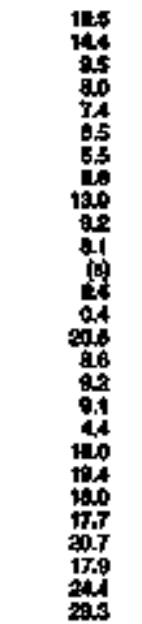 & $\begin{array}{l}21 \\
21 \\
30 \\
47 \\
49 \\
64 \\
80 \\
78 \\
71 \\
72 \\
72 \\
50 \\
40 \\
12 \\
21 \\
46 \\
47 \\
3.1 \\
59 \\
41 \\
41 \\
27 \\
27 \\
27 \\
21 \\
26\end{array}$ & 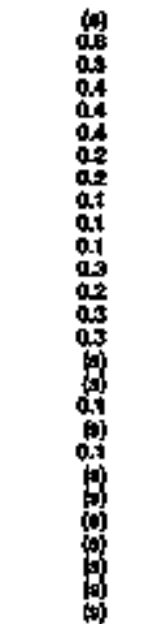 & 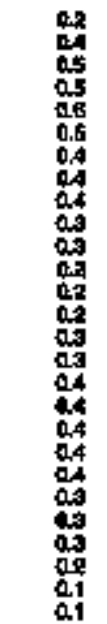 & $\begin{array}{l}1.5 \\
1.2 \\
1.1 \\
1.0 \\
1.0 \\
07 \\
1.1 \\
00 \\
10 \\
0.9 \\
0.0 \\
0.4 \\
0.5 \\
0.7 \\
0.4 \\
0.5 \\
0.5 \\
0.5 \\
0.5 \\
0.5 \\
0.4 \\
0.4 \\
0.1 \\
0.4\end{array}$ & 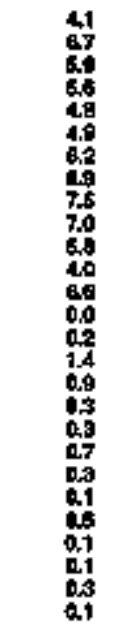 & 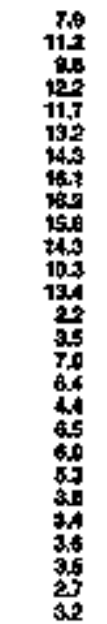 & 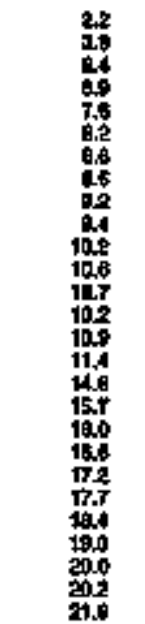 & 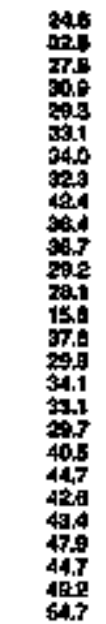 & 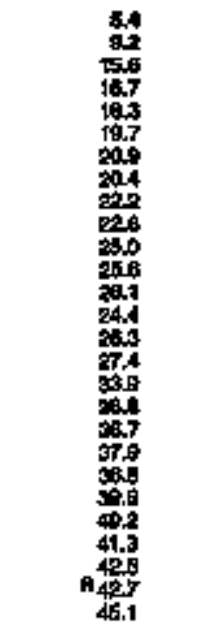 & 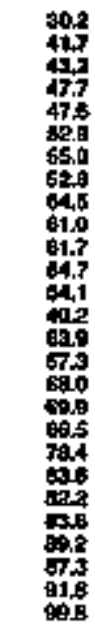 \\
\hline & of ${ }^{2} x$ & A polsts & is & libaled & $\operatorname{ling}$ & & & & & & $10 \mathrm{~m}$ & tit & 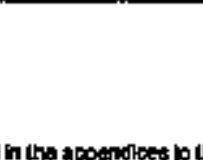 & \\
\hline
\end{tabular}


Tabte 294. Industriad Energy Consumpilon Estlmates, 1950, 1965, 1970-1894, Utzh

\begin{tabular}{|c|c|c|c|c|c|c|c|c|c|c|c|c|c|c|c|c|c|c|}
\hline \multirow[b]{3}{*}{ rer } & \multirow[b]{2}{*}{ ton } & \multirow[b]{2}{*}{ Manumen } & \multicolumn{9}{|c|}{ Potroken } & \multirow[b]{2}{*}{ 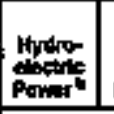 } & \multirow[b]{2}{*}{ Elathen | } & \multirow[b]{2}{*}{ otherent } & \multirow[b]{2}{*}{ Ementan" } & \multirow[b]{2}{*}{ 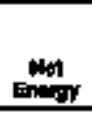 } & \multirow{2}{*}{ 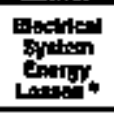 } & \multirow[b]{3}{*}{ Toth } \\
\hline & & & Nomend ond & portion & Kerotatol & LoG & 年 & moter & 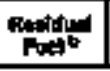 & Otwar & Tore & & & & & & & \\
\hline & Shations & ontion & \multicolumn{9}{|c|}{ Thovasad Bamit } & \multicolumn{6}{|c|}{ 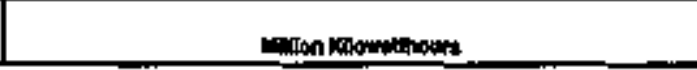 } & \\
\hline 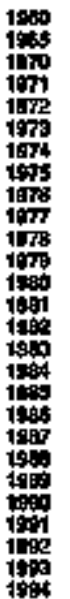 & 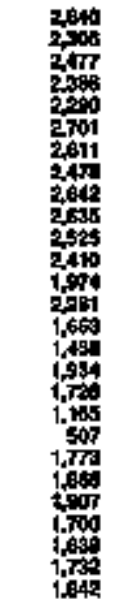 & 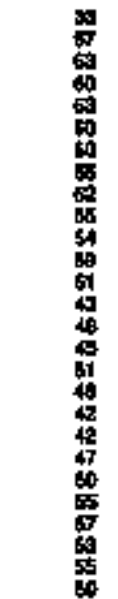 & 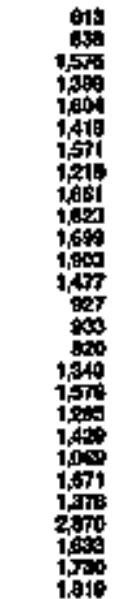 & 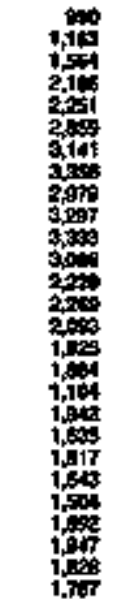 & 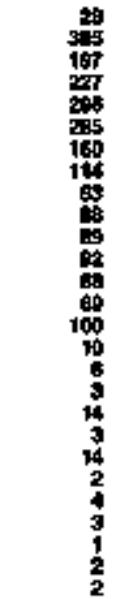 & 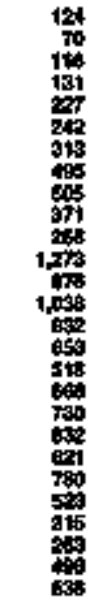 & 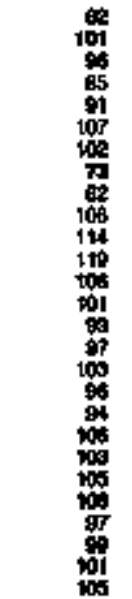 & 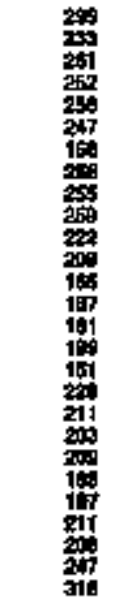 & 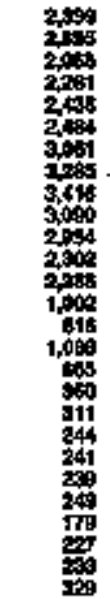 & 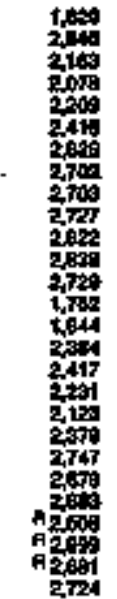 & 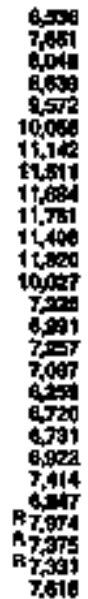 & 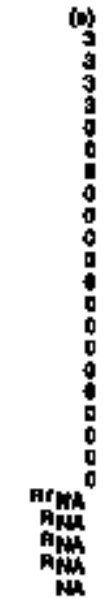 & 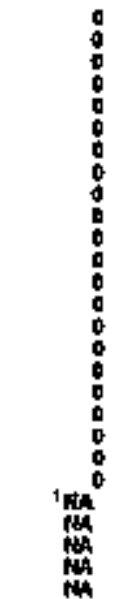 & 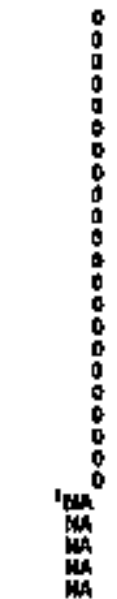 & 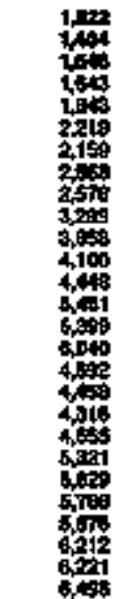 & $\begin{array}{l}\bar{z} \\
\bar{z} \\
\bar{z} \\
\bar{z}\end{array}$ & 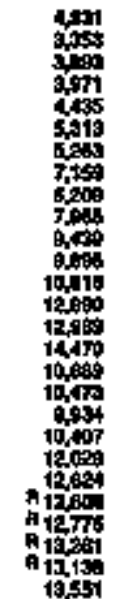 & \\
\hline
\end{tabular}

\begin{tabular}{|c|c|c|c|c|c|c|}
\hline 7.0 .5 & \multicolumn{2}{|l|}{ 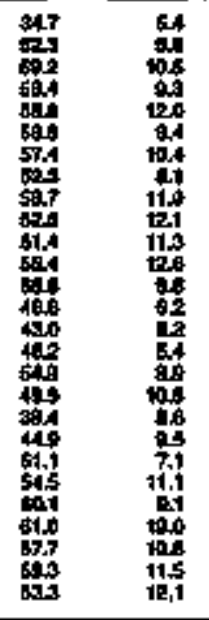 } & 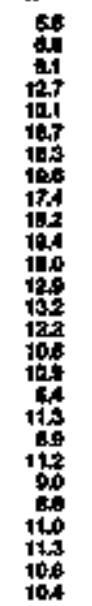 & 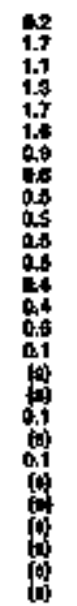 & 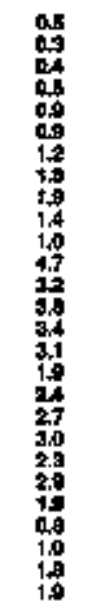 & 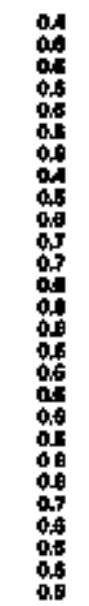 \\
\hline
\end{tabular}

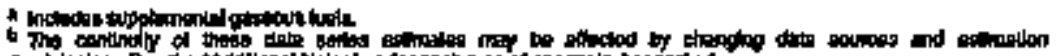

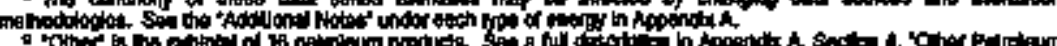

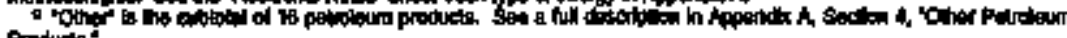

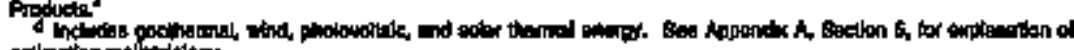

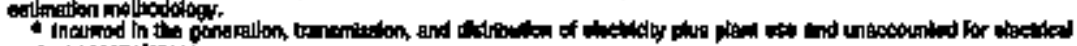

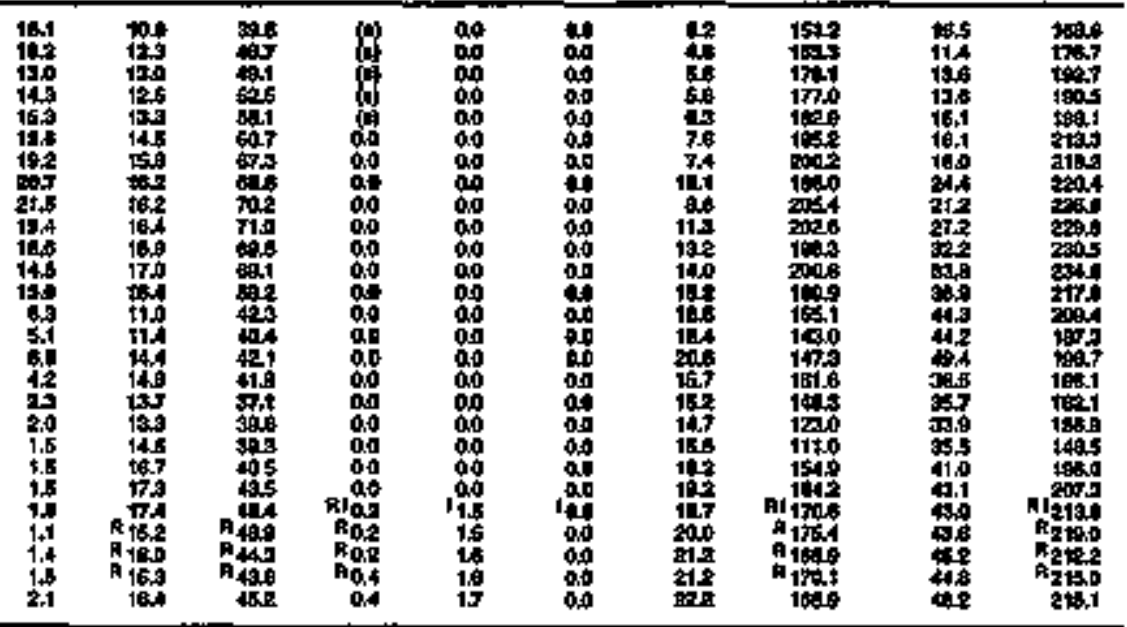

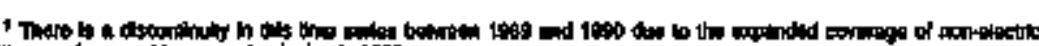

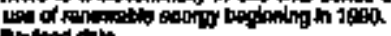

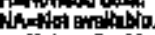

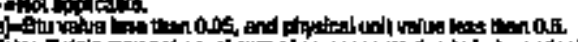

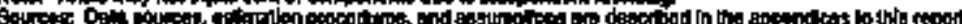

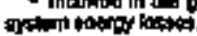


Tablo 285. Transpertation Energy Consumption Estinuales, 1960, 19s5, 1970-1994, Utah

\begin{tabular}{|c|c|c|c|c|c|c|c|c|c|c|c|c|c|c|c|}
\hline \multirow[b]{3}{*}{ Yow } & \multirow[b]{2}{*}{ Coesl } & \multirow[b]{2}{*}{ Mrafet } & \multicolumn{8}{|c|}{ Fetreloom } & \multirow[b]{2}{*}{ Bletuatia" } & \multirow[b]{2}{*}{ Dectithy } & \multirow[b]{2}{*}{ Enet } & \multirow[b]{2}{*}{ 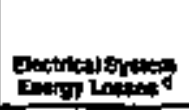 } & \multirow[b]{3}{*}{ Totes } \\
\hline & & & Anotitan & 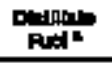 & Fiㅔ. & tept: & Lubdonitan= & rothen & $\begin{array}{l}\text { Mander } \\
\text { Fod }\end{array}$ & Yotu & & & & & \\
\hline & mowend & andien pen & \multicolumn{8}{|c|}{ 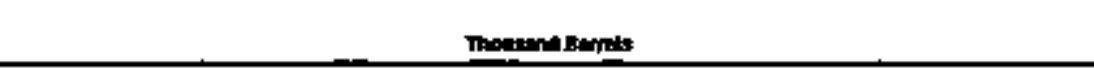 } & Thoment & \multicolumn{3}{|c|}{ 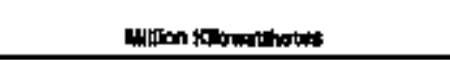 } & \\
\hline 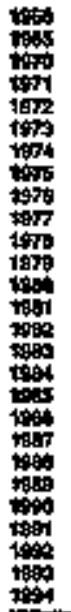 & 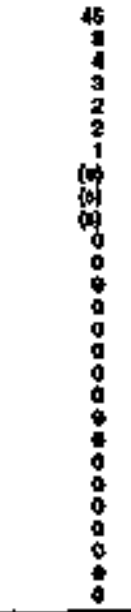 & $\begin{array}{l}\text { p) } \\
0 \\
\mathbf{3} \\
\mathbf{1} \\
\mathbf{1} \\
\mathbf{9} \\
\mathbf{4} \\
\mathbf{1} \\
\mathbf{1} \\
\mathbf{1} \\
\mathbf{1} \\
\mathbf{1} \\
\mathbf{1} \\
\mathbf{1} \\
\mathbf{1} \\
\mathbf{1} \\
\mathbf{1} \\
\mathbf{3} \\
\mathbf{3}\end{array}$ & 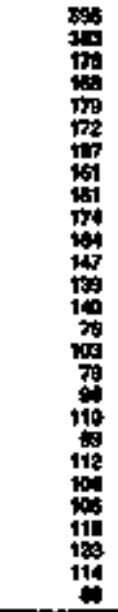 & 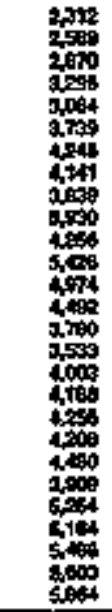 & 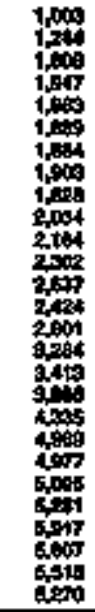 & 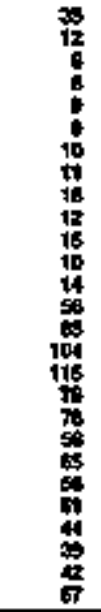 & 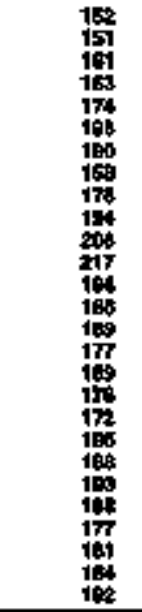 & 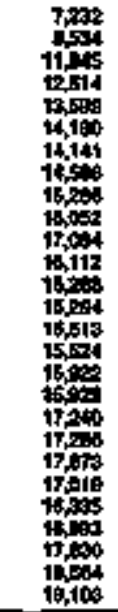 & 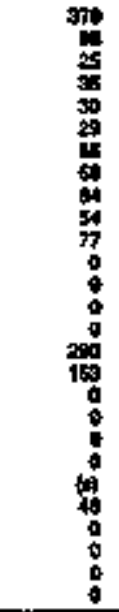 & 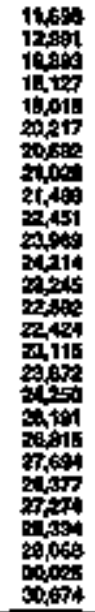 & $\begin{array}{l}0 \\
0 \\
0 \\
0 \\
0 \\
0 \\
0 \\
0 \\
0 \\
0 \\
0 \\
0 \\
0 \\
0 \\
0 \\
0 \\
0 \\
0 \\
0 \\
0 \\
0 \\
0 \\
0 \\
0\end{array}$ & $\begin{array}{l}0 \\
0 \\
0 \\
0 \\
0 \\
0 \\
0 \\
0 \\
0 \\
0 \\
0 \\
0 \\
0 \\
0 \\
0 \\
0 \\
0 \\
0 \\
0 \\
0 \\
0 \\
0 \\
0 \\
0 \\
0 \\
0 \\
0 \\
0 \\
0 \\
0 \\
0 \\
0\end{array}$ & $\begin{array}{l}z \\
z \\
z \\
z \\
z \\
z \\
z \\
z \\
z \\
z \\
z \\
z \\
z\end{array}$ & $\begin{array}{l}1 \\
1 \\
0 \\
0 \\
0 \\
0 \\
0 \\
0 \\
0 \\
0 \\
0 \\
0 \\
0 \\
0 \\
0 \\
0 \\
0 \\
0 \\
0 \\
0 \\
0 \\
0 \\
0 \\
0 \\
0\end{array}$ & $\begin{array}{l}= \\
= \\
= \\
= \\
= \\
= \\
= \\
= \\
= \\
= \\
= \\
= \\
= \\
= \\
=\end{array}$ \\
\hline
\end{tabular}

\begin{tabular}{|c|c|c|c|c|c|c|c|c|c|c|c|c|c|c|c|}
\hline 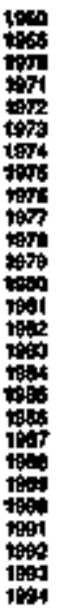 & 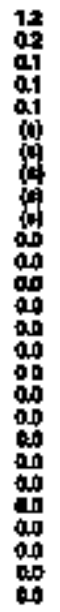 & 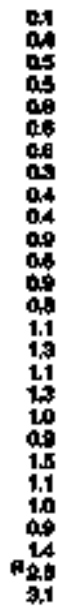 & 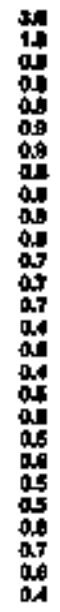 & 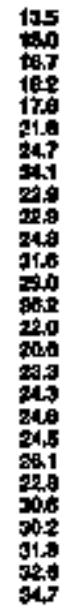 & 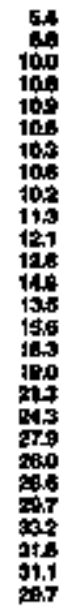 & 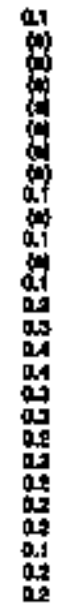 & 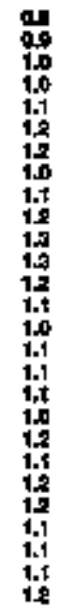 & 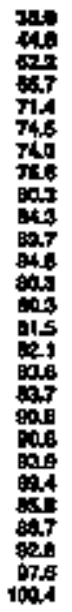 & 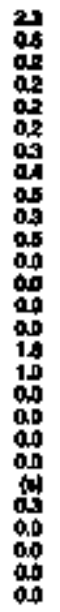 & 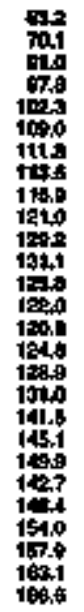 & 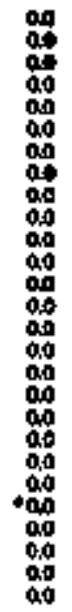 & $\begin{array}{l}00 \\
00 \\
00 \\
000 \\
00 \\
00 \\
00 \\
00 \\
00 \\
00 \\
00 \\
00 \\
00 \\
00 \\
00 \\
00 \\
00 \\
00 \\
00 \\
00 \\
00 \\
00 \\
00 \\
00 \\
00 \\
00 \\
00\end{array}$ & 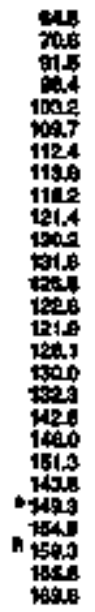 & 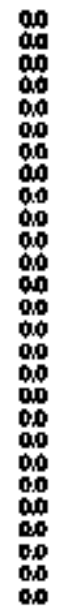 & 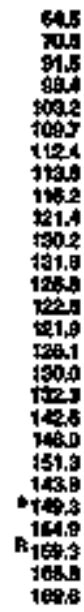 \\
\hline
\end{tabular}

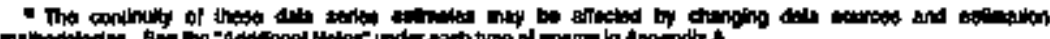

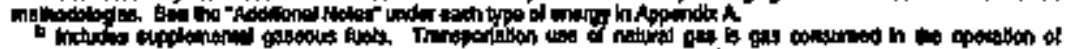

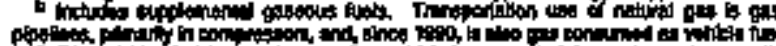

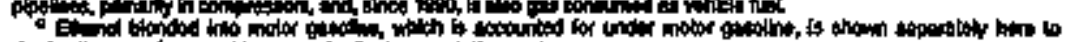

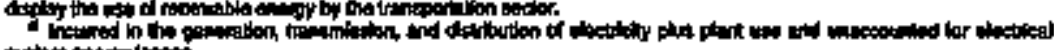

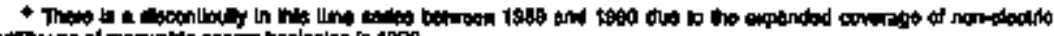

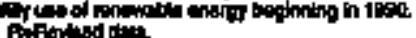

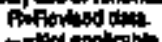

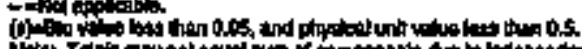

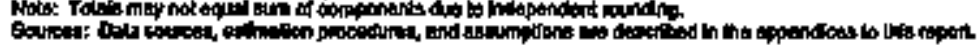

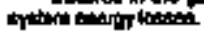


U Table 266. Estimates of Energy lnpul at Electric Utillies, 1960, 1965, 1970-1994, Utah

\begin{tabular}{|c|c|c|c|c|c|c|c|c|c|c|c|c|c|c|}
\hline \multirow[b]{3}{*}{$V_{n=0}$} & \multicolumn{3}{|c|}{ Ean } & \multirow{3}{*}{ 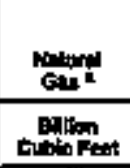 } & \multicolumn{4}{|c|}{ Antrotpon } & \multirow[b]{2}{*}{ 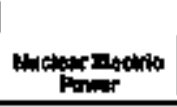 } & \multirow[b]{2}{*}{ Mraneleotolo } & \multirow[b]{2}{*}{ Olotang } & \multirow[b]{2}{*}{ Otoothem } & \multirow[b]{2}{*}{ Fine 401} & \multirow[b]{3}{*}{ Tow: } \\
\hline & 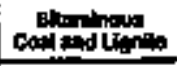 & Antarese & Tat & & ont & 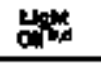 & Combing & Tot & & & & & & \\
\hline & \multicolumn{3}{|c|}{ Tronumend Shart Tons: } & & \multicolumn{4}{|c|}{ 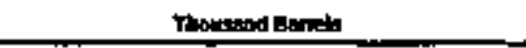 } & \multicolumn{5}{|c|}{ 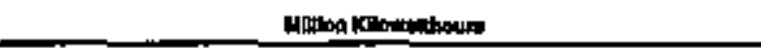 } & \\
\hline 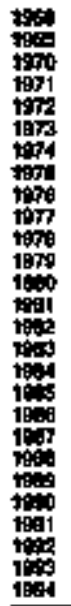 & 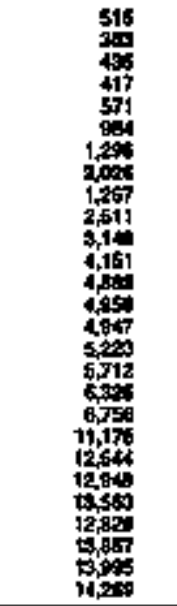 & $\begin{array}{l}0 \\
0 \\
0 \\
0 \\
0 \\
0 \\
0 \\
0 \\
0 \\
0 \\
0 \\
0 \\
0 \\
0 \\
0 \\
0 \\
0 \\
0 \\
0 \\
0 \\
0 \\
0 \\
0 \\
0 \\
0 \\
0 \\
0 \\
0\end{array}$ & 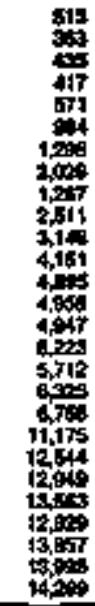 & 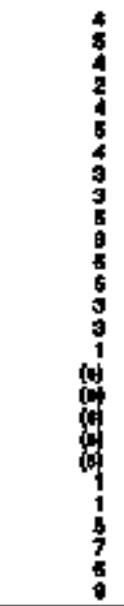 & 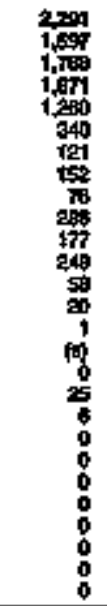 & 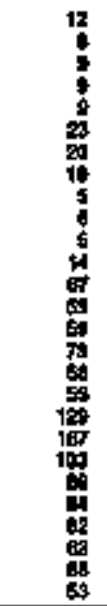 & $\begin{array}{l}0 \\
0 \\
0 \\
0 \\
0 \\
0 \\
: \\
: \\
0 \\
0 \\
0 \\
0 \\
0 \\
0 \\
0 \\
0 \\
0 \\
0\end{array}$ & 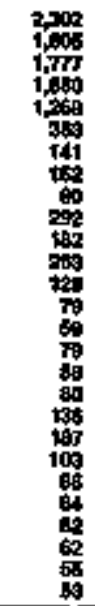 & $\begin{array}{l}0 \\
0 \\
0 \\
0 \\
0 \\
0 \\
0 \\
0 \\
0 \\
0 \\
0 \\
0 \\
0 \\
0 \\
0 \\
0 \\
0 \\
0 \\
0 \\
0 \\
0 \\
0 \\
0 \\
0 \\
0 \\
0 \\
0\end{array}$ & 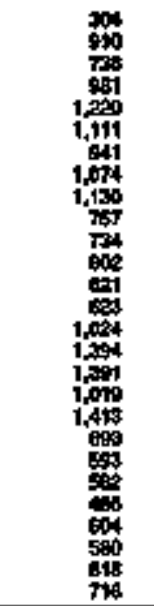 & $\begin{array}{l}0 \\
0 \\
0 \\
0 \\
0 \\
0 \\
0 \\
0 \\
0 \\
0 \\
0 \\
0 \\
0 \\
0 \\
0 \\
0 \\
0 \\
0 \\
0 \\
0 \\
0 \\
0 \\
0 \\
0 \\
0 \\
0 \\
0\end{array}$ & 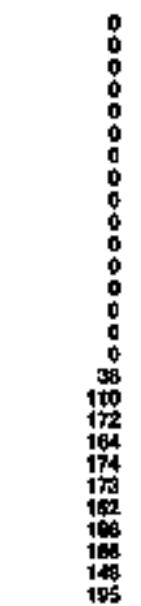 & $\begin{array}{l}0 \\
0 \\
0 \\
0 \\
0 \\
0 \\
0 \\
0 \\
0 \\
0 \\
0 \\
0 \\
0 \\
0 \\
0 \\
0 \\
0 \\
0 \\
0 \\
0 \\
0 \\
0\end{array}$ & $\begin{array}{l}z \\
z \\
z \\
z \\
z \\
z \\
z \\
z \\
z \\
z \\
z \\
z \\
z \\
z \\
z\end{array}$ \\
\hline \multicolumn{15}{|c|}{ Than the } \\
\hline
\end{tabular}

\begin{tabular}{|c|c|c|c|c|c|c|c|c|c|c|c|c|c|c|}
\hline 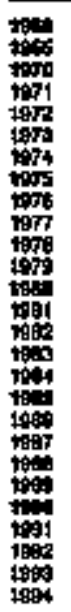 & 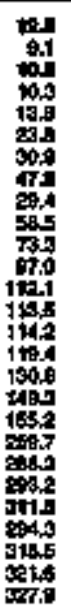 & 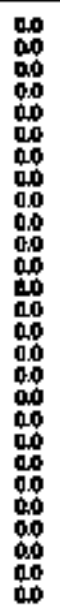 & 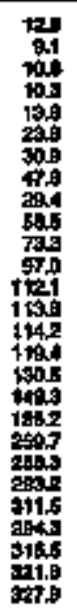 & 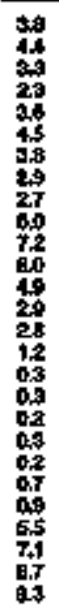 & 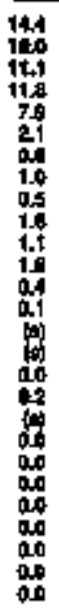 & 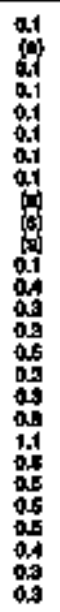 & 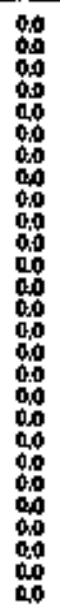 & 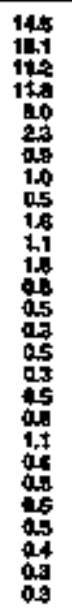 & 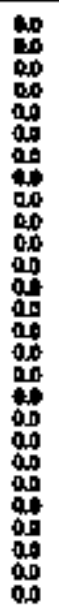 & 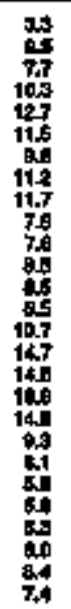 & 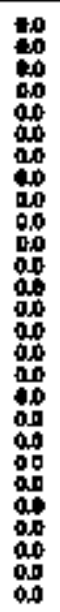 & 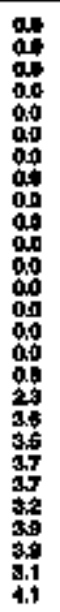 & 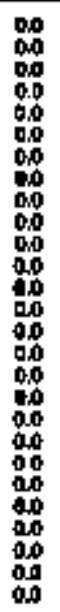 & 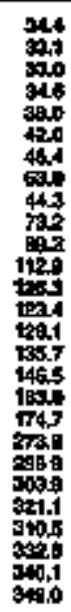 \\
\hline
\end{tabular}

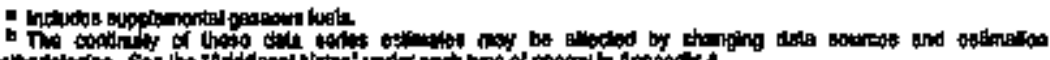

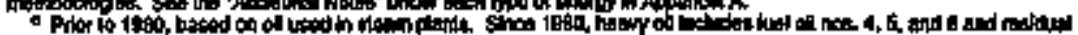

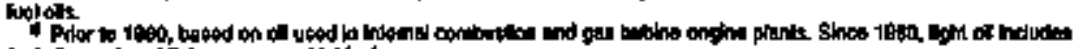

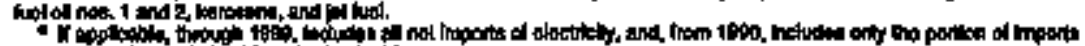

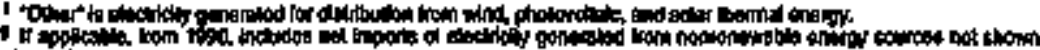

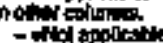

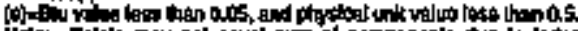

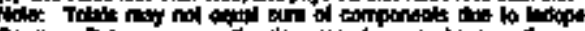

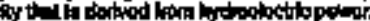


Tatbla 287. Energy Consumplian Estlmates by Source, 1960, 1965, 1970-1994, Yermont

\begin{tabular}{|c|c|c|c|c|c|c|c|c|c|c|c|c|c|c|c|c|c|c|c|}
\hline \multirow[b]{3}{*}{ Yent } & \multirow[b]{2}{*}{$\cos$} & \multirow[b]{2}{*}{ mintid } & \multicolumn{11}{|c|}{ Potsolatin } & \multirow[b]{2}{*}{ 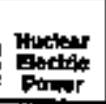 } & \multirow[b]{2}{*}{ 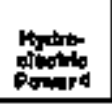 } & \multirow[b]{2}{*}{ Blotonge } & \multirow[b]{2}{*}{ Other is } & \multirow{3}{*}{ Hitions } & \multirow[b]{3}{*}{ Totaln } \\
\hline & & & Asphalinat & 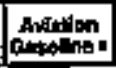 & 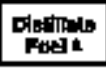 & Fet. & $\operatorname{kem}_{40,1}$ & tent: & 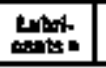 & 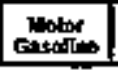 & Meathosil & ptowers & Totot & & & & & & \\
\hline & Trowend & conder & \multicolumn{11}{|c|}{ Thengand Banpoth } & \multicolumn{4}{|c|}{ 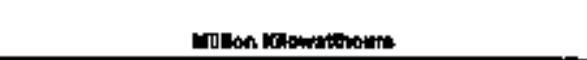 } & & \\
\hline 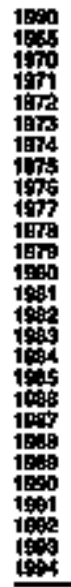 & 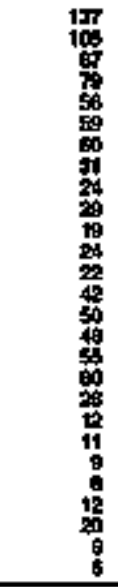 & $\begin{array}{l}0 \\
0 \\
3 \\
4 \\
4 \\
5 \\
4 \\
4 \\
4 \\
4 \\
4 \\
4 \\
4 \\
4 \\
5 \\
5 \\
5 \\
6 \\
8 \\
7 \\
7 \\
7 \\
7\end{array}$ & 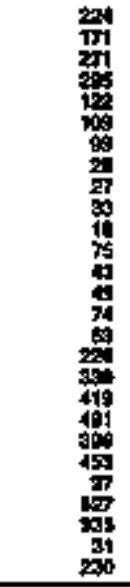 & 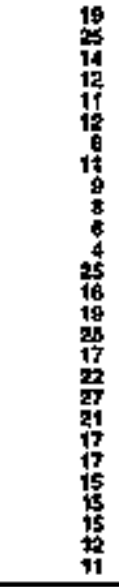 & 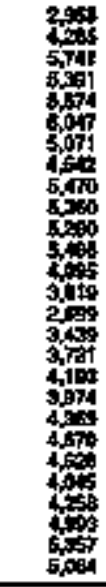 & 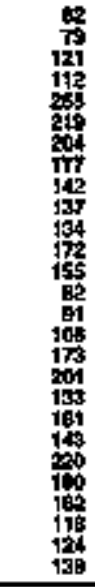 & 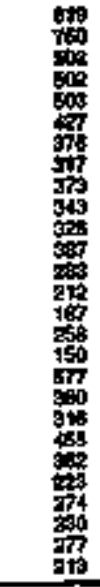 & 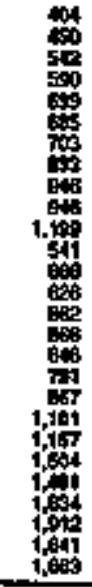 & 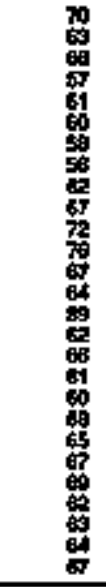 & 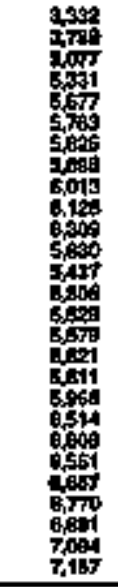 & 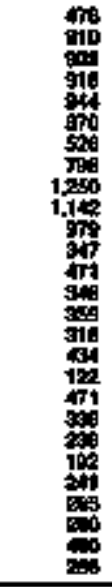 & 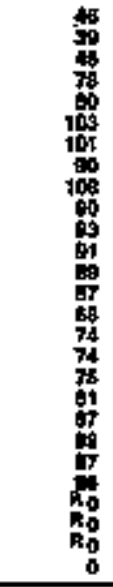 & 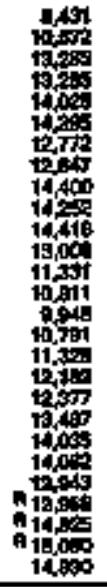 & 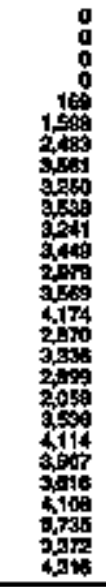 & 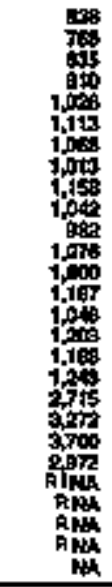 & 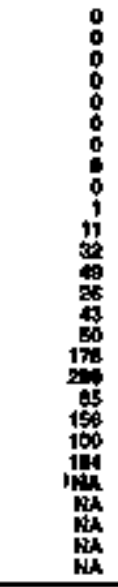 & 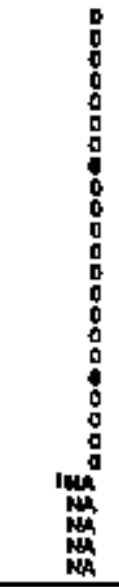 & 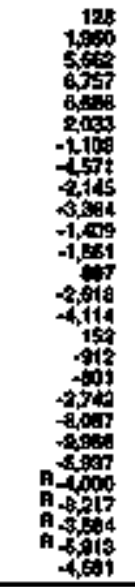 & $\begin{array}{l}z \\
\vdots \\
\vdots \\
\vdots \\
\vdots \\
\vdots \\
z \\
\vdots \\
\vdots \\
\vdots \\
z \\
z \\
z \\
z\end{array}$ \\
\hline \multicolumn{20}{|c|}{ Tiftan bis } \\
\hline 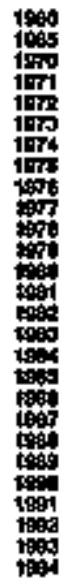 & 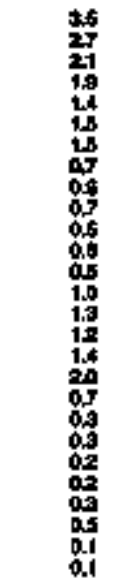 & 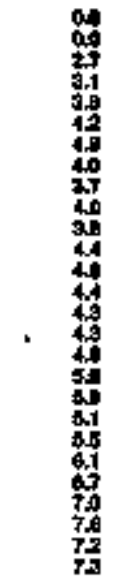 & 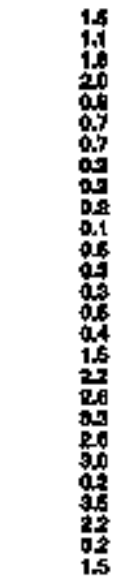 & 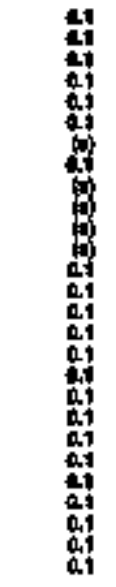 & 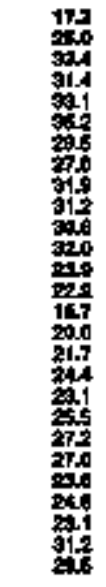 & 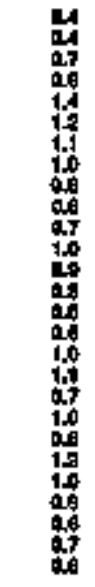 & 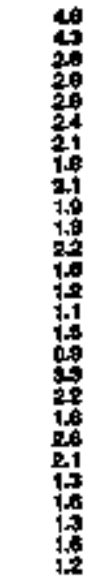 & 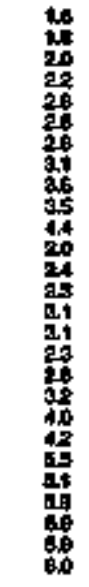 & 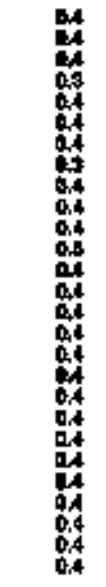 & 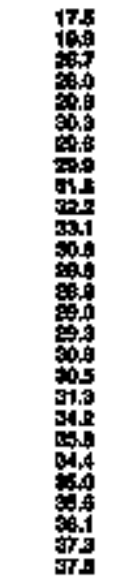 & 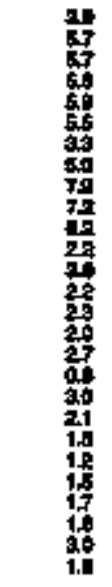 & 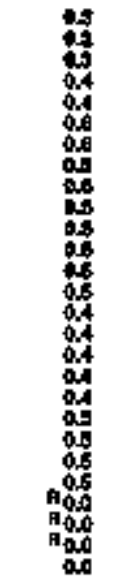 & 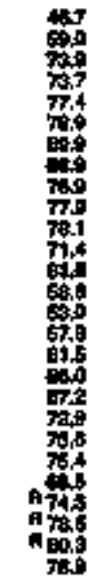 & 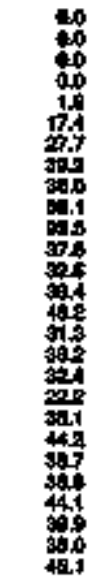 & 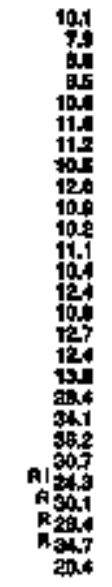 & 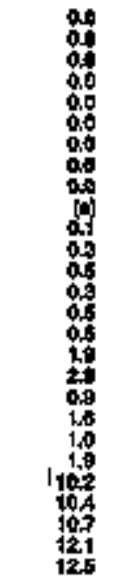 & 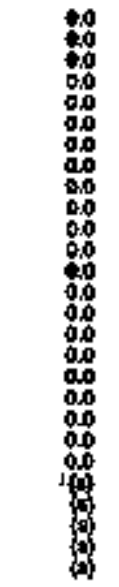 & 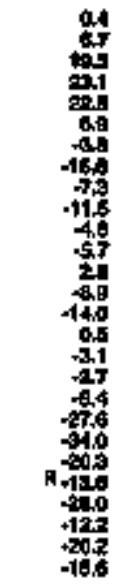 & 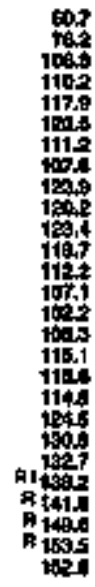 \\
\hline
\end{tabular}

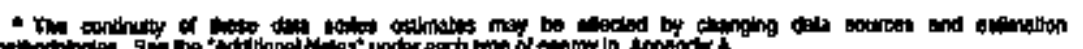

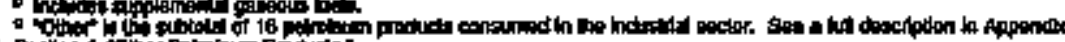

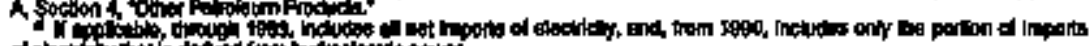

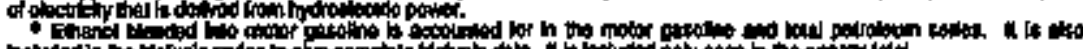

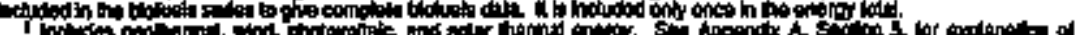

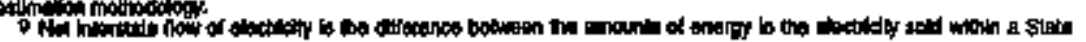

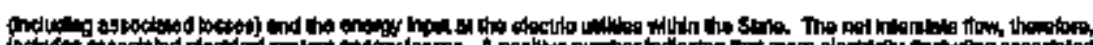

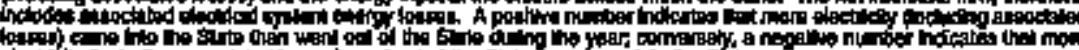

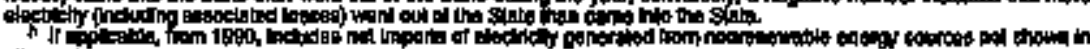

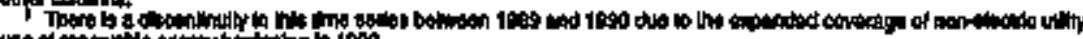

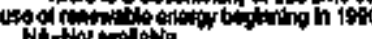

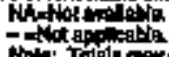

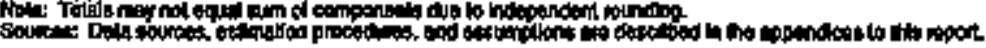


Table 298. Restdenllal Energy Consumption Estlmates, t960, 1965, 1970-1994, Vermont

\begin{tabular}{|c|c|c|c|c|c|c|c|c|c|c|c|c|c|c|}
\hline \multirow[b]{3}{*}{$\mathrm{YeE}$} & \multicolumn{3}{|c|}{ cont } & \multirow{3}{*}{ 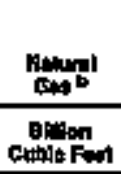 } & & & & & \multirow{3}{*}{$\begin{array}{l}\text { stofonts } \\
\text { Thewaents } \\
\text { cords }\end{array}$} & \multirow[b]{2}{*}{ Ooler" } & \multirow[b]{2}{*}{ Eloteristop } & \multirow[b]{2}{*}{ Enoty } & \multirow{3}{*}{ 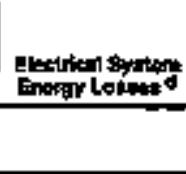 } & \multirow[b]{3}{*}{ Tolly } \\
\hline & 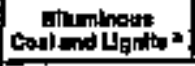 & Anthroetio" & Totel & & Ototalata & Korostane & LPG: & 7 Thtal & & & & & & \\
\hline & \multicolumn{3}{|c|}{ Thoonend Bhert Tons } & & \multicolumn{4}{|c|}{ Tpoutsond Bondts } & & \multicolumn{3}{|c|}{ 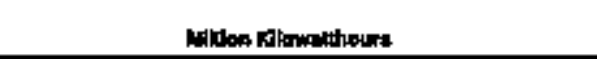 } & & \\
\hline 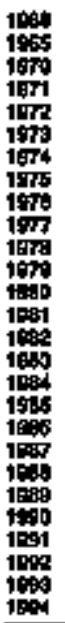 & $\begin{array}{l}0 \\
0 \\
0 \\
0 \\
0 \\
0 \\
0 \\
0 \\
0 \\
0 \\
0 \\
0 \\
0 \\
1 \\
0 \\
12 \\
000 \\
0 \\
0 \\
0 \\
0 \\
0 \\
0 \\
0 \\
2\end{array}$ & 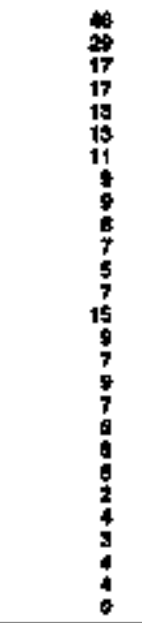 & 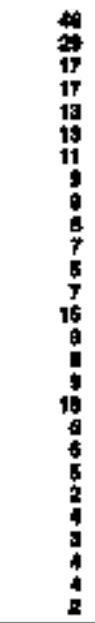 & $\begin{array}{l}\mathbf{1} \\
\mathbf{1} \\
1 \\
1 \\
1 \\
1 \\
1 \\
1 \\
1 \\
1 \\
1 \\
1 \\
1 \\
1 \\
2 \\
2 \\
2 \\
2 \\
2 \\
2 \\
3 \\
3 \\
2\end{array}$ & 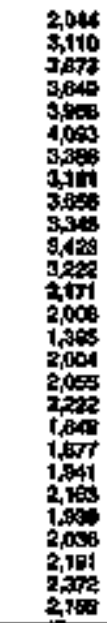 & 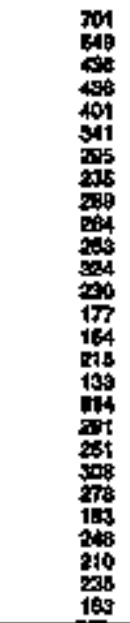 & 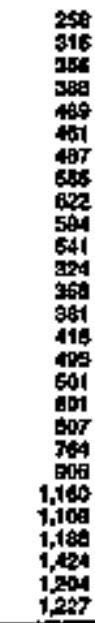 & 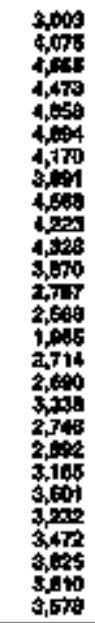 & $\begin{array}{l}: \\
0 \\
0 \\
0 \\
0 \\
0 \\
0 \\
0 \\
0 \\
0 \\
0 \\
0 \\
0 \\
0 \\
0 \\
0 \\
0 \\
0 \\
0 \\
0 \\
0 \\
09 \\
104 \\
110 \\
114 \\
111\end{array}$ & $\begin{array}{c}0 \\
0 \\
0 \\
0 \\
0 \\
0 \\
0 \\
0 \\
0 \\
0 \\
0 \\
0 \\
0 \\
0 \\
0 \\
0 \\
0 \\
0 \\
0 \\
0 \\
0 \\
0 \\
0 \\
0 \\
2 \\
2 \\
\mathbf{2} \\
3\end{array}$ & 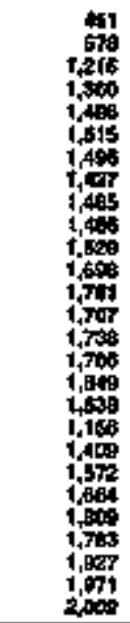 & $\begin{array}{l}= \\
= \\
= \\
= \\
= \\
= \\
= \\
= \\
= \\
= \\
= \\
= \\
= \\
= \\
= \\
=\end{array}$ & 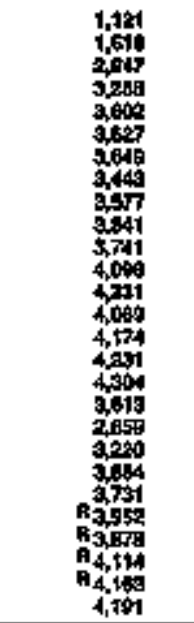 & 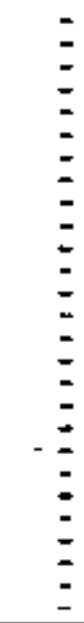 \\
\hline \multicolumn{15}{|c|}{ 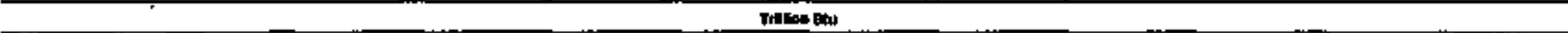 } \\
\hline 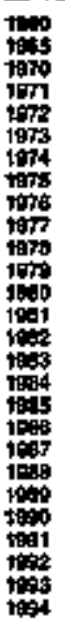 & 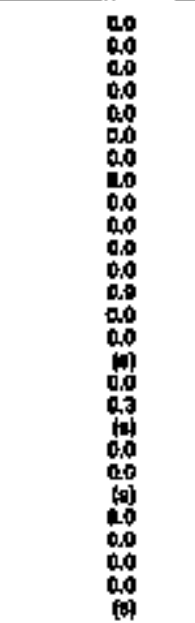 & $\begin{array}{l}1.1 \\
0.7 \\
04 \\
0.4 \\
0.3 \\
0.3 \\
0.2 \\
0.2 \\
0.2 \\
02 \\
0.2 \\
0.1 \\
01 \\
0.4 \\
0,0 \\
0.2 \\
0.2 \\
0.2 \\
0.1 \\
02 \\
01 \\
0.1 \\
0.1 \\
0.1 \\
0.1 \\
0.1 \\
0.0\end{array}$ & 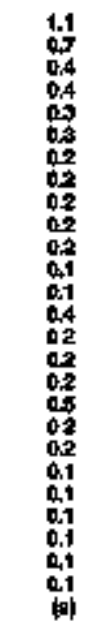 & $\begin{array}{l}6.4 \\
0.1 \\
1.1 \\
1.1 \\
12 \\
12 \\
1.1 \\
12 \\
1.2 \\
1.2 \\
1.4 \\
1.3 \\
1.3 \\
1.3 \\
1.3 \\
1.4 \\
1.6 \\
1.6 \\
21 \\
21 \\
22 \\
2.5 \\
2.5 \\
24\end{array}$ & 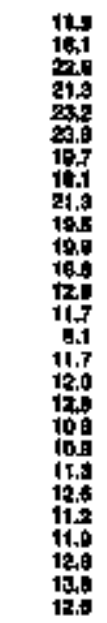 & 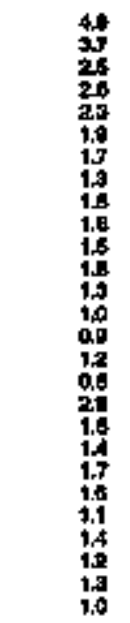 & 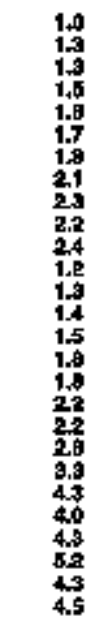 & 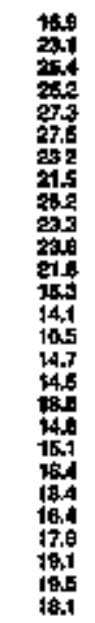 & 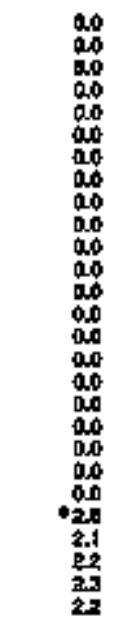 & 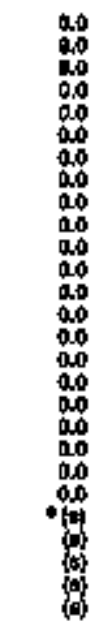 & 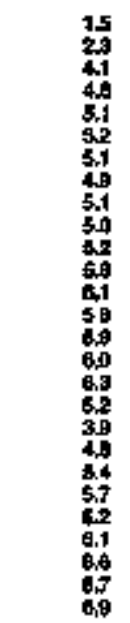 & 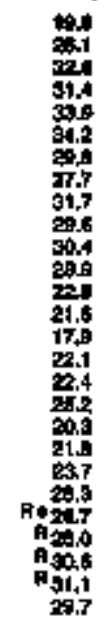 & 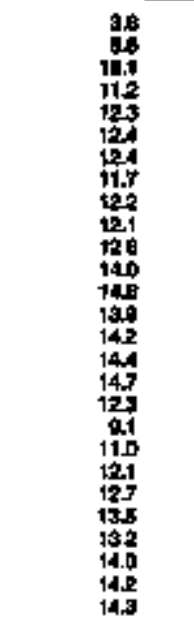 & 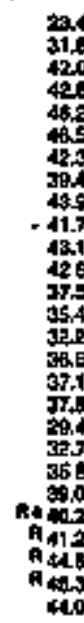 \\
\hline
\end{tabular}

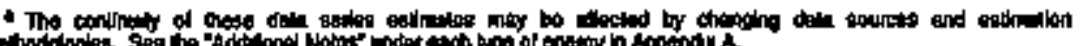

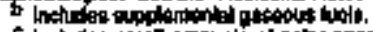
.

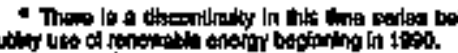
Wistion ding

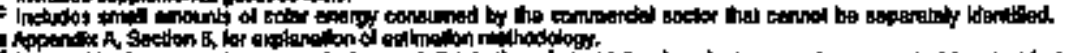

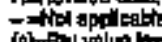

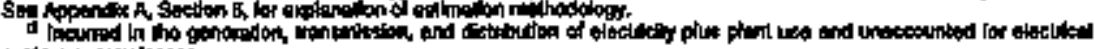


Table 299. Comunercial Energy Consumptlon Estlmales, 1960, 1965, 1970-1994, Vermont

\begin{tabular}{|c|c|c|c|c|c|c|c|c|c|c|c|c|c|c|}
\hline \multirow[b]{3}{*}{ Yerr } & \multicolumn{3}{|c|}{$\infty$} & \multirow{3}{*}{ 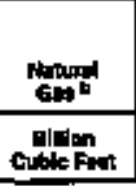 } & \multicolumn{6}{|c|}{ motrotoum } & \multirow[b]{2}{*}{ ancticky } & \multirow[b]{2}{*}{ Energy } & \multirow{3}{*}{ 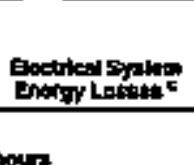 } & \multirow[b]{3}{*}{ Tot: : } \\
\hline & 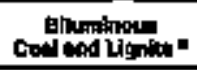 & Antrosolio $=$ & Tot & & 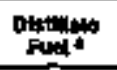 & Kenament & LPG: & 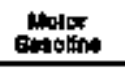 & Alotedur & Totat & & & & \\
\hline & \multicolumn{3}{|c|}{ moverand Short Ten: } & & \multicolumn{6}{|c|}{ Thowsand Burroh } & \multicolumn{2}{|c|}{ 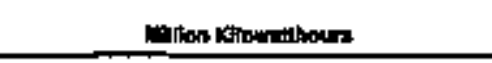 } & & \\
\hline 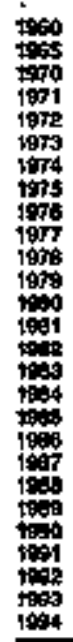 & $\begin{array}{l}0 \\
0 \\
0 \\
0 \\
0 \\
0 \\
0 \\
0 \\
0 \\
0 \\
0 \\
0 \\
0 \\
0 \\
0 \\
1 \\
0 \\
0 \\
1 \\
0 \\
0 \\
0 \\
0 \\
0 \\
0 \\
0 \\
0 \\
3 \\
\end{array}$ & 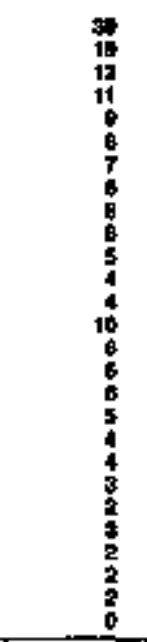 & 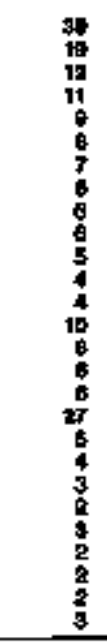 & $\begin{array}{l}0 \\
\vdots \\
1 \\
1 \\
1 \\
1 \\
1 \\
1 \\
1 \\
1 \\
2 \\
2 \\
2 \\
2 \\
2 \\
2 \\
2 \\
2 \\
2 \\
3\end{array}$ & 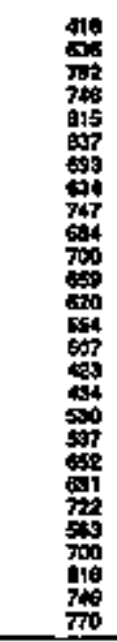 & 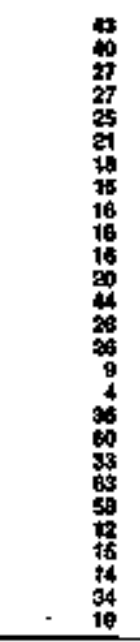 & 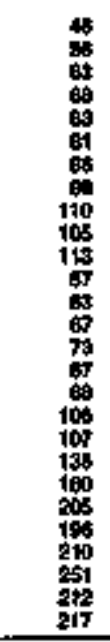 & 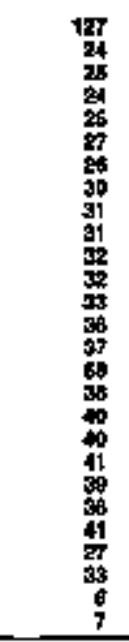 & 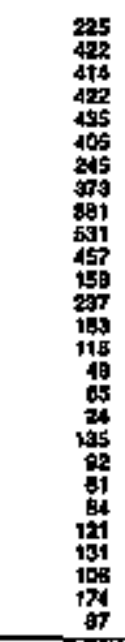 & 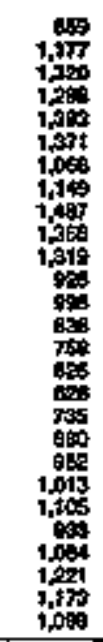 & 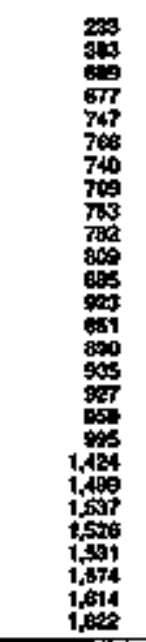 & $\begin{array}{l}\vdots \\
\vdots \\
\vdots \\
\vdots \\
\vdots \\
\vdots \\
\vdots \\
\vdots \\
\vdots \\
\vdots \\
\vdots \\
\vdots \\
\vdots \\
\vdots\end{array}$ & 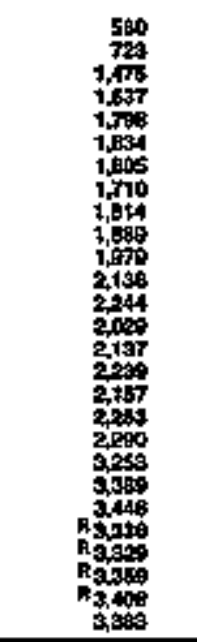 & $\begin{array}{l}\vdots \\
\vdots \\
\vdots \\
\vdots \\
\vdots \\
\vdots \\
\vdots \\
\vdots \\
\vdots \\
\vdots \\
\vdots \\
\vdots \\
\vdots\end{array}$ \\
\hline \multicolumn{15}{|c|}{ J7mpops } \\
\hline 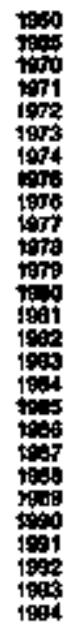 & 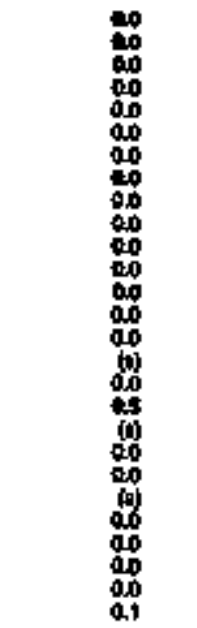 & 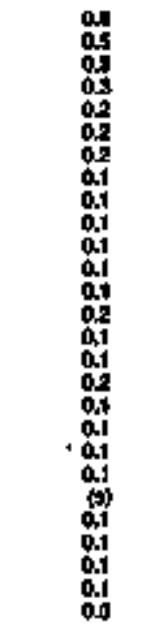 & 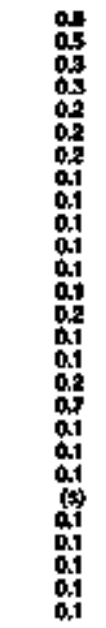 & 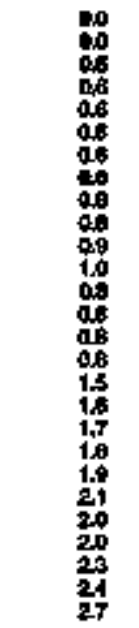 & 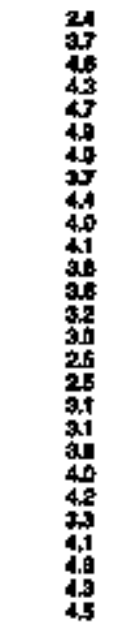 & 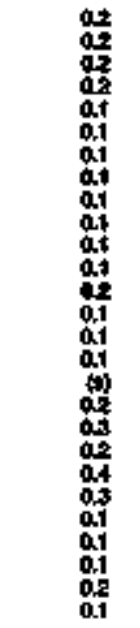 & 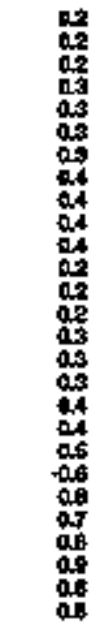 & 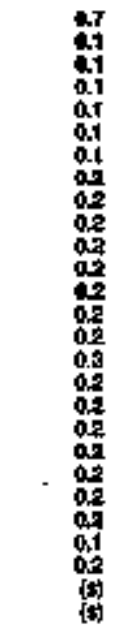 & 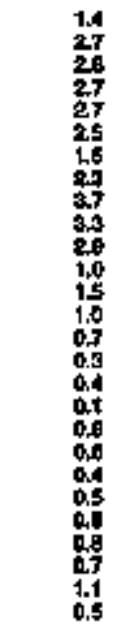 & 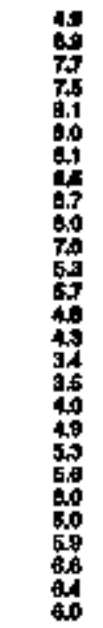 & 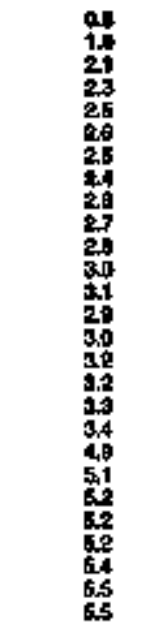 & 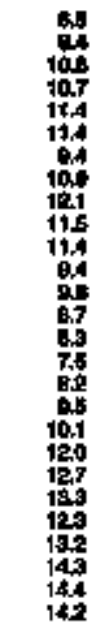 & 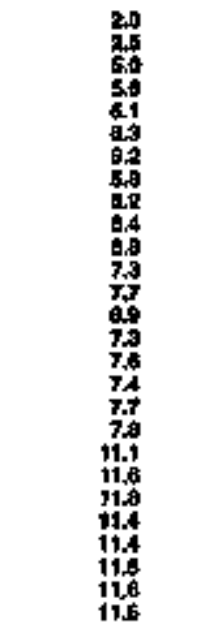 & 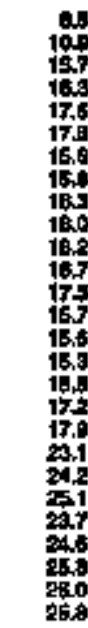 \\
\hline
\end{tabular}


Table 290. Industulal Energy Consumpition Estimates, 1960, 1995, 1970-1994, Vermonk

\begin{tabular}{|c|c|c|c|c|c|c|c|c|c|c|c|c|c|c|c|c|c|}
\hline \multirow[b]{2}{*}{ com } & \multirow[b]{2}{*}{ 4t } & \multicolumn{9}{|c|}{ Mrame } & \multirow[b]{2}{*}{ 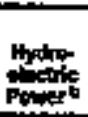 } & \multirow[b]{2}{*}{ moluests } & \multirow[b]{2}{*}{ 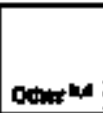 } & \multirow[b]{2}{*}{ Exeonongy of } & \multirow[b]{2}{*}{ entring } & \multirow{2}{*}{ 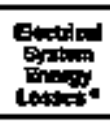 } & \\
\hline & & 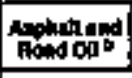 & ations & 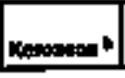 & Le日" & Lumpanst & mater & Anted & onestse & 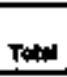 & & & & & & & \multirow[b]{2}{*}{$T$} \\
\hline Proutind & Miring & \multicolumn{9}{|c|}{ Droenumd Bunts } & \multicolumn{6}{|c|}{ 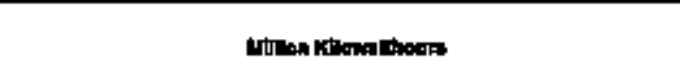 } & \\
\hline
\end{tabular}

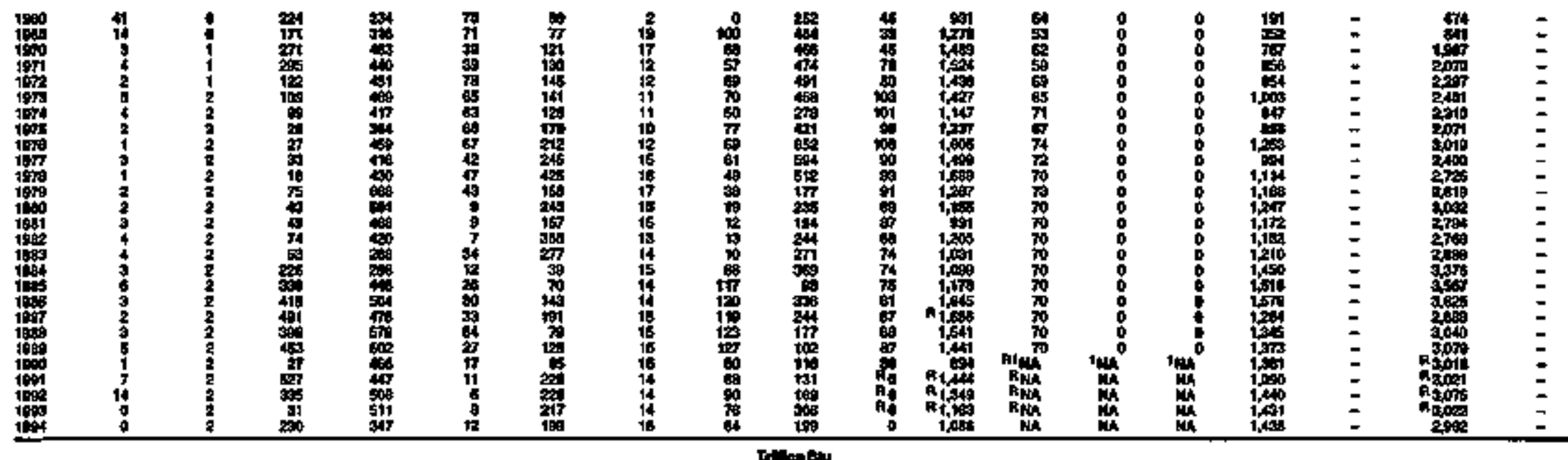

\begin{tabular}{|c|c|}
\hline 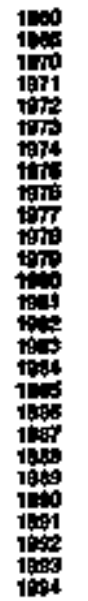 & 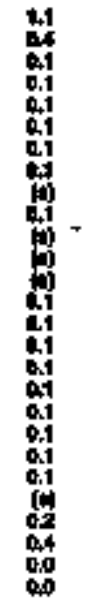 \\
\hline
\end{tabular}

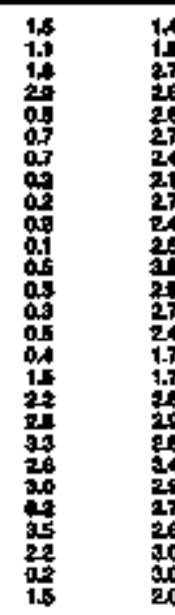

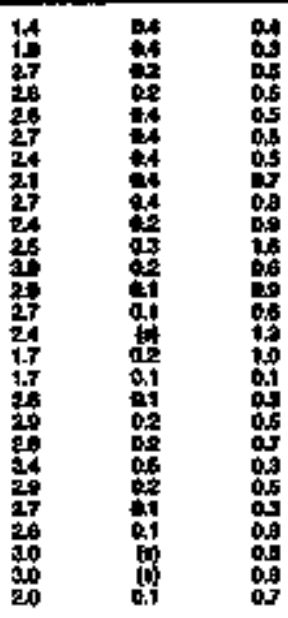

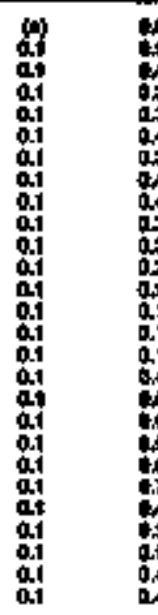

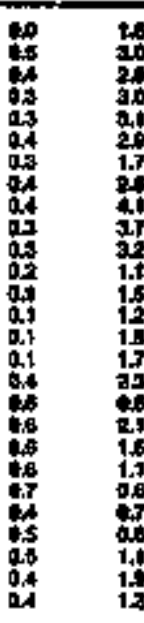

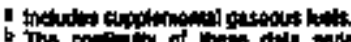

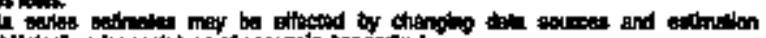

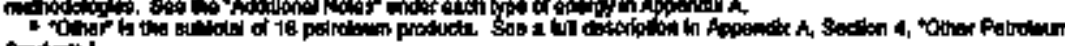

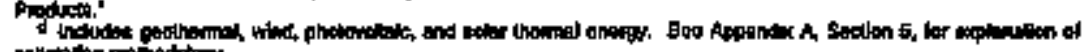

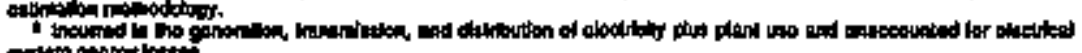

\begin{tabular}{|c|c|c|c|c|c|c|c|}
\hline 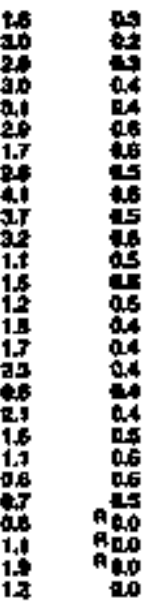 & 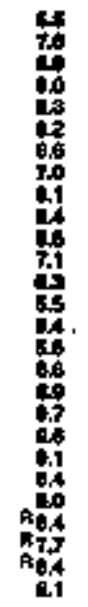 & 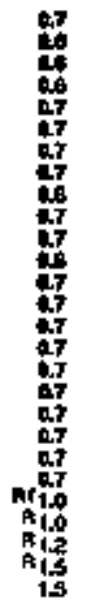 & 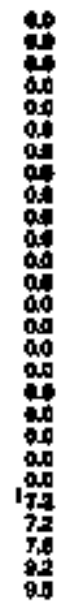 & 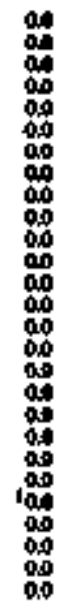 & 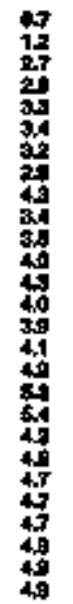 & 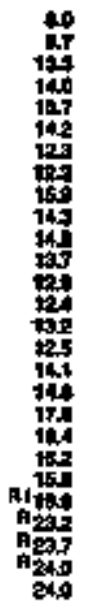 & 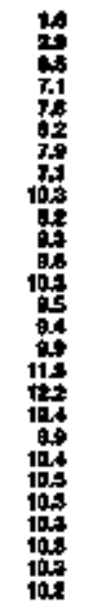 \\
\hline
\end{tabular}

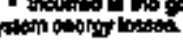

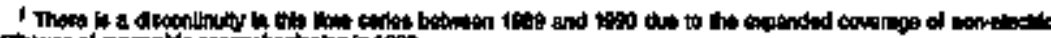

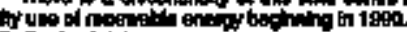

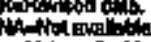

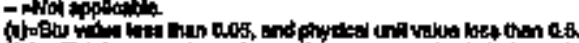

tem exp

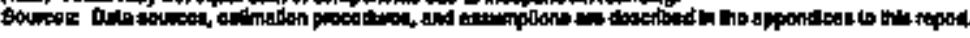


Table 291. Transportatton Energy Constimpition Estimatea, 1960, 1965, 1970-1994, Vermont

\begin{tabular}{|c|c|c|c|c|c|c|c|c|c|c|c|c|c|c|c|}
\hline \multirow[b]{3}{*}{$x$} & \multirow[b]{2}{*}{ cont } & \multirow[b]{2}{*}{ 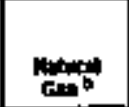 } & \multicolumn{8}{|c|}{ Potolown } & \multirow{3}{*}{ 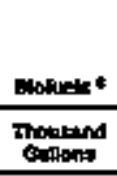 } & \multirow[b]{2}{*}{ Exermety 4} & \multirow[b]{2}{*}{ therst } & \multirow[b]{2}{*}{ 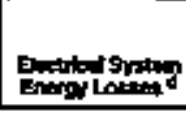 } & \multirow[b]{3}{*}{ Totol } \\
\hline & & & 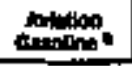 & 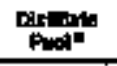 & 密. & Lmat & | whons: & molot & Fition & totor & & & & & \\
\hline & monest ind & alloforot & \multicolumn{8}{|c|}{ 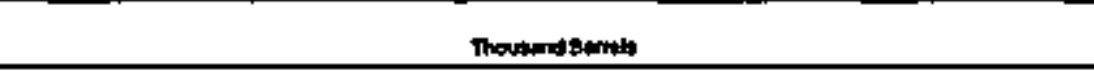 } & & \multicolumn{3}{|c|}{ Han Whomathoun } & \\
\hline 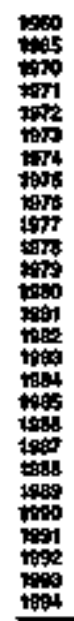 & $\begin{array}{l}1 \\
9 \\
0 \\
0 \\
0 \\
0 \\
9 \\
0 \\
0 \\
0 \\
0 \\
0 \\
0 \\
0 \\
0 \\
0 \\
0 \\
0 \\
0 \\
0 \\
0 \\
0 \\
0\end{array}$ & $\begin{array}{l}1 \\
: \\
0 \\
0 \\
0 \\
0 \\
0 \\
0 \\
0 \\
0 \\
0 \\
0 \\
0 \\
0 \\
0 \\
8\end{array}$ & 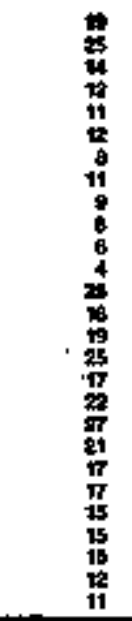 & 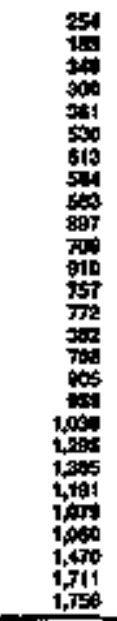 & 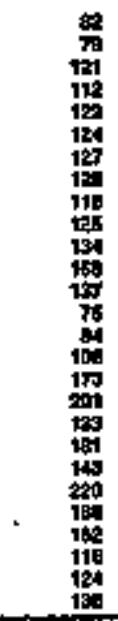 & 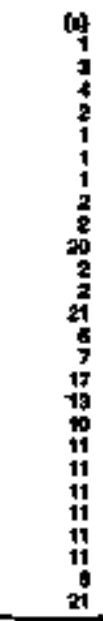 & 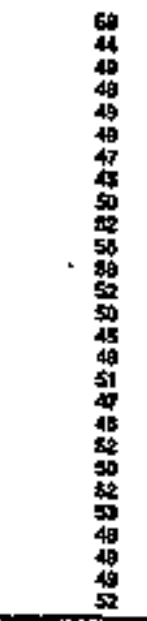 & 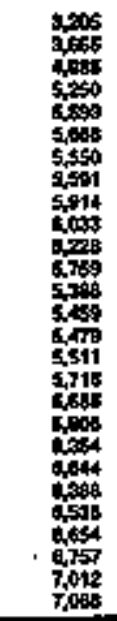 & $\begin{array}{l}: \\
0 \\
2 \\
2 \\
2 \\
2 \\
3 \\
3 \\
\vdots \\
\vdots \\
0 \\
0 \\
0 \\
\vdots \\
\vdots \\
0 \\
2 \\
0 \\
7 \\
3 \\
3 \\
0 \\
0 \\
0\end{array}$ & 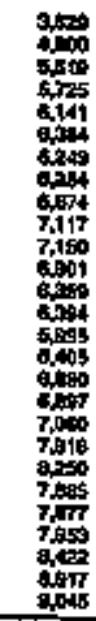 & $\begin{array}{l}: \\
0 \\
0 \\
0 \\
0 \\
0 \\
0 \\
0 \\
0 \\
0 \\
0 \\
0 \\
0 \\
0 \\
0 \\
0 \\
0 \\
0 \\
0 \\
0 \\
0 \\
0 \\
0 \\
0 \\
0\end{array}$ & $\begin{array}{l}\mathbf{0} \\
0 \\
0 \\
0 \\
0 \\
0 \\
\mathbf{0} \\
0 \\
0 \\
0 \\
0 \\
0 \\
0 \\
0 \\
0 \\
0 \\
0 \\
0 \\
0 \\
0 \\
\mathbf{0} \\
\mathbf{0} \\
\mathbf{0} \\
\mathbf{0} \\
\mathbf{0} \\
\mathbf{0}\end{array}$ & $\begin{array}{l}\vdots \\
\vdots \\
\vdots \\
\vdots \\
\vdots \\
\vdots \\
\vdots \\
\vdots \\
\vdots \\
\vdots \\
\vdots\end{array}$ & $\begin{array}{l}0 \\
\vdots \\
\vdots \\
\vdots \\
0 \\
\vdots \\
0 \\
0 \\
0 \\
\vdots \\
0 \\
0 \\
0 \\
0 \\
\vdots \\
\vdots \\
\vdots \\
\vdots \\
\vdots \\
0 \\
0 \\
0\end{array}$ & $\begin{array}{l}\vdots \\
\vdots \\
\vdots \\
\vdots \\
\vdots \\
\vdots \\
\vdots \\
\vdots \\
\vdots \\
\vdots \\
\vdots \\
\vdots \\
\vdots\end{array}$ \\
\hline & & & & & & & & Then ow & & & & & & & \\
\hline 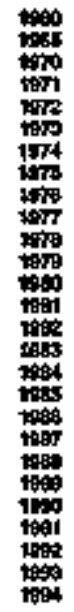 & 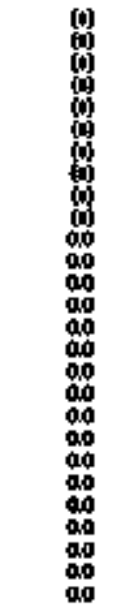 & 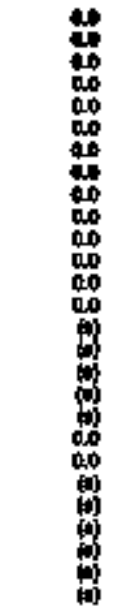 & 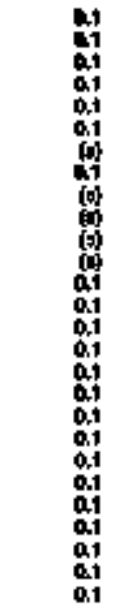 & 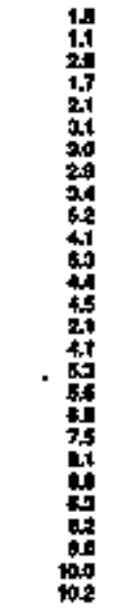 & 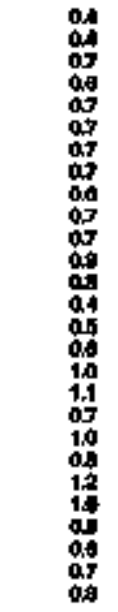 & 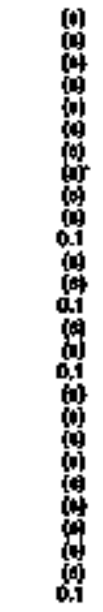 & 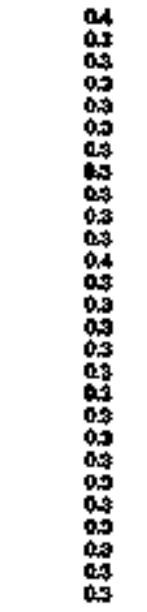 & 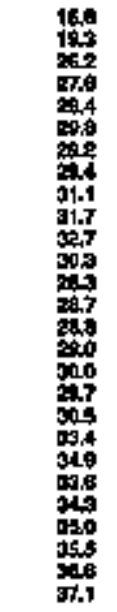 & 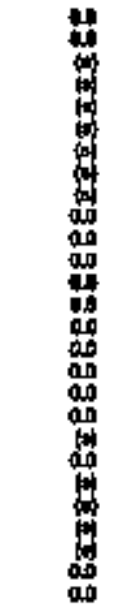 & 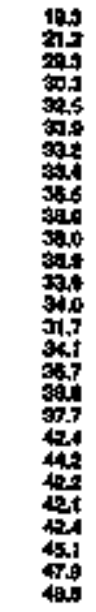 & 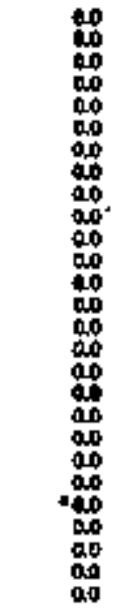 & 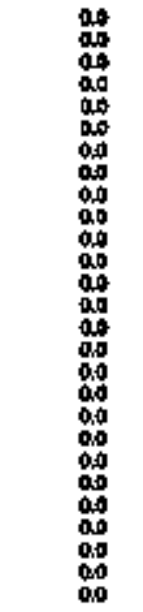 & 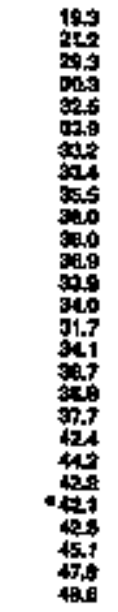 & 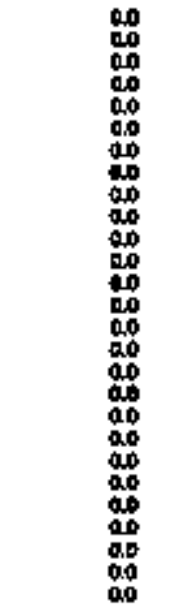 & 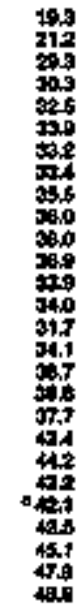 \\
\hline
\end{tabular}

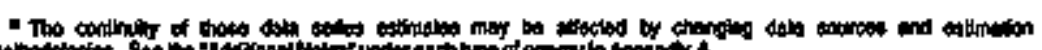
it

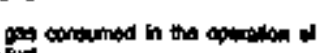

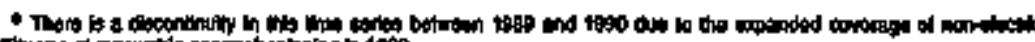

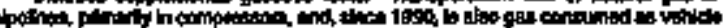

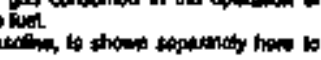

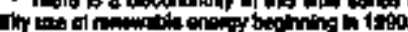

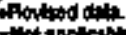

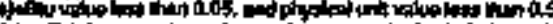

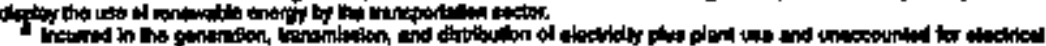

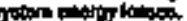


Teble 292. Estimates of Energy Input at Eethrie Utilitus, 1950, 1965, 1970-1994, Vermont

\begin{tabular}{|c|c|c|c|c|c|c|c|c|c|c|c|c|c|c|}
\hline \multirow[b]{3}{*}{ rew } & \multicolumn{3}{|c|}{ can } & \multirow[b]{2}{*}{ Mtang } & \multicolumn{4}{|c|}{ Putrovin } & \multirow[b]{2}{*}{ 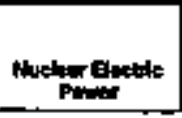 } & \multirow[b]{2}{*}{ 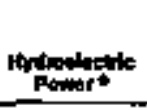 } & \multirow[b]{2}{*}{20} & \multirow[b]{2}{*}{ 年 } & \multirow{3}{*}{ onder } & \multirow[b]{3}{*}{ Trots: } \\
\hline & 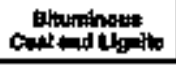 & 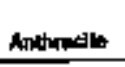 & זער & & 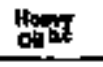 & 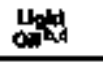 & 8 & Then & & & & & & \\
\hline & \multicolumn{3}{|c|}{ Thonenenstert Tom } & 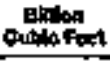 & \multicolumn{4}{|c|}{ Thoonend Buth } & \multicolumn{4}{|c|}{ 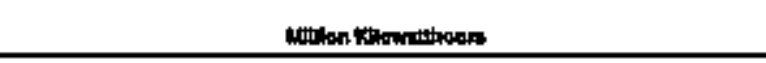 } & & \\
\hline 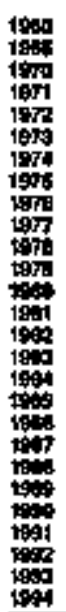 & 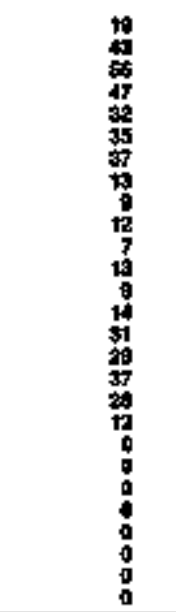 & $\begin{array}{l}0 \\
0 \\
0 \\
0 \\
0 \\
0 \\
0 \\
0 \\
0 \\
0 \\
0 \\
0 \\
0 \\
0 \\
0 \\
0 \\
0 \\
0 \\
0 \\
0 \\
0 \\
0 \\
0 \\
0 \\
0 \\
0 \\
0 \\
0\end{array}$ & 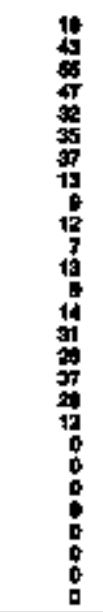 & 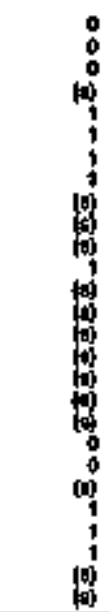 & 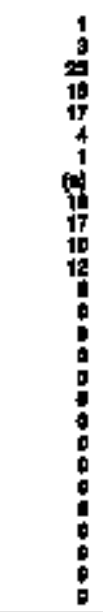 & 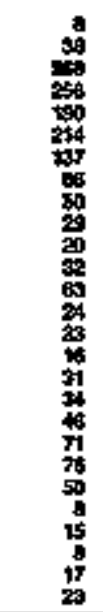 & 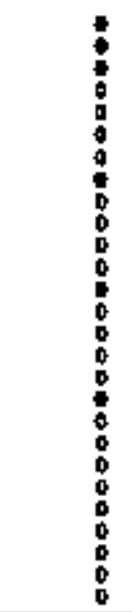 & 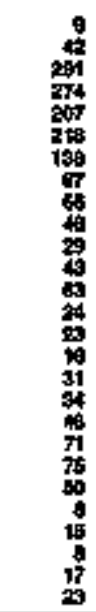 & 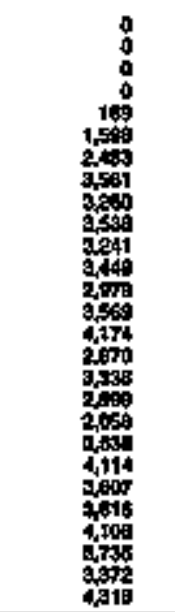 & 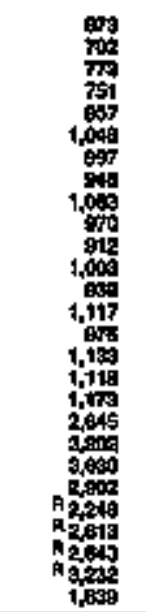 & 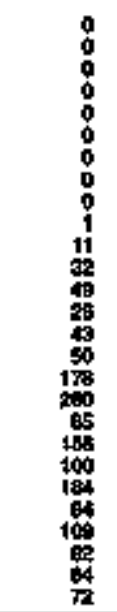 & $\begin{array}{l}0 \\
0 \\
0 \\
0 \\
0 \\
0 \\
0 \\
0 \\
0 \\
0 \\
0 \\
0 \\
0 \\
0 \\
0 \\
0 \\
0 \\
0 \\
0 \\
0 \\
0 \\
0 \\
0 \\
0 \\
0 \\
0 \\
0 \\
0 \\
0\end{array}$ & $\begin{array}{l}: \\
: \\
0 \\
0 \\
0 \\
0 \\
0 \\
0 \\
0 \\
0 \\
0 \\
0 \\
0 \\
0 \\
0 \\
0 \\
0 \\
0 \\
0\end{array}$ & $\begin{array}{l}\vdots \\
\vdots \\
\vdots \\
\vdots \\
\vdots \\
\vdots \\
\vdots \\
\vdots \\
\vdots \\
\vdots \\
\vdots \\
\vdots \\
\vdots\end{array}$ \\
\hline & & & & & & & & & & & & & & \\
\hline
\end{tabular}

\begin{tabular}{|c|c|c|c|c|c|c|c|c|c|c|c|c|c|c|}
\hline 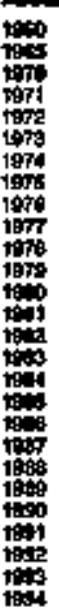 & 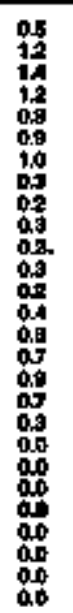 & 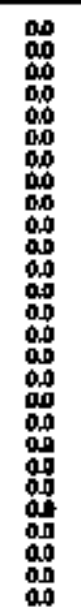 & 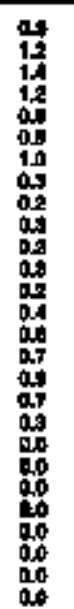 & 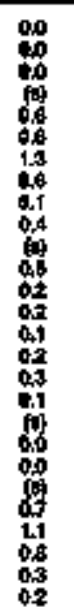 & 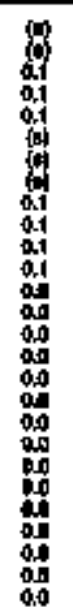 & 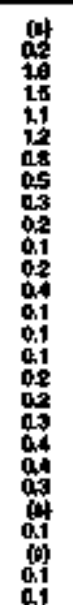 & 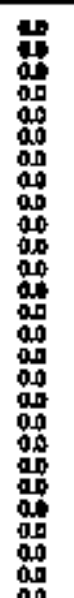 & 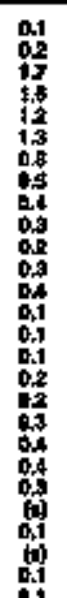 & 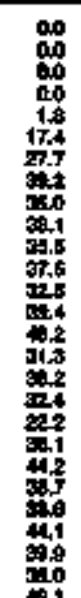 & 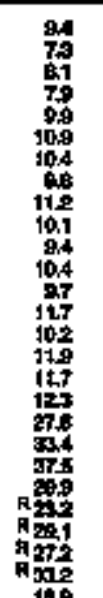 & 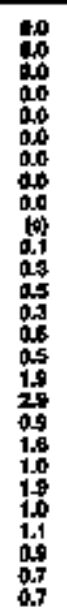 & 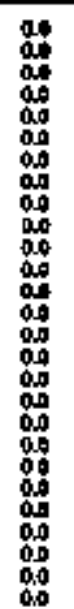 & 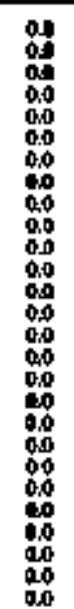 & 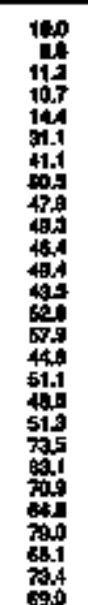 \\
\hline
\end{tabular}

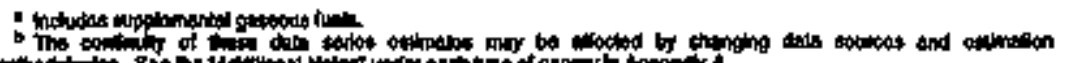
1. hes allat.

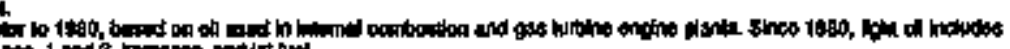

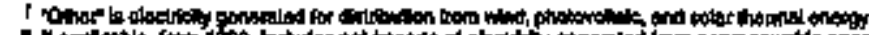

然

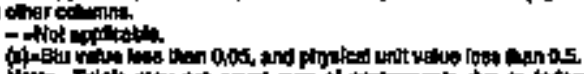

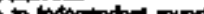

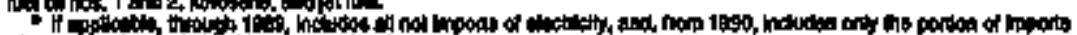

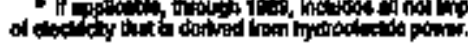


Table 299. Enengy Constumpilon Estimalas by Scurce, 1960, 1965, 1970-1994, Virginla

\begin{tabular}{|c|c|c|c|c|c|c|c|c|c|c|c|c|c|c|c|c|c|c|c|}
\hline \multirow[b]{3}{*}{$\begin{array}{r}- \\
y=1\end{array}$} & \multirow[b]{2}{*}{ tont } & \multirow[b]{2}{*}{ thents } & \multicolumn{11}{|c|}{ 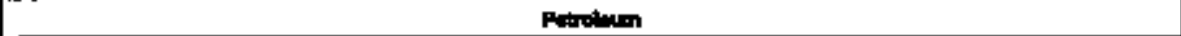 } & \multirow[b]{2}{*}{ 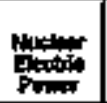 } & \multirow[b]{2}{*}{ Ponter } & \multirow[b]{2}{*}{ aliatueta 0} & \multirow[b]{2}{*}{ Catom in I } & \multirow{3}{*}{ 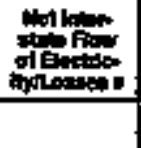 } & \multirow[b]{3}{*}{ Tont } \\
\hline & & & Apotitiond & Anstion. & (8) & Fit. & $\lim _{i \rightarrow \infty}$ & LPG: & \begin{tabular}{|l|} 
cotit. \\
wits \\
\end{tabular} & onsolinos & 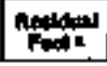 & Onem & Tot & & & & & & \\
\hline & 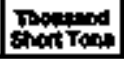 & cisiof & \multicolumn{11}{|c|}{ Thow: } & \multicolumn{4}{|c|}{ 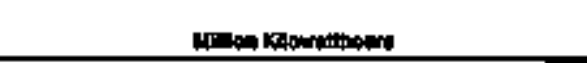 } & & \\
\hline 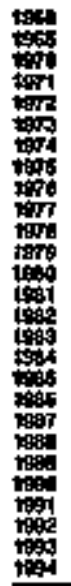 & 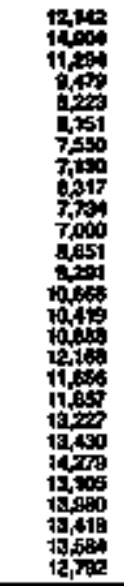 & 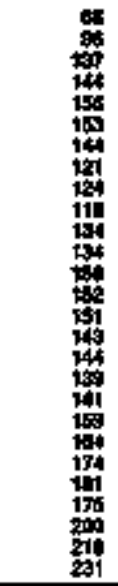 & 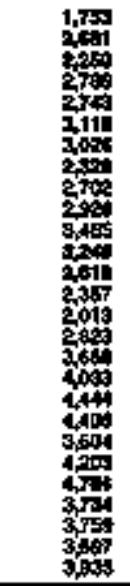 & 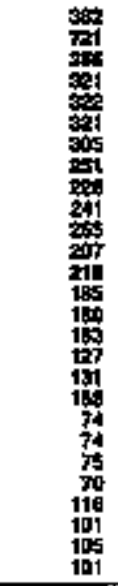 & 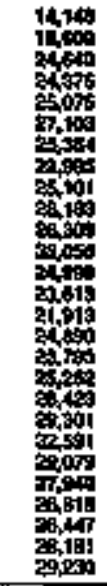 & 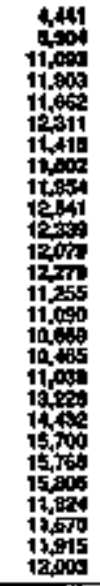 & 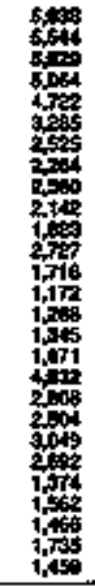 & 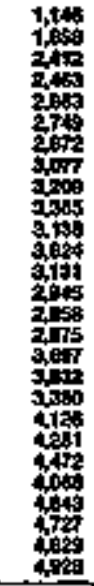 & 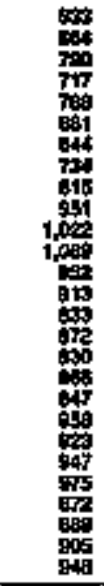 & 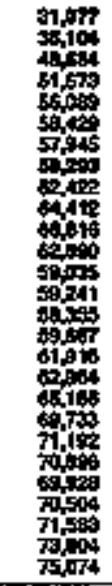 & 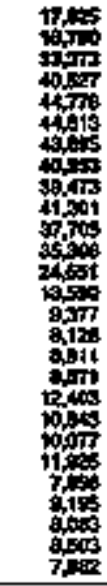 & 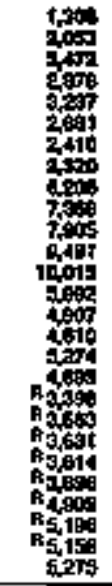 & 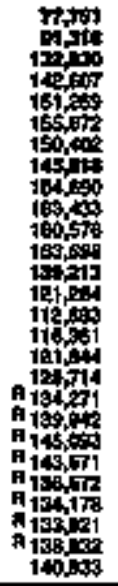 & 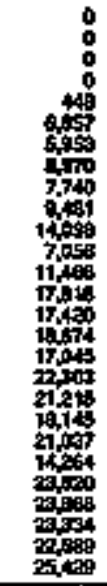 & 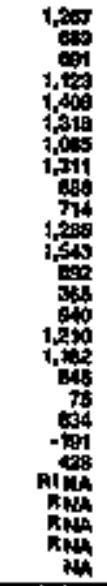 & 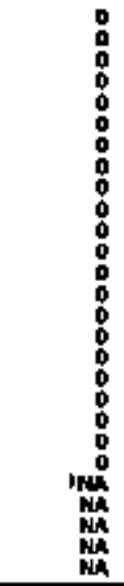 & 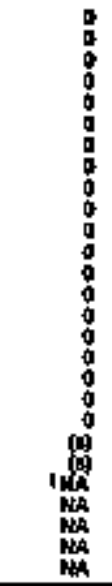 & 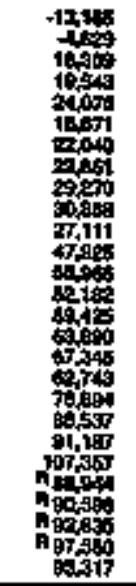 & $\begin{array}{l}z \\
z \\
z \\
z \\
z \\
z \\
z \\
z \\
z \\
z \\
z \\
z \\
z\end{array}$ \\
\hline \multicolumn{20}{|c|}{ Trimen 助 } \\
\hline 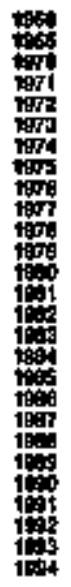 & 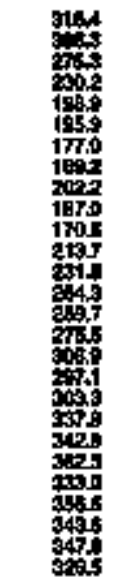 & 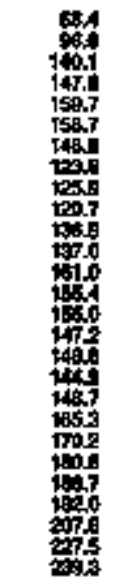 & 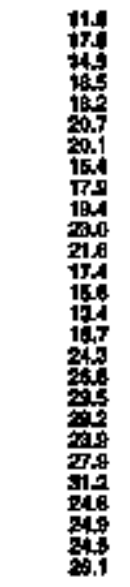 & 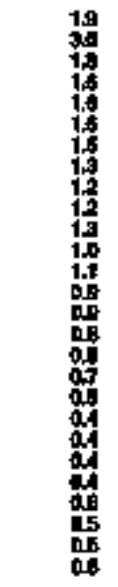 & 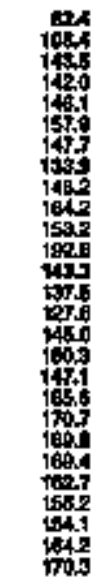 & 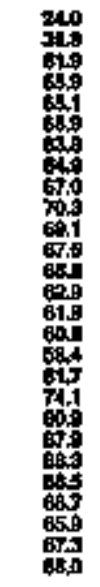 & 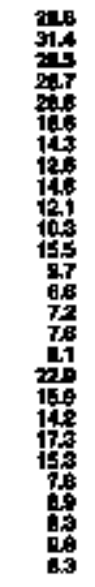 & 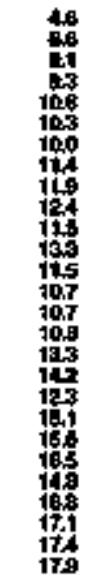 & 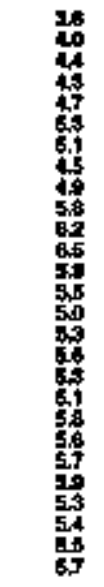 & 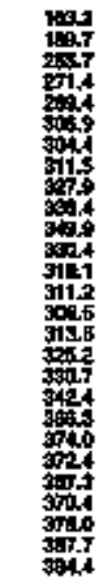 & 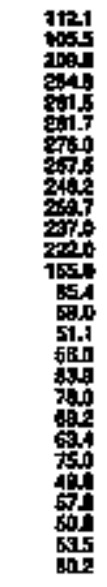 & 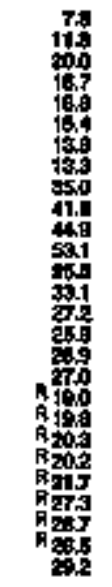 & 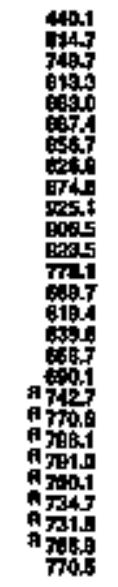 & 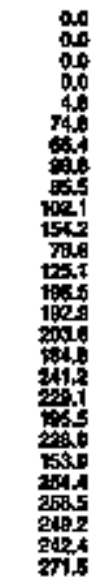 & 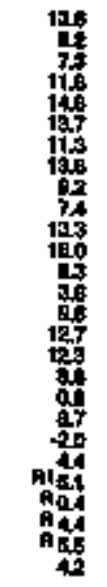 & 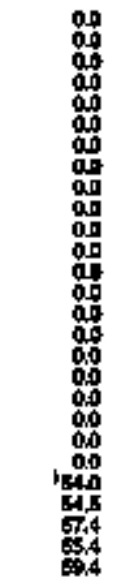 & 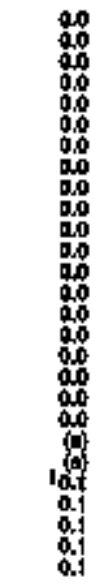 & 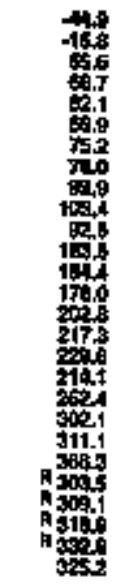 & 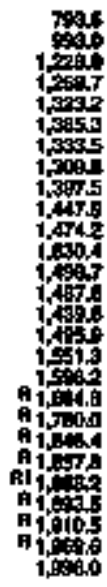 \\
\hline
\end{tabular}

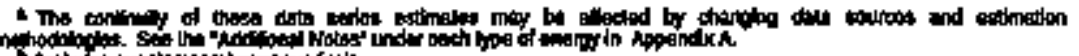

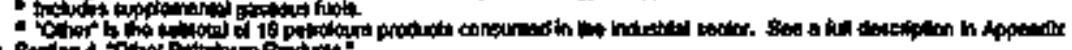

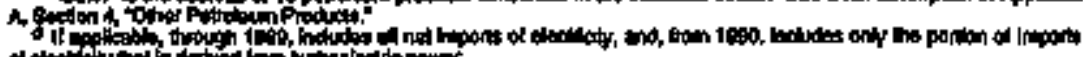

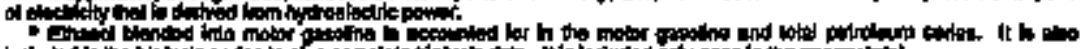

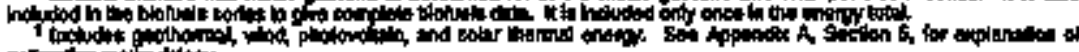

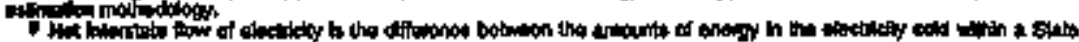

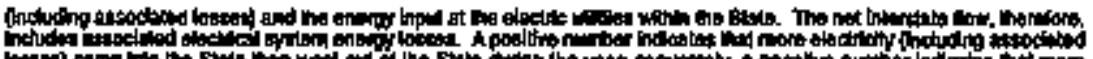

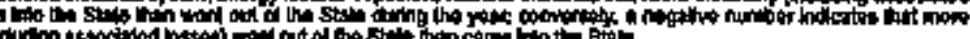

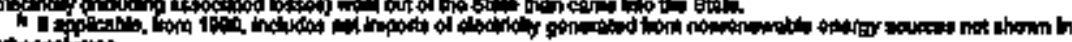

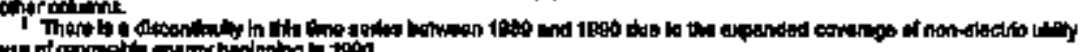
mif of

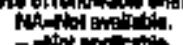

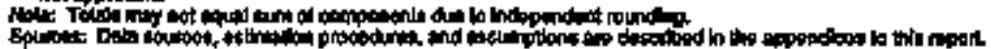


Tabjo 294. Rosidential Energy Constumption Estimates, 19150, 1955, 1970-1994, Virginia

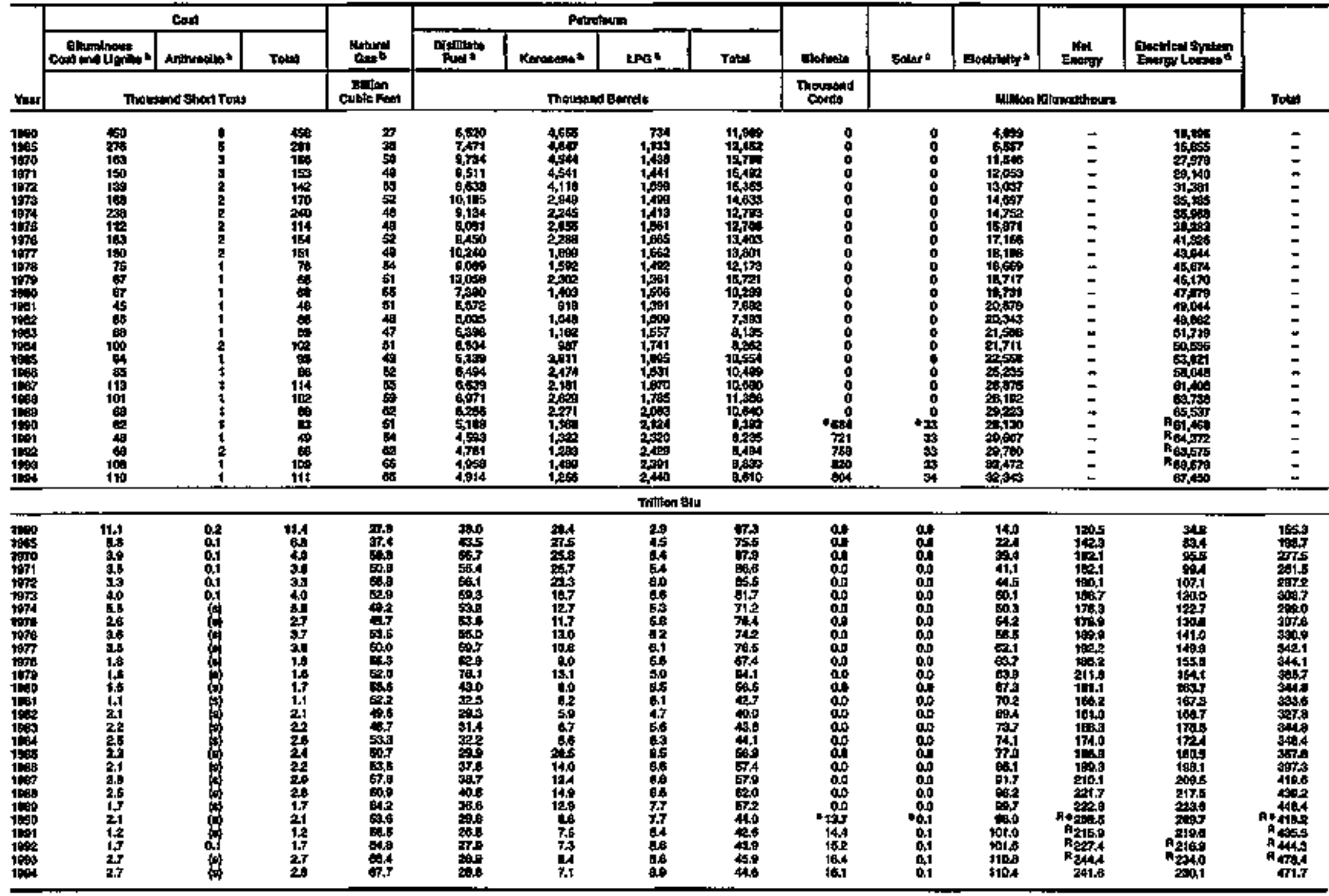

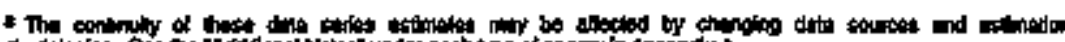

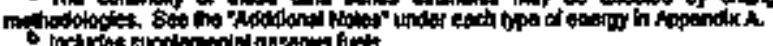

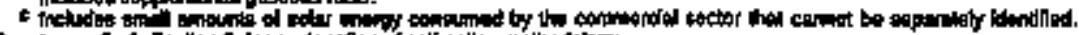

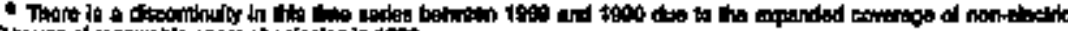

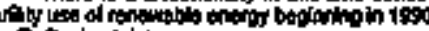
A flowhed dain

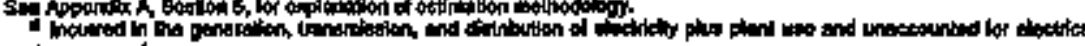

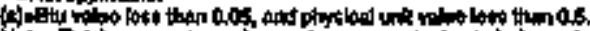

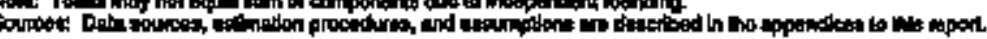

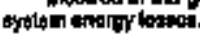


Table 295. Commerclal Ereigy Consumption Estimates, 1960, 1965, 1970-1994, Virginia

\begin{tabular}{|c|c|c|c|c|c|c|c|c|c|c|c|c|c|c|}
\hline \multirow[b]{3}{*}{ Yoent } & \multicolumn{3}{|c|}{$\cos 1$} & \multirow{3}{*}{ 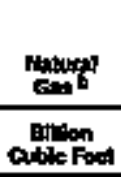 } & \multicolumn{6}{|c|}{ Potrodarn } & \multirow[b]{2}{*}{ Eemothly " } & \multirow[b]{2}{*}{ Envisy } & \multirow[b]{2}{*}{ 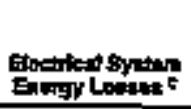 } & \multirow[b]{3}{*}{ Tatallo } \\
\hline & 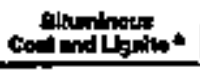 & Andination & Tated & & 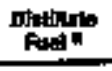 & Karowne" & trote & Motor & Fouldot & Tole & & & & \\
\hline & \multicolumn{2}{|c|}{ Thentend Phort tone } & & & \multicolumn{6}{|c|}{ Thoutand Buds } & \multicolumn{3}{|c|}{ Whon Krowathoum } & \\
\hline 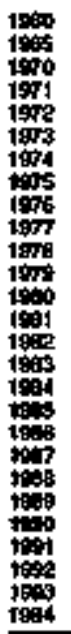 & 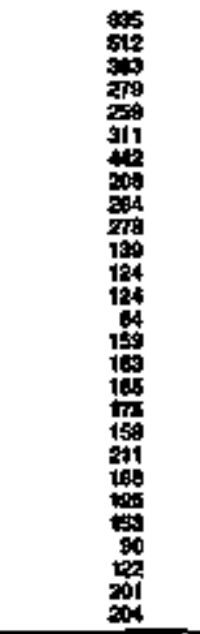 & 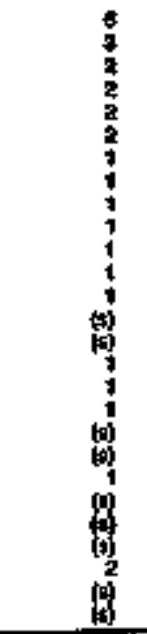 & 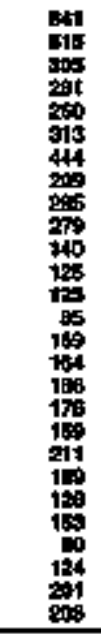 & 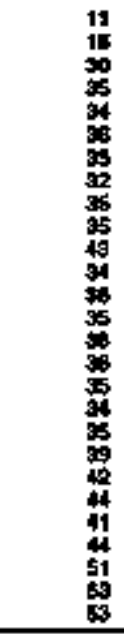 & 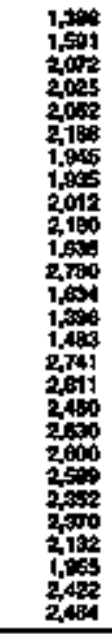 & 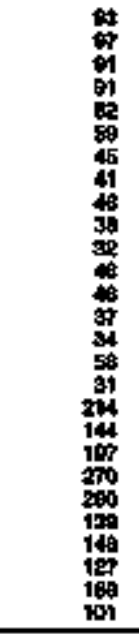 & 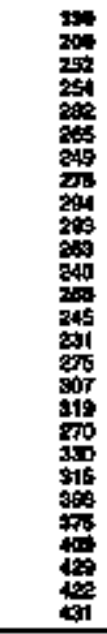 & 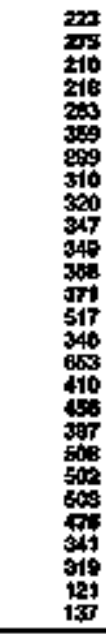 & 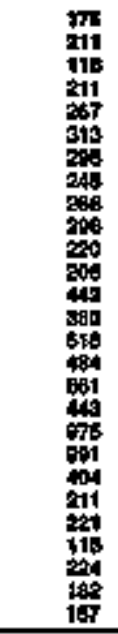 & 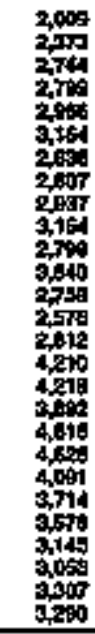 & 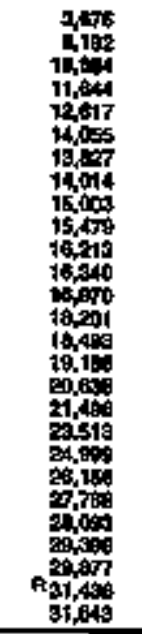 & $\begin{array}{l}= \\
= \\
= \\
= \\
= \\
= \\
= \\
= \\
= \\
= \\
= \\
= \\
= \\
=\end{array}$ & 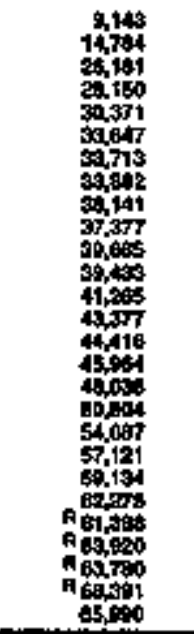 & $\begin{array}{l}= \\
= \\
z \\
z \\
z \\
z \\
z \\
z \\
= \\
z \\
z \\
= \\
z \\
z\end{array}$ \\
\hline \multicolumn{15}{|c|}{ Thillan Da } \\
\hline 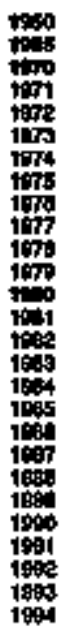 & 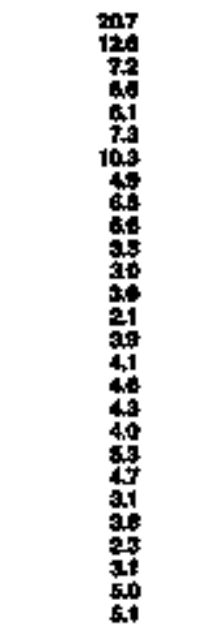 & 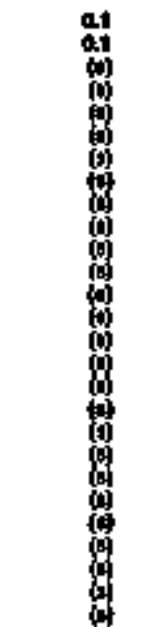 & 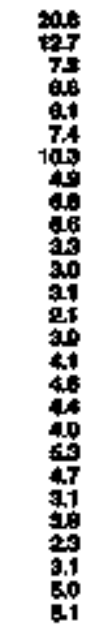 & 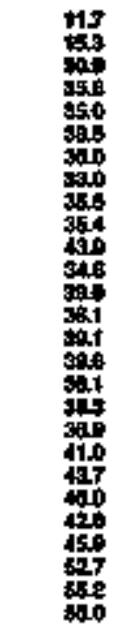 & 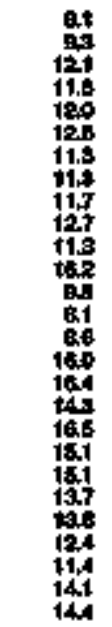 & 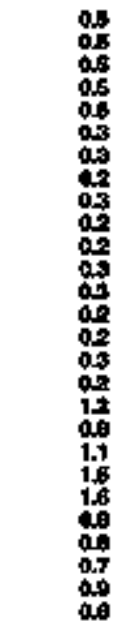 & 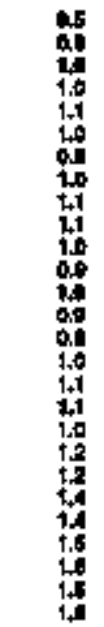 & 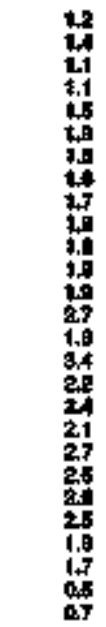 & 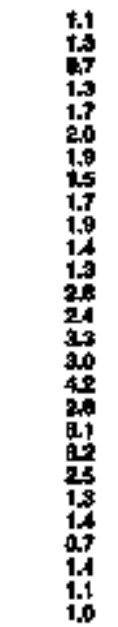 & 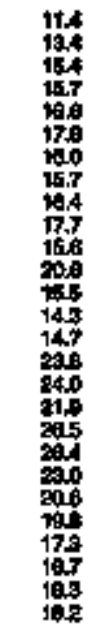 & 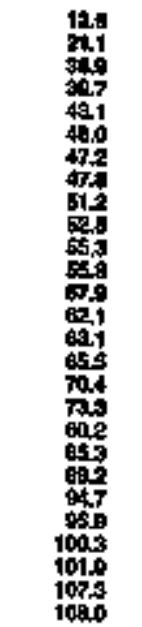 & 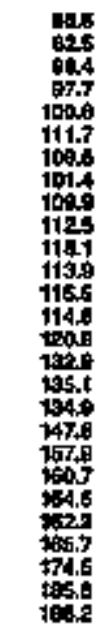 & 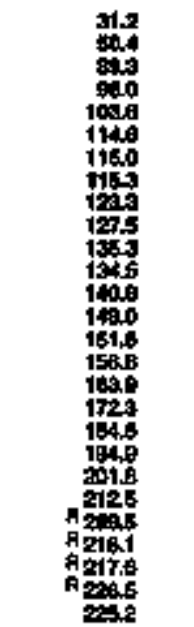 & 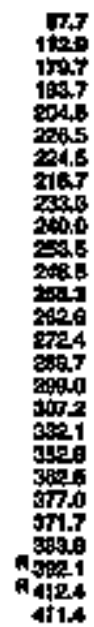 \\
\hline
\end{tabular}

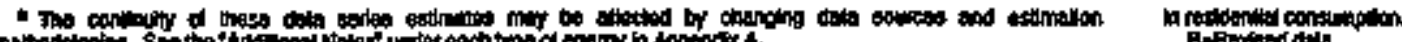

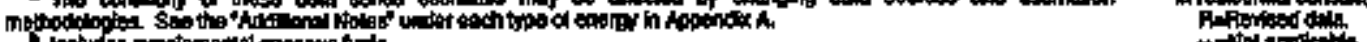

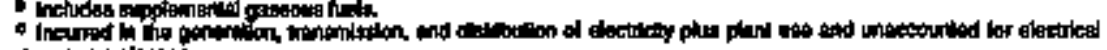

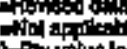

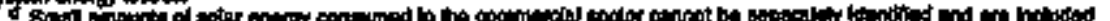


Table 296. Industubl Energy Consumption Esilmales, 1950, 1965, 1970-1994, Virginla

\begin{tabular}{|c|c|c|c|c|c|c|c|c|c|c|c|c|c|c|c|c|c|c|}
\hline \multirow[b]{3}{*}{ Yarr } & \multirow[b]{2}{*}{ Tod } & \multirow[b]{2}{*}{ 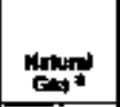 } & \multicolumn{9}{|c|}{ Pabrotarn } & \multirow[b]{2}{*}{ 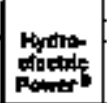 } & \multirow[b]{2}{*}{ Eiofices, } & \multirow[b]{2}{*}{ Oater } & \multirow[b]{2}{*}{ nopotithe } & \multirow[b]{2}{*}{ Entrin } & \multirow{2}{*}{ 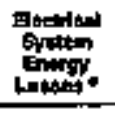 } & \multirow[b]{3}{*}{ Vow } \\
\hline & & & 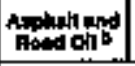 & Diplitionto & Koresone of & UPG & LLetember| & Cratoor & Patald & Otherper & Tous & & & & & & & \\
\hline & $\begin{array}{l}\text { Thonstand } \\
\text { Bthert Tono }\end{array}$ & cidite Fon & \multicolumn{9}{|c|}{ Thovasind Benteds } & \multicolumn{6}{|c|}{ 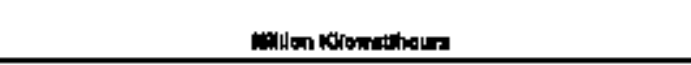 } & \\
\hline 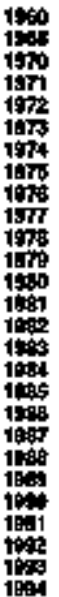 & 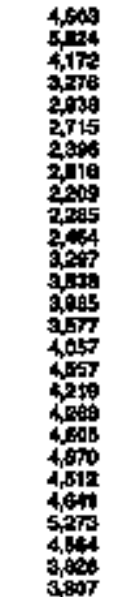 & 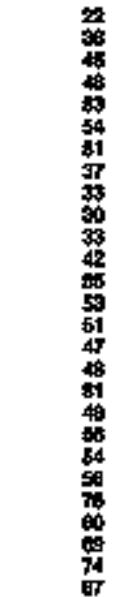 & 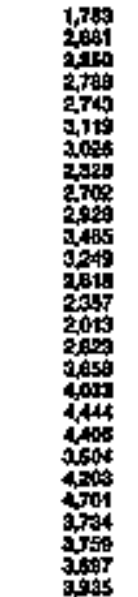 & 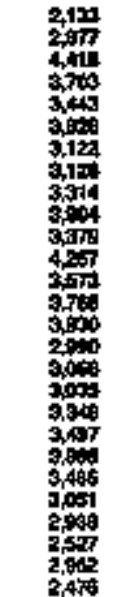 & 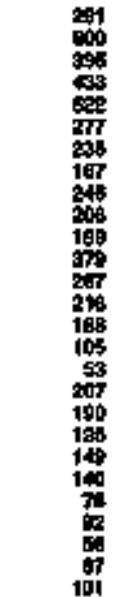 & 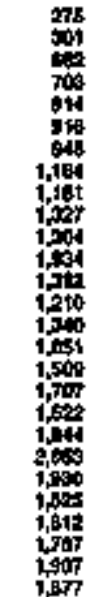 & 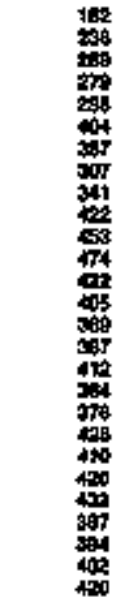 & 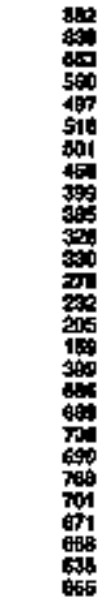 & 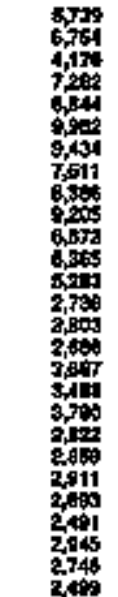 & 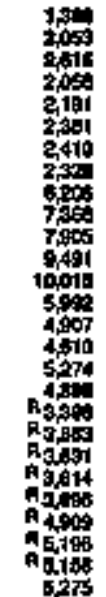 & 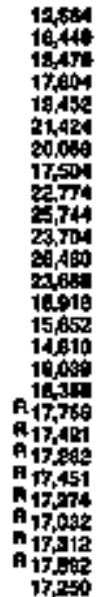 & 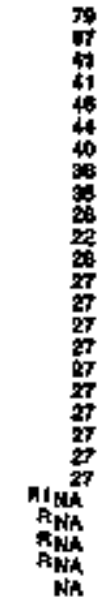 & 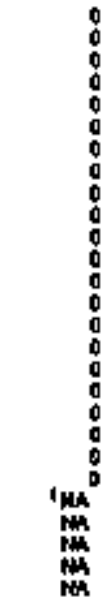 & 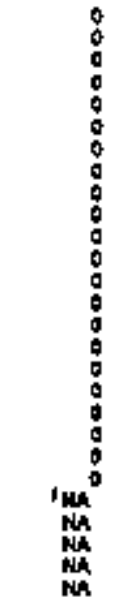 & 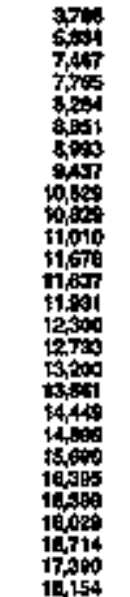 & $\begin{array}{l}= \\
z \\
z \\
z \\
z \\
= \\
= \\
= \\
z \\
z \\
z \\
z \\
z \\
z\end{array}$ & 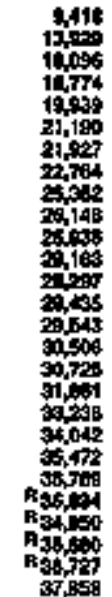 & 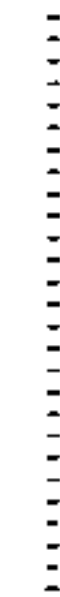 \\
\hline
\end{tabular}

\begin{tabular}{|c|c|c|c|c|c|c|c|c|c|c|c|c|c|c|c|c|c|c|}
\hline \multicolumn{19}{|c|}{ 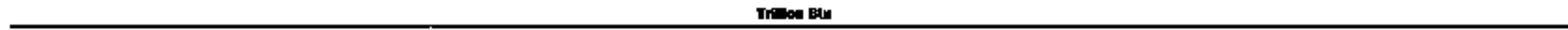 } \\
\hline 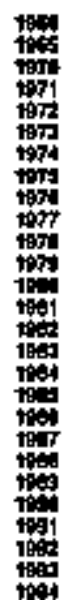 & 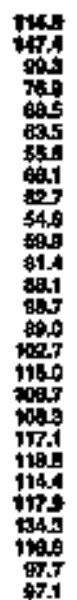 & 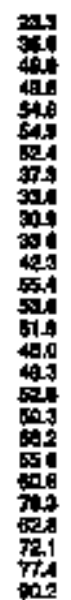 & 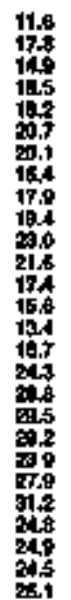 & 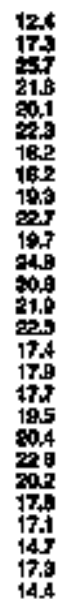 & 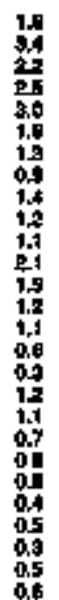 & 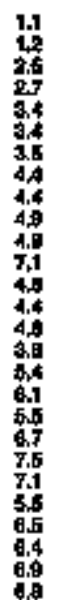 & 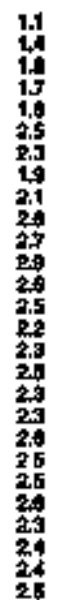 & 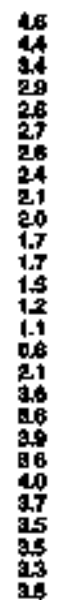 & 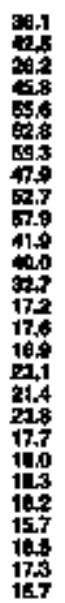 & 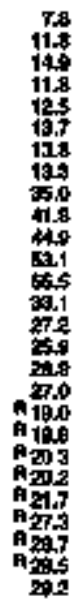 & 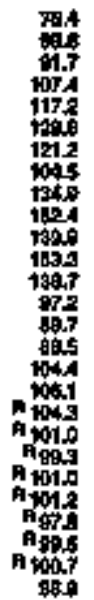 & 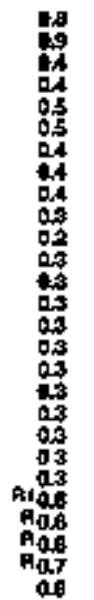 & 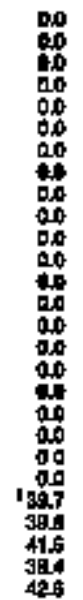 & 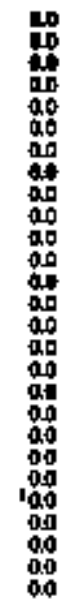 & 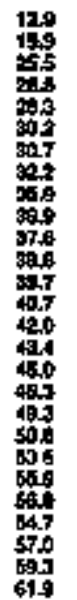 & 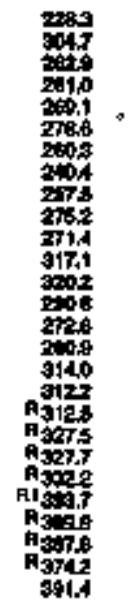 & 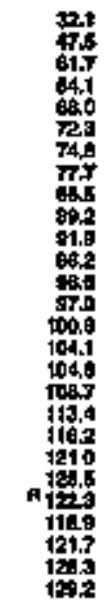 & 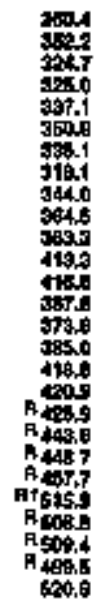 \\
\hline
\end{tabular}

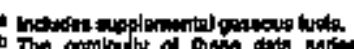

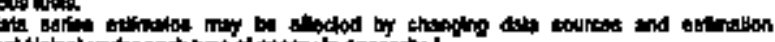

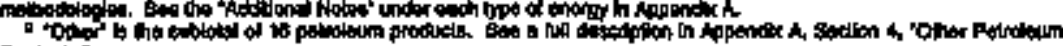
PToducta"

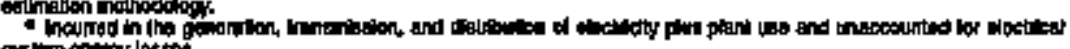

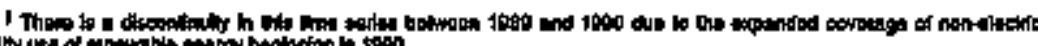

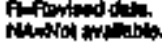

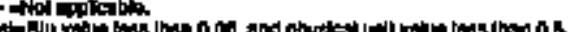

Tole

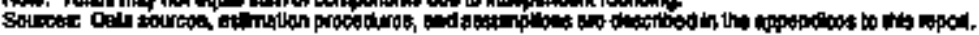


Table 297. Transpostation Energy Consumption Eslimaties, 1960, 1965, 1970-1984, Virginta

\begin{tabular}{|c|c|c|c|c|c|c|c|c|c|c|c|c|c|c|c|}
\hline \multirow[b]{3}{*}{ Yourt } & \multirow[b]{2}{*}{ cout } & \multirow[b]{2}{*}{ Mation } & \multicolumn{8}{|c|}{ 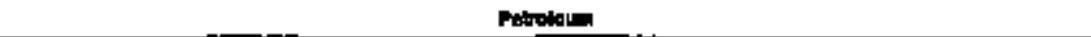 } & \multirow{3}{*}{ Blotuelé } & \multirow{2}{*}{\multicolumn{2}{|c|}{ 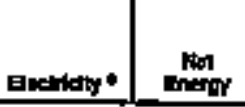 }} & \multirow[b]{2}{*}{ 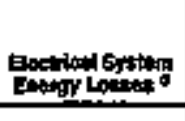 } & \multirow[b]{3}{*}{ Toter } \\
\hline & & & ramilan. & Populate & Fad. & LPo" & Lutalosint " & antion & Puetin & Toul & & & & & \\
\hline & 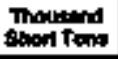 & Eision & \multicolumn{8}{|c|}{ 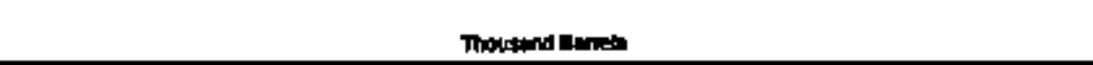 } & & \multicolumn{3}{|c|}{ 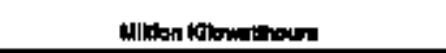 } & \\
\hline 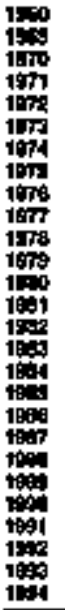 & $\begin{array}{c}7 \\
0 \\
7 \\
4 \\
3 \\
3 \\
2 \\
1 \\
0 \\
0 \\
0 \\
0 \\
0 \\
0 \\
0 \\
0 \\
0 \\
0 \\
0 \\
0 \\
0 \\
0 \\
0 \\
0 \\
0 \\
0 \\
0 \\
0 \\
0\end{array}$ & $\begin{array}{l}4 \\
7 \\
8 \\
7 \\
6 \\
4 \\
3 \\
3 \\
3 \\
3 \\
4 \\
6 \\
11 \\
7 \\
7 \\
7 \\
5 \\
8 \\
8 \\
7 \\
7 \\
8 \\
8 \\
6 \\
\end{array}$ & 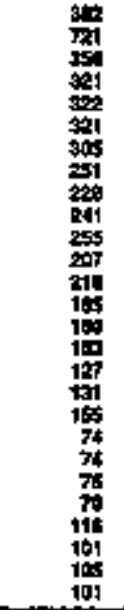 & 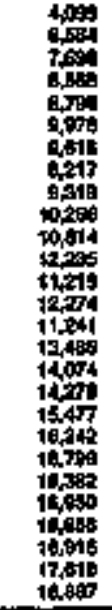 & 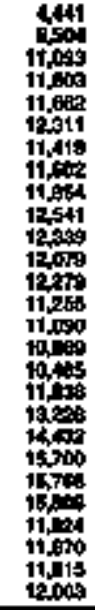 & 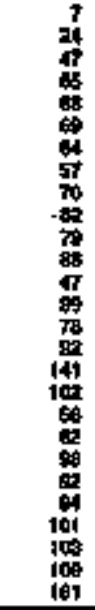 & 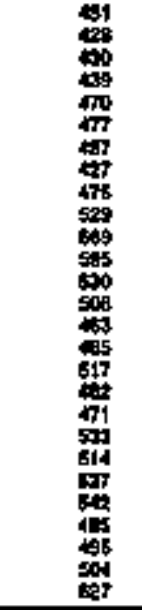 & 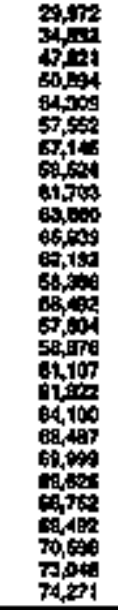 & 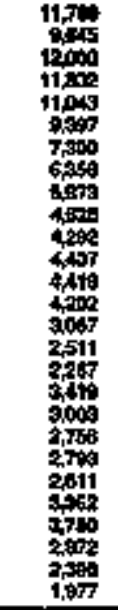 & 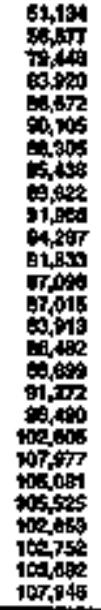 & 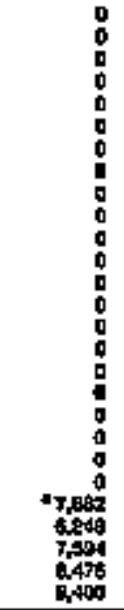 & 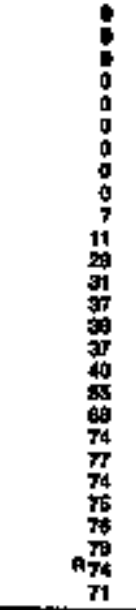 & $\begin{array}{l}z \\
= \\
z \\
z \\
z \\
z \\
z \\
z \\
z \\
z \\
z \\
z \\
z \\
z \\
z\end{array}$ & 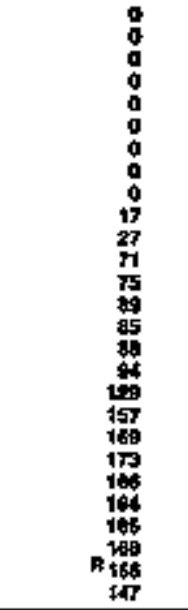 & 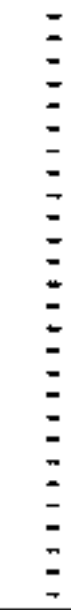 \\
\hline
\end{tabular}

\begin{tabular}{|c|c|c|c|c|c|c|c|c|c|c|c|c|c|c|c|}
\hline 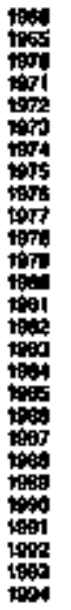 & 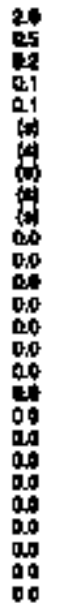 & 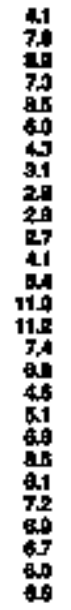 & 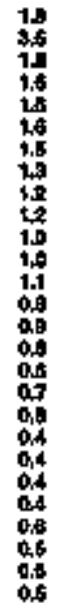 & 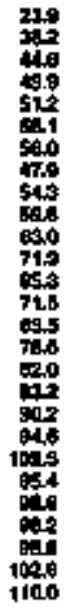 & 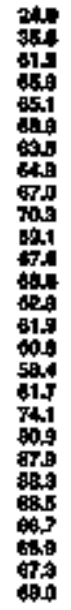 & 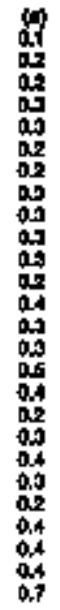 & 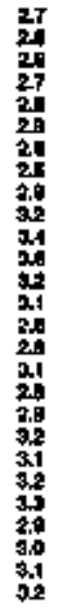 & 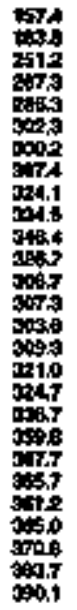 & 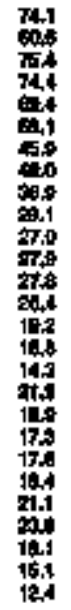 & 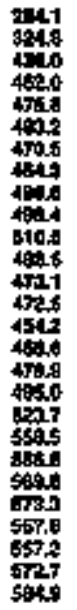 & 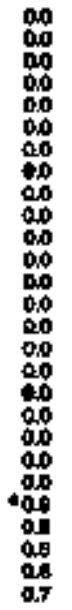 & 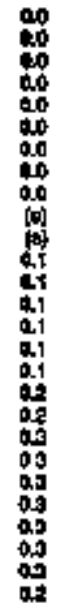 & 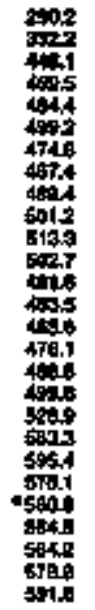 & 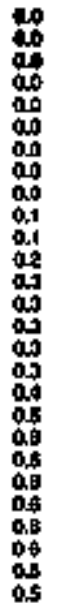 & 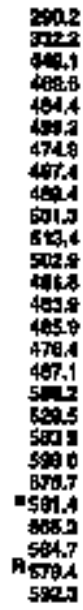 \\
\hline
\end{tabular}

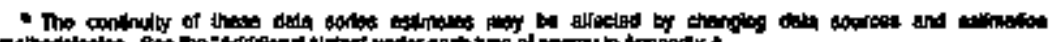

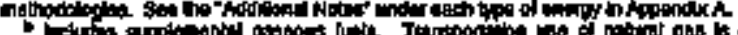

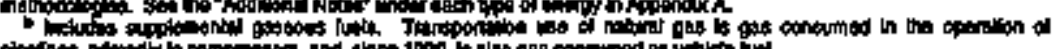

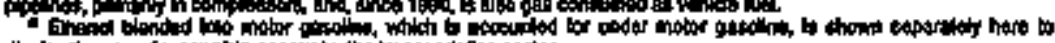

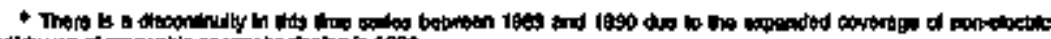

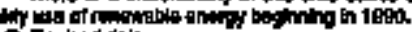
Finflations.

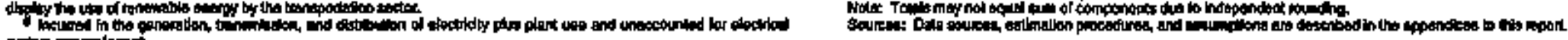

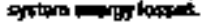


Table 2b8. Estimatos of Energy Input at Electrle Utillues, 1960, 1965, 1970-1994, Virglnla

\begin{tabular}{|c|c|c|c|c|c|c|c|c|c|c|c|c|c|c|}
\hline \multirow[b]{3}{*}{$y_{m}$} & \multicolumn{3}{|c|}{ لهort } & \multirow{3}{*}{ |ction } & \multicolumn{4}{|c|}{ Putadom } & \multirow[b]{2}{*}{ 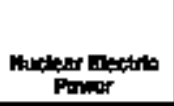 } & \multirow[b]{2}{*}{ Whotolectits } & \multirow[b]{2}{*}{ Bbahna } & \multirow[b]{2}{*}{ Feothmoll } & \multirow[b]{2}{*}{ otem W } & \multirow[b]{3}{*}{ Tous:! } \\
\hline & 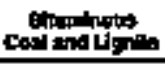 & Antaracion & Total & & tom & 和部 & $\begin{array}{l}\text { Putrotant: } \\
\text { colst }\end{array}$ & Tot & & & & & & \\
\hline & \multicolumn{3}{|c|}{ Thoustad Shart Tons } & & \multicolumn{4}{|c|}{ Thousand Bm min } & \multicolumn{5}{|c|}{ 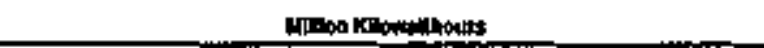 } & \\
\hline 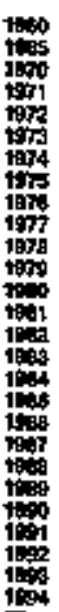 & 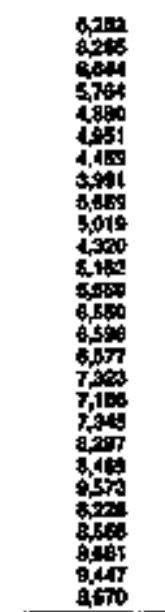 & $\begin{array}{l}0 \\
0 \\
0 \\
0 \\
0 \\
0 \\
0 \\
0 \\
0 \\
0 \\
0 \\
0 \\
0 \\
0 \\
0 \\
0 \\
0 \\
0 \\
0 \\
0 \\
0 \\
0\end{array}$ & 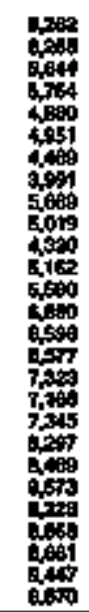 & 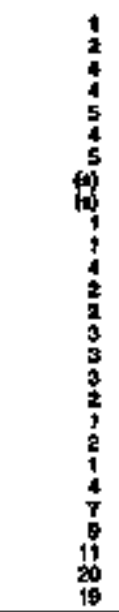 & 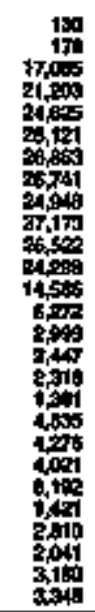 & 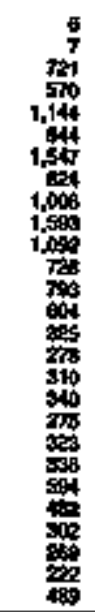 & 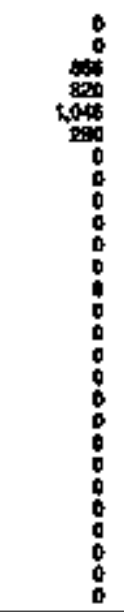 & 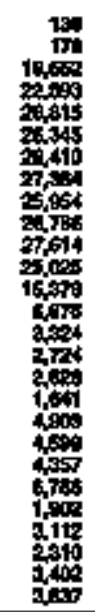 & 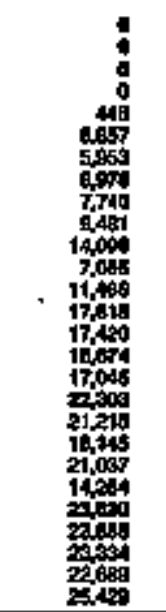 & 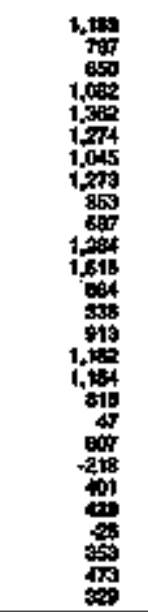 & $\begin{array}{l}0 \\
0 \\
0 \\
0 \\
0 \\
0 \\
0 \\
0 \\
0 \\
0 \\
0 \\
0 \\
0 \\
0 \\
0 \\
0 \\
0 \\
0 \\
0 \\
0 \\
0 \\
0 \\
0 \\
0 \\
0 \\
0 \\
0\end{array}$ & $\begin{array}{l}0 \\
0 \\
0 \\
0 \\
0 \\
0 \\
0 \\
0 \\
0 \\
0 \\
0 \\
0 \\
0 \\
0 \\
0 \\
0 \\
0 \\
0 \\
0 \\
0 \\
0 \\
0\end{array}$ & $\begin{array}{l}: \\
0 \\
0 \\
0 \\
0 \\
0 \\
0 \\
0 \\
0 \\
0 \\
0 \\
0 \\
0\end{array}$ & 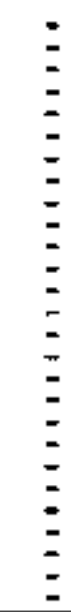 \\
\hline \multicolumn{15}{|c|}{ Thitlion Btor } \\
\hline
\end{tabular}

\begin{tabular}{|c|c|c|c|c|c|c|c|c|c|c|c|c|c|c|}
\hline 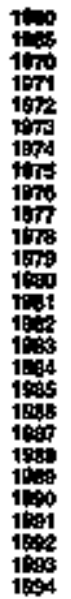 & 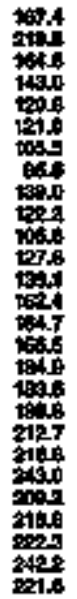 & 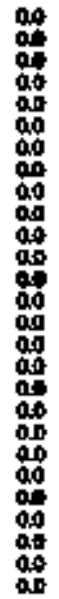 & 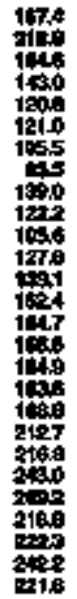 & 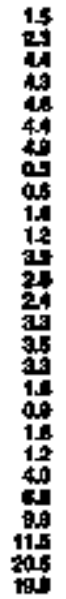 & 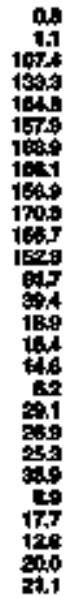 & 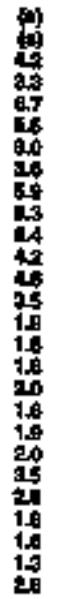 & 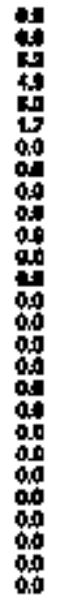 & 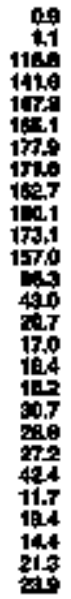 & 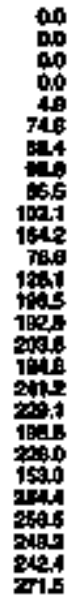 & 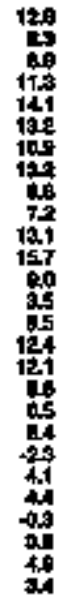 & 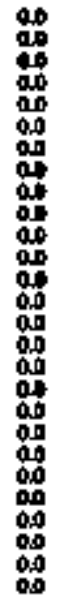 & 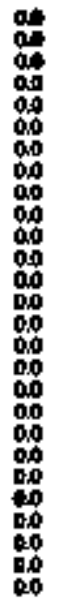 & 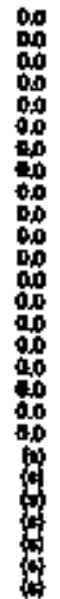 & 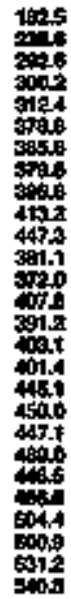 \\
\hline
\end{tabular}

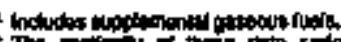

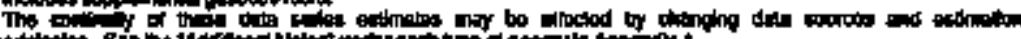

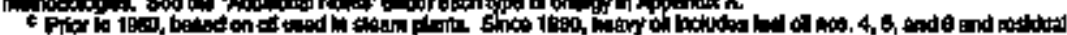
10.

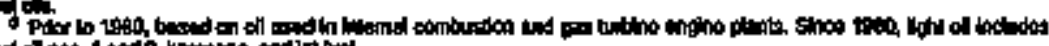

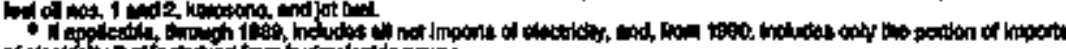

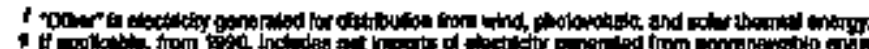

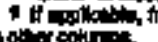

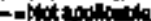

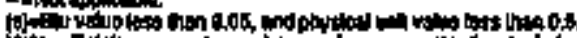

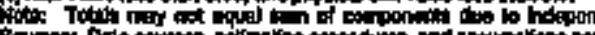


Tabie 299. Energy Consumpllon Estlmates by Souros, 1980, 1965, 1970-1994, Washlnglon

\begin{tabular}{|c|c|c|c|c|c|c|c|c|c|c|c|c|c|c|c|c|c|c|c|}
\hline \multirow[b]{3}{*}{$\mathrm{r}=\mathrm{E}$} & \multirow[b]{2}{*}{ cow: } & \multirow[b]{2}{*}{ Faners } & \multicolumn{11}{|c|}{ oxtritionan } & \multirow{2}{*}{ 然 } & \multirow{2}{*}{ Potistion: } & \multirow[b]{2}{*}{ 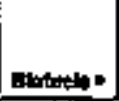 } & \multirow[b]{2}{*}{ Eitury at I } & \multirow{3}{*}{ 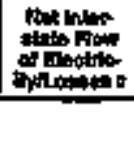 } & \multirow[b]{3}{*}{ Tetsth } \\
\hline & & & Aesphin and & Antersions: & 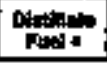 & Pant: & Xim. & Late. & cenot. & 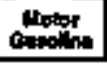 & Finglidul & Dower. & Tot: & & & & & & \\
\hline & Inowend & 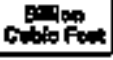 & \multicolumn{11}{|c|}{ 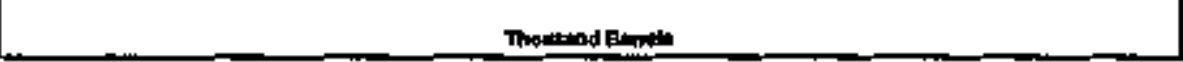 } & \multicolumn{4}{|c|}{ 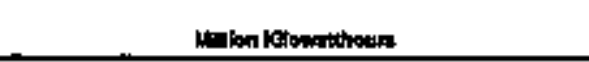 } & & \\
\hline 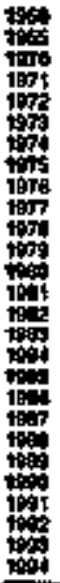 & 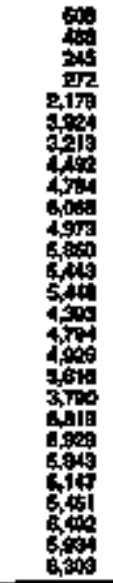 & 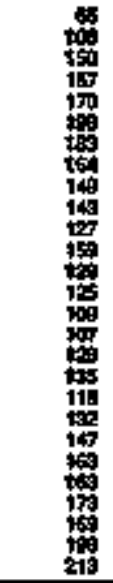 & 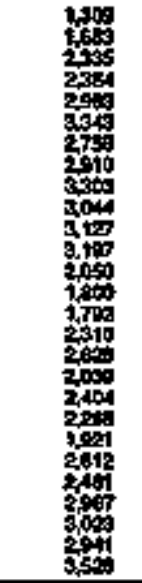 & 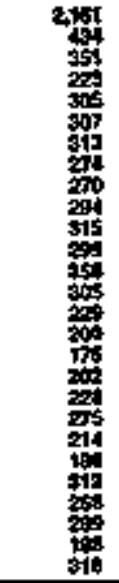 & 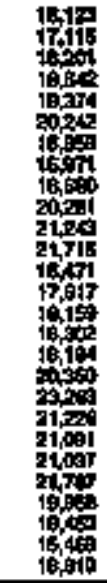 & 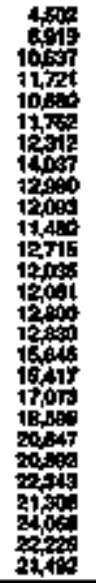 & 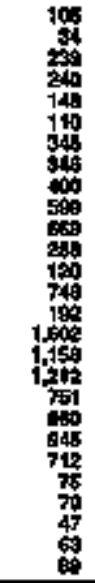 & 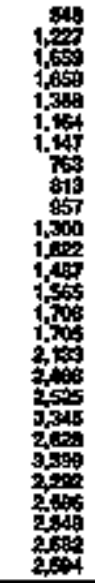 & 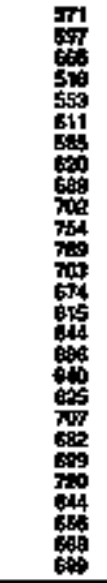 & 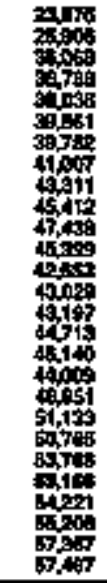 & 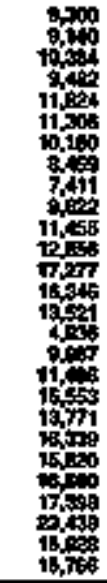 & 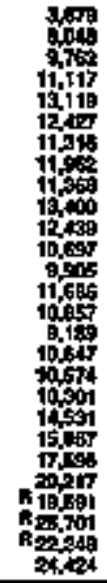 & 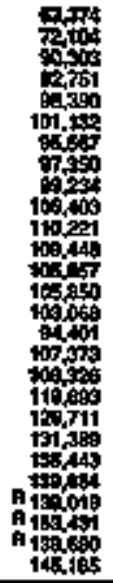 & 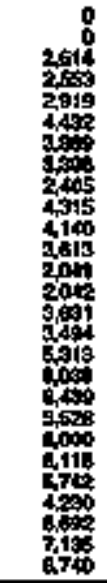 & 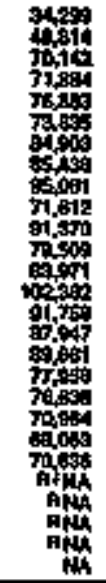 & 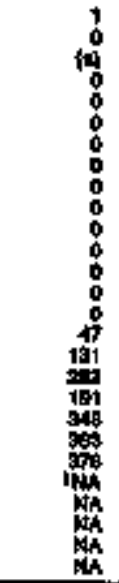 & 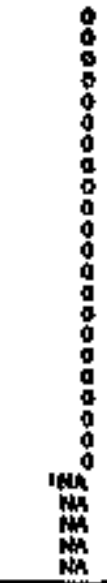 & 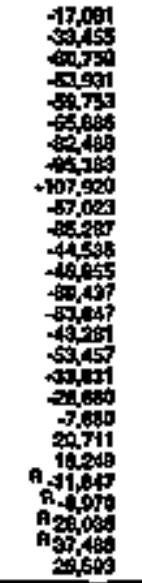 & $\begin{array}{l}z \\
z \\
z \\
z \\
z \\
z \\
z \\
z \\
z \\
z \\
z \\
z \\
z \\
z\end{array}$ \\
\hline & & & & & & & & & & Thenes Ex & & & & & & & & & \\
\hline
\end{tabular}

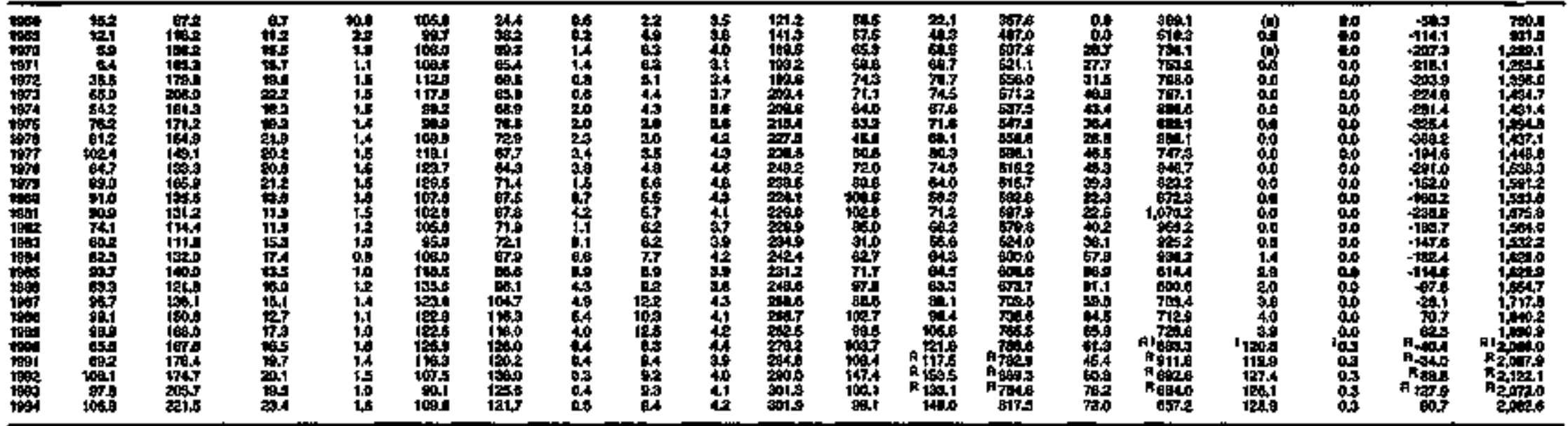

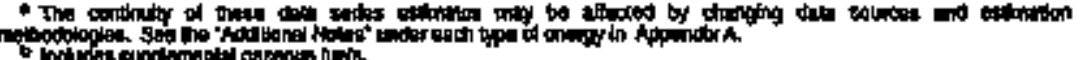

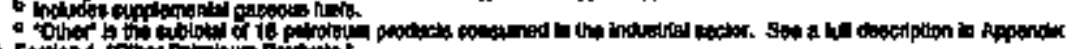

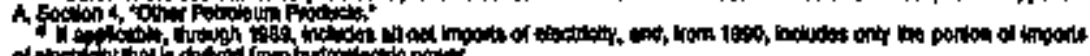

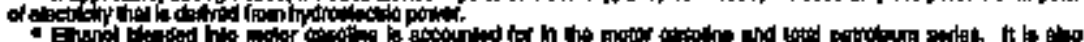

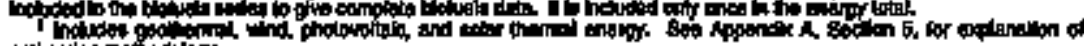

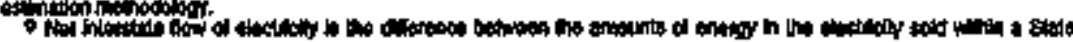

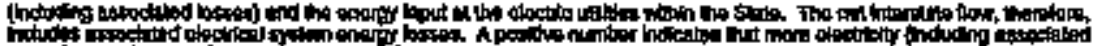

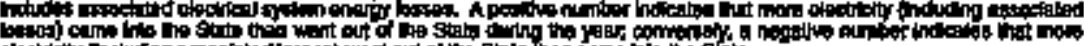

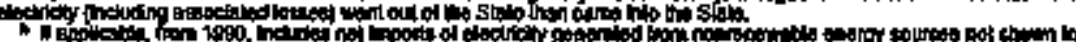

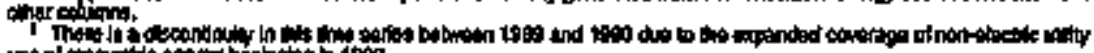

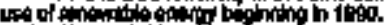
sion

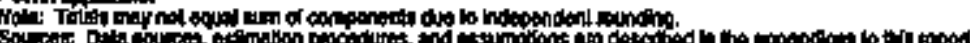


Table 300. Residential Enargy Consumption Estimatos, 1960, 1955, 1970-1994, Washington

$\mathbf{A}$
$\mathbf{S}$
$\mathbf{H}$
$\mathbf{N}$
$\mathbf{G}$
$\mathbf{T}$
$\mathbf{N}$

\begin{tabular}{|c|c|c|c|c|c|c|c|c|c|c|c|c|c|c|}
\hline & \multicolumn{3}{|c|}{ cons } & \multirow[b]{2}{*}{ 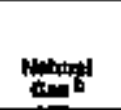 } & \multicolumn{4}{|c|}{ 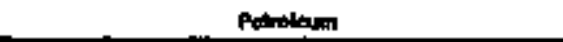 } & \multirow[b]{2}{*}{ Elonhente } & \multirow[b]{2}{*}{ Eolato } & \multirow[b]{2}{*}{ Exctiothy" } & \multirow[b]{2}{*}{ Entory } & \multirow[b]{2}{*}{ 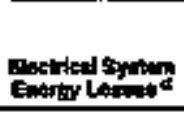 } & \multirow[b]{3}{*}{ Tots } \\
\hline & 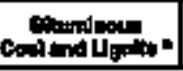 & 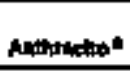 & Told & & Dhimente & Xerouting: & LPE: & Total & & & & & & \\
\hline$r=0$ & \multicolumn{3}{|c|}{ Mrovend short Tonth } & 最ion & \multicolumn{4}{|c|}{ 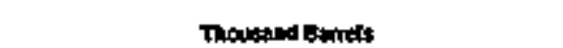 } & Theonitid & \multicolumn{4}{|c|}{ 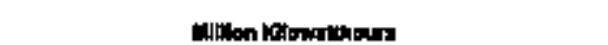 } & \\
\hline
\end{tabular}

\begin{tabular}{|c|c|c|c|c|c|c|c|c|c|c|c|c|c|}
\hline 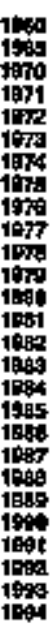 & 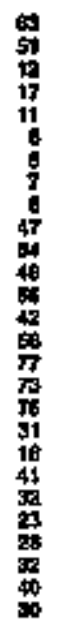 & $\begin{array}{l}0 \\
: \\
: \\
0 \\
0 \\
\vdots \\
0 \\
0 \\
0 \\
0 \\
0 \\
0 \\
0 \\
0 \\
0 \\
0 \\
0 \\
0 \\
0 \\
0 \\
0 \\
0 \\
0\end{array}$ & 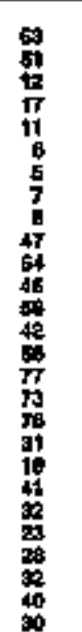 & 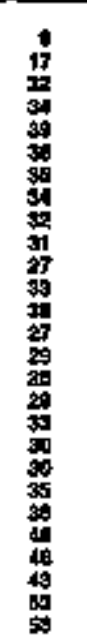 & 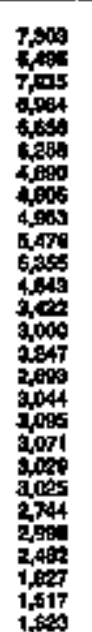 & 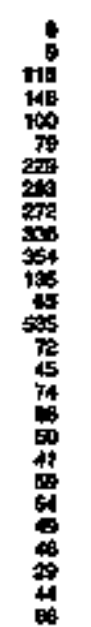 & 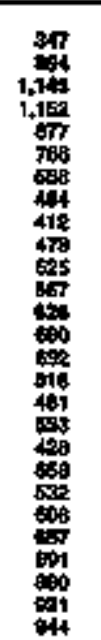 & 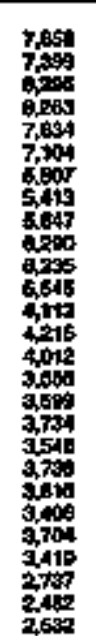 & 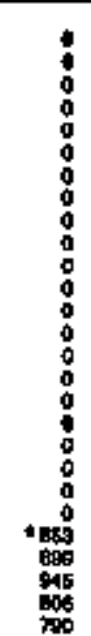 & 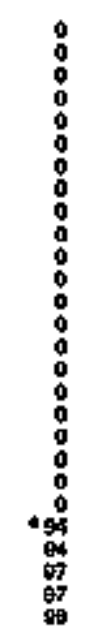 & 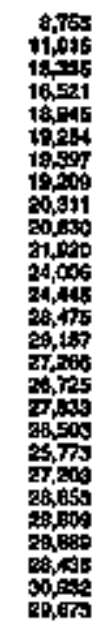 & - & 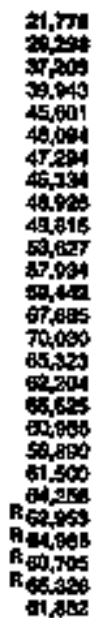 \\
\hline
\end{tabular}

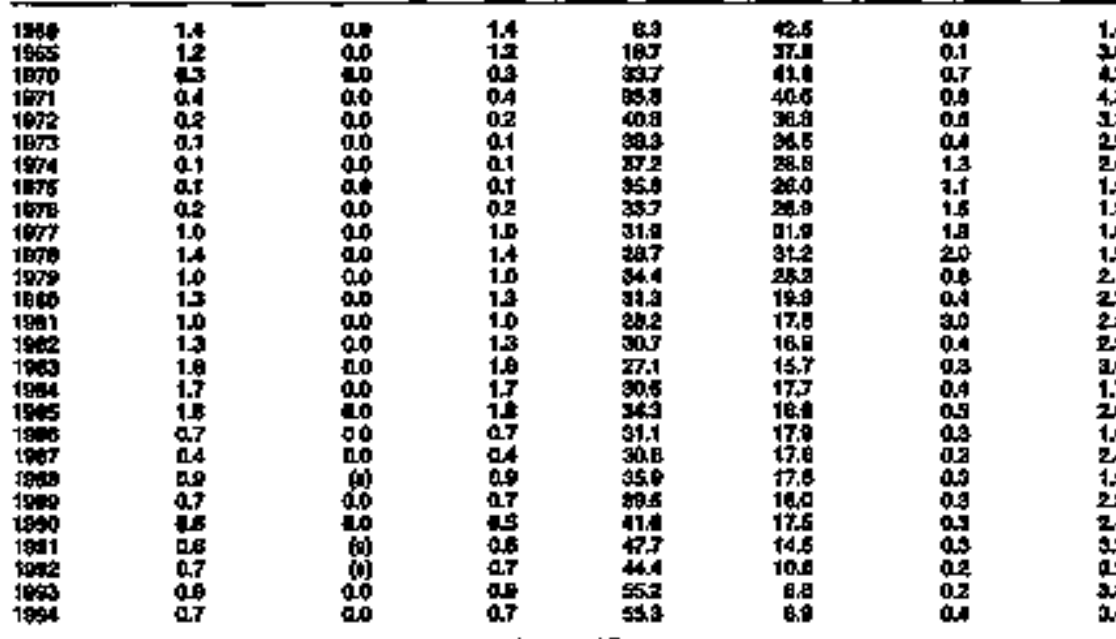

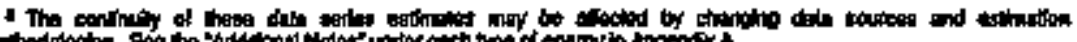

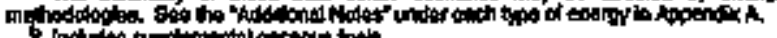

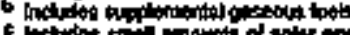

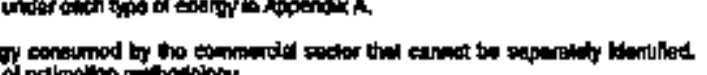

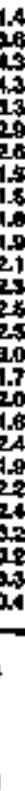

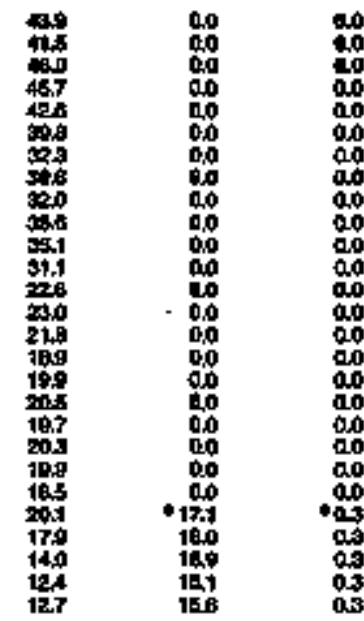

\begin{tabular}{|c|}
\hline 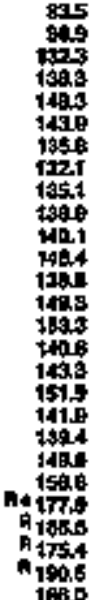 \\
\hline
\end{tabular}

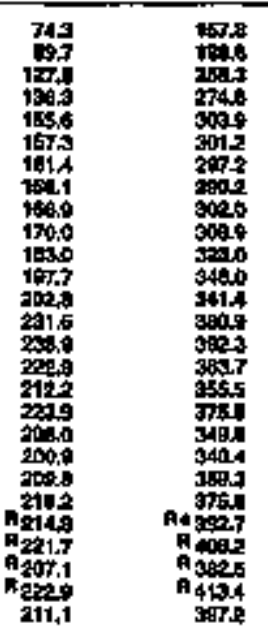

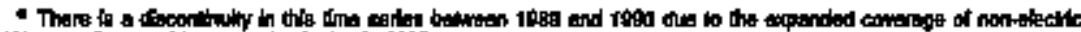

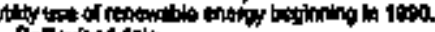
Finforted ath

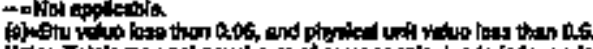

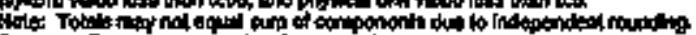

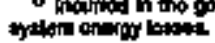

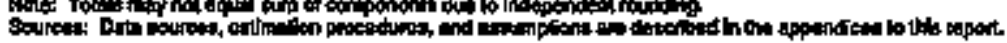

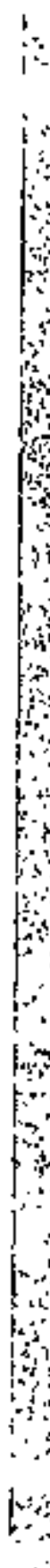


Table 301. Comwercial Energy Consumption Estinates, 1950, 1965, 1970-1994, Wasthington

\begin{tabular}{|c|c|c|c|c|c|c|c|c|c|c|c|c|c|c|}
\hline \multirow[b]{3}{*}{ Voar } & \multicolumn{3}{|c|}{$\infty$} & \multirow{3}{*}{ 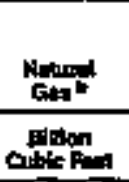 } & \multicolumn{6}{|c|}{ Patrotorn } & \multirow[b]{2}{*}{ anctidy " } & \multirow[b]{2}{*}{ that } & \multirow[b]{2}{*}{ 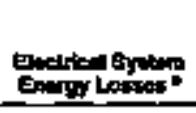 } & \multirow[b]{3}{*}{ tots } \\
\hline & 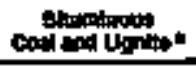 & Minnedo* & Totr & & 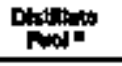 & Koroutent & ER⿴囗十 & motertion & Petigent & Toter & & & & \\
\hline & \multicolumn{2}{|c|}{ Thousand Enoth Twan: } & \multirow[b]{2}{*}{ 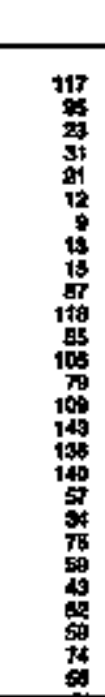 } & & \multicolumn{6}{|c|}{ 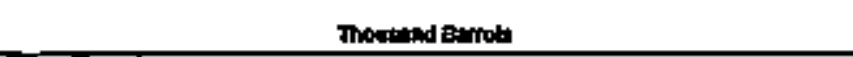 } & \multicolumn{3}{|c|}{ 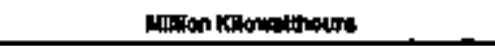 } & \\
\hline 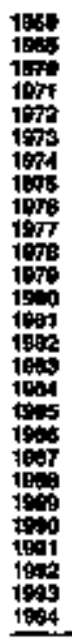 & 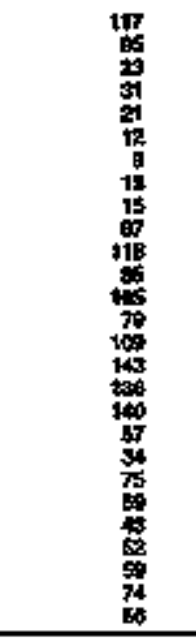 & $\begin{array}{l}0 \\
0 \\
0 \\
0 \\
0 \\
0 \\
0 \\
0 \\
0 \\
0 \\
0 \\
0 \\
0 \\
0 \\
0 \\
0 \\
0 \\
0 \\
0 \\
0 \\
0 \\
0 \\
0 \\
0 \\
0 \\
0 \\
0 \\
\end{array}$ & & 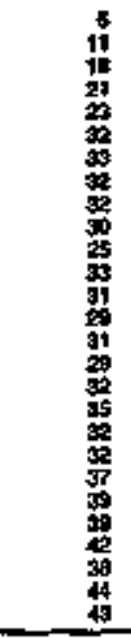 & 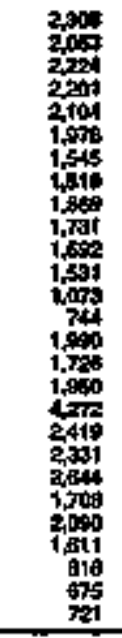 & 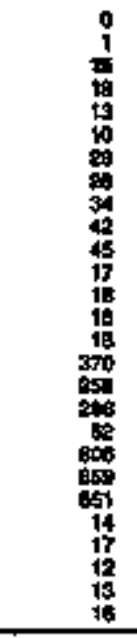 & 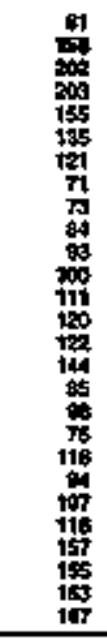 & 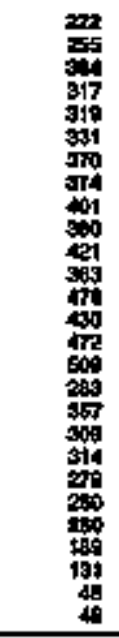 & 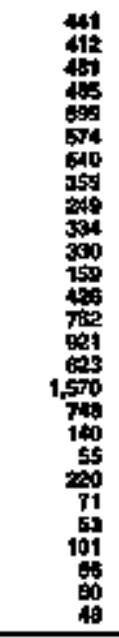 & 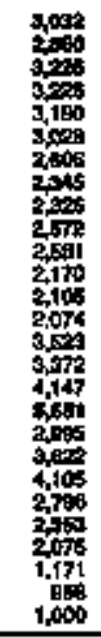 & 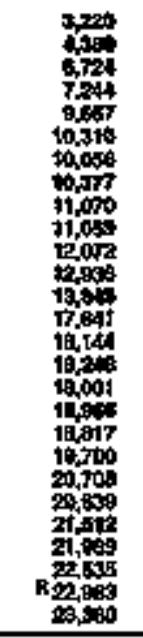 & $\begin{array}{l}= \\
= \\
= \\
= \\
= \\
= \\
= \\
= \\
= \\
= \\
= \\
= \\
= \\
=\end{array}$ & 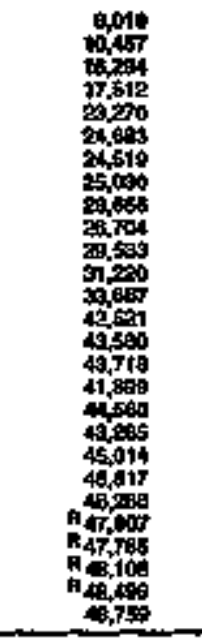 & $\begin{array}{l}= \\
z \\
z \\
= \\
z \\
z \\
= \\
= \\
= \\
z \\
z \\
= \\
z \\
z \\
\end{array}$ \\
\hline \multicolumn{15}{|c|}{ 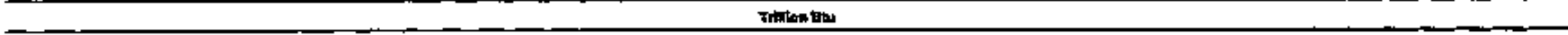 } \\
\hline 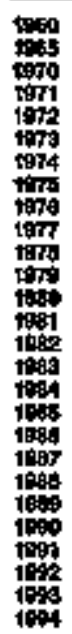 & 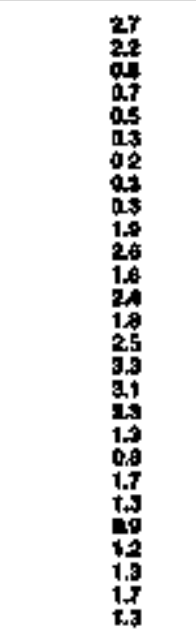 & 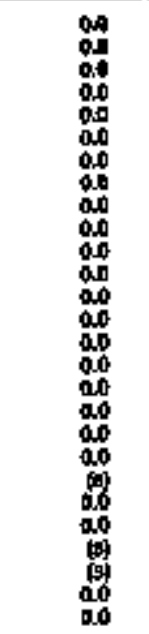 & 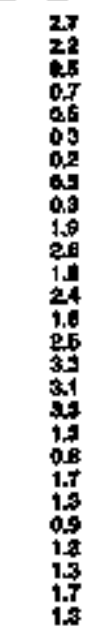 & 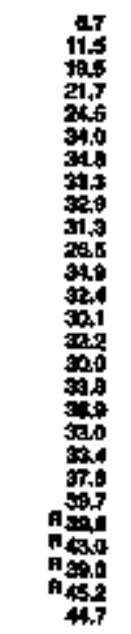 & 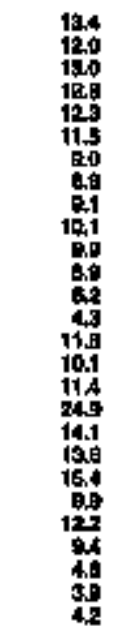 & 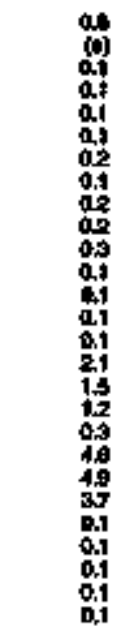 & 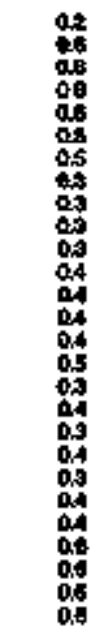 & 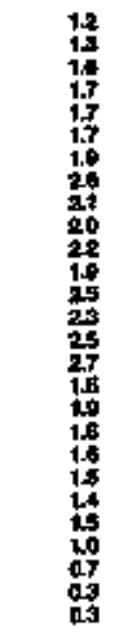 & 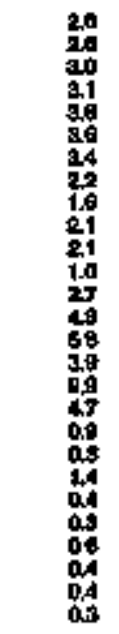 & 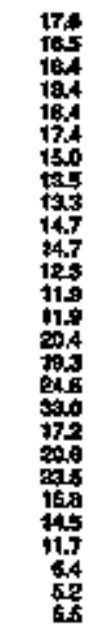 & 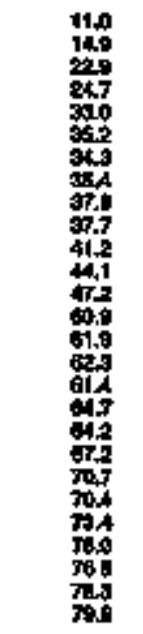 & 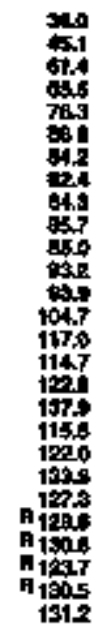 & 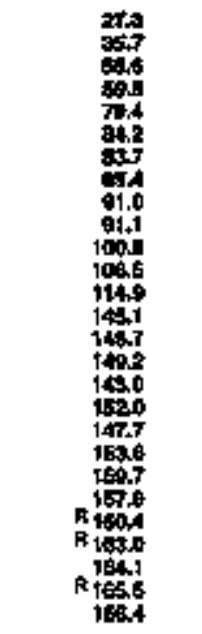 & 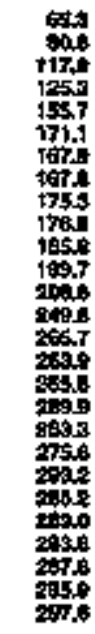 \\
\hline
\end{tabular}




\begin{tabular}{|c|c|c|c|c|c|c|c|c|c|c|c|c|c|c|c|c|c|c|}
\hline & \multirow[b]{2}{*}{ cont } & \multirow[b]{2}{*}{ atar: } & \multicolumn{9}{|c|}{ Parrolen } & \multirow{2}{*}{$\mid$} & \multirow[b]{2}{*}{ manumb } & \multirow[b]{2}{*}{ | } & \multirow[b]{2}{*}{ Enctions } & \multirow[b]{2}{*}{ Exty } & \multirow{3}{*}{ 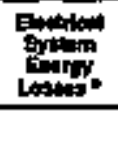 } & \multirow[b]{3}{*}{ Tol: } \\
\hline & & & Aaptiond & 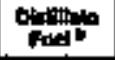 & 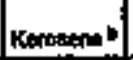 & LPa' & 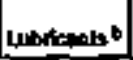 & Mdid & 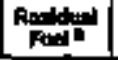 & Onter wo & Totat & & & & & & & \\
\hline 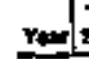 & 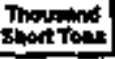 & 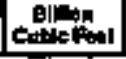 & \multicolumn{9}{|c|}{ 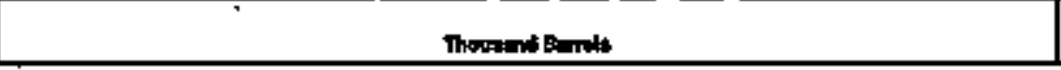 } & \multicolumn{5}{|c|}{ 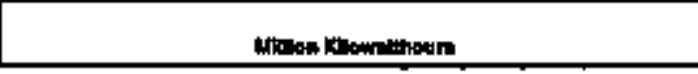 } & & \\
\hline 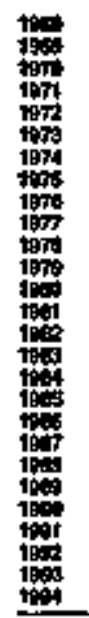 & 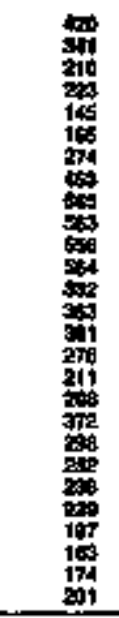 & 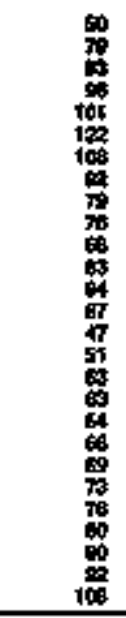 & 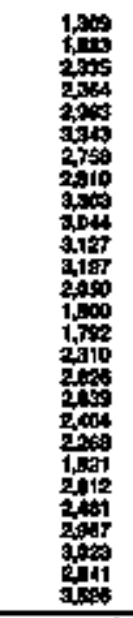 & 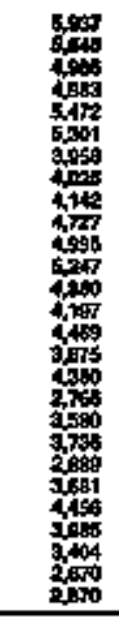 & 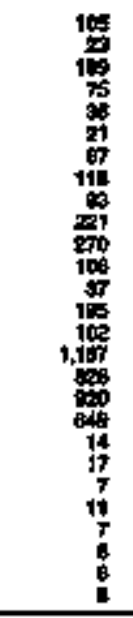 & 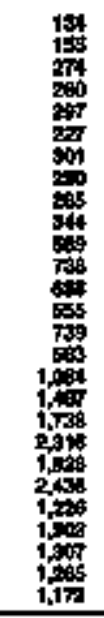 & 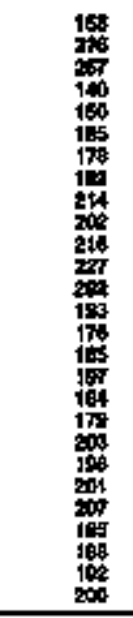 & 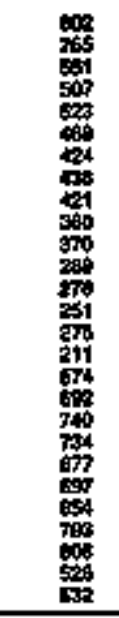 & 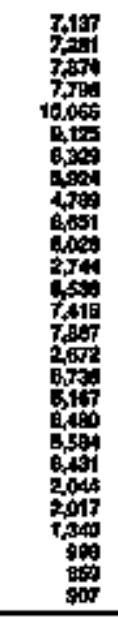 & 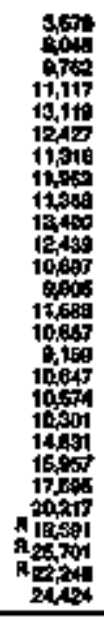 & 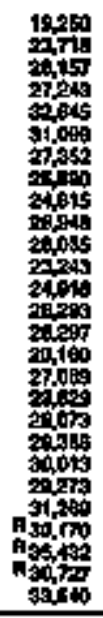 & 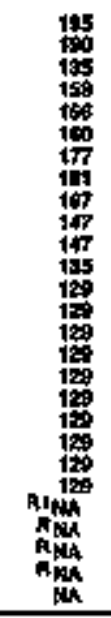 & 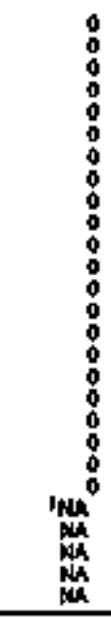 & 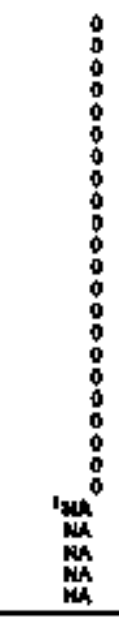 & 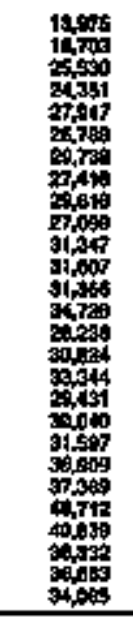 & 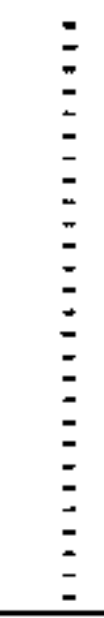 & 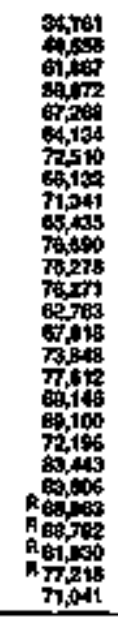 & $\begin{array}{l}z \\
\bar{z} \\
z\end{array}$ \\
\hline \multicolumn{19}{|c|}{$7 \mathrm{TH}$} \\
\hline 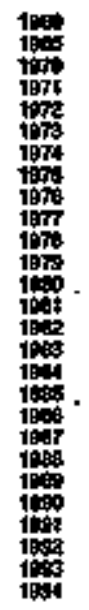 & 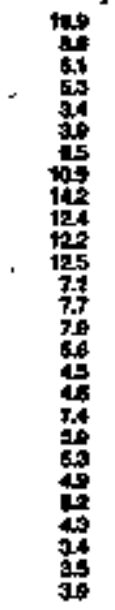 & 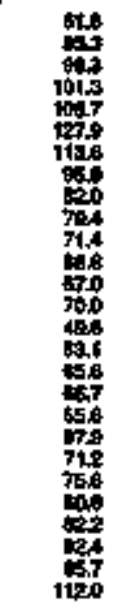 & 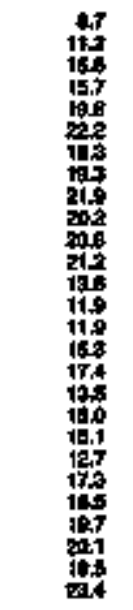 & 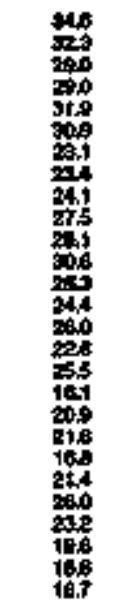 & 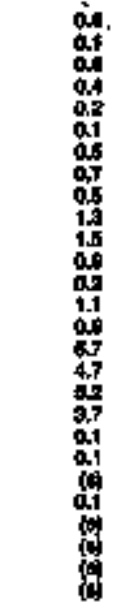 & 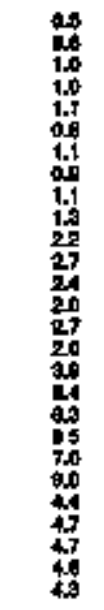 & 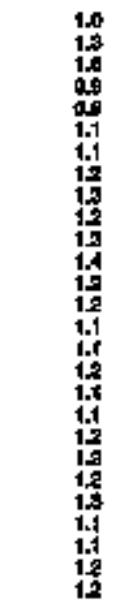 & 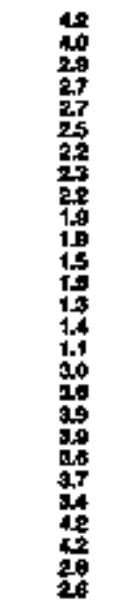 & 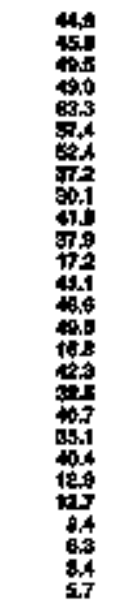 & 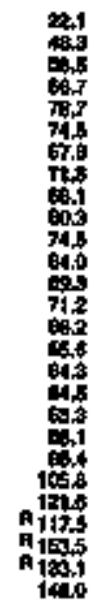 & 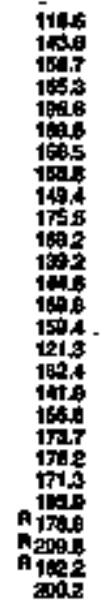 & 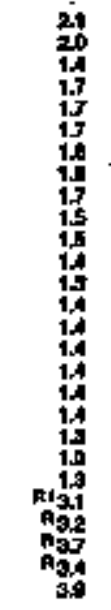 & 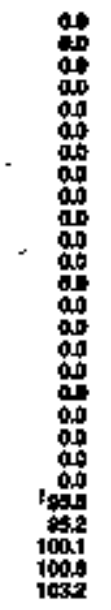 & 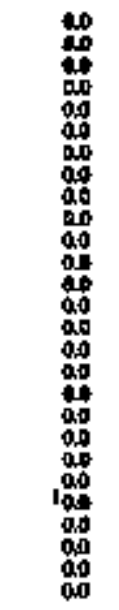 & 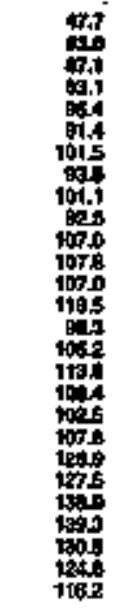 & 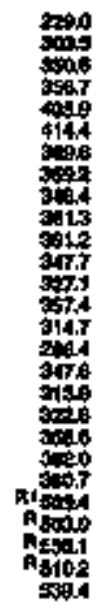 & 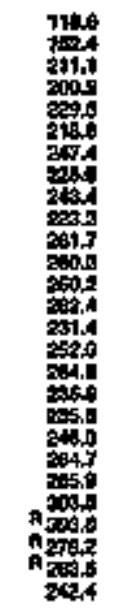 & 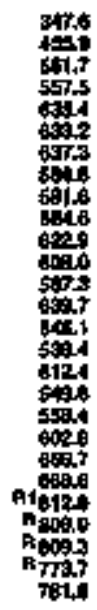 \\
\hline
\end{tabular}

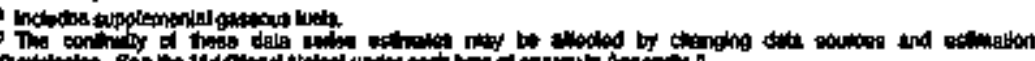

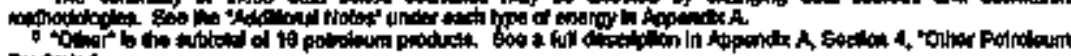

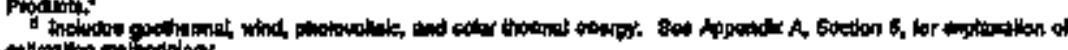

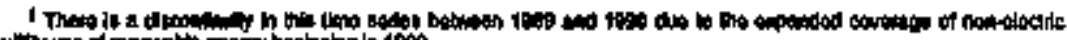
W

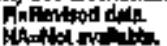
Chatham

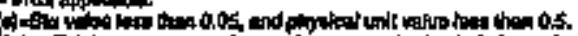

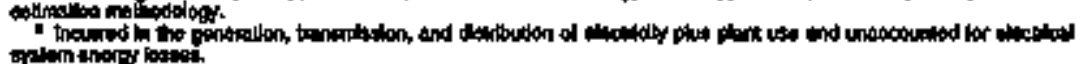

年

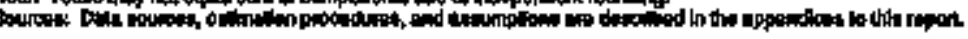


Table 303. Transportation Energy Constumption Estlmatet, 1960, to5s, t970-1994, Washingion

\begin{tabular}{|c|c|c|c|c|c|c|c|c|c|c|c|c|c|c|c|}
\hline & \multirow[b]{2}{*}{ ment } & \multirow[b]{2}{*}{ matis } & \multicolumn{8}{|c|}{ 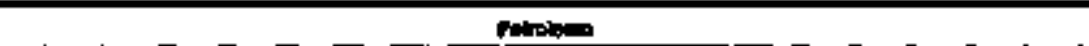 } & \multirow[b]{2}{*}{ Etanmestos } & \multirow[b]{2}{*}{ Empority" } & \multirow[b]{2}{*}{ Energr } & \multirow[b]{2}{*}{ 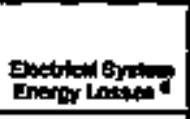 } & \multirow[b]{3}{*}{ Totot } \\
\hline & & & . & $\begin{array}{l}\text { Pnetmis } \\
\text { Pued" }\end{array}$ & int. & 5eon & Lobukman " & matrons & 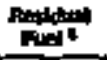 & Tots & & & & & \\
\hline$r \in$ & Thontiand & cultefinat & \multicolumn{8}{|c|}{ 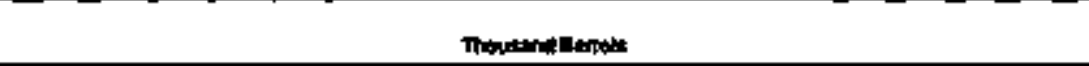 } & Thations & \multicolumn{3}{|c|}{ 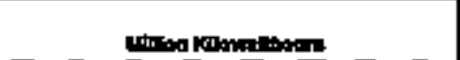 } & \\
\hline 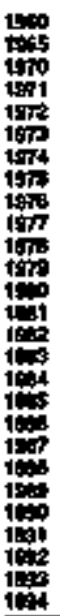 & $\begin{array}{l}7 \\
1 \\
0 \\
0 \\
0 \\
0 \\
0 \\
0 \\
0 \\
0 \\
0 \\
0 \\
0 \\
0 \\
0 \\
0 \\
0 \\
0 \\
0 \\
0 \\
0 \\
0 \\
0 \\
0 \\
0\end{array}$ & $\begin{array}{l}60 \\
1 \\
6 \\
6 \\
7 \\
6 \\
6 \\
6 \\
6 \\
7 \\
4 \\
2 \\
2 \\
8 \\
7 \\
2 \\
4 \\
4 \\
5 \\
5 \\
3 \\
4 \\
7\end{array}$ & 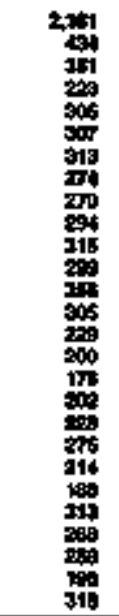 & 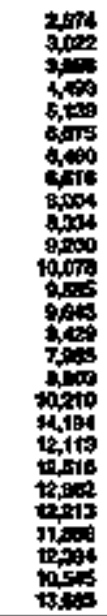 & 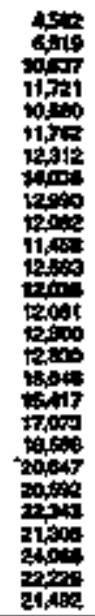 & 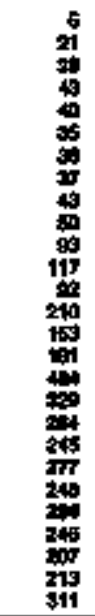 & 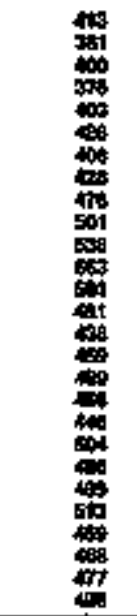 & 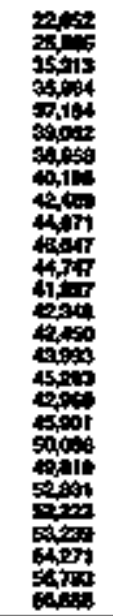 & 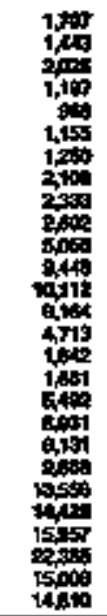 & 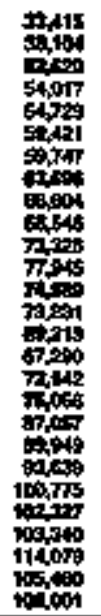 & 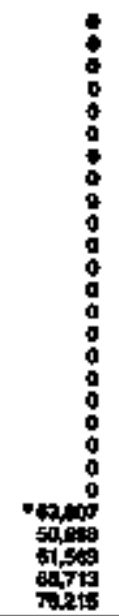 & $\begin{array}{c}\mathbf{t} \\
1 \\
\vdots \\
1 \\
1 \\
1 \\
1 \\
2 \\
2 \\
2 \\
1 \\
2 \\
2 \\
5 \\
5 \\
12 \\
12 \\
12 \\
12 \\
12 \\
12 \\
12 \\
15 \\
14 \\
75 \\
17 \\
15 \\
15\end{array}$ & $\begin{array}{l}= \\
= \\
= \\
z \\
= \\
= \\
= \\
= \\
= \\
= \\
= \\
= \\
z \\
= \\
=\end{array}$ & 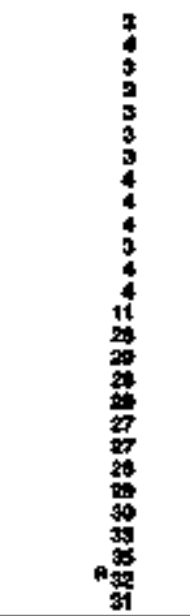 & 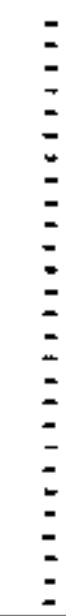 \\
\hline & & & & & & & & בים & & & & & & & \\
\hline
\end{tabular}

\begin{tabular}{|c|c|c|c|c|c|c|c|c|c|c|c|c|c|c|}
\hline 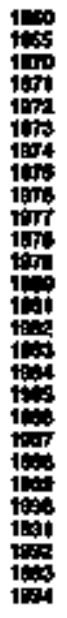 & 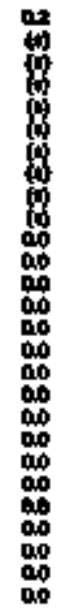 & 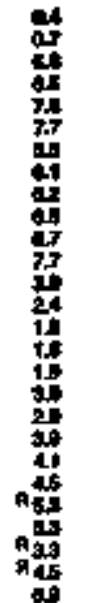 & 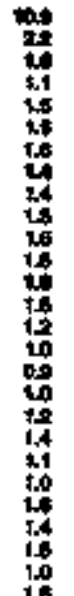 & 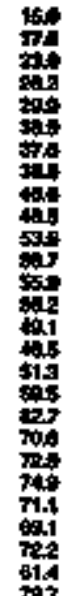 & 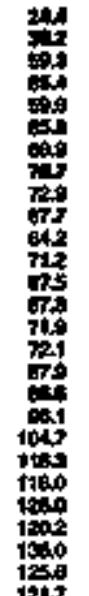 & 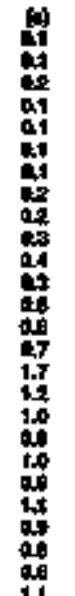 & 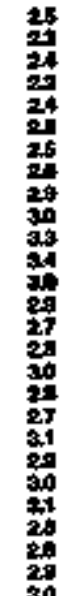 & 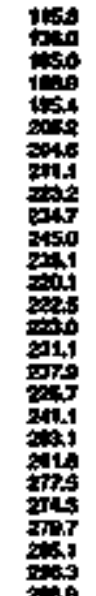 & 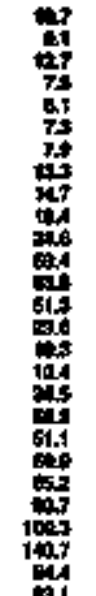 & 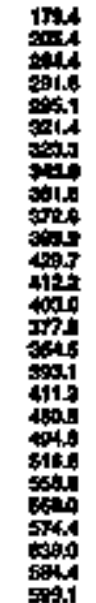 & 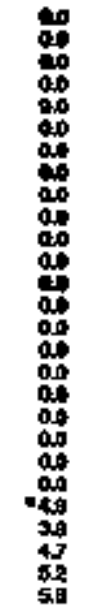 & 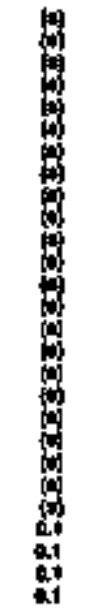 & 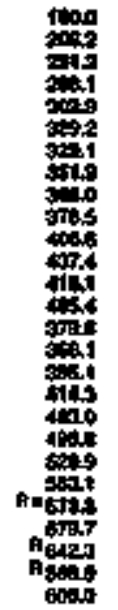 & 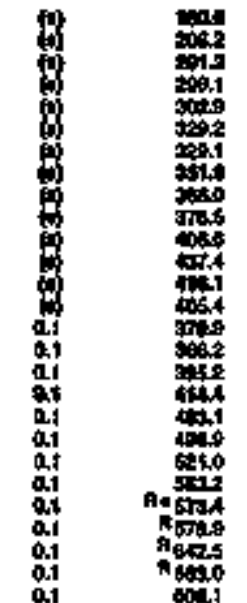 \\
\hline
\end{tabular}

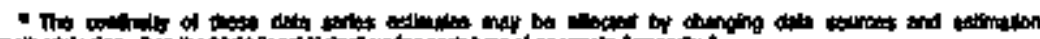

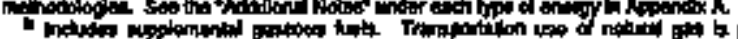

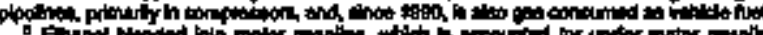

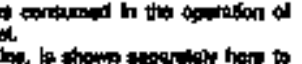

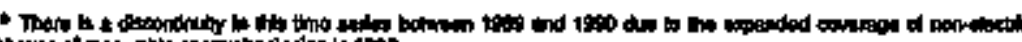

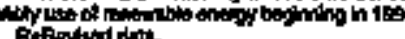



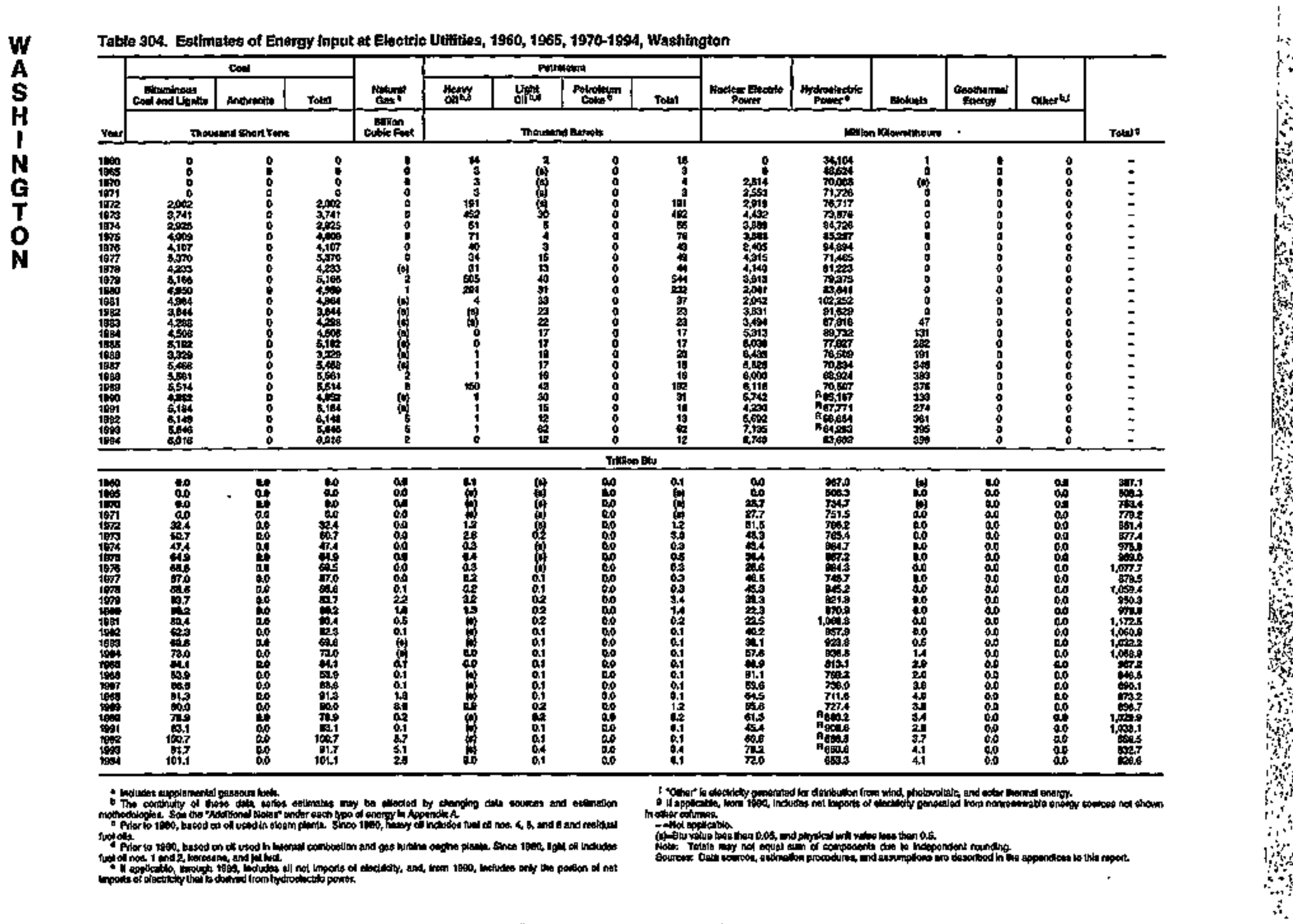
Table 303. Energy Consumption Estimates by Source, 1960, 1955, 1970-1994, West Vrrinle

\begin{tabular}{|c|c|c|c|c|c|c|c|c|c|c|c|c|c|c|c|c|c|c|c|}
\hline \multirow[b]{3}{*}{ Yer } & \multirow[b]{2}{*}{ Foment. } & \multirow[b]{2}{*}{ Mathrid } & \multicolumn{11}{|c|}{ Prtoloun } & \multirow[b]{2}{*}{ 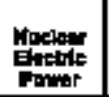 } & \multirow[b]{2}{*}{ 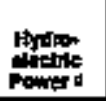 } & \multirow[b]{2}{*}{ Elohinget: } & \multirow[b]{2}{*}{ Dher of } & \multirow{2}{*}{ 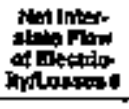 } & \multirow[b]{3}{*}{ Tot: $n$} \\
\hline & & & Asphe end & 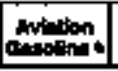 & Dathe & Fit. & "זיה & L연 & 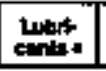 & antiof & mater & oneros? & Toll: & & & & & & \\
\hline & Dnesured Tont & citle foet & \multicolumn{11}{|c|}{ Thourind Empds: } & \multicolumn{5}{|c|}{ 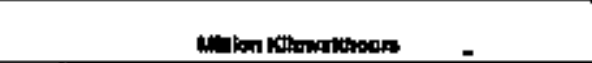 } & \\
\hline 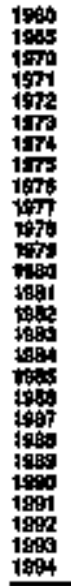 & 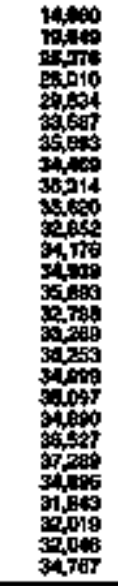 & 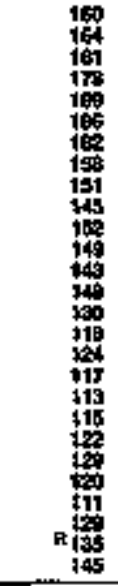 & 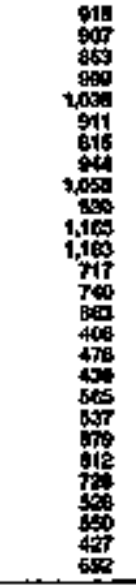 & 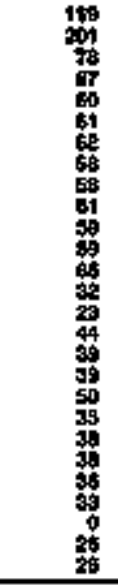 & 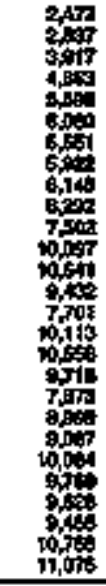 & 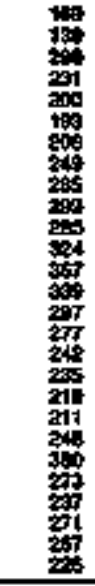 & 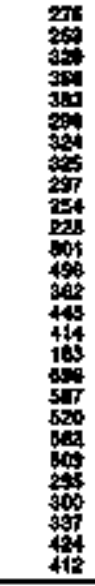 & 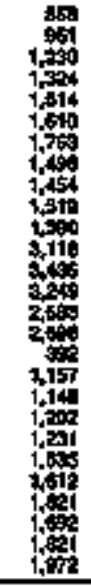 & 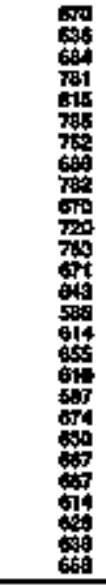 & 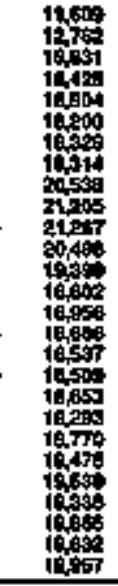 & 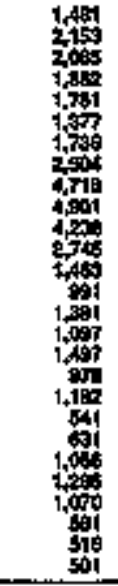 & 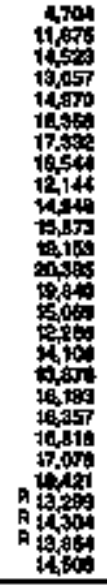 & 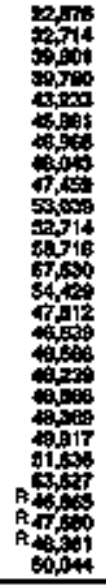 & $\begin{array}{l}0 \\
0 \\
0 \\
0 \\
0 \\
0 \\
0 \\
0 \\
0 \\
0 \\
0 \\
0 \\
0 \\
0 \\
0 \\
0 \\
0 \\
0 \\
0 \\
0 \\
0 \\
0 \\
0 \\
0\end{array}$ & 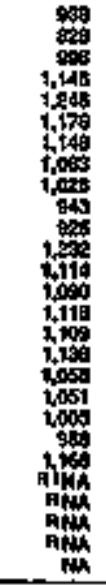 & 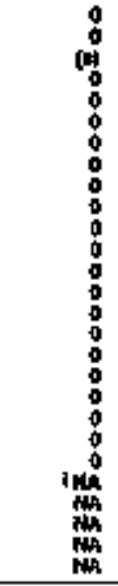 & 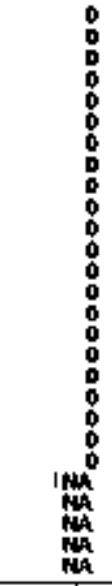 & 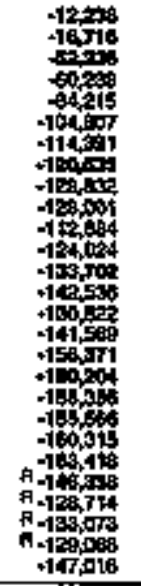 & 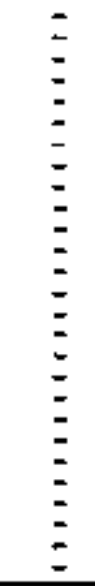 \\
\hline \multicolumn{20}{|c|}{ Thingopte } \\
\hline 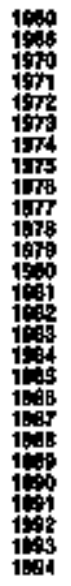 & 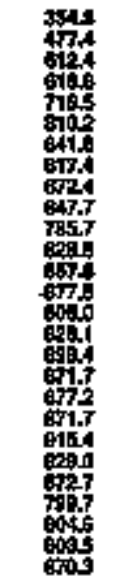 & 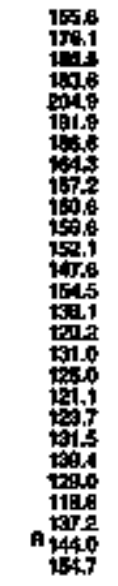 & 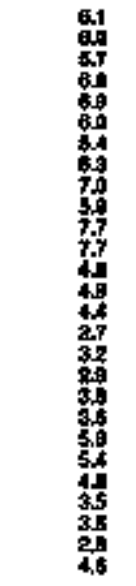 & 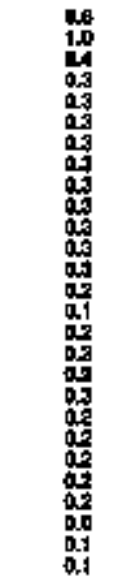 & 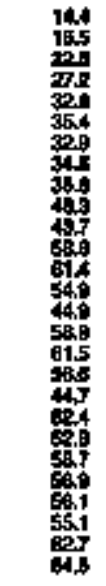 & 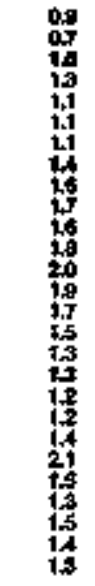 & 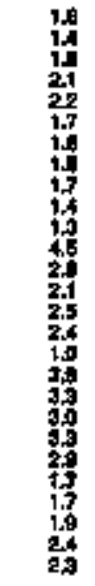 & 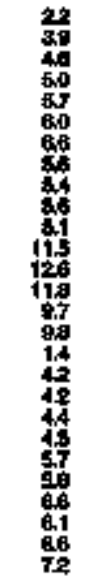 & 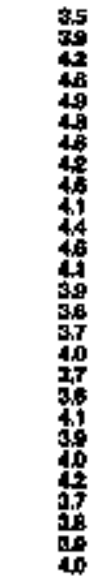 & 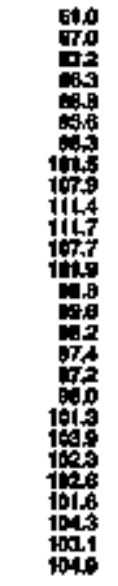 & 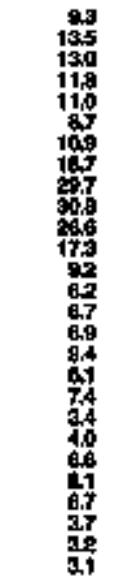 & 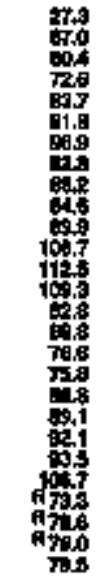 & 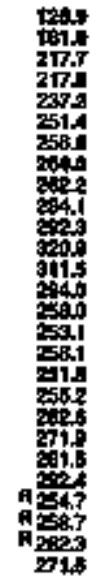 & 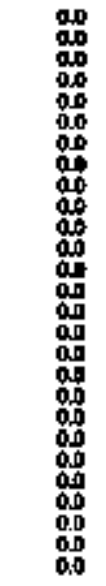 & 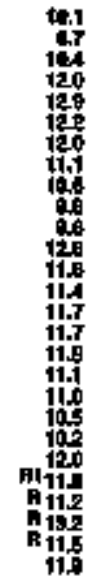 & 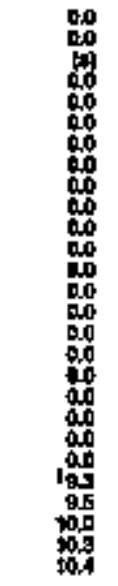 & 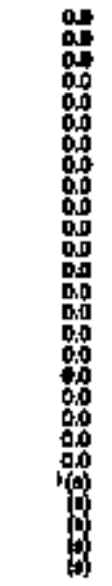 & 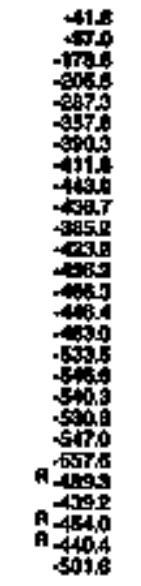 & 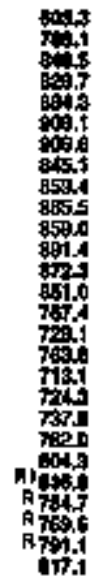 \\
\hline
\end{tabular}

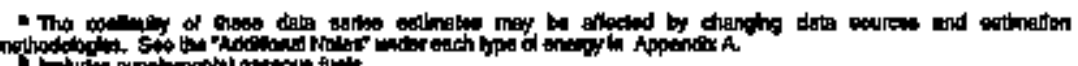

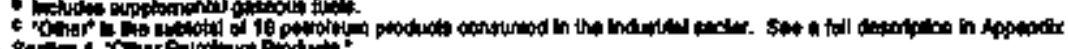

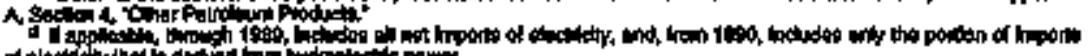

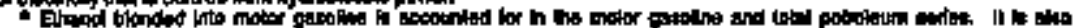

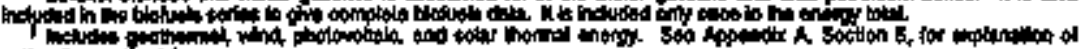

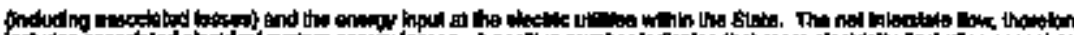

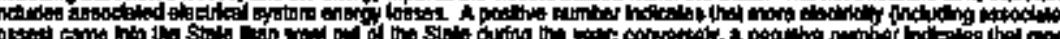

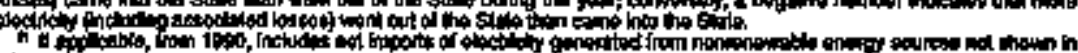

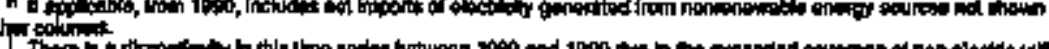
vos of Molotion

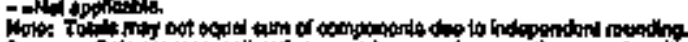

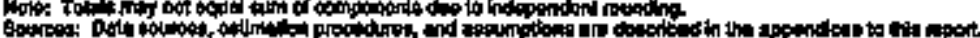

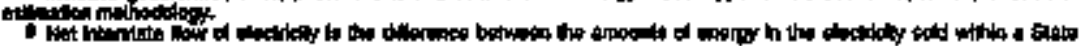


Table 306. Restidential Energy Consumption Estinates, 1960, 1965, 1970-1994, West Virginia

\section{The}

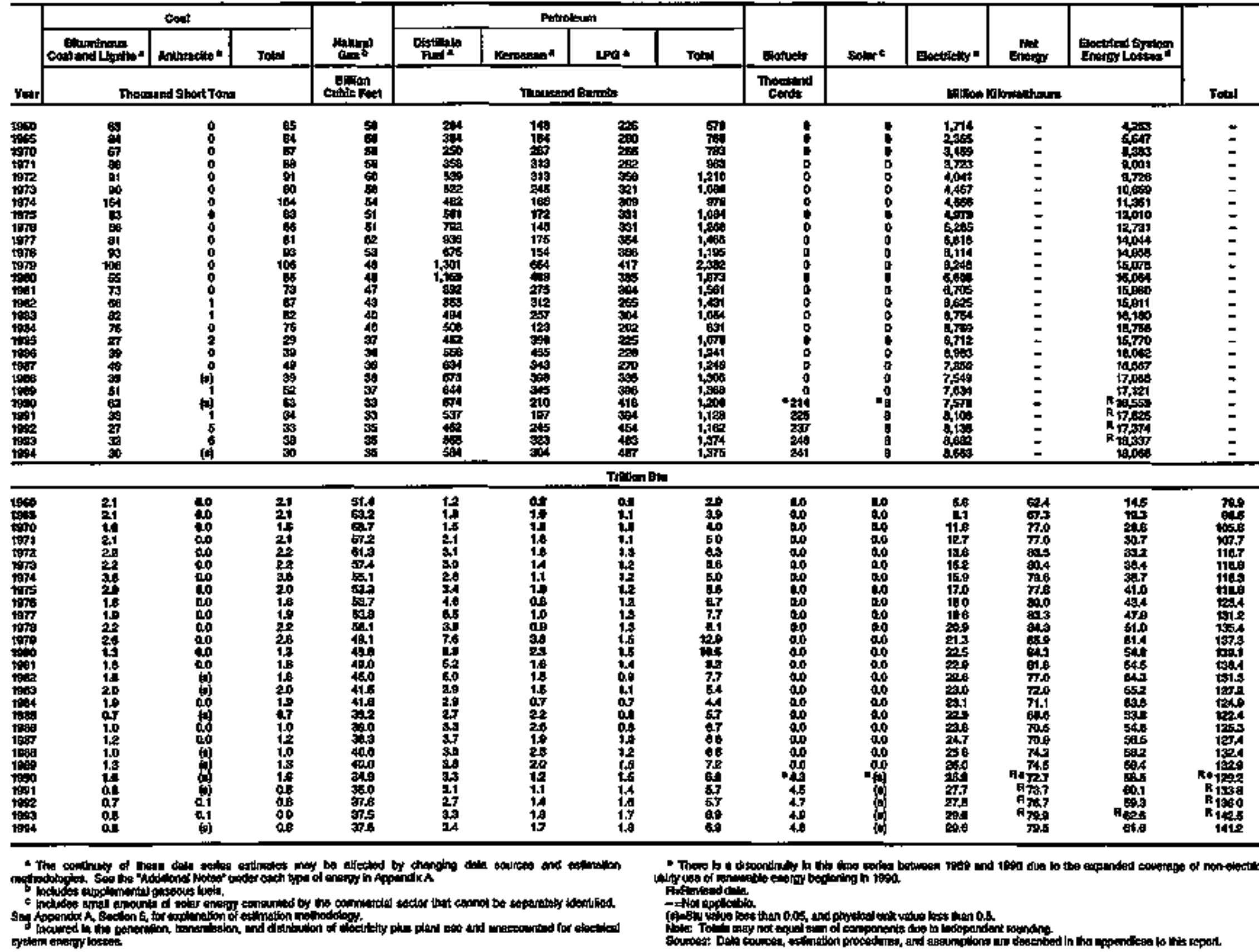


Table 307. Commerclal Energy Consumptlon Estlmates, 1960, 1965, 1970-1894, West Virginka

\begin{tabular}{|c|c|c|c|c|c|c|c|c|c|c|c|c|c|c|}
\hline \multirow[b]{3}{*}{ 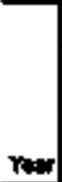 } & \multicolumn{3}{|c|}{$\cos$} & \multirow{3}{*}{ 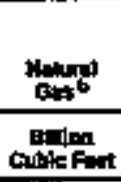 } & \multicolumn{6}{|c|}{ Putrolenen } & \multirow[b]{2}{*}{ Eloctikiny" } & \multirow[b]{2}{*}{ Ement } & \multirow[b]{2}{*}{ 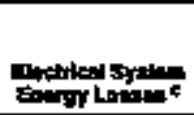 } & \multirow[b]{3}{*}{ Tolat d } \\
\hline & 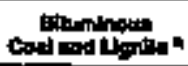 & Antuadio: & Totet & & 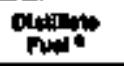 & 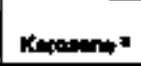 & LPB: & mition & Rosidined & Tot & & & & \\
\hline & \multicolumn{3}{|c|}{ Thowinund Spont Touip } & & \multicolumn{6}{|c|}{ Thontad Bing } & \multicolumn{3}{|c|}{ 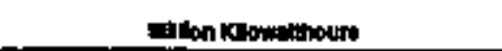 } & \\
\hline 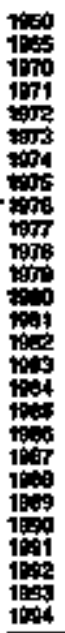 & 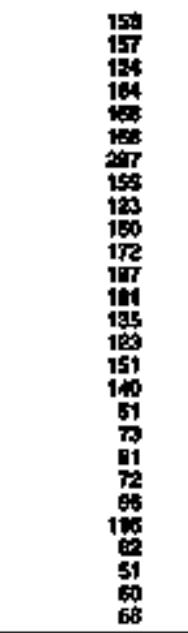 & 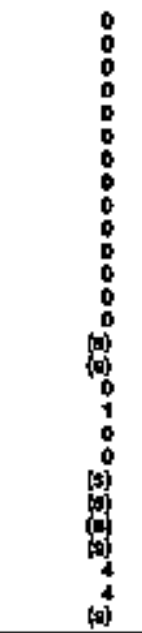 & 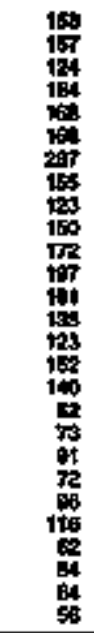 & 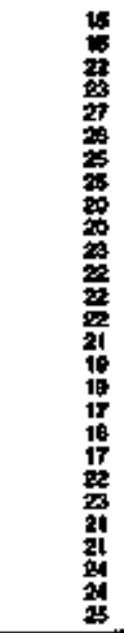 & 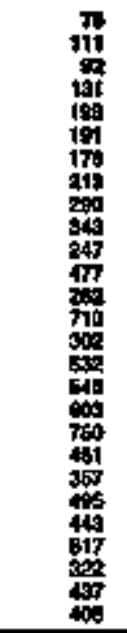 & 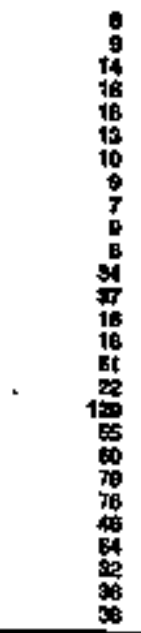 & 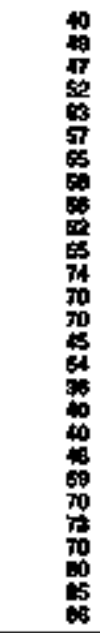 & 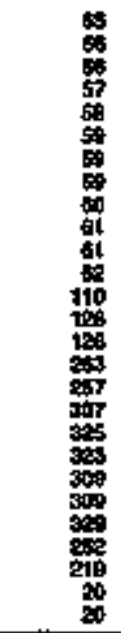 & 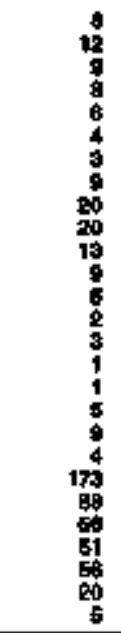 & 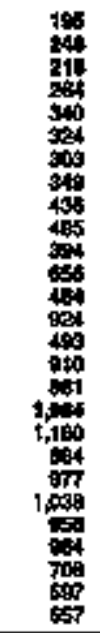 & 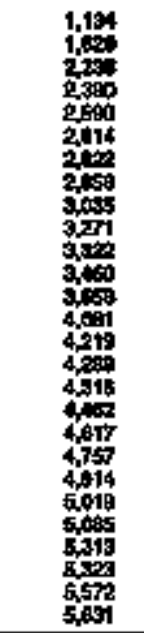 & $\begin{array}{l}= \\
= \\
= \\
= \\
= \\
= \\
= \\
= \\
= \\
= \\
= \\
= \\
= \\
=\end{array}$ & 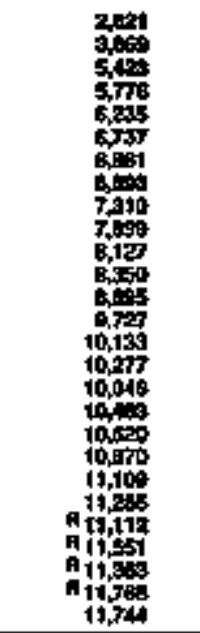 & $\begin{array}{l}= \\
= \\
z \\
z \\
= \\
= \\
= \\
= \\
z \\
= \\
= \\
z \\
z\end{array}$ \\
\hline \multicolumn{15}{|c|}{ Trimlon ou } \\
\hline 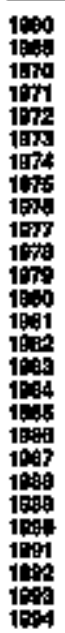 & 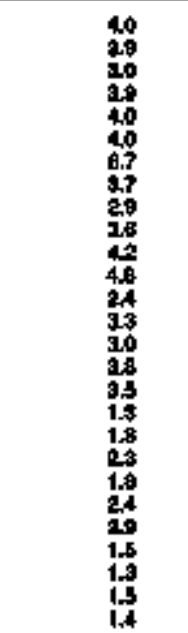 & 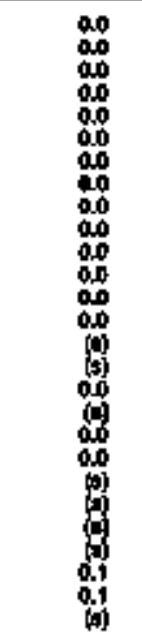 & 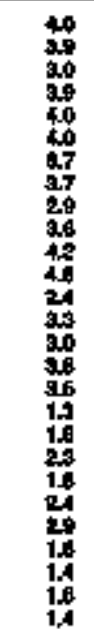 & 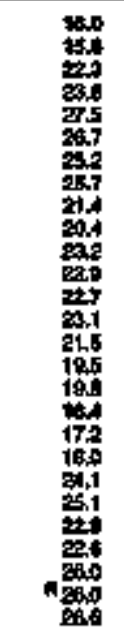 & 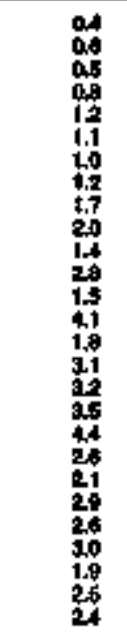 & 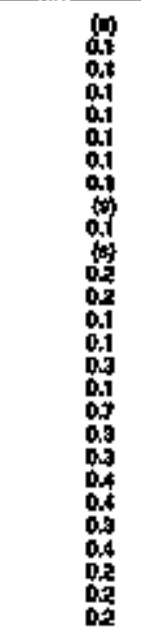 & 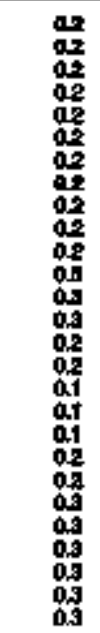 & 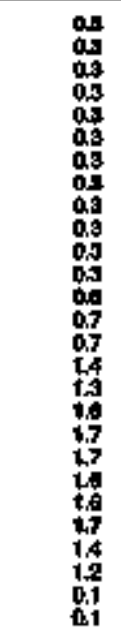 & 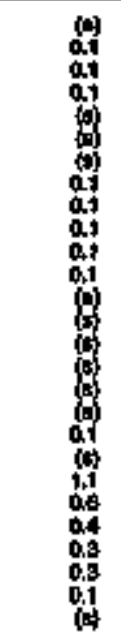 & 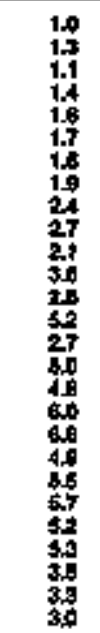 & 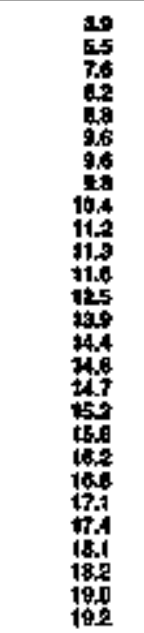 & 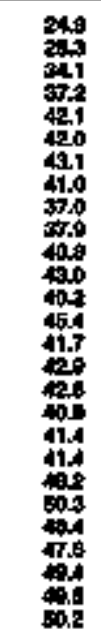 & 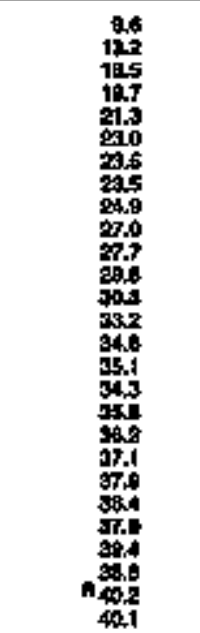 & 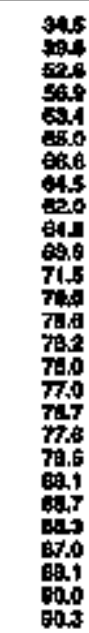 \\
\hline
\end{tabular}




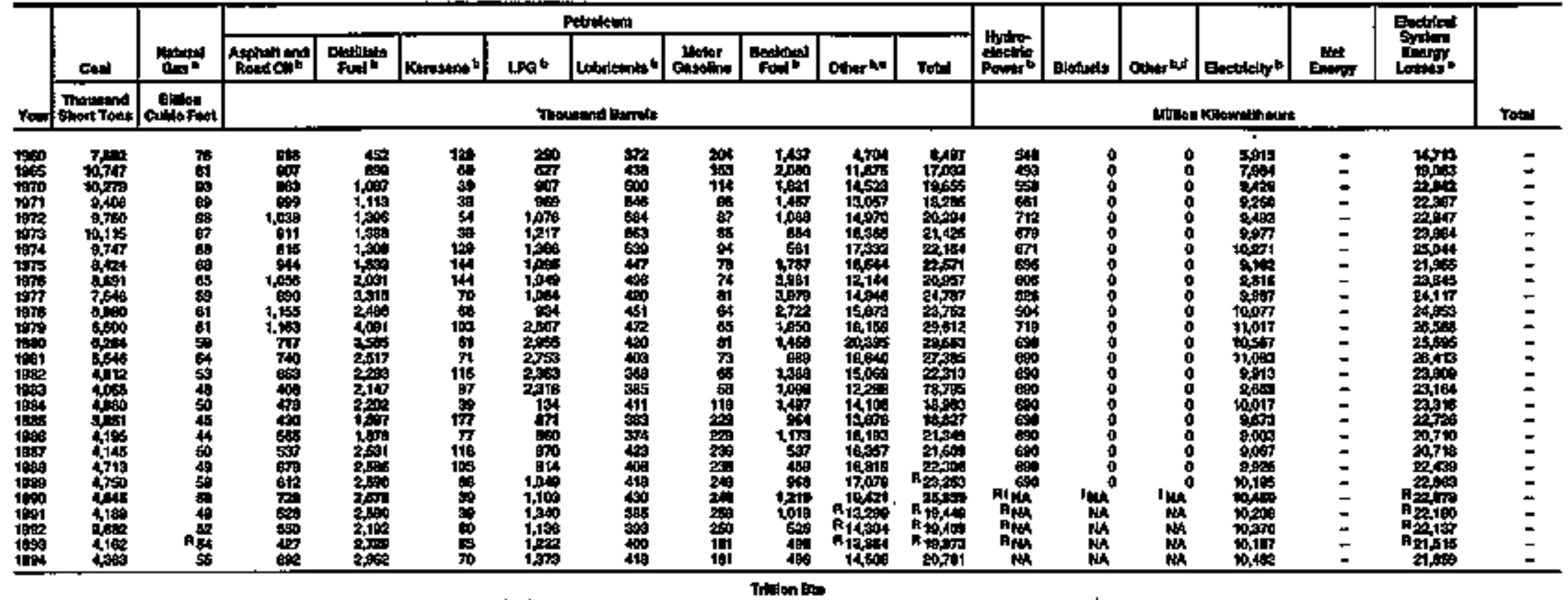

\begin{tabular}{|c|c|c|c|c|c|c|c|c|c|c|c|c|c|c|c|c|c|c|}
\hline 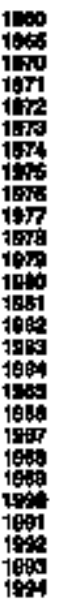 & 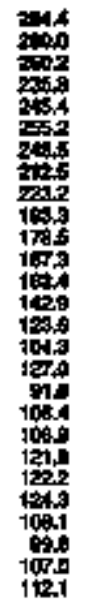 & 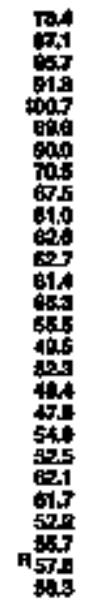 & 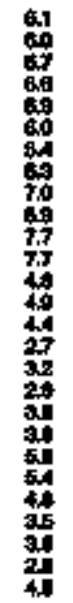 & 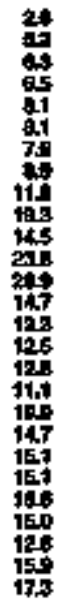 & 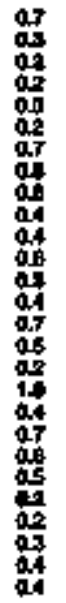 & 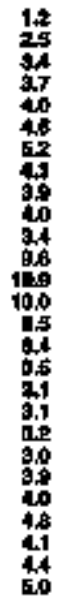 & 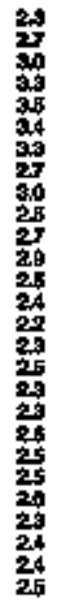 & 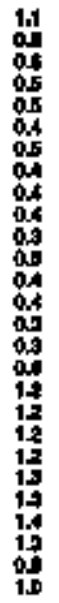 & 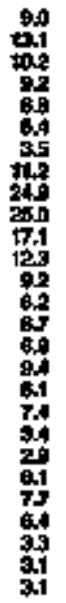 & 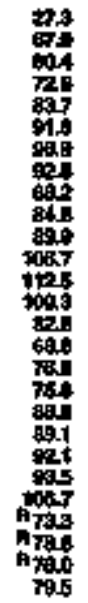 & 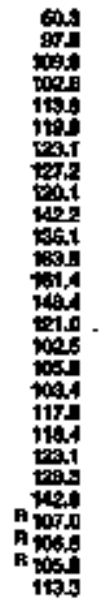 & 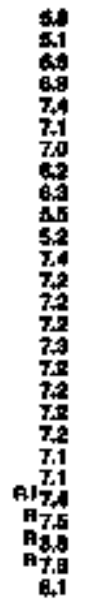 & 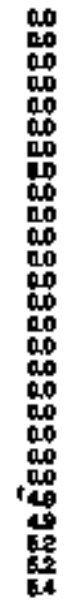 & 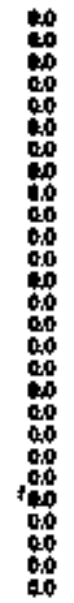 & 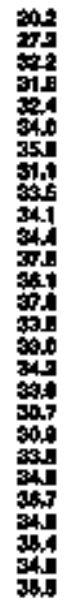 & 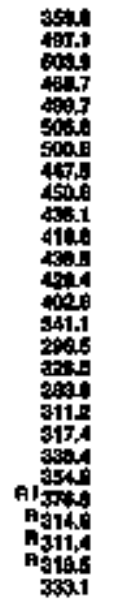 & 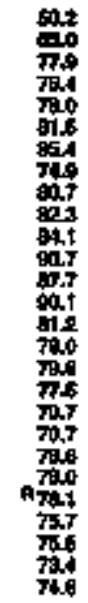 & 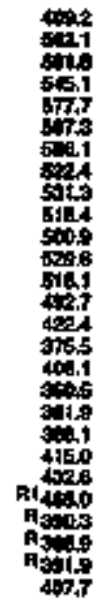 \\
\hline
\end{tabular}

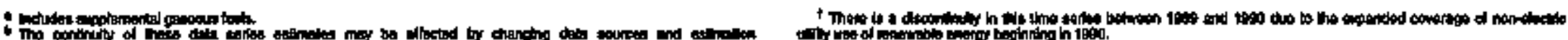

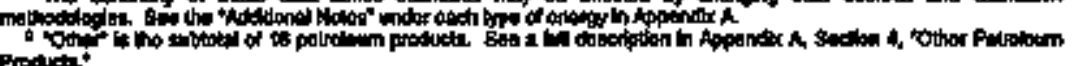

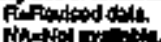

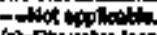

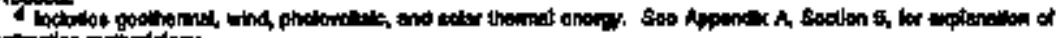

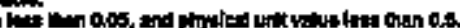


Thble sog. Transportafion Erergy Consumption Esalmales, 1960, 1965, 1970-1994, West Virginia

\begin{tabular}{|c|c|c|c|c|c|c|c|c|c|c|c|c|c|c|c|}
\hline \multirow[b]{3}{*}{ שו } & \multirow[b]{2}{*}{ Cod" } & \multirow[b]{2}{*}{ 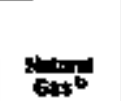 } & \multicolumn{8}{|c|}{ Patroten } & \multirow{3}{*}{ 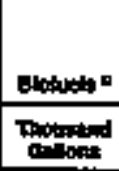 } & & \multirow[b]{2}{*}{ Int } & \multirow[b]{2}{*}{ Entolspang } & \multirow[b]{3}{*}{ Toth } \\
\hline & & & ation. & Dinthen & 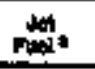 & tPG: & Unowants: & $\operatorname{mim}_{\text {andom }}$ & Pantherst & Told & & Exetidery & & & \\
\hline & thol...ed & 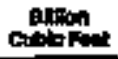 & \multicolumn{8}{|c|}{ 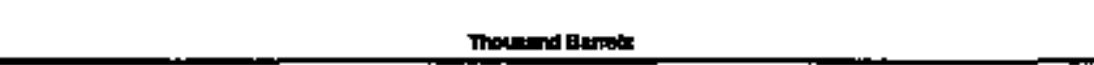 } & & \multicolumn{3}{|c|}{ 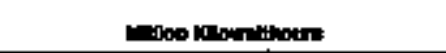 } & \\
\hline 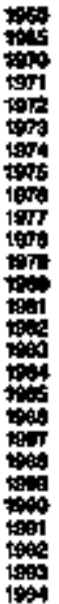 & 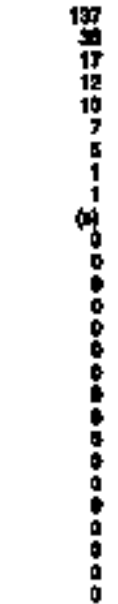 & 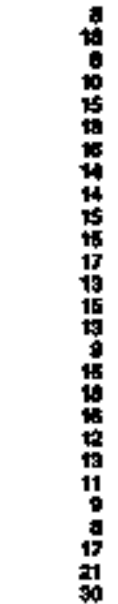 & 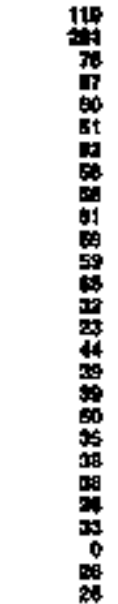 & 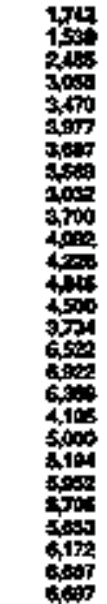 & 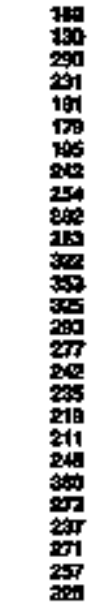 & 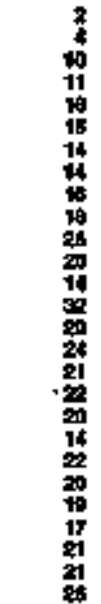 & 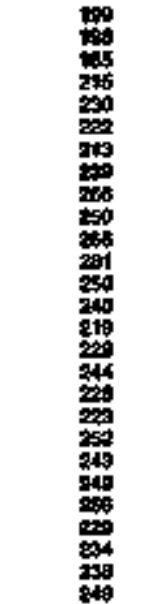 & 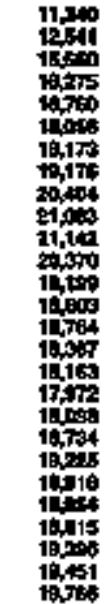 & $\begin{array}{l}3 \\
5 \\
: \\
0 \\
0 \\
0 \\
0 \\
0 \\
: \\
0 \\
0 \\
0 \\
0 \\
0 \\
0 \\
0 \\
0 \\
0 \\
0\end{array}$ & 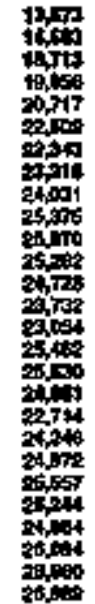 & 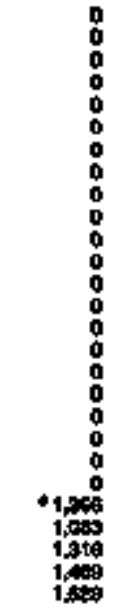 & $\begin{array}{l}0 \\
0 \\
0 \\
0 \\
0 \\
0 \\
0 \\
0 \\
0 \\
0 \\
0 \\
0 \\
0 \\
0 \\
0 \\
0 \\
0 \\
0 \\
0 \\
0 \\
0 \\
0 \\
0 \\
0 \\
0 \\
0 \\
0 \\
0 \\
0\end{array}$ & $\begin{array}{l}= \\
z \\
z \\
z \\
= \\
= \\
= \\
z \\
= \\
z \\
z \\
z \\
z \\
z \\
z\end{array}$ & $\begin{array}{l}0 \\
0 \\
0 \\
0 \\
0 \\
0 \\
0 \\
0 \\
0 \\
0 \\
0 \\
0 \\
0 \\
0 \\
0 \\
0 \\
0 \\
0 \\
0 \\
0 \\
0 \\
0 \\
0 \\
0 \\
0 \\
0 \\
0 \\
0\end{array}$ & $\begin{array}{l}z \\
z \\
z \\
z \\
z \\
z \\
= \\
= \\
z \\
z \\
z \\
z \\
z \\
z\end{array}$ \\
\hline
\end{tabular}

\begin{tabular}{|c|c|c|c|c|c|c|c|c|c|c|c|c|c|c|c|}
\hline 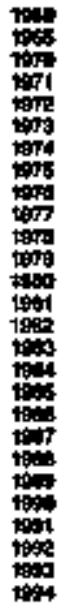 & 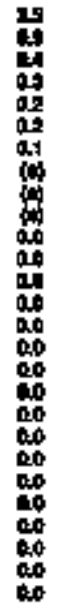 & 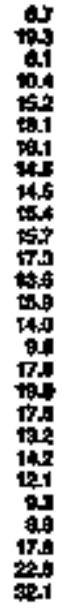 & 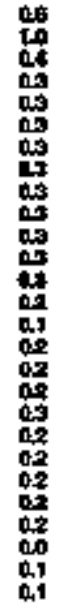 & 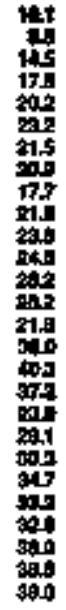 & 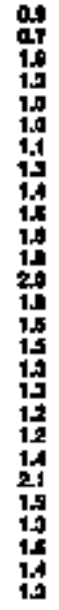 & $\begin{array}{l}y \\
g \\
y \\
0.1 \\
0.1 \\
0.1 \\
0.1 \\
0.1 \\
0.1 \\
0.1 \\
0.1 \\
0.1 \\
0.1 \\
0.1 \\
0.1 \\
0.1 \\
0.1 \\
0.1 \\
0.1 \\
0.1 \\
0.1 \\
0.1 \\
0.1 \\
0.1 \\
0.1 \\
0.1 \\
0.1\end{array}$ & 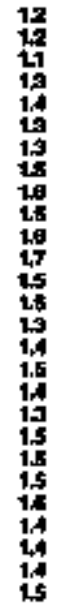 & 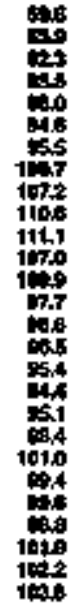 & $\begin{array}{l}60 \\
00 \\
000 \\
00 \\
00 \\
00 \\
00 \\
00 \\
00 \\
00 \\
00 \\
00 \\
00 \\
00 \\
00 \\
00 \\
00 \\
00 \\
00 \\
00 \\
00 \\
00 \\
00 \\
00 \\
00\end{array}$ & 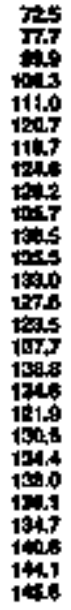 & 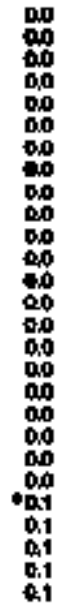 & $\begin{array}{l}00 \\
000 \\
000 \\
000 \\
000 \\
000 \\
000 \\
00 \\
00 \\
00 \\
00 \\
00 \\
00 \\
00 \\
00 \\
000 \\
00 \\
00 \\
00 \\
00 \\
00 \\
00 \\
00 \\
00 \\
00 \\
00\end{array}$ & 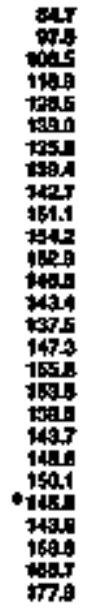 & 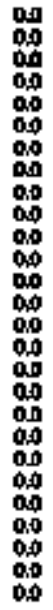 & 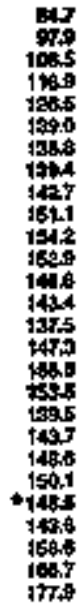 \\
\hline
\end{tabular}

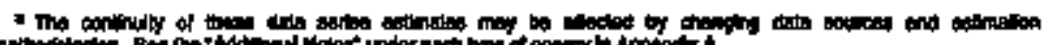

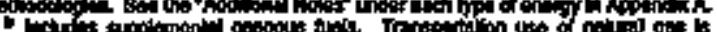

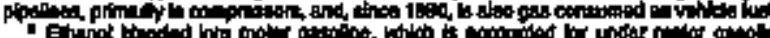

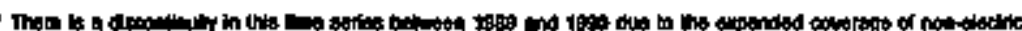

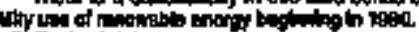

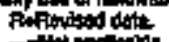

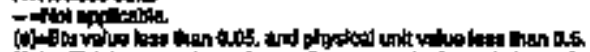

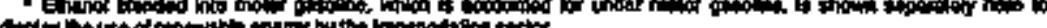

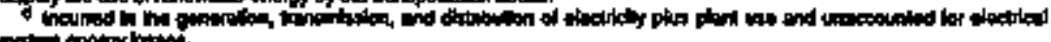


Tablo 310. Estimatos of Energy Input at Electrle Utintles, 1960, 1985, 1970-1994, West Virginle

\begin{tabular}{|c|c|c|c|c|c|c|c|c|c|c|c|c|c|c|}
\hline \multirow[b]{3}{*}{ Yes] } & \multicolumn{3}{|c|}{ 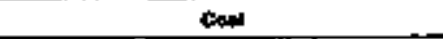 } & \multirow{3}{*}{ 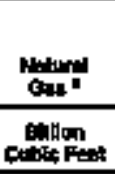 } & \multicolumn{4}{|c|}{ Potroten } & \multirow[b]{2}{*}{ 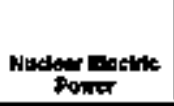 } & \multirow[b]{2}{*}{ 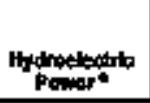 } & \multirow[b]{2}{*}{ Elotheds } & \multirow[b]{2}{*}{ 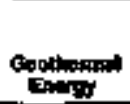 } & \multirow[b]{2}{*}{ olure to d } & \multirow[b]{3}{*}{$T$} \\
\hline & 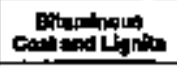 & Anhade & Total & & theng & 㟧的. & mromen & Total & & & & & & \\
\hline & \multicolumn{3}{|c|}{ 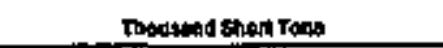 } & & \multicolumn{4}{|c|}{ 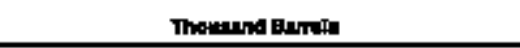 } & \multicolumn{5}{|c|}{ 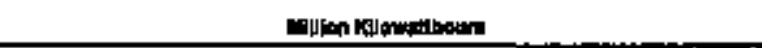 } & \\
\hline 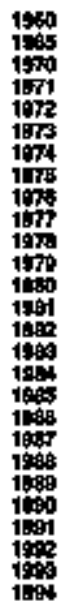 & 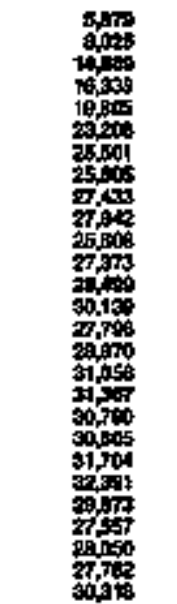 & $\begin{array}{l}: \\
: \\
0 \\
0 \\
: \\
: \\
: \\
: \\
: \\
: \\
: \\
0 \\
0 \\
0\end{array}$ & 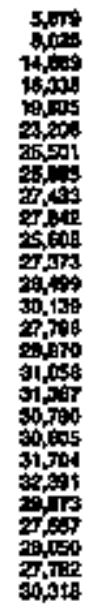 & 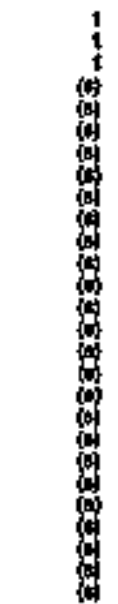 & 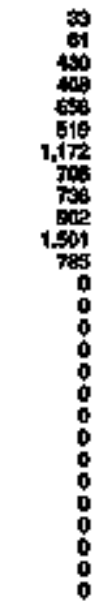 & 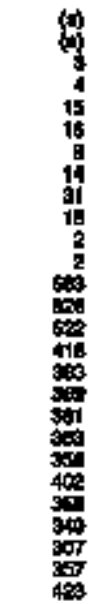 & $\begin{array}{l}0 \\
0 \\
0 \\
0 \\
0 \\
0 \\
0 \\
0 \\
0 \\
0 \\
0 \\
0 \\
0 \\
0 \\
0 \\
0 \\
0 \\
0 \\
0 \\
0 \\
0 \\
0 \\
0 \\
0 \\
0 \\
0 \\
0\end{array}$ & 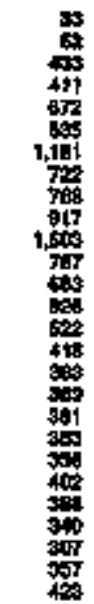 & $\begin{array}{l}0 \\
0 \\
0 \\
0 \\
0 \\
0 \\
0 \\
0 \\
0 \\
0 \\
0 \\
0 \\
0 \\
0 \\
0 \\
0 \\
0 \\
0 \\
0 \\
0 \\
0 \\
0 \\
0 \\
0\end{array}$ & 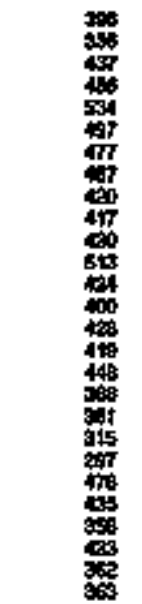 & $\begin{array}{l}0 \\
0 \\
0 \\
0 \\
0 \\
0 \\
0 \\
0 \\
0 \\
0 \\
0 \\
0 \\
0 \\
0 \\
0 \\
0 \\
0 \\
0 \\
0 \\
0 \\
0 \\
0 \\
0\end{array}$ & $\begin{array}{l}0 \\
0 \\
0 \\
0 \\
0 \\
0 \\
0 \\
0 \\
0 \\
0 \\
0 \\
0 \\
0 \\
0 \\
0 \\
0 \\
0 \\
0 \\
0 \\
0 \\
0 \\
0 \\
0 \\
0\end{array}$ & $\begin{array}{l}1 \\
0 \\
0 \\
0 \\
0 \\
0 \\
0 \\
0 \\
0 \\
0 \\
0 \\
0 \\
0 \\
0 \\
0 \\
0 \\
0 \\
0 \\
0 \\
0 \\
0 \\
0 \\
0 \\
0\end{array}$ & 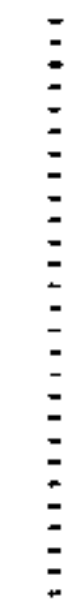 \\
\hline
\end{tabular}

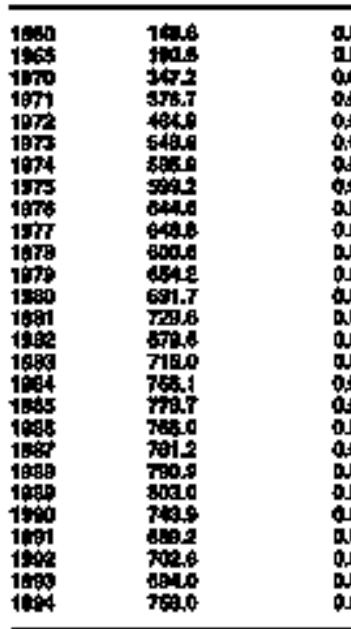

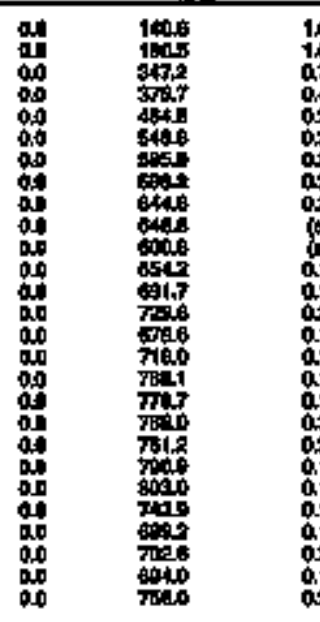

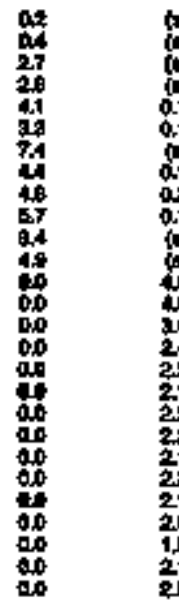

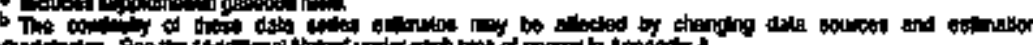

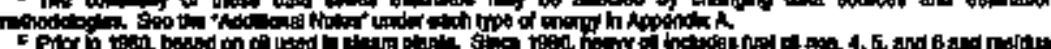

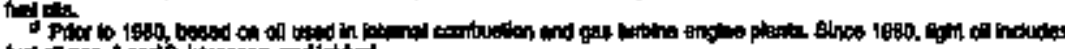

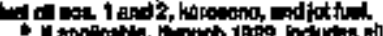

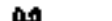

\begin{tabular}{|c|c|c|c|c|c|c|}
\hline 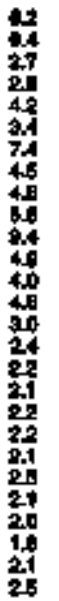 & 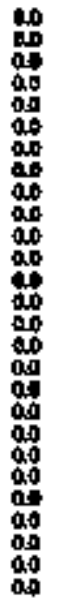 & 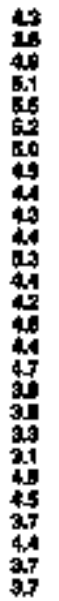 & 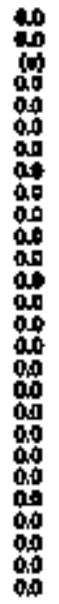 & 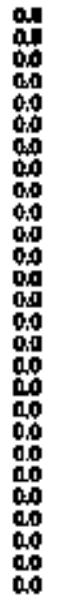 & 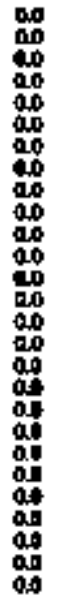 & 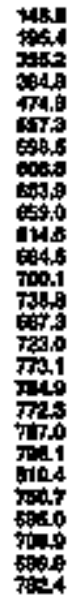 \\
\hline
\end{tabular}

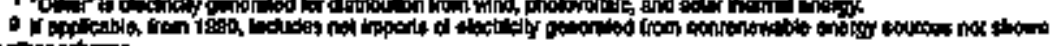
$-\infty$ tion

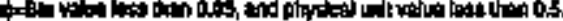

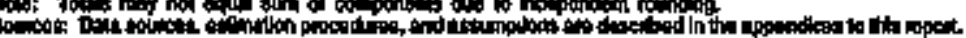

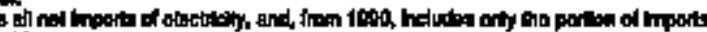


Tabla 311. Energy Consumption Estimates by Source, 1950, 1965, t970-1984, Wisconsin

\begin{tabular}{|c|c|c|c|c|c|c|c|c|c|c|c|c|c|c|c|c|c|c|c|}
\hline \multirow{2}{*}{\multicolumn{2}{|c|}{$\cos =$}} & \multirow[b]{2}{*}{ 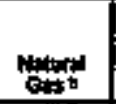 } & \multicolumn{11}{|c|}{ Pestrolas } & \multirow{2}{*}{ 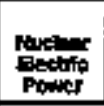 } & \multirow{2}{*}{ 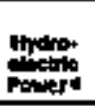 } & \multirow[b]{2}{*}{ Bhofmestse } & \multirow[b]{2}{*}{ Other on } & \multirow{2}{*}{ retinger } & \multirow[b]{3}{*}{ Totalh } \\
\hline & & & 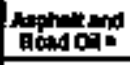 & | & 1. & Find. & $\operatorname{lom}$ & Les. & tontr. & Untror & Fintum & aner & Toter & & & & & & \\
\hline Ya: & Thoof tod & ching & \multicolumn{11}{|c|}{ 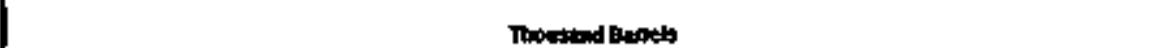 } & \multicolumn{5}{|c|}{ 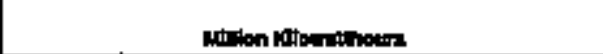 } & \\
\hline
\end{tabular}

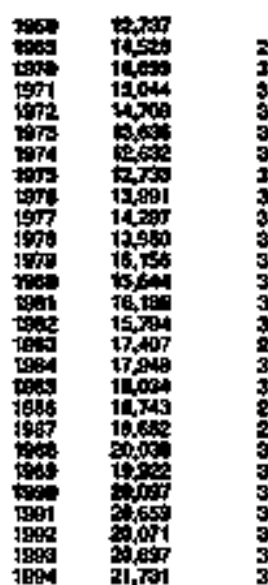

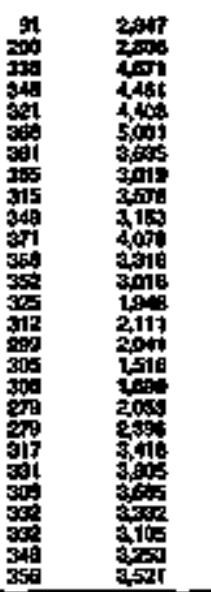

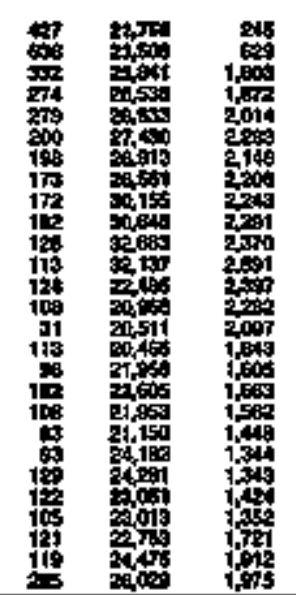

\begin{tabular}{|c|}
\hline 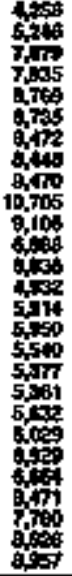 \\
\hline
\end{tabular}

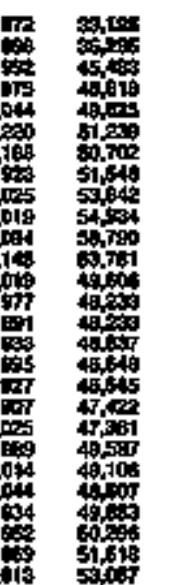

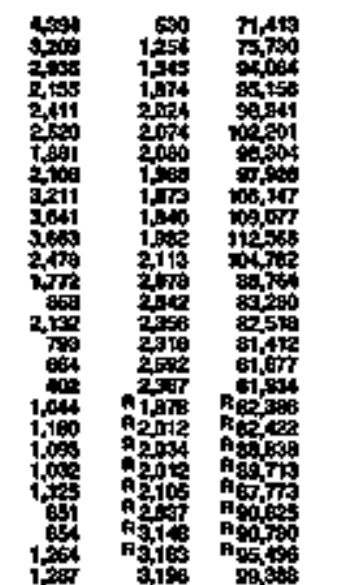

TH:日and

\begin{tabular}{|c|c|c|c|c|c|c|c|c|c|c|c|c|c|c|c|c|c|c|c|}
\hline 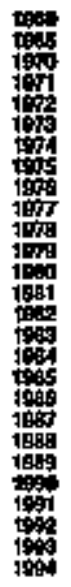 & 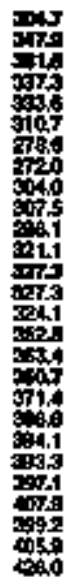 & 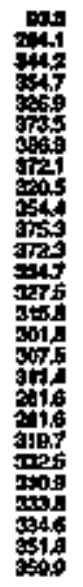 & 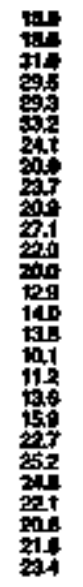 & 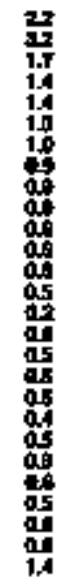 & 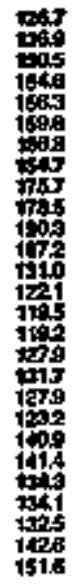 & 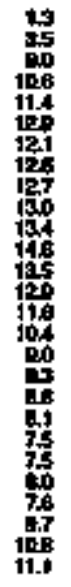 & 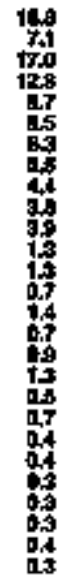 & 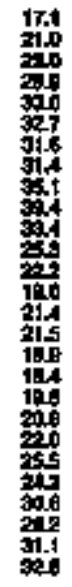 & 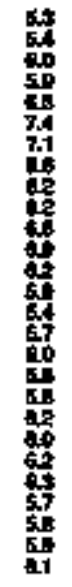 & 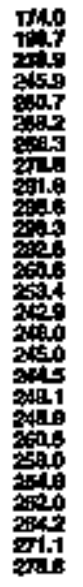 & 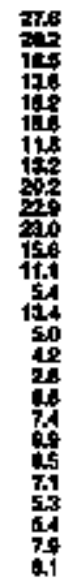 & 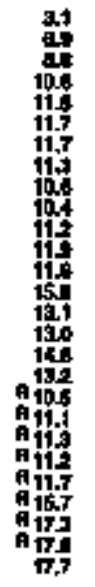 & 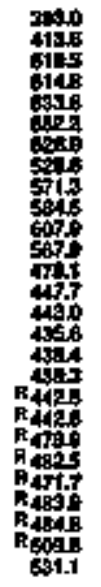 & 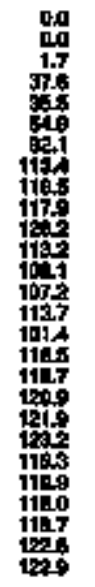 & 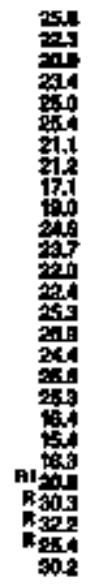 & 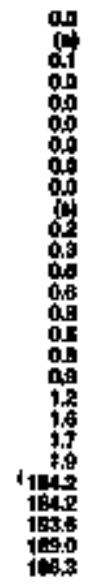 & 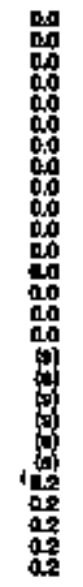 & 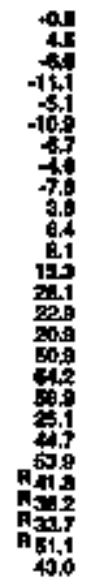 & 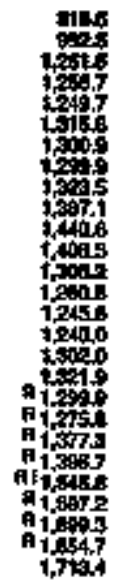 \\
\hline
\end{tabular}

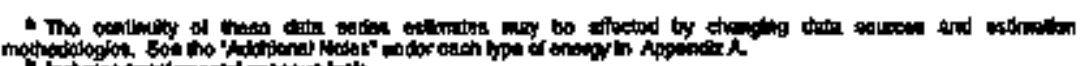

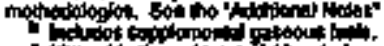

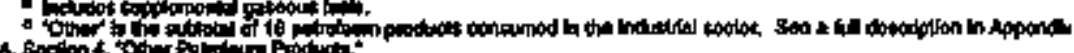

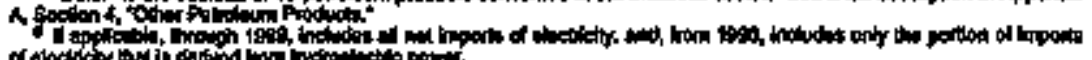

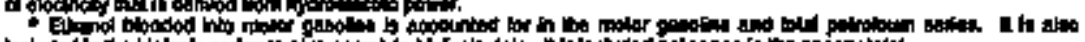

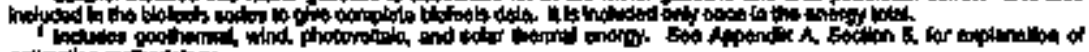

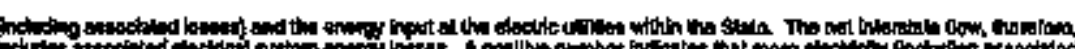

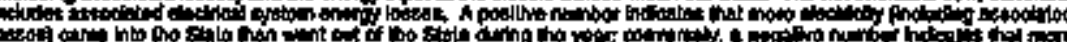

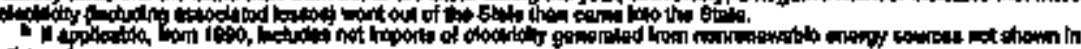
Hor cotimn:

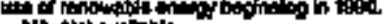

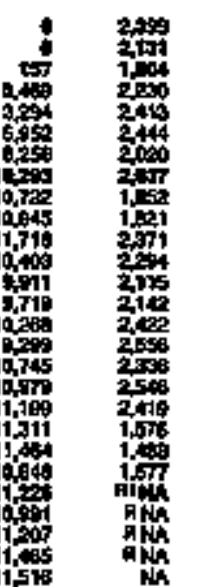

要

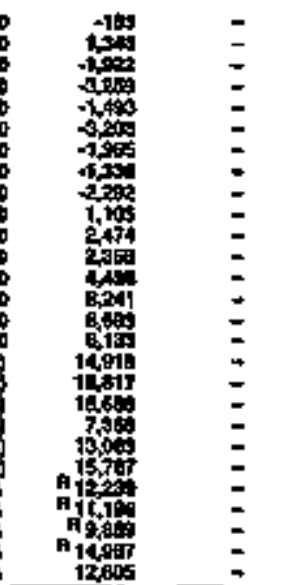

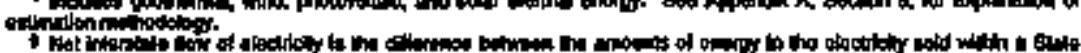

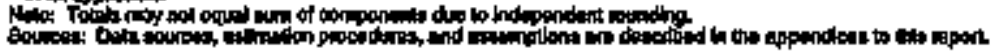


Table 312. Pesldential Energy Consumption Estimates, 1960, 1965, 1970-1994, Wisconsin

\begin{tabular}{|c|c|c|c|c|c|c|c|c|c|c|c|c|c|c|}
\hline \multirow[b]{3}{*}{ Yoar } & \multicolumn{3}{|c|}{ Conl } & \multirow{3}{*}{ 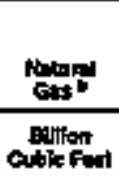 } & & Put: & & & \multirow{3}{*}{ 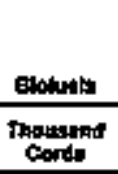 } & & \multirow[b]{2}{*}{ Excritity" } & \multirow[b]{2}{*}{ Entry } & \multirow{3}{*}{ 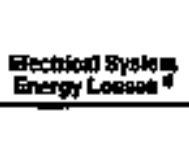 } & \multirow[b]{3}{*}{ Told } \\
\hline & 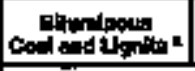 & Anthella & Totul & & 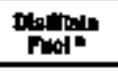 & Kerowone & LOOA & Tot I I & & Solato & & & & \\
\hline & \multicolumn{3}{|c|}{ Theosund Shoot Tome } & & \multicolumn{4}{|c|}{ Thoughed Ganrals } & & \multicolumn{3}{|c|}{ 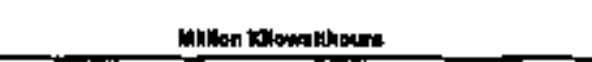 } & & \\
\hline 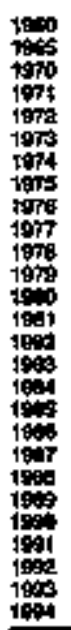 & 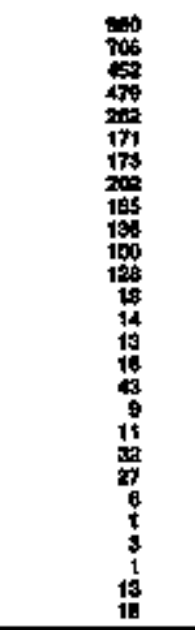 & 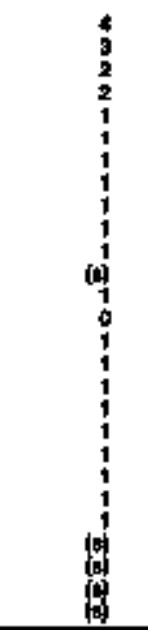 & 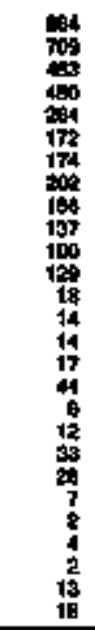 & 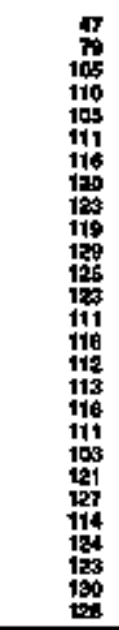 & 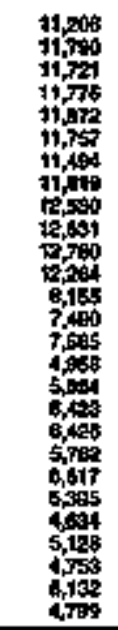 & 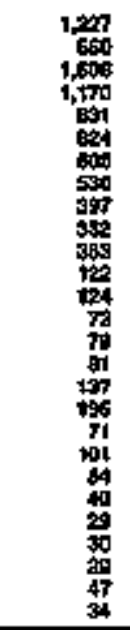 & 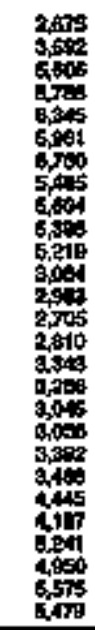 & 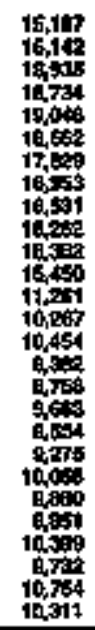 & 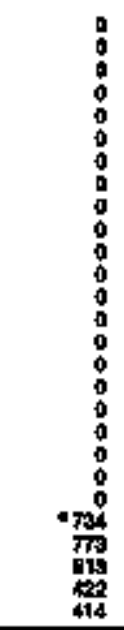 & 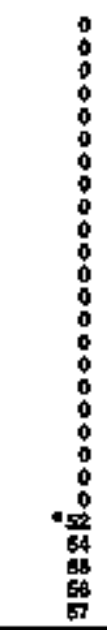 & 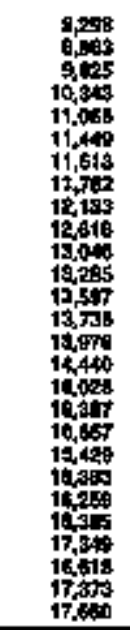 & $\begin{array}{l}= \\
= \\
= \\
= \\
z \\
= \\
= \\
= \\
z \\
z \\
z \\
z \\
z \\
z\end{array}$ & 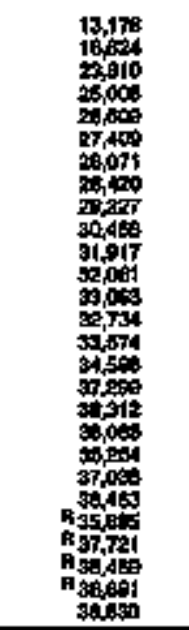 & $\begin{array}{l}= \\
z \\
= \\
z \\
= \\
= \\
z \\
z \\
z \\
z \\
z \\
z \\
z \\
z\end{array}$ \\
\hline \multicolumn{15}{|c|}{ Triluon Bu } \\
\hline 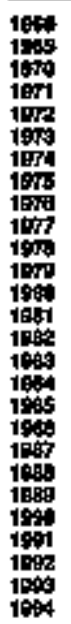 & 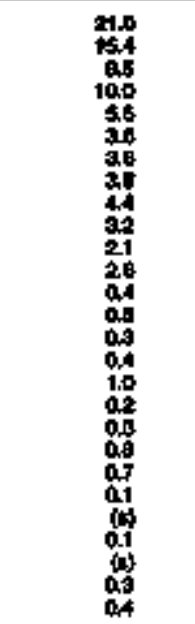 & 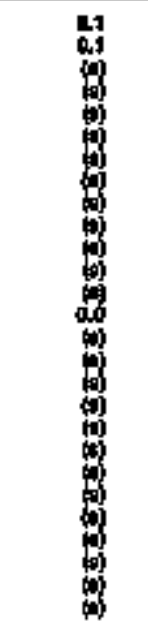 & 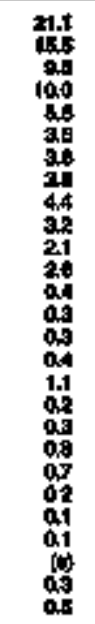 & 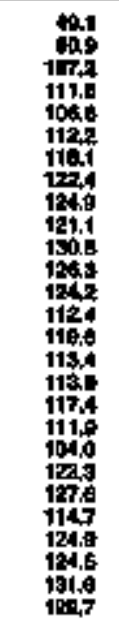 & 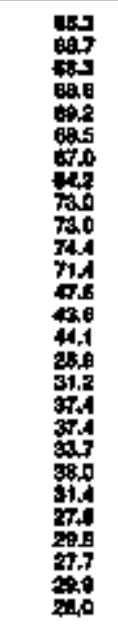 & 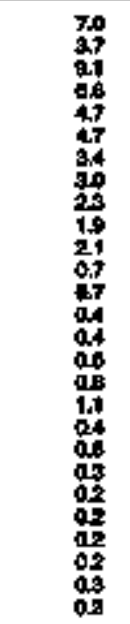 & 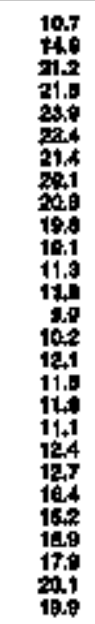 & 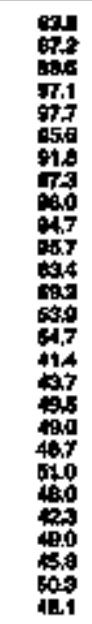 & 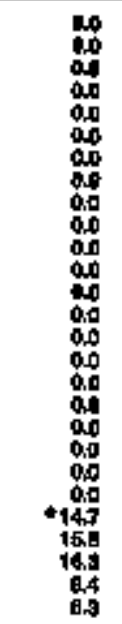 & 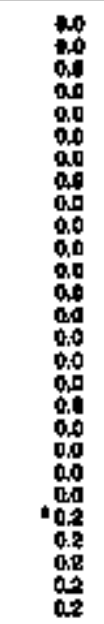 & 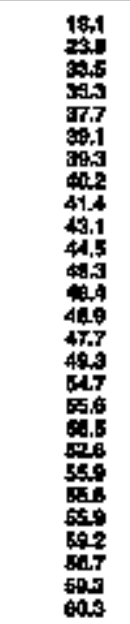 & 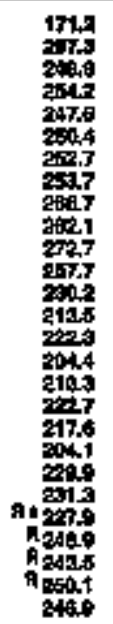 & 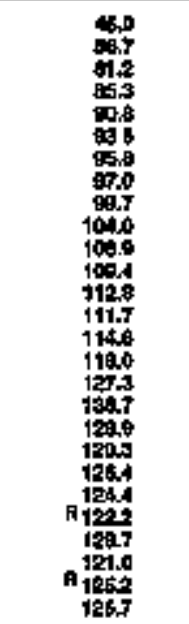 & 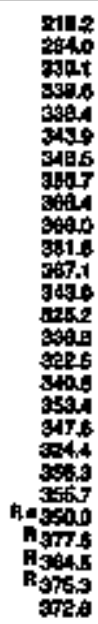 \\
\hline
\end{tabular}

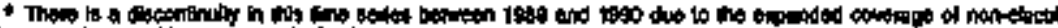

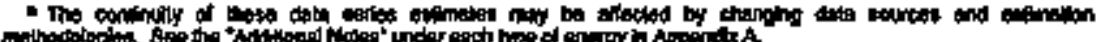

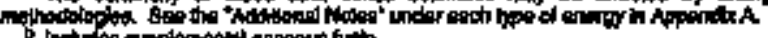

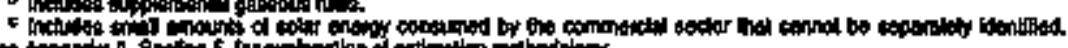

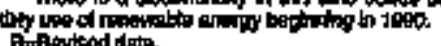

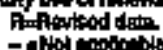

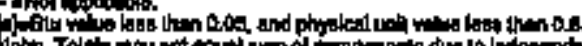

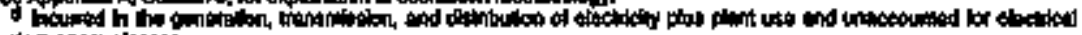

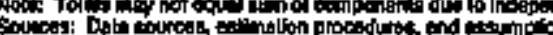

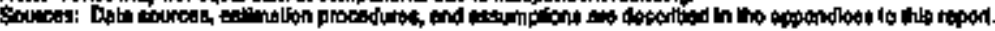

Energy Infommation Atminlotrailon 
Table 313. Comimarcial Energy Consumptlon Estimates, 1960, 1965, 1970-1994, Wisconsin

\begin{tabular}{|c|c|c|c|c|c|c|c|c|c|c|c|c|c|c|}
\hline \multirow[b]{3}{*}{$\operatorname{mex}$} & \multicolumn{3}{|c|}{ וas } & \multirow{3}{*}{ 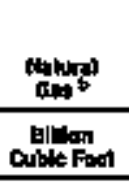 } & \multicolumn{6}{|c|}{ Potridex: } & \multirow[b]{2}{*}{ Eloctridty" } & \multirow[b]{2}{*}{ Enty } & \multirow[b]{2}{*}{ 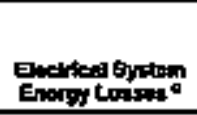 } & \multirow[b]{3}{*}{ Tota: } \\
\hline & 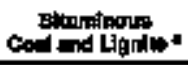 & Anthraches : & Totat & & Dallapho & Korotene $=$ & LPG* & Notion & Finolduil & Totol & & & & \\
\hline & \multicolumn{3}{|c|}{ Thorund Stard Toos: } & & \multicolumn{6}{|c|}{ Theorind Bumln } & \multicolumn{3}{|c|}{ Mon Krismathatas } & \\
\hline 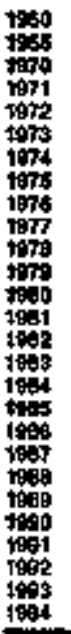 & 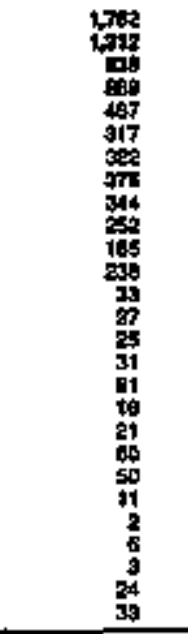 & 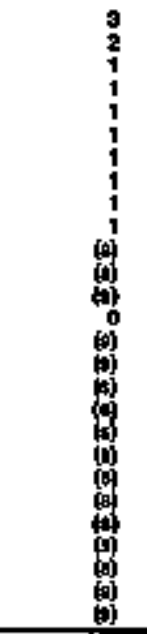 & 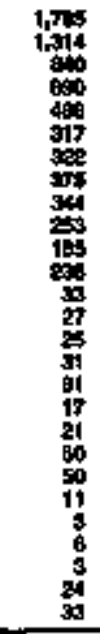 & 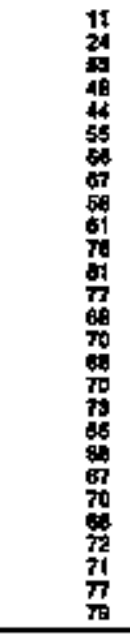 & 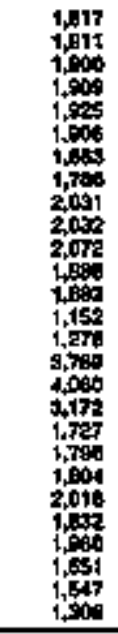 & 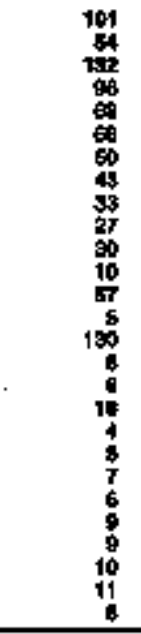 & 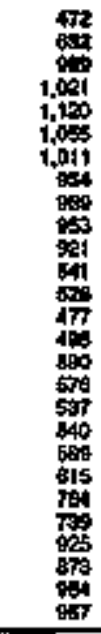 & 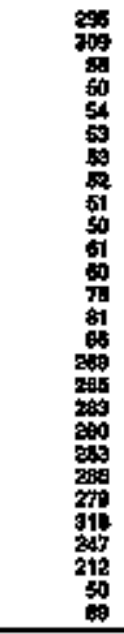 & 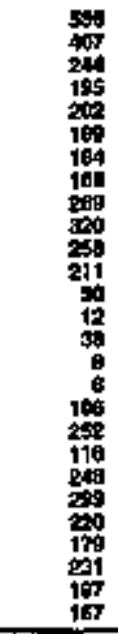 & 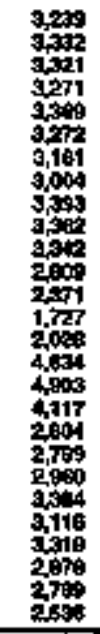 & 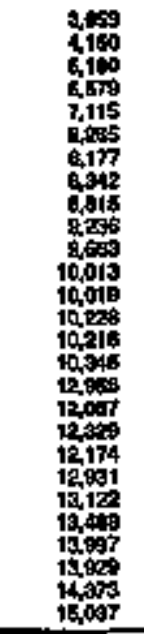 & 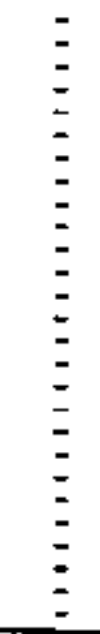 & 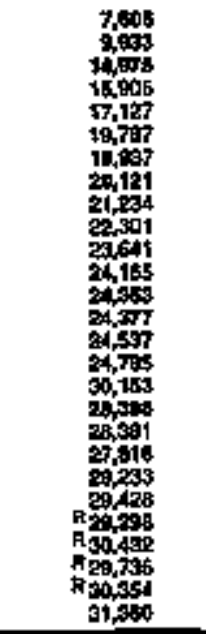 & $\begin{array}{l}= \\
= \\
= \\
= \\
= \\
= \\
= \\
= \\
= \\
= \\
= \\
= \\
= \\
= \\
\end{array}$ \\
\hline \multicolumn{15}{|c|}{ Thtion nitu } \\
\hline 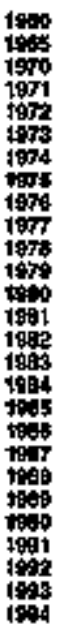 & 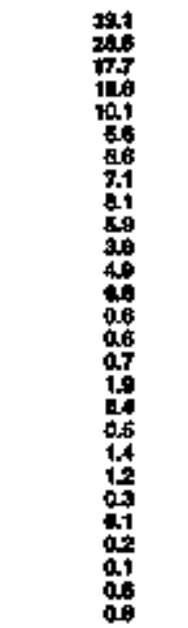 & 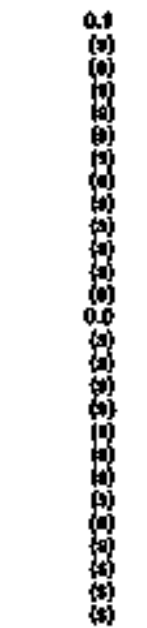 & 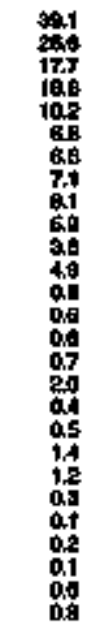 & 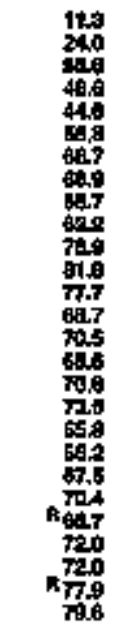 & 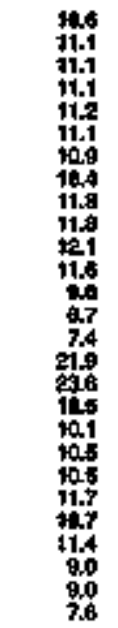 & 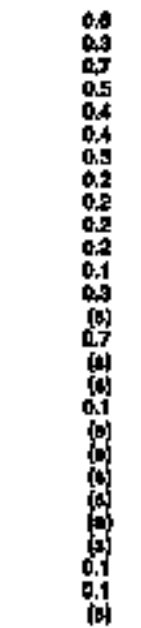 & 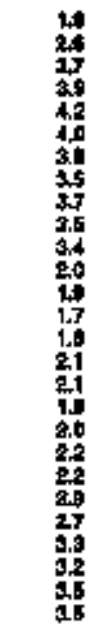 & $\begin{array}{l}1.3 \\
1.5 \\
0.4 \\
0.3 \\
0.3 \\
0.3 \\
0.3 \\
0.3 \\
0.3 \\
0.3 \\
0.3 \\
0.4 \\
0.4 \\
04 \\
0.5 \\
1.4 \\
1.5 \\
1.8 \\
1.5 \\
1.5 \\
1.5 \\
1.5 \\
1.1 \\
0.3 \\
0.5\end{array}$ & 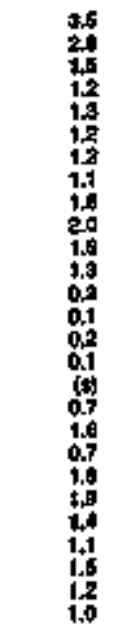 & 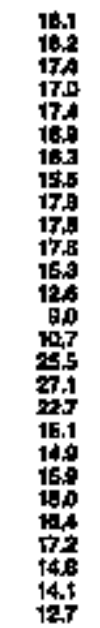 & 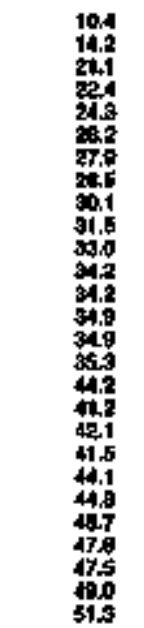 & 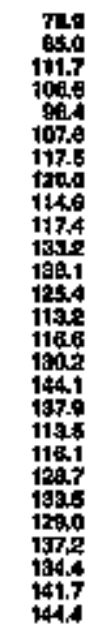 & 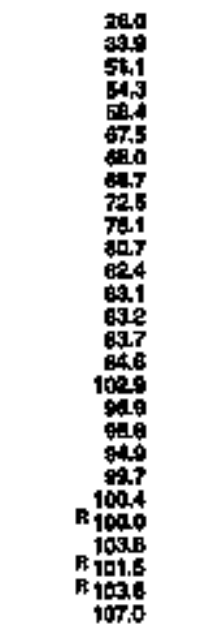 & 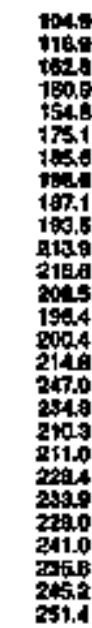 \\
\hline & 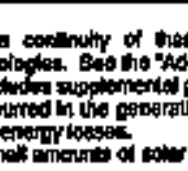 & do: & in & Iflactod & oing & & & 50 & rton. & & & 10 & 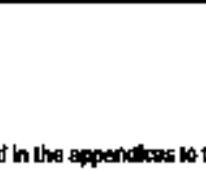 & \\
\hline
\end{tabular}

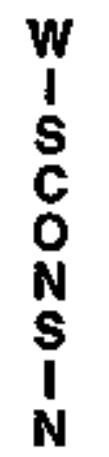


Tabie 314, Industrial Energy Cansumption Estimates, 1660, 1965, 1970-1994, Wroconsin

\begin{tabular}{|c|c|c|c|c|c|c|c|c|c|c|c|c|c|c|c|c|}
\hline \multirow[b]{2}{*}{$\cos$} & \multirow[b]{2}{*}{ intant } & \multicolumn{9}{|c|}{ 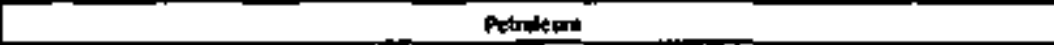 } & \multirow[b]{2}{*}{ Domb } & \multirow[b]{2}{*}{ 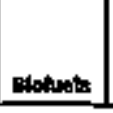 } & \multirow[b]{2}{*}{ ond } & \multirow[b]{2}{*}{ Geotindy' } & \multirow[b]{2}{*}{$\max _{x \rightarrow 0}$} & \multirow{2}{*}{ 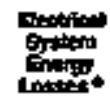 } \\
\hline & & 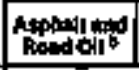 & 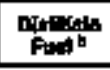 & Mansune : & LPab & Luthomest & 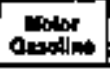 & Rostant & Onerde & tom & & & & & & \\
\hline Mnomed & 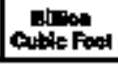 & \multicolumn{9}{|c|}{ 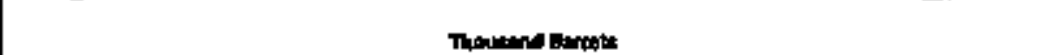 } & \multicolumn{6}{|c|}{ 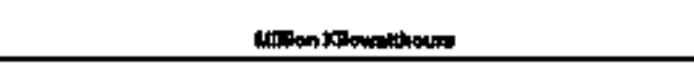 } \\
\hline
\end{tabular}

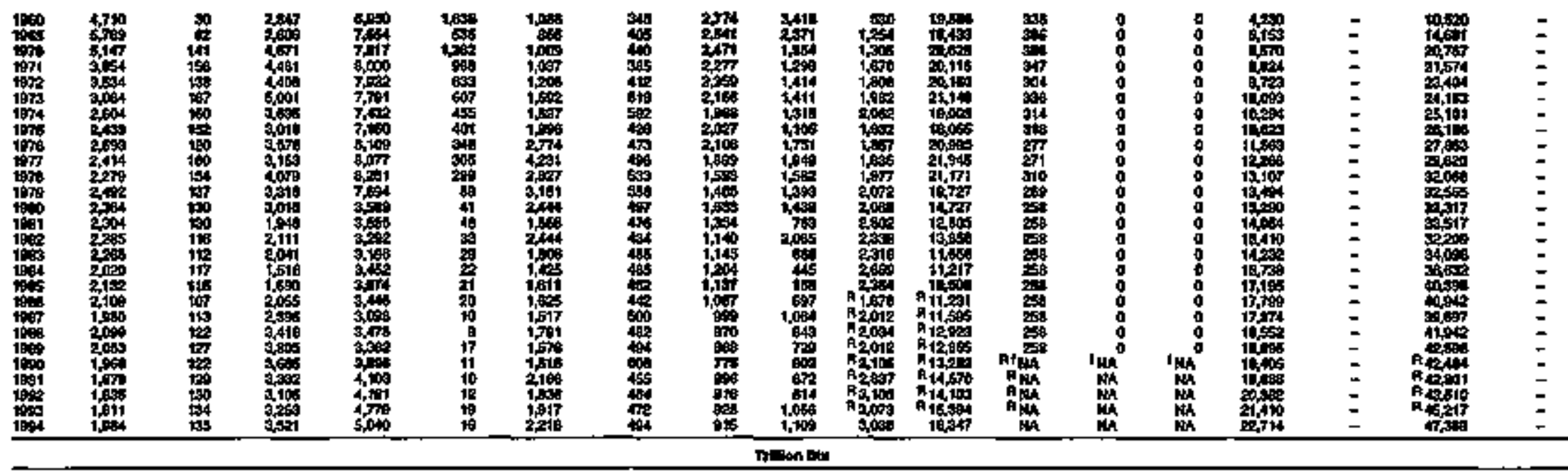

\begin{tabular}{|c|c|c|}
\hline & 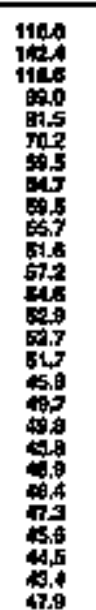 & 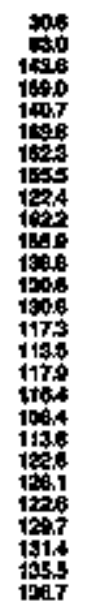 \\
\hline
\end{tabular}

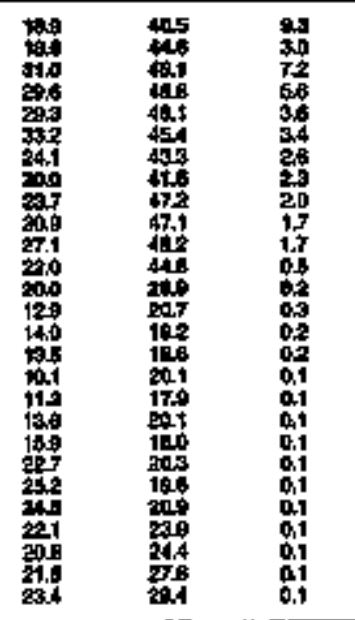

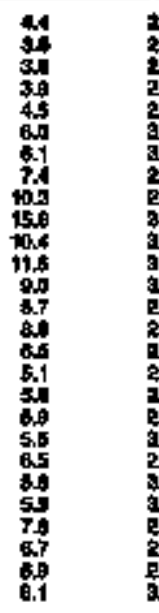

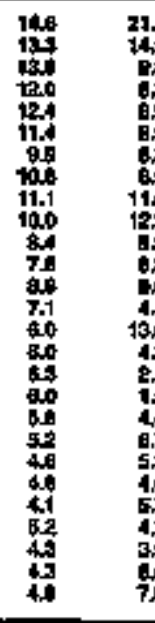

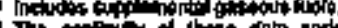
1.

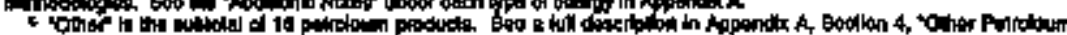
Fredeti:

\begin{tabular}{|c|c|c|c|c|c|c|c|c|}
\hline 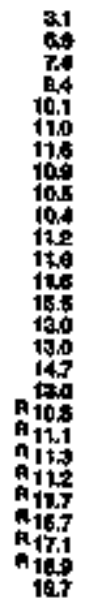 & 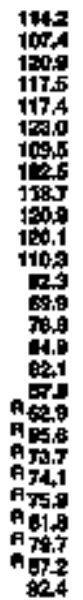 & 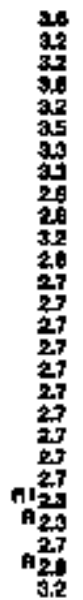 & 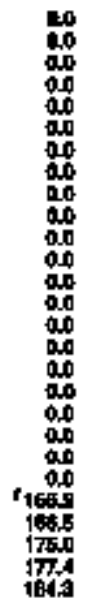 & 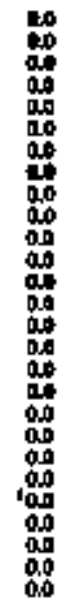 & 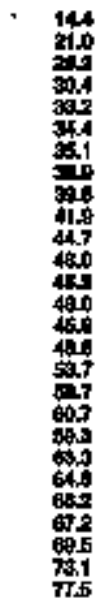 & 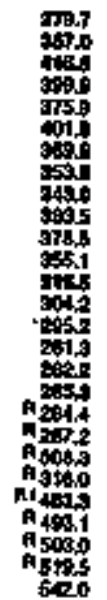 & 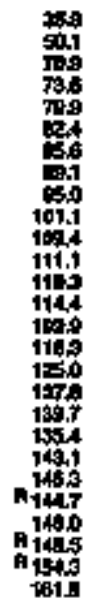 & 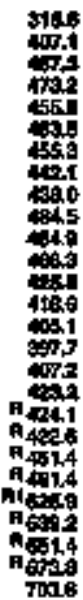 \\
\hline
\end{tabular}

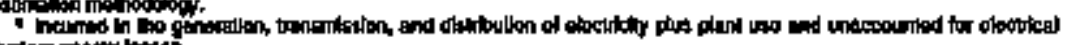

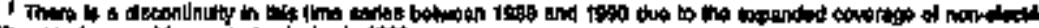

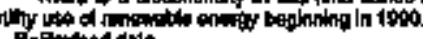

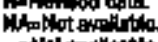

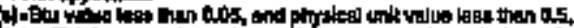

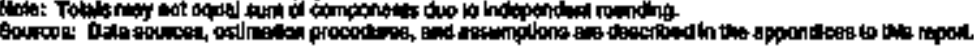


Table 315. Transportation Energy Corsumption Estimates, 1980, 1965, 1970-1994, Wisconsin

\begin{tabular}{|c|c|c|c|c|c|c|c|c|c|c|c|c|c|c|c|}
\hline \multirow[b]{3}{*}{$\mathrm{Yen}$} & \multirow[b]{2}{*}{ 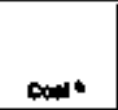 } & \multirow[b]{2}{*}{ 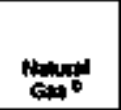 } & \multicolumn{8}{|c|}{ 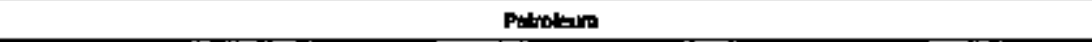 } & \multirow{3}{*}{ 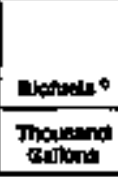 } & \multirow[b]{2}{*}{ 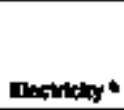 } & \multirow[b]{2}{*}{ thent } & \multirow[b]{2}{*}{ encrionsyntery } & \multirow[b]{3}{*}{ Tated } \\
\hline & & & Antillow. & 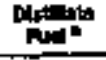 & twe & $\mathbf{H a n}$ & 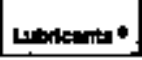 & antion & 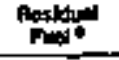 & Totes & & & & & \\
\hline & Thooting & 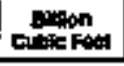 & \multicolumn{8}{|c|}{ 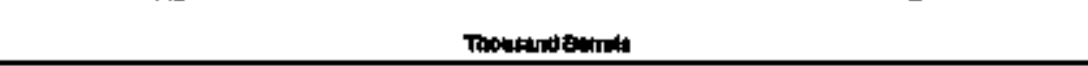 } & & \multicolumn{3}{|c|}{ 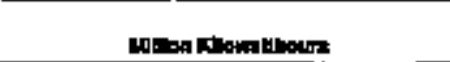 } & \\
\hline 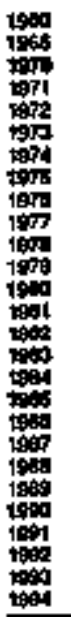 & $\begin{array}{l}0 \\
10 \\
5 \\
1 \\
2 \\
1 \\
0 \\
0 \\
0 \\
0 \\
0 \\
0 \\
0 \\
0 \\
0 \\
0 \\
0 \\
0 \\
0 \\
0 \\
0 \\
0 \\
0\end{array}$ & $\begin{array}{l}1 \\
2 \\
7 \\
6 \\
6 \\
5 \\
5 \\
5 \\
2 \\
2 \\
2 \\
4 \\
5 \\
4 \\
4 \\
3 \\
3 \\
4 \\
4 \\
4 \\
4 \\
4 \\
4 \\
4 \\
0\end{array}$ & 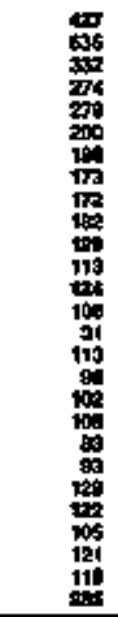 & 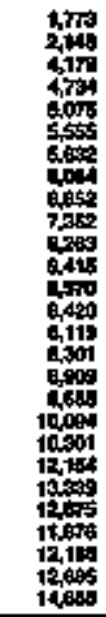 & 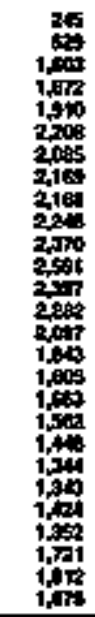 & 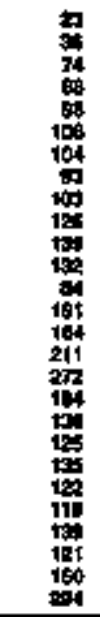 & 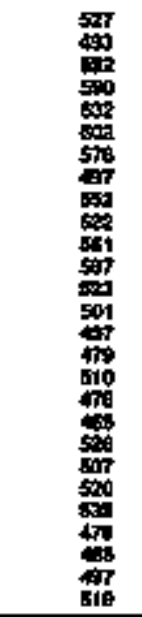 & 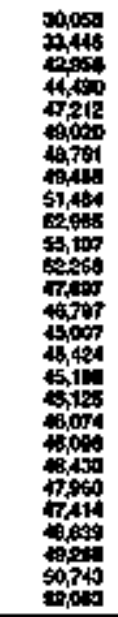 & 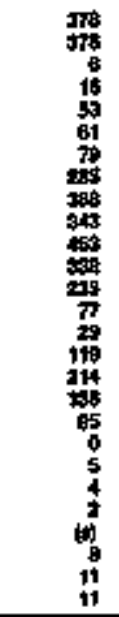 & 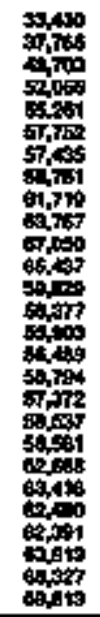 & 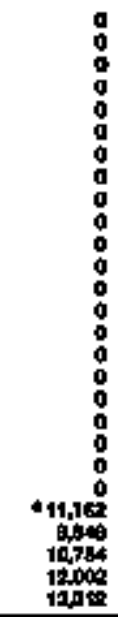 & $\begin{array}{l}0 \\
0 \\
0 \\
0 \\
0 \\
0 \\
0 \\
0 \\
0 \\
0 \\
0 \\
0 \\
0 \\
0 \\
0 \\
0 \\
0 \\
0 \\
0 \\
0 \\
0 \\
0 \\
0 \\
0\end{array}$ & $\begin{array}{l}= \\
z \\
z \\
= \\
z \\
= \\
= \\
= \\
= \\
= \\
= \\
= \\
=\end{array}$ & $\begin{array}{l}0 \\
0 \\
0 \\
0 \\
0 \\
0 \\
0 \\
0 \\
0 \\
0 \\
0 \\
0 \\
0 \\
0 \\
0 \\
0 \\
0 \\
0 \\
0 \\
0 \\
0 \\
0 \\
0 \\
0\end{array}$ & 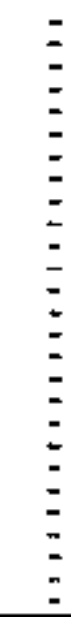 \\
\hline & & & & & & & & 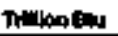 & & & & & & & \\
\hline
\end{tabular}

\begin{tabular}{|c|c|c|c|c|c|c|c|c|c|c|c|c|c|c|c|}
\hline 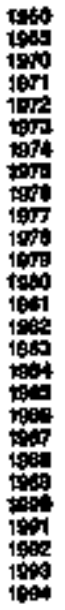 & 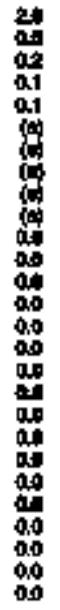 & 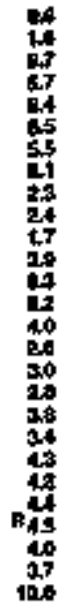 & 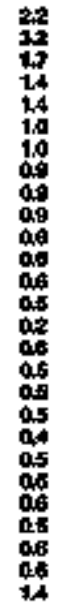 & 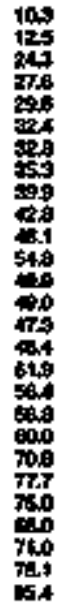 & 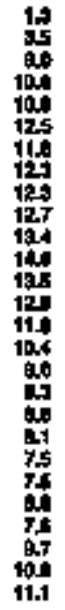 & 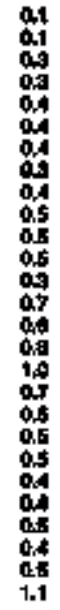 & 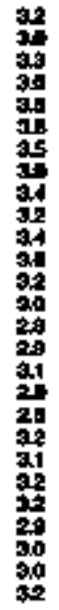 & 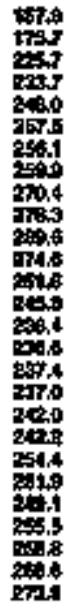 & 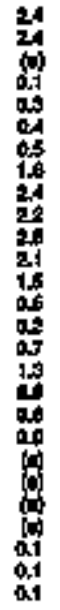 & 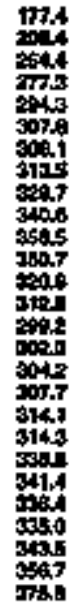 & 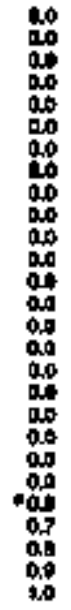 & $\begin{array}{l}00 \\
00 \\
000 \\
00 \\
00 \\
00 \\
00 \\
00 \\
00 \\
00 \\
00 \\
00 \\
00 \\
00 \\
00 \\
00 \\
00 \\
00 \\
00 \\
00 \\
00 \\
00 \\
000 \\
0.00 \\
00 \\
00\end{array}$ & 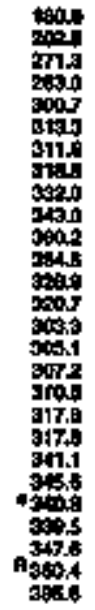 & 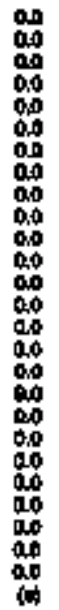 & 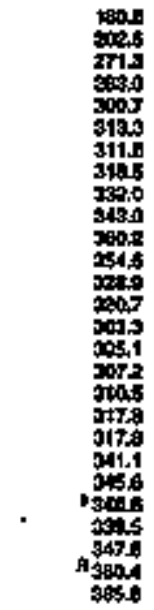 \\
\hline
\end{tabular}

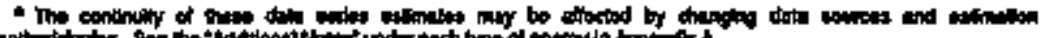

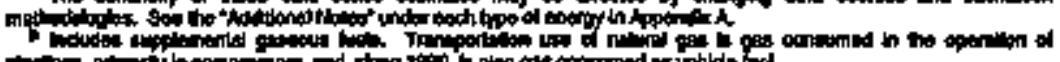

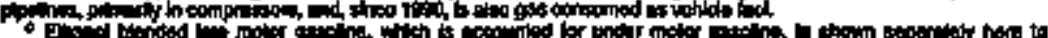

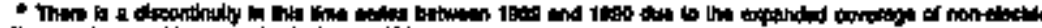

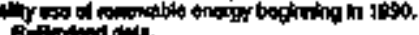

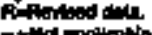

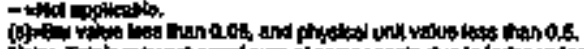

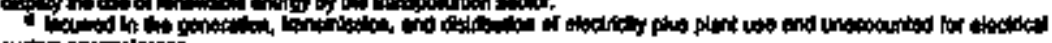

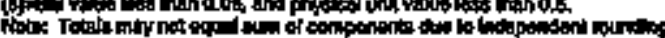

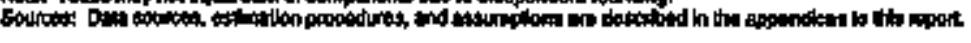




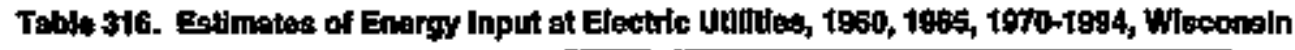

\begin{tabular}{|c|c|c|c|c|c|c|c|c|c|c|c|c|c|c|}
\hline \multirow[b]{3}{*}{ Yen } & \multicolumn{3}{|c|}{$\cos$} & \multirow{3}{*}{ 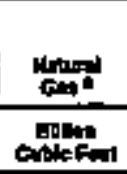 } & \multicolumn{4}{|c|}{ Patrolen } & \multirow[b]{2}{*}{ 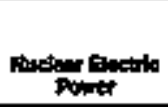 } & \multirow[b]{2}{*}{ 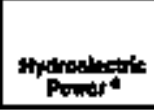 } & \multirow[b]{2}{*}{ elatents } & \multirow[b]{2}{*}{ 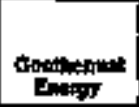 } & \multirow[b]{2}{*}{ Olwn } & \multirow[b]{3}{*}{ Tatal } \\
\hline & 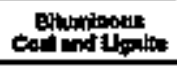 & Antanelo & Fote & & tand & 猋整 & Adrethen & Tot & & & & & & \\
\hline & \multicolumn{3}{|c|}{ 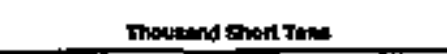 } & & \multicolumn{4}{|c|}{ Thousund Eom } & \multicolumn{5}{|c|}{ Hon followmitheires } & \\
\hline 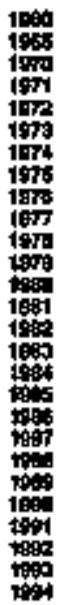 & 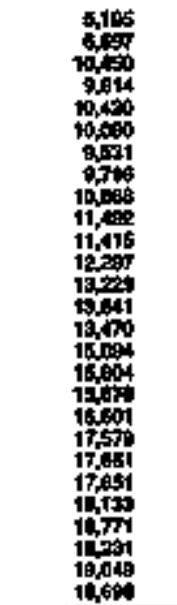 & $\begin{array}{l}0 \\
0 \\
0 \\
0 \\
0 \\
0 \\
0 \\
0 \\
0 \\
0 \\
0 \\
0 \\
0 \\
0 \\
0 \\
0 \\
0 \\
0 \\
0 \\
0 \\
0 \\
0 \\
0 \\
0 \\
0 \\
0\end{array}$ & 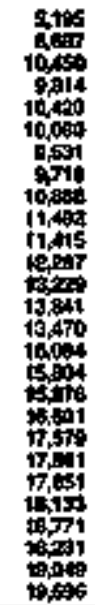 & 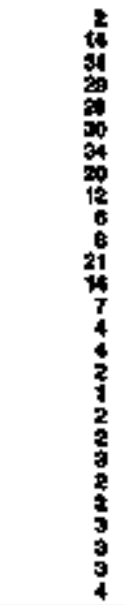 & 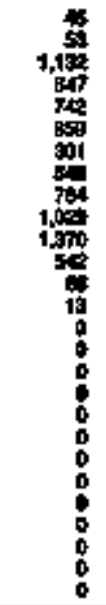 & 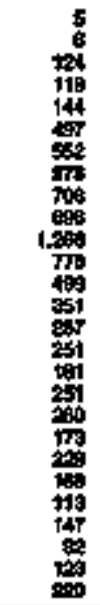 & 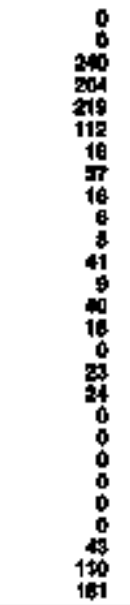 & 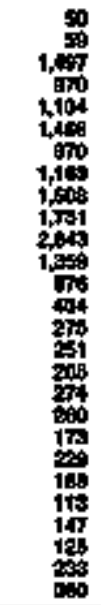 & 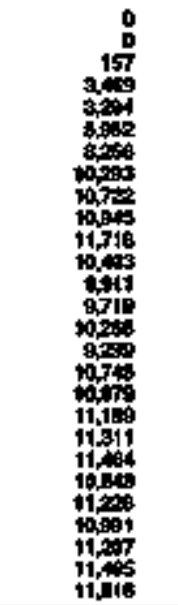 & 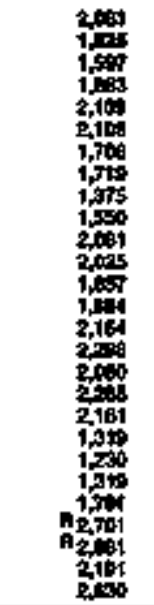 & 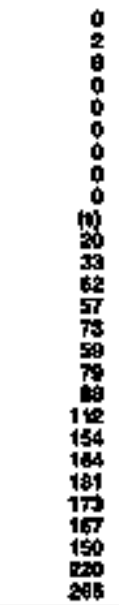 & $\begin{array}{l}0 \\
0 \\
0 \\
0 \\
0 \\
0 \\
0 \\
0 \\
0 \\
0 \\
0 \\
0 \\
0 \\
0 \\
0 \\
0 \\
0 \\
0 \\
0\end{array}$ & $\begin{array}{l}0 \\
0 \\
0 \\
0 \\
0 \\
0 \\
0 \\
0 \\
0 \\
0 \\
0 \\
0 \\
0 \\
0 \\
0 \\
0 \\
0 \\
0 \\
0 \\
0 \\
0 \\
0 \\
0 \\
0 \\
0 \\
0 \\
0 \\
0\end{array}$ & $\begin{array}{l}= \\
= \\
= \\
= \\
= \\
= \\
= \\
= \\
= \\
= \\
= \\
= \\
= \\
= \\
= \\
=\end{array}$ \\
\hline
\end{tabular}

\begin{tabular}{|c|c|c|c|c|c|c|c|c|c|c|c|c|c|c|}
\hline 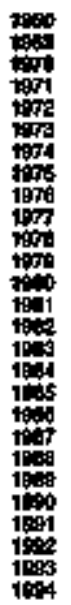 & 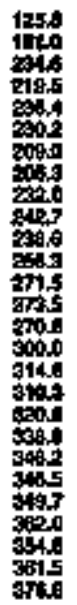 & 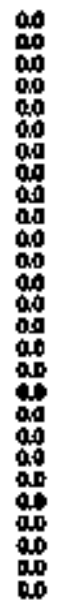 & 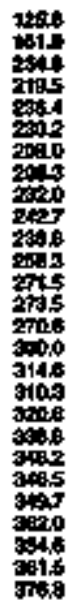 & 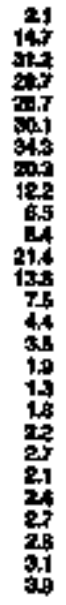 & 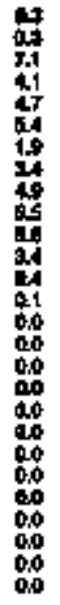 & 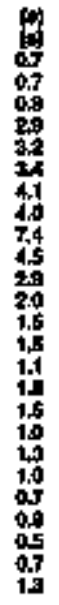 & 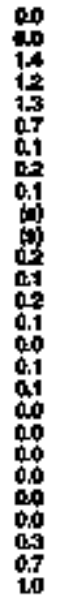 & 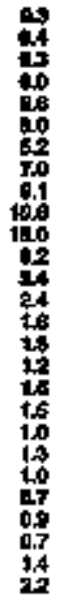 & 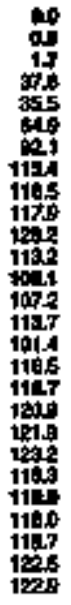 & 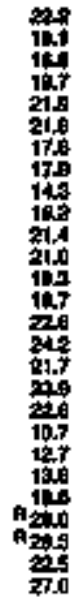 & 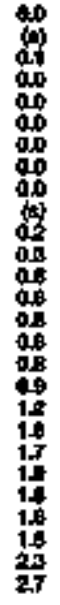 & 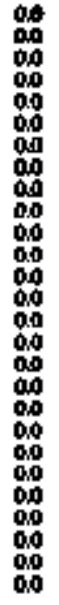 & 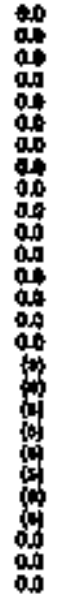 & 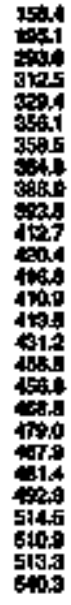 \\
\hline
\end{tabular}

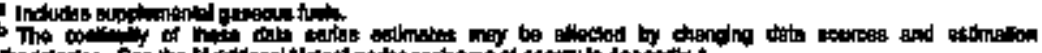

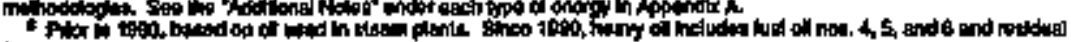

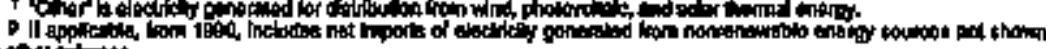

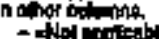

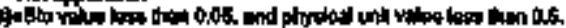

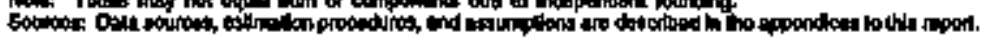

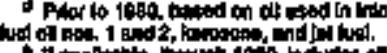

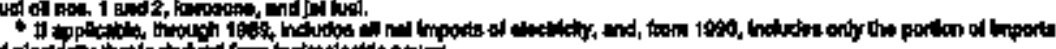


Table 317. Energy Consumplion Estimatos by Sounce, 1960, 1965, 1970-1994, Wyoming

\begin{tabular}{|c|c|c|c|c|c|c|c|c|c|c|c|c|c|c|c|c|c|c|c|}
\hline \multirow[b]{3}{*}{$\mathrm{rem}$} & \multirow[b]{2}{*}{ יםt } & \multirow[b]{2}{*}{$\frac{\operatorname{mon}}{\cos t}$} & \multicolumn{11}{|c|}{ Petrolpoun } & \multirow[b]{2}{*}{ 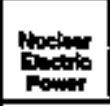 } & \multirow[b]{2}{*}{ 然 } & \multirow[b]{2}{*}{ Bimonneres } & \multirow[b]{2}{*}{ otherst } & \multirow{3}{*}{ 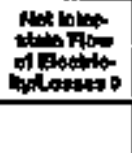 } & \multirow[b]{3}{*}{ Trobed } \\
\hline & & & 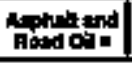 & 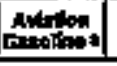 & Dention & Ave. & (nim & 2pac & chast. & Mroser & 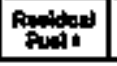 & | & Tob & & & & & & \\
\hline & Mnoventions & absersost & \multicolumn{11}{|c|}{ 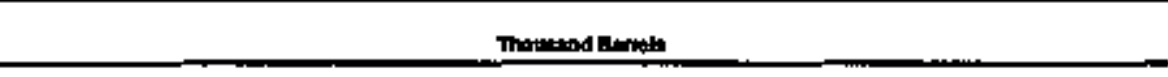 } & \multicolumn{4}{|c|}{ 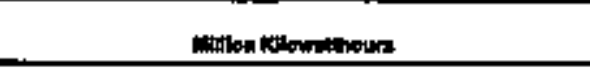 } & & \\
\hline 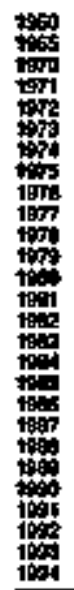 & 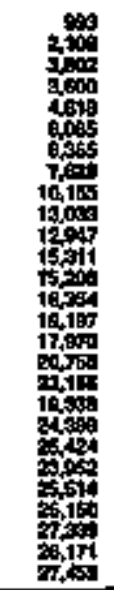 & 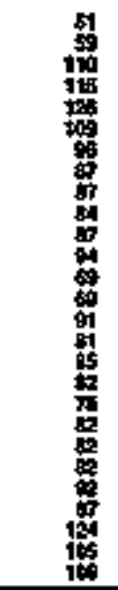 & 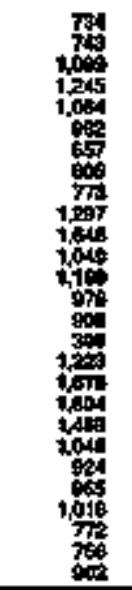 & 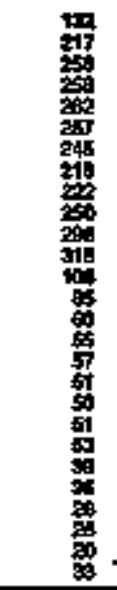 & 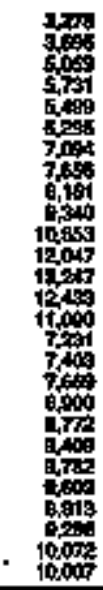 & 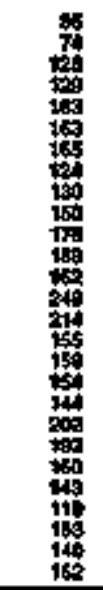 & 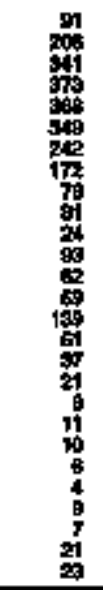 & 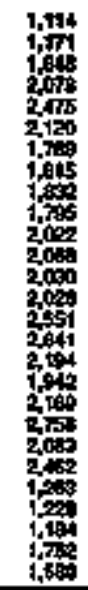 & 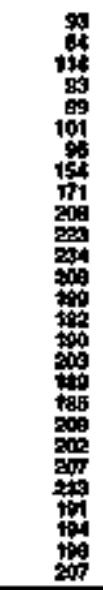 & 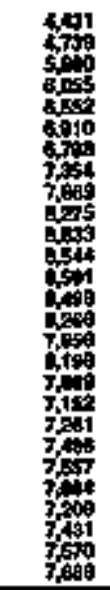 & 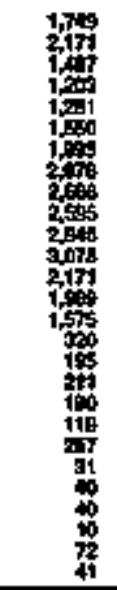 & 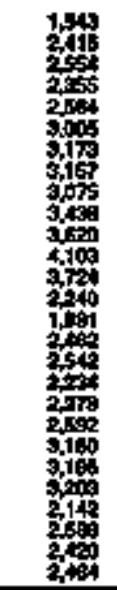 & 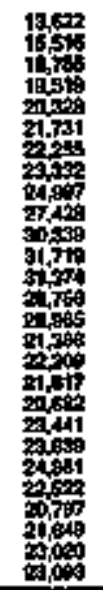 & $\begin{array}{l}0 \\
0 \\
0 \\
0 \\
0 \\
0 \\
0 \\
0 \\
0 \\
0 \\
0 \\
0 \\
0 \\
0 \\
0 \\
0 \\
0 \\
0 \\
0 \\
0 \\
0 \\
0 \\
0\end{array}$ & 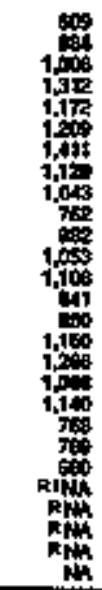 & 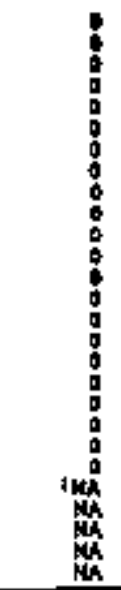 & 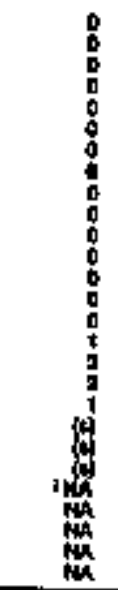 & 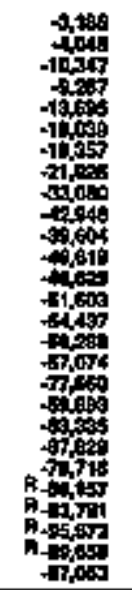 & \\
\hline \multicolumn{20}{|c|}{ Trimonen } \\
\hline 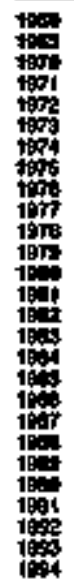 & 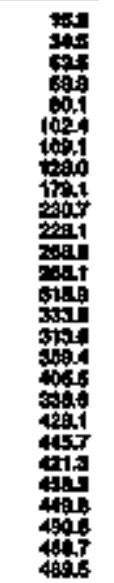 & 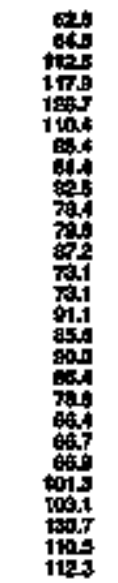 & 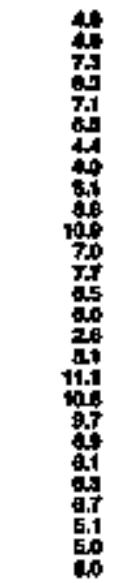 & 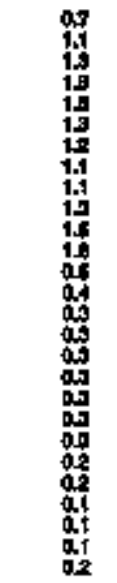 & 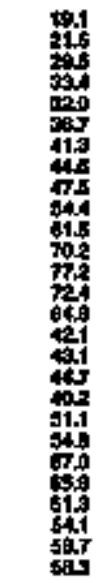 & 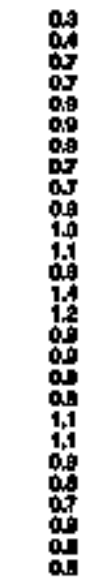 & 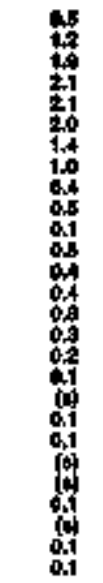 & 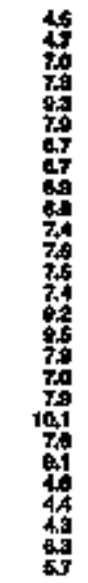 & 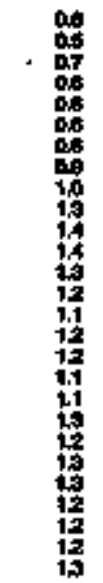 & 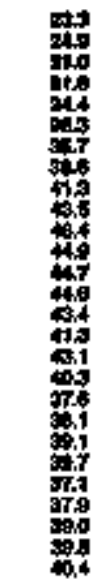 & 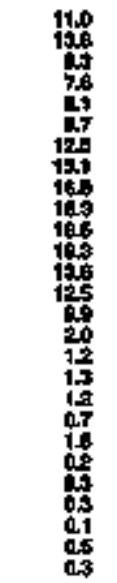 & 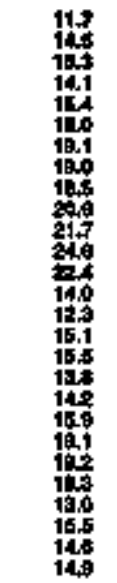 & 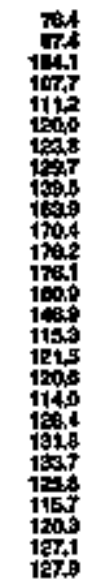 & 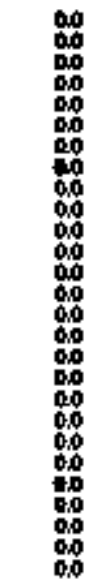 & 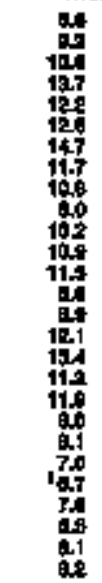 & 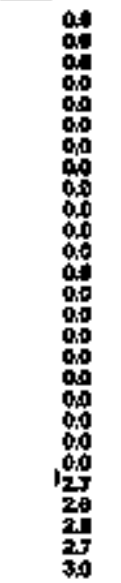 & 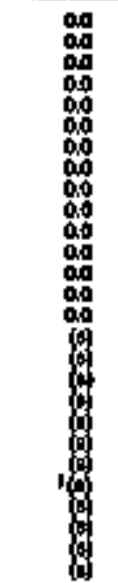 & 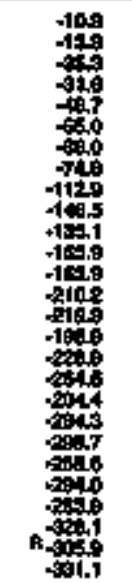 & 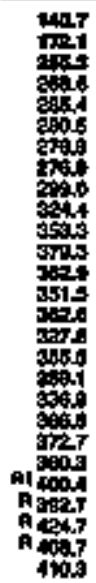 \\
\hline
\end{tabular}

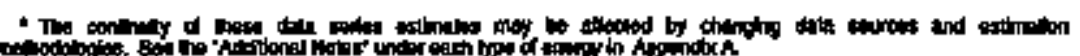

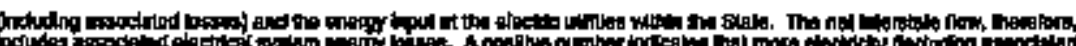

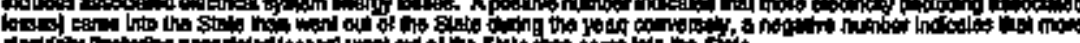

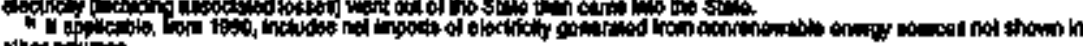

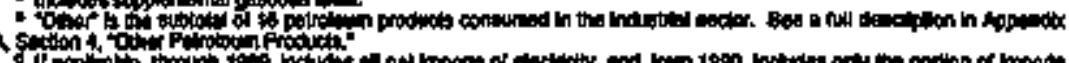

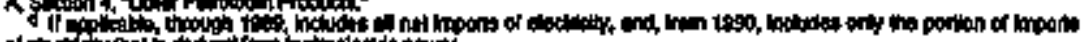

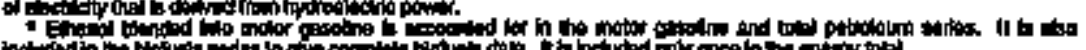

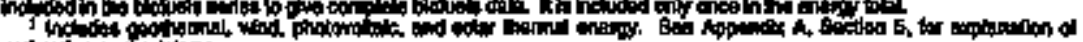

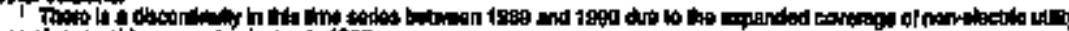

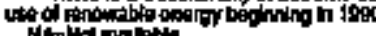

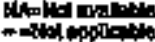

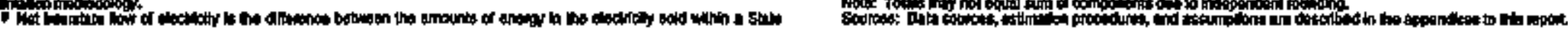


Table 318. Fegidential Energy Consumplon Estimates, 1860, 1965, 1970-1994, Wyoming

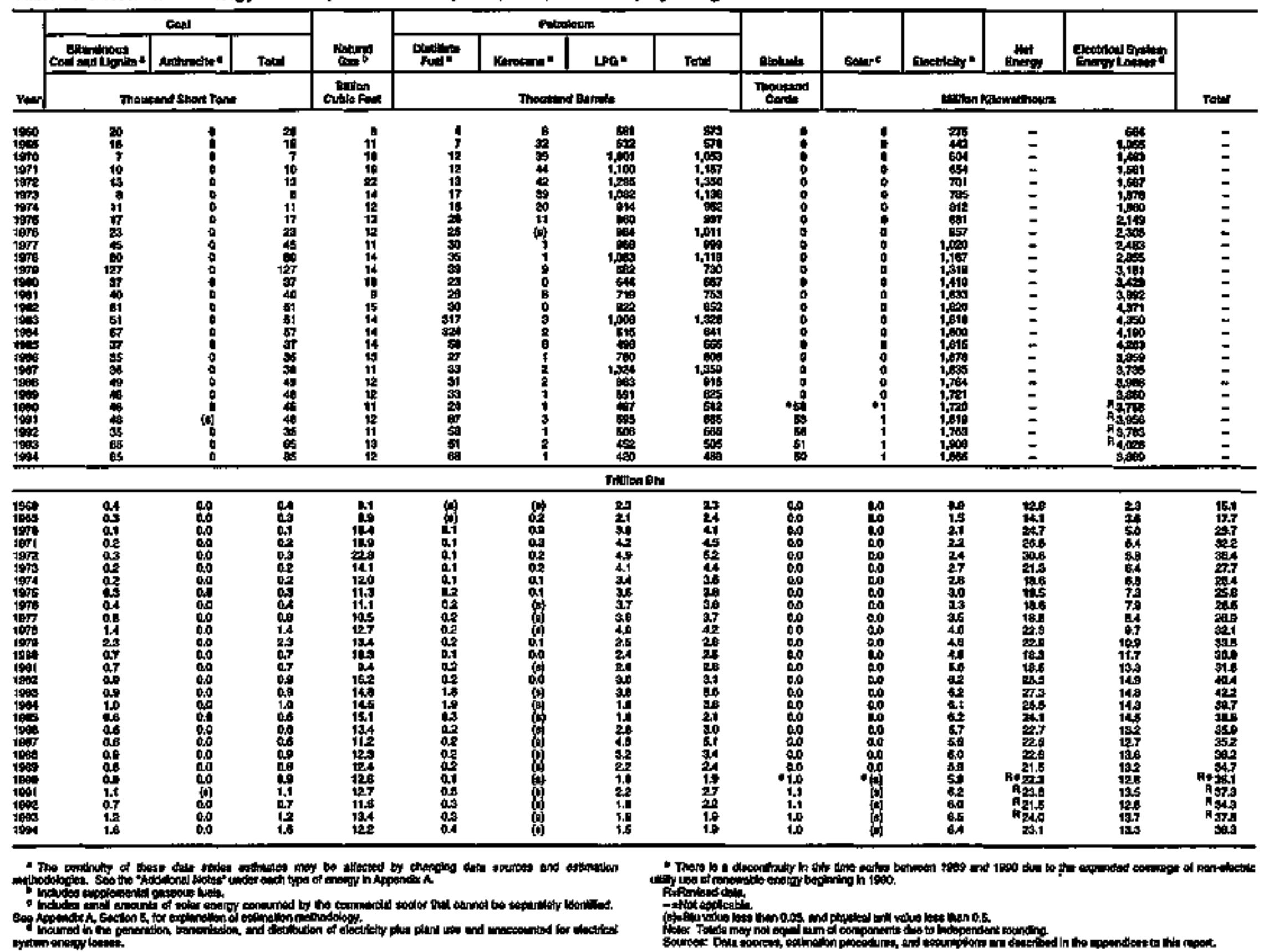


Table 319. Commeroial Energy Consumption Esulmates, t950, 1985, 1970-1994, Wyoming

\begin{tabular}{|c|c|c|c|c|c|c|c|c|c|c|c|c|c|c|}
\hline \multirow[b]{3}{*}{ Varr } & \multicolumn{3}{|c|}{ Dad } & \multirow{3}{*}{ 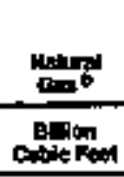 } & \multicolumn{6}{|c|}{ Potrolanm } & \multirow[b]{2}{*}{ Evotriking" } & \multirow[b]{2}{*}{ Enengr } & \multirow[b]{2}{*}{ 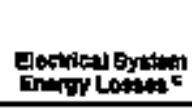 } & \multirow[b]{3}{*}{ Toteted 당 } \\
\hline & 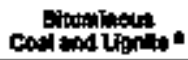 & Anthencline & Tot:ㅔ & & Diefilatis; & Mrovenas & LPQ" & Molor & Motidual & Toula & & & & \\
\hline & \multicolumn{3}{|c|}{ Thouland sthert Tonat } & & \multicolumn{6}{|c|}{ moutend Binole } & \multicolumn{3}{|c|}{ 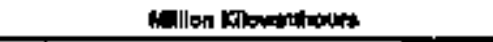 } & \\
\hline 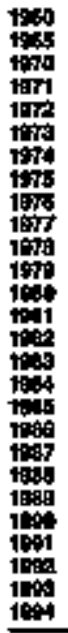 & 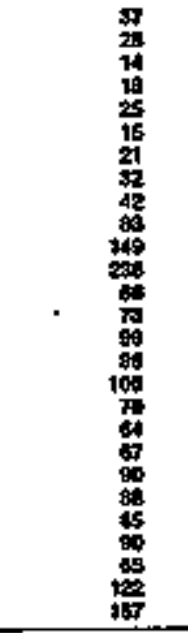 & $\begin{array}{l}0 \\
0 \\
0 \\
0 \\
0 \\
0 \\
0 \\
0 \\
0 \\
0 \\
0 \\
0 \\
0 \\
0 \\
0 \\
0 \\
0 \\
0 \\
0 \\
0 \\
0 \\
0 \\
0 \\
0 \\
0 \\
0 \\
0 \\
0 \\
0 \\
0\end{array}$ & 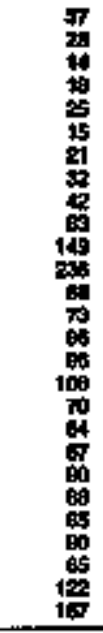 & 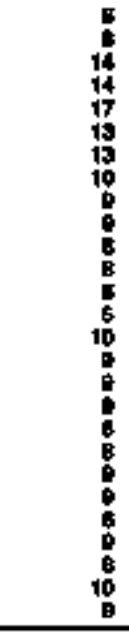 & 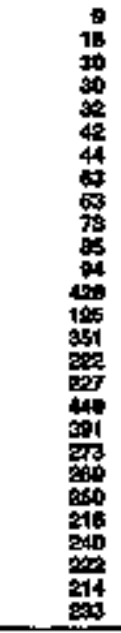 & 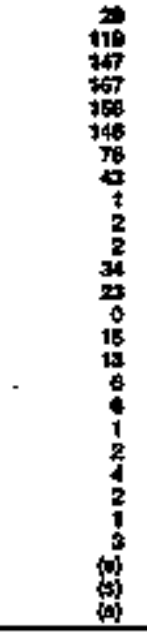 & 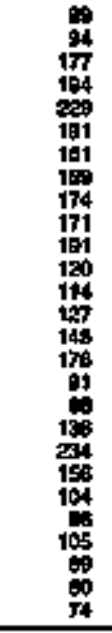 & 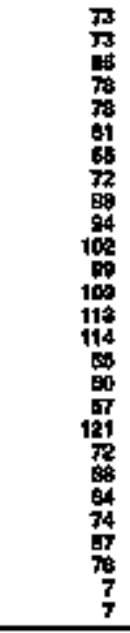 & 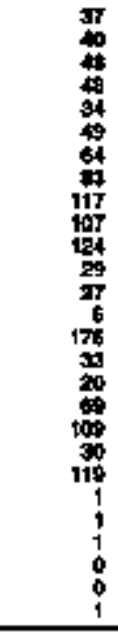 & 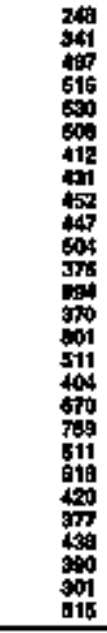 & 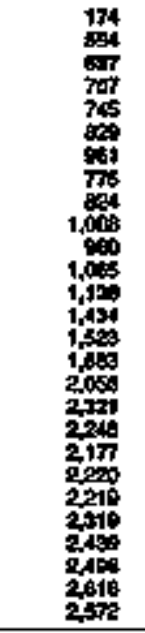 & $\begin{array}{l}= \\
= \\
= \\
= \\
= \\
= \\
= \\
= \\
= \\
= \\
= \\
= \\
= \\
= \\
=\end{array}$ & 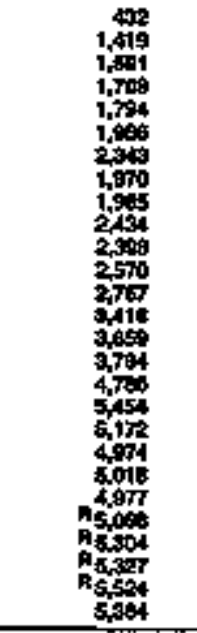 & $\begin{array}{l}= \\
= \\
= \\
= \\
= \\
= \\
= \\
= \\
= \\
= \\
= \\
= \\
= \\
= \\
=\end{array}$ \\
\hline \multicolumn{15}{|c|}{ Then } \\
\hline 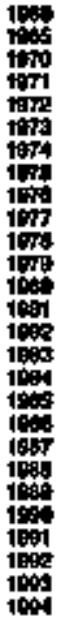 & 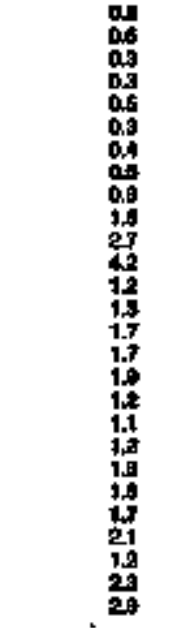 & 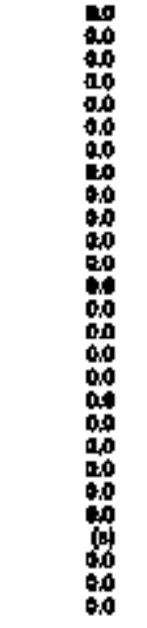 & 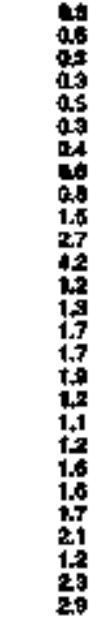 & 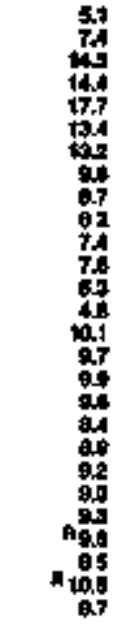 & 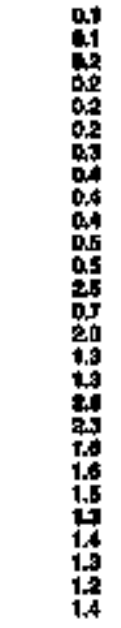 & 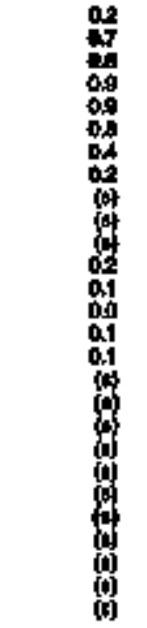 & 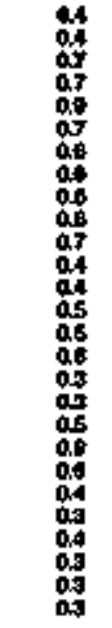 & 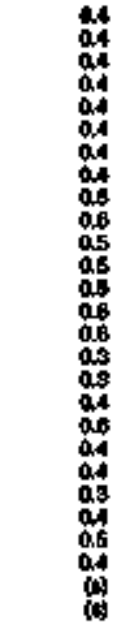 & 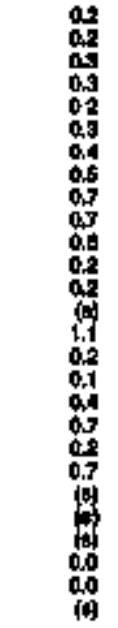 & 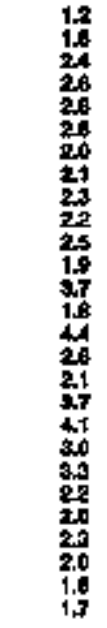 & 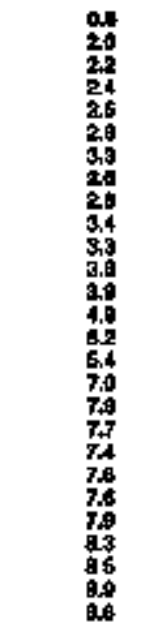 & 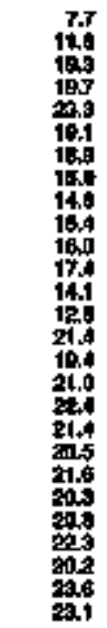 & 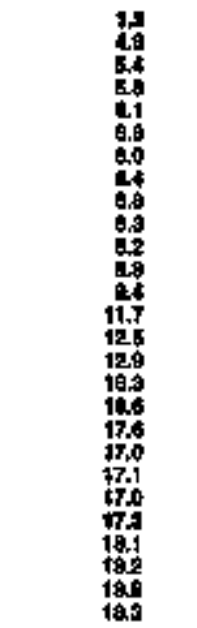 & 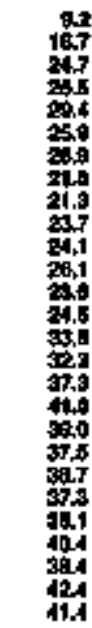 \\
\hline & 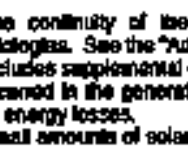 & 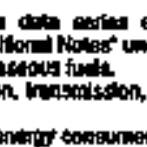 & 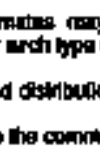 & thethed & $\log$ & & & & & & iv & & & \\
\hline
\end{tabular}


Toble 320. Induatrial Energy Consempiton Eelimates, 1980, 1985, 1970-1984, Wyoming

\begin{tabular}{|c|c|c|c|c|c|c|c|c|c|c|c|c|c|c|c|c|c|c|}
\hline \multirow{2}{*}{\multicolumn{2}{|c|}{ Cod }} & \multirow[b]{2}{*}{ 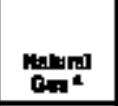 } & \multicolumn{9}{|c|}{ Potralower } & \multirow[b]{2}{*}{ 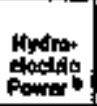 } & \multirow[b]{2}{*}{ Alohueds } & \multirow[b]{2}{*}{ ouner bes } & \multirow[b]{2}{*}{ 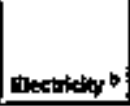 } & \multirow[b]{2}{*}{ Ginent } & \multirow{2}{*}{ 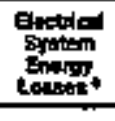 } & \multirow[b]{3}{*}{ اله内 T } \\
\hline & & & 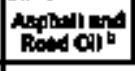 & Fut & Kerounente & $506^{\circ}$ & 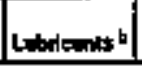 & cotor & Paspentions & Otherks & Toted & & & & & & & \\
\hline Vtar & Thoosund & cokitiog & \multicolumn{9}{|c|}{ Theretandermils } & \multicolumn{6}{|c|}{ 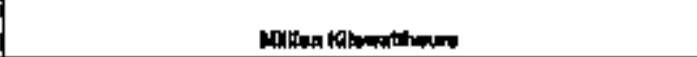 } & \\
\hline
\end{tabular}

\begin{tabular}{|c|c|c|c|c|c|c|c|c|c|c|c|c|c|c|c|c|c|c|}
\hline 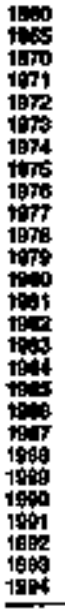 & 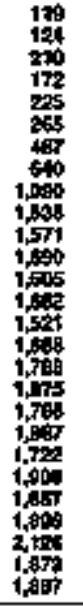 & 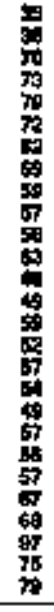 & 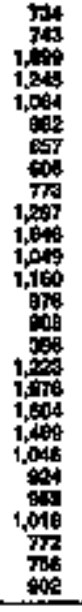 & 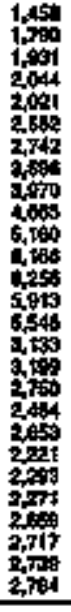 & 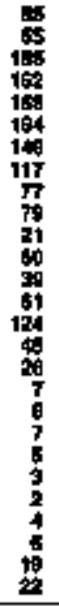 & 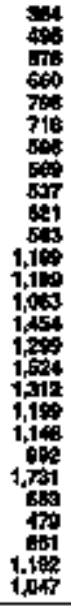 & 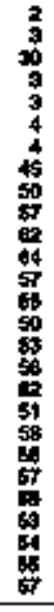 & 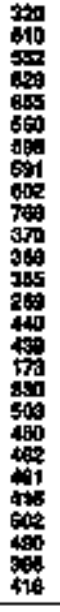 & 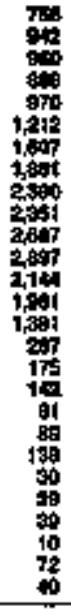 & 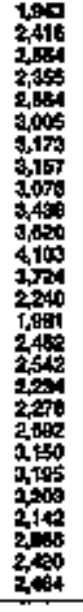 & 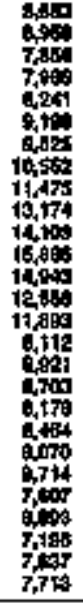 & 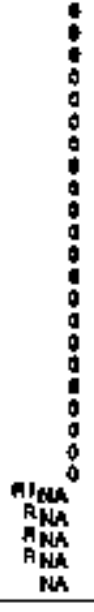 & 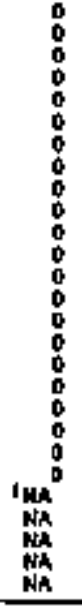 & 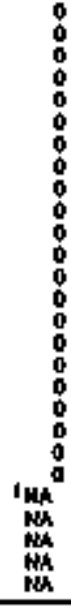 & 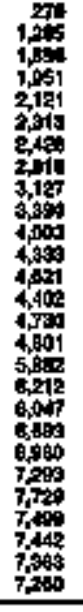 & $\begin{array}{l}z \\
= \\
= \\
= \\
= \\
= \\
= \\
= \\
= \\
= \\
= \\
= \\
= \\
= \\
z\end{array}$ & 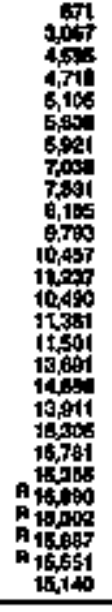 & $\begin{array}{l}5 \\
\\
\\
\\
\\
\\
\\
\\
\\
\end{array}$ \\
\hline
\end{tabular}

\begin{tabular}{|c|c|c|c|c|c|c|c|c|c|c|c|c|c|c|c|c|c|c|}
\hline 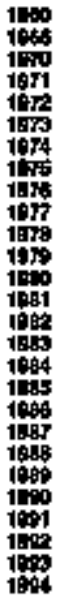 & 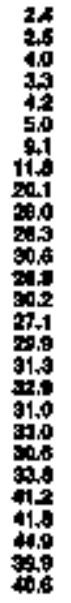 & 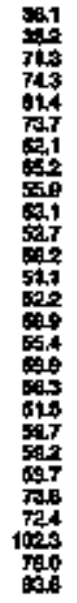 & 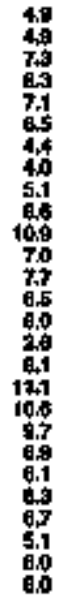 & 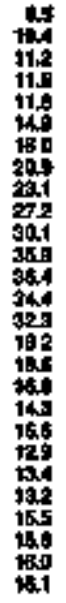 & 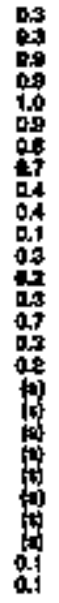 & 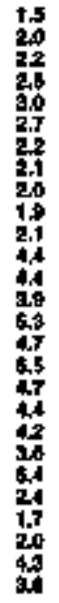 & $\begin{array}{l}0.8 \\
02 \\
0.9 \\
99 \\
90 \\
09 \\
0.3 \\
0.3 \\
0.4 \\
0.4 \\
0.3 \\
0.3 \\
0.3 \\
0.3 \\
0.3 \\
0.3 \\
0.4 \\
0.3 \\
0.3 \\
0.4 \\
0.3 \\
0.3 \\
0.3 \\
0.3\end{array}$ & 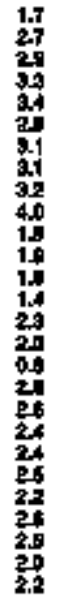 & 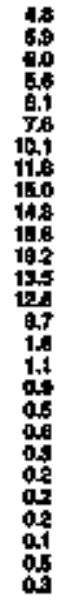 & 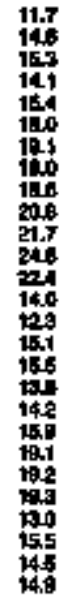 & 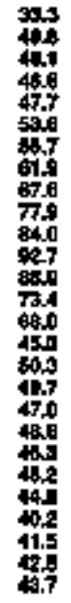 & 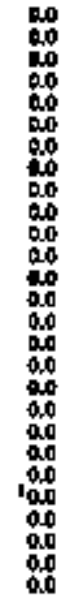 & 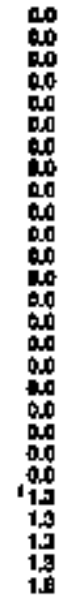 & 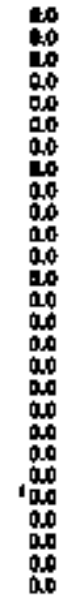 & 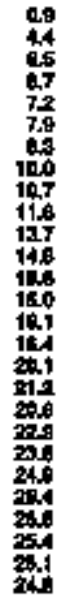 & 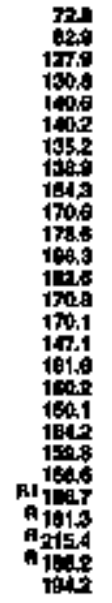 & 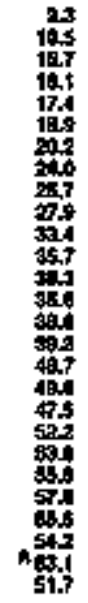 & 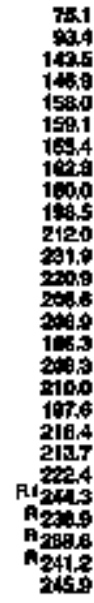 \\
\hline
\end{tabular}

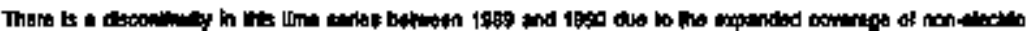

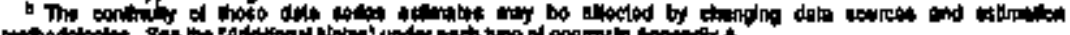

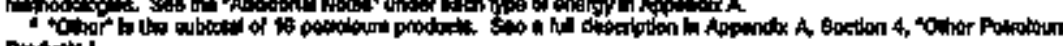

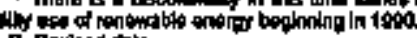

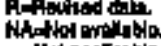

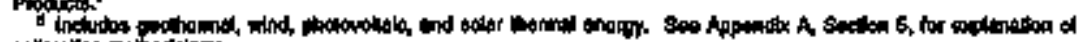

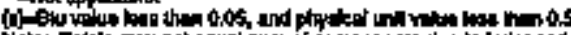

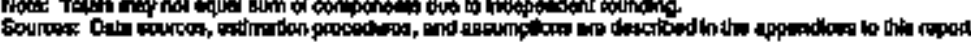

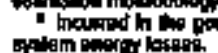


Tebla 321. Transportation Enorgy Constumptlon Estlmatas, 1950, 1965, 1970-1994, Wyanlng

\begin{tabular}{|c|c|c|c|c|c|c|c|c|c|c|c|c|c|c|c|}
\hline \multirow[b]{3}{*}{$\mathrm{rem}$} & \multirow[b]{2}{*}{$\cos \theta$} & \multirow[b]{2}{*}{ Finte } & \multicolumn{8}{|c|}{ 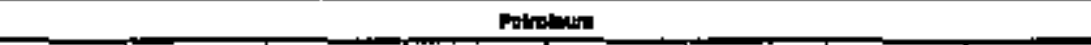 } & \multirow{3}{*}{ 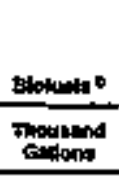 } & \multirow[b]{2}{*}{ Bactilety" } & \multirow[b]{2}{*}{ ratity } & \multirow[b]{2}{*}{ 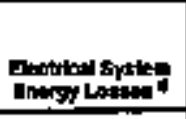 } & \multirow[b]{3}{*}{ Totat } \\
\hline & & & contallon. & 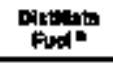 & rinet. & LPOE & 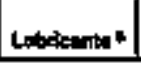 & Motor & $\begin{array}{l}\text { Maskithel } \\
\text { Fuel }\end{array}$ & Toth & & & & & \\
\hline & 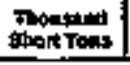 & aniren & \multicolumn{8}{|c|}{ Thoaltend Buncicts } & & \multicolumn{3}{|c|}{ 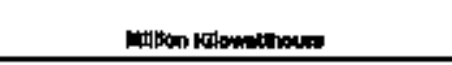 } & \\
\hline 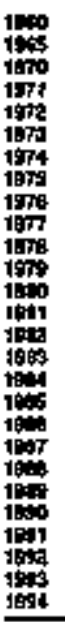 & $\begin{array}{l}0 \\
0 \\
9 \\
9 \\
0 \\
0 \\
0 \\
0 \\
0 \\
0 \\
0 \\
0 \\
0 \\
0 \\
0 \\
0 \\
0 \\
0 \\
0 \\
0 \\
0 \\
0 \\
0\end{array}$ & $\begin{array}{l}2 \\
2 \\
5 \\
6 \\
6 \\
6 \\
5 \\
7 \\
5 \\
7 \\
8 \\
8 \\
7 \\
8 \\
5 \\
5 \\
5 \\
6 \\
8 \\
5 \\
8 \\
7 \\
7 \\
\end{array}$ & 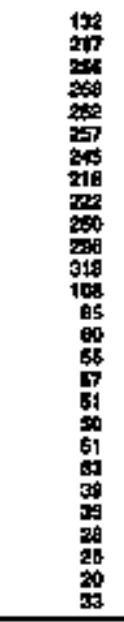 & 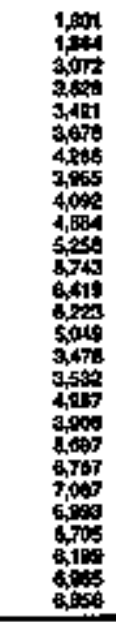 & 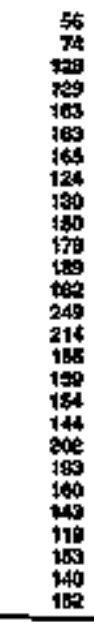 & 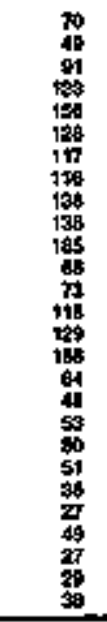 & 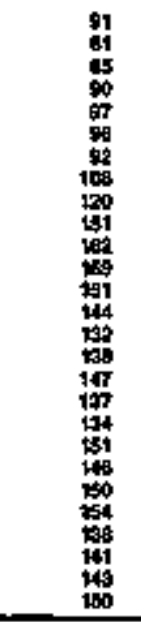 & 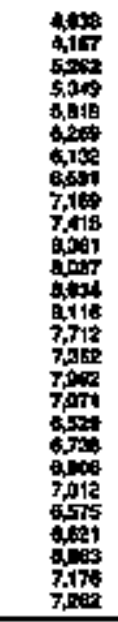 & 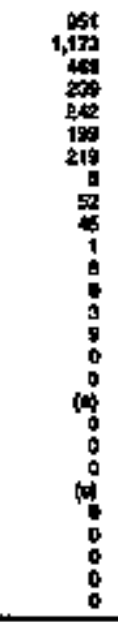 & 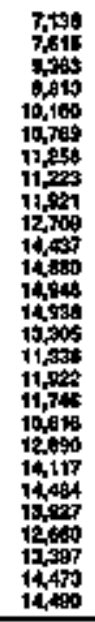 & 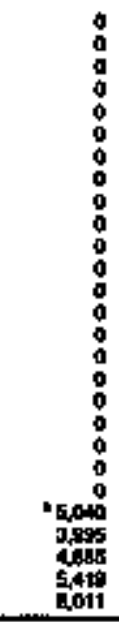 & $\begin{array}{l}0 \\
0 \\
0 \\
0 \\
0 \\
0 \\
0 \\
0 \\
0 \\
0 \\
0 \\
0 \\
0 \\
0 \\
0 \\
0 \\
0 \\
0 \\
0 \\
0 \\
0 \\
0 \\
0 \\
0 \\
0 \\
\end{array}$ & $\begin{array}{l}= \\
= \\
= \\
= \\
= \\
= \\
= \\
= \\
= \\
= \\
= \\
= \\
=\end{array}$ & $\begin{array}{l}0 \\
0 \\
0 \\
0 \\
0 \\
0 \\
0 \\
0 \\
0 \\
0 \\
0 \\
0 \\
0 \\
0 \\
0 \\
0 \\
0 \\
0 \\
0 \\
0 \\
0 \\
0 \\
0 \\
0 \\
0 \\
0\end{array}$ & 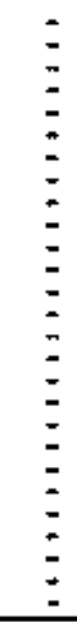 \\
\hline \multicolumn{16}{|c|}{ 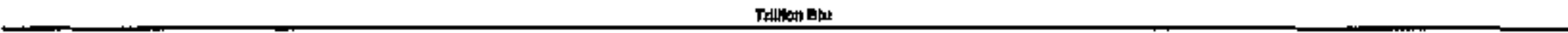 } \\
\hline 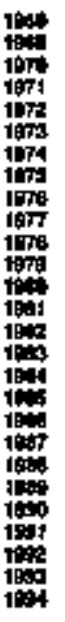 & 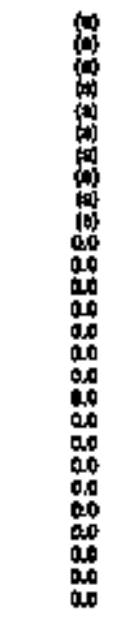 & 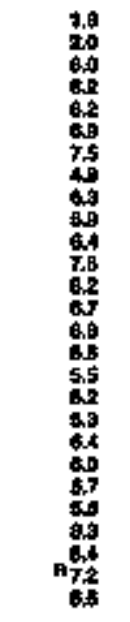 & 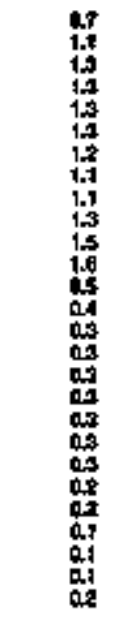 & 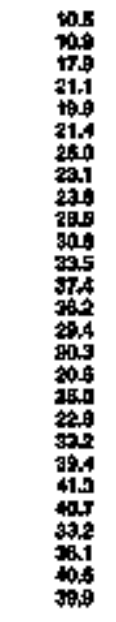 & 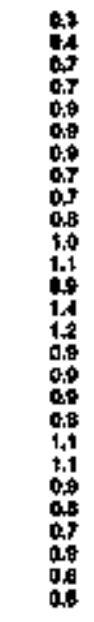 & 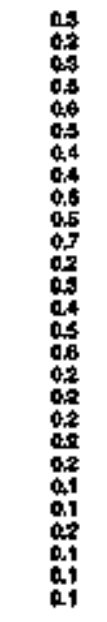 & 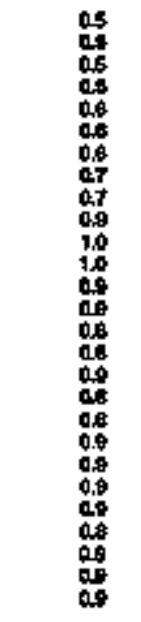 & 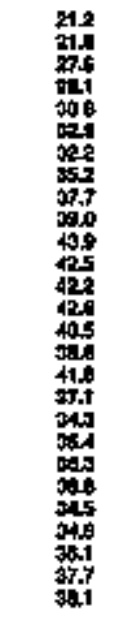 & 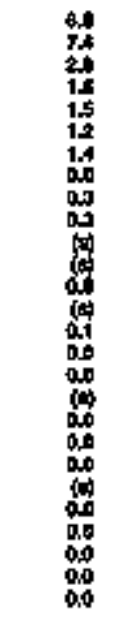 & 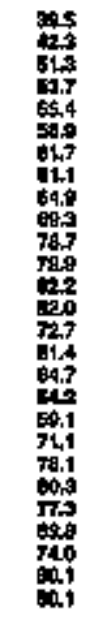 & 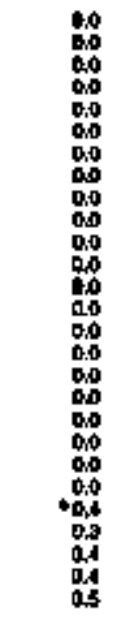 & $\begin{array}{l}00 \\
00 \\
000 \\
00 \\
00 \\
00 \\
00 \\
00 \\
00 \\
00 \\
000 \\
00 \\
00 \\
00 \\
00 \\
00 \\
00 \\
00 \\
00 \\
00 \\
00 \\
00 \\
00 \\
00 \\
00 \\
000 \\
000 \\
00\end{array}$ & 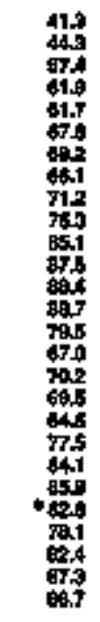 & $\begin{array}{l}00 \\
00 \\
00 \\
00 \\
00 \\
00 \\
00 \\
00 \\
000 \\
000 \\
00 \\
00 \\
00 \\
00 \\
00 \\
00 \\
000 \\
00 \\
00 \\
00 \\
00 \\
00 \\
00 \\
00 \\
00 \\
00 \\
00\end{array}$ & 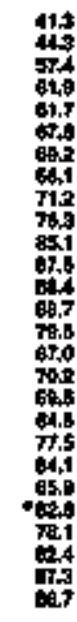 \\
\hline
\end{tabular}

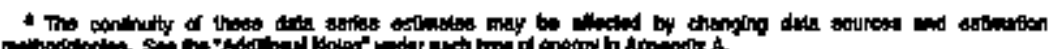

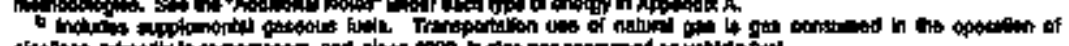

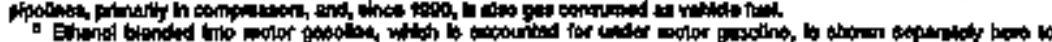

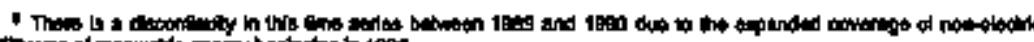

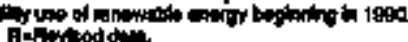

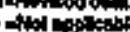

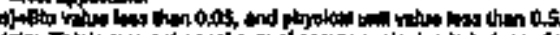

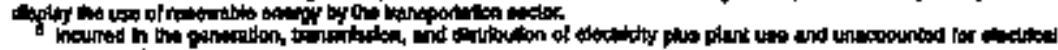

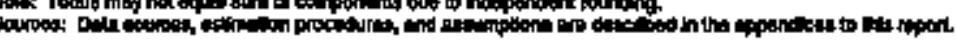

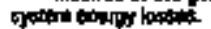


Tabio 322. Estidiates of Enocgy Input at Electric Utilities, 1960, 1985, 1970-1994, Wyoming

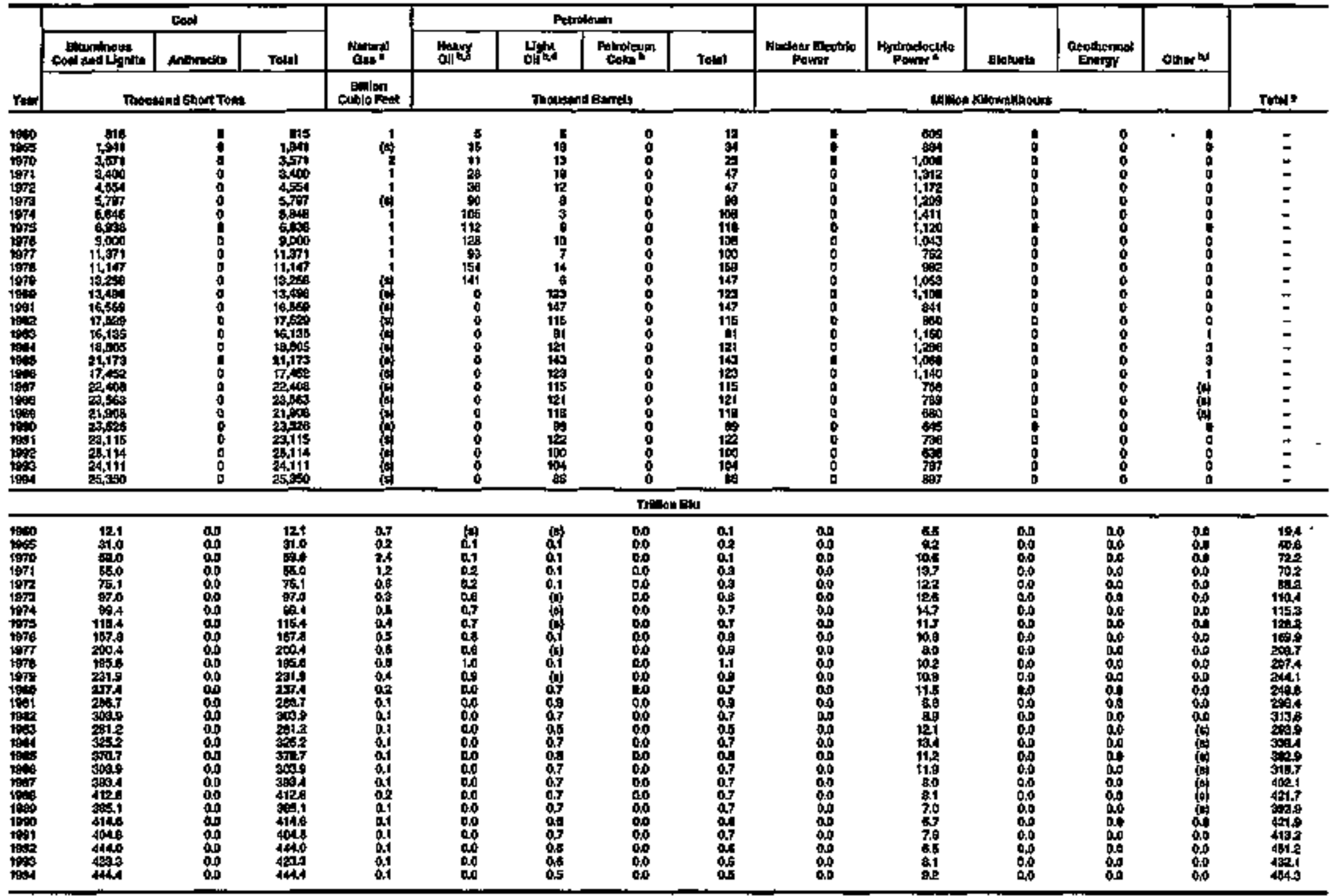

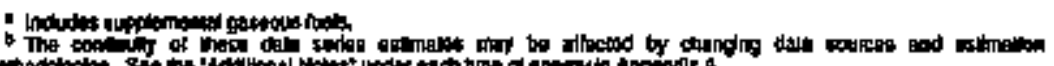

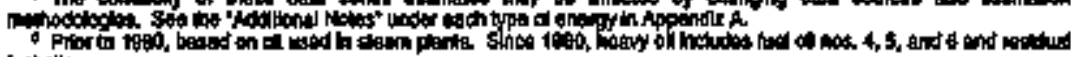

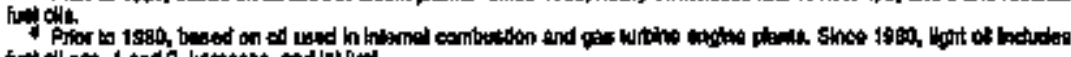

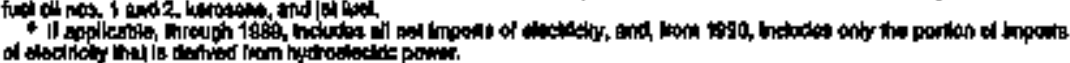

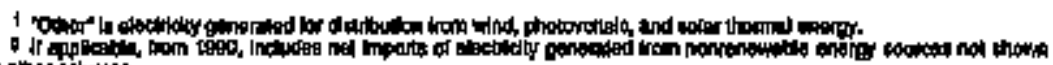

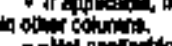

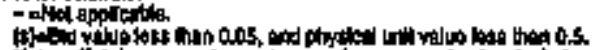

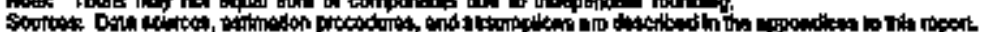


Energy Consumption Estimates by Sector and Source, Each State and the United States

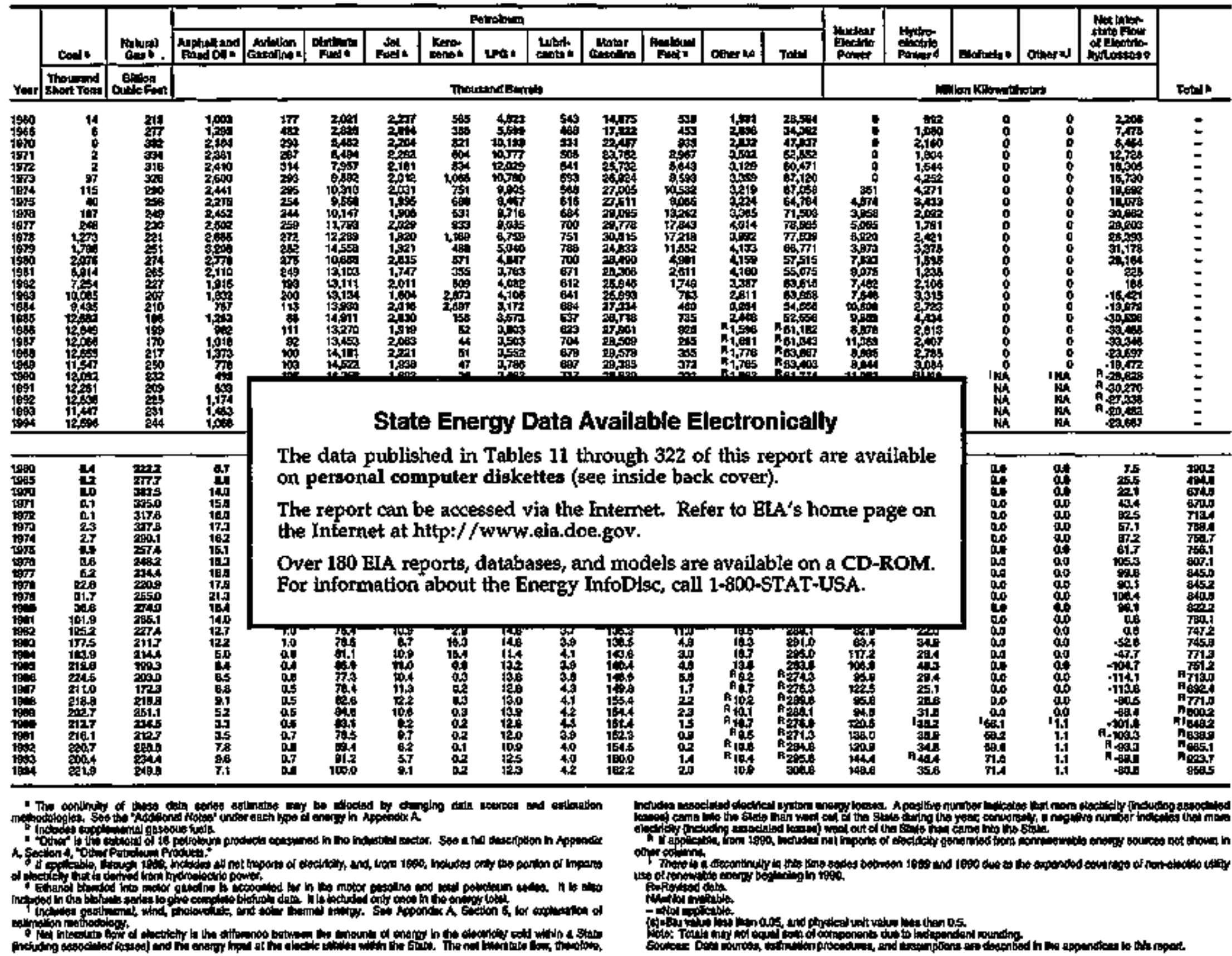



Appendix A

Documentation 


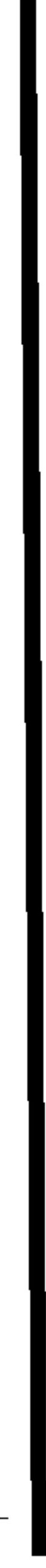




\section{Appendix A}

\section{Documentation}

\section{Section 1. Documentation Guide}

Appendix A of the State Energy Data Report describes how the estimates in the report were derived by the State Energy Data System (SEDS). The following five sections, one for each energy source, provide: descriptions of all the data series that are entered into SEDS; the formulas applied in SEDS for creating additional data series; and notes on special circumstances for any series.

Appendix B is an alphabetical listing of the variable names and formulas used in the system; Appendix $C$ lists the sources of all data series entered into SEDS; Appendix D lists the conversion factors used in SEDS to convert physical urits into British thermal units and gives the sources for those factors; Appendix $\mathrm{E}$ presents metric and other physical conversion factors for information, although they are not currently used in SEDS; Appendix F lists carbon dioxide emission factors for coal consumed by State for information, although they are not used in SEDS; Appendix $G$ is a summary of the changes made in SEDS since the last report, which was released in July 1995; and Appendix $H$ is a list of other Energy Information Administration reports containing State-level data.

Nearly 400 variables are used in SEDS to create the estimates in this report. All of the variables are identified by seven-letter names, such as MGTCPAL, somposed as follows:

\begin{tabular}{|c|c|c|c|c|}
\hline Characters: & MG & $\mathbf{T C}$ & $\mathbf{P}$ & AL \\
\hline $\begin{array}{l}\text { Positions: } \\
\text { Idently: }\end{array}$ & $\begin{array}{l}1 \text { and } 2 \\
\text { Type of } \\
\text { energy }\end{array}$ & $\begin{array}{l}3 \text { and } 4 \\
\text { Energy activity } \\
\text { or consumption } \\
\text { end-use sector }\end{array}$ & $\begin{array}{l}5 \\
\text { Type } \\
\text { of data }\end{array}$ & $\begin{array}{l}6 \text { and } 7 \\
\text { Geographic }\end{array}$ \\
\hline
\end{tabular}

In this example MGTCPAL is the identifying code for data on motor gasoline total consumption in physical units in Alabama.

The type of energy categories in SEDS, which are represented by the first two letters of the variable name, are:

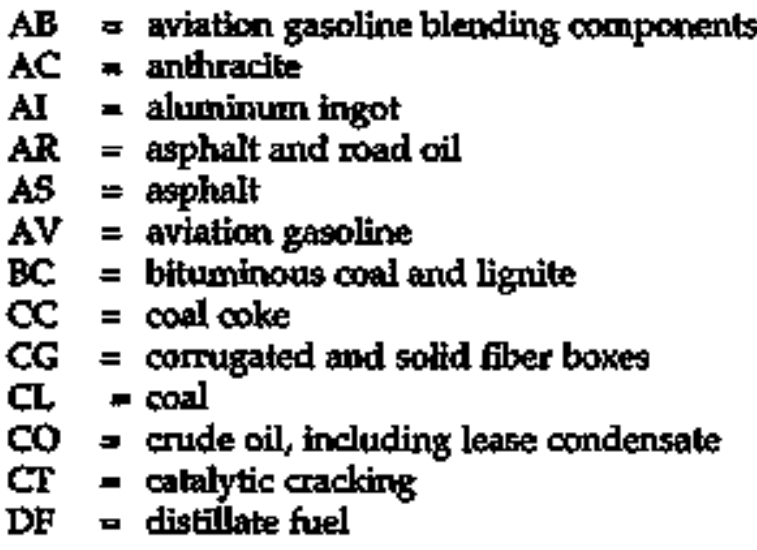



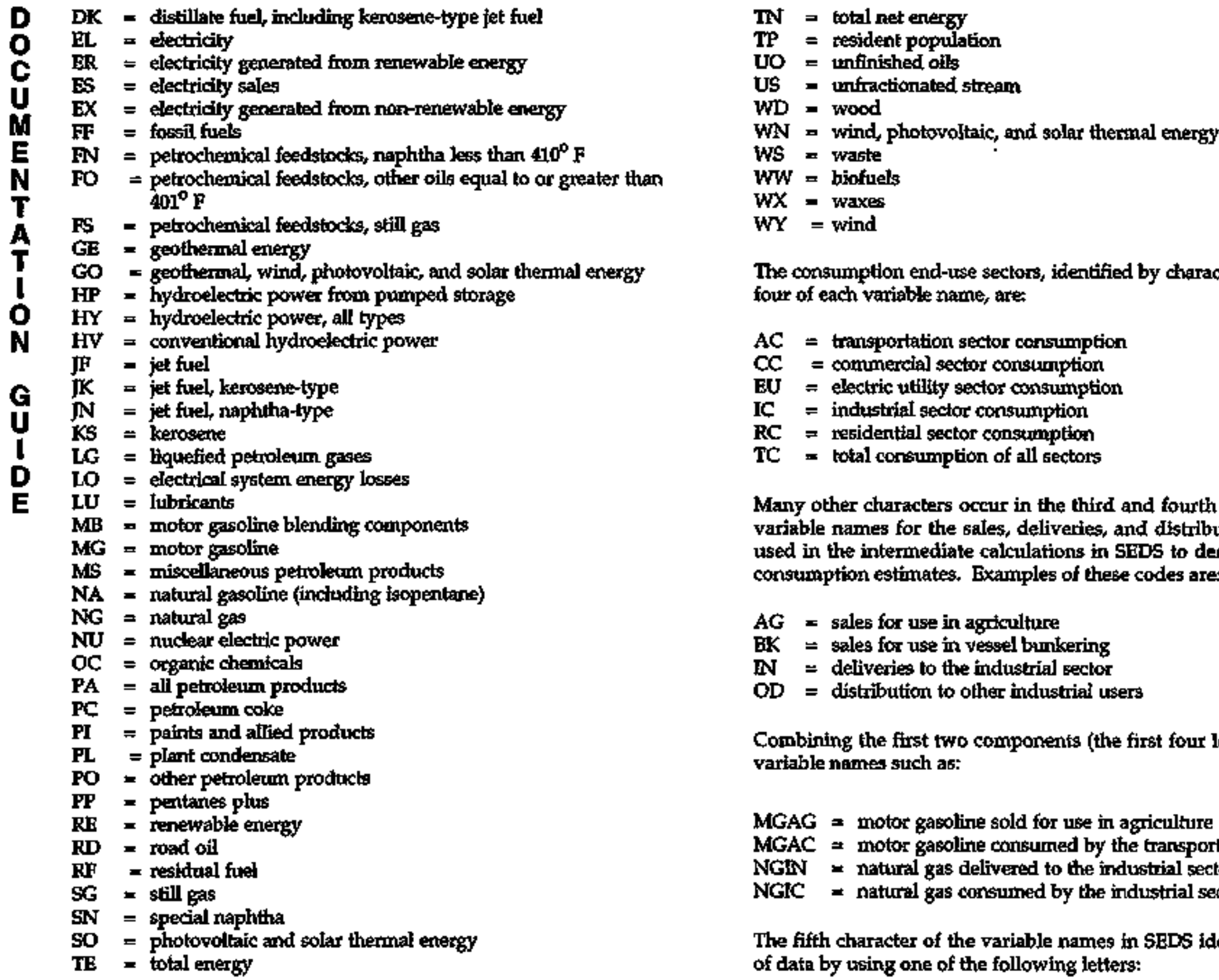

The consumption end-use sectors, identified by characters three and four of each variable name, are
$A C=$ transportation sector consumption
CC = commercial sector consuruption
EU = electric utility sector consumption
IC = industrial sector consumption
RC = residential sector constumption
TC = total consumption of all sectors

Many other characters occur in the third and fourth positions of the variable names for the sales, deliveries, and distribution data series used in the intermediate calculations in SEDS to derive the end-use consumption estimates. Examples of these codes are:
AG = sales for use in agriculture
BK $=$ sales for use in vessel bunkering
N = deliveries to the industrial sector
$O D=$ distribution to other industrial users

Combining the first two components (the first four letters) produces variable names such as:

MGAG a motor gasoline sold for use in agriculture

MGAC = motor gasoline consumed by the transportation sector

NGW = natural gas delivered to the industrial sector

NGIC = natural gas consumed by the industrial sector

The fifth character of the variable names in SEDS identifies the type of data by using one of the following letters: 
$K=$ factor for converting data frod physical urits to Btu

$\mathrm{M}=$ data in alternative physical units

$P$ = data in standardized physical units

$S=$ share or ralio expressed as a fraction

$V=$ value added in manufacture

Data entered into SEDS are in physical units, represented by a " $P$ " in the fffth chanacter: for example, coal data are in thousand short tons, petroleum data ate in thousand barrels, and natural gas data are in million cubjc feet. In a few cases, data are obtained from the source documents in different units, such as thousand gatlons instead of thousand barrejs, and are represented by an " $M$ " until converted in SEDS to the unit that is consistent with other vaxiables. Conversion factors, represented by a " $K$ " in the iffth character, are applied to the physical unit data to convert the data to British thermal units, a common unit for all forms of energy. The derived data series in thousand British thermal units are represented by " $\mathrm{B}^{\prime \prime}$ in the fifth character. In a few cases, consumption estimates are derived by calculating shares of aggregated consumption data. The fractions uged to calculate the consumption shares are identifled by an " $\mathrm{S}^{\prime}$ in the fifth character. The consumption estimates for some petroleum products are based or the value added in manufacture by related industries in each State. The data series for those industry activities are in dollars, and the variable names contain " $\mathbf{V}$ " in the fifth character.

The last two characters of each variable name are for geographic identification. Geographic areas used in SEDS are the 50 States and the Distritt of Columbin (represented by the U.S. Postal Service State abbreviations) and the United States as a whole. Some estimates of electricity sales and losses are derived by using only the contiguous 48 States and the District of Columbia, and the variables used in those calculations are identified by " 48 " in the last two characters of the names. Some data series have witheld data for States to avold disclosure of individual company proprietary data. For those data series the geographic code "OT" is used for the surn of the withheld State data. The geographic area codes used in SEDS are shown in Table A1.

Throughout this report, the term "State" includes the District of Columbia. Throughout this documentation, ${ }^{\prime} \mathrm{ZZ}^{\prime}$ is used as a geoSystem

\begin{tabular}{|c|c|c|c|}
\hline Code & State & Code & State \\
\hline AK & Alaska & NC & North Garolina \\
\hline AL & Alabarina & ND & North Dakota \\
\hline AR & Arkansas & NE & Nebraska \\
\hline $\mathbf{A Z}$ & Arizona & NH & New Hampshire \\
\hline CA & California & NJ & New Jersey \\
\hline Co & Colonado & NM & New Mexico \\
\hline CT & Comnecticut & NV & Nevada \\
\hline DC & Disirict of Colsmbia & NY & New York \\
\hline DE & Deleware & $\mathrm{OH}$ & Ohio \\
\hline FL & Forida & OK & Oklahoma \\
\hline GA & Georgia & OR & Oregon \\
\hline |ㅔ & Hawalt & PA & Pennsylvania \\
\hline IA & lowa & AI & Rhode lstand \\
\hline ID & tdaho & $\mathrm{sc}$ & South Carolina \\
\hline IL & ㅂlinols & so & South Dakcola \\
\hline IN & Indiang & TN & Tennessien \\
\hline Ko & Kanass & $T X$ & Texas \\
\hline KY & Kenlucky & UT & Utah \\
\hline LA & Loutslana & VA & Viglnfa \\
\hline MA & Masseanhuselts & vT & Vemont \\
\hline MD & Maryland & WA & Wasthington \\
\hline MAE & Maine & w & Wrsconsin \\
\hline MI & Mtchiggan & WV & West Vuginia \\
\hline MiN & Minnesola & Wr & Wyoming \\
\hline No & Missount & US & Unlled States \\
\hline MS & Mississippi & 48 & The contigurous 48 States \\
\hline \multirow[t]{2}{*}{ MT } & Montana & & and the District of Columbia \\
\hline & & OT & Olher States (widthiad data) \\
\hline
\end{tabular}

graphic identifier to represent the different State abbreviations that would be interchanged in that position of the variable name. 



\section{Section 2. Coal}

Two forms of coal一anthracite (AC) and bituminous coal and lignite (BC)-are added to provide coal totals (CL).

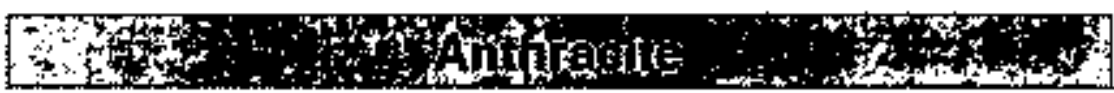

\section{Physical Units}

There are seven input data series used to estimate the State end-use consumption of anthracite, and all are in units of thousand short tons. " $\mathrm{ZZ}$ " in the variable names is used to represent the two-letter State code that differs for each State:

ACEUPRZ = anthraclte consumed by the electric utilities in each State;

ACHCPUS = anthracite consumed by the residential and commercial sectors in the United States;

ACHDPZZ = anthracite distributed to the residential and conumercial sectors in each State;

ACKCPUS = anthracite consumed by coke plants in the United States;

ACKDPZZ = anthracite distributed to coke plants in each State;

ACOCPUS $=$ anthracite consumed by other industrial users in the United States; and

ACODPZZ = anthracite distributed to other industrial users in each State.

The U.S. totals for the four State-level series, ACEUPZZ, ACHDPZZ, ACKDPZZ, and ACOCDPZZ, are calculated by summing the State data.

Estimates of anthracite consumed by the residential and commercial sectors combined are made by assuming that anthracite is consumed in proportion to the amount of anthracite distributed to the residential and commercial sectors in each State:

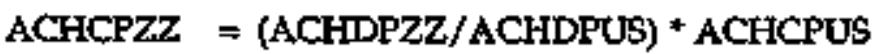

Little information is available regarding disaggregating the combined residential and commercial estimates. An estimate of 60 percent to the residential sector and 40 percent to the commercial sector is made for all States and years. Therefore, the residential sector consumption of anthracite, ACRCPZZ, is estimtated:

\section{$A C R C P Z Z=A C H C P Z Z * 0.60$}

and the commercial sector consumption, ACCCPZZ, is estingated:

\section{$\mathrm{ACCCPZZ}=\mathrm{ACHCPZZ} * 0.40$}

To gaim a perspective on these estimates, all anthracite consumed in the United States in 1994 accounted for less than 0.3 percent of total coal consumption, and the restdential and commercial use of anthracite was less than half of all anthrocite consumed.

The industrial sector consumption is estimated by State. An assumption is made that anthracite is consumed by coke plants in proportion to the amount of anthracite distributed to coke plants in each State. It is also assisted that the consumption of anthracite by industrial users other than coke plants is in proportion to the amount of anthracite delivered to the other industrial users in each State. The industrial sector consumption is the sum of anthracite consumed by coke plants and by other industrial users for each State:

$$
\begin{aligned}
& \text { ACKCPZZ }=\text { (ACKDPZZ/ACKDPUS) * ACKCPUS } \\
& \text { ACOCPZZ }=\text { (ACODPZZ/ACODPUS) *ACOCPUS } \\
& \text { ACICPZZ }=\text { ACKCPZZ + ACOCPZZ }
\end{aligned}
$$


Total anthracite consumption in each State is the sum of the sectors' consumption:

\section{$A C T C P Z Z=A C R C P Z Z+A C C C P Z Z+A C I C P Z Z+A C E U P Z Z$}

The U.S. anthracite consumption estimates for each of the sectors and the total are calculated as the sum of the States' values.

\section{British Thems Unlls (Blu)}

Two factors are used for converting anthracte consumption from physical units to Bta. The factors, in million Btu per short ton, are:

\section{ACEUKUS $=$ the factor for converting anthracite consumed in the} electric utility sector from short tons to Btu; and

ACNUKUS - the factor for converting anthracite consumed by all sectors ather than electric utilities from short tors to Btu.

D

The industrial sector Btu consumption is estimated in three steps in order to maintain separate series for anthracite used as coking coal (ACKCB) and anthracite consumed by other industrial users (ACOCB);

\section{ACKCBZZ = ACKCPZZ * ACNUKUS \\ $A C O C B Z Z=A C O C P Z Z * A C N U K U S$ \\ $A C I C B Z Z=A C K C B Z Z+A C O C B Z Z$}

The rematning end-use sectors are calculated for all States:

\section{ACEUBZZ $=$ ACEUPZZ * ACEUKUS \\ $A C R C B Z Z=A C R C P Z Z * A C N U K U S$ \\ $A C C C B Z Z=A C O C P Z Z * A C N U K U S$ \\ $A C T C B Z Z=A C R C B Z Z+A C C C B Z Z+A C I C B Z Z+A C E U B Z Z$}

Total U.S. end-use consumption estimates are calculated as the sum of the States' data.

\section{Additional Notes on Anthractie}

Anthracite consumption at the national level for the residential and commercial sectors (ACHCPUS), coke plants (ACKCYUS), and industries other then coke plants (ACOCPUS) are continuous data series. However, the total coal distribution and anthracite distribution data series used to develop State-level estimates are not continuous.

For 1960 through 1979 , State-level anthracite data are not available and the 1980 State data are used to apportion the U.S. totals to the States. From 1980 forward, the data in the distribution series variables-ACKDPZZ, ACODPZZ, and ACHDPZZ-are estimates of actual anthracite consumption rather than the distribution.

For 1980 forward, State-level total coal consumption data are available, but consumption by sector within many States is withheld. Estimates of the withheld sector consumption of total coal are derived by using the distribution series for the residential and commercial sectors to fill in withheld residential and commercial consumption. In most States, this leaves only one sector withheld and it can be derived by subtracting known sectors from the State total. This gives total coal consumption estimates for the end-use sectors that are compatible with State coal consumption data published in other EIA reports. Anthuacite consumption is then derived by using anthracite distribution data to estimate consumption within each sector and State. These estimates equal U.S. totals for anthracite consumption by sector contained in other BIA databases.

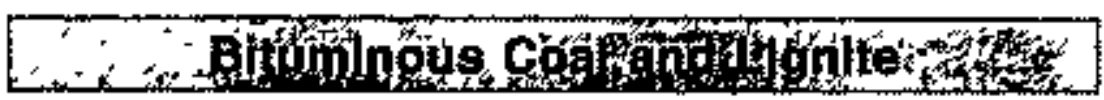

\section{Physical Unils}

Eight data series are used to estimate bituminous coal and lignite consumption. They are consumption and distribution data, and they are all in units of thousand short tons:

BCACPUS = bituminous coal and lignite consumed by the transportation sector in the United States; 
BCEUPZZ = bituminous coal and itgnitte consumed by the electric uttlities in each State;

BCHCPUS $=$ bituminous coal and lignite consumed by the residential and commerctal sectors in the United States;

BCHDPZZ = bituminous coal and ligrite distributed to the residential and commercial sectors in each State.

BCKCPUS = bituminous coal and ligrite consumed by coke plants in the United States;

BCKDPZZ = bituminous coal and lignite distributed to coke plants in each State;

BCOCPUS = bituminous coal and lignite consumed by other industrial users in the United States; and

BCODPZZ = bituminous coal and lignite distributed to other industrial users in each State.

The U.S. totals for the four State-level series, BCEUIZZ, BCHDPZZ, BCKDPZZ, and BCODPZZ, are calculated by summing the State data.

An assumption is made that bituminous coal and lignite are consumed by the residential and commercial sectors combined in proportion to the amount of bihuminous coal and lignite distributed to the residential and commercial sectors in each State:

\section{BCHCPZZ $=$ (BCHDPZZ / BCHDPUS $) *$ BCHCPUS}

Little information exists for disaggregating the combined residential and commercial estimates. An estimate of 35 percent to the residential sector and 65 percent to the commercial sector is made for all States and years. That is, the residential sector consumption, BCRCPZZ, is estimated:

BCRCPZZ $=$ BCHCPZZ 0.35

and the commercial sector consumption, BCCCPZZ, is estimated:

$\mathrm{BCCCPZZ}=\mathrm{BCHCPZZ} * 0.65$

To gain a perspective on these estimates, bituminous coal and iignite consumed by residential and commercial users in 1994 accounted for onty 0.5 percent of all bituminous coal and lignite consumed-that is,
5 million short tons out of the 927 million short tons consumed in 1994.

Consumption in the industrial sector is estimated by State. An assumption is made that bituminous coal and lignite is consumed by coke plants in proportion to the amount of bituminous coal and lignite distributed to coke plants in each State. It is also assumed that the consumption of bituminotus coal and lignite by industrial users other than coke plants is in proportion to the amount delivered to other industrial users in each State The industrlal sector consumption is the sum of bituminous coal and lignite consumed by coke plarts and by other industrial users for each State:

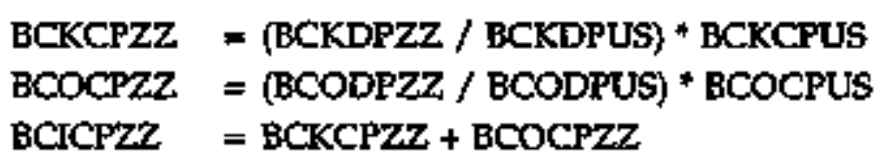

There are no data available for estimating the transportation sector's consumption of bituminous coal and ligrite by State. The quantity would be very small. The transportation sector accounted for only 1 percent of the national total consumption in 1960 and none since 1978. An assumption is made that when traxsportation sector consumption exists, the consumption by State, BCACPZZ, is in proportion to the share of the U.S. industrial sector attributed to each State:

\section{BCACPZZ = (BCICPZZ / BCICPUS $*$ BCACPUS}

Total consumption in each State, BCTCPZZ, is the sum of the sectors' consumption:

\section{BCTCPZZ = BCIRCPZZ + BCCCPZZ + BCICPZZ + BCACPZZ + BCEUPZZ}

The U.S. bituminous coal and lignite consumption estimates for each of the sectors and the total are calculated as the sum of the States' values. 


\section{Briltsh Thermal Units (Btu)}

Three factors are used for converting bituminous coal and lignite from physical units to Btu. The three factors, State-specific for each year, in units of million Btu per shont ton, are:

BCEUKZZ = the factor for converting bituminous coal and lignite consumed by the electric utility sector in each State from short tons to Btu;

BCHCKZZ = the factor for converting bituminous coal and fignite consumed by the residential and commercial sectors in each State from short tons to Btu; and

BCOCKZZ = the factor for converting bituminous coal and lignite consumed by other industrial tsers in each State from short tons to Btu.

The electric utility factor for each State is applied to estimate biturinous coal and lignite consumed by electric utilities in Btu:

BCEUBZZ = BCEUPZZ * BCEUKZZ

The residential and commercial sectors' State factor is applied to estimate bituminous coal and lignite consumed by the two sectors in Btu:

\section{BCRCBZZ = BCRCPZZ * BCHCKZZ \\ $\mathrm{BCCCBZZ}=\mathrm{BCCCPZZ} * \mathrm{BCHCKZZ}$}

The industrial sector Btu consumption is estimated in three steps. A constant conversion factor of $\mathbf{2 6 . 8 0}$ mitlion Btu per short ton is used for coking coal consumption for all years. The conversion factor for industrial users other than coke plants in each State is applied to other industrial users sector consumption. The industrial sector Btt consumption is then estimated by adding coking coal Btu constumption and other industrial users Btu consumption:
BCKCBZZ
BCOCBZZ
$=\mathrm{BCKCPZZ} * 26.60$
BCICBZZ
$=\mathrm{BCOCPZZ} * \mathrm{BCOCKZZ}$
$=\mathrm{BCKCBZZ}+\mathrm{BCOCBZZ}$

The transportation sector Btu consumption is estimated by applying the other industrial users sector State-factor to the transportation consumption:

$B C A C B Z Z=B C A C P Z Z * B C O C K Z Z$

Total consureption for each State is the sum of the sectors consumption:

BCTCBZZ D BCRCBZZ + BCCCBZZ + BCKCBZZ + BCACBZZ + BCEUBZZ

The U.S. consumption estimates in Btu are calctuated by stumming the State values for each of the data series.

\section{Adefitional Notes for Bituminous Coal and Lignile}

1. Bituminous coal and lignite consumption at the national level for the residential and commercial sectors (BCHCPUS), coke plants (BCKCPUS), and industries other than coke plants (BCOCPUS) are continuous data series. However, the digtribution data series used to develop State-level estimates by enduse sector ane not continuous.

For 1960 through 1979, State-level bituminous coal and lignite distribution data are used to apportion the U.S. consumption data to the States. From 1980 forward, the data in the distribution series variables-BCKDPZZ, BCODPZZ, and BCHDPZZ-are estimates of actual bituminous coal and lignite consumption rather than the distribution data used for the previouts years.

For 1980 forward, State-level total coal consumption data are available, but consumption by sector within many States is withheld. Estimates of the withheld sector consumption of total coal are derived by using the distribution series for the residential and conunercial sectors to fill in with held residential and commercial consumption. In most States, this leaves only one sector withheld and it can be derived by subtracting known sectors from the State total. This gives total coal consumption estimates for the end-use sectors that are compatible with State 
coal consumption data published in other FIA reports. Anthracite consumption is derived by using anthracite distribution data to estimate consumption within each sector and State that sum to the U.S. totals for anthracite consumption by sector contained in other EIA databases. Bituminous coal and lignite consumption for each sector and State is, then, the difference between the total coal consumption estimates and anthracite consumption estimates.

2. Prior to 1974, data for distribution of bituminous coal and lignite by State included several groupings of States for which separate State data were unavailable. These groupings were: (1) Maine, New Hampshire, Vermont, and Rhode Island; (2) North Dakota and South Dakota; (3) Delaware and Maryland; (4) Georgia and Florida; (5) Alabama and Mississippi; (6) Arkansas, Louisiana, Oklahoma, and Texas; (7) Montana and Idaho; (8) Arizona and Nevada; and (9) Washington and Oregon. Beginning with 1974, individual State distribution data became available. To estimate the 1960 through 1973 State distribution diata, the combined States were disaggregated in proportion to the individual States' shares of each similar State grouping in 1974.

3. Total coal consumption by State for 1980 thuough 1989 ptiblished in the EIA Quarteriy Coal Repport do not stum to the U.S. totals due to a quantity called "Unknown" in the source tables. This unknown coal consumption is assumed to be bituminous coal and lignite and is added to the residential, commercial, and "other industrial" sectors of Alabama, Ilinois, Kentucky, Penrsylvania, Tennessee, and West Virginia.

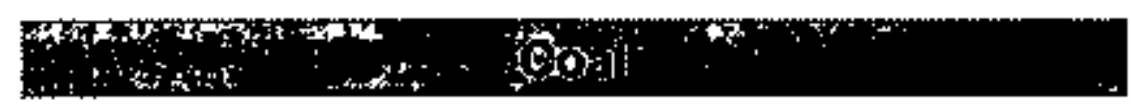

\section{Physical Unils}

All coal totals are the sum of the anthracite and bituminous coal and lignite estimates. It is assumed that no anthracite is consumed by the transportation sector. The calculations for each State and the U.S. total are:

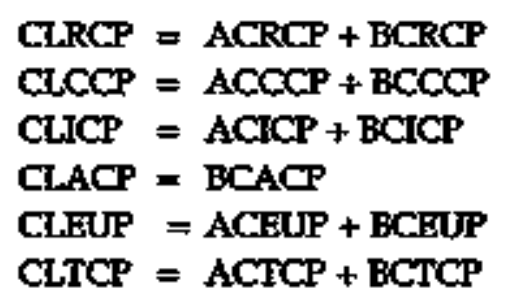

\section{Brilish Thermal Unils (Btu)}

Estimates of total coal consumption in Btu for each State and the U.S. are calculated:

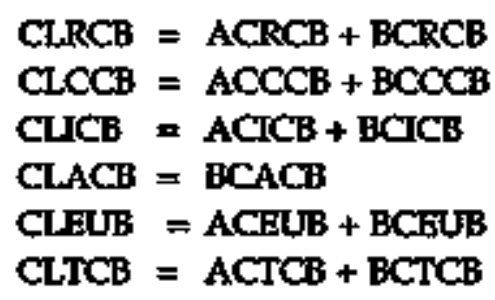

Additional calculations are performed to provide coal constumption estimates for the State Energy Price and Expenditure Data System (SEPEDS) from which the State Energy Price and Expenditure Report is produced. Cosl prices are available for two categories: coking coal (CLKCB) and all other types of coal, referred to as steam coal (CLSCB). Coking coal is a portion of the State Energy Data System (SEDS) industrial coal consumption. The remaining industrial portion, i.e, industrial steam coal, is the same as the other industutal coal consumption series in SEDS (CLOCB). The calculations for coking coal and steam coal State-level and U.S. data in Btu used by SEPEDS are:

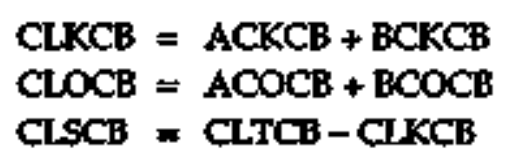




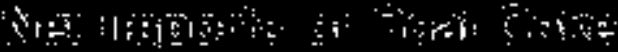

\section{Physficat Unils}

Net imports of coal coke is a component of total U.S. energy consumption. There is no attempt to estimate State allocations of this energy sounce. All of it is considered to be used by the industrial sector. In the State Energy Datt Report, net imports of coal coke is included in the U.S. data but not in the State-level data in all tables of total energy consumption and industrial sector energy consumption. Variables for net imports of coal coke into the United States are:

CCIMPUS = coal coke imported into the United States, in thousand short tons; and
CCEXPUS = coal coke exported from the United States, in thousand short tons.

Net imports is calculated:

CCNIPUS $=$ CCMMPUS - CCEXPUS

\section{Brilsh Thermel Units (Btw)}

The factor for converting coal coke from shork tons to Btu is 24.80 million Btu per short ton:
CCIMBUS
CCEXBUS
CCNIBUS
$=$ CCTMPUS * 24.80
$=$ CCBXTUS $* 24.80$
- CCMMBUS - CCEXBUS 


\section{Section 3. Natural Gas}

\section{Physical Units}

Six natural gas data series are used to derive the natural gas consumption estimates in the State Energy Data System (SEDS). Three of these data series are deliveries of natural gas to the end user by State and are used as consumption because actual consumption data at these levels are not available. The sources for the natural gas data are the reports in the Natural Gas Anntual series published by the Energy Information Administration (EIA) and its predecessors. These series, in million cubic feet, for each State are as follows (the two-letter State cade is represented by " $\mathrm{ZZ}$ " in the following variable names): cludes gas used by nonmanufacturing onganizations, such as hotels, restaumants, retail stores, laundres, and other service enterprises, and gas used in agriculture, forestry, and fisheries) plus natural gas delivered to other consumers (includes deliveries to municipalities and public authorities for institutional heating and street lighting);
NGCCPZZ = natural gas delivered to the commercial sector (in-
NGEUPZZ = natural gas consumed by electric utilities;
NGRPZZ = a portion of the natural gas delivered to the industrial sector (includes gas used as fuel and feedstock in chemical plants and to produce carbon black);
NGLEPZZ = natural gas consumed as lease fuel;
NGPLPZZ = natural gas consumed as plant fuel;
NGPZPZZ = natural gas consumed as pipeline furel;
NGRCPZZ \& natural gas delivered to the residential sector; and
NGVZPZZ = natural gas delivered for use as vehicle furel.

The U.S. totals of these independent variables are calculated as the sum of the States' values.
The data are combined into the four major end-use sectors used in SEDS as closely as possible. However, natural gas data are collected by using different aggregations of users. The industrial sector in SEDS is intended to contain energy used in agriculture, forestry, and fisheries. For natural gas, these categories are reported with commercial use of natural gas (the series called NGCCPZZ in SEDS) and cannot be separately identified. No adjustment for this end-use inconsistency could be made in SEDS.

The residential sector's consumption of natural gas is represented by the variable for deliveries to the restdential sector, NGRCPZZ.

The conwercial sector's consumption of natural gas is represented by the variable for deliveries to the commercial sector, NGCCPZZ.

The industrial sector's consumption of natural gas in SEDS, NGICPZZ, is estimated to be the sum of natural gas delivered to the industrial sector, NGINPZZ, natural gas consumed as lease fuel, NGLEPZZ, and natural gas consumed as plant fuel, NGPLPZZ. The source dacument reports lease and plant fuel combined for 1960 through 1992; the combined data series is stored as NGLEPZZ in SEDS.

\section{NGICPZZ = NGINPZZ + NGLEPZZ + NGPLPZZ}

The transportation sector's constumption of natural gas, NGACPZ2, is the sum of natural gas consumed it the operation of pipelines, primarily in compressors, NGPZPZZ, and natural gas delivered for wose as vehicle fuel, NGPVPZZ. Prior to 1990, the small amounts of natural gas consumed as vehicle fued are included in the commercial sector consumption and carnot be identified separately; therefore, NGPVPZZ is zero prior to I990 in SEDS.

NGACPZZ = NGPZPZZ + NGVZPZZ 
N Electric utilities' consumption of natural gas is represented by the A data series NGEUPZZ.

The total consumption of natural gas, estimated for each State, is the sem of the consumption by the end-use sectors and electric utilities;

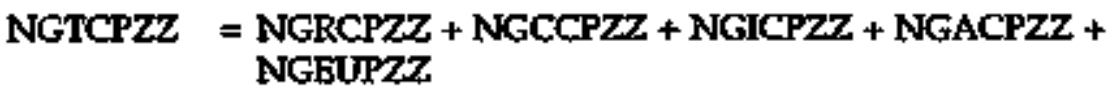
NGEUPZZ

The U.S. consumption estimates for each of the sectors and the U.S. total are calculated as the sum of the States' values.

\section{British Thermal Untts (Biu)}

Three factors for each State are used for converting the consumption of natural gas from its physical units of miltion cubic feet into thousand Btu per cubic foot. Two of these State-level factors are from sources listed in Appendix C:

NGEUKZZ - The factor for converting natural gas consumed by electric utilities from physical units to Btut and

NGTCKZZ = The factor for corverting natural gas consumed by all sectors from physical mitis to Btu.

These two factors are used to derive a thind factor, NGNUKZZ, for converting natural gas used by all sectors other than electric utilities from phystcal units to Btu:

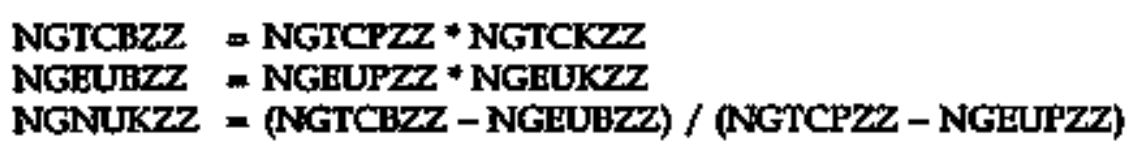

Natural gas consumption in Btu for the residential, commercial, industrial, and transportation sectors in tach State is calculated by multiplying the physical unit data by the factor NGNUKZZ, such as:

\section{NGACBZZ = NGACPZZ * NGNUKZZ \\ NGCCBZZ = NGCCPZZ * NGNUKZZ}

The U.S. consumption estimates in Btu for each of the sectors and the U.S. total are calculated as the sum of the States' Btu values.

Prior to 1972, conversion factors for natural gas consumed by electric utilities were not collected; therefore, the factor for all natural gas consumed (NGTCKZZ) is used for electric uttitites (NGEUKZZ) and for the other sectors (NGNUKZZ) for 1963 through 1971. Prior to 1969, State-level conversion factors for natural gas consumption were not collected and a standard factor of 1.035 thousand Btu per cubic fook is used for all sectors in all States for 1960 through 1962 .

\section{Additional Calculatione}

Although SEDS does not use U.S.-level conversion factors for calculating natural gas consumption, these factors are calculated by SEDS for reference and are shown in the natural gas tables in Appendix D:

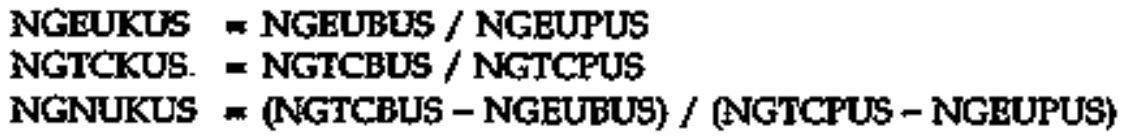

NGEUKUS - NGEUBUS / NGEUPUS

NGTCKUS. = NGTCBUS / NGTCPUS

NGNUKUS - (NGTCBUS - NGEUBUS) / (NGTCPUS - NGEUPUS) 


\section{Section 4. Petroleum}

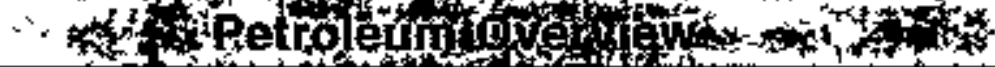

The 27 petroleum products included in the State Energy Data System (SEDS) are explained in this section. For 12 of these products, the means of estimating their individual consumption by State is described in individual sections. The 12 petroleum products are

- asphalt (AS)

- aviation gasoline (AV)

- distiliate fuel (DF)

- jet fuel, kerosene-type (JK)

- jet fuel, naphtha-type (0N)

- kerosene (KS)

- liquefied petroleum gases (LG)

- lubricants (LU)

- motor gasoline (MG)

- petroleum coke (PC)

- residual fuel (RF)

- road oil (RD)

The remaining 15 products are described in the section "Other Petroleum Products" and inclade the following:

- crude oil, including lease condensate (CO)

- miscellaneous petroleum products (MS)

- natural gasoline (NA) (including isopentane)

- petroleum feedstocks, naphtha less than $401^{\circ} \mathrm{F}$ (FN)

- petroleum feedstocks, other oils equal to or greater than $401^{\circ} \mathrm{F}$ (FO)

- petroleum feedstocks, still gas (FS)

- plant condensate (PL)

- pentanes plus (PP)

- special naphthas (SN)
- still gas (SG)

- unfractionated stream (US)

- waxes (WX)

- unfinished oils (UO)

- motor gasoline blending components (MB)

- aviation gasoline blending components $(\mathrm{AB})$

The last petroleum documentation section, "Petroleum Summaries," describes how the 27 petroleum products are combined for each major end-use sector's estimated consumption.

Table A2 summarizes the petroleum products' end-use assignments in SEDS. Shown in this table are the first four letters of the seven-letter variable names used to identify all energy sources. The first two letters identify the petroleum product and the next two letters identify the end-use sector. For example, the table shows that the aviation gasoline estimated to be consumed by the transportation sector is all aviation gasoline consumed, and that there is some estimated consumption of lubricants in the industrial and transportation sectors, while distillate fuel is consumed in every sector.

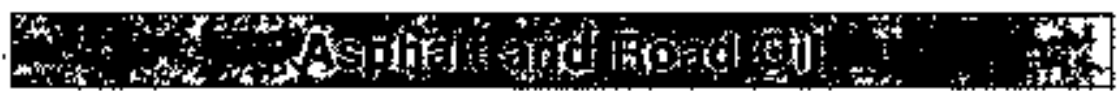

\section{Physiagl Units}

There are no State-level consumption data for asphalt and road oil available. Therefore, the State-level sales data are used to apportion the national consumption numbers to the States.

The asphalt and road oil sales data are in shore tons, while the consumption data are in thousand barrels. Because the sales data are 
P Table A2. Summary of Petroleum Products in the State Energy Data System

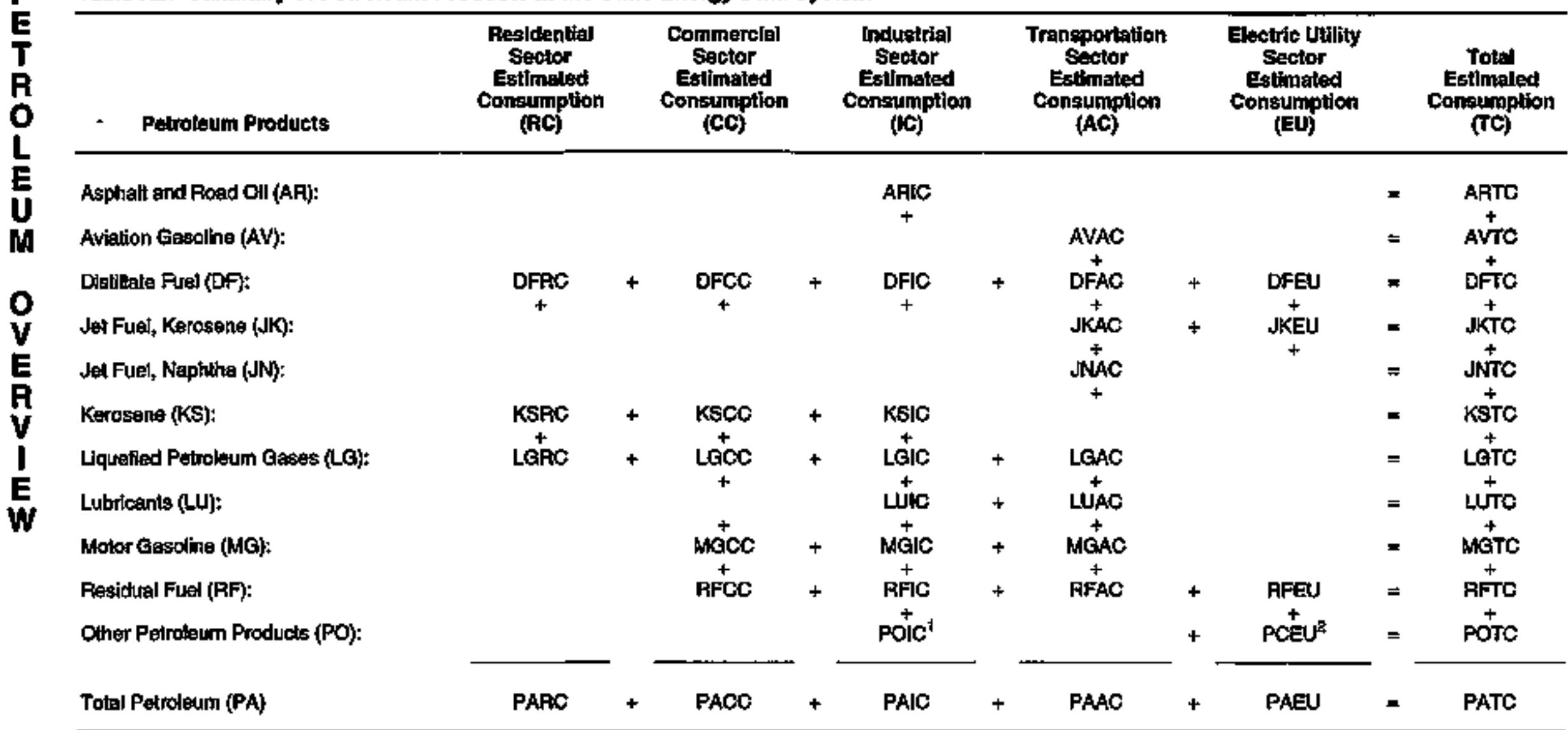

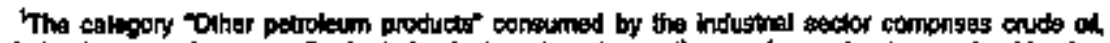

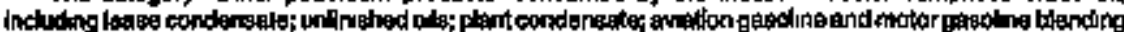

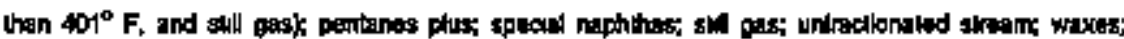

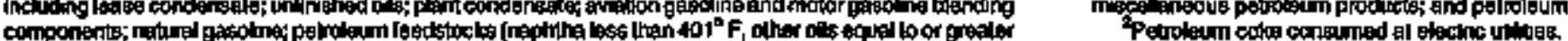

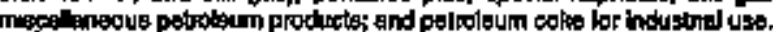


used only for apportioning the U.S. consumption data to the States, they do not need to be converted into thousand barrels.

The four data series that are used to estimate consumplion of asphalt and road ofl are (" $Z Z$ " in the variable name represents the two-letter State code that differs for each State):
ASINPZZ = asphalt sold for use in the industrial sextor of each State, in short tons;
ASTCPUS = asphalt total consumed in the United States, in thou- sand barrels;
RDINPZZ = road ail sold for use in the industrial sector of each State, in short tons; and
RDTCPUS - road oil total consumed in the United States, in thou- sand barrels.

All asphalt consumption is assigned to the industrial sector because it is used in construction activity. ASINPZZ represents all asphalt sold as paring products, as roofing products, and for all other uses.

ASTCPUS represents total U.S. consumption of asphalt, and RDTCPUS represents total U.S. consumption of road oil. Both are product supplied data series in the publication Peirolenom Supply $A x-$ nual, published by the Energy Information Administration (EIA). Beginning in 1983, asphalt product supplied includes road oil, and RDTCPUS is entered as zero in SEDS.

The source of the third variable, RDINPZZ, is the report series "Sales of Asphalt" for 1960 through 1980 , published by BIA. This sales seties was discontinued after the 1980 report. Values for 1981 and 1982 are estimated as described under "Additional Notes" in this section. Begirming with 1983 data, when road oil is included in asphalt product supplied data in the source publication, RDINPZZ is entezed as zero in SEDS.

To calculate State consumption estimates of asphalt, total sales of asphalt in the United States to the industrial sector is first calculated as the sum of the State data:

ASINPUS $=$ ¿ASINPZZ
Each State's consumption of asphalt in the industrial sector (ASICPZZ) is calculated to be in proportion to each State's sales:

\section{ASICPZZ = (ASINPZZ / ASINPUS) * ASTCPUS \\ ASICPUS $=$ EASICPZZ}

Since all consumption of asphalt is assumed to be in the industrial sector, the total consumption of asphalt in each State equals the industrial sector consumption:

$A S T C P Z Z=A S I C P Z Z$

The State sales of road oil are used to create an estimate of State consumption of road oil.

The U.S. total of all road oil sales to the industrial sector is calculated by adding all of the States' sales:

RDINPUS $=\Sigma$ RDINPZZ

Each State's consumption of road oil in the industrial sector (RDICPZZ) is calculated to be in proportion to each State's sales:

\section{RDICPZZ = (RDINPZZ / RDINPUS) * RDTCPUS \\ RDICPUS $=$ ERDICPZZ}

Since all road oil consumption is assumed to be in the industrial sector, the total consumption of road oil in each State equals the industrial sector consumption:

RDTCPZZ = RDICPZZ

Asphalt and road oil consumption are added together:
ARICPZZ
= ASICPZZ + RDICPZZ
ARICPUS
$=$ SARICPZZ
$A R T C P Z Z=A S T C P Z Z+R D T C P Z Z$
ARTCPUS
= $\triangle A R T C P Z Z$ 


\section{Brilish Thermal Units (Btu)}

Asphalt and road oil have a heat content value of approximately 6.636 million Btut per barrel. This factor is applied to convert asphalt and road oil estimated consumption from physical uribs to Btu:

$$
\begin{aligned}
\text { ARICBZZ } & =\text { ARICPZZ * } 6.636 \\
\text { ARICBUS } & =\text { EARICBZZ }
\end{aligned}
$$

Because all asphalt and road oil are assumed to be used by the indus trial sector, total asphalt and road oil consumption in each State and in the United States is assumed to equal the industrial sector corsumption:

\section{ARTCBZZ = ARICBZZ \\ ARTCBUS = AEICBUS}

\section{Additional Notes on Asphall and Foad OtI}

Because the Federal Government stopped collecting asphalt and road oil sales data in 1980, the source for these numbers in recent years has been reports published by the Asphalt Institute. There is an irherent problem in the methodology of using sales to estirnate consumption because asphalt and road oil sold by a producer in one State may be easily transported across State lines and consumed in a neighboring State. The Asphait Institute acknowledges this problem and estimates that, in any one year, about 15 States may have consumption estimates as much as 20 percent too high or too low.

Total U.S. consumption of asphalt and road oil are the product supplied data series from the ElA publication Petratem Supply Annual. Beginning with 1983 data, the road oil data series is no longer published separately but is included in the asphalt product supplied. The sum of the two series for all years in SEDS is a continuous series.

The EIA report series "Sales of Asphalt," which is the source for road oil sales by State (RDINPZZ) in SEDS for 1960 through 1980, was discontinued after the 1980 report. For 1981 and 1982, State estimates of road oil sales were created by first converting the amnual total U.S. road oil product supplied data into short tons (one short ton contains 5.5 bairels of road oil). Then, the U.S. total road oil product supplied, in short tons, was disaggregated to each State in proportion to the State's share of total U.S. asphalt sales as reported in the Asphalt Institute's Report on Sales of Asphalt in the U.S.

\section{"Aviation Gy}

\section{Phystoal Units}

The three data series used to extimate consumption of aviation gasoline are:
AVMIPZZ m aviation gasoline issued to the militacy in each Stake, in thousand barrels;
AVNMMKZ = aviation gasoline sold to nonmilitary users in each State, in thoustand gallons; and
AVTCPUS a aviation gasoline total consumed in the United States, in thousand barrels.

The U.S. Department of Transportation, Federal Highway Administration publishes the nonmilitary aviation gasoline sales data by State (AYNMMZZ) in Highwry Statistics.

AVMIPZZ is the issues of aviation gasoline to the military in each State and is obtained from the U.S. Department of Defense, Defense Loglstics Agency, Defense Fuel Supply Center.

Total U.S. consumption of aviation gasoline (AVTCPUS) is the product supplied data series in the publication Petroleum Strpply Annual published by the Rnergy Information Adminststration (EIA).

The State-level data series are summed to provide totals for the United States:

\section{AVMIIPS $=$ IAVMIIPZZ. AVNMMUS = EAVNMMZZ}

The State sales of nonmilitary aviation gasoline data are converted from thousand gallons to thousand barrels ( 42 gallons $\times 1$ barrel): 


\section{AVNMPZZ $=$ AVAMMZZ $/ 42$}

The U.S. norunilitary sales is the sum of the States' sales:

\section{AVNMPUS $=$ LAVNMPZZ}

The total sales of aviation gasoline is estimated as the sum of nonmilitary sales and military issues:

\section{AVTTPZZ = AVNMPZZ + AVMIPZZ \\ AVTTPUS $=$ IAVTTPZZ}

All aviation gasoline is assumed to be used by the transportation sector. An estimate of aviation gasoline consumption by the transportathon sector by State (AVACPZZ) is calculated by assuming that each State consumes aviation gasoline in proportion to the amount sold to that State:

$$
\begin{array}{ll}
\text { AVACPZZ } & =\text { (AVTTPZZ / AVTTPUS } * \text { AVTCPUS } \\
\text { AVACPUS } & =\text { IAVACPZZ }
\end{array}
$$

Total aviation gasoline consumption in each State, AVTCPZZ, equals the trarsportation sector consumption in each State:

\section{AVTCPZZ = AVACPZZ}

\section{Britlsh Thermal Units (Btw)}

Aviation gasoline has a heat content value of approximately 5.048 million Btu per barrel. This factor is applled to convert aviation gasoline estimated consumption from physical units to Bh:

$$
\begin{array}{ll}
\text { AVACBZZ } & =\text { AVACPZZ } \\
\text { AVACBUS } & =\text { IAVACBZZ }
\end{array}
$$

Becatuse all aviation gasoline is assumed to be used for transportation, aviation gasoline total consumption in each State and in the United States equals the transportation sector consiumption:
AVTCBZZ = AVACBZZ
AVTCBUS = AVACBUS

\section{Adtitional Notes on Aviation Gasolline}

Aviation gasoline issues to the military for each State (AVMIPZZ) are obtained from the U.S. Department of Defense, Defense Logistics A gency, Defense Fuel Supply Center. There are no data available for 1960 through 1974, and the data available for 1975 and 1976 are not consistent; therefore, the 1977 values are used for 1960 through 1976 in SEDS. The data are reported by fiscal year for 1977 through 1988 and taken from the Defense Energy Information System. For 1989 and 1990, fiscal-year data from two databases, Defense Fuel Automated Management System and the Into-Plane Database, are summed. For 1991 and 1992, data from the same two databases, reported by calendar year, are used.

\section{minger}

\section{Physical Units}

Since State-level and end-use consumption data for distillake fuel (except for that corsumed by electric utilities) are not available, sales of distillate fuel into or within each State, in thousand barrels, published by the Energy Information Administration (ELA) are used to estimate distillate fuel consumption. The sales data are adjusted to sum to the Petroleum Administration for Defense District subtotals of the EIA distillate fuel product supplied data series. Both the sales data and the adjusted sales series are published in the EIA FueI Oil and Kerosene Sales Report. The following variable names have been afsigned to the adjusted sales series (" $\mathrm{ZZ}$ " in the variable names represents the two-leiter State code that differs for each State):

DFBKPZZ = distillate fuel adjusted sales for vessel bunkering use (i.e., the fureling of commencial or private boats, such as pleasure craft, fishing boats, tugboats, and oceangoing vessels, including vessels operaked by oil companies, and fueling for other marine purposes), excluding that sold to the Armed Fonces;

DFOMPZZ = distillate fuel adjusted sales to commercial establishments for space heating, water heating, and cooking; 
DFIBPZZ a distillate fuel adjusted sales to industrial establishments for space healing and for other industrial use (i.e, for all uses to mines, smelters, plants engaged in producing manufactured products, in processing goods, and in assembling), including farm use;

DFMIIZZ = distillate fuel adjusted sales to the Armed Fonces, regardless of use;

DFOCPZZ = distillate fuel adjusted sales for oil company use, including all fuel oil, crude oil, or acid sludge used as huel at nefineries, by pipelines, or in field operations;

DFOFPZZ = distillate htel adjusted sales as diesel fuel for off-highway use in construction (i.e., earthmoving equipment, cranes, stationary generators, air compressors, etc.) and for offthighway uses other than construction (i.e., logging);

DFONPZZ = distillate fuel adjusted sales as diesel fuel for on-highway use (i.e., as engine fuel for trucks, buses, and automobiles);

DFOTPZZ = distillate fuel adjusted sales for all other uses not identified in other adjusted sales categories;

DFRRPZZ = distillate fuel adjusted sales to the railroads for use in fueling trains, operating railroad equipment, space heating of buildings, and other operations; and

DFRSPZZ = distillate fuel adjusted sales to the residential sector for space heating water heating, and cooking, excltoding farm houses.

Thure series are tnsed in SEDS for consumption data:

DKEUPZZ = distillate fuel consumed by electric utilities, in thousand barnels;

JKKUPZZ = kerosene-type jet fuel consumed by electric utilities, in thousand barrels; and

DFTCPUS - distillate fuel total constrmed in the United States, in thousand barrels.

Distillate fuel consumed by electric utilities (DKEUPZZ) is collected by EIA on Form EIA-759, "Monthly Power Plant Report," and predecessor forms. (See Note 4 at the end of this distillate furel section for hurther information on changes in this series' data definitions.) The series DKEUPZZ includes kerosene-type jet fuel consumed at electric utilities that is identified as JKEUPZZ. The kerosene-type jet fuel is subtracted from the distillate fuel data and accounted for in the jet fuel data described in a following section of this doctmentation.

Total consumption of distillate cuel in the United States, DFTCPUS, is the product supplied series in the EIA publication Petroleum Supply Annual.

To begin calculating distillate fuel State and end-use consumption, all of the State-level data series are summed to provide totals for the United States.

Next, the variables are combined as closely as possible into the major end-use sectors used in SEDS. The residential sector adjusted sales and the commercial sector adjusted sales contain only DFRSPZZ and DFCMPZZ, respectively.

The adjusted sales of distillate fuel to the industrial sector for each State, DFINPZZ, is the sum of the distillate fuel adjusted sales for industrial use, including industrial space heating and farm use (DFIBPZZ), for oil company trse (DFOCPZZ), for off-highway use (DFOFPZZ), and for all other uses (DFOTPZZ):

\section{DFINPZZ = DFIBPZZ + DFOCPZZ + DFOFPZZ + DFOTPZZ DFINPUS $\quad$ EDFINPZZ}

The adjusted sales of distillate fuel to the transportation sector for each State, DFTRPZZ, is the sum of the distillate fuel adjusted sales for vessel bunkering, military use, railroad use, and the diesel fuel used on-highway:

\section{DFTRPZZ = DFBKPZZ + DFMIPZZ $\div$ DERRPZZ + DFONPZZ DFTRFUS $=$ EDFTRPZZ}

Adjusted sales of distillate fuel oil to the residential, comunercial, industrial, and transportation sectors are added to create a subtotal of adjusted sales to all sectors other than the electric utility sector, DFNDPZZ:

DFNDPZZ = DFRAPZZ + DFCMPZZ + DFINPZZ + DFTRPZZ 
Consumption of distillate fuel by electric utilities (DFEUPZZ) is calculated by subtracting the kerosene-type jet fuel consamed by electric utilities from the inpuk series DKEUPZZ:

$$
\begin{array}{ll}
\text { DFEUPZZ } & =\text { DKEUPZZ }- \text { JKEUPZZ } \\
\text { DFEUPUS } & =\text { EDFEUPZZ }
\end{array}
$$

The estimated U.S. distillate fuel consumption by all sectors other than the electric ubility sector, DFNCPUS, is calculated by subtracting the distillate fuel consumption at electric utilities from the total U.S. distillate fuel consumption:

\section{DFNCPUS = DFTCPUS - DFEUPUS}

This U.S. subtotal of distillate fuel consumption by the four end-use sectors, DFNCPUS, is apportioned to the States by using the end-use sectors' State-level adjusted sales data. The assumption is made that each State consumes distillate fuel in proportion to the amount of adjusted sales to that State:

\section{DFNCPZZ $=$ (DFNDPZZ / DFNDPUS) * DFNCPUS}

The end-use sectors' subtotal for each State, DFNCPZZ, is further divided into estimates for the four end-use sectors in proportion to each sector's adjusted sales. The estimated residential sector consumption in each State, DFRCPZZ, is calculated:

$$
\begin{array}{ll}
\text { DFRCPZZ } & =(\text { DFRSPZZ } / \text { DFNDPZZ }) * \text { DFNCPZZ } \\
\text { DFRCPUS } & =\text { EDFRCPZZ }
\end{array}
$$

The commercial sector's estimated consumption in each State, DFCCPZZ, is calculated:

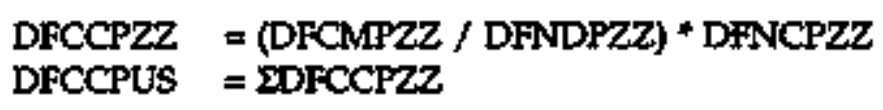

The industrial sector's estimated consumption in each State, DFICPZZ, is calculated:

$$
\begin{array}{ll}
\text { DFICPZZ } & =\text { (DFINPZZ / DFNDPZZ) } * \text { DFNCPZZ } \\
\text { DFICPUS } & =\text { DDFICPZZ }
\end{array}
$$

The transportation sector's estimated consumption in each State, DFACPZZ, is calculated:

$$
\begin{array}{ll}
\text { DFACPZZ } & =(\text { DFTRPZZ } / \text { DFNDPZZ }) * \text { DFNCPZZ } \\
\text { DFACPUS } & =\text { SDFACPZZ }
\end{array}
$$

Total State disillate fuel consumption is the sum of the end-use sectors' consumption subtotal and the electric utilities consumption:

DFTCPZZ = DFNCPZZ + DFEUPZZ

\section{Britsh Themal Unils (Btu)}

Distillate fuel has a heat content value of approximately 5.825 million Btu per barrel. This factor is applied to convert distillate fuel estimated consumption for the five consuming sectors from physical units to Btu as shown in the following examples:

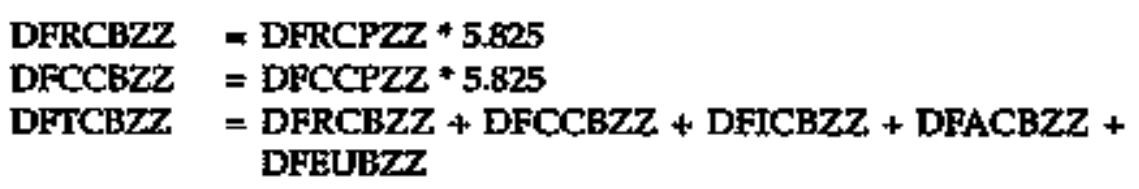

The U.S. level Btu consumption estimates are calculated as the sum of all the States' data.

In the State Entry Data Report tables, "Estimates of Energy Input at Flectric Utilities," the data used in the colunn headed "Light Oil" is the variable DKEUP (distillate fuel plus jet kerosene) in physical urits. The Btu variable, DKEUB, is calculated:

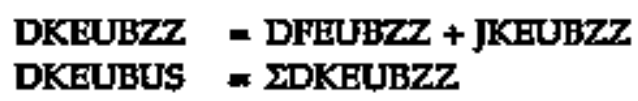

\section{Addhional Notes on Distitate Fued}

1. "Deliveries" data are actually called "shipments" in the source document for 1960 and 1961; "consumption" for 1962 through 1966; "shipments" for 1967; "sales" from 1968 through 1978; 
"deliveries" for 1979 through 1987; and "adjusted sales" for 1988 forwand.

2. State data for the variables DFONPZZ (on-highway use), DFOFPZZ (off-highway use), and DFOTPZZ (othex) for 1967 axe unavailable from published sources. These three variables compose the miscellaneous use category for distillate fuel, which is known for all years by State. State estimates of DFONPZZ and DFOFPZZ for 1967 were developed by dividing the 1966 values for DFONPZZ and DFOFPZZ by the 1966 total miscellaneous use for each State and applying these percentages to the 1967 total miscellaneous use for each State. The 1967 State estimates for DFOTPZZ ane the nemainder of the 1967 miscellaneous category after DFONPZZ and DFOFPZZ have been subtracted.

3. In 1979, ElA implemented a new survey form, EIA-172, to obtain deliveries of fuel oil and kerosene data and updated the list of respondents. (A detailed explanation is published in the Energy Datu Report, "Deliveries of Fuel Oil and Kerosene in 1979.") In the new survey form, certain end-use categories were redefined-in many cases to collect more disaggregated data. The reclassifications resulted in some end-use categories that were no longer comparable with those in previous surveys. Where discontinuities occurred, estimates for the pre-1979 years have been made in the State Energy Data System (SEDS) to conform with the 1979 fuel oil deliveries classifications. The pre1979 deliveries estimates are not published in this report, but are used in SEDS to disaggregate the known U.S. total product supplied (consumption) into State and major end-use sector consumption estimates.

For distillate fuel deliveries in 1979, the end-use categories called "residential," "commercial," "industrial," and "farm" are available. The pre-1979 deliveries categories ane called "heating" and "industrial" (which inchuded farm use). While the pre1979 categories individually are not contimuous with the 1979 categories, theitr subtotals are related. That is, a general comparison can be made between the sum of residential, commercial, industrial, and farm deliveries in 1979 and the sum of heating and industrial deliveries in the pre-1979 years. Therefore, the following method was applied to present a comparable series for distillate fuel delivered to the residential, commercial, and industrial sectors:

- For each of the pre-1979 years, a subtotal was created for each State by adding each State's heating and industrial de. liveries categories. A comparable $197 \%$ subtotal was created by adding each State's residential, commercial, industrial, and farm deliveries categories.

- Residential, commercial, and industrial (including farm) shares of the subtotal in 1979 were calculated for each State.

- These 1979 end-use shares were then applied to each pre1979 subtotal of distillate fuel deliveries in each State to create State estimates of enduse deliveries for 1960 through 1978.

The 1980 through 1982 distillate fuel deliveries data are based on the same survey as that used for 1979; therefore, the 1980 through 1982 data are directly comparable to 1979 data.

In 1984, ELA again updated the list of respondents for this survey, and the Form ELA-172 became the Form EIA-821, "Annual Fuel Otl and Kexosene Sales Report." EIA did not conduct a fuel oil and kerosene deliveries survey for 1983 . The 1983 estimates in SEDS are based on 1984 data obtained from the Form EIA-821. Statistical procedures and methodologies used for the Form ElA-821 differ from those used in previous years. Therefore, the 1983 and forward sales data may not be directly comparable to the pre-1983 data. (In the source document, the deliveries data for 1983 forward are reported in thousand gallons. These data are first converted to thousand barrels before being entered into SEDS.)

Some of the No. 2 diesel fuel neported as sold to the commercial and industrial sectors, DFCMPZZ and DFINPZZ, on the EIA forms may also be included in the on-highway data, DFONPZZ, obtained from the Federal Highway Administration. Included in the commencial sector ts some diesel fuel consumed by gov. emment vehicles and school buses, and included in the indus. trial sector is some diesel fuel consumed by fleets of trucks. 
Because the specific quantities involved are unknown, SEDS re flects the diesel fuel consumption as reported in the EIA Petroteum Morketing Monthly and no attempt has been made to adjust the end-use reporting.

4. The data on fuel oil consumed at electric utilities for all years and States are actual fuel oil consumption numbers collected from electric utilities on the ELA Form ELA-759, "Monthly Power Plant Report, and predecessor forms. Due to changes in fuel oil reporting classifications on the Form BLA-759 over the years, it is not possible to develop a thoroughty consistent series for all years. However, over time, data more accurately disaggregating fuel oil into distillate fuel and residual fuel have become available. For 1960 through 1969 , only data on total fuel oil consumed at electric utilities by State are available. For 1970 through 1979, fuel oil constmed by plant type (internal combustion and gas turbine plants combined and steam plants) by State are available. For 1980 forward, data on consumption of light oil at all plant types combined and consumption of heavy oil at all plant types combined are available by State. In SEDS, the following assumptions have been made:

- 1960 through 1969 - State timates of fuel bil consumption by plant type have been created for each year by applying the shares of steam plants (primarily residual fuel) and internal combustion and gas turbine plants (primarily distillate fuel phus small amounts of jet kerosene) by State in 1970 to each year's total fuel oil consumption at electric utilities for 1960 through 1969.

- 1970 through 1979 - fuel oil consumed by steam plants is asstumed to equal residual fuel consumption, and fuel oil consumed by intemal combustion and gas turbine plants is assumed to equal distillate fuel plus jet kerosene consumption.

- 1980 and forward - total heavy oil consumption at all plant types is assumed to equal residual futel consumption, and total tight oil consutuption at all plant types is assumed to equal distillate futel plus jet kerosene consumption.
The data series thus derived for SEDS for residual fuel and distillate fuel plus jet kerosene consumption at electric utilities is considered to be actual consurstion at electric utilities for each State and each year.

There are two types of jet fuel with different heat contents, kerosentetype jet fuel (JK) and naphtha-type jet fuel (JN), which are added in the State Energy Data System (SEDS) to give total jet fuel (JF). Jet fuel is used primarily for transportation, although, for 1972 through 1982, small amounts of the kerosene-type jet fuel were reported as used in the electric utility sector.

\section{Kerosene-Type Jet Fuel}

\section{Phyoleal Units}

Data series used to calculate kerosene-type jet fuel consumption estimates are ( ${ }^{\circ} \mathrm{ZZ}^{*}$ in the variable name represents the two-letter State code that differs for each Stake):

JKTCPUS = kerosene-type jet fuel total consumed, in thousand barrels;

JKEUPZZ = the electric utility sector consumption of kerosenetypo jet fuel in each State, in thousand barrels; and

JKTTPZZ = kerosene-type jet fuel total sold, in thousand gallons.

Total U.S. consumption of kerosene-type jet fuel, JKTCPUS, is the product supplied data series in the publication Petroleum Suppiy Anntual, published by the Energy Information Administration (EIA).

Kerosene-type jet fuel consumed by electric utilities, JKEUPZZ, is published by EIA in the Cost and Quality of Fuels for Electric Utility Plants. These data axe available for 1972 through 1982 only. Consumption in all other years is assumed to be zero. 
Kerosene-type jet fuel total soid, JKTTPZZ, was collected by the Ethy! Corporation, Petroleum Chemicals Division, for 1960 through 1983 and by ELA for 1984 forward. The Ethyl Corporation data are sales to commercial users and are used to represent total sales based on the assumption that there is little military use of kerosene-type jet fuel during 1960 through 1989. (See Note 1 in the "Additional Notes" section for the source reference for this assumption) The EIA data for 1984 forward include commercial and military sales.

U.S. totals for the two State series are calculated as the sum of the State data.

Most kerosene-type jet fuel is used by the transportation sector. The transportation sector consumption for the United States (IKACPUS) is estimated as the difference between the total kerosene-type jet fuel consumed and the electric utility consumption:

\section{JKACPUS = JKTCPUS - JKEUPUS}

It is assumed that kerosene-type jet fuel consumption in each State is in proportion to the atnount sold in each State:

JKACPZZ = (JKTTPZZ $/$ JKTTPUS $) *$ JKACPUS

Tótal kerosene-type jel fuel by State is estimated as:

$\mathrm{JKTCPZZ}=\mathrm{JKACPZZ}+\mathrm{JKEUPZZ}$

\section{Brttish Thermal UnIts (BIU)}

Kerosene-type jet fuel has a heat content value of approximately 5.670 million Btu per barrel. This factor is applied to convert kerosene-type fet fuel from physical units to Bta:
IKACBZZ $=$ JKACPZZ *5.670
JKACBUS $=$ EKACBZZ
JKEUBZZ = JKEUPZZ * 5.670
JKEUBUS $=$ I]KEUBZZ
IKTCBZZ = JKTCPZZ *5.670
IKTCBUS $=$ IJKTCBZZ

\section{Additional Notes on Kerosene-Type det Fuel}

1. An assumption is made that kerosene-type jet futel use by the military in 1960 through 1983 is negligible. This assumption is based on product definitions from the American Petroleum Institute's Standard Definitions for Pefroleum Statistics, Technical Report No. 1, Third Edition (1981), page 13, which states that kerosene-type jet fuel is used primarily by commercial aircraft engines.

2. Ethyl Corporation jet fuel sales to commercial users by State include some sales data that were improperiy allocated between the States of Illinois and Indiana for 1960 through 1973. To adjust for this error, the average relative proportions of Illinois and Indiana sales from 1974 through 1978 were applied to the sum of the Illinois and Indiana sales in 1960 through 1973. From 1974 through 1983, sales data were correctly allocated.

3. Jet fuel sales in llinois decreased sharply from 1984 forward, while sales in Indiana increased by about the same ancunt. It is possible that jet fuel for use at Chicago, Illinois, airports ntay have been purchased in Indiana. The same anomaly may have happened between New York and New Jersey beginning in 1981, when jet fuel for consumption at New York Ctty airports may have been purchased in New Jersey. This is an inherent problem with using sales data as an inctication of consumption, and no attempt has been made to adjust the numbers.

4. Prior to 1964, kerosene-type jet fuel was included in the total kerosene product supplied data in the source, the U.S. Department of the Interior, Burealu of Mines, Mineral Industry Sunveys, "Petroleum Statement, Annual," Table 2, "Salient Statistics of the Major Refined Petroleum Products in the United States," Table A3 summatizes the derivation of kerosene and jet fuel consumption estimates (columns 4 and 5) from data published in the source (colurins 1, 2, and 3) for 1960 through 1963. For 1964 and years following, kerosene and kerosene-type jet fuel are reported separately in the source documents.

5. Kerosene-type jet fuel consumed by electric utilities, JKEUPZZ, is published in the EIA Cost and Qunality of Fuels for Electric Utitity Plants. These data are available for 1972 through 1982 only. 
Tabla A3. Estimate of U.S. Consumption of Kerosene and Jet Fuel for 1960 through 1963 (Thousand barrels)

\begin{tabular}{|c|c|c|c|c|c|}
\hline Year & $\begin{array}{c}\text { (1) } \\
\text { Korosente Demand, } \\
\text { Including } \\
\text { Commtingial } \\
\text { Jet Fued }\end{array}$ & $\begin{array}{c}\text { (2) } \\
\text { Jet Fual Demand, } \\
\text { Uifitary } \\
\text { Uso Only }\end{array}$ & $\begin{array}{c}\text { (3) } \\
\text { sales of } \\
\text { Kerosene for } \\
\text { Commercial } \\
\text { Jet Fual Usa }\end{array}$ & $\begin{array}{l}\text { (4) } \\
\text { Estimated } \\
\text { Kenosent } \\
\text { Consumption } \\
\text { (1) }- \text { (3) }\end{array}$ & $\begin{array}{l}\text { (5) } \\
\text { Estimated } \\
\text { Totel Jet Fuel } \\
\text { Consumption } \\
(2)+(3)\end{array}$ \\
\hline $\begin{array}{l}1960 \\
1961 \\
1902 \\
1963\end{array}$ & $\begin{array}{l}132,499 \\
144,435 \\
164,167 \\
172,212\end{array}$ & $\begin{array}{l}102,803 \\
104,436 \\
112,401 \\
115,257\end{array}$ & $\begin{array}{l}33,159 \\
47,187 \\
66,134 \\
75,236\end{array}$ & $\begin{array}{l}99,340 \\
97,248 \\
98,003 \\
96,976\end{array}$ & $\begin{array}{l}135,969 \\
151,629 \\
178,585 \\
190,479\end{array}$ \\
\hline
\end{tabular}

Consumption in all other years is assumed to bero. Statelevel data for 1972 through 1974 are not available. The percentage of each State's consumption of the total U.S. consumption in 1975 was used to apportion the 1972 through 1974 national data to the States.

\section{Naphtha-Type Jet Fuel}

\section{Physical Units}

Two data series are used to estimate naphtha-type jet fuel consumption:

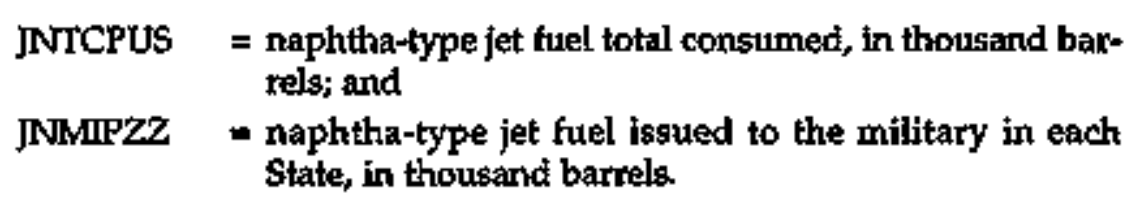

Total U.S. consumption of naphtha-type jet fuel, JNTCPUS, is the product supplied data series in the publication Petroleum Supply Annual, published by the Energy Information Administration (EIA).
Data on naphtha-type jet fuel issued to the military in each State, JNMIPZZ, are from the U.S. Department of Defense, Defense Logistics Agency, Defense Fuel Supply Center.

The total U.S. military issues is the sum of the State data:

$$
\text { JNMIPUS }=\text { EJNMIIPZZ }
$$

It is assumed that all naphtha-type jet fuel is used by military aircraft engines. (See the Additional Notes at the end of this section for the source reference for this assumption.) Therefore, an estimate of naphtha-type jet fuel consumption by State, JNTCPZZ, is calculated by assuming that each State consumes naphtha-type jet fuel in proportion to the amount issued to that State:

$$
\text { JNTCPZZ }=(\mathrm{NMMIPZZ} / \mathrm{JNMIPUS}) * \text { JNTCPUS }
$$

All naphtha-type jet fuel is assumed to be used for transportation purposes so the transportation consumption equals the estimated total consumption for each State and for the United States:

$$
\begin{array}{ll}
\text { JNACPZZ } & =\text { JNTCPZZ } \\
\text { JNACPUS } & =\text { JNTCPUS }
\end{array}
$$




\section{British Thermal Units (Bfu)}

Naphtha-type jet fuel has a heat content value of approximately 5.355 million. Btu per barxel. This factor is applied to convert naphtha-type jet fuel from physical units to Btu:
JNTCBZZ
$=\mathrm{JNTCPZZ} * 5.355$
INTCBUS
JNACBZZ
$=$ ENNTCBZZ
$=$ JNTCBZZ
INACEUS = JNTCBUS

\section{Adtitional Notes on Naphtha-Type det Fuel}

1. An assumption was made that the naphtha-type jet fuel is for military use only. This assumption was based on product defifinitions from the American Petroleum Institute's Standard Definitions for Patroletum Statistics, Technical Report No. 1, Third Edition (1981), page 13, which states that naphtha-type jet fuel is used primarily by military aircraft engines.

2. Data on naphtha-type jet fuel issued to the military for each State (INMIIZZ) are obtained from the U.S. Department of Defense, Defense Logistics Agency, Defense Fuel Supply Center There are no data available for 1960 through 1974, and the data available for 1975 and 1976 are not consistent; therefore, the 1977 values are used for 1960 through 1976 in SEDS. The data, are reported by fiscal year for 1977 through 1988 and are taken from the Defense Energy Information System. For 1969 and 1990, fiscal-year data from two databases, Defense Fuel Automated Management System and the Into-Plane; Database, are summed. For 1991 and 1992, data from the same two databases, reported by calendar year, are used.

\section{Jet Fuel Totals}

\section{Physical Unt}

The following calculations are used to provide total jet fuel consumption estimates by end use in physical units:
JEACPZZ
JFACPUS
JFEUPZZ
JFEUPUS
JFYCPZZ
JFTCPUS

\author{
$=\mathrm{JKACPZZ}+\mathrm{JNACPZZ}$ \\ $=$ IIFACPZZ \\ = JKEUPZZ \\ $=$ JKEUPUS \\ $=$ JFACPZZ + JPEUPZZ \\ - EJFTCPZZ
}

\section{Britssh Thermal Units (Btu)}

The following calculations are used to provide total jet fuel consumption estimates by end use in Btu:
JRACBZZ
$=$ JKACBZZ + JNACBZZ
JFACBUS
= $\mathrm{E}$ JACBZZ
JFEUBZZ
JFEUBUS
JFTCBZ2
- JKEUBZZ
- JKEUBUS
- JFACBZŻ + JFEUBŻZ
JFTCBUS = ITFTCBZZ

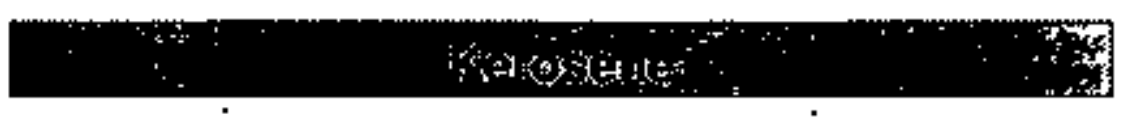

\section{Physkeal Unifs}

Because State-level and end-use consumption data for kerosene are not available, four data series published by EIA representing sales of kerosene into or within each State are used to estimate kerosene consumption. The fifth data series, the U.S. total consumption, is the product supplied series from the ETA Pelrolenm Supply Annual. The sales series are used to apportion the known U.S. total consumption into State-level estimates of end-use consumption. The following variable names have been assigned to the five data series ( $" \mathrm{ZZ}^{\prime}$ in the variable names represents the two-letter State code that differs for each State):

KSCMPZZ = kerosene sold to the commercial sector for heating, in thousand barrels;

KSIHPZZ = kerosene sold to the industrial sector for heating, in thousand barrels; 
KSOTPZZ = kerosene sold for all other uses, including fam use, in thousand barrels;

KSRSPZZ = kerosene sold to the residential sector for heating, in thousand barrels; and

KSTCPUS = kerosene total consumed in the United States, in thousand barrels.

U.S. sales totals for each of the four State-level series are created by summing the State values.

The variables are combined as closely as possible into the major enduse sectors used in SEDS. The residential and commercial sectors contain only KSKSPZZ and KSCMPZZ, respectively.

The sales of kerosene to the industrial sector, KSINPZZ, for each State is the sum of kerosene sold for industrial space heating (KSIHPZZ) and kerosene sold for all other uses (KSOTPZZ), including farm use. Sales of kerosene to the industrial sector are calculated:

\section{KSINPZZ = KSOTPZZ + KSIHPZZ \\ KSINPUS $=$ EKSINPZZ}

Total sales of kerosene in each State ts the sum of these three sectors' sales:

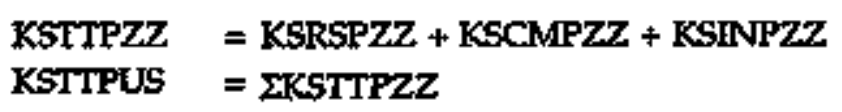

An estimate of each State's total consumption of kerosene is made by disaggregating the U.S. total consumption to the States in proportion to each State's sales share of the U.S. total sales:

\section{KSTCPZZ = (KSTTPZZ / KSTTPUS) * KSTCPUS}

Each State's residential sector sales percentage of total sales is applied to the State's estimated total consumption to create estimated residential sector consumption for the State, KSRCPZZ:

$$
\text { KSRCPZZ = (KSRSPZZ / KSTTPZZ }) * \text { KSTCPZZ }
$$

The commercial sector's estimated consumption in each State, KSCCPZZ, is calculated:

\section{XSCCPZZ $=($ KSCMPZZ / KSTTPZZ $) *$ KSTCPZZ}

The industrial sector's estimated consumption in each State, KSICPZZ, is calculated:

KSICPZZ - (KSINPZZ / KSTTPZZ) * KSTCPZZ

U.S. totals for the three sectore' consumption estimates are the sums of the States' estimated consumption.

\section{Britsh Thermal Units (Btw)}

Kerosene has a heat content value of approximately 5.670 million Btu per barrel. This factor is applied to convert kerosene estimated consumption from physical units to Btur:

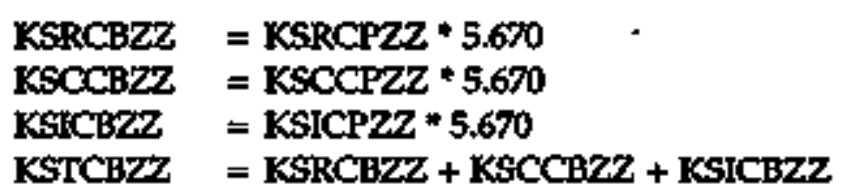

The U.S. Btu consumption estimates for the three consuming sectors and the U.S. total are calculated as the sum of the State-level data.

\section{Additional Moles on Kerosene}

1. See Note 4 at the end of the "Kerosene-Type Jet Fuel" section on page $\mathbf{3 6 0}$ for comments concerning the inclusion of kerosenetype jet fuel with the kerosene total product supplied prior to 1964 in the sourtce documents.

2. "Sales" data are actually called "shipments" in the source doctments for 1960 and 1961; "consumption" for 1962 through 1966; "shipments" for 1967; "sales" from 1968 through 1978; "deliveries" for 1979 through 1983; and "sales" for 1984 forward. 
3. In 1979, the Energy Information Administration (EIA) implemented a new survey form, EIA-172, to obtain deliveries of fuel oil and kerosene data and updated the list of respondents. (A detailed explanation is published in the Energy Datn Report "Deliveries of Fuel Oil and Kerosene in 1979.7) In the new survey form, certain end-use categories were redefined-in many cases, to collect more disaggregated data. The reclassifications resulted in some end-use categories that were no longer comparable with those in previous surveys. Where discontinuities occurred, estimates for the pre-1979 years have been made in SEDS to conform with the 1979 kerosene deliveries classifications. The pre-1979 deliveries estimates are not published in this report but are used in SEDS to disaggregate the known U.S. total product supplied (consumption) into State and major enduse sector consumption ertimates.

For kerosene deliveries in 1979, the end-use categories calted "residential," "commercial," and "industrial" ave avatlable. The pre-1979 deliveries category called "heating" is related to the sum of "residential," "commercial," and "industrbal" in 1979. Therefore, the following method was applied to present a comparable series for kerosene delivered to the residential, commerclal, and industrial sectors:

- A 1979 subtotal for heating was created by summing each State's residential, commercial, and industrial deliveries categortes, thereby creating a compavable deliveties subtotal for all years.

- Residential, commercial, and industrial shares of the heating subtotal in 1979 were calculated for each State.

- These 1979 end-use shares were then applied to each pre1979 heating subtotal in each State to create State estimates of end-use detiveries for 1960 through 1978.

The 1980 through 1982 kerosene deliveries data are based on the same survey as that used for 1979; therefore, the 1980 through 1982 data are directly comparable to 1979 data.
In 1984, EIA again updated the list of respondents for this survey, and the Form EIA-172 became the Form ELA-821, "Arunual Fuel Oil and Kerosene Sales Report." EIA did not conduct a fuel oil and kerosene sales survey for 1983 . The 1983 estimates in SEDS are based on 1984 data obtained from the Form ETA. 821. Statistical proceriures and methodologies used for the Form ELA-821 differ from those used in previous years. Therefore, the 1983 and forward sales data may not be directly comparable to the pre-1983 data. (In the source document, the sales data for 1983 forward are reported in thousand gallons. These data were first converted to thousand barrels before being entered into SEDS.)

4. In 1975 through 1977 , the industrial sector consumption of kerosene includes small quantities of kerosene-type jet fuel that were produced as jet fuel and sold as kerosene.

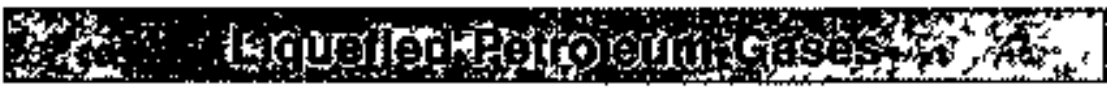

Liquefied petroleum gases (LPG) in SEDS include: ethane (including ethylene), propane (including propylene), norma? butane (including butylene), butane-propane mixtures, ethane-propane mixtures, and isobutane.

\section{Physteal Unils}

The following data series used in SEDS to estimate LPG consumption represent sales or estimated sales by State in thousand gallons. Sources for these variables are given in Appendix C.

\section{LGCBMZZ = LPG sold for internal combustion engine fuel use. In- cluded are sales for use in all kinds of highway vehi- cles, forklifts, industrial tractors, and for use in oil field drilling and production uses; \\ LGHCMZZ = LPG sold for residential and commercial use. In- cluded are sales for nonfarm private households for space heating, cooking, water heating, and other household uses, such as clothes drying and moinera- tion. Also included are sales to nonmanufacturing}


organizations, such as motels, restaurants, retail stores, laundries, and other service enterprises, primarily for use in space heating, water heating, and cooking; and

LGTTPZZ = LPG total sales for all uses.

Total U.S. consumption of LPG is the product supplied data seties in the publication Petrolezm Stuply Annund, published by the Energy mformation Administration (ELA):
LGTCPUS = LPG total consumed in the United States, in thousand barrels.

Another variable is used in SEDS to estimate LPG consumption by the transportation sector. It is described in detail in Note 2 at the end of this LPG section:
LGTRSUS = the transportation sector share of LPG internal com- bustion engine sales.

Since the LPG sales data are in gallons, they must be converted to barrels (42 U.S. gallons per U.S. barrel) to be comparable to total consumption estimates. The formulas for calculating State sales data are:

\section{LGCBPZZ = LGCBMZZ / 42 \\ LGHCPZZ = LGHCMZZ $/ 42$}

The U.S. totals for each of the State-level LPG sales data series are calculated as the sum of the State values.

An asstmption is made that 85 percent of the LPG sold for residential and commercial use (LGHCPZZ) is sold to the residential sector (LGRCPZZ), and 15 percent is sold to the commercial sector (LGCCPZZ) for all States and years. (See Note 3 at the end of this LPG section for the source reference for this assumption.) It is also assumed that LPG sales to the residential and commercial sectors are equal to the consumption in those sectors. The formulas used are:

LGRCPZZ LGHCPZZ *0.85

LGCCPZZ $=$ LGHCPZZ $* 0.15$
The LPG consumption by the transportation sector is estimated to be the transportation share of the sales for internal combustion engine fuel:

\section{LGACPZZ = LGCBPZZ * LGTRSUS}

An estimate of each State's totel LPG consumption (LGTCPZZ) is made by allocating the U.S. total consumption to the States in proportion to each State's sales share of the U.S. total sales:

\section{LGTCPZZ $=$ (LGTTPZZ / LGTTPUS) $*$ LGTCPUS}

The industrial (LGICPZZ) sector consumption of each State is the difference between the State's total LPG consumption and the sum of its residential, conmercial, and transportation sectors' constmption:

LGICPZZ = LGTCPZZ - (LGRCPZZ + LGCCPZZ + LGACPZZ)

U.S. totals for the four end-use sector constumption estimates are calculated as the sums of the State estimates.

\section{British Thermal Unfts (Btw)}

The factor for converting LPG from physical unit values to Britlsh thermal units, LGTCKUS, is calculated annually for 1967 forward by EIA as a weighted average by multiplying the quantity consumed of each of the component products (ethane, propane, butane, butanepropane, ethane-propane, and isobutane) by each product's conversion factor (listed in Appendix D, Thermal Conversion Factor Source Documentation) and dividing the sum of those heat contents by the sum of the quantities consumed. The consumption of each product is taken from the product supplied data series in the Entergy Dats Report, "Petroleum Statement, Annual" (1967 through 1960), and the Petrolewm Supply Annual (1981 forward), published by EIA. For 1960 through 1966, EIA adopted the Bureau of Mines thermal conversion factor of 4.011 million Btu per barrel as published in the Mintral Industry Suroeys, "Crude Petroleum and Petroleum Products, 1956," Table 4 footnote.

This factor is used to estimate consumption in Btu for all States and end uses: 


L. Table A4. Percentages Useat to Disaggregate Maryland and D.C.
Combined LPG \$ales Data

\section{LGRCBZZ = LGRCPZZ * LGTCKUS \\ LGCCBZZ $=$ LGCCPZZ * LGTCKUS \\ LGICBZZ = LGICPZZ * LGTCKUS \\ LGACBZZ = LGACPZZ * LGTCKUS}

U Total estimated consumption of LPG in Bhu is the sum of the end-use consumption estimates:

\section{LGTCBZZ = LGRCBZZ + LGCCBZZ + LGICBZZ + LGACBZZ}

The U.S. Btu consumption estimates for the four sectors and total LGP are calculated as the sum of the State data.

\section{Additional Notes on Liqupfied Petroleum Cases}

1. Sales data for Maryland and the District of Columbia are combined in the source documents. Sales data are published in six categories. The percentages shown in Table A4 are applied to disaggregate the combined State data in each of the sectors for all years.

2. The sales of LPG for internal combustion engine fuel use ane divided between the transportation sector and the industrial sector by using LGTRSUS, the transportation sector's share of internal combustion engine use. LGTRSUS is estimated from data on "special fuels used on highways," a category that includes orly LPG and diesel fuel. The special fuels data are published by the U.S. Department of Transportation, Federal Highway Administration (see MGSFPZZ in Appendix C). The quantity of LPG included in spectal futels is estimated each year (the LPG portion ranges from 8.4 percent in 1960 to 1.2 percent In 1990). LGTRSUS is then derived by dividing the quantity of LFG included in special fuels used on highways by the quantity of LPG sold for internal combustion engine use. This U.S. factor is applied to each of the States. LGTRSUS values are shown in Table A5.

3. Little information exists for allocating the residential and commencial tuse of LPG to the individual sectors. SEDS applies an 85 percent residential and 15 percent commercial split for all States and years based on figures published in the Federal Energy Administration Project Independence Blueprint Task Force Report, ${ }^{2}$ Residential and Commercial Energy Use Patterns, 1970 1990, November 1974, Table 1.A.1.

4. LPG sales data by State and end-use categories for 1960 through 1982 are from EIA's "Sales of Liquefied Petroleum Gases and

Table A5. Transportation Sector Share of LPC mternal Combuation Englie Use, 1960 Forward

\begin{tabular}{|c|c|c|c|c|c|}
\hline Year & LGTRSUS & Year & LGTRSUS & Yosr & LOTFBUS \\
\hline 1980 & 0.229 & 1972 & 0.392 & 1904 & 0.638 \\
\hline 1981 & 0258 & t973 & 0.394 & 1985 & 0.440 \\
\hline 1082 & 0.266 & 1974 & 0.981 & 1986 & 0.456 \\
\hline 196 & 0.273 & 1975 & 0.406 & 1967 & 0.374 \\
\hline 1964 & 0.259 & 1976 & 0.440 & foged & 0.437 \\
\hline 1985 & 0.290 & 1977 & 0.478 & 1989 & 0.427 \\
\hline 1966 & 0.325 & 1978 & 0.594 & 1990 & 0.475 \\
\hline 1967 & 0.368 & 1979 & 0.536 & 1991 & 0.426 \\
\hline 1968 & 0.389 & 1980 & 0.386 & 1992 & 0.426 \\
\hline 1909 & $0.34 t$ & 1961 & 0.671 & 1993 & 0.441 \\
\hline 1970 & 0.363 & 1982 & 0.579 & 1994 & 0.739 \\
\hline 1971 & 0.420 & 1983 & 0.579 & & \\
\hline
\end{tabular}


Ethane." In 1979, BLA modified the LPG sales survey, Form ELA-174, and changed the list of respondents. Because of the updated sampling frame, the 1979 through 1982 sales data may rot be directly comparable to the pre-1979 sales when a different estimation procedure was used. Explanation of the discon. tinuities caused by the change in the 1979 stampling frame are provided in EIA's Evergy Data Report, "Sales of Liquefied Petroleum Gases and Ethane in 1979."

Because of the change in survey techuiques used for measuring LPG sales, many States' data were withheld from publication in the 1979 through 1982 LPG sales reports to avoid disclosure of company-level data. The consumption estimates in SEDS use all data published in the 1979 through 1982 LPG sales reports and estimates prepared by EIA's Office of Oil and Gas for data that were withheld from pablication. (See Note 5 below for estimation procedures.) .

Some end-use categories changed in 1979 due to redefinition of the classifications. One of these changes, for example, occurred with LPG sold to farms for household heating and cooking. Prior to 1979 these sales were reported as part of the residential and commercial category, while in 1979 they were counted in the farm use category that goes into the industrial sector in SEDS. No attempt has been made to adjust for this type of inconsistency.

The Form EIA-174 was cancelled after collection of 1982 data. The 1983 LPG consumption estimates are based on the assumption that LPG end-use sector demand in 1983 occurred in the same proportion as 1982 sector demand within each State; i.e., the 1983 LPG product supplied figure was allocated to the States by using the distribution of volumes consumed for 1982.

5. The following procedures were used to estimate the State enduse sales that were withheld from publication in the 1979-1982 LFG sales reports:

- For each yeax, missing State total sales were estimated by allocating the sum of the missing State sales within each Petroleum Administration for Defense (PAD) District to the individual States, in proportion to the sum of the known end-use sales for those States.

- Missing PAD District end-use totals for 1979 and 1980 were obtained by using the 1980 and 1981 sales reports. Missing PAD District chemical sales were estimated by allocating the total missing volume of chemical stales to the PAD District in proportion to the number of chemical plants in each PAD District. The remaining PAD District end-use totals were

Table A6. State Shares of the Total U.S. LPG Sold for Chemleal Use, 1960 Through 1978

\begin{tabular}{|c|c|c|c|}
\hline Stale & Percent & Siate & Percent \\
\hline Alabaina & 0 & Montana & 0 \\
\hline Alaska & 0.589 & Nebraska & . \\
\hline Arizona & 0 & Nevada & 0 \\
\hline Arkansas & 0 & New Hampshive & 0 \\
\hline California & 2,667 & New Jensey & 2.040 \\
\hline Coloredo & 0.232 & Now Mexico & 0.600 \\
\hline Connecticut & 0.053 & New York & 0 \\
\hline Delaware & 0.811 & North Caxolina & 0.327 \\
\hline District of Columbia. & 0 & North Dakola & 0 \\
\hline Floricta & 0 & Ohio" & $1.10 \mathrm{~s}$ \\
\hline Georgta & 0.699 & Oklahoma & 0.309 \\
\hline Hawal & 0 & Oregon & 0 \\
\hline Idaho & 0 & Pennsylvanta & 0.354 \\
\hline IIInols & 7,066 & Ghode Islend & 0 \\
\hline Indianta & 0.243 & South Carolina & 0.021 \\
\hline lowa & 0.900 & South Dakota & 0 \\
\hline Kansas & 0.451 & Tennessee & 0 \\
\hline Kentucly & 2.548 & Texas & 57.425 \\
\hline Lotisiana & 20.566 & Utah & 0 \\
\hline Mane & 0.012 & Vermant & 0 \\
\hline Maryland & 0.050 & Virginia. & 0.025 \\
\hline Massachusetts & 0,009 & Washington & 0 \\
\hline Michtgan & 0.151 & West Virginia & 0.286 \\
\hline Minnesola & 0 & Wisconsin & 0 \\
\hline Mississippi & 0.315 & Wyoming & 0.091 \\
\hline Milssouri, & 0.054 & Unted States & 100.000 \\
\hline
\end{tabular}


obtained by subtraction. For 1981 and 1982, no PAD District estimations were necessary because all PAD District end-use totals are known.

- The published data and the estimated State and PAD District end-use totals were used to estimate missing State enduse sales volumes within a PAD District: missing State end-use sector values were estimated by allocating the missing volume for the State approximately proportional to the PAD District end-use sector totals.

6. Prior to 1979, State data for cheutical use of LPG were withheld from publication, although they were included in the U.S. total in the tables in ELA's "Sales of Liquefied Petroleum Gases and Ethane ${ }^{\prime \prime}$ reports. Begining in 1979, State-level chemical use data were published in the LPG sales reports, but data for seyeral States were withineld. Estimates for the withheld data for chemical use sales for 1979 and 1980 were created by using the estimation procedure described in Note 5 above. Then the published and the estimated State data for 1979 were used to create State shares of the totaI U.S. chemical use sales. These percentage shares (shown in Table A6) were applied to the total U.S. LPG chemical use sales in 1960 through 1978 to create State chemical use estimates. The chemical use estimates were added to the States' total LPG sales series, LGTTPZZ.

7. Beginning in 1984, the American Petroleum Institute (API), the Gas Processors Association, and the National LP-Gas Association jointly sponsored an LPG sales survey. The results are published in the API's report Sales of Natural Gas Liquids and Liquefied Refinery Gases. These data include sales of pentanes plus; the pentanes plus data were removed prior to use in SEDS.

The API report publishes total LPG sales for Alaska and Hawaii, but disaggregated data for those States are withheld. BIA estimates the withelid data for the "Residential and Commercial" and the "Internal Combustion Fuel" columns as follows:

- Alaska and Hawail are the only States of the seven States in PAD District $V$ for which data are withheld. Therefore, subtracting the available data for the other five States from the
PAD District V total gives the withheld data for Alaska and Hawaii combined.

- The withheld data are assigned to Alaska and Hawail in proportion to each State's share of their combined published total sales.

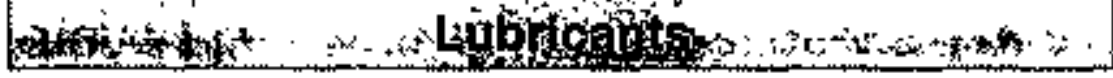

\section{Phystcal Units}

Three data series are used to estimate state consumption of lubricants. The two State-level sales data series ate used to apportion the U.S. total consumption data to the States and the end-use sectors within the States. " $\mathrm{ZZ}$ " in the varlable names represents the two-letter State code that differs for each State

\footnotetext{
LUINPZZ = lubricants sold to the industrial sector, in thousand barrels;

LUTRPZZ = lubricants sold to the transportation sector, in thousand barrels; and

LUTCPUS = lubricants total consumed in the United States, in thousand barrels.
}

Data for the first two variables are developed from the Bureau of the Census reports "Sales of Lubricating and Industrial Oils and Greases" in the Current industrial Reports series. These series were discontinued in 1977 and the method of estimation for 1978 forward is explained in Note 1 at the end of this "Lubricants" section. The third variable for lubricants is the product stupplied data series in the publication Petroleum Supply Annual, published by the Energy Information Administration (ELA). The first two variables are used for apportioning the third into State total consumption and State end-use consumption estimates by using the following calculations.

Total sales of lubricants for each Stake, LUTTPZZ, is created by adding the industrial and transportation sales:

LUTTPZZ = LUINPZZ + LUTRPZZ 
U.S. sales totals are calctilated by summing the State sales data.

Each State's proportion of total U.S. sales is used to calculate each State's estimated consumption of lubricants:

\section{LUTCPZZ = (LUTTPZZ / LUTTPUS) * LUTCPUS}

Each State's estimated total consumption of hubricants is further divided into end-use estimates in proportion to that State's sales by sector as a portion of total sales in the State. Lubricants consumed by State for industrial use, LUICPZZ, and for transportation use, LUACPZZ, are calculated:

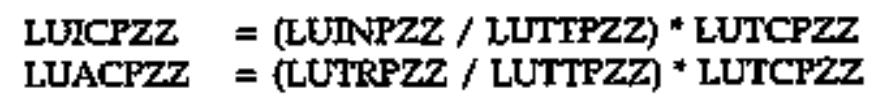

The consumption of lubricants in the United States by these two enduse sectors is created by summing the State estimates.

\section{British Thernsl Unils (Btw)}

Lubricants have a heat content value of approximately 6.065 million Btu per barrel. This factor is applied to convert lubricants estimated consumption from physical units to Btu:

\section{LUICBZZ = LUICPZZ* 6.065 \\ LUACBZZ $=$ LUACPZZ *6.065}

The State total consumption in Btu is the sum of the two sectors' consumption in Btu:

\section{LUTCBZZ = LUICBZZ + LUACBZZ}

The U.S. sector and total consumption estimates in Btu are calculated as the sum of the State data.

\section{Adelitional Noles on Lubrtoants}

I. The lubricants sales data (LUINPZZ and LUTRPZZ) were published approximately every other year by the Bureau of the
Table A7. Lubricants Sales Data Used in Consumption Estimates

\begin{tabular}{cc}
$\begin{array}{c}\text { Yeer of } \\
\text { Stles Data }\end{array}$ & $\begin{array}{c}\text { Year of } \\
\text { Consumption Estimutes }\end{array}$ \\
\hline 1960 & 1960 and 1961 \\
1968 & 1962,1963, and 1964 \\
1965 & 1965 and 1966 \\
1967 & 1967 and 1968 \\
1969 & 1969 and 1970 \\
1971 & 1971 and 1972 \\
1973 & 1973 and 1974 \\
1975 & 1975 and 1976 \\
1977 & 1977 fonward \\
\hline
\end{tabular}

Census until the discontinuation of the series after 1977. Each year's sales data have been used to calculate that year's and at least one other year's consumption estimates. Table A7 specifies which years of consumption estimates depend on which years of the sales data.

2. The saies data from the source document for LUINPZZ and LUTRPZZ are avaibable in incompatible units. The industrial series, LUINPZZ, is oils and greases sold for industrial lubricating and other uses measured in thousand gallons. The transportation series, LUTRPZZ, is oils and greases sold for automotive and aviation uses measured in thousand pounds. Prior to use in SEDS, these were converked to thousand barkefs by dividing the oil data by 42 gallons per barmel and dividing the greases data by 300 pounds per barrel. In the source document, some State data are not published to avoid disclosing figures for individual companies. The undisclosed data were entered as zero in SEDS. 
MGCUPZZ a motor gasoline sotd for construction use in each State. in thousand gallons;

\section{Physical Units}

Nine data series are used to estimate the State end-use consumption of motor gasoline. Eight of the series are from the US. Department of Transportation, Federal Highway Administration publication, Hightury Sfatistics, and represent sales of motor gasoline. The sales data are categorized as sales for highway and nonhighway use:

- Highway Use sales data (MGMFP) are from the Hightusey Statisfics Table MF-21; however, they are reduced by the amount of highway "special fuels" (MGSFP) used in each State each year as reported on Table MF-25 (prior to 2994) and Table MF21 (1994). Special fuels are primarily diesel fuels, not motor gasoline, and are included in the transportation sector of distillate fuel.

- Nonhighway Use sales are further subdivided into sales fort (1) public use by States, counties, and municipaltities (MGPNP) from Table MFF-21, and (2) private and commercial use as reported on MF-24. The private and commercial nonhighway use of motor gasoline has the following components: agricultural use (MGAGP), industrial and commercial use (MGTY), construction use (MGCUP), marine use (MGMRP), and miscellaneous and unclassified uses (MGMSP). Another component of the private and commercial nonhighway series is aviation gasoline (AVNMM), which is discussed under the "Aviation Gasoline" section of this doctumentation.

The ninth motor gasoline data series (MGTCPUS) is the total U.S. consumption of motor gasoline published in the product stupplied series in the EIA publication Petroleum Supply Amunal.

The nine motor gasoline data series are (" $\mathrm{ZZ}$ in the variable names represent the two-letter State code that differs for each State):

MGAGPZZ = motor gasolire sold for agricultural use in each State, in thousand gallons;

MGIYPZZ = motor gasoline sold for industrial and commercial use in each State, in thotsand gallons;

MGMFPZZ = motor fuel sold for highway use in each State, in thousand gallons;

MGMRPZZ = motor gasoline sold for marine use in each State, in thousand gallons;

MGMSPZZ = motor gasoline sold for miscellaneous and unclassified uses in each State, in thousand gallons;

MGPNPZZ = moter fuel sold for public nonhighway use in each State, in thoustand gallons;

MGSFPZZ = special fuels (primarily diesel fuel with small amounts of liquefied petroleum gases) sold in each State, in thousand gallons; and

MGTCPUS = motor gasoline total consumed in the United States, in thousand barrels.

U.S. totals for the eight State series named above are calculated as the sum of the State data.

The transportation sector accounts for most of the motor gasoline sales. Sales to the transportation sector is estimated to be the sum of motor fuel sales for marine use and for highway use (mintrs the sales of special fuels, whirh are primarily diesel fuels and are accounted for in the transportation sector of distillate fuel). Sales of motor gasoline to the transportation sector in each State (MGTRPZZ) is calctlated:

MGTRPZZ = MGMFPZZ + MGMRPZZ - MGSFPZZ

Two sales data series are added to estimate motor gasoline sales to the commercial sector: miscellaneous (including unclassified) and public nonhighway sales. Sales of motor gasoline to the conmercial sector in each State (MGCMPZZ) is calculated:

\section{MGCMPZZ $=$ MGMSPZZ + MGPNPZZ}

Sales of motor gasoline for use in the industrial sector in each State (MGINPZZ) is caiculated as the sum of the sales for agricultural use, for construction use, and for industrial and commercial use: 
MGINPZZ = MGAGPZZ + MGCUPZZ + MGTYPZZ

Total sales of motor gasoline in each State (MGTTPZZ) is calculated as the sum of the sales to the major sectors:

\section{MGTTPZZ = MGCMPZZ + MGNPZZ + MGTRPZZ}

U.S. totals for the three end-use sectors' sales and for total sales are calculated as the sum of the States' sales.

The motor gasoline sales data for the three end-use sectors in each State are used to apportion the U.S. total consumption of motor gasoline to the States and to the major end-use sectors within each State.

The estimated consumption of motor gasoline in each State is calculated according to each State's share of the total sales. Estimated consumption of motor gasoline in each State (MGTCPZZ) is calculated:

\section{MGTCPZZ = (MGTTPZZ / MGTTPUS) * MGTCPUS}

The commercial sector estimated consumption of motor gasoline (MGCCPZZ) is calculated:

\section{MGCCPZZ $=($ MGCMPZZ / MGTTPZZ $) *$ MGTCPZZ}

The industrial sector estimated consumption (MGICPZZ) is calculated:

\section{MGICPZZ = (MGINPZZ / MGTTPZZ $) *$ MGTCPZZ}

The teansportation sector estimated consumption (MGACPZZ) is calculated:

\section{MGACPZZ $=($ MGTRPZZ / MGTTPZZ) * MGTCPZZ}

The consumption of motor gatoline by major end-use sector in the United States is estimated by summing the States' estimated consumption.

\section{British Thermal Units (Bto)}

Motor gasoline has a heat content value of approximately 5253 million Btu per barrel. This factor is applied to convert motor gasoline estimated consumption from physical units to Btu;

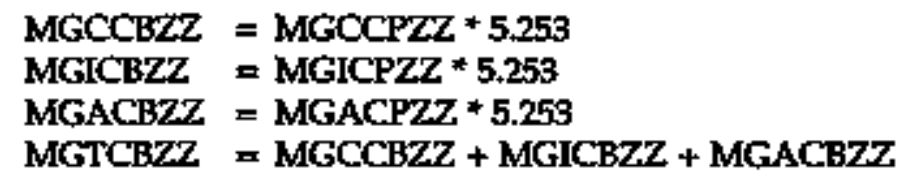

The U.S. level Btu consumption estimates are calculated by summing the State data.

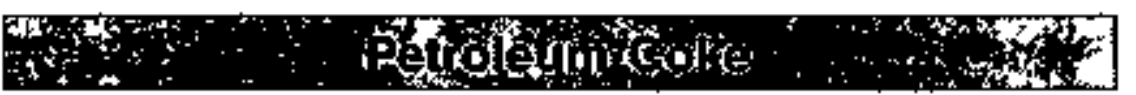

\section{Physical Units}

Five data series are used to estimate the consumption of petroleum coke. Three are measures of petroleum coke consumption and two are indicators of industrial activity used to apportion U.S. consumption to the States. "ZZ" in the variable name represents the two-letter State code that differs for fach State:

PCTCPUS = petroleum coke total consumed in the United States (electric utility and industrial sectors), in thousand barrels;

PCEUMZZ = petroleum coke consumed by electric utitities in each State, in thousand short torns;

PCCTPUS = petroleum coke used at refineries as both catalytic and marketable coke in the Untted States, in thousand barrels;

CTCAPZZ = catalytic cracking charge capacity of petroleum refinerles in each State, in barrels per calendar day (1960 through 1979) and barrels per stream day (1980 forward); and

AICAPZZ = ahuminum ingot production capacity in each State, in short tons. 
P The total consumption of petroleum coke in the United States E (PCTCPUS) is the product supplied series from the BIA publication T Petrolentin Supply Anntual.

Petroleum coke consumed at eiectric utilities, PCEUMZZ, is avallable from 1970 forward from the Energy Information Administration (EIA), Form EIA-759, "Monthly Power Plant Report," and predecessor forms. Prior to 1970, no data are available for this series and zero is used. These datz are in thousand short tons and are converted into
thousand barrels in SEDS by applying a conversion factor of 5 barrels per short ton:

\section{PCEUPZZ = PCEUMZZ * 5}

The source for petroletum coke used at refineries, PCCIPUS, is the EIA series of reports entitled "Petroleum Statement, Annual" and predecessor reports. For 1960 through 1980 , the data are provided in thousand short tons. For consistency with later years' data, the 1960 through 1980 data were first converted into thousand barrels before being used in SEDS.

The data for petroleum coke consumed by electric utilities are available by State. Other petroleum coke consumption data are available only on a U.S. level and are accredited to the industrial sector th SEDS, etther as petroleum coke used as catalyst coke at refineries in a process for increasing the yield of gasoline from crude oil (catalytic cracking) or as petroleum coke used for all other industrial uses (mainly for conversion into electrodes that are consumed in the production of aluminum). The industrial petroleum coke consumption series are apportioned to the States by using the data series that indicate the size of the refining industry and aluminum production industry in each State. The capacity of refineries' catalytic cracking equipment (CTCAPZZ) and the eluminum ingot production capacity (AICAPZZ) are not measured in thousand barrels, but since these data series are used only to apportion U.S. industrial consumption of petroleum coke to the States, they do not need to be converted into thousand barrels.

The U.S. totals for the State-level data series are calculated by summing the State data.
To estimate industrial consumption of petroleum coke, electric utility consumption is subtracted from the total U.S. petroleum coke product supplied:

$$
\text { PCICPUS = PCTCPUS - PCEUPUS }
$$

The petroleum coke used at refineries in the United States as catalytic coke is subtracted from the U.S. industrial sector consumption to derive consumption of petroleum coke by all other industrial users:

\section{PCOCPUS = PCICPUS - PCCTPUS}

State-level estimates of the catalytic coke portion of the industrial sector are calculated by assuming that each State consumes catalytic coke in proportion to the catalytic cracking charge capacity of the refineries in the State:

\section{PCCTPZZ = (CTCAPZZ / CTCAPUS $) *$ PCCTPUS}

State-level estimates of the petroleum coke consumed by the other industrial users are assumed to be in proportion to each State's aluminum ingot production capacity:

\section{PCOCPZZ = (AICAPZZ / AICAPUS $)$ PCOCPUS}

The State totals for the industrial sector use of petroleum coke are added:

\section{PCICPZZ = PCCTPZZ + PCOCPZZ}

Total petroleum coke consumption by State is industrial use plus electric utility use:

$$
\text { PCTCPZZ = PCICPZZ + PCEUPZZ }
$$

\section{British Thermal Units (Bto)}

Petroleum coke has a heat content value of approximately 6.024 million Btu per barrel. This factor is applied to convert petroleurn coke estimated consumption from physical units to Btu by State and at the US. level: 


$\begin{array}{ll}\text { PCICBZZ } & =\text { PCICPZZ * } 6.024 \\ \text { PCICBUS } & =\text { ZPCICBZZ } \\ \text { PCEUBZZ } & =\text { PCEUPZZ * 6.024 } \\ \text { PCEUBUS } & =\text { IPCEUBZZ } \\ \text { PCTCBZZ } & =\text { PCICBZZ + PCEUBZZ } \\ \text { PCTCBUS } & =\text { EPCTCBZZ }\end{array}$

\section{篗}

\section{Physioal Unils}

Since Stafe-level end-use constumption data for residual fuel (with the exception of electric utilities data) are not available, sales of residual fuel into or within each state, in thousand baxrels, published by the Energy Lnformation Administration (BIA), are used to estimate resid. ual fuel consumption. " $\mathrm{ZZ}$ " in the following variable names represents the two-letter State code that differs for each State:

RFBKPZZ = residual fuel sold for vessel bunkering use (i.e, the fueling of commercial or private boats, strch as pleasure craft, fishing boats, tugboats, and ocean-going vessels, including vessels operated by oil compantes, and fueling for other marine purposes), excluding sales to the Anmed Forces;

RFCMPZZ = residual fuzel sold to the commercial sector for heating; RFIBPZZ = residual fuel sold to industrial establishments for space heating and for other industrial use (1.e, for all uses to mines, smelters, plants engaged in producing mantufactured products, in processing goods, and in assembling);

RFMIPZZ = residual fuel sold to the Armed Forces, regardless of use;

RFMSPZZ - residual fuel sold for all other uses not identified in other sales categories;

RFOCPZZ = residual fuel sold for oil company use, including aft fuel oil, crude oll, or acid sludge used as fuel at refin-

RFRRPZZ eries, by pipelines, or in fleid operations; and residual fuel sold to the railroads for use in fueling trains, operating rallmoad equipment, space heating of buildings, and other opetations.

Two other data series that represent consumption of residual fuel are:

RFEUPZZ = residual fuel consumed by electric utilities in each State, in thousand barrels.

RFTCPUS = residual fuel total supplied in the United States, in thousand barrels.

Residual fuel oil consumed by electric utilities, RFEUPZZ, is collected by EIA on Form EIA-759, "Monthly Power Plant Report," and predecessor forms. (see Note 3 at the end of this residual fuel section for further information on changes in this series' data definitions.)

Total U.S. consumption of residual fuel, RFTCPUS, is the product supplied series in ELA's publication Petroletum Supply Anninal.

To begin calculating residual fuel state and and-use consumption estimates, all State-level data series are summed to provide totals for the United States.

Then the data series are combined as closely as possible into the major end-use sectors used in SEDS. No residual fuel is sold to the residential sector. Residual fuel sales to the commercial sector is the RFCMPZZ series.

The sales of residual fuel to the industrial sector in each State, RFINPZZ, is the sum of the residual fuel sold for industrial use, including industrial space heating (RFIBPZZ), for oil company use (RFOCPZZ) and for all other uses (RFMSPZZ):

\section{RFINPZZ = RFIBPZZ + RFOCPZZ + RPMISPZZ \\ RPINPUS $=$ ERFINPZZ}

The sales of residual fuel to the transportation sector in each State, RFTRPZZ, is the sum of the residual fuel sales for vessel burkering (RFBKPZZ), military use (RFMIPZZ), and railroad use (RFRRPZZ):

RFTRPZZ = RFBKPZZ + RFMIPZZ + RPRRPZZ 


\section{R RFTRPUS $=$ ERFTRPZZ}

Sales of residual fuel oil to the commercial, industrial, and transporEation sectors are added to create a subtotal of sales to all sectors other than the electric utility sector (RPNDPZZ):

\section{RFNDPZZ = RFCMPZZ + MFINPZZ + RFTRPZZ RFNDPUS $=$ XRFNDPZZ}

The estimated residual fuel consumption for the United States by all sectors other than the electric utility sector (RFNCPUS) is calculated by subtracting the total residual fuel consumption at electric utilities from the total U.S. residual fuel consumption:

\section{RFNCPUS = RFTCPUS - RFEUPUS}

This U.S. subtotal of residual fuel consumption by the end-use sectors combined (RFNCPUS) is apporttoned to the States using the States' end-use sector sales data. The assumption is made that each State consumes residual fuel in proportion to the amount sold in that State:

\section{RFNCPZZ = (RFNDPZZ / RFNDPUS $)$ * RFNCPUS}

The end-use sectors' subtotal for each State is further divided into estimates for each sector in proportion to each sector's sales. The estimated commercial sector consumption in each State, RPCCPZZ, is calculated:

RFCCPZZ = (RFCMPZZ / RFNDPZZ $) *$ RFNCPZZ

The industrial sector's estimaked consumption in each State, RFICPZZ, is calculated:

\section{RFICPZZ = (RFINPZZ / RFNDPZZ $) *$ RFNCPZZ}

The transportation sector's estimated consumption in each State, RFACPZZ, is calculated:

RFACPZZ = (RFIRPZZ / RFNDPZZ $) *$ RFNCPZZ
The consumption of residual fuel in the Urited States by the major end-use sectors is estimated by adding the States' estimated consumption.

3

Total State residual fuel consumption is the sum of the end-use sectors' consumption subtotal and the electric utilities consumption:

RFTCPZZ = RFNCPZZ +.RFEUPZZ

\section{Brtish Thermal Units (Btu)}

Residual fuel has a heat content value of approximately 6.287 million Btu per barrel. This factor is applied to convert residual fuel estimated consumption from phyaical units to Btu as shown in the following examples:

\section{RFCCBZZ = RFCCPZZ * 6.287 \\ RFICBZZ = RFICPZZ* 6.287 \\ RFTCBZZ = RFCCBZZ + RPICBZZ + RFACBZZ + RFEUBZZ}

The U.S. level Btu consumption estimates are calculated as the sum of the States' Btu consumptionon.

\section{Additional Notes on Residual Fuel}

1. "Salos" data are actually called "shipments" in the source documents for 1960 and 1961; "consumption" for 1962 through 1966; "shipments" for 1967; "sales" from 1968 through 1978; "deliveries" for 1979 through 1983; and "sales" for 1994 forward.

2. In 1979, the Energy Information Administration implemented a new survey form, ELA-172, to obtain deliveries of fuel oil and kenosene data and updated the list of respondents. (A detailed explaration is published in the Entergy Data Report "Deliveries of Fuel $\mathrm{Oit}$ and Kerosene in 1979.") In the new survey form, certain end-tuse calegories wese redefined-in many cases to collect more disaggregated data. The reclassifications resulted in some end-use categories that were no longer comparable with those in previous surveys. Where discontinuities occurred, estimates for the pre-1979 years have been made in SEDS to conform with 
the 1979 fuel oil deliveries classifications. The pre-1979 deliveries estimates are, not published in this report but are used in SEDS to disagrregate the known U.S. total product supplied (consumption) into State and major end-use sector consumption estimates.

For residual fuel deliveries in 1979, the end-use categories "commercial" and "industrial" are avallable. The pie-1979 deliveries categories are called "heating" and "industrial." While the pre-1979 categories individually are not continuous with the 1979 categories, their subtotals are related. That is, a general comparison can be made between the sum of commercial and industrial deliveries in 1979 and the sum of heating and industrial deliveries in the pre-1979 years: "Therefort, the following method was applied to present a comparable series for residual fuel delivered to the cormmencial and industrial sectors:

- For each of the pre-1979 years, a subtotal was created for each State by adding each State's heating and industrial deliveries cakegories. A comparable 1979 subtotal was created by adding each State's commerclal and industrial deliveries categories.

- Commercial and industrial shares of the subtotal in 1979 were calculated for each State.

- These 1979 end-use shares were then applied to each pre1979 subtotal of residual fuel deliveries in each State to create State estimateg of end-use deliveries for 1960 through 1978.

The 1980 through 1982 residual fuel deliveries data are based on the same survey as that ased for 1979 ; therefore, the 1980 through 1982 data are directly comparable to 1979 data.

In 1984, ELA again updated the list of respondents. for this survey, and the Form EIA-172 became the Form ElA-821, "Annual Fuel Oil and Kerosene Sales Report." EIA did not conduct a fuel oil and kerosene sales survey for 1983. The 1983 estimates in SEDS are based on 1984 data obtained from the Form EIA821. Statistical procedures and methodologies used for the
Form ELA-821 differ from those used in previous years. Therefore, the 1983 and forward sales data may not be directly com. parable to the pre-1983 data. (In the source document, the sales data for 1983 forward are reported in thousand gallons. These data were first converted to thousand barrels before being entered into SEDS.)

3. The fuel oil at electric utilities data for all years and States are actual fuel oil consumption numbers collected from electric utilities by EIA on Form EIA-759, "Monthy Power PIant Report," and predecessor forms. Due to changes in fuel oil reporting classifications, on the Form EIA-759 over the years, it is not possible to develop a thoroughly consistent-series for all years. However, over time, data more accurately disaggregating fuel oil into distillate, fuel and residual fuel have become available. For 1960 through 1969, only total fuel oil consumed at electric utilities by State is available. For 1970 through 1979 , fuet oil consumed by plant type (internal combustion arid gas turbine plarits combined and steam plants) by Stäte aro available. For 1980 forward, consumption of light oil at all plant types combined and consumption of heavy oil at all plant types combined are available by State. In SEDS, the following assumptions have been made:

- 1960 through 1969 - State estimlates of fuel oil consumption by plant type have been created for each year by applying the shares of steam plants (primarily residual fuel) and intemal combustion and gas turbine plants (primarily distiflate fuel plus small amounts of jet kerosene) by State in 1970 to each year's total fuel oil consumption at electric utilities for 1960 through 1969.

- 1970 through 1979 - fuel oil consumed by steam plants is assumed to equal residual fuel consumption, and fuel otl consumed by internal combustion and gas turbine plants is assumed to equal distillate fuel plus jet kerosene consumption.

- 1980 and forward - total heavy oil consumption at all plant types is assumed to equal residual fuel consumption, and 
total light oil consumption at all plant types is assumed to equal distillate fuel plus jet kerosene consumption.

The data series thus derived for SEDS for residual fuel and distitlate fuel plus jet kecosene consumption at electric utilities is considered to be actual consumption at electric utilities for each State and each year.

\section{Other Petroleuiníi:Próducts}

There are 16 petsoleum products that are summed and called "other petroleum products" in SEDS. These products, in thousand barrels, are;

M

$\mathbf{P}$

ABTCPUS = aviation gasoline blending components total consumed in the Untted States:

COTCPZZ = crude oil (including lease condensate) consumed in each State;

FNTCPUS $=$ petroletim feedstocks, naphtha less than $401^{\circ} \mathrm{F}$, total constumed in the United States;

FOTCPUS - petroleum feedstocks, other oils equal to or greater than $402^{\circ} \mathrm{F}$, total consumed in the United States;

FSTCPUS = petroleum feedstocks, still gas, total consumed in the United States;

MBTCPUS = motor gasoline blending components total consunted in the United States;

MSTCPUS = miscellaneous petroleum products total consumed in the United States;

NATCPUS = natural gasoline (including isopentane) total consumed in the United States;

PCTCPUS a petroleum coke total consumed in the United States;

PLTCPUS = plant condensate total consumed in the United States;

PFTCPUS = pentanes plus total consumed in the United States;

SGTCPUS = still gas total consumed in the United States;

SNTCPUS = special naphthas total consumed in the United States;

UOTCPUS = unfinished oils total consumed in the United States;

USTCPUS $*$ unfractionated stream total consumed in the United States; and

WXTCPUS = waxes total consumed in the United States.
The methods used to create State estimates for each of these products (except petrolettm coke, which was described earlier in the petroleum coke documentation) are explatined in the following sections. It is assumed that all of these products are used by the industrial sector, except for the small portion of petroleum coke consumed at electric utilities. State estimates are created for other petroleum products by using the following four variables to allocate the products to the States:

COCAPZZ - exude oil operating capacity at refineries in each State, in barrels per calendar day;

OCVAVZZ = value added in the manufacture of industrial organic chemicals in each $\mathbf{5 t a t e}$ in million dollarb;

PIVAVZZ = value added in the manufacture of paints and allied products tin each State, in million dotlars; and

CGVAVZZ = value added in the manufacture of corrugated and solid fiber boxes.

Value added by mamufacture is a measure of manufacturing activity that is derived by subtracting the cost of materials (which covers materials, supplies, containers, fuel, purchased electricity, and contract work) from the value of shipments. This difference is then adjusted by the net change in finished goods and work-in-process between the beginning and end-of-year inventories. Vaive added is considered to be the best value measure available for comparing the relative economic importance of manufacturing among industries and geo. graphic areas. The value added tata are from the Department of Commerce Census of Manufictures reports.

\section{Crude Oll}

\section{Physical Units}

State estimates for crude oil consumed in petroleum industry operations are the data series COTCPZZ. The U.S. total for this data series is summed:

\section{COTCPUS $=$ sCOTCPZZ}

Industrial consumption equals total consumption of crude oil: 


\section{COICPZZ = COTCPZZ \\ ColcPus $=$ COTCPUS}

\section{Eritsh Thermal Unils (Btu)}

Crude oil has a heat content value of approximately 5.800 million Btu per barrel. The calculations performed to estimate total Btu consumption and industrial use Btu consumption by State and for the United States are:

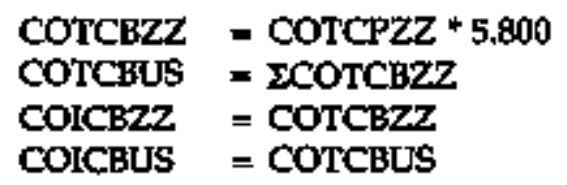

Aviation Gasoline Blending Components; Petroleum Feedstocks, still Gas; Motor Gasoline Blending Components; Still Gas; and Unfinished Ols

\section{Physical Units}

The five petroleum products in this category are consumed as refinery fuels. Begiruing in 1986, still gas for petrochemical feedstocks and still gas for other uses are reported together in the source document. State consumption estlmates of these products are created in proportion to each State's crude oil operating capacity at refineries (COCAPZZ). The U.S. total for this variable is summed:

\section{COCAPUS = ICOCAPZZ}

Aviation gasoline blending components State and U.S. consumption are estimated:

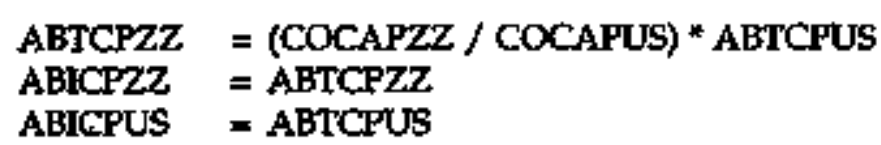

Petroleum feedstocks, still gas, State and U.S. consumption are estimated:

\section{FSTCPZZ = (COCAPZZ / COCAPUS $) *$ FSTCPUS \\ FSICPZZ = FSTCPZZ \\ FSICPUS $=$ FSTCPUS}

Motor gasoline blending components State and U.S. consumption are estimated:

$\begin{array}{ll}\text { MBTCPZZ } & =\text { (COCAPZZ } / \text { COCAPUS }) \text { MBTCPUS } \\ \text { MBICPZZ } & =\text { MBTCPZZ } \\ \text { MBICPUS } & =\text { MBTCPUS }\end{array}$

Still gas State and U.S. consumption are estimated:

$$
\begin{array}{ll}
\text { SGTCPZZ } & =(\text { COCAPZZ / COCAPUS }) \text { SGTCPUS } \\
\text { SGICPZZ } & =\text { SGTCPZZ } \\
\text { SGICPUS } & =\text { SGTCPUS }
\end{array}
$$

Unfinished oils State and U.S. consumption are estimated:

$$
\begin{array}{ll}
\text { UOTCPZZ } & =\text { (COCAPZZ / COCAPUS) * UOTCPUS } \\
\text { UOICPZZ } & =\text { UOTCPZZ } \\
\text { UOICYUS } & =\text { UOTCPUS }
\end{array}
$$

\section{British Thermal Units (Btu)}

\begin{tabular}{|c|c|}
\hline ABTCBZZ & $=$ ABTCPZZ * 5.048 \\
\hline ABTCBUS & $=$ EABTCBZZ \\
\hline $\begin{array}{l}\text { ABICBZZ } \\
\text { ABICBUS }\end{array}$ & $\begin{array}{l}=\text { ABTCBZZ } \\
=\text { ABTCBUS }\end{array}$ \\
\hline FSTCBZZ & $=$ FSTCPZZ * 6.000 \\
\hline FSTCBUS & $=$ EPSTCBZZ \\
\hline FSICBZZZ & $=$ FSrCBZZ \\
\hline FSICBUS & $=$ FSTCBUS \\
\hline
\end{tabular}

Btu estimates for the five products in this group are developed by multiplying the estimated consumption of each individual product in physical units by its respective heat content conversion factor. The calculations performed to estimate total Btu consumption and industrial use Btu consumption by State and for the Urited States are: 

MBTCBZZ = MBTCPZZ $* 5.253$
MBTCBUS = DMBTCBZZ
MBICBZZ = MBTCBZZ
MBICBUS = MBTCBUS
SGTCBZZ $=$ SGTCPZZ 6.000
SGTCBUTS = ESGTCBZZ
SGICBZZ = SGTCBZZ
SGICBUS = SGTCBUS
UOTCBZZ $=$ UOTCPZZ $* 5.825$
UOTCBUS $=$ sUOTCBZZ
UOICBZZ = UOTCBZZ
UOICBUS = UOTCBUS

Petroleum Feedstocks, Naphtha Less Than $401^{\circ}$ F; Petroleum Feedstocks, Other Oils Equal to or Greater Than 401 ${ }^{\circ}$ F; Wiscellaneous Petroleum Products; Natural GasoIIne (Including leopentaine); Plant Condensate; Pentanes Pilus; and Unfractlonaled Stream.

\section{Physical Unils}

The seven petroleum products in this category are allocated to the States in proportion to the value added in the manufacture of industrial organic chemicals in each State (OCVAVZZ).

The two petroleum feedstocks are consumed by the chemical industry in producing petrochemical "building blocks" that, in turn, are converted to such products as synthetic fibers, synthetic rubber, and plastics.

Miscellaneous products include such products as petrolatum, synthetic natural gas feedstocks, and speciatty oils (e.g, hydraulic oils insulating oils, medicinal oils, rust preventatives, and spray oils). Finished petrochemicals are usually the largest volume of miscellaneous product, and it is assumed that the chief consuming industry for this product line is the cherrical industry.
Natural gasoline (including isopentane), plant condensate, pentenes plus, and unfractionated stream are inchuded in this group because the chemical industry is the only one that could readify utitize these lighter liquid hydrocarbons (as petrochemical feedstocks). Beginning in 1984 in the source document, natural gasoline (including isopentane) and plant condensate are reported together as a new product, pentanes plus. At the same time, unfractionated stream was dropped because its components were reported separately as liquefied petroleum gases.

The U.S. total for the data sertes used to apportion these products to the States is stimmed:

\section{QCVAVUS = EOCVAVZZ}

Total petroleum feedstocks, naphtha less than $481^{\circ}$ F, State and U.S. consumption are estimated:

\section{FNTCPZZ = (OCVAVZZ / OCVAVUS) * FNTCPUS \\ FNICPZZ = FNTCPZZ \\ FNICPUS $=$ FNTCPUS}

Petroleum feedstocks, othex oils equal to or greater than $401^{\circ} \mathrm{F}$, State and U.S. consumptlon are estimated;

\section{FOTCPZZ = (OCVAVZZ / OCVAVUS $) *$ FOTCPUS \\ FOICPZZ = FOTCPZZ \\ FOICPUS $=$ FOTCPUS}

Miscellaneous petroleun products State and U.S. consumption are estimated:

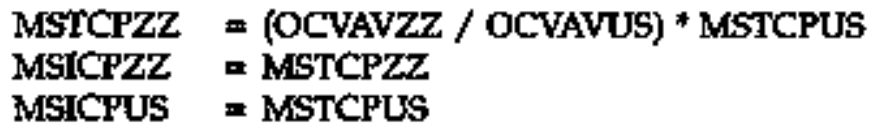

Natural gasoline (including tsopentane) State and U.S. consumption are estimated: 


\section{NATCPZZ = (OCVAVZZ / OCVAVUS) * NATCPUS \\ NAICPZZ = NATCPZZ \\ NAICPUS $=$ NATCPUS}

Plant condensate State and U.S. consumption are estimated:

$$
\begin{array}{ll}
\text { PLTCPZZ } & =\text { (OCVAVZZ / OCVAVUS) * PLTCPUS } \\
\text { PLICPZZ } & =\text { PLTCPZZ } \\
\text { PLICPUS } & =\text { PLTCPUS }
\end{array}
$$

Pentane plus State and U.S. consumption are estimated:

$$
\begin{aligned}
& \text { PPTCPZZ = (OCVAVZZ / OCVAVIS) * PPTCPUS } \\
& \text { PPICPZZ } \\
& \text { PPICPUS - PFTCPZZ } \\
& \text { - PPTCPUS }
\end{aligned}
$$

Unfractionated stream State and U.S. consumption are estimated:

$$
\begin{array}{ll}
\text { USTCPZZ } & =\text { (OCVAVZZ / OCVAVUS) * USTCPUS } \\
\text { USICPZZ } & =\text { USTCPZZ } \\
\text { USICPUS } & =\text { USTCPUS }
\end{array}
$$

\section{Brithoh Thermal Units (Btu)}

Btu estimates for the seven petroleum products in this group are de veloped by multiplying each individual product's estimated consumption in physical units by its respective approximate heat content conversion factor. The calculations performed to estimate total Btu consumption and industrial use Btu consumption by State and for the United States are:

$$
\begin{aligned}
& \text { FNTCBZZ = FNTCPZZ * } 5.248 \\
& \text { FNTCBUS }=\text { EFNTCBZZ } \\
& \text { FNICBZZ = FNTCBZZ } \\
& \text { FNICBUS = FNTCBUS } \\
& \text { FOTCBZZ = FOTCPZZ * 5.525 } \\
& \text { FOTCBUS }=\text { EFOTCBZZ } \\
& \text { FOICBZZ = FOTCBZZ } \\
& \text { FOICBUS }=\text { FOTCBUS }
\end{aligned}
$$

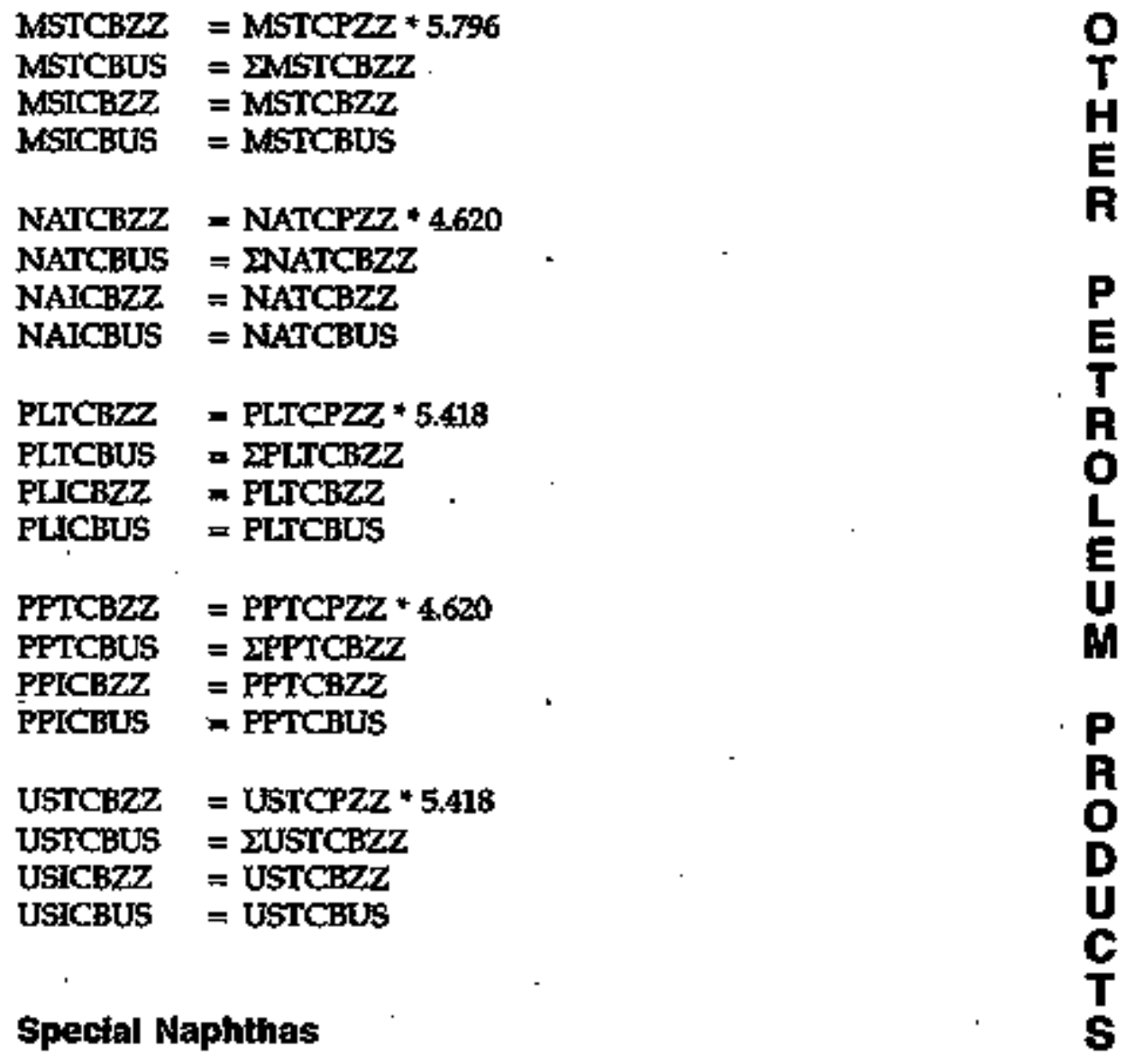

\section{Physical Unils}

Special naphthas are used as paint and varnish thinners and dry cleaning liquids or solvents. This petroleum product is allocated to the States in proportion to the value added in the manufacture of paints and allied products in each State (PTVAVZZ).

The U.S. total for the apportioning data series is calculated:

$$
\text { PIVAVUS }=\text { EPIVAVZZ }
$$

Special naphthas State and U.S. consumption are estimated: 


\section{SNTCPZZ = (PIVAVZZ / PTVAVUS) * SNTCPUS \\ SNICPZZ = SNTCPZZ \\ SNICPUS $\because$ SNTCPUS \\ Brtish Thermal Units (Btu)}

Special naphthas have a heat content value of approximately $\mathbf{5 . 2 4 8}$ million Btu per barrel. This factor is applied to convert special naphthas estimated consumption from physical urits to Bta by State and for the United States:

$\begin{array}{ll}\text { SNTCBZZ } & =\text { SNTCPZZ * } 5.248 \\ \text { SNTCBUS } & =\text { ENTCBZZ } \\ \text { SNCBZZ } & =\text { SNTCBZZ } \\ \text { SNICBUS } & =\text { SNTCBUS }\end{array}$

Waxes

\section{Physical unils}

Because petroleum waxes are very cost-effective moisture and gas barriers, food packaging is the largest market for petroleum waxes in the United States, accounting for more than 50 percent of petroleum wax consumption. Therefore, waxes are allocated to the States in proportion to the value added in the manufachure of corrugated and solid fiber boxes (CGVAVZZ).

The U.S. total for this variable is summed:

$$
\text { CGVAVUS = DCGVAVZZ }
$$

Stake and U.S. consumptlon are estimated:

$$
\begin{array}{ll}
\text { WXTCPZZ } & =\text { (CGVAVZZ / CGVAVUS }) * \text { WXTCPUS } \\
\text { WXICPZZ } & =\text { WXTCPZZ } \\
\text { WXICPUS } & =\text { WXTCPUS }
\end{array}
$$

\section{British Themat Units (Btu)}

Waxes have a heat content value of approximately 5.537 million Btu per barrel. This factor is applied to convert the estimated consumpton of waxes from physical units to Btu by State and at the U.S. level:

$$
\begin{array}{ll}
\text { WXTCBZZ } & =\text { WXTCPZZ } * 5.537 \\
\text { WXTCBIS } & =\text { EWXTCBZZ } \\
\text { WXICBZZ } & =\text { WXTCBZZ } \\
\text { WXICBUS } & =\text { WXTCBUS }
\end{array}
$$

\section{Total Other Petroleum Products}

\section{Physical Unils}

Total other petroleum products is the sum of the 16 "other petroleum products." All of these products are consumed by the industrial sector except for some petroleun coke consumed by electric utilities (PCEUP), which is calculated in SEDS with electric utility fuel consumption. Therefore, State and U.S. industrial use of these other petroleum products are calculated:

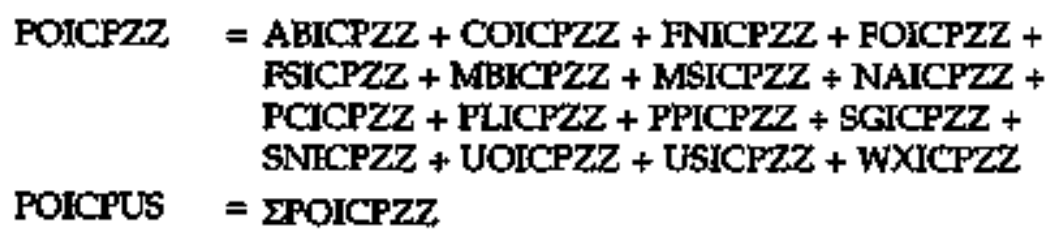

Total consumption of these products (including petroleum coke conBumption by electric utilities) is calculated:

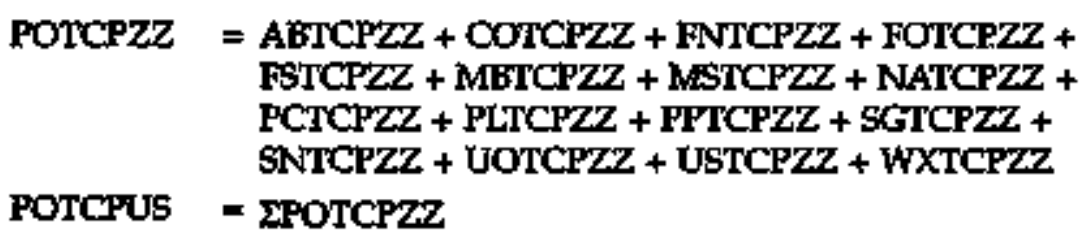




\section{British Thermal Units (Blu)}

Estimated corsumption of all 16 "other petroleum products" in Btu is the sum of the Btu consumption of each product by the industrial sector. The State and U.S. totals are calculated:

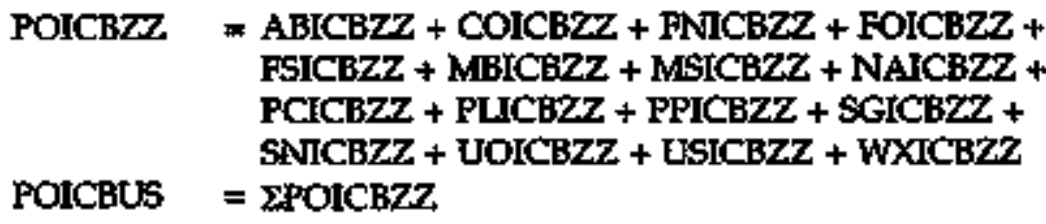

State and U.S. total consumption of these products, which includes electric utility consumption of petroleum coke, is calculated:

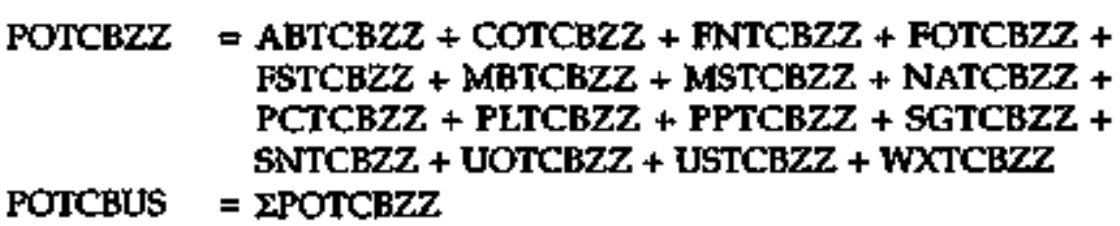

\section{Additional Notes on Other Petroleum Products}

1. In the "Energy Consumption Estimates by Source" tables in this report, a petroleum column called "Other" comprises the other products including petroleum coke consumed by electric utillities (POTCB and POTCP). In the "Industrial Energy Consumption Estimates" tables, the petroleum "Other" column is the other petroleum products consumption total for industrial use (POICB and POICP).

2. The value added by manufacture data used to allocate many of the other petroleum products are from the Department of Commerce, Census of Mantufactures reports. For all years, several States' data were withheld from publication to avoid disclosing operations of individual companies. The total withheld data was apportioned to the withheld States on the basis of those States' proportional values in the previous year.

In 1982, all respondents to the Census of Manufactures survey were requested to report their inventories at cost or market prior to accounting adjustments for "last in first out" cost. This is a change from prior years in which respondents were perritted to value their inventories by using any generally accepted accounting valuation method. Consequently, data for value added by manufacture for $\mathbf{1 9 8 2}$ ane not comparable to the prior years' data.

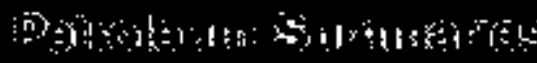

This section describes the method of estimating consumption by the major end-use sectors within the States for all petroleum data series. Table A2 on page 352 of this section indicates which petroleum products are consumed in each of the five major end-use sectors. In the preceding portions of this section, end-use consuription estimates have been derived for each petroleum product. These petroleum product subtotals are now surmed, in physical units of thousand barrels and in Btu, to create estimated end-use consumption for all petroleum products.

\section{Residentlal Sector}

Petroleum products consumed by the residential sector are: distillate fuel (DF), kerosene (KS), and liquefied petroleum gas (LS). For the residential sector, the State and U.S. totals in physical urits are:

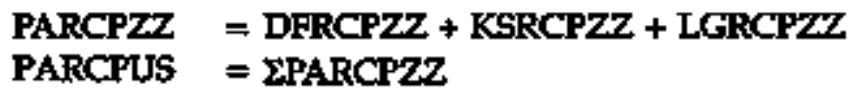

State and U.S. totals in Btu are:

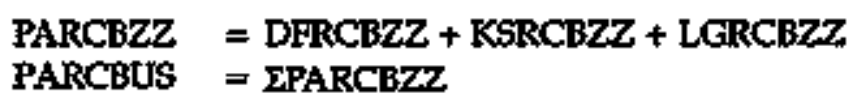

\section{Commerclal Sector}

The commercial sector's use of petroleum products includes: distillabe fuel (DF), kerosene (KS), liquefied petroleum gases (LG), motor 
P gasoline (MG), and residual fuel (RP). In physical urits, the State and the U.S. hotals for the commercial sector are calculated:

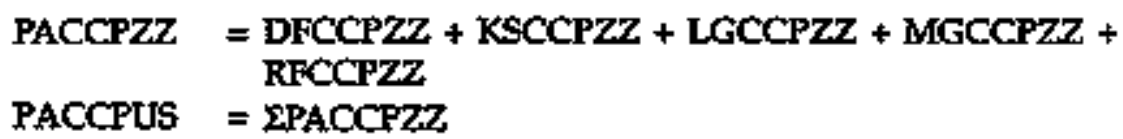

State and U.S. totals in Btu are-

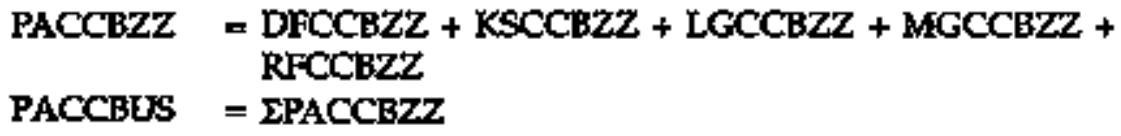

\section{Industrial Sector}

A Petroleum used in the industrial sector includes; asphalt and road oil R (AR); distillate fuel (DF); kerosene (KS); liquefied petroleum gases I (LG); lubricants (LU); motor gasoline (MG); residual fuel (RF); and E the 16 products that are already summed in the "other petroleum products" (PO) subtotal. The State and U.S. total estimates in physical tunits are:

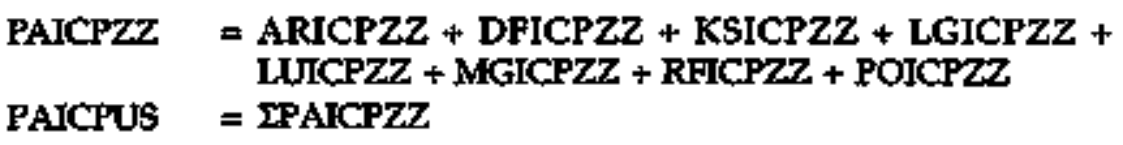

State and U.S. totals in Btu are:

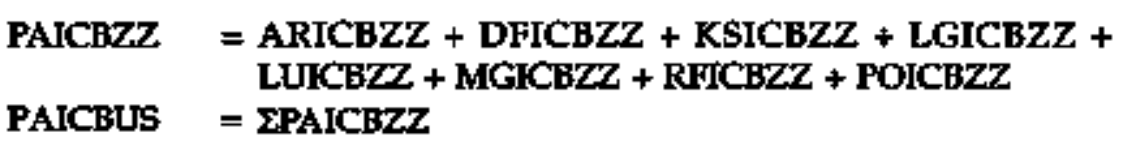

\section{Transportatlon Sector}

Petroleum products used in the transportation sector are: aviation gasoline (AV), distillate fuel (DF), kerosene-type jet fuel (IK), naph" tha-type jet fuel (JN), liquefied petroletum gases (LG), lubricants (LU), motor gasoline (MG), and residual fuel (RF). The State and U.S. totals in physical units are:

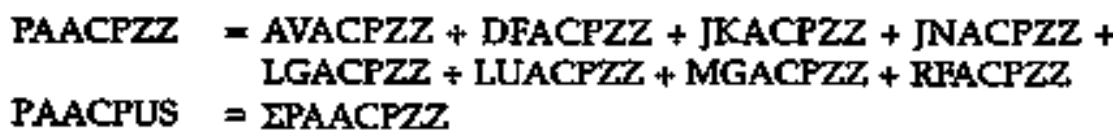

State and U.S. totals in Btu are:

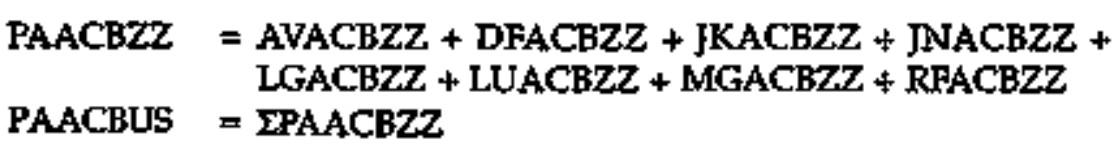

\section{Electric Utillty Sector}

Petroleumi products consumed by the electric utility sector are: distillate fuel (DF), kecosene-type jet fuel (TK), petroleum coke (PC), and residual fuel (RF). In physical units, the State and U.S. totals are:

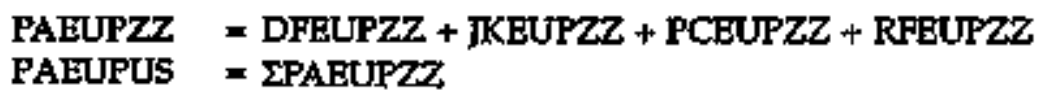

State and U.S. totals in Btu are:

$$
\begin{array}{ll}
\text { PAEUBZZ } & =\text { DFEUBZZ + JKEUBZZ + PCEUBZZ + RFEUBZZ } \\
\text { PAEUBUS } & =\text { EPAEUBZZ }
\end{array}
$$

\section{Total Consumption of Petroleum Products}

Total consumption of all petroleum products is the sum of all of the individual product totals. The State and U.S. physical urit totals are:

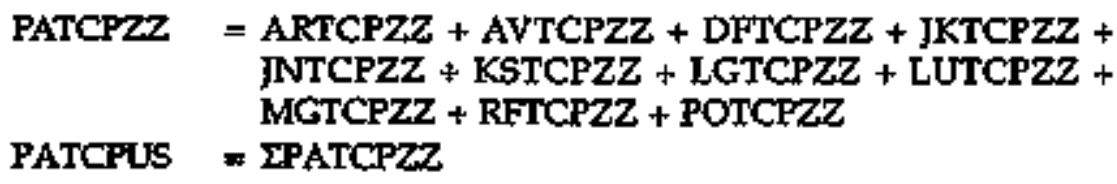

State and U.S. totals in Btu are:
PATCBZZ = ARTCBZZ + AVTCBZZ + DFTCBZZ + JKTCBZZ + JNTCBZZ + KSTCBZZ + LGTCBZZ + LUTCBZZ + MGTCBZZ + RFTCBZZ + POTCBZZ




\section{PATCBUS $=$ EPATCBZZ}

\section{Additional Calculations}

Additional calculations are performed by SEDS to provide data that are used in EIA's Anntuat Enery Review and published in the conversion factor section of EIA's Monthly Energy Reotew. Conversion factors for all petroleum products consumed by each sector as well as data for the residential and commercial sectors combined are calcu. lated by SEDS.

The conversion factor for all petroleum products consumed by the residential sector is calculated:

\section{PARCKUS = PARCBUS / PARCPUS}

The conversion factor for all petroleum products consumed by the corrmercial sector is calculated:

\section{PACCKUS = PACCBUS $/$ PACCPUS}

Consumption of all petroleum products by the residential and commercial sectors combined, in physical units, in Btu, and the average conversion factor are calculated:

\section{PAHCPUS = PARCPUS + PACCPUS \\ PAHCBUS $=$ PARCBUS + PACCBUS \\ PAHCKUS = PAHCBUS $/$ PAHCYUUS}

The conversion factor for all petroleum products consumed by the industrial sector is calculated:

\section{PAICKUS = PAICBUS / PAICPUS}

The conversion factor for all petroleun products consumed by the transportation sector is calculated:

\section{PAACKUS = PAACBUS / PAACPUS}

The conversion factor for all petroleum products consumed by electric utilities is calculated:

\section{PAEUKU'S = PAEUBUS / PAEUPUS}

The conversion factor for all petroleum products consumed by all sectors is colculated:

PATCKUS = RATCBUS / PATCPUS 
$7-6$ - 


\section{Section 5. Renewable Energy}

Renewable energy sources included in the State Energy Data System (SEDS) comprise biofuels (primarily wood, waste, and ethanol), geothermal, hydroelectric, wind, photovoltaic, and solar thermal energy sources. Extensive data collection for fuels used at electric utilities enables SEDS to include renewable energy tused to produce electricity for all years covered in the system, 1960 forward. Renewable energy consumption estimates for the residential, commercial, industrial, and transportation sectors are available for $\mathbf{1 9 9 0}$ forward.

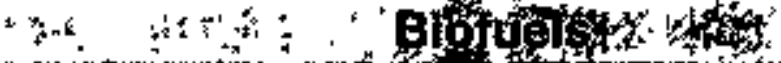

Different forms of biofuels are used by each consuming sector. The residentiai and commercial sectors burn wood for space heating. The industrial sector's primary biofuel source is combustible industrial by-products used for electricity generation and process steam, followed in importance by wood chips. The transportation sector uses ethanol as an additive to motor gasoline. Electric utilities use wood, industrial wood waste and waste gas, and municipal waste as cofiring or primary fuels to produce electricity. Consumption of biofuels in the residential, commercial, industrial, and transportation sectors is included in SEDS for 1990 forward. Biofuels consumption by electric utilities to produce electricity is included from 1960 forward.

\section{Residenttal and Commerclal Sectors}

Estimates of wood consumption in the residential sector by State are developed from data collected on the EIA triennial surveys, Residential Energy Consumption Survey (RECS) 1990 and 1993. The surveys provide data for the national total and for Census Divisions. The survey sample size of the 1993 RECS was large enough to provide data for California, Florida, New York, and Texas. Bstimates for the other
States are developed by allocating Division-level data to the States by using U.S. Department of Commerce, Bureau of the Census, number of housing units per State. Consumption for years between and following 1990 and 1993 is interpolated.

Commercial sector wood consumption is available only for 1993 and 1994 and only on the national level. The commercial sector data are combined with the residential sector in the State Energy Data Report (SEDR) tables.

The following data series and formulas are used to estimate biofuels consumption in the residential and commercial sectors. " $\mathrm{ZZ}$ " in the variable name represents the two-letter State code that differs for each State.

WDRCPZZ $=$ wood consumed in the restdential sector of each State, in thoussand cords; and

WDCCBUS $=$ wood consumed in the commercial sector in the United States, in billion Btu.

The State-level data are summed to a U.S. total:

WDRCPUS $=\Sigma W D R C P Z Z$

The residential sector data in cords are converted to Btu and the commercial sector data in Btu are converted to cords by using the conversion factor of $\mathbf{2 0}$ million Btu per cord:

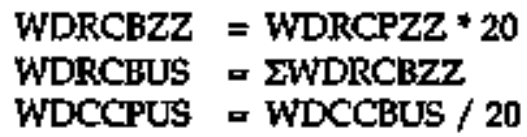

Residential and commercial sector consumption estimates are combined for display in the State Entrgy Data Report (SEDR) tables: 
R WWHCPZZ = WDRCPZZ

E $\quad$ WWHCPUS $=$ WDRCPUS + WDCCPUS

N $\quad$ WWHCBZZ $=$ WDRCBZZ

N WWHCBUS $=$ WDRCBUS + WDCCBUS

in

A

\section{Industrial Sector}

Industrial sector biofuels consumption estimates are based on national-level data published in the EIA, Renezoable Energy Annual 1995, Table 2; State-level date collected on the Form EIA-867, "Annual Nonutility Power Producer Report"; national data collected by Standard Industrtal Code (SIC) on the Form FIA-846, "1991 Maxufacturing Energy Consumption Survey"; and State data for the value added in manufacture from the U.S. Department of Commerce, Bureau of the Census, Census of Manufactures, Industry Series.

The State data for wood consumption and for waste consumption in British thermal units (Bhu) from the nonutility power producers report are summed, and the total is subtracted from the national value for industrial sector biofuels published in Btu in the Renezpable Energy Annurat. The difference is assumed to be used by the manufacturing sector. Based on information obtained from the EIA, "1991 Manufacturing Energy Consumption Survey," the assumption is made that biofuels use in the manufacturing sector occurs primarily in the industries included in SIC codes 2421 (sawmills and planing mills), 2541 (wood partitions and fixtures), and 2621 (paper mills). The State-level "Value Added in Manufacture" data series for these industries is used to allocate the derived U.S. total manufacturing biofuels consumption to the States. The State manufacturing biofuels consumption estimates are added to the nonutility State-level data to create State industrial sector biofuels estimates that equal the U.S. total published in the Renewable Energy Amual.

The etimates are entered into SEDS as State-level data in Btu. "ZZ" in the vaciable name represents the two-letter State code that differs for each State.
WWICBZZ = biofuels consumed by the industrial sector of each State, in billion Btu.

The U.S. total is calculated as the sum of the States' data:

\section{WWICBUS = EWWLBZZ}

There are no comparable physical units because industrial biofuels are measured in a variety of units (e.g., tons, cubic feet, and kilowatthours).

\section{Transportation Sector}

Biofuels are consumed in the transportation sector in the form of ethanol blended into motor gasoline. The data are collected from the States and reported in the U.S. Department of Transportation, Federal Highway Administration, Highway Statistics. The ethanol estimates are shown separately in SEDS and in the SEDR tables to reveal the renewable energy data, but they are already accounted for within the motor gasoline data series. WWACPZZ = ethanol for blending into motor gasoline by State, in
thousand gallons.

The U.S. value published in the EIA, Annual Energy Revieu is greater than the sum of the States' values in Highavay Staitsticts. The difference is entered in SEDS with the State code "OT" (other) that cannot be assigned to specific States. In SEDS, the U.S. value is calculated as the sum of the State-level values:

\section{WWACPUS $=$ IWWACPZZ}

Ethanol is converted to equivalent British themal units (Btu) by using a converston factor of 76,400 Btu per gallon.

WWACBZZ $=$ WWACPZZ * 0.0764

WWACBUS $=$ ZWWACBZZ

\section{Electric Unittles}

Electric utilities' generation of electricity from wood and waste energy, by State, are avatlable combined from 1960 through 1981 and separately from 1982 forward from the Form EIA-759, "Monthly Power Plant Report." The data identifiers in SEDS are: 
WDEOPZZ = electricity produced from wood energy sources at electric utilities in each state (included in waste energy for 1960 through 1981), in million kilowatthours; and

WSEOPZZ = electricity produced from waste energy sources at electric utilities in each State (includes wood energy for 1960 Erough 1981), in million kilowathours.

The U.S. totals are calculated as the sum of the States' data, and wood and waste are summed to provide a biofuels (WW) value:

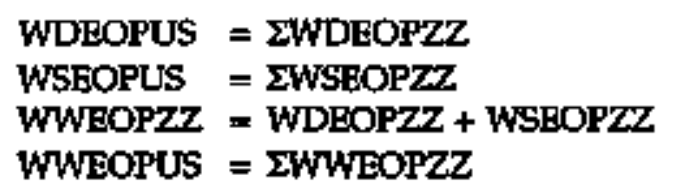

Electricity produced from wood and waste sources is converted into Btu by using a conversion factor that is the U.S. average heat content of fossil fuels burred at steam-electric power plants, FFEOKUS. The annual values for this factor ane shown in Appendix D, Table D1.

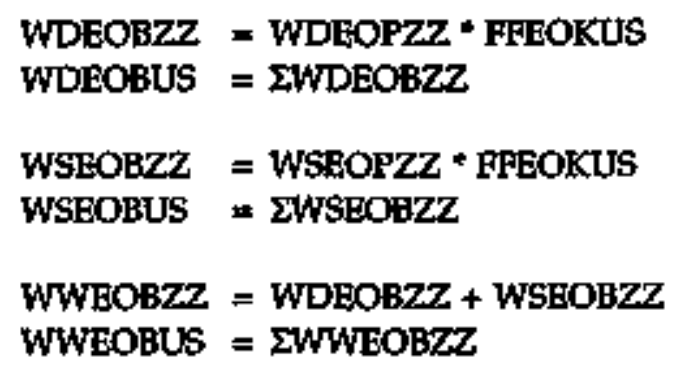

\section{Totals}

State total consumption of biofuels is calculated as the sum of the residential/commercial and industrial sectors' values plus consumption at electric utilities. The U.S. total is the sum of the State data:

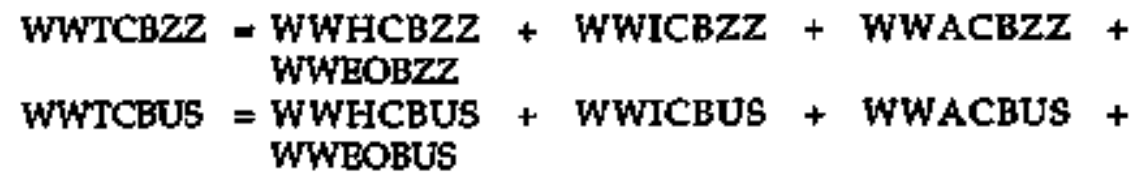

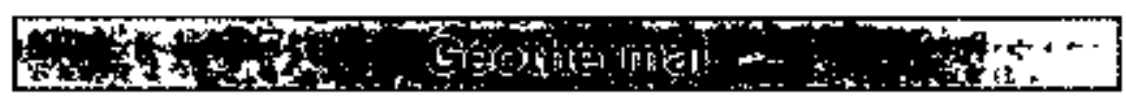

Electricity generation by electric utilities from geothermal energy is included in the State Energy Data System (SEDS) for all years, 1960 forward, as collected on the Form ELA-759, "Monthly Power Plant Report.". Data for electricity produced from geothermal energy sources and inported into the United States from Mexico are available from 1990 forward and are based on data from ELA's Office of Coal, Nuclear, Electric and Altemate Fuels. Geothermal energy used by nonutility power producers to produce electricity, which is included in SFDS industrial sector for 1990 forward, is not available in kilowatthours but is expressed in British thermal units (Bhu) and developed from data collected on the Form EIA-667 'Annual Nonutility Power Producers Report." These data series are identified in SEDS by the following names (" $\mathrm{ZZ}$ " in the variable name represerts the two-letter State code that differs for each State);

GEICBZZ = electricity produced from geothermal energy in the industrial sector by State, in billion Btu;

GEFOPZZ = electricity produced from geothermal energy at electric utlitities by State, in million kilowatthours; and

GEMPZZ = electricity produced from geothermal energy and imported into the United States, by State, in million kilowatthours;

The U.S. totals for the State-tevel sertes are calculated by summing the State data:

\section{GEICBUS $=$ XGEICBZZ \\ GEEOPUS = IGEEOPZZ \\ GEMMPUS $=$ SGEDMPZZ}

Electricity imports produced from geothermal energy are added to the electricity produced from geothermal energy at electric utilities to be shown in the "Ceothermal Enargy" column of the State Energy Data Report (SEDR) tables titled "Energy Input at Electric Utilities."

GEENPZZ $=$ GEEOPZZ + GEMMZZ
GEENPUS $=$ EGEIPNPZZ

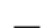

\section{$\mathbf{E}$}

$\mathbf{N}$ W

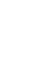 \\ $\mathbf{A}$}

E

.


To convert electricity produced from geothermal energy from kilowatthours into comparable Btu, a U.S. average factor that varies by year is used. The values for the factor, GEEOKUS, are shown in APpendix D, Table D1.

\section{GEEOKUS a factor for converting electricity produced from geo- themal energy from kilowatthours to Btu.}

The values for each sector within each State are converted to Btu:

$$
\begin{array}{ll}
\text { GEEOBZZ } & =\text { GEROPZZ * GEEOKUS } \\
\text { GEEOBUS } & =\text { УGEEOBZZ } \\
\text { GEMMBZZ } & =\text { GEMMPZZ * GEEOKUS } \\
\text { GEMMBUS } & =\text { SGEDMBZZ } \\
\text { GEENBZZ } & =\text { GEEOBZZ + GEDMBZZ } \\
\text { GEENBUS } & =\text { DGETCBZZ }
\end{array}
$$

The State totals for geothermal energy are the sum of the industrial sector geothermalbased generation, available only in Btu, and the elextric utilities' generation (including imports). The U.S. total is the sum of the State data.

\section{GETCBZZ - GEICBZZ + GEENBZZ \\ GETCBUS = IGETCBZZ}

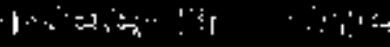

Electricity produced from hydropower in the industrial sector and by electric utilities is included in SEDS for all years. The industrial sector includes estimates of hydroelectricity generation by industries for their own use for all years, as well as generation by nonutility power producers for sale in 1990 forward as collected on the Form EIA-867, "Annual Nomutility Power Producers Report." Industrial data for 1990 forward are not available in kilowatthours, but are inchuded in SEDS in equivalent British thermal units (Btu). Electric utilities' use of hydropower is collected on the Form ELA-759, "Monthly Power Plant Report" and includes two types of hydropower-conventional and pumped storage. Conventional hydroelectric power'uses falling water to drive tubines to produce electricity. With pumped storage hydroelectricity, energy is used to pump water into higher storage areas during non-peak hours so that it can be released to drive turbines during times of peak electricity demand. Because pumped storage hydroelectricity uses energy, it is not considered a renewable energy souxce; however, it is discussed in this chapter with other hydropower.

The hydroelectric data series included in SEDS are identified by the following data series names ("ZZ" in the name represents the twoletter State code that differs for each State):

HVEOPZZ = electricity produced by conventional hydroelectric power at electric utilities, in million kilowathours;

FPEOPZZ - electricily prodinced by pumped storage hydroelectric power at electric utilitles, in million kilowatthours;

HYICPZZ = electricisy produced by hydroelectric power at industrial facilities, by State, in million kilowatthours (available for 1960-1989 only);

HYICBZZ = electricity produced by hydraelectric power at industrial facilities, by State, in billion Btu;

HYMMPZZ = electricity produced from hydroelectric power and imported into the United States, by State, in million kilowatthours; andi

HYEXPZZ w electricity produced from hydroelectric power and exported from the United States, by State, in million kilowathours.

The U.S. value for each of the serles is the sum of the State data.

Total electricity produced from hydropower at electric utilities is calculated as the sum of conventional and pumped storage hydroelectric power.

\section{HYEOPZZ - HVEOPZZ + HPEOPZZ HYEOFUS = Z्YHYEOPZZ}

Hydroelectric-based electricity that is imported and exported across U.S. borders is added to the electric utility hyctroelectric generation and shown in the "Flydroelectric Power" column of the Siate Energy Daka Report (SEDR) tables titled "Energy Input at Electric Utilities." 


\section{HYFNPZZ = HYEOPZZ + HYIMPZZ - HYEXPZZ}

HYENPUS $=$ IHYENPZZ

Additional calculations are done to estimate the renewable portion of hydroelectric power at electric utilities, i.e., excluding hydroelectricity produced from pumped storage:

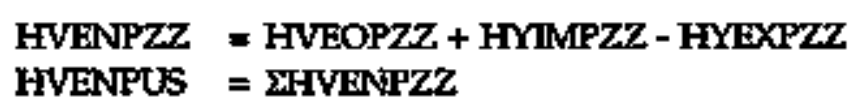

Electricity produced from hydroelectric power is converted from kilowatthours into Btu by using a conversion factor that is the U.S. average heat content of fossil fuels consumed at steam-electric power plants, FFEOKUS. The arnual values for this factor are shown in Appendix D, Table D1.

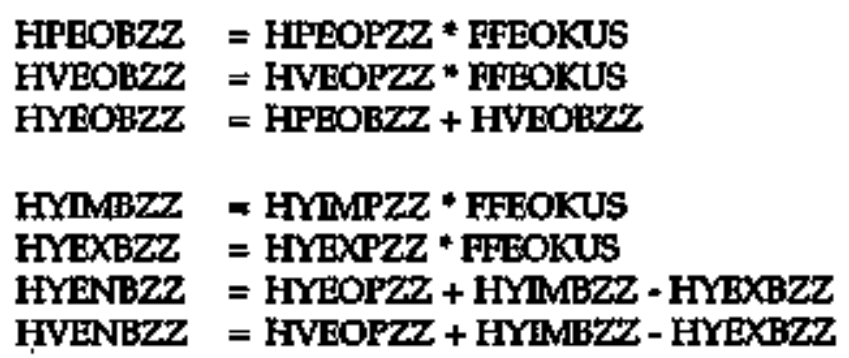

The U.S. value for each of the series is the sum of the State data.

Total hydroelectricity consumption for each State is the sum of the electric utilities generation (plus imports and minus exports) and the industrial sector generation:

HYTCBZZ $=$ HYENBZZ + HYTCBZZ
HYTCBUS $=$ IHYTCBZZ

\section{Sion}

Estimates of solar energy use for the residential and commercial sectors combined and the industrial sector are included in the State Energy Data System (SEDS) for 1990 forward. Generation of electric- ity by electric atilities from solar energy sources is included in SEDS for 1984 forwand.

\section{Aestidentallcommercial seator}

Solar thermal energy use in the residential and commercial sectors combined is estimated by using data on shipments of solar thermal collectors to State destinations, measured in thoussand square feet, as collected on the Energy Information Administration Form CE-63A, "Annual Solar Thermal Collector Manufacturers Survey," and predecessor surveys. The data are published for recent years in the ELA, Renezuable Energy Anural. The assamptions are that: (1) the retirement/replacement period for solar thermal collectors is 20 years and, therefore, the cumulative square footage of solar thermal collectors produced since 1974 are still in use; (2) the dally average energy output of all three categories of solar thermal collectors is 1,500 Btu per square foot; and (3) the average efficiency of the collectors is 50 percent. See Appendix $C$ for detailed source references.

SOHCBZZ = energy produced by solar thermal energy collectors in the residential and commercial sectors combined, in billion Btu.

The U.S. total is calculated as the sum of the State data:

\section{SOHCBUS = SOHCBZZ}

Solar thermal energy is converted to equivalent kilowathours by using the standard themal conversion factor for electricity of 3.412 thousand Btu per kilowatthour:

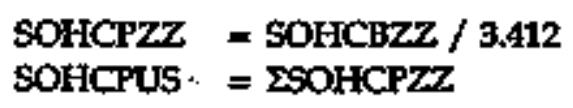

\section{Industrial Sector and Electric Uthilios}

Estimates of electricily produced from photovoltaic and solar thermal energy sources by nonutility power producers are included in the SRDS industrial sector for 1990 forward, in Btu, from data collected on the Form ElA-867, "Annual Nonutility Power Producers 
Report." Elextric ubilities" generation from solar sources are included for 1984 forward as collected on the Form EIA-759, "Monthly Power Plant Report." The data are identified in SEDS by the following names (" $\mathrm{ZZ}$ " in the varjable name represents the two-letter State code that differs for each State):

SOEOPZZ = electricity produced from photovoltaic and solar thermal energy sources at electric utilities, by State, in million kilowatthours; and

SOICBZZ = electritity produced from photovoltaic and solar thermal enersy sotarces by nonutitity power producers, by State, in billion Btu.

The U.S. totals for the State-level series are calculated as the sum of the State data:

\section{SOEOPUS $=$ XOOEOPZZ \\ SOICBUS $=$ 2SOICBZ7}

Electricity produced from photovoltaic and solar thermal energy at electric utilities is converted from kilowatthours to Btu by using a conversion factor that is the U.S. average heat content of fossil fuels consumed at steam-electric power plants, FFEOKUS. The annual values for this factor are shown in Appendix D, Table D1.

\section{SOEOBZZ = SOEOPZZ " FFEOKUS \\ SOEOBUS = ZSOEOBZZ}

\section{Totals}

Each State's total use of photovoltaic and solar thermal energy sources is the sum of the sectors' values, and the U.S. total is the sum of the States' totals:
SOTCBZZ
- SOHCBZZ + SOICBZZ + SOEOBZZ
SOTCBUS
$=$ SSOTCBZZ

\section{Wind}

Wind energy used to produce electricity by nonutility power pro. ducers is included in the SEDS industrial sector for 1990 forward in Btu from data collected on the Form EIA-867, "Annual Nonutility Power Producers Report." Electricity generation from wind energy by electric utilities is included for 1983 forward as collected on the Form BlA-759, "Monthly Power Plant Report." The data are identified in SEDS by the following names ( ${ }^{\prime \prime} Z^{\prime \prime}$ in the variable name represents the two-detter State code that differs for each State):

WYEOPZZ = electricity produced from wind energy at electric utilities, by state, in million kilowatthours; and

WYICBZZ Im electricity produced from wind energy in the industrial sector, by State, in billion Btu.

The U.S. totals are calculated as the sum of the State data:

\section{WYEOPUS = IWYEOPZZ \\ WYICBUS $=\Sigma$ WYKCBZZ}

Electricity produced from wind energy at electric utilities is converted from kilowathours to Btu by using a conversion factor that is the U.S. average heat content of fossil fuels consumed at steam-electric power plants, FFEOKUS. The aruual values for this factor are shown in Appendix D, Table D1.

\section{WYEOBZZ = WYEOPZZ * HFEOKUS \\ WYEOBUS $=\Sigma W Y E O B Z Z$}

The State and U.S. totals for wind energy are calculated:

$$
\begin{aligned}
& \text { WYTCBZZ }=\text { WYEOBZZ + WYICBZZ } \\
& \text { WYTCBUS }=\text { EWYTCBZZ }
\end{aligned}
$$

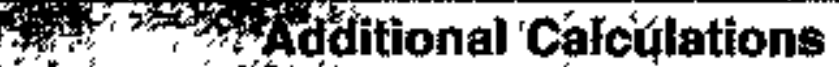

Additional calcutations are made in SEDS to aggregate some data series to be shown in table columns in this report. Geothermal, 
wind, photovoltaic, and solar thermal energy sources are combined to be shown in the "Other" column in tables titled "Energy Consumption Estimates by Source" and "Industrial Energy Consumption Estimates." The variables are calculated for each State and the United States in billion Btu as follows:

$$
\begin{array}{ll}
\text { GOICBZZ } & =\text { GEICBZZ + SOICBZZ + WYICBZZ } \\
\text { GOICBUS } & =\text { EGOICBZZ } \\
\text { GOTCBZZ } & =\text { GETCBZZ + SOTCBZZ + WYTCBZZ } \\
\text { GOTCBUS } & =\text { SKOTCBZZ }
\end{array}
$$

Renewable energy sources included in SEDS for 1960 through 1989 are only those used by the electric utitities to produce electrimty and hydroelectricity used in the industrial sector. These data are available in kilowatthours and are shown in both the physical unit portion of SEDR tables, as well as in the Btu portion. The calculations to provide the data in million kilowatthours for 1960 through 1989 only are.

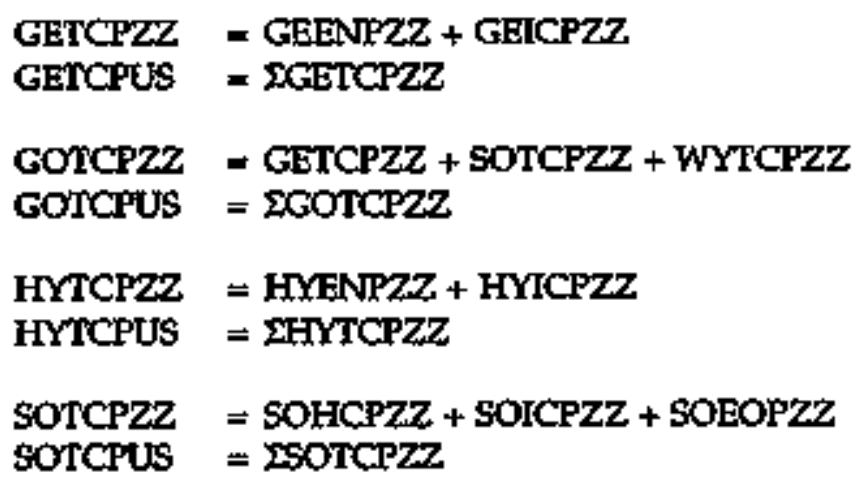

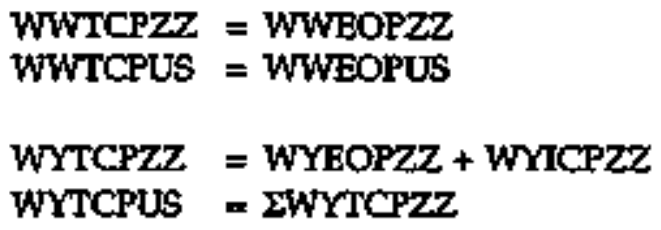

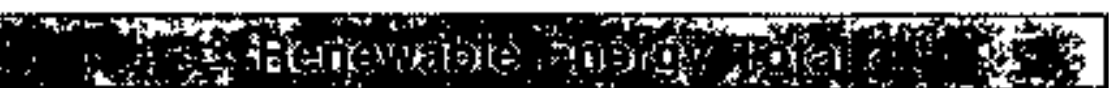

Renewable energy subtotals for each consuming sector in thousand Btu are calculated with the same formulas for each State and the U.S. totals. Renewable energy subtotals can also be calculated in physical units for the transportation sector (thousand gallons) and electric utilities (minlion kilowatthours).

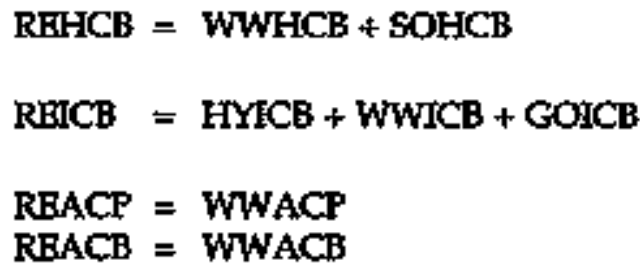

REEOP = HVENP + GEENP + WWEOP + WNEOP REEOB = HVEN + GEENB + WWEOB + WNEOB

RETCB = REHCB \& REICB + REACB + REEOB 


\section{Section 6. Electricity}

This section describes electrical energy sources; electricity consumed by end users (i.e., electricity sold to end users); estimates of the elec. trical system energy losses incurred in the generation, transmission, and distribution of electricity; and estimates of net interstate sales of electricity.

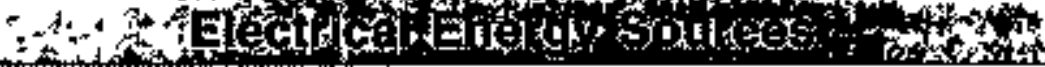

\section{Physical Units}

Electricity is produced from a number of energy sources. In the State Energy Data System (SEDS), coal, natural gas, and petroleum are measured in physical units of thousand short tons, million cubic feet, and thousand barreis, respectively, as they are consumed by the electric utilities. Because comparable measures in physical units for nuclear power, hydroelectric, biomass fuels, geothermal, wind, photovoltaic, and solar thermal energy sources axe not available, energy cutput in the form of electricity produced from these energy sources, in million kilowatthours, is used instead. The variable names for these data are as follows $\left({ }^{\mu} \mathrm{ZZ}^{\prime}\right.$ in the variable name represents the two-letter State code that differs for each State):

CLEUPZZ = coal consumed by electric utiltites (described in Section 2 of this report), in thousand short tons;

ELEXPZZ = electricity exported from the United States (assumed to be produced from hydroelectric power through 1989, in million kllowatthours;

ELIMPZZ = electricity imported into the United States (assumed to be produced from hydroelectric power through 1989), in million kilowatthours;
GEEOPZZ = electrictity produced from geothermal energy at electric utilities (described in Section 5), in million kilowatthours;

GEMMZZ = electricily produced from geothermal energy and imported into the United States (described in Section 5), in million kilowatthours;

HPEOPZZ = electricity produced from ptumped storage hydroelectric power at electric utilities (described in Section 5), in million kilowatthours:

HVEOPZZ a electricity produced from conventional hydroelectric power at electric utilities (described in Section 5), in million kilowatthours;

HYEXPZZ = electricity produced from hydroelectric power and exported from the United States (described in Section 5), in million kilowatthours;

HYMMPZZ = electricity produced from hydxoelectric power and imported into the United States (described in Section 5), in million kilowatthours;

NGEUPZZ = natural gas consumed by electric utilities (described in Section 3), in miltion cubic feet;

NUEOPZZ - electricity produced from nuclear power at electric utilities, in mitlion kilowatthours;

PAEUPZZ = petroleum consumed by electric utilities (described in Section 4), in thousand barrels;

SOEOPZZ = electricity produced from photovoltaic and solar thetmal energy sources at electric utilities (described in Section 5), in million kilowathours;

WDEOPZZ = electricity produced from wood energy sources at electrtc utilities (described in Section 5), in million kilowatthours;

WSEOPZZ = electricity produced from waste energy sources at electric utilities (described in Section 5), in miltion kilowatthours; and 

ties (described in Section 5), in million kilowatthours.

The U.S. totals for these series are calculated as the sum of the State data, with the exception of coal, which is the sum of the U.S. totals for each rank of coal as described in Section 2.

\section{British Thermal Unils (Btw)}

In order to total all the energy that is used to produce electricity, the energy sources are converted to the common unit of Btu. The methods for calculating the Btu content of coal, natural gas, petroleum, and renewable energy sources consumed by utilities are explained in their respective sections of this documentation. The following factors are used to convert the remaining components:

FFEOKLS = average factor for fossil fuels burned at steam-electric power plants, used to convert selected electricity series from kilowatthours to Btu, and

NUEOKUS = factor for converting nuclear electricity from kilowatthours to Btu.

These U.S. average factors, which vary from year to year, can be found in Appendix D, Table D1. The U.S. average conversion factor for fossil fuels burned at steam-electric power plants (FFEOKLS) is used to convert biofuels, hydroelectric power, wind, photovoltaic, and solar thermal energy sources (as described in Section 5) as well as electricity imports and exports derived from nonrenewable energy sources described in this section. The factor for converting electricity produced from nuclear energy (NUEOKUS) is developed from data collected from ruclear steam-electric power plants. The formulas for applying the nuclear factor are:

\section{NUEOBZZ $=$ NUEOPZZ * NUEOKUS \\ NUEEOBUS = ENUEOBZZ}

\section{Electricity Imports and Exports}

Inports and exports of electricity across U.S. borders prior to 1990 are assumed to be based on hydroelectric power. Beginning with
1990, traded electricity is identified in SEDS as derived from hydroelectric power, geothermal energy, or nonrenewable energy sources. Electricity imports and exports based on renewable energy sources are summed in million kilowathours and billion Btu and identified with "ER" as the source code in the variable name:

$\begin{array}{ll}\text { EREXPZZ } & =\text { HYEXPZZ } \\ \text { EREXPUS } & =\text { EEREXPZZ } \\ \text { ERMMPZZ } & =\text { HYMMPZZ + GEIMPZZ } \\ \text { ERIMIPUS } & =\text { EERMMPZ } \\ \text { EREXBZZ } & =\text { HXEXBZZ } \\ \text { EREXBUS } & =\text { XEREXBZZ } \\ \text { ERIMBZZ } & =\text { HYMMBZZ + GEIMBZZ } \\ \text { ERIMBUS } & =\text { EERIMBZZ }\end{array}$

Imports and exports of electricity produced from noncenewable energy sources (" $\mathrm{EX}$ "), in million kilowatthours, are calculated by subtracting renewable-based imports and exports from total electrictity tmports and exports :

$\begin{aligned} \text { EXIMPZZ } & =\text { ELIMPZZ }- \text { ERDMPZZ } \\ \text { EXIMPUS } & =\Sigma \text { EXMMZZ } \\ \text { EXEXPZZ } & =\text { ELEXPZZ - EREXPZZ } \\ \text { EXEXPUS } & =\Sigma \text { EXEXPZZ }\end{aligned}$

Nonrenewable-based electrtcity imports and exports are converted from million kilowathours to billion Btu using the average conversion factor for fossil fuels burned at steam-electric power plants (FFEOKUS):

$\begin{array}{ll}\text { EXMMBZZ } & =\text { EXIMPZZ * FFEOKUS } \\ \text { BXMBUS } & =\text { EBXMMBZZ } \\ \text { EXEXBZZ } & =\text { EXEXPZZ * HFEOKUS } \\ \text { EXEXBUS } & =\Sigma \text { EXEXBZZ }\end{array}$

Net imports of electricity produced trom nonrenewable energy sourses is calculated by subtracting exports from imports:
EXNIPZZ
$=$ EXIMPZZ $\cdot$ EXEXPZZ
EXNIPUS $=$ EEXNIPZZ 


\section{EXNIBZZ = EXIMBZZ - EXEXBZZ \\ EXNIBUS = EEXNIBZZ}

Net imports of renewable-based electricity are included in the "Total" column of SEDR tables titled "Energy Consumption Estimates by Source" and "Estimates of Energy Input at Electric Utilities" but are not shown separately in the tables' columns. Table A8 provides the data by State and year.

Total imports and exports of electricity are calculated in billion Btu by summing the renewable and nonrenewable components:

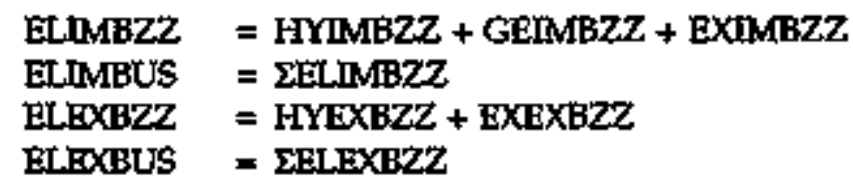

\section{Total Energy hout}

A total of all energy input at electric utilities, including imports and exports of electricity across U.S. borders, is calculated by the following formulas for each State and for the United States:

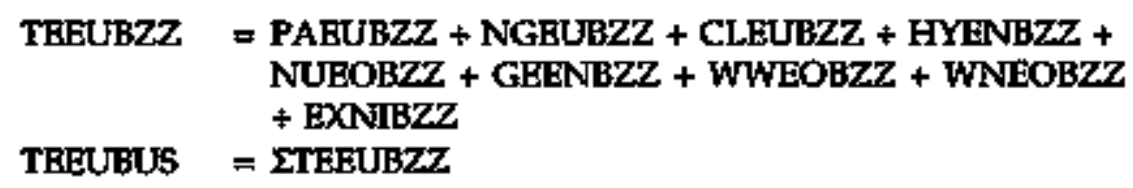

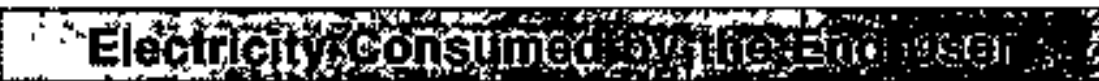

\section{Phystesl Units}

The amount of electricity sold to end users is considered to be the amount of electricity consumed by the end-use sectors. Five electricity sales data series, in physical units of million kilowathours, and one data series that represents the proportional share of an end-use sector are used to estimate consumption of electricity by end-use sector:
Table A8. Net Imports of Electricity Produced from Nonrenewabje Energy Soures, 1990-1994

(Trillion Btu)

\begin{tabular}{|c|c|c|c|c|c|}
\hline דוt. & 1800 & 1981 & $1 \mathrm{sen}$ & 1890 & 1004 \\
\hline 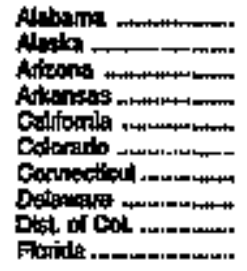 & $\begin{array}{l}0000 \\
0000 \\
00001 \\
0000 \\
0000 \\
16100 \\
0000 \\
0.304 \\
0000 \\
0000 \\
0000\end{array}$ & $\begin{array}{l}0.000 \\
0.000 \\
1+101 \\
0.000 \\
21.001 \\
0.000 \\
0.418 \\
0.000 \\
0.000 \\
0.000\end{array}$ & $\begin{array}{l}0.000 \\
0.000 \\
-0.000 \\
0.000 \\
17.40 \% \\
0.000 \\
0.304 \\
0.000 \\
0.000 \\
0.000\end{array}$ & $\begin{array}{r}0.000 \\
0,000 \\
-0,003 \\
0,000 \\
14,000 \\
0.000 \\
2,54 \\
0,000 \\
0.000 \\
0.000\end{array}$ & $\begin{array}{l}0.000 \\
0.0000 \\
-0.008 \\
0.000 \\
14.890 \\
0.000 \\
0.009 \\
0.000 \\
0.000 \\
0.000\end{array}$ \\
\hline 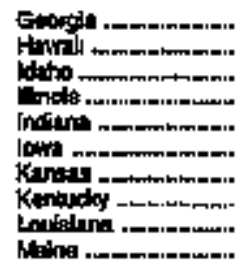 & $\begin{array}{l}0.000 \\
0,000 \\
0.190 \\
0.000 \\
0000 \\
0000 \\
0.000 \\
0,000 \\
0.000 \\
1.950\end{array}$ & $\begin{array}{l}0.000 \\
0.000 \\
0.300 \\
0.000 \\
0.000 \\
0.000 \\
0.000 \\
0.000 \\
0.000 \\
1.420\end{array}$ & $\begin{array}{l}0.000 \\
0000 \\
0607 \\
0000 \\
0.000 \\
0.000 \\
0.000 \\
0.000 \\
0000 \\
20001\end{array}$ & $\begin{array}{l}0.000 \\
0.000 \\
0.9+1 \\
0.000 \\
0.000 \\
0.000 \\
0.000 \\
0.000 \\
0.000 \\
0.040\end{array}$ & $\begin{array}{l}0.000 \\
0.000 \\
0.759 \\
0.000 \\
0.000 \\
0.000 \\
0.000 \\
0.000 \\
0.000 \\
2.998\end{array}$ \\
\hline 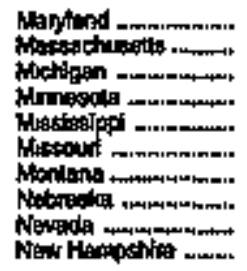 & 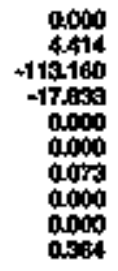 & $\begin{array}{r}0.000 \\
4.345 \\
-5.441 \\
+1114 \\
0.000 \\
0.000 \\
0.039 \\
0.000 \\
0.007 \\
2.043\end{array}$ & $\begin{array}{l}0.000 \\
4,109 \\
-2.092 \\
+0.750 \\
0.000 \\
0.000 \\
0.072 \\
0.000 \\
0.004 \\
2.304\end{array}$ & $\begin{array}{l}0.000 \\
4.360 \\
1,601 \\
+5,072 \\
0.000 \\
0.000 \\
0.048 \\
0.000 \\
0.009 \\
2.54\end{array}$ & 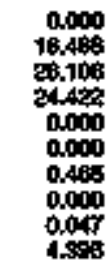 \\
\hline 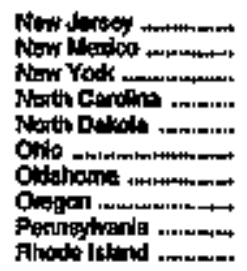 & $\begin{array}{r}0.000 \\
0,000 \\
+17.0030 \\
0.000 \\
0350 \\
0.000 \\
0.000 \\
4037 \\
0.000 \\
0.304\end{array}$ & $\begin{array}{r}0.000 \\
0.000 \\
+200 \\
0.000 \\
-2100 \\
0.000 \\
0.000 \\
6.270 \\
0,000 \\
0.418\end{array}$ & $\begin{array}{r}0.000 \\
0.000 \\
+1,722 \\
0.000 \\
+205 \\
0.000 \\
0.000 \\
+4.000 \\
0.000 \\
2.504\end{array}$ & $\begin{array}{l}0.000 \\
0.000 \\
0.153 \\
0.000 \\
0.216 \\
0.000 \\
0.000 \\
7.735 \\
0.000 \\
2544\end{array}$ & $\begin{array}{r}0.000 \\
0.000 \\
45.249 \\
0.000 \\
2350 \\
0.000 \\
0.000 \\
+8.005 \\
0.000 \\
2.005\end{array}$ \\
\hline 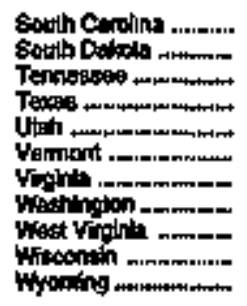 & $\begin{array}{l}0.000 \\
0.000 \\
0.000 \\
0,050 \\
0.000 \\
1.308 \\
0.000 \\
0.810 \\
0.000 \\
0.000 \\
0.000\end{array}$ & 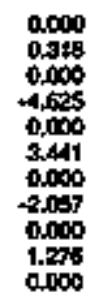 & $\begin{array}{l}0.000 \\
0.607 \\
0.000 \\
9.916 \\
0,000 \\
3.016 \\
0.000 \\
6.765 \\
0.000 \\
2.302 \\
0.000\end{array}$ & $\begin{array}{l}0.000 \\
0.000 \\
0.000 \\
0.235 \\
0.000 \\
3.151 \\
0.000 \\
-6.020 \\
0.000 \\
0.000 \\
0.000\end{array}$ & $\begin{array}{r}0.000 \\
1.429 \\
0.000 \\
+9.900 \\
0.000 \\
+2007 \\
0.000 \\
+4.001 \\
0.000 \\
4.750 \\
0.000\end{array}$ \\
\hline United Stroles . . mm & Atzsit & $24.3=1$ & $30 \pi 5$ & 7n.180 & 102000 \\
\hline
\end{tabular}

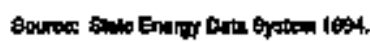


ESRCPZZ = electricity sold to the residential sector of each State; ESCMPZZ = a portion of the electrictity sold to the commercial sector of each State;

ESTCPZZ = electricity sold to the industrial sector of each State; .

ESOTPZZ = electricity sold to "Other" users (i.e., public street and highway lighting, other public authorities, railroads and railways, and interdepartmental sales) in each State;

ESTRPZZ - electricity constumed by transit systems, in each State; and

ESTRSUS = The stare of electricity sold to the "Other" users that is used for transportation.

U.S. totals for the five State-level series are calculated as the sum of the State data.

The sales of electricity to the residential and industrial sectors are used directly as consumption of electricity by these sectors.

The consumption estimates for the conunercial and transportation sectors are made, ftrst, by estimating the portion of sales to the "Other" sector that is used for transportation at the U.S. level, ESACPUS:

\section{ESACPUS = ESOTPUS * ESTRSUS}

The transportation share (ESTRSUS) of "Other" is calculated at the U.S. level because State information is not avaitable. (See Note 2 on page 398 for further information on this share) Next, State transpor tation use of electricity is estimated by assuming that each State consumes electricity for transportation in proportion to the amount of electricity consumed by transit systems tn each State (see Appendix C for the sources of the transit system esimates):

\section{ESACPZZ $=($ ESTRPZZ / ESTRPUS $) *$ ESACPUS}

Finally, the remaining portion of "Other" is then assigned to the commercial sector. The commercial sector consumption of electricity is represented by ESCCPZZ and is calculated:

\section{ESCCPZZ = ESCMPZZ + ESOTPZZ - ESACPZZ \\ ESCCPUS $=$ EESCCPZZ}

Total electricity consumed by the major end-use sectors is represented by ESTCPZZ and is calculated by adding the four major sector estimates:

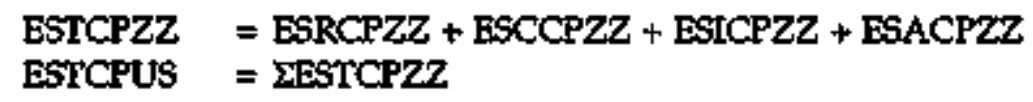

\section{British Thermal Units (Btw)}

Electricity consumption estimates are converted into Btu by applying a constant factor of $\mathbf{3 . 4 1 2}$ thoursand Btu per kilowatthour as illustrated in the formulas:

\section{ESRCBZZ = ESRCPZZ *3.412 \\ ESTCBZZ - ESTCPZZ * 3.412}

And U.S. totals in Btu are calculated by summing the States' Btu values.

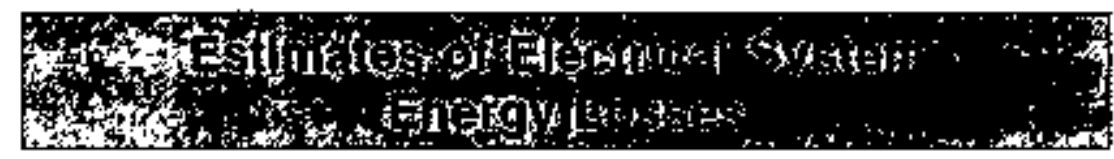

\section{British Thermal Units (Btu)}

Electrical system energy losses, identified by "LO," inchude all losses incurred in the generation, transmission, and distribution of electricity, including plant tree and unaccounted for quantities. Total losses for the United States, LOTCBUS, is assumed to be the difference between the total of all energy input at eiectric utilities (TEELBUS) and the total electricity sold to end users (ESTCBUS). Total losses for the United States is calculated in billion Btu as follows:

LOTCBUS a TEEUBUS - ESTCBUS 
Because Alaska and Hawaii have no exchanges of electricity with other States, their electrical system energy losses are estimated as the difference between the sum of all energy inputt at the State's electric utilities and the electricity sold within the State:

LOTCBAK = TEEUBAK - ESTCBAK

LOTCBHI = TEEUBHI - ESTCBHI

Individual State electrical system energy losses for the remaining States are estimated by a different method. The difference between each of the contiguous 48 States' (including the District of Columbia) TEEUB series and ESTCB is not only the losses but also any net interstate flow of electriclty that may occur between States. In some cases these net interstate flows are substantial. Therefore, an effort is made to estimate separately each State's losses and net interstate flow. The methodology is to calculate the contiguous-48-State subtotal of losses and subtotal of sales; to create annual losses-to-sales ratios for the ag-

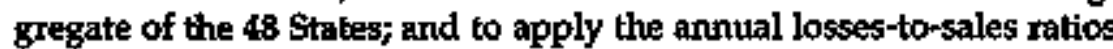
from the total 48 States to the individual 48 States' sales to estimate their losses.

The following steps axe performed to complete the losses estimates. A subtotal of losses in the contiguous 48 States, LOTCB48, is created by subtracting the Alaska and Hawaii losees from the total United States' losses:

\section{LOTCBA8 = LOTCBUS - (LOTCBAK + LOTCBHI)}

A similar subtotal of electricity sales in the 48 States only, ESTCB48, is calculated:

\section{ESTCB4B - ESTCBUS - (ESTCBAK * ESTCBHI)}

The losses-to-sales ratio for the contiguous 48 States only, ELLSS48, is cakculated:

\section{ELLSS48 = LOTCB48 / ESTCB48}

Over the 35-year period now covered in SEDS, the ratio is faitly constant, with a slight downward trend. For 1960, the ratio is 2.5; for 1961 through 1983 the ratio is 2.4; for 1984 through 1988 the ratio is
2.3; for 1989 through 1991 it is 22; and for 1992 forward the lossesto-sales ratio is 2.1. The decline in the ratio in recent years is partially attributed to the fact that electricity produced by nonutility power producers is included in the electricity sales data while the resources consumed to produce the nonutility electricity are not. When the nonutility production is subtracted from the electricity sales, the ratio remains at 2.3 for 1989 through 1993 and is 2.2 for 1994.

The U.S. ratios are applied to each State's sales to the major end-use sectors and total sales (temporarily including Alaska, Hawaii, and the 48-State subtotal for processing convenience);

LORCBZZ $=$ FSRCBZZ * ELISSSA
LOCCBZZ
LOICBZZ FSCCBZZ * ELLSS48
LOACBZZ ESICBZZ * ELLSS48
LOTCBZZ ESACBZZ * ELLSS48

Alaska, Hawaii, and the contiguous 48-State aubtotal are recalculated to their original estimates. The end-use losses for Alaska and Hawaii are created in proportion to each sector's share of the State's total electricity sales:

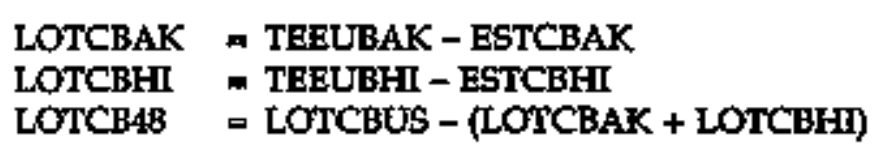

LORCBAK(FII) $=$ (ESRCBAK(HI) $/$ ESTCBAK(HI)) * LOTCBAK(HI) LOCCBAK(HI) $=(\operatorname{ESCCBAK}(\mathrm{HI}) / \operatorname{ESTCBAK}(\mathrm{HI}))^{*} \operatorname{LOTCBAK}(\mathrm{HI})$ LOICBAK(HI) = (ESICBAK(HI)/ ESTCBAK(HI) ${ }^{*}$ LOTCBAK(HI) LOACBAK(HI) $=(\text { ESACBAK(HI) } / \operatorname{ESTCBAK}(\mathrm{HI}))^{*}$ LOTCBAK(H)

Losses for the United Stakes, including Alaska and Hawaii, are the sums of all the States' losses.

\section{Physteal Units}

Estimates of losses in physical units of million kilowatthours are made by dividing the Btu estimtate by the constant 3.412 thoustand Btu per kilowathour as illustrated in the following formulas: 


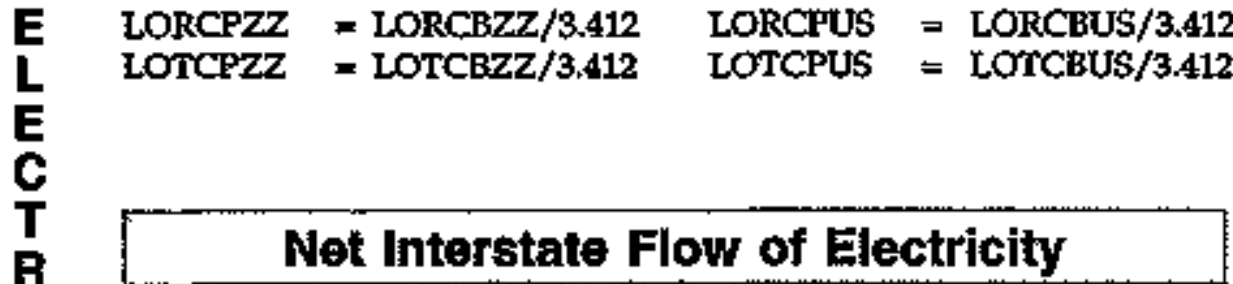

\section{Briftsh Thermal Units (Blu)}

An estimate of the net interstate flow of electricity is calculated as the difference between the total electricity sales and attributed losses and the sotal energy imput to the electric utilities within each State. The estimated net interstate flow of electricity (ELISB) for each State and the United States is calculated:

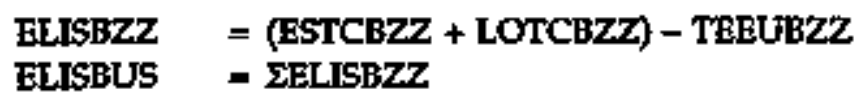

\section{Physical Units}

Estimates of net interstate flow of electricity in physical units of mitlion kilowatthours are calculated by dividing the Btu value by the constant 3.412 thousand Btu per kilowatthour:

\section{ELLSPZZ $=$ ELISBZZ / 3.412 \\ ELISPUS $=$ XELISPZZ}

Positive net interstate flow for a State means that the amount consumed within the State (including attributed losses) is greater than the amount of energy input at electric utilities in the State. That is, the State is using more electricity than it generates and, therefore, is a net buyer from other States.

A negative number indicates that the State's input into its electric utilities is greater than the requirements for electricity within its own bordens, and, therefore, it is a net seller of electricity to other States.

\begin{tabular}{cccccc}
\hline Year & ESTASUS & Year & ESTASUS & Year & ESTRSUS \\
\hline 1960 & 0.09867 & 1972 & 0.04497 & 1984 & 0.04134 \\
1961 & 0.10461 & 1973 & 0.04156 & 1985 & 0.04350 \\
1962 & 0.10506 & 1974 & 0.04524 & 1986 & 0.04390 \\
1963 & 0.09294 & 1976 & 0.04409 & 1987 & 0.04258 \\
1964 & 0.08959 & 1976 & 0.04984 & 1989 & 0.04453 \\
1965 & 0.08345 & 1977 & 0.04900 & 1989 & 0.04449 \\
1968 & 0.07785 & 1978 & 0.03563 & 1990 & 0.04486 \\
1967 & 0.06762 & 1979 & 0.04195 & 1991 & 0.04394 \\
1968 & 0.06008 & 1990 & 0.04226 & 1992 & 0.04286 \\
1969 & 0.05352 & 1981 & 0.03921 & 1998 & 0.04064 \\
1970 & 0.05177 & 1992 & 0.03736 & 1994 & 0.04051 \\
1971 & 0.04823 & 1993 & 0.03980 & & \\
\hline
\end{tabular}

\section{Additional Notesoso}

1. The source for the electricity sales data for 1960 through 1983 is the Energy Information Administration (EIA) Form EIA-826, "Electric Utility Company Monthly Statement," and predecessor forms. Electricity sales data for 1984 forward are from Form EIA-861, "Annual Electric Utility Report." At the national level, data from both forms correspond closely (within 3 percent) for all end-use sectors. However, differences in the number of survey respondents and the reporting of commercial and industrial sales caused inconsistencies between 1983 and 1984 data in those end-use sectors for some States. See the ELA's, Electric Potser Annual, 1991, DOE/EIA-0348(91), P. 130, and An Assessment of the Qualify of Selected ElA Data Series, Electric Power Daka, DOE/EIA-0292(87), Pp. 17-28, for detailed discussions of the reporting differences.

2. Annual estimates of the electricity sales to the transportation sector, which are a portion of "Other" sales in the source document, are made by using data published in Financial Statistics of Selected Intuestor-Ozoned Electric Utilities, DOE/EIA-0437. Sales to the "Other" category include: (I) public street and highway 
lighting; (2) sales to other public authorites; (\$) railroads and railways; and (4) interdepartmental sales.

ESTRSUS is the "Railroads and Railways" share of all four items in the "Other" category. The shares used in SEDS are shown in Table A9.

3. The source for the electricity sales data for 1960 through 1983 is the EIA Form BIA-826, "Electric Utility Company Monthly Statement," and predecessor forms. Electricity sales data for the District of Columbia and Maryland are combined on those forms. Estimates of separate sales for the District of Columbia and Maryland were created by using electricity sales data by end-use sector by conmunities from the FERC Form 1, "Annual Report of Major Electric Utilities, Licensees, and Others, " filed by the Potomac Electric Power Company (PEPCO). PEPCO sales to the District of Columbia were assumed to be total electricity sales in the Disttict of Columbia. The FERC Form 1 electricity sales to the District of Columbta by PEPCO were subtracted from the EIA-B26 District of Columbia and Maryland aggregate figures to obtain estimates of Maryland electricity sales by sector. Beginning with 1981 data, electric utilities were no longer required to report sales to specific communities. Therefore, sales data for the District of Columbia for 1981 through 1983 were abtained directly from PEPCO's accounting department.

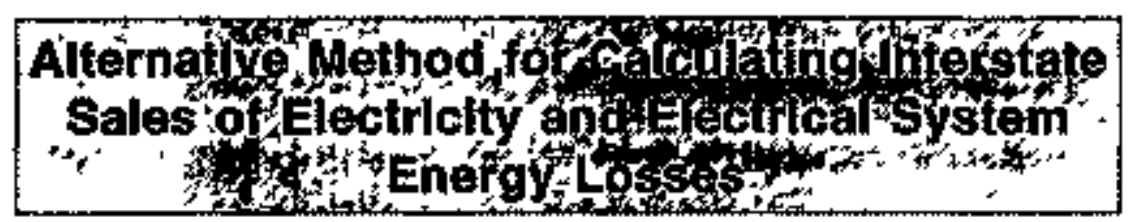

EIA is examining a method to disaggregate the estimates of net interstate sales of electricity and electrical system energy losses as shown in Tables A10 through A18.

The fuel consumed at electric utilities and the net generation of other energy sources by electric utilities collected by EIA on Form EIA-759, "Monthly Power Plant Report," represent the total energy input at electric utilities, TIEUB, shown in column 1 of Tables A10 through
A18. This series is equal to the SEDS series TEEUB, except TEEUB also contains net imports of electricity. The formulas for TIEUB for each State and the United States are.

\section{TIEUBZZ = PAEUBZZ + NGEUBZZ + CLEUBZZ + HYEOBZZ + TIEUBUS $=$ হTEUBZZ}

Another series collected on Form ElA-759 is the net generation of electricity by eiectric utilities (total generation minus plant use). This data series, in thousand kilowatthours, given the variable name ELEOP, represents the energy output of electric utilities. ELEOPZZ is converted to Btu by using the standard conversion factor of 3.472 thousand Btu per kilowatthour.

\section{ELEOBZZ = ELEOPZZ * 3.412 \\ ELEOBUS $=$ EBLEOBZZ}

Subtracting energy output by electric utilities, ELEOBZZ (shown in column 3 of Tables A10 through A18), from energy input at electric utilities, TIEUBZ.Z (shown in column I of those tables), gives an indication of energy losses that occtur at electric utility plants, ELPLBZZ (shown in column 2). These losses are primarily energy lost in the conversion of the energy sources to electricity. Plant use of electricity is also included in this number.

\section{BLPLBZZ = TIEUBZZ - ELEOBZZ \\ ELPLBUS $=$ EELPLBZZ}

Data for electricity imported or exported across U.S. borders, described earlier in "Electrical Energy Sources," are reported in thousand kilowathours. These data are converted to Btu by using the standard conversion factor and are shown in column 4 of Tables A10 through A18. Trade data are added to the net generation of each State involved in intemational exchanges of electricity to detive electricity transmitted for sale within the United States (shown in column 5 of the following tables).

$\begin{array}{ll}\text { ELIMBZZ } & =\text { ELIMPZZ *3.412 } \\ \text { ELEXBZZ } & =\text { ELEXPZZ *3.412 }\end{array}$ 


\section{ELNIBZZ = ELIMBZZ - ELEXBZZ \\ ELNIBUS $=$ EELNIBZZ}

\section{ELENBZZ = ELEOBZZ + ELNIBZZ \\ ELENBUS $=$ EELENBZZ}

Total electrical energy last in the transmission and distribution of electricity is reported by each electric utility. However, some electric utilities distribute electricity to more than one State. The EIA Electric Power Division has estimated these losses by State. The losses in million kilowathours are converted to billion Btu by using the standard conversion factor and are shown in column 6 in the following tables.

\section{ELLOBZZ = ELLOPZZ *3,412 \\ ELLOBUS = EELLOBZZ}

Subtracting the State estimates for transmisston and distribution losses (column 6) from the electricity transmitted (column 5) ylelds the electrictity available for sale within each State, ELFSBZZ, shown in column 7 .

\section{ELFGBZZ = EL.ENBZZ - ELLLOBZZ \\ ELFSBUS - EELFSBZZ}

Column 7, electricity available for sale, can be compared to column 8 , the actual reported sales to consumers within each State. The sales data are collected on Form EIA-861, "Annual Electric Utility Report," and are currently used in SEDS as variable ESTCBZZ. If column 7, the electricity available for sale, is larger than column 8 , the electric ity sold, the State would be a supplier of electricity to neighboring States. If the electricity available within the State is less than the amount needed to meet sales denand, the State would need to pur- chase electricity from other States. Column 9 of Tables A10 through A18 shows the difference between columns 8 and 7 for each State as calculated:

\section{ESISBZZ = ESTCBZZ - ELFSBZZ \\ ESISBUS $=$ EESISBZZ}

Negative values in columin 9 show the amount of electricity that flowed out of the State. Positive numbers show the amount of electricity that flowed into the State to meet the State's own requitrements. For comparison, data in column 10 are the "Net Interstate Hlow of Electricity and Associated Losses" (ELISBZZ) series now calculated by SEDS (as described earlier in "Net Interstate Flow of Electricity") and shown in the State tables of this report. Values in Column 9 are estimates of the net interstate flow of electricity alone and would be a subset of the tata in column 10, which include electrical system energy losses associated with that flow. .

There are some unresolved problerns with the separate estimate of electricity flow as shown in Column 9: Alaska and Hawait appear to have small purchases of electrtity from other States, and the total United States appears to have excess electricity. The current rtethodology used in SEDS avoids these discrepancies as shown in column 10. The U.S. vahue in column 9 may reflect, at least in part, the electricity that is generated by companies other than electric utilities that would be included in the electricity sales to end users (column 8) but not in the electricity available for sale (column 7). The U.S. value for electricity production by nonutilities is shown in the footnotes on Tables A10 through A18.

Comments on these methodologies would be appreciated. 
Tablo A10. Disaggregated Data for Net Interstate Flow and Electrical System Energy Losses, 1994 (B: lon Btu)

\begin{tabular}{|c|c|c|c|c|c|c|c|c|c|c|}
\hline 5tols & 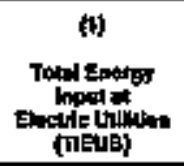 & 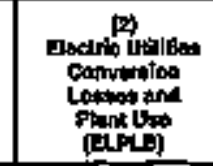 & 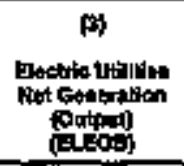 & 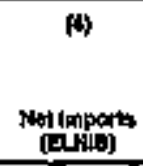 & 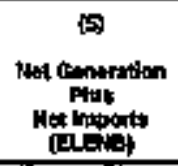 & 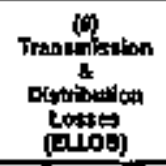 & 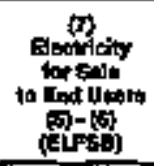 & 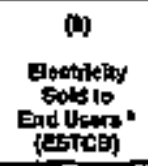 & 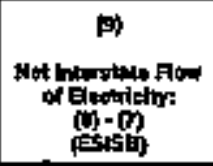 & 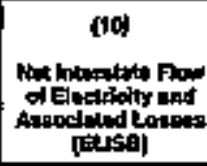 \\
\hline 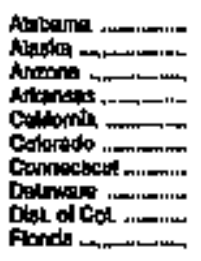 & 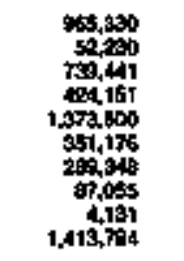 & 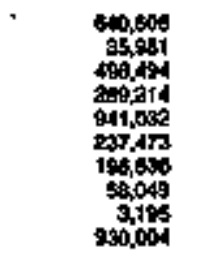 & 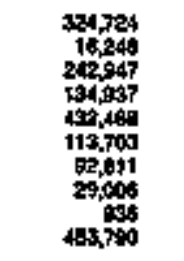 & 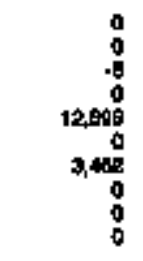 & 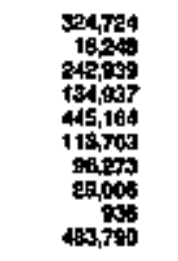 & 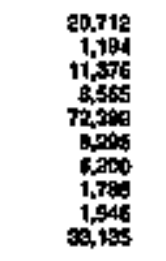 & 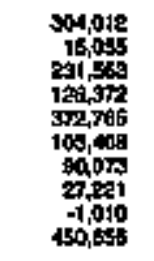 & 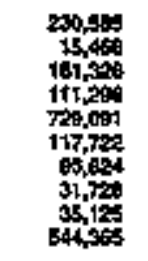 & 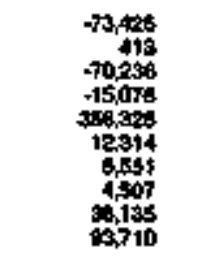 & 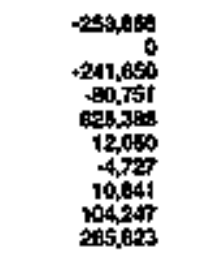 \\
\hline 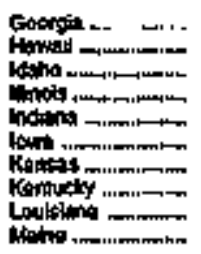 & 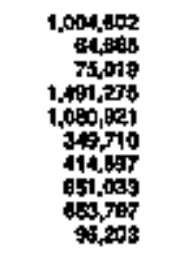 & 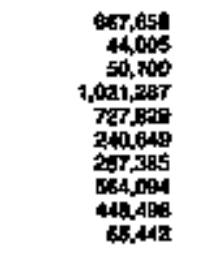 & 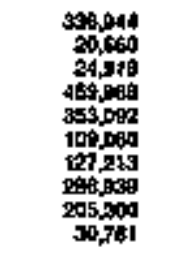 & $\begin{array}{r}0 \\
0 \\
040 \\
0 \\
0 \\
0 \\
0 \\
0 \\
0 \\
0 \\
10,695\end{array}$ & 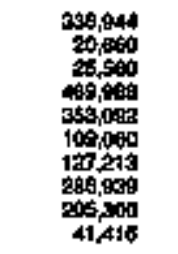 & 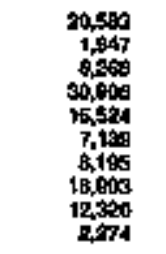 & 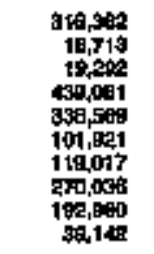 & 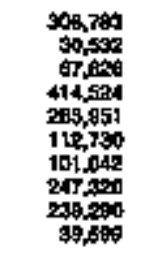 & 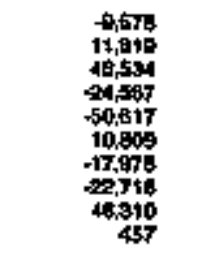 & 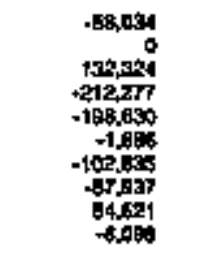 \\
\hline 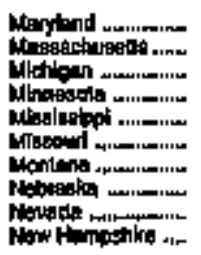 & 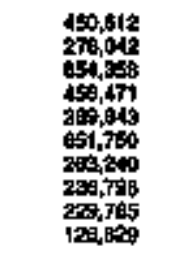 & 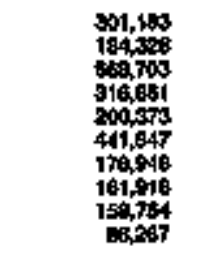 & 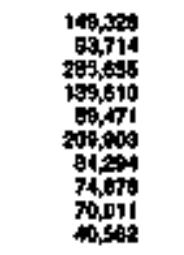 & 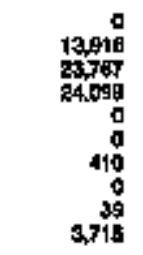 & 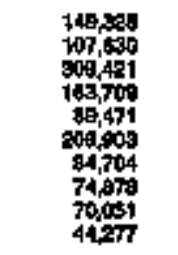 & 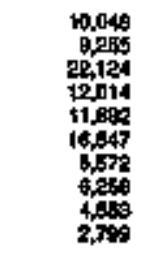 & 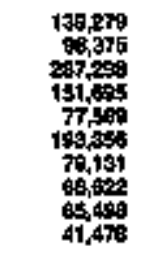 & 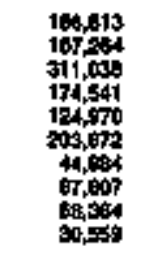 & 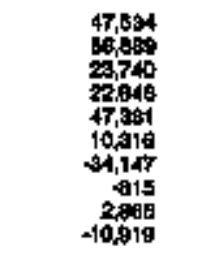 & 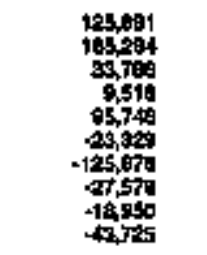 \\
\hline 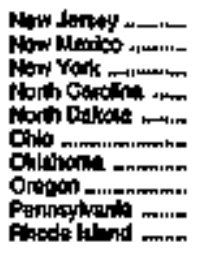 & 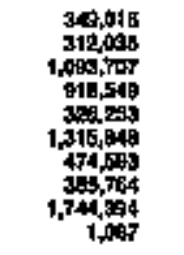 & 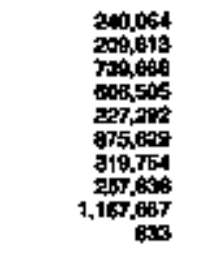 & 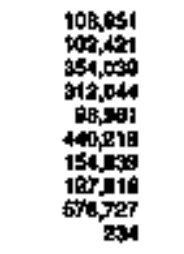 & 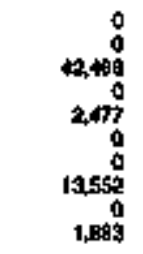 & 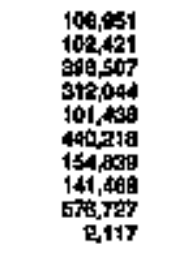 & 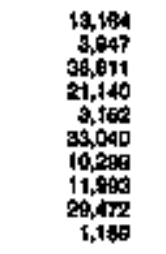 & 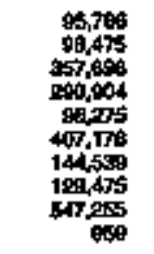 & 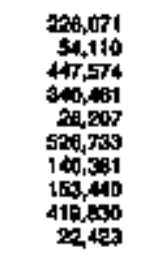 & 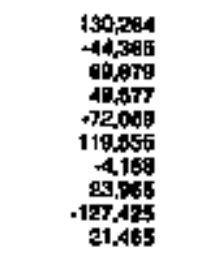 & 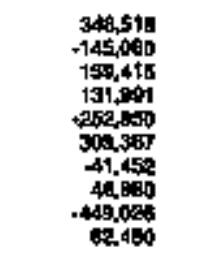 \\
\hline 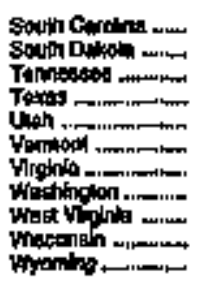 & 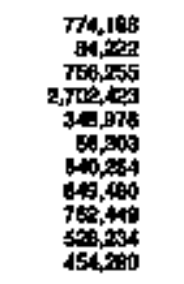 & 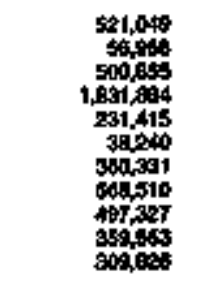 & 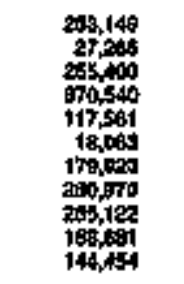 & 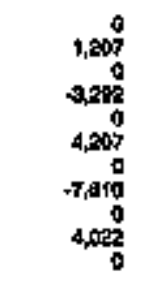 & 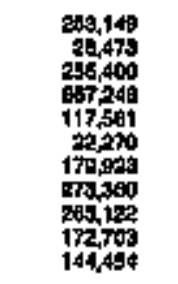 & 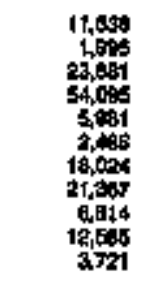 & 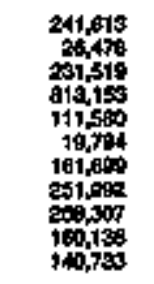 & 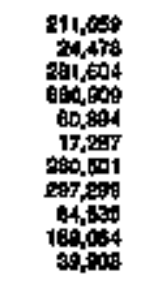 & 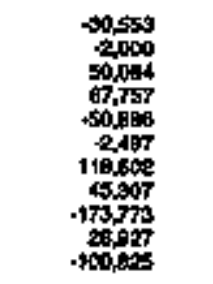 & 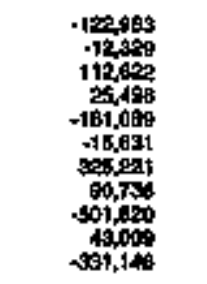 \\
\hline Whtlod sluken & 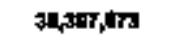 & $20,400,504$ & 9001,350 & 152,304 & $10,003,654$ & 719,360 & $0,205,205$ & $10,0+2,72$ & GLTAMA & - \\
\hline
\end{tabular}

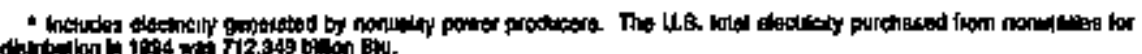

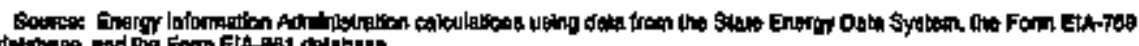




\begin{tabular}{|c|c|c|c|c|c|c|c|c|c|c|}
\hline stost & 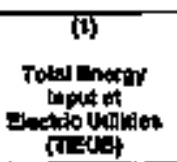 & 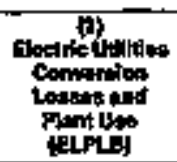 & 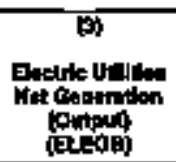 & 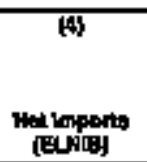 & 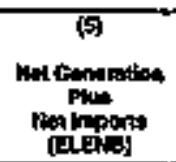 & 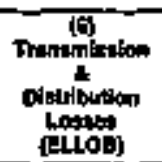 & 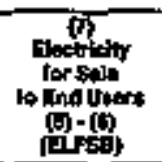 & 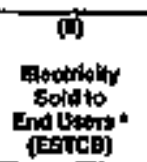 & 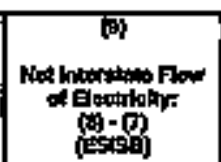 & 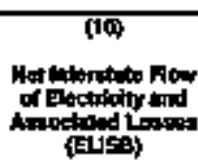 \\
\hline 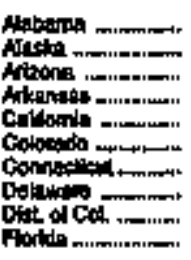 & 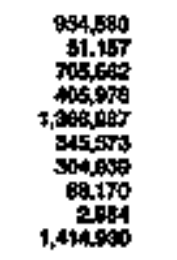 & 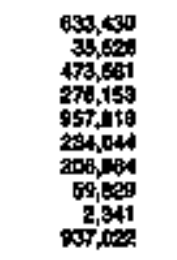 & 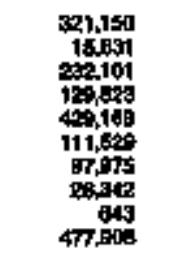 & $\begin{array}{r}0 \\
0 \\
0 \\
11,205 \\
0 \\
3,670 \\
0 \\
0 \\
0\end{array}$ & 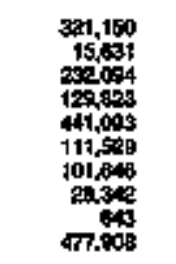 & 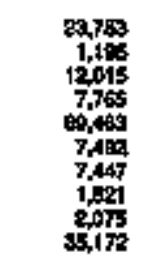 & 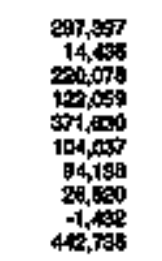 & 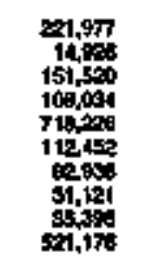 & 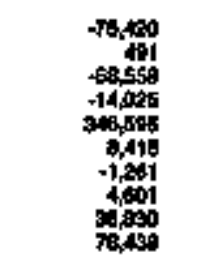 & 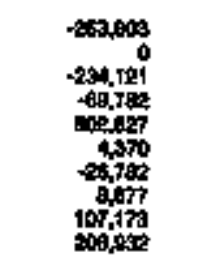 \\
\hline "ت, & 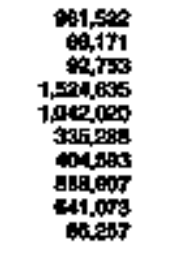 & 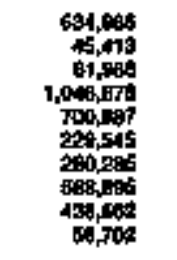 & 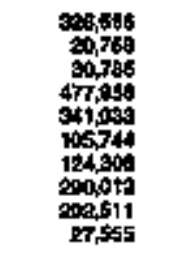 & $\begin{array}{r}0 \\
0 \\
40 \\
0 \\
0 \\
0 \\
0 \\
0 \\
0 \\
0 \\
7,3290\end{array}$ & 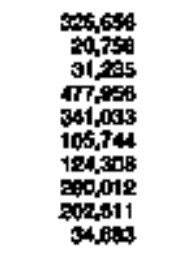 & 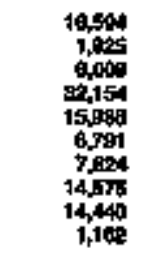 & 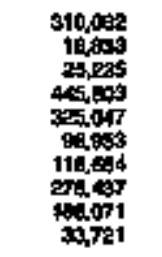 & 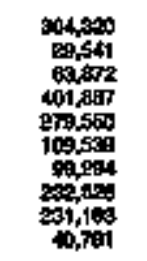 & 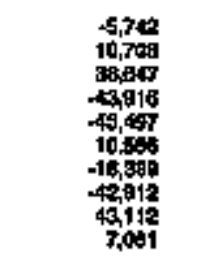 & 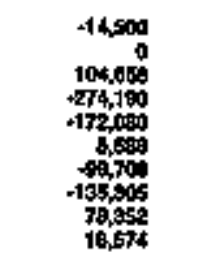 \\
\hline 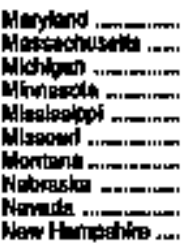 & 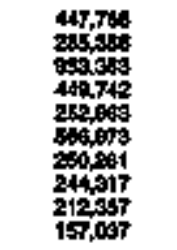 & 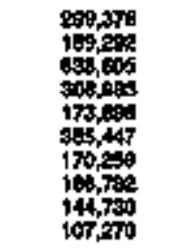 & 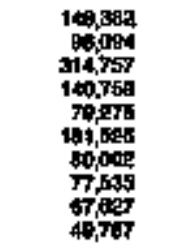 & 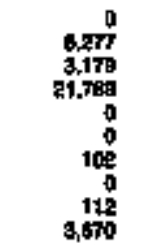 & 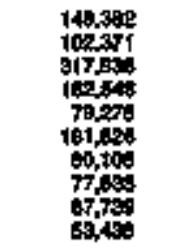 & 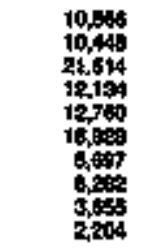 & 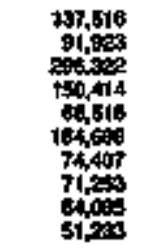 & 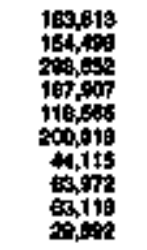 & 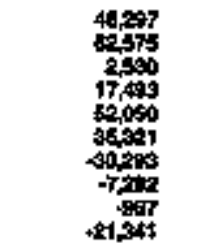 & 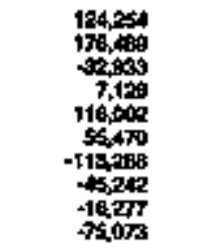 \\
\hline 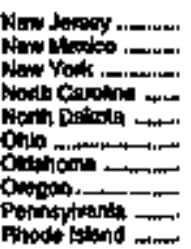 & 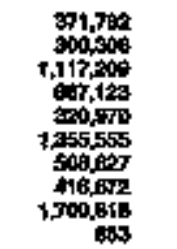 & 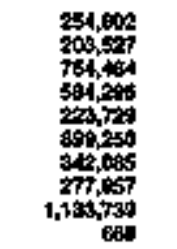 & 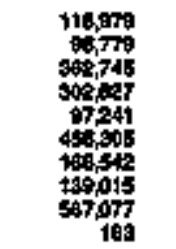 & 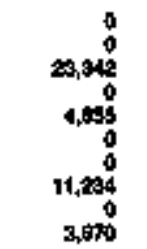 & 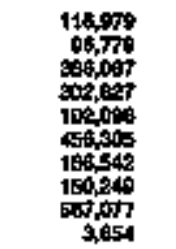 & 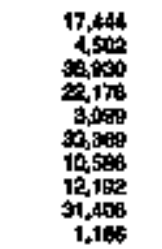 & 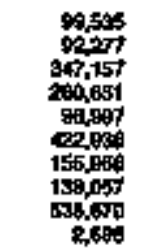 & 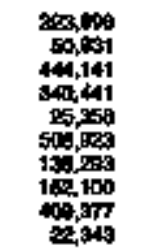 & 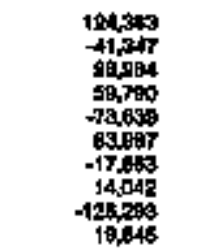 & 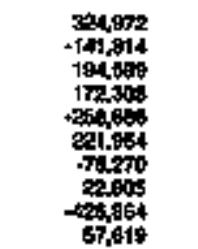 \\
\hline 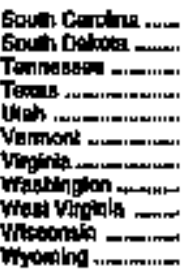 & 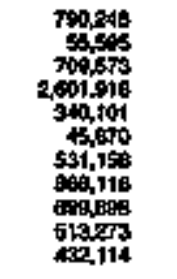 & 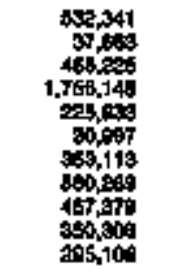 & 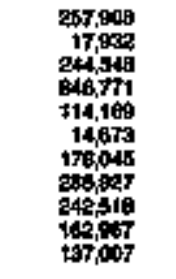 & $\begin{array}{r}0 \\
0 \\
0 \\
-2, \pi 3 \\
0 \\
0,2,3 \\
0 \\
-11,008 \\
0 \\
0 \\
0\end{array}$ & 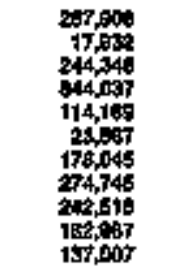 & 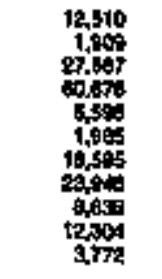 & 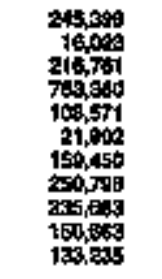 & 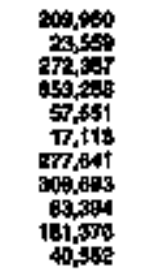 & 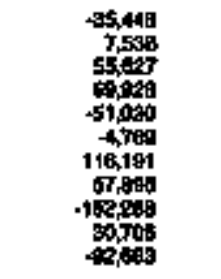 & 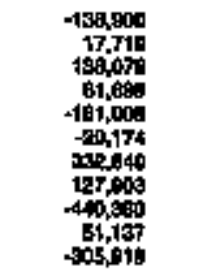 \\
\hline 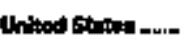 & $30,050,114$ & $20,224,838$ & Qeass 175 & ensor & $0,000,101$ & 230,500 & 0,793690 & Q,763,210 & $5 \% 961$ & 0 \\
\hline
\end{tabular}

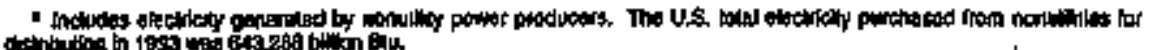

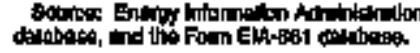


Table A12. Dlsaggregated Dała for Net interstate Flow and Electrical System Energy Losses, 1992 (Billion Btu)

\begin{tabular}{|c|c|c|c|c|c|c|c|c|c|c|}
\hline 6 & 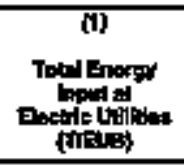 & 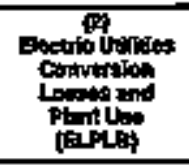 & 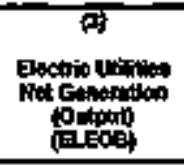 & 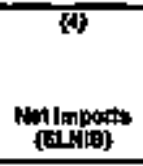 & 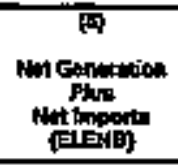 & 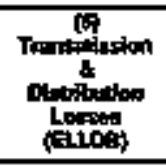 & 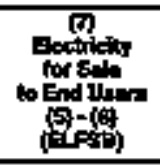 & 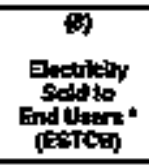 & 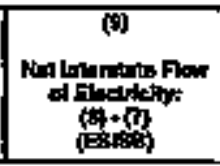 & 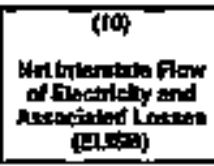 \\
\hline 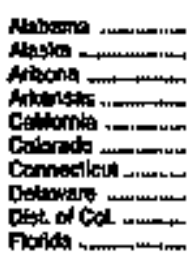 & 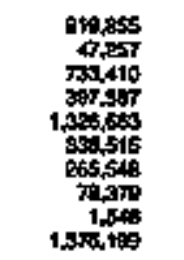 & 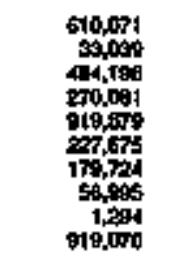 & 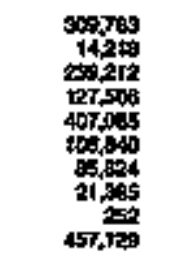 & $\begin{array}{r}0 \\
0 \\
-7 \\
0 \\
18,349 \\
0 \\
3,60 \\
0 \\
0 \\
0\end{array}$ & 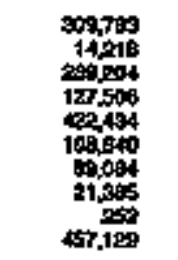 & 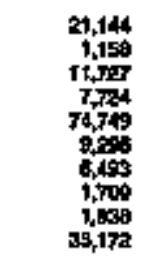 & 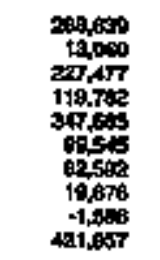 & 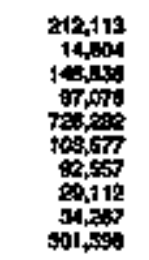 & 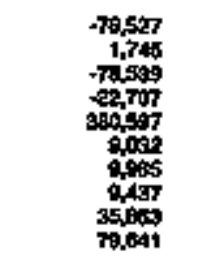 & 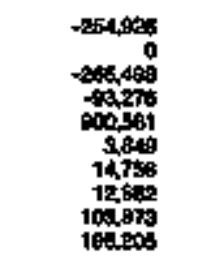 \\
\hline 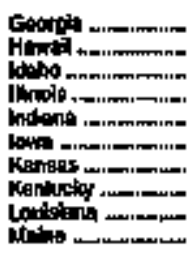 & 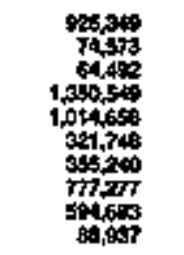 & 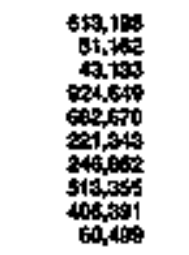 & 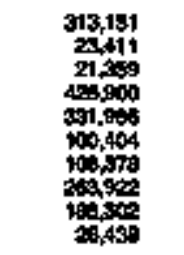 & $\begin{array}{r}0 \\
0 \\
08 \\
0 \\
0 \\
0 \\
0 \\
0 \\
0 \\
7,24\end{array}$ & 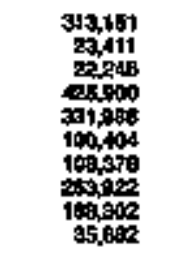 & 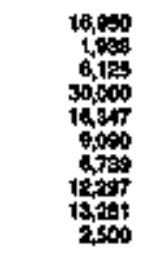 & 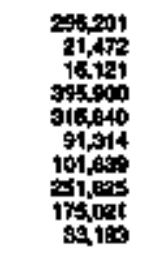 & 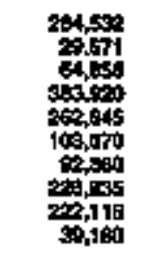 & 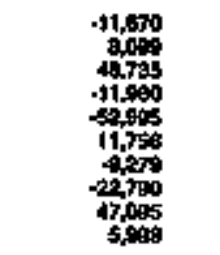 & 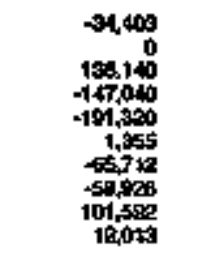 \\
\hline 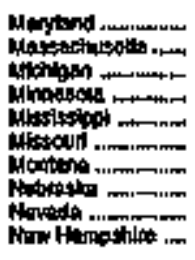 & 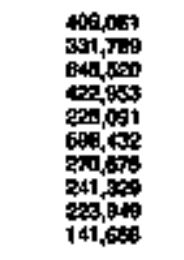 & 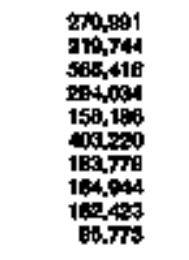 & 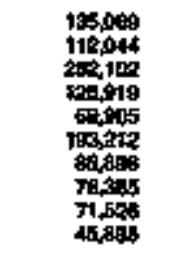 & 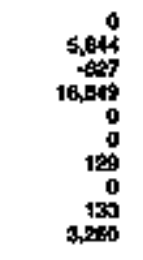 & 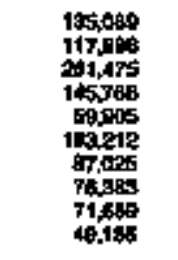 & 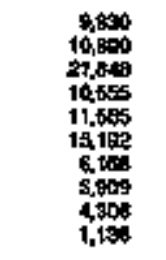 & 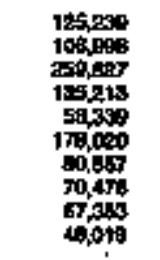 & 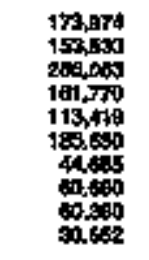 & 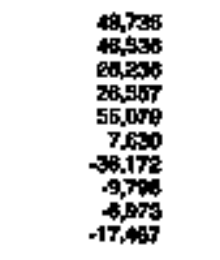 & 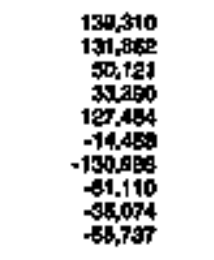 \\
\hline 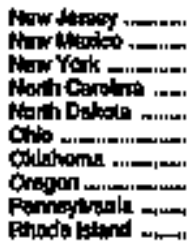 & 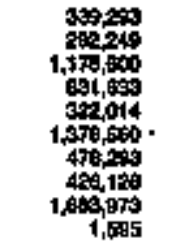 & 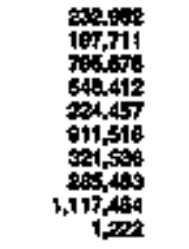 & 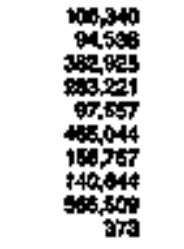 & 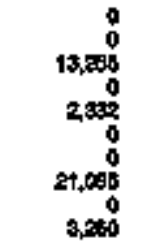 & 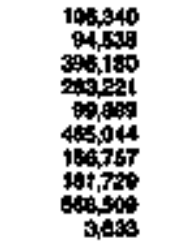 & 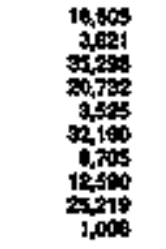 & 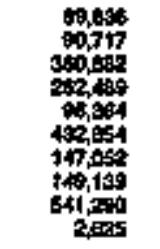 & 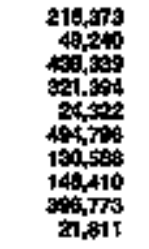 & 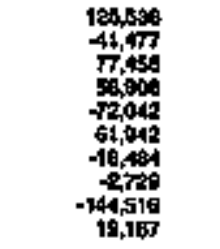 & 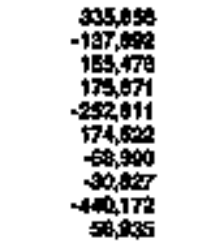 \\
\hline 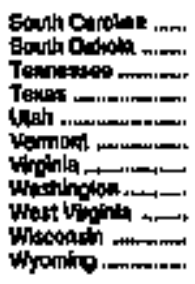 & 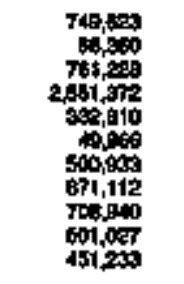 & 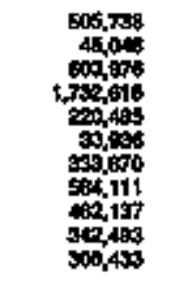 & 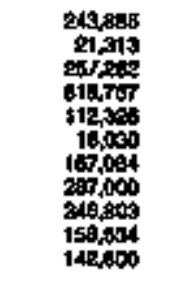 & 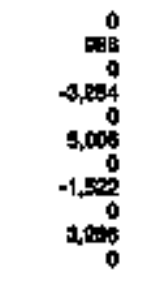 & 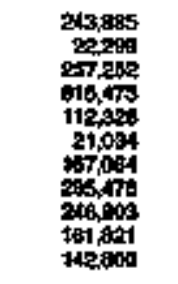 & 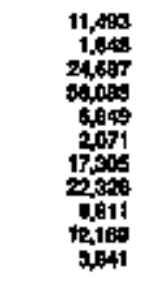 & 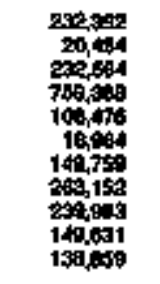 & 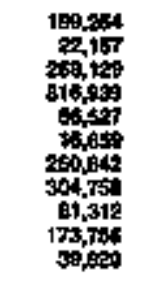 & 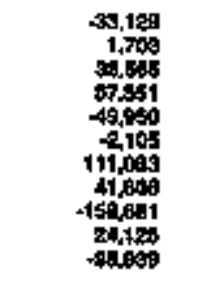 & 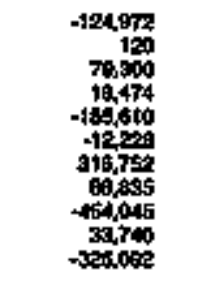 \\
\hline 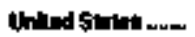 & $2,250,300$ & $19.90,79$ & $9,54,112$ & 96,724 & 9,040038 & T0,0T2 & $6,902,168$ & $0,425,600$ & 496,45 & 0 \\
\hline
\end{tabular}




\begin{tabular}{|c|c|c|c|c|c|c|c|c|c|c|}
\hline \$4 & 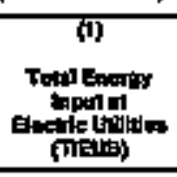 & 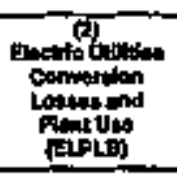 & 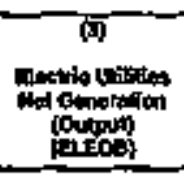 & 然 & 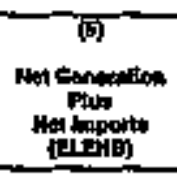 & 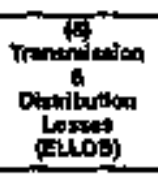 & 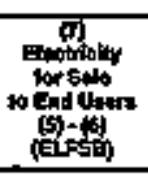 & 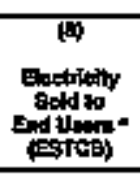 & 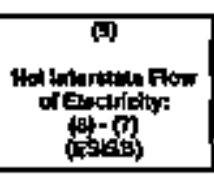 & 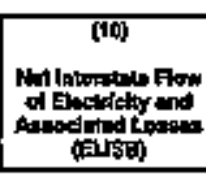 \\
\hline 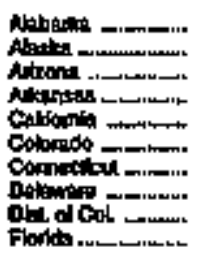 & 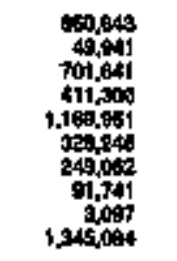 & 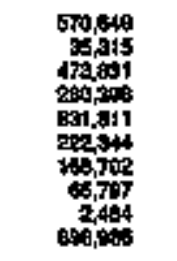 & 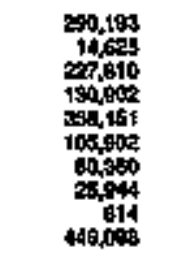 & 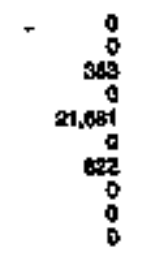 & 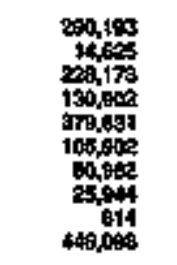 & 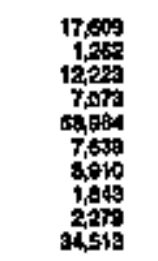 & 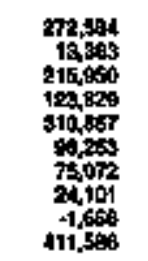 & 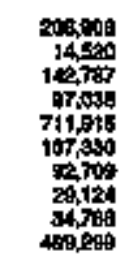 & 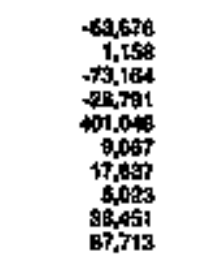 & 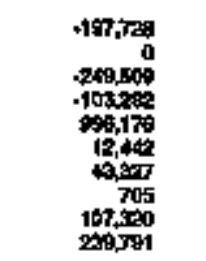 \\
\hline . & 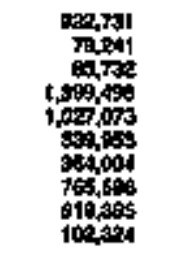 & 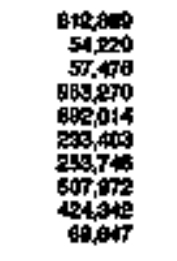 & 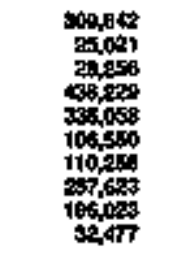 & $\begin{array}{r}0 \\
0 \\
458 \\
0 \\
0 \\
0 \\
0 \\
0 \\
0 \\
7,169\end{array}$ & 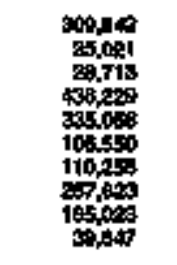 & 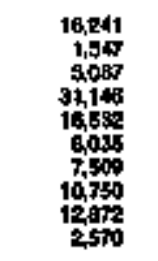 & 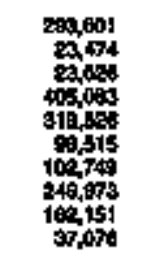 & 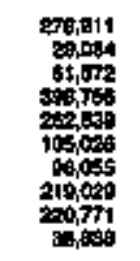 & 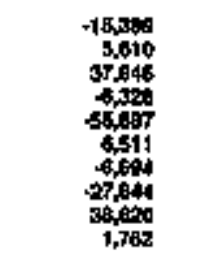 & 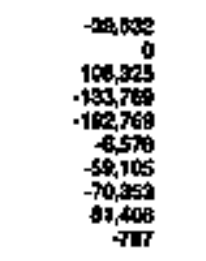 \\
\hline Mand & 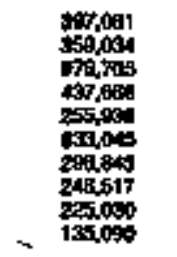 & 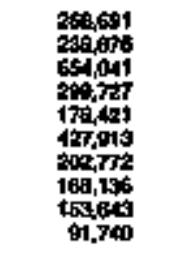 & 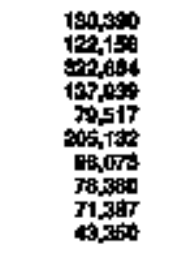 & 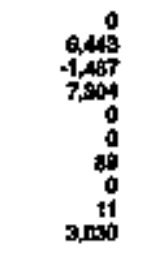 & 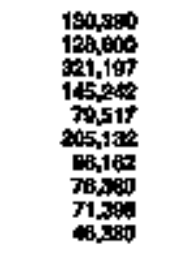 & 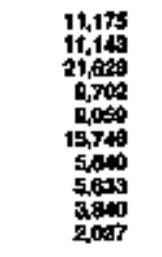 & 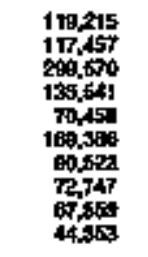 & 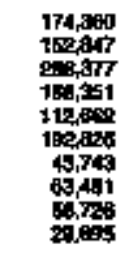 & 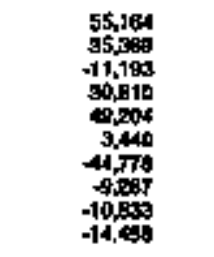 & 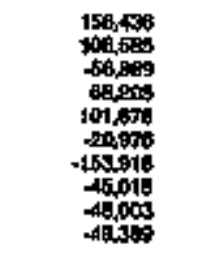 \\
\hline 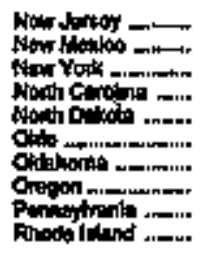 & 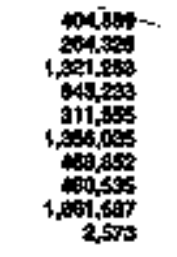 & 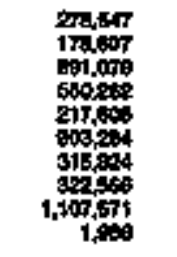 & 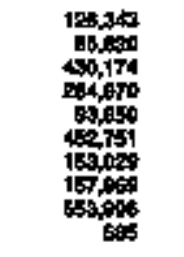 & 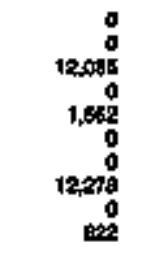 & 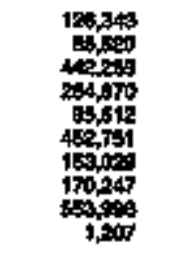 & 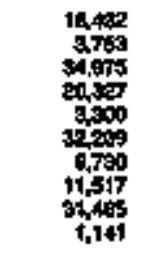 & 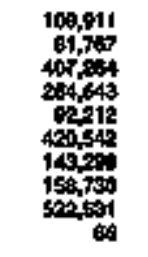 & 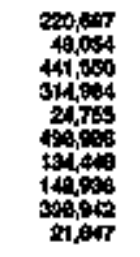 & 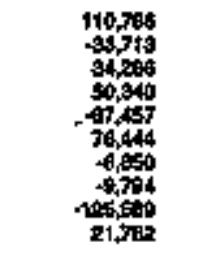 & 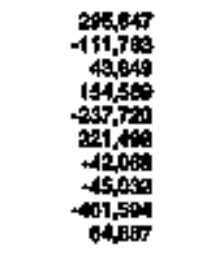 \\
\hline 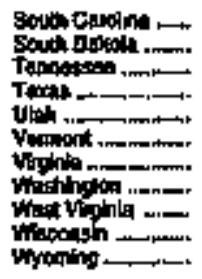 & 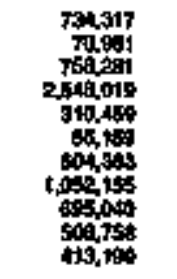 & 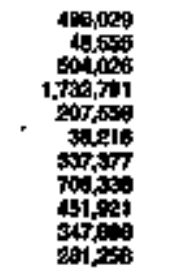 & 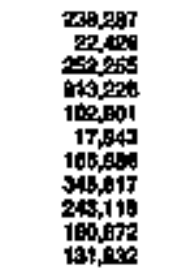 & 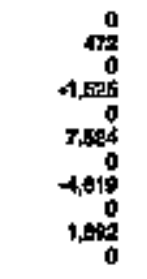 & 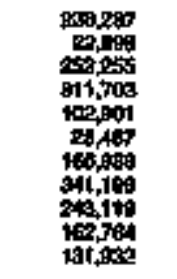 & 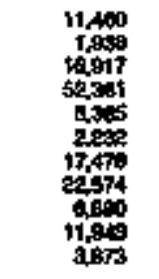 & 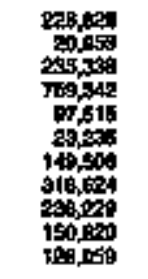 & 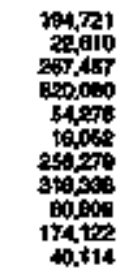 & 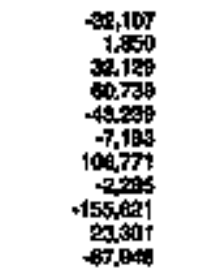 & 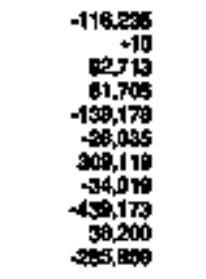 \\
\hline Undtod 5 tom & 20,000,0860 & 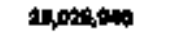 & 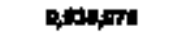 & $2 x+100$ & 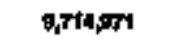 & Ans,094 & Bposm & $0,120,064$ & 304078 & 0 \\
\hline
\end{tabular}

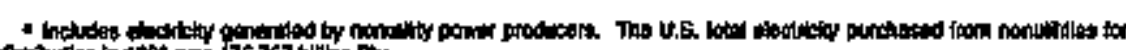

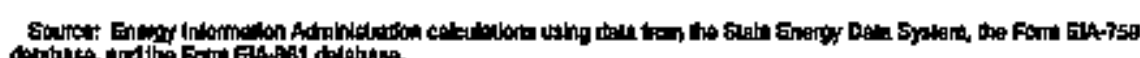


Table A14. Disaggregated Data for Net Interstate Flow and Electrical System Energy Losses, t990 (Bilion Bhi)

\begin{tabular}{|c|c|c|c|c|c|c|c|c|c|c|}
\hline stato & 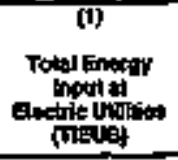 & 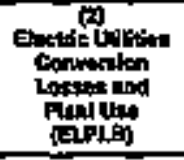 & 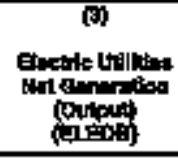 & 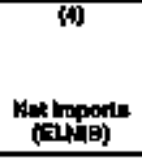 & 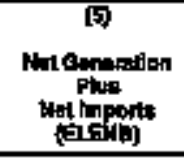 & 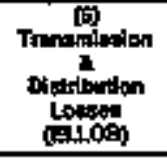 & 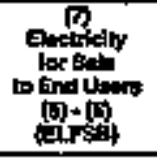 & 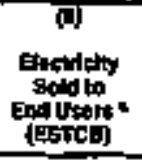 & 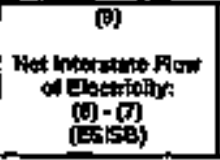 & 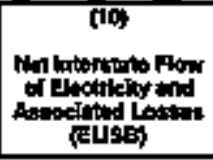 \\
\hline 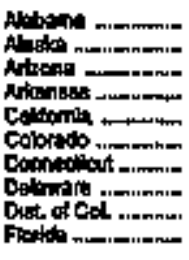 & 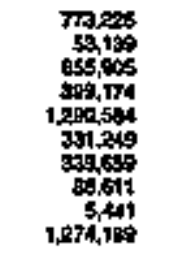 & 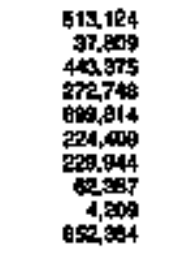 & 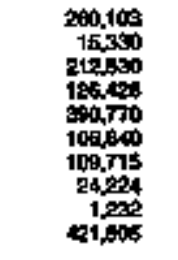 & $\begin{array}{r}0 \\
0 \\
-7 \\
0 \\
16,2 \% \\
0 \\
012 \\
0 \\
0 \\
0\end{array}$ & 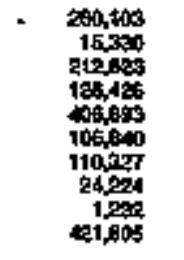 & 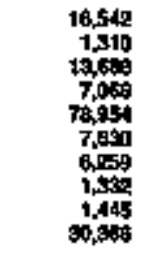 & 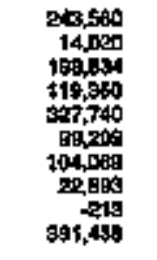 & 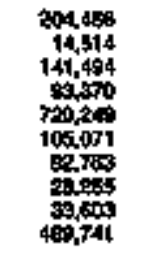 & 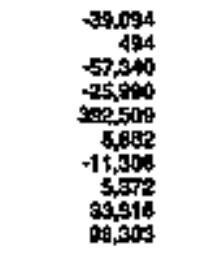 & 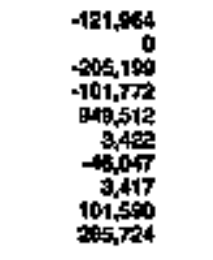 \\
\hline 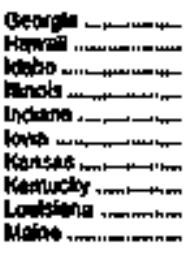 & 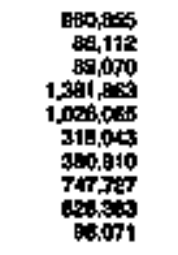 & 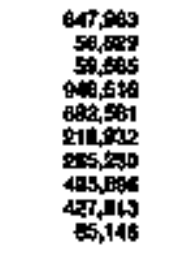 & 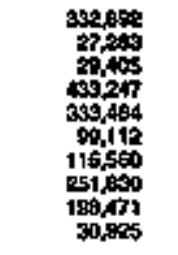 & $\begin{array}{r}0 \\
0 \\
310 \\
0 \\
0 \\
0 \\
0 \\
0 \\
0 \\
7,876\end{array}$ & 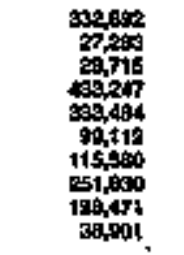 & 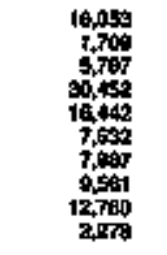 & 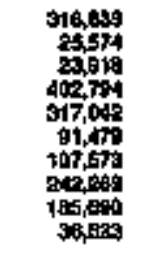 & 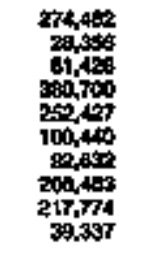 & 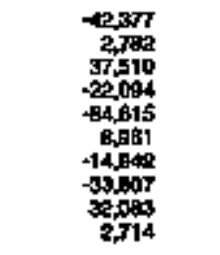 & 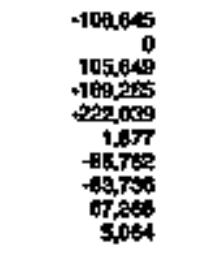 \\
\hline 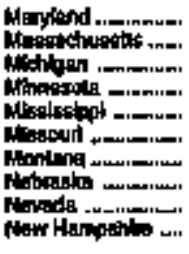 & 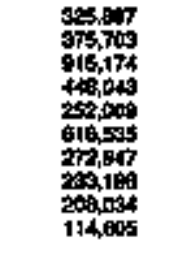 & 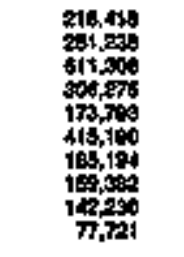 & 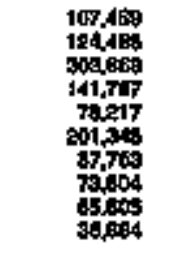 & $\begin{array}{r}0 \\
7.043 \\
37265 \\
7008 \\
0 \\
0 \\
165 \\
0 \\
0 \\
612\end{array}$ & 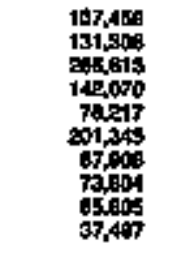 & 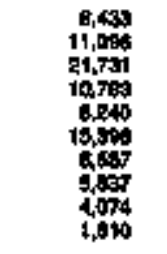 & 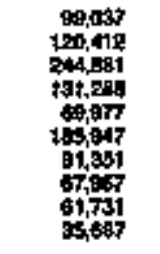 & 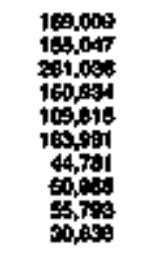 & 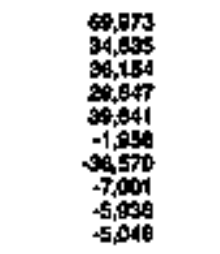 & 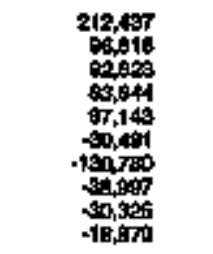 \\
\hline 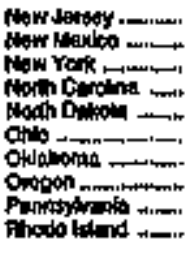 & 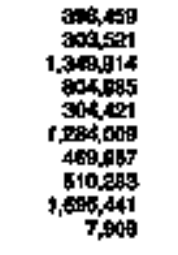 & 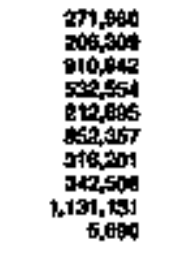 & 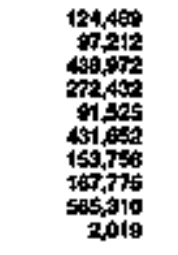 & $\begin{array}{r}0 \\
1,504 \\
2,2039 \\
0 \\
7,400 \\
0 \\
012\end{array}$ & 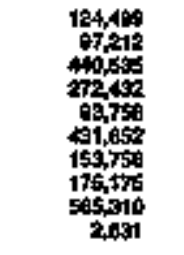 & 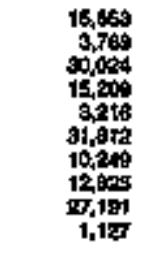 & 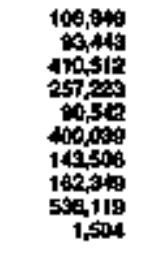 & 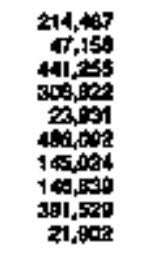 & 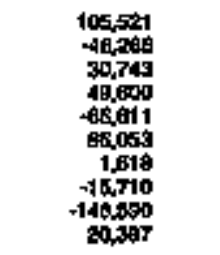 & 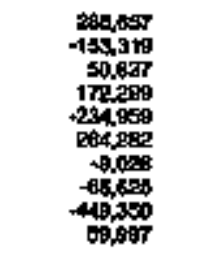 \\
\hline "..." & 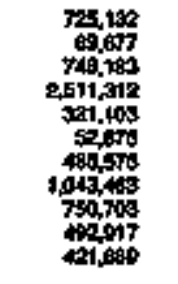 & 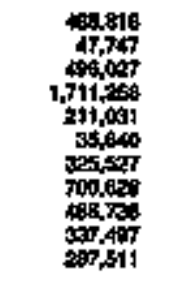 & 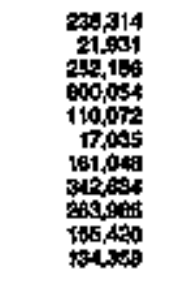 & $\begin{array}{r}0 \\
0 \\
0 \\
-217 \\
0 \\
040 \\
0 \\
0 \\
040 \\
0 \\
0 \\
0\end{array}$ & 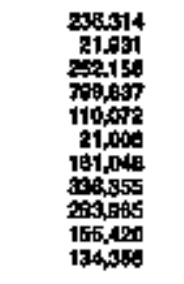 & 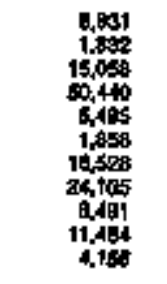 & 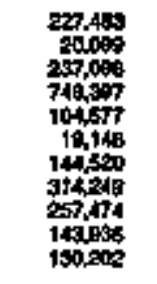 & 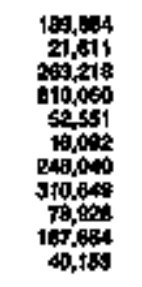 & 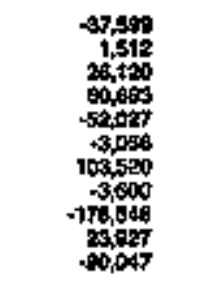 & 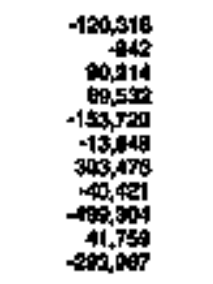 \\
\hline 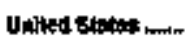 & $20,45+4001$ & $10,173,110$ & ostorss & $4 \sqrt{506}$ & 0.90169 & ans & notast & $0,250,257$ & 291, 190 & a \\
\hline
\end{tabular}

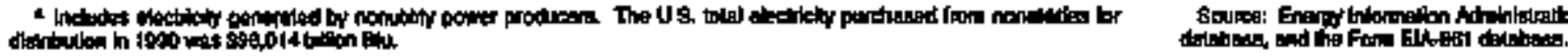




\begin{tabular}{|c|c|c|c|c|c|c|c|c|c|c|}
\hline sting & 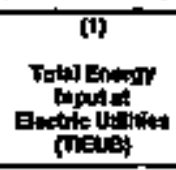 & 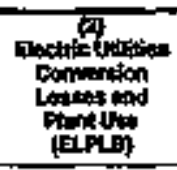 & 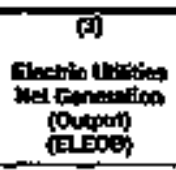 & (x) & 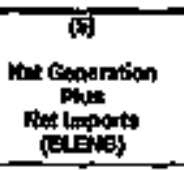 & 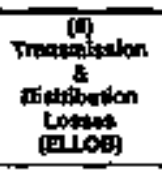 & 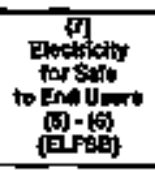 & 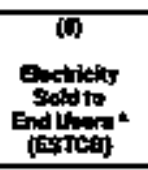 & 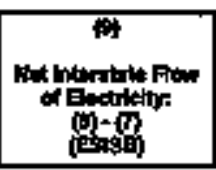 & 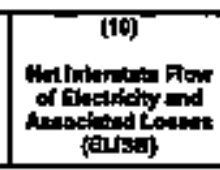 \\
\hline 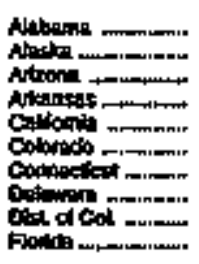 & 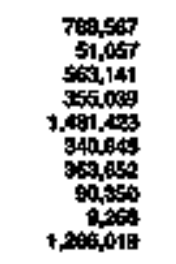 & 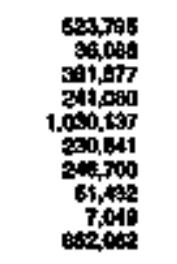 & 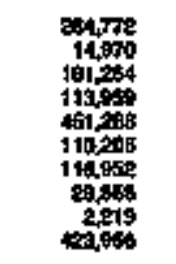 & $\begin{array}{r}0 \\
0 \\
-7 \\
0 \\
0 \\
0 \\
77 \\
0 \\
0\end{array}$ & 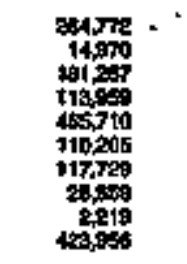 & 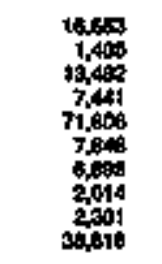 & 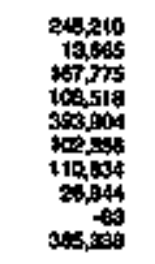 & 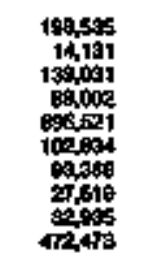 & 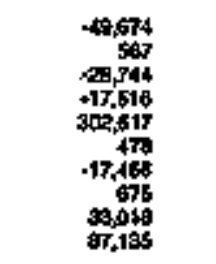 & 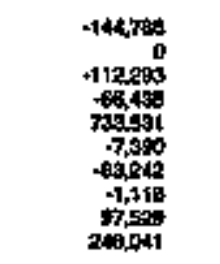 \\
\hline 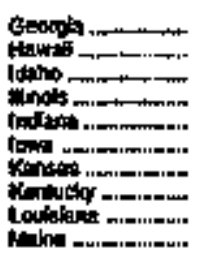 & 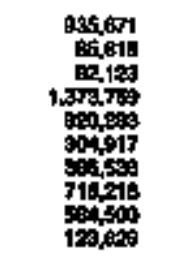 & 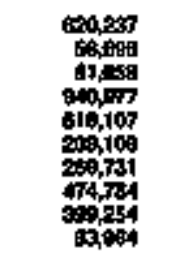 & 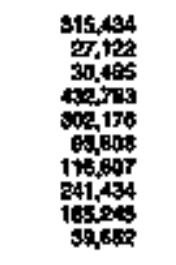 & $\begin{array}{r}0 \\
0 \\
97 \\
0 \\
0 \\
0 \\
0 \\
0 \\
0 \\
7.0109\end{array}$ & 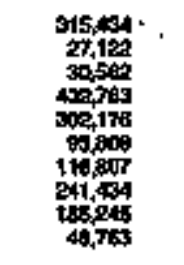 & 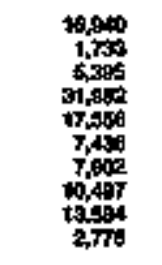 & 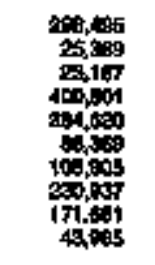 & 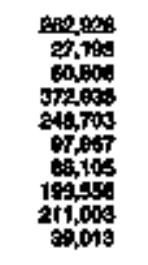 & 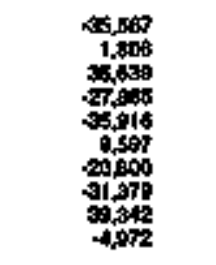 & 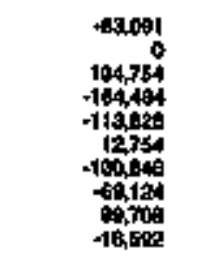 \\
\hline . & 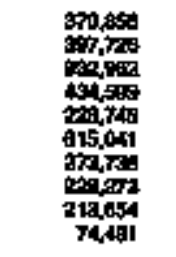 & 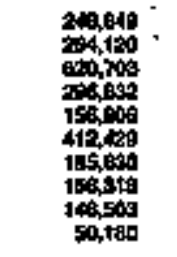 & 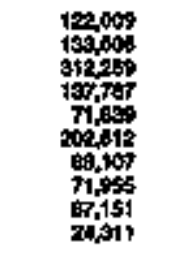 & 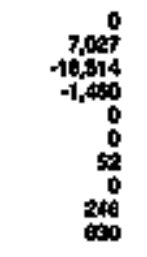 & 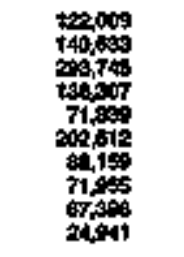 & 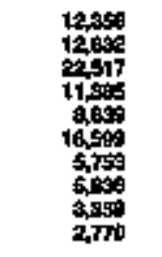 & 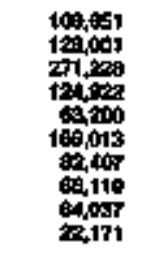 & 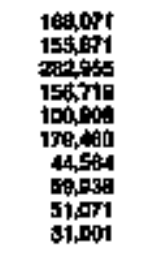 & 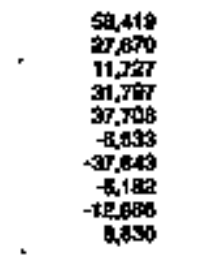 & 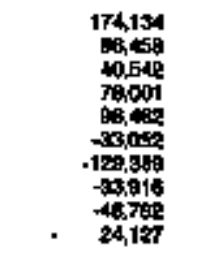 \\
\hline 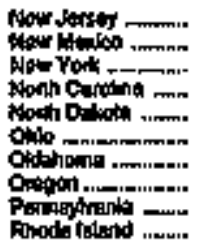 & 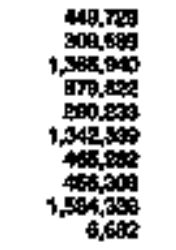 & 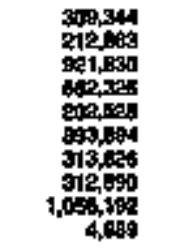 & 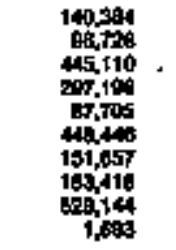 & 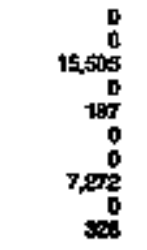 & 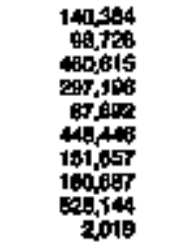 & 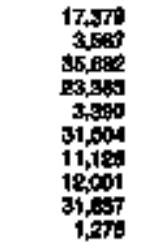 & 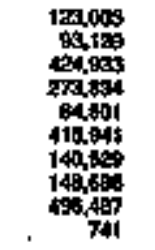 & 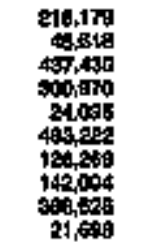 & 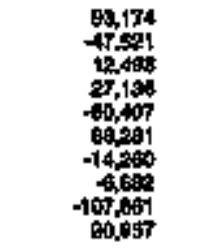 & 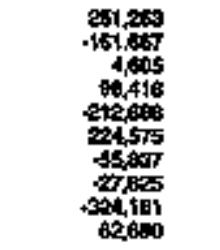 \\
\hline 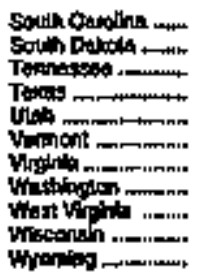 & 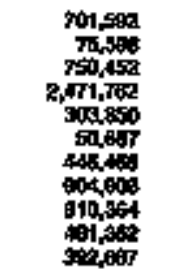 & 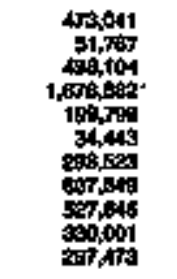 & 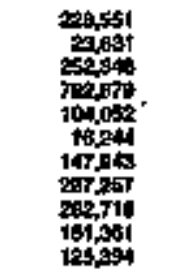 & 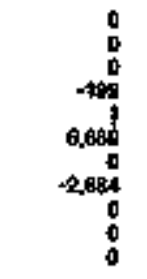 & 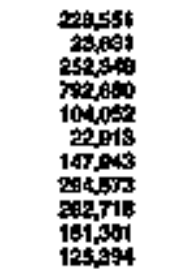 & 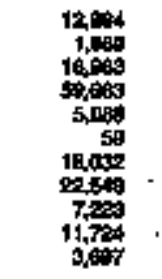 & 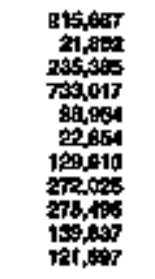 & 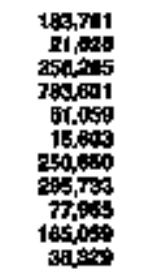 & 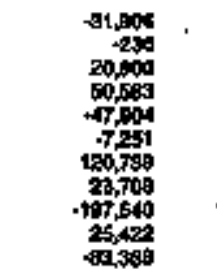 & 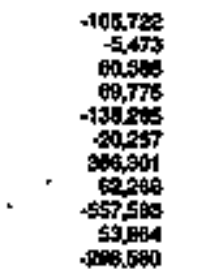 \\
\hline 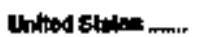 & $20,12,64$ & 18,6013677 & Q500,0n & 8,45 & 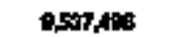 & TIGSAG & Lestesso & getponsts & t14203 & d \\
\hline
\end{tabular}

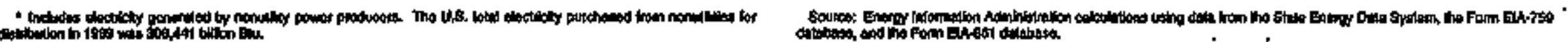


Table A16. Disaggregated Data for Net Interstate Flow and Electrical System Energy Losses, 1989 (Billion Btu)

\begin{tabular}{|c|c|c|c|c|c|c|c|c|c|c|}
\hline $\sin$ & 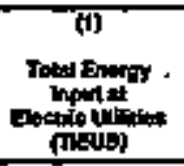 & 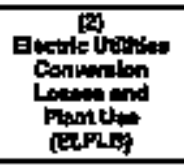 & 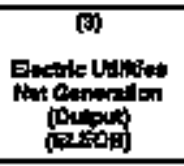 & 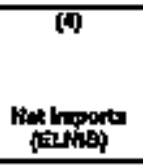 & 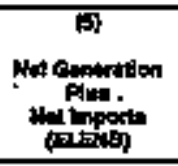 & 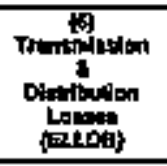 & 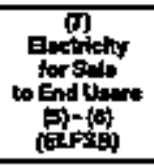 & 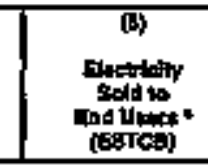 & 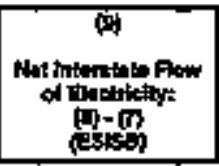 & 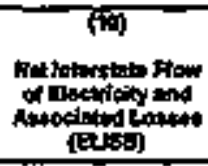 \\
\hline 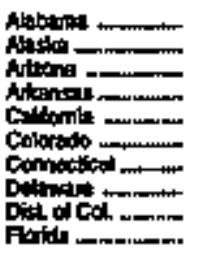 & 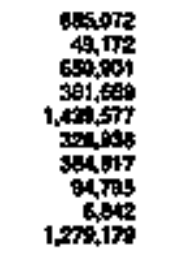 & 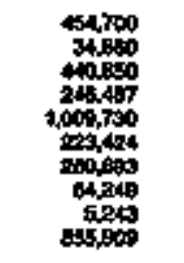 & 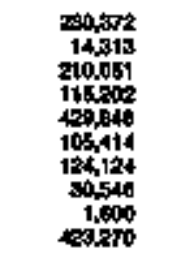 & $\begin{array}{r}0 \\
0 \\
6 \\
0 \\
0 \\
240 \\
290 \\
0 \\
0 \\
0 \\
0 \\
0\end{array}$ & 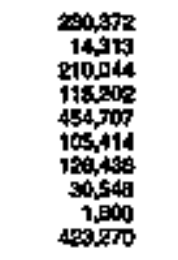 & 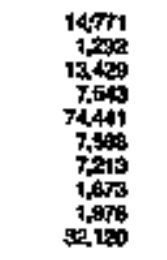 & 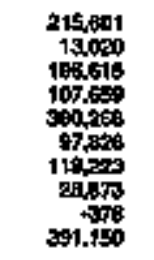 & 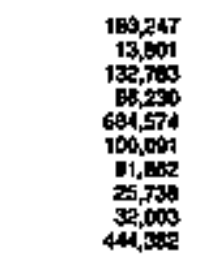 & 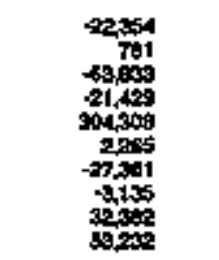 & 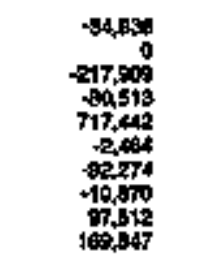 \\
\hline 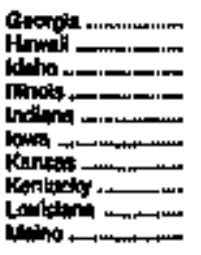 & 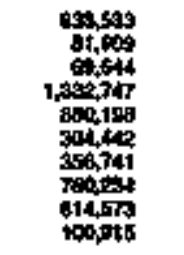 & 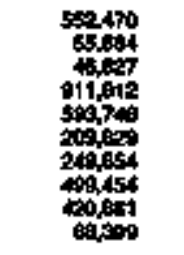 & 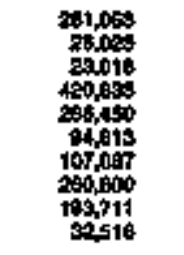 & $\begin{array}{r}0 \\
0 \\
30 \\
30 \\
0 \\
0 \\
0 \\
0 \\
0 \\
11,500\end{array}$ & 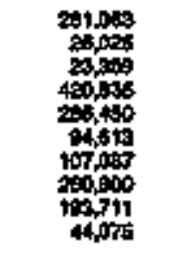 & 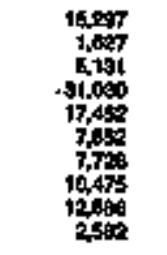 & 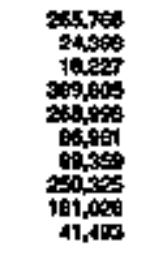 & 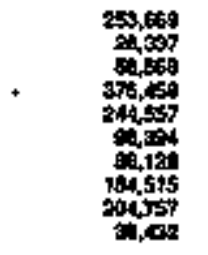 & 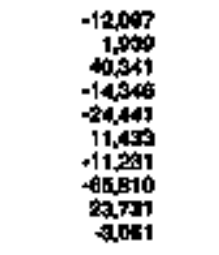 & 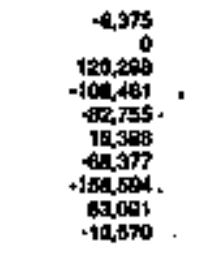 \\
\hline 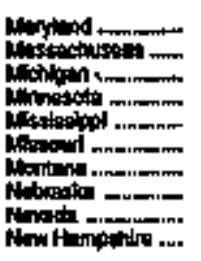 & 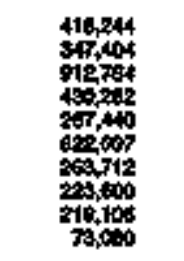 & 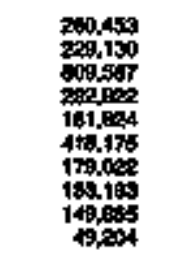 & 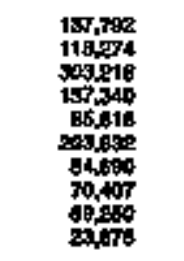 & 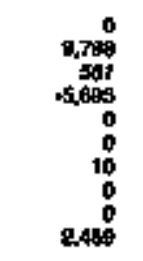 & 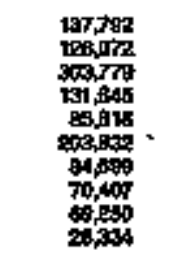 & 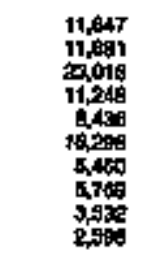 & 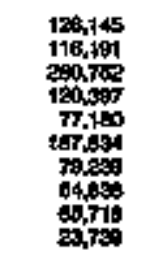 & 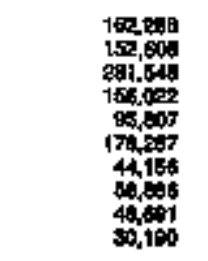 & 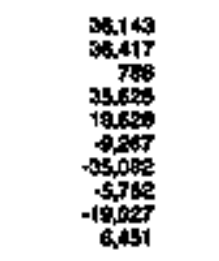 & 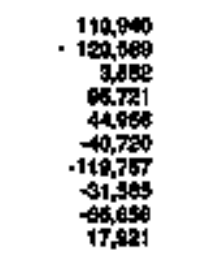 \\
\hline 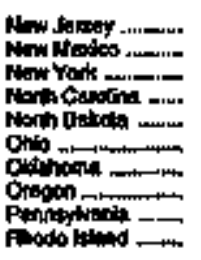 & 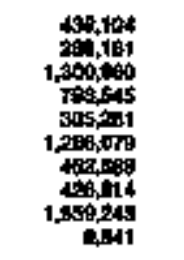 & 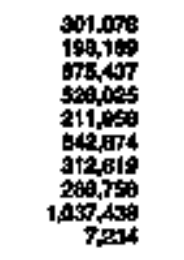 & 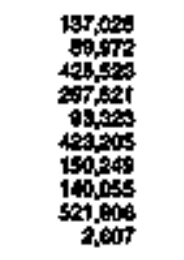 & 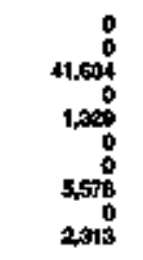 & 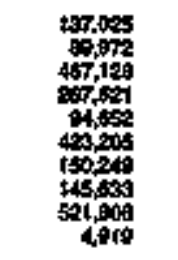 & 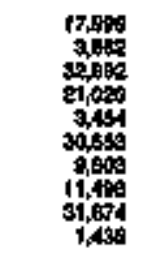 & 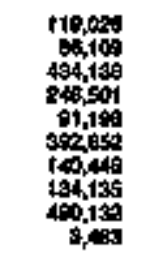 & 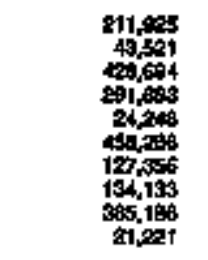 & 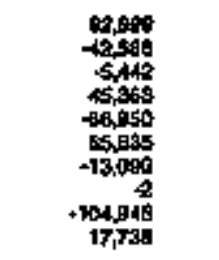 & 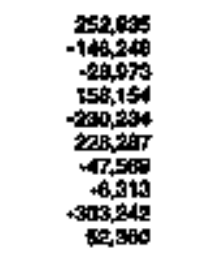 \\
\hline 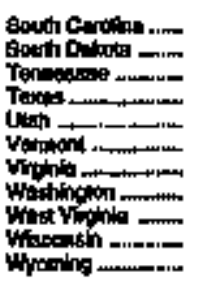 & 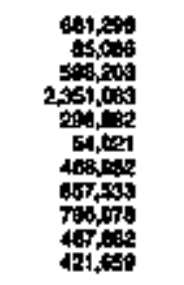 & 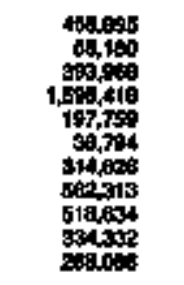 & 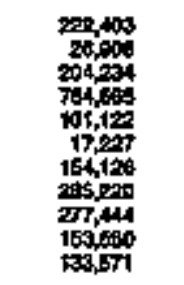 & 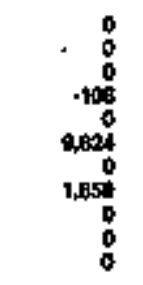 & 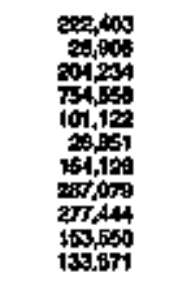 & 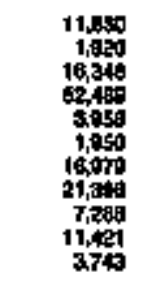 & 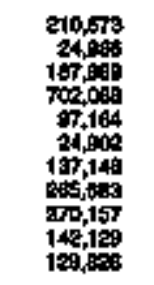 & 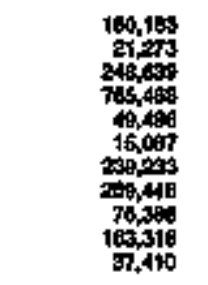 & 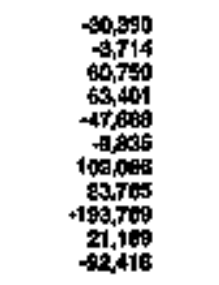 & 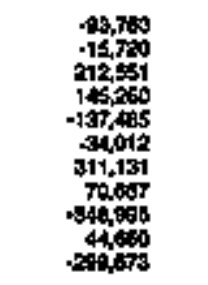 \\
\hline 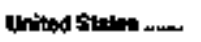 & 21050000 & t5,120,16 & satsont & 10000 & 9055,300 & $m, 201$ & 0,4as,000 & $0,760,40$ & 14tioss & 0 \\
\hline
\end{tabular}




\begin{tabular}{|c|c|c|c|c|c|c|c|c|c|c|}
\hline Stato & 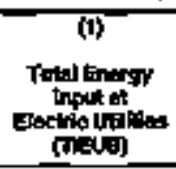 & 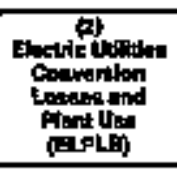 & 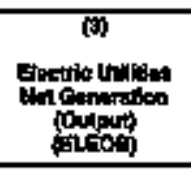 & 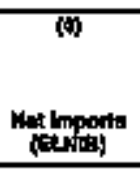 & 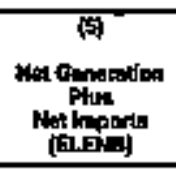 & 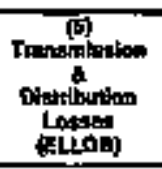 & 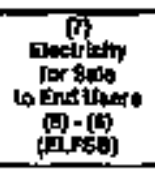 & 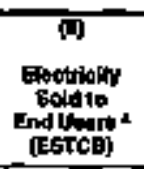 & 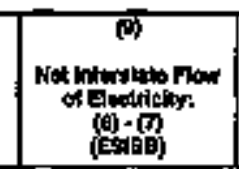 & 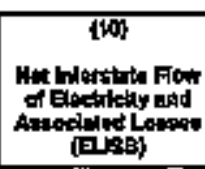 \\
\hline 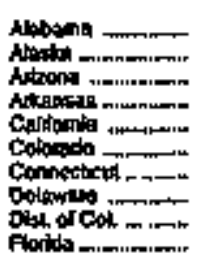 & 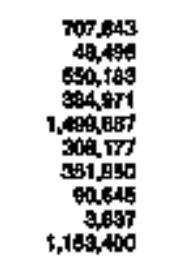 & 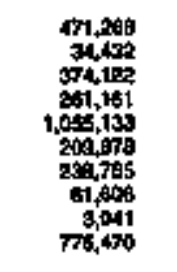 & 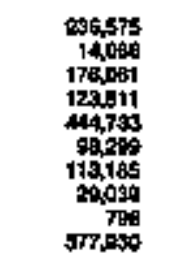 & $\begin{array}{r}\vdots \\
5 \\
0 \\
8,12 i \\
0.961 \\
0 \\
\vdots \\
0\end{array}$ & 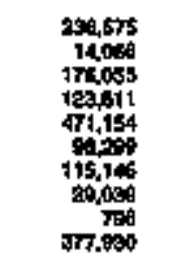 & 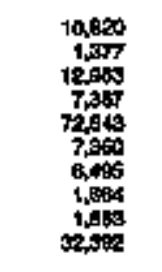 & 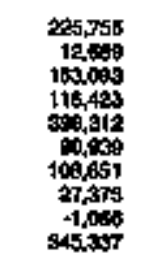 & 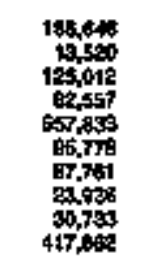 & 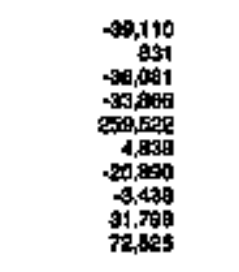 & 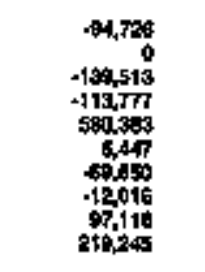 \\
\hline 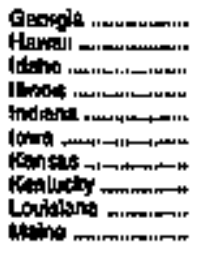 & 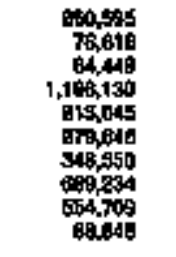 & 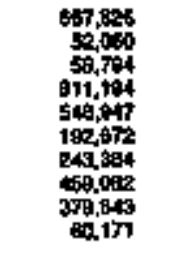 & 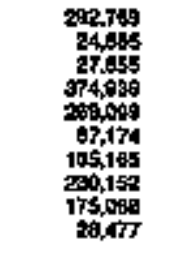 & $\begin{array}{r}0 \\
140 \\
0 \\
0 \\
0 \\
0 \\
0 \\
0 \\
+2 \%\end{array}$ & 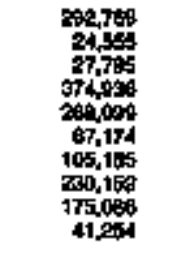 & 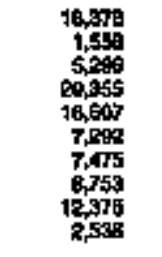 & 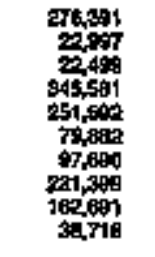 & 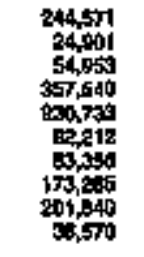 & 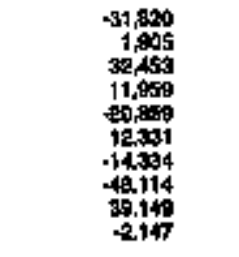 & 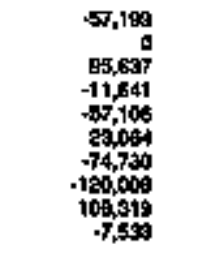 \\
\hline 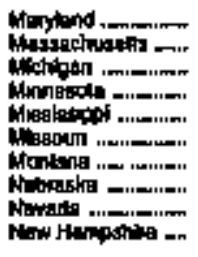 & 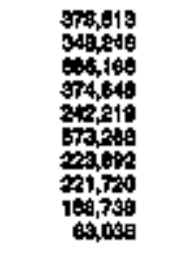 & 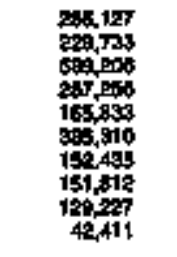 & 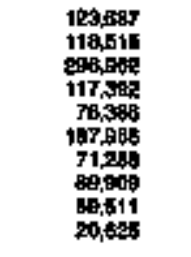 & 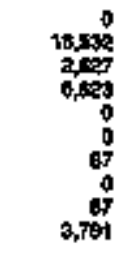 & 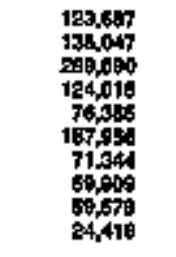 & 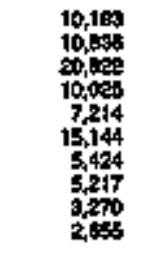 & 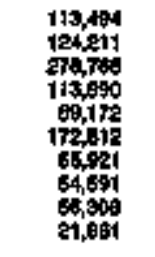 & 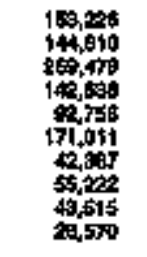 & 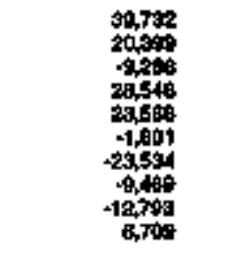 & 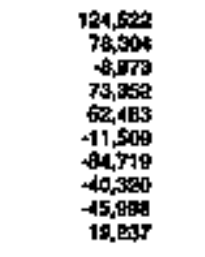 \\
\hline 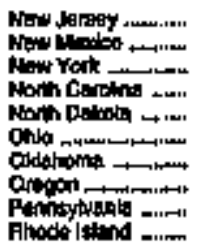 & 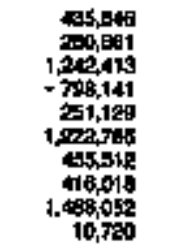 & 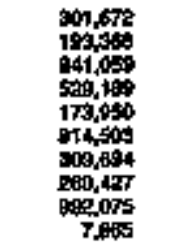 & 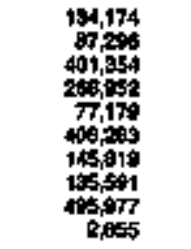 & 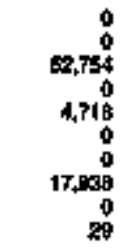 & 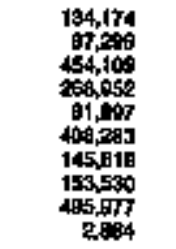 & 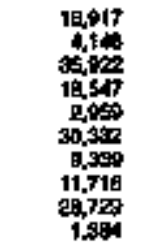 & 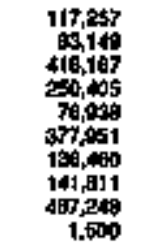 & 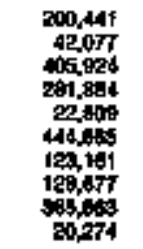 & 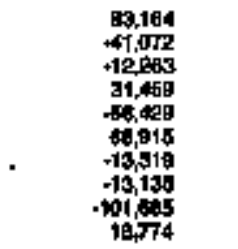 & 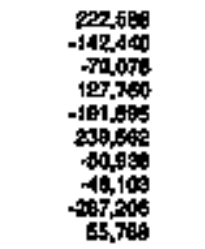 \\
\hline 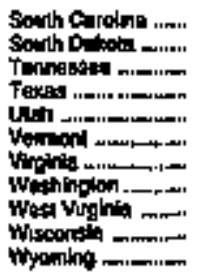 & 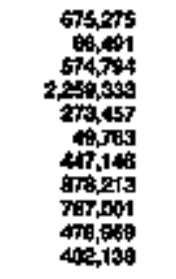 & 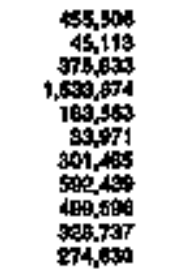 & 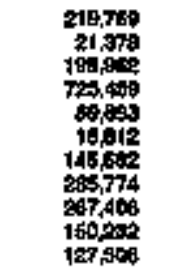 & 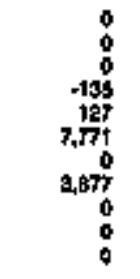 & 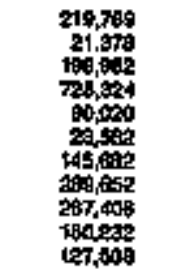 & 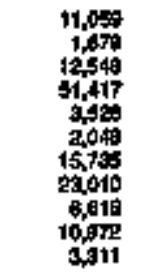 & 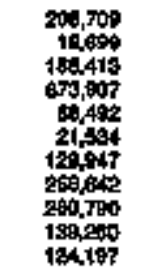 & 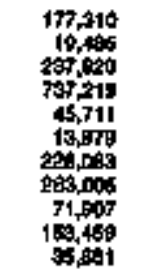 & 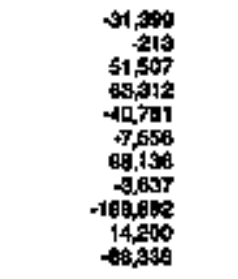 & 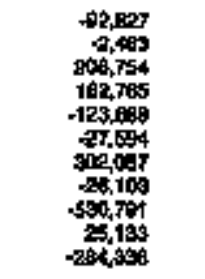 \\
\hline Undiad Gisha & 27,057,021 & 1Q,261,924 & $9, \pi / 4000$ & 180,101 & $8,834,197$ & 655,509 & G,279,644 & ASA,213 & 105,515 & 0 \\
\hline
\end{tabular}

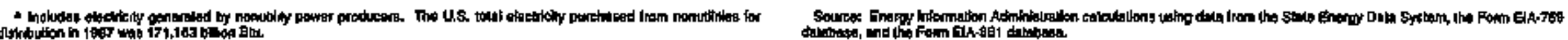


Table A18. Dlsaggregated Data for Net Interstate Flow and Electrical System Energy Losses, 1986 (Billion Btu)

\begin{tabular}{|c|c|c|c|c|c|c|c|c|c|c|}
\hline sitale & 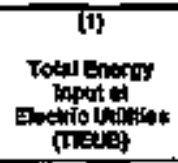 & 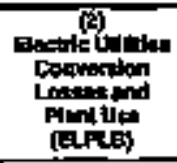 & 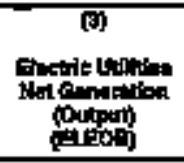 & 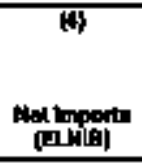 & 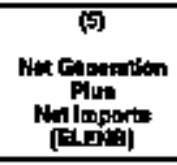 & 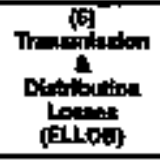 & 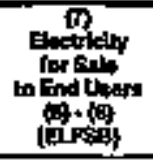 & 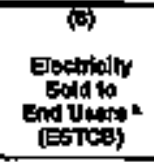 & 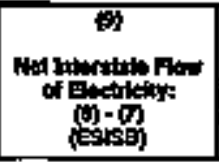 & 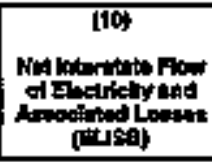 \\
\hline 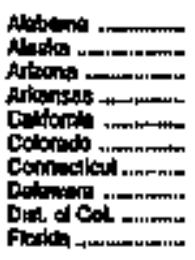 & 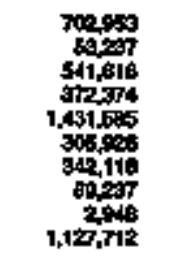 & 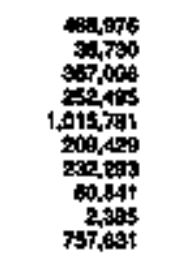 & 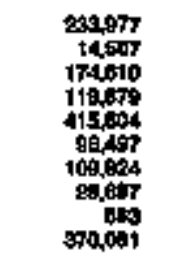 & $\begin{array}{r}0 \\
0 \\
1208 \\
0 \\
1,46 \\
0 \\
0 \\
0 \\
0\end{array}$ & 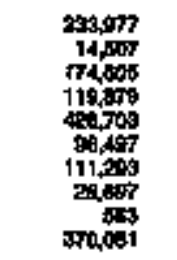 & 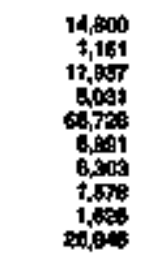 & 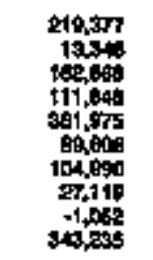 & 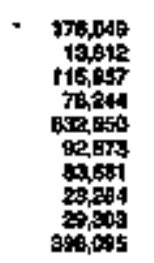 & 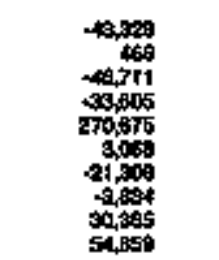 & 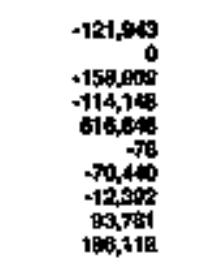 \\
\hline 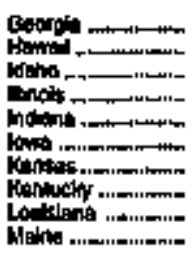 & 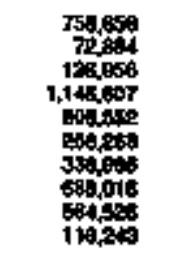 & 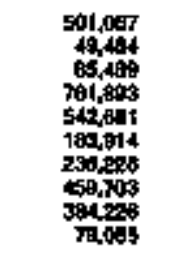 & 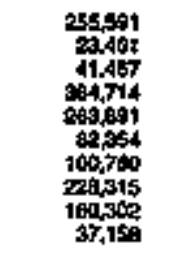 & $\begin{array}{r}0 \\
0 \\
0 \\
0 \\
0 \\
0 \\
0 \\
0 \\
0 . \pi 0\end{array}$ & 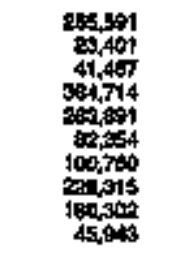 & 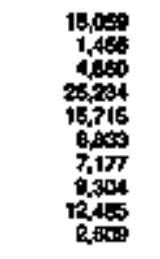 & 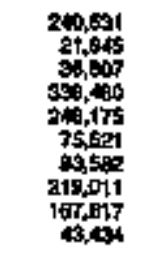 & 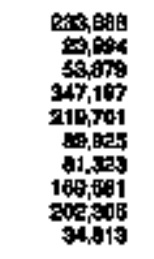 & 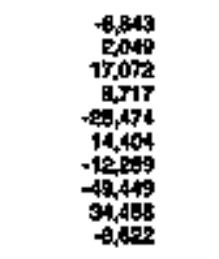 & 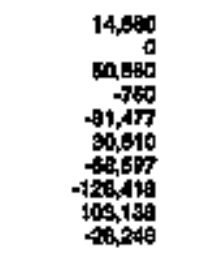 \\
\hline 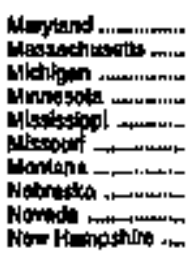 & 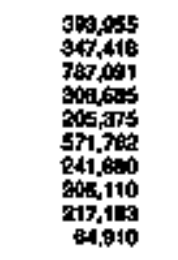 & 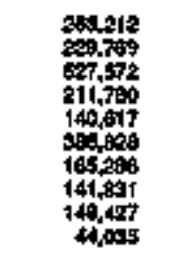 & 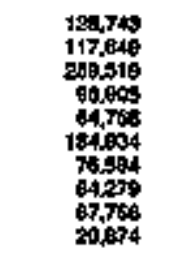 & $\begin{array}{r}1839 \\
2347 \\
23,407 \\
0 \\
0 \\
0 \\
0 \\
0 \\
0\end{array}$ & 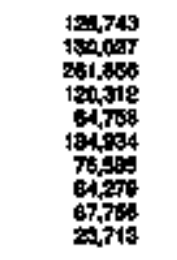 & 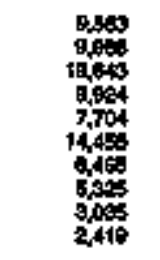 & 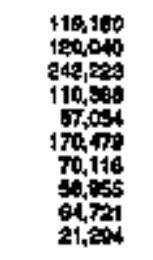 & 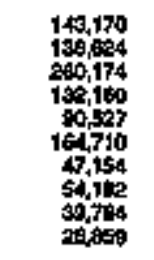 & 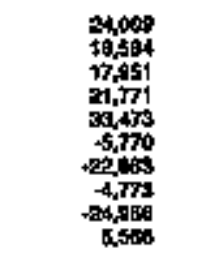 & 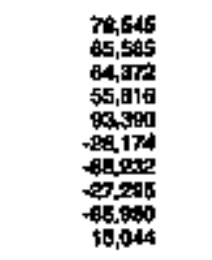 \\
\hline 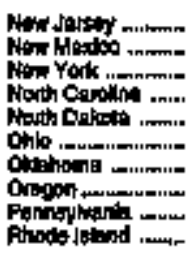 & 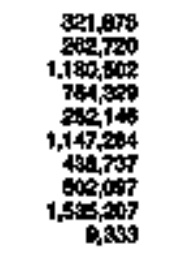 & 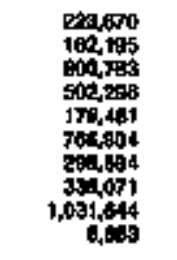 & 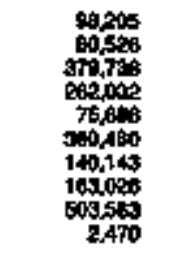 & 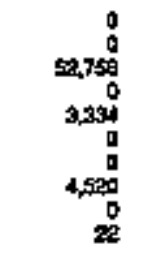 & 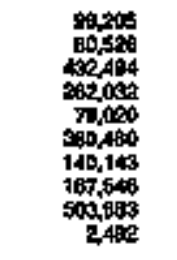 & 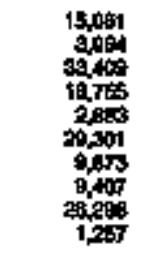 & 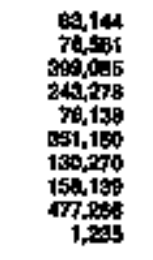 & 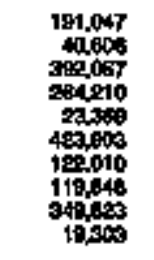 & 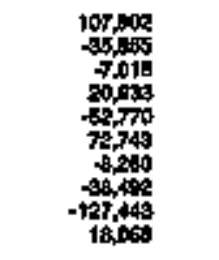 & 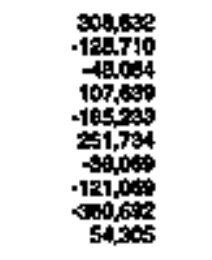 \\
\hline 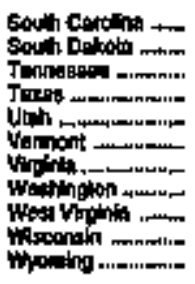 & 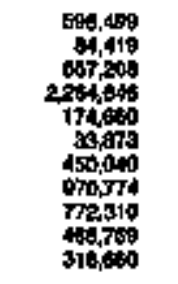 & 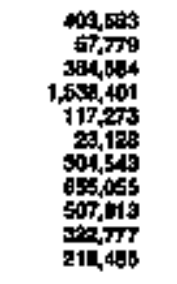 & 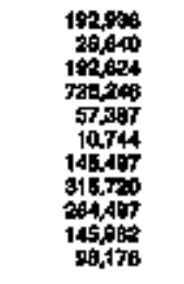 & $\begin{array}{r}0 \\
0 \\
0 \\
0 \\
0 \\
0,701 \\
0 \\
7,802 \\
0 \\
0 \\
0\end{array}$ & 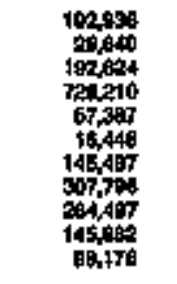 & 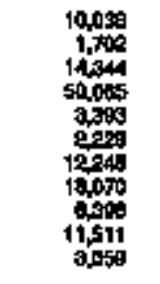 & 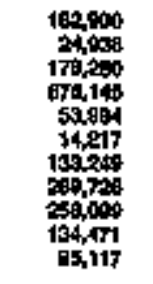 & 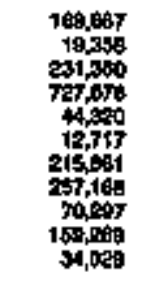 & 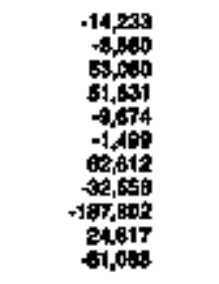 & 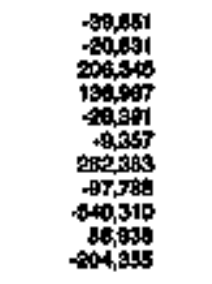 \\
\hline Uun & 28,20,13a & ท,612,192 & a,400,7mi & 122,491 & 6,1809,102 & otasy & $799010 \times 40$ & $9,0,105$ & 9.8\% & a \\
\hline
\end{tabular}

$E$
$\mathbf{L}$
$\mathbf{C}$
$\mathbf{T}$
$\mathbf{R}$
$\mathbf{J}$
$\mathbf{C}$
$\mathbf{I}$
$\mathbf{T}$
$\mathbf{Y}$
$\mathbf{N}$
$\mathbf{O}$
$\mathbf{T}$
$\mathbf{E}$
$\mathbf{S}$

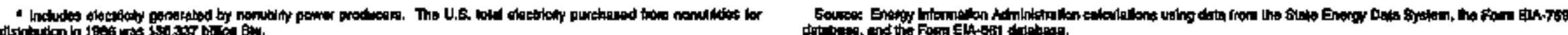





\section{Section 7. Total Energy}

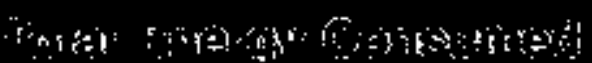

The preceding sections of this documentation describe how State end-use consumption estimates are made by individual energy source. This section describes how all energy sources are added in Btu to create end-use sector and total energy consumption estimates.

Energy consumption estimates for the residential sector include some wood and solar energy consumed in the commercial sector that cannot be identified separately. The code " $\mathrm{RC}^{\prime \prime}$ in the data identifier indicates residential sector and ${ }^{\circ} \mathrm{HC}^{\prime \prime}$ indicates residential and commercial sectors combined in the following calculation used for each State and the United States:

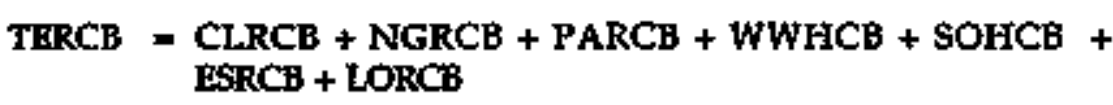

- The commercial sector calculations for each State and the U.S. total are:

\section{$\mathrm{TECCB} \cdot=\mathrm{CLCCB}+\mathrm{NGCCB}+\mathrm{PACCB}+\mathrm{ESCCB}+\mathrm{LOCCB}$}

For the industrial sector, the State calculations are slightly different from the U.S. calculation ("ZZ" in the vatiable name represents the twowteter State code that differs for each State). The industrial sector includes net imports of coal coke (CCNIBUS) in the U.S. total but not in the individual State estimates because no rellable means of allocating the U.S. amount to the States has been developed.

TEICBZZ = CLICBZZ + NGICBZZ + FAICBZZ + HYICBZZ + WWICBUS + GOICBUS + ESICBZZ + LOICBZZ
TEICBUS =

CLICBUS + NGICBUS + PAICBUS + HYICBUS + WWICBUS + GOICBUS + ESICBUS + LOICBUS + CCNIBUS

For the transportation sector, the calculations are:

$T E A C B=C L A C B+N G A C B+P A A C B+E S A C B+L O A C B$

Total energy consumed by all sectors is the sum of the consumption by the four end-use sectors:

TETCB $=$ TERCB + TECCB + TEICB + TEACB

\section{0}

A set of totals is calculated to estimate consumption in the four major end use sectors excluding each sector's share of all electrical system energy losses that are incurred in the generation, transmission, and distribution of electricity. This series is total net energy consumed and is represented by "TN."

Total net energy consumed by the residential, commercial, industrial, and transportation sectors are cakculated:

TNRCB. $=$ TERCB - LORCB

TNICB = TETCB - LOICB

TNCCB $=$ TECCB - LOCCB
TNACB $=$ TEACB - LOACB 


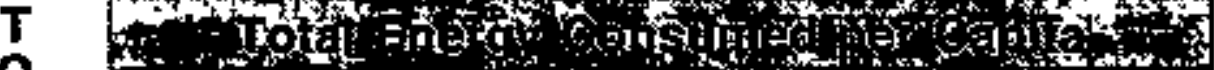

T

The energy consumed per person residing in each State and in the A United States is estimated by dividing the total energy series ("TE") L by the resident population as published by the U.S. Department of Commerce, Bureat of the Census. The U.S. total population published by the Bureau of the Census is based on tnxotunded numbers that are not available by State so that the sum of the States' population does not equal the U.S. total. Therefore, the U.S. total population is input to SEDS instead of being calculated as the sum of the States values. The Bureau of the Census series are estimated, in thousands of people, as of July $I$ of each year, except in 1960, 1970, 1980, and 1990, when the April 1 census data were used. The variable names for the series are:
TPOPPZZ = The resident population of each State; and TPOPPUS = The resident population of the United States.

Fstimated energy consumption per capita for each State and the United States, in million Btu, is represented by "TETP" and calculated:

TETPB $=$ TETCB $/$ TPOPP

The residential, commercial, industrtal, and transportation sectors' energy consumption per capita are estimated:
TERPB
TECPB $=$ TECCB $/$ TPOPP
TEIPB = TEEC $/$ TPOPP
TEAPB $=$ TEACB $/$ TPOPP 
Appendix B

\section{State Energy Data System} Variables 



\section{State Energy Data System Variables}

This is an alphabetical listing of all the variable names used in the State Energy Data System (SEDS). Provided for each variable on the system are: a brief description of the variable; units of the variable as found in SEDS; and the formulas used in SEDS to create the variable. If a variable is not one created by SEDS but is entered into the system, it is described as an independent variable. Formulas are provided for the State calculations (" $\mathrm{ZZ}^{*}$ in the variable name would be replaced by the two-tetter code for each State) and for the U.S. calculation (wherever appropriate).

Variables in the SEDS have seven-letter names that consist of the following components:

$\begin{array}{lllll}\begin{array}{l}\text { Character } \\ \text { Positions: }\end{array} & \mathbf{1} \text { and 2 } & \text { 3 and 4 } & 5 & 6 \text { and 7 } \\ \text { Identify: } & \begin{array}{l}\text { Type of } \\ \text { energy }\end{array} & \begin{array}{l}\text { Energy activity } \\ \text { or consumption } \\ \text { end-use sector }\end{array} & \begin{array}{l}\text { Type } \\ \text { of data }\end{array} & \begin{array}{l}\text { Geographic } \\ \text { arez }\end{array} \\ & & & \end{array}$

Characters 1 through 4 are explained in the description of each variable.

Character 5 is always one of the following:

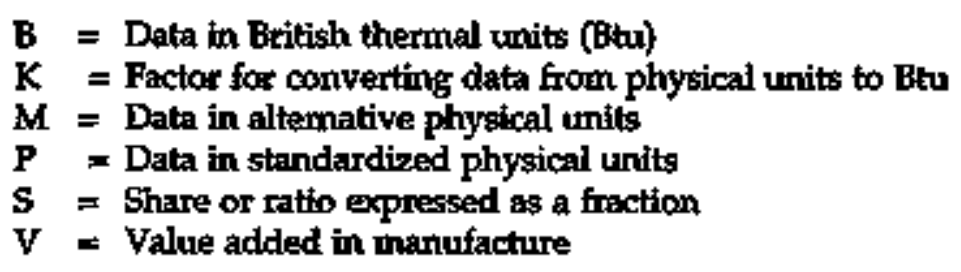

Characters 6 and 7 are two-letter U.S. Postal Service codes for the 50 States and the District of Columbia (represented by " $\mathrm{ZZ}$ " in the following variable names) and the United States ("US"). In this system, the United States means the 50 States and the District of Columbia. Some estimates of electricity sales and losses are derived by using only the contiguous 48 States and the District of Columbia. The variables used in those calculations are identified by " $48^{\prime \prime}$ as chatacters 6 and 7 in the variable names. 


\begin{tabular}{|c|c|c|c|}
\hline Variable & Description & Units & Fommulas \\
\hline ABICB & $\begin{array}{l}\text { Avtation gasoline blending components } \\
\text { total corsumed by the industrial sector. }\end{array}$ & Billion Btu & $\begin{array}{l}\text { ABICBZZ }=\text { ABTCBZZ } \\
\text { ABICBUS }=\text { ABTCBUS }\end{array}$ \\
\hline ABICP & $\begin{array}{l}\text { Avjatior gasoline blending components } \\
\text { total consumed by the industrial sector. }\end{array}$ & Thousand barrels & $\begin{array}{l}\text { ABICPZZ }=\text { ABTCPZZ } \\
\text { ABICPUS }=\text { ABTCPUS }\end{array}$ \\
\hline ABTCB & $\begin{array}{l}\text { Aviation gasoline blending components total } \\
\text { consumed. }\end{array}$ & Billion Btu & $\begin{array}{l}\text { ABTCBZZ }=\text { ABTCPZZ } * 5.048 \\
\text { ABTCBUS }=\text { इABTCBZZ }\end{array}$ \\
\hline ABTCP & $\begin{array}{l}\text { Aviation gasoline blending components total } \\
\text { consumed. }\end{array}$ & Thousand berrets & $\begin{array}{l}\text { ABTCPZZ = (COCAPZZ / COCAPUS) - ABTCPUS } \\
\text { ABTCPUS is independent. }\end{array}$ \\
\hline ACCCB & $\begin{array}{l}\text { Anthracite consumed by the commercial } \\
\text { sector. }\end{array}$ & Billion Btu & $\begin{array}{l}\text { ACCCBZZ }=\text { ACCCPZZ } \cdot \text { ACNUKUS } \\
\text { ACCCBUS }=\text { IACCCBZZ }\end{array}$ \\
\hline ACCCP & $\begin{array}{l}\text { Anthracite consumed by the commercial } \\
\text { sector. }\end{array}$ & Thousand short tons & $\begin{array}{l}\text { ACCCPZZ }=\text { ACHCPZZ } * 0.40 \\
\text { ACCCPUS }=\text { ¿ACCCPZZ }\end{array}$ \\
\hline ACEUB & Anthracite consumed by the electric utilities. & Billion Btu & $\begin{array}{l}\text { ACEUBZZ = ACEUPZZ } * \text { ACEUKUS } \\
\text { ACEUBUS }=\Sigma \text { IACEUBZZ }\end{array}$ \\
\hline ACEUKUS & $\begin{array}{l}\text { Factor for converting anthracite consumed by } \\
\text { the electric utilities from physical units to Btu. }\end{array}$ & Milition Btu per short ton & ACEUKUS is independent. \\
\hline ACEUP & $\begin{array}{c}\text { Anthracite consunted by the electric utilities. } \\
\text {. }\end{array}$ & Thausand short tons & $\begin{array}{l}\text { ACEUPZZZ is independent } \\
\text { ACEUPUS }=\text { IACEUPZZ }\end{array}$ \\
\hline ACHCP & $\begin{array}{l}\text { Anthracite consumed by the residential and } \\
\text { commercial sectors. }\end{array}$ & Thousend short tons & $\begin{array}{l}\text { ACHCPZZ = (ACHDPZZ / ACHDPUS) " ACHCPUS } \\
\text { ACHCPUS is independent. }\end{array}$ \\
\hline ACHDP & $\begin{array}{l}\text { Anthracite distributed to the residential and } \\
\text { commercial sectors. }\end{array}$ & Thousand short tons & $\begin{array}{l}\text { ACHDPZZ is tindependent, } \\
\text { ACHDPUS = EACHDPZZ }\end{array}$ \\
\hline ACICB & Anthracite consumed by the industrial sector. & Billion Bku & $\begin{array}{l}\text { ACICBZZ }=\text { ACKCBZZ }+A C O C B Z Z \\
\text { ACICBUS }=\Sigma A C I C B Z Z\end{array}$ \\
\hline ACICP & Anthracite consumed by the industrial sector. & Thoussand short tons & $\begin{array}{l}\text { ACICIZZ = ACKCPZZ + ACOCPZZ } \\
\text { ACICPUS }=\text { IACICPZZ }\end{array}$ \\
\hline ACKCB & Anthracite consumed at coke plants. & Billion Btu & $\begin{array}{l}\text { ACKCBZZ = ACKCPZZ * ACNUKUS } \\
\text { ACKCBUS = } \mathrm{\Sigma ACKCBZZ}\end{array}$ \\
\hline 416 & & $\begin{array}{l}\text { mlormation Aduinistration } \\
\text { Energy Data Report } 1994\end{array}$ & \\
\hline
\end{tabular}


ACKCP Anthracite consumed at coke plants.

ACKDP

Anthracite distributed to coke plants.

ACNUKUS Factor for converting anthracite consumed by all sectors other than the electric utility sector from physical units to Btu.

ACOCB

Anthracike consumed by other industrial users.

ACOCP

Anthracite consumed by other industrial users.

ACODP

Anthracte distributed to other industrial users.

ACRCB

Anthracite consumed by the restidential sector.

ACRCP

Anthracite consumed by the reidential seckor.

ACTCB

ACTCP

AICAP

ARICB

ARICP
Anthractte total consumed.

Aluminum ingot production capacity.

Anthracite total consumed.

Asphalt and road oil consumed by the industrial sector.

Asphalt and road oil consumed by the industrial sector.
Thousand short tons

Thousand short tons

ACKCPZZ \& (ACKDPZZ / ACKDPUS) " ACKCPUS ACKCPUS is independent.

ACKDPZZ is independent. ACKOPUS = $\mathrm{AACKDPZZ}$

Mitlion Btu per short ton

ACNUKUS is independent.

Billion Btu

Thousand short tons

Thousand short tons

Bsllion Btu

Thousand short tons

Billion Btu

Thotsend short tons

Short tons

Billion Btu

Thousand barrels
ACOCBZZ = ACOCPZZ * ACNUIKUS ACOCBUS' = IACOCBZZ ACOCPUS is independent.

ACODPZZ is independent. ACODPUS $=$ ₹ACODPZZ

ACRCBZZ - ACRCPZZ * ACNUKUS

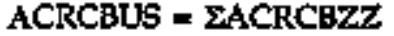

ACRCPZZ $=$ ACHCPZZ $* 0.60$ ACRCPUS = $\Sigma$ ACRCPZZ
$A C T C B Z Z=A C R C B Z Z+A C C C B Z Z+$ ACICBZZ + ACEUBZZ ACTCBUS = $\mathrm{AACTCBZZ}$

ACTCPZZ = ACRCPZZ + ACOCPZZ + ACTCPUS = $\mathrm{EACTCPZZ}$

AICAPZZ is indepertent. AlCAPUS $=$ EAICAPZZ

ARICBZZ $=$ ARICPZZ * 6.636 ARICBUS $=$ হARICBZZ

ARICPZZ = ASTCPZZ + RDICPZZ ARICPUS = EARICPZZ
ACOCPZZ = (ACODPZZ / ACODPUS) * ACOCPUS ACICPZZ + ACEUPZZ 


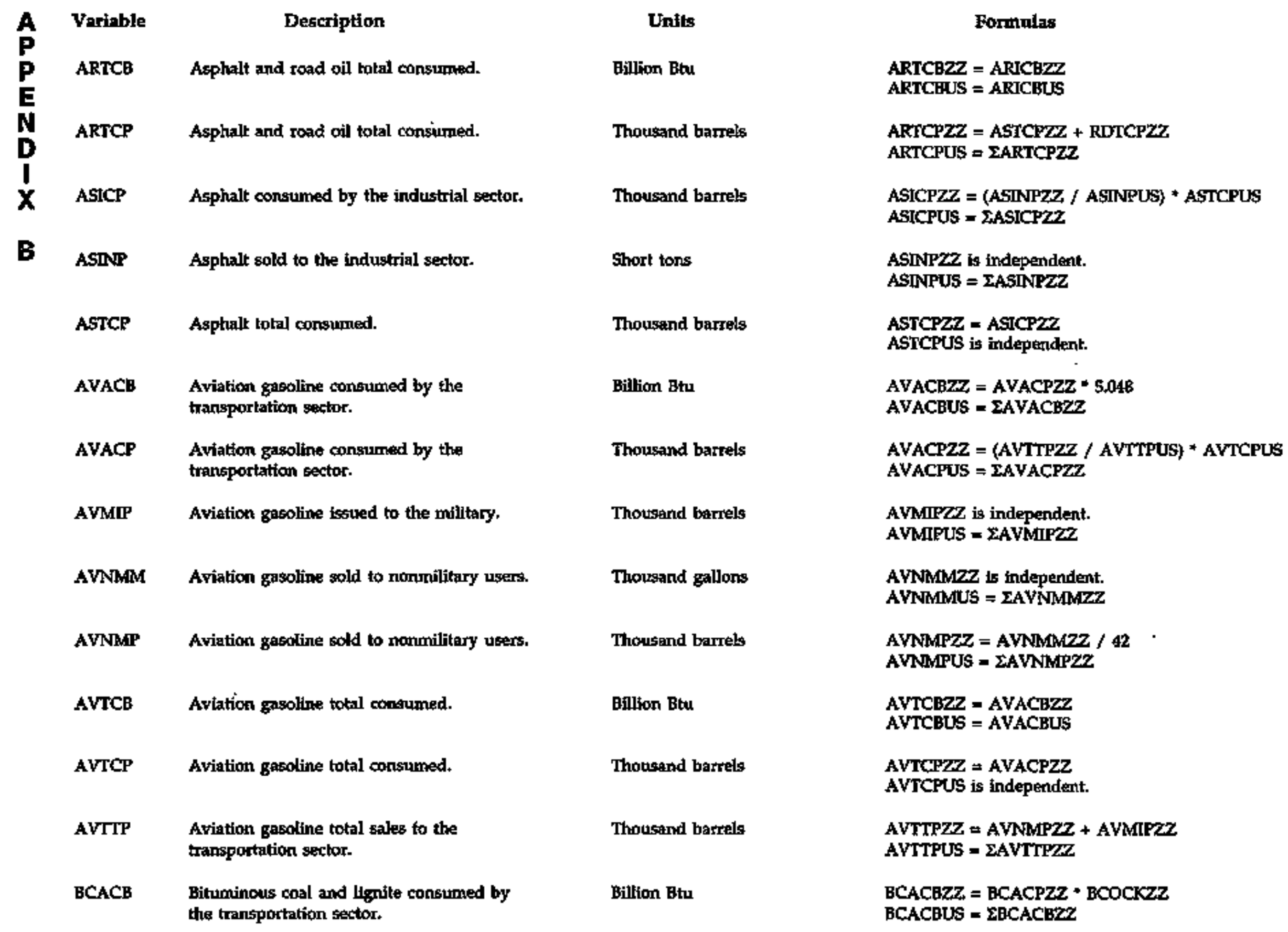


BCACP Bltuminous coal and lisnite consurned by the transportation sector.

BCCCB Bituminous coal and lignite consumed by the commercial sector.

BCCCP Bitaminous coal and lignite consumed by the commercial sector.

BCEUB Bituminous cool and lignite consumed by the elestric utilities.

BCEUKZZ Factor for converting bituminous coal and tignite corrsumed by the electric utilities from physical units to Bhu.

BCEUP Bituminous coal and lignilte consumed by the electric ntilities.

BCHCKZZ The factor for converting blthminous coal and lignite consumed by the residential and commercial sectors from physical units to Btu.

BCHCP Bituminous coal and tignite consumed by the residentlal and commercial sectors.

BCHDP Bituninous coal and lignite distributed to the restdentlal and commercial sectors.

BCICB Biturinous coal and lignite consumed by the industrfal sector.

BCICP Bituminous coal and ligrite consumed by the inderstrial sector.

BCKCB Bituminous cosl and tignite consumed by coke plants.

BCKCP Bituminous coal and tignite consumed by coke plants.
Thousand short tons

Billion Btu

Thousand short tons

Bitlion Btu

Million Btu per short ton

Thousand short tons

Million Btu per short ton

Thousand short tons

Thousand short tons

Billion Btu

Thousand short tons

Billion Bet

Thoussand short tons
BCACFZZ = (BCICPZZ / BCICPUS $)$ BCACPUS BCACFUS is independent.

$\mathrm{BCOCBZZ}=\mathrm{BCCCPZZ} * \mathrm{BCHCKZZ}$

BCCCBUS = $\Sigma$ BCCCBZZ

$\mathrm{BCCCPZZ}=\mathrm{BCHCPZZ} * 0.65$

BCCCPUS $=\mathbf{\Sigma B C C C P Z Z}$

BCELUZZ = BCEUPZZ * BCEUKZZ BCEUBUS - $\$$ BCEUBZZ

BCEUKZZ is independent.

BCEUPZZ is independent. BCEUPUS = इBCEUPZZ

BCHCKZZ, is independent.

BCHCPZZ = (BCHDPZZ / BCHDPUS) * BCHCPUS BCHCPUS is independent.

BCHDPZZ is independent.

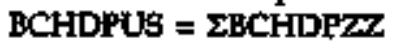

BCICBZZ = BCKCBZZ + BCOCBZZ BCICBUS $=\Sigma \mathrm{BCCICBZZ}$

BCICPZZ $=$ BCKCPZZ + BCOCPZZ BCICPUS $=$ ZBCICPZZ

BCKCBZZ $=$ BCKCPZZ $+26,80$ BCKCBUS = ₹BCKCEZZ

BCKCPZZ = (BCKOPZZ / BCKDPUS) * BCKCPUS BCKCPUS is indeppendent. 


\begin{tabular}{|c|c|c|c|}
\hline Variable & Description & Units & Eormulas \\
\hline BCKDP & $\begin{array}{l}\text { Bituminous coal and lignite distributed to } \\
\text { coke plants. }\end{array}$ & Thousand shott tons & 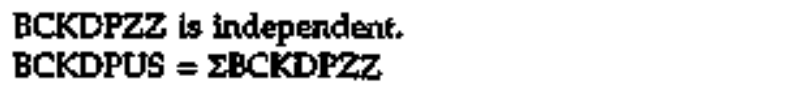 \\
\hline ICOCB & $\begin{array}{l}\text { Bituminous coal and lignite consumed by other } \\
\text { industrial wers. }\end{array}$ & Billion Btu & $\begin{array}{l}\text { BCOCBZZ = BCOCPZZ * BCOCKZZ } \\
\text { BCOCBUS = } \mathrm{BCCOCBZZ}\end{array}$ \\
\hline BCOCKZZ & $\begin{array}{l}\text { The factor for converting bituminous coal } \\
\text { and lignite consumed by other industriel } \\
\text { users from physical urits to Bku. }\end{array}$ & Million Btu per short ton & BCOCKZZ is independent. \\
\hline BCOCP & $\begin{array}{l}\text { Bituminous coal and figalte consumed by other } \\
\text { industrial users. }\end{array}$ & Thousend short tons & $\begin{array}{l}\text { BCOCPZZ = (BCODPZZ / BCODPUS) * BCOCPUS } \\
\text { BCOCPUS is independent. }\end{array}$ \\
\hline BCODP & $\begin{array}{l}\text { Bituminous coal and ltgnile distrlbuted to } \\
\text { other industrial users. }\end{array}$ & Thousand short tons & $\begin{array}{l}\text { BCODPZZ is independent. } \\
\text { BCODPUS = IBCODPZZ }\end{array}$ \\
\hline BCRCB & $\begin{array}{l}\text { Bthuminous coal and lignite consumed by the } \\
\text { residential sextor. }\end{array}$ & Billion Bhz & $\begin{array}{l}\text { BCRCBZZ, = BCRCEZZ } * \text { BCHCKZZ } \\
\text { BCRCBUS = IBCRCBZZ }\end{array}$ \\
\hline BCRCP & $\begin{array}{l}\text { Bituminous coal and lignite constumed by the } \\
\text { residential sertor. }\end{array}$ & Thousand short torts & $\begin{array}{l}\text { BCRCPZZ = BCHCPZZ *0.35 } \\
\text { BCRCPUS = EBCRCPZZ }\end{array}$ \\
\hline BCTCB & Bsturninous cold and lignite total consumed. & Billion Bat & $\begin{aligned} \text { BCTCBZZ }= & \text { BCRCBZZ + BCCCBZZZ * } \\
& \text { BCICBZZ + BCACBZZ + BCBUBZZ } \\
\text { BCTCBUS }= & \text { \&BCTCBZZ }\end{aligned}$ \\
\hline ВСТСР & Bituminous coal and lignite total consumed. & Thousand short tors & $\begin{aligned} & \text { BCTCPZZ }= \text { BCRCPZZ + BCCCPZZ + } \\
& \text { ACKCPZZ }+ \text { BCACPZZ + BCEUPZZ } \\
& \text { BCTCPUS = EBCTCPZZ }\end{aligned}$ \\
\hline CCEXBUS & Coal colke exported from the United States. & Billion Błu & CCEXBUS $=$ CCEXPUS $* 24.80$ \\
\hline CCEXPUS & Cosl colve exported from the Unnited States. & Thousand stort tons & CCEXPUS is independent. \\
\hline CCIMBUS & Coal coke imported into the United States. & Bitlion Btu & CCDMBUS = CCMMPUS * 24.90 \\
\hline CCIMPUS & Coal colke imported into the Unfted States. & Thousand short torns & CCMMPUS is independent. \\
\hline CCNIBUS & Coal coke net imports into the Undted States. & Billion Btu & CCNIBUS = CCMBUS - CCEXBUS \\
\hline CCNIPUS & Coal coke net inports into the United States. & Thoustand short tons & CCNIPUS = CCIMPUS - CCEXPUS \\
\hline \multicolumn{4}{|c|}{$\begin{array}{l}\text { Energy Informallon Administration } \\
\text { State Entwy Data Feport } 1894\end{array}$} \\
\hline
\end{tabular}

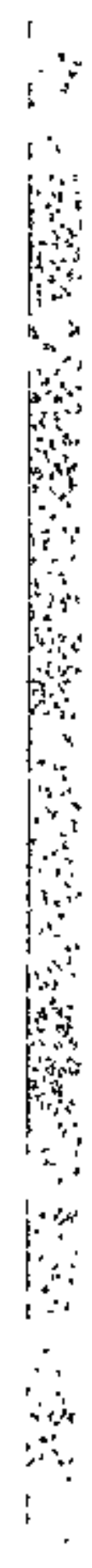




\section{Units}

Million dollars

Value added in the manufacture of corrugated and solid fiber boxes.

CLACB Coal consumed by the transportation sector.

CLACP

CLccB

CLCCP

Cleus

CLEUP

CLJCB

CLJCP

CLKCB

CLOCB

CLRCB

CLRCP

CLSCB
Coal consumed by the transportation sector.

Coal consumed by the commercial sector.

Coal consumed by the electric atilities.

Coal consumed by the electric atilities.

Coal consumed by the industrial sector.

Coal onsumsd by the industriat sector.

Coal consumed at coke pianks (coking coal).

Coal sonsumed by other industrial users.

Coal eonsumed by the residential seckor.

Coal consumed by the residential sector.

Coal consumed other than at coke planks (steàm coal).
Coal consumed by the commercial sector.

Billion Btux

Formulas

CGVAVZZ is independent. CGVAVUS $=$ ECGVAVZZ

Billian Btu

Thousand short tons

Billion Btu

Thousand short tons

Billion Btu

Thousand short tons

Billion Btu

Thousand short tons

Billion Btu

Billion Btu

Thousand short toms

Bitlion Btu
CLACBZZ = BCACBZZ

CLACBUS $=$ BCACBUS

CLACPZZ - BCACPZZ CLACPUS = BCACPUS

CLCCBZZ $=$ ACCCBZZ + BCCCBZZ CLCCBUS = ACCCBUS + BCCCBUS

CLCCPZZ = ACCCPZZ + BCCCPZZ CLCCPUS = ACCCPUS + BCCCPUS

CLEUTZZZ = ACEUBZZ + BCEUBZZ CLEUBUS = ACEUBUS + BCEUBUS

CLEUPZZ = ACEUPZZ + BCEUPZZ CLEUPUS = ACEUPUS + BCEUPUS

CLICBZZ = ACICBZZ + BCICBZZ CLICBUS = ACICBUS + BCICBUS

CLICPZZ = ACICPZZ + BCICPZZ CLYCPUS = ACICPUS + BCICPUS

CLKCBZZ = ACKCBZZ + BCKCBZZ CLKCCBUS $=$ ACKCBUS + BCKCBUS

CIOCBZZ = ACOCBZZ + BCOCBZZ CLOCBUS = ACOCBUS + BCOCBUS

CLRCBZZ = ACRCBZZ + BCRCBZZ CLRCBUS = ACRCBUS + BCRCBUS

CIRCPZZ = ACRCPZZ + BCRCPZZ CLRCPUS $=$ ACRCPUS + BCRCPUS

CLSCBZZ - CLTCBZZ - CLKCBZZ CLSCBUS $=$ CLTCBUS - CLKKCBUS 


\begin{tabular}{|c|c|c|c|}
\hline Variable & Description & Units & Formulas \\
\hline CLTCB & Coal total consumed. & Bultion Btu & $\begin{array}{l}\text { CLTCBZZ }=\text { ACTCBZZ + BCTCBZZZ } \\
\text { CLTCBUS }=\text { ACTCBUS + BCTCBUS }\end{array}$ \\
\hline CLTCP & Coal total consumed. & Thousand short tons & $\begin{array}{l}\text { CLTCPZZ }=\text { ACTCPZZ + BCTCPZZ } \\
\text { CLTCPUS }=\text { ACTCPUS + BCTCPUS }\end{array}$ \\
\hline COCAP & Crude oil operating capacity at refineries. & Barrels per calendar day & $\begin{array}{l}\text { COCAPZZ is independent. } \\
\text { COCAPUS = } 2 \text { COCAPZZ }\end{array}$ \\
\hline COICB & Crude oil cansumed by the industrial sector. & Billion Bhu & $\begin{array}{l}\text { COICBZZ }=\text { COTCBZZZ } \\
\text { COICBUS }=\text { COTCBUS }\end{array}$ \\
\hline COICP & Crude oil consumed by the industrial sector. & Thousand barrets & $\begin{array}{l}\text { COICPZZ = COTCPZZ } \\
\text { COICPUS = COTCPUS }\end{array}$ \\
\hline COTCB & $\begin{array}{l}\text { Crude oil consumed in petroleum industry } \\
\text { operations. }\end{array}$ & Billion Bhu & $\begin{array}{l}\text { COTCBZZ = COTCPZZ } * 5.800 \\
\text { COTCBUS }=\text { SCOTCBZZ }\end{array}$ \\
\hline COTCP & $\begin{array}{l}\text { Crude oil consumed in petroleum industry } \\
\text { operations. }\end{array}$ & Thousand barrels & $\begin{array}{l}\text { COTCPZZ Is independent. } \\
\text { COTCPUS = ICOTCPZZ }\end{array}$ \\
\hline CTCAP & $\begin{array}{l}\text { Catalytic cracking charge capacity of } \\
\text { petroleunm refineries. }\end{array}$ & $\begin{array}{l}1960 \text { through } 1979 \text { : } \\
\text { Barrals perr calemdar day } \\
1980 \text { forward: } \\
\text { Barrels per stream day }\end{array}$ & $\begin{array}{l}\text { CTCAPZZ is Independent. } \\
\text { CTCAPUS = ICTCAPZZ }\end{array}$ \\
\hline DFACB & $\begin{array}{l}\text { Distillate fuel consumed by the transportation } \\
\text { sector. }\end{array}$ & Bullion Btu & $\begin{array}{l}\text { DFACBZZ = DFACPZZ * 5.825 } \\
\text { DFACBUS }=\text { EDFACBZZ }\end{array}$ \\
\hline DFACP & $\begin{array}{l}\text { Distillate fuel consumed by the transportation } \\
\text { sector. }\end{array}$ & Thousand barrels & $\begin{array}{l}\text { DFACPZZ }=(\text { DFTRPZZ } / \text { DFNDPZZ }) * \text { DFNCPZZ } \\
\text { DFACPUS }=\text { EDFACPZZ }\end{array}$ \\
\hline DFBKP & $\begin{array}{l}\text { Distillate fuel adjusted sales for vessel burkering } \\
\text { use, excluding that sold to the Armed Forces. }\end{array}$ & Thousand barzels & $\begin{array}{l}\text { DFBKPZZ is independent. } \\
\text { DFBKPUS = IDFBKPZZ }\end{array}$ \\
\hline DFCCB & $\begin{array}{l}\text { Distillate fuel consumed by the commercial } \\
\text { sector. }\end{array}$ & Billion Btu & $\begin{array}{l}\text { DFCCBZZ = DFCCPZZ } * 5.825 \\
\text { DFCCBUS }=\text { IDFCCBZZ }\end{array}$ \\
\hline $\mathrm{DFCCP}$ & $\begin{array}{l}\text { Distillate fuet consunted by the commercial } \\
\text { sector. }\end{array}$ & Thousand barrels & $\begin{array}{l}\text { DFCCPZZ }=(\text { DFCMPZZ } / \text { DFNDPZZ }) * D F N C P Z Z \\
\text { DFCCPUS }=\text { EDFCCPZZ }\end{array}$ \\
\hline DFCMP & $\begin{array}{l}\text { Distillat\# fuel adiusted sales to the commercial } \\
\text { sector. }\end{array}$ & Thousind batrels & $\begin{array}{l}\text { DFCMPZZ is independente. } \\
\text { DFCMPUS = IDFCMPZZ }\end{array}$ \\
\hline
\end{tabular}


Distillate fuet consumed by the electric utilities.

Billion Btu

DFEUP

Disillate fuel (excluding keroseno-bye jet fuel) consumed by the electric utilities.

DFIBP Distillate fual adjusted sales for industrial space heating and other industrial use, including farm use

DFICB Distillate fuel consumed by the industrial sector.

DFICP Distillate hoel consurred by the industrial sector.

DFINP

Dhstillate fuel adjusted sales to the industrial sector.

DFMIP Distillate fuel adjusted sales to the Armed Forces, regardless of tse.

DFNCP Distillate fuel consumption by all sectors other than the electric ublity sector.

DFNDP Distillate fiel adjusted sales to all sectors other then the electric utility sector.

DFocP Distillate fuel adjusted sales for use by oil companies.

DFOFP Distillate fuel adjusted ales as diesel huel for off-highway ure.

DFONP Distillate fuel adjusted sales as diesel fuel for an-highway use.

DFOTP Distilate fuel adjusted sales for all other uses not ideatified in other adjusted sales categories.
Billion Btu

Thousand barrels

Thousend barrels

Thousand barrels

Thousand barrels

Thousand barrels

Thousend barrels

Thousand barrels

Thousand barrels

Thousand barrets

Thousand barrels

Thousand barrels
DFEUBZZ $=$ DFEUPZZ $\cdot 5.825$

DFEUBUS $=$ EDFEUBZZ

DFEUPZZ = DKEUPZZ - JKEUYZZ

DFEUPUS = $\Sigma$ DFEUPZZ

DFIBFZZ is independent.

DFIBPUS = IDFIBPZZ

DFICBZZ = DFICPZZZ * 5.825

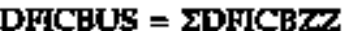

DEICPZZ = (DEINPZZ / DFNDPZZ $)$ - DFNCPZZ DFICPUS = EDFICPZZ

DFINPZZ $=$ DFIBPZZ + DFOCPZZ + DFOFPZZ + DFOTPZZ

DNNPUS = IDFINPZZ

DEMIPZZ is independent. DFMIPUS = EDFMIPZZ

DFNCPZZ - (DFNDPZZ / DFNDPUS) " DFNCPUS DENCPUS = DFTCPUS - DFEUPUS

DFNDEZZ = DFRSPZZ + DFCMPZZ + DFINPZZ + DFTRPZZ

DFNDPUS $=$ EDFNDPZZ

DFOCPZZ is independent. DFOCPUS = EDFOCPZZ

DFOFPZZ is independent. DFOFPUS $=\mathbf{E D F O T P Z Z}$

DFONPZZ is independent. DFONPUS = EDFONPZZ

DFOTPZZ is independent. DFOTPUS = EOFOTPZZ 


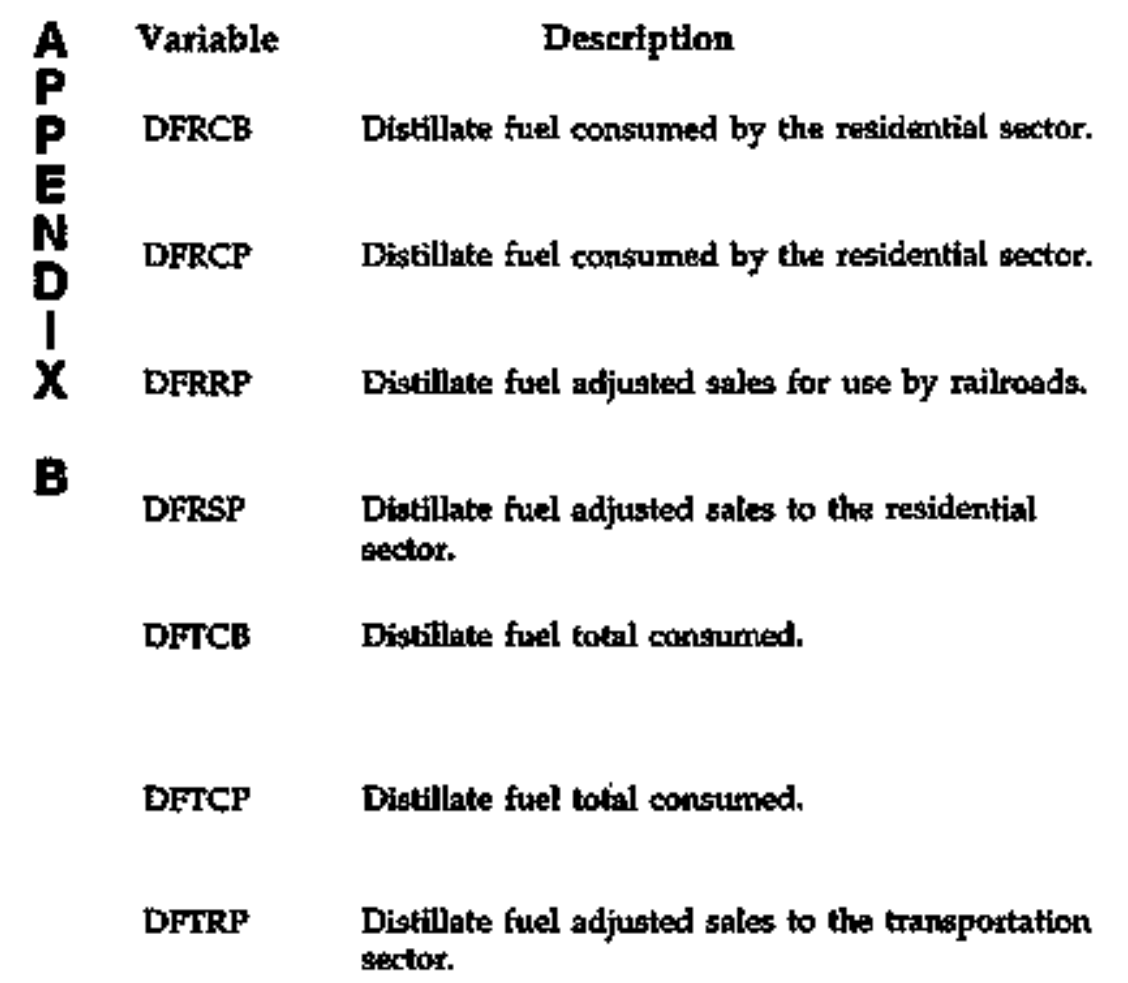

DKEU⿴囗 Distallate fuel and kerosene-type jet fuel consumed by the electric utilities.

DKBUP Distillate fuel and kerosene-type jet fupl consumed by the electric utilities.

ELEXB Flectricity exported from the United States (assumed to be produced from hydrcelectric power through 1989).

ELEXP

Electricity exported from the United States (assumed to be produced from hydroflectric power through 1989).

BLIMB

Electricity inported into the United States (assumed to be produced from hydroflectric power through 1989).
Units

Billion Btu

Thousand barrels

Thousand barrels

Thousand berrels

Billion Btu

Thousand barrels

Thousand barrels

Billion Btu

Thousand barrels

Billion Btu

Million kilowatthours

Billion Btu

Energy Intormallon Administration Stałe Energy Dale Peport 1994
DFRCBZZ $=$ DFRCPZZ * 5.825

DFRCBUS $=$ ¿DFRCBZZZ

DFRCPZZ * (DFFSPZZ / DFNDPZZ) * DFNCPZZ DFRCPUS = EDFRCPZZ

DFRRPZZ is independent. DFRRPUS = EDERRPZZ

DFRSPZZ is independent. DFRSPUS $=$ EDFRSPZZ

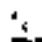

DFTCBZZ $=$ DFRCBZZ + DFCCBZZ + DFCBZZ + DFACBZZ + DFEUBZZ DFTCBUS $=$ EDFTCBZZ

DFTCPZZ = DFNCPZZ + DFEUPZZ DFTCPUS is independent.
DFTRPZZ = DFBKPZZ + DFMIIZZ + DFRRPZZ + DEONPZZ DFTRPUS $=$ XDFTRPZZ

DKEUBZZ $=$ DFEUBZZ + JKEUBZZ DKEUBUS $=$ \&DKEUBZZ

DKEUPZZ is independent. DKEUPUS = IDKEUPZZ

ELEXBZZ = HYEXBZZ + EXEXBZZ BLEXBUS $=$ EELEXBZZ

ELEXPZZ is independent. ELEXPUS $=$ EELEXPZZ

ELIMBZZ = HYMMBZZ + GEIMBZZ + EXMMBZZ ELIMBUS = $\Sigma$ ELIMEZZ 
Variable

Description

Units

Formulas

ELIMP

ELISB Net interstate sales of electricity- (Negative

ELISP Net interstate sales of electricity. (Negative

ELLSS48 The ratio of electrical system energy losses to electricity sold in the contiguous 48

EREXB sources and exported from the United States.

EREXP Electricity producpd from renewable energy sources and exported from the United Stafes.

ERDMB Electricity produced from xenewable enerrgy

ERIMP

ESACB Electricity consumed by (i.e., sold to) the transportation sector.

ESACP Electricity consumed by (i.e., sold to) the

ESCCB Electricity consumed by (t.e., sold to) the

ESCCP Electridty consumed by (t.e., sold to) the commercial sector.

ESCMP
Electricity imported into the United States

(assumed to be produced from hydroelectric power through 1989). indicates sales out of State; positive tndicates sales into Skake) indicates sates out of Stato; positive indicates sales into State.)

States and the District of Columbia.

Electricity produced from renewable energy sources and imported into the United Stakes.

Electricity produced from renewable energy sources and imported into the United States. transportation sector. commercial sector.

Million kilowatthours

Billion Btu

Million kilowatthours

Fraction

Esllion Bkx

Million kilowatthours

Billion Btu

Milison kilowatthours

Billion Bta

Million kilowattlours

Billtion Btu

Million kitlowatthours

Million kilowatthours sector.
ELIMPZZ is independert. ELEMPUS $=$ IELIMPZZ

ELISBZZ = (ESTCBZZ + LOTCBZZ) - TEEUBZZ ELISBUS = IELISBZZ

ELISPZZ = ELISBZZ / $\mathbf{3 . 4 1 2}$

ELISPUS $=\mathbf{\Sigma E L I S P Z Z}$

ELLSS48 = LOTCB48 / ESTCB48

EREXBZZ = HYEXBZZ

EREXBUS $=$ EEREXBZZ

EREXPZZ $=$ HYEXPZZ

EREXPUS = IFREXPZZ

ERIMBZZ = HYIMBZZ + GEIMBZZ ERJMBUS - SERIMBZZ

ERIMPZZ = HYIMPZZ + GEIMPZZ ERIMPUS $=$ ₹ERIMPZZ

ESACBZZ = ESACPZZ * 3.412 BSACBUS = $2 E S A C B Z Z$

ESACPZZ = (ESTRPZZ / ESTRPUS) * ESACPUS BSACPUS = ESOTPUS * ESTRSUS

ESCCBBZZ = ESCCPZZ * 3.412 ESCCBUS $=$ IESCCBZZ

ESCCPZZ = ESCMPZZ + ESOTPZZ - ESACPZZ ESCCPUS $=\Sigma$ EESCCPZZ

ESCMIZZ is independent. ESCMPUS = EESCMPZZ 

A Variable Description
$\begin{array}{ll}\mathbf{P} & \text { ESICR } \\ \mathbf{E} & \\ \mathbf{N} & \text { ESICP } \\ \mathbf{D} & \\ \mathbf{I} & \\ \mathbf{X} & \text { ESOTP }\end{array}$
E
Electricity consumed by (i-e, sold to) the industrial sector.
Electricity consumed by (i.e., sold to) the industral sector.
Electricity sold to the "Other" sector (1.e., public street and highway lighting, gales to other public authoritter, railroads and rallways, and interdepartmental sales).

ESRCB

Electricity consumed by (i.e., sold to) the residential sector.

ESRCP Electricity congumed by (i.e., sold to) the residential sector.

ESTCB Electricity total consumed (Le., sold).

ESTCP

Electricity tofal consumed (i.e, sold).

BSTRP

Electricity consumed by transit systems.

ESTRSUS

The share of electricity sold to the "Other" sector (ESOTP) that $t_{B}$ used for transportation.

EXEXB . Electricity produced from nonrenewable energy sources and exported from the United States.

EXEXP Electricity produced from nonrenewable energy sources and exported from the United States.

EXTMB

EXIMP

Electricity produced from nonrenewable energy sources and inported into the United States.

Electricity produced from nonrenewable energy sources and imported into the Uindted States.

\section{Units}

Billion Bikn

Million kilowatthours

Million kilowatthours

Billion Btu

Million kilowatthours

Bijtion Btu

Mitlion kilowatthours

Million kilowatthours

Fraction

Billion Btu

Million kilowatthours

Billion Bku

Million kllowatthours

Energy Intormation Admingetration State Energy Data Report 18BA

\section{Formulas}

ESICBZZ $=$ ESICPZZZ * 3.412

EMCBUS $=$ IESICBZZ

ESTCPZZ is independent.

ESICPUS $=$ IESICPZZ

ESOTPZZ is independent.

ESOTPUS = IESOTPZZ

ESRCBZZ = ESRCPZZ * 3.412

ESRCBUS = XESRCBZZ

ESRCPZZ is independent.

ESRCPUS = IESRCPZZ

ESTCBZZ $=$ ESTCPZZ $* 3.4 .12$

ESTCBUS $=$ EESTCBZZ

ESTCB48 = ESTCBUS - (ESTCBAK + ESTCBHI)

ESTCPZZ = ESRCPZZ + ESCCPZZ +

ESICPZZ + BSACPZZ

ESTCPUS = EESTCPZZ

ESTRPZZ is independent. ESTRPUS = IESTRPZZ

ESTRSUS is independent.

EXEXBZZ = EXEXPZZ * FFEOKUS

EXEXBUS $=$ IEXEXBZZ

EXEXPZZ = ELEXPZZ - EREXPZZ EXEXPUS $=$ ГEXEXPZZ

EXIMBZZ = EXMPZZ * FFEOKUS

EXIMBUS = EEXTMBZZ

EXIMPZZ = ELIMPZZ - ERJMPZZ EXIMPUS $=$ EEXMMPZZ 
EXNIIB Net imports of electricity into the United States produced from nonreniewable energy sources.

EXNIP

Net imports of electricity into the United States produced from nonrenewable energy sources.

FFEOKUS Fossil fuel steam-electric power plant conversion factor.

FNICB Petrochemical feedstocks, naphtha less than $01^{\circ} \mathrm{F}$, consumed by the industrial sector.

FNICP Petrochemical feedskocks, naphtha less than $401^{\circ} \mathrm{F}$, consumed by the industrial sector.

FNTCB Pertrochemical feedstocks, naphthe less than $401^{\circ} \mathrm{E}$, total consumed.

FNTCP Petrochemical feedstocks, naphtha less than $401^{\circ} \mathrm{F}$, total consumed.

FOICB Petrochemical feedstocks, other oils equal to or greater than $401^{\circ} \mathrm{E}$, consumed by the industrial sector.

FOICP Petrochemical feedstocks, other oils equal to or greater than $40 \mathrm{t}^{\circ} \mathrm{E}$, constumed by the industrial sector.

FOTCB Petrochemical feedstocks, other oils equal to or greater than $401^{\circ} \mathrm{F}$, total consurmed.

FOTCP Petrochemical feedstocks, other oils equal to or greater than $401^{\circ} \mathrm{F}$, total consumed.

FSICB Petrochemical fesdstocks, still gas, consumed by the industrial sector.

FSICP Petrochemical feedstacks, still gas, consumed by the industrial sector.

FSTCB Petrochemical feedstocks, still gas, tofal consumed.
Billion Btu

Million killowatthours

Thousand Btu per kilowatthour

Billion Btu

Thousend barrels

Billion Bta

Thousand barrets

Billion Błu

Thousand barrejs

Billion Btu

Thousand barrets

Billion Btu

Thousand barrels

Billion Btu
EXNIBZZ $=$ EXIMBZZ $\cdot$ EXEXBZZ

EXINIBUS = ZEXNIBZZ

EXNIPZZ = EXIMPZZ - EXEXXZZZ

EXNIPUS $=$ SEXNIPZZ

FFEOKus is independent.

FNICBZZ = FNTCBZZ

FNICBUS $=$ FNTCBUS

FNICPZZ \# FNTCPZZ

FNICPUS $=$ FNTCPUS

FNTCBZZ $=$ FNTCPZZ * 5.248

FNTCBUS = IFNTCBZZ

FNTCPZZ = (OCVAVZZ / OCVAVUS) * FNTCPUS FNICPUS is independent.

\section{FOICBZZ = FOTCBZZ}

FOICBUS $=$ FOTCBUS

FOICPZZ $=$ FOTCPZZ FOICPUS $=$ FONCPUS

FOTCBZZ - FOTCPZZ * 5.825

FOTCBUS $=$ IFOTCBZZ

FOTCPZZ = (OCVAVZZ / OCVAVUS) * POTCPUS FOTCPUS is independent.

FSICBZZ - FSTCBZZ

FSICBUS $=$ FSTCBUS

FSICPZZ = FSTCPZZ

FSICPUS $=$ FSTCPUS

FSTCBZZ $=$ FSTCPZZ * 6.000

FSTCBUS $=$ EFSTCBZZ 

A Variable
Description
FSTCP Petrochemical feedstocks, still gas, total consumed.
GEEOB Electrictty produced from geothermal energy at electric utilities.

GEEOKUS Factor for converting electricity produced from geothermal energy from physical units to Bhu.

B GEDOP Electricity produced from geothermal energy at electric utilities.

GECB Electricity produced from geothermal energy In the industrial sector.

GEIMB Electricity produced from gepthermal energy and imported into the United States

GEDMP Electriclity produced from geothermat energy and imported into the Unlted States.

GEENB Geothermal subtobal: electricity produced from geothermal energy at electric utiluties plus imports of electricity into the United Stater.

GEENP

Geothermal subtotat: electricity produced from gethermal energy at electric utilities plus imports of elextricity into the United States.

GETCB Geothermal total: electricity produced.

GETCP Geothermal total electriclty produced (available for 1960-1969 only).

GOICB Electricity produced from geothermal, wind, photovoltaic, and solar thermal energy coutres in the industral sector.
Units

Formulas

Thoussend barrels

Billion Btu

Thousand Btu per kilowatthour

Million kilowatthours

Bijtion Btu

Billion Btu

Million kilowatthours

Billion Btu

Million kilowathours

Bitlion Btu

Melllon kilowatthours

Billion Btu
FSTCPZZ = (COCAPZZ / COCAPUS $) *$ FSTCPUS FSTCPUS is independent.

GEEOHZZ = GEEOPZZ * GEEOKUS GEEOBUS = ZGEEOBZZ

GEEOKUS is independent.

GEEOPZZ is independent. GEEOPUS = LGEEOPZZ

GEICBZZ is independent. GEICBUS $\div$ EGEICBZZ

GEIMBZZ = GEIMPZZ * GEEOKUS GEDMBUS $=$ ZGEIMBZZ

GEDMPZZ is independent. GEIMPUS $=$ DGEIMPZZ

GEENBZZ $=$ GEEOBZZ + GEIMABZZ GEENRUS $=$ LGEENBZZ

GEENPZZ = GEBOPZZ + GEIMPZZ GEENPUS $=$ ХGEBNPZZ

GETCBZZ = GEICBZZ + GEENBBZZ GETCBUS = SGETCBZZ

GETCPZZ = GEICPZZ + GEENPZZ GETCPUS $=$ IGETCPZZ

GOICBZZ = GEICBZZ + \$OICBZZ + WYICBZZ GOICBUS $=$ EGOICBZZ 
GOTCB Electricity produced from geothermal, wind, photoviltaic and solar thermal energy sources; total produced.

GOTCP Electricity produced fron geothermal, wind, photovoltaic, and solar thermal energy sources; total produced (available for 1960-1989 only).

HPEOB Electricity produced from pumped storage hydroelectric power at electric tutlities.

HPEOP Electrictly produced from pumped storage hydroelectric power at electric utilities.

HVENB Renewable hydroelectric subtotal: electricity produced from conventional hydropower at electric utilitie plus net imports of electricity into the United States.

HVENP Renewable hydroelectric subtotal: electricity produced from conventional hydropower at electric utilities plus net imports of electricity into the United States.

HVEOB Electricity produced from conventional hydropower at electric utilities.

HVEOP Electricity produced from conventional hydropower at electric utilities.

HYENB Hydroelectric subtotal: electrictly produced from all types hydropower at electrte tailities plus net insports of electricity into the United States.

HYENP Hydroelectric subtotal: electricity produced from all types of hydropower at electric utilities plus not imports of electricity into the United States.

HYEOB
Electricty produced from all types of hydropower at electric utilities.
Bitlion Btu

GOTCBZZ = GETCBZZ + SOTCBZZ + WYTCBZZ GOTCBUS $=$ SGOTCBZZ

Millim kilnwatthours

GOTCPZZ = GETCPZZ + SOTCPZZ + WYTCPZZ GOTCPUS $=$ EGOTCPZZ

\section{Billion Btu}

Million kilowatthours

Sillion Btu

Million kilowatthours

Billion bis

Million kllowatthours

Billion Btu

Million kilowatthours

Billion Btu

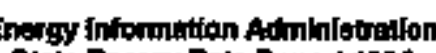
Stoto Eporgy Deto Repart 1994
HPEOBZZ = HPEOPZZ * FFEOKUS

HPEOBUS = IHPEOBZZ

HPEOPZZ is independent. HPEOPUS = УHPBOPZZ

HVENBZZ = HVEOBZZ + HYMMBZZ - HYEXBZZ HVENBUS $=$ నHVENBZZ

HVENPZZ = HVEOPZZ + HYMPZZ - HYEXPZZ HVENPUS $=$ इHVENPZZ

HVEOAZZ $=$ HVEOPZZ $*$ FFEOKUS HVEOBUS $=$ SHVEOBZZ

HVEOPZZ is independent. HVEOPUS - IHVEOPZZ

HYENBZZ = HYEOBZZ + HYIMBZZ - HYEXBZZ HYENBUS = DHYENBZZ

HYENPZZ = HYEOPZZ + HYMMPZZ - HYEXPZZ HYENPUS $=$ ZHYYENPZZ

HYEOBZZ = HPEOBZZ + HVEOBZZ HYEOBUS $=$ IHYEOBZZ 


\begin{tabular}{|c|c|c|c|}
\hline Variable & Description & Units & Formulas \\
\hline FYEOP & $\begin{array}{l}\text { Electricty produced from all types of hydropower } \\
\text { at electric utilities. }\end{array}$ & Million kilowatthours & $\begin{array}{l}\text { HYEOPZZ }=\text { HPEOPZZ }+ \text { HVEOPZZ } \\
\text { HYEOPUS = EHYEOPZZ }\end{array}$ \\
\hline HYEXB & $\begin{array}{l}\text { Electricity produced from hydroelectric } \\
\text { power and exported from the United States. }\end{array}$ & Billion Btu & $\begin{array}{l}\text { HYEXBZZ = HYEXYPZZ * FFEOKUS } \\
\text { HYEXBUS = EHYEXBZZ }\end{array}$ \\
\hline HYEXP & $\begin{array}{l}\text { Electricity produced from hydroelectric } \\
\text { power and exported from the United States. }\end{array}$ & Million kilowatthours & $\begin{array}{l}\text { HYEXPZZZ is independent. } \\
\text { HYEXPUS = EHYEXPZZ }\end{array}$ \\
\hline HYICB & $\begin{array}{l}\text { Electricity produced from hydroelectric power } \\
\text { at industrial facillties. }\end{array}$ & Billion Btu & $\begin{array}{l}\text { HYICBZZ is independent. } \\
\text { HYICBUS = ZHYICBZZ }\end{array}$ \\
\hline HYICP & $\begin{array}{l}\text { Electricity produced from hydzoelectric power } \\
\text { at industrial facilities (available for 1960-1989 } \\
\text { only). }\end{array}$ & Million kilowatthours & $\begin{array}{l}\text { HYICPZZ is independent. } \\
\text { HYICPUS = EHYICIPZZ }\end{array}$ \\
\hline HYMMB & $\begin{array}{l}\text { Electricity produced from bydroelectric } \\
\text { power and imported into the Uniked States. }\end{array}$ & Billion Ban & $\begin{array}{l}\text { HYMMBZZ }=\text { HYMMPZZ } * \text { FFEOKUS } \\
\text { HYTMBUS }=\text { IHYIMBZZ }\end{array}$ \\
\hline HYMMP & $\begin{array}{l}\text { Electricity produced from hydroelectric } \\
\text { power and improrted into the United States. }\end{array}$ & Milliton kilowatthours & $\begin{array}{l}\text { HYMAPZZ is independent. } \\
\text { HMDMPUS = IHYMAPZZ }\end{array}$ \\
\hline HYTCB & $\begin{array}{l}\text { Hydroelectric tokal: electricity produced from } \\
\text { hydropower at electric utilities (including net } \\
\text { imports of ejectricity) and at industrial facilities. }\end{array}$ & Billion Btu & $\begin{array}{l}\text { HYTCBZZ }=\text { HYENBZZ }+ \text { HYCBZZ } \\
\text { HYTCBUS }=\text { IHYTCBZZ }\end{array}$ \\
\hline HYTCP & $\begin{array}{l}\text { Hydroelectric total: electricity produced from } \\
\text { hydropower at electric utilities (Including net } \\
\text { imports of electricity) and at indastrial facilities } \\
\text { (nvailable for 1960-1989 only). }\end{array}$ & Million killowatthours & $\begin{array}{l}\text { HYTCPZZ }=\text { HYENPZZ }+ \text { HYICPZZ } \\
\text { HYTCPUS }=\text { SHYTCPZZ }\end{array}$ \\
\hline JFACB & let fuel consurned by the transportation sector. & Billion Btu & $\begin{array}{l}\text { JFACBZZ }=\text { JKACBZZ }+ \text { JNACBZZ } \\
\text { JFACBUS }=\text { EJEACBZZ }\end{array}$ \\
\hline JFACP & Jet fuel consumed by the transportation sector. & Throusand barrels & $\begin{array}{l}\text { JFACPZZ }=\text { JKACPZZ }+ \text { JNACPZZ } \\
\text { JFACPUS } \pm \text { IJFACPZZ }\end{array}$ \\
\hline JFEUB & Jet fuet consumed by electric utlibites. & 旺lion Btu & $\begin{array}{l}\text { JFEUBZZ }=\text { JKEUBZZZ } \\
\text { JFEUBUS }=\text { JKBUAUS }\end{array}$ \\
\hline FFEUP & Jet fuel consumed by electric utilitios. & Thoussand barrels & $\begin{array}{l}\text { JFEUPZZ }=\text { JKEUPZZ } \\
\text { JFEUPUS }=\text { JKEUPUS }\end{array}$ \\
\hline
\end{tabular}


JFTCP Jet fuel total consumed.

IKACB Kerasene-type jet fuel consumed by the transportation sector.

JKACP Kerosene-type jet fued consumed by the transportation sector.

JKEUB Kerosene-type jet fuel consumed by electric utalities.

JKEUP Kerosene-type jet fuel cansumed by electric utilities.

JKTTP Kerosente-kype jet fuel total sold.

JKTCB Kerosene-type jet fuel total consuned.

JKTCP Kerosene-type jet fuel total conisumed.

JNACB Nophtha-type jet fuel consumed by the transportation sector.

JNACP Naphtha-type fet fuel consumed by the transportation sector.

JNMIP Naphtha-type jet fuel issued to the military.

JNTCB Naphtha-type jet fuel total consumed.

JNTCP
Naphtha-type jet fued total consumed.
Thousand barrels
Billion Btu

Thousand barrels

JFTCBZZ = JFACBZZ + JFEUBZZ JFTCBUS $=$ IJFTCBZZ

JFTCPZZ = JEACPZZ + JFEUPZZ JFTCPUS \# EJFTCPZZ

Billion Btu

Thousand berrels

Billion Btu

Thousand bartsils

Thousand gallons

Billion Btu

Thousand berrets

Btllion Btu

Thoussand bartels

Btllion Btu

Thoupand barrels

JKEUPZZ is independent.

\section{JKACBZZ $=\mathrm{JKACPZZ} * 5.670$ \\ JKACBUS $=$ হJKACBZZ}

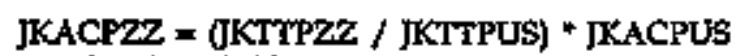
JKACPUS $=$ JKTCPUS - JKEUPUS

JKEUBZZ $=$ JKEUPZZ * 5.670

"JKEURUS = ZJKGUBZZZ JKEUPUS $=$ IJKEUPZZ

\section{JKTTPZZ is independent.} JKTTPUS = EJKTTPZZ

$\mathrm{JKTCBZZ}=\mathrm{JKTCPZZ} * 5.670$ JKTCBUS = IJKTCBZZ

$J K T C P Z Z=J K A C P Z Z$ + JKEUPZZ JKTCPUS is irdependent.

JNACBZZ $=$ JNTCBZZ JNACBUS = JNTCBUS

JNACPZZ $=$ JNTCPZZ JNACPUS $=$ JNTCPUS

JNMIFZZ is tindependent. INMIPUS = E]NMIPZZ

JNTCBZZ $=$ JNTCPZZ * 5.355 JNTCBUS $=\Sigma]$ NTCBZZ

JNTCPZZ = JNMIPZZ / JNMIPUS) * JNTCPUS JNTCPUS is independent. 


\begin{tabular}{|c|c|c|c|}
\hline Variable & Description & Units & Formulas \\
\hline $\mathrm{KSCCB}$ & Kerosene consumed by the commercial sector. & Billion Btu & $\begin{array}{l}\mathrm{KSCCBZZ}=\mathrm{KSCCPZZ} * 5.670 \\
\mathrm{KSCCBUS}=\Sigma \mathrm{KSCCBZZ}\end{array}$ \\
\hline KSCCP & Kerosene consumed by the commercial sector. & Thousand barxels & $\begin{array}{l}\text { KSCCPZZ = (KSCMPZZ / KSTTPZZ })^{*} \text { KSTCPZZ } \\
\text { KSCCPUS = IKSCCPZZ }\end{array}$ \\
\hline KSCMP & Kerosene sold to the comprexcial sector. & Thousand barrels & $\begin{array}{l}\text { KSCMPZZ is irdependent. } \\
\text { KSCMPUS = EKSCMPZZ }\end{array}$ \\
\hline KSSCB & Kerosene consumed by the industrial sector. & Billion Btu & $\begin{array}{l}\text { KSICBZZ = KSICPZZ * } 5.670 \\
\text { KSICBUS = IKSICBZZ }\end{array}$ \\
\hline KSICP & Kerosene consumed by the industrial sector. & Thousand batrels & $\begin{array}{l}\text { KSICPZZ = (KSINPZZ / KSTTPZZ) " KSTCPZZ } \\
\text { KSICPUS = EKSICPZZ }\end{array}$ \\
\hline KSIHP & Kerosene sold for industrial heating. & Thousand barteis & $\begin{array}{l}\text { KSHHPZZ is independent. } \\
\text { KSIHPUS = EKSIHIZZZ }\end{array}$ \\
\hline KSINP & Kerosene sold to the industrial sector. & Thousand barrels & $\begin{array}{l}\text { KSINPZZ = KSOTPZZ + KSIHPZZ } \\
\text { KSINPUS = EKSINPZZ }\end{array}$ \\
\hline KSOTP & $\begin{array}{l}\text { Karosene sold for all other uses, including } \\
\text { farm use. }\end{array}$ & Thousand barrels & $\begin{array}{l}\text { KSOTPZZ is independent. } \\
\text { KSOTPUS = EKSOTPZZ }\end{array}$ \\
\hline KSRCB & Kerosene constumed by the residential sector. & Bullion Btu & $\begin{array}{l}\text { KSRCBZZ = KSRCPZZ *5.670 } \\
\text { KSRCBUS }=\text { EKSRCBZZ }\end{array}$ \\
\hline KSRCP & Kerosene consumed by the residential sector. & Thousand batrels & $\begin{array}{l}\text { KSRCPZZ = (KSRSPZZ / KSTTPZZ) * KSTCPZZ } \\
\text { KSRCPUS = IKSRCPZZ }\end{array}$ \\
\hline KSRSP & Kerosene sold to the residential sector. & Thousand barrels & $\begin{array}{l}\text { KSRSPZZ is independent. } \\
\text { KSRSPUS = EKSRSPZZ }\end{array}$ \\
\hline KSTCB & Kerosene total consumed. & Billion Btu & $\begin{array}{l}\text { KSTCBZZ = KSRCBZZ + KSICBZZ + KSCCBZZ } \\
\text { KSTCBUS = IKSTCBZZ }\end{array}$ \\
\hline KSTCP & Kerosene total consumed. & Thousand barrels & $\begin{array}{l}\text { KSTCPZZ = (KSTTPZZ / KSTTPUS) " KSTCPUS } \\
\text { KSTCPUS is independent. }\end{array}$ \\
\hline KSTTP & Kerosene total sold. & Thousand barrels & $\begin{array}{l}\text { KSTTPZZ = KSRSPZZ + KSCMPZZ + KSINPZZ } \\
\text { KSTTPUS = EKSTTPZZ }\end{array}$ \\
\hline 432 & & Imormpy Data Report & \\
\hline
\end{tabular}


LGACB LPG consumed by the transportation sector.

LGACP

LPG consumed by the transportation sector.

LGCBM

LPG sales for interral combustion engire nse.

LGCBP

LPG consumed for internel combustion engine use.

LGCCB

LPG consumed by the commercial sector.

LGCCP

LPG consumed by the commercial sector.

LGHCM LPG sold for residential and commercial use.

LGHCP

LPG consumed by the restdental and contnercial ectors.

LGICE LFG consumed by the industrial sector.

LGICP LPG consumed by the Industrial sector.

LGRCB LPG consumed by the residential sector.

LGRCP LPG consumed by the residential sector.

LGTCB

LPG total corsumed.

LGTCKUS Factor for converting LPG from physical units to Bku.

Thausand barrels
Billion Btu

Thousand barrels

Thousand gallons

Billion Btu

Thousand barrels

Thousand gallons

Thousaryd barreis

Biltion Btu

Thousand barrels

Biliton Btu

Thousend barrels

Biltion Bku

Milition Btu per barzel

LGICBZZ = LGICPZZ * LGTCKUS
LGACBZZ = LGACPZZ * LGTCKUSS LGACBUS = ILGACBZZ

LGACPZZ = LGCBPZZ * LGTRSUS LGACPUS = ILGACPZZ

LGCBMZZ is independent. LGCBMUS $=$ LLGCBMZZ

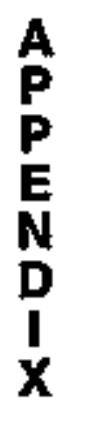

LGCBPZZ - LGCBMZZ / 42 LGCBPUS $=\Sigma \mathrm{LGCBPZZ}$

LGCCBZZ - LGCCPZZ * LGTCKUS LGCCBUS $=$ ILGCCBZZ

LGCCPZZ - LGHCPZZ * 0.15 LGCCPUS $=\Sigma L G C C P Z Z$

LGHCMZZ is independent. LGHCMUS = ILGHCMZZ

LGHCPZZ = LGHCMZZ $/ 42$ LGHCPUS $=$ \&LGHCPZZ LGICBUS $=\Sigma$ LGICBZZ

LGICPZZ = LGTCPZZ $-($ LGRCPZZ +

LGICPUS a ELGICPZZ

LGRCBZZ = LGRCPZZ " LGTCKUS LGRCBUS = ELGRCBZZ

LGRCPZZ = LGHCPZZ * 0.85 LGRCPUS $=$ \&LGRCPZZ

LGTCBZZ = LGRCBZZ + LGCCBZZ + LGICBZZ + LGACBZZ LGTCBUS = ILGTCBZZ

LGTCKUS is independent. 


\section{A Variable \\ $\mathbf{P}$

The transportatton sector's share of electrical system energy losses.

LOCCB

The commercial sector's share of electrical sybtem energy hosses.

LOCCP

The commercial sector's share of electrical syitem energy loss

LOICB

The industrial sector's share of tilectrical system energy losses.

LOICP

The industrial sector's share of electrical system enterigy losses.

LORCB The residential sector's share of electrital system energy losbes.

\section{Units}

Thousand barrels

Fraction

Thoussand gallons

Btllion Bku

Million kilowatthours

Billion Bku

Million kilowatthoum

Billion Btu

Million killowatthours

Bitlian Bku

LGTCPZZ = (LGTTPZZ / LGTTPUS) * LGTCPUS LGTCPUS is independent.

LGTRSUS is incependent.

LGTTPZZ is independent.

LGTTPUS = ELGTTPZZ

LOACBZZ = ESACBZZ - ELLSS48 Exceptions:

LOACBAK = (ESACBAK / ESTCBAK) * LOTCBAK

LOACBHI = (ESACBHI / ESTCBHI) * LOTCBHI LOACBUS = ILOACBZZ

LOACPZZ $=$ LOACBZZ / 3.412

LOACPUS = LOACBUS $/ 3.412$

LOCCBZZ * ESCCBZZ * ELLSS4S Exceptions:

LOCCBAK = (ESCCBAK / ESTCBAK) * LOTCBAK LOCCBHI $=$ (BSCCCBHI $/$ BSTCBHI $*$ LOTCBHI LOCCBUS = ELOCCBZZ

LOCCPZZ = LOCCBZZ / 3.412

LOCCPUS = LOCCBUS / 3.412

LOICBZZ = ESTCBZZ * ELLSSA8

Exceptions:

LOICBAK $=($ ESICBAK / ESTCBAK $)$ LOTCBAK

LOICBHI = (ESICBHI / ESTCBHI) " LOTCBHI

LOACBUS $=2$ LOICBZZ

LOICPZZ = LOICBZZ / 3.412

LOICPUS $=$ LOICBUS $/ \mathbf{3 . 4 1 2}$

LORCBZZ = ESRCBZZ - ELLSS48

Exceptions:

LORCBAK = (ESRCBAK / ESTCBAK $)$ * LOTCBAK

LORCBHI = (ESRCBHI $/$ ESTCBHII $)$ LOTCBHI

LORCBUS $=\Sigma$ LORCAZZ 
Variable

Deseription

LORCP

The residential sector's share of electrical system energy losses.

LOTCB . . Total electrical system energy losses.

LOTCP Total electrical system energy losses.

LUACB Lubricants consumed by the transportation sector.

LUACP Lubricants consumed by the transportation sector.

LUICB Lubricants consumed by the industrial sector.

LUICP

LUINP

LUTCB

LUTCP

LUTRP

LUTTP

MBICB

Motor basoline blending components consumed
Units

Million kilowatthours

Billion Btu

Million kilowatthours

Billion Btu

Thousand barrels

Btilion Btu

Thousand barrets

Thousand barrels

Etllion Btu

Thonsand barrels

Thousand basrels

Thousand barrels

Billion Btu by the industrial sector.
Formulas

LORCPZZ $=$ LORCBZZ / 3.412

LORCPUS = LORCBUS / 3.412

LOTCBZZ $=$ ESTCBZZZ * ELLLSAAB Exceptions:

LOTCBAK $=$ TEEUBAK - ESTCBAK

LOTCBHI $=$ TEEUBHI - ESTCBHI

LOTCBUS = TEEUBUS - ESTCBUS

LOTCB48 - LOTCBUS - (LOTCBAK + LOTCBHI)

\section{LOTCPZZ = LOTCZZZ / 3.412}

LOTCPUS = LOTCBUS / 3.412

LUACBZZ $=$ LUACPZZ * 6.065

LUACBUS $=$ ZLUACBZZ

LUACPZZ - (LUTRPZZ / LUTYPZZ) " LUTCPZZ LUACPUS = YLUACPZZ

LUICBZZ = LUICPZZ * 6.0065 LUICBUS $=$ ELUICBZZ

LUICPZZ = (LUINPZZ / LUTTPZZ) * LUTCPZZ LUICPUS $\approx$ ILUACPZZ

LUINPZZ is independent. LUINPUS = ZLUINPZZ

LUTCBZZ = LUICBZZ + LUACBZZ LUTCBUS = YIUTCBZZ

LUTCPZZ = (LUTTPZZ / LUTTPUS) * LUTCPUS LUTCPUS is independent.

LUTRPZZ is independent. LUTRPUS = ILUTRPZZ

LUTTPZZ = LUINPZZ + LUTRPZZ LUTTPUS $=$ ELUTTPZZ

MAICBZZ - MBTCBZZ

MBICBUSS $=$ MBTCBUS 


\begin{tabular}{|c|c|c|c|}
\hline Variable & Description & Units & Formulas \\
\hline MBICP & $\begin{array}{l}\text { Motor gasoline blending components consumed } \\
\text { by the industriel sector. }\end{array}$ & Thousand barrels & $\begin{array}{l}\text { MBICPZZ = MBTCEZZ } \\
\text { MBICPUS = MBTCPUS }\end{array}$ \\
\hline MBTCB & $\begin{array}{l}\text { Motor gasoline blending components total } \\
\text { consumed. }\end{array}$ & Billion Btu & $\begin{array}{l}\text { MBTCBZZ }=\text { MBTCPZZ *5253 } \\
\text { MBTCBUS }=\text { EMBTCBZZ }\end{array}$ \\
\hline MBTCP & $\begin{array}{l}\text { Motor gasoline blending components total } \\
\text { consumed. }\end{array}$ & Thousand barrels & $\begin{array}{l}\text { MBTCPZZ = (COCAPZZ / COCAPUS }) \text { MBTCPUS } \\
\text { MBTCPUS is independent. }\end{array}$ \\
\hline MGACB & $\begin{array}{l}\text { Motor gasoline consumed by the transportation } \\
\text { sector. }\end{array}$ & Billion Btu & $\begin{array}{l}\text { MGACBZZ = MGACPZZ * } 5.253 \\
\text { MGACEUS = DMGACBZZ }\end{array}$ \\
\hline MGACP & $\begin{array}{l}\text { Motor gasoline consumed by the transportation } \\
\text { sector. }\end{array}$ & Thousand barrels & $\begin{array}{l}\text { MGACPZZ }=\text { (MGTRPZZ } / \text { MGTTPZZ) } * \text { MGTCPZZ } \\
\text { MGACPUS }=\text { DMGACPZZ }\end{array}$ \\
\hline MGAGP & Motor gasoline sold for agricultural use. & Thousand gallons & $\begin{array}{l}\text { MGAGPZZ is independent. } \\
\text { MGAGPUS = IMGAGPZZ }\end{array}$ \\
\hline MGCCB & $\begin{array}{l}\text { Motor gasoline consumed by the commencial } \\
\text { sector. }\end{array}$ & Billtion Bku & $\begin{array}{l}\text { MGCCBZZ = MGCCPZZ } * 5.253 \\
\text { MGCCBUS }=\text { EMGCCBZZ }\end{array}$ \\
\hline MGCCP & $\begin{array}{l}\text { Motor gasoline consumed by the commercial } \\
\text { sector. }\end{array}$ & Thousand bartels & $\begin{array}{l}\text { MGCCPZZ = (MGCMPZZ / MGTTPZZ) * MGTCPZZ } \\
\text { MGCCPUS = EMGCCPZZ }\end{array}$ \\
\hline MGCMP & Motor gasoline sold to the commercial sector. & Thousand gallons & $\begin{array}{l}\text { MGCMPZZ = MGMSPZZ + MGPNPZZ } \\
\text { MGCMPUS = ZMGCMPZZ }\end{array}$ \\
\hline MGCUP & Motor gasoline sold for construction use. & Thousand gallons & $\begin{array}{l}\text { MGCUPZZ is independert. } \\
\text { MGCUPUS = IMGCUPZZ }\end{array}$ \\
\hline MGICB & $\begin{array}{l}\text { Mokor gasoling consumed by the industrial } \\
\text { sector. }\end{array}$ & Bullion Btu & $\begin{array}{l}\text { MGICBZZ }=\text { MGICPZZ } * 5.253 \\
\text { MGICBUS }=\text { 2MGICBZZ }\end{array}$ \\
\hline MGICP & $\begin{array}{l}\text { Motor gasoline cansumed by the industrial } \\
\text { eector. }\end{array}$ & Thousand barrels & $\begin{array}{l}\text { MGICPZZ = (MGINPZZ / MGTTPZZ) * MGTCPZZ } \\
\text { MGICPUS = DMGICPZZ }\end{array}$ \\
\hline MGNP & Motor gasoline sold to the industrial sector. & Thousand galkons & $\begin{array}{l}\text { MGINPZZ = MGAGPZZ + MGCUPZZ + MGYPZZ } \\
\text { MGINPUS = इMGINPZZ }\end{array}$ \\
\hline MGIYP & $\begin{array}{l}\text { Motor gasoline sold for tidustrial and comr- } \\
\text { mercial use (Federal Hightway Admiristration } \\
\text { terminology). }\end{array}$ & Thousand gailons & $\begin{array}{l}\text { MGIYPZZ is independent } \\
\text { MGIYPUS = ZMGIYPZZ }\end{array}$ \\
\hline
\end{tabular}


MGMFP Motor gasoline soid for highway use.

MGMRP Motor gasoline sold for marine use.

Motor gasoline soid for miscellaneous and unclassified user.

MGPNP

Motor gascline sold for publis nonlaighway use.

MGSFP

Motor gasoline special fueis sold (primarily diesel fuel with omall amounts of liquefied petroleum gases).

MGTCB Motor gasoline total consumed.

MGTCP Motor gasoline total consumied.

MGTRP

MGTTP

MSCB

MSICP

MSTCB

MSTCP

NAICB

by the industrial sector. by the industrial sector.

Miscellaneous petroleum products total consumed.

Miscellaneous petroleum prodicts total consumed.

Natural gasoline consumed by the industrial
Thousand gailons

Thousand gallons

Thousand gailons

Thousand gallons

Thousand gatlons

Btllion Btu

Thousand barrels

Thousand galtons

Thousand gallons

ginlion Bku

Miscellaneous petroleum products consumed sector.

Thous:and barrels

Bjllion Btu

Thousand barrels

Billion Błu
MGMFPZZ is independent. MGMFPUS = ₹MGMFPZZ

MGMRPZZ is independent. MGMRPUS = इMGMRPZZ

MGMSPZZ is independent. MGMSPUS = ZMGMSPZZ

MGPNPZZ is independent. MGPNPUS = УMGPNPZZ

MGSFPZZ is independent. MGSFPUS = IMGSFPZ

MGTCBZZ $=$ MGCCBZZZ + MGICBZZ + MGACBZZ MGTCBUS = \$MGTCBZZ

MGTCPZZ = (MGTTPZZ / MGTTPUS) * MGTCPUS MGTCPUS is independent.

MGTRPZZ = MGMFPZZ + MGMRPZZ - MGSFPZZ MGTRPUS = EMGTRPZZ

MGTTPZZ = MGCMPZZ + MGINPZZ + MGTRPZZ MGTTPUS = MGTTPZZ

MSICBZZ $=$ MSTCBZZ MSICBUS = MSTCBUS

MSICPZZ $=$ MSTCPZZ MSICPUS = MSTCPUS

MSTCBZZ = MSTCPZZ * 5.796 MSTCBUS $=$ ZMSTCBZZ

MSTCPZZ = (OCVAVZZ / OCVAVUS) * MSTCPUS MSTCPUS is independent.

NAJCBZZ $=$ NATCBZZ

NAICBUS $=$ NATCBUS 


\begin{tabular}{|c|c|c|c|}
\hline Varfable & Deseription & Unitls & Formulas \\
\hline NAICP & $\begin{array}{l}\text { Natural gaboline conseumed by the industrial } \\
\text { sector. }\end{array}$ & Thousand barrels & $\begin{array}{l}\text { NAICPZZ }=\text { NATCPZZZ } \\
\text { NAICPUS }=\text { NATCPUS }\end{array}$ \\
\hline NATCB & Natural gasoline tobal consumed. & Billion Btu & $\begin{array}{l}\text { NATCBZZ }=\text { NATCPZZ } * 4.620 \\
\text { NATCBUS }=\text { ENATCBZZ }\end{array}$ \\
\hline NATCP & Natural geasoline total consumed. & Thousand barrels & $\begin{array}{l}\text { NATCPZZ = (OCVAVZZ / OCVAVUS) * NATCPUS } \\
\text { NATCPUS is independent. }\end{array}$ \\
\hline NGACB & $\begin{array}{l}\text { Natural gas consumed by the transportation } \\
\text { sector. }\end{array}$ & Billion Btu & $\begin{array}{l}\text { NGACBZZ }=\text { NGACPZZ } * \text { NGNUTKZZ } \\
\text { NGACBUS }=\text { INGACBZZ }\end{array}$ \\
\hline NGACP & $\begin{array}{l}\text { Nahural gas consumed by the tratuportation } \\
\text { sector. }\end{array}$ & Million subic feet & $\begin{array}{l}\text { NGACPZZ }=\text { NGPZPZZ + NGVZPZZ } \\
\text { NGACPUS = ENGACPZZ }\end{array}$ \\
\hline NGCCB & $\begin{array}{l}\text { Natural gas detivered to the commercial sector, } \\
\text { used as consumption. }\end{array}$ & Billion Btu & $\begin{array}{l}\text { NGCCBZZ = NGCCPZZZ * NGNUKZZ } \\
\text { NGCCBUS = ENGCCBZZ }\end{array}$ \\
\hline NGCCP & $\begin{array}{l}\text { Natural gas delivered to the commencial sector, } \\
\text { used as consumption. }\end{array}$ & Million cubic feet & $\begin{array}{l}\text { NGCCPZZ is independent. } \\
\text { NGCCPUS = INGCCPZZ }\end{array}$ \\
\hline NGEUB & Natural gas consumed by the electric utilities. & Billion Btu & $\begin{array}{l}\text { NGEUBZZ = NGEUPZZ * NGEUKZZ } \\
\text { NGEUBUS = ENGEUBZZ }\end{array}$ \\
\hline NGEIJK & $\begin{array}{l}\text { Factor for convertini natural gats consumed by } \\
\text { the eflectric ufilities from physieal units to Btu. }\end{array}$ & Thoustand Bhu per cubic fook & $\begin{array}{l}\text { NGEUKZZ is independent. } \\
\text { NGEUKUS = NGEUBUS / NGEUPUS }\end{array}$ \\
\hline NGEUP & Natural gas consumed by the electric utilities. & Million cubic feet & $\begin{array}{l}\text { NGEUPZZ is independent. } \\
\text { NGEUPUS = ENGEUPZZ }\end{array}$ \\
\hline NGICB & Natural gas consumed by the industrial sector. & Billion Bkr & $\begin{array}{l}\text { NGICBZZ = NGICPZZ * NGNUKZZ } \\
\text { NGICBUS = ENGICBZZ }\end{array}$ \\
\hline NGICP & Natural gas consuned by the industrial sector. & Million cubic feet & $\begin{array}{l}\text { NGICPZZ = NGINPZZ + NGLEPZZ + NGPLPZZ } \\
\text { NGICPUS }=\text { ZNGICPZZ }\end{array}$ \\
\hline NGINP & $\begin{array}{l}\text { A portion of the natural gas delivered to the } \\
\text { industrial sector. }\end{array}$ & Mitlion cuble feet & $\begin{array}{l}\text { NGINPZZ ts independent. } \\
\text { NGINPUS = 2NGINPZZ }\end{array}$ \\
\hline NGLEP & Natural gas consurned as lease fuel. & Million cubic feet & $\begin{array}{l}\text { NGLEPZZ is independent. } \\
\text { NGLEPUS = ZNGILEPZZ }\end{array}$ \\
\hline 438 & \multicolumn{3}{|c|}{$\begin{array}{l}\text { Energy Information Admialstrallon } \\
\text { Stete Energy Dets Repport 1894 }\end{array}$} \\
\hline
\end{tabular}


Variable

NGNUK

Factor for converting natural gas consumed by all sectors other than the electric utility sector from phystical units to Btu.

NGPLP

Natural gas consumed as plant fuel.

Natural gas consumed as pipeline fuel.

Natusal gas delivered to the residential sector, used as consumption.

NGRCPP Natural gas delivered to the residential sector, used as consumption.

NGTCB Natural gas total consumed.

NGTCK Factor for converting natural gas total consurmed from physical usits to Btu.

NGTCP Natural gas total consumped.

NGVZP

Natural gas contrumed as vehicle fuel.

NUEOB

Bestricity produced from naclear power at electric utilities.

NUEOKUSS Factor for converting electricity produced from nuclear power from physical units to Btu.

NUEOP

Electrixity produced from nuclear power at electric utilities.

OCVAV

\section{Units}

Fomulas

Thousand Bta per cubic foot

Million cubic feet

Million cubic teet

Billion Btu

Million cubic feet

Bultion Btu

Thousand Btu per cubic foot

Milion cubic feet

Million cubic teet

Billion Btu

Thousand Btu per kilowatthour

Mullion kilowatthours

Million dollars

\section{NGNUKZZ = (NGTCBZZ - NGEUBZZ) / (NGTCPZZ - NGEUPZZ) NGNUKUS = (NGTCBUS - NGEUBUS) / (NGTCPUS - NGEUPUS)}

NGPLPZZ is independent. NGPLPLS = INGPLITZZ

NGPZPZZ is independent. NGPZPUS = INGPZPZZ

NGRCBZZ = NGRCPZZ * NGNUKZZ NGRCBLS = ENGRCBZZ

NGRCPZZ is independent. NGRCPUS $=\mathbf{N N G R C P Z Z Z}$

NGTCBZZ = NGTCPZZ * NGTCKZZ NGTCBUS = ENGTCBZZ

NGTCKZZ is independent. NGTCKUS = NGTCBUS / NGTCPUS

NGTCPZZ = NGRCPZZ + NGCCPZZ + NGICPZZ + NGACPZZ + NGEUPZZ NGTCPUS $=$ ENGTCPZZ

NGVZPZZ is independent. NGVZPUS = ENGVZPZZ

NUEOBZZ = NUEOPZZ * NUEOKUS NUEOBUS $=\mathbf{\Sigma}$ NUEOBZZ

NUEOKUS is independent.

NUEOPZZ is independent. NUEOPUS - DNUEOPZZ

OCVAVZZ is independent. OCVAVUS = sOCVAVZZ
Value added in manufacture of industrial organic chemicals. 


\begin{tabular}{|c|c|c|c|}
\hline Variable & Description & Units & Formulas \\
\hline PAACB & $\begin{array}{l}\text { All petroleum products consumed by the } \\
\text { transportation sector. }\end{array}$ & Bjtlion Btu & $\begin{aligned} \text { PAACBZZ = } & \text { AVACBZZ }+ \text { DFACBZZ + } \\
& \text { FKACBZZ + JNACBZZ }+ \text { LGACBZZ + } \\
& \text { LUACBZZ + MGACBZZ + RPACBZZ } \\
\text { PAACBUS = EPAACBZZ } & \text { EPAC }\end{aligned}$ \\
\hline PAACKUS & $\begin{array}{l}\text { Factor for converting all petroleum products } \\
\text { corsumed by the transportation sector } \\
\text { from physical units to Btr. }\end{array}$ & MIllion Btu per barref & PAACKUS = PAACBUS / PAACPUS \\
\hline PAACP & $\begin{array}{l}\text { All petroleum products consurmed by the } \\
\text { transportation sector. }\end{array}$ & Thausand barrets & $\begin{aligned} \text { PAACPZZ = } & \text { AVACPZZ + DFACPZZ + } \\
& \text { JKACPZZ + JNACPZZ + LGACPZZ + } \\
& \text { LUACPZZ + MGACPZZ + RFACPZZ } \\
\text { PAACPUS = } & \text { EPAACPZZ }\end{aligned}$ \\
\hline РАCCB & $\begin{array}{l}\text { All petroteum products consumed by the } \\
\text { commercial sector. }\end{array}$ & Billion Btu & $\begin{aligned} \text { PACCBZZ }= & \text { DFCCBZZ }+\mathrm{KSCCBZZ}+ \\
& \mathrm{LGCCBZZ}+\mathrm{MGCCBZZ}+\mathrm{RFCCBZZ} \\
\text { PACCBUS }= & \mathrm{YPACCBZZ}\end{aligned}$ \\
\hline PACCKUS & $\begin{array}{l}\text { Factor for converting all petroleum products } \\
\text { consumed by the commercial sector from } \\
\text { physical units to Btis. }\end{array}$ & Million Bta per barre] & PACCKUS = PACCBUS / PACCPUS \\
\hline PACCP & $\begin{array}{l}\text { All petroleum products consumed by the } \\
\text { commercial sector. }\end{array}$ & Thousand borrels & $\begin{array}{c}\text { PACCFZZ = DFCCPZZ + KSCCPZZ + } \\
\text { LGCCPZZ + MGCCPZZ + RFCCPZZ } \\
\text { PACCPUS = IPACCPZZ }\end{array}$ \\
\hline PAEUB & $\begin{array}{l}\text { All petroleum produrts consumed by the } \\
\text { electric utitities. }\end{array}$ & Bullion Btu & $\begin{array}{c}\text { PAEUBZZ = DFEUBZZ + \KEUBZZ + } \\
\text { PCEUEZZ + RFEUBZZ } \\
\text { PAEUBUS = IPAEUBZZ }\end{array}$ \\
\hline PAEUKUS & $\begin{array}{l}\text { Factor for converting all petroleum products } \\
\text { consumed by the electric utilities from } \\
\text { physical urits to Btu. }\end{array}$ & Mitlion Bfut per barrel & PAEUKUS = PAEUBUS / PAEUPUS \\
\hline PAEUP & $\begin{array}{l}\text { All petroleum producte consumed by the } \\
\text { electric utilities. }\end{array}$ & Thousand barrels & $\begin{array}{c}\text { PAEUPZZ - DFEUPZZ + JKEUPZZ + } \\
\text { PCEUPZZ + RFEUPZZ } \\
\text { PAEUPUS = EPAEUPZZ }\end{array}$ \\
\hline PAHCBUS & $\begin{array}{l}\text { All petroleum products consumed by the } \\
\text { residential and commercial sectors combined. }\end{array}$ & Billion Btu & PAHCBUS = PARCBUS + PACCBUS \\
\hline 440 & & $\begin{array}{l}\text { Mitormation Adninistradlon } \\
\text { Eneroy Data Report } 1994\end{array}$ & \\
\hline
\end{tabular}


PAHCKUS Factor for converting all petroleum products consumed by the residential and commercial sectors combined from physical untts to Bh.

PAHCPUS All petroleum products consumed by the residential and commercial sectors combined.

PAICB All petroleum products consumed by the indurtrial sector.

PAICKUS Factor for convering all petroleum products consumed by the industrial sector from physical units to Btu.

PAICP All petroleum products consumed by the industrital sector.

PARCB All petroleum products consumed by the residential sector.

PARCKUS Factor for converting all petroleum products consumed by the residential sector from physical units to Btu.

PARCP All petroleum products consumed by the residential sector.

PATCB Alt petroleum products consumed by all sectors.

PATCKUS Factor for converting alt petroleum products consumed by all sectors from physical units to Btu.

PATCP
All petroleum products consumed by all sectors.
Million Bhu per barrel

PAHCKUS $=$ PAHCBUS $/$ PAHCPUS

Thoušand berrels

Billion Btu

Million Btu per barrel

Thoussand batrels

Billion Btu

Million Btu per bortel

Thousend barrels

Billon Bta

Multion Btu per barrel

Thousand barrels
PAHCPUS = PARCPUS + PACCPUS
PAICBZZ = ARICBZZ + DFICBZZ + KSICBZZ + LGICBZZ + LUICBZZ + MGICBZZ + RFICBZZ + POICBZZ

PAICBUS = PAICBZZ

PAICKUS = PAICBUS / PAICPUS
PAICPZZ = ARICPZZ + DFICPZZ + KSICPZZ + LGICPZZ + LUICPZZ + MGICPZZ + REICPZZ + POICPZZ

\section{PAICPUS $=$ \&PAICPZZ}

PARCBZZ = DFRCBZZ + KSRCBZZ + LGRCBZZ PARCBUS = EPARCBZZ

PARCKUS $=$ PARCBUS $/$ PARCPUS

PARCPZZ = DERCPZZ + KSRCPZZ + LGRCPZZ PARCPUS = EPARCPZZ

PATCBZZ = ARTCBZZ + AYTCBZZ + DFTCEZZ + JKTCBZZ + INTCBZZ + KSTCBZZ + LGTCBZZ + LUTCBZZ + MGTCBZZ + RFTCBZZ + POTCBZZ

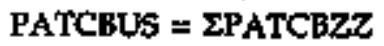

PATCKUS $=$ PATCBUS $/$ PATCPUS

PATCPZZ $=$ ARTCPZZ + AVTCPZZ + DFTCPZZ + JKTCPZZ + JNTCPZZ + 


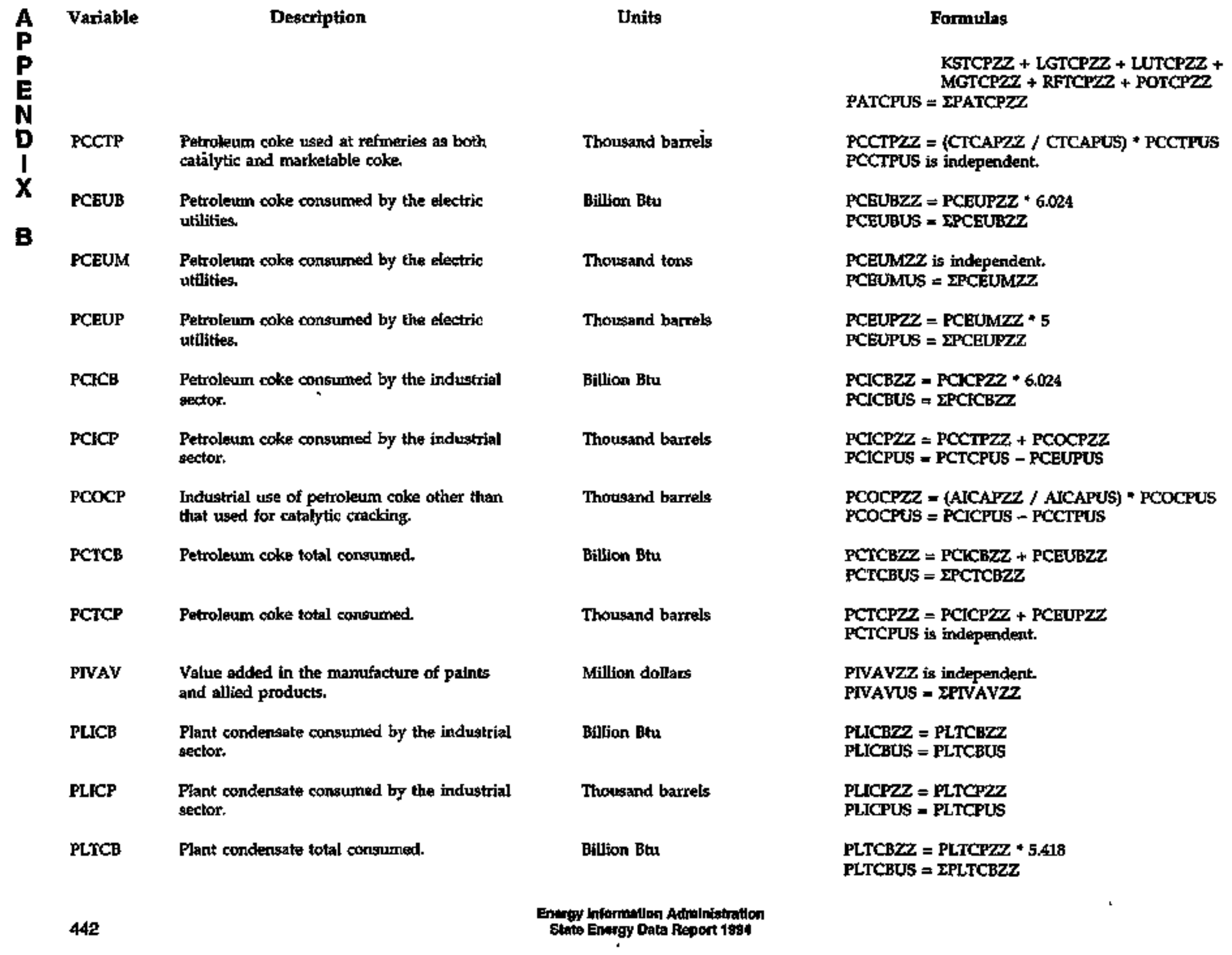


Variable

PLTCP Plant condenssate total consumed.

POICB Other pelroleam products consumed by the industrial sector.

Units

Thousand barrels

Billion Btu

Other petroleum products consumed by the industrial sector.

POrcB

Other petroleum products total consumed.

Billion Btu

Thousand barrels

POTCP Other petroleum products total consumed.

PPICB

Pentanes plus consumed by the industrial sector.

Billion Btu

PPICP

Pentanes plus consumed by the industrial sector.

PPTCB
Pentanes pius total consumed.
Thousand barrets

Billion Btu

\section{Formulas}

PLTCPZZ = (OCVAVZZ / OCVAVUS) * PLTCPUS PLTCPUS is independent.

POICBZZ = ABICBZZ + COICBZZ +

FNICBZZ + FOICBZZ + FSICBZZ + MBICBZZ + MSICBZZ + NAICBZZ + PCICBZZ + PLICBZZ + PPICBZZ + SGICBZZ + SNICBZZ + UOICBZZ + USICBZZ + WXICRZZ

POICBUS $=$ EPOICBZZ

$\mathbf{A}$
$\mathbf{P}$
$\mathbf{P}$
$\mathbf{E}$
$\mathbf{N}$
$\mathbf{D}$
$\mathbf{J}$
$\mathbf{X}$
$\mathbf{B}$

POICPZZ = ABICPZZ + COICPZZ +

FNICPZZ + FOICPZZ + FSTCPZZ + MBICPZZ + MSICPZZ + NAICPZZ + PCICPZZ + PLICPZZ + PPICPZZ + SGICPZZ + SNICPZZ + UOICPZZ + USICPZZ + WXICPZZ

POICPUS $=$ 乏POICPZZ

POTCBZZ = ABTCBZZ + COTCBZZ + FNTCBZZ + FOTCBZZ + FSTCBZZ + MBTCBZZ + MSTCBZZ + NATCBZZZ + PCTCBZZ + PLTCBZZ + PPTCBZZ + SGTCBZZ + SNTCBZZ + UOTCBZZ + USTCBZZ $\div$ WXTCBZZ

POTCBUS = ЕPOTCBZZ

POTCPZZ = ABTCPZZ + COTCPZZ + FNTCPZZ + FOTCPZZ + FSTCPZZ + MBTCPZZ + MSTCPZZ + NATCPZZ + PCTCPZZ + PLTCPZZ + PPTCPZZ + SCTCPZZ + SNTCPZZ + UOTCPZZ + USTCPZZ + WXTCPZZ POTCPUS $=$ \&POTCPZZ

PPICBZZ = PPTCBZZ PPICBUS = PFTCBUS

PPICPZZ = PPTCPZZ PPICPUS = PPTCPUS

PPTCBZZ $=$ PPTCPZZ * 4.620

PPTCBUS = IPPTCBZZ 


$\begin{array}{lll}\text { A } & \text { Variable } & \text { Description } \\ \text { P } & \text { PPTCP } & \text { Pentanes plus total consumed. } \\ \text { E } & & \\ \text { D } & \text { RDICP } & \text { Road oil consumed by the industrial sector. } \\ \text { D } & & \\ \mathbf{X} & \text { RDINP } & \text { Road oil sold to the industrial sector. } \\ \text { B } & \text { RDTCP } & \text { Road oil total consumed. }\end{array}$

REACB Renewable exergy sources consumed by the transportation sector.

REACP Renewable energy sources consumed by the transportation sector.

REEOB Renewable energy sources consurned by the electric utilities.

REEOP

Renewable energy sources consumed by the electric utalties.

REHCB Renewable energy sources consurned by the residential and conmmercial sectors.

REICB

Renewable energy sources consumed by the industrial sector.

RETCB

Renewable mergy sources total consumed.

RFACB

Residual fuel consumed by the transportation sector.

\section{Units}

Thousand barrels

Thousand barrels

Short tons

Thousand bairels

Billion Btu

Thousiand gallons

Billion Bku

Million kilowatthours

Billition Btu

Biflion Btu

Billion Btu

Billion Btu

Energy Information Adtanlistration State Energy Dqta Roport 1994
PPTCPZZ = (OCVAVZZ / OCVAVUS) * PPTCPUS PPTCPUS to independent.

RDICPZZ = (RDNPZZ / RDINPUS) * RDTCPUS RDICPUS = $\mathbf{R} D I C P Z Z$

RDINPZZ is independert. RDINIPUS $=$ ERDINPZZ

RDTCPZZ = RDICPZZ RDTCPUS is independent.

REACBZZ = WWACBZZ

REACBUS $=$ WWACBUS

\section{REACPZZ - WWACPZZ \\ REACPUS $=$ WWACPUS}

REEOBZZ = HVENBZZ + GEENBZZ + WWEOBZZ + WNEOBZZ

REEOBUS = HVENBUS + GEENBUS + WWEOEUS + WNEOEUS

REEOPZZ = HVENEZZ + GEENPZZ + WWBOPZZ + WNEOPZZ

REROPUS = HVENPUS + GEENPUS +

WWEOPLS + WNEOPUS

REHCBZZ = WWHCBZZ + SOHCBZZ REHCBUS = WWHCBUS + SOHCBUS

REICBZZ = HMCBZZ + WWICBZZ + GOICBZZ REICBUS a HYICBUS + WWICBUS + GOICBUS

RETCBZZ = REHCBZZ + REICBZZ + REACBZZ + REEOBZZ

RETCBUS $=$ REHCBUUS + REICBUS + REACBUS + REEOBUS

RFACBZZ $=$ RFACPZZ * 6.287

RFACBUS $=$ ERFACBZZ 
RFACP Residual fuel consumed by the transportation sector.

RFBKP Residual fuel sold for vessel bunkering use, excluding dellverles to the Armed Forces.

RPCCB Residual fuet consumed by the commertial sector.

RFCCP

Residual fuel consumed by the commercial sector.

RFCMP

Residual fuel sold to the commercial sector.

RFEUB

Residual fuel consumed by the electric utilities.

RFEUP

Residual fuel consumed by the electric utilities.

RFIBP

A portion of residual fuel sold for industrial use, including industrial space heating.

RFICB

RFICP

RFINP

RFMFP

RFMSP Restdual fuet sold for miscellaneous uses,

RFNCP
Residual fuel sold to the Armed Forces, regardless of use.

Residual fitel consumed by the Endustrial sector.

Residual fuel consumed by the industrial sector.

Residual huel sold to the industrial sector.

Restidual fuel consuruption by all sectors other than the electric utility sector.
Ballton Bhu

Thousand barrels

Thousand barrels

Bitlion Btu

Thousand barrels

Thousand barrels

Thousend barrels

Thuousand barrels

Billion Bhr

Thousand barrels

Thoussand barrels

Thousand barrels

Thousand barrels

Thoussand barrels

nex In formition A tointetrotion State Energy Dota Ruport 1004
RFACPZZ $=$ (RFIRPZZ / RFNDPZZ $)$ * RFNCPZZ RFACPUS $=$ IRFACPZZ

RFBKPZZ is independent. RFBKPUS = IRFBKPZZ

RFCCBZZ $=$ RFCCPZZ * 6.2B7

RFCCBUS $=$ IRFCCBZZ

RFCCPZZ = (RFCMPZZ / RFNDPZZ) * RFNCPZZ RECCPUS $=\mathbf{\Sigma K F C C P Z Z ~}$

RFCMPZZ is independent.

RFCMPUS' $=$ XRFCMPZZ

\section{RFEUA7Z = RFEUTPZ * 6.287 \\ RFEUBAUS $=\Sigma$ ERFEUBZZ}

RFEUPZZ is independent. RFHUFUS = ERPEUPZZ

RFIBPZZ is independent. RFIBPUS $=$ IRFIBPZZ

RFICBZZZ = RFICPZZ * 6.287 RFICBUS - YRFICBZZ

RFICPZZ - (RFINPZZ / RFNDPZZ) * RFNCPZZ RFICPUS = IRPICPZZ

RFINPZZ = RFHPZZ + RPOCPZZ + RFMSPZZ RFINPUS $=$ IREINPZZ

RFMIPZZ is independent. RFMIIPUS = IRFMIIZZZ

RFMSPZZ is independent. RFMSPUS = DRFMSPZZZ

RFNCPZZ = (RFNDPZZ / RFNDPUS $)$ * RFNCPUS RFNCPUS = RFTCPUS - RFEUPUS 

A Variable
Description
P
P RFNDP
Residual fuel sold to all sectors other than the electric ubility sector.
RFOCP
D.
$\mathbf{1}$
RFRRP Residual fuel sold for use by railroads.
B RFTCB Residual fuel total consumed.

RFTCP Residual fael total consumed.

RFTRP

Residual fued sold to the transportation sector.

SGICB

Still gas consumed by the industrial sector.

SGICP

Still gas consamed by the industrial sector.

SGTCB Stil gas total concumed.

SGICP Still gas total consumed.

SNICB

Special naphthas consumed by the industrial sector.

SNICP

Special naphthas consumed by the industrial sector.

SNTCB

Special naphthas tocal consumed.

SNTCP

Special naphthas total consumed.
Units

Thousand barrels

Thousend berrels

Thousand barrels

Bfllion Btu

Thousand barrels

Thousand barrets

Billion Btu

Thousand bartels

Bitlion Btu

Thousand barnels

Biltion Btu

Thousand barrels

Billion Btu

Thousand barrels
Fotmulas

RFNDPZZ = RFCMPZZ + RFINPZZ + RFTRPZZ RFNDPUS $=$ ₹RFNDPZZ

RFOCPZZ is independent. RFOCPUS = ERFOCPZZ

RFRRPZZ is independent. RFRRPUS = ERFRRPZZ

RFTCBZZ = RFCCBZZ + RFICBZZ + RFACBZZ + RFEUBZZ RFTCBUS $=$ ERFTCBZZ

RPTCPZZ = RFNCPZZ + RFEUPZZ RFTCPUS is independent. RFTRPZZ = RFBKPZZ + RFMIPZZ + RFRRPZZ
RFTRPUS = ERFTRPZZ SGICBZZ $=$ SGTCBZZ
SGICBUS $=$ SGTCBUS

SGICPZZ $=$ SGTCPZZ SGICPUS = SGTCPUS

SGTCBZZ $=$ SGTCPZZ *6.000 SGTCBUS = ESGTCBZZ

SGTCPZZ = (COCAPZZ / COCAPUS ) SGTCPUS SGTCPUS is independent.

SN3CBZZ $=$ SNTCBZZ SNICBUS - SNTCBUS

SNICPZZ $=$ SNTCPZZ SNICPUS = SNTCPUS

SNTCBZZ $=$ SNTCFZZ $* 5.248$ SNTCBUS $=$ ESNTCBZZ

SNTCPZZ $=$ (PIVAVZZ / PIVAVUS $) *$ SNTCPUS SNTCPUS is independent. 
SOEOP

SOHCB

SOHCP

SORCB

Electricity produced from photovoltalc and solar thermal energy by electric utilities.

Electricty produced from photovoltaic and solar thermal energy by electric utilities.

Solar thermal energy consumed by the residential and commercial sectors.

Solar thermal energy sonsumed by the residentital and commercial sectors.

Electrictly produced from photovoltaic and solar thermal entergy sources in the industrial sector.

SOTCB Photowoltaic and solar thermal energy sources total consumed.

SOTCP Photovoltaic and solar thermal energy sources tokal corsumed (available for 1960-1989 orly).

TEACB Total energy consumed by the transportation sectror.

TEAPB

The transportation sector's energy consumption per capita.

TECCB Total energy consumad by the commercial sector.

TECPB

The commercial sector's energy consumption perr capita.

TEEUB Total energy consumed by the electric utilities plus net imports of electrictly into the United States.
Billion Btu

Million kilowatthours

\section{Formulas}

SOEOBZZ $=$ SOEOPZZ * FFEOKUS SOEOBUS $\#$ DSOEOBZZ

SOEOPZZ is independent. SOEOPUS = 2SOEOPZZZ

Billion Btu

SOHCBZZ is independent. SOHCBUS = ZSOHCBZZ

Million kilowathours

Billian Btu

SOHCPZZ = SOHCBZZ / 3,412

SOHCPUS = ISOHCPZZ

SOICBZZ is independent. SOICBUS = ISOICBZZ

Biflion Btu

Mttlion kilowatthours

Billion Bhu

Million Btu

Billion Btu

Million Błı

Billion Btt

.

Energy Intomation Adminletration State Energy Daka Pepont 1894
SOTCBZZ = SOHCBZZ + SOICBZZ + SOEOBZZ SOTCBUS = ESOTCBZZ

SOTCPZZ = SOHCPZZ + SOICPZZ + SOEOPZZ SOTCPUS $=$ इSOTCPZZ

TEACBZZ = CLACBZZ + NGACBZZ + PAACBZZ + ESACBZZ + LOACBZZ

TEACBUS = CLACBUS + NGACBUS + PAACBUS + ESACBC'S + LOACBUS

TEAPBZZ = TEACHZZ / TPOPPZZ TEAPBUS = TEACBUS / TPOPPUS

TECCBZZ $*$ CLCCBZZ + NGCCBZZ + PACCBZZ + ESCCBZZ + LOCCBZZ TECCBUS $=$ CLCCEOUS + NGCCBUS + PACCBUS + ESCCBUS + LOCCBUS

TECPBZZ $=$ TECCBZZ $/$ TPOPPZZ TECPBUS $=$ TECCBUS $/$ TPOPPUS

TEEUBZZ = CLEUBZZ + NGELBZZ + PAEUBZZ + HYENBZZ + NUEOBZZ + GEENBZZ + WWEOBZZ + WNBOBZZ + EXNlBZZ

TEEUBUS = CLEUIBUS + NGEURUS + PAEUBUS + 

per capita.

TERCB

Total energy consumed by the residential sector.

Million Btu

Billion Btu

Million Btu per capita.

TETCB Total energy consumed by all sectors.

TETPB

TNACB

Total net energy consumed by the transportation sector excluding the sector's share of electrical system energy losges.

TNCCB

Total net energy consumed by the commercial sector excluding the sector's share of electrical system energy losses.

TNICB

Total net energy consumed by the indurtrial sector excluding the sector's share of electrical systern energy losses.

Billion Btu

Mijlion Bar

Bitlion Btu

Billion Bth

Bitlion Btu

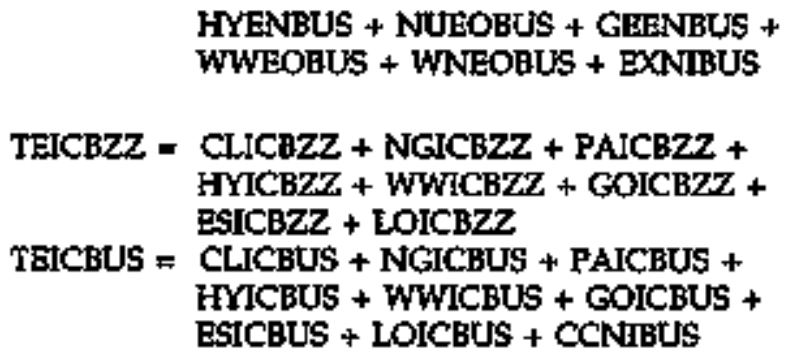

HYENBUS + NUEOBUS + GEENBUS + WWEOEUS + WNEOBUS + EXNIBUS

TEICBZZ $=$ CLICAZZ + NGICBZZ + PAICBZZ +
HYICBZZ + WWICBZZ + GOICBZZ +

TEICBZZ - CLICAZZ + NGICBZZ + PAICBZZ +
HYICBZZ + WWICBZZ + GOICBZZ + ESICBZZ + EOICBZZ

TEICBUS = CLICBUS + NGICBUS + PAICBUS + HYICBUS + WWICBUS + GOICBUS + ESICBUS + LOICBUS + CCNIBUS

TEIPBZZ = TEICBZZ / TPOPPZZ TEIPBUS = TEICBUS / TPOPPUS

TERCBZZ = CLRCBZZ + NGRCBZZ +

PARCBZZ + WWHCBZZ + SOHCBZZ + BSRCBZZ + LORCBZZ

TERCBUS = CLRCBUS + NGRCBUS +

PARCBUS + WWHCBUS + SOHCBUS + ESRCBUS + LORCBUS

TERPBZZ $=$ TERCBZZ $/$ TPOPPZZ TERPBUS $=$ TERCBUS $/$ TPOPPUS

TNCCBUS = TECCBUS - LOCCBUS

TNICBZZ = TEICBZZ - LOICBZZ TNICBUS $=$ TEICBUS - LOICBUS 
TNRCB

Total net energy consumed by the residential sector exccluding the sector's share of

Billion Btu electrical system energy losses.

TPOPP

The resident population including the Armed Forces residing in each State.

UOHCB

Uinfinished oils consumed by the industrial stector.

UOICF

Unfinished oils consumed by the industrial sector.

บOTCB

Unfirished oils total consumed.

UOTCP

Unfinisted oils total constmed.

USICB

Unfractionated stream constumed by the industrial sector.

USICP

Unfractionated stream consumed by the irdustrial sector.

USTCB

Unfractionated stream total consumed.

USTCP

Unfractionated stream total consumed.

WDCCBUS Wood energy consumed by the commercial sector.

WDCCPUS Wood emergy consumed by the commercial sector.

WDEOB

Electricity produced from wood energy sources at electric utilities.

WDEOP
Electricity produced from wood energy sources at electrlc utsitites.
Thousand

Billion Btu

Thousand barrets

Biltion Btu

Thousand barrels

Bitlion Btu

Thousand batrels

Bitlion Bto

Thousand barrels

Billion Btu

Thoussand cords

Billton Btu

Million kilowatthours

Endroy Mtormation Afint'alstration Stato Enesgy Dati Report 199 ;
TNRCBZZ - TERCBZZ - LORCBZZ

TNRCBUS $=$ TFRCBUS - LORCBUS

TPOPPZZ is independent. TPOPPUS is independent.

UoICBZZ = UOTCBZZ

LOICBUS = UOTCBUS

UOICPZZ = UDTCPZZ

UOICPUS = UOTCPUS

UOTCBZZ = UOTCPZZ * 5.825

UOTCBUS = EUOTCEZZ

UOTCPZZ = (COCAPZZ / COCAPUS * UOTCPUS UOTCFUS is independent.

USICBZZ = USTCBZZ

USICBUS $=$ USTCBUS

USICPZZ = USTCPZZ

USICPUS = USTCPUS

USTCBZZ = USTCPZZ * 5.418

USTCBUS $=\Sigma$ IUSTCBZZ

USTCPZZ = (OCVAVZZ / OCVAVUS) * USTCPUS

USTCPUS is andependent.

WDCCBUS is independent.

WDCCPUS a WDCCBUS / 20

WDEOBZZ = WDEOPZZ * FPEOKUS

WDEOBUS $=$ SWDEOBZZ

WDEORZZ is independent. WDEOPUS = EWDEOPZZ 


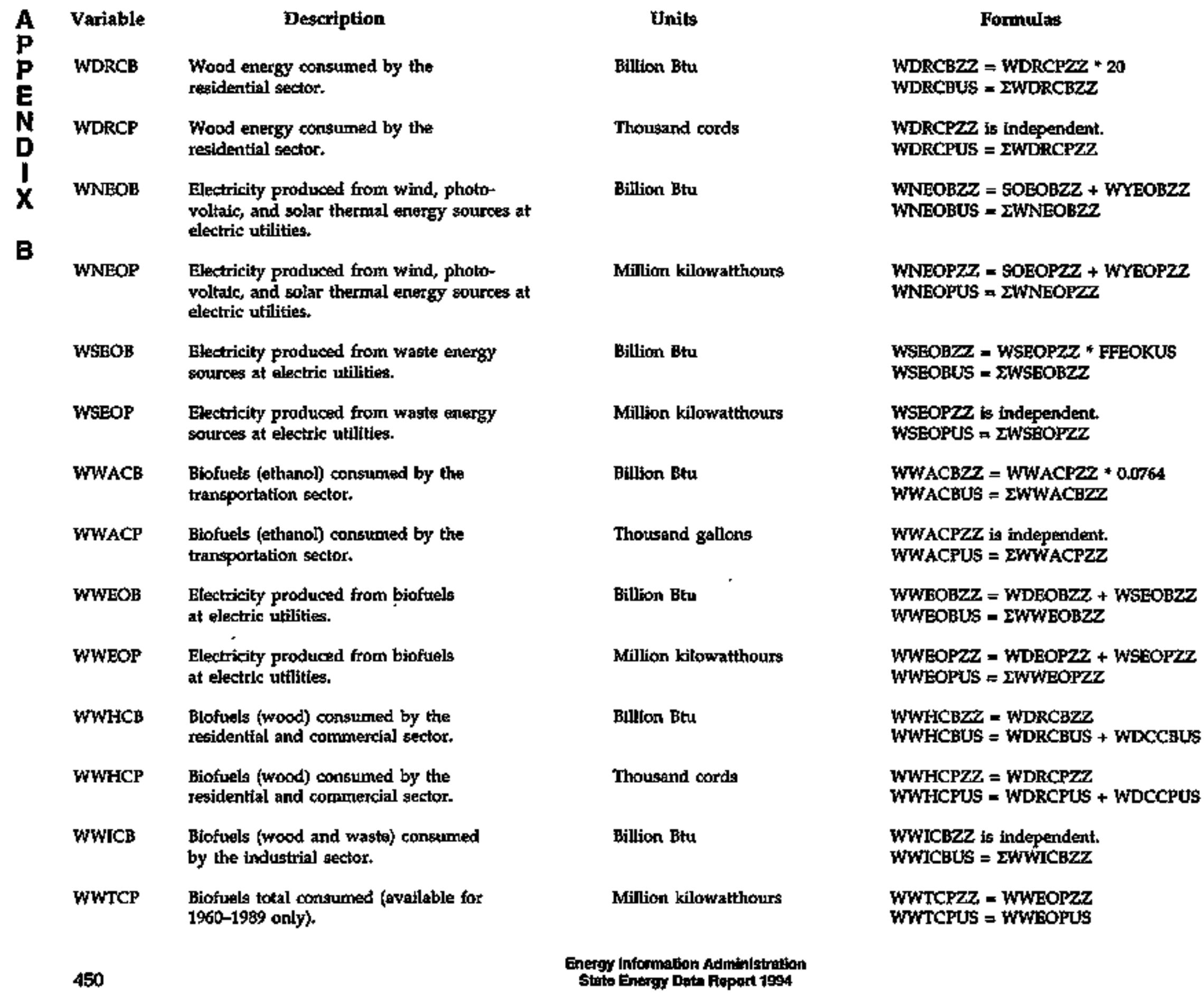


WXICB

Waxes consumed by the industrial sector.

WXCP

Waxes consumed by the industrial sector.

WXTCB

Waxes total consumed.

WXTCP

Waxes total consumed.

WYEOB

Electricty produced from wind energy at electric utilities.

WYEOP Electricity produced from wind energy at electric atilities.

WICB Electricity produced from wind energy by the industrial sector.

WYTCB

WYTCP
Electricity produced from wind energy total produced.

Electricity produced from wind energy total pxoduced (available for 1960-1989 only).
Billion Btu

\section{Formulas}

WWTCEZZ $=$ WWHCBZZ + WWICBZZ + WWACBZZ + WWEOBZZ

WWTCBUS $=$ WWHCBUS + WWICBUS + WWACBUS + WWLOBUS

Billion Bhy

Thousand barreis

Billion Btu

Thousand barrels

Billion Btu

Million kilowatthours

Billion Btu

Billion Btu

Million kllowatthours 

Appendix C

Sources of Independent Variables in the State

Energy Data System 



\section{Sources of Independent Variables In the State Energy Data System}

\section{Aluminum Ingot Production Capactiy}

AICAPZZ - Aluminum ingot production capacity in each State.

- 1960 bluough 1973: American Bureau of Metal Statistics, Year Baok.

- 1974 forward: American Bureau of Metal Statistics, Non-Ferrous Metal Data, table titled "Aluminum Ingot Production Capacity."

Note: Capacities for individual plants owned by one company have been withheld since 1986. The company's total capacity has been apportioned to the individual plants on the basis of their proportional capacities in 1985.

\section{Anthracile}

ACEUKUS - Factor for converting anthracite consumed by the electric utilities from physical units to Bhu.

- 1960 through 1972: Fnergy Information Administration (EIA) assumed that all anthracite consumed at electric utilities was recovered from culm banks and river dredging and was estimated to have an average heat content of 17.500 million Bto per short ton.

- 1973 forward: Calculated annually by EIA by dividing the heat content of anthracite receipts at electric utilities by the quantity of anthracite received at electric utilities. These data are reported on the Federal Energy Regulatory Commission (FERC) Form 423, "Monthly Report of Cost and Quality of Fuels for Electric Plants," and predecessor forms.
ACEUPZZ - Anthracite consumed by the electric utitities by State.

- EIA, Form EIA-759, "Monthly Power Plant Report," and predecessor forms.

ACHCPUS - Anthracite consumed by the residential and commercial sectors in the United States.

- 1960 through 1972: U.S. Department of the Interior, Bureali of Mines, Minterals Yeasbook, "Coal-Pennsylvania Anthracibe Annual,

- 1973 through 1984: EIA, Weekly Coat Production, August 9, 1986, Table 9 .

- 1985 through 1987: EIA, Weekly Coal Praduction, July 16, 1988, Table 8.

- 1988 forward: EIA, Unpublished data from Form ELA-6.

ACHDPZZ - Anthracite distributed to the residential and com mexcial sectors.

- 1960 through 1979: No data available. The 1980 State data are used for years 1960 through 1979.

- 1980 forward: Consumption estimates are used for this distribution series. Consumption of all types of coal by State is published in EIA, Quarterly Coal Report, October-December for each year. Data are from the report of the following year, t.e. 1982 final date are published in the Quarterty Coal Report, October-December 1983. The specific tables are:

- 1980. Unpublished data.

- 1981 through 1983: Table 27.

- 1984 throutgh 1990: Table 29.

- 1991 forward: Table 51. 
Withheld State values for consumption of all types of coal are estimated by using distributton data. When U.S. residenttal and commercial coal distribution does not equal U.S. residential and commercial coal consumption, the State distribution values are adjusted proportionaily until the sum of State distritbution values equals the U.S. consumption value published in the Quarterly Coal Report. The distribution data are published in:

- 1980 through 1984: EIA, Coal Distribution, January-December 1984, Table 21.

- 1985 through 1989: EiA, Coal Distribution, January-December 1989, Table 15.

- 1990 and 1991: EIA, Coal Distribution, January-December for each year, Table 16 .

- 1992 forwatd: EIA, Quarterly Coal Report, October-December for the following year, Table to.

Anthracite consumption is estimated by using distribution data published in EIA, Caal Distribution, January-December for each year. The speclfic tables are:

("District 24" represents all anthracite)

- 1980 through 1983: Tables 8 and 9.

- 1984: Tables 6 and 8.

- 1985 through 1989: Tables 6 and 3.

("Origin: Pennsylvania, Anthracite" represents all anthracite.)

- 1990 and 1991: Table 33.

- 1992 forward: Unpublished data from Form EIA-6.

State distribution data are increased or decreased proportion* ally until the sum of the States' distribution values equais the U.S. consumption (ACHCPUS).

ACKCPUS - Anthracite carbonized by coke plants in the United States.

- 1960 through 1972: U.S. Department of the Interior, Bureau of Mines, Minerals Yearbook, "Coal-Perunsylvania Anthracite Annual."

- 1973 through 1984: ELA, Weakly Coal Production, August 9, 1986, Table 9.

- 1985 through 1987; EIA, Weekly Coal Production, July 16, 1988, Table 8.

- 2988 forward: EIA, Unpublished data from Form EIA-5.
ACKDPZZ - Anthracite distributed to coke plants by State.

- 1960 through 1979: No data available. The 1980 State data are used for years 1960 through 1979.

- 1980 forward: Consumption estimates are used for this distribution series. Consumption of all types of coal by State is published in EIA, Quratherly Coal Report, October-Dectnber for each year. Data are from the report of the following year $t_{t}$ i.e, 1982 final data are published in the Quarterly Coal Report, October-Detember 1983. The specific tables are:

- 1980: Unpublished data.

- 1981 through 1983: Table 25.

- 1984, 1985, and 1987; Table 27.

- 1986, 1988, and 1989: Unpublished State revisions that are components of the U.S. revisions published in the Quarterly Coal Report, October-December 1991, Table 45.

- 1990: Table 27.

- 1991 forward: Table 48.

Withheld State values for consumption of all types of coal are estimated by using distribution data. After withheld residential and commercial coal consumption values have been estimated, withheld coke plant consumption is the difference between the sum of the published and estimated end-use sectors' consumption and the published State total consumption. Anthractte consumption is estimated by using distribution data published in EIA, Coal Distribution, January-December for each year. The specific tables are:

("District 24" represents all anthracite.)

- 1980 through 1983: Tables 8 and 9.

- 1984: Tables 6 and 8.

- 1985 through 1989: Tables 6 and 33.

("Origin: Pennsylvania, Anthracite" represents all anthracite.)

- 1990 and 1991: Table 33.

- 1992 forward: Unpublished data from Form BIA-6.

State distribution data are increased or decreased proportionally until the sum of the States' distribution values equals the U.S. consumption (ACKCPUS).

ACNUKUS - Factor for converting anthracite consumed by all sectors other than the electric utility sector from physical units to Btu.

- Calculated annually by EIA by dividing the heat content of anthracite produced less the heat content of the anthracite con- 
sumed at electric utilities, net exports, and shipments to U.S. Atmed Forces overseas by the quantity of anthracte consumption by all sectors other than the electric utility sector less the quantity of anthracite stock changes, losses, and "unaccounted for."

ACOCPUS - Anthracite consumed by industrial users other than coke plants in the United States.

- 1960 through 2972; U.S. Department of the Intertor, Burean of Mines, Minerals Yearbook, "Coal-Pennsylvania Anthracite, Annual."

- 1973 through 1984: EIA, Weekly Coat Production, August 9, 1986, Table 9.

- 1985 through 1987: BlA, Weekly Coal Production, July 16, 1988, Table 8.

- 1988 forward: EIA, Unpublished data from Forms EIA-3 and ELA-6.

ACODPZZ - - Anthracite distributed to industrial plants (other than coke plants) by State.

- 1960 through 1979: No data available. The 1980 State data are used for years 1960 through 1979.

- 1980 forward: Consumption estimates are used for this distribution series. Consumption of all types of coal by State is published in ELA, Qutarterly Coal Repori, October-December for each year. Data are from the report of the following year, i.e., 1982 final data are published in the Quarterly Conl Report, OctoberDecember 1983. The specific tables are:

- 1980; Unpublished data.

- 1961 through 1983: Table 26.

- 1984 through 1990: Table 28.

- 1991 forward: Table 49.

Witheld State values for consumption of all types of coal are estimated by using distribution data. After withheld residential and commercial coal consumption values have been estimated, withheld consumption by other industrial users is the difference between the sum of the published and estimated end-use sectors' consumption and the published State total consumption.
Anthracite consumption is estimated by using distribution data published in EIA, Coal Distribution, January-December for each year. The specific tables are:

("District 24" represents all anthracite.)

- 1980 through 1983: Tables 8 and 9.

- 1984: Tables 6 and 8 .

- 1985 through 1989: Tables 6 and 33.

("Origin: Pennsylvania, Anthracite" represents all anthracite.)

- 1990 and 1991: Table 33.

- 1992 forward: Unpublished data from Form ELA-6.

State distribution data are increased or decreased proportionally until the sum of the States' distribution values equals total U.S. consumption (ACOCPUS).

\section{Asphait}

ASINPZZ - Asphalt sold to the industrial sector by State.

- 1960 through 1977: U.S. Department of the Interior, Bureau of Mines, Mineral Industry Suroeys, "Sales of Asphalt," the spetific tables ane:

- 1960 through 1962: Table 6.

-1963 through 1977: Table 5 .

- 1978 through 1980: EIA, Energy Dota Reports, "Sales of Asphalt," Table 2.

- $198 \mathrm{~s}$ through 1986: The Asphalt Institute, Asphalt U6age 1987 United States and Cantada, Table B.

- 1987 and 1988: The Asphalt Institute, Aspinalt Usage 1988 Untited States and Conada, Tables A and B for State data. Assphalt Usage 1989 United States and Canada, page 2 for revised U.S. totals. The Asphalt Institute did not publish corresponding revised state data but did advise EIA on an estimation procedure to adjust 19 State values to sum to the revised U.S. totals.

- 1989 forward: The Asphalt Institute, Asphalt Usage United States and Canada, table titled "U.S. Asphalt Usage."

ASTCPUS - Asphalt total consumed in the United States.

- 1960 through 1975: U.S. Department of the Interior, Bureau of Mines, Mineral Industry Surveys, "Petroleum Statement, Annual," Table 1. 
- 1976 through 1980: EIA, Energy Dato Reports, "Petroleum Statement, Annual," Table 1.

- 1981 forward: EIA, Pefroleum Supply Annual, Table 2 (Beginning in 1983, this variable includes road oil)

\section{Avlation Gasoline}

AVMIPZZ - Aviation fuel issued to the military in the United States by State.

- 1960 through 1974: No data are available. The 1977 data are used for each year.

- 1975 and 1976: No consistent data series are available. The 1977 data are used for both years.

- 1977 through 1988: U.S. Department of Defense, Defense Logistics Agency, Defense Fuel Supply Center, Defense Energy Information System, military retail issues based on fiscal year data. The District of Columbia issues are assumed to be zero; therefore, values reported for the District of Columbla are added to Maryland.

- 1989 and 1990; U.S. Department of Deferse, Defense Logistics Agency, Defense Fuel Supply Center. State data for the fiscal year from two databases are summed: Defense Fuel Automated Management System (military wholesale issues) and Into-Plane Database (military purchases from commercial airports). Into-plane values reported for the District of Columbia are added to Virginia.

- 1991 forward: U.S. Department of Defense, Defense Logistics Agency, Defense Fuel Supply Center. State data for the calendar year from two databases are summed: Defense. Fuel Automated Management System (milltary wholesale issues) and Into-Plane Database (military purchases from commercial airports). Into-plane values reporbed for the District of Columbia are added to Virginla.

AVNMMZZ - Aviation gasoline sold to nonmilitary users by State.

- 1960 through 1964: U.S. Department of Commerce, Butreau of Public Roads, Highroty Statigtics, Table G-24.

- 1965 forward: U.S. Department of Transportation, Federal Highway Administration, Hightway Statistics, Table G-24 in 1965 and MF-24 in 1966 forwayd.
AVTCPUS - Aviation gasoline total consumed in the United States.

- 1960 through 1975: U.S. Department of the Interior, Bureau of Mines, Minteral Industry Suroeys, "Petroleum Statement, Annual," Table 1.

- 1976 through 1980: EIA, Energy Data Reports, "Petroleum Statement, Annual," Table 1.

- 1981 forward: ELA, Pefroleum Supply Annual, Table 2

\section{Aviation Gasoline Blending Components}

ABTCPUS - Aviation gasoline blending components total consumed in the United States.

- 1960 through 1980: No data available. Values are assumed to be zero.

- 1981 forward: EtA, Petroleunt Supply Annual, Table 2.

\section{Slofueis}

WDCCBUS - Wood constumed by the commercial sector in the United States.

- 1960 through 1992: No data available. Values are assumed to be zero.

- 1993 and 1994: ELA, Renearable Energy Annual 1995, Table 6.

WDEOPZZ - Electricity produced from wood energy sources at electric utilities by State.

- 1960 through 1981: Data included in waste energy sources.

- 1982 forward: EIA, Form EIA-759, "Monthly Power Plant Report.

WDRCPZZ - Wood energy consumed by the residential sector by State.

- 1960 through 1989: No data avatlable. Values are asstumed to be zero.

- 1990: Us. Census Division wood consumption data are from Form EIA-457, "1990 Residential Energy Consumption Survey." State-level estimates are derived by using the US.S. Department of Commerce, Bureau of the Census, American 
Housing Survey, Total Housing Units for 1990 by State, to allocate the division-level wood consumption to the States.

- 1991 and 1992: U.S. totals published in trillion Btu in the EIA. Rencuable Energy Antial 1935, Table 6, are converted to thousand cords and allocated to the States in proportion to the 1990 estimates.

- 1993: Data for U.S. Census Divisions and CA, FL, NY, and TX are from Form ELA-457, "1993 Residential Energy Consumption Survey." Data for other States are derived by using the U.S. Department of Commerce, Bureau of the Census, Amertcan Housing Survey, Total Housing Units for 1993 by State, to allocate the division-level wood consumption, minus known State consumption, to the remaining States in each Division.

- 1994: U.S. total published in trillion Btu in the BIA, Renenbable Entery Antual 1995, Table 6, is converted to thousand cords and allocated to the States in proportion to the 1993 estimates.

WSEOPZZ - Electricity produced from waste energy sources at electric utilities by State.

- 1960 forward: EIA, Form BlA-759, "Monthly Power Plant Report" (includes wood energy sources from 1960 through 1981).

WWACPZZ - Biofuels (ethanol) consumed by the transportation sector by State.

- 1960 through 1989: No data available. Vahues are assumed to be zero.

- 1990 through 1993: US. totals published in quadrillion Btu in the ELA, Retrewable Energy Anrual 2995, Table 2, are converted to gallons (by usting the conversion factor of $76,400 \mathrm{Btu}$ per gallon as documented in Appendix D) and allocated to the States in proportion to the 1994 estimates.

- 1994: U.S. Department of Transportation, Federal Highway Administration, Highroay Stakistics 1994, Table MF-33E, column "Total Ethanol Used in Gasohol."

WWICBZZ - Biofuels (wood and waste) consumed by the industrial sector by State.

- 1960 through 1989: No data available. Values are assumed to be zero.
- 1990 forward: ElA estimates are developed by using four data sources. U.S. totals for each year are from EIA, Renezouble Energy Anutual 1995, Table 2.

- A portion of the total for each year is allocated to the States by using State estimates of biofuels from the Form RIA867, "Annual Nonutility Power Producers Report."

- The remaining portion of the U.S. total for each year is assumed to be constumed by manufacturing industries. The Form EIA-846 "1991 Marufacturing Energy Consumption Survey (MECS)" identifies the largest manufacturing biofuels consumers to be industries in Standard Industrial Code (SIC) 2421 "Sawrills and Planing Mills," SIC 2541 "Wood Partitions and Fixtures," and SIC 2621 "Paper Mills." A State-level allocating data series is developed using MECS U.S. totals for the three series and the Statelevel "Value Added in Manufacture" series for each SIC from the U.S. Department of Commerce, Bureau of the Census, 1992 Census of Manufactures, Industry Series. (Data are available via Internet at http:/ www/census.gov/prod/manmin/92mmi/92manuff.html.) The weighted-average State allocator was applied to the U.S. mantfacturing portion for each year.

The two portions are summed by State and year to equal the original U.S. totals from the EIA, Rentewable Energy Awnupl 1995, Table 2.

\section{Bituminous God and Lignite}

BCACPUS - Bituminous coal and Hgntte consumed by the transportation sector in the Unitted States.

- 1960 through 1975: U.S. Department of the Interior, Bureau of Mines, Minerals Yeurbook, "Coal-Bituminous and Ltgnite."

- 1976 and 1977: EIA, Energy Data Regorts, "Coal-Bituminous and Lignite by Consumer and Retail Deliveries."

- 1978 forward: Small amounts of bituminous coll and lignite consumed by the transportation sector are included in the other industrial category (see BCOCPUS). Zero is entered for this variable.

BCEUKZZ - Factor for converting bituminous coal and lignite consumed by the electric utilfties from physical units to Bta by State. 
- 1960 through 1972: ELA adopted the average thermal conversion factor of the Bureau of Mtines, which used the National Coal Association (NCA) average thermal conversion factor for electric utilities calculated from the Federal Power Commission's (FPC) Form I and published in Steam Electric Plant Factors, an NCA annual report. The specific tables are:

- 1960 and 1961: Table 1.

- 1962 through 1972: Table 2.

- 1973 through 1982: The average heat content of coal received at steam electric plants $\mathbf{2 5}$ megawatts or greater from FPC Form 423 and published in Btu per pound in EIA, Cost and Quality of Fuels for Electric Utility Plants, tables titled "Destination and Origin of Coal 'Delivered to' (1973-1979) 'Receipts to' (1980) 'Received at' (1981-1982) Steam-Electric Plants 25-MW or Greater."

- 1983 forward: The average heat content of coal received at steam electric plants $\mathbf{5 0}$ megawatts capacity or larger from FERC Form 423 and published in Bta per pound in EIA, Casf antd Quality of Fuels for Electric Utility Plants. The specific tables ace:

- 1983 and 1984: Table 58.

- 1985 through 1989: Table 48.

- 1990 and 1991: Table 35.

- 1992: Table 22.

- 1993 and 1994: Table 4 or 22.

Notes: The State conversion factors for 1960 through 1972 were derived froun actual consumption data, while the conversion factors for 1973 to the present were based on receipts of coal. The factors for 1960 through 1972 may also have included some quantities of anthracite. These breaks in the series create some data discrepancies. Alaska and Hawaii were excluded from the NCA report, FPC Form 423, and FERC Form 423. However, Alaska reported consumption of bituminous coal and lignite at electric utilities for all years. An FPC heat rate for coal at electric utilities in Alaska was used for 1960 through 1978 as published in ELA, Federal Energy Data System (FEDS) Technical Documentation, June 1978, Table 21. The 1972 conversion factor (the last year for which a conversion factor was reported for Alaska) was used for 1972 through 1978. According to industry sources, new mines were opened in 1978 and a more representative factor was used for 1979 and following years. In instances where a
State had no receipts for a particular year but did report consump. tion, it was assumed that the coal received in one year was consumed durting the following year and the Btu value of the previous year's receipts was used.

BCEUPZZZ - Bituminous coal and lignite consumed by the electric utilities by State.

- EIA, Form ELA-759, "Monthly Power Plant Report," and predecessor forms.

BCHCKZZ - State factor for converting bituminous coal and lignite consumed by the residential and commercial sectors from physical units to Btu.

- 1960 through 1973: Estimated by ELA by adjustting the 1974 average heat value of bituminous coal and lipnite consumed in the residential and commercial sector by the ratios of 1960 through 1973 rational averages for the sector to its 1974 average.

- 1974 forward: Calculated by ELA by assuming that the bituminous coal and lignite consumed in the residenttal and commercial sector in each State contained heating values equal to those of bituminous coal and ligrite received at electric utiltties in each State from identified coal-producing districts as re ported on the Pederal Energy Regulatory Commission (FERC) Form 423, "Monthly Report of Cost and Quality of Fuels for Electric Plants." The average Btu content of coal delivered from each coal-producing district was applied to deliveries to the residential and commercial sector in each State and the sum total of the heat content was divided by total tonuages, yjelding a weighted average. The coal distribution data by coal-producing district are reported on Form EIA-6, ${ }^{\circ} \mathrm{Coal}$ Dis. tribution Report," and predecessor Bureau of Mines Form 61419-Q.

BCHCPUS - Bituminous coal and bigite consumed by the residential and commercial sectors in the United States.

- 1960 through 1972: U.S. Department of the Interior, Bureau of Mines, Mintrals Yearbook, "Coal-Bituminouts and Lignite," coluinn titled "Retail dealers" or "Retail sales."

- 1973 through 1984: EIA, Weekty Coal Production, August 9, 1986, Table 8. 
- 1985 through 1987: ELA, Weekly Coal Praduction, July 16, 1988, Table 7.

- 1988 forward: EIA, Unpablished data from Form ELA-6.

BCHDPZZ - Bituminous coal and Hignite distributed to the residential and commercial sectors by State.

- 1960 through 1976: U.S. Department of the Interior, Bureau of Mines, Minterals Yearbook, "Coal-Bituminous and Lignite," column titled "Retail dealers."

- 1977 through 1979: EIA, Energy Data Reports, "Coal-Bltuminous and Lisnite." The specific tables are:

- 1977: "Comparative Summary of Distribution of Bituminous Coal and Lignite Produced in the United States During the First Nine Months of 1977" and "Distribution of Bituminous Coal and Lignite Produced in the United States During October-December 1977, by Geographic Division and State Destimation," columns titled "Retail dealers."

- 1978: "Distribution of Bituminous Coal and Lignite Produced in the Lnited States," coluren titled "Retail sales."

- 1979: "Overall Summaxy of Distribution of Bituminous, Subbituminous, and Lignite Coal Produced in the United States," column titled "Retail sales."

- 1980 forward: Consumption estimates are used for this distribution series. Bituminous coal and lignite consumption is the remainder when estimated anthracite consumption is subtracted from all coal consumption in each State. (See ACHDPZZ for data sources and estimation procedures.) Consumption shown as "Unknown" is assumed to be bituminous coal and lignite and is allocated to six States (Alabama, Illinois, Kentucky, Pennsylvania, Ternessee, and West Virginia) in proportion to their total distribution of all coal.

BCKCPUS - Bituminous coal and lignite carbonized at coke plants in the United States.

- 1960 through 1972: U.S. Department of the Interior, Bureau of Mines, Minerals Yearbook, "Coal-Bituminous and Lignite," sum of columns "Beehive coke plants" and "Oven coke plants."

- 1973 through 1984: BIA, Weekly Corl Production, August 9, 1986 , Table 8 .

- 1985 through 1987: ElA, Weekly Coal Praduction, July 16, 1988, Table 7.
- 1988 forward: FIA, Unpublished data from Form EIA-5.

BCKDPZZ - Bikuminous coal and lignite distributed to coke plants, a portion of the industrial sector by State.

- 1960 through 1976: U.S. Deparment of the Interior, Bureau of Mines, Minerals Yearboak, "Coal-Bituminous and Lignite."

- 1977 through 1979: EIA, Energy Data Reporis, "Coal-Bitumlnous and Lignite." The specific tables are:

- I977: "Comparative Sumutary of Distribution of Bituminous Coal and Lignite Produced in the United States During the First Nine Months of $1977^{\prime \prime}$ and "Distribution of Bituminous Coal and Lignite Produced in the United States During October-December 1977, by Geographic Division and State Destination."

- 1978: "Distribution of Bituminous Coal and Lignite Produced in the United States."

- 1979; "Ovezall Summary of Distribution of Bituminous, Subbituminous, and Lignite Coal Produced in the United States.

- 1980 forward: Consumption estimates are used for this distribution series. Bituminous coal and lignite consumption is the remainder when estimated anthracite consumption is subtracted from all coal consumption in each State. See ACKDPZZ for data sources and estimation procedures.

BCOCKZZ - State factor for converting bituminous coal and lig rite consumed by other industrial users from physical urits to Btu.

- 1960 through 1973: Estimated by ELA by adjusting the 1974 average heat value of bituminous cond and lignite consumed by industrial users other than coke plants by the ratios of 1960 through 1973 national averages for the other industrial users to its 1974 average.

- 1974 forward: Calculated by EIA by assuming that the bituminous coal and lignite consumed by industrial users other than coke plants in each State contained heating values equal to those of bituminous coal and ligntte received at electric utilities in each State from identified coal-producing districts as reported on Federal Energy Regulatory Commission (FERC) Form 423, "Monthly Report of Cost and Quality of Fuels for Electric Plants." The average Btu content of coal delivered from each coal-producing district was applied to 
deliveries to other industrial users in each State and the sum total of the heat content was divided by total tomnages, yield. ing a weighted average. The coal distribution data by coalproducing district are reported on Form ELA-6, "Coal Distribution Report," and predecessor Bareau of Mines Form $6-1419-0$

BCOCPUS - Bituminous coal and lignite consumed by industrial users other than coke plants in the United States

- 1960 through 1972: U.S. Department of the Interior, Bureau of Mines, Mtnerals Yearbook, "Coal-Bituminous and Lignite," table titled "Consumption of bituminous coal and lignite, by consumer class, and retail deliveries in the United States." Sum of columns titled "Steel and rolling mills," "Cement mills," and "Other manufacturing and mining industries."

- 1973 through 1984: RIA, Weekly Cont Production, August 9, 1986, Table 8 .

- 1985 through 1987: ElA, Weekly Caal Praduction, July 16, 1988, Table 7.

- 1988 forward: ELA, Unyublished data from Forms EIA-3 and EIA-6.

BCODPZZ - Bituminous coal and lignite distributed to industrial plants (other than coke plants) by State-

- 1960 through 1976: U.S. Department of the Interior, Bureau of Mines, Minerals Yearbook, "Coal-Bituminous and Lignite."

- 1977 through 1979: ELA, Energy Defa Reporfs, "Coal-Bituminous and Lignite." The specific tables are:

- 1977: "Comparative Summary of Distribution of Bituminous Coal and Lignite Produced in the United States During the First Nine Months of $1977^{\text {N }}$ and "Distribution of Bitumirnous Coal and Lignite Produced in the United States During October-December 1977, by Geographic Division and State Destination."

- 1978: "Distribution of Bituminous Coal and Lignite Produced in the United States. ${ }^{\sigma}$

- 1979: "Overall Summary of Distribution of Bituminous, Subbituminous, and Lignite Coal Produced in the United Stakes,"

- 1980 forward: Constumption estimates are used for this distribution series. Bituminous coal and lignite consumption is the remainder when estimated anthracite consumption is subtracted from all coal consumption in each State. (See ACODPZZ for data sources and estimation procedures.) Consumption shown as "Unknown" is assumed to be bituminous coal and lignite and is allocated to six States (Alabama, Ilinois, Kentucky, Perursylvania, Ternessee, and West Virginia) in proportion to their total distribution of all coal.

\section{Calalytte Cracking Charge Capacity}

CTCAPZZ - Catalytic cracking charge capacity of petroleum refin* eries by State.

- 1960: Data are unavallable from published reports. The 1961 values are used for 1960 .

- 1961 through 1963: US. Department of the Interior, Bureau of Mines, Mineral Industry Surweys, "Petroleum Refineries in the United States." The specific tables are:

- 1961 and 1962: Table 7, under "Cracking Capacity" column heading "Charge."

- 1963: Table 6, under "Catalytic-Cracking Capacity" column heading "Charge."

- 1964 through 1976: U.S. Department of the Interlor, Bureau of Mines, Mtneral Industry Surozys, "Petroleum Refineries in the United States and Puerto Rico," Table 2, all entries next to "Cat. Ck" summed by State.

- 1977: ELA, Energy Data Reports, "Petroleum Refineries in the United States and Puerto Rico," Table 2, all entries next to "Cat. Ck." summed by State.

- 1978: EIA, Energy Dota Reports, "Tetroleum Refinecies in the United States and U.S. Territories," Table 2, all entries next to ${ }^{\text {"Cat }} \mathrm{Ck}^{*}$ summed by State.

- 1979 and 1980: ElA, Energy Data Reports, "Petroleum Refineries in the United States and U.S Territories." The spectific tar bles are:

- 1979: Table 2, sum of "Catalytic Cracking" columns, "Fresh" and "Recycle."

- 1980: Table 1, sum of "Catalytic Cracking (fresh)" and "Catalytic Cracking (recycle)" columns.

- 1981 forward: EIA, Petroleum Supply Atrual, sum of "Catalytic Cracking (Fresh)" and "Catalytic Cracking (Recycled)" columns in the following tables: 
- 1981 through 1983: Table I.

- 1984: Table 30.

- 1985 through 1989: Table 29.

- 1989 forward: Table 36.

\section{Cost Coke}

CCEXPUS - Coal coke exported from the United States.

- 1960 through 1975: U.S. Department of the Interior, Bureau of Mines, Mitrerals Yeurbook, "Coke and Coal Chemicals Annual."

- 1976 through 1979. EIA, Energy Data Reports, "Coke and Coal Chemicals Monthly."

- 1980 forward: EIA, Quarterly Caal Report (January-March of the following year). The specific tables are:

- 1980 through 1990: Table A1.

- 1991 forward: Table 2.

CCIMPUS - Coal coke imported into the United States.

- 1960 through 1975: U.S. Department of the Interior, Bureau of Mines, Minerals Yearbonk, "Coke and Coal Chemicals Annual"

- 1976 through 1979, ELA, Entrgy Data Reprorts, "Coke and Coal Chemicals Monthly."

- 1980 forward: EIA, Quarterly Conl Repork Uanuary-March of the following year). The specific tables are:

- 1980 through 1990: Table A1.

- 1991 forward: Table 2.

\section{Crude oll (heluding lease condensate)}

COCAPZZ - Crade oil operating capacity at refineries by State.

- 1960: U.S. Department of the Interior, Bureau of Mines, Petroleum Refinteries, Including Cracking Plants, in the United States, Table 3.

- 1961 through 1963: U.S. Department of the Interior, Bureaut of Mines, Mineral Indusiry Surveys, "Petroleum Refineries in the United States." The specific tables are:

- 1961 and 1962: Table 3.

- 1963: Table 1.
- 1964 through 1976: U.S. Department of the Interior, Bureau of Mines, Mineral Industry Suraeys, "Petroleum Refineries in the United States and Puerto Rico," Table 1.

- 1977: ElA, Energy Data Reports, "Petroleum Refineries in the United States and Puerto Rico," Table 1.

- 1978 through 1980: EIA, Energy Data Reports, "Petroleum Refineries in the United States and U.S. Territorfes, ${ }^{*}$ Table 1.

- 1981 forward: EIA, Petroleum Supply Annual. The specific tables are:

- Ig81 through 1983: Table 1.

- 1984: Table 30.

- 1985 through 1988: Table 29.

- 1989 forward: Table 36.

COTCPzZ - Crude oil consumed in petroleum industry operations by State.

- 1960 through 1982: Crude oil used directly was included in distillate and residual futel product supplied when reported to ELA. Zeros are entered for all years.

- 1983 forward: Data are available for Petroleum Administration for Defense (PAD) Districts, not by State. State estimates are calculated by allocating all crude oil consumption to the six States (Alaska, California, Colorado, Louisiana, Texas, and Utah) that reported distillate and residual fuels consumed by pipeline and leases in 1982. (Data on pipeline and lease consumption of fuels are not available after 1982) Each State's 1982 ratio of distillate and residual fuels consumed by pipe. line and leases to its respective 1982 PAD District total consumption of those fuels is calculated. This ratio is then applied to the 1983 forward PAD District totals of crude oil product supplied. The 1982 ratios are taken from the form EIA-90, "Crude Oil Stocks Report," and the crude oil product supplied data are taken from the ElA Petroleum Sipply Annual. The speciftc tables are:

- 1983 through 1988: Tables 2 and 4 through 8 .

- 1989 forward: Tables $2,4,6,8,10$, and 12 .

\section{Distillate Fuet}

DFBKPZZ - Distillate fuel adjusted sales for vessel bunkering use by State, excluding that sold to the Armed Forces. 
- 1960 through 1975: U.S. Department of the Interior, Bureau of Mines, Mineral Industry Suroeys, "Shipments of Fuel Oit and Kerosene." The specific tables are:

- 1960 and 1961: Table 17.

- 1962 and 1963: Table 16.

- 1964 and 1965: Table 15.

- 1966 through 1975: Table 11.

- 1976 through 1978: EIA, Entergy Data Reports, "Sales of Fuel Oil and Kerosene," Table 11.

- 1979 and 1980: ELA, Energy Data Reports, "Deliveries of Fuel Oil and Kerosene, ${ }^{\prime}$ Table 1.

- 1981 and 1982: EIA, Petroleum Supply Annual, Table 4.

Note: Data for 1983 forward were published in thousand gallons. They were converted to thousand barrels by dividing by 42 before being entered into SEDS.

- 1983 through 1987: EIA, Petroleum Marketing Monthly. The specific tables are:

- 1983 and 1984: July 1985 issue, Table A12.

- 1985 and 1986: July 1987 issue, Table A16.

- 1987: June 1988 issure, Table A16.

- 1988 forward: ELA, Fuel Oil and Kerosene Sites, Table 16.

DFCMPZZ - Distillate fuel adjusted sales to the commercial sector for space heating water healing, and cooking.

- 1960 through 1978: EIA estimates based on statistics of commercial sector deliveries of distillate fuel from the ELA, Energy Data Report, "Deliveries of Fuel Oil and Kerosene in 1979,"

Table 1. State ratios based on 1979 commencial sector deliveries were applied to each State's sum of heating plus industrial (including farm use) deliveries categortes from the fuel oil deliveries reports for each year 1960 through 1978. (See explanation in Appendix A, Note 3, on page 358.)

- 1979 and 1980: ELA, Energy Data Reports, "Deliveries of Fuel Oil and Kerosene," Table 1.

- 1981 and 1982: EIA, Petroleum Suppiy Annzal, Table 4.

Note: Data for 1983 forward were published in thousand gallons. They were converted to thousand barrels by dividing by 42 before being entered into SEDS.

- 1983 through 1987: BIA, Peiroleum Marketing Monthly. The specific tables are:

- 1983 and 1984: July 1985 jsoue, Table A12.
- 1985 and 1986: July 1987 issue, Table A16.

- 1987: June 1988 issue, Table A16.

- 1988 forward: ELA, Fuel Oil and Kerosene Sates, Table 16.

DFIBPZZ - Distillate fuel adjusted sales to industrial establishments for space heating and for other industrial use, inclucing farm use.

- 1960 through 1978: ElA estimates based on statistics of industrial sector deliveries of distillate fuel from the EIA. Energy Data Report, "Deliveries of Fuel Otl and Kerosene in 1979, "Table 1. State ratios besed on 1979 industrial sector deliveries were applied to each State's sum of heating plus industrial (including farm use) deliveries categories from the fuel oil deliveries reports for each year 1960 through 1978. (See explanation in Appendix A, Note 3, on page 358.)

- 1979 and 1980: EIA, Entergy Data Reports, "Deliveries of Fuel Oil and Kerosene," Table 1.

- 1981 and 1982: EIA, Petroleum Supply Anuzal, Table 4.

Note: Data for 1983 forward were published in thousand gallons. They were converted to thousand barrels by dividing by 42 before being entered into SEDS.

- 1983 through 1987: EIA, Petroleum Marketing Monthly. The specific tables are:

- 1983 and 1984: July 1985 issue, Table A12.

- 1985 and 1986: July 1987 issue, Table A16.

- 1987: June 1988 issue, Table A16.

- 1988 forward: BLA, Fuel Oit and Kerosene Sales, Table 16.

DFMIPZZ - Distillate fueI adjusted sales for military use (including imports for the military) by State.

- 1960 through 1975: U.S. Department of the Interior, Bureau of Mines, Mineral Industry Surweys, "Shipments of Fuel Oil and Kerosene." The specific tables are:

- 1960 and 1961: Table 18.

- 1962 and 1963: Table 17.

- 1964 and 1965: Table 16.

- 1966 through 1975: Table 12.

- 1976 through 1978: ELA, Energy Data Reports, "Sales of Fuel Oil and Kerosene," Table 12.

- 1979 and 1980: BIA, Energy Data Reports, "Deliveries of Fulel Oil and Kerosene," Table 1. 
- 1981 and 1982: EIA, Petroleun Supply Arnual, Table 4.

Note: Data for 1983 forward were published in thoustand gallons. They were converted to thousand barrels by dividing by 42 before being entered into SFDS.

- 1983 through 1987: EIA, Peiroletum Marketing Monthly. The specific tables are:

- 2983 and 1984: July 1985 issue, Table A12.

- 1985 and 1986: July 1987 issue, Table A16.

- 1987: June 1988 issue, Table A16.

- 1988 forward: ElA, Fuel Oil and Kerosene Sajes, Table 16.

DFOCPZZ - Distillate furel adjusted sales for use by oil companies by State.

- 1960 through 1975: U.S. Department of the Interior, Bureau of Mines, Mineral Industry Surveys, "Shipments of Fuel Oil and Kerosene." The specific tables are:

- 1960 and 1961: Table 14.

- 1962 and 1963: Table 13.

- 1964 and 1965: Table 12.

- 1966 through 1975: Table 9.

- 1976 through 1978: EIA, Energy Data Reports, "Sales of Fuel Oil and Kerosene," Table 9.

- 1979 and 1980: EIA, Energy Daia Reports, "Deliveries of Fuel Oil and Kerosene," Table 1.

- 1981 and 1982: EIA, Petroleum Supply Annual, Table 4.

Note: Data for 1983 forward were published in thousand gallons. They were converted to thousand barrels by dividing by 42 before being entered into SEDS.

- 1983 through 1987: ELA, Petrolewm Marketing Monthly. The specific tables are:

- 1983 and 1984: July 1985 issue, Table A12,

- 1985 and 1986: July 1987 issue, Table A16.

- 1987: June 1988 issue, Table A16.

- 1988 forward: BIA, Fuel Oil and Kerosene Sales, Table 16.

DFOFPZZ - Distillate fuel adjusted sales as diesel fuel for off-highway use by State.

- 1960 through 1975: U.S. Department of the Interior, Bureau of Mines, Mineral Inditstry Surougs, "Shipments of Fuel Oil and Kerosene." The specific tables are:

- 1960 through 1962; Table 19.
- 1969 and 1964: Table 18.

- 1965 through 1967: Table 17.

- 1968 thuough 1975: Table 14.

- 1976 through 1978: ELA, Energy Dafa Reporis, "Saies of Fuel Oil and Kerosene," Table 14.

- 1979 and 1980: EIA, Energy Data Reports, "Deliveries of Fuel Oil and Kerosene," Table 1.

- 1981 and 1982: ELA, Petroleum Supply Anmual, Table 4.

Note: Data for 1983 forward were published in thousand gallons. They were converted to thousand barrels by dividing by 42 before being entered into SEDS.

- 1983 through 1987: EIA, Petroieum Markeiting Monthly. The specific tables are:

- 1983 and 1984: July 1985 issue, Table A12.

- 1985 and 1986: July 1987 issue, Table A16.

- 1987: June 1988 issue, Table A16.

- 1988 forward: ELA, Fuel Oil and Kerosene Sales, Table 16.

DFONFZZ - Distillate fuel adjusted sales as diesel fuel for onhighway use by State.

- 1960 through 1975: U.S. Department of the Interjor, Bureau of Mines, Minetal Industry Suroeys, "Shipments of Fuel Oil and Kerosene*" The specific tables are:

- 1960 through 1962: Table 19.

- 1963 and 1964: Table 18.

- 1965 through 1967: Table 17

- 1968 through 1975; Table 14

- 1976 through 1978: ELA, Energy Data Reporis, "Sajes of Fuel Oil and Kerosene," Table 14.

- 1979 and 1980: ELA, Energy Data Reports, "Deliveries of Fuel Oil and Kerosene," Table 1.

- 1981 and 1982: ElA, Petroleum Supply Annual, Table 4.

Note: Data for 1983 forward were published in thousand gallons. They were converted to thousand barrels by dividing by 42 before being entered into SEDS.

- 1983 through 1987: BIA, Petroleum Marketing Monthly. The specific tables are:

- 1983 and 1984: July 1985 tssue, Table A12.

- 1985 and 1986: July 1987 issue, Table A16.

- 1987: June 1988 tosue, Table Ai6.

- 1988 forward: ELA, Fuel Oil and Kerasene Sales, Table 16. 
DFOTPZZ - Distlllate fuel adjusted saies for all other uses not identified in other adjusted sales categories.

- 1960 through 1975: U.S. Department of the Interior, Bureau of Mines, Mineral Industry Surozys, "Shipments of Fuel Oil and Kerosene." The spectfic tables are:

- 1960 through 1962: Table 19.

- 1963 and 1964: Table 18.

- 1965 through 1967: Table 17.

- 1968 through 1975: Table 14.

- 1976 through 1978: ELA, Energy Data Reports, "Sales of Fuel Oil and Kerosene," Table 14.

- 1979 and 1980: EIA, Energy Data Reports, Deliveries of Fuel Oil and Kerosene," Table 1.

- 1981 and 1982: ELA, Pefroleum Supply Annual, Table 4

Note: Data for 1983 forward were published in thousand gallons. They were converted to thousand barrels by dividing by 42 before being entered into SEDS.

- 1983 through 1987: ELA, Petroleant Marketing Monthly. The specific tables are:

- 1983 and 1984: July 1985 issue, Table A12.

- 1985 and 1986: July 1987 issue, Table A16.

- 1987: June 1988 igsue, Table A16.

- 1988 forward: ElA, Fud Oil and Kerosene Sales, Table 16.

DFRRPZZ - Distillate fuel adjusted sales for use by railroads by State.

- 1960 theotigh 1975: U.S. Deparment of the Interior, Bureau of Mines, Mineral' Industry Surzeys, "Shipments of Fuel Oil and Keroseme" The specific tables are:

- 1960 and 1961: Table 16.

- 1962 and 1963: Table 15.

- 1964 and 1965: Table 14.

- 1966 throughi 1975: Table 10.

- 1976 through 1978: ELA, Energy Data Reports, "Sales of Fuel Oil and Kerosene," Table 10.

- 1979 and 1980: FIA, Entrgy Dafa Reports, "Deliveries of Fuel Oil and Kerosene," Table 1.

- 1981 and 1982: ElA, Petroleum Supply Amnual, Table 4.

Note: Data for 1983 forward were published in thousand gallons. They were converted to thousand barrels by dividing by 42 before being entered into SEDS.
- 1983 through 1987: EIA, Petroleum Marketing Monthly. The specific tables are:

- 1983 and I984: July 1985 issue, Table A12.

- 1985 and 1986: July 1987 issus, Table A16.

- 1987: June 1988 issue, Table A16.

- 1988 forward: EIA, Fuel Oil and Kerosene Sales, Table 16.

DFRSPZZ - Distillate fuel adjusted sales to the residential sector for space heating, water heating, and cookinge

- 1960 through 1978: EIA estimates based on statistics of residextial sector deliveries of distillate fuel from the EIA, Energy Data Repost, "Deliveries of Fuel Oil and Kerosene in 1979," Table 1. State ratios bated on 1979 residential sector deliveries were applied to each State's sum of heating plus industrial (including farm use) deliveries categories from the fuel oil deliv. ertes reports for each year 1960 through 1978. (See explanation in Appendix A, Note 3, on page 358.)

- 1979 and 1980: ELA, Energy Data Reports, "Deliveries of Fuel Oil and Kerosene," Table 1.

- 1981 and 1982: EIA, Petroleum Supply Annual, Table 4.

Note: Data for 1983 forward were published in thousand gallons. They were converted to thousand barrels by dividing by $\mathbf{4 2}$ before being entered anto SEDS.

- 1983 through 1987: EIA, Petroleum Markating Monkhty. The Epecific tables are:

- 1983 and 1984: July 1985 issouse, Table A12.

- 1985 and 1986: July 1987 issue, Table A16.

- 1987: June 1988 issue, Table A16.

- 1988 forward: ElA, Fuel Oil and Kerosene Sales, Table 16.

DFTCPUS - Distillate fuel total consumed in the United States.

- 1960 through 1975: U.S. Department of the Interior, Bureau of - Mines, Minteral Industry Surreys, ${ }^{\circ}$ Petroleum Statement Annual, "Table 1.

- 1976 through 1980: EIA, Energy Data Reports, "Petroleum Statement, Arnual," Table 1.

- 1981 forward: EIA, Petrolenm Supply Annual, Table 2.

DKEUPZZ - Distillate fuel consumed by the electric utilities, including kerosene-type jet fuel. 
- BIA, Form EIA-759, "Monthly Power Plant Report," and predecessor forms. The following assiumptions have been made:

- 1960 through 1969: Only total fuel oil consumed at electric utilities by State is available. State estimates of distillate fuel consumption were created for each year by applying the shares of internal combustion and gas turbine plants (primarily distillate fuel plus small amounts of jet kerosene) by State from 1970 to each year's total fuel oil consumption at electric utilities for 1960 through 1969.

- 1970 through 1979: Fuel oil consumed by plant type by State is available. Fuel oil constumed by internal combus: tion and gas turbine plants combined is assumed to equal distillate and jet kerosene consumption.

- 1980 forward: Consumption of light and heavy oil at all plant types by State is available. Total light oil consumption at all plant types is assumed to equal distillate and jet kerosene consumption.

\section{Electriclty Exports and imports}

ELEXPZZ - Electricity exported from the United States (assumed to be produced by hydroelectric power through 1989) by State.

- 1960 through 1981: Econonic Regulatory Administration, Staff Regioris, "Report on Electric Energy Bxchanges with Canada and Mexico." Source data are arranged by the Regional Reliability Council Areas and then by the electric utility. State data were tabulated by aggregating the data of all electric utilities within each State.

- 1982 and 1983: EIA State estimates are based on data from Bconomic Regulatory Administration Form ERA-781R, "Annual Report of Electriçal Export/Import Data." State estimates are consistent with national and regional totals published in the BRA, Elestricity Exchanges Acrass International Borders.

- 1984 through 1987: EIA State estimates are based on data from Economic Regulatory Administration Form ERA-781R, "Annual Report of Electrical Export/Import Data," the Federal Energy Regulatory Commlssion Form 1, and the Bonneville Power Administration Annual Report. State estimates are consistent with national and regional totals published in the ERA, Electricty Transactions Across International Borders.
- 1988 forward: EIA State estimates are based on data from DOE, Fossil Fuels, Fuels Programs, Office of Coal and Electricity, Form FE-781R, "Annual Report of International Electrical Export/Hmport Data," and predecessor forms, the Federal Energy Regulatory Commission Form 1, the Bonneville Power Administration data, and the Canada National Energy Boand Annual Report.

ELIMPZZ - Electricity inported into the United States (assumed to be produced by hydroelectric power through 1989) by State.

- 1960 through 1981: Bconomic Regulatory Administration, Staff Reports, "Report on Electric Energy Exchanges with Canada and Mexico." Source data axe arranged by the Regional Reliability Council Areas and then by the electric utility. State data were tabulated by aggregating the data of all electric utilities within each State.

- 1982 and 1983: ELA State estimates are based on data from Economic Regulatory Administration Form ERA-781R, "Annual Report of Electrical Export/Import Data." State estimates are consistent with national and regional totals published in the ERA, Electricity Exchantiges Across International Borders.

- 1984 through 1987: FIA State estimates are based on data from Economic Regulatory Administration Form ERA-781R, "Annual Report of Electrical Export/Import Datz," the Federal Energy Regulatory Commission Form 1, and the Bonneville Power Administration Annual Report. State estimates are consistent with national and regional totals published in the ERA, Electricity Transactions Across Internationtal Borders.

- 1988 forward: EIA State estimates are based on data from DOE, Fossil Fuels, Fuels Programs, Office of Coal and Electricity, Form FE-7B1R, "Annual Report of International Electrical Export/Import Data, ${ }^{,}$and predecessor forms, the Federal Energy Regulatory Commission Form 1, the Bonneville Power Administration data, and the Canada National Energy Board Annual Report.

\section{Electricily Sales}

ESCMPZZ - A portion of the electricity sold to the commercial sector by State. 
A Note: Data for Maryland and the District of Columbia were comP bined for 1960 through 1989 . The method for disaggregating the data p is explained in Appendix A, Note 3 , on page 399 .

- 1960 through 1975: Federal Power Commission, Electric Potwer Statistics, "Sales of Electric Energy to Ultimate Consumers."

- 1976 through 1980: E[A, Electric Power Annual (November 1982), Table 125 . pany Monthly Statement, and predecessor forms. Published data rounded to gigawatthours in EIA, Electric Power Antnual
- 1981 through 1989: EIA, Form EIA-826, "Electric Utility Com1983, Table 51.

- 1984 through 1986: EIA, Form EIA-861, "Annual Electrit Uti2ity Report. ${ }^{\text {N }}$ Unpublished data.

- 1987: EIA, Form EIA-861, "Annual Electric Utility Report." Published data rounded to gigawatthours in ElA, Electric Power Annual 1988, Table 19.

- 1988 forward: EIA, Form EIA-861, "Annual Electric Utility Report." Published data rounded to gigawatthours in EIA, Electric Power Annual (Volume I for I994). The specific tables are:

- 1988 through 1990: Table 27.

- 1991 forward: Table 26.

ESICPZZ - Electricity consumed by the industrial sector by State.

Note: Data for Maryland and the District of Columbia were combined for 1960 through 1983 . The method for disaggregating the data is explained in Appentix A, Note 3, on page 399.

- 1960 through 1975: Federal Power Commission, Electric Power Statistics, "Sales of Electric Energy to Ultimate Consumens."

- 1976 through 1980: ELA, Electric Potwer Anmual (November 1982), Table 126.

- 1981 through 1983: EIA, Form EIA-826, "Electric Utility Company Monthly Statement," and predecessor forms. Published data rounded to gigawatthours in EIA, Electric Power Annual 1983, Table 51 .

- 1984 through 1986: ELA, Form ELA-861, "Armual Electric Utility Report." Unpublished data.

- 1987: BIA, Forn EIA-861, "Annual Electric Utility Report." Published data rounded to gigawatthours in EIA, Electric Power Anntwal 1988, Table 19.
- 1988 forward: ELA, Form EIA-861, “Annual Electric Utility Report." Published data rounded to gigawatthours in ELA Electric Poroer Anmual (Volume I for 1994). The specific tables are

- I988 through 1990: Table 27.

- 1991 forward: Table 26.

ESOTPZZ - Electricity sold to the "Other" sector (i.e., public street and highway lighting, sales to other public authorities, railroads and railways, and interdepartmental sales) by State.

Note: Data for Marylend and the District of Cohumbia were combined for 1960 through 1983. The method for disaggregating the data is explained in Appendix A, Note 3, on page 399

- 1960 through 1975: Federal Power Commission, Electric Power Statistics, "Sales of Electric Energy to UItimate Consumers."

- 1976 through 1980: ELA, Electric Posoer Annual (November 1982), Table 127

- 1981 through 1983: ELA, Form ELA-826, "Electric Utility Company Monthly Statement," and predecessor forms. Published data rounded to gigawatthours in EIA, Electric Ponder Annul 1983, Table 51.

- I984 through 1986: ELA, Form ELA-851, "Arnual Electric Utility Report." Unpublished data.

- 1987; BLA, Form EIA-861, "Annual Electric Utility Report." Published data rounded to gigawatthours in BIA, Electric Power Anntiol 1988, Table 19.

- 1988 forward: BIA, Form EIA-861, "Annual Blectric Utility Report." Published data rounded to gigawatthours in EIA, Electric Power Annual (Volume I for 1994). The specific tables are:

- 1988 through 1990: Tabls 27.

- 1991 forward: Table 26.

ESRCPZZ - Electricity consumed by the residential sector by State. Note: Data for Maryland and the District of Columbia were combined for 1960 through 1983. The method for disaggregating the data is explained in Appendix A, Note 3, on page 399.

- 1960 through 1975: Federal Power Commission, Electric Poarer Statistics, "Sales of Electric Enersy to Uttimate Consumers."

- 1976 through 1980: ELA, Electric Power Annual (November 1982), Table 124. 
- 1981 through 1983: ELA, Form FIA-826, "Electric Utility Company Monthly Statement," and predecessor forms. Published data rounded to gigawatthours in EIA, Electric Powor Anntual I983, Table 51.

- 1984 through 1986: ElA, Form ELA-861, "Annual Electric Utility Report." Unpublisbed data.

- 1987: EIA, Form EIA-861, "Annual Electric Utility Report." Published data rounded to gigawatthours in BLA, Electiric Power Annual 1988, Table 19.

- 1988 forward: EIA, Form EIA-861, "Annual Electric Utility Report." Published data rounded to gigawatthours in BLA, Electric Power Annual (Volume I for 1994). The specific tables are:

- 1988 throutgh 1990: Table 27.

- 1991 forward: Table 26.

ESTRPZZ - Electricity constumed by transit systems by State.

Note: The transit system data include electricity used to operate commuter rail, rapid rail, streetcars or light rail, cable cars, trolleybuses, motorbuses, aukomated guideways, inclined plane railways, and aerial tramways. These data do not include electricity used by Amtrak.

- 1960 through 1978: EIA estimates are based on data from:

- The American Public Transit Association (formerly the American Transit Association) annual operating reports.

- Pushkarev, Boris S. and others, Urban Rail in America. (Bloomington, IN: Indiana University Press, 1982.)

- U.S. Department of Transportation, $A$ Directory of Regularly Scheduled, Fixed Route, Local Public Transportation Service in LIrbanized Areas Over 5J,000 Population, 1980 and 1981.

- 1979 through 1989. U.S. Department of Transportation, Urban Mass Transportation Administration, Nationa! Urban Mass Trensportation Skatistics, Section 15 Anntal Repont, table titled "Fnergy Consumption: Details by Transit System."

- 1979 and 1980: Table 2,13.1.

- 1981 and 1982: Table 3.13.1.

- 1989 through 1989: Table 3.12.

- 1990 forward: U.S. Department of Transportation, Federal Transit Administration, Daka Tables for the Section 15 Report Yeatr.

- 1990: Table 2.12.
- 1991: Table 13.

- 1992 and 1993: Table 15.

- 1994: Prepublished data.

Data also available via internet

- 1993 and 1994: http://www.fta.dot.gov/fta/library/ reference/sec15.

Notes: These data are available on a fiscail year basis fuly 1 through June 30) for 1979 through 1982 and for calendar years 1983 forward. Some data for 1979 through 1983 were adjusted by EIA on the basis of an analysis of historical trends. Electricity consumption for the District of Columbia for 1976 forward is partially apportioned to Maryland and Virginia on the basis of electricity consumption data from the Washington Metropolitan Area Transit Atsthority.

ESTRSUS - The share of electricity soid to the "Other" sector (ESOTPZZ) that is used for transportation (i.e., by rallroads and xatrways) by State. (See further explanation of this share in Appendix A. Note 2, on page 398.) Based on kilowatthour sales from:

- 1960 through 1971: Federal Power Commisstion, Statistics of Privately Owned Electric Ublitties in the United States, 1971, Table 15.

- 1972 and 1973: EIA, Findncial Statistics of Selected Electric Litilitites 1976, Table 13.

- 1974 through 1983: EIA, Financial Statistics of Selected Electric Utilities 1983, Table 8.

- 1984 through 1987: ElA, Financial Statistics of Selected Electric Utilities 1987, Table 17.

- 1988 through 1992: ELA, Financial Statistics of Major U.S. Investor-Oroned Electric Utilities I992, Table 15.

- 1993 and 1994: ELA, Financial Statistics of Major U.S. IntestorOumed Electric Utilities 1994, Table 15.

\section{Petrochemical Feedstocks}

FNTCPUS - Petrochemical feedstocks, naphtha, less than $401^{\circ} \mathrm{F}$, total consumed in the United States.

- 1960 through 1975: U.S. Department of the Inferior, Burean of Mines, Mineral Industry Surveys, "Petroleum Statement, Annual," Table 1. 
- 1976 through 1980: ElA, Energy Data Reports, "Petroleum Statement, Annual," Table 1.

- 1981 forward: EIA, Petroleum Supply Amitul, Table 2.

FOTCPUS - Petrochemical feedstocks, other oils, equal to or greater than $401^{\circ} \mathrm{F}$, total consumed in the United States.

- 1960 through 1975: U.S. Department of the Interior, Bureau of Mines, Mineral Industry Surveys, "Petrolean Statement, Annual," Table 1.

- 1976 through 19B0: BIA, Energy Data Reports, "Petroleum Statement, Aruual," Table 1.

- 1981 forward: EIA, Petroleum Supply Annual, Table 2.

FSTCPUS - - Petrochemical feedstocks, still gas, total constumed in the United States.

- 1960 through 1975: U.S. Department of the Interior, Bureau of Mines, Mineral Industry Surdeys, "Petroleum Statement, Annual," Table 1.

- 1976 through 1980. EIA, Energy Data Reports, Petroletum Statement, Annual," Table 1.

- 1981 and 1982: ELA, Ptroleum Supply Annual, Table 14.

- 1983 through 1985: ELA, Petroleum Supply Anrual, Table 12.

- 1986 forward: ELA, Petroleton Supply Annual, Table 2, izcluded in "Still Gas."

\section{Fossill Futh}

FFEOKUS - Fossil fuel steam-electric power plant converstion factor.

- 1960 through 1991: Estimated by ELA as the weighted anurual average heat rate for fossil-fueled steamuelectric plants in the United States as published in the ELA, Electric Plant Cost and Poduer Production Expenses 1991, Table 9.

- 1992 forward; Unpublished factors calculated on the basts of data from Form BIA-767.

\section{Geothermal}

GEEOKUS - Factor for converting electricity produced from geothermal energy from physical units to Btu.
- 1960 through 1981: Calculated by EtA by weighting the annual average heat rates of operating geothermal units by the installed nameplate capacities as reported on Federal Power Corrurission Form 12.

- 1982 forwaxd: Estimated annually by the EIA on the basis of an informal survey of relevant plants.

GEEOPZZ - Electricity produced from geothermal energy at electric utilities by State.

- EIA, Form EIA-759, "Monthly Power Plant Report," and predecessor forms.

GEICBZZ - Electricity produced from geothermal energy in the industrial sector by State.

- 1960 through 1989: No data available. Values assumed to be zero.

- 1990 forward: ELA estimates based on data collected on Form BLA-867, "Arnual Nonutitity Power Producers Report."

GEMMPZZ - Electricity produced from geothermal energy and imported into the United States by State.

- 1960 through 1989: No data available. Values assumed to be zero.

- 1990 forward; ElA estimates based on data from U.S. Department of Energy, Fossit Energy, Form FE-781R, "Annual Report of international Electricity Export/Import Data."

\section{Hydroelectrtc Power}

HPEOPZZ - Electricity produced from pumped storage hydropower at electric utilites by State.

- 1960 through 1989: Included in conventional hydroelectric power.

- 1990 forward: EIA, Form EIA-759, "Monthly Power Plant Report."

HVEOPZZ, - Electricity produced from conventional hydropower at electric utilities (includes pumped storage hydroelectric power through 1989) by State. 
- 1960 through 1977: Federal Power Commission, News Release, "Power Production, Fuel Consumption, and Installed Capacity Data."

- 1976 through 1980: ELA, Entergy Data Reports, "Power Production, Fuel Consumption and Instailed Capacity Date."

- 1981 forward: EIA, Form ELA-759, "Monthly Power Plant Report, and predecessor forms. Published data rounded to gigawatthours in the following reports:

- 1981 through 1985: ElA, Electric Power Antmual 1985, Table 6.

- 1986 and 1987: ELA, Eleciric Power Annual 1987, Table 18. - 1988 and 1989: EIA, Electric Power Annual 1989, Table 14.

- 1990 forward: EIA, Form EIA-759, "Monthly Power Plant Report."

HYEXPZZ - Electricity produced from hydroelectric power and exported from the United States by State.

- 1960 through 1989: Assumed to be equal to total electricity exports (ELEXPZZ).

- 1990 forward: ElA estimates based on data from Natural Resources Canada, Electric Power in Cantado 1994, and National Energy Board of Canadà, Electrictiy Exports and Imports (Ottawa, Canada, 1994).

FMICBZZ - Electricity produced from hydropower at industrial facilities by State.

- 1960 through 1989: Calculated by ELA by multiplying the average factor for fossil fuels burned at steam-electric power plants (FFEOKUS) times the data in SEDS series HITCPZZ.

- 1990 forward: EIA estimates from data collected on Form EIA867, "Annual Nonutility Power Producers Report."

HYICPZZ - Flectricity produced from hydropower at industrial facilities by State (available for 1960 through 1989 only).

- 1960 through 1978: Federal Power Commission, Form 4, "Monthly Power Plant Report."

- 1979 and 1980: BIA estimates based on previous years' data.

- 1981 through 1989: No data available. The 1980 data are repeated for each year.
HYTMPZZ - Electricity produced from hydroelectric power and imported into the United States by State.

- 1960 throagh 1989: Assumed to be equal to total electricity imports (GLDMPZZ).

- 1990 forward: EIA estimates based on data from Natural Resources Canada, Electric Power in Canada 1994, and National Energy Board of Canada, Electricity Exports and Imports (Ottawa, Canada, 1994).

Jet Fuel

JKEUPZZ - Kerosene-type jet fuel consumed by electric utilities by State.

- 1960 through 1971: No dats available. Values are assumed to be zero.

- 1972 through 1974: U.S. Department of the Interior, Burean of Mines, Mineral Industry Surbeys, "Sales of Futel Oil and Kerosene," Table is footnote for U.S. value. These data were apportioned to the States by using the 1975 State proportions of the 1975 U.S. total from the source below.

- 1975 through 1979: Office of Electric Power Regulation, Federal Energy Regulatory Commission, Anntual Summary of Cost and Quality of Electric Utitity Plant Fuels, ${ }^{-}$Fuel Oil Deliveries for Combustion Turbine and Internal Combustion Units."

- 1980 through 1982: ELA, Cost and Quality of Fuel for Electric Utility Plants, Table 30.

- 1983 forward: Series discontinued; no data available. Values are assumed to be zero.

JKTTPZZ - Kerosere-type jet fuel total sold by State.

- 1960 through 1983: Ethyl Corporation, Petroleum Chemicals Division, Yearly Reporf of Gasoline Sales by States, "Aviation Turbine Fuel Sales.

- 1984 and 1985: EIA, Peiroleum Marketing Aंnuual 1985, Volume

- 1984: Table A6.

- 1985: Table 34.

- 1986 through 1988: EIA, Petroleum Marketing Annual, Table 46.

- 1989 forward: EtA, Petroleun Marketing Annual. - 1984 through 1993: Table 48. 
- 1994: EIA, Form EIA-782C, "Monthly Report of Prime Supplier Sales of Petroleum Products Sold for Local Consumpton." Data published in thousand gallons per day in ElA, Petroleum Marketing Annual 1994, Table 49. Withheld data were estimated by using averages of published months to fill in withheld months; subtracting published States from published PAD District totals; and assigning values based on previous years' quantitles.

JKTCPUS - Kerosenertype jet fuel total consumed in the United States.

- 1960 through 1975: U.S. Department of the Interior, Burean of

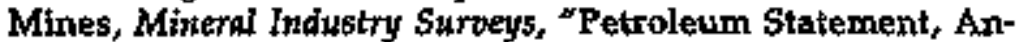
nual," Table 1.

- 1976 through 1980: ELA, Eniergy Dafa Reporis, "Petroleum Statement, Annual," Table 1.

- 1981 forward: BLA, Petroleum Supply Annual, Table 2.

JNMIPZZ - Naphtha-type jet fuel issuxed to the military in the United States.

- 1960 through 1974: No data are available. The 1977 data are used for each year.

- 1975 and 1976: No consistent data series are available. The 1977 date are used for both years.

- 1977 thurough 1987: The U.S. Department of Defense, Defense Logistics Agency, Defense Fuel Supply Center, Defense Energy Information System, military retail issues based on fiscal year data. The District of Columbia issues are assumed to be zero; therefore, values reported for the District of Columbia are added to Maryland.

- 1988: U.S. Department of Defense, Defense Logistics Agency, Defense Fuel Supply Center, average of 1987 data (see source above) and 1989 data (see source below).

- 1989 and 1990: U.S. Department of Defense, Defense Logistics Agency, Defense Fuel Supply Center, Defense Puel Atutomated Management System, military wholesale issues based on fiscal year data.

- 1991 forward: U.S. Department of Defense, Defense Logistics Agency, Defense Fuel Supply Center. State data for the calendar year from two databases are summed: Defense Fuel Automated Management System (military wholesale issues) and
Into-PIane Database (military purchases from commercial airports). Into-plane values reported for the District of Columbia are added to Virginia.

INTCPUS - Naphtha-type jet fuel total consumed in the United States.

- 1960 through 1975: U.S. Department of the Entertor, Bureau of Mines, Mineral Indusfry Surzeys, "Petroleum Statement, Annual," Table 1.

- 1976 through 1980; EIA, Energy Data Reports, "Petroleum Statement, Arnual," Table 1.

- 1981 forward: EIA, Petroleum Supply Annual, Table 2.

\section{Kerosene}

KSCMPZZ - Kerosene sold to the commercial sector for heating.

- 1960 through 1978: ELA estimates based on statistics of conmercial sector deliveries of kerosene from the EIA, Energy Data Report, "Deliveries of Fuel Oil and Kerosene, in 1979," Table 3. State ratios based on 1979 commercial sector deliveries were applied to each State's heating deliveries category from the fuel oil deliveries reports for each year 1960 through 1978. (See explanation in Appendix A, Note 3, on page 364.)

- 1979 and 1980: EIA, Enargy Data Report, "Deliveries of Fuel Oil and Kerosene," Table 3.

- 1981 and 1982: ElA, Petroleum Supply Amuual, Table 6.

Note: Data for 1983 forward were published in thousand gailons. They were converted to thousand barrels by dividing by 42 before being entered into SEDS.

- 1983 through 1987: BLA, Petroleum Marketing Monthly. The specific tables are:

- 1983: July 7985 isoue, Teble A14.

- 1984: July 1986 issue, Table A4.

- 1985 and 1986: July 1987 issue, Table A6.

- 1987: June 1988 issue, Table A6.

- 1988 forward EIA, Fuel Oil and Kerosene Sales, Table 6.

KSIHPZZ - Kerosene sold to the industrial sector for heating.

- 1960 through 1978: EIA estimates based on statistics of industrial sector deliveries of kerosene from the EIA, Energy Data Report, "Deliveries of Fuel Oil and Kerosene in 1979," Table 3. 
State ratios based on 1979 industrial sector dellverles were applied to each State's heating dellvertes category from the fuel oil deliveries reports for each year 1960 through 1978. (See explanation in Appendix $A$, Note 3, on page 364.)

- 1979 and 1980: EiA, Energy Data Reports, "Deliveries of Fuel Oil and Kerosene," Table 3.

- 1981 and 1982: EIA, Peiroleum Supply Anmual, Table 6.

Note: Data for 1983 forward were published in thousand gallons. They were converted to thousand batrels by dividing by 42 before being entered into SFDS.

- 1983 through 1987: ELA, Petroleum Marketing Monthiy. The specific tables are:

- 1989: July 1985 issue, Table A14.

- 1984: July 1986 issue, Table A4.

- 1985 and 1986: July 1987 issue, Table A6.

- 1987: June 1988 issue, Table A6.

- 1988 forward: ELA, Fuel Oil and Kerasene Sales, Table 6.

KSOTPZZ - Kerosene sold for all other uses, including farm use.

- 1960 through 1975: U.S. Department of the Interior, Bureau of Mines, Minteral Industry Surveys, "Shipments of Fuel Oil and Kerosene." The specific tables are:

- 1960 and 1961: Table 10.

- 1962 and 1963: Table 9.

- 1964 and 1965: Table 8.

- 1966 through 1975: Table 5.

- 1976 through 1978: EIA, Energy Data Reports, "Sales of Fuel Oil and Kerosene," Table 5.

- 1979 and 1980: BIA, Energy Data Reports, "Deliveries of Fuel Oil and Kerosene." Calculated as the sum of kerosene delivered for farm and other use from Table 3.

- 1981 and 1982: EIA, Petroleum Supply Antrual, Table 6.

Note: Data for 1983 forward were published in thousand gallons. They were converted to thousand barrels by dividing by 42 before being entered into SEDS.

- 1983 through 1987: EIA, Petroleum Marketing Monthly. The specific tables are:

- 1983: July 1985 issue, Table A14.

- 1984: July 1986 issue, Table A4.

- 1985 and 1986: July 1987 issue, Table A6.

- 1987: June 1988 issue, Table A6.
- 1988 forward: ELA, Fuel Oil and Kertsene Sales, Table 6.

KSRSPZZ - Kerosene sold to the residential sector for heating.

- 1960 through 1978: EIA, Energy Data Report "Deliveries of Fael Oil and Kerosene in 1979," Table 3. State ratios based on 1979 residential sector deliveries were applied to each State's heating deliveries category from the futel oil deliveries reports for each year 1960 through 1978. (See explanation ta Appen$\operatorname{dix}$ A, Note 3 , on page 364.)

- 1979 and 1980: EIA, Energy Data Report, "Deliveries of Fuel Oil and Kerosene," Table 3.

- 1981 and 1982: EIA, Petrolezm Stppty Aronual, Table 6.

Note: Data for 1983 forward were published in thousand gallons. They were converted to thotsand barrels by dividing by 42 before being entered into SEDS.

- 1983 through 1987: FIA, Petroleum Marketing Monthily. The specific tables are:

- 1983: July 1985 issue, Table A14.

- 1984: July 1986 issue, Table A4.

- 1985 and 1986: July 1987 issue, Table A6.

- 1987: June 1988 issue, Table A6.

- 1988 forward: ELA, Fuel Oil and Kerosene Sales, Table 6.

KSTCPUS - Kerosene total consumed in the United States.

- 1960 through 1975: U.S. Department of the Interior, Bureau of Mines, Mineral Industry Surbeys. "Petroleum Statement, Annual," Table 1.

- 1976 through 1980: BIA, Energy Data Reports, "Petroleum Statement, Annual," Table 1.

- 1981 forward: BIA, Petroleum Supply Annual, Table 2.

\section{Liquefied Petroleum Gases (LPG)}

LGCBMZZ - LPG sold for.internal combustion engine use by State.

Note: Data for Maryland and the District of Columbia were combined for all years. The method for disaggregating the data is explained in Appendix A, Note 1, on page 366.

- 1960 through 1967: U.S. Department of the Interior, Bureau of Mines, Minerul Industry Suroeys, "Shipments of Liquefied Petroleum Gases and Ethane." The speciffic tables are: 
- 1960 and 1961: Table 5 (data colled "Shipments")

- I962 througt 1966: Tabje 2 (data called "Consumption").

- 1967: Table 2 (data called "Shipments").

- 1968 through 1975: U.S. Department of the Interior, Burreau of Mines, Mineral Industry Surveys, "Sales of Liquefied Petroleum Gases and Ethane," Table 2.

- 1976 through 1980: EIA, Energy Data Reports, "Sales of Liquefied Petroleum Gases and Ethare," Table 2.

- 1981 and 1982: ElA, Petroletwm Supply Annuel, "Sales of Liquefied Petroleum Gases and Ethane," Table 3.

- 1983: EIA estimates.

Note: For 1984 forward, some data are adjusted and estimated by

EIA. (See explanation in Appendix A, Note 7, on page 368.)

- 1984 through 1988: American Petroleim Institute, 1990 Sales of Natural Gas Liquids and Liquefied Refinery Gases, pages 24 through 33 .

- 1989 through 1991: American Petroleum Institute, 1992 Sales of Natural Gas Liquids and Liquefied Refintry Gases, pages 4, 5, 18, and 19.

- 1992: American Petroleum Institute, 1993 Sales of Natural Gas Liquids and Liquefied Refineny Gases, Table 3.

- 1993 and 1994: American Petroleum Institute, 1994 Sales of Natural Gas Ligudds and Liquefied Refonery Gases, Table 3.

LGHCMZZ - LPG sold for residential and commerciel use by State. Note: Data for Mayyland and the District of Columbia were combined for all years. The method for disaggregating the data is explained in Appendix A, Note 1, on page 366 .

- 1960 through 1967: U.S. Depaxtment of the Interior, Bureau of Mines, Mineral Indtustry Suroeys, "Shipments of Liquefied Petroleurn Gases and Ethane." The specific tables are:

- 1960 and 1961: Table 5 (data called "Shipmenta").

- 1962 through 1966: Table 2 (data called "Consumption"), - 1967: Table 2 (data called "Shipments").

- 1968 through 1975: U.S. Diepartment of the Interior, Bureau of Mines, Mineral Industry Surveys, "Sales of Liquefied Petroleum Gases and Ethane," Table 2.

- 1976 through 1980: EIA, Energy Data Reports, "Sales of Liquefied Petroleum Gases and Ethane," Table 2.

- 1981 and 1982: ELA, Petrolentm Supply Annual, "Sales of Liquefied Petroleum Gases and Ethane," Table 3.
- 1983: ETA estimates.

Note: For 1984 forward, some data are adjusted and estimated by

EIA. (See explanation in Appendtx A, Note 7, on page 368.)

- 1984 through 1988: American Petroleum Institute, 1990 Sales of Natural Gas Liquiois and Liquefied Refinery Gases, pages 24 through 33 .

- 1969 through 1991: American Petroleum Institute, 1992 Sales of Natural Gas Liquitds and Liquefied Refinery Gases, pages 4, 5, 18 , and 19 .

- 1992: American Petroleum Irstitute, 1993 Sales of Nafural Gas Liquids and Liguefied Refinery Gases, Table 3.

- 1993 and 1994; American Petroleum Institute, 1994 Sales of Natiural Gas Liquids and Liquefied Refinery Gases, Table 3.

LGTCKUS - Factor for converting LPG from physical tuits to Btu.

- 1960 throughi 1966: U.S. Department of the Interior, Bureau of Mines, Mineral Industry Surveys, "Crude Petroleum and PetroFeum Products, 1956," Table 4 foothote, constant value of 4.011 million Btu per barrel.

- 2967 forward: Calculated annually by EIA as a weighted average by multiplying the quantity consumed of each of the component products by each product's conversion factor (given with soturce references in Appendix D) and dividing the sum of those heat contents by the sum of the quantities consumed. The component products are ethane (including ethylene); propane (including propylene), normal butane (including butylene), butane-propane mixtures, ethane-propane mixtures, and isobutane Quantities consumed are from:

- 1967 through 1980: EIA, Energy Data Reports, "Petroleum Statement, Annual, Table' 1.

- 1981 forward: ELA, Petroleum Supply Annual, Table 2.

LGTCPUS - LPG total consumed in the United States.

- 1960 through 1975: U.S. Department of the Interior, Bureau of Mines, Mineral Industry Surovys. "Petroleum Statement, An nual," Table 1 .

- 1976 through 1980: ELA, Energy Data Reports, "Petroleum Statement, Amnual," Table 1.

- 1981 forward: BLA, Peiroleum Stopply Annual, Table 2. 
LGTRSUS - The transpottation sector share of LPG internal combustion engine sales.

- EIA estimates based on the LPG portion of the special fuels used on highways published by the U.S. Department of Transportation, Federal Highway Administration (variable MGSFPUS in SEDS), as a percentage of the LPG sold for internal combustion engine use published by the American Petroleum Institute (variable LGCBMUS in SEDS). For an explanation of the estimation method, see Appendix A, Note 2, on page 366.

LGTTPZZ - LPG total sales for all uses by State.

Note: Data for Maryland and the District of Columbia were combined for all years. The method for disaggregating the data is explained in Appendix A, Noke 1, on page 366.

- 1960 through 1967: US. Department of the Interiox, Bureau of Mines, Mineral Industry Suroeys, "Shipments of Liquefled Petroleum Gases and Ethane." The specific tables are:

- 1960 and 1961: Table 5 (data called "Shipments")

- 1962 through 1966: Table 2 (data called "Consumption"). - 1967: Table 2 (data called "Shipments").

- 1968 through 1975: U.S. Department of the Interios, Bureau of Mines, Mineral Industry Surveys, "Sales of Liquefied Petroleum Gases and Ethane, Table 2.

- 1976 through 1980: EIA, Energy Dafa Reports, "Sales of Liquefied Petroleum Gases and Ethane," Table 2.

- 1981 and 1982. EIA, Petroleum Suppiy Annual, "Sales of Liquefied Petroleum Gases and Ethane, Table 3.

- 19B3: ElA estimates.

Note: For 1984 forward, some dats are adjusted and estimated by

EIA. (See explanation in Appendix A, Note 7, on page 368.)

- 1984 through 1988: American Petroleum Institute, 1990 Sales of Natural Gas Liquids and Liquefied Refintery Gases, pages 24 through 33.

- 1989 twrough 1991: American Petroleum Institute, 1992 Sales of Natural Gas Lipuids and Liquefied Refinery Gases, pages 4, 5, 18, and 19.

- 1992: American Petroleum Institute, 1993 Sales of Natural Gas Liquids and Liquefied Refintery Gases, Table 3.

- 1993 and 1994: American Petroleum Institute, 1994 Sajes of Natural Gas Liquids and Liquefied Refintery Grses, Table 3.

\section{Lubricants}

LUINPZZ - Lubricants sold to the industrial sector by State Calculated from:

- US. Department of Commerce, Bureau of the Census, Current Industrial Reports, "Sales of Lubricating and Industrial Oils and Greases," for 1960, 1962, 1965, 1967, 1969, 1971, 1973, 1975, and 1977. (See explanation in Appendix A, Notes 1 and 2, on page 369.)

LUTCPUS - Lubricants total consumed in the United States.

- 1960 through 1975: U.S. Department of the Interior, Burean of Mines, Mineral Industry Surweys, "Petroleum Statement, Annual, Table 1 .

- 1976 through 1980: EIA, Energy Data Reports, 'Petroleum Statement, Annual," Table 1.

- 1981 forward: ELA, Petroleum Supply Annual, Table 2.

LUTRPZZ - Lubricants sold to the transportation sector by State. Calculated from:

- U.S. Department of Commerce, Bureart of the Census, Current Industrial Reports, mSales of Lubricating and Industrial Oils and Greases," for 1960, 1962, 1965, 1967, 1969, 1971, 1973, 1975 , and 1977. (See explanation in Appendix A, Notes 1 and 2, on page 369.)

\section{Motor Gssoline}

MGAGPZZ - Motor gasoline sold for agricultural use by State.

- 1960 through 1964: U.S. Department of Commence, Bureau of Public Roads, Highray Statistics, Table G-24.

- 1965 forward: U.S. Department of Transportation, Federal Highway Administration, Highruay Statistics, Table G-24 in 1965 and MF-24 in 1966 forward.

MGCUPZZ - Motor gasoline sold for cotstruction use by State.

- 1960 through 1964: U.S. Department of Commerce, Bureau of Public Roads, Hitghtay Statistics, Table G-24. 
- 1965 forward: U.S. Department of Transportation, Federal Highway Administration, Highway Statistics, Table G-24 in 1965 and MF-24 in 1966 forward.

MGIYPZZ - Motor gasoline sold for industrial and commercial use by State.

- 1960 through 1964: U.S. Department of Connmerce, Bureau of Public Roads, Highwary Statistics, Table G-24.

- 1965 forward: U.S. Department of Transportation, Federal Highway Administration, Highway Statistics, Table G-24 in 1965 and MF-24 in 1966 forwand.

MGMPPZZ - Motor fuel sold for highway use by State.

- 1960 through 1964: U.S. Department of Comunerce, Bureau of Public Roads, Highwy Statsittcs, Table G-21.

- 1965 forward: US. Department of Transportation, Federal Highway Administration, Highroxy Statistics, Table G-21 in 1965 and MF-21 in 1966 forward.

- 1960, 1966 through 1971, 1975, and 1983 revisions: U.S. Department of Transportation, Federal Highway Administration, Highwany Statistics Summery to 1985, Table MF-221 gave revised U.S. totals. State revisions were calculated by adding data from Tables MF-225 and MF-226.

MGMRPZZ - Motor gasoline sold for matine use by State.

- 1960 through 1964: U.S. Department of Commerce, Bureau of Public Roads, Highosy Statistics, Table G-24.

- 1965 forward: U.S. Department of Transportation, Federal Highway Administration, Highwoxy Statistics, Table G-24 in 1965 and MF-24 in 1966 forward.

MGMSPZZ - Motor gasoline sold for miscellaneous uses by State.

- 1960 through 1964: U.S. Department of Commerce, Bureau of Public Roads, Hightoxy Statistics, Table G-24. Sum of the "Miscellaneous" column plus the "Unclassified" column minus the "Total Classified" column.

- 1965: U.S. Department of Transportation, Federal Highway Administration, Highway Statistics, Table G-24. Sum of the "Miscellaneous" column plus the "Unclassified" cotumn mitrus the "Total Classified" column.
- 1966 forward: U.S. Department of Transportation, Federal Highway Administration, Highway Statistics, Table MF-24. The specific columns are:

- 1966 through 1981: Sum of the "Miscellaneous" and "Unclassified" columns.

- 1982 forward: The "Miscellaneous" column.

MGPNPZZ - Motor futel sold for public nonhighway use by State.

- 1960 through 1964: U.S. Department of Conmerce, Bureat of Public Roads, Highxory Statistics, Table G-21.

- 1965 forward: U.S. Department of Transportation, Federal Highway Administration, Highrory Statistics, Table G-21 in 1965 and MF-21 in 1966 forward.

MGSFPZ7, - Motor gasoline special fuels sales by State (primarily diesel fuel with small amounts of liquefied petroleum gases).

- 1960 through 1985: U.S. Department of Transportation, Federal Fighway Administration, Hightway Statistics, Summary to 1985, Table MF-225.

- 1986 through 1993: U.S. Department of Transportation, Pederal Highway Administration, Highwary Statistick, Table MF-25.

- 1994: U.S. Department of Transportation, Federal Highway Administration, Highaxay Statistics, Table MF-21.

MGTCPUS - Motor gasoline total consumed in the United States.

- 1960 through 1975: U.S. Department of the Interior, Bureau of Mines, Mintral Industry Surpeys. "Petroleum Statement, Anntual, Table 1.

For 1960 through 1963, motor gasoline was combined with aviation gasoline and published as "gasoline" in the source table. Table 19 in the "Petroleum Statement, Annual" titled "Salient Statistics of Aviation Gasoline" provided separate data for aviation gasoline for those years. The aviation gascline data from the second table were subtracted from the gasoline data in the first table to derive the motor gasoline consumption series used in SBDS.

- 1976 through 1980: BIA, Energy Data Reporfs, "Petroleum Statement, Annual," Table 1.

- 1981 forward: EIA, Petroleum Supply Annual, Table 2. 


\section{Motor Gasoline Blending Components}

MBTCPUS - Motox gasoline blending components total consumed in the Urited States.

- 1960 through 1980. No data available. Values are assuned to be zero.

- 1981 forward: EIA, Petrolleum Supply Arnual, Table 2.

\section{Miscallaneous Petroleum Products}

MSTCPUS - Miscellaneous petroleum products consamed in the United States.

- 1960 through 1975: U.S. Department of the Interior, Bureau of Mines, Minerat Industry Suroys, "Petroleum Statement, Annual," Table 1.

- 1976 through 1980: EIA, Energy Data Reports. "Petroleum Statemtent, Arnual," Table 1.

- 1981 forward: EIA, Petroleum Supply Annual, Table 2.

\section{Natural Gasoline}

NATCPUS - Natural gasoline total consumed in the United States.

- 1960 through 1975: U.S. Department of the Interior, Bureau of Mines, Minteral Industry Surotys. "Petroleum Statement, Annual," Table 1.

- 1976 through 1980: ELA, Erergy Data Reports. "Petroleum Statement, Annual," Table 1.

- 1981 through 1983: ELA, Petroleum Supply Annual, Table 2.

- 1984 forward: RIA, Petroleum Stupply Arnulal, Table 2, included in "Pentanes Plus."

\section{Nalural Gas}

NGCCPZZ - Natural gas delivered to the commercial sector and to other consumers (municipalities and public authorities for institutional heating and street lighting), including natural gas consumed as vehicle fuel through 1989, by State.

- 1960 through 1966: U.S. Department of the Interior, Bureat1 of Mines, Mineral Industry Surveys, Nahural Gas Production and
Consumption," table titled "Number of consumers and volume of natural gas consumed by principal users in the Urited Stetes," column "Commencial-"

- 1967 through 1994: EIA, Natural Gas Antual 1994 Voltine II, Table 16.

Data also available via internet:

- 1967 through 1994.

ftp://ftp.ela.doe.gov/pub/natural.gas/data/annual/ ga94-v2.exe and

ftp://ftp.eia.doegov/pub/ratural.gas/pdf/nga4v22.pdf

The FTP files can be reached through http://www.eia.doe.gov (select "FTP Site" from the menu).

NGEUKZZZ - Factor for converting natural gas consumed by the electric utilities from physical units to Btu.

- 1960 through 1971: Assumed by the EIA to be equal to the thermal conversion factor for the consumption of natural gas by all usens (NGTCKZZ).

- 19/2 forwand: Calculated annually by ELA by dividing the total heat content of ratural gas consumed at electric utilities by the total quantity received at electric utilities. The heat contents and quantities received axe from the Federal Energy Regulatory Commission (FERC) Form 423, "Monthly Report of Cost and Quality of Fuels for Electric Plants," and predecessor formus. Data in Btu per cubic foot for 1994 are published in EIA, Cost and Quality of Fuels for Electric Utility Plants 1994, Table 14.

Note: For States that reported consumption on EIA-759 but were not large enough to report on FERC Form 423, factors were estimated by using previous years' factors or the factor for total natural gas consumption in the State.

NGEUPZZ - Natural gas consumed by the electric utilities by State.

- 1960 through 1975: Federal Power Commission, News Release, "Power Production, Fuel Consumption, and Installed Capacity Data," table titled "Consumption of Fut by Electric Utilities for Production of Electric Energy by State, Kind of Fuel, and Type of Prime Mover," sum of columms, "steam and 
gas turbine" and "internal combustion" under column heading "gas."

- 1976 through 1981: ELA, Eleckric Poture Annunl (1981), Table 67.

- 1982 forward: Untounded data as published in rounded form in the following teports:

- 1982 through 1986: EIA, Electric Power Antrual 1986, Table 14.

- 1987 and 1988: ELA, Electric Power Annal 1988, Table 13.

- 1989 and 1990: EIA, Electric Prover Annual 1990, Table 19.

- 1991: EIA, Electric Power Antual 1992, Table 20.

- 1992 and 1993: EIA, Electric Power Annual 1993, Table 20.

- 1994: EIA, Electric Power Annuth 1994 Voltome I, Table 18.

NGINPZZ - A portion of the natural gas delivered to the industrial sector by State.

- 1960 through 1966: U.S. Department of the Interior, Bureau of Mines, Mineral Industry Surbeys, "Natural Gas Production and Consumption," table thted "Number of constumers and volume of natural gas constumed by principal users in the United States." Sum of data in columns "Carbon black," "Refinery fuel," and "Other industrial fuel" (which includes electric utility fuel) minus data in column "Fuel used at electric utility plants."

- 1967 through 1994: EIA, Natural Gas Annual 1994 Volume Il, Table 16.

Data also atailable via internet:

- 1967 through 1994:

ftp: / /ftp.eia.doe.gov/pub/ratural.gas/data/annual/ nga94-v2.exe and

ftp://fp.eia.doe.gov/pub/natural.gas/pdf/nga4r22.pdf

The FTP files can be reached through hitp $/ /$ Www,eia.doe.gov (select "FIP Site" from the menu):

NGLEPZZ - Natural gas consumed as lease fuel by State (includes natural gas consumed as plant fuel in 1960 through 1992).

- 1960 through 1966: U.S. Department of the Interior, Burean of Mines, Minersis Yearbook, Natural Gas chapter. State data are not available from 1960 through 1966 , although U.S. totals are available. State estimates were calculated by apportioning the U.S. totals to the States on the basis of each State's share of the U.S. total in 1967.
- 1967 through 1992; EIA, Natural Gas Annual 1994 Volume It, Table 14.

- 1993 and 1994: ElA, Natrutal Gas Annual 1994 Volume II, Table 15.

Data also availzble via internet:

- 1967 through 1994:

ftp://ftp.eia.doe.gov/pub/natural.gas/data/annual// nga94-v2exe and

ftp://fip.eis.doe.gov/pub/natural.gas/pdf/nga4v22.pdf

The PTP files tan be reactied through http://www.eiadoe.gov (select "FTP Site" from the menu).

NGPLPZZ - Natural gas consumed as plant fuel by State.

- 1960 through 1992: Included with natural gas consumed as lease fuel (see NGLEPZZ).

- 1993 and 1994: EIA, Natura' Gas Anntal 1994 Volume II, Table 15.

Dala also available via intornet:

- 1967 through 1994:

ftp://ftp.eia.doe.gov/ptab/natural.gas/data/annual/ niga94-v2exe and

ftp: / /tp.eia.doe.gov/pub/natural.gas/pdf/nga4v22.pdf

The FIP files can be reached through http://www.eia.doegov (select "FTP Site" from the menu).

NGPZPZZ - Natural gas consumed as pipeline fuel by State.

- 1960 through 1966: U.S. Department of the Interior, Bureau of Mines, Mineral Industry Surpeys, "Natural Gas Production and Construption," table tikled "Number of consumers and volume of natural gas consumed by principal users in the United States," columin "Used as pipeline fuel."

- 1967 through 1992: ELA, Natural Gas Anitwal 1994 Volume II, Table 14.

- 1993 and 1994: ELA, Natural Gas Annual 1994 Volume 1, Table 15.

Data also available via internet:

- 1967 through 1994:

ftp://ftp.eia.doe.gov/pub/natural.gas/data/annual/ nga94-v2.exe and ftp://ftp.eia.doe.gov/pub/natural.gas/pdf/nga4v22.pdf 
The FTP flles can be reached through http://www.eia.doe.gow (select "FIP Site" from the menu).

NGRCPZZ - Natural gas delivered to the residential sector, used as consumption, by State.

- 1960 through 1966: U.S. Department of the Interior, Bureau of Mines, Mineral Industry Suroeys, "Natural Gas Production and Consumption," table titled "Number of consumers and volume of natural gas consumed by principal users in the United States," columu "Residential."

- 1967 through 1994: ELA, Natural Gas Antual 1994 Volume II, Table 16.

Data also available via internet:

- 1967 through 1994

ftp: / ftp.eia.doe.gov/pub/natural.gas/data/annual/ ngag4-V2.exe and

ftp://ftp.eia.doe.gov/pub/natural.gas/pdf/nga4p22.pdf

The FTP files can be reached through http: / /www.eia.doe.gov (select "FTP Stte" from the menu).

NGVZPZZ - Natural gas delivered for use as vehicle fuel by State.

- 1960 through 1989. Included in natural gas constumed by the commercial sector (See NGCCPZZ).

- 1990 through 1994: ELA, Natural Gas Annual 1994 Volume II, Table 16.

Data also available via internet:

- 1967 through 1994:

ftp://ftp.eia.doe.gov/ptab/natural.gas/data/annual/ nga94-v2.exe and ftp://ftp_eia.doe.gov/pub/natural.gas/pdf/nga4v22.pdf

The FTP files can be reached through http://www.eia.doe.gov (select "FTP Site" from the meru).

NGTCKZZ - Factor for cónverting natural gas consumed by all users from physical units to Btu.

- 1960 through 1962: EIA adopted the thermal converston factor of 1035 Btu per cubic foot as estimated by the Bureau of Mines and first published in the Petroleum Statement, Annual, 1956.

- 1963 through 1979: ELA adopted the thermal conversion factors calculated annually by the American Gas Association (AGA) and published in Gas Facts, an AGA annual.
- 1980 through 1994: EIA, Notural Gas Ammal 1994 Volume II. Table 16.

Data also avallable via internet.

- 1980 through 1994:

ftp://ftp.eia.doe.gov/pub/natural.gas/data/annual/ nga94-v2,exe and

ft://fp.eia.doe-gov/pub/natural.gas/pdf/ngafv22_pdf

The FTP files can be reached through hitp:/ / www.eia.doegov (seleat "FIP Site" from the menu).

Nuctear

NUEOKUS' - Factor for converting electricity produced from nuclear power from physical units to Btu.

- 1960 through 1991: Calculated annually by EIA by dividing the total heat content consumed in reactors at nuciear plants by the total (net) electricity generated by nuclear plants. The heat content and electricity generation are reported on FERC Form 1, "Annual Report of Major Electric Utilities, Licensees, and Others;" Form BLA-412, "Anrual Report of Public Electric Utilities;" and predecessor forms. The factors for 1982 through 1991 are published in the following:

- 1982: ElA, Historical Plant Cost and Annual Production Expenses for Selected Electric Plants, page 215.

- 1983 through 1991: EIA, Electric Plant Cost and Pawer Production Expenses 1991, Table 13.

- 1992 forward: Unpublished factors calculated annually by ELA by dividing the total heat content of the steam leaving nuclear generating units to generate electriclty by the total (net) electricity generated by.nuclear generating units. The heat content and electricity generation data are reported in the Nuclear Regulatory Commisston, Licensed Operating Reactors-Stafus Summary Repart.

NUEOPZZ - Electricity produced from nuclear power at electric utilities by State.

- 1960 through 1977: Federal Power Commission, News Release, Power Production, Fuel Consumption, and Installed Capacity Data," table tilled "Net Generation of Electric Utilities by State and Source." 
- 1978 turough 1980; EIA, Energy Datn Reparts, "Power Production, Fuel Consumption and Installed Capacity Data;" 1978: table titled "Net Generation of Electric Utilities by State and Source; 1979 and 1980: Table 36.

- 1981 forward: EIA, Form EIA-759, "Monthly Power Plant Report, " and predecessor forms. Published data rounded to gigawatthours in the following reports:

- 1981 through 1985: EIA, Electric Power Annual 1985, Table 6.

- 1986 and 1987: EIA, Electric Pozuer Annugl 1987, Table 19.

- 1988 and 1989. ELA, Electric Pouter Annual 1989, Table 14.

- 1990 forward: EIA, Electric Potoer Annual, Table 13.

\section{Pentanes Phus}

PPTCPUS - Pentanes plus total consumed in the United States.

- 1960 throutgh 1983: Data were reported separately as natural gasoline, isopentane, and plant condensate.

- 1984 forward: EIA, Petroleum Supply Annual, Table 2.

\section{Petroleum Coke}

PCCTPUS - Petroleum coke consumed at refineries (both catalyst and marketable) in the United States.

- 1960: No data available. The 1961 value is used for 1960.

- 1961 through 1975: U.S. Deparment of the Interior, Bureau of Mines, Mineral Industry Surogys, "Petroleum Statement, Annual. ${ }^{*}$ The specific tables are:

- 1961 and 1962: Table 18.

- 1962 through 1966: Table 19.

- 1967: Table 18.

- 1968: Table 19.

- 1969 through 1972: Table 18.

- 1973 and 1974: Table 21.

- 1975: Table 22.

- 1976 through 1980: EIA, Energy Data Reports, "Petroleum Statement, Annual." The specific tables are:

- 1976: Table 22.

- 1977: Table 21.

- 1978 through 1980: Table 20.
- 1981 forward: EIA, Petroleum Stpply Annual. The specific tables are:

- 1981 and 1982: Table 17.

- 1983: Table 15.

- 1984: Table 44.

- 1985: Table 43.

- 1986 through 1988: Table 38.

- 1989 through 1992: Table 45.

- 1993 and 1994: Table 47.

PCEUMZZ - Petroleum coke consumed by the electric utilities by State.

- 1960 through 1969: No data avajlable. Values are assumed to be zero.

- 1970 forward: EIA, Form EIA-759, "Monthly Power Plant Report," and predecessor forms.

PCTCPUS - Petroleum cake total consumed in the United States.

- 1960 through 1975: U.S. Department of the Intertor, Bureau of Mines, Mineral Industry Strveys. "Petroleum Statement, Annual, ${ }^{,}$Table 1.

- 1976 through 1980: EIA, Energy Data Report, "Petroleum statement, Armual," Table I.

- 1981 forward: ELA, Petroleum Supply Arnual, Table 2.

\section{Plent Condensate}

PLTCPUS - Plant condenșate total consumed in the United States.

- 1960 through 1975: U.S. Department of the Interior, Bureau of Mines, Mineral Industry Surpeys. "Petroleum Statement, Annual," Table 1.

- 1976 through 1980: ElA, Energy Data Reports, "Petroleum Statement, Annual," Table 1.

- 1981 through 1985: ELA, Petrojeum Supply Annudi, Table 2.

- 1984 forward: EIA, Petroleum Supply Annual, Table 2, included in "Pentanes Plus." 


\section{Population}

TPOPPUS - Resident population of the United States. April 1 census for 1960, 1970, 1980, and 1990, and July 1 estimates for all other years.

- U.S. Department of Commerce, Bureau of the Census, Current Population Reports, "Population Estimates and Projections," Series $\mathrm{P}-25$. Spectific publication numbers and table numbers:

- 1960 through 1969: Number 990, Table 4.

- 1970 through 1979. Number 957, Table 4.

- 1980 through 1989. Number 1058, Table 3.

- 1990 forward: Press Release Number CB96-10, January 26, 1996.

Data also available via intemet:

- 1990 forward:

ftp://ftp.census.gov/pub/population/estimates/state/ stintasr. zip

The FTP files can be reached through hitp://www.census.gov (select "FTP Files" from the menu).

TPOPPZZ - Resident population by State. April 1 census for 1960 1970, 1980, and 1990, and July 1 estimates for all other years.

- U.S. Deparment of Commerce, Bureau of the Census, Current Population Reports, "Population Estimates and Projections," Series $\mathrm{P}-25$. Specific publication numbers and table numbers:

- 1960 through 1969: Number 460, Table 1.

- 1970 through 1979. Number 957, Table 4.

- 1980 through 1989: Number 1058, Table 3.

- 1990 forward: Press Release Number CB96-10, January 26, 1996.

Data also available via internet

- 1990 forward:

ftp://ftp.census.gov/pub/population/estimates/state/ stintasr.zip

The FTP files can be reached through http://www.census.gov (select "FTP Files" from the menu),

\section{Residual Fuet}

RFBKPZZ - Residual fuel sold for vessel burkering use by State.
- 1950 through 1975: U.S. Department of the Interior, Bureau of Mines, Mineral Industry Surweys, "Shipments of Fuel oil and Kerosene." The specific tables are:

- 1960 and 1961: Table 17.

- I962 and 1963: Table 16.

- 1964 and 1965: Table 15.

- 1966 through 1975: Table 11.

- 1976 through 1978: ELA, Entergy Data Reports, "Sales of Fuel Oil and Kerosene, " Table 11.

- 1979 and 1980: ElA, Enetgy Data Reports, "Deliveries of Fuel Oll and Kerosene," Table 2.

- 1981 and 1982: EIA, Petroleum Supply Anumal, Table 5.

Note: Data for 1983 forward were published in thousand gallons. They were converted to thousand barrels by dividing by 42 before being entered into SEDS.

- 1983 through 1987: EIA, Petroleum Marketing Monthly. The specific tables are:

- 1983: July 1985 issue, Table AI3.

- 1984 and 1985: July 1986 issue, Table A3.

- 1986 and 1987: June 1988 issue, Table A5.

- 1988 forward: BIA, Fuel Oil and Kerosene Sales, Table 5.

RFCMPZZ - Residual fuel sold to the commercial sector for heating.

- 1960 through 1978: EIA estimates based on statistics of commercial sector deliveries of residual fuel from the EIA, Energy Date Report, "Deliveries of Fuel Oil and Kerosene in 1979," Table 2. State ratios based on 1979 commercial sector deliveries were applied to each State's sum of heating pltus industrial deliveries categories from the fuel oil deliveries reports for each year 1960 through 1978. (See explanation in Appendix A, Note 2, on page 374 .)

- 1979 and 1980; EIA, Energy Data Report, "Delivertes of Fuel Oil and Kerosene," Table 2.

- 1981 and 1982: ELA, Petroleum Supply Annual, Table 5.

Notes: Data for 2983 forward were publtshed in thousand gallons. They were converted to thousand barrels by dividing by 42 before being entered into SEDS. Data for Hawaii in 1986 through 1990 reflect unpublished revisions from an EIA internal memorandum from the Office of Oil and Gas to the Office of Energy Markets and End Use, "Revising Historical Petroleum Data," February 26, 1993. 
- 1983 through 1987: ELA, Petroleum Marketing Monthly. The specific tables are:

- 1983: July 1985 issue, Table A13.

- 1984 and 1985: July I986 issue, Table A3.

- 1986 and 1987: June 1988 issue, Table A5.

- 1988 forward: EIA, Fuel Oll and Kerosene Sales, Table 5.

RFEUPZZ, - Residual fuel consumed at electric utilities.

- EIA, Form EIA-759, "Monthly Power Plant Report," and predecessor forms. The following assumptions have been made. utilities by State is available. State estimates of residusal fuel consumption were created for each year by applying the shares of steam plants (primarily residual fuel) by State from 1970 to each year's total fuel oil consumption at electric utiflities for 1960 through 1969.

- 1970 through 1979: Fuel oil consumed by plant type by State is arailable. Fuel oil consumed by steam plants is assumed to equal residual fuel consumption.

- 1980 forward: Consumption of light and heavy oil at all plant types by State is available. Total heavy oil consumption at all plant types is asstuned to equal residual futel consumption.

RFIBPZZ - Residual fuel sold to industrial establishments for heating and for other industrial use.

- 1960 through 1978: BIA, estimates based on statistics of industrial sector deliveries of residual fuel from the EIA, Energy Data Report, "Deliveries of Fuel Oil and Kerosene in 1979," Table 2. State ratios based on 1979 industrial sector deliveries were applied to each State's sum of heating plus industrial deliveries categories from the fuel oil deliveries reports for each year 1960 through 1978. (See explanation in Appendix A, Note 2, on page 374.)

- 1979 and 1980: ELA, Energy Data Report, "Deliveries of Fuel Oil and Kerosene," Table 2.

- 1981 and 1982: EIA, Petrolerm Supply Armual, Table 5.

Note: Data for 1983 forward were published in thousand gallons. They were converted to thoustard barrels by dividing by 42 before being entered into SEDS.

- 1983 through 1987: ELA, Petroleum Marketing Monthly. The spectfic tables are:

- 1983: July 1985 issue, Table A13.

- 1984 and 1985: July 1986 issue, Table A3.

- 1986 and 1987: June 1988 issue, Table A5.

- 1988 forward: ElA, Fuel Oil and Kerosene Sales, Table 5.

RFMIPZZ - Restdual futel sold to the Armed Forces regardless of use by State.

- 1960 through 1975: U.S. Department of the Interior, Bureau of Mines, Mineral Industry Surzeys, "Shipments of Fuel Oil and Kerosene." The specific tables are:

- 1960 and 1961: Table 18.

- 1962 and 1963: Table 17.

- 1964 and 1965: Table 16.

- 1966 through 1975: Table 12.

- 1976 through 1978: BLA, Energy Doita Reports, "Sales of Fuel Oil and Kerosene," Table 12.

- 1979 and 1980. BIA, Energy Data Reports, "Deliveries of Futel Otl and Kerosene," Table 2 .

- 1981 and 1982: EIA, Petroleum Supply Annual, Table 5.

Note: Data for 1983 forward were published in thousand gallons. They were converted to thousand barrels by dividing by 42 before being entered into SEDS.

- 1983 through 1987: EIA, Petroleum Marketing Monthly. The specific tables are:

- 1983: July 1985 issue, Table A13.

- 1984 and 1985: July 1986 issue, Table A3.

- 1986 and 1987: June 1988 issue, Table A5.

- 1988 forward: EIA, Fuel Oil and Kerosene Sales, Table 5.

RFMSPZZ - Residual fuel sold for miscelianeous uses by State.

- 1960 through 1975: US. Department of the Interior, Bureau of Mines, Mineral industry Surveys, "Shipments of Fuel Oil and Kerosene." The specific tables are:

- 1960 throutgh 1962: Table 19.

- 1963 and 1964: Table 18.

- 1965 through 1967: Table 17.

- 1968 through 1975: Table 14.

- 1976 through 1978: EIA, Energy Dato Reports, "Sales of Fuel Oil and Kerosene," Table 14. 
- 1979 and 1980: EIA, Energy Dafa Reporls, "Deliveries of Fuel Oil and Kerosene," Table 2, column "Other."

- 1981 and 1982: ETA, Petroleum Supply Amnul, Table 5, column "All Other."

Note: Data for 1983 forward were published in thousand gallons. They were converted to thousand barrels by dividing by 42 before being entered into SEDS. The data series is tiled "All Other."

- 1983 through 1987: EIA, Petroleum Marketing Monthly. The specific tables are-

- 1983: July 1985 issue, Table A13.

- 1984 and 1985: July 1986 issue, Table A3.

- 1986 and 1987: Jume 1988 issue, Table A5.

- 1988 forward: EIA, Fued Oit and Kerosene Sales, Table 5.

RFOCPZZ - Residual fuel sold for use by oil companies by State.

- 1960 through 1975: U.S. Department of the Interlor, Bureau of Mines, Mineral Industry Surveys, "Shipments of Fuel Oil and Kerosene." The spectflc tables are:

- 1960 and 1961: Table 14.

- 1962 and 1963: Table 13.

- 1964 and 1965: Table 12.

- 1966 through 1975: Table 9.

- 1976 through 1978: EIA, Energy Data Reports, "Sales of Fuel Oil and Kerosene," Table 9.

- 1979 and 1980: EIA, Energy Data Reports, "Deliveries of Fuel Oil and Kerosene," Table 2.

- 1981 and 1962: EIA, Petralentm Supply Annual, Table 5.

Note: Data for 1983 forward were published in thousand gallons.

They were converted to thousand barrels by dividing by 42 before be ing entered into SEDS.

- 1983 through 1987: Ela, Petroleum Marketing Monthly. The specific tables are:

- 1983: July 1985 issue, Table A13.

- 1984 and 1985: July 1986 issue, Table A3.

- 1986 and 1987: June 1988 issue, Table A5.

- 1988 forward: EIA, Futel Oil and Kerosene Sales, Table 5.

RFRRPZZ - Residual fuel sold for use by railroads by State.

- 1960 through 1975: U.S. Department of the Interior, Bureau of Mines, Mineral Industry Suroeys, "Shipments of Fuel Oil and Kerosene." The specific tables are:
- 1960 and 1961: Table 16.

- 1962 and 1963: Table 15.

- 1964 and 1965: Table 14.

- 1966 through 1975: Table 10.

- 1976 through 1978: EIA, Entergy Datn Reports, "Sales of Furel Oil and Kerosere," Table 10.

- 1979 and 1980: EIA, Energy Data Reports, "Deliverties of Fuel Oil and Kerosene," Table 2.

- 1981 and 1982: EIA, Petroletom Supply Annual, Table 5.

Note: Data for 1983 forward were published in thousand gallons. They were converted to thousand barrels by dividing by 42 before being entered into SEDS.

- 1983 through 1987: EIA, Petroleum Marketing Monthly. The specific tables are:

- 1983: July 1985 issue, Table A13.

- 1984 and 1985: July 1986 issue, Table A3.

- 1986 and 1987: Jurne 1988 issure, Table A5.

- 1988 and 1989: EIA, Fvei Oil and Kerasene Sales 1989, Table 5.

- 1990 forward: EIA, Fuel Oil and Kerosene Sales, Table 5, included in the "All Other" data (RFMSPZZ in SEDS).

RFTCPUS - Residual fuel total consumed in the United States.

- 1960 through 1975: U.S. Department of the Interior, Burean of Mines, Mineral Industry Surveys, "Petroleum Statement, Annual," Table 1.

- 1976 through 1980: EJA, Energy Data Reports, "Petroleum Statement, Arnual," Table 1.

- 1981 forward: 'EIA, Pelroleum Supply Annudt, Table 2.

\section{Road ON}

RDINPZZ - Road oll sold to the industrial sector by State.

- 1960 through 1977: U.S. Department of the Interior, Bureau of Mines, Mineral Industry Surveys, "Sales of Asphalt." The specific tables aret

- 1960 through 1962: Table 6.

- 1963 through 1977: Table 5.

- 1978 through 1980: ELA, Energy Data Reports, "Sales of Asphalt," Table 2.

- 1981 and 1982: ELA estimates. (See explanation in Appendix A, under "Asphalt and Road Oil" on page 354.) 
- 1983 forwand: Road oil is included in asphalt data (see ASINPZZ).

RDTCPUS - Road oil total constumed in the United States.

- 1960 through 1975: U.S. Department of the Interior, Bureau of Mines, Mineral Industry Surveys, "Petroleam Statement, Annual," Table 1.

- 1976 through 1980: EIA, Energy Data Reports, "Petroleum Statement, Annual," Table 1.

- 1981 and 1982: ELA, Petrolerm Supply Annual, Table 2.

- 1983 forward: BLA, Petroleum Supply Ammul, Table 2, included In "Asphalt and Road Oil,"

\section{Solar Thermat and Photovoltaio Energy}

SOEOPZZ - Electricity produced from solar thermal entergy sources at electric utilities by State.

- 1960 through 1983: No data avajlable. Values are assumed to be zero.

- 1984 forward; EIA, Form EIA-759, "Monthly Power Plant Reprort."

SOHCBZZ - Electrictity produced from solar thermal energy sotuces in the residential and commercial sectors combined by State.

- 1960 through 1989: No data available. Values are assumed to be zero.

- 1990 forward: EIA estimates are developed by using the same method as used for the U.S. data published in the ELA, Renewabte Entergy Annual 1995, Table 11. Shipments of solar thermal collectors in the United States, in thousand square feet, for 1974 forward that are collected on the ELA Form CE-63A, "Annual Solar Thernal Collector Manufacturens Survey" are accumulated each year based on the assumption that the replacement/retirement period for solar thermal collectors is 20 years. The following factors affected the State estimates:

- Data for 1974 through 1985 are avaílable for the U.S. total only. U.S. values are allocated to the States by using an allocating series that is the simple average of each State's 1986 and 1987 data.

- The U.S. data are adjusted to remove Puerto Rico and the Virgin Islands. California data for 1986 forward are reduced by the number of high-temperature solar thermal collectors (used at an electric utility in Califorma) shown in the Renewable Energy Arnual 1995, Table 13.

- State data for 1986 through 1992 used in the accumulated data series are published in the EIA, Solar Collector Manufacturring Activity for each year. The table numbers are:

- 1986 through 1988: Table 5.

- I969: Table 4.

- 1990 through 1992: Table 13.

- State data for 1993 and 1994 used in the accumulated data series are from the EIA, Renetwable Energy Annual 1995, Table H13.

SOICBZZ - Electricity produced from solar thermal energy sources in the industrial sector by State.

- 1960 through 1989: No data available. Values are assumed to be zero.

- 1990 forward: ELA estimates based on data collected on Form ELA-867, "Annual Nonutility Power Producers Report."

\section{stit Gas}

SGTCPUS - Still gas total consumed in the United States.

- 1960 through 1975: US. Department of the Intertor, Bureau of Mines, Mineral Industry Suroeys, "Petroletm Statement, Annual," Table 1.

- 1976 through 1980: EIA, Energy Data Reports, "Petroleum Statement, Annual, ${ }^{\pi}$ Table 1.

- 1981 and 1982: EIA, Petrolerom Supply Annual, Table 14.

- 1983 through 1985: EIA, Petroleun Supply Annual, Table 12.

- 1986 forward: EIA, Petroletun Supply Amnusl, Table 2

\section{Speckat Naphthas}

SNTCPUS - Special naphthas total consuned in the United States.

- 1960 through 1969: Data included in motor gasoline.

- 1964 through 1975: U.S. Department of the Interior, Bureau of Mines, Mintenal Industry Suroeys, "Petroleum Statement, Annual, "Table 1.

- 1976 through 1980: EIA, Energy Data Reports, "Petroleun Statement, Amnual, Table 1. 
- 2981 forward: EIA, Pelroleum Supply Annutal, Table 2.

\section{Uminished Oibs}

UOTCPUS - Unfinished oils total consumed in the United States.

- 1960 through 1980. No data available. Values assumed to be zero.

- 1981 forward: EIA, Petroleum Supply Anrual, Table 2.

\section{Unfractlonatod Stream}

USTCPUS - Unfractionated stream total consumed in the United States.

- 1960 through 1978: EIA, Energy Data Reports, "Petroleum Statement, Armual," Table 1, included in "Plant Condensate."

- 1979 and 1980: EIA, Energy Data Reports, "Petroleum Statement, Arnual," Table 1.

- 1981 through 1983: ELA, Petroleum Supply Annual, Table 2.

- 1984 forward: ELA, Petroleum Supply Annual, Table 2, tndividual components are reported separately.

\section{Vatue Added by Manufacture}

CGVAVZZ - Value added by the manufacture of sanitary food containers by State. Beginning with 1992 data, this series became value added by the manufacture of corrugated and solid fiber boards by State.

- 1960 through 1965: U.S. Department of Commerce, 1963 Census of Mantffactures, Volume II, Part 1, SIC 2654. The 1963 State data are used for the years 1960 through 1965.

- 1966 throtsgh 1970: U.S. Department of Commerce, 1967 Census of Mamufactures, Volume II, Part 2, SIC 2654. The 1967 State data are used for 1966 through 1970 .

- 1971 through 1980: U.S. Department of Commerce, 1977 Census of Manufactures, Industry Series, SIC 2654. The 1972 State data are used for 1971 through 1975, and the 1977 Stake data axe used for 1976 through 1980.
- 1981 through 1990 U.S. Department of Commerce, 1982 Census of Manufactures (Final Report), Industy Series, SIC 2654. The 1982 State data are used for 1981 through 1990.

- 1991 forward: U.S. Department of Commerce, 1992 Census of Marufactures (Final Report), Industry Series, SIC 2653. The 1992 State data are used for 1991 forwand.

OCVAVZZ - Value added by the manufacture of industrial organic chemicals by State.

- 1960 through 1970: U.S. Department of Commerce, 1967 Census of Manufactures, Volume II, Part 2, SIC 281B. The 1963 State data are used for the years 1960 through 1965, and the 1967 State data are used for 1966 through $19 \% 0$.

- 1971 through 1980: U.S. Department of Commerce, $1977 \mathrm{Cen}-$ sus of Manufactures, Industry Series, STC 2869. The 1972 State data are used for 1971 through 1975, and the 1977 State data are used for 1976 through 1980 .

- 1980 through 1985: U.S. Department of Commerce, 1987 Censtus of Mannefackures (Final Report), Industry Series, SIC 2869. The 1982 State data are used for 1981 through 1985.

- 1986 forward: U.S. Department of Commence, 1992 Census of Manufactures (Final Report), Industry Series, SIC 2869. The 1987 State data are tused for 1986 through 1990, and the 1992 State data are used for 1991 forwrard.

PIVAVZZ - Value added by the manufacture of paints and allied produrts by State.

- 1960 through 1970: U.S. Department of Commerce, $1967 \mathrm{Cen}$. sus of Monnfactures, Volume II, Part 2, SIC 2851. The 1963 State data are used for the years 1960 through 1965, and the 1967 State data are used for 1966 through 1970.

- 1971 through 1980: U.S. Department of Commerce, 1977 Census of Manufactures, Industry Series, SIC 2851. The 1972 State data are used for 1971 through 1975, and the 1977 State data are used for 1976 through 1980 .

- 1981 through 1985: U.S. Department of Commerce, 1987 Census of Manufactures (Final Report), Industry Series, SIC 2851. The 1982 state data are used for the years 1981 through 1985.

- 1986 forward: U.S. Department of Commerce, 1992 Census of Monufactures (Final Report), Industry Series, SIC 2851. The 
1987 Siate data are used for the years 1986 through 1990, and the 1992 State data are used for 1991 forward.

\section{Wind Enargy}

WYEOPZZ - Electricity produced from wind at electric utilities by State.

- 1960 through 1982: No data available. Values are assumed to be zero.

- 1963 forward: EIA, Form Ela-759, "Monthly Power Plant Report."

WYCBZZ - Electricity produced from wind in the industrial sector by State.
- 1960 through 1989; No data available Values are assumed to be zero.

- 1990 forward: EIA estimates based on data collected on Form BIA-867, "Annual Nonutility Power Producers Report."

\section{Waxes}

WXTCPUS - Waxes total consumed in the United States.

- 1960 through 1975: U.S. Department of the Interior, Burealt of Mines, Mineral Industry Stroeys, "Petroleum Statement, Annutal, Table 1.

- 1976 through 1980: BLA, Energy Data Reports, "Petroleuma Statement, Annual," Table 1.

- 1981 forward: EIA, Petroleum Supply Annual, Table 2. 
Appendix D

\section{Thermal Conversion Factors}

Table D1. Approxlmate Heat Content of Petroleum and Coal and Heat Rates for Electricily

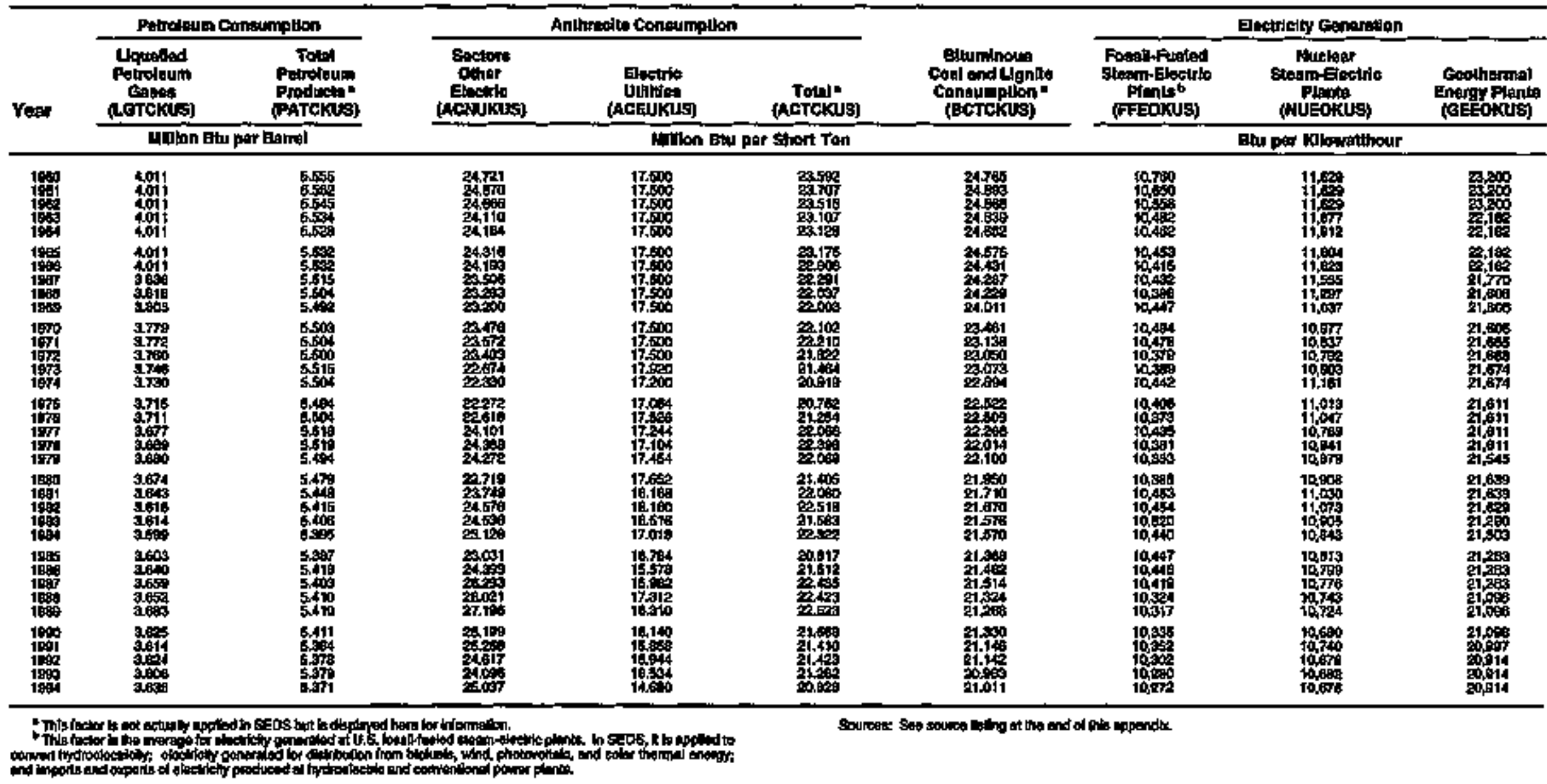


A Table D2. Approximate Heat Content of Natural Gas Consumed by Electule Utillites, 1960-1976 (Thousand Btu per Cubic Fock)

\begin{tabular}{|c|c|c|c|c|c|c|c|c|c|c|c|c|c|c|c|c|c|}
\hline stato & $+\infty 0_{0}$ & 19161 & 100 & 1000 & -1004 & 190 & 1006 & 1467 & 1960 & 5059 & $19 \pi$ & 161 & $19 m$ & 1973 & 1974 & 1075 & 1976 \\
\hline 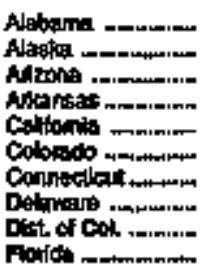 & 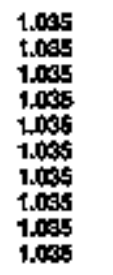 & 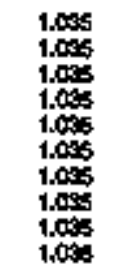 & 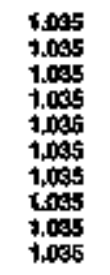 & $\begin{array}{l}1.007 \\
1.011 \\
1.077 \\
1.000 \\
1.060 \\
0.006 \\
1.002 \\
1.04 \\
1.009 \\
1.0026\end{array}$ & $\begin{array}{l}1.097 \\
1.0910 \\
1.078 \\
1.009 \\
1.071 \\
0.806 \\
1.090 \\
1.045 \\
1.024 \\
1.041\end{array}$ & $\begin{array}{l}1.004 \\
1.010 \\
1.070 \\
1.001 \\
1.073 \\
0.912 \\
1.002 \\
1.049 \\
1.004 \\
1.009\end{array}$ & $\begin{array}{l}1.003 \\
1.000 \\
1.078 \\
1.001 \\
1.072 \\
0.013 \\
1.002 \\
1.042 \\
1.004 \\
1.0000\end{array}$ & 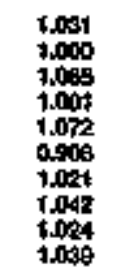 & $\begin{array}{l}1.033 \\
1.005 \\
1.078 \\
1.000 \\
1,090 \\
0,097 \\
1,016 \\
1.048 \\
1.028 \\
1,040\end{array}$ & $\begin{array}{l}1.038 \\
1.000 \\
1.076 \\
1.000 \\
1.069 \\
0.067 \\
1.016 \\
1.042 \\
1.028 \\
1.048\end{array}$ & 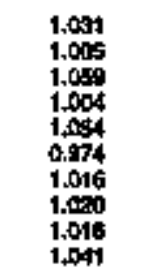 & $\begin{array}{l}1.001 \\
1.005 \\
1.050 \\
1.004 \\
1.004 \\
0.974 \\
1.016 \\
1.000 \\
1.016 \\
1.041\end{array}$ & $\begin{array}{l}1.058 \\
1.006 \\
1.070 \\
1.017 \\
1.06 \\
0.968 \\
1.016 \\
1.005 \\
.019 \\
1.019\end{array}$ & $\begin{array}{l}1.004 \\
1.006 \\
1.067 \\
1.005 \\
1.060 \\
0.679 \\
1.015 \\
1.04 \\
1.024 \\
1.04\end{array}$ & $\begin{array}{l}1.041 \\
1.000 \\
1.078 \\
1.011 \\
1.068 \\
0.061 \\
1.012 \\
1.001 \\
+ \\
1.009\end{array}$ & $\begin{array}{l}1.003 \\
1.006 \\
1.07 t \\
1.011 \\
1.006 \\
0.096 \\
1.006 \\
1.078 \\
-.009 .\end{array}$ & $\begin{array}{l}1.169 \\
1.000 \\
1.072 \\
1.019 \\
1.051 \\
0.092 \\
1.009 \\
1.006 \\
.1 .014\end{array}$ \\
\hline 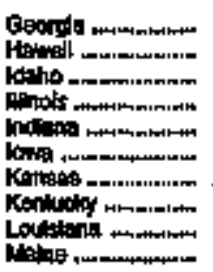 & 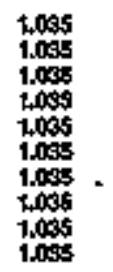 & 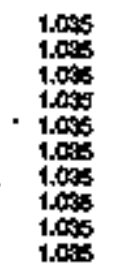 & 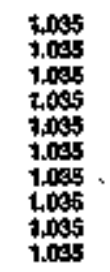 & $\begin{array}{l}1.040 \\
- \\
1.006 \\
1.0200 \\
1.000 \\
1.0007 \\
0.906 \\
1.045 \\
1.005 \\
-\end{array}$ & $\begin{array}{l}1.043 \\
-1.01 \\
1.030 \\
1.000 \\
1.004 \\
0.004 \\
1.009 \\
1.050 \\
-.\end{array}$ & $\begin{array}{l}1,000 \\
1,005 \\
1,009 \\
0,009 \\
1,010 \\
0,005 \\
1,000 \\
1,000\end{array}$ & $\begin{array}{l}1.042 \\
- \\
1.080 \\
1.030 \\
1.000 \\
1.059 \\
0.994 \\
.1024 \\
1.086 \\
1.025\end{array}$ & 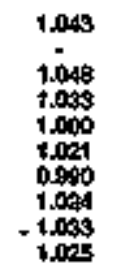 & 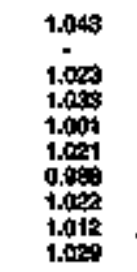 & 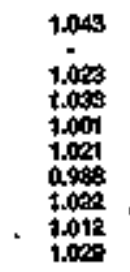 & 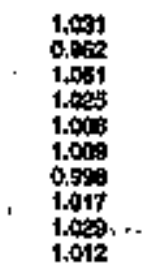 & 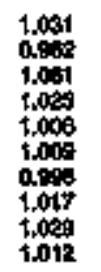 & 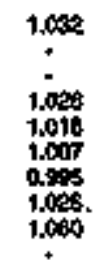 & $\begin{array}{c}1,000 \\
\vdots \\
1,025 \\
1.005 \\
1.004 \\
0.005 \\
1+025 \\
1+000 \\
4\end{array}$ & $\begin{array}{c}1.009 \\
1.007 \\
-1,090 \\
1,009 \\
-1.000 \\
0.008 \\
1,008 \\
1.089\end{array}$ & $\begin{array}{l}1.000 \\
1.000 \\
1.000 \\
1.000 \\
1.000 \\
0.901 \\
1.067 \\
1.600\end{array}$ & $\begin{array}{l}1.099 \\
1.009 \\
1.000 \\
1.003 \\
1.011 \\
0.400 \\
1.010 \\
1.061 \\
.\end{array}$ \\
\hline 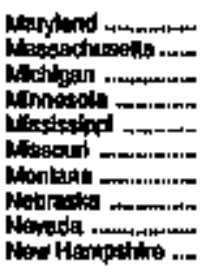 & $\begin{array}{l}1,085 \\
1.005 \\
1.055 \\
1.035 \\
1.005 \\
1.005 \\
1.005 \\
1.035 \\
1.055 \\
1.005\end{array}$ & 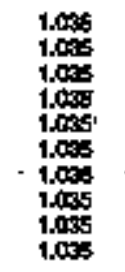 & 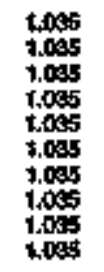 & $\begin{array}{l}1.020 \\
1.045 \\
1.012 \\
0.006 \\
1.002 \\
1.020 \\
1.000 \\
0.001 \\
1.072 \\
1.000\end{array}$ & 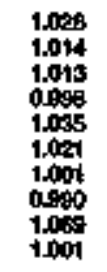 & $\begin{array}{l}1.005 \\
1.019 \\
1.014 \\
0.900 \\
1.009 \\
1.000 \\
1.001 \\
0.091 \\
1.000 \\
1.012\end{array}$ & 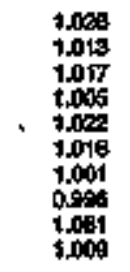 & 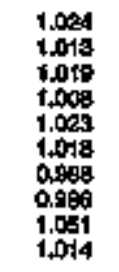 & $\begin{array}{l}1.094 \\
1.012 \\
1.013 \\
1.010 \\
1.024 \\
1.000 \\
0.999 \\
0.098 \\
1.005 \\
1.009\end{array}$ & $\begin{array}{l}t .024 \\
1.012 \\
1.011 \\
+.010 \\
1.024 \\
1.020 \\
0.909 \\
0.969 \\
1.050 \\
1.009\end{array}$ & $\begin{array}{l}1.028 \\
1.012 \\
1.018 \\
1.002 \\
1.005 \\
1.007 \\
\cdot 1.000 \\
1.000 \\
1.000 \\
1.010\end{array}$ & 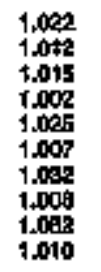 & $\begin{array}{l}0.000 \\
1.000 \\
0.790 \\
0.997 \\
1.042 \\
0.979 \\
1.168 \\
0.084 \\
1.071 \\
1.000\end{array}$ & $\begin{array}{l}0.090 \\
1.000 \\
0.505 \\
0.905 \\
1.093 \\
0.070 \\
1.470 \\
0.097 \\
1.066 \\
1.000\end{array}$ & $\begin{array}{l}0.090 \\
1.000 \\
0.761 \\
0.999 \\
1.045 \\
0.072 \\
1.750 \\
0.093 \\
1.000 \\
1.000\end{array}$ & $\begin{array}{l}0.048 \\
1.008 \\
0.091 \\
0.984 \\
1.000 \\
0.97 \\
1.149 \\
0.092 \\
1.009 \\
1.000\end{array}$ & $\begin{array}{r}0.918 \\
1,001 \\
0.767 \\
0.72 \\
1.018 \\
0.974 \\
1,192 \\
0.971 \\
-1,006 \\
1,000\end{array}$ \\
\hline 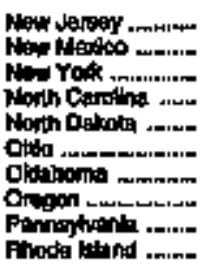 & 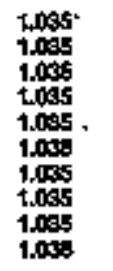 & 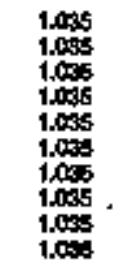 & $\begin{array}{l}1.005 \\
1.055 \\
1.005 \\
1.005 \\
1.005 \\
1.005 \\
1.004 \\
1.055 \\
1.005 \\
1.005\end{array}$ & 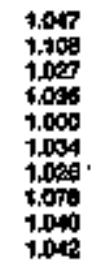 & 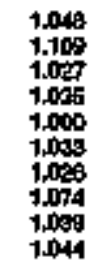 & $\begin{array}{l}1.045 \\
1.168 \\
1.009 \\
1.0035 \\
1.000 \\
1.035 \\
1.020 \\
1.000 \\
1.098 \\
1.048\end{array}$ & $\begin{array}{l}1.002 \\
1.109 \\
1.002 \\
1.001 \\
1.002 \\
1.003 \\
1,004 \\
1.05 \\
1.006 \\
1.000\end{array}$ & $\begin{array}{l}1.000 \\
1.097 \\
1.020 \\
1.000 \\
1.006 \\
1.039 \\
1.084 \\
1.009 \\
1.004 \\
1.041\end{array}$ & 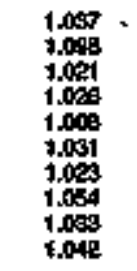 & 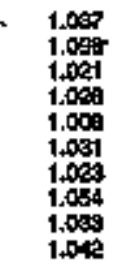 & $\begin{array}{l}1.003 \\
1.003 \\
1.021 \\
1.004 \\
1.001 \\
1.002 \\
1.008 \\
1.045 \\
1.035 \\
1.001\end{array}$ & $\begin{array}{l}1.000 \\
1.003 . \\
1.021 . \\
1.004 \\
1.001 \\
1.003 \\
1.002 \\
1.045 \\
1.003 \\
1.021\end{array}$ & $\begin{array}{l}1.000 \\
1.050 \\
1.040 \\
1.009 \\
1.050 \\
0.9049 \\
1.051 \\
1.084 \\
1.007 \\
1.042\end{array}$ & 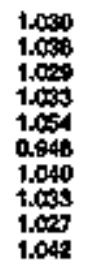 & 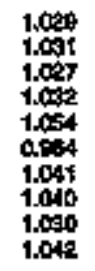 & 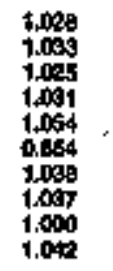 & 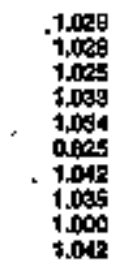 \\
\hline 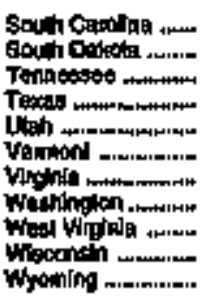 & 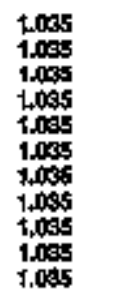 & $\begin{array}{l}1.005 \\
1.055 \\
1.005 \\
1.005 \\
1.005 \\
1.005 \\
1.005 \\
1.005 \\
1.005 \\
1.055 \\
1.005\end{array}$ & $\begin{array}{l}1.005 \\
1.005 \\
1.005 \\
1.005 \\
1.005 \\
1.005 \\
1.005 \\
1.055 \\
1.053 \\
1.005 \\
1.005\end{array}$ & 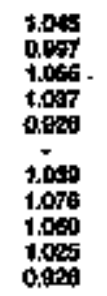 & 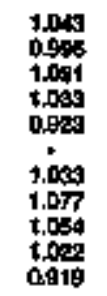 & 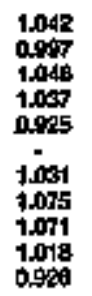 & $\begin{array}{l}1.008 \\
1.000 \\
1.04 \\
1.045 \\
0.929 \\
1.009 \\
1.000 \\
1.008 \\
1.040 \\
1.029 \\
0.050\end{array}$ & $\begin{array}{r}1.000 \\
1.004 \\
1.002 \\
1.044 \\
0.924 \\
1.009 \\
1.000 \\
1.008 \\
1.042 \\
-1.002 \\
-0.000\end{array}$ & $\begin{array}{l}1.080 \\
1.005 \\
1.0089 \\
1.009 \\
0.05 \\
1.000 \\
1.031 \\
1.000 \\
1.000 \\
1.005 \\
0.000\end{array}$ & 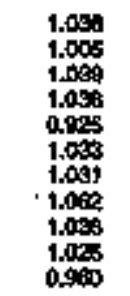 & 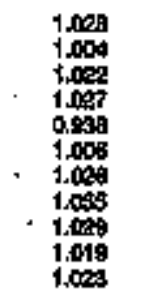 & 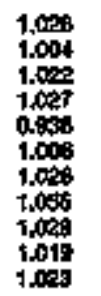 & $\begin{array}{l}1.002 \\
1.001 \\
1.045 \\
1,002 \\
0.040 \\
1.000 \\
1.065 \\
0.000 \\
1.000 \\
0.063\end{array}$ & $\begin{array}{l}1.000 \\
0.000 \\
5.058 \\
1.019 \\
0.049 \\
1.000 \\
1.059 \\
0.505 \\
0.5015 \\
1.015 \\
0.053\end{array}$ & 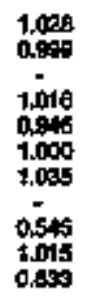 & $\begin{array}{l}1.008 \\
1.000 \\
- \\
1.016 \\
0.941 \\
1.000 \\
1.0 \% 5 \\
0 \\
0.075 \\
1.046 \\
0.045\end{array}$ & $\begin{array}{l}1,000 \\
0.000 \\
1.000 \\
1.010 \\
0,000 \\
1.000 \\
1.091 \\
- \\
.0083 \\
1.014 \\
0.043\end{array}$ \\
\hline U.S. Averagy & t.0s5 & 1.000 & 1005 & 1005 & 1.0007 & $1.08 \mathrm{a}$ & 1.059 & 1.0050 & 1,097 & 1.085 & 1.09 & 1.029 & 1.027 & 1.021 & 1,000 & 1.020 & 1.003 \\
\hline
\end{tabular}

\section{-nNat applonble.}

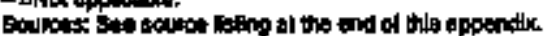


Table D3. Approximale Heat Content of Wahural Gas Consumed by Elactric Utilities, 1977-1994 (Thousand Btu per Cubic Foot)

\begin{tabular}{|c|c|c|c|c|c|c|c|c|c|c|c|c|c|c|c|c|c|c|}
\hline stant & 197 & $19 \%$ & 1970 & 1090 & tosit & 1002 & 1000 & 1004 & tos & 1908 & 螨 & 7068 & 19 gوg & סוקו & 19 & $19 \% 2$ & T993 & $\operatorname{tas} \theta$ \\
\hline 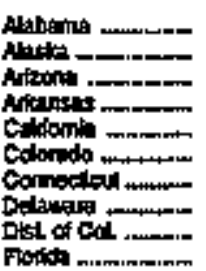 & $\begin{array}{l}1.182 \\
+000 \\
1.058 \\
1.051 \\
1.069 \\
0.088 \\
1.109 \\
.019\end{array}$ & $\begin{array}{l}1.126 \\
1.006 \\
1.064 \\
1.053 \\
1.060 \\
0.002 \\
-.00 \\
1.070 \\
-.024\end{array}$ & 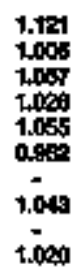 & $\begin{array}{l}1.120 \\
1.006 \\
1.007 \\
1.050 \\
1.050 \\
0.001 \\
1.042 \\
-.015\end{array}$ & $\begin{array}{l}1.154 \\
1.005 \\
1.049 \\
1.029 \\
1.056 \\
0.076 \\
1.006 \\
1.018\end{array}$ & $\begin{array}{l}1.125 \\
1.005 \\
1.051 \\
1.052 \\
1.053 \\
0.004 \\
1.039 \\
1.014\end{array}$ & 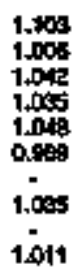 & 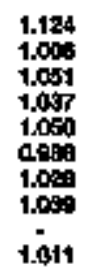 & 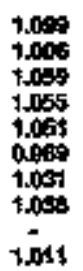 & $\begin{array}{l}1.001 \\
1.006 \\
1.044 \\
1.053 \\
1.005 \\
0.094 \\
1.089 \\
1.046 \\
1.006\end{array}$ & $\begin{array}{l}1.054 \\
1.006 \\
1.0054 \\
1.051 \\
1.038 \\
0.088 \\
1.081 \\
1.098 \\
- \\
1.008\end{array}$ & $\begin{array}{l}1.039 \\
1.009 \\
1.034 \\
1.039 \\
1.039 \\
0.098 \\
1.091 \\
1.072 \\
-.068\end{array}$ & $\begin{array}{l}1.000 \\
1.000 \\
1.035 \\
1.099 \\
1.040 \\
0.977 \\
1.030 \\
1.075 \\
1.040\end{array}$ & $\begin{array}{l}1.000 \\
1.006 \\
1.034 \\
1.018 \\
1.099 \\
0.099 \\
1.054 \\
1.094 \\
1.041\end{array}$ & $\begin{array}{l}1.009 \\
1.001 \\
1.007 \\
1.020 \\
1.020 \\
0.095 \\
1.0099 \\
1.0002 \\
1.014\end{array}$ & $\begin{array}{l}1.001 \\
1.000 \\
1.091 \\
1.005 \\
1.005 \\
1.000 \\
1.011 \\
1.007 \\
1.011\end{array}$ & 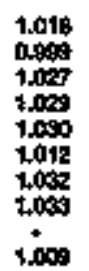 & 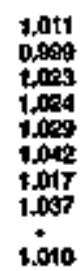 \\
\hline 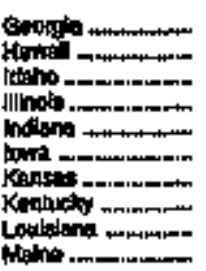 & 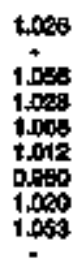 & 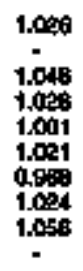 & 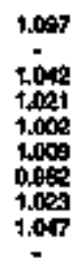 & $\begin{array}{l}1.005 \\
1.054 \\
1.004 \\
1.004 \\
1.009 \\
0.900 \\
1.024 \\
1.041 \\
-4\end{array}$ & 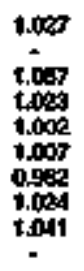 & $\begin{array}{l}1.020 \\
= \\
1.0 \% 5 \\
1.024 \\
1.004 \\
1.019 \\
0.058 \\
1.024 \\
1.040 \\
-\end{array}$ & $\begin{array}{l}1.05 \\
1.097 \\
1.009 \\
1.000 \\
1.007 \\
0.003 \\
1.000 \\
1.000 \\
-\end{array}$ & 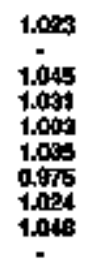 & 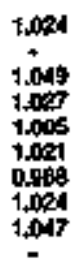 & $\begin{array}{l}1,004 \\
1,001 \\
1,008 \\
1.008 \\
1.017 \\
0.998 \\
1.02 \\
1.044 \\
-\end{array}$ & $\begin{array}{l}1.003 \\
- \\
1.017 \\
1.009 \\
1.000 \\
1.000 \\
0.008 \\
1.021 \\
1.043 \\
-\end{array}$ & 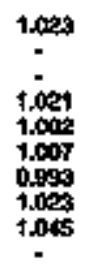 & $\begin{array}{l}1.004 \\
: \\
-017 \\
1.000 \\
1.007 \\
0.971 \\
1.021 \\
1.044 \\
-\end{array}$ & $\begin{array}{l}1.024 \\
\vdots \\
1.021 \\
1.000 \\
1.006 \\
0.000 \\
1,000 \\
1,045\end{array}$ & $\begin{array}{l}1.025 \\
\vdots \\
1.010 \\
1.010 \\
1.004 \\
0.000 \\
1.0020 \\
1.040 \\
-\end{array}$ & $\begin{array}{l}1.004 \\
\vdots \\
1.016 \\
1,001 \\
1.004 \\
0.970 \\
1.000 \\
1,040\end{array}$ & 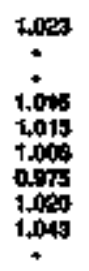 & 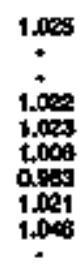 \\
\hline 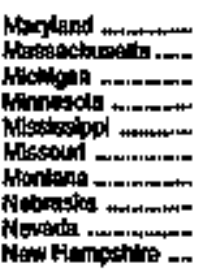 & $\begin{array}{l}0.098 \\
\$ .000 \\
0.508 \\
0.972 \\
1.020 \\
0.973 \\
1.173 \\
0.967 \\
1.069 \\
1.000\end{array}$ & 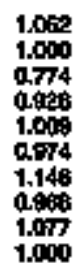 & 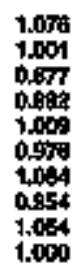 & $\begin{array}{l}1.009 \\
1.000 \\
0.750 \\
0.094 \\
1.017 \\
0.97 \\
1.049 \\
0.060 \\
1.074 \\
1.025\end{array}$ & 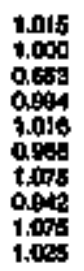 & 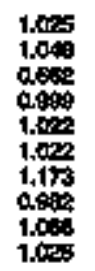 & $\begin{array}{l}1.006 \\
1.064 \\
0.213 \\
1.011 \\
1.009 \\
0.0 \% 5 \\
1.197 \\
0.949 \\
1.006 \\
1.025\end{array}$ & $\begin{array}{l}1.005 \\
1.050 \\
0.092 \\
1.001 \\
1.097 \\
0.900 \\
1.170 \\
0.948 \\
1.000 \\
1.003\end{array}$ & 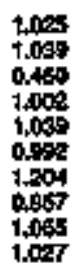 & 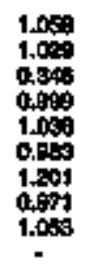 & $\begin{array}{l}1.043 \\
1.008 \\
0.404 \\
0,009 \\
1.004 \\
0.000 \\
1.008 \\
0.077 \\
1.045 \\
1.007\end{array}$ & $\begin{array}{l}1.002 \\
+.020 \\
0.151 \\
1.000 \\
1.006 \\
0.094 \\
1.200 \\
0.054 \\
1.027 \\
1.027\end{array}$ & $\begin{array}{l}1.015 \\
1.018 \\
0.100 \\
1.005 \\
1.055 \\
1.016 \\
1.213 \\
0.050 \\
1.007 \\
1.027\end{array}$ & 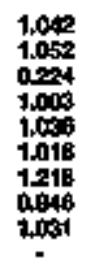 & $\begin{array}{l}1.048 \\
1.049 \\
0.090 \\
1.005 \\
1.0605 \\
1.014 \\
1.194 \\
0.042 \\
1.04 \\
-\end{array}$ & $\begin{array}{l}1.045 \\
1.000 \\
0.414 \\
1.000 \\
1.009 \\
1.000 \\
1.200 \\
0.009 \\
1.005 \\
-\end{array}$ & $\begin{array}{l}1.041 \\
1.084 \\
0.079 \\
1.000 \\
1.002 \\
1.006 \\
1.765 \\
0.075 \\
1.029 \\
1.016\end{array}$ & $\begin{array}{l}1.003 \\
1.007 \\
0.000 \\
1.000 \\
1.000 \\
1.000 \\
1.008 \\
0.007 \\
1.008 \\
1.015\end{array}$ \\
\hline 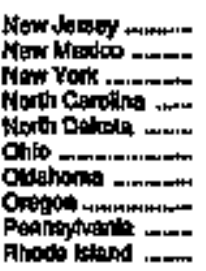 & 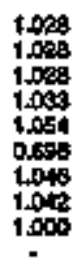 & 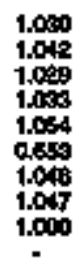 & 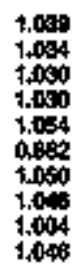 & $\begin{array}{l}1.034 \\
1.009 \\
1.035 \\
1.054 \\
1.094 \\
1.004 \\
1.046 \\
0.098 \\
1.020 \\
1.028\end{array}$ & 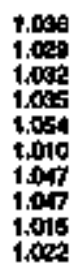 & $\begin{array}{l}1.003 \\
1.001 \\
1.000 \\
1.005 \\
1.054 \\
1.014 \\
1.045 \\
0.000 \\
1.000 \\
1.000\end{array}$ & $\begin{array}{l}1.097 \\
0.092 \\
1.000 \\
1.000 \\
1.034 \\
1.011 \\
1.061 \\
0.000 \\
1.000 \\
1.000\end{array}$ & $\begin{array}{l}1.0058 \\
0.095 \\
1,000 \\
1.003 \\
1.054 \\
1.014 \\
1.000 \\
0,000 \\
1,000 \\
1,0030\end{array}$ & $\begin{array}{l}1.040 \\
1.015 \\
1.096 \\
1.030 \\
1.054 \\
1.014 \\
1.040 \\
0.090 \\
1.000 \\
1.094\end{array}$ & 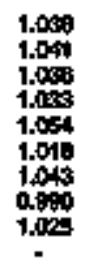 & 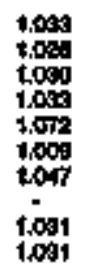 & $\begin{array}{l}1.005 \\
1.006 \\
1.031 \\
1.083 \\
1.065 \\
1.012 \\
1.005 \\
1.035 \\
1.0032\end{array}$ & 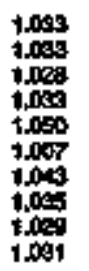 & 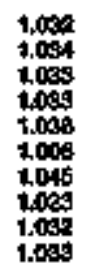 & 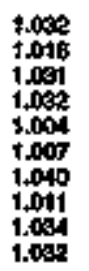 & 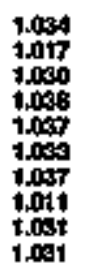 & 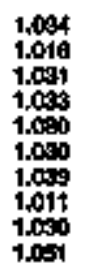 & 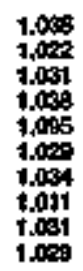 \\
\hline 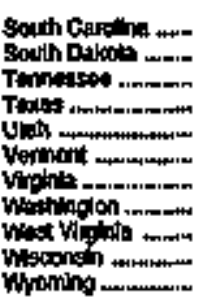 & $\begin{array}{l}1.028 \\
0.900 \\
1.008 \\
0.045 \\
1.000 \\
1 . t 74 \\
1.000 \\
1.044 \\
0.054\end{array}$ & 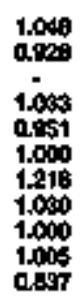 & 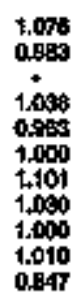 & 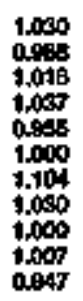 & $\begin{array}{l}1,009 \\
0.093 \\
1.010 \\
1.000 \\
0.002 \\
1.000 \\
1.097 \\
1.001 \\
1.000 \\
1.009 \\
0.035\end{array}$ & $\begin{array}{l}1.000 \\
0.948 \\
1.000 \\
0.040 \\
1.000 \\
1.004 \\
1.000 \\
1.000 \\
1.012 \\
0.047\end{array}$ & 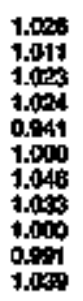 & $\begin{array}{l}1.002 \\
1.05 t \\
1.000 \\
1.000 \\
1.000 \\
1.04 t \\
1,009 \\
1.000 \\
0,000 \\
1.047\end{array}$ & $\begin{array}{l}1.009 \\
1.010 \\
1.000 \\
1.075 \\
1.000 \\
1.040 \\
1.090 \\
1.000 \\
1.000 \\
1.049\end{array}$ & $\begin{array}{l}1.003 \\
1.000 \\
1.000 \\
1.000 \\
1.000 \\
1.000 \\
1.000 \\
1.000 \\
1.000 \\
1.002\end{array}$ & $\begin{array}{l}1.097 \\
1.019 \\
- \\
1.065 \\
1.070 \\
1.009 \\
1.000 \\
1.000 \\
0.9 \% 8 \\
1.010\end{array}$ & $\begin{array}{l}1.002 \\
1.000 \\
1.001 \\
1.039 \\
1.078 \\
1.004 \\
1.039 \\
1.0000 \\
1.000 \\
1.004\end{array}$ & 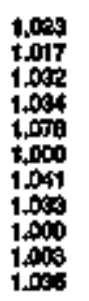 & 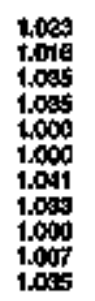 & $\begin{array}{l}1.005 \\
1.000 \\
1.003 \\
1.000 \\
1.065 \\
0.080 \\
1.004 \\
1.050 \\
1.000 \\
1.000 \\
1.001\end{array}$ & 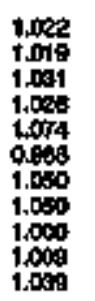 & 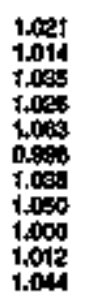 & $\begin{array}{l}1.029 \\
0.972 \\
1.009 \\
1.029 \\
1.044 \\
0.94 \\
1.007 \\
1.000 \\
1.000 \\
1.014 \\
1.069\end{array}$ \\
\hline 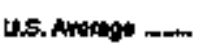 & 1.089 & 1.083 & 1.003 & 1.003 & 1,039 & 1, (10\% & 1.0es & 1,000 & 1.097 & 1.003 & 1.00e & 1.027 & 1.60 & $1.02 \pi$ & 1.020 & 1.023 & 1.009 & $1.00 \mathrm{~s}$ \\
\hline
\end{tabular}

- chlot appocable.

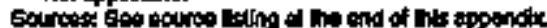


A

$\mathbf{P}$

E

N

D

$\mathbf{3}$

D
Table D4. Approximate Heat Content of Natura: Gas Consumed by Sectors Other Than Electric Utilities, 1960-1976 (Thousand Btu per Cublc Foot)

\begin{tabular}{|c|c|c|c|c|c|c|c|c|c|c|c|c|c|c|c|c|c|}
\hline Statos & 1000 & 1961 & +102 & $1 \% 0$ & 1964 & 1903 & 1900 & 106 & 1900 & 196: & 1970 & $m$ & 1072 & $10 \mathrm{ma}$ & 1974 & 1975 & 1976 \\
\hline 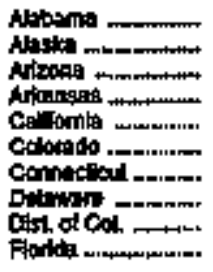 & $\begin{array}{l}1.038 \\
1.035 \\
1.005 \\
1.005 \\
1.055 \\
1.055 \\
1.009 \\
1.025 \\
1.045 \\
1.005\end{array}$ & $\begin{array}{l}1.005 \\
1.005 \\
1.065 \\
1.055 \\
1.005 \\
1.055 \\
1.085 \\
1.065 \\
1.045 \\
1.005\end{array}$ & 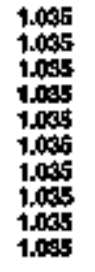 & $\begin{array}{l}1.008 \\
1.011 \\
1.077 \\
1.000 \\
1.000 \\
0.000 \\
1.004 \\
1.043 \\
1.002 \\
1.028\end{array}$ & $\begin{array}{l}1.097 \\
1.010 \\
1.073 \\
1.003 \\
1.071 \\
0.090 \\
1.030 \\
1.040 \\
1.024 \\
1.041\end{array}$ & $\begin{array}{l}1.034 \\
1.010 \\
1.078 \\
1.001 \\
1.073 \\
0.912 \\
1.002 \\
1.043 \\
1.002 \\
1.089\end{array}$ & $\begin{array}{l}1.003 \\
1.000 \\
1.076 \\
1.001 \\
1.072 \\
0.018 \\
1.022 \\
1.042 \\
1.004 \\
1.00\end{array}$ & 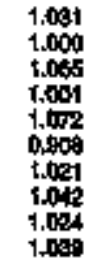 & 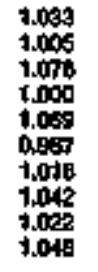 & $\begin{array}{l}1.008 \\
1.005 \\
1.076 \\
1.000 \\
1.009 \\
0.997 \\
1.016 \\
1.042 \\
1.027 \\
1.046\end{array}$ & $\begin{array}{l}1,001 \\
1,005 \\
1,009 \\
1,004 \\
1.054 \\
0.94 \\
1.019 \\
1.000 \\
1016 \\
1.041\end{array}$ & $\begin{array}{l}1.001 \\
1.005 \\
1.069 \\
1.004 \\
1.054 \\
0.974 \\
1.016 \\
1.000 \\
1.016 \\
1.011\end{array}$ & $\begin{array}{l}1.001 \\
1.006 \\
1.005 \\
1.000 \\
1.001 \\
1.000 \\
1.016 \\
1.016 \\
1.016 \\
1.070\end{array}$ & $\begin{array}{l}1.029 \\
1.011 \\
1.052 \\
0.986 \\
1.050 \\
0.980 \\
1.015 \\
1.0000 \\
1.018 \\
1.065\end{array}$ & 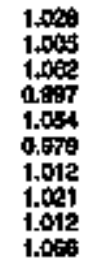 & 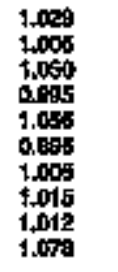 & 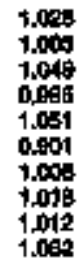 \\
\hline 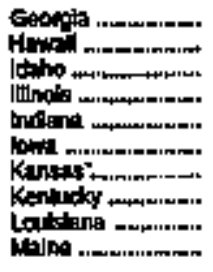 & 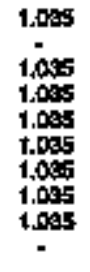 & $\begin{array}{l}1.035 \\
1.0055 \\
t .035 \\
1.0055 \\
1.035 \\
1.0856 \\
1.0055 \\
1.035 \\
-\end{array}$ & 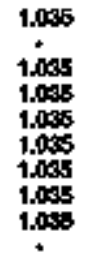 & 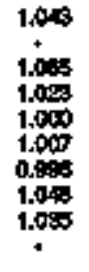 & $\begin{array}{l}1,040 \\
1.071 \\
1,000 \\
1.000 \\
1,000 \\
0.904 \\
1,000 \\
1.050 \\
+\end{array}$ & $\begin{array}{l}1.040 \\
1.005 \\
1.000 \\
0.090 \\
1.010 \\
0.000 \\
1.002 \\
1.00\end{array}$ & $\begin{array}{l}1,042 \\
1,060 \\
1,005 \\
1,000 \\
1,019 \\
0,094 \\
1,024 \\
1,005 \\
1,025\end{array}$ & 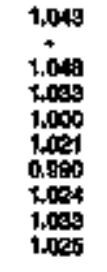 & $\begin{array}{l}1.043 \\
1.000 \\
1.003 \\
1.009 \\
1.041 \\
0.060 \\
1.002 \\
1.012 \\
1.009\end{array}$ & $\begin{array}{l}1.040 \\
1.0003 \\
1.050 \\
1.001 \\
1.021 \\
0.985 \\
1.028 \\
1.012 \\
1.020\end{array}$ & 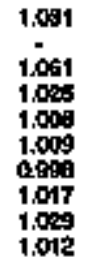 & $\begin{array}{l}1.001 \\
+ \\
1.061 \\
+005 \\
1.000 \\
1009 \\
0.009 \\
1.017 \\
1.009 \\
1.012\end{array}$ & $\begin{array}{l}1.031 \\
1.061 \\
1.005 \\
1.000 \\
1.000 \\
0.009 \\
1.017 \\
1.001 \\
1.012\end{array}$ & $\begin{array}{l}1.030 \\
- \\
1.056 \\
1.023 \\
0.000 \\
1.014 \\
0.065 \\
1.010 \\
1.003 \\
1.011\end{array}$ & $\begin{array}{l}1.029 \\
.002 \\
1.020 \\
0.997 \\
1.012 \\
0.092 \\
1.015 \\
1.020 \\
1.011\end{array}$ & 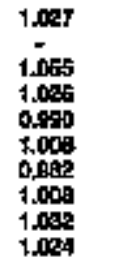 & $\begin{array}{l}+.027 \\
1.067 \\
1.005 \\
0.900 \\
1.008 \\
0.081 \\
1.011 \\
1.003 \\
1.029\end{array}$ \\
\hline 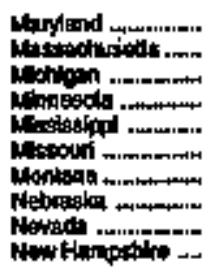 & $\begin{array}{l}1.095 \\
1.005 \\
1.035 \\
1.035 \\
1.005 \\
1.036 \\
1.005 \\
1.035 \\
1.0038 \\
1.055\end{array}$ & $\begin{array}{l}1.005 \\
1.034 \\
1.035 \\
1.0035 \\
1.035 \\
1.035 \\
1.035 \\
1.035 \\
1.035 \\
1.035\end{array}$ & 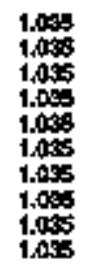 & 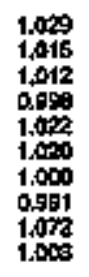 & 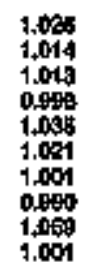 & $\begin{array}{l}1.0 .5 \\
1.013 \\
1.014 \\
0.095 \\
1.005 \\
1.000 \\
1.001 \\
0.991 \\
1.008 \\
1.012\end{array}$ & $\begin{array}{l}1.005 \\
1.013 \\
1.017 \\
1.005 \\
1.023 \\
1.016 \\
1.001 \\
0.05 \\
1.095 \\
1.000\end{array}$ & 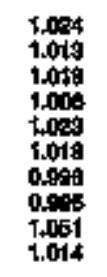 & 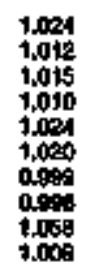 & $\begin{array}{l}1.024 \\
1.012 \\
1.015 \\
1.010 \\
1.024 \\
1.020 \\
0.900 \\
0.006 \\
1.050 \\
1.000 .\end{array}$ & $\begin{array}{l}1.002 \\
1.012 \\
1.015 \\
1.002 \\
1.025 \\
1.007 \\
1.002 \\
1.000 \\
1.008 \\
1.010\end{array}$ & $\begin{array}{l}1,002 \\
\text { t,018 } \\
1,015 \\
1,002 \\
1,005 \\
1,007 \\
1.002 \\
1,009 \\
1,002 \\
1.010\end{array}$ & $\begin{array}{l}1.009 \\
1,012 \\
1,000 \\
1,000 \\
1,019 \\
1,012 \\
1,060 \\
1.015 \\
1,097 \\
1.010\end{array}$ & 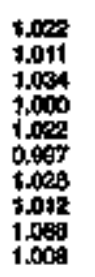 & $\begin{array}{l}1.005 \\
1.009 \\
1.009 \\
1.000 \\
1.004 \\
1.000 \\
1.000 \\
1.007 \\
\pm .004 \\
1.000\end{array}$ & 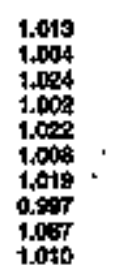 & $\begin{array}{l}1.014 \\
1.008 \\
1.003 \\
0.099 \\
1.004 \\
1.005 \\
1.012 \\
0.897 \\
1.008 \\
1.010\end{array}$ \\
\hline 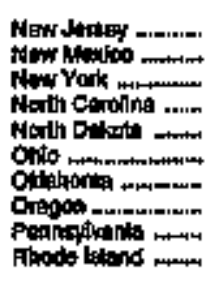 & $\begin{array}{l}1.005 \\
1.005 \\
1.055 \\
1.035 \\
1.008 \\
1.005 \\
1.005 \\
1.005 \\
1.0085 \\
1.0035\end{array}$ & 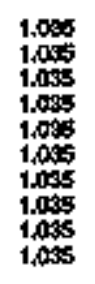 & 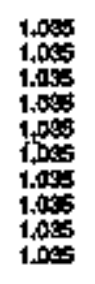 & $\begin{array}{l}1.00 \% \\
t, 100 \\
1.027 \\
1.066 \\
1.000 \\
1.034 \\
1.028 \\
1.076 \\
1.040 \\
1.042\end{array}$ & 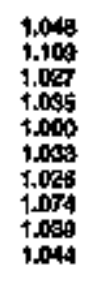 & $\begin{array}{l}1.000 \\
1.108 \\
1.025 \\
1.038 \\
1.000 \\
1.003 \\
1.028 \\
1.070 \\
1.086 \\
1.042\end{array}$ & 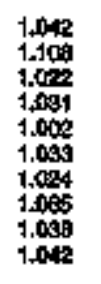 & 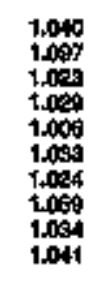 & 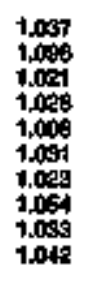 & $\begin{array}{l}1.097 \\
1.096 \\
1.021 \\
1.028 \\
1.000 \\
1.001 \\
1.023 \\
1.094 \\
1.035 \\
1.042\end{array}$ & 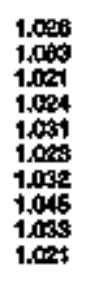 & 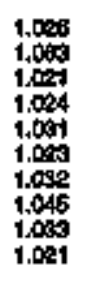 & 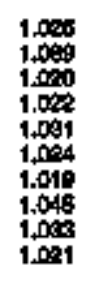 & $\begin{array}{l}1.007 \\
1.006 \\
1.080 \\
1.007 \\
1.004 \\
1,060 \\
1.010 \\
1.000 \\
1.006 \\
1.016\end{array}$ & $\begin{array}{l}1.005 \\
1.073 \\
1.002 \\
+.025 \\
1.002 \\
1.002 \\
1.005 \\
1.04 \\
1.004 \\
1.010\end{array}$ & $\begin{array}{l}1.051 \\
1.076 \\
1.045 \\
1.045 \\
1.001 \\
1.004 \\
0.964 \\
1.039 \\
1.006 \\
1.0014\end{array}$ & 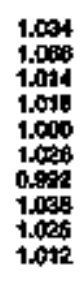 \\
\hline 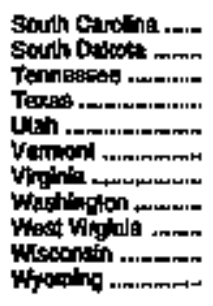 & $\begin{array}{l}1.005 \\
1.005 \\
1.0035 \\
1.005 \\
1.005 \\
1.005 \\
1.005 \\
1.005 \\
1.005 \\
1.0055 \\
1.0036\end{array}$ & 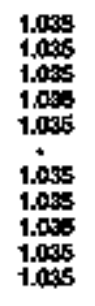 & $\begin{array}{l}1.055 \\
1.055 \\
1.055 \\
1.050 \\
1.055 \\
1.055 \\
1.056 \\
1.055 \\
1.055 \\
1.055\end{array}$ & $\begin{array}{l}1.065 \\
0.097 \\
1.006 \\
1.00 \% \\
0.020 \\
1.090 \\
1+076 \\
1.000 \\
1.005 \\
0.020\end{array}$ & 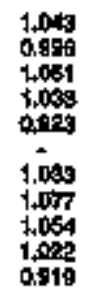 & 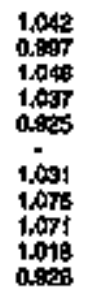 & 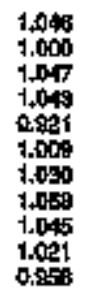 & 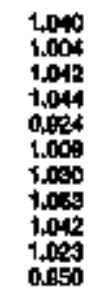 & 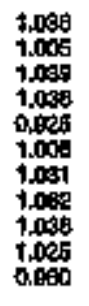 & $\begin{array}{l}1.098 \\
1.005 \\
1.039 \\
1.036 \\
0.950 \\
1.053 \\
1.051 \\
1.098 \\
1.058 \\
1.005 \\
0.900\end{array}$ & $\begin{array}{l}1.088 \\
1.004 \\
1.022 \\
1.027 \\
0.938 \\
1.006 \\
1.028 \\
1.056 \\
1.029 \\
1.019 \\
1.003\end{array}$ & 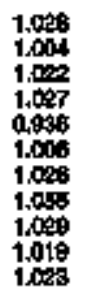 & 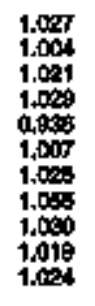 & 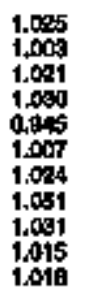 & $\begin{array}{l}1.028 \\
0.099 \\
1.020 \\
1.082 \\
0.652 \\
1.011 \\
1.021 \\
1.047 \\
1.024 \\
1.015 \\
0.097\end{array}$ & $\begin{array}{l}1.029 \\
1.000 \\
1.001 \\
1.030 \\
0.050 \\
1.009 \\
1.019 \\
1.042 \\
1.009 \\
1.000 \\
0.035\end{array}$ & $\begin{array}{l}1.009 \\
0.009 \\
1.000 \\
1.009 \\
0.049 \\
1.000 \\
1.018 \\
1.041 \\
1.041 \\
1.017 \\
0.051\end{array}$ \\
\hline U.3. Awergige & 1.035 & 1.0ss & 1.003 & 1.082 & 1.032 & 1.000 & 1,020 & 6.032 & 1.000 & 1.020 & 1.005 & 10020 & 1.000 & 1.005 & 1.005 & 1.028 & 1.081 \\
\hline
\end{tabular}

- Not appilcalo.

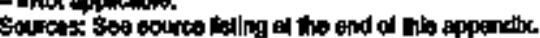

Enorgy intorimation Adminlstration

Evate Energy Dala Repart 1994
烈 
Table D5. Approximate Heat Content of Natural ties Consumed by Sectors Other Than Electric Utidites, 1977-1994 (Thousand Btu per Cabic Foot)

\begin{tabular}{|c|c|c|c|c|c|c|c|c|c|c|c|c|c|c|c|c|c|c|}
\hline sting & $10 \pi$ & tgre & $19 \%$ & $\operatorname{tg} 0$ & 19001 & 1002 & 190 & 1904 & 1806 & 1989 & $100 \%$ & tgrag & 1839 & 1950 & 1991 & 1092 & 1093 & 1994 \\
\hline 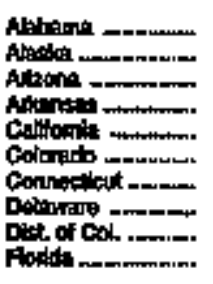 & $\begin{array}{l}1.009 \\
1.005 \\
1.054 \\
1.0020 \\
1.050 \\
0.090 \\
1.010 \\
1.024 \\
1.018 \\
1.067\end{array}$ & 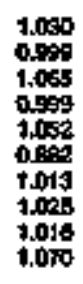 & 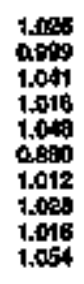 & $\begin{array}{l}1.005 \\
1.002 \\
1.046 \\
0.94 \\
1.044 \\
0.055 \\
\text { t.002 } \\
1.005 \\
1.003 \\
1000\end{array}$ & $\begin{array}{l}1.005 \\
1.004 \\
1.005 \\
0.0005 \\
1.040 \\
0.005 \\
1.005 \\
1.005 \\
1.014 \\
1.100\end{array}$ & 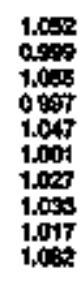 & $\begin{array}{l}1.008 \\
t .001 \\
1.048 \\
1.001 \\
1.041 \\
1.008 \\
1.000 \\
1.0015 \\
6.010 \\
t .097\end{array}$ & 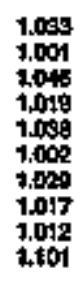 & $\begin{array}{l}1.039 \\
1.008 \\
1.040 \\
1.017 \\
1.030 \\
0.099 \\
1.090 \\
1.0909 \\
1.015 \\
1.109\end{array}$ & 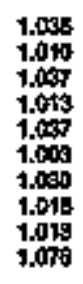 & 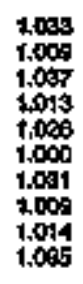 & $\begin{array}{l}1.009 \\
1.001 \\
1.004 \\
1.001 \\
1.009 \\
1.000 \\
1.008 \\
1.018 \\
1.001 \\
1.000\end{array}$ & $\begin{array}{l}t .0050 \\
0.000 \\
1.000 \\
1.004 \\
1.008 \\
1.012 \\
1.004 \\
1.014 \\
1.010 \\
1.006\end{array}$ & 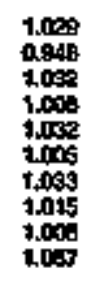 & 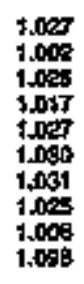 & $\begin{array}{l}1.008 \\
1.000 \\
1.001 \\
1.007 \\
t .007 \\
1.003 \\
1.008 \\
+.004 \\
1.007 \\
1.100\end{array}$ & 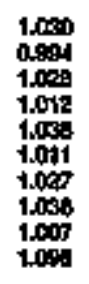 & $\begin{array}{l}1.000 \\
1.001 \\
1.000 \\
1.002 \\
1.021 \\
1.004 \\
1.091 \\
1.005 \\
1.011 \\
1.124\end{array}$ \\
\hline 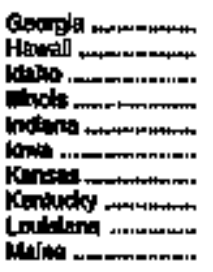 & $\begin{array}{l}1.007 \\
1.000 \\
1.028 \\
0.9000 \\
1.004 \\
0.991 \\
1.011 \\
1.025 \\
1.024\end{array}$ & 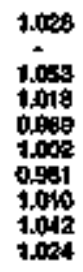 & 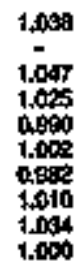 & $\begin{array}{l}1.008 \\
0.003 \\
t .058 \\
1.002 \\
0.060 \\
1.000 \\
0.004 \\
1,008 \\
1.008 \\
1.004\end{array}$ & 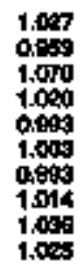 & $\begin{array}{l}1.030 \\
0.009 \\
1.007 \\
1.002 \\
1.011 \\
1.000 \\
1,007 \\
1.014 \\
1.047 \\
1.005\end{array}$ & 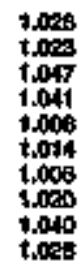 & $\begin{array}{l}1.002 \\
1.006 \\
1.045 \\
1.040 \\
1.000 \\
1.015 \\
0.094 \\
5.002 \\
1.041 \\
1.032\end{array}$ & $\begin{array}{l}1.029 \\
1.002 \\
1.009 \\
1.040 \\
1.000 \\
1.011 \\
1.000 \\
1.000 \\
1.039 \\
1.036\end{array}$ & 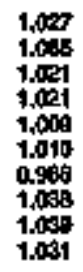 & 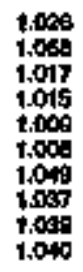 & $\begin{array}{l}1.005 \\
1.078 \\
1.020 \\
1.018 \\
1.018 \\
1.007 \\
0.008 \\
1.037 \\
1.041 \\
1.007\end{array}$ & $\begin{array}{l}1.008 \\
1.000 \\
1.007 \\
1.002 \\
1.018 \\
1.011 \\
0.099 \\
1.090 \\
1.040 \\
1.003\end{array}$ & 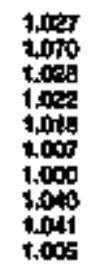 & $\begin{array}{l}1,007 \\
1.060 \\
1.039 \\
1.019 \\
1,00 t \\
1.000 \\
1.011 \\
1.047 \\
1,040 \\
1.000\end{array}$ & 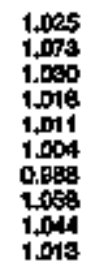 & 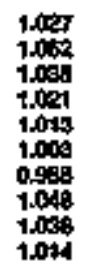 & 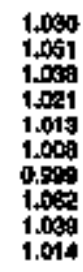 \\
\hline 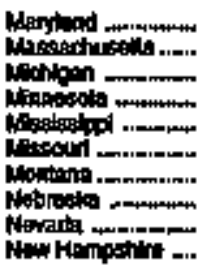 & 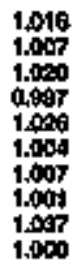 & $\begin{array}{l}1.000 \\
1.000 \\
1.007 \\
0.097 \\
1.004 \\
1.000 \\
0.009 \\
1.000 \\
1.000 \\
1.005\end{array}$ & 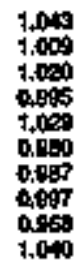 & $\begin{array}{l}1.000 \\
1.018 \\
1.000 \\
0.00 \\
1.004 \\
1.018 \\
1.000 \\
0.000 \\
1.000 \\
1.000\end{array}$ & 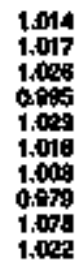 & $\begin{array}{l}1.018 \\
1.002 \\
1.000 \\
1.005 \\
1.091 \\
1.095 \\
1.000 \\
0.001 \\
1.074 \\
1.000\end{array}$ & $\begin{array}{l}1.004 \\
1.001 \\
1.005 \\
1.003 \\
1.002 \\
1.002 \\
1.007 \\
0.002 \\
1.007 \\
t .021\end{array}$ & $\begin{array}{l}1.006 \\
1.005 \\
1.009 \\
1.000 \\
1.001 \\
1.097 \\
1.004 \\
0.091 \\
1.009 \\
1.087\end{array}$ & $\begin{array}{l}1.094 \\
1.004 \\
1.029 \\
1.004 \\
1.005 \\
1.017 \\
0.099 \\
0.902 \\
1.001 \\
1.007\end{array}$ & 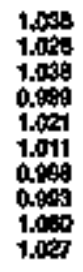 & 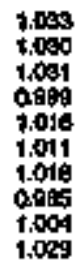 & $\begin{array}{l}1.008 \\
1.030 \\
1.040 \\
1.001 \\
1.015 \\
1.000 \\
1.004 \\
0.009 \\
0.004 \\
1.025\end{array}$ & $\begin{array}{l}1.001 \\
1.006 \\
1.000 \\
1.000 \\
1.001 \\
1.000 \\
1.019 \\
0.019 \\
1.002 \\
1.019\end{array}$ & 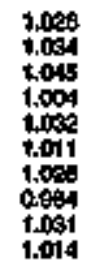 & 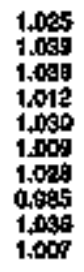 & $\begin{array}{l}1.009 \\
1.038 \\
1.007 \\
1.011 \\
1,062 \\
1.002 \\
1.002 \\
0.479 \\
1.009 \\
1.006\end{array}$ & 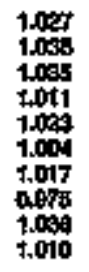 & 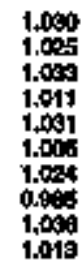 \\
\hline 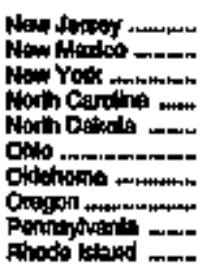 & 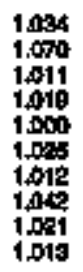 & $\begin{array}{l}1.0045 \\
1.000 \\
1.012 \\
1.002 \\
1.000 \\
1.004 \\
1,004 \\
1.0045 \\
1.002 \\
1.013\end{array}$ & $\begin{array}{l}1.005 \\
1.065 \\
1.013 \\
1.0192 \\
1.000 \\
1.009 \\
1.005 \\
1.045 \\
1.021 \\
1.000\end{array}$ & $\begin{array}{l}t .003 \\
1.045 \\
1.083 \\
7.012 \\
1.068 \\
1.018 \\
1,008 \\
1.04: \\
1.002 \\
1.021\end{array}$ & $\begin{array}{l}1,000 \\
1,004 \\
1.0016 \\
1.012 \\
1.002 \\
1.009 \\
1.000 \\
1.040 \\
1.002 \\
1.002\end{array}$ & 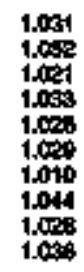 & 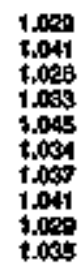 & $\begin{array}{l}2.000 \\
1.001 \\
1,005 \\
1.034 \\
1.049 \\
1.037 \\
1.097 \\
1.096 \\
1.034 \\
1.000\end{array}$ & $\begin{array}{l}1.002 \\
1.009 \\
1.097 \\
1.094 \\
1.002 \\
1,044 \\
1.000 \\
1.090 \\
1,034 \\
1.099\end{array}$ & 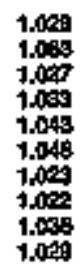 & 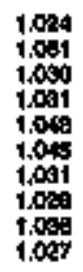 & 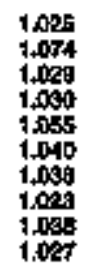 & $\begin{array}{l}1.025 \\
1,000 \\
1,009 \\
1.091 \\
1.040 \\
1.042 \\
1.002 \\
1.035 \\
1.009 \\
1.027\end{array}$ & $\begin{array}{l}1.005 \\
1.000 \\
1.029 \\
1.009 \\
1.000 \\
1.040 \\
1.000 \\
1.004 \\
1.008 \\
1.027\end{array}$ & $\begin{array}{l}1.095 \\
1.042 \\
1.027 \\
1.092 \\
1.045 \\
1.044 \\
1.019 \\
1.091 \\
1.095 \\
1.028\end{array}$ & $\begin{array}{l}1.005 \\
1.000 \\
1.009 \\
1.034 \\
1.045 \\
1.008 \\
1.008 \\
1.060 \\
1.038 \\
1.016\end{array}$ & 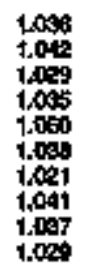 & 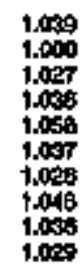 \\
\hline 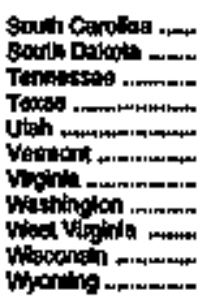 & 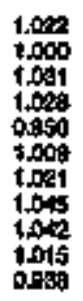 & $\begin{array}{l}1.031 \\
0.098 \\
1.006 \\
1.006 \\
0.057 \\
1.011 \\
1.010 \\
1.018 \\
1.037 \\
1.014 \\
0.914\end{array}$ & 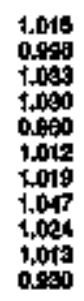 & 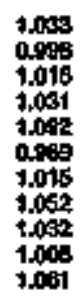 & 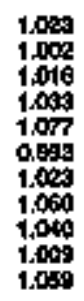 & $\begin{array}{l}1.000 \\
0.999 \\
1.004 \\
1.009 \\
0.030 \\
0.003 \\
1.003 \\
1.050 \\
1.047 \\
1.012 \\
1.002\end{array}$ & $\begin{array}{l}1.007 \\
1.011 \\
1.008 \\
1.002 \\
1.007 \\
0.908 \\
1.0030 \\
1,043 \\
1.038 \\
t .000 \\
1.000\end{array}$ & 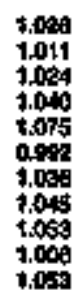 & $\begin{array}{l}1.020 \\
1.010 \\
1.094 \\
1.099 \\
1.075 \\
0.902 \\
1.039 \\
1.040 \\
1.097 \\
1.010 \\
1.051\end{array}$ & 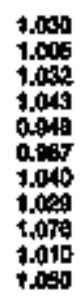 & 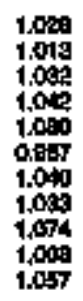 & $\begin{array}{l}1.027 \\
1.000 \\
1.001 \\
1.040 \\
1.001 \\
0.070 \\
1.041 \\
1.008 \\
1.077 \\
1.000 \\
1.058\end{array}$ & 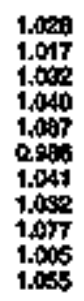 & $\begin{array}{l}1.000 \\
1.016 \\
1.005 \\
1.042 \\
1.009 \\
0.000 \\
1.002 \\
1.000 \\
1.091 \\
1.000 \\
1.000\end{array}$ & 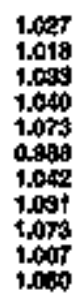 & $\begin{array}{l}1.007 \\
1.015 \\
1.031 \\
1.060 \\
t .018 \\
0.096 \\
1.008 \\
1.038 \\
1.005 \\
1.000 \\
1.008\end{array}$ & 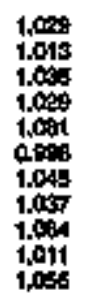 & 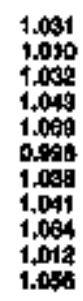 \\
\hline US. Aratoge & 9002 & 1.000 & 1.020 & 1.004 & 1.0020 & 10 织 & t.031 & 9,030 & 1.002 & 1.030 & 1,091 & 10030 & 1.088 & 1.031 & 1.001 & t.0ose & 1 1000 & 1.0930 \\
\hline
\end{tabular}

- Hint apalosible.

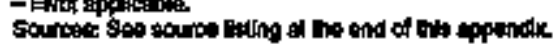




\begin{tabular}{|c|c|c|c|c|c|c|c|c|c|c|c|c|c|c|c|c|c|}
\hline sonin & 1000 & I\$s; & Jape & 1969 & 1864 & 1965 & 1989 & 167 & 1869 & $\operatorname{sen}$ & 1990 & 1975 & 1 1952 & $19 \mathrm{Ts}$ & 1574 & 195 & 1978 \\
\hline 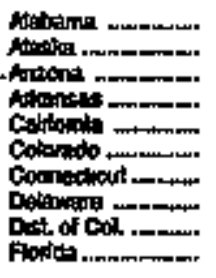 & 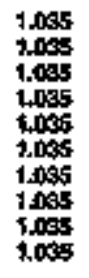 & $\begin{array}{l}1.005 \\
1.055 \\
1.035 \\
1.035 \\
1.035 \\
1.055 \\
1.035 \\
1.065 \\
1.0035 \\
1.086\end{array}$ & 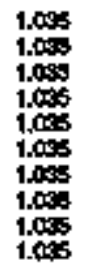 & $\begin{array}{l}1.007 \\
1.011 \\
1.07 \\
1.000 \\
1.000 \\
0.000 \\
1.000 \\
1.003 \\
1.005 \\
1.006\end{array}$ & 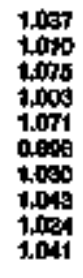 & $\begin{array}{l}1.004 \\
1.010 \\
1.076 \\
1.001 \\
1.007 \\
0.12 \\
1.002 \\
1.003 \\
1.024 \\
1.007\end{array}$ & 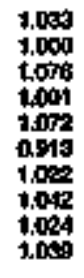 & $\begin{array}{l}1.031 \\
1.000 \\
1,000 \\
1.001 \\
1.007 \\
0.000 \\
1.001 \\
1.000 \\
1.004 \\
1.000\end{array}$ & 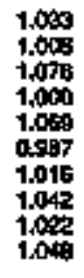 & $\begin{array}{l}1.009 \\
1.006 \\
1.070 \\
1.000 \\
1.000 \\
0.997 \\
1.016 \\
1.048 \\
1.002 \\
1.046\end{array}$ & $\begin{array}{l}1.001 \\
1.005 \\
1.009 \\
1.004 \\
1.064 \\
0.074 \\
1.016 \\
1.000 \\
1.016 \\
1.041\end{array}$ & $\begin{array}{l}1.061 \\
1.005 \\
1.009 \\
1.004 \\
t .054 \\
0.974 \\
1.018 \\
1.000 \\
1.018 \\
1.041\end{array}$ & $\begin{array}{l}1.091 \\
1.005 \\
1.059 \\
1.004 \\
1.054 \\
0.074 \\
1.018 \\
1.000 \\
1.016 \\
1.040\end{array}$ & $\begin{array}{l}1.009 \\
1.010 \\
1.008 \\
0.098 \\
1.058 \\
0.062 \\
1.015 \\
1.000 \\
1.013 \\
1.043\end{array}$ & $\begin{array}{l}1.028 \\
1.005 \\
1.065 \\
0.908 \\
1.058 \\
0.967 \\
1.012 \\
1.040 \\
1.012 \\
1.045\end{array}$ & $\begin{array}{l}1.099 \\
1.005 \\
1.052 \\
0.997 \\
1.057 \\
0.013 \\
1.005 \\
1.000 \\
1.012 \\
1.049\end{array}$ & $\begin{array}{l}1.030 \\
1.005 \\
1.058 \\
0.997 \\
1.069 \\
0.014 \\
1.006 \\
1.08 \\
1.012 \\
1.049\end{array}$ \\
\hline 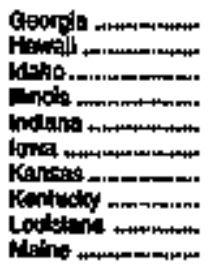 & 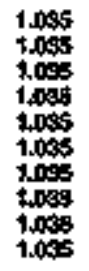 & 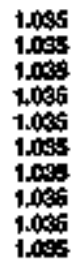 & 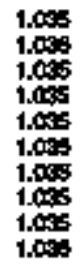 & $\begin{array}{c}1.005 \\
5.006 \\
1.003 \\
1.000 \\
1.009 \\
0.906 \\
1.045 \\
1.005 \\
-\end{array}$ & $\begin{array}{l}1.009 \\
1.001 \\
1.000 \\
1.000 \\
1.000 \\
0.04 \\
1.00 \\
1.050 \\
-\end{array}$ & $\begin{array}{l}1.040 \\
-0005 \\
1.000 \\
0.969 \\
1.010 \\
0.95 \\
1.005 \\
1.00\end{array}$ & $\begin{array}{l}t .002 \\
.000 \\
1.006 \\
t .000 \\
1.019 \\
0.994 \\
t .024 \\
1.096 \\
1.085\end{array}$ & $\begin{array}{l}1.013 \\
- \\
1.048 \\
1.009 \\
1.000 \\
1.001 \\
0.990 \\
1.0024 \\
1.003 \\
1.0025\end{array}$ & $\begin{array}{l}1.043 \\
1.000 \\
1.003 \\
1.001 \\
1.021 \\
0.09 \\
1.00 \\
1.014 \\
1.000\end{array}$ & 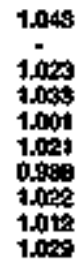 & 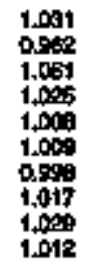 & $\begin{array}{l}1.081 \\
0.989 \\
1,081 \\
1,006 \\
1,000 \\
1.000 \\
0.908 \\
1.017 \\
1.009 \\
1.012\end{array}$ & 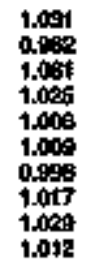 & 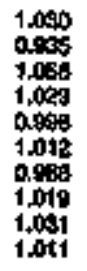 & 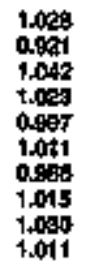 & 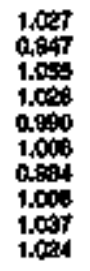 & $\begin{array}{l}1.027 \\
0.011 \\
1.057 \\
1.004 \\
0.090 \\
1.000 \\
0.891 \\
1.091 \\
1.0088 \\
1.004\end{array}$ \\
\hline 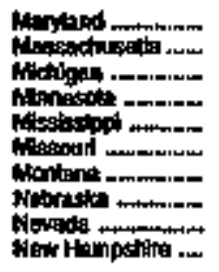 & 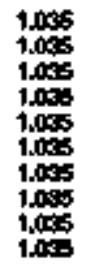 & 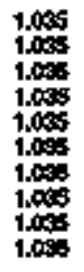 & 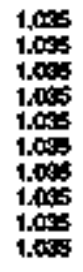 & $\begin{array}{l}1.009 \\
1.015 \\
1.018 \\
0.000 \\
1.000 \\
1.000 \\
1.000 \\
0.901 \\
1.092 \\
1.000\end{array}$ & 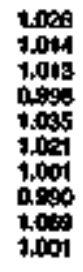 & $\begin{array}{l}1.005 \\
1.013 \\
1.014 \\
0.909 \\
1.009 \\
1.000 \\
1.001 \\
0.901 \\
1.000 \\
1.012\end{array}$ & 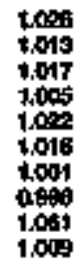 & $\begin{array}{l}1.004 \\
1.093 \\
1.019 \\
1.000 \\
1.000 \\
1.016 \\
0.098 \\
0.900 \\
1.051 \\
1.014\end{array}$ & $\begin{array}{l}1.004 \\
1.012 \\
1.015 \\
1.010 \\
1.004 \\
1.000 \\
0.900 \\
0.006 \\
1.008 \\
1.009\end{array}$ & $\begin{array}{l}1.004 \\
1.0012 \\
1.015 \\
1.010 \\
t .004 \\
1.000 \\
0.090 \\
0.906 \\
1.060 \\
1.009\end{array}$ & $\begin{array}{l}1.002 \\
1.012 \\
1.015 \\
1.000 \\
1.005 \\
1.000 \\
1.002 \\
1.000 \\
1.002 \\
1.000\end{array}$ & $\begin{array}{l}1.002 \\
1.012 \\
1.015 \\
1.002 \\
1.005 \\
1.007 \\
1.002 \\
1.009 \\
1.008 \\
1.010\end{array}$ & $\begin{array}{l}1,002 \\
1,012 \\
1,015 \\
1,000 \\
1,005 \\
1.000 \\
1.000 \\
1,000 \\
1,002 \\
1.010\end{array}$ & $\begin{array}{l}1.000 \\
1.011 \\
1.010 \\
0.990 \\
1.094 \\
0.995 \\
1.002 \\
1.005 \\
1.067 \\
1.006\end{array}$ & $\begin{array}{l}1.020 \\
1.009 \\
1.007 \\
1.001 \\
1.000 \\
1.005 \\
1.002 \\
1.002 \\
1.007 \\
1.007\end{array}$ & $\begin{array}{l}1.003 \\
1.004 \\
1.0012 \\
1.001 \\
1.003 \\
1.006 \\
1.021 \\
0.054 \\
1.067 \\
1.010\end{array}$ & $\begin{array}{l}1.014 \\
1,000 \\
1.000 \\
0.985 \\
1,023 \\
1.009 \\
1.014 \\
0.094 \\
1,066 \\
1.010\end{array}$ \\
\hline 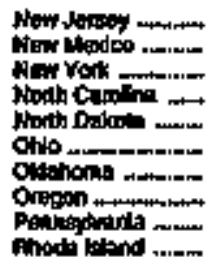 & 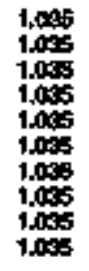 & $\begin{array}{l}1.005 \\
1.005 \\
1.055 \\
1.005 \\
1.005 \\
1.005 \\
1.005 \\
1.085 \\
1.005 \\
1.0035\end{array}$ & $\begin{array}{l}1.005 \\
1.005 \\
1.005 \\
1.005 \\
1.005 \\
1.008 \\
1.005 \\
1.055 \\
1.005 \\
1.006\end{array}$ & 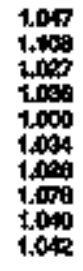 & 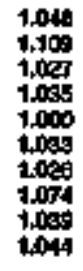 & $\begin{array}{l}1.005 \\
1.100 \\
1.050 \\
1.003 \\
1.000 \\
1.000 \\
1.0045 \\
1.090 \\
1.006 \\
1.042\end{array}$ & 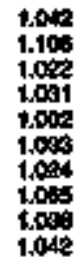 & 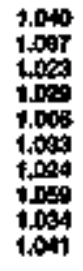 & $\begin{array}{l}1.008 \\
0.000 \\
1.021 \\
1.006 \\
1.006 \\
0.031 \\
1.002 \\
1.004 \\
1.083 \\
1.042\end{array}$ & 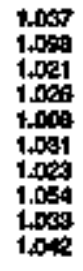 & 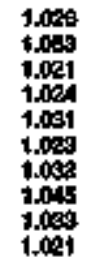 & 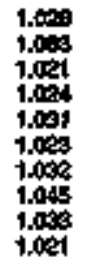 & $\begin{array}{l}1.000 \\
1.005 \\
1.001 \\
1.404 \\
1.001 \\
1.000 \\
1.004 \\
1.045 \\
1.008 \\
1.041\end{array}$ & 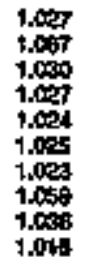 & 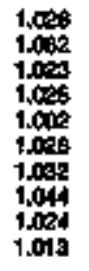 & $\begin{array}{l}1.091 \\
1.004 \\
1.016 \\
1.018 \\
1.005 \\
1.008 \\
1.018 \\
1.000 \\
1.005 \\
1.014\end{array}$ & $\begin{array}{l}1.004 \\
1.059 \\
1.014 \\
1.018 \\
1.000 \\
1.005 \\
1.014 \\
1.066 \\
1.025 \\
1.013\end{array}$ \\
\hline 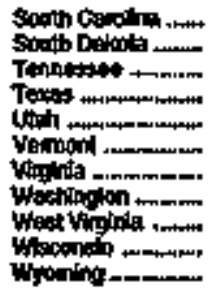 & 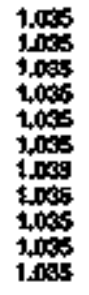 & 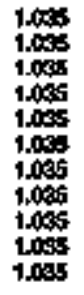 & 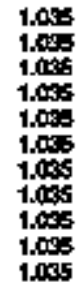 & 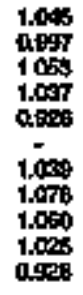 & 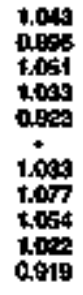 & 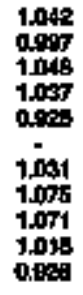 & $\begin{array}{l}1.046 \\
1.000 \\
1.047 \\
1.045 \\
0.021 \\
1.009 \\
1.000 \\
1006 \\
1.045 \\
1.021 \\
0.058\end{array}$ & $\begin{array}{l}1.000 \\
1.004 \\
1,042 \\
1.044 \\
0.024 \\
1.009 \\
1.000 \\
1.003 \\
1.042 \\
1.000 \\
0.050\end{array}$ & 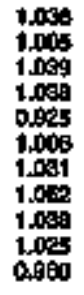 & $\begin{array}{l}1.000 \\
1.000 \\
1.009 \\
1.009 \\
0.005 \\
1.003 \\
1.001 \\
1.000 \\
1.000 \\
1.000 \\
0.900\end{array}$ & $\begin{array}{l}1.000 \\
1.004 \\
1.002 \\
1.007 \\
0.000 \\
1.000 \\
1.000 \\
1.060 \\
1.009 \\
1.019 \\
1.003\end{array}$ & 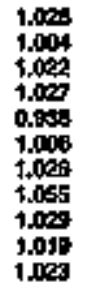 & $\begin{array}{l}1.028 \\
1.004 \\
1.004 \\
1.007 \\
0.050 \\
1.008 \\
1.006 \\
1.065 \\
1.005 \\
1.010 \\
1.023\end{array}$ & $\begin{array}{l}1.000 \\
1.002 \\
1.002 \\
1.00 \% \\
0.015 \\
1.006 \\
1.005 \\
1.001 \\
1.005 \\
1.015 \\
1.017\end{array}$ & 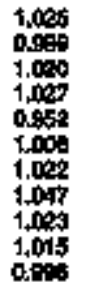 & 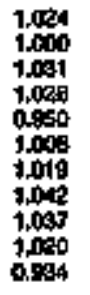 & 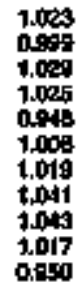 \\
\hline US. Avtases . . & 1.035 & 1.085 & 1.005 & 1000 & 1.009 & 1,000 & 1.030 & 1,038 & 1.001 & 1.000 & $1+000$ & $t .069$ & $1.02 \mathrm{~B}$ & $1+0 \mathrm{es}$ & 1,024 & $\$, 022$ & $t, 022$ \\
\hline
\end{tabular}

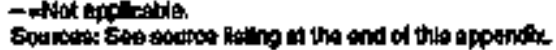


Table D7. Approximate Heat Content of Natural Gas Total Consumption, 1977-1994 (Thousand Blu per Cubic Foot)

\begin{tabular}{|c|c|c|c|c|c|c|c|c|c|c|c|c|c|c|c|c|c|c|}
\hline state & $\mathrm{knn}$ & 1970 & $18 \mathrm{Pi}$ & 90 & 1801 & 1600 & roes & 19:04 & 1005 & 18006 & $19 \mathrm{sor}$ & 1908 & g.9 & 1890 & 1991 & 1902 & 1909 & $\operatorname{tse} 4$ \\
\hline 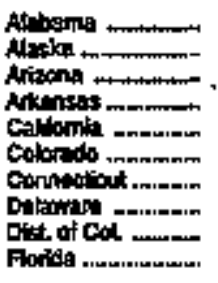 & $\begin{array}{l}1.091 \\
1.005 \\
1.066 \\
1.001 \\
1.059 \\
0.900 \\
1.010 \\
1.000 \\
1.016 \\
1.045\end{array}$ & $\begin{array}{l}1.035 \\
1.000 \\
1.005 \\
1.001 \\
1.003 \\
0.068 \\
1.006 \\
1.051 \\
1.0016 \\
1.017\end{array}$ & $\begin{array}{l}1,009 \\
1,000 \\
1.045 \\
1,017 \\
1,060 \\
0.090 \\
1,012 \\
1.031 \\
1.018 \\
1.097\end{array}$ & $\begin{array}{l}1.004 \\
1.009 \\
1.006 \\
1.001 \\
1.040 \\
0.900 \\
1.002 \\
1.065 \\
1,000 \\
+.041\end{array}$ & $\begin{array}{l}1,005 \\
1.004 \\
1.050 \\
1,001 \\
1.008 \\
0.091 \\
1.026 \\
1.006 \\
1.014 \\
1.06\end{array}$ & $\begin{array}{l}1.008 \\
1.000 \\
1.054 \\
1.000 \\
1.049 \\
1.000 \\
1.027 \\
1.033 \\
1.017 \\
1.044\end{array}$ & 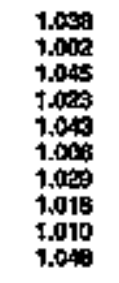 & $\begin{array}{l}1.003 \\
1.000 \\
1.017 \\
1.001 \\
1.042 \\
1.002 \\
1.006 \\
1.002 \\
1.012 \\
1.040\end{array}$ & $\begin{array}{l}1.038 \\
1.006 \\
1.050 \\
1.019 \\
1.043 \\
0.099 \\
1.090 \\
1.025 \\
1.015 \\
1.003\end{array}$ & $\begin{array}{l}1.006 \\
1.009 \\
1.050 \\
1.010 \\
1.059 \\
1.000 \\
1.000 \\
1.010 \\
1.013 \\
1.098\end{array}$ & $\begin{array}{l}1.068 \\
1.009 \\
1.008 \\
1.010 \\
1.030 \\
1.000 \\
1.031 \\
1.018 \\
1.014 \\
1.044\end{array}$ & 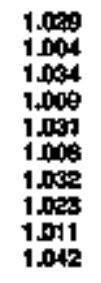 & $\begin{array}{l}1.050 \\
0.099 \\
1.040 \\
1,006 \\
1.007 \\
1.001 \\
1.034 \\
1.005 \\
1,010 \\
1.042\end{array}$ & $\begin{array}{l}1.089 \\
1.954 \\
1.002 \\
1.009 \\
1.0032 \\
1.005 \\
1.039 \\
1.026 \\
1.000 \\
1.040\end{array}$ & $\begin{array}{l}1.027 \\
1.009 \\
1.065 \\
1.017 \\
1.027 \\
1.029 \\
+.031 \\
1.034 \\
1.005 \\
1.040\end{array}$ & $\begin{array}{l}1.028 \\
1.002 \\
1.001 \\
1.009 \\
1.069 \\
1.023 \\
1.009 \\
1.009 \\
1.007 \\
1.040\end{array}$ & $\begin{array}{l}1.030 \\
0.994 \\
1.020 \\
1.014 \\
1.030 \\
1.011 \\
1.027 \\
1.085 \\
1.007 \\
1.462\end{array}$ & $\begin{array}{l}1.0000 \\
1.009 \\
1.007 \\
1.002 \\
1.0020 \\
1.005 \\
1.000 \\
1.005 \\
1.011 \\
1.008\end{array}$ \\
\hline 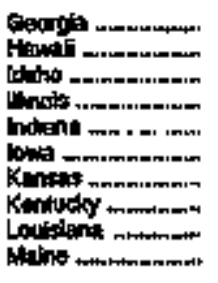 & $\begin{array}{l}1.097 \\
0.949 \\
1.060 \\
1.028 \\
0.900 \\
1.004 \\
0.991 \\
1.011 \\
1.039 \\
1.002\end{array}$ & 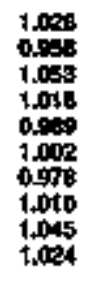 & 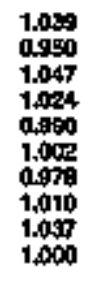 & 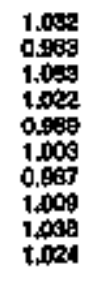 & $\begin{array}{l}1.027 \\
0.059 \\
1.070 \\
1.0720 \\
0.903 \\
1.009 \\
0.097 \\
1.014 \\
1.097 \\
1.005\end{array}$ & $\begin{array}{l}1.030 \\
0.999 \\
1.072 \\
1.009 \\
1.018 \\
1.009 \\
0.999 \\
1.014 \\
1.047 \\
1.025\end{array}$ & $\begin{array}{l}1.025 \\
1.023 \\
1.047 \\
1.044 \\
1.000 \\
1.014 \\
0.099 \\
1.009 \\
1.044 \\
1.006\end{array}$ & 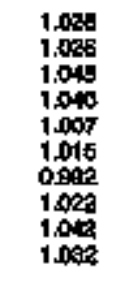 & $\begin{array}{l}1.028 \\
1.082 \\
1.049 \\
1.040 \\
1.908 \\
1.011 \\
0.99 \\
1.0901 \\
1.040 \\
1.005\end{array}$ & $\begin{array}{l}1.027 \\
1.088 \\
1.002 \\
1.002 \\
1.009 \\
1.010 \\
0.585 \\
1.038 \\
1.040 \\
1.091\end{array}$ & $\begin{array}{l}1.068 \\
1.050 \\
1.017 \\
1.045 \\
1.000 \\
1.009 \\
1.049 \\
1.007 \\
1.040 \\
1.040\end{array}$ & $\begin{array}{l}1.005 \\
1.078 \\
1.000 \\
1.018 \\
1,018 \\
1,007 \\
0.068 \\
1,00 \% \\
1,002 \\
1,004\end{array}$ & $\begin{array}{l}1.085 \\
1.080 \\
1.087 \\
1002 \\
1.016 \\
1.011 \\
0.008 \\
1.096 \\
1.043 \\
1.008\end{array}$ & $\begin{array}{l}1.027 \\
1.070 \\
1.020 \\
1.020 \\
1.010 \\
1.007 \\
0.999 \\
1,040 \\
1.042 \\
t .000\end{array}$ & $\begin{array}{l}1.027 \\
1.000 \\
1.008 \\
1.019 \\
1.014 \\
1.008 \\
1.007 \\
1.047 \\
1.047 \\
1.006\end{array}$ & $\begin{array}{l}1.005 \\
1.079 \\
7.030 \\
1.011 \\
1.011 \\
1.004 \\
0.095 \\
1.059 \\
1.044 \\
1.013\end{array}$ & $\begin{array}{l}1.057 \\
1.062 \\
1.003 \\
1.021 \\
1.015 \\
1.003 \\
0.987 \\
1.048 \\
1.037 \\
1.014\end{array}$ & $\begin{array}{l}1.050 \\
1.051 \\
1.098 \\
1.021 \\
1.013 \\
1.008 \\
0.998 \\
1.062 \\
1.040 \\
1.014\end{array}$ \\
\hline 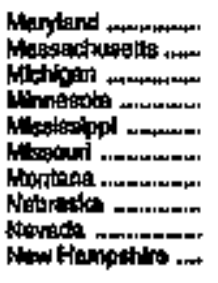 & $\begin{array}{l}1.016 \\
1.007 \\
1.006 \\
0.907 \\
1.006 \\
1.002 \\
1.006 \\
0.9 \% 9 \\
1.049 \\
1.000\end{array}$ & $\begin{array}{l}1.030 \\
1.000 \\
1.006 \\
0.987 \\
1.007 \\
1.004 \\
1.007 \\
0.908 \\
1.006 \\
1.007\end{array}$ & $\begin{array}{l}1.046 \\
1.009 \\
1.005 \\
0.995 \\
1.024 \\
0.980 \\
0.990 \\
0.994 \\
1.015 \\
1.040\end{array}$ & $\begin{array}{l}1.000 \\
1.010 \\
1.011 \\
0.997 \\
1.007 \\
1.014 \\
1.012 \\
0.978 \\
1.001 \\
1,000\end{array}$ & $\begin{array}{l}1.014 \\
1.016 \\
1.017 \\
0.985 \\
1.025 \\
1.010 \\
1.011 \\
0.978 \\
1.078 \\
1.029\end{array}$ & $\begin{array}{l}1.016 \\
1.024 \\
1.022 \\
1.005 \\
1.029 \\
1.010 \\
1.011 \\
0.991 \\
1.070 \\
1.000\end{array}$ & $\begin{array}{l}1.02 t \\
1.025 \\
1.024 \\
1.000 \\
1.027 \\
1.027 \\
1.008 \\
0.002 \\
1.006 \\
1.004\end{array}$ & $\begin{array}{l}1.009 \\
1.000 \\
1.017 \\
1.009 \\
1.030 \\
1.017 \\
1.005 \\
0.901 \\
1.080 \\
1.027\end{array}$ & $\begin{array}{l}1.039 \\
1.027 \\
1.015 \\
1.004 \\
1.002 \\
1.017 \\
1.001 \\
0.902 \\
1.002 \\
1.027\end{array}$ & $\begin{array}{l}1.036 \\
1.026 \\
1.028 \\
0.909 \\
1.025 \\
1.011 \\
1.000 \\
0.008 \\
1.006 \\
1.009\end{array}$ & $\begin{array}{l}1.094 \\
1.029 \\
1.021 \\
0.099 \\
1.019 \\
1.011 \\
1.090 \\
0.0995 \\
1.009 \\
1.009\end{array}$ & $\begin{array}{l}1.008 \\
1.000 \\
1.000 \\
1.009 \\
1.017 \\
1.000 \\
1.005 \\
0.003 \\
1.003 \\
1.000\end{array}$ & 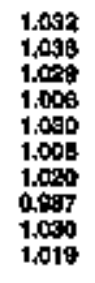 & $\begin{array}{l}1.009 \\
1.030 \\
1.0024 \\
1.004 \\
1.003 \\
1.013 \\
1.008 \\
0.003 \\
1.001 \\
1.004\end{array}$ & $\begin{array}{l}1,008 \\
1,009 \\
1,000 \\
1.002 \\
1.000 \\
1.008 \\
1.009 \\
0.984 \\
1.092 \\
1.007\end{array}$ & $\begin{array}{l}1.000 \\
1,007 \\
1,000 \\
1.011 \\
1.047 \\
1.0002 \\
1.0009 \\
0.979 \\
1.001 \\
1.000\end{array}$ & 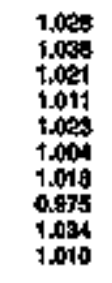 & 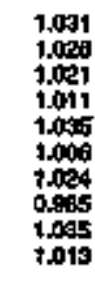 \\
\hline 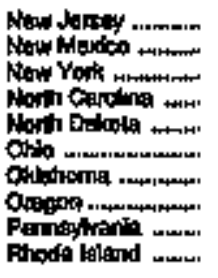 & $\begin{array}{l}1.034 \\
1.067 \\
1.011 \\
1.019 \\
1.000 \\
1.004 \\
1.007 \\
1.042 \\
1.021 \\
1.018\end{array}$ & $\begin{array}{l}1.058 \\
1.056 \\
1.012 \\
1.001 \\
1.000 \\
1.005 \\
1.004 \\
1.041 \\
1.002 \\
1.013\end{array}$ & $\begin{array}{l}1.039 \\
1.050 \\
1.010 \\
1.002 \\
1.000 \\
1.005 \\
1.024 \\
1.045 \\
1.021 \\
1.011\end{array}$ & $\begin{array}{l}1.003 \\
1.040 \\
1.005 \\
1.012 \\
1.008 \\
1.016 \\
1.002 \\
1.046 \\
1.002 \\
1.0021\end{array}$ & $\begin{array}{l}1.034 \\
1.047 \\
1.020 \\
1.012 \\
1.048 \\
1.023 \\
1.055 \\
1.044 \\
1.0022 \\
1.022\end{array}$ & 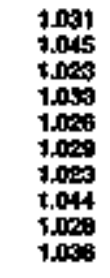 & $\begin{array}{l}1.091 \\
1.089 \\
1.067 \\
1.003 \\
1.045 \\
1.004 \\
1.042 \\
1.041 \\
1.009 \\
1.005\end{array}$ & 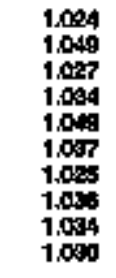 & 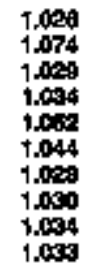 & 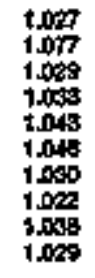 & 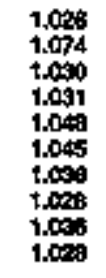 & 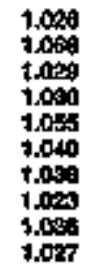 & $\begin{array}{l}1.007 \\
1.049 \\
1.009 \\
1.091 \\
1.049 \\
1.042 \\
1.000 \\
1.005 \\
1.007 \\
1.007\end{array}$ & 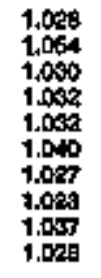 & 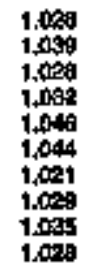 & $\begin{array}{l}1,026 \\
1,040 \\
1,029 \\
1,004 \\
1,045 \\
1,008 \\
1,008 \\
1,095 \\
1,098 \\
1,018\end{array}$ & 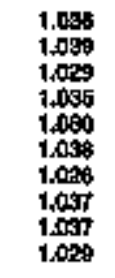 & 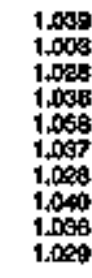 \\
\hline 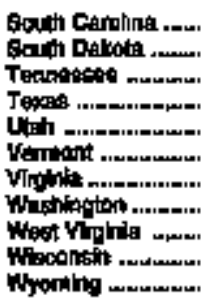 & $\begin{array}{l}1.002 \\
1.000 \\
1.031 \\
1.007 \\
0.000 \\
1.000 \\
1.003 \\
1.045 \\
1.042 \\
1.015 \\
0.078\end{array}$ & $\begin{array}{l}1.008 \\
0.005 \\
1.008 \\
1.008 \\
0.098 \\
1.014 \\
1.004 \\
1.048 \\
1.001 \\
1.012 \\
0.914\end{array}$ & $\begin{array}{l}1.018 \\
0.996 \\
1.050 \\
1.023 \\
0.900 \\
1.010 \\
1.021 \\
1.047 \\
1.024 \\
1.013 \\
0.902\end{array}$ & 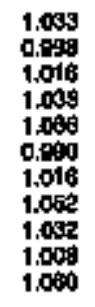 & $\begin{array}{l}1.023 \\
1.002 \\
1.016 \\
1.002 \\
1.073 \\
0.993 \\
1.004 \\
1.000 \\
1.040 \\
1.009 \\
1.050\end{array}$ & $\begin{array}{l}1.030 \\
0.009 \\
1,004 \\
1.082 \\
0.039 \\
0.093 \\
1,04 \\
1.003 \\
1.047 \\
1.012 \\
1.008\end{array}$ & 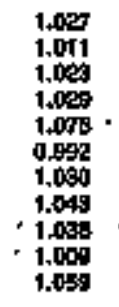 & $\begin{array}{r}1.020 \\
1.011 \\
1.024 \\
1.003 \\
1.076 \\
0.992 \\
1.098 \\
.1 .045 \\
1.053 \\
1.009 \\
1.050\end{array}$ & $\begin{array}{l}1.029 \\
1.010 \\
1.004 \\
1.039 \\
1.078 \\
0.092 \\
1.089 \\
1.040 \\
1.067 \\
1.070 \\
1.054\end{array}$ & $\begin{array}{l}1.000 \\
1.009 \\
1.002 \\
1.040 \\
0.048 \\
0.078 \\
1.000 \\
1.009 \\
1.079 \\
1.010 \\
1.090\end{array}$ & $\begin{array}{r}1.008 \\
1.013 \\
1.002 \\
1.040 \\
1.000 \\
0.097 \\
1.040 \\
1.065 \\
1.004 \\
1.006 \\
1.057\end{array}$ & $\begin{array}{l}1.027 \\
1.020 \\
1.034 \\
1.059 \\
1.099 \\
0.090 \\
1.041 \\
1.008 \\
1.07 \\
1.008 \\
1.053\end{array}$ & $\begin{array}{l}1.008 \\
1.017 \\
1.082 \\
1.009 \\
1.087 \\
0.098 \\
1.041 \\
1.002 \\
1.007 \\
1.005 \\
1.058\end{array}$ & $\begin{array}{l}1.020 \\
1.016 \\
1.035 \\
1.040 \\
7.008 \\
0.987 \\
1.042 \\
1.050 \\
1.071 \\
1.006 \\
1.006\end{array}$ & $\begin{array}{l}1.007 \\
1.018 \\
1.008 \\
1.007 \\
1.073 \\
0.008 \\
1.042 \\
1.001 \\
1.075 \\
1.007 \\
1.000\end{array}$ & 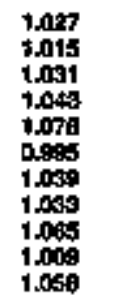 & 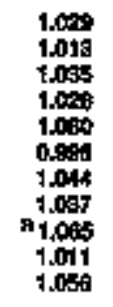 & 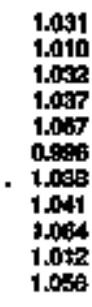 \\
\hline US,Angapge & 1,009 & 1.002 & $\$ .022$ & 1.008 & 1.027 & 1.02: & 1.030 & $t .0 s t$ & 1,003 & 1.091 & 1.031 & 1.060 & 1.091 & 1030 & 1,000 & 1,090 & 1,009 & 1,000 \\
\hline
\end{tabular}

Rifrentopd diate.

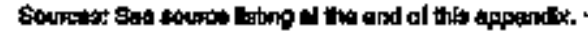


A Table D8. Approximete Heat Content of Bituminous Cos and Lignite Consumed by the Residertial end Commercial Sector, 1960-1976

$P \quad$ (Million Bku per Short Ton)

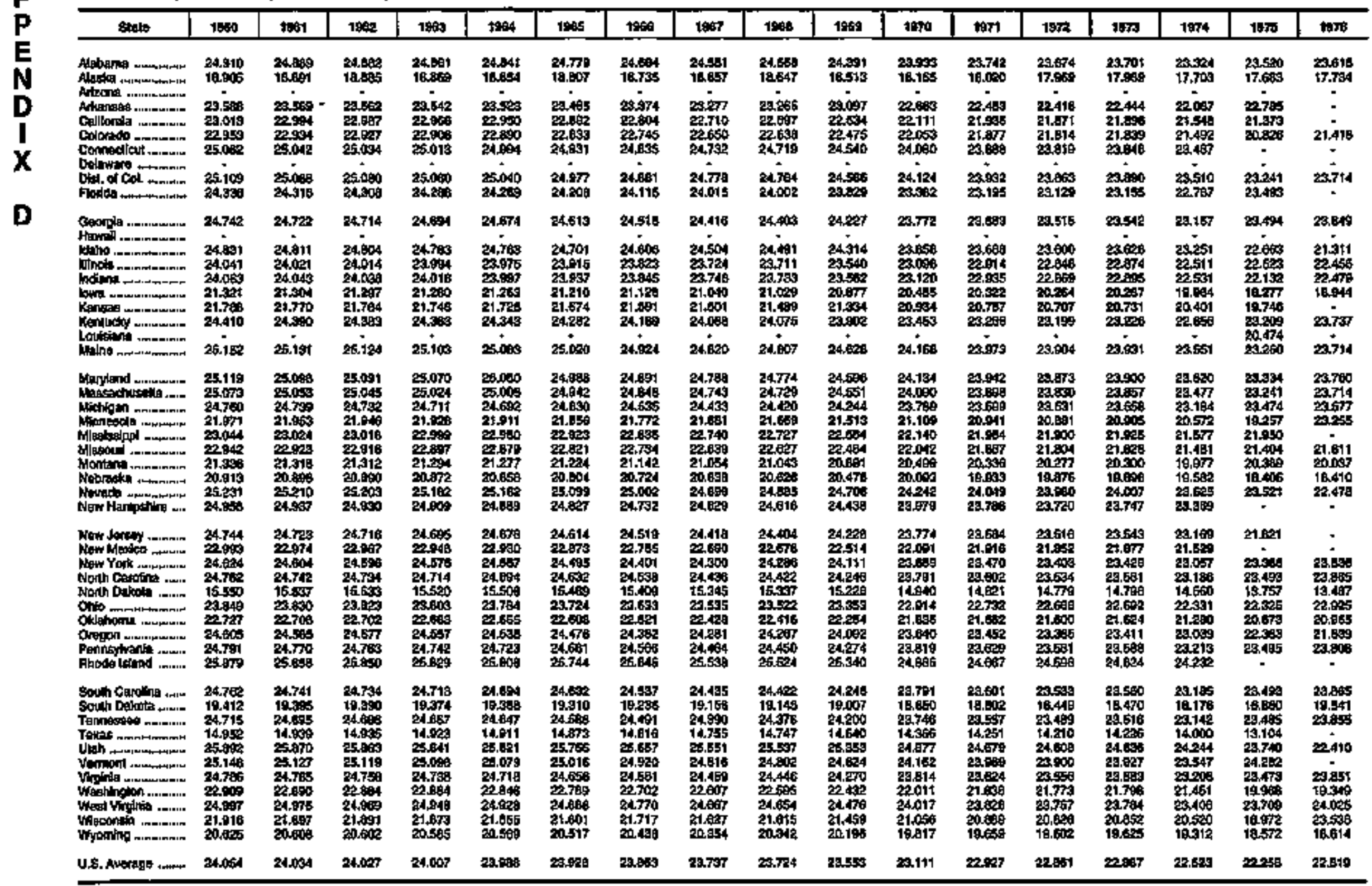

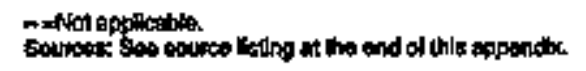


Table D9. Approximate Heat Content of Bituminous Coal and Lignite Consumed by the Residential end Commercial Sector, $1977-1994$ (Million Btu per Short Ton)

\begin{tabular}{|c|c|c|c|c|c|c|c|c|c|c|c|c|c|c|c|c|c|c|}
\hline Stant & $\pi$ & $\mathrm{r}$ & 79 & 90 & 191 & 4 & 9tot & 904 & 48 & 1985 & 867 & 19\$\$\$\$ & 19 & 196 & 1991 & tim & a & 1904 \\
\hline 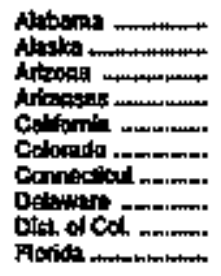 & 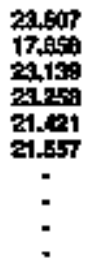 & 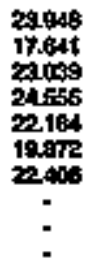 & $\begin{array}{l}- \\
20.581 \\
21.735 \\
21.054 \\
24,148 \\
24.006\end{array}$ & 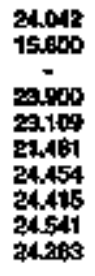 & 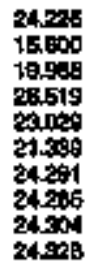 & 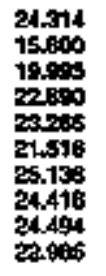 & 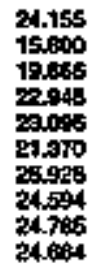 & 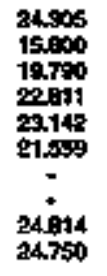 & 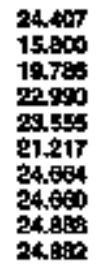 & 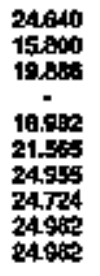 & & $\begin{array}{l}0 \\
0 \\
4 \\
0 \\
9 \\
6 \\
4 \\
7 \\
9 \\
4\end{array}$ & 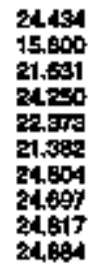 & & & & & 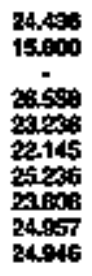 \\
\hline . & 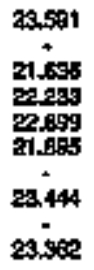 & 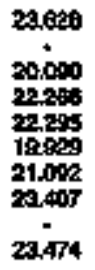 & $\begin{array}{l}24.100 \\
19.148 \\
28.204 \\
02.384 \\
21.334 \\
20.508 \\
28.912 \\
24.187\end{array}$ & 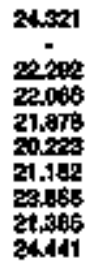 & 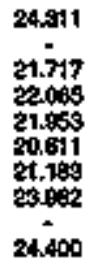 & 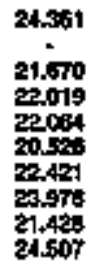 & 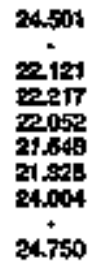 & 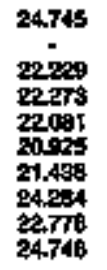 & 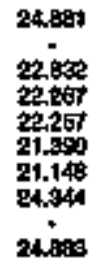 & $\begin{array}{l}24.950 \\
22.550 \\
22.340 \\
22.394 \\
21.129 \\
21.378 \\
24.357 \\
25 i 113\end{array}$ & 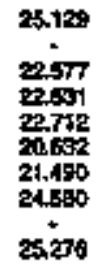 & $\begin{array}{l}25.210 \\
-5 \\
22.582 \\
22.452 \\
22,589 \\
20.475 \\
21.920 \\
24.406 \\
25.160\end{array}$ & 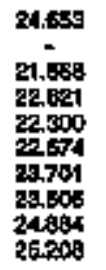 & $\begin{array}{l}25.148 \\
22478 \\
22.498 \\
22.449 \\
28.947 \\
24.260 \\
24.448 \\
24.632\end{array}$ & 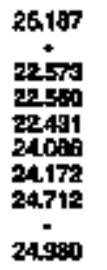 & $\begin{array}{l}25.196 \\
22.490 \\
22.691 \\
22.440 \\
22.694 \\
21.410 \\
21.79 \\
= \\
28.094\end{array}$ & 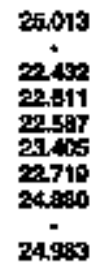 & 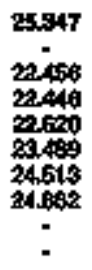 \\
\hline . & 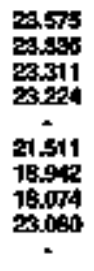 & $\begin{array}{l}23.006 \\
29.59 \\
29099 \\
20.699 \\
23.315 \\
21.446 \\
18.492 \\
17.997\end{array}$ & 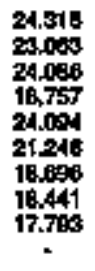 & & 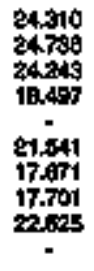 & 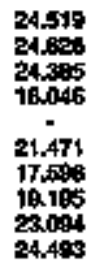 & 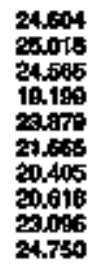 & 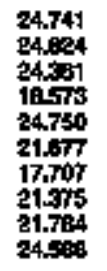 & . & $\begin{array}{l}24.099 \\
24.862 \\
16.978 \\
24.968 \\
22.616 \\
17.079 \\
20.800\end{array}$ & & & & 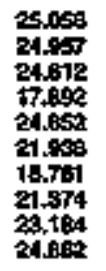 & $\begin{array}{l}5157 \\
4.984 \\
4.85 \\
7.726 \\
1.949 \\
1.949 \\
0.544 \\
1.5146 \\
5028\end{array}$ & 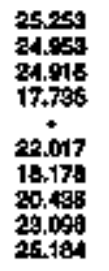 & 2 & 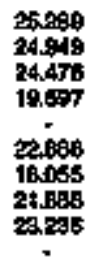 \\
\hline 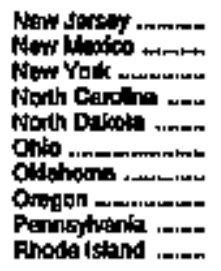 & 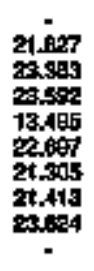 & $\begin{array}{l}19.972 \\
23.074 \\
23.409 \\
13.289 \\
22.659 \\
21.691 \\
20.447\end{array}$ & $\begin{array}{l}24.007 \\
24.012 \\
24.100 \\
13.451 \\
22.077 \\
25.72 \\
19560\end{array}$ & 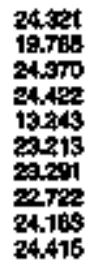 & 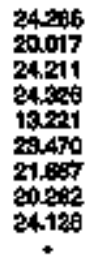 & 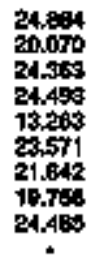 & $\begin{array}{l}18.668 \\
24.660 \\
24.749 \\
13.167 \\
23.746 \\
21.318 \\
20.240 \\
24.908\end{array}$ & 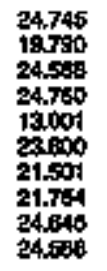 & & 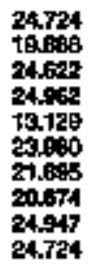 & & 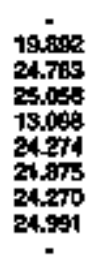 & & & & & & 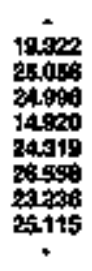 \\
\hline 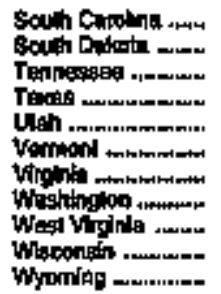 & 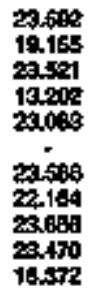 & $\begin{array}{l}23.5 \% \\
21.0 \% 7 \\
24.169 \\
20.015 \\
18050\end{array}$ & $\begin{array}{l}24044 \\
24.603 \\
24.148 \\
20.454 \\
17.840\end{array}$ & 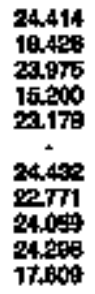 & 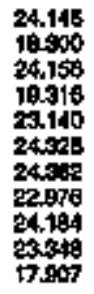 & $\begin{array}{l}16005 \\
24.005 \\
17.763 \\
28.279 \\
25.165 \\
24.566 \\
29.039 \\
24.718 \\
23.423 \\
17.564\end{array}$ & 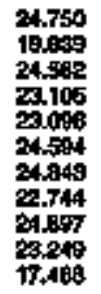 & $\begin{array}{l}24.279 \\
23.148 \\
24.743 \\
24.797 \\
22.789 \\
24.620 \\
24.798 \\
17.913\end{array}$ & & 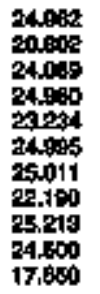 & 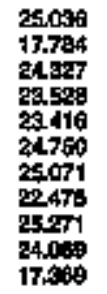 & $\begin{array}{l}16.940 \\
24.79 \\
23.448 \\
23.048 \\
+ \\
2.175 \\
22.022 \\
25.47 \\
24.400 \\
17.690\end{array}$ & & 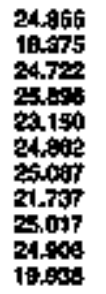 & & 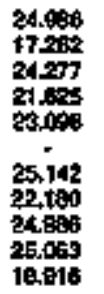 & & 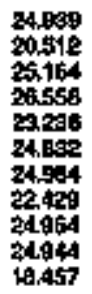 \\
\hline 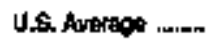 & 594 & $10 \mathrm{OP}$ & ogd & $48 B$ & 2010 & 2,220 & $4 d$ & 2006 & 569 & 斿 & 600 & 2,135 & 917 & 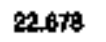 & 2065 & & $7 A 9$ & \\
\hline
\end{tabular}


A Tabje D10. Approximate Heat Content of Bltuminous Coal and Lignite Consumed by Other Industrlal Users, 1960-1976 (Mimon Bts per Shart Ton)

\begin{tabular}{|c|c|c|c|c|c|c|c|c|c|c|c|c|c|c|c|c|c|}
\hline Btoto & 196. & 1951 & 102 & $\mathrm{I968}$ & 934 & 966 & 1908 & MT & 1908 & 1969 & 970 & 1971 & 1972 & t979 & 1997 & 1005 & 196 \\
\hline 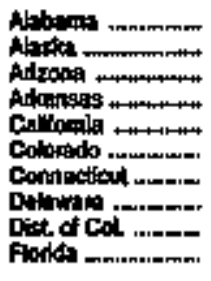 & 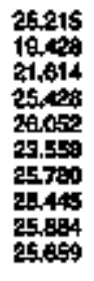 & 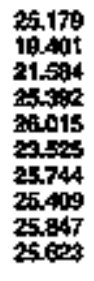 & 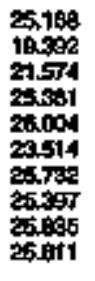 & 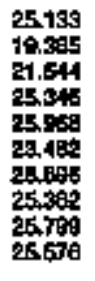 & 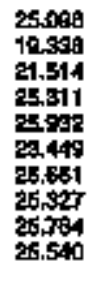 & 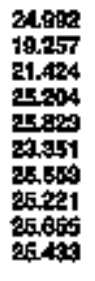 & 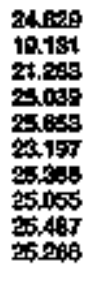 & 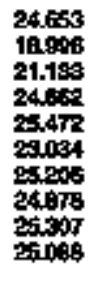 & 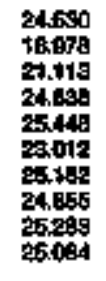 & $\begin{array}{l}243263 \\
19743 \\
20.052 \\
24532 \\
25.139 \\
22727 \\
24071 \\
24.547 \\
24.971 \\
24754\end{array}$ & 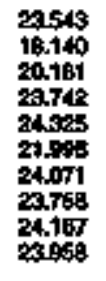 & 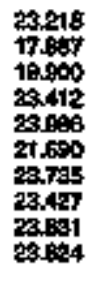 & 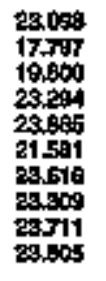 & 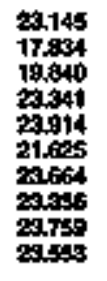 & 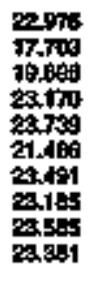 & 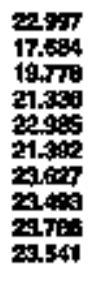 & 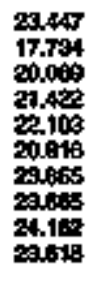 \\
\hline 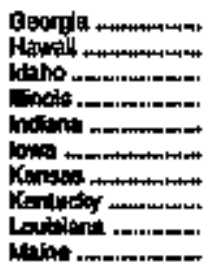 & 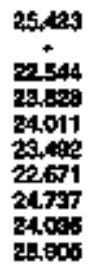 & 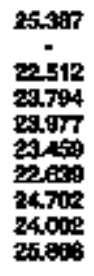 & 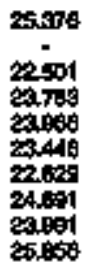 & 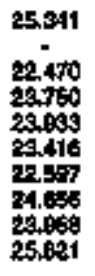 & 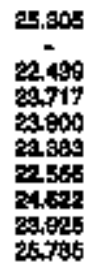 & 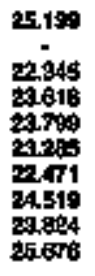 & 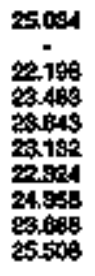 & 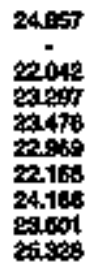 & 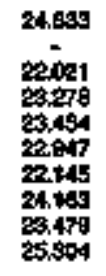 & 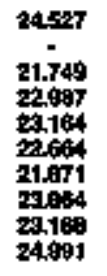 & 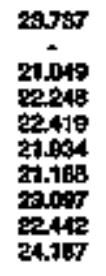 & 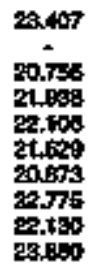 & 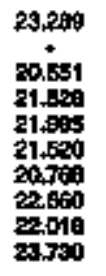 & 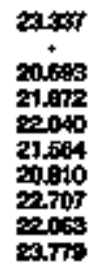 & $\begin{array}{l}22.100 \\
20.502 \\
21.712 \\
21.679 \\
21.400 \\
00.609 \\
22.540 \\
21.902 \\
21.605\end{array}$ & $\begin{array}{l}23.600 \\
+ \\
19.855 \\
21.694 \\
21.024 \\
21.291 \\
20.400 \\
20.948 \\
21.034 . \\
24.105\end{array}$ & 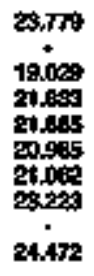 \\
\hline 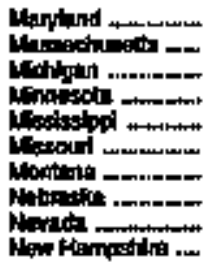 & 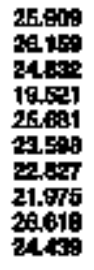 & 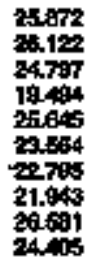 & 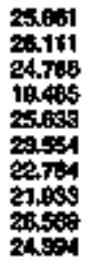 & 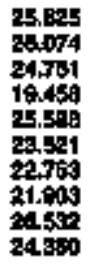 & 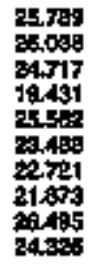 & 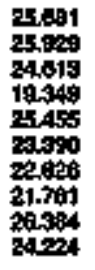 & 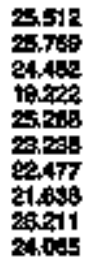 & 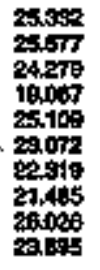 & 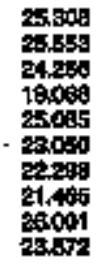 & 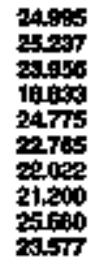 & 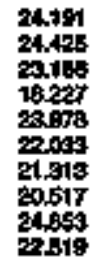 & 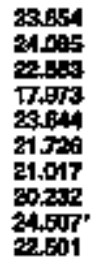 & 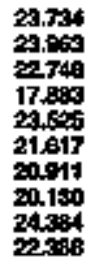 & 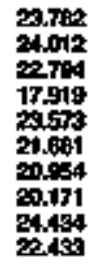 & 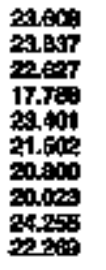 & 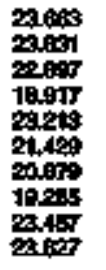 & 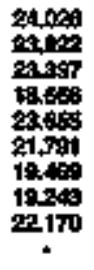 \\
\hline 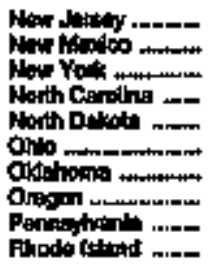 & 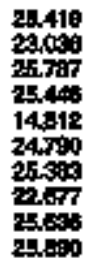 & 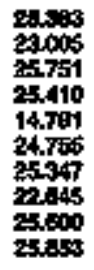 & 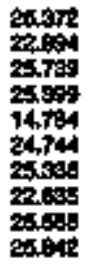 & 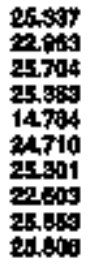 & 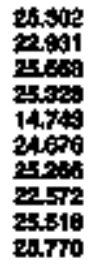 & 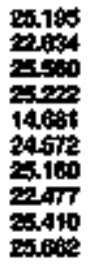 & 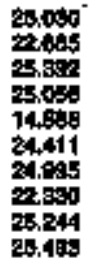 & 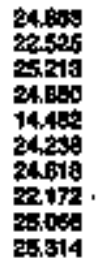 & 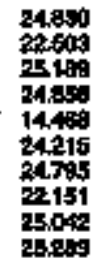 & 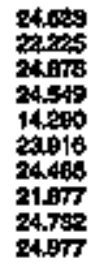 & 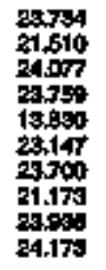 & 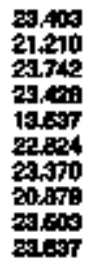 & 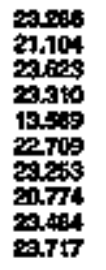 & 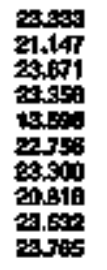 & 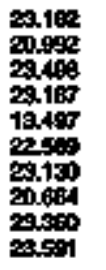 & 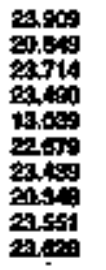 & 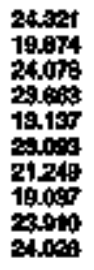 \\
\hline 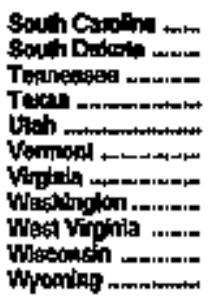 & 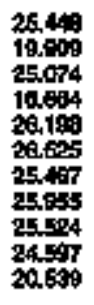 & 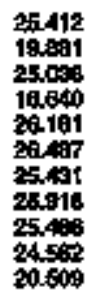 & 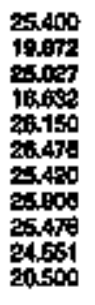 & 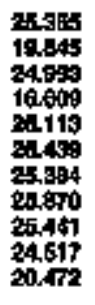 & 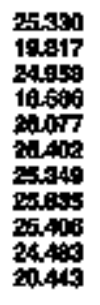 & 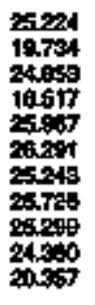 & 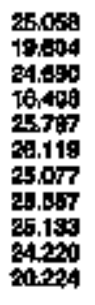 & 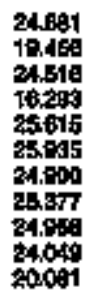 & 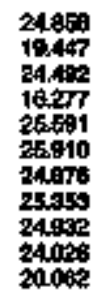 & 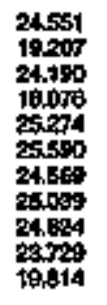 & 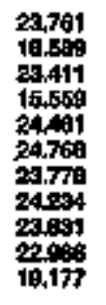 & 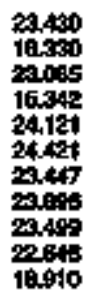 & 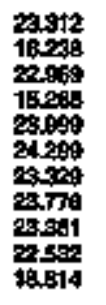 & 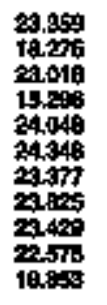 & 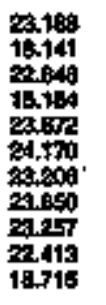 & 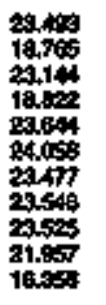 & 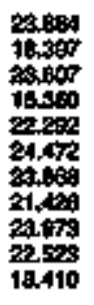 \\
\hline US. Alaropg & 24004 & $24-60$ & 24550 & $24: 524$ & $4+90$ & 24300 & 24247 & 22050 & 24034 & 23,737 & 208 & 2053 & 22.509 & 22585 & 22.420 & 22439 & 22158 \\
\hline
\end{tabular}

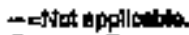

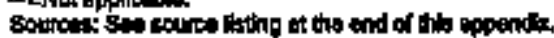


Table D11. Approximate Heat Content of Bltuminous Coal and Lignite Consumed by Other Industrial Users, 1977-1994 (Miion Btu per short Ton)

\begin{tabular}{|c|c|c|c|c|c|c|c|c|c|c|c|c|c|c|c|c|c|c|}
\hline & $m$ & $\mathrm{ama}$ & $\mathrm{mo}$ & 0.80 & 1: & 19 & 803 & 84 & كin & كים: & דה: & 98: & ens & $\sin$ & 991 & 189 & $\sin$ & $\operatorname{tag}$ \\
\hline 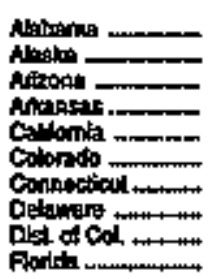 & 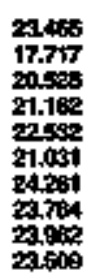 & 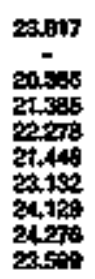 & 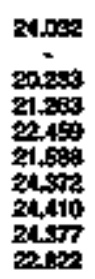 & 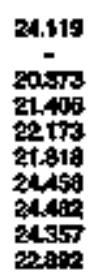 & 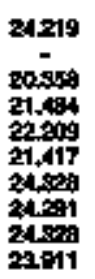 & 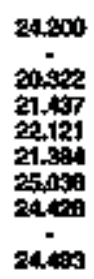 & 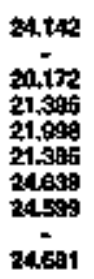 & 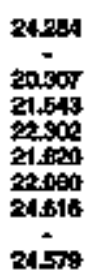 & 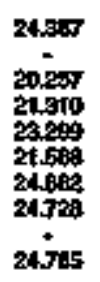 & 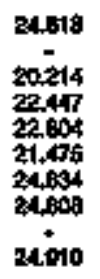 & 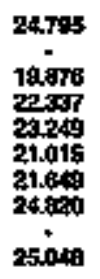 & 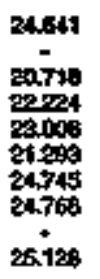 & 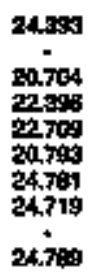 & $\begin{array}{l}24.679 \\
20.070 \\
22.808 \\
22.502 \\
21.105 \\
24.098 \\
25.004\end{array}$ & $\begin{array}{l}24.531 \\
15942 \\
124.198 \\
22751 \\
21.091 \\
24.843 \\
25.033 \\
25.131\end{array}$ & $\begin{array}{l}4.049 \\
0.017 \\
4009 \\
2.970 \\
0.107 \\
4.906 \\
5.203 \\
\text { sione }\end{array}$ & 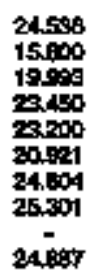 & 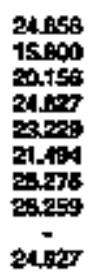 \\
\hline . & 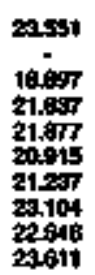 & 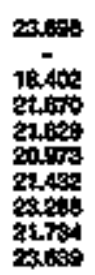 & 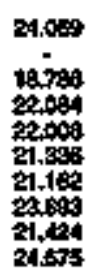 & $\begin{array}{l}24331 \\
17.694 \\
22450 \\
22,254 \\
21.499 \\
21.540 \\
24119 \\
22159 \\
2475\end{array}$ & 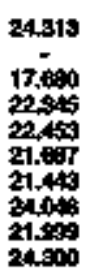 & 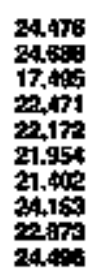 & 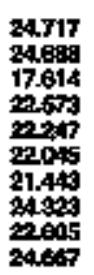 & 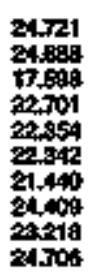 & 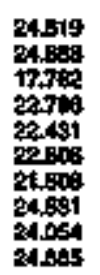 & 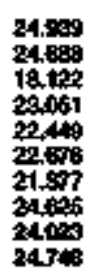 & 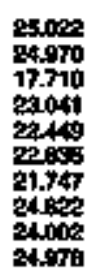 & 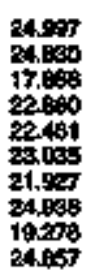 & 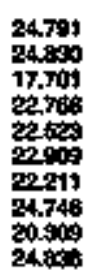 & 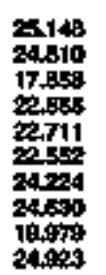 & 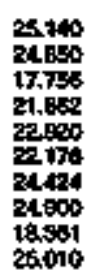 & 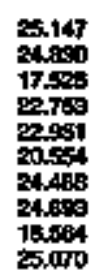 & 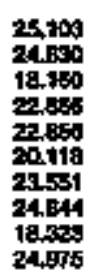 & 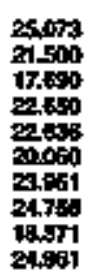 \\
\hline mand & 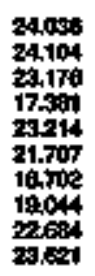 & 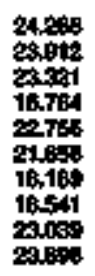 & 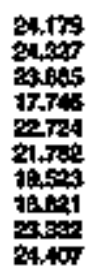 & 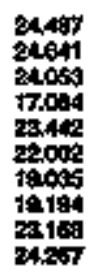 & 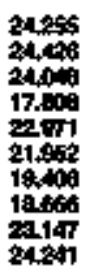 & 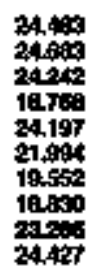 & 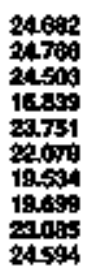 & 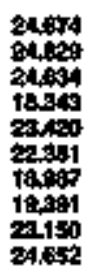 & 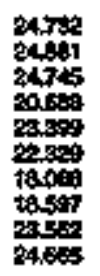 & 2 & 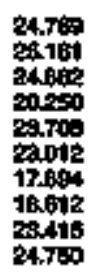 & & & & & & & 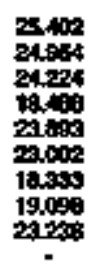 \\
\hline 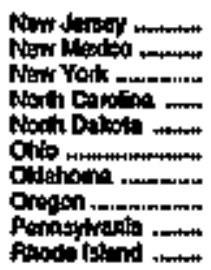 & 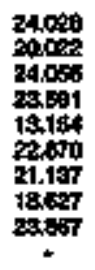 & 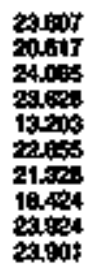 & 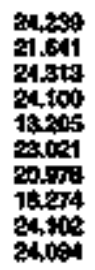 & 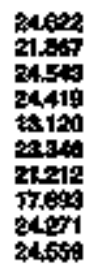 & $\begin{array}{l}21.54 \\
24381 \\
21340 \\
13140 \\
22,940 \\
21909 \\
19000\end{array}$ & 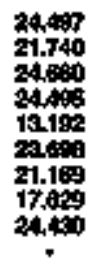 & $\begin{array}{l}21.400 \\
24608 \\
24767 \\
12111 \\
2481 \\
21.598 \\
17.954 \\
24.711\end{array}$ & $\begin{array}{l}21.644 \\
24.765 \\
24750 \\
13.159 \\
24.929 \\
21.275 \\
19.799\end{array}$ & 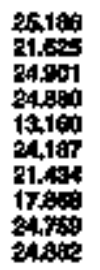 & & $\begin{array}{l}00 \\
05 \\
03 \\
03 \\
03 \\
08 \\
0\end{array}$ & & & & & & & 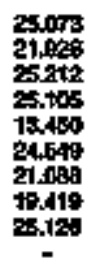 \\
\hline "........ & 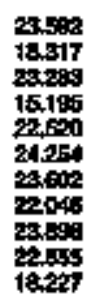 & 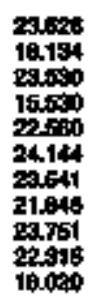 & 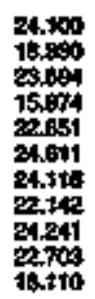 & 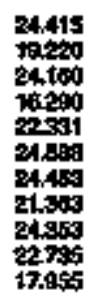 & 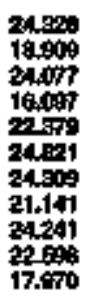 & 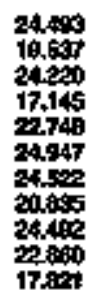 & 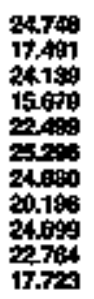 & & $\begin{array}{l}17 \\
24 \\
15 . \\
22 \\
24 \\
24 \\
21 \\
24 \\
23 \\
17\end{array}$ & 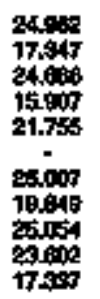 & & 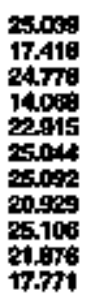 & & 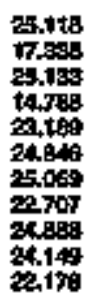 & & & 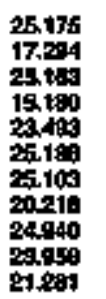 & 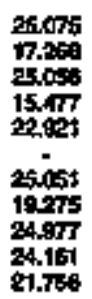 \\
\hline 3. Arowdpg +4 & 22.290 & 2.175 & 49 & 2000 & 2072 & 1065 & 2060 & 1525 & L13 & 2165 & 22360 & 2354 & 1324 & 244 & 240 & $2: 342$ & 2.111 & 2.048 \\
\hline
\end{tabular}

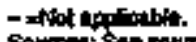

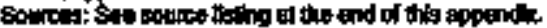


A Table 012. Approximate Heat Content of Bituminous Cosl and Lignite Consumed by Electrlo Utifites, $1950-1976$ (Mition Btup per Shart Ton)

\begin{tabular}{|c|c|c|c|c|c|c|c|c|c|c|c|c|c|c|c|c|c|}
\hline sento & 90 & 1901 & $\Leftrightarrow 2$ & $\infty$ & tast. & 1965 & tass & 1967 & 969 & 100 & 1970 & 471 & 1972 & 1903 & t974 & 1975 & 虾 \\
\hline 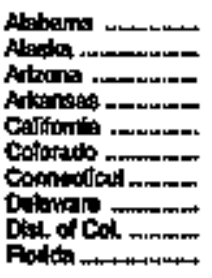 & 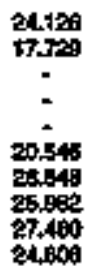 & $\begin{array}{c}29.052 \\
17.704 \\
\vdots \\
\vdots \\
21.204 \\
28.542 \\
26.504 \\
27.440 \\
24.204\end{array}$ & 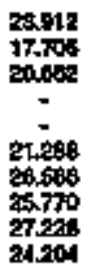 & 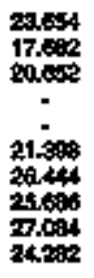 & $\begin{array}{c}28.542 \\
17.306 \\
20.766 \\
\vdots \\
21.5006 \\
26.154 \\
25014 \\
26.712 \\
23.902\end{array}$ & 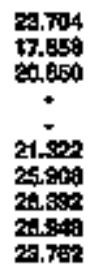 & 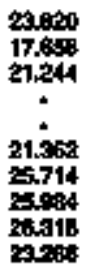 & 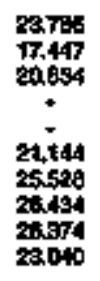 & 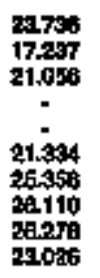 & 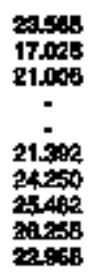 & $\begin{array}{c}23.514 \\
17.000 \\
21.259 \\
\vdots \\
21.590 \\
23.549 \\
24.100 \\
25.020 \\
22.740\end{array}$ & 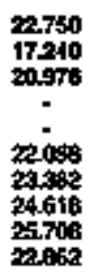 & 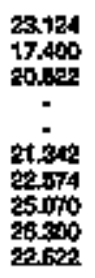 & $\begin{array}{c}23,068 \\
17.400 \\
20.456 \\
\vdots \\
21.295 \\
29,488 \\
24.736 \\
20.260 \\
22,978\end{array}$ & $\begin{array}{c}2.991 \\
17.100 \\
2+.005 \\
\vdots \\
12.099 \\
23.904 \\
24207 \\
25009 \\
22009\end{array}$ & $\begin{array}{c}22.164 \\
17.400 \\
21.090 \\
\vdots \\
19.800 \\
23.504 \\
34.634 \\
25.619 \\
23.009\end{array}$ & $\begin{array}{c}22.138 \\
17.400 \\
21.100 \\
\vdots \\
19.594 \\
20.904 \\
24.938 \\
25.619 \\
\$ 3.294\end{array}$ \\
\hline 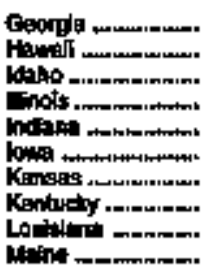 & 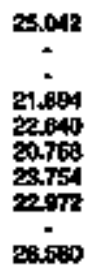 & 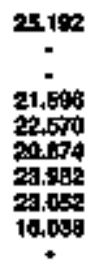 & 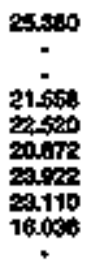 & 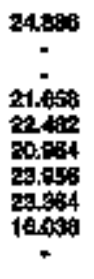 & 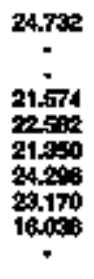 & 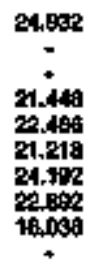 & $\begin{array}{c}24.596 \\
\vdots \\
21.376 \\
22450 \\
21.246 \\
24170 \\
22.810 \\
\vdots\end{array}$ & 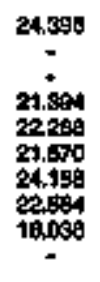 & $\begin{array}{c}24.70 \\
\vdots \\
21.368 \\
22.200 \\
21.499 \\
24074 \\
2.50 \\
\vdots\end{array}$ & 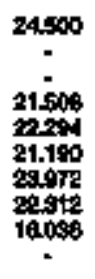 & 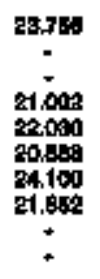 & $\begin{array}{c}20206 \\
\vdots \\
\vdots \\
20.714 \\
21.720 \\
20.8989 \\
24.216 \\
21.684 \\
\vdots\end{array}$ & 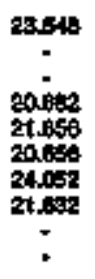 & $\begin{array}{c}20.051 \\
\vdots \\
- \\
20.680 \\
21.012 \\
21.049 \\
20.789 \\
81.739 \\
\vdots \\
+\end{array}$ & 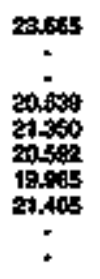 & 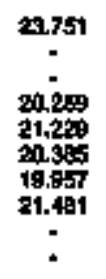 & $\begin{array}{c}23.767 \\
\vdots \\
20.483 \\
21,472 \\
20.255 \\
20.365 \\
21.603 \\
\vdots\end{array}$ \\
\hline 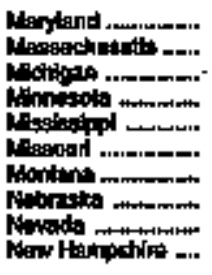 & 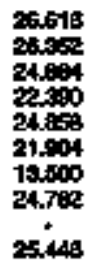 & 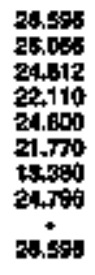 & $\begin{array}{l}28.542 \\
25.918 \\
24.972 \\
22006 \\
24.128 \\
22008 \\
13401 \\
24.04 \\
28.58\end{array}$ & 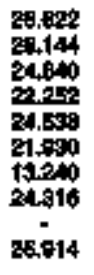 & 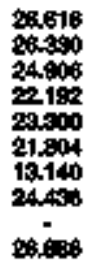 & 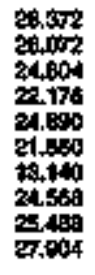 & 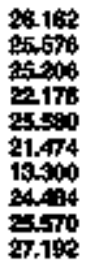 & 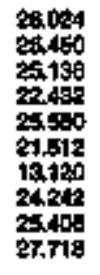 & 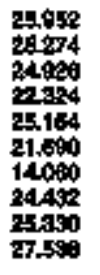 & 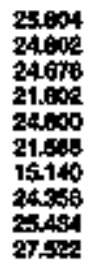 & 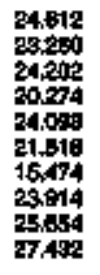 & 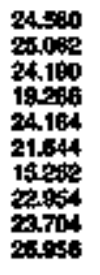 & 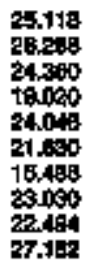 & 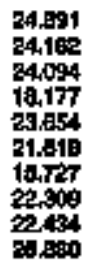 & 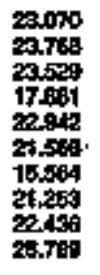 & $\begin{array}{l}24.528 \\
24.347 \\
23.652 \\
17.940 \\
20.164 \\
21.404 \\
18.909 \\
20.954 \\
20.396 \\
26.704\end{array}$ & 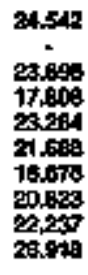 \\
\hline 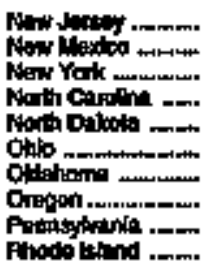 & $\begin{array}{l}26.72 \\
25.000 \\
26.066 \\
26.242 \\
19.986 \\
23.70 \\
25.942 \\
24.446 \\
29.152\end{array}$ & 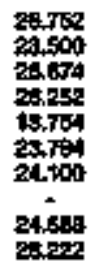 & 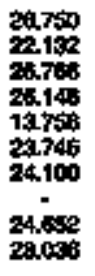 & 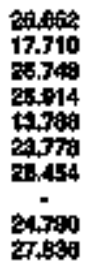 & 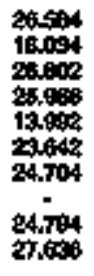 & 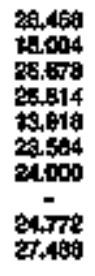 & 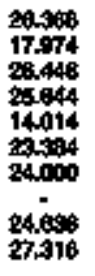 & 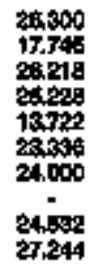 & $\begin{array}{l}20.254 \\
17.269 \\
20240 \\
25.254 \\
13210 \\
23.342 \\
25.950 \\
24.440 \\
27.226\end{array}$ & 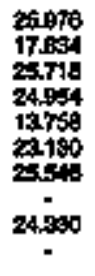 & 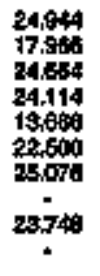 & 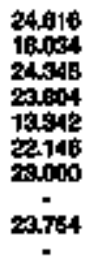 & 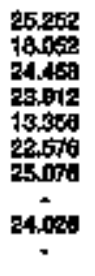 & 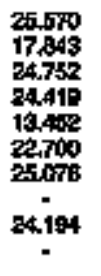 & $\begin{array}{l}24.640 \\
17,771 \\
23.504 \\
28.689 \\
13.380 \\
21,609 \\
25.076 \\
28.710 \\
24.6=2\end{array}$ & $\begin{array}{l}29.401 \\
17.849 \\
24.050 \\
20.798 \\
13.344 \\
21.919 \\
25.976 \\
20.760 \\
-\end{array}$ & 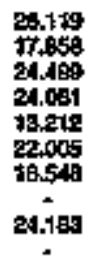 \\
\hline 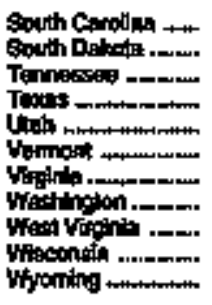 & $\begin{array}{l}24734 \\
17.108 \\
24040 \\
24.940 \\
27.760 \\
24720 \\
2900 \\
24.200 \\
14.940\end{array}$ & 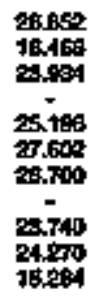 & 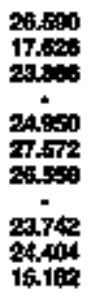 & 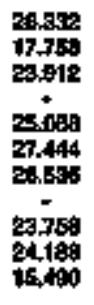 & 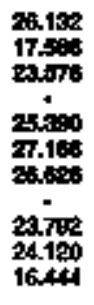 & 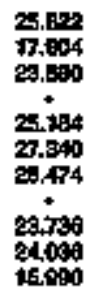 & 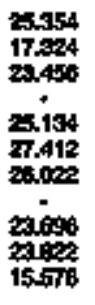 & 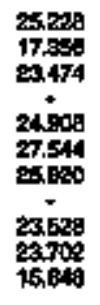 & 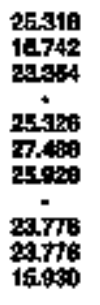 & 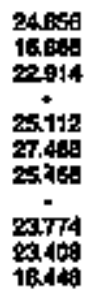 & 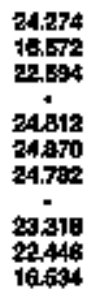 & 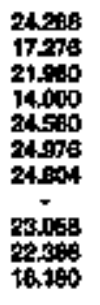 & 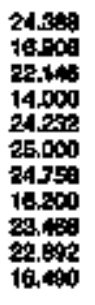 & 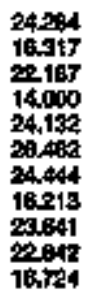 & 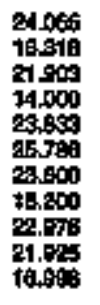 & 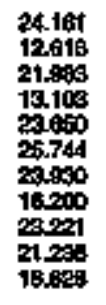 & $\begin{array}{l}24.389 \\
12.605 \\
22.491 \\
19.282 \\
29.199 \\
26.744 \\
21.509 \\
16.200 \\
2.496 \\
21.344 \\
17.582\end{array}$ \\
\hline U. Alerdge & 24009 & 25.000 & 2896 & 28,462 & 24940 & 6.5693 & 20000 & 28554 & 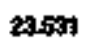 & 20.274 & 22603 & 22.325 & 22.225 & 22202 & $21+790$ & 21 , & $21.6 \mathrm{se}$ \\
\hline
\end{tabular}

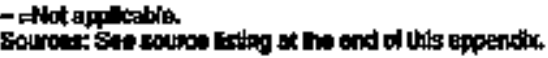


Table D13. Approximate (teat Content of Bituminous Coal and Llgnite Consumed by Electrie Utilitles, 1977-t994 (Milition Błu per Short Ton)

\begin{tabular}{|c|c|c|c|c|c|c|c|c|c|c|c|c|c|c|c|c|c|c|}
\hline $8 \log$ & vin & 190r. & 97 & 80 & tola1 & 1992 & 1998 & $\cos$ & 085 & 90 & $10 \%$ & 1890 & 1809 & 5800 & 1091 & 1992 & Ja泊 & L994 \\
\hline 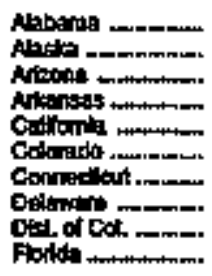 & 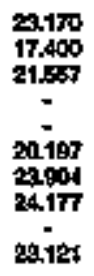 & 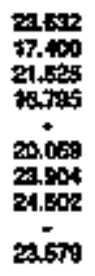 & 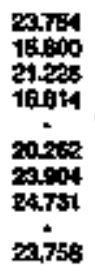 & 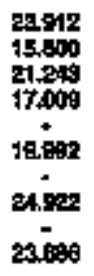 & 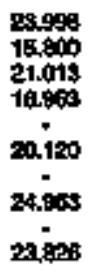 & 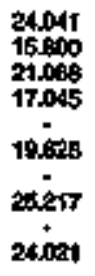 & 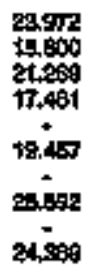 & 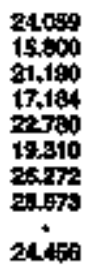 & $\begin{array}{l}24111 \\
16600 \\
20696 \\
17207 \\
- \\
19497 \\
20.317 \\
25.924 \\
2400\end{array}$ & 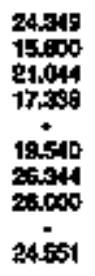 & 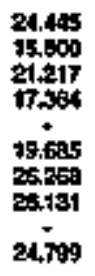 & 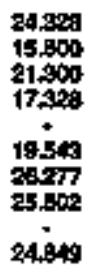 & $\begin{array}{l}24045 \\
13.000 \\
21.195 \\
17.459 \\
19.497 \\
20616 \\
20.697 \\
24.75 \%\end{array}$ & $\begin{array}{l}24.100 \\
15.000 \\
20.965 \\
17.400 \\
19.616 \\
28.406 \\
25.070 \\
24.700\end{array}$ & $\begin{array}{l}24.214 \\
15.000 \\
20.712 \\
17.469 \\
19.776 \\
6.777 \\
2.100 \\
24.701\end{array}$ & $\begin{array}{l}24.122 \\
15.500 \\
20.007 \\
17.440 \\
19.840 \\
26.985 \\
24.129 \\
24.740\end{array}$ & $\begin{array}{l}24.194 \\
15.000 \\
20.509 \\
17.329 \\
19.775 \\
19.798 \\
20.059 \\
24.664\end{array}$ & 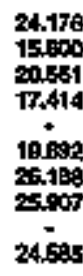 \\
\hline . & 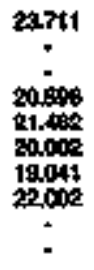 & 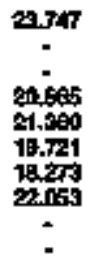 & 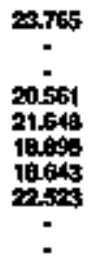 & 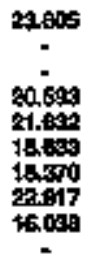 & 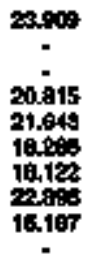 & 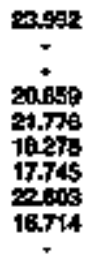 & 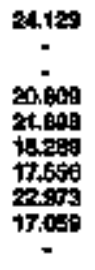 & 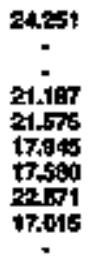 & 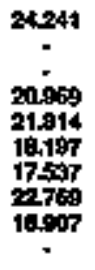 & 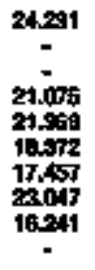 & 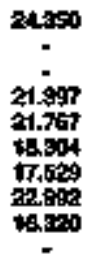 & $\begin{array}{c}21.271 \\
21.069 \\
10.492 \\
17.968 \\
22050 \\
16595 \\
-\end{array}$ & $\begin{array}{c}21.41 \% \\
21.9 \% 7 \\
17.7 \% \\
17.75 \\
22.96 \\
16374 \\
-\end{array}$ & 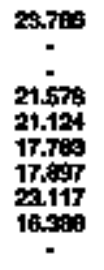 & $\begin{array}{c}21.442 \\
21.190 \\
17.799 \\
17.908 \\
2.109 \\
16.449 \\
-\end{array}$ & 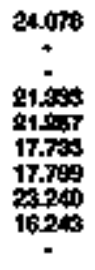 & 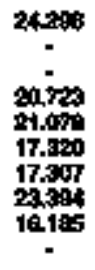 & 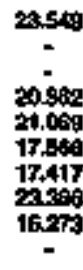 \\
\hline 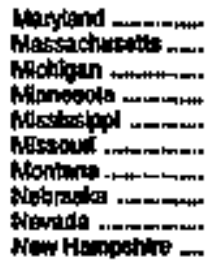 & 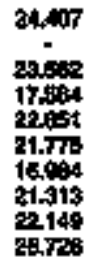 & 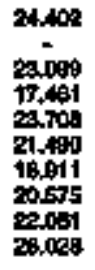 & 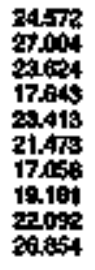 & 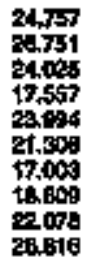 & 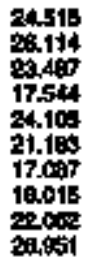 & 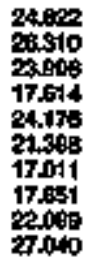 & 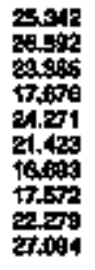 & 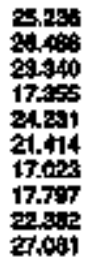 & 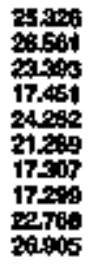 & 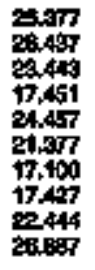 & 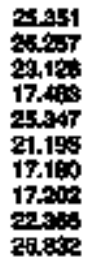 & 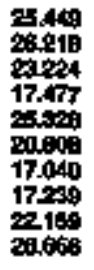 & 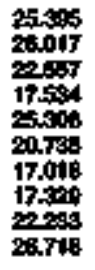 & 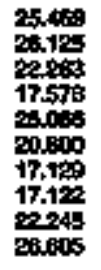 & 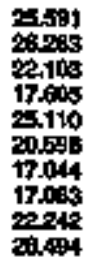 & 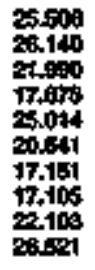 & 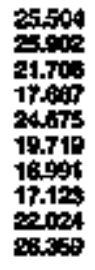 & 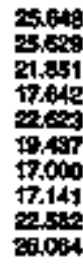 \\
\hline 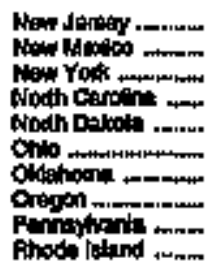 & 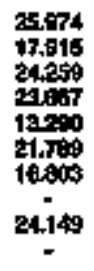 & 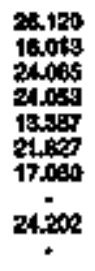 & 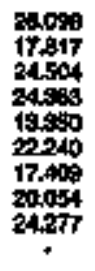 & 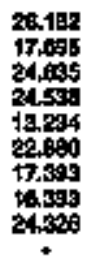 & 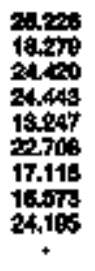 & 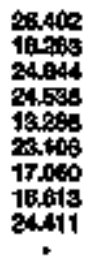 & 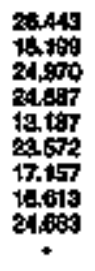 & 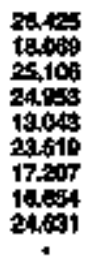 & 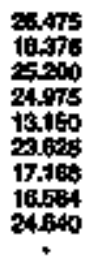 & 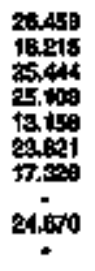 & 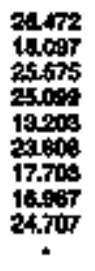 & 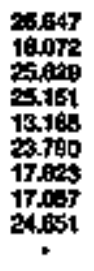 & 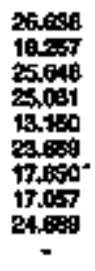 & 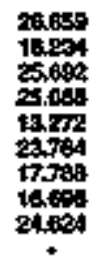 & 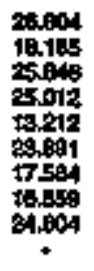 & 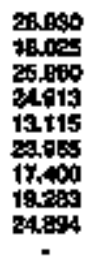 & 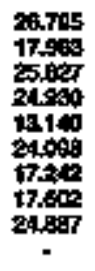 & 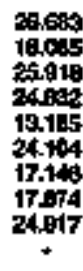 \\
\hline 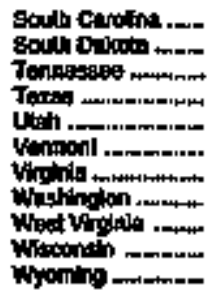 & 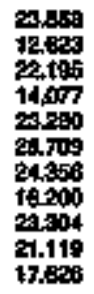 & $\begin{array}{l}24.175 \\
12.450 \\
22.568 \\
14.225 \\
20.204 \\
35.709 \\
24.451 \\
16.200 \\
24.492 \\
20.001 \\
17.650\end{array}$ & 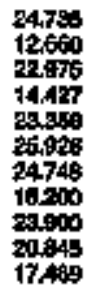 & 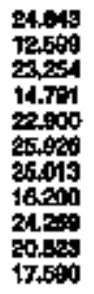 & 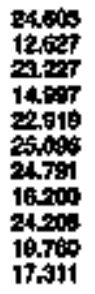 & 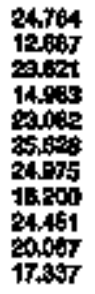 & 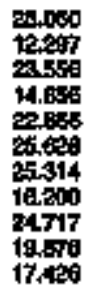 & 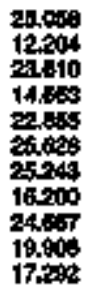 & 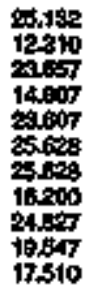 & 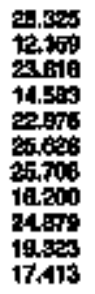 & 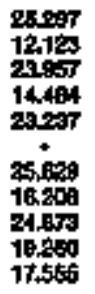 & 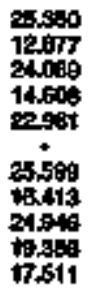 & 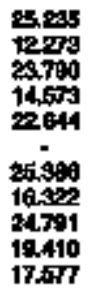 & 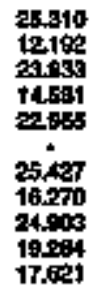 & 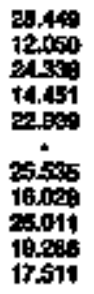 & 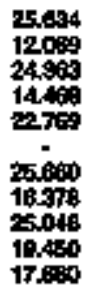 & 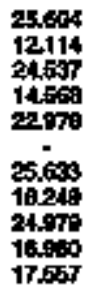 & 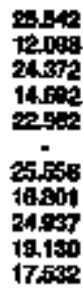 \\
\hline 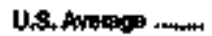 & 21.521 & 21.284 & 21.972 & 21,301 & 21.009 & .200 & .141 & 11.108 & 1.566 & 1.09 & 2t.143 & 200905 & 20.854 & 20.985 & 20.761 & eares & 20,630 & 20:661 \\
\hline
\end{tabular}

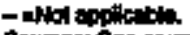

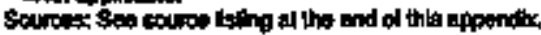




\section{Thermal Conversion Factor Source Documentation}

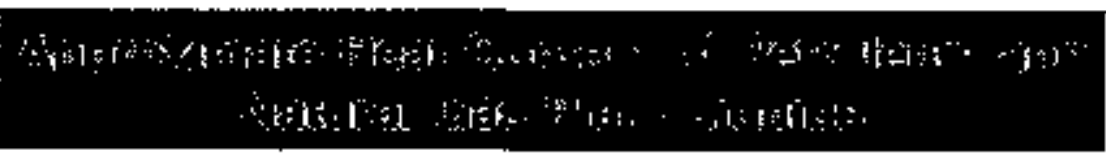

A6phalt. EIA adopted the thermal conversion factor of 6.636 million British thermal units (Btu) per barrel as estimated by the Bureau of Mines and first published in the Petrolenem Statement, Annual, 1956.

Aviation Gasoline. EIA adopted the Bureau of Mines thermal conversion factor of 5.048 million Btn per barrel for "Gasoline, Aviation" as published by the Texas Eastern Transmisston Corporation in Appendix V of Competition and Growth in American Energy Markets 19471985, a 1968 release of historical and projected statistics.

Butane. ElA adopted the Bureau of Mines thermal conversion factor of 4326 million Btu per barrel as published in the Calffornta Otl World and Petraleum Industry, First Issue, April 1942.

Butane-Propane Mixture. EIA adopted the Bureau of Mines calculation of 4.130 million Btu per barrel based on an assumed mixture of 60 percent butane and 40 percent propane. See Butane and Propane.

Crude Oil (Including Lease Condensate) Used Directly. EIA adopted the thermal conversion factor of 5.800 million Btu per barkel as reported in a Bureau of Mines internal memorandum "Bureau of Mines Standard Average Heating Vahue of Various Fuels, Adopted January 3, 1950."

Distlllate Fuel O1l. FIA adopted the thermal conversion factor of 5.825 million Btu per barrel as reported in a Bureau of Mines internal memorandim "Bureau of Mines Standard Average Heating Value of Vartious Fuels, Adopted Jantuary 3, 1950."
Fthane. EIA adopted the Bureau of Mines thermal conversion factor of 3.082 million Btu per barrel as published in the California Oil World and Pekroleuin Industry, First Issue, April 1942.

Ethane-Propane Mixture. EIA calculated 3.308 million Btu per barrei based on an assumed mixture of 70 percent ethane and 30 percent propane. See Ethane and Propane.

Isobutane. ElA adopted the Bureau of Mines thermal conversion factor of 3.974 million Btu per barrel as published in the Colifformia onl World and Petralewn Industry, First Isstre, April 1942.

Jet Fuel, Kerosene Type. ELA adopted the Bureau of Mines thermal conversion factor of 5.670 million Btu per barrel for "Jet Fuel, Commercial" as published by the Texas Eastem Transmission Corporation in Appendix $\mathrm{V}$ of Competition and Growth in American Entergy Morkets 1967-1985, a 1968 release of historical and projected statistics.

Jet Fuel, Naphtha Type. EIA adopted the Bureau of Mines thermal conversion factor of 5.355 million Btu per barrel for "Jet Fuel, Military" as published by the Texas Eastern Transmission Corporation in Appendix $\mathrm{V}$ of Competition and Growth in American Energy Morkets 1947-1985, a 1969 release of historical and projected statistics.

Kerosene. EIA adopted the thermal conversion factor of 5.670 million Btu per barrel as reported in a Buresu of Mines intenal memorandum "Bureau of Mines Standard A verage Heating Values of Various Fuels, Adopted Jantrary 3, 1950."

Liquefied Petroleum Gabes. (LGTCKUS) - 1960 through 1966; ELA adopted the Bureau of Mines thermal conversion factor of 4.011 mitlion Btu per barrel as published in the Mineral Industry Surotys, "Crude Petroleum and Petroleum Products, 1956," Table 4 footnote. 
- 1967 forward: Calculated anurually by EIA as a weighted average by multiplying the quantity consumed of each of the component products by each product's conversion factor and dividing the sum of those hest contents by the sum of the quantities consumed. The component products are ethane (including ethylene), propane (including propylene), normal butane (including butylene), butane-propane mixtures, ethane-propane mixtures, and isobutane.

Uubricanta. FIA adopted the thermal conversion factor of 6.065 million Btu per barrel as estimated by the Bureau of Mines and first published in the Petroleutn Statement, Antuat, 1956.

Misecllnneous Products. BIA adopted the thermal canversion factor of 5.796 million Btu per barrel as estimated by the Bureau of Mines and first published in the Petrotenon Statement, Amual, 1956.

Motor Gasoline. ELA adopted the Bureau of Mines thermal conversion factor of 5.253 million Btu per barrel for "Gasolime, Motor Futel" as published by the Texas Eastern Transmission Corporation in Appendix V of Competition and Growth in American Energy Markets 19471985, a 1968 release of historical and projected statistics.

Natural Gasoline. BIA adopted the thermal conversion factor of 4.620 million Btu per barrel as estimated by the Bureau of Mines and first published in the Petroleum Statement, Amnual, 1956.

Pentanes Plus. BIA assumed the thermal conversion factor to be 4.620 million Btu per barrel, equal to that for natural gasoline. See Natural Gasoline.

Petroctemical Feedstocks, Naphtha Less Than $401^{\circ} \mathrm{P}$. EIA assumed the thermal conversion factor to be 5.248 million Btu per barrel, equal to that for special naphthas. See Special Naphthas.

Petrochemical Feedgtock, Other Oils Equal to or Greater Than 401 ${ }^{\circ}$ F. ELA assumed the themal conversion factor to be 5.825 million Bu per barrel, equal to that for distillate fuel oil. See Distillate Fuel Oil.
Petroshemical Feedstock, Still Gas. Assumed by EIA to be 6.000 million Btu per barrel, equal to the thermal conversion factor for still gas. See Still Gas.

Petroleum Coke. EIA adopted the thermal conversion factor of 6.024 million Btu per barrel as reported in Btu per short ton in the Bureau of Mines internal memorandum "Bureau of Mines Standard Average Heating Value of Various Fuels, Adopted January 3, 1950." The Bureau of Mines calculated this factor by dividing $30,120,000$ Btu per short ton, as given in the referenced Bureau of Mines internal memorandum, by 5.0 barrels per short ton, as given in the Bureau of Mines Form 6-1300-M and successor EIA forms.

Petroleum Producti, Total Consumption. Calculated annually by EIA as the average of the thermal conversion factors for all petroleum products consumed, weighted by the quantity of each petroteum product consumed.

Flant Condensate. Estimated to be $\mathbf{5 . 4 1 8}$ million But per barrel by EIA from data provided by McClanahan Consultants, Inc., Houston, Texas.

Propane. ELA adopted the thermal conversion factor of 3.836 million Btu per barrel as published in the Califarnia Oil World and Petrolewon Industry, First Issue, April 1942.

Residual Fuel Oil. EIA adopted the thermal conversion factor of 6.287 million Btu per barrel as reported in the Bureau of Mines internal memorandum "Bureau of Mines Standard Average Heating Values of Various Fuels, Adopted January 3, 1950."

Road OIL. EIA adopted the Bureau of Mines thermal conversion factor of 6.636 million Btu per barrel, equal to that of asphalt and first published by the Bureau of Mines in the Petroleum Statement, Annuat, 1970. See Asphalt.

Special Naphthas. EIA adopted the Bureau of Mines thermal conversion factor of $\mathbf{5 . 2 4 6}$ million Btu per barrel, equal to that of total gasoline (aviation and motor) factor and first published in the Petroletum Statement, Annual, 1970. 
A Still Gas. ELA adopted the Bureau of Mines estimated thecrnal conP version factor of 6.000 million Btu per barrel and first published in P the Petroteum Statement, Annual, 1970.

Unfinished Oit. EIA assumed the thermal conversion factor to be 5.825 million Btu per barrel, equal to that for distillate fuel ot] and first published in the Annual Report to Congress, Volime 3, 1977. See Diatitlate Fue] Oil.

Unfractionated Stream. BtA assumed the thermal conversion factor to be 5.418 million Btu per barrel, equal to that for plant condensate and first published in the Anntual Report to Congress, Volume 2, 1981. See Plant Condensate.

Waxes. FIA adopted the thermal conversion factor of 5.537 millton Btu per barrel as estimated by the Bureau of Mines and first published in the Petroleum Statement, Annual, 1956.

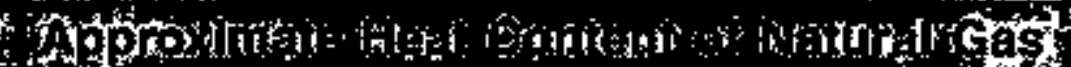

Natural Gas, Tatal Consumption. (NGTCKZZ) * 1960 through 1962: EIA adopted the thermal conversion factor of 1,035 Btu per cubic foot as estimated by the Bureau of Mines and first published in the Petroleum Statement, Annual, 1956. - 1963 through 1979: EIA adopted the thermal conversion factors calculated annually by the American Gas Association (AGA) and published in Gis Facts, an AGA anmual. 1980 through 1994: EIA, Natural Gas Anntual 1994 Volume II, Table 16. - Data for 1980 through 1994 are also available via internet. From the EIA homepage, http://ela.doe.gov, select "FTP Site" to reach ftp://ftp.eid.doegov/pub/naturalgas/data/annual/ga94-v2.exe and ftp://ftp.eia.doe.gov/pub/natural.gas/pdf/rga4v22.pdf.

Natural Gas, Contsumption by Electric Utilities. (NGEUKZZ) - 1960 through 1971: Assumed by EIA to be equal to the thermal conversion factor for the consumption of natural gas. See Natural Gas, Total Consumption. 1972 forward: Calculated annually by EIA by dividing the total heat content of natural gas received at electric utilities by the total quantity received at electric utilities. The heat contents and receipts are from Federal Energy Regutlatory Com- mission (FERC) Form 423 and predecessor forms. Data in Btu per cubic foot are published in the BtA, Cost and Quality of Futs for Elect. iric Utility Plants. For States that reported consumption on Form EIA-759 bat were not large enough to report on FERC Form 423, factors were estimated by using previous years' factors or the factor for total natural gas consumption in the State.

Natural Gas, Consumption by Sectors Other Than Electric Util;ties. (NGNUKZZ) - 1960 through 1972: Assumed by EIA to be equal to the thermal conversion factor for the consumption of natural gas. See Natural Gas, Total Consumption. - 1973 forward: Calculated annually by EIA by dividing the heat content of all natural gas consumed less the heat content of natural gas consumed at electric utilities by the quantity of all natural gas consumed less the quantity of electric utility consumption. Data are from FERC Form 423, Forms EIA-176 and EIA-759, and predecessor forms.

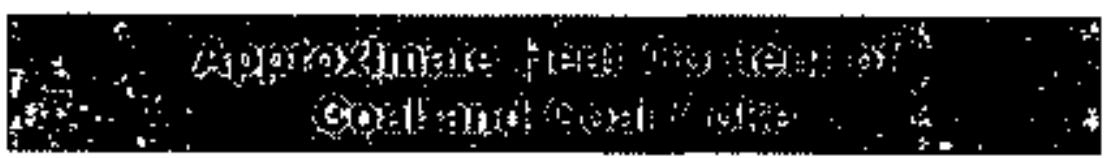

Anthracite, Total Consnmption. Calculated annually by BIA by dividing the sum of the heat content of anthracite consumed by electric utilities and by all other sectors combined by the total quantity of anthracite consumed.

Anthracite, Consumption by Electric Utilities. (ACEUKUS) - 1960 through 1972: Assumed by ELA that all anthracite consumed at electric utilities was recovered from culm banks and river dredging and estimated to have an average heat content of 17.500 million Btu per short ton. - 1973 forward: Calculated annually by ElA by dividing the heat content of anthractite receipts at electric utilities by the quantity of anthracite received at electric utilities, as reported on FBRC Form 423 and predecessor forms.

Anthracite, Consumption by Sectors Other Than Electric Utilities. (ACNUKUS) Calculated annually by EIA by dividing the heat content of anthracite produced less the heat content of the anthracite consumed at electric utilities, net exports, and shipments to U.S. Armed Forces overseas by the quantity of anthracite corsumed by 
all sectors other than electric utilities less the quantity of anthracite stock changes, losses, and "uraccounted for."

Bituminous Coal and Ifignite, Total Consumption. Calculated annually by EIA by dividing the sum of the heat content of bituminous coal and lignite consumed by electric utilities, coal coke plants, other industrial plants, the residential and commercial sector, and the transportation sector by the sum of their respective tornages.

Bituminous Coal and Lignite, Consumption by Coke Plants, Estimated by EIA to be 26.800 million Btu per short ton based on an input-output analysis of coal carbonization.

Bituminous Coal and Lignite, Consumption by Electric Utilities. (BCEUKCZ) - 1960 through 1972: EIA adopted the average thermal conversion factor of the Bureau of Mines, which used the National Coal A5sociation (NCA) average thermal conversion factor for electric utilities calculated from the Federal Power Commission's (FPC) Form 1 and published in Stern Electric Plant Factors, an NCA arnual report. * 1973 through 1982: The average heat content of coal received at steam electric plants 25 megawatts or greater from FPC Form 423 and published in Btu per pound in ElA, Cost and Qutlity of Fuels for Electric Utitity Planks, "Destination and Origin of Coal Delivered to' (1973-1979) Receipts to' (1980) 'Received at' (1981-1982) Steam-Electric Plants 25-MW or Greater." - 1983 forward: The average heat content of coal received at steam electric plants having 50 megawatts capacity or larger from FERC Form 423 and published in Btu per pound in EIA, Cost and Quality of Fuels for Electric Utility plants.

Notes: - The State conversion factors for 1960 through 1972 were derived from actual consumption data, while the conversion factors for 1973 to the present were based on receipts of coal. The factors for 1960 through 1972 may also have included some quantities of anthria cite. These breaks in the serles create some data discrepancies. Alaska and Hawaii were excluded from the NCA report, FPC Form 423 and FERC Form 423. However, Alaska reported consumption of bituminous coal and lignite at electric utilities for all years. An FPC heat rate for coal at electric utilities in Alaska was used for $\mathbf{1 9 6 0}$ through 1978 as published by ElA in Federal Energy Data Sysiem (FEDS) Techical Documentation, June 1978, Table 21. The 1972 con- version factor (the last year for which a conversion factor was reported for Alaska) was used for 1972 through 1978. According to in. dustry sources, new mines were opened in 1978 and a more representative factor was used for 1979 and following years. - In instances where a State had no receipts for a particular year but did report consumption, it was assumed that the coal received in one year was consumed during the following year and the Btu value of the previous year's receipts was used.

Bftuminous Coal and Lignite, Consumplion by Other Industrial Users. (BCOCKZZ) - 1960 through 1973: Estimated by EIA by adjusting the 1974 average heat value of bituminous coal and lignite consumed by industrial users other than coke plants by the ratios of 1960 through 1973 national averages for the other industrial users to its 1974 avetage. - 1974 forward; Estimated by BLA by assuming that the bltuminous coal and lignite consumed by other industrial users in each State contained heating values equal to those of bituminous coal and lignite recelved at electric utilities in each State from identified coal-producing districts as reported on FRRC. Form 423 . The average Btu content of coal delivered from each coal-producing district was applied to deliveries to other industrial users in each State and the sum total of the heat content was divided by total tonnages, yielding a weighted average. The coal distribution data by coal-producing district are reported on Form KlA-6 and predecessor Bureau of Mines Form 6-1419-Q.

Bituminous Coal and Lignite, Consumption by Residential and Commercial Users. (BCHCKZZ) - 1960 through 1973; Estimated by EIA by adjusting the 1974 average heat value of bituminous coal and Hgnite consumed in the residential and commercial sectors by the ratios of 1960 through 1973 national averages for the sectors to its 1974 average. - 1974 forward: Calculated by EIA by assuming that the bituminous coal and lignite consumed in the residential and commercial sectors in each State contained heating values equal to those of bituminous coal and lignite received at electric utilities in each State from identiffed coal-producing districts as reported on FERC Form 423. The average Btu content of coal delivered from each coal-producing district was applied to deliveries to the residential and commercial sectors in each State and the sum total of the heat content was divided by total tonnages, yielding a weighted av. erage. The cosl distrlbution data by coal-producing district are 
A reported on Form EIA-6 and predecessor Bureau of Mirtes Form 6P 1419-0.

Bituminous Coal and Lignite, Consumption by Transportation Users. Asstumed by ELA to be equal to the Btu conveision factor for bituminous coal and lignite consumption by other industrial users. See Bituminous Coal and Lignite, Consumption by Other Industrial Users.

Coal Coke, Inports and Exports. EIA adopted the Bureau of Mines estiviate of 24.800 million Btu per short ton.

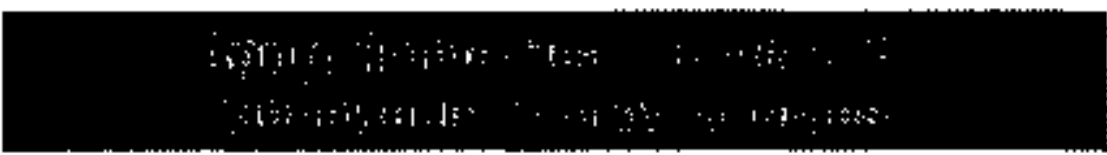

Ethanol, Consumption by the Transportation Sector. Ethanol, which is accounted for under motor gasoline, is shown separately in SEDR to display the use of renewable energy in the transportation sector. The data in thousand gallons are converted to billion Btu by using the conversion factor of 76,400 Btu per gallon as reported in the EIA, Estimates of U.S. Biomass Energy Consumption 1992, page 42.

Solar Energy, Consumption by the Residential and Commencial Sectors. Fhotovoltaic and solar thermal energy sources consumed by the residential and commencial sectors are estimated in Btu and converted to kilowatthours by using the standard conversion factor for a kilowatthour of electricity produced, regardless of the generation process, of 3,412 Btu per kilowatthour.

Wood, Consumption by the Residential and Commercial Sectors. Estimated by EIA to be 20 million Btu per cond of wood. This rolugh average factor takes into account a number of variables, such as moisture content and species of woad, as explained in the EIA, Household Energy Consumption and Expenditures 1993, page 314.
Fossil-Fueled Steam-Electric Plant Generation. (FFEOKUS) There is no generally accepted practice for measuring the thermal conversion rates for power plants that generate electricily from hydroelectric, biomass fuels, wind, photovoltaic, or solar thermal energy sources. Therefore, EIA uses data from Form ELA-767 to calculate a rate factor that is equal to the prevailing anuual average heat rate factor for fossil-fueled steam-electric power plants in the United States. By using that factor, it is possible to eraluate fossil fuel requirements for replacing those sources during periods of interruption, such as droughts. The heat content of a kilowatthour of electricity produced, regardless of the generation process, is 3,412 Btu per kilowatthour. - 1960 through 1991: The weighted annual average heat rate for fossil-fueled steam-electric power plants in the United States, as published by ELA in Electric Plant Cost and Pouter Production Expenses 1991, Table 9. - 1992 forward: Unoublished factors calculated on the basis of data from Form BLA-767.

Geothermal Energy Plant Generation. (GEEOKUS) - 1960 through 1981: Calculated annually by ELA by weighting the annizal average heat rates of operating geothermal units by the installed nameplate eapacities as reported on FFC Form 12. - 1982 forward: Estimated annually by ELA based on an informal survey of relevant plants.

Nuclear Steam-Electric Plant Generation. (NUEOKUS) * 1960 thoutgh 1991: Calculated aruually by ELA by dividing the total heat content consumed in nuclear generating units by the total (net) electricity generated by nuclear generating units. The heat content and electricity generation data are reported on FRRC Form 1, Form EIA412, and predecessor forms. The factors, beginning with 1982 data, are published in the following EIA reports-1982: Historical Plant Cost and Ammual Production Expenses for Selected Electric Planis 1982, page 215; 1983 through 1991: Electric Plent Cost ond Power Production Expenses 1991, Table 13. - 1992 forward: Unpublished factors calculated annually by EIA by dividting the total heat content of the steam leaving nuclear generating units to generate electricity by the total (net) electricity generated by nuclear generating units. The heat content and electricity generation data are reported in the Nuclear Regulatory Commission, Licensed Operating Reactors-Status Summary Report. 
Appendix E

Metric and Other Physical Conversion Factors 


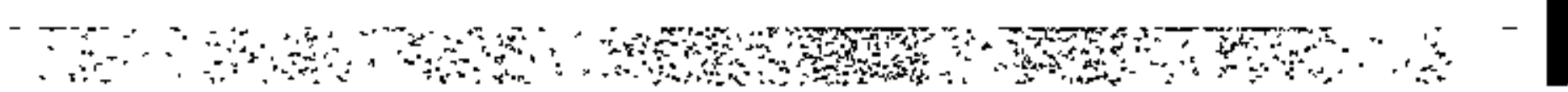




\section{Metric and Other Physical Conversion Factors}

Data presented in the State Energy Data Report and in other Energy Information Administration publications are expressed predominately in units that historically have been used in the United States, such as British thermal units, barrels, cubic feet, and short tons. However, because U.S. commerce involves other nations, most of which use metric units of measure, the U.S. Government is committed to the transition to the metric system, as stated in the Metric Conversion Act of 1975 (Public Law 94-168), amended by the Omunibus Trade and Competitiveness Act of 1988 (Public Law 100-418), and Bxecutive Order 12770 of July $25,1991$.

The metric conversion factors presented in Table B1 can be used to calculate the metric-unit equivalents of values expressed in U.S. customary units. For example, 500 short tors are the equivalent of $\mathbf{4 5 3 . 6}$ metric tons (500 short tons $\times 0.9071847$ metric tons $/$ short ton $=453.6$ metric tons).

In the metric system of weights and measures, the names of multiples and subdivisions of any unit may be derived by combiring the name of the unit with prefixes, such as deka, hecto, and kilo, meaning respectively, 10, 100, 1,000, and deci, centi, and millk, meaning respectively, one-tenth, one-hundredth, and one-thousandth. Common metric prefixes can be found in Table E2.

The conversion factors presented in Table $\mathrm{ES}$ can be used to calculate equivalents in various physical units commonly used in energy analyses. For example, 10 barrels are the equivalent of $\mathbf{2 0}$ U.S. gallons ( 10 barrels $\times 42$ gallots $/$ barrel $=420$ gallons). 
A Table E1. Motric Comversion Factors

\begin{tabular}{|c|c|c|c|c|c|c|c|c|c|}
\hline U.S. لإ"t & $\begin{array}{l}\text { mutipligod } \\
\text { by }\end{array}$ & $\begin{array}{l}\text { Conversion } \\
\text { Factor }\end{array}$ & equals & Metrits Unit & U.S. Unit & $\begin{array}{c}\text { moltipitad } \\
\text { by }\end{array}$ & $\begin{array}{l}\text { Corversion } \\
\text { Factor }\end{array}$ & equals & retrie Unit \\
\hline 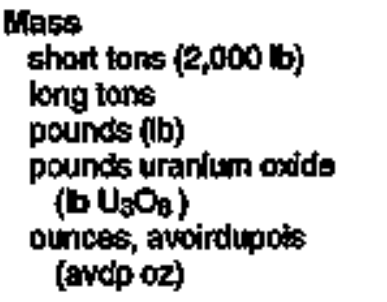 & $\begin{array}{l}\mathbf{x} \\
\mathbf{x} \\
\mathbf{x} \\
\mathbf{x} \\
\mathbf{x}\end{array}$ & $\begin{array}{l}0.9071847 \\
1.016047 \\
0.45969237^{4} \\
0.384647^{\circ} \\
28.34962\end{array}$ & $\begin{array}{l}= \\
= \\
= \\
=\end{array}$ & $\begin{array}{l}\text { motic tons (t) } \\
\text { moltic tons (l) } \\
\text { lohigrams (kg) } \\
\text { kilograms } \\
\text { uranitim (kg) } \\
\text { grams (g) }\end{array}$ & 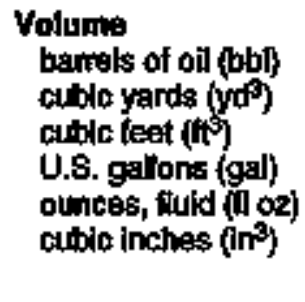 & $\begin{array}{l}x \\
x \\
x \\
x \\
x \\
x\end{array}$ & $\begin{array}{l}0.1589873 \\
0.764555 \\
0.02831685 \\
3.795412 \\
29.57353 \\
16.39706\end{array}$ & $\begin{array}{l}= \\
= \\
F \\
= \\
= \\
=\end{array}$ & 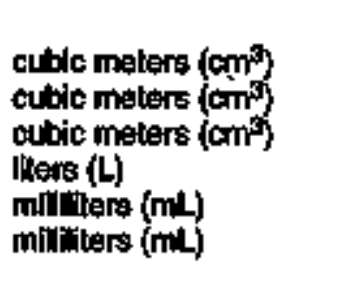 \\
\hline $\begin{array}{l}\text { Lenisth } \\
\text { miles (mi) } \\
\text { yand (yol) } \\
\text { fest (h) } \\
\text { inches (in) }\end{array}$ & $\begin{array}{l}\mathbf{x} \\
\mathbf{x} \\
\mathbf{x} \\
\mathbf{x}\end{array}$ & $\begin{array}{l}1.609 .344^{\mathrm{a}} \\
0.9144^{\mathrm{a}} \\
0.3948^{\mathrm{a}} \\
2.54^{\mathrm{a}}\end{array}$ & $\begin{array}{l}= \\
= \\
\mathbf{a} \\
\mathbf{a}\end{array}$ & $\begin{array}{l}\text { Gilometers (km) } \\
\text { meters (m) } \\
\text { moters (m) } \\
\text { centimelers (cm) }\end{array}$ & 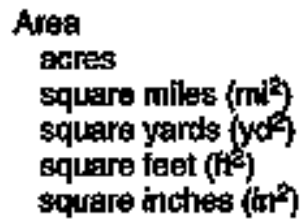 & $\begin{array}{l}\mathbf{x} \\
\mathbf{x} \\
\mathbf{x} \\
\mathbf{x} \\
\mathbf{x}\end{array}$ & $\begin{array}{l}0.40469 \\
2.509989 \\
0.8961274 \\
0.099290904^{9} \\
6.4516^{a}\end{array}$ & $\begin{array}{l}= \\
= \\
= \\
= \\
=\end{array}$ & $\begin{array}{l}\text { hectares (ha) } \\
\left.\text { square kitomelers ( } \mathrm{km}^{2}\right) \\
\text { square meters }\left(\mathrm{m}^{2}\right) \\
\text { square maters }\left(\mathrm{m}^{2}\right) \\
\text { equare centimeters (cm }\end{array}$ \\
\hline $\begin{array}{l}\text { Envity } \\
\text { Bringh Themat Units (Blu) } \\
\text { catories (cal) } \\
\text { kilowatthours (kWh) }\end{array}$ & $\begin{array}{l}\mathbf{x} \\
\mathbf{x} \\
\mathbf{x}\end{array}$ & $\begin{array}{c}1,055.05585262^{30} \\
4.1668^{\circ} \\
3.6^{a}\end{array}$ & $\begin{array}{l}= \\
= \\
=\end{array}$ & $\begin{array}{l}\text { jotles }(\mathfrak{J}) \\
\text { joules }(\mathfrak{J}) \\
\text { megajoules (Mل) }\end{array}$ & $\begin{array}{l}\text { Temperature } \\
\text { degrees } \\
\text { Fetrentoll (of) }\end{array}$ & $\begin{array}{r}\mathbf{5} \\
\mathrm{s}\end{array}$ & $\begin{array}{l}\text { B/9 (after } \\
\text { subiracting ae) } \text { a,d }^{2}\end{array}$ & $=$ & $\begin{array}{l}\text { degroes } \\
\text { Celsulus ( }\end{array}$ \\
\hline
\end{tabular}

\section{Eoger compagion.}

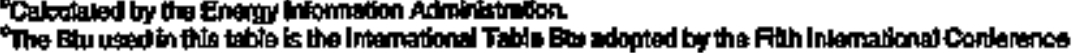

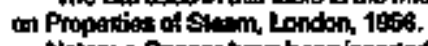

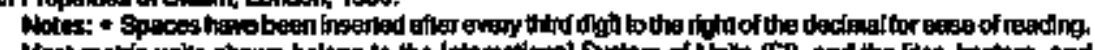

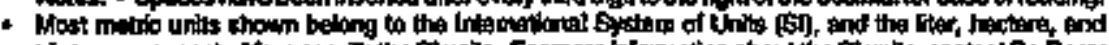

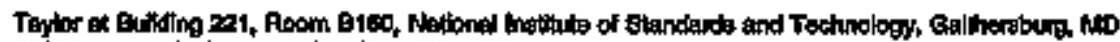

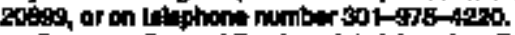

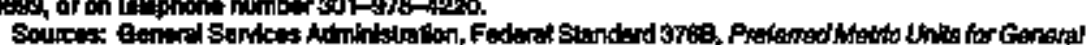

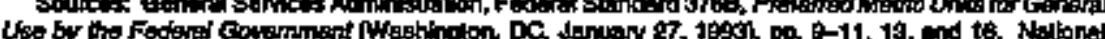
The of

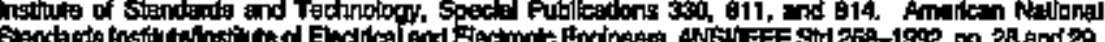

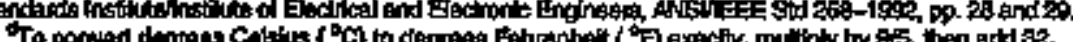


Tabie E2. Metric Prefixes

\begin{tabular}{|c|c|c|c|c|c|}
\hline $\begin{array}{c}\text { Unt } \\
\text { Auntiple }\end{array}$ & Preflx & Symbot & $\begin{array}{c}\text { Unit } \\
\text { subulavision }\end{array}$ & Prefix & Symbo \\
\hline $\begin{array}{l}10^{2} \\
10^{2} \\
10^{3} \\
10^{6} \\
10^{9} \\
10^{12} \\
10^{15} \\
10^{18} \\
10^{21} \\
10^{24}\end{array}$ & $\begin{array}{l}\text { deka } \\
\text { hecto } \\
\text { kilo } \\
\text { mega } \\
\text { giga } \\
\text { tera } \\
\text { pata } \\
\text { exa } \\
\text { zelta } \\
\text { yotta }\end{array}$ & $\begin{array}{l}\text { da } \\
h \\
k \\
M \\
G \\
T \\
P \\
E \\
Z \\
Y\end{array}$ & $\begin{array}{l}10^{-1} \\
10^{2} \\
10^{-3} \\
10^{6} \\
10^{-9} \\
10^{-12} \\
10^{-15} \\
10^{-18} \\
10^{-21} \\
10^{-24}\end{array}$ & $\begin{array}{l}\text { deci } \\
\text { cenid } \\
\text { mili } \\
\text { micro } \\
\text { nano } \\
\text { pico } \\
\text { feitito } \\
\text { atto } \\
\text { zeplo } \\
\text { yocto }\end{array}$ & $\begin{array}{l}d \\
0 \\
m \\
1 \\
n \\
p \\
f \\
a \\
z \\
y\end{array}$ \\
\hline
\end{tabular}

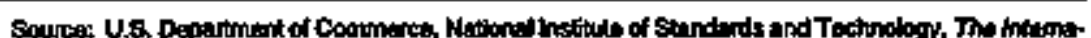

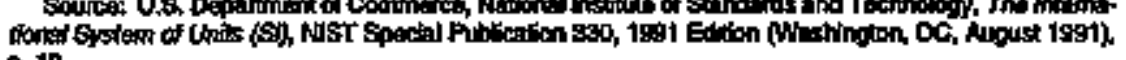

Tabłe Ex. Other Physleal Conversion Factors

\begin{tabular}{|c|c|c|c|c|c|}
\hline Energy Soumbe & Orig"inal Uni & & $\begin{array}{c}\text { Conversion } \\
\text { Factor }\end{array}$ & & Flnal Untt \\
\hline Poinoloum & barrel's (bbl) & $\mathbf{x}$ & $42^{2}$ & $=$ & U.S. gallons (gal) \\
\hline Co:t & $\begin{array}{l}\text { short kons } \\
\text { long tons } \\
\text { metric tons (t) }\end{array}$ & $\begin{array}{l}\mathbf{x} \\
\mathbf{x} \\
\mathbf{x}\end{array}$ & $\begin{array}{l}2,000^{n} \\
2,240^{\mathrm{a}} \\
1,000^{\mathrm{a}}\end{array}$ & $\begin{array}{l}= \\
= \\
=\end{array}$ & $\begin{array}{l}\text { pounds (b) } \\
\text { pounds (b) } \\
\text { klograms (kg) }\end{array}$ \\
\hline Wopd & $\begin{array}{l}\text { cords }(c-d) \\
\text { cords }(c+d)\end{array}$ & $\begin{array}{l}x \\
x\end{array}$ & $t^{1.25^{h}}$ & $\begin{array}{l}= \\
=\end{array}$ & $\begin{array}{l}\text { short tons } \\
\text { cubic foet }\left\langle\mathrm{t}^{3}\right)\end{array}$ \\
\hline
\end{tabular}

\section{Eratal conversion.}

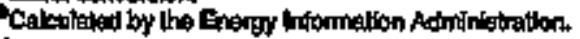

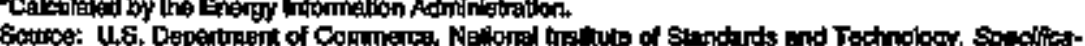

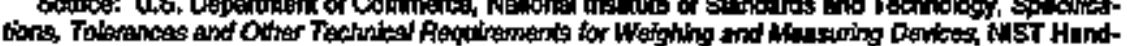

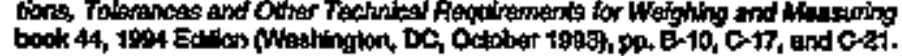

$\mathbf{A}$
$\mathbf{P}$
$\mathbf{P}$
$\mathbf{E}$
$\mathbf{N}$
$\mathbf{D}$
$\mathbf{D}$
$\mathbf{X}$
$\mathbf{E}$ 

Appendix $\mathbf{F}$

Carbon Dloxide Emission Factors for Coal 


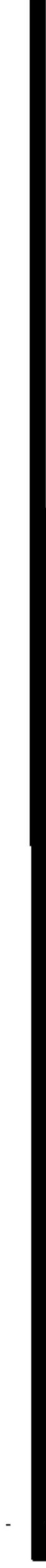


The need for accurate estimates of carbon dioxide emissions produced during the combustion of coal has led the Energy Information Administration (EIA) to develop basic emission factors. Basic emission factors reflect the carbon-to-heat-content ratio of coal, a ratio which measures cartoon dioxide emissions per unit of energy (pounds per million Bta), assuming complete combustion. These basic factors are derived from 5,426 sample analyses maintzined in EIA's Coal Analysis File. Variations in the carbon-to-heat-content of different coals were observed to follow conl rank and geographic origin, leading EIA to develop basic entission factors specific to the rank and the State of origin of the coal.

On the basis of these rank- and State-spectific basic emission factors for coal, ELA has also developed emission factors by sector. These sectoral emission factors waight the coal consumed in a given sector by its rank and State of origin. Tables F1 through 75 present the U.S. average carbon dioxide entission factors for coal by sector. Bmission factors differ among sectoss and within a given sector over time for a number of reasons:
- A higher average emission factor in the residential and commercial sector can be attributed to the steady consumption of bituminous coal and anthracite (presumably for home heating).

- Virtually all of the coal consumed by coke plants comes from only a few States in the Appalachian Coal Basin (West Virginia, Virginia, and eastem Kentucky). Hence, the emission factors for this sector have remained fairly constant.

- Other industrial users of coal (not coke plants) increased consumption of low-rank, high-emission western coals, which has contributed to a rise in their average emission factor.

- Electric utilities, which account for most U.S. coal consumption, have shifted over time away from high-rark, low-enission bituminous coal to low-rank, high-emission subbituminous coal and lignite as reflected in a gradually itsing weighted-average carbon dioxide envission factor. 


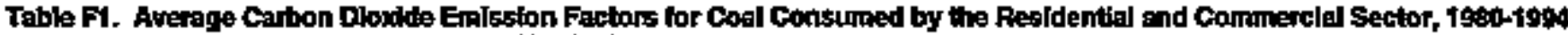
(Pounds of Carben Dloxffe per Million Etu)

\begin{tabular}{|c|c|c|c|c|c|c|c|c|c|c|c|c|c|c|c|}
\hline $8+6$ & 1900 & 19001 & 160 & 1800 & 1984 & 1905 & 1000 & $10 \%$ & 168 & 1609 & 1000 & 1891 & 1902 & 1893 & t994 \\
\hline 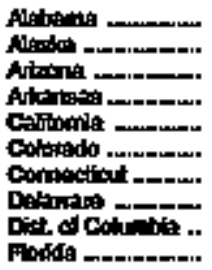 & $\begin{array}{l}205.4 \\
- \\
- \\
2050 \\
2045 \\
212.5 \\
202.1 \\
221.0 \\
2055 \\
205.0\end{array}$ & $\begin{array}{l}2055 \\
2 \neq 10 \\
209.7 \\
211.3 \\
2053 \\
242.0 \\
2057 \\
2210 \\
2049 \\
20050\end{array}$ & 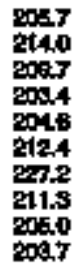 & 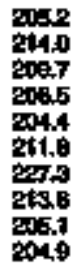 & $\begin{array}{l}205.4 \\
201.0 \\
20.7 \\
202.5 \\
204.1 \\
212.0 \\
203.4 \\
20.4 \\
200.3 \\
204.1\end{array}$ & 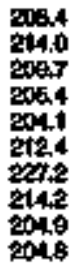 & $\begin{array}{l}208.4 \\
2140 \\
200.9 \\
272.4 \\
2097 \\
212.5 \\
208.9 \\
213.9 \\
2052 \\
204.8\end{array}$ & 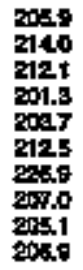 & $\begin{array}{l}200.9 \\
2+1.0 \\
210.6 \\
200.6 \\
201.2 \\
212.5 \\
201.5 \\
201.1 \\
20.3 \\
214.3\end{array}$ & 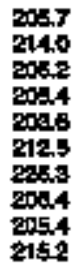 & $\begin{array}{l}2060 \\
2140 \\
2003 \\
2050 \\
204.1 \\
2124 \\
206.7 \\
2067 \\
206.4 \\
207.5\end{array}$ & $\begin{array}{l}2056 \\
2140 \\
2123 \\
2053 \\
2044 \\
2124 \\
2273 \\
2050 \\
2055 \\
207.4\end{array}$ & 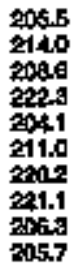 & $\begin{array}{l}2057 \\
2140 \\
2120 \\
2099 \\
204.9 \\
2120 \\
2050 \\
2000 \\
200.4 \\
205.1\end{array}$ & $\begin{array}{l}205.7 \\
214.0 \\
227.4 \\
207.2 \\
204.1 \\
211.2 \\
206.3 \\
203.4 \\
2045 \\
205.0\end{array}$ \\
\hline 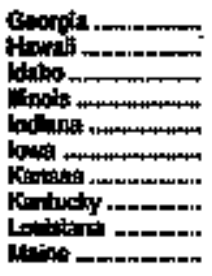 & 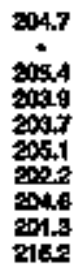 & 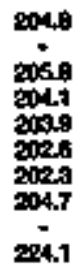 & 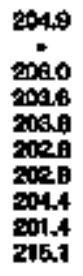 & 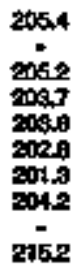 & 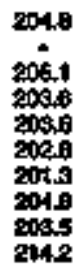 & $\begin{array}{l}205.3 \\
20148 \\
20046 \\
20036 \\
20029 \\
2001.9 \\
201.2 \\
211.0\end{array}$ & $\begin{array}{l}205.2 \\
2045 \\
2030 \\
203.6 \\
200.0 \\
201.3 \\
20.4 \\
20.4 \\
210.1\end{array}$ & 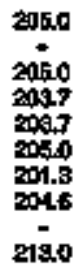 & 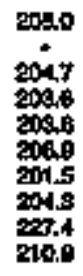 & 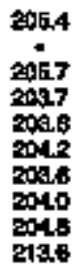 & $\begin{array}{l}205.1 \\
204.9 \\
20085 \\
209.7 \\
2041 \\
2032 \\
204.2 \\
2120\end{array}$ & 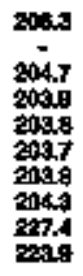 & $\begin{array}{l}2049 \\
2050 \\
20090 \\
20018 \\
2042 \\
2002 \\
2010 \\
2130\end{array}$ & $\begin{array}{l}206.1 \\
204.9 \\
200.9 \\
204.1 \\
204.5 \\
20039 \\
205.1 \\
227.4 \\
212.2\end{array}$ & $\begin{array}{l}2080 \\
2060 \\
20040 \\
2041 \\
204.6 \\
2030 \\
2050 \\
- \\
2280\end{array}$ \\
\hline 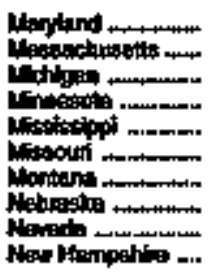 & 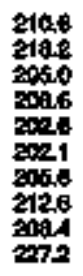 & $\begin{array}{l}218.0 \\
2200 \\
2051 \\
211.6 \\
227.4 \\
201.6 \\
2191 \\
242.6 \\
207.7 \\
207.4\end{array}$ & 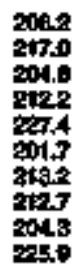 & 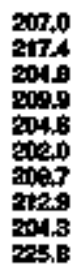 & 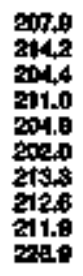 & $\begin{array}{l}200.8 \\
20.4 \\
204.6 \\
210.8 \\
200.2 \\
204.4 \\
2014.3 \\
212.6 \\
2041 \\
227.4\end{array}$ & $\begin{array}{l}\text { 207.1 } \\
219.9 \\
2 \times 47 \\
210.8 \\
2048 \\
2048 \\
2039.9 \\
2121 \\
2127 \\
2041 \\
2272\end{array}$ & $\begin{array}{l}2008 \\
2297 \\
2019 \\
211.8 \\
2040 \\
201.8 \\
2131 \\
212.7 \\
2041 \\
227.4\end{array}$ & 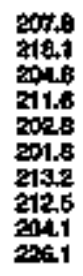 & 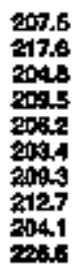 & $\begin{array}{l}207.8 \\
2138 \\
2049 \\
2120 \\
2094 \\
202.7 \\
211.7 \\
2127 \\
212.3 \\
226.4\end{array}$ & 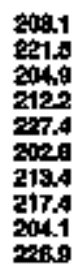 & $\begin{array}{l}211.7 \\
214,1 \\
2050 \\
212.4 \\
227.4 \\
20034 \\
2123.3 \\
219.2 \\
204.1 \\
226.4\end{array}$ & 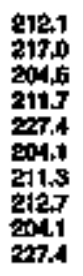 & $\begin{array}{l}200.6 \\
2000 \\
200,3 \\
2004 \\
203.5 \\
213.4 \\
212.7 \\
2041 \\
227.4\end{array}$ \\
\hline 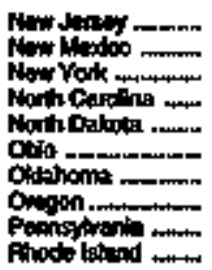 & 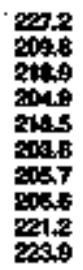 & 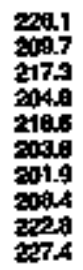 & 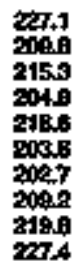 & 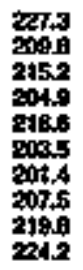 & 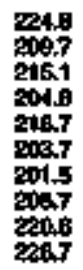 & 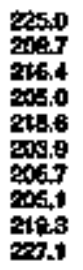 & 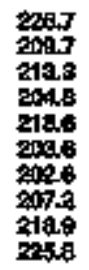 & 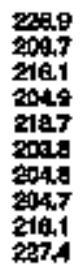 & $\begin{array}{l}227.4 \\
2007 \\
214.6 \\
2046 \\
210.8 \\
2043 \\
2123 \\
2047 \\
2197 \\
227.4\end{array}$ & 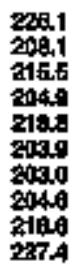 & $\begin{array}{l}2972 \\
2057 \\
2140 \\
200.7 \\
217.0 \\
2042 \\
20032 \\
0041 \\
2100 \\
2073\end{array}$ & $\begin{array}{l}2289 \\
2058 \\
2154 \\
20,1 \\
217.6 \\
204.4 \\
205.9 \\
204.1 \\
218.2 \\
227.4\end{array}$ & $\begin{array}{l}227.1 \\
200.3 \\
2100 \\
200.2 \\
216.0 \\
205.5 \\
207.0 \\
2004.1 \\
219.7 \\
227.4\end{array}$ & 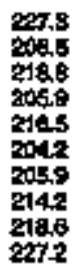 & $\begin{array}{l}227.0 \\
205.6 \\
214.8 \\
200.0 \\
216.4 \\
204.5 \\
206.5 \\
213.4 \\
220.1 \\
227.4\end{array}$ \\
\hline 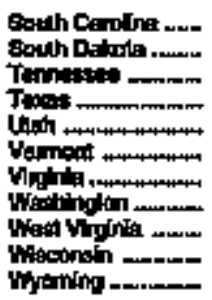 & 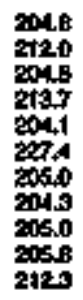 & $\begin{array}{l}204.5 \\
2120 \\
204.6 \\
2006 \\
204.1 \\
227.3 \\
205.1 \\
20.6 \\
205.2 \\
200.7 \\
211.9\end{array}$ & $\begin{array}{l}204.8 \\
241.7 \\
204.1 \\
245.9 \\
2001.1 \\
207.3 \\
203.2 \\
204.4 \\
20.5 \\
205.1 \\
212.5\end{array}$ & $\begin{array}{l}204.0 \\
209.1 \\
204.6 \\
246.3 \\
2001.1 \\
201.3 \\
200.3 \\
204.4 \\
205.6 \\
204.4 \\
212.0\end{array}$ & $\begin{array}{l}204.8 \\
205.0 \\
201.2 \\
20.4 \\
201.1 \\
203.0 \\
205.4 \\
204.5 \\
200.7 \\
200.2 \\
20.9\end{array}$ & 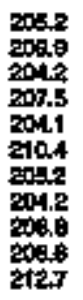 & 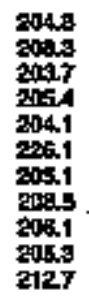 & $\begin{array}{l}2049 \\
211.9 \\
3040 \\
2043 \\
2041 \\
2072 \\
20052 \\
2019 \\
2005 \\
2039 \\
2127\end{array}$ & $\begin{array}{l}204 a \\
2122 \\
2044 \\
2040 \\
2042 \\
2074 \\
2054 \\
2054 \\
205.3 \\
2053 \\
2127\end{array}$ & $\begin{array}{l}204.8 \\
2129 \\
2040 \\
206.6 \\
204.2 \\
227.1 \\
200.1 \\
20049 \\
200.9 \\
205.8 \\
212.3\end{array}$ & 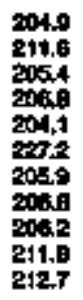 & 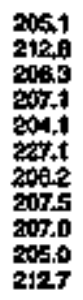 & 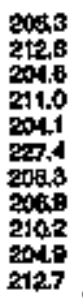 & 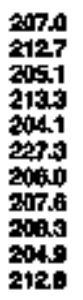 & $\begin{array}{l}207.0 \\
2099 \\
2050 \\
227.0 \\
204.1 \\
207.2 \\
2010.4 \\
2082 \\
207.0 \\
204.9 \\
212.0\end{array}$ \\
\hline U.S. Avaragen - - & 240.6 & 2120 & 210.4 & $\operatorname{ang}$ & 209.8 & 2003 & 2009 & 20094 & 202.1 & 209.7 & 200.5 & 210.2 & 211.2 & 2000 & 200.5 \\
\hline
\end{tabular}


Table F2. Average Carbon Dioxida Émisetion Factors for Coal consumed a by coke Plants, 1980-1994 (Poundis of Carbon Cioxide per Mallon Btu)

\begin{tabular}{|c|c|c|c|c|c|c|c|c|c|c|c|c|c|c|c|}
\hline othe & toss & tist & 1902 & 1968 & 1984 & 100s & 18006 & ts:s & 1958 & 1989 & 1900 & 1991 & 1902 & 1983 & 1984 \\
\hline 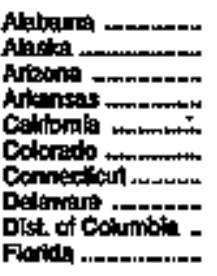 & $\begin{array}{c}205.5 \\
= \\
= \\
2197 \\
2126 \\
= \\
=\end{array}$ & $\begin{array}{c}2055 \\
\vdots \\
\vdots \\
207+8 \\
\vdots \\
\vdots\end{array}$ & $\begin{array}{c}205.4 \\
\vdots \\
2007.4 \\
2125 \\
\vdots \\
\vdots\end{array}$ & $\begin{array}{c}2054 \\
\vdots \\
\vdots \\
\vdots \\
\vdots \\
\vdots\end{array}$ & $\begin{array}{l}\cos 4 \\
\vdots \\
= \\
= \\
\vdots \\
:\end{array}$ & $\begin{array}{l}205.4 \\
: \\
= \\
= \\
=\end{array}$ & $\begin{array}{c}2008 \\
\vdots \\
\vdots \\
\vdots \\
:\end{array}$ & $\begin{array}{c}205.3 \\
\vdots \\
\vdots \\
\vdots \\
\vdots \\
\vdots\end{array}$ & $\begin{array}{c}2053 \\
\vdots \\
\vdots \\
\vdots \\
\vdots\end{array}$ & $\begin{array}{c}200.4 \\
\vdots \\
\vdots \\
\vdots \\
\vdots \\
\vdots\end{array}$ & $\begin{array}{c}2000 \\
\vdots \\
\vdots \\
= \\
= \\
=\end{array}$ & $\begin{array}{c}2009 \\
= \\
\vdots \\
= \\
= \\
=\end{array}$ & $\begin{array}{c}206.1 \\
= \\
= \\
: \\
= \\
=\end{array}$ & $\begin{array}{c}2062 \\
= \\
\vdots \\
\vdots \\
= \\
=\end{array}$ & $\begin{array}{c}2062 \\
= \\
= \\
: \\
= \\
=\end{array}$ \\
\hline 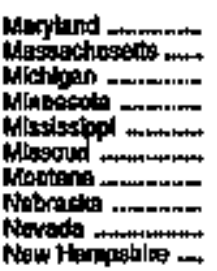 & $\begin{array}{c}2060 \\
205 \\
\vdots \\
205 \\
\vdots \\
\vdots\end{array}$ & $\begin{array}{c}206.1 \\
205.4 \\
\vdots \\
206.6 \\
= \\
=\end{array}$ & $\begin{array}{c}205.7 \\
205.4 \\
\vdots \\
205.7 \\
\vdots \\
=\end{array}$ & $\begin{array}{c}205.5 \\
205.35 \\
\vdots \\
205.1 \\
\vdots\end{array}$ & $\begin{array}{c}205.5 \\
205.4 \\
\vdots \\
2005.4 \\
\vdots \\
\vdots\end{array}$ & $\begin{array}{c}2055 \\
205.4 \\
= \\
205.4 \\
\vdots \\
=\end{array}$ & $\begin{array}{c}205.5 \\
205.5 \\
\vdots \\
20 i s \\
\vdots \\
:\end{array}$ & $\begin{array}{c}205.5 \\
200.0 \\
\vdots \\
2000 \\
\vdots \\
\vdots\end{array}$ & $\begin{array}{c}205.9 \\
205.4 \\
\vdots \\
= \\
=\end{array}$ & 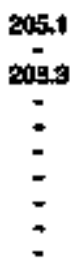 & $\begin{array}{l}200.2 \\
-0.4 \\
=\end{array}$ & $\begin{array}{c}205.9 \\
2007 \\
\vdots \\
\vdots \\
\vdots\end{array}$ & $\begin{array}{c}\dot{2} \\
\dot{207} \\
\vdots \\
= \\
\vdots \\
-\end{array}$ & $\underset{:}{20786}$ & $\frac{-}{205.7}$ \\
\hline 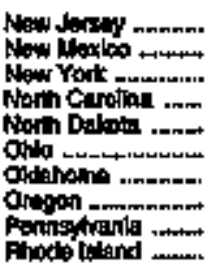 & 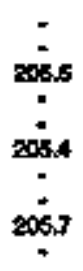 & 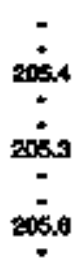 & $\begin{array}{c}\vdots \\
2054 \\
\vdots \\
2053 \\
\vdots \\
2035\end{array}$ & 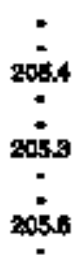 & $\begin{array}{c}\vdots \\
2086 \\
\vdots \\
0092 \\
\vdots \\
2056\end{array}$ & 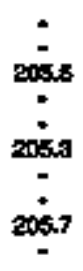 & $\begin{array}{c}\vdots \\
2005 \\
\vdots \\
203 \\
\vdots \\
200 \\
-\end{array}$ & 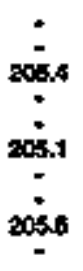 & $\begin{array}{c}\vdots \\
2058 \\
205 \\
20.5\end{array}$ & $\begin{array}{c}: \\
20058 \\
\vdots \\
205 \\
20 \\
20\end{array}$ & 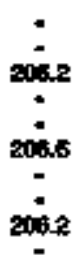 & $\begin{array}{c}\vdots \\
208.1 \\
208.4 \\
\vdots \\
206.2\end{array}$ & 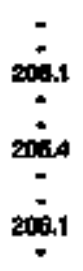 & $\begin{array}{c}* \\
2000 \\
200 \\
20\end{array}$ & 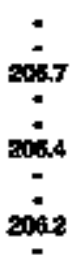 \\
\hline 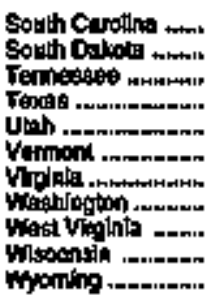 & $\begin{array}{l}: \\
2102 \\
2028 \\
2100 \\
2062 \\
- \\
2054 \\
2053\end{array}$ & $\begin{array}{l}= \\
207.1 \\
212.2 \\
210.8 \\
200.2 \\
205.4 \\
205.3 \\
-\end{array}$ & $\begin{array}{l}: \\
2053 \\
212.3 \\
211.3 \\
200.3 \\
2093 \\
2014\end{array}$ & $\begin{array}{c}: \\
2051 \\
2127 \\
2124 \\
2062 \\
2065 \\
\vdots\end{array}$ & $\begin{array}{c}- \\
205.3 \\
2127 \\
211.7 \\
2052 \\
\overline{2053} \\
\vdots\end{array}$ & 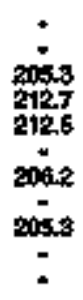 & $\begin{array}{c}\vdots \\
20 ; 2 \\
2127 \\
2019 \\
2002 \\
2050 \\
\vdots\end{array}$ & $\begin{array}{c}: \\
20.1 \\
200.3 \\
20032 \\
\dot{205.1} \\
\vdots\end{array}$ & $\begin{array}{l}= \\
2040 \\
200.7 \\
200.2 \\
204.9 \\
=\end{array}$ & $\begin{array}{l}= \\
2040 \\
200.0 \\
206.2 \\
205.1 \\
=\end{array}$ & $\begin{array}{c}: \\
2007.6 \\
200.2 \\
2060.2 \\
206.7 \\
\dot{-}\end{array}$ & 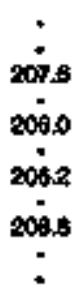 & $\begin{array}{l}= \\
200.2 \\
008.8 \\
200.2 \\
200.7 \\
=\end{array}$ & 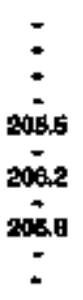 & 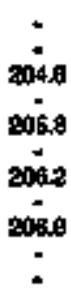 \\
\hline 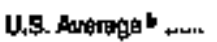 & 2050 & 2nsa & 205.7 & 2005 & 20056 & 205.6 & 2005 & 2052 & 20159 & 2053 & 2062 & 206.2 & 20062 & 2002 & 206.9 \\
\hline
\end{tabular}

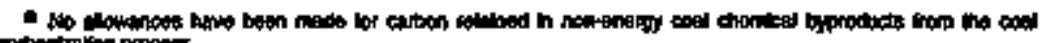

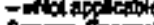

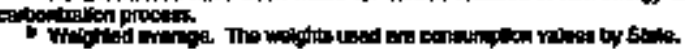


Table F3. Average Carbon Dloxite Emission Factors for Cota Gonstamed by Other Industrial Users, 1980-1994 (Founds of Cabon Dioxide per (ulition Eu)

\begin{tabular}{|c|c|c|c|c|c|c|c|c|c|c|c|c|c|c|c|}
\hline state & 1980 & 1901 & 1002 & tous & te64 & 1906 & $10 \% 6$ & $109 \%$ & 1969 & 1969 & toser & tgat & 1992 & 1998 & 194 \\
\hline 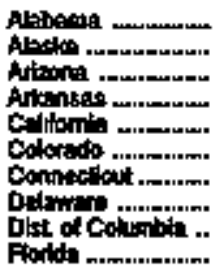 & $\begin{array}{l}205.5 \\
- \\
201.2 \\
201.4 \\
205.6 \\
212.6 \\
2015.4 \\
2005.9 \\
205.0 \\
204.2\end{array}$ & $\begin{array}{l}2055 \\
20 \\
2010.4 \\
2014 \\
2059 \\
212.7 \\
209.2 \\
2050 \\
2040 \\
204.8\end{array}$ & $\begin{array}{l}205.3 \\
210.3 \\
201.4 \\
200.4 \\
212.4 \\
20.9 \\
200.6 \\
204.9\end{array}$ & $\begin{array}{l}205.2 \\
210.3 \\
201.4 \\
200.4 \\
2127 \\
2076 \\
205.8 \\
204.8\end{array}$ & $\begin{array}{l}208.5 \\
- \\
210.0 \\
201.5 \\
205.6 \\
212.7 \\
216.9 \\
205.0 \\
204.8\end{array}$ & $\begin{array}{l}2065 \\
210.1 \\
201+6 \\
3047 \\
2127 \\
200.2 \\
205.9 \\
205.9\end{array}$ & $\begin{array}{l}205.5 \\
500.9 \\
200.7 \\
205.0 \\
20.7 \\
200.2 \\
205.0 \\
205.0\end{array}$ & $\begin{array}{l}205.4 \\
- \\
210.1 \\
208.2 \\
200.4 \\
212.1 \\
212.0 \\
205.9 \\
206.0\end{array}$ & $\begin{array}{l}205.4 \\
200.4 \\
200.4 \\
204.8 \\
202.3 \\
200.5 \\
205.9 \\
205.2\end{array}$ & $\begin{array}{l}205.4 \\
-09.6 \\
2003.6 \\
204.7 \\
212.6 \\
204.8 \\
205.9 \\
20.49\end{array}$ & $\begin{array}{l}205.5 \\
-5 \\
207.5 \\
205.3 \\
204.6 \\
2120 \\
207.4 \\
206.1 \\
205.4\end{array}$ & $\begin{array}{l}205.6 \\
- \\
207.0 \\
205.1 \\
204.8 \\
212.6 \\
205.9 \\
20.0 \\
-5.0 \\
205.2\end{array}$ & $\begin{array}{l}205.7 \\
- \\
208.7 \\
200.2 \\
204.2 \\
212.5 \\
204.7 \\
207.4 \\
205.4\end{array}$ & $\begin{array}{l}205.4 \\
-7 \\
200.9 \\
2065.9 \\
204.1 \\
212.7 \\
907.1 \\
200.0 \\
205.2\end{array}$ & $\begin{array}{l}205.4 \\
227.4 \\
207.2 \\
200.8 \\
204.1 \\
213.1 \\
207.1 \\
207.7 \\
205.1\end{array}$ \\
\hline 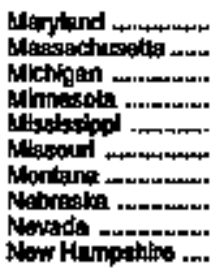 & $\begin{array}{l}206.1 \\
2004.3 \\
2040 \\
211.6 \\
204.6 \\
200.8 \\
211.2 \\
212.3 \\
2045 \\
2075\end{array}$ & 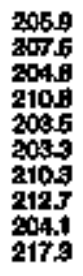 & 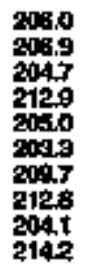 & $\begin{array}{l}205.3 \\
2003.3 \\
204.9 \\
213.0 \\
204.5 \\
2003 \\
2009.7 \\
2127 \\
204.1 \\
201.7\end{array}$ & $\begin{array}{l}205.9 \\
201.6 \\
205.0 \\
211.8 \\
200.2 \\
20.5 \\
210.5 \\
213.1 \\
204.1 \\
206.9\end{array}$ & $\begin{array}{l}205.1 \\
200.4 \\
200.0 \\
200.9 \\
204.0 \\
200.5 \\
212.6 \\
213.4 \\
204.1 \\
219.5\end{array}$ & 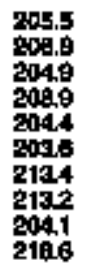 & 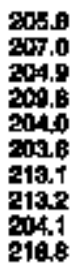 & $\begin{array}{l}205.6 \\
207.1 \\
204.9 \\
210.9 \\
204.0 \\
2001,7 \\
211.9 \\
219.1 \\
204.1 \\
218.6\end{array}$ & $\begin{array}{l}205.9 \\
20074 \\
204.8 \\
210.3 \\
2040 \\
20313 \\
211.8 \\
212 . \\
206.7 \\
207.6\end{array}$ & $\begin{array}{l}207.8 \\
2000 \\
204.9 \\
211.8 \\
203.7 \\
204.1 \\
217.7 \\
213.3 \\
204.1 \\
216.2\end{array}$ & $\begin{array}{l}207.8 \\
2007 \\
204.9 \\
211.1 \\
204.2 \\
204.1 \\
211.6 \\
213.3 \\
204.1 \\
206.8\end{array}$ & $\begin{array}{l}208.4 \\
207.0 \\
209.3 \\
211.8 \\
204.8 \\
204.5 \\
211.4 \\
213.1 \\
204.1 \\
207.1\end{array}$ & 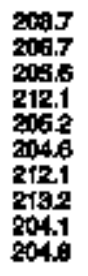 & 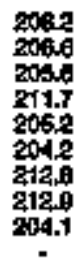 \\
\hline 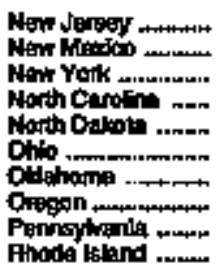 & 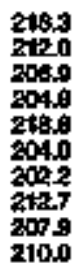 & $\begin{array}{l}2246 \\
2727 \\
200.7 \\
2187 \\
2089 \\
2019 \\
211.3 \\
2039 \\
210.3\end{array}$ & $\begin{array}{l}219.8 \\
21213 \\
207.0 \\
204.8 \\
219.7 \\
2040 \\
2010 \\
211.9 \\
207.0 \\
=\end{array}$ & $\begin{array}{l}212.8 \\
212.7 \\
207.1 \\
2048 \\
219.7 \\
2099 . \\
2093.3 \\
2120 \\
207.0 \\
204.8\end{array}$ & $\begin{array}{l}213.4 \\
212.7 \\
201.8 \\
204.8 \\
210.5 \\
204.4 \\
2010.8 \\
20.0 \\
200.8 \\
204.8\end{array}$ & $\begin{array}{l}217.5 \\
212.5 \\
206.8 \\
204.9 \\
210.6 \\
204.9 \\
202.4 \\
211.8 \\
207.3 \\
20.57\end{array}$ & $\begin{array}{l}210.9 \\
212.7 \\
209.9 \\
204.8 \\
219.6 \\
204.3 \\
2002.5 \\
211.9 \\
207.4 \\
200.9\end{array}$ & $\begin{array}{l}210.0 \\
212.7 \\
207.1 \\
204.8 \\
212.4 \\
204.2 \\
200.3 \\
211.7 \\
207.1 \\
219.8\end{array}$ & $\begin{array}{l}200.1 \\
20.7 \\
207.4 \\
204.6 \\
218.6 \\
204.4 \\
200.1 \\
212.7 \\
200.8 \\
204.8\end{array}$ & $\begin{array}{l}207.5 \\
2127 \\
2069 \\
2048 \\
2196 \\
2043 \\
205.7 \\
202 \\
20128 \\
204.8\end{array}$ & 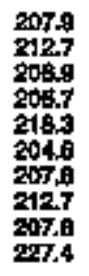 & 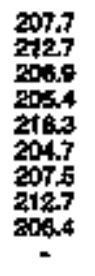 & $\begin{array}{l}207.3 \\
212.7 \\
207.0 \\
205.7 \\
216.3 \\
204.5 \\
207.6 \\
271.5 \\
200.5 \\
-\end{array}$ & 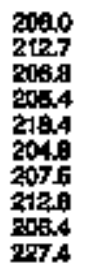 & $\begin{array}{l}210.3 \\
212.7 \\
207.0 \\
205.6 \\
219.2 \\
204.9 \\
209.1 \\
210.4 \\
209.8 \\
-.9\end{array}$ \\
\hline 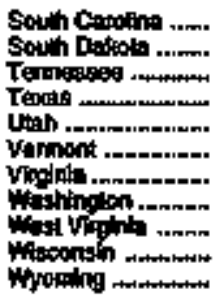 & 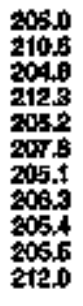 & $\begin{array}{l}2050 \\
2107 \\
204.9 \\
212.9 \\
203.9 \\
2070 \\
205.0 \\
207.1 \\
205.3 \\
204.9 \\
212.2\end{array}$ & 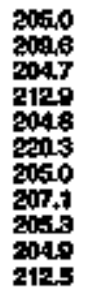 & 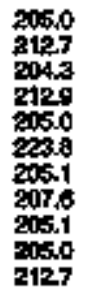 & 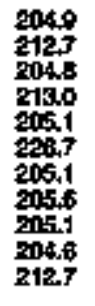 & $\begin{array}{l}2050 \\
212.7 \\
204.7 \\
213.1 \\
2015.6 \\
210.3 \\
20,1 \\
208.1 \\
205.5 \\
205.0 \\
2127\end{array}$ & 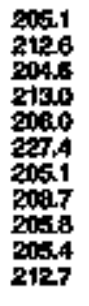 & 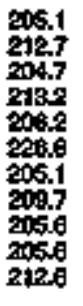 & $\begin{array}{l}205.1 \\
2012.7 \\
204.7 \\
2113.3 \\
204.9 \\
202.8 \\
200.1 \\
200.9 \\
201.4 \\
200.3 \\
212.4\end{array}$ & $\begin{array}{l}205.0 \\
212.7 \\
204.9 \\
218.2 \\
204.6 \\
218.6 \\
20.4 .1 \\
200.7 \\
205.4 \\
206.0 \\
212.1\end{array}$ & 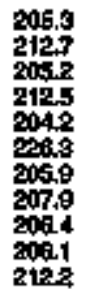 & $\begin{array}{l}205.3 \\
212.6 \\
206.2 \\
212.1 \\
204.1 \\
200.1 \\
205.4 \\
200.6 \\
200.6 \\
200.6 \\
212.3\end{array}$ & $\begin{array}{l}206.3 \\
212.7 \\
20.5 \\
218.3 \\
204.1 \\
212.2 \\
200.2 \\
206.5 \\
206.6 \\
200.1 \\
212.5\end{array}$ & $\begin{array}{l}205.9 \\
214.7 \\
205.4 \\
212.1 \\
204.5 \\
20.5 .7 \\
200.5 \\
206.5 \\
200.5 \\
200.5 \\
212.5\end{array}$ & $\begin{array}{l}205.5 \\
2127 \\
205.2 \\
2121 \\
208.1 \\
- \\
208.0 \\
2010.1 \\
200.8 \\
206.0 \\
212.6\end{array}$ \\
\hline U,S Averages & 205.9 & 2059 & 208.0 & 205.9 & 20.2 & 208.4 & 206.5 & 20084 & 2064 & $\mathbf{2 0 8 . 8}$ & 2008 & 200.9 & 207.1 & 207.0 & 2072 \\
\hline
\end{tabular}


Table F4. Average Carbon Dloxłde Emission Factors for Coal Consumed by Electric Utilities, 1980-1994 (Pounds of Carbon Dtoxide per Million Btu)

\begin{tabular}{|c|c|c|c|c|c|c|c|c|c|c|c|c|c|c|c|}
\hline Enth & 1500 & ts:s & 1000 & tots & 1064 & 1995 & 1800 & 1060 & 1060 & 1500 & 1990 & 1901 & 190 & 1998 & 1894 \\
\hline 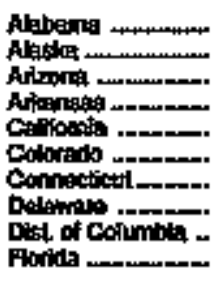 & $\begin{array}{l}2050 \\
214.0 \\
2010 \\
2123 \\
2115 \\
-5 \\
2050 \\
2040\end{array}$ & $\begin{array}{l}204.9 \\
2140 \\
200.1 \\
212.7 \\
-5 \\
2095 \\
- \\
208.1 \\
204.1\end{array}$ & $\begin{array}{l}2049 \\
2140 \\
2000 \\
212.7 \\
\vdots \\
2097 \\
200.2 \\
2040\end{array}$ & $\begin{array}{l}205.0 \\
214.0 \\
2079 \\
212.7 \\
211.0 \\
200.4 \\
204.5\end{array}$ & $\begin{array}{l}2050 \\
2140 \\
2070 \\
212.7 \\
212.1 \\
204.6 \\
206.5 \\
204.4\end{array}$ & $\begin{array}{l}2049 \\
2140 \\
2078 \\
212.7 \\
2120 \\
20048 \\
206.5 \\
204.3\end{array}$ & $\begin{array}{l}2050 \\
2140 \\
2079 \\
212.7 \\
211.9 \\
204.8 \\
200.1 \\
204.3\end{array}$ & $\begin{array}{l}2049 \\
2140 \\
2080 \\
2127 \\
201 \\
2048 \\
2060 \\
204.4\end{array}$ & 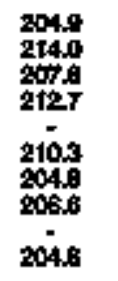 & $\begin{array}{l}204.9 \\
2140 \\
207.9 \\
212.7 \\
210.9 \\
204 . \\
200.7 \\
204.5\end{array}$ & $\begin{array}{l}205.1 \\
214.0 \\
297.7 \\
211.0 \\
-1.0 \\
209.4 \\
201.4 \\
200.7 \\
204.5\end{array}$ & $\begin{array}{l}205.3 \\
2140 \\
207.7 \\
212.7 \\
200.9 \\
204.6 \\
20.9 \\
20.4 .4\end{array}$ & $\begin{array}{l}2053 \\
2140 \\
207.7 \\
212.7 \\
2009 \\
204,9 \\
200.9 \\
204,4\end{array}$ & $\begin{array}{l}205.3 \\
214.0 \\
207.5 \\
212.7 \\
209.9 \\
205.0 \\
200.9 \\
204.5\end{array}$ & $\begin{array}{l}205.3 \\
207.5 \\
212.7 \\
209.7 \\
20.5 \\
207.4 \\
201.8\end{array}$ \\
\hline 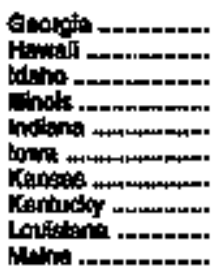 & $\begin{array}{l}204.3 \\
= \\
207.1 \\
2016 \\
207.2 \\
209.2 \\
204.0 \\
212.7\end{array}$ & 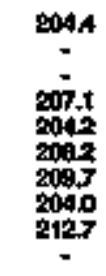 & $\begin{array}{c}2044 \\
\vdots \\
2015 \\
2042 \\
210.3 \\
210.8 \\
2040 \\
212.7\end{array}$ & $\begin{array}{c}204.4 \\
\vdots \\
206.5 \\
2042 \\
210.5 \\
210.9 \\
2040 \\
212.6\end{array}$ & $\begin{array}{c}204.7 \\
\vdots \\
205.9 \\
204.3 \\
210.8 \\
2106 \\
204.0 \\
212.7\end{array}$ & $\begin{array}{l}204.7 \\
\vdots \\
20196 \\
2040 \\
200 \\
2105 \\
2003 \\
212.7\end{array}$ & $\begin{array}{c}204.7 \\
: \\
200.6 \\
2047 \\
210.1 \\
210.7 \\
2041 \\
212 .\end{array}$ & $\begin{array}{c}604.7 \\
: \\
206.1 \\
201.5 \\
210.4 \\
210.6 \\
204.0 \\
212.7\end{array}$ & $\begin{array}{c}204.0 \\
\vdots \\
206.3 \\
204.8 \\
209.9 \\
210.7 \\
204.4 \\
212.7\end{array}$ & $\begin{array}{c}2040 \\
\vdots \\
205.8 \\
20050 \\
210.5 \\
2104 \\
2040 \\
212.7 \\
-\end{array}$ & $\begin{array}{c}200.2 \\
- \\
- \\
206.6 \\
205.4 \\
210.7 \\
210.7 \\
204.1 \\
212.7 \\
-\end{array}$ & $\begin{array}{c}205.2 \\
\vdots \\
205.9 \\
205.8 \\
210.6 \\
210,8 \\
204.3 \\
212.9 \\
=\end{array}$ & $\begin{array}{c}204.9 \\
- \\
- \\
208,2 \\
000,6 \\
211.1 \\
210,8 \\
204.1 \\
212.9 \\
-\end{array}$ & $\begin{array}{c}205.3 \\
\vdots \\
20.5 \\
200.8 \\
211.9 \\
211.9 \\
204.3 \\
212.9\end{array}$ & $\begin{array}{l}200,2 \\
\vdots \\
2073 \\
2000.0 \\
211.5 \\
211.4 \\
204.5 \\
212,0\end{array}$ \\
\hline 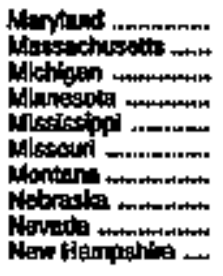 & $\begin{array}{l}200.6 \\
20.4 \\
200.0 \\
212.9 \\
204.7 \\
204.5 \\
213.9 \\
21.7 \\
209.2 \\
206.5\end{array}$ & $\begin{array}{l}200.7 \\
206.5 \\
200.6 \\
213.0 \\
204.9 \\
2046 \\
213.9 \\
212.2 \\
207.9 \\
207.5\end{array}$ & 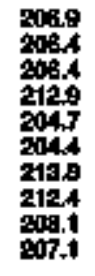 & $\begin{array}{l}207.2 \\
20.5 \\
207.1 \\
213.0 \\
204.9 \\
204.2 \\
214.0 \\
219.8 \\
207.8 \\
207.4\end{array}$ & 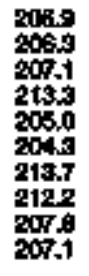 & $\begin{array}{l}206.9 \\
200.4 \\
2007.0 \\
213.0 \\
205.2 \\
2004.4 \\
2128 \\
2124 \\
203.3 \\
2068\end{array}$ & $\begin{array}{l}207.4 \\
2015 \\
207.5 \\
212.0 \\
204.9 \\
204.4 \\
213.6 \\
212.4 \\
207.6 \\
206.6\end{array}$ & 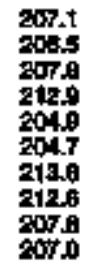 & $\begin{array}{l}207.9 \\
205.7 \\
207.9 \\
213.0 \\
204.6 \\
205.4 \\
213.5 \\
212.8 \\
209.2 \\
206.9\end{array}$ & $\begin{array}{l}207.0 \\
20.4 \\
201.5 \\
213.0 \\
204.2 \\
20.5 \\
213.6 \\
212.0 \\
207.9 \\
201.7\end{array}$ & $\begin{array}{l}207.2 \\
200.5 \\
200.7 \\
2100 \\
204.9 \\
205.7 \\
213.6 \\
212.7 \\
207.7 \\
206.7\end{array}$ & $\begin{array}{l}207.2 \\
206.7 \\
2008.9 \\
2130 \\
204.5 \\
208.2 \\
213.6 \\
212.7 \\
200.0 \\
20.2\end{array}$ & 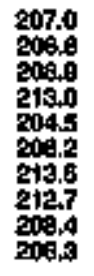 & $\begin{array}{l}2068.8 \\
203.6 \\
209.4 \\
213.0 \\
204.8 \\
209.4 \\
219.8 \\
212.7 \\
202.4 \\
201.3\end{array}$ & $\begin{array}{l}207.0 \\
208.7 \\
209.0 \\
213.0 \\
20.9 \\
209.5 \\
213.5 \\
212.8 \\
200.4 \\
20.1\end{array}$ \\
\hline 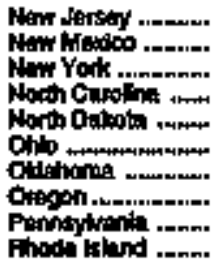 & $\begin{array}{l}200.6 \\
205.7 \\
205.7 \\
205.6 \\
219.6 \\
20.4 .4 \\
210.5 \\
212.7 \\
203.1\end{array}$ & $\begin{array}{l}200.8 \\
200.7 \\
20.58 \\
20.68 \\
213.8 \\
204.3 \\
212.5 \\
212.7 \\
200.0 \\
*\end{array}$ & $\begin{array}{l}200.7 \\
205.7 \\
2058 \\
2008 \\
20.8 \\
210.8 \\
204.5 \\
2127 \\
212.7 \\
200.4 \\
7\end{array}$ & $\begin{array}{l}200.8 \\
207.7 \\
2058 \\
200.0 \\
210.8 \\
204.8 \\
212.7 \\
2127 \\
200.4 \\
-\end{array}$ & 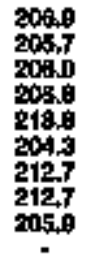 & 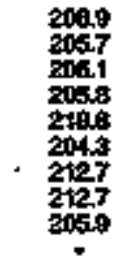 & $\begin{array}{l}200.9 \\
205.7 \\
20.1 \\
205.8 \\
210.6 \\
204.4 \\
212.5 \\
205.0 \\
-\end{array}$ & $\begin{array}{l}200.0 \\
205.7 \\
201.2 \\
205.8 \\
219.8 \\
204.4 \\
212.1 \\
212.7 \\
200.0 \\
7.0\end{array}$ & $\begin{array}{l}200.6 \\
205.7 \\
200.9 \\
205.9 \\
216.9 \\
2045 \\
212.2 \\
212.7 \\
200.1 \\
-.\end{array}$ & $\begin{array}{l}200.7 \\
206.7 \\
200.7 \\
20,7 \\
211.3 \\
202.5 \\
2120 \\
212.7 \\
200.1 \\
?\end{array}$ & $\begin{array}{l}206.7 \\
206.9 \\
206.3 \\
205.9 \\
214.9 \\
204.5 \\
212.1 \\
212.7 \\
208.2 \\
.\end{array}$ & $\begin{array}{l}201.7 \\
205.7 \\
200.2 \\
205.9 \\
210.9 \\
204.4 \\
212.4 \\
212.7 \\
200.2\end{array}$ & 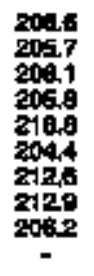 & $\begin{array}{l}206.7 \\
205.7 \\
200.0 \\
206.8 \\
216.9 \\
204.5 \\
212.7 \\
212.4 \\
200.0 \\
-\end{array}$ & 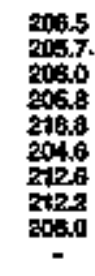 \\
\hline 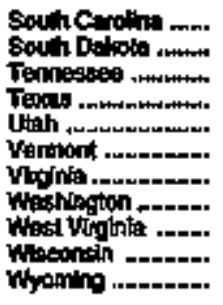 & $\begin{array}{l}204.9 \\
219.4 \\
204.0 \\
213.0 \\
204.1 \\
205.3 \\
205.9 \\
209.7 \\
206.9 \\
201.0 \\
212.7\end{array}$ & $\begin{array}{l}204.9 \\
216.1 \\
2040 \\
212.9 \\
204.1 \\
205.7 \\
200.7 \\
200.7 \\
201.9 \\
20010 \\
2127\end{array}$ & $\begin{array}{l}2050 \\
2190 \\
2040 \\
2120 \\
204.1 \\
205.7 \\
205.8 \\
200.7 \\
207.0 \\
207.7 \\
2+2.7\end{array}$ & $\begin{array}{l}2050 \\
2198 \\
204.0 \\
2120 \\
204.1 \\
204.7 \\
200.0 \\
200.7 \\
207.0 \\
207.8 \\
207.7\end{array}$ & 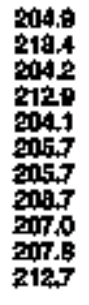 & $\begin{array}{l}204.9 \\
2+29 \\
204.8 \\
2+2.9 \\
204.1 \\
206.7 \\
205.0 \\
200.7 . \\
200.0 \\
200.0 \\
212.7\end{array}$ & $\begin{array}{l}205.0 \\
211.9 \\
204.0 \\
212.9 \\
204.5 \\
206.7 \\
200.0 \\
20.7 \\
207.1 \\
20.3 \\
212.7\end{array}$ & $\begin{array}{l}205.0 \\
219.8 \\
204.1 \\
212.9 \\
204.3 \\
20.9 \\
209.8 \\
207.4 \\
20.9 \\
212.7\end{array}$ & $\begin{array}{l}205.0 \\
218.3 \\
204.1 \\
213.5 \\
204.2 \\
= \\
206.1 \\
209.0 \\
207.2 \\
209.3 \\
212.7\end{array}$ & 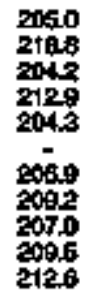 & 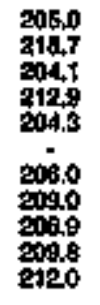 & $\begin{array}{l}205.0 \\
216.8 \\
2040 \\
212.9 \\
204.3 \\
-50 \\
200.0 \\
200.7 \\
207.1 \\
200.7 \\
212.0\end{array}$ & $\begin{array}{l}206.0 \\
218.8 \\
2040 \\
212.9 \\
204.3 \\
-5 \\
20000 \\
20093 \\
207.0 \\
200.0 \\
218.0\end{array}$ & $\begin{array}{l}206.0 \\
218.0 \\
201.3 \\
212.0 \\
204.3 \\
205.9 \\
209.4 \\
207.4 \\
210.7 \\
212.0\end{array}$ & $\begin{array}{r}205.0 \\
218.0 \\
204.1 \\
213.1 \\
204.0 \\
205.9 \\
.209 .2 \\
207.4 \\
210.4 \\
211.0\end{array}$ \\
\hline U.S. Averagen ..-. & 208.7 & 2069 & 207.0 & 2007.1 & 2017,1 & $200 \pi$ & 2073 & 207.3 & 207.6 & 207.6 & 207.6 & 207.7 & 207.7 & 2078 & 207.0 \\
\hline
\end{tabular}

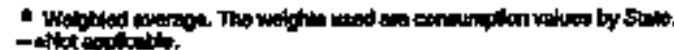

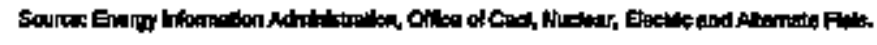


Table F5. Average Carbon Dibxide Emiscion Factora for Total Cos' Consumed, 1980-1994

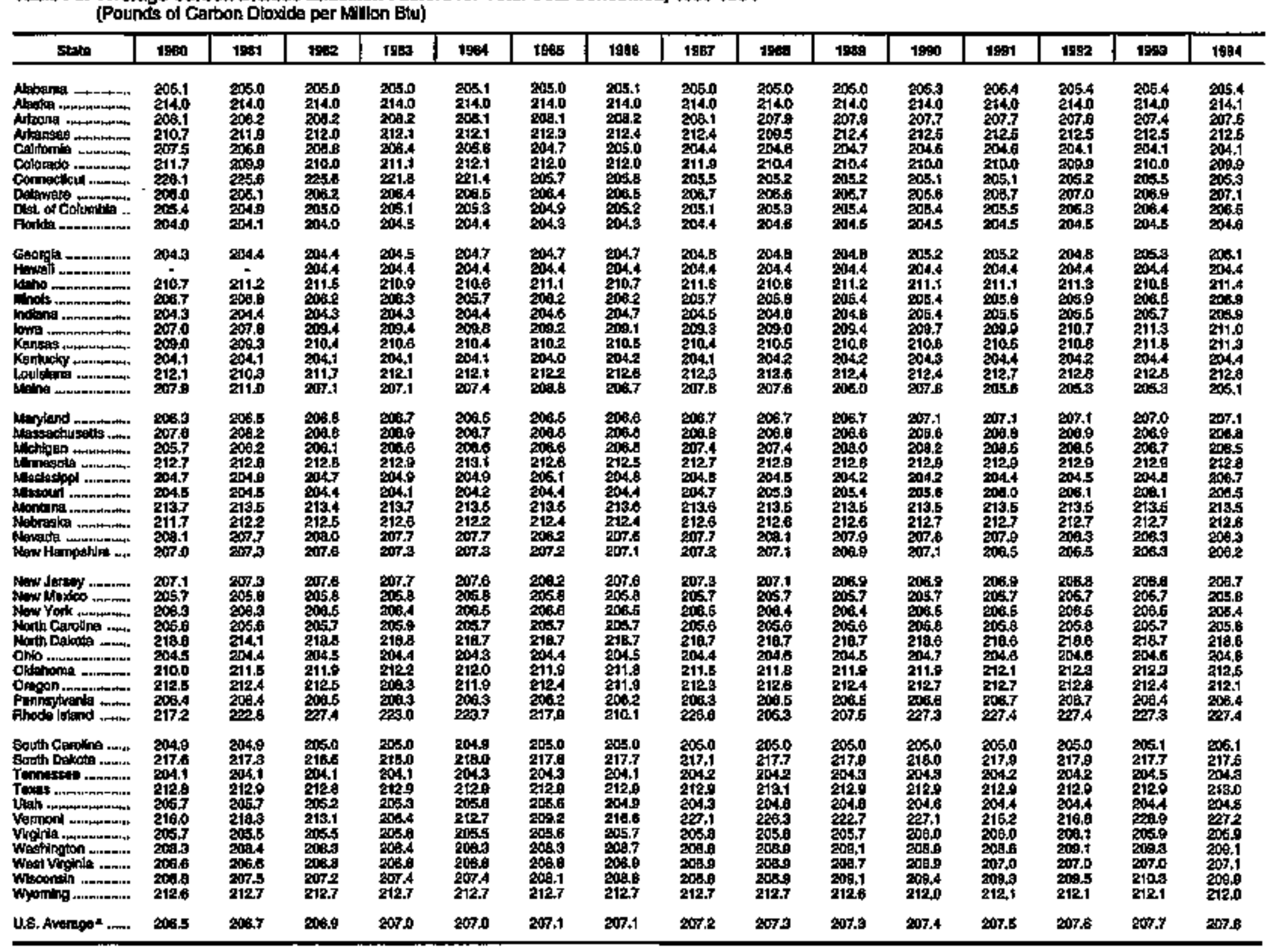


Appendix G

Summary of Changes Since the State Energy Data Report 1993 



\section{Summary of Changes Since the State Energy Data Report 1993}

\begin{abstract}
Modifications to the State Fnergy Data System (SPDS) that are incorporated into this edition of the Sinte Eretgy Data Report (SEDR) are summarized in this appendix. The constraint of page stze in SEDR does not allow for all 35 years of SEDS data to be included in the published tables. Data for 1961 through 1964 and 1966 through 1969 are not shown in the tables. Those data are inchuded on the personal computer diskettes and would be covered by this section of documentation, although no changes were made to those data in this year's update.
\end{abstract}

The majority of the changes in methodology and to the variable rames in this ectition of SEDS are due to the incorporation of renewable energy sources in the residential, commercial, industrial, and transportation sectors and the augmentation of revewable data in the electric utility sector for 1990 through 1994. The additional data added approximately 3 quadrillion Btu to the national total consumption each year.

\section{Renewable Energy Sources, 1990 through 1994}

\section{Biotuels}

Residential and Commersial Sectors. Wood consumption in the residential and commercial sectors combined for 1990 through 1994 are shown in SEDR residential sector tables and axe identified in the SEDS system with the mnemonics WWHCP (in thousand cords) and WWHCB (in billion Btu). All States are estimated to have some residential and commercial wood consumption; California and New
York, are the largest State consumers, together accounting for 19 percent of the U.S. total each year.

Industrial sectox. Wood and waste energy used in the industrial sector is included in SEDS in billion Btu for 1990 through 1994. The corresponding data in physical units are not available because of the variety of energy forms used (e.g., tons, cubic feet, and kilowatthours). All States are estimated to have some industrial use of biofueis, with the exception of the District of Columbia. North Dakota registered a small amount for the first time in 1994. Wisconsin was the largest industrial biofuels consumer followed, in differing order, by Alabama, California, Louisiana, Maine, and Washington during the 5-year period. The U.S. total ranged from 1,943 trillion Btu in 1991 to 2,152 trillion Beu in 1994. Wisconsin's consumption of 166 trillion Btu in 1991 to 184 trillion Btu in 1994 was the largest State industrial sector use of biofuels each year.

Transportation Sector. Ethanol used as an additive to motor gasoline is added to SEDS and shown in SEDR tables. The quantities of ethanol consumed are already contained in the motor gasoline data; therefore, the values for ethanol are not added into the transportation sector or total energy consumption totals, and they cause no data revisions.

Electric Utllities. Estimates of wood and waste consumption at electric utilities are now available separately in SEDS from 1982 forward. The data identified as WWEO in SEDS, which had been input into the system, are now the sum of data identified with two new mnemonics, WDEOP, for wood consumption, and WSEOP, for waste 
consumption, at electric utilities. Access to the data from the Form EIA-759 database made it possible to obtain additional decimal places of accuracy in 1982, and recalculating the data for 1982 through 1993 caused revisions in the last decimal place for a few States in several years.

\section{Geothermal}

Industrial Sector. Geothermal energy used by nonutility power producers to produce electricity is incluted in SEDS industrial sector consumption in billion Btu for 1990 through 1994. The corresponding date in million kilowatthours are not available. California, Hawaii, and Nevada are the three States with industrial geothermal energy use. Each State's geothermal-based electricity production has increased each year, causing the U.S. total to increase from 146 trillion Btu in 1990 to 212 trillion Btu in 1994. Although Califorria's portion was the largest, that share decreased other the period from 86 percent of the U.S. total in 1990 to 82 percent in 1994 .

Electricity Imports. Prior to 1990, all electricity imports and exports are assumed to be hydraelectric in origin. Beginning with the 1990 data, imports of electricity based on geothermal energy in Mexico and imported into California are identified separately. These quantilies range from 538 thousend kilowatthours in 1990 to 1,172 thousand kllowatthours in 1994.

\section{Hydroelectric Power}

Industrial. Data on the industrial use of hydroelectric power in SEDS for 1960 through 1978 are collected by the Federal Power Commission. The data for 1979 and 1980 are estimated on the basis of the 1978 data. The estimates for 1980 are repeated each year for 1981 through 1989. In this edition of SEDS, beginning with the 1990, data on industrial hydroelectric power use are collected from nonutility power producers on Form ELA-867. The data are entered in SEDS in billion Btu and the comparable values in million kilowatthours are not available. For 1990 through 1993, California, Maine, and New York were the'three largest users of hydroelectric power in the indurstriel sector among the 32 States reporting some industrial use. In 1994, North Carolina reported the largest industrial electricity generation from hydropower, 20 trillion Btu of the U.S. total of 136 trillion Btu. Nonutility power production from hydroelectric power in the nation as a whole has grown each year from 82 trillion Btu in 1990 .

Electric Uttlitles. Although estimates of electricity produced from hydroelectric power at electric utilities are not changed in SEDS, the portion of the hydroelectricity that is generated from purnped storage hydroelectric power is now identified separately from conventonal hydroelectric power for 1990 forward. The data series identified as HYEO in SEDS, which had been input into the system, is now the sum of two data ereries identified as HPEO (hydroelectricity from pumped storage) and HVEO (conventional hydroelectricity). Recakulating the data for 1990 through 1993 caused revisions in the last decimal place for a few States in several years.

Electricity Imports and Exports. Prior to 1990, all electricity imports and exports are assumed to be hydroelectric in origin. Beginning with the 1990 data, electricity produced from hydropower is identified separately from geothermal and nonrenewable energy sources. This change caused revisions to hydinelectricity net im ports data for 18 to 22 States in 1990 through 1993 . The largest percentage changes occurred in Michigan, Minnesota, and Texas, where large exports of electricity produced from norrenewable energy sources are no longer being included in the hydroelectricity net imports data.

\section{Solar}

Residential and Commercial Sectors. All States and the District of Columbia are estimated to have some solar energy use in the residential and commercial sectors combined for 1990 through 1994. The largest tser is estimated to be Florida, with a range of 23 trillion Btr in 1990 to 26 trillion Btu in 1994. Florida's solar energy consumption accounts for 47 percent of the U.S. total in 1990 through 1992 and 48 percent in 1993 and 1994. The next largest user of residential and commercial solar energ $y^{\prime}$ is California, consuming trom 16 trillion Btu in 1990 to 18 trillion Btu in 1994. Arizona was the third largest residential/commercial solar energy consumer, using about 3 trillion Btu in each of the 5 years. 
Industrial. California was the only State with solar energy use reported by the nonutility power producers in the industrial sector for 1990 through 1994. The values, reported in billion Btu because comparable million kilowatthour data are not available, ranged from 7,000 billion Bta in 1990 to a high of 9,219 billion Btu in 1993 and decreased to 8,464 billion Btu in 1994.

Electric Utilities. Although electricity generation from photovolbic and solar thermal energy at electric utilities has not changed, the data, which were previously combined with wind energy in SEDS, are now available separately from 1984 forward and are identified with the mnemonic SORO. California was the first State with solar energy use at electric utilities, joined by Virginia in 1988 and by Texas in 1989. Electric utilities in California produced over 80 percent of the solar-based electricity consumed in the United States each year.

\section{Wind}

Industrial. Wind energy used by nonutility power producers to produce electricity is included in the industrial sector consumption in billion Btu for 1990 through 1994. The corresponding data in million kilowatthours are not available. Callfornia and Hawail were the conly States recording industrial wind use for all 5 years; nonutilities in Oregon reported some wind use in 1990 and 1991; and Minnesota began industrial use of wind energy in 1994. Each State's wind-based electricity production has increased each year, causing the U.S. total to ixcrease from 24 trillion Btu in 1990 to 36 trillion Btu in 1994. California's portion was the largest, accounting for 99 percent of the U.S. total in 1990 through 1993 and 98 percent of the total in 1994.

Electric Uttlities. Although electricity generation from wind energy at electric utilities has not changed, the data, which were previously combined with photovoltaic and solar thermal energy in SEDS, are now available separately from 1983 forward and are identified with the muemonic WYEO. California, Oregon, and Wyoming were the first States with wind energy use at electric utilities. By 1994, Iowa, Kansas, and Minnesota were the only States with electric utilities using wind energy. During the intervening years, electric utilities in Alaska, Montana, North Dakota, Vermont, and Wisconsin tried some use of wind energy for electricity production. Wind energy use at electric utilities peaked at 68 billion Btu in 1984 and decreased to 3 billion Btu by 1994.

\section{Renewable Energy Totals}

Renewable energy sources account for about 6 quadrillion Btu of the total energy consumption in SEDS for 1990 through 1994. Of the renewables, approximately 3 quadrillion Btu have been included in the electric utility and transportation sectors in previous editions of SEDS. In this edition, the additional 3 quadrillion Btu, over 75 percent of which are in the industrial sector, are included by energy source. Although estimated consumption of renewable energy for the residential and commercial sectors combined during the 5 yearperiod peaked in 1992 at 697 trillion Bhu, total tuse of renewables in the U.S. was at the lowest point that year, at 6,097 trillion Btu. Total U.S. consumption of renewable energy peaked in 1993 at 6,397 trillion Btu. Industrial sector renewable use grew steadily from 2,207 trillion Btu in 1990 to 2,544 trillion Btu in 1994.

California ranked the largest in total renewable energy use, having the greatest use of xenewrables among the States' residential $/ \mathrm{com}-$ mercial and industrial sectors. The additional data on renewables consumption caused Calfornia's total energy consumption estimates to increase by 5 percent in 1990 and 1991 and 6 percent in 1992 and 1993. Washington was the.second largest consumer of renewable energy sources during the 1990-through-1994 period, with its electric utilities using at least 50 percent more than the second-place electric utilities in California. Washington's total energy consumption estmates increased by 6 percent or 7 percent in 1990 through 1993 with the addition of the renewables date. New York was the third-largest consumer of renewable energy sources during the 5 -year period because of its residential/commercial sector use, which was second to the residential/commercial sector use in California. New York's total energy consumption was increased by 4 percent each year in the period 1990 through 1993.

The additional renewables consumption data from nonutility power producers caused Maine 's total energy consumption estimates to increase by 40 percent to 42 percent in 1990 through 1993, the largest State increase due to the new renewables data. New Hampshire's reaidential, commercial, and industrial sectors' additional 
renewables data contributed to that State's tolal energy consumption increase of 14 percent in 1990 and 1991 and 15 percent in 1992 and 1993, the second largest State percentage increase in SEDS. The additional renewables data from nonutility power producers in Wisconsin caused Wisconsin's total energy consumption estimates to increase by 13 percent or 14 percent in 1990 through 1993.

\section{Electricity Sales, 1992 and 1993}

All Sectors. Small revisions in electricity sales to all end-use sectors in 1992 and 1993 were made for a few States as published in the BIA Electric Power Arnual (EPA) 1993 and EPA 1994, respectively. In 1992, commercial sector sales were revised in Nebraska, New York, and Oklahoma by less than 1 million kilowatthours. In 1993, electricity sales to the commercial sector were revised for nine States, the largest revision being a shift of 4 million kilowatthours from Kentucky to Tennessee. Maryland's commercial electricity sales decreased by 3 million kilowathours, while New Jersey's increased by 2 million kilowatthours and revisions to the other five States were by less than 1 million kilowatthours in 1993. These revisions affected the SEDS calculations of commercial and transportation sector electricity consumption estimates for all States by amounts too small to be seen in SEDR tables, but they are noticeable in the full precision data on the SEDS personal computer diskettes. Industrial electricity consumption was revised for 7 States in 1992, the largest being a 12million kilowatthour increase in Tennessee, followed by a 5-million kilowatthour increase in Alabama and 3-million kilowatthour increases in Mississippi and Kentucky. Data on industrial sales of electricity were revised in 5 States in 1993, most notably the 9-million kilowathour shift from Kentucky to Temessee, a 7-million kilowatthour increase in Alabama, and a 6-inillion kilowatthour increase in Maryland. Residential sector sales in 1992 were revised in Nebraska, New York, and Oklahoma by less than 1 million kilowatthours. In 1993, residential electricity consumption was revised for 10 States by 3 million kilowatthours or less, with the exception of the 5-midition kilowathour increase in Alabama.

In addition to the revisions in EPA data, preliminary estimates of electricity sales to transit systems in 1993 were finalized by the U.S. Departinent of Transportation, Uxban Mass Transportation Admini- stration. Those changes caused revisions to the transportation and commercial sector in the District of Columbia and the 18 States with transportation electricity use, but the U.S. totals for the two sectors were not affected. The most noticeable revisions were a 16-million kilowathour shift from the transportation sector to the commercial sector in Pennsylvania and 12-million kilowatthour shifts from the commercial sector to the transportation sector in California and Maryland. The other States' revisions were by 6 million kilpwatthours or less.

These revisions to the estimates of electricity use in all end-use sectors caused proportional revisions to the estimated electrical system energy losses for the sectors and total losses and net interstate flow for the affected States.

\section{Electriclty Imports and Exports, 1990 through 1994}

Flectricity imports and exports are assumed to be produced from hydroelectric power in SEDS from 1960 through 1989 and are included in the "Fydroelectric Power" columns in the SEDR tables. Beginning with the 1990 data, electricity imports and exports produced from geothermal energy, hydropower, and nonrenewable energy souxces are available separately in SEDS. Although the data for total net imports, measured in million kilowatthours, has not changed, the geothermal-based portion is added to data shown in the "Geothermal" columns, the hydropower-based portion is shown in the "Hydroelectric Power" columus, and the remaining nonrenewablebased portion is added into the "Total" columns in the SEDR tables. The corresponding values in billion Bhu are also combined with data in the three columns, and since the geothermal net imports are converted from million kilowathours to billion Btu by using the geow thermal conversion factor, the sum of the three portions in Btu for each year is slightly different from the previous total Btu value. The electricity imports and exports data are not shown separately in SEDR tables or on the personal computer diskettes, but they are available on request. The use of the geothermal conversion factor increases the US. total net imports of electricity by amounts ranging from 6 trillion Btu in 2990 to 12 trillion Btu in 1994. 


\section{Petroleum Produots}

\section{Aviation Gasoline, 1953}

A data entry erfor for aviation gasoline consumption by the military in 1993 was cotrected in SEDS. The change revises consumption in Alaska from 79 barrels to zero and consumption in Alabama from zero to 79 barrels. The recalculation caused rounding differences in other State datz in the last decimal place and all revisions are too small to be seen in the SEDR tables due to the level of rounding, but may be noticed in the full precision data on the personal conputer diskettes.

\section{Kerosene-Type Jet Fuel, AN Vears}

Although no data changed for kerosene-type jet fuel in SEDS, the name of the State-level data series used to alkcate the U.S. total consumption to the States was changed to more accurately reflect the data. JKNMP (kerosene-type jet fuel non-military consumption) was changed to JKTTP (kerosene-type jet fuel total sales). The use of kerosene-type jet fuel by the military has always been included in the data and, as military use increases, the previous name is less representative of the data.

\section{Lqueffed Petroloum Gases, 1998}

The American Petroleum Institute revised industrial sector sales of liquefied petroleum gases (LPG) for seven States for 1993. The sales increases in Iowa, llinois, Louisiana, and Mississippi offset the decreases in Kansas, Michigan, and Texas so that the U.S. industrial sector subtotal and the U.S. total sales did not change. The revisions were as large as 79 percent in Illinois and 69 percent in fowa and as small as 2 percent in Louisiana and 3 percent in Texas.

\section{Other Petroletw Products}

Six petroletum products that are components of the "Other" cohumn in the "Petroleum" portion of SEDR tables are estimated by using State-level data from the U.S. Department of Commerce, Buresu of the Census, Census of Manufactures, Industry Series. The 1992 Census data, recently available, is used to replace 1987 Census data in the estimation calculations for 1991 through 1993 in SEDS and revistons to the 1987 Census data were incorporated into the calculations for 1986 through 1990 data in SEDS.

U.S. total consumption of petroleum feedstocks, both naphtha and other oils, pertanes plus, and the miscellaneous petroleum products category of numerous products is allocated to the States by using the Census Bureau data for the value added in the manufacture of organic chemicals (OCVAV in SEDS). U.S. total consumption of special naphthas is allocated to the States by using the Census series for the value added in the manufacture of paints and vamishes (PIVAV in SEDS). The Census series used to allocate waxes was redefined to include manufacturing activities not related to waxes. A different Census series considered to be equally representative of waxes consumption is used as the allocating variable CGVAV in SEDS for 1991 through 1994.

The revisions to the 1987 Census allocating seties for OCVAV and PIVAV caused revisions to all 33 States in 1986 through 1990 with industrial consumption of these petroleum products. The revisions in 1986 through 1991 were consistent among the States-15 States experienced a 21-percent decrease and the remaining States' revisions were 2 percent or less.

The revisions to estimlates for 1991 through 1993, caused by the replacement of the 1987 Census data with the 1992 Census and the use of a different series for allocating waxes, were lerger than the revisions for earlier years for the 45 States affected. Three States-ldaho, Maine, and South Dakota- which previously were estimated to have no consumption of these six petroleum products in their industrial sectors, now are estimated to consume small amounts. Vermont, which previously had a small amount, is now estimated to have none. Estimates of consumption in Arizona more than doubled and Kentucky's increased by over 84 percent for 1991 through 1993. Other petroleum products industrial consumption in Nebraska was decreased by 93 percent in the 3 years, while Missouri's consumption decreased by approximately 50 percent and lowa's decreased by about 40 percent. 


\section{Natural Gas}

Residential, Commercial, and Industrial Sectors, 1993. The EIA Natural Gas Anntal 1994 published revisions to the 1993 data in the residential, commercial, and industrial sectors. The revisions to Florida and Iowa residential natural gas consumption were less than 1 billion cubic feet. Commercial sector utse of natural gas in Florida, Iowa, Kansas, Nebraska, South Carolina, Texas, and West Virginia was revised by less than 1 percent, except for the 22-percent decrease in Texas. Industrial use of natural gas was revised for 1993 in Florida, Iowa, Louisianta, and Nebraska by less than 1 percent. West Virginia's 1993 industrial natural gas consumption was decreased 10 percent, while South Dakoka's industrial consumption was increased by 5 percent and use in Texas was ircreased by 2 percent. The revisions to West Virginia's natural gas consumption caused the estimated average heat content to be revised from 1.064 Bto per cubic foot to 1.065 Btu per cubic foot.

Industrial Sector, 1993. Beginning with the 1993 data, the EIA Nattrul Gas Avnutul (NGA) publishes the separate components of the previous data series "Lease and Plant Fuel." SEDS 1994 is modified to assign different data identifiers to the separate series, "NGLE" (natural gas consumed as lease fuel) and "NGPL" (natural gas consumed as plant fuel). The combined data for 1960 through 1992, previously identified in SEDS as "NGLP," are now stored under the lease fuel mnemonic "NGLE." NGA revisions to the 1993 lease fuel data for Louisiana, Sotth Dakota, and West Virginia were also incorporated into SEDS 1994
Commercial and Transportation Sectors, 1990 through 1998. Beginning with the 1990 data, the EIA Natural Gas Annual published natural gas consumed as vehicle fuel separately. Prior to 1990, vehicle fuel use was reported with commercial sector consumption and could not be separately identified. In this edition of SEDS, the vehicle fuel data, identified as NGVZP, ate removed from the commerctal sector and are added to the transportation sector for 1990 through 1993. The shift of 255 million cubic feet or less in both sectors of the 37 States reporting natural gas vehicle fuel use may be noticed in the date on the SEDS personal computer diskettes, but they are too small to be seen in SEDR tables due to the level of rounding.

\section{Population (TPOPP), 1990 through 1993}

The U.S. Department of Commerce, Bureau of the Census resident population estimates for 1990 through 1993 were revised and are available via Internet in greater precision than in previous SEDS for all States, the District of Columbia, and the U.S. total. The incorporation of the new data caused revisions of 0.2 percent or less for all States, the District of Columbia, and the U.S. in all 4 years. These data do not appear in SEDR tables but they are included for 1960 through 1994 on the personal computer diskettes and are used in the calculation of the data shown in the "Total Energy per Capita" ranking column of Table 9. 
Appendix $\mathrm{H}$

State Data in Other EIA Reports 


\section{State Data in Other EIA Reports}

Readers of the State Energy Dula Report may be interested in the following ELA reports containing State-level data on these subjects:

\section{Multiple Energy Sources}

State Energy Price and Expenditure Report 1993, DOE/ELA-0376(93). Energy prices and expenditures by energy sources within consuming sectors based on the consumption values estimated in the State Entergy Duta Report 1993.

\section{Petroleum}

Petroleum Supply Monthly, DOE/RIA-0109. Production of crude oil; refinery, bulk terminal, and natural gas plant stocks of selected petroleum products; and tmports of residual fuel by State of entry and stilfur content.

Petroletm Supply Annual, Voitumse 1, DOE/EIA-0340/1. Anmual data on production of cude oil; imports of residual fuel by State of entry and sulfur content; refinery, bulk terminal, and natural gas plant stocks of selected petroleum products; number, capacity and production capacity of operable refineries; working storage and shell storage capacities at refineries; and number and production capacity of operable oxygenate plants.

Petroletna Staply Annual, Voltume 2, DOE/EIA-0340/2. Data series as in Volume 1 shown by month for production of crude oil; refinery, bulk terminal, and natutal gas plant stocks of selected petroleum products; and imports of residual fuel oil by State of entry and sulfur content.

Petroleunt Marketing Montihly, DOE/ELA-0380. Prices of No. 2 distillate to residences; domestic crude oil first purchase price; refiner/reseller sales prices for conventional, oxygenated, reformulated and unleaded regular, midgrade, premium, and all grades motor gasoline by type of seller; refiner sales prices to end users and for resale of aviation gasoline, kerosene-type jet fuel, kerosene, No. 1 distillate, No. 2 distillate, and No. 4 fuel oil; prices of No. 2 distillate for selected States by seller type and end user; prices of No. 2 fuel oil for selected States by end user; residual fuel oil prices for selected States by sulfur content; refiner sales volumes of motor gasoline by seller and by grade; refiner sales volumes of aviation gasoline, kerosene-type jet fuel, propane, kerosene, No. 1 and No. 2 distillates, and No. 4 and residual fuel oils; and prime supplier sales volumes of motor gasoline by prades, aviation gasoline, kerosene-type and naphtha-type jet fuels, propane, residual fuel oil (by sulfur content), kerosene, No. 1 and No. 2 distllates, and No, 4 fuel oil. The explanatory notes contain Federal and State motor fuel taxes.

Petroleum Marketing Amrual, DOE/EIA-0487. Annual and monthly data for prices of No. 2 distillate to residences; domestic crude oil first purchase prices; refiner/reseller sales prices for conventional, oxygenated, reformulated, and urleaded regular, midgrade, premium, and all grades motor gasoline by sellex type; refiner sales prices and volumes to end users and for resale of aviation gasoline, 
A kerosene-type jet fuels, keroseme, No. 1 distillate, No. 2 distillate, and P No. 4 fuel oil; sales No. 2 distillate prices for velected States by seller P fype and end user; sales prices of No. 2 distillate for selected States by E end taser; residual huel oil prices for selected States by sulfur content; prime supplier sales volumes of motor gasoline by seller tope, grade, and formulation, aviation gasoline, kerosene-type and naphtha-type jet fuels, propane, residual fuel oils (by sulfur content), kerosene, distillate fuel oils, and No. 4 fuel oil. The explanatory notes contain Federal and State motor fuel taxes.

Fuel Oit and Kerosene Sales, DOE/EIA-0535 (Annual). Sales and ad-

justed sales of distillate fuel oil, residual fuel oil, and kerosene by enduse sector.

\section{Maturaf Gas}

U.S. Crude Oil, Natural Gas, and Natural Gas Liquids Reseroes, DOE/EIA0216 (Arunual). Crude oil proved reserves and indicated addittonal reserves, reserves changes, and production; total, nonassociated, and associated-dissolved natural gas proved reserves, reserves changes, and production (wet after lease separation); coalbed methane proved reserves and production; reported reserves of natural gas, wet after lease separation, in nonproducing reservoirs; dry natural gas and natural gas liquids proved reserves, reserves changes, and production; and natural gas plant liquids and lease condensate proved reserves and production. Appendix D contains historic reserves statistics, 1977 forward.

Natural Gas Monthly, DOE/ETA-0130. Markefed production of natural gas; gross withdrawals and marketed production; net withdrawals from tunderground storage; activities of underground storage operators; delfveries and average prices to residential, commercial, industrial, electric utility consumers; deliveries to all consumers; average ctty gate prices; and percentage of total deliveries represented by onsystem sales.

Natural Gas Arnural 1994, Volume I, DOE/ELA-0131(94)/1. Natural gas production, transmission, and consumption balance table; gross withdrawals and marketed production; offshore withdrawals; number of producing wells and gas condensate wells; estimated total dry natural

gas proved reserves; wellhead value and marketed production; natural gas processed, liquids extracted, and estimated extraction loss; interstate movements and movements acsoss U.S. borders; additions to and withdrawals from gas storage; tunderground storage capacity; supplemental gas supplies; consumption of nakural gas; number of consumers and quantity of natural gas delivered to consuming sectors, and heat content of total natural gas delivered; natural gas delivered for the account of others to commercial and industrial customers, and electric utilities; firm and interruptible deliveries to consuming sectors; average city gate price; average price of natural gas delivered to consuming sectors including average firm and intertuptible prices; average consumption and annual cost per customer for the residential sector; and summary statistics tables for each State. Appendix A contairs a comparison of electric utilities consumption data from forms EIA-176 and EIA-759; volumes of natural gas "unaccounted for," and natural gas processing plant volumes and composition of liquids extracted, extraction losses, and estimated heat content of extraction losses.

Naiural Gas Annual 1994, Volume 2, DOE/EJA-0131(94)/2, available electronically only. Data for 1967 through 1994 for gross withdrawals and inacketed production; number of producing gas and gas condensate wells; average wellhead price, marketed production, and imputed wellhead value; interstate movements and movements across U.S. borders; changes to underground and LNG storage; supplemental gas supplies 1980 through 1994; production, transmission, and consumption balence table; consuniption of nataral gas; firm and intertuptible deliveries to consumers, 1993 and 1994; quantity and number of consumers of natural gas delivered by sector, and heat content of total natural gas delivered; average price and heat content price of natural gas delivered to consuming sectors; and average consumption end annual cost per consumer in the residential, commercial, and industrial sectors.

\section{Coal}

U.S. Cal Reseroes: An Update by Heat and Sulfur Content, DOE/EIA. 0529. Updates and revisions to the demonstrated reserve base and total inventory of coal sample analyses. Appendix B contains inaccessible and recoverable coal resource factors. Appendix $C$ 
contains estimates of demonstrated, accessible, and recoverable reserve base coal by ranges of sulfur content per Btu and type of mining.

Wekty Coal Production, DOE/EIA-0218. Weekly coal production by region and State.

Quarterly Cool Report, DOE/EIA-0121. Coal production; coke and breeze production; domestic distribution by State of origin, to end-use sectors within State of destination, and by method of transportation; foreign distrlbutlon by State of origin; coal receipts at electric utility plants-quantities at avexage price, contract price and spot price, average cost, and sulfur content; destination of coal received at electric utilities by origin; origin of coal received at electric utilities by destination; coal receipts and average price as coke plants and other industrial plants; total coal receipts and consumption; receipts and consumption by residential and commercial sectors, and other industiral plants; coal carbonized at coke plants, and consumed by electric utilities; total coal stocks; stocks at electric utilltes, coke plants, other industrial plants, and at producers and distributors; and stocks of coke and breeze at coke plants.

Coal Industry Annual, DOE/ELA-0584. Coal production and number of mines by type of mining, and mine production range; bituminous, subbituminous, lignite, and anthracite production by coal group; acreage, production and royalties from federal and indian leases; underground production by mining method; production by type of mining; production and average mine price and real mine price by type of mining, disposition, and coal rank; underground and surface mining productivity; average number of miners working daily by type of mining; recoverable coal reserves and average recovery percentage by type of mining; average daily production, daily productive capacity, and capacity utilization; average price of coal delivered by end-use sector; average quality of coal received by electric utilities and manufacturing and coke plants; status of recoverable reserves from producing federal leases; year-end producer and distributor stocks; imports received by electric utilities and manufacturing and coke plants; consumption and stocks by end-use sector; estimated emissions from electric generation; coal production trends; and rankings for coal consumption, reserves, and production.
State Conl Profiles, DOE/EIA-0576. Coal deposits, production, and use in the 27 coal-producing States. Estimates of reserves by mining method and sulfur content; production, number of mines, miners, productivity, average mine price of coal, disposition, and consumption for selected years. Appendix A contains production and consumption rankings of States and percent of U.S. total.

\section{Electric Power}

Electric Potoer Monthly, DOE/ELA-0226. Net generation by energy source; consumption of fossil fuels by type of fuel; coal and petroleum stocks; receipts and average cost of coal by type; receipls and average cost of petsoleum products by type and sulfur content; receipts of natural gas by type for steam-electric plants with capacity of 50 megawatts or larger; sales of electricity to consuming sectors; revenute from sales to ultimate consumers by sector; and average revemue per kilowatthour by sector.

Electric Power Anmual Volume I, DOE/BLA-10348/1. Number of electricity generators and generating capability, by fuel source and by type of generating unit; planned capability additions at electric utilities by energy source; net generation by type of generating unit and by energy source; consumption and receipts of fossil fuels; stocks of coal and petroleum; average cost of fossil fuel receipts; sales of electricity and number of consumers by end-use sector; revenue from sales and average revenue per kilowatthour by consuming sector.

Electric Power Annual Volume II, DOE/EIA-0348/2. Sulfur dioxide, nitrogen oxides, and carbon dioxide emisstons by type of fossil fuel; number and capacity of generators with environmental equipment by type of equipment for coal-fired generators and for petroleum-and gas-fired generators combined; average quality of fossil fuels burned; average flue gas desulfurization costs; and nonutility electricity supply and disposition for facilities with an installed car pacity of 1 megawatt or larget.

Cost and Quality of Fuels for Electric Utilty Plants, DOE/BIA-0191 (Annual). Data for steam-electric plants with a capactty of 50 megawatts or larger: total heating value and cost of fossil fuels; 
receipts of coal and average cost by sulfur content, origin and destintation of coal receipts; receipts by type of coal; petroleum receipts by product type; gas receipts by type of gas; average cost of coal receipts by type of purchase and type of mining, of petroleum teceipts by type of purchase and product, and of gas receipts by type of ptorchase; coal and petroleum recejpts and average cost by stalfur content and type of purchase; average sulfur content of coal shipped to electric utilities by State of origin; and origin and destination of coal receipts.

Inpentory of Power Plonts in the Lurited Stafes, DOE/FIA-0095 (Annual). Number of generating units, operable capacity, and planned capacity additions by energy source. Information grouped by State (no State totals) on generating wnits that started operation and retired during report year; generating units' capacity, type, energy source, and year of initial operation by company and plant; and projected generating turt changes and additions, 1995-2004, by company and plant; operable renewable generating urits. Appendix C contains fointly owned generating urits by company and plant.

Electric Sales and Reoenute, DOE/ELA-0540 (Annual). Electric sales, revenues, and number of consumers by sector and by utibity class of ownership; and average revenue per kilowatthour by sector and utility class of ownership.

Urantitur Industry Anmual, DOE/ElA-0478. Surface drilling; distribution of reserves by forward-cost category; mine production of uranium; uranium concentrate production; and employment in the uranium industry.

\section{Renewable Energy}

Solar Coltector Manufacturing Activity, DOE/EIA-0174 (Annual). Top five in thermal collector mantufacturing; destinations of shipments of solar thermal collectors.

Geothermal Energy in the Western United States and Hawnit: Resources and Projected Electricity Gemeration Supplies 1991, DOE/EIA-0544. Net capacity, generation, and initial date of operation of geothermal generating plants and utilities planned capacity additions and announced projects. Appendtx B contains projected capacities of hydrothermal rescurce sites. 


\section{Glossary}





\section{Glossary}

Anthracite: A hard, black, hustrous coal containing a high percentage of fixed carton and a low percentage of volatile matter. Often referred to as hard coal. It conforms to ASTM Specification D388-84 for anthuacite, meta-anthracite, and semianthracite.

Asphalt: A dark-brown-to-black cement-like material contatning bitumens as the predominant constltuents. It is obtained by petroleum processing. The definition includes crude asphalt, as well as the following finished products: cements, fluxes, the asphalt content of emulsions (exclusive of water), and petroleum distillates blended with asphalt to make cutback asphalts.

\section{ASTM: The American Society for Testing and Materials.}

Aviation Gasoline: All special grades of gasoline for use in aviation reciprocating engines, as given in ASTM Specification D910 and Military Specification MIL-G-5572. Aviation gasoline includes blending components.

Aviation Garoline Blending Components: Naphthas that are used for blending or compounding into finished aviation gasoline (e.g., straight-run gasoline, alkylate, and reformate). Excluded are oxygenates (alcohols and ethers), butane, and pentanes plus.

Barkel (petrolemum): A unit of volume equal to 42 U.S. gallons.

Barrels per Calendar Day (operable refinery capacity): The maximum number of barrels of input that can be processed during a 24hour period after making allowances for the following limitations: the capability of downstream facllities to absorb the output of crude oil processing facilities of a given refinery (no reduction is made when a planned distribution of intermediate streams through other than downstream facilities is part of a refinery's normal operation); the types and grades of inputs to be processed; the types and grades of products to be manufactured; the environmental constraints associated with refinery operations; the reduction of capacity for scheduled downtime, such as routine inspection, mechanical problems, maintenance, repairs, and turnaround; and the reduction of capacity for unscheduled downtime, such as mestanical problems, repairs, and slowdowns.

Barrele per Stream Day (operable refinery capacity): The maximum number of barrels of input that can be processed in an atmospheric distillation facility running at full capacity under optimal crude and product slate conditions with no allowance for downtime.

Biofuels: Nonfossil biomass energy sources and biomass-derived fuels, which together encompass all energy sources from recent-term organic (plant and animal) matter. Nonfossil biomass energy sources are essentially unprocessed; they are burmed or gasified, as received, to produce thermal energy or electricity. Examples are fuelwood, waste wood, garbage, and crop waste Biomass-derived fuels, on the other hand, result from the processing of blomass energy sources. They may be by-products of industrial or agricuttural processes or they may be fuels made from biomass feedstocks. Biomassderived fuels generally have concentrated energy density and are more easily transported and used. Examples are wood by-products (such as wood chips and dewatered wood liquors), pellets, briquettes, refuse-derived fuel (made from garbage), ethanol (made from crops such as com), and methanol (made from wood). Diffexent mixes of biofuels are used by each consuming sector. The residential and commercial sectors burn wood and pellets for space heating. The industrial sector's largest biofuel soturce is combustible by-products used for electricity generation and process steam, 
followed in importance by wood chips. The transportation sector uses ethanol as an additive to motor gasoline. Some electric utilities use wood, industrial wood waste, and municipal waste as cofiring or primary fuels.

Bituminous Coal: A dense black coal, often with well-defined bands of bright and dull material, with a moisture content usually less than 20 percent. Often referred to as soft coal. It is the most common coal and is used primarily for generating electricity, making coke, and space heating. It conforms to ASTM Specification D383-84 for bituminous coal. In this report, bituminous coal inchudes subbituminous coal.

British Themal Unit (Btu): The quantity of heat needed to raise the temperature of 1 pound of water by $1^{\circ} \mathrm{F}$ at or near $39.2^{\circ} \mathrm{F}$. See Heat Content of a Quantity of Fuet, Gross, and Heat Content of a Quantity of Fuel, Net.

Butane: A normally gaseous straight-chain or branched-chain hydrocarbon $\left(\mathrm{C}_{4} \mathrm{H}_{10}\right)$. It is extracted from natural gas or refinery gas streams. It includes isobutare and normal butane and is designated in ASTM Specification D1835 and Gas Processors Association Specifications for commercial butane.

- Isobutane: A normally gaseous branched-chain hydracarbon. It is a colorless paraffiric gas that boils at a temperature of $10.9^{\circ} \mathrm{F}$. It is extracted from natural gas or refinery gas streams.

- Normal Butane: A normally gaseous straight-chain hydrocarbon. It is a colorless paraffinic gas that boils at a temperature of $31.1^{\circ} \mathrm{F}$. It is extracted from natural gas or refinery gas streans.

Butylene: An olefinic hydrocabon $\left(\mathrm{C}_{4} \mathrm{H}_{5}\right)$ recovered from refinery processes.

Catalytic Cracking A refining process that consists of using a catalyst and heat to break down the heavier and more complex hydrocarbon molecules into lighter and simpler molecules.

Coal: A black or brownish-black solid, combustible substance formed by the partial decomposition of vegetable matter without access to air. The rank of coal, which indudes anthractite, bituminous coal, stubbituminouts coal, and lignite, is based on fixed carbon, volathle matter, and heating value. Coal rank indicates the progressive alteration, or coalification, from lignife to anthracite. Lignite contains approximately 9 to 17 million Btu per ton. The heat contents of subbituminous and bituminous coel range from 16 to 24 million Btu per ton, and from 19 to $\mathbf{3 0}$ mitlion Btu per ton, respectively. Anthracite contains approximately 22 to 28 million Btu per ton.

Coal Coke: A hard, porous product made from bakòng bituminous coal in ovens at temperatures as high as $2,00^{\circ} \mathrm{F}$. It is used both as a fuel and as a reducing agent in smelting iron ore in a blast firnace.

Coke Plants: Plants where coal is carbonized in slot or beehive ovens for the menufacture of coke.

Commercial Sector: The commercial sector, as defined economically, consists of business establishments that are not engaged in transportation or in manufacturing or other types of industrial activity (agriculture, mining, or construction). Commercial establishuments include hotels, motels, restaurants, wholesale businesses, retail stores, laundries, and other service enterprises; religious and nomprofit organizations; health, social, and educational institutions; and Federal, State, and local governments. Street lights, pumps, bridges, and public services are also included if the establishment operating them is considered commercial.

Conversion Factor A number that translates units of one system into corresponding values of another system. Conversion factors can be used to translate physical units of measure for various fuels into Btu equivalents.

Crude Oil (Including Lease Condenate): A mixture of hydrocarbons that exists in tiquid phase in underground reservoirs and remains liquid at atmospheric pressure after passing through surface separating facilities. Included are lease condensate and liquid hydrocarbons produced from tar sands, gilsonite, and oil shale. Drip gases are also included, but topped crude oil (residual oil) and other unfinished oils are excluded. Where identifiable, liquids produced at natural gas processing plants and mixed with crude oil are likewise excluded. 
Crude Ofl Uned Direclly: Crude oil consumed as fuel by crude oll pipelines and on crude oil leases.

Cubic Foot (ratural gas): A unit of volume equal to 1 cubic foot at a pressure base of 14.73 pounds standard per square inch absolute and a temperature base of $60^{\circ} \mathrm{F}$.

Diesel Fuel: Fuel used for internal combustion in diesel engines; usually that fraction of crude oil that distills after kerosene. See Distillate Fuet Oit.

Distillate Foel Oil: A general classification for one of the petroletm fractions produced in conventional distillation operations. Included are products known as No. 1, No. 2, and No. 4 fuel oils and No. 1, No. 2 , and No. 4 diesel fuels. It is used primarily for space heating. on-and off-highway diesel engine fuel (including railroad engine fuel and fuel for agticultural machínery), and electric power generation.

Electrical System Energy Losses: The amount of energy lost during generation, transmission, and distribution of electricity, inciuding plant and unaccounted-for uses.

Electricity Production: Net generation of electricity (gross output measured at generator tenminals mintus power plant use) by publicly and privately owned electric utilities. Excludes industrial generation of electricity (except autogeneration of hydroelectric power).

Electricity Sales: The amount of kilowatthours sold in a given period of time; usually grouped by classes of service, such as residential, commercial, industrial, and other. "Other" sales include sales for public street and highway lighting and other sales to public authorities, sales to railroads and railways, and interdepartmental sales.

Electric Power Plant: A station containing prime movers, electric generators, and auxiliary equipment for converting mechanical, chemical, and/of fission energy into electric energy.

Electric Utility: A corporation, person, agency, authority, or other legal entity or instrumentality that owns and/or operates facilities for the generation, transmission, distribution, or sale of electric energy, primarily for tuse by the public, and that files forms listed in the Code of Federal Regulations, Title 18, Part 141. Facilities that qualify as cogenerators or small power producers tunder the Public Uility Regulabory Policies Act are not considered electric utilities.

Electric Utility Sector. The electric utility sector consists of privately and publicly owned establishments that generate, transmit, distribute, or sell electricity primarily for vese by the public and that meet the defintition of an edectric utility. Nonutlity power producers are not included in the electric utility sector.

End-Use Sectors: The residential, commercial, industrial, and transportation sectors of the economy.

Energy: The capacity for doing work as measured by the capability of doing work (potential energy) or the conversion of this capability to motion (kinetic energy). Energy has several forms, some of which are easily convertible and can be changed to another form useful for work. Most of the world's convertible energy comes from fossil fuels that are bumed to produce heat that is then used as a transfer medium to mechanical or other means in order to accomplish tasks. Electrical energy is usually measured in kilowatthours, while heat energy is usually measured in British thermal units.

Energy Consumption: The use of energy as a source of heat or power or as an input in the manufacturing process.

Energy Congumption, End-Use: The strm of fossil fuel consumption by the four end-use sectors (residential, commercial, industrial, anr transportation) plus electric utility sales to those sectors and generation of hydroelectric power by nonelectric utilities. Net end-use energy consumption exchudes electrical system energy losses. Total end-use energy consumption includes electrical system energy losses.

Energy Consumption, Total: The sum of fossil fuel consumption by the five sectors (residential, commercial, industrial, transportation, and electric utility) plus hydroelectric power, nuclear electric power, net imports of coal coke, and electricily generated for distribution from biofuels, geothermal, wind, photovoltaic, and solar thermal energy. 
Ethane: A normally gaseous straight-chain hydrocatbon $\left(\mathrm{C}_{2} \mathrm{H}_{6}\right)$. It is a colorless, paraffinic gas that boils at a temperature of $-127.46^{\circ} \mathrm{F}$. It is extracted from natural gas and refinery gas streams.

Ethanol: An anhydrous, denatured aliphatic alcohol $\left(\mathrm{C}_{2} \mathrm{H}_{5} \mathrm{OH}\right)$ intended for motor gasoline blending.

Ethylene: An olefinic hydrocarbon $\left(\mathrm{C}_{2} \mathrm{H}_{4}\right)$ recovered from refinery processes or petrochemical processes.

Exports: Shipments of goods from the 50 States and the District of Columbia to foreign countries and to Pterto Rico, the Virgin Islands, and other U.S. possessions and territories.

Federal Energy Regulatory Commigsion (FERC): The Federal agency with jurisdiction over interstate electricity sales, wholesale electric rates, hydroelectric licensing, natural gas pricing, oil pipeline rates, and gas pipeline certification. FERC is an independent regulatory agency within the Department of Energy and is the successor to the Federal Power Commission.

Federal Power Commission (FPC): The predecessor agency of the Federal Energy Regulatory Commission. The Federal Power Commission was created by an Act of Congress under the Federal Water Power Act on Jume 10, 1920. It was charged originally with regulating the electric power and natumal gas inhustries. It was abolished on September 30, 1977, when the Department of Energy was created. Its functions were divided between the Department of Energy and the Federal Energy Regulatory Commission, an independent regulatory agency.

Fiscal Year: The U.S. Govenment's fiscal year nuns from October 1 through September 30. The fiscal year' is designated by the calendar year in which it ends; e.g. fiscal year 1992 begins on October 1, 1991, and ends on September 30, 1992.

Fossil Fuel: Any naturally occurring fuel, such as petroleum, coal, and natural gas, formed in the Earth's crust from long-term organic matter.
Fossil-Fueled Steam-Electric Power Plant: An electricity generation plant in which the prime mover is a hurbine rotated by high-pressure steam produced in a boiler by heat from burning fossil fuels.

Gasohol: A blend of finished motor gasoline (leaded or unleaded) and alcohol (generally ethanol but sometimes methanol) limited to 10 percent by volume of alcohol. Gasohol is included in finished leaded and unleaded motor gasoline.

Gas-Turbine Electric Power Plant: A plant in which the prime mover is a gas turbine. A gas turbine typically conststs of an axialflow air compressor, one or more combustion chambers where liquid or gaseous fuel is burned and the hot gases expand to drive the generator and then are used to run the compressor.

Geothermal Energy: Hot water or steam extracted from geothermal reservoirs in the Earth's crust and supplied to steam turbines that drive generators to produce electricity.

Heat Content of a Quantity of Fuel, Gross: The total amount of heat released when a fuel is burned. Coal, crude oil, and natural gas all include chemical corapounds of carbon and hydrogen. When those fuels are burned, the carbon and hydrogen combine with oxygen in the air to prodtace carton dioxide and water. Some of the energy released in burning goes into transforming the water into steam and is usually lost. The amount of heat spent in transforming the water into steam is counted as part of gross heat content but is not counted as part of net heat content. Also referred to as the higher heating value. Btu conversion factors typically used in ELA represent gross heat content.

Heat Content of a Qwantity of Fuel, Net: The amount of usable heat energy released when a fuel is burned under conditions similar to those in which it is normally wsed. Also referred to as the lower heating value. Btu conversion factors typically used in EIA represent gross heat content.

Heavy Oil: The fuel oils remaining after the lighter oils have been distilled off during the refining process. Except for start-up and flame stabilization, virtually all petrolemm used in steam-electric power plants is heavy oil 
Hydrolectric Power: The production of electricity from the kinetic energy of falling water.

Hydroelectric Power Plant: A plant in which the turbine generators are driven by falling water.

Imports: Receipts of goods into the 50 States and the District of Columbia from foreign countries and from Puerto Rico, the Virgin Islands, and other U.S. possessions and territories.

Industrial Sector The industrial sector comprises manufacturing industries, which make up the largest part of the sector, along with mining, construction, agriculture, fisheries, and forestry. Establishments in this sector range from steel mills to small farms to companies assembling electronic components.

Intemal Combustlon Electric Power Plant A power plant in which the prime mover is an intemal combustion engine. Diesel or gasfired engines are the principal types used in electric power plants. The plant is usually operated during periods of high demand for electricity.

Isopentane: A saturated branched-chain hydrocatbon $\left(C_{5} \mathrm{H}_{12}\right)$ obtained by fractionation of natural gasoline or isomerization of normal pentane.

Jet Fuel: The term includes kerosene-type jet fuel and naphtha-type jet fuel. Kerosene-type jet fuel is a kerosene-quality product used primarily for commercial turbojet and tuboprop aircraft engines. Naphtha-type jet fuel is a fuel in the heavy naphthas range used primarily for military turbojet and turboprop aireraft engines.

Kerosene: A petroleum distillate that has a maximum distillation temperature of $401^{\circ} \mathrm{F}$ at the 10-percent recovery point, a final boiling point of $572^{\circ} \mathrm{F}$, and a minimum flash point of $100^{\circ} \mathrm{F}$. Included are the two grades designated in ASTM D3699 (No. 1-K and No. 2-K) and all grades of kerosene called range or stove oil. Kerosene is used in space heaters, cook stoves, and water heaters; it is suitable for wise as an illuminant when burned in wick lamps.
Lease and Plant Fael: Natural gas used in well, field, and lease operations (such as gas used in drilling operations, heaters, dehydrators, and field compressors), and as fuel in natural gas processing plants.

Lease Condensater A nahrral gas liquid recovered from gas well gas (associated and non-associated) in lease separators or natural gas field facllities. Lease condensate conslsts primarily of pentanes and heavier hydrocarbons.

Light Oil: Lighter fuel oils distilled off during the refining process. Virtually all petroleum used in intemal combustion and gas-turbine engines is light oil.

Ligniter A brownish-black coal of low rank with a high content of moisture and volatile matter. Often referred to as brown coal. It is used almost exclusively for electric power generation. It conforms to ASTM Specification D398-84 for lignite.

Liquefied Petroleam Gases (LPG): Ethane, ethylene, propane, propylene, normal butane, butylene, and isobutane produced at refineries or natural gas processing plants, including plants that fractionate new natural gas plant liquids.

Lubricants: Substances used to reduce friction between bearing surfaces or as process materials either incorporated into other materials used as processing aids in the manufacturing of other products or as carriers of other materials. Petroleum lubricants may be produced either from distillates or residues. Other substances may be added to impart or improve certain required properties. Excluded are byproducts of lubricating oil tefining, such as aromatic extracts derived from solvent extraction or tars derived from deasphalting. Included are all grades of lubricating oils from spindle oil to cylinder oil and those used in greases. Lubricants cakegories are paraffinic and naphthenis.

Methanol: A light, volatile alcohol $\left(\mathrm{CH}_{5} \mathrm{OH}\right)$ eligible for motor gasoline blending.

Mfacellaneous Pelroleum Products: All finished petroleum products not classified elsewhere-for example, petrolatum, lube refining 
C byproducts (aromatic extracts and tars), absotption oils, ram-jet fuel, L petroleum rocket fuels, synthetic natural gas feedstocks, and speQ cialty oils.

Motor Gasoline: A complex mixture of relatively volatile hydrocarbons, with or without small quantities of additives, obtained by blending appropriate refinery streams to form a fuel suitable for use in spark-ignition engines. Motor gasoline includes both leaded and unleaded grades of finished motor gasoline, reformulated motor gasoline, oxygenated motor gasoline, other finished motor gasoline, blending components, and gasohol.

Motor Gasoline Blending Components: Naphthas that will be used for blending or compotunding into finished motor gasoline (e.g. straight-run gasoline, alkylate, reformate, benzene, tolutene, and xylene). Excluded ane oxygenates (alcohols and ethers), butane, and pentanes plus.

Natural Gasa A mixture of hydrocarbons (principally methane) and small quantities of various nonhydrocarbons existing in the gaseous phase or in solution with crude oil in underground reservoirs.

Natural Gaboline: A mixture of hydrocarbons, mostly pentanes and heavier, extracted from natural gas that meets specifications for natural gasoline set by the Gas Processors Association. Natural gasoline inclutides isopentane.

Net Interetate Flow of Electricity: The difference between the sum of electricity sales and losses within a State and the total amount of electricity generated within that State. A positive number indicates that more electricity (including associated losses) came into the State than went out of the State during the year; conversely, a negative numiber indicates that more electricity (including associated losses) went out of the State than came into the State.

\section{Nonutilities: See Nonutility Power Producer.}

Nontutility Power Producer: A corporation, person, agency, authority, or other legal entity or instrumentality that owns electric generating capacity and is not an electric atility. Nonutility power prodtrcers include qualifying cogenerators, qualifying small power producers, and other nonutility geverators (including independent power producers) without a designated, franchised service aren and that do not file forms listed in the Code of Federal Regulations, Title 18, Part 141.

Nuclear Electric Power: Electricity generated by an electric power plant whose turbines are driven by steam generated in a reactor by heat from the fissioning of nuclear fuel.

Nuclear Electric Power Plant: A single-unit or multiunit facility in which heat produced in one or more reactors by the fissioning of ntrclear fuel is used to drive one or more steam turbines.

Pentanes Plus: A mixture of hydrocarbons, mostly pentanes and heavier, extracted from natural gas. Included are isopentane, natural gasoline, and plant condensate.

Petrochemical Feedstocks: Chemical feedstocks derived from petroleum principally for the mantufacture of chemicals, synthetic nubber, and a variety of plastics. The categories reported are naphthas less than $401^{\circ} \mathrm{F}$ endpoint and other oils equal to or greater than $401^{\circ} \mathrm{F}$ endpoint.

Petroleum: A generic term applied to oil and oil products in all forms, such as crude oil, leaze condensate, unfinished oils, petroleum products, natural gas plant liquids, and nonhydrocarbon compounds blended into finished petroleum products.

Petroleum Coke: A residue that is the final product of the condensation process in cracking. The product is either marketable petroleum coke or catalyst petroleum coke.

Petroleum Coke, Catalyst. The carbonaceous residue that is deposited on and deactivates the catalyst used in many catalytic operations (e.g. catalytic cracking). Carbon is deposited on the catalyst. thus deactivating the catalyst. The catalyst is reactivated by buming off the carbon, which is used as a fuel in the refining process. That carbon or coke is not recoverable in a concentrated form.

Petroleum Coke, Marketable: Those grades of coke produced in delayed or fluid cokers that may be recovered as relatively pure 
carbon. Marketable petroleam coke may be sold as is or further puttfied by calcining.

Petrolenm Consumption: The sum of all refined petroletun products supplied. For each refined petroleum product, the amount supplied is calculated by adding production and imports, then subtracting changes in primary stocks (net withdrawals are a plus quantity and net additions are a minus quantity) and exports.

Petroleum Products: Products obtained from the processing of crude oil (including lease condensate), natural gas, and other hydrocarbon compounds. Petroleum products include unfirished oils, liquefied petroleum gases, pentanes phus, aviation gasoline, motor gasoline, naphtha-type jet fuel, kerosene-type jet fuel, kerosene, distillate fuel oil, residual fuel oil, petrochemical feedstocks, special naphthas, hubricants, waxes, petroleum coke, asphalt, road oil, still gas, and missellaneous products.

\section{Petroleum Products Supplied: See Petroleun Consumption.}

Photovoltaic and Solar Thermal Energy: Energy radiated by the sun as electromagnetic waves (electromagnetic radiation) that is converted into electricity by means of solar (photovoltaic) cells or concentrating (focusing) colfectors.

Plant Condensatei One of the natural gas liquids, mostly pentanes and heavier hydrocarbons, recovered and separated as liquids at gas inlet separators or scrubbers in processing plants.

Propme: A normally gaseous straight-chain hydrocarbon ( $\left.\mathrm{C}_{3} \mathrm{H}_{38}\right)$. It is a colorless paraffinic gas that boils at a temperature of $-43.67^{\circ} \mathrm{F}$. It is extracted from natural gas or refinery gas streams. It includes all products designated in ASTM Specification D1835 and Gas Processors Association Specifications for commercial propane and HD-5 propane.

Propylene: An olefinic hydrocarbon $\left(\mathrm{C}_{3} \mathrm{H}_{6}\right)$ recovered from refinery or petroritemical processes.
Refineny (petroleum): An installation that manufactures finished petroleum produrts from crude oil, unfinished oils, natural gas liquids, other hydrocarbons, and alcohol.

Residential Sector: The residential sector is considered to consist of all private residences, whether occupted or vacant, owned or rented, including single-family homes, multifamily housing units, and mobile homes. Secondary hones, such as summer homes, are also included. Institutional housing, such as school dormitories, hospitals, and military barracks, generally are not included in the residential sector; they are included in the commercial sector.

Residual Fugl Oil: The heavier oils that remain after the distillate fuel oils and lighter hydrocarbons are distilled away in refinery operations and that conform to ASTM Specifications D396 and D975. Included are No. 5, a residual fuel ofl of medium viscosity; Navy Special, for use in steam-powered vessels in government service and in shore power plants; and No. 6, which includes Bunker $C$ fuel oil and is used for commercial and industrial heating, electricity generation, and to power ships. Imports of residual fuel oil include imported crude oil bumed as fuel.

Road Oil: Any heavy petroleum oil, including residual asphaltic oil, used as a dust palliative and surface treatment on roads and highways. It is generally produced in six grades, from 0 , the most liquid, to 5 , the most viscous.

Short Ton (coal): A unit of weight equal to 2,000 pounds.

SIC See Standard Industrial Classification.

Solar Energy: The radiant energy of the sun that can bs converted into other forms of energy, such as heat or electricity.

Special Naphthas: All finished products within the naphtha boiling range that are used as paint thinners, cleaners, or solvents. Those producbs are refined to a specified flash point. Special naphthas in" clude all commercial hexane and cleaning solvents conforming to ASTM Specifications D1836 and D484, respectively. Naphthas to be blended or marketed as motor gasoline or avlation gasoline, or that 
G are to be used as petrochemical and synthetic natural gas (SNG) feedstocks, are excluded.

Standard Industrial Classification (SIC): A set of codes developed by the Office of Management and Budget which categorizes industries into groups with similar economic activities.

Stean-Electric Power Plant: A plant in which the prime mover is a steam turbire. The steam used to drive the turbine is produced in a boiler where fossil fuels are burned.

Sttll Gas (refinery gas): Any form or mixture of gas produced in refineries by distillation, cracking, reforming, and other processes. The principal constiturents are methane, ethane, ethylene, normal butane, butylene, propane, and propylene. It is used primarily as refinery fuel and petrochemical feedstock.

Sabbituminous Coal: A dull, black coal of rank intermediate between lignite and bituminous coal. It conforms to ASTM Specification D388-84 for subbituminous coal. In this report, subbituminous coal is included in bituminous coaI.

Supplemental Gaseous Fuels: Ary gaseous substance that, introduced into or commingled with natural gas, increases the volume available for disposition. Such substances include, but are not limited to, propane-air, refinery gas, coke oven gas, still gas, marufactured gas, biomass gas, and air or inert gases added for Btu stabilization.

Transportation Sector: The transportation sector consists of private and public vehicles that move people and commodities. Inchuded are automobiles, trucks, buses, motorcycles, railroads and railways (including streetcars), aircraft, ships, barges, and natural gas pipelines.

Unfinished Oils: All oils requiring further refinery processing, except those requiring only mechanical blending. Included are naphthas and lighter oils, kerosene and light gas oils, heavy gas oils, and sesiduum.

Unfractionated Streams: Mixtures of unsegregated natural gas liq. uid components, excluding those in plant condensate. This product is extracted from natural gas.

\section{United States: The 50 States and the District of Columbia.}

Value Added by Manufacture: A measure of manufactuxing activity that is derived by subtracting the cost of materials (which covers materials, supplies, containers, fuel, purchased electricity, and contract work) from the value of shipments. This difference is then adjusted by the net change in finished goods and work-in-progress between the begtuning and end-of-year inventories.

Waxes: Solid or semisolid materials detived from petroleutn distillates or residues. Waxes are light-colored, more or less translucent crystalline masses, slightly greasy to the touch, consisting of a mixture of solid hydracarbons in which the paraffin series predominates. Included are all marketable waxes, whether crude scale or fully refined. Waxes are used primarily as industrial coating for surface protection.

Wind Einergy: The kinetic energy of wind converted into mechanical energy by wind turbines (i.e., blades rotating trom a hub) that drive generators to produce electricity for distribution. 


\section{Introducing the Energy InfoDisc ...}

How much oll does the U.S. import from the Perslan Guif?

How many U.S. households use personal computers?

What's the long range outlook for nuclear power, and how will it affect other fuols?

How are clean alr regulations affecting emissions in the electric power industry?

Find answers to all your energy questions with Energy InfoDlsc, the new CD-ROW providing the most cornpre. hensive collection of energy information and analysis tools available today. Energy InfoDisc is a valuable resource for industry and government analysts, forecasters, joumalists, and academic researchers- almost anyone needing access to curtent or histortcal energy informatton.

The Energy InfoDise comes with advanced search capability to find the infomation you need in seconds from over 180 of the most cutrent periodicals and analytical publications of the Energy Information Administration (EIA), the indeperdent statistical and analytical agency within the U.S. Department of Energy. With Energy InfoDlsc, you also have instant access to ElA's wide range of national energy databases and special analysts applications. Don't be without this infportant resourte; subscribe today.

\section{STAT-USA Order Form}

Yes, enter my order for the Energy InfoDise co-ROM:

Number of coples:

Dingta issue (U.S., Ganada, B Mexico): 546

$\square$ single issue outside the U.S., Canada a Mexlco: $\$ 60$

Quarterly subseriptlon in the U.S+4 Canada, \& Mexlep; 5100

Q Quarterfy subscription outside the U.S., Canada 8. Nexica: $\$ 160$

Contact or personal name (please type or print)

Company

Street address

Additional address

city, State, ZlP Code

Daytime phorie

Fax number
Charge your order, It's easy!

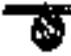

To fax yout orders: (202) 482-2164

To phone your orders: (202)482-1986

For privacy protection, check the box below:

$\square$ Do not make my name avallable to requesters.

Check method of payment:

a AMEX $\square$ Dlscover $\square$ Mastercard $\square$ VISA

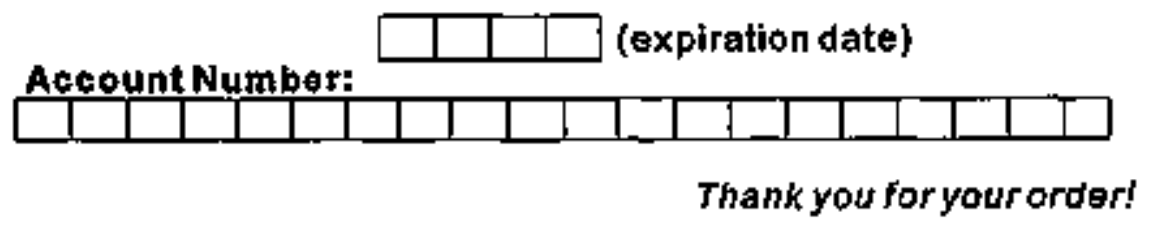

Authortzing signature

Date

Gheck payable to STAT-USA

Mail to: U.S. Department of Commerce

STAT-USA, ROOM H-4BES

Washington, DC 20230 